\title{
Precision Measurement and Calibration
}

Qlectricity and Electronics

Mandbooks $7 \%$ - Volume I

United States Department of Commerce

National Bureau of Standards 




\title{
Precision Measurement and Calibration
}

\author{
Selected Papers on \\ Electricity and Electronics
}

\begin{abstract}
A compilation by Sherman F. Booth of previously published technical papers by the staff of the National Bureau of Standards.

Issued in three volumes*

I. Electricity and Electronics.

II. Heat and Mechanics.

III. Optics, Metrology, and Radiation.
\end{abstract}

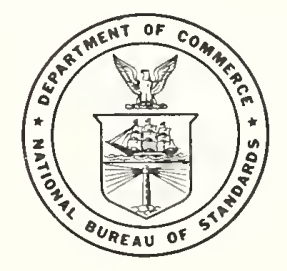

National Bureau of Standards, Handbook 77 - Volume I

Issued February 1, 1961

Reavinet re sp $190^{\circ}$

For sale by the Superintendent of Documents, U.S. Government Printing Office, Washington 25, D.C.

"Prices: Volume I $\$ 6.00$, Volume II $\$ 6.75$, Volume III $\$ 7.00$. The three volumes at $\$ 19.75$ a set. 


\begin{abstract}
This Handbook is a three-volume compilation of approximately 150 selected papers previously published by the staff of the National Bureau of Standards on precision measurement, calibration, and related subjects. It was prepared to meet the urgent need of newly established standards laboratories for a "textbook" and reference source in these fields. Volume I contains papers in electricity and electronics; Volume II, heat and mechanics; and Volume III, opties, metrology, and radiation. Each volume contains a complete index of the entire Handbook by author, subject, and title.
\end{abstract}




\section{Foreword}

The National Bureau of Standards is charged with the responsibility of establishing and maintaining the national standards of physical measurement, and of providing means for their effective utilization. This responsibility carries with it the mission of providing the central basis for a complete, consistent system of physical measurement, adequate for national growth in research and technology.

The recent tremendous increase in industrial activity, particularly in the missile and satellite fields, has led to an unprecedented demand for precision measurement, which, in turn, is bringing about the establishment of hundreds of new standards laboratories. Many of these new laboratories must cover the entire field of measurement, and must do so with a staff not previously trained in work on standards of precision measurement.

To aid these laboratories in transmitting the accuracies of the national standards to the shops of industry, the Bureau has prepared this three-volume Handbook. It is a compilation of publications by the Bureau staff that have been found of value to those who are establishing and operating new standards laboratories. Omitted are some extended works, as well as a few shorter papers that are otherwise readily available.

It is hoped that this compilation will serve both as a "textbook" and a reference source for the many scientists and engineers who must be trained in the shortest possible time to fill responsible positions in this critical area.

A. V. Astin, Director. 


\section{Preface}

Because of the urgent need for this Handbook, it has been reproduced by a photoduplication process. As a result, the individual publications that make up the compilation will be found to vary in such details as style, size of type, and method of pagination.

Each paper reproduced for the compilation is essentially complete as originally published and retains its original page numbering. All pages have also been numbered in regular sequence throughout the three volumes. Thus, the volume page number and the original page number are combined, for example $100 / 10$.

Because of the short time available, a complete review of each paper included was not possible. However, some efforts were made to bring the older papers up to date, and only those papers have been included that are of current value. Nevertheless, users should be cautioned that the state of the art may have advanced beyond that represented in some of the older papers.

These three volumes, extensive as they are, include only a fraction of the published work of the National Bureau of Standards relating to standards. However, many of the reprinted papers contain extensive bibliographies that will enable the user who is confronted with a special problem to locate additional information.

The papers that appear or are cited in this three-volume Handbook were originally published over a period of several years as circulars, research papers, chapters of books, and as articles in scientific and technical periodicals. Thus individual copies of many papers are no longer readily available. More recent Bureau publications, and in some cases the older papers for which prices are given, may still be obtainable by purchase from the Superintendent of Documents, U.S. Government Printing Office, Washington 25, D.C. Other papers may often be obtained directly from the authors or from the publishers of the Journals in which the papers appeared. The papers referred to in the various lists, if not generally available as stated above, are usually available for reference in technical, university, Government depository, and public libraries. 


\section{Contents}

\section{Electricity}

Suggested practices for electrical standardizing laboratories _....... 2

Irage

Establishment and maintenance of the electrical units _............ 12

Extension and dissemination of the electrical and magnetic units by

the National Bureau of Standards.

Measurement of curlent with a Pellat-type electrodynamometer

Measurement of current with the NBS current balance -

Precision resistors and their measurement

Methods, apparatus, and procedures for the comparison of precision standard resistors

Testing and performance of volt boxes . . . . . . . . . . . . . . . . .

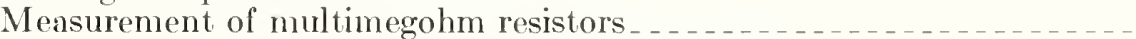

Standard cells and the unit of electromotive force

Fffect of service temperature conditions on the electromotive force of unsaturated portable standard cells

The measurement of high voltage $\ldots \ldots \ldots \ldots$

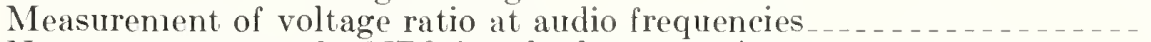

New apparatus at the NBS for absolute capacitance measurement

Capacitor calibration by step-up methods

Variable capacitor calibration with an inductive voltage divider bridge

Thermal converters as ac-dc transfer standards for current and voltage measurements at audio frequencies.

Thermal voltage converters for accurate voltage measurements to 30 megacycles per second .

AC-DC transfer instruments for current and voltage measurements

A wide-range volt-ampere converter for current and voltage measurements .

Phase angle master standard for 400 o/s

Standard electrodynamic wattmeter and ac-de transfer instrument $\ldots$

Precision comparison method of testing alternating-current wathour meters

The testing of electrical instruments $\ldots$

Testing electrical instruments

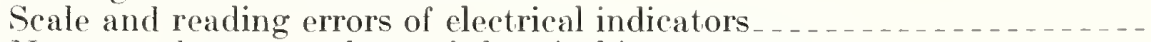

Notes on the care and use of electrical instruments . . . . . . . . . . . .

A camera obscura for instrument reading .......................

Power supplies for 60-cycle tests of electrical instruments and meters _.

Electrical mits, instruments, and measurements, list of publications on

\section{Electronics}

High-frequency standards of the Electronic Calibration Center, NBSB

National standards of time and frequency in the United States

Standard frequencies and time signals, WWV and WWVH

Adjustment of high-precision frequency and time standards . . . . . . .

Accurate microwave wavemeters with convenient calibration tables _. -

Short-time stability of a quartz-crystal oscillator as measured with an ammonia maser

An evaluation of a cesium beam frequency standard $\ldots \ldots$

Precise time synchronization of widely-separated clocks $\ldots \ldots \ldots \ldots$

A UHF and microwave matching termination ....................

High-frequency voltage measurement

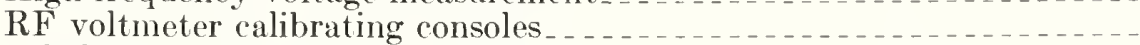

A bolometer bridge for standardizing radio-frequency voltmeters

Application of RF micropotentiometers for calibration of signal generators to $1,000 \mathrm{Mc}$ 
Development of very-high-frequency field-intensity standards $\ldots$

Influence of the ground on the calibration and use of VHF field-intensity

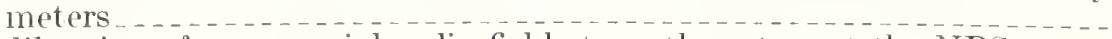

Calibration of commercial radio field-strength meters at the NBS

High-frequeney impedance standards at the N'BS

Recently developed microwave impedance standards and methods of

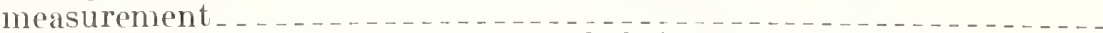

Coaxial radio-frequency connectors and their electrical quality _......

Tables of frequency, VSWR, and $|\Gamma|$ for selected half-round inductive obstacle impedance standards in WR-90 (X-band) rectangular waveguide

Tables of frequency, VSWR, and $|\Gamma|$ for selected half-round inductive obstacles impedance standards in WR-280 (S-band) rectangular waveguide

Tables of frequency, VSWR, and $|\Gamma|$ for selected half-round inductive obstacle impedance standards in WR-187 rectangular waveguide_...

Half-round inductive obstacles in rectangular waveguide .............

An adjustable sliding termination for rectangular waveguide _.........

A new technique for the measurement of microwave standing-wave

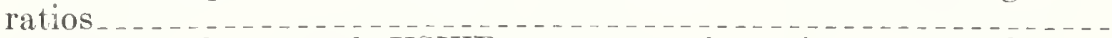

Magnified and squared VSWR responses for microwave reflection coefficient measurement _.....................................

Microwave reflectometer techniques _. . . . . . . . . . . .

Precision millimeter wave interferometry at the U.S. National Bureau

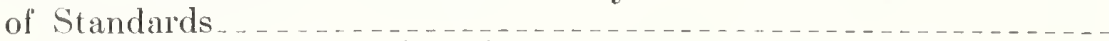

A standard of attenuation for microwave measurements .............

Determination of attenuation from impedance measurements . . . . . . .

Microwave attenuation of measurements with accuracies from 0.001 to 0.06 decibel over a range of 0.01 to 50 decibels

Mismatch errors in the measurement of ultrahigh-frequency and microwave variable attenuators . . . . . . . . .

Mismatch errors in cascade-comnected variable attenuators

A method for measuring the directivity of directional couplers _. . _ _.

Recent developments in the field of microwave power measurements at the NBS

A dry, static calorimeter for $\mathrm{RF}$ power measurement

A self-balancing direct-current bridge for accurate bolometric power

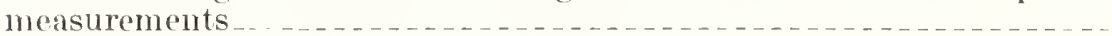

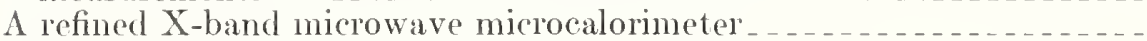

High frequeney power measuring bridge cireuit

A technique for reducing errors in permeability measurements with coils

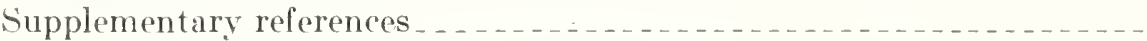

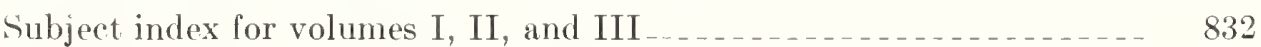

Author index for volumes I, II, and III $\ldots \ldots \ldots$

Publications program of the NBS $\ldots \ldots \ldots$ 
Selected Papers on Electricity

(Contents on page v) 



\title{
Suggested Practices for \\ Electrical Standardizing Laboratories
}

\author{
Francis B. Silsbee
}

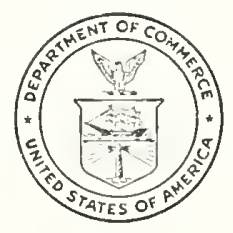

National Bureau of Standards Circular 578

Issued August 30, 1956 


\section{Preface}

Since World War II there has been a very marked increase in the number and scope of private standardizing laboratories throughout the United States. The function of each such laboratory is to maintain the accuracy and uniformity of the measuring instruments and apparatus used by the organization or organizations that the laboratory serves. A basic pliase in this process is necessarily to correlate the reference standards of the laboratory with those of the National Bureau of Standards. This Circular has been prepared to suggest techniques and principles that experience has shown to be useful in such operations. Although this Circular covers explicitly only the field of electrical measurements, many of the principles involved are equally applicable in other kinds of measurement.

\section{A. V. Astin, Director.}

\section{Contents}

Preface _.

1. Introduction

2. Creneral principles . . . . . . . . . . . .

2.1. Competence of persomel . . . . . . . . . . . . . . . . . . . . . . .

2.2. Position in organization $\ldots \ldots \ldots$

2.3. Appropriate scope and accuracy $\ldots \ldots$

2.4. Laboratory atmosphere

2.5. Care of standards . . . . . . . . . . . . . . . . .

2.6. Timing of celibrations . . . . . . . . . . . .

3. Reference standards $\ldots \ldots$

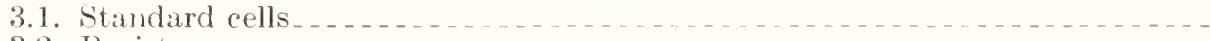

3.2. Resistors . . . . . . . . . . . . . . . . . . . . . . . . . .

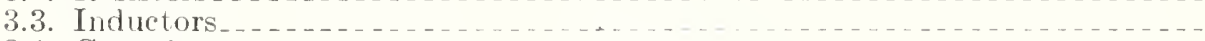

3.4. Capacitors $\ldots$

3.5. Volt boxes

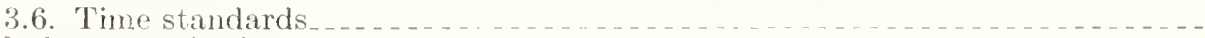

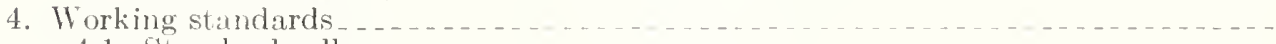

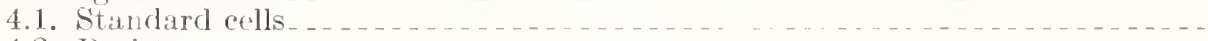

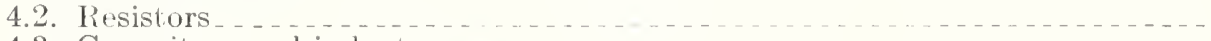

4.3. Capacitors and inductors

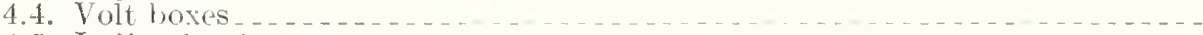

4.5. Indicating instruments . . . . .

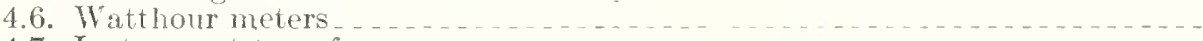

4.7. Instrument transformers $\ldots \ldots \ldots$

5. Comparison apparatus

5.1. Consoles $5 . \ldots$ current potentiometers . . .

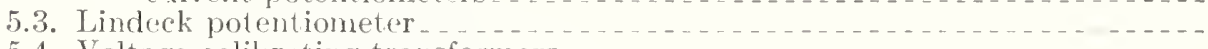

5.4. Voltage-calibrating transformers . . . . . . . . . . . . . . . . . . . .

5.5. Instrument-transfomer test sets

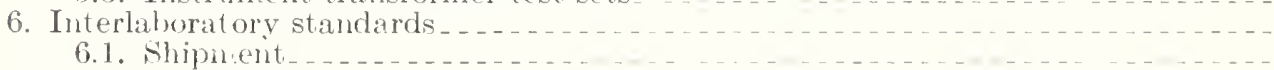

6.2. Standard cells.

6.3. Resistors, capacitors, and inductors

6.4. Instrument transformers

6.5. Indicating instruments . .

7. Summary.

8. References 


\title{
Suggested Practices for Electrical Standardizing Laboratories
}

\author{
Francis B. Silsbee
}

\begin{abstract}
A number of basic principles are given that experience has shown to be important in the operation of private standarding laboratories. Types of stambard apparatus are classified and schedules appropriate for their intercomparison and for their checking at the National Bureau of Standards are suggested.
\end{abstract}

\section{Introduction}

The purpose of this Cireular is to indicate recommended procedures and sehedules for the calibration testing of elcetrical instruments and measuring apparatus used in the range of frequency from 0 to 30 kilocyeles per second.

For many year's the clectric-power companies have maintained large and efficient laboratories $[1,2]^{1}$ suited to their particular needs, and certain universities have similarly supplied services [3] to their teaching and research staffs. More recently, laboratories are being established in many newly founded manufacturing plants, in firms concemed with research and development, and in Government agencies (particularty in the military services). Although the particular problems that confront the various agencies may differ greatly, their standardizing laboratories have a great many common functions in their work of maintaining and disseminating the units of measurement.

The process of measurement always involves a succession of steps in which the units in terms of which the measurement of any quantity is expressed are transmitted by using measuring apparatus that has been standardized, by comparisoll with standards of a higher level, to calibrate other measuring apparatus which is to serve as a standard at a lower level. At the source is the National Burean of Standards, authorized by Congress to establish and maintain the basic standards of the nation $[4,5]$. Next, in any organization, may come a primary group of laboratory workers. which maintains its basic reference standards and in turn calibrates the working standard apparatus used by the next lower, or secondary, group, which in turn calibrates the instruments used in making measurements in the plant or shop. In some cases these latter two operations are merged in a single

\footnotetext{
1 Figures in brackets indicate the litcrature references at the cnd of this Circular.
}

group, while, at the other extreme, a single primary group maty serve many secondary groups located in different cities.

It is the rest of the organization that uses the shop instruments standardized by the workingstandards group, and carries on the day-to-day work in the power systems, manufacturing operiations, research studies, or aceeptance tests. This use insures proper billing, adequate quality, and valid criteria for the acceptance of manufactured articles. In this Circular the terms "laboratory" or "standardizing laboratory" will be used to indicate the segregated part of the complete organization that is charged with the duty of supplying calibration services, at either the reference or the working'-standard level, to the measuring appariatus used by the rest of the organization, which will liere be designated as "plant" or "shop," even though it may actually be more correctly described as a research or testing laboratory.

For clarity in the rest of this Circular, the measuring instruments and apparatus used in an industrial organization will be referred to under the following categories:

(a) Reference standards, which embody fixed electrical quantities and serve to maintain their respective units during the intervals between checks with a laboratory at a higher echelon.

(b) Working standards, calibrated in terms of the reference standards and reserved for use in the calibration of shop instruments and apparatus. Working standards should never be used directly for measurements in the plant or shop.

(c) Comparison equipment such as bridges, potentiometers, ete., and consoles used in comparing shop instruments with working standards.

(d) Interlaboratory standards, often similar in construction to reference standards, used for transport to NBS and return in the periodic checking of the local reference standards.

(e) Shop instruments and measuring apparatus calibrated in the standardizing laboratory and used in the day-to-day operation of the plant. 


\section{General Principles}

The following general principles have been found by experience to be applicable to many of the problems involved in the maintenance of accuracy in laboratory work and any program of calibration service testing should conform to them so far as possible.

\subsection{Competence of Personnel}

The person in direct charge of any standardizing laboratory must be thoroughly familiar with the fundamentals of electricity and magnetism and with the principles of operation of all types of electrical measuring apparatus $[8,9]$. To be competent to carry on within the laboratory the various sequences of calibration testing outlined below, he must have taken a college-level course in electrical measurements or have had laboratory experience equivalent thereto. It is rery desirable that he be a graduate in physics or electrical engineering from an aceredited college. He must. be mentally alert to detect discrepancies and inconsistencies in internal check tests and to recognize abnormal behavior of any part of the apparatus or circuits. Unless persons of this type are arailable in a standardizing laboratory, both its calibration service and the measurement work that depends upon it throughout the plant is certain to become unsatisfactory, regardless of how often particular pieces of apparatus may be tested by other persons in some higher echelon or at NBS.

\subsection{Position in Organization}

Experience through the years has shown that the most satisfactory results are obtained by maintaining a definite separation of the standardizing laboratory from the rest of the organization. Responsibilities for arcuracy are thereby made definite, and team spinit can be developed in the stafl of the standardizing laboratory that keeps them interested in their work, which might otherwise scem routine and unimportant. This separation protects them from pressures that demand inproper laste and sloppy workmanship, and gives them authority dincetly delegated from a high level in management.

Closely related to the foregomg is the valuable principle that team spirit can be developed and maintained by recognizing that the quality of the roference stanclards of a laboratory are to a consideralde "xtent a measure of the competence of its stall" "a worker is known by the sharpness of his tools"). Honce, in cases where a given large organization mantains a plurality of standardizing laboratorios in its plants in different cities, it is very desirable that areh reforence and intertaboratory standare should be assigned permanently to an individual standardizing laboratory, and returned to it following periodic checks at any laboratory of a higher cohelon. If this is not done, it becomes difficult to fix responsibility for errors that may be discovered subsequently, and the laboratory workers lose the basis for much of their pride in workmanship.

\subsection{Appropriate Scope and Accuracy}

The nature and accuracy of the work done in a standardizing laboratory may vary considerably with the size of the plant it serves and the nature of the output of the plant. However, there are very few modern activities in which some phases do not require measurements of rather high accuracy. A standardizing laboratory must, of course, be equipped for calibration measurements of a higher accuracy than any used elsewhere in the plant, because the accuracy of measurement can never lise above that of its source.

Many such local standardizing laboratories provide service in many scientific fields, e. g., mechanies, heat, optics, and chemistry, in addition to their standardizing work in electricity and magnetism, which alone is the subject of this Circular. The direction and extent of such activities, as well as the degree of specialization in particular branches of electrical science, may greatly affect the types and ranges of the electrical standards required.

\subsection{Laboratory Atmosphere}

Atmospheric conditions are of considerable importance in an electrieal standardizing laboratory. If the relative humidity exceeds 65 percent, a conducting film of noisture is adsorbed on the surface of many trpes of insulating material. Leakage over such surfaces, particularly to the cireuit of a sensitive galvanometer, may introduce serious errors.

Certain types of apparatus, for example some wire-wound resistors of high value, show changes of several parts per $10^{2}$ with prolonged changes in lamidity. Such variations ran be detected by comparing calibrations made near the middle of the winter heating season, during which indoor humidities are normally very low, with those made near the middle of the summer when the ambient humidity is high.

Most electrical apparatus is compensated for temperature to some extent. Howerer, if the ambient temperature is subject to changes, the response of the various components may not be equally prompt. In such circumstances the compensation may be temporarily ineffective. It is therefore important that the Iaboratory temperature be held fairly constant $( \pm 2$ deg $F)$ at all times. Such room control also makes casier the task of the thermostats that control the baths in which more temperature-sensitive apparatus, such as standard cells or standard conductivity samples, are kept. The particular temperature chosen is of less importanee. The conditions specified by the American society for Testing Materials for its 
"Standard Laboratory Atmosphere" are a relative humidity of $50 \pm 2$ percent and a temperature of $23^{\circ} \pm 1.1 \mathrm{deg} \mathrm{C}\left(73.4^{\circ} \pm 2 \mathrm{deg} \mathrm{F}\right)$. This combination is widely used in tests of dielectrics and other materials, and, in addition, is very close to the maximum of comfort for the workers. Hence it is a good target to aim at in designing a new laboratory.

Freedom from vibration and dust are also important. A shop in which electrical instruments may be opened for repair should be scrupulously clean and dust free. Magnetic particles are attracted to the air gaps of permanent magnet instruments and must be constantly guarded against. Effective air filtering or electrostatic precipitation are very desirable.

\subsection{Care of Standards}

In many categories, a good standard piece of apparatus improves with age but deteriorates with handling and shipping. The shipping of primary and working standards and of comparison equipment can be minimized and the transfer of the units be achieved best by the use of interlaboratory standards of particular types that are as rugged as practicable.

The adjustment of a piece of measuring equipment to be close to its nominal value is very often desirable in the shop instruments used in the plant, because of the great saving in time and reduction in possible erroneous applications of corrections that result. On the other band, the exact opposite is usually true of working and reference standards. In general, such standards should not be readjusted, even if their values have gradually drifted materially away from their nominal values. Any standardizing laboratory naturally maintains records of the values of its reference standards [10]. These are preferably kept on an individual eard or record sheet for each standard, so that its history is apparent at a glance. Each such record provides a valuable indication of the quality of the standard, and gives warning of deterioration and the need for replacement toward the end of its useful life. Frequent adjustments tend to interfere with such records and increase the probability of improper corrections, as the proper corrections before and after a slight readjustment may not be conspicuously different. Even more important is the risk that the adjustment may initiate a progressive drift in value of the standard as a result of introducing mechanical strain, or local heating.

\subsection{Timing of Calibrations}

The most desirable sequence of steps and the frequency with which comparisons of measuring apparatus should be made depend, of course, upon a great number of cireumstances, such as the volume of equipment to be tested, the delicacy of the apparatus, and particularly the type of personnel by which it is to be handled. (For measurements made by inexperienced students or by military recruits, the likelihood of damage is much greater than when a laboratory is staffed by experts.) In every laboratory a real possibility is always present that an abrupt change may oceur in some standard as a result of an unrecognized accident or abuse. Fairly frequent checks made locally are therefore an indispensable supplement to any program of calibrations to a higher echelon.

Cooperative arrangements with a similar laboratory in the same city may allow frequent checks to be made against an independently maintained group of standards. This may permit a much less frequent checking schedule between either laboratory and one of higher echelon.

In any laboratory, the accuracy needed in measurement may differ widely on different projects. Hence the frequency with which the working instruments should be checked may vary considerably from project to project, even though instruments of the same accuracy class are used $[2,6,7]$. The time intervals suggested elsewhere in this Circular for check tests represent in most cases the considered opinion of persons who have had long experience with standard apparatus of the types now commonly used. Similar test intervals are listed in the American Standards Association Code for Electricity Meters [2]. When a new type comes into use, it must be appraised by being checked at frequent intervals during its first few years of service. After its good stability has thus been demonstrated, the interval between tests may safely be made longer. Several types of standard capacitors and inductors are currently in this probational stage.

A procedure that is often of value, particularly for the checking of consoles and complex equipment such as a-c bridges, is to use an interlaboratory standard that is measured in one laboratory and shipped to a laboratory of lower echelon for a repeat measurement, the lower laboratory not initially being aware of the value obtained at the higher. This procedure can detect significant defects in the wiring or insulation of the test console, improper procedure and careless operations at the lower-lerel laboratory, as well as changes or deterioration in the reference or rorking standards of the lower laboratory.

When systematic local intercomparisons indicate changes or abnormal performance in primary ol' working standards, clrecks with a higher echelon are in order regardless of whether any formal scheduled time for such a check has been reached. If the local periodie checks uncover unsteadiness of operation, liarge sudden changes in value, or other indication of a constructional defect in the reference or working standard, this standard should be sent to its manufacturer for repail before it is submitted to NBS for calibration. 


\section{Reference Standards}

The function of the reference standards of a laboratory is to maintain locally a continuity of value in the units of measurement that they embody. Successive comparisone with the higherechelon standards of NBS by means of in terlaboratory standards shipped back and forth will give an indication of any slow drift of the reference standard. When this cumulative drift exceeds the confidence interval of the values derived bv the comparisons with the interlaboratory standards, the value assigned to the reference standard is corrected accordingly. The reference standards are used primarily to calibrate periodically the working standards of a lower echelon, such as indicating instruments. Also, on occasion they may be used as working standards themselves.

$\dot{I}$ t is evident that both reference standards and working staridards should be as permanent and reliable in construction as possible. Definiteness and repeatability are of major importance, whereas sensitivity, low losses, and freedom from extraneous influences are relatively much less important. Closeness of adjustment to nominal value is of decidedly minor importance. An undetected change in a reference standard may easily initiate a chain of error that will propagate throughout the plant and cause losses in time and material exceeding manyfold the original cost of the standard. Hence, these reference standards should be purchased only on very strict specifications, and from manufacturers of high reputation and of long experience in producing shop instruments and apparatus of demonstrated permanence.

\subsection{Standard Cells}

A large and important laboratory, which is expected to need a standard of voltage with an accuracy of 0.002 percent and to certify the emf of unsaturated standard cells used in its plant or by its subcontractors, normally maintains as a reference standard a group of 5 or 6 cadmium standard cells of the saturated type. These cells are relatively permanent but have a temperature coefficient of about 0.005 percent per degree Celsius (centigrade) at room temperature. They must, therefore be kept in a bath thermostatted to $\pm 0.01 \mathrm{deg} \mathrm{C}[11,12]$. A highly refined, watelwhite, acid-free mineral oil, having a viscosity of about 0.25 poise at $25^{\circ} \mathrm{C}$ and a flash point of $170^{\circ}$ $\mathrm{C}$, has been found suitable for baths for standard rells and for resistors. Such a group of saturated cells should preferably be checked initially at NBS and its value reassigned annually for the first 3 years and biemnially thereafter, by using a second group of 2 or 3 saturated cells as an interlaboratory standard. The individual cells of the reference group can be intercompared monthly. The reference group is used to check the laboratory's working-standard cells of the unsaturated type and, on occasion, shop-standard cells also.
For laboratories requiring an accuracy of not over 0.01 percent in their standard of voltage, the reference standard often consists of a group of three cadmium standard cells of the unsaturated type. The individual cells are intercompared weekly by connecting them by pairs in series opposition and measuring the differential emf. These cells have a very small temperature coefficient and can be shipped safely by parcel post (if carefully packed). However, they are less constant than saturated cells and their emf usually decreases at a rate ranging from 40 to 120 microvolts per year. Therefore, their values should be reappraised at least annually on the basis of a periodic check from a higher echelon. A second group of 2 or 3 unsaturated cells can be used as an interlaboratory standard for such checks. When the emf of an unsaturated standard cell has fallen below 1.0183 volts, it is approaching the end of its useful life and can no longer be used as a reference or an interlaboratory standard. Following a moderate change in temperature, many cells tend to show a very considerable change in emf, which may persist for several days. This thermal "hysteresis" must be guarded against by allowing cells to stand for some time after shipment before taking readings. Temperature troubles with unsaturated standard cells can be minimized by keeping them in a thermally lagged copper-lined box. This reduces temperature fluctuations and differences in temperature between the two electrodes. It is important that the leads be brought out through very high grade insulation to an external terminal board. [13,14].

\subsection{Resistors}

If the laboratory possesses at leave two standard resistors of each decimal value covering the range over which it expects to make accurate measurements, one of these can be submitted to NBS every 2 year's as an interlaboratory standard, while the other resistor of each pair remains undisturbed as a reference standard in its laboratory.

\subsection{Inductors}

Many laboratories find it useful to possess two fixed standard inductors of each decimal value over the range it expects to cover with accurate measurements. In recent years the quality of standard inductors has been greatly improved, and this Bureau does not yet have sufficient data on the stability of the newer types to make a definite estimate of their expected stability. It is suggested therefore that one inductance standard of each denomination be submitted annually to the NBS, until a sufficient history is obtained to predict its performance.

\subsection{Capacitors}

Reference standards of capacitance include small fixed air-dielectric units, precision-variable air capacitors, and solid-dielectric capacitors using mica or an equivalent dielectric. Fixed standards 1,000 picofarads (micromicrofarads) or less in 
value must be of three-terminal construction in order to avoid uncertainties due to stray capacitances. Precision-variable air capacitors are preferably hand carried, although shipment of the capacitor in its wooden container within a padded carton is usually satisfactory. Variable capacitors with noticeable backlash must be adjusted or repaired before submission to NBS for calibration. Capacitors with worm reduction gear are calibrated at the cardinal points corresponding to each whole revolution of the worm wheel. If the worm is eccentric, capacitance increments between cardinal points will depart from linearity, but it is usually not necessary to calibrate the vernier dial for every turn.

Each laboratory should possess two capacitance standards of each decimal value and type needed to cover the range of concern. After its initial check at NBS, one standard of each value can be kept as a reference standard while the other is submitted to .NBS at regular intervals as an interlaboratory standard. This interval should initially be 1 year for any one pair of standards. After the first 3 or 4 calibrations, an examination of the record will indicate the appropriate future frequency of calibration, taking into account the actual accuracy demands made on particular standards. On variable air capacitors the eccentricity correction ordinarily will be determined only during the initial calibration; thereafter, calibration at a few cardinal points is usually sufficient.

\subsection{Volt Boxes}

Each laboratory should have one volt box with a plurality of ratios, which can serve as a reference standard. This can be checked initially at NBS. Unless the laboratory is rather large, this same volt box may be rechecked at NBS at intervals of 2 years, and thus serve as an interlaboratory standard also.

\subsection{Time Standards}

Although time is not an electrical quantity, many electrical laboratories require reference standards of time or of frequency. A high-grade seconds pendulum clock with photoelectric pickup or a standard crystal-controlled oscillator may form the reference standard. Either can be calibrated by reference to the standard-frequency radio signals emitted by NBS stations WWV or WWVH. ${ }^{2}$ By the use of a multivibrator in combination with the reference-standard oscillator, the frequency of working-standard oscillators can be calibrated over a wide range. Such an oscillator can be used to control a standard frequency circuit to which, in turn, synchronous timers can be connected to serve as working standards for measuring time intervals. The use of the frequency of local electric-power circuits as a time standard, while very convenient, may be subject

\footnotetext{
2 For information on this service, consult Radio Standards Division, National Bureau of Standards, Boulder Laboratories, Boulder, Colo.
}

to errors approaching 1 percent for short periods of time, even though the average frequency over a longer time as shown by a clock is very high. These short-time fluctuations in frequency are materially less if the supply is tied in synchronism with a large power system.

\section{Working Standards}

The working standards constitute the principal tools of the standardizing laboratory. They are calibrated at intervals by comparison with the reference standards, and used in the daily work of checking shop instruments. The number needed of any one kind and range will depend upon the volume of testing service demanded in that range. If the volume is very small, and also in special cases where extreme accuracy is needed, a reference standard can be used as a working standard also.

\subsection{Standard Cells}

Most laboratories will need a number of unsaturated cadmium standard cells to serve as working standards and relieve their reference cells of excessive use and of the hazard of accidental abuse. Such working-standard cells should be checked against a reference standard at intervals of 1 or 2 weeks.

\subsection{Resistors}

Depending upon the nature of the work in the laboratory, there will probably be required, in addition to sets of fixed standard resistors, a number of dial-type resistance boxes, perhaps including resistors in the megohm and multimegohm ranges, and also resistors or shunts capable of carrying larger currents than are appropriate to the reference standards. These working standards may initially be tested at NBS to determine the effects, if significant, of current and temperature on their resistance. Thereafter these working standards need to be checked, using a moderate current, at intervals of about 6 months by comparison with the reference standards of resistance, using a direct reading ratio set, Wheatstone bridge, or double ratio set [15], or the potentiometer method. If it is believed that a resistor has been overloaded, it should be checked against the appropriate reference standard without delay, and if a significant change in value has occurred since the last regular check, the overloaded resistor should be checked at frequent intervals until its resistance again becomes steady.

Most standard resistors are made of manganin. This alloy has the valuable property of showing low thermal electromotive force to copper and of changing relatively little in resistance with change in temperature. The resistance, $R_{t}$, at a temperature $t^{\circ} \mathrm{C}$ is related to that, $R_{25}$, at $25^{\circ} \mathrm{C}$ by the formula

$$
R_{t}=R_{25}\left\{1+\alpha(t-25)+\beta(t-25)^{2}\right\} .
$$

Here the coefficient $\alpha$ is usually less than $10 \times 10^{-6}$ and $\beta$ usually lies between $-3 \times 10^{-7}$ and $-6 \times 10^{-7}$. 


\subsection{Capacitors and Inductors}

Each laboratory should compare all of its working standards with its reference standards once a year, and also immediately after an interlaboratory group has been calibrated at NBS. Working standards that are used frequently, or upon which great dependence is placed, may be compared with the laboratory reference standards whenever an important series of plant calibrations is undertaken.

\subsection{Volt Boxes}

A small laboratory may find it sufficient to use its reference volt box as a working standard also. This can be checked initially and at intervals of 2 years at NBS. It is well to measure and record the resistance of each section of each volt box initially, and monthly thereafter, as a means for detecting possible changes such as might be caused by inadvertently overloading one of the lowervoltage ranges. More often other working-standard volt boxes will be used as auxiliaries to potentiometers for the calibration of both workingstandard indicating instruments and shop instruments. These can be compared monthly with the reference-standard volt box by a null method [16].

\subsection{Indicating Instruments}

Direct-current and alternating-current ammeters, voltmeters, and wattmeters of either the 0.1 -percent or the 0.25-percent class [17] will be needed for the range of current, voltage, and power over which the shop instruments used in the plant are to be checked. The a-c instruments must be of the electrodynamic, electrostatic, or electrothermal (thermocouple) types, which can be used on direct as well as on alternating current $[6,8,18]$. The usual a-c instrument of the moving-iron type is not suitable as a transfer standard. The working-standard indicating instruments can be submitted to NBS for an initial test, and the a-c instruments for the additional determination, on appropriate ranges, of the ac-de differe: ce $_{\text {ce }}$ comparison with a transfer instrument. This ac-de difference test should cover the full range of frequency over which the instrument is likely to be used. After this initial test, they can be retained in the laboratory and checked in terms of a standard cell and standard resistor, using a potentiomenter. Preferably, the frequency of these checks ranges from 2 weeks to 2 months, depending upon the frequency of use of the working standards and their reliability as indicated by earlier check tests. Direct-current working-standard indicating instruments, when built in to a console, usually have terminals so arranged that the instrument and its range-cxtending resistors can be checked by using a potentiometer and a bridge. Alternatingcurrent instruments may have to be checked by comparison with interlaboratory standard instruments of a multirange type.

\subsection{Watthour Meters}

Laboratories that have occasion to test large numbers of watthour meters, as do those of power companies, are best guided by the Electrical Metermen's Handbook [1]. This book was prepared by a committee of experienced meter engineers for the instruction of meter-laboratory personnel.

If the laboratory has occasion to test watthour meters only rarely, it is sufficient to have available an electrodynamic wattmeter of suitable range and a standard of time accurate to the degree needed in the energy measurement. The wattmeter can be calibrated by using direct current and a potentiometer, and then used to hold a known constant power while the revolutions of the watthour meter are timed.

In intermediate cases it may be desirable to install a group of three working-standard watthour meters. These can be calibrated as indicated in the preceding paragraph, and each can be used to check a number of other meters. To minimize friction they should be used without register mechanisms but with photoelectric pickups. The complete but time-consuming calibration against the wattmeter need be made only at intervals of a month or so, provided quick intercomparisons among the three working-standard meter's shows no relative change in their rates. Provision can be made by means of suitable precision current transformers so that the standard meter can be operated always at about its full-load speed, even when the meter under test is at light load.

\subsection{Instrument Transformers}

Many laboratories will need a set of multirange current and voltage transformer's covering the range of current and voltage over which other shop transformers are to be calibrated. An initial calibration at NBS, using the burden of the standard circuit of the transformer testing set (plus ammeter or voltmeter), can be made at 60 cycles per second. The errors of current transformers in general are smaller at higher frequencies, but the initial tests should include tests at $400 \mathrm{or}^{\circ} 800$ cycles per second if the transformer is to be used at such frequencies. Subsequent tests at NBS need be made only at intervals of 5 , or even 10 , years.

\section{Comparison Apparatus}

The term "comparison apparatus" includes equipment by means of which the calibration of a shop instrument or standard is checked by comparing it with an appropriate working standard of the laboratory. In many cases the working standard is substituted for the device under test in the same circuit of the comparison equipment, and the change in its indication is taken as the measure of their difference. Such substitution methods are in general capable of very high accuracy, and should be used wherever practicable. 
In other cases a working standard is in effect built in as part of the comparison equipment. Examples of the latter are (1) the working standard indicating instrument built into an instrument testing console and (2) the rheostat arm of a Wheatstone bridge when used directly (as contrasted with its use by substitution).

\subsection{Consoles}

These devices, containing appropriate sources, adjusting transformers and rheostats, and panel instruments for approximate adjustment, will provide the circuits for the comparison of shop indicating and recording instruments with workingstandard instruments $[19,20]$. In some types of console the working-standard instruments and their auxiliary range-extending apparatus also are permanently built into the equipment [20]. If suitable special terminals are available, these working standards can be calibrated like portable standards. If not, some secondary procedure must. be set up by which the console in effect tests other working standards that have previously been checked over the full range. The console can be shipped direct from the manufacturer to the laboratory. The person in charge of calibration work at the laboratory can then satisfy himself, by appropriate measurements of insulation resistance and of circuit resistance, that the connections are correct and that the leakage and lead resistance are not such as to introduce errors. In general, these particular hazards are less if the leads can be run directly in the open between the working standard and the instrument under test.

The working-standard indicating instruments are sometimes mounted at an angle of $45^{\circ}$ for greater convenience in reading. This arrangement, however, tends to introduce additional pivot friction. 'Care must also be taken to insure that no ferromagnetic material is located near enough to either the instrument under test or the standard instrument to affect its calibration. Even nonmagnetic metal supports can cause trouble by providing eddy-current circuits which can affect unshielded a-c instruments. Stray magnetic fields from supply transformers and rheostats (particularly those wound on enameled steel tubes) must be eliminated. The familiarity that the superrisor will obtain by carrying on this acceptance test forms an essential part of his training for the job. His success in it can be verified adequately by the use of a group of indicating instruments as interlaboratory standards in arnual tests. The local checks of insulation and of lead resistance should be repeated every 6 months.

5.2. Direct-Current Bridges, Direct-Reading Ratio Sets, Universal Ratio Sets, Direct-Current Potentiometers

These can be tested initially at NBS and in most cases need only to be resubmitted at intervals of 3 years. During the interim, local checks can be made at intervals of 6 months by using the bridge or ratio set to measure or compare interlaboratory-standard resistors. A potentiometer can be given a rough check at least annually at one point by using it to measure a standard "cell of known emf or, alternatively, by using a single cell first as the standard by which to adjust the potentiometer current and then as the unknown to be measured.

\subsection{Lindeck Potentiometer}

Any laboratory will find very useful a combination of a standard resistor and milliammeter (of the $0.25 \%$ class) to use as a low-range Lindeck potentiometer for the purpose of intercomparing the various standard cells in the laboratory by measuring the differences between them in pairs. The resistor and milliammeter can then be checked on the same schedule as the workingstandard resistors and the working-standard instruments, respectively $[12,21,22]$. Care must be taken to minimize thermal enf in such a circuit.

\subsection{Voltage-Calibrating Transformers}

The ratios of the voltages of the various tapped sections of the secondary winding to the voltage of the tertiary winding to which the standard voltmeter is connected can be determined initially at each operating frequency. This should be done with no load and with rated load on the secondary while the standard voltmeter, or an impedance duplicating it, is connected to the tertiary. Subsequent tests need be made only at intervals of 5 or even 10 years.

\subsection{Instrument-Transformer Test Sets}

The instrument-transformer test sets can easily be checked annually at the 100-percent point and also at one other point. ${ }^{3}$ These checks can be supplemented by using the test set and workingstandard instrument transformer to measure once a year the ratio and phase angle of an interlaboratory-standard instrument transformer of the same range, which is checked at NBS every 5 years. These test sets are currently available for use at 60 and 25 cycles per second. only. Radical modifications in procedure or in component values are required if they are to be used at other frequencies.

\section{Interlaboratory Standards}

Interlaboratory standards in general are similar in nature and inherent accuracy to reference standards and working standards. The smaller laboratories may well use some of their reference standards to serve as interlaboratory standards also. These should, so far as possible, be of rugged construction to minimize clamage and change of value in shipment. On this account they usually are standards of fixed value rather than continuously adjustable devices.

${ }^{3}$ Simple methods for such one-point eheeks on instrument-transformer test sets are now being developed at the National Bureau of Standards. 
The function of an interlaboratory standard is to transmit some one of the electrical units of measurement from a laboratory of higher echelon, such as the NBS, to the local laboratory. It is sent systematically to NBS for a calibration and is compared before and after this operation with the appropriate reference standard of the local laboratory. In those cases in which a large organization maintains a plurality of separate standardizing laboratories under its authority, a single set of interlaboratory standards may profitably be circulated to give a "round-robin test" by being sent to 3 or 4 of the company's laboratories in suecession between trips to NBS. Such a prosram provides a check both on the comparison apparatus and on the operating competence of the personnel at the rarious laboratories. It also performs the function of maintaining the assigned ralues of the reference standards of each laboratory in conrordance with NBS standards.

\subsection{Shipment}

Electrical measuring instruments such as ammeters, voltmeters, wattmeters, and watthour meters contain extremely delieate jewels and pivots, upon which the operation of each instrument depends. These delicate parts must be carefully protected from mechanical shocks and jars during shipment. Sensitive instruments will not arrive in satisfactory operating condition unless great care is taken in packing. Every offort is made to handle and to repack these instruments carefully at the Bureau, and whenever possible the return shipment is made in the original container.

Before each instrument is packed, all binding posts should be tightened, and any externally operated clamping device for the moving systen should be switched to the "clamp" or "transit" position. Plugs and other small accessories should be enclosed in a small separate container tied to the instrument. Glass windows of instruments lacking protective cases should be protected by pieces of thin wood or heavy cardboard beforc wrapping. Each instrmment should then be wrapped in heavy manila paper or similar covering and sealed with gummed tape to exclude dust and excelsior.

Boxes in which instruments are packed should be strong, preferably of wood, with screwed-on tops to avoid damage to pivots or jewels, which may be caused by a hammer or nail puller.

Clean, fresh excelsior or its equivalent in special packaging material should be used as the shockahsorbing material. A layer of excelsior at least 3 to 4 inches deep, pressed down firmly, should surround each wrapped instrument. Instruments having pivoted components should be packed upside down.

High-grade pivoted instruments of the laboratory-standard type, which have comparatively heavy moving systems without clamping devices, should be packed with special care and should always be individually shipped in wooden boxes with 4 to 6 inches of excelsior around the wrapped instrument. Portable standard watthour meters (rotating standards) should also be individually packed.

Certain heavy accessories used with instruments, such as ammeter shunts, current transformers, and voltage (potential) transformers, should be packed in separate boxes to avoid possible damage to the instruments. Heavy pieces should always be shipped in wooden boxes and held in place, if necessary, by cliecks or cleats. Large transformers, especially those having oilfilled iron cases, should be crated singly, and arranged whenever possible so that the terminals can be made accessible for tests without removing the entire crate.

The tops of boxes and crates must be marked "This Side Up." Boxes containing delicate instruments should be marked "Fragile, Handle With Care." Those containing any glass parts should be marked "Glass." Failure to use such markings precludes recourse in the event of loss or damagn in shipping.

\subsection{Standard Cells}

A laboratory having leterence-standard cells of the saturated type would logically provide itself with a group of about three saturated standard cells which can be sent to the NBS annually, while the reference cells are new, but biennially thereafter. Cells of this type must be kept upright at all times and protected as far as possible from shock and temperature changes. This necessitates hand carrying and arrangements at each end for installing them in a thermostatted bath $[11,12]$. Smaller laboratories having unsaturated reference standards may use 2 or 3 msaturated cadmium standard cells as interlaboratory standards. These can be shipped by parcel post. Shipments in extremely cold or hot weather should be avoided. If each interlaboratory ecll is compared with the cells of the laboratory reference group before and after their transport to NBS, a very desirable check is obtained on any changes that may have oceurred during transport. If any one call shows the same value relative to the local group, before and after its travels, it is highly probable that its emf did not change and recover by an equal amount. If all cells are unchanged, the probability of the comparison being valid is greatly increased.

\subsection{Resistors, Capacitors, and Inductors}

Fixed standard resistors, capacitors, and inductors, whether of the fixed-unit type or groups of these combined in dial-type boxes, are satisfactory as interlaboratory standards and can be used at intervals of 1 to 2 years for comparison with NBS, depending on the stability of the reference standards that they serve. Intercomparison between the laboratories of a single organization at intervals of 1 year may prove useful in cases where the volume of testing at the individual laboratories is large and the working standards at these laboratories are therefore un- 
isually liable to deterioration or accidental damage. The values of the interlaboratory standards of this group should be such as to cover the range of measurements with which the laboratory is concerned.

\subsection{Instrument Transformers}

Standard multirange current and voltage transformers can be obtained which are of quite rugged construction and give reliable performance for long periods of time. For a small laboratory, a single set of such transformers covering the complete range may be tested initially at NBS and resubmitted at intervals of 5 or even 10 years for verification. A larger laboratory in which the program of transformer testing cannot be interrupted will need a duplicate set of standardinstrument transformers, one set being used as interlaboratory standards at intervals of 5 years, while it and the other set both serve as working standards the rest of the time.

\subsection{Indicating Instruments}

The use of indicating instruments as interlaboratory standards is often of great value as an over-all check on the comparison equipment and on personnel and procedures. On the other hand, in general, the actual transport of the units of measurement from a higher to a lower echelon is done more accurately by standard cells and resistors. The transfer of the electrical units from d-c to a-c standards is based on the initial tests at NBS [23] of suitable 0.1-percent or 0.25-percent wattmeters, ammeters, and voltmeters. It may be found desirable, as a guard against accidental changes, to verify the performance of the transfer standards by comparing them with similar interlaboratory standards at 5-year intervals. A group of multirange a-c instruments may be used as interlaboratory standards to check the orer-all accuracy of the a-c working standards built permanently into some types of consoles.

\section{Summary}

In the foregoing sections, some of the basic principles on which the operations of an electrical standardizing laboratory should be based have been listed; the types of standard equipment needed have been classified; and the intervals at which these pieces of equipment should be intercompared locally and checked by comparison with a laboratory of higher echelon have been suggested.

The most important considerations in such an enterprise are:

(1) The leader must have a high degree of technical knowledge and competence in the specialized field of electrical measurements;

(2) The measuring apparatus must be adequate and chosen specifically to fit the kinds of measurement and level of accuracy demanded;

(3) The checking procedures must be definite and followed carefully, but should be flexible enough to meet emergencies;
(4) The laboratory must accept responsibility for the internal consistency of its measurements. and should look to a higher echelon (such as NBS) only for its initial calibration and for periodic checks to detect drifts in the values of its reference standards.

\section{References}

[1] 'The meter laboratory, Electrical metermen's handbook, 6th ed., chap. 12 (Edison Electric Inst., New York, N. Y., 1950).

[2] American standard code for electricity meters, Am. Standards Assoc. C12-1941 (Edison Electric Inst., New York, N. Y.)

[3] H. N. Hayward, The calibration, checking and testing of electrical instruments in an education laboratory, Eighth Natl. Conf. Instr. Soc. Amer., paper 53-2-2 (1953)

[4] F. B. Silsbee, Establishment and maintenance of the electrical units, NBS Circ. 475 (1949).

[5] F. B. Silsbee, Extension and dissemination of the electrical and magnetic units, NBS Circ. 531 (1952).

[6] F. L. Hermach, The testing of electrical instruments, Eighth Natl. Conf. Instr. Soc. Amer., paper 53-2-3 (1953)

[7] F. D. Weaver, Notes on the care and use of electrical instruments, Instruments 23, 1236-1239 (1950).

[8] F. K. Harris, Electrical measurements (John Wiley \& Sons, Inc., New York, N. Y., 1952).

[9] F. A. Laws, Electrical measurements (McGraw-Hill Book Co., Ine., New York, N. Y., 1938).

[10] J. B. Dowden, Organizing an electrical instrument standardizing laboratory, Weston Engineering Notes 2, 6 (June 1947); 4, 3 (Feb. 1949).

[11] E. F. Mueller and H. F. Stimson, A temperaturecontrol box for saturated standard cells, J. Research NBS 13, 699 (1934) RP739.

[12] A. W. Spinks and F. L. Hermach, Portable potentiometer and thermostatted container for standard cells, Rev. Sci. Instr. 26, 770 (1955).

[13] J. H. Park, Effect of service temperature conditions on the clectromotive force of unsaturated portable standard cells, BS J. Research 10, 89 (1933) RP518.

[14] G. WV. Vinal, Primary batteries, chap. 6, Standard cells (John Wiley \& Sons, Inc., New Fork, N. Y. 1950).

[15] J. L. Thomas, Precision resistors and their measurement, NBS Cire. 470 (1948)

[16] F. B. Silsbee and F. J. Gross, Testing and performance of volt boxes, J. Research NBS 27, 269 (1941) RP1419.

[17] American standard, Electrical indicating instruments, ASA C39.1-1955 (American Standards Association, New York, N. Y.)

[18] F. L. Hermach and E. S. Williams, Multirange audiofrequency thernocouple instruments of high accuracy, J. Research NBS 52, 227 (1954) RP2494.

[19] F. D. Weaver, An easily assembled console for rapid testing of electrical indicating instruments, Instruments 2\%, 396-399 (1949).

[20] E. A. Gilbert, Equipment for instrument calibration, Elec. Eng. 68, 1065 (1949)

[21] H. B. Brooks, The standard cell comparator, a specialized potentiometer, BS J. Research 11, 211 (1933) RP5S6.

[22] J. H. Mliller, Simplified standard cell comparator, Weston Engineering Notes 9, 1 (Dec 1954) Trans. Am. Inst. Elec. Engrs. 73, (Pt. 1), 413 (1954).

[23] J. H. Park and A. B. Lewis, Standard electrodynamic wattmeter and ac-de transfer instrument, J. Research NBS 25, 545 (1940) RP1344.

[24] S. C. Richardson and F. A. Ludewig, A solution to the problem of electrical instrument standardization service, First Intern. Conf. Instr. Soc. Amer., paper $54-21-2(1954)$.

Washington, May 10, 1956. 


\section{Establishment and Maintenance of the}

\section{Electrical Units}

by F. B. Silsbee

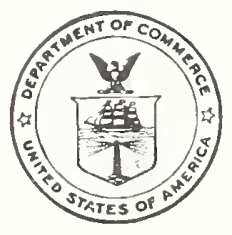

National Bureau of Standards Circular 475

Issued June 30, $19+9$ 


\section{Preface}

January 1, 1948, marked the end of an era in the evolution of the science of electrical measurements. On that date the system of so-called "international" electrical units was abandoned, and a new system of measurement, using what are commonly called "absolute" units, was officially instituted. It is the purpose of this Circular to put on record an account of the working of the international system of electrical units, with particular reference to the maintenance of the international units in the 37 years (January 1, 1911, to January 1, 1948) during which the system was in its final form, to point out the trends of development that made them obsolete, to record the official steps by which they were superseded, and to describe briefly the methods used in the measurements that now form the basis for the new absolute units.

In this record many of the illustrative data will be quoted from the records of the National Bureau of Standards, merely because such data were the most readily accessible to the author. It is to be presumed that a similar examination of the records in any of the other national laboratories would show a similar picture.

E. U. Condon, Director. 


\section{Contents}

Preface _. . . . . .

I. Historical _............... 1

II. Maintenance of Units _... 5

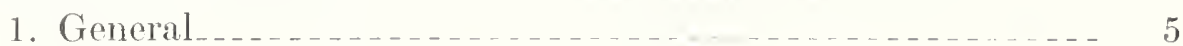

2. Maintenance of the olim at the National Bureau of Stand-

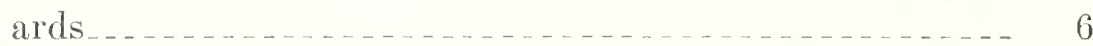

3. Maintenance of the volt at the National Burcau of Stand-

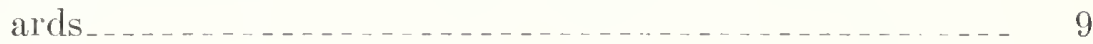

4. Maintenance of units at the International Bureau of Weights and Measures_...................... 11

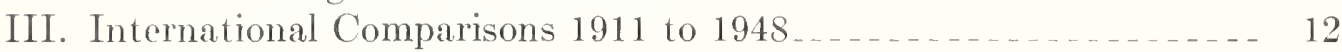

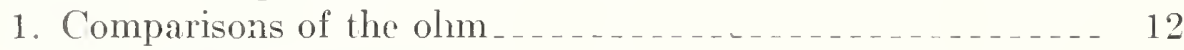

2. Comparisons of the volt _...

IV. Developments 1911 to $1948 \ldots \ldots \ldots \ldots$

1. Enlargement of scope of International Committee on Weights and Measures _....................... 17

2. Trend toward absolute units _...................... 18

3. Official actions leading to the adoption of the absolute units _._.

V. Absolute Measurements__._.

1. General _._.

2. Absolute measurement of resistance _._. $\ldots \ldots \ldots \ldots \ldots \ldots$

3. Absolute measurement of current _... . . . . . . . . . . _ 27

VI. Summary _ 30

VII. References _... 30

VIII. Appendix 1

IX. Appendix 2 $\ldots \ldots \ldots \ldots$

X. Appendix 3

XI. Appendix $4 \ldots 35$

XII. Appendix 5 $5 \ldots$

XIII. Appendix 6 36

XIV. Appendix 7_ 37 


\title{
Establishment and Maintenance of the Electrical Units
}

\begin{abstract}
By F. B. Silsbee
Abstract

A history is given of the establishment of the "international" system of electrical units, its operation during the interval 1911 to $\mathbf{3} 947$, inclusive, and of the developments that caused it to be superseded. It includes a record of the international comparisons, which indicated that the units of some countries have at times drifted nearly 0.01 percent from the mean, an account of the maintenance procedures used at the National Bureau of Standards, and brief descriptions of the methods currently available for the absolute measurement of resistance and of current, which will be used in the future as a check on the maintenance of the units.
\end{abstract}

\section{Historical}

The early workers in electrical measurements set up arbitrary standards of resistance by specifying the use of pieces of wire of a definite material, length, and cross section (or weight), and used the Daniell (zinc-copper) cell as a standard of electromotive force. The work of Gauss (1833) and Weber (1851) showed, however, the possibility of measuring electric and magnetic quantities in terms of mechanical units. In 1861 the British Association for the Advancement of Science established a committee on electrical units and standards [1] ${ }^{1}$ with Maxwell as chairman, which for over 50 years played a leading part in the development and coordination of electrical standards. This committee recognized the fundamental value of correlating the electrical and mechanical units and, in 1863, recommended the particular set of decimal multiples of the centimeter-gramsecond electromagnetic units that form the "absolute practical" units. In this designation the word "absolute" signifies that the unit is defined directly in terms of the mechanical units by a numerically simple relation. The work "practical' signifies that the unit is (except for the unit of capacitance) of a size that is convenient in practical engineering work. The particular factors chosen are given in the following tabulation.

1 absolute coulom $b=0.1 \mathrm{cgs}$ electromagnetic unit of charge.

1 absolute ampere $=0.1 \mathrm{cgs}$ electromagnetic unit of current.

\footnotetext{
1 Figures in brackets indicate the literature referenees at the end of this 1 Figures
paper.
}

1 absolute volt $=10^{8}$ cgs electromagnetic units of electromotive force.

1 absolute ohm $=10^{9}$ cgs electromagnetic units of resistance.

1 absohute joule $=10^{7}$ ergs.

1 absolute watt $=10^{7}$ ergs per second.

These factors were given international status by the Paris Electrical Congress of 1881.

Experiments made under the auspices of the British Association Committee, and by others, led to the assigning of numerical values to the resistance of wire standards and to the electromotive force of certain types of standard cells, which at the time were adequately close approximations to the absolute values. Numerous copies of this "B. A. Ohm" were distributed to laboratories in several countries by the committee as a means of securing international consistency.

In the early use of electricity for communication, the principal need for quantitative results was in the field of resistance measurements, but in the 1880's, with the beginning of the application of electric energy to light and power, the need for measurements of other electrical quantities became acute. Numerous power stations, factories for building electric machinery, and electrical laboratories were rapidly being established in many countries. At that time there were no national standardizing laboratories, and the procedures for making accurate absolute measurements were (as now) both difficult and timeconsuming. There was, therefore, a very definite need for more convenient and universally accepted 
procedures by which the electrical units could be reproduced in an isolated laboratory. To satisfy these needs, much experimental work was carried on to develop appropriate reproducible standards, and a number of international electrical congresses were held to promote their acceptance.

As a reproducible standard of resistance, the most promising seemed to be the resistance of a column of pure mercury of specified dimensions at a specified temperature. A pair of such "mercury cell has electrodes of zinc amalgam and of mercury with an electrolyte of mercurous and zinc sulfates. Its emf is 1.434 volts at $15^{\circ} \mathrm{C}$. Somewhat later Weston (1893) [42] introduced the use of cadmium in place of zinc. This change eliminated a number of the difficulties experienced with the Clark cell, and the Weston cell has come into universal use. The positive electrode is mercury and the negative an amalgam containing about 10 percent of cadmium. A paste of mixed mercurous-

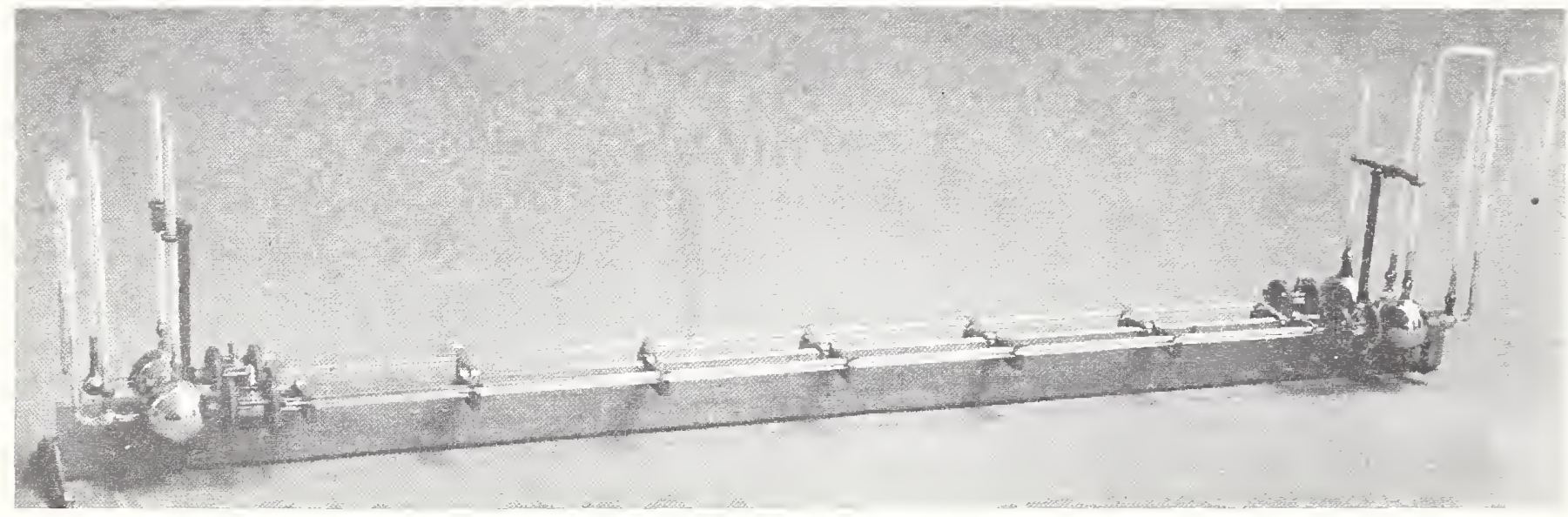

Figure 1. Mercury ohm tubes.

Two are shown, each provided with a large spherical bulb at either end. The potential leads are sealed through the bulb walls at the top, and the current leads connect through the filling tubes along the axis. The ends of the main tube are cut to be flush with the inner surfaces of the bulbs, and a small eorrection is applied to allow for the effective resistances in the bulbs.

ohms" is shown in figure 1. Mercury is easily purified and, at room temperature, has a much higher resistivity and lower temperature coefficient than any other pure metal. One such standard - the old Siemens Einheit - had been the resistance at $0^{\circ} \mathrm{C}$ of a column of mercury $1 \mathrm{~m}$ long and $1 \mathrm{sq} \mathrm{mm}$ in cross section. An international commission, appointed in accordance with a resolution of the International Congress of Electricians, Paris, 1881, adopted as the "legal olım" a mercury column having a length of 106 $\mathrm{cm}$ and a cross section of $1 \mathrm{sq} \mathrm{mm}$. This unit was never officially "legalized" by any government action, and by the time of the Chicago Congress of 1893 a length of $106.3 \mathrm{~cm}$ was recognized as much closer to the correct value.

As a reproducible standard of current, the work of Lord Rayleigh, Kohlrausch, and others gave promise that high accuracy might be obtained with the silver voltameter, in which the current is measured by the rate at which silver is deposited electrolytically from a silver-nitrate solution on the interior surface of a platinum cup. At a meeting of the British Association in Edinburgh in 1892 , a rate of deposition of $0.001118 \mathrm{~g}$ per second under specified conditions was adopted as that corresponding to 1 ampere.

A reproducible standard of voltage had been developed by Latimer Clark (1873) [41] in the type of standard cell that bears his name. This sulfate and cadmium sulfate crystals is placed over the mercury electrode, and the electrolyte is a solution of cadmium sulfate. Cadmium standard cells are made in two types. In one of these,

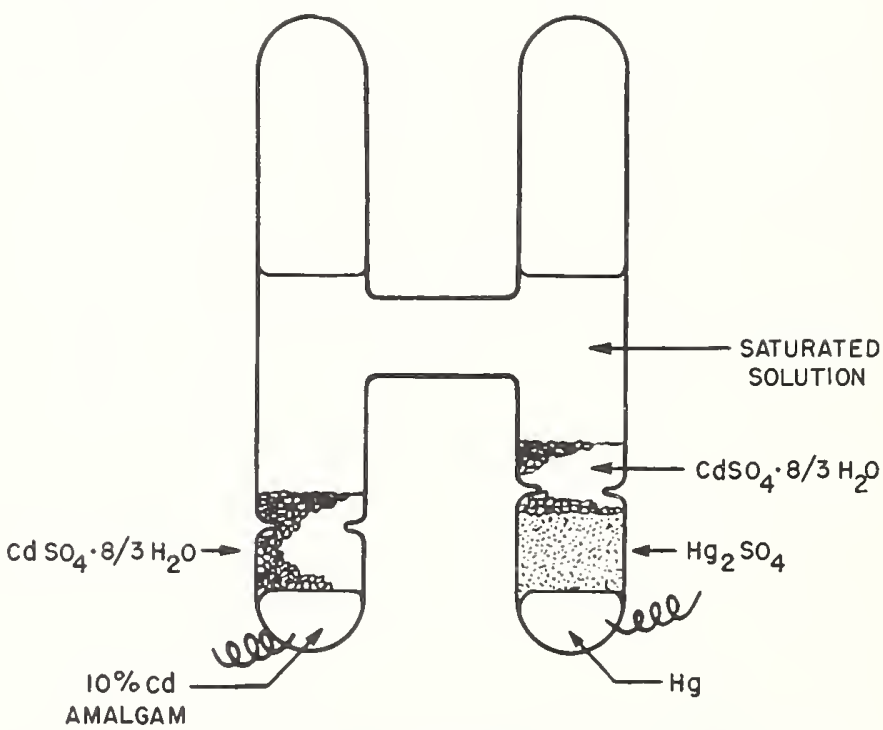

FIGURE 2. Cross section of a saturated cadmium standard cell.

The primary reaction in such a cell is

$\mathrm{Cd}(2$-phase amalgam $)+\mathrm{Hg}_{2} \mathrm{SO}_{4}(s)+\frac{8 / 3}{m-8 / 3} \mathrm{CdSO}_{4} m \mathrm{H}_{2} \mathrm{O}(l)=\frac{m}{m-8 / 3} \mathrm{CaSO}_{4}$ $8 / 3 \mathrm{H}_{2} \mathrm{O}(s)+2 \mathrm{Hg}(l)$. 


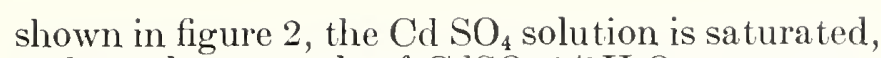
and surplus crystals of $\mathrm{CdSO}_{4} \cdot 8 / 3 \mathrm{H}_{2} \mathrm{O}$ are present at any ordinary temperature. Such cells show a very remarkable constancy of emf with time but have a temperature coefficient of about -0.004 percent per degree C. It is this type that is used for all work of high accuracy, such as maintaining or comparing the national units of emf. Often cells of the saturated type are made using 0.05normal or 0.1-normal sulfuric acid. Such cells are also very permanent but have an emf that is lower by $30 \mu \mathrm{v}$ and $60 \mu \mathrm{v}$, respectively. The second, or unsaturated type, has such a concentration of $\mathrm{CdSO}_{4}$ as to make the temperature coefficient of emf so small as to be negligible for all engineering purposes. This particular concentration corresponds to saturation at $4^{\circ} \mathrm{C}$. This unsaturated cell is the type generally preferred in ordinary laboratory work in the United States because of its independence of temperature. It is, however, less constant in enf.

The term "Weston normal cell" is often used to designate any individual cadmium standard cell of the saturated type described above. Such cells may differ from one another in emf by 10 or $20 \mu \mathrm{v}$. When written "Weston Normal Cell," the term takes on a slightly different and almost mystical significance as a cell that embodies the essential features, namely, emf and temperature coefficient, shown by the average of an infinite population of Weston normal cells. In the light of measurements reported below, an alternative definition for "The Weston Normal Cell" would be a standard cell the emf $E$, of which at a temperature of $t^{\circ} \mathrm{C}$ is given in international volts by the equation

$E=1.018300-0.0000406(t-20)$ $-0.00000095(t-20)^{2}+0.00000001(t-20)^{3}$.

In the literature many instances will be found in which the author has chosen to express the emf of a particular cell or group of cells as differing by a specified amount from the Weston Normal Cell as defined in this way.

The International Electrical Congress held in Chicago in 1893 recognized the fundamental value of the absolute units based on the cgs system of mechanical units and confirmed the decisions of eartier congresses as to the particular integral powers of 10 by which the "practical" units should be related to the cgs units. However, the Chicago congress also recognized the utility of the three reproducible standards and formally recommended the legal adoption of units of both systems as being mutually equivalent.

In accordance with these recommendations the United States Congress passed, on July 12, 1894, Public Bill No. 105, making the practical units, based upon the cgs system and embodied in the reproducible standards, the "legal units of elec- trical measure in the United States." See Appendix 1.

In the years that followed, national standardizing laboratories (Physikalisch-Technische Reichsanstalt 1887, National Physical Laboratory 1899 , National Bureau of Standards 1901), were created in the more highly industrialized nations. The results of work at these laboratories made it clear that, to the higher accuracy then desired, the units of the two sets recommended at Chicago were not equal in magnitude but differed by amounts that were large enough to be measured definitely, and which would, if ignored, cause confusion in future measurements.

Another unfortunate feature of the Chicago recommendations was the specification on independent bases of all three units (the ohm, the ampere, and the volt). In the case of the absolute practical units this is permissible, because an inherent feature of the cgs electromagnetic system of units leads as a theoretical consequence to the relation that an absolute volt is the difference of potential produced between the terminals of an absolute ohm by the presence of an absolute ampere. However, with independent definitions for all three units, Ohm's Law becomes a relation to be experimentally determined, and its expression requires a numerical coefficient, which, in general, will not turn out to be exactly unity. The obvious procedure to avoid such an awkward cocfficient is to define only two units independently in terms of separate standards. There was general agreement that one of the two primary units should be the ohm, but opinion was divided as to whether the other should be the ampere or the volt. The discussion [3] waxed violent, one group emphasizing the (apparent) simplicity of the voltameter, while their opponents, pointing out that the unit had to be ultimately maintained by a standard cell, urged the simple process of specifying the construction of a cell and thus saving one experimental step.

After preliminary discussions at international gatherings in St. Louis, 1904, and Berlin, 1905, an International Conference on Electrical Units and Standards was held in London in October 1908. The resolutions drawn up at this conference were in such form (appendix 2) as to distinguish clearly between (a) the "fundamental units," ohm, ampere, etc., which are exact decimal multiples of the cgs electromagnetic units, and (b) a "system of units representing the above and sufficiently near to them to be adopted for the purpose of electrical measurements and as a basis for legislation . . . the International Ohm, the International Ampere, and the International Volt . . " The "international" ohm was defined in terms of a mercury column, which was specified in full detail, and the "international" ampere was defined in terms of the silver" voltameter, the specifications for which, however, 
were incomplete. The "international" volt was then defined as "the electrical pressure which, when steadily applied to a conductor whose resistance is one International $\mathrm{Ohm}$, will produce a current of one International Ampere." The volt thus became a derived unit and the standard cell became a secondary standard, the emf of which was to be determined in terms of the two primary international units.

The previous practice of setting up cells as standards was still strong in the minds of the committee so that as a sort of byproduct it stated in its Schedule C, "The Weston Normal Cell may be conveniently employed as a standard of electric pressure. . . ," and gave fairly definite specifications for its construction. A provisional value of 1.0184 volts was assigned, but it was expected that this value would be revised from time to time as a result of future experiments. differed by only $10 \mathrm{ppm}$ (parts per million), the German unit being the larger. Preliminary results of work then in progress at the National Bureau of Standards [13] were also in agreement, and the committee therefore "recommended to all countries for general use [5] as the international ohm the mean of the values of the units realized at the P'TR (Physikalisch-Technische Reichsanstalt) and the NPL (National Physical Laboratorv)."

The basis for the ampere was found to be much less satisfactory. In the operation of the silver voltameter one complication arises from the formation at the silver anode of "anode slime" that must be prevented from getting to the cathode where it might cause an error in the weight of the deposit. To avoid such an error a number of arrangements have been used, two of which are shown in figure 3 . In the Rayleigh form of

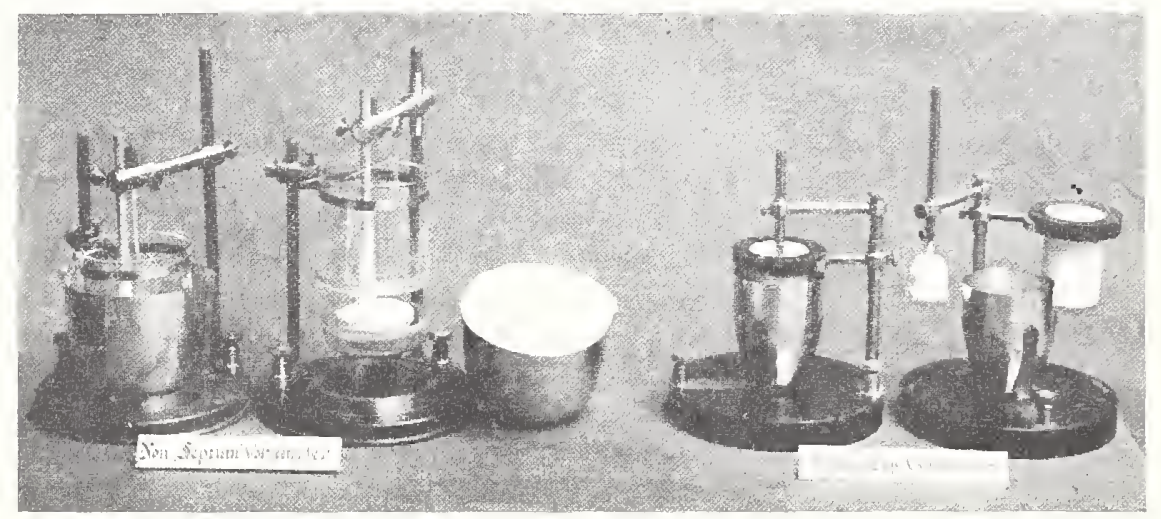

Figure 3. Two forms of voltameter.

The nonseptum voltameter is of the Smith form and the porous-cup voltameter is the Richards form. Each form is shown assembled at the left and disassembled at the right.

As a sequel to the London Conference, a special International Technical Committee met in Washington at the Bureau of Standards in April and May 1910 to carry out work that the Conference had recommended. The three foreign members ${ }^{2}$ of the Committee brought from their respective British, French, and German national laboratories standard resistors and cells that had been evaluated in terms of their national units.- 'They made careful intercomparisons of these coils and cells and those in use at the National Bureau of Standards and also carried out an elaborate series of experiments with silver voltameters of various types. As a basis for the value of the ohm, it happened that both the Reichsanstalt and the National Physical Laboratory had each recently completed an elaborate program of comparisons of the resistance of mercury columns with wire standard resistors $[11,12]$. The comparisons of their standards at Washington showed that they

${ }^{2}$ The International Technical Committee consisted of Dr. E. B. Rosa (NBS), chairman: Dr. W. Jaeger (PTR); Prof. F. Laporte (LCE); Mr. F.E. Smith (NP1); and Dr. F. A. Wolft (NBS). voltameter the anode is wrapped in filter paper. In the Richards (or porous pot) form a porous, unglazed porcelain pot surrounds the anode and catches the slime while permitting the current to flow in the electrolyte in the pores. In the early Kohlrausch form, a shallow glass cup is placed below the anode to catch the slime, and in the later Smith for'm, a glass cylinder can be lowered at the completion of the run so as to fit tightly on the upper edge of the cup and prevent any spilling of material when the voltameter is disassembled. A siphon form, in which the two electrodes are in separate vessels, has also been used.

The work of the committee showed clearly that voltameters of various types might give consistently different results. For instance, the deposits from solutions in which filter paper was used were heavier by about $150 \mathrm{ppm}$. The committee was therefore "of the opinion that the specifications for the silver voltameter should not be completed until further experiments shall be made . . .". A great deal of work was 
done later, particularly at the National Bureau of Standards, [21] in response to this recommendation. A number of sources of error were tracked down and definite operating procedures for the silver voltameter were formulated, but the specifications were never officially adopted.

In spite of the incompleteness of the specifications for the voltameter, however, the committee used the results of the voltameter experiments, together with the values in international ohms assigned to various coils, to derive a value for the international volt and to assign values in terms of this for the emf of the various standard cells used in the work.

A further result of the work of the International Technical Committee was a revision of the value tentatively assigned in 1908 for the emf of the Weston Normal Coll in terms of the International Volt. The committee took as a value representing the Weston Normal Cell the mean of the separate group means of the four groups of cells that had originated in the four different countries, and which had had time to settle down to constant values. A total of 104 cells were included, the number in a group ranging from 15 to 40 cells. The a verage deviations for the emf of an individual cell from the mean of its group was $\pm 12 \mu \mathrm{v}$, while the groups deviated by $\pm 5 \mu \mathrm{v}$ from the mean of the four. The voltameter experiments, excluding the most doubtful results such as those with filter paper present, gave for the final mean the value of 1.0183 international volts.

On the completion of the work of the International Technical Committee [5], each delegate carried back to his respective laboratory its standard coils and standard cells each with its newly assigned value in terms of a common system of units. The system of "international" electrical units was thus successfully launched with universal agreement on the magnitude of the units as of January 1, 1911.

\section{Maintenance of Units}

\section{General}

The general procedure followed at the National Bureau of Standards to preserve through the years the value of a primary electrical unit of measurement is to keep under observation a large group, $N$, of standards of the highest grade. A selected group, $n$, of these standards is regarded as the primary reference group, and the assumption is made that in the interval between intercomparisons the mean value of all the standards in the primary group, $n$, has remained unchanged. The intercomparisons are made at intervals, usually once a month for standard cells and seldom exceeding a year for standard resistors. Such comparisons are often preliminary to important checks on the standards of other laboratories.

Following each set of measurements, the value of each of the standards of group $n$ relative to the new mean is compared with its previous value relative to the old mean, and its apparent change is compared with those of the other standards of group $n$. If these changes are reasonably small and well distributed statistically it is assumed that all is in order. If however one standard shows a change markedly out of line with the others, it is assumed that some unsuspected accident has occurred and the value of this standarel is omitted from the mean. In its place is inserted the value of one of the other standards, from the larger group, $N$, which has shown a reasonable performance during the preceding periods. Thus the final decision as to which standards carry the unit for a given period is made after the data obscrved at the end of the period have been scrutinized.

After this process has been in operation for a number of periods a new criterion for the rejection from group $n$ of a questionable standard becomes available. A standard that shows only a moderate change during any one interval may, when earlier data are examined, be found to show a progressive drift in one direction and may be rejected for that reason. In such a case two procedures are possible: (1) The value assigned to the mean of the new group may be taken as that which it had at the beginning of the last interval between comparisons; or (2) A new value of the unit can be derived on the basis that the suspected standard had been rejected at the time it first began its drift and that thereafter the unit had been carried by the remainder of the group. The latter process is called "recapturing the unit." Procedure (2) results in a formal admission of an abrupt change in the value of the unit at the time it is "recaptured," because measurements of the same quantity made just before and just after" the readjustment will differ by the amount of the adjustment. With procedure (1) the cliange in the unit is distributed over the period of drift and is not recognizable though still present. Procedure (2), of course, is the more suceessful in the long run in eliminating the cumulative effects of drifts in one direction. At the National Bureau of Standards, procedure (2) is regularly used in the maintenance of the volt because the tendency of defective standard cells is always toward a drop in emf. In the maintenance of the ohm, procedure (1) has been followed. With either procedure, the intervals are so short and the accuracy of the measurement is so high that any defective standard can be detected and weeded out beiore its effect is large enough to be 
significant. The use of a fairly large number $(n=10$ or 25$)$ of standards in the primary group contributes very materially to reducing the effect of a drift in any one of them.

\section{Maintenance of the Ohm at the National Bureau of Standards}

Following the legalization of the electrical units by Act of Congress in 1894, the Office of Standards, Weights and Measures, then part of the Treasury Department, was authorized in 1897 to appoint a rerifier. As fast as the very limited appropriations permitted, standard resistor's of various denominations were purchased in Germany and standardized at the PhysikalischTechnische Reichsanstalt. When the National Bureau of Standards was set up, following the passage of its organic act in 1901, it took over this equipment and the personnel that had been operating it. The unit of resistance during this period was therefore that of the German institution. At that time resistance apparatus made in Berlin by the firm of Otto Wolff was recognized as being of the highest quality available, and the Reichsanstalt, the oldest of the national laboratories, was currently active in setting up mercury columns. Resistance standards and other resistance apparatus of Wolff's manufacture were purchased by the Bureau in considerable quantity, submitted to the PTR for standardization in terms of the unit of that institution, and then shipped to Washington to equip the new laboratory.

In 1907 a group of four recently certified 1-ohm resistors of the Reichsanstalt type were received from Berlin. The values of these resistances formed the basis for assigning values to a larger group consisting of ten 1-ohm and seven 0.1-ohm standard resistors. The mean of this group was assumed to remain constant from that time until May 1909, and thus carricd the unit forward.

In the meantime, experimental work at Washington had shown that open standards, such as those of the Reichsanstalt type [31], underwent a marked seasonal fluctuation in resistance, which, in the case of some 1,000-ohm resistor's, amounted to as much as 200 parts per million in 3 months. This effect, reported by Rosa and Babcock [32], was traced to seasonal variations in atmospheric humidity. The effect is much less with 1-ohm resistor's but, to avoid these fluctuations, Rosa [33] designed a new type of standard resistor. In this the insulated manganin coil is enclosed in a hermetically sealed metal container. The container is nearly filled with refined mineral oil, and the terminals are brought out through a hard rubber top. A considerable number of standards of this construction were manufactured both in the instrument shop at the NBS and also by Otto Wolff.
In May 1909, values of resistance were assigned to a group of 1-ohm standards of this new construction, on the basis of the unit that had been carried until then by the seventeen open-type coils. From May 1909 until October 1932 the ohm was maintained at the NBS by a "primary" group of 10 resistors selected from these 1-ohm standard resistors of the Rosa sealed type. The unit thus maintained is that which later came to be designated as the "Ohm-certified NBS".

Comparisons made in 1910 , in connection with the work of the International Technical Committee on electrical units, showed that at that time this unit was larger by $2 \mathrm{ppm}$ than the unit then used by the PTR, and larger by $7 \mathrm{ppm}$ than the mean value of the British and the German units. This mean value was adopted by the Committee for purposes of international uniformity and is commonly designated as the "Washington unit". (It is this Washington unit that is represented in figure 8.) The slight difference of 7 ppm was entirely negligible in the practical conduct of ordinary electrical measurements at that time, and therefore the "book values" assigned to the several resistance standards on the earlicr basis were not changed.

Between 1909 and 1932, careful comparisons of the relative resistances of the entire group of 1-ohm coils were made at somewhat irregular intervals, averaging 6 months. In computing the results of each intercomparison, it was assumed that the average resistance of 10 selected coils of the highest grade had remained unchanged since the prior intercomparison. On this basis a new value was assigned to each of the coils of the entire group. An examination of the data shows that the average difference (without regard to sign) between the two values thus successively assigned to a resistor was only \pm 1.3 microhms, and that in 95 percent of the cases the difference in the successive values was less than \pm 3 microhms.

In general, the same resistor's were kept in the standard group from year to year unless there was some reason for a change. Also, five of the standards that at one time had been removed from the primary group were later used in it again, so that during the whole period of 23 years twenty standards were involved. During the period, standards were removed from the primary group on 15 oceasions. On six of these occasions there was no suspicion of a change or defect in the standard, but the removal was occasioned by the use of the standard for some other purpose, frequently for shipment abroad for use in an international intercomparison. Of the remaining nine removals, one was oceasioned by the development of a loose telminal, which obviously caused the hermetic seal to be broken; five were occasioned by an abnormally large increase in the resistance of the coil over its value at the preceding intercomparison. These increases ranged from a minimum of 5 to a 
maximum of 12 microhms. Three were oceasioned by decreases in resistance, the maximum of which was less than nine microhms. Two of the standard resistors remained in the primary group for the entire period, and six resistors were in the group for more than 15 years.

Examination of the data shows that the resistances of five of the resistors drifted at a slow and fairly steady rate over the period while they were in the primary group. The rates of drift of their resistances ranged from a decrease of 1 ppm per year to an increase of 4 ppm per vear. One standard that was used for a short period showed a decrease at a rate of 6 ppm per year. 'The coil that approximated most closely to the mean showed a decrease of 0.3 ppm per year. Several coils showed a dift that changed from an increase of 0.6 ppm per year when first included to a decrease of 2 ppm per year after several years had elapsed.

A comparison of the individual readings made on one of the stamdards, which showed a nearly linear decrease of 1 ppm per year, with a least square straight line adjusted through these points shows a standard (root mean square) deviation of $\pm 2.8 \mathrm{ppm}$. It is probable that the lack of precision in the electrical measurements involved in cach interomparison could have contributed only a very small part of this varuation. Most of it probably results from minor secular variations, and perhaps some from meertainties in the temperature of the standard itself.

Begimning in 1928, Thomas [34] had constructed a number of standard resistors of a new type sometimes referred to as "dlouble-walled." One out through a shellac seal. A gioup of such resistors showed such excellent behavior that two of them were used in the primary group in Octobel 1930 and a third in April 1932. Beginning in October 1932, the use of the Rosa sealed standards in the primary group was discontinued, and 10 resistors of the 'Thomas type made with a coil diameter of $6 \mathrm{~cm}$ formed the primary group until Mareh 1939.

During this period the average difference observed between successive measurements made at intervals avelaging 6 months was only 0.3 microhm, and 80 percent of these differences were less than 0.8 microhm. A least square line adjusted to fit the data over this period of 6 years, for the worst of these resistors, showed an average slope of less than 0.5 microhm per year and a standarcl deviation from a straight line of only \pm 0.4 microhm. It is thus evident that this type of construction produces a standard resistor the relative stability of which is better by an order of magnitude than that of the Rosa type.

In 1933, work was completed on the construetion of an additional group of 'Thomas-type standards of somewhat larger dimensions in which the coil had a cliameter of $8 \mathrm{~cm}$, with a correspondingly greater cooling surface. These standards were compared with the smaller double-walled standards between 1932 and 1939, and from March 1939 to the present time a group of 10 of the large Thomas-type standards has been used to carry the unit forward. The average difference observel between successive measurements in individual coils is \pm 0.2 microhm, and 95 percent of the differences do not exceed \pm 0.5 microhm.

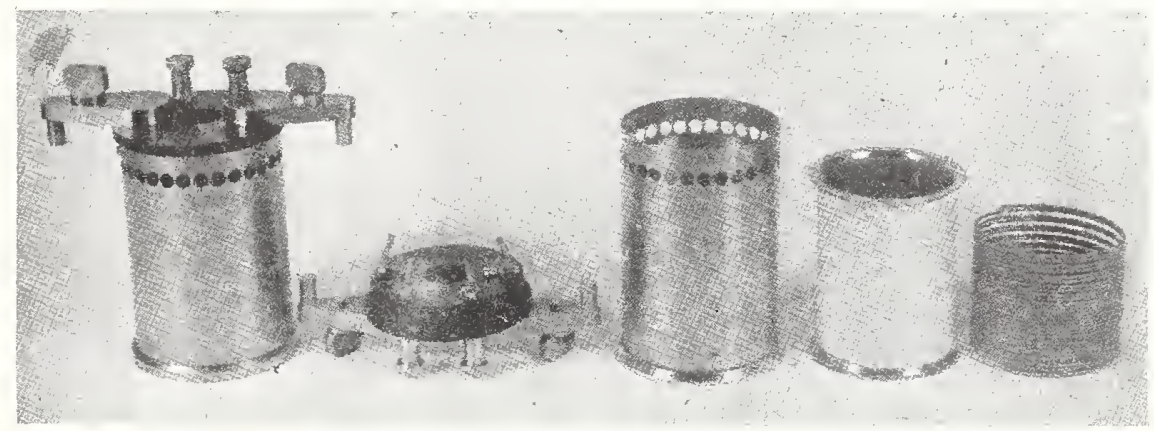

FICURE 4. Double-walled (Thomas type) standard resistor.

The manganin wire shown at the extreme right is annealed in vacuun. It is tied down on the inner form shown next to it and then sealed retween the inner and outer brass eylinders.

of them is shown in frgure 4 . In these the bare manganin wire is annealed in a vacuum at a temperature of $500^{\circ} \mathrm{C}$. or above, while wound on a manchel. It is then slipped on an insulated brass form, spaced with linen thread, tied down, and fastened with shellac, and then baked for about an hour at $80^{\circ} \mathrm{C}$. The form is tubular and constitutes the inner wall of a container that is sealed by soldering its end flanges to an outer, shightly larger, tube. Dry air at about atmospherie pressure fills the container, and the leads are brought
The slope of a least-square line passed through the points observed, in the case of the worst of these standards, is 0.1 microhm per vear, and the standard deviation of an individual point is 0.1 microhm.

One methor attempted at the NBS as a means for independently corroborating the maintenance of the ohm was the use of standards formed by wires of pure metals. It would not be expected that such standards would drift at the same rate and in the same direction as those of the metallur- 
gically ḿnore complex manganin alloy. In 1933, a platinum and a gold standard were made by winding wires of these metals on insulated brass tubes and enclosing each in a sealed metal container. When in use the container was immersed in an ice bath. Because of the high temperature coefficient of resistance of pure metals, a change of $0.00025 \mathrm{deg} \mathrm{C}$ will change the resistance by $1 \mathrm{ppm}$. This accuracy in temperature seems to be about at the limit to which the ice-point can be reproduced. Measurements at intervals of about a year, between 1933 and 1939, in which these pure metal standards were compared with the small Thomas-type primary group, showed for the gold coil an apparent decrease in its resistance of only $0.13 \mathrm{ppm}$ per year, but the platinum coil showed an apparent increase of nearly $0.7 \mathrm{ppm}$ per year. The standard deviation of either of these rates of drift is $\pm 0.2 \mathrm{ppm}$ per year. When used in 1947 it was found that the insulation on both of these standards had deteriorated to such an extent that precise measurements could not be made. It is doubtful, therefore, whether much weight should be assigned to the results from the coils of pure metals.

Perhaps the best indication of the constancy of the ohm as maintained in Washington is found in the results of comparisons with the standards of other national laboratories and with the mercury columns set up occasionally by these other institutions. The situation is discussed in section III and summarized by figure 8 .

It seems evident from the foregoing lata that the apparent constancy of the unit of resistance as maintained between 1909 and 1932, while Rosatype standards were used, was in considerable measure the result of good luck in the use of standards, some of which increased while others de- creased in resistance. The tendency to increase was presumably the result of oxidation or corrosion of the resistance alloy, while the tendency to decrease was perhaps the result of a slow release of strains, or of more obscure metalhurgical processes. Of course, the use of a considerable number of standards in the primary group decidedly increased the probability that the group should contain standards drifting in both directions, but even with as many as ten standards in the group the drift in the mean value night well have been much greater than it apparently was. The basis for these self-congratulatory remarks is found, of course, not in the data resulting from intercomparisons within the group but from the international intercomparisons shown in section III, 1 , figure 8 . Both the German and the British laboratories used a smaller number (four) of standard resistors and apparently were unfortunate in choosing coils that tended to increase in resistance, but their painstaking work with mercury columns enabled them to detect these drifts before they became significant in the industrial field and to make corresponding adjustments in their units. The various deterininations of the olm in absolute units made prior to 1925 are probably not accurate enough to be of much significance in insuring its maintenance but give no indication of any continuous drift. As indicated below, it is probable that from 1939 onward absolute measurements of higher precision may prove to be very valuable for this purpose as well as for the primary establishment of the unit.

The comparisons of the 1-ohm standard resistors have been made since 1918 by use of the highprecision bridge shown in figure 5 [36]. It is used as a Kelvin double bridge and the main ratio arms are of the Waidner-IVolff type [37]. The

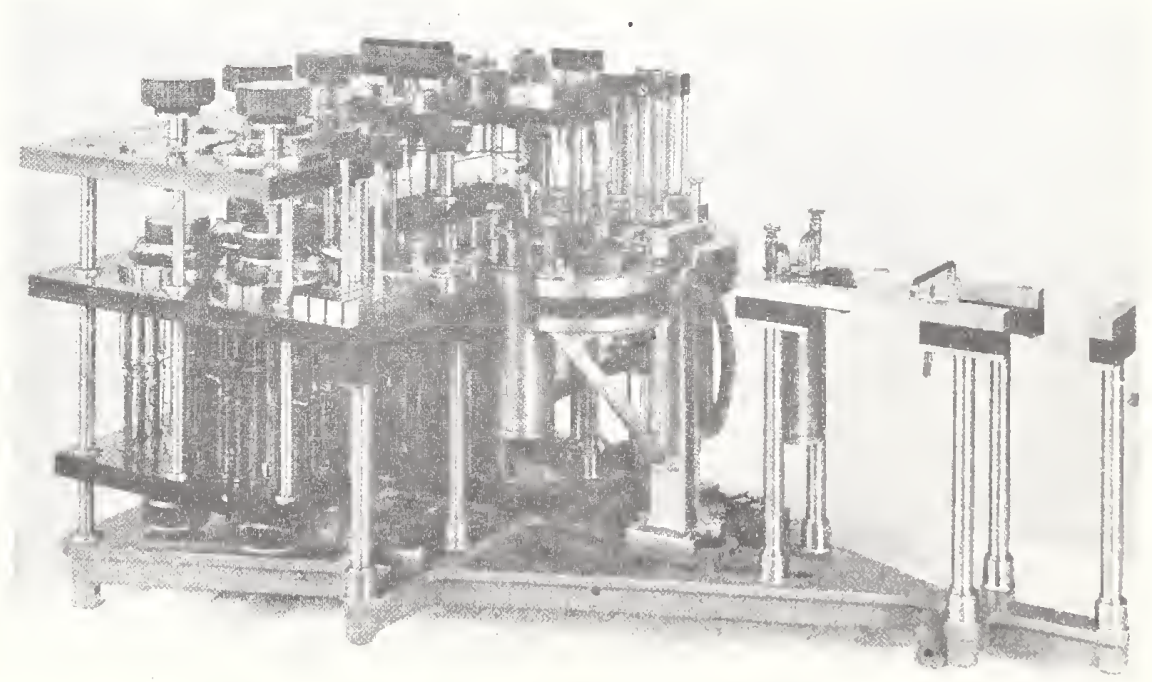

Figure 5. IIigh-precision bridge.

In use, the whole structure is immersed in a temperature-controlled oil hath to a level above that of the intermediate insulating slab. All resistors and contacts are therefore at a uniform constant temperature. It may be connected as a Wheatstone or as a Kelvin bridge. The nominal ratio may be $1: 1$ or 10 . The standards under test are connected through amalgamated contacts. 
contacts are only in adjustable shunt circuits of relatively high resistance in parallel with the main resistor's, which are connected permanently. The lowest dial corresponds to $1 \mathrm{ppm}$ per step and the galvanometer is sufficiently sensitive to permit of extrapolation to the next significant figure.

\section{Maintenance of the Volt at the National Bureau of Standards}

The Chicago Electrical Congress of 1893 and the resulting law passed in 1894 had specified 1.434 volts at $15^{\circ} \mathrm{C}$ as the emf of the Clark cell. A number of Clark cells were made up prior to 1906 at this Bureau using specially purified materials. After correcting for temperature and subtracting 0.0003 volt to allow for the higher purity of the mercurous sulfate used, a value of 1.42110 volts was decided upon as the mean value at $25^{\circ} \mathrm{C}$ to be assigned to the new Clark cells. Some saturated Weston cadmium cells, made in May 1906, were then compared with the Clark cells, and as a result of the measurements, a mean value of 1.01890 volts at $25^{\circ} \mathrm{C}$ was derived as the mean of a group of 12 of the Weston cells. This group, with certain losses and replacements, served to carry the unit until the close of the meeting of the International Technical Committee in May 1910.

The experimental work of this committee with silver voltameters of various types fixed the International Ampere. Earlier measurements in England and Germany on mercury columns had served to fix the values of wire standard resistors in terms of the International Ohm. The combination of these units by the committee in its voltameter experiments, which involved the NBS reference cells, led to the conclusion that the volt, as then maintained at the NBS, was smaller by 852 microvolts than the "International Volt" as derived from the International Ohm and the International Ampere. Accordingly, effective January 1, 1911, new values, smaller by this amount, were assigned to all the cells, and the volt and the other electrical units derived from it were changed abruptly on that date by corresponding amounts. This change was announced in Bureau of Standards Circular C29 [4], which contained a brief history of the subject and a summary of the reasons for making the change.

As a byproduct of the work of the International Technical Committee, the Bureau came into possession of a group of 36 cells, which had been included in the large group used to assign a value to the Weston Normal Cell, and which included cells of British and German as well as American origin. Until January 1912, this group was used to maintain the unit of emf, and the mean of these 36 cells was assumed to remain at 1.018300 volts at $20^{\circ} \mathrm{C}$. By that time it became evident that some of the cells had drifted rather badly. A smaller group of 19 cells, which had shown steady and nearly equal increases on the new basis, and four of which had been in the reference group in 1908, was therefore used instead to carry the unit forward from 1910 and continued to serve (with two eliminations) as the primary group until 1914. Several changes were then made, and a group of eighteen cells carried on until July 1919 when three were dropped and five added. These 20 cells carried on, with the loss of four, until April 1937 when nine cells of the 0.05-normal acid type, which had been made in December 1932 , were added. These 25 cells were in use through 1947.

As an indication of the mutual consistency of the neutral, saturated cadmium cells, data taken between 1919 and 1933 on the primary reference group showed that 18 of these cells had changed relative to their mean by less than $15 \mu \mathrm{v}$ (microvolts). Nine cells showed a net increase, while the other nine had decreased, the average net change during the 14 year's, taken without regard to sign, being $\pm 5.0 \mu \mathrm{v}$. This corresponds to an average drift rate of $\pm 0.25 \mu \mathrm{v}$ per year; the greatest mean drift rate being $1 \mu \mathrm{v}$ per year.

Superposed on these net drifts the cells showed slight fluctuations in emf. Successive values for the same cell measured after the lapse of about a year differed on the average by $\pm 2 \mu v$. The precision of each comparison as indicated by repeat measurements made a few hours or a few days apart was about $\pm 0.1 \mu \mathrm{v}$, so that these secular fluctuations must be attributed to random changes in the cells. None of the differences between the annual values exceeded $10 \mu \mathrm{v}$, and 83 percent of them were less than $4 \mu \mathrm{v}$.

In contrast to this behavior of "good" cells, certain cells after showing similar good behavior for many years suddenly begin to drop in emf at a rate of from 3 to $10 \mu \mathrm{v}$ per year. Since 1919 four such cases have developed and after the "delinquency" of each cell was definitely established by the continuance of its drift, the cell was removed from the primary group. The unit was then "recaptured" by going back to a time before the abnormal drift began and computing forward again on the basis of the remaining cells only. 'This process resulted in introducing three abrupt increases of $4.6,0.8$, and $1.1, \mu \mathrm{v}$, respectively, in the volt on September 19, 1933, April 10, 1937, and September 7,1944 . 'The changes in the group between January 1912 and July 1919 resulted from shorterperiod troubles, which involved three broken cells. These did not involve a shift in the unit.

Of the 16 neutral cells in the group in 1947, 13 had been in the primary group since 1914 and five since 1908. The nine acid cells had been in the group since 1937.

Theoretically, the procedure of maintaining a unit of measurement solely by the use of a large group of standards is subject to the fundamental limitation that a simultaneous progressive drift 
of all the standards cannot be detected by the intercomparisons and will result in an equally rapid drift of the unit. It is therefore important to seek external corroborative evidence of the constancy of the unit.

One such line of evidence derives from the construction of new groups of cells from freshly prepared batches of pure materials. One example of this is a batch of six cells constructed in 1925 the mean value of which was found to be greater in terms of the 1925 unit by only $12 \mu \mathrm{v}$ than the mean of the large number of cells set up 15 years earlier by the International Technical Committee. Very similar evidence results from the comparisons shown below (see figure 9 section III, 2) between the British and American volts over a long time. At the British National Physical Laboratory fresh groups of cells are made up every few years and the older groups are discarded at intervals so that their unit has been carried forward by cells that range in age from 1 to 20 years averaging about 6 years. Since 1912 most of the American neutral cells ranged in age from 6 to 41 years, averaging over 20 years. Another difference arises because the British laboratory uses 0.1-normal acid cells, while until 1937 the American cells were all neutral. Yet since 1925 the difference between the two volts has oscillated only over a range of $\pm 7 \mu \mathrm{v}$ from a mean difference of $24 \mu \mathrm{v}$, the British volt being the larger. The reason for the earlier drift of $18 \mu \mathrm{v}$ between 1911 and 1925 (that is 1.3 $\mu \mathrm{v} /$ year) is not known.

Another line of evidence lies in the comparison of acid and neutral cells. The emf of a 0.05normal acid cell is about $30 \mu \mathrm{v}$ lower than that of a neutral cell, but the tendency for the mercurous suliate to hydrolyze and for constituents of the glass container to be dissolved by the electrolyte are definitely less. On the other hand, acid cells sometimes develop objectionable gaseous films on the amalgam electrode. Because of these chemical differences, it seems reasonable to expect that ii either type of cell showed a characteristic consistent drift in emf its rate would be somewhat different than that for cells of the other type. Over the 7 years from 1937 to 1944 , the average of the nine acid cells $(0.05 \mathrm{~N})$, which had been made in 1932, increased relative to the 16 old neutral cells, which were at least 24 years old, at a rate of only $0.9 \mu \mathrm{v}$ per year.

$\dot{A}$ better check on drift can be obtained by the use of the silver voltameter. Even yet there are no authoritative specifications that enable the voltameter to be used unambiguously as a means for reproducing the international ampere. How-

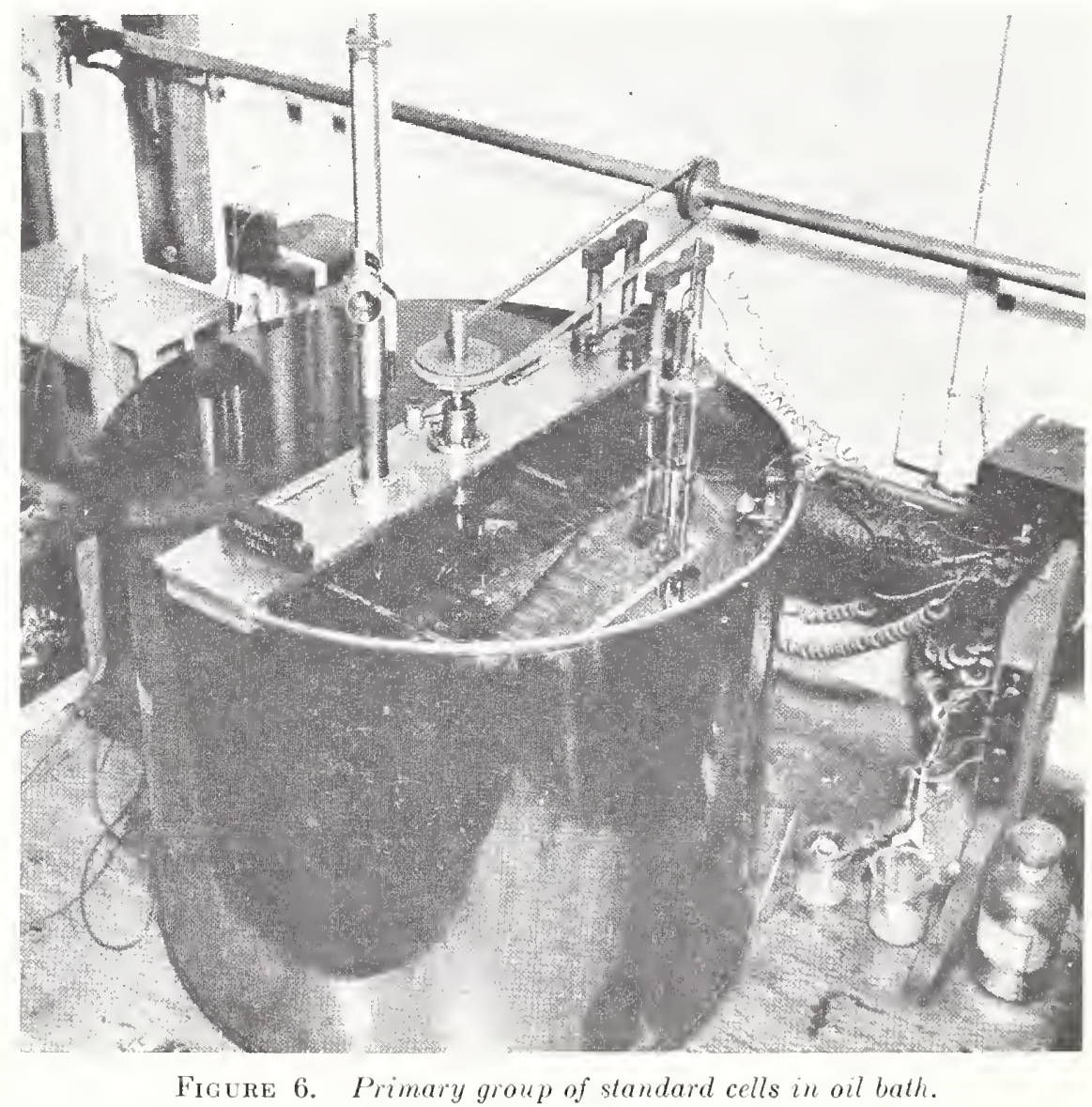

The stired bath is thermostatieally controlled at $28 \pm 0.01^{\circ} \mathrm{C}$. Conneetions are made through mercury cups under the on to the platinum leads sealed through the glass $\mathrm{H}$-tubes. 
ever, experiments repeated with a given type under given conditions after the lapse of a number of years are adequate to reproduce the same value of current to a high accuracy. In 1931 a series of measurements using the Smith form of voltameter were carried out at the Reichsanstalt by Vinal [52], Vigoureux [53], and Von Steinwehr [54]. These indicated that the volt as maintained at the National Bureau of Standards was the same in 1931 within $10 \mathrm{ppm}$ as it had been when similar experiments with the Smith form of voltameter were made in 1912 at Washington. Hence the average drift could not have exceeded $0.6 \mathrm{ppm}$ per year.

Figure 6 shows the oil bath that contains the primary group of cells. Its temperature is controlled automatically to $0.01^{\circ} \mathrm{C}$. As a precaution against loss of the unit by fire or other catastrophe, two supplementary reference groups of 10 cells each are housed in other buildings. Although these groups are not held at a constant temperature, they have shown little tendency to drift as compared with the primary group.

The intereomparisons of the cells in the primary group are made by opposing the emf of the cell being measured to that of a selected reference cell and measuring the small difference in emf. Because the difference in emf is small, only a. moderate percentage accuracy in its determination is required to give very high accuracy in the final result. Since 1933 the instrument used in this work at the NBS has been the Brooks Standard Cell Comparator [43] shown in figure 7. It com- tional emf developed at its termınals. By an arrangement of slots in the covers of the dials, the instrument serves also as a computing device, one part of which is set to read the known emf of the chosen reference cell, while the other part, when a balance is obtained, indicates directly the emf of the unknown.

The greatest source of error in such measurements is the presence in the eireuit of spurious emfs, such as those from thermoelectric action. The principal features in the design and operation of the comparator are devoted to minimizing such emfs. Each division on the milliammeter corresponds to $1 \mu \mathrm{v}$, and the galvanometer is sufficiently sensitive to make readings to 0.1 division significant.

\section{Maintenance of Units at the International Bureau of Weights and Measures}

Additional information on the accuracy with which electrical units can be maintained at a properly equipped laboratory is afforded by the performance of the reference groups of resistors and standard cells kept at the International Bureau. The report by M. Romanowski [58] lists the results of intercomparisons between 1939 and 1946 .

Data are given on the four resistors that form the primary group, $\mathrm{GO}_{3}$, and on five other standard resistors. Romanowski in his report has made an extrapolation on the assumption that the the resistances of these standards were increasing

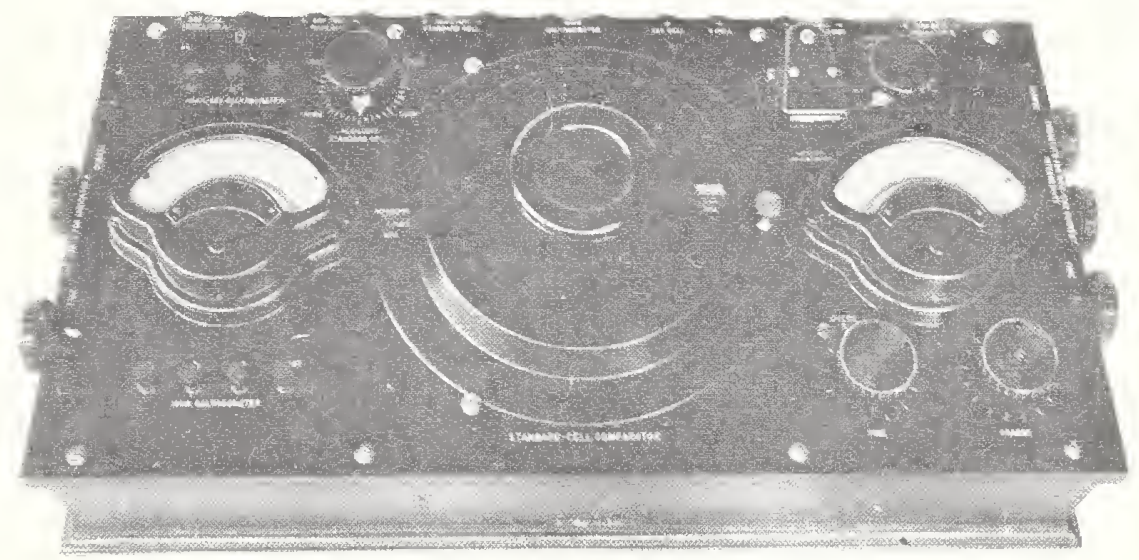

Figure 7. Brooks standard-cell comparator.

The cover and the left-hand milliammeter are adjusted to read the known emf of the reference cell. The main galvanometer (not shown) is balanced hy turning the central handle and adjusting the eurrent in the right-hand milliammeter. When a balance is reaehed, the emf of the unknown cell is read from the dizits in the right-hand slot and the right-hand milliamneter.

prises a specialized form of potentiometer in which tho bulk of the emf to be measured is balanced against the IR drop in an adjustable precision resistor but the last two digits of the result are obtained by reading a milliammeter, which measures the current in a small additional resistor in series in the cireuit and thus indieates the addi- in value by 0.95 ppm per year. As will be shown below, such a drift was probably not real but was a result of the assumption that the mean of the ohms of the six national laboratories had not drifted between 1936 and 1939 . If his data are reduced on the alternative assumption that the mean value of $\mathrm{GO}_{3}$ remained constant over the 
7-year period, all the standards except the one open-type coil (designated $\mathrm{R} 2836$ ) show a very excellent performance. The mean difference taken without regard to sign between successive annual values of a given standard relative to $\mathrm{GO}_{3}$ is only $\pm 0.4 \mathrm{ppm}$. For the one open coil it is $\pm 5.1 \mathrm{ppm}$, and the net drift of this one coil in the 7 years is $+35.6 \mathrm{ppm}$. None of the other coils drifted relative to the mean more than 1.3 ppm in the 7 years. Of the eight good coils three had been made at Teddington, one at Tokyo, one at Philadelphia, and three at Washington. The British coils were sealed in oil, and the others were sealed in gas.

The International Bureau is custodian of a primary group of 47 standard cells made up at various times in six different laboratories. Of this group eight are neutral cells, six are 0.05normal acid cells, and 33 are $0.1-\mathrm{N}$ acid cells. They are divided into nine subgroups according to their origin and time of manufacture.

The available data on these cells are less uniformly distributed in time than those on the resistors, the five sets of observations being spaced at intervals of $3,2,1$, and 1 years. If this fact is disregarded, a fair index of the constancy of the cells can still be obtained by averaging, without regard to sign, the differences between successive readings on the same cell. Another measure of quality is the net drift of a cell in 7 years relative to the mean of the whole group. The following table 1 contains the average value of these two indices for the cells of each subgroup.

It will be seen that the constancy of these coils and cells is about the same as that of the primary
TABLE 1. Performance of standard cells at International Bureau 1939 to 1946

\begin{tabular}{|c|c|c|c|}
\hline $\begin{array}{l}\text { Sub- } \\
\text { group }\end{array}$ & Origin & $\begin{array}{c}\text { Average } \\
\text { difference } \\
\text { of succes- } \\
\text { sive val- } \\
\text { ues (aver- } \\
\text { age inter- } \\
\text { val } 1.8 \\
\text { years) }\end{array}$ & $\begin{array}{l}\text { Average } \\
\text { net drift of } \\
\text { individual } \\
\text { cells (in } \\
7 \text { years) }\end{array}$ \\
\hline $\begin{array}{l}R_{1-\ldots} \\
E_{-\ldots} \\
M_{1-\ldots} \\
C_{2 \ldots-} \\
S_{-\ldots} \\
I_{B-\ldots} \\
I_{A} \ldots \\
I_{1-\ldots} \\
S_{2 \ldots}\end{array}$ & $\begin{array}{l}\text { PTR (Germany) } \\
\text { ETI (Japan) } \\
\text { IMS (USSR) } \\
\text { LCE (France) } \\
\text { NBS (USA) neu- } \\
\text { tral. } \\
\text { BIPM (Sevres) } \\
\\
\text { NBS (USA) acid. }\end{array}$ & $\begin{array}{l}\mu v \\
\pm 15.2 \\
\pm 6.5 \\
\pm 5.1 \\
\pm 2.7 \\
\pm 2.1 \\
\pm 1.7 \\
\pm 1.4 \\
\pm 1.2 \\
\pm 0.8\end{array}$ & $\begin{array}{r}\mu v \\
+9.0 \\
+0.1 \\
-8.1 \\
+5.4 \\
-3.3 \\
-0.6 \\
+0.2 \\
-0.2 \\
+0.8\end{array}$ \\
\hline
\end{tabular}

- The small net drift listed for subgroup $E$ is the difference between an in. crease of $36.6 \mu v$ for one cell and a nearly equal combined decrease in the other four cells of the subgroup.

groups of the NBS, on which more detailed data are given in the two preceding sections. In the case of most of the standards, the performance is amply adequate to meet the current needs in the field of precise measurements and to serve as a reference basis for international comparisons. However, it must be expected that even the small residual drift of such groups will ultimately, over a period of years, accumulate to an excessive error unless some check such as the absolute measurements discussed later is available.

\section{International Comparisons 1911 to 1948}

\section{Comparisons of the $\mathrm{Ohm}$}

Following the meeting in Washington, April 4 to May 25, 1910, of the International Technical Committee, the members of that committee carried back to their respective national laboratories standard resistors to which values had been assigned on the basis of the mean between the British and the German units. This mean value was smaller by $7 \mathrm{ppm}$ than the unit that had been used previously in the United States. This difference was so small that it was felt sufficient to continue the use of the older unit in certifying the values of standard resistors in the United States. This older unit is referred to as the "international olm (certified NBS)." On the other hand, for purposes of international comparison, the value $7 \mathrm{ppm}$ smaller than the older unit has been used consistently and is commonly referred to as the "Washington unit."

The term "Washington unit" has been used by various foreign laboratories to designate the ohm as maintained in their respective laboratories on the basis of manganin wire coils, the values of which were initially assigned in 1910 following the conclusion of the work of the International Technical Committee. To distinguish between this unit and the units that in later years were determined in certain other national laboratories as a result of later experimental realizations of the mercury ohm, these latter units have been commonly designated as "international ohms" of the respective countries.

In the years immediately following 1910, comparisons of the units used in different countries were made only sporadically. When an American scientist crossed the Atlantic for some other purpose, he was frequently persuaded to carry standard resistors and standard cells from one national laboratory to another, but it was not until the International Bureau of Weights and Measures was authorized to enter the field of electrical measurements that systematic international comparisons were begun. In a comparison between two laboratories, coils are first carefully measured in terms of the unit of their country of origin. They are then taken to the second laboratory where they are measured in terms of the unit of 
that laboratory and are watched (preferably for some months) to be sure that no changes have been stimulated by the transportation. They are then carried back to the first laboratory and again measured. If any differences are obtained between the first and last measurements it is assumed that the change is a result of a gradual drift, and an intermediate value is assigned by linear interpolation to represent the value in terms of the units of the first laboratory at the time when the measurements in the second laboratory were made. The difference between this value and the value measured in the second laboratory then constitutes a measure of the difference in the units of the two countries involved. The amount of the drift is, of course, an indication of the reliability of the intercomparison and has ranged from changes of less than $1 \mathrm{ppm}$, in some instances, to values as high as $80 \mathrm{ppm}$ in cases where the coils have been carried to many intermediate laboratories and ultimately half around the world.

Although, according to the decision of the London Conference in 1908, the mercury column was by definition the ultimate standard of resistance, in actual practice relatively little use has been made of it. At the National Bureau of Standards mercury ohm tubes were set up and careful measurements made in 1911 and 1912
[13]. The results were found to agree with the Washington unit within the experimental error estimated for the work with the mercury columns, and therefore no adjustment was made in the American unit.

At the National Physical Laboratory, Smith in 1912 [14] and Hartshorn [15] in 1924 made careful determinations with mercury columns and found their Washington unit to be greater than that derived from the mercury column by $16 \mathrm{ppm}$ and by $41 \mathrm{ppm}$, respectively, on the two occasions. In view of this, in the international comparisons of 1931, the National Physical Laboratory decided to abandon the Washington unit which it had used until then and to use a unit smaller by 30 ppm.

Between 1923 and 1925 Von Steinwehr and Schultze [16] at the Reichanstalt carried out extensive determinations with mercury columns. As a result, in the comparisons with the Japanese standards brought to them in 1927 by Professor Jimbo, and in the comparisons with British and American standards in 1931 and thereafter, they used a new unit smaller by $33 \mathrm{ppm}$ than the Washington unit that they had used previously. The results of the work with mercury columns in the three laboratories are indicated by the isolated points marked " $\mathrm{Hg}$ " in figure 8.

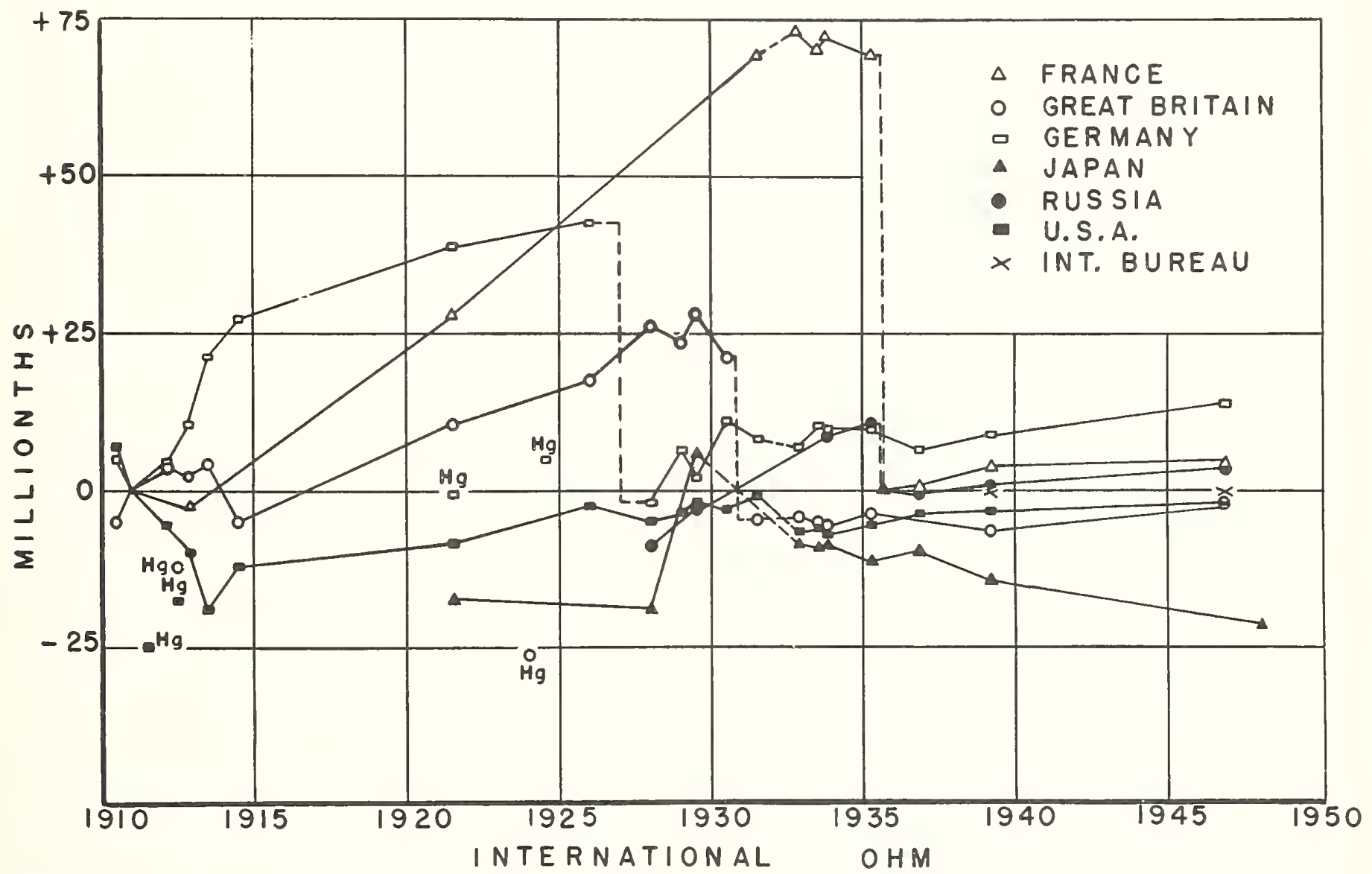

FIGURE 8. Differences from the adjusted mean of the values of the International Ohm, as maintained by various national laboratories, 1911 to 1948.

Points marked "Hg" indicate the results of measurements on mercury columns. 
Between 1932 and 1939 frequent and systematic intercomparisons were made by the staff of the International Bureau of Weights and Measures to determine the relative values of the units maintained in a number of countries. Standards from France, Germany, Great Britain, Japan, Russia, and the United States of America were used in almost all of these intercomparisons.

Following World War II comparisons at the International Bureau were resumed. Although the standards from the various countries could not be assembled in Paris simultaneously, by the middle of 1948 data had been obtained on the units of all six countries.

The results of these intercomparisons are shown in figure 8. These data are, for the most part, taken from the proceedings of the International Committee on Weights and Measures [51]. In this figure, for various epochs, the relative values of the ohm as recognized by the various countries have been plotted to a very open scale. Many of the apparent small changes in value of the national units may well be the result of errors introduced by the hazards of transportation. It is probable that the actual uncertainty of the electrical measurements by which any two standard resistor's are intercompared is usually less than 1 $\mathrm{ppm}$, and therefore not appreciable in the figure.

It should be kept in mind that, in the very nature of the case, the location of the point indicating the value of the unit of a given country at a later time can be related to the position of the corresponding point at an earlier date only by making some arbitrary assumption as to the constancy of some unit between the two dates. Therefore, too much significance should not be given to the slope of the lines, which, in the diagram, have been arbitrarily drawn to connect successive points. In the plotting of figure 8, for convenience, three different conventional procedures have been used to relate the data at successive epochs, as follows.

From 1910 to 1932 only data derived from measurements at the NPL, PTR, and NBS were used to carry forward a common base from epoch to epoch. This convention was chosen because data from other laboratories were very fragmentary. The mean of the three values of the Washington unit as maintained during this period at the three laboratories has been assumed to have drifted upwards at the rate of $1.1 \times 10^{-6}$ per year. The justification for this somewhat arbitrary assumption is as follows. In 1931 the NPL, as a result of earlier measurements on mercury columns, decided that its Washington unit had increased by $30 \mathrm{ppm}$ in the course of the 21 years since the unit was established at Washington. This corresponds to a drift of $1.4 \times 10^{-6}$ per year. Similarly, the PTR, in 1927, decided that its Washington unit had drifted upwards by $33 \mathrm{ppm}$ in the preceding 17 year's, or at a rate of $1.9 \times 10^{-6}$ per year. The NBS has seen no reason to change its unit, and it is assumed that its rate of drift was 0 . The mean of these three drift rates is the $1.1 \times 10^{-6}$ assumed above.

Between 1932 and 1939 the work of MM. Pérard and Romanowski at the International Bureau provides a much better basis to give continuity between successive intercomparisons. In 1935 the Advisory Committee on Electricity recommended that there be taken as the mean international ohm the mean obtained that year of the units of Germany, Great Britain, Japan, Russia, and the United States of America. The French unit, which had been used in this and in some earlier intercomparisons, was recognized as being out of line with the others. Therefore it was not included, and the Laboratoire Central d'Electricité (LCE) decided to adjust its unit by decreasing it by $69.5 \mathrm{ppm}$ to coincide with the new mean. The Russian laboratory also readjusted its unit by reducing it by $10.6 \mathrm{ppm}$. This latter readjustment, however, was made after the international mean value for 1935 had been fixed. In plotting figure 8, this 1935 mean value has been used as a reference corresponding to 0 on the scale of ordinates. In other words, it has been assumed to be the same as the mean value chosen in 1910 as a result of the intercomparisons at Washing. ton. In projecting the 1935 mean value forward or backward in time it has been assumed that tke mean of the units of all six countries remained unchanged. In plotfing the points for December 1932 and July 1933, when data on the Russian unit were not available, it was assumer that the ratio of the Russian unit to the mean of the other five nations was the same as in November 1933.

It will be noted that this procedure of equating the mean international ohm of 1935 with the mean Washington unit of 1910 , when combined with the rate of drift postulated during the earlier years, gives a very satisfactory correlation between the 1931 data on the older basis and the 1932 data on the newer basis. This correlation would seem to justify the apparently arbitrary assumption of drift used in plotting the earlier data.

When the international comparisons of the ohm were resumed, following World War II, a question arose as to the proper basis for carrying forward the mean international ohm from 1933 to 1947. The problem was unfortunately complicated by a strange coincidence that illustrates the importance of having some absolute standard as a fundamental basis for preserving units of measurement. The staff of the International Bureau had originally assumed that the Mean International Ohm was the same in 1939 as it had been in 1936. On this assumption it appeared that the resistances of the coils in their reference group $\mathrm{GO}_{3}$ were increasing at the rate of $0.95 \mathrm{ppm}$ per year. An extrapolation of this rate over the war period would predict an accumulated increase of $6.6 \mathrm{ppm}$ by 1946 . The 
comparisons in 1946 between $\mathrm{GO}_{3}$ and some American standards showed only a very slight increase $(0.9 \mathrm{ppm})$ in the American unit relative to $\mathrm{GO}_{3}$. If this were combined with the predicted drift in $\mathrm{GO}_{3}$ it would imply an increase of $6.6+0.9=7.5 \mathrm{ppm}$ in the American unit. Strangely enough, comparisons in 1946 between British and American standards indicated that the provisional value of the British ohm was then $19.3 \mathrm{ppm}$ smaller than the American ohm, although in 1939 it had been only $3.3 \mathrm{ppm}$ smaller. A splitting of the difference in this shift would imply an increase of $8 \mathrm{ppm}$ in the American unit and an equal decrease in the British. In spite of the apparent agreement between these two figures 7.5 and $8 \mathrm{ppm}$, such an increase in the American ohm seemed improbable in view of the high quality of the standards in the American group. The National Physical Laboratory therefore made further comparisons of its reference group with other British standards of high quality and found a number of divergencies which led them to "recapture" their unit by using as a basis for its maintenance other coils of probably better stability. On this final basis the British ohm in 1946 was larger than the provisional value by $18.2 \mathrm{ppm}$. If as an alternative basis the international reference group $\mathrm{GO}_{3}$ is assumed to have remained constant from 1939 to 1946, it follows that the British and the American ohm have increased by only 4.8 and $0.9 \mathrm{ppm}$, respectively [57]. This latter basis has therefore been accepted by all concerned and was used in plotting the 1947 value in figure 8.

\section{Comparisons of the Volt}

In general the procedures used in the maintenance and in the intercomparisons of the volt at the various national laboratories have been similar to those used for the ohm. For many years the comparisons were rather sporadic, and they have been interrupted by the two World Wars. With the entry of the International Bureau of Weights and Measures into the field of electrical measurements, the intercomparisons became much more frequent and systematic. The records of many of the earlier intercomparisons, as well as of the later ones, will be found in the Procès Verbaux of the International Committee for Weights \& Measures and in their various Amnexes.

The standard cell is a much more delicate device than a standard resistor, and therefore more susceptible to changes during shipment. Consequently, in general, groups of from 4 to 10 such cells have been used for important intercomparisons. For these precise comparisons, as well as for maintaining the value of the unit in the national laboratories, cells of the "saturated" type are used. This practice introduces some difficult problems of temperature control. The temperature coefficient of such cells is about $40 \mathrm{ppm}$ per $\operatorname{deg} \mathrm{C}$, which is several times as large as that of a manganin resistor. Also, it is the net difference of the separate temperature coefficients of the potentials developed at each of the electrodes. Each of these half-cells has a temperature coefficient of over 300 ppm per deg $\mathrm{C}$ but the two are of opposite sign. Consequently, temperature gradients in the enclosing oil bath may introduce serious error. Most national laboratories maintain their standard cells at about $20^{\circ} \mathrm{C}$, while some foreign laboratories make measurements at temperatures as low as $16^{\circ} \mathrm{C}$, but the climate at Washington requires that the bath be held at $28^{\circ} \mathrm{C}$ if condensation of moisture is to be avoided. The situation is further complicated because the temperature coefficient of a standard cell varies somewhat with its age and perhaps with its acidity.

As one indication of the probable precision of such international comparisons, it may be noted that in 1929 six comparisons were reported, each based on a different group of cells. The standard deviation from the mean value of the six observed differences between the units at the NPL and the NBS was 4 ppm. Among these comparisons were those involving Russian and Japanese cells, which had been transported long distances, and which had been handled many times in different laboratories. In seven different years, comparisons between the same two laboratories were made by using more than one group of cells. A second measure of the accuracy of these comparisons is obtaimed by noting for each of these seven years the highest and the lowest value of the observed ratio of the units of the two countries. The difference between each pair of extreme values indicates the uncertainty for that year. The average difference for the seven years was $9 \mathrm{ppm}$. In contrast with such differences that appear when transportation of cells is involved are the results of work done in 1931 [53] at the Reichsanstalt, when a group of eight British cells was compared with a group of 10 American cells on eight different days. The observations were scattered over an interval of 5 weeks, but the cells were not moved between measurements. On only two of the eight days did the difference in the units reported by the two groups differ by $1 \mathrm{ppm}$ from the mean value of 30 ppm; on one day the difference was 29 , and on another, it was 32.

The results of the various comparisons of the volt througl the period from 1911 to 1948 are shown in figure 9. In this figure, the plotted points show at a given epoch the relative values of the volt as maintained by the various national laboratories. The correlations between the values at one epoch, and those at another, have been made on the basis of the following somewhat arbitrary assumptions. From 1911 to 1932, inclusive, it has been assumed that the mean value of the units at the NPL and NBS was constant, and this mean is made the axis of abscissas. The 


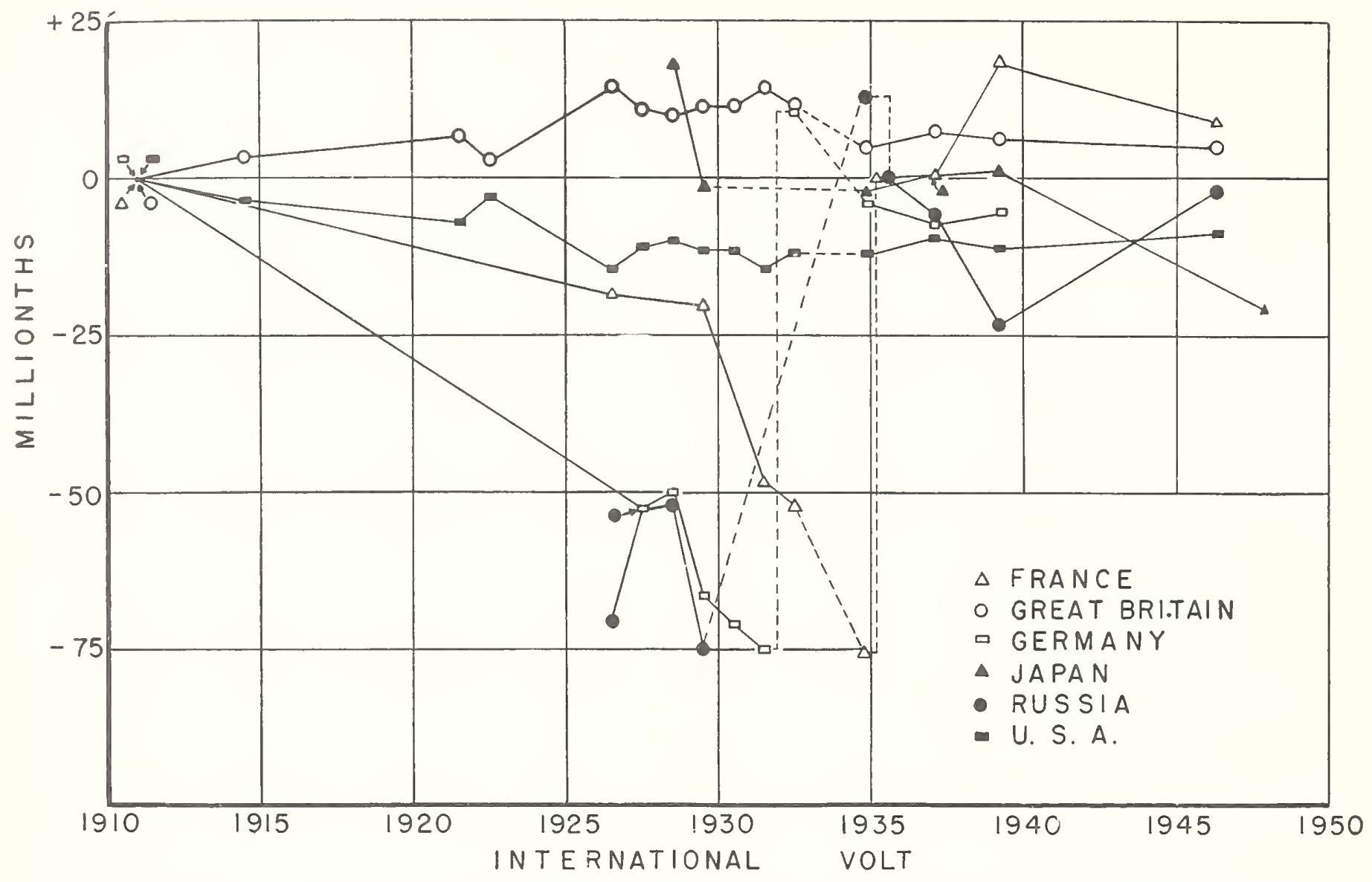

FIGURE 9. Differences from the mean of the values of the International Volt, as maintained by various national laboratories, 1911 to 1948 .

reason that data from the PTR were not included is, first, that during the early ycars relatively few comparisons were made with the German laboratory, and, second, that in 1931, the PTR recognized that its standard had drifted by $82 \mathrm{ppm}$. It would be difficult to decide when the major part of this drift occurred and to make a reasonable correction for it in the graph. After 1934, the data in figure 9 have been plotted on the assumption that the "Mean International Volt", $V_{m}$, as defined in 1935, by the Consultative Committee on Electricity [56], has remained constant. During World War II, this is equivalent to assuming that the large groun of standard cells maintained at the International Bureau and originating in various laboratories has also remained constant in enf.

In 1931 it became evident that there was an increasing discrepancy between the unit as maintained at the PTR, and that at the British and Amcrican laboratories. Consequently, at the invitation of the Reichsanstalt, arrangements were made to have an international committce carry out experiments with the silver voltameter, and, thus, to reestablish the international volt. P. Vigoureux of the NPL and G. W. Vinal of the NBS took to Berlin standard cells and standard resistors, the values of which had been carefully determined at their respective laboratories. In Berlin, these standards were compared with those of the PTR and an extensive series of silver deposits were made using several types of voltameters. The results have been reported by all three laboratories $[52,53,54$.$] Following this$ work the P'TR increased its unit by $82 \mathrm{ppm}$.

The large change in the Russian unit between 1929 and 1934 was presumably the result of extensive work with the silver voltameter reported by Kolossov [22].

Comparisons at the International Bureau began in 1932 [55] with measurements on standard cells from four countries, and in December 1934 data were obtained for cells from all six national laboratories. The LCE, finding its unit to be out of line with the others, increased its volt by $76 \mathrm{ppm}$ in 1935. The Consultative Committce on Electricity suggested [56], in view of the vel'y small differences between the units of the other five laboratorics and the mean of the units, that each should, if possible, adopt this mean value as its unit and thus obtain an immediate international unification. However, only the Russian laboratory followed this suggestion and reduced its volt by $13 \mathrm{ppm}$. In the intercomparisons of 1937 and 
1939, the International Bureau has carried forward the mean value of $V_{m}$ as defined prior to the change in the Russian unit by assuming the mean of the six national units to be the same (that $\overline{\mathrm{is}}$, $\left.1-2.1 \times 10^{-6}\right)$ as it was in 1935 , after the Russian adjustment.
Between July 1945 and May 1948, standard cells were received at the International Bureau from five of the six national laboratories (The Reichsanstalt did not submit any). The results are plotted in figure 9 on the basis that the mean emf of the reference group $G V_{2}$ of 47 cells at the International Bureau has remained constant.

\section{Developments 1911 to 1948}

\section{Enlargement of Scope of International Committee on Weights and Measures}

The London Conference of 1908 envisaged, as shown by its schedule D (appendix 2), the ultimate establishment, by the governments interested, of a permanent International Commission for Electrical Standards. Pending such action, its president, Lord Rayleigh, appointed an interim scientific committee of 15 members. The only action of this committee seems to have been to select the delegates who constituted the special International 'Technical Committee, which carried on the experimental work in Washington in 1910 , and to officially promulgate its results. The work of the technical committee was so satisfactory that during the next few year's little need was felt for further improvement in the situation. In 1914 World War I started and rendered international cooperative action, in other than military matters, quite impractical.

Following the war, there was a wave of interest in new international cooperative organizations, and older organizations began to renew their activities. One of the oldest international scientific organizations is that set up in 1875 by the treaty commonly designated The Convention of the Meter. This provided for the establishment of a permanent International Bureau of Weights and Measures, which is located on a plot of ground set aside as international territory in the Parc de St. Cloud at Sevres, near Paris. The functions of that Bureau included the custody and preservation of the metric standards of length and mass and comparison of the several national standards with the international prototypes. The work of the Bureau is supervised by an International Committee of 18 scientists, appointed by reason of their individual competence in the field of measurements, but with the proviso that only one member shall be appointed from any one nation. This Committee normally meets at the Bureau every 2 years to review the work of the Bureau and lay out its future program. At intervals, normally of 6 years, a General Conference on Weights and Measures, to which all nations signatory to the Convention of the Meter may send delegates, meets to review the work of the Committee and of the Bureau and to give official authority to their actions. The Conference is a diplomatic rather than a scientific body, and many of the delegates come from the embassies of the various countries.

At the 1920 meeting of the International Committee for Weights and Measures, after the close of the war, the American and Belgian members suggested that the scope of the International Bureau of Weights and Measures might well be enlarged to include the electrical units and the determination of important physical constants.

The proposition was formally presented at the Sixtl General Conference on Weights and Measures (1921) [6]. There was evidently a very real advantage to be gained in keeping under the control of a single international body as many fields of exact measurement as possible, because, thereby, duplications of effort and conflicts of a uthority would be minimized. On the other hand, the large amounts of expensive equipment and specially trained personnel required to make real advances in the science of precise electrical measurement seemed to be beyond the very meager financial resources that the various signatory nations had hitherto been willing to contribute to the International Bureau. The British delegates in particular opposed any major enlargement of the laboratory facilities of the Bureau such as would be needed for an independent primary establishment of the units and pointed out that such a procedure would duplicate services already available in the national laboratories. However, all felt that the Intermational Committee could perform a very valuable function by. coordinating the work of the national laboratories, comparing the units established by them, and giving official status to properly weighted mean values.

The Sixth Conference finally voted unanimously to amend the Convention of the Meter (Appendix 3) so as to give to the International Committee and Bureau authority for the "establishment and conservation of the standards for the electrical units ...". This action of the General Conference was duly ratified by the United States Senate in 1923.

With the assuniption of its new duties it seemed to the International Committee that it should secure (1) the cooperation of experts in the field of electrical measurements and (2) a closer and more official connection with the national standardizing laboratories where the primary electrical measurements were being developed. To achieve 
these objects it recommended the formation of an Advisory Committee on Electricity that should advisc the International Committee on questions relating to electrical standards and systems of measurement. This recommendation was approved by the Seventh General Conference held in 1927. The Advisory Committee on Electricity is limited to 10 members, including a representative appointed by each of the national laboratories designated by the International Committee, and also specialists named by the Committee. The cliairman of the Advisory Committee is elected by the International Committee from among the membership of the latter.

The national laboratories designated were the National Physical Laboratory of Great Britain, the Laboratoire Central d'électricité at Paris, the Physikalisch-Technische Reichsanstalt of Germany, the Central Chamber of Weights and Measures of the USSR, the Electrotechnical Laboratory of the Department of Communications of Japan; and the National Bureau of Standards of the United Statcs. Two individual specialists, Prof. L. Lombardi, Director of the Electrotechnical Laboratory of the Royal School for Engineers, Rome, and M. Ch.-Ed. Guillaume, Director of the International Bureau, were appointed in 1928 .

At its mceting in 1929, the International Committee asked the Advisory Committee to consider also problems rclated to photometric measurements and standards, and specialists in photometry were invited to attend the meetings. At this time the committee was often referred to as the "Advisory Committee on Electricity and Photometry." However, in view of the differences between the two fields of work, a separate Advisory Committee on Photometry organized along parallel lines was authorizcd by the International Committee at its 1935 meeting.

The increase in the scope of work of the International Bureau required increased laboratory space, and in November 1928 the International Education Board contributed 900,000 francs and the French government relcased an additional small strip of land that made it possible to add sufficient laboratory space to house the electrical apparatus. Another assistant was added to the staff in 1929 to carry the additional work.

\section{Trend Toward Absolute Units}

In the decades following the London Conferencc of 1908, the developments of science and of the elcctrical art materially changed the situation that had led to the establishnient of the system of international units. The genius of Lord Rayleigh, who served as President of the Conference, may have enabled him to foresee thesc trends, for even when he was in the act of establishing the international system by formally putting to a vote the resolution on the mercury ohm, he could not refrain from saying, "Before putting the resolution, I should like to say a word or two. I have no doubt this proposition will commend itself to a large majority of the Conference, but in my own mind I feel some doubt as to whether the introduction of the mercury column is not what we call a fifth wheel to the coach ... to define it (the ohm) as the resistance of a column of mercury seems to me-I will not say illogical, but hardly in accord with the precision that absolute measurements made now obtain ... I only wish to liberate my conscience by these few remarks . . . I will put the resolution to the Conference, that The International Ohm shall be defined as the resistance of a specified column of mercury. . . ."

A number of factors cooperated in the ensuing years to demonstrate the wisdom of Lord Rayleigh. One of these was the fact that the need for standards that were conveniently reproducible vanished witl the growth of testing service by the national laboratories. A standard cell or resistor could be shipped from San Francisco to Washington, tested, and returned with a tiny fraction of the time and expense involved in setting up a voltameter or mercury column experiment. Laboratories in the smaller countries could obtain similar service from the large national laboratories or, more recently, from the International Bureau.

A second factor in the diminishing utility of the international units was the discovery of isotopes. Mercury has seven isotopes, of which five occur in considerable amounts, and which have masses covering a range of 2 percent. Silver has two isotopes of almost equal abundance, which differ in mass by about 2 percent. The volume resistivity of mercury is presumably nearly the same for all isotopes, but the Chicago Congress, in an endeavor to make the definition more precise, changed the earlier specification for a column $1 \mathrm{sq} \mathrm{mm}$ in cross section by specifying a column having a mass of $14.4521 \mathrm{~g}$. With this definition a change in isotopic composition would be expected to makc a cliange in the resistance of the column proportional to the change in density. This difficulty is probably more academic than real because specimens of mercury from widely different sources have been found to liave very closely the same density.

The difficulty from the silver isotopes is less academic, because the process of electrolysis may well introduce an appreciable change in isotopic composition. In fact, electrolysis is the most common process for concentrating the heavy isotope of hydrogen. If, in the deposit, the ratio of the light to the heavy isotope content is greater by 1 percent than it is in the solution, the deposit will be $100 \mathrm{ppm}$ lighter than if no separation had occurred. Moreover the extensive work from 1911 to 1914 witl the silver voltameter 
revealed still other complications such as effects from colloidal silver, spurious inclusions in the deposit, etc., which could cause appreciable errors in work of the highest accuracy.

At first sight it might appear that the conventional choice of a fixed number, namely 0.00111800 grams of deposit per international coulomb, to define the unit of electric charge would have a theoretical advantage by eliminating one experimental step in the determination of the Faraday. This pliysical constant would then be given by $F=A / 0.00111800$, where $A$ is the atomic weight of the silver deposited. For the standard atomic weight $107.880, F$ is 96,494 international coulombs per gram equivalent, and the only experimental determination involved is that of the atomic weight. However, in the numerous theoretical uses of the Faraday, as in the determination of Avogadro's number, it is combined with other physical constants at least some of which involve the electromagnetic forces on elementary charges, which in turn are expressible primarily in absolute units. The "international Faraday" is therefore useful only when the ratio of the international to the absolute coulomb is also known. Moreover, the complications from inclusions, colloidal silver and possible isotopic separation constitute serious sources of error when the silver voltameter is used to determine the Faraday. On the other hand, they drop out when a single particular type of voltameter operating under closely controlled conditions. is used to reproduce the same average current in a repeat experiment even after the lapse of many years.

The third and perhaps most important factor was the tremendous increase in the scope of application of electrical methods of measurement in many fields of science and engineering and the simultaneous increase in the accuracy required. Electrical methods are used to measure temperatures, pressures, strains, acidity, concentrations, and a myriad of other physical and chemical quantities. In some of these, particularly in the measurement of energy and in the calibration of calorimeters, the desired precision has long been so high that corrections had to be be applied for the difference between the international and the absolute units. Electrical effects are fundamental in the experiments by which a large number of basic physical constants such as the Faraday, electronic charge, mechanical equivalent of heat, Bohr magneton, and Zeeman displacement constant are measured. It soon became evident that the bother and confusion already experienced in the field of thermochemistry would soon become serious in many other fields if the independently

fined international units continued in use.

\section{Official Actions Leading to the Adoption of the Absolute Units}

The possibility of shifting back to the absolute system of units had doubless been in the minds of many scientists for a long time, and indications of it are evident in some of the discussions in 1921 regarding the extension of the authority of the International Committee. Perhaps the first official action in this direction was a set of resolutions formulated by the Committee on Instruments and Measurements of the American Institute of Electrical Engineers and formally adopted June 27, 1928, by its Board of Directors. This (see appendix 4) pointed out the unsatisfactory aspects of the situation then existing and urged the national laboratories to undertake the additional researches necessary to make it feasible to legalize the absolute units.

At the same time Dr. G. K. Burgess, then Director of the National Bureau of Standards and its representative on the international Advisory Committee, called together an American Advisory Committee on Electrical Units and Standards. The membership included representatives of the National Academy of Sciences, American Institute of Electrical-Engineers, the American Physical Society, the National Electric Light Association, the Association of Edison Illuminating Companies, the National Electrical Manufacturers Association, and the American Telephone and Telegraph Company. In 1930, two members, representing the Illuminating Engineers Society and the Optical Society of America, were added when photometric questions were under consideration. This advisory committee met first in Washington on June 16, 1928, and discussed the functions that it was desirable to have the International Bureau undertake in connection with the electrical units, and the consensus was as follows:

(1) A central secretariat to arrange for systematic exchange of standards and compilation of results of intercomparisons thus made among the national laboratories.

(2) A laboratory to which concrete standards representing the results obtained in the different countries may be brought for precise comparisons.

(3) A repository for international reference and working standards with the necessary equipment so that other standards may be compared with these standards on request.

The committee then voted the following resolution:

That in the opinion of this Committee, in view of improvements which are being made in absolute measurements, electrical standards should in future be based upon the absolute system of units.

At almost the same time Dr. D. W. Dye, who represented the National Physical Laboratory on 
the international Advisory Committee, laid the same questions before the Electrical Units and Standards Committee of the NPL. ${ }^{3}$ The opinions of this committee as to the desirable functions of the International Bureau were very closely concordant with those of the American Advisory Committee. They also regarded it "as of the utmost importance that the use of the absolute units should be introduced at the earliest possible moment. *** It is felt that this change can be made now (1928), but that the difficulties involved will increase with each year of delay."

The German position at this time, as set forth in a memorandum by Dr. von Steinwehr, was much less favorable to the proposed change. While admitting the theoretical advantages of the absolute system, they emphasized the inconveniences that they feared would develop in practice during the transition. They proposed that much additional work should be carried out first to determine the ratios of the international to the absolute units. After these ratios were definitely established, they would then urge that the definitions in absolute terms be used as the basis for defining the units, but that the established ratios should then be applied so as to keep the magnitudes of the units actually used at the same values as the current international ones.

On the other hand, a similar committee of Italian scientists under the chairmanship of Prof. L. Lombardi favored the ultimate transition to absolute units, although they felt that considerable further experimental work in absolute measurements should be done first. Professor D. Konovalov of the Central Chamber of Weights and Measures, USSR, sent in proposals that had been approved by a group representing several central scientific and technical institutions in Russia. These proposals were closely parallel to those of the American and British members. Similar responses came from analogous committees of the Société Française des Electriciens and from the Japanese Electrotechnical Laboratory.

In view of this preponderance of opinion, the Advisory Committee, on June 8, 1929, adopted a resolution translated as follows:

In view of the great importance of unifying the systems of electrical measurement upon a basis free from arbitrary characteristics, the absolute system, derived from the centimeter-gram-second system, should replace the international system of units for all measurements in science and industry.

This was officially approved by the International Committee at its 1929 meeting and widely publicized.

As might have been expected, a few individuals disagreed with the proposed change in units, and felt that the change in the ohm would involve excessive inconveniences in laboratory work.

3 The members of this Committee were Sir Richard Glazebrook, Chairman, Sir William Bragg, Dr. D. W. Dye, Dr. Alexander Russell, Sir Arthur Schuster, Dr. F. E. Smith, and Sir Joseph Petavel.
G. A. Campbell, in a paper entitled, "A definitive system of units," which he presented at a meeting in Chicago on June 24, 1933, of the Committee for Symbols, Units, and Nomenclature (SUN Committee) of the International Union of Pure and Applied Physics, suggested that the ohm be fixed by a material standard resistor and that all other electrical units be derived from this and from the meter, the kilogram, and the second. On this basis, the "permeability of space," $\mu_{v}$, would become a derived quantity to be determined by experiment and would have a.value of about $0.9995 \times 10^{-7}$ henry per meter. According to this plan the prototype ohm was to be preserved at the International Bureau in the same manner as the meter and the kilogram. It would follow that if this standard resistor should drift in time as did those whose values are shown in figure 8 , the experimentally determined value of $\mu_{r}$ would also be found experimentally to drift and to have different values at different epochs. This proposal was formally rejected by the SUN Committee.

A similar suggestion was proposed by. Prof.-Ing G. Giorgi and endorsed by F. Emde and by A. Sommerfeld. A great deal of confusion has arisen because the name Giorgi thus became attached to a second system of units, which was, in effect, almost a special case of an earlier system of units that he had proposed. As early as 1901 Giorgi [2] had made the constructive suggestion that the incomplete "practical absolute" system of eight electrical units (coulomb, ampere, volt, ohm, henry, farad, joule, watt) could be extended into a complete system that would include mechanical and magnetic units as well. By the artifice of assigning to $\mu_{i}$ the value $10^{-7}$ in place of the value 1 , which is chosen in the centimeter-gram-second electromagnetic system, Giorgi found that the meter, the kilogram, and the second became automatically the mechanical units in his new complete system, and it is therefore usually called the "MKS System". 'The unit of force in this system has a magnitude of $10^{5}$ dynes and the name "newton" has been proposed for it. This valuable suggestion has met with growing but rather belated approval.

In 1935 the International Electrotechnical Commission meeting at Scheveningen gave its formal approval to this MKS system, recommended its general use for enginecring purposes, and suggested that it be called the "MKS-Giorgi" system. In engineering uses, the distinction between the international units and the absolute units is negligible and was probably not given any consideration by the IEC. Theoretically, if the absolute units are used everything will be inherently consistent. On the other hand, with the suggestion of Campbell or the later suggestion of Giorgi, which was not approved by the IEC, the joule and watt would have retained their absolute values, the ohm would by definition have kept its inter- 
national value, and the ampere and the volt would have had neither their absolute nor their international values, but would have been respectively less and greater than their absolute values by the square root of the factor by which the international olmm is greater than the absolute olm. Later, at its meeting in Torquay in 1938, the IEC explicitly showed its preference for the absolute system by recommending ". . . as the comnecting link between the clectrical and mechanical units the permeability of free space with the value $\mu_{0}=10^{-\gamma}$ in the unrationalized system or $\mu_{v}=4 \pi \cdot 10^{-\gamma}$ in the rationalized system."

The alternative suggestions of Campbell and of Giorgi were duly considered by the International Committee, but at its meeting in 1935 the decision to shift to the absolute units was reaffirmed.

It should be emphasized that the action of the International Committee for Weights and Measures in authorizing the change from the international to the absolute electrical units has no bearing whatever on the question also now under diseussion as to the relative merits of the MhS and cgs systems. The absolute olim, ampere, and volt may be regarded equally well either (1) as units of the absolute MKS system (either rationalized or umrationalized), or (2) as convenient decimal multiples of the corresponding units of the egs system. Moreover, the action of the IEC in specifically assigning a value to $\mu_{\tau}$ as the link between electrical and mechanical units should not be interpreted as giving any particular fundamental significanee to permeability. The IEC Committee on Electrie and Magnetic Magnitudes and Unit's (EMMU) explieitly stated "The Committee recognizes that any one of the following practical units, olim, ampere, volt, henry, farad, eoulomb, weber already in use may equally serve as the fourth fundamental unit, because it is possible to derive each unit and its dimensions from any four others mutually independent."

At the Eighth General Conference on Weights and Measures, meeting in 1933, the change to the absolute units was definitely confirmed (see appendix 5). The Conference delegated to the International Committee full authority to fix the ratios of the new to the old units and to set the date for their adoption. It was hoped that the change might be made in 1935 .

However, absolute measurements of high aecuracy are very time-consuming, and although work was pushed vigorously at several national laboratories, it became evident that reliable values of adequate accuracy for the ratios of the absolute to the international units could not be obtained so quickly. At the 1935 meeting, the International Committee [8] postponed the date of adoption to January 1, 1940, asked the Advisory Committee to meet early in 1939 to fix the ratios, and announced that only experimental work reported before the end of 1938 would be eonsidered in fixing the values. It published provisional values for the ratios expressed to parts in 10,000 based on

1 international $\mathrm{ohm}=1.0005$ absolute ohms.

1 international rolt $=1.0004$ absolute volts.

The Adrisory Committee met in June 1939, and the several members reported the status of the work in their respective laboratories on determining the ratios of the international to the absolute units. The German, Japanese, and Russian laboratories had not yet completed their programs. However, the Committee felt that the good agreement between the British and American data, and the inereasing ineonvenienee that the transition would entail if too long postponed justified adhering to the proposed date of January 1, 1940. Resolutions were drawn up transmitting this opinion to the International Committee, fixing provisionally the ratios to be announced, and offering suggested wordings for exaet theoretieal definitions of the new absolute units, whieh could if desired be incorporated in the legislation that might be enacted by the several adherent nations to put the new units into full effect.

The meeting of the International Committee has been scheduled for September 1939, but the outbreak of World War II brought all aetivity in this field to a standstill.

Following a brief preliminary session in November 1945, the International Committee met in plenary session in October 1946. $A$ poll of the Iaboratories represented on the Advisory Committee on Electrieity showed that, with the exeeption of the Reichsanstalt, all still eoncurred in the opinion expressed by the Committee in 1939 that the transition to the new units should be made promptly. The Germans still would have preferred to postpone the change until they could verify by their own experiments the eorreetness of the new units. It was evident however that their flight from Berlin to Weida to escape the bombings must have deranged the laboratory to such an extent that they would not be in position to make an effective eontribution for a very long time. Accordingly the International Committee formally voted resolutions (see appendix 6) based on the recommendations made in 1939 by the Advisory Committee. These (1) set the date January 1, 1948, for the change; (2) fixed the ratios

1 mean international ohm $=1.00049$ absolute ohms. 1 mean international volt $=1.00034$ absolute volts.

as those governing the change on that date; and (3) gave offieial formulations of definitions for the new units.

\footnotetext{
4 The membership of the International Committee at this time was: I. de
Broglie (France); G. Cassinis (Italy); M. Chattelain (USSR); E. C. Crittenden (USA); M. Dehalu (Belgium); W. J. de Has (Holland); E. S. Johansen (Denmark); C. Kargatchin (Yugoslavia); W. Kösters (Germany); H. Na. (Denmark); C. Kargatchin (Yugoslavia); W. Kösters (Germany); H. Na,
gaoka (Japan); Z. Rauszer (Poland); M. Ros (Switzerland); J. E. Sears gaoka (Japan); Z. Rauszer (Poland); M. Ros (Switzerland); J. E. Sears
(Great Britain); M. Siegbahn (Sweden); C. Statescu (Rumania); A. Pérard.
} 
This action was given ex post facto approval by the Ninth General Conference when it met in October 1948.

Conformance with this formal action of the International Committee was of course desirable on the part of the United States as a signatory nation. Accordingly the National Bureau of Standards proceeded to prepare for the impending change. Bureau Circular C459, "Announcement of change in electrical and photometric units", was published in May 1947 and given wide circulation. Excerpts appeared in most of the technical magazines concerned, and oral announcements were made at various scientific meetings. During 1947 all certificates issued by the Bureau giving precise electrical values contained a supplementary statement of what the values were going to be in the new units. Similarly, during 1948 such certificates contained not only the values in the new units but also a supplementary statement of what the values would have been in terms of the old units.

Because of the differences, shown in figures 8 and 9 , between the mean international units as defined by the intercomparisons at the International Bureau and the Washington units as maintained at the National Bureau of Standards, the ratios of the units as certified by the NBS prior to January 1,1948 to the absolute units used after that date are as given in the tabulation below. (It should be noted that the U. S. "Washington ohm" was $7 \mathrm{ppm}$ smaller than the ohm as certificd for use in the USA).

$$
\begin{aligned}
& 1 \text { international ohm }=1.000495 \text { absolute ohms. } \\
& 1 \text { international volt }=1.000330 \text { absolute volts. } \\
& 1 \text { international ampere }=0.999835 \text { absolute ampere. } \\
& 1 \text { international coulomb }=0.999835 \text { absolute coulomb. } \\
& 1 \text { international henry }=1.000495 \text { absolute henries. } \\
& 1 \text { international farad }=0.999505 \text { absolute farad. } \\
& 1 \text { international watt }=1.000165 \text { absolute watts. } \\
& 1 \text { international joule }=1.000165 \text { absolute joules. }
\end{aligned}
$$

The legal status of the new units in the United States is, of course, exactly the same as that of the older ones because the law of 1894 (appendix 1) mentions both sets of units on an equivalent basis. However, in order to remove the ambiguities of the old act, and also to provide a statutory basis for photometric units that have hitherto been based merely on the common consent of those interested, new legislation has been proposed. Substantially identical bills, HR4113 and S1850 were introduced in the United States Congress in July 1947 and December 1947, respectively, but died with the expiration of the 80th Congress in December 1948. Bill S441 (see appendix 7) was introduced in the 81st Congress in January 1949.

\section{Absolute Measurements}

\section{General}

The many interactions between electrical and mechanical phenomena have offered a considerable variety of possible methods for deriving electrical quantities from mechanical measurements $[61,62$, 63]. However, experience has shown that relatively few of these give prospects of yielding an accuracy as high as a few parts in 100,000 .

One such relation is that between the inductance of a circuit and its geometrical size and shape. Many formulas [69] have been worked out for such relations. By constructing an inductor of simple shape in such a way that all its significant dimensions can be measured with sufficient accuracy, and by ensuring that all matcrials used in its construction or located in its neighborhood are nonmagnetic, a standard is obtained, the inductance of which can be computed in absolute henries. For results of high accuracy, extreme care and attention to detail are required in the construction of the inductor. The mechanical measurements involve a tedious multiplicity of measurements of diameter and length of various parts of the structure, all of which must be of the highest accuracy. An example of the effort to avoid systematic crrors is the use by one group at the NBS of end-length standards only, while the workers using another method used line standards only.
As a second step, the inductance can be used in onc of several forms of measuring circuit at a known frequency to determine the value in absolute ohms of the resistance of a resistor. Because of the fact that resistors rather than inductors are commonly used for the maintenance of the unit, most experimenters have combined the two steps so that the final result of their experiment is the determination of the value of a resistance in absolute units. Hence such experiments are referred to as absolute-ohm determinations, although the absolute henry is really the first product.

It might appear that this single elcetrical determination of the ohm should suffice because the ampere can thcoretically be defined as the current that dissipates energy at the rate of 1 watt when flowing in a resistance of $1 \mathrm{ohm}$, and the watt is purcly a mechanical unit. In practice, however, the mechanical measurement of cnergy or power with high accuracy is difficult. It lias been found preforable to make use of a sccond type of absolute measurement in which the current in a pair of coils derelops a mechanical force of attraction or repulsion between them. The force, $F_{x}$, in the $x$ direction developed in such a circuit by a current, $I$, is giren by

$$
F_{x}=I^{2} \frac{d M I}{d_{x} r}
$$


where $d M / d X$ is the rate of increase of the mutual inductance between the fixed and the moving coils as the dimension is increased. By arranging the coils so that $F_{x}$ is vertical, it may be measured by balancing it against the gravitational force, $M g$, on a mass, $M$. The force is then readily obtained and the major problem is the computation of $d M / d X$ from the known proportions of the circuit. Such an apparatus is commonly called a "current balance."

Because of the transitory nature of an electric current, the customary procedure is to connect in series with the coils of the balance a resistor of resistance, $R$. The voltage drop, $I R$, in this resistor is opposed to the emf, $E$, of a standard cell. When the current, $I$, is held at such a value that $I R$ balances $E$ and at the same time the mass, $M$, of the weight has been adjusted to balance the electrodynamic force, the relation

$$
E=R \sqrt{m g / \frac{d M}{d x}}
$$

holds. If $R$ is known in absolute ohms, $E$ can be computed in absolute volts. An experiment of this type is usually called a determination of the ampere in absolute measure, although the final result is the determination of the emf of a standard cell.

\section{Absolute Measurement of Resistance}

In recent year's, four distinct methods of comparable and very high accuracy for the absolute measurement of resistance have been used in the national standardizing laboratories of Great Britain and of the United States. The following paragraphs give a brief description of each.

An early method employs the "Lorenz apparatus," [64], which has been used successfully several times at the National Physical Laboratory. Figure 10 shows a schematic cross section of the

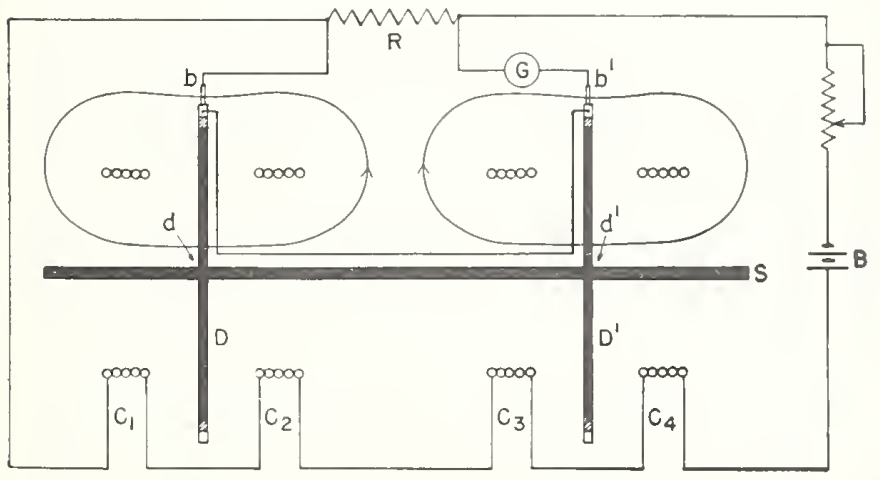

FiguRE 10. Lorenz apparatus.

This schematic cross scetion shows how the $I R$ drop in the resistor, $R$, which is being measured, is balanced against the emf, induced between the brushes $b$ b1 of the homopolar generator. The generator field is formed by the coils $C_{1} C_{2} C_{3} C_{4}$, coaxial with the shaft and connected in series with $R_{2}$, while its earried by disks $D$ and $D^{1}$. apparatus. $D$ and $D^{\prime}$ are disks mounted on the rotating shaft, $S$. Brushes $b$ and $b^{\prime}$ make contact with the periphery of the disks. Each pair of fixed coils, for example, $C_{1}$ and $C_{2}$, or $C_{3}$ and $C_{4}$ may be considered the primary windings of a mutual inductor, $M$, of the Campbell type, the secondary of which is formed by the periphery of a slip-ring carried on a disk. When there is a current $I$ in the coils, the magnetic flux linking the periphery of one ring is $\phi=M I$. When the shaft, $S$, is rotated, each conducting path $\left(b d d^{\prime} b^{\prime}\right)$ from a point on the periphery of one ring through the disk and shaft to a corresponding point on the periphery of the other ring will cut the total flux embraced by the peripheries of the two rings, and this flux is given by $\phi+\phi^{\prime}=\left(M+M^{\prime}\right) I$. The emf generated between the brushes is therefore proportional to the current and to the spe:d of rotation.

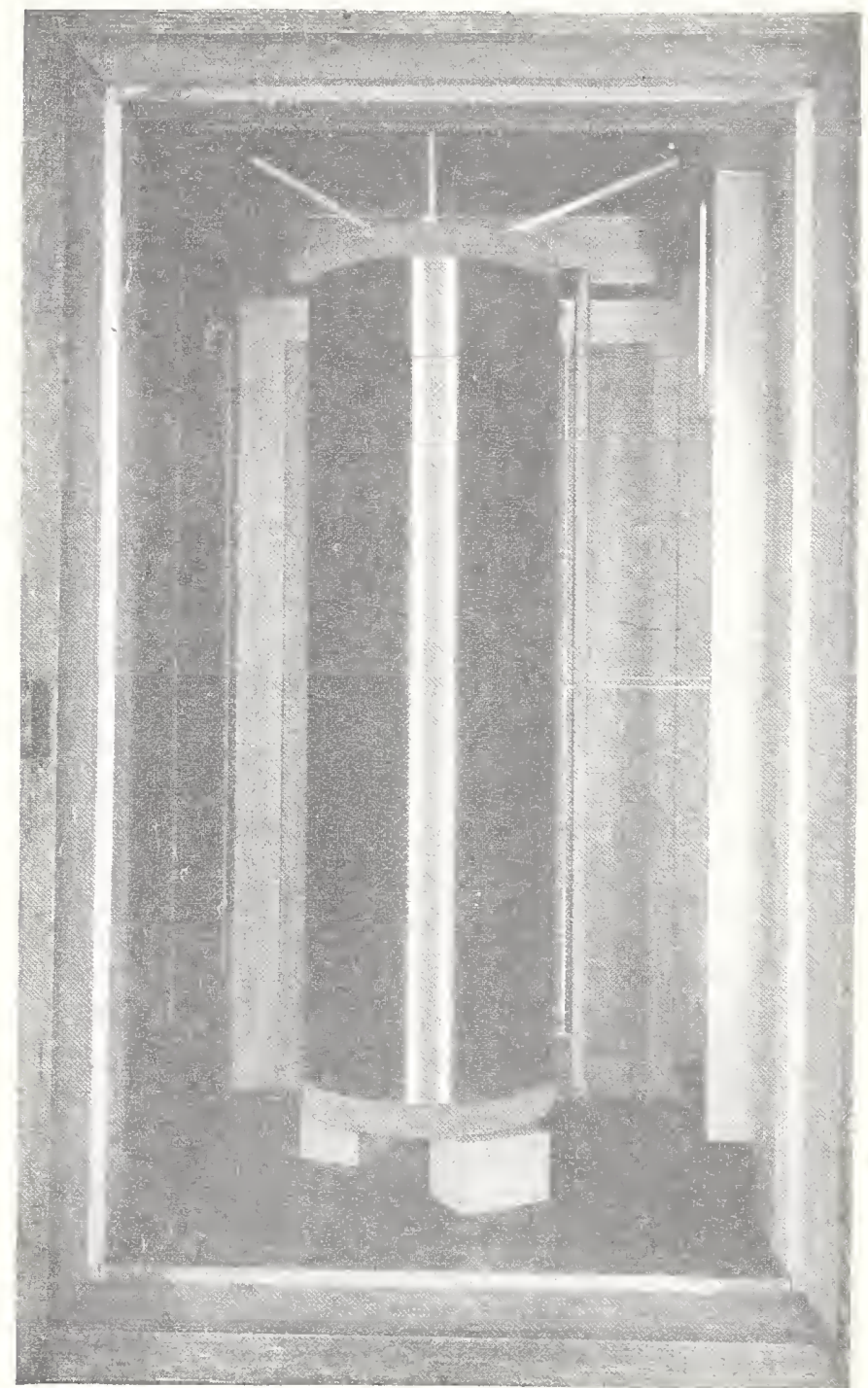

FIGURE 11. Self-inductor wound in lapped helical groove in pyrex glass form.

The coil has 1,000 turns, is about $35 \mathrm{~cm}$ in diameter and $100 \mathrm{~cm}$ long. Its inductance is $103 \mathrm{mh}$. The enclosing eabinet for temperature control is made of wood and glass with no metal fastenings. 
This. emf is balanced against the $I R$ drop of the same current through the resistor, $R$, which is being measured. When a balance is obtained, $R=n(M+M)^{\prime}$. Here $R$ is in ohms if $n$ is the speed in revolutions per second and $M$ and $M^{\prime}$ are in henries.

One method used at the NBS by Curtis, Moon, and Sparks [65] was to construct a large self inductor of copper wire wound in a carefully lapped groove on the outer cylindrical surface of a form of pyrex glass or of fused quartz. The inductor on the pyrex form is shown in figure 11. The computation of the inductance of such a coil is made by the formulas developed by Snow. Figure 12 shows the circuits used in the electrical

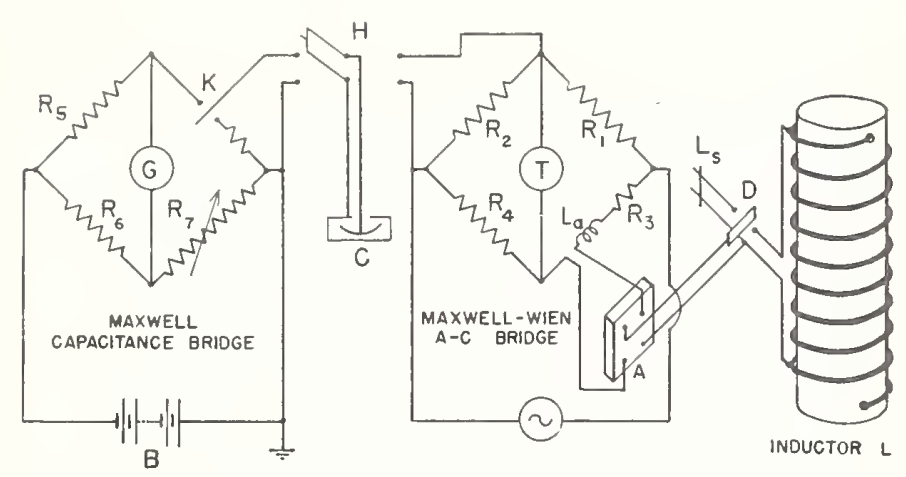

Figure 12. Schematic diagram of circuits used by Curtis, Moon, and Sparks for the absolute measurement of resistance.

experiment by which, in two stages, the measurement is made in terms of inductance and time. The first stage consists in an experiment at a frequency of $24 \mathrm{c} / \mathrm{s}$ using a Maxwell-Wien bridge to establish with high precision the relation between the values of the inductor, $L$, a large air capacitor, $C$, and two resistance arms, $R_{1}$ and $R_{4}$. The switch, $D$, serves to give a base reading in which a link of small and computable inductance, $L_{\mathrm{s}}$, is substituted for the large inductor. The capacitor, $C$, is then connected into a Maxwell absolute capacitance bridge circuit in which its value is determined in terms of the resistances $R_{5}, R_{6}$, and $R_{7}$ and the number (100) of charges and discharges per second produced by the contactor, $K$. Additional comparisons among the resistors serve to express the final results in terms of the resistance of any one of them. In all, four different inductor's wound on forms of different materials and dimensions have been used in this work.

The Campbell method used by Hartshorn and Astbury [66] at the British National Physical Laboratory involves the construction of a mutual inductor of the Campbell type [67]. A schematic cross section of such an inductor is shown in figure 13. 'This type of mutual inductor has the

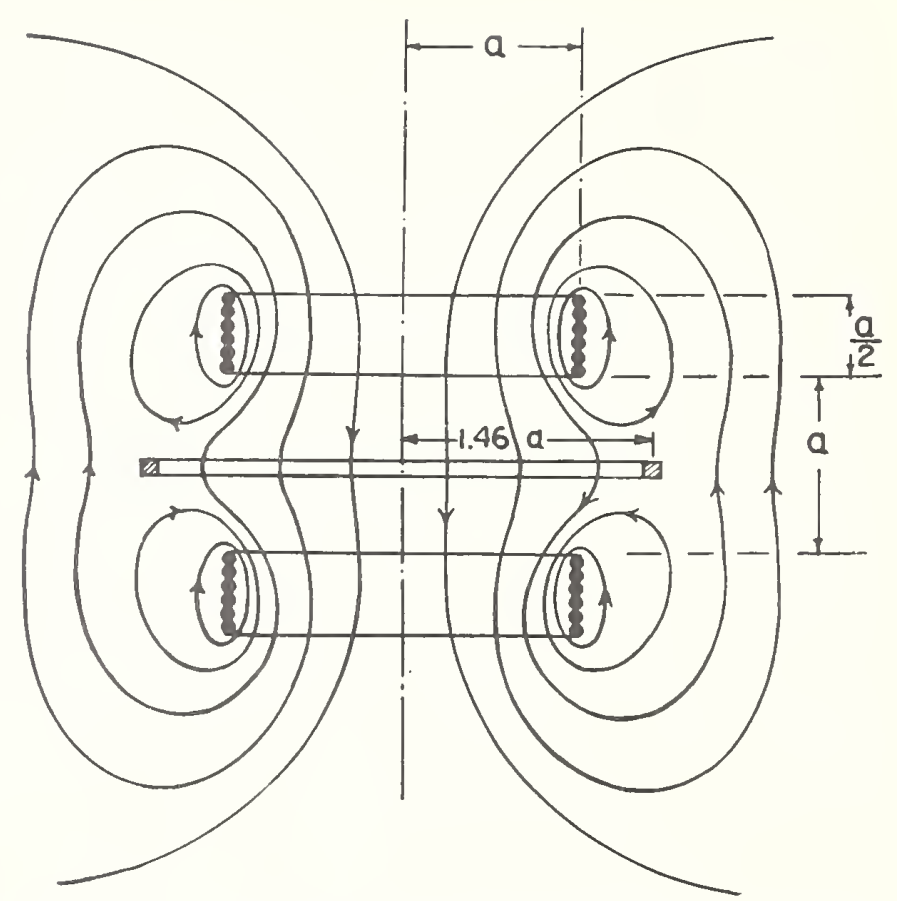

Figure 13. Schematic cross section of Campbell mutual inductor.

For a primary winding in two sections of the proportions shown, the magnetic field is almost zero in the annular space occupied by the secondary coil. Hence an error in the measured dimensions or location of the secondary coil will have little effect on the calculat ad mutual inductance.

advantage that the secondary circuit is located in an annular space in which the magnetic field is very weak. Hence the location of the secondary turns need be known with an accuracy less by about three orders of magnitude than the accuracy that is required in the location of the primary turns. This annular region of weak magnetic field results from the presence of the gap between the upper and lower halves of the primary winding as indicated. The mutual inductance is calculated from the dimensions of the coils.

The inductances of two working mutual inductors, $M_{1}$ and $M_{2}$, are then compared successively with that of the single computable inductor of the Campbell type, of the same nominal value, by directly opposing the secondary emf's to that of the standard when the primary coils are carrying a suitable current at a frequency of about 10 $\mathrm{c} / \mathrm{s}$. The circuit shown in figure 14 is then used with a frequency of about $100 \mathrm{c} / \mathrm{s}$ to compare the inductances with the resistances $r$ and $R$. Here $R$ indicates the resistance of the entire circuit formed by the secondary coil of $M_{1}$ and the primary coil of $M_{2}$, together with the auxiliary resistor, $S$. When a balance exists the constants of the circuit satisfy the relations:

$$
\begin{aligned}
\omega^{2} M_{1} M_{2} & =R r \\
M_{1} S & =\left(L_{1}+L_{2}\right) r
\end{aligned}
$$




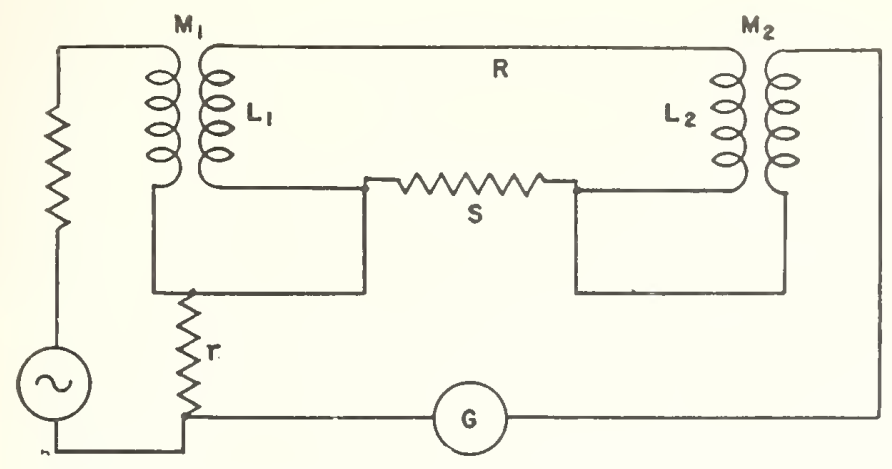

FigurE 14. Campbell method for the absolute measurement of resistance.

The values of the two mutual inductors are derived by comparison with a computable standard inductor. The sccondary emf of $M_{2}$ is nearly equal in magnitude and opposite in phase to the drop of the primary current in $r$.

The first of these equations, together with the measured ratio of $R$ to $r$, gives a value for $R$ (or $r$ ) in terms of $M$ and $\omega$.

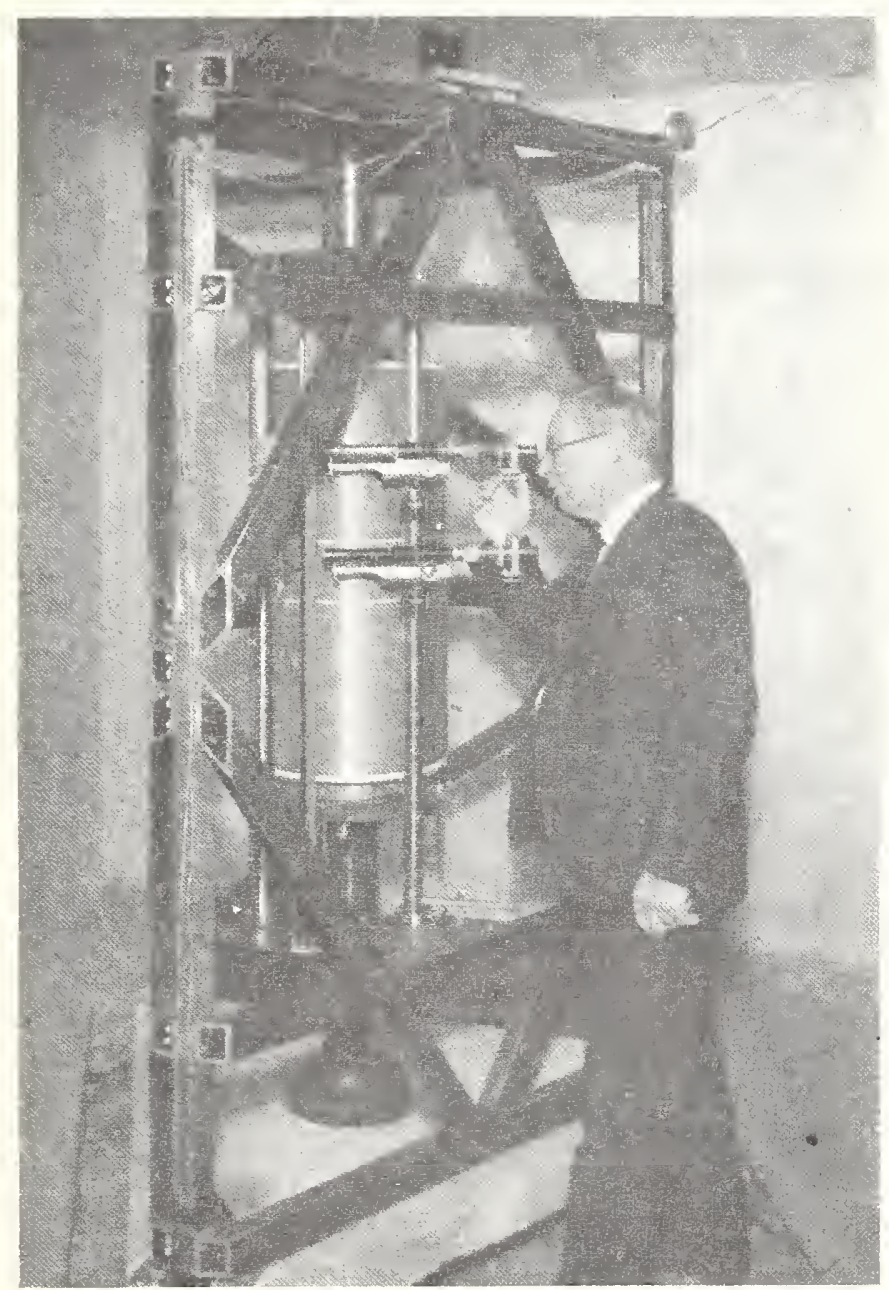

Figure 15. Modified Campbell inductor used in Wenner method. Here Dr. F. Wenner is shown measuring the average pitch of a section of
the primary winding. For the electrical measurements the coil is removed from this steel comparator frame and mounted with its secondary in a nonfrom this steel cornp
metallic housing.
A second method used at the NBS is the Wenner method [68], which combines certain desirable features of several of the preceding methods. The mutual inductor, the primary of which is shown in figure 15, is a modification of the Campbell type with two gaps in the primary winding so placed as to produce two annuli of zero field. By proper design, the resulting annular space within which the field is very small and in which the secondary winding is to be located can be made relatively large. The circuit used is shown in figure 16 . A direct current flowing through the four-terminal resistance, $R$, which is being measured (here shown with an adjustable shunt, $S_{h}$, across its potential terminals) also flows in the primary winding, $P$, of the mutual inductor $M$, but is reversed in direction cyclically by the commutator, $C_{p}$. The emf induced in the secondary of the mutual inductor is rectified by the commutator, $C_{s}$, and opposed through the galvanometer, $G$, to the $I R$ drop in the resistor, $R$. The two commutators are mechanically alined with such relative phases that the secondary connections are reversed after the primary current has had time to settle to a steady value. The current flowing in the secondary at the time of secondary commutation is negligibly small. When a balance is obtained the resistance is given by the equation

$$
R=4 n M I_{r} / I_{a},
$$

where $n$ is the frequency of commutation in cycles per second, $I_{a}$ is the average current through the resistor, $R$, and $I_{\tau}$ is the current flowing in the primary of the inductor at the moment the secondary connections are reversed. The primary current is supplied from two storage batteries and the circuit is so adjusted that battery, $B_{1}$, supplies energy as far as the commutator, $C_{P}$, while battery, $B_{2}$, supplies the energy needed for the rest of the circuit. With this adjustment the voltage between points $a$ and $c$ is always zero, and no change in the current occurs when commutator $C_{P}$ makes its connection between $a$ and $c$ at the beginning of the process of commutation. The resistance network, $R_{G}$; associated with battery, $B_{2}$, is connected to a series of contact segments similar to the commutator of a d-c motor, and these contacts are mechanically driven by the same shaft as that which drives the primary and secondary commutators. The resistances in the network are so chosen that the wave form of the primary current approximates a trapezoid, whereas the wave form of the secondary voltage consists of a sequence of approximately square-topped pulses. The magneto generator, $J$, inserts into the galvanometer circuit an alternating emf, the average value of which is strictly zero, which serves to maintain an approximate balance of the emf's throughout the cycle. The inductors, $K_{s}$ and $K_{p}$, serve to greatly reduce the fluctuations in current in the two circuits. 


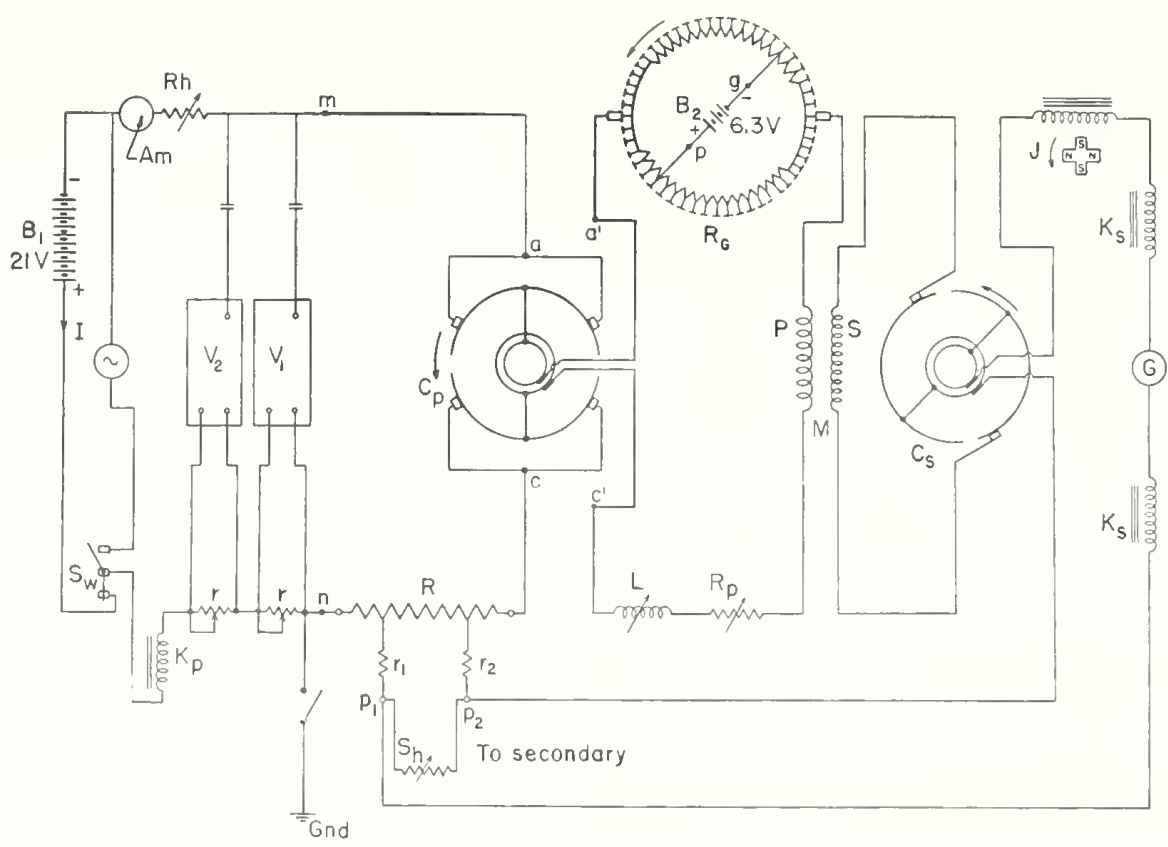

FIGURE 16. Schematic circuit diagram for the Wenner method.

Resistor $R$ is measured in terms of mutual inductor $M$, and frequency of reversal of primary and secondary commutators $C_{\mathrm{p}}$ and $C_{\theta}$. E lectronic amplifier $r_{1}$ and $r_{2}$ hold the current in $P$ constant, while that in $P$ reverses cyclically.

Two electronic amplifiers, $V_{1}$ and $V_{2}$, of the phaseinverter type that have a "gain" of $1: 1$, give further regulation and reduce any cyclic and irregular fluctuations in the potential across $a$ and $c$ by a factor of 400 . The current in the resistor, $P$, is thus kept constant to better than $1 \mathrm{ppm}$ throughout the cycle. At the time of each secondary commutation, the resistor is directly in series with the primary winding of the mutual inductor. Therefore $I_{r}$ equals $I_{a}$ to a high accuracy, and

$$
R=4 n M \text {. }
$$

This method has been found so convenient to operate that it is planned to kecp the apparatus in commission continuously and to measure the resistance of the 1-ohn standards in the primary group at intervals of not longer than 1 year. A continued agreement with earlier values will be a very strong indication that neither the resistance standards nor the mutual inductor has drifted in value. The chance that two such different structures should show simultaneous and equal drift in the same direction is very small. If discrepaneies develop, the laborious process of measuring the mechaniral dimensions of the inductor will have to be repeated. It is hoped that this will not be necessary more often than once every 10 or 20 years.

Table 2 summarizes results obtained by these four methods for the absolute measurement of resistance and shows that the agreement is highy satisfactory in spite of the very considerable differenees between the methods. It therefore seems probable that the systematic errors have becu largely eliminated from all of them.
TABLE 2. Absolute measurements of resistance

\begin{tabular}{|c|c|c|c|}
\hline Date & Authors & Method & Value ${ }^{1}$ \\
\hline 1937 & \multirow{5}{*}{$\begin{array}{l}\text { Vigoureux } \\
\text { Hartshorn and } \\
\text { Astbury. } \\
\text { Curtis, Moon, } \\
\text { and Sparks. } \\
\text { Thomas, Peter- } \\
\text { son, Cooter, } \\
\text { and Kotter. } \\
\text { Mean. }\end{array}$} & Lorenz_... & 1. 000499 \\
\hline 1937 & & Campbell__. & 1. 000505 \\
\hline $1936-38$ & & $\begin{array}{l}\text { Self-induc- } \\
\text { tor. }\end{array}$ & \\
\hline \multirow[t]{2}{*}{1948} & & Wenner _... & 1. 000484 \\
\hline & & & 1. 000490 \\
\hline
\end{tabular}

1 Number of absolute ohms in 1 mean international ohm.

Many sourecs of error are common to all of the methods, although they would be expected to appear to a rery different extent in different methods. One of these is the uncertainty in the dimensions and the uniformity of the windings. Perhaps the best inductors are those of Curtis, Moon, and Sparks, but even in these the outside diameter of the helix has a maximum variation of 4 microms, although the rariations in pitch correspond to axial irregularities certainly less than a few tenths of a micron. Errors may arise from the change in dimensions resulting from the temperature rise caused by the heating effect of the current if the current used in the measurement is increased to obtain higher sensitivity. Still another sounee of crror is the unrertainty in the permeability of the form on wheh the induetor is wound and the offects from magnetie material in the neighborhood. For instance, to aroid surle effects at the NBS, the

Tational Burean of Standards Circular 485 
end of the building assigned for absolute measurements was constructed with bronze plumbing, reinforcing rods of special nonmagnetic steel, lead window weights, etc.

The frequency used in the main balance in the second method, or in the Maxwell bridge in the third method, and the speed of rotation of the generator or commutator shafts in the other two methods must be measured to the full accuracy desired in the final result. 'This, however', has not constituted a serious limitation because the average speed over a considerable time can readily be determined to high accuracy by comparison with circuits timed by crystal oscillators. A more subtle source of error may be the uncertainty in the location of the current within the cross section of the primary winding. If the material were homogeneous one would expect the center of gravity of the current distribution to lie somewhat inside the center of the wire because of the shorter circumference of the inner edge of each turn. The problem is complicated, however, because in the process of winding, parts of the material are strained even beyond their elastic limits, and these strains may cause small changes in the resistivity, which in turn will affect the distribution of current. Such errors, are, of course, greater when the cross section of wire is made large to permit the use of large currents.

Other sources of error applicable to particular methods that may be mentioned are: Imperfect integration of the current by the galvanometer used in the Maxwell bridge; changes in the resistance of the intermediate circuit of the Campbell method as a result of heating of the copper coils that form part of this circuit; the low voltage developed in the Lorenz apparatus, which makes thermoelectric and contact trouble at the brushes rather significant, and the possibility of residual emf in the secondary of the inductor at the time of commutation of the secondary circuit in the Wenner method.

In spite of all these possible error's, the great effort expended on these methods has led to results which, as shown in table 2 , differ by only a very few parts in 100,000 .

\section{Absolute Measurement of Current}

The only methods $[70,71,72]$ used in recent times for the absolute measurement of current with high precision involve a current balance somewhat like that shown schematically in figure 17. The current to be measured is passed in series

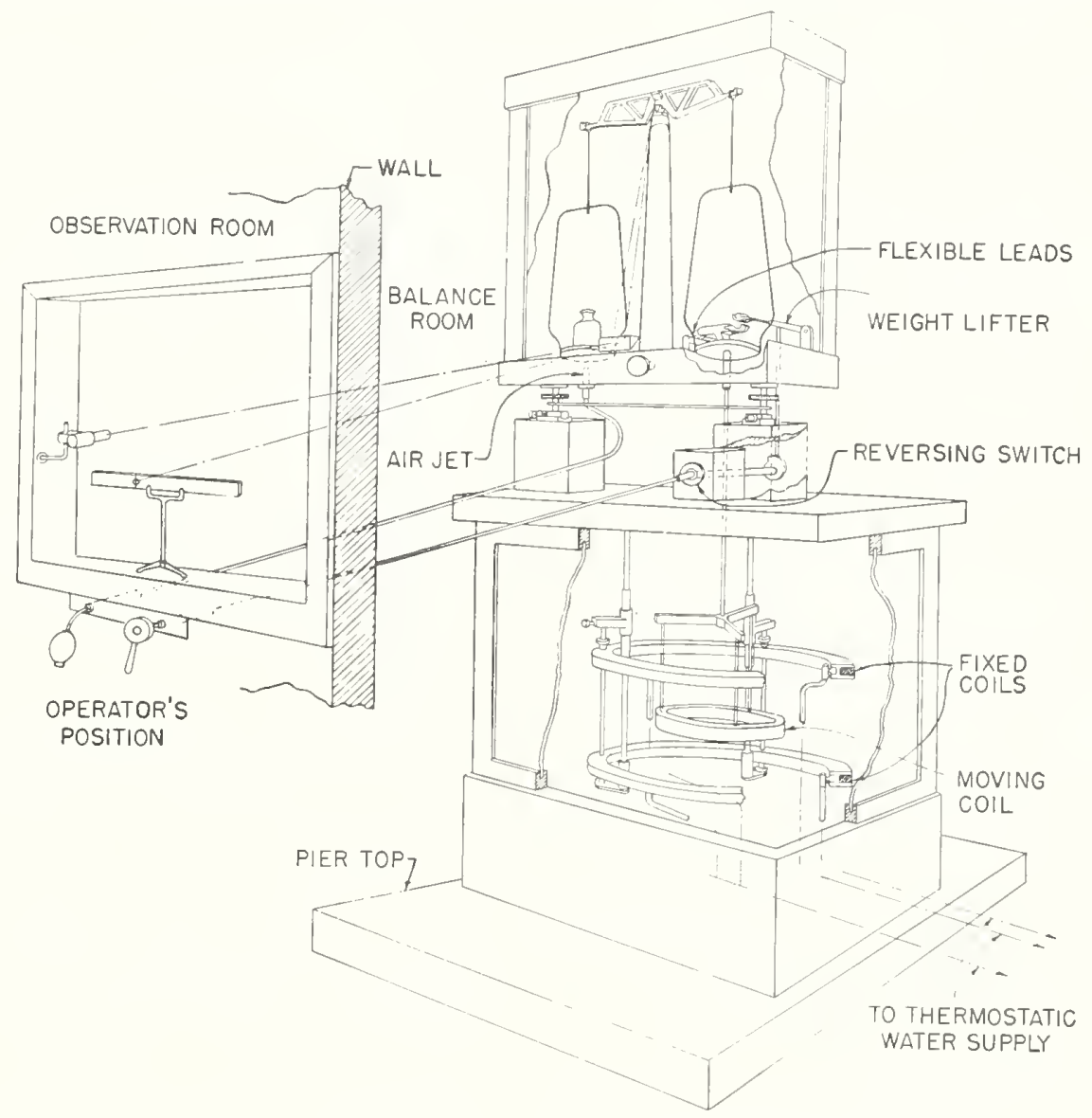

FIGURE 17. Schematic drawing of Rayleigh current balance.

With the weight lifted, the direction of currents is such that the moving coil is attracted hy the lower and repelled by the upper fixed coil. When the switch is reversed, the weight is lowered on to the balance pan and the electrodynamic forces are in the opposite direction.

Establishment and Maintenance of the Electrical Units 
through the two fixed coils and the moving coil. The direction of the windings is such that the magnetomotive forces of the two fixed coils are in opposition so that they conspire to force the moving coil in the same direction. The current balance at the NBS uses coils the cross section of which is small relative to their radius and is commonly designated as of the Rayleigh type. At the NPL all three coils are wound with single-layer windings that have considerable axial length, and the balance is designated as the Ayrton-Jones type. The NPL balance has two sets of coils, one moving coil being supported from either end of the balance. This has the advantage of cloubling the force and of giving some compensation between the disturbing forces due to convection currents arising from the heating developed by the current in the moving coils. In operation, the rest point of the balance is observed before and after reversal of current in the fixed coils. A compensating mass of known weight, nearly equal to the change in electromagnetic force, is also added during one observation and removed during the other.

The formulas for computing the force in terms of current are complicated, but in the Rayleigh for'm involve principally the ratio of the effective radii of the fixed and moving coils. It has been found possible to measure this ratio of the effective radii by an electrical method with a higher

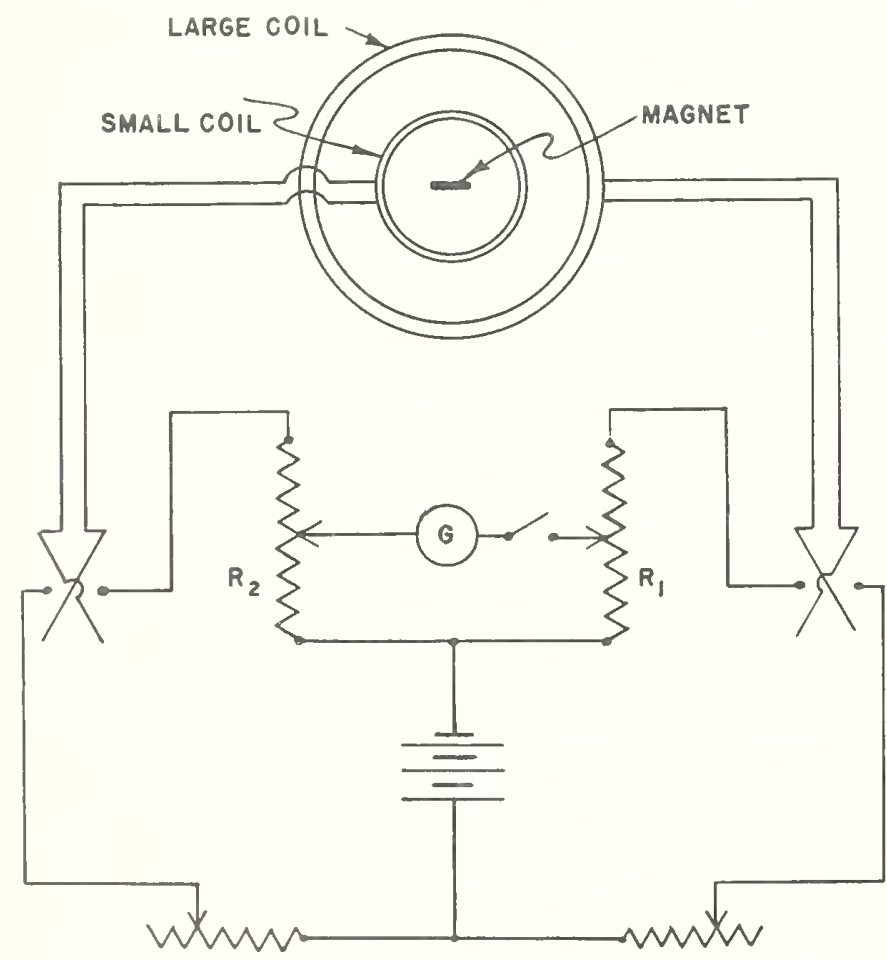

FIGURe 18. Circuit for measuring ratio of effective radii of current-balance coils.

If the currents are adjusted to have equal and opposite effects on the small magnet, their ratio is equal to that of the effective radii of the coils. If the galvanometer is also balanced, this ratio is equal to that of the resistance $R_{1}$ to $R_{2}$. precision than can be obtained by purely mechanical measurements. This is done with the circuit shown in figure 18. The two coils concerned are mounted concentrically with their planes in the magnetic meridian and are energized with currents of such magnitude that the net magnetic field at the common center of the coils is zero. This condition is indicated by a sensitive magnetometer at the center. When the magnetometer shows no deflection as a result of a reversal of both currents, the ratio of the radii is equal to the ratio of the currents. The ratio of the currents is then equal to the ratio of the resistances, $R_{1}$ and $R_{2}$, when the galvanometer, $G$, also shows a balance.

The principal limitation in the absolute measurement of current seems to be in the uncertainty in the location of the coils, and it has seemed better to sacrifice the greater mechanical forces that can be obtained by using multilayer coils of round insulated wire and to use instead single layer solenoids of bare wire or coils wound of metallic ribbon to form a coil of many layers but of one turn per layer. Aluminum ribbon insulated, by an anodizing process has been found convenient for such coils.

Figure 19 shows the combination of a helical moving coil with a pair of spiral fixed coils as mounted at the NBS. A comparison of the results obtained when different coil combinations are in use is shown in table 3 . The values listed in this table are the amounts in ppm by which the absolute ampere, as derived by the use of various combinations of fixed and moving coils in the current balance at the NBS, exceeds the NBS international ampere as certified in the United States. It will be seen that when the larger (25-cm diam) moving coil was used with the smaller (40-cm diam) of the fixed coils, the difference, $26 \mathrm{ppm}$, was $145 \mathrm{ppm}$ smaller than the value, $171 \mathrm{ppm}$, observed when the ribbon-wound (25-em diam) moving coil was used with the fixed single-layer solenoids (46-cm diam). These systematic difference are definitely larger than the random errors of observation and are still unexplained.

TABLE 3. Results with different coils in current balance

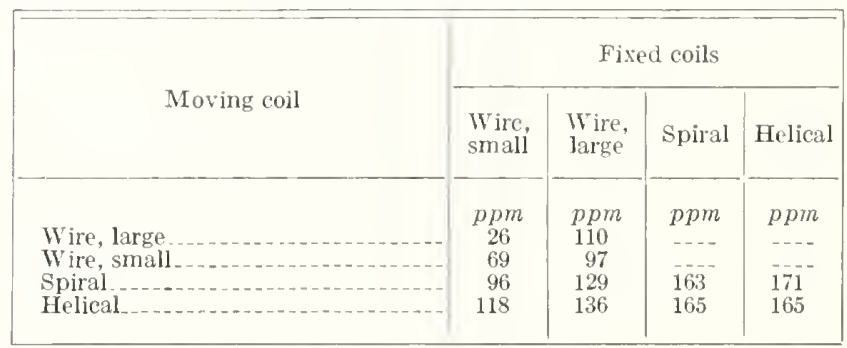

A furtler uncertainty arises from possible errors in the value, $g$, used for the acceleration of gravity in computing the gravitational force on a known 


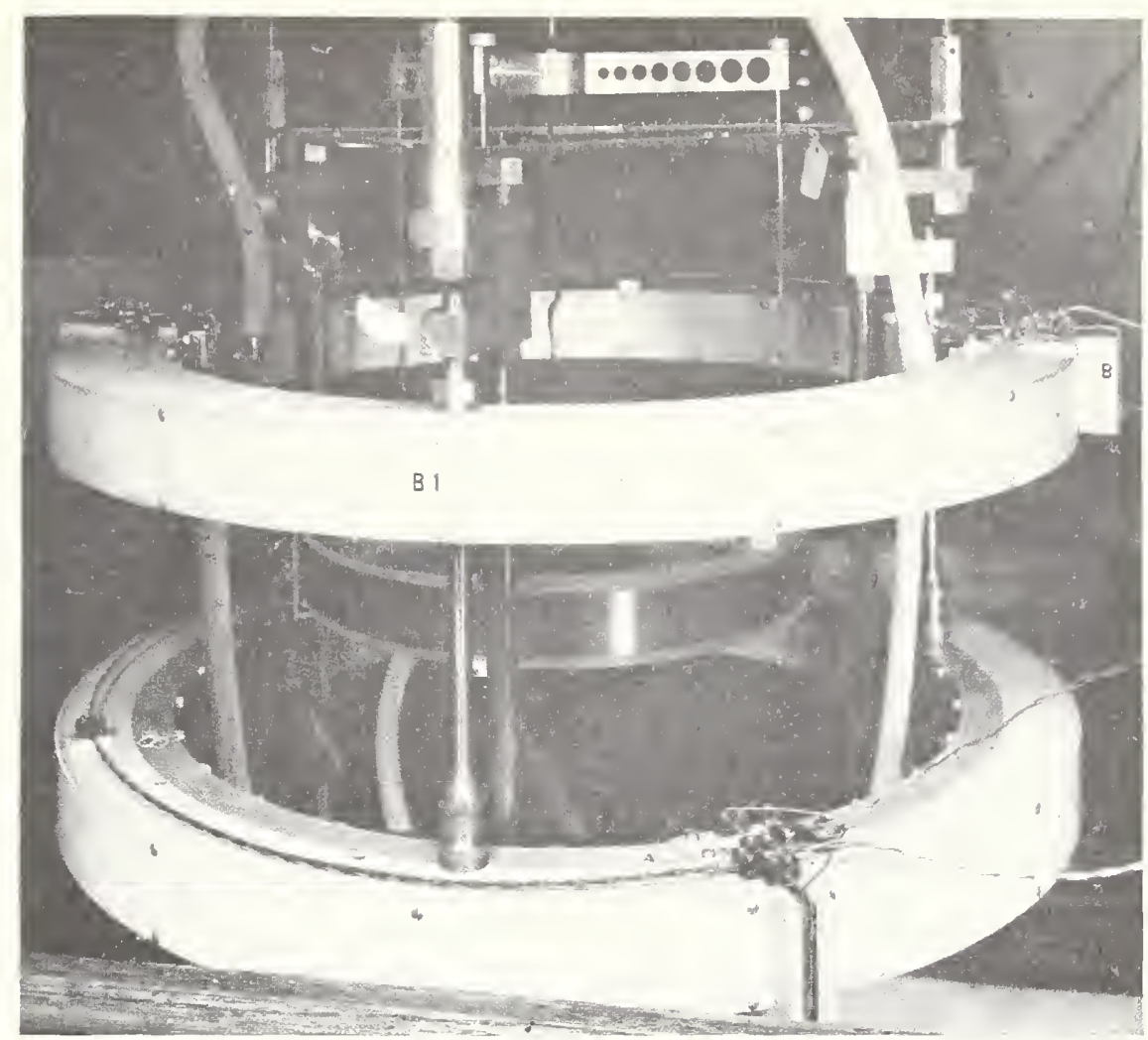

Figure 19. Coils of current balance.

Here the two spiral fixed eoils are wound of anodized aluminum strip on the ontside of the water jaeket and enelosed in a proteetive ease. The moving eoil

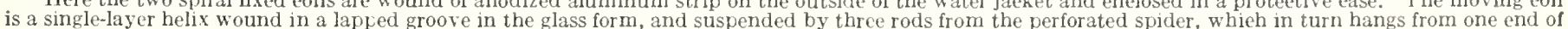
the balance.

mass. The values reported from earlier work and given in table 4 are based on the 1906 Potsdam determination. In the light of more recent measurements [75], it appears that the best value of $g$ is probably less by $15 \mathrm{ppm}$ than that derived from Potsdain.

The results of the best recent absolute measurements of current are summarized in table 4 . [62].

TABLE 4. Absolute measurement of current

\begin{tabular}{|c|c|c|c|}
\hline Date & Authors & Method & Value a \\
\hline 1936 & Vigoureux _ & A y $r$ to $n-$ & 0. 999863 \\
\hline 1939 & Curtis, Curtis, & Rayleigh .... & 0. 999.860 \\
\hline \multirow[t]{2}{*}{1942} & $\begin{array}{c}\text { Curtis, Driscoll, } \\
\text { and Critchfield. }\end{array}$ & $\underset{\text { tion }}{\text { Com in }}$ & 0. 999856 \\
\hline & Mean_ & & 0. 999860 \\
\hline
\end{tabular}

a Number of absolute amperes in 1 mean international ampere, using values of gravity derived from the Potsdam determination.
On the basis of these results, the International Committee chose the reduced value 0.99985 for the value of the mean intermational ampere in absolute amperes. Hence the international ampere as certified by the National Bureau of Standards is 0.999835 absolute ampere.

It is evident from table 3 , however, that the certainty of the final result is not as high as night be concluded from the (perhaps fortuitous) agreement of the independent values listed in table 4 .

There is an evident need for the development of some radically different method for measuring current in absolute amperes that will not be subject to the same types of systematic error. The Pellat balance [73] in which the current in two coils placed with their axes at right angles produces a mechanical torque, seems to offer such a possibility. The construction of such a balance was begun at the NBS some time ago, and the mathematical formulas needed in its use have been worked out by Snow [74]. It is hoped that this project can be resumed in the near future. 


\section{Summary}

The preceding record has shown that for the 37 years from 1911 to 1947 , inclusive, the national standardizing laboratories, with valuable cooldinating service from the International Bureau of Weights and Measures; succeeded in maintaining throughout the civilized world a system of electrical units that did not vary in time among any of the six participating nations by more than 0.01 of 1 percent. The units in the United States apparently never departed from the desired ideal by more than 20 ppm.

The ideal pursued during these years was the system of international electrical units, formulated by the London Conference of 1908, and experimentally realized by the Technical Committee in Washington in 1910 . The basic definitions in terms of mercury columns and silver deposits were brought into play apparently on only four occasions: To readjust the British ohm in 1927, the German olim and the volt in 1932, and the Russian volt in 1930 , when each of these nations changed its unit to offset the effect of cumulative drift in the standards it lad been using to maintain the unit. The fact that such drifts did accumulate makes it clear that in the future as in the past some fixed definitions must be set up by which the standards can be checked periodically. The results of absolute measurements by a varicty of methods have shown that such absolute measurements can be used to serve as a check against cumulative drift with an aceuracy at least as great as that attainable in the discarded international standards.

The rllange of January 1, 1948, involved both a change in the size of each unit by the factor shown on page 22 and a change in the type of experi- ment by which, at fairly long intervals, checks will be made by one or another of the national laboratories to detect any incipient progressive drifts in its standards. The older procedures for maintaining the ohm and the volt by groups of standard resistors and cells of the highest quality will continue unchanged. The biennial intercomparisons at the International Bureau are being resumed and will serve both to detect relative drifts between the standards of different countries and to maintain accurate values for the standards deposited at Sèvres, which in turn serve as primary standards for the laboratories of countries that do not have national laboratories of their own.

It is to be expected that the International Advisory Committee on Electricity will meet only at fairly long intervals as additional experimental realizations of the absolute units become available, or if an undue drift in the value of one or more nation's unit relative to the mean indicates a need for consideration by the International Committee on Weights and Measures. Any readjustment of values resulting from such situations will presumably be very small.

However, the adoption of the new ideal, which is inherently consistent with the mechanical units, has automatically eliminated all future need of correcting precise scientific data to allow for the artificial distinction between the international and the absolute units. Future absolute rneasurements can be depended upon to keep the units as maintained amply close to the ideal, because with adequate attention they may be expected to increase in accuracy in parallel with any possible future increases in the accuracy of the scientific measurements that they are to correlate.

\section{References}

[1] Historical Reports of the Committee on Electrical Stanclards appointed by the British Association for the Advancement of Scicnce (reprinted 1913 by (ambridge Univ. Press).

[2] G. Giorgi, Proposal concerning elcetrical and physical mits, Trans. Int. Electrical Congress 1, 136 (St. Louis, Mo., 1904).

[3] F. A. Wolff, The prineiples involved in the selection and definition of the fundamental electrical units to be proposed for international adoption, Bul. BS 5 , 243 (1908-9) \$102.

[4] Annomecment of a change in the value of the international volt. NBS Circular C29 (1910).

[5] Report to the International Committec on Elcetrical Units and Standards of a special technical committee appointed to investigate and report on the concrete standards of the international electrical units and to recommend a value for the Weston Normal Cell, NBs Nisc. Pub. MI6 (1912).

[6] Comptes Rendus des Sćance de la Sixième Conférence Générale des Poids et Mesures, pp) 31 to 42 (Gauthicr-Villars, Paris, 1921).

[7] Procès Verbaux, Comité International des Poids et Mesures 13, 58 (1929).
[8] Procès Verb. Comité International des Poids et Mesures 1\%, 74 (1935).

[9] Announcement of changes in electrical and photometric units, NBS Circular C459 (1947).

\section{Mercury Resistance Standards}

[11] W. Jaeger, K. Kahle, and H. Diesselhorst, Mercury standards of the Reichsanstalt, Wiss. Ablandl. physik, tcch. Reichsanstalt 4, 117 (1905).

[12] F. E. Smith, Mercury standards and resistance temperature coefficient of mercury, Phil. Trans. 204, 57 (1904).

[13] F. A. Wolff, M. P. Shoemaker, and C. A. Briggs, Construction of primary mercurial resistance standards, Bul. BS 12, 375 (1915-16) S256.

[14] F. E. Smith, Nat. Plys. Lab. Report for the Icar 1912 , p. 40.

[15] L. Hartshorn, Nat. Phys. Lab. Report for the year 1925 , p. 93.

[16] von Steinwchr and Schulze, Die Quecksilbernormale der P'TR für das Ohm, Wiss. Abhandl. physik. tech. Reichsanstalt 11,75 (1927).

Tational Bureau of Standards Circular 475 


\section{Silver Voltameter}

[21] E. B. Rosa and G. W. Vinal, Summary of experiments on the silver voltameter at the Bureau of Standards and proposed specifications, Bul. BS 13, 479 (1916-17) S285.

[22] A. C. Iiolossov, Determination by the method of the silver voltameter of the electromotive force of the International Weston Normal Cell, Procès Verbaux, Comité International des Poids et Mesures 16, 150 (1933) Annexe No. 19.

\section{Standard Resistors}

[31] W. Jaeger and S. Lindeck, Reichsanstalt type resistance standards, Ann. phys. 65, 572 (1898); Electrician 59, 626 (London, 1907).

[32] E. B. Rosa and H. D. Babcock, On the variation of resistances with atmospheric humidity, Bul. BS 4, $121(1907-08)$ S73

[33] E. B. Rosa, A new form of standard resistance, Bul. BS 5, $413(1908-09)$ S107.

[34] J. L. Thomas, A new design of precision resistance standard, BS J. Research 5, 295 (1930) RP201.

[35] J. L. Thomas, Stability of double-walled manganin resistors, J. Research NBS 36, 107 (1946) RP1692.

[36] F. Wenner, methods, apparatus and procedures for the comparison of precision standard resistors, J. Research NBS 25, 229 (1940) RP1323.

[37] F. Wenner and E. F. Mueller, The Waidner-Wolff and other adjustable electrical resistance elements, J. Research NBS 15, 477 (1935) RP842.

\section{Standard Cells}

[41] L. Clark, On a standard voltaic battery, Phil. Trans. Roy. Soc. 164, 1 (i 874 ).

[42] The Weston Standard Cell, Electrician 30, 742 (London, 1893)

[43] H. B. Brooks, The standard-cell comparator, a specialized potentiometer, BS J. Research 11, 211 (1933) RP586.

\section{International Comparisons of Units}

[51] Procès Verbaux, Comité International des Poids et Mesures: D. W. Dye, 13, 161 to 166 (1929); K. Takatsu \& S. Jimbo, 14, 192 (1931); M. K. Malikov and A. C. Kolossov 14, 199 to 207 (1931); G. K. Burgess, 14, 312 (1931); A. Pérard and M. Romanowski, 17, 288 (1935).

[52] G. W. Vinal. International comparisons of electric standards, BS J. Research 8, 729 (1932) RP448.

[53] P. Vigoureux, International determination of the electromotive force of the Normal Weston Cell in International Volts, Coll. Res. Nat. Phys. Lab. 24, 79 (1932).

[54] H. von Steinwehr and A. Schulze, Neubestimmung der EMIK des Internationales Westonelements, Z. Instrumenk. 52, 249(1932).
[55] M. Romanowski and M. Roux, Premiers Essais de Comparison des Etalons Nationaux de Force Electromotrice, Procès Verbaux, Comité International des Poids et Mesures, 16, 141 (1933).

[56] Procès Verbaux, Comité International des Poids et Mesures 17, 94 (Resolution 4). (1935).

[57] Procès Verbaux, Comité International des Poids et Mesures 21, (1948)

[58] M. Romanowski, Conservation of the electrical units at the International Bureau during the vears 1939 to 1946 , Procès Verbaux, Comité International des Poids et Mesures 20, 179 (1946).

\section{Absolute Measurements}

[61] H. L. Curtis, Electrical measurements (McGraw Hill Book Co., Ine. New York, N. Y., 1937).

[62] H. L. Curtis, Review of recent absolute determinations of the ohm and the ampere, J. Research NBS 33, 235 (1944) RP 1606.

[63] H. L. Curtis, A review of the methods for the absolute determination of the ohm, J. Wash. Acad. Sci., 32, 40 (1942).

[64] P. Vigoureux, Determination of the olnm by the method of Lorenz, Nat. Phys. Lab. Collected Researches 24, 277 (1938); 11, 209 (1914).

[65] H. L. Curtis, C. Moon, and C. M. Sparks, A determination of the absolute ohm using an improved self-inductor, J. Research, NBS 21, 375 (1938) RP1137.

[66] L. Hartshorn and N. F. Astbury, The absolute measurement of resistance by the method of Albert Campbell, Phil. Trans. Roy. Soc. (London) [A] 236, 423 (1937)

[67] A. Campbell, On a standard of mutual inductance, Proc. Roy. Soc. (London) [A] 79, 428 (1907).

[68] J. L. Thomas, C. Peterson, I. I. Cooter, and F. R Kotter, An absolute measurement of resistance by the Wenner method, unpublished.

[69] E. B. Rosa and F. W. Grover, Formulas and tables for the calculation of mutual and self-inductance (3d ed. revised), Bul. BS 8, 1 (1912) S169.

[70] P. Vigoureux, An absolute determination of the ampere, Phil. Trans. Roy. Soc. (London) [A] $\mathbf{2 3 6}$ 133 (1937).

[71] H. L. Curtis, R. IV. Curtis, and C. L. Critchfield, An absolute determination of the ampere, using improved coils, J. Research NBS 22, 485 (1939) RP1200.

[72] R. W. Curtis, R. L. Driscoll, and C. L. Critchfield, An absolute determination of the ampere, using helical and spiral coils, J. Research NBS 28, 133 (1942) RP1449.

[73] H. Pellat, An absolute electrodynamometer, J. phys. (2d series) 6, $172(1887)$.

[74] C. Snow, Mutual inductance and torque between two concentric solenoids, BS J. Pesearch 1, 686 (1928) RP24.

[75] H. L. Dryden, A reexamination of the Potsdam absolute determination of gravity, J. Research NBS 29, 303 (1942) RP1502.

\section{Appendix 1}

U. S. Law of 1894, 53d Congress, 28 Stat., Ch. 131, p. 102

(Public-No. 105)

An Act To define and establish the units of electrical measure

Be it enacted by the Senate and House of Representatives of the United States of American in Congress assembled, That from and after the passage of this Act the legal units of electrical measure in the U'nited States shall be as follows:

First. The unit of resistance shall be what is known as the international ohm, which is substantially equal to one thousand million units of resistance of the centimetergram-second system of electro-magnetic units, and is represented by the resistance offered to an unvarying electric current by a column of mercury at the temperature of melting ice fourteen and four thousand five hundred and twenty-one ten-thousandths grams in mass, of a constant cross-sectional area, and of the length of one hundred and six and three tenths centimeters. 
Second. The unit of current shall be what is known as the international ampere, which is one-tenth of the unit of current of the centimeter-gram-second system of electromagnetic units, and is the practical equivalent of the unvarying current, which, when passed through a solution of nitrate of silver in water in accordance with standard specifications, deposits silver at the rate of one thousand one hundred and eighteen millionths of a gram per second.

Third. The unit of electro-motive force shall be what is known as the international volt, which is the electromotive force, that steadily applied to a conductor whose resistance is one international ohm, will produce a current of an international ampere, and is practically equivalent to one thousand fourteen hundred and thirty-fourths of the electro-motive force between the poles or electrodes of the voltaic cell known as Clark's cell, at a temperature of fifteen degrees centigrade, and prepared in the manner, described in the standard specifications.

Fourth. The unit of quantity shall be what is known as the international coulomb, which is the quantity of electricity transferred by a current of one international ampere in one second.

Fifth. The unit of capacity shall be what is known as the international farad, which is the capacity of a condenser charged to a potential of one international volt by one international coulomb of electricity.

Sixth. The unit of work shall be the Joule, which is equal to ten million units of work in the centimeter-gramsecond system, and which is practically equivalent to the energy expended in one second by an international ampere in an international ohm.

Seventh. The unit of power shall be the Watt, which is equal to ten million units of power in the centimetergram-second system, and which is practically equivalent to the work done at the rate of one Joule per second.

Eighth. The unit of induction shall be the Henry, which is the induction in a circuit when the electro-motive force induced in this circuit is one international volt while the inducing current varies at the rate of one Ampere per second.

SEc. 2. That it shall be the duty of the National Academy of Sciences to prescribe and publish, as soon as possible after the passage of this Act, such specifications of details as shall be necessary for the practical application of the definitions of the ampere and volt hereinbefore given, and such specifications shall be the standard specifications herein mentioned.

\section{Appendix 2}

\section{International Conference on Electrical Units and Standards, 1908}

\section{REPORT}

The Conference on Electrical Units and Standards for which invitations were issued by the British Government, was opened by President of the Board of Trade, the Right Hon. Winston S. Churchill, M. P., on Monday, 12th October 1908, at Burlington House, London, S. W.

Delegates were present from twenty-two countries, and also from the following British Dependencies, namely, Australia, Canada, India and the Crown Colonies.

It was decided by the Conference that a vote each should be allowed to Australia, Canada and India, but a vote was not claimed or allowed for the Crown Colonies.

The total number of delegates to the Conference was forty-ix, and their names are set out in schedule A to this Report.

The officers of the Conference were;

\section{President}

The Right Hon. Lord Rayleigh, O. M., President of the Royal Society.

Vice-Presidents

Professor S. A. Arrhenius. Dr. N. Egoroff.

Dr. Viktor Edler von Lang.

Mr. M. J. Collins.

Mr. W. Duddell, F. R. S.

\section{Secretaries}

M. Jippmann

Dr. S. W. Stratton.

Dr. E. Warburg.

Mr. C. W. S. Mr. F. E. Smith.

The Conference elected a Technical Committee to draft specifications and to consider any matter which might be referred to the Committee and to report to the Conference.

The Conference and its Technical Committee each held five sittings.

As a result of its deliberation the Conference adopted the resolutions and specifications attached to this report and set out in Schedule B, and requested the Delegates to lay them before their respective Govermments with a view to obtaining uniformity in the legislation with regard to Electrical Units and Standards.

The Conference recommend the use of the Weston Normal Cell as a convenient means of measuring both electromotive force and current when set up under the conditions specified in Schedule C.

In cases in which it is not desired to set up the Standards provided in the resolutions Schedule B, the Conference recommends the following as working methods for the realisation of the International Ohm, the Ampere, and the Volt.

1. For the International Ohm.

The use of copies, constructed of suitable material and of suitable form and verified from time to time, of the International Ohm, its multiples and submultiples.

2. For the International Ampere.

(a) The measurement of current by the aid of a current balance standardized by comparison with a silver voltameter;

or (b) The use of a Weston Normal Cell whose electromotive force has been determined in terms of the International $\mathrm{Ohm}$ and International Ampere, and of a resistance of known value in International Ohms.

3. For the International Volt.

(a) A comparison with the difference of electric potential between the ends of a coil of resistance of known value in International Ohms, when carrying a current of known value in International Amperes;

or (b) The use of a Weston Normal Cell whose electromotive force has been determined in terms of the International Ohm and the International Ampere.

The duties of specifying more particularly the conditions under which these methods are to be applied has been assigned to the Permanent Commission, and pending its appointment to the Scientific Committee to be nominated by the President (see Schedule D), who will issue a series of Notes as Appendix to this Report.

The Conference has considered the methods that should be recommended to the Governments for securing uniform administration in relation to Electrical Units and Standards, and expresses the opinion that the best method of securing uniformity for the future would be by the establishment of an International Electrical Laboratory with the duties of keeping and maintaining International Electrical Standards. This I aboratory to be equipped entirely independently of any National Laboratory. 
The Conference further recommends that action be taken in accordance with the scheme set out in Schedule D.

Signed at London on 21st October, 1908.

By the Delegates of the Countries above written. For the United States of America:

\section{For Austria:}

For Belgium:

For Brazil:

For Chile:

For Colombia:

S. W. Stratton. Henry S. Carhart.

Edward B. Rosa.

Victor Von Lang.

Ludwig Kusminsky.

P. Clément.

Leopold J. Weiss.

Victor Eastman.

Jorge Roa.

For Denmark and Sweden: Svante Arrhenius.

For Ecuador:

For France:

C. Nevares.

G. Lippmann

J. Réne Benoît.

T. De Nerville.

For Germany:

E. Warburg.

IV. Jaeger.

St. Lindeck.

For Great Britain:

Rayleigh.

J. Gavey.

R. T. Glazebrook.

IV. A. J. O'Meara.

A. P. Trotter.

J. J. Thomson.

Francisco de Arce.

Harsánvi Desiré.

Vater Joisef

Antonio Ròiti.

Osuke Asano.

Shigeru Kíndo.

Alfonso Castelló.

Dr. H. Haga.

Max. F. Croskey.

N. Egoroff.

L. Swentorzetzky.

Jose Ma. de Madariaga.

A. Montenegro.

Dr. H. F. Weber.

P. Chappuis.

Jean Landry.

C. W. Darley.

Threlfall.

Ormond Higman.

P. Cardew.

II. G. Simpson.

M. J. Collins.

W. Duddell.

C. IV. S. Crawley.

F. E. Smith.

Secretarics.

\section{Schedule B}

\section{RESOLUTIONS}

1. The Conference agrees that as heretofore the magnitudes of the fundamental electric units shall be determined on the electro-magnetic system of measurement with reference to the centimetre as the unit of length, the gramme as the mit of mass and the second as the unit of time.

These fundamental units are (1) the Ohm, the unit of electric resistance which has the value of $1,000,000,000$ in terms of the centimetre and second; (2) the Ampere, the unit of electric current which has the value of one-tenth (0.1) in terms of the centimetre, gramme, and sccond; (3) the Volt, the unit of electromotive force which has the value $100,000,000$ in terms of the centimetre, the gramme, and the second; (4) the Watt, the Unit of Power which has the value $10,000,000$ in terms of the centimetre, the gramme, and the second.
II. As a system of units representing the above and sufficiently near to them to be adopted for the purpose of electrical measurements and as a basis for legislation, the Conference recommends the adoption of the International Ohm, the International Ampere, and the International Volt defined according to the following definitions:

III. The Ohm is the first Prinary Unit.

IV. The International Ohm is defined as the resistance of a specified column of mercury.

$V$. The International Ohm is the resistance offered to an unvarying electric current by a column of mercury at the temperature of mclting ice, 14.4521 grammes in mass, of a constant cross sectional area and of a length of 106.300 centimetres.

To determine the resistance of a column of mercury in terms of the International Ohm, the procedure to be followed shall be that set out in Specification I attached to these Resolutions.

VI. The Ampere is the second Primary Unit.

VII. The International Ampere is the unvarying electric current which, when passed through a solution of nitrate of silver in water, in accordance with Specification II attached to these Resolutions, deposits silver at the rate of 0.00111800 of a gramme per second.

VIII. The International Volt is the electrical pressure which, when steadily applied to a conduct or whose resistance is one International Ohm, will produce a current of one International Ampere.

IX. The International Watt is the energy expended per second by an unvarying electric current of one International Ampere under an clcetric pressure of one International Volt.

\section{Specification I.}

Specification Relating to Mercury Standards of Resistance

The glass tubes used for mereury standards of resistance must be made of a glass such that the dimensions mav remain as constant as possible. The tubes must be wcll annealed and straight. The bore must be as nearly as possible uniform and circular, and the area of cross-section of the bore must be approximately one square millimetre. The mercury must have a resistance of approximately one ohm.

Each of the tubes must be accurately calibrated. The correction to be applied to allow for the area of the crosssection of the bore not being exactly the same at all parts of the tube must not exceed 5 parts in 10,000 .

The mercury filling the tube must be considered as bounded by plane surfaces placed in contact with the ends of the tube.

The length of the axis of the tube, the mass of mercury the tube contains, and thc electrical resistance of the mercury are to be cletermined at a temperature as near to $0^{\circ} \mathrm{C}$, as possible. The measurements a re to be corrected to $0^{\circ} \mathrm{C}$.

For the purpose of the electrical measurements, end vessels carrying connections for the current and potential terminals are to be fitted to the tube. These end vessels are to be spherical in shape (of a diameter of approximately four centimetres) and should have cylindrical pieces attached to make connections with the tubes. The outside edge of each end of the tube is to be comcident with the inner surface of the corresponding spherical end vessel. The leads which make contact with the mercury are to be of thin platinum wire fused into glass. The point of entry of the current lead and the end of the tube are to be at opposite ends of a diameter of the bulb; the potential lead is to be midway between these two points. All the leads must be so thin that no error in the resistance is introduced through conduction of heat to the mercury. The filling of the tube with mercury for the purpose of the resistance measurements must be carried out under the same conditions as the filling for the determination of the mass. 
The resistance which has to be added to the resistance of the tube to allow for the effect of the end vessels is to be calculated by the formula,

$$
A=\frac{0.80}{1063 \pi}\left(\frac{1}{r_{1}}+\frac{1}{r_{2}}\right) \text { ohm, }
$$

where $r_{1}$ and $r_{2}$ are the radii in millimetres of the end sections of the bore of the tube.

The mean of the calculated resistances of at least five tubes shall be taken to determine the value of the unit of resistance.

For the purpose of the comparison of resistances with a mercury tube the measurements shall be made with at least three separate fillings of the tube.

\section{Specification II}

\section{Specification Relating to the Deposition of Silver}

The electrolyte shall consist of a solution of from 15 to 20 parts by weight of silver nitrate in 100 parts of distilled water. The solution must only be used once, and only for so long that not more than 30 percent of the silver in the solution is deposited.

The anode shall be of silver, and the kathode of platinum. The current density at the anode shall not exceed $1 / 5$ ampere per square centimetre and at the kathode $1 / 15$ ainpere per square centimetre.

Not less than 100 cubic centimetres of electrolyte shall be used in à voltameter.

Care must be taken that no particles which may become mechanically detached from the anode shall reach the kathode.

Before weighing, any traces of solution adhering to the kathode must be removed, and the kathode dried.

\section{Schedule C}

\section{Weston Normal Cell}

The Weston Normal Cell may be conveniently employed as a standard of electric pressure for the measurement both of E. M. F. and of current, and when set up in accordance with the following Specification, may be taken, provisionally, $*$ as having, at a temperature of $20^{\circ}$ C., an E. M. F. of 1.0184 volts.

\section{Specification Relating to the Weston Normal Cell}

The Weston Normal Cell is a voltaic cell which has a saturated aqueous solution of cadmium sulphate $\left(\mathrm{CdSO}_{4} 8 / 3 \mathrm{H}_{2} \mathrm{O}\right.$ ) as its electrolyte.

The electrolyte must be neutral to Congo Red.

The positive electrode of the cell is mercury.

The negative electrode of the cell is cadmium amalgam consisting of 12.5 parts by weight of cadmium in 100 parts of amalgam.

The depolariser, which is placed in contact with the positive electrode, is a paste made by mixing mercurous sulphate with powdered crystals of cadmium sulphate and a saturated aqueous solution of cadmium sulphate.

The different methods of preparing the mercurous sulphate paste are described in the notes.t One of the methods there specified must be carried out.

* See duties of the Scientific Committee, Schedule D.

†Notes on methods pursued at various standardising laboratories will be issued by the Scientific Committee or the Permanent Commission, as an Appendix to this Report.
For setting up the cell, the $\mathrm{H}$ form is the most suitable. The leads passing through the glass to the electrodes must be of platinum wire, which must not be allowed to come into contact with the electrolyte. The amalgam is placed in one limb, the mercury in the other.

The depolariser is placed above the mercury and a layer of cadmium sulphate crystals is introduced into each limb. The entire cell is filled with a saturated solution of cadmium sulphate and then hermetically sealed.

The following formula is recommended for the E. M. F. of the cell in terms of the temperature between the limits $0^{\circ} \mathrm{C} . \& 40^{\circ} \mathrm{C}$.

$$
\begin{aligned}
\mathrm{E}_{t}=\mathrm{E}_{20}- & 0.0000406\left(t-20^{\circ}\right) \\
& -0.00000095\left(t-20^{\circ}\right)^{2}+0.00000001\left(t-20^{\circ}\right)^{3}
\end{aligned}
$$

\section{Schedule D}

1. The Conference recommends that the various Governments interested establish a permanent International Commission for Electrical Standards.

2. Pending the appointment of the Permanent International Commission the Conference recommends( $\left.{ }^{1}\right)$ that the President, Lord Rayleigh, nominate for appointment by the Conference a scientific Committee of fifteen to advise as to the organisation of the Permanent Commission, to formulate a plan for and to direct such work as may be necesary in connection with the maintenance of standards, fixing of values (2), intercomparison of Standards and to complete the work of the Conference $\left(^{3}\right)$. Vacancies on the Committee to be filled by co-optation.

3. That Laboratories equipped with facilities for precise electrical measurements and investigations should be asked to cooperate with this Committee and to carry out, if possible, such work as it may desire.

4. The Committee should take the proper steps forthwith for establishing the Permanent Commission, and are empowered to arrange for the meeting of the next Conference on Electrical Units and Standards, and the time and place of such meeting should this action appear to them to be desirable.

5. The Committee or the Permanent International Commission shall consider the question of enlarging the functions of the International Commission on Weights and Measures, with a view to determining if it is possible or desirable to combine future Conferences on Electrical Units and Standards with the International Commission on Weights and Measures, in place of holding in the future Conferences on Electrical Units and Standards. At the same time it is the opinion of the Conference that the Permanent Commission should be retained as a distinct body, which should meet at different places in succession.

(1) In accordance with the above, Lord Rayleigh has nominated the follow ing Committee, which has been approved by the Conference, viz.:

Dr. Osuke Asano. Prof. G. Lippmann.

M. R. Bepoit.

Dr. N. Egoroff.

Prof. Eric Gérard.

Prof. E. B. Rosa.

Dr. H. Haga.

D. L. Kusminsky.

Prof. St Lindeck.

(2) This will include the reconsideration from time to time of the E. M. F of the Weston Normal Cell.

(3) With this object the Committee are authorised to issue as an Appendix to the Report of the Conference Notes detailing the methods which have been adopted in the Standardising Laboratories of the various countries been adopted in the standardising Laboratories of the various countries up the Weston Normal Cell. 


\section{Appendix 3.}

Metric Convention. Article 7, as amended 1921

ART. 7. After the committee shall have proceeded with the work of coordinating the measures relative to electric units and when the general conference shall have so decided by a unanimous, vote, the bureau will have charge of the establishment and keeping of the standards of the electric units and their test copies and also of comparing with those stanclards, the national or other standards of precision.
The bureau is also charged with the duty of making the determinations relative to physical constants, a more accurate knowledge of which may be useful in increasing precision and further insuring uniformity in the provinces to which the above-mentioned units belong (article 6 and first paragraph of article 7).

It is finally charged with the duty of coordinating similar determinations effected in other institutions.

\section{Appendix 4.}

Resolutions Adopted by the Board of Directors, American Institute of Electrical Engineers, at its Meeting in Denver, Colorado, June 27, 1928

Whereas, there is conclusive evidence that there are discrepancies between the statutorily established international electrical units (ohm, ampere, and volt) and the fundamental ohm, ampere and volt which the international units were intended to represent, these discrepancies in the case of the ohm and the volt amounting to approximately one-twentieth of one per cent; and

Whereas, differences of this magnitude are objectionably large in comparison with the precision required and 110 if being attained in the construction and use of standards fundamental to all electrical measurements: therefore be it

Resolved, that the American Institute of Electrical Engineers hereby urges the Bureau of Standards and foreign national standardizing laboratories to undertake, as soon as possible, the additional researches necessary in order that legislation to reduce these discrepancies to within acceptable limits may be enacted in the near future.

And whereas, the present electrical units are defined by statute in terms of material standards, namely, the mercury ohm and the silver voltameter, which it is now known only approximately represent the absolute ohm and ampere and which experience has shown to have serious limitations, and

Whereas, such progress has been made in recent years in the art of making absolute electrical measurements in terms of the fundamental units of length, mass, time and space permeability that the accuracy and reproducibility of a system of electrical units realized by such absolute measurements would seem to be adequate for commercial. industrial and scientific purposes; and

Whereas, the legalization of the absolute ohm and ampere and the units derived from them (these units to be realized by the national standardizing laboratories) would avert the recurring proposals for revision of the values of the legalized units, and would establish the electrical units on a permanent legal basis; therefor be it

Resolved, that the American Institute of Electrical Engineers hereby urges the Bureau of Standards and foreign national standardizing laboratories to undertake as soon as possible, the additional researches necessary in order that the absolute ohm and absolute ampere based on the centimeter-gram-second electromagnetic system, with the absolute volt, watt and other units derived from them, may be legalized in place of the international ohm and ampere and their derived units.

Resolved, further, that in order to avoid the confusion which would result from an interim use of new empirical units based on corrected values of the international units, the international electrical units should be continued in effect without any readjustment of values until such time as the practicability of legalizing the above-mentioned absolute units shall have been determined.

Resolved, further, that copies of these resolutions be communicated to the various national standardizing laboratories and other interested bodies, by the Standards Committee.

\section{Appendix 5.}

Translation of Resolution No. 10 adopted October 6, 1933, by the 8th General Conference on Weights and Measures

Resolution No. 10, Substitution of the absolute electrical units for the units called "international".

In accord with the first resolution relative to the electrical units proposed by the Advisory Committee and approved by the International Committee on Weights and Measures;

The Conference approves the principle of the substitution of the absolute system of electrical units for the inter- national system:

Considering, however, that a number of national laboratories have not yet completed the measurements necessary to relate the international units to the absolute units;

It decides to postpone to the year 1935 the provisional fixing of the ratio between each international unit and the corresponding absolute unit:

For this purpose it gives to the International Committee the necessary authority to fix at that time and without waiting for another Conference, these ratios as well as the date of adoption of the new units. 


\section{Appendix 6.}

Translation of Resolutions adopted October 29,1946 by the International Committee on Weights and Measures

\section{RESOLUTIONS}

\section{Concerning the Change in Electrical Units}

\section{Resolution 1}

The International Committee on Weights and Measures meeting officially for the first time since 1937, adopts in principle the resolutions which were submitted to it by the Advisory Committee on Electricity in June, 1939 To adapt these resolutions to the current situation, which has resulted from the developments and scientific progress accomplished since 1939, it decides that:

1) The date for putting in effect the absolute units shall be January 1,1948 .

2) The relations for passing between the mean international units and the absolute units are:

1 mean international ohm $=1.00049$ absolute ohm.

1 mean international volt $=1.00034$ absolute volt .

The precision of the two relations given above will permit laboratories and industries to express all electrical quantities in terms of the new units, without introducing in this conversion an error exceeding two units in the last place. This error is hardly larger than that estimated as being the accuracy obtained in the national laboratories for their absolute measurements.

\section{Resolution 2}

1) Definitive Substitution of Absolute Electrical Units for the International System.-In virtue of authority which was conferred on it by the General Conference on Weights and Measures in 1933, the International Committee on Weights and Measures announces at this time its decision that the substitution of the system of practical absolute electrical units for the international system shall be put into effect beginning January 1, 1948.

The present resolutions constitute the "new announcement" which the Circular Letter of January 1, 1940 , signed by the President and Secretary of the International Committee on Weights and Measures, requested the various countries to await, before proceeding with any change in units.

2) Historic Continuity of the System.-The first definition of the practical absolute svstem of electrical units adopted by the Committee was enunciated by the London Conference of 1908 in the following manner:

(Here follows Schedule B of the London Conference, see A ppendix 2.)

3) Ceneral Considerations. - The definitions of the absolute electrical and magnetic units rest on the generally recognized electromagnetic laws, which lead to a system of inter-dependent relations among the various quantities which are to be measured. Consequently the units can be defined in various ways, according to the starting point chosen.

In order to formulate legal decisions which are concerned solely with the size of the units and not with the processes experimentally employed for their realization in accordance with the theory on which they are based, it is convenient to have a set of definitions, adequate for this purpose, expressed in as simple and easily understood language as is possible.

In response to questions which have been addressed to it concerning a text intended to serve as a guide for the wording of legislation, the Committee recommends consequently the adoption of the sequence of definitions given in paragraph 4. The Magnitudes of the units: ohm, ampere, volt and watt, thus defined, are identical with those which were adopted at the London Conference of 1908.

The procedure to be followed for the establishment and the conservation of the standards of reference required for certain chosen units is indicated in paragraphs 6 and 8 , which are likewise equally intended to serve as a guide for legislation

4) Theoretical Magnitudes of the Units.-A. Defrnition of Mechanical Units Used in the Following Text:

I. Unit of Force--The unit of force (in the system M. K. S. (meter, kilogram, second)) is the force which gives to a mass of 1 kilogram an acceleration of 1 meter per second per second.(1)

II. The Joule (unit of energy or work).- The joule is the work done when the point of application of the M. K. S. unit of force is displaced a distance of 1 meter in the direction of the force.

III. The Watt (unit of power).-The watt is the power which gives rise to the production of energy at the rate of 1 joule per second.

B. Definition of the Electrical Units. The Committee offers the following proposals defining the theoretical magnitudes of the electrical units.

IV. The Ampere (unit of electric current).-The ampere is the constant current which, if maintained in two straight parallel conductors of infinite length, of negligible circular sections, and placed 1 meter apart in a vacuum, will produce between these conductors a force equal to $2 \times 10^{-7}$ M. K. S. unit of force per meter of lengch.

$\mathrm{V}$. The Volt (unit of difference of potential and of electromotive force).-The volt is the difference of electric potential between two points of a conducting wire carrying a constant current of 1 ampere, when the power dissipated between these points is equal to 1 watt.

VI. The Ohm (unit of electric resistance).-The uhm is the electric resistance between two points of a conductor when a constant difference of potential of 1 volt, applied between these two points, produces in this conductor a current of 1 ampere, this conductor not being the seat of any electromotive force.

VII. The Coulomb (unit of quantity of electricity).The coulomb is the quantity of electricity transported in 1 second by a current of 1 ampere.

VIII. The Farad (unit of electric capacitance).-The farad is the capacitance of a capacitor between the plates of which there appears a difference of potential of 1 volt when it is charged by a quantity of electricity equal to 1 coulomb.

IX. The Henry (unit of electric inductance).-The henry is the inductance of a closed circuit in which an electromotive force of 1 volt is produced when the electric current in the circuit varies uniformly at a rate of 1 ampere per second.

$\mathrm{X}$. The Weber (unit of magnetic flux). - The weber is the magnetic flux which, linking a circuit of 1 turn, produces in it an electromotive force of 1 volt as it is reduced to zero at a uniform rate in one second.

5) Object of These Definitions. - The definitions given in paragraph 4) are intended solely to fix the magnitude of the units and not the methods to be followed for their practical realization. This realization is effected in accord with the well-known laws of electro-magnetism. For example, the definition of the ampere represents only a particular case of the general formula expressing the forces which are developed between conductors carrying electric currents, chosen for the simplicity of its verbal expression. It serves to fix the constants in the general formula which has to be used for the realization of the unit.

(1) It has been proposed to give the name "newton" to the M. K. S. unit of force. 
The foregoing text refers to the M. K. S. system. It is naturally possible to translate it into another system (C. G. S., M. T. S., etc.) by appropriate modification by powers of ten.

6) Material Standards.-For practical comparisons the electrical units are represented by material standards for the ohm and the volt to which are assigued appropriate values expressed in absolute units. The standards for the ohm at the present time take the form of resistance coils and those for the volt of voltaic cells (Weston cells for example.)

7) International Standards of Reference.-The values which are to be assigned to the standards of reference maintained at the International Bureau of Weights and Measures will be fixed from time to time by the International Committee, on the recommendation of the Advisory Committee on Electricity, in accord with the results of comparisons made between these standards and the national standards the values of which will have been determined directly by absolute measurements.

8) National Standards of Reference.-The values to be assigned to the national standards of reference will be determined in accordance with the results of comparisons made with the standards of reference of the International Bureau.

9) Ratio Retween the Absolute Units and the Units of the International Systems. - These ratios are indicated in Resolution 1 above.

For the standards of each nation, or of particular laboratories, it will be necessary to take account, not only of the values indicated above for the ratios between the "mean international units" $\Omega_{M}, A_{M}$, and $V_{M}$ (which have been accepted by the International Committee) and the absolute units, but also of the difference between the units of the international system maintained by each laboratory and the corresponding "mean international units."

\section{RECOMMENDATION}

For the purpose of avojding confusion as far as possible the Committee advises that, during the period of transition, the adjective "international" (abbreviation: "int.") be applied to the names of the electrical units for the units formerly in use and the adjective "absolute" (abbreviation: "abs.") for the units which the Committee has decided to adopt.

\section{Annex to Resolution 1}

In anticipation of the meeting of the International Committee in October, 1946, the International Bureau of Weights and Measures sent a circular letter to a number of national laboratories requesting their opinion on the question of the absolute electrical units. The replies received showed that on the whole the opinion was clearly favorable to the immediate adoption of these units.
The National Bureau of Standards transmitted to the International Committee a formal recommendation requesting that the date of introduction of the absolute units in practical use should be fixed as January 1, 1948.

Moreover, this laboratory has made a very exhaustive study including an objective and impartial discussion of all the experimental work carried out in the majority of the large scientific institutions of the world. The result of this study together with the results of later work done at the National Bureau of Standards is such that it seems proper to consider that the 5 th decimal place has been obtained in the measurement of the ratios between the international and the absolute units. The ratios proposed by the National Bureau of Standards are therefore:

1 mean international ohm $=1.00049$ absolute ohms.

1 mean international ampere $=0.99985$ absolute ampere.

1 mean international volt $=1.00034$ absolute volts.

the 6 th decimal being zero.

The National Physical Laboratory declared itself in accord with the proposals of the National Bureau of Standards and merely remarked that it would be preferable not to give the results of the standardization to more than five decimal places and to reserve strictly the use of the 6th decimal to the work of coordination between the national laboratories and their relations with the International Burean of Weights and Measures.

It is on the basis of the above documents and on the consensus of the replies received from members of the Advisory Committee on electricity, that the International Committee has adopted Resolution 1.

The difference in opinion shown by the PhysikalischTechrische Reichsanstalt has not appeared such as to justify a further delay in the introduction of the absolute units which are so much desired by the electrical workers in the majority of the nations. By force of circumstances, the Physikalisch-Technische Reichsanstalt has not been able to participate in the recent discussions and its objection appears to be the result of a certain doubt on the part of its physicists as to the accuracy which one should attribute to the ratios that appear in Resolution 1. The reply of the Physikalisch-Technische Reichsanstalt expresses the fear that the results stated by the Committee may have too provisional a character and may have to be corrected in the future.

The International Committee points out that in any event the values for the ratios appearing in its Resolution 1 will be applied only on the present occasion. Any work on the absolute units will lead to slight future adjustment of the values assigned to the national standards and to those of the International Bureau. The concept of a ratio between the absolute units and the international units will itself lose all interest in the future with the disappearance of the latter.

\section{Appendix 7}

81st Congress, 1st Session.

\section{S. 441}

\section{In the Senate of the United States January 13, 1949}

Mr. JoHnson of Colorado (by request) introduced the following bill; which was read twice and referred to the Committee on Interstate and Foreign Commerce.

\section{A BILL}

To redefine the units and establish the standards of electrical and photometric measurements.

Be it enacted by the Senate and IIouse of Representatives of the United States of America in Congress assembled, That from and after the date this Act is approved, the legal units of electrical and photometric measurement in the United States of America shall be those defined and established as provided in the following sections.

SEc. 2. The unit of electrical resistance shall be the ohm, which is equal to one thousand million units of resistance of the centimeter-gram-second system of electromagnetic units.

Sec. 3. The unit of electric current shall be the ampere, which is one-tenth of the unit of current of the centimeter-gram-second system of electromagnetic units.

SEc. 4. The unit of electromotive force and of electric potential shall be the volt, which is the electromotive force that, steadily applied to a conductor whose resistance is one ohm, will produce a current of one ampere. 
SEc. 5. The unit of electric quantity shall be the coulomb, which is the quantity of electricity transferred by a current of one ampere in one second.

SEc. 6. The unit of electrical capacitance shall be the farad, which is the capacitance of a capacitor that is charged to a potential of one volt by one coulomb of electricity.

SEc. 7. The unit of electrical inductance shall be the henry, which is the inductance in a circuit such that an electromotive force of one volt is induced in the circuit by variation of an inducing current at the rate of one ampere per second.

SEC. 8. The unit of power shall be the watt, which is equal to ten million units of power in the centimetergram-second system, and which is the power required to cause an unvarying current of one ampere to flow between points differing in potential by one volt.

SEC 9. The units of energy shall be (a) the joule, which is equivalent to the energy supplied by a power of one watt operating for one second, and (b) the kilowatt-houl, which is equivalent to the energy supplied by a power of one thousand watts operating for one hour.

SEC. 10. The unit of intensity of light shall be the candle, which is one-sixtieth of the intensity of one square centimeter of a perfect radiator, known as a "black body", when operated at the temperature of freezing platinum.

SEc. 11. The unit of flux of light shall be the lumen which is the flux in a unit of solid angle from a source of which the intensity is one candle.

SEc. 12. It shall be the duty of the National Bureau of Standards to establish the values of the primary electric and photometric units in absolute measure, and the legal values for these units shall be those represented by, ox derived from, national reference standards maintained by the National Bureau of Standards.

SEc. 13. The Act of July 12, 1894 (Public Law Numbered 105, Fifty-third Congress), entitled "An Act to define and establish the units of electrical measure", is hereby repealed.

Washington, March 1, 1949 


\title{
Extension and Dissemination of the Electrical and Magnetic Units by the National Bureau of Standards
}

\author{
Francis B. Silsbee
}

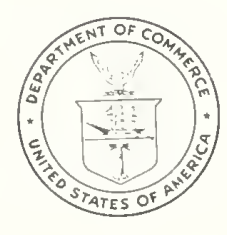

National Bureau of Standards Circular 531

Issued July 14, 1952 


\section{Preface}

One of the primary functions of the National Bureau of Standards is the establishment, maintenance, and dissemination of units of measurement that shall be uniform throughout the Nation and constant through the years. To meet the needs of industry in the electrical field, which is developing so rapidly in extent and in complexity, this duty has involved a continuous growth of staff and equipment and the improving of old methods of measurement and the inventing of new ones.

A report of the stewardship of the National Bureau of Standards with respect to the establishment and maintenance of the fundamental electrical units of electromotive force and resistance is given in the Bureau's Circular 475, which describes both the older international system of electrical units and the present absolute system, which was put into effect to replace the older system on January 1, 1948. The present Circular carries the report further by showing how the other electrical and magnetic units are derived from these two, how the range of measurement is extended, and how the Bureau serves the electrical industry by determining the corrections for apparatus submitted for test. It is hoped that these brief descriptions of the methods that have been found effective in the laboratories of the Bureau will be a useful guide in the development of other laboratories that may have the duty of extending the measurement process. This general picture of the procedures by which accurate measurements are actually carried out should be helpful also to educators who wish to give their students a definite and correct understanding of the practical application of their teachings in the theory of electrical measurements.

A. V. Astin, Director. 


\section{Contents}

Prefice -

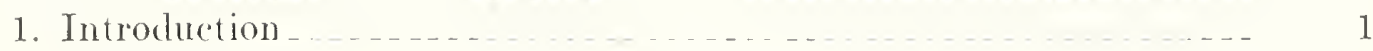

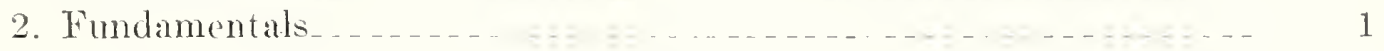

3. Derivation of other units _. 3

3.1. Capacitance _... . . .

3.2. Inductance $\ldots \ldots \ldots \ldots \ldots$

3.3. Current, power, and energy

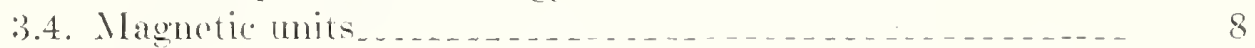

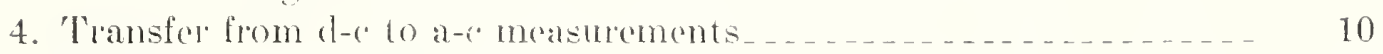

5. Extension of measurement langes....................... 13

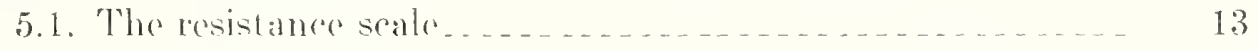

5.2. 'The scale of direet voltage_.......

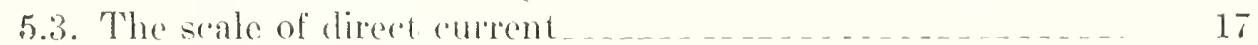

5.4. The scale of altermating current . . . . _ .

5.5. The seale of alternating voltage

5.6. Measurement of crest and surge voltage ............ 24

6. Dissemination of units ........ 26

7. International relations .............. 27

8. Bibliography 28

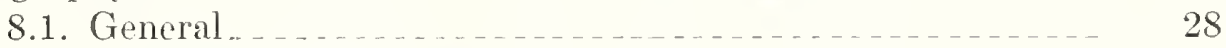

8.2. Absolute measurements _.................. 29

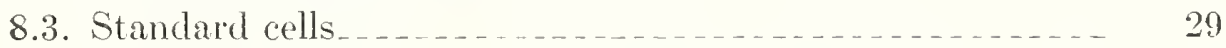

8.4. Standard resistors and resistance measurements _....... 29

8.5. Potentiometers, bridges, etc___. 30

8.6. Capacitance 30

8.7. Inductance (construction and measurement) ........ 30

8.8. Inductance (computations) ................. 31

8.9. Galvanometers and detectors _... _.............. 31

8.10. Instruments and meters _... 31

8.11. Instrument transformers _._.

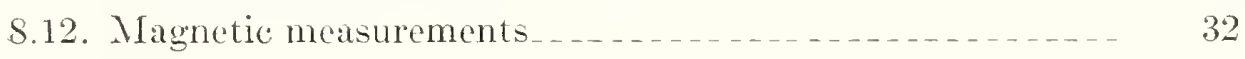




\title{
Extension and Dissemination of the Electrical and Magnetic Units by the National Bureau of Standards
}

\author{
By Francis B. Silsbee
}

\begin{abstract}
Starting with the ohm and the volt as maintained by groups of standard resistors and cells, this paper describes the experimental processes by which the other electric and magnetic units, e.g., farad, henry, ampere, watt, joule, gauss, and oersted are derived. It also describes the series of steps by which the scales of measurement of resistance, direct and alternating current, and voltage are derived experimentally. Brief mention is made of the procedures for the dissemination of these standards of measurement throughout the world by the calibration of standard electrical measuring apparatus. An extensive bibliography lists papers describing the measurement procedures in greater detail and serves as a historical report of the work of the National Bureau of Standards in the field of electrical measurements during its first 50 years.
\end{abstract}

\section{Introduction}

It is the purpose of this Circular to give an overall picture of the sequence of measuring processes by which a self-consistent system of electrical units is built up in the laboratories of the National Bureau of Standards and thence disseminated throughout the Nation. The branching chains of measurement thus initiated may be considered as extending outward without a break from the groups of primary standard cells and standard resistors by which the units of voltage and of resistance are maintained from year to year, and as ending finally in the myriad measuring operations throughout the Nation, on the basis of which scientific research is conducted, manufactured articles are adjusted and inspected, electric energy is bought and sold, and industrial processes are precisely controlled. It is only as a result of this self-consistency that scientists in different laboratories can talk in the same terms, that apparatus formed by components manufactured in different places at different times can be expected to function properly, that competing sources of energy can be fairly compared, and that complex indus- trial operations will consistently yield a product of constant quality.

Although many links in this network of measurement are welded in remote laboratories, the $\mathrm{Na}$ tional Bureau of Standards has exerted for the past 50 years a very significant influence on the development of the system, both directly by its testing service and by the invention of new methods of measurement, and indirectly by example and precept. The particular experimental methods described herein are primarily those used in the Bureau laboratories, but to a large extent the same or closely similar methods are used elsewhere. The following paragraphs show the points of contact of the Bureau with the industrial world in more detail. The extensive bibliography gives references in the field of electrical measurements, not only to technical papers originating with the Bureau staff, but also, on occasion, to other noteworthy publications in this field.

The extension of measurements into the radio field has not been included in this Cireular because the methods used at these higher frequencies constitute a quite distinct discipline.

\section{Fundamentals}

By international agreement $[4,6]^{1}$ the official basis for electrical measurements throughout the world is the system of practical absolute electrical units. These units developed historically as convenient decimal multiples of the units of the centimeter-gram-second electromagnetic system, but they also form the electrical part of the self-

\footnotetext{
I Figures in brackets indicate the literature references at the end of this paper.
}

consistent meter-kilogram-second-ampere, or Giorgi, system of units.

The experimental processes by which these units are established in terms of length, mass, time, and the conventionally assumed constant $\mu_{v}$, usually designated "permeability of space" are called "absolute measurements."

Because of the importance in such measurements of ready access to highly accurate standards of length, mass, and time, and because of the 
long, tedious, and therefore expensive, observational programs required if significant accuracy is to be attained, the burden of periodic determinations of this nature rests almost entircly on the few larger national standardizing laboratories of the world. Of the many theoretically possible forms of absolute electrical measurement, two types of experiment have so far shown the greatest possibilities for high accuracy and, except in certain very special circumstances that will be noted later, are universally used $[17,36]$.

The first of these experiments involves the construction of a standard of self- or of mutual in(luctance, the geometrical configuration of which is such that (1) its significant dimensions can be directly and accurately measured $[45,46,47]$ and (2) its inductance can be calculated precisely from these measured values $[142,148,149,47]$. As a preliminary for such procedures, Dr. E. B. Rosa and his colleagues, in the early vears of the Burcau, carried on an extensive theoretical examination of the various available formulas for inductance and published a number of paper's [132 to 141] in this ficld, the major results of which are summarized in [135]. A large volume of later work was done by Dr. Chester Snow and others [142 to 159], the results of which are, of course, applicable to the computation of the inductances of various eircuit configurations for many other purposes, as well as for absolute measurements. After the value of the induetanee of a standard inductor in absolute hemries has been computed, an experiment is performed by which the value in absolute ohms of the resistance of a standard resistor is obtained in terms of the reactance of the standard inductor at the known frequency used in the experiment. The net result of the whole process is usually called an "absolute mocasurement of resistance." At the National Burcau of Standards two completely independent procedures have been used for this purpose, one $[38,39]$ starting with a self inductor and the other [47] with a mutual induetor. The two final results differed by only $12 \mathrm{ppm}$ (parts per million). The second of these procedures has proved so convenient that it is planned to make fairly frequent (perhaps ammual) clectrical romparisons between the standard resistor's and the standard mutual inductor. It is hoped that any secular changes in the dimensions of the inductor will be so slow that the tedious mechanical measurements of its dimensions need be repeated only at intervals of ten years or more.

The second type of experiment is the absolute measurement of eurrent by the use of a eurrent balance $[40,41,42]$. This is an instrument by which it is possible to weigh accurately the mechanical force that is exerted between two coils as a result of the electric enrrents in them. The relation between the foree and the rurrent can be ealculated by fundamental eleetromagnetie theory and is expressible by formulis that involve only the geometrical shape, but not the absolute size, of the coil system. After such a current balance has been constructed and its shape determined by suitable measurements, it is connected in scries with a resistor, the resistance of which is known in absolute units by reference to the former type of experiment.

The current is adjusted until the change in force that results when the current in one coil is reversed is balanced by the change in loading when a known weight is added to one pan of the balance. At the same time the potential drop produced by the current in the known resistor is compared with the clectromotive force of a standard cell. Br this process, a value in absolute volts is obtained for the electromotive fore of the cell. The cell then serves to preserve the result of the experiment for future use. Such an experiment is usually called an "absolute measurement of current," although the final result is the assigmment of a value in absolute rolts to a standard cell. Coils of a considerable variety of shapes and construction have been used in the current balance at the National Burean of Standards, and, as a further check against systematic errors, it is planned to use a radically different form, the Pellat $[44,145]$ balane, in the neal future.

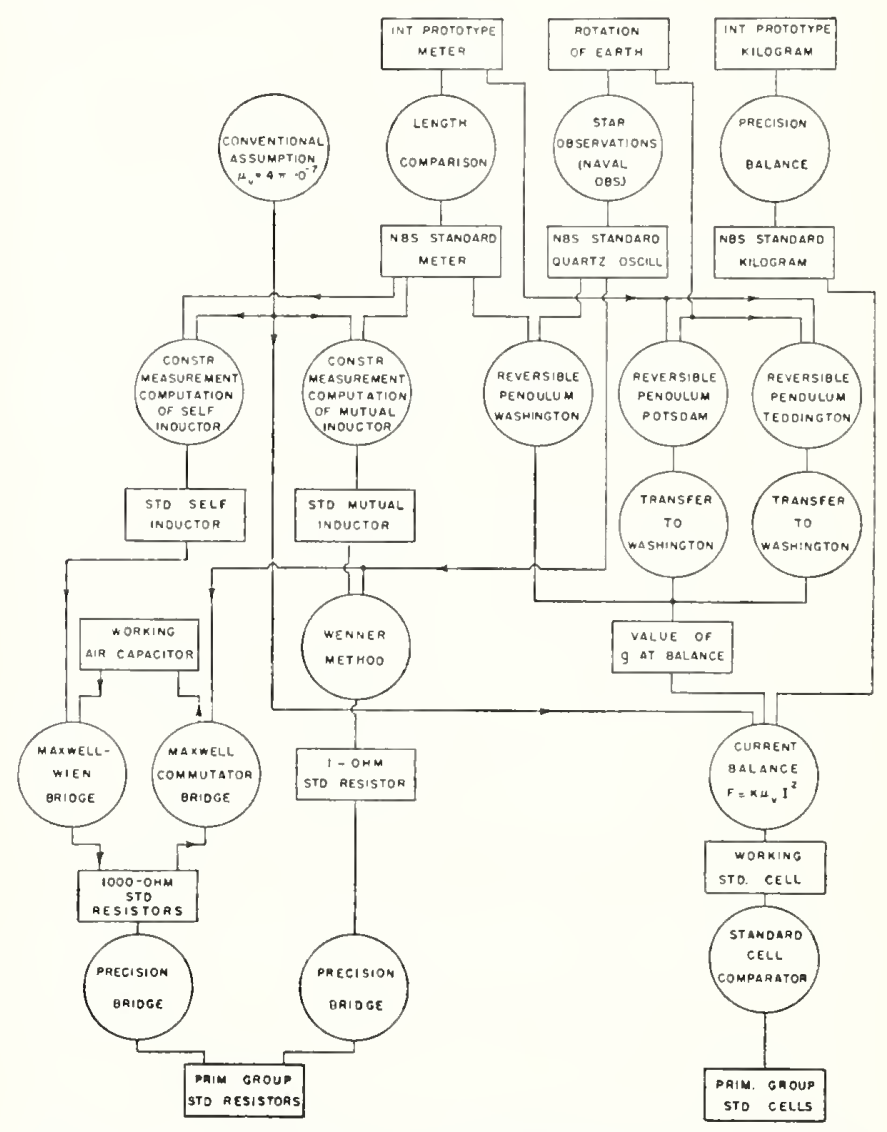

Frgure 1. Experimental establishment at the National Burean of Standards of the primary electrical units by absolute incasurements.

The rectangles represent physical standards embodying the corresponding units, the circles represent experimental procedures with their associated thits, the cir in the passated apparatts tisnt int he meastirements. The lines indieate the passage of

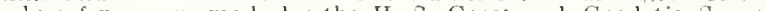
ralues for 9 were mate by the U. S. Coast and freodetic Survey, using 
The relations of these absolute measurements to the basic mechanical standards are indicated by the block diagram of figure 1 . It will be noted that the local value of the acceleration of gravity, $g$, enters into the computation of force from mass, and that the value used is based upon three indèpendent determinations $[31,32]$ made at Potsdam, Washington, and Teddington.

These absolute measurements give results in electromagnetic units. An early research was devoted to a precise determination of the ratio of the units of this system to those of the electrostatic system. For this purpose Rosa and Dorsey $[28,29,30]$ used a group of capacitors of such shapes that their capacitances could be computed in electrostatic units from théir mechanical dimensions. They then measured their capacitances by electromagnetic methods at frequencies of a few hundred cycles per second. The ratio was found to be in very satisfactory agreement (at least to 1 in $10^{4}$ ) with the velocity of light measured at optical frequencies.

The units realized by these absolute measurements are preserved by a group of 10 manganin standard resistors of the Thomas double-walled type $[66,67]$ and a group of 25 saturated cadmium standard cells ( 16 neutral and 9 of the 0.05 -normal acid type). The standards of either group are intercompared at intervals, and following each intercomparison slightly revised values are assigned to the individual standards, on the assumption that the mean value of the group has not changed during the interval [6]. Certain of the standards are used during the intervals as the reference standards for the calibration of other resistors and cells that constitute the Bureau's working standards. As shown in the following sections, the values of the other electrical units are derived from the ohm and the volt as thus maintained.

\section{Derivation of Other Units}

The many electric and magnetic quantities used in science and engineering, such as current, inductance, capacitance, and magnetic flux, are all connected with each other and with mechanical quantities by the science of electromagnetism to form a closely linked structure of exact mathematical relations. Similarly, the various units, such as the ampere, henry, farad and weber, in terms of which these quantities are measured, are also linked by a parallel structure of exact definitions. Starting with any group of independent units, chosen as fundamental, it is possible to derive definitions for all the other units by successively applying the appropriate mathematical relations. Both the selection of the fundamental units and of the particular order in which the various derived units are successively defined can be made arbitrarily. The choice is determined by taste or convenience and may well differ according to the purpose for which the definitions are to be used. Thus the sequence chosen by a college professor for teaching a course in electrical measurements may well differ from the sequence offered by the International Committee on Weights and Measures "as a guide for the wording of legislation" $[6, \mathrm{p} 36]$.

The limitations and exigencies of practical experience have led to the use of still other sequences in the laboratory. In nearly all electrical measurements, resistance and voltage are among the fundamental quantities chosen, because their units can be readily embodied and preserved in standard resistors and standard cells. In this section of the Circular, the particular, and sometimes rather devious, sequences normally used in the electrical testing work at the Bureau are described in some detail. For some quantities, different parts of the full range of measurement are derived independ- ently by different sequences from the fundamental units.

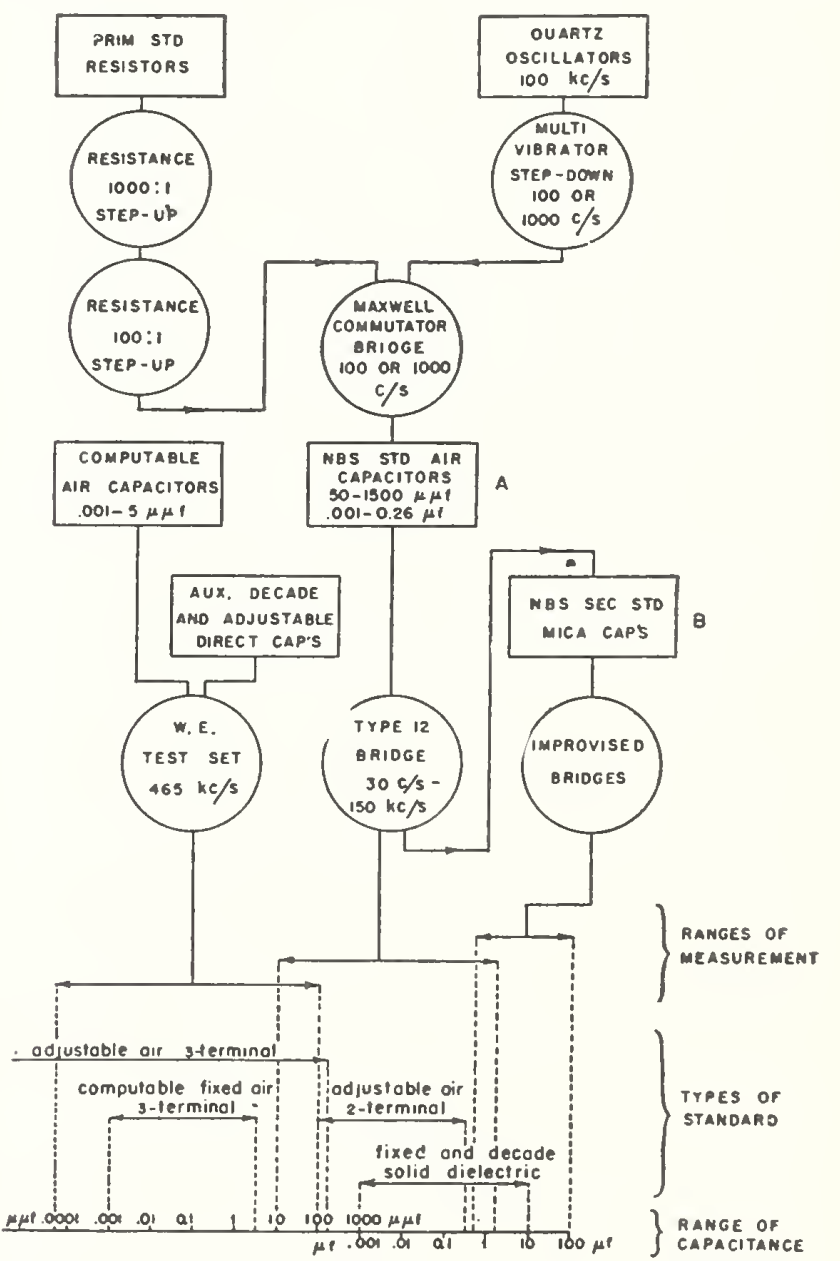

Figure 2. Establishment and extension of capacitance measurements.

The dotted lines indicate the approximate limits of the range over which the severel types of standards and methods of measurement are useful. The total range from $0.0001 \mu \mu$ f to $190 \mu$ f involves a factor of $1,000,000,000,000$. 


\subsection{Capacitance}

The unit of eapacitance is derived from the ohm and the second as indicated by the block diagram in figure 2. Standards of capacitance are measured by a Maxwell commutator bridge [108]. Its circuit is shown schematically in figure 3.

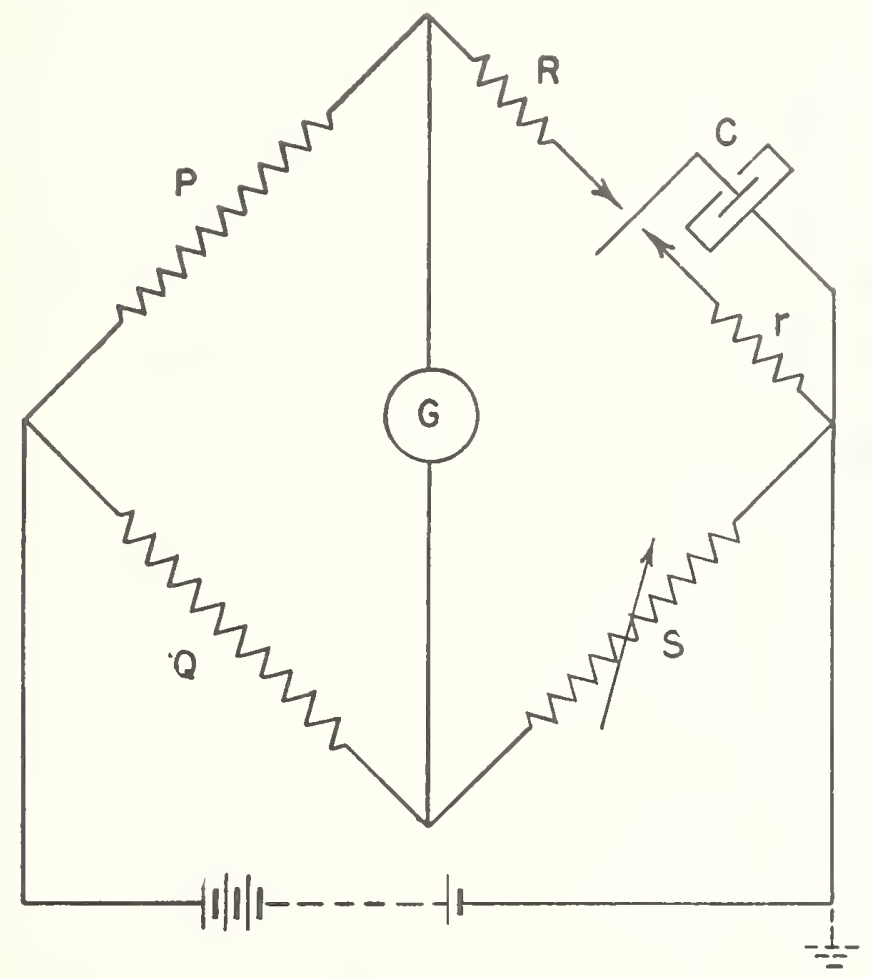

Figure 3. Maxwell commutator bridge.

The capacitance, $C$, is measured in terms of the resistances of the bridgc arms and the known frequency of the vibrating contactor, which alternately arms and the kiown frequency of the vibrating contactor, which alt
charges $C$ by way of the resistance, $P$, and discharges it through $r$.

The contactor, which alternately charges and discharges the capacitor, is operated by a tunimg fork driven at frequencies of 100 or $1,000 \mathrm{c} / \mathrm{s}$ by a multivibrator. 'This multivibrator' is coupled to the $100 \mathrm{kc} / \mathrm{s}$ quart $z$ oscillators which constitute the NBS standard of frequency [25]. The three resistance arms of the bridge may range in value from 10 to $10^{5}$ ohms, depending on the value of the air capacitor to be measured. This process is used to assign values of capacitance to a group of fixed air capacitors shown in figure 4 , which can be connected in parallel with each other and with an adjustable capacitor which has a range from 50 to $1,500 \mu \mu \mathrm{f}$. 'This combination of air' capacitor's can be used as a standard for any ralue of capacitance from $50 \mu \mu \mathrm{f}$ to $0.26 \mu \mathrm{f}$. They are useful over a wide range of frequency, provided certain corrections for the self-inductance of the capacitors and their leads are made at the higher frequencies.

Occasionally an independent check of the unit of capacitance is obtained by using a MaxwellWien a-c bridge to determine capacitance in terms of resistance and the primary computable standards of self- or of mutual inductance. 'The values derived by the two methods agree within 0.002

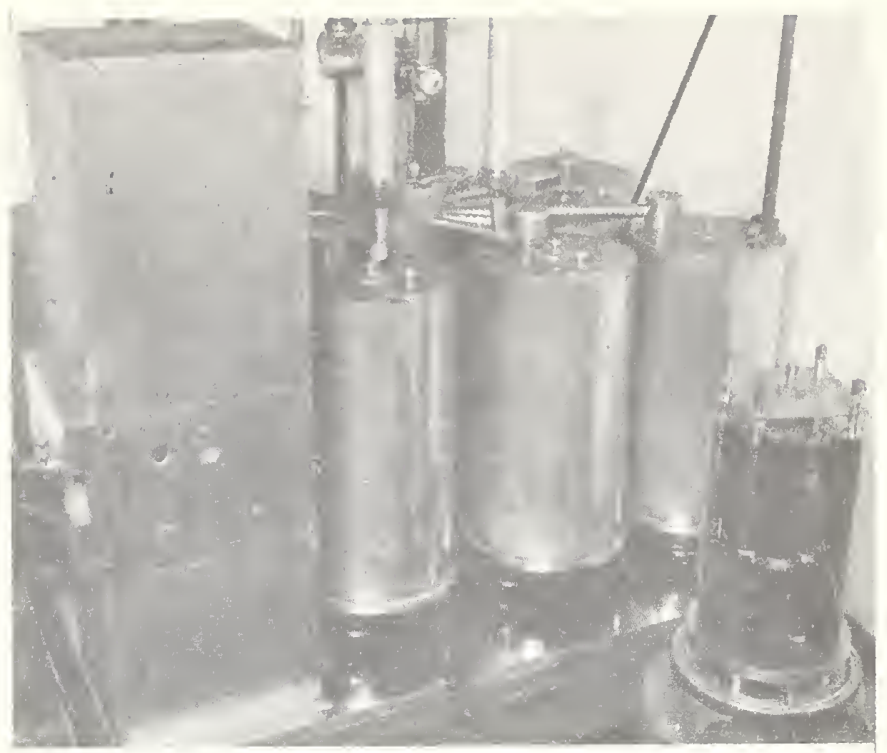

Figure 4. Standard air capacitors.

Unit at left has a continuously adjustable section. Cover of sparc mit is removed to show interleaved scuare plates and supporting rods. Only "a few of the 21 fixed units are shown.

percent. This operation is the inverse of the use shown for the Maxwell-Wien bridge at the loft in figure 1.

A commercial highly precise capacitance-conductance bridge [116] used in connection with these standard air capacitors serves to measure other capacitors having either air or solid dielectric in the range $10 \mu \mu \mathrm{f}$ to $1.11 \mu \mathrm{f}$. This completely shielded bridge has two equal fixed resistance arms and an adjustable admittance arm reading directly in capacitance up to $1.11 \mu \mathrm{f}$ ond in conductance up to 1,111 micromhos. This bridge may be used either as a comparison bridge, in which case its calibration is checked against the standard air capacitors, or it may be used in a substitution method using the air capacitors as standards. Normally frequencies from $200 \mathrm{c} / \mathrm{s}$ to $150 \mathrm{kc} / \mathrm{s}$ are used, but with special external detectors, measurements are possible down to $30 \mathrm{c} / \mathrm{s}$. A number of fixed and decade groups of mica capacitors with a total capacitance of $10 \mu \mathrm{f}$ [106, 109] are calibrated from time to time at a particular frequency by the type 12 bridge, and are occasionally used at the same frequency in an improvised Schering bridge for measurements of other solid-dielectric capacitors up to $100 \mu \mathrm{f}$.

To cover the range of extremely low capacitanees, such as the interelectrode capacitance of electron tubes, an independent procedure has been developed $[112,115]$, based on the construction of capacitors of such shape and size that their capacitance can be computer accurately from their measured dimensions. These capacitors are either parallel-plate units of the conventional Kelvin guard-ring type or of a form referred to as the "guard-well" type, in which the guarded "island" electrode lies at the bottom of a crindrical opening, or "well", in the guard ring (see fig. 5). They range in value from 0.001 to $\tilde{5} \mu \mu \mathrm{f}$. 


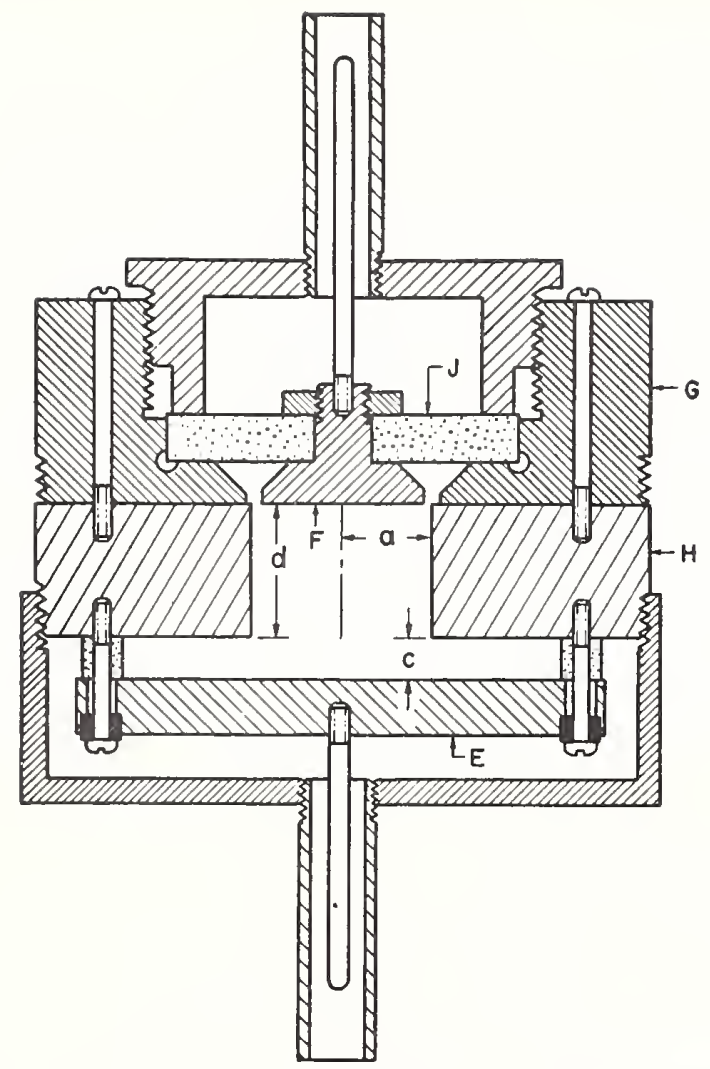

Finure 5. Cross section of standard capacitor of the guardwell type.

$F$. Guarded electrode, supported by an insulator of low expansion glass, $J$ : $E$, high-voltage cleetrode; $G, H$, parts of guard ring. If the depth, $d$, of the well is greater than its radius, $a$, the direct eapaeitance between $E$ and $F$ is very mueh smaller than if $F$ is flush with the lower surface of $H$.

Auxiliary standards used in this range include (1) an adjustable capacitor of the guard-well trpe, in which the island electrode can be adjusted by a micrometer serew to any desired depth in the well, thus securing a working range $0.1 \mu \mu$ down to $0.001 \mu \mu f .(2)$ an adjustable capacitor of a modified Zichner type with a movable septum between the electrodes by which the direct capacitance can be varied from $0.3 \mu \mu$ f down to zero when the aperture in the septum is elosed; (3) a decade group of $0.1 \mu \mu \mathrm{fmits}$, the direct capacitance of any unit of which can be reduced to zero by inserting a grounded septum between its electrodes; and (4) a similar group of $0.01 \mu \mu \mathrm{f}$ units.

By the substitution of these standards in the $X$-arm of a commereial direct-capacitanee test set having inductive ratio arms with a fixed 1:1 ratio, and a fixed fourth arm, ther may be intercompared at $465 \mathrm{kc} / \mathrm{s}$, and other standards of direct capacitance up to $5 \mu \mu$ f can be measured. It is also possible to step down from standards of higher value with a shickded Sehering bridge to $5 \mu \mu f$, and at this level the two procedures have been found to agree within 0.05 pereent. The value derived br extending this step-down process to a $0.1-\mu \mu f$ apacitor was found to differ from the value derived independently from the computable standards by less than 0.1 pereent. Because of these close agreements, in the range of overlap, no significant inconsistencies can arise anywhere in the range of capacitance, in spite of the independent bases on which the two ends rest.

The unit of dielectric constant for solid dielectries is derived from the standards of capacitance by combining the electrically measured capacitance of a specimen with its mechanical dimensions. The capacitance is measured at frequencies from $60 \mathrm{c} / \mathrm{s}$ to $200 \mathrm{ke} / \mathrm{s}$ in a shielded Schering bridge (sec fig. 6) that has been calibrated in terms of the standard air capacitors. I major complication arises from the fringing of the electric ficld at the edges of the specimen, and a number of approximate working formulas have been dereloped for correcting for such effects [114]. With these edge-correction formulas, the accuracy is about 1 percent. For higher aceuracy, the guardring technique is used. The area of the specimen is readily computed from its sealed dimensions, and the accuracy is usually limited by the measurement of thickness. When feasible, this is deduced from measurements of the mass, density, and area of the samples. Under the best conditions, results are repeatable to 0.1 pereent.

Inother method for minimizing edge effects [117] is that of placing a 2-inch-diameter disk of the dielectric in a micrometer electrode holder. A capacitance measurement is made with the specimen in place and another with it removed. Either the electrodes may be moved eloser together by a measured amount to give the same value of capacitane for the second measurement, or they may be left at the same spacing and the new value of the capacitance measured.

Standards for the measurement of loss factor in capacitors and dielectries are usually air capacitors. Studies have shown that such apparatus may have a loss factor as large as $1 \times 10^{-4}$, and that if accuracies of $1 \times 10^{-6}$ are needed, a more precise standard is required. By combining

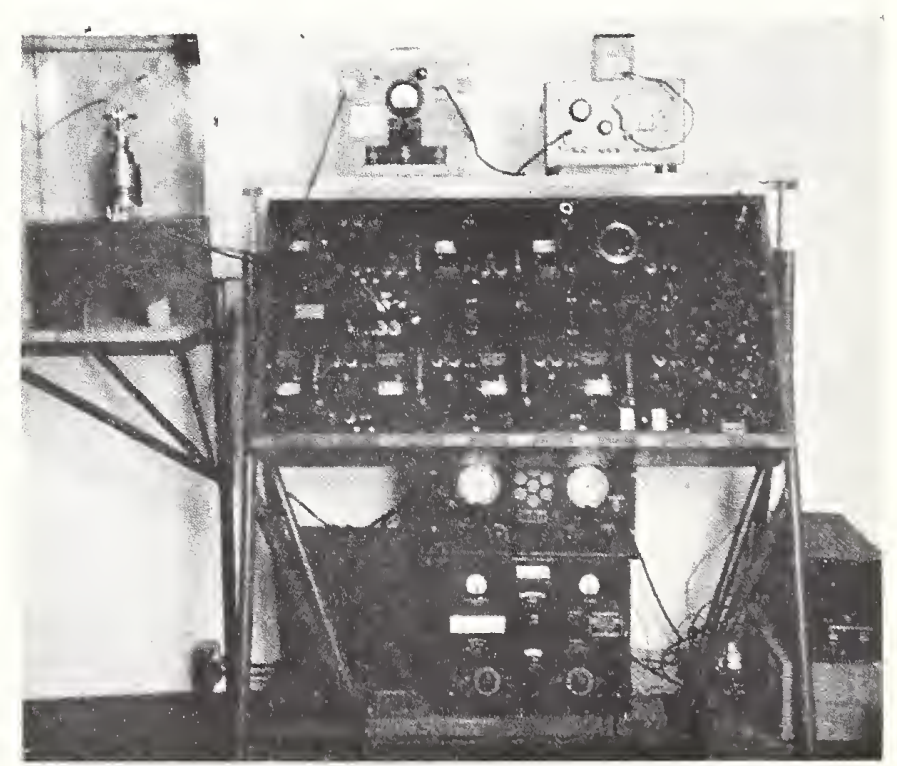

FIgURE 6. Modified Schering bridge and accessories for measuring dielectric constant and loss factor $u p$ to $200 \mathrm{kc} / \mathrm{s}$.

Oseillators are below, detectors above, mierometer eleetrode holder at left. 
data obtained with (a) a capacitor of constant spacing and adjustable area and (b) a capacitor of constant area and adjustable spacing, a valid determination of the true zero of power factor can be obtained $[110,111]$.

Capacitors have recently come into use for measuring the very small direct currents encoumtered in ionization measurements. Here very low absorption and leakage are essential qualities. The nsual calibration procedure is to allow a known current to charge the eapacitor for a measured time and to note the resulting roltage with an electrometer. The current is chosen so as to make the total charging time and the final voltage approximately the same as they will be in the future use of the capacitor.

\subsection{Inductance}

In the practical work of inductance measurement at the National Bureau of Standards it usually has been found more expedient to derive the lienry from the ohm and the farad than to use directly the values computed from the dimensions of the large computable inductors constructed for absolute measurements. These inductors are very bulky and hence have relatively large capacitance to ground and considerable electromagnetic coupling to other circuits. Ther are wound with thick wire $(0.7 \mathrm{~mm})$ and would show appreciable skin effect if used at high frequencies. How ever, they are very valuable for use occasionally to give an independent rheck on the accuracy

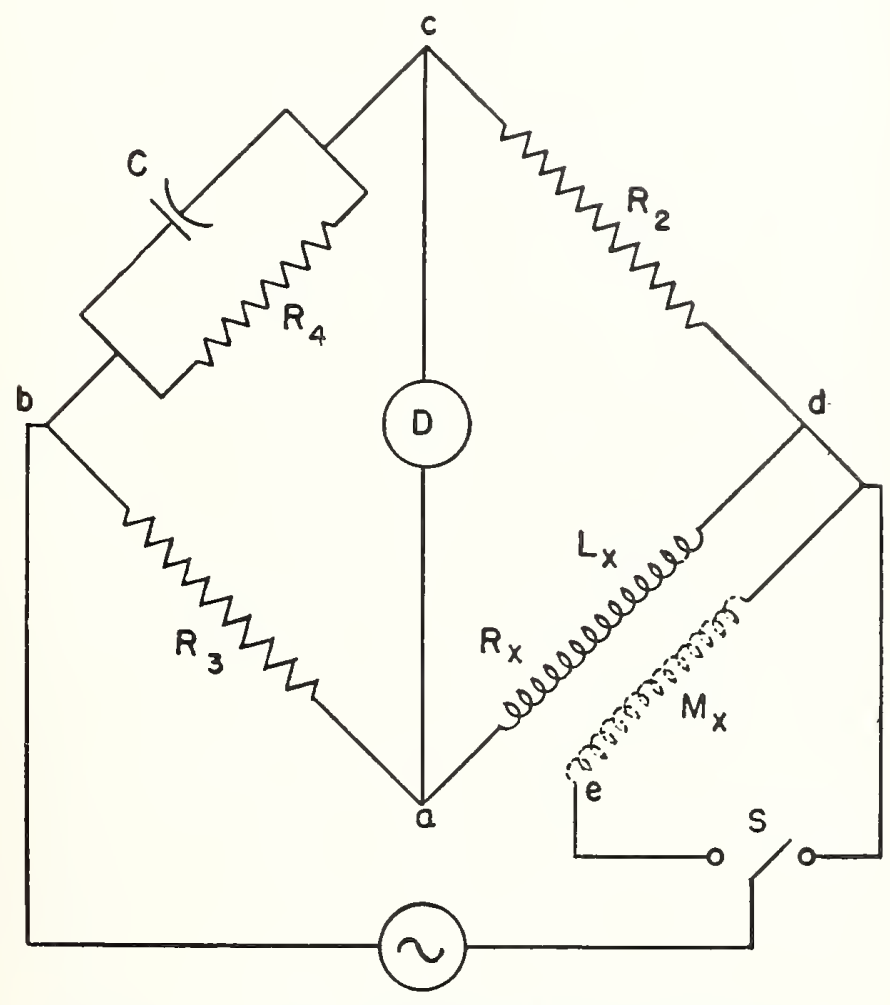

Figure 7. Maxwell-Wien bridge for inductance measurement. of measurement of inductances in the MaxwellWien bridge.

The circuit regularly used to measure induetance is that of the Maxwell-Wien bridge [17, p.113] shown in figure 7 . The a-c source is connected across bd and the detector (a vibration galvanometer for $60 \mathrm{c} / \mathrm{s}$, a telephone receiver with suitable amplifier for $1,000 \mathrm{c} / \mathrm{s}$, or an electronic null (letector) is connected across ca. The adjustable eapacitor, $C$ ', may be either the group of air apacitors shown in figure 4, which has been (alibrated directly by the Maxwell commutator bridge ( $A$, figure 2$)$ or a mica capacitor $(R$, figure 2) calibrated by the trpe-12 bridge at the particular frequener at which the inductor is to be tested, usually $60 \mathrm{c} / \mathrm{s}$ or $1,000 \mathrm{c} / \mathrm{s}$. This procedure is convenient for inductors from $1 \mu \mathrm{h}$ to $10 \mathrm{~h}$, and gives results good to 0.01 pereent over the middle of this range.

Mutual inductances for use with alternating current and of ranges up to about $50 \mathrm{mh}$ are regularly measured by the Curtis method $[17$. p. 117] with the circuit as shown, including the dotted lines in figure 7 . With switch $S$ closed to the right, capacitor $C$ is adjusted to a value $C_{L}$, which is a measure of $L_{x}$. Switch $S$ is then thrown to the left and $C$ readjusted to a new balanee at $C_{y}$. The mutual inductance is then computerl from the relation

$$
M=\left(C_{L}-C_{M}\right) R_{2} R_{3} R_{4} /\left(R_{3}+R_{4}\right)
$$

with further correction terms involving the residual inductances of the resistance arms.

For inductors of larger value, or at higher frequencies where the effects of distributed capacitance are larger, this method becomes impractical. In such eases, the primary and secondary windings are connected in series, first aiding and then opposing, and the self-inductance of each combination is measured with a Maxwell-IIIen bridge. If the observed values are $L_{-A}$ and $L_{O}$, respectively, and if $L_{P}$ and $L_{S}$ are the inductances of the primary and secondary windings measured separately,

$$
M=\frac{L_{A}-L_{O}}{4}=\frac{L_{A}-L_{P}-L_{S}}{2}=\frac{L_{P}+L_{S}-L_{O}}{2} .
$$

Any inconsistencies among these equations give a valuable indieation of the order of magnitude of the effects of stras capacitance between the coils. It has been found that the values br the Curtis method agree most closely with those computed as $\left(L_{A}-L_{O}\right) / 4$.

In independent basis for the moasurement of mutual inductance is the use of a computahle standard $(K=6.6 \mathrm{mh})$ mutual inductor formed by two interwound single-laver heliees wound in a double thread on the surface of a porcelain form. With the two windings connected in series. "opposing" measurements may be made with good aceurac $(0.01 \%)$ up to $1 \mathrm{kc} / \mathrm{s}$. 
Mutual inductors of the types used to calibrate the ballistic galvanometers employed in measuring the $d-c$ properties of ferromagnetic materials (see sect. 3.4) are tested ballistically. For this purpose such inductors are compared with the large computable mutual inductor that was constructed primarily for the absolute measurement of resistance by the Wenner method [47]. 'To so this, the primary windings of the standard and test indurtor' are connected in series. 'Their secondary windings are connected in opposition and with two resistaner boxes also in the series circuit. A galvanometer is bridged across between the junction of the resistance boxes and the junction of the two secondary windings. The resistances are then adjusted until the galvanometer remains balanced as the primary current is reversed. For this condition, the mutual inductances are proportional to the total resistance of the two parts of the secondary cireuit. 'This method avoids any complications that might enter an a-c measurement because of capacitance in the windings.

At low values of induetance, errors from the residual pliase defeets of the bridge arms may become appreciable. Hence, for the measurement of the residual inductances of nominally "noninductive" two-terminal resistors $[125,126]$ it becomes essential to use a substitution method. In this a standard, the inductanes of which can

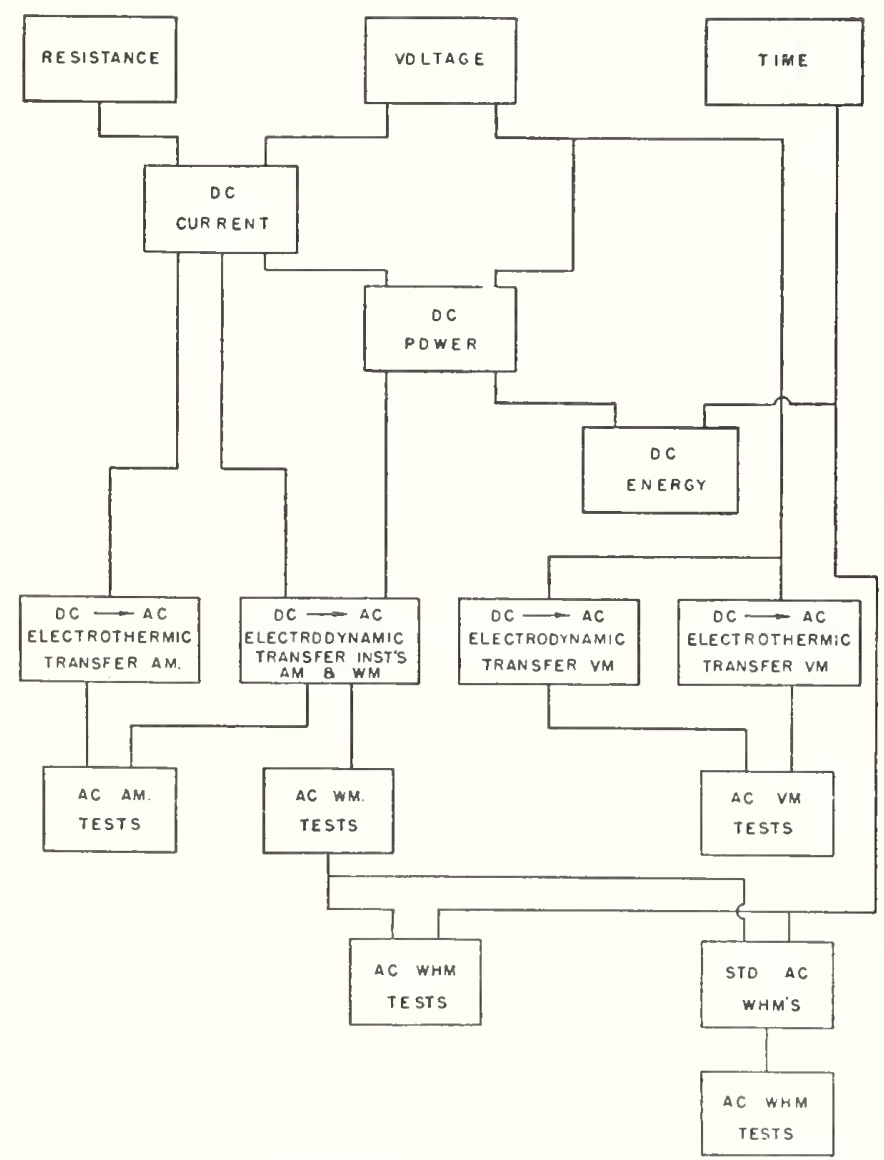

FIGURE 8. Development of units and standards for current, power, and energy on direct and on alternating current. be computed and the resistance of which is nominally equal to that of the impedance under test, is substituted for the unknown in the $X$-arm of the bridge. Each of the computable standards for 10,000 and $1,000 \mathrm{ohms}$ consists of a fine wire stretched down the axis of a brass tube about 5 cm in diameter. For 100- and 10-ohm standards, parallel wires are used. The time constants $(L / R)$ of these standards can be computed to $1 \times 10^{-8}$ sec. For still lower resistances fourterminal standards are used, either of the parallelwire, reflexed-strip, or coaxial-tube type [128, 129]. Some of the coaxial tube trpe have their potential leads inside the inner tube, whereas, others have them outsiche the outer tube. 'These four-terminal standards of inductance range in resistance from 0.1 to 0.0002 olim and in current rating from 10 to $2,500 \mathrm{amp}$.

\subsection{Current, Power, and Energy}

The block diagram of figure 8 shows the derivation of the units of current, power, and encrgy from those of resistance, voltage, and time. An electric current (if one excepts the persistent current in a supereonducting (ireuit) is in the nature of things, a transitory affair, and a "standard ampere" "annot be preserved on a laboratory shelf as can a standard ohm. For the precise measurement of direct current the normal procedure is to insert a known resistance, $R$, in the circuit and to compare the potential drop produced in this resistance by the unknown current with the emf of a standard coll. This comparison is usually made with a potentiometer, but in rare cases, where extreme accuracy is needed, special resistors are used of such value that the IR drop at the desired current is very closely equal to the enf of the standard cell. "Thus the circuit becomes very simple, and errors from contacts, thormal electromotive forces, and drift in an auxiliary potentiometer current are minimized or climinated. With a potentiometer and suitable standard resistor, it is easily possible to measure a direct current much more accurately than with an ammeter. Thus indicating ammeters of the

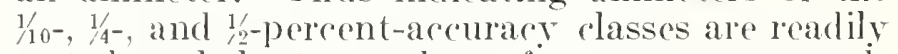
tested, and the true values of current corresponding to a number of scale points are determined. Such ammeters can in turn be used to check ammeter's of lower aceuracy and also to check recording instruments.

The measurement of power in a d-c eircuit, as for instance, in calibrating a calorimeter, ean be made most aceulately by using a potentiometer with an aceessory shime and volt box to measure the current ant the voltage of the cirenit separately and by then taking their product. The checking of a wattmeter is donc in the same way, except that in this case separate storage batieries are used to supply the eurreut and the voltage eincuits, as shown in figure 9 . With switch S. Sev closed in either direction and with switeh $S_{A D}$ 


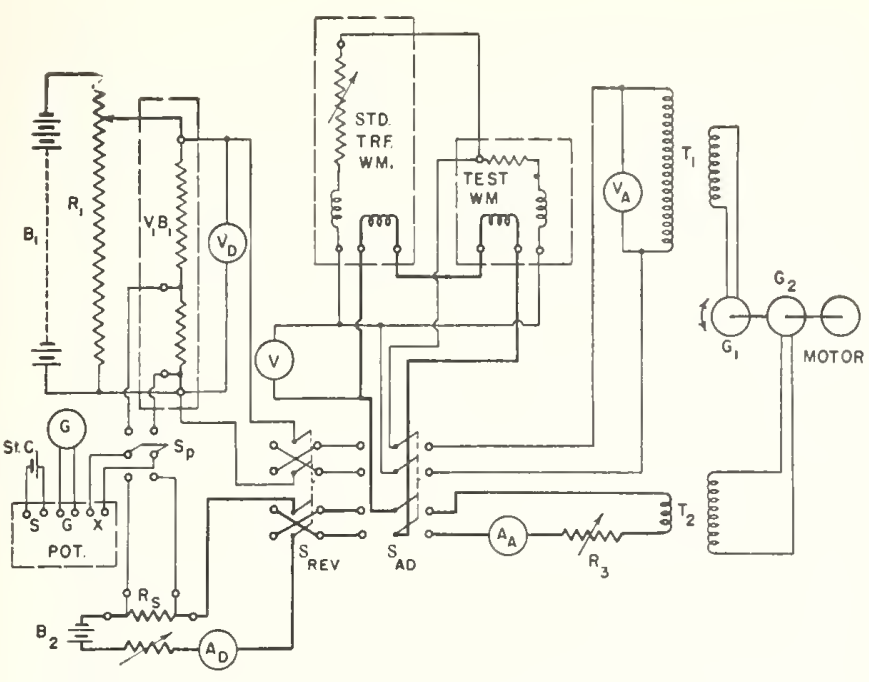

FIGURE 9. Circuits for transfer testing a wattmeter.

With switch $S_{A D}$ closed to the left, both the test wattmeter and the standard transfer wattmeter are energized with direct currents from batteries$B_{1}$ and $B_{2}$. With switch $S_{A D}$ closed to the right, both wattmeters are energized with alternating currents from the two coupled generators, $G_{1}$ and $G_{2}$. Ammeters and voltmeters $A A, A_{D}, T_{A}, T_{D}$ indicate approximate values of current and voltage for control and for setting power factor. Potentiomete ives more exact measures of $d-c$ voltage and current for $d-c$ calibrations. Switch $S_{\text {reo }}$ by reversing direction of currents in wattmetel circuits permit elimination of errors from the earth's magnetic field. Voltmeter Y serves as electrostatic tie and as indicator of stray leakage between batteries and ground.

closed to the left, the voltage circuits of both the test wattmeter and the standard transfer wattmeter are energized by battery $B_{1}$, and the voltage is measured both by voltmeter $V_{D}$ and more accurately by the combination of the volt box, $V B$, and potentiometer (with switch $S_{p}$ closed upward). 'The current circuit of the test wattmeter and of the standard transfer wattmeter are energized by battery $B_{2}$, and the current is measured by ammeter $A_{D}$ and more accurately by the combination of the standard resistor, $R_{s}$. and the potentiometer. By this use of separate sources, it becomes possible to test a wattmeter of high range (many liklowatts) under its normal conditions of use, while consuming only a few watts in each of the two measuring circuits. A worthwhile precaution is to connect the two (otherwise insulated) circuits at one point, near the lowvoltage end of the voltage circuit, to eliminate any possibility of electrostatic forces affecting the position of the movable system. The use of a voltmeter ( $V$, fig. 9) as such an "electrostatic tie" gives warning of any serious leakage from one battery to the other via the ground.

Electric energy is, of course, the integral of power with respect to time, and the normal way to test a $d-c$ watthour meter is to maintain, by using a potentiometer, a known and constant current and voltage at its terminals for a measured time. In the testing of portable watthour meters ("rotating standards") the voltage and current are held constant, and the voltage circuit is closed by a relay and opened automatically by a stepping relay after the lapse of a predetermined number of seconds (usually 100). For a "house-type" meter a photocell pickup counts the passages of the black spot on the meter disk and opens the circuit of a svnchronous timer after a predetermined integral number of revolutions. The timer, or in the case of the portable meter, the stepping relay, derives its time signals from a multivibrator operating in synchronism with the 100-ke standard quartz oscillators.

The test of an a-c watthour meter is similar except that not only the magnitudes of the current and the voltage, but also the phase angle between them must be held constant. This is accomplished by using the special astatic electrodynamic transfer wattmeter described in section 4. 'This wattmeter is first standardized for a particular value of power using direct current and voltage, which are measured with a potentiometer. It is then connected with its current coil in series with the current circuit of the a-c watthour meter under test and with its voltage circuit in parallel with that of the watthour meter. The two circuits are then energized from separate transformers that are supplied from two coupled generators driven by a common motor. The current and voltage measured by an auxiliary ammeter and voltmeter are adjusted separately to the desired values by control of the generator fields, and the stator of one generator is then shifted in angular position until the desired relative phase of current and voltage is secured. This condition is indicated by the reading of the transfer wattmeter, which then gives the same reading as on the earlier d-c calibration. One observer then holds the transfer wattmeter at this constant reading by occasional slight readjustments of the field rheostats while a second observer counts and times the revolutions of the watthour meter as described above for d-c wathour meters. At the end of each "run" the d-c standardization of the wattmeter is repeated, and a small correction is applied if any drift in its calibration has occurred.

This procedure is much more elaborate and timeconsuming than those used by power companies in their routine testing of large numbers of meters. Its use is justified (a) because the NBS normally receives for test only master standards of high quality, by means of which many other meter's are standardized, and (b) because the relatively small number but considerable variety of meters submitted would not justify a large investment in special automatic test equipment.

In a project, currently active, it is planned to set up a group of standard a-c watthour meters that are to be standardized at long intervals by the method just described and used as working standards for the more convenient testing of other meters. No definite data are ret arailable on the practicability of this procedure.

\subsection{Magnetic Units}

The unit of magnetic flux, the weber, is derived from the henry and the ampere, the latter in turn being derived from the volt and the ohm. The 
procedure is to change, by a known amount, the current in the primary winding of a mutual inductor and to note the resulting deflection of a ballistic galvanometer, which, together with the test coil that is to be used in later measurements, is connected to the secondary winding of the mutual inductor. The value of the mutual inductance in henries is obtained by comparing the working inductor with a large standard computable mutual inductor. This computable standard has an inductance of about $11 \mathrm{mh}$, and the working standard inductors range from about 2 to $50 \mathrm{mh}$. The value in amperes of the change in current is determined by a potentiometer, which compares the emf of a standard cell with the change in $I R$ drop in a known resistor. The change, $N \Delta \phi$, in flux linkages with the $N$ turns of the secondary winding of the working standard inductor, which results from the change, $\Delta I$, in the primary current is given simply by

$$
N \Delta \phi=M \Delta I .
$$

For convenience, the primary current is merely reversed so that $\Delta I=2 I$. If $I$ is in amperes and $M$ is in henries, $N^{\top} \Delta \phi$ is in webers. Normally the resistance of the complete secondary cireuit (including all test coils) is adjusted so as to make the galvanometer scale direct reading [244].

The weber as thus established is used to measure the flux in test specimens in various types of permeameter. The test specimen in a permeameter' is surrounded by a "B-coil," of a known number of turns, which is comnerted in series with the secondary winding of the calibrating mutual inductor in the galvanometer circuit. A measured change in the magnetizing current in the permeameter produces a change in the flux linking the galvanometer circuit, and the resulting deflection measures the flux change.

A measurement of the cross-sectional dimensions of the test specimen then permits the computation of the change in magnetic induetion, $\Delta B=\Delta \phi / A$. If $A$ is in square meters, $\Delta B$ is in webers/meter ${ }^{2}$ (myriagausses). This i'esult multiplied by $10^{t}$ gives the induction in gausses. Normally the area of the specimen and number of turns in the $B$-coil are lumped with the other factors in the calibration of the galvanometer, so that the induction is read directly.

Two distinct methods are used for establishing the unit of magnetizing for'e. In the older method, exemplified by the Burrows Permeameter [236, 249], the magnetomotive f-rce is distributed around the magnetie cireuit in proportion to the reluetanee of the various portions so that all portions carry the same flux and are at substantially the same magnetic potential. It this condition is attained, the magnetizing force, $H$, at any section, such as the central portion of the test specimen, is directly computable from the currentturns per unit length at that section. At the surface of the specimen the tangential component of $H$ is, of course, the same inside the metal as outside. Hence, the value thus computed applies to the interior of the specimen also. To approximate the desired distribution of magnetizing forces, the currents in auxiliary coils near the joints and rokes as well as the current in the main magnetizing coils are adjusted until the flux in the central part of the specimen is equal to the average of the fluxes at two sections at the quarter points of its length. For high accuracy it is essential that the test specimen be very uniform throughout its length [237].

The second method for realizing the unit of magnetizing force, $H$, is to measure the tangential component of magnetic induction, $B$, in the air closely adjacent to a test specimen. This is numerically equal, in the entimeter-gram-secondelectromagnetic sistem, to $H$ in the air, and by a fundamental theorem the tangential component of $H$ is continuous across the boundary and into the specimen. The measurement of $I^{j}$ in the ail is preferably made by quickly rotating a pair of "flip coils" of known area and number of turns through $180^{\circ}$. The axes of these coils are parallel to the axis of the specimen at the beginning and end of the rotation. This method is used in the High-II $[241,242]$ and the $M I I$ permeameters [243]. The latter is shown in figure 10 .

Because the value of $I$ may vary with distance out from the surface of the specimen, by reason of leakage flux from other parts of the eireuit, it is necessary to apply a suitable correction to the value observed by the "flip coil" nearest the surface. The difference between the flux linked by this flip eoil and the second located at a known greater distane from the surface of the specimen gives a basis for extrapolating to the value at the surface. Such multiple coils are provided in the High- $I I$ and $M I I I$ pelmeameters. Br applying such corrections and by careful calibration of the galvanometer, accuracies of 0.5 pereent in $B$ or $I I$ can be obtained. In ordinary testing practice, the accuracy is about 1 pereent for citler $B$ or $I I$.

Alternatively a fixed $I I$-coil, adjacent to the specimen may be used and the magnetizing force

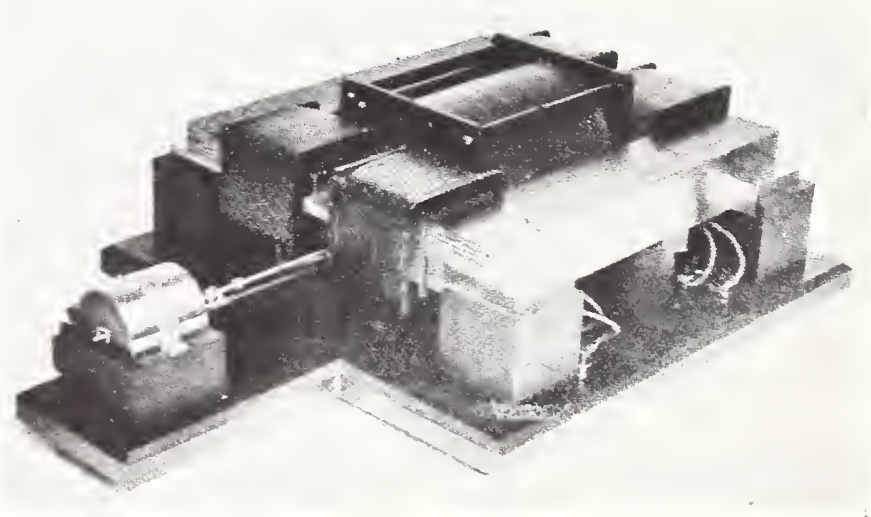

Figure 10. The MII permeameter.

The bar specimen lies along the axis of the central coil. The fonr auxiliary colls supply magnetomotive force for the joints and yokes. The plunger of the iron-clad solenoid at the left "flip.
below the specimen near its center. 
deduced from the change in flux linkage in this coil when the magnetizing current of the permeameter is reversed. This is the normal procedure with the widely used Fahy Simplex Permeaneter $[248,250,252]$.

Here the $H$-coil extends between two blocks of soft iron clamped to the ends of the specimen and responts to the average value of the magnetic potential gradient between the blocks. For accurate work a correction factor must be applied to relate this to the true magnetizing force acting on the specimen. This factor is determined br the use of standard test bars that have been first calibrated in an absolute permeameter.

With permeameters of these types, measurements of the normal induction curve, hysteresis loops, and of residual induction and coercive force [244] are regularly made on sample bars submitted for test by the laboratories of steel companies and other organizations. The circulation of such bars insures uniformity and accuracy in this branch of industry. Also by such comparisons the performances of permeameters of other types have been studied in detail [246 to 252]. A closely related service is the calibration of search coils to determine magnetically their effective areaturns. This is done by placing the coil near the center of a long, uniformly wound, single-layer solenoid with the axis of the search coil parallet to that of the solenoid. The mutual inductanee, $M$, between the coil and the solenoid winding is measured ballistically. The area-turns, $A N$, of the coil are then computed by the equation

$$
A N=M I / B \text {. }
$$

The ratio of the central flux density, $B$, to the 'urrent, $I$, is a constant that is computed from the measured dimensions and pitch of the solenoid winding.

Measurements of magnetic propertics with alternating currents involve either power measurements with wattmeters or the use of a-c bridges or potentiometers. The maximum crelic change in flux is measured by using a roltmeter of high resistance (400 ohms/v) or more with a copperoxide rectifier to indicate the arerage value of the induced voltage. The derivation of the corresponding units is given elsewhere.

Recent work at the National Bureau of Standards has made possible an alternative method for measuring with extremely high accuracy the magnetic induction in fields above 1,000 gausses. The studies of Bloch [253] and of Purcell and his colleagues [254] have shown that if a substance is placed in a strong fixed magnetic field of intensity $B_{0}$, on which is superposed a weak radio-frequency field, the mat erial will show a resonance absorption at a particular frequency, $f_{0}$. This resonant frequeney is given by $f_{0}=\gamma R_{0} / 2 \pi$, where $\gamma$ is the grvomagnetic ratio of the atomic nucle of the substance used. At the Bureau, Thomas, Driscoll, and Hipple [255] have performed this experiment with great care, using a material (water) rich in protons and have determined the grromagnetic ratio of the proton to be

$$
\gamma=(2.67528 \pm 0.00006) \times 10^{4} \mathrm{sec}^{-1} \text { gauss }^{-1} \text {. }
$$

In this work the gauss was established by observing the mechanical force exerted by the field on the conductors that formed one side of a rectangular "force coil" hanging in the field and carrying a measured current. The current was measured in terms of a standard cell and standard resistor. The force was measured by balancing it against the weight of a known mass that could be added to or removed from the pan of the precision balance from which the foree coil was suspended. The effective length of the conductors in the bottom side of the coil was measured by a special micrometer in comparison with a knowir end standard.

$\mathrm{Br}$ using this value of $\gamma$ and a resonance probe similar to the one developed for this work, other experimenters can measure the induction in an unknown magnetic field by a simple determination of the resonant frequency in terms of the standard radio-frequencr emissions from NBS radio station WWT [25]. Such a resonance detector can also be used (1) to study the distribution of flux density in a magnetic fiekd and (2) with suitable additional eircuits as a monitor to hold constant the induction at a given place in the field.

\section{Transfer from D-C to A-C Measurements}

A standard cell and the units of voltage and of current realized from it directly or in conjunction with a standard resistor are applicable to the measurement of d-c quantities only. On the other hand, by far the more frequent and important measurements in the power and communication fields involve alternating currents. The procedure used to transfer from the fundamental $l-c$ standards to a-c measuring apparatus, as indicated in the middle of figure 8, therefore constitutes an essential link in the chain of electrical measurement.

Of the three fundamental methods for relating the root-mean-square value of the alternating current to an equivalent direct current, viz: electrodynamic, electrostatic, or thermal, the NBS normally uses an electrodynamic method for wattmeters for frequencies up to about $1,000 \mathrm{c} / \mathrm{s}$. The same instrument can be used with a shunt as an ammeter up to $10 \mathrm{amp}$. Methods using thermal converters, which have been confirmed by comparisons with this instrument, are normaliy uscel in testing ammeters and voltmeters at freyuencies up to $20 \mathrm{kc} / \mathrm{s}^{2}$

\footnotetext{
2 It may be noted that at the British National Physieal Lahoratory [1- $x$ ] an electrostatic method is used as fundamental. A very carefully construeted quadrant electrometer itself calibrated on direct eurrent serves to calibrate in turn other voltmeters direetly on alternating current. It is also used to hold a known alternating voltage at the terminais of a noninductive resistor and a known alternating voltage at the terminals of a noninductive resist or, and i second highly precise quadrant electrometel conneeted so as to funetion as a wattmeter measures the power dissipation in the resistor. With the electrostatic wattmeter thus ealibrated at unity power factor, it ean be used
ealibrate ordinary wattmeters at any desired power factor and frequeney.
} 


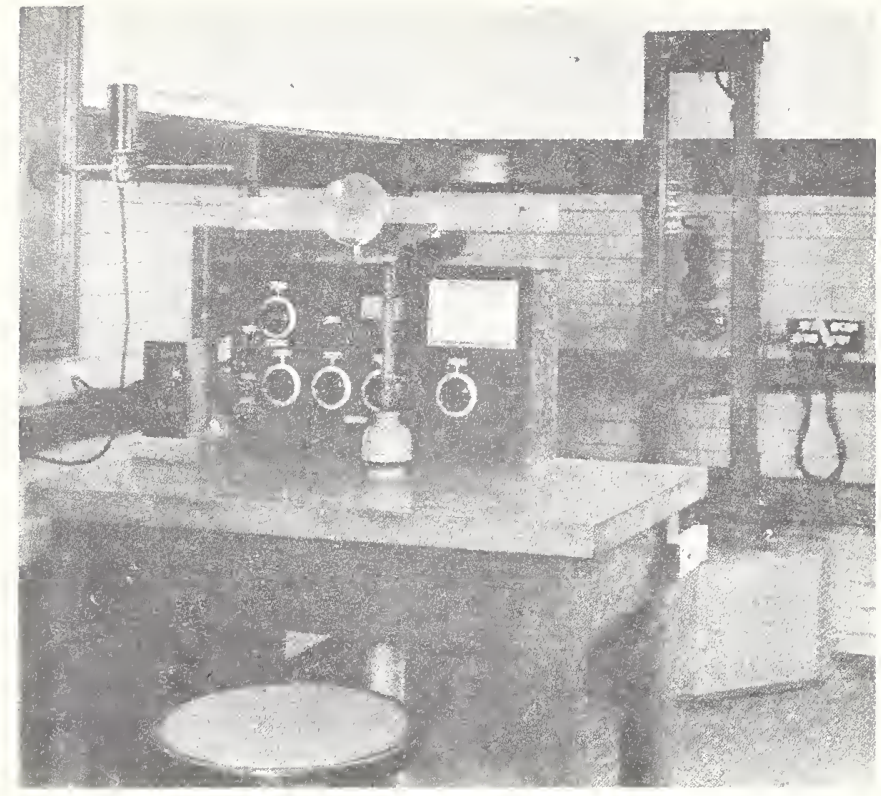

FIrURE: 11. Electrodynamic ac-de transfer instrument.

The links besule the instrunent uroper wermit eonneeting the fixed coil in varallel or sertes. The dials below the seale arljust the mestanee is serin with the moving coil.

The basic edectrodynamic transfer instrument for eurrent and power at power frequeneies $[177]$ is shown in fixure 11. It is an astatic electrodynamic wattmeter, the moving coils of which are carried by a taut suspension of phosphor-bronze strip. The fixed coils are rated at $2.5,5$, or $10 \mathrm{amp}$, aceorling as they are comnected in series, seliesparalled, or' parallel. 'They are supported in slabs of plate class to which adjustable supperts and a torsion head for the suspension are firmly at tached. 'The angular position of the pair of movable coils is read by a mirror and scale at a radius of $2 \mathrm{~m}$. The movable coils are used in the position where the mutual inductance between them and the fixed coils is substantially zero. 'The deflection is usually about $60^{\circ}$. A capacitor shunts part of the series resistor and thus compensates for the sedfinductance of the movable coils. $\Lambda$ change in deflection of $1 \mathrm{~mm}$ corresponds to 0.025 percent in power and (with the fixed coils in series) to a change of $2 \times 10^{-6}$ (amp) $)^{2}$ in the product of the currents in the fixed and moving coils.

Even though the electrodynamie transfor instrument was carefully designed to be as free as practicable from such sources of a-c error as eddy currents, capacitance, and inductance, a great many rhecks were made on its performance before it was put into commission as a transfer instrument. These included (1) a comparison up to $3,000 \mathrm{c} / \mathrm{s}$ when connected as an ammeter, with a Hartman and Braun expansion-type hot-wire ammeter, (2) a comparison at $60 \mathrm{c} / \mathrm{s}$ and full volt-amperes but zero power factor with a sensitive quadrant electrometer, and (3) the measurement at 500, 1,000, and $2,000 \mathrm{c} / \mathrm{s}$ of the loss in a $10-\mu \mathrm{f}$ mica capacitor, the phase defeet of which had been previously measured in an a-c bridge. These check tests showed the existence of two small departures from an ideal electrodynamic instrument. The first was a spmious torque due to eddy currents in the aluminum damping vane. This effect is less than $2 \mathrm{ppm}$ at $60 \mathrm{c} / \mathrm{s}$ but incleases as the square of the frequency and has to be corrected for at frequencies above $1,000 \mathrm{c} / \mathrm{s}$. The second is an effective phase lefect that increases as the first powre of the frequency and at $60 \mathrm{c} / \mathrm{s}$ amounts to osly 0.38 minute, i. e., to an error of 0.011 pereent of the volt-amperes, even at zero power factor.

'This transfer' instrument is used principally in (hecking the accuracy of electrodynamic wattmoters of the 1/10-percent or the 1/2-pereent class, which in turn are used as standard transfer instruments in other laboratories for the checking of other wattmeters or watthour meter's using alternating emrent. In the usual test, the unknown and the standard transfer wattmeter are connected with their current circuits in series and their voltage cireuits in parallel, as shown in figure 9. With switch $S_{A D}$ closed to the right, alteruating current and voltage are applied from separate transformers, $T_{2}$ and $T_{1}$, and their relative phase is adjusted by shifting the stator of $G$ to give the desired power factor (usually 0.5 ). This can be calculated accurately enough from the readings of the wattmeter under test and of the auxiliary ammeter, $A_{A}$, and voltmeter, $\Gamma_{A}$. The series resistor of the standard instrument is redued until the spot of light comes to a convenient position near the center of the scale, where the axes of the moving coils are at right angles to those of the fixed coils and the mutual inductance between the two circuits is zero. One obselver then adjusts the current to bring the pointer of the instrument under test as exactly as possible on some scale mark, usually using either a projection equipment or a magnifying glass. "The other observer reads as $A_{1}$ the position of the spot of light on the instrument scale. The four-pole double-throw switch, $S_{-1 D}$, is then closed to the left to substitute previous!y adjusted direct current and voltage in place of the a-c quantities. Current is again adjusted mil the instrument under test rearls the same, and the standard instrument reading, $D_{1}$, is recorded for this setting: Both 'urrent and voltage are then quickly reversed by switch $S_{\text {rer }}$ and a second d-c reading, $D_{2}$, is obtained. The original connections are then restored and a check a-c reading. $A_{2}$, is recorded. The average of $D_{1}$ and $D_{2}$ is free from any effect of the earth's magnetic ficld. 'The a verage of $A_{1}$ and $A_{2}$ corresponds to substantially the same time of observation as does the mean of $D_{1}$ and $D_{2}$. Hence any linear drifts like the effects of spring fatigue or of heating in either instrument (ancel out, and their difference, when divided by the mean equivalent deflection $\left({ }^{3}\right), B$,

3 the standard transfer instrument is used with a uniform millimeter seale, the divisions of whieh do not exaetly eorrespond with equal increments in the ineasured power over the $w$ hole range Henee to obtain relative elange the per the in power from the observed ehanges in defleetion, it is eollvenient to divide $d D / d P$ is the slope of the grapl of defleetion, $D$, versus power, $P$, at the part of the seale used 


$$
\frac{A_{1}+A_{2}-D_{1}-D_{2}}{B}
$$

is a measure of the real difference in the performance of the instrument under test on alternating current as compared with direct current. In practice three such sets of four readings each are taken in rapid succession, and with a wattmeter of good quality the mean difference thus obtained and divided by the effective deflection rorresponding to the d-c reading will be found repeatable to \pm 0.02 percent.

The performance of many wattmeters on alternating-current can often be represented approximately by an equation of the form

$$
P_{a}=P_{d}(1+a)+b E I \sin \theta,
$$

where $P_{a}$ is the reading on alternating current, and $P_{d}$ is the reading on direct current for the same true power, $E$ and $I$ are voltage and current, and $\cos \theta$ is the power factor. A transfer test first made at unity power factor $(\sin \theta=0)$ gives a measure of $a$, which is usually very small. 'This combined with a second test at $\cos \theta=0.5$ ( $\sin$ $\theta=0.866$. gives a measure of $b$.

If eq (1) represented completely the performance of a wattmeter, it would be more convenient to make the second test on alternating current at zero power factor only. This can be done by adjusting the phase difference until the wattmeter under test indicates zero with rated current and voltage and then noting the reading, $P_{a}^{\prime}$, of the standard instrument, after which the latter is calibrated using direct current. This procedure gives

$$
b=\frac{P_{a}{ }^{\prime}}{E I} .
$$

For such a test at zero power factor the torsion head of the transfer instrument is shifted so as to bring the zero of the instrument near the center of the scale.

However, wattmeters are frequently found not to obey eq (1). This condition is best revealed by a pair of tests at low power factor with the current first leading and then lagging by the same angle. If the formula applies, the changes in error when going from unity power factor to the other two conditions should be equal in amount and opposite in sign. Half of the algebraic difference in such a pair of relative ac-de differences, when divided by $\tan \theta$, is a good measure of the cffective phase defect coefficient, $b$, but half their algebraic sum is often quite different from the value of a derived from the test at unity power factor. The reasons for such departures of performance from eq (1) are obscure, but in part may be associated with small vibrations in the moving system.

The same standard electrodynamic instrument serves also as the basis for the transfer testing of ammeters at power frequencies. For this purpose it is used as a wattmeter to measure the rate of energy dissipation in a noninductrve standard resistor that is in series with the ammeter under test. Readings are made with alternating and direct current alternately, as described above. It has been found more convenient, however, in the normal testing of a-c ammeters to use, as an intermediate standard, an ammeter of the composite-coil type [180]. The a-c performance of this instrument has been carefully compared with that of the fundamentally simpler astatic wattmeter, and it is regularly used on alternating current, often with a standardized current transformer, to check ammeters on alternating current.

The basic instrument for the transfer of voltage from direct current to alternating current at power frequencies is a suppressed-zero electrodynamic voltmeter [179] in which the movable system is carried by a taut suspension and its position is read with a telescope and scale at a scale distance, as now installed, of $3 \mathrm{~m}$. A short boom attached to the moving system is free to play between two stops, only a few degrees apart and insures that the suspension is held at all times with substantially the same twist as that present when readings are made. As normally used in the transfer testing of voltmeters, the double optical lever and temperature compensating coils described in [179] have been found unnecessary. An adjustable noninductive resistance in series with the instrument is set at such a value as to bring the cross hair somewhere near the center of the scale when the operating voltage is applied. Sets of alternately $d-c$ and $a-c$ readings are then obtained as described above for wattmeter testing. A measurement of the instrument sensitivity is also made by applying two successive values of direct voltage and noting the change in deflection which results.

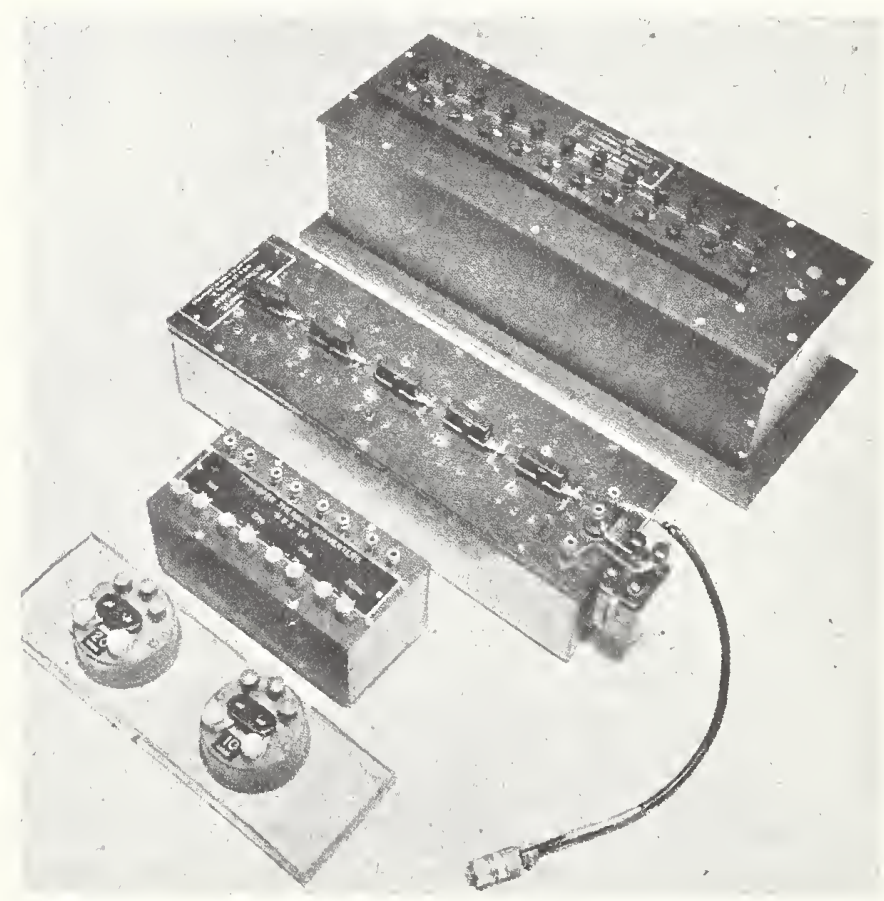

Figure 12. Group of thermal ac-de transfer instruments. 
An alternative procedure for transferring from $\mathrm{d}-\mathrm{c}$ to $\mathrm{a}-\mathrm{c}$ standards is the use of electrothermic instruments. Such apparatus of high precision has recently been installed $[182,207]$ and is regularly used for testing voltmeters from 0.2 to 750 . $\mathrm{v}$ and ammeters from $1 \mathrm{ma}$ to $50 \mathrm{amp}$ at frequencies up to $20,000 \mathrm{c} / \mathrm{s}$. Figure 12 shows some of these instruments. In spite of the fundamental simplicity of this method, a number of possible sources of error exist such as Peltier temperature shifts in the thermal converter, losses in the insulating bead and resistance in common between heater and couple circuits. With some individual thermal converters, these effects may not be negligible when an accuracy of 0.01 percent is sought. As finally worked out, however, the electrothermic transfer for current and for voltage has been found to agree with the electrodynamic transfer to an accuracy of 0.01 percent at frequencies up to $500 \mathrm{c} / \mathrm{s}$, and it is believed to be superior to the latter and to be accurate to 0.02 percent for higher frequencies up to $20 \mathrm{kc} / \mathrm{s}$.

Still another unit, definable only in a-c circuits, is the var, the unit of reactive power. It is the reactive power corresponding to the maintenance of $1 \mathrm{amp}$ in quadrature with a potential difference of $1 \mathrm{v}$. The test of a single-phase varmeter is normally made by supplying the instrument with sinusoidal current and voltage from two generators coupled to a common driving motor. The power factor is adjusted to zer'o by mechanically shifting the stator of one generator relative to the other until a wattmeter indicates zero The reartive power in vars is then equal to the product of the voltage and current, as measured by a voltmeter and ammeter. A further check at unity power factor by connecting the varmeter at the terminals of a load of substantially nonreactive resistors, gives an indication of any phase defect in the varmeter circuit.

The calibration of polypliase varmeters and the use of varmeters with nonsinusoidal currents introduces theoretical complications that are, in general, more serious than the purely instrumental errors. The Bureau has endeavored to bring some order out of the earlier confused practices by cooperating in the formulation of standard definitions for the numerous concepts involved [205].

\section{Extension of Measurement Ranges}

The procedures and apparatus described in the foregoing sections have indicated how the various electric and magnetic units are derived from the primary volt and ohm. It is not enough, however, to be able to measure one ampere or one watt. Methods must be developed for extending the range of all electrical quantities to cover the magnitudes encountered in science and industry. Such methods commonly involve the use of bridge circuits having unequal ratio arms, multirange instruments having different values of series, or parallel, resistances, or other procedures that utilize resistances of widely different values. The establishment of the scale of resistance values is therefore of fundamental importance.

\subsection{The Resistance Scale}

The primary tool used at the National Bureau of Standards for the measurement of resistance is a precision bridge built in the Bureau shops in 1918, which incorporates many features developed as a result of the Bureau's first 15 years of experience in the field of precise measurements [79]. The more important resistance sections consist of hermetically sealed coils; the dial adjustments are of the Waidner-Wolff type, [75] made by changing the shunting around fixed resistors so that the effects of contact resistance and emf are greatly reduced; all resistors and contacts operate under the oil of a thermostatically controlled bath; and the circuit is shielded from stray currents. The bridge may be used either as a Wheatstone or as a Kelvin bridge, and is commonly used with a ratio that is nominally either $1: 1$ or $10: 1$. This ratio can be adjusted over a limited range $( \pm 0.5$ percent $)$ in the vicinity of the nominal value emploved.
The adjustment is mode by means of four decade dials, one step on the lowest dial corresponding to a shift of $1 \mathrm{ppm}$ in ratio.

The step that is fundamental to extending the resistance scale is the establishment of an accurate $10: 1$ ratio between ratio arms of this bridge. This is done by using a special assemblage of resistors [79] (figure 13). Six of these resistors are nominally of $150 \mathrm{ohms}$ each, and the seventh is of $50 \mathrm{ohms}$. By successive substitution in the precision bridge, the relative values of the six resistors can be measured. Also by substitution the values of the resistances of nominally $50 \mathrm{ohms}$ each, formed by connecting in parallel each of the two sets of three 150 -ohm units, is determined relative to the $50-\mathrm{ohm}$ resistor with high accuracy. The three 150-ohm units of one set are then connected in series with each other and with the 50ohm unit to form a 500-ohm group. The three $150-0 h m$ units of the other set are connected in parallel to form a 50-ohm group.

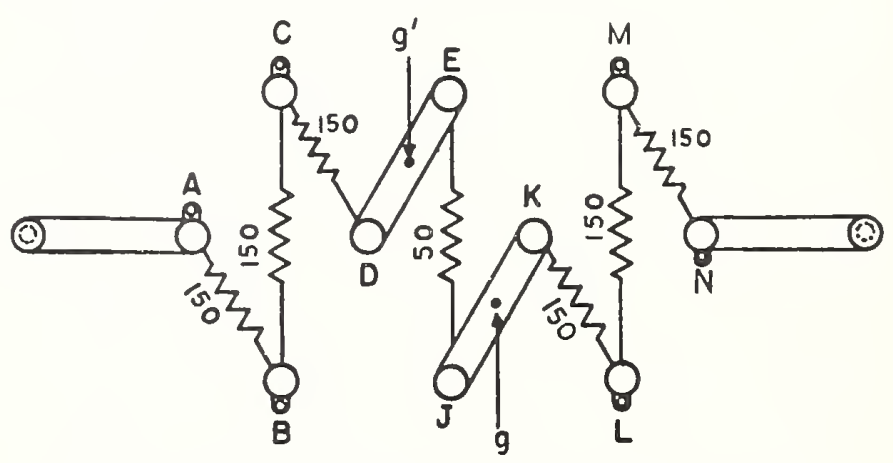

FIGURE 13. Circuil for establishing 10:1 ratio.

With links joining $A$ to $C$ and $B$ to $D$, the tap at $g^{\prime}$, ratio is 1:10. With links joining $K$ to $M$ and $L$ to $N$, and tap at $g$, ratio is $10: 1$. 
These two groups, having a ratio close to $10: 1$ are connected to form two arms of the highprecision bridge, the remaining two arms being the adjustable ratios referred to above. The bridge is then batanced by manipulating the four dials and their setting, $\hat{N}_{1}$, is noted. The paralleling links are then shifted so that the 150 -ohm coils of the first set are put in parallel and form a 50 -ohm group, and those of the second sot are in series, and, with the central 50-ohm coil, form a 500-ohm group. These groups are turned end for' end and again connected in the bridge, and a second dial setting, $N_{2}$, at balance is noted. The mean of the two observed ratio settings, $N_{1}$ and $N_{2}$, is then adjusted by applying a small correction derived from the observed clifferences between the 50ohm coil and the means of the groups of three in parallel. $A$ further correction for the resistance of the paralleling links is applied, and after these several corrections are taken into account, the settung of the bridge dials for which the ratio is exactly 10:1 is known within about 1 - part in 3 million.

An alternative, but more laborious, procedure sometimes used for obtaining an accurate 10:1 ratio, is to use a group of six 100 -olm standard resistors. Each in turn is used as one arm of a bridge; the other five in series constitute an adjacent arm. The other two arms are the adjustable 100-ohm arm of the precision britlge and a fixed $20-\mathrm{ohm}$ arm. The average of the six successive settings on the adjustable arm then corresponds to an exact 100:20 ratio to an orker of accuracy given by the sum of the squar's of the departures of the individual 100-ohm standards from their mean, provided proper corrections are applied for the resistances of the contacts and connectors. This ratio is then used to compare each of the two 50 -ohm sections of a divided $100-$ ohm standard resistor with a $10-0 h m$ standard, and from these two results the ratio of the divided $100-$ ohm to the 10-ohm is conputed. The results obtained by this procedure al'e usually found to agree with those by the first-mentioned procednle to a few parts in $10^{\text {? }}$.

By using the bridge, thus calibrated, it is easy by successive steps to measure the values of wirewound standard resistors of $10,100,1,000, \mathrm{Or}$ 10,000 ohms, and similarly to step lown to 0.1-, $0.01-, 0.001-$, and $0.0001-0 h m$ standarels. Resistances of intermediate value are oceasionallymeasured by using analogous series or parallel combinations of nominally equal resistors to evaluate other bridge ratios that involve small integers.

In contrast to the foregoing methods for testing individual standard resistor's while immersed in a thermostated oil bath, the procedure normally used for calibrating a decade dial of a bridge arm or resistance box is to substitute a known standard resistor suecessively for each of the steps of the dial [80]. 'This is done, as shown in figure 14, by connecting in series (1) a resistance box that las

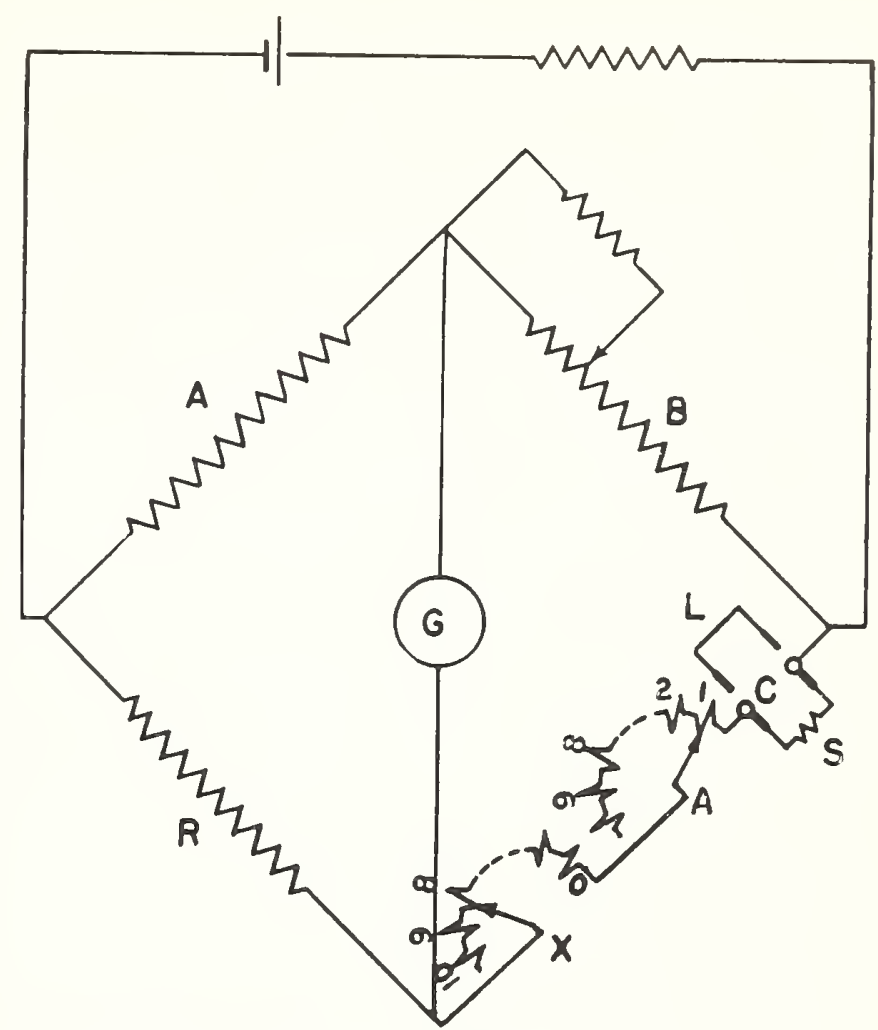

Ficure 14. Circuit for calibrating resistance decades.

the dial $X^{r}$ to be tested, (2) an auxiliary box that lias a dial $A$ of the same nominal value as $X$, and (3) a pair of mercury cups, $C$, into which may be placed either (a) a short-circuiting link, $L$, or (b) a known standard resistor, $S$, of the same nominal value as one step of dial $X$ or $A$. The series combination is comnected to form one alm of a Wheatstone bridge, the other arms of which are formed by a direct-reading latio set $A-B$ and al

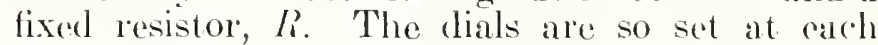
stage in the process that the total resistance of the arm is nominally 10 times the resistance of one dial step. Successive pairs of bridge balances arre then made. In the first balance of each pair the standard resistor is in the circuit. In the second balance it is replaced by the link, and the $X$ dial is at a setting one step higher than during the first balance. This constitutes in effect a substitution of the dial step for the standand resistor, and their difference is read from the small changer required in the balancing an'm of the bridece. The auxiliary dial is then shifted one step, and a second pair of similar balances gives a caliblation for the next step of $X$. Incidentally, the datar thus obtained suffice for the calibration of the auxiliary dial also.

When making precise neasurements with losistors of 1 ohm or less, it is essential to $11 \mathrm{se}$ losistors of the four-terninal repe to eliminate the eflects of contad resistance. The Kolvin bridge or sone equivalent circuit has proved to be in general the most suitable for the comparison of such resistors [76]. Of the various procedures [77] that ean be used to balance out the effects of 
the resistance in the potential leads, that which involves 'applying a short-circuit across the ends of the main ratio arms is commonly used.

The Kelvin double bridge is usually employed at the NBS in all measurements of low resistance, but remarkably good results can often be obtained with simpler apparatus, provided appropriate methods and precautions are used [78].

At the other extreme the measurement of high resistance merges into the measurement of the resistivity of insulating materials [81]. Highgrade wire-wound resistance elements are available up to $1,000,000$ ohms. Groups of such 1-megohm resistors may be measured in a high-grade Wheatstone bridge while connected in parallel and can be used singly or in series to form a 10-megohm standard. Units of higher individual value consisting of heterogenous compositions or of very thin film are likely to show changes in resistance with changes in temperature, voltage, and, unless very carefully protected, in ambient humidity. Great precision in their measurement is therefore not required, and a simple direct deflection method using a shunted galvanometer of high current sensitivity $\left(10^{5} \mathrm{~mm} / \mu \mathrm{a}\right)$ is often satisfactory.

If the voltagte is limited to a few volts, this procedure fails at resistances exceeding $10^{9} \mathrm{ohms}$. For higher resistances the charge carried by the test resistor is accumulated on one electrode of an adjustable air capacitor. The rate of accumulation is measured by either changing the capacitance or the potential applied to the other electrode while using an electrometer as a null indicator. When higher voltages can be used, correspondingly higher resistances can be measured $[82,86]$. An accuracy of 0.2 percent can be obtained up to $10^{13}$ ohms at $1.5 \mathrm{v}$.

\subsection{The Scale of Direct Voltage}

The starting point of the voltage scale is of course the primary group of saturated cadmium standard cells [61], which maintain the volt during the long intervals between absolute measurements of current. This primary group [6] is picked from a much larger stock of saturated cadmium cells, batches of which are made up at the Bureau from time to time, using very carefully purified materials [48 to 58].

The members of the group are intercompared every few months by means of the standard cell comparator [91]. This is a highly specialized form of potentiometer that measures the difference in electromotive force between the cell under test and a standard reference cell. A simple automatic computing device incorporated in the apparatus enables the observer to read the electromotive force of the unknown cell directly. One division on the instrument corresponds to $1 \mu \mathrm{v}$. Great pains were taken in the design of this comparator to minimize spurious thermoelectric effects, which usually constitute the most serious source of error in this type of measurement. It is used regularly in testing the unsaturated standard cells that are sent to the Bureau periodically for check from a large number of scientific and industrial laboratories, as well as for the comparison of saturated cells.

When an unsaturated standard cell is received at the Bureau for test it is placed with others in a large box that is thermally insulated, but not thermostatically controlled, and its electromotive force is measured daily for at least 10 days. If, as is the usual case, the emf soon settles down and remains within $\pm 50 \mu \mathrm{v}$ of a steady value, a certificate is issued giving this mean value to the nearest $10 \mu v$. However, if the fluctuations continue to exceed 0.01 percent or if the cell has an abnormally low value, below $1.0183 \mathrm{v}$, or if it shows an abnormally high internal resistance, no formal "certificate" is issued, but instead the data observed are sent to the customer in the form of a "report." If properly used, unsaturated cadmium standard cells can constitute very accurate and reliable standards of voltage. They are, however, easily damaged by excessive current drain, and their electromotive force may be temporarily affected by sudden changes in temperature and also by any temperature difference that may exist between the two electrodes [52]. Modern- cell containers are made with copper liners to minimize this latter effect.

The extension of the $d-c$ voltage scale.to lower values is usually made by the use of a potentiometer. For the normal precision testing of lowrange voltmeters and millivoltmeters at the Bureau a well-seasoned null potentiometer of the Feussner type is used. The lowest dial has steps of $10 \mu \mathrm{v}$. For mole rapid and less exacting calibrations a Brooks deflection potentiometer [96 to 99], developed at the Bureau primarily for the rapid calibration testing of electric indicating instruments, and for incandescent lamp testing, is used. It is, however, not suited for measurement of emf in circuits of high internal resistance. In this apparatus the bulk of the. voltage to be ineasured is balanced by the IR drop in the resistance of the main dial. Any unbalanced residue causes current to flow in the calibrated galvanometer and produce a corresponding deflection. The value of the total voltage is then indicated directly by the one (or two) digits on the dial followed by three digits read from the galvanometer scale, the last digit corresponding to 0.1 scale division.

A number of other types of potentiometer have been developed at the Bureau for special purposes. The Wenner type $[94,95]$ is quite generally used for precise temperature measurement with thermocouples. On its lower range, one step on the lowest dial corresponds to $0.1 \mu \mathrm{v}$. A special multirange potentiometer [90] has proved convenient for measuring small temperature differences. For the measurement of very small intensities of radiation by a physical photometer, 
another special potentiometer [93] reading to 0.001 $\mu \mathrm{v}$ is used.

Potentiometers, in effect, measure merely the ratio of the unknown electromotive force to that of the standard cell. Their calibration therefore needs involve no direct measurement of resistance, but merely the determination of ratios of resistances. Hence, for the testing of potentiometers [87] and also for other applications, it has been found convenient to use a universal ratio set, which is equivalent to a slicle wire having a total resistance of $2111.1 \mathrm{ohms}$, with a tap adjustable in steps of $0.01 \mathrm{ohm}$.

In the measurements of voltages of extremely low values, as well as in the highly precise measurement of resistance, one limiting factor may be the sensitivity of the galvanometer. This was recognized early, and considerable effort has been devoted to theoretical studies and practical designs of galvanometers for both direct $[168,169$, 92] and alternating $[163,164,165,166]$ current. The galvanometers used at the Bureau for precision resistance measurements are of a special Bureau design, with taut suspensions and with the center of gravity slightly out of line with the suspensions. With this construction, it is possible to adjust the effective restoring torque by properly tilting the instrument. With a 10 -second period, these have a sensitivity of $40 \mathrm{~mm} / \mu \mathrm{v}$. In most of the other electrical measurements, commercial galvanometers of appropriate design are used. The more complex amplifying combinations, which can push the sensitivity to the limit set by Brownian motion $(0.001 \mu \mathrm{v}$ for a $10-0 h \mathrm{~m}$ circuit and 2-second period), are seldom needed. The Coblentz [167] moving-magnet galvanometer has been superseded by moving-coil galranometers, as they are not similarly affected by external magnetic fields.

The extension of the scale of direct voltage to higher values while still using the potentiometer is made by using instrument voltage dividers (niore commonly called "volt boxes") [100]. Such a divider consists of two resistors, $R_{a}$ and $R_{b}$, which are connected in series, across the voltage to be measured. A potentiometer is connected to measure the voltage, $E_{b}$, at the terminals of $R_{b}$, and the total voltage, $E_{a+b}$, is then calculated by the relation

$$
E_{a+b}=E_{b} \frac{\left(R_{a+} R_{h}\right)}{R_{b}}
$$

In principle the ratio of resistances might be computed from measurements of $R_{a}$ and of $R_{b}$ separately, such measurements being based on the 10:1 resistance ratio described in section 5.1. This method is not desirable, however, because of the two principal sources of error that arise in the use of volt boxes: (1) the possibility that at the high values of voltage applied and hence larger values of current in $R_{a}$, it may be so heated that its value becomes significantly different from that observed in a resistance-bridge measurement at smaller current, and (2) the possibility that leakage currents in the insulating supports may bypass part or all of $R_{a}$ but flow through $R_{b}$, thus invalidating the assumption implicit in eq (3) that the same current exists throughout the entire series circuit. To minimize such errors, the extension of the voltage scale at the Bureau is made by using an especially constructer standard volt box rated for $750 \mathrm{v}$, but satisfactory for use as high as $1,500 \mathrm{v}$ [100]. In the design of this apparatus (1) the resistance has been chosen so ligh $\left(333^{1 / 3} \mathrm{ohms} / \mathrm{v}\right)$ that heating effects are negligible, and (2) guard plates have been provided to intercept any leakage paths over the insulating supports and bushings. The guard plates are connected to taps on an auxiliary guard circuit so that the potential difference aeross any insulator is nevel more than $75 \mathrm{v}$. and at most bushings is nominally zero.

The measuring circuit is composed of groups of five coils of nominally equal resistances alt crirating with single coils, each having a l'esistance nominally equal to the total resistance between it ard the grounded end of the circuit. To determine the ratio of the divider with high accuracy, it is necessary merely to substitute its successive equal coils or groups of coils in one arm of a sensitive and stable, but uncalibrated, Whea tstone bridge. The relative values of the coils are noted, and the value of the total resistance to any tap point is computed from these relative values, and an assumed nominal value for the lowest step, on the assumption that the resistances are truly additive. In taking the ratio of any total resistance to the resistance up to a tap point, the assumed nominal value cancels out leaving only the ratio. With reasonable care, an aceuracy of 0.005 pereent can be obtained.

Jll use the rolt box under test is ammected in parallel with the standarel volt box, using on the latter a tap giving the sanre nominal ratio. 'The difference in voltage, if any exists, betweon the tap points of the two divider's is measuror with a small potentiometer of the Lindeck type. 'The ratio of this difference to the total applied voltage, moasured with a voltmeter, gives the ratio erior of the volt box under test relative to the standard volt, box. Corrections for lead resistanee, cte., are derived by auxiliary measurements with the poten-

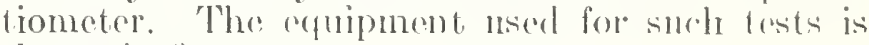
shown in figure 15 .

In the measmement of the higher dirent voltages nsed in X-ray genorators a new somrer of error enters the pieture. 'This is the coroma dischanere from points and edges that are at a considerabile potential above their surromendings. This "an result in a signifieant enrent hakiage to eroumel directly through the air and can also cause frouble from the rhemieal acetion of the o\%one ald oxides of nitrogen formed and from the clectrostatic precipitation of dust. 'To obviate these trombles, a construction has been developed as the Burau [102] in which the resistance, $R_{n}$, is subdivided into units of $5 \times 10^{6}$ ohms. Each is strrounded with a 


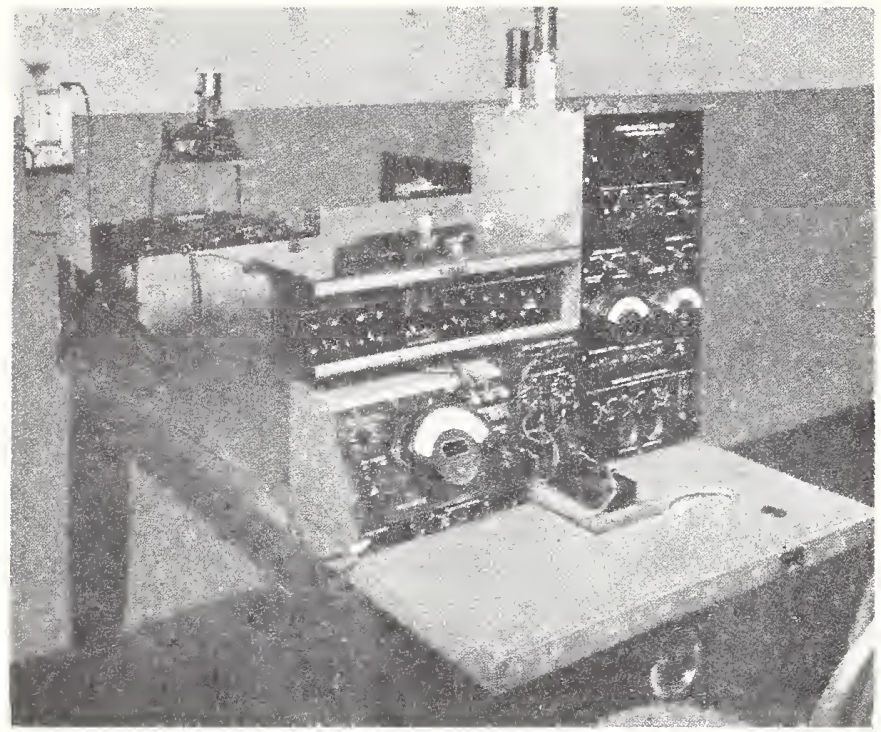

Figure 15. Equipment for testing volt boxes.

The standard wolt box is under the galvanometer at the back. The Lindeek potentioneter with sloping panel is in the foreground, and above it is the selector panel, which ermneets the potentiometer and its guard eircuits at ibny desired voltage level.

metal shield formed with surfaces of such large radius of enrvature that no external corona develops. Each shield is comnected to one and of the group of five 1-megohm resistor's that it surrounds, and the voltage of 7,500 between resistor and case is so low that no corona occurs inside the shicld. A divider of this construction, having 20 units of 5 megohms each, serves to measure direct voltages up to $150 \mathrm{kv}$ with an accuracy of 0.01 percent.

For measurements in connection with X-rays of still higher voltage, a voltage divider has been constructed for 1,400 lilovolts. It is contained in the left-hand column shown in figure 16. It consists of 10 sections, each enclosed in an outer shield which is connected to an appropriate tap on the cascarle transformer-rectificr set of 10 mits, which produces the high direct voltage. Within each of these 10 sections the divider consists of 14 subsections, each of 10 megohms. Each subsection is enclosed by an inner corona shicld, which is conneeted to one end of the subsection, but insulated from the outer shickd. 'The normal current is 1 ma. If this current is measured by a potentiomcter, and if corrections for self-heating are applied, the total voltage ean be determined with an aceuracy of about $1 / 10$ pereent.

\subsection{The Scale of Direct Current}

The measurement of a current by measuring with a potentiometer, the voltage drop in a resistor" in which the eurrent is flowing suffices to 'over' a very wide lange of direct currents. At the low current end of the seale the accuracy begins to be limited by the current sensitivity of the galvanometer when the resistance of the resistor materially exeecds that of the potentiometer and galvanometer. For a galvanometer sensitivity

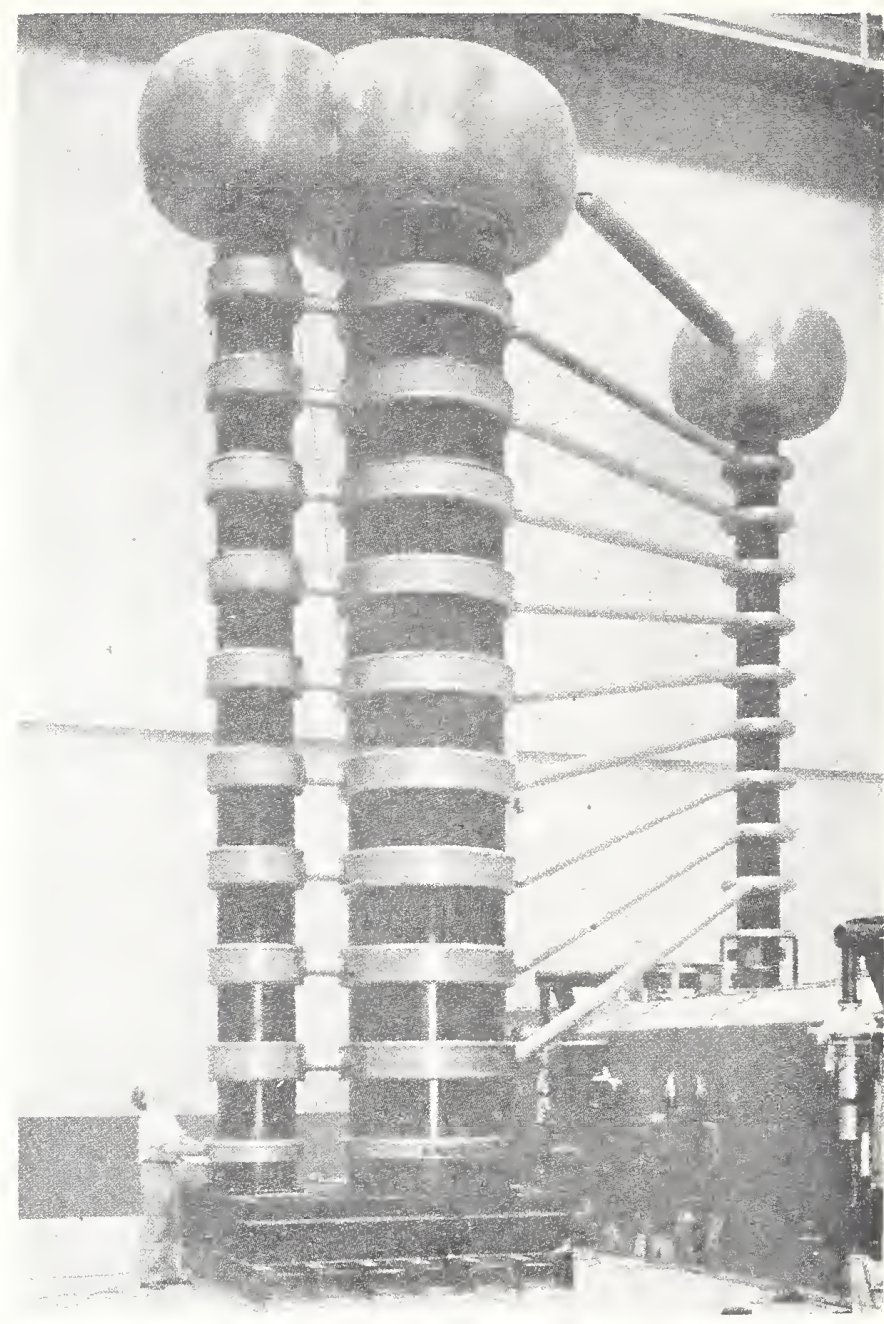

FICURE 16. Equipment for the generation and measurement of $1,400 \mathrm{kv}$ direct voltage.

Large central colunn eontains easeade of transformers, rectifiers, and cupacitors; left colmm contains sectionalized shielded resistor; distant colunin at right encloses $\mathrm{X}$-ray tube.

of $1 \mathrm{~mm}$ for $10^{-8}$ amp $1 \mu$ could be nreasured in this way to 1 percent. 'To calibrate instruments at lower currents it is necessary only to connect a large resistance, $R_{1}$, in series with the instrument and to shunt the combination with a smaller resistance, $R_{2}$. 'The total current can then be measured as before, and the range of possible calibration is thus extended by the ratio of $R_{1}$ to $R_{2}$. 'This procedure, however, is not applicable to the measurement of an unknown small current, and this latter problem is identical with that of measuring very high resistances, already discussed. 'The techniques ised for this are equivalent to the measurement of currents down to $10^{-15} \mathrm{amp}$.

At the high-current end of the scale the limiting factors are the hoating and thermoclectric effects in the series resistor. At the National Burcau of Standards a Wolff precision resistor of $0.0001 \mathrm{ohm}$ is available for measurements at high currents. This is immersed in stirred oil, which in turn is cooled by a coil of tubing through which water is circulated. At currents up to 1,000 amp the resistance of this resistor is a definite and repeatable function of the cooling-oil temperature. 
The, function has the usual parabolic form characteristic of manganin, with a maximum resistance at $32^{\circ} \mathrm{C}$. At higher currents, even though the temperature is held at the same value by vigorous cooling, the resistor shows an increase in resistance. This effect amounts to $100 \mathrm{ppm}$ at 3,000 amp, and if proportional to the square of the current, would be 0.1 percent at $10,000 \mathrm{amp}$. It seems probable that the effect is the result of mechanical strain set up in the resistance alloy sheets by their differential expansion relative to the cooler rigid copper terminal blocks. Pending the construction of an improved high-current resistor free from such effects, the upper end of the precise scale of direct currents must be considered as tapering off from an accuracy of 0.01 percent at $3,000 \mathrm{amp}$ to 0.1 percent at $10,000 \mathrm{amp}$. It apperrs that there would be no serious difficulty in constructing a high-current resistor that could extend the highaccuracy limit well above 10,000 amp, if a need for such measurements should arise. The capacity of the storage batteries presently available at the National Bureau of Standards permits a current to be held at this latter value for about $1 / 2$ hour. Figure 17 shows this high-current circuit.

\subsection{The Scale of Alternating Current}

The electrothermic transfer ammeter's offer a convenient means for measuring with high accuracy alternating currents down to 1 ma at frequencies up to $20 \mathrm{kc} / \mathrm{s}$. Lower values are readily derived by using a resistive shunting network, the ratio of which is deduced from the resistance of its parts. With such networks very low currents can be produced and used to calibrate a-c amplifiers. The amplifiers in turn can be used to measure unknown currents of low value. 'The principal limitations in such work arise from superposed noise and instability in amplifier gain rather than from any difficulty in correlating the results with the ampere.

In the early years of the Bureau the scale of alternating current was extended upward by using a group of astatic electrodynamic instruments [175] having suspended moving coils and fixed coils of cable formed of insulated strands of fine wire (litzendraht). 'The highest rated (5,000 amp) instrument was a "tubular" electrodynamometer" [176] in which the fixed "coil" consisted of two coaxial copper tubes. With the coming into general use of current transformers [227], the number of highrange, self-contained ammeters, wattmeters, and watthour-meters submitted for test has fallen to almost zero. The old high-range standard instruments have been discarded, and when occasionally a high-range instrument is tested, the measurements are made with the 5 -amp standard transfer instrument and a carefully calibrated current transformer.

The ratio and phase angle of a current transformer depend to a very appreciable extent on the conditions of burden and frequency at which it is operated and, to a much less extent, on the arrangement of the primary leads. The extension of accurate measurements to large alternating cur-

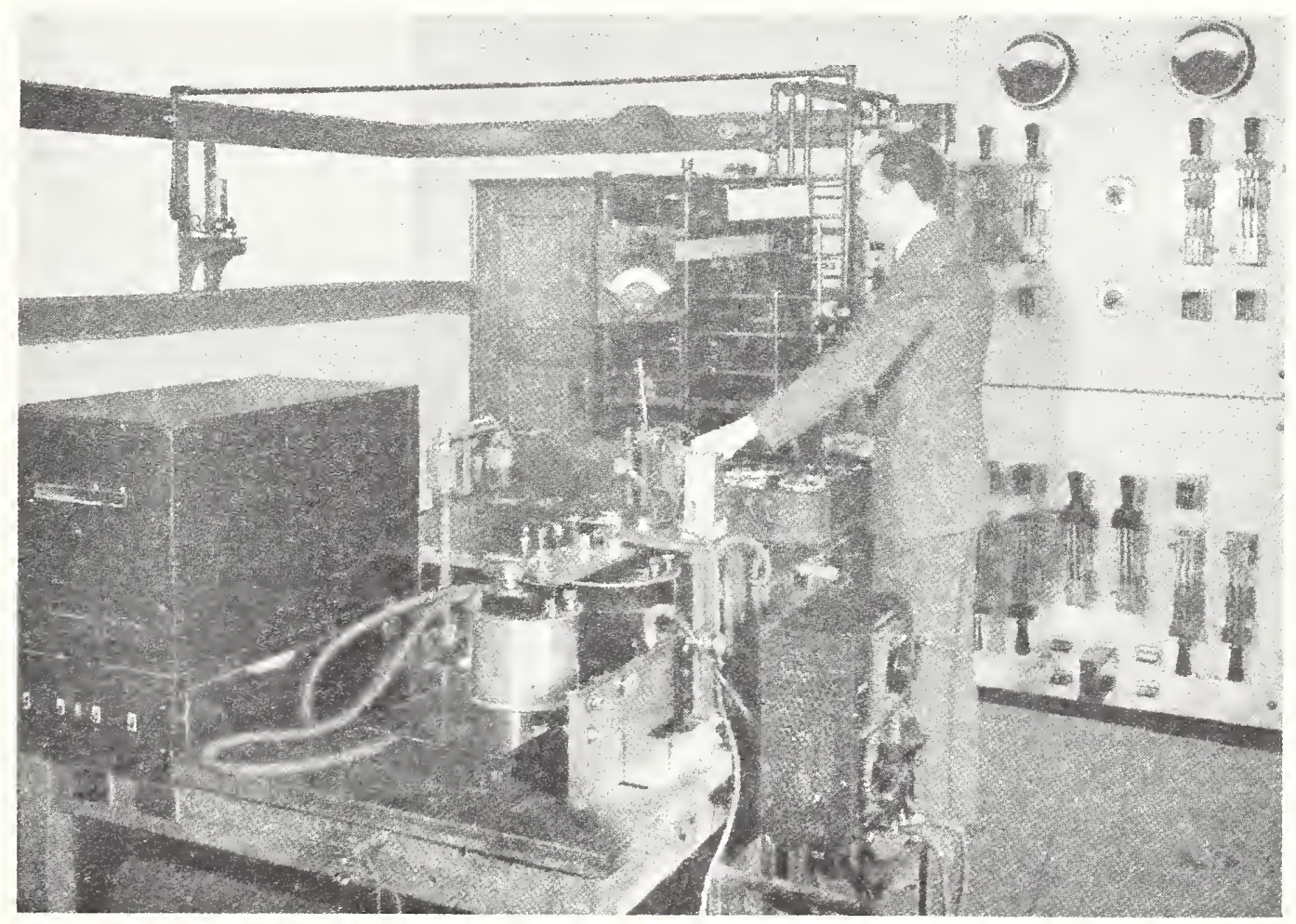

Figure 17. Equipment for large direct currents.

Large switehes eonnect cell groups in series or parallel. Thr water-cooled rheostat in baekground and fine rheostat in front adjust eurrent. Standard resistors are in cylindrical oil tanks. Black transite box at left is used for tests at elevated temperatures. 
rents has therefore required the careful study of the performance of current transformers [211, $225,226]$ and the development of methods for determining their ratio and phase angle with high accuracy $[210,213,217]$. It happens that it is much easier to compare the relative performances of two transformers of the same nominal ratio than it is to determine the ratio and phase angle of a single transformer [215, 216]. Hence many public utility companies and manufacturers of electric machinery submit to the National Bureau of Standards for test groups of transformers of assorted current ratings which they expect to use as standards for the testing of other transformers of the corresponding ratings which they regularly use for measurement purposes.

The circuit used in testing current transformers [224] up to primary ratings of $2,500 \mathrm{amp}$ is shown

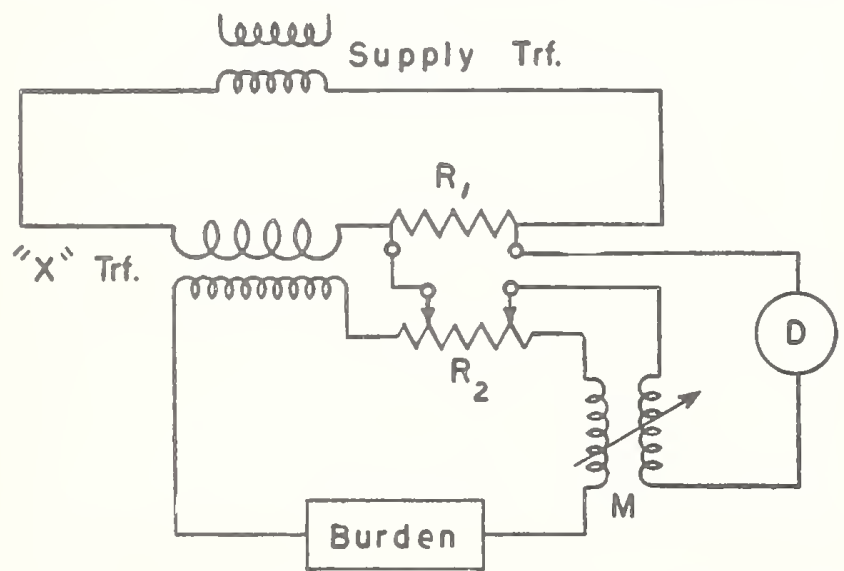

FIGURE 18. Circuit for testing a current transformer by the resistance method.

in figure 18. Here $R_{\mathbb{1}}$ is a resistor of high currentcarrying capacity and of low and known inductance; $R_{2}$ is a four-terminal resistor adjustable from 0.01 to $0.1 \mathrm{ohm}$ of 5 -amp rating and known low inductance; $M$ is an adjustable mutual inductor continuously adjustable to $50 \mu \mathrm{h}$ that serves to measure the phase-angle between the primary and secondary currents. When the detector, $D$, shows a balance the ratio of the transformer is equal to $R_{2} / R_{1}$, and its phase angle can be computed from the setting of $M$. A limiting factor in this method has been the determination of the effective four-terminal self-inductances of the resistors used as $R_{1}[128,129]$. The oil-cooled resistors rated at 2,500 amp and at 1,000 amp are of tubular construction, and their inductance can be computed from their dimensions. The air-cooled resistors for currents of 500 amp and less are formed of flat strips and have their potential leads so placed that their mutual coupling to the strip largely compensates for the self-inductance of the latter. The small residual inductances are measured by a substitution process that compares them with those of a group of special resistors constructed so as to attain accurately computable inductances, although at the sacrifice of current capacity and stability of resistance.
For primary currents exceeding 2,500 amp use is made of a general principle in instrument transformer design, namely, if the primary winding of an instrument transformer consists of two or more sections, all having the same number of turns, then at the same frequency, secondary burden, and secondary current (or voltage) the ratio correction factor (i. e., the quotient of true ratio divided by nominal ratio) and the phase angle will be the same, to a high degree of precision, whether the primary sections are connected in series or in parallel. In other words, the core and secondary winding cannot distinguish between the additional magnetomotive force caused by the same current linking the core in an additional series turn and that caused by additional current linking the core in a parallel turn. Deviations from this principle will arise, for a current transformer, only in proportion to the product of two factors each of which is unlikely to be large: (a) the relative difference in the distribution of current among the several primary sections when they are in parallel, and (b) the relative difference in the coupling (mutual inductance) between each of the several primary sections and the secondary winding. If their relative differences do not exceed 1 percent each, the principle will be valid to 0.01 percent.

To apply this principle, a special high-range standard current transformer having eight primary ranges has been constructed. This has 24 sections of primary winding, and with these in series, can be calibrated by the usual resistance method at ratings from 500 to $2,500 \mathrm{amp}$. It is then used with its primary sections in parallel as a standard up to 12,000 amp. A careful study [224] of its performance has shown that the equality of the coupling of the various sections of the secondary and the equality of the division of current among the primary sections are both satisfactorily close. When the transformer is measured with only 500 secondary turns (i. e., 2,500 amp turns at full current) and with each of the eight possible connections of its primary turns, its ratio correction factor at any secondary current was constant to 0.02 percent and its phase angle to 1 minute, and when operating at its normal 12,000 amp turns, the performance of the transformer is presumably even better. Even if the flux distribution is greatly distorted by omitting one of the primary turns, the ratio correction factor is affected by less than 0.01 percent.

It seems probable that serious difficulty will be encountered in an attempt to construct noninductive primary resistors for sustained currents exceeding 2,500 amp because the lack of chemical uniformity in the resistance alloy will affect the distribution of current and thus the inductance. However, there is every indication that the measurement range at power frequencies can be extended well above 12,000 amp by the use of multirange standard transformers. 


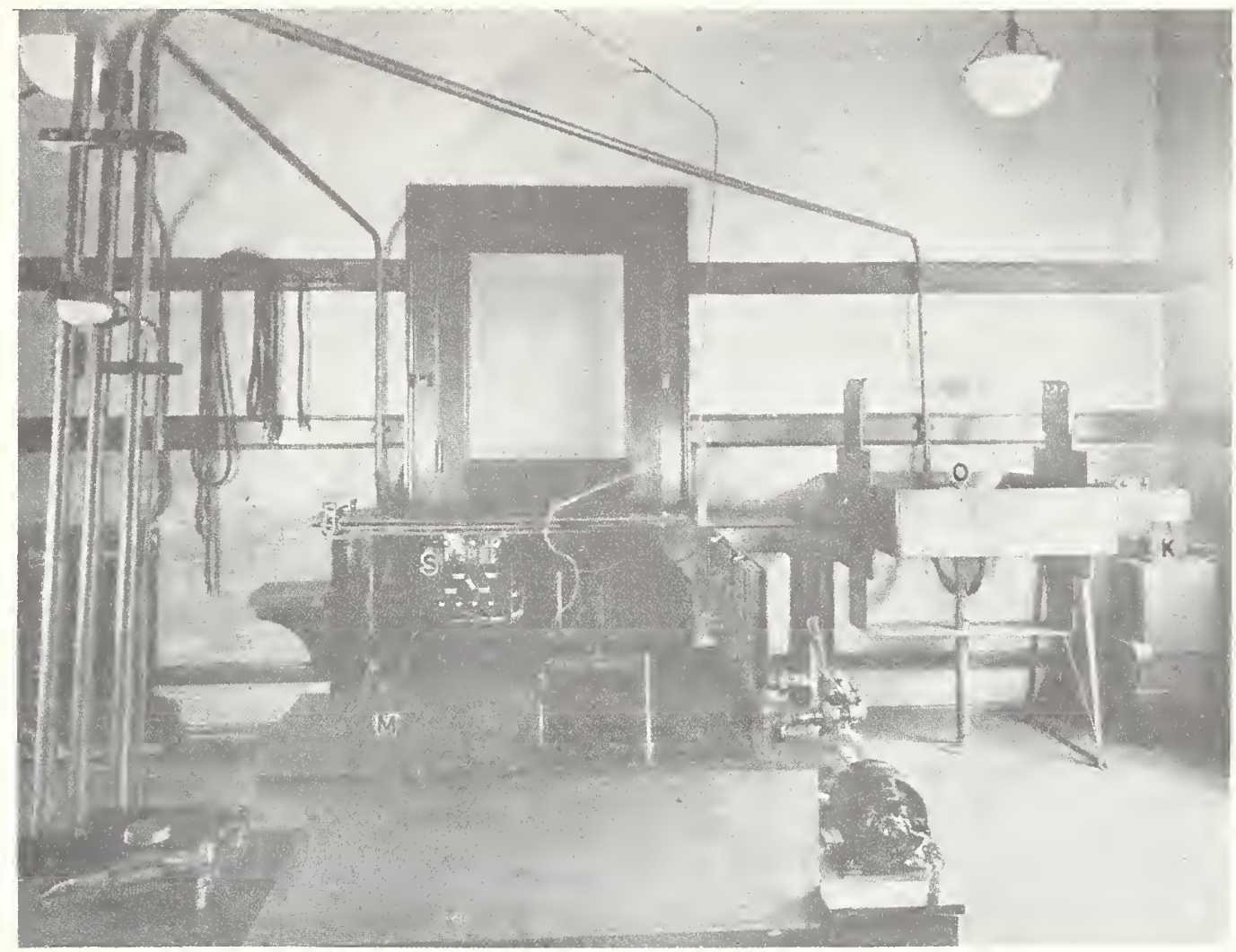

Figure 19. Equipment for testing high-range current transformers.

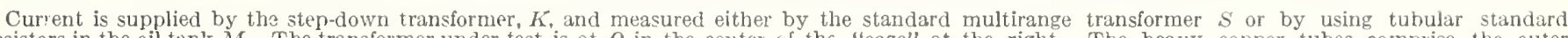

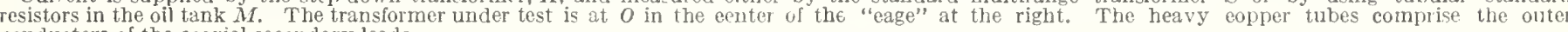
conduetors of the coaxial secondary leads.

Figure 19 shows the circuit used in measurcments from 500 to $12,000 \mathrm{amp}$. The transformer' under test is placed at the right in the "cage" formed by the four 10 - by $1 / \frac{1}{4}$-inch copper bars, which, in parallel, constitute the "return" lead of the circuit. The central "outgoing" lead either" passes through the window of the transformer or is bolted to the transformer primary terminals if it is of the bar type. This circuit geometry [187] offers two advantages. The first is that the components of magnetic field at the transformer' in the center, produced by the action of the four return bars cancel one another so that the resultant effect is closely the same as if the return circuit were at infinity. This situation constitutes a logical basis with which the effects of other configurations can be compared $[225,226]$. The second advantage is that the resultant magnetic field of the entire circuit drops off very rapidly with distance from the axis (roughly as $r^{-5}$ ) and is negligible in those parts of the laboratory where the measuring circuits are located.

Most of the transformers submitted for test are intended to be used as standards with which to compare the performance of other current transformers. Such comparisons are usually made by means of the Silsbee current transformer testing set, the circuit of which was developed at the Bureau in 1918 [216]. In the use of this set the measuring elements are required to measure merely the difference in magnitude and phase of the secondary currents of the two transformers being compared. 'Therefore, a high accuracy can be obtained in their relative ratios, although only moderate accuracy is required in the adjustment of the circuit constants. Nevertheless, as a check on possible incorrect adjustment at the factory or on major drifts in calibration, such transformer test sets are frequently submitted to the National Bureau of Standards for test. The test consists in the measurement of the resistance of the slide wire at 13 points on the ratio scale and of the mutual inductance of the inductor at 15 points on the phase-angle scale. The test set is also experimentally checked in actual operation at midscale and one other point. The true values of transformer ratio and phase angle are then computed and tabulated against the corresponding settings used in the test.

\subsection{The Scale of Alternating Voltage}

Between $1 \mathrm{v}$ and $300 \mathrm{v}$, two alternative bases are available for the measurement of alternating voltages. The first, applicable at power frequencies, is to use the electrodynamic transfer voltmeter, described in section 4, to transfer from direct to alternating voltage at the $100-$ or $200-\mathrm{v}$ level, and then to use a three-winding step-down transformer. 'The ratios of the voltages of the various sections of the two secondary windings to each other are checkerl by comparison with a 
special resistive voltage divider. Normally, one winding supplies the standard transfer voltmetèr, already calibrated with direct voltage, and the other tapped secondary supplies the a-c voltmeter under test. Such a transformer has a very constant ratio over a wide range of primary voltages.

The second procedure, applicable also at audio as well as at power frequencies, is to use the electrothermic transfer voltmeters directly to fill in the scale from 1 to $750 \mathrm{v}$.

Below $1 \mathrm{v}$, known voltages ean be obtained by using resistive voltage dividers or attenuators that have been checked at somewhat higher voltages against the standard volt box or by measuring the resistances of their component parts. "The low voltages thus derived, down to about $20 \mu \mathrm{v}$, can be used to check low-range electronic voltmeters and as stantard signals for testing amplifier gain. To measure an unknown voltage in this low range, the known low voltage with properly adjusted phase, can be bucked against the unknown. The difference, after amplification, is applied to a detector, and the known low voltage is adjusted to balance the unknown.

From about 300 up to $250,000 \mathrm{v}$ almost all precise a-c voltage measurements make use of step-down voltage (potential) transformers [227]. The Bureau has contributed to the establishment of this alternating-voltage seale by developing methods and equipment for measuring the ratio and phase angle of voltage transformers, by suggesting methods for the comparison of the performance of a voltage transformer with a stantard transformer of the same nominal rating $[214,215]$, and by its testing service for calibrating such standard transformers and comparison test sets [229].

The use of standard voltage transformers as a basis for the checking of others of the same nomiral ratio is even more desirable than the analogous use of standard current transformers both (1) because very simple apparatus suffices for the comparison $[214,228]$ and (2) because of the greater safety when the operator is isolated from the high-voltage circuit by the transformer insulation and need handle only cireuits conneeted to the low-voltage secondary windings.

It is almost universal practice to use voltage transformers with one end of the primary winding at ground potential. However, some unpublished experimental studies at the Bureau have indicated that, with transformers of normal construction, the ratio and phase angle are affected very little, even if the transformer is used with its primary across the limes of a three-phase, grounded neutral system while its secondary is kept grounded.

It is common practice in the electric-power industry to use portable commereial voltagetransformer test sets [228] to compare the secondary voltages of the standard and test transformers. These sets in turn are often submitted to the National Bureau of Standards for test to verify

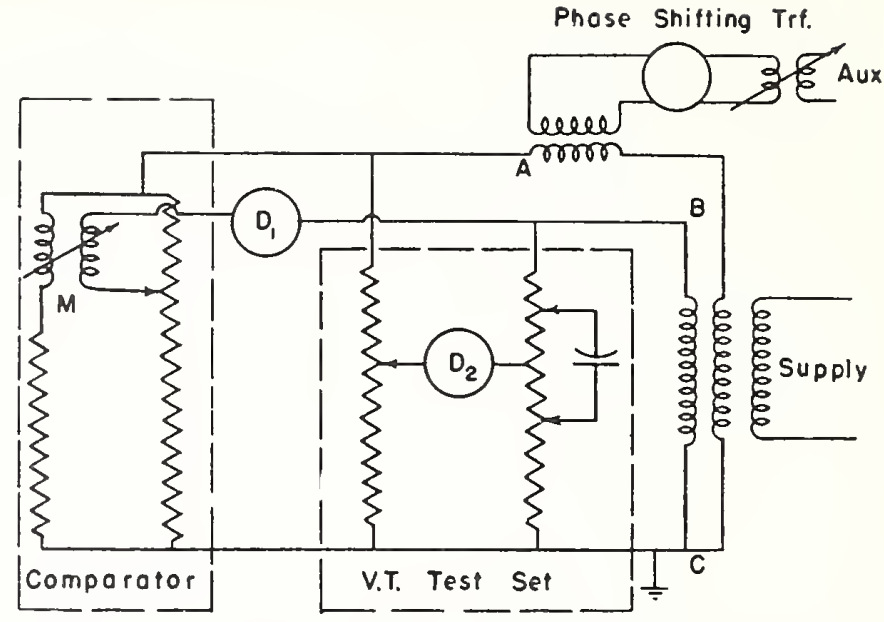

Ficure 20. Circuit for testing voltage-transformer test sets.

Voltage $A C$ is supplied to both the comparator and the "Standard" cir. cuit of the test set. Voltage $E C$ is applied to the " $X$ " circuit of the test set By adiustmelt of the magnitude and phase of the auxiliary supply, voltage $A C$ may be set with any desired plase and magnitude relative to voltage $R C$.

the accuracy of their adjustment. This is done by supplying both the set under test and a special voltage transformer comparator from a pair of 60 -cycle voltages (a-c and $d-c$ ) which differ in magnitude and phase by adjustable amounts (see fir. 20). The set under test is set on a succession of 11 points on its ratio scale and 13 points on its phase-angle scale, and also as a check at the four "corner" points correspond ling to combinations of the extreme settings of the two seales. The true voltage relations are read on the comparator for each point, and the resulting colrections are reported in tabular form.

The comparator is also used to compare transformers submitted for test with one of the standard transformers owned by the Bureau. 'These seven standard transformers have the ratings shown in table 1. Their ratios and phase angles are determined with great care at intervals of about a year by the methods outlined in the following paragraphs, and have been found to be very stable. By using them as intermediate working standards, the need for operations involving direct metallic connections between the observer and the highvoltage circuit is limited to the annual performanee checks.

TABLE 1. Ratings of standard vollage transformers

\begin{tabular}{|c|c|c|c|}
\hline Disignation & Primary volts & $\begin{array}{c}\text { Secondary } \\
\text { rolts }\end{array}$ & $\begin{array}{l}\text { Kilovolt- } \\
\text { ampere } \\
\text { rating }\end{array}$ \\
\hline 21,213 & $50 M 0 / 2500 / 1250$ & $255 / 127,5$ & 2 \\
\hline 34.313 & $25,000 / 12,500 / 6,250$ & $255 / 127.5$ & 3 \\
\hline $10 \mathrm{~A}, 10 \mathrm{~B}$ & $100,0000 / 50,0(0) / 25,000$ & $255 / 127.5$ & 10 \\
\hline $100 \ldots$. & $250,0006 / 125,000$ & $255 / 127.5$ & 100 \\
\hline
\end{tabular}

Up to $30 \mathrm{kv}$ the standard transformers are measured by the use of a shielded resistor [220] that has a total resistance in its working circuit of 512,000 ohms. All, or part, of this may be connected in parallel with the primary of the transformer under test, as shown in figure 21. The 


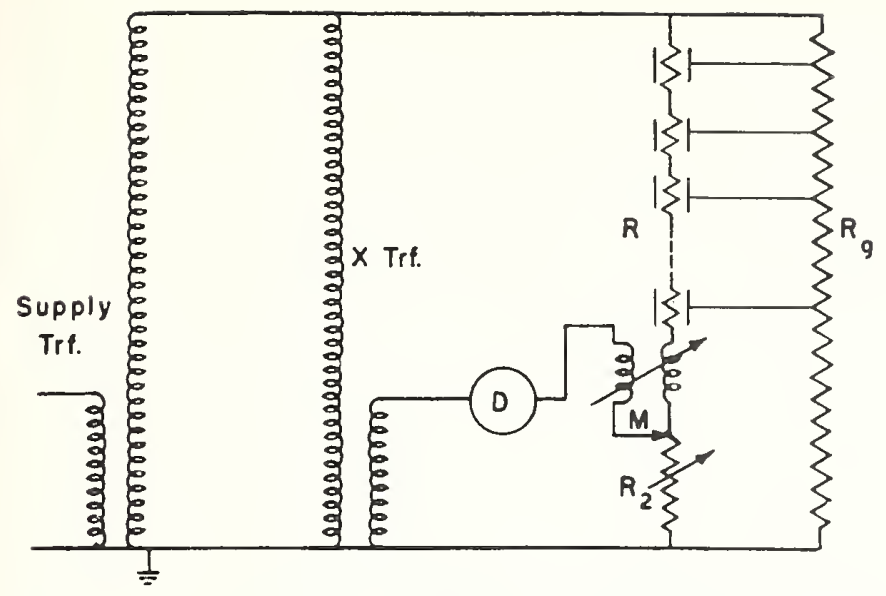

Figure 21. Circuit for testing a vollage transformer by the use of a shielded resistor.

secondary roltage of the transformer is the'n balanced by the potential diop in an adjustable portion, $R_{2}$, of the resistor cirenit at its grounded encl. A mutual induetor, $M$, which has its prinary in series witle the resistor and its seeondary in series with the detector, serves to balanee out any phase displacement between primany and secondary voltages. The prineipal souree of erros in sucle in method is the capacitance to ground of the various sections of the resistor'. To minimize this error, the working cirenit is divided into sections of 20,000 olms cach, and cach section is enclosed in a metal shicled. Earde shiclel is connected to an appropriate tap point on an auxiliary high-resistance guard circuit, $R_{g}$. This guard circuit, also of about 500,000 olms, is in parallel with the working eircuit, and its taps are adjusterl to be at the same potentials as the midpoints of the sections of the working circuit they shielel. The capacitance eurrents from the shields to ground therefore flow in the guard eireuit only and produen only a second-order error in the working circuit. With such an arrangement the secondorder error increases roughly as the fourth power of the primary voltage, with the result that the method becomes rather impractical above $30 \mathrm{kv}$.

Above $30 \mathrm{kv}$ several procedures are possible. One is the use of a capacitance voltage divider as used by Sharp and Crawford [222] and in more convenient form, by Bousman and Ten Broeck [223]. Such a circuit is now under development at the National Bureau of Standards as a further check on the performance of the standard multirange voltage transformers that are currently in use to carry the testing service to $250 \mathrm{kv}$.

These multirange voltage transformers constitute another'application of the general principle enunciated earlier, which forms the basis for extending the range of alternating current. Each is made with its high-voltage winding in several sections that can be connected in parallel for calibration and in series for use as a high-voltage standard transformer. Ideally, such a transformer

\footnotetext{
4 By using an autotransformer of high rating (75 kva) to supply the shields
} Weller [221] found it possible to push this method to $132 \mathrm{kv}$. would have the same phase angle on all ranges, and its ratio would be strictly proportional to the number of turns in series on each range. Departures from this ideal might result eithel": (1) from inequalities in the magnetic coupling from the several primiry sections to the secondary or (2) from the presence of capacitance currents flowing between portions of the primary eircuit in a different manner when different connections are used. The applieability of this principle has been demonstrated in a large number of multirange transformers in which both the higher and the lower ranges coukl be tested by the use of the $30-\mathrm{kr}$ shielded resistor [193, p. 327]. Auxiliary measurements of equality of seetions and of eapacitance between sertions indicate that the departures from the ideal are negligible in the two higher-lange transformers used in the extemsion of the Bureatu's seale of alternatine voltage. The first of these transformer's is calibrated with its four sections in pallallel up to $30 \mathrm{kr}$ and used with them in series up to $120 \mathrm{kr}$. The second is eompared with the first up to $120 \mathrm{kr}$ and used with its two sortions in series up to $240 \mathrm{kr}$. Figure 22 shows some of the standard transformers and the shidded resistor.

The chain of measurement of voltage from the

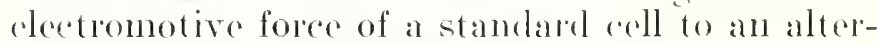
nating voltage of 240,000 is a long one of many links, and it was folt clesirable to check the result by some independent method. For this purpose

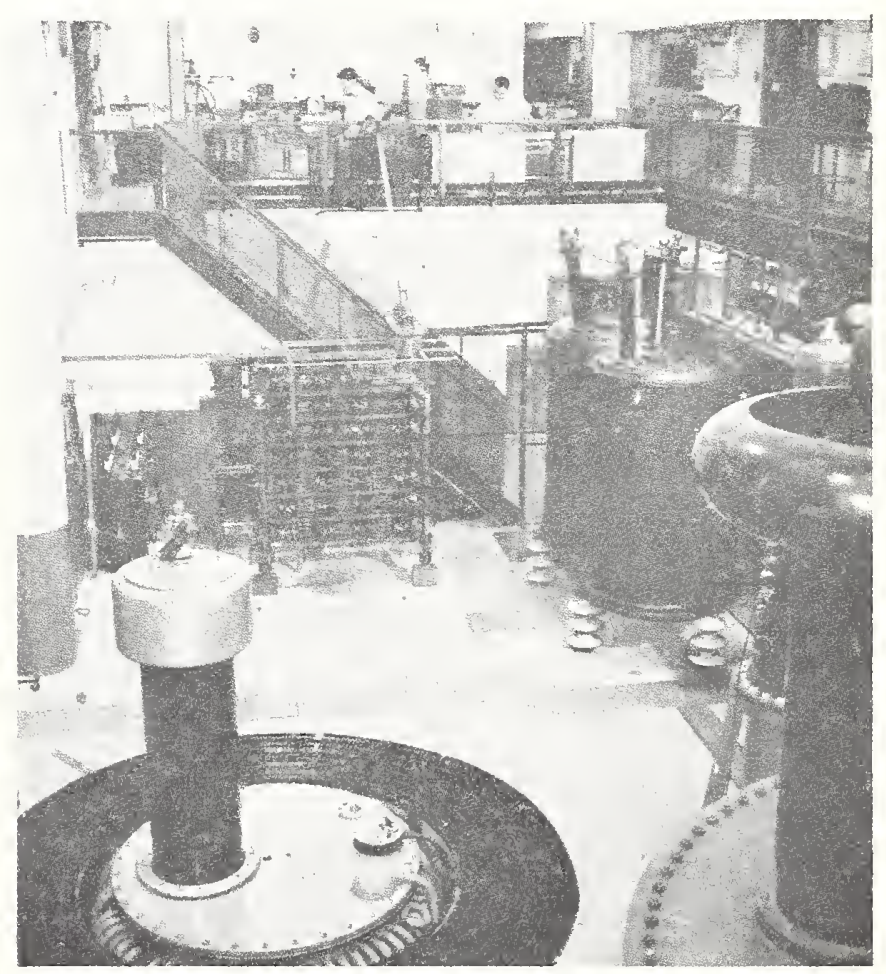

Figure 22. Ioltage-transformer testing bay in National Bureau of Standards High-Toltage Laboratory.

The $30-\mathrm{kv}$ shielded resistor is in the center at the back; standard trans The $30-\mathrm{kV}$ shielded lesistor is in the center at the back; standard trams-
formers for $25 \mathrm{kv}$ are at the left and for $100 \mathrm{kv}$ at the right (on insulating supformers for $25 \mathrm{kv}$ are at the left and for $100 \mathrm{kv}$ at the right (on insulating sup-
ports). The observers on the mezzanine are setting the burden and balancing the secondary voltage against the $1 \mathrm{R}$ drop in the grounded section of the resistor. The large $350-\mathrm{kv}$ transfcrmer in the pit in the left foreground can be used as a supply. One bushing of the $125 / 250-\mathrm{k} v$ standard transformer shows at the right edge. 
an absolute electrometer of the attracted-disk type suitable for voltages up to $275 \mathrm{kv}$ was designed and constructed [192]. In this instrument a light Duralumin disk (16 cm in diameter) hangs from one arm of a sensitive balance and is centered with small clearance in an opening in the center of a large guard ling. A flat circular plate is supported below the disk and guard ring, parallel to them and at a distance that can be adjusted over a range from $2 \mathrm{~cm}$ for low voltages $(10 \mathrm{kv})$ to a maximum of $110 \mathrm{~cm}$ for $275 \mathrm{kv}$. The rms value of the voltage applied between the disk and the lower plate can be computed in electrostatic units from the meas. ured diameter of the disk, the spacing between it and the plate, and the force of attraction, which is weighed by the balance. Figure $2: 3$ shows the general arrangement of the electrometer, and figure 24 shows some details at the balance.

A long series of experiments [193] was performed in 1936 in which an alternating voltage (usually of $60 \mathrm{c} / \mathrm{s}$ ) was measured simultaneously with the olectrometer and with a sensitive electrodynamic voltmeter fed by one of the calibrated standard voltage transformer's. 'The conditions were varied from one experiment to another by changes that

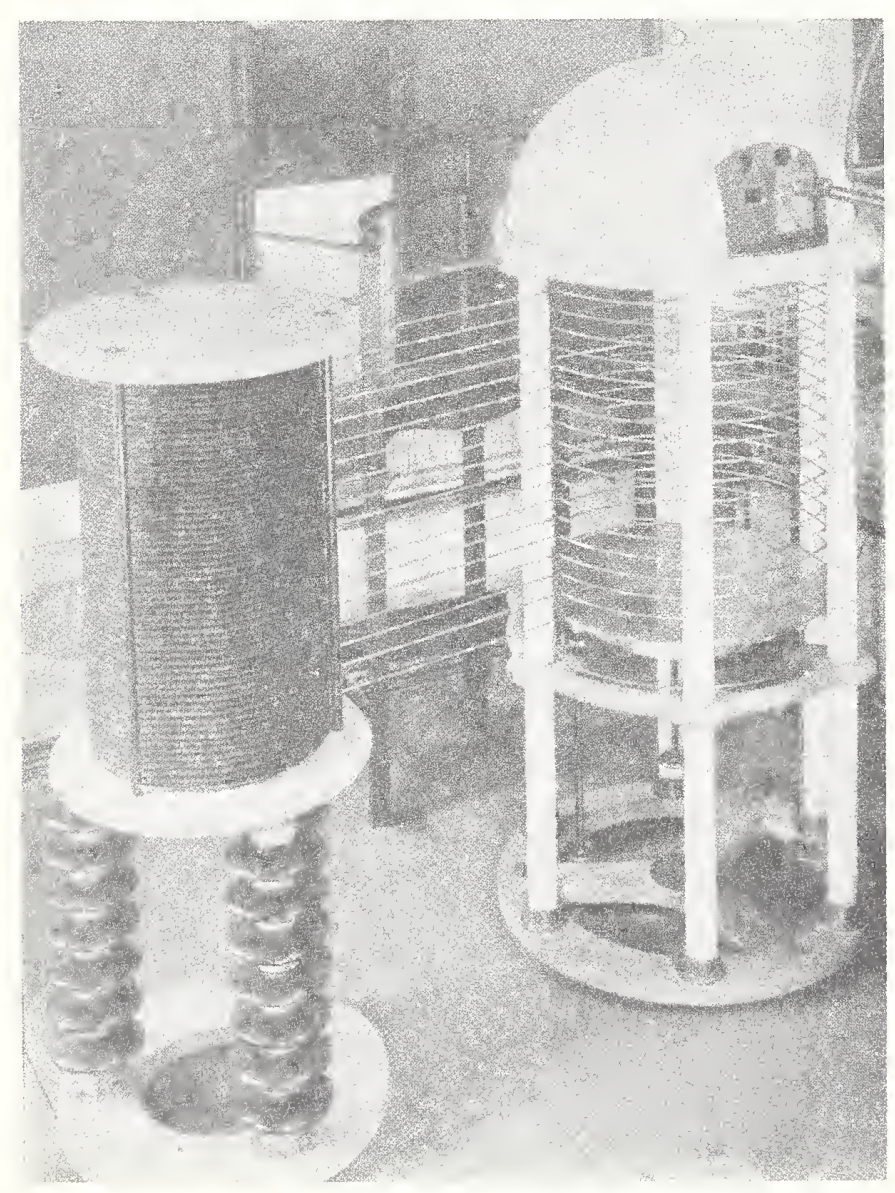

FIGURE 23. The Brooks absolute electrometer.

The attracted disk hangs flush with the lower surface of the upper plate. It and the halance are shielded from extraneous forces by the dome. The lower plate is carried by three rods from the intermediate ring and can be adjusted for any desired vertical spacing. The hoops are suppcrted by pegs inserted into the fused silica pillars and are cnergized by taps from the stack of micanite capacitors at the left. As shown, only cne-third of the normal complement of hoops are in place. involved different transformer connections, electrometer spacings and disk diameters, frequencies $(60 \mathrm{c} / \mathrm{s}$ and $25 \mathrm{c} / \mathrm{s})$, wave forms and magnitudes $(10,000$ to $100,000 \mathrm{r})$ of the voltage measured, and changes in the potentiometer circuit used to calibrate the voltmeter. The two methods were found to agree with an average discrepancy without regard to sign of only 0.01 percent. The correctness of the extension of the a-c voltage scale by the use of voltage transformers is thus confirmed by an entirely independent method. Because of the much greater convenience of the transformer method, it will be used almost exclusively in the future. It is planned, however, to reassemble the absolute electrometer again and to make a similar cross check between the two methorls at the 275-kv level.

The interrelations of the two methorls in ter'ms of the present absolute svstems of units is shown in figure 25. It will be seen that both methods use a mass subject to local gravity as the measure of foree (at $\mathrm{F}$ ) and that the electrometer and the ("mrent balance oecupy corresporiding positions, each involving only the ratios of its significant dimensions.

In the electrostatic method. the conventional constant, $\epsilon_{v}$, usually designated as "the permittivity of space," is arbitrarily chosen as 1 (in the centimeter-gram-second-electrostatic system). On the other hand, in the electromagnetic method, it is the conventional constant, $\mu_{v}$, usually designated as "the permeability of space," that is chosen arbitrarily either as 1 (in the cgs-emu system) or as $4 \pi \cdot 10^{-7}$ (in the rationalized mlisa system).

The absolute onm is evaluated by a separate process, from the units of length and time and the chosen value of $\mu_{v}$. Combining the absolute ohm with the absolute ampere, evaluated by the current balance, fixes the absolute volt, in terms of which values are assigned to NBS standard cells. The long chain of step-up and d-c to a-c transfer procedures then yields the electromagnetic value for a high alternating voltage shown at $A$. The rlectrometer yields directly at $B$ the value of the same voltage in statrolts. Aceording to electromagnetic theory $\epsilon_{v}$ and $\mu_{v}$ are not independent, but are connected (as indicated by the dot-dash lines in figure 25) by the relation

$$
\mu_{v} \epsilon_{r}=1 / c^{2},
$$

where $c$ is the velocity of light. This velocity has been measured by optical experiments at very high frequencies and at the NBS by Rosa and Dorsey $[28,29,30]$ at low frequency. The two very different types of experiment gave results in satisfactory agreement and are indicated by the vertical dashed line in figure 25. Accordingly, in the verification of the high-voltage scale, the electrometer results (at $B$ ) were multiplied by this factor (299.805 megameters/sec) and compared with the transformer results (at $A$ ). The optical experiments are presumably of materially higher 


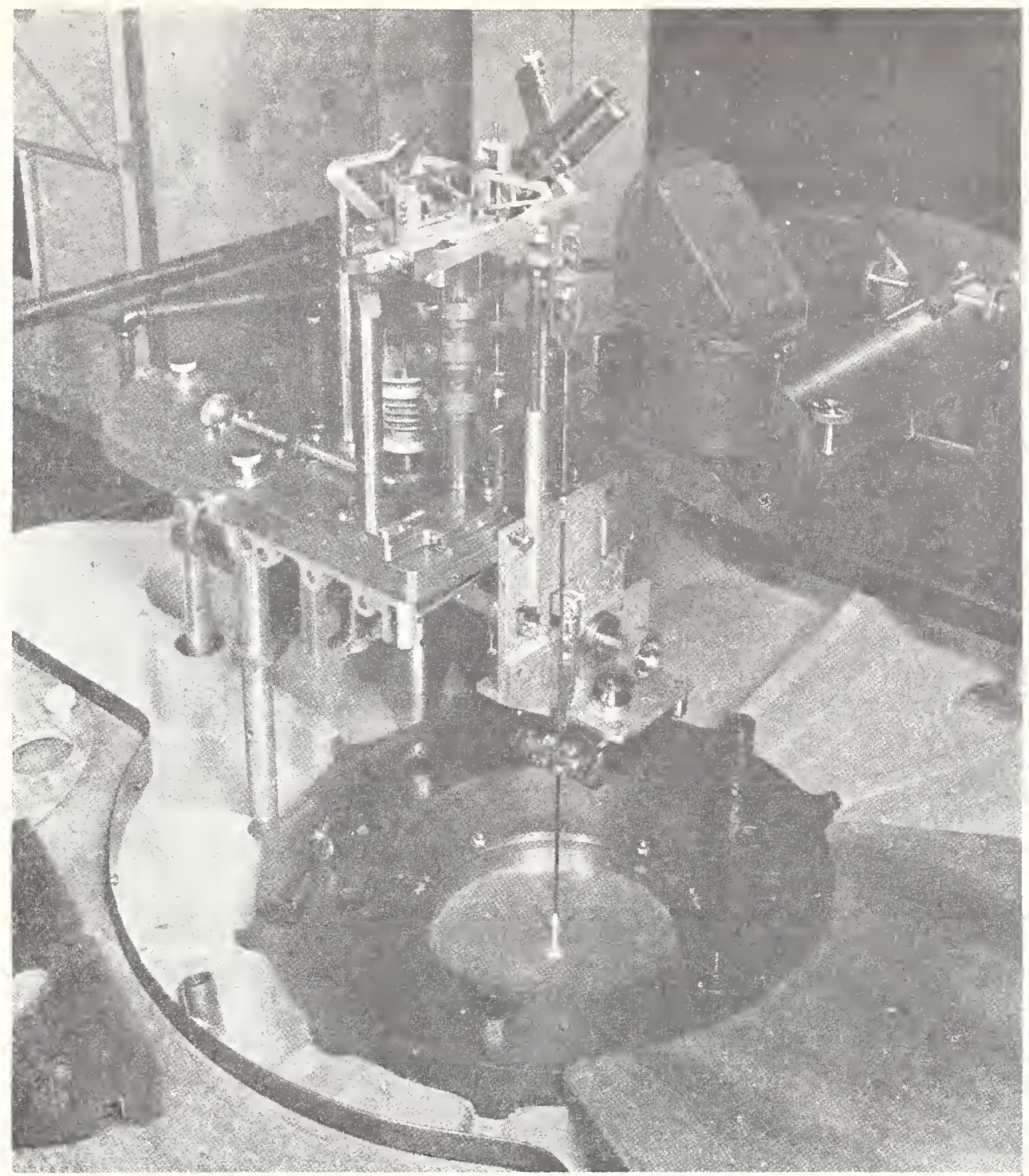

FIGURE 24. Balance and attracted disk of the Brooks absolute electrometer.

Disk is shown with draft cover removed; above it are the Poynting clamp and the stirrup for the measuring weights; the compensated counterweight and the "chainomatic" chain hang from the other end of the beam; the optical path is enclosed in the black tubing and prism boxes.

accuracy, but the slight observed residual difference in the voltage measurements might be regarded as an independent determination of $c$ as being 299.783 .

\subsection{Measurement of Crest and Surge Voltage}

In the higher voltage range, engineers are usually more concerned with crest values than with rms values of alternating voltages. This is because the breakdown of insulation is to a large extent dependent upon the crest value of the voltage applied to it, and the testing of insulation is the principal application of voltages above the normal operating levels. The commonly used measurement technique in this field is to note the sparkover voltage between metal spheres of known diameter and spacing in air under standard test conditions. The standard currently recognized in the United States is that of the American Institute of Electrical Engineers [202], which is based on the pioneer work of the large electric manufacturing companies. The values in it differ somewhat from those in the corresponding standard of the International Electrotechnical Commission, which is generally used in Europe. One of the projects still ahead of the National Bureau of Standards is a study of the spark-over voltage of standard sphere gaps with a view to reconciling the differences and perhaps improving the accuracy of measurement. Preliminary experiments have indicated the feasibility of determining the crest factor of an alternating voltage by using a capacitance potential divider and a point-by-point measurement of wave form with a quadrant electrometer as a detector. This method is slow but capable of an accuracy better than 0.1 percent; it will form the basis for calibrating other techniques.

An alternative method for measuring cresit volt- 

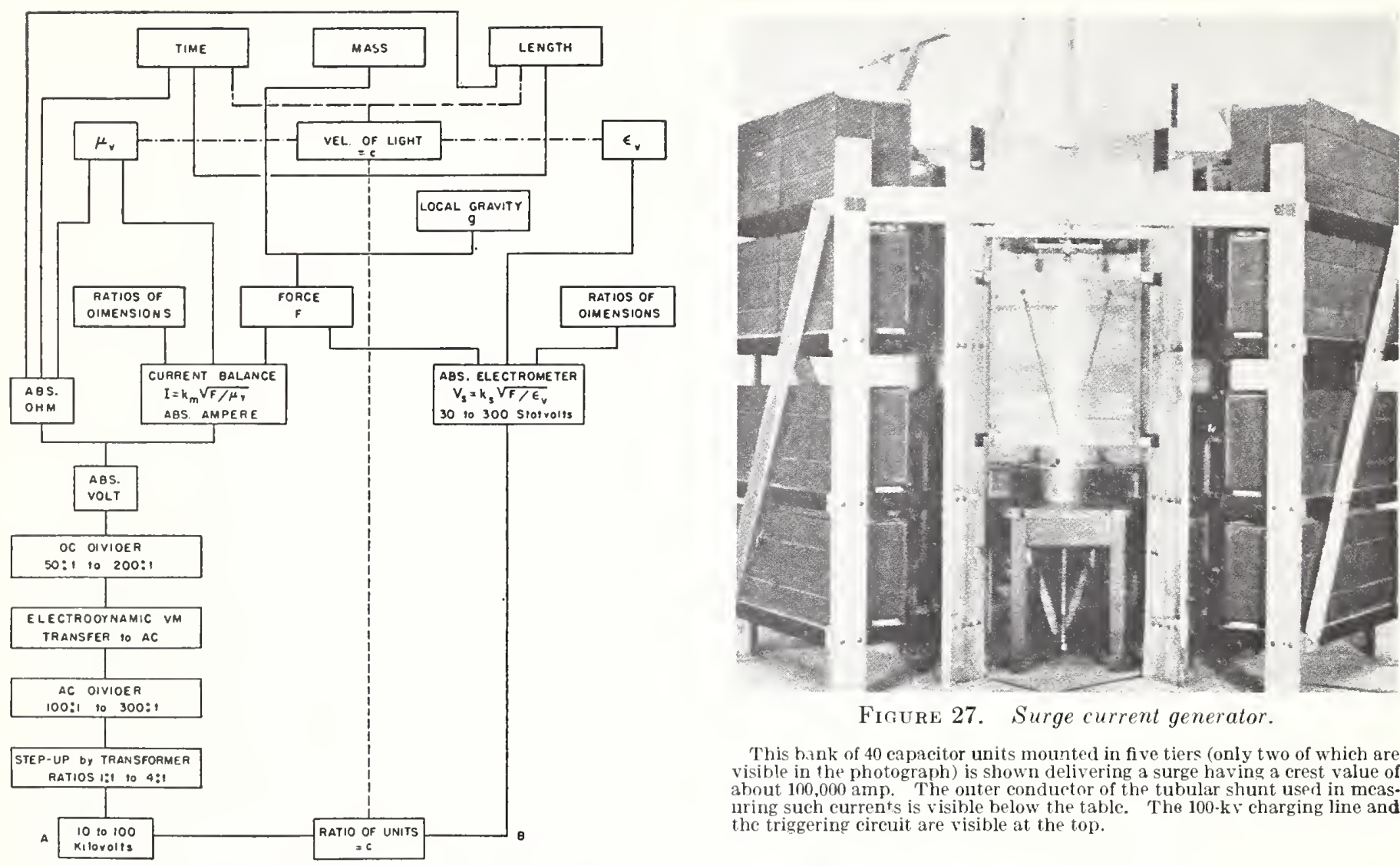

Figure 27. Surge current generator.

This bank of 40 capacitor units mounted in five tiers (only two of which are visible in the photograph) is shown delivering a surge having a crest value of about $100,000 \mathrm{amp}$. The onter conducter of the tubular shunt used in meas. uring such currents is visible below the table. The 100-kv charging line and the triggering circuit are visible at the top.

FIGURE 25. The high-voltage scale and its confirmation by the absolute electrometer.

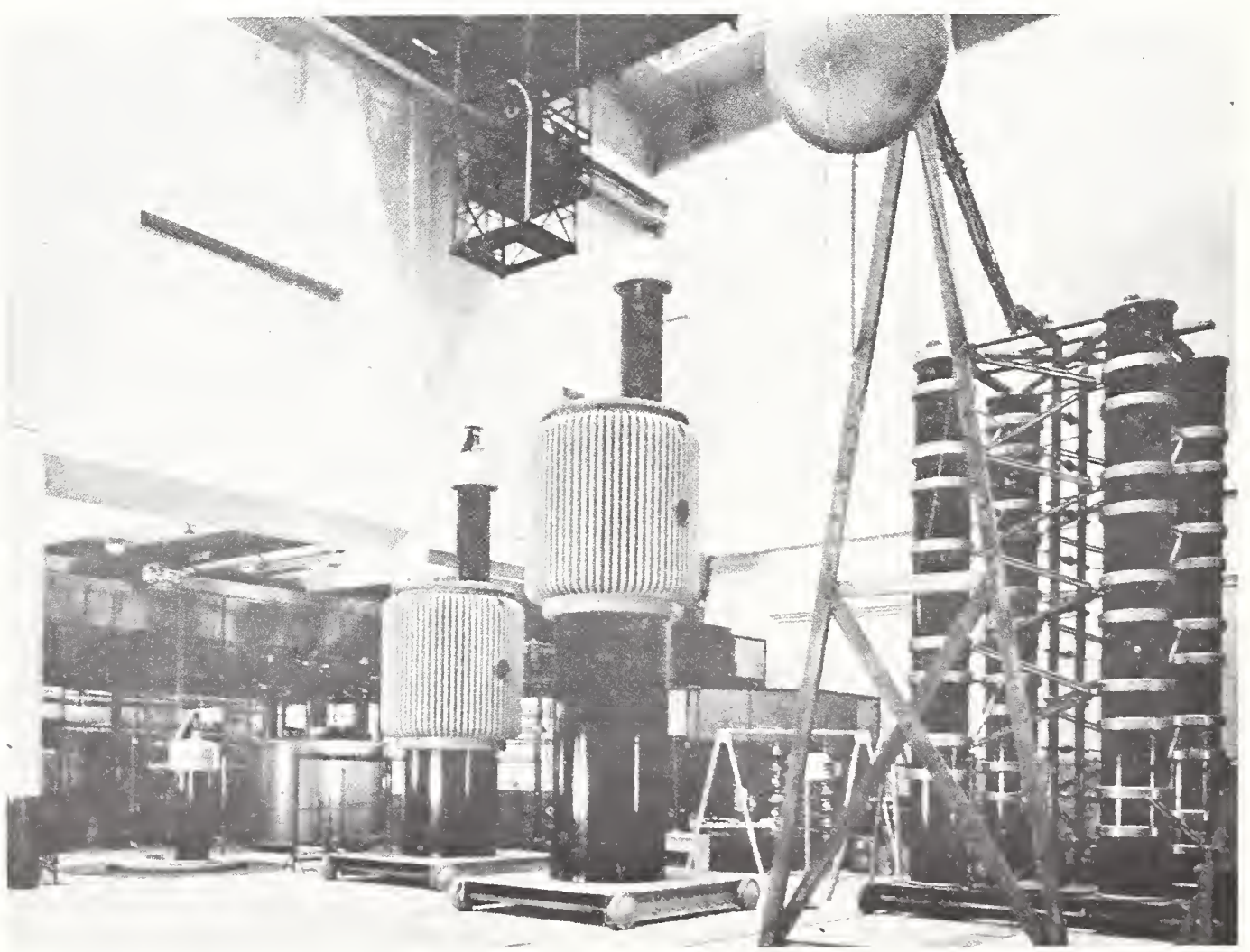

Figure 26. View in Iligh-Voltage Laboratory at National Bureau of Standards.

The four columns of capacitors at the right constitute the 2,000,000-volt surge-voltage gencrator; the three 60 -cycle 350,000 -v transformers (one is in

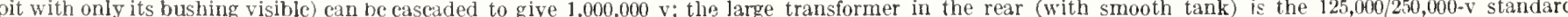
voltage transformer. 
age is the corona voltmeter of Whitehead. This was studied by the Bureau in 1928 and its possibilities and limitations examined [194].

A closely related field is the measurement of surge voltages and currents. In recent years there has been a decided trend toward the use of transient voltage surges ("artificial lightning") for the proof-testing of electric apparatus. The reasons for this are twofold. First, the duration of such a test and the distribution of electric stress within the apparatus during the test can be made to approximate the extreme conditions to which the equipment will be exposed in actual service, and the results are therefore more significant than those of a test at an abnormally high value of sustained alternating voltage. Second, in spite of its complicated construction, the cost of such test equipment is usually less than that of an equivalent conventional transformer. Figure 26 shows the 2,000,000-volt 33,000-joule surge-voltage gen- erator now in use at the National Bureau of Standards for studying methods of surge-voltage measurement, and figure 27 shows the 50,000 -joule surge-current generator. This has a rating of 10 $\mu \mathrm{f}$ at $100,000 \mathrm{v}$ and has delivered currents of 200,000 amp.

The complications which arise from inductance and skin effect in measuring transient currents are closely related to those encountered on the precise measurement of the phase angle of current transformers. By applying similar procedures, standard forms of shunt and of inductor have been developed [130] for measuring surge currents and the rates of change of such currents, respectively. The design of a voltage divider that will be accurate for rapidly changing volt age, especially within the first microsecond of the surge, constitutes a difficult problem, and the Bureau's work on it has just bigun.

\section{Dissemination of Units}

The unit of time as represented by standard radio carrier and modulation frequencies can be disseminated conveniently and with extreme accuracy by broadcasting. National Bureau of Standards radio stations $W W^{r} V$ and WWVH perform this service [23 to 26], and anyone interested can just tume in. The electrical units cannot be transmitted so easily, and hence the values have to be disseminated by the more laborious physical transportation of electric instruments, meters, and other.measuring apparatus. Electric measuring apparatus to be standardized is sent to the Bureau from manufacturers of electric instruments and machinery, public utility companies, State public utility commissions. university laboratories, industrial rescarch laboratories, as well as from Federal agencies and private individuals.

Some organizations send in groups of saturated standard cells and 1-ohm standard resistors and carry on the rest of the measurement chain in their own laboratories. A number of foreign governments that are currently in the process of founding or expanding their national standardizing agencies come in this class. More often, unsaturated standard cells, sets of standard resistors, capacitors, inductors, and instrument transformers covering a considerable range of values are submitted. Many power companies submit their standard transfer wattmeters for comparison with the NBS instrument to verify the relation of its a-c performance to that on d-c, although they are well equipped to calibrate it on direct eurrent themselves. Manufacturers of electric measuring devices submit for test their own "laboratory tools," by the use of which they adjust their product, and thus disseminate the units to their ultimate customers, who therefore may not need any direct contact with the Bureau. Indicating instruments are submitted not only by small laboratories that use then as primary standards, but also by larger organizations as a check on the steps in the measurement sequence as performed in their own laboratories. As a result a stream of about 2,000 high-grade electric instruments and pieces of related apparatus flows through the electrical laboratories of the Bureau annually.

The Bureau is required by law $[1,2]$ to charge appropriate fees for such testing service unless it is for a branch of the Federal or State Government. Regular fee schedules [5] list these charges for the more usual types of test, and arrangements can often be made by correspondence for special unlisted tests at appropriate fees.

A considerabie fraction of the apparatus submitted to the Bureau for test is newly manufactured, and in some cases (e. g., standard resistors) it is desirable to have it held in the laboratory for several months to enable successive measurements to insure that secular drifts in value, originating in the process of manufacture, have steadied down to a negligible rate. Other standard apparatus, however, is usually submitted at regularly scheduled intervals to insure the sustained accuracy of measurements based on it. The desirable frequency of stoch periodic checks depends on a great number of factors, including (1) the accuracy required, (2) the extent to which intercomparisons are made among the standards in the local laboratory. (3) the ruggedness and stability of the particular type of apparatus, and (4) the carefulness and skill of those handling the apparatus.

A standard cell is relatively delicate and can be ruined in a few seconds by an excessive drain of current, yet if a group of at least three cells is available and if its nembers are intercompared frequently, a change of any one relative to the rest is quickly evident, and the volt as fixed by the mutually consistent remaining cells may be trusted to an accuracy of 0.01 percent for 6 months or a year. Most standard cells of the unsaturated 
type show a gradual decrease in emf, which on the average is about $80 \mu \mathrm{v} /$ year.

By contrast, a standard resistor, intended for the measurement of currents of several thousand amperes, is a very rugged structure. and is not at all likely to be damaged by carelessness in the laboratory. Barring corrosion from acids in its cooling oil, if this is allowed to become rancid, or very slow metallurgical changes, such a resistor may be trusted to an accuracy of 0.01 percent for 5 or even 10 years. Resistors made with fine wires are decidedly more prone to change as a result of corrosion and mechanical strains, and should be rechecked every 2 or 3 years. This periodicity also applies to resistance boxes, measurement voltage dividers, bridges, and potentiometers.

In many cases an initial test at the Bureau may be very desirable, and further periodic checks may be unnecessary. An example is the transfer test of a high-grade wattmeter, which determines the difference, if any, between its a-c and its d-c performance. This difference depends upon such factors as eddy currents, inductance, and capacitance in its windings and is very unlikely to change appreciably with time or use. The d-c calibration as a wattmeter, which depends also on its springs and the dimensions and relative positions of its coils, may show secular changes.
This d-c performance, however, can be adequately checked by measurements made at the local laboratory with a potentiometer and standard cell.

Recognizing the desirability of having precise apparatus checked initially at the Bureau, several manufacturers of electric measuring apparatus make it a practice to submit for test groups of usually 10 or 20 similar standards. After the test these are returned to the maker's stock and later sold "with a National Bureau of Standards Certificate" at an increase in price to cover the Bureau's fees and the handling costs. By this process, the necessary seasoning time does not introduce a delay between the sale of the standard and its ultimate use.

The results of calibration tests of measuring instruments and apparatus are issued in one of two forms: "Certificates" and "Reports." A Certificate is usually issued if the results have relatively permanent validity, and if the apparatus tested meets certaiin standards of precision and tolerance.

Reports are issued when unusually long and complex measurement programs are involved, when the apparatus is such that it can be too readily thrown out of adjustment, or when it shows symptoms of instability.

\section{International Relations}

By the Convention of the Meter, as amended in 1921 [6], the International Bureau of Weights and Measures at Sèvres, France, is authorized to coordinate the work of the various national standardizing laboratories in the electrical field. The National Bureau of Standards cooperates actively with the International Bureau and sends groups of standard cells and of standard resistors to Sèvres every 2 years for comparison with the groups of standards kept at the International Bureau. Other nations do the same, and in this way the relative values of the units as maintained in the different countries are derived. Graphs of these values are given in $[6$, p. 13, 16]. In abstract theory, all electrical units are supposed to be derived by an unbroken chain from those maintained at the International Bureau. In the final analysis, however, because of errors in comparison, changes during transportation, and drifts with time, the unit actually used in practice in any individual laboratory is bound to differ somewhat from the ideal. The relative differences between countries seldom exceed \pm 20 parts per million. The international comparisons of high precision serve to give early warning to any laboratory in case its units should show a drift relative to those of the other laboratories. The appearance of such relative drifts in the German standards led in 1931 to cooperative experiments in Berlin in which representatives of the British National Physical Laboratory and the National Bureau of Standards took part [12]. As a result of these experiments the German laboratory changed the values of its electrical units [6]. Also, the results of any new and improved absolute measurements can be disseminated with high precision to the rest of the world by these biennial intercomparisons.

Most countries derive their electrical units by sending standards to the International Bureau for certification. As a matter of practical convenience, however, a number of national laboratories have preferred to have their standards tested at the National Bureau of Standards. This is particu.. larly expedient when the standard in question is manufactured in the United States and can readily be standardized in Washington before shipment abroad.

Occasionally, when a standard capacitor or indicating instrument of extreme accuracy happens to be shipped, say, from England to the United States, it is found feasible to arrange to have it tested at the National Physical Laboratory before shipment and again at the National Bureau of Standards on its arrival in this country [109]. The agreements found in such comparisons are usually not as precise as those based on the shipment of standard resistors and cells, but give a very valuable assurance that the methods used in the two laboratories for deriving other units from the more fundamental ones lead to results that are consistent to an accuracy well within the needs of commercial operations.

The dissemination of scientific units of measurement throughout the world offers an almost unique example of harmonious international relations which have persisted for many years. The gain 
to society from world-wide uniformity in the field of measurement is very great, and the sacrifices required to maintain uniformity, at least in the electrical fields, are small. The workers in the national standardizing laboratories, confronted as they are with similar problems, have learned a mutual respect for their confreres. The inter- national organization which deals with weights and measures, established in 1875, has filled such a definite need and was devised with such farsighted wisdom that it has survived two world war's and has grown in prestige, scope, and influence. The precise unification of electrical measurements is one of its outstanding accomplishments.

\section{Bibliography}

The following list gives references to a large number of technical papers, standards, and books originating for the most part at the National Bureau of Standards and dealing more or less directly with the extension and development of methods for electrical and magnetic measurements. A number of papers on absolute measurements have also been included because the references listed in Bureau Circular 475 included only a few selected papers in this field. Many NBS papers that give the results of applying these methods of measurement in various fields, such as to the properties of insulating and of magnetic materials or to the performance of engineering devices, have not been included. A few of the references are included for their historic value only, but in those cases where the content of an older paper has been included as part of a later publication, the older reference has been omitted.

The following letter-symbol designations are used in combination with the Bureau publication numbers, and these letters should be included with the numbers in all references to Bureau publications: S, Scientific Papers; T, Technologic Papers; RP, Research Papers; C, Cireulars; H, Handbooks; M, Miscellaneous Publications; LC, Letter Circulars (mimeographed).

When a price is stated, the publication may be purchased from the Superintendent of Documents, Government Printing Office, Washington 25, D. C. Many of the older papers are now out of print, but can be consulted in large public or university libraries which maintain sets of Bureau publications.

A complete catalog of Bureau publications covering the period from 1901 to June 30, 1947, with author and subject indexes, NBS Circular C460, is available from the Superintendent of Documents for $\$ 1$. A mimeographed supplement to this is included. Copies of articles published in other technical journals are not obtainable from the Government. The standards mentioned may be purchased from the American Standards Association, 70 East 45th Street, New York. 17, $\mathrm{N}$. Y., or from the American Institute of Electrical Engineers, 30 West 39th Street, New York 18, N. Y.

In the following references, to save space, the code letters listed below have been used preceding the volume number as abbreviations to indicate the corresponding series of Bureau publications: B, Bulletin of the Bureau of Standards; S, Scientific Papers of the Bureau of Standards; BJ,
Bureau of Standards Journal of Research; J. Journal of Research of the National Bureau of Standards; C, Circular (of the National Bureau of Standards).

\subsection{General}

[1] 31 Stat., ch 872, p 1449 (1901) Establishment of the National Bureau of Standards.

[2] Public Law 619-81st Congress, (ch 486-2d Session) (S2201). Amendment of section 2 of the Act of March 3, 1901 (31 Stat. 1449)

[3] Public Law 617-81st Congress (ch 484-2d session) An act to redefine the units and establish the standards of electrical and photometric measurements.

[4] Announcement of changes in electrical and photometric units, C459 (1947) 5 é.

[5] Testing by the National Bureau of Standards (policy, general information, fee schedules) C483, $25 \%$.

[6] F. B. Silsbee, Establishment and maintenance of the electrical units, C475 (1949) $25 \dot{\phi}$.

[7] Frank A. Wolff, The so-called international electrical units, B 1, 39 (1904-05) S3.

[8] Frank A. Wolff, The principles involved in the selection and definition of the fundamental electrical units to be proposed for international adoption, B 5, 243 (1908-09). S102.

[9] E. C. Crittenden, Present status of the international electrical units, Trans. AIEE 40, 990 (1927).

[10] G. W. Vinal, Units of electrical measurement, Trans. Am. Electrochem. Soc. 55, 43 (1929).

[11] H. B. Brooks, The unit of electrical resistance, Past history and impending change, Trans. AIEE 50, 1318 (1931).

[12] G. W. Vinal, International comparison of electrical standards, BJ 8, 729 (1932) RP 448.

[13] E. C. Crittenden, The Work of the Bureau of Standards in electricity and radio, Sci. Monthly $\mathbf{3 7}, 405$ (1933).

[14] E. C. Crittenden, Revision of Electrical Units, Elec. Engin. (April 1940)

[15] J. H. Dellinger, International svstem of electric and magnetic units, B 13, 599 (1916-17) S292.

[16] Electric Units and Standards, C60, 2d ed. (1920).

[17] H. L. Curtis, Electrical measurements, McGrawHill Book Co., New York (1937).

[18] F. B. Silsbee, Standards for electrical measurement, Elec. Engin. 70, 202 (Narch 1951).

[19] Radio instruments and measurements, C74, $2 \mathrm{~d}$ ed. (1918). $\$ 1.50$.

[20] P. G. Agnew, W. H. Stannard, J. L. Fearing, A system of remote control for an electric testing laboratory, B 13, 581 (1916-17). S291.

[21] A. B. Lewis, Calculations of electrical surge-generator circuits, J 17, 585 (1936) RP929.

$[22]$ H. L. Curtis, Shielding and guarding electrical apparatus, Trans. AIFE 48, 1263 (1929).

[23] G. H. Lester, Experimental standard-frequency transmitting station, WWVH, Communications 29, 20 (1949).

[24] John M. Shaull, Adjustment of high-precision frequency and time standards, Proc. IRE 28, 6-15 $(1950)$. 
[25] Standard frequencies and time signals, WWV and WWVH, NBS Letter Circular LC974.

[26] J. M. Shaull and C. M. Kortman, Comparing outputs from precision time standards, Electronics 24, 102 (1951).

[27] E. B. Rosa and G. W. Vinal, Summary of experiments on the silver voltameter at the Bureau of Standards and proposed specifications B 13, 479 (1916-17) S285. (This includes a summary of the results of 10 earlier papers on the voltameter.)

\subsection{Absolute Measurements}

[28] E. B. Rosa and N. E. Dorsey, A new determination of the ratio of the electromagnetic to the electrostatic unit of electricity, B 3, 433 (1907) S65.

[29] E. B. Rosa and N. E. Dorsey, A comparison of the various methods of determining the ratio of the electromagnetic to the electrostatic unit of electricity, B 3,605 (1907) S66.

[30] H. L. Curtis, Note on the ratio of the electromagnetic to the electrostatic unit of clectricity as compared to the velocity of light, BJ 3,63 (1929) RP83.

[31] Paul R. Hevl and Guy S. Cook, The value of gravity at Washington, J 17, 805 (1936) R P946.

[32] H. I. Dryden, A reexamination of the Potsdam absolute determination of gravity, J 29, 303 (1942) RP1502, 5e.

[33] H. L. Curtis, The determination of the electrical units by mechanical measurements, Jour. Wash. Acad. Sci. 22, 193 (1932).

[34] H. I. Curtis, Principles involved in determining the absolute values of the electrical units, Am. Physics Teacher 5, 7 (1937):

[35] H. L. Curtis, A review of methods for the absolute determination of the ohm, Jour. Wash. Acad. Sci. 32, 40 (1942)

[36] H. I. Curtis, Review of recent absolute determinations of the ohm and the ampcre, J 33, 235 (1944) RP1606, $10 \notin$.

[37] H. L. Curtis, Absolute electrical measurements, Sci. Monthly 69, 9 (1949).

[38] H. L. Curtis, Charles Moon, and C. M.'Sparks, An absolute determination of the ohm, J 16, 1 (1936) RP857.

[39] H. L. Curtis, Charles Moon, and C. M. Sparks. A determination of the absolute ohm using an in proved self-inductor, J $\mathbf{2 1}, 375$ (1938) RP1137, $15 \phi$.

[40] E. B. Rosa, N. E. Dorsey, and J. M. Miller, A determination of the international ampere in absolute measure, B 8, 269 (1912) S171.

[41] H. L. Curtis and Roger W. Curtis, An absolute determination of the ampere, BJ 12, 665 (1934) RP685.

[42] H. L. Curtis, Roger W. Curtis, and C. L. Critchfield, An absolute detcrmination of the ampcre using improved coils, J 22, 485 (1939) RP1200, 10 \&.

[43] R. W. Curtis, R. L. Driscoll, and C. I. Critchfield, An absolute determination of the ampere using helical and spiral coils, J 28, 133 (1942) RP1449 10\&.

[44] H. Pellat, An absolute clectrodynamometer, Jour. de Physique, 2d Series, 6, 172 (1887).

[45] Charles Moon, Apparatus for comparison of length of gages, BJ 10, 249 (1933) RP528.

[46] Charles Moon, A precision cathetometer, J 14, 363 (1935) RP774.

[47] J. L. Thomas, Chester Peterson, I. L. Cooter, and Ralph Kotter, An absolute mcasurcment of resistance by the Wenner method, J 43, 291 (1949) RP2029, 30ф.

\subsection{Standard Cells}

[48] F. A. Wolff and C. E. Waters, Prcliminary specifications for Clark and Weston standard cells, B 3, 623 (1907) S67.
[49] F. A. Wolff and C. E. Waters, Clark and Weston standard cells, B 4, 1 (1907-08) S70.

[50] F. A. Wolff and C. E. Waters, The electrode equilibrium of the standard cell, B4, 81 (1907-08) S71.

[51] F. A. Wolff, The temperature formula of the Weston standard cell, B5, 309 (1908-09) S104.

[52] J. H. Park, Effect of service temperature conditions on the electromotive foree of unsaturated portable standard cells, BJ 10, 89 (1933) RP518.

[53] E. F. Mueller and H. F. Stimson, A temperaturecontrol box for saturated standard cells, J 13, 699 (1934) RP739.

I54] G. W. Vinal and M. Langhorne Howard, Effect of glasss containers on the electromotive force of Weston normal cclls, BJ 11, 255 (1933) RP588.

[55] D. N. Craig, G. W. Vinal and Francis E. Vinal, Solubility of mercurous sulphate in sulphuric-acid solutions, J 17, 709 (1936) RP939.

[56] G. W. Vinal and I. H. Brickwedde, Metastability of cadmium sulfate and its effect on clectromotive force of saturated standard cells, J 26, 455 (1941) RP1389, 5c.

[57] L. H. Brickwedde and G. W. Vinal, Electromotive force of saturated Wcston standard cells containing deuterium oxide, J 20, 599 (1938) IRP1094, 5\&.

[58] L. H. Brickwedde and G. W. Vinal, Relation of electromotive force to the concentration of deuterium oxide in saturated standard cells, J. 27, 479 (1941) RP1435, 5e.

[59] G. W. Vinal, D. N. Craig, and L. H. Brickwedde, Standards of electromotive force, Trans. Electrochem. Soc. 68, 139 (1935).

[60] G. W. Vinal, Maintenance of the volt, Trans. Am. Electrochem. Soc. 54, 247 (1928).

[61] G. W. Vinal, Primary batteries (John Wiley \& Sons, 1950 , ch 6).

[62] G. W. Vinal, Standard cells and the change from international to absolute electrical units, Trans. Electrochem. Soc. 93, 95 (1948).

[63] G. W. Vinal, I. H. Brickwedde, and W. J. Hamer, New quartz containers for standard cells at the National Bureall of Standards, C. R. 15th Conference, Intl. Union of Pure and Applied Chemistry, p 92 (1949).

\subsection{Standard Resistors and Resistance Measurements}

[64] E. B. Rosa and H. D. Babcock, On the variation of resistances with atmospheric humidity, B 4, 121 (1907-08) S73.

[65] E. B. Rosa, A new form of standard resistance, B 5, 413 (1908-09), S107.

[66] J. I. Thomas, A new design of precision resistance standard, BJ 5, 295 (1930) RP201.

[67] J. L. Thomas, Stability of double-walled manganin resistors, $J$ 36, 107 (1946) RP1692, 5c.

[68] J. I. Thomas, Some experiments with pure-metal resistance standards, BJ 1\%, 313 (1934) RP657.

[69] J. L. Thomas, Reproducibility of the icc point, BJ 12, 323 (1934) RP658

[70] J. L. Thomas, Gold-chromium resistance alloys, J 13, 681 (1934) RP737.

[71] T. B. Godfrey, Further data on gold-chromium resistance wire, J 22, 565 (1939) RP1206, 5 \%.

[72] J. I. Thomas, Gold-cobalt resistance alloys, J 14, 589 (1935] RP789.

[73] J. L. Thomas, Elcetrical-resistance alloys of copper, manganese and aluminum, J 16, 149 (1936) RP863.

[74] Frank Wenner and J. L. Thomas, A method of adjusting the temperature cocfficicnt and resistance of low-valued resistance standards, BJ 12, 147 (1934) RP639.

[75] E. F. Mueller and Frank Wenner, The Waidner-Wolff and other adjustable electrical-resistance clements, J 15, 477 (1935) RP842. 
[76] Frank Wenner, The four-terminal conductor and the Thomson bridge, B 8, 559 (1912) S181.

[77] Frank Wenner and Ernest Weibel, Adjustments of the Thomson bridge in the measurement of very low resistances, B 11, 65 (1915) S225.

[78] Frank Wenner and Alva Smith, Measurement of low resistance by means of the wheatstone bridge, S $\mathbf{1 9}$, 297 (1923-24) S481.

[79] Frank Wenner, Methods, apparatus, and procedures for the comparison of precision standard resistors, J 25, 229 (1940) RP1323, 15 \&.

[80] J. L. Thomas, Precision resistors and their measurement, C470 (1948) 20 .

[81] H. L. Curtis, Insulating properties of solid dielectries, B 11, 359 (1915) S234.

[82] L. S. Taylor, Accurate measurement of small electric charges by a null method, BJ 6, 807 (1931) RP306.

[83] H. B. Brooks, Accuracy tests for meggers, Elec. World, 85, 973 (1925)

[84] Master test code for resistance measurements, AIEE Std. No. 550 (1947).

[85] Copper wire tables, C31, 3d ed. (1914) 30\%.

[86] P. J. Higgs, A method for measuring high insulation resistance, Jour. Sci. Instr. 10, 169 (1933).

\subsection{Potentiometers, Bridges, etc.}

[87] Frank Wenner and Ernest Weibel, Tlie testing of potentiometers, B 11, 1 (1915) S223.

[88] C. W. Waidner, H. C.Dickinson, E. F. Mueller, and D. R. Harper, 3d, A Wheatstone bridge for resistance thermometry, B 11, 571 (1915) \$241.

[89] E. F. Mueller, Wheatstone bridges and some accessory apparatus for resistance thermometry, B $\mathbf{1 3}, 547$ (1916-17) S288.

[90] H. B. Brooks and A. W. Spinks, A multirange potentiometer and its application to the measurement of small temperature differences, BJ 9, 781 (1932) RP506.

[91] H. B. Brooks, The standard-cell comparator, a specialized potentiometer, 13.J 11, 211 (1933) R P586.

[92] Frank Wenner, A method of reducing the effect of disturbances in the galvanometer branch of a potentiometer circuit, J 2\%, 425 (1939) RP1194, 56.

[93] R. P. Teele and Shuford Schuhmann, A potentiometer for measuring voltages of 10 microvolts to an accuracy of 0.01 microvolt, J $\mathbf{2 2 ,} 431$ (1939) RP1195, 106.

[94] F. Wenner, Note on potentiometer design, Phys. Rev. 31, 94 (1910).

[95] Leo Behr, The Wenner potentiometer, IRev. Sci. Instr., 3, 109 (1932).

[96] H. B. Brooks, A new potentiometer for the measurement of electromotive force and current, B 2, 225, (1906) S33.

[97] H. B. Brooks, A deflection potentiometer for voltmeter testing, B 4, 275 (1907-08) S79.

[98] H. B. Brooks, Deflection potentiometers for current and voltage measurements, B 8, 395, (1912) S172.

[99] H. B. Brooks, Outline of design of deflection potentiometers with notes on the design of moving-coil galvanometers, B 8, 419 (1912) S173.

[100] F. B. Silsbee and F. J. (iross, Testing and performance of volt boxes, $J \mathbf{2 7}, 269$ (1941) RP1419, 10e.

[101] Melville F. Peters, George F. Blackburn, and Paul T. Hamnen, Theory of voltage dividers and their use with cathode ray oscillographs, BJ 9, 81 (1932) RP 460.

[102] L. S. Taylor, Apparatus for the measurement of high constant or rippled voltages, BJ 5, 609 (1930) RP217.

\subsection{Capacitance}

[103] E. B. Rosa and F. W. Grover, The absolute measurement of capacity, B 1, $153(1904-05)$ S10.

[104] E. B. Rosa, Wattmeter methods of measuring power expended upon condensers and circuits of low power factor, B 1, $383(1904-05)$ S18.
[105] F. W. Grover, The simultaneous measurment of the capacity and power factor of condensers, B 3, 371 (1907) S64.

[106] H. L. Curtis, Mica condensers as standards of capacity, B 6, 431 (1909-10) S137.

[107] F. W. Grover, The capacity and phase difference of paraffined paper condensers as functions of temperature and frequency, B $\%, 495$ (1911) S166.

[108] H. L. Curtis and Charles Moon, Absolute measurement of capacitance by Maxwell's method, S 22, 487 (1927-28) S564.

[109] H. L. Curtis, C. M. Sparks, L. Hartshorn, and N. F. Astbury, Capacitance and power factor of a mica capacitor as measured at the National Bureau of Standards and the National Physical Laboratory, BJ 8, 507 (1932) RP431.

[110] A. V. Astin, Measurement of relative and true power factors of air capacitors, J 21, 425 (1938) RP1138, $10 \phi$.

[111] A. V. Astin, Nature of energy losses in air capacitors at low frequencies, J 22, 673 (1939) RP1212.

[112] C. Moon and C. M. Sparks, Standards for low values of direct capacitance, $J \mathbf{4 1}, 497$ (1948) RP1935, $10 e^{c}$.

[113] J. H. Dellinger and J. L. Preston, Methods of measurement of properties of electric insulating materials, S 19, 39 (1923-24) S471.

[114] A. H. Scott and H. L. Curtis, Edge correction in the determination of dielectric constant, J 22, 774 (1939) RP1217, 5 \%.

[115] Chester Snow, A standard of small capacitance, J 42, 287 (1949) RP1970, 15c.

[116] W. D. Voelker, An improved capacitance bridge for precision measurements, Bell Lab. Record 20, 133 (1942).

[117] L. Hartshorn and W. H. Ward, Measurement of the permittivity and power factor of dielectrics at frequencies from $10^{4}$ to $10^{8}$ cycles per second, J. Inst. Elec. Engrs. (London) 79, 597 (1936).

\subsection{Inductance (Construction and Measurement)}

[118] E. B. Rosa and F. W. Grover, Measurement of inductance by Anderson's method, using alternating currents and a vibration galvanometer, B 1, $291(1904-05)$ S14.

[119] E. B. Rosa and F. W. Grover, Use of serpentine in standards of inductance, B 1, 337 (1904-05) S15.

[120] J. C. Coffin, Construction and calculations of absolute standards of inductance, B 2,87 (1906) S29.

[121] H. B. Brooks and F. (. Weaver, A variable self and mutual inductor, B 13, 569 (1916-17) $\$ 290$.

[122] H. B. Brooks and A. B. Lewis, Improved continuously variable self and mutual inductor, J 19, 493 (1937) RP1040, 10

[123] H. B. Brooks, Design of standards of inductance, and the proposed use of models in the design of air-core and iron-core reactors, BJ 7, 289 (1931) RP342.

[124] H. L. Curtis and Leon Wr. Hartman, Dual bridge for the measurement of self-inductance in terms of resistance and time, $J \mathbf{2 5}, 1$ (1940) RP1310.

[125] F. W. Grover and H. L. Curtis, The measurement of the inductances of resistance coils, B 8, 455 (1912) S175.

[126] H. L. Curtis and F. W. Grover, Resistance coils for alternating current work, B 8, 495, (1912) S177.

[127] Frank Wenner, Ernest Weibel and F. B. Silsbee, Methods of measuring the inductances of lowresistance standards, B 12, $11(1915-16)$ S246.

[128] F. B. Silsbee, A study of inductance of four-terminal resistance standards, B 13, 375 (1916-17) S281.

[129] F. B. Silsbee, Notes on the design of four-terminal resistance standards for alternating currents, BJ 4, 73 (1930) RP133.

[130] J. H. Park, Shunts and inductors for surge-current measurements, J 39, 191 (1947) RP1823. 25\%. 
[131] B. Hague, Alternating current bridge methods (Sir Isaac Pitman \& Sons, Ltd., 5th ed., 1946).

\subsection{Inductance (Computations)}

[132] E. B. Rosa, The self-inductance of a coil of any length and any number of layers of wire, B $\mathbf{4}, 369$ (1907-08) S83.

[133] E. B. Rosa and Louis Cohen, The mutual inductance of two circular coaxial coils of rectangular section, B 2, 359 (1906) S42.

[134] Louis Cohen, The self-inductance of a solenoid of any number of layers, B $\mathbb{4}, 383(1907-08)$ S84.

[135] E. B. Rosa and F. W. Grover, Formulas and tables for the calculations of mutual and self-inductance, B 8, 1 (1912) S169, 55 d.

[136] F. W. Grover, Additions to the formulas for the calculations of mutual and self-inductance, B 14, 537 (1918-19) S320.

[137] F. W. Grover, Calculation of the maximum force between two coaxial circular currents, B 12,317 (1915-16) S255.

[138] F. W. Grover, Tables for the calculation of the inductance of circular coils of rectangular cross sections, S 18, $451(1922-23)$ S455.

[139] F. W. Grover, Formulas and tables for the calculation of the inductance of coils of polygonal form, S 18, 737 (1922-23) S468.

[140] H. L. Curtis and C. M. Sparks, Formulas, tables, and graphs for computing the mutual inductance of two coaxial circles, S 19, 541 (1923-24) S492.

[141] F. W. Grover, Tables for the calculation of the mutual inductance of circuits with circular symmetry about a common axis, S 20, 1 (1924-26) S498.

[142] Chester Snow;, Formula for the inductance of a helix made with wire of any section, $\mathrm{S} \mathbf{2 1}, 431$ (1926-27) S537.

[143] F. W. Grover, Methods for derivation and expansion of formulas for the mutual inductance of coaxial circles and for the inductance of single-layer solenoids, BJ 1, 487 (1928) RPr6.

[144] Chester Snow; Mutual inductance of any two circles, BJ 1, 531 (1928) RP18.

[145] Chester Snow, Mutual inductance and torque between two concentric solenoids, BJ 1, 685 (1928) R.P24.

[146] F. W. Grover, A comparison of the tormulas for the calculation of inductance of coils and spirals wound with wire of large cross section, BJ 3, 163 (1929) RP90.

[147] Chester Snow, The mutual inductance of two parallel circles, BJ 3, 255 (1929) RP94.

[148] Chester Snow, The effect of small variations in pitch upon the inductance of a standard solenoid, B $\mathbf{6}$, 777 (1931) RP304.

[149] Chester Snow, A simplified precision formula for the inductance of a belix, with corrections for the lead-in-wires, BJ 9, 419 (1932) RP479.

[150] Chester Snow, Mutual inductance and force between two coaxial helical wires, J 22, 239 (1939) RP1178, $15 \%$.

[151] Chester Snow, Theory of a single-layer, bifilar absolute standard of mutual inductance, $J$ 24, 597 (1940) RP1302, 10ф.

[152] Chester Snow, Mutual inductance of two helices whose axes are parallel, J 25, 619 (1940) RP1346, $10 \notin$.

[153] F. W. Grover, Inductance calculations (D. Van Nostrand Co., Inc., New York, N. Y., 1946).

[154] J. G. Coffin, Influence of frequency upon the selfinductance of coils, B 2, 275 (1906) S37.

[155] Louis Cohen, The influence of frequency on the resistance and inductance of solenoidal coils, B 4, 161 (1907-08) S76.

[156] John M. Miller, Effective resistance and inductance of iron and bimetallic wires, B 12, 207 (1915-16) S252.
[157] H. L. Curtis, An integration method of deriving the alternating-current resistance and inductance of conductors, S 16, 93 (1920) S374.

[158] C. N. Hickman, Alternating-current resistance and inductance of single-layer coils, S 19, 73 (1923-24) S472.

[159] Chester Snow, Alternating current distribution in cylindrical conductors, S 20, 277 (1924-26) S509.

[160] Walter Bartky, Numerical calculation of generalized complete elliptic integrals, Rev. Modern Physics 10, 264 (1938).

\subsection{Galvanometers and Detectors}

[161] L. W. Austin, Detector for small alternating currents and electrical waves, B 1, 435 (1904-04) S22.

[162] M. G. Lloyd, Function of a periodic variable given by the steady reading of an instrument; with a note on the use of the capillary electrometer with alternating voltages, $B$ 4, 525. (1907-08) S90.

[163] F. Wenner, A theoretical and experimental study of the vibration galvanometer, B 6, 347 (1909-10) S134.

[164] P. G. Agnew, A new form of vibration galvanometer, S 16, 37 (1920) S370.

[165] H. L. Curtis, A vibration electrometer, B 11, 535 (1915) S239.

[166] Ernest Weibel, A study of electromagnetic moving coil galvanometers for use in alternating-current measurements, B 14, 23 (1918-19) S297.

[167] W. W. Coblentz, Sensitivity and magnetic shielding tests of a thomson galvanometer for use in radiometry, B 13, 423 (1916-17) S282.

[168] F. Wenner, General design of critically damped galvanometers, B 13, 211 (1916-17) S273.

[169] H. B. Brooks, Sensitivity of a galvanometer as a function of its resistance, BJ 4, 297 (1930) RP150.

[170] L. S. Taylor, Accurate measurement of small electric charges by a null method, BJ 6, 807 (1931) RP306.

[171] L. F. Curtiss, A resistance-coupled amplifier for measuring ionization currents, BJ 10, 583 (1933) RP550.

[172] W. W. Nicholas, An electrostatic voltmeter, BJ 8, 111 (1932) RP404.

[173] P. G. Agnew, A device for measuring the torque of electrical instruments, B \%, 45 (1911) S145.

[174] H. B. Brooks, Temperature compensation of millivoltmeters, J 17, 497 (1936) RP926.

[175] E. B. Rosa, The compensated two-circuit electrodynamometer, B 3, 43 (1907) S48.

[176] P. G. Agnew, A tubular electrodynamnometer for heavy currents, B 8, 651 (1912) S184.

[177] J. H. Park and A. B. Lewis, Standard electrodynainic wattmeter and ac-de transfer instrument, J 25, 545 (1940) RP1344, 10 c.

[178] Paterson, Rayner, and Kinnes, The use of the electrostatic method for the measurement of power, J. Inst. Elec. Engrs. (London) 51, 294 (1913).

[179] F. K. Harris, A suppressed-zero electrodynamic voltmeter, BJ 3, 445 (1929) RP105.

[180] F. B. Silsbee, Composite-coil electrodynamic instruinents, BJ 8, 217 (1932) RP411.

[181] J. H. Dellinger, High-frequency ammeters, B 10, 91 (1914) S206.

[182] F. L. Hermach, A precision electrothermic voltmeter for measurements between 20 and $20,000 \mathrm{c} / \mathrm{s}$, Trans. AIEE 6\%, 1224 (1948).

\subsection{Instruments and Meters}

[183] E. B. Rosa, M. G. Lloyd and C. E. Reid, Influence of wave form on the rate of integrating induction wattmeters, 1, 421 (1904-05) S21.

[184] T. T. Fitch and C. J. Huber, A comparative study of American direct-current watthour meters, B 10, 161 (1914) S207. 
[185\} T. T. Fitch and C. J. Huber, A comparison of American direct-current switchboard voltmeters and ammeters, B 7, 407 (1911) S163.

[186] A. E. Peterson, Performance of portable electrical instruments in magnetic fields, Trans. AIEE 6\%, 1228 (1948).

[187] F. B. Silsbee, Precautions against stray magnetic fields in measurements with large alternating currents, Trans. AIEE 48, 1301 (1929).

[188] P. G. Agnew, An approximate experimental method for the analyses of EMF waves, B 6, 95 (1909-10) S119.

[189] F. W. Grover, Analyses of alternating-current waves by the method of Fourier, with special reference to methods of facilitating the computations, B 9, 567 (1913) S203.

[190] F. K. Harris, A new cathode-ray oscillograph and its application to the study of power loss in dielectric materials, BJ 12, 87 (1934) RP636.

[191] Chester Snow, Effect of clearance and displacement of attracted disk and also of a certain arrangement of conducting hoops, upon the constant of an electrometer, BJ 1, 513 (1928) RP17.

[192] H. B. Brooks, F. M. Defandorf, and F. B. Silsbee, An absolute electrometer for the measurement of high alternating voltages, J $\mathbf{2 0}, 253$ (1938) RP1078, 15e.

[193] F. B. Silsbee and F. M. Defandorf, A transformer method for measuring high alternating voltages and its comparison with an absolute electrometer, J 20, 317 (1938) RP1079, 10 .

[194] H. B. Brooks and F. M. Defandorf, An experimental study of the corona voltmeter, BJ 1, 589 (1928) RP21.

[195] F. M. Defandorf, The measurement of high roltage, Jour. Wash. Acad, Sci. 38, 33 (1948).

[196] F. D. Weaver, An easily assembled console for rapid testing of electrical indicating instruments, Instruments 22, 396 (1949).

[197] F. D. Weaver, Notes on the care and use of electrical instruments, Instruments 23, 1236 (1950).

[198] Electrical measuring instruments, C20, 2d ed. (1915).

[199] H. B. Brooks, Accuracy of commercial electrical measurements, Trans. AIEE 39, 495 (1920)

[200] H. B. Brooks, Standardization of electrical measuring instruments, 'Trans, AIEE 42, 894 (1923).

[201] Am. standard for electrical indicating instruments, (ASA Std. C39.1-1951).

[202] Am. standards for measurement of test voltage in dielectric tests (ASA Std. C68.1-1942, AIEE No. 4).

[203] Code for electricity meters, ASA Std. No. C12 (4th ed. 1941).

[204] Electrical metermen's 'handbook, (Edison Electric Institute, 420 Lexington Ave., New York, 6th ed., 1950 )

[205] H. L. Curtis and F. B. Silsbee, Definition of power and related quantities, Trans. AIEE 54, 394 (1935).

[206] F. B. Silsbee, Power factor of polyphase systems, Proc. AIEE 39, 59 (1920).

[207] F. L. Hermach, Thermal co vverters as ac-de transfer standards for current and voltage measurements at audio frequencies, $J \mathbf{4 8}, 121$, (1952) RP2296.

\subsection{Instrument Transformers}

[208] E. B. Rosa and M. G. Lloyd, The determination of the ratio of transformation and of the phase relations in transformers, B 6, 1 (1909-10) S116.

[209] M. G. Lloyd and P. G. Agnew, The regulation of potential transformers and the magnetizing current, B 6, $273(1909-10)$ S 129.

[210] P. G. Agnew and T. T. Fitch, The determination of the constants of instrument transformers, B $\mathbf{6}$, 281, (1909-10) S130.

[211] P. G. Agnew, A study of the current transformer with particular reference to iron loss, B \%, 423 (1911) S16t.
[212] P. G. Agnew and F. B. Silsbee, Accuracy of the fofmulas for the ratio, regulation and phase angle or transformers, B 10, 279 (1914) S211.

[213] P. G. Agnew and F. B. Silsbee, The testing of instrument transformers, Trans. AIEE 31, 1635 (1912).

[214] H. B. Brooks, Testing potential transformers, B 10, 419 (1914) $\mathrm{S} 217$

[215] P. G. Agnew, A wathour meter method of testing instrument transformers, B 11, 347 (1915) S233.

[216] F. B. Silsbee, A method for testing current transformers, B 14, $317(1918-19)$ S 309 .

[217] F. B. Silsbee, Methods for tsiting current transformers, Trans. AIEE 43, 282 (1924).

[218] F. B. Silsbee, Léad resistance for current transformers, Elec. World 81, 1082 (1923).

[219] H. B. Brooks and F. C. Holtz, The two-stage current transformer, Trans. AIEE 41, 382 (1922).

[220] F. B. Silshee, A shielded resistor for voltage transformer testing, S $\mathbf{2 0}, 489$ (1924-26) S516.

[221] C. T. Weller, A $132-\mathrm{kv}$ shielded potentiometer for determining the accuracy of potential transformers, Trans. AIEE 48, 790 (1929).

[222] C. H. Sharp and W. W. Crawford, Some recent developments in exact alternating-current measurements, Trans. AIEE 29, 1517 (1910).

[223] H. W. Bousman and R. L. Ten Broeck, A capacitance bridge for determining the ratio and phase angle of potential transformers, Trans. AIEE 62, 541 (1943).

[224] F. B. Silsbee, Ray L. Smith, Nyna L. Forman and J. H. Park, Equipment for testing current transformers, BJ 11, 93 (1933) RP580.

[225] J. H. Park, Accuracy of high-range current transformers, J 14, 367 (1935) RP775.

[226] J. H. Park, Effect of wave form upon performance of current transformers, J 19, 517 (1937) RP1041.

[227] B. Hague, Instrument transformers (Sir Isaac Pitman \& Sons, Ltd. 1936).

[228] Leeds \& Northrup Co., Potential transformer test set, Cat. E-50-501(2) 1942.

[229] F. B. Silsbee, New high-voltage laboratory at the National Bureau of Standards, Elec. Engin. 59 (June 1940).

\subsection{Magnetic Measurements}

[230] Charles W. Burrows, The best method of demagnetizing iron in magnetic testing, B 4, 205 (190708) $\mathrm{S} 78$.

[231] M. G. Lloyd and J. V. S. Fisher, An apparatus for determining the wave form of magnetic flux, B 4, 467, (1907-08) S87.

[232] M. G. Lloyd, Dependence of magnetic hysteresis upon the wave form, B 5, 381 (1908-09) S106.

[233] M. G. Lloyd. Errors in magnetic testing with ring specimens, B 5, 435 (1908-09) S108.

[234] M. G. Lloyd and J. V. S. Fisher, The testing of transformer Steel, B 5, 453 (1908-09) S109.

[235] C. W. Burrows, Uniformity in magnetic testing and in the specification of magnetic properties, Proc. ASTM, 8, 658 (1908).

[236] Charles W. Burrows, The determination of the magnetio induction in straight bars, B 6, 31 (1909-10) S117.

[237] R. L. Sanford, Determination of the degree of uniformity of bars for magnetic standards, B 14, 1 (1918-19) S295.

[238] W. L. Cheney, Magnetic testing of straight rods in intense fields, S 15, 625 (1919-20) S361.

[239] W. L. Cheney, Measurement of hysteresis values from high magnetizing forces, S 16, 281 (1920) S383.

[240] M. F. Fischer, Apparatus for the determination of the magnetic properties of short bars, S 18, 513 (1922-23) S458.

[241] R. L. Sanford and Evert G. Bennett, An apparatus for magnetic testing at high magnetizing forces, BJ 10, 567, (1933) RP548. 
[242] R. L. Sanford, and E. G. Bennett, An apparatus for magnetic testing at magnetizing forces up to 5,000 oersteds, J 23, 415 (1939) RP1242.

[243] R. L. Sanford and P. H. Winter, A permeameter for magnetic testing at magnetizing forces up to 300 oersteds, BJ 45, 17, (1950) RP2109, 10 \&.

[244] R. L. Sanford, Magnetic testing, C456 (November 1946) $10 \%$.

[245] R. L. Sanford, Permanent magnets, C448 (August 1944) $10 \phi$.

[246] Charles IV. Burrows, An experimental study of the Koepsel permeameter, B 11, 101 (1915) S228.

[247] Charles W. Burrows and R. L. Sanford, An experimental study of the Fahy permeameter, B 14, 267 (1918-19) S306.

[248] R. L. Sanford and James M. Barry, Determination of the magnetic induction in sheet steel, S 21,727 $(1926-27) \mathrm{S} 545,10 \phi$.
[249] R. L. Sanford, Standards for testing magnetic permeameters, B.J 4, 177 (1930) RP140.

[250] R. L. Sanford, Performance of the Fahy simplex permeameter, BJ 4, 703 (1930) RP174.

[251] R. L. Sanford, A method for the standardization of permeameters at high magnetizing forces, BJ 6, 355 (1931) R P279.

[252] R. L. Sanford and Evert G. Bennett, Determination of magnetic hysteresis with the Fahy simplex permeameter, J 15, 517 (1935) RP845.

[253] F. Bloch, Nuclear induction, Phys. Rev. 70, 460 (1946).

[254] E. M. Purcell, H. C. Torrey, and R. V. Pound, Resonance absorption by nuclear magnetic moments in a solid, Phys. Rev. 69, 37 (1946).

[255] H. A. Thomas, R. L. Driscoll, and J. A. Hipple, Measurement of the proton moment in absolute units, J 44, 569 (1950) RP2104. 


\title{
Measurement of Current with a Pellat-Type Electrodynamometer
}

\author{
R. L. Driscoll
}

\begin{abstract}
The value of an electric current has been determined in absolute measure by means of an electrodynamometer, and simultaneously by standard cells and standard resistors as currently maintaimed. 'The electrodynamometer used was of the Pellat type, and featured a fused silica balance beam and single laver helical coils.

The relation of the NBS ampere to the absolute ampere, from this determination, maybe expressed as
\end{abstract}

\section{$1 \mathrm{NBS}$ ampere $=1.000013$ absolute amperes.}

The uncertainty in this result from all known sources is estimated to be eight parts per million.

\section{Introduction}

The important units in electrical measurements are the ampere, the ohn, and the volt. Since these units are connected by Ohm's law, any one of the three is fixed when the values of the other two are settled upon. These units are all defined in terms of the mechanical units, but the accuracy of their physical realization depends upon experimental approximations to unattainable ideal conditions. In the electromagnetic system, the ampere is defined as $10^{-1} \mathrm{cgs}$ units, the olim $10^{9} \mathrm{cgs}$ units and the volt $10^{8} \mathrm{cgs}$ units. The two units so far found susceptible of accurate independent evaluation in terms of the mechanical units are the olım and ampere. On account of the transitory nature of electric current the ohm and the volt are the two units maintained in the form of concrete standards. It is necessary therefore to assign a value to the standard of electromotive force obtained from a combination of the results of primary standardization of the ohm and ampere. On account of the implicit relation of the electrical units to other physical constants, it is desirable that these units be close to their defined values. In order to detect drifts in the standards, repetition of absolute measurements from time to time has been the accepted practice. Experiments now being conducted at this Bureau on the precession frequency of protons in the magnetic field of a coil carrying a current, show promise of providing a precise method for determining the constancy of the current over long periods of time. An account of the last work on the ampere at this Bureau was published in 1942 [1]. ${ }^{1}$ In that investigation a current balance ${ }^{2}$ was employed; in an effort to avoid possible svstematic errors in the current balance, a Pellat-type electrodynamometer [4] (sometimes referred to as a Pellat balance) has been used in this work. In the current balance the force measured is one of translation and in the electrodynamometel it is one of rotation. This paper describes the important physical measurements that were made ancl assesses the accuracy of the determination of a current by the latter absolute method.

1 Figures in brackets indicute the literature references at the end of this parer.

2 A companion paper to follow in this Journal will present values from recent mieasulements.

\section{Value of a Current in Terms of Mechanical Effects}

The torque between two coils in which the currents $i_{1}$ and $i_{2}$ are held constant has been worked out in a general way by Maxwell [2]. In the particular case of the Pellat electrodynamometer, the two coils are adjusted to be concentric and to have an angle, $\theta$, of $90^{\circ}$ between their axes. With this adjustment the torque, $\mathscr{T}$, is a maximum and is given by

$$
\tilde{T}=i_{1} i_{2}\left[\frac{\partial M}{\partial \theta}\right]_{\theta=\frac{\pi}{2}}
$$

where $M$ is the mutual inductance of the coils. In absolute measurements the geometrical factor, $\partial M / \partial \theta$, is computed from measured values of the linear dimension of the coils. The mathematical contributions of Snow [3] make possible a second order calculation of this factor for the electrodynamometer that includes several small corrections made necessary by the fact that the coils are helical wires having small measurable irregularities and are not uniform current sheets.

Another factor affecting the absolute measurement of current is the accuracy realized in the evaluation of the torque produced. When single layer helical windings are enployed, the torque produced is relatively small and more effort is required for its accurate evaluation than that demanded in the case where multikyer windings are used. The advantage gained in the accuracy of the geometrical factor, $\partial M / \partial \theta$ when single layer helical windings are enployed more than offsets the disadvantage of the smaller torque.

\section{Electrodynamometer}

This instrument has a long stationary solenoid (fig. 1) with its axis horizontal; the centered inner coil, mounted on the beam of a balance (fig. 2), is a short solenoid with its axis vertical. The balance beam (fig. 7) is equipped with conventional knife edges and supports the inner coil which thus becomes rotatable about the central knife edge.

With a steady current in both coils in series, the balance is put into equitibrium by means of a suitable 


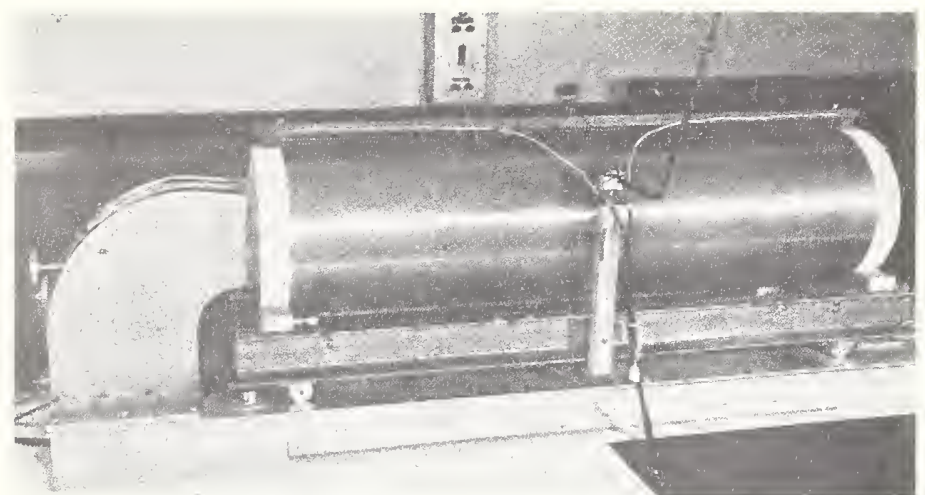

Frgure 1. Side view of the clectrodynamometer.

counterweight; on reversing the current in the stationary coil, the equilibrium of the balance is restored by placing a weight on the arm of the balance. From the known value of the balancing weight, the length of the balance arm, and the geometry of the windings, the value of the current can be calculated by eq 1 .

The rersion of the Pellat electrodynamometer used in this work employs single-layer helical windings of bare wire the dimensions of which can be checked at any time. In his instrument Pellat employed multilayer windings. These windings produced a large torque with permissible currents but the large uncertainty in their lincar dimensions would be intolerable according to present requirements. The other important modification in the present instrument is the use of a fused silica balance bcam instead of the brass beam of Pellat. The construction of this type of current measuring device was proposed by Harvey $\mathrm{L}$. Curtis as long ago as 1927. Niaterials for the bcam and stationary coil were purchased around 1930. Through the efforts of Roger W. Curtis murel of the support for this balance was completed by 1940. At the end of World War II a project was set up for the continuation of the primary standardization of current and it has been pursued without much interruption since 1950. Much encouragement and help on the finishing of the balance beam and the coils was obtained from Charles Moon before his death in 1953.

An over-all vicw of the electrodrmamometer with the stationary coil in place is shown in figure 1 ; in figure 2 the stationary coil has been moved to the side to expose the interior of the instrument. The supports of the balanee are mostly of aluminum alloy. A small amount of brass and phosphor bronze was used in the arrestment mechanism. Each pice was tested before finishing to assure that it hacl low magnetic susceptibility. The agate planes and knife aloes of the balance wore finished by the Optical Shop of this Bureau, the material for these boing donated from the collection of Earl F. W (abb). E] cotrical connections to the rotatable coil are eflected by means of two sets of flexible wires. Fach set consists of ten 1 mil bare copper wires, in parallel, having a length of about 3 inches. Slack in the wires was adjusted to make their restraint on the balance tolerable.

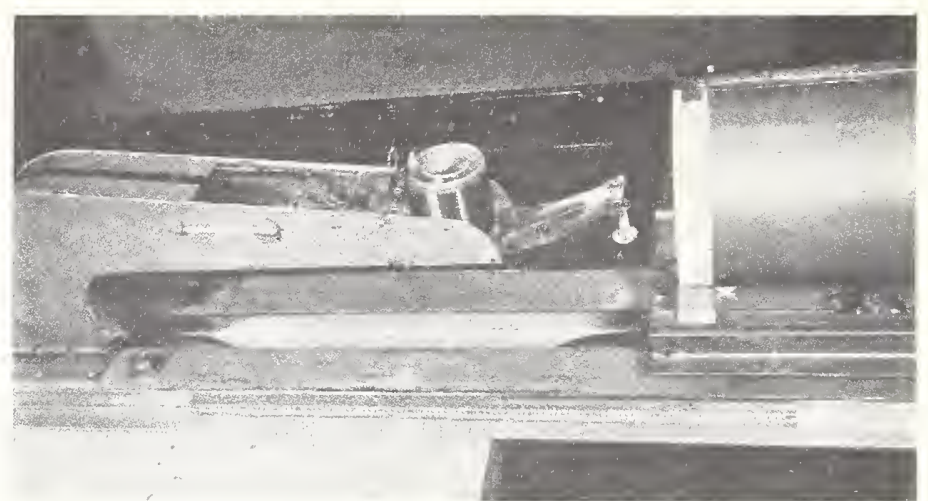

Figure 2. Rotatable coil and balance mechanism.

\section{Construction of the Coils}

Both the stationary and rotatable coils were constructed by the method of Moon that has been described in some detail br Curtis, Moon, and Sparks [5]. Following MLon's method the wire was wound in a helical groove that was produced in the coil form by the process of lapping. The stationary coil form is of fused silica and the rotatable coil form is of Pyrex glass. Oxygen-free copper wire is used in the windings of both coils. Sappline dies were used to reduce the wire to the desired diameter. After each of the preliminary drawing operations, the wire was spooled on smooth drums in a single layer to avoid kinking. After final drawing, the wire on leaving the die was wound directly in the groove on the coil form.

\section{Measurement of the Dimensions of the Coils}

As far as the stationary coil is concerned, the pitel is the parameter of primary importance, the diameter is of less importance as it appears only in a correction term to take account of the finite length of this coil. On the other hand, the diameter of the rotatable coil is extremely important but the value of its pitch is necded with relatively lit te aceuracy.

\subsection{Pitch Measurement}

The pitch of the rotatable coil was taken as the nominal value of the pitch of the lapped groove which was $2 / 3 \mathrm{~mm}$.

The pitch of the stationary coil was measured by the micrometer method described by Curtis, Moon. and Sparks [5]. Longitudinal readings for the measurement of pitch were taken at several places along the coil in each of six angular positions around the winding. Measurements were made with the coil supported at its ends. It was demonstrated that the bending of the tube when supported in the manner did not affect the measured pitch by as much as $1 \mathrm{ppm}$. The results are summarized in 1atale 1.

\subsection{Diameter of Coils}

The importance of the diameter of the rotatable 
coil has already been mentioned. Although the diameter of the stationary coil was not needed with the highest accuracy, considerable pains were taken on account of its potential use as a standard of self inductance, and the values obtained will be recorded here. The method used in measuring both coils was similar to that described by Curtis, Moon, and Sparks [6]. The experimental arrangement of apparatus used in the measurement of the rotatable coil is shown in figure 3 and that used in the case of the stationary coil is given in figure 4 . The results are given in tables 2 and 3 and in figures 5 and 6 . The correction given in table 2 to the diameter of the

TABLE 1. Axzal length and pitch of stationary coil

\begin{tabular}{|c|c|c|c|c|c|}
\hline Date, 1952 & $\begin{array}{l}\text { Azimuth } \\
\text { position }\end{array}$ & End std. & $\begin{array}{l}\text { Interval } \\
\text { measured }\end{array}$ & $\begin{array}{l}\text { Intervals } \\
\text { examined }\end{array}$ & $\begin{array}{l}\text { Pitch at } \\
25^{\circ} \mathrm{C}\end{array}$ \\
\hline $\begin{array}{l}21 \mathrm{Mar} \\
24 \mathrm{Mar}- \\
25 \mathrm{Mar}- \\
25 \mathrm{Mar} \\
26 \mathrm{Mar}\end{array}$ & $\begin{array}{c}\text { Degrees } \\
0 \\
90 \\
180 \\
270 \\
300\end{array}$ & $\begin{array}{c}\text { NBS No. } \\
6,827 \\
6,827 \\
6,827 \\
6,827 \\
6,827\end{array}$ & $\begin{array}{l}\text { Turns } \\
454 \\
454 \\
454 \\
454 \\
454\end{array}$ & $\begin{array}{c}\text { Number } \\
8 \\
8 \\
8 \\
8 \\
8\end{array}$ & $\begin{array}{l}\mathrm{mm} / \mathrm{tur} \\
1.00008 \\
1.00008 \mathrm{1} \\
1.00008 \\
1.000088 \\
1.000088\end{array}$ \\
\hline $\begin{array}{r}27 \mathrm{Mar} \\
4 \mathrm{Apr} \\
4 \mathrm{Apr} \\
9 \mathrm{Apr} \\
9 \mathrm{Apr}\end{array}$ & $\begin{array}{l}120 \\
300 \\
300 \\
300 \\
120\end{array}$ & $\begin{array}{l}6,827 \\
1,993 \\
1,992 \\
6,827 \\
6,827\end{array}$ & $\begin{array}{l}454 \\
281 \\
274 \\
454 \\
454\end{array}$ & $\begin{array}{l}8 \\
7 \\
6 \\
8 \\
8\end{array}$ & $\begin{array}{l}\text { 1. } 00008 \\
1.000093 \\
1.00009 \\
1.000087 \\
1.00008\end{array}$ \\
\hline \multirow[t]{2}{*}{$\begin{array}{l}10 \mathrm{Apr} \\
10 \mathrm{Apr}\end{array}$} & \multirow[t]{2}{*}{$\begin{array}{r}30 \\
210\end{array}$} & \multirow[t]{2}{*}{$\begin{array}{l}6,827 \\
6,827\end{array}$} & \multirow[t]{2}{*}{$\begin{array}{l}454 \\
454\end{array}$} & $\begin{array}{l}8 \\
8\end{array}$ & $\begin{array}{l}\text { 1. } 00008 \mathrm{f} \\
\text { 1. } 000087\end{array}$ \\
\hline & & & & Average & 1.000087 \\
\hline
\end{tabular}

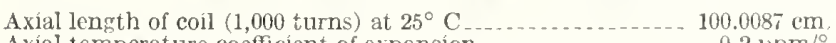

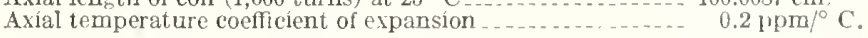

TABIE 2. Diameter and temperature coefficient of rotatable coil

\begin{tabular}{|c|c|c|c|}
\hline l'osition & \multicolumn{2}{|c|}{ Diameter, over-all } & Temperature \\
\hline$\underset{0}{\text { Degrees }}$ & $\begin{array}{c}m m \text { at } 25.0^{\circ} C \\
116.4014\end{array}$ & $\mathrm{~mm}$ at $35.7^{\circ} \mathrm{C}$ & $p p m /^{\circ} \mathrm{C}$ \\
\hline $\begin{array}{l}45 \\
90\end{array}$ & $\begin{array}{l}116.4009 \\
116.4011\end{array}$ & 116.4068 & 47 \\
\hline 135 & 116.4012 & 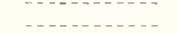 & \\
\hline Average & 116.4011 & $\cdots$ & 4.7 \\
\hline
\end{tabular}

Diameter of wre

\begin{tabular}{|c|c|c|}
\hline \multirow{2}{*}{$\begin{array}{c}\text { Sample } \\
\text { No. }\end{array}$} & \multicolumn{2}{|c|}{ Origin of sample } \\
\hline & $\begin{array}{l}\text { Peginning } \\
\text { of wirnding }\end{array}$ & $\begin{array}{l}\text { End of } \\
\text { winding }\end{array}$ \\
\hline $\begin{array}{l}1 \\
2 \\
3\end{array}$ & $\begin{array}{c}\mathrm{mm} \\
0.5613 \\
5619 \\
5617\end{array}$ & $\begin{array}{c}m m \\
0.5620 \\
.5619 \\
.5620\end{array}$ \\
\hline Avelage & & \\
\hline
\end{tabular}

Average over-all diameter at $25.0^{\circ} \mathrm{C}$

$116.4011 \mathrm{~mm}$

Mean diameter of winding, $2 \bar{a}$, at $25.0^{\circ} \mathrm{C}$

Force on wire during windin

Initial strain in wile,

$.5618 \mathrm{~mm}$

$115.8393 \mathrm{mr}$

12 ib

$21.4 \times 10^{-4}$

-7.6 ppm

$57.9192 \mathrm{~mm}$ 3.6570 ohm

$0.0135 \mathrm{oghm}^{\circ} \mathrm{C}$

$0.8 \mathrm{~cm}$

$-1 \times 10^{-6}$

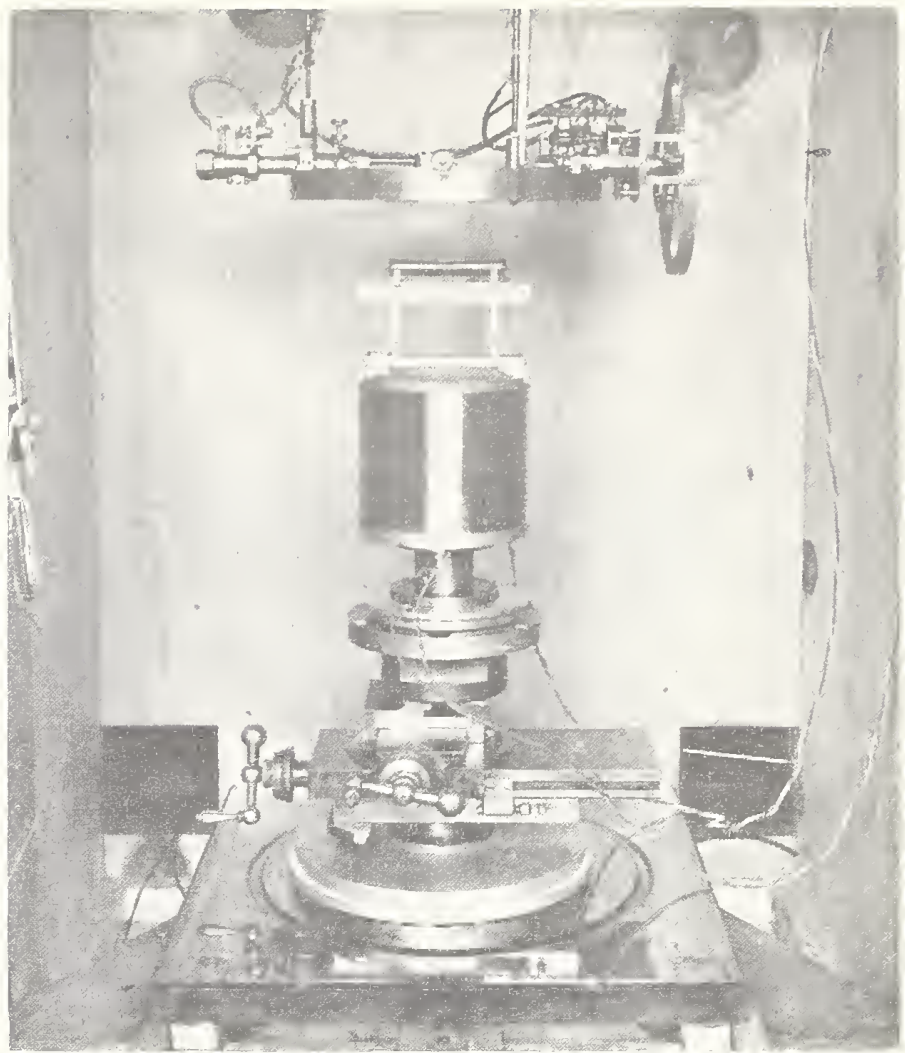

Figure 3. Equipment used in measuring the diameter of the rotatable coil.

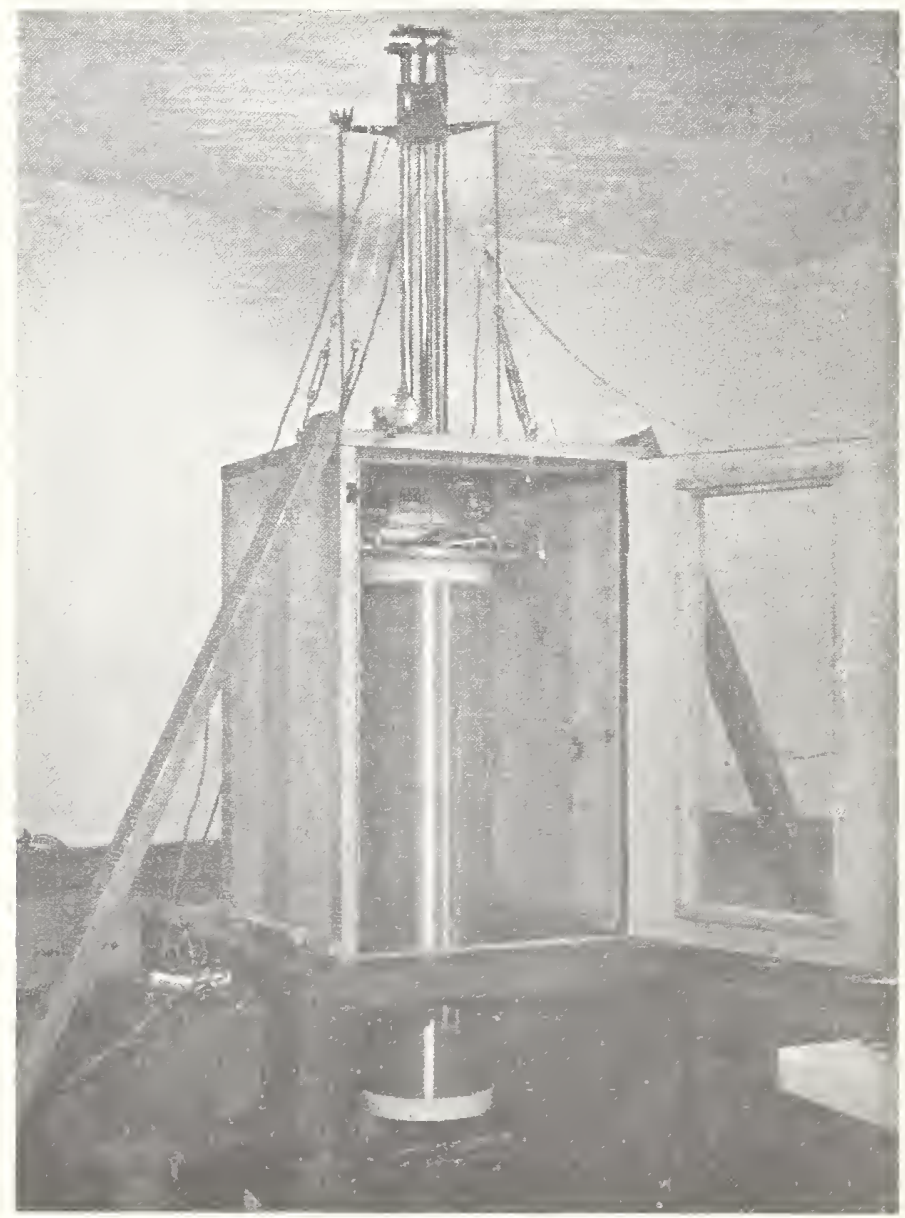

FIGURE t. Stationary coil as mounted for the measurement of diameter. 
rotatable coil to take account of the current distribution is based upon the theory of Chestel Snow and the experiments of Thomas Wells [7] on the resistivity-strain coefficient of the wire used in this coil as discussed in section 7 .

TABLE 3. Diameter and temperature coefficzent of stationary coil

\begin{tabular}{|c|c|c|c|c|c|}
\hline \multirow{2}{*}{ Position } & \multicolumn{4}{|c|}{ Diameter, over-all } & \multirow{2}{*}{$\begin{array}{l}\text { Tempera- } \\
\text { ture coeff- } \\
\text { cient }\end{array}$} \\
\hline & Top half & $\begin{array}{c}\text { Bottom } \\
\text { half }\end{array}$ & $\begin{array}{l}\text { Bottom } \\
\text { half }\end{array}$ & $\begin{array}{c}\text { Bottom } \\
\text { half }\end{array}$ & \\
\hline Degrees & $m$ m at $25^{\circ} \mathrm{C}$ & mmat $25^{\circ} \mathrm{C}$ & $\operatorname{mmat} 28.9^{\circ} \mathrm{C}$ & $\operatorname{mmat} 34.4^{\circ} \mathrm{C}$ & $p p m /{ }^{\circ} C$ \\
\hline 0 & 280.3236 & 280.3235 & -...... & $\ldots$ & - n \\
\hline $\begin{array}{l}45 \\
90\end{array}$ & $\begin{array}{l}280.3243 \\
280.3242\end{array}$ & $\begin{array}{l}280.3231 \\
280.3246\end{array}$ & - & & $-\cdots$ \\
\hline 135 & 280.3235 & 280.3241 & 280.3253 & 280.3266 & +1.0 \\
\hline Average & 280.3239 & 280.3238 & & & \\
\hline
\end{tabular}

Diameter of wire

\begin{tabular}{|c|c|c|}
\hline \multirow{2}{*}{$\begin{array}{c}\text { Sample } \\
\text { No. }\end{array}$} & \multicolumn{2}{|c|}{ Origin of sample } \\
\cline { 2 - 3 } & $\begin{array}{c}\text { Beginning } \\
\text { of winding }\end{array}$ & $\begin{array}{c}\text { End of } \\
\text { winding }\end{array}$ \\
\hline & $m m$ & $m m$ \\
1 & 0.6995 & 0.6990 \\
2 & .6992 & .6989 \\
3 & .6992 & .6990 \\
Average & .6993 & .6990 \\
\hline
\end{tabular}

A verage over-all diameter at $25.0^{\circ} \mathrm{C}$

Wire diameter..

Mean diameter of winding, $2 \bar{a}_{1}$, at $25^{\circ} \mathrm{C}$

Force on wire during winding

lnitial strain in wire, $\mathrm{K}_{1} \ldots$

Correction for current distribution, $\frac{\Delta \bar{a}_{1}}{\bar{a}_{1}}$ (see section 7 ) ...... -2.1 ppm

Effective radius, $a_{1}=\bar{a}_{1}+\Delta \bar{a}_{1}$.

Thickness of fused silica form.

Magnetic susceptibility of form
Resistance of winding at $25^{\circ} \mathrm{C}$ -

Change in winding resistance with temperature.............. $0.15 \mathrm{ohm} /{ }^{\circ} \mathrm{C}$

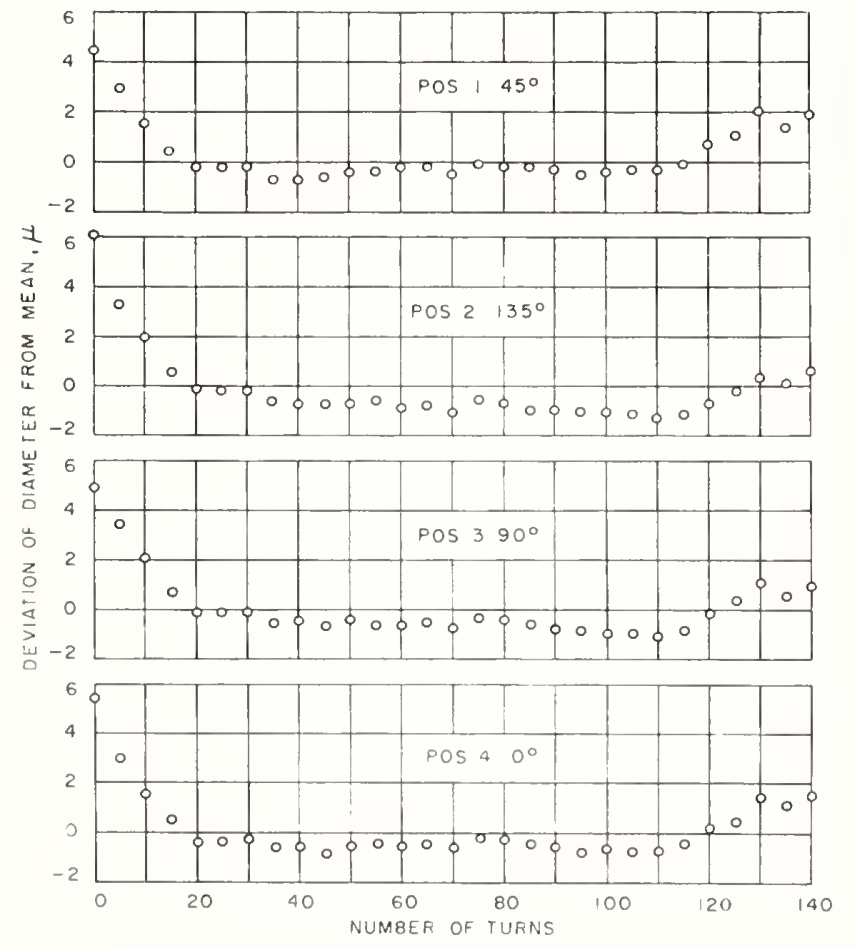

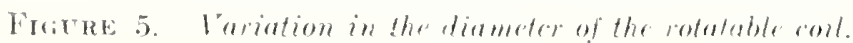

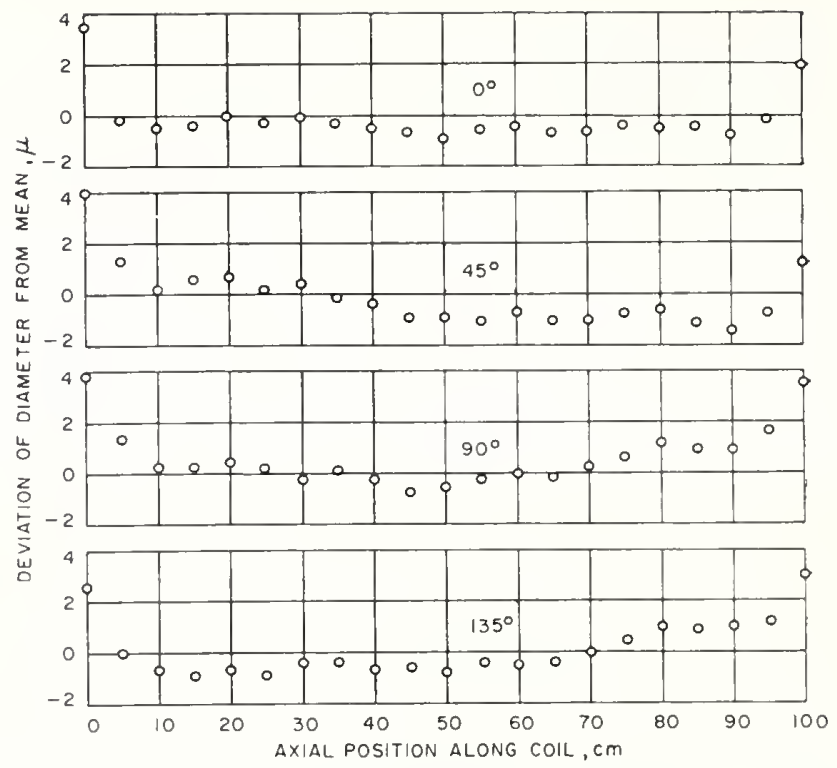

FICURE 6. Tariation in the diameter of the stationary coil.

\section{Length of the Balance Arm}

The distance between the outer knife edges of the beam, figure 7 , was evaluated by comparison with a calibrated interval on a line standard. The balance beam was mounted on a comparator intended originally for the purpose of comparing line standards. In this instrument the unknown and standard are placed side by side on separately adjustable mountings. These mountings were attached to a carriage that could be moved to bring either the standard or unknown into the field of two rigidly supported microscopes. It was found possible to set the cross hairs of the microscopes over the apexes of the knife edges on the beam in the same manner as on a graduation of a line standard. The difference between the spacing of the outer knife edges and a known interval on the line standard was obtained from the readings of the filar micrometer erepieces of the microscopes. Through the cooperation of L. V. Judson and B. L. Page of the Metrology Section, facilities were provided for making this measurement.

The ratio of the arin lengths was determined by weighing known masses. As indicated in table 4 no significant difference was found in this ratio over a period of 3 years whether 20-gram or 3-gram masses were used. "The masses were transposed as far as possible but since the arm lengths differed by 1.5 percent a number of small masses had to be left on one pan. Dividing the unloaded length $\left(l_{1}+l_{2}\right)$ by $\left(1+l_{2} / l_{1}\right)$, where $l_{2} / l_{1}$ is the ratio of the arms when symmetrically loaded, gives a first approximation to the length, $l_{1}$, of the arm chosen to receive the balancing mass. As pointed out by R. D. Cutkosky a further correction inust be applied to allow for the increase in the length of the arm when the pan and balancing mass are applied. To measure this distortion, the beam was mounted on a surface plate, the four bosses under the central part of the beam (fig. 7) boing secured to the plate with beeswax. 


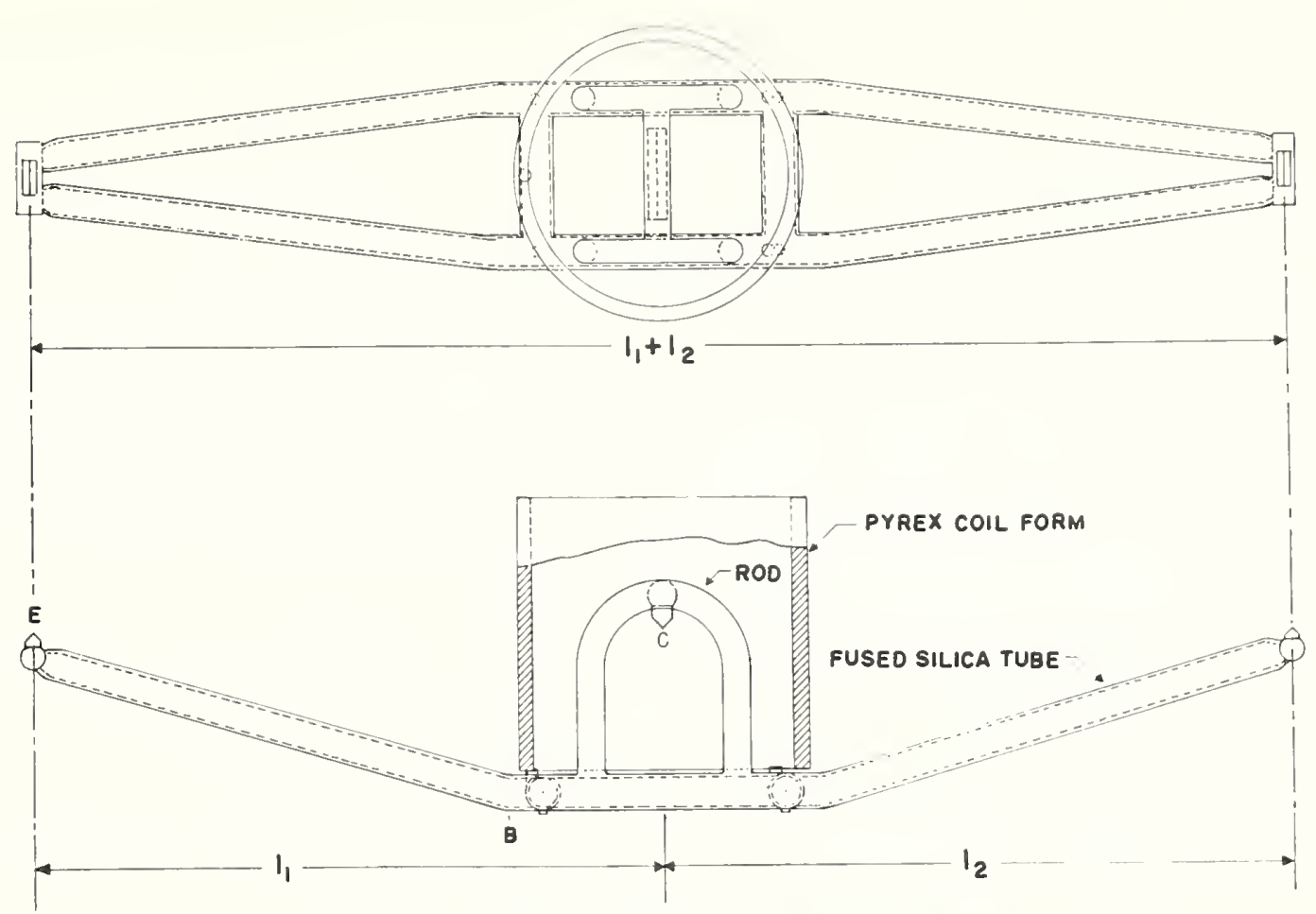

FiguRE 7. Balance beam and rotatable coil form.

TABLE 4. Length of balance arm

Listance between end knife edges, $\left(l_{1}+l_{2}\right)$

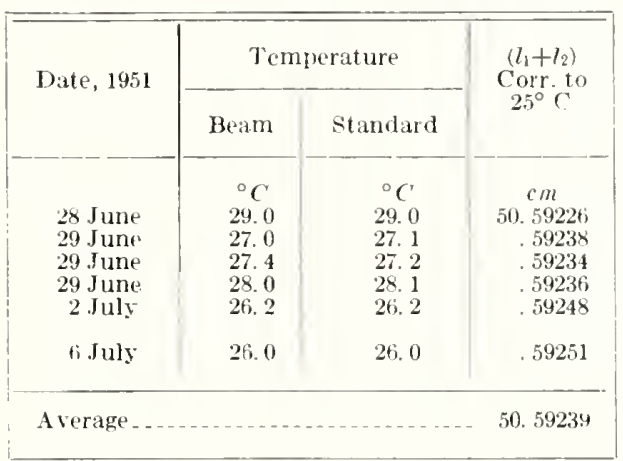

Above values based upon calibrated interval 390 to $896 \mathrm{~mm}$ on meter bar $153-\mathrm{R}$.

Ratio of balance arms, $l_{2} / l_{1}$

\begin{tabular}{|c|c|c|}
\hline Datc & $l_{2} / h_{i}$ & Condition of weighing \\
\hline $\begin{array}{r}\text { 19-20 Aug. } 1952 \\
15 \text { Dec. } 1953 \\
22 \text { July } 1955 \\
23 \text { July } 1955\end{array}$ & $\begin{array}{r}0.984546 \mathrm{f} \\
.984548 \\
.984550 \\
.984547\end{array}$ & $\begin{array}{l}\text { 20-gram masses } \\
\text { 3-gram inasses transposed } \\
\text { 3-g masses transposed } \\
\text { 3-g masses transposed }\end{array}$ \\
\hline Mrall & 0. 984548 & \\
\hline
\end{tabular}

Unloaded length $l_{1}$ of halance arm ........ $25.49316 \mathrm{~cm}$

Correter (3.1 ppm)

(It was on these bosses that the beam rested during the measurement of the distance between the end knife edges.) By means of a microscope equipped with a filar micrometer eyepiece the endwise movement of the knife edge at $\mathrm{E}$ figure 7 was measured when a load equal to the scale pan was applied to that end of the beam. A further complication arises when the standard mass is added to the scale pan and the current in the stationary coil is reversed, that is, there is then an additional increase in the length of the balance arm. Both distortions affect the position of all the load on the end knife edge including, though to a lesser extent, the distributed mass of the balance arm.

In order to discuss these effects, let the following notation be used:

$P=$ Mass of scale pan,

$m=$ standard balancing mass,

$y=$ acceleration of gravity,

$i_{1}=$ unloaded length of balance arm.

$\delta l_{P}=$ change in $l_{1}$ on adding load $P$,

$\delta \cdot \widetilde{J_{P}}=$ change in torque to allow for distortion of the distributed mass of beam on adding load $P$,

$\widetilde{s_{1}}=$ torque on rotatable coil with mass $m$ removed and stationary coil current in forward direction,

$\tau_{2}=$ torque on rotatable coil with mass $m$ on scale pan and stationary coil current reversed.

If the standard mass $m$ is adjusted so that the balance is in equilibrium for both directions of the stationary coil current, the change in torque on the rotatable coil is given by $\pi_{2}-\pi_{1}$,

where

$$
\widetilde{T_{1}}=P !\left(l_{1}+\delta l_{P}\right)+\delta \cdot \widetilde{\mathcal{J}_{P}}
$$

and

$$
. \widetilde{\gamma_{2}}=(P+m) g\left(l_{1}+\delta l_{P+r l}\right)+\delta \cdot \widetilde{T}_{P+m} .
$$

Subtracting the above expressions 


$$
\widetilde{T_{2}}-\mathscr{T}_{1}=m g l_{1}\left[1+\frac{P}{m} \frac{\delta l_{m}}{l_{1}}+\frac{\delta l_{P+m}}{l_{1}}\right]+\delta \mathscr{T}_{m} .
$$

The quantity $\delta \mathscr{T}_{m}$ is the change in torque resulting from the additional distortion of the distributed mass of the arm on adding the balancing mass $m$. Its value was estimated from the observed distortion, $\delta l_{m}$, measured at the end knife edge, the dimensions of the various parts, and the density of the material (assumed to be 2.2 for fused silica). Referring to figure 7 it is assumed that the portion of the beam between $\mathrm{C}$ and $\mathrm{B}$ would contribute nothing to $\delta \mathscr{T}_{m}$ on account of the relative stiffness of that part. The part of the arm between $\mathrm{B}$ and the cross rod at $\mathrm{E}$ is assumed to bend as a cantilever beam fixed at $\mathrm{B}$ with concentrated load $m$ at $\mathrm{E}$. The total mass $M_{r}$ of the cross rod and knife edge at $\mathrm{E}$ is considered to be concentrated at $\mathrm{E}$. Under these assumptions $\delta \mathscr{T}_{m}$ is given by

$$
\delta \mathscr{T}_{m}=\left[M_{\tau}+3 / 8 M_{l}\right] \delta l_{m},
$$

where $M_{t}$ is the mass of the arm between $\mathrm{B}$ and the cross rod at $\mathrm{E}$. With this value of $\delta \mathscr{T}_{m}$ eq. (2) becomes

$\mathscr{T}_{2}-\mathscr{T}_{1}=m g l_{1}\left[1+\frac{\left(P+M_{\tau}+3 / 8 M_{t}\right)}{m} \frac{\delta l_{m}}{l_{1}}+\frac{\delta l_{P+m}}{l_{1}}\right]$.

Numerical values obtained for the quantities in the brackets of eq $\left(2^{\prime}\right)$ are as follows:

$$
\begin{array}{rlrl}
m & =1.481 \text { grams } & M_{t}=31.5 \mathrm{~g} \\
P & =18.3 \text { grams } & \delta l_{m} / l_{1}=1.0 \times 10^{-6} \\
M_{r} & =4.4 \text { grams } & \delta l_{P+m} / l_{1} & =13.8 \times 10^{-6}
\end{array}
$$

When these numerical values are applied in eq $\left(2^{\prime}\right)$ the corrected torque is

$$
\mathscr{T}_{2}-\mathscr{T}_{1}=m g l_{1}\left[1+37.1 \times 10^{-6}\right] .
$$

In effect the unloaded length $l_{1}$ of the balance arm is increased by $37.1 \mathrm{ppm}$ as a result of the distortion under load. The correction for this is applied in table 4.

\section{Geometrical Factor of the Electro- dynamometer}

The maximum torque, $T$, produced by unit currents in concentric helical wires is given by Snow [3] as follows:

$$
\begin{aligned}
& T=\left[\frac{\partial M}{\partial \theta}\right]_{\theta=\pi / 2} \\
&=4 \pi^{2} \frac{N_{1}}{L_{1}} a_{2}^{2} N_{2} \mu_{1}\left\{1+S+S_{1}+\frac{2}{L_{2} a_{2}} \int_{-L_{2} / 2}^{L_{2} / 2} U_{a}\left(x_{2}\right) d x_{2}\right. \\
&+\frac{a_{1}}{2 \mu_{1}} \int_{-L_{1} / 2}^{L_{1} / 2}\left[\frac{\left(2 x_{1}^{2}-a_{1}^{2}\right)}{\left(x_{1}^{2}+a_{1}^{2}\right)^{5 / 2}} U_{a}\left(x_{1}\right)-\right. \\
&\left.\left.\frac{3 a_{1} x_{1}}{\left(x_{1}^{2}+a_{1}^{2}\right)^{5 / 2}} U_{x}\left(x_{1}\right)\right] d x_{1}\right\}
\end{aligned}
$$

The subscripts 1 and 2 refer to the stationary coil and the rotatable coil respectively, and

$a=$ radius of coil,

$L=$ length of winding,

$N=$ total number of turns in a winding,

$x=$ axial distance from center of a winding,

$\mu=$ cosine of the angle between the diagonal of a coil and its axis,

$U_{x}(x), U_{a}(x)=$ observed displacement of any part of a winding in an axial or radial sense respectively from the position it would have if the winding were uniform.

The series $S$ is given by:

$S=\left(1-\mu_{1}^{2}\right) \sum_{n=1}^{\infty}(-1)^{n+1}\left(\frac{r_{2}}{r_{1}}\right)^{2 n} C_{n}\left(\mu_{1}\right) C_{n+1}\left(\mu_{2}\right)\left(n+\frac{1}{2}\right) A_{n}$,

where

$$
\begin{aligned}
A_{n} & =\frac{1 \cdot 3 \cdot 5 \cdots(2 n-1)}{2 \cdot 4 \cdot 6 \cdots 2 n} \\
C_{n}(\mu) & =\frac{P_{2 n}^{\prime}(\mu)}{n(2 n+1) \mu}, \\
r & =\sqrt{a^{2}+L_{/}^{2} / 4}
\end{aligned}
$$

$P_{n}(\mu)$ is Legendre's polynomial of degree $n$, and $P_{n}^{\prime}(\mu)$ is $(d / d \mu) P_{n}(\mu)$.

Also

$$
\begin{aligned}
S_{1}=\frac{\left(1-\mu_{1}^{2}\right)\left(1-\mu_{2}^{2}\right)}{6 a_{2}^{2}} \sum_{n=1}^{\infty}(-1)^{n+l}\left(\frac{r_{2}}{r_{1}}\right)^{2 n} & \\
& \cdot C_{n}\left(\mu_{1}\right) C_{n}\left(\mu_{2}\right) n\left(n+\frac{1}{2}\right) \mathcal{A}_{n}\left[n \frac{L_{1}^{2}}{N_{1}^{2}}-\left(n+\frac{1}{2}\right) \frac{L_{2}^{2}}{N_{2}^{2}}\right]
\end{aligned}
$$

The radius of the rotatable coil, $a_{2}$, in eq (3) is the effective radius rather than the mean radius, $\bar{a}_{2}$, of the wmding, determined by mechanical measurement. The difference between the effective and mean radii depends on the variation of current density over the cross section of the wire. The "natural" distribution of current density (inversely proportional to distance from the axis of the coil) would certainly apply if the resistivity were constant everywhere in the wire. Some measurements by Wells [7] indicate that for a wire in simple tension, the resistivity-strain coefficient can be represented empirically by

$$
\frac{\Delta \sigma}{\sigma_{0}} / \frac{\Delta l}{l_{0}}=\beta+\gamma\left(\frac{\Delta l}{l}\right)^{2},
$$

where, $\sigma_{0}$, is the resistivity and $l_{0}$ is the length of the unstrained specimen. The constants $\beta$ and $\gamma$ were determined by Wells on oxygen-free copper wire to have the values $\beta=1.13$ and $\gamma=-2.5 \times 10^{4}$. To the extent that the strain at any point in the wire can be 
determined from the initial tension and bending, the resistivity-strain relation can be incorporated in an expression for the current density as a function of the distance from the axis of the coil. A section in the wire cut by a plane through the axis of the coil is shown in figure 8 . The coordinates of a point, $P\left(x, y^{\prime}\right)$, are shown as functions of $r$ and $\theta$ in the wire. The current density, $i\left(y^{\prime}\right)$, is assumed to be inversely proportional to $\left(\bar{a}_{2}+y^{\prime}\right)$ and the resistivity, $\sigma\left(y^{\prime}\right)$, and is independent of $x$. The initial strain, $K_{2}$, is known from the winding tension and is taken to be independent of $x$ and $y^{\prime}$. 'The strain due to bending around the form is assumed to be equal to $y^{\prime} / \bar{a}_{2}$ and independent of $x$. On the basis of these assumptions, the current in a filament of area $r d \theta d r$ is given by

$$
i\left(y^{\prime}\right) r d \theta d r=\frac{C r d \theta d r}{2 \pi\left(\bar{a}_{2}+y^{\prime}\right) \sigma\left(y^{\prime}\right)} .
$$

On setting the strain $\Delta l / l_{0}=\left(K_{2}+\frac{y^{\prime}}{\bar{a}_{2}}\right)$ in eq 4 , the resistivity at any $y^{\prime}$ can be written

$\sigma\left(y^{\prime}\right)=\sigma_{0}\left(1+\beta K_{2}+\right.$

$$
\left.\gamma K_{2}^{3}\right)\left[1+B_{1} \frac{y^{\prime}}{\bar{a}_{2}}+B_{2}\left(\frac{y^{\prime}}{\bar{a}_{2}}\right)^{2}+B_{3}\left(\frac{y^{\prime}}{\bar{a}_{2}}\right)^{3}\right]
$$

where

$$
\begin{aligned}
& B_{1}=\frac{\beta+3 \gamma K_{2}{ }^{2}}{1+\beta K_{2}+\gamma K_{2}{ }^{3}}, \\
& I_{2}=\frac{3 \gamma K_{2}{ }^{2}}{1+\beta K_{2}+\gamma K_{2}{ }^{3}}, \\
& B_{3}=\frac{\gamma}{1+\beta K_{2}+\gamma K_{2}{ }^{3}} .
\end{aligned}
$$

On combining eqs (5) and (6) and neglecting terms of higher order than the third in $\left(y^{\prime} / \bar{a}_{2}\right)$, the current density is given by:

$$
\begin{aligned}
i\left(y^{\prime}\right)=\frac{C}{2 \pi \bar{a}_{2} \sigma_{0}\left(1+\beta K_{2}+\gamma K_{2}^{3}\right)}\left[1-C_{1} y^{\prime} / \bar{a}_{2}+\right. \\
\\
\left.C_{2}\left(y^{\prime} / \bar{a}_{2}\right)^{2}-C_{3}\left(y^{\prime} / \bar{a}_{2}\right)^{3}\right]
\end{aligned}
$$

where

$C_{1}=B_{1}+1$,

$\mathrm{C}_{2}=-\left(B_{2}-B_{1}-B_{1}{ }^{2}-1\right)$, and

$C_{3}=\left(B_{3}-B_{2}+B_{1}+B_{1}^{2}+B_{1}^{3}-2 B_{1} B_{2}+1\right)$.

The constant $C / 2 \pi \bar{a}_{2} \sigma_{0}\left(1+\beta K_{2}+\gamma K_{2}^{3}\right)$ must be $i(o)$ and its value, by the integral $\int_{0}^{\rho_{2}} \int_{0}^{2 \pi} i\left(y^{\prime}\right) r d \theta d r$ that

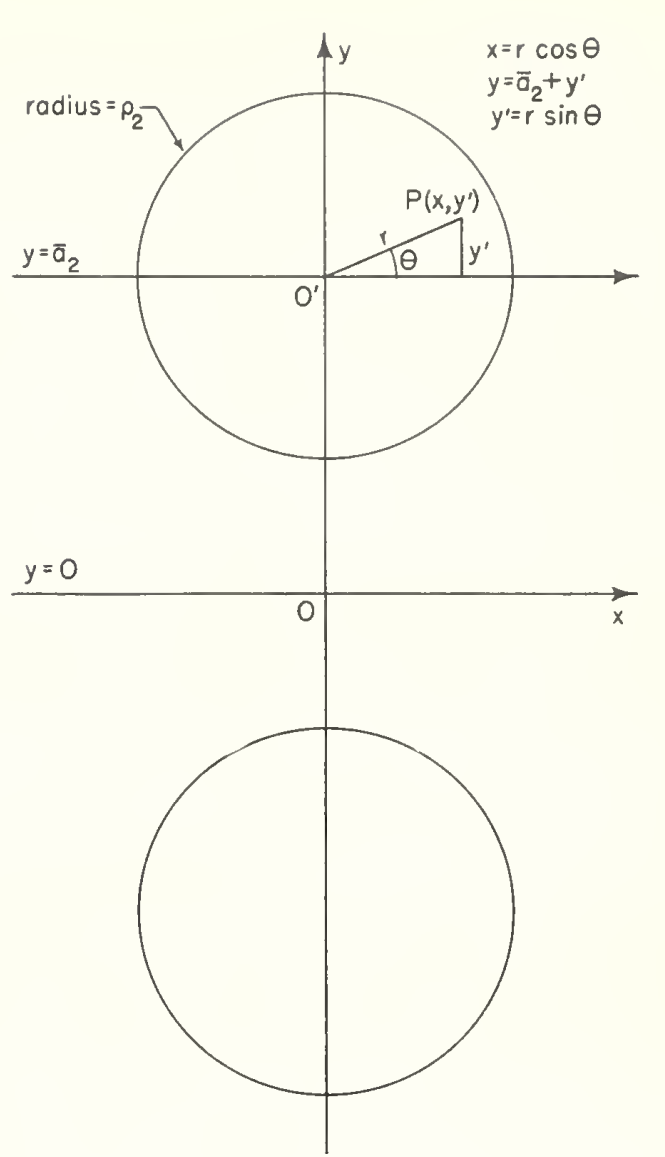

FIGURE 8. Section through one turn of rotatable coil cut by an axial plane.

makes unit current in the wire is

$$
\frac{1}{\pi \rho_{2}^{2}}\left[1-\frac{C_{2}}{4}\left(\frac{\rho_{2}}{\bar{a}_{2}}\right)^{2}\right]
$$

If this value be placed in eq 7 one gets

$$
\begin{aligned}
i\left(y^{\prime}\right)= & \frac{1}{\pi \rho_{2}^{2}}\left[1-C_{1}\left(\frac{y^{\prime}}{\bar{a}_{2}}\right)+\right. \\
& \left.C_{2}\left\{\left(\frac{y^{\prime}}{\bar{a}_{2}}\right)^{2}-\frac{1}{4}\left(\frac{\rho_{2}}{\bar{a}_{2}}\right)^{2}\right\}-C_{3}\left(\frac{y^{\prime}}{\bar{a}_{2}}\right)^{3}\right] .
\end{aligned}
$$

The current function, eq (8), is of the same form as that considered by snow [3] who found that in effect this current distribution in the wire changes its radius from $\bar{a}_{2}$ to $\left(\bar{a}_{2}+\Delta \bar{a}_{2}\right)$ where $\Delta \bar{a}_{2}=\frac{1}{8}\left(\rho_{2}^{2} / \bar{a}_{2}\right)(1-$ $\left.2 C_{1}\right)$, terms in $\left(\rho_{2} / \bar{a}_{2}\right)^{t}$ and higher being neglected. Accordingly one can write to a good approximation, since the constants $\beta, \gamma$, and $K_{2}$ are not known with an accuracy better than a few percent.

$$
\Delta \bar{a}_{2}=-\frac{1}{8} \frac{\rho_{2}^{2}}{\bar{a}_{2}}\left(1+2 \beta+6 \gamma K_{2}^{3}\right)
$$

Likewise it is found that

$$
\Delta \bar{a}_{1}=-\frac{1}{8} \frac{\rho_{1}^{2}}{\bar{a}_{1}}\left(1+2 \beta+6 \gamma K_{1}^{3}\right) .
$$


Numerical values of $\Delta \bar{a}_{2}$ and $\Delta \bar{a}_{1}$ are given in tables 2 and 3.

A summary of the computations on the constant $T$ is given in table 5. The following expression, obtained by differentiating the main term of eq (3), was used to take account of small changes in the linear dimensions of the coils:

$$
\frac{\delta T}{T}=2 \frac{\delta a_{2}}{a_{2}}-\frac{4 a_{1}^{2}}{\left(L_{1}^{2}+4 a_{1}^{2}\right)} \frac{\delta a_{1}}{a_{1}}-\frac{L_{1}^{2}}{\left(L_{1}^{2}+4 a_{1}^{2}\right)} \frac{\delta L_{1}}{L_{1}}
$$

The constant $T$ can be set equal to $F l_{1}$ where $F$ is the force constant referring to the scale pan on balance $\operatorname{arm} l_{1}$. From the results of tables 4 and 5 the value of $F$ is

$$
F=\mathrm{T} / l_{1}=70029.91 \text { dynes } / \overline{\operatorname{cgsu}}^{2} .
$$

The acceleration of gravity $g$ at the scale pan, on the basis of the Dryden reduction [8] and a gravity survey made at this Bureau in 1948 by the Geological Survey, is $980.081 \mathrm{~cm} / \mathrm{sec}^{2}$. The required balancing mass per unit current squared is

$$
F / g=71.45318 \text { grams } / \overline{\operatorname{cgsu}}^{2} .
$$

On reversing the current in the stationary coil the change in torque on the rotatable coil is doubled. After applying a correction to the torque of $-3 \mathrm{ppm}$ on account of the magnetic susceptibility of the coil forms and changing to the practical unit of current, the calculated balancing mass per ampere squared is

$$
2 F\left(1-3.0 \times 10^{-6}\right) / 100 g=1.429059 \mathrm{grams} / \overline{\mathrm{amp}}^{2} .
$$

The correction applied above to the electrodynamometer constant on account of the magnetic susceptibility of the coil forms was estimated in the following manner. From the known susceptibility, $k$, of each coil form, the area $A$ of its cross section, and the mean axial component of field $h$ existing in the form, the quantity, $q$, of free magnetism existing on its ends was calculated by the expression $q=k A H$.

TABLE 5. Computation of balance constant

\begin{tabular}{|c|c|}
\hline Quantity & Numerical value \\
\hline $\begin{array}{l}\mu_{1}-\ldots+1 \\
\mu_{2}\end{array}$ & $\begin{array}{c}0.963064 \\
.6274\end{array}$ \\
\hline $4 \pi^{2} \frac{N_{1}}{L_{1}} a_{2}^{2} N_{2 \mu 1} \ldots$ & $1.785461 \times 10^{6}$ \\
\hline $\begin{array}{l}C_{1}\left(\mu_{1}\right) \\
C_{2}\left(\mu_{1}\right) \\
C_{2}\left(\mu_{2}\right) \\
C_{5}\left(\mu_{2}\right) \\
\left.S_{1}\right)\end{array}$ & $\begin{aligned} & 1.0 \\
& 0.873111 \\
- & .0612 \\
- & .212 \\
-63.0 \times 10^{-6} & \\
& .0 \times 10^{-8}\end{aligned}$ \\
\hline$\frac{a_{1}}{2} \int^{L_{1} / 2} \underline{\left(2 x_{1}^{2}-a_{1}^{2}\right)} U_{-}\left(x_{1}\right) d x_{1}$ & $+10 \times 10^{-6}$ \\
\hline$T=[\partial M / \partial \theta]_{\theta=\frac{\pi}{2}}$ & $1.785350 \times 10^{6}$ dyne-cm \\
\hline
\end{tabular}

[Value used for the independent variables:

$a_{1}=13.98121 \mathrm{~cm} . \quad L_{1}=100.0087 \mathrm{~cm} . \quad N_{1}=1000$.

$a_{2}=5.79192 \mathrm{~cm} . \quad L_{2}=9.3333 \mathrm{~cm} . \quad N_{2}=140$.]
The magnetic charge distributed over each end of the stationary coil with one ampere flowing was thus estimated to be $1.5 \times 10^{-3}$ pole. The contribution of the field of these charges to the field at the center of the stationary coil is found to be positive and of the order of one part in $10^{7}$ even if the charges are regarded as point charges on the axis of the coil.

The magnetic charges on the ends of the rotatable coil induced by its own magnetic field are estimated to be 0.56 pole when one ampere is flowing. These charges contribute to the magnetic moment of the coil in the amount of $-5 \times 10^{-3}$ dyne $\mathrm{cm} /$ gauss. The magnetic moment of the rotatable coil in the absence of the form is $1,400 \mathrm{dyne} \mathrm{cm} / \mathrm{gauss}$. The correction to the torque on account of the rotatable coil form is thus estimated to be $-3.0 \mathrm{ppm}$. An attempt was made to ascertain experimentally the effect of the aluminum alloy used in the balance support by placing more of this material in the opposite end of the stationary coil. No significant change in the measured torque was observed when approximately twice the original amount of aluminum alloy was added. It must be admitted that it would be difficult by this kind of test to guarantee that the effect was not as large as 1 ppm.

\section{Measurement of the Torque}

The electrical connections of the balance are shown in figure 9. During the measurement of the torque, the current was held constant at about 1.02 amp as determined by the standard cell and standard resistor. These standards were maintained at a constant temperature and compared from time to time with this Bureau's primary standards. When the current in the stationary coil was reversed, the change in torque was compensated by placing a mass on the balance arm. The mass (a platinumiridium rod) was adjusted by trial and later evaluated by comparison with known standards. Small corrections to the balancing mass were made from readings on the calibrated scale of the balance. The sensitivity of the balance was $1.8 \mathrm{~cm} / \mathrm{mg}$ and the corresponding half period was about 10 sec. The contribution of the current in the leads to the torque was determined separately and subtracted from the total torque. A series of runs, table 6 , shows the fluctuations in the experimental result after all known corrections have been applied. The result of each run is derived from ten resting points of the balance each of which is calculated from nine observed turning points. Resting points were obtained at intervals of about $4 \mathrm{~min}$ with the current in the stationary coil alternately in the forward and reversed direction.

\section{Results}

On combining the results of the absolute current measurement with the value of the same current as 'derived from the NBS electrical standards, the final result of table 7 is obtained. 


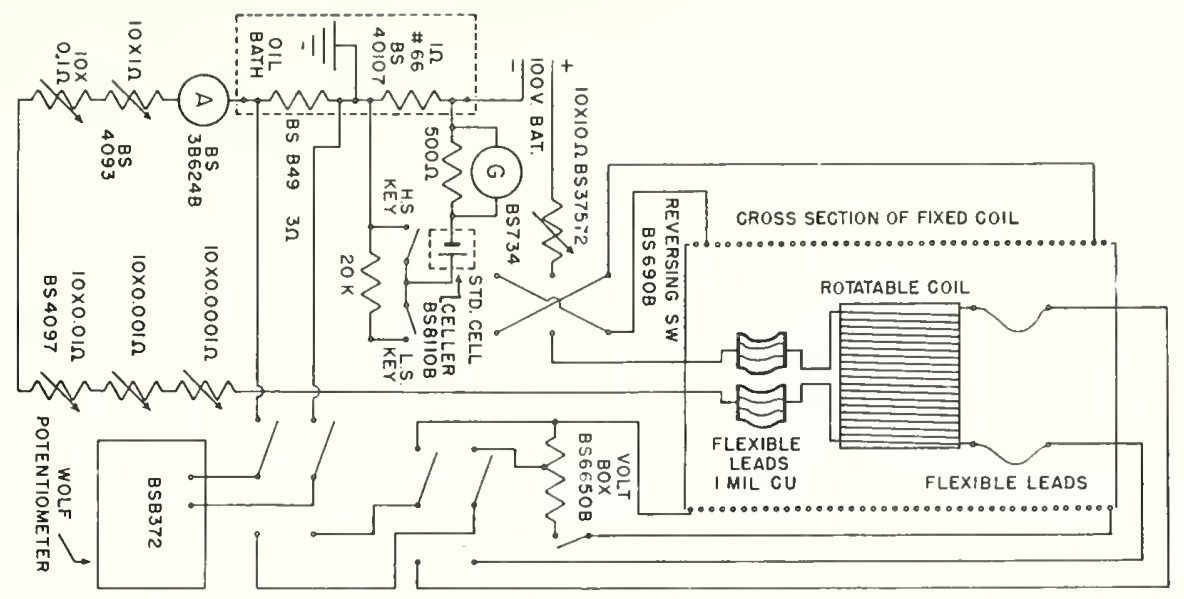

Figure 9. Electrical connections of the electrodynamometer.

TABLE 6. Determination of balancing mass

[Standard mass (Pt-Ir cylinder) - $1481.146 \mathrm{mg}$. Buoyancy correction (air density 0.001164 gram/ $\mathrm{cm}^{3}$ ) -0.080 mg. Standard mass corrected-1481.066 mg.

\begin{tabular}{|c|c|c|c|c|c|c|c|c|c|}
\hline \multirow{2}{*}{ Date, 1954} & \multirow{2}{*}{\multicolumn{2}{|c|}{$\begin{array}{l}\text { Difference in rest } \\
\text { points of balance }\end{array}$}} & \multicolumn{6}{|c|}{ Corrections to individual trials for departures from $25^{\circ} \mathrm{C}$} & \multirow{2}{*}{$\begin{array}{l}\text { Add to } \\
\text { standard } \\
\text { mass }\end{array}$} \\
\hline & & & \multicolumn{2}{|c|}{ Rot. coil } & \multicolumn{2}{|c|}{ Stat. eoil } & \multicolumn{2}{|c|}{ Beam } & \\
\hline $\begin{array}{l}23 \mathrm{Apr} \\
24 \mathrm{Apr} \\
25 \mathrm{Apr}- \\
26 \mathrm{Apr} \\
27 \mathrm{Apr}\end{array}$ & $\begin{array}{c}c m \\
0.002 \\
.012 \\
.011 \\
.016 \\
.013\end{array}$ & $\begin{array}{l}m g \\
0.001 \\
.006 \\
.005 \\
.009 \\
.007\end{array}$ & $\begin{array}{l}\circ C \\
36.9 \\
36.9 \\
36.8 \\
36.7 \\
36.9\end{array}$ & $\begin{array}{l}m g \\
-0.166 \\
-.166 \\
-.164 \\
-.163 \\
-.166\end{array}$ & $\begin{array}{c}\cdot \circ C \\
30.8 \\
30.8 \\
30.7 \\
30.6 \\
30.7\end{array}$ & $\begin{array}{l}m q \\
0.002 \\
.002 \\
.002 \\
.002 \\
.002\end{array}$ & $\begin{array}{l}{ }^{\circ} C \\
29.0 \\
29.0 \\
29.0 \\
29.0 \\
29.0\end{array}$ & $\begin{array}{c}m g \\
0.002 \\
.002 \\
.002 \\
.002 \\
.002\end{array}$ & $\begin{array}{c}m q \\
-0.161 \\
-.156 \\
-.154 \\
-.150 \\
-.155\end{array}$ \\
\hline $\begin{array}{l}23 \mathrm{Apr} \\
28 \mathrm{Apr} \\
29 \mathrm{Apr} \\
30 \mathrm{Apr} \\
1 \mathrm{May}\end{array}$ & $\begin{array}{l}.002 \\
.002 \\
.007 \\
.010 \\
.006\end{array}$ & $\begin{array}{l}.001 \\
.001 \\
.001 \\
.005 \\
.003\end{array}$ & $\begin{array}{l}37.0 \\
37.1 \\
36.7 \\
36.9 \\
37.0\end{array}$ & $\begin{array}{l}-.167 \\
-.168 \\
-.163 \\
-.166 \\
-.167\end{array}$ & $\begin{array}{l}30.9 \\
31.0 \\
30.6 \\
30.8 \\
30.9\end{array}$ & $\begin{array}{l}.002 \\
.002 \\
.002 \\
.002 \\
.002\end{array}$ & $\begin{array}{l}29.0 \\
29.0 \\
29.0 \\
29.0 \\
29.0\end{array}$ & $\begin{array}{l}.002 \\
.002 \\
.002 \\
.002 \\
.002\end{array}$ & $\begin{array}{l}-.162 \\
-.163 \\
-.155 \\
-.157 \\
-.160\end{array}$ \\
\hline $\begin{array}{l}2 \text { May } \\
10 \text { May ... } \\
2 \text { May... }\end{array}$ & $\begin{array}{l}.004 \\
.002 \\
.002\end{array}$ & $\begin{array}{l}.002 \\
.001 \\
.001\end{array}$ & $\begin{array}{l}37.3 \\
36.6 \\
36.8\end{array}$ & $\begin{array}{l}-.171 \\
-.161 \\
-.161\end{array}$ & $\begin{array}{l}31.1 \\
30.5 \\
30.7\end{array}$ & $\begin{array}{l}.002 \\
.002 \\
.002\end{array}$ & $\begin{array}{l}29.0 \\
29.0 \\
29.0\end{array}$ & $\begin{array}{l}.002 \\
.002 \\
.002\end{array}$ & $\begin{array}{l}-.165 \\
-.156 \\
-.159\end{array}$ \\
\hline $\begin{array}{l}\text { Averagc } \\
\text { Rota } \\
\text { Stati }\end{array}$ & $\begin{array}{l}\text { able coil } \\
\text { nary coil }\end{array}$ & d effect. & & & & & & & $\begin{array}{c}-0.158 \\
-.111 \\
\text { Negligible }\end{array}$ \\
\hline
\end{tabular}

TABLE 7. Summary of results

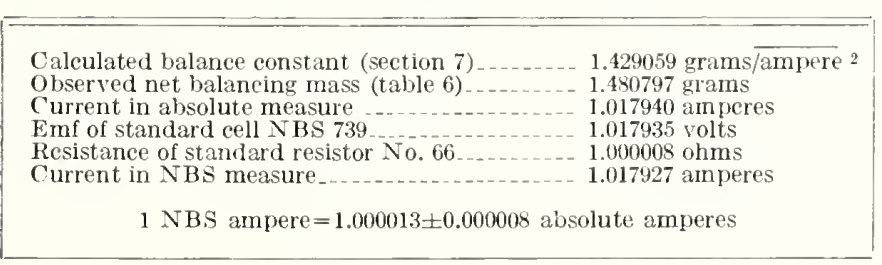

Known sources of error affecting the final result in table 7 are enumerated in table 8 . The values given are intended to represent a 50-percent confidence interval in the measured current caused by the estimated uncertainty in the mean values of the quantities listed. The limiting factor in the accuracy of this electrodynamometer at present is the uncertainty in the radius of the rotatable coil. After increasing the radius by a factor of 1.5 and making use of a fused silica coil form it is estimated that the uncertainty in the radius of the rotatable
TABLE 8. Error in the final result

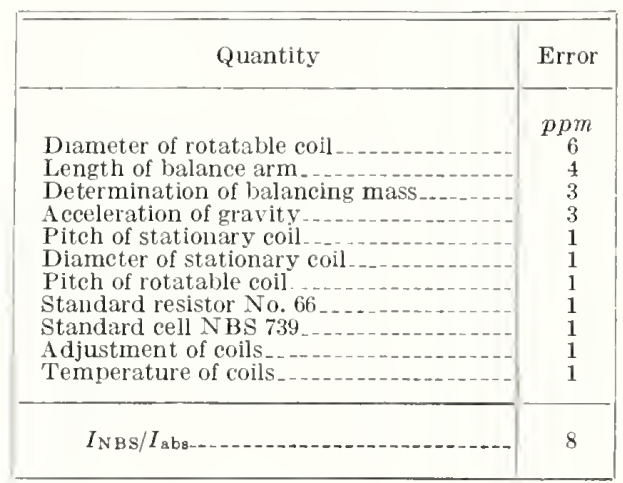

coil could ultimately be cut in half. The relatively large uncertainty in the length of the balance arm is not inherent in this method but reflects the need of a more rigid balance beam. 
This undertaking extending over a number of years has accumulated the contributions of many other staff members. In addition to those already named, the author wishes to thank the following: R. D. Cutkosky for valuable support in verifying the results by careful experimental checks; L. P. Slivka for assistance in the design and construction of the balance arrestment mechanism; Catherine Law for the calibration of the standard cell; W. H. Wood for the calibration of the standard resistor; Eleanor Clinton for the calibration of the standard mass; and A. G. Strang for the calibration of the length standards. Helpful suggestions were received from J. L. Thomas and F. B. Silsbee.

\section{References}

[1] R. W. Curtis, R. L. Driscoll, and C. L. Critchfield, J. Research NBS, 28, 133 (1942) RP1449.

[2] J. C. Maxwell, Treatise on Electricity and Magnetism, Clarendon Press, Oxford, Vol. II, Third Ed., Section 583.

[3] Chester Snow, Torque between concentric single layer coils, NBS J. Research 22, 607 (1939) RP1208.

[.t] M. H. Pellat, J. Phys. 6, 175 (1887); Bull. Soc. Intern. des Electriciens 2d Series 8, 573 (1908).

[5] H. L. Curtis, Charles Moon, and C. Matilda Sparks, J. Research NBS 21, 375 (1938) RP1137.

[6] H. L. Curtis, Charles Moon, and C. Matilda Sparks, J. Research NBS 16, 1 (1936) RP875.

[7] T. E. Wells, Proc. Instr. Soc. of Am., Vol. II, (1956).

[8] H. L. Dryden, J. Research NBS 29, 303 (1942) RP1502.

Washington, May 13, 1957. 


\title{
Measurement of Current with the National Bureau of Standards Current Balance
}

\author{
R. L. Driscoll and R. D. Cutkosky
}

\begin{abstract}
Prior to the adjustment of the electrical units in 1948, the value of a current had been determined in absolute units by means of a current balance and simultaneously measured in NBS amperes by comparison with standard resistors and standard cells. This work was reported in RP1449. Similar measurements made recently with an electrodynamometer indicate a possible change in the values of the standards. The present paper reports a repetition of the work described in RP1449. The purpose of this remeasurement was to determine whether or not the standards had changed. Only minor changes were made in the equipment in order that factors which might have introduced small systematic errors in the results would remain unchanged.

According to the work described in this paper, 1 NBS ampere $=1.000008$ absolute amperes. Recent work with the Pellat electrodynamometer gave the result 1 NBS ampere $=1.000013$ absolute amperes. The weighted mean of these two values is
\end{abstract}

\section{NBS ampere-1.000010 \pm 0.000005 absolute amperes}

The results given above for the current balance differ by $6 \mathrm{ppm}$ from those obtained in 1942. This indicates, in view of the uncertainties of measurement, that any change in the ampere as maintained by standard resistors and standard cells does not exceed a few parts in a million.

\section{Introduction}

The accuracy to which the electrical units as maintained at the National Bureau of Standards are known is under a continual process of improvement. A history of the development of the electrical units up to the adoption of the absolute units in 1948 [1] ${ }^{1}$ has been presented by Silsbee [2]. Since the 1948 revision, two absolute determinations of electric current have been made at the Bureau.

The recent determination of current with a Pellat-type electrodynamometer [3] led to the result that the NBS unit of current was larger than the absolute ampere by $13 \mathrm{ppm}$ (parts per million). The difference was not much more than the estimated uncertainty of the absolute measurement; but, since the values assigned to the NBS primary standard cells depend largely upon an earlier determination of current with the NBS current balance [4], it was thought necessary to repeat the earlier work in order to determine whether an appreciable drift in the electrical standards had taken place. This work was done as soon as possible after the completion of the measurement using the electrodynamometer, to assure as far as possible that both sets of absolute measurements were referred to the same electrical standards.

Photographs of the current balance used in 1942 and again in this determination appear in figures 1, 2, and 3. Briefly, the equipment consists of a helical fixed coil designated $H_{1}$ (fig. 6) in which current flows into the coil through a lead in the center of the helix, and out through leads on each end. A smaller helical coil designated $P_{1}$ hangs from an arm of a

\footnotetext{
1 Figures in brackets indicate the literature references at the end of this paper.
}

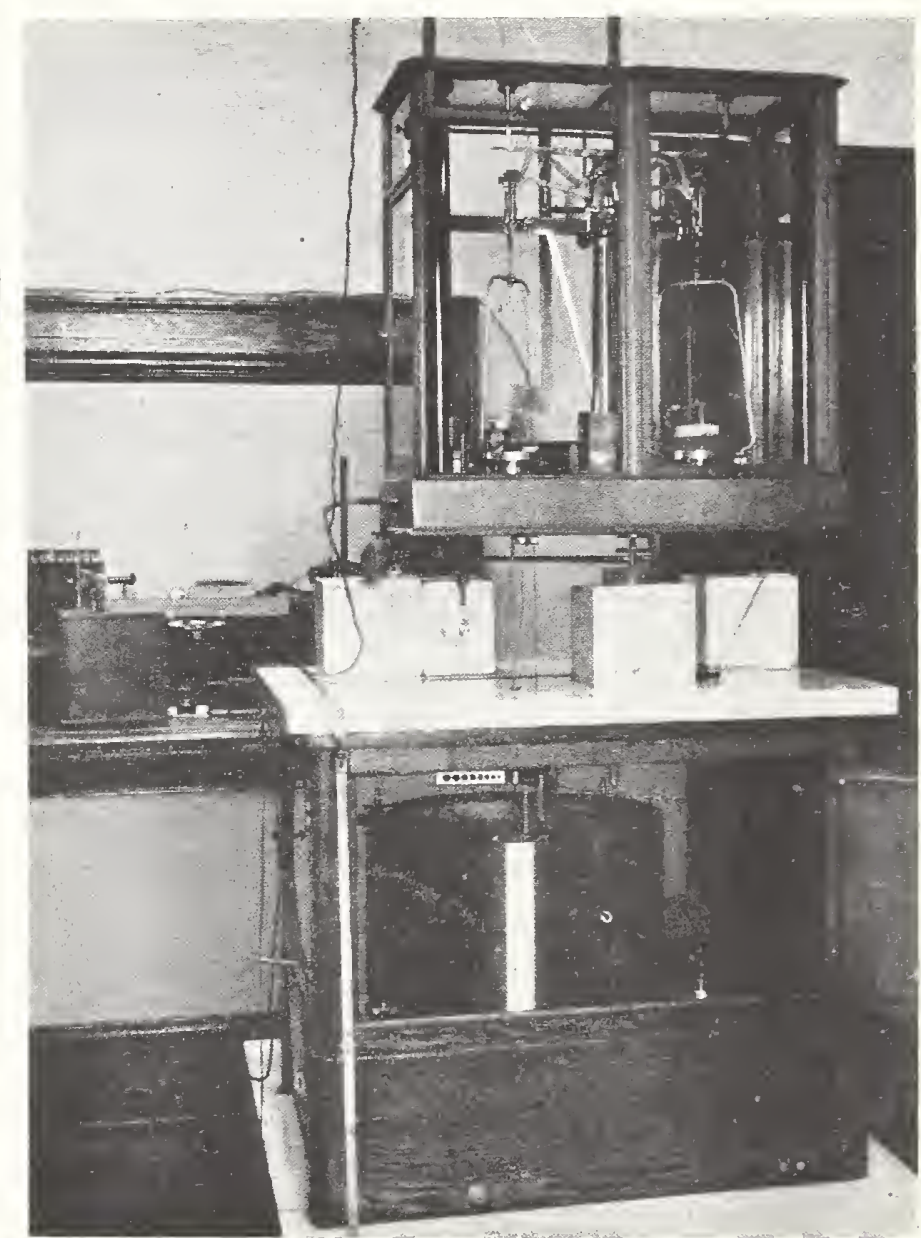

Figure 1. Rear vew of current balance showing fixed coil in operating position and operatıng room in background. 


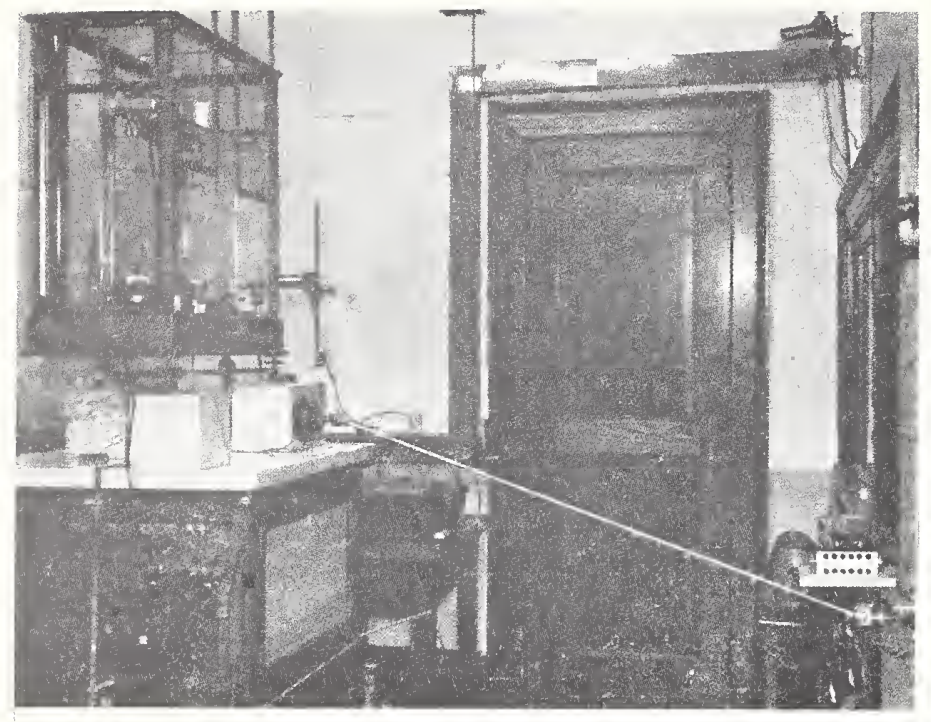

FIrilRE 2. Sile view of current balance shoumg recersing switches and operating rod.

sensitive balance so as to be concentric and coaxial with the fixed coil. A current flowing in the movable coil produces a foree between the two coils, and tends to deflect the beam.

In practice, the current is held constant and evaluated in NBS units by comparing the potential drop it produces across a known resistance with the emf of a standard cell which is known with reference to the NBS primary standard cells. The balance is adjusted to equilibrium with this current flowing in both coils. Then, the eurrent in the fixed coil only is reversed, and simultaneously a weight is placed on the balance pan. The weight is adjusted to equal as closely as possible the change in force caused by reversing the current. The small difference between the forces is observed as a change in the rest point of the balance. A switch for reversing the current is mounted on the coil case. A rod extends from this switch to the operating room; a cam and other connecting linkages enable the observer by turning this rod to raise and lower the weight on the balance pan and reverse the eurrent at the same time.

The change in force caused by reversing the current is measured by comparison with the force exerted by gravity on the mass placed on the balance pan. This force is equal to the square of the current times a calculable function of the physieal dimensions of the coils. From these equivalent expressions for the force, the current flowing can be determined in the mechanical mits of length, mass, and time.

\section{Changes in Equipment}

Inasmuch as the redetermination of the ampere by means of the current balance was intended primarily as a check on the stability of the NBS standards, the principal features of the equipment were kept intact. The only geometrical change in the arrangement of the coils was a change in $\alpha$, the angle between the movable and fixed coil leads, which has only a very small effect on the mutual force.

The standard cells were moved from the underground compartment to a "standard celler" [5] where

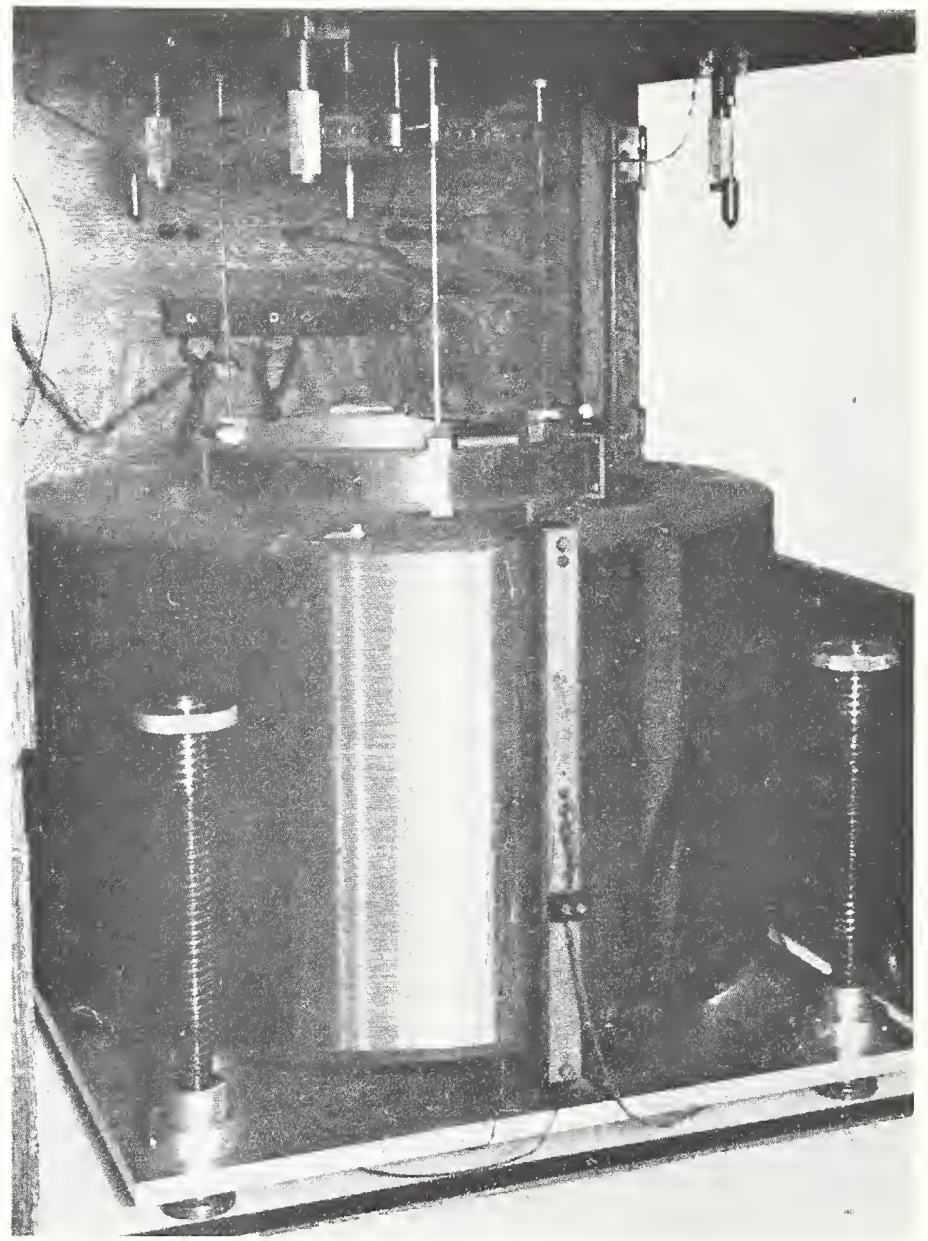

Figure 3. Coil case with ficed coil lowered to show movable coil.

their temperatures were thermostatically controlled near $34^{\circ} \mathrm{C}$. This arrangement was used also for the Pellat electrodynamometer, and made it possible to regulate the cell temperatures and hence the cell voltages more precisely than had been possible before.

Changing the temperatures of the cells also changed their voltages, and made it necessary to decrease the size of the platimum weight that had been used with the balance in the earlier work.

The turning points of the balance are observed on the scale in the operating room by a beam of light reflected to the scale from a mirror mounted on the balance beam. A scheme in which the beam of light was reflected twice from a moving prism had been used before, in order to increase the balance sensitivity. We preferred to use a singly refleeting mirror instead of the doubly reflecting prism, because the hair line at the light source could be focused more sharply at the balance scale. The sensitivity of the balance dropped from $1.21 \mathrm{mg} / \mathrm{cm}$ to $2.33 \mathrm{mg} / \mathrm{cm}$, but the reliability of the readings was improved.

During the preliminary measurements it was noticed that throwing the reversing switch mounted on the coil case gave the case a push that changed the apparent rest point of the balance as observed on the scale in the operating room. It was decided that the coil case was too shaky to be reliable, so copper straps were bound around it to make it more 
rigid. 'These can be seen in the photographs. Also, a sliding joint was put into the switch rod. The performance of the balance was then checked with no current in the coils, and it was found that the position of the reversing switch had no effect on the rest point of the balance.

The turning points of the current balance have always been subject to random fluctuations. These are attributed to fluctuations in the air flow around the movable coil. Much experimentation has beea done with ventilation of the coil case in an effort to steady the swings of the balance. 'The most satisfactory arrangement found was used for the final runs. 'This consisted of a honeycomb baffle under' the movable coil and a fan to draw air from the top of the coil case. .The fan was located about 20 feet from the coils and was connected with the coil case by means of a tube.

Reversing the current and changing the weight sometimes gives the balance an impulse which, if unchecked, would make the balance amplitude unsatisfactory. Previously the balance had been steadied after reversing the current by injecting short blasts of air under the balance pans. It was found that the turning points of the balance were more regular if the adjustments in balance amplitude were made by changing briefly the current through the coils. Two switches were installed in the operating room, one to increase, and one to decrease the current. All of the runs reported in this paper were obtained without the use of air jets.

\section{Mechanical Dimensions}

The mechanical dimensions of the coils were remeasured, using for the most part the methods that had been used in 1942. The end standards used to measure the diameters of the coils were re-evaluated by the NBS Gage Section. Summaries of the coil dimensions appear in table 1.

\subsection{Diameter}

Figures 4 and 5 illustrate the coherence between the earlier and recent measurements of the diameters

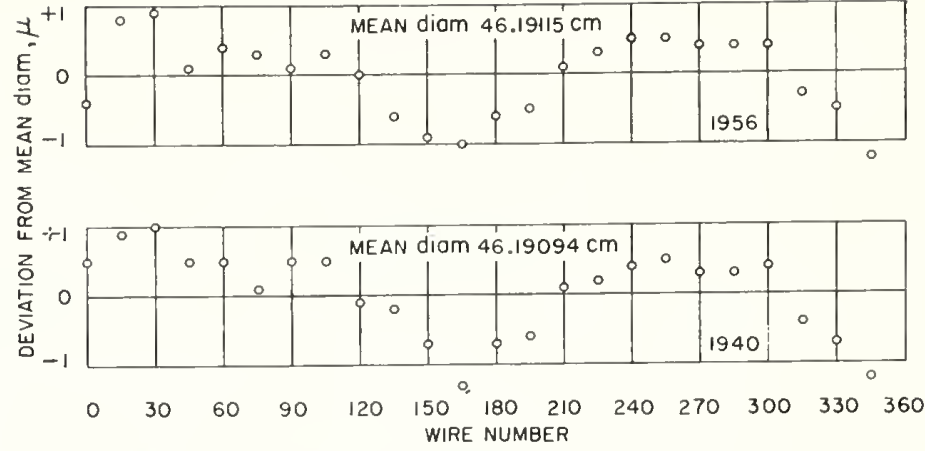

Figire 4. Coherence between 1942 and 1956 measurements of fixed coil wire diameters.

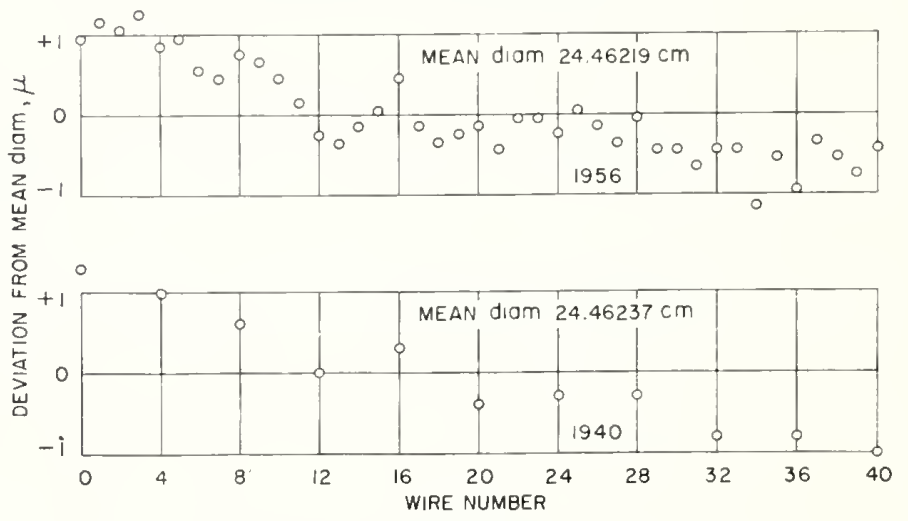

Figure 5. Coherence between 1942 and 1956 measurements of movable conl wire diameters.

of the two coils at $30^{\circ} \mathrm{C}$. It can be seen that apparently the fixed coil became larger and the movable coil smaller. Some clianges in dimensions are to be expected, and could be caused by a gradual relaxation of the strains in the wires or forms.

The diameter and electrical resistance of each coil were measured at three temperatures: near $25^{\circ}$, $30^{\circ}$, and $35^{\circ} \mathrm{C}$. From these measurements it was possible to estimate, from measurements of the resistances of the wires, the diameters of the coils when they were in the balance case under different ambient conditions.

The newly determined temperature coefficients of expansion agree very well with the values found in

TABLE 1. Constants of the fixed helix $H_{1}$ and the movable helix $P_{1}$

[All values if duced to $30^{\circ} \mathrm{C}$ ]

\begin{tabular}{|c|c|c|c|c|}
\hline & \multicolumn{2}{|c|}{$H_{1}$} & \multicolumn{2}{|c|}{$P_{1}$} \\
\hline & 1942 & 1956 & 1942 & 1956 \\
\hline A verage outside diameter of coil ................. & 46.26090 & 46. 26111 & 24.51360 & 24.51342 \\
\hline Diameter of wire & 0.06996 & 0.06996 & 0.05123 & 0.05123 \\
\hline Mean dianeter of coil ............. & 46.19094 & 46.19115 & 24.46237 & 24.46219 \\
\hline Current distribution correction & -0.00003 & -0.00003 & -0.00003 & -0.100003 \\
\hline Weighting correction & +.00002 & +.00002 & .0000 & .0 \\
\hline Effeetive mean diameter. ..... & 46. 19093 & 46. 19114 & $24 \quad 46231$ & 24. 46216 \\
\hline$A$ vial length of coil . . . . . . . . . & 27.51654 & 27.51612 & 2. 60050 & 2. 6649 \\
\hline 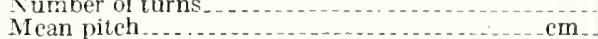 & ${ }^{344} 0.0799899$ & 0.0799896 & $\begin{array}{r}41 \\
0.065000\end{array}$ & 0.061998 \\
\hline & 11.8016 & 11. 7806 & 2.8220 & 2.8169 \\
\hline Resistance-temperature $\{$ Upper & $\begin{array}{r}11.7974 \\
0.0443\end{array}$ & $\begin{aligned} 11.7805 \\
0.0436\end{aligned}$ & $2.022 \mathrm{C}$ & 2.0103 \\
\hline $\begin{array}{l}\text { relationship } \\
\text { Temperature cocflicient of expansion }\end{array}$ & 3.64 & 3.54 & 3.9 & 3.7 \\
\hline Winding tension & & & & \\
\hline
\end{tabular}


the old measurements. The temperaturre coefficients of resistance do not agree, but this is because they were assumed, not measured, in the earlier work. At that time the temperature coefficients were taken from tables of copper-wire characteristics. Since the wires are under considerable strain, it is not surprising that the measured temperature coefficients differ from the values assumed in RP1449.

A new measurement of the diameter of the wire on the movable coil was made, and the result agreed with the previous measurement. In view of the excellent agreement it was felt unnecessary to remeasure the fixed coil wire diameter.

The current distribution corrections contained in table 1 correct for the variation of current density over the cross section of each wire; no corresponding corrections were made in the work reported in RP1449 because the net effect based on Snow's assumption of the "natural" distribution [6] was small. Recently Wells [7] has measured the resistance-strain relation in copper wire, making it now possible to give further expression to the variation of current density over the cross section of the wires. U'sing Snow's formula for the helix equivalent to a helical wire, we have

$$
\bar{r}_{1}=r_{1}+\frac{\rho_{1}^{2}}{8}\left(\frac{1}{r_{1}}+2 A_{1}\right)
$$

where $\bar{r}_{1}=$ the effective coil radius,

$$
\begin{aligned}
r_{1} & =\text { the mean coil radius, } \\
\rho_{1} & =\text { the wire radius, } \\
A_{1} & =\frac{\left[\frac{d u\left(r_{1}^{\prime}\right)}{d r_{1}^{\prime}}\right]_{r_{1}^{\prime}=r_{1}}}{u\left(r_{1}\right)}
\end{aligned}
$$

and $u_{1}\left(r_{1}^{\prime}\right)$ is the volume density of current in the wire as a function of the distance $r_{1}^{\prime}$ from the $x$ axis. A relationship similar to the above holds for the novable coil, whose coefficients will be denoted in what follows by the subscript "2." Snow has shown that the radius corrections fo not depend upon the second derivative of $u(r)$. This means that a first order expansion of $u(r)$ will lead to a radius correction which is correct to second order.

The current density at a given point in the fixed coil is given by $u_{1}\left(r_{1}^{\prime}\right)=G / \sigma r_{1}^{\prime}=\left(G / \sigma r_{1}\right)\left[1-\left(y_{1} / r_{1}\right)\right]$ to first order in $\dot{y}_{1} / r_{1}$, where $\sigma$ is the resistivity of the copper, $y_{1}=r_{1}^{\prime}-r_{1}$, and $G$ is a constant. The analrsis to follow is carried out for the fixed coil only, wire 1 , but it is to be understood that the equations are valid for the movable coil, wire 2 , also.

The resistivity of the copper has been measured in terms of the strain by Wells [7]. He finds that

$$
\frac{\frac{\Delta \sigma}{\sigma_{0}}}{\frac{\Delta l}{l_{0}}}=\beta+\gamma\left(\frac{\Delta l}{l_{0}}\right)^{2},
$$

where $\beta=1.13$ and $\gamma=-2.5 \times 10^{4}$;

or

$$
\sigma=\sigma_{0}\left[1+\beta \frac{\Delta l}{l_{0}}+\gamma\left(\frac{\Delta l}{l_{0}}\right)^{3}\right] .
$$

$\frac{\Delta l}{l_{0}}$ at a point in wire 1 is related to the initial strain in the wire, $K_{1}$, and the position of the point, $y_{1}$, by $\Delta l / l_{0}=\left(y_{1} / r_{1}\right)+K_{1}$, where $\left.K_{1}=2.5 \times 10^{-3}\right)$ (and $K_{2}=$ $\left.1.4 \times 10^{-3}\right)$. We then have to first order in $y_{1} / r_{1}$

$$
\sigma=\sigma_{0}\left(1+\beta K_{1}+\gamma K_{1}^{3}\right)\left[1+\frac{y_{1}}{r_{1}} \frac{\left(\beta+3 \gamma K_{1}^{2}\right)}{\left(1+\beta K_{1}+\gamma K_{1}^{3}\right)}\right],
$$

and

$$
\frac{1}{\sigma}=\frac{1}{\sigma_{0}\left(1+\beta K_{1}+\gamma K_{1}^{3}\right)}\left[1+\frac{y_{1}}{r_{1}} \frac{\left(\beta+3 \gamma K_{1}^{3}\right)}{\left(1+\beta K_{1}+\gamma K_{1}^{2}\right)}\right]
$$

leading to the result

$$
\begin{aligned}
u_{1}\left(r_{1}{ }^{\prime}\right)= & \frac{G}{r_{1} \sigma_{0}\left(1+\beta K_{1}+\gamma K_{1}^{3}\right)}[1- \\
& \left.\frac{y_{1}}{r_{1}} \frac{\left(1+\beta K_{1}+\gamma K_{1}^{3}+\beta+3 \gamma K_{1}^{2}\right)}{\left(1+\beta K_{1}+\gamma K_{1}^{3}\right)}\right]
\end{aligned}
$$

We then have

$$
A_{1}=-\frac{1+\beta+3 \gamma K_{1}^{2}+\beta K_{1}+\gamma K_{1}^{3}}{1+\beta K_{1}+\gamma K_{1}^{3}},
$$

and similarly for $A_{2}$. For the fixed coil, we have $A_{1}=-0.07, \Delta r_{1}=-1.7 \times 10^{-5} \mathrm{~cm}$; and for the movable coil, $A_{2}=-0.13, \quad \Delta r_{2}=-1.5 \times 10^{-5} \mathrm{~cm} . \quad \Delta r_{1}$ and $\Delta r_{2}$ are doubled and applied in table 1 as diameter corrections.

An attempt has been made to determine higher order corrections to the effective diameter based on Wells' resistivity determinations, but for the coils used here such corrections are negligible.

\subsection{Pitch}

The pitch of $H_{1}$ was measured as described in RP1449, and found to be insignificantly different from the earlier value. The pitch of $\dot{P}_{1}$ was last measured in 1934, and was not remeasured for the 1942 work. A new determination was folt to be in order for the completeness of this determination, even though the force constant is not strongty dependent upon the pitch of the movable coil.

For the measurement of the movable-coil pitch a meter bar was set up vertically, parallel to the coil axis. A telescope was clamped to a vertical bar which was free to pivot in such a way as to swing the telescope from the meter bar to horizontal grachuations ruled on the wires of the coil. The telescope was equipped with a filar micrometer eyepiece. A reading was made of the distance between a graduation ruled on a wire and a graduation on the meter bar. Then, the telescope was raised to measure the position of another wire. The distance between the 
two wires is given by the distance between the two meter bar graduations plus the difference between the readings of the filar micrometer eyepiece.

The accuracy of the measurement depended upon how well the coil and meter bar remained fixed with respect to each other, and upon the repeatability of the pivot of the vertical bar. The measurement is not as good as that used to measure the fixed-coil pitch, which used two telescopes, both of which were mounted on the vertical bar. It is felt, though, that the method is better than that used in 1934, which used a single telescope mounted on a carriage with a calibrated screw movement. The two-telescope method is better than either method used, but the movable coil was too short to be viewed by both telescopes at the same time. The result of the pitch measurements is that the changes found were too small to make any change in the balance constant as large as $1 \mathrm{ppm}$.

\section{Calculation of the Force Constant}

The force between the two helices is computed from the formula given by Snow [6]. With the notation of RP1449,

$r_{1}=$ mean radius of fixed helix.

$r_{2}=$ mean radius of movable helix.

$l_{1}=$ axial length of fixed helix (pitch $\times$ number of turns).

$l_{2}=$ axial length of movable helix (pitch $\times$ number of turns).

$N_{1}=$ number of turns on fixed coil.

$N_{2}=$ number of turns on movable coil.

$\alpha=$ angle between movable coil and fixed coil leads.

$$
\begin{aligned}
& X_{1}=\frac{l_{2}}{2} . \\
& X_{2}=\frac{l_{1}-l_{2}}{2} . \\
& X_{3}=\frac{l_{1}+l_{2}}{2} .
\end{aligned}
$$

The force in dynes between the movable coil and the upper half of the fixed coil with unit cgs current flowing in each of them is given by

$$
f=2 \omega^{\prime}\left(X_{1}\right)+\omega^{\prime}\left(X_{2}\right)-\omega^{\prime}\left(Y_{3}\right),
$$

where

$$
\omega^{\prime}(X)=\omega_{\theta}^{\prime}(X)+\omega_{a}^{\prime}\left(Y^{2} ; \alpha\right)+\bar{\omega}_{X}^{\prime}\left(X^{\prime}, \alpha\right) .
$$

Also,

$$
\begin{aligned}
& \omega_{\theta}^{\prime}(\mathrm{X})=\frac{2 \pi \Lambda_{1} N_{2}}{l_{1} l_{2}}\left\{X \sqrt{\Gamma^{2}+\left(r_{1}+r_{2}\right)^{2}}[\mathrm{~K}-\mathrm{E}]+\right. \\
& \left.\frac{\mathrm{X}\left(r_{1}-r_{2}\right)^{2}}{\sqrt{\mathrm{X}^{2}+\left(r_{1}+r_{2}\right)^{2}}}[\mathrm{~K}-\Pi]\right\} ;
\end{aligned}
$$

$$
\begin{aligned}
& \omega_{a}^{\prime}(X, \alpha)=\frac{\pi X}{6 \sqrt{X^{2}+\left(r_{1}+r_{2}\right)^{2}}}\left[\frac{2-k^{2}}{1-k^{2}} \mathrm{E}-2 \mathrm{~K}\right] \\
& +\frac{\pi X}{4 \sqrt{r_{1} r_{2}}}\left(\cos \frac{\alpha}{2}\right) \sin ^{-1}\left(k \cos \frac{\alpha}{2}\right)-\frac{k\left(2-k^{2}\right)}{2\left(1-k^{2}\right)} \sqrt{1-k^{2} \cos ^{2} \frac{\alpha}{2}} \\
& +\left(\sin \frac{\alpha}{2}\right) \log _{e}\left[\frac{k \sin \frac{\alpha}{2}+\sqrt{1-k^{2} \cos ^{2} \frac{\alpha}{2}}}{\sqrt{1-k^{2}}}\right] \\
& \bar{w}_{X}^{\prime}(X, \alpha)=\frac{X}{\sqrt{X^{2}+\left(r_{1}+r_{2}\right)^{2}}} \log _{e} \frac{r_{1}}{\sqrt{r_{1}^{2}+r_{2}^{2}-2 r_{1} r_{2} \cos \alpha}} ; \\
& k^{2}=\frac{4 r_{1} r_{2}}{X^{2}+\left(r_{1}+r_{2}\right)^{2}} ; \text { and } k_{0}^{2}=\frac{4 r_{1} r_{2}}{\left(r_{1}+r_{2}\right)^{2}} .
\end{aligned}
$$

$\mathrm{K}, \mathrm{E}$, and $\Pi$ are the complete elliptic integrals of the first, second, and third kind, respectively, to the modulus $k$ and parameter $k_{0}$.

As in RP1449, the force in dynes between the helices with one ampere in the wires, $F_{H H}$, taking account of both halves of the fixed helix and of reversal of the current, is $F_{H H}=4 f / 100$.

The formula for the calculation of the force constant assumes that the diameter of each coil is uniform throughout its length. Clearly some turns affect the force constant more strongly than others. Use of the mean diameter in the calculations attaches undue importance to certain turns of wire, such as those near the center of the fixed coil, which have little effect upon the force constant. A plot was made of the calculated force, $f(x)$, between the movable coil and a turn of the fixed coil, as a function of the distance $x$ between the center of the moving coil and the turn. It was decided to weight the radius of the turn at position $x$ with the factor $f(x)$.

Let $r(x)=$ the radius of a wire as a function of its axial position, $\bar{r}=$ the average radius of the coil, and $r_{\text {eff }}=$ the weighted mean radius. Then, summing over all the turns,

$$
r_{e f f}=\frac{\sum_{n} f(x) r(x)}{\sum_{n} f(x)}
$$

and,

$$
\Delta r=r_{\text {eff }}-\bar{r}=\frac{\sum_{n} f(x) r(x)-\sum_{n} f(x) \bar{r}}{\sum_{n} f(x)}=\frac{\sum_{n} f(x)[r(x)-\bar{r}]}{\sum_{n} f(x)}
$$

$r(\ell)-\bar{r}$ for the fixed coil is plotted in figure 4. $\Delta r$ is found by simple summation to be +0.1 micron, and the eflect is entered in table 1 as a diameter correction. A similar correction for the movable coil would be much smaller, and was not considered worth calculating.

Table 2 summarizes the calculations of the force constant $F_{H H}$. Comparison with table 7 of RP1449 shows the difference in $F_{H H}$ to be close to that calculated with the variation coefficients $\partial F / \partial r_{1}, \partial F / \partial r_{2}$, etc. 
TABLE 2.-Summary of computations on force due to unit currents

The following values of the independent variables were used in the computation:

\begin{tabular}{|c|c|c|c|}
\hline \multirow{2}{*}{ Quantities used in computing functions } & \multicolumn{3}{|c|}{ Values of terms for-- } \\
\hline & $X_{1}=1.33245$ & $X_{2}=12.42576$ & $X_{3}=15.09066$ \\
\hline 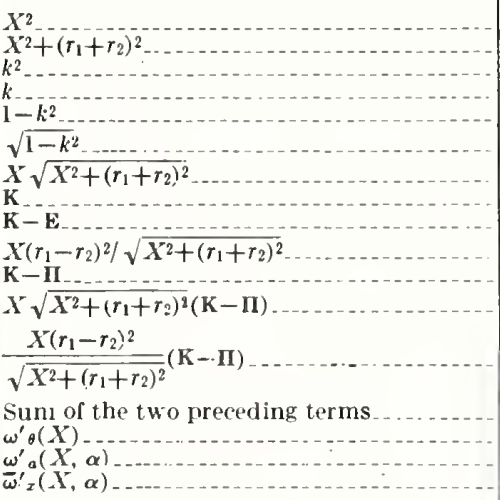 & $\begin{array}{c}1.775423 \\
1249.847623 \\
0.904130939 \\
.950858001 \\
.095869061 \\
.309627294 \\
47.1044656 \\
2.59795964 \\
1.49658483 \\
4.44894310 \\
-8.98674363 \\
70.4958287 \\
-39.9815111 \\
30.5143176 \\
36.876 .779 \\
0.0696 \\
.1191\end{array}$ & $\begin{array}{c}154.399512 \\
1402.37171 \\
0.805731806 \\
.897625649 \\
.194268194 \\
.440758658 \\
465.323014 \\
2.27042996 \\
1.09582099 \\
39.1659333 \\
-6.95142649 \\
509.910727 \\
-272.259106 \\
\\
237.651621 \\
287.747 \\
0.2542 \\
.1048\end{array}$ & $\begin{array}{c}227.782019 \\
1475.70022 \\
0.765694466 \\
.875039694 \\
.234305534 \\
.484051168 \\
579.705346 \\
2.18562183 \\
0.984555860 \\
46.3688424 \\
-6.46910960 \\
570.752295\end{array}$ \\
\hline
\end{tabular}

Principal term: $\left[2 \omega_{\theta}^{\prime}\left(X_{1}\right)+\omega_{\theta}^{\prime}\left(X_{2}\right)-\omega_{\theta}^{\prime}\left(X_{3}\right)\right]=33,708.998$ dynes.

Azimuthal eorreetion term: $\left[2 \omega_{a}^{\prime}\left(X_{1}, \alpha\right)+\omega_{a}^{\prime}\left(X_{2}, \alpha\right)-\omega_{a}^{\prime}\left(X_{3}, \alpha\right)\right]=+0.1600$.

Axial correetion term: $\left[2 \bar{\omega}_{x}^{\prime}\left(X_{1}, \alpha\right)+\bar{\omega}_{z}^{\prime}\left(X_{2}, \alpha\right)-\bar{\omega}_{x}^{\prime}\left(X_{3}, \alpha\right)\right]=+0.0045$.

$$
\begin{aligned}
& f=33,709.163 \text { dynes. } \\
& F_{H H}=1348.3665 \text { dynes. }
\end{aligned}
$$

The computation of the coefficients in the variation formula for these two helices gave the equation:

\section{Experimental Determination of the Force Between the Coils}

The experimental determination of the force between the coils was made as described in RP1449. The wiring diagram is shown in figure 6 for more convenient reference.

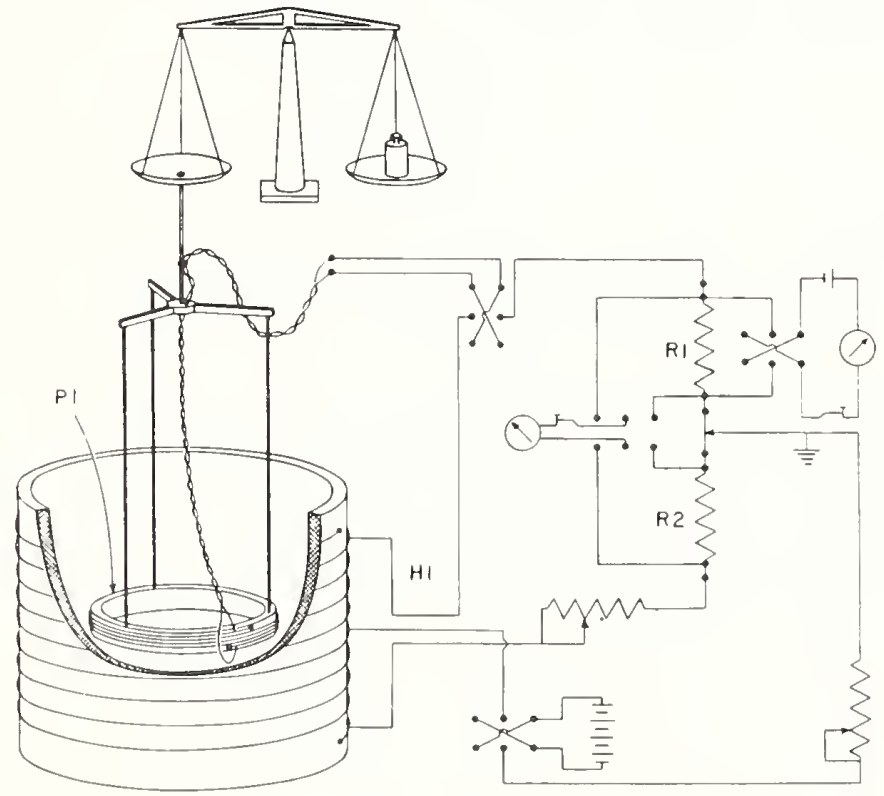

Figrae 6. H'iring diagram of current balance.
The following adjustments were made for differences hetween the values of dimensions used in the above computation and the dimensions given in table 1 : Fixed coil diameter $\Delta r_{1}=+0.1$ micron; $\Delta F=-0.0016$ dynes.

Movable coil diameter $\Delta r_{2}=-0.1$ micron; $\Delta F=-0.0030$ dynes.

Total force adjustment $=-0.0046 \mathrm{dynes}$.

llence for $I_{1}$ and $P_{1}$ at the dimensions of the coil corresponding to $30^{\circ} \mathrm{C}$, $F_{H H}=1348.3619$ dynes.

Readings were made of nine turning points of the balance with the eurrent in one direction, then the current in the fixed coil was reversed and the measurement of turning points repeated. A set of ten measurements involving nine reversals of current was averaged and entered in table 3 as one determination.

The force between the fixed-coil leads and the movable coil was measured by removing the fixed coil from the cireuit without changing the lead-wire configuration. The movable-coil lead effect was measured in a similar way. These forces must be subtracted from the total force between the coils, and appear in table 4.

It was found that the mechanical dimensions of the coil supports were not as stable as had been hoped. Even with the straps around the ease as described earlier, the vertical position of the movable coil with respect to the fixed coil changed about 0.2 $\mathrm{mm}$ in one month. The change was ascribed to dimensional changes in the wooden ease due to a change in humidlit $\mathrm{y}$, and the observed balancing mass was corrected for this change under the assumption that the shift was proportional to time. The numbers given in table 3 are corrected for the effect, which was never more than $4 \mathrm{ppm}$ in the current.

The temperatures listed in table 3 are the temperatures of the wires, computed from their resistances and measured temperature coefficients. Because of temperature gradients in the coil forms, the mean 
TABLE 3. Results of measurememts of force

Observed difference in rest points equals avcrage difference in scale reading of rest points of the balance, $\delta$, corresponding to "on" and "off" positions of weight, multiplied by the sensitivity of the balance $(2.33 \mathrm{mg} / \mathrm{cm})$. Mass of weight (a platinum cylinder): $1.425569 \mathrm{~g}$.

\begin{tabular}{|c|c|c|c|c|c|}
\hline \multirow{3}{*}{$\begin{array}{c}\text { Date (May } \\
1956)\end{array}$} & \multicolumn{3}{|c|}{ Temperature ${ }^{\circ} \mathrm{C}$} & \multirow{3}{*}{$\begin{array}{l}\text { Observed } \\
\text { difference } \\
\text { in rest } \\
\text { points }\end{array}$} & \multirow{3}{*}{$\begin{array}{l}\text { Difference } \\
\text { in rest } \\
\text { points } \\
\text { corrected } \\
\text { to } 30^{\circ} \mathrm{C}\end{array}$} \\
\hline & \multirow{2}{*}{$P_{1}$} & \multicolumn{2}{|c|}{$H_{1}$} & & \\
\hline & & Upper & Lower & & \\
\hline $\begin{array}{l}18 \ldots \\
21 \ldots \\
21 \ldots \\
22 \\
22 \ldots\end{array}$ & $\begin{array}{l}24.79 \\
29.45 \\
29.55 \\
29.54 \\
29.59\end{array}$ & $\begin{array}{l}29.84 \\
29.45 \\
29.56 \\
29.56 \\
29.64\end{array}$ & $\begin{array}{l}28.60 \\
28.26 \\
28.37 \\
28.37 \\
28.42\end{array}$ & $\begin{array}{r}m q \\
-0.041 \\
-.001 \\
-.002 \\
+.004 \\
-.029\end{array}$ & $\begin{array}{c}m y \\
-0.049 \\
-.003 \\
-.010 \\
-.001 \\
-.037\end{array}$ \\
\hline $\begin{array}{l}23 \ldots \\
23 \ldots \\
23 \ldots \\
23 \ldots \\
24 \ldots\end{array}$ & $\begin{array}{l}29.88 \\
29.92 \\
29.96 \\
29.99 \\
29.95\end{array}$ & $\begin{array}{l}29.93 \\
29.95 \\
30.00 \\
30.05 \\
30.05\end{array}$ & $\begin{array}{l}28.71 \\
28.73 \\
28.78 \\
28.80 \\
28.82\end{array}$ & $\begin{array}{l}-.049 \\
+.014 \\
-.063 \\
-.053 \\
-.046\end{array}$ & $\begin{array}{l}-.057 \\
+.006 \\
-.071 \\
-.071 \\
-.053\end{array}$ \\
\hline $\begin{array}{l}24 \\
24 \\
24\end{array}$ & $\begin{array}{l}29.95 \\
29.96 \\
29.97\end{array}$ & $\begin{array}{l}30.05 \\
30.06 \\
30.07\end{array}$ & $\begin{array}{l}28.82 \\
28.82 \\
28.82\end{array}$ & $\begin{array}{l}-.019 \\
-.002 \\
-.007\end{array}$ & $\begin{array}{l}-.026 \\
-.009 \\
-.014\end{array}$ \\
\hline
\end{tabular}

Average $\delta$ times sensitivity of the balance.

$-0.031$

TABIE 4. Calculation of final results

[Value of acceleration of gravity $980.081 \mathrm{~cm} / \mathrm{sec}^{2]}$ ]

\begin{tabular}{|c|c|c|}
\hline & 1942 & 1956 \\
\hline Standard mass (a platinum cylinder) & & \\
\hline $\begin{array}{ll}\text { grams.- }\end{array}$ & 1. 427655 & $1+25569$ \\
\hline Buoyance correction .......... grams. & $-0.00007 \pi$ & -0.000079 \\
\hline Lead corrections \{ Moving coil . grains.- & -.000068 & -000044 \\
\hline$\delta$ times sensicity of balance & -.000018 & -.000022 \\
\hline $\begin{array}{l}\delta \text { times sensicity of balance } \ldots \text { grams } \\
\text { Correction for temperature gradients. }\end{array}$ & +.000222 & -.000031 \\
\hline in coll forms & -.000014 & -.0000014 \\
\hline Net compensating mass $=\boldsymbol{M}$ & 1. 427700 & 1. 425379 \\
\hline $\begin{array}{l}\text { Measured force (M times gravity) }= \\
F_{M} \text { dynes } \\
\text { Calculated force for unit current at } 30^{\circ}\end{array}$ & 1399.262 & $1391) .987$ \\
\hline $\mathrm{C}=F_{C} \ldots$ & 1348.394 & $134 \searrow 3619$ \\
\hline Equivalent current, $I_{\mathrm{ab}, s}=\sqrt{\frac{F_{M}}{F_{C}}}$ & & \\
\hline Emf of standard alosolute amperes _- & 1.018688 & 1. 0178.714 \\
\hline $\begin{array}{l}\text { Emf. of standard cell } \\
\text { Resistance of standard resistor, } R_{1}\end{array}$ & 1.018702 & 1. 0178703 \\
\hline Resistance of standard resistor, $R_{2}$ & 1. 000015 & 1. 0000076 \\
\hline ohms & 1. 000019 & 1.0000056 \\
\hline $\begin{array}{l}\text { Equiralent current .... NBS amperes. } \\
I_{\text {abs }}\end{array}$ & & 1.0178630 \\
\hline$\overline{I_{\mathrm{NB} B}} \ldots \ldots \ldots$ & 1.000002 & 1.000008 \\
\hline
\end{tabular}

temperatures of the coils are slightly different from the wire temperatures. A measure of this effect was made and applied to the work reported in RP1449. The 1942 temperature gradient measurements were corrected by the better resistance measurements made recently; and it was found that under equilibrium conditions with one ampere through the roils, the mean fixed toil form temperature was $1.1^{\circ}$ C'below the fixed coil wire temperature, and the mean morable coil form temperature was $0.1^{\circ} \mathrm{C}$ below the movable coil wire temperature. Application of the romputed force-diameter variation coefficient from table 2 and the temperature coefficients of expansion from table 1 leads to $-0.014 \mathrm{mg}$ as the required correction. This will be found applied in table 4.

For the comparison of the present work with the work of 1942 in table 4 , both of these determinations have been referred to the same electrical standards and to the same value of the acceleration of gravity. This makes it possible to interpret the results directly as an apparent change in the electrical standards. The measured values of the currents are expressed in "NBS amperes," which is taken in this paper to mean the current with reference to the present NBS standards of resistance and electromotive force, which went into effect in 1948 [1]. The value of the acceleration of gravity is based on the Dryden reduction [8] and a gravity survey made at the National Bureau of Standards by the Geological Survey. To make the comparison complete, the new diameter weighting, current distributions, and temperature gradient corrections are applied in this paper to both the 1942 and 1956 work. It may be pointed out here that these last three corrrections tend to cancel, and do not change the 1942 result by more than 1 ppm.

\section{Permeability of the Forms}

It was assumed in the earlier work on the current balance that the permeability of the coil forms had a negligible effect on the force constant. Inasmuch as the susceptibility of each form was only $-1 \times 10^{-6}$, the correction would certainly be small; but an order of magnitude calculation was felt desirable.

In the following computation, the permeabilities of the movable coil and of the fixed coil are treated separately. Unit current (1 amp) is assumed flow$\mathrm{mg}$ in each of the coils. It is necessary with the method used to compute the magnetic fieds of the solenoids at various points. This can be done in all cases by means of formulas given in a paper by Snow [9].

The field of the movable coil serves to induce magnetic poles on the ends of the movable eoil form, whose magnitude can be computed through the relation $m=(\bar{H} \chi V) / l$, where $\bar{H}=3.5$ oersteds is the mean field intensity in the form, $\chi=-1 \times 10^{-6}$ is the susceptibility, $I^{\top}$ is the rolume of the coil form, and $l$ is its length. $m$ is from this approximately $-3.0 \times 10^{-4}$ pole. The axial component of the fixed coil field intensity, $I_{X}$, at the end of the movable coil form is computed to be $I_{X}=0.65$ ocrsted. The force on each end of the coil form is then $F^{\prime}=$ $-0.65 \times 3.0 \times 10^{-4}$ drne. Since the total force between the coils is $F=1348 / 2=674$ dynes without reversal of the current, the permeability of the movable coil has an effect of $-\left[\left(2 \times 2.0 \times 10^{-4}\right) / 674\right]=$ $-0.6 \mathrm{ppm}$ in the force, considering both ends of the form.

Because of the complicated field distribution inside the fixed coil form due to current in the fixed coil, a rather elaborate calculation was made of the fixed coil permeability effect. A rough estimate indicated that the form, although diamagnetic, would cause an increase in the radial component of field at the morable coil, which is not what one would at first expect.

The magnetic charge distributed over the surface of the form was calculated using the normal component of fickl given by Snow's formulas, and the 
form susceptibility. The distribution was broken up into a series of rings of charge one centimeter wide extending around the form, and the total charge per ring was determined. This charge was then assumed concentrated on a circle located at the center of the ring. A formula for the potential of a circle of charge has been given by smythe [10], in terms of Legendre polynomials, but this did not converge sat isfactorily for our purposes. A solution was found in terms of elliptic integrals, which leads to an easier numerical calculation.

It can be shown that the potential of a circle of charge at a point a distance $r$ from the axis of the circle and a distance $d$ from the plane of the circle is given by $\Gamma^{2}=[2 Q K(k)] /(\pi \mathscr{R}), \quad k^{2}=(4 r R) / \mathscr{R}^{2}, \quad \mathscr{R}^{2}=$ $(r+R)^{2}+d^{2}, Q$ is the total charge on the circle, $R$ is the radius of the circle, and $K$ is the complete elliptic integral of the first kind. From this the radial component of field at the point is given by

$$
H_{r}=-\frac{2 Q}{\pi \cdot R^{3}}\left\{\left[2 R-\frac{4 r R(r+R)}{R^{2}}\right] \frac{B}{1-k^{2}}-K(r+R)\right\}
$$

where $B=K\left[1-\left(1 / k^{2}\right)\right]+\left(E / k^{2}\right)$ and $E$ is the romplete elliptic integral of the second kind.

This cxpression allows one to sum the contributions of the separate circles of charge to the field at the movable coil. One finds the total contribution to bo $H_{T}^{(1)}=1.25 \times 10^{-6}$ ocisted.

The radial field at the movable coil due to the fixed coil itself ean be calculated from the force $F$ between the two coils, using the relation $F=$ $I \int d s \times T^{(\theta)}$, where $B^{(\theta)}$ is the radial romponent of magnetic indurtion at the movable coil due to the fixed coil and $I$ is the current in the movable coil, with the path of integration going around the movable coil. We then have, snce the current is not reversed, $F=1348 / 2=674=(0.1 \times 41) \times(2 \times 12.2) F^{(o)}$. or $J^{(0)}=2.2$ gauss. The effect of the permenbility of the fixed coil is thus $\left(1.25 \times 10^{-6}\right) / 2.2=+0.6 \mathrm{ppm}$ in the foree.

A detailed calculation shows that because of the way in which the radial ficld of a circle of charge drops off at points away from the plane of the circle, those charees near the center of the form have the greatest effeet on the field. Since the charges on the outside of the form are concentrated at the center of the coil and the charges on the inside are spreacl out, the charges on the outside have a slightly lareer influence on the radial field at the movable coil. For this reason the fixed coil susceptibility causes an increase in the foree.

The effects of the fixed and movable coil forms are in the opposite direction, and are seen to cancel. The calculations were made to a degree of precision which could cause an error of only a fraction of a part per million in the current.

\section{Uncertainties}

Table 5 contains estimates of known uncertainties in the current. The numbers given are probable errors for those measurements which can be treated statistically, and "50-percent-error estimates" for"
TABLE 5. Sources of error causing an uncertainty, e, in ratio $I_{a b s} / I_{N B S}$ as large as $1 \mathrm{ppm}$

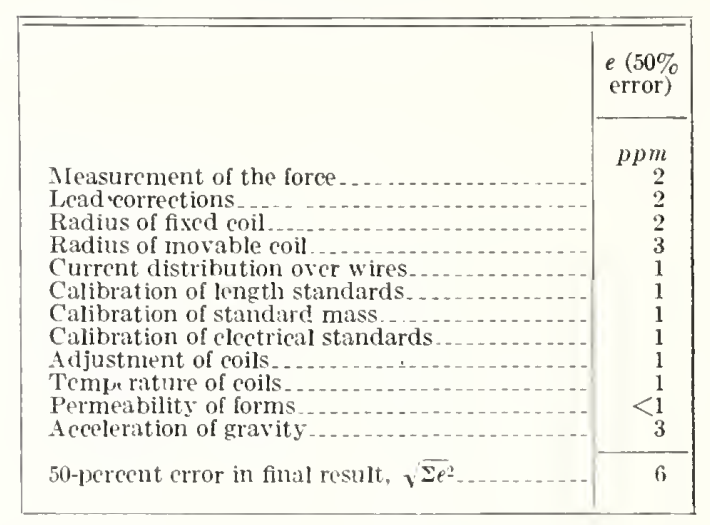

those cases in which no statistical information is available. Both of these measures of precision will be referred to as 50-percent elrors. The estimated total meertainty in the measurement can be determined by the usual procedure of taking the square root of the sum of the squares of the indiridual 50pereent errors.

Huntoon and MeNish [11] have estimated in an as ret unpublished paper that the probable eror of the mean of three gravity determinations, those of Kühnen and Furtwängler (revised), Heyl and Cook, and of Clark is about $2 \mathrm{ppm}$.

They also estimate that systematic errors could be as great as $15 \mathrm{ppm}$. We have estimated the total 50 -perecnt error in these gravity determinations to be $6 \mathrm{ppm}$, which is equivalent to $3 \mathrm{ppm}$ in the eurrent.

Several laboratories have recently completed or are now working on new determinations of the ac. celeration of gravity. If the presently accepted value for the acceleration of gravity is revised as a result of such work, this paper sliould be rerised accordingly.

\section{Comparison with the Pellat Balance}

According to the work described in this paper, the ratio of the absolute ampere to the ampere as presently maintained at the Bureau is, as given in table 4 ,

\section{NBS ampere $=1.000008 \pm 0.000006$ absolute amperes.}

Aceording to the work done on the Pellat clectrodyriamometer,

$$
\begin{gathered}
1 \text { NBS ampere }=1.000013 \pm 0.000008 \text { absolute } \\
\text { amperes. }
\end{gathered}
$$

Since the acceleration of gravity is a common factor in these two determinations, it must be taken out before averaging, and reentered after averaging. Doing this and using the appropriate weighting factors, we have

\section{$1 \mathrm{NBS}$ ampere $=1.000010 \pm 0.000005$ absolute amperes.}


The observed difference between the absolute and NBS units of current could be ascribed to a change in the electrical standards, to inaceuracies in the measurements which were used to define the present standards, or to the uncertainty in the present measurements. The present standards were defined by rounding off the arerage of sereral ampere determinations made in various countries to the nearest 10 ppm. The rounding off process com.. bined with the uncertainties of the individual measurenents could have alused an crror large enough to explatin our difference within the estinialed 50 -percent error. The 6-ppm difference between the results of the present work and the work using the same coils reported in RP1449 is also small enough to be interpreted as a combination of rantom errors.

It cannot be stated with certainty whether or not the standards have drifted. Our results indicate only that they have not diffed more than a frow parts per million and that the NBS mnit of current is greater than the absolute ampere by $(10 \pm 5) \mathrm{ppm}$.

Washingtox, May 1:3. $195 \%$.

\section{References}

[1] Announcement of changes in electrical and photometric units, NBS Cire. 459 (1947).

[2] F. B. Silsbee, Establishment and maintenance of the electrical units, NBS (ire. 475 (1949).

[3] R. L. Driscoll, Measurement of current with the Pellat electrodynamometer, J. Rese arch NBS 60, 287 (1958) R P $28+5$.

[4] R. W. C'urtis, R. L. I)riscoll, and C. L. Critchfield, An absolute determination of the ampere, using helical and spiral coils, J. Rwearch X'BS 28, 133 (1942) RP1449.

[5] A. W. Spinks and F. I. Hernach, Portable potentiometer and thermostatted container for stanclard cells, Rev. Siei. Instr. 26, 770 (1955).

[6] C'hester snow, Mutual induetance and foree betwen two coaxial hehical wires, J. Restarch NBS 22, 239 (1939) RPl178.

[i] T. E. Wells. Measurenent of the resistance-strain rekat tion and Poisson's ratio for copper wires, Proc. Instr. Soc. An. 11, Paper 56-8-3 (1956).

[81 H. L. Dryden, A reexamination of the Potsdam absolute detemination of qravity, J. Researeh XBS 29, (1942) RP P150).

[9] Chester Snow, Magnetic fields of eylindrical coils and annular coils, NBS Appliech Math. Series 38 (1953).

[10] II. R. Snvthe, static and dynamic electricity, 2d Ed. (McGraw-Hill P'ublishing Co., Now York, N. I. $(1950)$.

[11] R. D. Huntoon and A. (i. MeNish, Present status of reserarch on the phrsical constants at the (lnited Statess)- National Bureatr of Standards, Paper presented before the Avogadro Festival in Italy (1956). To be publishere in Nuovo Cimento. 
UNITED STATES DEPARTMENT OF COMMERCE - Charles Sawyer, Secretary NATIONAL BUREAU OF STANDARDS - E. U. Condon, Director

\section{Precision Resistors and}

\section{Their Measurement}

by James L. Thomas

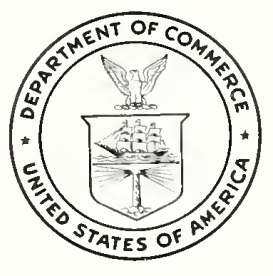

National Bureau of Standards Circular 470

Issued October \&, 1948 


\section{Preface}

There are few fields of scientific investigation in which accurate measurements of electrical resistance are not required. For this purpose the Wheatstone bridge, in one of its several forms, is almost universally used. This instrument is comparativcly simple to use and at the same time has a very high sensitivity. Unfortunately, however, sensitivity and accuracy are not synonomous, and some knowledge of the practical limitations of resistance-mcasuring bridges is nceded by all users. This circular is intended to supply such information. Although the prescntation is essentially nontechnical, it is believed that the subject matter will be of valuc to any onc interested in the accurate measurement of resistance

In addition to information about the usc of resistance bridges, this circular presents methods for their calibration. The subject matter is limited to directcurrent calibrations, and the methods discussed arc those regularly used at this Bureau when an accuracy of 0.01 percent or better is required. No attempt is made to present a complete discussion of methods of resistance measurement or to consider the relative merits of various methods. Those presented are comparatively simple, bcing based largely on substitution procedures, yet they are capable of yielding results of high accuracy.

E. U. Condon, Director. 


\section{Contents}

Preface

I. Introduction $\ldots \ldots \ldots$

1. Definition of resistance $\ldots \ldots \ldots$

2. Importance of resistance measurements _............... 1

3. Types of resistors $\ldots \ldots \ldots \ldots$

II. Resistance Materials and Construction Methods _............... 2

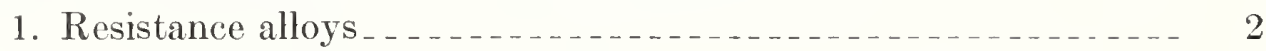

2. Spools, winding and adjustment $\ldots 4$

3. Sheet-metal resistor's . . . . .

4. Accelerated aging $\ldots$

5. Annealed resistors

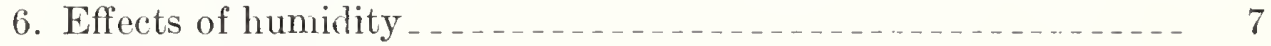

7. Load coefficients $\ldots \ldots \ldots$

8. Stability of resistors with time

1II. Methods of Comparison of Resistors

1. Ammeter-voltmeter methods _............... 10

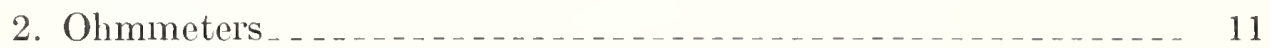

3. Potentiometer methơd _._.

4. Differential-galvanometer method _... 12

5. Bridge methods _... 13

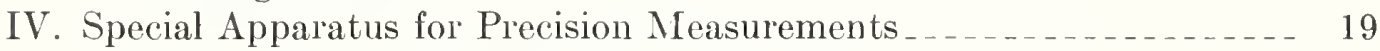

1. Direct-reading ratio set_._.

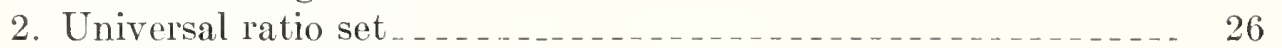

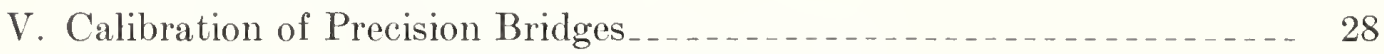

1. Calibration of Wheatstone bridges . . . $\ldots 28$

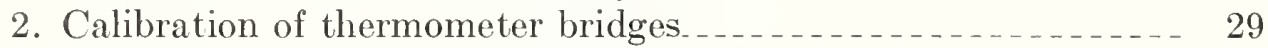

VI. Resistivity of Solid Conductors

1. Resistivity, definition and units $\ldots \ldots \ldots$

2. Measurement of resistivity $\ldots \ldots \ldots \ldots \ldots \ldots$

VII. References _._. 


\title{
Precision Resistors and Their Measurement
}

\author{
By James L. Thomas
}

\begin{abstract}
This circular contains information on the construction and characteristics of wire-wound resistors of the precision type. There are also included descriptions of the methods used at this Bureau for the test of precision resistors and the calibration of precision resistance measuring apparatus. Although the presentation is nontechnical, there is a considerable amount of information on the characteristics and limitations of apparatus of this type that should be of interest to any one making accurate measurements of electrical resistance.
\end{abstract}

\section{Introduction}

\section{Definition of Resistance}

The modern concept of electrical resistance is based largely on the work of G. S. Olmm, who in 1826 published an equation that was formulated on the basis of his experiments with direct-current circuits. In modern terminology this equation is

$$
I=\gamma^{+4} \frac{4}{l} E,
$$

where $E$ is the potential difference across a conductor of length $l$ and cross-sectional area $A$ when a current, $I$, flows. The factor $\gamma$ depends upon the material of the conductor and is now called conductivity (see sec. VI). The above equation was abbreviated by Ohm as

$$
I=\frac{E}{\mathscr{L}}
$$

where $\mathscr{Z}$ is the length of a hypothetical wire having unit conductivity and cross-sectional area. $\mathscr{Z}$. was then the length of a wire across which unit current would produce a unit of potential difference, that is, $\mathcal{L}$ was Ohm's unit of resistance and his experimental equation can be abbreviated as

$$
E=R I \text {. }
$$

This equation is almost universally referred to as Olmn's Law, although some writers contend that the longer form should be so designated. Although experimentally determined for individual conductors, Olım's Law was soon applied to the entire circuit if $E$ designates the net electromotive force in the circuit.

\section{Importance of Resistance Measurements}

Although Ohm found that the ratio of potential difference across a conductor to the current flow- ing in it is dependent on the material and dimensions of the conductor, more precise experiments showed it to depend upon temperature and even upon the presence of stress in the conductor. Instead of incorporating such quantities in our equation for the current, we say that the resistance is a function of temperature and stress, and for a given conductor we must state the values of temperature and stress for which a value of resistance is given.

The fact that resistance is a function of temperature is made use of in temperature measurements, the resistance of a wire being measured at known temperatures or fixed points and then at the unknown temperatures. Also, changes in resistance with dimensions are utilized for measuring small displacements, and change in resistance with stress is utilized for the measurement of liquid pressures. In addition to such phenomena, a large number of physical and chemical phenomena are investigated by means of measurements of electromotive forces, and the measurement of electromotive force is customarily carried out by measurements of resistance ratios. Electric current is readily measured in terms of the potential difference across a known resistor. In fact, a large proportion of electrical quantities is measured by methods that involve the measurement of resistance.

In some cases the resistance of a conductor depends upon the magnitude of the current flowing through it. That is to say, Olm's law is not applicable, and the resistance must be determined under the conditions of use. Also, the resistance of all conductors is to some extent a function of frequency, and the resistance must often be determined in such a way as to allow for the effect of frequency. However, the content of this circular is limited largely to direct-current measurements of resistors which follow Ohm's law, with special emphasis on precision measurements, i. e., measurements to 0.01 percent or better. 


\section{Types of Resistors}

Resistors are used for many purposes with a correspondingly large range of types and accuracies of adjustment. They are used as electric heaters, as current-limiting devices such as motor starters, for component parts of radio, telephonic and similar equipment, and in electrical instruments of greater or less precision. For some applications adjustments must be made to perhaps 10 to 20 percent, whereas in otliers the resistors must be within 0.01 percent or less of their nominal values.

For use as heaters, resistors are usually made of special alloy wire that will witlistand high temperatures for long periods of heating. The most used alloy for this purpose is of nickel and chromium, with or without the addition of a considerable amount of iron. These alloys can be kept at a "red heat" in air for long periods of time without damage from oxidation. High-resistance units for' radio circuits are often made from a nonconducting binder, such as clay, with which is mixed sufficient powdered graphite to make the material slightly conducting; or from a nonconducting rod on the surface of which is deposited a conducting film. For resistor's to be used in precision instruments the important quality desired is stability with time and temperature, and special alloys have been developed, which are discussed in section II.
Stability with time and temperature are also the important characteristics required for resistors that are to be used as reference standards for the calibration of other resistors.

Standard resistors are usually commercially available only in decimal multiples or fractions of the unit, usually from $0.0001 \mathrm{ohm}$ to $10,000 \mathrm{ohms}$. These are of two general types of construction, either two-terminal or four-terminal types. For values of resistance where the resistance of the contacts, made in connecting to the resistor, is not negligible, it is customary to use four-terminal resistors. Whether or not the contact resistances are negligible depends upon the accuracy desired, but in general standard resistors of 1-ohm and less are of the four-terminal type, higher valued resistors needing only two terminals. Special standard resistors are required if an accuracy of better than 0.01 percent is required. These are usually sealed to protect the resistors from the effects of oxygen and moisture in the atmosphere, whereas resistors for an accuracy of 0.1 percent do not require such protection. Although precision resistors are ordinarily adjusted to their nominal values to 0.01 percent or better, they gradually change in resistance with time. For work to this accuracy it is desirable to have standards recalibrated occasionally, applying if necessary corrections corresponding to their departure from nominal values.

\section{Resistance Materials and Construction Methods}

\section{Resistance Alloys}

Manganin. Since their introduction in about 1890 , alloys of copper, manganese, and nickel have come into almost universal use as resistance materials for precision resistors and for resistance measuring apparatus. The most common of these alloys is "manganin" which has the nominal proportions of 84 percent of copper, 12 percent of manganese and 4 percent of nickel. This material has a resistivity of 45 to 50 microhm-cm, a thermoelectromotive force against copper of 2 or 3 $\mu \mathrm{v} /{ }^{\circ} \mathrm{C}$, and, when properly heat treated, is very stable in resistance with time.

'The electrical properties of alloys of copper, manganese, and nickel over a large range of proportions were investigated in 1925 by Pilling [1] ${ }^{1}$ and later for alloys made of high-purity ingredients by Dean and Anderson [2]. Botli of these investigations showed that an alloy having a tempcrature coefficient that averaged zero over the interval 0 to $100^{\circ} \mathrm{C}$. would be obtained with approximately 10 percent cach of manganese and nickel, the remainder being copper. I series of alloys also having zero temperature coefficients could be obtained by increasing scparately either the manganese or the nickel up to as much as

\footnotetext{
Figures in brackets indicate the literature references at the end of this paper.
}

20 or 30 percent, with a corresponding reduction in copper.

On the basis of small temperature coefficients there would appear to be a wide choice of compositions for alloys of the manganin type. However, in order to kecp the thermoclectric power against copper as low as possible it is necessary to keep the nickel content low, as the thermoelectric power increases rapiclly in proportion to the amount of nickel above 2 or 3 percent. Also, if the nickel is kept constant and the percentage of manganese is increased the curvature of the resistance-temperature curve increases. This means that although the resistance might have the same value at $0^{\circ}$ and $100^{\circ} \mathrm{C}$, , the departure from this value at intermediate temperatures increases witl the manganese content. It is therefore desirable for general use to liave an alloy that is as low as possible in both nickel and manganese.

The published data on copper-manganesenickel alloys give average temperature coefficients over a temperature interral of some 80 to $100^{\circ} \mathrm{C}$. These data do not show the best compositions for use at ordinary laboratory temperatures. If it is desired to keep the thermoelectric power against copper as small as possible, the alloy should have a content of about 2 percent of nickel and 14 percent of manganese in order to obtain at the 
same time a small temperature coefficient of resistance at ordinary room temperatures. On the other hand, if the thermoelectric power is of no importance the resistance of an alloy of about 20 percent of nickel and 10 percent of manganese would be most constant with temperature in the ordinary range of laboratory temperatures. The accepted composition of manganin, 84 percent of copper, 12 percent of manganese and 4 percent of nickel is reasonably close to the optimum for a general purpose resistance alloy. Fortunately the proportions need not be exact, as the melting losses make the composition somewhat difficult to control.

If the resistance of a sample of manganin is plotted against temperature, the curve will be found to be of the general shape of that shown in figure 1 .

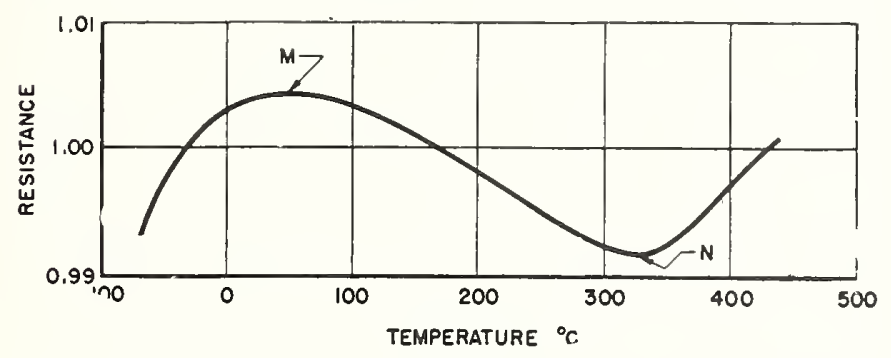

Figure 1. Resistance-temperature curve for manganin.

The maximum, $M$, of this curve is in the neighborhood of room temperature, being generally between $20^{\circ}$ and $50^{\circ} \mathrm{C}$, while the minimum, $N$, is at about $350^{\circ} \mathrm{C}$. Somerville [3] found the difference in resistance between the maximum and the minimum to be about $1 \frac{1}{2}$ percent of the value at the maximum.

For an interval at least $15^{\circ}$ to $20^{\circ} \mathrm{C}$ on each side of the maximum, the resistance-temperature curve for manganin is symmetrical with respect to a vertical axis. Its equation can be accurately represented by

$$
R_{t}=R_{25}\left[1+\alpha(t-25)+\beta(t-25)^{2}\right],
$$

where $R_{t}$ is the resistance at $t^{\circ} \mathrm{C}$ and $R_{25}$ is the resistance at $25^{\circ} \mathrm{C}$. The coefficient $\alpha$ is the slope of the resistance-temperature curve at $25^{\circ} \mathrm{C}$, and for manganin of good quality $\alpha$ has a value of $10 \times 10^{-6}$ or less. The value of $\beta$, which determines the curvature at any point in the interval, is usually between $-3 \times 10^{-7}$ and $-6 \times 10^{-7}$. This means that $10^{\circ} \mathrm{C}$ either side of the maximum the resistance is less than at the maximum by from 30 to 60 parts per million (ppm).

The temperature at which the maximum of resistance occur's is a function of the thermal and mechanical treatment of the manganin as well as of its composition. Different size wires drawn from the same melt will have maxima at different temperatures, that is, at any given temperature their coefficients of resistance will not be the same. From the ingot, manganin is usually worked cold with an occasional softening by heating to a "red heat." If this annealing is done in ail there is a selective oxidation of the surface that breaks down the alloy and leaves a coating with a relatively high conductivity. This surface layer may be removed by "pickling" in an acid, but again the action of the acid is somewhat selective, and the surface layer is left with a slightly different composition from that of the interior. These complications add to the difficulty of the control of the quality of manganin wire. In addition, the completed resistance coils are usually baked at about $150^{\circ} \mathrm{C}$ for 24 to 48 hours in order to stabilize the resistance. This baking also affects the temperature coefficient of the wire by amounts depending upon the size of the wire and the length of time of the baking. All of these factors make it difficult to produce resistors with the maximum at the best temperature, usually between $20^{\circ}$ and $25^{\circ} \mathrm{C}$. However, precision resistors of good quality usually can be obtained with temperature coefficients in that interval of not more than $10 \mathrm{ppm} /{ }^{\circ} \mathrm{C}$.

Temperature coefficients of resistance change little with time, and if changes do occur in their values there is very probably an accompanying large change in resistance. Standards that are stable in resistance need not have redeterminations of their temperature coefficients. Although the data are meager and only the order of magnitude is known, it appears that for manganin resistors a change in resistance of one part in a thousand will be required to change the slope of the temperature-resistance curve at room temperature by one or two parts in a million per degree centigrade.

Constantan.-A series of alloys of nickel and copper containing 40 to 60 percent nickel, with a small amount of manganese to improve their mechanical properties, all have practically the same electrical properties. These alloys are sold as "constantan," or under various trade names, for use as thermocouple materials, and have thermoelectric powers against copper of about $40 \mu \mathrm{v} /{ }^{\circ}$ C. However, except for their large thermoelectric powers the electrical properties of these alloys are remarkably similar to those of manganin.

The resistance-temperature curve for constantan is similar to that for manganin shown in figure 1. Its maximum is at or near room temperature with the minimum around $500^{\circ} \mathrm{C}$. The difference in resistance between the maximum and minimum is somewhat less than for manganin, and the curvature in the neighborhood of the maximum is also less. As a consequence, constantan clianges somewhat less in resistance over the ordinary range of atmospheric temperatures than does manganin. Its stability with time is about the same as that of manganin. At room temperature the resistivity 
of constantan is 45 to 50 microhm-cm as it is for manganin.

Resistance coils of constantan are sometimes used instead of manganin in values of 1,000 ohms and above. They may also be used in smaller denominations in cases where no difficulty will arise from the large thermal emf's, as for example in alternating current circuits.

Therlo.-When manganin was first developed a small amount of nickel was added to the copper and manganese in an attempt to reduce the thermoelectric power of the alloy against copper. With the proportion of nickel now used the thermoelectric power against copper at room temperature is almost the same, although of opposite sign, as when the nickel is omitted. However, the nickel improves the mechanical properties of the alloy and probably reduces the surface action during forging and annealing.

The development of therlo was another attempt to reduce the thermoelectric power of coppermanganese alloys against copper. Instead of nickel, an equal percentage by weight of aluminum was added to the copper and manganese. As its name implies, the resulting alloy had a very small thermoclectric power against copper at room temperatures, less than $1 \mu \mathrm{v} /{ }^{\circ} \mathrm{C}$. Its other electrical properties are almost identical with those of manganin. However, as the thermoclectric power of manganin against copper is only of the order of 2 or $3 \mu \mathrm{v} /{ }^{\circ} \mathrm{C}$, the improvement was of little significance.

At the National Bureau of Standards [4] an investigation has been made of copper manganesealuminum alloys of the therlo type. There it was found that for a resistance alloy the best composition is 85 percent of copper, 9.5 percent of manganese, and 5.5 percent of aluminum. This alloy has nearly the same resistivity as manganin, its temperature coefficient at $25^{\circ} \mathrm{C}$ ean be brought to zero by a suitable heat treatment, and the change in the temperature coefficient with temperature is about half that of manganin, at least in the ordinary range of room temperatures. Its thermoelectric power against copper at $25^{\circ} \mathrm{C}$ is only about 10 percent of that of manganin, and this thermoelectric power may be further reduced by the addition of a very small percentage of iron, without materially affecting the other properties of the alloy. The stability of such alloys with time was found to be equal to that of manganin. Except for unusual applications, the difference between manganin and therlo is of little importance and the alloys may be used interchangeably.

Gold-chromium.-An alloy of recent introduction, which appears to be very promising for some applications, is gold with slightly over 2 percent of chromium [5]. This alloy has a resistivity at room temperatures of about 20 times that of copper. By baking at fairly low temperatures the temperature coefficient can be made extremely small. Resistors of this material have been produced such that the total change in resistance in the interval $20^{\circ}$ to $30^{\circ} \mathrm{C}$ did not exceed a few parts in ten million. The thermoelectric power of this gold-chromium, alloy against copper is several times that of manganin, being 7 or $8 \mu \mathrm{v} /{ }^{\circ} \mathrm{C}$ at $25^{\circ} \mathrm{C}$. The stability of this alloy with time has not been thoroughly tested but preliminary results were promising.

For many applications the extremely small temperature coefficient of gold-chromium alloy makes its use desirable. However, the temperature coefficient must be adjusted for each coil by baking, and the cost of this adjustment limits the use of the material. Although the tenperature coefficient may be made small at room temperature, the interval over which the cocfficient is small is not more than $20^{\circ}$ or $30^{\circ} \mathrm{C}$. The temperature-resistance curve is similar to that for manganin, as shown in figure 1 , but with $M$ and $N$ nuch closer together in temperature and in resistance.

Other Alloys:- It is probable that all resistance alloys that have small tem.perature cocfficients at room temperature have temperature-resistance curves that are cubies, similar to that of manganin shown in figure 1. These curves are nearly straight in the neighborhood of the inflection points between the maxima and minima. The ideal resistance alloy for use in instruments would have this inflection point at room temperature with a zero slope. Moreover, the maximum and minimum should be at widely separated temperatures so that the zero slope would be obtained over the usual range of atmospherie temperatures. Of the alloys already discussed, only gold-chromium has the inflection point in the neighborhood of ordinary room temperatures, the others having small slopes because of use near the point of maximum resistance. None of these have small coefficients over a very large temperature interval.

Alloys of nickel and chromium. are commercially available that have practically linear temperatureresistance curves over an interval of several hundred degrees centigrade, which interval includes ordinary atmospheric temperatures. Although the temperature cocfficient is constant, it is too large for use in apparatus where the highest accuracy is required. Recent attem.pts to reduce the coefficients of these alloys by the addition of comparatively small amounts of other materials, such as copper and aluminum, appear ver'y promising. It is quite possible that an alloy and beat tr eatment will be developed such that no correction for temperature will need be made, at least throughout the range of laboratory temperatures.

\section{Spools, Winding and Adjustment}

In the beginning of the electrical instrument industry, wire coils were wound on wooden spools 
like those that are still used for thread. However, because of the demand for increased accuracy, these wooden spools have been entirely replaced by metal spools for resistors of high quality. The reason for the change to metal has been twofold. In the first place, the wooden spools absorb moisture in amounts dependent upon the humidity in the air, and expand or contract therefrom. This results in varying stresses applied to the wire, with accompanying changes in resistance.

A more important reason for the use of metal spools is the fact that they more readily dissipate the heat produced in a coil by the passage of the current. The wire is wound in rather intimate thermal contact with the metal spools, and the heat is readily transferred to the spools. The entire surface of the spools, both inside and outside, is effective in dissipating heat to the surrounding air. However, for wooden spools the area that is effective in dissipating heat is largely the exposed outer surface of the resistance wires. When metal spools are used, the temperature rise for a given heat dissipation depends primarily upon the size of the spool and only to a minor extent upon the size of the wire. However, the wire size should be selected so as to cover the spool as completely as possible, and if necessary the turns should be spaced to prevent bunching of the coil at one end of the spool.

For many alternating-current applications, the use of metal spools is undesirable or even out of the question. For such applications, when wirewound coils are required, wooden spools may be used, although for these purposes ceramic spools have come into rather general use. The objections to ceramic spools for resistors of high precision are their poor heat conductivity and the fact that their temperature coefficients of linear expansion are very much smaller than for the resistance wire.

Metal spools are ordinarily of brass, which has a coefficient of thermal expansion nearly the same as that of the resistance alloys. This avoids large changes in stress in the coils because of temperature changes. The spools are ordinarily mounted with their axes vertical, and both ends should be left at least partially open in order to allow a ready flow of convection currents of air through the spools. Before being wound, the spools are enameled or covered with a single layer of silk, which is impregnated with shellac varnish and allowed to air dry.

The resistance wire is generally double-silk or silk and cotton covered, and often the wire is enameled before these coatings are applied. 'The correct length of resistance wire is cut, doubled at its center, and the center is attached by means of a thread near one end of the insulated metal spool. The two halves are then wound side-by-side (bifilarly) after which the free ends are tied down with silk thread.

High-quality resistors are wound with only one layer of wire. Although this requires the use of smaller wire than for multilayer coils, there are several advantages. In the first place, the heat dissipation is more satisfactory for a single-layer coil, since a considerable temperature rise may be obtained in the center of a multilayer coil as a result of the passage of the current through the coil, and the load coefficients are usually large. Moreover, multilayer coils are more subject to change in resistance because of changes in atmospheric humidity (see section II, 6), and are usually found to be less stable in resistance with time.

After being wound, the coils are artificially aged by baking in air at about $150^{\circ} \mathrm{C}$ for 48 hours, after which they are kept for a considerable period of time before final adjustment. Some manufacturers impregnate coils before baking with a shellac varnish, while others impregnate them after baking with special waxes. The final adjustment is usually accomplished in two steps. The excess wire is cut off in order to make the resistance just slightly less than the nominal value. Copper lead-wires, which are usually somewhat larger in diameter than the resistance wire, are then silver-soldered to the ends of the coil. Final adjustment is made hy filing or scraping the resistance wire near the end, care being taken to see that the metal cuttings are not forced into the insulation. Any filed part is finally painted with shellac varnish, which is allowed to air dry. For coils of the highest precision the interval between baking and final adjustment should be as long as practicable, an entire year being desirable.

\section{Sheet-Metal Resistors}

Precision resistors having values of $0.1 \mathrm{ohm}$ or below are usually made of sheet manganin brazed or silver-soldered to heary copper terminal posts.

Potential leads are attached as shown in figure 2. Here $C$ and $C$ are copper rods, with binding posts at the top, attached to the sheet of resistance material, $S$. 'The resistance material may be a single sheet of manganin $\mathrm{or}^{\mathrm{r}}$ several sheets in parallel. The sheets are often not straight but are bent in an S-shape in order that greater lengths can be used. Additional binding posts $P, P$ are used as potential terminals and are connected by copper wires soldered at some point on the copper terminal bars. For a resistor of this type the resistance is measured between the two branch points. This is to say, the resistance is equal to the ratio of the potential difference between the potential terminals $P, P$ to the current flowing in and out the current terminals $C, C$. For standards, sheet-metal resistors are mounted in perforated containers with hard rubber or bakelite tops. These are often used in oil baths in order to facilitate the dissipation of the heat 


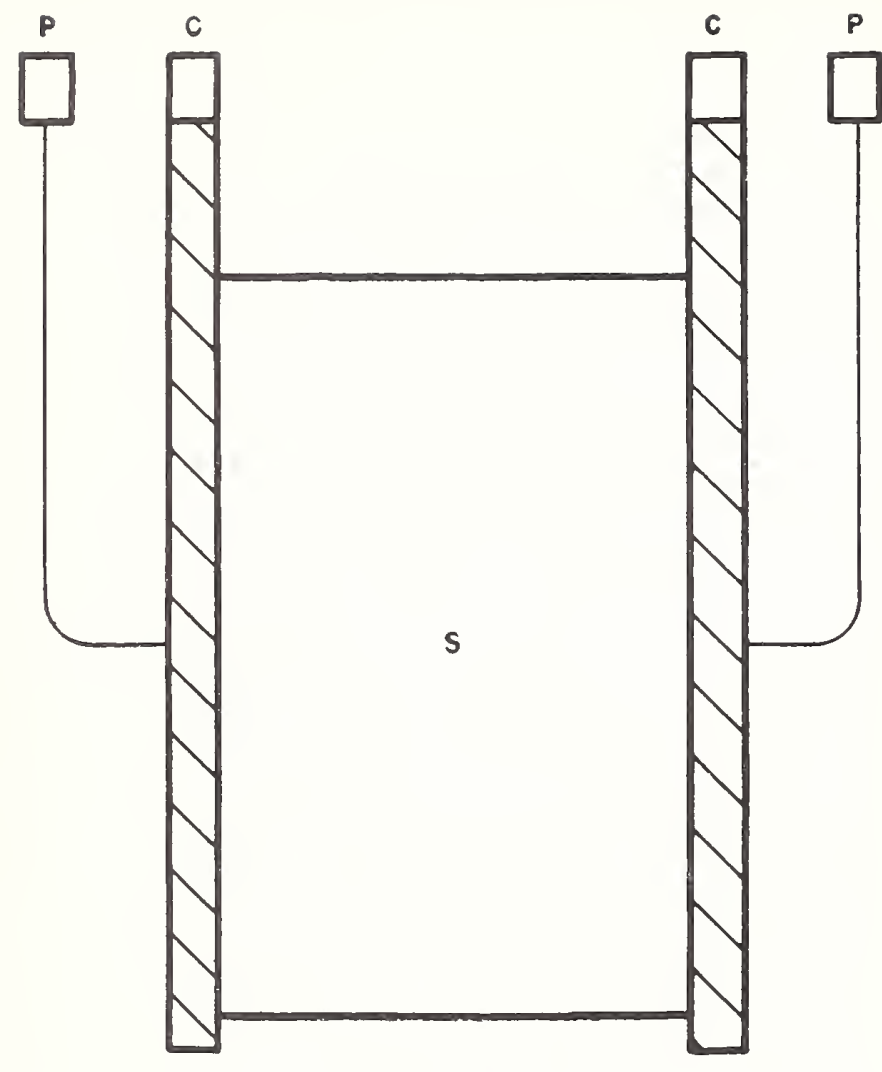

FIGURE 2. Sheet-metal resistor.

caused by the flow of current through the resistance element.

An accelerated aging of these resistors is ob. tained by heating in air at $150^{\circ} \mathrm{C}$ for 48 hours, as is done for wire-wound resistors. Final adjustment of the resistance may be accomplished by changing the points of attachment of the potential leads, the resistance decreasing as the points of attachment are lowered. Increases in resistance may be obtained by scraping or by drilling holes in the resistance material. Small changes can be obtained by filing the copper current posts, but the direction of the change is dependent upon the location of the potential leads.

Adjustment of sheet metal resistors is often accomplished by making $U$-shaped saw cuts in the sheet, with the open ends of the U's pointing in the direction of flow of the current. The Usliaped tabs so formed are bent outward and to them are attached the potential leads. Adjustment is made by increasing the length of the $U$ cut. Lengthening one cut will increase the foulterminal resistance, whereas lengthening the other cut will decrease the resistance.

Not only may the resistance of a four-terminal resistor of the type shown be altered by changes in the points of attacliment of the potential leads, but it is possible to change the temperature coefficient of resistance in the same way. In fact, the temperature coefficient is lowered at the same time as the resistance if the points of attachment are lowered on the current posts [6]. This effect is large when the resistance of the copper terminal posts is high in proportion to that of the resistance element. For standards of very low resistance, large temperature coefficients may result unless the potential branch points are carefully located.

\section{Accelerated Aging}

After being wound, resistance coils are impregnated with a sliellac varnish and given an accelerated aging by baking. The temperature for baking is limited by the silk insulation, which should not be lieated above about $150^{\circ} \mathrm{C}$, and the coils are usually baked at this temperature for 48 liours. As a result of the baking the resistance of a coil may decrease, sometmes as much as 1 or 2 percent, and a sufficient length of wire must be used to compensate for this change. After being baked, the resistance of the coils is much more stable with time than is that of unbaked coils. This aging process is often called "annealinge", but it is loubtful that the improvement in stability results from the relieving of internal stresses in the wire, as happens cluring true annealing. In the two or three months immediately following their baking, resistance coils will ordinarily increase in resistance by an amount that is usually of the order of 0.01 percent. They then are ready for final adjustment.

Sheet-metal resistor's are usually painted with a lacquer or shellac varnish as a protection of the surface. This coating limits the temperature to which these resistor's can be raised during aging, and they are usually treated in the same way as insulated wire-wound coils, being baked at $150^{\circ} \mathrm{C}$ for 48 hours. Sheet resistors may be heated at high temperatures before being lacquered, but such treatment is apparently no improvement over baking at $150^{\circ} \mathrm{C}$, as 'far as subsequent stability is concerned.

\section{Annealed Resistors}

As has already been stated, the usual baking of wire-wound and sheet-metal resistors is not done at a sufficiently high temperature to anneal the resistance material. As baking at $150^{\circ} \mathrm{C}$ improves the stability with time, it would be logical to expect greater stability if the heating were carried on at a sufficientiv high temperature to obtain actual annealing. In the case of manganin this takes place between $500^{\circ}$ and $600^{\circ} \mathrm{C}$.

The annealing of metal is a complicated process, but the first step is probably a reforming of the metallic crystals to the shape they had before being cold-worked. This results in the relieving of many of the internal stresses that resulted from the distortions of the crystals. The amount of this restoration of the metal to its preworked condition depends upon both the annealing tem- 
perature and the time. In general, the higher the temperature the shorter the time required for a given annealing. If the annealing is continued after crystals have been restored to their original condition, there may result an actual uniting of adjacent crystals, with an accompanying decrease in the mechanical strength of the material.

For base-metal alloys, annealing should take place in a vacuum or in an inert atmosphere to avoid a reaction between the metal and the surrounding air. Such reaction might take place inside the metal at the intercrystalline boundaries as well as at the surface of the metal. These reactions do not necessarily decrease the stability of resistance with time if the products are stable. There may, however, be a selective reaction that makes the material inhomogeneous, and this might have a considerable effect on the resistivity and temperature coefficients.

When a resistor is made by winding wire on a spool, the wire usually will not straighten if it is removed from the spool. This means that parts of the wire have been stressed past their elastic limit and a permanent deformation has taken place. For such a bent wire the portions farthest from the center of the spool have been elongated past their elastic limit, while the filaments nearest the center of the spool have been compressed beyond the elastic limit. Intermediate filaments are subjected to stresses that depend upon the changes in their length, which resulted from the bending. The stress distribution is from a maximum in tension to zero and then to a maximum in compression. These stresses are superimposed upon the stresses that were produced in the wire as it was being fabricated.

These internal stresses in a wire result in a slight change in the shape of its cross section. In addition to this change there is a cliange in resistivity, which results from the presence of the stress [7]. The resistivity is therefore not uniform across the wire, and the difference between parts of the wire may amount to nearly 1 percent in the case of manganin, and perhaps more for other alloys. Although the resistivity changes considerably when a wire is bent, there is not necessarily much change in resistance, as the change in resistance of the parts under compression may compensate for the change in the parts under tension.

The effect of annealing of a coil of wire is to reduce the internal stresses, thus making the resistivity more nearly uniform in the wire. It is probable that a slow annealing takes place at room temperatures, and the accompanying reduction in the internal stresses may be one reason for the change with time of the resistance of a coil of wire. Another cause for change might well be some reaction between the wire and the surrounding atmosphere. Both of these sources of instability would be avoided or reduced if a coil were annealed and mounted in a vacuum.

Annealed resistors mounted in vacuo have been tested at the National Bureau of Standards and found to be very stable. It is difficult, however, to seal the coils in suitable containers for high evacuation. Equally good results have been obtained with annealed coils mounted in sealed metal containers filled with dry air. Whatever the effect of the air, an equilibrium condition is soon obtained when the supply of air is limited. Probably the most stable resistors that have been made are a group of annealed 1-ohm manganin resistors mounted in double-walled air-filled containers, now being used at the National Bureau of Standards [8] for maintenance of the unit of resistance.

Although good annealing improves the stability of sealed resistors, it is apparently of no special value for unsealed coils. When mounted in open containers, annealed resistors cannot be expected to be any better than, if as good as, those baked at $150^{\circ} \mathrm{C}$. This is true even if the resistors are varnished or enameled after the annealing. None of these coatings is impervious to the atmosphere, and they merely retard any reaction between the air and the resistance material.

\section{Effects of Humidity}

It has long been known that wire-wound resistors undergo seasonal variations in resistance, being higher in resistance in summer and lower in winter. This effect is most noticeable in high resistance coils of small wire, and even in highgrade resistors may amount to several hundredths of a percent of the resistance. The effect is to a large extent a result of changes in average humidity, and is greatest in climates where there is a large difference in humidity between winter and summer. This seasonal change was first observed in the case of manganin resistors made with silkcovered wire. The accepted explanation was that the resistance changes resulted from dimensional changes of the shellac with which the coils were impregnated, as the shellac absorbed or gave off water vapor.

The effect of moisture on resistors has been thoroughly investigated by Dike [9], who came to the conclusion that the effect of changes in humidity is to change the tension in the silk with which the wire is customarily insulated. This change in tension changes the pressure transmitted to the wire by the insulation and hence changes the resistance. He also found that the effect of humidity on cotton insulation is opposite to that on silk, and by using a mixture of cotton and silk fibres for insulating the wire he was able to eliminate most of the seasonal changes in resistance that result from changes in humidity.

For standard resistors the effect of humidity 


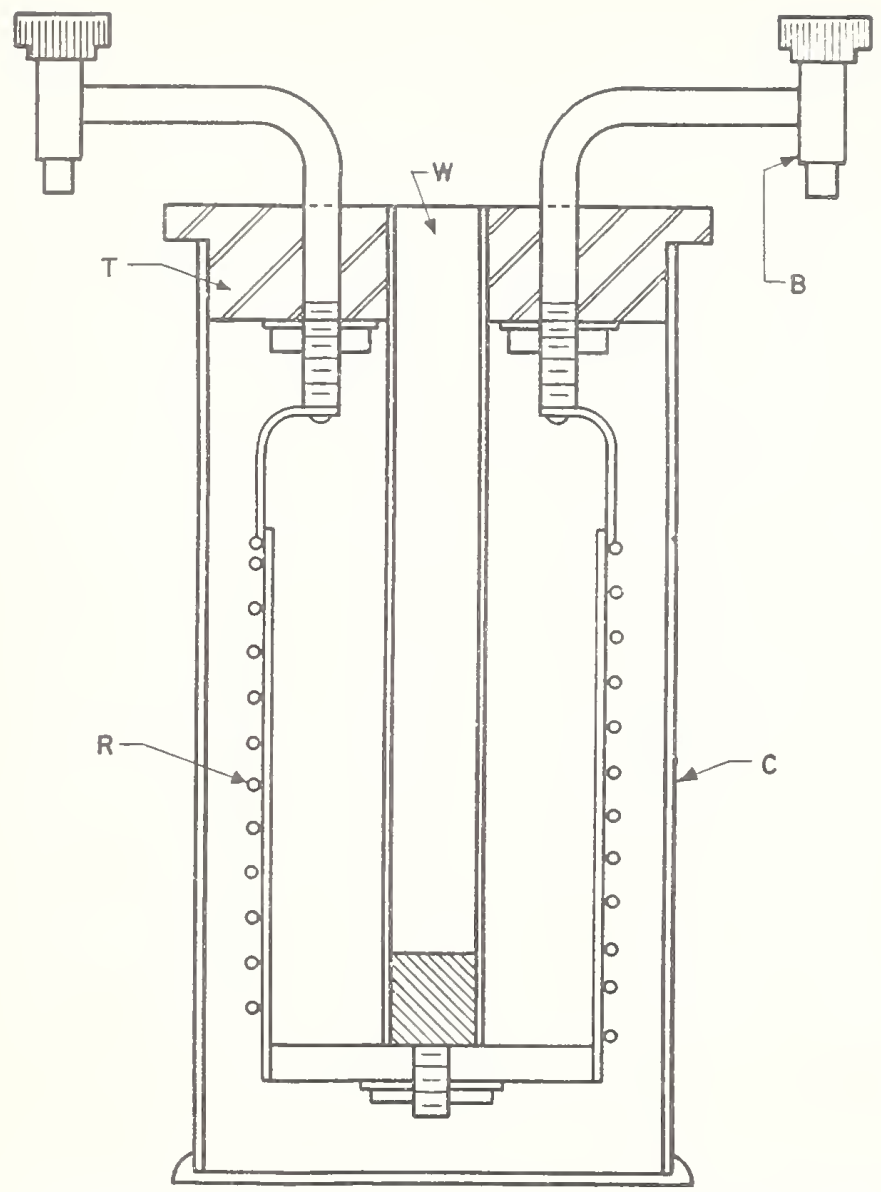

Figure 3. National Bureau of Standards standard resistor.

may be also eliminated by mounting the coils in scaled containers. This procedure was first advocated by Rosa [10] who designed the resistor shown in figure 3 , known as the NBS type of standard resistor. In this figure, $R$ represents the manganin coil mounted on a silk-insulated brass spool and baked as described in section II, 4. This coil is supported from the hardrubber top, $T$, by means of the thcrmometer tube, $W$, which is so arranged that a thermometcr can be inserted from the outside. The copper lead wires, which are silver-soldered to the ends of the resistance coil, are in turn soft-soldered to the copper binding posts, $B$. The hard-rubber top is screwed into the metal container, $C$, which is filled with a good quality light mincral oil. The binding posts, thermometer well and the threads by which the hard-rubber top is connected to the container are all sealed with shellac, which is not soluble in oil.

The purpose of the oil is to give good thermal contact between the resistor and the case and to facilitate the dissipation of the heat developed in the resistor by the current through it. In addition, the oil in effect increases the heat capacity of the resistor, thus increasing the current that it can carry temporarily without over- heating. The objection to the oil is the fact that it may in time become somewhat acid, and the acid may corrode the resistance wire or injure the insulation.

The advantage of ready dissipation of heat combined with the advantage of hermetic sealing is found in the double-walled type of standard resistor [8] developed at the Bureau. In this type the container is made of coaxial cylinders only slightly different in diameter with the space between the cylinders sealed. The resistance element is mounted in this sealed space in good thermal contact with the smaller cylinder, which serves as the inside wall of the container. One of these resistor's is shown in figure 4 . The outside diamcter of the container is $9 \mathrm{~cm}$ and its length $13 \mathrm{~cm}$. The series of holes near the top are just above the double-walled part and are intended to increase the facilities for cooling, and the containers are left open at the bottom for the same purpose. These double-walled resistors readily give up heat to an oil bath yet are not affected by humidity changes. The sealed space in which the coil is mounted is filled with dry air, and no oil comes in contact with the resistance material.

The seasonal changes in resistance of standard resistors that arise from changes in humidity are readily eliminated, as has just been discussed, by sealing in metal containers. This arrangement is not satisfactory for large instruments and measuring apparatus, which are not readily sealed. If the cases of such equipment are reasonably tight a drier, such as calcium chloride, may be kept inside the case. This procedure is somewhat hazardous, since if not replaced with a sufficient frequency the drier may become dissolved in

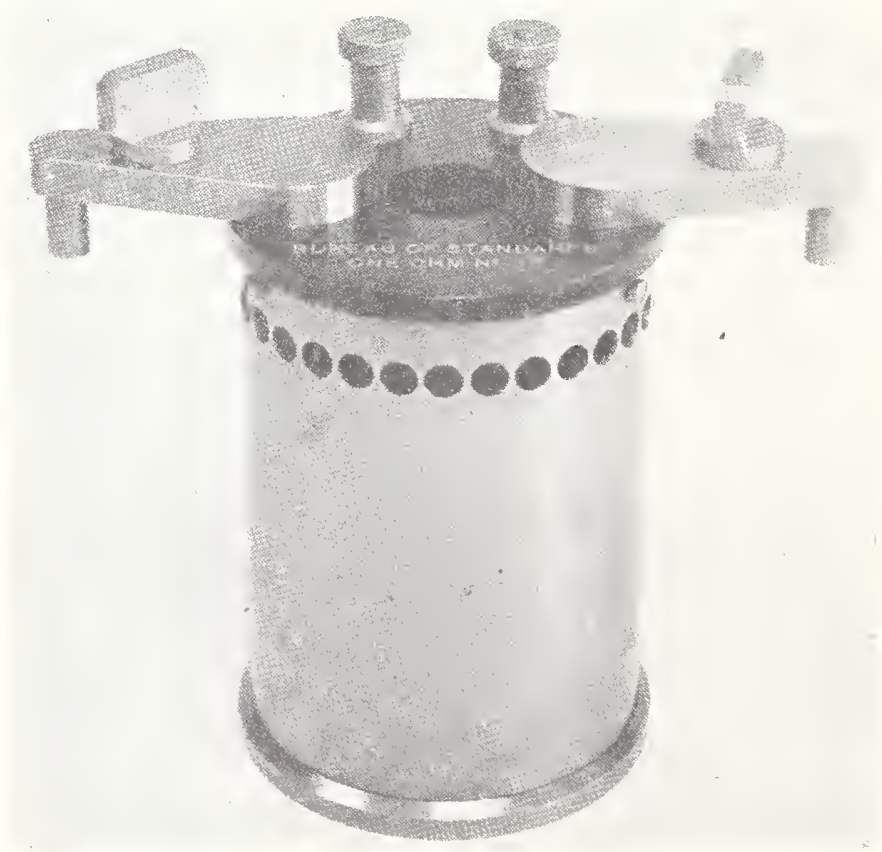

Figure 4. Double-walled standard resistor. 
adsorbed water and be spilled on the coils. The use of silica gel avoids this danger. A more satisfactory method is to mount a heater in the apparatus and, by means of a thermostat, maintain the temperature of a metal box containing important resistors at a constant value, above that of the laboratory. In this way the relative humidity is kept always low and seasonal variations may be reduced. Additional advantages are that no temperature corrections need be applied when calibration is made under conditions of use, and that resistors will usually be more stable in resistance if kept at a constant temperature instead of being allowed to follow variations in laboratory temperatures.

\section{Load Coefficients}

The load coefficient of a resistor is defined as the proportional change in resistance caused by the production of heat in the resistor at the rate of one watt. This change, however, is a function of time. When a current starts flowing in a wire-wound resistor the wire very quickly takes up a temperature above that of the spool on which the wire is mounted. The amount of this change depends upon the thermal contact between the wire and the spool. If the current continues to flow, this initial difference in temperature is maintained, but the coil and spool continue to rise in temperature together. The maximum temperature that will then be attained depends upon the facilities for dissipation of heat by the spool, and often upon the facilities for cooling the material to which heat from the spool flows.

Suppose we have a standard resistor of the NBS type resting on a table and we send through it a sufficient current to liberate one watt in the coil. Within about 30 seconds the temperature of the coil will rise to a steady value about $1^{\circ} \mathrm{C}$ above that of its spool. Spool and coil will then continue to rise in temperature at the rate of about $10^{\circ}$ or $15^{\circ}$ $\mathrm{C}$ per hour, and if allowed to continue will in about 2 hours reach a steady temperature of some $10^{\circ}$ to $20^{\circ} \mathrm{C}$ above that of the room. This may cause a permanent change in resistance, which, however, ordinarily amounts to only a few parts in a million. If, instead of being mounted in an oil-filled container, the same resistor had been left open in the air of the room, its final rise in temperature would probably have been less by some 50 percent and would have been reached in 15 to 20 minutes.

The rise in temperature of the resistance coil will be accompanied by a change in resistance, whose amount depends upon the temperature coefficient of resistance of the wire and also upon changes in stress in the wire because of dimensional changes of the coil and its support. For single-layer manganin coils mounted on brass spools the relative changes in dimensions are small, and the change in resistance results primarily from temperature changes of resistivity. For such resistors the effect of the heating may usually be taken into account by measuring the temperature change and calculating the change in resistance from the temperature coefficients of the resistor. For sheet-metal resistors the effect of stress changes may be important and the calculated change cannot be relied upon.

It should be evident from the above discussion that the load coefficient of a resistor is a rather indefinite quantity, varying with time of flow of the current and with the environment of the resistor. To be of value it should be measured under conditions of use. Measurement is usually made by passing the desired current through the resistor and a second resistor connected in series, measuring the ratio of resistance both with negligible and with the required heating. This ratio may be measured by means of a bridge or a potentiometer, and the comparison resistor should be one that is not appreciably affected by the test current. A resistor of one-tenth or less the resistance of that under test should be used, and its load will be one-tenth or less than for the resistor under test. For the reference lower-valued resistor one that is known to have a small load coefficient should be chosen. If such a resistor is not available one with a low temperature coefficient should be selected, on the assumption that its load coefficient is correspondingly low, and its load coefficient should be roughly determined to make sure that it is small. This can be done by balancing the resistor in any bridge using a small test current. From an external source a large current is then sent through the resistor under test for several minutes, after which the extra circuit is disconnected and the bridge circuit is again balanced. This last balance must be made quickly before the heat from the large current is dissipated.

If a resistor is being used under conditions where the load changes its resistance, the amount of the change may often be determined experimentally. To do this the heating may be doubled, by increasing the current by 40 percent, and the resultjng change noted. To a first approximation this doubling of the heating doubles the change in resistance, and twice the change should be subtracted algebraically from the final value. Such a procedure is especially satisfactory in the case of Wheatstone bridges, as the procedure will correct back to zero test current, whichever branch or branches are being changed by the current.

\section{Stability of Resistors With Time}

In applications where stability with time is of importance, as for instance for standard resistors or precision measuring apparatus, manganin is used almost exclusively. In such applications low temperature coefficients of resistance and small thermal emf's against copper are usually also required, 
and few alloys other than manganin are suitable. Consequently, a discussion of the stability of resistors is largely a discussion of the stability of manganin.

Most national standardizing laboratories keep a selected group of manganin liesistors, which are regularly intercompared and used for maintenance of the unit of resistance. The relative values of such standards are remarkably constant, the individual resistances not ehanging by more than one or two parts in a million per year with reference to one another and for some groups very much less. It is supposed that the average of a group remains constant to a high degree. but such stability can be only assumed. No method of measurement has been used that would detect with certainty changes of less than 10 or 20 parts in a million in the group as a whole. The international ohm. was defined as the resistance of a mercury column of specified dimensions at the temperature of melting ice. However, such resistors have not been constructed with sufficient accuracy to demonstrate the performance of manganin resistors used to maintain the unit. Likewise, absolute ohm determinations have not been sufficiently reproducible to give such information. If the unit as maintained by means of manganin resistors were tested every ten years by comparing against mereury olime or absolute olim, determinations, the apparent clange would probably not exceed 10 or 20 parts per million. This could be just as well attributed to errors in realizing the unit experimentally as to changes in the unit as maintained by the manganin resistors.

The a verage user of standard resistors is interested in the stability that may be expected from standards available commercially. In this connection, an analysis made in 1941 at this Bureau is relevant. Of nearly 600 standard resistors that had been submitted more than once to this $\mathrm{Bu}$ reau for test, the average yearly change in resistance, without regard to sign, was found to be $8 \mathrm{ppm}$. Of the total only 2 pereent averaged greater than $60 \mathrm{ppm}$ per year, and for nearly 90 pereent of all standards tested the annual change was 10 ppm or less. If signs were neglected there was no significant difference between the a verage yearly change of sealed and unsealed resistors. This would appear to mean that sealing merely reduces seasonal variations without improving the long-time stability. However, if regard is taken of sign, the performanee of sealed and unsealed resistors was quite different. In this case the average yearly change was about $-.3 \mathrm{ppm}$ for scaled and about $+5 \mathrm{ppm}$ for unsealed standards. That is to say, scaled standards about as often decrease as increase in resistance with time, whereas the change in unsealed standard resistors is predominantly upward. In connection with the sealed resistors it is interesting to note that practically the same result would have been obtained if the unit of resistance had been maintained by supposing the average value of the 400 sealed resistors had remained constant, as was obtained by assuming the average of a group of 10 selected resistors of special construction to be constant.

\section{Methods of Comparison of Resistors}

\section{Ammeter-Voltmeter Methods}

Precise measurements of electrical resistance are made with comparative ease with bridge methods, an aceuracy of a few parts in a million being readily obtained in the comparison of nominally equal resistances of say 10 or 100 ohms. Although the actual measurements are rather simple, special apparatus is required. For many types of resistor's high arcuracy is not desired and the measurements may be made with deflecting instruments, which are usually arailable in electrical laboratories. 'The most common method, where an aceuracy of 1 or 2 pereent is sufficient, is the ammeter-voltmeter method. In this method a moasured current is passed through the resistor under test, and the potential difference across its terminals is also measured. The current is measured with an ammeter and the potential difference by means of a voltmeter, the resistance in ohms being the ratio of the roltmeter reading in volts to the ammeter reading in anperes, in accordance with Olım's Law.
The accuracy that may be attained by the ammeter-voltmicter method depends upon the accuracy of the two instruments. However, there are a few precautions that must be observed. Referring to figure 5 , if the voltmeter, $V$, is con-

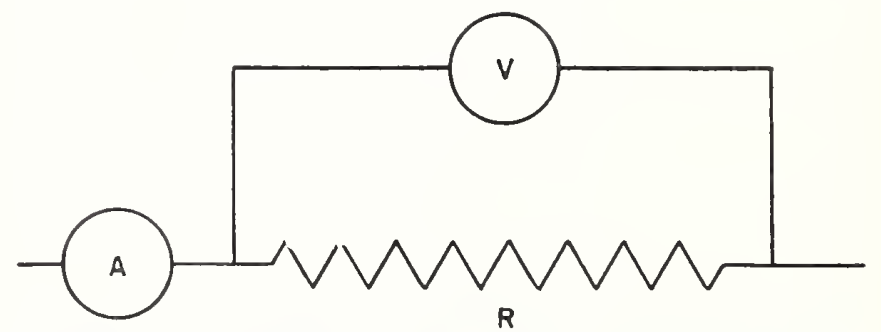

Fictre 5. Connection for ammeter-vcltmeter measurement of resistance.

nected as shown across the resistor, $R$, the current read by the ammeter, $A$, is the sum of the current through $R$ and the current through the voltmeter. If the resistance of the voltmeter is large as compared with $R$, the current through the voltmeter 
may be neglected and $R$ may be calculated as the ratio of the instrument readings. If the resistance of the voltmeter is not large as compared with $R$, the latter may be calculated from the equation

$$
R=\frac{E}{I\left(1-E / I R_{v}\right)}
$$

where $R_{v}$ is the resistance of the voltmeter, its reading being $E$ volts, and the ammeter reading $I$ amperes. If the resistance of the ammeter is known or if it is negligible as compared with the unknown resistance, the voltmeter may be connected across both the resistance and the ammeter, as shown in figure 6 . In this case the ratio of

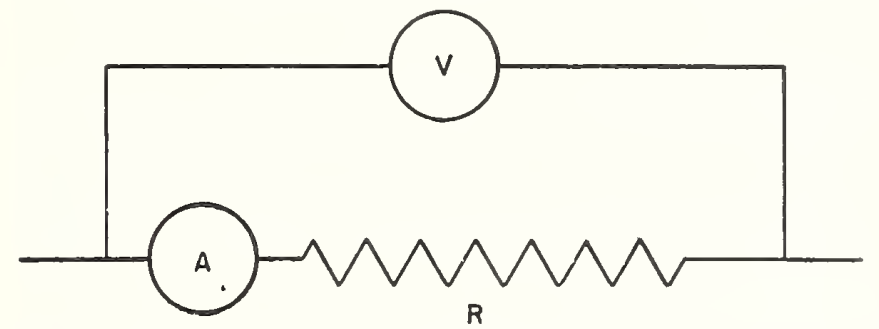

Figure 6. Alternate connection for ammeter-voltmeter measurement of resistance.

voltmeter to ammeter readings gives the total resistance between the points of attachment of the voltmeter, that is, the resistance of the ammeter and of the connecting leads is included. That is

$$
R=\frac{E}{I}-R_{A}
$$

where $R_{A}$ is the resistance of the ammeter and of all lead wires between the points of attachment of the voltmeter leads.

With suitably calibrated.instruments it is possible to measure resistance to 0.1 or 0.2 percent provided the instruments are of such ranges that large deflections are obtained. For this accuracy it is usually necessary to calibrate the voltmeter with the same leads as those that are to be used in the resistance measurements.

\section{Ohmmeters}

Ohmmeters are instruments for indicating directly on a scale, with a minimum of manipulation or computation, the resistance of the circuit connceted across their terminals. They are available in a wide range from milliohmmeters reading to $0.001 \mathrm{ohm}$ to megohmmeters reading to 50,000 megohms. Their accuracy is limited both by the calibration and reading of the indicating instrument and in the simple olimmeter by their dependence upon a fixed value of voltage. They are, therefore, in general, not suited for applications requiring high precision such as the determination of tcmperature rise or of the conductivity of line conductors. When used with circuits that are highly inductive or capacitive, the precautions appropriate for such resistance measurements should be observed.

Ohmmeters may be classified according to either their principles of operation, their source of energy, or their range.

The principles commonly used are: Simple ohmmeter, ratio ohmmeter, Wheatstone bridge.

In the simple ohmmeter a source, the voltage of which is assumed to be definite and to correspond to the calibration of the instrument, is applied to the unknown resistor and the resulting current causes an indicating instrument to deflect over a scale. This scale is so graduated that the pointer indicates directly the resistance in ohms (or megohms). In many cases provision is made by a magnetic or electric shunt to adjust the instrument at one point, usually at zero resistance, to fit the existing value of the voltage. In some of these instruments the final indication is by a vacuum-tube voltmeter, which measures the drop produced in a very high resistance by the current through the specimen.

In the ratio meter or "crossed-coil" type of ohmmeter, the current through the unknown resistor flows in one of the coils, while the other carries a current that is proportional to the voltage. The current is led to the coils by ligaments, which exert a negligible torque so that the moving system takes up a position that depends on the relative magnitude of the currents in the two coils. The scale can therefore be laid off to indicate resistance directly, and the indication will be independent of the voltage used, provided that the resistor under test obeys Ohm's Law.

The designation "ohmmeter" is also applied (though perhaps incorrectly) to certain forms of the Wheatstone bridge in which the dial that adjusts the balance is calibrated to read directly the value of the unknown resistance.

\section{Potentiometer Method}

It was pointed out in section III, 1 that the ammeter-voltmeter method for measuring resistance is complicated by the current drawn by the voltmeter. Such complications may be avoided by using a voltmeter of some type that requires no current from the circuit being measured, i. e., electrostatic or vacuum. tube voltmeters.

Potentiometers are also suitable for the measurement of potential difference when it is desired to avoid drawing a current from the source of potential difference. They are especially good in cases where an accuracy of 0.1 percent or better is required, as such accuracy is difficult to attain with deflecting instruments. Where a potentiometer is available, the ammeter-voltmeter method may 
be modified so as to use the potentiometer to measure the current through the unknown resistor as well as the potential difference across it. This requires the replacement of the ammeter with a standard resistor, and the measurement of the potential difference across the standard resistor yiclds the value of the current if this potential difference is divided by the value of the resistance of the standard.

Actually, if a suitable standard resistor is available, it is unnecessary to calculate the current through the unknown resistor. If the same curlent flows through the known and the unknown resistor, the ratio of the potential differences across the two is the same as the ratio of the resistances. Hence

$$
X=S \frac{T_{r}^{T}}{T_{s}}
$$

where $X$ and $S$ are the values of the unknown and stanclard resistances, $V_{x}$ and $V_{s}$ are the measured potential differenees across $X$ and $S$ respectively. Care must be exereised in using this method to insure that the current through $X$ and $S$ remains constant during measurement. This may be verified by measuring the potential differences alterinately sereral times.

High aceuracy in the measurement of resistance ean be attained with the potentiometer method if a good potentiometer and good standard resistors are used. It has an advantage over the ordinary Wheatstone bridge in that the resistance in terms of which the unknown is measured may be that of an actual standard resistor instead of one of the coils of the bridge. Stanclard resistors are so mounted that they are ordinarily more constant in resistanee and henee more aceurately known than are unsealed coils usually used in bridges. The potentiometer method, however, is more difficult to use as the currents through the potentioneter and in the measuring circuit must both be kept constant, whereas the balance of a Wheatstone bridge is independent of the current flowing through it. In comparing resistors, the accuracy of a potentiometer is not dependent upon the accuracy of calibration of the standard cell used with the instrument.

A type of measurement for which the potentiometer method is especially well suited is for the measurement of four-terminal resistors, which are parts of complicated networks, where connections to the resistors must be made through other resistors. The resistances in the potential leads, which connect to the potentiometer, have no effect upon the balance of the potentiometer. There way be, however, a reduction in sensitivity unless the damping resistor for the galvanometer may be changed to allow for these extra resistances.

\section{Differential-Galvanometer Method}

The differential galvanometer was formerly used rather extensively for the comparison of equal resistances. Such a galvanometer has two separate windings made as nearly the same as possible, so that when equal emf's are applied to the terminals of the windings, equal and opposite torques are produced on the deflecting element. The windings are constructed with two wires side-by-side, wound at the same time and as nearly as possible symmetrically with respect to the magnetic circuit.

For the moving-magnet galvanometer the field coils are wound in duplicate, and a small movable coil is usually connected in series with one winding. This moving coil is adjusted in position to compensate for any lack of equality of the ficlds produced by the two windings. Unfortunately this adjustment is different for different conditions of use. Moreover the differential galvanometer of the moving-magnet type has the same handicaps as others of the moving-magnet type. That is to say, the damping is difficult to control and claborate precautions must be taken to avoid magnetic disturbances from external sourees.

Many of the troubles of the moving-magnet galvanometer are avoided in the D'Arsonval, or. moving-coil galvanometer, and this coil may be made in duplicate for differential use. However, this requires two sets of leads from the moving element, which are difficult to arrange, and which also in effect stiflen the suspensions and lower the sensitivity. With the need for greater and greater precision, the differential galvanometer has been gradually discarded, but it still is very satisfactory for some types of measurements.

In theory, the use of the differential galvanometcr is very simple. A current, $I$, is passed through the standard, $S$, and the unknown, $X$, comnected in serins, and one winding, $G$, of the galvanometer is connected to the terminals of each resistor, as shown in figure 7 . If the galvanometer circuits were exactly alike in resistance and opposite in their magnetic effects there would be no deflection if $X^{2}$ and $S$ were equal. These conditions on resistance and magnetic effect are difficult to

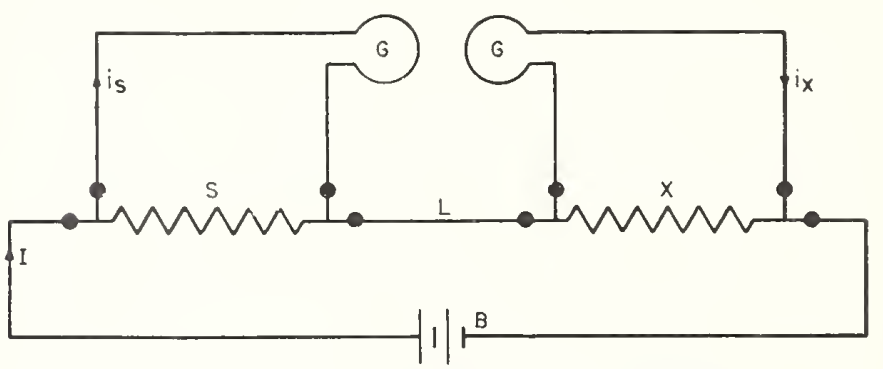

Figure 7. Connections of differential galvanometer. 


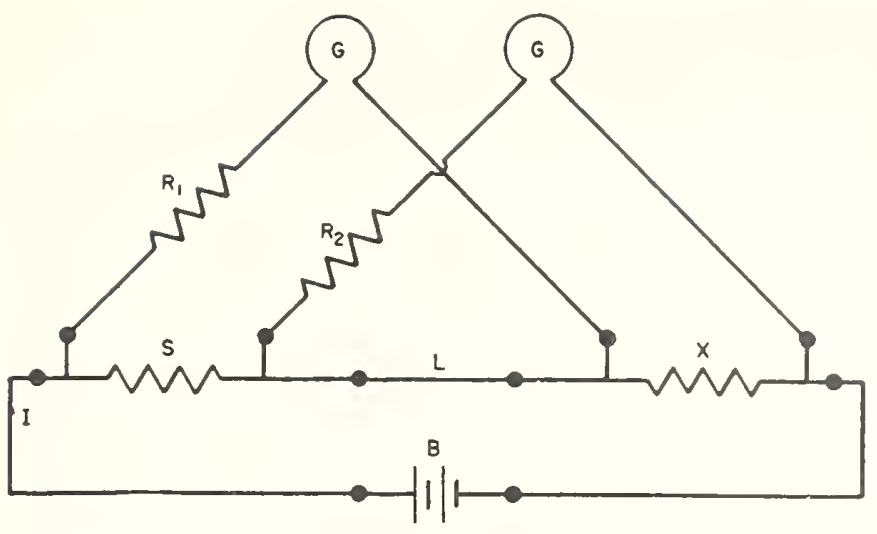

FIGURE 8. Kohlrausch's connection of differential galvanometer.

meet, and it is necessary to devise methods of use for which the conditions need bc only approximately met, if high accuracy is to be attained. Such is that due to Kohlrausch [11], and known as his method of overlapping shunts.

For Kohlrausch's method the circuit is as shown in figure 8. The two galvanometer windings, $G$, are connected respectivcly across $X$ and $S$, and in addition each shunt bridges the resistance, $L$, which is used to connect $X$ and $S$ in series. The resistance of $L$ is usually small as compared with the other resistances. In series with the galvanometer windings are connceted the resistors, $R_{1}$ and $R_{2}$, one or both of which are adjustable. In addition, a special switch not shown must be used, which will interchange the battery $B$ and the lead resistance $L$. This must be done without materially changing the current furnished by the battery. Thic effect of the interchange is to reverse the current through both galvanometer windings. The values of the adjustable standard, $S$, and the rheostat, $R_{1}$ or $R_{2}$, are adjusted until there is no deflection of the galvanometer for either position of the battery, or until the deflection is the same in magnitude and direction for both battery positions. Under either condition of adjustment, $S$ and $X$ are equal.

In actual practice, where $S$ and $X$ are standard resistors under comparison, the larger is made adjustable by a precision rheostat connected in parallel, and the amount of the shunt required to make $X$ and $S$ equal allows an easy calculation of the difference in resistance between the two. The balance is cxact even if the two circuits of the galvanometer are not exactly alike electrically or magnetically.

\section{Bridge Methods}

By far the largest proportion of measurements of electrical resistance are made by means of bridge metlods. For resistors abovi 1 ohm the simple Wheatstone bridge is used, whereas for 1-ohm resistors anel below the Kelvin double- bridge is more suitable. The choice between the simple and the double bridge is usually made on the basis of the required accuracy.

It is difficult to attach a copper lead wire to a resistor by means of binding posts or other clamped connections without introducing an unknown contact resistance of the order of 0.0001 ohm. For resistors above $1 \mathrm{ohm}$ such an uncertainty is usually negligible, whereas for a resistor of say $0.01 \mathrm{ohm}$ the uncertainty is 1 percent. For standarel resistors the contact resistance is often recluced by amalgamating the contact surfaces. For clean well-fitterl contacts the resistance then amounts to only a few microhms, but the resistance of such contacts will rise with time as the copper combines with the mercury to form a granular material. This material shoukd be removed every few months by scraping and wiping the surfaces.

Where accuracy is required, low-valued resistors are usually of the four-terminal type. For these, two leads are soldered or brazed to each enel of the resistance material, as shown in figure 9. The

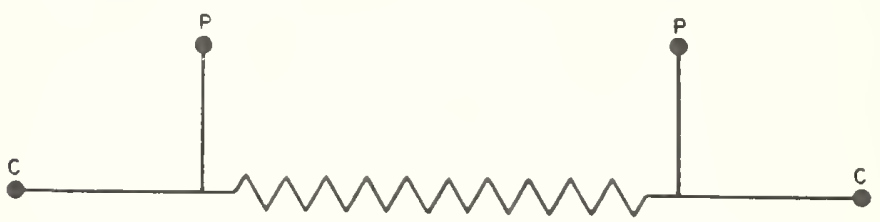

Firtate 9. Four-terminal resistor.

resistance in question is that between the branch points at the two ends. That is to say, the resistance is the ratio of the potential difference between the terminals $P, P$ to the current flowing in and out the current terminals, $C^{\prime}, C$. Mothods of measurement are used such that any effect from the lead resistances is aroided or reduced to a negligible amount. For such a purpose the potentiometer method is suitable as no appreciable current is drawn through the potential leads, and the potential drop between branch points is independent of the magnitude of the lead resistances in the current circuit. Double-bridge methods balance out the lead resistane's or connect them in high-resistance branches whore they are negligible.

Simple Wheatstone bridge.-The simple Wheatstone bridge is primarily a group of four resistor's connected in series-parallel as shown in figure 10 . A current, $I$, is passed through the two parallel branches, and $G$ is a detector connected to the junctions of the resistors as shown. It may be readily shown that if there is no potential difference across the detcetor, $G$, the relation between the resistances of the four anms is as follows:

$$
\frac{Y}{S}=\frac{1}{B}
$$

Or

$$
I=s \frac{-1}{B}
$$




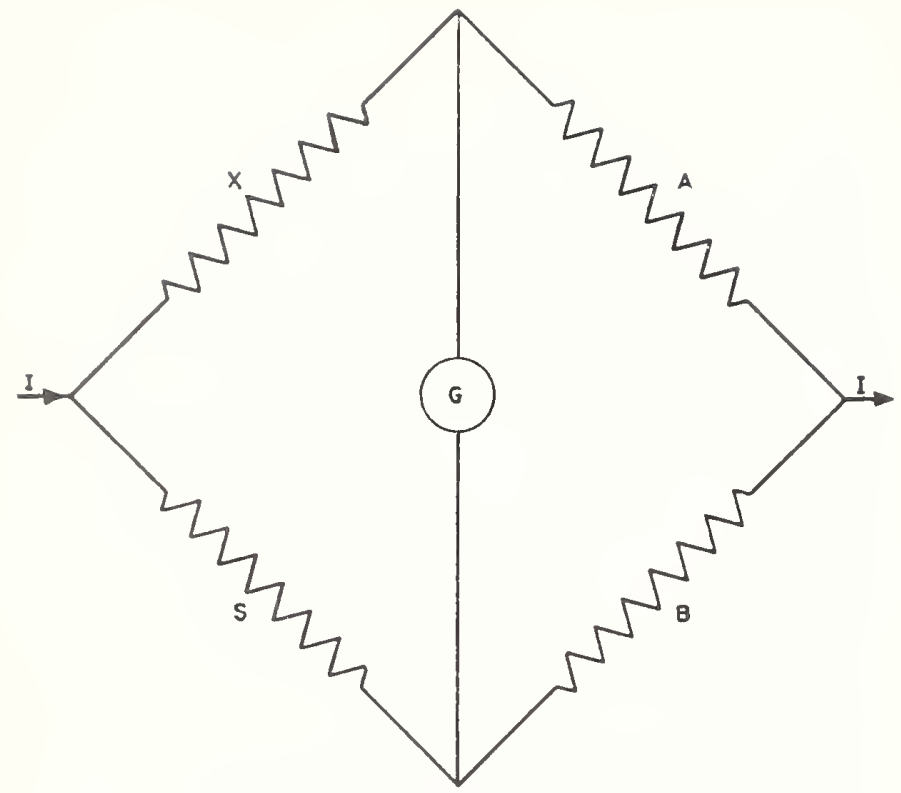

Figure: 10, Wheatstone bridge.

The detector, $G$, is usually a galvanometer, and the lack of a potential difference across it is evidenced by a lack of motion of the galvanometer coil if a switch or key in series with $G$ is opened or closed, or better, if the battery circuit is opened or closed. Opening or closing of the battery. cir"uit should be aroided, however, if there is inductance or capacitance in the circuit of the unknown resistance or in any arm of the Wheatstone bridge. In measuling the resistance of a field winding of an electric motor, for example, the galvanometer may be clamaged if its eirenit is left closed and the battery cir'uit of the bridge is opened. In this case the eurrent should be left on until a steady state is reached before testing the bridge balance by opening and closing the galvanometer cireuit.

From the above equation it is erident that the resistance of any one of the four arms, say $Y$, ean be ealculated if the resistanees of the other three are known, or if one of the three and the ratio of the other two are known. The usual general purpose Wheatstone bridge is made in such a way that any one of several known resistors may be selected for use as either $A$ or $B$ and hence their ratio may have any one of a number of values. $S$ is then it known resistor whose ralue may be adjusted in small steps orer a wide range of resistance.

In commercial instruments the ratio arms $A$ and 1) are usually coils that are connected into the bridge by inserting suitable plugs. As there are resistances in these plug contacts, which may be rather variable, it is desirable to use relatively high values of resistance for $A$ and $B$ in order to reduce the uncertainty in the resistance of the ratio arms. On the other hand, high-resistance coils are more affected by humidity and are less stable in resistance with time and therefore retain their calibra- tion for a shorter time. Other things being equal, ratio coils of from 10 to 100 ohms are therefore about the best compromise.

The choice of resistance for the adjustable arm of a Wheatstone bridge is influenced by the same factors as the choice of resistances for the ratio arms. That is to say, the lower the value the greater the stability in resistance but the more troublesome the contact resistances become, and in the adjustable arm several contact resistances are required. Steps smaller than $0.1 \mathrm{ohm}$, which allow readings of the adjustable arm to about 0.01 ohm by interpolation from galvanometer deflections, are seldom used as it is not wise to rely upon the combined effect of the several contacts of the arm to be definite to much better than $0.01 \mathrm{ohm}$.

Wheatstone bridges for the measurement of resistance to 0.1 percent are available commercially at moderate prices. To this accuracy these can usually be relied upon without the application of corrections to the readings. However, for measurements to 0.01 percent, corrections to the readings of the ratio arms and of the rheostat arm must usually be applied, and the bridge must be maintained at the temperature of calibration within a few degrees. Primarily because of the effects of changes in humidity, calibrations of a Wheatstone bridge must be made rather frequently if an accuracy of 0.01 percent is to be attained. This is especially true where there is a marked change in ambient conditions. For example, between winter' and summer the ratio coils and the rheostat arm may rach change by 0.01 pereent or more, and the errors may be additive rather than compensating. The use of air-conditioned laboratories improves the performance markedly, but even then it is advisable to make occasional spot checks by measuring standard resistors. Whenerer possible, ratio arms below 10 olıms or above $1,000 \mathrm{ohms}$ should be avoided, as should rheostat readings in excess of $1,000 \mathrm{olmms}$. This means that the Wheatstone bridge is best suited for the measurement of resistance in the range 10 to $10,000 \mathrm{ohms}$.

When an accuracy greater than 0.01 percent is required, special bridges are required or special techniques are used. One of the best of the special methods is that of substitution, in which the unknown resistor is replaced with a standard resistor or resistors having the same nominal resistance as that of the unknown. The bridge that is being used is then relied upon to determine only the difference between the standard and the unknown, and this difference need not be accurately measured. If the unknown and standard differ by 0.1 pereent, the difference need be determined to only 1 percent to give the unknown to 10 parts in a million in t八ms of the standard. Nearly any good bridge can be so used without calibration, provided contact resistances are sufficiently constant that readings can be repeated to the desired precision. It is erident that the calibration of the measuring 
bridge is of least importance when the standard and unknown resistances are nearest equal. Standard resistors are usually available only in decimal multiples or submultiples of an ohm and for many resistance measurements the lack of a suitable standard prevents the use of the substitution method.

Mueller bridge.-For the accurate measurement of odd-sized resistances, one of the best bridges is that designed by E. F. Mueller [12] of the National Bureau of Standards for use in resistance thermometry. This is a special bridge intended for the accurate measurement of resistances up to about $110 \mathrm{ohms}$. The effects of humidity changes are greatly reduced by mounting the resistors in a compartment that is electrically heated and whose temperature is maintained at $35^{\circ} \mathrm{C}$ by means of a tlrermostat. As this is a temperature to which the laboratory. temperature seldom rises, the relative humidity in the compartment housing the resistors is kept low and variations in the low humidities lave little effect on the resistances of the coils.

For the Mueller bridge the ratio arms are equal. and the arms can be interchanged to test the equality. A small slide wire is connected between the ratio coils, and the sliding contact is used as the branch point for the bridge as shown in figure 11. The operator can set the ratio arms to equality by properly proportioning the resistance of the slide wire between the two ratio arms by changing

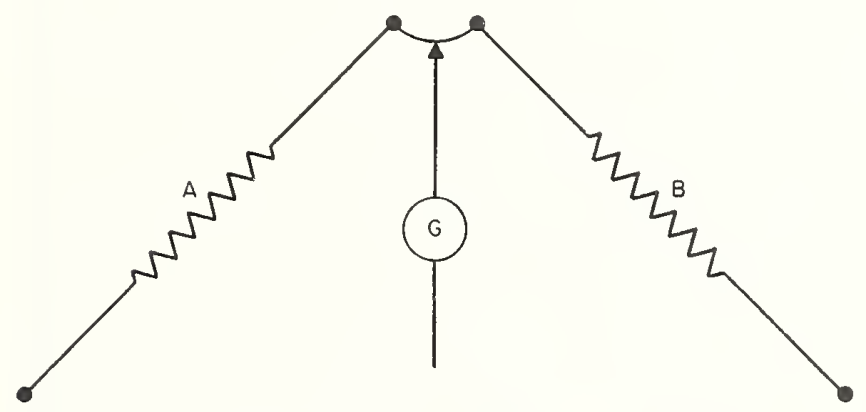

Figure 11. Ratio arms of Mueller bridge.

the setting of the slide contact. This setting is correct if no change in the bridge results when the arms $A$ and $B$ are interchanged. The remaining two arms are the adjustable rheostat and the unknown resistor, which must be of the four-terminal type. Resistance thermometers are usually of the four-lead type, and other resistors should be provided with four leads when they are to be measured on a bridge of this type. 'The method of connection of the unknown resistance is as shown in figure 12. $R$ is the adjustable rheostat arm that will be discussed in detail later. $X$ is a four-terminal r'sistor with lead wires $L_{1}, L_{2}, L_{3}$, and $L_{4}$ commected to the binding posts $1,2,3$, and 4 , respectively. If the galvanometer is connected to binding post 2,

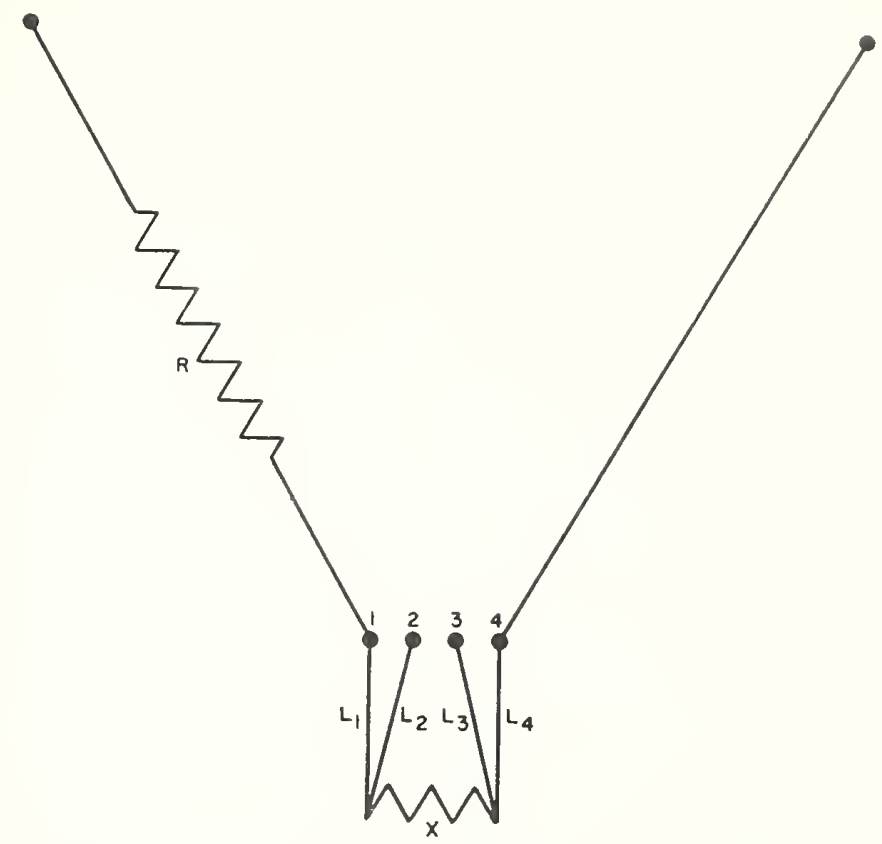

Figtre 12. Connection of bridge to 4-lead thermometer.

the resistor $X$ and the lead resistor $L_{4}$ are connected in to the right-hand arm, and the lead resistor $L_{1}$ is in the adjustable arm, $R$. With equal ratio arms, $R$ and $X$ would be equal for a balance of the bridge only if $L_{1}$ and $L_{4}$ are equal. Instead of adjusting $L_{1}$ and $L_{4}$ to exact equality, their connections are interchanged and a second bridge balance is obtained, the average of the two balances being that which would be obtained with $L_{1}$ and $L_{4}$ equal. When $L_{1}$ and $L_{4}$ are interchanged, it is necessary to shift the branch point from lead 2 to lead 3 in order to keep $X$ in the right-hand arm. In actual use, instead of the lead wires being interchanged, the internal connections of the bridge arms to the binding posts 1 and 4 are interchanged to obtain the same result. This interchange is effected by means of an amalgamated switch for which the uncertainty in contact resistance is only a few microhms.

The rheostat arm of the Mueller bridge is adjustable in steps as small as $0.0001 \mathrm{ohm}$. In order that such steps should not be masked by changes in contact resistance, special types [13] of decades are used. The 0.0001-ohm-per-step decade is approximately as shown in figure 13 . With the

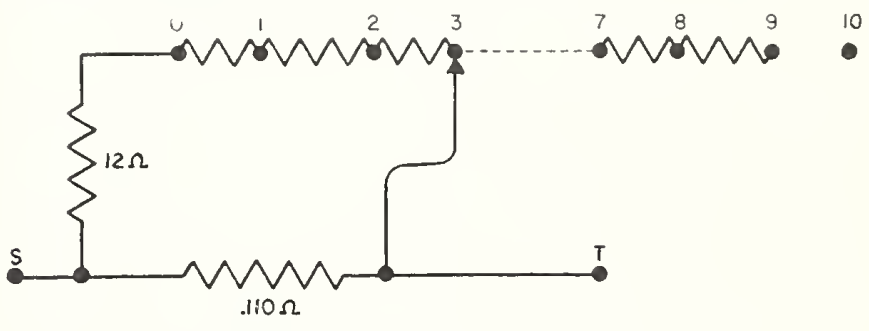

Figtre 13. Shunt-type decade. 
switch contact on the 0 stud, the resistances of 12 ohms and $0.110 \mathrm{ohm}$ are connected in parallel, and the resistance between the terminals $S$ and $T$ is about $0.1090 \mathrm{ohm}$. When the switch contact is moved to stud 1, the 12-ohm branch is increased sufficiently to increase the parallel resistance from 0.1090 to $0.1091 \mathrm{ohm}$, an increase of $0.0001 \mathrm{ohm}$. Likewise when the switch is moved to 2 the parallel resistance increases to $0.1092 \mathrm{ohm}$, etc. Thus the resistance between the terminals $S$ and $T$ may be increased in steps of $0.0001 \mathrm{ohm}$, although the value is not zero when the switch is set to read zero.

The 0.001- and 0.01-ohm-per-step decades are made in the same way as the $0.0001-0 h m$ decade. When these three decades are connected in series and all set to zero, there is a series resistance of about $1.6 \mathrm{ohms}$. However, since the bridge has equal ratio arms an equal resistance of $1.6 \mathrm{ohms}$ may be included in the $X$-arm, and the value of $X$ is measured by observing the increase in the resistance of the rheostat arm when $X$ is inserted in its arm. A short-circuiting plug is provided with the bridge that connects together the terminals 1 and 4 of figure 12 . The bridge is then balanced with and without this shorting plug, and the difference in the readings of the rheostat arm gives the resistance of $X$, and leads.

The purpose of the shunted type of decade that was described above is to reduce the effect of variations in contact resistances and transient emf's in the switch. Referring again to figure 13, it is seen that the switch contact is in the high resistance branch. The ratio of resistance for the two branches is about 100 to 1 , and it may be readily shown by differentiation that the effect of a variation of switch contact resistance is reduced by the square of this ratio. Thus a contact variation as high as $0.01 \mathrm{ohm}$ would vary the resistance from $S$ to $T$ by only 1 microhm, which is negligible in the rheostat arm where the minimum steps are 100 microhms. For the 0.001 and 0.01 decades, the ratio of currents in the branches is less than 100 to 1 , and less variation in the switch-contact resistance can be tolerated.

The 1-ohm and 0.1-ohm steps of the rheostat arm are not made of the shunt type, as the resistance with the dial set on zero would be rather large. The contact resistances for the 1-ohm dial are thrown into the ratio arm $A$, as shown in figure 14 . The bridge current is introduced through the $101-0 h m$ coils of this decade, and the switch merely changes the point of connection of the ratio arm, without opening the circuit of the 1-obm decade. In this case a variation of the switch contact resistance changes the resistance of ratio $\operatorname{arm} A$. This effect is reduced by using high resistance ratio arms, $A$ and $B$ each being 1,000 ohms. The 0.1-ohm-per-step dial is arranged in the same way as the 1-ohm-per-step dial, with the switch contact in series with the $B$ ratio arm. With the 0.1 -ohm dial set at zero, all 10 of the

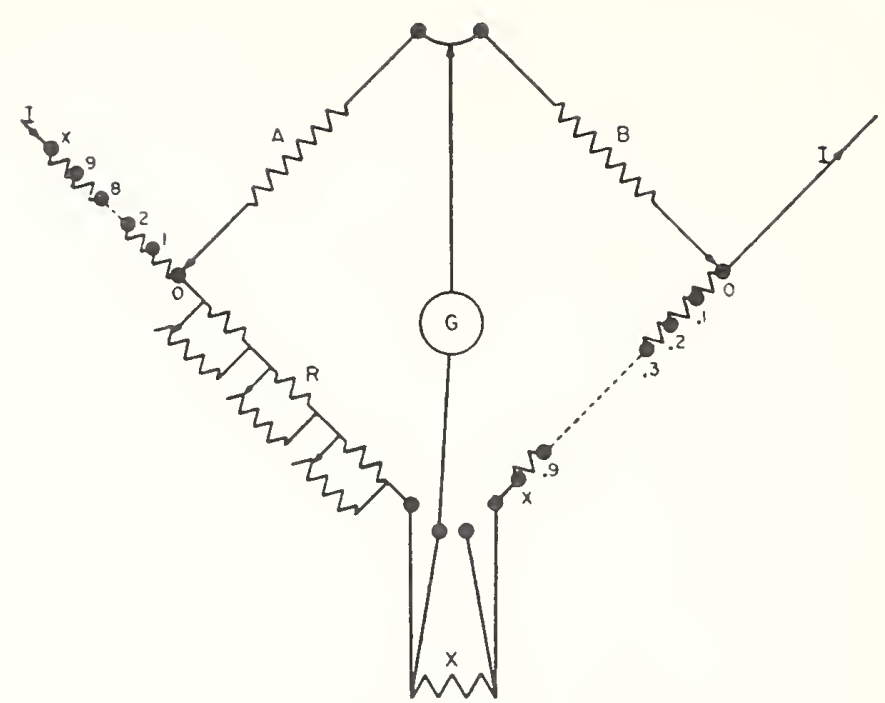

Figure 14. Switch connections for Mueller bridge.

0.1 -ohm resistors are in series in the $X$-arm. When the dial reading is increased, the resistance is removed from the $X$-arm, the effect being the same as if equal resistance were added to the rheostat arm, $R$, since the bridge has equal arms. This may be seen from the following consideration. Assume that the bridge is balanced with the unknown resistor and the 10 steps of the $0.1-\mathrm{ohm}$ dial all connected in the $X$-arm. If now $X$ is increased by $0.1 \mathrm{ohm}$, the $X$ - and $R$-arms may be brought again to equality by either increasing $R$ by $0.1 \mathrm{ohm}$ or by decreasing the resistance in series with $\mathrm{X}$ by $0.1 \mathrm{ohm}$. The readings of the dial in the $X$-arm are such that increases in readings of the dial correspond to decreases in the resistance in the $X$-arm.

The 10-ohm-per-step decade of the rheostat, not shown, is the only one that has contact resistances directly in series with the arm. A special type of dial that has amalgamated contacts is used for this purpose. Such contacts are uncertain by only a few microhms, which may be tolerated in the rheostat arm where the minimum steps are 100 microhms. For Mueller bridges available commercially, the galvanometer and battery are interchanged from the positions assumed in the above discussion. This in no way affects the validity of the conclusions.

Adjustable-ratio bridges. As has already been stated, the Wheatstone bridge shown in figure 10 is balanced if

$$
X=S \frac{A}{B}
$$

and this balance may be realized by keeping $A$ and $B$ fixed and adjusting $S$, or by keeping $S$ fixed and adjusting the ratio $A / B$. In most commercial Wheatstone bridges the balance is obtained with $S$. However, for comparing nominally equal resistances, bridges are sometimes constructed in which the ratio is adjustable. 


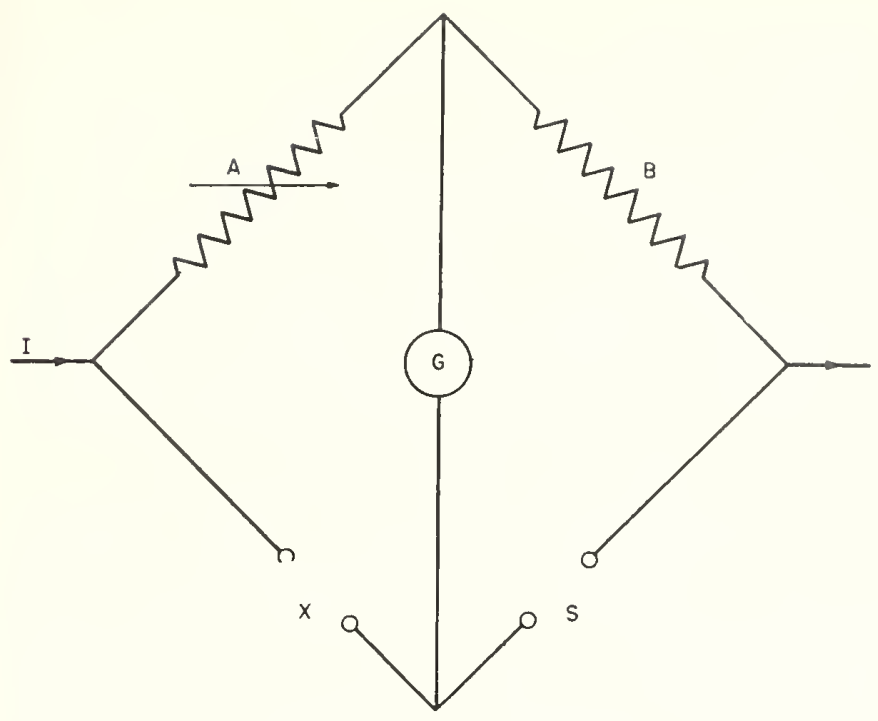

Figure 15. Limit bridge.

An adjustable-ratio bridge is very convenient for use in sorting large numbers of resistors that must be equal within given tolerances. Such a bridge may be constructed as shown in figure 15, where $A$ and $B$ are the two arms of a resistance ratio that is adjusted by changing $A$. A resistor is connected to the binding posts $S$, and the resistors that are to be compared with it are connected in turn to the $X$ terminals. If the resistors are to be the same to, say, 0.1 pereent, the $\operatorname{arm} A$ is made to have either of two values, which give ratios, $A / B$, of 0.999 or 1.001 . This could be realized by making $B 100$ ohms and $A$ either 99.9 ohms or $100.1 \mathrm{ohms}$, the latter accomplished, for instance, by making $A$ equal to 100.1 ohms and having a plug to short-circuit $0.2 \mathrm{ohm}$ of this total. The procedure is then to observe the direction of deflection of the galvanometer with either ratio setting and then change to the other ratio. If this causes a reversal of the direction of deflection of the galvanometer, the correct balance of the bridge is between the two ratio settings. In other words the ratio of $X / S$ is between the two ratios 0.999 and 1.001 , i. e., the two are equal within \pm 0.1 percent.

Besides this use in limit bridges, adjustable ratio bridges may be used for precise measurements of resistors in terms of nominally equal standards. Such bridges when used in conjunction with the substitution method permit very accurate measurements of resistance. Such an adjustable ratio is the "direet-reading ratio set" described in section IV, 1, where the appropriate techniques are also discussed.

Kelvin double-bridge.-As has been already stated, four-terminal resistors are used in order to avoid uncertainties arising from variations in contact resistances. For precision work, resistors of $1 \mathrm{ohm}$ or below are usually of the four-terminal type.
For measurement, a four-terminal resistor is usually connected in series with a four-terminal standard. The ratio of the potential differences across the two resistors is then determined when a current flows through the two. This ratio may be determined by means of a potentiometer $\mathrm{Or}^{\circ}$ in terms of a resistance ratio by means of the Kelvin double-bridge.

The eircuit of the Kelvin double bridge is shown in figure 16 , where $X$ and $S$ denote the unknown and standard resistor's, each with both current and potential terminals. The two are connected by means of a conductor $L$, preferably of low resistance as compared with $X^{\prime}$ or $S$. The resistors $A$ and $B$ and also $a$ and $b$ provide resistance ratios that must be known, as must also be the value of $S$. This bridge is balanced if there is no change in the deflection of the galvanometer when the current circuit is opened or closed. When balanced the following relation holds between the values of the resistance:

$$
X=S \frac{A}{B}+\frac{b\left(\frac{A}{B}-\frac{a}{b}\right)}{a+b+L} L .
$$

This equation would be exactly the same as that for a simple Wheatstone bridge if the last term on the right side were zero or negligibly small as compared with the term $S A / B$. That last term can be made zero by making $a / b=A / B$, irrespective of the value of the link resistance, $L$. However, the smaller the value of $L$, the less important is any lack of equality between the ratios $A / B$ and $a / b$.

In actual use two methods are employed for balancing the double-bridge. The resistors $A$ and $B$, and also $a$ and $b$ may be fixed ratio coils so chosen that $A / B=a / b$ and the bridge balanced by varying $S$, which is an appropriate adjustable standard; or $S$ may be a fixed standard resistor and the bridge balanced by adjusting the ratios $A / B$ and $a / b$, keeping these ratios at all times equal.

When a double-bridge is to be balanced by means

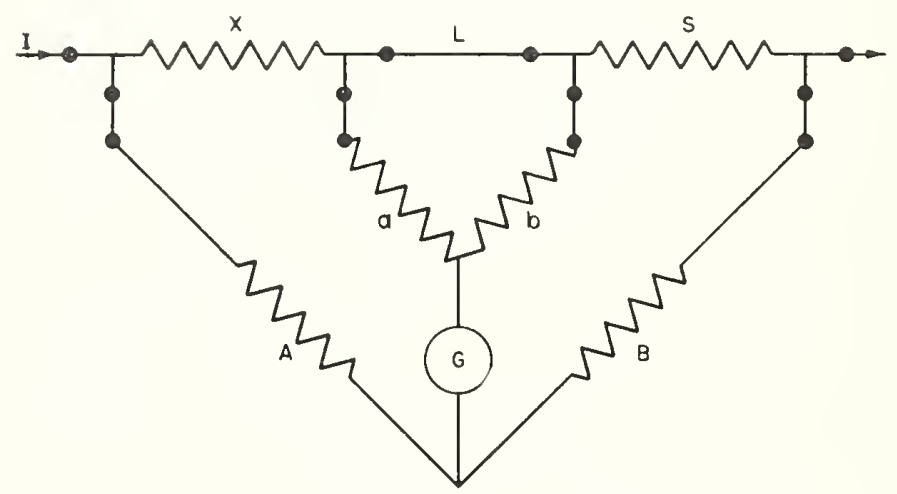

Figure 16. Kelvin double-bridge. 
of an adjustable standard, care should be taken in connecting up such a standard in order to keep the resistance small between it and the unknown. Adjustable standards are usually made such that the position of one or both af the potential terminals is adjustable. As a result, varying amounts of resistance are left at the ends in series with the used part of the resistor. The adjustable standard should be connected in the circuit in the way that connects the larger part of unused resistance in the external circuit rather than between resistors so as to form part of the link resistance, $L$.

When a fixed standard is used for $S$, and the bridge is balaneed by adjusting the ratios $A / B$ and $a / b$, the most convenient arrangement is to have the ratios adjustable together. This is accomplished by having the same dial handle operate two dials together so that both ratios are changed simultaneously. This arrangement requires special apparatus, but such double-ratio sets are conmercially available.

If not "ganged" together, the two ratios are changed separately but always by the same amount. Actually, however, it is not necessary to be able to adjust the auxiliary ratio, $a / b$, in known steps, and any convenient adjustable ratio, such as a slide wire, may be used. In this case the balance of the double bridge is by successive approximation. It is first balanced by adjusting the main ratio, $A / B$, after which the circuit is opened at the link, $L$, and a balance now obtained by varying the auxiliary ratio $a / b$. The link circuit is again closed and a new balance obtained with the main ratio. The procedure is repeated until the same balance is obtained with or without the link circuit's being open. Ordinarily the final balance is obtained after only a few sets of adjustments, and aceurate results may be so attained. Since only one ratio need be known any apparatus having such a ratio, as for example a simple Wheatstone bridge, may be used in conjunction with a slide wire and a fixed standard resistor to make good measurements by the double-bridge method.

In work of the highest accuracy, account must be taken of the resistance of the leads that are used to connect the ratio arms to the unknown and standard resistors. There is also lead resistance in these resistors, between the branch points and the terminal binding posts, which is not necessarily negligible. One way of taking these lead resistances into account is to use such high resistanees in the ratio arms that the connecting leads have a negligible effect. Usually, however, this reduees the sensitivity of balance of the bridge. A better arrangement is to make the lead resistances adjustable and sclect their ratio in such a way as to halance out their effects. A convenient method of adjusting the lead resistances is as follows: Reforing to figure 17 , the

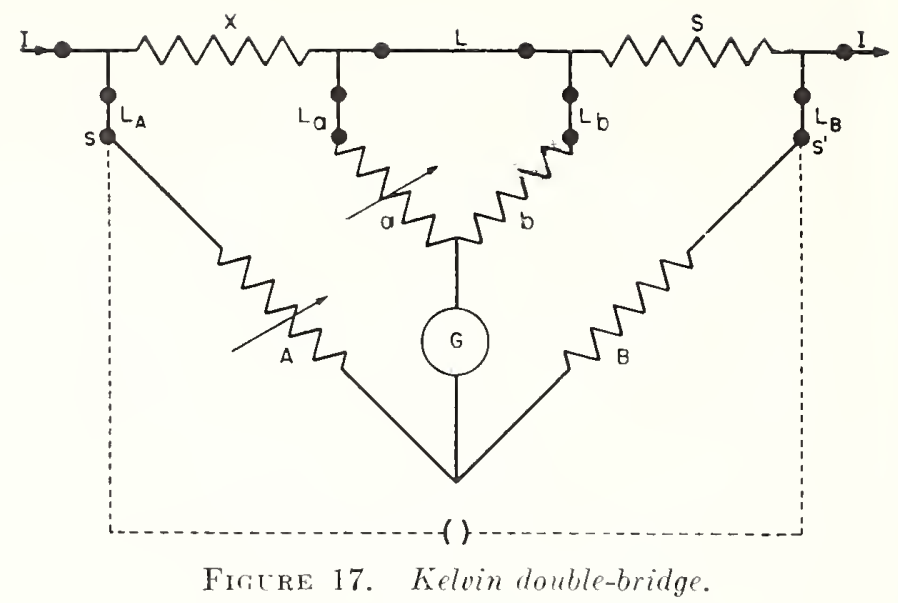

ratio $A$ and $B$ are such that their ratio is known only for the resistance between the two binding posts $s$ and $s^{\prime}$. The lead resistors $L_{A}$ and $L_{B}$ and the internal leads in $X$ and $S$ add to the resistance of $A$ and $B$. This ordinarily prevents the ratio of the bridge arms from being the same as the known ratio of $A$ to $E$. In other words

$$
\frac{A+L_{A}}{B+L_{B}} \neq \frac{A}{B}
$$

except under the condition that $L_{A}$ and $L_{B}$ are respectively negligible as compared with $A$ and $B$, or under the condition that the ratio of the leads is the same as the ratio of $A$ to $B$. That is

$$
\begin{gathered}
\frac{A+L_{A}}{B+L_{B}}=\frac{A}{B}, \\
\frac{L_{A}}{L_{B}}=\frac{A}{B} .
\end{gathered}
$$

If the lead resistances are small as compared with $A$ and $E$, the second equation need be only approximately satisfied.

The same considerations apply to the lead resistance $L_{a}$ and $L_{b}$ in the auxiliary ratio arms. Their effect may be made negligible by making their ratio the same as the ratio $a / b$.

The following procedure for balancing the bridge and eliminating the effects of the leads by adjusting their ratio may be followed. Arrangement is marle for readily opening and closing the circuit at $L$ and for short-circuiting the main ratio arms by comnecting together $s$ and $s^{\prime}$. Shorting $s$ to $s^{\prime}$ is the cquivalent of reducing $A$ and $B$ both to zero, leaving only the lead resistances in the arms. If the ratios are ganged and nominally equal, the bridge is balanced in the following steps:

1. With the circuit as shown, the ratios $A / B$ and $a / b$ are adjusted until the galranometer indicates a balance. This gives

$$
\frac{A+L_{A}}{B+L_{B}}=\frac{T}{S} \text { approximately. }
$$

2. The main ratio $A / B$ is now short-circuited 
and the bridge is again balanced, by changing the ratio of the lead resistances. This may be done by adjusting the length of one lead wire, or a small rheostat may be used in series with one of the leads. This step makes the ratio of the lead resistances the same as $X / S$ and hence to a first approximation also to $A / B$.

3. The short is removed from the main ratio and the link opened. The bridge then becomes the simple Wheatstone bridge shown in figure 18. To a first approximation

$$
X / S=A / B=a / b=L_{A} / L_{B},
$$

and the simple bridge is unbalanced only if the resistances of the leads $L_{a}$ and $L_{b}$ are not in this same ratio. They are made in this ratio by adjusting $L_{a}$ or $L_{b}$ until the simple bridge shows a balance.

4. The link, $L$, is restored, giving again the double-bridge, and a second balance is obtained by varying the ratios $A / B$ and $a / b$. This balance is more nearly correct than the first, as the leads have been approximately adjusted to their proper ratio.

5. The entire procedure is repeated until no further change is required in the settings of the main ratio, under which condition

or

$$
X / S=A / B=a / b,
$$

$$
X=S A / B=S a / b \text {. }
$$

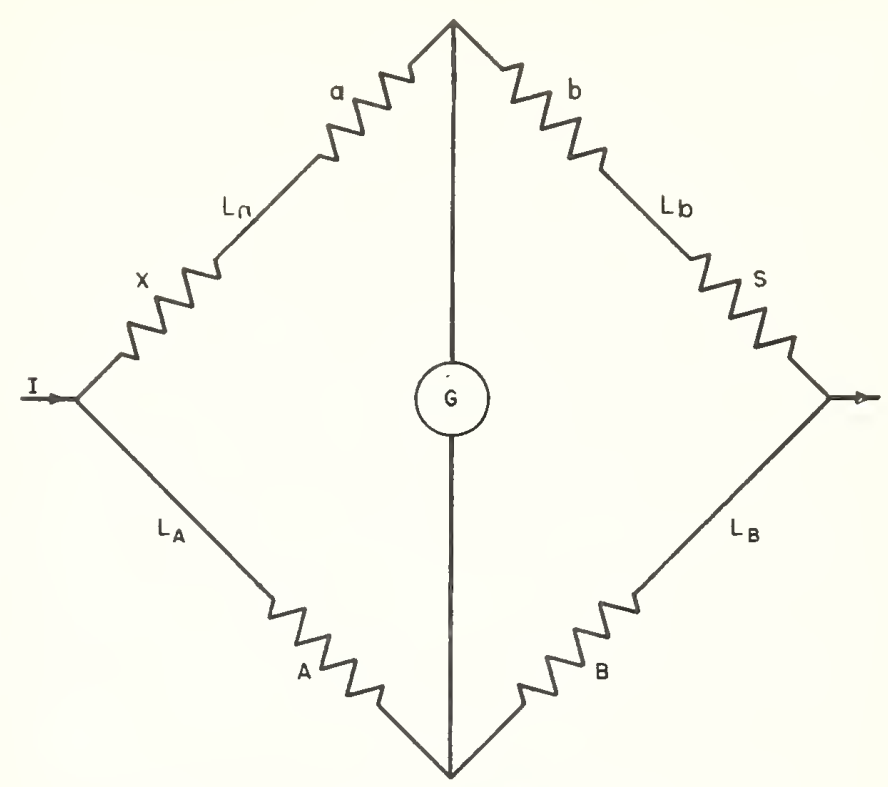

Figure 18. Equivalent Wheatstone bridge.

In case $a / b$ is obtained from a slide wire, or a ratio that is adjustable but not known, the same procedure is followed as that outlined above. In this case, however, the balance for step 3 is obtained by varying the ratio $a / b$, if necessary making a fine adjustment of the ratio by varying the lead resistances $L_{a}$ and $L_{b}$.

\section{Special Apparatus for Precision Measurements}

\section{Direct-Reading Ratio Set}

The problem of calibrating precision resistance apparatus usually involves the comparison of resistors in the instrument with standard resistors of the same nominal value. This is most readily done by some substitution method, which is usually a method for determining differences between the resistances of the unknowns and of the standards. For well-adjusted instruments, the differences are small and need to be determined only approximately. For example, if the difference between the standard and unknown is 0.01 percent, the difference need be determined to only 1 percent to give the value of the unknown to one part in a million in terms of the standard.

One of the most convenient instruments for the measurement of such differences in ratio is the "direct-reading ratio set." With this comparatively inexpensive instrument and a group of standard resistors, it is possible to calibrate accurately most types of resistance apparatus such as Wheatstone bridges, potentiometers, resistance boxes, etc.

The direct-reading ratio set is merely an adjustable resistance ratio with which bridges may be assembled, the remainder of the bridges being ordinary laboratory equipment. In its simplest form the ratio set is as shown in figure 19 . The resistor $B$ is a $100-\mathrm{ohm}$ coil, which constitutes the fixed arm of the ratio. The adjustable arm consists of a fixed resistor of $99.445 \mathrm{ohms}$ and three dials, $D_{1}, D_{2}$, and $D_{3}$. The dial $D_{1}$ consists of ten steps of $0.1 \mathrm{ohm}$ each, and $D_{2}$ and $D_{3}$ have

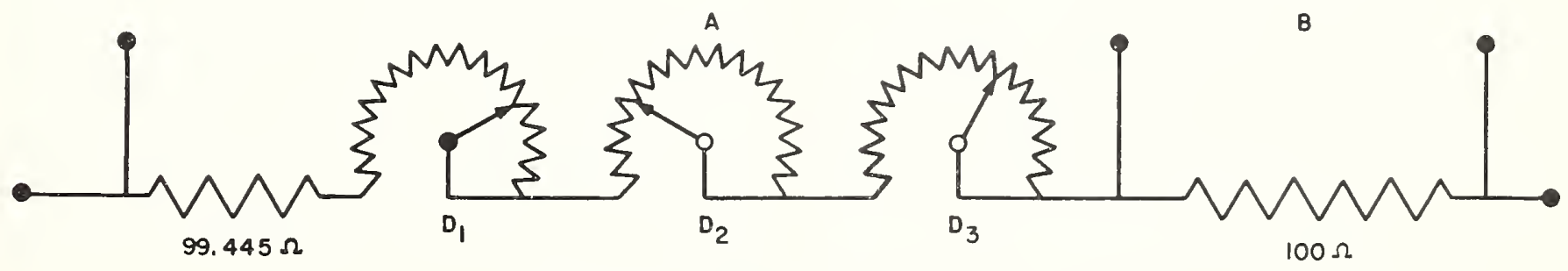

Figure 19. Direct-reading ratio set. 
ten steps each of 0.01 and $0.001 \mathrm{ohm}$ respectively. When at their center positions, the resistance of the three dials is $0.555 \mathrm{ohm}$, and with the fixed coil of 99.445 ohms the total of the arm is 100 ohms. Starting from these center positions, a step on $D_{1}$ will either mise or lower the $A$-arm by $0.1 \mathrm{ohm}$. The ratio of $A$ to $B$ is then changed from $100 / 100$ to $100.1 / 100$ or to $99.9 / 100$, that is to say, it is raised or lowered by 0.1 percent. Likewise, steps on $D_{2}$ and $D_{3}$ change the ratio by steps of 0.01 and 0.001 pereent respectively. By interpolation of the steps on the lowest dial, changes of 0.0001 percent may be determined.

Although the ratio set described above is correct in theory, variations in the resistances of the contacts of the dial switches would make the readings uncertain by several steps on the lowest dial when ordinary dial switches are used. The use of mercury switches will reduce the variations to a few microhms, but such switches are somewhat difficult to operate and keep in condition.

To avoid difficulties from valiations in switchcontact resistances the design is usually modified so as to reduce their effect. This is done by placing the switches in high-resistance shunt circuits that require comparatively large changes in resistance to obtain small changes in the parallel resistance, as was done in the rheostat of the Mueller bridge (sce section III, 5). The shunt circuits may be made sufficiently high that switch contact variations are negligible even when switches of moderate quality are used. An example of a shunted dial for obtaining steps of $0.1 \mathrm{olm}$ is shown in figure 20. With the dial set at 0 the resistance of the shunt arm totals 133.636 ohms, and this in parallel with the $30-0 h m$ branch gives a total resistance of $24.5 \mathrm{ohms}$. When the dial is moved to stud 1 , the resistance of the parallel combination increases by $0.1 \mathrm{ohm}$ to $24.6 \mathrm{ohms}$, which requires that the shunt arm be 136.666 ohms. That is to say, between studs 0 and 1 there is a resistance of 3.030 ohms. Likewise

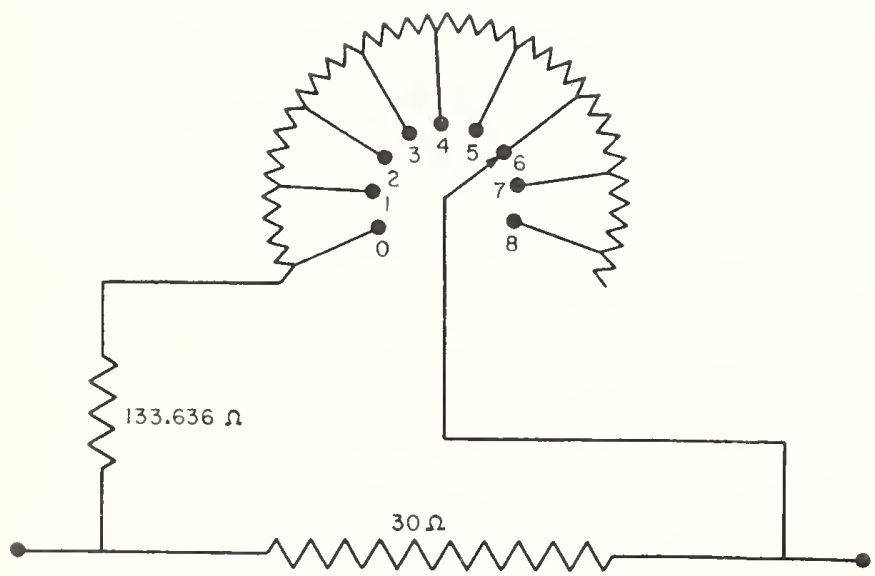

Figure 20. Shunt-type decade. between studs 1 and 2 there is a resistance of 3.145 olhms so that when the dial is at 2 , the shunt arm totals $139.811 \mathrm{ohms}$ and the parallel combination is $24.7 \mathrm{ohms}$. The successive steps of the dial are not of equal magnitude, but the parallel resistance may be changed by 0.1 -ohm steps from 24.5 to 25.5 ohms. For this the steps on the dial average about $3.6 \mathrm{ohms}$ each.

Since a change in the high-resistance arm of about $3.6 \mathrm{ohms}$ is required to change the parallel resistance by $0.1 \mathrm{ohm}$, it is obvious that switch contact variations in the high resistance arm will have their effect reduced by a factor of about 36 to 1. Their effect could be still further reduced by increasing the resistance of the shunt, the effect being reduced as the square of the ratio of the current in the high resistance branch to the total current.

In the same way, it is possible to make a decade for changing in 0.01-ohm or 0.001-ohm steps by means of a 150-ohm shunt in parallel with a 30 -ohm resistor, the 150 -ohm shunt to be changed in steps that average about 0.36 or 0.036 ohm: respectively. Three decades with appropriate shunts and a 25-ohm series resistor could be used to obtain the equivalent of the $A$-arm. of figure 19, but with the ratio not appreciably affected by normal variations in switch contact variations. An equivalent instrument is available commercially and, together with a group of standard resistor's, is one of the most useful pieces of apparatus for the measurement of electrical resistance, especially for the calibration of resistance apparatus. Some of the procedures will now be described.

Comparison of two-terminal resistance standards, substitution methods.-In nearly all measurements where the highest possible accuracy is desired, a substitution method is used. That is, the change required to restore balance after replacing a standard with an unknown is measured. In comparing two-terminal standard resistors by substitution, the direct-reading ratio set is very rapid and convenient, and accurate results may be obtained. For this comparison a Wheatstone bridge is set up as shown in figure 21. $A$ and $B$ are the two arms of the ratio set, and $Y$ is an auxiliary resistance of the same nominal value as the standard resistors under comparison. The two resistors are in turn placed in the mercury eups $Q$, and the bridge balanced by varying the ratio $A / B$. The difference in the ratio for the two balances gives the pereentage difference between the two standard resistors. Thus, if the difference in the ratio for the two balances is one step on the 0.001 dial, one resistor is 0.001 percent higher than the other. If we are comparing 1,000-ohm. coils, for example, the difference is 0.001 pereent of $1,000 \mathrm{ohms}, \mathrm{i}$. e., $0.01 \mathrm{ohm}$. Which coil is the larger is determined by observing whether the ratio is increased or decreased when the standard resistor is replaced by the unknown resistance. Actually the dif- 


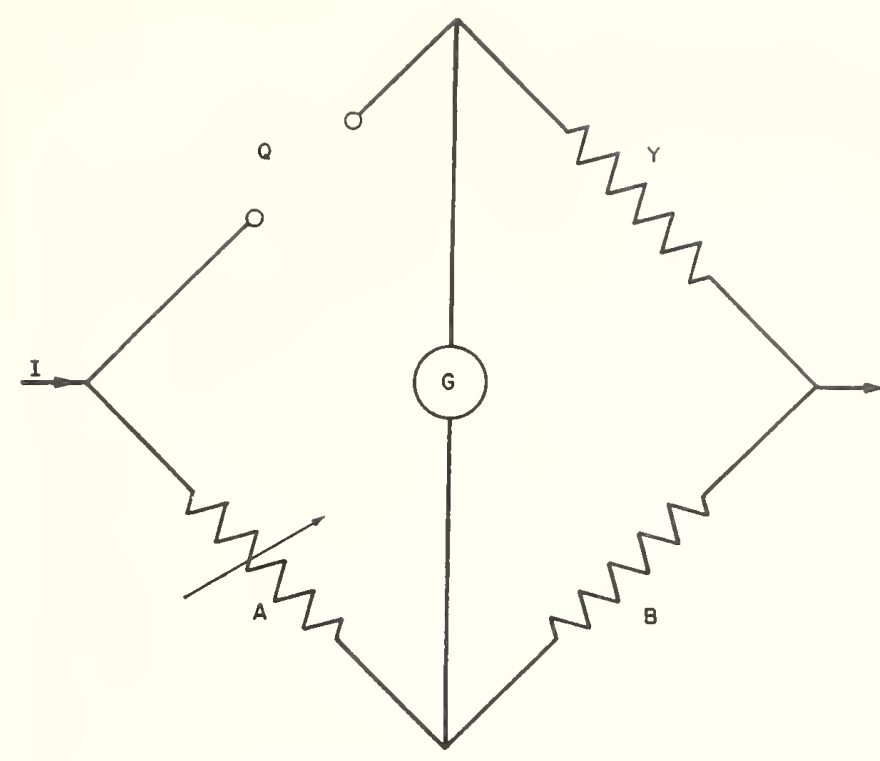

Figure 21. Wheatstone bridge for substitution method.

ference is determined in percentage of the arm $Y$, but for small differences any departure of $Y$ from its nominal value is of no consequence.

Although the dials of a direct-reading ratio set change the ratios in decimal parts of a percent, it is desirable to mark the dials in parts per million. Thus the steps on the 0.001-percent dial would be marked, 0, 10, 20, etc., and the 0.01-percent dial would be $0,100,200$, etc. Readings of the instrument and data taken from it are then whole numbers of moderate sizes rather than decimals. In. the discussion that follows, it is assumed that the ratio set is marked in parts per million, and that corrections to resistor's are also expressed in parts per million. Bridge readings and corrections to standards are then readily added and subtracted and calculations are greatly simplified.

In comparing nearly equal resistances by substitution, the lack of correct adjustment of the coils of a well-made ratio set will cause errors of much less than 1 part in a million. The variation in the contact resistance of the dial switches may be appreciable unless they are kept clean. The switches should be frequently cleaned and lubricated with a little good-quality light oil. When kept in good condition, the resistance of the three switch contacts should not vary as much as a thousandth of an ohm. From the values of the coils on the 0.001 percent dial, we see that a variation of a thousandth of an ohm will cause variations in the ratio of $1 / 3.6$ of a part in a million.

In comparing resistances by the substitution method, the two balances of the ratio set should be made quickly so that temperature changes will not cause variations in the ratio set between readings. It is best to have all coils of the ratio set made from the same lot of resistance material. If this is done and manganin coils are used, a change in temperature of 1 leg $\mathrm{C}$ should not change the ratio more than 2 or 3 parts in a million.

In spite of these sources of error, it is probably possible to compare standard resistors ranging from about 10 ohms to 1,000 ohms to within 1 or 2 parts in a million by the use of this ratio set. This, of course, requires that the set be well constructed, that a fairly sensitive galvanometer be used and that the resistances differ by not more than about a tenth of a percent. With resistances less than 10 ohms, the resistance of the mercury cup contacts may cause trouble. For this reason standard resistors smaller than 10 ohms are now almost always four-terminal resistors. Methods of comparing such resistors will be discussed later.

Another method for comparing nominally equal two-terminal standard resistors, which is practically equivalent to the preceding substitution method, is what might be called a double substitution method. In this latter arrangement, the two resistors under comparison are used to form two arms of a Wheatstone bridge, the ratio set forming the other two arms. The resistors are both mounted in mercury cups so that they may be interchanged without affecting lead resistances in series with them. After a balance is obtained by adjusting the ratio set, the resistors are interchanged, and a new balance is obtained. The percentage difference between the two resistor's is half the difference between the two readings of the ratio set. This method is used for the same range of resistances as those measured by the simple substitution method, and about the same accuracy may be attained.

Comparison of four-terminal resistances with twoterminal resistance.- The direct-reading ratio set is convenient for the comparison of two-terminal with four-terminal resistances. Although the occasion seldom arises for the comparison of two-terminal with four-terminal standard resistors, it is often necessary to compare a standard resistor of one type with a resistance coil of the other type. Thus, in measuring the coils of many pieces of electrical apparatus it is impossible to make connection with the coils except through comparatively larec connecting resistances. However, it is gencrally possible to make potential comnections to the two ends of the coils and measure them as four-terminal conductors. In doing so, it is often convenient to compare them with two-terminal standard resistors.

In figure $22, X$ is a four-terminal resistor to be measured, having the current terminals $T_{1}$ and $T_{2}$, and potential terminals $P_{1}$ and $P_{2}$. The potential leads may contain considerable resistance in addition to that of the leads. $A$ and $B$ are the two arms of the direct-reading ratio set, and $I_{1}$ and $M_{2}$ are mercury cups into which either a twoterminal standard resistor or a short cireuiting link may be placed.

Suppose we start with the galvanometer con-

\section{Precision Resistors and Their Measurement}


nected at $P_{1}$, a standard resistor nominally equal to $X$ inserted in $M_{1}$, and with $M_{2}$ shorted. We then have a simple Wheatstone bridge, which is balanced by varying the setting of the ratio set. After this balance is obtained, the galvanometer connection is shifted to $P_{2}$, and the standard resistor is placed in $M_{2}, M_{1}$ being shorted with the link. We again have a Wheatstone bridge but with the standard resistor and unknown interchanged. This interchange has been obtained without making any change in the resistance of the leads of the measuring circuit, except for possible variations in the resistances of the mercury cup contacts, which will be small if the mercury contacts are clean. The bridge is now again balanced by means of the ratio set. The percentage difference between the unknown and the standard resistor is half the difference in reading of the ratio set for the two balances. Unless the resistances under comparison are fairly large, it will be necessary to take into account the resistance of the short-circuiting link. This is done by subtracting the link resistance from the resistance of the standard resistor and considering that the resistor has this new value and is being interchanged with a link of zero resistance.

The resistance of the link can be measured as follows: Connect the link between two 1-ohm resistors to form two arms of a Wheatstone bridge, using the direct-reading ratio set for the other two arms, as shown in figure $23 . L$ is the link, and $A$ and $B$ are the arms of the ratio set. Two balance readings are taken, first with the galvanometer connected at one end of $L$ and then at the other. Half the difference in the readings is the value of the link resistance in percentage of the 1-ohm arms. If the link resistance is large, it may be necessary to use larger resistances in place of the 1-ohm coils. This method is very convenient for the measurement of small resistances such as links, connecting wires, switch contact resistances, etc. It is not a precision method but usually is sufficiently accurate for the measurement of resistances such as those just mentioned, which are to be used in series with larger resistances.

Substitution method for decades. - In the calibration of precision rheostats the occasion often arises for the measurement of a series of resistors of the same nominal value. This is readily done by the substitution method, using a standard resistor of the same value as the steps of the rheostat, reading differences on a direct-reading ratio set. The procedure is illustrated in figure 24. In this figure $P R$ is the precision rheostat to be calibrated, and let us assume that the 10ohm-per-step dial is to be checked. $P B$ is then a plug box or any decade with 10-ohm steps, and $M$ is a pair of mercury cups in which is placed a standard 10 -ohm resistor. The arm $I$ is a 100 ohm resistor whose value need not be accurately

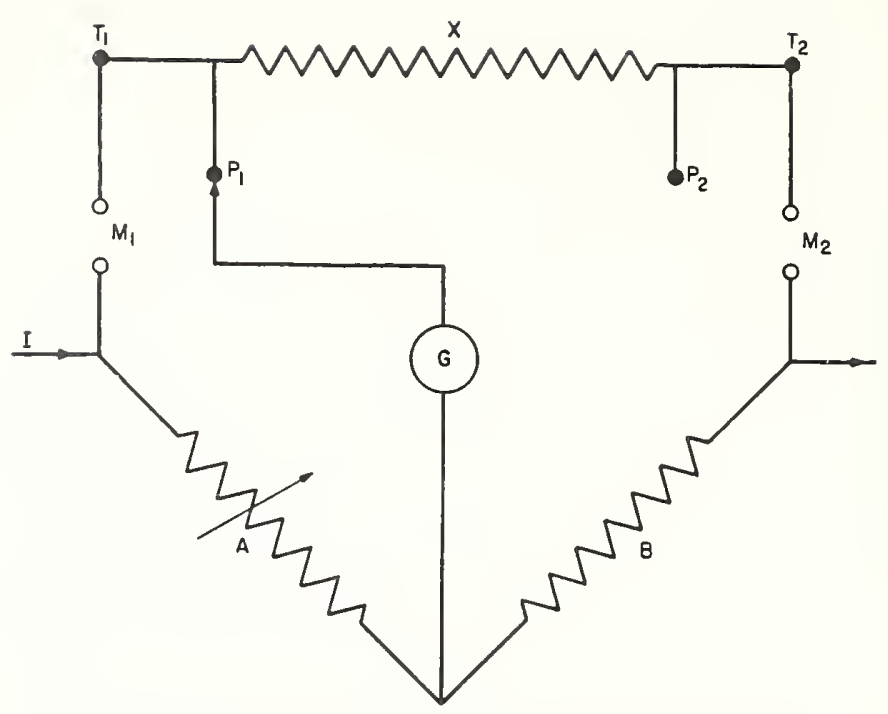

Figure 22. Measurement of 4-terminal resistor.

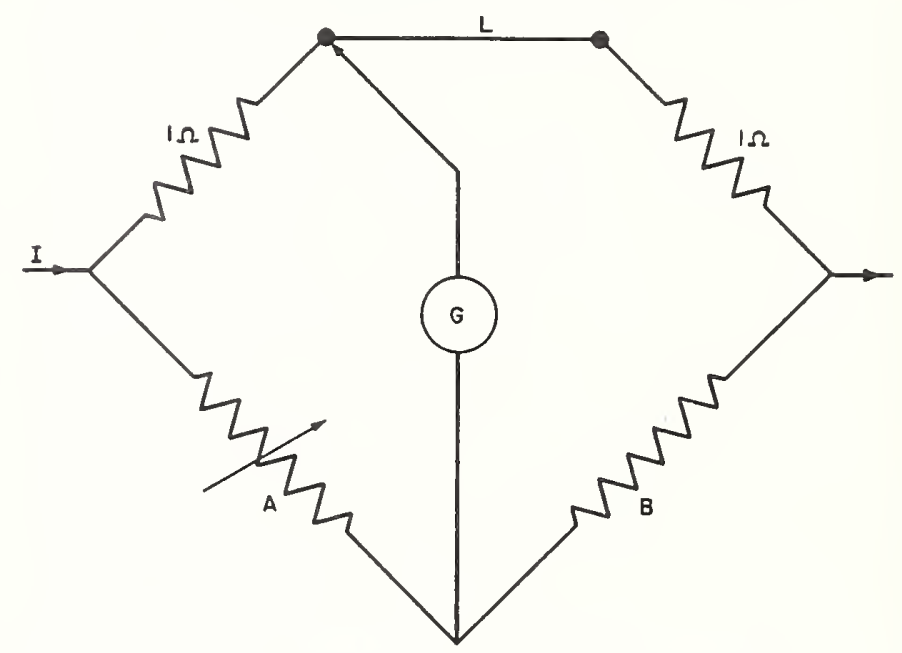

Figure 23. Measurement of lead resistance.

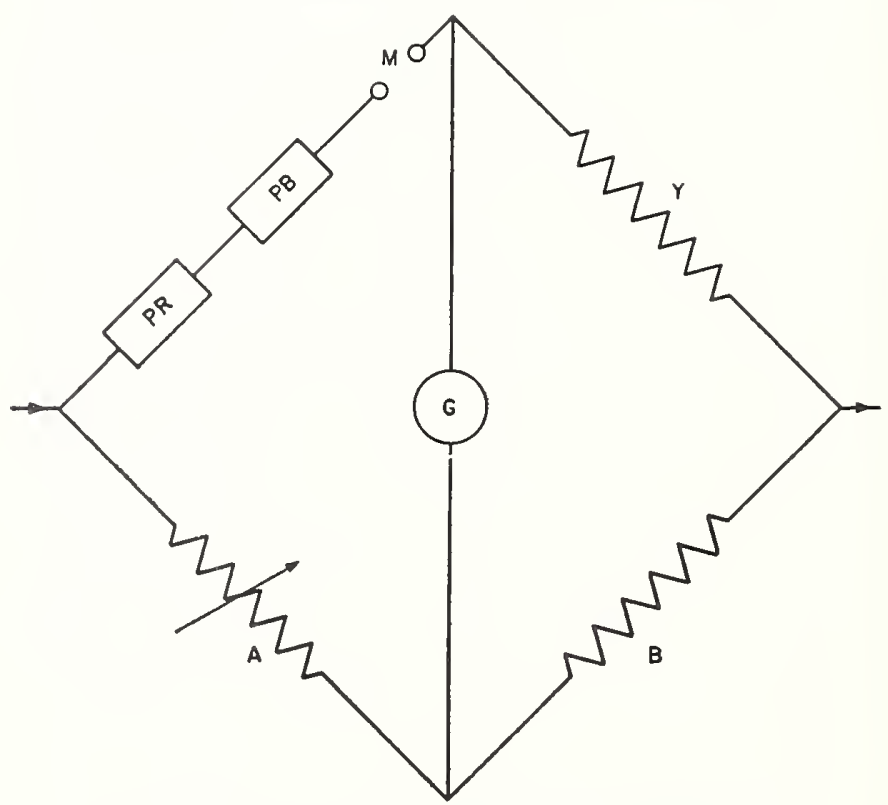

Figure 24. Test of decades by substitution. 
known, and $A$ and $B$ are the two arms of a direct reading ratio set. The reading of the rheostat under calibration is set at zero, the plug box is set at 90 and the $10-0 h m$ resistor is placed in $M$, making the total nominal resistance of the arm 100 ohms.

After the bridge has been balanced by changing the setting of $A$, the standard resistor is removed and the mercury cups shorted with an amalgamated copper link, and also the reading of $P R$ is changed from 0 to 10 ohms. This has in effect substituted a 10-ohm step of $P R$ for the 10 -olim standard resistor. The change in the reading of $A$ required to again obtain a balance of the bridge is a measure of the difference between the step on the rheostat and the standard resistor, such difference being a percentage of $100 \mathrm{ohms}$. The second $10-o h m$ step on the rheostat is obtained by leaving $P R$ at its $10-\mathrm{ohm}$ reading, again placing the standard resistor in $M$ and reducing the PB resistance to $80 \mathrm{ohms}$. These three resistors still total 100 ohms, nominally. The standard is now again replaced by a short-circuiting link, and.PR is set to read 20 ohms, the resulting change being read from $A$. The change was produced by the substitution of the second $10-\mathrm{ohm}$ step of the precision rheostat for the 10-ohm standard.

The procedure is continued, the steps of $P R$ being successively substituted for the standard resistor. It should be noted that the steps of the auxiliary plug box, $P B$, are also being replaced by the standard resistor as $P R$ is being increased and $P B$ decreased in reading, the standard being cut in and out of the circuit. Data are therefore obtained for calibration of both $P R$ and $P B$ in terms of the standard resistor. Hence by this method, two precision rheostats may be calibrated simultaneously.

When 10 -ohm steps are calibrated in a $100-\mathrm{ohm}$ arm, the accuracy is reduced by one order. That is to say, differences in readings of the ratio set must be obtained to 0.0001 percent if the 10 -ohm steps are to be determined to 0.001 percent. The method has the advantage that changes in the over-all resistance of the rheostat are determined under the conditions of use, which is often not the

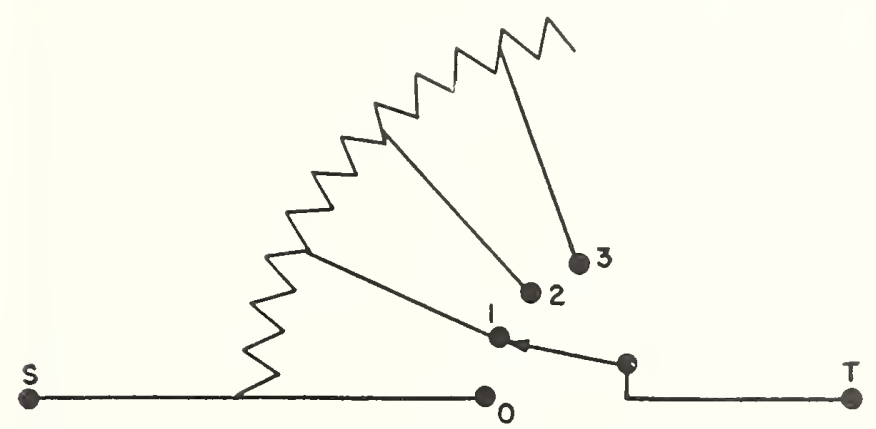

Figure 25. Dial connections.

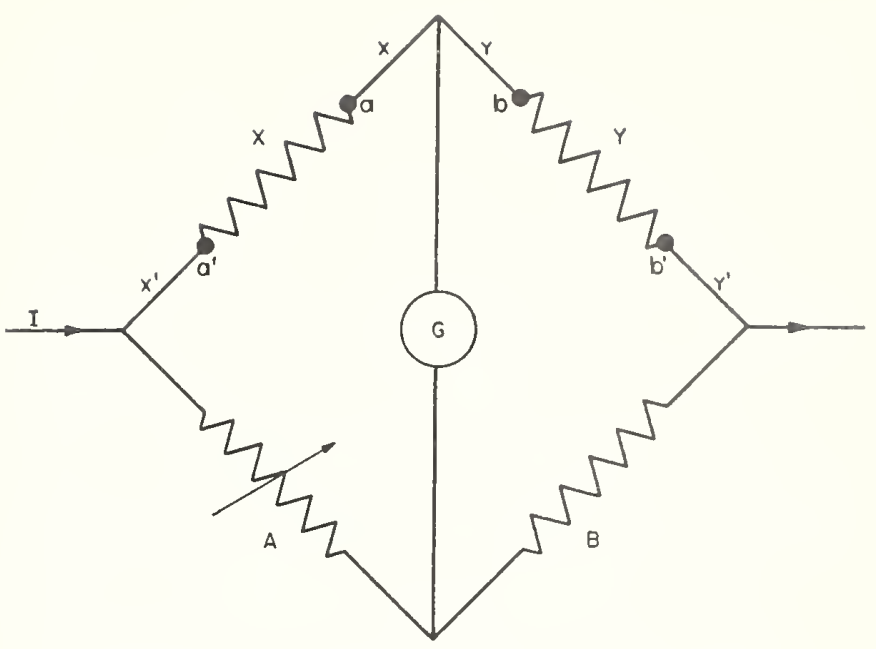

FIGURE 26. Lead resistances in Wheatstone bridge.

case when the individual resistors are measured directly. This may be seen by reference to figure 25 , which represents the connections to one dial of the rheostat. The switch contact may be set on the contact studs marked $0,1,2,3$, etc., thus connecting $0,1,2$ or more resistors between the dial terminals $S$ and $T$. It should be noted that the resistors are usually connected in series and lead wires are connected from the junctions of the resistors to the switch contact studs. If the resistors are calibrated by measuring batween studs 0 and 1,1 and 2,2 and 3 , etc., two of these leads are included with each resistor. In actual use all the leads to the coils in use are not in the circuit, but only one lead is used for any setting of the dial. The above method of calibration determines the step as in actual use, the only additional data required being the resistance between terminals with the dial set at zero. This is readily measured by the method just described for the determination of link resistances. For any setting, this "zero resistance" must be added to the sum of the steps as determined by substitutions.

Comparison of four-terminal resistance standards.-Before taking up the question of the comparison of four-terminal standard resistors, let us consider briefly the effect of the lead wires of a simple Wheatstone bridge. In figure $26, X$ and $I$ are nominally equal resistances, and $A$ and $B$ are the two arms of a direct-reading ratio set. The conductors $x, y, x^{\prime}$, and $y^{\prime}$ are used to connect up the bridge, and we will also denote their resistances by $x, y, x^{\prime}$, and $y^{\prime}$, respectively. The ratio of the resistances of the two arms containing $X$ and $Y$ is not in general the same as the ratio of $X$ to $Y$ because of the resistance of these connecting leads. If we could select leads such that the ratios $x / y$ and $x^{\prime} / y^{\prime}$ were the same as $X / Y$, the ratio of the two arms would be independent of the actual values of the lead resistances. That is, the balance would be the same as if the resistances of

\section{Precision Resistors and Their Measurement}


the leads were negligibly small. It is possible to make the leads adjustable and make the ratios $x / y$ and $x^{\prime} / y^{\prime}$ the same as $X / Y$. In fact, such an arranement is used at the National Bureau of Standards to reduce the effect of the leads when using the Kotrin clouble bridge. Instead of adjusting the leads, it is possible to balance the bridge with fixed leads, and then find what this balanee would have been with the proper ratio of lead resistances, or with negligibly small lead resistances.

$I$ and $Y^{r}$ were assumed to haxe practically equal resistances. Instrad of connecting the galvanomcter as shown, suppose we balance the bridge with the upper galvanometer comection first at $a$ and then at $b$. The arrage of the two readings is the value that would have been obtained had the conductors $x$ and y been equal or negligibly small in resistance. Thus, by taking two readings we ran take into aceount the effect of these two connecting resistances. This average balance reading is not the correct reading, howerer, unless also $x^{\prime}=y^{\prime}$, which is probably not true. We must now find what this balanee would have been with $x^{\prime}=y^{\prime}$. To do this suppose we shift the current connections from the points shown to the points $a^{\prime}$ and $b^{\prime}$. In doing so we remore the lead resistances $x^{\prime}$ and $y^{\prime}$ from the $X$ and $Y^{r}$ arms and fonnect them in series with the ratio arms $A$ and $B$. If $x^{\prime}$ and $y^{\prime}$ are not equal, we will change the balanee of the bridge by removing them from. the armes $X$ and $Y$. Wo will have still further changed this balance by adding them. to the ratio arms $A$ and $B$. Which of these changes is the greater depends upon the relative sizes of the two pairs of arms. If $A$ and $B$ are equal to $X$ and $Y$, the changes will be equal. That is, with all arms nominally equal the balanee would be changed a certain amount if the battery connections were shifted to $a^{\prime}$ and $b^{\prime}$. This change is twice that which would have been obtained had we onty removed $x^{\prime}$ and $y^{\prime}$ from the $X$ and $Y^{Y}$ arms. The average of the readings before and after ehanging the battery connections is then the reading that woukl have been obtained with $x^{\prime}=y^{\prime}$ or both necligibly small.

Suppose, howerer, that $A$ and $B$ are ten times as large as $X$ and $Y^{\prime}$. Then comecting $x^{\prime}$ and $y^{\prime}$ in series with $A$ and $B$ produces only a tenth as large a change as is caused by their removal from. $X$ and $Y$. Then ten-elevenths of the change in balance when the battery connections are shifted is due to the removal of $x^{\prime}$ and $y^{\prime}$ from the $X$ and $Y^{\prime}$ arms, and the remaining one-cleventh is due to the connection of the leads in the ratio arms. This enables us to ealculate what the balance would be with $x^{\prime}$ and $y^{\prime}$ equal or negligibly small. As an example, suppose $X$ and $Y$ are each 10 ohms, and the arms of the direct-reading ratio set are 100 ohms. Assume that with the battery connected as shown in figure 26 and the galvanometer at $a$, the reading of the ratio set with the bridge balanced is 5497 millionths, and with the galranometer connection changed to $b$, the reading is 5613. Let us further assume that when we change the battery leads to $a^{\prime} b^{\prime}$, leaving the galvanometer comnected at $b$, the balance reading changes to 5835. Then with leads of the proper ratio, or leads with negligibly small resistances, the balance would have been at $(5,613+5.497) / 2+$ $10 / 11(5,835-5,613)$; i. e., at 5,757 .

As a matter of fact, this scheme for taking into account the connecting lads is practically nerer used in comparing two-terminal resistors. As the substitution method requires no consideration of the lead resistanees, except to see that they are reasonably small, it is generally used. However, in comparing four-terminal resistances with a simple Wheatstone bridge we follow exactly the steps outlined above. Figure 27 shows a bridge set up for this purpose. $A$ and $B$ are the two arms of the direct-reading ratio set. $X$ and $Y$ are the four-terminal resistors under comparison, with current terminals $T_{1}, T_{2}, T_{1}^{\prime}$, and $T_{2}^{\prime}$, and potential terminals $P_{1}, P_{2}, P_{1}^{\prime}$, and $P_{2}^{\prime}$. 'The bridge is balanced when connected as shown, and a second balance is obtained after shifting the galvanometer connection to $P_{2}^{\prime}$. The thired balance is obtained after now changing the current connections from $T_{1}$ and $T_{1}^{\prime}$ to $P_{1}$ and $P_{1}^{\prime}$, and calculations are made as above. The two alms $X$ and $I$ are now interchanged and the three readings again obtained. From these two sets of readings, we get two balance points on the ratio set. Half the differenee betwoen these two balanee rearlings is the pereentage difference between the two four-terminal resistors.

Although the direct-reading ratio set was developed for use in the comparison of nominally equal resistanees, it can be readily adapted for the comparison of resistances of any ratio plovided some independent means is availuble for aceurately

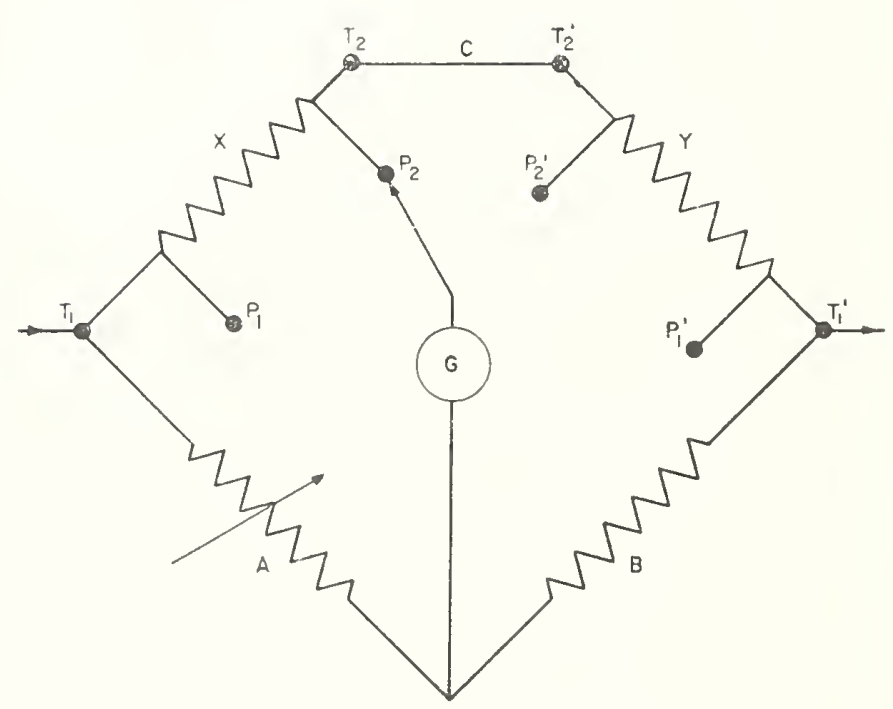

Figure 27. Comparison of 4-terminal resistors. 
realizing the same resistance ratio. As an example of such a use let us consider the calibration of a 25-ohm resistor by comparison with a 100-ohm standard.

As has been described above, the direct-reading ratio set consists of the arm $A$, which is adjustable in small steps from values slightly below to slightly above 100 ohms. The $B$-arm is ordinarily a fixed $100-$ ohm arm. Suppose we change the $B$-arm by connecting an additional 300 ohms in series. The ratio $A / B$ then becomes $100: 400$, which is adjustable in the same percentage steps as was the $100: 100$ ratio. Using two sets of mereury cups, let us set up the bridge shown in figure 28 . The 25 -ohm resistor is placed at $X$ and the $100-$ ohm standard at $S$, and the bridge is balanced by adjusting $A$, and let us call this reading $A$. Also let $A_{0}$ be the reading $A$ would have had if the $X$ and $S$ arms had been exactly in the ratio 25:100. If $c_{x}$ and $c_{s}$ designate the proportional corrections of $X$ and $S$, the actual balance $A$, of the bridge, will be

$$
A=A_{0}+c_{x}-c_{\mathrm{s}} .
$$

Since $A$ is obtained experimentally and $c_{s}$ is known, this equation could be solved for $c_{x}$ if $A_{0}$ were also known. To obtain $A_{0}$, the arms $X$ and $S$ must be replaced by a resistance ratio of exactly $1: 4$.

To realize a $1: 4$ ratio it is necessary merely to have five resistors that are reasonably nearly equal. If one of these five is connected in place of $X$ and the other four in series in place of $S$, the ratio will be only approximately $1: 4$. However, if the five resistors are placed one after the other in $X$, the remaining four each time being connected in $S$, the average of the five ratios will be $1: 4$ to a very high accuracy. In other words, the average of the five readings of $A$ with the five resistors in turn at $X$, the remaining four in series in the $S$-arm, will be $A_{0}$, the reading of $A$ for an exact $1: 4$ ratio. This value of $A_{0}$ can be substituted in eq 19 to allow the calculation of $c_{x}$, the proportional correction to the unknown 25 -ohm resistor.

Another method of obtaining $A_{0}$ would be to balance the ratio set with one resistor at $X$ and the other four at $S$, thus obtaining $A$ of eq 19 . The five resistors could then be substituted in any bridge and their differences determined. The terms $c_{x}$ and $c_{z}$ are then the amounts in proportional parts that the resistor in $X$ and those in $S$ differ respectively from the average value of the five.

To get an accurate value of the $1: 4$ ratio, the five resistors should have large enough resistances that the lead resistances of the $X$ and $S$ arms are negligible, or the lead resistances should be in the ratio $1: 4$. They may usually be so set with a sufficient accuracy by shorting $X$ and $S$ and aljusting the bridge circuit so obtained to a balance by changing the length of the learl wires. The five

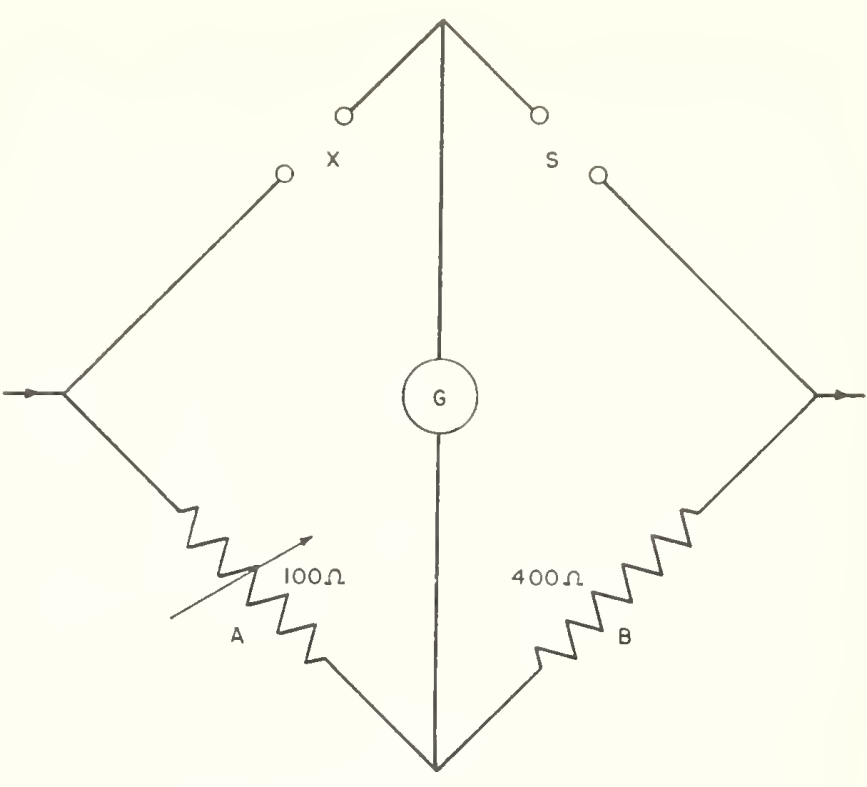

Figure 28. Bridge with 1:4 ratio.

resistors do not need to be equal to a very high precision. However, they must not differ by more than 0.1 percent if the average of the five ratios is to be correct to one part in a million.

A procedure analogous to this method of obtaining a ratio of $1: 4$ may be followed to determine any ratio $1: n$, where $n$ is an integer, by taking the average of $n+1$ ratios. It may also be used to determine any ratio $r: n$, where both $r$ and $n$ are integers. Starting with $r+n$ equal resistors, $r$ in one arm and $n$ in the other, the resistors are rotated in a cyelic order until each resistor occupies each position once. The average of the $r+n$ readings is the reading that would be obtained if all resistors were equat. Moreover, the resistors in either arm may be in parallel rather than in series.

In the calibration of hich resistances, use may be made of the fact that the proportional correction to a group of nominally equal resistors is the same when they are connected in parallel as when connected in series. For eximple the ten 100,000ohm sections of a megohm box may be connected in parallel and measured against a 10,000-ohm standard resistor. If the parallel group is high in resistance by 0.01 perent, the series resistance of 1 megohm will also be high by 0.01 percent. Here it is assumed that the ten sections are sufficiently near to equality that in the expansion

$$
\frac{1}{1+c}=1-c+c^{2}-c^{3} \text {, etc. }
$$

the second and higher powers of $c$ are negligible, where $c$ is the proportional amount by which the resistance of any section differs from the average of all. If this condition is satisfied, the ratio of 
the resistance of $n$ resistors in parallel to their resistance in series is exactly $1: n^{2}$.

The methods that have just been presented assume the use of a direct-reading ratio set. These methods, however, are entirely satisfactory when use is made of any ratio set that has small and definite steps. Such a set may be assembled from ordinary laboratory apparatus. For example, the adjustable 100-ohm arm might be made of a $105-0 h m$ coil with a parallel decade box reading about 2,500 ohms. A change in reading of the decade box by $0.1 \mathrm{ohm}$ would change the $100-\mathrm{ohm}$ arm by about $1 \mathrm{ppm}$. The changes in the parallel resistance are not directly proportional to the change in the high resistance arm, but they may be readily calculated. This adjustable 100-ohm arm, together with a fixed 100-ohm resistor, constitutes an adjustable ratio set.

\section{Universal Ratio Set}

Precision standard resistors are usually made only in integral multiples or submultiples of an ohm. Consequently odd-valued resistors usually cannot be measured by a substitution mothod, except in a few cases where standard resistors can be combined to give a resistance nearly that of the unknown. The comparison of odd-valued resistors with standards is then not possible with a direct-reading ratio set, but a ratio set is required that is accurately adjustable over a wide range, at least from a $1: 1$ to a $5: 1$, or preforably to a $10: 1$ ratio.

A very convenient wide-range ratio set is one used at the National Bureau of Standards and called a "universal ratio set" [14]. This instrument is one having a constant resistance, between two external terminals, of about 2,111 ohms. An arrangement of dials is such that in effect a potential connection may be made at any point of the 2,111 olims to the nearest $0.01 \mathrm{olm}$. The ratio of the resistance between the potential point and one terminal of the set to the resistance between the potential point and the other terminal is therefore adjustable in small steps over a very large range. The device is the equivalent of a long slide wire with a movable contact, and its use's are analagous.

Suppose it were desired to measure a resistance of say 6.8 olnms by comparing it with a $10-$ ohm standard resistor. The two resistors could be connected in series and the combination connected across a slide wire as slown in figure $29, X$ being the unknown, $S$ the standard, and II a slide wire. If a galvanometer, $G$, is conneeted to terminal $a$ of the unknown and to the slide wire, a Wheatstone bridge is obtained that will balance with the slide wire at some point, 1 , near the end. If now the galvanoneter connection is changed successively to $b, c$, and $d$, successive balances will be obtained with the slide wire at 2,3 , and 4 , respectively. The ratio $X / S$ is then the same as

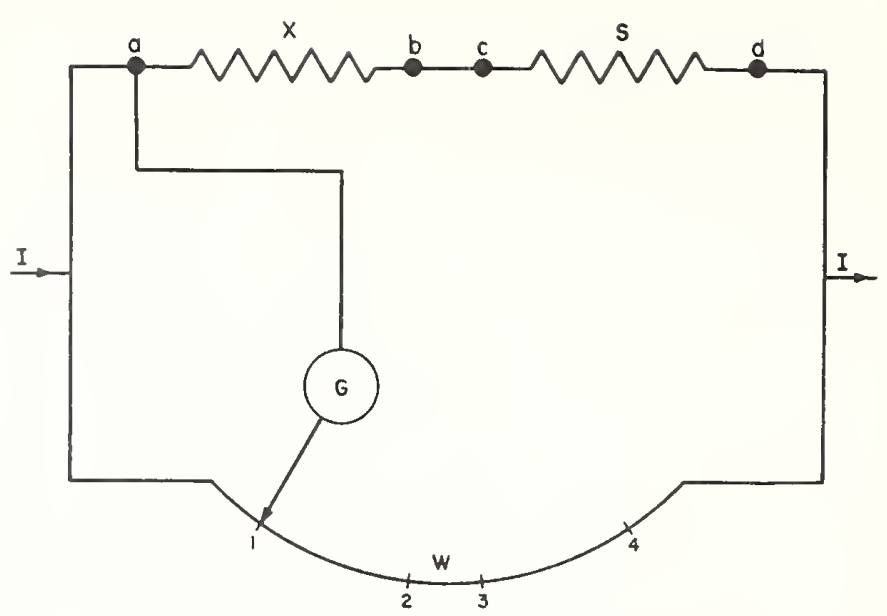

Figire 29. Comparison of resistors with slide wire.

$R_{1,2} / R_{3,4}$ where $R_{1,2}$ and $R_{3,4}$ are the resistances of the slide wire between the points 1 and 2 , and 3 and 4 , respectively.

It is very difficult to make a wire of sufficient length and uniformity that the ratio $R_{1,2} / R_{3,4}$ can be aecurately determined. Instead of a slide wire, the miversal ratio set makes use of a gromp of wire wound resistors so that the resistanees between the balance points 1, 2, 3, and 4 can be acenrately known. The arrangentent of its dials is as follows. The highest dial consists of twenty 100-ohm resistors in series, with the dial contact acting as the potential comnection to the instrument, as shown in figure 30 . As the dial is rotated in a clockwise direction, the $100-\mathrm{ohm}$ resistor's are successively changed from the right to the left side of the contact. To change resistance from the right to left side in 10-ohm steps, two more dials are used, each having ten 10-ohm steps as seen in figure 31 . These two dials are operated by the same handle but with one dial increasing as the other decreases in resistance. The total resistance between $S$ and $T$ remains constant for any setting of the 10-ohm decades,

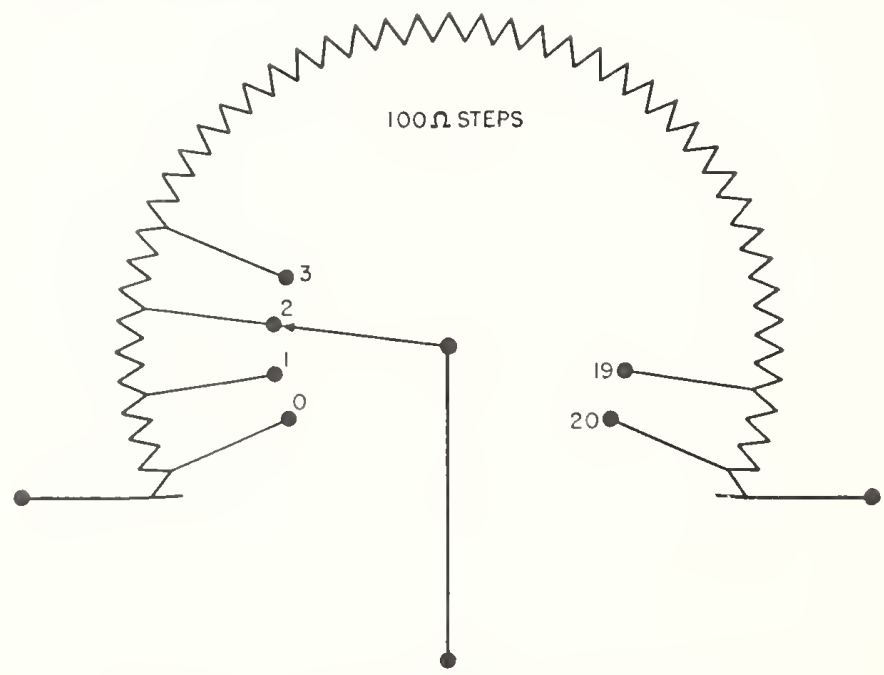

Figcre 30. 100-ohm dial of universal ratio set. 


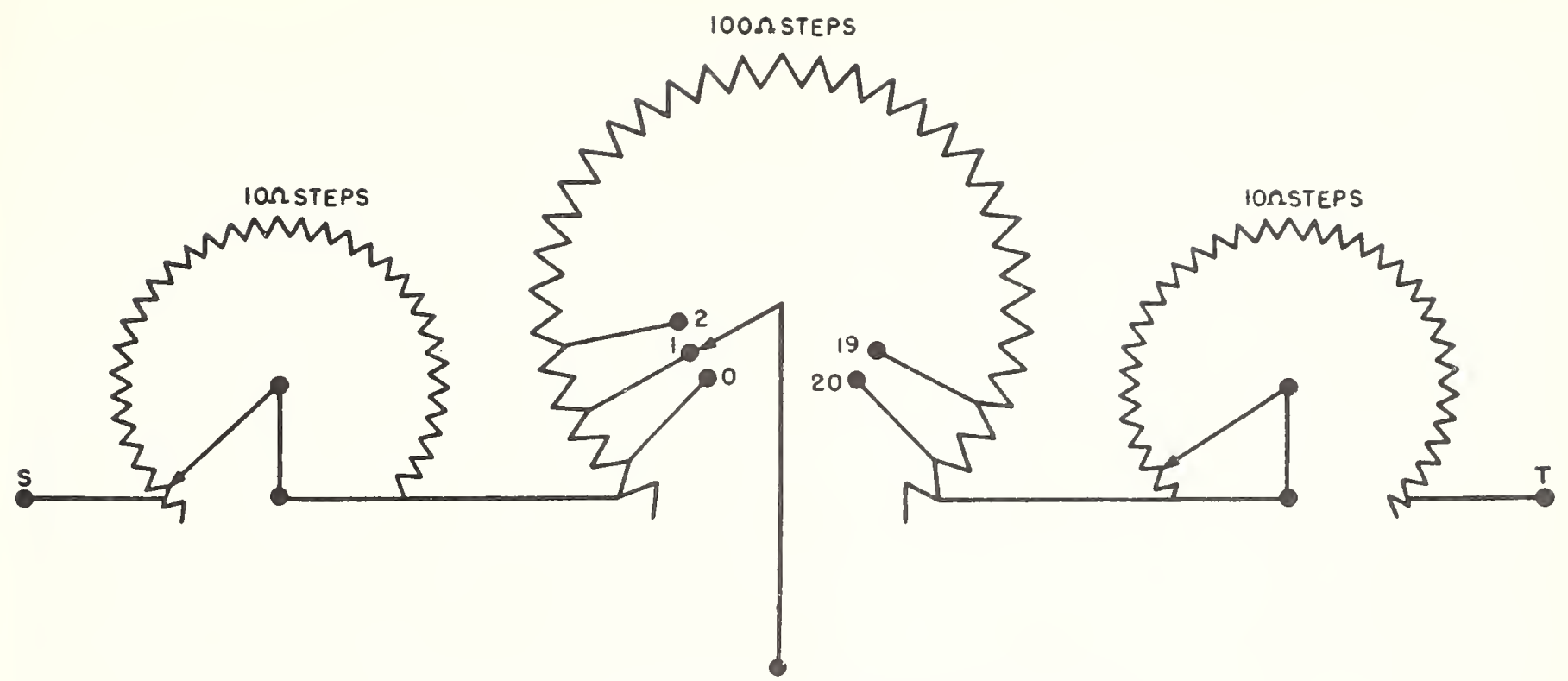

Figure 31. 100- and 10-ohm dials of universal ratio set.

and of course it is unaffected by the position of the $100-0 h m$ dial switch that is merely a potential contact.

Steps of $1,0.1$, and 0.01 ohm-per-step are obtained in the same way as the 10-ohm steps, that is with two decades of each denomination operating together, one on each side of the 100-ohm dial. This scheme for the change of the potential point along a fixed resistance is essentially the same as that of the Feussner type potentiometer. The large number of contacts in series limits its use for precision work to ratio sets of fairly high resistance.

The universal ratio set is used only for the determination of resistance ratios and hence may be calibrated in terms of any unit. It is most conveniently calibrated in terms of an average step on the highest or lowest dial. This is done by comparing each step of a decade with the ten steps of the preceding dial starting with the smallest dials. Only the steps on one side of the $100-\mathrm{ohm}$ dial need be tested, as the function of the other group is to keep the total resistance constant. A check of the constancy of the over-all resistance needs to be made for all readings of the double dials.

The use of a universal ratio set is the same as that of a slide wire as described in conjunction with figure 29, for the measurement of odd-sized resistances. It may be used in the measurement of four-terminal resistors and is especially convenient for the tests of potentiometers. The test of a potentiometer consists in the measurement of the ratio of the emf-dial resistance to the standard-cell resistance for all settings of the emf and standard-cell dials. These resistors are of the four-terminal type with potential connections brought out to einf and standard-cell binding posts. The method is satisfactory even when some resistance is common to both the standardcell and main-dial resistance. The arrangement for such a test is shown in figure 32 . The ratio set, URS, which is shown as a slide wire, is connected in parallel with the potentiometer, the connection. to the latter being made to the battery binding posts, $B A+$ and $B A-$. Readings on URS are made with the galvanometer connected successively to the $S C$ and emf binding posts, for all settings of the emf dials. It should be noted that changes in the emf dials are merely changes in potential points and do not affect the readings obtained for the $S C$ dial.

Potentiometers are provided with rheostats in the battery circuit for adjusting the potentiometer current. A change in this battery rheostat will change the differences in readings obtained on the universal ratio set but not the ratio of the differences. It is possible to make the ratio set directreading by adjusting the battery rheostat until the difference on the ratio set for the standard-cell posts is a decimal multiple, preferably 1,000 , of the reading of the standard-cell dial. Corrections for the emf dials may then be read directly from differences across the emf terminals.

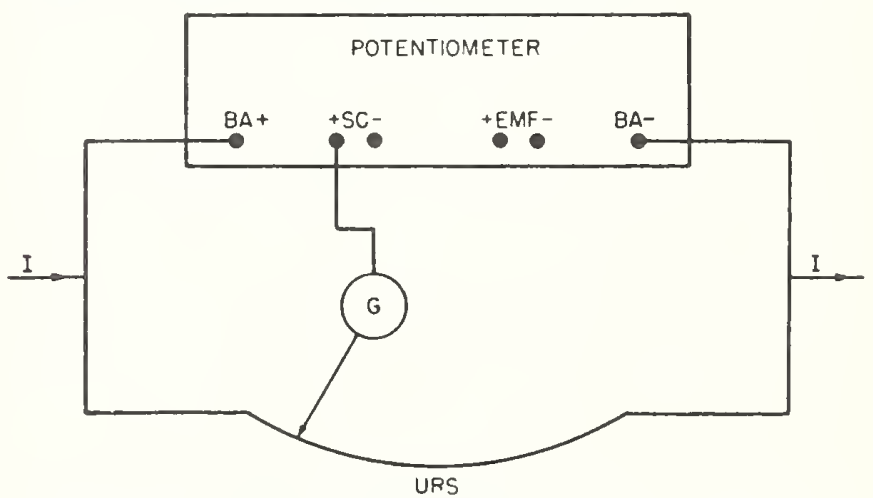

Figure 32. Check of potentiometer with universal ratio set. 


\section{Calibration of Precision Bridges}

\section{Calibration of Wheatstone Bridges}

The circuit of a commercial precision Wheatstone bridge is usually as shown in figure 33 . The two ratio arms $A$ and $B$ may have any one of several values, the choice being made by inserting a plug in series with the desired resistor. The rheostat arm, $R$, consists of four to six decades of not less than $0.1 \mathrm{ohm}$ nor more than 10,000 -ohm steps. The unknown resistor is connected to heavy binding posts, $X$, and a battery and galvanometer are connected to the external binding posts $B A$ and $G A$. The switches $S_{B}$ and $S_{G}$ open and close the battery and galvanometer circuits.

In calibrating a bridge of this type, it is necessary to determine the resistance of the ratio arms between the branch points 2 and 3 or 3 and 4 rather than between the external binding posts, as the resistances from the branch points to the binding posts are usually not negligible. Also it is necessary to find the resistance between 1 and 4 with the rheostat dial, $R$, set at zero, as well as the corrections to the readings of the rheostat dials themselves. It is also necessary to measure the lead resistances between the branch points 1 and 2 , as these are in series with the unknown resistance connected at $X$. The bridge balance determines the entire resistance of the $X$-arm, and the leads must be subtracted in order to obtain $X$ itself. These four types of resistance measurements are made by application of some of the general principles previously discussed.

To measure the ratio arm $B$, for example, it is necessary to determine the four-terminal resistor having branch points 3 and 4 . This is easily done by comparing it with a two-terminal standard of the same nominal value, making application of the method outlined in section IV, 1 and using the circuit shown in figure 34 . The resistors $a$ and $b$ are the arms of a directreading ratio set, or any two ratio arms that may be adjusted in small known steps. As shown, one side of the galvanometer is in effect connected to the branch point 4, through the rheostat arm, $R$, which may be set at zero. The standard resistor is placed in the mercury cups, $M_{1}$, and a short-circuiting link is placed across the other mercury cups, $M_{2}$. After a balance is obtained by adjusting arm $a$, the standard and shorting link are interchanged and the galvanometer connection is shifted in effect to point 3 by connecting to the other $X$ binding post. Half the change in the ratio that results gives the difference between the $B$-arm and the standard resistor.

The resistance of the 1 ratio arm ninay be measured in exactiy the same way as for the $B$ arm by malsing connection to the other $G A$ terminal instead of the one shown. Some difficulty may arise from variations of the contact

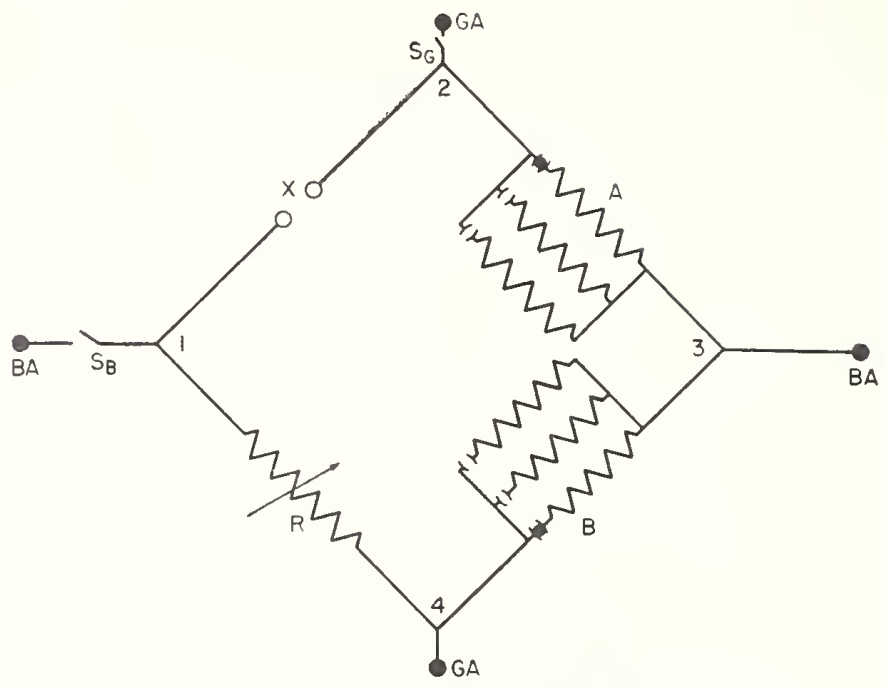

FIGURE 33. Wiring arrangement of Wheatstone bridge.

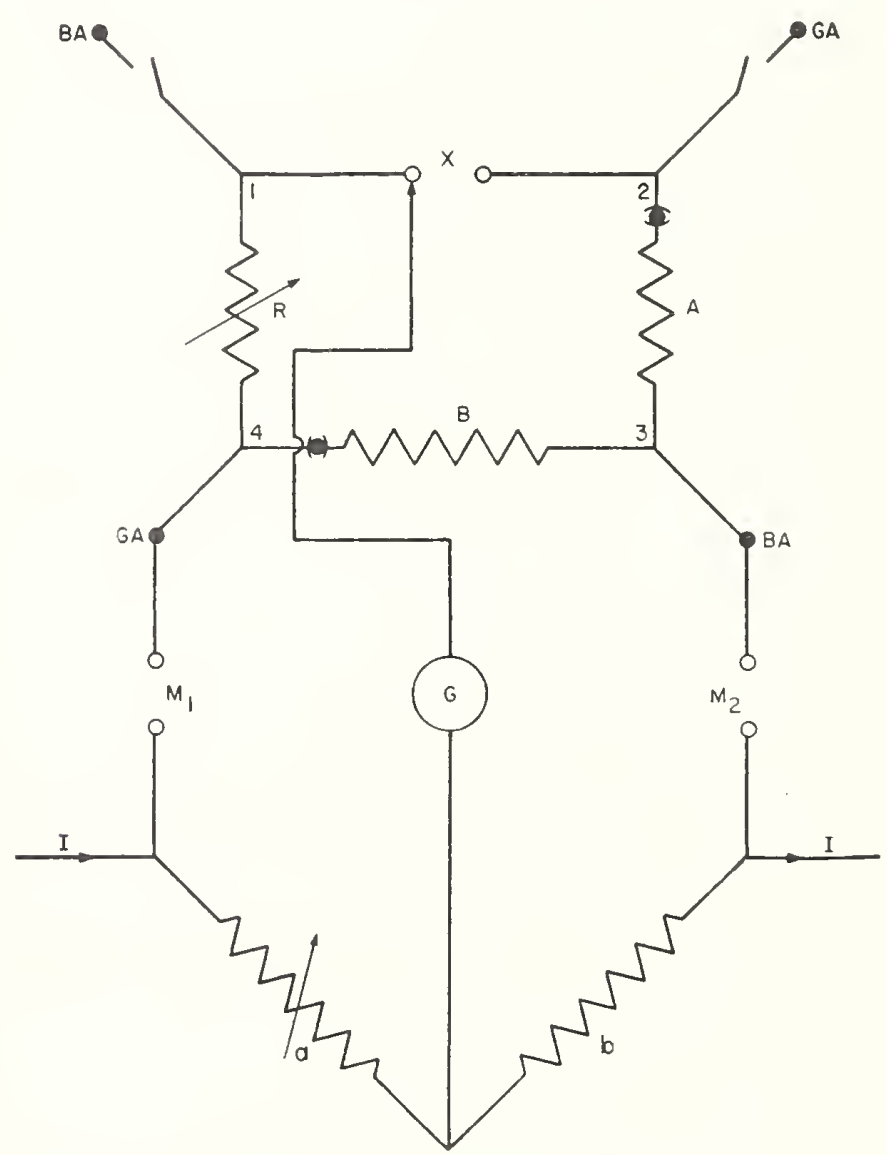

Figure 34. Check of ratio arm of Wheatstone bridge.

resistance of the galvanometer key, which wil] now be in the measuring circuit. This may be avoided by interehanging the adjacent $X$ terminal and the GA terminal. The galvanometer would then be shifted from one $X$ binding post to the $G A$ binding post instead of from one $X$ binding 
post to the other. That is to say, the current and potential connections at point 2 may be interchanged. This will place the variable galvanometer switch resistance in series with the galvanometer where it will not affect the bridge balance.

The rheostat arm, $R$, may be calibrated like any other precision rheostat as described in section IV, 1. Connection should be made through the appropriate $X$ and $G A$ terminals. The resistance of the rheostat arm with all dials set at zero may be measured by connecting the arm between two equal resistors, using a direct-reading ratio set to connect up a Wheatstone bridge. The change in the reading of the ratio set is determined when the galvanometer connection is changed from one branch point of the rheostat arm to the other. This is the same as the method given in section IV, 1 for the measurement of lead resistances. In a similar way, the lead resistances in the $X$ arm may be determined, taking readings with the galvanometer connection to branch points 1 and 2 and to the external $X$ binding posts, with the latter connected by a shorting wire. The calculation must be made in two parts in order to exclude the resistance of the shorting wire.

For calculating $X$ from the calibration data, it is convenient to express the corrections to the readings of the rheostat arm in ohms and to express the corrections to the ratio arms in proportional parts. The calculation in proportional parts involves merely a division of the correction in ohms by the nominal value in olims. The value of $X$ for a given balance is calculated from the equation

$$
X=\frac{A}{B}(1+a-b)\left(R+r+r_{0}\right)-X_{0} .
$$

In this equation $a$ and $b$ are the proportional corrections to the ratio arms $A$ and $B$ respectively, $R+r$ is the sum of the dial readings and corrections, and $r_{0}$ is the resistance of the rheostat arm with all dials at zero. The term $X_{0}$ is the resistance in ohms of the lead wires in the $X$-arm of the bridge. The factor $(1+a-b)$ is an approximation for $(1+a) /(1+b)$ and is accurate if $a$ and $b$ are small as compared with unity. If neither $a$ nor $b$ exceeds 0.001 , the error from neglecting the second-order terms does not exceed two parts in a million.

\section{Calibration of Thermometer Bridges}

Because of the space limitations and structural difficulties, platinum resistance thermometers usually have resistances of less than $100 \mathrm{ohms}$. The common values are about 2.5 or 25 ohms, the actual values being so chosen that the change in resistance is very close to 0.01 or $0.1 \mathrm{ohm}$ per degree centigrade change in temperature. In order to read to $0.001^{\circ} \mathrm{C}$, it is necessary to read these thermometers respectively to 0.00001 or $0.0001 \mathrm{ohm}$. For such measurements, bridges of special design are usually used.

In this country thermometer bridges for precision work are usually made with equal ratio arms. These arms are interchangeable, so that the use of an average value eliminates errors from lack of equality of the ratio arms, or the ratio arms may be interchangeable and adjustable so that they may be made equal at any time. With such interchangeable ratio arms, actual calibrations of them are unnecessary. The calibration of a thermometer bridge requires only a calibration of the rheostat arm.

In order to measure platinum resistance thermometers, which as stated above are usually not very high in resistance, with a bridge having equal ratio arms, it is necessary to have the rheostat arm adjustable in small steps. In fact, steps as low as 0.0001 or 0.00001 ohm are needed for work of the highest precision. In order to obtain such small steps, contact resistances cannot be used directly in the rheostat arm. Hence recourse is had to decades of the Waidner-Wolff type described above in section III, 5 , in which the changes in resistance result from changes in the values of high-resistance shunts on comparatively small resistances. Switches are placed in the high-resistance shunts, where variations in their resistance will have a negligible effect. Decades of the Waidner-Wolff type cannot be set to have zero resistance, but the small changes start from an appreciable minimum value. However, for equal-arm bridges, compensating resistance may be placed in the $X$-arm. The value of the unknown is then measured by the increase in reading of the rheostat arm when the unknown is connected into the circuit.

The use of a resistance thermometer to measure temperature involves merely the determination of resistance ratios. Hence in calibrating thermometer bridges it is necessary to determine only relative values of resistance, which does not require the use of standard resistors. This calibration is readily made by the user, especially for bridges having equal ratio arms.

The calibration of such thermometer bridges requires as auxiliary equipment only an adjustable resistor that has the same range as that of the bridge rheostat. This resistor needs to be accurately adjustable, although the resistance need not be known for any position. Such an adjustable resistor may be assembled, for example, from a decade box with minimum steps of $0.1 \mathrm{ohm}$ in series with an $0.1-$ or $1-0 h \mathrm{~m}$ resistor, which is in turn shunted by a slide wire or a rheostat of fairly high resistance. This adjustable resistor is connected across the $X$-terminals of the bridge and is used to balance the bridge after the rheostat arm is set to certain required readings. The shunted 0.1 - or $1-\mathrm{ohm}$ should be attached to the X-termi- 
nals so as to be adjacent to the rheostat arm. If a slide wire is used for the shunt, the sliding connection should be used as the galvanometer branch point so that its variable resistance will be in the galvanometer branch where it will not affect the balance. This, in effect, throws the shunted resistance partly in the $X$ and partly in the rheostat arm. We then have the equivalent of the bridge shown in figure 35 , the ratio arms $A$ and $B$ being

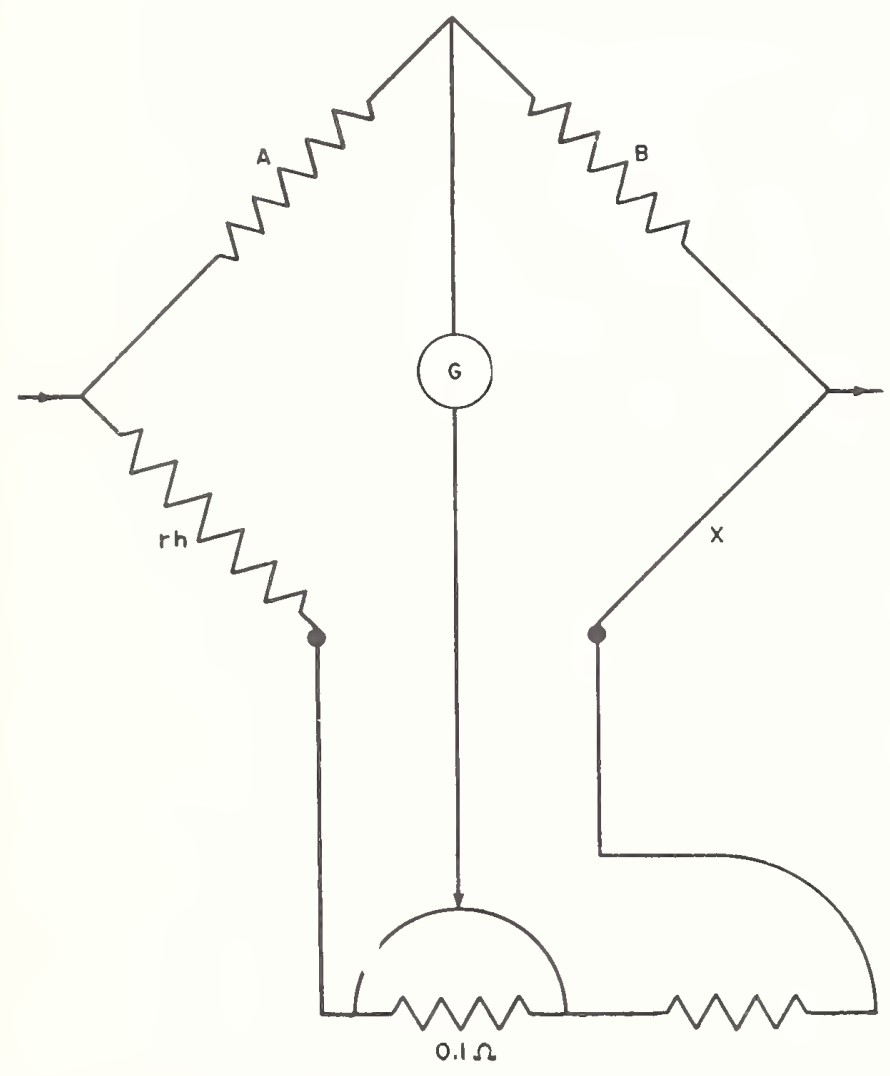

Figure 35. Calibration of rheostat arm of thermometer bridge.

accurately adjusted to equality. For a balance the rheostat arm, $r h$, and the $X$-arm must be also equal. If then $X$ is changed by any amount, as by the insertion of a resistance thermometer in series, the rheostat would have to be changed by an equal amount to again balance the bridge. Thus, actual values of the rheostat arm need not be known if changes in the arm are accurately determined.

The procedure for checking the rheostat arm is usually as follows. The resistances of all steps in the arm are determined in terms of an average step on the smallest dial. The steps of the smallest dial are first intercompared to see that they are equal within 0.1 step, which is as accurately as the steps may be readily read by interpolation from galvanometer deflections. An easy way to intercompare the steps on the lowest clial is to set it at 0 , the other dials being at any convenient setting, and balance the bridge by adjusting $X$. The galvanometer deflection is now read when the lowest dial reading is changed from 0 to 1 . Leaving this dial on 1, the bridge is again balanced with the $X$-rheostat, and the galvanometer deflection then again read for a shift of the lowest rheostat dial from 1 to 2 . These alternate balances with $r h$ and $X$ are continued until the value of all the steps on the smallest dial of $r h$ are determined in terms of galvanometer deflections. Unless one of the steps is defective, these should be the same to the nearest 0.1 step.

The steps on the second dial of $r h$ should each equal the 10 steps of the lowest dial. They are measured in terms of the lowest dial by setting the Jowest dial at 10 and the second dial at 0 , the other dials being at any convenient value, usually small in order to obtain good sensitivity. With the lowest dial at 10 and the next larger dial at 0 , the bridge is balanced by adjusting the value of $X$, after which the smallest dial is turned to 0 and the other dial shifted from 0 to 1 . If there is now any galvanometer deflection, it is because the first step on the second dial is not the same as 10 steps on the smallest. The amount that they differ, in terms of steps of the lowest dial, is determined by reading the galvanometer deflection and evaluating this deflection by reading the additional change in deflection resulting from a change of the smallest dial setting from 0 to 1 .

To measure the second step on the second dial its reading is left at 1 and the lowest dial now set at 10 , a balance being obtained with $X$. The lowest dial js now set back to 0 and the other increased to 2, thus substituting the second step of the higher dial for 10 steps on the lower. The lack of balance is again translated into fractions of a step on the lowest dial by interpolation, using galvanometer deflections. This procedure is continued, cach step of the second dial being compared in turn with the 10 steps of the lowest dial. A table is now made showing the value, in terms of steps of the lowest dial, of the first step of the second dial, the sum of the first two steps, sum of the first three steps, etc. This table will give the resistance corresponding to any reading of this dial in terms of steps on the smallest dial, which we might call "bridge units".

The values of the steps on the next higher dial are now determined in the same way but in terms of the 10 steps on the second dial, the unit agairi being a step on the smallest dial. The continuation of this process gives the resistance of each step of each dial in terms of the 10 steps on the preceding dial, from which finally is calculated the resistance for each setting of each dial in terms of bridge units.

The resistances as determined may be used with any resistance thermometer without converting their values to ohms. It is sufficient to standardize the resistance thermometer on this 
same bridge, by measuring its resistanee at known temperatures. However, it is often desirable to convert the values to ohms in order that the bridge may be used for the measurement of resistance other than that of thermometers. This is done by measuring the resistance of a standard by balaneing the bridge with the standard resistor connected to the $X$-terminals. All readings of the rheostat should then be multiplied by the ratio of the resistance of the standard in ohms (rather, the difference between its resistanee and that of the shorting connector) to its resistance in bridge units in order to convert the rheostat ealibration to ohms.

The above procedure may be used for the calibration of the rheostat arm of any Wheatstone bridge, in terms of steps of the lowest decade and then in ohms, by comparison with a standard resistor. When used with equal ratio arms, the "zero" resistance of the rheostat need not be determined, if two bridge balances are obtained, the first with the unknown conneeted to the $X$ terminals and the seeond with the unknown resistance short-circuited.

Many precision thermometer bridges provide a shorting plug for short-circuiting the resistor conneeted to the $X$-terminals. The galvanometer connects to the center of the shorting connector so that equal amounts of its resistance are inserted in the $X$ and rheostat arms thus giving the same balanee as if the shorting eonnector had a negligible resistance. With this method it is unnecessary to know the resistance of the shorting connector, although sueh resistanee may usually be estimated with sufficient accuraey from its length and gauge size.

\section{Resistivity of Solid Conductors}

\section{Resistivity, Definition and Units}

In the experimental work that led to the formulation of his law, Ohm found that the resistance, $R$, of a eonductor is directly proportional to its length, $l$, and inversely proportional to its crosssectional area, $A$. These experimental faets may be written in the form of an equation as

$$
R=\rho \frac{l}{A},
$$

where $\rho$ is a constant of proportionality whose value depends upon the material of the conductor and upon the units used in measuring $l$ and $A$. This constant of proportionality is called resistivity.

The above equation, which defines resistivity may be written

$$
\rho=R \frac{A}{l} \text {. }
$$

No name has been assigned to the unit of resistivity, and consequently the unit is specified by stating the units used in measuring $R, A$, and $l$. This has resulted in the use of a large number of units, as each of $R, A$, and $l$ may be expressed in more than one unit or subunit. From the above equation for $\rho$, it is seen that the value of $\rho$ is numerically equal to that of $R$ for a conductor lraving unit length and unit eross-sectional area. A cube is such a conduetor, and this has led to the rather common expressions for the unit of resistivity "ohms per eubic ineh" or "mierohms per cubie centimeter". These expressions are undesirable, because they imply that resistivity is the ratio of resistance to volume. It is logieally better to say "ohms times square inches per inch", "microhms times square centimeters per eentimeter" or more bricfly "ohm-inches" and "microhm-cen timeters".

\section{Measurement of Resistivity}

For a uniform conductor, it is merely necessary to measure the resistance of a known length of the eonductor and then measure its eross-sectional area in order to determine its resistivity. For conductors of small cross seetion, it is usually possible to use a sufficient length that the resistance may be aceurately measured with a simple Wheatstone bridge. For conductors of large eross section, it is customary to measure the resistance with a Kelvin double bridge in order to a void errors from contact resistances. Whorever possible, the eross-sectional area is calculated from mierometer measurements. For conduetors of small or irregular eross section, micrometer measurements are not sufficiently accurate, and the average area is determined from mass and density measulements.

For a uniform conductor the cross section, $A$, is

$$
A=\frac{V}{l}
$$

where $V$ is the rolume and $l$ the length of the conductor. But since, from the definition of density, $D$,

$$
V=\frac{m}{D}
$$

where $m$ is the mass, the preceding equation may be written

$$
A=\frac{m}{l D^{\circ}}
$$

For many purposes it is sufficient to assume the density as that given in tables. If this is not sufficiently accurate, the specifie gravity is determined from weighings in air and in water, and from these data and the density of the water, the 
density of the conductor is determined by the equation

$$
D=\frac{w_{a}}{w_{a}-w_{w}} D_{w}
$$

where $\mathrm{w}_{a}$ and $w_{w}$ are the weights of the specimens in air and water, respectively, and $D_{w}$ is the density of the water. At a temperature of $21^{\circ} \mathrm{C}$, the density of water is $0.998 \mathrm{gm} / \mathrm{cm}^{3}$ and this value decreases uniformly to $0.997 \mathrm{gm} / \mathrm{cm}^{3}$ at about $25^{\circ} \mathrm{C}$.

Instead of calculating $A$ separately, the value of $A$ from eq 26 may be substituted into eq 23 , giving

$$
\rho=\frac{R}{l} \times \frac{m}{l} \times \frac{1}{D}
$$

From this equation it is seen that the resistivity, $\rho$, equals the product of resistance per unit length and mass per unit length, divided by density. The length need not be the same for the resistance and mass measurements if the material is uniform. For two conductors having the same density, the ratio of their resistivities is the same as the ratio of their values for the product $R / l \times m / l$. This product is called "mass resistivity", and is a constant that is characteristic of the material of a conductor, being $D$ times as large as the ordinary, or volume, resistivity. The mass resistivity is often specified in the purchase of conductors for electrical uses, and $i^{t}$ is sometimes more readily measured than is volume resistivity. However, it is doubtful that this advantage is ufficient to compensate for the confusion that rises from the use of two types of resistivity.

It is usual commercial practice to specify percentage conductivity rather than resistivity, especially in the purchase of copper conductors. Conductivity is the reciprocal of resistivity, and percentage conductivity is obtained by dividing the resistivity of the giren sample into that of the standard and multiplying by 100 .

By international agreement, the resistivity of annealed copper is taken as 1.7241 microhm-cm at $20^{\circ} \mathrm{C}[15]$. This value was selected from measurements of the resistivity of a large number of samples of high-purity commercial copper wire from both American and European refiners. The value was agreed upon as a standard for reference and was not intended to be the value for absolutely pure material. Copper has been produced with a conductivity of several pereent greater than 100, which probably indicates a material of higher purity than that of the standard.

Some effort has been made to secure international agreement for a standard for the resistivity of aluminum, but so far copper is the only metal for which such a value has been adopted.

The measurement of the resistivity of a liquid may often be made by comparison with another liquid of known resistivity. In this case measurements of dimensions may be avoided, as the known and unknown are given the same dimensions by placing them in turn in the same container or "conductivity cell." The ratio of their resistivities is then the same as the ratio of their resistances. Mercury is often used as the liquid of known resistivity, since its value is accurately known.

Although the resistivity of mercury is known to a high accuracy, its use as a liquid of known resistivity may lead to errors of considerable magnitude. In comparing the resistivity of liquids by placing them in turn in the same cell and measuring their resistance, it is tacitly assumed that the current distribution through the cell is the same for both liquids. This may be incorrect unless the liquids have very nearly the same resistivity, as the distribution of current in the cell depends to some extent upon the resistance of the metal electrodes. For high-resistivity liquids the resistance of the clectrodes may play a negligible part in determining the current distribution, but in the case of mercury, which is a relatively good conductor, the current distribution may depend to a considerable degree upon the dimensions of the electrodes and upon the resistivity of the metal of which they are made.

In determining the resistance of a liquid, it is necessary to use an alternating-current bridge. When a dircet current is used, the ions in the liquid will drift towards the electrodes thus making the density nonuniform. Moreover, polarization will often be produced by the liberation of gases at the electrodes. When measured with alternating current a conductivity cell is found to be electrically the equivalent of a resistor and a capacitor in parallel. This requires a balance of reactance as well as of resistance when the cell is measured in an alternating current Wheatstone bridge.

\section{References}

[1] N. B. Pilling, Trans. Am. Electrochem. Soc. 48, 171 (1925).

[2] R. S. Dean and C. T. Anderson, Trans. Am. Soc. Metals 29, 899 (1941).

[3] A. A. Somerville, Phys. Rev. 31, 261 (1910).

[4] J. L. Thomas, J. Research NBS 16, 149 (1936) RP863.

[5] J. L. Thomas, J. Research NBS 13,681 (1934) RP737.

[6] F. Wenner and J. I. Thomas, BS J. Research 12, 147 (1934) RP639.

[7] P. H. Bridgman, Proc. Am. Acad. Arts Sci. 52, 573 (1917).

[8] J. I. Thomas, J. Research NBS 36, 107 (1946) RP1692.

[9] P. H. Dike, Rev. Sci. Inst. 7, 278 (1936).

[10] E. B. Rosa, IBS Bul. 5, 413 (1908-09) S107.

11] F. Kohlrauch, Wied. Ann. 20, 76 (1883).

[12] E. F. Mucller, 13S Bul. 13, 547 (1916) S288.

[13] E. F. Mucller and F. IVemner, J. Research NBS 15, 48. (1935) RP842.

[14] F. Wenner and E. Weibel, BS Bul. 11, 27 (1914) S223.

[15] BS Circular C31 (1914).

Washington, March 30, 1948. 
RESEARCH PAPER RP1323

Part of Journal of Research of the National Bureau of Standards, Volume 25, August 1940

\title{
METHODS, APPARATUS, AND PROCEDURES FOR THE COMPARISON OF PRECISION STANDARD RESISTORS
}

\author{
By Frank Wenner
}

ABSTRACT

Reference is made to some of the more important contributions that have been made to the subject of precise measurements of electrical resistance. The sensitivity of bridges when used with the modern high-sensitivity moving-coil galvanometer is discussed rather fully. Special consideration is given to the methods and apparatus used and to the procedures followed in the National Bureau of Standards in those comparisons in which the precision desired is of the order of 1 part in a million.

The more important factors limiting the precision of the comparisons, such as load coefficients, terminals, and contacts, thermoelectromotive forces, insulation, and the optical system of the galvanometer, are discussed rather fully. A method of analysis of networks containing both linear and nonlinear four-terminal conductors is given, and the theoretical basis for the experimental procedure used in determining the effect of slight defects in the insulation is pointed out. This is followed by a brief discussion of Ohm's law from the standpoint of precise resistance measurements and by a brief discussion of units of resistance. Finally, reference is made to more than 100 publications having a more or less direct bearing on the subject of resistance comparisons.

\section{CONTENTS}

I. Introduction $\quad$ Page

II. Review of earlier work

III. Sensitivity of bridges

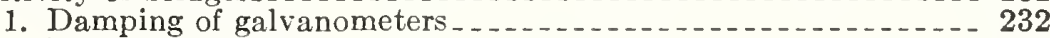

2. Wheatstone bridge

3. Thomson bridge

IV. Load coefficients _.....

V. Galvanometer used with NBS precision bridge

VI. NBS precision bridge....

VII. Bridge equations........ 248

1. Substitution method

2. Interchange method $\ldots \ldots \ldots$

3. Known ratio method

VIII. Establishment of known ratios

IX. Adjustments of Thomson bridge.....

X. Precision and accuracy

XI. Appendices

Appendix 1. Terminals and contacts

(a) Standard resistors

(b) Terminal blocks of bridge. $\ldots \ldots \ldots \ldots \ldots$

(c) Terminal blocks of auxiliary apparatus

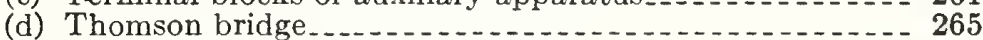

Appendix 2. Thermoelectromotive forces........... 266

(a) Thermoelectric balances...

(b) Thermal shunts_.......... 267 
XI. Appendices-Continued.

Appendix 3. Insulation

(a) Theory in case of defective insulation

(b) Procedure in case of defective insulation $\ldots \ldots \ldots$

(c) Test of insulation $\ldots \ldots \ldots \ldots$

(d) Wagner branches............. 272

Appendix 4. Optical systems of galvanometers

(a) Description of optical system _............... 274

(b) Quality of optical systems and sensitivity of bridges ... 275

Appendix 5. Methods of analyses (three expedients) _._._._._. 277

(a) Classical form of solution of unbalanced Wheatstone

bridge _...

(b) First expedient (hypothetical battery of zero

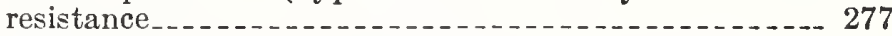

(c) Second expedient (hypothetical battery of high electromotive force and high resistance)

(d) Third expedient (separation and synthesis of the effects of the six independent resistances of a fourterminal conductor)

Appendix 6. Ohm's law

Appendix 7. Units of resistance

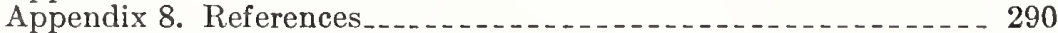

(a) Authors .......... 290

(b) Subjects

XII. Index _......... 294

\section{INTRODUCTION}

Over a period of many years information pertinent to the comparisons of the resistances of precision standard resistors has been accumulating in this Bureau. This paper presents in a connected form the more important parts of this information not adequately explained in previous publications.

The paper is intended to present:

1. For those having a general interest in electrical measurements, a somewhat comprehensive discussion of the Wheatstone bridge method and of the Thomson bridge method, and a brief description of the apparatus used and procedures followed in this Bureau in making resistance comparisons to a precision of 1 part in 1 million.

2. For those making precise resistance comparisons, a discussion of various factors having a bearing on the precision of such comparisons.

To serve this twofold purpose the work of others previous to 1910 is reviewed briefly, and in this review it is pointed out that the conclusions reached by Heaviside, Maxwell, and others were based on assumptions which do not conform to present conditions. Fundamentals are considered in the earlier parts of the paper, and these are explained rather fully, while highly technical discussions are, for the most part, placed in appendices. The manner of presentation makes it possible for those whose interest is only general to familiarize themselves with some of the fundamentals of precision resistance comparisons without reading more than approximately the first third of the paper, and for those who may be interested only in some particular phase of the problem, such as thermoelectromotive forces or the analyses of networks, to find readily (by referring to the index at the end of the paper) what is given on the subject. 


\section{REVIEW OF EARLIER WORK}

The first measurements, by a null method, of what later was recognized as a definite property of a conductor, called the resistance, were made by Becquerel [3]. ${ }^{1}$ Using a differential galvanometer, he obtained the first definite proof of the relation between the resistance, length, and cross section of wires. His results were published in 1826 . It was in the same year that Ohm [66] published his most important paper, though it has hitherto been generally considered that Ohm's law is of a somewhat later origin. In 1833 Christie [12], who was not familiar with the work of Ohm, described an arrangement of wires which later became known as the Wheatstone bridge. With this connection he verified the results obtained by Becquerel and also determined the relative conductivities of a number of metals. As the significance of Ohm's work was not understood until later, Christie was in the position of having devised one of the best methods of measuring resistance before the concept of resistance had become definitely established.

In 1843 Wheatstone $[117,118]$ presented a paper before the Royal Society of London, in which he defined resistance and referred to "standard of resistance" and "resistance coils."

In 1862 Thomson [94] published a paper in which he described what he called a "New Electrodynamic Balance for the resistances of short bars and wires." This later became known as the Thomson bridge, the Thomson double bridge, the Kelvin bridge, and the Kelvin double bridge. Thomson seems to have been the first to attempt measurements of the highest precision attainable with the apparatus then available and the first to have even an approximate understanding of the factors limiting the precision of measurement.

Since then the sensitivity of bridges has been a subject of much discussion. Of the more important of the earlier contributions, mention should be made of a paper by Schwendler [79] published in 1866, of a paper by Heaviside [32] published in 1873, a paper by $T$. Gray [26] published in 1881, and the second and third editions [58] of Maxwell's Electricity and Magnetism, 1892. 'The conclusions given in these publications were based on an assumption that the battery used was not capable of supplying all of the power desired. Obviously, this assumption would seldom be valid at the present time. Furthermore, no account was taken of the electromotive force developed by the relative motion of the winding and magnet of the galvanometer. This constitutes another reason why the conclusions given are not applicable in case a modern high-sensitivity moving-coil galvanometer is used. However, not all of the earlier writers on this subject were of the opinion that the then available sources of electric power constituted a limiting factor in the attainable sensitivity, since as early as 1862 Thomson, in the paper to which reference has been made, said "I shall conclude by remarking that the sensibility of the method which has been explained, as well as of Wheatstone's balance, is limited solely by the heating effect of the current used for testing." In 1889 Paalzow and Rubens [68], in connection with a study of bolometers, made a rather thorough investigation of the effects of heating by the current in a Wheatstone bridge, but their conclusions are not

\footnotetext{
1 Figures in brackets indicate literature references at the end of this paper.
} 
directly applicable to resistance measurements. In 1892 Guye [28], in a further study of bolometers, pointed out that the effect of heating by the test current is proportional to the temperature coefficient of the material from which a bridge arm is constructed, proportional to the square of the current in it, and inversely proportional to its facilities for dissipating heat. He also pointed out that by making all, or pairs, of the bridge arms alike in all respects, the effects of heating by the test current could be almost completely compensated, unless the power dissipation in the bridge arms were unusually large.

In 1893 Glazebrook [23] gave the results of a series of measurements of standard resistors, using different test currents. He found the resistance of $10-\mathrm{ohm}$ standard resistors increased perceptibly as the test current was increased from 0.05 to 0.15 ampere, but he concluded that since the increase in resistance was proportional to the square of the test current, the effect of the smaller test current was extremely small.

In 1895 this was again pointed out by Schuster [78], who evidently was not familiar with the work of Thomson, though he was familiar with that of Guye. Schuster's conclusion was that "The highest percentage accuracy with which a given resistance can be measured is directly proportional to the square root of the maximum electric work which can be done on it without overheating." In 1906 Jaeger [40] and Smith [82, 83, 84], independently, and more recently Von Steinwehr [86], discussed the subject from the same point of view. Nevertheless, the conclusions of Heaviside, Gray, and Maxwell still persist.

The effect of the electromotive force developed by the relative motion of the winding and magnet of the galvanometer is less obvious. However, it has been taken into consideration by Jaeger [40] and by Von Steinwehr [86], both of whom give a different formula for the sensitivity of the Wheatstone bridge (also the Thomson bridge), according to the use of a moving-magnet galvanometer or a suitably damped moving-coil galvanometer.

\section{SENSITIVITY OF BRIDGES}

\section{DAMPING OF GALVANOMETERS}

In the modern high-sensitivity moving-coil galvanometer the electromotive force generated in the galvanometer during the time the deflection is changing at its maximum rate may be of the same order of magnitude as the impressed electromotive force. Therefore, during this time it has a marked effect on the current and consequently on the motion of the coil. What is observed is a damping of the motion of the coil, and, among other factors, this depends upon the resistance of the complete galvanometer circuit, that is, the resistance to an electromotive force in the galvanometer branch of the bridge. If this resistance is much less than that which results in critical damping, the movement of the coil toward any new equilibrium position is very sluggish. If, on the other hand, this resistance is considerably more than that which results in critical damping, the coil continues for some time to oscillate about any new equilibrium position. Neither condition is conducive to rapid nor accurate measurements. To obtain a satisfactory performance, either a magnetic shunt or an auxiliary resistance is used for adjusting the damping. As the latter 
is more convenient and is more generally used, it only will be considered in deriving expressions for the sensitivity. However, it should be pointed out that in general somewhat higher sensitivities may be obtained by the use of an adjustable shunt on the magnet of the galvanometer.

It is convenient to think of the resistance to an electromotive force in the galvanometer branch as consisting of two parts, namely, the resistance of the galvanometer and the resistance external to the galvanometer. In case the resistance of the bridge between its galvanometer terminals, with the galvanometer branch open, is less than the external resistance which gives a desired damping the auxiliary resistance, $U$, is connected in series with the galvanometer, as shown in figure 1, and adjusted so as to give the desired damping. In case the resistance of the bridge between the galvanometer terminals is so high as to give insufficient damping, the auxiliary resistance is placed in parallel with the bridge and galvanometer, and so adjusted as to give the desired drmping. Some prefer to have the damping critical in all cases. However, measurements can be made somewhat more quickly when the damping is approximately two-thirds critical, which results in an "overshoot" of 6 percent. Also in some cases the sensitivity is higher with the damping less than critical, while in

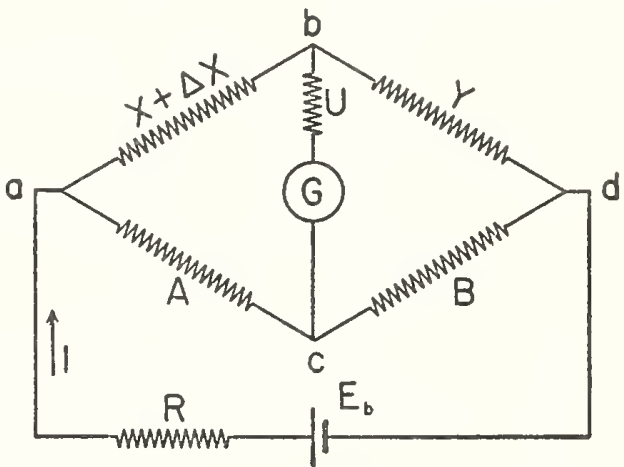

FIGURE 1.-Circuit diagram of unbalanced Wheatstone bridge.

Here $X, Y, A$, and $B$ represent values of the resistances of the four arms for which the bridge would be balanced.

others it is higher with critical damping. Consequently, it is not desirable to use the same damping in all cases. However, to avoid undue complications, it will be assumed, for the present, that the auxiliary resistance will be so adjusted as to give the same damping in all cases, and this will be referrèd to as the specified damping.

\section{WHEATSTONE BRIDGE}

In what follows, the arms of the Wheatstone bridge will be designated $X, Y, A$, and $B$. When the bridge is balanced, the resistances of the arms will also be designated $X, Y, A$, and $B$. To indicate that a reduction of the resistance of the $\dot{X}$ arm by an amount $\Delta X$ would establish a balance of the bridge, the resistances of all arms of the bridge will be designated as shown in figure 1.

Therefore,

$$
X / Y=A / B
$$

With the galvanometer branch open, let

$E=$ the potential drop from $a$ to $d$,

$E_{x}=$ the potential drop from $a$ to $b$,

$\mathrm{E}_{\mathrm{a}}=$ the potential drop from $a$ to $c$, and

$E_{\mathrm{g}}=$ the difference between the potential of $b$ and $c$. 
Obviously then

$$
\begin{aligned}
& E_{x}=\frac{E(X+\Delta X)}{X+\Delta X+Y}, \\
& E_{a}=\frac{E A}{A+B}, \text { and } \\
& E_{g}=\mp \frac{E(X+\Delta X)}{X+\Delta X+Y} \pm \frac{E A}{A+B} .
\end{aligned}
$$

Since the direction of $E_{g}$ taken as positive is immaterial, to avoid complications in what follows the $F$ sign will be taken as positive, and the \pm . sign will be taken as negative. Then, since from eq 1 it follows that

eq 4 takes the form

$$
\frac{A}{A+B}=\frac{X}{X+Y}
$$

$$
\begin{aligned}
& E_{o}=\frac{E X}{X+Y}\left[\frac{1+\Delta X / X}{1+\Delta X /(X+Y)}-1\right] \text { or } \\
& E_{0}=\frac{E X}{X+Y}\left[(1+\Delta X / X)\left(1-\frac{\Delta X}{X+Y}+\left(\frac{\Delta X}{X+Y}\right)^{2} \text {-etc. }\right)-1\right] .
\end{aligned}
$$

If the bridge is nearly balanced, second- and higher-order terms may be neglected, in which case eq 7 reduces to

$$
E_{0}=E Y \Delta X /(X+Y)^{2}
$$

Letting $d X$ represent the proportional decrease in the resistance of the $X$ arm, which would establish a balance of the bridge,

Consequently,

$$
d X=\Delta X / X
$$

$$
E_{g}=E X Y d X /(X+Y)^{2} \text {. }
$$

Since the potential difference which would appear across a break were a branch of a network opened, may be considered as an electromotive force acting in that branch, $E_{g}$ may be considered as an electromotive force in the galvanometer circuit.

Now let $D$ be the change (in scale divisions) of the deflection of the galvanometer resulting from unit change of the electromotive force in the galvanometer circuit when the resistance $V$ connected in series with the galvanometer is that which gives the specified damping. Hereafter $D$ will be referred to as the sensitivity of the galvanometer. Also let $W$ be the resistance of the bridge between its galvanometer terminals, $b$ and $c$ of figure 1 , with the galvanometer branch open. The case in which $W$ is less than $V$ will be considered first. Then $U$ is placed in series with the galvanometer, as shown in figure 1, and so adjusted that

$$
U+W=V
$$

Consider that the zero of the galvanometer scale is at an end of the scale, that $Q$ is the scale reading with the battery branch of the bridge open, that $Q_{1}$ is the scale reading with the battery connected 
as shown in figure 1 , that $Q_{2}$ is the scale reading with the leads to the battery interchanged, and that the galvanometer is so connected that, with $d X$ positive, $Q_{1}>Q>Q_{2}$. Then from eq 10 it follows that

and

$$
Q_{1}-Q=D E X Y d X /(X+Y)^{2} \text {, }
$$

$$
Q_{2}-Q=-D E X Y d X /(X+Y)^{2} \text {. }
$$

Subtracting eq 12 from eq 11 eliminates $Q$ and gives

$$
d Q=2 D E X Y d X /(X+Y)^{2},
$$

where $d Q\left(=Q_{1}-Q_{2}\right)$ is the change in the deflection of the galvanometer following a reversal of the connections to the battery.

Now let $S$ represent the combined sensitivity of the bridge and galvanometer, that is, define $S$ by the equation

$$
S=d Q / d X
$$

Then it follows from eq 13 and 14 that

$$
S=2 D E X Y /(X+Y)^{2} .
$$

Since from eq 1 it follows that

$$
X Y /(X+Y)^{2}=A B /(A+B)^{2},
$$

another expression for the sensitivity is

$$
S=2 D E A B /(A+B)^{2} .
$$

However, if the resistance $W$ of the bridge between its galvanometer terminals is greater than the resistance $V$, which gives the specified damping of the galvanometer, the resistance $U$ is placed in parallel with the galvanometer and adjusted so that

$$
U W /(U+W)=V
$$

From eq 10 it follows that with breaks in both the $U$ and galvanometer branches the potential drop across each of the breaks

$$
E_{o u}=E X Y d X /(X+Y)^{2} .
$$

With the galvanometer branch only open, the current in the $U$ branch is $E_{g u} /(U+W)$, while the potential drop across the break in the galvanometer branch, $E_{g}$, is $U$ times this current, or $E_{g u} U /(U+W)$. Therefore, since $U /(U+W)=V / W$.

$$
E_{g}=E X Y d X V / W(X+Y)^{2} .
$$

That is, the effect of the resistance in parallel with the galvanometer (in parallel with the bridge, if considered from the standpoint of the electromotive force developed in the galvanometer coil as a result of its motion), when of such value as to give the specified damping of the galvanometer, is a reduction of the electromotive force in the galvanometer circuit, and consequently of the combined sensitivity 
of the bridge and galvanometer by the ratio of $V$ to $W$ [101]. Therefore, instead of the relations given by eq 15 and 17 ,

$$
S=2 D E \frac{X Y V}{(X+Y)^{2} W}
$$

and

$$
S=2 D E \frac{A B V}{(A+B)^{2} W}
$$

With reference to these equations it should be noted: (1) That although they are first-order approximations, for the purpose at hand they may be considered as exact, and it is immaterial whether the potential drop across the bridge is measured with the galvanometer branch (and its parallel branch) open or closed. (2) That if they are to apply with the resistance used in adjusting the damping of the galvanometer either in series or in parallel with the galvanometer, in all cases in which the ratio of $V$ to $W$ is greater than one it is to be taken as one.

In what follows, the relative positions of the battery and galvanometer shown in figure 1 (and fig. 2) will be considered as their normal positions. With the position of the galvanometer and battery and their respective rheostats interchanged,

and

$$
S=2 D \epsilon \frac{A X V}{(A+\bar{X})^{2} W}
$$

$$
S=2 D \epsilon \frac{B Y V}{(B+Y)^{2} W^{\circ}}
$$

Here $\epsilon$ is the potential difference between branch points $b$ and $c$, which now are the battery terminals of the bridge.

Except for limitations imposed by the heat developed in the bridge arms by the test current, eq 20 and 21 are in convenient form for use. However, if $E$ (or $\epsilon$ ) exceeds a certain magnitude, the heating in the bridge will result in a change of the resistance of one or more of the bridge arms by an amount in excess of that permissible or in excess of that corresponding to the precision sought in the measurement. There is, therefore, a fairly definite upper limit to the sensitivity which may be used in any particular case. This will be referred to as the permissible sensitivity. The permissible sensitivity depends on the sensitivity of the galvanometer to an electromotive force in a circuit giving the specified damping, the resistance external to the galvanometer which gives the specified damping, the relative magnitudes of the bridge arms, the resistance of the bridge between its galvanometer terminals (factors which have been considered above), the precision sought in the measurement, and the load coefficients of the bridge arms.

The load coefficient of a conductor will be defined as the ratio of the proportional change in its resistance to the power dissipated in it. It would be logical therefore to develop formulas for the sensitivity of bridges based explicitly on the power dissipated in each bridge arm. However, there is some advantage in using either the current [40] or 
the potential drop in each bridge arm, and here the potential drop will be used. How these factors, especially the precision desired in the measurement and the load coefficients, limit the permissible sensitivity may be seen by considering the following example.

Assume that

$$
\begin{aligned}
& \mathrm{D}=10 \text { millimeters per microvolt, } \\
& V=15 \text { ohms, } \\
& X=10 \text { ohms, } \\
& Y=5 \text { ohms, } \\
& A=50 \text { ohms, and } \\
& B=25 \text { ohms. }
\end{aligned}
$$

Assume that the bridge is to be balanced to 1 part in a million and that investigation of the load coefficients has shown that a change of 1 part in a million occurs

in $X$ when $E_{x}$, the potential drop in $X$, is 0.75 volt,

in $Y$ when $E_{y}$, the potential drop in $Y$, is 0.75 volt,

in $A$ when $E_{a}$, the potential drop in $A$, is 2 volts, and

in $B$ when $E_{b}$, the potential drop in $B$, is 1.5 volts.

Obviously, if the resistance of $X$ is to be calculated from values assigned to $A, B$, and $Y$, the potential drop in none of the four resistances can be permitted to exceed the value just stated, and preferably it should not exceed two-thirds this value. In the absence of known compensating effects, it will be assumed that the maximum permissible sensitivity is obtained when the potential drop in the bridge is as high as possible, without that in any arm of the bridge being higher than two-thirds that which results in a proportional change in the resistance equal to the precision sought in the measurement.

With the battery and galvanometer in their normal positions, it is readily seen that $W=20 \mathrm{ohms}$, also that as $E$ is increased $E_{x}$ is the first to reach the maximum permissible value and that $E_{x}=E X /(X+Y)$. Therefore, eq $20 a$ may be written

$$
S=2 D E_{x} \frac{Y V}{(X+Y) W^{\prime}}
$$

and taking $E_{x}=0.5$ volt gives

$$
S=\frac{2 \times 10 \times 10^{6} \times .5 \times 5 \times 15}{15 \times 20}=2.5 \times 10^{6} .
$$

That is, the change in deflection of the galvanometer following a reversal of the connections to the battery is $2.5 \mathrm{~mm}$ per part per million lack of balance of the bridge.

With the positions of the battery and galvanometer interchanged, it is readily seen that $W$ is less than $V$; that, as $\epsilon$ is increased, $E_{b}$ is the first to reach the maximum permissible value; and that $E_{b}=\epsilon B /(B+Y)$. Therefore, eq $21 b$ may be written

$$
S=2 D E_{b} \frac{Y V}{(B+Y) W}
$$

Taking $E_{b}=1$ volt and $V / W=1$ gives

$$
S=\frac{2 \times 10 \times 10^{6} \times 1 \times 5 \times 1}{30}=3.3 \times 10^{6},
$$


or a deflection of $3.3 \mathrm{~mm}$ per part per million lack of balance of the bridge.

It will thus be seen that either arrangement of battery and galvanometer gives a permissible sensitivity more than ample for establishing balances of the bridge to 1 part per million, and that there is not much choice between the two.

If the bridge were to be balanced to 1 part in 4 million, the permissible potential drop in each bridge arm, and consequently the permissible sensitivity, would be only half as large, while the precision sought is higher by a factor of 4 . Consequently, the permissible residual deffection of the galvanometer could be only one-eighth of what it might equally well be in making a balance to 1 part in a million. On the other hand, if the load coefficients were smaller by a factor of 4 , the permissible potential drops would be higher by a factor of 2 , and consequently the permissible sensitivity would be higher by a factor of 2 .

Returning now to a general consideration of the sensitivity of the Wheatstone bridge, it should be noted that with the battery and galvanometer in their normal positions,

$E=E_{x}(X+Y) / X=E_{y}(X+Y) / Y=E_{a}(A+B) / A=E_{b}(A+B) / B$,

and with the positions of the battery and galvanometer interchanged, $\epsilon=E_{x}(A+X) / X=E_{y}(B+Y) / Y=E_{a}(A+X) / A=E_{b}(B+Y) / B$,

Therefore, it follows from eq 20 and 24 that

and

$$
\begin{gathered}
S=2 D E_{x} \frac{Y V}{(X+Y) W}, \\
S=2 D E_{y} \frac{X V}{(X+Y) W}, \\
S=2 D E_{a} \frac{B V}{(A+B) W},
\end{gathered}
$$

and from eq 21 and 25 that

$$
S=2 D E_{b} \frac{A V}{(A+B) W}
$$

and

$$
\begin{aligned}
& S=2 D E_{x} \frac{A V}{(A+X) W}, \\
& S=2 D E_{y} \frac{B V}{(B+Y) W}, \\
& S=2 D E_{a} \frac{X V}{(A+X) W},
\end{aligned}
$$

Now, if it is understood that $E_{x}, E_{y}, E_{a}$, and $E_{b}$ each represents the maximum permissible potential drop in $X$, in $Y$, in $A$, and in $B$, each of eq 26 and each of eq 27, in general, gives a different sensitivity. However, that one of eq 26 which gives the lowest sensitivity gives the maximum permissible sensitivity with the battery and galvanometer in their normal positions; while that one of eq 27 which gives 
the lowest sensitivity gives the maximum permissible sensitivity with the positions of the battery and galvanometer interchanged. This statement concerning the maximum permissible sensitivity is based on the assumption that the resistance of one arm of the bridge is to be calculated from known values of the resistances of the other arms. Later it will be shown that measurements may be made in such a way as to largely eliminate the effect of heating by the test current in one or more of the bridge arms, and that the effect of heating in all bridge arms, if not excessive, may be determined experimentally and an appropriate correction applied. In either case, a somewhat higher sensitivity may be permissible.

It will be noted that by keeping the galvanometer branch closed and reversing the connections to the battery, the permissible sensitivity is twice that which would be obtained by first closing the battery branch and then the galvanometer branch. In addition this eliminates the effects of thermoelectromotive forces and leakage from power circuits, insofar as these remain constant over a time corresponding to a few periods of the galvanometer. Furthermore, a few reversals of connections to the battery, at intervals corresponding approximately to the period of the galvanometer, with the galvanometer branch closed eliminate the effect of gradual drifts and hysteresis in the galvanometer deflections. This is of great importance, since usually in measurements of the highest precision, adjustments must be carried to a point at which the changes in the deflection of the galvanometer resulting from a lack of perfect balance of the bridge are much less than the changes in the deflection resulting from disturbing influences.

\section{THOMSON BRIDGE}

Referring to figure 2, a material simplification in the analysis may be brought about by using Kennelly's [48] $\Delta$ to $Y$ transformation, that

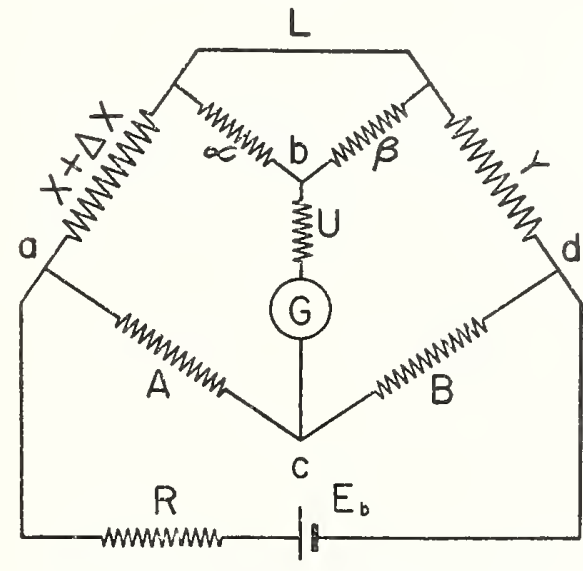

FiguRe 2.-Circuit diagram of unbalanced Thomson bridge.

Here $X, Y, A, B, \alpha$, and $\beta$ represent resistances of the six arms for which the bridge would be balanced.

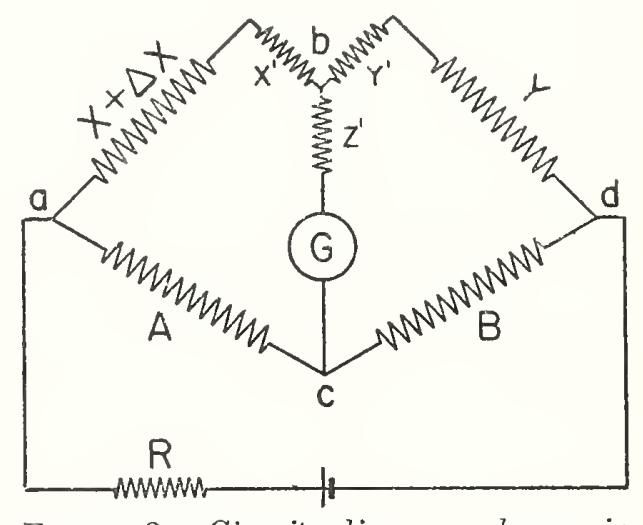

TIGURw 3.-Circuit diagram shown in figure 2 , with the della connected resistances, $\alpha, \beta$, and $L$, replaced by the equivalent star-connected resistances, $X^{\prime}, Y^{\prime}$, and $Z$ '.

is, by considering the three $\Delta$ connected conductors, $L, \alpha$, and $\beta$, as replaced by three $Y$ connected conductors, $X^{\prime}, Y^{\prime}$, and $Z^{\prime}$, as is shown in figure 3. 
This is the same as the Wheatstone bridge, except that there is a resistance $X^{\prime}$ in series with $X, Y^{\prime}$ in series with $Y$, and $Z^{\prime}$ in series with the galvanometer.

The procedure followed in deriving eq 26 and 27 then leads to

$$
\begin{aligned}
& S=2 D E_{x} \frac{\left(Y+Y^{\prime}\right) V}{\left(X+X^{\prime}+Y+Y^{\prime}\right) W^{\prime}} \\
& S=2 D E_{y} \frac{\left(X+X^{\prime}\right) V}{\left(X+X^{\prime}+Y+Y^{\prime}\right) W^{\prime}} \\
& S=2 D E_{a} \frac{B V}{(A+B) W^{\prime}}
\end{aligned}
$$

and

$$
S=2 D E_{b} \frac{A V}{(A+B) W^{\prime}}
$$

if the positions of the battery and galvanometers are as shown in figure 2 , and

and

$$
\begin{aligned}
& S=2 D E_{x} \frac{A V}{\left(A+X+X^{\prime}\right) W}, \\
& S=2 D E_{y} \frac{B V}{\left(B+Y+Y^{\prime}\right) W}, \\
& S=2 D E_{a} \frac{\left(X+X^{\prime}\right) V}{\left(A+X+X^{\prime}\right) W^{\prime}}
\end{aligned}
$$

$$
S=2 D E_{b} \frac{\left(Y+Y^{\prime}\right) V}{\left(B+Y+Y^{\prime}\right) W^{\prime}}
$$

if the relative positions of the battery and galvanometer are interchanged.

With reference to the differences between eq 26 and 28 and eq 27 and 29 , it should be pointed out that for the $Y$-connected conductors to be equivalent to the $\Delta$-connected conductors it is necessary that

and

$$
\begin{aligned}
& X^{\prime}=L \alpha /(L+\alpha+\beta), \\
& Y^{\prime}=L \beta /(L+\alpha+\beta),
\end{aligned}
$$

$$
Z^{\prime}=\alpha \beta /(L+\alpha+\beta) \text {. }
$$

In all cases, $X^{\prime}$ and $Y^{\prime}$ are each less than $L$, and usually $L$ is very small in comparison with $A+X$ and $B+Y$. Consequently, there are relatively few cases in which $X^{\prime}$ and $Y^{\prime}$ may not be omitted from eq $29 x$ and $29 y$, which then become identical with eq $27 x$ and $27 y$. In few if any cases is the permissible sensitivity determined by the power dissipated in $A$ or $B$. Therefore there is no need for drawing conclusions from either eq $29 a$ or eq $29 b$. The Thomson bridge method, with the positions of the battery and galvanometer interchanged, is not used in the comparison of precision standard resistors. However, the Thomson bridge method may be used in resistance 
thermometry, and then there are advantages in interchanging the positions of the galvanometer and battery.

With reference to eq $28 x$ and $28 y$, which apply when the battery and galvanometer are in their normal positions (see figs. 2 and 9), as a balance is approached by the procedure to be described later, $\left(Y+Y^{\prime}\right) /\left(X+X^{\prime}+Y+Y^{\prime}\right)$ approaches $Y /(X+Y)$ and $\left(X+X^{\prime}\right) /(X+$ $\left.X^{\prime} Y+Y^{\prime}\right)$ approaches $X /(X+Y)$. Equations 28 may, therefore, be considered to be the same as eq 26 . However, with the battery and galvanometer in their normal positions and with $A, B, \mathrm{X}$, and $Y$ the same in both the Thomson bridge and the Wheatstone bridge, $W$ is higher in the Thomson bridge than in the Wheatstone bridge. In the comparisons of precision standard resistors made in this Bureau by the Thomson bridge method, this is of no consequence, since $W$ is less than $V$. Under these conditions, the sensitivity of the Thomson bridge is the same as that of the Wheatstone bridge.

It is improbable that a case might arise in which the power dissipated in the $\alpha$ arm or the $\beta$ arm of the bridge would limit the permissible sensitivity, whether the positions of the battery and galvanometer are normal or interchanged. Therefore, no equation containing the potential drop in $\alpha$ or in $\beta$ is given.

\section{LOAD COEFFICIENTS}

Reference has already been made to load coefficients defined as the ratio of the proportional increase in the resistance to the power dissipated. This definition requires some amplification, since, in all cases, time and the medium surrounding the resistor are involved. In the following discussion it will be assumed that the resistors are immersed in oil of low viscosity and that this oil is kept in fairly rapid circulation. Fortunately, most precision resistors come to an approximate temperature equilibrium with the oil in a fairly short time, usually less than 1 minute. With resistors of the type developed in this Bureau about 30 years ago [76], which are sealed in cases containing oil, the change in resistance is rapid at the start of the measuring current and later is very gradual. These resistors apparently have two thermal-time constants, one of about 30 seconds and one of about 30 minutes. Stated in another way, the difference in temperature between the resistance element and the oil in the sealed container becomes nearly constant in a minute, while the difference in temperature between the oil in the container and the oil of the bath becomes nearly constant in an hour. The load coefficients stated for standards of this type apply when the current has been passing from 1 to 2 minutes, the time usually required for making a measurement.

From what has just been said it might be assumed that load coefficients are proportional to temperature coefficients and inversely proportional to the facilities provided for dissipating heat. Experience shows that, in general, this is so only if the design is such that the heating by the test current results in no marked mechanical strain. Consequently, in measuring load coefficients it may be better, when possible, to use a procedure such that this assumption is reduced from a first to a second or third order of importance.

As an illustration of this point consider that it is desired to determine the load coefficient of a 1-ohm standard. In that case the procedure might be as follows: 
(1.) From among the available 0.1- and 0.01-ohm standards select from each denomination one of the better from the standpoint of facilities for dissipating heat and low temperature coefficient.

(2.) Inspect these two standards and make an estimate of their relative facilities for dissipating heat.

(3.) From the estimate of their relative facilities for dissipating heat and their known temperature coefficients make an estimate of the ratio of the load coefficient of the 0.01-ohm standard to the load coefficient of the $0.1-0 h m$ standard, and designate this ratio $k$.

(4) Place the $0.1-\mathrm{ohm}$ standard in the $X$ arm and the $0.01-\mathrm{ohm}$ standard in the $Y$ arm of a bridge, and balance the bridge by adjustments of the $A \mathrm{arm}$, first with 0.1 -watt and then with 0.5 -watt power dissipation in the $0.1 \mathrm{ohm}$-standard. Assuming that the power dissipated in the $A$ and $B$ arms of the bridge has no appreciable effect on their resistances, the load coefficient of the $0.1-\mathrm{ohm}$ standard (that is, the change in the resistance of the $0.1-\mathrm{ohm}$ standard resulting from the dissipation of 1 watt in it) is taken as $23 \%$ times the proportional increase in the resistance of the $A$ arm divided by (1$0.1 \mathrm{k})$.

(5) Place this $0.1-0 h m$ standard in the $Y$ arm of the bridge and a 1-ohm standard in the $X$ arm, and balance the bridge first with one and then another potential drop across the bridge. The load coefficient of the $1-\mathrm{ohm}$ standard is taken as equal to the proportional increase in the resistance of the $A$ arm of the bridge divided by the increase in power dissipation in the $1-\mathrm{ohm}$ standard plus 0.1 , the load coefficient of the $0.1-o h m$ standard as determined in (4). It will be noted that the result obtained involves the initial estimate of the load coefficients of the $0.01-\mathrm{ohm}$ standard relative to that of the $0.1-0 \mathrm{~km}$ standard to the extent of only 1 percent. The value thus obtained for the load coefficient of the 1-ohm standards may, therefore, be presumed to be somewhat more precise than the value obtained for the load coefficient of the 0.1-ohm standard.

Load coefficients of standards of higher nominal values may be determined as outlined above, but for standards of the lowest nominal value used a different procedure is required. If among these there are two standards of such construction that it may be assumed that the temperature rises for equal power dissipations are equal and that the temperature inequalities cause little or no mechanical strain, and if these two standards have markedly different resistance-temperature coefficients, their load coefficients may be determined from their resistance-temperature coefficients and the difference of their load coefficients obtained by direct comparison.

Having determined the load coefficients of one or more standard resistors, the load coefficients of others of the same nominal value are readily determined by direct comparisons, whether or not their performances are normal.

Investigation of wire standard resistors of the Physikalisch-Technische Reichsanstalt design having winding areas of approximately $40 \mathrm{~cm}^{2}$ has shown that the proportional change in their resistance resulting from the dissipation of 1 watt in their windings is about the same as the proportional change in their resistance resulting from increasing the temperature of the oil bath $1^{\circ} \mathrm{C}$. It may be concluded therefore that the temperature rise of the resistance material above the temperature of the oil is about $1^{\circ} \mathrm{C}$ per watt power dissipation. 
On the same basis it has been concluded that for the sealed standard resistors developed in this Bureau about 1907 and the double-walled type constructed prior to 1930 [89] the temperature rise is also about $1^{\circ} \mathrm{C}$ per watt power dissipation. For standard resistors of lower denominations in which the resistance material is in the form of sheets, the temperature rise per watt dissipation is less. For those of the Physikalisch-Technische Reichsanstalt design, of the smaller size, the temperature rise is about $0.2^{\circ} \mathrm{C}$ for the $0.01 \mathrm{ohm}, 0.4^{\circ} \mathrm{C}$ for the 0.001 $\mathrm{ohm}$, and $0.3^{\circ} \mathrm{C}$ for the $0.0001 \mathrm{ohm}$ per watt dissipation.

Since most of the temperature coefficients are less than 20 parts per million, errors resulting from heating will, in general, be less than 1 part in 2 million if the potential drops are limited to the following values:

0.15 volt for 1 -ohm, .5 volt for $10-\mathrm{ohm}$,

1.5 volts for $100-\mathrm{ohm}$,

5. volts for $1,000-\mathrm{ohm}$,

15. volts for $10,000-\mathrm{ohm}$,

0.05 volt for $0.1-\mathrm{ohm}$,

.035 volt for $0.01-\mathrm{ohm}$,

.0075 volt for $0.001-0 h m$, and

.0025 volt for $0.0001-\mathrm{ohm}$ standard resistors.

The temperature rises, and consequently the load coefficients, depend on the viscosity and other factors affecting the circulation of the oil, the design of the standards, and their temperature coefficients, so there must of necessity be large variations. However, the potential drops stated above are about the maxima permissible in measurements to 1 part per million, unless the load coefficients are known to be abnormally low, or a procedure is followed for eliminating the error which otherwise would result from the heating by the test current.

In routine testing the schedule of potential drops given above is followed approximately in the comparisons of resistors having nominal values of $1 \mathrm{ohm}$ and less. In the comparisons of resistors having nominal values of $10 \mathrm{ohms}$ and more the potential drops used are much less than those given in the schedule. In special cases the criterion used for the maximum permissible potential drops, without an application of a correction for the heating, is a very small but definitely noticeable change in the balance of the bridge on increasing the potential drop across the bridge by a factor of 2 .

If there is occasion to use larger potential drops, if there is reason to suspect one or more abnormally large load coefficients, or if the load coefficient of one or more of the bridge arms is not known to the accuracy necessary for obtaining the desired precision, a valid correction for the effect of the heating may be obtained by balancing the bridge first with what is presumed to be a suitable potential drop across the bridge and second with a somewhat larger potential drop across the bridge. If the second potential drop is twice the first, the effects of the temperature rise in each arm of the bridge will be 4 times as large as with the first potential drop. Consequently, if the balances are established by adjustments of the $A$ arm (or $A$ and $\alpha$ arms) of the bridge and the value taken for $A$ is four-thirds the first minus one-third the second, a correction is applied for the effects of heating by the test current in all arms of the bridge. 


\section{GALVANOMETER USED WITH NBS PRECISION BRIDGE}

The galvanometer which has been and still is being used in most of the precision resistance measurements made in the National Bureau of Standards was designed and constructed especially for the purpose about 1914 [116]. It has an adjustable shunt on the magnet, allcopper circuit, and taut suspensions with the center of gravity of the moving system slightly off the axis of rotation. This latter feature makes it possible by tilting to adjust the period; $T$, over a range from about 5 to about 15 seconds. In cases in which really high sensitivity is desired the performance seems to be most satisfactory with a period of about 10 seconds: The shunt of the magnet is so adjusted that with a period of 10 seconds the external resistance giving critical damping is 35 ohms. This adjustment once made is seldom changed as the shunt is not readily accessible. The galvanometer is used with a scale distance of $1.5 \mathrm{~m}$. With critical damping the operating constants then are

$$
\begin{aligned}
& T=10 \text { seconds, } \\
& D=30 \mathrm{~mm} / \mu \mathrm{v}, \\
& V=35 \mathrm{ohms},
\end{aligned}
$$

or with two-thirds critical damping, which results in a 6 percent overshoot and gives about the maximum speed of operation with the 10 second period, the operating constants are

$$
\begin{aligned}
& T=10 \text { seconds, } \\
& D=20 \mathrm{~mm} / \mu \mathrm{v}, \\
& V=60 \mathrm{ohms} .
\end{aligned}
$$

It may be of interest to see what the permissible sensitivity is in the comparison of $10-\mathrm{ohm}$ standards. when the $A$ and $B$ arms of the bridge are each $25 \mathrm{ohms}$, the battery is in the normal position, and the galvanometer is used critically damped. In this case the resistance, $W$, of the bridge between galvanometer terminals is 1732 ohms. With $E_{x}$ or $E_{y}=0.5$ volt, it follows from eq $26_{x}$ or $26_{y}$ that $S=2 \times 30 \times .5 \times 10 / 20=15 \mathrm{~mm}$ per part per million lack of balance of the bridge. This is not only much higher than is needed but is higher than it is desirable to use. In some cases, however, the permissible sensitivity is none too high, and in a very few cases it is not quite sufficient for the establishment of balances to 1 part per million. In these the potential drop may be increased to the point at which errors resulting from henting (calculated from a knowledge of the load coefficients of the particular standards used) and from lack of sensitivity are approximately equal, or even beyond this point, and then a correction for the heating is determined and applied. In a very few cases there would be an advantage in using a galvanometer of higher sensitivity. However, for a considerable part of the measurements the galvanometer is adjusted so as to have a period of from 6 to 8 seconds. Then both the sensitivity and the external resistance giving a specified damping are less than with the 10-second period.

\section{NBS PRECISION BRIDGE}

The bridge now in use in the comparisons of precision standard resistors was designed and constructed in this Bureau in 1918. All parts of the bridge arms and the adjustable resistor used in regulating 
the damping of the galvanometer are immersed in oil. The ammeter, voltmeter, and rheostats used in regulating the test current and other auxiliary equipment are conveniently located outside the oil bath. The entire bridge circuit is shielded against leakage from directcurrent power circuits. The oil bath is thermostatically controlled at a temperature of $25^{\circ} \mathrm{C}$, and during the time the apparatus is in use the oil is kept in vigorous circulation. During the summer the normal dew point is occasionally very nearly $25^{\circ} \mathrm{C}$. To prevent the condensation of moisture in the oil bath and to maintain good insulation of the battery, galvanometer, and other parts of the circuit outside the oil bath, the air of the laboratory is dried by refrigeration.

The more important resistance sections of the bridge are of the double-walled sealed type developed jointly with others [98] of the Bureau's staff. The cases contain no oil, since the use of oil in permanently sealed resistors has long been considered inadvisable. To obtain low load coefficients the cases were made considerably larger than those first described, and the resistance wire was selected on the basis of low temperature coefficient. The primary of a well-insulated variable mutual inductor is connected in series with one of the battery leads, and its secondary is connected in series with one of the galvanometer leads. This inductor (not shown in fig. 5) serves to

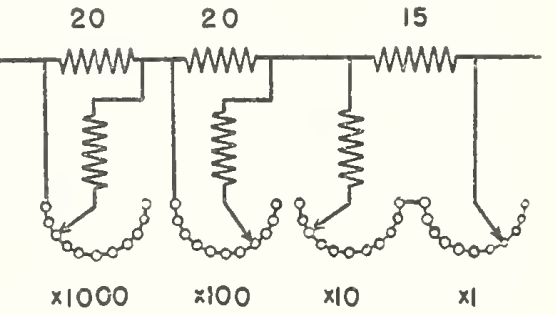

Figure 4.-The part of the $A$ arm of the NBS precision bridge which is adjustable in steps of 0.0001 ohm over the range from approximately 49.4445 ohms to approximately 50.5555 ohms.

balance the electromotive force induced in the galvanometer circuit on reversing the test current. ${ }^{2}$ The $A$-arm of the bridge is of the adjustable direct-reading type, such as is discussed in a recent publication [63] of this Bureau. It consists of five resistance sections, three of which are adjustable by means of dial switches. These three sections are shown in figure 4 . With each of the dial switches set at its mid-position, the current in each shunt is one-tenth of that in the section shunted, and the sum of the resistances of the three

2 The lack of an inductive halance manifests itself as hallistic deflection of the galvonometer, following a reversal of the test current. If this hallistic deffection is large, it limits, somewhat, the prccision of the resistive halance. However, there is a more important reason why the hallistic deflections should he kept small. Most sensitive galvanometers when deflected alternately in onc and then in the other direction, by equal amounts, have their rest points shifted slightly in the direction of the last deflection. For the by equal amounts, have their rest points shirted slightly in the direction of the last deflection. For the galvanometer used with the NBS precision hridge, if the rest point is ohserved after a defection in one
direction and again after an equal deflection in the opposite direction, the difference between the two direction and again after an equal deflection in the opposite direction, the difference between the two
ohserved rest points is from 1 to 2 percent. of the amplitude of the defiections. lf, therefore, systenlatic errors from this source are to he insignificant, inductive halances must he such that the ballistic deflections are less than 50 times the change in deflection resultizg from lack of resistive balances correspond. ing to the precision sought in the comparisons.

In the comparisons of standard resistors of the usual construction, having resistances in the range from 0.1 to 100 ohms, usually this condition is realized without a special device for making incluctive balances and without spccial precautions on the part of the ohserver. However, if the resistors in the $X^{\prime}$ and $Y^{r}$ arms of the bridge have low resistances of different nominal values (such, for example, as 0.001 and 0.0901 ohm), and if a high precision is desired a mens for compensating the effect of the difference hetween their time conf a high stants and of mutuninductances hetween different parts of the bridge circuit is necessary. Likewise, ir tl resistors in the $X$ and $Y$ arms of the hridge are of the usual bifilar construction and have high resistances of different nominal values (such, for example, as 10,000 and 1,000 ohms), and if a high precision is desired, provision should he made for compensating the effect of the distributed capacitances. The effects of sel and mutual inductances and of distributed eapacitances may be compensated by a mutual inductor having one of its windings connected in a galvanometer lead and the other winding connected in a battery lead hetween the current-rcversing switch and a current terminal of the bridge, provided the inductance is adjustahle over a suitable range of positive and negative values. 


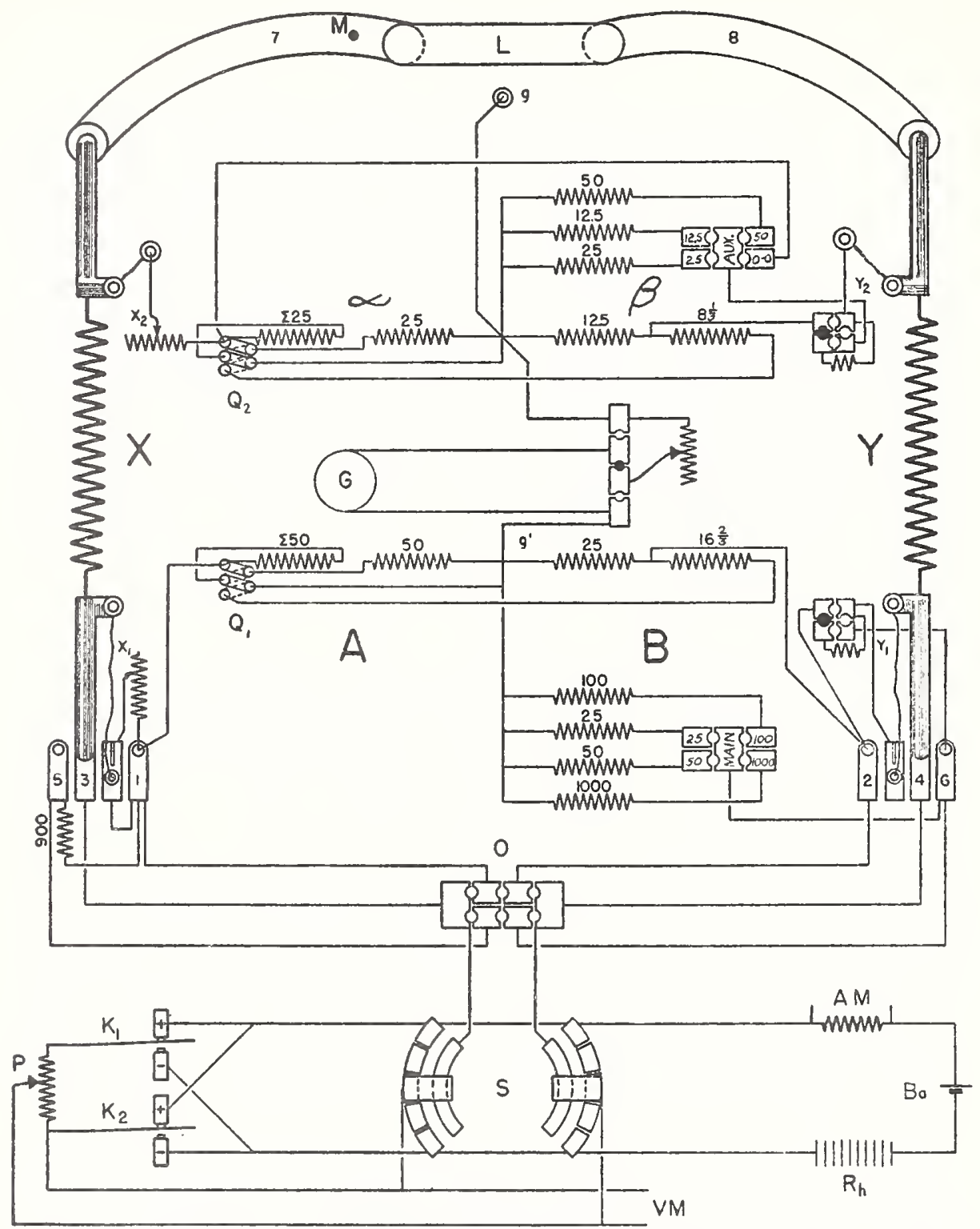

FIGURE 5.-Diagram of essential features of the NBS precision bridge--connected as a Thomson bridge.

In general, the notation here is the same as $\ln$ figures 2,9 , and 16 . Here 1 and 2 represent main terminal Iu general, the notation here is the same as ln tgures 2,9 , and 5 and 6 , terminal blocks for the less freblocks (shown in detail in fig. 11); 3 and 4, current-terminal blocks; 5 and 6 , terminal blocks for the less irequently used ratios of $A$ to $B ; 7$ and 8 , binged terminal blocks to accommodate standard resistors with different spacing of terminals; $\Sigma 50$, the three adjustable resistance sections shown in 1 gure $4, R_{\mathrm{b}}$, compression carbonplate rheostat for adjusting large test currents; $P$, potential divider for adjusting small test currents; $A M$ ammeter shunt; $V M$, voltmeter leads; $k_{1}, k_{2}$, and $S$, keys and switch for closing battery branch and reversing connections to battery leads, $k_{1}$ and $k_{2}$ for small test currents, $S$ for large test currents; $Q_{1}$ and $Q_{2}$, copper links and terminal blocks with amalgamated contacts for connecting two sections of the $A$ arm and of the arm either in serics or In parallel. For the Wheatstonc bridge method usually terminals 1 and 2 are used Instead of 3 and 4 , and a connection between $M$ and 0 . 
sections with their shunts is $50 \mathrm{ohms}$. The resistance sections of the shunts are so chosen that the steps of the dial switches change the combined resistances of sections and shunts respectively by 0.0001 , $0.001,0.01$, or by $0.1 \mathrm{ohm}$. The positions of each dial switch are numbered from 0 to 10 . When the three shunted sections designated $\Sigma 50$ in figure 5, are connected in series, or in parallel, with the $50-\mathrm{ohm}$ section of the $A \mathrm{arm}$, and any section of the $B$ arm of the bridge used, differences in the readings of the dial switches correspond to differences in the ratio of $A$ to $B$ in parts per million of the nominal ratio of $A$ to $B$. When the three shunted sections are connected in series with the 50 -ohm section and the $900-0 h m$ section, differences in the readings of the dial switches correspond to differences in the ratio of $A$ to $B$ in parts in 10 million of the nominal ratio of $A$ to $B$. In all cases the $A$ arm has its nominal resistance when the reading of the dial switches is approximately 5555 .

The dial switches may be rotated indefinitely in either direction, while the complete range of adjustment is covered by a rotation of slightly less than $120^{\circ}$. The brushes, three in number for each switch, are of the multiple-leaf type, such as are used by Otto Wolff. They are mounted on what amounts to the feet of a rigid tripod, and the contact pressure is supplied by three coiled steel springs. This arrangement largely eliminates rocking of the brushes as the switch is rotated and gives a nearly constant pressure of the switches against the contact blocks. With the switch in any position, one of the brushes rests on an insulated segment and the circuit is through the other two brushes in series.

The dial switches are of good quality and operate under oil. Presumably, therefore, variation of the resistance of the contacts of each dial switch never exceeds $0.001 \mathrm{ohm}$. Since the resistance of each shunt is 10 times the resistance of the section shunted, a variation of $0.001 \mathrm{ohm}$ in each of the four dial switches in the same direction results in a variation of the combined resistance of sections and shunts of $0.004 / 121 \mathrm{ohm}$. This is 1 part in 3 million in case $A$ is nominally either 25 or $100 \mathrm{ohms}$, and 1 part in 30 million in case $A$ is nominally 1,000 ohms. However, the probable effects of the variations of the resistances of the dial switches are approximately one order smaller than this.

The $B$ arm (see fig. 5) has resistance sections of $16 \% 3,25,50,100$, and $1,000 \mathrm{ohms}$, most of which may be used singly or in combinations. Therefore, a number of values for the ratios of $A$ to $B$ may be had, such as 1 to 1,2 to 1,4 to 1,10 to 1,1 to 2,1 to 4 , and others seldom used. Each of these ratios may be varied by changing the readings of the dial switches of the $A$ arm, and for the most part the range of variation is from 0.5 percent below to 0.5 percent above the nominal ratio.

Adjustments are such that differences in the readings of the dial switches, not in excess of 500 , correspond to differences in the ratio of $A$ to $B$ well within 1 part in a million of the nominal ratio providing no one of the readings differs by more than 500 from that for which $A$ has its nominal value. In exceptional cases corrections must be applied to the readings to give differences accurate to 1 part in a million of the ratio.

The $\alpha$ and $\beta$ arms (see fig. 5) are similar to the $A$ and $B$ arms, except that the resistance of corresponding sections is half as large, the $\alpha$ arm 
does not have a 450-ohm section corresponding to the 900 -ohm section of the $A \mathrm{arm}$, and the $\beta$ arm does not have a 500 -ohm section corresponding to the $1,000-\mathrm{ohm}$ section of the $B$ arm. The dial switches of $A$ and $\alpha$ arms are mechanically connected so as to always have the same reading.

A general view of the bridge removed from the oil is shown in figure 6. A general view of the bridge and oil bath is shown in figure 7. Two features of the oil bath which should be mentioned are the means employed for securing a reasonably uniform temperature throughout the bath and of maintaining the desired temperature. The oil bath has a false bottom supported about $3 \mathrm{~cm}$ above the true bottom of the bath and extending to within about $1 \mathrm{~cm}$ of the side walls, which are of nickel-plated copper. Near the center of the false bottom there is an opening about $8 \mathrm{~cm}$ in diameter, and the circulating propeller is located beneath this opening and concentric with it. The circulation of the oil in the central part of the bath is downward, through this opening, outward between the false and true bottoms, upward next to the side walls to near the surface of the oil, and inward on and under the oil surface. The rate of the circulation is approximately 3 liters per second, so that the volume of oil passing through the central opening in 1 minute is approximately the same as the volume of oil contained in the bath. The heat for maintaining the temperature is supplied by small carbon-filament lamps located slightly above and near the opening in the false bottom. By means of radiation, a part of the heat developed in the lamp filaments is distributed through the oil almost instantly. That part of the radiation from the lamp filaments absorbed in and near the lamp bulbs is carried quickly to the side walls of the bath and surface of the oil, the parts from which heat is being lost by radiation and air convection.

\section{BRIDGE EQUATIONS}

In the usual discussion of the Wheatstone bridge it is assumed that if $X$ represents the unknown resistance, its magnitude is to be calculated from the equation

$$
X=Y A / B
$$

That is, it is assumed that magnitudes of $Y, A$, and $B$ are each known to an accuracy at least as high as that expected for $X$, or that either the magnitude of $Y$ or $A$ and the ratio of $A$ to $B$ or $Y$ to $B$ are known to this accuracy. In measurements of the type under consideration neither of these assumptions can, in general, be made. It is therefore necessary to use some procedure which avoids the use of these assumptions.

\section{SUBSTITUTION METHOD}

If a number of nominally equal resistances $X_{1}, X_{2}, X_{3}, X_{4}$, etc. are to be measured and the resistances of two or more of these are known, for example $X_{2}$ and $X_{4}$, usually a standard of the same nominal value as those being measured is placed in the $Y$ arm, and the $B$ arm is made nominally equal to the $A$ arm. Then all of the $X$ 's may be substituted one after the other in the $X$ arm, and the bridge balanced in each case by an adjustment of the $A$ arm. If $a_{1}, a_{2}, a_{3}, a_{4}$, etc., are the read- 


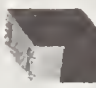

$\frac{1}{2}=$

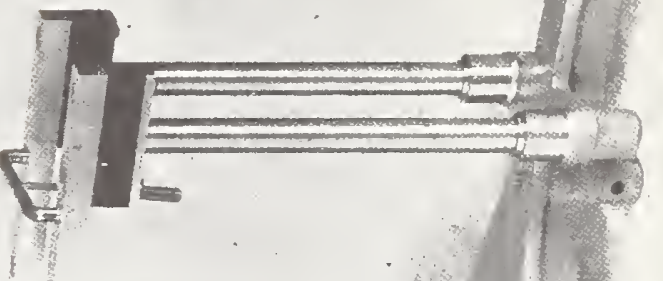

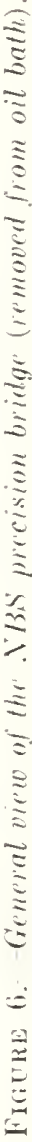




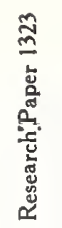

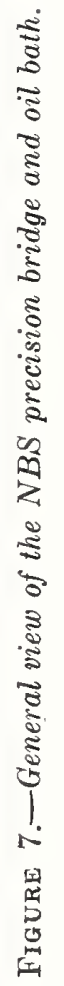


ings of the dial-switch settings, $A_{n}$ is the nominal value of $A$, and $a_{n}$ is the reading of the dial-switch settings for which $A=A_{n}$,

$$
\begin{aligned}
X_{1} & =\frac{Y}{B} A_{n}\left(1+a_{1}-a_{n}\right) \\
X_{2} & =\frac{Y}{B} A_{n}\left(1+a_{2}-a_{n}\right) \\
X_{3} & =\frac{Y}{B} A_{n}\left(1+a_{3}-a_{n}\right) \\
X_{4} & =\frac{Y}{B} A_{n}\left(1+a_{4}-a_{n}\right)
\end{aligned}
$$

etc.

Letting $N$ represent the nominal value of $X$ and of $Y A_{n} / B$, and $x, y$, and $b$ represent the amounts in proportional parts by which $X, Y$, and $B$ exceed their nominal values, eq 33 may be written in the form

$$
N(1+x)=N(1+y)\left(1+a-a_{n}\right) /(1+b) .
$$

Expanding eq 34 and neglecting $3 \mathrm{~d}$ and higher-order terms gives

$$
x=y+\left(a-a_{n}\right)-b+y\left(a-a_{n}\right)-b\left(a-a_{n}\right)+b^{2}-b y .
$$

Letting $z$ represent that part of eq 35 which, for the series of measurements, is a constant, and, for the present, leaving out of consideration the second-order term $(y-b)\left(a-a_{n}\right)$ gives

etc.

$$
\left.\begin{array}{l}
x_{1}=a_{1}+z \\
x_{2}=a_{2}+z \\
x_{3}=a_{3}+z \\
x_{4}=a_{4}+z
\end{array}\right\}
$$

Since $X_{2}$ and $X_{4}$ are known, $x_{2}$ and $x_{4}$ are known. Furthermore, all $a$ 's will be read from the bridge. Therefore, $z$ may be determined from the second or fourth of eq 36; or what is better, from both of these equations and the mean value used. Substituting the mean value of $z$ in each of eq 36 gives a value for each of the $x^{\prime} s$ and consequently for $X_{1}, X_{2}, X_{3}, X_{4}$, etc. in terms of the unit in which values of $X_{2}$ and $X_{4}$ are expressed.

To see to what extent the values thus found are in error as a result of the approximations involved in eq 36 , it is necessary to consider the second-order term $(y-b)\left(a-a_{n}\right)$ of eq 35 , the magnitudes of $x_{1}, x_{2}, x_{3}, x_{4}$, etc. and what constitutes nominal values of each of the bridge arms. It was intended that resistances of the sections of the $A$ arm be so proportioned that differences of readings of the dials equal differences in resistance in parts per million of the resistance the $A$ arm has when the reading of the dials is 5555 . This resistance (instead of 25,100 , or 1,000 ohms, depending on what sections are used and the way they are connected) should be taken as the nominal value of $A$. The appropriate multiple of the unit used is taken as the 
nominal value of the $X$ 's, and nominal values of $B$ and $Y$ are so chosen that

$$
B_{n} / Y_{n}=A_{n} / X_{n}
$$

As a result of the way in which the eq 36 are used $z$ includes all of $(y-b)\left(a-a_{n}\right)$ except the part equal to $(y-b)\left[a-\left(a_{2}+a_{4}\right) / 2\right]$, while the nominal value chosen for $A$ makes

$$
y-b=5555-a+x, \text { approximately. }
$$

Therefore $(5555-a+x)\left[a-\left(a_{2}+a_{4}\right) / 2\right]$ may be taken as the error in or a correction term for any one of eq 36 . From the data obtained in a series of measurements it is therefore possible to determine, almost at a glance, the correction which, if significant, should be applied to one or more of eq 36 . The first factor in this correction term is a constant for the series of measurements and depends upon the relative departures of $Y$ and $B$ from their nominal values. By a selection of the standard resistor to be used in the $Y$ arm, usually it is possible to make this factor less than 0.02 percent. If the first factor is less than 0.02 percent and the resistance of no one of the standard resistors substituted one after the other in the $X$ arm differs from the mean of $X_{2}$ and $X_{4}$ by more than 0.05 percent, in no case will the error or correction term exceed 1 part in 10 million.

Returning now to a further consideration of the sensitivity of bridges, the substitution method not only obviates the requirement of an accurate knowledge of the resistances of the $A, B$, and $Y$ arms of the bridge, but, for the most part, eliminates the effects of heating by the test current in these arms. Therefore, if the battery and galvanometer are in their normal positions, it is permissible to use in the $Y$ arm a standard resistor having a higher nominal value than those being substituted alternately in the $X$ arm and thus realize a somewhat higher permissible sensitivity, as may be seen by reference to eq $26 x$. If, on the other hand, the positions of the battery and galvanometer are interchanged, the use in the $Y$ arm of a standard of lower nominal value than those being substituted in the $X$ arm gives a higher permissible sensitivity only in case $W$ is larger than $V$, as may be seen by reference to eq 27x. Furthermore, it is not the load coefficients of the resistors being compared, but their differences or spread which limits the permissible potential drop in these resistors.

Equation 36, and others which follow under the heading "Bridge Equations," are applicable if the Wheatstone bridge method is used. They are also applicable if the Thomson bridge method is used, provided the adjustment is made in the manner which will be described later (or its equivalent), and provided further that the resistance of the potential lead which then constitutes a part of the $A$ arm of the bridge is not so large but that differences in readings of the dials may be taken as differences in the ratio of $A$ to $B$ in parts per million of its nominal value. If $x_{1}$ is the resistance of this lead, including the adjustable rheostat (see fig. 9), the effect of this resistance is taken into account by the addition to the right-hand members of eq 36 the second-order term $\left(x_{1} / A_{n}\right)\left[a-\left(a_{2}+a_{4}\right) / 2\right]$. Usually $x_{1} / A_{n}$ does not exceed 0.05 percent, and $a-\left(a_{2}+a_{4}\right) / 2$ does not exceed 0.05 percent, so usually this second-order term amounts to less than 1 part in 1 million. 
A point which should be brought out here is that if a change in the unit of resistance is made, no change will be required either in the bridge or the procedure followed in the reduction of the observational data. A change in the unit will change $x$ in the correction term

$$
[5555-a+x]\left[a-\left(a_{2}+a_{4}\right) / 2\right],
$$

but an approximately equal change in $a, a_{2}$, and $a_{4}$ may be made by the selection of a different standard resistor for use in the $Y$ arm of the bridge.

\section{INTERCHANGE METHOD}

If two nominally equal resistances, $X_{1}$ and $X_{2}$, are to be compared, the bridge may be balanced by an adjustment of the $A$ arm, first with $X_{1}$ in the $X$ arm and $X_{2}$ in the $Y$ arm and then with $X_{2}$ in the $X$ arm and $X_{1}$ in the $Y$ arm. This gives two relations, which may be written as follows:

$$
\begin{aligned}
& X_{1}=X_{2} \frac{A_{n}}{B}\left(1+a_{1}-a_{n}\right) . \\
& X_{2}=X_{1} \frac{A_{n}}{B}\left(1+a_{2}-a_{n}\right) .
\end{aligned}
$$

From eq 38 and 39 it follows that

$$
X_{2}=X_{1} \sqrt{1+a_{2}-a_{1}+\left(a_{2}-a_{n}\right)\left(a_{n}-a_{1}\right)+\left(a_{1}-a_{n}\right)^{2}}
$$

If, therefore, conditions are such that the second-order terms under the radical and terms of the order of $\left(a_{2}-a_{1}\right)^{2}$ may be neglected,

$$
X_{2}=X_{1}\left[1+\left(a_{2}-a_{1}\right) / 2\right] \text {. }
$$

If, in addition, $X_{1}$ has so nearly its nominal value that $x_{1}\left(a_{2}-a_{1}\right) / 2$ may be neglected,

$$
x_{2}=x_{1}+\left(a_{2}-a_{1}\right) / 2,
$$

where $x_{2}$ and $x_{1}$ are the departures of $X_{1}$ and $X_{2}$ from their nominal values.

\section{KNOWN RATIO METHOD}

In the comparison of two resistances, $X_{1}$ and $X_{2}$, whose nominal values differ by a factor such as $2,3,5$, or 10 , different procedures may be used, but most of these only in special cases. The following is almost universally applicable and most frequently used in this Bureau.

The ratio of the bridge is set nominally equal to the ratio of the resistances of the two standards, which are placed one in the $X$ arm and the other in the $Y$ arm. Then, if $X_{2}$ is known and it is placed in the $Y$ arm of the bridge,

$$
X_{1}=R X_{2}\left(1+a_{1}-a_{r}\right)
$$

where $R$ is the nominal ratio of $X_{1}$ to $X_{2}, a_{1}$ is the reading of the dials of the $A$ arm of the bridge, and $a_{r}$ is the reading (as yet undetermined) 
of the dials of the $A$ arm, for which the ratio of $A$ to $B$ has the nominal ratio of $X_{1}$ to $X_{2}$. From eq 43 it follows that

$$
x_{1}=x_{2}+a_{1}-a_{r} \text {, approximately, }
$$

where $x_{1}$ and $x_{2}$ are the departures of $X_{1}$ and $X_{2}$ from their nominal values.

If, however, $X_{2}$ is known and it is placed in the $X$ arm of the bridge

$$
x_{1}=x_{2}+a_{\tau}-a_{1} .
$$

If $A_{n} / B$ differs from the nominal ratio of $X_{1}$ to $X_{2}$ (or $X_{2}$ to $X_{1}$ ) by less than 3 parts in 10,000 , and $X_{1}$ and $X_{2}$ differ from their nominal valute by less than 3 parts in 10,000, the approximations are not likely so exceed 1 part in 10 million. The procedures followed in determining $a_{r}$ will be considered in the next section.

\section{ESTABLISHMENT OF KNOWN RATIOS}

The principle underlying one of the procedures followed in finding the reading of the $A$ arm (that is, $a_{\tau}$ of eq 43) of the bridge for which the ratio of $A$ to $B$ is accurately an integer may be illustrated as follows. If there are at hand $n$ standards or coils having nearly equal values, $M_{1}, M_{2}, M_{3}$, etc., and $S$ is their resistance when connected in series,

$$
S=M_{1}+\dot{M}_{2}+M_{3}+\text { etc. }
$$

Also, if $P$ is their resistance when connected in parallel,

$$
\frac{1}{P}=\frac{1}{M_{1}}+\frac{1}{M_{2}}+\frac{1}{M_{3}}+\text { etc. }
$$

Now, let $M$ be their mean resistance and $m_{1}, m_{2}, m_{3}$, etc. be the departures of each in proportional parts from the mean of all. Then

$$
S=M\left(1+m_{1}+1+m_{2}+1+m_{3}+\text { etc. }\right)
$$

and

$$
\frac{1}{P}=\frac{1}{M\left(1+m_{1}\right)}+\frac{1}{M\left(1+m_{2}\right)}+\frac{1}{M\left(1+m_{3}\right)}+\text { etc. }
$$

Assuming that the resistances of the standards or coils are so nearly equal that the third and higher powers of $m$ may be neglected, expansion by the binomial theorem of the terms $1 /\left(1+m_{1}\right), 1 /\left(1+m_{2}\right)$, $1 /\left(1+m_{3}\right)$, etc. in eq 49 gives

$$
\frac{1}{\bar{P}}=\frac{1}{M}\left(1-m_{1}+m_{1}{ }^{2}+1-m_{2}+m_{2}{ }^{2}+1-m_{3}+m_{3}{ }^{2}+\text { etc. }\right) .
$$

Since by definition $m_{1}+m_{2}+m_{3}+$ etc. equals zero,

$$
S=n M,
$$


and

$$
\frac{1}{P}=\frac{n}{M}\left(1+\frac{1}{n} \sum m^{2}\right)
$$

where $\Sigma m^{2}=m_{1}{ }^{2}+m_{2}{ }^{2}+m_{3}{ }^{2}+$ etc. It follows, therefore, from eq 51 and 52 that

$$
\frac{S}{P}=n^{2}\left(1+\frac{1}{n} \sum m^{2}\right)
$$

There is no difficulty in adjusting a group of standard resistors or coils so that the resistance of no one differs from the mean of all by more than a few, or even 1 part in 10,000, in which case $\Sigma m^{2} / n$ would be not more than 1 part in 10 million or 1 part in 100 million, so usually $\Sigma \mathrm{m}^{2} / n$ may be neglected. However, the conductors used in making the series and parallel connections will in general have resistances which cannot be neglected.

Presumably Lord Rayleigh was the first to use the same coils connected alternately in parallel and in series [72] in building up from unit standards. Steps of $4,9,-16,25$, etc. are readily obtained simply by changing the connections of the appropriate numbers of coils of approximately equal resistances from parallel to series. Unfortunately, the square root of 10 is not an integer, so a step of 10, which is most frequently needed, cannot be obtained directly in this way.

In this Bureau an auxiliary apparatus constructed in 1912 is used for finding the reading of the dial switches of the $A$ arm of the bridge for which the ratio of $A$ to $B$ is accurately 10 to 1 . The circuit of this apparatus is shown in figure 8. It will be observed that there are seven resistance sections, six of $150 \mathrm{ohms}$ each, and one of 50 ohms, all connected in series. Two amalgamated copper links not shown in the figure serve to connect either group of three of the 150-ohm sections in parallel. Also other amalgamated copper links are provided FIGURE 8-Circuit diagram of the auxiliary for use in determining the relative resistances of the sections. The resistances of the links are relatively low and definite and

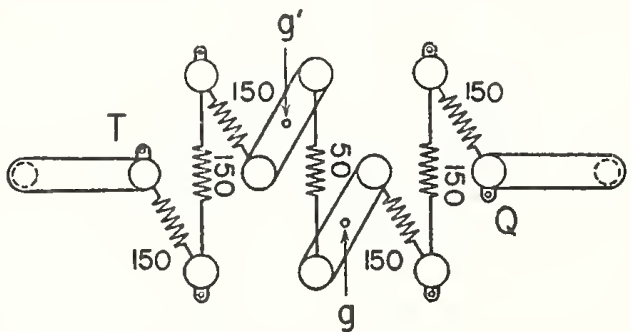
FIGURE 8.-Circuit diagram of the auxiliary
apparatus used for determining the reading of the dial switches of the NBS precision bridge, for which the ratio of the resistance of the $A$ arm to the resistance of the B arm is 10 to 1 .

the arrangement is such that the

resistance of each link, including two amalgamated contacts and the appropriate portion of each of two terminal blocks may readily be measured. In the appendix on terminals and contacts, it will be shown that the effert of the resistances of parts of terminal blocks not included as parts of links or as parts of resistance sections is negligibly small. All other connectors are so arranged that the effect of their resistance is eliminated in the procedure followed in the use of the apparatus.

When this apparatus is placed in the bridge with a galvanometer connection made at $g$, it constitutes the $X$ and $Y$ arms of the bridge. With the three sections on the right connected in parallel and all other sections in series, the ratio of the resistance of the series sections 
to the resistance of the parallel sections, that is, the ratio of $X$ to $Y$, is very nearly 10 to 1 . With $A$ set at a nominal value of $100 \mathrm{ohms}$, $B$ set at a nominal value of $10 \mathrm{ohms}$, and with no part of the auxiliary apparatus in the $A$ or $B$ arms, the bridge is balanced by an adjustment of the $A$ arm and the reading of the dial switches recorded. Then with the battery leads transferred to the points $T$ and $Q$, the bridge is again balanced by an adjustment of the $A$ arm and the reading of the dial switches recorded.

In the first balance, the resistance of the connection between the normal junction of the $X$ and $A$ arms and the point $T$ is in the $X$ arm, while in the second balance this resistance is in the $A$ arm. Likewise, in the first balance the resistance between the normal junction of $Y$ and $B$ and the point $Q$ is in the $Y$ arm, while in the second it is in the $B$ arm. Since the ratio of $A$ to $X$ and $B$ to $Y$ is 1 to 5 , one-sixth of the difference between the two readings [111] of the dial switches applied to the first in the direction which moves it towards the second corrects for the error which otherwise would result from the resistances of the end connectors of the auxiliary apparatus. Now the auxiliary apparatus is turned through $180^{\circ}$, the parallel connectors are transferred to what is now the right side, and the galvanometer connection is made at $g^{\prime}$, so as to again place the 50 -ohm section in the $X$ arm of the bridge. Then two additional balances of the bridge are established and weighted as just described, giving a second reading of the dial switches, corrected for the effect of the resistances of the end connectors. Taking the mean of these two readings corrects for the difference in the mean resistance of the three sections on the right and the three sections on the left, as shown in figure 8 . It is therefore the reading of the dial switches for which the ratio of $A$ to $B$ is accurately 10 to 1 , excepting a correction to account for the lack of strictly proper adjustment of the 50 -ohm section and a further correction to account for the resistances of the paralleling connectors. The first of these is -0.1 , the amount expressed in proportional parts by which the resistance of the 50-ohm section exceeds the mean resistance of the other six sections taken three at a time in parallel, and corrected for the resistances of the paralleling connections, while the second is $+8 / 9$ the mean resistance of the paralleling connections expressed in proportional parts of $50 \mathrm{ohms}$. These corrections (which amount to only a few parts in 1 million and are easily determined to 1 part in 10 million) applied to the mean of the two readings, referred to above, give the reading $a_{r}$ of eq 43,44 , and 45 , corresponding to a. ratio of $A$ to $B$ equal to 10 to 1 , with a probable error not in excess of 1 part in 3 million. However, to obtain this precision requires interpolation from galvanometer deflections, since the apparatus reads directly only to 1 part in 1 million. The reading of the dial switches of the $A \mathrm{arm}$ of the bridge for which the ratio of $A$ to $B$ is 10 to 1 must be known to a high accuracy, since (starting with 1 -ohm standards, which are used in maintaining the unit) any error in this reading enters once in the evaluation of the 10 -ohm standards, twice in the evaluation of the $100-\mathrm{ohm}$ standards, three times in the evaluation of the 1,000-ohm standards, and four times in the evaluation of the 10,000-ohm standards. It also enters once in the evaluation of the $0.1-\mathrm{ohm}$ standards, twice in the evaluation of the 0.01 -ohm standards, three times in the evaluation of the $0.001-\mathrm{ohm}$ standards, and four times in the evaluation of the 0.0001-ohm standards. 
When it is desired to find the reading of the $A$ arm for which the ratio of $A$ to $B$ is 2 to 1 , the procedure is as follows:

1. The $A \mathrm{arm}$ is connected so that its nominal resistance is 100 ohms, the $B$ arm is connected so that its nominal resistance is 50 ohms, the $X$ arm is arranged to receive two standard resistors connected in series, and the $\bar{Y}$ arm is arranged to receive one standard resistor.

2. Three standard resistors having the same nominal value (preferably $100 \mathrm{ohms}$ ) are used.

3. The bridge is balanced by an adjustment of the $A$ arm with each of the three standard resistors placed one after the other in the $Y$ arm, the other two being in the $X$ arm.

4. The mean of the three readings of the $A$ arm, after a correction is applied to account for the resistance of connectors, is taken as the reading of the $A$ arm for which the ratio of the resistance of the $A$ arm to the resistance of the $B$ arm is 2 to 1 .

A similar procedure is followed in determining the reading of the $A \mathrm{arm}$ for which the ratio of $A$ to $B$ is 3 to 2,3 to 1,2 to 3,1 to 3 , 1 to 2 , or involves other small integers.

\section{ADJUSTMENTS OF THOMSON BRIDGE}

A simplified diagram of the bridge circuit when the Thomson bridge method is used, is shown in figure 9. Here, $x_{1}$ represents one of the

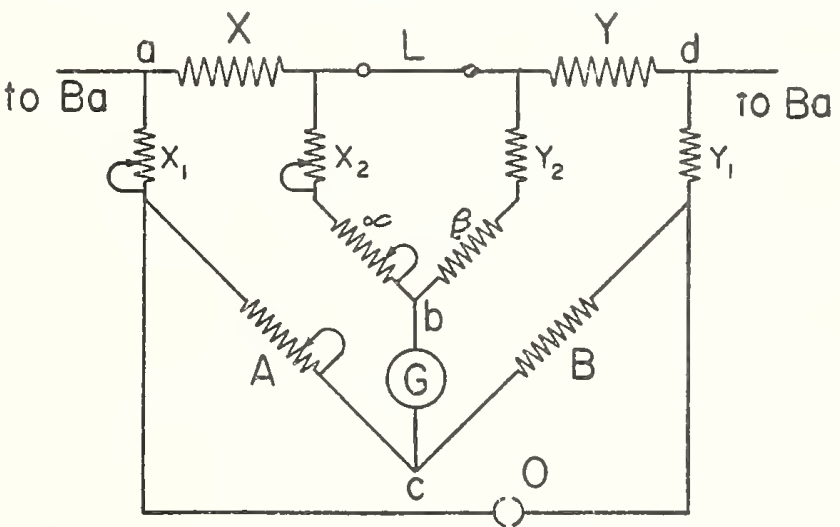

FIGURE 9.-Circuit diagram of the Thomson bridge arranged for explanation of the procedure followed in establishing balances of the bridge in such a way as to make $A / B=X / Y$.

potential terminals of $X$, the potential lead, and a rheostat continuously adjustable through a range of about $0.01 \mathrm{ohm} ; y_{1}$ represents one of the potential terminals of $Y$, the potential lead, and a resistance which for the purpose at hand may be considered as fixed, though actually it is adjustable in steps; $x_{2}$ is similar to $x_{1}$; and $y_{2}$ is similar to $y_{1}$. L and $O$ represent connectors of rather low resistance, which may be opened or closed at will.

The adjustments are carried out as follows:

(1) With the connector $L$ closed and the connector $O$ open, the direct-reading arms, $A$ and $\alpha$ are so adjusted as to give a balance of the bridge. This makes

$$
\frac{A+x_{1}}{B+y_{1}}=\frac{X}{Y} \text { approximately. }
$$


(2) With the connectors $L$ and $O$ both closed, the bridge is balanced by an adjustment of $x_{1}$. These two adjustments make

$$
\frac{x_{1}}{y_{1}}=\frac{X}{Y} \text { approximately. }
$$

(3) With the connectors $L$ and $O$ both open, the bridge is balanced by an adjustment of $x_{2}$ [74]. This makes

$$
\frac{X+x_{2}+\alpha}{Y+y_{2}+\beta}=\frac{A+x_{1}}{B+y_{1}}
$$

(4) With $L$ closed and $O$ open, the bridge is again balanced by an adjustment of the direct-reading arms which, as stated above, are mechanically connected so always read the same.

These four adjustments give

$$
\frac{X}{\bar{Y}}=\frac{A}{\bar{B}}
$$

usually to the precision desired or attainable. The process is really one of successive approximations, but the observer soon learns to judge from the magnitude of the change in the reading of the dials in making the fourth adjustment and the known precision required in the various adjustments, if the adjustments need be repeated. Equation 56 is the same as the Wheatstone bridge equation. Therefore, in making a series of measurements, the data recorded and all calculations are the same as though the Wheatstone bridge method had been used. That is, only the readings of the dials for each of the final balances of the bridge are recorded, and these are substituted in the appropriate equation $36,42,44$, or 45 considered above.

Since $x_{1} / A$ and $y_{1} / B$ seldom exceed 0.001 , while in the final adjustment $x_{1}$ is in series with $A$ and $y_{1}$ is in series with $B$, the precision required in the second adjustment may be two or three orders lower than that sought in the measurement. Furthermore, the precision required in the third adjustment need not be high, and in many cases this adjustment may be omitted. An important feature of the procedure is that the adjustments result in making $X / Y=A / B$, whether or not the four-terminal conductors, $X$ and $Y$, are linear.

The simplicity of the adjustments results more from having suitably designed and well-constructed apparatus than from the procedure followed, which differs only in a minor detail from that described by Jaeger and Diesselhorst [45]. Procedures for determining the correction terms [P] of the Thomson bridge equation or for making these terms negligibly small have been discussed by several of those who have had occasion to use the Thomson bridge method.

\section{PRECISION AND ACCURACY}

The expected precision of the measurements may not be fully realized on account of slightly faulty insulation in the bridge or in one of the resistances being measured, static electrical effects, rapidly varying thermoelectromotive forces, etc. Whether or not it is actually obtained depends to a large extent on the temperament and skill of the observer. To be properly qualified he should have a desire to do 
the job well, be neither easily fatigued nor perturbed, recognize slight disturbances promptly and be able to locate their source and correct the difficulty, and, above all else, be able to differentiate between that which is essential and that which is not essential in each of eight orders of magnitudes.

At present the resistance of no standard resistor can be presumed to be known (either in international ohms ${ }^{3}$ or in absolute ohms) to an accuracy within one order of the precision to which two or more nominally equal resistances can be compared. (if within the range considered here) or quite to the precision to which an 0.0001-ohm standard may be compared with a 10,000-ohm standard (provided a sufficient number of intermediate steps is used). It might seem, therefore, that the precision considered here is unnecessarily high. However, the precision of the resistance measurements is a factor contributing somewhat to the accuracy to which units of resistance may be realized and is an important factor in selecting the particular standard resistors used in maintaining a unit of resistance. For judging the relative quality of standard resistors, a precision of 1 part in 1 million is really needed, and it is a great convenience that for the most part this is readily obtained. As an indication of a limited need for a somewhat higher precision, it may be pointed out that the resistance of each of a fairly large group of 1-ohm standard resistors (of the double-walled type constructed by Thomas in 1933) is remaining so constant relative to their mean resistance that only by making the comparisons to a precision of about 1 part in 10 million can the changes occurring during a few months be detected.

In the above discussions a stated precision represents twice the probable error of a single measurement. The precision is estimated from the extent of the agreement of results on repetitions of the measurement under different conditions, or the consistency of results obtained in case more than the minimum required number of measurements is made.

For example, if five 1-ohm standards are to be intercompared, they may be substituted one after another in the same arm of the bridge. The five balances of the bridge thus obtained give data from which the resistance of each standard may be calculated in terms of the resistance of any one or the mean resistance of any two or.more. Repetitions of these measurements under conditions giving different distributions of the systematic errors furnish data for determining the probable error of a single measurement by the substitution method, that is, the probable error of each of the five results obtained from five balances of the bridge.

The same five standards may be compared by interchange between the $X$ and $Y$ arms of the bridge in all possible combinations. The data obtained from the 20 balances of the bridge then serve in calculating the resistance of each standard in terms of the resistance of any one or the mean resistance of any two or more, the probable error in the results and the probable error in a single measurement by the interchange method. If all balances of the bridge were equally reliable, the probable error in the results obtained from the 20 balances of the bridge would be smaller than that obtained from five balances

\footnotetext{
3 Here by "international ohm" is meant a unit of resistance realized by the use of a column of mercury under specified conditions, as disting uished from the unit of resistance of this country or the mean of the units of resistance of several countries.
} 
of the bridge; also the probable error of a single measurement by the interchange method would be smaller than by the substitution method.

In the course of time many sets of measurements are made and repeated, so fairly definite estimates may be made of the precision obtained in individual measurements under various conditions. In repetitions of measurements after one or more days, the agreement of results occasionally is not as good as would be expected from the estimated precision of the measurements. In such cases, extending the measurements over longer periods of time usually leads to the conclusion that changes in the standard resistors are mainly responsible for the lack of agreement of results. Consequently, there is little increase in the accuracy of the measurements other than the detection of a possible error in recording a bridge reading, by making more than the minimum required number of balances of the bridge. 'Therefore, in comparing a number of standard resistors, usually this is done by the substitution method, making the minimum required number of balances of the bridge, and then repeating this series of measurements one or more days later, under conditions giving different readings of the $A$ arm of the bridge. In case the results obtained from the two series of measurements are as consistent as should have been expected, means of the values thus obtained are taken as the results of the measurements. In case of a lack of a reasonable agreement in the results, further measurements are made to determine the cause and to remedy the difficulty should it be found to be a defect in the measuring apparatus instead of changes in the resistance of one or more of the standard resistors used in the series of measurements. In certificates for standard resistors, usually the stated accuracy of the values given is determined not by the precision of the measurements but by the uncertainty of the values in international ohms of the standard resistors used in maintaining units of resistance and the estimated changes with time of the resistances of the standard resistors being certified. More frequently than otherwise, the stated accuracy is 0.005 percent.

\section{APPENDICES}

In the above discussion a number of important points have been passed over with little or no consideration. Some of these will now be considered somewhat in detail under the headings: Terminals and Contacts; Thermoelectromotive Forces; Insulation; Optical System; Methods of Analyses; Ohm's Law; and Units of Resistance; while others will be found discussed in publications to which reference is made.

\section{APPENDIX 1. TERMINALS AND CONTACTS}

(a) STANDARD RESISTORS

For the resistance of a conductor to be definite, one of several requirements is that the current always enter and leave the conductor in such a way as to give always the same or an equivalent distribution of the current density in that part of the conductor between the particular equipotential surfaces which serve in limiting or defining the resistance. In addition, the potential drop must always be taken between the same two or equivalent equipotential surfaces. Each time a standard resistor is removed from and replaced in a circuit in which there is an electromotive force sume change occurs in the current distribution over the surfaces through which the current enters and leaves the standard, and the potential drop cannot always be taken between exactly the same two or equivalent equipotential surfaces. It will therefore be of interest to see what conditions are neces- 
sary in order that the resistance be definite to 1 part in 1 million, considering only the effects of contacts and current and potential distributions in terminals. But first it will be necessary to state more precisely what is to be understood by the resistance, or rather the resistances, of a conductor.

Since the more important resistances of a four-terminal conductor are more definite than is the resistance of a two-terminal conductor, four-terminal conductors will be considered first. Referring to figure 10, the heavy lines designated $1,2,3$, and 4 will be considered as representing surfaces on which connections to other conductors may be made, that is, they will represent terminals. In case 1 and 4 are normally used as current terminals and 2 and 3 are normally used as potential terminals, the resistance may be defined as the ratio of the drop in potential from 2 to 3 to the current entering on 1 and leaving on 4 . This will be designated the resistance (1234). In case 2 and 4 are used as current terminals and 1 and 3 as potential terminals, the resistance may be defined as the ratio of a drop in potential from 1 to 3 to the current entering on 2 and leaving on 4 . This will be designated the resistance (2134).

It will thus be seen that a four-terminal conductor has as many four-terminal resistances as there are permutations of four numbers. However, only two of

2

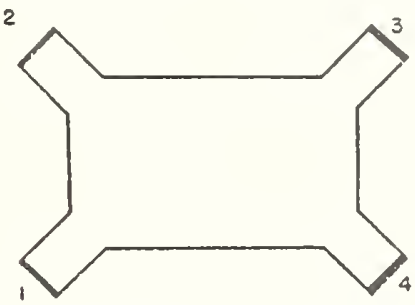

FIGURE 10.-Generalized four-terminal conductor. these resistances are independent of each other, and the relations between all are rather simple. Since there are so many resistances, it is a convenience to divide them into groups and to attach distinctive names to these groups. The four equal resistances (1234), (2143), (4321), and (3412) will be called the direct resistance. The four equal resistances (1432), (4123), (2341), and (3214) will be called the cross resistance, and the four equal resistances (1243), (2134), (3421), and (4312) will be called the diagonal resistance. When defined in this way, the direct resistance minus the cross resistance equals the diagonal resistance, as was pointed out in a previous paper [101]. Furthermore, any one of the 12 remaining four-terminal resistances is equal in magnitude but opposite in sign to either the direct, the cross, or the diagonal resistance.

It is also a convenience to divide four-terminal conductors into groups in accordance with the relative magnitudes of the resistances and attach names to these groups. Conductors for which the cross resistance is less than a millionth part of the direct resistance will be called linear, ${ }^{*}$ and all others will be called nonlinear.

If variations in the current and potential distribution on terminals are to affect the direct resistance by certainly less than 1 part in a million, the terminals must be so designed that the sum of the following potential ratios will be less than 0.000001 . With 1 and 4 used as current terminals, (1) the potential difference between any two points on 2 to the potential difference between 2 and 3 ; (2) the potential difference between any two points on 3 to the potential difference between 2 and 3 , and with 2 and 3 , used as current terminals; (3) the potential difference between any two points on 1 to the potential difference between 1 and 4; and (4) the potential difference between any two points on 4 to the potential difference between 1 and 4 . This condition is generally realized in standard resistors designed for precise measurements, but is seldom realized in standard resistors designed for use with large currents. In many cases the resistance depends upon the manner of connecting the current leads to the current terminals to the extent of 0.01 percent, and in exceptional cases to the extent of 1 percent.

The resistance of a two-terminal resistor may be defined as the ratio of the potential drop to the current, taken between two equipotential surfaces lying partially in the terminals of the resistor and partially in the conductors through which the current enters and leaves the resistor. From an experimental standpoint, it is better to define the resistance of a two-terminal resistor as the difference in resistance of two four-terminal conductors which are the same in all respects, except that one does and the other does not include the two-terminal resistor.

4 It should be noted that the distinction made here between a linear and nonlinear conductor does not involve a proportionality and lack of proportionality of the potential drop to the current. 
To illustrate what is meant by this statement and to show that the resistance so defined is not strictly a constant, let it be assumed that there is at hand a short copper rod $1 \mathrm{~cm}$ in diameter and having plane end surfaces which are amalgamated and that the resistance of this rod is to be measured. Also let it be assumed that there are at hand two sets of cylindrical copper terminal blocks having plane amalgamated end surfaces, the diameter ot one set of blocks being $1 \mathrm{~cm}$ and the diameter of the other set of blocks being $2 \mathrm{~cm}$. Each terminal block has a flexible current lead and a flexible potential lead. If (1) the copper rod is placed between the amalgamated surfaces of the terminal blocks having the smaller diameters and the resistance of the four-terminal conductor thus constituted is measured; if (2) the copper rod is removed, the amalgamated surfaces of terminal blocks are placed in contact, and the resistance of the four-terminal conductor thus constituted is measured; and if (3) the latter measured value is subtracted from the former measured value, a value is obtained for the resistance, as defined, of the copper rod. This resistance will in general be slightly higher than that which may be calculated from the known resistivity and dimensions of the copper rod. Now, if this procedure is repeated, using the terminal blocks having the larger diameter, a still higher value will be obtained for the resistance of the copper rod, provided the contacts between the rod and terminals are equally good in the two cases, and provided also that when terminal blocks are placed together, the contact is such as to give a substantially uniform current density over the contact area. In the first case, one amalgamated contact is included in the measured resistance. In the second case, there is in addition a nonuniformity of current density in the vicinity of the contacts, which in effect increases the measured resistance.

Obviously, therefore, the resistance of a two-terminal standard resistor depends to a greater or less extent on the manner in which it is connected into a circuit. For example, when a two-terminal standard resistor is placed in an arm of a Wheatstone bridge, the bridge arm, which is a four-terminal conductor, consists of the standard and the end connectors. If the terminals of the standard and of the end connectors are amalgamated, the end connectors consist of two amalgamated surfaces or mercury cups and two terminal blocks of the bridge. With end connectors of the usual type, that is, having deep mercury cups, the resistance of the bridge arm may depend on the amount of the mercury in the cup and the position of the terminals of the standard in the cup to the extent of a few microhms. This difficulty is obviated to a considerable degree by the use of plane amalgamated surfaces, instead of mercury cups.

What is of more importance than the form of the contact surfaces is the gradual accumulation of copper amalgam in the solid phase on the terminals of standard resistors and on end connectors or contact blocks. Cases have bcen observed in which the removal of the amalgam in the solid phase has resulted in a lowering of the resistance by more than 10 microhms (though a definite reason for so large a change has not been found). Even when the amalgamated surfaces of the terminals of the standard resistor and of the terminal blocks with which it may be used are apparently in good condition, the resistance may be expected to depend on the resistances of the amalgamated contacts and the distribution of the current density in their vicinity to the extent of a few microhms.

\section{(b) TERMINAL BLOCKS OF BRIDGE}

The more important terminal blocks of the NBS precision bridge and all terminal blocks of the auxiliary apparatus used in establishing known resistance ratios have four or more terminals.

A diagram of a main terminal block of the bridge which is used when the $A$ arm is 25 or $100 \mathrm{ohms}$ is shown in figure 11 (a). The block is constructed of copper, and terminals 1,2,4, and 6 are copper wires soldered in holes drilled in the under side of the block. Terminal 3 is a plane amalgamated surface (not a mercury cup), and terminal 5 is a small mercury cup. Terminals 1 and 5 are so located with respect to the other terminals that they may be considered as equivalent, unless both are used at the same time. Terminal 6 is connected to the $900-0 \mathrm{hm}$ section of the $A$ arm. The main terminal block normally used with the $B$ arm of the bridge, when this arm has a resistance of $25 \mathrm{ohms}$ or $10 \mathrm{ohms}$, is similar to that shown in figure 11 (a) except that it has no terminal 6 . When the Wheatstone bridge method is used, terminals 1 (or 5), 3 and 4 are used as shown in figure 11 (b). When the Thomson bridge method is used, terminals 1,2 , and 4 are used as shown in figure 11 (c). Consequently, the current distributions in these blocks are not the same in the two cases, and as a result the contributions of one terminal 
block to the resistance of the $A$ arm and of the other terminal block to the resistance of the $B$ arm are not the same. When the Thomson bridge method is used the resistance contributed to the $A$ arm or to the $B$ arm by its terminal block is higher than when the Wheatstone bridge method is used, by the four-terminal resistance (1234).

Since the reading of the $A$ arm of the bridge for which the ratio of $A$ to $B$ is 10 to 1 is determined by the Wheatstone bridge method and this reading is used with both the Wheatstone and the Thomson bridge methods, the effect of these resistances should be known. Measurements of these resistances give values ranging from -0.3 to $+0.3 \mathrm{microhm}$, depending upon the point on the amalgamated

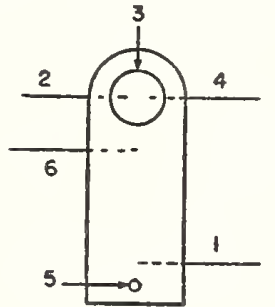

a

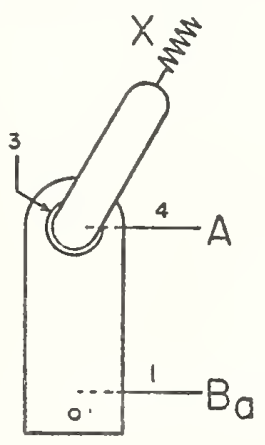

b

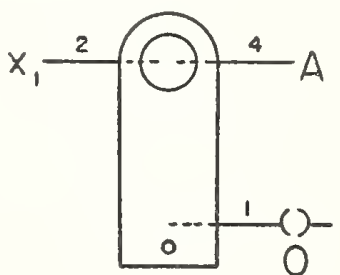

C

FIGURE 11.-A main terminal block of the NBS precision bridge.

$a$ shows the six terminals of the terminal block, $b$ shows the three terminals used with the Wheatstone bridge method, and $c$ shows the three terminals used with the Thomson bridge method.

surface serving as terminal 3. However, as used, the connection is distributed somewhat uniformly over the central portion of the surface, so probable values lie in the range from -0.1 to $+0.1 \mathrm{microhm}$. Therefore, since the lowest resistance used in the $A$ arm is 25 ohms and the lowest resistance used in the $B$ arm is 10 ohms, changes in the current distribution resulting from a change from one bridge method to another and from variations in contacts on the amalgamated surfaces affect the ratio of $A$ to $B$ only a few parts in 100 million.

The contribution of these terminal blocks to the resistances $x_{1}$ and $y_{1}$ when the Thomson bridge method is used is of no consequence, since adjustments make $\left(A+x_{1}\right) /\left(B+y_{1}\right)=(A / B)=(X / Y)$. In addition to the resistance of an amalgamated surface, these terminal blocks contribute approximately 1 microhm to the resistance of the $X$ arm and $Y$ arm of the bridge when the Wheatstone bridge method is used. However, if a precision of 1 part in 1 million is expected, the resistances of the $X$ and $Y$ arms must be $10 \mathrm{ohms}$ or more, or the conductors must be of the four-terminal type. When the conductors are of the four-terminal type, all connecting conductors can be changed from one to another of two adjacent arms of the bridge [111], and this makes it possible to apply a correction to account for the resistances of connectors, including amalgamated contacts.

\section{(c) TERMINAL BLOCKS OF AUXILIARY APPARATUS}

In the previous discussion of the auxiliary apparatus, used in determining the reading of the dial switches of the $A$ arm of the bridge for which the ratio of $A$ to $B$ is 10 to 1 , very little was said concerning corrections to account for the effect of the resistances of the terminal blocks. This matter will now be considered in detail.

Each of these terminal blocks is a four-terminal conductor and consequently has two independent four-terminal resistances. One of the end terminal blocks is shown in elevation in figure 12 and in plan in figure 8 . Here terminal 3 is an amalgamated surface on which a copper link rests when the three resistance sections adjacent to the terminal block are connected in parallel, terminal 4 is a part of one of the 150-ohm resistance sections, terminal 2 is a small mercury cup which is used as a potential (or current) terminal in measuring the resistance of the paralleling connection, that is, of the copper link, the two amalgamated con- 
tacts and a portion of each of two terminal blocks. Terminal 2 is also used as a current or potential terminal, both when the three 150 -ohm resistance sections adjacent to it are connected in series and when they are connected in parallel. Terminal 1 serves in connecting the apparatus into the bridge. With the parallel connection two-thirds of the current is through terminal 3, one-third of the current is through terminal 4 , and all of the current is through terminal 2 or terminal 1. In measuring the resistance of the paralleling connection, all of the current is through terminal 3 and all through terminal 1 or terminal 2 . With the series connection, all the current is through terminal 1 or terminal 2, and all through terminal 4.

One of the terminal blocks to which two 150-ohm sections are connected is shown in elevation in figure 13 and in plan in figure 8 . Here terminal 3 is an amalgamated surface on which a copper link rests when the resistance sections terminating in the block are connected in parallel, and 2 is a small mercury cup used as a terminal in measuring the resistance of the paralleling connection.

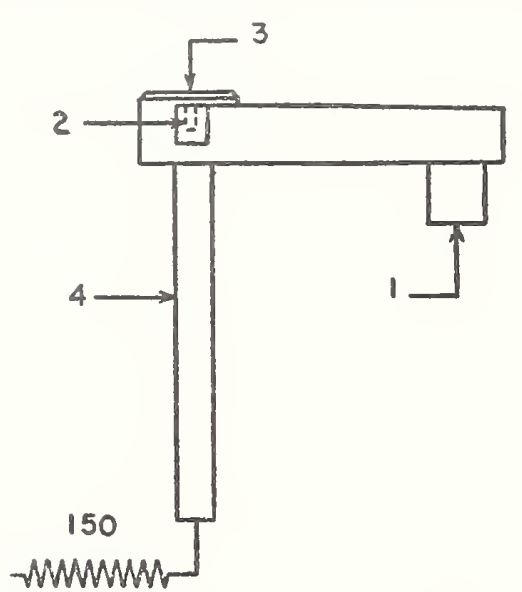

Figdre 12.-An end terminal block of auxiliary apparatus.

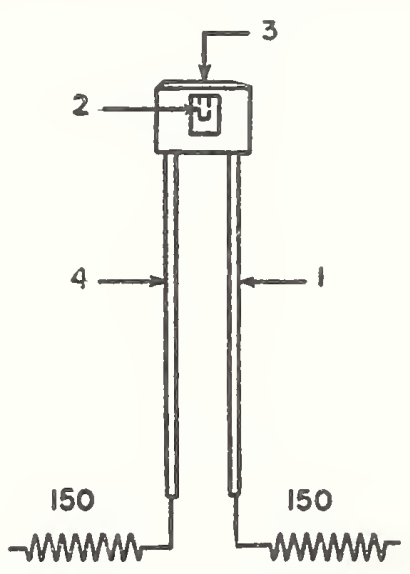

FIgURe 13.-A terminal block of auxiliary apparatus to which two 150-ohm resisfance sections are connected.

Terminal blocks of this type are also used with three different current distributions.

Consequently, the effects of the four-terminal resistances of these terminal blocks are more complicated than the effects of the four-terminal resistances of the main terminal blocks of the bridge, in which it was necessary to consider only two current distributions in each block.

In the use of the auxiliary apparatus, what is of most importance is the ratio of the four-terminal resistances of each of the two groups of three 150-ohm resistance sections when connected in series, $R_{s}$, to their four-terminal resistances when connected in parallel, $R_{\nu}$. The effect of slight inequalities in the resistances of the 150-ohm sections of each group has already been discussed, and here it will be assumed that this effect is too small to require consideration. Since the problem is complicated, only the results which have been obtained will be given here, while the analysis will be given in the appendix on methods of analyses. With the notation shown in figure 14 , the ratio

$$
\begin{aligned}
& R_{\delta} / R_{p}=9\left\{1-4\left[L_{1}+L_{2}+\right.\right.(1234)_{a}-(1432)_{a} / 2-(1234)_{b}+ \\
&\left.\left.(1432)_{b}-(1234)_{c}+(1432)_{c}+(1234)_{d}-(1432)_{d} / 2\right] / 450\right\}
\end{aligned}
$$

Also the resistance of terminal 1 of terminal block $a$, that is, the potential drop from 1 to 2 of $a$, to the current entering on 1 of $a$ and leaving on 1 of $d$, and which will be designated the resistance $\left(1_{a} 1_{a} 2_{a} 1_{d}\right)$, is higher by two-thirds the resistance $(1432)_{a}$, and the resistance of terminal 1 of terminal block $d,\left(1_{d} 1_{d} 2_{d} 1_{a}\right)$, is higher by two-thirds the resistance (1432) $)_{d}$ with the parallel connection than with the series connection. The former is taken into account by the procedure followed in the use of the apparatus. However, the latter represents the increase in resist- 


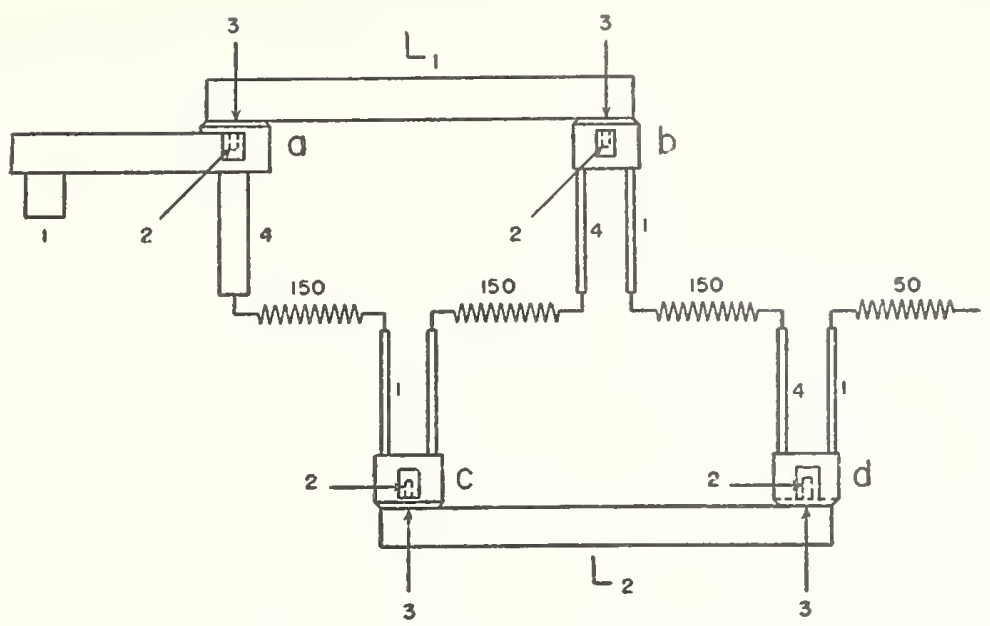

FIGURE 14.- Parallel connection of three 150-ohm resistance sections of the auxiliary apparatus.

This connection involves the four terminal blocks, disignated $a, b, c$, and $d$, and two paralleling connectors, designated $L_{1}$ and $L_{2}$. The series connection is made by removing the paralloling connectors.

ance of the $50-\mathrm{ohm}$ section resulting from the change of the connection of three 150 -ohm sections from series to parallel. In eq 57, $\mathrm{L}_{1}$ represents the four-terminal resistance of the paralleling connection between terminal blocks $a$ and $b$, using 1 and 2 of terminal block $a$, and 1 and 2 of terminal block $b$, as the terminals (the measurement being made with the other paralleling connection open). A similar statement applies to $L_{2}$, and $(1234)$ represents the direct resistance of terminal block $a,(1432)_{a}$ the cross resistance of terminal block $a$, etc. A similar solution applies to the other group of three $150-$ ohm sections. To this point, three different current distributions in each of the eight terminal blocks of the auxiliary apparatus have been considered.

However, the relative values of resistance sections must be determined, and measurements of these require additional current distributions in at least some of the terminal blocks. Of these additional current distributions, the two in each of the central terminal blocks, $d$ and $d^{\prime}$, which are of the type shown in figure 15, are of most importance. The additional current distributions in the central terminal blocks are involved in a determination of the ratio of the resistance of the 50-ohm section to the resistance of the six 150 -olim sections connected in series. The measurements used in this determination

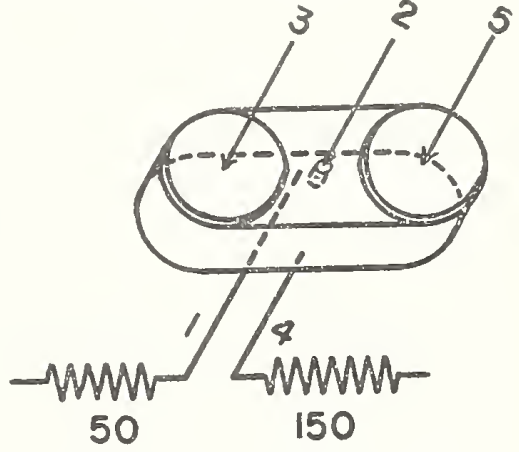

Figure 15.-A central terminal block of the auxiliary apparatus. consist in comparisons of the resistance of the 50 -ohm section with the resistance of one and then of the other of the groups of three 150-ohm sections connected in parallel.

In the measurement of the resistance of one group of three 150 -ohm sections connected in parallel, 1 and 2 of terminal block $a$ and 5 and 2 of terminal block $d$ are used as the four terminals. In the measurement of the resistance of the other group of three 150-ohm sections connected in parallel, 1 and 2 of terminal block $a^{\prime}$ and 5 and 2 of terminal block $\mathrm{d}^{\prime}$ are used as the four terminals. These resistances will be referred to as the resistances $\left(1_{a} 2_{a} 2_{d} 5_{d}\right){ }_{p}$ and $\left(1_{a^{\prime}} 2_{a^{\prime}} 2_{d^{\prime}} 5_{d^{\prime}}\right)_{p}$. The resistance of the 50 -ohm section is measured, using 5 and 2 of terminal block $d$ and 5 and 2 of terminal block $d^{\prime}$ as the four terminals. This will be referred to as the resistance $\left(5_{d} 2_{d^{\prime}} 2_{d^{\prime}} 5_{d^{\prime}}\right)$. The addition of the resistance $\left(2_{a} 5_{d} 1_{d} 2_{d}\right)_{p}$ to the resistance $\left(1_{a} 2_{a} 2_{d} 5_{d}\right)_{p}$ gives the resistance $\left(1_{a} 2_{a} 2_{d} 1_{d}\right)_{p}$, which, when multiplied by the ratio given by eq 57 , gives the resistance $\left(1_{a} 2_{a} 2_{d} 1_{d}\right)_{s}$, that is, the four-terminal resistance of the three $150-0 h m$ sections in series and with the normal distribution 
of current in each of the four-terminal blocks involved. The resistances of the other group of three $150-\mathrm{ohm}$ sections in series is obtained in the same way. The addition of the resistances $\left(2_{d} 4_{d} 5_{d} 2_{d^{\prime}}\right)$ and $\left(2_{d^{\prime}} 4_{d^{\prime}} 5_{d^{\prime}} 2_{d}\right)$ to the resistance $\left(5_{d} 2_{d} 2_{d^{\prime}} 5_{d^{\prime}}\right)$, the measured resistance of the 50 -ohm section, gives the resistance $\left(4{ }_{d} 2_{d} 2_{d^{\prime}} 4_{d^{\prime}}\right)$, that is, the resistance of the 50 -ohm section with what may be considered as the normal distribution of current in the its terminal blocks, $d$ and $d^{\prime}$. The result of these measurements may be expressed in the form

$$
\frac{18 R_{50}}{R_{150}+R_{150}+R_{150}+R_{150}+\mathrm{R}_{150}+R_{150}}=1+a \text {. }
$$

Here $a$ is the amount, expressed in proportional parts, by which the resistance of the $50-\mathrm{ohm}$ section exceeds the resistance of the six 150 -ohm sections in series divided by 18 , with what may be considered as the normal current distribution in all terminal blocks. As has previously been pointed out, $-a / 10$ is a correction which must be applied in the use of the apparatus.

In the design of these terminal blocks and in the selections of values for the resistances of sections, an effort was made to make the effects of the different current distributions in the terminal blocks negligibly small. The extent to which this aim was realized may be seen from the relations stated above and the fact that measurements of the four-tcrminal resistances of the terminal blocks give values for each ranging from about -0.5 to +0.5 microhm, depending mainly on the part of the amalgamated surface serving as terminal 3 . However, in use, each connection is distributed somewhat uniformly over the entire amalgamated surface, and under these conditions it is hardly probable that any of the four-terminal resistances lie outside the range from -0.3 to +0.3 microhm. Presumably, therefore, changes in current distribution in terminal blocks and systematic errors in the determination of the resistances of paralleling connections of the auxiliary apparatus contribute not more than 1 part in 50 million to the urcertainty of the reading of the $A$ arm of the bridge, for which the ratio of $A$ to $B$ is 10 to 1 .

Consequently, mcasurements of the relative values of the resistance sections and of the parelleling connections of the auxiliary apparatus may be made with any connection found convenient, provided only that each resistance section and each paralleling connection is considered as a four-terminal conductor.

What is of more importance than the resistances of the terminal blocks is the resistance of the amalgamated contacts included in the paralleling connections. Each of these contacts has a surface on the terminal block and another surface on the connecting link. The surfaces of the terminal blocks lie substantially in the same plane, and each has an area of approximately $1.25 \mathrm{~cm}^{2}$. The under side of each connecting link is a plane, and the dimensions are such that when two terminal blocks are connected by a link, the entire contact areas of each of the two terminal blocks are effective. A few weeks, at most, previous to the use of the apparatus, the surfaces of terminal blocks and connecting links are polished with fine emery paper backed by a piece of plate glass or other plane surface having an area of not less than $300 \mathrm{~cm}^{2}$ until the copper may be seen distinctly, indicating the removal of practically all of the accumulated copper amalgam in the solid phase. The surfaces are then reamalgamated, using the sodiumamalgam process. In placing links on the terminal blocks, they are moved back and forth parallel to the amalgamated surfaces until they slide freely indicating the removal from between the surfaces of any loose solid copper-amalgam or other solid material. With these precautions, a link may be removed and replaced by another of the same dimensions without changing the resistance of the parallcling connection by more than a few tenths of a microhm. In addition, the measured resistance of each parallel connector is in agreement with the value estimated from the dimensions and the resistivity of copper. The liberal design of these contacts, the possibility of removing the copper-amalgam in the solid phase without impairing the mechanical fit, and the exceptional precautions taken in their use result in a very much better performance than is realized with the contacts between standard resistors and terminal blocks of the NBS precision bridge.

Furthermore, if the average resistance of all contacts used in the paralleling connections were 1 microhm higher, or lower, at the time of use than at the time of measurement, this would result in an error in the determination of the reading of the $A$ arm of the bridge, for which the ratio of $A$ to $B$ is 10 to 1 , of less than 1 in 25 million. Consequently, it is not necessary that the resistances of the paralleling connectors be measured when and as used. 


\section{(d) THOMSON BRIDGE}

When the Thomson bridge method is used with the battery and galvanometer in their normal positions there is current through each of the four terminals of each of the two four-terminal conductors designated $X$ and $Y$ in figures 9 and 16 . There may therefore be some question as to which if any of the four-terminal resistances of $X$ and $Y$ are compared.

If the bridge is balanced according to the procedure outlined above or any other giving the same result, the connection through $L$ may be opened or elosed without changing the balance of the bridge; also the connection through $O$ may be opened or closed without changing the balance of the bridge. That is, the balance of the bridge is independent of the resistance of the branch $L$ and independent of the resistance of the branch $O$. As will be shown in the appendix on methods of analysis, a conductor having a resistance, $R$, and in which there is a current, $I$, may

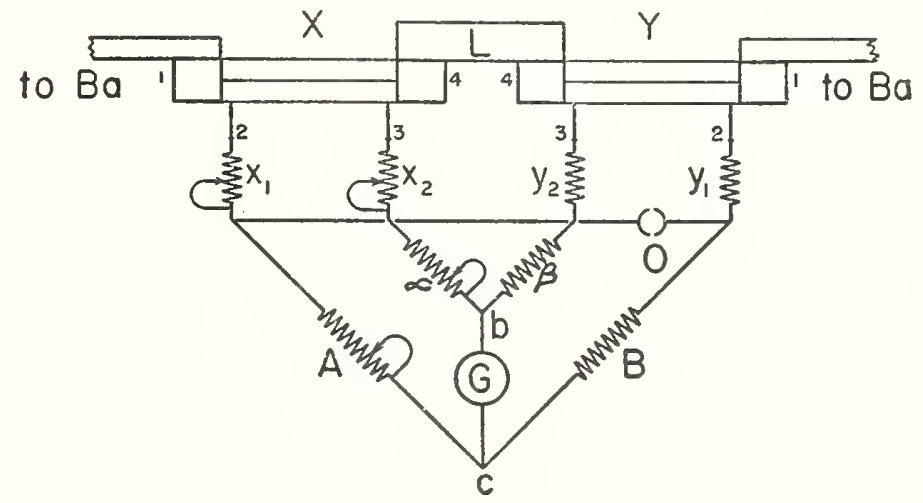

FIGURE 16 - Thomson bridge circuit so arranged that with a suitable procedure in establishing a balance, the ratio of the resistance of $A$ to the resistance of $B$ equals the ratio of the direct resistance of $X$ to the direct resistance of $Y$

It should be noted that when $X$ and $Y$ are nonlinear there are no definite branch points eorresponding to $a$ and $d$ of figure 9 .

be replaced by a battery having an electromotive foree $E=-R I$. Hence a battery may be placed in each of the branches $L$ and $O$, as well as in the normal battery branch of the bridge. Since the balance of the bridge is independent of the electromotive force in the battery branch, and the bridge is so adjusted that the balance is independent of the resistances in the $L$ and $O$ branches, the balance is independent of the electromotive force of each of the three batteries. It is convenient to consider that these three branches are closed alternately through a battery. The stage is now set for the application of Kirchhoff's reciprocal theorem [49], from which it follows that in any balanced bridge the position of the battery and galvanometer may be interchanged without disturbing the balance. Therefore, if a battery is placed in the normal position of the galvanometer and the galvanometer is connected alternately in the normal battery branch, the $O$ branch and in the $L$ branch, a balance will be indicated in each case. This is the Matthiessen and Hockin bridge arrangement, and according to the Mathiessen and Hockin bridge equation [114],

$$
\frac{A}{B}=\frac{x_{1}}{y_{1}}=\frac{(2143)_{x}}{(2143)_{y}}=\frac{x_{2}+\alpha}{y_{2}+\beta}
$$

Obviously, therefore, the procedure followed in adjusting the bridge establishes a relation between the various resistances which is independent of the proportional part of the current in each of the three branches of the bridge. Furthermore, in this relation but one of the four-terminal resistances of $X$ and but one of the fourterminal resistances of $Y$ appear explicitly. When all terminals of $X$ and $Y$ normally used as potential terminals (or normally used as current terminals) are connected to ratio arms of the bridge,

$$
\frac{A}{B}=\frac{\text { the direct resistance of } X}{\text { the direct resistance of }} \bar{Y} \text {. }
$$


If, for example, terminal 1 of $X$ were connected to the $A$ arm of the bridge, terminal 2 were used as a connection to the battery, and other connections were as shown in figure 16 , the adjustment would establish the relation

$$
\frac{A}{B}=\frac{\text { the diagonal resistance of } X}{\text { the direct resistance of } Y} \text {. }
$$

The conductors, $X$ and $Y$, might be connected into the bridge in such a way that the adjustment would make the ratio of $A$ to $B$ equal to any one of several ratios of a four-terminal resistance of one, to a four-terminal resistance of the other. Finally, it should be noted that whether the Thomson bridge method, the potentiometer method, the Matthiessen and Hockin bridge method, or the Kohlrausch differential galvanometer metlod is used the same value will be found for the ratio of a four-terminal resistance of $X$ to a four-terminal resistance of $Y$, provided proper adjustments are made in each case and equal precisions are attained in these adjustments.

\section{APPENDIX 2. THERMOELECTROMOTIVE FORCES}

In measurements to a precision of 1 part in 1 million, the potential drops in the bridge arms resulting from the test current must be balanced in many cases to 0.01 microvolt and in exceptional cases to 0.001 microvolt. However, conditions under which the measurements must be made are such that unless careful consideration were given to the thermoelectromotive forces they would often amount to a few or even several microvolts and change rather rapidly. As pointed out above, keeping the galvanometer circuit closed and judging the balance of the bridge from the changes in the deflection of the galvanometer following reversals of the test current eliminate the effect of the thermoelectromotive forces insofar as these remain constant during time intervals corresponding to a few periods of the galvanometer. While it is very desirable that the thermoelectromotive forces be kept constant, experience shows that a thermoelectromotive force which is changing slowly and at a fairly uniform rate does not preclude accurate balances of the bridge by a skillful observer. The principal means used for steadying the thermoelectromotive forces, most of which also reduce their magnitudes, are mentioned in the following numbered paragraphs:

1. Bridge arms, terminal blocks, the rheostat used in regulating the damping of the galvanometer, and a part of the leads between the bridge and galvanometer are placed under oil which is kept at very nearly a uniform temperature throughout by vigorous circulation.

2. The galvanometer is mounted on an inside wall, where it is fairly free from erratic changes in temperature.

3. A tight metal case completely surrounds the galvanometer, cxcept that there is a glass window of sufficient size to permit of a view of the coil and a reflection of a light beam from the mirror.

4. The coil, coil terminals, suspensions, and terminals of the galvanometer, and the leads between galvanometer and connections in the oil bath are of copper, but the necessary connections between them are soldered.

5. That part of the galvanometer circuit outside the oil bath is thermoelectrically balanced insofar as this is practicable.

6. Most of the soldered connections outside the oil bath are thermally shunted, at least to some extent.

7. Thermal resistances are made high in parts of the circuit in which this is an advantage.

8. All soldered connections are protected from direct exposure to the general circulation of the air of the laboratory.

9. Soldered connections outside both the galvanometer case and the oil bath are kept to a minimum number.

10. Soldered connections outside the oil bath are made with the copper parts as nearly in direct contact as is practicable over areas equal to or larger than the cross section of the conductors and with only a sufficient amount of solder to produce a good thermal contact.

11. Abrupt changes in the thermal capacity per unit length of conductor in the vicinity of possible sources of changing thermoelectromotive force are avoided to as great an extent as is practicable.

12. Masses of material having relatively large thermal capacities are placed in the vicinity of more probable sources of changing thermoclectromotive force for the purpose of reducing the rate of change of the temperature. 
13. The dial switches serving to adjust balances of the bridge operate in the oil bath, so are well lubricated, and this reduces the heat developed in the contacts by the operation of the switches.

14. The resistances in series with the dial switches are high relative to the resistances of the sections shunted. Consequently, variations of the thermoelectromotive force, introduced in to the $A$ arm of the bridge by the dial switches, are fully one order smaller than the variations of the thermoelectromotive forces in the dial switches.

Since thermoelectric balances and thermal shunts are not generally used elsewhere, an explanation of each may be in order.

\section{(a) THERMOELECTRIC BALANCES}

If a complete electric circuit is made up in part from one kind of metal and in part from another kind of metal, there must be at least two and in any case an even number of junctions between dissimilar metals. That is, the junctions occur in pairs. When these junctions are at different temperatures usually there is a thermoelectromotive force in the circuit. However, it is not necessary that all junctions be at the same temperature to avoid a thermoelectromotive force. The thermoelectromotive force would be zero if the two junctions constituting one pair were at one temperature, the two junctions constituting another pair were at another temperature, etc. This is the end sought in making thermoelectric balances. In the simplest case a thermoelectric balance consists in making the two junctions of a pair of similar construction and of placing these two junctions in such relative positions that both will be exposed to very nearly the same ambient temperature. More generally, a thermoelectric balance consists of constructions and arrangements of parts such that some type of symmetry both from the thermal and from the thermoelectric points of view is realized for two or more of several junctions in an electric circuit. For a circuit to be thermoelectrically balanced, each junction between dissimilar metals must be taken into consideration.

For the purpose of illustrating a method of realizing a thermoelectric balance, consider a soldered connection between two copper wires of the same size which are exposed to air currents of varying temperature. The connection is made similar in the two directions parallel to the circuit. The conductor is supported from opposite walls of the laboratory, and the connection is placed at some distance from either support. Consequently, air currents heat and cool the wires on opposite sides of the connection at very nearly the same rate, and keep them at very nearly the same temperature. Therefore, the two junctions, solder to copper and copper to solder, always assume very nearly the same temperature, so both the magnitude and the rate of change of the thermoelectromotive force are very small. In this case, one of the junctions is balanced against the other.

For the purpose of illustrating another method of realizing a thermoelectric balance, consider two sections of a circuit in each of which there is a soldered connection between a copper wire $1 \mathrm{~mm}$ in diameter, and a copper wire $0.5 \mathrm{~mm}$ in diameter. The two soldered connections are made similar and so located with respect to each other that in case of a changing temperature, the rate of heat transfer from a larger to a smaller wire is the same through one as through the other of the soldered connections. As a consequence, the difference in temperature of two junctions, copper to solder and solder to copper, of one connection, is very nearly the same as that of the other connection. Therefore, the two soldered connections, each constituting a thermocouple, develop very nearly the same electromotive force. Further more the arrangement is made such that in case of a current in the circuit, this current is from the larger to the smaller wire through one of the soldered connections, and from the smaller to the larger wire through the other connection. Therefore, the electromotive force developed in one of the soldered connections is in opposition to that developed in the other. In this case, one of the thermocouples is balanced against the other.

\section{(b) THERMAL SHUNTS}

A thermal shunt consists of an electrically insulated or insulating heat conductor which serves to equalize the temperature of two sections of a circuit between which there are possible sources of thermoelectromotive force. As an illustration, consider a soldered connection between a relatively large and a relatively small insulated copper wire that is exposed to air currents of varying temperature. Only sufficient insulation is removed from the ends of the wires to permit of soldering, after which the smaller wire is wrapped a few times around 
the larger wire, and this portion thoroughly impregnated with shellac. Then, although air currents may heat and cool the smaller wire more rapidly than the larger, the heat transfer between the two is mainly through the electrical insulation. The result is a material reduction in the temperature difference and rate of change of the temperature difference between the two junctions, copper to solder and solder to copper, and consequently, a material reduction in the thermoelectromotive force and its rate of change.

Thermoelectric balances and thermal shunts constitute two of the simpler and more generally applicable of the effective means of reducing troubles from thermoelectromotive forces. Furthermore, each may be made to supplement the other and sundry means employed for this purpose, such as a thermal shield the circulation of air in an enclosed space, the blocking of air currents, etc.

With the arrangement used and procedure followed in establishing balances of the bridge, thermoelectromotive forces seldom constitute a limiting factor on the precision of measurement, even when there is forced circulation of the air from the general heating system at a temperature $10^{\circ} \mathrm{C}$ above the temperature of the bridge and $15^{\circ} \mathrm{C}$ above the general temperature of the laboratory or from the refrigerating system at a temperature $15^{\circ} \mathrm{C}$ below the temperature of the bridge and laboratory. Only in exceptional cases is it necessary to place temporarily an obstruction to the normal circulation of the air in the vicinity of the galvanometer, the terminals of standard resistors, or the connectors extending above the surface of the oil.

\section{APPENDIX 3. INSULATION}

The maintenance of sufficiently good insulation constitutes a troublesome factor, especially in the measurement of higher resistances. Referring to figure 1, it will be seen that the battery and galvanometer leads are at different potentials. Consequently, at least a small part of the current supplied by the battery passes through the insulation between them, and as a result each arm of the bridge is shunted by a resistance of the order of the resistance of the insulation between a battery lead and a galvanometer lead. In case the resistance of one arm of the bridge is 10,000 ohms, the resistance of the insulation between the battery lead and the galvanometer lead connected to it must be in excess of the $10^{10} \mathrm{ohms}$, or be compensated in some way, such for example, as by the use of a substitution method, if the error from this source is to be less than 1 part in a million. Resistances of the insulation between the battery and galvanometer leads in excess of $10^{10} \mathrm{ohms}$ are not readily realized, and having been realized, it is not safe to assume that they are being maintained unless certain precautions are taken, and then only in case occasional checks justify the assumption. Usually it is assumed that a Wagner branch [96, 97] if properly designed and properly used, constitutes an effective means of avoiding errors which otherwise might result from slightly defectıve insulation. Originally, the bridge was equipped with a Wagner branch, but it was removed for reasons, the more important of which will be discussed later under the heading "Wagner branches." Since the removal of the Wagner branch, efforts have been made to maintain the resistance of the insulation between the battery branch and the galvanometcr branch sufficiently high that the leakages between these branches do not constitute a limiting factor in the precision of measurement. However, if the insulation were to be sufficiently good to obviate errors which might arise as a result of passage of leakage current from direct current power circuits through the bridge, its resistances would have to be two or possibly three orders higher. While errors from this source are eliminated for the most part by the reversal of the connections to the battery used with the bridge, and this is the regular practice followed in balancing the bridge, a grounded metal guard is placed under all mechanical supports of the bridge, the battery and its leads, and the galvanometer and its leads. This is a very effective means of isolating the bridge from power circuits. This guard also serves in testing the insulation of battery and galvanometer leads. Furthermore, the power uscd in regulating the temperature and driving the stirring motor is supplied from an alternating current circuit, one side of which is grounded.

The means employed for securing good insulation are as follows: (1) The air of the laboratory is dried by refrigeration when this is necessary to keep the dew point well below the temperature of the oil bath and laboratory, not only while the measurements are being made but during a sufficient time in advance to thoroughly dry all insulating material exposed to the air. Incidentally, drying the air of the laboratory reduces the moisture content of the oil, and this helps in the maintenance of its insulating property. (2) Care is taken never to reduce 
the temperature of the oil below the dew point of the air of the laboratory, as this would result in the condensation of water in the oil. (3) The oil is selccted on the basis of original freedom from acids and tendency to develop acids, is protected from strong light, is kept covered when the apparatus is not in use, and is renewed at intervals of about 2 years. (4) Most of the insulating material is protected from strong light, especially when not in use. (5) Care is taken not to permit the accumulation of excessive amounts of dust or hygroscopic sulfur compounds on the surfaces of the insulators of the hard-rubber type. (6) The bridge proper and the rheostat used in regulating the damping of the galvanometer are kept under oil. (7) The galvanometer leads have but few supports and these are of amber. (8) The galvanometer case is supported on amber posts, and the galvanometer circuit is insulated from the case. (9) The battery is supported on blocks of paraffin, and the battery leads are well insulated.

However, the resistance of the insulation of the battery and its leads, the rheostats for regulating the test current, the ammeter-voltmeter, keys, etc., generally is from two to four orders lower than the resistance of the insulation of the galvanometer and galvanometcr leads. It should be noted that it is not necessary that the insulation of both the battery branch and the galvanometer branch be exceptionally good. Nevertheless, when the humidity is very low, the insulation of the entire bridge circuit is so good that there may be trouble from electrostatic actions. To avoid electrostatic effects arising from the stirring of the oil, the circulating propeller is electrically connected to the guard, and the belt driving the circulating propeller is located beneath the metal tank containing the oil bath. The more serious of the remaining sources of electrostatic effects arise from movements of the observer. In case these movements cause trouble, the guard is temporarily extended under the observer so he becomes a part of it, the observer is thoroughly insulated from the floor, or a galvanometer lead is connerted to the galvanometer case. Should slight defects in the insulation be suspected, in any case, their presence or absencc may be confirmed easily. If present, but not very scrious, usually it is a simple matter to carry out the mcasurement in such manner as to avoid an error from this cause.

\section{(a) THEORY IN CASE OF DEFECTIVE INSULATION}

The correction for the effect of slightly defective insulation will be designated as $f$. Then, following the notation used in the paper, the Wheatstone bridge equation may be written

$$
X=\frac{Y}{B} A_{n}\left[1+a-a_{n}+f\right]
$$

In cases in which the resistances of the battery leads and galvanometer leads are negligibly small in comparison witl the resistances of the bridge arms, as is usually assumed in discussions of this subject, figure 17 represents the bridge circuit with sufficicnt exactness for the purpose at hand. Here $a, b, c$, and $d$ represent branch points of the bridge; $e$ represents the guard extending under all mechanical supports of the apparatus; and $m, n, o$, and $p$ represent the resistances of the insulation between leads, or branch points of the bridge and the guard. For this somewhat ideal arrangement, if the bridge is balanced, the relation between $A, B, X, Y, m, n, o$, and $p$ is

$$
\frac{X(1+Y m p / F)}{Y(1+X o p / F)}=\frac{A(1+B m n / F)}{B(1+A n o / F)}
$$

where

$$
F=m n o+n o p+o p m+p m n \text {. }
$$

Equation 63 is readily obtained by the use of Rosens [77] star-polygon transformation. If put in the form of eq 62 , and if $F$ is sufficiently large relative to $Y p m$, $X o p, B n m$, and Ano, that terms divided by the second and higher power of $F$ may be neglected,

$$
X=\frac{Y}{B} A_{n}\left[1+a-a_{n}+(X o p+B m n-A n o-Y m p) / F\right]
$$


Therefore, to a first order approximation,

$$
f=\frac{X o p+B m n-A n o-Y m p}{m n o+n o p+m o p+m n p} .
$$

Equation 66 shows the effect of slight defects in the insulation and the relation between these defects necessary for them to compensate each other. However, it is of little use in the solution of the particular problem at hand, since, in general, $m, n, o$, and $p$ will not be known. Furthermore, it is not sufficiently general to be applicable in all cases.

Frequently a resistance is placed in one or both battery leads to reduce the potential drop across the bridge to a value-less than the electromotive force of the battery and in one or both galvanometer leads or in parallel with the galvanometer

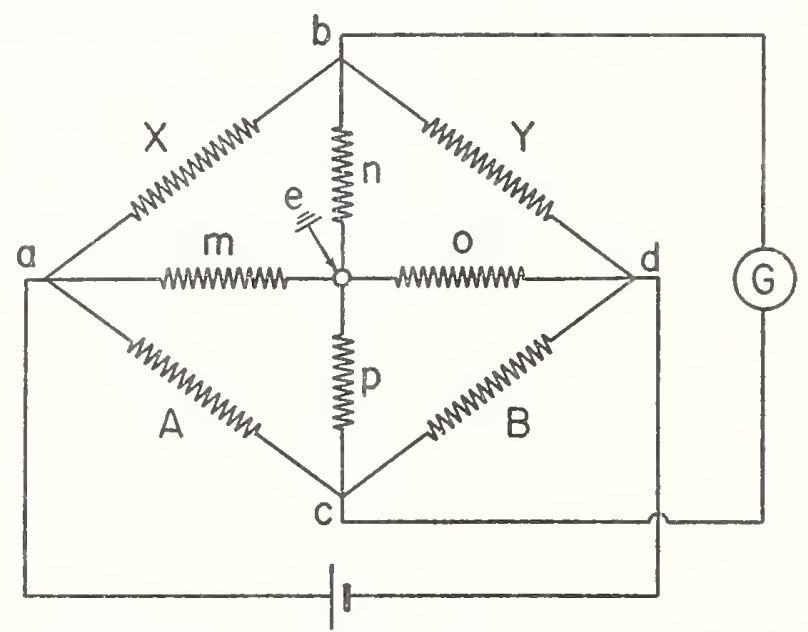

Figure 17. - Wheatstone bridge circuit arranged to show the effect of slightly dejective insulation of the battery leads and of the galvanometer leads.

to adjust the damping of the galvanometer. Also major parts of the leakage through or over insulating material may be distributed near the battery and galvanometer. When this is the case, resistances in the leads make slight defects in the insulation more serious.

\section{(b). PROCEDURE IN CASE OF DEFECTIVE INSULATION}

For such a bridge circuit as shown in fig. 18 or the simpler one (shown in fig. 17), to a first-order approximation, the correction term, $f$, of eq 62 may be considered to be the product of two factors. ${ }^{5}$ One of these, which will be designated $J$, comprises two or more resistance ratios involving only the resistances of bridge arms, galvanometer leads, and leakage paths between galvanometer leads and the guard. The other, which will be designated $K$, is the potential drop from the guard to the branch point $b$ (or branch point c) divided by the potential drop from $a$ to $d$.

Following the notation used above and replacing $f$ by the product of $J$ and $K$,

$$
X=Y \frac{A_{n}}{B}\left[1+a-a_{n}+J K\right] \text {. }
$$

With branch point $a$ connected to the guard

so

$$
K_{n}=X /(X+Y),
$$

$$
X=Y \frac{A_{n}}{B}\left[1+a_{a}-a_{n}+J X /(X+Y)\right] \text {. }
$$

With branch point $d$ connected to the guard,

so

$$
K_{d}=-Y /(X+Y) \text {, }
$$

$$
X=Y \frac{A_{n}}{B}\left[1-i \cdot a_{d}-a_{n}-J Y /(X+Y)\right] .
$$

8 The basis of the discussion given here will be explained in the appendix on methods of analysis, first expedient. 
From eq 69 and 71 it follows that

$$
J=a_{d}-a_{a} .
$$

From eq 69 and 72 , or 71 and 72 , it follows that

$$
X=Y \frac{A_{n}}{B}\left[1+\left(a_{a} Y+a_{d} X\right) /(X+Y)-a_{n}\right] .
$$

In like manncr it may be shown that if balances of the bridge are made with branch points $b$ and $c$ alternately connected to the guard

$$
X=Y \frac{A_{n}}{B}\left[1+\left(a_{b} A+a_{c} X\right) /(A+X)-a_{n}\right] .
$$

Referring to eq 73 or eq 74 , it will be seen that by making two balances of the bridge instead of the usual one, the effect of slight defects in the insulation of battery and galvanometer leads is taken into account. For eq 73 or 74 to give results accurate to 1 part in 1 million, $a_{a}-a_{d}$ or $a_{b}-a_{c}$ must lie in the range 0.001 to -0.001 . However, these two equations are independent of each other, so usually one or the other will be applicable.

It should be pointed out that the situation is somewhat more consplicated than would appear from the above discussion or an inspection of figure 18. For reasons which are stated on page 239 , the balance of the bridge is judged from changes in the deflection of the galvanometer following reversals of connections to the leads of the battery. These reversals are made at points at solne distances from the battery terminals. As a consequence, the direction of the potential gradient in the insulation may be reversed in some and not in others of the leakage paths, so the correction term may be different in the two cases. Consequently, when eq 73 or 74 is used an appropriate procedure should be followed in establishing the extra balances of the bridge.

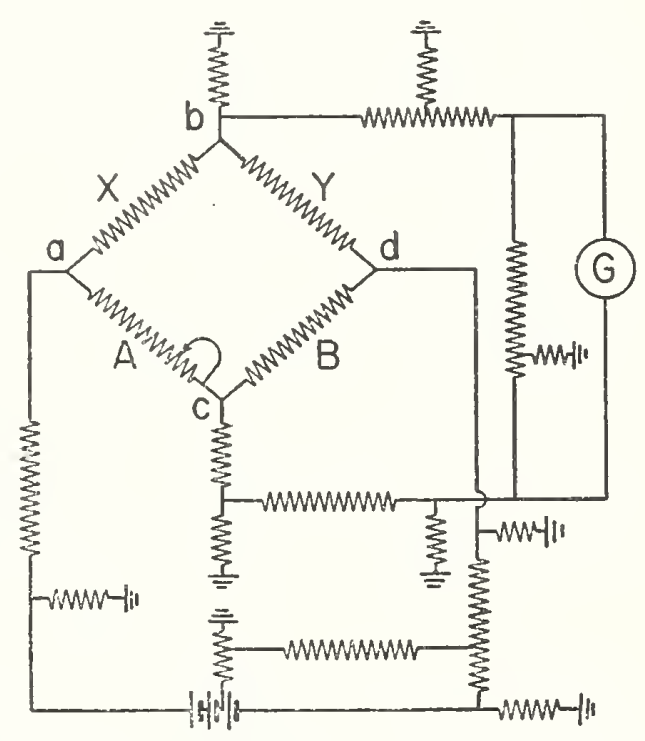

FIGURE 18.- Wheatstone bridge circuit with leakage paths between battery leads and the guard, and between the galvanometer leads and the guard.

\section{(c) TEST OF INSULATION}

However, what is desired is not an evaluation of a correction term but an assurance that it is so small that it may be neglected without reducing the precision of the measurements. Keeping in mind the relations given above, it is a simple matter to more or less definitely locate defects in the insulation if these defects are really serious, although it nay or may not be a simple matter to remedy the difficulty. Since the insulation of the galvanometer, galvanometer leads and rheostats used in regulating the damping of the galvanometers is generally much better than the insulation of the battery, battery leads, switches, and rheostats used in adjusting the current through the bridge, the insulation may be tested by making $K$ of eq 67 have first a positive and then a negative value somewhat larger than the value it can possibly have as the bridge is used, and noting the resulting changes, if any, in the balances of the bridge.

The value $K$ naturally assumes presumably will never lie outside the range from plus to minus the electromotive force of the battery, supplying the test current, divided by the potential drop from $a$ to $d$. Usually, therefore, an appropriate 
change in $K$ may be made by connecting an auxiliary battery (having an electromotive force of about one order higher than the electromotive force of the battery supplying the test current) between the guard and branch point $a$ (or $d$ ) and then reversing connections to this auxiliary battery. This should be done with the reversing switch in the battery branch of the bridge in one position and then repeated with the reversing switch in the other position, the battery branch being closed in both cases.

Of the locations not so far considered, at which slightly defective insulation might introduce errors, the more probable is in the auxiliary apparatus used in finding the reading of the dial switches of the $A$ arm of the bridge for which the ratio of $A$ to $B$ is 10 . At the time this apparatus was constructed the importance of providing a means of testing the ins ilation was not appreciated. For example, should the resistance of the insulation between one of the end terminal blocks and one of the central terminal bloclis be as low as $10^{3} \mathrm{ohms}$, the reading obtained would correspond to a ratio differing from 10 by about 2 parts in 1 million. However, checks by a number of standard resistors singly and then in series have not indicated any significant error from this source.

It should be pointed out that when nominally equal resistances are being compared, either by substitution one after another in the same arm of the bridge or by interchange between arms, errors which otherwise would result from slightly defective insulation are automatically eliminated to the extent that the shunting effects remain constant during a series of measurements. Also, that as the apparatus is used, leakages within the $A$ and $B$ arms of the bridge are automatically taken into account to the extent that they remain constant during a series of ineasurements.

In general, leakages within resistance standards have the effect of lowering their resistances, rather than limiting the precision of the measurements. In exceptional cases in which the conduction through the insulation is sufficient to result in noticeable electrolytic polarization the usual precision of measurement cannot be obtained.

It has been assumed that direct leakage from any part of the bridge circuit to points within $X$ or $Y$ is negligible. Also that direct leakage between battery and galvanometer leads is negligible. The bridge is kept under oil of good quality, and the battery and galvanometer leads are brought out, so that there is little possibility of a current from one to the other except through bridge arms or by way of the guard. So presumably these assumptions are justifiable.

\section{(d) WAGNER BRANCHES}

It was mentioned that originally the bridge was equipped with a Wagner branch. Usually when a Wagner branch is used with a Wheatstone or other four-arm bridge, it is attached as shown in figure 19. Here $W_{a}$ and $W_{b}$ represent the arms of the Wagner branch. The bridge and the Wagner branch are adjusted by successive approximations. First, the bridge is adjusted with the key $k_{w}$ open, so that there is no current in the galvanometer. Second, with the galvanometer connected between branch point $b$ (or $c$ ) and the guard or with the galvanometer in its normal position, and the key, $k_{w}$, closed, the Wagner branch is so adjusted that there is no current through the galvanometer. This second adjustment, if it involves any marked changes in the ratio of $W_{a}$ to $W_{b}$, disturbs the first adjustment to some extent. Therefore, the first adjustment is repeated and this disturbs the second adjustment slightly, etc. However, successive adjustments require smaller and smaller changes, and after the changes become insignificant, the galvanometer branch is at the potential of the guard. Then there is no leakage current through the leakage path, $q$, or any arm of the bridge. Therefore, since there is no current through the galvanometer, the Wheatstone bridge equation gives the relation between $X, Y, A$, and $B$.

If this adjustment were valid with the key $k_{1}$ against the + battery terminal block and the key, $k_{2}$, against the - battery terminal block, the relation between the resistances would be as follows:

$$
\frac{W_{a}\left(1+W_{b} / q\right)}{W_{b}}=\frac{X}{Y}=\frac{A}{B} .
$$

However, with this adjustment the galvanometer branch will not be at the potential of the guard with both keys $k_{1}$ and $k_{2}$ against + battery terminal blocks, as shown in figure 18 , or with $k_{1}$ against $a-$, and $k_{2}$ against a + battery terminal 
block. More specifically, when a switch, such as is shown in figure 19, is used in reversing the current through the bridge and this switch is located between the bridge and a leakage path between a battery lead and the guard, no adjustment can be inade such that the galvanometer branch will be at the potential of the guard for all permutations of the settings of the keys $k_{1}, k_{2}$, and $k_{w}$. If then there is a leakage path from a galvanometer lead to the guard, no adjustment can be made such that there is no current through the galvanometer with the eurrent through the bridge alternately in one and then in the other direction, when the key $k_{w}$ is open and also when the key $k_{w}$ is closed.

Notwithstanding this difficulty it is possible, in some cases only, to make an adjustment such that the relation between $X, Y, A$, and $B$ is that given by the

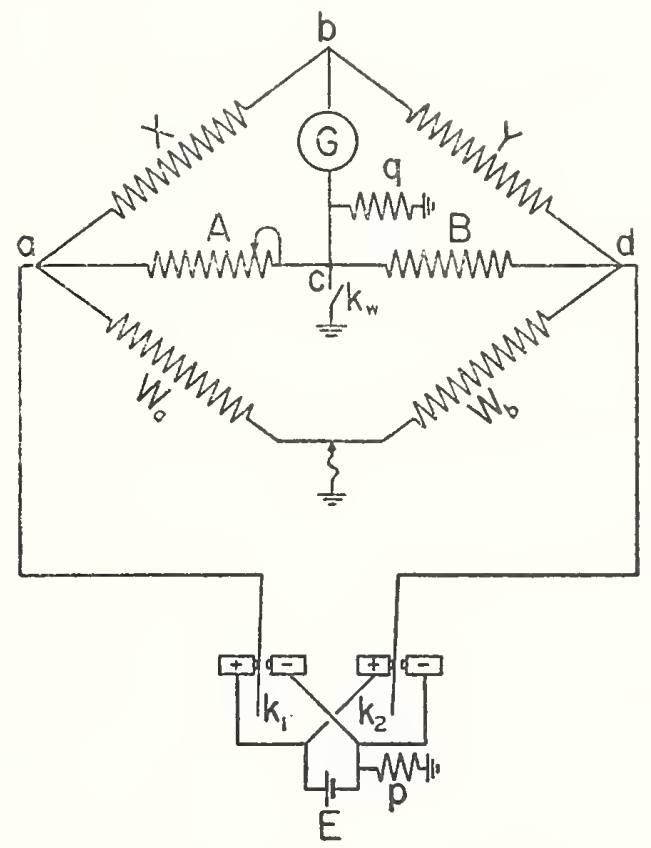

FIGURE 19.-A Wagner branch connected in parallel with the battery branch of a Wheatstone bridge.

$W_{a}$ and $W_{b}$ represent the arms of the Wagner branch, and $p$ and $q$ represent leakage paths between the bridge circuit and the guard.

Wheatstone bridge equation. Adjustments of the bridge and of the Wagner branch are made by successive approximations, judging balances by changes in the deflection of the galvanometer following reversals of the test current. In adjusting the bridge, the key $k_{w}$ is left open, while in adjusting the Wagner branch, it is kept closed. When proper adjustments have been realized there may be a fairly large current through the galvanometer with the key $k_{w}$ open and a different farrly large current through the galvanometer with the key $k_{w}$ closed. However, the magnitudes and directions of these currents are independent of the direction of the current through the bridge.

The situation is not nearly so complicated if the Wagner branch is attached to branch points $b$ and $c$ of the bridge, as is shown in figure 20 . With this arrangement adjustment by successive approximations leads to a condition such that there is no current through the galvanometer with any possible combination of the settings of the keys $k_{1}, k_{2}, k_{w}$, and $k_{w}$. While this adjustment does not bring the galvanometer to the potential of the guard or prevent a leakage between a galvanometer lead and the guard, it nevertheless leads to the relation between $X, Y, A$, and $B$ given by the Wheatstone bridge equation.

This discussion of the Wagner branch is by no means comprehensive. It should however serve to show that an attachment of a Wagner branch to the NBS precision bridge would not constitute a simple and convenient means of obviating the need of good insulation. 


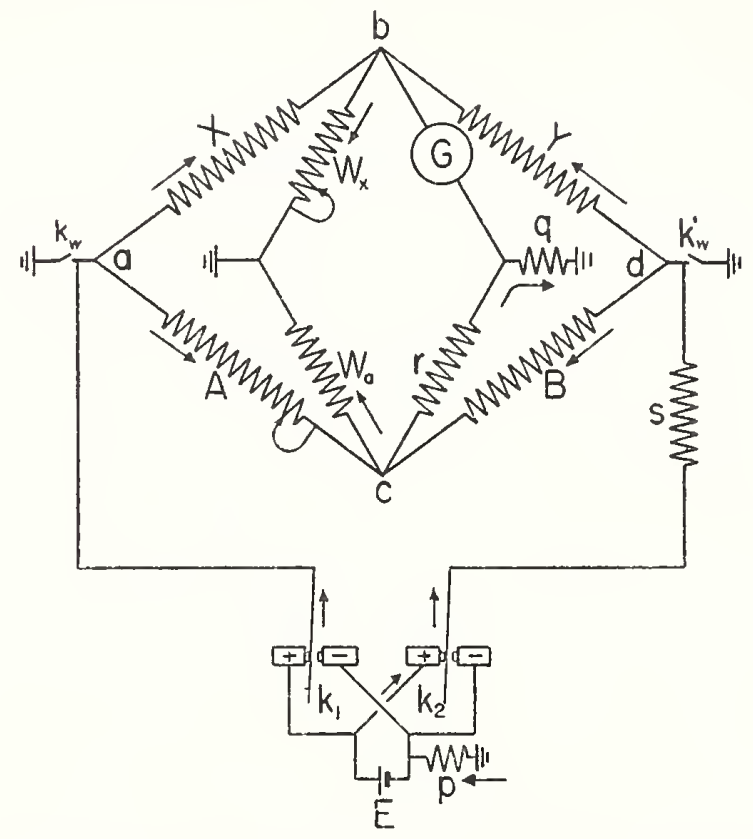

FIGURE 20.-A Wagner branch connected in parallel with the galvanometer branch of a Wheatstone bridge

Here the arrows indicate the direction of the leakage current in each branch of the network when the potential of all bridge arms is higher than the potential of the guard.

\section{APPENDIX 4. OPTICAL SYSTEMS OF GALVANOMETERS}

While balances of the bridge are being established, the deflection of the galvanometer is ever changing, so it requires the concentrated attention of the observer. Watching the deflection and separating those componeuts of the deflection resulting from a lack of balance of the bridge from those resulting from other causes constitute the most fatiguing part of a series of measurements. As fatigue is a factor affecting the precision of a series of measurements the optical system should be as suitable for the purpose as can be obtained without excessive refinements. What constitutes the best obtainable optical system depends on the skill of the available optician, personal preference, and physiological factors.

\section{(a) DESCRIPTION OF OPTICAL SYSTEM}

In the system used in the precision measurements of resistance made in this Bureau an image of a line source of light is focused on a ground-glass scale. The scale is placed at a distance of about $1.5 \mathrm{~m}$ in front of the galvanometer mirror and is adjustable in height so as to accommodate it to different observers while standing. 'The graduations and ground surface are on the observer's side of the scale, which is a matter of very considerable importance, since it serves to almost completely eliminate direct refiections from the scale.

The light source is a $35-\mathrm{w}$ straight flament lamp with a cylindrical bulb. However, it is operated far below normal intensity for reasons which will be explained presently. The lamp is enclosed in a cylindrical metal case with openings at the top and bottom for ventilation and an opening on the side which can be so adjusted as to exclude from the light beam reflected from the galvanometer mirror, images resulting from reflections within the lamp bulb. The galvanometer mirror is $1 \mathrm{~cm}$ in diameter and about $0.5 \mathrm{~mm}$ in thickness. The quality of the mirror and galvanometer window is such that practically theoretical resolving power is realized.

All direct illumination back of the ground-glass scale is avoided. Consequently, the only marked contrasts arise from the graduations and the image of the light source. The grinding is rather fine but not so fine but that both the graduations and the image are seen distinctly when viewed at an angle of as much as $20^{\circ}$ with respect to the normal to the scale. This permits the use of 
both eyes and does not require that the observer keep his head in a very definitely fixed position. The graduations are fine, distinct, black lines with $1-\mathrm{mm}$ spacing.

The lamp and scale are supported from a side wall and the ceiling, both of which are masonry. Also the galvanometer is supported from a side wall. Consequently, mechanical vibrations of the scale and image are seldom troublesome.

With the lamp operated at normal intensity, the image appears rather wide and excessively bright. Therefore, the power supplied to the lamp is reduced somewhat below the point at which, on dull days and with the window shades open, the diffraction fringes do not contribute to the apparent width of the image and a suitable contrast is obtained. As the outside illumination changes the intensity of the light source is not changed but occasionally one or more of the window shades are closed or the lights of the laboratory turned on. The general illumination is so adjusted that the contrast between the graduations and ground glass is approximately the same as the contrast between the ground glass and image.

In cases in which the combined sensitivity of the bridge and galvanometer are barely sufficient for obtaining the desired precision, repetitions of series of measurements show that an exceptionally skillful observer can so nearly balance the bridge that that component of the electromotive force resulting from the lack of balance of the bridge does not exceed 0.001 microvolt. Since the sensitivity of the galvanometer then would be $30 \mathrm{~mm} / \mathrm{microvolt}$, it follows that 0.001 microvolt corresponds to changes in the deflection of $0.06 \mathrm{~mm}$, following reversals of the direction of the test current. To associate a change in the deflection that is only slightly larger than this with a change in the direction of the test current (while the deflection is changing gradually as a result of one or more of several causes, abruptly on reversals of the test current because of a lack of a perfect inductive balance, and erratically as a result of one or more of several possible causes); requires that the deflection be observed continuously while the test current is reversed several times, at intervals corresponding approximately to the period of the galvanometer. However, a balance of the bridge to 0.001 microvolt can be made only when the thermoelectromotive forces are remaining reasonably constant, the inductances of the bridge are reasonably well balanced, local and microseismic vibrations are not troublesome, electromotive forces induced by changes of the magnetic field in the laboratory are not troublesome, and conditions otherwise are favorable.

\section{(b) QUALITY OF OPTICAL' SYSTEMS AND SENSITIVITY OF BRIDGES}

Since the optical system of the galvanometer is the last of the factors affecting the sensitivity of bridges to be discussed at this time, it is in order to give an illustration of how a consideration of most of these factors leads to a fairly definite conclusion. For this purpose let the reader assume that he has the problem of comparing a number of 10 -ohm standard resistors, that he has at hand a bridge suitable for the purpose, and that he has prepared a specification for a galvanometer, but before purchasing a galvanometer wishes to know if the specification is adequate and satisfactory for the realization of a precision of 1 part in 1 million in the comparisons.

Let it be assumed that:

1. The period of the galvanometer is to be 7 seconds.

2 . The galvanometer is to be critically damped with an external resistance of 60 ohms.

3. The sensitivity of the galvanometer is to be such that with 1 microvolt, in the circuit giving critical damping, the deflection is five scale divisions, with the distance between the mirror and scale that which is to be used.

4. The optical system of the galvanometer is to be of such quality that a change of the deflection of 0.1 scale division is readily detectable.

5. It is estimated that local disturbances will cause random change in the apparent equilibrium position of the galvanometer of not more than 0.1 scale division during time intervals of 7 seconds.

6. The resistance between the galvanometer terminals of the bridge is $60 \mathrm{ohms}$.

7. The comparisons are to be made by substituting the 10-ohm standard resistors successively in the $X$ arm of the bridge.

8. A 10 -ohm standard resistor is to be placed in the $Y$ arm of the bridge and is not to be disturbed during the series of measurements.

9. Balances of the bridge are to be checked by a single observation of the change in the deflection of the galvanometer following a reversal of the test current, with no provision for establishing inductive balances. 
10. The divergence of the load coefficients of the series of 10-ohn standard resistors, to be compared, is 10 parts in 1 million per watt dissipation.

As previously pointed out (eq $26 x$ ), the change in the deflection of the galvanometer following a reversal of the test current is

$$
2 D E_{x} Y /(X+Y)
$$

provided, as according to 2 and 6 , the specified damping is realized without the use of a shunt. Here $E_{x}$, the potential drop in the $X$ arm, is not assumed to be known but is to be determined from the data given above. Since the sensitivity of the galvanometer, $D$, is five scale divisions per microvolt and $X$ and $Y$ are equal, the change in the deflection of the galvanometer resulting from 1 part in 1 million lack of balance of the bridge is five $E_{x}$ scale divisions, provided $E_{x}$ is expressed in volts.

From 1, 2, 4, 5, and 9 it follows that the bridge may be out of balance nearly by an amount corresponding to a change of the deflection of the galvanometer of 0.2 scale division without being detected. Consequently, the minimum discernible

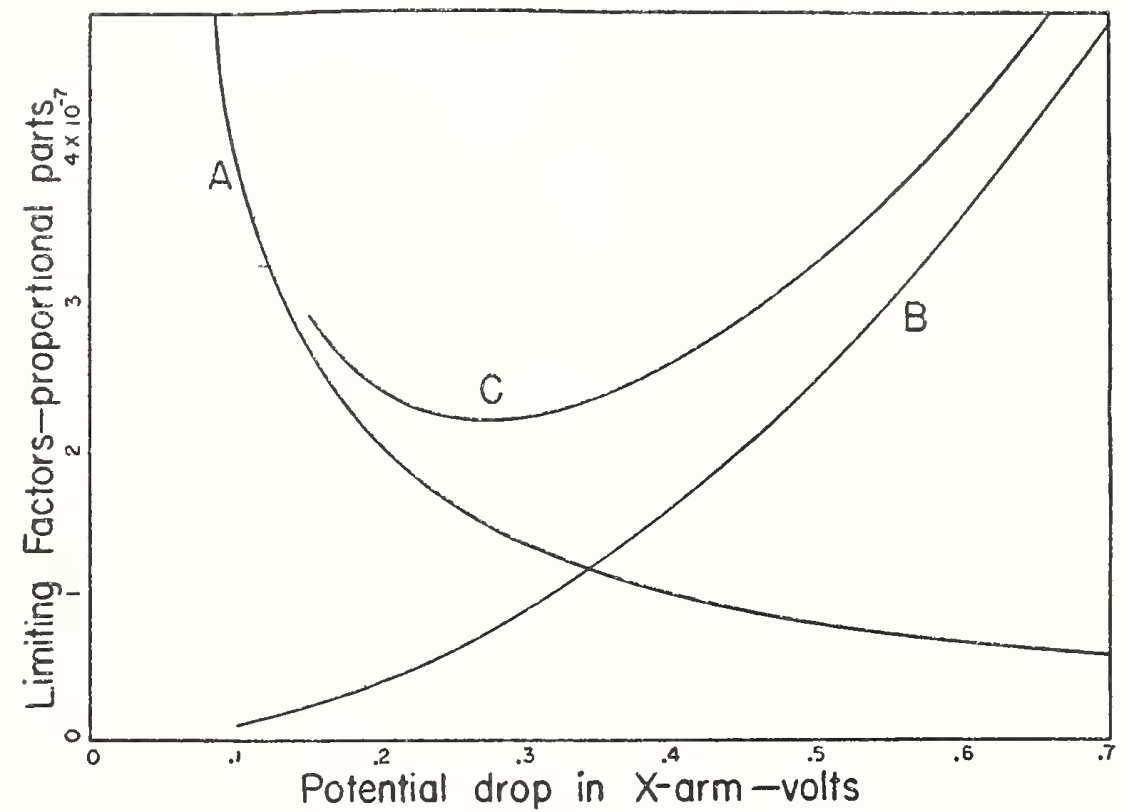

FrGURE 21.- Sensitivity of bridge and galvanometer (an illustrative p:oblem).

Curve $A$ shows minimum discernible lack of balance of the bridge; curve $B$, effect of heating; and curve $C$, sum of factors limiting the sensitivity.

lack of balance of the bridge may be taken as 1 in 25 million $E_{x}$. Plotting the minimum discernible lack of balance of the bridge as ordinates and $E_{x}$ as abscissas gives curve $A$ of figure 21 .

From 7 and 10, since the resistance in the $X$ arms is $10 \mathrm{ohms}$, it follows that for the series of measurements the divergence of the effects of heating is $E_{x}^{2} \times 10^{-6}$. Plotting $E_{x}{ }^{2}$ as ordinates and $E_{x}$ as abscissas gives curve $B$. Adding the ordinates of curves $A$ and $B$ gives curve $C$, the sum of the various factors limiting the sensitivity. It will be observed that the maximum of what may be called the effective sensitivity is 2.2 in 10 million, or about 1 in $4 \frac{1}{2}$ million, and that this sensitivity is obtained with a potential drop of approximately 0.27 volt in the $X$ arm of the bridge. Obviously this sensitivity is somewhat more than that required for a precision of 1 part in 1 million.

In this analysis it has been assumed that the combined effect of the various factors limiting the effective sensitivity is obtained by the addition of their individual effects, that is, the analysis is based on the worst, not the probable, of the assumed conditions. It may be concluded, therefore, that a galvanometer having the operating characteristics specified would be suitable for obtaining the desired precision in the series of measurements, and inasmuch as there would be no difficulty in obtaining a galvanometer having these operating constants, it may be concluded that the specification is satisfactory. 


\section{APPENDIX 5. METHODS OF ANALYSES (THREE EXPEDIENTS)}

Instead of Kirchhoff's laws or Maxwell's "mesh currents" simpler expedients have been used in this paper in the solution of network problems. For the most part these are so obvious or so well known that no explanation need be given, or reference is made to publications in which they are discussed. However, three of the expedients used deserve special mention. The first of these consists in the substitution of a battery in place of a resistance in which there is a current, the second consists in the substitution of a very high resistance and battery of very high electromotive force in place of a low (or zero) resistance and battery of low electromotive force, while the third consists in a separation and synthesis of the effects of the independent resistances of a nonlincar four-terminal conductor. This is accomplished by the substitution of first four, then five, and finally another five linear conductors in place of the nonlinear four-terminal conductor. These substitutions present no experimental difficultics, since they are made not in an actual circuit but in a diagram representing the circuit or merely in a mental picture.

\section{(a) CLASSICAL FORM OF SOLUTION OF UNBALANCED WHEATSTONE BRIDGE}

Before considering any one of these three expedients a few of the many possible expressions for the electromotive force or current in the galvanometer circuit of an unbalanced Wheatstone bridge will be stated, for the purpose of comparison with corresponding expressions, one of which will be derived by the use of the first and another by the use of the second of these expedients.

The expression given by Maxwell and others, and which will be referred to as the "classical solution," is

$$
\begin{gathered}
I_{q}=\frac{E_{b} B \Delta X}{R G(A+B+X+\Delta X+Y)+R(A+X+\Delta X)(B+Y)+} \\
+G(A+B)(X+\Delta X+Y)+X Y(A+B)+A B(X+\Delta X+Y)+\Delta X Y(A+B) .
\end{gathered}
$$

Here $I_{g}$ is the current in the galvanometer branch and $G$ is the resistance of the galvanometer branch. The significance of the other characters are as explained in connection with figure 1 .

It has been shown in this paper (eq 6 ) that

$$
E_{g}=\frac{E X}{X+Y}\left[\frac{1+\Delta X / X}{1+\Delta X /(X+Y)}-1\right]
$$

from which it follows $[30,71,88,108]$ that

$$
I_{g}=\frac{E X}{X+Y}\left[\frac{1+\Delta X / X}{1+\Delta X /(X+Y)}-1\right] / R_{9},
$$

where $R_{g}$ is the resistance to an electromotive force in the galvanometer branch, that is, the resistance of the galvanometer branch plus the resistance of the bridge between its galvanometer terminals with the galvanometer branch open and the battery branch closed.

\section{(b) FIRST EXPEDIENT (HYPOTHETICAL BATTERY OF ZERO RESISTANCE)}

For a battery having no resistance to be equivalent to a resistance in which there is a current, the electromotive force of the battery must be equal to minus the product of the current and resistance. As simple illustrations of the application of this expedient, consider the circuit shown in figure 1 . Here the current distribution in the bridge arms and galvanometer branch may be considered to depend on the potential drop from $a$ to $d$, regardless of how this potential drop is produced. We are therefore at liberty to consider that the potential drop is produced in any way which suits our convenience. One of the ways in which the potential drop might be produced would be by replacing the resistance, $R$, in the battery branch in which there is a current, $I$, by a sccond battery having no resistance and an electromotive force equal to $-R I$, or by replacing the entire battery branch by a battery having no resistance and an electromotive force $E_{1}=E_{b}-R I$. If this were done, the potential drop from $a$ to $d$ would not change on opening the galvanometer branch. The potential drop across the break in the galvanometer branch is given by eq 6 , if $E_{1}$ is substituted for $E$. Another simplification accomplished by this expedient is that from the standpoint of the 
resistance to an electromotive force in the galvanometer branch, branch points $a$ and $d$ are connected by a conductor of zero resistance. Consequently, an expression for the resistance to an electromotive force in the galvanometer branch can be written from an inspection of figure 1, and is

$$
G+\frac{A B}{A+B}+\frac{(X+\Delta X) Y}{X+\Delta X+Y}
$$

Consequently,

$$
I_{g}=\frac{E_{1} X}{X+Y}\left[\frac{1+\Delta X / X}{1+\Delta X /(X+Y)}-1\right] /\left(G+\frac{A B}{A+B}+\frac{(X+\Delta X) Y}{X+\Delta X+Y}\right)
$$

This equation or its equivalent was communicated to me orally by Leo Behr, without an analytical proof. However, his original analysis was the same as that given here.

This expedient, in some cases, is helpful in the solution of complicated network problems as well as simpler problems, such as that just considered. As an

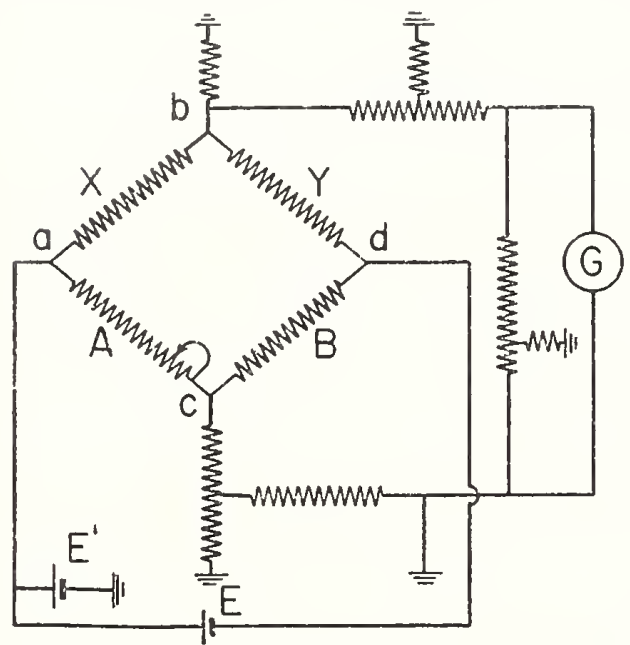

Figune 22. - The battery, battery leads, and leakage paths (belween battery leads and the guard only) shown in figure 18 replaced by two batteries. ilustration, consider the bridge circuit shown in figure 18 and consider tlat. the bridge is balanced. Hypothetically, the battery, battery leads, and leakage paths between the guard and battery, and guard and battery leads may be replaced by two or more batteries (each having the appropriate electromotive force) connected as shown in figure 22 , or otherwise, without changing the current or potential relative to the guard at any point in the bridge arms, galvanometer leads, and leakage paths between the guard and galvanometer leads.

Obviously, if $E^{\prime}=E X /(X+Y), 110$ culrent is drawn from the battery whose electromotive force is $E^{\prime}$ and the usual Wheatstone bridge equation gives the relation between $X, Y, A$, and $B$. However, in gencral the relation betweeli $X, Y, A$, and $B$ will depart from the Whoatstone bridge relation proportionally to $\left(E^{\prime} / E\right)-$ $\left(X /\left(X+Y^{\prime}\right)\right)$, while the proportionality factor depends upon the relative resistances of the bridge arms, leakage paths from the galvanometer leads to the guard, and sections of the galvanometer leads. By deliberatcly changing $\left(E^{\prime} / E\right)-(X /(X+Y))$ first to one and then to another known value and noting the resulting changes in the balance of the bridge, both the initial value of $\left(E^{\prime} / E\right)-$ $(X /(X+Y))$ and the proportionality factor may be found. This is the basis of the discussion given in the appendix on insulation.

\section{(c) SECOND EXPEDIENT (HYPOTHETICAL BATTERY OF HIGH ELECTROMOTIVE FORCE AND HIGH RESISTANCE)}

For a high resistance and battery of high electromotive force to be equivalent to a low (or zero) resistance and battery of low electromotive force, it is necessary that the higl electromotive force minus the product of the high resistance and current be equal to the low electromotive force minus the product of the low resistance and current, and that the current be the same in the two cases. As a simple illustration of the application of this expedient, consider again the circuit shown in figure 1. Here the current distribution in the briage arms and galvanometer branch may be considered as dependent on the current in the battery branch regaldless of how this current is produced. We are therefore at liberty to consider that the current is produced in any way which suits our convenience.

One of the ways in which the current $I$ in the battery branch might be produced would be by increasing the resistance $R$ indefinitely and increasing the 
elcctromotive force $E_{b}$ sufficiently to give this current. If this were done the galvanometer branch could be opened without changing the current $I$ in the battery branch. Furthermore, from the standpoint of an electromotive force in the galvanometer branch, the resistance of the battery branch would re infinite. Consequently, an expression for the resistance to an electromotive force in the galvanometer branch could be written from an inspection of figure 1. With the galvanometer branch open it is readily seen that the current in the $X$ arm is $I(A+B) /(X+\Delta X+Y+A+B)$ and in the $A$ arm is $I(X+\Delta X+Y) /$ $(X+\Delta X+Y+A+B)$. These currents multiplied by the resistances $(X+\Delta X)$ and $A$ give the potential drop from $a$ to $b$ and from $a$ to $c$, while the difference between these rwo potential drops is the electromotive force appearing in the galvanometer circuit when the galvanometer branch is closed. With the galvanometer branch closed it is readily seen that the resistance to an electromotive force in the galvanometer branch is $G+(X+\Delta X+A)(Y+B) /(X+\Delta X+Y+A+B)$. Consequently, the current in the galvanometer branch

$$
I_{g}=\frac{I[(X+\Delta X)(A+B)-A(X+\Delta X+Y)] /(X+\Delta X+Y+A+B)}{G+(X+\Delta X+A)(Y+B) /(X+\Delta X+Y+A+B)},
$$

which reduces to

$$
I_{g}=\frac{I B \Delta X}{G(A+B+X+\Delta X+Y)+(A+X+\Delta X)\left(B+Y^{r}\right)} .
$$

This is an equation given without proof by Jaeger [39].

Equations 76, 77, 78, 79, and 81, are exact, and two or more of them might be applied to the same bridge at the same time. That is, each of these equations is a different form of the solution of the same prolilem. To reduce them to a common form, eq 76 for example, would requirc a determination of the relation between $E, E_{1}, I$, and $E_{b}$, and of an expression for $R_{g}$ in terms of $G, X+\Delta X, Y$, $A, B$, and $R$. A comparison of the derivation of eq 76 either by the method used by Heaviside or by the method used by Maxwell with that used in deriving eq 79 and that used in deriving eq 81 would serve to show the relative simplicity of the methods used here.

(d) THIRD EXPEDIENT (SEPARATION AND SYNTHESIS OF THE EFFECTS OF THE SIX INDEPENDENT RESISTANCES OF A FOUR-TERIMINAL CONDUCTOR)

Before considering the separation and synthesis of the cffects of the independent resistances of a nonlinear four-terminal conductor, it is important to know the number and nature of these resistances and the purpose of the separation and synthesis. In the appendix on terminals and contacts it was pointed out that a four-terminal conductor has but two independent four-terminal resistances. These are the direct resistance and the cross resistance, which here will be designated $D$ and $C$. The remaining independent resistances are the terminal resistances. which are of the thrce-terminal typc. These are

$$
\left.\begin{array}{l}
R_{1}=(1124)=(1142)=(4211)=(2411) \\
R_{2}=(2231)=(2213)=(1322)=(3122) \\
R_{3}=(3342)=(3324)=(2433)=(4233) \text { and } \\
R_{4}=(4431)=(4413)=(1344)=(3144) .
\end{array}\right\}
$$

(See appendix on terminals and contacts for notation)

The purpose of the separation and synthesis of the effects of these six resistances is to obtain analytical solutions of complicated networks composed of linear conductors and nonlinear four-terminal conductors. Normally, little or nothing would be gained by separating the effect either individually or collectively of the terminal resistances of the four-terminal conductors from the effect of the resistances of the linear conductors connected to the terminals. Therefore the only separations which will be considered are (a) of the effect of the direct resistances from the combined effect of the cross resistances, the terminal resistances, and the resistances of the linear conductors; and (b) of the effect of the cross resistances from the combined effect of the direct resistances, the terminal resistances, and the resistances of the linear conductors. Since the solution must. contain the combined effect of the terminal resistances and the resistances of linear conductors, in geneial it is necessary to determine three effects. These three effects may be determined by considering (1) that each nonlinear fourterminal conductor, constituting a part of a network, has four linear resistances arranged as shown in figure 23 (a), (2) that each nonlinear four-terminal conductor 
has five linear resistances arranged as shown in figure 23 (b), and (3) that each nonlinear four-terminal conductor has five linear resistances arranged as shown in figure 23 (c).

When in the analysis of a network problem any of the well-known procedures is followed in the treatment of the linear conductors, the first consideration gives a partial solution which is complete, except that it contains neither the effect of the direct resistances nor the effect of the cross resistances of the nonlinear fourterminal conductors. The second consideration gives a partial solution which is complete, except that it does not contain the effect of the cross resistances of the nonlinear four-terminal conductors. The third consideration gives a partial solution which is complete, except that it does not contain the effect of the direct resistances of the nonlinear four-terminal conductors.

The second partial solution minus the first partial solution gives the effect of the direct resistances, that is, the separation (a); while the third partial solution minus the first partial solution gives the effect of the cross resistances, that is, the separation (b). The addition of the effect of the direct resistances and the effect of the cross resistances to the combined effect of the terminal resistances and resistances of linear conductors constitutes a synthesis of these three effects, and consequently gives a complete solution of the network problem. Obviously, the complete solution may be obtained by the addition of the second and third, and

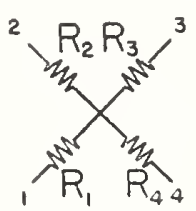

a

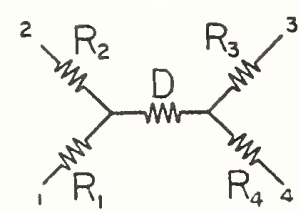

b

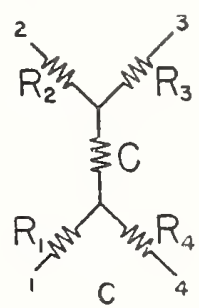

Figure 23.-Hypothetical arrangements of linear resistances for separating the effect of the direct resistance and the effect of the cross resistance from the effect of the terminal resistances of a nonlinear four-terminal conductor.

$a$, four linear resistances equivalent to the four terminal resistances of a nonlinear four-terminal conductor; $b$, five lincar resistances equivalent to the direct resistance and the four terminal resistances of a nonlinear four-terminal conductor; $c$, five linear resistances equivalent to the cross resistance aud the four terminal resistances of a nonlinear four-terminal conductor.

subtraction of the first of the partial solutions. If the second or third of these partial solutions contains the effect of the direct resistances or of the cross resistances as additive terms only, that is, if the separation (a) or the separation (b) occurs more or less automatically, the transfer of the additive terms occurring in one of these partial solutions to the other of these partial solutions gives the complete solution, so the first partial solution is not required. In cases in which the combined resistances of terminals of the nonlinear four-terminal conductors and linear conductors connected to the terminals are sufficiently high relative to the four-terminal resistances of the nonlinear four-terminal conductors that first order approximations of the effects of the four-terminal resistances are sufficient, the effect of the direct resistances can always be obtained as additive terms in the second partial solution, and the effect of the cross resistances can always be obtained as additive terms in the third partial solution.

To show how this method of analysis works out in practice, consider that three linear conductors having resistances of $0.43,1$, and $1.37 \mathrm{ohms}$ respectively, are connected to a nonlinear four-terminal conductor, $Q$, as shown in figure 24 (a), that the six independent resistances of $Q$ are

$$
\begin{aligned}
& R_{1}=.07 \mathrm{ohm}, \\
& R_{2}=.13 \mathrm{ohm}, \\
& R_{3}=.05 \mathrm{ohm}, \\
& R_{4}=.03 \mathrm{ohm}, \\
& D=.08 \text { ohm, and } \\
& C=.03 \text { ohm, }
\end{aligned}
$$

and that the problem is to find first the potential drop from $M$ to 3 with 1 ampere in the circuit and second the resistance of that part of the circuit included between $M$ and $N$. To more definitely visualize these problens, it may be assumed that $Q$ consists of a circular piece of sheet metal to which terminal posts are soldered. 
The first partial solution is obtained from the arrangement of linear conductors in figure 24 (b). For this arrangement the resistance between $M$ and the junction $R_{1}, R_{2}, R_{3}$, and $R_{4}$ (see fig. $\left.23, \mathrm{a}\right)$ is $(.5 \times 1.5) / 2$, or $.3750 \mathrm{ohm}$. Therefore, since the current is 1 ampere, the potential drop from $M i$ to $3, E_{1}=.3750$ volt.

The sccond partial solution is obtained from the arrangement of linear conductors shown in figure 24 (c). If $E_{2}$ represents the potential drop from $M$ to 3 for
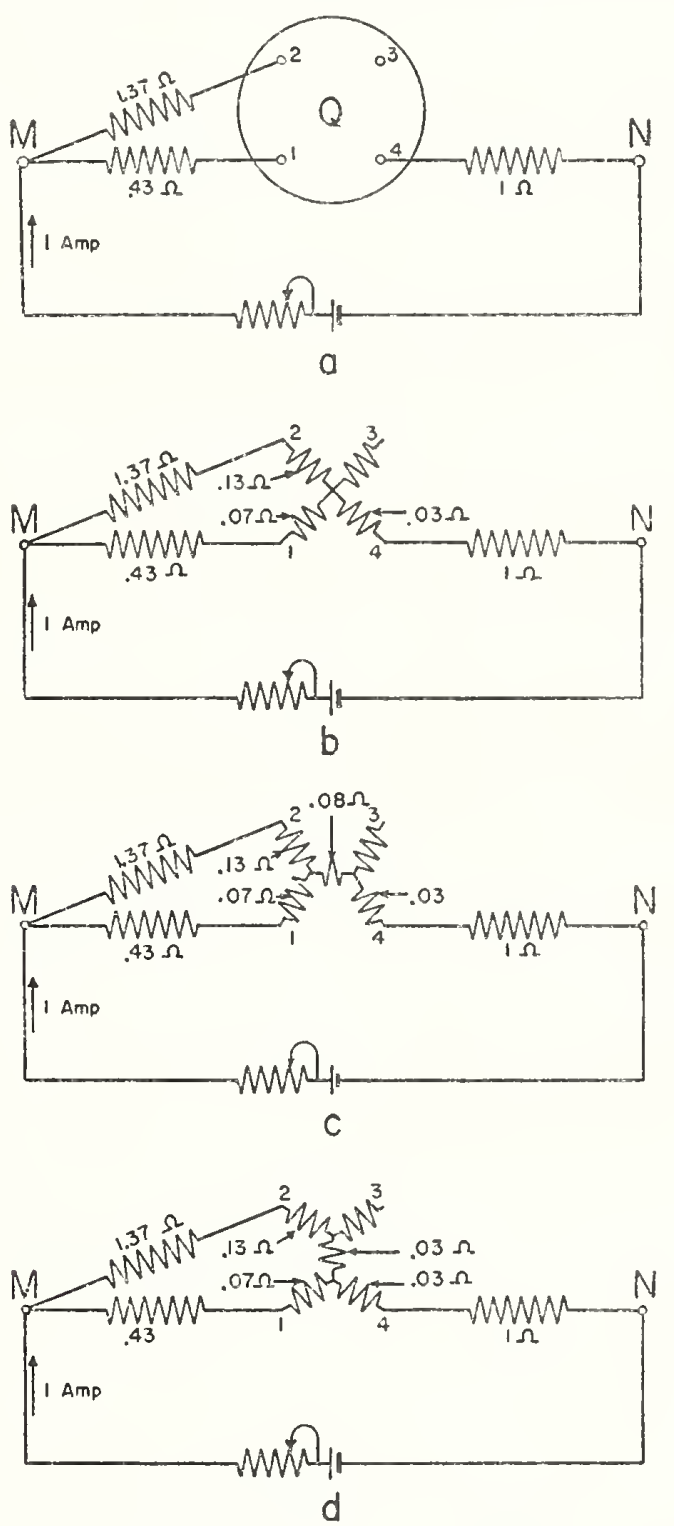

Figure 24.-A simple network containing one nonlinear four-terminal conductor.

$a$, a complcte circuit in which $Q$ represents the nonlinear four terminal conductor; $b$, a circuit showing the tcrminal resistances of $Q$ as linear resistances; $\boldsymbol{c}$, a circuit showing the terminal resistances and the direct resistance of $Q$ as linear resistances; and $d$, a circuit showing the terminal resistances and the cross resistance of $Q$ as linear resistances.

this arrangement, obviously, $E_{2}$ is then larger than $E_{1}$ by 0.08 volt, so $E_{2}=.4550$ volt.

The third partial solution is obtained from the arrangement shown in figure 24 (d). If $E_{3}$ represents the potential drop from $M$ to 3 for this arrangement, 
it is readily seen that the current in the upper branch is $(.43+.07) /(.43+.07+.03+$ $.13+1.37)=.2463$ ampere. This current multiplied by 1.5 , the resistance of the upper branch between $M$, and junction of $R_{2}, C$, and $R_{3}$ (see fig. 23, c) gives $E_{3}=.3695$ volt. Therefore, the potential drop from $M$ to 3 of the actual circuit, $E_{i}=E_{2}+E_{3}-E_{1}=.4495$ volt.

Sirce in the second partial solution the effect of the direct resistance of $Q$ appeared as an additive term, namely 0.08 volt, the first partial solution might have been omitted and the complete solution obtained by transferring this additive term to the third partial solution. This would have given $E=E_{3}+.08=.4495$ volt.

Considering now the second problem and letting $r_{1}, r_{2}$ and $r_{3}$ be the resistances of that part of the circuit between $M$ and $N$ for the arrangements shown in figures $23 b, c$, and $d$, it will be seen that the effect of the direct resistance, which is 0.08 ohm, will appear in the solution for $r_{2}$ as an additive term with a coefficient of unity. Therefore, it is not necessary to solve for $r_{1}$ or $r_{2}$.

Referring to figure 23 (d), it is readily seen that

$$
r_{3}=\frac{(1.37+.13+.03)(.43+.07)}{1.37+.13+.03+.43+.07}+.03+1=1.4069 \text { ohms. }
$$

Adding to this the resistance contributed by the direct resistance of $Q$, namely $.08 \mathrm{ohm}$, gives for the resistance of that part of the actual circuit between $M$ and $N$ of figure 23 (a)

$$
r=1.4069+.0 S=1.4869 \text { ohms. }
$$

The values stated for the six independent resistances of the nonlinear fourterminal conductor, $Q$, may be realized by the use of eight linear conductors

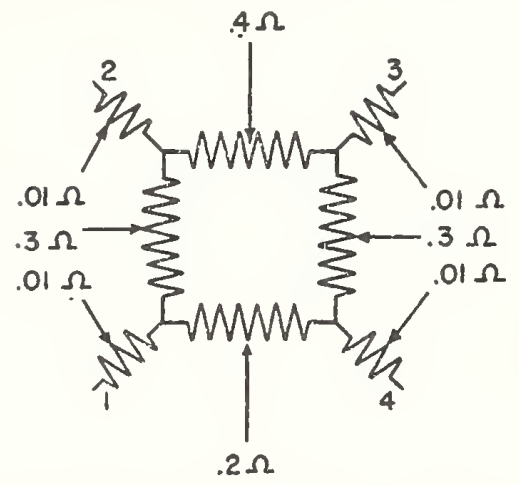

FIGURE 25. - Eight linear conductors so arranged and having such resistances as to be equivalent to the nonlinear four-terminal conductor, $Q$, of figure $24(a)$.

having the resistances and arrangement shown in figure 25 . If therefore these eight linear conductors are substituted for the nonlinear four-terminal conductor, the problems just considered become problems in linear networks, and the solutions given may be checked by well-known methods.

A somewhat more complicated problem is a determination of the effects of the three different current distributions in each of the four terminal blocks involved in changing three resistance coils from a series to a parallel connection and in measuring the resistances of the paralleling connections. A solution of this problem would serve to give a better idea as to how the method of analysis under consideration works out in practice, and at the same time give an answer to a question that frequently arises in connection with precise resistance comparisons.

Referring to figure 14, the problem is to find the ratio of the four-terminal resistance of the three 150 -ohm resistance sections with the paralleling connectors $L_{1}$ and $L_{2}$ removed to their four-terminal resistance with these connectors as shown, using in each case terminals 1 and 2 of terminal block $a$ and terminals 1 and 2 of terminal block $d$ as the four terminals. The resistances of the paralleling connections $L_{1}$ and $L_{2}$ are to be measured as four-terminal conductors. In the measurement of $L_{1}, L_{2}$ is removed and terminals 1 and 2 of terminal block $a$ and terminals 1 and 2 of terminal block $b$ are used as the four terminals. In the measurement of the resistance of $L_{2}, L_{1}$ is removed and terminals 1 and 2 of terminal block $c$ and terminals 1 and 2 of terminal block $d$ are used as the four terminals.

It will be assumed that all conductors are linear except the terminal blocks $a, b, c$, and $d$; and that each of these is a nonlinear foul-terminal conductor. Aside from what is obvious, the problen resolves itself into a determination of the effects of the direct and cross resistances of the terminal blocks $a, b, c$, and $d$.

With reference to the terminal resistances of the terminal blocks, it will be seen by reference to figure 14 that $R_{3}$ of each terminal block is included in the measurement of $L_{1}$ or $L_{2}, R_{1}$ of terminal blocks $a$ and $d$ are outside the resistances 
under consideration, $R_{1}$ of terminal blocks $c$ and $d$ may be considered as a part of a 150-ohm resistance section, and in each case $R_{4}$ may be considered as a part of a $150-0 h m$ resistance section. Consequently, none of the terminal resistances of any of the terminal blocks need appear explicitly in the analyses of the problem. Furthermore, the effects of the direct resistances and of the cross resistances of the terminal blocks must be small relative to the effects of the 150 -ohm resistance sections, so there is no need of making the first partial solution.

Considering that the terminal blocks are replaced by linear conductors representing the direct resistances of the terminal blocks gives the arrangement shown in figure 26 (a), and considering that the terminal blocks are replaced by linear conductors representing the cross resistances gives the arrangement shown in figure 26 (b). Therefore, the second partial solution may be obtained from a

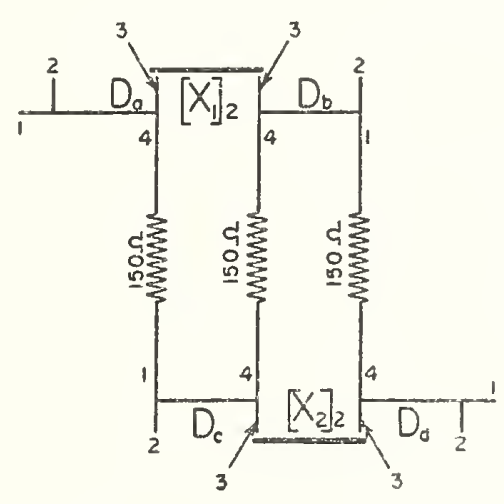

0

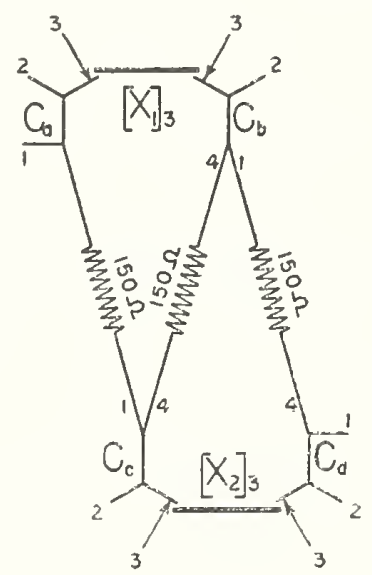

b

FIGURE 26. - Two arrangements of linear resistances for determining the the effect of three different current distributions in each of the four nonlinear four-terminal blocks shown in figure 18.

consideration of the arrangement shown in figure 26(a), and the third partial solution may be obtained from a consideration of the arrangement shown in figure $26(\mathrm{~b})$. Here $D_{a}, D_{b}, D_{c}$, and $D_{d}$ represent the direct resistances and $C_{a}, C_{b}, C_{c}$, and $C_{d}$ represent the cross resistances of the terminal blocks, $a, b, c$, and $d$. Let

$\left[R_{s}\right]_{2}=$ the four-terminal resistance of the entire combination with the series connection, excepting that part contributed by the cross resistances $C_{a}, C_{b}, C_{c}$, and $C_{d}$ of the terminal blocks.

$\left[R_{p}\right]_{2}=$ the four-terminal resistance of the entire combination with the parallel connection, excepting that part contributed by the cross resistances.

$\left[X_{1}\right]_{2}$ and $\left[X_{2}\right]_{2}=$ the resistances of the paralleling connections, excepting that part contributed by the cross resistances.

$\left[R_{s}\right]_{3}=$ the four-terminal resistance of the entire combination with the series connection, excepting that part contributed by the direct resistances, $D_{a}, D_{b}, D_{c}$, and $D_{d}$.

$\left[R_{D}\right]_{3}=$ the four-terminal resistance of the entire combination with the parallel connection, excepting that part contributed by the direct resistances.

$\left[X_{1}\right]_{3}$ and $\left[X_{2}\right]_{3}=$ the resistances of the paralleling connections, excepting that part contributed by the direct resistances.

$L_{1}$ and $L_{2}=$ the four-terminal resistances of the paralleling connections.

$R_{s}=$ the four-terminal resistance of the entire combination with the series connection, and

$R_{p}=$ the four-terminal resistance of the entire combination with the parallel connection. 
Referring to figure 26(a), it is readily seen that

$$
\begin{aligned}
& {\left[R_{s}\right]_{2}=450+D_{a}+D_{b}+D_{c}+D_{d},} \\
& {\left[R_{p}\right]_{2}=50+\left(9 D_{a}+4\left[X_{1}\right]_{2}+D_{b}+D_{c}+4\left[X_{2}\right]_{2}+9 D_{d}\right) / 9,} \\
& {\left[X_{1}\right]_{2}=L_{1}-D_{a}-D_{b}, \text { and }} \\
& {\left[X_{2}\right]_{2}=L_{2}-D_{c}-D_{d} .}
\end{aligned}
$$

From eq 83 it follows that

$$
\left[R_{\mathrm{s}}\right]_{2}=450\left[1+\left(D_{\mathrm{a}}+D_{\mathrm{b}}+D_{\mathrm{c}}+D_{\mathrm{d}}\right) / 450\right],
$$

and from eq 84,85 , and 86 it follows that

$$
\left[R_{p}\right]_{2}=50\left[1+\left(4 L_{1}+4 L_{2}+5 D_{a}-3 D_{b}-3 D_{c}+5 D_{d}\right) / 450\right] .
$$

Referring to figure 26 (b), it is easily seen that

$$
\begin{aligned}
& {\left[R_{s}\right]_{3}=450,} \\
& {\left[R_{p}\right]_{3}=50+\left(4\left[X_{1}\right]_{3}+4\left[X_{2}\right]_{3}-6 C_{a}-6 C_{d}\right) / 9} \\
& {\left[X_{1}\right]_{2}=L_{1}+C_{a}+C_{b}, \text { and }} \\
& {\left[X_{2}\right]_{3}=L_{2}+C_{c}+C_{d \cdot} .}
\end{aligned}
$$

From eq 89, 90, 91, and 92 it folldws that

$$
\begin{aligned}
& {\left[R_{\mathrm{s}}\right]_{3}=450, \text { and }} \\
& {\left[R_{p}\right]_{3}=50+\left(4 L_{1}+4 L_{2}-2 C_{a}+4 C_{b}+4 C_{c}-2 C_{d}\right) / 9 .}
\end{aligned}
$$

Transferring the additive terms of eq 88 which contain the direct resistances to eq 94 gives

$$
\begin{aligned}
R_{p}= & 50\left[1+\left(4 L_{1}+4 L_{2}+5 D_{a}-2 C_{a}-3 D_{b}+4 C_{b}-3 D_{c}+4 C_{c}+\right.\right. \\
& \left.\left.5 D_{d}-2 C_{d}\right) / 450\right] .
\end{aligned}
$$

Transferring the additive terms of eq 87 which contain the direct resistances to eq 93 gives

$$
R_{s}=450\left[1+\left(D_{a}+D_{b}+D_{c}+D_{d}\right) / 450\right] .
$$

From eq 95 and 96 it follows

$$
R_{s} / R_{p}=9\left[1-4\left(L_{1}+L_{2}+D_{a}-C_{a} / 2-D_{b}+C_{b}-D_{c}+C_{c}+D_{d}-\right.\right.
$$

which is eq 57 in the appendix on terminals and contacts.

It is readily seen that the resistance $R_{1}$, of terminal block $a$ is higher by $2 C_{a} / 3$ and the resistance $R_{1}$, of terminal block $d$ is higher by $2 C_{d} / 3$ with the parallel connection than with the series connection. In fact, this is the reason why $C_{a}$ and $C_{d}$ appear in eq 90.

Obviously the effects of the different current distributions in each of the fourterminal terminal blocks depend on their four-terminal resistances. Furthermore, by design or adjustment, these four-terminal resistances may be made exceedingly small. As an illustration of a design having exceedingly small fourterminal resistances consider an equilateral tetrahedron, using the apices as the terminals. Also, by design, it is possible to make the four-terminal resistances definite to almost any extent desired. In cases in which the four-terminal resistances are sufficiently small to be neglected, and are equally definite, a nonlinear four-terminal conductor may be considered as equivalent to four linear conductors connected in star.

In the solution of network problems, it is frequently possible to select for the independent variable any one of two or more quantities which are dependent on each other. Making different sclections leads to different forms. Then, by making approximations, still other forms are obtained of the solution. As an illustration of exact expressions for the current in the galvanometer branch of an unbalanced bridge consider eq $76,78,79$, and 81 . The second of these involves the drop in potential across the bridge with the galvanometer branch open, the third the drop in potential across the bridge with the galvanometer branch 
closed, the fourth the current through the bridge, while the first, that is the usual classical solution, involves the electromotive force in the battery branch. The second of these involves the resistance of the battery branch in a rather complicated way, as does also the classical solution. Therefore, if only these four were available it is conceivable that cases might arise in which it would be more convenient to use either the third or the fourth rather than the second or the classical form of solution. In many cases approximate solutions may be obtained in forms much more convenient to use than any of the forms of exact solutions, yet giving all the accuracy required.

\section{APPENDIX 6. OHM'S LAW}

The work of Ohm contributed materially to our concepts of current, electronotive force, and potential difference; originated our concepts of resistance and resistivity; gave the generally used relation between current, electromotive force, and resistance; and led to the formulas used in expressing the resistances of combinations of conductors in terms of their individual resistances and to various laws and theorems pertaining to the distribution of current in systems of conductors. However, more than 50 years before Ohm published his more important papers, Cavendish very probably had concepts of the quantities now called potential difference, curient, resistance, and of the property now called resistivity. Furthermore, from experiments with electrolytic conductors he reached the conclusion that the current is proportional to the first power of the potential difference.

During 1825,1826 , and 1827 , Ohm published a number of papers in which he described measurements of the electric conductance of metal wires, drew some general conclusions from his experimental data, and made an analysis of the electric circuit. These papers led to those general ideas and relations which taken collectively may be considered to be Ohm's law. From the standpoint of resistance comparisons, the most important of these general ideas is that the resistance of a conductor is independent of the current in it, while the more important of these relations are the equations used in expressing the resistances of combinations of conductors in terms of the resistances of the individual conductors.

The various conclusions reached by Ohm were not accepted unless or until verified by others. However, by 1843, when Wheatstone published an important paper, they were more or less generally accepted. Yet the feeling persisted that more accuiate measurements would show Ohm's law to be merely an approximation. In the early 1870's it was thought that definite departures from the law had been found. Accordingly, the British Association appointed a committee to investigate and report on the matter. Maxwell was the chairman of this committee, and presumably he devised the methods of test, but the measurements were made by Chrystal. A conclusion reached from an analysis of the experimental data obtained in the tests and reported by Maxwell in 1876 was that "If a conductor of iron, platinum or German silver one square centimeter in cross section has a resistance of one ohm for infinitely small currents its resistance when acted upon by an electromotive force of one volt (provided the temperature is kept the same) is not altered by so much as $1 / 10^{12}$ part." These tests have usually been considered as proof that Ohm's law is exact, at least to the extent likely to be significant in electrical measurements. Nevertheless, the subject is deserving of some discussion.

An expression for the potential drop between the terminals of a standard resistor (in which there is a current) that presumably is more exact than that generally used is

where

$$
e=R I+x+y+z
$$

$e=$ the potential drop between the potential leads of the standard resistor, $R=$ the direct resistance of the standard lesistor,

$I=$ the component of the current under the control of the operator and which enters and leaves the standard resistor through the current terminals,

$x=R i$, where $i$ is the current not under the control of the operator,

$y=$ the component of the potential difference having its origin in the standard resistor and potential leads and which is not caused by the current $I$, and

$z=$ the component of the potential drop in the standard resistor and potential leads caused by changes in the magnetic field. 
Letting $E=$ that component of the potential drop caused by the current, $I$, it follows from eq 98 that

$$
E=R I \text {. }
$$

Pertinent questions concerning the application of these and other equations, based on Olim's law, to standard resistors and resistance coils under normal conditions of use are:

1. To what extent is $R$ independent of $I$ ?

2 . To what extent is eq 99 reliable?

3. To what extent are the formulas used in expressing the resistance of combinations of conductors in terms of the resistances of the individual conductors reliable?

4. To what extent can $E$ be separated from $e$ ?

Question 1.-A precision method of determining the extent to which $R$ is independent of $I$ consists in the use of a bridge (either Wheatstone or Thomson) and observing the changes in balance with changes in the magnitude of the test current and the changes in balance with the time following the establishment or reversal of the test current. The procedure followed in establishing balances must be such as to give a separation of $E$ and $e$. Questions 2 and 3 are not involved.

Obviously only relative effects of the test current upon the resistances of the conductors constituting the arms of the bridge can be determined in this way. Therefore, a quantitative conclusion as to the effect upon some particular standard resistor can be reached only after a number of observations have been made, and this conclusion must involve some assumption such as that the effect for a selected group of standard resistors is, on the average, zero, except to the extent that causes are found and the effects of these causes are taken into consideration.

In the normal use of standard resistors the test current causes the resistance material to assume a temperature above that of the surrounding medium. This results in what may be considered as the normal change in the resistance of the conductor corresponding to the difference in temperature. In addition, the difference in temperature between the resistance material and its mechanical support may cause strains in the conductor, and if so, these strains result in a further change in the resistance. These changes in resistance are at least approximately proportional to the square of the current and reach their full magnitude only after the current has been maintained a sufficient time for the difference in temperature to become substantially constant. ${ }^{6}$ These will be referred to as the primary efiects of the current upon the resistance. A procedure for determining and applying a correction for the primary efiects of the test current has been considered under the heading "Load Coefficients."

In addition, the current affects the resistance, in some cases at any rate, in other ways. When a number of standard resistors are substituted one after another in the $X$ arm of the bridge and balances are established by the procedure outlined above, it not infrequently happens that the precision attainable for these balances is less with some of the standards than with others, although all may have low temperature coefficients and equal facilities for dissipating the heat developed by the test current. In cases in which the precision attainable in establishing a balance of the bridge is well below the average, it not infrequently happens that the resistance of the resistor under investigation is low immediately following a reversal of the test current and then increases more or less gradually attaining a practically constant value in a time ranging from a few seconds to a minute or more. It happens less frequently that the resistance changes in an irregular way and does not come to a practically constant value.

A comparison of the performance characteristics with the details of construction, properties of the materials used in the construction, insulation resistance, etc., frequently give a definite clue as to the cause of the changes in resistance. In some cases it appears that following a change in the current the Peltier effect, especially at junctions between copper and the resistance material, causes a local increase and a local decrease in the temperature, which results in a thermoelectromotive force. This electromotive force, after it reaches a constant value, is proportional to the current and reverses sign following a reversal of the direction of the current. In other cases, it appears that an appreciable part of the current passes through the insulation, and that this part decreases for a time (relative to

${ }^{6}$ It is conceivable (in cases in which the conductor is not rigidly supported) that the forces resulting from electromagnetic action might produce strains of sufficient magnitude to cause perceptible changes in the resistance. If so, these changes would appear almost instantly following a change in the magnitude of the current. 
the total current) following the establishment or reversal of the test current. In still other cases it appears that there is a gradual mechanical yielding of the insulating material under the stresses caused by the temperature difference between the resistance material and its mechanical support. In such cases the effect of the test current upon the resistance may be larger at the end of 15 seconds than at the end of a longer time.

Changes in resistance resulting from the test current, whether or not the way in which they are brought about is known, and provided they are not proportional to the square of the current, will be referred to as the secondary effects of the current upon the resistance. There are many possible causes of secondary effects, so it is not possible in all cases to obtain as complete information concerning them as might be desired.

However, correlation of the secoudary effects with details of construction of many resistors makes it possible to predict with some degree of certainty their magnitudes in others in which they are too small to be observed. For relatively few resistance coils and standard resistors under normal conditions of use is it probable that the secondary effects of the current upon the resistance amount to less than 1 in 100 million. On the other hand, for relatively few standard resistors having resistances in the range from 0.0001 to $10,000 \mathrm{ohms}$, if of the precision type and of good quality, are the secondary cffects of the normally used test currents in excess of 1 in 100,000 .

Question 2.-It appears that the first precise measurements having a direct bearing on the relation between a change in current from one steady value to another steady value and the resulting change in potential drop were made by F. A. Wolff of this Bureau. At a meeting of the American Association for the Advancement of Science in the summer of 1899 he presented a paper entitled "Experimental Test of the Accuracy of Ohm's Law." Unfortunately, no further record of this investigation has been found either in the form of a published paper or unpublished notes or data. The circuit used was essentially that shown in figure 27. Here $x_{1}, x_{2}, x_{3}$, and $x_{4}$ represent the four arms of a balanced Wheatstone bridge. This bridge constitutes the $X$ arm of a second Wheatstone bridge, the other arms of which are designated $Y, A$, and $B$. This bridge is also balanced. Dr. Wolff refers to the

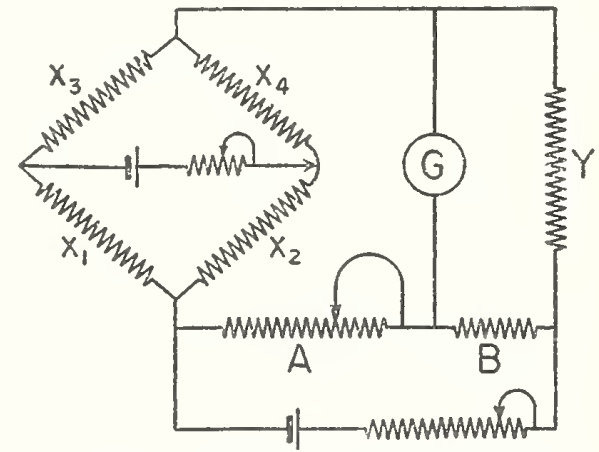

FIGURE 27.-Wolff's circuit for testing the accuracy of Ohm's law. bridge whose arms are designatcd $x_{1}, x_{2}, x_{3}$, and $x_{4}$ as the auxiliary bridge, the other bridge as the main bridge, and the circuit as a bridge within a bridge.

This method was tried about 1920 [105], when it gave promise of reasonably satisfactory results, though some difficulties were encountered. These difficulties were, for the most part, obviated by replacing the battery of the auxiliary bridge by a source of alternating current and omitting a provision for balancing the auxiliary bridge. Standard resistors having nominal values of $10 \mathrm{ohms}$ were used as the $x_{1}, x_{2}, x_{3}$, and $x_{4}$ arms of the auxiliary bridge and the $Y$ arm of the main bridge. The direct current in the $Y$ arm was adjusted to a value somewhat larger than that regularly used in testing 10 -ohm standard resistors and the alternating current in the supply branch of the auxiliary bridge was adjusted to a root mean square value equal to approximately twice the value of the direct current in the $Y$ arm of the main bridge.

Since $x_{1}, x_{2}, x_{3}$, and $x_{4}$ were approximately equal in resistance very little of the alternating current passes through the $A, B$, and $Y$ arms, or through the battery or galvanometer branch of the main bridge. Presumably, therefore, the alternating current had no appreciable effect except in $x_{1}, x_{2}, x_{3}$, and $x_{4}$, the arms of the auxiliary bridge. Following the usual procedure in balancing the main bridge, the precision of the balances was about 1 part in 10 million. On opening the connection to the source of the alternating current, the balance was changed by an amount not perceptibly different from that corresponding to the change in resistance of the series parallel combination of $x_{1}, x_{2}, x_{3}$, and $x_{4}$ resulting from the reduced power dissipation. 
A conclusion to be drawn from this experiment, for standard resistors such as were used and currents having values over a limited range, including values normally used in testing such standards, is that part of the potential drop caused by the current is equal to the product of the resistance and current to an cxtent at least somewhat beyond that to which the resistance may be considered as independent of the current. It appears that if apparatus were designed especially for the purpose and the most suitable resistance wire obtainable were used, the method might be expected to yield a precision of a few parts in 100 million.

Question 3. - The test made by Chrystal, using "the first method which occurred to the Committee," might have given directly an answer as to the reliability of one of the formulas used in expressing the resistance of a combination of conductors in terms of the resistances of the individual conductors. In this method five resistance coils of equal resistance are compared with each other, four in a 2-and-2 series-parallel combination. According to Ohm's law, the resistance of each coil is the same as the resistance of the other four in the series-parallel combination, and on the average this would be true, even if there were slight differences in the resistances of the five coils. However, the quality of the resistors used by Chrystal was not sufficiently good to permit of measurements to what now would be considered a high accuracy.

An insignificant modification of the method consists in the comparison of the resistances of four standard resistors (of nominally equal resistance) with each other and with the four in the series-parallel combination, by alternate substitution in the same arm of a Wheatstone bridge. In a test made about 1920 [105], using 10-ohm standard resistors of good quality, this method gave reasonably satisfactory results, when corrections were applied to take into account the effects of the resistances of terminals and contacts, but not sufficiently satisfactory to be considered as a definite answer as to the reliability of one of the formulas used in expressing the resistance of a combination of conductors in terms of their individual resistances. However, there seems to be no direct or indirect experimental evidence (such as that given in the answer to question 2) not in conformity with the supposition that the resistances of combinations of conductors, as expressed in terms of their individual resistances, are reliable to the extent that the resistances of the individual conductors are definite. But to realize this accuracy it may be necessary to take into account not only the resistances of connectors but also the effects of the different current distributions in terminal blocks or the different current distributions through or over the surface of the insulation.

Question 4.- The extent to which the potential drop $E$ resulting from the current $I$ and the actual potential drop $e$ can be separated may be judged by deliberately introducing into a bridge circuit a disturbing electromotive force and noting the resulting change in the balance and precision of the balance. In cases in which the disturbing electromotive force amounts to only a few microvolts and is reasonably constant or is only two or three orders higher and alternating at frequencies of power distribution systems (or higher) neither the balance nor the precision of the balance is affected by amounts corresponding to as much as 0.01 microvolt. This question has been discussed more fully in appendix 2 , Thermoelectromotive Forces.

This brief discussion of Ohm's law has been limited mainly to questions arising in precision resistance comparisons. It represents an attempt to coordinate one of several classes of information obtained more or less incidentally in the design, construction, and testing of standard resistors, bridges, potentiometers, etc.

\section{APPENDIX 7. UNITS OF RESISTANCE}

A comprehensive discussion of units of resistance is not pertinent to the subject of this paper. However, some reference should be made here to the unit now known as the NBS International Ohm and the relations between this unit and some of the other units now being used or whose use is contemplated.

The International Conference at London in 1008 established an International Committee on Electrical Units and Standards to formulate a plan for and direct such work as may be necessary in connection with maintenance of standards, fixing of values--intercomparison of standards and to complete the work of the conference. This International Committee met in Washington in the spring of 1910 , and one of its decisions was "to accept (for the present) as the International ohm the mean of the units of the Physikalisch-'Technische Reichsanstalt and the National Physical Laboratory" which had been realized in these laboratories from the so-called mercury ohm. 
Standard resistors from Germany, France, and England were compared with standard resistors of the United States from time to time during a period of about 6 weeks. Values were then assigned to all the standard resistors used in the intercomparisons in terms of the new unit. In assigning the new values, it was assumed that the mean value of one of the standard resistors from the National Physical Laboratory during the time of the intercomparisons was the same as the value previously assigned in the National Physical Laboratory, and that the mean of the values of the resistances of the two standard resistors from the Physikalisch-Technische Reichsanstalt during the time of the intercomparion was the same as the mean of the valnes previously assigned in the PhysikalischTechnische Reichsanstalt. Equal weights were given to the previously assigned value in the National Physical Laboratory and the mean of the previously assigned values in the Physikalisch-Technische Reichsanstalt.

Following the assignment of new values to the standard resistors of this Bureall used in the intercomparison, they were intercompared either directly or indirectly with miany other standard resistors of this Bureau. From time to time since then, selected groups (usually of 20 or more) 1-ohm standard resistors have been intercompared, and on the basis of relative changes and previous good performances a group of 10 standards has been selected from the larger group. In assigning new values to all the standard resistors of the larger group and to others, it has been assumed that the mean resistance of the group of 10 had not changed since the previous similar intercomparison. This in general is the procedure by which the unit established in 1910 has been maintained in this Bureau.

From 1910 to 1929 only standard resistors of the sealed type developed by Rosa [76] were included in the groups of 10 . A total of 20 different standards have been included in the groups of 10 , while 3 of these 20 have been included in every group of 10 . From 1932 to 1938 only standards of the smaller doublewalled type [89] were included in the groups of 10. More recently, only standards of the larger double-walled type constructed by Thomas in 1933 have been included in the groups of 10 .

The unit established in 1910 and maintained in this Bureau in the way described above has been referred to by different names. The name now being used is the NBS International Ohm.

Obviously, a unit of resistance maintained in this way changes with time to the extent of the proportional decrease (or increase) of the resistances of the standard resistors used in its maintenance. Therefore, it is not to be presumed that the NBS International Ohm has remained constant during the time since its establishment. To obtain information on this point various investigations have been made in this Bureau. Most of those since 1927 have been made by Thomas. In addition, groups of standard resistors have been sent abroad for measurements of their resistances in other national laboratories, and standards of other national laboratories have been measured in this Bureau. More recently these international intercomparisons have been made at the International Bureau of Weights and Measures: Comparisons of the values found for the resistances of the same standard resistors in different national laboratories have given the relations between the units of resistance of the different countries. From information as to the manner in which the units of the different countries were established and have been maintained, the results of the international intercomparisons and the investigations made in this Bureau, it may be concluded that the probable change in the NBS International Ohm during the 30 years since its establishment has been less than 30 parts in 1 million. Since the standard resistors now being used in this Bureau in the maintenance of the units are fully one order better than those formerly used for this purpose, it is reasonable to presume that the present rate of change of the NBS International Ohm is less than 1 part in 1 million per year.

The relation between the NBS International $\mathrm{Ohm}$, the mean international ohm, and the units of resistance of other countries is given in table 1, which contains data taken from the 1933,1935 , and 1937 reports of the International Committee of Weights and Measures (Comité International des Poids et Mesures, ProcésVerbaux des Séances) and Comptes Rendus, page 24, volume 209, 1939. 
TABLE 1.-Units of resistance of various countries, as determined from comparisons of standards at the International Bureau of Weights and Measures ${ }^{1}$

[Expressed as departures in microhms from the Mean International Ohm]

\begin{tabular}{|c|c|c|c|c|}
\hline Unit of- & $\begin{array}{c}\text { Third compari- } \\
\text { son, November } \\
1933\end{array}$ & $\begin{array}{c}\text { Fourth com- } \\
\text { parison, March } \\
1935\end{array}$ & $\begin{array}{l}\text { Fifth compari- } \\
\text { son, December } \\
1936\end{array}$ & $\begin{array}{c}\text { Sixth compari- } \\
\text { son, February } \\
1939\end{array}$ \\
\hline $\begin{array}{l}\text { Germany } \\
\text { United States. } \\
\text { France. } \\
\text { Great Britain } \\
\text { Japan. } \\
\text { Russia }\end{array}$ & $\begin{array}{r}+10.6 \\
-6.4 \\
+7.3 \\
-5.2 \\
-8.3 \\
+9.5\end{array}$ & $\begin{array}{r}+9.8 \\
-5.5 \\
+69.5 \\
0.0 \\
-3.6 \\
-11.2 \\
+10.6 \\
0.0\end{array}$ & $\begin{array}{r}+6.6 \\
-3.7 \\
+0.9 \\
-3.9 \\
-10.0 \\
-0.4\end{array}$ & $\begin{array}{r}+9.1 \\
-3.2 \\
+3.7 \\
-6.5 \\
-14.4 \\
+0.8\end{array}$ \\
\hline
\end{tabular}

I In this table a plus sign signifies that the unit of the country was larger than the Mean International Ohm.

The Mean International Ohm was defined as the mean of the units of electrical resistance, in 1935, of Germany, United States, Great Britian, Japan, and Russia. After this mean was obtained, it was taken by France as the new French unit and by Russia as the new Russian unit. The data given in the table are based on the assumption that units of resistance are being maintained with equal fidelity in each of the six countries.

The unit of the United States is the unit of resistance referred to above as the NBS International Ohm. Therefore, in the early part of 1939,

1 NBS International Ohm=0.999 997 Mean International Ohm.

From measurements made in this Bureau by Wenner, Thomas, Cooter, and Kotter, the results of which were reported to the International Bureau of Weights and Measures in December 1938,

1 NBS International $\mathrm{Ohm}=1.000485$ absolute ohm.

From measurements made in this Bureau by Curtis, Moon, and Sparks, the results of which were reported to the International Bureau of Weights and Measures in December 1938,

1 NBS International $\mathrm{Ohm}=1.000484$ absolute ohm. In each case the uncertainties in the measurements were estimated to be 2 parts in 100,000 .

In 1937 the International Committee on Weights and Measures adopted provisionally the relation

1 Mean International $\mathrm{Ohm}=1.00048$ absolute ohm.

From this relation and the data given in table 1 , it follows that provisionally

1 NBS International $\mathrm{Ohm}=1.000477$ absolute ohm as of February 1939.

\section{APPENDIX 8. REFERENCES}

This list of references is by no means comprehensive, but an effort has been made to include most of the little known publications containing contributions of importance at the time. In cases in which original publications are not readily available, references are made to later publications or to text and handbooks.

\section{(a) AUTHORS}

The letters included in brackets at the end of each reference indicate the subjects discussed. The key to these letters is given under the heading "Subjects" p. 293.

[1] R. Appleyard, Proc. Phys. Soc. (London) 14, 243 (1895-96) [P].

[2] W. E. Ayrton and T. Mather, Electrician 32, 627 (1893) [G].

[3] A. C. Becquerel, Ann. Chim. 32, 425 (1826) [D].

[4] A. Blondel, Ann. phys. [9] 10, 219 (1918) [G].

[5] P. W. Bridgeman, Proc. Am. Acad. Arts Sci. 5y, 129 (1922) [I].

[6] H. B. Brooks, BS J. Research 4, 297 (1930) RP150 [F].

[7] H. B. Brooks, BS J. Research 11, 211 (1933) RP586 [O].

[8] H. B. Brooks and A. W Spinks, BS J. Research 9, 781 (1932) RP506 [O].

[9] H. L. Callendar, Proc. Phys. Soc. (London) 22, $220(1909-10)$ [K].

[10] G. Carey-Foster, Teleg. Eng. J. 1, 196 (1872-13) [S].

[11] C. D. Child and O. M. Stewart, Phys. Rev. 4, 502 (1897) [K]. 
[12] S. H. Christie, Trans. Roy. Soc. (London) 123, 95 (1833) [U].

[13] G. Chrystal, Brit. Assn. Adv. Sci. Rep. 46, 38 (1876) [I].

[14] E. Dibbern, Z. Instrumentenk. 31, 105 (1911) [F].

[15] H. Diesselhorst, Z. Instrumentenk. 31, 250 (1911) [F].

[16] P. H. Dike, Rev. Sci. Instr. 2, 379 (1931) [Q, T].

[17] I. R. Edler, Proc. Am. Acad. Arts Sci. 40, 549 (1904-05) [S].

[18] H. Edwards, Electrotech. u. Maschinenbau 33, 165 (1915) [G].

[19] W. Esmarch, Ann. Physik [4] 69, 255 (1922) [K, P, S].

[20] J. Fischer, Z. Instrumentenk. 54, (137) 1934) [K].

[21] J. A. Fleming, Handbook for the Electrical Laboratory and Testing Room (Electrician, London, 1901) [K, P].

[22] R. T. Glazebrook, Phil. Mag. [5] 11, 291 (1881) [0].

[23] R. T. Glazebrook, Brit. Assn. Adv. Sei. Rep. 63, 136 (1893) [K].

[24] R. T. Glazebrook, Phil. Mag. [5] 50, 410 (1900) [L].

[25] A. Gray, Absolute Measurements in Electricity and Magnetism, vol. I (Macmillan, London, 1888) [E, K, P, S].

[26] T. Gray, Phil. Mag. [5] 12, 283 (1881) [K].

[27] E. H. Griffiths, Trans. Roy. Soc. (London) 184, 391 (1893) [O].

[28] C. E. Guye, Arch. Sci. phys. nat. 27, 26 (1892) [F, K].

[29] B. Hague, Alternating Current Bridge Methods (Pitman \& Sons, London, 1930) [C, K, O, T, U].

[30] T. R. Harrison and P. D. Foote, Trans. Am. Inst. Elec. Eng. 39, 390 (1920) [C].

[31] H. Hausrath, Ann. Physik. [4] 16, 134 (1905) [D].

[32] O. Heaviside, Phil. Mag. [4] 45, 114 (1873) [K].

[33] O. Heaviside, Phil. Mag. [4] 45, 245 (1873) [D].

[34] O. Heaviside, Phil. Mag. [4] 46, 469 (1873) [D].

[35] H. Helmholtz, Pogg. Ann. 89, 211 (1853) [F].

[36] R. Hieck, Electrotech. u. Maschinenbau 40, 158 (1922) [P].

[37] A. V. Hill, J. Sci. Instr. 11, 309 (1934) [F].

[38] W. Jaeger, Z. Instrumentenk. 23, 261 and 353 (1903) [F].

[39] W. Jaeger, Z. Instrumentienk. 24, 288 (1904) [D, K].

[40] W. Jaeger, Z. Instrumentenk. 26, 69 (1906) [D, F, J, K].

[41] W. Jaeger, Ann. Physik [4] 21, 64 (1906) [F].

42] W. Jaeger, Z. Instrumentenk. 26, 360 (1906) [K].

[43] W. Jaeger, Z. Instrumentenk. 28, 206 (1908) [F].

[44] W. Jaeger, Elektrischemesstechnik (3d ed.) (Johann Ambrosius Barth, Leipzig, (1928) [F. G. H].

[45] W. Jaeger and H. Diesselhorst, Wiss. Abhandl. physik-tech. Reichsanstalt 4, 119 (1904) [B. P].

[46] W. Jaeger, St. Lindeck and H. Diesselhorst. Z.' Instrumentenk. 23, 33 (1908) $[\mathrm{H}, \mathrm{P}]$.

[47] G. Jones, J. Am. Chem. Soc. 50, 1049 (1928) [Q, T, U].

[48] A. E. Kennelly, Elec. World 34, 413 (1899) [N].

[49] G. Kirchhoff, Pogg. Ann. 72, 497 (1847) [C].

[50] G. Kirchhoff, Phil. Mag. [5] 11, 81 (1881) [D].

[51] F. Kohlrausch, Pogg. Ann. 7\%, 497 (1847) [C].

[52] F. Kohlrausch, Ann. Physik 20, 76 (1883) [D].

[53] K. Kuepfmueller, Arch. Electrotech. 12,160 (1923) [N].

[54] F. A. Laws, Electrical Measurements (McGraw-Hill Book Co., New York, N. Y., 1917) [D, F, P, S].

[55] M. G. Lloyd, Proc. Eng. Soc. West. Pa. (Oct. 1903) [H, M, P].

[56] T. Mather, Phil. Mag. [5] 29, 434 (1890) [F].

[57] J. C. Maxwell, Brit. Assn. Adv. Sci. Rep. 16, 36 (1876) [I].

[58] J. C. Maxwell, Electricity and Magnetism, 3d ed. (Clarendon Press, Oxford, 1892) [E, K, S].

[59] Michel, Compt. rend. 189, 1257 (1929) [F].

[60] M. F. Molikov, Mémoirs de la Chambre Centrale des Poids et Mesures de j'U. R. S. S. Présentés au Comité. Consultatif d'Électricité et Photométrie. p. 5 (1932) [J].

[61] W. J. H. Moll, Proc. Phys. Soc. (London) 35, 253 (1923) [F].

[62] W. J. H. Moll and H. C. Burger, Phil. Mag. [6] 50, 626 (1925) [F].

[63] E. F. Mueller and F. Wenner, J. Research NBS 15, 477 (1935) RP842 [A].

[64] F. H. Nalder, Brit. Assn. Adv. Sci. Rep. 63, 702 (1893) [S].

[65] E. F. Northrup, Methods of Measuring Electrical Resistance. (McGrawHill Book Co., New York, N. Y., 1912) [D, F, G, K, P, S].

[66] G. S. Ohm, Schweiggers J. 46, 137 (1826) [I].

[67] G. S. Ohm, Gesammelte Abhandlungen (Johann Ambrosius Barth, Leipzig, 18.92) 17, $25[\mathrm{I}]$. 
[68] A. Paalzow and H. Rubens, Ann. phys. [3] 37, 529 (1889) [K].

[69] H. A. Perkins, Phvs. Rev. 18, 131 (1921) [I].

[70] H. A. Perkins, J. phys. [6] 4, 246 (1923) [I].

[71] Lord Rayleigh, Proc. Poy. Soc. (London) 49, 203 (1891) [C, K].

[72] Lord Rayleigh, Trans. Roy. Soc. (London) 173, 697 (1882) [L].

[73] E. H. Rayner, Proc. Phys. Soc. (London) 27, 384 (1914-15) [M].

[74] J. H. Reeves, Proc. Phys. Soc. (London) 14, 166 (1895) [B, P].

[75] H. Rohmann, Physik. Z. 14, 203 (1913) [F].

[76] F. B. Rosa, Bul. BS 5, 413 (1908) S107 [R].

[77] A. Rosen, J. Inst. Elec. Fing. 62, 916 (1924) [N].

[78] A. Schuster, Phil. Mag. [5] 39, 175 (1895) [K].

[79] L. Schwendler, Phil. Mag. [4] 31. 364 (1866) [K].

[80] G. F. C. Searle, Electrician 66, 999 (1911) [F].

[81] G. F. C. Searle, Electrician, 67, 12 (1911) [M, P].

[82] F. E. Smith, Electrician, 57, 976 (1906) [B, D, H, J, K, L, S, U].

[83] F. E. Smith, Electrician, 57, 1009 (1906) [K, L, P, S].

[84] F. E. Smith, Dictinnary of Applied Physics, R. Glazebrook edition (Macmillan, London, 1922) 2, 718 (1922) [K, I, M, P, S].

[85] S. G. Starling, Electricity and Magnetism (Longmans, London, 1912) $[\mathrm{K}, \mathrm{S}]$.

[86] H. von Steinwehr, Handbuch der Physik (Springer, Berlin, 1927) 16, 445 $[\mathrm{D}, \mathrm{F}, \mathrm{J}, \mathrm{K}, \mathrm{U}]$.

[87] F. Streintz, Ann. Physik [4] 3, 1 (1900) [S].

[88] L. Thevenin, Comptes rendus 97, 159 (1883) [C].

[89] J. L. Thomas, BS, J. Research 5, 295 (1930) RP201 [R].

[90] J. L. Thomas, BS., J. Research 12, 313 (1934) R P657 [R].

[91] J. L. Thomas, J. Research NBS 13, 681 (1934) RP737 [R].

[92] J. L. Thomas, J. Research NBS 14, 589 (1935) RP789 [R].

[93] J. L. Thomas, J. Research NBS 16, 149 (1936) RP863 [R].

[94] W. Thomson, Phil. Mag. [4] 24, 156 (1862) [K, P].

[95] W. Volkman, Ann. Physik [4] 10, 217 (1903) [G].

[96] K. W. Wagner, Elek trotech. Z. 32, 1001 (1911) [T].

[97] K. W. Wagner, Elektrotech. Z. 33, 635 (1912) [T].

[98] C. W. Waidner, H. C. Dickinson, F. F. Mueller, and D. R. Harper, 3d. Bui. BS 11. 575 (1915) RP241 [RU].

[99] F. Wenner, Phys. Rev. 17, 384 (1903) [S].

[100] F. Wenner, Bul. BS 6, 347 (1909) RP134 [C, F, K].

[101] F. Wenner, Bill. BS 8.559 (1912) RP181 [B, E, F, K, P, S].

[102] F. Wenner, J. Wash. Acad. Sci. 2, 114 (1912) [R].

[103] F. Wenner, BS Sci. Pap. 12, 469 (1915) S258 [S].

[104] F. Wenner, Bul. BS 13, 211 (1916) S273 [F].

[105] F. Wenner, Phys. Rev. [2] 15, 531 (1920) [I].

[106] F. Wenner, Phys. Rev. [2] 23, 780 (1924) [I].

[107] F. Wenner, J.Opt. Soc. Am. 11, 495 (1925) [G].

[108] F. Wenner, BS Sci. Pap. 21, 191 (1926) S531; Proc. Phys. Soc. 39, 124 (1927) [Cं].

[109] F. Wenner, N. L. Forman, and A. R. Lindberg, Phys. Rev. [2] 20, 589 (1922) [I].

[110] F. Wenner and J. L. Thomas, BS J. Research 12, 147 (1934) RP639 [R].

[111] F. Wenner and A. Smith, BS Sci. Pap. 19, 297 (1924) S481 [H].

[112] F. Wenner, E. H. Smith and F. M. Soule, BS J. Research 5, 711 (1930) R P223 [U].

[113] F. Wenner and F. M. Soule, Phys, Rev. [2] 23, 780 (1924) [I].

[114] F. Wenner and F. Weibel. Bul. BS 11, 1 (1914) S 223 [S].

[115] F. Wenner and F. Weibel, Bul. BS 11, 65 (1914) S225 [B, S].

[116] F. Wenner, E. Weibel, and F. C. Weaver, Phys. Rev. [2] 3, 497 (1914) [F].

[117] C. Wheatstone, Trans, Roy. Soc. (London) 133, 303 (1843) [U].

[118] C. Wheatstone, Pogg. Ann. 62, 535 (1844) [U].

[119] W. P. White, Phys. Rev. 19, 305 (1904) [F].

[120\} W. P. White, Z. Instrumentenk. 34, 71 (1914) [A, G, O].

[121] F. A. Wolff, M. P. Shoemaker, and C. A. Briggs, Bul. BS 12, 449 (1915-16) $\mathrm{S} 256[\mathrm{~B}, \mathrm{H}, \mathrm{P}]$.

[122] A. Zeleny, Phys. Rev. 32, 297 (1911) [F].

[123] F. Zernike, Koninkl. Akar. Wetenschappen Amsterdam 24, 239 (1922) [F].

[124] L. A. Zurcher and A. C. Seletzky, Trans. Am. Inst. Elec. Engrs., 1939 [K]. 


\section{(b) SUBJECTS}

The numbers included in brackets at the end of each subject refer to publications in which the subject is discussed. The key to these numbers is given under the heading "Authors."

[A] Adjustable resistance elements. $[63,120]$.

[B] Balancing of resistances of connectors. [45, 74, 82, 101, 115, 121].

[C] Current distribution in a linear network. $[29,30,40,100,108]$.

[D] Differential galvanometer method. $[3,31,33,34,39,40,50,51,52,54,65$, $82,86]$.

[E] Four-terninal conductors. [25, 35, 58, 80, 101$]$.

[F] Galvanometers. $[6,14,15,28,37,38,40,41,43,44,54,56,59,61,62,65$, $75,86,100,101,104,116,119,122,123]$.

[G] Galvanometer shunts. $[2,4,17,44,65,95,107,120]$.

[H] Measurement of resistances of connectors. [44, 46, 55, 82, 111, 121].

[I] Ohm's law. [5, 13, 57, 66, 67, 69, 70, 105, 106, 109, 113].

[J] Potentiometer method. [40,60, 82, 86].

[IK] Sensitivity of bridge's. [9, 11, 19, 20, 21, 23, 25, 26, 28, 29, 32, 39, 40, 42, 51, $58,65,68,71,78,79,82,83,84,85,86,94,100,101,125]$.

[L] Series-parallel build up. [24, $72,82,83,84]$.

[M] Shunting of standard resistors. $[55,73,81,84]$.

[N] Star-polygon transformations. $[48,53,77]$.

[O] Thermoelectromotive forces. $[7,8,22,27,29,120]$.

[P] Thomson bridge. $[1,19,21,25,36,45,46,54,55,65,74,81,83,84,94,101$, 1211 .

[Q] Shielding. $[16,47]$.

[R] Standard resistors. [76, 89, 90, 91, 92, 93, 98, 102, 110].

[S] Various methods and their modifications. [10, 18, 19, 25, 54, 58, 64, 65, 82, $83,84,85,87,99,101,103,114,115$ ].

[T] Wagner hranch. $[16,29,47,96,97]$.

[U] Wheatstone bridge. $[12,29,47,82,86,98,112,117,118]$. 


\section{INDEX}

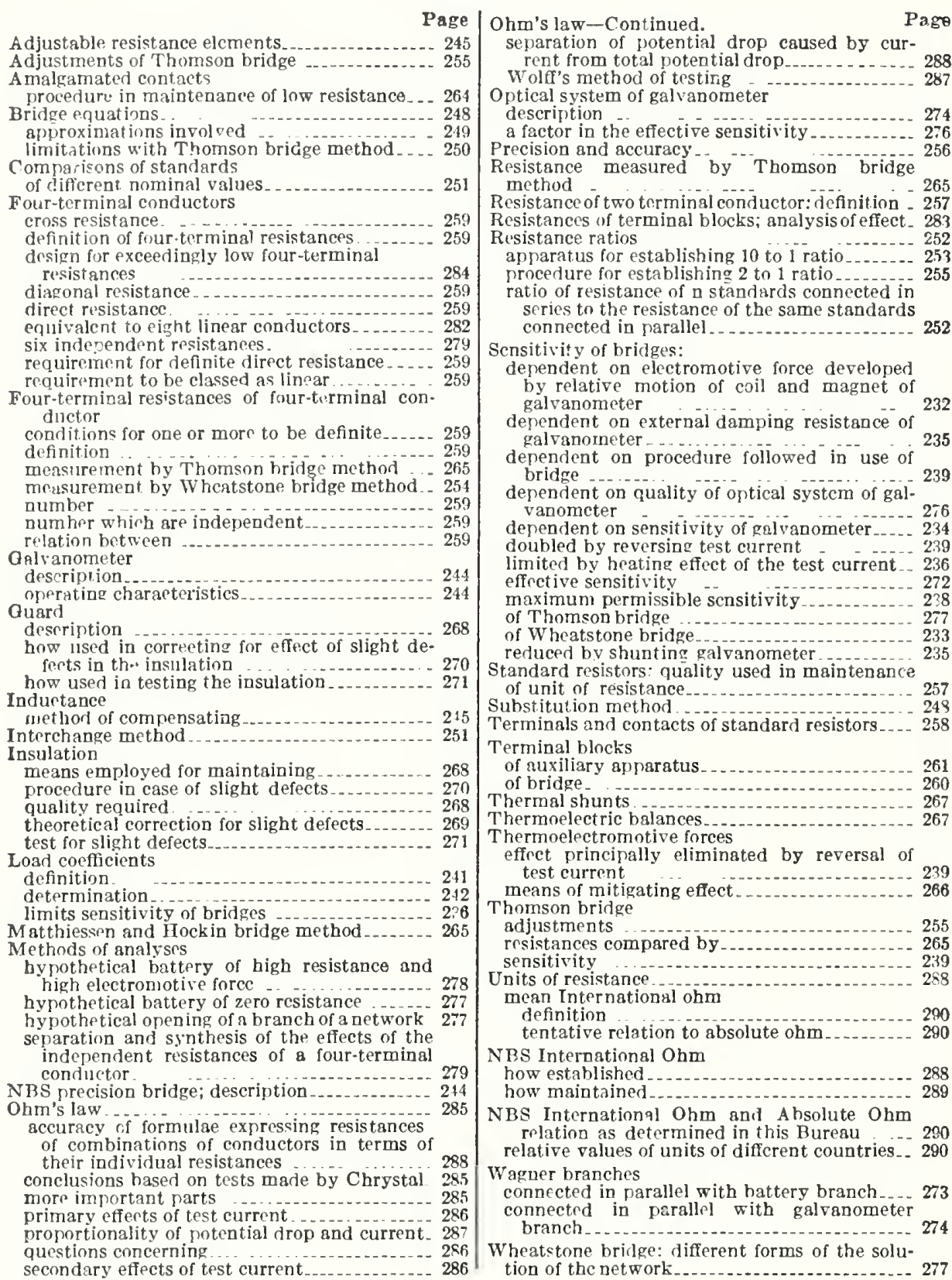

Washington, April 30, 1940. 
RESEARCH PAPER RP1419

Part of Journal of Research of the National Bureau of Standards, Volume 27, September 1941

\title{
TESTING AND PERFORMANCE OF VOLT BOXES
}

\author{
By Francis B. Silsbee and Francis J. Gross
}

\section{ABSTRACT}

The apparatus and method used in the precise measurement of the ratio of volt boxes at the National Bureau of Siandards are described in detail. The method is applicable with rated voltage applied to the volt box and has an accuracy approaching one part in 100,000. The results of such measurements on a number of volt boxes under various conditions of humidity and temperature are given as a guide to their use and an index of their accuracy.

I. Introduction $\ldots \ldots \ldots \ldots$

II. Method

III. The standard volt box

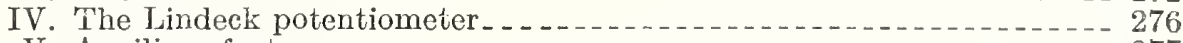

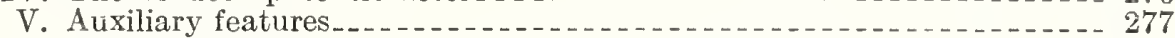

VI. Test procedure $\ldots \ldots \ldots \ldots \ldots$

VII. Typical performance characteristics of volt boxes . . . . . . . . . . . 278

1. Accuracy of manufacturer's adjustment of ratio .......... 279

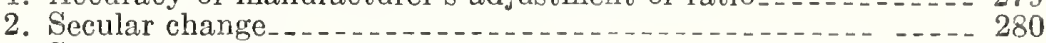

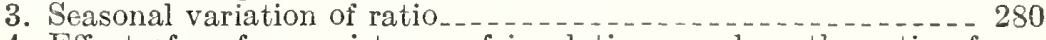

4. Effect of surface resistance of insulacing panel on the ratio of

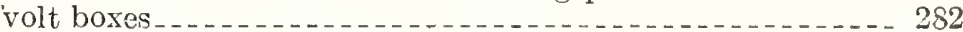

5. Effect of self-heating

VIII. Appendix I. Alternative test method

IX. Appendix II. Derivation of test schedule for calibrating the standard volt box.

\section{INTRODUCTION}

A volt box, more appropriately called a "resistance potentiai divider," is a device commonly used to extend the range of a potentiometer by a definite factor so as to permit of the precise measurement of direct voltages which exceed the limited range of the potentiometer. Any error in this factor will of course enter directly into the measurement of voltage, and in cases where the volt box is used in connection with the testing of wattmeters or watthourmeters, into the measurement of the commercially valuable quantities, electric power and energy.

A volt box consists of a number of resistors connected in series and provided with binding posts so that the unknown voltage to be 
measured ean be conneeted to the whole of the series eireuit (or to some relatively large fraction thereof), while the potentiometer is eonnected to measure the potential drop between the terminals of a smaller part of the eireuit thus energized. In the absenee of eleetric leakage, the ratio of the unknown voltage to the potential drop measured by the potentiometer is evidently equal to the ratio of the resistance of the whole cireuit to that of the portion conneeted to the potentiometer. An accurate determination of this ratio thus constitutes a test of the volt box.

It will be seen that such a test involves only the ratio of two resistances and not their absolute values. Consequently a test of a volt box does not neessarily involve any measurements of resistance in terms of the International Ohm, but only the mueh easier proeess of relative measurement. A further result of this faet is that certain types of variation which affeet all parts of the eireuit equally, such as changes in room temperature, tend to caneel their effeets leaving only very small residual errors. Theoretically it would be dosirable if all parts of the circuit were formed of wire of the same sizo, eomposition, and treatment. Practical diffieulties in making adjustments may arise if the length of fine wire required for any of the low ranges beeomes too short. Sometimes this diffieulty can be met by using a short length of larger manganin wire at one end of the small fine-wire coil and making; the final adjustment on this larger wire. Also, the eonstruetion of eoils of very different resistanee often produces differences in conling surfaces and thus in load coeffieient.

Volt boxes are, therefore, subject to a number of sources of error, two of whieh depend upon the applied voltage. First, the heat developed in the resistor's when subjeeted to the operating voltage (sometimes as mueh as 1,500 volts) may ereate temperature differences and henee vary the relative resistanee of the component parts. Second, any leakage over the insulating supports of the individual resistors will make the current in some of them differ from that in others, so that the ratio of voltages will not be the same as the ratio of resistances. It is evident that a design of volt box in which selfheating trouble has been made negligible by the use of a circuit of very high resistanee will be particularly liable to leakage trouble, and vice versa. In view of the facts that in general the resistance of leakage paths does not obey Ohm's law, and that the resistanee of manganin does not vary linearly with temperature, it is obviously very desirable that a volt box be tested under conditions as nearly as possible the same as those under whieh it is used, and in partieular, that it be tested at operating voltages.

It is the purpose of this paper to describe the equipment used at the National Bureau of Standards for making such tests, and also to give typical data showing the performanee of present commereial volt boxes, as a guide to other laboratories which may be called upon for similar testing work.

\section{IMETHOD}

The method of test here used is based on a comparison of the ratio of the volt box under test with that of a standard volt box of the same nominal range, the ratio of which has been determined by methods to be described. Of the various possible methods for making the intereomparison, the following has been found most flexible and eonvenient. 
The two volt boxes are connected in parallel between junction points $A$ and $B$, as shown in figure 1, and supplied with rated voltage, $V$. The corresponding terminals, such as $S_{3}$ and $X_{3}$ or $S_{2}$ and $X_{2}$, are then at very nearly equal potentials. If the small potential differences between these terminals are measured and denoted by $v_{1}, v_{2}, v_{3}$, and $v_{4}$, respectively, then the ratio, $F_{X}$, of the unknown is given by the equation

$$
F_{X}=F_{S}\left(1+\left[\left(v_{4}-v_{1}\right) / V\right]-F_{S}\left[\left(v_{3}-v_{2}\right) / N\right]+\ldots .\right),
$$

in which terms of the second and higher order in $F_{S}\left(v_{3}-v_{2}\right) / V$ and $\left(v_{4}-v_{1}\right) V$ have been neglected. Here any $v$ is to be taken as positive if the $X$ terminal is positive with respect to the $S$ terminal when $B$

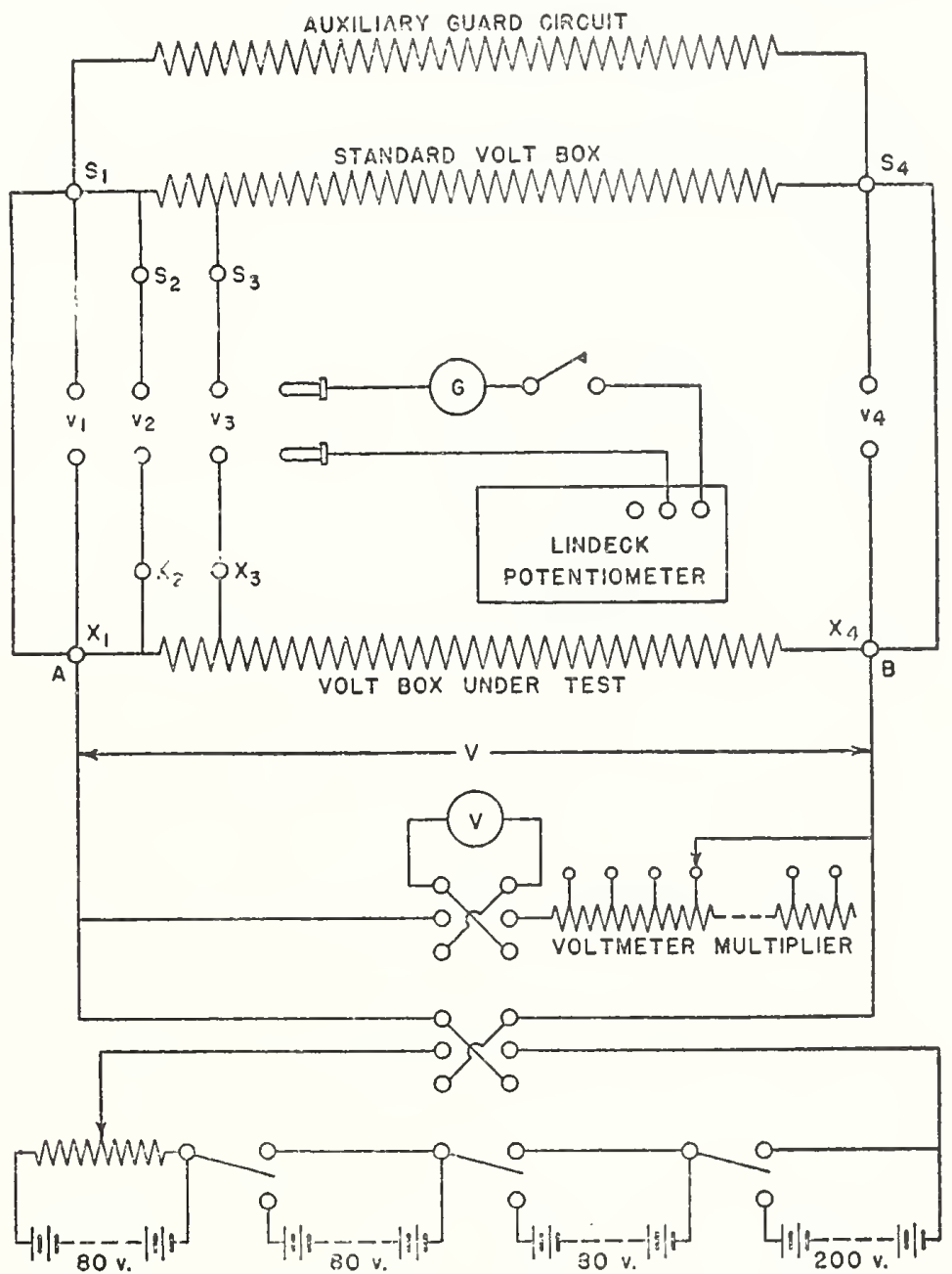

Figure 1. - Diagram of cireuit used to intercompare volt boxes by the first method.

is positive with respect to $A$. The quantities $v_{4}$ and $v_{1}$ arise only from the resistance of leads and contacts and are always small (seldom more than 250 microvolts at rated voltage); $v_{2}$ is also usually small, and differs from $v_{1}$ only as the result of the resistance of connectors within the volt boxes. Futhermore $v_{3}$ is small (seldom exceeding 
1,000 microvolts) unless the volt box under test is scriousiy out of adjustment. Consequently in measuring the small potential differences, $v$, and the applied voltage, $V$, only a moderate relative accuracy is sufficient to give a high precision in $F_{X}$. The applied voltage, $V$, is conveniently measured with a voltmeter, but the small potential differences, $v$, should be measured with a low-range potentiometer.

The three sections which follow describe in some detail the main components of the equipment used in this method at the National Bureau of Standards.

\section{THE STANDARD VOLT BOX}

The apparatus used at the National Bureau of Standards as a standard volt box is shown in figure 2, and its ranges are listed in column 8 of table 1 (p. 287). It was designed so as to minimize the two sources of error mentioned above and at the same time to provide for its own calibration in as simple a manner as possible. The self-heating error is kept low by the use of a high-resistance working circuit. This normally carries only 3 milliamperes (that is, its resistance corresponds to $3331 \frac{1}{3}$ ohms per volt) so that at its rated 750 volts, the power dissipated is only 2.25 watts, which corresponds to 0.25 milliwatt per square centimeter of coil area. The total resistance is 250,000 ohms, and with a box of normal construction an error of 0.1 percent might be caused if the insulation resistance of the effective leakage path fell below 250 megohms. To reduce the error which could be caused by such leakage, the working circuit is divided into 11 parts no one of which has a resistance of more than 25,000 olums. Nine of these parts consist of ten 2,500-ohm coils and the tenth of nine such coils. The coils of each part are carried by a panel of hard rubber. Each of these ten panels in turn is hung by metal blocks from the under side of the Bakelite top of the box, as shown in figure 3. The eleventh part consists of coils of lower resistance which are hung from a portion of the Bakelite top which is separated by a metal guard from the rest of the box. The hard-rubber panels for adjacent parts are spaced with a clearance of $5 \mathrm{~mm}$, and any surface leakage from one panel to the next is intercepted by the supporting blocks. An auxiliary or "guard" circuit, corresponding sections of which can be connected in parallel with the working circuit, is provided with taps at appropriate points to which the metal supporting blocks are electrically connected. Each of the binding posts of the working circuit is also provided with a surrounding metal guard ring connected to a point on the guard circuit which has the same potential as that of the post. The result is that there can be no leakage paths which are normally subject to more than 75 volts or which shunt more than 25,000 ohms, and most of the insulation of the working circuit is subjected to only the very small differences of potential which result from lack of perfect adjustment of the guard circuit. The leakage errors on the high ranges are thus reduced by a factor of more than 100. The guard circuit has a nominal resistance of $266 \% 3$ ohms per volt.

The calibration of the standard volt box requires the determination of the resistances of various parts of the working circuit relative to one another. The process consists in, first, the intercomparison of a group of sections of nominally equal resistance and then the connection 


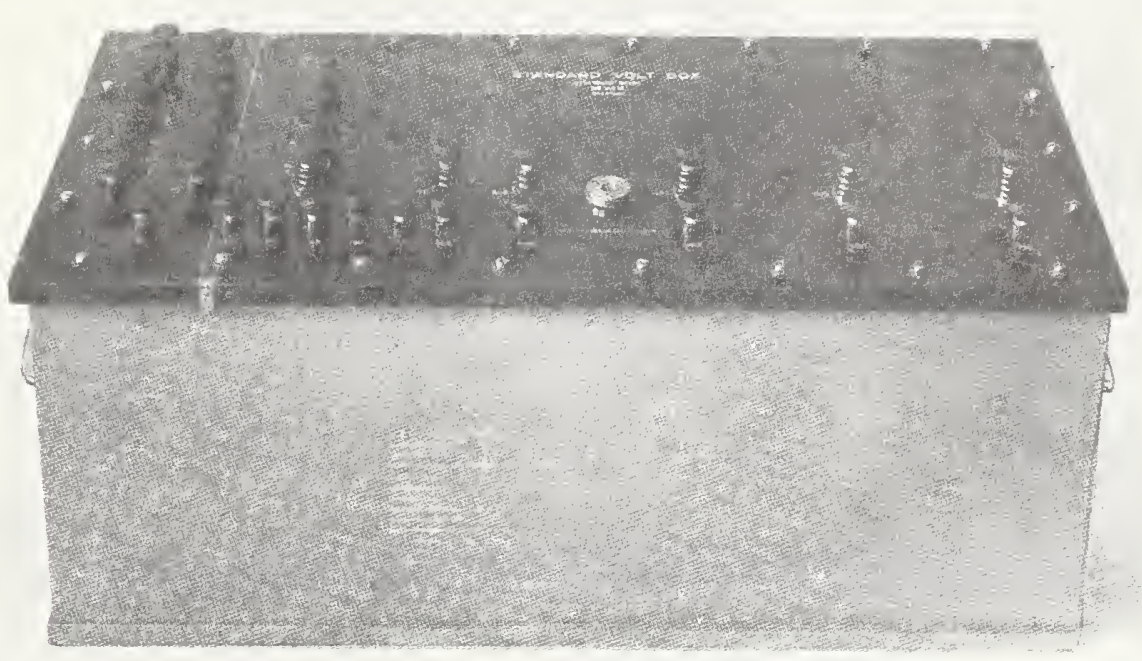

Figure 2. The standard volt box. 
of these equal sections in series to form the first section of a group of larger denomination. If the resistance of the first section of a group of five is arbitrarily taken as a base, the first four intercomparisons give the separate resistances of the next four sections relative to this base. The addition of these values gives the resistance relative to the base of the entire group connected in series. This group itself constitutes the first section of the next group, the resistances of which are thus determined in terms of the original base. To minimize the number of individual sections while retaining the series of nominal ratios used in most commercial volt boxes, it was found desirable to use

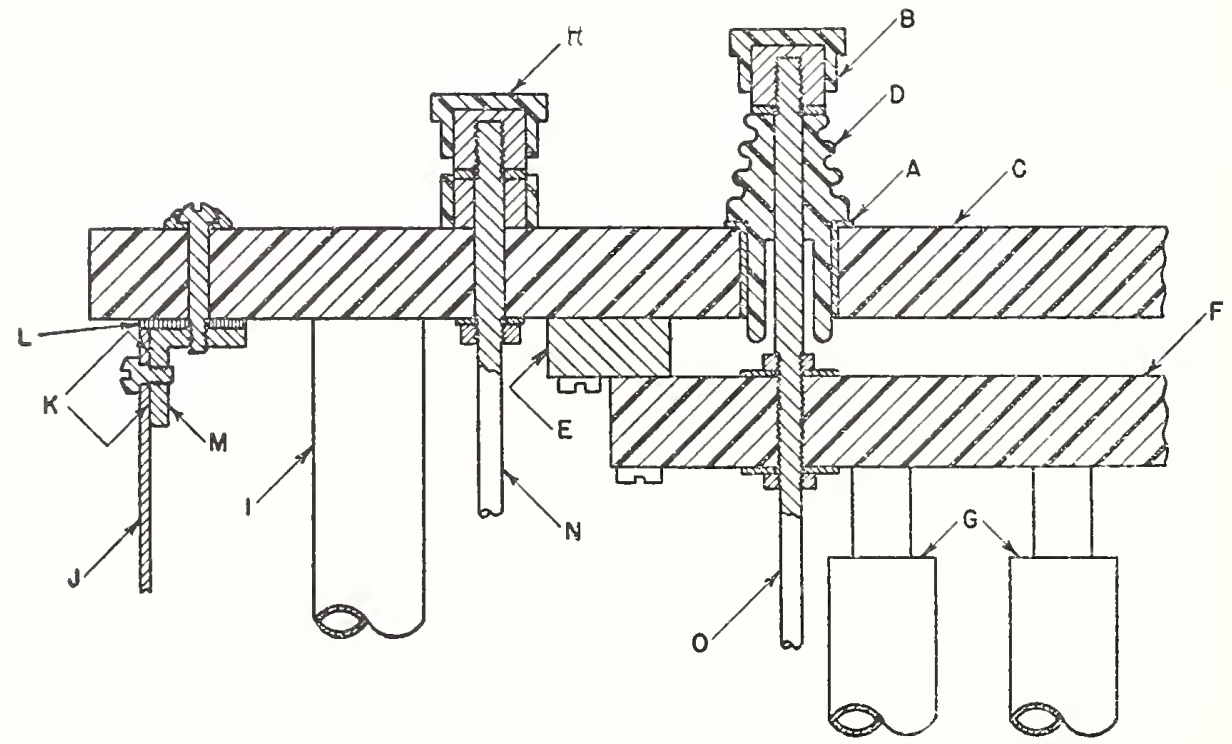

FIGORE 3.-Cross section through a portion of the standard volt box.

A, Mctal guard ring connected to guard circuit. $B$, A terminal of the standard volt box.

$C$, Bakelite top panel.

C, Bakelite top panel.

$E$, Brass support for panel $F . \quad E$ is connected to the Buard circuit.

$F$, Hard rubber panel.

G. Two resistance coils of the working circuit.

$I_{1}$ A guard-circuit terminal.
I. A resistance coil of the guard circuit.

$J$, Air-tight metal box.

$K$, Sweated air-tight joint.

$L$, Rubber gasket.

$M$, Brass rod, which cxtends to bottom of $I$. A tap

0 , Brass of guard cxtending to bottom of $G$. A tap from the working circuit connects to bot tom of $O$.

groups containing alternately five and two nominally equal sections. Thus the first group consists of five sections of $50 \mathrm{ohms}$ each which are rated at 150 millivolts each. These sections connected in series form the first 250-ohm section of the second group of two sections. These two 250 -ohm sections in series form in turn the first 500-ohm section of the third group, which again consists of five sections. This scheme is carried along to the highest sections of 50,000 ohms each, which are rated for a potential drop of 150 volts.

The most convenient way of intercomparing the resistance of the sections of any one group is to substitute one after another in the unknown arm of a Wheatstone bridge as shown in figure 4 ( $A$ and $B$ ). This bridge circuit must be definite and sensitive to the full percentage accuracy desired in the volt-box calibration, but the corrections to its arms and the lead resistances ordinarily need not be known, provided they remain the same during the intercomparison of the sections of each group. 


\author{
DEPARTMENT OF COMMERCE \\ NATIONAL BUREAU OF STANDARDS \\ Washington $25, \mathrm{D}$. C. \\ Errata to Accompany \\ NBS Handbook 77 - Volume I \\ Precision Measurement and Calibration \\ Electricity and Electronics
}

\begin{abstract}
Printed on the reverse side of this sheet is a supplementary page numbered 216a/274 for inserting in NBS Handbook 77, Volume I, Electricity and Electronics.

This page was omitted in the preparation of this volume for printing.
\end{abstract}

May 15, 1961

USCOMM-NBS-DC 
The intercomparison of the lower voltage sections by this substitution method can readily be made with full rated voltage applied to the section under test. To apply full voltage (150 volts) to the higher

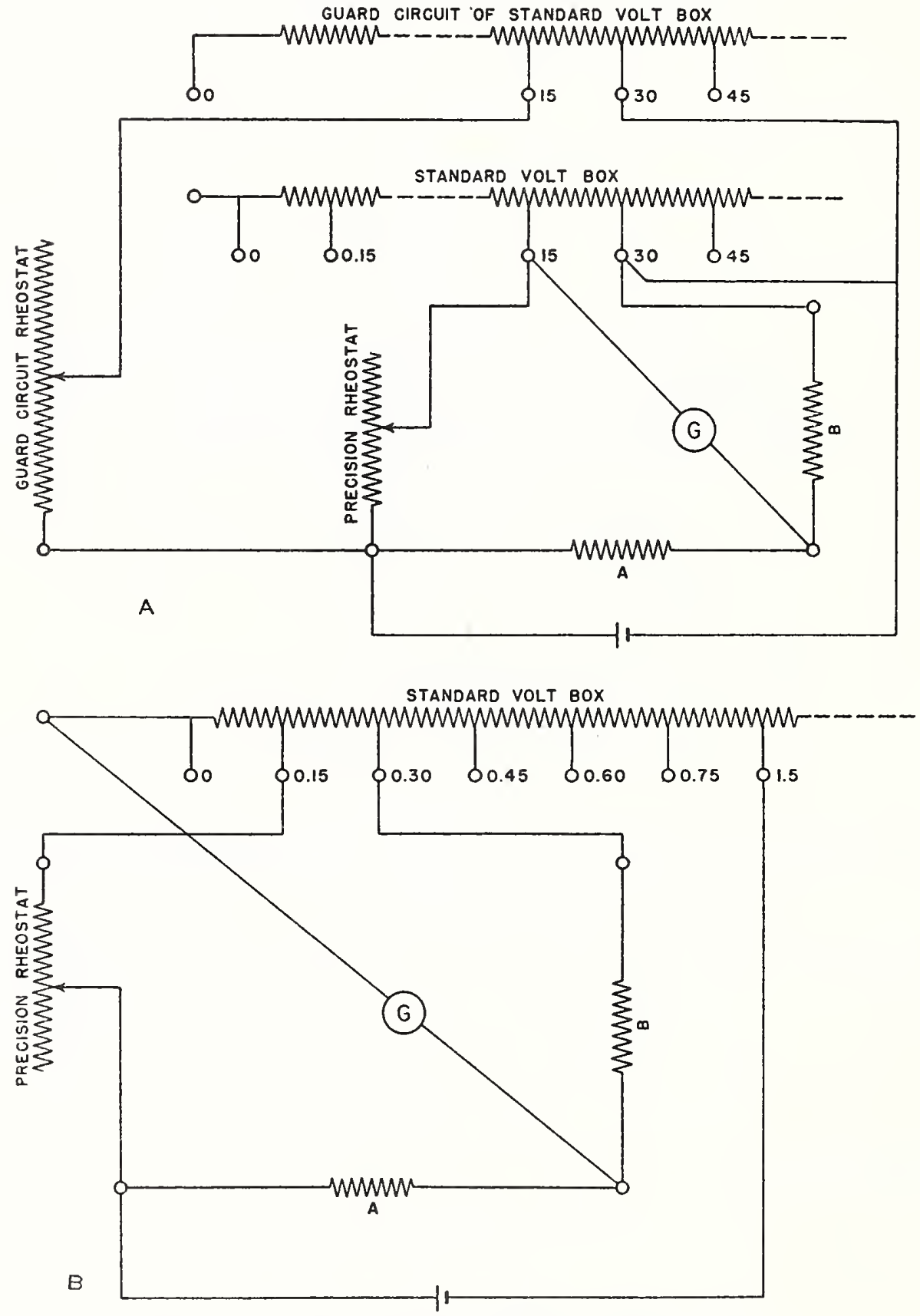

FIgURe 4.-Bridge circuits for calibrating standard volt box.
A, Higher sections.
$B$, Lower sections.

voltage sections with the bridge arrangement of figure $4(A)$ would require the use of a bridge arm of about $10,000 \mathrm{ohms}$, the resistance of which remains steady when dissipating 2.25 watts. Experiments 
using such an improvised arm have shown that the ratio of the 50,000 ohm sections to the $500-\mathrm{ohm}$ section is the same at 300 volts and at 150 volts as at 30 volts, within 3 parts per million. Routine calibrations are therefore made at only 30 volts. In the case of the high-voltage sections, the measurement should be made with the corresponding section of the guard circuit energized by connecting it to the battery which supplies the bridge current, as shown in figure $4(A)$.

Table 1 (see p. 287) is the schedule used for computing the final volt-box corrections from the bridge readings. In the table each value of $p$ is the observed resistance of the precision rheostat in the bridge circuit of figure $4(A$ and $B$ ). The computed values of $s$ in column 7 are the corrections in parts per million which, when applied to the nominal ratio of the resistance of the range in column 8 to the resistance of the 0 to 0.15 range, will give the actual ratio. To obtain the actual ratio for any range in column 8 to any other range listed in that column, subtract the value of $s$ for the second range from that of the first and apply this difference as a correction in parts per million to the nominal ratio of the two ranges. The mathematical derivation of the equations of table 1 is given in appendix II.

The actual carrying out of a test by this procedure is very rapid, a complete test and computation for all ranges being obtainable in less than 3 hours. The sole theoretical error in the procedure as described arises from the resistance of the rods which lead from the junction points of the sections to the binding posts. The resistance of each rod is only $0.0006 \mathrm{ohm}$. If the rods are equal in resistance, the crror will be equal to the resistance of two rods divided by the total resistance of the volt-box range used. Differences in rod resistance will cause errors equal to these differences divided by the resistance of the section measured. To eliminate this latter error in the measurements of the $50-\mathrm{ohm}$ sections, the galvanometer and battery connections of the bridge circuit are marle through the sections next adjacent to the one under test, as shown in figure $4(B)$, and thus the rods are put into arms of higher resistance. The effect of the rod resistance on the voltbox ratio is less than 3 parts per million.

As a measure of the precision readily attainable in this process of calibration, data obtained over a period of 12 years were examined and 27 successive independent determinations of the ratio of two sections of identical construction, namely that between the 450- and 600-volt taps and that between the 600- and 750-volt taps, were tabulated. The standard deviation of these values from their mean was found to be 7 parts per million, the gieatest individual deviation being 19 parts per million. This deviation, of course, includes any drift in the relative values of the coils involved as well as the errors in the setting up of their ratio.

The individual coils are wound with a single layer of silk-covered manganin wire, all coils being wound with wire from the same spool so as to minimize differences in the temperature coefficient of resistance of the various parts. Nevertheless, early data showed a tendency to a cyclical annual fluctuation in the ratio of the 750 -volt to the 1.5 -volt section amounting to about 30 parts per million. This is apparentiy the result of changing atmospheric humidity. To minimize such effects, a small air pump is arranged to maintain the average pressure within the standard volt box at $15 \mathrm{~mm}$ of water above atmospheric. Air from the pump passes through a drying vessel containing $\mathrm{CaCl}_{2}$ 
and is thus thoroughly dried before entering the box. A rubber gasket betwcen the metal box and the Bakelite top seals the joint sufficiently so that an air flow of about $0.1 \mathrm{~m}^{3}$ per 24 hours is enough to maintain the pressure differential. Since the installation of this dry-air supply, the cyclic fluctuation has been very small.

\section{THE LINDECK POTENTIOMETER}

The potentiometer generally used for mensuring the small voltages, $v_{1}, v_{2}, v_{3}$, and $v_{4}$, referred to in section II is of the Lindeck type. ${ }^{1}$ Figure 5 is the circuit diagram of this instrument. In this device a current

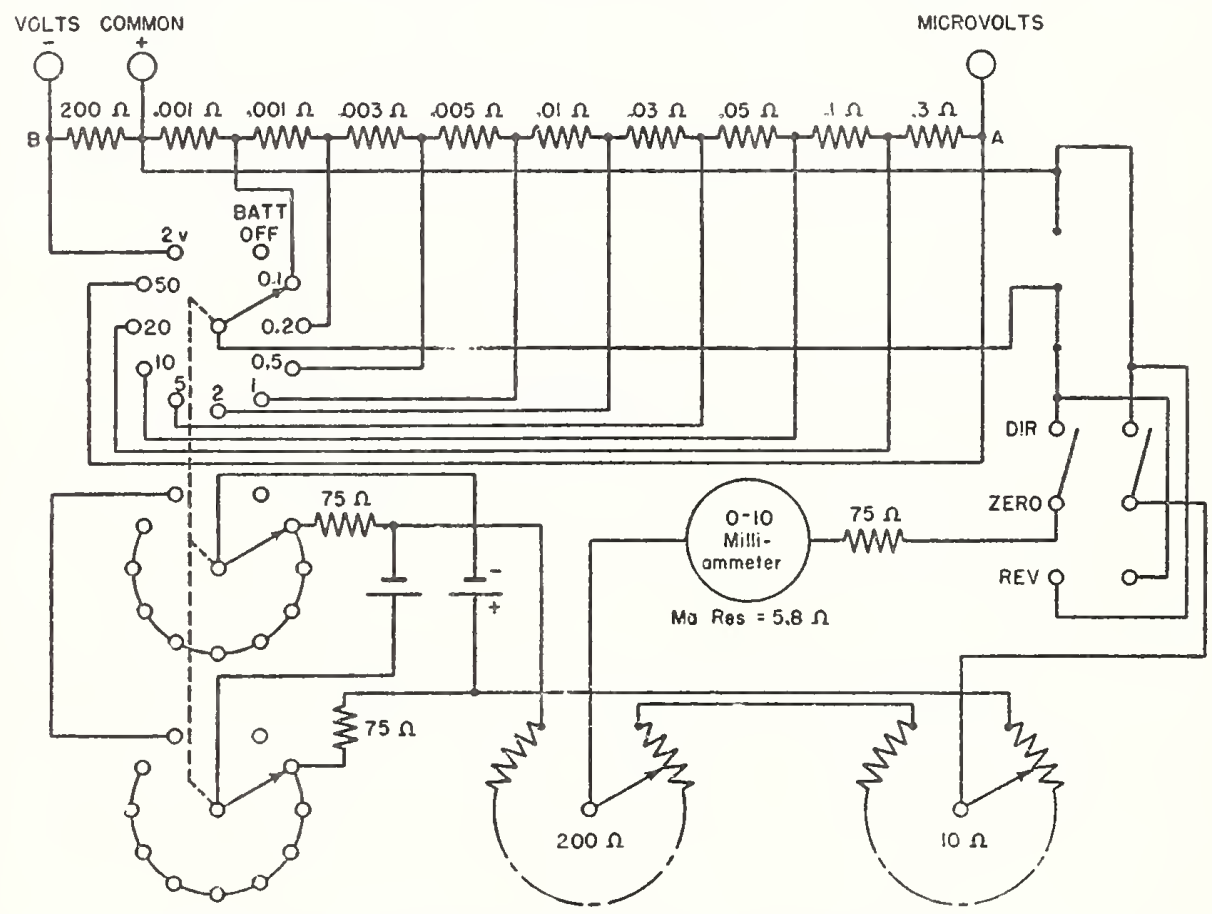

FIGURE 5. - Circuit of the Lindeck potentiometer.

When intercomparing volt boxes, the terminals labeled "common" and "microvolts" are used. When Interchecking the calibrations of the potentiometer and the voltmeter, the terminals labelled "common". interchecking the calibs

mcasured by the milliammeter passes through a selected portion of the standard resistor, $A B$, and this current is adjusted until the voltage drop in the standard resistor is exactly equal and opposite to the voltage to be measured, as indicated by the external galvanometer (shown at $G$ in fig. 1), which is used as a null indicator.

The milliammeter has a scale length of approximately $15 \mathrm{~cm}$ and is equipped with a parallax mirror. It requires $10 \mathrm{ma}$ for full-scale deflection.

The standard resistance sections between $A-B$ in figure 5 are made of manganin and are so constructed that the tcmperature risc is negligiblc even with the current of 8 amperes which is used to calibrate them on a Kelvin double bridge. Their resistance should be adjusted

1 J. C. Poggendorff, Ann. Phys. Chem. 54, 161 (1841); Lindcck and Rothe, Z. Instrumentenk. 20, 293 (1900); H. B. Brooks and A. W. Spinks, BS J. Research 9, 781 (1932) RP506. This last paper contains a brief summary of the two references above and a discussion of basic potentiometer circuits. 
to a precision somewhat better than that to which the milliammeter can be read.

Since the resistance of the galvanometer circuit may vary from a low value for measurements of $v_{1}, v_{2}$, and $v_{4}$ up to several hundred ohms in the measurement of $v_{3}$, the time required to obtain a reading can be shortened by providing manganin rheostats in series and in parallel with the galvanometer, so that the instrument can be critically damped in all four measurements. In operation, the voltage applied to the volt boxes, and the potentiometer current are reversed simultaneously, so that the effect of any constant thermal emf in the galvanometer circuit or elsewhere is eliminated. By moderate care in using only copper and materials, such as manganin, which have a low thermal eme against copper, the residual emf is so small that fluctuations in it cause only negigible errors.

The external galvanometer has a sensitivity of $3 \mathrm{~mm}$ per microvolt at $1 \mathrm{~m}$ distance, with a critical external damping resistance of 200 obms and a period of 5.8 seconds.

\section{AUXILIARY FEATURES}

All of the wires in the measuring circuits are run in shielded cable, and each shield is maintained at the same potential as the wire inside it by means of the auxiliary "guard" circuit of the standard volt box mentioned in section III above. The galvanometer and the Lindeck potentiometer are mounted on metal guard plates which are so located as to intercept any possible leakage currents. When voltage $v_{1}, v_{2}$, or $v_{3}$ is being measured, these guard plates are connected to the low-voltage terminal of the auxiliary guard circuit of the standard volt box; but when $v_{4}$ is measured, these plates are connected to the high-voltage terminal of the guard circuit. A special plug-connector panel is used to connect the Lindeck potentiometer circuit to measure $v_{1}, v_{2}, v_{3}$, and $v_{4}$, and this panel is designed so that guard plates intercept leakage currents between the different measuring circuits.

For test voltages from 400 to 750 volts the batteries shown in figure 1 are replaced by a motor-driven d-c generator. The driving motor and the field of the generator are energized by means of batteries.

With this equipment, 750 volts is the nommal limit at which volt boxes are tested. However, experiment has shown that the calibration of the standard volt-box can be relied upon up to 1,500 volts, and by special arrangements the generator can be connected in series with a group of batteries to obtain this voltage.

Since this method of calibrating a volt box involves the ratio of the small voltages measured by the potentiometer to the larger voltage measured by the voltmeter, it is necessary that the readings of these two instruments be mutually consistent, though neither need be accurate. Means were therefore provided for readily intercomparing them. This is done by applying the same voltage to the voltmeter and the standard volt box and measuring the voltage drop across the 0 to 1.5 -volt range of the standard rolt box with the Lindeck potentiometer. The "volts" and "common" terminals (see fig. 5) of the potentiometer are used. Knowing the volt-box ratio, the agreement of the two instruments can be readily checked. Since the volt- 
meter is provided with a shunt circuit around the moving element to provide temperature compensation in the manner described by Brooks, ${ }^{2}$ it was found convenient to incorporate in this shunt circuit a small rheostat which can be used to adjust the current through the moving element, so that the calibration of the voltmeter may be made consistent with that of the potentiometer in spite of any small changes in either instrument.

\section{TEST PROCEDURE}

When a volt box is received for test at the National Bureau of Standards, the resistances between its terminals are first measured with a Wheatstone bridge, in order to detect any defective resistance coils before full voltage is applied to them. Data from these measurements can also be used to compute the approximate ratio. The volt box is then connected into the circuit of figure 1, and voltage is applied. Voltages $v_{1}, v_{2}, v_{3}$, and $v_{4}$ are then measured successively, by adjusting the Lindeck potentiometer until the simultaneous reversal of polarity of the applied voltage, $V$, and of the potentiometer does not change the galvanometer deflection. This latter procedure makes negligible those errors that might be caused by any constant thermal emf in the galvanometer circuit. Because of the self-heating effect, to be described in section VII-5, the volt box is then allowed to warm up until the measured value of $v_{3}$ becomes reasonably constant. If the volt box has more than one range, the highest range is measured first and then the other ranges are measured in order of decreasing ratio. This procedure requires only one warming-up period for the entire box.

A very effective check on the accuracy of the measurements is obtained by making two independent measurements of each ratio of the volt box under test, using two different standard volt-box connections which give the same ratio. Examples of standard volt-box terminal arrangements which give the same ratio are: $0-1.5-150,0-3-300$, 0-6-600, and 0-7.5-750.

Ratios of the tested volt box are computed by means of the equation given in section II. If for any range the total change in ratio caused by sclif-heating is greater than the precision $(0.01$ percent) to which results are reported, then the observed change and the time required to complete it are reported.

\section{TYPICAL PERFORMANCE CHARACTERISTICS OF VOLT BOXES}

Although the technical literature abounds wh articles on potentiometers and much has been done to improve their accuracy, rolt boxes are mentioned only incidentally and with the exception of manufacturer's guarantees, no literature appears to have been published which discusses the factors affecting the accuracy of the voltage ratio of volt boxes. Perhaps the reason for this lack of published information is that up to the present time the accuracy of the measurements in which volt boxes have been used has not been noticeably limited by any variation in the voltage ratio of the latter. In view of this situation, a somewhat detailed discussion is given below of the

2H. B. Brooks, J. Research NBS 17, 523 (1936) RP926. 
factors which affect the voltage ratio so that those who use volt boxes may know what factors may affect measurements of a given precision.

At the time of the construction of a volt box the departure of its actual voltage ratio from the nominal value will depend upon the care with which the manufacturer adjusts the resistances of the various coils. Thereafter the departure of the voltage ratio from the intial value may depend upon (a) secular change of the resistance of the coils, (b) ambient temperatire, (c) temperature changes caused by self-heating, (d) slow seasonal variations in ratio caused by seasonal changes in relative humidity, and (e) changes in insulation resistance between terminals and between the coil studs.

\section{ACCURACY OF MANUFACTURER'S ADJUSTMENT OF RATIO}

Figure 6 indicates the accuracy of the manuiacturer's adjustment of voltage ratio on 86 volt boxes tested at the National Bureau of

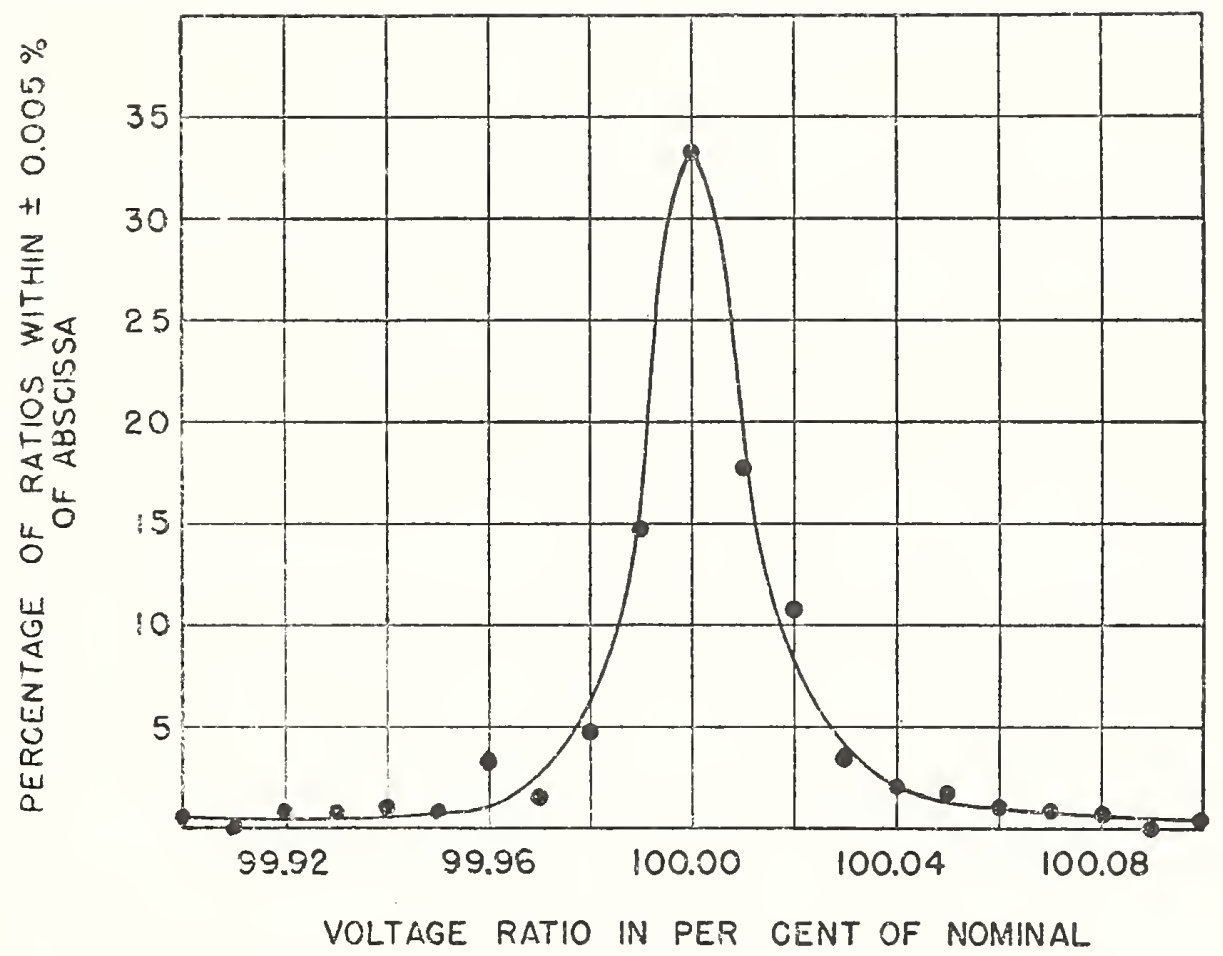

FIGURE 6. - Accuracy of the manufacturer's adjustment of voliage ratio.

This curve represents data on 86 volt boxes tested at the National Bureau of Standards between 1927 and 1940. In computing the curve each range of a multiple-range voll box was considered separately. so that there were 413 ranges tested.

Standards between the years 1927 and 1940. For each plotted point in this figure the ordinate represents the percentage of volt-box ranges tested the ratio of which was within \pm 0.005 percent of the ratio indicated by the abscissa. If the volt box was tested on more than one occasion, only the results of the initial test were used in locating this curve. Curves of this type for individual ranges had the same 
general shape and distribution of points as the composite curve shown here. All of the volt boxes represented in this curve were for 1.5-volt potentiometers. However, a curve of this type based on tests of a small number of volt boxes used with 150-millivolt deflection potentiometers displayed a similar shape and distribution of points.

\section{SECULAR CHANGE}

One would expect that the secular change of ratio would be less than the secular change of resistance of the individual coils, provided that all of the coils are of similar construction. Data are available on seven volt boxes which have been sent in to the National Bureau of Standards for annual test over periods of from 6 to 10 years. Of these seven volt boxes, the voltage ratio of the 150 -volt to the 1.5 -volt range has varied 0.01 percent, or less, in six of them, and the ratio of the seventh has increased uniformly by a total of 0.04 percent in a 10 -year period. The other ranges of each volt box in this group varied in the same manner as the 150 -volt to the 1.5 -volt range. It may be supposed that this performance is typical-of high-grade volt boxes.

\section{SEASONAL VARIATION OF RATIO}

It has long been known that resistance coils of silk-covered manganin wire, such as those used in many volt boxes, are subject to seasonal variations in resistance which are primarily due to seasonal variations in relative humidity. In 1936 Dike $^{3}$ published the results of his investigation of the phenomenon. He found that the silk threads

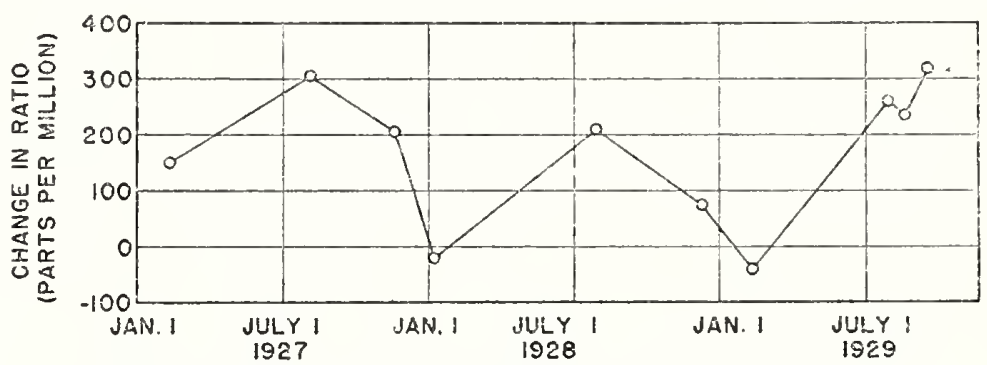

FIgURE 7.-Seasonal variation of ratio for volt box $A$.

Construction features of $A$ are described under figure 8.

used to insulate the manganin wire had a greater length at high relative humidity than at low, and he concluded that this property of the silk was responsible for the fact that the resistance of silk-insulated manganin wire coils is greater at the higher humidities. He also found that if such coils were coated with certain waxes and suddenly changed from an atmosphere of one relative humidity to that of another, the resistance change would require approximately 3 weeks to complete, but that coils not coated with wax required less than 24 hours although the magnitude of the change in resistance was the same. The percentage change of resistance is greater the finer the wire.

Figure 7 shows the seasonal variation in ratio for a volt box in which the wire size is much larger on the low-voltage coil than on the coils nearer the high-voltage end. Dike's theory explains this curve very well, because from that theory we would expect the humid summer

\footnotetext{
3 P. If. Dike, Rev. Sci. Instr. 7, 278 (1936),
} 
air to produce a greater percentage increase in the resistance of the fine wire coils than in the coarser wire of the low-voltage coil and hence make the voltage ratio higher during the summer months than in the drier winter months. It should be noted that artificial heating in winter causes the relative humidity indoors to be materially lower than that of the outside air. This curve should not be interpreted

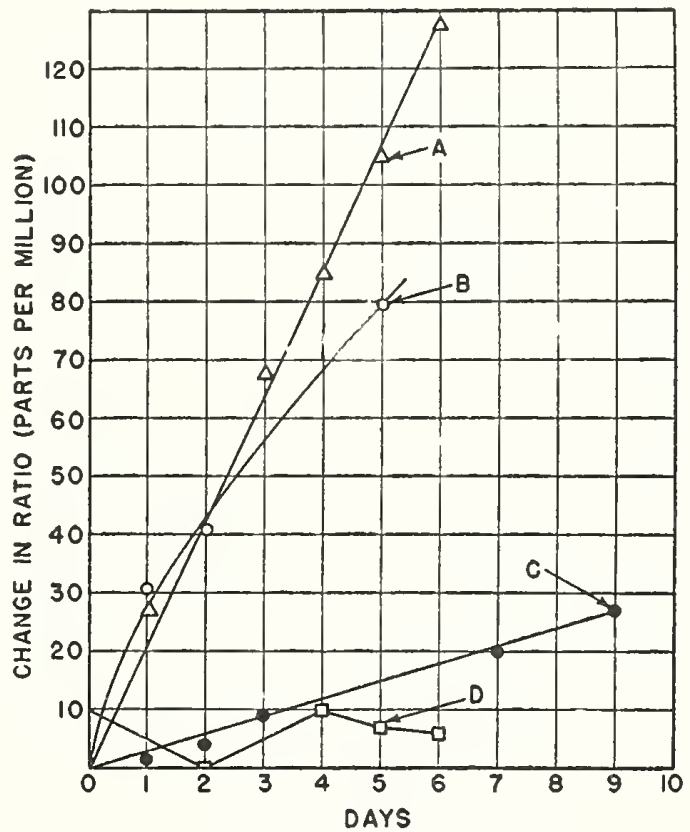

FIGURE 8.-Drift of voltage ratio after a sudden increase in relative humidity.

Each volt box stood for several weeks in a room having a relative humidity hetween 25 and 40 percent, and was then placed in a closed cabinet in which a saturated solution of sodium chloride maintained a relative was then placed in a closed cabinet in which a saturated solution of sodium chloride maintained a relative humidity of 75 percent. Each curve represents measurements made on the $150-\nabla$ olt to the 1.5 -volt range of
a volt hox, with the exception of the curve for volt hox $A$ on which the 150 -volt to the 0.15 -volt range was measured.

molt hox $A$ has a resistance of 40 ohms per volt, its wood spools are wound with silk-insulated wire, and the diameter of the wire on the low-voltage coils is approximately 10 times that of the wire on the hifhvoltage coils.

Volt box $\dot{B}$ has a resistance of 200 ohms per volt, its metal spools are wound with silk-covered wire, and the ratio of the diameter of the wire in the low-voltage coil to that of the wire in the high-voltage coil is in termediate between that of volt hoxes $A$ and $C$.

Volt box $C$ has a resistance of 2633 ohms per volt, its wood spools a re wound with silk-covered wire, and the diameter of the wire in the low-voltage coil is twice that of the wire in the high-voltage coils.

Volt box $D$ has a resistance of 333 ohms per volt; its metal spools a re wound with enameled wire which is insulated with a patented mixture of silk and cotton, and the ratio of the diameter of the wire in the low. voltage coils to that of the wire in the high-voltage coils is approximately the same as that of $C$.

The coils of all four volt hoxes are wax-coated.
Theltage coils to that of the wire in the high-voltage

as a typical curve for all volt boxes, since it probably represents an extreme type of construction among volt boxes built by reputable manufacturers.

To obtain additional data on the effects of humidity, an air-tight cabinet was built in which a volt box could be placed. A tray of saturated sodium chloride solution kept the relative humidity constant at 75 percent. Shielded leads were brought out so that the ratio of the volt box could be measured without opening the cabinet. Data for each of the curves in figure 8 were obtained by placing in this cabinet a volt box which for several weeks previously had been in an atmosphere having a relative humidity of less than 40 percent, and then periodically measuring the voltage ratio without removing 
the volt box'from the cabinet. These ratio measurements were made on the 150 - volt range of each volt box with only 30 volts applied so as to make any self-heating effects negligible.

Volt boxes $A, B$, and $C$ have coils wound with silk-covered manganin wire. The manganin wire of volt box $D$, however, is insulated with a patented ${ }^{4}$ combination of silk and cotton, which is claimed to cause much less seasonal valiation of resistance with humidity. The improvement is obvious from the curves.

These curves for volt boxes in which silk-covered manganin is used bear out two implications from Dike's paper. First, for a given voltage ratio the effect of a change in humidity will be least for the volt box having the fewest ohms per volt, since such a box will usually have thicker wire; this would explain the small effect on the ratio of $C$ in figure 8. Second, the greater the difference between the wire diameters of the low-voltage and high-voltage coils, the greater will be the effect of humidity. This is shown by the curves for $A$ and $B$, where $A$ has one-fifth of the olmms per volt of $B$ but the greater difference in wire size.

After the data for figure 8 were obtained, volt box $A$ was kept in the cabinet for 4 weeks, and its ratio was found to have increased 0.048 percent in that time. Upon removing it from the cabinet to the dry air of the room, the ratio decreased exponentially, with a change of 0.029 percent the first week. Although the experiments reported above were made on a limited number of volt boxes, the results probably indicate the magnitude and character of the effects that would be experienced in the actual use of volt boxes.

\section{EFFECT OF SURFACE RESISTANCE OF INSULATING PANEL ON THE RATIO OF VOLT BOXES}

Some of the coil studs and binding post terminals of volt box $C$, which had the customary hard-rubber top, were disconnected from the coils and the insulation resistance between them was measured. Computations based on these measurements showed that surface leakage between coil studs and between terminals of a volt box would cause variations in the ratio of less than 5 parts per million for a relative humidity of 75 percent or less, provided the resistance was less than 400 ohms per volt, terminal spacings were $1 \frac{11}{4}$ inch or more for 150 volts, and the coil stud spacings were 1 inch or more.

However, it is probable that at some relative humidity higher than 75 percent the surface resistivity of the panel of a volt box would become low enough to have a serious effect on the ratio.

\section{EFFECT OF SELF-HEATING}

For most of the volt boxes tested at the National Bureau of Standards during the past 13 years, it has been found that the ratio decreases during the first hour or two that rated voltage is applied, and remains nearly constant thereafter until the voltage is removed. The decrease is an exponential one, and the magnitude of the chonge for the 300 -volt range in volt boxes having a resistance of $200 \mathrm{ohms}$ per volt has been found to range from 0.005 percent to 0.03 percent, the average value being about 0.02 percent. One-half of this change occurs in about 20 minutes for most volt boxes.

\footnotetext{
${ }^{4}$ P. H. Dike. U. S. Patent 2,026,616, issued Jan. 7, 1936.
} 
Two volt boxes having a resistance of $333 \mathrm{ohms}$ per volt and a maximum resistance per coil of $5,000 \mathrm{ohms}$ were found to show no measurable change in ratio even after several hours at rated voltage.

The 750-volt range of a volt box having $100 \mathrm{ohms}$ per volt showed a change in ratio of 0.04 percent in 2 hours at rated voltage.

Two factors are responsible for this self-heating effect. First, the resistance wire has an appreciable temperature coefficient of resistance; and second, for reasons of economy in construction the resistance coils in the higher voltage sections are usually on spools of approximately the same physical dimensions as those between the potentiometer taps, but have a much greater resistance per spool and hence a much greater temperature rise. The grade of manganin usually used in volt boxes has its maximum resistance near room temperature; and hence, as the higher resistance coils heat up, their resistance becomes a smaller percentage of the cold value than does that of the coils between the potentiometer taps. Hence, the voltage ratio of the volt box decreases.

Thermocouples were attached to the coils of a volt box of the usuar construction having $200 \mathrm{ohms}$ per volt. It was found that for the 9,000 - and 10,000-ohm coils the temperature rise was $10^{\circ} \mathrm{C}$ as compared with $4^{\circ} \mathrm{C}$ for the 300-ohm coil. The ratio decreased with time on a smooth exponential curve for both the 150-volt range and the 300-volt range, the total change being 0.005 percent.

For volt boxes of normal construction the temperature rise of the coils is negligible at 20 percent of rated voltage, so that if the ratio of a volt box at rated voltage difiers from that at 20 -percent voltage by an amount that is negligible for a given measurement then the heating effect will also be negligible for that measurement.

\section{APPINDIX I}

\section{Alternative Test Method}

A method which is simpler than that described in section II and which is for many purposes amply accurate for comparing the ratio $F_{X}$, of an unknown volt box with that, $F_{S}$, of a standard volt box is shown in figure 9 . Here $H_{X}$ and $P_{X}$ are the resistances of the high-voltage side between $b$ and 4 and of the potentiometer side between $a$ and $b$, respectively, of the box under test, and $H_{S}$ and $P_{S}$ are the corresponding resistances of the standard. Hence, by definition (provided the resistances of the connections from 1 to $a$ and from $1^{\prime}$ to $d$ are negligible in comparison with $H_{X}+P_{X}$ and $H_{S}+P_{S}$, respecively),

$$
F_{x}=\frac{H_{X}+P_{x}}{P_{X}} \text { and } F_{S}=\frac{H_{s}+P_{s}}{P_{X}} \text {. }
$$

The galvanometer is first connected between the terminals 2 and $2^{\prime}$ and the slider beiween 1 and $1^{\prime}$ is adjusted to give a balonce. This assures that the resistances of the two sides of the rheostat, together with the leads to $a$ and to $d$, are in the ratio

$$
\frac{L_{x}}{L_{s}}=\frac{P_{x}+H_{X}+R_{x}}{P_{s}+I_{s}+R_{s}}=\frac{i_{s}}{i x} \text {. }
$$

The galvanometer is then shifted to join 3 and $3^{\prime}$, anc sue vi tave auxiliary resistors $R_{\mathcal{S}}$ or $R_{X}$ as needed is increased until a balance is again reached. This gives the relation

$$
\frac{L_{x}+P_{x}}{L_{s}+P_{s}}=\frac{I_{x}+R_{x}}{H_{s}+R_{s}}=\frac{i_{s}^{\prime}}{i_{x}^{\prime}}
$$


When by successive adjustment a balance is obtained for both positions of the galvanometer

so that

$$
\frac{i_{X}}{i_{s}}=\frac{i_{x}^{\prime}}{i_{s}},
$$

Whence

$$
\begin{gathered}
\frac{P_{X}}{P_{s}}=\frac{H_{X}+P_{X}+R_{X}}{H_{s}+P_{s}+R_{s}} \\
F_{X}=F_{s}\left[\frac{\left(1+\frac{R_{s}}{H_{s}+P_{s}}\right)}{\left(1+\frac{R_{X}}{H_{X}+P_{X}}\right)}\right] .
\end{gathered}
$$

In actual practice $R_{X}$ or $R_{S}$ is left zero according as $F_{X}$ is greater or less than $F_{S}$, so that the formula becomes very simple. This procedure does not fully eliminate the effect of the connections between points 1 and $a$ and between $1^{\prime}$ and $d$, but such

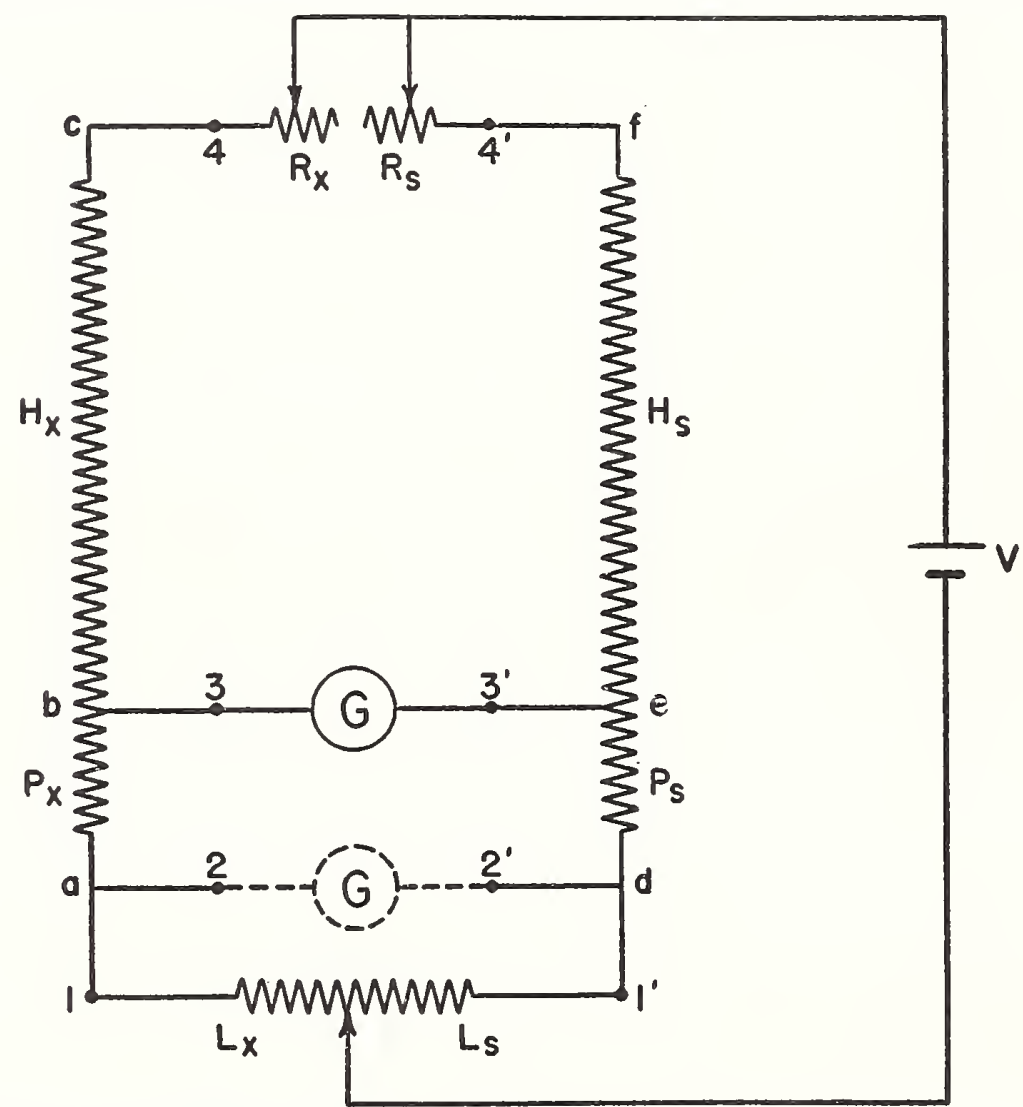

FIGURE 9.-Schematic diagram of circuit for intercomparison of volt boxes by the alternative method.

lead resistances are usually quite negligible. It also requires the measurement, though with only very moderate precision, of $H_{S}+P_{S}$ (or $H_{X}+P_{X}$ ). For boxes of different resistances a wide range of values of $R_{X}$ (or $R_{S}$ ) is required. 


\section{APPENDIX II}

\section{Derivation of Test Schedule for Calibrating the Standard Volt Box}

The working circuit of the standard volt box can be considered as made up of a succession of groups each made up of a number of sections. The first five sections of any one group have nominally equal resistances, and the last section has a resistance nominally five times this value. The first (counting from the low-potential end) section of each group is formed by all the preceding groups connected in series. Any section therefore can be identified by specifying two integers, the first, $m$, indicating the group of which the section is part, and the second, $k$, indicating the position of the section in its group.

If the Wheatstone bridge used in the successive measurements of the first five sections of the $m$ th group has the fixed ratio $B_{m} / A_{n}$, then the resistance of the $k t h$ section is given by

$$
R_{m, k}=\frac{B_{m}}{A_{m}} p_{m}, k,
$$

where $p_{m, k}$ is the resistance of the rheostat arm when measuring section $(m, k)$. Hence

$$
R_{m, k}=R_{m, 1}+\frac{B_{m}}{A_{m}}\left(p_{m, k}-p_{m, 1}\right)=R_{m, 1}+\frac{B_{m}}{A_{m}}\left(n_{m}, k\right),
$$

where $n_{m, k}$ is defined as $\mathrm{p}_{m, k}-p_{m, 1}$. The total resistance of the first five sections of the group is given by

$$
\sum_{k=1}^{k=5}\left(R_{m, k}\right)=5 R_{m, 1}+\frac{B_{m}}{A_{m}} \sum_{k=1}^{k=5}\left(n_{m, k}\right) .
$$

The bridge ratio is now changed to $A^{\prime}{ }_{m} / B^{\prime}{ }_{m}$ and the bridge is balanced first across the combination of the first five sections in series giving

$$
\sum_{k=1}^{k=5}\left(R_{m, k}\right)=\frac{B_{m}{ }^{\prime}}{A_{m}{ }^{\prime}} p_{m, s}
$$

and then across the sixth section giving

Hence

$$
R_{m, t}=\frac{B_{m}^{\prime}}{A_{m}^{\prime}} p_{m, t}
$$

$$
R_{m, t}-\sum_{k=1}^{k=5}\left(R_{m, k}\right)=\frac{B_{m}^{\prime}}{\Lambda_{m}{ }^{\prime}}\left(p_{m, t}-p_{m, s}\right) .
$$

We may write $p_{m, t}-p_{m, s}=n_{m, t}$ and combine eq 6 with eq 3 to get

$$
R_{m, t}=5 R_{m, 1}+\frac{B_{m}}{A_{m}} \sum_{k=1}^{k=5} n_{m, k}+\frac{B_{m}^{\prime}}{A_{m}^{\prime}} n_{m, t} \text {. }
$$

The resistance of the entire $m$ th group is therefore

$$
\sum_{k=1}^{k=5} R_{m, k}+R_{m, t}=10 R_{m, 1}+2 \frac{B_{m}}{A_{m}} \sum_{k=1}^{k=5} n_{m k}+\frac{B_{m}{ }^{\prime}}{A_{m}{ }^{\prime}} n_{m, t}
$$

A very convenient choice of bridge ratios, which keeps $p$ nominally at 5,000 or 2,500 ohms, is to make

$$
\frac{B_{m}}{A_{m}}=10^{m-3} \text { and } \frac{B_{m}{ }^{\prime}}{A_{m^{\prime}}}=10^{m-2}
$$

Also, as an abbreviation, we may write

$$
q_{m, b}=\sum_{k=1}^{k=5} n_{m, k}
$$


Inserting these values and noting that this total resistance of the $m$ th group is identical with the resistance of the first section of the $(m+1)$ th group we get

$$
R_{(m+1,1)}=10 R_{m, 1}+2 \times 10^{m-3} q_{m, b}+10^{m-2} n_{m, t}
$$

as the equation connecting resistance values in successive groups.

If this relation is applied successively, beginning with the first group $(m=1)$ we get for the resistance of the first section of the $m$ th group in terms of the resistance of the first section of the first group and the observed differences

$$
\begin{aligned}
R_{m, 1}=10^{m-1} R_{1,1} & +10^{m-3} n_{m-1, t}+2 \cdot 10^{m-4} q_{m-1,5} \\
+ & 10^{m-4+1} n_{m-2, t}+2 \cdot 10^{m-5+1} q_{m-2,5} \\
+ & 10^{m-5+2} n_{m-3, t}+2 \cdot 10^{m-6+2} q_{m-3,5}
\end{aligned}
$$

Simplifying this we get

$$
\begin{aligned}
& R_{m, 1}=10^{m-1} R_{1,1}+2 \cdot 10^{m-4}\left(q_{1, b}+q_{2,5} \ldots q_{m-1,5}\right) \\
& +10^{m-3}\left(n_{1, t}+n_{2, t}+\ldots n_{m-1, t}\right) .
\end{aligned}
$$

The total resistance $S_{m, k}$ up to the kth tap in the $m$ th group is derived from eq 2

$$
S_{m, k}=\sum_{k^{\prime}=1}^{k^{\prime}=k} R_{m, k^{\prime}}=k R_{m, 1}+\frac{B_{m}}{A_{m}} \sum_{k^{\prime}=1}^{k^{\prime}=k} n_{m, k^{\prime}}
$$

Defining $q_{m, k}$ as $\sum_{k^{\prime}=1}^{k^{\prime}=k} n_{m, k^{\prime}}$ and inserting the values of ratio from eq 9 , we get

$$
S_{m, k}=k R_{m, 1}+10^{m-3} q_{m, k}
$$

or by eq 11

$$
\begin{aligned}
S_{m, k} & =k \cdot 10^{m-1} R_{1,1}+2 \cdot 10^{m-4} k\left(q_{1,5}+q_{2,5} \cdots q_{m-1,5}\right) \\
& +10^{m-3} k\left(n_{1, t}+n_{2, t} \cdots n_{m-1, t}+\frac{q_{m, k}}{k}\right)
\end{aligned}
$$

If the circuit is used as a volt box with the first section as the low-voltage side and the total resistance to the $k$ th tap of the $m$ th group as the high side, the actual ratio is $S_{m, k} / R_{1,1}$ and the nominal value is $k \cdot 10^{m-1}$. The difference between these two values expressed in parts per million is, from eq 14

$$
\begin{gathered}
s_{m, k}=\frac{10^{6}}{k \cdot 10^{m-1}}\left(\frac{S_{m}, k}{R_{1,1}}-k \cdot 10^{m-1}\right)=\frac{2,000}{R_{1,1}}\left(q_{1,5}+q_{2,5} \cdots q_{m-1,5}\right) \\
\quad+\frac{10,000}{R_{1,1}}\left(n_{1, t}+n_{2,5} \cdots n_{m-1, t}+\frac{q_{m, k}}{k}\right) .
\end{gathered}
$$

The nominal value of $R_{1,1}$ is 50 ohms, so that

$$
s_{m, k}=40\left(q_{1,5}+q_{2,5} \cdots q_{m-1,5}\right)+200\left(n_{1, t}+n_{2, t} \cdots n_{m-1, t}\right)+\frac{200}{k} q_{m, k} .
$$

When the standard volt box is calibrated, it is usually desirable to obtain the corrections for all the taps in a single process. For this purpose the schedule of computation indicated in table 1 has been derived by, in efiect, applying eq 16 to consecutively increasing values of $k$ and of $m$. The quantities designated as $r_{m, k}$ constitute the last term in eq 16, and the summations indicated by the earlier terms are automatically made by the successive steps in computation. 
TABLE 1.- Schedule for computing corrections for the standard volt box

\begin{tabular}{|c|c|c|c|c|c|c|c|}
\hline $\begin{array}{l}\text { Section in } \\
\text { bridge arm }\end{array}$ & $\begin{array}{l}\text { Bridge } \\
\text { ratio? }\end{array}$ & $\begin{array}{l}\text { Ad- } \\
\text { just- } \\
\text { able } \\
\text { arm }\end{array}$ & $n^{\prime} \mathrm{s}$ & q's & $r$ 's & Correction & Range \\
\hline 1 & 2 & 3 & 4 & 5 & 6 & 7 & 8 \\
\hline $0-0.15$ & & $\left(p_{1,1}\right.$ & $n_{1,1}=p_{1,1}-p_{1,1}$ & & & $s_{1,1}=0$ & $0-0.15$ \\
\hline $0.15-0.30$ & $100 / 10,000$ & $p_{1,2}$ & $n_{1,2}=p_{1,2}-p_{1,1}$ & $q_{i, 2}=n_{1,2}$ & $r_{1,2}=100 q_{1,2}$ & $s_{1,2}=r_{1,3}$ & $0-0.30$ \\
\hline $0.30-0.45$ & $100 / 10,000$ & $p_{1,3}$ & $n_{1,3}=p_{1,3}-p_{1,1}$ & $q_{1,3}=q_{1,2}+n_{1,3}$ & $r_{1,3}=67 \eta_{1,3}$ & $\varepsilon_{1,3}=r_{1,2}$ & $0-0.45$ \\
\hline $0.45-0.60$ & & $p_{1,4}$ & $n_{1,4}=p_{1,4}-p_{1,1}$ & $\eta_{1,4}=\eta_{1,3}+n_{1,4}$ & $r_{1,4}=50 q_{1,4}$ & $\varepsilon_{1,4}=r_{1,4}$ & $0-0.60$ \\
\hline $0.60-0.75$ & & $p_{1,5}$ & $n_{1,5}=p_{1,5}-p_{1,1}$ & $q_{i, 5}=\eta_{1,4}+n_{1,5}$ & $\tau_{1,5}=40 q_{1,5}$ & $s_{1,5}=r_{1,5}$ & $0-0.75$ \\
\hline $0-0.75$ & \multirow{7}{*}{$1,000 / 10,000$} & $\left(p_{1, \epsilon}\right.$ & & & & & \\
\hline $0.75-1.5$ & & $p_{1, t}$ & $n_{1, t}=p_{t, t}-p_{1,8}$ & $q_{1, t}=n_{1, t}$ & $r_{1, t}=200 q_{1, t}$ & $s_{2,1}=8_{1,5}+r_{1,1}$ & $0-1.5$ \\
\hline $0-1.5$ & & $p_{2,1}$ & & & & & \\
\hline 1. 5-3. 0 & & $p_{2,2}$ & $n_{2,2}=p_{2,2}-p_{i, 1}$ & $q_{2,2}=n_{2,2}$ & $r_{2,2}=100 g_{2,2}$ & $s_{2,2}=s_{2,1}+r_{2,2}$ & $0-3.0$ \\
\hline $3.0-4.5$ & & 132,3 & $n_{2,3}=p_{2,3}-p_{2,1}$ & $a_{2,3}=q_{2,2}+n_{2,3}$ & $r_{2,3}=6 q_{2,3}$ & $\Omega_{2,3}=s_{2,1}+-r_{2,3}$ & $0-4.5$ \\
\hline $4.5-6.0$ & & $p_{2,4}$ & $n_{2,4}=p_{i, 4}-p_{i, 1}$ & $q_{2,4}=q_{2,3}+n_{2,4}$ & $r_{2,4}=50 q_{2,4}$ & $\varepsilon_{2,4}=s_{2,1}+r_{2,4}$ & $0-6.0$ \\
\hline $6.0-7.5$ & & $p_{2,5}$ & $n_{2,5}=p_{2,5}-p_{2,1}$ & $q_{2,5}=q_{2,4}+n_{2,5}$ & $r_{\lambda, 3}=10 q_{2,5}$ & $s_{2,5}=s_{2,1}+r_{2,5}$ & $0-7.5$ \\
\hline $0-7.5$ & \multirow{6}{*}{$1,000 / 1,000$} & $\left(p_{2,3}\right.$ & & & & & \\
\hline $\begin{array}{r}7.5-15 \\
0-15\end{array}$ & & $\left\{\begin{array}{l}p_{2,8} \\
p_{3,1}\end{array}\right.$ & $n_{2, t}=p_{2, t}-p_{2, s}$ & $q_{2,1}=n_{2,1}$ & $r_{2, l}=200 q_{2, l}$ & $s_{3,1}=s_{2,5}+r_{2, l}$ & $0-15$ \\
\hline $15-30$ & & $\left\{\begin{array}{l}P 0,1 \\
p_{3,2}\end{array}\right.$ & $n_{3,2}=p_{3,2}-p_{3,1}$ & $q_{3: 2}=n_{3,2}$ & $r_{3.2}=100 q_{3,2}$ & $s_{3,2}=s_{3,1}+r_{3,2}$ & $0-30$ \\
\hline $30-45$ & & $D_{3,3}$ & $\left.\eta_{3,3}=p_{3,3}-p\right)_{3,1}$ & $q_{13,3}=q_{3,2}+n_{3,3}$ & $r_{3.3}=67 / 3.3$ & $s_{3,3}=s_{3,1}+r_{3,3}$ & $0-45$ \\
\hline $45-60$ & & $p_{3.4}$ & $n_{3,4}=p_{3,4}-p_{3,1}$ & $q_{3,4}=q_{3,3}+n_{3,4}$ & $r_{3.4}=50 q_{3.4}$ & $\varepsilon_{3,4}=s_{3,1}\left\{-r_{\mathbf{3}, 4}\right.$ & $0-60$ \\
\hline $60-75$ & & $\left(p_{3,5}\right.$ & $n_{3.5}=p_{3.5}-p_{3.1}$ & $q_{3.5}=q_{2.3 .4}+n_{3.5}$ & $\tau_{3.5}=40 q_{3.5}$ & $s_{3,6}=s_{3,1}+r_{3,5}$ & $0-75$ \\
\hline $0-75$ & \multirow{7}{*}{$10,000 / 1,000$} & $p_{3,8}$ & & & & & \\
\hline $75-150$ & & $D 3,2$ & $n_{3, t}=n_{3, t}-p_{3,3}$ & $\eta_{3, t}=n_{3, t}$ & $T_{3, t}=200 q_{3.1}$ & $s_{4,1}=s_{3,5}+r_{3,1}$ & $(1-150$ \\
\hline $\begin{array}{r}0-150 \\
150-300\end{array}$ & & $p_{1,1}$ & & & & & \\
\hline $150-300$ & & $p_{4,2}$ & $\eta_{!}, 2=p_{b, 2}-p_{!}, 1$ & $Q_{4,2}=n t, 2$ & $r_{i, 2}=10 n_{1,2}$ & $s_{4,2}=s_{4,1}+r_{4,2}$ & $0-300$ \\
\hline $300-450$ & & $P, 3$ & $n_{1,3}=p_{4,3}-p_{1,1}$ & $q_{4,3}=q_{4,2}+n_{4,3}$ & $r_{4,3}=6 r_{1,3}$ & $s_{4,3}=s_{1,1}+\tau_{i, 3}$ & $0-150$ \\
\hline $450-600$ & & $p_{4,4}$ & $n_{i, 4}=p_{i, 4}-p_{4,1}$ & $q_{4,4}=q_{i, 3}+n_{i, 4}$ & $r_{4,4}=-0 q_{4,4}$ & $s_{4,4}=s_{4,1}+r_{4,4}$ & $0-600$ \\
\hline $600-750$ & & $p_{4,5}$ & $n_{4,5}=p_{!, 5}-p_{1,1}$ & $\eta_{4,5}=q_{4,4}+n_{1,5}$ & $r_{4,5}=40_{14,5}$ & $s_{1,5}=s_{4,1}+r_{1,6}$ & $0-750$ \\
\hline
\end{tabular}

an figure 4 ( $A$ and $B$ ) the bridge ratio is $B / A$.

Washington, June 6, 1941. 


\title{
Measurement of Multimegohm Resistors
}

\author{
Arnold H. Scott
}

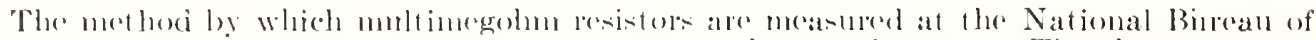

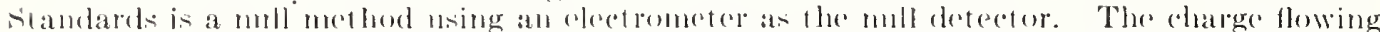

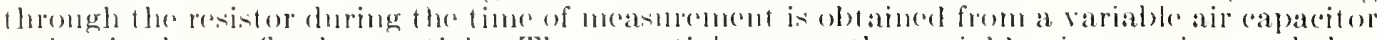

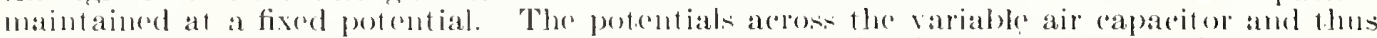

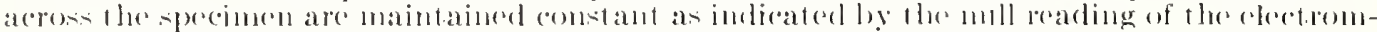

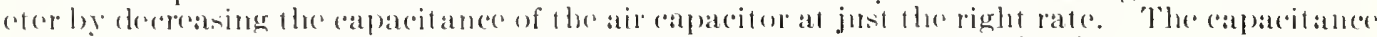

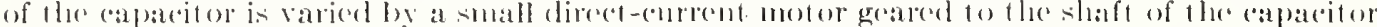

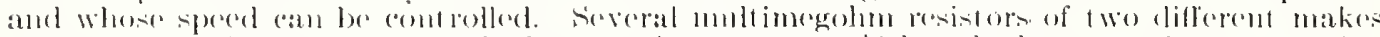

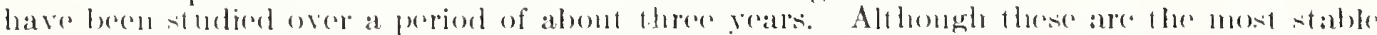

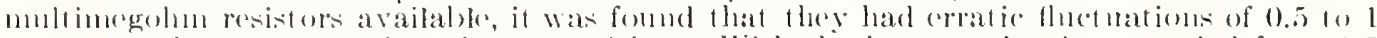

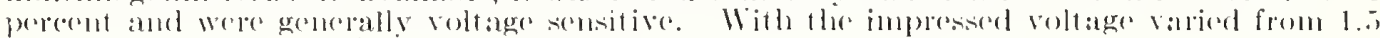
to 180 volls, valions resistors showed resistance changes ranging from 0.4 to $2(i .9)$ pereent.
\end{abstract}

\section{Introduction}

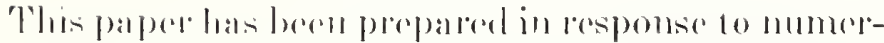

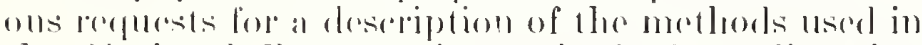

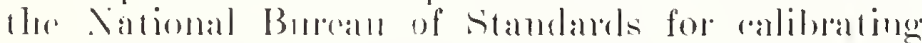
resistors latrine values alowe those convernienty allel recomomically a valiable in wire-wound form. A prantial limit for wire-mound resistors may hr taken as 10$)^{7}$ ollms.

Bridgess argupped with suitable detectors and capable of bring balaneed with a precision of a tew tenthe of a pereent are commererally avalable for morastrements in the range $10^{7}$ to $10^{13}$ olmos. All of these repuipmontes alde rompalrison devions and involve

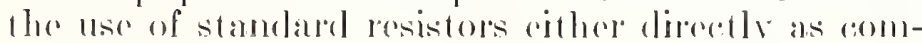

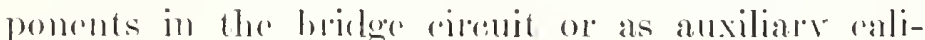

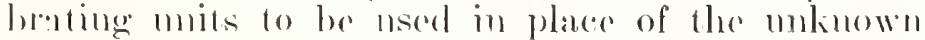
resistor. It is am mofortumate fact that cven the lost multimexohm resistors produred today do not possesse strfliciont stability to serve as reliable stamelateds in the latere montionerl aloove. A later sertion of this paper will present the results of a

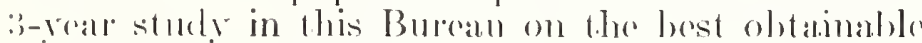
millimomohim resistors.

In the absenere of suitable standards in this lange, resistops must lor avaluated by the applieation of other primedples of moasuremiont. These msually

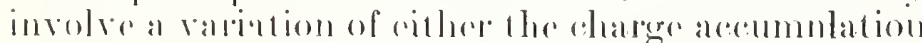
or the loss-of-ehatrere method. For rither mothod

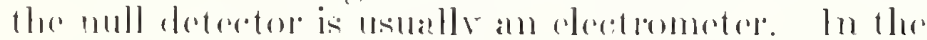

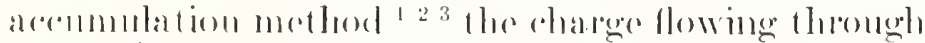

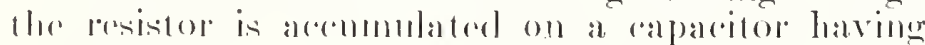
enool insulation. 'Tlas potential from one sisle of the

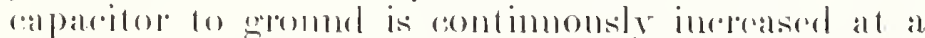

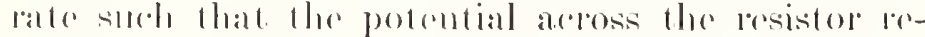

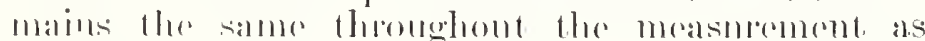

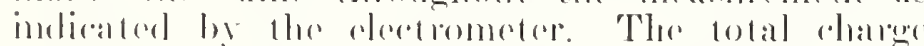
that flows theoreh the resistor in a kuown time is

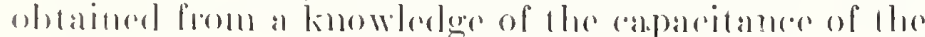

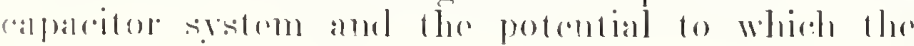

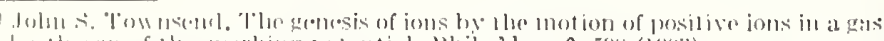

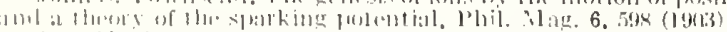

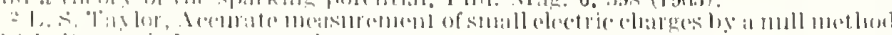

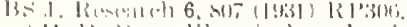

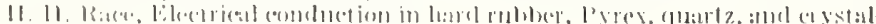

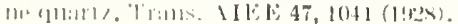

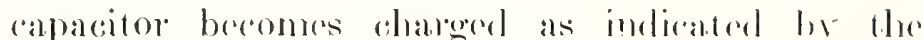
potontiomeres. "The disalvalutages of this molhod

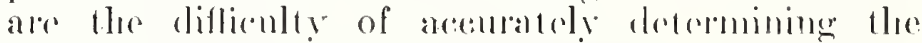

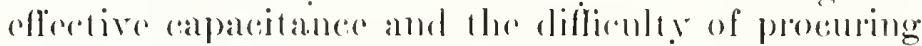
a eontinuously variable potentiometer having the range and precision of setting desired.

A method for morasuring small arrents deseribed

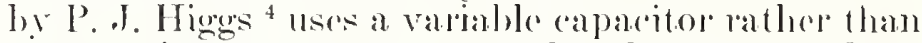
a potentiomeder to modsume the charege that flows through the resistol. In this asse the loss-of-rharege method is mosed. 'The ehatrere that flows through the

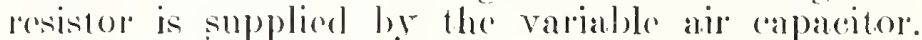
Constant potential areoss the resistor as inclicated hy the olectrometer is obtained by antimously

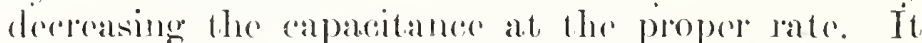
is not meessary to know the total rapalcitanere of the system, only the amonnt by which the alparitance lias berm alimuged duringe monsuremont.

\section{Method of Measurement}

A slightly modified version of the Higers' method has beren developed by the present author. $A$ diagram of the eirente is slown in figure 1. $\Lambda$ potential divider is nsed to provide the polentials for the speeimen and variable air capacitor rather than dieret batery eomoctions. This permits the atio of the voltages to be more assily determined with the desired acemracy than when the voltages alre measured. A small il-e: motor is erenered to the

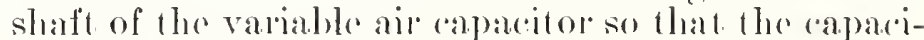
tamere can be ahanged more smoothly that by hand.

The hasie principle of this methorl may be molerstood from the following enensiderations: Bufore the starl of a moraterment the switel, s, across the

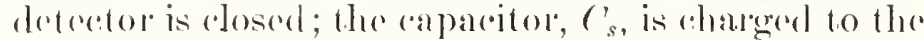

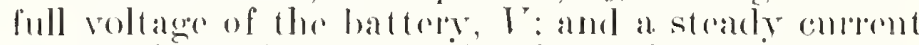
of magmitude $i=\left(r_{x} / r_{s}\right)\left(I^{*} / X\right)$ is flowne through the

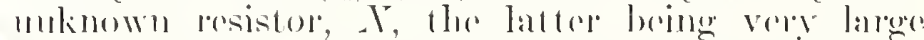

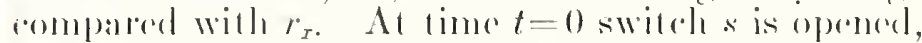

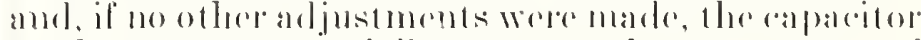
dischalderes expomentially to somo lower voltager of

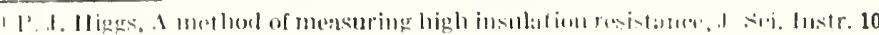
lain (.111110 1 1933). 


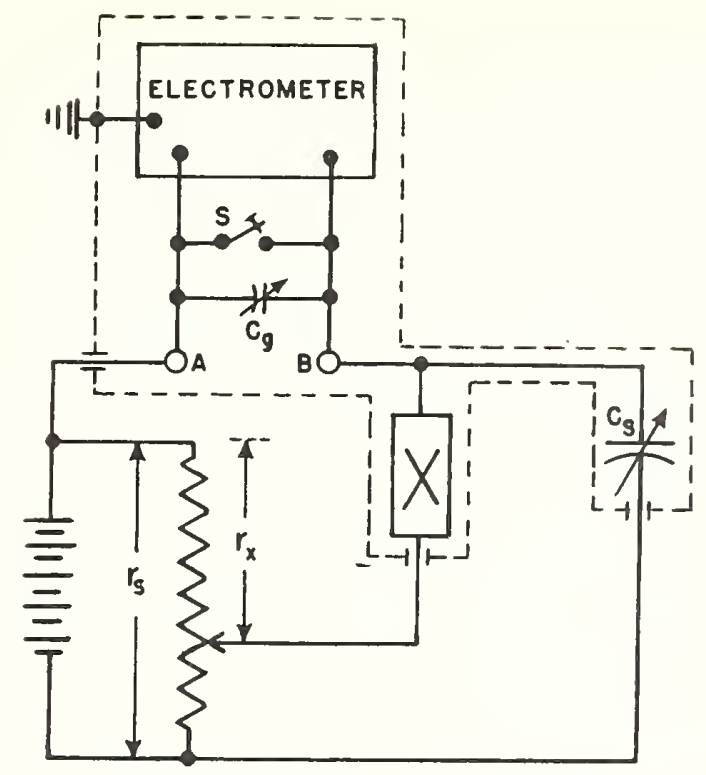

Figure 1. Circuit used to measure multimeghom resistors.

magnitude dependent upon the setting of tne contact $P$. The proeedure is to deercase the value of $C_{s}$ eontinuously and smoothly in such a manner that the potential differenee aeross it remains constant at its initial value as its eharge diminishes. The rate at whieh $C_{s}$ is decreased is such that the potential differenee aeross open switeh $s$ remains zero as indieated by the detector. It will be obvious, therefore, that eurrent through $X$ is being maintained eonstant at its initial value $i$, with the difference that the energy dissipated in $X$ is now fumished by the capaeitor rather than the battery. Under these eonditions the pertinent equation is:

$$
R=\frac{V_{x}}{V_{s}} \frac{\Delta t}{\Delta C}
$$

where $R$ is the resistance of the specimen, $\Delta C$ is the ehange in eapaeitance during time interval $\Delta t$ and $V_{x}$ and $V_{s}$ are the potentials aeross the spceimen and variable air eapaeitor, respeetively. Sinee the eurrent through the speeimen is negligibly small eompared to the current in the potential divider, the ratio $V_{x} / V_{s}$ may be taken equal to $r_{x} / r_{s}$ and eq (1) bceomes:

$$
R=\frac{r_{x}}{r_{s}} \frac{\Delta t}{\Delta C}
$$

\section{Description of Equipment}

Any good electrometer having the required sensitivity may be used as the null instrument to indieate when points $A$ and $B$ of figure 1 are at the same potential. 'The insulation between the terminals of the eleetrometer must be of such quality that the leakage current will be negligible eompared to the eurrent flowing through the spceimen. 'Two types of eleetrometers have been used, a Compton quad-

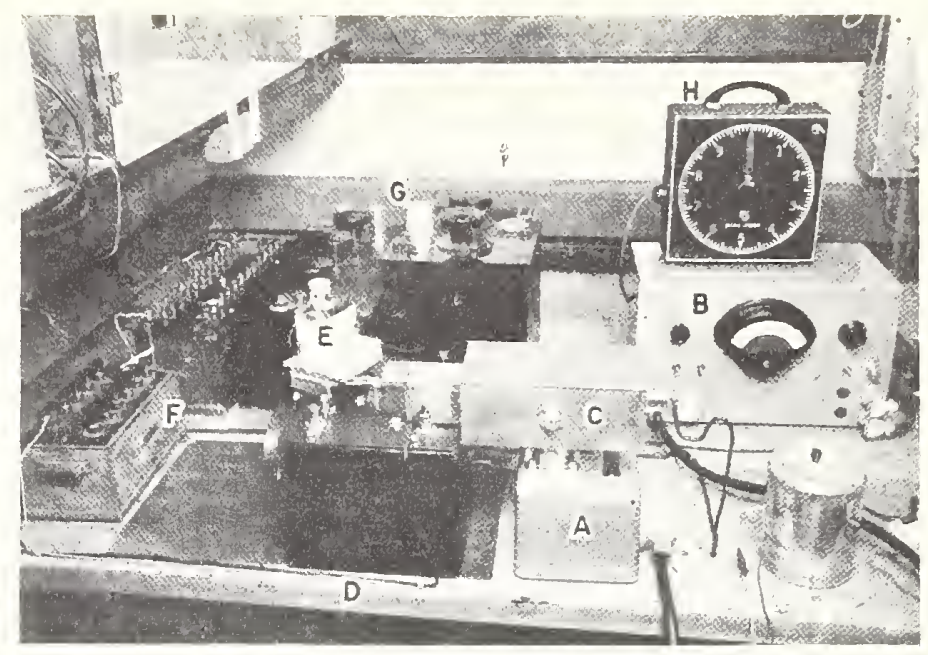

FIGURE 2. Equipment used to measure multimegohm resistances when the vibrating-reed electrometer is the null indicating instrument.

A, Electrometer head; B, electrometer controls; C, specimen shield; $D$, variable air capacitor, $C_{s} ; \mathrm{E}$, motor drive for variable air capacitor; $\mathrm{F}$, voltage divider; $G$, variable capacitor, $C_{\mathrm{g}}$.

rant electrometer and a vibrating-reed electrometer. ${ }^{5}$ The equipment used in the latter case is shown in figure 2 .

When the Compton quadrant eleetrometer is used, the point $A$ is directly connected to ground. The quadrant electrometer has a sensitivity such that a potential of $1 \mathrm{mv}$ produces a reflection of about 1.5 $\mathrm{mm}$ at a scale distance of $2 \mathrm{~m}$. Its period is about $11 \mathrm{sec}$, and this relatively long interval is a serious disadvantage when trying to observe potential ehanges and to compensate for them.

Of the two, the vibrating-reed electrometer is both more sensitive and more rapid in its response. Its sensitivity is suel that a potential of $1 \mathrm{mv}$ produces a meter deflection of 50 s'ale divisions. The response is almost instantaneous. A disadvantage is that neither of the measuring terminals may be conneeted to ground. This requires that all equipment be insulated from erround. Another disadvantage is that on the nost sensitive lange it has a zero drift that must be determined and for which a eorrcetion must be applied. 'The vibrating-reed electrometer is, however, generally more satisfactory than the quadrant clectrometer.

The capacitor', ('s, must have neglierible lakage between its plates. A three-terminal variable air capaeitor is used. Each set of plates is insulated from the frame and case. 'There is no dielectric dircetly between the plates. Conductance to ground on the high-potential sicle auses no error because the leakage emrent returns to the battery without affeeting the electrometer. 'There is no difficulty' in keeping the leakage to yround on the low side suffieiently low because this part of the eircuit is maintained at, or near, zero potential at all times, and the leakage current is therofore newligible.

5 This electrometer converts a d-e potcutial into :ul il-c potential by means of a reed vibrating near an anvil on which the d-c potential appears. The a-c potential is then amplified and rectifier. A negative leethark stabilizes the circuit. A full (lescription of this type of electunneter is given hy H. Palevsky, R. K. Swank, and 18. Grenchik, i Jesign of dynamic condenser electrometers, Rev. Sci. Instr. 18, 298-314 (May 1947). 
It has been found that when the relative humidity rises above about 40 percent appreciable conduction does occur directly between the plates of the capacitor. This is probably due to very fine fibers that are gcnerally floating in the air and which settle onto the plates, occasionally bridging them. When a drying agent is placed in the capacitor, this conductance disappears.

Any one of three capacitors having ranges of 9.5 to $20.5,15$ to 125 , and 100 to 1,100 picofarads (micromicrofarads) are availablc for use in the measurements of resistance. With these capacitor's, and with proper potentials applied to them, measurements can be made involving currents from $10^{-9}$ to $10^{-13}$ amp. For instance, if a $10^{9}$-ohm resistor is being measured at $1.5 \mathrm{v}$, it will be seen from eq (1) that a potential of $500 \mathrm{v}$ will be required on the variable air capacitator for a capacitance change of 900 picofarads in $300 \mathrm{sec}$. If a $10^{13}$-ohm resistor is being measured at $1.5 \mathrm{v}$, a potential of $15 \mathrm{v}$ will be required on the variable air capacitor for a capacitance change of 9 picofarads in 900 sec.

It is necessary to decrease the capacitance of the capacitor, $C_{s}$, at a specific uniform rate. For this purpose, a small d-c motor is geared to the shaft of the capacitor, as shown in figure 3 . When using the vibrating-reed electrometer, the motor must bc insulated from the shaft and frame of the capacitor, so a fiber gear and insulating supports are used. The speed of the motor is controlled by a series resistancc. This resistance is adjusted so that the speed of the motor is just below the proper speed, and small changes in the speed are made by shorting part of the resistance by means of a key. By closing this key momentarily with varying regularity, it is possible to keep the potential between points $A$ and $B$ within plus or minus $1 \mathrm{mv}$ as indicated by the electrometer.

The potential divider is a plug box having a total resistance of 1 megohm. This resistance is low compared to the resistances being measured, and yet is high enough to prevent undue drain on the battery used for potential source. The potential source must be very steady. The voltage on the variable air capacitor must not change sufficiently during the period of measurement to cause a change of charge on the capacitor, which is appreciable compared with the total charge that flows through the specimen. The charge $Q_{x}$ that flows through the specimen during measurement is given by $Q_{x}=V_{s} \cdot \Delta C$, and the charge change due to voltage change is given by $Q_{s}=C \cdot \Delta V$. If the ratio $Q_{s} / Q_{x}$ is to be less than the limit of error $L$, then $\Delta V$ must be less than $L(\Delta C / C) V_{s}$. If the limit of desired error is 0.1 percent, $\Delta C$ is 9 picofarads, and $C$ is 20 picofarads, then $\Delta V$ must not be more than 0.045 pereent of $V_{s}$. It has been found that heavyduty radio $B$ batteries will satisfy this requircment when connected to a potential divider that causes a current drain not cxceeding $0.5 \mathrm{ma}$.

The time is measurcd by means of an electric timer that indicates to tenths of a sccond. This timer is started by a switching mechanism that opens switch

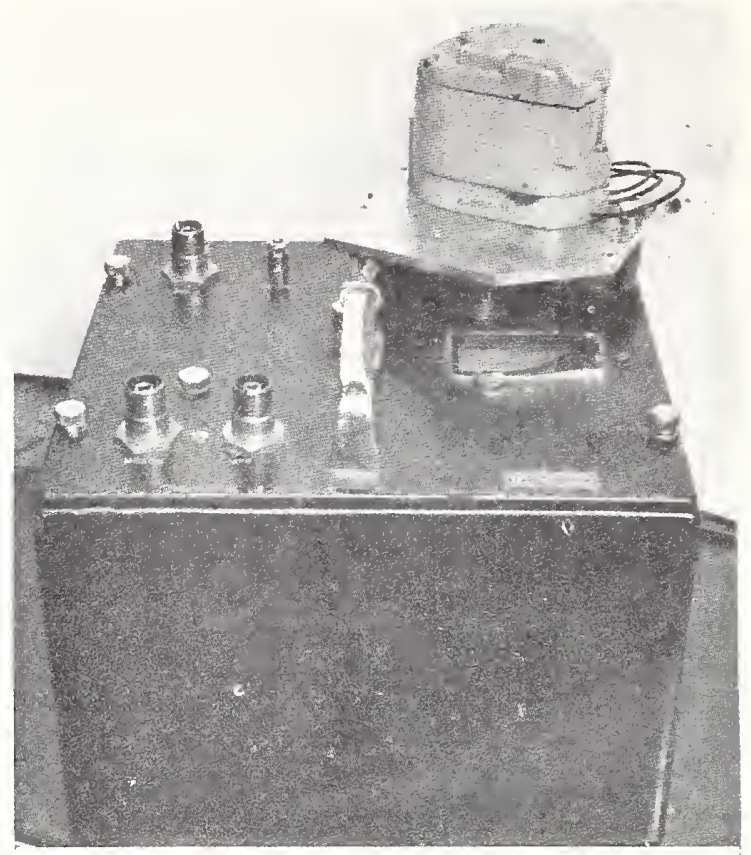

Figure 3. Motor drive for variable air capacitor

$S$ and starts the motor driving the capacitor $C_{s}$ so that all three events occur simultaneously.

For attainment of the desired accuracy (limit of error of 0.1 percent in the present case), the following requirements must, be met. (1) The capacitance change, $\Delta C$, during measurement must be large enough so that it can be determined with the required accuracy. This depends upon the range of the capacitor being used. (2) The time of measurement must be long enough so that $\Delta t$ can be determined with the required accuracy. The timer used in this work can be read to 0.1 sec, so a time of measurement of about 300 sec is generally used. (3) The sensitivity of the electrometer to voltage must be such that in combination with proper regulation, the deviations of the potential between points $A$ and $B$ from zero arc negligible in comparison to the voltage applied to the specimen.

\section{Measurement Procedure}

The measurement procedure can be best described by an example such as the following. Suppose that a resistor having a nominal value of $10^{10} \mathrm{ohms}$ is to be measured at $1.5 \mathrm{v}$ : A time of measurcment of 300 sec is chosen. If the capacitor having a range of 1,000 picofarads is used, a $\Delta C$ of 900 picofarads is chosen. As secn from eq (1), choicc of the above three valıes will require that the voltage on the capacitor be $50 \mathrm{v}$. As this is also the value of the voltagc across the voltage divider, the battery connections are adjustcd to give that value as nearly as possible. The battery pack is so constructed that its voltage output can be adjusted in steps of $1.5 \mathrm{v}$. As $0.5 \mathrm{ma}$ is the current chosen for the potential divider, the resistance of the potential divider is adjusted to $100,000 \mathrm{ohms}$. The tap for the specimen is placed at 3,000 ohms to give $1.5 \mathrm{v}$. The value of the voltage across the potential divider is watched by means of a potentiometer connected across 300 
ohms. To keep the sensitivity of the rireuit within a practical working range, the yalue of $C_{g}$ is adjusted until the potential difference across the open sivitch, $s$, changes at the rate of about $20 \mathrm{mv} / \mathrm{sec}$ when the capacitancé, $C$, remains fixerl. An appreciably higher rate than this makes it difficult to adjust thic speed of the driving motor on the variable air capacitor quickly enough to keep the potential difference across the open switeh, $S$, from fluctuating more than $1 \mathrm{mr}$. 'The equipment is now adjuster for' measurement.

To make a measurement, switch $s$ is closed, the caparitor is set at its highest value, and the battery is connected to the potential divider. At time $t=0$, switch $s$ is opened at the same time that a switch is closed, which starts both the motor drive on the capacitor and the timer. The potential difference across the open switch, $s$, is kept less than $1 \mathrm{my}$ (with practice it can be kept less than $0.4 \mathrm{mv}$ ) by slightly changing the speed of the driving motor. At the end of about 300 see the switch is opened, which stops the motor and timer. Switch $s$ is then closed. The capacitance difference is determined from its original and final readings, and the time interval is read from the timer. The resistance of the specimen is computed by eq (2). The capacitor is then reset to its highest value, the timer reset to zero, the battery to the potential divider is reversed, and anöther measurement is made, as deseribed above. An average of this pair of measurements is considered a single determination. Two determinations are made on each specimen.

\section{Accuracy and Precautions}

A limit of error of 0.1 percent can be obtained with this equipment if the following precautions are observed. The capacitance difference, $\Delta C$, should be large enough so that it can be determined with the required accuracy. The variable air capacitors used are calibrated to 0.01 percent of their maximum range. Because only about nine-tenths of the maximum range is used in a measurement and inaccuracies due to stopping the motor are involved, $\Delta C$ cannot be determined with an accuracy greater than about 0.04 percent.

The time interval, $\Delta t$, must be of such duration that it can be determined with sufficient accuracr. Because the timer reads to $0.1 \mathrm{sec}, 300 \mathrm{sec}$ is chosen. Care must be taken to assure that the timer is started at the same instant switch $s$ is opened. Under present procedure, the error due to lack of the sinchronization is not greater than 0.1 sec. Thus jt can be determined with an accuracy of 0.03 pereent.

The resistances in the potential divider can be readily determined to 0.01 percent, so these present no problen regarding limit of error.

It is not possible to keep the potential difference across the open switch, s, always at zero. The appearance of this potential causes an error in two wavs. It changes the potential across the specimen and across the capacitor. Because a voltage of 1.5 $r$ is applied to the specimen, a voltage of $0.5 \mathrm{mv}$ across switch, s, would change the voltage on the specimen and hence the current by about 0.033 percent. The potential that appears across the switch, $s$, is random in nature, being both positive and negative, so the average effect is less than 0.03 percent.

The appearance of a potential across switch $s$ also changes the potentials across the capacitors, $C_{s}$ and $C_{g}$. The appearance and disappearance of these random potentials do not affect the measurement except as they produce a leakage of charge over the insulation from the low-potential side of the capacitor, $r_{s}$, to the shield or through the insulation of the capacitor, $C_{g}$. If the insulation is good (10 ${ }^{10} \mathrm{ohms}$ or greater), this leakage will be neglibible. However, if the measurement is terminated at a time when the potential across the switeh $s$ is a maximum of $0.5 \mathrm{mv}$, then the resistance of the specimen as computed by eq (2) will be in error because part of the charge that flowed through the specimen came from a change in potential of the capacitors, $C_{s}$ and $C_{g}$, rather than from a change in capacitance of capacitor $C_{s}$. The error due to this can be estimated by recalling that $C_{g}$ is adjusted so that the rate of change of potential across switch $s$ is about $20 \mathrm{mv} / \mathrm{sec}$ when the capacitance, $C_{s}$, remains fixed. This is equivalent to about 6,000 $\mathrm{mv}$ in the 300 sec during which the measurement is made, so an error of $0.5 \mathrm{mv}$ at the end of the measurement would cause an error of about 0.01 percent.

In those cases where the current flow is less than $10^{-12}$ amp (resistance greater than $10^{12}$ olıms at $1.5 \mathrm{v}$ ), the capacitance desired for $C_{g}$ becomes less than the minimum capacitance of the system and cannot be realizerl. Therefore, the measurement time must be increased correspondingly if the same acurracy is to be obtained.

Because of the small currents involved, it was found necessary to take special precautions in shielding to climinate spurious effects. It was found that contact-potential differences of different metals in the shielding and conductor can cause a drift of the electrometer even though no potential is applied. It was not practical to make all parts of the equipment from the same metal. However, the effect was virtually eliminated by the proper selection by a cut-and-try process of metals for the various parts. Correction for the residual can be made by making measurements with the battery connecter direct and reversed. An average of these two measurements gives a value from which the elror is eliminated, provided the effect does not change during the time of measurement. The terminals on some of the resistors are especially barl in this respect. The trouble from specimen terminals may be eliminater by surrounding the terminal with a sheath of metal that does not prorluce a drift of the electrometer. A bare copper wire wound into at tight helix makes an excellent sheath.

There is a drift in the vibrating-reed electrometer that is equivalent to a current of about $10^{-16}$ amp. This is not significant when measuring resistances below $10^{12}$ olms, but becomes appreciable for 
resistances above this value. This drift is sufficiently constant over the period of measurement, so that an average of two measurements with reversed battery practically eliminates this effect.

To test the equipment for spurious currents, a measurement is made with the resistor removed from the circuit. When the circuit is set for measuring resistances in the range $10^{9}$ up to $10^{11}$ ohms, and when it is operating properly, no detectable change in potential (equivalent to less than 0.02 percent of the resistance for which the circuit is set to measure) is observed across open switch $s$ in 300 sec. When the circuit is set for measuring resistances of the order of $10^{13} \mathrm{ohms}$, the readings are appreciable and somewhat erratic. The potential appearing across open switch $s$ always has the same polarity, regarelless of the polarity of the battery across the potential divider and is great enough to produce an error of from 0.1 to 0.5 percent Averaging a pair of measurements with the battery reversed tends to cancel this effect, but because this effect is somewhat er'ratic, the limit of error for measurements of $10^{13}$ ohms is 0.2 to 0.3 percent.
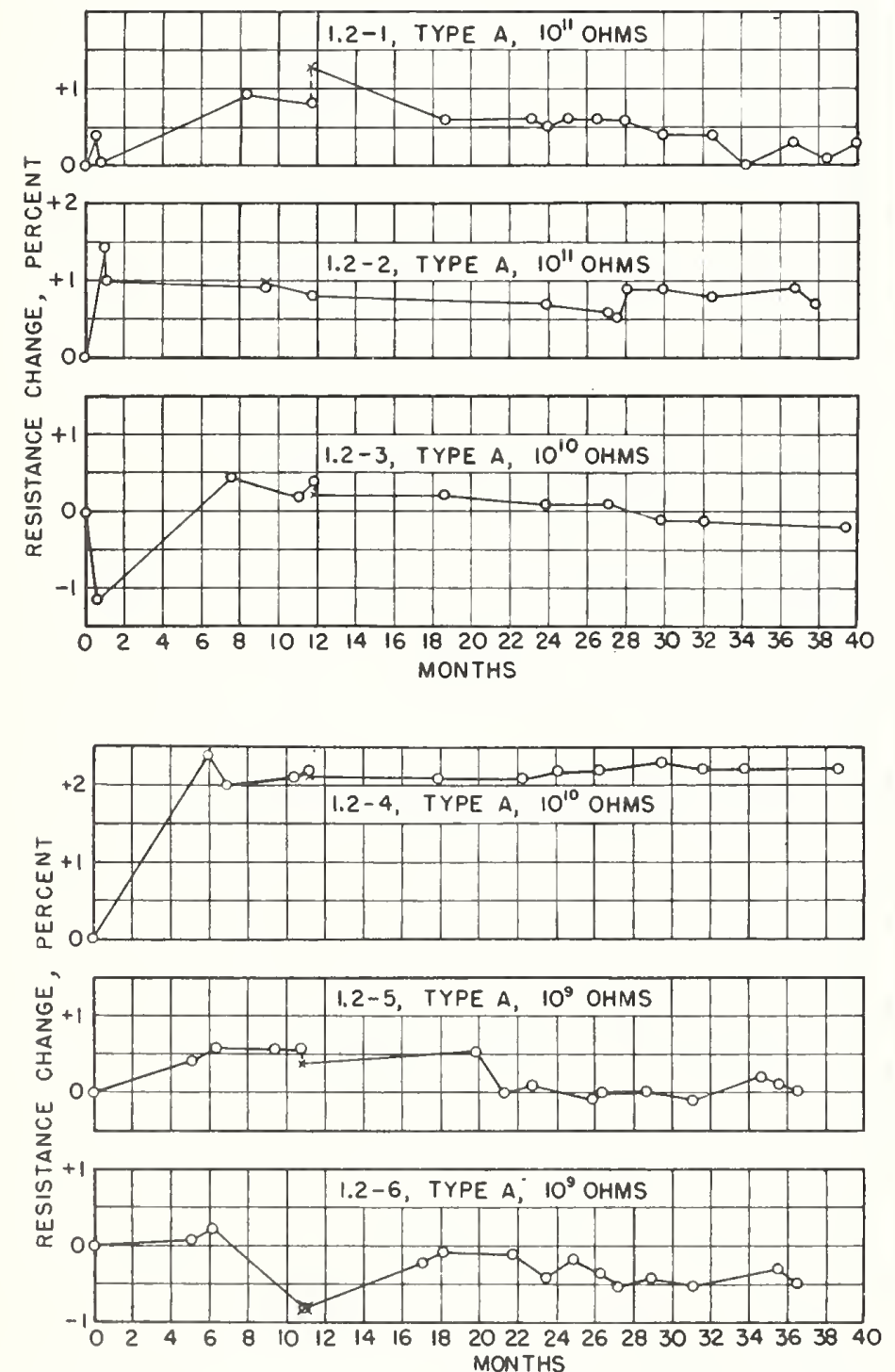

\section{Agging and Voltage Coefficient of Certain Commercial Resistors}

A number of multimegohm resistors have been studied over the past 3 vears, during which time they have been kept under standard laboratory conditions $\left(23^{\circ} \mathrm{C}\right.$ and less then 50 percent relative humidity). Most of these resistors have been measured by the method described in this paper, but a few of these resistol's had resistances too low to be measured in this manner. These latter resistors were measured either by the "comparison method, using a galvanometer" described in ASTM 'Tentative methods of test for electrical resistance of insulating materials D257-52'T, or more lately br means of the Wheatstone bridge. The study included two trpes of resistors. One type (1.2-1 through 1.2-9) consisted of a small coated rod sealed in a glass envelope. "The. glass envelope had been treated to reduce surface conductance. The other type (1.2-10 through 1.2-14) consisted of molded composition material.

The values of resistance fluctuated with time, as shown in figure 4 and were a function of the voltage
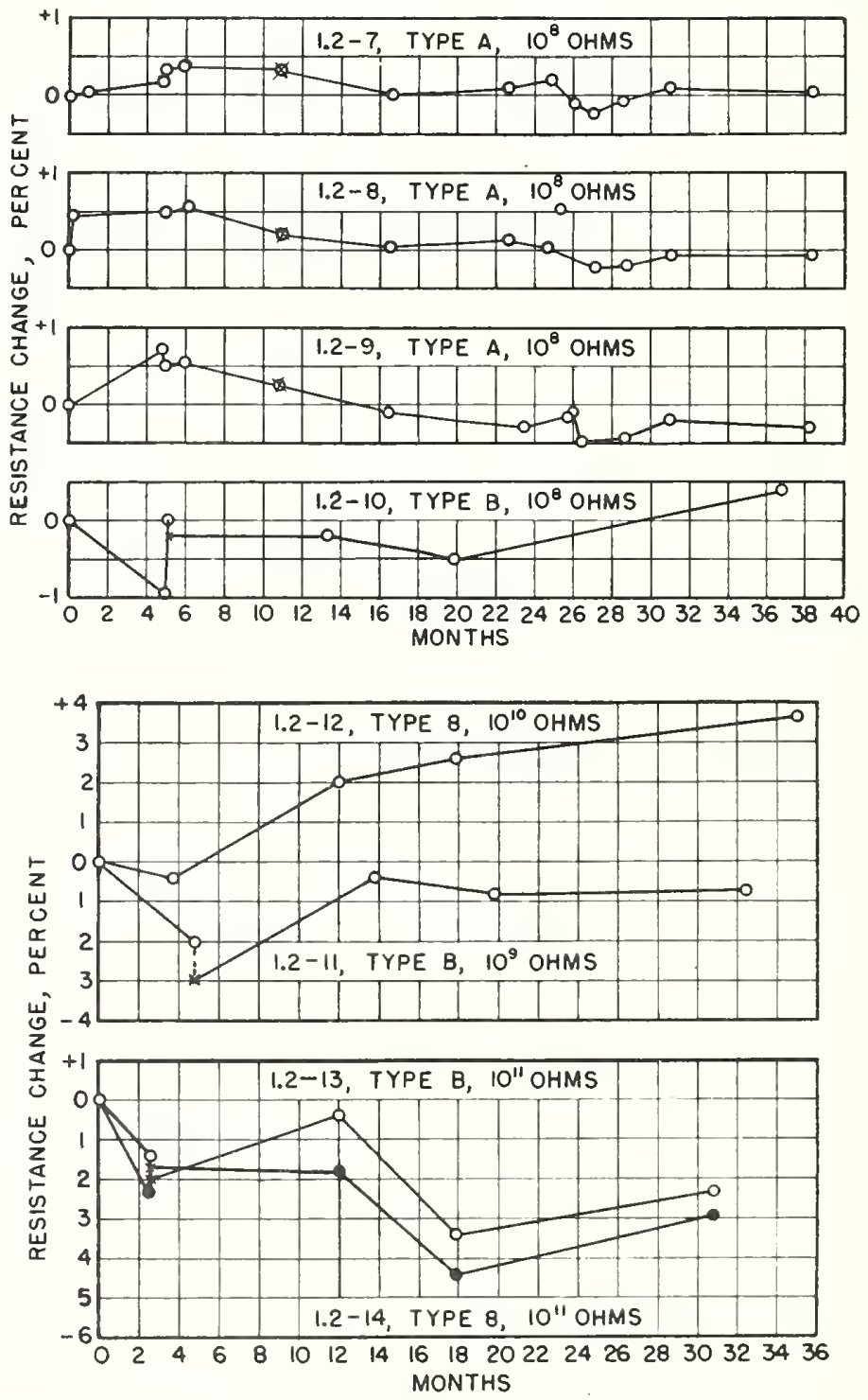

Faril RE 4. Change of resistance of multimegohm resistors with time.

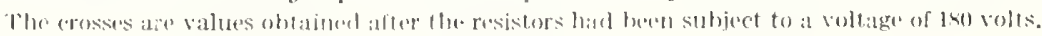


at which they were measured, as shown in table 1. The values of the molded composition resistors (1.2-10 to 1.2-14) fluctuate more with time than do the glasssealed resistors (1.2-1 to $1.2-9)$, which may be due to humidity effects. One of the molded composition resistors (1.2-12) now has over twice the resistance that is stamped on it. When originally received about 12 years ago, it was measured by a moderately accurate method (limit of error probably about 2 percent) and found to have approximatcly the value of resistance stamped on it. 'This means that in the intervening years, the resistance had doubled. This is the resistor that shows the greatest voltage coefficient.

Three other types of resistors have been tested, but thcir stability was so much poorer than the two mentioned above that no extended study has becn made of them.

There is some indication that the fluctuations in resistance of some of the sealed resistors are decreasing with age. But even the best of these resistors have erratic fluctuations of resistance of the order of 0.5 percent.

The effect of change in voltage is often very different for different resistors, even when they are the same make (sce table 1). The percentage changes between 1.5 - and $180-\mathrm{v}$ range from 0.4 to 26.9 percent. There appears to be no significant difference between the two types of resistors as regards effect of voltage.

Although the null-clectrometer method described above is capable of measuring resistances up to $10^{12}$ ohms at $1.5 \mathrm{v}$, with a limit of crror of 0.1 percent, no multimegohm resistors $\left(10^{9} \mathrm{ohms}\right.$ or above) have
TABLE 1. Change of resistance of multimegohm resistors with voltages above 1.5 volts

\begin{tabular}{|c|c|c|c|}
\hline \multirow{2}{*}{ Resistor } & \multicolumn{2}{|c|}{ Volts } & \multirow{2}{*}{$\begin{array}{l}\text { Nominal } \\
\text { value }\end{array}$} \\
\hline & 1.5 to 45 & 1.5 to 180 & \\
\hline $\begin{array}{l}1.2-1 \\
1.2-2 \\
1.2-3 \\
1.2-4 \\
1.2-5\end{array}$ & $\begin{array}{c}\% \\
-2.0 \\
-0.4 \\
-.4 \\
-.4\end{array}$ & $\begin{array}{l}\% \\
-8.3 \\
-3.4 \\
-2.5 \\
-2.5 \\
-2.7\end{array}$ & $\begin{array}{c}\text { Ohms } \\
10^{11} \\
10^{11} \\
10^{10} \\
10^{10} \\
10^{9}\end{array}$ \\
\hline $\begin{array}{l}1.2-6 \\
1.2-7 \\
1.2-8 \\
1.2-9 \\
1.2-10\end{array}$ & $\begin{array}{l}+.7 \\
0 \\
-.5 \\
-3.9 \\
-0.1\end{array}$ & $\begin{array}{r}-1.5 \\
-1.9 \\
-3.0 \\
-12.4 \\
-1.1\end{array}$ & $\begin{array}{l}10^{9} \\
10^{8} \\
10^{8} \\
10^{8} \\
10^{8}\end{array}$ \\
\hline $\begin{array}{l}1.2-11 \\
1.2-13 \\
1.2-14 \\
1.2-12\end{array}$ & $\begin{array}{c}+.6 \\
-0.3 \\
-10.4\end{array}$ & $\begin{array}{r}-0.4 \\
-0.4 \\
+2.0 \\
-26.9\end{array}$ & $\begin{array}{r}10^{8} \\
10^{11} \\
10^{11} \\
2 \times 10^{10}\end{array}$ \\
\hline
\end{tabular}

bcen noted that are stable with timc to that accuracy. They all have voltage cocfficients. All these things considered, the presently available resistors may not be relied upon to maintain their values closer than 0.5 to 1.0 percent. There is great need for a more stable resistor for use as a standard in the multimegohm range.

The author is indebted to J. F. Richardson for his aid in making many of the measurements and to the late Dr. Charles Moon for his suggestions and advice.

Washington, Deccmber 15, 1952. 
Reprinted from NBS Circular 524, Electrochemical Constants. Issued August 14, 1953.

\section{Standard Cells and the Unit of Electromotive Force}

By Walter J. Hamer, Langhorne H. Brickwedde, and Phyllis R. Robb ${ }^{1}$

\section{Introduction}

One of the legal responsibilities of the National Bureau of Standards is the maintenance of standards of electromotive force (emf) for the United States including certification of standards of emf for industrial concerns, Government agencies, universities, and standardizing laboratories. The national standard of emf, the unit of which is the volt, is maintained at the National Bureau of Standards by a group of saturated cadmium cells ${ }^{2}[1]^{3}$ consisting of a cadmium-amalgam negative, a mereury-mercurous sulfate positive in a saturated aqueous solution of cadmium sulfate. Part of the present NBS primary group of standard cells was constructed in the years 1906 to 1913 and another part in 1932. Other cells made annually or biennially, with the war years excepted, supplement the primary group.

The volt, although an important unit, is a secondary one. Its value is established experimentally through Ohm's law and the absolute ohm and ampere. Early in this century the practical standard of resistance was a $\mathrm{Hg}$ column of specified dimensions, and the practical standard of current was defined by the amount of $\mathrm{Ag}$ deposited in a specified time under specified conditions. At the present time (since January 1,1948 ) the ohm is evaluated in absolute units in terms of inductance and frequeney $[2,3,4,5]$ and the ampere in absolute units by the measurement of a force produced by the current in a current balance $[6,7,8]$. These absolute measurements are involved, require painstaking work and, therefore, are unsuitable for determinations of the volt as frequently as such determinations are demanded. To circumvent the necessity of frequent absolute measurements, standard cells are constructed, their emf are determined in relation to absolute current and resistance, and these cells are then used to maintain the volt in the interim.

The validity of this procedure rests on the assumption that the emf of standard cells are independent of time. Obviously, all of a group of identical cells may increase or decrease in emf without evident departures from an assigned mean. Today we have arrived at a state where we believe that the precision of cell measurements and the agreement of cells within the group indicate that the standard is not drifting by more than $0.6 \mu \mathrm{v}$, or 6 parts in 10 million, per year. This does not imply, however, that absolute determinations, which are the only proof of the constancy of the cells, are so precise.

\footnotetext{
I National Bureau of Standards, Washington, D.C

2 Historically, these cells are referred to as Weston cells after Edward Weston, who first proposed them in 1892. The term "Weston Normal Cell" refers to the so-called "neutral" type of cell, in which a saturated aqueous solution of cadmium sulfate is used.

3 Figures in brackets indicate the literature references on p. 113.
} 


\section{History of the United States Unit of Electromotive Force}

In 1893 the Chicago International Electrical Congress ${ }^{4}$ chose the Clark cell 5 as the standard of emf, and assigned to it a value of $1.434 \mathrm{v}$ at $15^{\circ} \mathrm{C}$, based on units of current and resistance then accepted and in conformity with the report of November 1892 of the Committee of the Board of Trade of Great Britain. By an act of the United States Congress, July 12, 1894, definitions were adopted for electrical units substantially equivalent to those adopted at Chicago. The National Academy of Sciences, acting on authority of the United States Congress, prepared and published on February 9, 1895, detailed specifications for realizing the international ampere and the international volt by the silver coulometer and the Clark standard cell. Thus, in $1895^{\circ}$ the Clark cell was the legal standard of emf in the United States. It was assigned a value of $1.434 \mathrm{v}$ at $15^{\circ} \mathrm{C}$.

In the years immediately following 1893, most countries adopted $1.434 \mathrm{v}$ for the Clark cell at $15^{\circ} \mathrm{C}$. Iater work showed that cells made with specially purified $\mathrm{Hg}_{2} \mathrm{SO}_{4}$ had an emf of $1.4337 \mathrm{v}$ at $15^{\circ}$ $\mathrm{C}$, or an emf $0.0003 \mathrm{v}$ lowre than the cells made prior to the meeting of the Chicago Intermational Electrical Congress. Germany, however, recommended and adopted $1.4328 \mathrm{v}$ at $15^{\circ} \mathrm{C}$ for the Clark cell in 1898. 'Their" lower value was based on different values of the ohm and ampere. Although the German value was not universally accepted, it was a more nearly correct value as later experiments showerl. Also, during the years 1893 to 1905, the Weston eell (see footnote 2) was found to have many arlvantages over the Clark cell and at an informal international conference ${ }^{6}$ at Charlottenburg in October 1905, the Weston normal cell was first proposed for adoption' in maintaining the volt. 'This informal recommendation, however, was not officially adopted until 1908 (sce below).

In 1906 the United States standard of omf was defined in terms of the Clark cell made with specially purified $\mathrm{Hg}_{2} \mathrm{SO}_{4}$ and of the Weston normal cell, whose emf was expressed in terms of its measured emf difference from the Clark cell. 'The Clark cells were assigned a value of $1.42110 \mathrm{v}$ at $25^{\circ} \mathrm{C}$. This was based on the value $1.434 \mathrm{v}$ at $15^{\circ} \mathrm{C}$ adopted by the Chicago International Electrieal Congress and leogalized by the U. s. Congress, less the correction of $0.0003 \mathrm{v}$ for the use of specially purified $\mathrm{Hg}_{2} \mathrm{SO}_{4}$, and less the temperature correction calculated from the emf-temperature coefficient determined by Callendar and Barnes [10], i. e., 1.434$0.0003-0.0126=1.421 \mathrm{i}$ v. The mean of the Weston normal cells made at NBS, and then on hand, was $1.01890 \mathrm{v}$ at $25^{\circ} \mathrm{C}$ in terms of the Chark cells at $25^{\circ} \mathrm{C}$. 'Thus in 1906 the standard of emf in the United States was a group of Weston normal standard cells, whose mean emf was taken to be $1.01890 \mathrm{v}$ at $25^{\circ} \mathrm{C}$.

In 1908 the International Conference on Electrical Units and Standards [11] met in Isondon and officially accepted the Weston

4 This Congress was composed of delegates from Austria, Canada, France, Germany, Great Britain, Italy, Mexico, Sweden, Switzerland, anc the United States.

5 The Clark cell was devised by Latiner Clark in 1872 [9]. It consists of a Zn-imalgam negatire, a llg$11 \mathrm{~g}_{2} \mathrm{SO}_{4}$ positive in a saturated solution of $\mathrm{ZnSO}_{4}$

6 The luternational Eleetrieal Congress in St. Louis in 1904 recommended that the various governments coneerned appoint members to an International Commission to consider inequalities known to exist in the electrical units as maintaired in the various countries. In aceordance with this recommenclation, the I'hysikalisch-'Technische Reichsanstalt invited representatives of the various standardizing Iaboratories to a eonference at Charlottenburg. 
Normal Cell on the recommendation of the Charlottenburg Conferenee of 1905. The London Conference further adopted provisionally $1.0184 \mathrm{v}$ as the emf of the Weston Normal Cell at $20^{\circ}$ $C$, and reeommended for the emf-temperature coefficient of the Weston Normal Cell the formula based on the measurements of Wolff [12] at NBS. By this formula, the Weston Normal Cell at $25^{\circ} \mathrm{C}$ had an emf of $1.018174 \mathrm{v}$, which is $0.000726 \mathrm{v}$ lower than the value then aceepted in the United States. This diserepaney was eaused largely by the fact that the United States had accepted $1.4337 \mathrm{v}$ for the Clark cell at $15^{\circ} \mathrm{C}$, whereas the German value of $1.4328 \mathrm{v}$ at $15^{\circ} \mathrm{C}$ had proved to be a more nearly eorreet value. 'The London Conferenee still felt, however, that further work was needed and recommended that additional experiments be made.

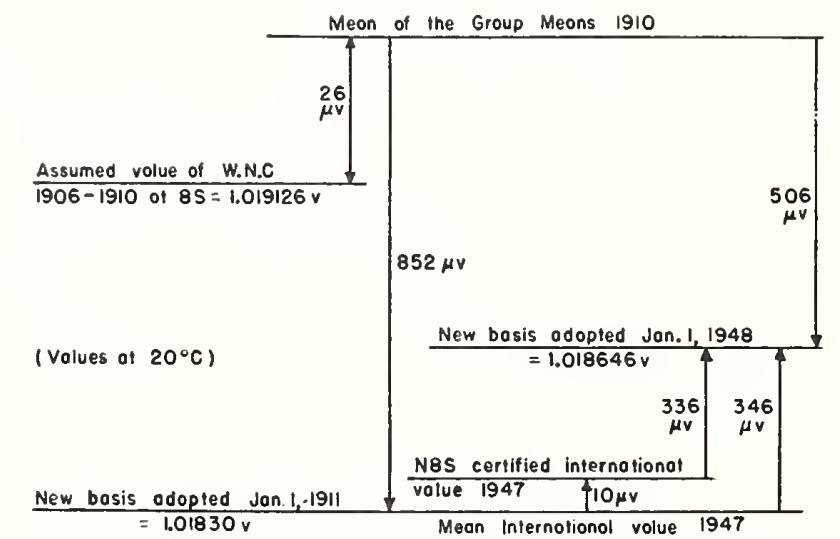

Figure 12.1. Effect of changes in, the volt for the Uniled States from 1906 to 1951, inclusive.

(Not drawn to scale.)

Aeeordingly, scientists from England, France, and Germany met with American seientists at the National Bureau of Standards in 1910, and as a result of their experiments with a large number of Weston Normal Cells and silver-eoulometer determinations adopted $1.01830 \mathrm{v}$ as the emf of the Weston Normal Cell at $20^{\circ} \mathrm{C}$. "The faet that this was nceessarily only an approximation to the "true" or "absolute" value was indicated by prefixing the adjeetive "international," and 1.01830 was taken by mutual agreement as the emf of the Weston Normal Cell in "international volts." This is the value familiar to most seientists and found in nearly all textbooks. This value stood until 1948 when the United States and other eountries went over to absolute units. On the new absolute basis the value of the Weston Normal Cell at $20^{\circ} \mathrm{C}$ beeomes 1.018646 $\mathrm{v}$. 'This value is now of historieal interest only.

In figure 12.1, the effect of ehanges in the value of the United States unit of emf in international and absolute units is given from 1906 to 1951. 'The values are all given for a typieal Weston Normal Cell at $20^{\circ} \mathrm{C}$, the temperature ehosen as referenee by the London International Eleetrieal Conferenee. In 1906 the NBS primary group of 12 eells had an enf of $1.019126 \mathrm{v}$ at $20^{\circ} \mathrm{C}$, based on the ealeulations and experiments deseribed above. Early in 1907, however, one coll was broken, and in 1908 several of the remaining eells were accidentally damaged in the tempcrature-coeffieient work. Seven 
other cells made in 1906, which had shown exceptionally good chalacteristics, were made the Bureau's reference cells in Februm 1908. The mean of this group of seven cells was $14 \mu \mathrm{r}$ betow the group of 1906. The group of seven cells served officially as the standard of emf from 1908 to 1911 and had a moan valus of $1.019112 \mathrm{r}$. In 1910 the mean of the group means of the cells submitted by England, France, Germany, and the United States was $40 \mu \mathrm{r}$ higher than the mean of the NBS older cells, or $26 \mu \mathrm{r}$ higher than the ralue assmund by NBS in 1906 for the primary group. This differenee of $40 \mu \mathrm{r}$ $(0.04 \mu \mathrm{r})$ was later found to be largely caused by an anging offect of these cells, the duration of which was about 2 rears. In other words, the difference between the Burcaurs older group of calls, and the new cells made by the four countries in 1910 decreased by $37 \quad \mu \mathrm{r}$, and hene became insignificant within about 2 years. As the Bureau's older group was then 6 years old (4 years at start of comparison), the new cells made by the four comitries were judged to decrease in emf with time; an increase in emf of the older colls was decmed extremely unlikely.

Those who met in Washington in 1910 still realized, howerer, that the $\mathrm{Hg}$ ohm and the $\mathrm{Ag}$ coulometer were not fundamental standards for resistance and current, and bolieved that absolute mits based on the egs system were more lesirable. Aceordingly, the standardizing laboratories of several nations continued their absolute measurements and determinations of the relation between absolute units and the international units then in use. " It was not until 1948, however, that the absolute seale based on mechanieal units become a reality. Two World Wars had caused delays in the necessary experimental work. As a result of determinations of the relation between absolute units and the international units in various comntries, it was found that the mean ratio was 1.000490 for the olm and 0.99985 for the ampere [13]. Therefore, through Ohm's law the ratio for the volt is 1.00034. As international comparisons of the ampere are not feasible, the conversion factors in transportable units were chosen, and the International Committee on Weights and Measures in October 1946 announced [14]:

\section{1 mean international $\mathrm{ohm}=1.00049$ absolute ohms. 1 mean international volt $=1.00034$ absolute volts.}

The mean international units were the averages of units maintained in national laboratories of France, Germany, Great Britain, Japan, USSR, and the USA that took part in the international comparisons before the outbreak of Workl War II.

Specifically, the units maintained in the United States differed from the above averages by a few parts in a million, and the conversion factors were

\section{1 international ohm (USA) $=\mathbf{1 . 0 0 0 4 9 5}$ absolute ohms. 1 international volt $(\mathrm{USA})=1.00033$ absolute volts.}

The International Committee set the date January 1, 1948, for the

\footnotetext{
7 This does not imply that methods for absolute measurements were not known in 1910, but that sufficient accuracy of the measurements had not been attained. Actually the work of Gauss in 1833 and of 1 eber in 1851 showed the possibility of measuring electric and magnetic quantities in terms of mechanical units. [13].
} 
change to absolute units. ${ }^{8}$ Likewise, other countries had their own conversion fartors. In essence, therefore, on January 1, 1948, the absolute volt and absolute ohm were identical in all countries.

Between 1906 and 1951, the progress in the science of electrical measurements, as formally recognized by international agreements, had led to a number of changes in the unit of emf in the United States. The magnitude and significance of these changes are summarized in table 1. The emf of a typical saturated cadmium cell and the standard electrode potential of the $\mathrm{Ag}-\mathrm{AgCl}$ electrode at $25^{\circ} \mathrm{C}$ in terms of the national standard at various periods in the history of the United States are listed. The effect of the conversion from international to absolute units is shown in terms of a quantity well known to chemists. Although the changes are not large, care should be exercised in making comparisons of literature data prior to 1948 with data reported since January 1, 1948.

TABLE 1.-Effect of changcs in the unit of the United States standard of electromotive force on the electromotive force of saturated cadmium standard cells at $20^{\circ}$ $C$ and on the standard electrode potential of the silver-silver chloride electrode at $25^{\circ} \mathrm{C}$ as certified by NBS.

\begin{tabular}{|c|c|c|}
\hline Year & $\begin{array}{l}\text { Standard } \\
\text { cell }\end{array}$ & $\begin{array}{l}\text { Silver- } \\
\text { silver } \\
\text { chloride } \\
\text { eleetrode }\end{array}$ \\
\hline $\begin{array}{l}1906 \\
1910 \\
1911 \\
1912 \\
1913 \\
1947 \\
1948 \\
1951\end{array}$ & $\begin{array}{r}\text { Tolts } \\
1.019152 \\
1.019152 \\
1.018300 \\
\mathrm{a} 1.018337 \\
1.018300 \\
1.018310 \\
1.018646 \\
1.018646\end{array}$ & $\begin{array}{r}\text { Tolts } \\
0.22258 \\
.22258 \\
.22239 \\
.22240 \\
.22239 \\
\text { b } 22239 \\
.22246 \\
.22246\end{array}$ \\
\hline
\end{tabular}

a Effect of aging of reference group. Correction for this was made and incorporated by 1913.

b Value of $0.22239 \mathrm{v}$ is that given by 11 . S. Harned and R. W. Ehlers [15].

The change from international to absohute volts causes no change, however, in many electrochemical and physical chemical constants, for example, activity coefficients obtained from emf measurements. Activity coefficients of $\mathrm{HCl}$ determined from measurements of the cell:

$$
\mathrm{H}_{2}(\mathrm{~g})|\mathrm{HCl}(\mathrm{aq})| \mathrm{AgCl}(\mathrm{s}), \mathrm{Ag}(\mathrm{s})
$$

are given by the equation

$$
\frac{\left(E-E^{0}\right) \text { volts }}{(R T / \mathbf{F}) \text { volts }}+\ln m_{\mathrm{H}} m_{\mathrm{Cl}}=\ln \gamma_{\mathrm{H}} \gamma_{\mathrm{Cl}}
$$

and the rolt cancels on the left side of this equation. Therefore, the value of the activity coefficient of $\mathrm{HCl}$ is the same whether the emf of the cell is measured in international $\mathrm{or}^{\circ}$ in absolute volts. Of course, $E, E^{0}$, and $R T / \mathbf{F}$ must be measured or expressed in the same units. The same holds for pH values, ionization constants, and like quantities. Conversion from international to absolute units does

\footnotetext{
${ }^{8}$ It had been suggested that absolute volts be used to designate the new unit to distinguish it from the older unit. The National Bureau of Standards, however, took the view that it was preferable to drop the adjeetive "absolute" and use only "volt." It was deemed advisable to speak of only one kind of volt and to do so from the time of the change. When the National Bureau of Standards uses the term "volt," "absolute
volt" is therefore implied.
} 
affect the values of the faraday, standard electrode potentials, redox potentials, electron charge, electron volt, free-energy changes, and ionic mobilities. Conversion factors for these and other quantities are given elsewhere [16].

\section{Modifications in Standard Cells}

In maintaining the standard of emf, it is assumed that between absolute determinations the emf of the cells in the reference group remains constant. As pointed out above, it is obvious that a group of identical cells may increase or decrease so uniformly that departures from the assigned mean may not be evident. Modifications in the type of cells used for a primary standard are desirable. An alternative type of standard cell using an entirely different electrochemical system would be most valuable, for if changes of emf with time in two different cell systems occurred, they would not be likely to follow the same pattern. However, the prime requirement of constancy has not been as well realized in other systems as in the carmium-mercury one. Further, the measurement of emf rlifferences of a half volt or

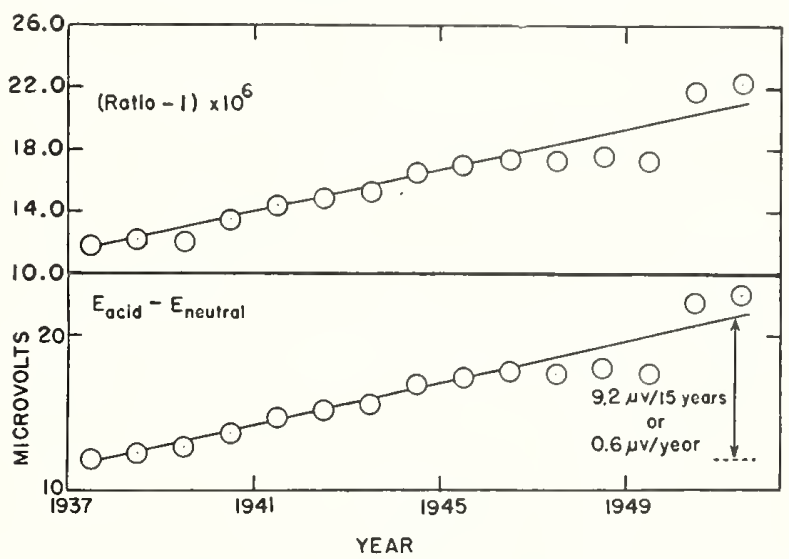

Figure 12.2. Ratios and differences in acid and neutral saturated cadmium cells.

thereabouts with the precision required imposes a problem, though not an insurmountable one.

In order to have difterent kinds of cells in its primary standard, the NBS has effected in recent rears sereral modifications in Weston normal cells. These modifications include (1) acid cells, (2) acid cells with deuterium oxide, and (3) substitution of quartz for glass as container material for both acid and neutral cells. 'Thus, including neutral cells in glass, 10 ratios may be obtained, and these ratios may be followed over a period of year's.

The first morlification that was effected was the construction of acid cells. In the so-called "neutral" Weston normal cell, $\mathrm{Hg}_{2} \mathrm{SO}_{4}$ hydrolyzes to form basic mercurous sulfate and $\mathrm{H}_{2} \mathrm{SO}_{4}$ of an equilibrium concentration. Although these cells are stable in emf and basic mercurous sulfate, once formed, appears to have no effect on the stability of the cell, many experimenters have felt that better cells would be obtained if $\mathrm{H}_{2} \mathrm{SO}_{4}$ were added in sufficient amount to prevent the formation of the basic salt. Accordingly, in 1932 a group of cells were constructed that were $0.04 \mathrm{~N}$ in $\mathrm{H}_{2} \mathrm{SO}_{4}$. Higher concentrations of acid cause the formation of appreciable quantities of gas at the carl- 
mium amalgam. These cells were studied for a number of years and then incorporated into the primary group in 1937.

In figure 12.2, the ratio of the emf of acid and neutral cells in the NBS primary group is slown as a function of time for the past 15 years (in the figure (Ratio-1) $\times 10^{6}$ is plotted for convenience).. This ratio has varied with the neutral cells decreasing and the aeid cells increasing in emf in terms of the group mean. In the lower part of the figure, the differences in emf between acid and neutral cells are shown as a function of time. Over a 15-year period this difference has shifted by $9.2 \mu \mathrm{v}$, or 9.2 ppm, or 6 parts in 10 million $(0.0006 \mathrm{mv})$ per year. This shift is very much beyond the precision and aceuracy of most seientific investigations, but is a real effect.

Of course, we cannot decide from these measurements which. type of cell is changing in emf with time, or which type is more stable. Recourse, therefore, has been made to measurements of the temperature coefficient and effect of age on the stability of the two types. In table 2 , the differences of the emf at $20^{\circ} \mathrm{C}$. from those at $25^{\circ}, 28^{\circ}$, and $30^{\circ} \mathrm{C}$. are given for a group of 46 cells of different ages and acidities but of similar construction to the cells in the NBS primary group.

TABLE 2. Differences in the electromotive forces of slandard cells of different acidities and ages at $20^{\circ} \mathrm{C}$ from the electromotive forces at $25^{\circ}, 28^{\circ}$, and $30^{\circ} \mathrm{C}$

\begin{tabular}{|c|c|c|c|c|c|c|c|}
\hline \multicolumn{8}{|c|}{ Neutral (21 cells) } \\
\hline Age & $\begin{array}{l}\text { Number } \\
\text { of cells }\end{array}$ & $(20-25)^{\circ} \mathrm{C}$ & $\Delta^{\mathrm{a}}$ & $(20-28)^{\circ} \mathrm{C}$ & $\Delta \Delta$ & $(20-30)^{\circ} \mathrm{C}$ & $\Delta^{\mathrm{a}}$ \\
\hline $\begin{array}{l}\text { Years } \\
10 \\
20 \\
40\end{array}$ & $\begin{array}{l}7 \\
7 \\
7\end{array}$ & $\begin{array}{c}\mu v \\
227.5 \\
228.6 \\
231.0\end{array}$ & $\begin{array}{l}\mu v \\
3.2 \\
4.2 \\
4.5\end{array}$ & $\begin{array}{c}\mu v \\
382.4 \\
384.2 \\
386.2\end{array}$ & $\begin{array}{l}\mu v \\
4.4 \\
4.9 \\
5.5\end{array}$ & $\begin{array}{c}\mu v \\
494.6 \\
495.9 \\
501.7\end{array}$ & $\begin{array}{l}\mu l) \\
5.2 \\
8.3 \\
5.0\end{array}$ \\
\hline Mean..... & & 229.0 & 4.0 & 384.3 & 4.9 & 497.4 & 6.2 \\
\hline \multicolumn{8}{|c|}{ Acid (25 cells) } \\
\hline $\begin{array}{l}3 \ldots \ldots \\
9 \\
15 \\
18\end{array}$ & $\begin{array}{l}7 \\
7 \\
9 \\
2\end{array}$ & $\begin{array}{l}222.5 \\
221.2 \\
219.7 \\
222.1\end{array}$ & $\begin{array}{r}0.4 \\
.3 \\
2.5 \\
0.1\end{array}$ & $\begin{array}{l}372.9 \\
372.9 \\
371.9 \\
373.6\end{array}$ & $\begin{array}{r}0.8 \\
.5 \\
3.7 \\
0.0\end{array}$ & $\begin{array}{l}484.5 \\
483.6 \\
480.1 \\
483.5\end{array}$ & $\begin{array}{r}0.9 \\
.7 \\
2.7 \\
0.2\end{array}$ \\
\hline Mean...... & & 221.1 & 0.8 & 372.6 & 1.3 & 482.6 & 1.1 \\
\hline
\end{tabular}

a Mean deviation.

It will be noted that the average deviation from the mean is much larger for neutral cells than for acid cells, being approximately $5 \mu \mathrm{v}$ for the former and $1 \mu \mathrm{v}$ for the latter. Therefore, acid cells exhibit less variation in behavior than neutral cells and are probably more reliable. It will also be noted that the emf-temperature coefficients of acid and of neutral cells differ and that the emf-temperature coefficient for acid cells is more constant with age. This observation also suggests that acid cells are more reliable. Our observations on acid eclls are not very different from those given by Vigoureux and Watts [17].

'The second modification that was effected was the substitution of deuterium oxide for ordinary water as the electrolyte solvent. An account of these cells is given in paper's by Brickivedde and Vinal $[18,19]$. In figure 12.3 , the relation between the emf of saturated standard cells and the mole pereent of deuterium oxide is shown. 'The total change in going from pure-water' solution to pure deuterium- 
oxide solution is only $395 \mu \mathrm{v}$, which is well within the range of $2,100 \mu \mathrm{v}$ of the specialized potentiometer [20] used in the intercomparison of primary-group cells. The cause of the change in emf resulting from substitution of eleuterium oxide for water has been given in the papers of Brickwedde and Vinal [18, 19].

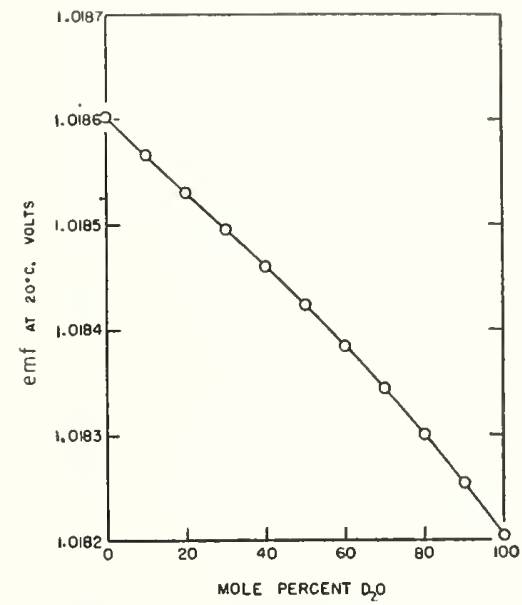

FIGURE 12.3. Effect of deuterium oxide on the electromotive force of saturated cadmium standard cells at $20^{\circ} \mathrm{C}$.

In 1949 four groups of standard cells containing either deuterium oxide or water were prepared. Each cell was made $0.02 \mathrm{~N}^{\top}$ in $\mathrm{H}_{2} \mathrm{SO}_{4}$. In figure 12.4 the arerage 2 -year change in enf for each group of cells is given in terms of the standard deviation. The average change is greater for the $\mathrm{H}_{2} \mathrm{O}$ groups than for the $\mathrm{D}_{2} \mathrm{O}$ groups, but the standard

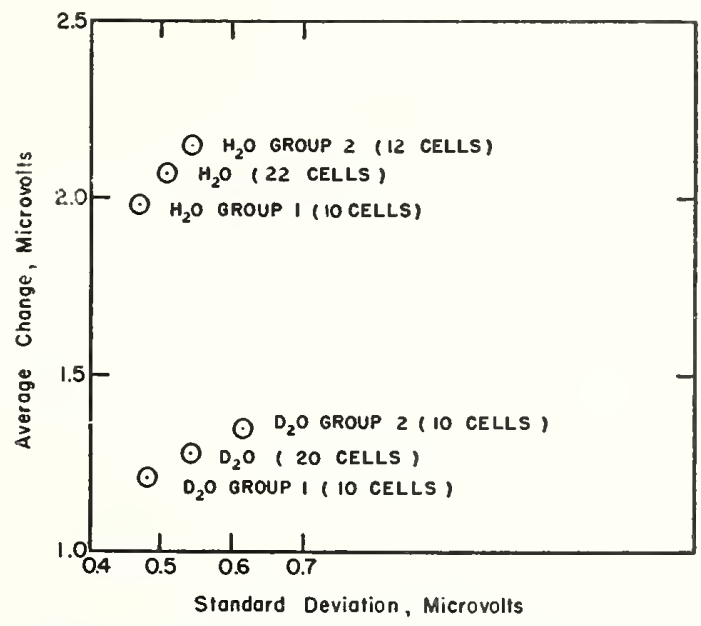

Figure 12.4. Statistics on the changes in the electromotive forces at $28^{\circ} \mathrm{C}$ from 1949 to 1951 of standard cells made with water or deuterium oxide.

deviations are the same. Unfortunately, these observations cover only a 2-year period, a period during which standard cells are known to age, and, therefore, no definite conclusions may be drawn at this time as to the relative stability of water and deuterium-oxide cells. At this time, these observations could be considered to reflect eflects 
of experimental technique rather than differences inherent in the chemistry of the cells. Definite conclusions will be possible only after more extensive observations.

The third modification that was effected was the substitution of a fused-quartz cell container [21] for the Kimble standard flint glass container that has been used at the National Bureau of Standards for many years. It has been realized for a long time that the alkali of glass may neutralize the acid in acid standard cells (neutral cells should not be affected as $\mathrm{Hg}_{2} \mathrm{SO}_{4}$ should hydrolyze to maintain the equilibrium concentration of $\mathrm{H}_{2} \mathrm{SO}_{4}$ ), thereby affecting the stability of the cell $[22,23,24]$. It was felt that fused quartz may, therefore, be a better container. Details of their construction have been given elsewhere [21] and will not be repeated here.

Seven satisfactory saturated standard cells were made in these containers. Eight control cells in Kimble glass were made at the same time. Five acid cells $\left(0.02 \mathrm{~N}\right.$ in $\left.\mathrm{H}_{2} \mathrm{SO}_{4}\right)$ were made in both types of containers and two neutral cells in quartz and three neutral cells in glass. Readings of the emf were taken at intervals during 1949,1950 , and 1951 . If we take the cell average for 1951 and subtract the average for the same cell in 1949, we obtain an enf difference that is a measure of the change in emf of that cell over a 2 -year period. These em!f differences at $28^{\circ} \mathrm{C}$ are given in table 3 .

TABLE 3. Differences in the electromotive force of standard cells in quartz and glass containers between 1951 and 1949 (1951 value minus 1949 value)

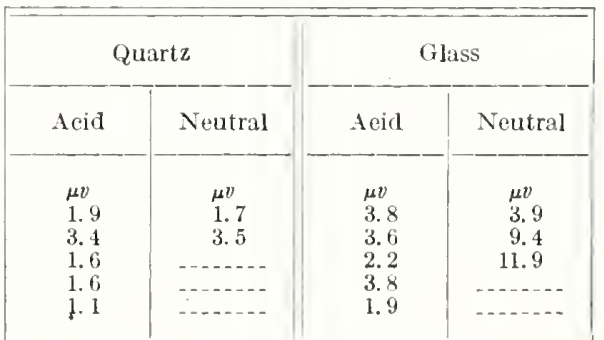

Average change for quart $=2.1$. A verage ehange for glass $=5.06$,

The average change in emf for glass containers is higher than for quartz containers. In addition, the changes in emf vary less in the quartz group than in the glass. Greater average change and greater variability in emf seem to be the case for glass in both neutral and acid cclls. The sample size, however, was too small to draw definite conclusions between the behaviors of acid and neutral cells in the two types of containers. Additional cells will be obtained and further studies nade.

\section{International Comparisons}

Annually or biennially, the National Bureau of Standards transports by messenger saturated standard cells that have been compared with the NBS primary standard cells to the Bureau International des Poids et Mesures ${ }^{9}$ for comparisons against the international group maintained there. The National Bureau of Standards measures the emf of its cells immediately prior to transport to the International

${ }^{2}$ For a brief history of the International Bureau, see the discussion of F. B. Silsbee under standard cells at the end of this paper. 
Bureau and immediately after their return. Except on very rare occasions, the two measurements have agreed within a microvolt, showing that transportation by messenger had hot affected the cells.

Comparisons with the International Bureau (BIPM) since World War II are shown in figure 12.5. Differences between the NBS and BIPM values reported for the NBS cells are given. Reported differences are represented by the shaded circles. In 1946, 1948, and 1950 the values for the NBS cells differed by $+10.0,+9.0$, and $-0.7_{g} \mu \mathrm{V}$ from the BIPM values for the same cells. However, BIPM reported their results in international volts in 1946 and 1948 and in absolute volts in 1950. If their international values of 1946 and 1948 are converted to absolute volts, using their conversion factor of 1.00034 , the differences between the BIPM and NBS values for the same cells

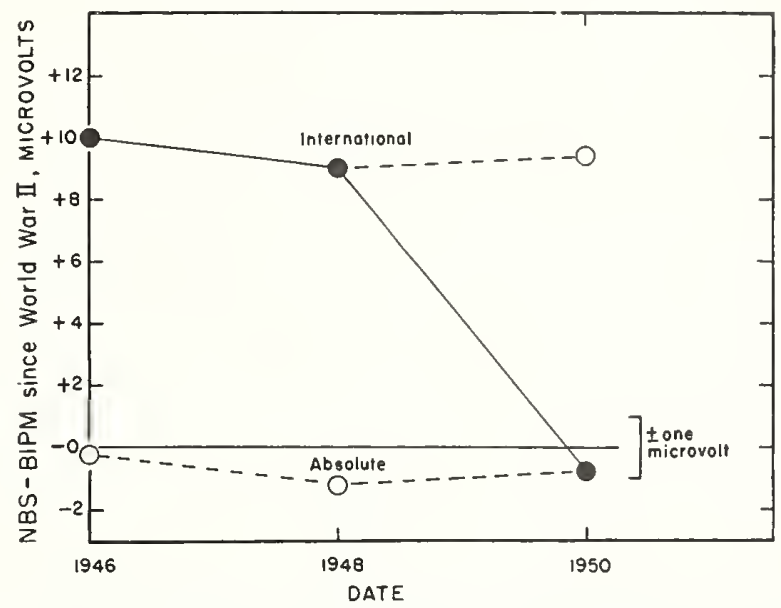

Figure 12.5. Comparisons of electromotive forces certified by the National Bureau of Standards with the Bureau International des Poids et Mesures since World War II.

- Reported; O, calculated. Conversion factors, 1.00033 (United States), 1.00034 (international).

are $-0.2,-1.2$, and $-0.7_{9}$ for 1946,1948 , and 1950 , respectively. The difference between the two groups of cells, those at NBS and those at BIPM, each maintained differently and by different staffs, has not varied on the average by as much as $1 \mu \mathrm{v}$ since World War II. Of course, in arriving at the respective conversion factors, cognizance had been taken of the 10-ppm difference between the BIPMI and NBS units that existed prior to January 1, 1948.

In table 4, comparisons made in $1950^{\circ}$ with BIPM and other countries are listed. In column 2 are shown the difference in the volt from BIPM, of the National Physical Laboratory (England), Laboratoire Central des Industries Electriques (France), the Electrotechnical Laboratory (Japan), the Institut de Metrologie d'U. R. S. S. (Russia), and the Deutsches Amt fur Mass and Gewicht (Germany), who submitted cells to BIPM in 1950. The mean of the differences from the BIPM unit for the countries contributing to international comparisons is $-2.02 \mathrm{ppm}$. If we omit the Russian and the German values, the mean difference is only $+0.16 \mathrm{ppm}$. The numbers represent the relative changes in the unit since the comparison of 1947 , on which were based the various conversion factors that equalized the absolute units adopted in the several countries on January $1,1948$. 
In column 3 of table 4 , the differenees of the units of other eomntries from the U. S. unit are listed. The mean differenee for the participating countries is only $-1.25 \mathrm{ppm}$. Comparisons hawe been carried out at the National Bureau of Standards for the national laboratories of Sweden, Israel, and the Union of South Africa.

TABLE 4. Results of international comparisons of varions units of electromotive force in 1950

\begin{tabular}{|c|c|c|}
\hline Country & $\begin{array}{c}\text { BII'M unit } \\
\text { minus national } \\
\text { unit }\end{array}$ & $\begin{array}{c}\text { NHS unit } \\
\text { minus national } \\
\text { unit }\end{array}$ \\
\hline $\begin{array}{l}\text { United States... } \\
\text { England .... } \\
\text { France... } \\
\text { Japan } \\
\text { Russia.......... } \\
\text { Germany .... }\end{array}$ & $\begin{array}{l}p p m \\
-0.77 \\
-2.20 \\
+0.09 \\
+3.54 \\
-23.0 \\
+10.2\end{array}$ & $\begin{array}{c}p p m \\
-1.43 \\
+0.8_{6} \\
+4.31 \\
-22.2 \\
+11.0\end{array}$ \\
\hline Mean_... & -2.02 & \\
\hline
\end{tabular}

It may be conclurled, therefore, that the United States volt is in excellent agresment with the average unit maintained in the other standardizing laboratories of the world. It may also bo concluded that the methorls employed at the National Bureau of Standards, especially for the past 10 years, in maintaining the volt are souncl. In the future, studies of standard colls made acid with $\mathrm{H}_{2} \mathrm{SO}_{4}$, standard cells with solvents of deuterium oxide, and standarl eells made in quartz containers shonid make possible the maintenanee of the volt with even more confidence than was possible in the past.

'The authors express their appreciation to Georece W. Vinal, who for 25 years was eharged with responsibility of maintaining the Uniter States unit of emf, for his suggestions and cncouragement during the initial phases of these studies.

\section{References}

[1] Wdward Weston, German Patent 75,194 (Jan. 5, 189)2); The Electrician 30, $741(1893)$.

[2] J. L. Thomas, C. Peterson, I. L. Cooter, and F. R. Kíoter, J. Research N13S 43, 2!) (1949) RR'2029.

[3] II. I. Curtis, C. Moon and C. M. Sparks, J. Research NiBS 21, 375 (1938) RP'1 137.

[4] P. Vigourenx, Nat. Phys. Lab. Collected Researches 24, 277 (1938); F. E. Smith, 11, 209) (1914).

[5] I. Hartshorn and N. F. Astbury, Phil. Trans. Roy. Soc. (London) [A] 236, 42:3 (1937)

[6] H. I. (urtis, R. W. Curtis, and C. L. Critchfield, J. Research N I3S 22, 485 (1939) RRP1200.

[7] R. W. Curtis, R. L. Driscoll, and C. L. Critchficld, J. Research N BS 28, 133 (1942) RP144!.

[8] P. Vigourcux, Phil. Trans. Roy. Soc. (Iondon) [A] 236, 133 (1937).

[9] Latimer Clark, Proc. Roy. Soc. (I London) 20, 444 (1872).

[10] II. L. Callendar and H. T. Barnes, I'roc. Roy. Soc. (London) 62, 117 (1897).

[11] Report of International Conference on Electrical Units and Standards, 1908 (refer to appendix 2 of reference [13] for reprint of this report).

[12] F. A. Wolff, 'Trans. Am. Electrochem. Soe. 13, 187 (1908).

[13] F. B. Silsbee, NIBS Circular 475 (June 30, 1949).

[14] N BSS Circular C459 (1947); see also appendix 6 of reference [13]. 
[15] 1H. S. Harned and R. IV. Ehlers, J. Am. Chem. Soe, 55, 2179 (1933).

[16] (i. W. Vinal, J. Wash. Acart, Sci. 38, 265 (1948).

[17 P. Vigourenx and S. Watts, Proc. Phys. Soc. 45, l'art 2, 172 (193:3).

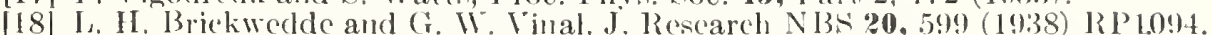

[1!)] I. H. Brickwedde and G. 'W. Vinal, J. Research NIBS 27, 479 (1941) RP'1435.

[20] 11. 13. Brooks, J. Research NBS 11, 22:3 (1933) RlP586.

[21] (i. IV. Tinal, I, II. Brickwedrle, and W. J. IIamer, Compt. Rend. de la Quinzieme Conforence te l'Union Inteluationale de C'hemic lure et Ipplieuere, Nmsterdam, 92 (1949).

[22] 11. Von Steinwehr, Proe. Congres lnt. d'blectricite, Paris, 2d section, Report No. $7, !)(1932)$

[23] 1. N. Shaw, II. I. Reiller, and R. J. Clark, Phil. Trans. Royal Soc. I,ondon 29:9, 1:34 (1930).

[24] (i. W. Vinal and M. I. Howard, BS J. Research 11, 255 (193:3) R P588.

\section{Discussion}

Dr. D. A. Marinnes (Rorkefoller Jnstitute for Medieal Resegredh): Could your tell us something alount the International Burean?

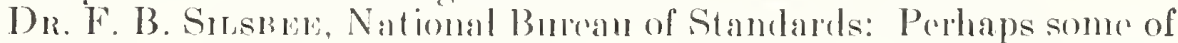
the andience may not know that hack in 1875 there was an international agreomente called the ('onvention of the Meter, to which some 17 mations subseribed and moder that treaty there were sot up throes oreanizations: first, an official lntermational Conferener on Wrierhts

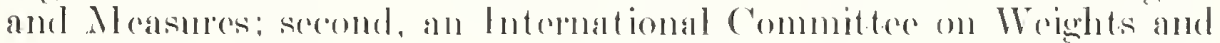
Measures (a body of 15 or 17 individual experts, no two of which ean

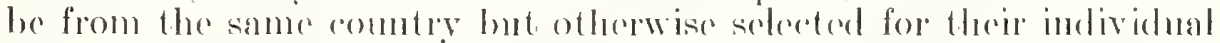

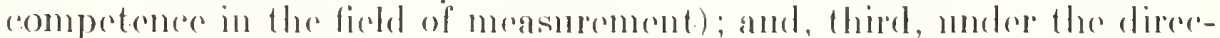
tion of the lattere, there was ereated an Intrernational Burean of Wrights

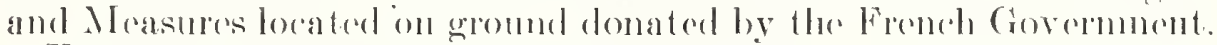

For many years the Burean and commitere had furiselietion only over length and mass, but in 1921 this jurisdiction was extended hy an amendment to the treaty to cover electriedty, photomedry. and the thermometrie seale. In 1921 the Intermational Burean sot up sat isfactory apparatus for the comparison of standard colls and onc-ohm resistors and several of the more highty industrialized countries donated to the Intomational Burean standard colls and standard resistors, which have been kept there "roverince.

Beginning about 1935 a serites of hiomial comparisons was initiated; standards were sent to Paris by the various national aborateries and then returned to the origimating aboratory at the ond of alde commparison. 'The mean value that I)r. Hamer showed on the slide was the average for each comparison of the mits of all four mations active in the comparisons. It was assumed to be ronstant. (On two ocensions the Freneh and the Germans have vohntarily changed there unit berause they were convineed they had drifted materially.

During the war period there wore no comparisons, and since then there have not been complete romparisons with Russia. There are two German laboratories now, but comparisons with them have becm rather sporalic. The International Bureau has a croup of some 47 cells, and the average of this group serms to have remained rery closely the same as that of omr gromp here at the National Burenu of Standards and as that of the National Physieal Laboratory in England. I think that none of those three mits have changed materially, and the final slide of Dr. Hamer showed that agreement as it stands now.

Dr. A. J. Rergers, Eniversity of Ghent, Belgium: When you want to kecp this fundamental group of ecells very constant some things may 
change- $-\mathrm{H}_{2} \mathrm{SO}_{4}$ may come in. Would it not be advisable to add the acid in the form of a buffer solution?

Dr. W. . . HAmer: I think that is a very good suggestion. I believe some work with buffer solutions has been done by Vosburgh. When you add a buffer solution you are, of course, adding more materials that could cause harm. Nevertheless, I think your suggestion is a good one.

Dr. H. H. Uhuig, Massachusetts Institute of Technology, Cambridge, Mass.: I should like to ask why heavy water changes the potential of a standard cell.

Dr. HAmer: In a standard cell, water takes part in the cell reaction. The cell reaction is

$$
\begin{aligned}
& \mathrm{Cl}(\mathrm{s})+\mathrm{Hg}_{2} \mathrm{SO}_{4}(\mathrm{~s})+\frac{8 / 3}{m-8 / 3}\left(\mathrm{CdSO}_{4} \cdot m \mathrm{H}_{2} \mathrm{O}\right)(\mathrm{l}) \\
& \quad=\frac{m}{m-8 / 3}\left(\mathrm{ClSO}_{4} \cdot \frac{8}{3} \mathrm{H}_{2} \mathrm{O}\right)(\mathrm{s})+2 \mathrm{Hg}(\mathrm{l}),
\end{aligned}
$$

where $m$ is the number of moles of water associated with 1 mole of $\mathrm{CdSO}_{4}$ in the saturated solution. Mrs. Brickwedde, in making up the cells, did not only change the solvent but used cadmium sulfate with heary water of hydration. The difference in enf between a cell with ordinary water and one with heavy water is related merely to the differences in the free-energy changes of the cell reactions and involves both the solvent and the solid phases.

Dr. Uhlat: I asked the question because I wondered whether, in the standard cell, one might not be measuring the polarized $\mathrm{Hg}$ potential. This means that if $\mathrm{Cd}$ is added to $\mathrm{Hg}$, we measure essentially the potential of an alloy, with IIg polarized by small corrosion currents to the same potential as the alloyed Cd. The potential, therefore, is of $\mathrm{Hg}$ on which hydrogen atoms are adsorbed, and would change the potential to an active value almost but not exactly equal to the reversible potential of $\mathrm{Cd}$ in $\mathrm{Hg}$. In heavy water, deuterium is adsorbed instead of hydrogen, which polarizes $\mathrm{Hg}$ to a slightly different potential, because the overvoltage value differs from that of hydrogen. I wonder if this might he a possible explanation.

1). HAmer: I would not think so. I think it is mostly just the differcnce in the cell reactions. In other words, we could change the solvent to ethyl alcohol and get a difference in emf corresponding to a difference in free-encrgy changes, or a change in the solid phases.

Dr. W. F. K. Wynne-Jones, University of Durham, Neweastle, England: I agree with Dr. Hamer. Surely in any cell of this sort the thermodynamies should show quite clearly that one is dealing simply with the diflerent phases present. The only possible effect is the one Dr. Hamer mentioned, and that is the change in the solid phase. Dr. Uhlig's iclea is interesting, but it just ean't be right. We certainly liaven't the wrong chemical process in the cell.

Dr. UHuic: Wouldn't it be worthwhile to check this by arranging a cell where water of hydration does not enter? It seems to me there woukl be a smaller effect of heavy water if no water of hydration were involverl.

Dr. Hamer: It could be checked in an unsaturated cell, but $\mathrm{CdSO}_{4}$ is less soluble in heavy water than in ordinary water, and a comparicon based on equal ronesntrations (or solubility) would show that the emf difference woulcl be larger rather than smaller, as the emf increases on dilution of electrolyte. 
Dr. Y. K. LaMer, Columbia University, New York, N. Y.: This is one of the most reversible cells we ever had, isn't it?

Dr. M. Eppler, The Eppley I aboratory, Inc., Newport, R. I.: Tes, but a reversible cell owes its reversibility to the maintenance of the original conditions. If you come to other conditions, the cell is no longer reversible. This cell comes as nearly to being rerersible as a cell can be.

Dr. LHuig: This is what I am driving at. The separate opencircuit potentials of $\mathrm{Hg}$, and Cd in mereury, are represented as shown in figure $\mathrm{A}$. Upon alloying $\mathrm{Hg}$ with $\mathrm{Cd}$ and immersing in an electrolyte a small corrosion current is set up between $\mathrm{Hg}$ and $\mathrm{C}$ (l atoms, which cathodically polarizes $\mathrm{Hg}$ to the potential $E_{\text {obs }}$ (corrosion potential). similarty, $\dot{C} d$ is anodically polarized from its reversible value also to

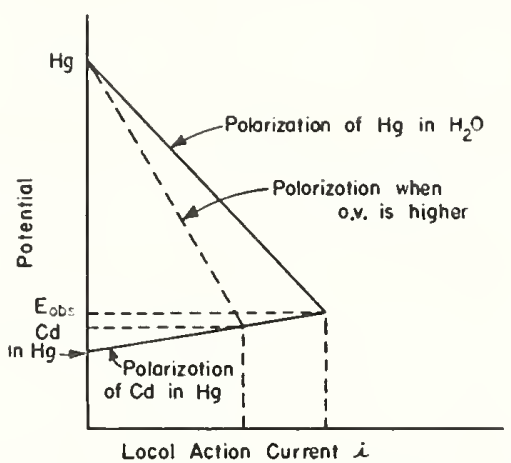

FiguRE A. Fffect of local-action currents on obsered potential (Fobs) of Heston Standard Cell.

$E_{\text {obs }}$ (neglecting small IR drops in the electrolyte and amalgam). Cal is polarized much less than is Hg, but some polarization must oceur, sinee current flows. Evidence for this current comes from the accumulation of hydrogen in standard cells on long-time aging. Fince the overvoltage of deuterium on $\mathrm{Hg}$ differs from that of hydrogen on $\mathrm{Hg}$, it follows that the corrosion currents differ in the two solvents, and therefore the polarized potential of $\mathrm{Cd}$ in $\mathrm{Hg}$ differs. 'Therefore, heary water, in addition to the usuatly considered thermodynamic effect, can influence the potential by changing the local-action currents.

(Dr. Thlig communicated the following comments for insertion in the discussion:)

The effect of local-action currents presented above loads to an increase in the potential of the Weston cell when $\mathrm{D}_{2} \mathrm{O}$ is substituted for $\mathrm{H}_{2} \mathrm{O}$, because the $O$. $I^{\text {r o }}$. $\mathrm{D}_{2}$ is lareer than for $\mathrm{H}_{2}$. The effect, of course, is much smaller than the thermodynamic effect mentioned by Dr. Hamer, which produces ehange of potential in the opposite direction. 'The local-action eurrents, however, on the Cd amalgam surface very likely explain the slight change of potential on aging of standard cells. A measurable shift in potential can conceivably occur through effects of impurities on overvoltage or polarization of $\mathrm{IJg}$, in this way altering the local-action currents. Impurities that increase the overvoltage lead to a larger measured potential for the cell, whereas those that decrease overvoltage have the opposite effect.

The magnitude of the local-action current can be calculated from values for the activities of Cd amalgam and dissolved ions, and the 
hydrogen overvoltage on $\mathrm{Hg}$. This approach makes use of the observations of Wagner and Traud (Z. Elektrochem. 44, 391 (1938)) that corrosion of $\mathrm{Zn}$ amalgam in hydrochloric acid can be expressed quantitiatively as an equivalent current that polarizes $\mathrm{Hg}$ to the potential of the amalgam in the same acid environment. Their investigation constituted proof that amalgams, like most metals, corrode by reason of short-circuited local-action cells, even though $\mathrm{Hg}$ cathodes and $\mathrm{Cd}$ anodes of the amalgam are on an atomic scale.

The activity of the amalgam electrode can be calculated from the potential of the Weston Cell $\left(1.0181 \mathrm{v}\right.$ at $\left.25^{\circ} \mathrm{C}\right)$, the $E^{0}$ values for $\mathrm{Cd} \rightarrow \mathrm{Cd}^{++}+2 \epsilon(0.402 \mathrm{v})$ and $2 \mathrm{Hg} \rightarrow \mathrm{Hg}_{2}^{++}+2 \epsilon(-0.799 \mathrm{v})$, and the activity of $\mathrm{Hg}_{2}^{++}$in saturated $\mathrm{CdSO}_{4}$. The expression for the emf of the cell at $25^{\circ} \mathrm{C}$ is the following:

$$
1.0181=0.402+0.799-\frac{0.0591}{2} \log \frac{\left(\mathrm{Cd}^{++}\right)}{(\mathrm{Cd})}-\frac{0.0591}{2} \log \frac{1}{\left(\mathrm{Hg}_{2}^{++}\right)},
$$

where $\left(\mathrm{Hg}_{2}^{++}\right)=4.8 \times 10^{-6}$.

The activity of $\mathrm{Hg}_{2}^{++}$above is calculated from the activity of $\mathrm{SO}_{4}^{--}$ in saturated $\mathrm{CdSO}_{4}$ and the solubility product of $\mathrm{Hg}_{2} \mathrm{SO}_{4}\left(6.2 \times 10^{-7}\right.$, Corrosion Handbook, p. 1138, edited by H. H. Uhlig (John Wiley \& Sons, Inc., 1948)). The activity of $\mathrm{SO}_{4}{ }^{--}$is obtained from the solubility of $\mathrm{CdSO}_{4} \cdot \frac{8}{3} \mathrm{H}_{2} \mathrm{O}$, namely, 0.06627 mole $\mathrm{CdSO}_{4} / \mathrm{mole}_{2} \mathrm{O}$ (L. H. Brickwedde, J. Research NBS 36, 377 (1946)), or 3.68 molal, and the activity coefficient for 3.5 molal $\mathrm{CdSO}_{4}$ equal to 0.035 (The Physical Chemistry of Electrolytic Solutions, H. Harned and B. Owen, 2d ed, p. 427, Reinliold Pub. Co., 1950). Therefore, the log ratio of the activity of $\mathrm{Cd}^{++}$to activity of $\mathrm{Cd}$ in $\mathrm{Hg}$ is given by $\log \left(\mathrm{Cd}^{++} / \mathrm{Cd}\right)=0.88$. With this $\log$ ratio, it is possible to calculate the potential difference $E_{\mathrm{H}}$ of the hydrogen electrode and Cd-amalgam electrode in saturated $\mathrm{CdSO}_{4}$. The latter contains about 0.03 mole $\mathrm{H}_{2} \mathrm{SO}_{4}$ /litre (L. H. Brickwedde and G. W. Vinal, J. Research NBS 27, 479 (1941) $\mathrm{RP} 1435$ ), the $\mathrm{pH}$ of which is assumed for present purposes to be 1.5 , and the partial pressure of hydrogen is equal to 1 atmosphere. Then

$$
E_{\mathrm{H}}=0.402-\frac{0.0591}{2} \log \frac{\left(\mathrm{Cd}^{++}\right)}{(\mathrm{C} d)}-0.059 \mathrm{pH}=0.29 \mathrm{v} .
$$

If the pressure of $\mathrm{H}_{2}$ is less than 1 atmosphere, as is expected, the value for $E_{\mathrm{H}}$ would be somewhat larger.

The above potential is available to polarize $\mathrm{Hg}$ bevond the equilibrium potential for evolution of hydrogen. The accompanying current, equal to the local-action current, can be calculated from the hrdrogen overvoltage values $\left(O . I^{\top}\right.$.) for $\mathrm{Hg}$. Kortüm and Bockris (Electrochemistry, II, 759, Elsevier Pub., 1951) give the following value for $\mathrm{Hg}$ in $0.2 N \mathrm{H}_{2} \mathrm{SO}_{4}$

$$
\mathrm{O} . \mathrm{V} .=\frac{2.303 R T}{0.52 F} \log \frac{i}{6 \times 10^{-12}},
$$

where $i$ is in amp $/ \mathrm{cm}^{2}$. Setting the overvoltage above equal to 0.29 and solving for $i$, one obtains $2.1 \times 10^{-9} \mathrm{amp} / \mathrm{cm}^{2}$. This is equivalent to the liberation of $\left(0.008 \mathrm{ml} \mathrm{H}_{2} / \mathrm{cm}^{2}\right) / \mathrm{sr}$. 
Hence for the Weston Cell, the reversible potential $E$ (therm.) can be expressed in terms of the polarized or observed potential $E$ (obs.) as follows: $E$ (therm.) $=E$ (obs.) $+f(i)$, where $f(i)$ is the additive term to the potential resulting from polarization of the $\mathrm{Cd}$ electrode in accord with the local-action current, $i$. Hence, the thermodynamic potential $E$ (therm.) is always greater than the observed value, the difference in general being small, although presumably measurable. From Wagner and Traud's data on anodic polarization of $\mathrm{Zn}$ amalgam, $f(i)$ is in the order of $(13 \mathrm{mv} / \mathrm{ma}) / \mathrm{cm}^{2}$; hence, the corresponding potential shift for $2.1 \times 10^{-9} \mathrm{amp} / \mathrm{cm}^{2}$ is $3 \times 10^{-8} \mathrm{v}$.

In this regard, the value $2.1 \times 10^{-9} \mathrm{amp} / \mathrm{cm}^{2}$ should be looked upon as a minimum value, with the actual value probably being higher. An increase, in some instances, by a factor of 100 is not inconceivable. For one thing, metallic impurities in the amalgam would tend in general to decrease hydrogen overvoltage, thereby increasing corrosion and local-action currents and producing hydrogen far in excess of the calculated $\left(0.008 \mathrm{ml} / \mathrm{cm}^{2}\right) / \mathrm{rr}$, as is sometimes observed. Even if the amalgam were pure, the overvoltage on either solid or liquid $\mathrm{Cd}$ amalgam may be lower than that for pure Hg. Dissolved oxygen and depolarizers from cork, possibly contaminating the electrolyte, would tend to increase the local-action currents above the calculated value without accompanying accumulation of hydrogen gas, but with the expected effect on the polarized potential of $\mathrm{Cd}$ and of the cell.

All these effects point to local-action currents on the surface of $\mathrm{Cd}$ amalgam as an important if not primary source of potential drift in Weston standard cells.

Dr. Hamer: I think rour discussion refer's to the stability of the cell rather than to the question of the difference in the emf (opencircuit voltage) of standard cells made with water or deuterium oxide.

Dr. J. O. M. Bockris, Imperial College of Science and Technologr, London, England: I don't fully understand Prof. Uhlig's idea. The cell must be working reversibly if no actual change in the concentration of reactants is detectable over a period of years. It would not suffice to test the explanations for the effect of heavy water on the emf of the cell merely by removing the water of hydration from the reaction. Change of medium changes the solvation energy of the ions and hence the emf of the cell. It would be relativcly easy to calculate the change in emf of the cell to be expected upon change from water to deuterium oxide throughout, assuming the usual cell reaction stated and complete reversibility. Then we could see if this new figure was in agreement with the experimental one.

Dr. Eppley: I tried to measure the potential of $\mathrm{Hg}$ in a $\mathrm{CdSO}_{4}$ solution. There is no definite potential that I have ever been able to find. Of course, rou would expect there would be practically no $\mathrm{Hg}$ ion there because the $\mathrm{Cd}$ would be forcing it the other way. That is the explanation I have given. Is that a correct explanation? Now the depolarizer, so-called, is not a depolarizer but is a device whereby you maintain a concentration of $\mathrm{Hg}$ ions over the $\mathrm{Hg}$. Of course, if we use it up, everything goes wrong.

Dr. G. W. Vinal, Princeton, N. J.: I agree.

Dr. Hamer: Your explanation for the potential of $\mathrm{Hg}$ in solutions of $\mathrm{CdSO}_{4}$ appears plausible. We have made no studies of this type at the Bureau. The $\mathrm{Hg}-\mathrm{Hg}_{2} \mathrm{SO}_{4}$ electrode is one of the second kind, but ceases to be of this type when $\mathrm{Hg}_{2} \mathrm{SO}_{4}$ becomes depleted. 


\title{
EFFECT OF SERVICE TEMPERATURE CONDITIONS ON THE ELECTROMOTIVE FORCE OF UNSATURATED PORTABLE STANDARD CELLS
}

\author{
By J. H. Park
}

ABSTRACT

The effect of external tempcrature disturbances such as might occur in practice on the electromotive force (emf) of unsaturated portable standard cells of the models in general use as secondary standards, was investigated. The experiments included unequal heating of different parts of the cell and sudden changes of temperature. Temporary changes in cell emf, causcd by these disturbances, in some cases as large as $1.5 \mathrm{mF}$ (nearly 0.2 of 1 per cent), were measured and the results are shown by curves. During all tests the cell elcments were left in the cases furnished with them. Methods of protecting the cells from external tomperature disturbances wcre tried and thcir effectiveness is shown.

\section{CONTENTS}

I. Introduction and purpose $\ldots \ldots \ldots \ldots \ldots \ldots$

II. Effect of temperature inequality of the limbs

III. Hysteresis effects . . .

IV. Simmary

\section{INTRODUCTION AND PURPOSE}

Nearly all persons who regularly use the unsaturated portable cadmium cell (also called the TVeston cell) as a standard of emf have been cautioned (1) to keep all parts of their cells at the same temperature, (2) to avoid sudden changes in the temperature of the cell, (3) not to submit the cells to temperatures below $4^{\circ} \mathrm{C}$. or above $40^{\circ} \mathrm{C}$., and (4) not to draw more than 0.0001 ampere from a cell. Changes in the emf of a cell due to deviations from these rules have been investigated and briefly summarized in a rery comprehensive discussion of standard cells by Eppley. ${ }^{1}$ However, results were not given for the cell alements in their cases, as they are generally used, and temperature effects depend upon the thermal insulation which envelops the cell element as well as the external temperature conditions.

The purpose of this investigation was to determine the effect on the emf of an unsaturated cell (1) when the limbs of the cell are at unequal temperatures as a result of conditions simulating those which might exist in practice, and (2) when the cell is subjected to a sudden change in temperature. All experiments were performed on the models of cells in general use and the glass elements of these cells

\footnotetext{
1 Eppley, Trans. of the A. I. E. E., vol. 50, No. 4, p. 1293, December, 1931.
} 
were thermally insulated by the cases furnished with them. In some experinents additional thermal shielding was used to determine its eflectiveness in protecting the cells from temperature disturbances.

The general nature of the eflects here described has been known for a long time, but it is believed their magnitude has often been underestimated. The present paper is intended therefore to show these magnitudes and to direct attention to the importance of taking suitable precautions in order to obtain the precision of which standard cells are capable. Fur ther studies are being made of the causes underlying these effects.

\section{EFFECT OF TEMPERATURE INEQUALITY OF THE LIMBS}

The temperature coefficients of unsaturated portable cells are negligible ${ }^{2}$ for practical purposes. However, the over-all temperature coefficient is the alrebraic sum of the coefficients of the two limbs of the cell. The temperature coefficient of each limb taken separately ${ }^{3}$ is quite large, but the two are opposite in sign. Thus, when the temperatures of the two limbs of a cell are changed by an equal amount the resulting change in the final steady value of the emf of the cell will usually be very small, but when the temperatures of the two limbs are changed by unequal amounts the resulting change in the emf of the cell will be relatively large, its magnitude depending upon the amount of temperature inequality. Inequalities of temperature are liable to occur in practice (1) if the cell is placed near a hot radiator, a resistor carrying current, a lighted lamp, or a cold wall; (2) if the cell is placed where the sun's rays will strilie it; (3) if the cell is held in the bare hand; or (4) if the cell is placed where hot or cold air will strike it (such as in the draft from a register or cold-air duct or near an open window in the winter time). The above are the more obvious causes of irregular heating of cells, but must not be considered to be every possibility.

Several models of cells were subjected to most of the temperature conditions just mentioned, the procedure being as follows: The emf of the cell under test was first noted for a sufficient time to make sure it was steady. Then the cell was subjected to the temperature condition and its emf was measured every 10 minutes until it had reached a steady value. Next, the source of heat was removed and the emf was again measured every 10 minutes until it was steady and very close to its original value. In one experiment the temperature difference between limbs was also measured by placing a thermocouple inside the cell case with each junction in contact with one of the limbs.

The emf of the cell under test was found by comparing it with the emf of a reference cell, the small difference being measured by means of a simple potentiometer of the Lindeck-Rothe type. An unsaturated cell in good condition, placed in an additional container (shown in fig. 1) for protection against temperature inequality, was used as the reference cell.

Three models (designated here as A, B, and C) of unsaturated portable standard cells which are in general use as secondary standards of emf, were tested. Cells of models $\mathrm{A}$ and $\mathrm{C}$ were in bakelite cases,

Weston, The Electrician, vol. 30, p. 741, 1892. Vosburgh and Eppley, J. Opt. Soc. of Am., vol. 8, p. 72,1924

3 F. E. Smith, Phil. Mag., vol. 19, p. 272, 1910. 
and cells of model B were in metal cases. Several cells of each model were tested, but since all cells of the same model gave practically the same performance, data are given here for only one cell of each model.

The results of the experiments to determine the effect of temperature inequalities are shown by the curves in Figures 2 to 6 . Curves in Figure 2 show the results on a standard cell of model $A$, when it was placed near a 16 -inch slide rheostat dissipating 288 watts. The inserted diagram shows the position of the cell with respect to the rheostat. Curves $A$ and $B$ show the effect on emf when the cell was placed 10 inches from the heated rheostat. When the positive limb is nearer to the rheostat (curve $A$ ), its temperature is higher
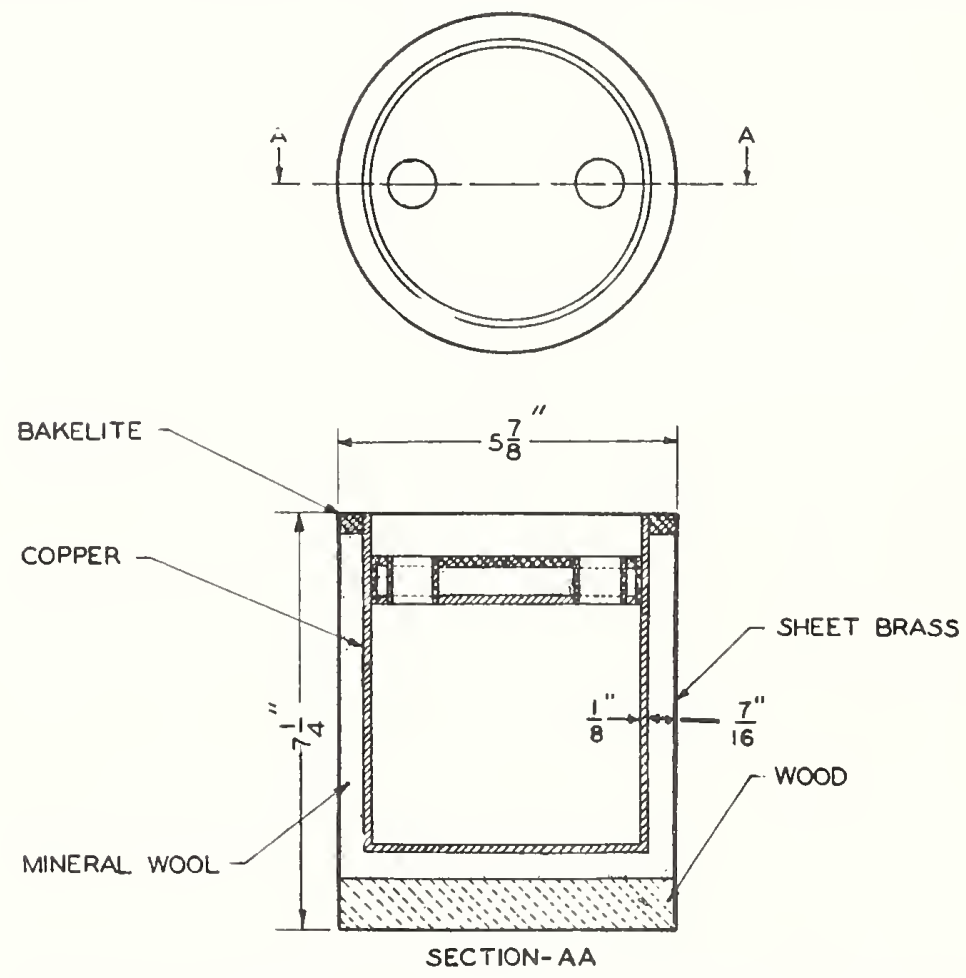

FIGURE 1.-Standard cell container for protection against temperature inequality

Designed at the Bureau of Standards in 1927.

than that of the negative limb, and the cell emf increases. When the negative limb is nearer to the rheostat (curve $B$ ), its temperature is higher than that of the positive limb, and the cell emf decreases. These curves illustrate the fact that the temperature coefficients of the two limbs of the cell taken separately have the opposite effect on the cell emf, also that the effects are about the same per degree difference in temperature. In curve $A$ the emf of the cell does not return to its normal value until about 36 hours after removing the source of heat; this and other slight irregularities which may occur in the cooling part of the curves are due to the hysteresis effect which will be taken up later. Curve $E$ shows the temperature difference between the limbs of the cell under the same heating conditions as for curves $A$ and $B$. As seen from curves $A, B$, and $E$, a temperature difference 
of $2.7^{\circ} \mathrm{C}$. between the limbs of the cell causes a change in emf of about $1 \mathrm{mv}$.

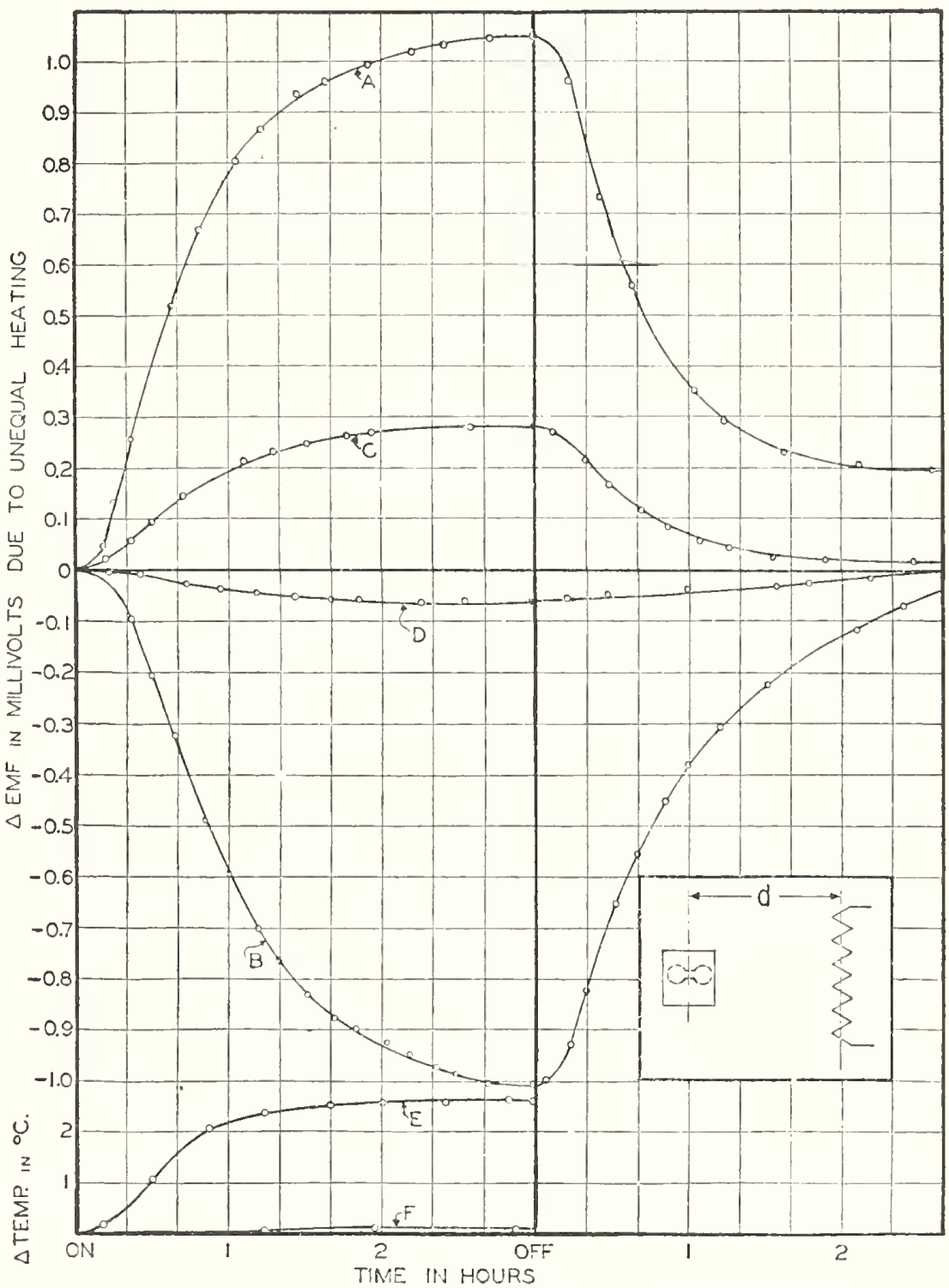

FIGURE 2.-Temperature inequality effects on standard cell model $A$, caused by heat from a 16-inch slide rheostat dissipating 288 watts

Curve $A$, effect on emf when $d=10$ inches, positive limb nearer to rbeostat. Curve $B$, effect on emf when $d=10$ inches, negative limb nearer to rheostat. Curve $C$, ellect on emf when $d=20$ inches, positive limb nearer to rhcostat. Curve $D$, same as curve $B$ with cell in special container. Curve $E$, temperature difference between limbs when $d=10$ inches. Curve $F$, same as curve $E$ with cell in spccial container

Curve $C$ (fig. 2) shows the effect on the emf when the cell was placed 20 inches from the heated rheostat. By comparing this curve with curve $A$, it is seen that the change in emf due to unequal heating happens to vary approximately inversely as the square of the distance from the rheostat. 
Curves $D$ and $F$ (fig. 2) show the results when the cell was placed in a protecting container (shown in fig. 1) and submitted to the same heating conditions as for curves $A, B$, and $E$. As seen from curve $F$, a container of this sort practically eliminates temperature inequality in the cells. The change in the emf of the cell, shown by curve $D$, is partially due to the fact that the temperatures of both limbs of the cell were increased about $5^{\circ} \mathrm{C}$. due to the radiation of heat from the rheostat.

The curves in Figure 3 show the results obtained when the experiments just described were repeated, using a standard cell of model B.

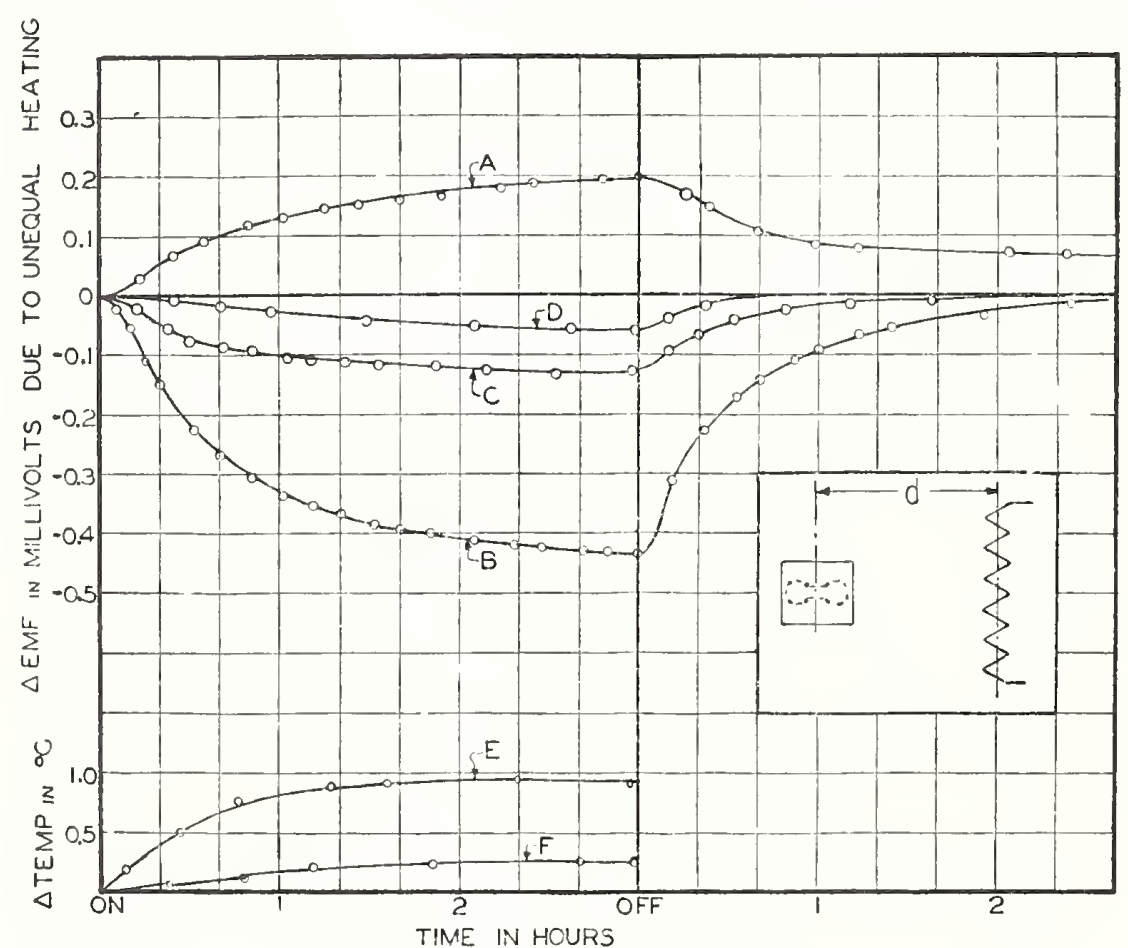

FIGURE 3.-Temperature inequality effects on standard cell model $B$, caused by heat from a 16-inch slide rheostat dissipating 288 watts

Curve $A$, effect on emf when $d=10$ inches, positive limb cearer to rheostat. Curve $B$, effect on emf when $d=10$ inches, negative limb nearer to rheostat. Curve $C$, effect on emf when $d=20$ inches, negative limb nearer to rheostat. Curve $D$, same as eurve $B$, with cell in speeial container. Curve $E$, temperature difference between limbs when $d=10$ inehes. Curve $F$, same as eurve $E$, with cell in speeial container

The metal case of this model tends to equalize the temperatures of the two limbs, and the curves show that the same heating condition causes a much smaller temperature difference and therefore a much smaller change in emf than for a cell in a bakelite case. The difference in maximum values between curves $A$ and $B$ may have been due to a difference in the cotton and wood thermal insulation between the metal case and the limbs of the cell.

The curves in Figure 4 are the results of similar experiments performed on a standard cell of model $C$. The case of this cell was too large to fit into the container used for the previous cells in obtaining data for curves $D$ and $F$. However, there was sufficient space inside the bakelite case to allow the insertion of a copper lining one-sixteenthinch thick. The results indicate this method of equalizing cell tem- 
perature to be as effective for a cell of this model as the use of a special container was for cells of models $A$ and $B$.

The curves in Figure 5 show the change in the emf of the cell due to the heat of the hand, held continuously on one side of the cell case for over two hours. The curves in Figure 6 show the change in emf

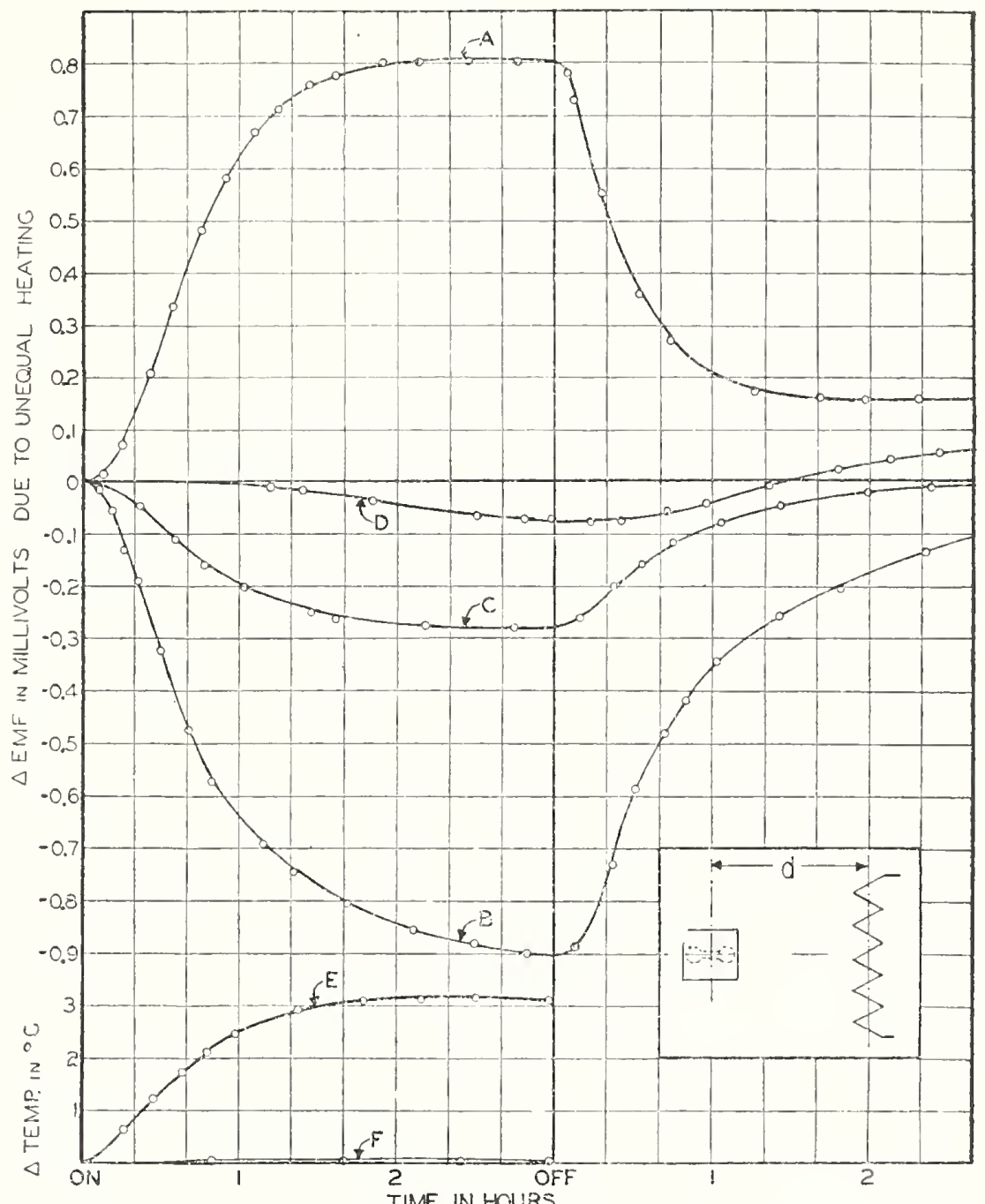

FIGURE 4.-Temperature incquality effects on standard cell model $C$, caused by heat from a 16-inch slide rheostat dissipating 288 watts

Curve $A$, effect on emf when $d=10$ inches, positive limb nearer to rheostat. Curve $B$, effect on emf when $d=10$ inches, negative limb nearer to rheostat. Curve $C$, effect on emf $w h c n d=20$ inches, negative limb nearer to rheostat. Curve $D$, same as curve $A$, with cell case copper lined. Curve $E$, temperature difference between limbs when $d=10$ inches. Curve $F$, same
as curve $E$, with cell case copper lined

due to the heat of the sun's rays, shining continuously on one side of the cell case for over two hours. For both of the above conditions the cell and the source of heat were arranged to give the maximum opportunity for temperature inequality of the two limbs of the cell. Curves are shown for the three models of cells in their usual cases and the differences between the behavior of these cells under the same conditions are largely due to the differences of their cases. 
Change in the emf of a cell due to unequal heating caused by radiation from a 40-watt lamp placed 1 foot from the cell was found to be under $50 \mu \mathrm{v}$.

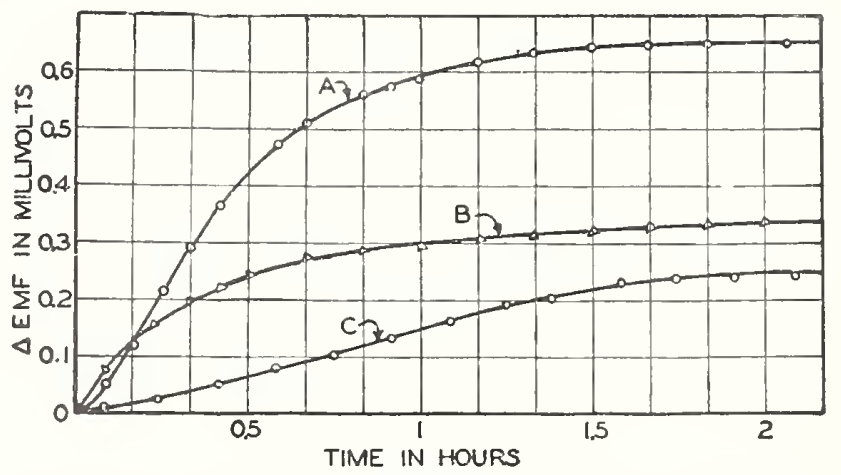

FIGURE 5.-Change in standard cell emf due to the heat of the hand, held continuously on one side of the cell case

Curve $A$, standard cell model $A$. Curve $B$, standard cell model B. Curve $C$, standard cell model C

The results of the above experiments should not be applied quantitatively as a correction to other cells of the same model under like temperature disburbances; because variables such as room tempera-

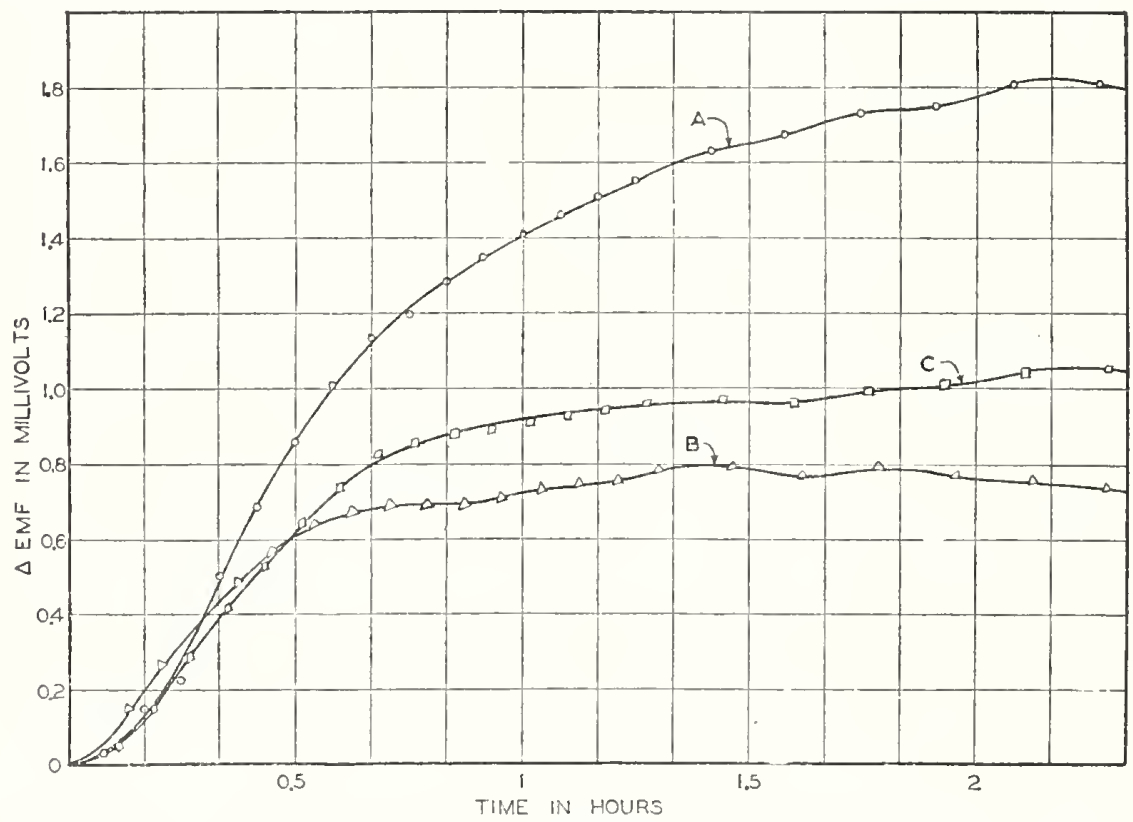

FIGURE 6.-Change in standard cell emf due to exposure of one side of cell case to sunlight

Curve $A$, standard cell model A. Curve $\underset{\text { model C }}{B \text {, standard cell model B. Curve } C \text {, standard cell }}$

ture or condition of the cell may cause cells of the same model to have different changes in emf for the same external temperature disturbance. However, the results shown may be used as a guide for the user to determine whether protection against temperature inequality is 
needed for the accuracy required. The methods mentioned here for protection against temperature inequality are probably sufficient for all practical work. However, where high precision (0.01 of 1 per cent or better) is required, more effective methods of protection against temperature disturbances should be used.

\section{HYSTERESIS EFFECTS}

When the temperature of an unsaturated standard cell is changed, all parts of the cell being held at equal temperatures throughout the change, the emf of the cell will change rapidly until a steady temperature is reached. Then the emf will slowly return to approximately its original value. This effect is known as hysteresis.

Several different theories have been advanced to explain the causes of this hysteresis effect. However, it was not the purpose of this investigation to explain any of these theories or to obtain data in support of them. It is well known that hysteresis is present in most cells to a greater or less extent, and that the effect is more pronounced with a declining temperature than with a rising temperature. The purpose of the experiments to be described was to find within what limits the hysteresis error lies for the unsaturated portable cells which are in general use, also to determine what means might be employed to reduce these errors.

Six cells, of various models and ages, taken from a group in regular laboratory use, were tested for hysteresis. In order to duplicate as nearly as possible the conditions in practice, the cells in their usual cases were placed in a temperature-controlled air bath. ${ }^{4}$ The air was circulated by a blower and its temperature was held constant to within $\pm 0.1^{\circ} \mathrm{C}$. by a thermostat which operated a heater in the air stream. The bath temperature was first held at $23^{\circ} \mathrm{C}$. until the cell emf's became constant. Then it was changed to $27^{\circ} \mathrm{C}$. (time required being about two hours) and held there, measurements of the emf of all cells being made at frequent intervals until the equilibrium values for $27^{\circ} \mathrm{C}$. were reached. Next the air temperature was changed back to $23^{\circ} \mathrm{C}$. (in about two hours) and held there, measurements again being made until equilibrium was reached. These measurements were made in the same way as in the temperatureinequality experiments except that a saturated cell ${ }^{b}$ in a temperaturecontrolled oil bath was used as a reference standard.

The results of the hysteresis experiments are shown by the curves in Figure 7. Time after starting to change the ambient temperature was plotted as abscissas and change in the emf of the cell as ordinates. Curve $A$ shows the effect on the emf of Cell II (cell with the least hysteresis) when the temperature was raised from $23^{\circ}$ to $27^{\circ} \mathrm{C}$. Curve $B$ shows the effect on the emf of the same cell when the temperature was lowered from $27^{\circ}$ to $23^{\circ} \mathrm{C}$. Curves $C$ and $D$ show the effect on the emf of Cell IV (cell with the most hysteresis) under the same temperature changes as for Cell II. Hysteresis curves of Cells I, III, V, and VI lie between those of Cells II and IV, but are not shown. The results of measurements on these six cells did not show any correlation between cell emf, model of cell, or age of cell and hysteresis effect. One of the newest cells with a high emf showed

4 This temperature-controlled cabinet was designed by A. E. Peterson

5 The emf of this cell was checked by Miss M. L. Howard at least once a week against the average of the 20 colls which constitute the bureau's reference standard of emf. 
much greater hysteresis than one of the oldest cells with a low emf. Therefore, it is very difficult, if not impossible, at the present time to predict just what the hysteresis error of any given cell will be. However, from the curves in Figure 7, an approximation may be had as to within what limits the hysteresis error of any unsaturated portable cell in good condition ${ }^{6}$ might fall. Eppley ${ }^{7}$ found that when an unsaturated cell was suddenly cooled from $30^{\circ}$ to $25^{\circ} \mathrm{C}$. the average hysteresis error was from 0.02 to 0.027 per cent. This average error lies within the limit shown here.

A method of protecting cells from hysteresis errors was applied to Cell IV, which was chosen because of its large hysteresis. It was placed inside a container (such as shown in fig. 1) and this container

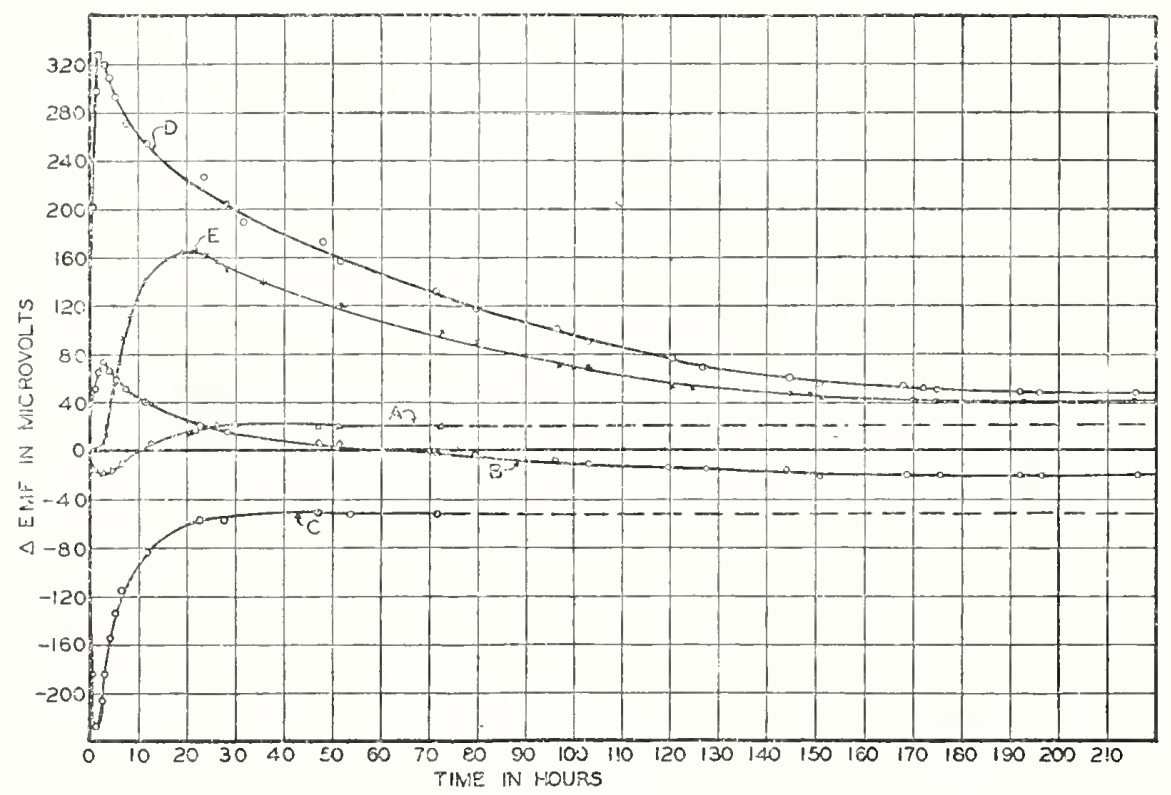

Figure 7.-Hysteresis effect on portable unsaturated standard cells

Curve $A$, effect on emf of Cell 11, when temperature was changed from $23^{\circ}$ to $27^{\circ} \mathrm{C}$. Curve $B$, effect on emf. of Cell 11, when temperature was changed from $27^{\circ}$ to $23^{\circ} \mathrm{C}$. Curve $C$, effect on emf of Cell $1 \mathrm{~V}$, when temperature was changed from $23^{\circ}$ to $27^{\circ} \mathrm{C}$. Curve $D$, effect on emf of Cell IV. when temperature was changed from $27^{\circ}$ to $23^{\circ} \mathrm{C}$. Curve $E$, same as curve $D$ with cell in insulated box

was placed in a wooden box which was sufficiently large to leave about 3 inches of space between the container and the box on all sides, mineral wool being loosely packed in this space. The cell, thus thermally insulated, was placed in the constant-temperature cabinet, set for $27^{\circ} \mathrm{C}$. and maintained at this temperature until the emf of the cell became steady. Then the air temperature was lowered to $23^{\circ} \mathrm{C}$. and held there, readings of the emf being taken regularly until it again became constant. The results of this experiment are shown by curve $E$ in Figure 7. By comparing this curve with curve $D$ it may be seen that thermally insulating the cell decreases the hysteresis effect by about 50 per cent in the case of a sustained temperature change. However, under ordinary laboratory conditions, where the average temperature over a period of 24 hours does not change much from day

${ }_{6}^{6}$ Cells which are not in good condition may show much greater hysteresis errors.

7 See reference 1, p. 1299. 
to day, thermally insulating a cell would be of great benefit in smoothing out the hour-to-hour temperature changes, thus greatly reducing the hysteresis error.

In instances where large sustained temperature changes occur, the only means of eliminating hysteresis errors is to keep the cell in a container whose temperature can be thermostatically controlled. However, as noted from curves $A$ and $B$ (fig. 7) some cells have very low hysteresis errors. In cases where high accuracy is required and the necessary means for eliminating hysteresis are not feasible, it would be advisable to use a particular cell whose hysteresis has been observed and found to be small.

\section{SUMMARY}

Temperature inequality between the two limbs of a standard cell, which may occur (1) when the cell is placed near a heat source, such as a hot radiator or a rheostat carrying current; (2) when the cell is placed where the sun's rays will strike it; (3) when the cell is placed where cold air will strike it; or (4) when the cell is held in the hand, caused an error in the emf of the cell of about $300 \mu \mathrm{v}$ per ${ }^{\circ} \mathrm{C}$. difference in temperature. The temperature inequality between limbs can be practically eliminated by placing the cell in a container such as shown in Figure 1, or, where possible, by lining the cell case with sheet copper one-sixteenth inch thick.

The hysteresis error, which occurs in portable unsaturated cells when their temperature is changed, varies widely among different cells, and the only way to determine this error for a given cell is to check its emf immediately after its temperature has been changed. Errors due to hysteresis may be reduced by sufficiently insulating the cell against changes in temperature; but the only way to eliminate these errors is to keep the cell in a box whose temperature is thermostatically controlled. Under normal laboratory conditions precautions against hysteresis need be taken only if accuracies of 0.03 per cent or better are required.

Washington, October 12, 1932. 


\section{PHYSICS.-The measurement of high voltage. ${ }^{1}$ F. M. Defandorf, National Bureau of Standards.}

\section{INTRODUCTION}

This paper presents an outline of the basic principles used in measuring high voltages. It appraises the accuracies attained by some of the methods and clevices currently in use, rather than attempts to give historical credit for discovery and invention, although numerous references are cited which should prove useful to one interested in tracing such matters. The expression "high voltage," in the title of this paper, will be construed to include voltages of the order of 100 kilovolts and higher. It will be convenient to leave out of consideration many voltage measuring devices useful in the neighborhood of $100 \mathrm{kv}$ and lower but of doubtful value in extending the range to much higher voltage by increasing the proportions of the device. This demarcation also serves to exclude from this discussion the methods of measurement of voltage at extremely high frequencies because voltages of this magnitude are nonexistent at those frequencies. The first portion of this paper will logically be deroted to a discussion of certain reliable methods of extension in range from low voltage standards.

It is proper to ask why one should be interested in the accurate measurement of high roltage. One must be interested because many phenomena depend on voltage. For instance, the hardness limit of $\mathrm{X}$-radiation emitted by an X-ray tube depends upon the maximum value of the voltage across the tube including the ripple or any superposed surge. Similarly the sparkover voltage of a sphere gap and the a-c flash-

\footnotetext{
${ }^{1}$ Address of the Retiring President of the Plilosophical Society of Washington, delivered at the 127 th meeting of the Society on January 4, 1947. Received July 28, 1947.
}

over values for a string of line insulators depend upon the crest of the alternating voltage applied to the gap or string. In those cases it is the maximum voltage arising from the combination of all voltage components in which we are interested. In the surge-voltage breakdown test of a lightning arrester it is the crest of the applied surge that is fundamental, although the form of the surge, if it rises rely rapidly, may be important. This is because with surges of increasingly steep wave front the crest breakdown voltage actually increases because it requires an appreciable though short time to establish the mechanism of breakdown. On the other hand, in the commercial sale of energy it is the effective value of the voltage and its phase with respect to the current that is important. Thus the measurement of high voltage in the cases just mentioned presupposes some knowledge not only of the magnitude of roltage, say from thousands to several millions of volts, but also a knowledge of the rariation of the voltage with time. This rariation of voltage with time, which may have the form of a wave, pulse, ripple, or some other shape in no way resembling our everyday conception of a wave, is called wave form.

From the foregoing discussion one may conclude that the significant characteristics of voltage to be measured, illustrated in Fig. 1, may be listed as:

1. Direct voltage (d-c voltage).

2. Effective alternating voltage (effective a-c voltage).

3. Average alternating voltage (average a-c voltage).

4. Crest or maximum voltage of a rippled d-c-, alternating-, or surge-voltage.

5 . The wave form of a surge- or alternatingvoltage. 
For the measurement of voltage it has been found convenient to atopt as a primary standard, the voltage of the standard cell. The unsaturated standard cell, the voltage of which is approximately $1.0186 \pm$ volts. is almost universally used as a laboratory reference standard for voltage measurements. When kept in a thermostated enclosure and used under favorable conditions. it exhibits a remarkable constancy of voltage. The small differences that may arise in a thermostated group of cells from year to year are of the order of microvolts. Unfortunately, similar electrochemical derices haring $100,1,000$, or $1,000,000$ times the roltage of a standard cell do not exist. Therefore, in the usual measurement of higher voltages, it has been necessary to develop derices which ultimately refer back to the standard cell.

Resistance methods of extending the range of roltage upward from the value of the voltage of the standard cell make use of the fact that br selecting suitable alloys and heat treating them properly resistors can be constructed to have a resistance, or a ratio of resistance, that remains constant to within a few parts in 100,000 over a satis-
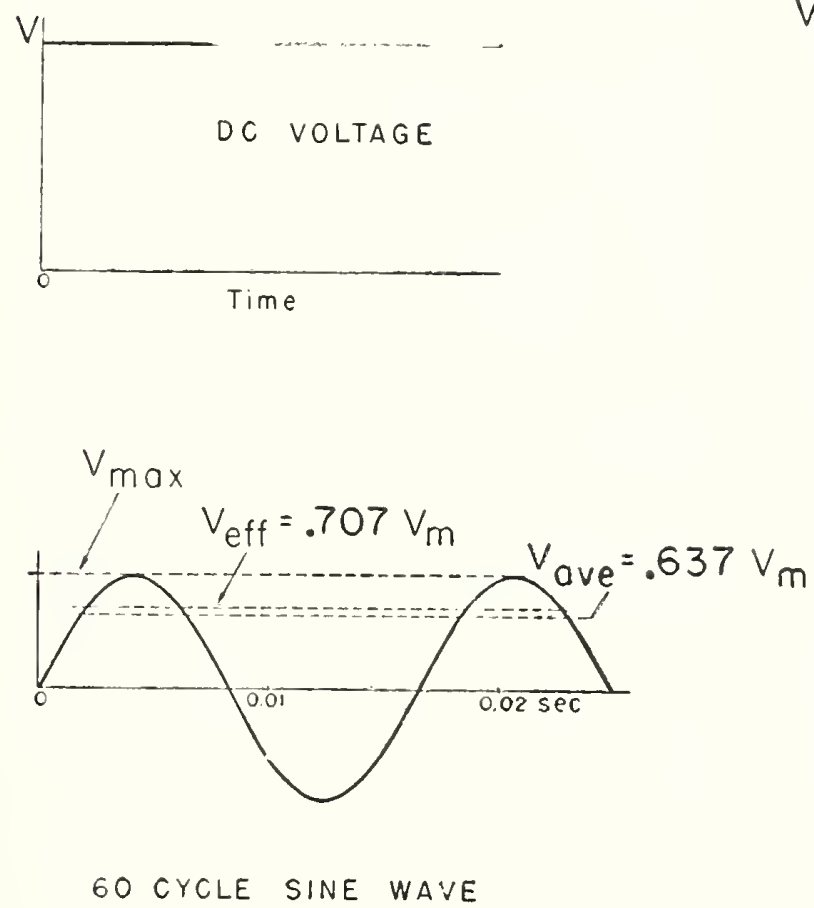

factory temperature range. The use of a potentiometer or of a tap resistor (volt box) is the simplest method for extending the range of measurements of direct voltages by reference to the standard cell. If $r$ is the resistance from one end to the tap point and $R$ is the total resistance including $r$, then the voltage $e$ across $r$ ean be measured with reference to a standard reil by using a potentiometer and the voltage $i$ across $R$ is then $r=e \cdot R / r$.

Although this simple resistance method is adequate for small steady direct voltages it requires modification for the precise measurement of the high rarying direct-, alternating-, surge-, and pulse-roltages that one encounters in practice. The manner in which the voltage at any instant raries with the time is a fundamental factor in the problem of measurement. It is necessary to express alternating roltage in terms of direct voltage which ean be evaluated by reference to the standard cell. Instruments designed to read the same on the effective value of alternating voltage as on direct voltage are called "transfer instruments." As transfer instruments, electrostatic instruments (1) are favored in Creat Britain,

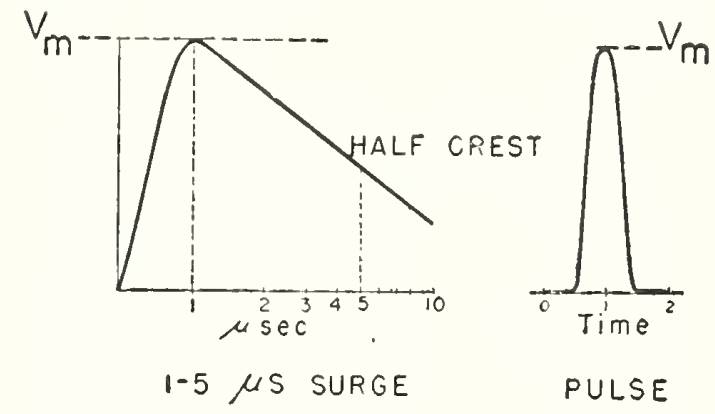

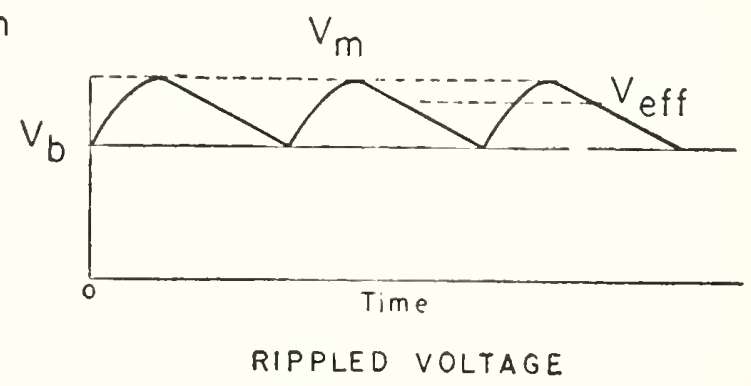

Fig. 1.-Wave forms. 
while electrodynamic instruments (2) find favor in this country. An electrostatic voltmeter, for instance, should give the same indication for a direct roltage $V$ as it does in the case of an alternating roltage of pure sine-wave form for which the crest or maximum voltage $V_{m}=\backslash 2 \mathrm{~V}$.

If rectified alternating roltage is applied to a direct current voltmeter the instrument will read the average value of voltage applied to it, i.e., $T_{\text {are }}=(1 / t) \int_{0}^{t} v d t$.

If, however, completely rectified alternating roltage is applied to a good electrostatie voltmeter the voltmeter will read the crest roltage. Crest voltage can also be determined from the length of a spark gap arcross which it will just ause a dischatrge, or it can be readily evaluated from the wave form cletermined by use of a calibrated os(illograph.

A more detailed knowledge of the variation of the roltage with time such as is given by an oscillograph becomes of special interest in the case of pulse and surge voltages. Thus it would seen appropriate to appraise the value of the several devices used in the delineation of wave form, and to mention each trpe under the particular voltage divider or device with which it is generally associated.

1 consideration of high-voltage measuring devices appears to lend itself better for diseusion under a classification of methods of measurement or types of devices rather than under the classification of characteristies of voltage previously outlined. The devices which are useful in measuring high roltage may be conveniently classified as to trpe by considering whether the method of measurement employs:

1. A high series impedance with a low-imperance instrument to indicate current through the impedance.

2. A potential divider in which a fraction of the total voltage is measured across tip points of the imperlitice.

3. A voltage transformer that per mits measurement of a low voltage having a direct ratio to the high voltage.

4. I generating voltmeter in which a voltage propertional to the field intensity in the region of the instrument is indientere

5. A spouts discheure in which the length of the spark gives a measure of the voltage.

6. "The cooling effect of an "eleretrie wind" as in the ionic wind voltmeter.
7. Force arising from the attraction or repulsion of electrostatic charges on electrodes.

$\mathrm{S}$. The deflection of a stream of charged particles by means of a known field, either electrostatic or magnetic, after their acceleration in vacuo by the voltage to be measured.

The devices used in measuring high roltage will be considered in accordance with the above classification and in the order listed.

\section{SERIES IMPED INCE METHOD}

The simplest method of measuring high voltage would appear to be to eomnect a high impedance in series with a sufficiently sensitive current measuring instrument hasing a negligible imperlance compared to the vitue of the high series imperdance, $Z$. Vitlues of the indicated current. $i$, would then give the high voltage, $V=i Z$. The impedance $Z$ may, of course, be primarily resistive, inductive, or capacitive or combinations of these elements.

Series resistors. - This basically simple metlod has been widely used in nuelear disintegration work for direct voltage meatsurements, wherein the impedance $Z$ is buit of many high resistance units in series (diagram at $A$ in Fig. 2), care being taken to insure that the current entering at the high roltage end of the resistor is the same as that leaving through the deflecting instrument at the low roltage end. For reliable measurements it is necessary to be sure that the electrical leakage across insulating supports of the resistor and from section to section is negligible and that there is negligihle corona current from the umits. Changes arising from self-heating must be made negligibly small or must be allowed for. The design of such a series resistor should be -ublantially the same as for the potential divider tye to be discussed later in detail.

Series reaclors.-For alternating voltage measuremonts, series reactors have bern used as the series impedance (3). They have the drawback, howerer, of requiring iron cores at lower frequencies if the induetive reactance is to be mate large in comparison with the resistance of the windings. Stray

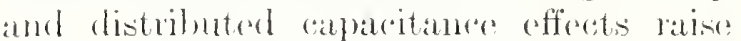
addilional objections so that series reactors have been little used in high voltage moasurements. 


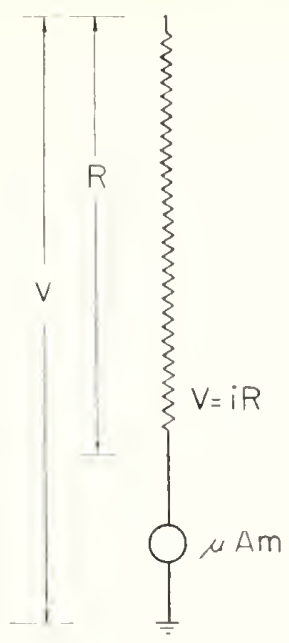

A

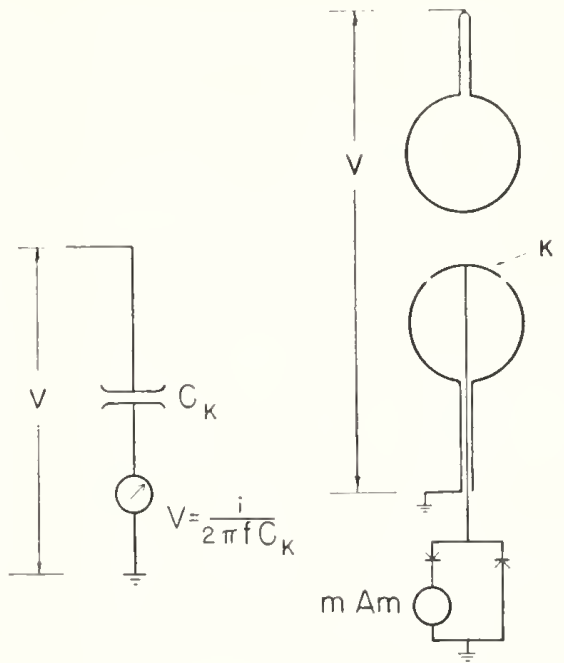

B

FIG. 2.-Diagrams of series impedors.

Series capacitors.-For the measurement of high alternating voltage, capacitance may be used in series with the low voltage indicating instrument, as indicated at B and $\mathrm{C}$ in Fig. 2. The capacitor, which must be relatively free from losises, corona, and brush discharges, magnifies the effect of harmonics present in the voltage wave form unless the low voltage instrument is of the capacitance type. ${ }^{2}$ However, if harmonics are present, corrections can be applied. This device in a form for measuring highcrest voltage was originally described by Chubb (4) who employed the two spheres of a sphere gap as the capacitor, the lower and grounded sphere being insulated to permit rectification of the capacitance current to it. Such devices $(5,6,7)$ appear to have been used more generally in foreign laboratories than in this country. The alrangement is indicated at $\mathrm{C}$ in Fig. 2. Haefely \& Co. patented $(\gamma)$ such a device which employs as one electrode of the high-voltage capacitor, a large insulated circular segment of the lower'sphere. The remainder of the sphere serves as the grounded guard for this segmental electrode. The upper sphere serves as the high-voltage electrode.

${ }^{2}$ Here the combination serves as a potential divider and a low voltage clectrostatic voltmeter across a large capacitance gives effective values of voltage independent of wave form.
These devices generally permit only a rough computation of capacitance and therefore need to be calibrated against some other voltage standard, but they have the advantage over the sphere spark gap of giving a continuous rather than transient indication of voltage when used with an electrostatic voltmeter or rectifier-milliammeter combination. Although the early device of Chubb gave the crest value ${ }^{3}$ of voltage, later similar arrangements (9) were devised that permit the determination of both crest-and effective-values of voltage, and when supplemented with a cathode ray oscillograph or synchronous commutator (8) give the high voltage wave form as well. For relative measurements, this series capacitance method should be good to a few tenths of one percent.

\section{POTENTIAL DIVIDERS}

The potential divider is essentially some form of impedance with one or more tap points permitting the measurement of the voltage drop between tap points by a method which preferably does not change appreciably the current flowing through the divider. The potential divider is connected across the voltage to be measured. The

\footnotetext{
${ }^{3}$ Except in the case of altrinating voltages of unusual wave form in which there are several $\operatorname{maxima}(8)$.
} 
value of voltage measured between taps, when multiplied by the ratio of the value of the total impedance to that of the tapped section then gives the value of voltage across the total impedance. Potential dividers are commonly constructed of sections of resistance, of resistance shunted by capacitance, or of capacitance. Types of each of these will be described.

Corona shiclded resistor.- $\mathrm{F}_{\mathrm{Or}}$ a resistive divider one may use $n$ resistors, each of resistance $r$, all exactly alike (or nearly so), connected in series, or one resistor $(n-1)$ times as large as the smaller one connected in series with it, and across which the voltage drop is measured. For use at higher voltages, both the electrical and mechanical designs of the physical device require care. Improvements in his earlier designs (10) have been incorporated by L. S. Taylor in a resistor, not yet described, which is in use in the measurement of the high rectified voltage supply for a 1.4-millionvolt $\mathrm{X}$-ray tube in the $\mathrm{X}$-ray Laboratory of the National Bureau of Standards. As seen in Fig. 3, the column at the left side contains the corona-shielded resistor, which is installed immediately adjacent to the column of 10 large cascaded-rectifier tanks, each of which at maximum voltage contributes $140 \mathrm{kv}$ to the total voltage of 1.4 million. This arrangement provides double shielding. The external system seen in Fig. 3 has tubular connections from the spun-metal corona shields to each section of the cascaded supply. The internal resistor assembly, also separately corona shielded, is thoroughly insulated from this outer system. Thus the currents required by the outer shiclds are supplied as a sectionalized direct load on the supply independently of the measuring circuit. This outer shield therefore takes care of current flow arising from external ionization, migratory dust particles. and minor surface discharges as well as surface leakage across the insulating columnar supports, and relieves the internal resistor of the resulting irregularly varying effects. In addition, the separate outer shields provide mutual field grading and the large top shield is effective in shaping the general electrostatic field. The internal resistor as- sembly is sectionalized, each section consisting of uniformly spaced wire-wound resistors located between toroidally shaped corona shields, which are electrically connected to the end of each group of resistors. Isolantite insulation is used for supporting the resistor's and the uniformly spaced corona shiclds. The shields were constructed of smooth copper-tubing of circular section in order to provide satisfact ory field grading along the resistor and thus to insure freelom from corona discharge currents at the highest intended operating voltage. The design provides for adequate insulation, mechanical support, and centering of this

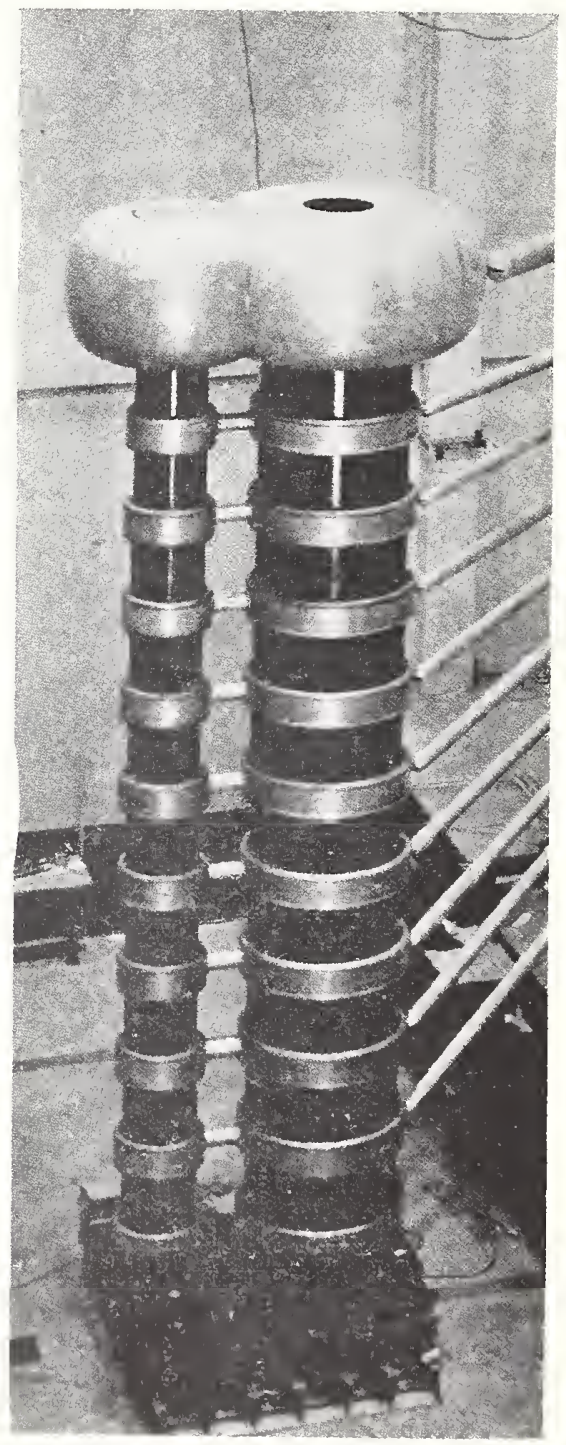

FIG. 3.-1.t-million-volt rectifier column with shiched resistor column on left side. 
coluinn inside the outer corona shield. Each section was adjusted and thoroughly tested at rated voltage before assembly and subsequent measurements of individual sections show their resistance to be practically free from drift. These precautions were taken to make" sure that the current throughout the resistor will be the same as at its low voltage end where measurement of the current is niade; i.e., in order that the high voltage, $V$, being measured is equal to nri where $n r$ is the total resistance of the stack of $n$ shielded resistors. Actually the current, $i$, is often determined by adding a small resistor, $r_{o}$, and measuring $v=i r_{o}$ by means of a potentiometer.

Manganin wire-wound resistors and a relatively large current of 1 milliampere were chosen as design values in order to minimize parasitic effects of leakage, absorption, and corona currents, and thus to insure a resistor that would permit measurements to 0.1 percent or better. A current of 1 milliampere corresponds to a load of 1.4 kilowatts, so that the resistor was designed to dissipate a corresponding amount of heat. Carbon or the so-called "metallized" radio-type resistors have a much higher temperature coefficient and are in general less stable than wire-wound resistors. Considerable care must therefore be taken in their use to insure freedom from thermal and voltage effects. Although reduction in self-heating and in energy loss in such resistors may be secured by reducing the current say to 0.1 milliampere, the effects of leakage currents become relatively more important and may contribute to some extent in reducing the attainable accuracy to the order of 0.5 percent.

Still another consideration that should not be overlookerl is the shunting effect on the resistance of the capacitance of the shields. In the case of a resistor of the high resistance type, sudden surges are easily transmitted through the shunting capacitances so that the measuring instrument, which must be correspondingly more sensitive, requires increased surge-protection. Thus there are drawbacks to either increasing the current in the measuring resistor to values greatly exceeding one milliampere because of increased energy loss, or decreasing it to values much below 0.05 milliampere because of the instability of the resistance insulation, and of other troubles. For much of the pioncering nuclear disintegration work (11) carbon resistors a vailable in units of much ligher resistance per unit length than the wire-wound type have proved both economical and useful.

It should be observed that the relatively high shunting capacitance of the corona shields of this type of resistor limits its usefulness as a resistor for alternating current measurements.

Capacitance shielded and guarded resistors for alternating current.-The resistor divider, in high favor for the measurement of direct voltage, has certain specific defects in addition to its relatively large consumption of porrer. It necessarily has residual inductance and stray capacitance associated with its resistance. As shown at $\mathrm{A}$ in Fig. 4, each section $r_{1} \cdots r_{n}$ may be considered as having at its terminals a lumped shunting capacitance of value $C_{1} \cdots C_{n}$, and between its upper terminal and ground a capacitance $C_{a} \cdots C_{m}$. If the value of capacitive reactance $1 / 2 \pi f C_{1}$ is $\gg r_{1}$ and $1 / 2 \pi f C_{a} \gg n r_{1}$ and if the impedance used in the measuring circuit shunting $r_{n}$ is $\gg r_{n}$, the voltage division by resistance will be trustworthy. As a result of the increase in shunting effect of the stray capacitances with frequency, these inequalities grow less until the division of the applied voltage is no longer proportional to resistance.

The shielded a-c resistor (12) minimizes the effects of the unavoidable ground capacitances at the cost of additional energy dissipation, by employing a second or guard resistor in parallel with the first or "working" resistor. This guard resistor is so connected to the shields that it supplies current to the ground capacitances that otherwise would have to be charged through and along portions of the working resistor. In an a-c shielded resistor the working, or shielded resistor, is composed of sections of value $r$ each contained within a metal shield or box as indicated at B in Fig. 4. Each shield is maintained at a potential corresponding to the midpoint of its enclosed resistor by comnecting it to a tap point on the proper section $R_{n}$ of the guard 
resistor. Thus this arrangement makes the value of cach shunting capacitance definite and supplies the ground capacitance current from the guard circuit. This is accomplished at the cost of a slight increase in the shunting capacitance of the individual resistors because of the capacitance to the shield in which they are located, since one end of the enclosed resistor is above and the other below the potential of the shield. However, because the potential difference across the capacitance of either end of the resistor dement to its shield is only one-half the voltage drop in this section of resistance $r$, the shunting effeet need not be excessive at low frequencies, say $2500^{\circ} 60$ rycles. Such a resistor, composed of atbout 25 shiclded sections, is used in a voltage transformer testing setup for phase-angle measurements and ratio measurements to 0.01 percent at the National Bureau of Standards. This resistor is rated at 0.05 amperes in each of the two (working and guard) 500,000-ohm circuits. In this device, which at $25 \mathrm{kv}$ absorbs $2.5 \mathrm{kw}$, the individual sections of the rorking resistor are enclosed in metal shield boxes, which are filled witl oil in order to improve thermal characteristies. This resistor is arranged in tiers, each tiel consisting of four working resistor boxes with corresponding sections of the guard resistor supported on a mahogany framework. Tiers are assembled one above the other by means of porcelain bus-type insulators, which serve to provide additional insulation and to permit bolting together of the resistorsupporting frameworks to form a mechanically stable asscmbly. Each box contains 20 flat miea cards wound unifilarly with manganin wire. It is not feasible to extend the range of a-e shielded resistors and retain the same order of aceuracy (0.01 percent) by this procerdure much above $30 \mathrm{kv}$ because of the effects of the capacitances which shunt the guard resistances. The resulting error increases as the fourth porrer of the voltage.

An a-c shielded resistor of this type is also satisfactory for use as a series resistor in conjunction with an indicating voltmeter or sensitive oscillograph. It may be used equally well on direct current but would ordinarily not be used because its
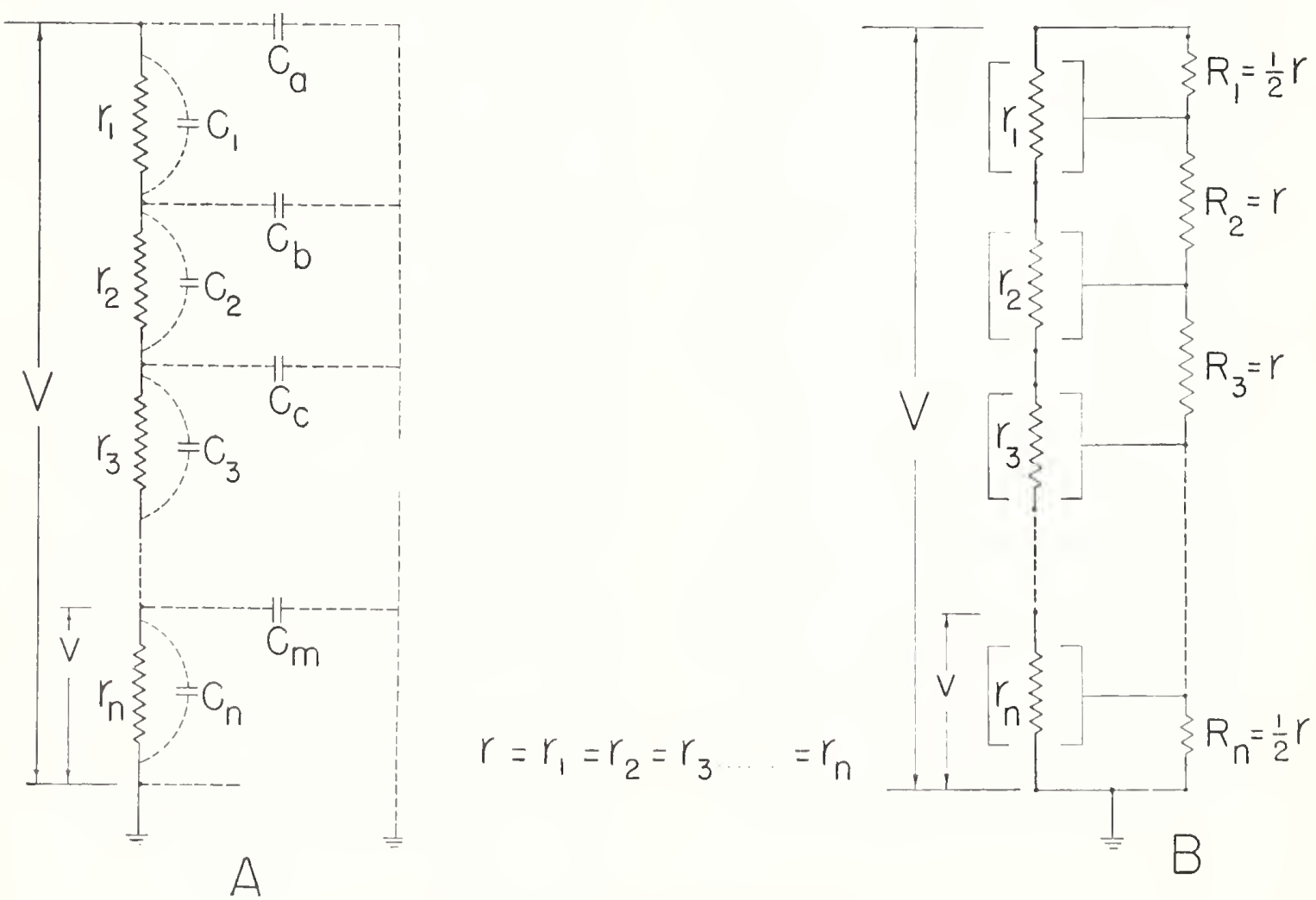

FIG. 4.-A-C shiclded resistor. 

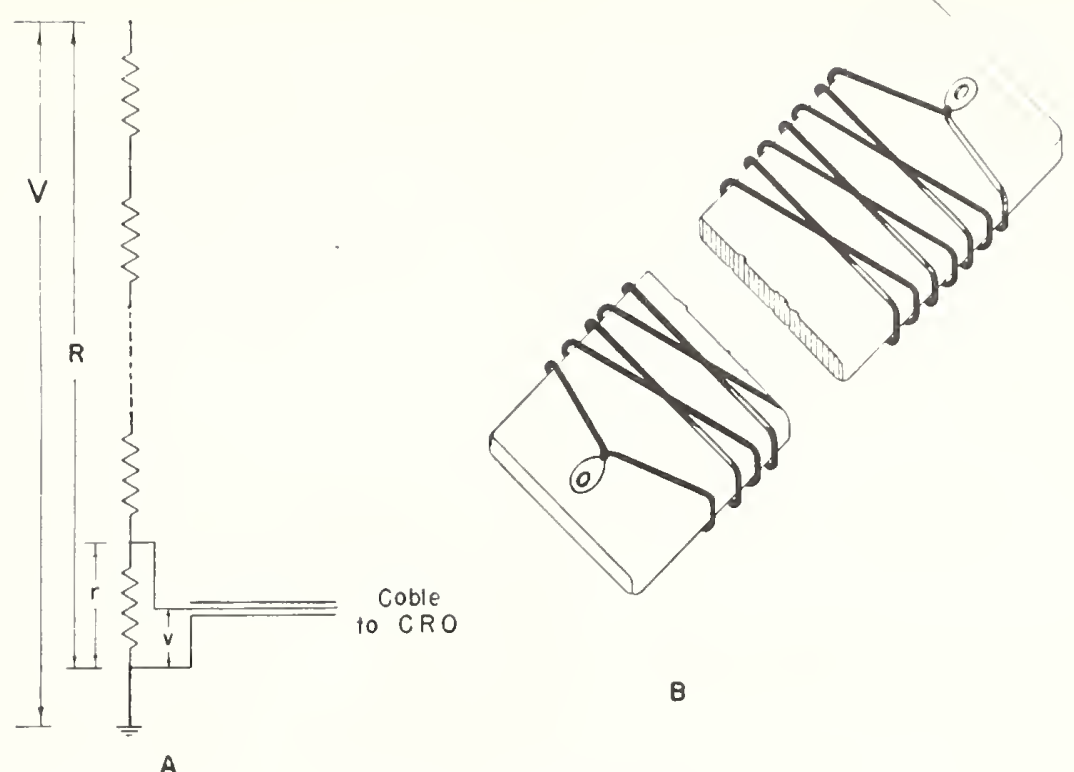

FIG. 5.-Surge resistor.

load on the source is greater than that for the simpler corona shielded resistor.

Weller (13) devised a shielded a-c resistor for use in transformer testing up to $132 \mathrm{kv}$ in which the shield current was supplied by auto-transformers rated at $75 \mathrm{kva}$, instearl of by a tapped resistor.

Surge resistors.-In surge voltage testing equipment used for studies of surge effects on electric power and transmission equipment, the fundamental component of voltage in a $1 \frac{1}{2} \times 40$ microsecond wave $^{4}$ is of the order of $10^{5}$ cycles per second. Hence components as high in frequency as $10^{7}$ cycles per second are of significance in fixing the wave form. The cathode ray oscillograph used to delineate such surge wave forms in conjunction with a potentialdividing resistor is generally located at some distance from the resistor and main discharge circuit in order to avoid induction effects from the large surge currents. Fig. 5 indicates the common method used for connecting the resistor tap point through a coaxial cable to the deflecting plates of the oscillograph. The resistor is composed of a series of resistance cards. Each card has two similar windings wound in opposite directions and connected in parallel to reduce

\footnotetext{
${ }^{4}$ I.e., one that rises to crest in $1 \frac{1}{2}$ mieroseconds and falls again to one-half crest value in 40 microseconds.
}

inductance. The cable connection to the cathode ray oscillograph has a surge impedance usually of the order of $50 \mathrm{ohms}$, whereas the full-scale sensitivity of the cathode ray oscillograph is of the order of 2,000 volts for a cold cathode-type and 200 volts for a hot cathode-type cathode-ray oscillograph. Thus for full-scale deflection the currents to a cable considered as a resistance would be of the order of 40 or 4 amperes, respectively.

Resistance dividers for high-voltage surge measurements are not provided with corona shields because they would add excessive stray capacitance but are often so located adjacent to the surge generator itself that some degree of shielding and field grading along the divider is secured. Since a resistor for a 2,000,000-volt surge measurement must be at least $700 \mathrm{~cm}$. long to provide adequate longitudinal insulations and avoid flashover troubles in air, its distributed and stray capacitances introduce disturbing effects unless the resistance is held proportionately low. Thus a divider resistor for a 2,000,000-volt surge may have a resistance as low as $5,000 \mathrm{ohms}$ and, if wire-wound, the wire must be of sufficient diameter to carry high momentary currents without damage. $\Lambda$ s a rule, accuracies of the order of one percent are all that are required. If it is desired to study surge com- 
ponents of the order of $10^{8}$ or $10^{9}$ eycles per second the residual inductance of each element and the distributed and particularly the non-uniform stray capacitance effects, assume especial significance in surge resistor dividers. For those frequencies, supplementary capacitance elements (14) may be added to the resistance elements of the divider in such a manner as to make uniform the capacitance shunting effect on each element and thus insure proper division of the surge voltage and its correct delineation by the oscillograph. The present interest in better voltage measurements of surge wavefronts of duration less than one microsecond should lead to improved designs of surgevoltage resistor dividers.

The cathode ray oscillograph plays such an important role in the field of surgevoltage (and surge-current) measurements that its contribution should be mentioned at this point. Although its value for use at lower frequencies should not be underestimated, it stands alone in versatility for use in high-frequency, surge, and pulse measurements. It owes this versatility to the small inertia of its electron beam, which serves at the same time as the moving element and pointer. The cathode-ray oscillograph, with a suitable sweep circuit, amplifiers, shunts, and potential dividers, has been developed into one of the most useful pieces of electrical laboratory equipment and cover's a range from a few cycles per second to frequencies of millions of cycles per second. Developments in electron optics and of new phosphors are constantly enlarging the place of the cathode-ray oscillograph as a useful precision device in spite of the complication of its accessory equipment. In the future an order of accuracy better than 1 percent may be expected. For most measurements, and especially those of high voltage surges, its high effective impedance results from the low capacitance between its deflection plates. The energy loss, arising largely from stray ions and electrons within the tube, is so small as to be of little concern. Thus in the visual or photographic delineation of wave, surge, and pulse shape the starting point now seems to be a calibrated cathode-ray oscillograph except in very special instances in the low frequency range where a higher order of accuracy is required.

Capacitor dividers.-Because residual inductance and residual capacitance effects in a resistance divider are unavoidable, not only in the resistance elements themselves but also in their electrical connections, it is natural to turn to the use of capacitance elements for alternating voltage division. The capacitance divider generally consists of a single high-voltage guarded-electrode capacitor in series with a low-voltage capacitor of very much higher capacitance. The

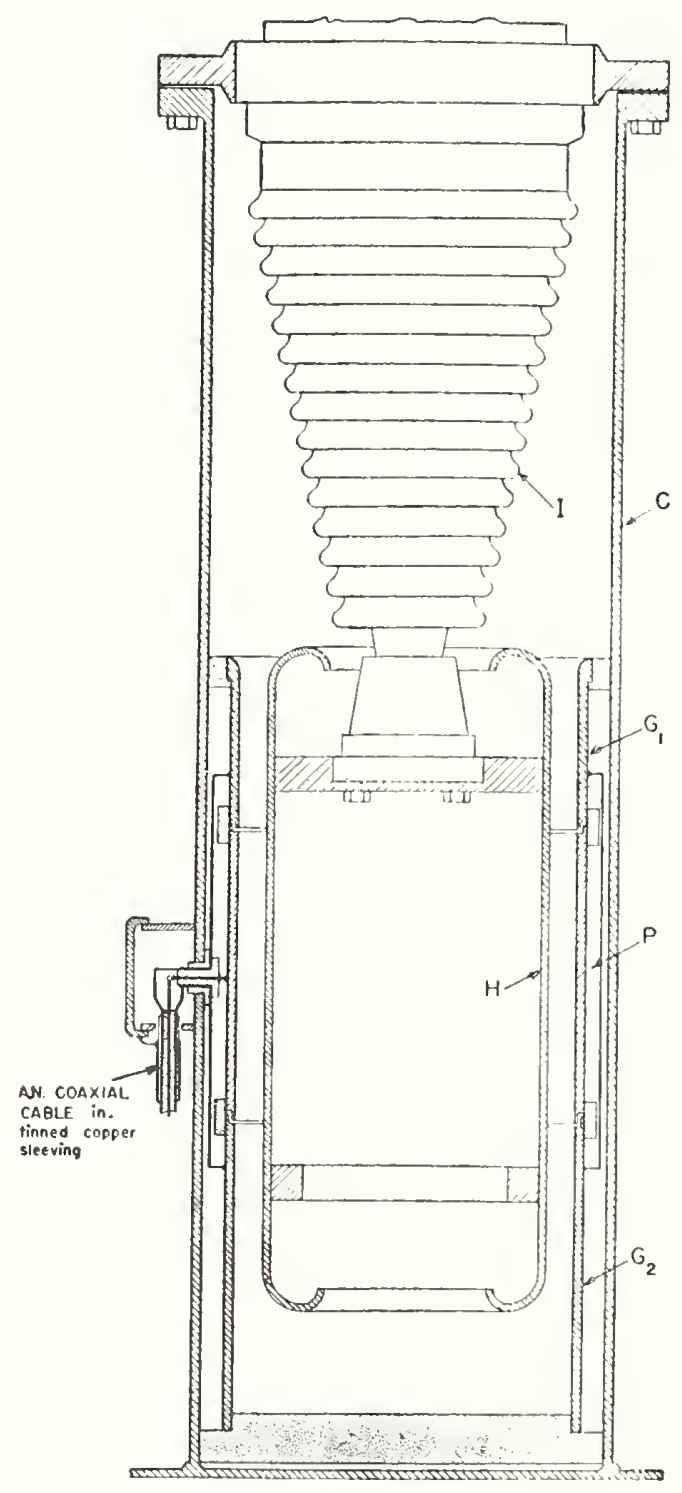

Frg. 6.-Compressed-gas capacitor. Diagram of Bousman and Ten Broeck capacitor altered to include complete shiclding of the working electrode. 
residual inductance effects in capacitors are minimized by properly shaping their sizable conducting parts. The residual resistance effects are minimized (1) by using high grade solid insulation for the electrode supports in order to keep electrical leakage through and across the surface of the insulation low; (2) by using free-air or compressed gases $(15,16)$ as the dielectric because of their low diclectric loss; and (3) by using electrodes with well-rounderl contours and smooth surfaces to insure freedom from corona discharge. It is customary to support the guard electrode on its own insulation opposite the high-voltage electrode and in turn to support the guarded low-voltage electrode on the guard. The insulation between the guard and the guarded electrode is arranged to lie outside the high electrostatic field so that dielectric losses as well as surface and volume leakage to the working capacitance are minimized.

The range of various types in free air is limited by the breakdown voltage of air to a practical value of about $15,000 \mathrm{v} / \mathrm{cm}$ gradient. Churcher (17) has described a capacitor for use at $300 \mathrm{kv}$, cylindrical in form, with an over-all diameter of 2 meters and a height of 7 meters. This unit is to some extent an absolute standard as it permits computation of its capacitance from dimensions measured under normal working conditions. To that extent it is preferable to a smaller compressed gas type (16) shown in Fig. $6^{5}$ for use up to 300 $\mathrm{kv}$, having an outside diameter of less than 1 meter and height over the bushing of 3 meters, and a shielded capacitance to the high-voltage electrode of 50 micromicrofarads. However, when the space available is limited, the compressed-gas equipment may be built to occupy about one-fourth the space of a free air unit of the same rating. The one advantage, which may be in part psychological, of constructing a capacitor whose capacitance can be calculated from its dimensions lies in the care and precision demanded in its design and manufacture.

5 In this figure the original design of Bousman and Ten Broeck has been modified to show eomplete shielding of the outer guarded cylindrical section by the guard.
I rather recent and desirable cireuit anrangement using a compressed-gas shielded capacitor for testing potential transformers is described by Bousman and Ten Broeck (16). Their circuit arrangement is similar to a Schering bridge, i.e., a bridge in which the $\mathrm{A}$ and $\mathrm{X}$ arms are capacitances instead of resistances. The difference lies primarily in the mode of supplying the voltage to the bridge, the high-voltage arm (A) being supplied by connection to the ungrounded end of the high-voltage winding of the transformer, and the low-voltage arm $(\mathrm{X})$ being connected to the ungrounder end of the low-voltage winding. Thus if this bridge is balanced when supplying power to one winding of the transformer, one may determine from the settings of the bridge arms both the voltage ratio and phase angle of the transformer. A simple reconnection of the bridge arms to a suitable supply permits quickly checking the constancy of the bridge arm components before and aiter ratio and phase angle measurements. Thus in this bridge reliance for the ratio measurement is placed primarily not on capacitances but on the constancy of resistance coils which are more suitable as reference standards because of their stability, while the phase angles are based on the air capacitors. An extension in range to higher voltages might logically follow the arrangement of Bousman and Ten Broeck without serious reduction in the accuracy of 0.1 percent claimed for their equipment.

The subject of high-voltage wave form should also be considered in connection with capacitance dividers. Offhand, capacitance dividers would appear to be ideal for use with the cathode-ray oscillograph, as its impedance is essentially capacitive reactance. In surge measurements, however, it is usually both desirable and convenient to locate the cathode-ray oscillograph at some distance from the surge circuit in order to minimize inductive effects. This involves the use of a fairly long high-quality cable (preferably coaxial) connecting the divider to the oscillograph so that the surge impedance of the cable, primarily resistive, rather than the capacitive impedance of the oscillograph plates assumes the major role in the measurement 
circuit. Thus, as suggested earlier, a surge resistor is to be preferred although surge capacitor dividers (18) have been used. For lower-frequency measurements this objection does not exist to the same extent and capacitor dividers with eathode-ray oscillographs as well as with amplifiers supplying electromagnetic oscillographs (string or loop in use up to several thousand rycles per second) have been found uscful in delineating wave form with an accuracy of a few percent.

A capacitance-divider method yielding a high order of precision in delineating lowfrequency wave form is due to Silsbee (19) who, in line with the early work of Rosa, used a point-by-point method. In his device a potentiometric balance by means of a quadrant electrometer is obtained across the low voltage portion of a capacitance potential divider for as many points in a repeated voltage wave as may be desired, thus permitting the evaluation of crest-, average-, and effective-values of the wave form. This method requires a synchronously driven contactor which may be set aceurately for each balance point selected for delineating the wave. The accuracy of measurement is very high and appears to be limited primarily by the steadiness of the alternating voltage source, say to a few parts in ten thousand.

\section{TRANSFORMER METHODS}

Toltage transformer.- The method of measuring high alternating voltage in common use in the United States employs a step-down transformer termed a voltage or "potential" transformer by the manufacturer in order to designate its intended use in voltage and power measurement. The high-voltage winding is connected across the terminals of the voltage source to be measured, a voltmeter is connected across the low-voltage winding and its reading is multiplied by the ratio of transformation to obtain the value of the high voltage. The ratio of voltage of the high-voltage winding to that of the low-roltage winding of a well-designed transformer remains nearly constant over a wide range of roltage. The measured values of ratio of well-buitt transformers kept under normal laboratory conditions have been found to remain remarkably constant over long periods of time, variations in ratio requiring for their detection measurements having an accuracy of the order of 0.01 percent. Departures from nominal ratio have been determined by resistance bridge methods (20) up to 30 $\mathrm{kv}$ with a precision of 0.01 percent and by capacitance bridge methods up to $132 \mathrm{kv}$ with a precision of 0.1 percent or better (16).

Where symmetrical multiple high-voltage windings are provided in a roltage transformer the following "series-parallel principle" has been found valid for extrapolating measurements of ratio factor at low voltage to permit their use at higher voltage. Within the voltage limits of a highvoltage shielded resistor or capacitor, and with the high-voltage windings in parallel, measurements of ratio factor are marle to cover the voltage per coil range of the lowvoltage winding. The nominal ratio for series connection is then multiplied by the measured ratio factor at the same rolts per coil for the parallel connection. Reliable measurements of effective alternating voltage by this method can be made to better than 0.1 percent (20) and are in use up to $250 \mathrm{kv}$. The cost of transformers with such symmetrical multiple windings increases rapidly with voltage. Other less expensive and less accurate ( 1 percent to 0.5 percent) transforming devices such as (1) a number of small chain-connected or cascaded transformers (21, 22) and (2) a high-voltage resistor (23), reactor (24), or capacitor (25) in series with a small transformer, have been introduced in Europe for measuring high voltage but have not met with general favor in the United States.

Supply transformer with high-roltage winding tap or with voltmeter coil. - Although the use of a voltage transformer with only an instrument connected as the burden on the low-rultage winding represents the ideal arrangement, it should be mentioned that adecfuately precise values of high roltage may often be deduced from roltage measurements made on the low-voltage input windings. This is particularly true if the resistance and leakage reactance of the high-voltage winding are low and the cur- 
rent drawn by the load on the high-voltage winding is small. Better still, an instrument connected between ground and a tap point on the high-voltage winding (26) near its grounded end may serve for measuring a relatively low voltage which will be proportional to the high voltage except in so far as the voltage across the tapped section of winding is affected by distributed and stray capacitance currents which flow through it from the rest of the high-voltage winding. Fig. 7 shows three such $350 \mathrm{kv}$ $60 \mathrm{c} / \mathrm{s} 1,000$ kva transformers in the High Voltage Laboratory of the National Bureau of Standards. These units are shown connected in cascade to give $1,000,000$ volts. Each unit has a high-voltage winding tap connection giving an accuracy of voltage measurement of about 2 percent at full load and correspondingly better accuracy at lighter loads. Still another arrangement consists in providing a third winding or voltmeter coil $(27)$ so located relative to the low- and high-voltage windings that the magnetic flux linked by it automatically takes into account any voltage drop in the high voltage winding arising from the load connected to it. An accuracy of one-half of 1 percent at full-load leading current and better accuracy at smaller loads is claimed for a good design. These latter arrangements are not considered to be as trustworthy as the use of a separate voltage transformer.

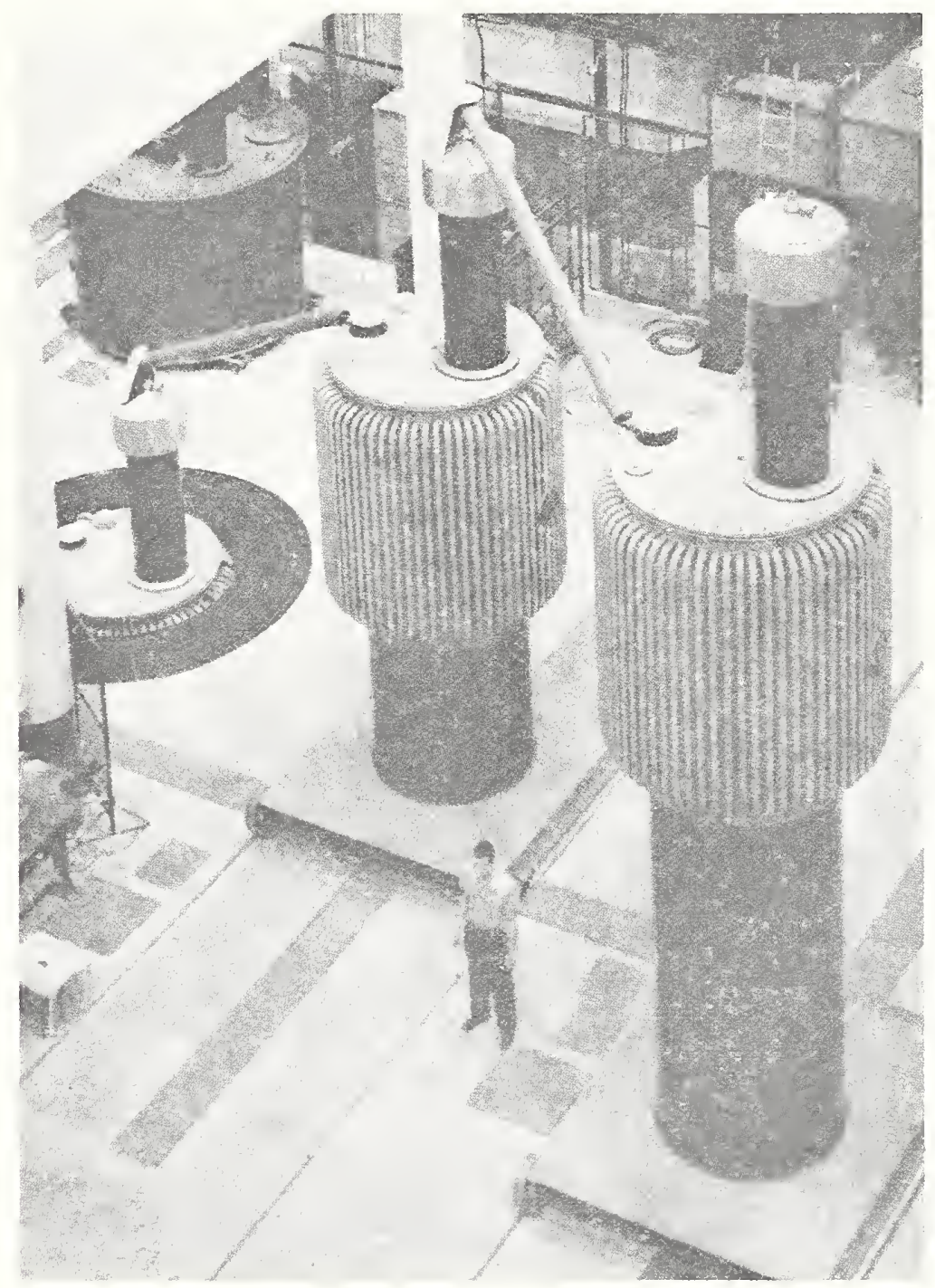

Fic. 7.- Three transformers connected in cascade to give 1,000,000 volts. 

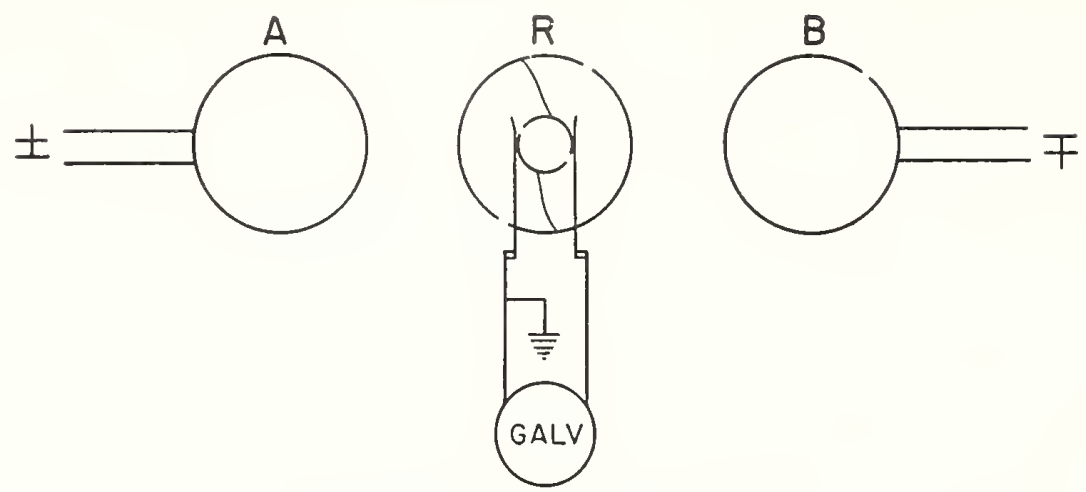

FIG. 8.- Bipolar generating voltmeter.

\section{GENERATING VOLTMETER}

The generating (rotary) voltmeter (28) first described by Kirkpatrick has been useful in estimating local potential gradients and as an auxiliary control device. It has been likened to a d-c generator, but instead of currents induced by moving wires in a magnetic field it employs a configuration of electrodes which permits the commutation of charges induced on plates alternately exposed to and shielded from an electrostatic field. Fig. 8 shows the diagram of a bipolar generating voltmeter. Fig. 9 shows a ceiling-mounted type designed by Behr that employs a sector disk as the rotating element.

The fundamental idea has been incorporated in many designs. Because of the necessity of alternately exposing and shielding the active plates (electrodes) the gen- erating voltmeter appears to offer greater promise for use in relative measurements than in absolute measurements. Thus it is usually first calibrated in terms of other satisfactory low-voltage standards, prior to use at higher voltage, but it may be used in those cases not requiring a high order of accuracy and thus amenable to simplifying assumptions for the purpose of computing the high voltage from dimensions. By so shaping the high voltage electrodes that discharges and consequent space charges are avoided it may be used by extrapolation techniques to extremely high voltages both on direct and alternating voltage. Without an incorporated high-voltage electrode it is essentially a gradient measuring device. It has been useful in a study of atmospheric electric charge and field phenomena responsible for lightning (29), and as a voltage

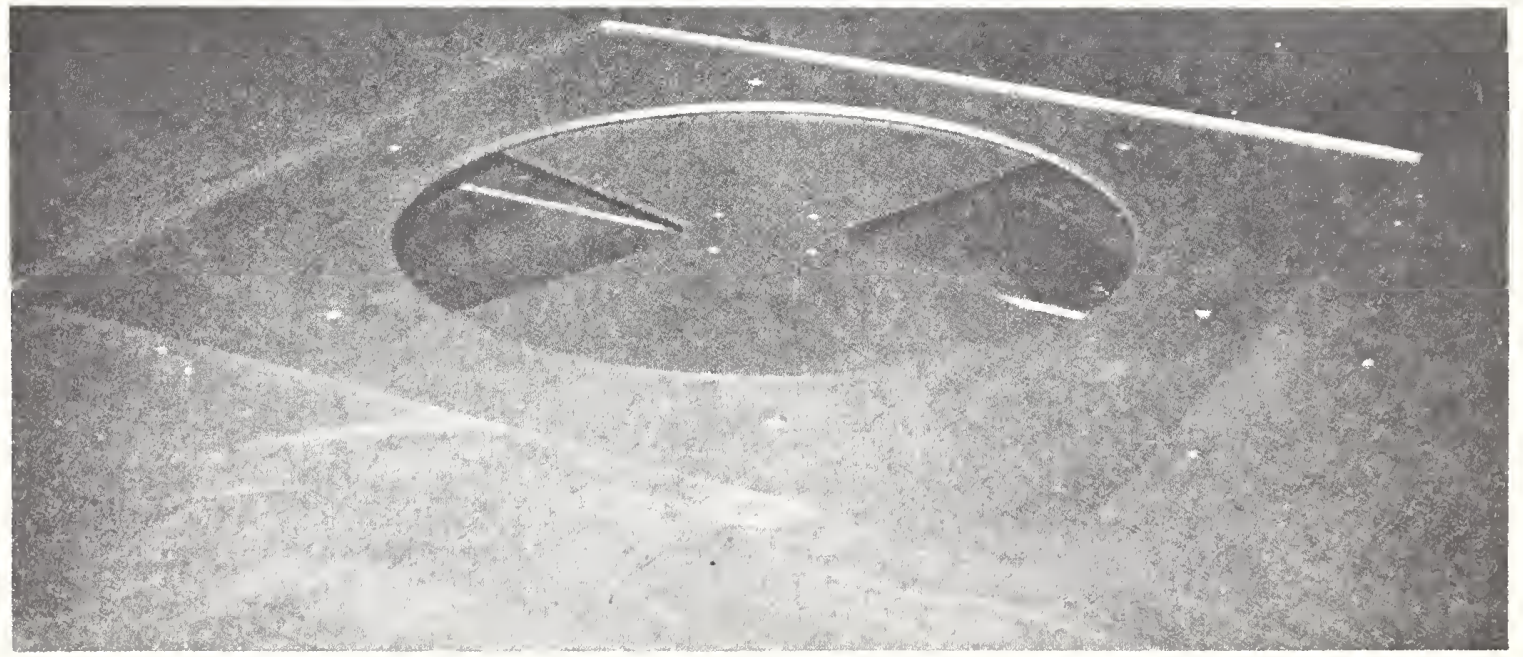

FIG. 9.- Rotating segments of Behr generating voltmeter. 
measuring device associated with highvoltage generators of the Van de Graaff type.

When the generating voltmeter is used as a voltage measuring component (i.e., as an instrument for determining a value of gradient to be multiplied by a constant factor in order to obtain the total voltage difference) it is not sufficient that the fields in the immediate neighborhood of the instrument shall be below corona or discharge-forming values, but it is equally necessary that the gradients at surfaces in the neighborhood of the sample ficld as well as those at the high-voltage electrode be below discharge-forming values and that there be no nearby relatively large sources of air ionization or dust. The effect of dust and charged particles may indirectly produce serious distortions in high fields by being deposited and aligned on otherwise smooth polished metal surfaces so as to form the necessary points for initiating discharges and consequent space charges that would otherwise not exist. If the charge on the surface of the segments alternatoly exposed to the electric field is to be in definite ratio to the total field, then at no place in the field may the gradient exceed the approximate breakdown gradient, $30 \mathrm{kv}$ crest/em, in air at NPT. The practical limit is actually much lower. For instance, the vahue of average gradient at which self-propagating discharges occur in the case of thmnderstorms appears to be about $10 \mathrm{kv} / \mathrm{cm}$. In the case of the Brooks electrometer (30) discharges have been known to oecur between the parallel faces of the electrometer plates when the voltage applied divided by the spacing had a vahue of $5 \mathrm{kv}$ eff $/ \mathrm{cm}$. Thus in air at NPT it is doubtful that for a generating voltmeter an avrrage value of gradient exceeding $7 \mathrm{kv}$ $\mathrm{max} / \mathrm{cm}$ with an upper limit of $20 \mathrm{kv} / \mathrm{cm}$ at the electrode surfaces can be employed continuously without some error in measurement arising from the alove canses. Presumably this practical limit arises from the almost ever-present small particles of dust (insulating or semi-conducting) which when deposited on the surface upset the ideal condition of pointless smooth surfaces one wishes to assume after the instrument maker has done his best to produce them. With reasonable care and cleanliness in assembly, dust has not been found to be a problem in generating voltmeters operating within a pressure chamber at higher gradients (31).

When used with suitable precautions, the generating voltmeter offers a means of obtaining information not readily available in other ways. For instance when its commutating device is provided with a phaseshifting mechanism and the rotor is synchronously driven it provides a means of determining wave form (32) at high voltage with practically no load on the source being investigated. It is effective on a rippled direct voltage as well as on alternating voltage. When used for determining wave form it may be located in an undisturbed and readily accessible portion of the high voltage field. The generating voltmeter method of determining wave form should be good to a fer tenths of 1 percent.

\section{AIR SPARK-GAP BRE. KDOWN}

Sphere and rod gaps.-If an accuracy of the order of 3 percent in determining crest voltage is sufficient, as in the case of insulator testing, the sphere spark gap in air is usefnl as a crest voltage measuring device. For voltages above $17 \mathrm{kv}$ spheres of 6.25 $\mathrm{cm}$ diameter and larger (up to 2 meter diameter for approximately $2,000,000$ volts) operated under controlled conditions serve as voltage standards for electrical breakdown measurements of dielectries (33). Earlier theories of breakdown of sphere gaps assumed that pure air, as well as other gases, has a definite breakdown strength or breakdown gradient at normal pressure and temperature. Pasehen's law relating the length of the breakdown gap with air density permits correction for usual temperature and pressure variations. Russel, Dean, Peek, and others have given empiricill irrlationships based in part on electroilativ. field theory for both sphere and cylindrical gaps, which. although they fit well in a limited domain of pressure and temperatture, are not so satisfying as relations (34) developed at a later date on the basis of the newer atom-physieal batckground. The detailed work on discharges in gases by Loeb) 
(35) and his collaborators as well as much other valuable work in this field has provided a fairly satisfactory explanation of some of the scattering of breakdown values of spark gaps. Meek (36) suggests that in the case of the shorter gaps the electron avalanche initiated at the cathode constitutes the usual initial process whereas in the case of larger gaps the mechanism is more akin to the positive streamer discharge of lightning and originates within the gap at some distance from the electrode. For gaps of intermediate length there lies a. domain in which the initiation may be of either variety and such gaps show a larger scattering of breakdown values. The probability of a free electron existing in the right location to initiate a discharge is, of course, an important factor in the scattering of the initial breakdown voltage when the time of application of voltage is extremely short. Irradiation (3\%) of small electrocles of the gap with radium or with ultraviolet light to produce photoelectrons is helpful in reducing this type of seattering but appears not to be necessary in the case of large spheres because of the much higher probability, in the larger volume of air between the spheres of the presence of an initiating ion or electron.

An idea of the effectiveness of ultraviolet irradiation in reducing scatt ring may be cited in the case of $12.5 \mathrm{~cm}$ diameter brass spheres. Irradiation of the spheres by an open carbon are reduced the scattering of indiridual 60-cycle sparkover values by a factor of 5 as compared with the results obtained without irradiation. On the other hand the average values of sparkover voltage were lower by from two to five percent in the irradiated case, the amount of lowering being dependent on the intensity of radiation.

Basing his work on the detailed information now available on the mechanism of spark formation Ver Planek (38) appears to have successfully correlated the enormous amount of data on sphere spark gaps.

From the very nature of the spark between spheres and of corona on cylinders, voltage measurements based on sphere gaps and corona cylinders (34), because of their dependence on surface shape, cleanli- ness of the surface, and cleanliness of the air, appear to be limited to a rather low and questionable accuracy, say from 1 to 3 percent. When extreme precautions are used by following a ritual of cleansing, the use of ultraviolet irradiation of the spheres, limitation by resistance of surface pitting by the spark current, and by insuring "cleanup" of the sparking surfaces of the sphere gap through preliminary sparking, a series of 10 or more sparkover values often may be observed to agree to within \pm 0.1 percent. However, this apparent high precision, equal to that of the high-grade indicating roltmeter emplover as part of the equipment in making such observations, is deceptive. Painstaking observations made the following day under seemingly identical conditions may agree among themselves to the same precision but their average value will almost invariably differ by several tenths of one percent, and sometimes by more than 1 percent from those made on the previous day. In spite of its low order of accuracy the sphere gap serves as a commercial standard (33) for high alternatingand surge-voltage measurements apparently because of its basic simplicity. In larger sizes it gives a useful measurement of the maximum value of surge voltage and essentially serves as a voltage limiter when it is used in parallel with a device undergoing voltage-withstand tests.

Simple needle gaps, as a matter of historical interest, were once accepted as alternative standard voltage measuring gaps, but as a result of the inherently high scattering of values of sparkover voltage, variation in sparkover values with changes in humidity, and large scattering in sparkover roltage when used for measuring surge voltage, they have been discarded as standards. Rod gaps (39) in which the electrodes are used repeatedly as contrasted with the formerly prescribed use of new \#00 needles for each sparkover of the needle gap, have sparkover values which are also affected by humidity to about the same extent as the flashover of porcelain insulation and appear to have replaced needle (or point) gaps for those uses where gaps are desired for correlating the flashover voltage of insulators. 


\section{IONIC WIND VOLTMETER}

The Ionic Wind Voltmeter described by Thornton, Waters, and Thompson (40) merits some mention because it represents a unique application of a thermal method to the measurement of high alternating voltage. Use is made of the cooling effect on a heated filament arising from "electric wind." (See Fig. 10.) The heated filament, with a suitable grounded shield, is located at a distance from the high-voltage electrode in such a position that, although in the electric field, it will always be below corona-forming voltage. Ions that may be present move back and forth as a result of the alternating electric field and in striking neutral molecules increase the general molecular motion. This results in an increase in cooling effect on the heated filament proportional to the electric field. A filament that has a high temperature coefficient of resistance is connected in one arm of a Wheatstone bridge. The bridge out-of-balance indicator is then calibrated in terms of the high voltage applied to the Ionic Wind Voltmeter. Although this device may be constructed to have good sensitivity and is useful as a control device or relay, its indications are affected by change in wave form and an accuracy of only \pm 2 percent is claimed for it.

\section{ELECTROSTATIC VOLTMETERS AND ELECTROMETERS}

Electrostatic roltmeters and electrometers basically depend for their indication on a measurement of the force of attraction between charges on the movable portion of one electrode surface and charges of opposite sign on another fixed electrode surface. By arranging the movable portion of the electrode to be part of a suitable geometric surface-sphere, ellipsoid, or plane-it is possible to devise an instrument in which the voltage applied can be computed theoretically from measured dimensions and the measured force of attraction. An electrometer designed to approximate quite closely the theoretical assumptions as to the conductor shape and relative dimensions required for simple theoretical computations, and thus to permit computation of the value of applied voltage from dimensions and the resulting force, is termed an absolute electrometer. This is in contradistinction to the term electrostatic voltmeter, which signifies an instrument that may be used for relative measurements but that requires calibration by means of some other standard of voltage measurement. A number of designs of high-voltage electrostatic voltmeters $(41-51)$ have been constructed. Such instruments require much less electrical energy for their operation than an electrodynamic instrument with a series resistor. Corner's rounded sufficiently to avoid electric discharges, highquality insulation, and electrode spacing adequate to prevent discharges are prerequisites in the construction of both clectrostatic voltmeters and electrometer's.

Electrostatic voltmeters. With a few exceptions $(46,47,49)$ the high roltage electrostatic voltmeters follow the pattern of the Kelvin (52) guard-ring electrometer by having a guard ring for the attracted disk or movable electrode while placing less emphasis on the flatness of the movable electrode. Provision is usually made for

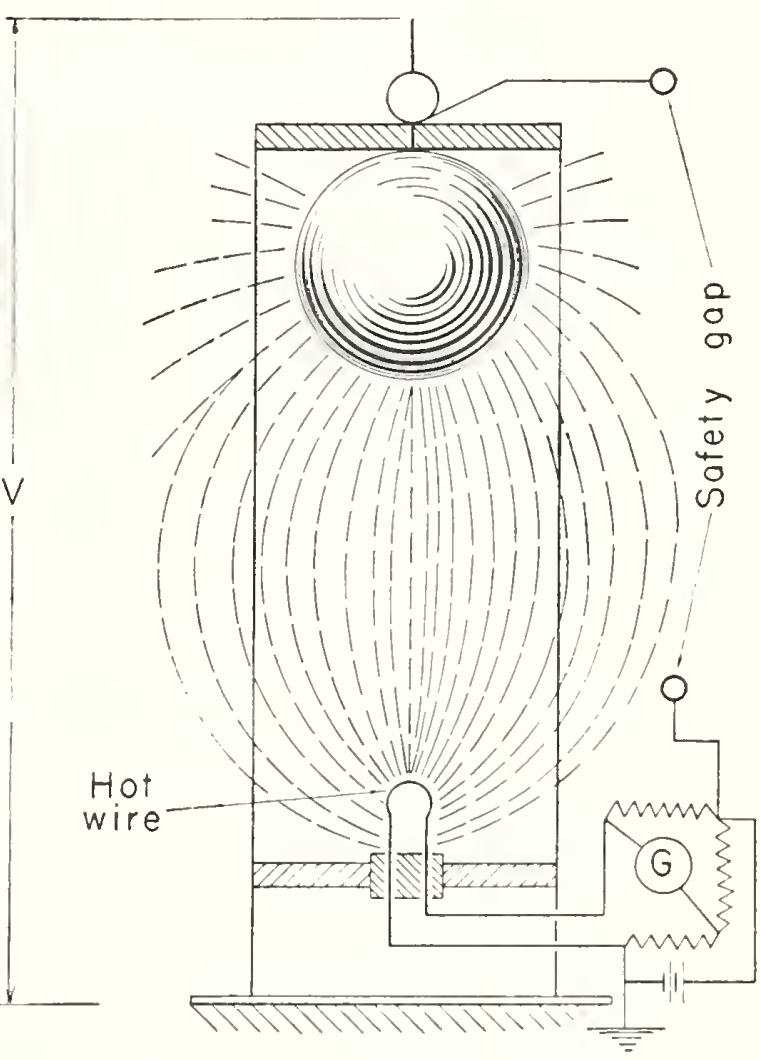

Frg. 10.--Ionic wind voltmeter. 
change in range by adjusting the spacing between the high-voltage electrode and grounded electrode of which the moving element usually forms a part. It is interesting to consider the developments in these instruments over a few years as shown in Figs. 11, 12, and 13. In the early design of Abraham and Villard (41) (1911), Fig. 11, the curvature of the disk and guard is

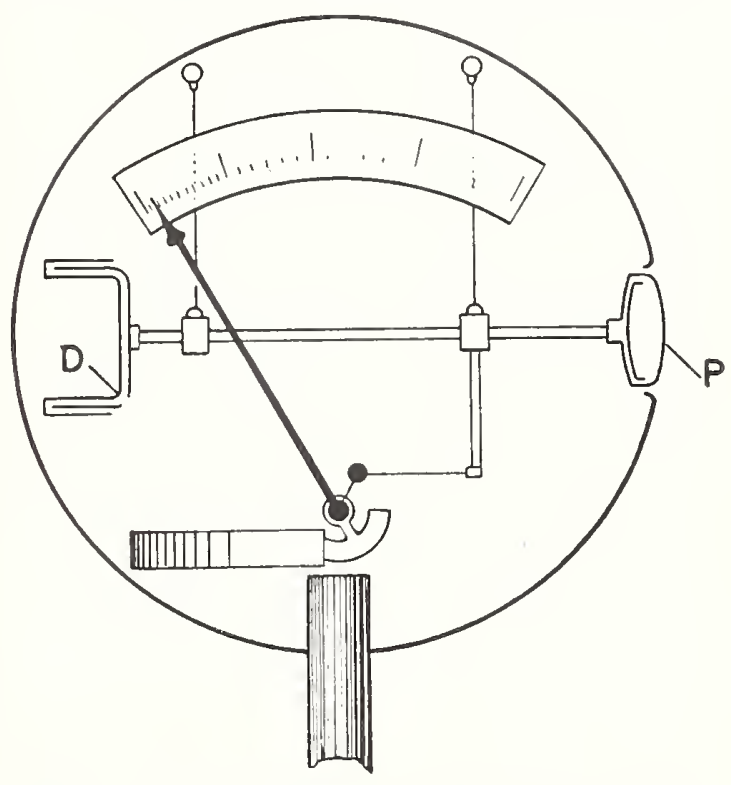

FIG. 11. - Abraham and Villard electrostatic voltmeter.

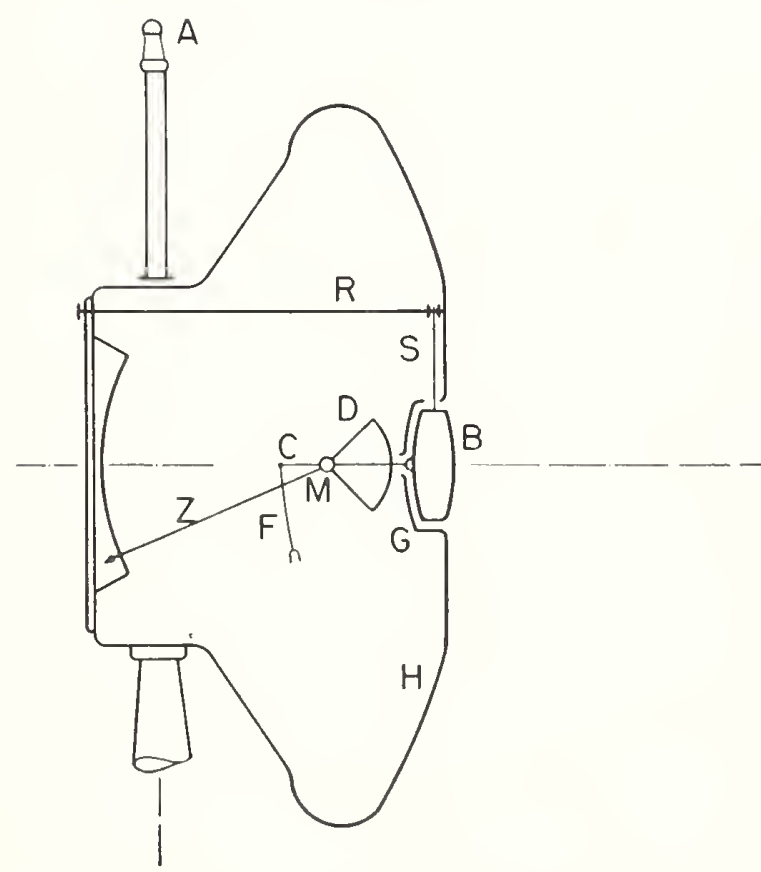

nearly spherical. The moving system is rather massive with a separate damping chamber within the guard. The Imhof (44) (1926) design, Fig. 12, provides a flattened guard ring and fits the light-weight attracted electrode into its own damping chamber. Starke and Schroeder (43) (1928) in one model, Fig. 13, employed a relatively larger flat-guarded electrode and a flatstrip suspension for the movable rectangular flat electrode, $P$, providing it with a mirror instead of a mechanical pointer, as well as with a balanced damping chamber arrangement. Nearby objects would be increasingly less effective in producing deflection errors at equivalent spacings in the later voltmeters. These voltmeters may be read to closer than 1 percent but unless calibrated in place, especially when used at large spacings at maximum rated voltage, are likely to be affected by nearby objects.

Ellipsoidal voltmeter.-The ellipsoidal voltmeter of Thornton and Thompson (53), illustrated in Fig. 14, is of the nature of an electrometer and satisfactory theoretical equations have been developed for it. It, like the electrometer, depends on a relatively undistorted axial field if it is to be used as an absolute instrument.

The moving element consists of a metallic ellipsoid of revolution carried on a bifilar silk suspension and is provided at its lower end with a reflecting mirror and damping

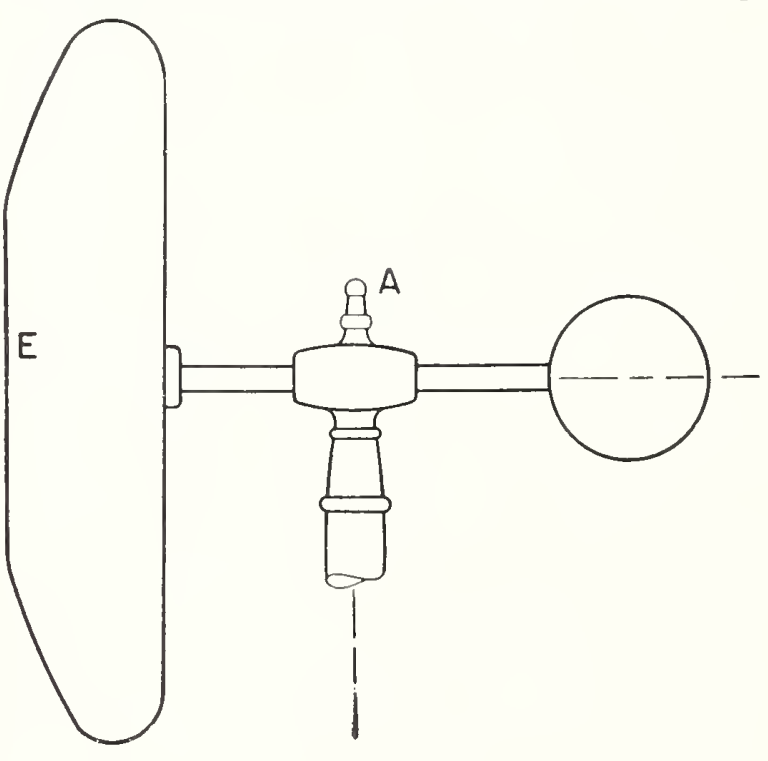

Frci. 12.-Imhof electrostatic voltmeter. 


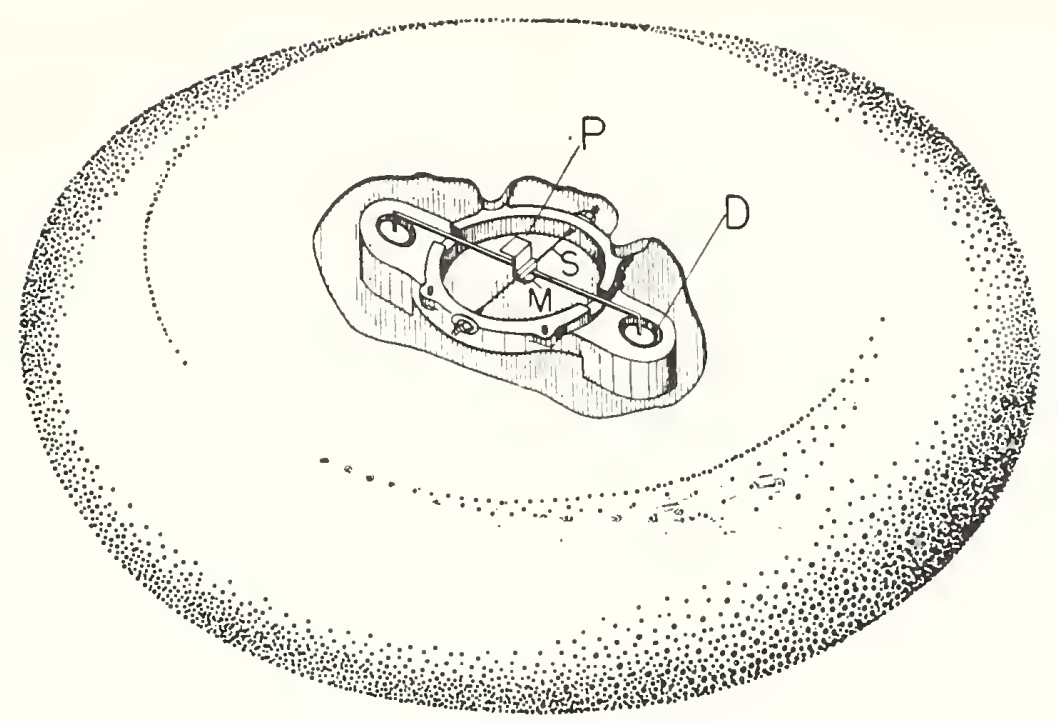

FIG. 13.-Starke and Schroeder electrostatic voltmeter.

vane. The ellipsoid is suspended with its long axis horizontal and is centrally located between two vertical circular plates facing each other and at an adjustable distance apart. Polarization of the ellipsoid by the electric field established between the plates by the source of voltage to be measured, gives rise to a mechanical couple tending to align the ellipsoid, which is initially set at an angle to the horizontal axis perpendicular to both plates. Although the change in the angular deflection of the ellipsoid.may be used as a measure of the voltage applied, a more sensitive and rather interesting method of voltage indication has been contrived which involves measuring the increase in frequency of its swings when voltage is applied. Thus:

$$
E=k\left(n^{2}-n_{o}^{2}\right)^{1 / 2}
$$

where $E$ is the electric field strength, $k$ is a constant found from dimensions, $n$ is the number of swings per second with the voltage on, and $n_{o}$ is the number of swings per second with the voltage off.

Sufficiently large plates are used and at such a separation that the electric gradient $E$ throughout a considerable volume along the axis would be substantially uniform if the ellipsoid were not present. Since the disturbance effected by the ellipsoid is small, the voltage applied to the plates is

$$
V=E d
$$

where $d$ is the plate separation.
The instrument described was designed for measurements up to $200 \mathrm{kv}$. As show11 schematically in Fig. 14 the plates were $140 \mathrm{~cm}$ in diameter and were spaced as much as $100 \mathrm{~cm}$ apart (i.e., average gradient of $2 \mathrm{kv}$ per $\mathrm{cm}$ ). An exploration of the field indicated that it was quite uniform at the midpoint between the plates within a radial distance of $25 \mathrm{~cm}$; however, no study of its longitudinal variation appears to have been made. Great care was taken to insure that the ellipsoids of revolution were accurately shaped so that the theoretical relationships would apply. One ellipsoid of duralumin was $3.9692 \mathrm{~cm}$ long and 0.5970 $\mathrm{cm}$ in diameter and weighed 2.0496 grams.

The electric gradients at the tips of the ellipsoids are considerably higher than the average gradient between the plates. These gradients must be kept well below coronaforming values if disturbing effects from electric wind are to be avoided. In its practical form where it is to be used as a deflection instrument for laboratory or shop measurements an insulating enclosure is provided for the suspension, ellipsoid, mirror, and damping mechanism to shield them against wind and dust. At the bottom end of the moving-system assembly the damping vane is suspended in a damping chamber attached to a tube whose upper end is cemented to the bottom of a hollow glass sphere in which the ellipsoid is centered. The upper end of the sphere is cemented to a second tube housing the bifilar 
suspension and an adjustable suspension control for changing the period of swing. The control mechanism and the torsion head are mounted at the supported upper end of the tube. A spherical shape was used for the hollow glass sphere enclosure for the ellipsoid so as to permit a theoretical evaluation of the effect of the dielectric of the sphere on the electric field $E$ at the ellipsoid. The theoretical correction derived for this spherical glass enclosure agreed well with experimental results when the relative humidity was not high enough to cause electrical surface leakage.

The ellipsoidal voltmeter is saicl to be accurate to 0.1 percent and to be only slightly affected by humidity. Because of low average gradient, $2 \mathrm{kv}$ eff $/ \mathrm{cm}$, it appears to be more bulky than other electrostatic voltmeters and is of interest mainly because it is a unique arrangement permitting absolute measurements.

Sparkless sphere-gap voltmeter.-Large spheres ordinarily used as sphere spark-gap voltmeters in measuring high crest-voltage have been modified to permit their use as electrostatic voltmeters for measuring the effective value of voltage at spacings slightly in excess of sparking distances. This arrangement has been called a sparkless sphere-gap voltmeter (47).

Hueter (46) employed a vertical arrangement of 1 -meter spheres. The upper high-voltage sphere was supported on a spring whose additional extension as a result of the electrostatic force was magnified by a lamp-mirror-scale arrangement. The spring and the mirror optical-lever arrangement were mounted within the sphere shank which was provided with a small window. An external arc-lamp and scale were mounted on an adjacent wall in the laboratory and gave satisfactory readings in daylight. The vertically adjustable lower sphere was grounded and its driving screw mechanism was arranged to indicate the gap length. The weight of the upper one-meter sphere was $60 \mathrm{~kg}$ and for a $75 \mathrm{~cm}$ gap the electrostatic attraction was approximately 800 grams at $1,000,000$ volts. In order to minimize effects of changing gap length, only small displacements (less than 0.5 percent of the gap length) of the spring-suspended sphere were used. An oil-cup damper made the sphere motion nearly aperiodic. An accuracy of 1 percent was claimed.

Sorensen $(47,48)$ employed a horizontal arrangement of 1-meter spheres with rather long slender shanks presumably in order to reduce effects of attraction arising from the shanks. The electrostatic attraction of the grounded sphere could be readily measured as it was supported by suspending its shank by four ropes tied to the shank at the apices of the two thus-formed $V$-suspensions. The upper ends of the ropes were attached to ceiling members. This laterally stable suspension possessed only a small longitudinal stability so that differences in longitudinal electrostatic forces of several hundred grams could be measured to better than one gram. A small wire in line with and attached to the end of the grounded sphere shaft ran over the rim of a bicycle wheel thus insuring low friction. A small weight pan attached to the end of the wire permitted weighing the force of attraction.

These two sphere-electrometer devices represent useful laboratory tools for they can be calibrated and used as voltmeters for measuring effective voltage. They can

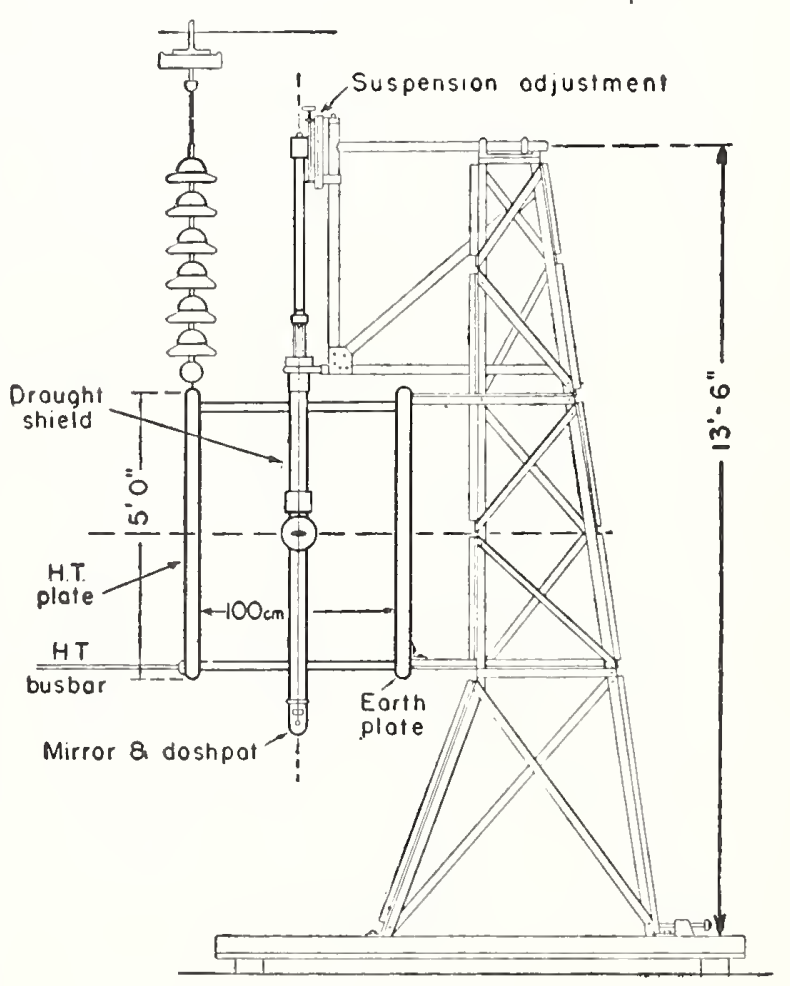

FIG, 14,-Elliosoidal voltmeter. 
also be employed in the usual mammer as sphere spark-gaps for the measurement of crest voltage. If one is satisfied with the accuracy of the sphere spark-gap as a high-voltage standard, measurement of the voltage wave form permits calibration of these devices as electrometer's for the measurement of effective voltage without recourse to other methods. To a very limited extent they may be considered to be absolute electrometers, particularly if adequate relative clearances to the floor, walls, leads and other conductor's and insulators are maintained so that approximate corrections can be made for the presence of these objects. Simple theoretical calculations of the force of attraction can be made by the method of image charges in the case of two insulated or one grounded and one insulated sphere. For a 25-cm spacing of his 1-meter spheres Sorensen considered that no correction was necessary, and at $35 \mathrm{~cm}$ the effect of shanks and other nearby objects was said not to exceed 1.5 percent.

One may hope for an accuracy approaching 0.5 percent with the design of Hueter in making relative measurements up to one million volts. This would involve ample clearance for the spheres and first calibrating by an accurate lower voltage method using a separation of the spheres adequate for one million rolts and making certain that corona-free electric field conditions exist in the neighborhood.

Disk electrometers.-About 1880 Lord Kelvin made an outstanding contribution by incorporating a guard ring for the disk of the attracted disk electrometer. The guard ring not only validified the use of the simplifying mathematical assumptions in computing the electrostatic force of attraction of the disk but it established the basis for a design whose readings were less effected by nearby objects. When coplanar with the guard ring the force

$$
F=\frac{V_{s}^{2} A}{8 d^{2}},
$$

where $V_{s}$ the voltage is in rectrostatic units, $A$ is the area of the disk, and $d$ theseparation from the opposite gromuled plate.

For deflections of a guarded disk away from coplanarity, Snow (54) has developed a mathematical solution which takes into account the change in force arising from change in position of the disk. This change in force might at first appear only to vary inversely with the separation $d$ (shown by a simple differentiation of equation (3)). However, Snow has evaluated the additional change in force arising from the redistribution of the charges, which in the case of a protruding disk results in an increase in concentration of charge at the edge of the disk at the expense of the charge on the adjacent edge of the guard plate and vice versa in the case of a retracted disk. Troublesome instability in disk electrometer's arising from this latter component of force has for a long time been recognized as a reakness in electrometers designed to cover a wide range of voltage measurement by adjusting the spacing $d$. One may either elect to provide a linear restoring force adequate for all spacings $d$ at a considerable sacrifice in sensitivity at large spacings or provide for some adjustment of the restoring fores with change in spacings at nearly constant maximum allowable gradient. The restoring force required to balance the electrostatic attraction in high voltage electrostatic voltmeter's and electrometer's has been provided by the following devices:

(1) Suspension of the moving electrode on one arm of a gravity balance, i.e., change of restoring force secured by adjustment of $\mathrm{c}$.g. of balance relative to central knife edges $(52,30)$.

(2) Suspension of moving clectrode on a coiled spring $(46,61)$.

(3) Pendulous suspension of electrode (49).

(4) Suspension of disk electrode assembly on a flat-strip (torsion) suspension $(4,3,55)$.

(5) Suspension of the disk from a metallic membrane $(50,51)$.

(6) By combining the torque provided by at flat-strip supporting suspension with that produced in a current-balance arrangement of coils which permits adjustment of the restoring force (55) by change in current.

The last three types merit special mention because of the novelty of their alrangement. Fig. 13 illustrates the flat-strip suspension arrangement of Starke and Sehroceler. The sixtl of the above arrangements, used by Nacken (55), employs two pairs of "current balance" coils in a con- 
nection that permits adjustment of stability as well as of sensitivity. The restoring force arises in part from the vertical strip suspension which carries the disk with its plane vertical as well as the two similarly mounted astatically connected current balance coils. One pair of field coils with current $i_{f}$ is connected in series aiding one moving coil and provides a torque proportional to $i_{f}$ for balancing the electrostatic force on the disk; the other pair of coils with current $i_{m}$ is connected in series opposing so that by reversing $i_{m}$ and adjusting its value relative to the current in the moving coil the restoring force of the strip suspension toward the null or coplanar position of the disk may be either opposed or aided thus altering the stability of this system by the simple adjustment of the current in this pair of coils. Thus this arrangement permits a desirable adjustment toward higher sensitivity at large spacings of the electrodes where the deflecting force decreases.

The fifth of the above arrangements is exemplified in the devices of Rogowski and Böcker $(50,51)$ (illustrated in Fig. 15) in which an elastic diaphragm $D$ provides the restoring force for the attracted disk. The diaphragm supports an iron-cored coil (above) which is actually part of a current transformer ("Messdose") constructed with two air gaps in its iron core. The fixed coil with its core form the other part of the current transformer which is supplied from a voltage-regulated alternating-current supply. Very small changes in the air gap (moving coil position) suffice to produce full scale deflections of an ammeter connected across the moving coil and this ammeter is calibrated to read the high voltage. As part of this particular moving system, arranged for use in a compressed gas enclosure, a force coil (Druckspüle) $K$ is suspended below in an iron-clad solenoid especially constructed to permit a measurement of force in terms of solenoid current. This arrangement permits, prior to assembly of the unit in the compressed gas chamber, a direct calibration with known weights on the disk for current in the solenoid against deflections of the diaphragm as indicated by the ammeter. This preliminary calibration with known weights against current is made in order to permit subsequent checking of the calibration of the electrometer by means of the current instead of weights after it has been filled with compressed gas.

Brooks absolute electrometer.-The absolute high voltage electrometer of Brooks (30) (Figs. 16 and 17) is illustrative of what can be accomplished in the way of precision when the attracted disk is supported on one arm of a gravity-type balance. Brooks's modification of the Kelvin electrostatic attracted disk electrometer with guard ring was designed for use in free air up to 275 $\mathrm{kv}$ on alternating voltage and was arranged to allow a step by step experimental evaluation of errors not readily calculable. In addition to a guard ring, it employed guard

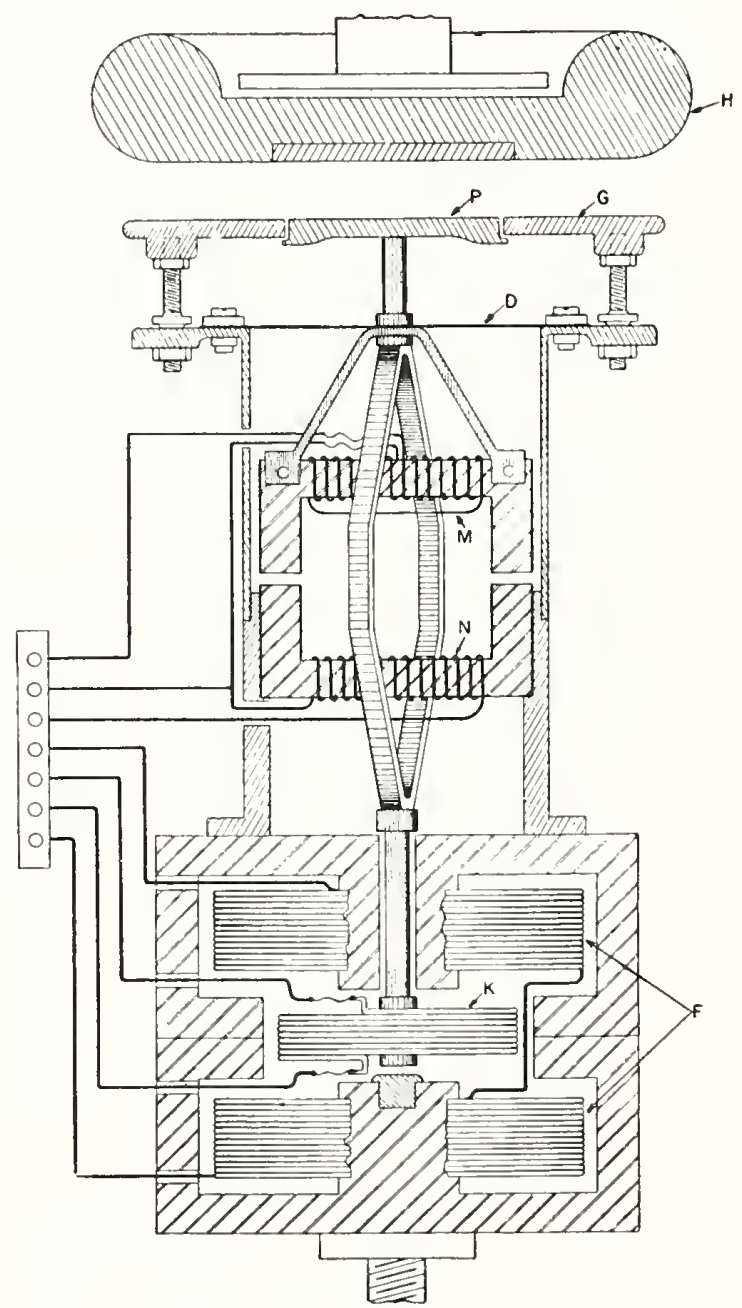

FıG. 15.-Rogowski and Böcker compressedgas electrostatic voltmeter. 
hoops equally spaced between the guarded and grounded electrodes. These guard hoops, spaced $2 \mathrm{~cm}$ apart, are connected to tap points on a potential dividing capacitor also across the voltage source. Thus the equally spaced hoops are maintained at equally spaced potentials corresponding to the roltage applied between the high-voltage (upper) and grounded (lower) plates of the electrometer. The hoops not only screen

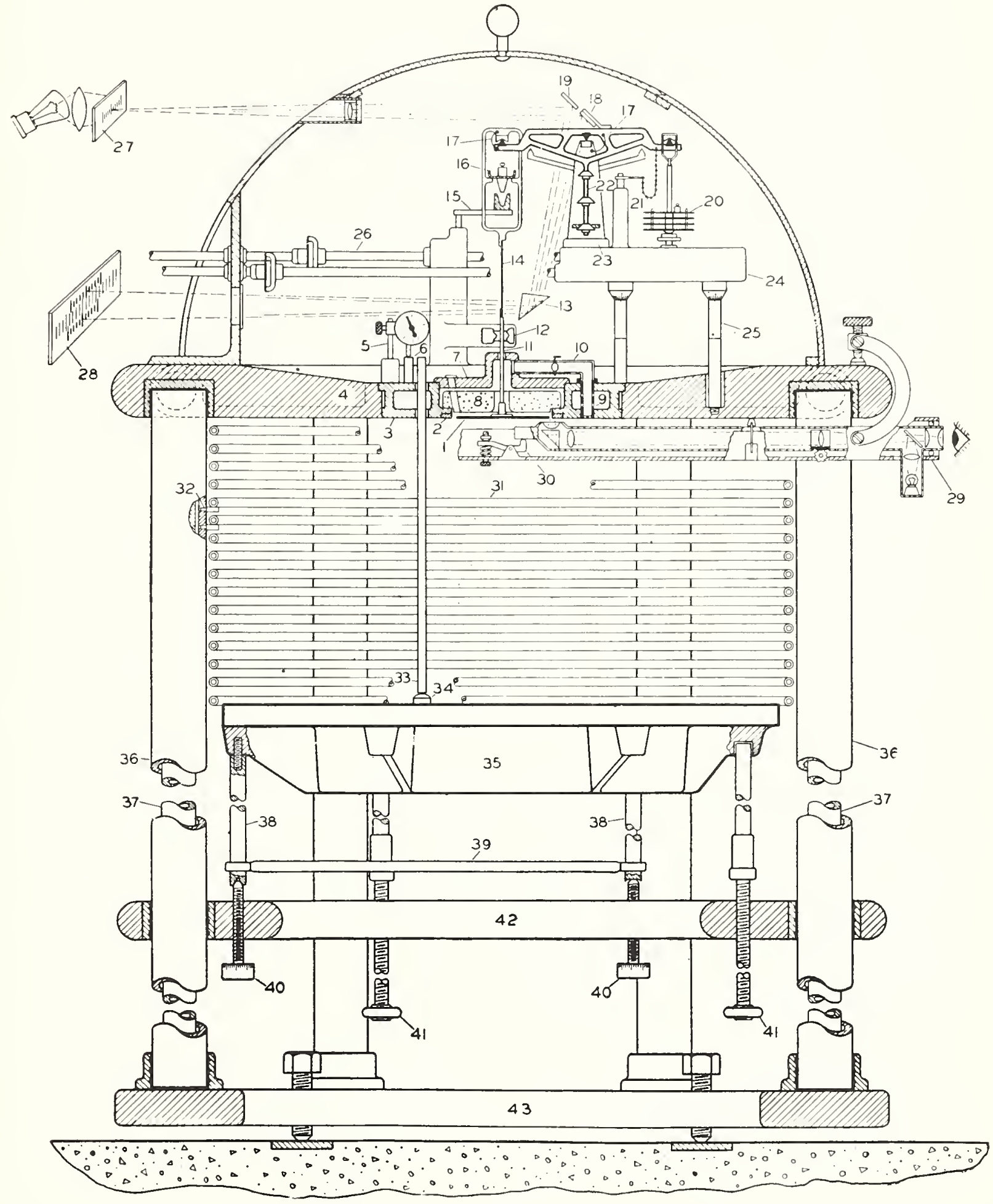

FIG. 16.-Brooks attracted disk electrometer (schematic). 
the disk from external fields, but by their potential distribution tend to correct, within their diameter, electric field distortion that would otherwise arise from edge effects at the upper guard ring and lower plate. A mathematical solution of the contribution to force on the disk arising from these hoops has been earried through by Snow (54) and Silsbee (30) and has been demonstrated to be adequate by suitable experimental tests. ${ }^{6}$ For instance by omitting hoops, or electrically shorting various sets of hoops, their potential distribution was changed drastically. For such conditions when the corresponding corrections were applied based on the mathematical solution and experimentally measured potential distribution, very good agreement in the measured values of voltages was obtained.

As a result of the work on the Brooks Absolute Electrometer, which was compared with the transformer voltmeter method of measuring voltage, it was concluded that this instrument is reliable for absolute determinations to about 0.01 percent. This work was limiterl to 100,000 volts-approximately one-third its rated voltage-because the clearances in the space in which the equipment was housed were inadequate. Results up to full rated value, $275 \mathrm{kv}$, are not yet available. (With the cessation of the war it is expected to extend the measurements to higher values of voltage in the modern High Voltage Laboratory of the National Bureau of Standards.)

${ }^{6}$ The simple equation (3) may be rearanged $V_{s}=2 d \sqrt{2 F / A}$. This equation may be thougit of as being satisfartory for mowsurements yicld-

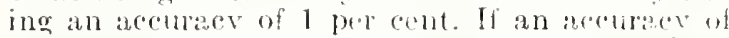
0.01 per rent is desired, correspondinghy refined experimental techniques must be emplored and additional physical measmements mest he mitie as indicated by the larger number of terms in the complete rguation for the voltage in electromagnetir unts of the Broolss alectrometert,

$$
\begin{aligned}
V_{M}= & \frac{4 v\left(c+h_{d}\right) \sqrt{2 M_{F} g}}{\left(r_{b}+r_{d}\right)}\left[1+\frac{A_{v}-A_{o}}{2 M_{F} g}\right. \\
& +\frac{\gamma}{M_{F}}\left(S_{v}-S_{0}\right)-\frac{f\left(S_{v}-S_{m}\right)}{2 q}+\frac{f h_{m}}{2} \\
& \left.-\sum_{k=1}^{k=\infty} C_{k} G_{k}-\frac{\epsilon-1}{2}+\phi_{1}\left(\frac{h_{d}}{r_{d}}\right)+\phi_{2}\left(\frac{s}{r_{h}}\right)\right] .
\end{aligned}
$$

Compressed gas electrometer.-A consideration of the compressed gas electrometers of Tschernyscheff (56), Palm (57), Rogowski, Böcker $(50,51)$, and others who attained accuracies approaching 0.1 percent leads one to consider whether additional design features might be incorporated in the compressed gas type in order to give an accuracy approaching that of the Brooks electrometer in free air.

Actually the matter of precise weighing of the forces (less than 2.5 grams) on a 16-cm diameter disk, involved in the Brooks electrometer uffers no serious problem in free air aside from the necessity of providing a carefully thermostated enclosure to avoirl air currents. The possibility of having correspondingly higher forces to measure has its appeal. In the Rogowski and Böcker voltmeter the maximum force of attraction of the disk may reach 250 grams and is measured to 0.2 grams. Thus the forcer employed in measurements are (ne hundred times as large as in the Brooks electrom. eter, however, the relative accuracy of measurement of this larger force is less in their device.

At the outset one would have to provide a force-measuring device approaching the accuracy and repeatability of a high-grade gravity balance, i.e., something better than that incorporated in the Rogowski and Böcker electrometer. Smaller-scale length moasurement would have to be made with about the same relative accuracy and the flat metal surfaces of the plates would require a high quality optical finish. The greatest loss involved in the use of compressed gas rather than free air, as in the Brooks electrometer, lies in the relative difficulty of making and checking mechanical measmements under pressure before and after voltage observations.

The immerliate gain from the use of compressed air (15) or other gases (51B) is to increase sparkover voltages nearly in proportion to pressure. For instance in the design for use to $400 \mathrm{kv}$ alternating, Böcker used carbon dioxide at 15 atmospheres pressure which permits a gradient of 100 $\mathrm{kv}$ eff $/ \mathrm{cm}$ as compared with $2.5 \mathrm{kv}$ eff $/ \mathrm{cm}$ in the Brooks electrometer as limited by the present potential dividing capacitor con- 
nected to the hoops. Gaseous "freon" (58) (dichlorodifluoromethane) has between smooth electrodes about 2.3 times the breakdown strength of air at pressures up to six atmospheres where it still remains gaseous. In the case of points or sharp edges the relative breakdown strength of freon is still higher. Freon has the disadvantage of breaking down into highly corrosive products if corona or other discharges actually take place in it, making it less desirable than carbon dioxide on that account. Sulfurhexafluoride (58C) appears to offer some advantages over freon because of its greater chemical stability and higher equilibrium pressure at normal temperatures.

The greater size of the "free air" as compared with the "compressed gas" electrometer appears to be its chief drawback, making it too cumbersome at the highest voltages.

DEFLECTION OF FREE-MOVING CHARGED PARTICLES AND THE HIGH VOLTAGE SCALE

The cathode ray oscillograph and electrostatic (and magnetic) analyzers are examples of devices that employ the deflection of free-moving charged particles. Employing for this discussion the relations given by Hanson and Benedict (59), if a slowly moving stream of charged particles is accelerated in vacuo along an electrostatic field of total voltage $T_{a}$ then

$$
V_{\mathrm{a}} e=\frac{1}{2} m v^{2}
$$

where $m$ is the mass of the particle, $v$ its velocity, and $e$ the charge on the particle. When a stream of particles with the velocity $v$ is directed between parallel plates perpendicular to the electric field established by the voltage $V_{d}$ between the plates then for the idealized arrangement of plates at the far edge the deflection

$$
d=T_{d} l^{2} / 2 S m v^{2}
$$

where $l$ is the length and $S$ is separation of the plates. For nonrelativistic velocities if $L$ is the distance to and $D$ the deflection at the screen or receiver

$$
D=L d /(1 / 2)=V_{d} e L l / S m l^{2}
$$

so that

$$
V_{a} / V_{d}=L l / 2 S D,
$$

and if the relativistic velocities are taken into account (59)

$$
V_{a} / V_{a}\left(1-\frac{V_{a} e}{2\left(E_{o}+V_{a} e\right)}\right)-L l / 2 S D,
$$

where the rest energy $E_{0}=m_{0} c^{2}$ and $m_{0}$ is the rest mass and $c$ the velocity of light.

For relation (8) it may be shown that the relativistic correction is about 1 percent for electrons accelerated by a voltage $\mathrm{V}_{a}=$ $10.5 \mathrm{kv}$ and increases to about 14 percent at $200 \mathrm{kv}$. The existence of a relativistic correction of such magnitudes on account of high electron velocity may be looked on as somewhat of a nuisance and as a limitation of the cathode-ray oscillograph when used for the direct measurement of high voltages. Applications of the cathode-ray oscillograph therefore seem to have been limited to the measurement of lower voltages. As is well known, the beam-accelerating voltage source for $T_{a}$ is generally maintained as constant as possible in order to preserve the sharpness of the cathode spot. The voltage to be measured (or a fraction of it from the voltage divider) is applied as the voltage $V_{d}$ to the deflecting plates. $V_{d}$ is kept sufficiently low to leave the deflection $D$ materially unaffected by the relativistic mass correction for velocity of the electrons.

At first thought the fundamental simplicity of this method of measuring voltage is decidedly appealing. It applies the accelerating voltage field directly to the elementary charge of the electron unhampered by additional matter. As indicated above and by equation (8) there is a disadvantage because the resulting high electron velocities even for relatively low-voltage accelerating fields become so large that relativistic corrections for moving charges must be introduced. Thus on second thought, the method involving the acceleration of electrons appears far from ideal but an examination of equation (5) indicates the advantage to be gained by using charged particles of greater mass than the electron because of the resulting lower velocity. Although what one might consider a practical 
device for everyday use in the measurement of high voltage by deflecting a stream of free-moving positively charged particles has not been developed, the nuclear physicist has used such a device in his work for a number of years. To help him on his way in measuring high voltages he has estab- lished the High Voltage Scale. A short digression in explanation of how this was accomplished seems in order prior to $\cdot a$ presentation of the contribution of the free-moring charged-particle deflectionmethod in this work.

The study of atom physical phenomena

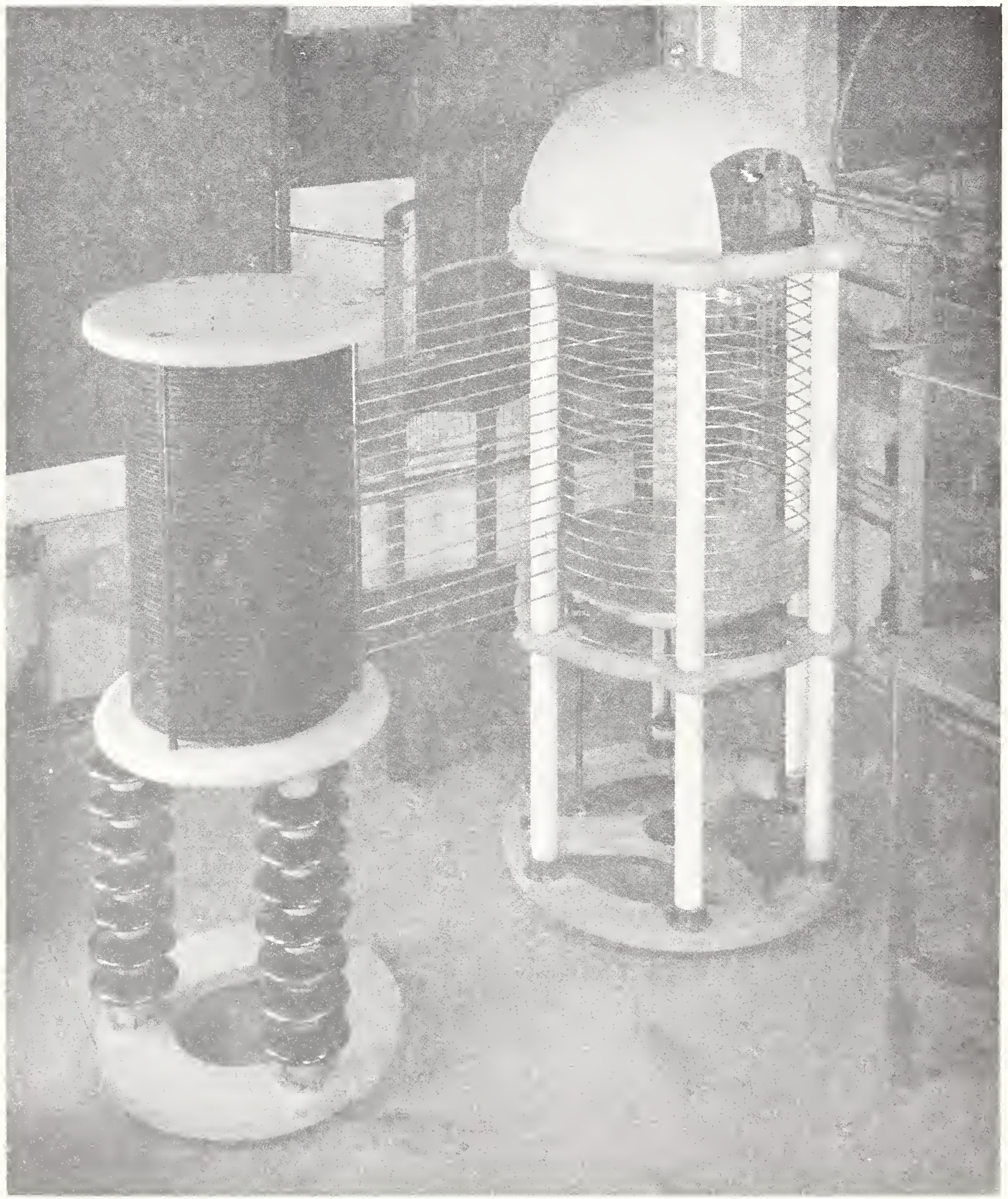

FIG. 17.-Brooks attracted disk electrometer. 
led to the physicists' need for a voltage reference standard of the order of $1,000,000$ times the voltage of the standard cell.

Historically, one may go back to Planck's equation $h \nu=T^{\prime} e$ wherein $h$ is Planck's constant, $\nu$ is the maximum frequency corresponding to the maximum accelerating voltage $V$, and $e$ is the ralue of the elementary charge. Duane and Hunt (60) used a steady high-rcltage storage battery in order to evaluate $h$ from a careful measurement of the accelerating voltage $V$ and the corresponding maximum frequency limit of the continucus X-ray spectum produced. Since those measurements were made the value of $h$ has been well established by other methods. Thus this relation affords a method of determining $V$ by a measurement of the maximum frequency of emitted continunus X-radiation. Iittle use has been made of this method as a highvoltage reference standard largely because of the accuracy required in evaluating the frequency. With the advent of work on nuclear disintegration a pressing need for high voltage reference standards became apparent as the usual extrapolation methods of measuring $V$, the particle accelerating voltage, were both. cumbersome and none too reliable. In early bombardment work it was found th at resunance radiation of gamma rays occurred within a rather narrow range of the voltage used in accelerating the protons. The early careful measurements by Tuve, Hafstad, and Heydenburg (11) of the voltage at which these radiations occurred provided the basis for their adoption of certain values of voltage corresponding to the resonance gamma radiation for selected elements as fixed points on the High Voltage Scale.

The measurement process for establishing values for the fixed points on the High Voltage Scale consists in:

(1) Providing a source of protons which are accelerated by a carefully measured adjustable voltage $\mathrm{V}$.

(2) Directing this beam of protons on a target of a selected element such as lithium or one of its salts.

(3) Measuring the gamnia-ray resonance radiation effects by means of Geigel-Mueller counters or similar devices which permit a quantitative measurement of this radiation as a function of the accelerating voltage.
From plotted curves of gamma radiation against accelerating voltage (proton energy) the rather sharp maximum of gamma ray effect fixes the value of voltage chosen as the resonance voltage. By selecting elements for these reactions in which a single sharp maximum occurs and by carefully determining the corresponding voltages in terms of the standard cell, the voltages at which particular reactions occur were established as reference points on the High Voltage Srale. In a similar manner but using a boron fluoride ionization chamber (paraffin surrounding the target tube and nearby chamber) instead of the GeigerMueller counter, neutron counts may be made for similar reactions in which neutrons are ejected from the bombarded target by voltage accelerated protons. In this case points for the scale are determined by gradually reducing the accelerating voltage $V$ and choosing that voltage at which there is an abrupt decrease in ionization chamber current as the limiting voltage for ejection of neutrons is reached.

Early experimental values of voltage corresponding to the resonant radiation in the bombardment of lithium by protons was fixed at $440 \mathrm{kv}$ for $L i(p \gamma)$ with a probable error of 2 percent and a relative accuracy of 1 percent by Tuve, Hafstad, and Heydenburg (11) of the Carnegie Department of Terrestrial Magnetism. A redetermination of voltage values for some of the fixed points was leported in 1944 by Hanson and Benerlict (59), of the University of Wisconsin. Their electrostatic analyzer (a device for determining the value of the electrostatic field at right angles to a beam of charged particles that will deflect the beam a given amount) was carefully constructed so that its deflection constant could be computed from dimensions as a check on values determined experimentally. Their experimental method employed an electron beam in place of the proton beam used later in their evaluation of the fixed points. The use of a low-voltage electron beam (accelerating voltages of 8 to 20 $\mathrm{kv}$ ) and of small deflecting voltages (150 tc 360 volts) permitted precise voltage measurements and higher precision for determining the deflection constant of their 
analyzer than the absolute method eonsisting of a computation of the deflection constant using carefully measured dimensions. Tarious refinements including the use of a highly stabilized source for the deflecting roltage and automatie regulation of the ion-aceelerating voltage reduced fluctuations and contributed to highly precise measurements of the fixed points on the High Voltage Scale. Hanson and Benedict consicler the following values expressed in terms of the Million Eleetron Volt scale to be accurate to 0.3 pereent: $\operatorname{Li}(p \gamma)$ $0.4465, F(p \gamma)$ 0.877, Li $(p n)$ 1.883, Be(pn) 2.058. Their apparatus gave relative values agreeing to better than 0.1 pereent. Their values are seen to agree within the tolerances given for the values of Tuve, Hafstad, and Heydenburg although they are approximately 1.5 percent higher.

The establishment of the High Voltage suale baserl on a phenomenon unaffeeted by temperature, pressure, and humidity except as their ahnormalities plague the collateral work of the investigator, thus represents a distinet step forward in the process of better measurements and standards for high voltatges.

\section{SLMAARY AND CONCLUSION}

The principal methods in use for measuring high roltages hare been outlined through the discussion of a number of devices. Mention was first made of the correlation of different methods of highroltage measurements through extrapolation teehniques in which the standard eell was used as the primary standard of voltage. Absolute high-voltage eleetrometers were later diseussed and the good agreement between their independently determined values when compared with a standard-cell voltage-extrapolation method was noted. Concluding remarks eited the use of hoth the standard-eell voltage extrapolation-technique and a less preeise absolute method in establishing the voltage values for certain fixed points on the High Voltage Scale.

It the moment it appears that high roltages as we know them in the laboratory stop with values of the order of $10,000,000$ rolts. On the other hand, charged particles come to us from spaee or may be aeeelerated in the laboratory by resonance techniques to have electron velocity equivalents approaching one hundred times that value. Presumably the future holds in store some new insulating arrangement for the colleetor of the high energy particles that man is able to produee-an arrangement that will permit the colleetor to build up to the unbelievable potentials we think of when we hear of a new machine in the rumor or blueprint stage that will produce 1,000 million electron-volt partieles. Just what such voltages will be used for and how they will be measured I wish to leave as part of your field of eonjecture.

\section{REFERENCES}

(1) RaYnen. Journ. Inst. Electr. Enu. 59: 13s. 1921.

(2) A. Rosa. Bull. Bur. Stand. 3: 43. 1906.

B. Harris. Bur. Stand. Journ. Res. 3 : 445. 1929.

(3) Keinath. Die Technik elektrischer Messgerëte 2: 17.1928.

(4) Chuвb. Proc. Inst. Electr. Eng. 35: 121. 1916.

(5) PALM. Elektrotechn. Zeitschr. 47: \$73, 904. 1926.

(6) König. IJely. Phys. Acta 1929: 357.

(7) Haefely et Cie, Basle. German Patent No. 394014. 1923.

(8) Van Cauwenberghe and Landsberg. Bull. Techn. Assoc. Ing. Bruxelles 4. 1929.

(9) Davis, Bowlder, and Staxdring, Journ. Inst. Electr. Eng. 68: 1222. 1930.

(10) Taytor. Bur. Stand. Journ. Res. 5: 609. 1930.

(11) A. Tuve, Hafstad, and Heydenburg. Phys. Rev. 50: 504. 1936.

B. Hafstad, Heydenburg, and Tuve. Phys, Rev. 53: 230. 1938.

C. Heydenburg, Hafstad, and Tuve. Phys. Rev. 56: 1078. 1939.

(12) A. Straber. Bur. Stand. Sci. Pap. 20: 489. 1925.

B. D Avis. Journ. Inst. Electr. Eng. 69: 1028, 1931 .

C. de la Gorce. Rev. Gen. d'Electr. 29: 427. 1931 .

(13) Weller. Trans. Amer. Inst. Electr. Eng. 48: 790. 1929.

(14) A. Elsner. Arch. für Elektr. 36: 329. 1942.

B. HöHL. Arch. für Elektr. 35: 663. 1941.

(15) Palm. Zeitschr. für techn. Physs. 14: 390. 1933. 
(16) Bousman and Ten Broeck. Trans. Amer. Inst. Electr. Eng. 62: 541. 1943.

(17) Churcher and Dannatt. Journ. Inst. Electr. Eng. 69: 1019. 1931.

(18) Beldaschi. Trans. Amer. Inst. Electr. Eng. 52: 544. 1933.

(19) Referred to in Bur. Stand. Journ. Res. 1: 610. 1928.

(20) Silsbee And Defandorf. Bur. Stand. Journ. Res. 20: 317. 1938.

(21) Pfiffnkr. Elektrotechn. Zeitschr. 47: 44. 1926.

(22) A. Kénath. Siemens Zeitschr. 8: 629 1928.

B. Wrrz. Arch. für Elektr. 21: 563. 1929.

C. Camilli. Gen. Electr. Rev. 39: 95. 1936.

(23) Iиноғ. Schweiz. Electr. Ver. Bull. 19: 741. 1928.

(24) KÜCHLER. Elektrotechn. Zeitschr. 58: 203. 1937.

(25) A. Wellings and Mortuoch. Journ. Inst. Electr. Eng. 79: 577. 1936.

B. Pronst. F.lektrotechn. Zeitschr. 28: 750. 1924.

(26) Hendricks, Hubbard, and Vallin. Gen. Electr. Rev. 42: 420. 1939.

(27) A. Cahall. U. S. Patent No. 1979096. 1934.

B. Hendricks. Gen. Electr. Rev. 46: 477. 1943.

(28) Kirkpatrick. Electr. Eng. 51: 863. 1932.

(29) Workman and Holzer. Rev. Sci. Instr. 10: 160.1939.

(30) Brooks, Defandorf, and Silisbee. Bur. Stand. Journ. Res. 20:253. 1938.

(31) Herb, Parkinson, and Kerst. Rev. Sei. Instr. 6: 261. 1935. Phys. Rev. 51: 75.1937.

(32) Kirkpatrick. Rev. Sci. Instr. 3: 1, 430. $1932 ; 5: 33.1934$.

(33) ASA American Standards for Measurement of Test Voltage in Dielectric Tests, C68.1-1942.

(34) Threlanck. Amer. Inst. Electr. Eng. Techn. Pap. 41-4.

(35) Lowr. Fundamental processes of electrical discharge in gases. New York, 1939.

(36) Mrek. Journ. Franklin Inst. 230: 229. 1940.

(37) Mék. Journ. Inst. Electr. Eng. 93 (II-32): 97.1946.

(38) VerPlaxck. Trans. Aner. Inst. Electr. Eng. 57: 45. 1938.
(39) Bellaschi and Teague. Electr. Journ. 32: 56.1935

(40) Thorntox, Waters, and Thompsos. Journ. Inst. Electr. Eng. 69: 533. 1931.

(41) Abraham and Villard. Journ. de Phịs. $1911: 525$.

(42) Schumaxy and Kewm. Arch, für Eletetr. $12: 553 . \quad 1923$.

(43) STARKe and Schroeder. Arch. fiir Elektr. 20: 115. 192s.

(44) IмноF. Arch. für Elektr. 23:25я. 1929.

(45) Th. Wulf. Phys. Zeitschr. 31: 315. 1930.

(46) Huterer. Elektrotechn. Zeitschr. 55: 833. $1934 ; 56: 1319$. 1935.

(47) Sorensen, Hobson, and Ramo. Electr. Eng. 54: 651. 1935.

(48) Sorensen and RAmo. Electr. Fin.. 55: 444. 1936.

(49) Rogowski. Arch. für Elektr. 25: 52l. 1931.

(50) Rogowskr and Böcker. Arch. für Elektr. 32: 44. 1938. Elektrotechn. Zeitschr. 59: 123. 1938.

(51) Böcker. Arch. für Elektr. 33: 801. 1939. Elektrotechn. Zeitschr. 61: 729. 1940.

(52) Kulvin. Papers on electrostatics and electromagnetism, ed. 2 , sect. $360: 287$. London, 1884.

(53) A. Thornton and Thompson. Journ. Inst. Electr. Eng. 71: 1. 1932.

B. Bruce. Journ. Inst. Electr. Eng. 94 (2): 129. 1947. (Published subsequent to delivery of this paper.)

(54) Snow. Bur. Stand. Journ. Res. 1: 513. 1928.

(55) NACKEN. Arch. für Elektr. 33: 60. $1939 ; 36: 678.1942$.

(56) Tschernyscheff. Phys. Zeitschr. 11: 445. 1910.

(57) Palm. Zeitschr. für techn. Physik 1: 137. 1920

(58) A. Charlton and Cooper. Gen. Electr. Rev. 40: 439. 1937.

B. Trump, Safford, and Cloud. Trans. Aner. Inst. Electr. Eng. 60: 132. 1941.

C. Schumb. Phys. Rev. 69: 692. 1946.

(59) Hanson and Benedict. Pliys. Rev. 65 : 33. 1944

(60) Duane and Hunt. Phys. Rev. 6: 166. 1915.

(61) Winkelibrandt. Arch. für Elektr. 31: 672. 1937. 


\section{Measurement of Voltage Ratio at Audio Frequencies}

\author{
WILBUR C. SZE \\ MEMBER AIEE
}

$\mathbf{H}^{2}$ IGH orders of accuracy in voltageratio measurements are becoming increasingly important in computer fields. Accuracies of $0.01 \%$ or better are desired. Equipment such as precision ratio transformers are used in these applications. However, methods have been lacking for verifying the ratios of this equipment to that accuracy at the desired frequencies of 100 to $10,000 \mathrm{cps}$ (cycles per second).

The purposes of this paper are: 1. to describe the use of a resistance-capacitance voltage divider in the measurement of voltage ratio and phase angle at audio frequencies, 2. to describe the special equipment used for the construction of the circuit, 3 . to analyze various factors affecting the accuracy with which precision measurements can be made, and 4 . to show the test results obtained from 100 to 10 ,$000 \mathrm{cps}$ for a ratio transformer.

\section{Description of Method}

The measuring circuit, shown in Fig. 1 (A), consists of a symmetrically arranged resistance-capacitance network $\left(R_{1} C_{1} R_{2}\right.$ and $C_{2}$ ) and the guard circuit consisting of a similarly arranged network $\left(R_{g l} C_{b 1} R_{g 2}\right.$ and $C_{q^{2}}$ ). The measuring circuit is balanced by adjusting either $R_{1} C_{1}$ or $R_{2} C_{2}$ uritil a null is indicated on the detector $D$. Similarly the guard circuit is balanced by means of adjusting $R_{g 1} C_{g 1}$ or $R_{g 2} C_{g 2}$. These procedures of balancing are continued successively until the detector $D$ indicates a null in both the test and the guard circuit.

In addition to these two circuits there are two auxiliary circuits: 1. the Schering bridge, Fig. 1(B), used for the precise measurement of the phase angles and 2 . the Wheatstone bridge, Fig. 1(C), used for the precise determination of the voltage ratios.

The transfer from one circuit to another is accomplished by the arrangement of switches shown in Fig. 2, described later.

The guard circuit, for both the transformer test circuit and the Schering bridge, is arranged to eliminate the effect of ground admittances by completely shielding the detector, detector circuit, and associated apparatus.

\section{Theoretical Relations}

The equations for the ratio-correction factor and phase angle were developed as follows: By definition, the voltage ratio of a transformer or divider is the ratio of the rms primary or input terminal voltage $\left|V_{p}\right|$ to the rms secondary or output terminal voltage $\left|V_{s}\right|$ so that

$$
\frac{\left|V_{p}\right|}{\left|V_{s}\right|}=N F
$$

where $N$ is the nominal ratio and $F$ is the ratio-correction factor derived from the measured quantities. The phase angle $\gamma$ is the difference in phase between the primary and the secondary voltage of the transformer or divider.

The circuit diagram for the derivation of the following equations is shown in Fig. 1(A).

$$
\begin{aligned}
& Z_{1}=\frac{R_{1}}{1+j \omega C_{1} R_{1}} \\
& Z_{2}=\frac{R_{2}}{1+j \omega C_{2} R_{2}}
\end{aligned}
$$

When detector $D$ indicates a null, then $I_{s}=0, \quad V_{p}=I_{p}\left(Z_{1}+Z_{2}\right)$, and $V_{s}=I_{p} Z_{1}$. Let

$$
\begin{aligned}
& V_{p}=\left|V_{p}\right|(\cos \gamma-j \sin \gamma) \\
& V_{s}=\left|V_{s}\right|=\left|V_{p}\right| / N F
\end{aligned}
$$

Then

$$
\begin{aligned}
N F(\cos \gamma-j \sin \gamma)=\frac{V_{p}}{V_{s}}=\frac{Z_{1}+Z_{2}}{Z_{1}} \\
=\frac{R_{1}+R_{2}+j \omega R_{1} R_{2}\left(C_{1}+C_{2}\right)}{R_{1}\left(1+j \omega R_{2} C_{2}\right)}
\end{aligned}
$$

From equation 1 equate the real terms to get

$F=\frac{1}{N}\left(\frac{R_{2}}{R_{1}}+1\right)\left(\frac{1}{\cos \gamma+\omega R_{2} C_{2} \sin \gamma}\right)$

Since the a-c resistors $R_{1}$ and $R_{2}$ are not stable to the degree of accuracy desired, the ratio of the divider $R_{2} / R_{1}$ is deter-

Paper \$7-648, recommended by the AIEE Special Instruments and Auxiliary Apparatus Committee and approved by the AIEE Technical Operations Department for presentation at the AIEE Summer General Meeting, Montreal, Que., Canada, June 24-28, 1957. Manuscript submitted March 22 1957; made available for printing April 17, 1957.

WrLBUR C. SzB is with the National Bureau of Standards, Washington, D. C.

The author wishes to acknowledge the valuable help and advice received from F. L. Hermach of the National Bureau of Standards. 


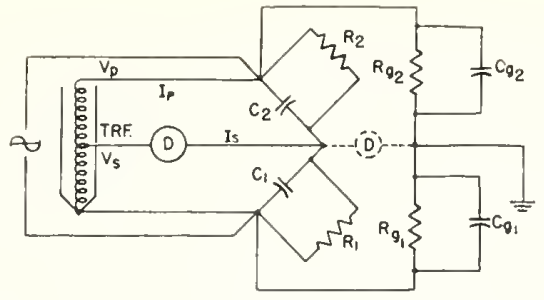

(A)

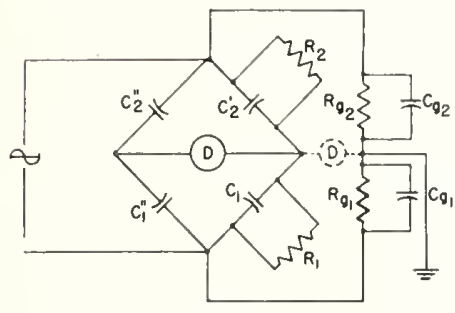

(B)
Fig. 1. Circuil diagrams cuit

B-Schering bridge circuit C-Wheatstone bridge circuit
A-Transformer calibration cir-

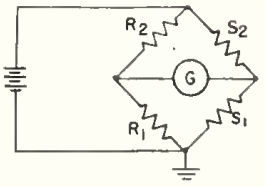

(C) mined immediately after each measurement by using the highly accurate $d-c$ resistance bridge shown in Fig. 1(C). Then

$F=\frac{1}{N}\left(\frac{S_{2}}{S_{1}}+1\right)\left(\frac{1}{\cos \gamma+\omega R_{2} C_{2} \sin \gamma}\right)$

However, in order to obtain the desired accuracy, a-c/d-c corrections for $R_{2}$ and $R_{1}$ must also be added. The previous equation therefore needs to be modified as follows

$$
\begin{array}{r}
F=\frac{1}{N}\left[\frac{S_{2}}{S_{1}}\left(1+\mu_{R^{2}}-\mu_{R^{1}}\right)+1\right] \times \\
\left(\frac{1}{\cos \gamma+\omega R_{2} C_{2} \sin \gamma}\right)
\end{array}
$$

where $\mu_{R 2}$ and $\mu_{R 1}$ are the a-c/d-c corrections for resistors $R_{2}$ and $R_{1}$ respectively. Although these corrections are very small they cannot be neglected. They arise from skin effect, dielectric loss, and the residual reactances which are inevitably associated with all resistors. These effects become increasingly important as the test frequency is raised, because they change the terminal resistance values from their low-frequency or d-c values. Measurements to determine $\mu_{R 1}$ and $\mu_{R 2}$ are described in a later section.

Again from equation 1 equate the imaginary terms to get

$j N F\left(\omega R_{2} C_{2} \cos \gamma-\sin \gamma\right)=j \omega R_{2}\left(C_{1}+C_{2}\right)$

Since $\gamma$ is usually very small, it can be assumed that in this expression

$N F \approx\left(R_{1}+R_{2}\right) / R_{1}, \cos \gamma \approx 1, \sin \gamma=\gamma$

Therefore

$\gamma=\frac{\omega R_{2}}{R_{1}+R_{2}}\left(C_{2} R_{2}-C_{1} R_{1}\right)$ radians

Because it is nearly impossible to measure accurately the stray capacitances of the test circuit which are in parallel with the capacitors $C_{1}$ and $C_{2}$, errors are introduced in the determination of the phase angle $\gamma$ if equation 3 is used. A much more elaborate substitution method was therefore developed to eliminate or minimize the aforementioned sources of error so that they do not affect the desired accuracy of the results. For the connection shown in Fig. 1(A), a null balance is determined. The values of $R_{1}$, $R_{2}$, and $C_{2}$ are then left fixed, and $C_{1}{ }^{\prime \prime}$ and $C_{2}{ }^{\prime \prime}$ are connected in place of the transformer, as shown in Fig. 1(B). This is the well-known Schering bridge circuit with the special requirement that the phase defect angles of capacitors $C_{1}{ }^{\prime \prime}$ and $C_{2}{ }^{\prime \prime}$ must be negligible. These must be very stable capacitors but their values of capacitance need not be known with great accuracy. Three-terminal air capacitors having phase defect angles of less than 2 seconds (and therefore negligible) were constructed as will be described in a later section.

A new null balance of the bridge is obtained by varying $C_{1}{ }^{\prime \prime}$ or $C_{2}{ }^{\prime \prime}$ and by making a slight adjustment of $C_{1}$ to a new value $C_{1}{ }^{\prime}$. Then

\section{$R_{2} / R_{1}=C_{1}{ }^{\prime} / C_{2}=C_{1}{ }^{\circ} / C_{2}{ }^{\prime \prime}$}

In this particular physical arrangement, the values of the capacitors $C_{1}$ and $C_{2}$ and of resistors $R_{1}$ and $R_{2}$ remain essentially fixed with respect to each other except for a very small adjustment in $C_{1}$.

Because the phase angle $\gamma$ will be determined as a slight difference in setting from $C_{1}$ to $C_{1}{ }^{\prime}$, the exact values of $C_{1}$ and $C_{2}$ may remain undetermined as long as these capacitors are stable and do not shift in value. Similarly the stray capacitances of the circuit may remain undetermined as long as they remain fixed and may therefore be considered as included in the settings of $C_{1}$ and $C_{2}$.
Substitute $R_{2}=R_{1}\left(C_{1}^{\prime} / C_{2}\right)$ in equation 3 to obtain

$\gamma=\frac{R_{1} R_{2}}{R_{1}+R_{2}} \omega\left(C_{1}^{\prime}-C_{1}\right)$ radians

By the same token, $C_{2}$ may be adjusted to $C_{2}^{\prime}$ rather than varying $C_{1}$. The following is then obtained

$\gamma=\frac{R_{2}{ }^{2}}{R_{1}+R_{2}} \omega\left(C_{2}-C_{2}{ }^{\prime}\right)$ radians

\section{Shielding and Grounding}

The use of the Wagner ground is an easy and simple way of eliminating the effect of ground admittances. Consider the impedance bridges of Figs. 1(A) and (B), consisting of the usual 4-arm network, but having in addition the auxiliary arms $Z_{g 1}$ and $Z_{g 2}$ with a ground at their junction. The latter forms the auxiliary impedance bridge with the detector circuit shown by dashed lines.

Although the entire detector circuit is at ground potential when both test and guard circuits are balanced and all groundadmittance effects have been eliminated, a potential difference exists between the detector circuit and the leads from the supply. To eliminate inductive pick-up loops, these supply leads must be placed as close as possible to the detector circuit; thus the effect of leakage between them may be considerable. Therefore, the entire detector circuit must be shielded (shields shown by dashed lines in Fig. 2) to eliminate or reduce this effect to such an extent that it does not affect the accuracy of the results.

\section{Wiring Diagram}

Fig. 2 shows the actual wiring diagram of the setup and the arrangement of the switches in the transfer switch box. It is important to note that this transfer switch box is separated into two shielded compartments. The smaller compartment contains the entire detector circuit which, when properly balanced, is at ground potential. The larger compartment contains the a-c/d-c power selector switch and Schering bridge, transformer test selector switches.

Every precaution must be taken to ensure that: 1 . components of the entire circuit be arranged as symmetrically as possible, 2. all leads in the test circuit be kept as short as possible, 3 . the connecting leads, between the switch box and any group of apparatus, be tightly twisted together, 4 . all the shields be electrically connected, and 5 . that there be only a single ground point in the whole circuit, i.e., the Wagner ground. 


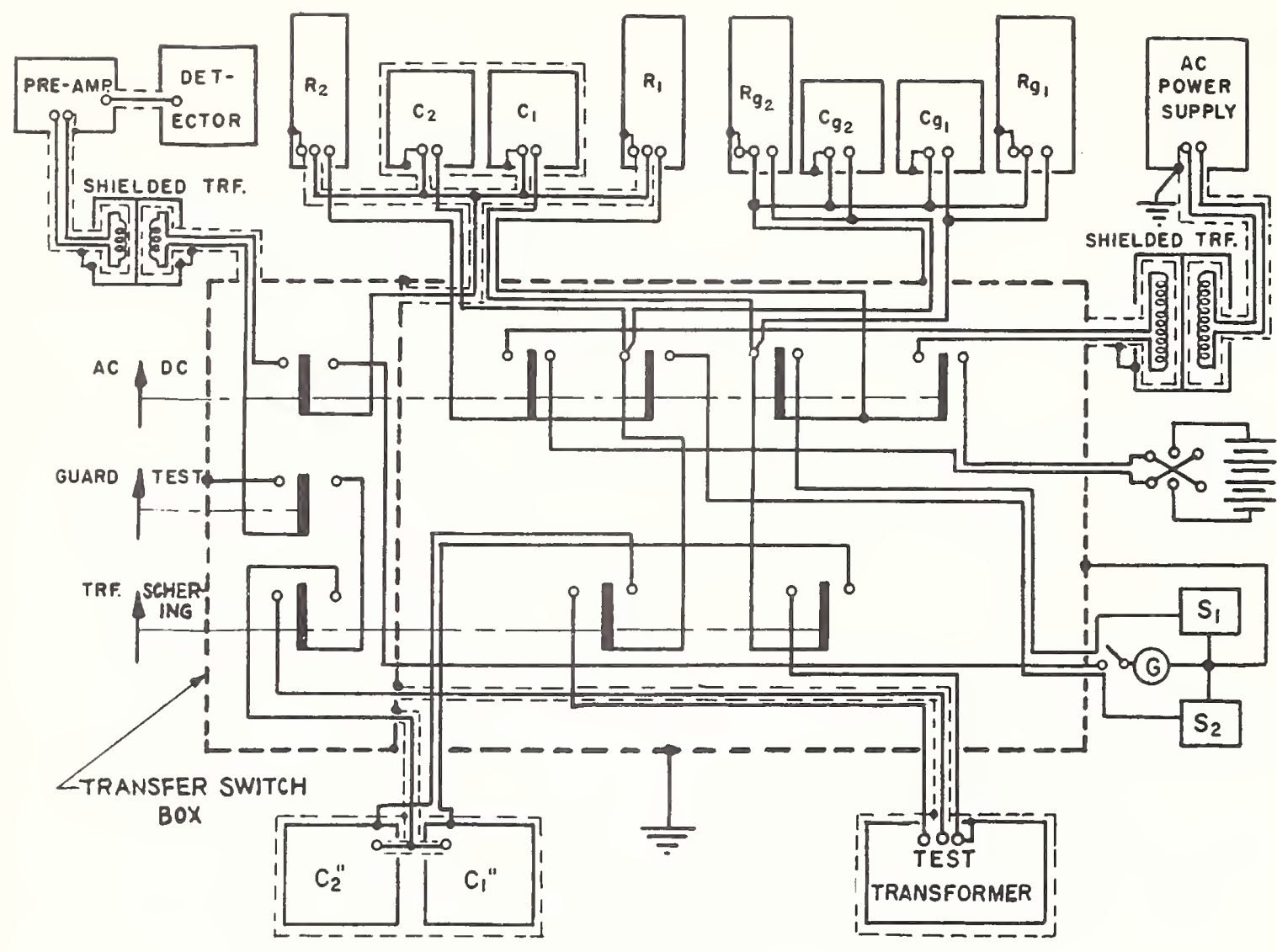

Fig. 2. Wire diagram

\section{Test Procedure}

The procedure in testing a voltage transformer or voltage divider with this circuit arrangement consists of three separate steps.

Step 1. Connect the test transformer in the circuit and set the selector switches in Fig. 2 to marks $A C$, Test, and Trf, essentially giving the circuit shown in Fig. 1(A). Next select the proper values of settings for all the apparatus in the circuit, so that

$$
\begin{aligned}
S_{2} / S_{1} \approx R_{2} / R_{1} \approx R_{g 2} / R_{g 1} & \approx C_{1} / C_{2} \approx \\
C_{1}{ }^{\prime \prime} / C_{2}{ }^{\prime \prime} & \approx C_{g 1} / C_{g 2} \approx N-1
\end{aligned}
$$

Vary either $C_{1}$ and $R_{1}$ or $C_{2}$ and $R_{2}$ to obtain a null indication on the detector. Now set the center switch to Guard and adjust $C_{g 1}$ and $R_{g 1}$ or $C_{g 2}$ and $R_{g 2}$ to obtain a null indication on the detector. This procedure must be repeated until no further adjustment is required for a balance indication of the detector with the switch set to either the Test position or the Guard position.

Step 2. Turn the top selector switch in Fig. 2 to $D C$; this essentially gives the same circuit as shown in Fig. 1(C). Close the galvanometer switch and vary $S_{1}$ or $S_{2}$ for a zero-current indication of the galvanometer $G$, with the battery connections reversed.

Step 3. Reset the selector switches to
$A C$, Test, and Schering. This gives the circuit of Fig. 1(B). Obtain a null indication on the detector by varying $C_{2}$ " and $C_{2}$ or $C_{1}{ }^{\prime \prime}$ and $C_{1}$. Again the guard circuit must be balanced as in step 1 .

Steps 1,2, and 3 should follow one another with as little delay as possible between each step, so that the variation in resistance values of $R_{1}$ and $R_{2}$ due to temperature change is small.

With the values of settings recorded from the procedure, the voltage-ratio correction factor and the phase angle of the test transformer can be computed by substituting the proper values into equations 2 and 4 or 5 , depending upon whether $C_{2}$ or $C_{1}$ was varied.

\section{Description of Apparatus}

The two low-loss variable air capacitors $C_{1}{ }^{\prime \prime}$ and $C_{2}{ }^{\prime \prime}$ are NBS-type, parallel-plate, 100 to 5,000 picofarads (micromicrofarads), modified for use as shielded 3 terminal capacitors. Their general construction is shown in Fig. 3. One set of the plates is mounted on the central shaft and may be rotated by the shaft relative to the fixed set. The central shaft is electrically connected to the case. The other set is stationary and supported on three guarded quartz-rod insulators. The guards are arranged to shield completely the quartz insulation from the active field between the working electrodes. One of these supporting structures is shown in Fig. 4. The dielectric loss angle of these capacitors was measured and found to be about 1 second at 1,000 cps.

$C_{1}$ and $C_{2}$ are two precision variable air capacitors specially developed and designed for this setup, 20 to 120 picofarads and 50 to 1,100 picofarads respectively, used as shielded 3 -terminal capacitors. The dielectric loss angles were measured and found to be approximately 2 seconds and 5 seconds respectively at 1,000 cps. Such small values are relatively unimportant in balances of the circuit as long as the corresponding conductance values are very small in comparison with the resistors $R_{1}$ and $R_{2}$.

Resistors $R_{1}$ and $R_{2}$ are two precision a-c decade resistors, 11,111 ohms total in steps of $0.1 \mathrm{ohm}$. Their resistance elements have very small residual reactances. However, in precise a-c measurements, especially at higher audio frequencies, the effect of these reactances shouid be taken into account. A special bridge described later was therefore set up to evaluate the change of the effective resistance over the range from direct current to $10,000 \mathrm{cps}$.

The null detector is connected to the several bridge arrangements through a preamplifier and an impedance-matching 

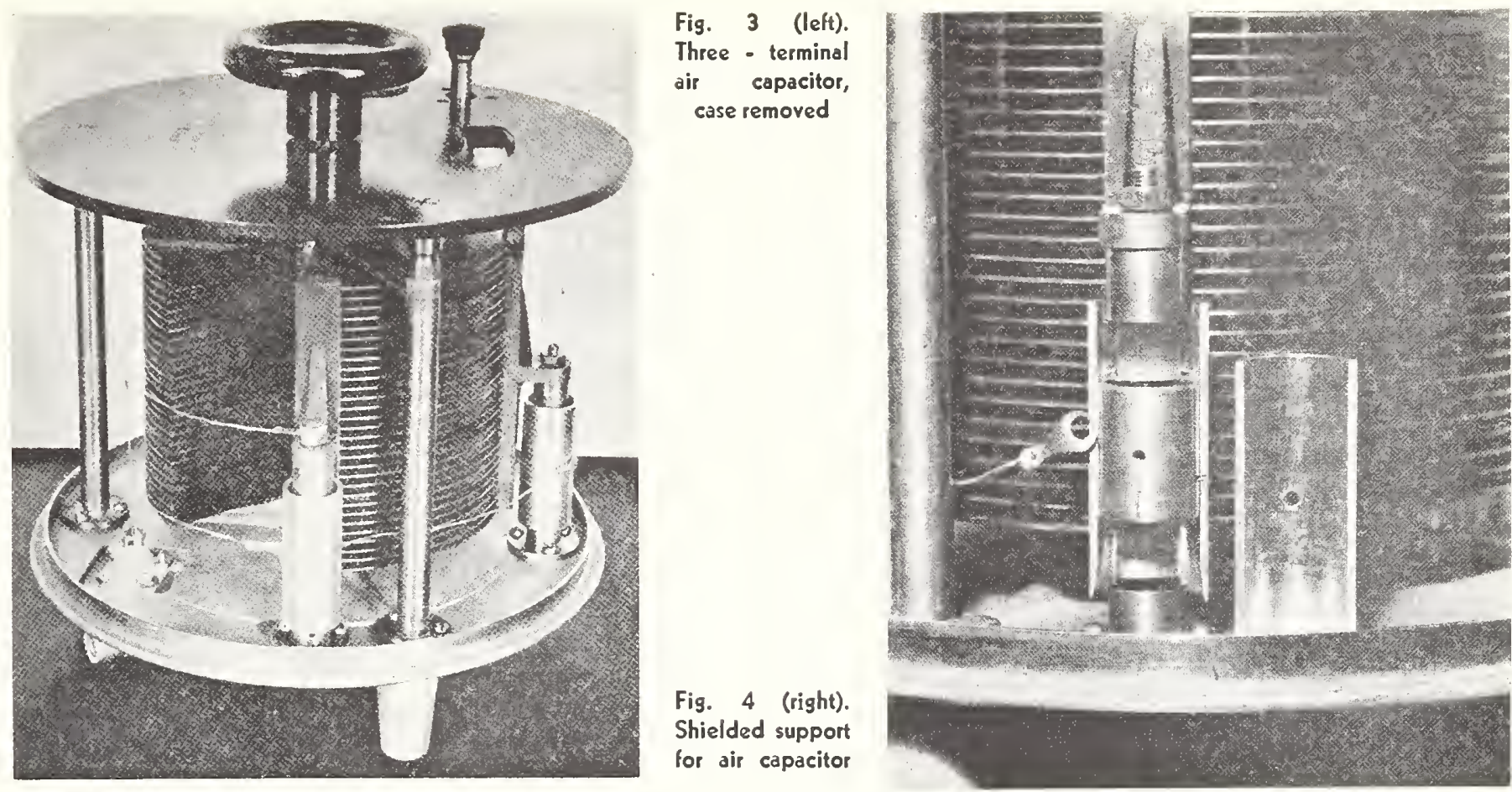

shielded transformer; see Fig. 2. The battery-operated preamplifier is a single pentode amplifier. It is completely shielded by its metal container connected to the shields for the detector leads. The impedance-matching shielded transformer had individually shielded windings.

A commercial wave analyzer was used in balancing the test and guard circuits. The over-all resolution of the complete detecting system is better than 1 microvolt.

\section{D-C Wheatstone Bridge}

Only those special features of the $d-c$ Wheatstone bridge that are essential to a high order of accuracy of measurement are described

Precision standard resistors were used for the $S_{1}$ and $S_{2}$ arms of the Wheatstone bridge. The resistance elements are hermetically sealed in oil to eliminate effects from the humidity variations in the laboratory.

The arrangement of standard resistors in the ratio arms $S_{2} / S_{1}$ are determined by the ratio $R_{2} / R_{1}$. These standard resistors are connected in series-parallel arrangements so that

${ }_{2} / S_{1} \approx N-1$

An adjustable resistance box which is not hermetically sealed is connected in one arm, preferably in the arm with large resistance values, for the fine adjustments of the d-c bridge. The lowest dial increment on the resistance box must give sufficient adjustment for the accuracy of the results desired. Corrections for exact value, temperature coefficient, and lead resistances must be applied to all the resistors. Speical precautions must be taken to ensure that the leakage currents across the ratio arms are insignificant. With this procedure, the uncertainty of ratio $S_{2} / S_{1}$ should not exceed $20 \mathrm{ppm}$ (parts per million) or $0.002 \%$.

Changes in value of these standard resistors, since their initial measurements, have been trivial. As a guard against any significant unpredicted changes in resistance, a schedule has been established whereby these resistors and the resistance box can be intercompared to $10 \mathrm{ppm}$ after about 2 hours of systematic work.

\section{A-C Tests of Resistors}

Since the voltage ratio is derived from measurements with $\mathrm{d}-\mathrm{c}$ resistance standards, it is necessary to know the change of effective resistance of the a-c resistors with frequency. In this connection Berberich developed an excellent substitution method for determining the phase angle of a resistor. ${ }^{1}$ By means of a bridge circuit he compares a resistor with a parallel-wire reference standard of computable reactance which has approximately the same resistance value as that of the resistor. The substitution method he developed makes it simple to minimize the effect of parasitic influences in the circuit, does not require special apparatus, is easy to use, and gives highly accurate results. It is readily adaptable for measuring the effective resistance of a resistor with change in frequency at the same time the phase angle is measured, without altering the test setup.

Fig. 5 shows a diagram of the circuit used for measuring the phase angle and effective resistance of the resistors $R_{1}$ and $R_{2}$. Capacitors $C_{W 2}$ and $C_{W 1}$ and a selector switch were added to the Wagner arms in order to provide for balancing the circuits at frequencies up to $10 \mathrm{kc}$. In this figure $R_{s}{ }^{\prime}$ and $L_{s}{ }^{\prime}$ represent the resistance and equivalent inductance of the parallel-wire standard. $R_{x}{ }^{\prime}$ and $L_{x}{ }^{\prime}$ represent the resistance and equivalent inductance of the resistance boxes whose phase angles are to be measured.

To determine the a-c/d-c difference of the resistance boxes the ohmic value of the unknown resistance box is set equal to that of the parallel-wire standard at a low base frequency, $400 \mathrm{cps}$ or less, then without changing the original settings of the box, the frequency is increased in successive steps and the bridge circuit is rebalanced with arm $C$ for both the standard and the box at each step.

The proportional change of the effective resistance of the computable parallel-wire standard $\alpha$ is computed from

$\alpha=\left(2 \omega^{2} L C / 3\right)-\left(2 \omega^{2} C^{2} R^{2} / 15\right)$

The change $\beta$ of the effective resistances of the resistance box due to frequency is computed from the difference of the resistance settings of $R_{x}$ and $R_{s}$ dials at sucessive increasing frequency, i.e.,

$\beta=\left(R_{x}-R_{8}\right) / R_{3}$

However, the effective resistance and 


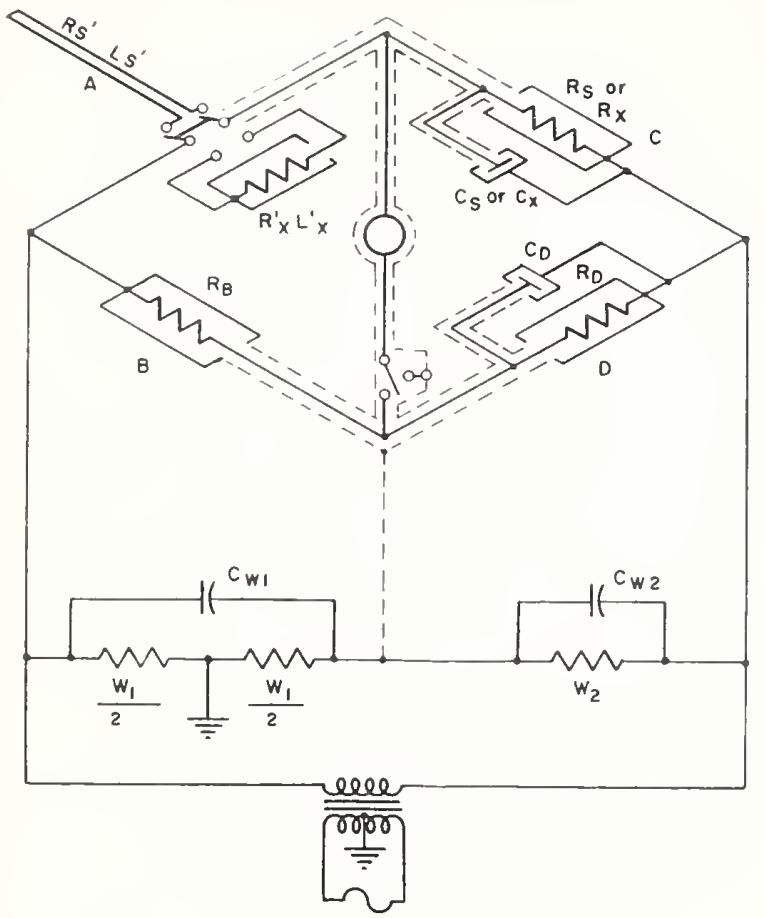

Fig. 6 (right). Frequency correction for $G R$. type 1432-N decade resistor Fig. 5 (left).
Modified Ber-
berich's bridge

effective reactance of the box, $R^{\prime}+j x^{\prime}$, as determined by Berberich's method, are based on equivalent series circuit. When the box is actually connected and used in the ratio-measuring circuits (Figs. 1(A) and (B)), the effective parailel resistance and reactance enter into the formulas. Therefore, to the foregoing correction $\beta$ must be added the term

$\lambda^{2}=\omega^{2} C_{\mathrm{par}}^{2} R_{\mathrm{par}}^{2}$

where $C_{\text {par }}$ is the measured equivalent parallel capacitance and $R_{\mathrm{par}}$ is the equivalent parallel resistance; see the Appendix.

The effective resistance of the resistance box at any frequency may be expressed in the following equation

$R_{x}^{\prime \prime}=R_{x 1}{ }^{\prime}\left(1+\alpha+\beta+\lambda^{2}\right)=R_{x 1}{ }^{\prime}(1+\mu)$

The correction $\mu$ is expressed in proportional parts. $R_{x 1}{ }^{\prime}$ is the resistance value of the resistance box at base frequency. $\omega$ is shown as $\mu_{R 1}$ for corrections $R_{1}$ and $\mu_{R 1}$ for correction to $R_{2}$ in equation 2 , corresponding to measured values that were read from Fig. 6.

\section{Results of Tests}

Test results of one ratio transformer at an average ambient temperature of 26 degrees centigrade and with 50 volts rms applied to the primary are given in Table I. The transformer has input-to-output ratio settings covering the range from 0.000000 to 1.000000 , adjustable by means of 5 -decade switches and one rheostat.

\section{Conclusions}

A setup has been developed for measuring the ratio and phase angle of precision ratio transformers or alternating voltage dividers at audio frequencies. An overall accuracy of within about $0.005 \%$ in ratio and 0.2 minute in phase angle at frequencies from 100 to $10,000 \mathrm{cps}$ is achieved. To obtain this accuracy in ratio, corrections must be applied for the $a-c$ and d-c difference of the decade resistance boxes and for the differences between true and nominal values of ohmic resistance of the standard resistors. The accuracy of phase-angle measurements relies on the constancy of the small loss angle of the precision air capacitors, which should not change appreciably except at high relative humidities and because of exceptional surface or air contamination. Ordinary care is sufficient to avoid bothersome changes.

A simple circuit is arranged symmetrically with the detector always at earth potential. Identical apparatus is provided on either side the detector circuit so that it can be interchanged electrically. In order to ensure that the circuit is electrically as well as physically symmetrical, the test transformer or divider

Table 1. Results of Test

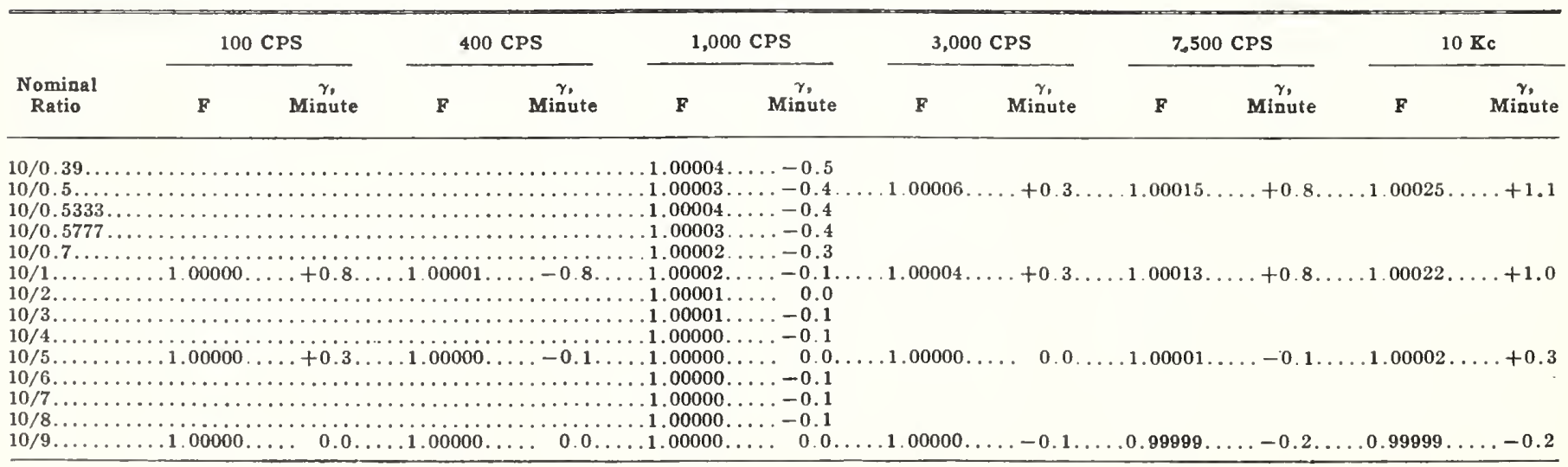


can also be inverted in relation to the rest of the circuit.

The equipment has been used in checking transformers and a-c dividers for voltage ratio up to 1,000 to 1 . The flexibility of the setup is considerable. Fractional ratios may be measured as readily as integral ratios. Test results for high and low ratios are obtained with a slight reduction in the accuracy $( \pm 0.005 \%)$ attainable for ratios between 1 to 1 and 100 to 1 .

\section{Appendix. Derivation of Equations for Correction $\mu$}

Correction $\mu$ is the sum of $\alpha, \beta$, and $\lambda^{2}$. $\alpha$ is the correction due to the change of effective resistance of the parallel-wire standard with change in test frequency. As derived by Harris, it is, to the desired approximation, ${ }^{2}$

$\alpha=\frac{2 \omega^{2} L C}{3}-\frac{2 \omega^{2} C^{2} R^{2}}{15}$

$\beta$ is the correction due to the change of effective resistance of the resistance box by the change in test frequency. Consider Fig. 5, with the standard connected in arm $A$ of the bridge, then at any frequency

$R_{s}^{\prime}=R_{s}\left(\frac{R_{B}}{R_{D}}\right)$

With the same frequency, substitute a re-
$R_{x}^{\prime}=R_{x}\left(\frac{R_{B}}{R_{D}}\right)=\frac{R_{x} R_{s}{ }^{\prime}}{R_{\delta}}$

since $R_{x}-R_{s}<<1$. Then

$R_{x}^{\prime}-R_{x 1}^{\prime}=\frac{R_{x} R_{s}^{\prime}}{R_{s}}-\frac{R_{x 1} R_{s 1}{ }^{\prime}}{R_{s 1}}$

where subscript 1 indicates the base frequency. Since $R_{x 1}=R_{s 1}$ was set, and $R_{s}{ }^{\prime}=$ $R_{s 1}{ }^{\prime}(1+\alpha)$, then

$R_{x}^{\prime}-R_{x 1}^{\prime}=R_{s 1}{ }^{\prime}\left(1+\frac{R_{x}-R_{s}}{R_{s}}\right)(1+\alpha)-R_{s 1}{ }^{\prime}$

Let

$\beta=\frac{R_{x}-R_{s}}{R_{s}}$

Then

$R_{x}{ }^{\prime} \approx R_{x 1}{ }^{\prime}+R_{s 1}{ }^{\prime}(\alpha+\beta)$

$R_{x}^{\prime} \approx R_{x 1}{ }^{\prime}\left[1+\frac{R_{31}{ }^{\prime}}{R_{x 1}{ }^{\prime}}(\alpha+\beta)\right]$

$R_{x}^{\prime} \approx R_{x 1}^{\prime}(1+\alpha+\beta)$

since $R_{s 1}{ }^{\prime} / R_{x 1}{ }^{\prime} \approx 1 . \quad R_{x}{ }^{\prime}$ is ohmic resistance expressed as in the equivalent series circuit.

The term $\lambda^{2}$ is the changeover factor from the equivalent series to equivalent parallel connections:

$R_{\mathrm{ser}}+j X_{\mathrm{ser}}=\frac{R_{\mathrm{pax}}+j \frac{R_{\mathrm{par}}{ }^{2}}{X_{\mathrm{par}}}}{1+\frac{R_{\mathrm{par}}{ }^{2}}{X_{\mathrm{par}}{ }^{2}}}$
$R_{\mathrm{par}} / X_{\mathrm{par}}=\lambda \ll 1$

Then

$R_{\mathrm{ser}}+j X_{\mathrm{ser}}=\frac{R_{\mathrm{parr}}(1+j \lambda)}{1+\lambda^{2}}$

from which $R_{\mathrm{par}}=R_{\text {ser }}\left(1+\lambda^{2}\right)$ and $\lambda=X_{\text {ser }} /$ $R_{\mathrm{ser}}$. Therefore, the effective resistance for equivalent parallel circuit at any frequency is

$R_{x}^{\prime \prime}=R_{x}{ }^{\prime}\left(1+\lambda^{2}\right)=R_{x 1}{ }^{\prime}(1+\alpha+\beta)\left(1+\lambda^{2}\right)$

$R_{x}^{\prime \prime} \approx R_{x 1}{ }^{\prime}\left(1+\alpha+\beta+\lambda^{2}\right)=R_{x 1}{ }^{\prime}(1+\mu)$

$R_{x 1}{ }^{\prime}$ is the resistance value at base frequency.

\section{References}

1. The Measurements of the Phase Angles of Shinlded Resistors, L. J. Berberich. Physics, New York, N. Y., vol. 3, Dec. 1932, pp. 296-313.

2. Electrical Measurements (book), F. K. Harris. John Wiley \& Sons, Inc., New York, N. Y., 1952, pp. 217-20.

3. Nature of Enhrgy Losses in Air Capacitors At Low Frequencies, A. V. Astin. Journal of Research, National Bureau of Standards, Washington, D. C., vol. 22, 1939, pp. 673-95.

4. Design, Construction and Use of Resistors of Calculable Reactance, N. F. Astbury. Journal, Institution of Electrical Engineers, .London, England, vol. 76, 1935, p. 389.

5. A.C. Bridge Methods (book), B. Hague. Isaac Pitman and Sons, Ltd., London, England, 1946.

6. Instrument Transformer (book), B. Hague. Isaac Pitman and Sons, Ltd, 1936. 
Reprinted from IRE TRANSACTIONS

ON INSTRUMENTATION

Volume I-7, Numbers 3 \& 4, December, 1958

PRINTED IN THE U.S.A.

\title{
New Apparatus at the National Bureau of Standards for Absolute Capacitance Measurement*
}

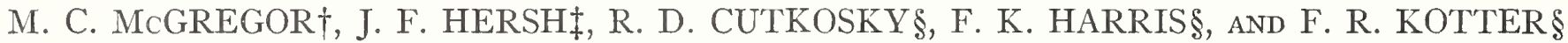

\section{INTRODUCTION}

T THE use of tightly coupled inductive ratio arms rather than resistive ratio arms in a 4 -arm bridge for the comparison of impedances was suggested by Blumlein ${ }^{1}$ in 1928, and the use of a 3 -winding transformer in such a bridge circuit was described by Starr $^{2}$ in 1932. Other bridges using Blumlein's principle have been described by several workers ${ }^{3}$ in the past 30 years. Historically, it is of interest to note that conjugate bridges making use of 3-winding transformers were described by Elsas 4 in 1888 for resistance comparison and by Trowbridge ${ }^{5}$ in 1905 for capacitance and inductance comparisons.

Thus the basic principle of operation and the general arrangement of transformer bridges have been known for many years. However, the possibilities of such bridges for the precise comparison of very low value capacitors had never been fully exploited before the

* Manuscript received by the PGl, August 14, 1958.

$\uparrow$ National Standards Lab., Chippendale, N.S.W., Australia.

F General Radio Co., Concord, Mass.

$\$$ National Bureau of Standards, Washington, D. C.

1 British Patent No. 323037.

2 A. T. Starr, "A note on impedance measurement," W. Eng. and Exp. W., vol. 9, pp. 615-617; November, 1932.

${ }^{3}$ C. H. Young, "Measuring inter-electrode capacitances," Bell Labs. Rec., vol. 24, pp. 433-438; December, 1946.

H. A. M. Clark and P. B. Vanderly"n, "A. C. bridges with inductively coupled ratio arms," Proc. IEE, vol. 96, pp. 365-378; May, 1949.

C. W. Oatley and J. G. Yates, "Bridges with coupled inductive ratio arms for the comparison of standards of resistance or capacitance," Proc. IEE, vol. 101,pp. 91-100; March, 1954.

A. M. Thompson, "A bridge for the measurement of permittivity," Proc. IEE, vol. 103, pt. B, pp. 704-707; November, 1956.

${ }^{4}$ A. Elsas, "Ueber Widerstandsmessungen mit dem Differentialinductor," Ann. Phys., vol. 35, pp. 828-833; 1888 .

5 A. Trowbridge, "On the differential transformer," Phys. Rev. vol. 20 , pp. $65-76 ; 1905$. work of Thompson and his group at the National Standards Laboratory of Australia. By combining the best techniques for constructing ratio transformers, completely shielded 3-terminal capacitors and detectors of high sensitivity, together with a cylindrical cross capacitor as a calculable standard, there is now promise of being able to assign values to capacitance standards comparable with, or perhaps even better than, the accuracy assigned to our present standards of electromotive force and resistance.

The present paper describes a transformer bridge constructed at the National Bureau of Standards for measuring the direct capacitance of 3-terminal capacitors ranging in values up to $1 \mu \mathrm{f}$ and having a least count of $1 \mu \mathrm{pf}$. Although the transformers and network components described below were designed specifically for operation at $1 \mathrm{kc}$, the operation is by no means limited to this frequency. Voltage output of the ratio transformers constitutes the most serious limitation at lower frequencies, but it is reasonable to suppose that, with relatively minor modifications, satisfactory operation should be possible over the audio-frequency range to at least $10 \mathrm{kc}$.

While some of the present bridge components differ substantially from their counterparts at NSL, it should be understood that no more is involved generally than modifications and in some cases improvements of designs already proven by Thompson and his group in Sydney.

There has been little detailed information published up to now concerning these components, and the present paper must be considered primarily as a discussion of the constructional details and performance of the NBS transformer-ratio bridge. 


\section{Balance Conditions}

The transformer bridge with closely coupled ratio arms may be considered as an arrangement of two lowimpedance generators supplying EMF's of opposite phase, and of a known ratio, to two completely shielded 3-terminal capacitors as shown schematically in Fig. 1(a). The capacitances $C_{l_{1}}, C_{l_{2}}$ between the enclosing shields and the line terminals $l_{1}, l_{2}$ of capacitors $C_{1}, C_{2}$ will be in shunt across one or the other of the two generators, as shown in Fig. 1(b). The capacitances $C_{d_{1}}, C_{d_{2}}$ between shields and detector terminals $d_{1}, d_{2}$ of $C_{1}, C_{2}$ will be in shunt across the detector. Hence, when the bridge is balanced and there is no current through the detector, the currents through the direct capacitances $C_{1}, C_{2}$ must be equal in magnitude, and the balance relation must be

$$
\frac{V_{1}}{V_{2}}=\frac{C_{2}}{C_{1}} .
$$

If now the impedances $Z_{1}, Z_{2}$ are negligible compared to

$$
\frac{1}{\omega C_{l_{1}}}, \frac{1}{\omega C_{l_{2}}},
$$

then, to a very close approximation,

$$
\frac{e_{1}}{e_{2}}=\frac{C_{2}}{C_{1}} .
$$

\section{Transformer Design and Construction}

By careful design of the transformer, the impedances $Z_{1}, Z_{2}$ of its ratio arms can be made very small, and the ratio of the induced voltages $e_{1} / e_{2}$ can be made stable and precise. In a 3-winding transformer, arranged as in Fig. 2, the sole function of the primary winding $P$ is to provide flux in the common core, linking the secondary windings $S_{1}, S_{2}$. The ratio of the terminal voltages $V_{1} / V_{2}$ of the ratio arms under load is affected by the resistances and leakage reactances of the secondary windings, but the resistance and leakage reactance associated with the primary will in no way affect the bridge balance relation. If the two secondary windings could be so constructed that a common flux were confined entirely within the windings, the ratio of their induced voltages would depend only upon their turns ratio, and this ratio would be stable and exact. If, in addition, the elements of the two windings $S_{1}, S_{2}$ could be brought into exact coincidence, turn by turn, so as to link identical flux at any loading, the series impedances, producing changes in the terminal voltages $V_{1}, V_{2}$ when loads are applied, would be ${ }^{*} \quad$ sistances of the windings.

The loading error of the bridge ratio can be made small by decreasing the size of the resistances of the secondary windings and by reducing as far as possible their effective leakage inductances. It should be observed that the self-capacitance of the secondary windings of the transformer constitutes a load which is always present, so that the terminal voltages of the transformer differ from the induced voltages even on
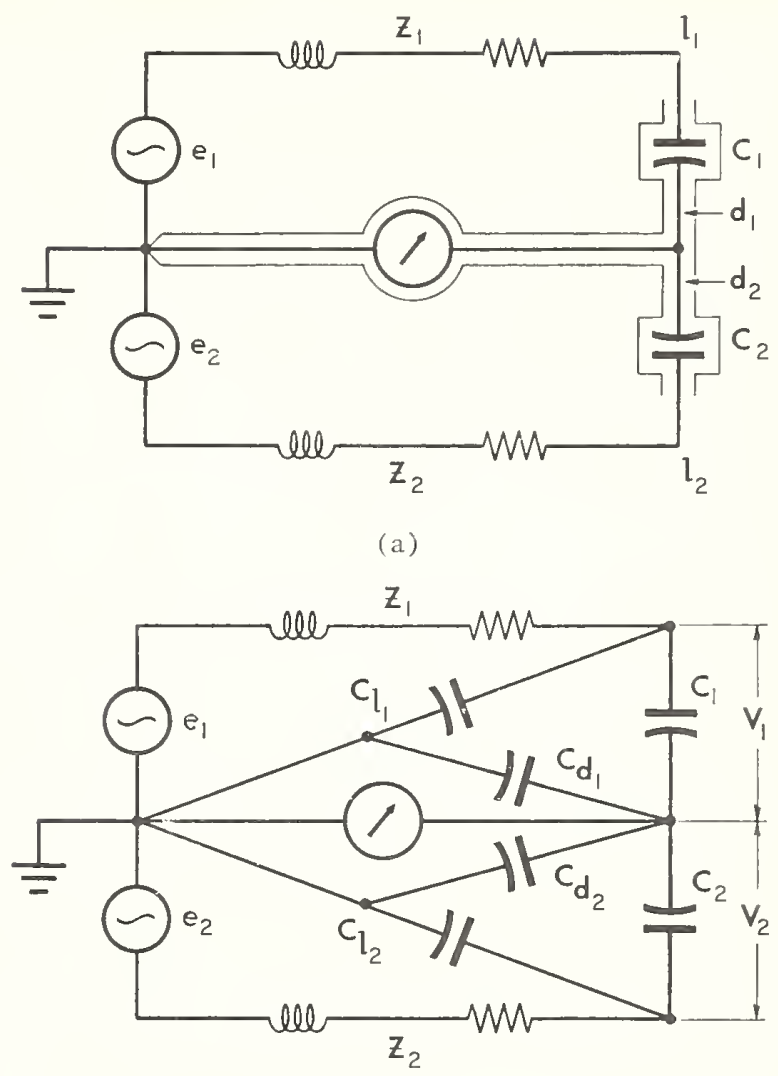

(b)

Fig. 1-Transformer-ratio bridge schematic.

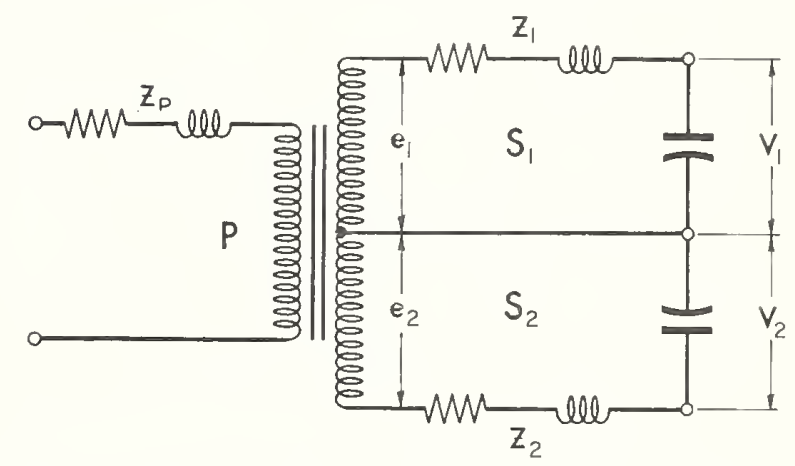

Fig. 2-Equivalent circuit of a loaded 3-winding transformer.

open circuit. The amount of this difference and, even more important, the amount by which the open-circuit ratio differs from the turns ratio will depend on the magnitudes and distribution of these self-capacitances. The transformer design should be such as to keep the self-capacitances of the secondaries as small as possible, and ideally these capacitances should be symmetrically distributed.

In the design and construction of the transformers used for ratio arms of the bridge, the features favorable to good performance have been carefully considered. Very small ratio errors, even for large loads, have been achieved by special construction. A good approximation to unity coupling has been obtained by the use of a large toroidal core of high permeability. Voltages from sources other than the core flux have been excluded by carefully 


\section{McGregor, et al.: New Apparatus at the NBS for Absolute Capacitance Measurement}

shielding the secondary from the primary and from external fields, and the effect of leakage flux on the ratio has been minimized by a uniform distribution of each winding completely around the toroid. The loading error resulting from the resistance of the windings has been kept low by using copper strip rather than wire for the secondaries.

Two bridge transformers of similar design have been built, differing in some details and in the ratios of their secondary voltages. The first of these was a $10 / 1$ ratio transformer and its construction will be considered in detail. Its core is a toroid, wound of continuous 1-mil Supermalloy tape, ${ }^{6}$ and has an OD of 4.5 inches, an ID of 3 inches, and a height of 1.5 inches. This Supermalloy has an initial dc permeability of about $75 \times 10^{3}$, and the thinness of the tape permits operation at frequencies of the order of $1 \mathrm{kc}$ without serious diminution of its permeability. The large cross-sectional area of the core, $1 \frac{1}{8}$ square inches, enables the required secondary voltage to be obtained with few turns and, hence, with low winding resistance. When operated at $1 \mathrm{kc}$ in its region of maximum permeability (flux density about 5 kilogausses) it produces about 1.6 volts per turn. A reasonable bridge voltage is of the order of 100 volts, which is obtained with a secondary winding of 70 turns. This gives good sensitivity without excessive voltage on the capacitors.

The primary winding is of rectangular copper wire 0.036 inch $\times 0.104$ inch so that 73 turns just fill the inner circumference of the toroid. This heavy wire was sellected, not from any concern for low primary resistance, but to insure a uniform and nearly continuous distribution of magnetomotive force along the toroid. Small flexible leads are connected with minimum separation to the ends of the primary winding, and one of the leads is returned around the circumference of the toroid in such a direction as to cancel the field produced by the "single-turn" effect of the toroidal winding.

It has already been pointed out that the ratio of voltages induced in the secondary windings, which is of interest in a bridge transformer, must be as completely independent of the primary as possible. To insure this independence, the secondary is electrostatically shielded from the primary by two copper shields. The inner shield is a toroidal cup of 20 -mil copper fitting snugly over the primary and closed by a copper lid soldered to the cup around the outer circumference: An insulated gap along the inner edge prevents a shorted turn. One of the primary leads is connected to this shield, and the other is brought out through a small copper tube soldered to the lid. This tube and the wire inside constitute a coaxial pair of leads to the primary winding. After insulation with Teflon tape the inner shield is fitted into another toroidal cup which is closed with a similar lid having an insulated gap around its inner cir-

- The authors greatly appreciate the help of J. E. Mitch, chief engineer of the Arnold Eng. Co., Marengo, Ill., in selecting and making available the core material. cumference. Connection to the outer shield is by a tube coaxial with the primary-lead tube but insulated from it. The outer shield is covered with an impregnated glass-fiber insulation which can withstand the heat and mechanical abrasion involved in assembling the secondary windings. The use of two separate shields between primary and secondary makes it possible to separate the grounds so that, even with considerable capacitance current from the primary to the inner shield, the outer shield, next to the secondary, can be.kept at the ground potential of the bridge. In addition the double copper shield provides some magnetic shielding as a result of eddy current action and so assists in confining the primary flux, with the result that all the secondary turns more nearly link all the primary flux than would be the case without this shielding.

The two secondaries which provide bridge voltages of precise and stable ratio are wound around the shielded and electrostatically isolated primary. As noted above, a winding of about 70 turns provides the desired 100 -volt output. For a $1: 1$ ratio the secondary, winding would consist of two sections of 70 turns each. The coupling of these sections to the primary could be made nearly identical by the familiar use of a bifilar winding in which corresponding turns of the two sections are made to occupy, as nearly as possible, the same position relative to the core. Any nonuniformity in flux distribution around the core would then influence both sections alike, and the ratio of secondary voltages would not be affected. For ratios other than unity the winding cannot be truly bifilar, but the turns of the two sections can be distributed to effect a similar sampling of the flux around the core by both. The secondary windings in the $10: 1$ ratio transformer are distributed in the following manner. The secondary is divided into twenty 7 -turn sections. The seven turns of each section are wound as a uniform spiral around the toroid so that each section samples the flux around the entire toroid and ends at a point adjacent to its beginning. The ends of all $20 \mathrm{sec}-$ tions are brought out at the outer circumference in such a manner that 10 sections can be connected in series to form a 70-turn winding, and the other 10 sections in parallel to form a 7 -turn winding. Further, the,connections are such that the turns of the 70 -turn winding are always separated by turns of the 7 -turn winding as they progress around the toroid, thus approximating the uniform flux sampling of a bifilar winding.

The requirement of low error in the secondary ratio, when the transformer is heavily loaded, necessitates a winding resistance of only a few milliohms. To achieve this, the cross section of the copper must be large, so that edge-wound strips rather than wire are used in the secondary to utilize best the limited space inside the toroid. The requirement that 140 turns of minimum resistance separated by 2 -mil mica strips must be fitted in to the toroid leads to dimensions of 0.040 inch $\times 0.255$ inch for the cross section of the strips inside the toroid. It is convenient to use stock of 40-mil thickness for all 
the winding strips, but the width of the other three sides of the turn can be increased to $\frac{1}{2}$ inch to reduce the resistance. The turns are constructed from this copper strip by using a $C$-shaped piece to enclose the top, inside, and bottom of the toroid and curved strips fitting the contour of the outer circumference to space the C's at intervals of $\frac{1}{7}$ of the circumference (see Fig. 3). The strips are connected by soldering with a special clamptype resistance heater having graphite jaws thin enough to fit the small gaps between turns. After assembly the strips inside the toroid are separated by insulating strips of 2-mil mica and on the outer circumference by strips of 6-mil impregnated glass-fiber insulation.

The transformer is enclosed in a Mu-metal can with the ends of all 20 sections of the secondary, and the coaxial primary and shield leads, brought outside the can through slots. The connections of the secondary sections in series and in parallel are made with additional copper strip outside the can, where the stray field of the transformer should be negligible. An outer brass case covers the Mu-metal shieid and connections and is used to mount the $\mathrm{BPO}^{7}$ connectors through which the transformer leads are brought out to the bridge and driving amplifier.

The characteristics of the transformer can be determined in the capacitance bridge by the intercomparison and summation of a group of closely matched capacitors, as described by Thompson. ${ }^{8}$ The ratio error (tor light loads) was measured using 100-pf (picofarad) capacitors. The ratio, defined as $V_{10} / V_{1}=10(1+\alpha+j \beta)$, has a magnitude correction $\alpha=03 \times 10^{-6}$ and a phase correction $\beta=2.7 \times 10^{-6}$. The effective leakage inductance and series resistance of the two secondary windings have been determined by measuring the ratio change when known loads are connected across one section. The equivalent circuit thus determined at the BPO connectors to the transformer is shown in Fig. 4.

A second transformer, having a toroidal core of a somewhat smaller cross section $\left(0.94 \mathrm{in}^{2}\right)$ and with an 80 -turn primary, was built in a similar manner. This transformer has two equal 80-turn secondaries in 8-turn sections around the toroid, which are intermingled as described above. The ratio of this transformer at $1 \mathrm{kc}$ has a magnitude correction at light loading of $0.1 \times 10^{-6}$, and a phase angle of $0.8 \mu \mathrm{r}$. The leakage inductance of each secondary winding is about $1 \mu \mathrm{h}$ and its resistance is $0.030 \mathrm{ohm}$.

\section{Design and Construction of Capacitors}

An unknown capacitor supplied by one secondary of the transformer (see Fig. 1) may be balanced in the bridge by a capacitance of appropriate magnitude supplied by the other secondary. This is accomplished through the use of two 3 -terminal decade capacitance

7 These coaxial connectors are of British manufacture and may be described as British Post Office Pattern 8.

${ }^{8}$ A. M. Thompson, "The precise measurement of small capacitances," this issue, pp. 245-253.

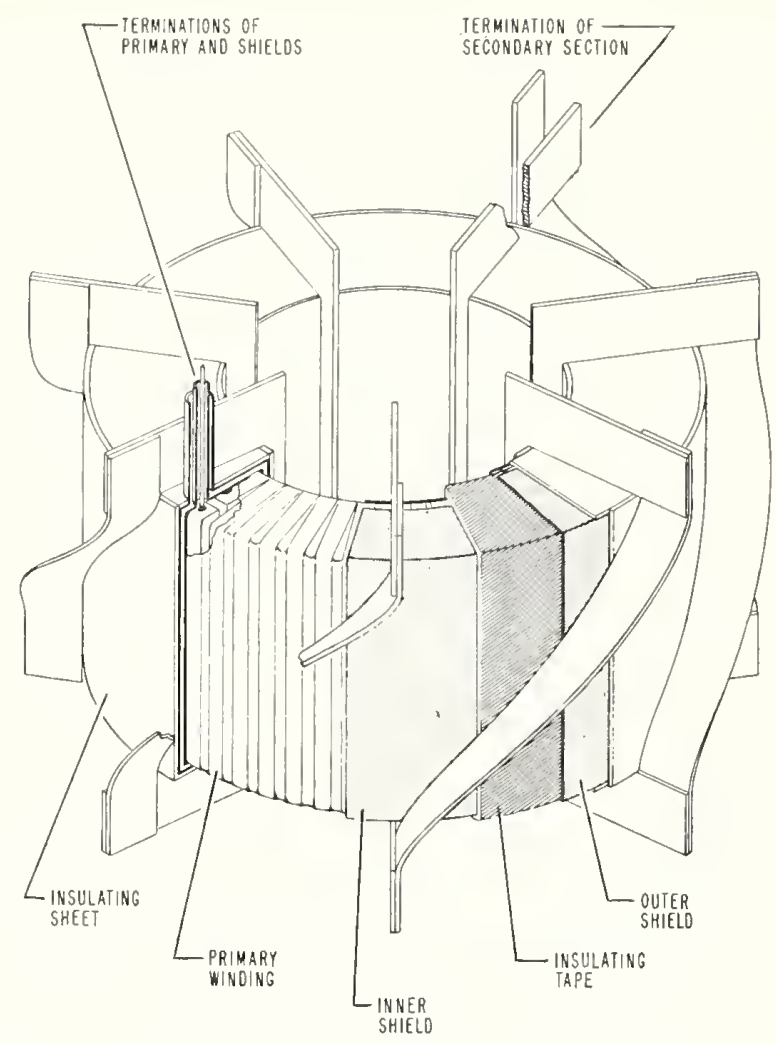

Fig. 3-Transformer construction details.

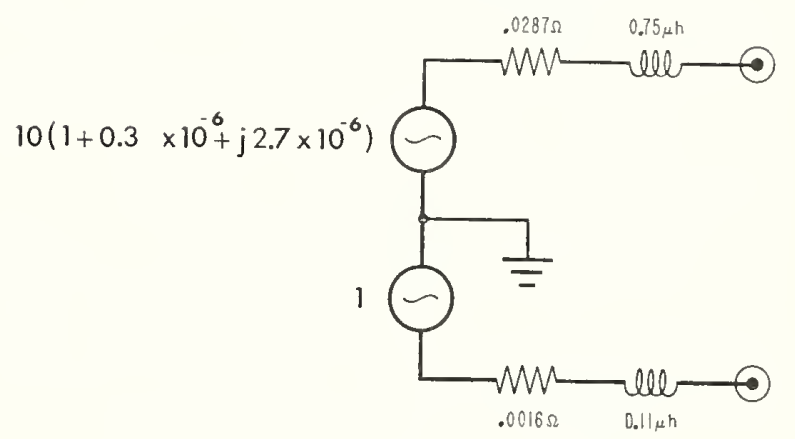

Fig. 4-10/1 transformer characteristics.

boxes covering a range of 12 orders of magnitude from $10^{-6} \mathrm{pf}$ to $1 \mu \mathrm{f}$.

The range from $1 \mathrm{pf}$ to $1 \mu \mathrm{f}$ is covered in a 6 -decade box having a special switching system that allows the selection of any individual capacitor separately for calibration and the addition of the capacitors in parallel to balance an arbitrary unknown. The switching system is such that each capacitor is either connected directly between line and detector, or is grounded at both terminals. The switch decks controlling line and detector connections, respectively, are completely shielded from each other, so that switch and lead capacitances are connected to the shield system rather than to the other active electrode of the capacitor [see Fig. 1(b)]. With this system and with the complete shielding incorporated in the design, the residual direct capacitance with all switches on zero is not observable and is certainly less than $10^{-6}$ pf. Each decade consists of ten equal capacitors, permitting quick intercomparison of 
the various units in the decade. The arrangement is also convenient in measuring the ratios of transformers used in the bridge itself, or in other ratio devices.

The upper three decades of the high-range box consist of precision-quality silvered-mica capacitors, covering the range 0.001 to $1 \mu \mathrm{f}$. The lower three decades, covering the range $1 \mathrm{pf}$ to $10^{3} \mathrm{pf}$, are air capacitors of a cylindrical design of McGregor.

The 10-pf unit is typical of the air capacitors and is shown in cross section in Fig. 5 . The center post $A$ is grounded, and the entire assembly is fastened in a hole in a grounded brass block by means of the flange. The brass block serves both as a shield and as a heat sink to minimize the rate of temperature variation in the capacitor. Cylinder $B$ is connected by means of a wire through a hole in the ground post to the detector junction of the bridge. It is mounted on the ground post by means of two high-density polyethylene washers between the detector electrode $B$ and the ground post. These washers are machined about 0.002 inch oversize, making an interference fit which compresses the washers and results in a rigid and mechanically stable assembly. Electrode $C$ is also supported on polyethylene washers and is connected to a line terminal of the bridge. It should be noted that no solid insulation appears in the fieldbetween the electrodes $B$ and $C$ of the direct capacitance measured. In addition, the electrodes are goldplated to reduce losses arising from oxide films on their surfaces. In the absence of both solid dielectric and surface oxide films, the phase defect of these capacitors should be stable and quite small.

Fine adjustment of the capacitance between electrodes $B$ and $C$ is accomplished by drilling two holes on a diameter of electrode $C$. Field from electrode $B$ passes through these holes and terminates on the outer shield, decreasing the direct capacitance between $B$ and $C$ and slightly increasing the shield capacitance to $B$. A sleeve with matching holes fits over electrode $C$, and rotation of this sleeve with respect to the electrode opens or closes the effective aperture through which electrode $B$ "sees" the outer shield, thus changing the capacitance $B-C$ by a small amount depending on the size of the holes.

The 1-pf unit has essentially the same construction but is shorter. The means of fine adjustment of its value is similar. The 100-pf unit makes use of a group of nested coaxial cylinders of close spacing in order to obtain the larger capacitance within approximately the same physical volume. Here the fine adjustment is accomplished by a sliding skirt, fitted to the shield, which approaches the outer active electrode and intercepts field, that would otherwise contribute to the direct capacitance, at its end. Thus the direct capacitance of each of the air capacitors is smoothly and continuously adjustable over a small range, making it possible to match closely the values of all the units in a decade. Because of the symmetrical design, small departures from radial alignment have very little effect on the ca-

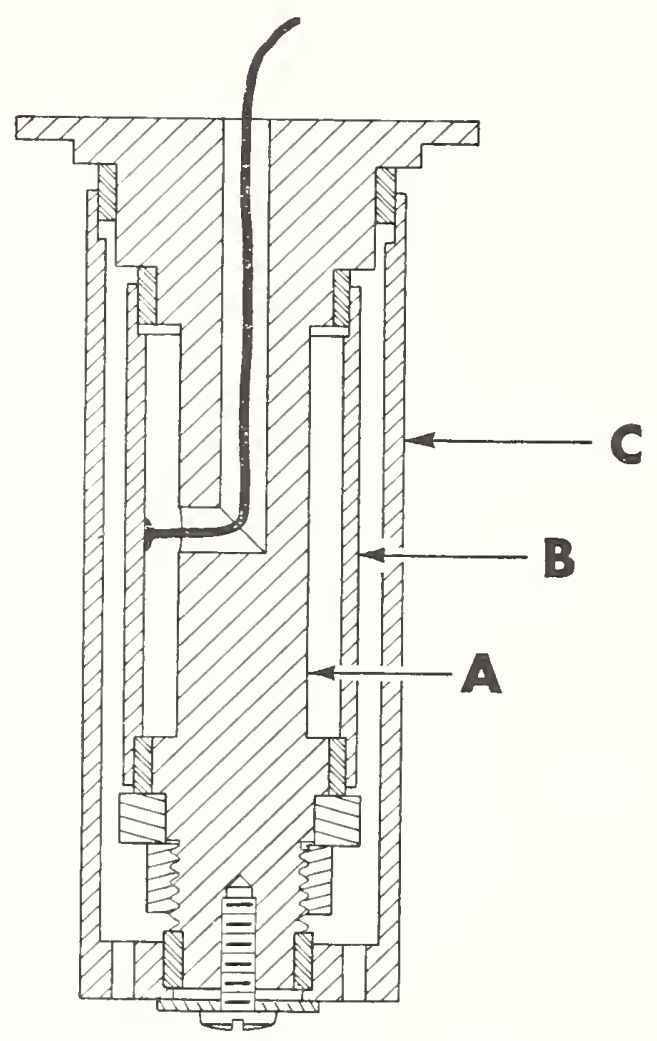

Fig. 5-Section of 10-pf capacitor.

pacitance of the unit, and the capacitors can be taken apart and reassembled without appreciable change in their values.

The low-range decade box $\left(10^{-6} \mathrm{pf}\right.$ to $\left.1 \mathrm{pf}\right)$ differs from the higher decades in that it uses fixed value capacitors connected to a variable voltage. This voltage is obtained from a tapped inductor, which is connected across one side of the bridge transformer to divide the transformer voltage into 10 equal steps. This tapped inductor is constructed by winding 50 turns of 10 -wire tape of 20 AWG wire on a small Supermalloy core, and its impedance is high enough to impose negligible loading on the bridge transformer. If a capacitor of a given value is connected between the first tap of the divider and the detector junction point, the current which it injects into the junction will be only $1 / 10$ of the value it would have been had the capacitor been connected directly across the transformer secondary. On the $n$th tap, its effect on the bridge balance will be $n / 10$ of its actual value. The low-range (or micropicofarad) capacitance box consists of six separate capacitors of values, $10^{-5}, 10^{-4}, 10^{-3}, 10^{-2}, 10^{-1}$, and $1 \mathrm{pf}$, switched independently along the tapped inductor. The entire range of 6 decades, from $10^{-6}$ pf to 1 pf, thus consists of a single tapped inductor, 6 switches, and 6 capacitors. The arrangement with its shielding is shown in Fig. 6.

The six capacitors have cylindrical electrodes spaced with polyethylene washers. The $1-p f$ unit is like the 1 -pf capacitors in the high-range box. All the lower valued capacitors are of the Zichner diaphragm type, having a grounded cylindrical shield extending between the two 


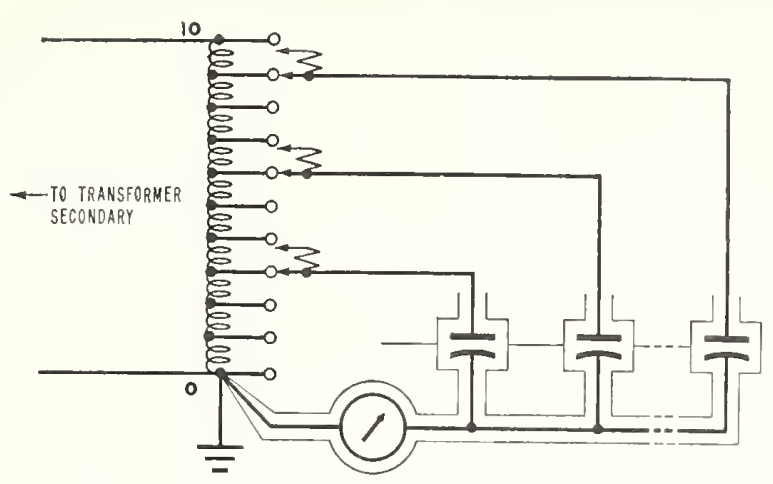

Fig. 6--Schematic of low-range decade capacitor.

active electrodes. If this diaphragm were solid, the direct capacitance would be zero since it would intercept all the field between the electrodes. However two diametrically opposite holes of appropriate size are drilled in the diaphragm, and the field which penetrates these holes and continues from the inner to the outer electrode produces the direct capacitance of appropriate magnitude. Another larger pair of holes, drilled in the outer electrode, in line with the diaphragm holes, allows some of the field from the inner electrode, which penetrates the diaphragm openings, to continue on through the openings in the outer electrode and terminate on an outer grounded shield. This decreases the value of the direct capacitance. Rotation of the outer electrode with respect to the diaphragm moves the sets of holes with respect to each other and constitutes the fine adjustment of capacitance for the unit.

The switches for the low-range decades deserve special mention because of their linear rather than rotary motion and because of the special movingcontact brushes that are used. A linear motion was preferred over the usual rotary motion for these selector switches to facilitate their manipulation either singly or in groups. This feature is particularly useful in the lower decades, as it makes possible a more rapid approach to the bridge balance. The use of these switches with an inductive voltage divider requires that special consideration be given the problem of making and breaking contact. Clearly, whatever switch is used for this application should not be of the shorting type, or a section of the inductor will be momentarily shorted during switching; nor should it be of a nonshorting type, or the detector will be momentarily floating, causing the indicating device to go off scale and perhaps causing the preamplifier to overload. The switches can be considered as nonshorting with an added moving contact brush adjacent to the main brush, the two being connected by a resistor of appropriate value (see Fig. 6). In the normal position the auxiliary brush is floating. When the switch is advanced to another position, the auxiliary brush touches the adjacent switch stud before the main contact is broken, and the main brush touches the new stud before the auxiliary contact is broken. Thus there is always a connection either direct or through the resistor, and the inductor section is at no time shunted by an impedance less than that of the resistor.

The capacitors in the units so far described are sealed to reduce changes in value resulting from variations of humidity and atmospheric pressure. Under these circumstances the principal cause of short-term drift in their values is variation in ambient temperature, as their temperature coefficients of capacitance are all nominally equal to the coefficient of lincar expansion of brass, approximately $20 \times 10^{-6} /{ }^{\circ} \mathrm{C}$.

\section{Temperature-Compensated CapaCitors}

Standards for maintaining the unit of capacitance must be independent of environmental conditions. The first stable capacitors completed at $N$ BS were a set of four temperature-compensated modifications of the 1-pf design used in the decade boxes. The cylindrical structure of this design was lengthened, and grounded Duralumin ${ }^{9}$ sleeves were inserted between the active electrodes from either end of the assembly, leaving a gap in the center that determined the active length of the capacitor. The remainder of the brass capacitor structure had a linear temperature coefficient substantially less than that of the Duralumin. The length of the sleeves were so chosen that the capacitance was independent of temperature. The structure of the capacitor is shown in section in Fig. 7.

The completed capacitors have temperature coefficients in the neighborhood of 1 ppm (part per million) per ${ }^{\circ} \mathrm{C}$. They are quite sensitive to temperature gradients and must be well isolated from rapid temperature changes to exhibit this low coefficient. In the final assembly these capacitors are mounted in a massive brass container with $\frac{3}{4}$-inch walls and sealed in an atmosphere of diry nitrogen. This container is surrounded with heatinsulating material and is mounted in a box. During the first month after their completion, the values of three of these capacitors did not drift relative to each other by as much as $1 \mathrm{ppm}$. The behavior of the fourth unit has been somewhat less satisfactory. It has changed by nearly 3 ppm during the same period.

Stable capacitors with low temperature coefficients covering the range from 10 to $10^{4} \mathrm{pf}$ are being built but have not yet been completed.

\section{Conductance-Balance Control}

At a single frequency any physically realizable capacitor may be represented by the parallel combination of pure capacitance and pure resistance. The current through it, in response to an impressed voltage, may then be treated as the resultant of a major component which leads the voltage by $\pi / 2$ radians and a minor component in phase with the voltage. Even for airdielectric capacitors the in-phase component of current

${ }^{9}$ Duralumin is a class of copper-bearing aluminum alloys. A typical analysis is $\mathrm{Cu}-4.5$ per cent, $\mathrm{Mn}-0.8$ per cent, $\mathrm{Mg}-0.4$ per cent, $\mathrm{Si}-0.8$ per cent. 


\section{McGregor, et al.: New Apparatus at the NBS for Absolute Capacitance Measurement}

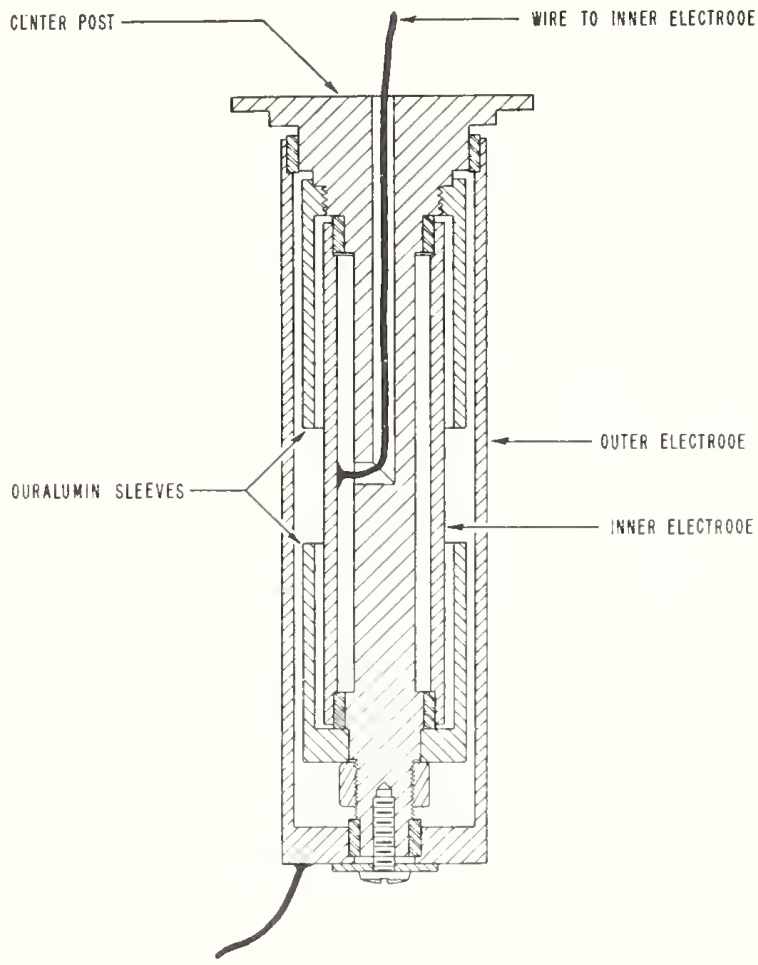

Fig. 7-Section of temperature compensated 1-pf capacitor

may be only four or five orders of magnitude smaller than the quadrature component, and it is necessary therefore that any device for the precise comparison of capacitors be provided with a means of compensating their loss components of current.

The balance condition for the bridge network is satisfied when the current from one capacitor to the detector junction is equal in magnitude and phase to that from the detector junction to the other capacitor. Hence an unbalance which results from lack of equality between the in-phase components of these currents may be corrected by injecting an in-phase current of proper sign and magnitude at the detector junction. This is accomplished as shown in Fig. 8.

The reactance of the parallel combination of $C_{1}$ and $C_{2}$ is so small relative to $R$ that the current $i_{d}$ is very nearly in phase with the voltage $e_{1}$. It is brought exactly in phase by means of a small trimming capacitor $C_{r}$ connected from the midpoint of $R$ to ground. The magnitude of $i_{d}$ is determined by the voltage applied to $R$, the value of $R$, and the relative values of $C_{1}$ and $C_{2}$, which act as a current divider. ${ }^{10}$

The decade voltage divider is a tapped inductor with four separate windings connected to linear switches of the type described in the preceding section. This is shown in Fig. 9. High accuracy of voltage division is

10 The value of $C_{t}$ is so small relative to the sum of $C_{1}$ and $C_{2}$ that its effect is negligible on the magnitude of the current injected at the detector branch point. It should be noted that the use of a $0.0889-\mu$ f capacitor as the final step of the capacitance current divider results in a multiplying factor on the highest range which departs by 10 per cent from its nominal value. In a later construction a $0.1-\mu$ f capacitor has been used and the values of $R$ modified so that the nominal ratio of 10 is preserved for all multiplying factors.

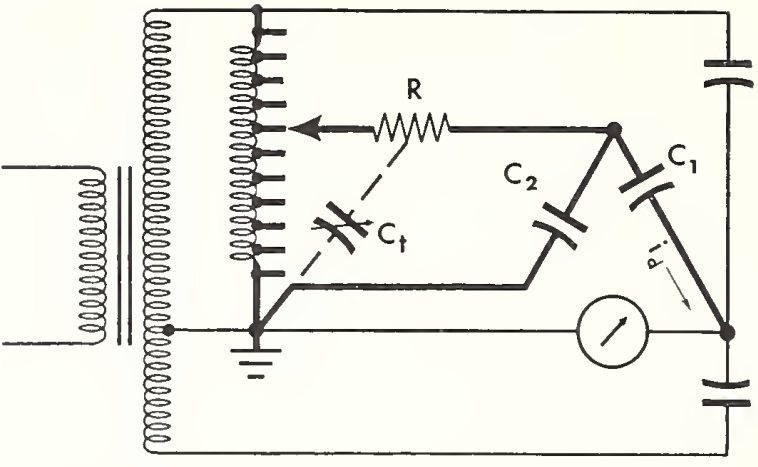

Fig. 8-Basic conductance balance control circuit.

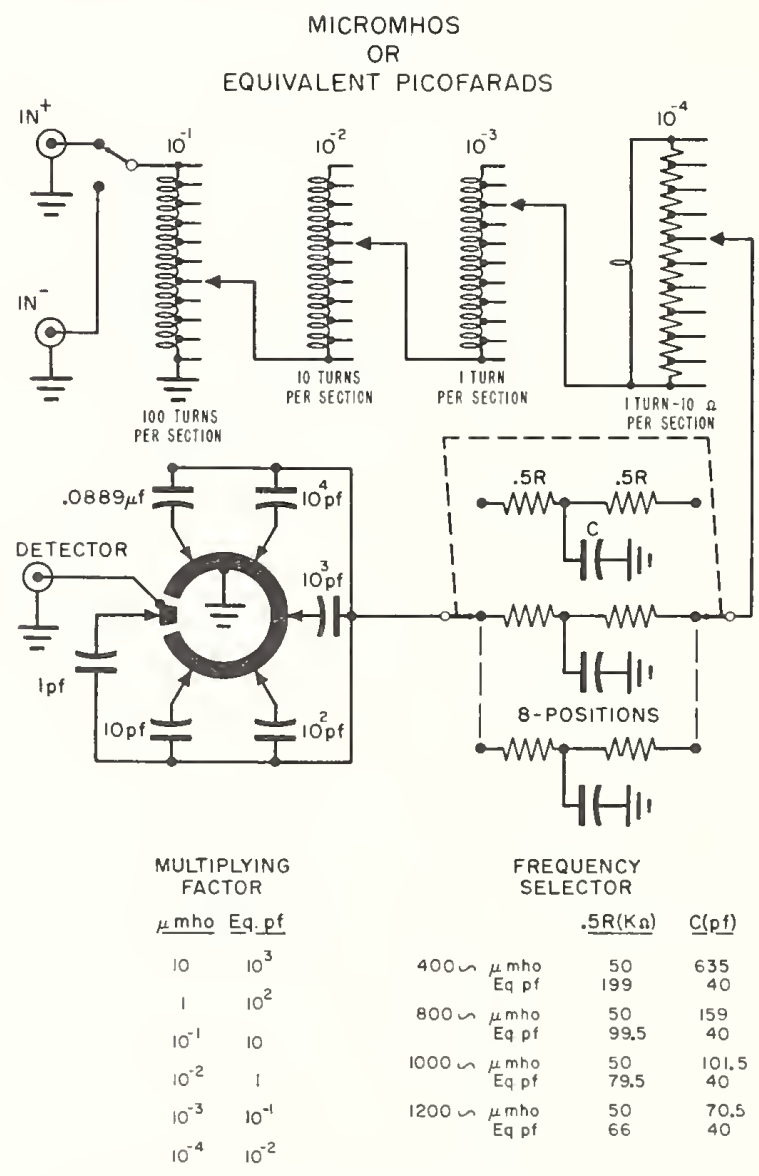

Fig. 9-Details of conductance balance control circuit.

obtained by winding all turns on the same high permeability core. The Supermalloy 1-mil tape-wound core has a section of $0.25 \mathrm{in}^{2}$ and an outside diameter of 2.5 inches. AWG no. 26 copper wire was used for all windings.

If the conductance balance control were to provide a measure of the loss characteristics of capacitors expressed in the common unit of conductance, the micromho, small changes in the phase trimming capacitor $C_{t}$ would suffice to adapt the unit for use with various frequencies. However, it is often convenient to express the loss characteristic of a capacitor in "equivalent capacitance" units. As the conductance and "equivalent capacitance" are related by the equation $C_{\mathrm{eq}}=G / \omega$, a change in frequency changes the relative magnitudes of 
their increments. The desired flexibility is incorporated in to the unit by providing frequency selection switching which changes the value of the resistor if the reading is desired in equivalent picofarads and the phase trimming capacitor if the reading is desired in micromhos. The unit is calibrated in the bridge by comparison with resistors having known or negligible residual reactances.

\section{Computable Standard of Capacitance}

The sensitivity and range of the bridge, the accuracy of its ratio transformers, and the stability of the air capacitors described above require a better low-value standard of capacitance than has been available heretofore at NBS.

Parallel plate and guard-well capacitors ${ }^{11}$ have been used as low-value calculable standards. Plans for materially improving such capacitors by using optical interference techniques in determining their mechanical dimensions were abandoned when it became apparent that a better computable capacitor could be constructed using the electrode configuration suggested by Thompson and Lampard. ${ }^{12}$ Lampard $^{13,14}$ had shown that an electrode assembly made up of equal right circular cylinders, located at the corners of a square, fulfilled the necessary conditions of symmetry, and that for certain types of small departures from symmetry, the mean value of the cross capacitances was not significantly affected. Further, measurements by Thompson and one of the authors ${ }^{15}$ on such an assembly had shown that the re-entrant nature of the gaps between the cylinders made possible substantial separation of the electrodes without appreciable effect on the measured capacitance. In fact, these measurements indicated that the principal source of uncertainty in the value of a cylindrical cross capacitor made from an assembly of right circular cylinders resulted from the use of insulated end sections as guards. Although the mechanical length of the guarded cylinder forming the defined capacitor could be accurately determined, its electrical length extended into the insulated gaps between it and the guarding end sections.

Any departure from exact colinearity of the cylindrical surfaces adjacent to the gap would shift the electrical length and hence the value of the capacitor in the direction of the lower surface. To attain the desired accuracy in computing the value of such a capacitor, a

${ }^{11}$ C. Moon and C. M. Sparks, "Standards for low values of direct capacitance," J. Res. NBS, vol. 41, pp. 497-507; November, 1948.

${ }_{12}$ A. M. Thompson and D. G. Lampard, "A new theorem in electrostatics and its application to calculable standards of capacitance," Nature, vol. 177, p. 888; May, 1956.

13 Lampard's theorem may be generalized as follows: If four infinite cylindrical conductors of arbitrary cross sections are assembled with their generators parallel to form a completely enclosed hollow cylinder in such a way that the internal cross capacitances per unit length are equal, then in vacuum these cross capacitances are equal to $\ln _{e} 2 / 4 \pi^{2}$ esu $/ \mathrm{cm}$

${ }_{14}$ D. G. Lampard, "A new theorem in electrostatics with applications to calculable standards of capacitance," Proc. IEE, vol. 104, pt. C, pp. 271-280; September, 1957.

is Informally communicated from NSL, Sydney, Australia. method was required that would eliminate this end effect from the calculation.

Cylindrical gauge bars ("reference-grade" end standards) of high quality were available in a variety of lengths. The added requirements of uniformity and equality of diameter among the various bars in the assembly could be met within one or two ten-thousandths of an inch. ${ }^{16}$ The cylindrical cross capacitor, designed as a computable standard for the present work, is constructed of such gauge bars. The end sections are 2 -inch gauge bars having an axial hole for bringing shielded connections through to the central guarded electrode. A 2 -inch gauge bar is permanently bolted to, but insulated from, each of the guard bars to form a portion of the defined central electrode. The inner bars can either be wrung together mechanically to form a 4 -inch central electrode, or each can be wrung to an end of a 10-inch bar to form a 14-inch central electrode. If the guard assembly is not disturbed, the difference in values of the 4 -inch and 14-inch capacitors should equal the computed value of a 10-inch capacitor without the need for end corrections. The attainable accuracy should, under favorable circumstances, ${ }^{17}$ very nearly equal the accuracy to which the mechanical length of the 10-inch gauge bar can be determined.

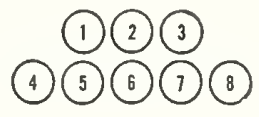

(a)

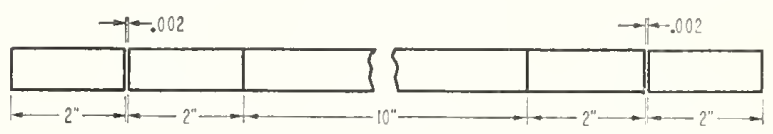

(b)

Fig. 10-Arrangement of cylindrical cross capacitor. (a) Bar arrangement, (b) gauge bar assembly.

The assembly is shown schematically in Fig. 10 (a) which is an end view of the bar arrangement and Fig. 10 (b), a side view of the guarded electrode assembly. Bars 2 and 6 have insulated end sections. The remaining bars $(1,3,4,5,7,8)$ are continuous cylinders of nominal 18 -inch lengths but with diameters equal as nearly as possible to the gauge bars in 2 and 6 . A capacitance balance for either the 4- or 14-inch assembly can be made with the central section of 2 connected to the detector branch point of the bridge, bars 5 and 7 connected in parallel to one side of the ratio transformer, and the remaining bars, $1,3,4,6$, and 8 , as well as the guard (end) sections of 2, connected to ground. Capacitances between electrodes $2-5$ and $2-7$ are in parallel and add directly, doubling the value computed from Lampard's

${ }_{16}$ The assistance of E. J. Schneider of the Engis Equipment Co., Chicago, Ill., and of his principals, the Coventry Gage and Tool Co., Coventry, Eng. in constructing these special gauge bars, is gratefully acknowledged.

${ }_{17}$ It will be apparent that small variations may be introduced by residual misalignment when taking apart and reassembling the end standards forming the defined electrode. 
1958 MCGregor, et al.: New Apnriratus at the NBS for Absolute Capacitance Measurement

formula for the given length. A second bridge balance can be made using 6 as the detector bar, 1 and 3 in parallel as the line bars, and the remaining bars, 2, 4, 5, 7, 8, together with the end sections of 6 grounded. In this instance the capacitances of 1-6 and 3-6 add in parallel.

The difference between the balance readings taken with 2 and 6 alternatively used as detector bars is a measure of any lack of symmetry in the assembly. The mean of the values is very closely equal to the value computed for the capacitance. ${ }^{18} \mathrm{~A}$ close approximation to symmetry is accomplished by using bars of equal and uniform diameter and by separating them with equal insulating spacers near their ends. The spacers are arranged in the end sections of the assembly so that there is no solid dielectric in the electric field of the calculable central portion. The entire assembly rests on a granite surface plate and is enclosed in a steel housing. This container can be evacuated to a pressure below $0.1 \mathrm{~mm}$ $\mathrm{Hg}$, eliminating the need for an air correction to the computed value of capacitance.

A sample set of measurements on the computable capacitor is given in Table I. The individual observations are stated in terms of an arbitrary unit, the mean of the four temperature-compensated capacitors. The value of the cross capacitance is computed in pf, from the measured lengths of the two 10-inch gauge bars. The difference between this computed capacitance and the measured value gives the departure of the arbitrary unit from the absolute unit of capacitance. The uncertainty of this value is believed to be less than $3 \mathrm{ppm}$.

\section{CONCLusion}

A transformer-ratio bridge for the precise comparison of 3-terminal capacitors has been described. The construction of a cylindrical cross capacitor as a computable standard has also been described. This absolute standard of capacitance is of such a nature as to make full use of the sensitivity and precision available in the bridge. It is expected that the combination of the bridge and

${ }^{18}$ For example, if the difference in readings amounts to 0.1 per cent, it can be shown that the mean value of the capacitors differs from the computed value by less than $0.1 \mathrm{ppm}$; more precisely

$$
\frac{C_{1}+C_{2}}{2 C_{0}}=1+\frac{\ln \epsilon}{8}\left(\frac{C_{1}-C_{2}}{C_{0}}\right)^{2}
$$

where $C_{1}, C_{2}$ are the measured values and $C_{0}$ is the computed value of the capacitor.
TABLE I

Sample Data on Computable Cross Capacitor

Mean Cross Capacitance, Corrected to $20^{\circ} \mathrm{C}$, Referred to Mean of Temperature-Compensated Capacitors

\begin{tabular}{c|c|c}
\hline \hline Date & \multicolumn{1}{|c|}{$14-$ Inch Assembly } & 4-Inch Assembly \\
\hline $7-11-58$ & 1.3897864 Arbitrary Units & \\
$7-11-58$ & 1.3897860 & \\
$7-11-58$ & 1.3897855 & \\
$7-11-58$ & 1.3897855 & \\
$7-14-58$ & 1.3897859 & \\
$7-14-58$ & 1.3897866 & \\
$7-14-58$ & 1.3897862 & \\
& 1.3897860 (Average) & 0.3973392 \\
& & 0.3973395 \\
$7-15-58$ & & 0.3973400 \\
$7-15-58$ & & 0.3973400 \\
$7-16-58$ & & 0.3973391 \\
$7-16-58$ & & 0.3973399 \\
$7-16-58$ & & 0.3973402 \\
$7-16-58$ & & \\
$7-16-58$ & & \\
& & \\
\hline $7-17-58$ & 1.3897864 & \\
$7-17-58$ & 1.3897863 & \\
$7-21-58$ & 1.3897866 & \\
$7-21-58$ & 1.3897862 & \\
$7-23-58$ & 1.3897856 & \\
$7-23-58$ & 1.3897864 & \\
$7-25-58$ & 1.3897875 & \\
& 1.3897864 (Average) & \\
\hline & & \\
\hline-5 & & \\
\hline
\end{tabular}

Difference between 14 -inch and 4 -inch assemblies $=0.9924465$. Computed cross capacitance at $20^{\circ} \mathrm{C}=0.9924127 \mathrm{pf}$.

True mean of temperature compensated capacitors

$=1(1-0.0000338) \mathrm{pf}$.

the computable standard will make possible assignment of more accurate values to capacitors in the range below $10^{4} \mathrm{pf}$ than has been possible in the past at the National Bureau of Standards.

\section{ACKNOWLEDGMENT}

It is almost invariably true that the performance of measurement apparatus of high precision depends on the skill and ingenuity of the men who make its mechanical parts. The present bridge is no exception to this rule. The authors wish to acknowledge their indebtedness in this essential matter to the shop personnel working under the direction of D. Kennedy, and especially express their appreciation of the skills and patience of Chidester, Guatney, Graef, Matwey, Pararas, and Stadler. 


\title{
Capacitor Calibration by Step-Up Methods
}

\author{
Thomas L. Zapf \\ (October 8, 1959)
}

\begin{abstract}
Step-calibration methods are used in many physical laboratories for the extension of measurements to quantities far removed from the magnitude of greatest accuracy at which absolute determinations are made. The excellent precision of repetitive substitution procedures is exploited by step-up or step-down methods to extend measurements to higher or lower magnitudes without serious degradation of accuracy. The application of step-up techniques to the calibration of variable air capacitors is described in this paper as a practical example of the method.
\end{abstract}

\section{Introduction}

One of the important statutory functions of the National Bureau of Standards is the calibration of physical standards of measurement used in science and industry: The chain of measurements connecting this calibration service to the national prototype standards of length, mass, and time is complex, and for electrical measurements involves meticulous experiments to assign numerical values to calibration standards and corrections to standard instruments. These devices, designed for excellent stability and definitude, serve as comparison standards basic to the calibration services rendered by the Bureau. Equally important to accurate scientific work is the proper use of these standards to overcome their inherent limitations. Frequently an appropriate choice of method and the employment of suitable techniques are as important as the judicious selection of equipment. The close association between methods, techniques, and equipment is particularly evident when, in the course of calibration activities, it is necessary to obtain accurate measurements at magnitudes far removed from that at which absolute determinations are made. The extension of range of eleetrical measurements is sometimes accomplished by the establishment of aceurately known ratios. For example, ratios very nearly equal to the squares of integers may be obtained through the successive measurement in series and parallel of resistors having nearly equal values [1]. ${ }^{\mathrm{I}}$ Resistance ratios of approximately 10:1 may be realized by the successive measureinent of 11 resistors in arbitrary units and the use of these as the 10:1 ratio arms of a bridge. A unique 10:1 ratio apparatus used with a special resistance bridge is described by Wenner [2].

The building-up to ratios larger than $10: 1$ is particularly well exemplified by the procedure followed in calibrating the standard volt box at the Bureau [3] in which a group of sections of nominally

${ }^{1}$ Figures in brackets indicate the literature references at the end of this paper. equal resistance is intercompared. These sections, connected in series, form the first section of a group of larger denomination. The buildup to large ratios is rapid and exact. The standard rolt box was designed specially for self-ealibration by this method.

The calibration of resistance decade boxes and the resistance decades of bridges by the stepsubstitution or step-up method illustrates yet another technique of obtaining accurate measurements over wide ranges [4]. A similar process is used by the Bureau for the calibration of the capacitance bridges that are used daily to measure standards of capacitance.

In order to obviate the concel'n over connection errors and avoid the detailed consideration of connectors, it is customary and convenient to use as standards of low grounded capacitance such devices as variable air capacitors and capacitance decade boxes which may be calibrated accurately for capacitance difference from some arbitrary setting. The calibration of such variable capacitor's may be accomplished quite effectively by the step-up method emploxing fixed standards or standards of eapacitance difference.

An excellent description of a step-up method applied to the calibration of deeade capacitors for both eapacitance and dissipation factor has been described by Ford and Astbury of the British National Physieal Laboratory [5].

\section{Equipment}

Very little special equipment is needed to calibrate a variable capacitor by step-up methods. If the variable air capacitor, $X$, having a range from 100 to $1,100 \mathrm{pf}$, is to be calibrated at every 100-pf division mark, it is necessary to have a fixed air capacitor, $S$, of approximately $100 \mathrm{pf}$ that can be connected in parallel with the variable capacitor under test in a precisely repeatable manner. This can be achieved if the mating connectors introduce no significant uncertainties to the capacitance added to the circuit and if the connectors are designed to couple quickly and easily to either the variable capacitor or the 
bridge that will be used, or if a capacitor can be connected or disconnected by a precise switching arrangement. The 100-pf capacitor should be adjusted close to the nominal value, but it need not be calibrated. It must, however, be free from significant drift over the period of a quarter-hour or so during which the test is being run.

A calibrated 1,000-pf air capacitance standard, $S^{\prime}$, is needed to relate the results of the step-up test to the national reference standard of capacitance.

The bridge used for this step calibration need not have great accuracy but must be stable, for it is used with a sensitive detector for substitution measurements. A small variable capacitor, $T$, is required, having a least count (smallest readable increment) one-tenth that of $X$ or smaller. It is advantageous to choose the smallest possible precision variable capacitor, $T$, consistent with other limitations so that the corrections to $V$ are negligibly small relative to the corrections to $\mathrm{X}$. The total range of $V$ niust be at least a little larger than the range of errors in the capacitor to be calibrated. The readable accuracy of this capacitor, if expressed in percent of total range, need not be very great.

The equipment described is assembled as shown in figure 1. It is most important that the cables used to connect components be shielded and rigid, or if flexible cables are used, it should be ascertained that variations in cable capacitance are negligible. The cables must be fixed in position and must not be disturbed during the entire calibration. This precaution is intended to emphasize the importance of particular care to one of those sources of systematic error that could impair good calibration accuracy. The operator must have a good technical appreciation of the apparatus and quantities measured, gained through study and experience.

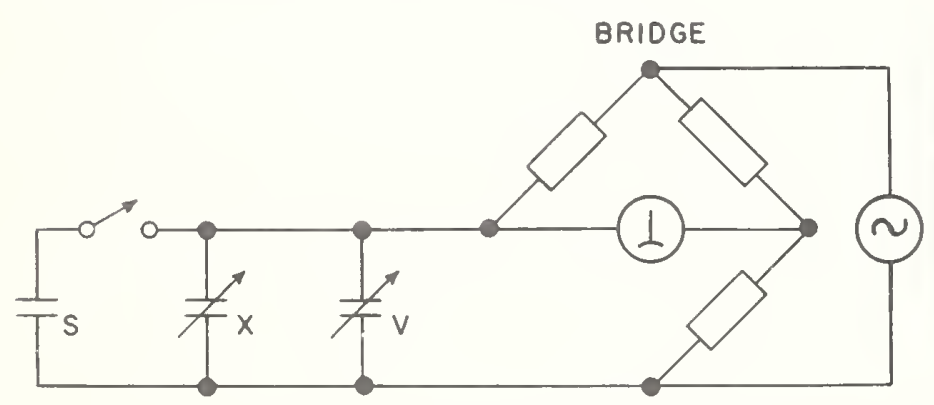

Figure 1. The variable capacitor under text, $\mathbf{X}$, is calibrated by a step-up method employing a fired capacitor, S, and a small variable capacitor, $\mathrm{V}$.

\section{Procedure and Computations}

'The true value of each of the capacitors involved in the calibration may be clefined as the nominal value plus a correction; thus, the true capacitance of the uncalibrated 100-pf capacitor is $S=S_{n}+s$, where $S_{n}=100$ (exactly) and $s$ is the small correction. Similarly the calibrated 1,000-pf standard has the value $S^{\prime}=S_{n}^{\prime}+s^{\prime}$, and the variable air capacitor to be calibrated may be represented by $X^{\prime}=X_{n}+x$, where $x$ is the correction to the reading (or setting) $X_{n}$. The calibration will consist of the determination of the relatively small correction, $x$, to each 100-pf division mark, $X_{n}$, and since only capacitance differences are of interest, the observer is free to choose any one of the division marks as a reference point. It is frequently convenient to choose as a reference the first marked point on the dial. In this case the 100-pf mark is considered as a reference and a correction of 0.00 is arbitrarily assigned to it. For this example it will be assumed that the small variable capacitor, $T$, has corrections that are negligibly small.

The capacitor under test is first carefully set to the 100-pf mark ( $X_{n 1}=100 \mathrm{pf}$ ) avoiding backlash errors by approaching the mark in the direction of increasing dial readings. The small variable capacitor, $V$, is set to any convenient mark near the center of its range. The 100-pf capacitor, $S$, is connected in parallel with $\mathrm{X}$ and $V$. Now the bridge must be balanced using the controls on the bridge itself If a balance cannot otherwise be obtained, $V$ may be used to attain balance. When the bridge is balanced the reading $V_{A}$ is recorded. The fixed capacitor, $S$, is then removed and $X$ set to the 200-pf mark $\left(X_{n 2}=200 \mathrm{pf}\right)$, again approaching the mark in the same direction. Without changing any other component the circuit is rebalanced by changing $V$ alone, and the reading $V_{B}$ recorded as before. In the first balance the external bridge arm consisted of $S+Y_{i}+V_{A}$, and for the sacond balance, with the bridge unchanged, the external arm consisted of $\mathrm{I}_{2}+V_{B}$. These can be equated to yield

$$
S+X_{1}+V_{A}=X_{2}+V_{B}
$$

The cable and connector capacitance, as well as residuals within the bridge, contribute equally to both balances and are therefore deliberately disregarded.

It is convenient to work with small numbers, and eq (1) can be expressed as

$$
S_{n}+s+X_{n 1}+x_{1}+V_{A}^{r}=X_{n 2}+x_{2}+V_{B}
$$

and since

$$
\begin{gathered}
S_{n}=X_{n 2}-X_{n 1} \text { and } x_{1}=0 \\
x_{2}=\left(V_{A}-V_{B}\right)_{2}+s
\end{gathered}
$$

where the subscript is appended to the difference $\left(V_{A}-V_{B}\right)$ to distinguish this set of data from-other sets and to appropriately correlate the difference with the setting of $X$ in the second balance of each set.

The quantity $x_{2}$ is the desired correction to $Y_{n}$ when $Y_{n}=200$ pf. The difference $\left(V_{A}-V_{B}\right)_{2}$ is easily computed from the recorded data.

Without changing $T, S$ is reconnected and the bridge rebalanced using the bridge controls and $V$, if necessary, to attain exact balance. The reading, $T_{A}{ }^{2}$, is then recorded. Capacitor $S$ is then removed and $X$ set to the $300-p f$ mark $\left(X_{n}=300\right)$. The bridge is rebalanced using $T$ alone and the reading, 
$V_{B}$, recorded. When the first balance is equated to the second balance

$$
S+X_{2}+V_{A}=X_{3}+V_{B}
$$

or

$$
S_{n}+s+X_{n 2}+x_{2}+V_{A}=X_{n 3}+x_{3}+V_{B}
$$

and. since

$$
\begin{gathered}
S_{n}=X_{n 3}-X_{n 2} \\
x_{3}=x_{2}+\left(V_{A}-V_{B}\right)_{3}+s .
\end{gathered}
$$

Substituting eq (4) in eq (8)

$$
x_{3}=\left(V_{A}-V_{B}\right)_{2}+\left(V_{A}-V_{B}\right)_{3}+2 s .
$$

Continuing this process step-by-step, in general for the $m$ th step

and finally

$$
x_{m}=\sum_{2}^{m}\left(V_{A}-V_{B}\right)+(m-1) s
$$

$$
x_{11}=\sum_{2}^{11}\left(V_{A}-V_{B}\right)+10 s
$$

A tabulation of the differences and the cumulative sum of the differences is shown in table 1 , which shows the data and computations for a typical calibration.

\begin{tabular}{|c|c|c|c|c|c|c|}
\hline$S$ & $X$ & $V$ & $V_{A}-V_{B}$ & $\Sigma\left(V_{A}-V_{B}\right)$ & $n 8$ & $x$ \\
\hline 100 & 100 & 5. 00 & \multirow{2}{*}{0.81} & \multirow{2}{*}{0.31} & \multirow{2}{*}{-0.14} & \multirow{2}{*}{0.17} \\
\hline 0 & 200 & 4. 69 & & & & \\
\hline 100 & 200 & 4.84 & \multirow{2}{*}{-.02} & \multirow{2}{*}{.29} & \multirow{2}{*}{-.29} & \multirow{2}{*}{.00} \\
\hline $\begin{array}{r}0 \\
100\end{array}$ & $\begin{array}{l}300 \\
300\end{array}$ & $\begin{array}{l}\text { 4. } 86 \\
4.99\end{array}$ & & & & \\
\hline $\begin{array}{r}0 \\
100\end{array}$ & $\begin{array}{l}400 \\
400\end{array}$ & $\begin{array}{l}4.93 \\
4.92\end{array}$ & .06 & .35 & -.43 & -.08 \\
\hline $\begin{array}{r}0 \\
100\end{array}$ & $\begin{array}{l}500 \\
500\end{array}$ & $\begin{array}{l}4.51 \\
4.65\end{array}$ & .41 & .76 & -.58 & .18 \\
\hline $\begin{array}{r}0 \\
100\end{array}$ & $\begin{array}{l}600 \\
600\end{array}$ & $\begin{array}{l}4.64 \\
4.81\end{array}$ & .01 & .77 & -.72 & .05 \\
\hline $\begin{array}{r}0 \\
100\end{array}$ & $\begin{array}{l}700 \\
700\end{array}$ & $\begin{array}{l}4.76 \\
4.87\end{array}$ & .05 & .82 & -.86 & -.04 \\
\hline $\begin{array}{r}0 \\
100\end{array}$ & $\begin{array}{l}800 \\
800\end{array}$ & $\begin{array}{l}\text { 4. } 60 \\
4.73\end{array}$ & .27 & 1.09 & -1.01 & .08 \\
\hline $\begin{array}{r}0 \\
100\end{array}$ & $\begin{array}{l}900 \\
900\end{array}$ & $\begin{array}{l}\text { 4. } 42 \\
\text { 4. } 57\end{array}$ & .31 & 1.40 & -1.15 & .25 \\
\hline $\begin{array}{r}0 \\
100\end{array}$ & $\begin{array}{l}1000 \\
1000\end{array}$ & $\begin{array}{l}\text { 4. } 53 \\
4.67\end{array}$ & .04 & 1.44 & -1.30 & .14 \\
\hline 0 & 1100 & 4. 12 & .55 & \multirow[t]{2}{*}{1.99} & -1.44 & .55 \\
\hline 1000 & 100 & 5.04 & & & & \\
\hline 0 & 1100 & 4. 12 & 0.92 & & & \\
\hline
\end{tabular}
It remains to determine the value of $s$ so that the corrections $x_{2}$ through $x_{11}$ can be evaluated.

The 1,000-pf standard capacitor, $S^{\prime}$, accurately calibrated for insertion capacitance, is now connected

Table 1. Observations and calculations All values in picofarads in parallel with $X$ and $V$. With $X$ set at $100 \mathrm{pf}$, the bridge is balanced with the bridge controls and $V$, if necessary, and the reading, $V_{A}$, recorded. $S^{\prime}$ is removed, $X$ is set to the 1,100 -pf mark, the bridge rebalanced using $V$ alone, and the reading, $V_{B}$, recorded. Then

or

$$
S^{\prime}+X_{1}+V_{A}=X_{11}+V_{B}
$$

and since

$$
S_{n}^{\prime}+s^{\prime}+X_{n 1}+V_{A}=X_{n 11}+x_{11}+V_{B}
$$

$$
\begin{gathered}
S_{n}^{\prime}=X_{n 11}-X_{n 1} \\
x_{11}=\left(V_{A}-V_{B}\right)_{T}+s^{\prime}
\end{gathered}
$$

where the subscript $T$ denotes the $V$ difference obtained when the capacitor, $S^{\prime}$, is used.

In this way $x_{11}$ is determined accurately in terms of a small difference reading of the variable capacitor, $V$, and the known correction, $s^{\prime}$, to the standard capacitor, $S^{\prime}$. The correction, $s$, to the fixed capacitor, $S$, can now be computed from eq (11)

$$
10 s=x_{11}-\sum_{2}^{11}\left(V_{A}-V_{B}\right)
$$

or

$$
10 s=s^{\prime}+\left(V_{A}-V_{B}\right)_{T}-\sum_{2}^{11}\left(V_{A}-V_{B}\right)
$$

The quantity $10 \mathrm{~s}$ is then added algebraically to the sum of the $V$ differences corresponding to the test of the 1,100-pf mark, the result being the correction to this reading. Similarly $9 s$ is added to the sum of the $V$ differences corresponding to the 1,000-pf mark, and so on, until only $s$ is added to the $V$ differences corresponding to the 200-pf mark. These small corrections are listed in table 1 under the heading $n s$.

The observations can be made rapidly and the computations are simple, since only small differences appear. A second complete calibration, preferably by another observer, enables one to appraise the precision of the measurements including the stability and resetability of the capacitor under test, and serves to reveal measurement and arithmetic errors that might otherwise remain undetected.

In the procedure described above the fixed increment of calibration was $100 \mathrm{pf}$. It is well to point out that other increments can be accommodated as .well. A 50-pf capacitor, if used as a fixed step, would permit calibration at 50-pf intervals. Although the procedure has been described using a fixed capacitor as a step, a continuously variable capacitor or decade capacitor would also be satisfactory if it were used in such a manner as to provide a repeatable difference of capacitance. Care would be necessary to avoid setting errors caused, for example, by backlash in the control mechanism, or by careless setting to the index.

A variable capacitor calibrated for capacitance difference by this method can be used as a standard for extending the method to capacitance calibrations of still lower magnitudes. 


\section{Dual Calibration}

Reviewing the calibration described above, it is noticed that for each set of two balances, one balance is obtained with the bridge controls and $V$, if necessary. The fact that the change in the bridge reading is always an amount approximately equal to $S$ (or $S^{\prime}$ ) leads to the consideration of calibrating two variable capacitors having the same range with practically no extra work.

If $X$ and $U$, the variable capacitors to be calibrated, are connected as shown in figure 2 , the procedure is similar to that described above except that the bridge need not be changed after the initial setting. The settings of $X$ and $U$ are listed in table 2. Care must be taken to apply the proper sign to the differences and to cumulatively add the differences for the calibration of $U$ beginning at the bottom of the table rather than the top.

Lower range capacitors can be calibrated similarly, but extreme attention must be paid to good mechanical rigidity in all parts of the circuit.

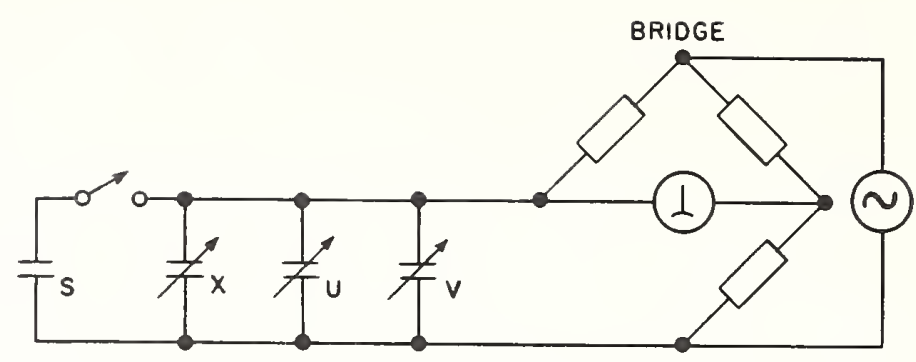

FIgURe 2. Two variable capacitors, $\mathrm{X}$ and $\mathrm{U}$, may be calibrated simultaneously by a step-up method.

\section{Discussion}

If attention is confined to the calibration of twoterminal variable air capacitors having a capacitance range from several picofarads to about $1,000 \mathrm{pf}$, the procedure outlined in this paper demonstrates the ability of step-calibration methods to provide accurate calibrations of capacitors at levels at which good accuracy is otherwise difficult to obtain.

TABLE 2. Observations and calculations (dual calibration)

\begin{tabular}{|c|c|c|c|c|c|c|c|c|c|c|c|}
\hline$S$ & $x$ & $U$ & $v$ & $V_{A}-V_{B}$ & $\Sigma \underset{V_{B}}{\Sigma\left(V_{A}-\right.}$. & $n s$ & $x^{\mathrm{a}}$ & $V_{B}-V_{A}$ & $\underset{\Sigma\left(V_{B}-\right.}{\left(V_{A}\right)}$ & $n s$ & $u$ ब \\
\hline 0 & 100 & 1100 & 5. 00 & \multirow{3}{*}{0.91} & \multirow{3}{*}{0.91} & \multirow{3}{*}{-0.14} & \multirow{3}{*}{0.17} & \multirow{2}{*}{0.34} & \multirow{2}{*}{2.05} & \multirow{2}{*}{-1.38} & \multirow{2}{*}{0.67} \\
\hline 100 & 100 & 1000 & 5. 34 & & & & & & & & \\
\hline 0 & 200 & 1000 & 5. 03 & & & & & \multirow{2}{*}{.04} & \multirow{2}{*}{1.71} & \multirow{2}{*}{-1.24} & \multirow{2}{*}{.47} \\
\hline 100 & 200 & 800 & 5.07 & \multirow{2}{*}{-.09} & \multirow{2}{*}{.28} & \multirow{2}{*}{-.28} & \multirow{2}{*}{.00} & & & & \\
\hline 0 & 300 & 900 & 5. 10 & & & & & \multirow{2}{*}{.07} & \multirow{2}{*}{1.67} & \multirow{2}{*}{-1.10} & \multirow{2}{*}{.57} \\
\hline 100 & 300 & 800 & 5.17 & \multirow{2}{*}{.06} & \multirow{2}{*}{.34} & \multirow{2}{*}{-.41} & \multirow{2}{*}{-.07} & & & & \\
\hline 0 & 400 & 800 & 5.11 & & & & & \multirow{2}{*}{.24} & \multirow{2}{*}{1.60} & \multirow{2}{*}{-0.97} & \multirow{2}{*}{.68} \\
\hline 100 & 400 & 700 & 5. 35 & \multirow{2}{*}{.87} & \multirow{2}{*}{.71} & & & & & & \\
\hline 0 & 500 & 700 & 4.98 & & & -.00 & .10 & $0 c$ & 100 & 109 & -0 \\
\hline 100 & 500 & 600 & 5. 34 & 0 & $\gamma_{8}$ & fo & a & & & -.85 & -1.63 \\
\hline 0 & 600 & 600 & 5. 32 & .02 & .60 & -.09 & .044 & $\$ 1$ & 100 & -69 & 31 \\
\hline 100 & 600 & 500 & 5.63 & & $\sim q$ & 80 & -05 & .01 & 1.00 & -.69 & .01 \\
\hline 0 & 700 & 500 & 5. 58 & .05 & .18 & -.85 & -.00 & & $0 \rho 0$ & 55 & 11 \\
\hline 100 & 700 & 400 & 5.84 & & & & & .20 & 0.09 & -.00 & .144 \\
\hline 0 & 800 & 400 & 5. 58 & .20 & 1.04 & -.97 & .07 & & & & \\
\hline 100 & 800 & 300 & 5.87 & & & & & .29 & .43 & -.41 & $.0 z$ \\
\hline 0 & 900 & 300 & 5. 56 & .31 & 1.35 & -1.10 & .25 & 01 & $x$ & 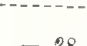 & u \\
\hline 100 & 900 & 200 & 5.57 & & & -191 & 11 & .01 & .144 & -20 & -1.14 \\
\hline 0 & 1000 & 200 & 5. 54 & .03 & 1.38 & -1.24 & .14 & & 18 & -11 & 0.01 \\
\hline 100 & 1000 & 100 & 5.67 & & 10 & $1>0$ & -1 & & .15 & -.14 & -.01 \\
\hline 0 & 1100 & 100 & 5. 13 & .34 & 1.92 & -1.38 & .54 & & & & \\
\hline 1000 & 100 & 1100 & 5. 37 & 91 & & & & & & & \\
\hline 0 & 1100 & 1100 & 4.46 & .01 & & & & 101 & & & \\
\hline 1000 & 1100 & 100 & 5. 50 & & & & & 1.04 & & & \\
\hline-0.3 & & & & $10 s=-1$. & $8 \mathrm{pf}$ & & & $10 s=-$ & & & \\
\hline
\end{tabular}

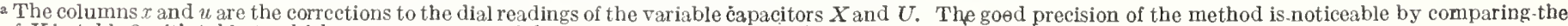
test of $X$ in table 2 with table 1 , which represents a test of the same capacitor about an hour earlier. 
Standards of grounded capacitance, often called twoterminal capacitors, are characterized by having one of the capacitor electrodes connected to the case, in contrast with standards of direct capacitance (threeterminal capacitors) having both capacitive electrodes insulated from the case. The direct capacitance, $C_{D}$, between the two active electrodes, as shown in figure 3 , is definite to the extent that the separate terminals and associated leads are shielded from. each other. Adequate shiclding that does not interfere with the direct capacitance is relatively easily obtamed, and excellent accuracy in direct capacitance measurements is possible to a fraction of a micropicofarad $\left(10^{-18} \mathrm{f}\right)$.
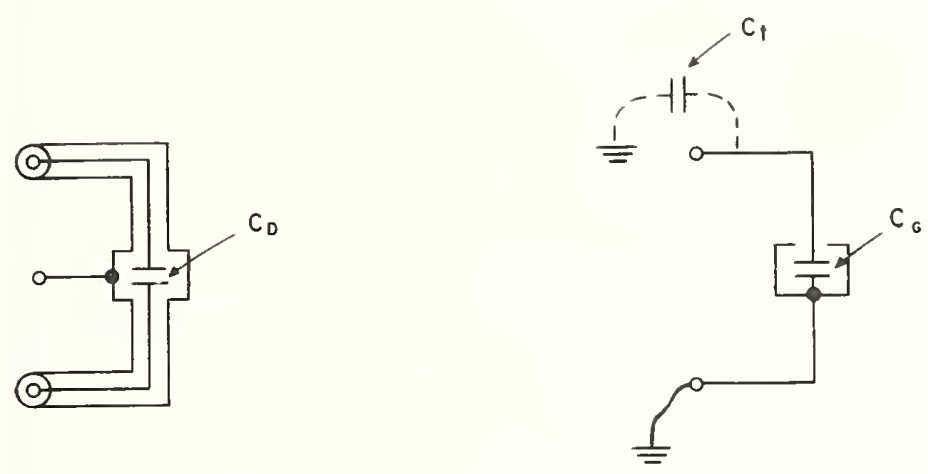

FIGURE 3. The direct (threcterminal) capacitance, $\mathrm{C}_{\mathrm{D}}$ is made definite by complete shiclding. The grounded (twoterminal) capacitance, $\mathrm{C}_{\mathrm{G}}$, is indefinite because of variations in the stray capacity.

The gromded capacitance, $C_{G}$, shown in figure 3 , is more difficult to define in a precise manner, because the capacitance between the ungrounded terminal and all grounded objects, $C_{t}$, is indistinguishable from. $C_{G}$ unless a separate "zero-balance" of the measuring apparatus is made with the leads attached but the capacitor disconnected. Even this procedure will not insure good precision unless care is used to connect the capacitor to the measuring apparatus in identically the same way every time. The best precision in practical measurements of grounded capacitance is possible only if the method of connection to the measurement circuit is well defined. An adequately shichled rigid adapter or connector is necessary as an auxiliary part of the capacitor and must be used with it for every measurement. If the capacitor is to be used as a standard for accurate capacitance measurements, the same connector must be used with the capacitor when it is calibrated, and the assembly becomes a standard of, capacitance added to a circuit, or in other words, capacitance difference. Thus, good precision of repeated measurements is simply obtained in any laboratory if the successive measurements are accomplished using rigid wiring and the same connectors every time. The best accuracy in terms of the national reference standards can be obtained only for magnitudes sufficiently large that negligible errors result from differences between the connectors used in the several laboratories involved. Accurate calibration of small fixed capacitors can only be accomplished if mating connectors are submitted.

The accuracy of measurements on fixed standards of grounded capacitance having electrodes terminated in binding posts or unshielded plugs is limited by the variation in the geometrical design of the instrument panels, cables, and connectors to which the standard can be attached. Differences as large as several tenths of a picofarad are possible with present commercially a vailable standards and apparatus with which they may be used. It is understandable that differences of several tenths of a percent are to be expected if a fixed 100-pf standard of this type is measured in several laboratories or on different equipment, while if measurements are performed on 1,000-pf standards the uncertainties at the connectors would be only several hundredths of a percent of the quantity measured. In the step-up procedure described in this paper it is evident that the precision of repeated measurements, the freedom from the effects of residuals in test apparatus obtainable by substitution methorls, and the accuracy of measurements at magnitudes closer to optinum, are combined in a manner favorable to the accurate calibration of the capacitance differences of variable capacitor's. The method is quite applicable at any frequeney although at higher frequencies residuals in components can be troublesome and may require special attention. For example, it may be necessary to apply corrections for errors introduced by residual inductance in the cables comnecting the apparatus.

\section{Conclusion}

Step-calibration methods can be employed for the calibration of variable capacitors. The few necessary items of equipment are generally available in any electrical measurements laboratory. Reference to the national electrical standards is made through the use of a single fixed capacitance standard that can be transported to other standardizing laboratories more easily, and calibrated less expensively than variable capacitors.

\section{References}

(1) Lord Rayleigh, Phil. Trans. 183, 661 (1882).

[2] F. Wenner, J. Research X゙BS $25,252(1940)$. RP 1323.

[3] F. B. Silsbee and F. J. (iross, J. Research NBS 27, 269 (1941). RP1419.

[4] J. L. Thomas, NBS ('ire. t70 (1!)+8).

[5] I. H. Ford and N. F. A-thury, I. Nei. Inntr. 15, 122 (1938).

Boulder, Colo.

(Paper 64C1-27) 


\title{
Eechnical Note
}

\author{
57 \\ May, 1960
}

VARIABLE CAPACITOR CALIBRATION WITH AN

INDUCTIVE VOLTAGE DIVIDER BRIDGE

by

Thomas L. Zapf

NBS Technical Notes are designed to supplement the Bureau's regular publications program. They provide a means for making available scientific data that are of translent or limited interest. Technical Notes may be listed or referred to in the open literature. They are for sale by the Office of Technical Services, U. S. Depart ment of Commerce, Washington 25, D. C.

\section{DISTRIBUTED BY \\ UNITED STATES DEPARTMENT OF COMMERCE OFFICE OF TECHNICAL SERVICES}

WASHINGTON 25, D. C.

Price $\$ .50$

$310 / i$ 
ABSTRACT

1. INTRODUCTION 1

2. EQUIPMENT 1

3. THE BRIDGE CIRCUIT 2

4. TWO-TERMINAL CAPACITANCE MEASUREMENTS 3

5. CONCLUSION 4

REFERENCES

\section{LIST OF ILLUSTRATIONS}

Figure 1. A simple transformer capacitance bridge for direct capacitance measurements 6

Figure 2. A modification of the bridge shown in Figure $1 \quad 6$

Figure 3. Connections for two-terminal capacitance measurements

Figure 4. Connections to a dual-range, two-terminal variable air capacitor showing guard cap over unused terminal 


\section{VARIABLE CAPACITOR CALIBRATION WITH AN \\ INDUCTIVE VOLTAGE DIVIDER BRIDGE \\ Thomas L. Zapf}

\section{ABSTRACT \\ The use of an inductive voltage divider bridge for the calibra- tion of three-terminal and two-terminal variable air capacitors is discussed.}

\section{INTRODUCTION}

The accurate calibration of variable air capacitors can be ac complished by several methods, one of which has been described as a step-up or step-substitution method (1). Another method, that is particularly useful for the calibration of three-terminal capacitors, is presented in this paper.

Very simple inductive ratio arm bridges may be assembled for the calibration of direct capacitance of three-terminal capacitors or capacitance differences of two-terminal variable air capacitors using fixed three-terminal capacitors as reference standards.

\section{EQUIPMENT}

Commercially available inductive voltage dividers can be used as irductively-coupled ratio arms of a capacitance bridge. The accuracy of ratio of the arms thus formed often far exceeds that needed for the calibration of variable air capacitors, even if the nominal ratio is used without correction.

Because of the inherent freedom from variations of their direct capacitance, it is desirable to use three-terminal capacitors fnow commercially available; as fixed standards, particularly with bridges having inductively-coupled ratio arms. The stray capacitances to ground are then across either the detector or the ratio arms. A small capacitance across the detector generally does no 
more than reduce the sensitivity, and the effect on accuracy of loading the closely coupled ratio arms, which have very small equivalent series impedance, is negligible.

\section{THE BRIDGE CIRCUIT}

Figure 1 shows an oscillator-amplifier power supply connected to the extremities of an inductive voltage divider, to one terminal of the fixed standard capacitor, $C_{S}$, and to one terminal of the variable capacitor under test, $C$. The other terminal of each of the capacitors is connected to one input terminal of a sensitive ac detector by means of well-shielded cable with shielded connectors. The shields of these cables, the other terminal of the detector, and the variable tap on the inductive voltage divider are connected together and to ground. The variable capacitor is set to the calibration point and the inductive voltage divider adjusted until the bridge is balanced. If $\mathrm{A}$ is the reading of the inductive voltage divider, $C=\left(\frac{1}{A}-1\right) C_{S}$. For best precision it is suggested that $\mathrm{C}_{\mathrm{S}}$ be approximately equal to the maximum capacitance of $\mathrm{C}$.

In Figure 2, a modification is shown in which the inductive voltage divider is grounded at a fixed tap, $A_{T}$, by means of a separate wire connected to one contact of the first decade switch. When the bridge is balanced, $C=\left(\frac{A}{A_{T}}-1\right) C_{S}$, and if $A_{T}=0.5$, $\mathrm{C}=2(\mathrm{~A}-0.5) \mathrm{C}_{\mathrm{S}}$. This particular modification is rather convenient for routine calibrations because computations are simplified.

If good quality air capacitors are used in the above bridge circuit, it may not be necessary to provide for the conductance balance of the circuit. If better resolution is desirable, however, a small adjustable resistor of several hundred ohms or less may be placed 
In series with one or the other (as needed) of the capacitors in the unshielded leads to the inductive voltage divider as.shown in Figure 2. Resistors, so placed, may permit a considerable improvement in the precision of the balance.

It should be evident that one three-terminal variable capacitor, set to a known capacitance by means of a larger fixed standard, serves excellently as a temporary standard to extend measurements to smaller values. Several orders of magnitude can be covered by this means. If extension of measurements to larger capacitance is contemplated, consideration must be given to the effect of inductance in the leads and in the resistor mentioned above.

4. TWO-TERMINAL CAPACITANCE MEASUREMENTS

The electrical connections to a single-range, two-terminal, variable air capacitor are shown in Figure 3. It is evident that the case of the capacitor is not grounded. The admittance from case to ground is merely a load on the inductive voltage divider and is generally of no consequence. If the voltage applied to the extremities is kept low, there is no danger to the operator, but to avoid small but disconcerting changes in the balance of the bridge resulting from changing capacitance at the terminals, the observer should avoid touching the case of the capacitor during the balancing operation, and should keep away from the terminals. The uncertainties of capacitance at the terminals of two-terminal capacitors is discussed in detail in reference (2). If the unused terminal of a dual-range, two-terminal, variable air capacitor is normally left unconnected. then, when calibrating the capacitor by this method it will be necessary to place a guard cap over, but not touching, the unused terminal. The guard cap must be electrically connected to the case of the 
capacitor as shown in Figure 4. Capacitance differences corresponding to two settings of a variable air capacitor can be measured with excellent accuracy by this method.

\section{CONCIUSION}

Inductive voltage dividers can be used as ratio arms of a transformer capacitance bridge for two-terminal capacitance difference measurements as well as three-terminal capacitance measurements. 
(1) T. L. Zapf, Capacitor calibration by step-up methods, J. Res. NBS, 64C, 75 (1960)

(2) J. F. Hersh, A close look at connection errors in capacitance measurements, General Radio Experimenter. 33, (No. 7), 3 (1959)

The two following papers contain references to prior literature on bridges having inductively-coupled ratio arms.

(3) A. M. Thomson, The precise measurement of small capacitances, IRE Trans. on Instrumentation. I-7, 245 (1958)

(4) M. C. McGregor, J. F. Hersh, R. D. Cutkosky, F. K. Harris, and $F$. R. Kotter, New apparatus at the National Bureau of Standards for absolute capacitance measurement, IRE Trans. on Instrumentation. I-7, 253 (1958) 


\author{
DEPAR TMENT OF COMMERCE \\ NATIONAL BUREAU OF STANDARDS \\ Washington $25, \mathrm{D}$ 。C. \\ Errara to Accompany \\ NBS Handbook 77 - Volume I \\ Precision Measurement and Calibration \\ Electricity and Electronics
}

Printed on the reverse side of this sheet is a supplementary page numbered $316 \mathrm{a} / 6$ for inserting in NBS Handbook 77 , Volume I, Electricity and Electronics.

This page was omitted in the preparation of this volume for printing。

May 15, 1961

USCOMM-NBS- DC 


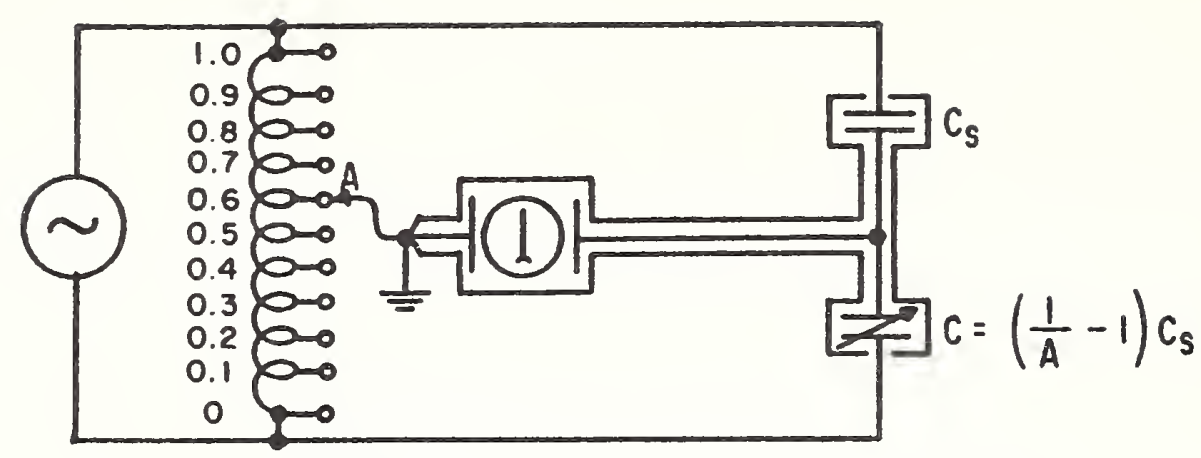

Figure 1. A simple transformer capacitance bridge for direct capacitance measurements.

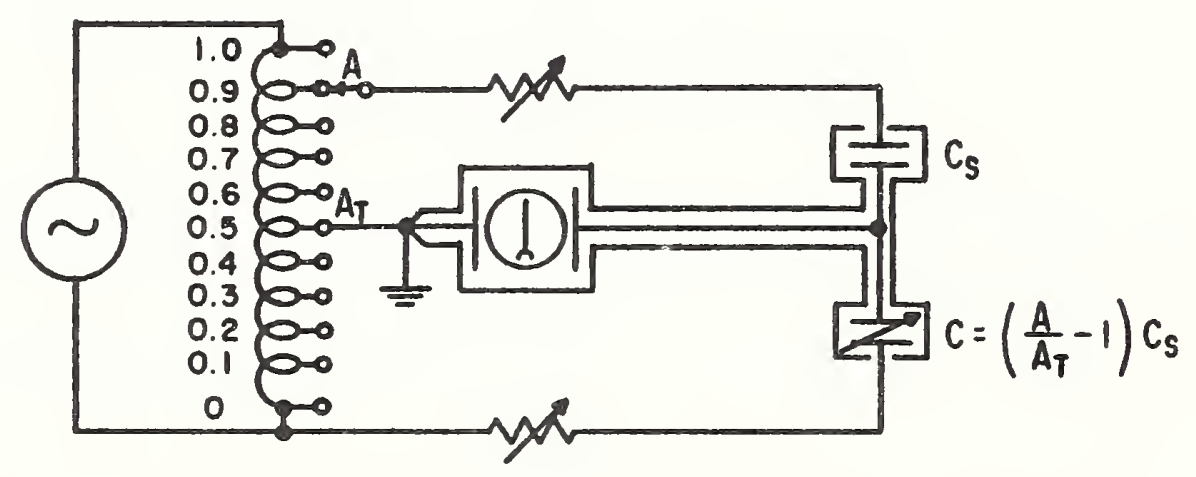

Figure 2. A modification of the bridge shown in Figure 1.

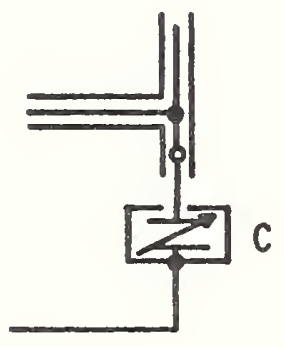

Figure 3. Connections for two-terminal capacitance measurements.

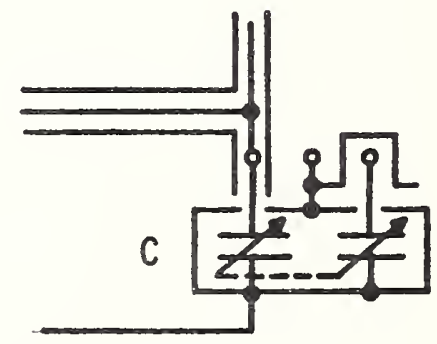

Figure 4. Connections to a dual-range, two-terminal variable air capacitor showing guard cap over unused terminal. 


\title{
Thermal Converters as AC-DC Transfer Standards for Current and Voltage Measurements at Audio Frequencies
}

\author{
Francis L. Hermach
}

\begin{abstract}
Thermal converters and associated equipment that are used as ac-de transfer standards at the National Bureau of Standards for the precise measurement of current and voltage at power and audio frequencies are described. The standards and the equipment are primarily used to standardize a-c ammeters and voltmeters submitted to the Bureau for certification. The ac-de transfer may be made with these thermal converters at currents from 1 milliampere to 50 amperes, voltages of 0.2 to 750 volts, with an accuracy of 0.01 percent at frequencies from 25 to 20,000 cycles per second.

The special tests to insure the required accuracy of the transfer standards are described, and the results are presented. A number of factors that limit the transfer accuracy of thermal converters have been discovered, and the results of special tests and theoretical work to evaluate these factors are discussed. The solutions, by an approximation method, of certain pertinent nonlinear differential equations governing the heating of a conductor by an electric current are given.
\end{abstract}

\section{Introduction}

The increasing use of electric energy for aircraft, induction furnaces, and induction heating, and the greater accuracy required in measurements in electronics, have led to increasing demands for the accurate standardization of ammeters and voltmeters at frequencies extending upward from power frequencies through the cntire audio-frequency range. To meet these demands, special instruments have been developed at the National Bureau of Standards for the measurement of current and voltage over rather wide ranges. They make use of thermal converters ${ }^{1}$ (often called thermoclements) like those incorporated in ordinary thernocouple instruments, but differ in the manner of reading and use. They may be used either directly to measure the ac-de differences of ammeters and voltmeter's, or with a suitable potentiometer and accessories to measure alternating currents and voltages. They were designed and are used primarily for testing electric instruments, at currents from $1 \mathrm{ma}$ to $50 \mathrm{amp}$ and voltages from 0.2 to $750 \mathrm{v}$, with an accuracy of 0.01 percent at frequencies from 25 to $20,000^{\circ} \mathrm{c} / \mathrm{s}$.

\section{Transfer Principle}

The basic electrical units are defined in terms of a concordant system of mechanical units and are realized by absolute electrical measurements carricd out at national standardizing laboratories to $\mathrm{fix}$ the value of groups of standard cells and resistors. These standards are used in conjunction with a potentiometer to make measurements of direct voltage, current, and power. It has been known for a long time, but not sufficiently realized, that the measurement of the corresponding alternating-

I Proposed AIEE definition 30.89.040: A thermal converter is a device that consists of one or more thermojunctions in thermal contact with an elcetric heater or integral therewith, so that the electromotive force developed at its output terminals by thermoelectric action gives a measure of the input current in its heater. current quantities depends fundamentally on certain standard types of electric transfer instruments that ideally have the same response on direct and alternating current. If the instruments are calibrated on direct current at the time of each use and if the precision of reading is suitably increased, long-time stability, freedom from drift, small temperature influence, low losses, and other normally desirable characteristics become of secondary importance, and the instruments may be primarily designed and constructed to have the best possible frequency characteristic. For difference measurements such instruments need not have long scales in the ordinary sense, but rather may be designed for increased precision of reading by methods that would not be applicable to instruments intended for general selvice.

Special electrodrnamic transfer instruments have long been used at the Bureau for accurate a-c measurements. These transfer' standards are used largely to standardize (test) other instruments submitted to the Bureau for certification. There are two distinct types of such tests. For the "straight a-c test", the instrument under test and a transfer standard are connected to measure the same alternating electrical quantity (current, voltage, or power), which is adjusted to produce the desired deflection of the test instrument. The response of the standard instrument is observed, then the standard is transferred to direct current. The direct quantity is adjusted to give the same response of the standard and is then measured with a suitable potentiometer and accessories. For the so-called "ac-dc difference test", both instruments are connerted to measure the same quantity first on alternating and then on direct current, which is in each case adjusted to give the same deflection of the test instrument. From the averaged difference in the response of the transfer standard, the ac-dc difference of the test instrument is computed. The second type of test can be made with somewhat higher accuracy than the first, and in conjunction 
with a test on direct current gives more information about the performance of the test instrument. Because the ac-de difference of an instrument depends upon geometrical factors that are relativelypermanent, subsequent tests for checking the constancy of calibration need generally be made only on direct current. Oceasionally transfer tests are made by using a selected low frequener (such as $60 \mathrm{c} / \mathrm{s})$ as the reference in place of direct current.

An example of the accuracy with which such tests are made, laboratory standard instruments, with seales $12 \mathrm{in}$. long, are regularly standardized and certificd at the Bureau to 0.05 of a scale division. For a 150-division instrument this requires an accuracy of measurement of 0.03 percent or better.

\section{Development and Description of Electro- thermic Transfer Standards}

\subsection{Choice of Standards}

Several types of instruments were considered in choosing transfer stanclarels to cover the full aucliofrequener range. Eleetrodynamie instruments have been highly developed $[1,2,3]^{2}$ at the Burcau for measurements at the commereially important power frequencies and can be used with proper colrections, up to about 2,000 c/s. However, unavoidable inductance anel stray capacitance errors limit their usceful frequencr range and present little hope for the desired extension. Electrostatic instruments have hoenl carefully studiod $[4,5]$ at the National Phrsical Laboratory. 'Lher are useful orer wide frequeney ranges. but because of their low-torque-weight ratio at fow roltage are not reaclily adaptable for either the low voltager or the current ranges desired in this application. Elecetrothermic instruments, which use an effect produced by the heating of a conductor carring a current to be measured, secmed more promising. Of the several kinds, thermocouple insiruments, in which the temperature rise of the con(huctor (heater) is measured by a thermocouple, sermed most foasible. Straight-wire heaters allow a wide frecgurency lange, and the thermocouple measurements permit high precision of reading. The ustal disadvantages of poor stability and large temperature influenec are not important in their use as transfer instruments, and their low orertoad rapareit $\mathrm{r}$ is not a scrious limitation in carculul laborator work, Fortumately, good thermal ronverters are commercially available. They have beem used for measurements at frequencies up to about $65 \mathrm{Mc}$, with a d-c millivoltmeter as the indierator, and are generally considered in the $1_{2-}$ to 2 -pereent aceuracy classes. Sio far as is known, they have not bech preriously studied for use as transfer instruments at the frecpionedes and the aceuracies needed in this application.

For these reasons, commereial thermal converters were selected for study to determine their value as ar-dle standalds in a wide-range audio-fregurney

\footnotetext{
Figures in torackets indicate the literature references at the end of this pajer.
}

transfer voltmeter, A careful experimental and theoretical study of their performance led to the purchase of additional commercial thermal converters of suitable ranges and the design and construction of equipment for using them as transfer standards for voltage and eurrent.

\subsection{Description of Transfer Standards}

Each thermal converter purchased in the milliampere ranges is of the vacuum type, mounted in an "vacuated glass bulb with supports of copper wire embedded in the glass. The conductor, heated by the current to be measured, is a short straight wire gencrally less than $1 / 2 \mathrm{~cm}$ long and often less than $0.001 \mathrm{ir}$. in diameter. The heater allor and dimensions are chosen to give a temperature rise of about $200^{\circ} \mathrm{C}$ at the centel where the hot junction of the thremocouple is fastened by a ceramic bead. This bead provides electrieal but not thermal insulation hetwern the heater and thermocouple circuits, with a coupling capacitance less than $1 \mu \mu \mathrm{f}$ and an insulation resistance greater than 50 megolms at $25^{\circ} \mathrm{C}$.

Becrase high current sensitivity is not required, the thermal converter's in the 1 - to 50 -amp ranges are not raacuated. 'The hot junction of the thermocouple is wededed chectly to the midpoint of a thinwalled tubular heater. 'These thermal converters aremperature compensated [6].

For current measurements, thermal converters in the series 1, 2, 5, 10, etc., with rated output electrometive forers of $10 \mathrm{mr}$ were purchased, with ranges from 1 ma to 50 amp. Thermal converters of 7.5and 30-ma ratings, with appropriate series resistors, are uscel for roltage measurements. Figures 1 and 2 show some of the thermal converters and the model $A$ and model B voltmeter elements. It should be cmphasized that the caption NBS on the nameplate shown in figure 2 signifies only that the equipment was assembled for use at the National Bureau of standards. All the thermal converters were purrhased commereially, as were the components of the roltmeters. It shoukd also be emphasized that the thermal converters shown in these fienres are the transfer clements only. An indicator (to be dis"ussod in the next section) is necessarr for" all measurements, and a potentioneter and accessories are also necessary, exeept for transfer tests of other instrumonts.

The moded A roltmeter was dereloped as a prototrpe instrument to meret an immerliate need for roltage measurements. It comsists of a 30-ma thermal converter commertad with its hater in sories with a commonecial decate resistance box: modified to have two l.0(0)-ohm-per-step derades. and a 100 -, a $10-$ and a 1 -ohm-por-step decade, all in series. As shown in figure:3, one cond of the heater of the thermat ronverter is annereded to the how-side terminal of the box, with the shichel of the box conmereted to the othere and of the heater and to the shickd of the roaxial cable romecting the instrument to the circuit. The center lead of this cable is ronnecterl direstly to the high-side terminal of the box. 


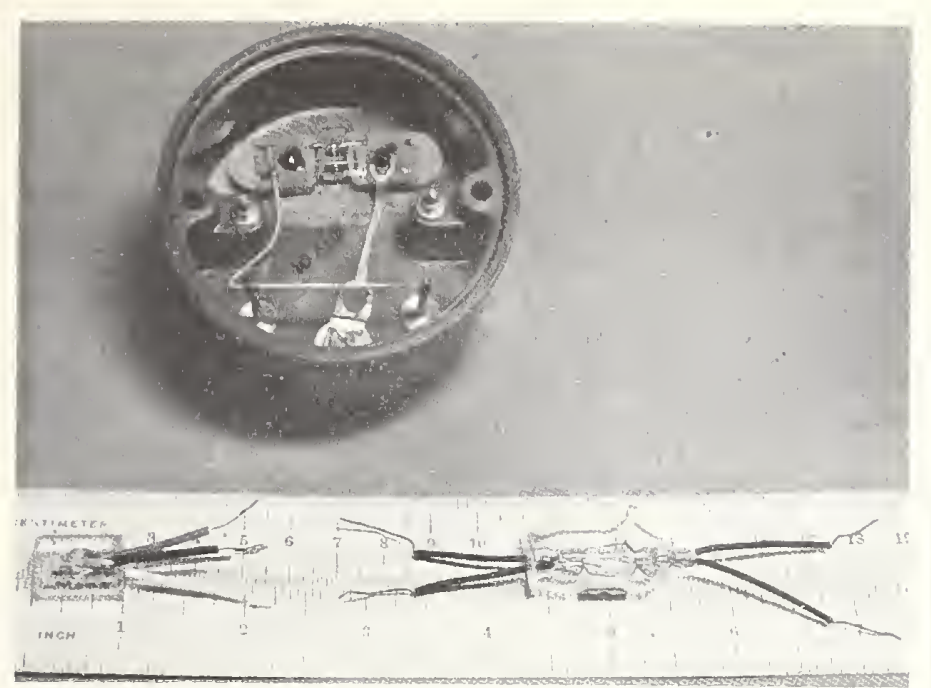

Figure 1. Typical thermal converters.

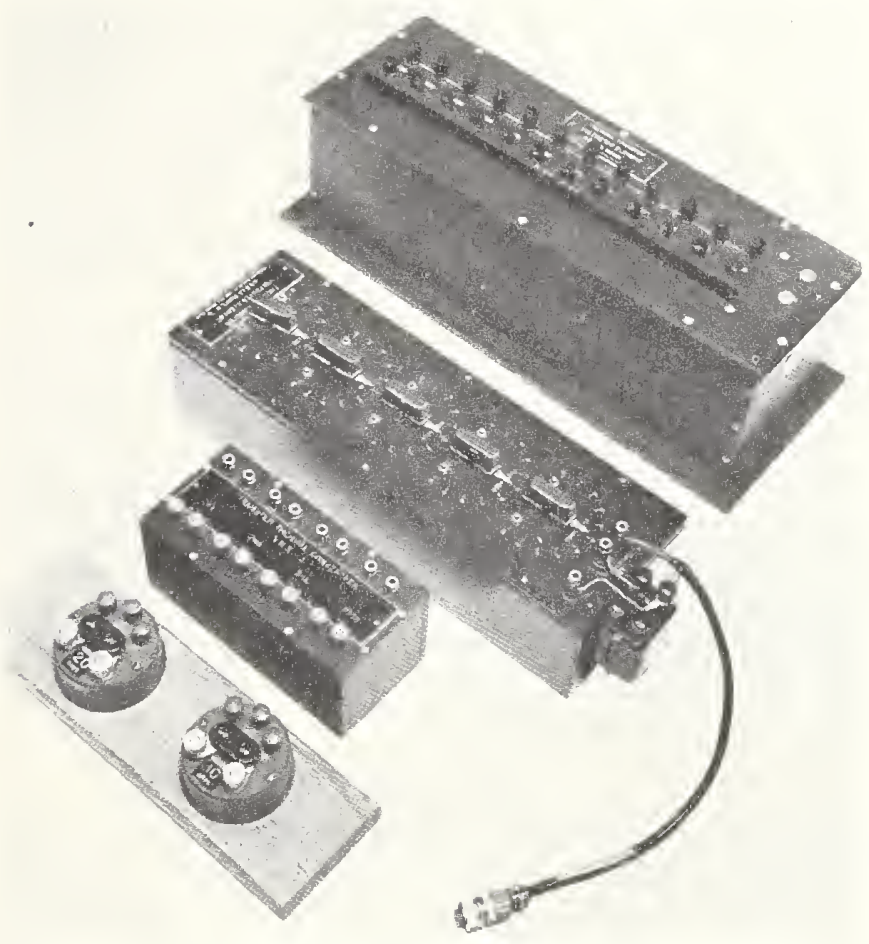

Firitin 2. Nounted thermal converters and the model $A$ and model $B$ voltmeter elements.

like the electrodymamic transfer standards used at lower frequencies, the series resistor is adjusted in use to give the same nominal current level for each measured voltage. This was chosen at $20 \mathrm{ma}$, resulting in a voltmeter having a constant $50 \mathrm{ohms} / \mathrm{v}$ and an upper lange of $400 \mathrm{v}$. The model A voltmeter. described in some detail in an earlier paper $[\tau]$ is now no longer used for routine measurements.

For the model B roltmeter, a built-in $7.5-\mathrm{ma}$ thermal ronverter was used, with a fixed resistor having taps to give voltage ranges of $1.5,3,6,7.5$, $15,30,60,75,150,300,600$, and $750 \mathrm{v}$. The resistance cards for this instrument were purehased rommoreially, and the cards and thermal ronverter wore mounted in a suitable shielded box with the shield connected to the low-side terminal of the instrument, as shown in figure 3. For both instruments the effects of capacitance currents and of the selfinductance of the resistors were carefully considered; the limiting factors are discussed in another section of this paper.

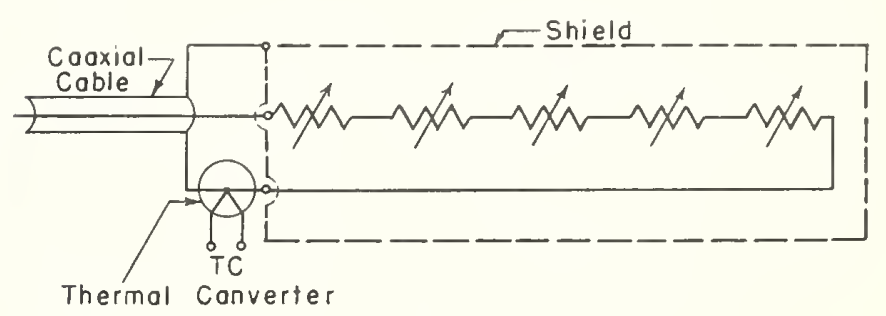

A

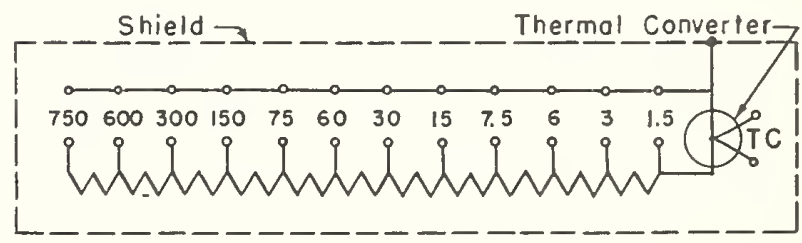

B

Figure 3. Circuit diagrams of the model $A$ and model $B$ voltmeter elements.

A, Model A voltmeter element; B, model B voltmeter element.

\subsection{Indicator}

Because of its convenience and portability, a millivoltmeter is generally used for measuring the emf in the usual thermocouple instrument. The precision of reading of the usual millivoltmeter is of course far too low for this application. For increased accuracy a potentiometer can be used to measure the thermocouple emf. However, if the thermal converter is used only as a transfer standard, the emf need not be measured in a "straight a-c" test; and in ac-de difference tests it is only the small change in emf between the a-c and d-c settings that is significant. The change and the full emf with which it is compared need be measured with relatively low accuracy, provided the change is referred to a highly stable base value. Thus high sensitivity and high stability but only moderate accuracy in the measurement of emf are required of the indicator. For these requirements, a lindeck potentiometer for providing the base value, used in conjunction with a suitable galvanometer, the deflection of which indicates the change, forms the ideal indicator. In a lindeck potentiometer, based on Poggendorf's second principle [8], the emf to be measured is balanced by an adjustment of the current through a fixed resistor. At balance the voltage drop across the resistor, which is the product of the potentiometer current and the resistance, is equal to the measured emf. The enrent is ordinarily measured with a milliam- 
meter, which generally sets the limit on the accuracy obtainable with this form of potentiometer.

Such a potentiometer has been incorporated in a panel constructed for the audio-frequency testing. It was designed to have adequate stability, freedom from changing extraneous electromotive force and the required ranges, with sufficient precision of reading. Its circuit is shown in the central portion of figure 4. The resistor, $R$, is made of manganin, and a special thermofree key [8] and copper binding posts are used in the electrothermally sensitive emf circuit. The components are mounted in the central part of the panel, as shown in figure 5, and are enclosed by a

\subsection{Use of Instruments}

These transfer standards are used almost solely for standardizing other a-c instruments. The necessary switching and control circuits for such tests have been incorporated in the panel shown in figure 5 . In addition to these circuits and the Lindeck potentiometer, the panel contains the impedance-matching transformers for the ligh-roltage circuits used in testing voltmeters and the high-current circuits used in testing ammeters. The present transformers provide ranges up to $50 \mathrm{amp}$ and $800 \mathrm{v}$, with a nominal input roltage of $150 \mathrm{v}$ and a power level up to $100 \mathrm{w}$.

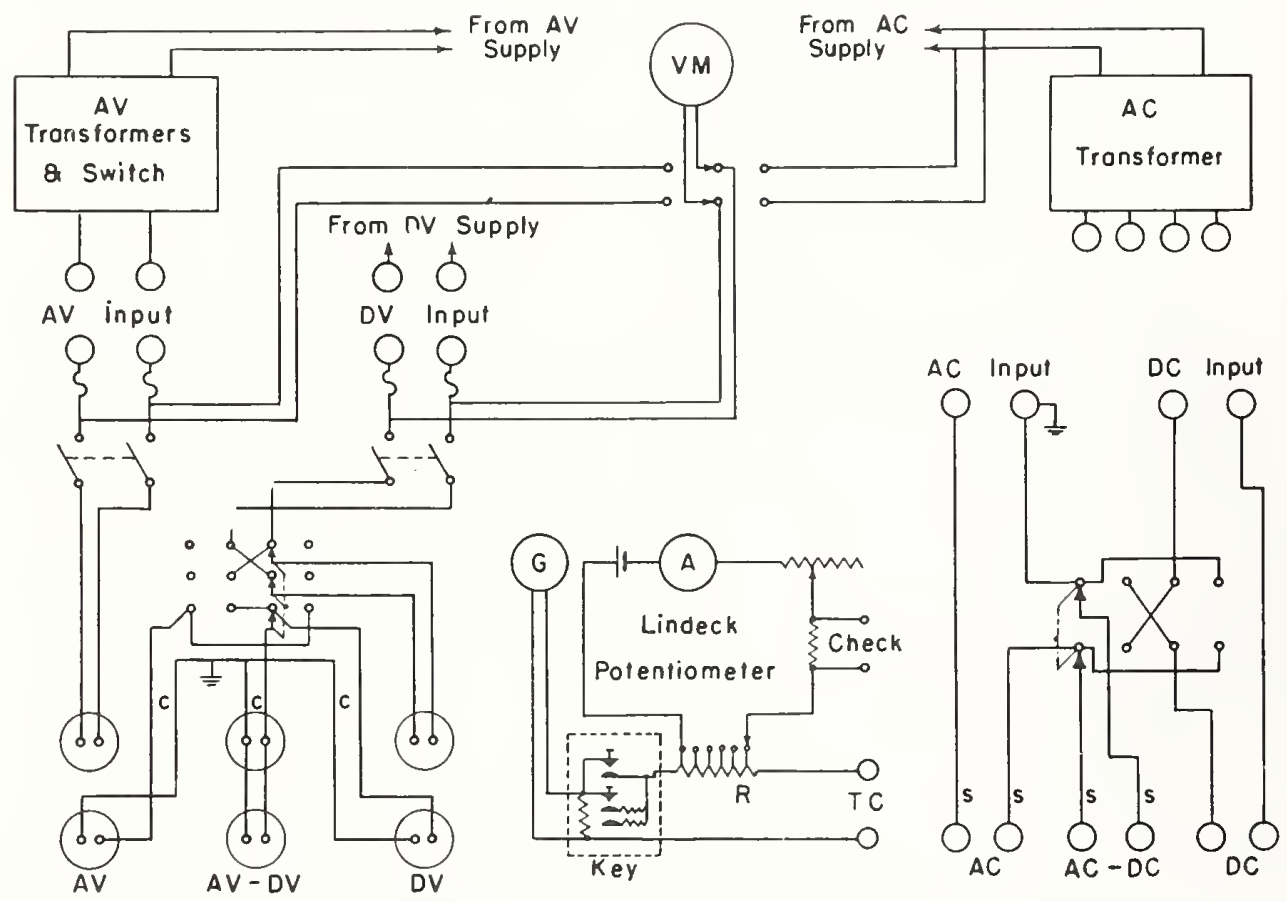

Figure 4. Schematic diagram of circuits used for testeng instruments. C signifies coaxial lead with shield grounded; s signifies shielded leak with shield grounded.

grounded metal shicld. No other thermal precautions were found necessary. A reflecting wallmounted galvanometer is used, with a voltage sensitivity (in the circuit) of $1 \mathrm{~mm} / \mu \mathrm{v} . \quad 1 \mathrm{No} .6$ dry ecll supplies the potentiometer current, which is adjustable from 0.2 to $1.2 \mathrm{ma}$. At these current lovels, repeated tests have shown that the steady drift in the voltage across the four terminal resistor is less than 0.02 pereent per hour, and that superposed fluctualtions are much less than 0.01 percent. The fourterminal mangmin resist or has taps at $0.1,0.5,2,5$, 5 , 10, and 25 ohms, giving milivolt ranges, at al current of $1 \mathrm{ma}$, of these same numerical values. The lowest range is used in transfer tests as a quick cherk of the potentiometer. With the thermocouple of the thermal converter connected to the "TC" posts of the potentiometer, but with no current through its heater, a galvanometer deffection of 10.0 $\mathrm{cm}$ to the loft on the scale with the Lindeck set for 0.1 mv serves as a raluable partial check of the required accuracy and correctness of the Lindeck circuit.
Motol-generator sets and voltage stabilizers are available for tests at $60 \mathrm{c} / \mathrm{s}$ and audio-frequency oscillators and power amplifiers for tests at other frequencies. Batteries, controlled by suitable adjustable resistance voltage dividers and series resistor's, alre used for the necessary direct-current sources. The potentiometer, standard cells, volt boxes, and resistors alr those normally used for instrument testing and ane periodically standardized to insure a rontinted areverat'y of rensiderably better than 0.01 perecolle.

A schematic diagram of the major eircuits and equipment on this panel is shown in figure 4 . For an a-c test of an anmeter, the transfer themoclement is connecterl to the AC-DC binding posts and its thermocouple to the TC posts on the pancl. 'The test instrument is connected to the $\mathrm{AC}$ posts, and a suitable four-terminal standard resistor is connected to the DC posts for use in measuring the d-c curlent by means of a potentiometer. For low currents at the higher audio frequencies, shiekded leads with the 
shields grounded are used to minimize errors due to stray capacitance currents. The alternating current is adjusted for the desired deflection of the test instrument, and the Lindeck potentiometer is adjusted for an "on-scale" deflection of the galvanometer. The standard instrument is then switched to direct current, which is adjusted to give the same galvanometer deflection and is then measured with the external potentiometer. The direct current through the heater of the thermoelement is then reversed and the d-c measurement repeated. ${ }^{3}$

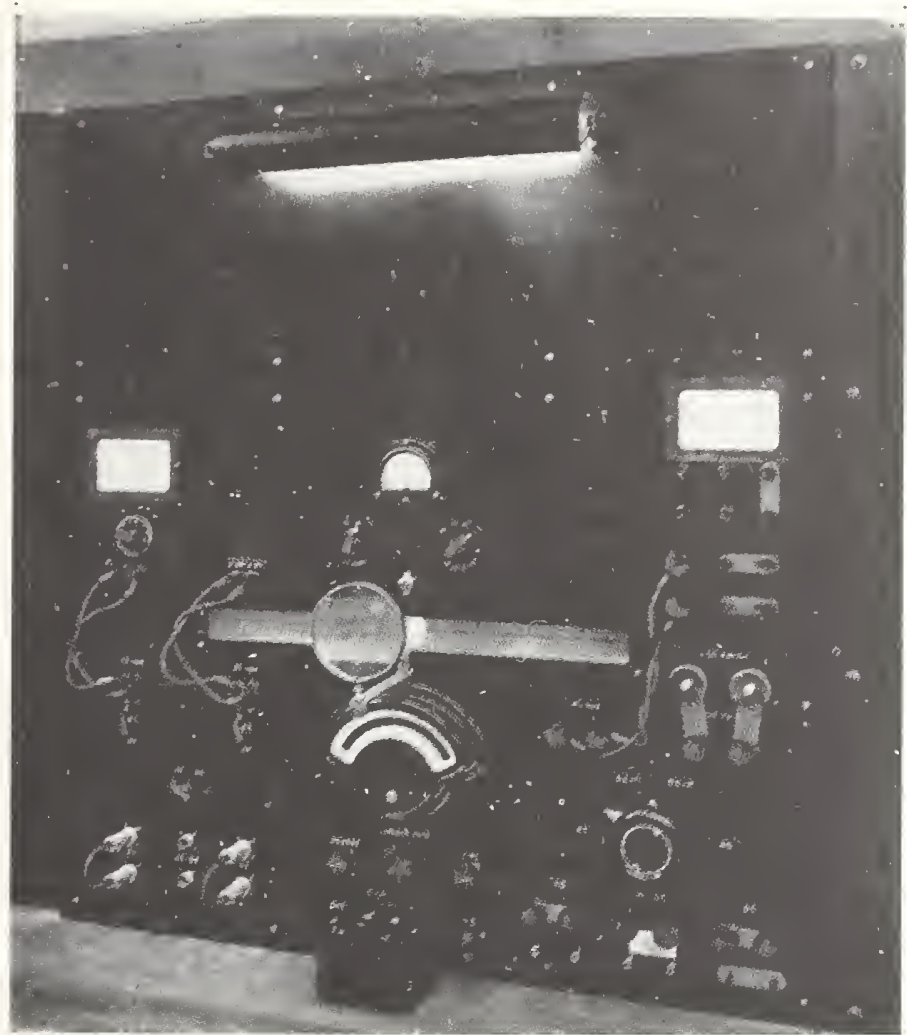

FIGURE 5. Instrument testing panel, including Lindeck element.

For an a.e test of a voltmeter, a similar procedure is used. The transfer standard is connected to the AV..DV receptacle on the panel with a coaxial lead, with due care to connect its thermal converter to the grounded shield of this lead, and the thermocouple is connected to the $\mathrm{TC}$ posts on the panel. The test instrument is connected to the circuit with a special twin lead consisting of two coaxial lines permanently paralleled at the instrument and connecting to the two AV receptacles on the panel. Similar leads of the same length are used to connect the high side of a volt box for use with the external poten.. tiometer to measure the direct voltage, to the two DV receptacles. The use of these leads eliminates the effect of any stray magnetic field or lead resistance on the measurements.

This simple procedure is reliable only if the dririt of the response of the standard instrument is negligibly small in the time taken for a series of such

${ }^{3}$ Such reversed d-c readings are common practice for highest accuracy with all types of ac-de instruments. readings. This is fortunately true for each of the thermal converters used. After an initial warm-up period of a minute or so, the maximum observed drift under those laboratory conditions of any of the thermal converters tested was less than 0.5 percent per hour, and the average less than 0.05 percent per hour. If it were necessary, the effect of significant drift could be practically eliminated by using a deflection method as outlined for transfer tests, calibrating the standard instrument on direct current at the nominally correct current or voltage before and after each a-c setting.

For ac-de difference or transfer tests, which are more frequent and usually more important than straight a-c tests, the test and standard instruments are connected in series to the $\mathrm{AC}-\mathrm{DC}$ posts ${ }^{4}$ in the case of ammeters, or in parallel with suitable coaxial leads to the $A V-D V$ receptacles in the case of voltmeters. Suitable short leads are used to connect the two $A V$ receptacles together and to connect the two DV receptacles together. The current or voltage is adjusted for the desired deflection of the test instrument, and the Lindeck potentiometer is adjusted for a deflection near the null position at the center of the galvanometer scale. The two instruments are then in quick succession connected to alternating, direct, reversed direct, and then alternating current. The current is adjusted to give the same deflection of the test instrument in each case. The resulting deflections of the galvanoneter of the Lindeck potentiometer are observed with the potentiometer controls undisturbed. This procedure practically eliminates any effect of drift in either instrument, and errors in repeating the settings of the instrument under test can be made very small by using a lowpower microscope and setting the image of the instrument pointer in coincidence with a cross-hair in the eyepiece. The control circuits have been arranged to facilitate switching, and with two observers the procedure is rapid even with moderately fluctuating voltages as only one of the two instruments is set to a given deflection.

From the average of the deflections on alternating and on direct current, the ac-de difference of the test instrument is readily computed by a simple formula. ${ }^{5}$

\section{Evaluation of Transfer Performance}

The ac--de difference of each transfer standard must be known to the full accuracy desired in the measurements to be made. As, in a sense, there are no absolute transfer standards, such an evaluation essentially consists of four steps.

1. Choice of a type of instrument that theory indicates is suritable over the desired ranges.

2. Study of all known effects that can cause such an instrument to depart from this ideal.

3. Construction and use of an instrument in such a way that these effects, by computation on direct tests of the separate components, are negligible or known.

${ }_{4}^{4}$ One terminal of the heater is connected to the post that is grounded on alternating current.

5 The formulas used in computing the results of the a-c and transfer tests are developed in appendix 1 . 
4. Comparison of actual transfer performance of the standard with that of some other instru.. ment, preferably of a quite different type, whose performance has also been evaluated.

The evaluation of these electrothermic transfer standards was considered of primary importance because of the high order of accuracy demanded in this application. The unique facilities of the Bureau made this quite feasible, particularly for step 4, which is necessary to guard against elrors from un. suspected causes. Prototype instruments such as these, in which previously available ranges are greatly extended, cannot be directly compared with other types of standards over their full range. The evaluation therefore consisted of the study, computations, and special tests of steps 2 and 3 during the design and construction of these instruments and their panel, the intercomparisons of thermal converters of adjacent current ranges in the series, the comparison at low frequencies of selected thermal converters with the electrodynamic transfer stand.. ards at the Bureau, and the comparison of these thermal converters with certain portable hot-wire and electrostatic instruments also available.

A thermal converter can be used as a transfer standard for alternating..current measurements over the range of frequencies for which the response char.. acteristic, $E=f(I)$, is the same as with direct current, where $E$ is the output emf, and $I$ the current through the heater of the thermal converter. For a volt.. meter element consisting of a resistor in series with a thermal converter, the nagnitude of the effective impedance $Z=V / I$ must also be equal to the effective resistance on direct current, where $V$ is the applied voltage. In addition to these two requirements for instrument testing, the fundamental principle that the standard and the instrument under test "see" exactly the same quantity must be carefully observed.

The response characteristic of the types of thermal converters used were believed to be suitable at frequencies far higher than those used in this applica- cation, and their direct-current and low-frequency responses were verified by special tests described in other sections. The effective impedance of each voltmeter element was approximately computed from inductance and capacitance data supplied by the manufacturer of the resistance cards, and was verified by special tests to be described later. The capacitance and resistance of the Lindeck potentiometer to ground were measured and found to be $1,600 \mu \mu \mathrm{f}$ and 10,000 megohms at an ambient relative humidity of 40 percent. 'Thus the computed stray currents are sufficiently small so that no appreciable fraction of the currents measured will be diverted through the thermocouple at audio frequencies when one end of the heater is grounded. The use of shielded leads where necessary, the avoidance of a ground between the test instrument and the standard in the current circuits, and the use of the special coaxial lines in the voltage circuits assure that the fundamental principle is observed even at the smallest current and voltage in the present ranges.

The comparison tests of step 4 were planned to give maximum possible assurance against error's due to unsuspected causes. Each comparison consisted of repeated careful transfer (ac-dc difference) tests, as described in section 3.4. The 1-, 2- and 5-amp thermal converters were compared with the NBS electrodynamic transfer ammeter at selected frequencies from 25 to $200 \mathrm{c} / \mathrm{s}$. The model A voltmeter was compared with the NBS suppressed-zero electrodynamic voltmeter at frequencies of 30 and $60 \mathrm{c} / \mathrm{s}$ and voltages from 20 to 240 v. Both of these electrodynamic instruments have been carefully studied $[1,2]$. They can be read with a precision of better than 0.005 percent. The results of these comparisons, corrected for the small known transfer errors of the electrodynamic instruments, are shown in table 1. They are estimated to be accurate to 0.005 percent or better. The results of these tests disclosed that the model $A$ voltmeter and the electrodynamic voltmeter were in excellent agreement at these frequencies.

TABLE 1. Results of comparisons of thermal converters with other types of instruments

\begin{tabular}{|c|c|c|c|c|c|c|c|c|c|c|c|}
\hline \multirow{2}{*}{$\begin{array}{c}\text { Thermal converter } \\
\text { range }\end{array}$} & \multirow{2}{*}{ Other instrument } & \multirow{2}{*}{$\begin{array}{c}\text { Test } \\
\text { current } \\
\text { or } \\
\text { voltage }\end{array}$} & \multicolumn{9}{|c|}{ Comparative ac-de difference } \\
\hline & & & $\begin{array}{l}25 \\
\mathrm{c} / \mathrm{s}\end{array}$ & $\begin{array}{l}30 \\
\mathrm{c} / \mathrm{s}\end{array}$ & $\begin{array}{l}60 \\
\mathrm{c} / \mathrm{s}\end{array}$ & $\begin{array}{l}100 \\
\mathrm{c} / \mathrm{s}\end{array}$ & $\begin{array}{l}200 \\
\mathrm{c} / \mathrm{s}\end{array}$ & $\begin{array}{c}1,000 \\
\mathrm{c} / \mathrm{s}\end{array}$ & $\begin{array}{c}10,000 \\
\mathrm{c} / \mathrm{s}\end{array}$ & $\begin{array}{c}15,000 \\
\mathrm{c} / \mathrm{s}\end{array}$ & $\begin{array}{c}20,000 \\
\mathrm{c} / \mathrm{s}\end{array}$ \\
\hline $\begin{array}{l}\quad a m p \\
1 \\
1 \\
1 \\
2 \\
2 \\
5 \\
5\end{array}$ & $\begin{array}{l}\text { Electrodynamic ammeter } \\
\text { do do do do do } \\
\text { Hot-x ire ammeter } \\
\text { do do }\end{array}$ & $\begin{array}{c}a m p \\
0.4 \\
1 \\
1.2 \\
0.8 \\
2 \\
2.4 \\
4 \\
5 \\
6 \\
4 \\
5\end{array}$ & $\begin{array}{c}\% \\
-0.001 \\
-.053 \\
-.067 \\
-.022 \\
-.115 \\
-.154 \\
-.004 \\
-.010 \\
-.011\end{array}$ & $\begin{array}{c}\% \\
\\
\\
\end{array}$ & $\begin{array}{r}\% \\
+0.001 \\
-.013 \\
-.001 \\
-.025 \\
-.035 \\
-.006 \\
-.018 \\
\end{array}$ & $\begin{array}{r}\% \\
-0.001 \\
-.008 \\
-.008 \\
+.001 \\
-.009 \\
-.015 \\
-.005 \\
-.005 \\
-.008 \\
-\end{array}$ & $\begin{array}{r}\% \\
-0.002 \\
-.001 \\
-.005 \\
+.001 \\
+.001 \\
-.002 \\
\end{array}$ & $\begin{array}{c}\% \\
\\
\\
-007 \\
-07 \\
\end{array}$ & $\begin{array}{c}\%_{0} \\
\\
\\
-0.004\end{array}$ & $\begin{array}{c}\% \\
\\
\end{array}$ & $\begin{array}{c}\% \\
\\
\\
\end{array}$ \\
\hline $\begin{array}{l}\text { Model A voltmeter } \\
\text { Do } \\
\text { Do } \\
\text { Do } \\
\text { Do Do } \\
\text { Do }\end{array}$ & $\begin{array}{l}\text { Electrodynamic voltmeter } \\
\text { do do }\end{array}$ & $\begin{array}{r}r \\
50 \\
50 \\
120 \\
150 \\
240 \\
150 \\
300\end{array}$ & (n) & $\begin{array}{l}-0.002 \\
+.004 \\
+.004\end{array}$ & $\begin{array}{r}-.004 \\
-.005 \\
-.003 \\
-.002 \\
\end{array}$ & $\begin{array}{r}-1.2 \\
-.002 \\
-.004\end{array}$ & 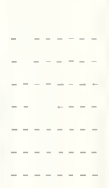 & $\begin{array}{l} \\
-\ldots . . \\
+.004 \\
-.010\end{array}$ & $\begin{array}{c}\ldots \\
\ldots \\
\cdots \\
-.004 \\
\cdots\end{array}$ & -0.008 & $\begin{array}{l} \\
-.002 \\
+.009\end{array}$ \\
\hline
\end{tabular}


They disclosed significant disagrecments between the ampere-range thermal converters and the electrodynamic ammeter, which were considered to be due to a low-frequency error in the 1-and 2-amp thermal converter's and a small error independent of frequency in the 5-amp thermal converter.

'The model A voltmeter was also compared with two new high-grade portable pivoted clectrostatic voltmeters, having scales over 5 in. long, at selected frequencies from 30 to $20,000 \mathrm{c} / \mathrm{s}$. For increased precision a low-power microscope was focused on the pointer of the electrostatic instrument under test. This instrument was always set to the same deflection and was tapped lightly before cach reading. Repeated sets of careful readings were taken at each frequency, and all tests were at the full-scale deflection of the electrostatic instrument. Because of these repeated readings and the favorable conditions, the averaged results are estimated to be accurate to 0.01 percent. The results, as shown in table 1 , verify the absence of capacitance error's in the thermal voltmeter. Direct comparisons at the higher frequencies could not be made at lower voltages, but calculations indicate that skin effect and the effect of inductance should be considerably less than 0.01 percent at $20,000 \mathrm{c} / \mathrm{s}$.

Similarly, the 5-amp thermal converter was compared with a high-quality portable 5-amp hot-wire ammeter at frequencies from 25 to $20,000 \mathrm{c} / \mathrm{s}$. The results, as shown in table 1 , indicate excellent agreement at frequencies of $1,000 \mathrm{c} / \mathrm{s}$ and above. Tests at lower frequencies showed very puzzling discrepancies, which, in a separate investigation, were traced to vibration errors ${ }^{c}$ in the hot-wire instrument. For that reason the results at frequencies less than $1,000 \mathrm{c} / \mathrm{s}$ are not shown in this table.

A plus sign in table 1 indicates that with equal response of the thermal converter on direct and alternating currents the response of the other instrument was greater on alternating than on direct curlent.

Each thermal converter was compared with the next higher- and lowel-range thermal converter in the series at two curents and at selected frequencies from 25 to $20,000 \mathrm{c} / \mathrm{s}$. The results of each of these comparisons at the highes of the two currents at which tests were made are shown in table 2 . 'They' are estimated to be accurate to somewhat bettel than 0.01 percent, with very little possibility of systematic error. A plus signi in the table indieates that with equal response of the lower-range thermal ronverter on alternating and on direct currents, the response of the higher-lange thermal converter was greater on alternating than on direct currents. This series of tests was made to extend stepwise the results of low-frequency comparisons with the electrodynamic ammeter, and to indicate any highfrequency offects that might well be expected to differ in different thermal converters. ${ }^{7}$ The group

\footnotetext{
Elretromagnetic forees between the hot wire and its elosely adjacent iron mounting plate resulted in vibration of the wire because the plate was magnetized
by the damping magnet of the instrument. This vibration eaused additional cooling and thus an crror that was as larye as 0.2 percent at certain frequencies. cooling and thus an crror that was as large as 0.2 percent at certain frequencies. to be independent of the current level and could thus be evaluatcd by these tests
}

TABLE 2. Results of intercomparisons of thermal converters

\begin{tabular}{|c|c|c|c|c|c|c|c|}
\hline \multirow{2}{*}{$\begin{array}{l}\text { Thermal converter } \\
\text { ranges }\end{array}$} & \multirow{2}{*}{$\begin{array}{l}\text { Test } \\
\text { cur- } \\
\text { lent }\end{array}$} & \multicolumn{6}{|c|}{ Comparative ac-de difference } \\
\hline & & $\begin{array}{l}25 \\
\mathrm{c} / \mathrm{s}\end{array}$ & $\begin{array}{l}100 \\
\mathrm{c} / \mathrm{s}\end{array}$ & $\begin{array}{l}400 \\
\mathrm{c} / \mathrm{s}\end{array}$ & $\begin{array}{c}1,000 \\
\mathrm{c}_{l}^{\prime} \mathrm{s}\end{array}$ & $\begin{array}{c}10,000 \\
e / s\end{array}$ & $\underset{\mathrm{c} / \mathrm{s}}{20,000}$ \\
\hline $\begin{array}{l}1 \text { to } 2 \mathrm{ma} \ldots \ldots . . . \\
2 \text { to } 5 \mathrm{mi} \ldots \\
5 \text { to } 10 \mathrm{ma} \ldots \\
10 \text { to } 20 \mathrm{ma} \ldots \\
20 \text { to } 30 \mathrm{ma} \mathrm{a}_{\text {a. }}\end{array}$ & $\begin{array}{c}m a \\
1.2 \\
2.4 \\
6 \\
12 \\
24\end{array}$ & $\begin{array}{r}\% \\
.0 .000 \\
+.006 \\
+.004 \\
.000 \\
+.001\end{array}$ & $\sigma_{c}$ & -0.003 & $\begin{array}{r}\% \\
0.000 \\
+.003 \\
.000 \\
+.004 \\
-.001\end{array}$ & $\%$ & $\begin{array}{r}\% \\
+0.006 \\
-.003 \\
-.000 \\
-.006 \\
.000\end{array}$ \\
\hline $\begin{array}{l}30^{a} \text { to } 50 \mathrm{ma} \\
50 \text { to } 100 \mathrm{ma} \\
100 \text { to } 200 \mathrm{ma} \\
200 \text { to } 506 \mathrm{ma} \\
500 \text { to } 1.000 \mathrm{ma}\end{array}$ & $\begin{array}{r}36 \\
60 \\
100 \\
240 \\
600\end{array}$ & $\begin{array}{r}+.004 \\
+.024 \\
-.046 \\
+.026 \\
-.002\end{array}$ & +0.028 & & $\begin{array}{r}.000 \\
+.036 \\
-.044 \\
-.002\end{array}$ & $\begin{array}{r}-0.050 \\
-.002\end{array}$ & $\begin{array}{r}.000 \\
+.027 \\
+.049 \\
+.039 \\
+.002\end{array}$ \\
\hline $\begin{array}{l}1,000 \mathrm{ma} \text { to } 1 \mathrm{amp} \\
\text { 1 to } 2 \mathrm{amp}- \\
2 \text { to } 5 \mathrm{amp}- \\
5 \text { to } 10 \mathrm{amp} \\
10 \text { to } 20 \mathrm{amp} \\
20 \text { to } 50 \mathrm{amp}\end{array}$ & $\begin{array}{l}a m p \\
1.2 \\
1 \\
2 \\
5 \\
10 \\
20\end{array}$ & $\begin{array}{l}+.034 \\
-.028 \\
-.123 \\
-.001 \\
-.010 \\
-.014\end{array}$ & $\begin{array}{l}-.002 \\
-.011 \\
-.006 \\
-.010 \\
-.015\end{array}$ & $\begin{array}{l}-. . \\
-.0 ! 4\end{array}$ & $\begin{array}{l}-.065 \\
-.002 \\
+.003 \\
-.006 \\
-.008 \\
-.018\end{array}$ & $\begin{array}{r}-.056 \\
-.002 \\
+.004 \\
-.007 \\
-.012 \\
-.035\end{array}$ & $\begin{array}{r}-.065 \\
+.001 \\
+.009 \\
-.004 \\
-.009\end{array}$ \\
\hline
\end{tabular}

a This 30 -ma thermal converter was the one used in the model A voltmeter.

of tests disclosed small discrepancies independent of frequency in a few thermal converters, and verified the absence of any high-frequency errors in the thermal converters tested. However, they could not be used to assign numerical values to the errors of individual thermal converters in the milliampere range as the differences were found to depend upon the current. The concluding series of tests to evaluate these crrors were comparisons of a shunted thermal converter with each of those pairs of thermal converter's that showed discrepancies in the previous intercomparisons. The model A voltmeter was shunted with a bifilar resistor of computed skin effect and time constant for tests at currents from 1 to 40 amps, and with a suitable high-quality audio-frequeney decade resistance box for tests at lower currents. The results are shown in table 3 and are estimated to be accurate to better than 0.01 percent. $A$ positive sign in this table indicates that with equal

TABLE 3. Results of comparisons of selected thermal convcrters with shunted model $A$ thermal voltmeter

\begin{tabular}{|c|c|c|c|c|}
\hline \multirow{2}{*}{$\begin{array}{c}\text { Thermal converter } \\
\text { range }\end{array}$} & \multirow{2}{*}{$\begin{array}{l}\text { Test } \\
\text { current }\end{array}$} & \multicolumn{3}{|c|}{ Comparative ac-de difference } \\
\hline & & $25 \mathrm{c} / \mathrm{s}$ & $100 \mathrm{c} / \mathrm{s}$ & $400 \mathrm{c} / \mathrm{s}$ \\
\hline \multirow[t]{2}{*}{$m a t$} & \multirow{6}{*}{$\begin{array}{r}m a \\
60 \\
70 \\
100 \\
1<0 \\
240 \\
200 \\
400 \\
600 \\
400 \\
600 \\
800\end{array}$} & $\begin{array}{c}\% \\
-0.003\end{array}$ & $\%$ & $\%$ \\
\hline & & - & $\begin{array}{r}-0.043 \\
-.062\end{array}$ & -....... \\
\hline 200 . & & $\begin{array}{r}-.065 \\
-.010\end{array}$ & +.002 & $\begin{array}{r}-0.065 \\
+.00^{2}\end{array}$ \\
\hline $500 \ldots$ & & $\begin{array}{l}-.044 \\
-.056\end{array}$ & - & $\begin{array}{l}-.042 \\
-.054\end{array}$ \\
\hline & & $\begin{array}{l}-.068 \\
-.041\end{array}$ & -.070 & -.069 \\
\hline $1,000 \ldots \ldots$. & & -.061 & $\begin{array}{l}-.060 \\
-.059\end{array}$ & (n) \\
\hline$a m p$ & $a m p$ & -.004 & & -.006 \\
\hline $10 \ldots$ & $\begin{array}{l}10 \\
12\end{array}$ & -018 & -.014 & $\begin{array}{l}-.015 \\
-.020\end{array}$ \\
\hline & 10 & -.003 & ........... & -.006 \\
\hline $20 \ldots$ & 20 & $\cdots-\cdots$ & -.015 & -.017 \\
\hline & 20 & -.008 & (n........ & -.010 \\
\hline & 40 & -.010 & -.010 & -.010 \\
\hline
\end{tabular}


response of the thermal converter on alternating and on direct current the response of the shunted model A voltmeter was greater on alternating than on direct current. The results of tests of the 1-, 2* and 5 -amp thermal converters, duplicating those with the electrodynamic ammeter, are not shown in the table. The average difference between eight such duplicate results obtained with this ammeter and with the shunted model A voltmeter was 0.004 percent, and the largest difference was 0.013 percent. A few additional check tests of these thermal converters with the shunted voltmeter were made at other frequencies but are not tabulated.

The results of all these tests were combined to assign reliable values of ac-dc difference, $\delta$, to each thermal converter in the series from $1 \mathrm{ma}$ to $50 \mathrm{amp}$ and to the model A voltmeter. These values were less than 0.01 percent for the model $\mathrm{A}$ voltmeter and for all the thermal converters of ranges less than 100 ma. The values for the other converters (rounded off to the nearest $0.005 \%$ ) are shown in table 4 .

TABLE 4. Observed percentage ac-dc differences of thermal converters (to the nearest $0.005^{\circ}$ percent)

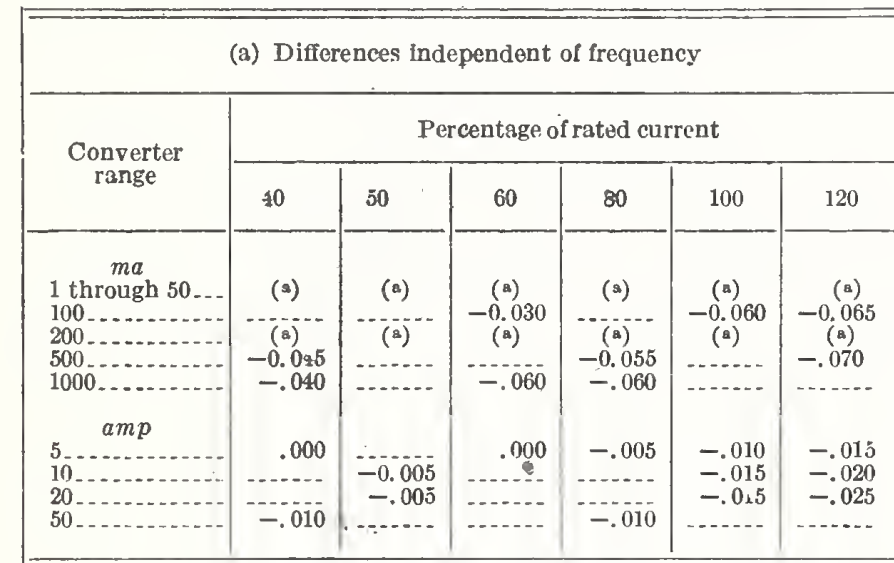

(b) Differences dependent upon frequency

\begin{tabular}{|c|c|c|c|c|c|}
\hline \multirow{2}{*}{$\begin{array}{c}\text { Converter } \\
\text { range }\end{array}$} & \multirow{2}{*}{$\begin{array}{c}\text { Test } \\
\text { current }\end{array}$} & \multicolumn{4}{|c|}{ Frequency (cycles per sccond) } \\
\hline & & 25 & 60 & 100 & 200 \\
\hline " $a m p$ & $\left\{\begin{array}{l}0.4 \\
1.0 \\
1.2\end{array}\right.$ & $\begin{array}{r}-0.010 \\
-.055 \\
-.065\end{array}$ & $\begin{array}{r}0.000 \\
-.015\end{array}$ & $\begin{array}{r}0.000 \\
-.010 \\
-.010\end{array}$ & $\begin{array}{r}0.0 C 0 \\
-.000 \\
-.005\end{array}$ \\
\hline $2 \ldots \ldots$ & $\left\{\begin{array}{l}0.8 \\
2.0 \\
2.4\end{array}\right.$ & $\begin{array}{l}-.020 \\
-.120 \\
-.155\end{array}$ & $\begin{array}{r}.000 \\
-.025 \\
-.035\end{array}$ & $\begin{array}{r}.000 \\
-.010 \\
-.015\end{array}$ & $\begin{array}{l}.000 \\
.000 \\
.000\end{array}$ \\
\hline
\end{tabular}

$\triangle$ Less than 0.01 percent throughout.

The model B voltmeter, which was developed to give a wider voltage range and greater convenience of use than the model A, was compared with the model A voltmeter at rated voltage on each range up through the $300-\mathrm{v}$ range and at $400 \mathrm{v}$ on the higher ranges, at selected frequencies from 30 to $20,000 \mathrm{c} / \mathrm{s}$. The results are shown in table 5 and represent directly the ac-dc difference, $\delta$, of the model B voltmeter. They disclosed significant but not unexpected errors for the higher voltage ranges at the higher frequencies.
TABLE 5. Results of comparisons of model $A$ and model $B$ thermal voltmeters

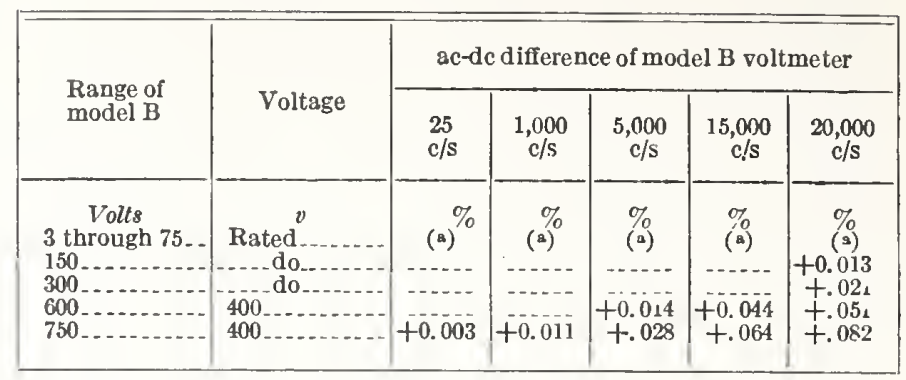

a Less than 0.01 percent, 25 to $20,000 \mathrm{c} / \mathrm{s}$ each range,

As a result of these extensive series of tests, the transfer performance of each of this first set of thermal converters and of each of the two voltmeters was uniquely determined. Small but significant acde differences of both known and unsuspected causes were found. Corrections for these differences can readily be applied when these thermal converters are used as transfer standards. However, an investigation of such differences is an especially important objective of a development such as this. As a result of such an investigation, a number of factors that limit the transfer accuracy of an electrothermic instrument were discovered and evaluated, with the results given in the following sections.

\section{D-C Response}

In all measurements with a transfer standard the average of the $d-c$ response of the standard for the two directions of current is taken as the reference upon which the a-c measurements are based. For all three types of such standards, the reversed $d-c$ average gives a better basis than does the response for one direction alone as effects that lead to small differences in response for the two directions of direct current are reduced to the second order of smalhness when the average is taken.

Reversal differences may be expected in a contact thermal converter because of the flow of heater current through the hot junction of the thermocouple. This can cause a voltage drop in the thermocouple circuit, which may lead to second-order error's because of the nonlinearities of the thermal and electric circuits. This cause of reversal difference is minimized by the special constructions used in most high-range thermal converters, and is eliminated by the use of the small insulating bead between the heater and the thermocouple in the low-range thermal converters. Surprisingly, however, early tests of the first thermal converters purchased for the model A voltmeter showed appreciable reversal differences (up to $0.2 \%$ ) even in these insulated-heater ther'mal converters. It was soon realized that this was prol-ably due to thermoelectric effects in the heater circuit. Peltier and Thomson heating of the heater can cause dissymmetry in the temperature rise of the heater, and thus cause differences in the emf of the thermocouple unless the hot junction is exactly at the 
midpoint of the heater. ${ }^{8}$ This supposition was verified by an analysis of the data, which showed that the differences were larger in those thermal converters with heaters having larger thermoelectric effects. Further verification was secured by tests of special thermal converters, constructed by one of the manufacturers ${ }^{9}$ at the Bureau's request, with the hot junction of each deliberately and considerably offcenter. The reversal differences were much larger, and the signs and magnitudes were found to be roughly in agreement with calculations based on thermoelectric data for the alloys used.

Early intercomparisons of these few thermal converters indicated that no significant ac-dc differences should be expected, even with rather large reversal differences from this cause, provided that the average for the two directions of current is taken as the $\mathrm{d}$-c response. However, intercomparisons and tests of the much larger number of thermal converters later purchased for current measurements showed that a good proportion had measurable ac-dc differences, all of the same sign and independent of frequency. This suggested a d-c error, for which these thermoelectric efforts seemed the most likely cause. This was investigated theoretically (see appendix 2) by considering the temperature rise of a homogeneous conductor heated by an electric current and cooled solely by conduction to two relatively massive terminals; i. e. by neglecting, for simplification, radiation and convection losses and the heat abstracted by the attached thermocouple.

With only the normal joule, or resistance, heating, and with the terminals of the conductor at equal and fixed temperature, the temperature distribution along the conductor is parabolic, and the temperature rise at the midpoint is, as Goodwin showed, ${ }^{10}$ equal to

$$
\theta_{d}=\begin{gathered}
V_{c}^{2} \\
8 \rho k
\end{gathered}
$$

where $V_{c}$ is the voltage drop across the conductor, and $\rho$ and $k$ are the electrical resistivity and thermal conductivity, respectively. In addition, however, there is Peltier heating or cooling at the junctions of dissimilar metals (the two junctions between the heater and its two supports) and Thomson heating along each half of the wire. Unlike joule heating, these are dependent upon the direction of the current flow and can thus cause a dissymmetry of the temperature distribution along the conductor, which reverses when the direct current through the conductor is reversed. On alternating current, even at the low frequencies in which we are interested, the reversal of current occurs so rapidly that the thermal inertia of the wire prevents any such dissymmetry, and the temperature dis-

\footnotetext{
${ }^{8}$ This dissymmetry has actually been utilized to measure the Thomson effect.

See [9]. the reversal differences were dependent upon the type of heater material.

to See [6]. In addition to this elassic paper, Goodwin has written an excellent series of artieles on "Thermal problems relating to measuring and control services", which appeared in Weston Eng. Notes, 1948-50 (Weston Electrical Instrument Corp., Newark, N. J.).
}

tribution is unchanged by these thermoelectric effects. Calculations show (see appendix 2) that with direct current the temperature rise at the midpoint of the conductor, where the hot junction of the thermocouple is attached, is unaffected by the Peltier heating, for completely symmetrical construction of a thermoelement. They show, however, that it is changed by Thomson heating, and becomes, approximately, ${ }^{11}$

$$
\theta_{t}=\theta_{d}\left[1-\frac{4}{3}\left(\frac{\sigma \theta_{d}}{V_{c}}\right)^{2}\right]
$$

where $\theta_{t}$ is the midpoint temperature rise in the presence of Thomson heating, in ${ }^{\circ} \mathrm{C} ; \theta_{d}$, the rise without Thomson heating; $\sigma$, the Thomson voltage coefficient in volts-degree ${ }^{-1}, V_{c}$, the voltage across the conductor in volts.

This was derived by considering $\sigma$ a constant, whereas both experimental results and the electron theory of metallic conduction indicate that $\sigma=B T$, where $B$ is a constant and $T$ is the absolute temperature. However, a solution by a perturbation method, ${ }^{12}$ of the nonlinear equation that results when $\sigma=B T$, shows that the above results give a sufficiently close approximation for the temperature rise of about $200 \mathrm{deg} \mathrm{C}$ encountered in typical thermal converters. Thus eq 2 shows that an ac-dc difference, or transfer error, should be expected in a thermal converter, and enables the approximate calculation of this error. Such calculations have been made for some common metals and some of the alloys used as heaters in thermal converters, on the assumption of a $200 \mathrm{deg} C$ rise at the midpoint, and a voltage drop of $0.2 \mathrm{v}$ at rated currents. ${ }^{13}$ The results, converted to ac-dc difference for the same temperature rise, rather than temperature difference for the same current, are shown in table 6 . For the computation of these results, the Thomson coefficient $\sigma$ was considered to be equal to $B\left(\theta_{0}+2 / 3 \theta_{d}+273\right)$, where $B$ is the second derivative of the characteristic curve of emf versus temperature for a thermocouple composed of the indicaied metal and lead. ${ }^{14} \quad \theta_{0}$ is the ambient temperature in $\operatorname{deg} \mathrm{C}$. The results show approximately the errors to be expected for these materials. TABLE 6. Calculated $x c$-dc difference at rated current due to
Thomson effects in thermal converter heaters

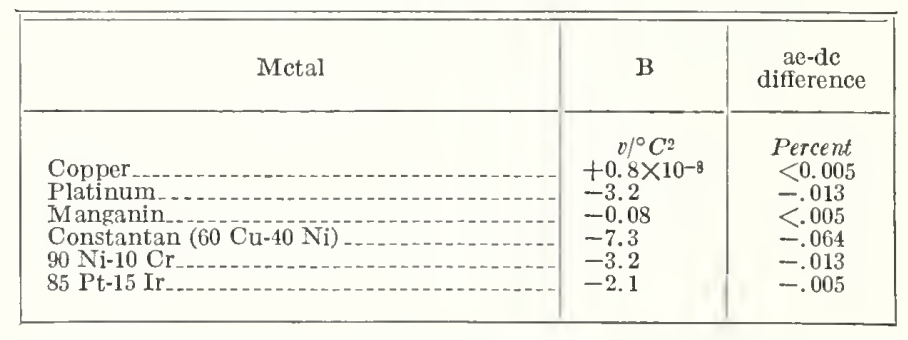

11 At other points along the eonductor the average temperature rise for the two direetions of direct eurrent is also ehanged by Thomson heating.

${ }^{12}$ This method was suggested by Chester Snow.

${ }^{13}$ From eq 1, a series of thermal converters having similar thermoeouples and the same rated output emf at rated current, and having heaters that obey the Weidemann-Franz law will all have the same temperature rise and the same voltage drop aeross the heater. The temperature rise is about $200 \mathrm{deg} C$, and the voltage drop about $0.2 \mathrm{v}$ at rated current for most thermal converters.

14 This weighted average absolute temperature gives results in closer agreement with those eomputed by the more exact method than docs the unweighted average,
$\theta_{0}+1 / 2 \theta_{d}+273$. 
The results may be compared with the observed ac-de differences shown in table 4 . From information supplied by the manufacturers, the 1-, 2-, 5-, 10-, 20-, $50-$, and 200-ma thermal converters have heaters of either carbon, nickel-chromium, or platinumrhodium-ruthenium alloys. The observed ac-rle differences of these converters were less than 0.01 percent. The 100-, 500- and 1,000-ma thermal converters had heaters of constantan (Advance). The observed differences for these converters (at rated current) were close to the calculated value of -0.06 percent. The 1- and 2-amp converters, of platinumiridium, had observed differences (from table 4,b) less than 0.005 percent, except at low frequencies, also close to the calculated values. The 5-, 10-, 20and 50 -amp converters, of a special platinum-3percent-copper alloy, had differences of -0.010 to -0.015 percent. Thus the agreement between the computed and observed values serves as excellent verification of this source of error in transfer thermal converters. Very fortunately, the error is significantly large only for materials having unusually large thermoelectric effects, such as constantan. However, this does lead to the interesting conclusion that for operation at a low temperature a thermal converter should liave large thermoelectric effects in the thermocouple, but that for the highest transfer accuracy it should have very small thermoelectric effects in the heater.

Additional experimental verification of this cause of transfer error was secured by constructing ${ }^{15}$ at the Bureau two 5-amp thermal converters differing only in that one had a heater of manganin and the other of constantan. These were 5 -amp thermal converters with a small Borax bead between the hot junction of a copper-constantan thermocouple and the center of the heater wire, to eliminate contact effects. Careful ac-dc difference tests with the standard electrodynamic ammeter as the reference established agreement within 0.005 percent for the manganin element and indicated an ac-dc difference of -0.06 percent for the constantan element at an emf of about $5 \mathrm{mv}$.

As a result of this work, a second set of thermal converters in the 1- to 1,000-ma range was purchased under specifications prohibiting heaters of constantan or similar alloys. All of these converters were found to have ac-de differences less than 0.01 percent at audio frequencies.

\section{Low-frequency Response}

It has long [10] been known that there is a lowfrequency limit to the correct rms response of a thermal converter, for reasons analogous to those causing a similar low-frequency limit in all other types of transfer instruments. Below this limit the response characteristic $\mathrm{E}=f(\mathrm{I})$ is not independent of frequency (except for symmetrical square waves), and may be expected to be in error by an amount that is inversely proportional to the square of the frequency. As table $4, \mathrm{~b}$, shows, such an error was observed for the 1 - and 2-amp thermal converters.

\footnotetext{
15 These thermal eonverters were construeted by E. S. Williams.
}

When a constant direct current is applied, the heater of a thermal converter reaches a constant temperature. When an alternating current is applied (after the transient state is over) the temperature of the heater varies cyclically about a mean value, with an amplitude that decreases with increasing frequency because of the thermal capacity of the element. At frequencies high enough so that the cyclic variations are negligible, the temperature rise of the heater is independent of frequency (provided that at very high frequencies the skin effect and electromagnetic radiation are negligible and that standing waves do not alter the temperature distribution). However, at lower frequencies the mean value, to which the $d$-c instrument or potentiometer connected to the thermocouple responds, may not be the same because of nonlinearities in the thermal and electric circuits. These nonlinearities are caused principally by radiation losses, the dependence upon temperature of the electrical resistivity and the thermal conductivity of the heater, and the curvature of the emf versus temperature characteristic of the thermocouple. A theoretical evaluation of these effects for the conditions in a thermal converter, in which most of the cooling is by conduction to the heater, is difficult, but does serve as a guide in determining the approximate limiting frequencies for an arbitrarily chosen error and for determining which factors influence that error. Such an approximate analysis has been carried out by a perturbation method (see appendix 3 ). The results show that the average (time average) temperature rise at the midpoint of a conductor heated by a sinusoidal electric current and cooled solely by conduction to massive terminals is approximately

$$
\theta_{a c}=\theta_{d c}\left[1+\frac{h}{2} \theta_{d c} q^{2}\right]
$$

and that the percentage $a c-d c$ difference of a thermal converter having the hot junction of a thermocouple fastened to the midpoint of such a heater is approximately

where

$$
\delta=-25 H \theta_{d c} q^{2}
$$

$\theta_{a c}=$ the midpoint temperature rise in degrees centigrade on alternating current of rms value $I$ amperes

$\theta_{d c}=$ the midpoint temperature rise in degrees centigrade for the same value of direct current.

$h=\alpha-\beta-2 N T_{0}^{2} l^{2}$, where $\alpha$ is temperature coefficient of electrical resistivity.

$\beta=$ the temperature coefficient of thermal conductivity of the conductor,

$N=p \zeta K / a k$.

$p$ and $a=$ the perimeter and area of the cross section of the conductor, respectively.

$\zeta=$ the emissivity.

$K=$ the Stefan-Boltzmann constant in watts-cm ${ }^{-2}-(\operatorname{deg} K)^{-4}$

$k=$ the thermal conductivity in watts$\mathrm{cm}^{-1}-\mathrm{deg}^{-1}$ 
$T_{0}=$ the ambient temperature in degrees Kelvin

$q=d / \omega l^{2}$ and is (approximately) the ratio of the crest value of the cyclic fluctuation of temperature at the midpoint to the average temperature rise at the midpoint

$\omega=2 \pi f$, where $f$ is the frequency of the applied current in cycles per second.

$d=$ the thermal diffusivity of the heater material in $\mathrm{cm}^{2}-\mathrm{sec}^{-1}$.

$l=$ one-half the length of the conductor in centimeters.

$H=h+B / 2 A$, where $A$ and $B$ are the constants in the emf-temperature characteristic for the thermocouple itself, that is, in the expression, $E=A \theta+(B / 2) \theta^{2}$

These equations indicate the factors upon which the low-frequency error depends. They show that the temperature rise can correctly be used as a measure of the rms alternating current, at frequencies high enough so that the second term in the bracket of eq 3 is negligible for the accuracy desired; in other words, at frequencies for which the heater integrates reasonably well. This is true even if the thermal and electrical conductivities of the heater are dependent upon temperature. As a corollary, these equations show that a thermal converter correctly indicates rms response, except at low frequencies, even though its response characteristic $E=f(I)$ is not quadratic.

The above equations are valid only at frequencies for which the second term in the brackets of eq 3 is small compared to 1 .

Equation 4 may be used to calculate approximately the frequencies for which ac-dc differences may be expected to cause an error of an arbitrarily chosen value. This has been done for some typical materials for an error of 0.1 percent, a temperature rise of $200^{\circ} \mathrm{C}$, and a typical heater length of $0.4 \mathrm{~cm}$. The results are shown in table $7 .{ }^{16}$

TABLE 7. Frequencies for which the computed ac-dc difference is 0.1 percent

[T C-A signifies a thermoeouple of Chromel $P$ and Alumel, and TC-B a thermocouple of copner and constantan, for whieh $\mathrm{B} / 2 \mathrm{~A}=-0.1 \times 10^{-3}$ and $+0.9 \times 10^{-3}$ respectivcly?

\begin{tabular}{|c|c|c|c|c|c|}
\hline \multirow{2}{*}{ Heater material } & \multicolumn{2}{|c|}{$\begin{array}{l}\text { A pproximate temperature } \\
\text { coefficient }\end{array}$} & \multirow{2}{*}{$\begin{array}{l}\text { Thermal } \\
\text { diffusivity }\end{array}$} & \multicolumn{2}{|c|}{$\begin{array}{l}\text { Frequeney for } \\
\qquad \delta=0.1^{\mathrm{s}}\end{array}$} \\
\hline & $\begin{array}{l}\text { Electrical } \\
\text { resistivity }\end{array}$ & $\begin{array}{l}\text { Thermal } \\
\text { eonductivity }\end{array}$ & & $\mathrm{TC}-\mathrm{A}$ & $\mathrm{TC}-\mathrm{B}$ \\
\hline $\begin{array}{l}\text { Copper } \\
\text { Platinum } \\
\text { Manganin } \\
\text { Colistantan } \\
80 \text { Ni-20 Cr... } \\
\text { Carbon }\end{array}$ & $\begin{aligned} & \% /{ }^{\circ} \mathrm{C} \\
&+ 3.9 \times 10^{-3} \\
&+ 3.5 \\
&< .1 \\
&<.1 \\
& .1 \\
&-.5\end{aligned}$ & $\begin{array}{c}\% /^{\circ} \mathrm{C} \\
-0.2 \times 10^{-3} \\
+.5 \\
+2.5 \\
+2.3 \\
\mathrm{~b}+3.5 \\
+.4\end{array}$ & $\begin{array}{c}\mathrm{cm}^{2}-\mathrm{sec}-1 \\
1.1 \\
.25 \\
.075 \\
.064 \\
.038 \\
.2\end{array}$ & $\begin{array}{r}c / s \\
60 \\
12 \\
3 \\
3 \\
2 \\
5\end{array}$ & $\begin{array}{c}c / s \\
70 \\
14 \\
2 \\
2 \\
2 \\
1\end{array}$ \\
\hline
\end{tabular}

s For a heater $0.4 \mathrm{~cm}$ long with a temperature rise of $200^{\circ} \mathrm{C}$.

b Estimated from Lorenz' Law.

16 The effect of radiation josscs has been neglected in preparing this table. In general, this effeet is signifieant only in the very low and very high range thermal converters.
It is evident that the most significant factor in the error term of eq 4 is the length of the heater as the error is dependent upon the fourth power of this quantity. ${ }^{17}$ It is of interest to note that the lengths, $2 l$, of the heater of the 1- and 2-amp thermal converters are only 0.28 and $0.19 \mathrm{~cm}$, respectively, and that the length of each of the higher-range thermal converters is about $0.5 \mathrm{~cm}$. The value of $H$ can be determined from the departure from "square law" of the characteristic equation $E=f(I)$ of a thermal converter. For these thermal converters it was considerably less than the factor given for platinum in table 7 . 'The estimated frequencies for a 0.1-percent error, on the assumption that the diffusivity of the platinum-iridium alloy used in these thermal converters is the same as that of platinum, were about one-half the observed values.

\section{High-Frequency Response}

As these thermal converters are inherently suitable at frequencies up to $100 \mathrm{Mc}$ or more, the upperfrequency limit is set by the circuits in which they are used. The precautions indicated in section 4 are sufficient to control capacitance and leakage currents in tests of ammeters at frequencies considerably higher than the $20,000 \mathrm{c} / \mathrm{s}$ established as the present upper limit by the demand for this testing service. The actual upper-frequency limit has not been established.

The magnitude of the effective impedance sets the upper frequency limit for the model $\mathrm{A}$ and the model $B$ voltmeters. For an accuracy of 0.01 percent at $20,000 \mathrm{c} / \mathrm{s}$, a time constant of less than $1 \times 10^{-7}$ and a skin effect less than 0.01 percent are necessary for the impedance of each step of the model $A$ and each range of the model $B$ instruments. The time constant of each decade of the model $A$ voltmeter was computed from the inductance and direct-capacitance values furnished by the manufacturer of the resistance box used. This was less than $5 \times 10^{-8}$ for each decade. The skin effect was also negligible for each decade. The resistance box is connected as a three-terminal resistor, with the shield connected to the grounded side of the line. For this connection the errors caused by the capacitance currents to the shield are much less than those that would occur if the shield were connected to a resistor terminal. They are significant only for the two 1,000-ohmsper-step decades. The effects of the capacitance of the resistance cards and switch studs of these decades to the shield were computed from the manufacturer's capacitance figures of about $10 \mu \mu \mathrm{f}$ per decade by considering the capacitance as distributed along a uniform transmission line, composed of resistance elements and short-circuited at the end. From the usual transmission line formulas, the magnitude of

17 For a given heater alloy, the low-frequeney range ean be extended by using longer heaters, with proportionatcly greater cross-scetional area to give the same temperature 1 ise for the same eurrent. If solid heaters are used, the improved low-frequeney performanee will be accompanied by increased error due to skin effeet at high frequeneies. 
the current at the short-circuited end was computed as approximately

$$
|I|=\frac{V}{R}\left[1-0.0056(\omega C R)^{2}\right],
$$

wherc

$V=$ the applied voltage

$I=$ the current at the short-circuited end

$R=$ the resistance in the circuit

$C=$ the total capacitance to shield of the $\omega=2 \pi f$. windings in use

At $20,000 \mathrm{c} / \mathrm{s}$ for a capacitance of $30 \mu \mu \mathrm{f}$ this is changed by less than 0.005 pcrcent at 20,000 ohms, the maximum setting. 'The effect of the capacitancc (about $10 \mu \mu \mathrm{f}$ ) of each rather large switch blade to the shield was separately computed, and found to be less than 0.005 percent at $20,000 \mathrm{c} / \mathrm{s}$. Thus it was concluded that the errors of this voltmeter should be a maximum of 0.01 percent at the highest settings and frequencies used, and less at lower settings and frequencies. The performance at the top settings was verified by the tests outlined in section 4 .

The impedance of the model B voltmeter was similarly computed, and only the capacitance to the shield was found to be significant. As a rough but adequate approximation this may be considered the same for each step, which lcads to the approximation of considering the capacitance as distributed along a nonuniform transmission line composed of resistance elements and short-circuited at the end. The effective impedance was computed by a perturbation method, ${ }^{18}$ on the rough assumption that the resistance per unit length was proportional to the square of the electrical distance from the output end. The magnitude of the current at the short-circuited end was found to be approximately

$$
|I|=\frac{V}{R}\left[1-0.0022(\omega C R)^{2}\right] .
$$

From the measured capacitance of $50 \mu \mu \mathrm{f}$ and the total resistance of $100,000 \mathrm{ohms}$ for the $750-\mathrm{v}$ range, the change in current was computed as -0.09 percent at $20,000 \mathrm{c} / \mathrm{s}$, which is in very good agreement with the observed results of table 5. Moderately good agrcement for the other ranges was also obtained, but the change in current was not observed to increase in proportion to the square of the frequency as indicated by eq 6 . However, the agrcement is good enough to show that the ac-de difference of this instrument is due to capacitance currents to the shield. These currents cannot easily be reduced in a shielded multirange instrument of reasonable size. (A shicld is desirable to control the currents.) They could be compensated by connecting appropriatc capacitors across sections of the resistors, but this seems less desirable than the procedure of applying small corrections and has not been donc in the model $B$ voltmeter. Much of the capacitance is that between the binding posts of each range (see fig. 2)

\footnotetext{
${ }^{18}$ Sce appendix"4.
}

and between the high-side binding post and the top shield. This shield is brought as close as $2.5 \mathrm{~cm}$ to the row of high-side posts.

\section{Other Effects}

The intercomparisons of insulated-heater thermal converters disclosed an unsuspected ac-dc difference that was dependent upon the voltage between the heater and ground and upon the heater current of each of these elements. This was traced to a very small leakage current flowing through the ceramic bead between the heater and thermocouple and then through the stray capacitance of the Lindeck potentiometer to ground. This current caused additional heating of the bead and thus an error as on direct current the insulation resistance of the Lindeck was much higher than the capacitive reactance, even at $20 \mathrm{c} / \mathrm{s}$. In addition, the ceramic bead of each of these thermal converters exhibited marked dielectric absorption, so that its a-c resistance was much less than its d-c resistance. For a typical thermal converter at rated current a leakage current of $0.7 \mu \mathrm{a}$ rcsulted from a difference of $50 \mathrm{v}$ between the heater and ground, resulting in a power dissipation of $35 \mu \mathrm{w}$ in the bead. 'This was sufficient to cause a 0.2-percent error, which was proportional to the squarc of the voltage difference and was very markedly dependent upon the heater current. ${ }^{19}$ The reactance of a capacitance as small as $100 \mu \mu f$ is less than the computed bead resistance, even at a frcquency as low as $50 \mathrm{c} / \mathrm{s}$, so that in almost any application this source of error makes it necessary to maintain the heater near ground potential.

\section{Other Applications}

This equipment was designed for the testing of other instruments. Portability and the ease of making other a-c measurements were not considered to be important. However, the thermal converters and voltmeter elements are rcadily portable and have bcen used, with a small portable millivolt potentiometer as the indicator, for measurements at other locations. For mcasurements of current or voltage to 0.01 percent, it is necessary to use a transfer method, calibrating the thermal converter or voltmeter on direct current at the time of use. For a more moderate accuracy of about 0.1 percent, it should be feasible to use a low-range thermal converter, shunting it for current measurements and adding a series resistor for voltage measurements. Only the thermal converter itself need be transferred to direct current if the resistances of the shunts and the series resistor are properly adjusted. Thus d-c sources of extended range, volt boxes, etc., should be unnecessary. The whole apparatus could be built in a convenient case, containing a small potentiomcter for the measurcments of the current through. or the voltage across the thermal converter heater.

19 This was due to the marked deerease in the resistanee of the eeramie bead with an inerease in temperature. With a fixed voltage aeross the head, the error was in some eases proportional to the tenth power of the heater eurrent. 
A suitable low-range thermal converter should also make feasible an a-c potentiometer with an inherent accuracy of 0.01 percent. The basic circuit for a polar form of such a potentiometer (to measure the magnitude but not the components or phase angle of an alternating voltage) is shown in figure 6 . With the switch thrown to direct-current the potentiometer is standardized in the usual manner, and the deflection of the indicator of the thermal converter is observed. The switch is then thrown to alternating current, which is adjusted for the same deflection of this indicator. The circuit is surely not new, but a proved thermal converter makes possible a convenient transfer with accuracies comparable to those of a d-c potentiometer. The other basic limitation remains that any a-c potentiometer measures only the fundamental component of the unknown voltage. However for an alternating quantity having a harmonic.content as large as 2 percent, the rms value differs from the fundamental by only 0.02 percent. Audio-frequency oscillators and amplifiers with amplitude distortion much less than this are now available. Such an oscillator could be used to supply the potentiometer directly and to supply the test circuit through an amplifier preceded by a suitable phaseshift network.

\section{Summary}

The electrothermic transfer standards used at the Bureau for the precise measurement of voltage and current at audio frequencies, and designed primarily for the standardization of electric instruments, have been described. The extensive tests to establish the transfer performance of the standards have been outlined, and the results have been given. The causes of the observed ac-dc differences have been outlined and the errors evaluated. The equations governing the temperature rise of a conductor heated by an electric current and cooled by conduction to relatively massive terminals have been solved to establish the steady-state midpoint temperature rise on direct current with Peltier and Thomson heating, as well as the ordinary resistance heating, in order to obtain the d-c elror of a thermal converter. 'The equations have been solved to establish the average midpoint temperature rise on alternating current, in order to obtain the low-frequency error of a thermal converter in which the electrical and thermal conductivities of the heater are temperature dependent, in which some loss of heat occurs by radiation, and in which the characteristic of the thermocouple is nonlinear. Possible applications and modifications of the equipment for measurements under less stringent conditions have been suggested, and the application to an a-c potentiometer of excellent inherent accuracy has been diagrammed.

The results of this work establish the excellence of thermal converters as transfer standards of highest accuracy, comparable over wide ranges with that obtainable with other types of standards at much narrower ranges. 'The requirements for' such standards are feasible and the manner of use to insure such accuracy not unreasonable. The cost of the required number of standards and the equipment is not prohibitive. Thus electrothermic instruments may be ranked on a par with electrodynamic and electrostatic instruments for a-c measurements of the highest accuracy.

As a result of this work, thermal converters of negligible or small known transfer error are now available as transfer standards with which the transfer performance of other thermal converters may be evaluated at the NBS to 0.01 percent at audio frequencies.

The author acknowledges the help of Murray Blitz, who made most of the intercomparisons described in section 4 of this report and checked all the tables and equations, and of Earl Williams, who constructed all the equipment and assisted with the measurements.

\section{References}

[1] F. K. Harris, A suppressed-zero electrodynamic voltmeter, BS J. Research 3, 445 (1929) RP105.

[2] J. H. Park and A. B. Lewis, Standard electrodynamic wattmeter and ac-dc transfer instrument, J. Research NBS 25, 545 (1940) RP1344.

[3] F. B. Silsbee, Composite-coil electrodynamic instruments, BS J. Research 8, 217 (1932) RP411.

[4] C. C. Pattersolı, E. H. Rayner, and A. Kinnes, The use of the electrostatic method for the measurement of power, J. Inst. Elec. Engrs. (London) 51, 294 (1913).

[5] R. S. J. Spilsbury and A. Felton, The electrostatic voltmeter as a dc-ac transfer instrument, J. Inst. Elec. Engrs. (London) 89-II, 129 (1942).

[6] W. N. Goodwin, Jr., The compensated thermocouple ammeter, Trans. Am. Inst. Elec. Engrs. 55, 23-33 (1936).

[7] F. L. Hermach, A precision electrothermic voltmeter for measurements between 20 and 20,000 cycles, Trans. Am. Inst. Elec. Engrs. 6\%, 1224 (1948).

[8] H. B. Brooks and A. W. Spinks, A multi-range potentiometer and its application to the measurement of small temperature differences, BS J. Research 9, 781 (1932) RP506.

[9] G. Borelius, Bestimmung des Thomsoneffektes aus dem thermischen Gleichgewicht in einem strömdurchflossenen Drahte, Ann. Physik 63, 845 (1920).

[10] M. G. Lloyd, Elec. Rev. \& Western Elec. 58, 65 (Jan. 14, 1911).

\section{Appendix 1. Development of Working Equations}

By definition, the percentage ac-de difference of an instrument is the percentage difference in the quantity required to give the same response on alternating and direct current. It is

$$
\delta=100 \frac{Q_{a c}-Q_{d c}}{Q_{d c}},
$$

where $\mathrm{Q}$ is the quantity (current, voltage, or power) that the instrument measures.

In a "straight a-c test" the direct current or voltage is adjusted to give the same response of the transfer standard observed on alternating current. The direct current or voltage is measured with the potentiometer and its accessories. Therefore, from the above definition,

$$
Q_{a c}=Q_{d c}\left(1+\frac{\delta_{s}}{100}\right)
$$


where $O_{d c}$ is the quantity measured with the potentiometer, $\delta_{s}$ is the ac-dc difference of the transfer standard, and $Q_{a c}$. is the a-c value required for the observed deflection of the test instrument.

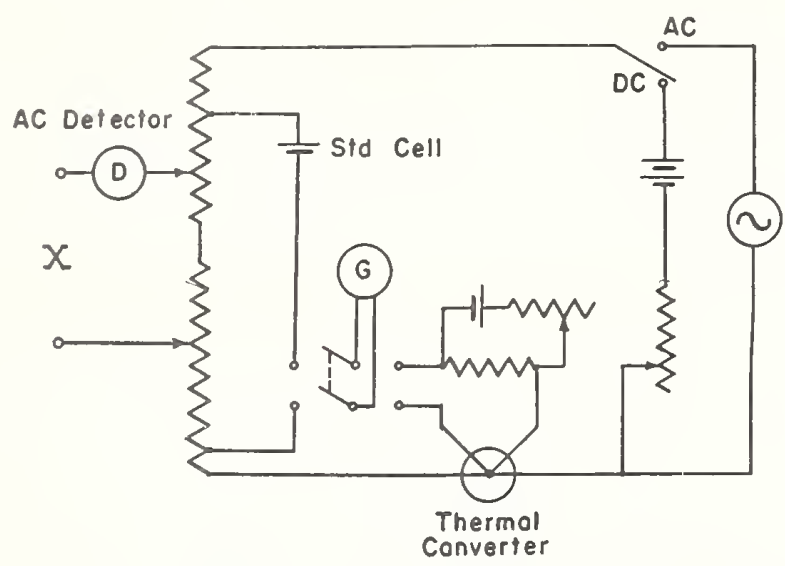

Figure 6. Elementary form of Polar a-c potentiometer.

When a thermal converter is used for transfer tests the small percentage difference, $\delta_{t}$, between the values required for the same indication of the test instrument on alternating and direct current is determined from the resulting deflection of the Lindeck galvanometer. Therefore, for each transfer thermal converter, the small percentage difference in output emf resulting from a small percentage difference in heater current must be evaluated. This relationship may be expressed as

$$
\frac{\Delta E}{E}=n \frac{\Delta I}{I}
$$

where $E$ is the output voltage, $I$ the heater current, and $n$ a number that is generally close to 2 , but may range from from 1.5 to 2.2 . The characteristic, $n$, may be evaluated on direct current by measuring with a potentiometer the small percentage differences in $\mathrm{E}$ resulting from small percentage differences in heater current successively set with a potentiometer at selected current levels, then computing $n$ by the above expression and plotting $n$ versus $E$. The characteristic need not be determined with an accuracy better than 1 percent and is relatively permanent.

If the Lindeck potentiometer shown in figure 3 is used to measure $E$ and $\Delta E$ in millivolts in a transfer test, then at balance $E=I_{p}{ }^{20}$ the voltage drop across the four-terminal resistor. If the galvanometer deflection $D$, is directly proportional to the current through the galvanometer 21 and if the potentiometer voltage is unchanged during a determination,

$$
\Delta E=\frac{\Delta D R_{p}}{S},
$$

where $\Delta D$ is the difference in galvanometer deflection corresponding to a difference $\Delta E$ in the applied emf, $R_{p}$ is the resistance of the galvanometer circuit, and $S$ is the current sensitivity of the galvanometer in centimeters per milliampere on the scale. In the Lindeck potentiometer used in this setup, the galvanometer sensitivity is adjusted so that numerically

$$
\frac{100 R_{p}}{S}=1
$$

These relationships may be combined with eq 9 to give

$$
100 \frac{\Delta I}{I}=\frac{\Delta D}{n V_{p}} .
$$

20 An accuracy of 1 percent is sufficient for this messurement

21 The use of a straight instead of cylindrical scale introduces an error that can be shown to be less than 1 percent if the galvanometer-to-scale distance is at least three times the scale length.
Therefore, for a transfer test of an ammeter with a thermal converter that has no transfer error, the required ac-dc difference of the test instrument is by eq 7 and 10

$$
\delta_{T}=100\left(\frac{I_{a c}-I_{d c}}{I_{d c}}\right)=\frac{D_{a c}-D_{d c}}{n V_{p}},
$$

where the alternating and direct currents are adjusted for the same response of the test instrument, and the resulting galvanometer deflections are $\mathrm{D}_{a c}$ and $\mathrm{D}_{d \varepsilon}$, respectively.

If the transfer thermal converter has a percentage ac-dc difference, $\delta_{s}$, at the current and frequency at which the test is made, then the ac-dc difference of the instrument under test is, to the desired order of approximation,

$$
\delta_{T}=100\left(\frac{I_{a c}-I_{d c}}{I_{d c}}\right)=\frac{D_{a c}-D_{d c}}{n V_{p}}+\delta_{s} .
$$

Similarly, for a voltmeter test,

$$
\delta_{T}=100\left(\frac{V_{a c}-V_{d c}}{V_{d c}}\right)=\frac{D_{a c}-D_{d c}}{n V_{p}}+\delta_{s},
$$

in which $\delta_{s}$ is the percentage ac-de difference of the range (or setting) of the standard voltmeter used.

In this deflection method of using the Lindeck potentiometer for rapid transfer tests, the resistance of the circuit seen by the galvanometer must be constant to better than 1 percent. The resistance of the thermocouple of each thermal converter has been adjusted to the same value so that the simplified formulas may always be used. The temperature coefficient of the resistance of the Lindeck circuit has been computed as less than 0.1 percent per $\operatorname{deg} \mathrm{C}$, and computations show that the resistance seen by the galvanometer is changed by less than 0.2 percent by the adjustable battery circuit, for all ranges of the potentiometer except the topmost.

\section{Appendix 2. Effect of Peltier and Thomson Heating}

The problem is to find the steady-state temperature rise of a uniform conductor of length $2 l$ and uniform cross-sectional area, $a$, carrying a constant current, $I$, and cooled solely by conduction to its terminals. The joule heating of a length $\tilde{d x}$ occurs at the time rate $I^{2} \rho d x / a$ watts, where $\rho$ is the electric resistivity in ohm-cm. The difference in the heat conducted per second across the two ends of this differential element is $a k\left(d^{2} \theta / d x^{2}\right) d x$ watts where $k$ is the thermal conductivity in watts-cm ${ }^{-1}$-degrees ${ }^{-1}$, and $\theta$ is the temperature of the element in deg C. Equating these and dividing by $d x$ gives the differential equation governing the temperature of the conductor

$$
a k \frac{d^{2} \theta}{d x^{2}}+\frac{I^{2} \rho}{a}=0
$$

which is of the form

$$
\theta^{\prime \prime}=-b \text {, }
$$

where $b=I^{2} \rho / a^{2} k$.

If the terminals remain at equal temperatures $\theta_{0}$, and if the origin is at the midpoint of the conductor, the boundary conditions are $\theta=\theta_{0}$ at $x= \pm l$. The solution, by direct integration and application of these conditions, is

$$
\theta-\theta_{0}=\frac{b}{2}\left(l^{2}-x^{2}\right)
$$

and at the midpoint

$$
\theta_{m}-\theta_{0}=\frac{b l^{2}}{2}=\theta_{d}
$$

If the terminals are of different metal than the conductor, Peltier heating and cooling (proportional to the first power of the current) may occur at the junctions of the conductor with the terminals. These will be equal and opposite in sign at the two terminals, and, if we can assume complete thermal symmetry, their effect will be to raise the tempertaure of 
one terminal by a small amount $\Delta \theta$ and to lower the other by an equal amount. The differential equation then has as its boundary conditions, $\theta=\theta_{0}+\Delta \theta$ at $x=+l$ and $\theta=\theta_{0} \cdots \Delta \theta$ at $x=-l$. The solution is "

$$
\theta-\theta_{0}=\frac{b}{2}\left(l^{2}-x^{2}\right)+\frac{\Delta \theta x}{l},
$$

and is therefore unchanged at the midpoint.22 Of course, complete thermal symmetry is never attained, so that Peltier heats may cause a change in the midpoint temperature However, for massive terminals the temperature changes, $\Delta \theta$, are very small, so that Peltier heating need not be considered significant.

-Thomson heating occurs in homogeneous conductors having a temperature gradient in the direction of the current flow. For the one-dimensional case here considered, the Thomson heat in an element of length $d x$ having a temperature difference $d y$ is $\sigma I d \theta$ wat ts where $\sigma$ is the Thomson voltage coefficient. Experiment and theory indicate that $\sigma$ is proportional to the absolute temperature, but, as the Thomson heating is small compared to the joule heating, we might, as a first approximation, consider $\sigma$ constant. Equation 14 then becomes

$$
a k \frac{d^{2} \theta}{d x^{2}}+\frac{I^{2} \rho}{a}+\sigma I \frac{d \theta}{d x}=0,
$$

which is of the form

$$
\theta^{\prime \prime}+c \theta^{\prime}+b=0
$$

with the boundary conditions $\theta=\theta_{0}$ at $x= \pm l$, where $c=\sigma I / a k$. The steady-state solution of this eq is

$$
\theta-\theta_{0}=\frac{\mathrm{b} l}{c}\left(\frac{1}{\tanh q}-\frac{e^{-c x}}{\sinh q}\right)-\frac{b x}{c},
$$

where $q=c l<<1$. At the midpoint, where $x=0$

$$
\theta_{m}-\theta_{o}=\frac{b l^{2}}{q}\left(\frac{\cosh q-1}{\sinh q}\right)
$$

Expanding the hyperbolic functions gives, by neglecting higher-order terms,

$$
\theta_{m}-\theta_{0}=\theta_{\imath}=\frac{b l^{2}}{2}\left(1-\frac{q^{2}}{12}\right)=\theta_{d}\left(1-\frac{q^{2}}{12}\right)
$$

$B_{y}$ substituting the values for $b$ and $c$ and noting that $2 I \rho l / a=V$, the vortage drop across the conductor, eq 1 and 2 of the text can be obtained. They show that Thomson heating causes a small (second order of smallness) decrease in the temperature rise at the midpoint of the conductor, and can thus lead to a transfer error in a thermal converter. However, the approximation of considering $\sigma$ a constant is a drastic one, and a more accurate approach will be to consider $\sigma=B T$, where $B$ is a constant, ${ }^{23}$ and $T$ is the absolute temperature. Fquation 17 then becomes

$$
a k \frac{d^{2} T}{d x^{2}}+\frac{I^{2} \rho}{a}+B I T \frac{d T}{d x}=0
$$

which is of the form

$$
T^{\prime \prime}+w T T^{\prime}+b=0
$$

with the boundary conditions $T=T_{0}$ at $x= \pm l$, where $w=B I / a k$

This is a nonlinear equation and very difficult to solve Chester Snow showed that it could be reduced to a Ricatti and then to a Bessel equation, but suggested an approximation or perturbation method to give the desired results more simply and directly. The answer is not significantly different from

${ }^{22}$ Since Peltier heating depends upon the junction temperature, a secondorder change in midpoint temperature may occur.

${ }_{23} B=d^{2} E / d T^{2}$, where $E$ is the emf of a thermocouple composed of the metal in question and a metal such as lead, which has very small Thomson heating. that of eq 18. The method is powerful, however, and was used later in evaluating other perturbing effects, so that it will be outlined here. The Thomson heating, which is much smaller than the Joule heating, can be considered as perturbing the temperature distribution of the conductor. Its effect can be approximated by solving the differential equation without the perturbing term, then substituting the result, and its derivatives as necessary, in the perturbing term only, and solving the resulting equation.

Without the Thomson heat term, eq 19 becomes

$$
T^{\prime \prime}=-b
$$

with the boundary condition given, the solution of which is

$$
T_{1}-T_{0}=\frac{b}{2}\left(l^{2}-x^{2}\right)
$$

where the subscript 1 indicates the first approximation. Placing this value of $T_{1}$ and $T_{1}^{\prime}$ in the perturbing term of 19 , reduces this to a linear equation,

$$
T_{2}^{\prime \prime}=-b-\frac{w b^{2} x^{3}}{2}+w b x\left(T_{d}+T_{0}^{\prime}\right)
$$

where $T_{d}=b l^{2} / 2$, the solution of which is

$$
\begin{aligned}
T_{2}-T_{0}= & \frac{b}{2}\left(l^{2}-x^{2}\right)-\frac{w b^{2} x^{5}}{40}+\frac{w b}{6}\left(T_{d}+T_{0}\right) x^{3}- \\
& w T_{d}\left(\frac{7}{30} T_{d}+\frac{T_{0}}{3}\right) x
\end{aligned}
$$

At $x=0$ this reduces to $T_{1}-T_{0}$ as only first-order terms are given, so that the process must be repeated to find the secondorder term indicated by eq 18 . The algebra rapidly becomes very lengthy and tedious, but the procedure is straightforward, and the resulting temperature rise at the midpoint is found to be approximately

$T_{3}-T_{0}=T_{d}\left[1-3.4 \times 10^{-2} z_{d}^{2}-10.5 \times 10^{-2} z_{0} z_{d}-8.3 \times 10^{-2} z_{0}^{2}\right]$,

where $z_{0}=w T_{0} l$, and $z_{d}=w T_{d} l$

\section{Appendix 3. Low-Frequency Error of a Thermoelement}

The problem is to find the steady-state average (over an integral number of cycles) temperature rise at the center of a uniform conductor of length, 2l, and cross-sectional area, $a$, carrying a sinusoidal current and cooled chiefly by conduction to massive terminals that are at fixed and equal temperatures. Assume that the current remains sinusoidal and that only Joule heating of the conductor occurs. If all the cooling is by conduction to the terminals, the differential equation governing the temperature of the conductor is, under these assumptions,

$$
a k \frac{\partial^{2} \theta}{\partial x^{2}}+\frac{i^{2} \rho}{a}=a m s \frac{\partial \theta}{\partial t}
$$

with the boundary condition $\theta( \pm l, t)=0$, where

$\theta=$ temperature in $\operatorname{deg} \mathrm{C}$

$i^{2}=$ the square of the instantaneous value of the current $=$ $I^{2}(1-\cos 2 \omega t)$

$I=$ rms value of the current

$\omega=2 \pi f$, and $f$ is the frequency of the current in cycles per second

$t=$ time in seconds

$m=$ density of the conductor in grams- $\mathrm{cm}^{-3}$

$s=$ thermal capacity of the conductor in joules-grams ${ }^{-1}$ $-\operatorname{deg} \mathrm{C}^{-1}$. 
The procedure will be to consider $\rho$ and $k$ constant and to solve for the periodic steady-state temperature rise, then to approximate $\rho$ and $k$ as $\rho=\rho_{0}(1+\alpha \theta)$ and $k=k_{0}(1+\beta \theta)$, to introduce radiation loss, and to solve the resulting equation by the perturbation method just outlined. For the cases considered $\alpha \theta$ and $\beta \theta$ are considerably less than 1, and radiation loss is much less than the Joule heating of the conductor.

With $\alpha$ and $\beta=0$, eq 21 is the form

$$
\frac{\partial^{2} \theta}{\partial x^{2}}-g \frac{\partial \theta}{\partial t}=-b(1-\cos 2 \omega t)
$$

where $g=m s / k_{0}$. and $b=I^{2} \rho_{0} / a^{2} k_{0}$.

Let $\theta=u(x, t)+\psi(x)$. Then

$$
\frac{\partial^{2} \theta}{\partial x^{2}}=\frac{\partial^{2} u}{\partial x^{2}}+\frac{\partial^{2} \psi}{\partial x^{2}}
$$

Let $\partial^{2} \psi / \partial x^{2}=-b$. Then $\psi=-\left(b x^{2}\right) / 2+c_{1} x+c_{2}$ Applying boundary conditions gives

$$
\begin{gathered}
\theta(+l, t)=u(+l, t)+\psi(l)=0 \\
\theta(-l, t)=u(-l, t)+\psi(-l)=0 .
\end{gathered}
$$

We may take $\psi(l)=\psi(-l)=0$.

Then $\psi=b / 2\left(l^{2}-x^{2}\right)$ and eq 22 becomes

$$
\frac{\partial^{2} u}{\partial x^{2}}-g \frac{\partial u}{\partial t}=b \cos 2 \omega t
$$

with $u( \pm l, t)=0$.

From complex number theory if $u$ is harmonic in time, it can"be represented by the real part of $U e^{i 2 \omega t}$, where $U$ is a complex function of the real variable $x$, and $j=\sqrt{-1}$. Similarly, $b \cos 2 \omega t$ can be represented by the real part of $b e^{j \omega t}$, where $b$ is a real number. Substituitng these quantities and the appropriate derivatives in eq 23 and dividing by $e^{j 2 \omega t}$ results in

$$
\frac{d^{2} U}{d x^{2}}-j 2 \omega g U=b,
$$

with $\mathrm{U}=\mathrm{O}$ when $x= \pm l$. The solution of this is

$$
U=-\frac{b}{\mu_{2}}\left[1-\frac{\cosh \mu x}{\cosh \mu l}\right]
$$

where $\mu=\sqrt{j 2 \omega g}$. At the midpoint

$$
U=-\frac{b}{\mu^{2}}\left[1-\frac{1}{\cosh \mu l}\right] \approx j \frac{b}{2 \omega g},
$$

as at the frequencies we are interested in $\cosh \mu l>>1$ Therefore, $u=$ real part of $U e^{i 2 \omega t}=$ real part of

$$
\left\{\frac{j b}{2 \omega g}(\cos 2 \omega t+j \sin 2 \omega t)\right\} \approx-\frac{b}{2 \omega g} \sin 2 \omega t .
$$

So that, to this approximation, at the midpoint

$$
\theta_{m 1}=\frac{b l^{2}}{2}\left(1-\frac{1}{\omega g l^{2}} \sin 2 \omega t\right)=\theta_{d}(1-q \sin 2 \omega t),
$$

where $\theta_{d}=b l^{2} / 2$ and $q=1 / \omega g l^{2}$. Note that $q$ is the crest value of the cyclic temperature fluctuation divided by the average temperature rise at midpoint.

In general, $U=U_{a}+j T_{b}$, where $U_{a}$ and $U_{b}$ are functions of $x$, so that $u=U_{a} \cos 2 \omega t-U_{b} \sin 2 \omega t$ and

$$
\theta_{1}=\frac{b}{2}\left(l^{2}-x^{2}\right)+U_{a} \cos 2 \omega t-U_{b} \sin 2 \omega t .
$$

Thus the average value over an integral number of cycles of the temperature rise is the same as that found in appendix 2 for the same numerical value of direct current, so that under these conditions the temperature rise of the conductor can be used as a measure of the current without ac-de error.

We shall now consider that $\rho=\rho_{0}(1+a \theta)$ and $k=k_{0}(1+\beta \theta)$ and that some small fraction of the total heat is lost by radiation. If $p$ is the perimeter of the heater in centimeters, then the heat radiated per second from the surface of a differential length, $d_{x}$, of the heater is

$$
d H_{r}=p \zeta K\left(T^{4}-T_{n}^{4}\right) d x,
$$

where $\zeta$ is the emissivity of the heater material, a numerical constant, and $K$ is the Stefan-Boltzmann constant in watts$\mathrm{cm}^{-2}(\operatorname{deg} K)^{-4}$. $\quad T$ is the absolute temperature of the element; $T_{0}$ is the absolute temperature of the surroundings. Introducing these terms in eq 22 gives, as $\beta \theta<1$,

$$
\frac{\partial^{2} \theta}{\partial x^{2}}-g(1-\beta \theta) \frac{\partial \theta}{\partial t}=-b(1-\cos 2 \omega t)(1+\eta \theta)+\frac{p \zeta K}{a k}\left(T^{4}-T_{0}^{4}\right)
$$

where $\eta=\alpha-\beta$. The perturbing terms are

$$
\begin{gathered}
+\beta g \theta \frac{\partial \theta}{\partial t} \\
-b(1-\cos 2 \omega t) \eta \theta \\
\frac{p \zeta K}{a k}\left(T^{4}-T_{0}^{4}\right)
\end{gathered}
$$

As these perturbing terms are relatively small, the solution will differ by only a small amount from that given by eq 27 . Hence a sufficient expression for these small terms will be given by substituting the known values of the dependent variables $y$ from eq 27, and its derivatives as necessary, in these perturbing terms. When this is done, it is found that the first of these terms reduces to functions of $x$, each multiplied by $\sin n \omega t$ or $\cos n \omega t$, where $n=2,4$. The second reduces to similar harmonic terms plus the terms $-b[\eta P-$ $\left.\left.{ }_{\eta} U_{a} / 2\right)\right]$, where $P=\left(l^{2}-x^{2}\right) b / 2$. The third term becomes unnecessarily complex. It can be simplified by making the further approximation that for this term

and

$$
T-T_{0}=\theta_{r}=P(1-q \sin 2 \omega t),
$$

$$
T^{4}-T_{0}^{4}=\left(T_{0}+\theta_{r}\right)^{4}-T_{0}^{4} \approx 4 \theta_{r} T_{0}^{3}+6 \theta_{r}^{2} T_{0}^{2}, \theta_{r}<T_{0}
$$

and also that $k=k_{0}$. With these approximations, this term reduces to harmonic terms plus the terms

$$
4 N T_{0}^{3} P+6 N T_{0}^{2} P^{2}\left(1+\frac{q^{2}}{2}\right)
$$

where $N=p \xi K / a k_{0}$.

Thus eq 28 becomes

$$
\begin{aligned}
\frac{\partial^{2} \theta}{\partial x^{2}}-g \frac{\partial \theta}{\partial t}= & -b\left(1+\eta P-\frac{\eta U_{a}}{2}\right)+2 N T_{0}^{2} P\left[2 T_{0}+3 P 1+\left(\frac{q^{2}}{2}\right)\right]+ \\
& \sum_{n=2}^{n=4}\left(F_{n} \cos n \omega t+G_{n} \sin n \omega t\right),
\end{aligned}
$$

where $F_{n}$ and $G_{n}$ are known functions of $x$ alone. We again let $\theta(x, t)=u(x, t)+\psi(x)$ and set $\partial^{2} \psi / \partial x^{2}$ equal to the terms independent of $t$ in eq 29 , so that

$$
\frac{\partial^{2} u}{\partial x^{2}}-g \frac{\partial u}{\partial t}=\sum_{n=2}^{n=t}\left(F_{n} \cos n \omega t+G_{n} \sin n \omega t\right)
$$


For each frequency of, the harmonic driving terms in the right-hand side of this equation, the solution of the equation that vanishes at the end points will be of the form $U_{a} \cos$ $n \omega t-U_{b} \sin n \omega t$, where $U_{a}$ and $U_{b}$ are functions of $x$. The average value, over an integral number of cycles of the lowest frequency, will therefore vanish. Since this is a linear equation, the solution is the sum of such solutions and will therefore also vanish. Thus the average value of $\theta$, which we are interested in, is equal to $\psi(x)$.

Evaluation $\psi(x)$ in the same manner as before gives for the value of $\psi$ at the midpoint where $x=0$,

$$
\psi(0)=\theta_{d}\left[1+\frac{5}{6} \eta \theta_{d}-\frac{\eta U_{a 2}(l)}{l^{2}}-\frac{5}{3} N T_{0}^{3} l^{2}-\frac{11}{5} N T_{0}^{2} \theta_{d} l^{2}\left(1+\frac{q^{2}}{2}\right)\right]
$$

where

$$
U_{a 2}(x)=\int_{0}^{x} d x_{2} \int_{0}^{x_{2}} U_{a}\left(x_{1}\right) d x_{1}
$$

and on the assumptions that $U_{a 2}(-l)=U_{a 2}(l)$ and $U_{a 2}(0)=0$.

The required expression for $U_{a}$ can be evaluated from eq 25 and 27 by noting that

$$
\mu x=\sqrt{j 2 \omega g} x=\gamma x+j \gamma x \text { and } \mu l=\mu l+j \gamma l,
$$

where $\gamma=\sqrt{\omega g}$, so that $\cosh \mu x=\cosh \gamma x \cos \gamma x+j \sinh \gamma x \sin$ $\gamma x$, and $\cosh \mu l=\cosh \gamma l \cos \gamma l+j \sinh \gamma l \sin \gamma l$. At the frequencies we are interested in $\mu l>5$, so that

$$
\cosh \mu l \approx \frac{e^{\gamma l}}{2}(\cos \gamma l+j \sin \gamma l) .
$$

Since $u=$ real part of

$U e^{i 2 \omega t}=\operatorname{re}\left\{\frac{j b}{2 \omega g}\left[1-\frac{\cosh \mu x}{\cosh \mu l}\right] e^{j 2 \omega t}\right\}=U_{a} \cos 2 \omega t-U_{b} \sin 2 \omega t$,

it will be found that

$$
U_{a}=\frac{b}{\omega g e^{\gamma l}}[\sinh \gamma x \sin \gamma x \cos \gamma l-\cosh \gamma x \cos \gamma x \sin \gamma l] .
$$

Performing the desired integration results in

$U_{a 2}=-\frac{b}{2 \omega g \gamma^{2} e^{\gamma l}}(\cos \gamma l \cosh \gamma x \cos \gamma x+\sin \gamma l \sinh \gamma x \sin \gamma x)$.

From which

$$
U_{a 2}(l)=U_{a 2}(-l) \approx-\frac{b}{4 \omega^{2} g^{2}} \text { and } U_{a 2}(0)<<U_{a 2}(l) .
$$

Therefore, eq 31 becomes

$$
\begin{aligned}
\psi(0)=\theta_{a c}=\theta_{d}\left[1+M+\left(\frac{\eta}{2}-\frac{11}{10} N T_{0}^{2} l^{2}\right) \theta_{d} q^{2}\right]= \\
\theta_{d}\left[1+M+\frac{h}{2} \theta_{d} q^{2}\right]
\end{aligned}
$$

where $M$ is equal to the remaining terms in eq 31 and $h \approx \eta-$ $2 N T_{0}^{2} l^{2}$.

By the same procedure, eq 28 can be solved for the d-c case by considering $\partial \theta / \partial t=0$ and $i=I$, a constant of the same numerical value as the rms value of the alternating current. The temperature rise at the midpoint will be found to be

$$
\theta_{a c}=\theta_{d}[1+M] \text {. }
$$

Thus the temperature rise on direct current is the same as on alternating current when the frequency is high enough so that the third term in the brackets of eq 32 is sufficiently small compared to unity. Therefore the temperature rise at the midpoint on alternating current is

$$
\theta_{a c}=\theta_{d c}\left[1+\frac{h \theta_{d c} q^{2}}{2(1+M)}\right] \approx \theta_{c c c}\left[1+\frac{h}{2} \theta_{d c} q^{2}\right]
$$

if $M$ is small compared to unity.
If the hot junction of the thermocouple is fastened to the midpoint of the heater, the emf of the couple may be represented by

$$
E=A \theta_{m}+\frac{B}{2} \theta_{m}^{2}
$$

where $\theta_{m}$ is the temperature of the hot junction in $\operatorname{deg} \mathrm{C}$, and $A$ and $B$ are constants to be evaluated at the temperature of the cold juncion. On direct current $\theta_{m}=\theta_{d c}$ and

$$
E=A \theta_{d c}\left[1+\frac{B}{2 A} \theta_{d c}\right] \text {. }
$$

On alternating current of the same rins value, $\theta_{m}$ may be considered to be closely enough for these purposes

$$
\theta_{m}=\theta_{d c}\left[1+\frac{h}{2} \theta_{d c} q^{2}\right](1-q \sin 2 \omega t) .
$$

The average value of emf over an integral number of cycles of altemating current is then (to the desired accuracy, and if $(B / 2 A) \theta_{d c}$ is small compared to unity)

$$
E_{A}=A \theta_{d c}\left(1+\frac{B}{2 A} \theta_{d c}\right)\left[1+\left(\frac{h}{2}+\frac{B}{4 A}\right) \theta_{d c} q^{2}\right]
$$

From these expressions

where $H=h+B / 2 A$.

$$
\frac{E_{a}-E_{d}}{E_{d}}=\frac{H}{2} \theta_{d c} q^{2}
$$

For table 7 , it is desired to compute the frequency at which the ac-dc difference of a thermal converter due to this effect is equal to 0.1 percent. From eq 7 and 9 and for $n=2$ the difference in current for the same emf is one-half the difference in emf for the same current, and is opposite in sign. Therefore the percentage ac-dc difference, $\delta$, is approximately

$$
\delta=-25 H \theta_{d c} q^{2}=\frac{-25 H \theta_{d c} d^{2}}{(2 \pi f)^{2} l^{4}}
$$

where in addition to the symbols already defined, $d=1 / g=$ $k_{0} / m s$ and is the thermal diffusivity in appropriate units.

\section{Appendix 4. Effect of Shield Capacitance on the Model B Voltmeter}

We may approximate the effects' of capacitance to the shield of this voltmeter by considering the voltmeter as consisting of a transmission line of $r_{x}=r_{0} x^{2}$ ohms per unit length, having a constant capacitance $c_{0}$ farads per unit length to the shield, witha sinusoidal voltage of rms value $r$ volts applied to the high-resistance end. The other end may be considered short-circuited, since the thermal converter, which is connected between the low-resistance end and the ground post, is of low resistance. The shield is also connected to this ground post. We wish to find the magnitude of current, $I_{0}$, at the short-circuited end. For an element of length $d x$ at a distance $x$ from this end, we have

$$
\frac{d V}{d x}=I r_{x}=I r_{0} x^{2} \text { and } \frac{d I}{d x}=j \omega c_{0} V \text {, }
$$

where $d T$ is che rms voltage drop across the element, and $d I$ is the rms current from the element to the shield. Differentiating the first of these and substituting the values of $I$ and $d I / d x$ in the resulting expression gives

$$
\frac{d^{2} V}{d x^{2}}-\frac{2}{x} \frac{d V}{d x}-\lambda^{2} x^{2} V=0,
$$

subject to the boundary conditions $V=0$ at $x=0$ and $V=V_{a}$ at $x=l$ and where $\lambda^{2}=j \omega c_{0} r_{0}$.

When $\lambda=0$ this becomes, with the same boundary conditions,

$$
\frac{d^{2} V}{d x^{2}}-\frac{2}{x} \frac{d V}{d x}=0
$$


the solution of which is

$$
T_{1}=\frac{V_{a} x^{3}}{l^{3}}
$$

We now put this value of $\mathrm{I}$ in the perturbing term containing $\lambda^{2}$ in eq 36 , to get

$$
\frac{d^{2} V}{d x^{2}}-\frac{2}{x} \frac{d V}{d x}=\frac{\lambda^{2} V_{a} x^{5}}{l^{3}}
$$

The solution of this, with the same boundary conditions, is

$$
\Gamma_{2}=\frac{V_{a} x^{3}}{l^{3}}\left(1-\frac{\lambda^{2} l^{4}}{28}\right)+\frac{\lambda^{2} T_{a} x^{i}}{28 l^{3}} .
$$

$A=\lambda^{2}=j \omega c_{0}{ }^{2}$, we will need to repeat this process to secure terms in $\lambda^{4}$, for we are interested in the magnitudes of $I^{\circ}$ and $I$. Putting the above value of $\mathrm{T}_{2}$ in the last term of ey 36 and solving as before gives

$$
r_{3}=\frac{r_{a^{2}} x^{3}}{l^{3}}\left(1-\frac{\lambda^{2 r^{7}}}{28}+8.8 \times 10^{-4} \lambda^{2 l^{4}}\right)+\frac{\lambda^{2} T_{a} x^{7}}{28 l^{3}}\left(1-\frac{\lambda^{2 / 4}}{28}\right)+\frac{\lambda^{4} T_{a}{ }_{a} x^{11}}{2+64 l^{3}}
$$

From eq 35

$$
I=\frac{1}{r_{0} x^{2}} \frac{d V}{d x}
$$

Performing the indicated differentiation of eq 37 and substituting in 38 , and noting that the total resistance is $R=r_{0} l^{3} / 3$ leads to

$$
I=\frac{V_{a}}{R}\left[1-\frac{\lambda^{2} l^{4}}{28}+8.8 \times 10^{-4} \lambda^{2 / 4}+\frac{\lambda^{2} x^{4}}{12}\left(1-\frac{\lambda^{2} l^{4}}{28}\right)+\frac{\lambda^{4} x^{8}}{672}\right] .
$$

Solving for $I_{0}$ at $x=0$, and noting that the total capacitance is $C^{\prime}=c_{0} l$ gives

$$
I_{0}=\frac{r_{a}}{R}\left[1-j 0.107 \omega C R-7.9 \times 10^{-3}(\omega C R)^{2}\right] .
$$

Since the terms in the bracket are small compared with unity

$$
\left|I_{0}\right|=\frac{V_{a}}{R}\left[1-0.0022(\omega C R)^{2}\right] .
$$

Washington, August 16, 1951. 
F. L. Hermach

This is a publication of the American Institute of Electrical Engineers SYNOPSIS

Thermal voltage converters, each consisting of a resistor in series with a thermoelement in a coaxial line, have been developed for measurements of rms voltages of 1 to 200 volts at frequencies from $3 \mathrm{cps}$ to $30 \mathrm{mc}$. An accuracy of $0.1 \%$ or better may be obtained by ac-dc transfer techniques up to at least $10 \mathrm{mc}$ and $0.2 \%$ at $30 \mathrm{mc}$.

\section{INTRODUCTION}

Thermal voltage converters* containing thermoelements in series with wire-wound resistors can be used to make highly accurate voltage measurements at audio and ultrasonic frequencies.1,2 The frequency range of such voltage converters is limited primarily by the residual reactances of their wire-wound resistors, but tests have indicated that for some of them good. performance might be expected to frequencies approaching $1 \mathrm{mc}$. This paper describes thermal voltage converters of 1 ow and computable reactance that have been developed at the National Bureau of Standards to meet the need for determining the frequency limit of such instruments. Single-range converters with depositedcarbon resistors have been constructed with ranges of 1 to 200 volts. Each has a frequency influence less than $0.1 \%$ to $10 \mathrm{mc}$ and less than $0.4 \%$ at $30 \mathrm{mc}$. These rms voltage converters may also be used to calibrate $1 / 2 \%$ r-f thermocouple voltmeters which are now commercially available, and, with sine wave generators, could be used to calibrate electronic voltmeters as well.

\section{DESCRIPTION AND CONSTRUCTION OF CONVERTERS}

The r-f thermal voltage converters make use of the transfer principle, 1 in which a $d-c$ voltage is substituted for the a-c voltage to be measured. The d-c voltage is adjusted to give the same output emf of the thermoelement that was obtained with the a-c voltage applied, and is measured with a potentiometer or other suitable means. Thus only good short-time stability, high precision of reading and small known frequency influence are required of the transfer standard.

Each converter consists of a cylindrical deposited-carbon resistor in series with a UHF thermoelement having a short straight heater in line with its supports, coaxially mounted in a brass cylinder, as shown schematically in Fig. 1. The residual reactances are much smaller than those of wire-wound, resistors, and can be computed at least approximately, so that the frequency errors can be estimated. The frequency errors of the UHF thermoelements as current converters are believed to be small to over $100 \mathrm{mc}$.

The dimensions of the converters and additional data are given in the caption of Fig. 1. Each converter is assembled by first soldering the resistor, $R$, to the thermoelement, $T E$, and to the coaxial input connector, $A$, which is mounted in disc, $D_{1}$. This disc is then fastened to the cylinder, F, and the two thermocouple leads are soldered to the 2-pin output connector, B. Disc, $D_{2}$, which has a small hole with a cross wire at $G$, is then fastened to $F$ and the free end of the heater of the thermoelement is

* A thermal voltage converter is a thermoelement of low current input rating with an associated series impedance or transformer, such that the emf developed at the output terminals gives a measure of the voltage applied to the input terminals. (Definition 3.11.3 ASA Standards C39.1 - Electrical Indicating Instruments) 
soldered to the cross wire. For clarity both thermocouple leads are shown in the figure. However the plane of the thermocouple leads and the pins of the output connector is actually at right angles to the axis of the cylinder so that minimum emf is induced in the output circuit. In use $F$ must be grounded through the input connector.

The 100- and 200-volt converters contain two resistors and two cylindrical inner shields, $\mathrm{S}_{1}$ and $\mathrm{S}_{2}$, as shown in $\mathrm{Fig}$. 2, to reduce the effect of the distributed capacitance from the resistors to the outer cylinder. This considerably extends their frequency range. Shield, $S_{2}$, is centered in $F$ by a narrow ring, and is fastened to $F$ by 3 equally spaced screws. Slots in $F$ make axial adjustment of $S_{2}$ possible. The endplate of $\mathrm{S}_{1}$ is soldered to the input lead and to the resistor in assembly and this shield is fastened to $\mathrm{D}_{1}$ by small insulators. The assembly is otherwise similar to that described for the other converters. The 50-volt converter has a single resistor and a fixed inner shield like $S_{1}$ in a cylinder of the dimensions given in the caption of Fig. 1.

Additional information on the voltage converters is given in the first three columns of Table 1. The commercially available deposited carbon resistors are $1 / 8$ inch in diameter and 2 inches long, and are vacuum sealed within a $3 / 16$ inch diameter glass cylinder. (Some resistors of a different construction showed unaccountable ac-dc errors above 10 mc when used in earlier converters.) The 5- and 10-ma UHF thermoelements, each with colinear supports and heater wire, are also commercially available, with an output voltage of about $7 \mathrm{mv}$ at rated current. Each is in an acorn-shaped evacuated glass bulb, and has a small bead between the heater and hot junction of the thermocouple to electrically but not thermally insulate them.

A Lindeck potentiometer rather than a millivoltmeter is connected to the output emf of the converter to provide high resolution. A circuit diagram is shown in Fig. 3 . The potentiometer has ranges of $0.15,0.75,7.5$ and $15 \mathrm{mv}$. The two lowest ranges are used for sensitivity checks. With a galvanometer having a sensitivity of $200 \mathrm{~mm} / \mu \mathrm{a}$ it provides a selected resolution of either 2 or $10 \mu \mathrm{v}$ per mon deflection. The potentiometer is completely shielded, and a 2-conductor shielded cable connects the output of the converter to the terminals marked $\mathrm{E}$ in the figure. The advantages of this potentiometer are its low thermal emf (less than $1 \mu \mathrm{v}$ ) and its freedom from drift (much less than $0.01 \% / m i n u t e$ under ordinary laboratory conditions).

\section{THEORY}

Because of the geometry, the resistor, $\mathrm{R}$, in the cylinder of each low-voltage converter (without inner shields) may be represented as a transmission line of length, $\ell$, with a uniformly distributed series impedance of $z$ ohms per unit length and a uniformly distributed shunt admittance of $y$ mhos per unit length. The line is terminated by the heater of the thermoelement. We are interested in the magnitude of the trans-impedance $\mathrm{Z}_{c}=\mathrm{V}_{\mathrm{i}} / \mathrm{I}_{0}$ where $\mathrm{V}_{\mathrm{i}}$ is the input voltage being measured and $\mathrm{I}_{0}$ is the current through the heater. If the heater resistance, $R_{h}$, is much less than that of the resistor, $R$, we may to a first approximation consider this a short circuited line. (It will be apparent later that this is not a drastic approximation at low frequencies since we are concerned only with the effect of the line reactance on $Z_{c}$.) Then by ordinary steadystate transmission-line theory,

$$
Z_{c}=\frac{Z}{\sqrt{Z Y}} \sinh \sqrt{Z Y}
$$

where $Z=z l=R+j \omega L$, and $Y=y l=j \omega C$ and $R, L$, and $C$ are the total resistance, series inductance and shunt capacitance of the line. We are interested in the low frequency range in which the effects of the reactances are small. 
We may define the parameters $a=\omega C R$ and $b=\omega L / R$. If each of these is less than unity the hyperbolic function may be approximated by the first 3 terms of its series expansion, so that

$$
Z_{c}=Z\left(1+\frac{Z Y}{6}+\frac{(Z Y)^{2}}{120}+\cdots\right)
$$

Then if all terms in $a^{n^{m}}$ for which $m+n \geq 3$ are discarded we find that

$$
z_{c} \approx R(1+j b)\left(1-\frac{a b}{6}-\frac{a^{2}}{120}+\frac{j a}{6}\right)
$$

and, to the same order,

$$
\left|z_{c}\right| \approx R\left(1+\frac{a^{2}}{90}+b^{2}-\frac{a b}{3}\right)^{1 / 2} \approx R\left(1+\frac{a^{2}}{180}+\frac{b^{2}}{2}-\frac{a b}{6}\right)
$$

The ac-dc difference of a voltage converter is defined as

$$
\mathrm{s}=\frac{\mathrm{v}_{\mathrm{ac}}-\mathrm{V}_{\mathrm{dc}}}{\mathrm{V}_{\mathrm{dc}}}
$$

where $V_{a c}$ and $V_{d c}$ are the rms alternating and direct voltages required to obtain the same response (output emf) of the converter. If the thermoelement has no ac-dc difference and if we define $\mathrm{k}=\mathrm{b} / \mathrm{a}$, this becomes

$$
S_{O}=\frac{\left|z_{c}\right|-R}{R} \approx \frac{a^{2}}{2}\left(k^{2}-\frac{k}{3}+\frac{1}{90}\right)
$$

where $S_{O}$ signifies that other possible causes of ac-dc differences are neglected.

The relation makes it possible to estimate $S_{O}$ (to this approximation) very simply. For the cylindrical construction of figure 1 with a cylindrical unspiraled resistor, $\mathrm{L}=0.012 \mathrm{lM}$ microhenries and $\mathrm{C}=0.61 \mathrm{l} / \mathrm{M}$ picofarads, where $\mathrm{M}=\log _{10}(\mathrm{~g} / \mathrm{h})$ and $\mathrm{g}$ and $\mathrm{h}$ are the diameters of the eylinder and resistor, respectively, and $\ell$ is in inches. Thus numerically, $\mathrm{k}=1.9 \times 10^{4}(\mathrm{M} / \mathrm{R})^{2}$ and $\mathrm{a}=3.8 \times 10^{-12} \mathrm{f} \ell \mathrm{R} / \mathrm{M}$, where $\mathrm{f}$ is the frequency.

For example if $R=2000$ ohms, $g$ and $h$ are 2 in. and $1 / 8 \mathrm{in.}$, and $\ell=2.0$ in., $\mathrm{S}_{\mathrm{O}}=+0.001(+0.1 \%)$ at $40 \mathrm{mc}$.

It is apparent from eq 4 that to this approximation certain values of $k$ should make $\mathrm{S}_{\mathrm{O}}=0$. These are $\mathrm{k}_{1}=0.038$ and $\mathrm{k}_{2}=0.30$. The numerical values of resistance required are $R_{1}=710 \mathrm{M}$ and $R_{2}=250 \mathrm{M}$. Unfortunately the logarithmic relationship for $\mathrm{M}$ permits little range in $R$ for reasonable values of $g$ and $h$, for if $g$ is greater than the length of the resistor, end effects may become pronounced.

It is of interest to extend the analysis to higher values of the parameter, $a$. This has been done by solving eq 1 for $z_{c}$ with the aid of Kennelley's tables and graphs of hyperbolic functions having complex arguments, ${ }^{3}$ and then computing $S_{O}=\left(\left|Z_{c}\right|-R\right) / R$. The results are shown in Fig. 4. It is rather striking that the first solution of eq 4, $\mathrm{k}_{1}=0.038$, results in reasonably small ac-dc differences $(<5 \%)$ to $a=10$, a range much greater than that permitted by the initial assumptions.

For low voltage converters the effect of the heater resistance $R_{h}$, cannot be neglected. To the next approximation the heater can be considered as a lumped resistance termination for the transmission line. Then from steady-state transmission-1ine formulas, 


$$
Z_{c}=R_{h} \cosh \sqrt{Z Y}+\frac{Z}{\sqrt{Z Y}} \sinh \sqrt{Z Y}
$$

By expanding the hyperbolic functions and discarding higher.order terms as before (for $a<1$ and $b<1$ ) we find that if $R_{h}<R$

$$
S_{O}=\frac{\left|Z_{c}\right|-R_{t}}{R_{t}} \approx \frac{1}{2}\left(\frac{a}{1+m}\right)^{2}\left(k^{2}-A k+\frac{B}{6}\right)
$$

where $R_{t}=R+R_{h}, R_{h} / R=m, A=m^{2}+2 m / 3+1 / 3$ and $B=m^{2}+2 m / 5+1 / 15$.

The values of $S_{O}$ for the 1- to 20-volt converters have been calculated by eq 6 at a frequency of $40 \mathrm{mc}$ (at which $a$ and $b$ are still less than unity). The results are given in the last column of Table 3 .

When $k<0.038$, distributed capacitance from the resistor to the cylinder predominates. At a given frequency and geometry, the resultant error is proportional to the square of the resistance. It can be minimized by the construction shown in Figure 2 in which two inner cylindrical shields are used, with one connected to the input lead. Thus there are now two transmission lines in series. If $R_{h} \ll R$, application of transmission line formulas, with careful attention to signs, gives for the desired trans-impedance,

$$
\mathrm{Z}_{c}=\sqrt{\frac{\mathrm{Z}_{1}}{\mathrm{Y}_{1}}} \tanh \sqrt{\mathrm{Z}_{1} \mathrm{Y}_{1}} \cosh \sqrt{\mathrm{Z}_{2} \mathrm{Y}_{2}}+\sqrt{\frac{\mathrm{Z}_{2}}{\mathrm{Y}_{2}}} \sinh \sqrt{\mathrm{Z}_{2} \mathrm{Y}_{2}}
$$

where the subscripts 1 and 2 refer to the left and right hand lines (resistors and shields) of figure 2, and the resistance of the thermoelement is neglected.

If

$$
\begin{aligned}
& Z_{1}=Z_{2}=Z \text { and } Y_{1}=Y_{2}=Y \text { we have } \\
& Z_{c}=\frac{2 Z}{\sqrt{2 Y}} \sinh \sqrt{Z Y}
\end{aligned}
$$

Thus the trans-impedance is twice that of either line, and the ac-dc difference, $S_{0}$, will be that of half the total line. The parameter, a, of eq. 4 will then be $1 / 4$ th that for a line of the same dimensions without inner shields. Thus at a given frequency for which $a<l$ the use of the double shield rather than a single cylinder of the same diameter should reduce the frequency error (for resistors of the same value and dimensions) by a factor of 16 .

However more detalled analysis indicates that even further improvement may be possible. For these converters $k<0.038$, so that the series inductance of each line may be neglected. By expanding the hyperbolic functions of eq 7 and discarding higher order terms it can be shown by lengthy and rather tedious algebraic manipulation that $s_{0} \approx 0$ when $\ell_{1} / \ell_{2} \approx 1.032$, where $\ell_{1}$ and $\ell_{2}$ are the lengths of the left and right hand lines of fig. 2 , and $a<1$.

For the conventent dimensions shown in figure 1, the parameter, a, of each lowvoltage converter now being used (up through 20 volts) is low enough that it has not been necessary to proportion the converters for minimum error in accordance with eq 6 . The higher voltage elements are constructed with dual shields, with the grounded shield axially adjustable. This shield is set to give minimum frequency error as explained in the next section. 
A useful theoretical study of coaxial lines, terminated by resistors, was published by Crosby and Pennypacker. ${ }^{4}$ They show conditions for minimizing the input reactance.

\section{TESTS}

Although long-time stability is not required of these converters, fluctuations or drifts in emf for the short time between the a-c use and the d-c calibration must be less than the desired accuracy. Such changes can arise from self heating effects and ambient temperature changes, and from thermal emfs and other changes in the Lindeck potentiometer. Tests have shown that the self-heating of the carbon resistors (which have a load coefficient of about $1 \%$ per watt) is the largest source of drift. The change in resistance is very nearly exponential, with a time-constant of about 2 minutes. Since d-c calibrations can easily be made within 30 seconds of the a-c readings, this self heating error is not significant in a-c tests if a short warm-up period is allowed. It is almost completely eliminated by the procedure used for ac-dc transfer tests. The effect of ambient temperature changes (the thermoelements have temperature coefficients up to $0.1 \% /{ }^{\circ} \mathrm{C}$ ) should also be insignificant in a laboratory with reasonable temperature control.

A great advantage of thermoelements for ac-dc transfer measurements is the almost complete electrical isolation of the input and output circuits. The UHF thermoelements have a small electrically insulating bead between the heater and hot junction of the thermocouple to eliminate conductive coupling. To minimize mutual inductance the plane of the thermocouple leads is at right angles to the heater and its colinear supports. Tests showed that at $4 \mathrm{mc}$ the induced a-c voltage in the output circuit of each thermoelement was less than 20 microvolts (the resolution of the detector used). Induced currents in the thermocouple circuit can cause errors by joule heating of the thermocouple, but calculations indicate this should not be significant if the induced voltage is less than a few millivolts.

The shield of the Lindeck potentiometer provides reasonab]e immunity from induced fields. In some tests at $10 \mathrm{mc}$ a current of 2 ma from the shield to ground through the lead from the potentiometer to the converter caused no significant error.

For transfer measurements the most important requirement is that the ac-dc difference of each of these converters be known to. the full accuracy desired. The general principles on which such determinations are based have been given. 1 In the frequency range studied, the major error of each of these converters is caused by the reactance. For a given converter this error should be independent of voltage level. Thus it was feasible to evaluate the relative errors by intercomparing converters of adjacent voltage ranges to determine their differences in frequency response. A complete series of such comparisons was made at 2 voltage levels for each paix of converters at frequencies up to $40 \mathrm{mc}$. In each comparison the two converters were connected in parallel to a coaxial lead through a tee fitting (GR 874), and a shielded potentiometer was connected to each output. Each potentiometer was adjusted for zero deflection at the test voltage. The converters were then supplied in succession with alternating, direct, reversed-direct, and alternating voltage. Each voltage was adjusted to produce the same emf of the higher range converter and the deflection of the galvanometer connected to the other converter was observed. From the differences in emf (directly determined from these differences in deflection) the difference in the frequency response of the two converters was determined. 1 In these tests the movable inner shields of the 100- and 200-volt converters were adjusted for best performance over the desired frequency range, by comparison with the 20- and 50-volt converters. The errors of these adjustable converters were found to be complicated functions of the frequency and shield position. 
The results of these intercomparisons from 0.1 to $40 \mathrm{mc}$ are given in Table 2 . They show that for each pair of converters the relative ac-dc differences were independent of the applied voltage to $0.02 \%$ or less, and were less than $0.4 \%$ up to $30 \mathrm{mc}$. For the 1 owvoltage converters they were 1 ess than $0.05 \%$ to $20 \mathrm{mc}$, without exception and without evidence of systematic errors.

The relative ac-dc differences of most of the thermoelements used in the voltage converters were also determined (before the thermoelements were installed) by making similar ac-dc intercomparisons (as current-measuring elements) at $40 \mathrm{mc}$. For these tests the two thermoelements were connected in series along the axis of a brass cylinder having the same dimensions as in fig. 1 but with a coaxial input connector at each end and with two 2-pin output connectors. (A split cylinder with one part readily removable facilitated changing thermoelements.) The input was applied to one connector with the other shorted. Rough calculations indicated that at $40 \mathrm{mc}$ the current should change by about $0.5 \%$ along the transmission line formed by the heaters of the thermoelements in this cylinder, but that the distribution should not be greatly dependent on the resistance of the heaters (less than $100 \mathrm{ohms}$ each). Therefore two determinations of relative ac-dc differences were made with each pair of thermoelements, with first one end then the other of the cylinder shorted. For each pair the two determinations differed by about $0.4 \%$ at $40 \mathrm{mc}$, but in each case their algebraic average was less than $0.02 \%$.

These calculations and tests also provide assurance that even at $40 \mathrm{mc}$ the current along the heater of a single thermoelement terminating a voltage converter is well within 0.1 percent of the value at the mid-point of the heater.

The 1-volt converter was compared at rated voltage with a wire-wound thermal voltage converter of known ac-dc difference at $3 \mathrm{cps}$ and $20 \mathrm{kc}$, with observed differences of $+0.02 \%$ and $0.00 \%$ respectively.

The response of the 1-volt converter, with a shielded d-c millivoltmeter connected to its output, was determined to better than 1\% at frequencies from 1 to $400 \mathrm{mc}$ by the bolometer bridge of Selby \& Behrent5, with the results shown in Table 3 . It is evident that the frequency influence is very small to $100 \mathrm{mc}$. It increases rapidly at higher frequencies, becoming $-5.2 \%$ at $300 \mathrm{mc}$. The calculated ac-dc difference at $300 \mathrm{mc}$ by equation 6 is only $-0.9 \%$ (this converter has a $1 / 4$ in. diameter unsealed resistor). Some of the discrepancy may be accounted for by the voltage rise in the connector (a type N-UG58/U input connector was substituted for the type 874 for these tests).

As a check on eq 6 at larger ac-dc differences, the 50-volt converter was tested before its inner shield was installed. The measured ac-dc difference at 40 mc was $+1.3 \%$ and was accurately proportional to the square of the frequency. The computed values were $40 \%$ smaller, indicating that the effective length of the resistor (end effect) was 1.2 times the actual length. At $40 \mathrm{mc}$ the measured ac-dc difference was changed by less than $0.02 \%$ when the resistor was mounted $3 / 32$ inch off the axis of the cylinder, indicating that exact centering is not critical even when the errors are large.

The intercomparisons of Table 2 show that the ac-dc differences of the five lowvoltage converters without inner shields (1- to 20-volt ranges) are all equal to better than $0.05 \%$ to more than $20 \mathrm{mc}$. At $40 \mathrm{mc}$ they agree to $0.1 \%$ with the values calculated by eq 6. (Values cannot readily be computed for the higher voltage elements with the inner shields.) This unanimity between converters having such a wide range of resistors and different thermoelements gives good assurance against unknown sources of error. It is quite unlikely (but not impossible) that each converter would have the same ac-dc error. However the test of the 1-volt converter with the bolometer bridge provides most valuable additional 
assurance. The large errors of this converter above $100 \mathrm{mc}$ decrease rapidly with decreasing frequency, well within the stated accuracy of the bridge measurements. For converters without inner shields almost all known causes of such errors (such as the effect of reactance, eq 6 , skin effects in the resistor or thermoelement, etc.) should cause ac-dc differences approximately proportional to the square of the frequency, over the range for which the errors are small. Thus the authors believe that the large measured errors above $100 \mathrm{mc}$ can be extrapolated downward to lower frequencies to indicate with considerable confidence that the ac-dc difference of this converter is less than $0.2 \%$ at $40 \mathrm{mc}$ and less than $0.1 \%$ below $30 \mathrm{mc}$.

Based on the above consideration the authors assigned a value of zero to the ac-dc difference of the 1 volt converter to $40 \mathrm{mc}$, and then determined the ac-dc differences of all the other converters from the intercomparison data in Table 1 . The results, rounded to the nearest $0.05 \%$ to $10 \mathrm{mc}$ and $0.1 \%$ to $40 \mathrm{mc}$, are given as observed values in Tab1e 4 .

\section{$\underline{\mathrm{USE}}$}

These converters are most conveniently used to measure the ac-dc difference or frequency influence of other rms instruments such as thermocouple voltmeters (which are now available with accuracies of $1 / 2 \%$ to $10 \mathrm{mc}$ ). A typical set-up for this is shown in Fig. 5. The scale calibration of the voltmeter can then easily be checked on reversed direct current. The ac-dc difference tests are similar to the intercomparisons already described, and d-c calibration of the converter is not necessary once the scale factor of the Lindeck potentiometer is determined for each converter. This is the percent change in input voltage per centimeter change of galvanometer deflection. 1 Either direct or low-frequency alternating current may be used as the reference frequency and the test may be made rapidly and accurately. The results are only slightly affected by drifts in either instrument. At frequencies above about $20 \mathrm{mc}$ a small lead correction may be necessary if the connectors between the junction plane and the two instruments are not electrically equal, but this is readily determined to the required accuracy.

For a-c measurements a d-c potentiometer of $0.1 \%$ accuracy or better is required to measure the $d-c$ reference voltage. A deflection potentiometer and volt box, or an automatic self-balancing potentiometer ("digital voltmeter") should be convenient for this. For testing electronic voltmeters frequency-response measurements (differences in reading for the same voltage at the test and reference frequencies) should be particularly convenient. The form factor and crest factor of the a-c sources must be within $0.1 \%$ of the values for a sine wave ( 1.111 and 1.414 respectively) since most electronic voltmeters respond essentially to the average or crest (peak) values. Since these factors depend upon the phase angle as well as the magnitude of each harmonic it would ordinarily be necessary to make sure that the ratio of the magnitude of the nth harmonic to the fundamental does not exceed $0.1 \mathrm{xn} \%$ when an average-reading instrument is tested and $0.1 \%$ when a crest-reading instrument is tested. The results should then differ from those obtained with a sine wave by less than $0.1 \%$.

An $r-f$ generator of at least 5 watts output and good voltage stability is required but only moderate frequency accuracy and stability are needed. It is very difficult to construct broad-band generators of good wave form at this power level. However, a simple adjustable LC tuned circuit, with the instruments connected across the capacitor, can be used to simultaneously improve the wave form, match the impedance of the instruments to that of usual 50-ohm source, eliminate the capacitance loading of the instruments on the source, and provide the higher voltages often required. For these combined purposes the values of inductance and capacitance should be chosen so that $\omega \mathrm{L}=1 / \omega \mathrm{C} \approx \sqrt{R_{1} R_{2}}$, where

$R_{1}$ and $R_{2}$ are respectively the resistances of the source (including the inductor) and the load (instruments). For low voltages a 50-ohm resistor can be connected in series with the LC circuit, and the instruments and attenuators as needed can be connected in parallel with this resistor for improved wave form. 
Cylindrical film resistors in series with a thermoelement in a coaxial line makes possible single-range thermal voltage converters that are useful as ac-dc transfer instruments over a very wide frequency range, $3 \mathrm{cps}$ to at least $30 \mathrm{mc}$, with unusually high accuracy. They are inexpensive and easy to construct. Their frequency influence may be estimated by reasonably simple formulas, with results which agree well with the measured values up to $40 \mathrm{mc}$. The voltage converters may be useful to considerably higher frequencies. The higher range converters require considerable power (up to 2 watts) and have a marked but short warm-up drfft. These disadvantages could be reduced by using metal-film resistors of higher resistance (with a 5-ma thermoelement) but this would very probably reduce the frequency range for the same attainable accuracy. The good performance of these converters was obtained with deposited-carbon resistors of a new type. Earlier converters with resistors of different construction showed discrepancies up to $0.5 \%$ at $40 \mathrm{mc}$. Further work is planned to determine the cause of these differences.

These voltage converters can be used quickly and easily to make ac-dc difference tests to determine the frequency influence of other rms instruments to $0.1 \%$ or better to at least $10 \mathrm{mc}$ and to $0.2 \%$ at $30 \mathrm{mc}$. Direct a-c measurements are made by the transfer technique, which in most cases can be arranged for reasonable simplicity. With sources of suitable wave form, average-reading and crest-reading instruments could also be calibrated if desired. In all of these applications the applied frequency need not be closely determined or held because of the flat frequency response of these voltage converters.

\section{ACKNOWLEDGEMENTS}

It is a pleasure to acknowledge the help of R. Richardson and J. Scofield who checked the equations and carried out the computations which made Fig. 4 and the last column of Table 3 possible. J. E. Hill assisted with some of the measurements. Sincere thanks are also extended to M. C. Selby and L. Behrent for their careful calibrations of the 1-volt converter.

\section{REFERENCES}

1. Thermal converters as AC-DC Transfer Standards for Current and Voltage Measurements at Audio Frequencies, F. L. Hermach, J. Research NBS, vol. 48, pp. 121-138, 1952.

2. Wide Range Vo1t-Ampere Converters for Current \& Voltage Measurements, F. L. Hermach and E. S. Williams, Communications \& Electronics (AIEE) 1959 (paper 59-161).

3. Tables and Chart Atlas of Complex Hyperbolic \& Circular Functions, A. E. Kennelly, Harvard University Press, 1921.

4. Radio-Frequency Resistors as Uniform Transmission Lines, D. R. Crosby and C. H. Pennypacker, Proc. IRE, vol. 34, pp. 62-66, 1946.

5. A Bolometer Bridge for Standardizing Radio-Frequency Voltmeters, M. C. Selby and L. F. Behrent, J. Research NBS, vol. 44, pp. 15-30, 1950.

TABLE 1. VOLTAGE CONVERTERS

\begin{tabular}{|c|c|c|c|}
\hline \multirow{4}{*}{$\begin{array}{c}\text { Rated } \\
\text { Voltage } \\
\text { Volts } \\
\end{array}$} & \multicolumn{2}{|c|}{ Thermoelemen $\tau$} & \multirow{4}{*}{$\begin{array}{l}\text { Series } \\
\text { Resistor } \\
\text { Kilohms } \\
\end{array}$} \\
\hline & Rated & Heater & \\
\hline & Current & Resistance & \\
\hline & ma & Ohms & \\
\hline 1 & 5 & 90 & 0.2 \\
\hline 3 & 5 & 90 & 0.5 \\
\hline 5 & 5 & 90 & 1 \\
\hline 10 & 5 & 90 & 2 \\
\hline 20 & 10 & 25 & 2 \\
\hline 50 & 10 & 25 & 5 \\
\hline 100 & 10 & 25 & $10(2)$ \\
\hline 200 & 10 & 25 & $20(2)$ \\
\hline
\end{tabular}


TABLE 2. COMPARATIVE AC-DC DIFFERENCES OF VOLTAGE CONVERTERS

Voltage Range

\begin{tabular}{|c|c|c|c|c|c|c|c|c|c|}
\hline Converter & Converter & Applied & Com & arative & $A C-D C$ & Differ & nce, D & (Perce & $n t)$ \\
\hline $\mathrm{A}$ & B & Volts & $0.1 \mathrm{mc}$ & $1 \mathrm{mc}$ & $5 \mathrm{mc}$ & $10 \mathrm{mc}$ & $20 \mathrm{mc}$ & $30 \mathrm{mc}$ & $40 \mathrm{mc}$ \\
\hline 1 & 3 & 1 & +0.01 & 0.00 & & & & & -0.08 \\
\hline 1 & 3 & 1.5 & & +0.01 & -0.01 & 0.00 & -0.02 & -0.03 & -0.06 \\
\hline 3 & 5 & 2 & & 0.00 & & & & & -0.05 \\
\hline 3 & 5 & 3 & 0.00 & 0.00 & 0.00 & -0.01 & -0.01 & -0.03 & -0.04 \\
\hline 5 & 10 & 3 & & 0.00 & & & & & +0.14 \\
\hline 5 & 10 & 5 & 0.00 & 0.00 & -0.01 & +0.01 & +0.02 & +0.07 & +0.15 \\
\hline 10 & 20 & 6 & & 0.00 & & & & & -0.02 \\
\hline 10 & 20 & 10 & & 0.00 & & 0.00 & 0.00 & -0.01 & -0.02 \\
\hline 20 & 50 & 15 & 0.00 & 0.00 & & & & & -0.04 \\
\hline 20 & 50 & 20 & & & & 0.00 & 0.00 & -0.01 & -0.04 \\
\hline 20 & 100 & 20 & 0.00 & 0.00 & +0.02 & +0.07 & +0.24 & $+0.33^{*}$ & +0.17 \\
\hline 50 & 100 & 50 & & 0.00 & & & & +0.33 & \\
\hline 50 & 200 & 50 & +0.01 & 0.00 & -0.02 & -0.06 & -0.04 & $+0.19^{*}$ & +0.57 \\
\hline 100 & 200 & 90 & & 0.00 & & & & -0.14 & \\
\hline
\end{tabular}

NOTE: $D=100\left(S_{B}-S_{A}\right)$, where $S$ is defined by eq 3, and the subscripts refer to converters $A$ and $B$.

* Additional comparisons made at 25 and $35 \mathrm{mc}$.

TABLE 3. RESULTS OF TEST OF 1-VOLT VOLTMETER

$\begin{array}{cc}\begin{array}{c}\text { Frequency } \\ \text { mc }\end{array} & \begin{array}{c}\text { Applied Voltage * } \\ \text { Volts }\end{array} \\ 1 & 0.997 \\ 30 & 0.998 \\ 100 & 0.998 \\ 200 & 0.999 \\ 300 & 0.982 \\ 400 & 0.945 \\ \text { For same reading of millivoltmeter at } \\ \text { Fach frequency. }\end{array}$

TABLE 4. AC-DC DIFFERENCES OF VOLTAGE CONVERTERS

\begin{tabular}{r} 
Rated \\
Voltage \\
Volts \\
\hline 1 \\
3 \\
5 \\
10 \\
20 \\
50 \\
100 \\
200
\end{tabular}

Percent AC-DC Differences (100S)

\begin{tabular}{|c|c|c|c|c|c|}
\hline \multicolumn{5}{|c|}{ Observed } & Calculated \\
\hline to $5 \mathrm{mc}$ & $10 \mathrm{mc}$ & $20 \mathrm{mc}$ & $30 \mathrm{mc}$ & $40 \mathrm{mc}$ & $40 \mathrm{mc}$ \\
\hline$<0.05$ & $<0.05$ & 0.0 & 0.0 & 0.0 & 0.0 \\
\hline$<0.05$ & $<0.05$ & 0.0 & 0.0 & -0.1 & 0.0 \\
\hline$<0.05$ & $<0.05$ & 0.0 & -0.1 & -0.1 & 0.0 \\
\hline$<0.05$ & $<0.05$ & 0.0 & 0.0 & 0.0 & +0.1 \\
\hline$<0.05$ & $<0.05$ & 0.0 & 0.0 & 0.0 & +0.1 \\
\hline$<0.05$ & $<0.05$ & 0.0 & 0.0 & 0.0 & - \\
\hline$<0.05$ & +0.05 & +0.2 & +0.3 & +0.1 & - \\
\hline$<0.05$ & -0.05 & 0.0 & +0.2 & +0.5 & - \\
\hline
\end{tabular}




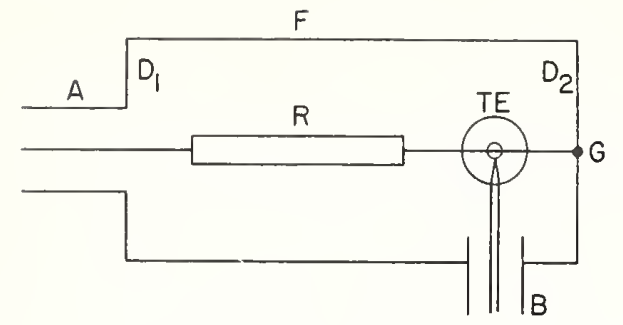

Fig. 1. Thermal Voltage Converter

R Film-type resistor $1 / 8$ in. diam., . 2 in. 1ong.

TE Thermoelement

A Coaxial input connector, GR874PB

B Two-pin output connector, AN10SL

$\mathrm{D}_{1}, \mathrm{D}_{2}$. Circular brass discs, $3 / 16$ in. thick, secured to $F$ with 4 2-56 brass screws.

F Cylindrical brass shield, 2 in. diam. 4.25 in. long.

$G$ Cross wire at center of $D_{2}$ (see text)

NOTE: Plane of thermocouple leads at right angles to axis of $F$.

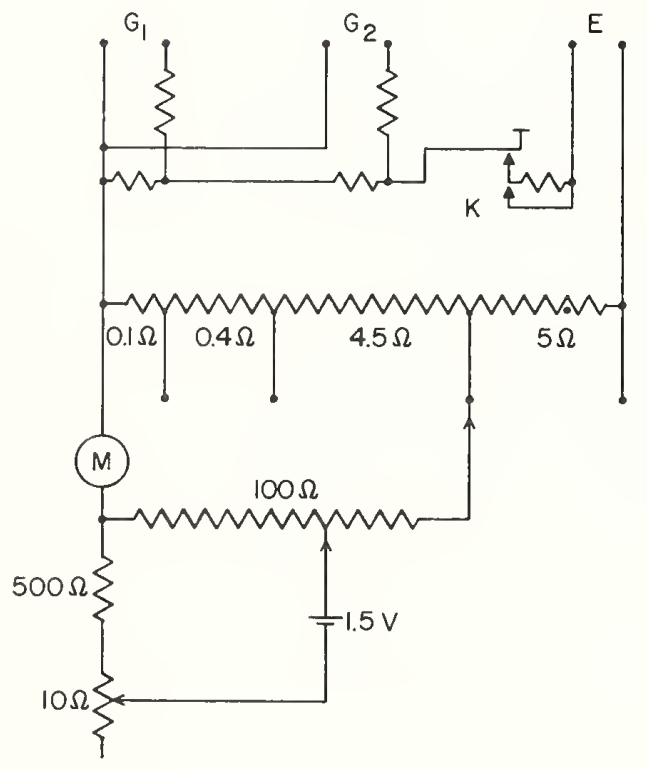

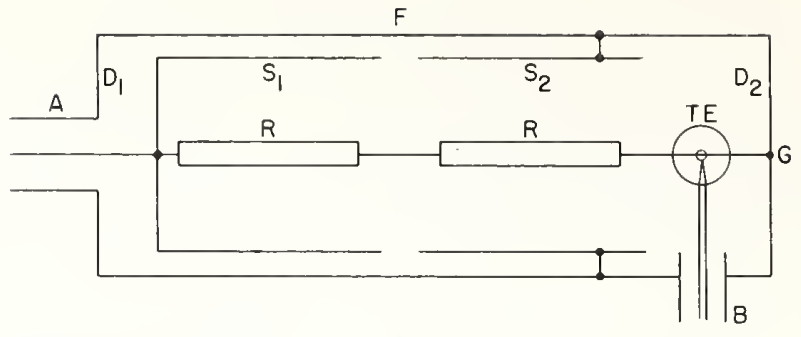

Fig. 2. Voltage Converter with Compensating Shields

$\mathrm{S}_{1}$ Cylindrical brass shield, 2 in. diam. with one brass end-plate.

$\mathrm{S}_{2}$ Cylindrical brass shield, 2 in. diam. both ends open, axially adjustable, (see text)

Other symbols same as Fig. 1 except $F$ is $2.5 \mathrm{in.} \mathrm{diam.,} \mathrm{and} 7.5 \mathrm{in.}$ long. NOTE: Plane of thermocouple leads at right angles to axis of $\mathrm{F}$.
Fig. 3. Circuit of Lindeck Potentiometer M 1.5 ma d-c milliammeter

$\mathrm{K}$ Thermofree key

E emf terminals, AN10SL

$G_{1}, G_{2}$ galvanometer termina1s, AN10SL NOOTE: All fixed resistors of manganin, accuracy $\pm 0.2 \%$. 


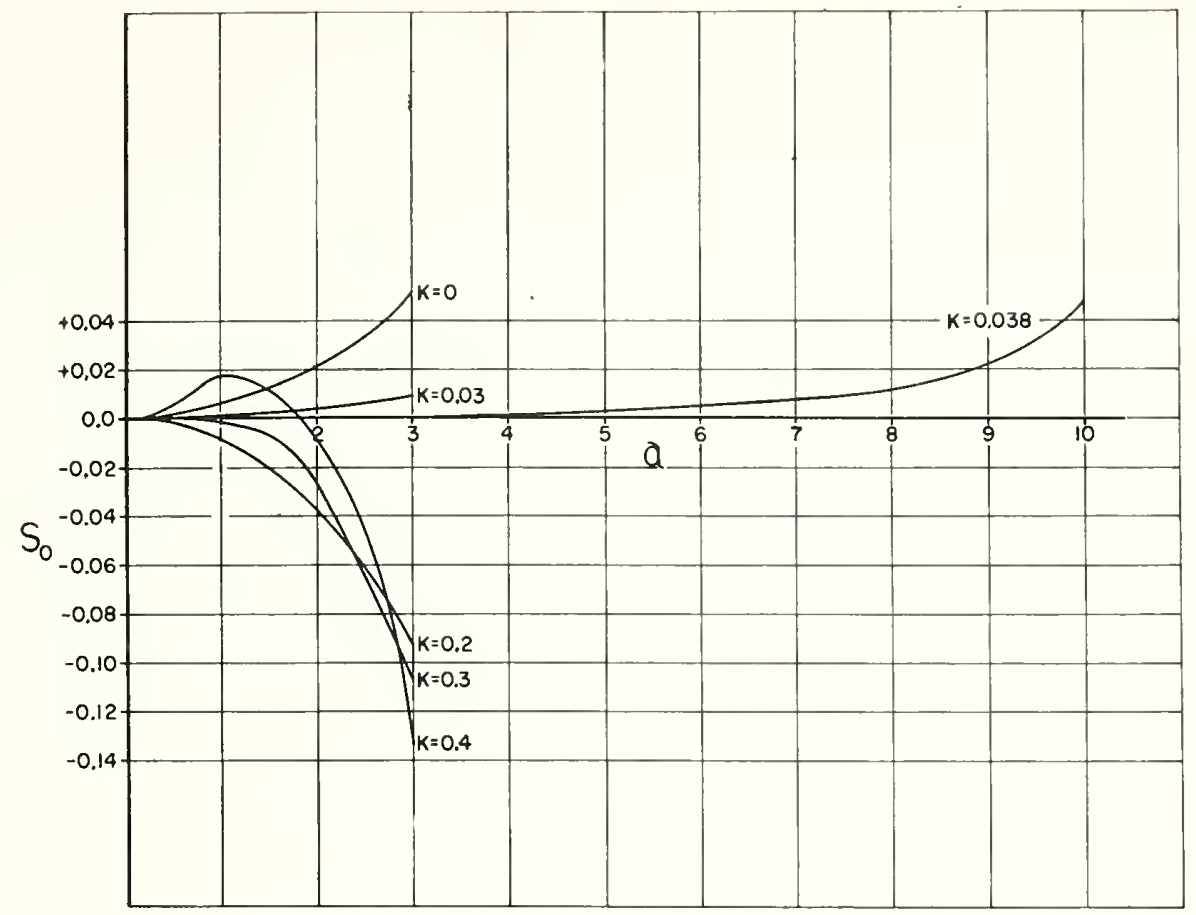

Fig. 4. Computed AC-DC Difference as a function of the parameters a and $k$.

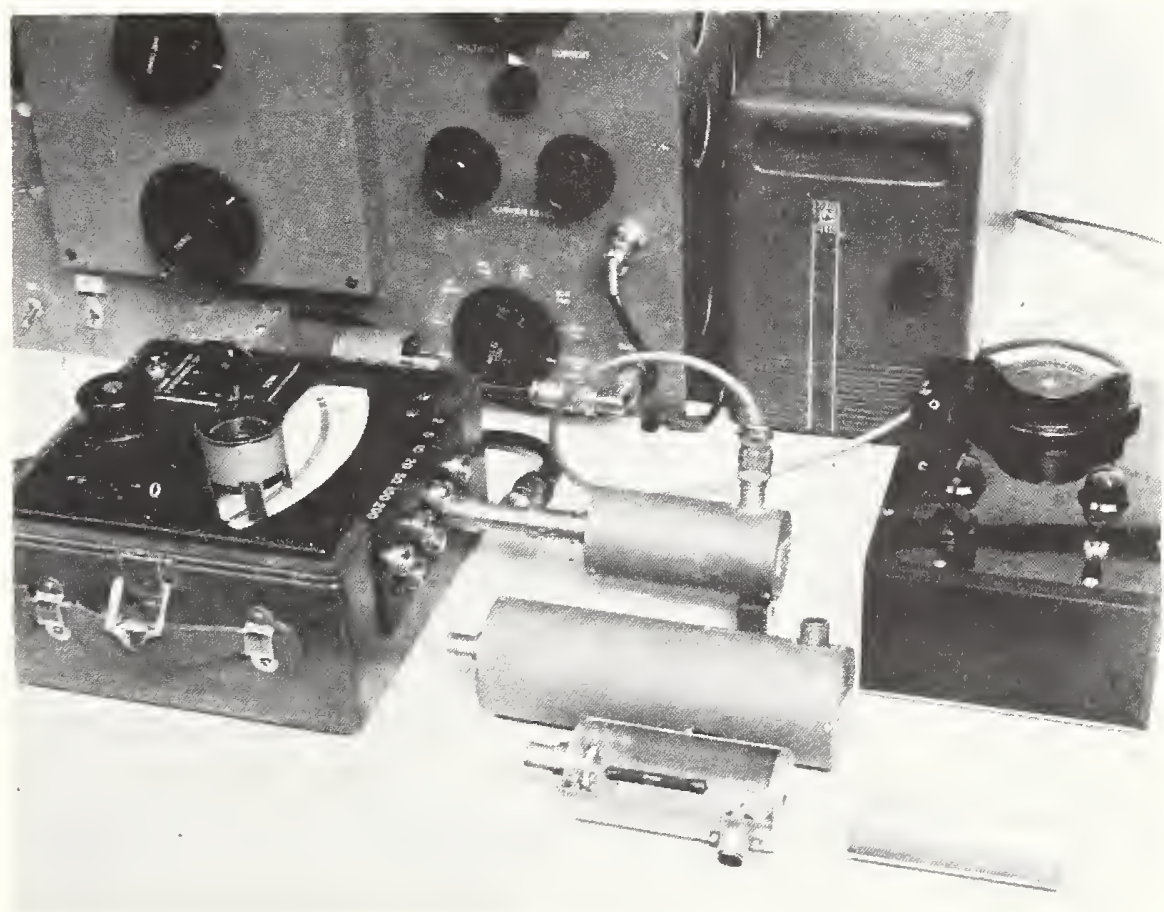

Fig. 5. 5-Volt Converter set Up for AC-DC Test of Experimental Thermocouple Voltmeter.

(Lindeck potentiometer at right, doubleshield converter and experimental splitshield element in foreground, ac-dc switch not shown) 


\title{
AC-DC Transfer Instruments for Current and Voltage Measurements*
}

\author{
FRANCIS L. HERMACH $\dagger$
}

\section{INTRODUCTION}

$\mathbb{T}$ $\checkmark \mathrm{HE}$ basic electrical units are maintained by groups of standard cells and resistors. With these standards and a dc potentiometer, measurements of direct voltage and current are readily made with an accuracy of 0.01 per cent or better. Corresponding measurements of alternating voltage and current at power and audio frequencies depend, at the present time, upon ac-dc transfer instruments which have nearly equal ac and dc response. Such instruments may be calibrated on direct current and then used on alternating current, or alternatively, may be used directly to make ac-dc difference measurements, as in the certification of other instruments.

In addition to the instantaneous values as determined by an oscilloscope or point-by-point methods, there are at least seven other quantities that may be determined for a periodic ac wave in which all values separated by the periodic time, $T$, are equal. These are: 1) the rms or effective value, $I$, defined by

$$
I^{2}=\frac{1}{T} \int_{0}^{T} i^{2} d t
$$

where $i$ is the instantaneous value; 2 ) and 3 ) the positive and negative average values, i.e., the average of all the positive or all of the negative values during one cycle, defined by

$I_{a+}=\frac{1}{2 T} \int_{0}^{T}(|i|+i) d t$ and $I_{a-}=\frac{1}{2 T} \int_{0}^{T}(|i|-i) d t$

where $|i|$ is the magnitude of $i ; 4)$ the rectified fullwave average, defined by

$$
I_{a r}=\frac{1}{T} \int_{0}^{T}|i| d t
$$

5) and 6) the positive and negative crest or peak values; and 7) the crest-to-crest or peak-to-peak value.

Instruments for measuring each of these values of an ac voltage or current wave are commercially available (with widely varying accuracies). Ordinarily, however, only one of these values is really required in a measurement. Unfortunately, it is difficult to deduce accurately one value from the measurement of another, except for a few waveforms (which, of course, must be known). In most cases the rms value is the one really desired, since the rate of transformation of electrical to other forms of energy (which is governed by the heating produced by a current and by the electromagnetic force produced by currents acting on each other) is a function of the square of this value.

The three general types of ac-dc transfer instruments which are at present suitable for rms measurements of better than 0.1 per cent accuracy are dependent upon these same laws of the interchange of energy. They are: 1) electrodynamic instruments, which depend upon the force between current-carrying conductors; 2) electrostatic instruments, which depend upon the force between charged conductors; and 3) electrothermic instruments, which depend upon some effect produced by the heating of a current-carrying conductor. The first and third respond essentially to current and the second to voltage, but series or shunt resistors make all three types suitable for both measurements, while other circuit arrangements make power measurements almost equally feasible.

\section{Response Equations}

The instantaneous torque of an electrodynamic instrument in which two sets of conductors, one fixed and one rotatable about an axis, carry the same current, $i$, is $^{1}$

$$
\tau=i^{2} \frac{\dot{d} M}{d \theta}
$$

where $M$ is the mutual inductance and $\theta$ some welldefined angle between the two sets of conductors.

In its simplest form such as instrument has an opposing torque, $U \theta$, so that, with direct current applied, the rest position, $\theta_{f}$, is defined by

$$
\left.U \theta_{f}=B_{1} I^{2} \quad \text { where } \quad B_{1}=\frac{d M}{d \theta}\right]_{\theta=\theta f}
$$

The differential equation governing the angular deflection is ${ }^{1}$

$$
P \theta^{\prime \prime}+A \theta^{\prime}+U \theta=B i^{2}
$$

where $P$ and $A$ are the inertial and damping constants, respectively. If the mechanical inertia is sufficiently great that with alternating current applied the periodic fluctuations of $\theta$ are negligible compared with the average value, termwise integration over an integral number of cycles gives 


$$
U \theta_{f}=\frac{B_{1}}{T} \int_{0}^{T} i^{2} d t=B_{1} I^{2} .
$$

Thus if $U$ and $B$ each have the same value on direct as on alternating current, the instrument has the same response, $\theta_{f}$.

The instantaneous torque of the usual two-element electrostatic instrument with an applied voltage, $v$, is

$$
\tau=\frac{v^{2}}{2} \frac{d C}{d \theta}
$$

where $C$ is the capacitance between the elements. Similar considerations lead again to identical expressions for the rest position with direct or alternating voltage applied, viz.,

$$
\left.U \theta_{f}=B_{2} V^{2} \quad \text { where } \quad B_{2} \doteq \frac{1}{2} \frac{d C}{d \theta}\right]_{\theta=\theta \rho}
$$

With reasonable restrictions, the temperature rise, $\theta$, of a thin homogeneous conductor carrying a current $i$ in the $x$ direction is governed by the differential equation

$$
\frac{\partial^{2} \theta}{\partial x^{2}}-\frac{H \theta}{K}+\frac{i^{2} R}{K}=\frac{1}{D} \frac{\partial \theta}{d t}
$$

where $D$ is the thermal diffusivity and $R, K$, and $H$ are the electrical resistance, thermal conductance, and rate of surface heat loss per degree rise of a unit length of the material. Again, if the "thermal inertia" is sufficiently great that periodic fluctuations in temperature are negligible, the average temperatures are equal with equal direct and rms alternating currents.

In each instrument the equality holds even if the "constants" such as $U, B, R, D, H$, and $K$ in the equations are dependent upon the response, $\theta$. This important advantage stems from the property that each instrument combines in a single measuring element a function proportional to the square of the instantaneous current or voltage, a restraining function, and an inertial function or "flywheel effect" to enable it to integrate so that the time-average value of the response is proportional to the square of the rms current or voltage. For this reason high accuracy may be more easily attained with these instruments than with other squaring devices or circuits, which must synthesize exact and equal square-law responses in two quadrants (or in one quadrant with an accurate rectifier) and provide a separate integrator. However, each of these three types of instruments has a lower limit of frequency below which the average value of the response is incorrect if the constants are dependent upon $\theta$.

\section{TRANSFER INSTRUMENTS}

It is easier in an actual instrument to insure that the constants in the equations are indeed equal on direct and alternating current than it is to measure them separately and compute the response of the instrument. Thus with few exceptions ${ }^{2}$ absolute instruments, such as the electrodynamic current balance, ${ }^{3}$ are used solely for dc measurements, and most ac instruments of high accuracy are calibrated in terms of dc standards. In the usual instruments, this calibration is preserved on a scale, and the instrument is tested or standardized periodically to guard against changes in the factors which affect the response. However, if the instrument is calibrated at the time of each use, long-time stability, extremely low temperature coefficients, and other normally desirable characteristics become of secondary importance, and the instrument may be designed and constructed to have high resolution and the best possible frequency characteristic. Indeed, in a standardizing laboratory in which the standard is used primarily to test other instruments, a dc calibration may not be necessary because most instruments which are submitted to such laboratories can also be used on direct as well as alternating current. The scale calibration of such instruments may be verified on direct current with a potentiometer (taking the mean of the values required to obtain the same deflection for the two directions of applied current or voltage). The ac-dc difference or frequency influence may then be determined by connecting the instrument under test and the transfer-instrument so that they respond to the same quantity (current, voltage, or power) and successively switching both to alternating-, direct-, reversed-direct, and alternating current. In each case the measured quantity is adjusted to obtain the same deflection of the test instrument and the differences in deflection of the standard instrument are observed. From these differences and the scale factor of the standard instrument (per cent change per division) the ac-dc difference of the test instrument may be determined.

This procedure has been used for over 40 years in the certification of instruments at the National Bureau of Standards, because it separates several sources of errors and can be carried out with great accuracy. Since the ac-dc differences of a well-designed instrument are small within its working frequency range and are relatively permanent, they need not ordinarily be redetermined, so that the periodic tests of the instrument need be made only with direct current.

For either a direct ac measurement or an ac-dc difference determination, however, the ac-dc difference of the standard instrument must be known to the full accuracy of the measurement. The determination of this difference has been recognized as a major problem in

${ }^{2}$ H. B. Brooks, F. M. Defandorf, and F. B. Silsbee, "An absolute electrometer for the measurement of high alternating voltages," J. Res. NBS, vol. 20, pp. 253-316;1938.

${ }^{3}$ R. L. Driscoll and R. D. Cutkosky, "Measurement of current with the NBS current balance," J. Res. NBS, vol. 60, pp. 297-305; 1958. 
most national standardizing laboratories. In general, there are three rather distinct steps in a complete determination. These are: 1) choice of two different types of instruments that are inherently suitable as ac-dc transfer instruments, and a theoretical study of all known effects that can cause ac-dc differences in each type; 2) construction of instruments of each type in such a way that each of these effects by computations and tests can be evaluated over the required ranges; and 3) comparison of the actual transfer performance of the two instruments to guard against unknown sources of error. The tests of step 2) will of ten take three forms. These are: a) tests of components of the instrument for the effects of known factors (such as magnetic susceptibility, for example; b) intercomparisons of instruments of the same type but of different ranges, in which some of the effects (such as residual reactance) may be expected to be very different; and c) repeated intercomparisons, in which each known source of error in one instrument is accentuated by definite amounts (such as by the deliberate introduction of known reactance). Normally the comparisons of step 3) can be made over only part of the useful ranges of frequency, voltage, etc., and the performance must also be judged by the results of steps 1 ) and 2). Normally also one of the two instruments will have better-known characteristics, wider or more suitable ranges, or better-behaved ac-dc differences than the other, and thereafter is used as the principal transfer instrument for that laboratory, but both are important.

The accuracy of ac measurements is often limited by the stability of the source rather than the instrument. For the calibration of instruments with a transfer instrument, stabilized sources free from short-time fluctuations are required, but long-time stability and good load regulation are of less importance., ${ }^{4.5}$ Repeated sets of readings make it possible to determine the ac-de difference to a precision considerably better than the fluctuations in the source, and the procedure described tends to eliminate drifts in both the test and standard instruments. If both instruments inherently respond to the same values (rms for example), low-order harmonics up to several per cent ordinarily cause the test to be in error by much less than 0.1 per cent.

\section{Electrodynamic Instruments}

The electromagnetic force between two conductors with reasonable geometry and currents is so small that to obtain satisfactory torque the effect is magnified by coiling the conductors. The torque-weight ratios thus obtained are still considerably less than those enjoyed by dc instruments with permanent magnets, but rea-

4. H. M. Arnold, "Alternating-current instrument testing equipment," Proc. IEE, vol. 101, pp. 121-133; 1954.

5 F. L. Hermach, "Power supplies for 60 -cycle tests of instruments and meters," Proc. ISA, vol. 11, paper 56-21-3; 1956. sonably rugged and portable pointer-and-scale instruments of the 0.1 per cent accuracy class, based on IVeston's designs, have been available for a number of decades. For increased resolution and accuracy, recently developed commercial instruments, following Silsbee's composite-coil design, ${ }^{6}$ now use a taut-suspension system with the moving coil of a second instrument rigidly fastened to it to provide an electromagnetic restoring torque. ${ }^{7-9}$ This torque can be precisely determined by measuring the current through the second instrument with a resistor and dc potentiometer.

As the torque equations indicate, an electrodynamic instrument responds to the square of the current, the scale being marked to indicate current directly. As an unshunted ammeter (limited to about 0.1 ampere because of the springs or ligaments which must carry the current to the moving coil), it will be useful as a transfer instrument over the range of frequencies for which the average torque, $T=f(I)$, is the same as with direct current. The chief factors which affect this in an actual instrument are: 1) capacitances between turns and between coils, which alter the currents; 2) eddy currents in neighboring metals, which alter the field that links with the moving coil; and 3 ) electrostatic torque, caused by differences in potential between the fixed and moving coils. In general, these set an upper limit of about 1 kc for such instruments. The frequency ranges of ammeters, which use shunts across the moving coils, and voltmeters, which use series resistors to limit the current, are more sharply limited by the inductances of the coils. The manufacturer must insure that the ratio of the impedances of the two parts of the divided circuit of the ammeter is equal to the ratio of resistances, to the full accuracy of the measurement over the frequency range of interest. Similarly he must insure that the magnitude of the impedance of the voltmeter (defined as the ratio of the applied voltage to the current through the coils) is equal to its dc resistance. By connecting resistors in series with the coils of the ammeter and capacitors across portions of the voltmeter, the upper frequency limit for 0.1 per cent error in commercial instruments can be raised from $100 \mathrm{cps}$ to about $1000 \mathrm{cps}$, and for 0.25 per cent error to about $2500 \mathrm{cps}^{10}$

Because the mutual inductance between the coils changes with scale position (the operating torque depends upon this) complete compensation cannot be attained at all scale positions. Mutual inductance intro-

${ }^{\circ}$ F. B. Silsbee, "Composite coil electrodynamic instruments," J. Res. NBS, vol. 8, pp. 217-264; 1932

${ }^{7}$ G. F. Shotter and H. D. Hawkes, "A precision ac/dc comparator for power and voltage measurements," Proc. IEE, vol. 93, pp. 314$324 ; 1946$

${ }^{8} \mathrm{~J}$. Sorge, "A new precision instrument for ac power measurements," VDE Fachberichte, vol. 17, pp. 27-30; 1953.

${ }^{9}$ R. F. Estoppey, "Inductronic Electrodynamometer," Conference on Electronic Standards and Measurements, paper 35; August, 1958.

$10 \mathrm{~J}$. H. Miller, "Frequency compensation of ac instruments," Trans. $A I E E$, vol. 70, pp. 217-221; 1951. 
duces other errors as well, so that the electrodynamic ac-dc transfer instruments designed and constructed at the U.S. National Bureau of Standards ${ }^{11}$ and the National Physical Laboratory of South Africa ${ }^{12}$ are normally operated only over a narrow range of deflections about the position of zero mutual inductance, by the use of a continuously adjustable external multiplier for the first instrument and a torsion-head for the second. In the NBS instrument (see Fig. 1), two sets of coils are astatically arranged to eliminate the effect of uniform external fields, and a strip suspension and light-beam pointer with a scale 2 meters from the instrument insure definite readings and permit a scale factor at 0.01 per cent $/ \mathrm{mm}$ at currents from 0.1 to 20 amperes. The instrument is normally used as a wattmeter or ammeter, and a similar but older companion instrument as a voltmeter (10 to 600 volts). ${ }^{13}$ The NPLSA instrument is quite similar, but may be connected as a voltmeter as well $(0.05$ to 5 amperes and 25 to 500 volts). The ac-dc difference of each of these instruments is believed to be known to better than 0.01 per cent at power frequencies and to perhaps 0.1 per cent up to $3 \mathrm{kc}$ (above 50 volts), verified by careful study and by comparison with electrothermic and electrostatic instruments.

\section{Electrostatic Instruments}

The electrostatic force between two conductors at reasonable spacings is very low at normal line voltages. The resultant torque can be multiplied, as in the Kelvin electrostatic voltmeter, by interleaving the fixed and moving conductors (plates), but unfortunately only on a one-to-one basis, so that torque-weight ratio remains low. Thus electrostatic instruments, as distinct from electrodynamic instruments where the fixed coil may be designed to have more ampere-turns than the moving coil, seem rather delicate except at voltages above about $1 \mathrm{kv}$, and are rarely available commercially for precision measurements. However, taut-suspension, light-beampointer constructions, using improved optical systems and modern materials, have begun to appear commercially in Europe and may presage a renaissance of this type of instrument.

Electrostatic instruments inherently respond to voltage but may be adapted for current measurements by measuring the voltage across a resistor carrying the current. However, the voltage must be rather high ( 50 to 100 volts) for adequate accuracy and the shunt must then dissipate considerable power at high currents, or an instrument transformer must be used (either a current transformer with a resistor in its secondary, or a voltage transformer between a resistor and the instrument).

$11 \mathrm{~J}$. H. Park and A. B. Lewis, "Standard electrodynamic wattmeter and ac-dc transfer instrument," J. Res. NBS, vol. 25, pp. 545$579 ; 1940$.

i2 J. W. Whittaker, "A precision electrodynamometer standard and ac/dc transfer instrument," Proc. IEE, vol. 101, pp. 11-20; 1954.

${ }^{13} \mathrm{~F}$. K. Harris, "A suppressed zero electrodynamic voltmeter," J. Res. NBS, vol. 3, pp. 445-457;1929.

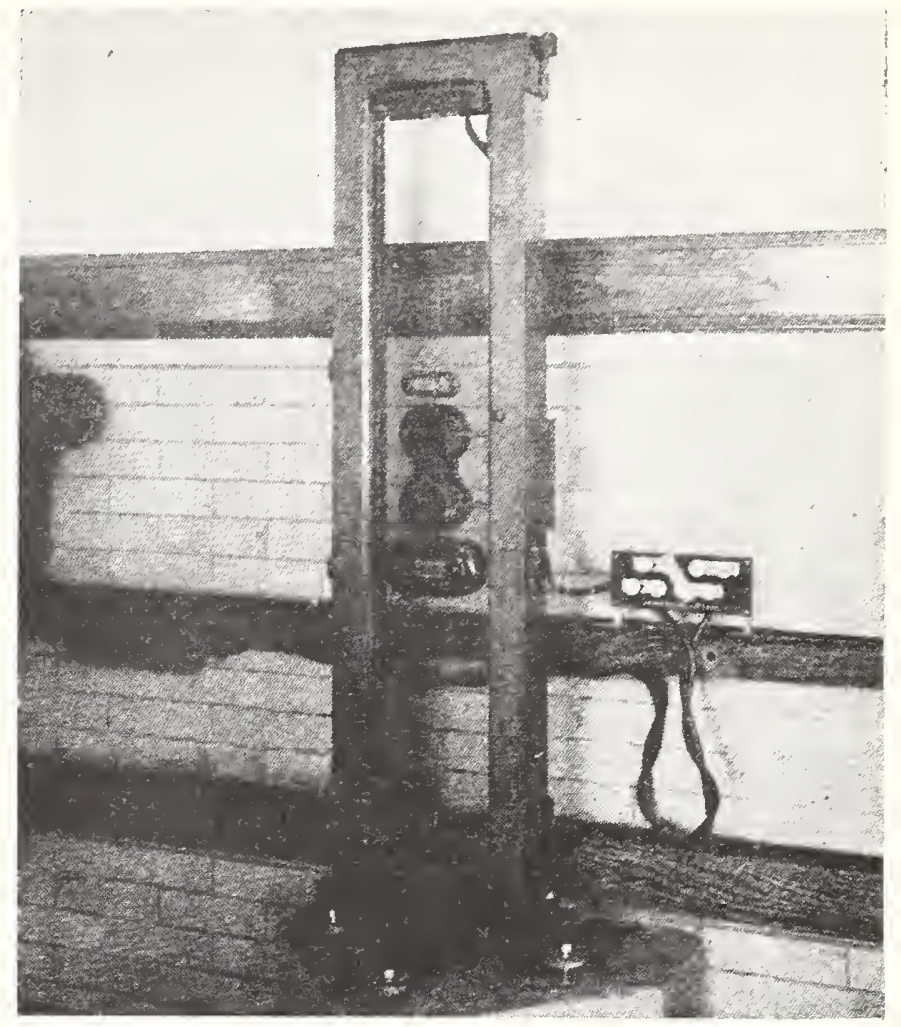

Fig. 1-NBS electrodynamic ac-dc transfer instrument (external multiplier and scale not shown).

Electrostatic instruments also have a number of sources of ac-dc differences. Among these are: 1) the series impedance of the suspension or springs, 2) surface charges on insulation in the electric field, which can modify the field with direct voltage applied, and 3) imperfectly conducting films on the plates which can cause the field strength between the plates to differ on direct and alternating voltage. ${ }^{14}$ None of these limit the frequency range as sharply as does the inductance of electrodynamic instruments, and electrostatic voltmeters may be accurate to perhaps 1 mc. However, when an electrostatic instrument is used as an ammeter the impedance of the 4-terminal resistor must be equal to its dc resistance. (If a transformer is used, it is no longer an ac-dc transfer instrument.) This is difficult to achieve above about $20 \mathrm{kc}$.

Electrostatic voltmeters with ranges from 50 to 160 volts have been the principal ac instruments for over 40 years at the National Physical Laboratory in England $^{14}$ and somewhat modified instruments of the same basic design are similarly used at the National Standards Laboratory in Australia. The ac-de differences of the NPLE instruments are known to 0.01 per cent at power frequencies and to better than 0.05 per cent up to $100 \mathrm{kc}$ (verified by calculations of the known sources of error and by comparison with electrodynamic and electrothermic instruments and with other electrostatic

${ }^{14}$ R. S. J. Spilsbury and A. Felton, "The electrostatic voltmeter as a dc/ac transfer instrument," J. IEE, vol. 89, pp. 129-137; 1942 . 
instruments having noble-metal conductors). Special transformers and resistors have been constructed and tested to permit the measurement of current and of voltage below $20 \mathrm{vglts}$, and resistance voltage dividers have been evaluated for voltage measurements to 1000 volts (at audio frequencies). ${ }^{4}$

\section{ELECTROTHERMiC INSTRUMENTS}

Easily measurable effects are produced by the heating of short straight metallic conductors or very small beads of semiconductors, having very low electrical timeconstants. These effects can thus be independent of frequency up to $100 \mathrm{mc}$ or more. In addition, the effects can be measured electrically, rather than mechanically, with high precision and freedom from mechanical resonances. These are the chief advantages of electrothermic instruments.

Because their output is electrical, and because of their low reactance, thermocouple instruments and bolometer bridges are the only two forms now generally used. In commercial thermocouple instruments the temperature rise of a conductor (heater) is measured with a thermocouple and millivoltmeter. The combination of the heater and thermocouple is called a thermal converter. Many commercial bolometer bridges now contain bead thermistors (temperature-sensitive resistors) and are unbalanced by the temperature rise caused by the heating of the applied current. The unbalance voltage is measured or balance is restored by additional currents of a different frequency, which are measured. Thermocouple instruments of the $\frac{1}{2}$ to 2 per cent class have long been commercially available for current and voltage measurements, while most commercial bolometers are used for power measurements (absorbing all of the measured power). In either case undesired thermal effects, such as high ambient temperature coefficients, accelerated aging of the heater at the elevated temperature, and transfer of heat to other parts of the measuring element have limited the sustained accuracy obtainable. However, by using such instruments solely as ac-dc transfer instruments, calibrating them before and after each reading, these effects can largely be eliminated. Studies at the National Bureau of Standards ${ }^{15}$ have shown that properly designed thermal converters may be used as ac-dc transfer instruments at currents from 1 ma to 50 amperes with an accuracy approaching 0.01 per cent at power and audio frequencies and very probably up to $200 \mathrm{kc}$. Their low reactance also makes possible transfer voltmeters from 0.2 to 600 volts (at 133 ohms per volt) with wire-wound series resistors that have ac-dc differences less than 0.03 per cent at audio frequencies. Voltmeter elements with film resistors in coaxial lines terminated by thermal converters have been constructed and intercompared to 0.05 per cent to

${ }^{15} \mathrm{~F}$. L. Hermach, "Thermal converters as ac-dc transfer standards for current and voltage measurements at audio frequencies," J. Res. NBS, vol. 48, pp. 121-138; February, 1952.
10 mc. ${ }^{16}$ The calculated ac-dc differences of the converters and of the voltmeter elements were verified by intercomparisons of different ranges and by comparisons (over limited ranges) with electrodynamic and electrostatic instruments. A Lindeck deflection potentiometer is used at NBS with these converters and voltmeter elements to provide high resolution $(0.005$ per cent $/ \mathrm{mm}$ at rated output emf) and rapid reading.

Similarly it has been shown that a properly selected and mounted pair of indirectly heated thermistors may be used in a bridge as an ac-dc transfer instrument for an audio-frequency potentiometer to better than 0.02 per cent at frequencies from 0.2 to $20,000 \mathrm{cps}^{17}$ Thermistors are also used for ac measurements at the Physikalisch-Technische Bundesanstalt in Germany. ${ }^{18}$ Their rather long thermal time constants (about 10 seconds) provide good low-frequency response but make highly stable sources necessary.

The small ac-dc differences of thermal converters and thermistor bridges at audio frequencies are caused almost solely by: 1) thermoelectric effects in the heater, which affect the temperature rise on direct current, but because of thermal inertia do not affect it on alternating current; 2) integration errors at low frequencies, which are inversely proportional to the square of the frequency; and 3) small reactance effects in the voltmeter elements, such as capacitance currentś between parts of the multiplier, and from the multiplier to its surrounding shield. Fortunately, it is possible to obtain thermal converters with heaters of manganin or other alloys of low thermoelectric effects and of sufficient length to make the ac-dc differences 0.01 per cent or less from 20 cps to $200 \mathrm{kc}$.

A single 10-ma thermal converter is used with multirange shunts and series resistors for ac measurements in the U.S.S.R., both in a central standardizing laboratory and in convenient consoles at branch laboratories. ${ }^{19}$ Series and shunt resistors are also used in the laboratories of the Germany Authority for Weights and Measures, but with two converters in a differential circuit. ${ }^{20}$ As a further modification of the transfer principle, the heater of the thermal converter alone (without the shunt or series resistors) can be switched to direct current for the dc calibration. ${ }^{21}$ Only a small dc source is

${ }^{16} \mathrm{~F}$. L. Hermach, "Electrothermic instruments for the measurement of alternating current and voltage," Proc. NPL Symposium on Precision Electrical Measurements, HMS Stationary Office, London, Eng., paper No. 15; 1955.

17 F. C. Widdis, "The indirectly heated thermistor as a precise ac-dc transfer device," Proc. IEE, vol. 103, pt. B, pp. 693-703; 1956.

${ }^{18} \mathrm{H}$. J. Schrader, "A-C potentiometer measurements with indirectly heated NTC resistors," Electrotech. Z., vol. 73-A, pp. 547$549 ; 1952$.

${ }^{19} \mathrm{~K}$. P. Shirokov, "An installation for calibrating ammeters and voltmeters at high frequencies," Proc. Russ. Inst. Meteorology, vol. 24, p. 24,1954 .

${ }_{20} \mathrm{~W}$. Rump, "On the exact absolute measurement of ac voltages and a potentiometer for testing ac instruments," Elektrotechnik, vol. 5 , pp. $64-67 ; 1951$.

${ }_{21}$ F. L. Hernach and E. S. Williams, "Multirange audio-frequency thermocouple instruments of high accuracy," J. Res. NBS, vol. 52 , pp. $227-234 ; 1954$. 
required, making possible a convenient portable accessory" to a dc potentiometer, which, in a recently improved model (see Fig. 2) provides ranges of $7.5 \mathrm{ma}$ to 20 amperes and 0.5 to 600 volts with an accuracy of 0.05 per cent at frequencies from 5 to $50,000 \mathrm{cps}$.

\section{Other Instruments}

A transfer instrument is an essential part of any accurate ac potentiometer. Such potentiometers have been used to some extent in Europe but rarely in the United States, except for special measurements. They require phase as well as magnitude balance, and with the usual tuned detector, measure only the fundamental component rather than the rms value. An electrodynamic milliammeter is most commonly used with a standard cell to standardize the current through the resistance network, but a thermal converter or thermistor bridge could serve equally well and over a wider range of frequencies. $^{15,17}$ Recent studies ${ }^{22}$ have shown that some converters have excellent long-time stability if they are maintained at a constant ambient temperature.

A method of determining the equality of a dc voltage and the crest-to-crest value of an ac voltage to better than 0.005 per cent has been developed at the National Standards Laboratory of Australia ${ }^{23}$ as well as a method of generating an ac voltage wave with no harmonic greater than 0.001 per cent of the fundamental. The equipment has been used to verify the ac-dc performance of the NSL electrostatic voltmeter to better than

${ }^{22} \mathrm{~J}$. J. Hill, "A precision thermo-electric wattmeter for power and audio frequencies," Proc. IEE, vol. 105, pt. B, pp. 61-68; January, 1958.

${ }^{23}$ W. E. Smith and W. K. Clothier, "Determination of the dc/ac transfer error of an electrostatic voltmeter," Proc. IEE, vol. 101, pp. $465-469 ; 1954$.

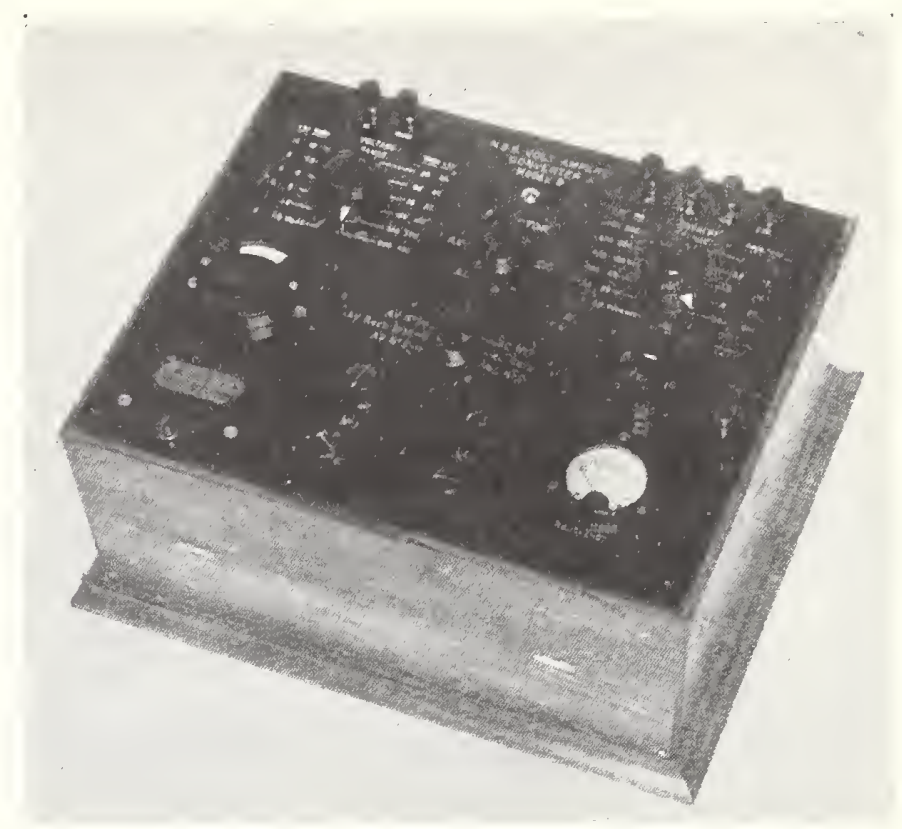

Fig. 2-Volt-ampere converter for ac current and voltage measurements.

0.01 per cent at $50 \mathrm{cps}$ and may be considered to be a crest-value ac-dc transfer instrument.

High-gain amplifiers with a rectifier in a feedback loop, and driven rectifiers based on the ring modulator, may be used with a potentiometer to measure the average value of an ac voltage wave to 0.1 per cent or better, provided the galvanometer or other detector properly integrates the rectified output. Their accuracy must also be verified by the procedure outlined for ac-dc transfer instruments. At present this is most easily done by comparison with rms instruments in circuits with voltages of known waveforms.

Reprinted from IRE TRANSACTIONS

ON INSTRUMENTATION

Volume I-7, Numbers 3 \& 4, December, 1958 


\section{A Wide-Range Volt-Ampere Converter for Current and Voltage Measurements}

\author{
F. L. HERMACH \\ MEMBER AIEE
}

T ECHNOLOGICAL developments have brought increasing needs for convenient, portable instruments for measuring current and voltage with high accuracy over a wide range of frequencies. A "universal" volt-ampere (VA) converter has been developed for making such measurements to $0.05 \%$ with alternating current at frequencies from $5 \mathrm{cps}$ (cycles per second) to $50 \mathrm{kc}$ and with direct current. The new model is based on an earlier, much more limited prototype converter, ${ }^{1}$ and, like it, is used with a null-type d-c potentiometer. With this single, self-contained, portable, 44-range instrument, the 1.5 -volt range of a suitable potentiometer can be used to make a-c (rms) and d-c measurements from 7.5 milliamperes (ma) to 20 amperes and from 0.5 volt to 600 volts. With a more sensitive external galvanometer, the instrument can also be used as an a-c-d-c transfer standard for a-c-d-c difference

Paper 59-161, recommended by the AIEE Indicating and Integrating Instruments Committee and approved by the AIEE Technical Operations Department for presentation at the AIEE Winter General Meeting. New York, N. Y., February 1-6, 1959. Manuscript submitted November 1, 1957; made available for printing December 1, 1958.

F. L. Hermach and E. S. Williams are with the National Bureau of Standards, U. S. Department of Commerce, Washington, D. C.

\section{E. S. WILLIAMS \\ NONMEMBER AIEE}

measurements to $0.02 \%$ over the same frequency range.

\section{Method}

For a-c measurements, a single thermal converter (often called a thermoelement or thermocouple) serves as an a-c-d-c transfer standard. Its heater is connected in series with an a-c resistor for voltage measurements and in parallel with an a-c shunt for current measurements, as shown in Fig. 1. The electromotive force (emf) of the converter is manually balanced by the voltage from a built-in d-c source, with a galvanometer as the indicator (not shown in the figure). The heater of the converter is then switched to a second $\mathrm{d}-\mathrm{c}$ source, which is adjusted to rebalance the galvanometer. The voltage across a portion of this circuit is then measured with the potentiometer and multiplied by a simple number to obtain the alternating voltage or current. For d-c measurements, the same multiplier is connected as a volt box and the same shunts are used, without the thermal converter.

From Fig. 1(A), with the thermal converter connected to $R_{m}, V_{a}=I_{a}\left(R_{m}+R_{f}\right)$. With the converter connected to the d-c circuit, the current through the heater is $I_{h}=I_{d} R_{m}{ }^{\prime} /\left(R_{h}+R_{m}{ }^{\prime}\right)=V_{p} R_{m}{ }^{\prime} / R_{p}\left(R_{h}+\right.$ $R_{m}{ }^{\prime}$ ). If the a-c-d-c difference of the converter is negligible, $I_{a}=I_{h}$, and if $R_{m}{ }^{\prime}=R_{m}, \quad V_{a}=V_{p} R_{m} / R_{p}$. The a-c voltage to be measured is thus the product of the measured potentiometer voltage and a ratio of resistances, and is independent of both the emf-versus-current characteristic and the heater resistance of the converter.

From Fig. 1(B), with the converter connected to $R_{s}$, the current through the heater is $I_{h}=I_{a} R_{s} /\left(R_{h}+R_{s}\right)$. With the converter in the d-c circuit, $V_{p}=I_{d}\left(R_{h}+\right.$ $R_{s}{ }^{\prime}$ ). If $I_{h}=I_{d}$ and $R_{s}{ }^{\prime}=R_{s}$, and if the a-c impedance of the heater is equal to its d-c resistance, $I_{a}=V_{p} / R_{s}$, again desirably independent of the emf-versus-current characteristic and the resistance of the converter.
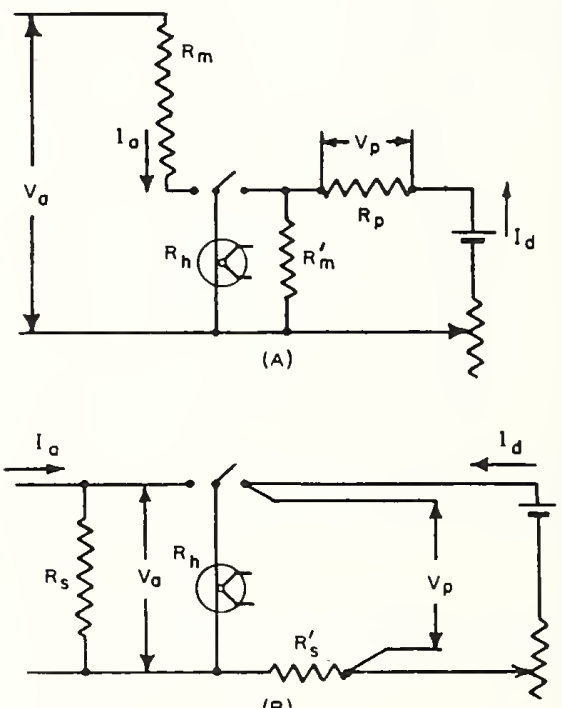

(B)

Fig. 1. Schematic diagram of VA converter 


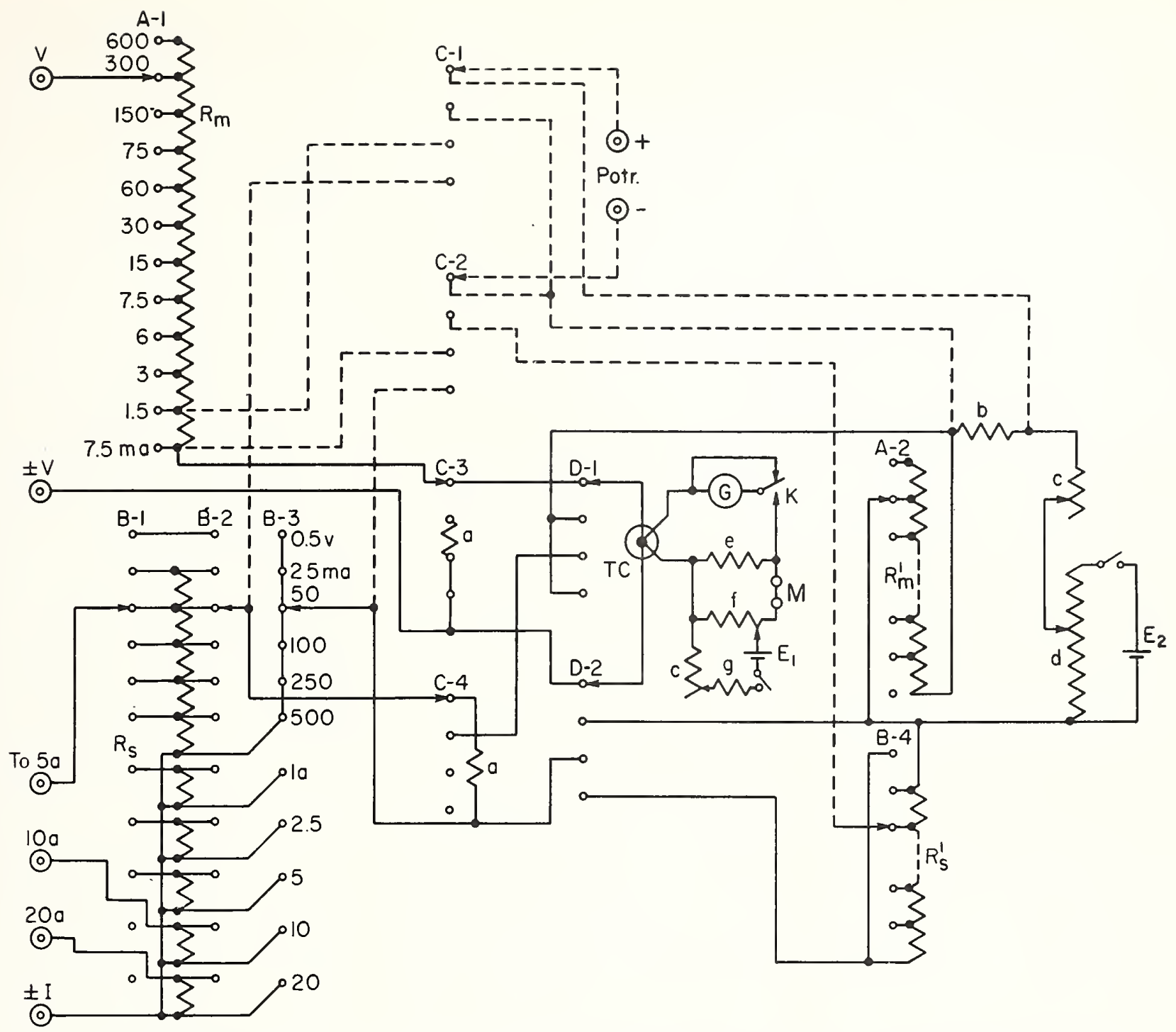

Fig. 2. Wiring diagram of VA converter. Switch-position markings from top to bottom on diagram: switch $C, A V, A C, D V, D C$, switch D, $A \vee$ No. 1, AV No. 2, AC No. 1, AC No. 2. Dotted lines signify potential leads except at A-2 and B-3 where they indicate intermediate switch positions are not shown

$A-1$, etc. $=$ pole 1 of switch $A$

$A, B=$ voltage and current range switches

$C, D=$ selector and transfer switches

$\mathrm{TC}=$ thermal converter, $7.5 \mathrm{ma}$

$\mathrm{G}=$ galvanometer, 0.3 microampere per centimeter

$K=$ galvanometer key

$a=66-2 / 3$ ohms, manganin

$b=(R p) 200$ ohms, manganin

$$
\begin{aligned}
& c=10 \text { ohms, } 3 \text {-turn voltage divider } \\
& d=1,000 \text { ohms, } 3 \text {-turn voltage divider } \\
& e=10 \text { ohms manganin } \\
& f=100 \text { ohms, } 3 \text {-turn voltage divider } \\
& g=700 \text { ohms, manganin } \\
& E_{1}=1.5 \text { volts, no. } 6 \text { dry cell } \\
& E_{2}=3 \text { volts, no. } 6 \text { dry cells } \\
& M=\text { binding posts for external milliammeter }
\end{aligned}
$$

For direct voltage measurements, the heater of the thermal converter is disconnected, the multiplier, $R_{m}$, is connected directly to the voltage to be measured, and a 1.5-volt section of it is connected to the potentiometer. If this section is designated as $R_{a}, V_{d c}=V_{p} R_{m} / R_{a}$, and if $R_{a}=R_{p}$ the nominal multiplying factor, $F_{y}=R_{m} / R_{a}$, for the volt box thus formed is the same as that for the corresponding a-c voltage range. For d-c measurements the potential terminals of the shunt $R_{s}$ are connected directly to the potentiometer, so that $I_{d c}=V_{p} / R_{s}$, and again the nominal multiplying factor, $F_{t}=1 / R_{s}$, is the same as that for the corresponding a-c range.

If the resistors $R_{p}, R_{s}, R_{m}, R_{m}{ }^{\prime}$, and $R_{s}{ }^{\prime}$ have small proportional corrections of $a_{p}, a_{s}, a_{m}, a_{m}{ }^{\prime}$, and $a_{s}{ }^{\prime}$ respectively, more detailed analysis shows that, to a sufficient approximation,

$$
\begin{array}{r}
V_{a}=\frac{V_{p} R_{m}}{R_{p}}\left[1-a_{p}+\frac{a_{m}+a_{m}{ }^{\prime} R_{h} / R_{m}}{1+R_{h} / R_{m}}\right] \\
=V_{p} F_{v}(1+v)
\end{array}
$$

and

$$
\begin{aligned}
I_{a}=\frac{V_{p}}{R_{s}}\left[1-\frac{a_{s}+a_{s}{ }^{\prime} R_{s} / R_{h}}{1+R_{s} / R_{h}}\right] & \\
& =V_{p} F_{\imath}(1+w)
\end{aligned}
$$

where the capital letters now signify the nominal values. For the useful ranges, $R_{m}>R_{h}>R_{s}$, so that the accuracy required of the compensating resistors $R_{m}$. and $R_{s}{ }^{\prime}$ is roughly inversely proportional to the current or voltage range, while essentially full accuracy is required of $R_{m}, R_{s}$, and $R_{p}$. In practice the over-all correction factors, $v$ and $w$, are directly 


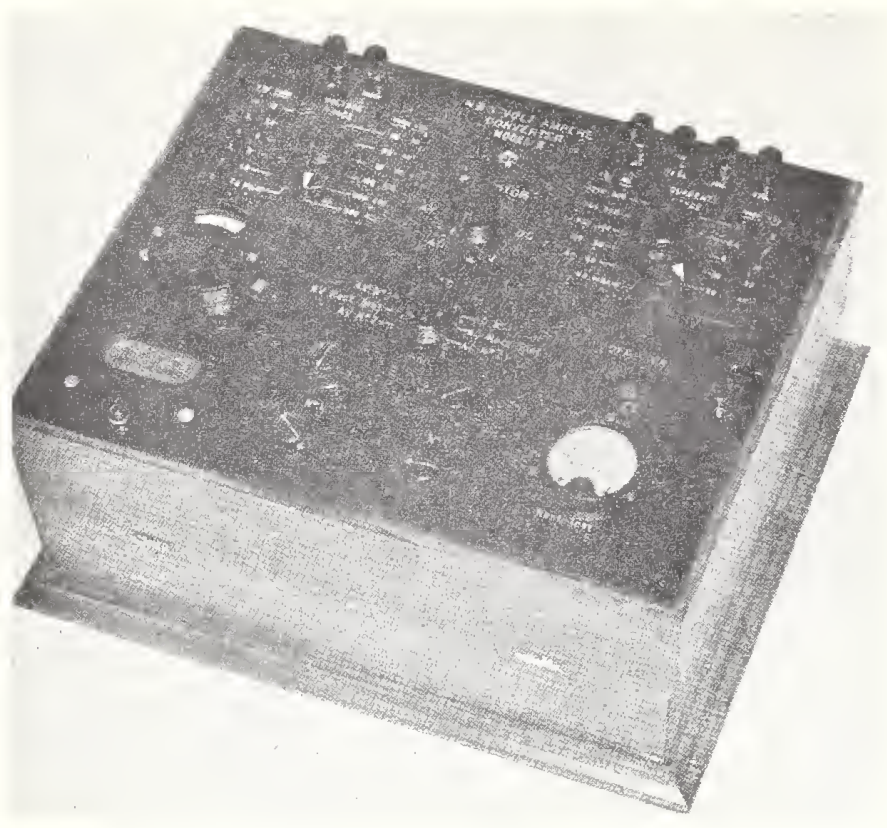

Fig. 3. Volt-ampere converter

evaluated by d-c tests of each range of the instruments.

An instrument which responds to both direct and alternating current is often best tested by evaluating its scale corrections on direct current and then determining its $a-c-d-c$ difference at the desired frequencies ${ }^{2}$ (defined as the difference between the direct and alternating current or voltage required to produce the same deflection). This difference is usually small and relatively permanent. Such a-c-d-c difference tests can readily be made with the VA converter by connecting the two instruments together and to a source of the desired voltage or current, making a preliminary setting of the test instrument, and then balancing the emf of the thermal converter, $E$, to give zero deflection of the galvanometer. The instruments are then successively switched to a-c, d-c, reversed d-c, and a-c sources, which are adjusted to give the same deflection of the test instrument, and the resulting galvanometer deflections are observed, without rebalancing. The percentage a-c-d-c difference of the test instrument, $T$, is computed as $T=\Delta D / K E$ where $\Delta D$ is the difference between the mean of the two a-c and the two d-c galvanometer deflections, and $K$ is a sensitivity factor. This factor is readily determined by observing the changes in galvanometer deflection resulting from small measured differences in applied direct current or voltage at several different levels of heater current, and plotting $K$ versus $E$. Tests show that only two such curves are necessary, one of which applies to all current ranges and the other to all voltage ranges. (They differ because of the dependence of heater resist- ance upon temperature and therefore current.)

\section{Description}

A wiring diagram of the VA converter is shown in Fig. 2 and a photograph of the instrument in Fig. 3. The ranges and the nominal shunt and series resistances are listed in the first two columns of Tables I and II, respectively. There are separate input binding posts for the current and voltage circuits, with separate range switches, $A$ and $B$ in Fig. 2. The instrument can thus be used to measure, in succession, the voltage and current in a d-c or a-c circuit without reconnections. It may also be useful for wattmeter testing on direct current or at unity power factor on alternating current (more strictly at the power factor of maximum deflection of a wattmeter) by the familiar "phantom loading method."

Switch $C$ selects the quantity to be
* Number of months after completion of the instrument. measured and switch $D$ is the a-c-d-c transfer switch for the thermal converter. The switch markings are identified in the note on Fig. 2. For a d-c measurement, the position of switch $D$ is immaterial, and it is only necessary to set the range and selector switches and connect the potentiometer. For an a-c measurement, after these are set switch $D$ is rotated to AV No. 1 or AC No. 1 , connecting the thermal converter to the desired a-c source, and the $E_{1}$ circuit is adjusted with controls marked "No. 1" (coarse and fine) for zero deflection of the built-in galvanometer (or any other convenient deflection). Switch $D$ is then rotated to $\mathrm{AV}$ No. 2 or AC No. 2 connecting the thermal converter to the $E_{2}$ circuit which is adjusted with controls marked "No. 2" to rebalance the galvanometer to the same deflection. The external potentiometer is then read in the usual way. In switch positions marked "Rect. Inst.," (rectifier instrument) two additional poles of switch $D$ (not shown in Fig. 2) connect a panel-type rectifier instrument to the a-c input in place of the thermal converter. This is useful for preliminary settings and for checking to make sure that the thermal converter will not be overloaded in the No. 1 position.

A 7.5-ma thermal converter having a heater resistance of $66^{2} / 3 \mathrm{ohms}$ is used in the instrument. Because of the compensating resistors, the actual heater resistance need not be closely adjusted. High-quality a-c resistance cards are used for the series and shunt resistors of 1 ohm or more. Shunts of lower resistance, for the higher current ranges, were specially made in our laboratories of bifilar strip, ranging from 2 to 20 mils in thickness, of nickel-chromium-aluminum-iron alloy, with insulation of 1-mil polyester film. The dimensions were chosen as a compromise between the requirements of large area for cooling and of thin strip for

Table I. Results of D.C Tests of Current Ranges

\begin{tabular}{|c|c|c|c|c|c|}
\hline \multirow{3}{*}{$\begin{array}{l}\text { Current } \\
\text { Range, } \\
\text { Amperes }\end{array}$} & \multicolumn{5}{|c|}{ Per-Cent Correction } \\
\hline & \multirow[b]{2}{*}{$\begin{array}{c}\mathbf{R}_{s_{3}} \\
\text { Ohms }\end{array}$} & \multicolumn{2}{|c|}{ D-C Ranges, at 9 Months* } & \multicolumn{2}{|c|}{ A-C Ranges, at Rated I } \\
\hline & & Rated I & $\begin{array}{c}20 \% \\
\text { Rated I }\end{array}$ & $\begin{array}{c}\text { At } 6 \\
\text { Months* }\end{array}$ & $\begin{array}{c}\text { At } 18 \\
\text { Months }\end{array}$ \\
\hline \multicolumn{6}{|c|}{$20 \ldots \ldots \ldots \ldots+0.025 \ldots \ldots \ldots \ldots+0.05 \ldots \ldots \ldots-0.07 \ldots \ldots \ldots \ldots+0.03 \ldots \ldots \ldots+0.05$} \\
\hline $10 \ldots$ & 0,05 . & $\ldots \ldots \ldots \ldots-0.01$. & $\ldots-0.09$. & $\ldots+0.01$. & $\ldots+0.02$ \\
\hline $5 \ldots$ & 0.1 & $\ldots \ldots \ldots-0.02$. & $.0 .01 \ldots$ & $\ldots+0.04$. & $\ldots+0.03$ \\
\hline $2.5 \ldots$ & 0.2 & $\ldots \ldots \ldots \ldots+0.02$. & +0.03. & $\ldots+0.03$. & $\ldots+0.05$ \\
\hline & 0.5 & $\ldots \ldots \ldots \ldots 0.00$. &.$+0.03 .$. & $\ldots+0.03$. & $\ldots+0.05$ \\
\hline $500 \mathrm{ma}$. & . 1 & $\ldots \ldots \ldots \ldots-0,09$. & .0 .08$. & $\ldots-0.07$ & $\ldots-0.02 t$ \\
\hline $250 \mathrm{ma}$. & . 2 & $\ldots \ldots \ldots .-0.04$. & $.0,03$. &..-0.02 . & .. 0.00 \\
\hline $100 \mathrm{ma}$. & 5 & $\ldots \ldots \ldots \ldots-0.01$. & $-0.01 \ldots$ & $\ldots-0.01$. & .. 0.00 \\
\hline $50 \mathrm{ma}$. & .10 & $\ldots \ldots \ldots \ldots-0.04$. & -0.04 & $\ldots-0.04$. & $\ldots-0.03$ \\
\hline 25 ma. & & $\ldots \ldots \ldots-0.03$. & -0.03 & $\ldots-0.02$. & $\ldots-0.02$ \\
\hline $7.5 \mathrm{ma}$. & None & $\ldots \ldots \ldots \ldots$ & $\ldots \ldots$ & $\ldots+0.01$ & \\
\hline
\end{tabular}

t Readjusted before this test; adjustment affects lower-current ranges. 
(NBS) standard volt box. The reversed d-c tests were made with a potentiometer and auxiliary shunts and volt boxes. The $a-c-d-c$ difference tests were made with the NBS standard thermal converters, ${ }^{5}$ which have recently been evaluated down to $2 \mathrm{cps}$, and with a newly constructed transfer voltmeter element, which has been evaluated to $100 \mathrm{kc}$.

The results of the d-c tests show generally satisfactory performance, adjustment, and stability of the instrument, except for the 10- and 20-ampere ranges in which much larger shunts should have been used. Separate correction curves have been prepared for these ranges to show the change in resistance caused by the self-heating of the measured current.

The a-c $-\mathrm{d}-\mathrm{c}$ differences, defined as $\left(I_{a c}-I_{d c}\right) / I_{d c}$ or $\left(V_{a c}-V_{d c}\right) / V_{d c}$ for the same thermocouple emf with alternating and direct current, are very small. They depend upon the ratios of small reactances to resistances in the instrument and may be expected to remain constant to the accuracy desired. Thus, unless the components are changed, future periodic tests of the instrument need normally be made with direct current only, with a-c-d-c checks made only occasionally. This greatly simplifies the standardization of the instrument.

Additional low-frequency tests of the
0.5 -volt and 7.5-ma ranges showed that the $a-c-d-c$ difference of the converter itself is not more than $0.01 \%$ at frequencies down to $5 \mathrm{cps}$. Both theory and experiment indicate that the difference should also be negligible on all other ranges of the instrument.

The small discrepancies in Tables I and II between the corrections for the corresponding $d-c$ and a-c ranges are caused by the d-c reversal difference of the thermal converter, the difference between $R_{a}$ and $R_{p}$, and the influence of the compensating resistors $R_{m}{ }^{\prime}$ and $R_{s}{ }^{\prime}$. While these are small (the cause of the relatively large discrepancy $[0.06 \%]$ between the corrections for the $d-c$ and the a-c 5ampere ranges is unknown), they are difficult to evaluate separately, so that future $d-c$ tests must probably be made on both a-c and $d-c$ ranges. In most laboratories a volt box and shunt box could be reserved as standards for these and other tests and could be sent to a suitably equipped standardizing laboratory periodically, or the instrument itself could be sent to such a laboratory.

\section{Conclusions}

This portable, VA converter can serve as an accessory to a null-type d-c potentiometer to make possible d-c and a-c meas- urements of very high accuracy over truly wide ranges of voltage, current, and frequency. Its accuracy depends primarily upon wire-wound resistors and strip shunts of excellent stability and lowtemperature coefficient. After initial a-cd-c difference tests of the instrument, routine periodic tests need ordinarily be made only with direct current, thus simplifying the standardization of the instrument.

The VA converter should be particularly suitable for testing other instruments, since all three types of tests, (d-c, a-c, and a-c-d-c transfer tests) may be made with it.

\section{References}

1. Multirange, AUdiofrequency Thermo COUPLE INSTRUMENTS OP HIGH ACCURACY, F. L Hermach, E. S. Williams. Journal of Research National Bureau of Standards, Washington, D. C. vol. 52,1954, p. 227.

2. The Testing of Electrical InStrument: F. L. Hermach. Proceedings, Instrument Societ of America, Pittsburgh, Pa., vol. 8, 1953, Pap 53-2-3.

3. Electrical Measurements (book), F. Harris. John Wiley \& Sons, Inc., New Yor N. Y. 1952, chap. 11.

4. Notes on the DEsign OF Four TERMis Resistance Stanoaros for Alternating C RENT, F. B. Silsbee, Journal of Research, Natic Bureau of Standards, vol. 4, 1930. p. 73.

5. Thermal Converters as ac-DC Tran: Standaros for Current ano Voltagb Mbas Ments at Audio Frequencibs, F. L. Hern Ibid., vol. 48,1952 , p. 121 . 


\title{
Phase Angle Master Standard for 400 Cycles per Second
}

\author{
J. H. Park and H. N. Cones
}

\author{
(January 25, 1960)
}

\begin{abstract}
A continuously variable, 0 - to 180 -degree, phase shift standard for 400 cycles per second is described in detail. It consists of a $\pi$-section line made up of twelve 14.6 degree and three 4.3 degree sections to provide for two sizes of coarse steps and an $R C$ circuit at the input to the line to provide for fine steps and a continuous fine control. A method for accurately adjusting the characteristic impedance of all $\pi$-sections to the same value, which is used as the termination, was devised. Under these conditions it is shown that the phase shift introduced by each $\pi$-section can be accurately computed from a measured value of inductance. The phase shift of each $\pi$-section was also determined by an experimental procedure dependent upon a 180-degree phase shift introduced by a toroidal transformer. The values obtained by these two independent methods agree to within 0.01 degree.
\end{abstract}

\section{Introduction}

Phase angle in this paper is defined as the quantity used to measure the time phase relation between two sinusoidal voltages of the same frequency. Highly accurate measurements of small angles, i.e., less than $3^{\circ}$, have long been made in connection with instrument transformer calibration testing and phase angle defect measurements of capacitors. Impedance and power factor measurements involving somewhat larger phase shifts but usually with less accuracy have also been performed for some time. However, until quite recently the accurate measurement of phase angle as such over a wide range and at frequencies higher than $60 \mathrm{cps}$ has not received much attention.

Interest in precise phase angle measurements has been aroused as a result of computer and guidance problems. Various methods and devices for measuring phase shift have been developed in solving these problems. In order to obtain accurate comparisons and coordination of the test data obtained in various standardizing laboratories, reference standards must be available which will maintain their calibration when shipped from one laboratory to another. Also, at least one laboratory (probably the National Bureau of Standards) must be capable of checking the absolute calibration of such reference standards.

The calibration of reference standards is most conveniently carried out if a master standard is available which has been thoroughly investigated and accurately calibrated. The purpose of this paper is to describe such a master standard which has been designed and constructed for use at $400 \mathrm{cps}$ at the National Bureau of Standards and to explain the methods used in arriving at its calibration.

\section{Requirements of a Master Standard}

Conferences with various persons concerned with accurate phase angle measurements indicated that the greatest current need was for measurements from $0^{\circ}$ to $180^{\circ}$ at $400 \mathrm{cps}$ with an accuracy of $0.01^{\circ}$, if possible. There are numerous methods of obtaining phase shift in this range, and measurement accuracies to within a degree or even a few tenths of a degree can be attained without great difficulty. However, to covel the range from $0^{\circ}$ to $180^{\circ}$ with a $0.01^{\circ}$ accuracy at any point, a master standard must contain several different magnitudes of phaseshift steps which can be switched in or out by selector switches and a cont inuousl $y$ variable fine control. To obtain a usable crlibration, the phase shift put in by each step on any selector switch or by any given change in the fine dial must be independent of the settings on all other switches and dials. In the case of a resistance decade box a similar requirement is nearly always automatically fulfilled, but for most phase shifting devices this is not true. For a master standard phase shifter it should also be possible to compute phase shift for each step from the measured values of resistance, capacitance, and inductances.

\section{Description of Design Used}

Resistance-capacitance circuits are commonly used to obtain phase shifts, and the phase shifts inserted by such circuits can, under certain conditions, be accurately computed from measured values of resistance and capacitance. Howerer for such circuits phase shift is proportional to change in capacitance or resistance only up to a few degrees, and above that a fixed change in resistance or capacitance gives a different phase shift depending upon the total values of resistance and capacitance. 'Thus, decades of resistance or capacitance cannot be used to make up a calibrated phase shifter for large angles but fortunately they can be used for fine control up to about $5^{\circ}$.

To obtain coarse steps of $5^{\circ}$ and larger, $L-C^{\prime}$ lumped delay lines (multisection ladder networks) were tried and found to be suitable. The main advantage of such a delay line is that the phase shift added by any individual section is ahwys the same no matter how many sections are already in use, provided the characteristic or surge impedance of all sections is accurately adjusted to the same value and the line is terminated with this same impedance. 
Under these conditions the phase shift of each section can be computed from a measured value of inductance. Also the input impedance is independent of the number of sections so that the input can be part of an $R-C$ circuit used to get fine control of phase angle,

The final design consisted of twelve $14.6^{\circ} \pi$-sections and three $4.3^{\circ} \pi$-sections with switching arrangements so that any desired combination of sections could be connected as a delay line. The input to this line was used as part of an $R-C$ network which incorporated 10 capacitor steps giving $0.44^{\circ}$ plase shift each and a variable air capacitor for continuous fine control.

Several circuit connections and switching arrangements for the complete phase shifter were considered and tried. The one finally selected is shown schematically in figure 1. The "input" or voltage to be shifted is connected to a 4,000-ohm noninductive resistor, shunted by a capacitance-remove type variable air capacitor (1,100 to 100 pf), in series with the input to the delay line. A decade set of mica capacitors (0.004 to $0.044 \mu \mathrm{f}$ ) and a zero adjusting capacitance are connected in parallel with the delay line input. A set of 13 single-pole, two-position, mercury-contact switches are arranged to connect any number from 0 to 12 of the $14.6^{\circ} \pi$-sections into this delay line. Also a set of 4 switches is arranged to connect any number from 0 to 3 of the $4.3^{\circ}$ $\pi$-sections into the delay line. The end of the delay line is connected to a resistor of 1,000 ohms shunted by a capacitor of $0.0021 \mu \mathrm{f}$ which was chosen to be equal to the characteristic impedance of the line. The terminated end of the line is connected to the "output" binding posts of the phase shifter through the "test-check" switch when it is in the "test" position.

\section{Theory and Method of Measurements on $\pi$-Sections}

The circuit diagram in figure 2 shows a generalized $\pi$-section delay line which can be used as a phase shifter. To eliminate reflections so that the shift introduced by any one section is constant irrespective of other sections in the line, the terminating impedance, $Z_{0}$, must be made equal to the input impedance, $Z_{\text {in }}$. Considering one section only and solving for $Z_{0}$ when $Z_{0}=Z_{\text {in }}$ gives

$$
Z_{0}^{2}=\frac{Z_{1} Z_{2}}{1+\frac{Z_{1}}{4 Z_{2}}} .
$$

Also,

$$
e_{0}=\frac{e}{1+\frac{Z_{1}}{2 Z_{2}}+\frac{Z_{1}}{Z_{0}}} \text {. }
$$

Equation (2) shows that for each section the output voltage lags the input voltage by the angle represented by the vector, $1+\left(Z_{1} / 2 Z_{2}\right)+\left(Z_{1} / Z_{0}\right)$.

For a properly terminated line made up of pure inductors and capacitor's the phase shift per section

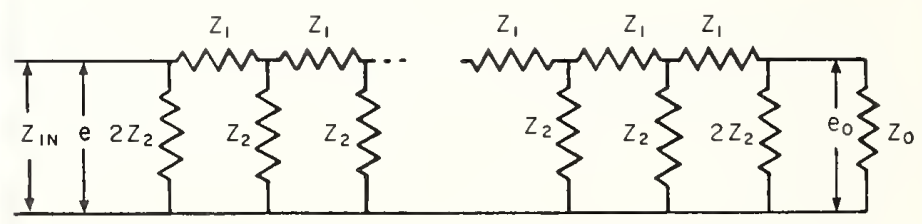

Frgure 2. Generalized $\pi$-section line.

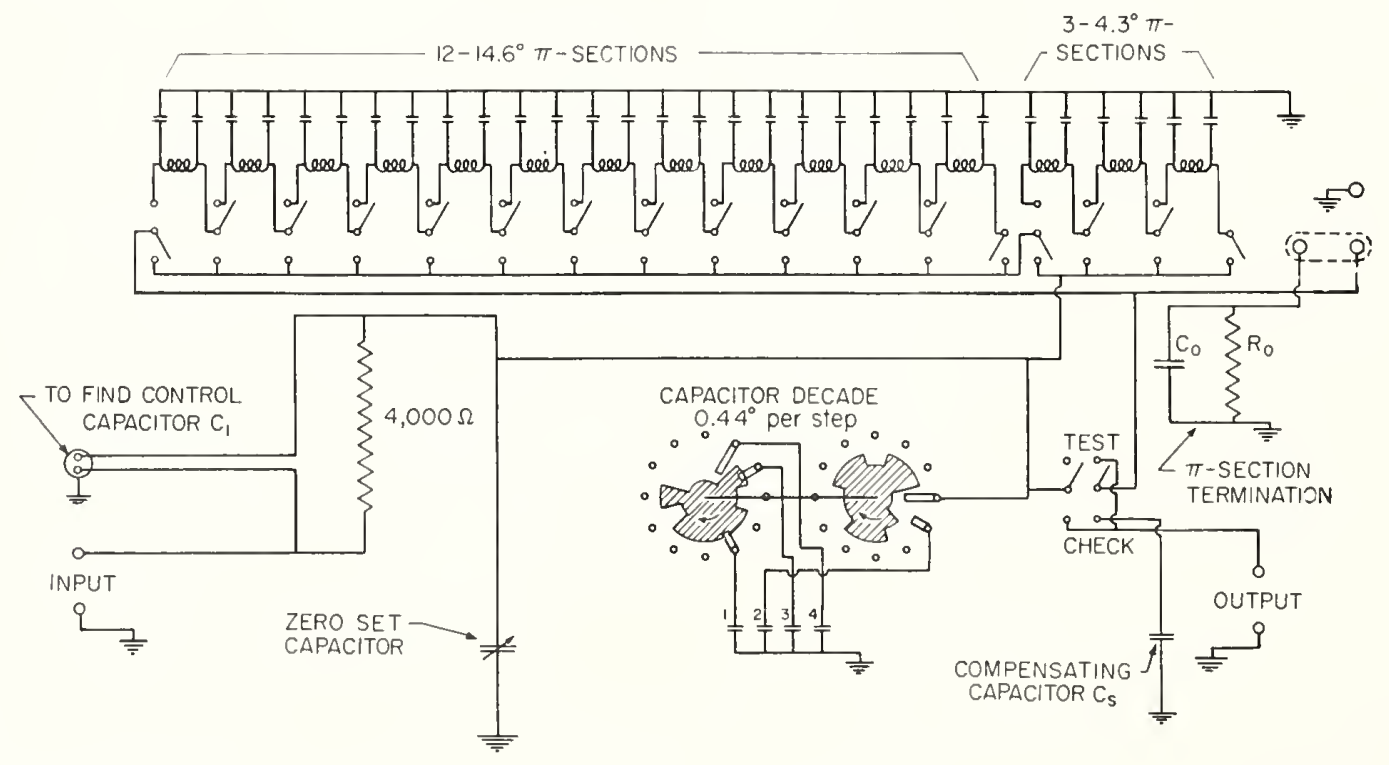

FIGURE 1. Schematic wiring diagram for 0 to 180 degree reference standard phase shifter $400 \mathrm{cps}$. 
could be readily computed from the known values of inductance and capacitance. However, there are several practical considerations which tend to reduce the accuracy of the computed value. For $Z_{1}=j \omega L$ and $Z_{2}=1 / j \omega C$, the terminating impedance as determined from eq (1) must be

$$
Z_{0}^{2}=\frac{L}{C} \frac{1}{1-\frac{\omega^{2} L C}{4}} .
$$

Thus $Z_{0}$ is real (made up of resistance only), but its value depends to some extent upon frequency. If sections with different values of inductance are to have the same $Z_{0}$, then $C$ must be determined from the above equation and becomes

$$
C=\frac{L}{Z_{0}^{2}}\left(1+\frac{\omega^{2} L^{2}}{4 Z_{0}^{2}}+\frac{\omega^{4} L^{4}}{8 Z_{0}^{4}} \cdots\right)
$$

Another practical consideration is the resistance of the inductors. To get a suitable value of terminating resistance (say $1,000 \mathrm{ohms}$ ) the inductance for a $15^{\circ}$ section must be $100 \mathrm{mh}$. An air-core inductor of this magnitude would either have too much resistance if wound on a toroidal shaped core or would be too susceptible to nearby magnetic fields if made in the form of a circular coil for optimum ${ }^{1}$ time constant. The best practical solution is a toroidal winding on a high permeability core. However, since both the inductance and effective resistance of such a high- $Q$ coil would be dependent to some extent on the current through it, the current must be held to a nearly constant value-chosen to be considerably below that giving saturation of the core. Measurements of resistance to about 1 percent and inductance to a much higher accuracy must also be made at the same current, and these values should be stable.

\subsection{Method Used To Measure Inductors}

An accurate measurement of both the inductance and the resistance of the toroidal high- $Q$ inductors was obtained by using a Maxwell-Wien bridge with a Wagner ground as shown in figure 3 . Here $R_{2}$ and $R_{4}$ are precision woven wire resistance standards (300 ohms each) for which the time constant is less than $10^{-8}$ sec. $C_{1}$ consists of a 0 - to $1.11-\mu \mathrm{f}$ threedecade mica capacitor in parallel with a 50 - to 1,100 pf variable air capacitor, both of which are completely shielded and accurately calibrated. $R_{1}$ is a fourdecade resistance box ( 0 to 100,000 ohms) of low time constant woven wire resistors. The bridge balance is obtained by adjusting $C_{1}$ and $R_{1}$. When both the Wagner arm and the bridge are balanced the following relations hold for $L_{3}$ and $R_{3}$ (neglecting the second-order term):

$$
L_{3}=C_{1} R_{2} R_{4} \quad \text { and } \quad R_{3}=\frac{R_{2} R_{4}}{R_{1}}
$$

1 H. B. Brooks, Design of standards of inductance and the proposed use of models in the design of air-core and iron-core reactors, BS J. Research (1931) RP342.

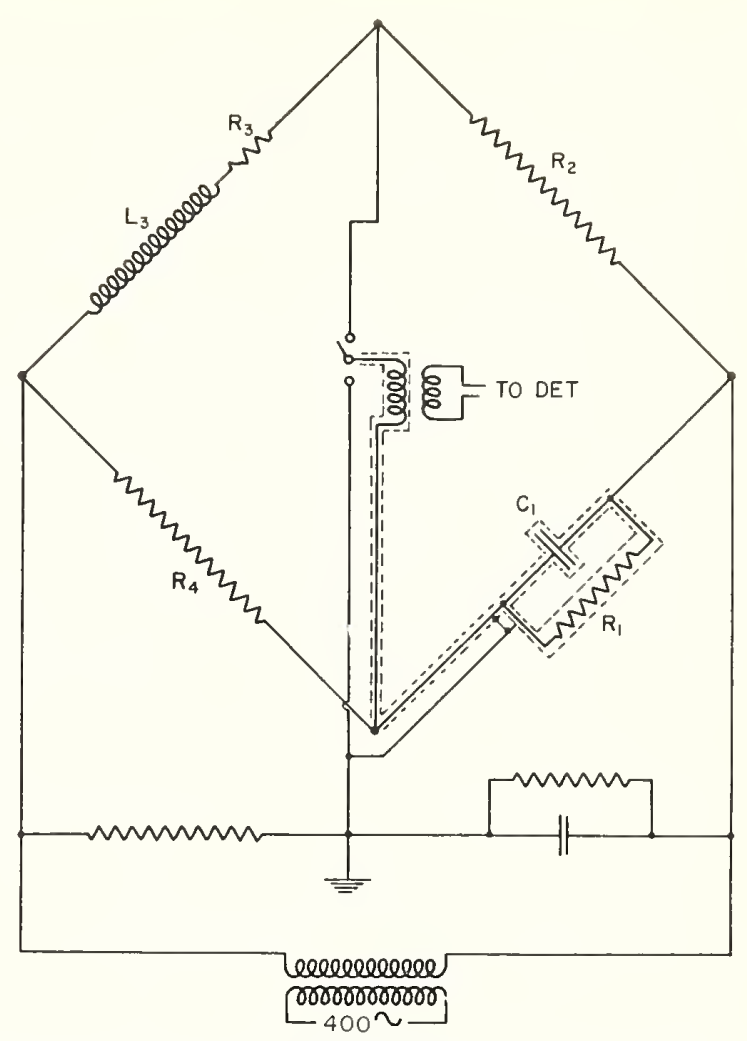

Figure 3. Maxwell-Wien bridge with Wagner ground used to measure inductance and resistance of inductors.

For the bridge components used, the second order corrections are negligible for an accuracy of 0.01 percent in $L_{3}$ and 1 percent in $R_{3}$. The values used for $R_{2}$ and $R_{4}$ should include any lead resistance up to the bridge corners.

An oscillator having an output waveform with total distortion less than 0.1 percent was used to supply this bridge. The frequency was accurately set to $400 \mathrm{cps}$ by forming a Lissajous pattern with a $100-c p s$ standard frequency signal. The voltage appled to the inductor was set by using a voltmeter connected from ground to the $L_{3} R_{4}$ bridge corner. The actual values of $L$ and $r$ for the inductors used in the $\pi$-sections are listed in table 1 . These are the

\begin{tabular}{|c|c|c|c|c|c|}
\hline \multicolumn{4}{|c|}{400 cps measurements using Maxwell-Wien Bridge } & \multirow{2}{*}{$\begin{array}{l}\text { Phase shift } \\
\text { computed } \\
\text { from meas- } \\
\text { ured } L \text { in } \\
\text { degrees }\end{array}$} & \multirow{2}{*}{$\begin{array}{l}\text { Phase shift } \\
\text { from ex- } \\
\text { pcrimental } \\
\text { calibration } \\
\text { in degrees }\end{array}$} \\
\hline Inductor no. & $\begin{array}{l}\text { Volts across } \\
\text { inductor }\end{array}$ & $\mathrm{mh}$ & ohms & & \\
\hline & $0.25 \ldots$ & $\begin{array}{l}100.290 \\
100.340 \\
100.199 \\
100.15_{4} \\
100.51_{3} \\
100.15_{2}\end{array}$ & $\begin{array}{l}2.79 \\
2.82 \\
2.78 \\
2.80 \\
2.80 \\
2.85\end{array}$ & $\begin{array}{l}14.600 \\
14.606 \\
14.587 \\
14.581 \\
14.631 \\
14.580\end{array}$ & $\begin{array}{l}14.600 \\
14.609 \\
14.589 \\
14.581 \\
14.636 \\
14.581\end{array}$ \\
\hline 10 & 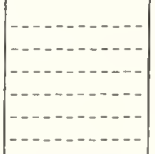 & $\begin{array}{r}100.085 \\
99.89_{9} \\
100.15_{4} \\
99.70_{6} \\
100.407 \\
100.66_{0}\end{array}$ & $\begin{array}{l}2.79 \\
2.87 \\
2.95 \\
2.87 \\
3.02 \\
3.10\end{array}$ & $\begin{array}{l}14.571 \\
14.546 \\
14.580 \\
14.518 \\
14.616 \\
14.651\end{array}$ & $\begin{array}{l}14.571 \\
14.542 \\
14582 \\
14.515 \\
14.620 \\
14.658\end{array}$ \\
\hline 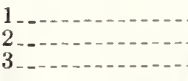 & $\left|\begin{array}{c}.075 \\
-0 .\end{array}\right|$ & $\begin{array}{l}29.876 \\
29.852 \\
29.891\end{array}$ & $\begin{array}{l}1.025 \\
1.020 \\
0.986\end{array}$ & $\begin{array}{l}4.307 \\
4.304 \\
4.309\end{array}$ & $\begin{array}{l}4.308 \\
4.304 \\
4.310\end{array}$ \\
\hline
\end{tabular}
values used in computing phase shift for each section.

TABLE 1. Measured and computed data for inductors 
Because of the procedure used in matching characteristic impedance for each $\pi$-section, as described in the following paragraphs, this computation does not require that the exact value of capacitance for the $\pi$-section be known. It does require an exact knowledge of the terminating impedance.

\subsection{Procedure Used in Matching the Characteristic Impedance of Each $\pi$-Section}

By arranging a potentiometer-bridge circuit with the $\pi$-section termination $\left(C_{0}\right.$ and $P_{0}$ in parallel $)$ as one of the arms and using a sensitive detector to obtain a balance, any small variation in this terminating impedance can readily be detected. If all circuit elements are kept the same after getting an initial balance, except that one $\pi$-section is addech ahead of the termination, the detector would remain in balance if the characteristic impedance of the $\pi$-section were exactly equal to $C_{0}$ and $R_{0}$ in parallel. If the characteristic impedance is off slightly the detector can be rebalanced by a fine adjustment on the two capacitor legs of the $\pi$-section-thus experimentally making the impedance of the $\pi$-section exactly equal to $C_{0}$ and $R_{0}$ in parallel.

The circuit arrangement actually used in making. these adjustments is shown schematically in figure 4 . Most of the circuit elements shown in this diagram either represent or correspond directly to those used to make up the $\pi$-section phase standard (as shown in detail in fig. 1). $R_{1}$ is the 4,000- $\Omega$ resistor. $C_{1}$ is the fine control capacitor. $C_{2}$ represents the capacitor decade and the zero set capacitor. The single $\pi$-section is used to represent the entire delay line. $C_{0}$ and $R_{0}$ are the $\pi$-section termination. The only additional circuit elements required are the ratio transformel and detector (the detector transformer only being shown). The procedure used was to first balance the detector with the $\pi$-section discomnected (switch A up and B open) by adjusting the ratio transformer and capacitor $C_{2}$. 'Then with a $\pi$-section comnected ahead of $C_{0}$ and $R_{0}$ (switch $\mathrm{A}$ down and $\mathrm{B}$ closed) the detector was balanced by adjusting

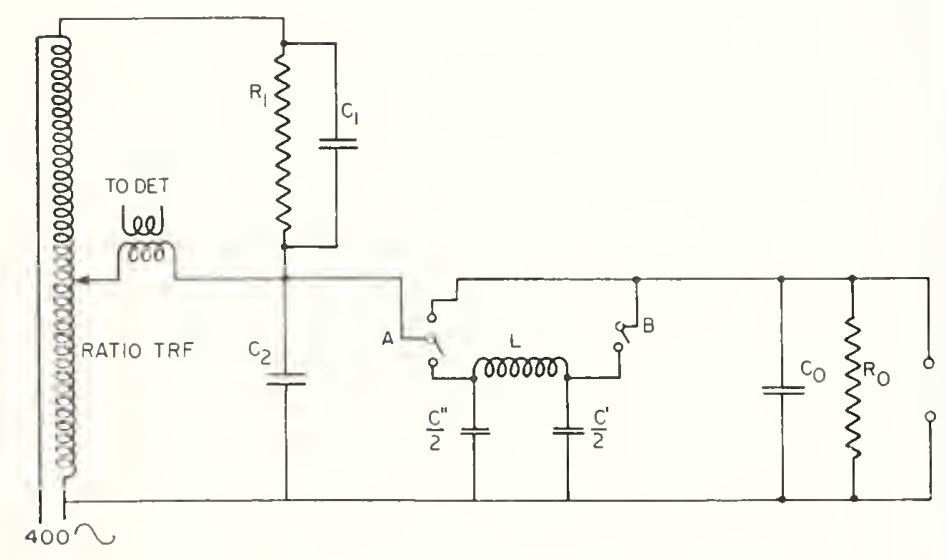

FIGURE 4. Schematic diagram of phase standard with ratio transformer and detector as connccted for adjustment of capacitor trimmers on each $\pi$-section.
$C^{\prime} / 2$ and $C^{\prime \prime} / 2$, using fine trimmers in parallel with the main capacitance. This last balance is somewhat "tricky" to obtain because the two unbalanced voltages put in by $C^{\prime} / 2$ and $C^{\prime \prime} / 2$ respectively are not $90^{\circ}$ apart in phase-but after a little practice it can be obtained quite rapidly. By using a lighly selective tuned amplifier as detector this method gives a very sensitive way to adjust the characteristic impedance of the $\pi$-section to be exactly equal to $R_{0}$ and $C_{0}$ in parallel.

The derivation of the relations between the $\pi$-section cirsuit constants and the terminating impedance, for which the input and terminating impedance are equal, is given in appendix 1 . The procedure used was to derive an expression for the input impedance of the $\pi$-section (terminated by $C_{0}$ and $R_{0}$ in parallel) and equate this to $C_{0}$ and $R_{0}$ in parallel, giving a complex equation in circuit parameters. The two separate equations obtained from the complex equation can then be used to determine any two of the circuit parameters in terms of the others. First, it is assumed that the capacitors in the two legs of the $\pi$-section are equal, i.e., $=C^{\prime} / 2=C^{\prime \prime} / 2=C / 2$. 'The expressions derived for $C_{0}$ and $C / 2$ are given by eqs $(9)$ and (10), respectively, in appendix 1. These values of $C_{0}$ and $C / 2$ can now be put in the two original equations by letting $C^{\prime \prime} / 2=C / 2\left(1+\lambda^{\prime \prime}\right)$ and $\Gamma^{\prime} / 2=C / 2\left(1+\lambda^{\prime}\right)$. Equations (11) and (12) obtained in this manner can be solved for actual values of $\lambda^{\prime}$ and $\lambda^{\prime \prime}$ which are measures of the adjustments required in the capacitance legs to maintain the same characteristic impedance for slight changes in other $\pi$-section parameters. 'To help clarify the above procedure an example using actual values for the $14.6^{\circ} \pi$-section is worked out in appendix 1.

The values of $C^{\prime} / 2$, i.e., $C / 2\left(1+\lambda^{\prime}\right)$, obtained in this manner, together with the known or assigned values of the other circuit parameter's can be used to compute phase shift as explained in the following section.

\subsection{Computation of Phase Shift for a $\pi$-Section}

The phase shift of a $\pi$-section, i.e., phase difference between voltage in and out, does not depend upon the input capacitance leg because it is in parallel with the input voltage. It does depend on all other circuit constants including the terminating impeckance. A derivation for phase shift is given in appendix 2, and eq (19) gives an expression for tan $\theta$ as a function of these circuit constants. Actually for any $\pi$-section whose values of $C^{\prime \prime} / 2$ and $C^{\prime} / 2$ have been adjusted experimentally (as described above) so that its characteristic impechance is equal to the termination ( $R_{0}$ and $C_{0}$ in parallel) the phase shift can be computed from the measured value of inductance $L$ only provided some limits are set for values of $r, R^{\prime}$, and $R^{\prime \prime}$. This can best be illustrated by using a specific example.

Take first the average $14.6^{\circ} \pi$-section values as given in appendix 1 , i.e., $L=100 \mathrm{mh}, \quad r=2.8 \Omega$, 
$R^{\prime}=10^{7} \Omega . \quad$ For $R_{0}=1,000 \Omega$ and $C^{\prime} / 2=C^{\prime \prime} / 2=C / 2$, $C_{0}=0.002099 \times 10^{-6}$ and $C / 2=0.0508191 \times 10^{-6}$ (as computed in appendix 1). Putting these values in eq (19), the phase shift angle $\theta$, as shown in appendix 2 , can be expressed as $\tan \theta=0.25968$. For the maximum changes in $r, R^{\prime}$ and $R^{\prime \prime}$ encountered in the twelve $14.6^{\circ} \pi$-sections as determined from actual measurements, the changes in $\tan \theta$, as computed in appendix 2 , are given in the following table.

\begin{tabular}{c|r|r|r}
\hline$r$ & \multicolumn{1}{c|}{$R^{\prime}$} & \multicolumn{1}{c|}{$P^{\prime \prime}$} & \multicolumn{1}{c}{$\operatorname{Tan} \theta$} \\
\cline { 2 - 3 } 2.80 & $10^{7}$ & $10^{7}$ & 0.25968 \\
3.10 & $10^{7}$ & $10^{7}$ & .25968 \\
2.80 & $10^{8}$ & $10^{7}$ & .25967 \\
2.80 & $10^{7}$ & $10^{5}$ & .25969 \\
2.80 & $10^{8}$ & $10^{5}$ & .25968 \\
\hline
\end{tabular}

Since $\tan \theta$ is very nearly the same for all of these changes in $r, R^{\prime}$, and $R^{\prime \prime}$, an expression for $\tan \theta$ in terms of $L$ only, which holds for each of the $14.6^{\circ}$ $\pi$-sections can be derived. As shown in appendix 2 this expression is

$$
\tan \Theta=\frac{L}{R_{0}} 2596.7_{8}
$$

Using the same procedure, as described for the $14.6^{\circ} \pi$-section, an expression for $\tan \theta$ which holds for each of the $4.3^{\circ} \pi$-sections was also derived. This expression is

$$
\tan \theta=\frac{L}{R_{0}} 2520.5
$$

\section{Theory and Calibration of R C Network}

By using the special measuring techniques and theoretical relations described in the previous section, a computed value of phase shift for each of the $\pi$-sections can be derived, and such values are listed in table 1. 'The phase shift inserted by the $R-C$ network, connected ahead of the $\pi$-sections as shown in figures 1 and 4 , may also be computed using known values of $R$ and $C$. Appendix 3 shows how this can be done, and actual phase shifts for various values of $C_{1}$ and $C_{2}$ are given in table 2 .

One requirement for a standard phase shifter is that the phase shift introduced by any given change in the continuous fine control be independent of the setting of other phase shift dials. From the fourth column in table 2 it can be seen that the phase shift put in by a 500-pf change in the fine control capacitor is the same to within $0.0005^{\circ}$ for all settings on $r_{2}$ (the $0.46^{\circ}$ steps) up to $43,000 \mathrm{pf}$. 'Thus, if the $\pi$-sections are all matehed so that the impedance looking into the line is the same no matter how many sections are connected, the phase shift put in by a given change in the setting of the fine control capacitor will be essentially the same irrespective of phase shift put in by the "capacitor decade" and
TABLE 2. Phase shift for various settings of the capacitors in the $R-C$ network

\begin{tabular}{|c|c|c|c|c|}
\hline \multicolumn{5}{|c|}{$R_{1}=4,000$ ohms; $\quad R_{0}=1,000$ ohms } \\
\hline$C_{\mathrm{t}}$ & $C_{2}$ & $\begin{array}{l}\text { Phase } \\
\text { shift, } \theta\end{array}$ & $\begin{array}{l}\Delta \theta \text { for } 500 \\
\text { pf change } \\
\text { in } C_{1}\end{array}$ & $\begin{array}{l}\Delta \theta \text { for } 3,900 \\
\text { pf ehange } \\
\text { in } C_{2}\end{array}$ \\
\hline $\begin{array}{l}p f \\
1,200 \\
700 \\
200\end{array}$ & $\begin{array}{l}p f_{4,000} \\
\end{array}$ & $\begin{array}{l}d e g \\
+0.0921 \% \\
-.1382_{3} \\
-.36862\end{array}$ & $\begin{array}{l}\text { deg } \\
0.2303_{4} \\
.2303_{9}\end{array}$ & deg \\
\hline $\begin{array}{r}1,200 \\
700 \\
200\end{array}$ & 7,900 & $\begin{array}{l}-.3570_{3} \\
-.5874_{2} \\
-.8178_{4}\end{array}$ & $\begin{array}{l}.2303_{9} \\
.2304_{2}\end{array}$ & $\begin{array}{r}0.4491_{8} \\
.4491_{9} \\
.4492_{2}\end{array}$ \\
\hline $\begin{array}{r}1,200 \\
700 \\
200\end{array}$ & 11,800 & $\begin{array}{r}-.80609 \\
-1.03650 \\
-1.26693\end{array}$ & $\begin{array}{l}.2304_{1} \\
.2304_{3}\end{array}$ & $\begin{array}{l}.4490_{6} \\
.4490_{8} \\
.4490_{9}\end{array}$ \\
\hline $\begin{array}{r}1,200 \\
700 \\
200\end{array}$ & 15,700 & $\begin{array}{l}-1.2549_{6} \\
-1.4853_{9} \\
-1.7158_{5}\end{array}$ & $\begin{array}{l}.2304_{3} \\
.2304_{6}\end{array}$ & $\begin{array}{l}.4488_{7} \\
.4488_{9} \\
.4489_{2}\end{array}$ \\
\hline $\begin{array}{r}1,200 \\
700 \\
200\end{array}$ & 19,600 & $\begin{array}{l}-1.70359 \\
-1.9340_{6} \\
-2.16455\end{array}$ & $\begin{array}{l}.23047 \\
.23049\end{array}$ & $\begin{array}{l}.44863 \\
.4486_{7} \\
.44870\end{array}$ \\
\hline $\begin{array}{r}1,200 \\
700 \\
200\end{array}$ & 23,500 & $\begin{array}{l}-2.1519_{3} \\
-2.3824_{4} \\
-2.6129_{\mathrm{s}}\end{array}$ & $\begin{array}{l}.2305_{1} \\
.2305_{4}\end{array}$ & $\begin{array}{l}.4483_{4} \\
.44839 \\
.4484_{3}\end{array}$ \\
\hline $\begin{array}{r}1,200 \\
700 \\
200\end{array}$ & 27,400 & $\begin{array}{l}-2.5999_{2} \\
-2.83049 \\
-3.0610_{5}\end{array}$ & $\begin{array}{l}.23057 \\
.23056\end{array}$ & $\begin{array}{l}.44799 \\
.44805 \\
.44807\end{array}$ \\
\hline $\begin{array}{r}1,200 \\
700 \\
200\end{array}$ & 31,300 & $\begin{array}{l}-3.0475_{0} \\
-3.27813 \\
-3.5087_{6}\end{array}$ & $\begin{array}{l}.2306_{3} \\
.2306_{3}\end{array}$ & $\begin{array}{l}.44753 \\
.44764 \\
.4477 \mathrm{I}\end{array}$ \\
\hline $\begin{array}{r}1,200 \\
700 \\
200\end{array}$ & 35,200 & $\begin{array}{l}-3.49464 \\
-3.7253_{3} \\
-3.9560_{0}\end{array}$ & $\begin{array}{l}.23069 \\
.2306_{7}\end{array}$ & $\begin{array}{l}.4471_{4} \\
.44720 \\
.4472_{4}\end{array}$ \\
\hline $\begin{array}{r}1,200 \\
700 \\
200\end{array}$ & 39,100 & $\begin{array}{l}-3.9412_{6} \\
-4.1720_{1} \\
-4.402 \overline{7}_{6}\end{array}$ & $\begin{array}{l}.23075 \\
.2307_{5}\end{array}$ & $\begin{array}{l}.44662 \\
.4466 \\
.44676\end{array}$ \\
\hline $\begin{array}{r}1,200 \\
700 \\
200\end{array}$ & 43,000 & $\begin{array}{l}-4.3873_{3} \\
-4.6181_{4} \\
-4.84898\end{array}$ & $\begin{array}{l}2308_{1} \\
.2308_{4}\end{array}$ & $\begin{array}{l}.44607 \\
.44613 \\
.4462\end{array}$ \\
\hline
\end{tabular}

(See figure 8)

the $\pi$-section line. As shown in table 2 the average value of this phase shift is $0.2305^{\circ}$ for a change in $C_{1}$ of $500 \mathrm{pf}$. $\quad\left(0.0046^{\circ}\right.$ for $10 \mathrm{pf}$ or $0.01^{\circ}$ corresponds to $21.7 \mathrm{pf})$.

The last column in table 2 indicates that the phase shift put in by a 3,900-pf change in $C_{2}$, i.e., by one step on the decade capacitor dial, depends to a slight extent upon the total values for $C_{1}$ and $C_{2}$. For any given setting of $C_{2}$ the change in this phase shift for settings of $C_{1}$ from 1,200 to $200 \mathrm{pf}$ is less than $0.00015^{\circ}$. Thus, the effect of the fine control capacitor setting on the decade capacitor steps is negligible. $\quad A_{s} C_{2}$ is increased the phase shift for a 3,900-pf change in $C_{2}$ decreases somewhat, the maximum change being about $0.003^{\circ}$. The calibration for each step on the capacitor decade can take this change into account, but this does indicate that another capacitor decade could not be added. The phase shift for each step on the decade capacitor could be computed if the value of capacitance added for each step were known to a high accuracy (to about 1 pf for $0.0001^{\circ}$ ), but it was decided that the experimental method as explained in the following section would be casier to apply. 


\section{Experimental Calibrations}

An experimental calibration can be devised for any phase shifter which fulfills the requirements given in section 2. In brief, the procedure is first to obtain the phase shift of each step of each dial in terms of settings on the other dials (including the fine control). These relative values can then be converted to absolute values either by using a known reference phase shift such as $180^{\circ}$ or by assuming that the calibration of the fine dial is correct. In the procedure actually followed the computed values for the fine control dial were used to get a calibration on each of the decade capacitor steps. Using these, a preliminary calibration of first the small and then the large $\pi$-sections was obtained. Then the $180^{\circ}$ point was checked using a special toroidal core transformer. Details of the method used will now be described.

A potentiometer-bridge circuit arrangement plus an auxiliary phase shifter (see fig. 5) were used to compare the phase shift settings on the various dials of the $\pi$-section standard. The auxiliary phase shifter, consisting of an $R-C$ circuit supplied through a ratio transformer, need not be accurately calibrated, but it must be stable and continuously variable from $0^{\circ}$ up to at least $15^{\circ}$. A sensitive amplifier with shielded input transformer was used to indicate when the phase shifts put in by standard and auxiliary phàse shifters were equal. The ratio transformer was used to give a magnitude balance.

The phase shift put in by the fine control capacitor as computed in section 5 was assumed to be exactly correct, and then each step on the capacitor decade switch was measured in terms of the fine control capacitor, as follows. All $\pi$-sections were disconnected. With the fine control capacitor set on

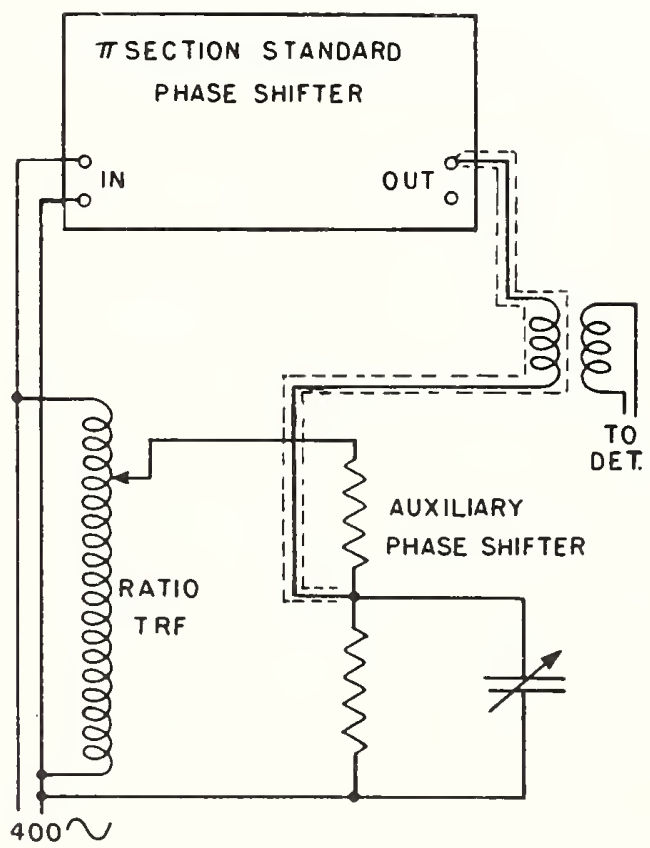

Figure 5. Setup used in experimental calibration.
0, i.e., maximum capacitance and the capacitor decade switch on step one, the auxiliary phase shifter was adjusted (together with the ratio transformer) until the detector showed a balance. 'Then, with the auxiliary phase shifter setting held constant and the decade capacitor switch set back to zero the detector was balanced by adjusting the fine control capacitor and ratio transformer. Since the auxiliary phase shifter remains unchanged for these two balances the phase shift put in by the first step on the decade capacitor must equal that read on the fine control dial after the second balance. The change in phase shift when the decade capacitor switch is changed from 2 to 3,3 to 4 , etc., can be measured in the same manner. The actual values as measured are given in table 3.

TABLE 3. Phase shift for each step of capacitor decade switchas determined by comparison with fine control dial

\begin{tabular}{|c|c|c|c|c|}
\hline Decade capacitor step & $\begin{array}{l}\text { Phase shift } \\
\text { of each step }\end{array}$ & $\Sigma$ & $\begin{array}{l}\text { Phase shift } \\
\text { corrected } \\
\text { for } 180^{\circ} \\
\text { check }\end{array}$ & $\Sigma$ \\
\hline $\begin{array}{l}1 \\
1 \\
3 \\
4 \\
5\end{array}$ & $\begin{array}{l}\text { deg } \\
0.4468 \\
.4429 \\
.4454 \\
.4340 \\
.4436\end{array}$ & $\begin{array}{l}0.8897 \\
1.3351 \\
1.7691 \\
2.2127\end{array}$ & $\begin{array}{r}0.4470 \\
.4431 \\
.4456 \\
.4342 \\
.4438\end{array}$ & $\begin{array}{l}0.8901 \\
1.3357 \\
1.7699 \\
2.2137\end{array}$ \\
\hline 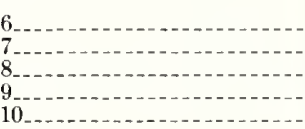 & $\begin{array}{l}.4440 \\
.4344 \\
.4447 \\
.4406 \\
.4428\end{array}$ & $\begin{array}{l}\text { 2. } 6567 \\
\text { 3. } 0911 \\
3.5358 \\
3.9764 \\
4.4192\end{array}$ & $\begin{array}{l}.4442 \\
.4346 \\
.4449 \\
.4408 \\
.4430\end{array}$ & $\begin{array}{l}2.6579 \\
3.0925 \\
3.5374 \\
3.9782 \\
4.4212\end{array}$ \\
\hline
\end{tabular}

To measure the phase shift of each small $\pi$-section, the switching arrangement was modified slightly from that shown in figure 1, so that any one of the three sections could be connected or disconnected individually. 'Then by using the method just described for the capacitor decade steps, the phase shift of each small $\pi$-section was measured in terms of the decade capacitor switch and the fine control dial. Another modification of the switching arrangement made it possible to connect any individual large $\pi$-section in by itself, and the phase shift of each of these sections was measured in terms of the three small sections plus the setting on the capacitor decade and the fine control dial. Before measuring each $\pi$-section its impedance was checked and adjusted to be equal to $R_{0}$ and $C_{0}^{\prime}$ in parallel by the method described in section 4.2 .

The experimental calibration values obtained by the method just described and those computed for the fine control dial (given in table 2) were actually carried out to $0.0001^{\circ}$. The estimated error for each step of the capacitor decade is $0.0005^{\circ}$. If this error is not completely random it would be accumulative and might add up to an appreciable value for the large $\pi$-sections. Consequently the values assigned to the $14.6^{\circ} \pi$-sections based entirely on the computed phase shift for the fine control capacitor were found to differ from computed values based on measured values of inductance of each section by as much as $0.02^{\circ}$. Variations in these differences upon repeated calibrations were usually found to be less 
than $0.005^{\circ}$. In order to correct for these fairly large errors in the $14.6^{\circ} \pi$-sections introduced by very small errors in the calibration of the capacitor decade, an independent check of the $180^{\circ}$ point was required.

This was done by connecting the primary of a uniformly wound toroidal transformer in parallel with the input to the standard phase shifter. The secondary of the toroidal transformer was used to get an exact $180^{\circ}$ phase shift by (1) reversing polarity or (2) using its two coils as a center tap winding with the center grounded. The phase shifter zero was set with the secondary polarity giving zero phase shift, and then with the opposite polarity the $180^{\circ}$ point was measured on the phase shifter. The magnitude was balanced by connecting a ratio transformer either at the primary or at the secondary of the toroidal transformer. The $180^{\circ}$ point was measured using five different arrangements for the windings of the toroidal transformer and with the two locations of ratio transformer for each of the five arrangements. Since the results obtained on all measurements were the same to within $0.01^{\circ}$ it was concluded that the phase shift being measured was exactly $180^{\circ}$. Using the preliminary experimental calibration, based on computed values of phase shift for the fine control capacitor, the measured value came out to be $179.92^{\circ}$. Thus a correction factor of $180 / 179.92$ or $(1+0.000417)$ was applied to the preliminary calibration values to get the correct values as listed in table 1 for the 14.6 and 4.3 degree $\pi$-sections and in columns 4 and 5 of table 3 for the capacitor decade steps. As indicated in table 1 these measured values agree quite well with the values computed from measured values of induetance for each $\pi$-section. Although the change in the capacitor decade calibration introduced by the $180^{\circ}$ check is quite small, as seen from table 3 , it should be included for accurate measurements.

\section{Requirements for Use of Phase Standards}

The master phase angle $\pi$-section standard described in this paper is a continuously variable phase shifter whose absolute calibration has been accurately determined. It is primarily intended to be used to establish corrections for other continuously variable phase shifters and phasemeters used as standards. Herein, a phasemeter is defined as an instrument capable of measuring the phase shift between two alternating voltages of the same frequency. Throughout this kind of calibration testing the two factors most likely to affect accuracy are (1) the impedance of the instrument under test and (2) waveform distortion in supply voltage. These two points are of sufficient importance to be considered separately

\subsection{Compensation for Impedance of Instrument Under Test}

The connections used for calibrating a phasemeter are shown in the block diagram in figure 6 . The

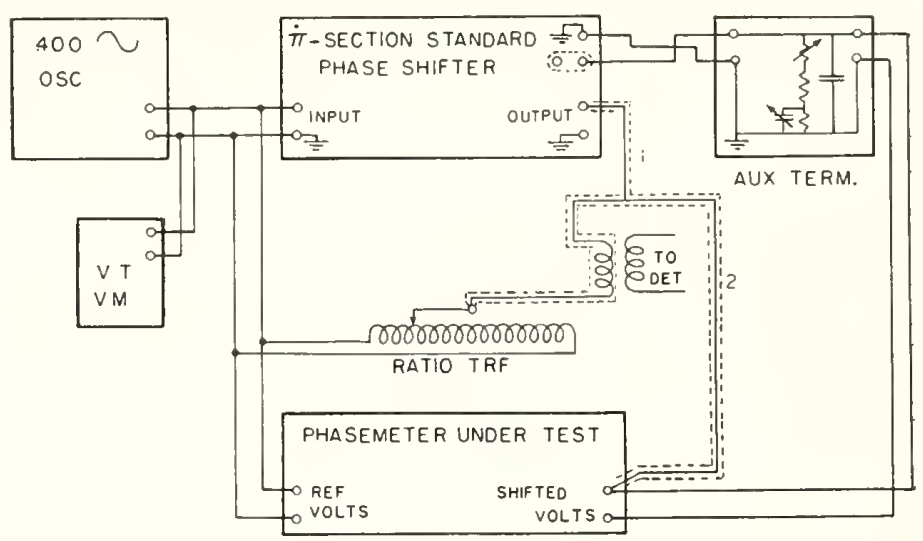

Figure 6. Selup used for calibrating a phasemeter.

"input" to the $\pi$-section phase standard is supplied by a 2 -w low distortion oscillator with a regulated output of $5 \mathrm{v}$. This value of voltage was chosen because it puts $0.25 \mathrm{v}$ across each $14.6^{\circ} \pi$-section, at which value the change of inductance with voltage is minimum for the iron core inductors. It was found that a 5-percent change in voltage at this level was required to produce a detectable change in inductance. Actually the voltage is maintained constant to within 2 percent. The reference or unslifted voltage input terminals of the meter under test are connected in parallel with the standard "input." At this point the value of impedance of the instrument under test is not critical because it merely acts as an additional load on the oscillator, and its only possible effect would be a slight change in waveform of the supply voltage. This effect would be entirely negligible for most instruments used as reference standards. If a value of voltage other than that required for the $\pi$-section standard $(5 \mathrm{v})$ is required by the meter under test, a transformer would normally be used. In such a case the phase shift introduced by the transformer must be known so that a correction for it can be applied.

If the impedance looking into the phasemeter through the shifted voltage binding posts were infinitely higl, the output of the $\pi$-section standard could be connected directly to these "shifted voltage" binding posts without affecting the terminating impedance of the $\pi$-section line. Under this condition the "auxiliary termination" would not be needed. However, since the performance of the $\pi$ section line is sensitive to small changes in its termination, $Z_{0}$, some compensation will normally be required for the impedance at the "shifted voltage" binding posts. This compensation is provided for by two insulated binding posts at the output end of the $\pi$-section phase standard, (see figs. 6 and 1), one being connected to the output end of the $\pi$-section line and the other to $R_{0}$ and $C_{0}$ (in parallel) which form the normal line termination. When these two binding posts are connected by a copper link the $\pi$ section line is terminated inside the $\pi$-section standard case and the normal output terminals can be used. By removing the link the internal line ter- 
mination is disconnected and the "auxiliary termination" may be comnected as shown in figure 6 . This auxiliary termination consists of a stable adjustable $R-C$ network which is connected in parallel with the leads from the $\pi$-section standard and arranged so that with the "shifted volts" leads also connected in parallel, the total impedance thus formed can be adjusted to be equal to $Z_{0}$ (the $\pi$ section impedance).

In order to make this adjustment a ratio transformer and a sensitive detector with a shielded input transformer are connected as shown in figure 6. First the leads from the $\pi$-section standard to the auxiliary termination are disconnected, the link replaced on the $\pi$-section standard and the switch put in "check" position (see fig. 1). Under these conditions the $\pi$-section line termination is the same as it was when the capacitor legs were adjusted, i.e., it consists of $C_{0}, R_{0}$, and the compensating capacitor $C_{s}$ in parallel. With the detector transformer lead in position " 1 " and all $\pi$-section standard dials set on "zero," the detector is balanced by adjusting the ratio transformer and the "zero set capacitor." Next the line termination $C_{0}, R_{0}, C_{s}$ is replaced by the "auxiliary termination." 'This is done by removing the link from the $\pi$-section standard binding posts, putting the switch in the "test" position, and connecting the auxiliary termination to the $\pi$-section standard and the phasemeter under test as shown in figure 6 . The detèctor is then balanced by adjusting the impedance of the auxiliary termination. To correct for any phase shift introduced by the "auxiliary termination" and its leads, the detector lead position is changed from " 1 " to " 2 " and the zero set capacitor is adjusted to balance the detector. Any correction for the phase shift in the ratio transformer can be made during this balance by actually setting this phase shift on the $\pi$-section standard fine control dial before making the final balance. The phasemeter under test can now be calibrated for any phase shift angle by first disconnecting all leads from the phase shifters to the detector transformer, then setting the required angle on the $\pi$-section phase standard and reading the corresponding value on the phasemeter.

If a voltage higher than the $1 \mathrm{v}$ availabie from the $\pi$-section standard output must be applied to the phasemeter under test, an amplifier can be used between the auxiliary termination and the "shifted volts" binding posts. The only requirements are (1) the amplifier must have low distortion and (2) the three balances as described in the previous paragraph must be made with the amplifier and its leads in position and the amplifier gain set to the same voltage as to be used during the calibration. The phase shift introduced by the amplifier may be fairly large, thus requiring considerable change in the zero set capacitor during the final detector balance before starting actual calibration.

For calibrating another continously variable phase shifter the procedure would be essentially the same as that just described for a phasemeter except that an external detector must be used to indicate when the phase shift put in by the phase shifter being tested is equal to that of the standard. For a given setting of the phase shifter being tested the detector would be balanced by adjusting the phase shift put in by the $\pi$-section standard and the magnitude of the output voltage from the phase shifter being lested by using a ratio transformer.

\subsection{Effect of Waveform Distortion}

At the very beginning of this paper phase angle was defined to be the quantity used as a measure of the time phase relation between two voltages having pure sine waveform. So far the effects of distortion have not been mentioned. However, since it is impossible to obtain pure sine waves in practice, such effects must be considered. For a distorted waveform, phase angle is herein defined as the time phase relation between the fundamental sine-wave components of the two voltages. Thus, there is no change in definition but a definite basis is established for a discussion of the effects of unwanted harmonics. If all the circuit elements were linear (i.e., no iron cores or lossy dielectrics were used) and if measuring and detecting instruments were only sensitive to the fundamental, then the use of a distorted waveform for making the measurements would make no difference. Unfortunately, these postulations cannot be met in practice even though very selective amplifier detectors are available.

Since the $\pi$-section standard as described in this paper is made up of iron core inductors the voltage drop across each inductor is always kept low enough so that flux density in the core is well below saturation. Yet it is possible that distortion could be introduced by the inductors even though the supply voltage has a pure sine wave form. To investigate this possibility, first an oscillator of very low distortion was used to supply the phase shifter "input," and distortion was measured at the "output" posts for various phase angle settings, i.e., for different numbers of $\pi$-sections in the "line." The waveform with phase shifter set on " 0 " i.e., no iron core inductor's connected contained 0.02 percent $2 \dot{0}$ harmonic, 0.01 percent $3 \mathrm{~d}$ lrarmonic, and 0.01 percent of higher harmonics. As inductors were connected all harmonics remained at about the same value except the $3 \mathrm{~d}$ which gradually increased to about 0.04 percent with all twelve $14.6^{\circ} \pi$-sections in circuit. Thus for a nearly pure sine-wave input the inductors in the $\pi$-sections slightly increase the $3 \mathrm{~d}$ harmonic distortion.

Similar tests were made using an oscillator with somewhat greater distortion, i.e., about 0.03 percent $2 d, 0.5$ percent $3 d$, and 0.16 percent 4 th. For this imput voltage the effect of adding $\pi$-section inductors on the distortion was too small to be significant (less than about 0.02 percent). In order to check for possible effects of this distortion on measurements involving only the fundamental component, the $180^{\circ}$ point was checked as described in section 6 using each of the oscillators whose distortions have just been given. The results using the oscillator with 0.5 percent $3 d$ harmonic agreed with those using the 
nearly pure sine wave oscillator to within about $0.005^{\circ}$. Thus it was concluded that for accuracies within $0.01^{\circ}$ the total wave form distortion should be less than 0.5 percent. It should be borne in mind that in making these tests a highly selective, feed-back tuned, amplifier was used in the detector circuit.

Waveform may also have an effect on or may be affected by the phase shifter or phasemeter being calibrated. For testing another phase shifter of the same design as the $\pi$-section standard the restrictions on distortion should be the same as those given above. For other types of phase shifters or phasemeters the effects of wave-form distortion should be determined by repeat tests using two oscillators of significantly different waveform. From a limited number of such tests it is believed that in general for all oscillators with less than 0.5 percent total distortion the phaseangle test results would be the same to within about $0.01^{\circ}$.

\section{Summary and Conclusions}

A continuously variable, $0^{\circ}$ to $180^{\circ}$ phase shifter for $400 \mathrm{cps}$ has been described in detail. By using a special procedure for matching the characteristic impedance of the $L-C \pi$-sections used in this phase shifter, a method for experimentally measuring the phase shift of each section has been devised. This phase shift has also been computed from measured values of inductance for each $\pi$-section. The results obtained by these two methods agree to within $0.01^{\circ}$. These results indicate that this phase shifter can be used as a master standard in calibrating other phase shift devices.

Similar phase angle standards for higher audiofrequencies could be designed, but on the basis of the experience gained it is thought that the upper limit in frequency would be about $20 \mathrm{kc}$. At higher frequencies, stray capacitance introduced by connecting and switching leads might prove troublesome. It might be feasible to use the same master standard over a frequency range of about 2 to 1 by some readjustments on the $\pi$-sections and termination for each frequency.

\section{Appendix 1. Computation of Characteris- tic Impedance of $\pi$-Section-Losses Con- sidered}

A single terminated $\pi$-section with losses in both inductor and capacitor considered, is shown schematically in figure 7 . To keep the derivation general, the

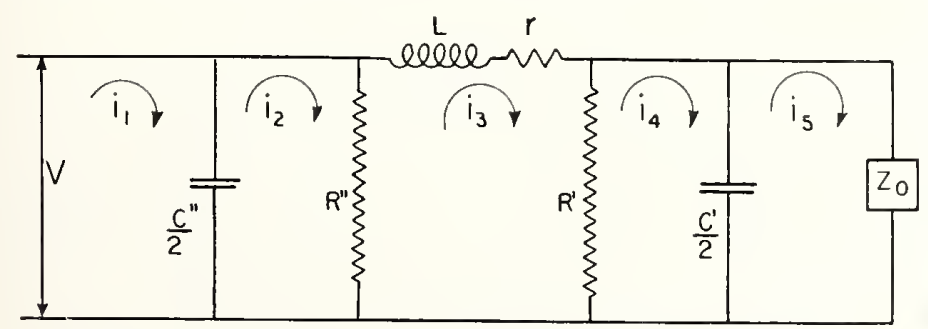

Figure 7. A terminated $\pi$-section with losses considered. two capacitor legs are not assumed to be equal. $R^{\prime \prime}$ represents the total losses in $C^{\prime \prime} / 2$ expressed as a parallel resistance and likewise $R^{\prime}$ for $C^{\prime} / 2$. The total losses in the inductor are represented by $r$, as a series resistance. Five circuit equations may be written by inspection of figure 7:

$$
\begin{aligned}
& i_{1} a-i_{2} a=V, \\
& -i_{1} a+i_{2} b-i_{3} R^{\prime \prime}=0, \\
& -i_{2} R^{\prime \prime}+i_{3} d-i_{1} R^{\prime}=0, \\
& -i_{3} R^{\prime}+i_{4} f-i_{5} g=0, \\
& -i_{4} g+i_{5}\left(Z_{0}+g\right)=0,
\end{aligned}
$$

where

$$
\begin{gathered}
a=\frac{1}{j \omega \frac{C^{\prime \prime}}{2}}, \quad b=R^{\prime \prime}+\frac{1}{j \omega \frac{C^{\prime \prime}}{2}}, \quad d=R^{\prime \prime}+r+R^{\prime}+j \omega L \\
f=R^{\prime}+\frac{1}{j \omega \frac{C^{\prime}}{2}}, \quad \text { and } \quad g=\frac{1}{j \omega \frac{C^{\prime}}{2}} .
\end{gathered}
$$

Using these equations and putting $1 / Z_{0}=\left(1 / R_{0}\right)+$ $j \omega C_{0}$, the following expression for input admittance $Y_{\mathrm{n}}$ is obtained:

$$
\begin{gathered}
Y_{i n}=j \omega \frac{C^{\prime \prime}}{2}+\frac{\left[1+j \omega R_{c}\left(C_{0}+\frac{C^{\prime}}{2}\right)\right]\left[1+\frac{r}{R^{\prime \prime}}+j \omega \frac{L}{R^{\prime \prime}}\right]}{\left[1+j \omega R_{0}\left(C_{0}+\frac{C^{\prime}}{2}\right)\right](r+j \omega L)} \\
\frac{+R_{0}\left[\frac{1}{R^{\prime}}+\frac{r}{R^{\prime} R^{\prime \prime}}+\frac{1}{R^{\prime \prime}}+\frac{j \omega L}{R^{\prime} R^{\prime \prime}}\right]}{+R_{0}\left[1+\frac{r}{R^{\prime}}+\frac{j \omega L}{R^{\prime}}\right]}
\end{gathered}
$$

To obtain a relation which requires the characteristic impedance of the $\pi$-section to be $R_{0} /\left(1+j \omega C_{0} R_{0}\right)$ equate the above value of $Y_{\text {in }}$ to $\left(1 / R_{0}\right)+j \omega C_{0}$. This gives a complex equation from which two equations involving circuit parameters only can be formed. By equating the real parts of the complex equation the following is obtained:

$$
\begin{gathered}
\omega^{2} R_{0} r C_{0}^{2}-\left[\omega^{2} R_{0} r\left(\frac{C^{\prime \prime}}{2}-\frac{C^{\prime}}{2}\right)-2 \omega^{2} L\right] C_{0}+\omega^{2} L\left(\frac{C^{\prime}}{2}\right. \\
\left.-\frac{C^{\prime \prime}}{2}\right)-\omega^{2} R_{0} r \frac{C^{\prime} C^{\prime \prime}}{4}-\frac{r}{R_{0}}+m=0,
\end{gathered}
$$

where

$$
\begin{array}{r}
m=\frac{R_{0}}{R^{\prime}}\left[1+\frac{R^{\prime}}{R^{\prime \prime}}-\frac{r}{R_{0}}+\frac{r R^{\prime}}{R_{0} R^{\prime \prime}}+\frac{r}{R^{\prime \prime}}-\frac{\omega^{2} L R^{\prime}}{R^{\prime \prime}}\left(C_{0}+\frac{C^{\prime}}{2}\right)\right. \\
\left.+\omega^{2} L\left(C_{0}-\frac{C^{\prime \prime}}{2}\right)\right] .
\end{array}
$$


By equating the imaginary parts of the complex equation the following is obtained:

$$
\begin{gathered}
\omega r\left(2 C_{0}+\frac{C^{\prime}}{2}-\frac{C^{\prime \prime}}{2}\right) \\
+\frac{\omega L}{R_{0}}\left[1-\omega^{2} R_{0}^{2}\left(C_{0}^{2}+\frac{C_{0} C^{\prime}}{2}-\frac{C_{0} C^{\prime \prime}}{2}-\frac{C^{\prime} C^{\prime \prime}}{4}\right)\right] \\
+\omega R_{0}\left(-\frac{C^{\prime \prime}}{2}-\frac{C^{\prime}}{2}\right)+\frac{n}{R_{0}}=0
\end{gathered}
$$

where

$$
\begin{aligned}
n / R_{0}=\omega L\left(\frac{1}{R^{\prime}}-\frac{1}{R^{\prime \prime}}-\frac{R_{0}}{R^{\prime} R^{\prime \prime}}\right) & \\
& +\omega r R_{0}\left[\frac{C_{0}-\frac{C^{\prime \prime}}{2}}{R^{\prime}}-\frac{C_{0}+\frac{C^{\prime}}{2}}{R^{\prime \prime}}\right]
\end{aligned}
$$

For $C^{\prime} / 2=C^{\prime \prime} / 2=C / 2$ these two equations can be used to solve for $C / 2$ and $C_{0}$ when $L, R_{0}, r R^{\prime}, R^{\prime \prime}$ are known. Equation (6) is used to solve for $C_{0}$. Putting $C / 2=\left(L / 2 R_{0}^{2}\right)(1+\delta)$ where $\delta<1$, this equation becomes:

$$
\omega^{2} R_{0} r C_{0}^{2}+2 \omega^{2} L C_{0}-\frac{r \omega^{2} L^{2}}{4 R_{0}^{3}}(1+\delta)^{2}-\frac{r}{R_{0}}+m=0 .
$$

Solving it for $C_{0}$ gives

$$
C_{0}=\frac{r}{2 \omega^{2} L R_{0}}(1+\alpha),
$$

where

$$
\begin{gathered}
1+\alpha=\left[1+\frac{\omega^{2} L^{2}}{4 R_{0}^{2}}(1+\delta)^{2}-\frac{R_{0}}{r} m\right]\left(1-\frac{\Delta}{4}+\frac{\Delta^{2}}{8}-\frac{5 \Delta^{3}}{64}+\cdots\right), \\
\Delta=\frac{r^{2}}{\omega^{2} L^{2}}\left[1+\frac{\omega^{2} L^{2}}{4 R_{0}^{2}}(1+\delta)^{2}-\frac{R_{0}}{r} m\right] .
\end{gathered}
$$

An estimated value is used first for $\delta$. The exact value is determined later from eq (10) and a recomputation made if necessary. Using this value for $C_{0}$ and $C^{\prime} / 2=C^{\prime \prime} / 2=C / 2$, eq ( 7 ) becomes

$\omega^{2} L\left(\frac{C}{2}\right)^{2}-2 \frac{C}{2}+\frac{L}{R_{0}^{2}}\left[1+\frac{3 r^{2}}{4 \omega^{2} L^{2}}\left(1+\frac{2 \alpha}{3}-\frac{\alpha^{2}}{3}\right)+\frac{n}{\omega L}\right]=0$.

Solving for $C / 2$ gives

$$
\frac{C}{2}=\frac{L}{2 R_{\mathrm{C}}^{2}}\left[1+\gamma+\frac{\omega^{2} L^{2}}{4 R_{0}^{2}}(1+\gamma)^{2}+\frac{\omega^{4} L^{4}}{8 R_{0}^{4}}(1+\gamma)^{3}+\ldots\right]
$$

where

$$
\gamma=\frac{3 r^{2}}{4 \omega^{2} L^{2}}\left(1+\frac{2 \alpha}{3}-\frac{\alpha^{2}}{3}\right)+\frac{n}{\omega L} .
$$

Now going back to eq (6) and putting $C^{\prime} / 2=C / 2$ $\left(1+\lambda^{\prime}\right)$ and $C^{\prime \prime} / 2=C / 2\left(1=\lambda^{\prime \prime}\right)$, where $C / 2$ is the value from eq $(10)$, it follows that

$$
\begin{gathered}
\lambda^{\prime}-\lambda^{\prime \prime}+\frac{C_{0} R_{0} r}{L}\left(\lambda^{\prime}-\lambda^{\prime \prime}\right)-\frac{r R_{0} C}{2 L}\left(\lambda^{\prime}+\lambda^{\prime \prime}+\lambda^{\prime} \lambda^{\prime \prime}\right) \\
=\frac{r R_{0}}{L} \frac{C}{2}-\frac{r R_{0} C_{0}^{2} 2}{L C}-\frac{2 C_{0} 2}{C}+\frac{r 2}{\omega^{2} L R_{0} C}-\frac{m 2}{\omega^{2} L C}
\end{gathered}
$$

Likewise eq (7) gives

$$
\begin{array}{r}
\lambda^{\prime}+\lambda^{\prime \prime}-\frac{r}{R_{0}}\left(\lambda^{\prime}-\lambda^{\prime \prime}\right)+\omega^{2} L C_{0}\left(\lambda^{\prime}-\lambda^{\prime \prime}\right) \\
-\omega^{2} L \frac{C}{2}\left(\lambda^{\prime}+\lambda^{\prime \prime}+\lambda^{\prime} \lambda^{\prime \prime}\right)=\frac{2 r C_{0} 2}{R_{0} C}+\frac{L 2}{k_{0}^{2} C} \\
-\frac{\omega^{2} L C_{0}^{2} 2}{C}+\frac{\omega^{2} L C}{2}-2+\frac{2 n}{\omega R_{0}^{2} C} .
\end{array}
$$

Equations (11) and (12) can be used to compute actual changes in $C^{\prime} / 2$ and $C^{\prime \prime} / 2$ that are made experimentally when adjusting the characteristic impedance of a given $\pi$-section to exactly match the terminating impedance ( $R_{0}$ and $C_{0}$ in parallel). First the values for $C / 2$ and $C_{0}$ are computed using eqs (10) and (9) by assuming average values for $L, r, R^{\prime}$ and $R^{\prime \prime}$. A value for $R_{0}$ must also be chosen. Then if these values of $R_{0}$ and $C_{0}$ are kept the same the effect of small changes in $r, R^{\prime}$, and/or $R^{\prime \prime}$ on values of $C^{\prime \prime} / 2$ and $C^{\prime} / 2$ can be computed using eqs (11) and (12). The actual value of $C^{\prime} / 2$ can then be used to accurately compute the phase shift for each individual $\pi$-section as indicated in appendix 2 .

The above procedure may be best illustrated by taking actual values for the $14.6^{\circ} \pi$-section:

$$
\begin{aligned}
& \mathrm{L}=100 \mathrm{mh}, \quad r=2.8 \Omega, \quad R^{\prime}=R^{\prime \prime}=1 \times 10^{7} \Omega, \\
& R_{0}=1,000 \Omega, \quad \omega=2513.27, \quad \omega^{2}=6.31653 \times 10^{6} \text {. }
\end{aligned}
$$

Putting these values in eqs (9) and (10) gives

$$
\begin{aligned}
C_{0} & =0.002099 \times 10^{-6}, \\
C / 2 & =0.050819_{1} \times 10^{-6} .
\end{aligned}
$$

When these values are put in eqs (11) and (12) they give $\lambda^{\prime}=\lambda^{\prime \prime}=0$ for the values of $r, R^{\prime}$, and $R^{\prime \prime}$ given above. For small changes in $r, R^{\prime}$, and $R^{\prime \prime}$ these equations give the corresponding values for $\lambda^{\prime}$ and $\lambda^{\prime \prime}$ required to" keep the characteristic impedance the same.

First keep all values the same as above except change $r$ to 3.1 ohms. Putting values in eq (11) gives

$$
0.99849 \lambda^{\prime}-1.00164 \lambda^{\prime \prime}=0.00952 \text {. }
$$

Putting values in eq (12) gives

$$
0.96613 \lambda^{\prime}+0.96967 \lambda^{\prime \prime}=0.000065 \text {. }
$$

Solving eqs (13) and (14) for $\lambda^{\prime}$ and $\lambda^{\prime \prime}$ gives

$$
\lambda^{\prime}=0.00480 \quad \text { and } \quad \lambda^{\prime \prime}=-0.00472 \text {. }
$$

Next keep all values the same as assumed above except put $R^{\prime}=10^{8}$. Then eq (11) becomes:

$$
0.99864 \lambda^{\prime}-1.00148 \lambda^{\prime \prime}=0.00275 \text {. }
$$


Equation (12) becomes

$$
0.96643 \lambda^{\prime}+0.96937 \lambda^{\prime \prime}=-0.000137
$$

Solvings eqs (15) and (16) for $\lambda^{\prime}$ and $\lambda^{\prime \prime}$ gives:

$$
\lambda^{\prime}=0.00131 \quad \text { and } \quad \lambda^{\prime \prime}=-0.00144
$$

If the same computation is made for $R^{\prime \prime}$ changed to $10^{8}$ the values of $\lambda^{\prime}$ and $\lambda^{\prime \prime}$ will be very nearly the same because $m$ is the same and the effect of the change in $n$ is quite small.

Now keep all values as originally assumed except $R^{\prime}=R^{\prime \prime}=10^{8}$. Then eq (11) becomes

$$
0.99864 \lambda^{\prime}-1.00148 \lambda^{\prime \prime}=0.00544 \text {. }
$$

Equation (12) becomes

$$
0.96643 \lambda^{\prime}+0.96937 \lambda^{\prime \prime}=0.000040 \text {. }
$$

Solving (17) and (18) for $\lambda^{\prime}$ and $\lambda^{\prime \prime}$ gives

$$
\lambda^{\prime}=0.00274 \quad \text { and } \quad \lambda^{\prime \prime}=-0.00270 .
$$

These values of $\lambda^{\prime}$ and $\lambda^{\prime \prime}$ were worked out so that they could be used to compute phase shift under these particular assumed conditions, as will be done in appendix 2 .

\section{Appendix 2. Computation of Phase Shift of a $\pi$-Section From Value of Inductance}

For computing phase shift the first capacitor leg of the $\pi$-section need not be considered because it is in parallel with the supply voltage or " $V_{\mathrm{ln}}$." Thus the circuit diagram is as shown in figure 8 .

The purpose of this derivation is to obtain an expression for the phase shift between $V_{\text {In }}$ and $V_{\text {out }}$ when the complete $\pi$-section (see fig. 7) has been adjusted experimentally so that its impedance as seen from the input end is equal to $Z_{0}$.

Three circuit equations may be written. by in spection of figure 8:

$$
\begin{gathered}
i_{1}\left(R^{\prime}+r+j \omega L\right)-i_{2} R^{\prime}=V_{1 \mathrm{n}}, \\
-i_{1} R^{\prime}+i_{2}\left(R^{\prime}+\frac{1}{j \omega \frac{C^{\prime}}{2}}\right)-i_{3} \frac{1}{j \omega \frac{C^{\prime}}{2}}=0, \\
-i_{2} \frac{1}{j \omega \frac{C^{\prime}}{2}}+i_{3}\left(\frac{1}{j \omega \frac{C^{\prime}}{2}}+Z_{0}\right)=0 .
\end{gathered}
$$

For $Z_{0}=R_{0} / 1+j \omega C_{0} R_{0}$, these equations give the relation

$$
\frac{V_{\text {out }}}{V_{\mathrm{ln}}}=\frac{1}{1+\frac{r}{R_{0}}+\frac{r}{R^{\prime}}-\frac{\omega L A}{R_{0}}+\frac{j r A}{R_{0}}+\frac{j \omega L}{R^{\prime}}+j \frac{\omega L}{R_{0}}}
$$

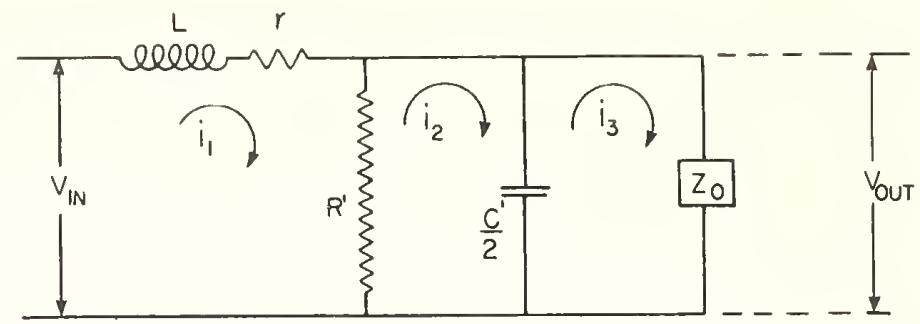

Figure 8. A terminated $\pi$-section without first capacitor leg.

where

$$
A=\omega R_{0}\left(C_{0}+\frac{C^{\prime}}{2}\right)
$$

The phase shift, $\theta$, is then the angle of the vector in the denominator, thus,

$$
\tan \theta=\frac{\omega L / R_{0}+\omega L / R^{\prime}+r \omega\left(C_{0}+\frac{C^{\prime}}{2}\right)}{1+r / R_{0}+r / R^{\prime}-\omega^{2} L\left(C_{0}+\frac{C^{\prime}}{2}\right)} .
$$

This equation can be used to compute $\tan \theta$ for various values of $L, r, C^{\prime} / 2, R^{\prime}, C_{0}$, and $R_{0}$. First using average values ( $L=100 \mathrm{mh}, r=2.8 \Omega, R^{\prime}=R^{\prime \prime}=$ $10^{7} \Omega$, and $R_{0}=1,000 \Omega$ ) from eqs. (9) and (10), $C / 2=$ $C^{\prime} / 2=0.0508191 \mu \mathrm{f}$ and $C_{0}=0.002099 \mu \mathrm{f}$. These values give

$$
\tan \theta=0.25968 \text {. }
$$

For the next set of values assumed in appendix 1, i.e., $L=100 \mathrm{mh}, R^{\prime}=R^{\prime \prime}=10^{7} \Omega, r=3.1 \Omega, R_{0}=1,000$ $\Omega, C_{0}=0.002099 \mu \mathrm{f}, \quad C^{\prime} / 2=0.051063 \mu \mathrm{f}$ (from $\lambda^{\prime}$ $=0.00480$ ).

$$
\tan \theta=0.25968 \text {. }
$$

For $L=100 \mathrm{mh}, \quad r=2.8 \Omega, \quad R_{0}=1,000 \Omega, \quad C_{0}$ $=0.002099 \mu \mathrm{f}, R^{\prime \prime}=10^{7} \Omega, \quad R^{\prime}=10^{8} \Omega, \quad C^{\prime} / 2=0.05089$ $\mu$ f $\left(\right.$ from $\left.\lambda^{\prime}=0.00131\right)$,

$$
\tan \theta=0.25967 \text {. }
$$

For the same values as above, except $R^{\prime \prime}=10^{8} \Omega$ and $R^{\prime}=10^{7} \Omega, C^{\prime} / 2$ still equals $0.05089 \mu \mathrm{f}$ and

$$
\tan \theta=0.25969 \text {. }
$$

Now for all values the same, except $R^{\prime}=R^{\prime \prime}=10^{8} \Omega$, giving $C^{\prime} / 2=0.05095 \mu \mathrm{f}$ (from $\lambda^{\prime}=0.00274$ ) and $C_{0}+C^{\prime} / 2=0.05305 \mu \mathrm{f}$

$$
\tan \theta=0.25968 \text {. }
$$

The changes in $r, R^{\prime}+R^{\prime \prime}$ as assumed above are the maximum to be encountered among the twelve $14.6^{\circ}$ sections, as found from actual measurements. Since $\tan \theta$ is very nearly the same for all of these assumed values, the average values based on the actual measurements may be used, i.e., $r=2.8 \mathrm{ohm}$, $R^{\prime}=R^{\prime \prime}=10^{7}$. ohm. For $R_{0}=1,000$ ohms these 
values grve $C / 2=0.0508191 \mu$ and $C_{0}=0.002099 \mu \mathrm{f}$. When these values are put in eq (19) it reduces to a constant times $L$ and for $f=400$ cps this gives

$$
\tan \theta=2.59678 L \text {. }
$$

Thus for the actual inductors used, if the terminating impedance is held constant $\left(R_{0}=1,000 \mathrm{ohms}\right.$ and $\mathscr{C}_{0}=0.002099 \mu \mathrm{f}$ ) and if the trimmers on each capacitance leg are adjusted so that the impedance looking into the line equals the terminating impedance (by the method explained in section 4.2), the phase shift introduced by each section is equal to a constant times its inductance.

\section{Appendix 3. Computation of Phase Shift of the $\mathrm{R}-\mathrm{C}$ Network}

Figure 9 is a simplified circuit diagram of the $R-C$ network. $\quad R_{1}$ is a precision resistor accurately adjusted to 4,000 ohms. $C_{1}$ is a precision air capacitor with a capacitance remove dial variable from 1,050 to $50 \mathrm{pf}$. The change in $C_{1}$ can be read from the dial setting to within $1 \mathrm{pf}$ so that it can be used as a fine control of phase shift. $R_{0}$ is the resistance component of the $\pi$-line input impedance $(1,000$ ohms). $\quad C_{2}$ inclucles the capacitance component $C_{0}$ of the $\pi$-line together with the decade capacitor (ten steps of $0.44^{\circ}$ each), the zero setting capacitor and any lead capacitance. The purpose of the following derivation is to get an expression for phase shift between voltages $E$ and $e$ in terms of the circuit constants.

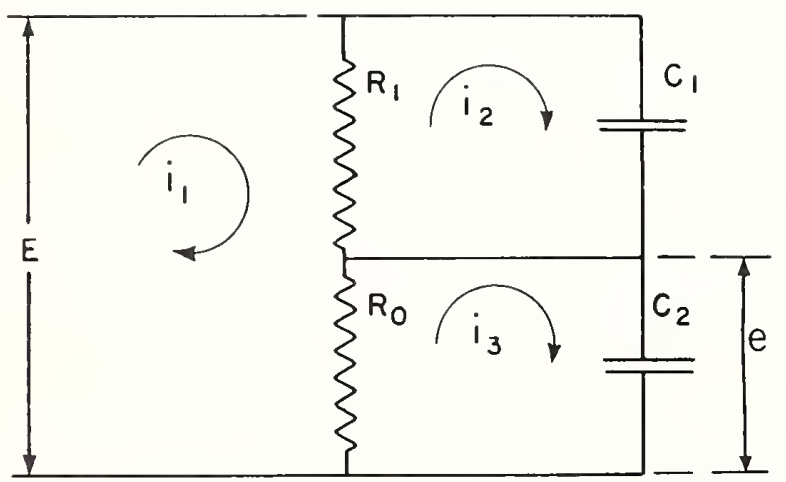

FIGURE 9. Schematic diagram of the $R C$ circuit in the phase standard.
The following circuit equation may be written by inspection of figure 9:

$$
\begin{aligned}
& i_{1}\left(R_{1}+R_{0}\right)-i_{2} R_{1}-i_{3} R_{0}=E, \\
& -i_{1} R_{1}+i_{2}\left(R_{1}+\frac{1}{j \omega C_{1}}\right)=0, \\
& -i_{1} R_{0}+i_{3}\left(R_{0}+\frac{1}{j \omega C_{2}}\right)=0 .
\end{aligned}
$$

These equations yield the following relation:

$$
\frac{e}{E}=\frac{R_{0}}{R_{1}+R_{0}} \frac{1+j \omega C_{1} R_{1}}{1+\frac{j \omega R_{1} R_{0}}{R_{1}+R_{0}}\left(C_{1}+C_{2}\right)} .
$$

By clearing the $j$ term from denominator the following expression for $\tan \theta$ is obtained where $\theta$ is the angle by which $e$ leads $E$ :

$$
\tan \theta=\frac{\omega R_{1}\left(C_{1} \frac{R_{1}}{R_{1}+R_{0}}-C_{2} \frac{R_{0}}{R_{1}+R_{0}}\right)}{1+\frac{\omega^{2} R_{1}^{2} R_{0}}{R_{1}+R_{0}} C_{1}\left(C_{1}+C_{2}\right)} .
$$

For

$$
\begin{aligned}
R_{1}=4,000 \mathrm{ohms}, & R_{0} \\
= & 1,000 \mathrm{ohms}, \text { and }\left\{\begin{array}{l}
\omega=2513.27 \\
\omega^{2}=6.3165_{3} \times 10^{6}
\end{array}\right.
\end{aligned}
$$

the expression becomes

$$
\tan \theta=\frac{2.01062 \times 10^{6}\left(4 C_{1}-C_{2}\right)}{1+20.213 \times 10^{12} C_{1}\left(C_{1}+C_{2}\right)} .
$$

Using this expression, phase shifts, $\theta$, were computed for three values of $C_{1}(200,600$, and 1,200 pf) and for values of $C_{2}$ from $0.004 \mu \mathrm{f}$ to $0.043 \mu \mathrm{f}$ in steps of $0.0039 \mu \mathrm{f}$. The results which were carried out to $0.0001^{\circ}$ in order to show small changes are presented in table 2. 
RESEARCH PAPER RP1344

Part of Journal of Research of the National Bureau of Standards, Volime 25, November 1940

\title{
STANDARD ELECTRODYNAMIC WATTMETER AND AC-DC TRANSFER INSTRUMENT
}

\author{
By John H. Park and Arthur B. Lewis
}

\section{ABSTRACT}

A description is given of the design and construction of the standard instrument used at the National Bureau of Standards in testing wattmeters and watthour meters.

The investigation to determine the accuracy of this instrument for alternatingcurrent testing at frequencies up to 2,000 cycles per second is described and the results are given.

\section{CONTENTS}

Fage

I. Introduction

II. Design and construction...

1. Criteria of design

2. General features of design ........... 548

3. Details of design and construction . . _ .

(a) Supporting framework

(b) Fixed coils .................. 550

(c) Moving system

(d) Preliminary tests . . .

(e) Series resistor

(f) Shielding ...

(g) Wattmeter constants

(1) Fixed coils

(2) Moving system

III. Performance tests . . _..

1. Direct-current accuracy _...........

2. Analysis of ac-dc errors

3 . Errors depending on $E$ or $I$ separately $" \Delta \alpha "$

(a) Interaction between parts of voltage circuit....... 563

(b) Magnetic impurities in the moving system ....... 563

(c) Induced and capacitance currents in the moving system - 564

4. Errors depending upon $E I$-test at unity power-factor...... 567

(a) Method ........ 567

(b) Procedure

(c) Results

5. Errors depending upon phase angle $\theta$-tests at zero power-

factor

(a) At 60 cycles per second, using quadrant electrometer.. 569

(1) Method ........ 569

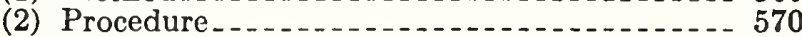

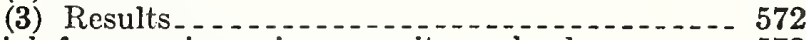

(b) At high frequencies, using capacitance load..... 573

(1) Method and procedure............... 573

(2) Derivation of equation................ 574

(3) Results $\ldots$

IV. Summary _.

V. Appendix I. Computation of the effect of variation in wattmeter sensitivity

VI. Appendix III. Phase difference between the fixed-coil current and the quadrant voltage on the electrometer and the relation of this phase difference to $\theta$, the phase angle between $E$ and $I \ldots \ldots . . . . . . . . . .578$ 


\section{INTRODUCTION}

The basic units for electrical measurements are maintained at the National Bureau of Standards by the use of standard cells and standard resistors. The corrections to be applied to the indications of d-c measuring instruments can therefore be readily and accurately determined by using secondary standards, which have been directly compared with these primary standards, in conjunction with potentiometers and volt boxes. Since the primary standards are maintained by means of d-c measurements, the determination of the corrections to be applied to the indications of a-c instruments must involve at some point the use of a secondary standard or transfer instrument. This transfer instrument must be such that its reading or indication is the same with direct current as with alternating current, or differs by a known amount. The standard instrument is calibrated ${ }^{1}$ on reversed direct current ${ }^{2}$ and this calibration is then transferred to its a-c indications, hence the name.

Such transfer instruments are used in two separate and distinct kinds of tests: (1) in the transfer testing of a-c ammeters, voltmeters, and wattmeters which are of types that can be used on either alternating or direct current, and (2) in the straight testing with alternating current of voltmeters, ammeters, wattmeters, and watthour meters of types that are not suitable for use on direct current. In making the transfer test, the instrument under test is first tested, or calibrated, using the average of the reversed d-c values. The instrument under test and the standard transfer instrument are then so connected as to respond to the same current, voltage, or power. The current on both instruments is then adjusted until the instrument under test comes to the desired scale point and the standard instrument is read. A series of readings, using alternately direct and alternating current, is obtained, and the difference between the mean of the readings obtained with alternating current and that of those with direct current is computed from these data. This procedure very completely eliminates all sources of error-such as heating, shift of zero, and reading error of either instrument-and leaves in the net difference only those errors which arise from sources such as inductance, capacitance, and eddy currents, in which the effect on alternating current differs from that on direct current. The transfer instruments have been carefully studied and any such effects in them are small and definite and can readily be allowed for, so that the net error of the instrument under test on alternating current relative to its performance on direct current is thus determined.

In a straightforward test with alternating current the transfer instrument is calibrated by applying reversed direct current to it alone. It is then used as a standard, and the readings of the instrument under test are compared with it, using alternating current. The standard transfer instrument is calibrated on reversed direct current before and after it is compared at any scale point with the instrument under test. This procedure has to be used when the a-cinstrument is of such nature

1 "Calibrate" es used in this paper means the determination of the relation between the indications of, for example, a wattmeter and the true watts.

"The expresslon "reversed direct current" is used to desigate the regular procerlure in the use of Instruments of this kind on direct current in order to a void error caused hy the local megnetic field. For example, in using an electrodynamic wattmeter on direct current, two readings are taken, the direction of current flow through the instrument belng reversed before taking the second reading. The mean of the two readings gives a result independent of the local megnetic field. 
that it does not respond to direct current, and with all instruments the range of which exceeds the range (10 amp and $300 \mathrm{v}$ ) of the standard transfer instruments. In this latter case instrument transformers must be used to extend the range of the transfer instrument. It may be noted that any self-heating or shift of zero which occurs in the instrument under test during the course of this straight a-c test will be included in the final result, and the accuracy of a test of this type is therefore definitely less than that of the transfer test.

The transfer instruments usually used at the National Bureau of Standards are of the electrodynamic type. The voltmeter in use at the present time has been described in a previous publication. ${ }^{3}$ The present paper describes the wattmeter now being used. Although designed primarily as a wattmeter, this instrument may also be used as an ammeter if it is connected to measure the power consumed in an external shunt.

\section{DESIGN AND CONSTRUCTION}

\section{CRITERIA OF DESIGN}

A transfer instrument to be satisfactory for the type of testing work done at the National Bureau of Standards should possess, so far as possible, the following desirable characteristics:

(1) The frame of the instrument should be made from a nonmagnetic, nonconducting material. This material should lend itself to a sufficiently sturdy and permanent construction to avoid future difficulties resulting from warping of the instrument frame and consequent sticking of the moving system.

(2) The zero drift of the instrument on steady deflection should be as small as possible.

(3) The sensitivity of the instrument should be such that a change of 0.01 percent of the total deflection can be detected readily and with certainty on all ranges.

(4) The ac-dc differences of the instrument, that is, the differences between the indications of the instrument on alternating current and the average of its indications on reversed direct current, should be negligible for power frequencies and as low as possible for somewhat higher frequericies.

(5) The instrument should be astatic in construction.

(6) The damping of the moving system should be slightly less than critical.

(7) The response time ${ }^{4}$ of the instrument should be reasonably short, preferably not greater than $10 \mathrm{sec}$.

(8) The two moving coils, the reflecting mirror, and the damping vane should be rigidly interconnected in order to avoid forced vibrations of these parts with respect to each other.

(9) The indication of the instrument should be read easily and with a minimum of eyestrain.

(10) The moving system should be suitable for use on voltages up to $300 \mathrm{v}$, and the fixed-coil system should have a primary current range of $5 \mathrm{amp}$ and such additional ranges as can be obtained without undue difficulty.

\footnotetext{
3 F. K. Harris, A suppressed-zero electrodynamic voltmeter, BS J. Research 3, 445 (1929) RP105.

4 The response time of an instrument is the time required for the polnter of the Instrument to come to apparent rest after a change in the value of the measured quantity.
} 
These desirable characteristics are listed in their approximate order of relative importance as determined largely by the previous experience of members of the staff in the construction and use of similar instruments. It was proposed to incorporate these characteristics in the construction of the new instrument so far as was compatible with their sometimes conflicting requirements.

\section{GENERAL FEATURES OF DESIGN}

Since pivot friction could seriously limit the sensitivity attainable, pivots are not used but the moving system is suspended from top and bottom on two phosphor-bronze ribbons. The deflection of the instrument is read on a ground-glass scale by means of a beam of light reflected from a small mirror attached to the moving system. The distance from mirror to scale is $2 \mathrm{~m}$, giving the instrument an effective scale length, at full-scale deflection, of about $4 \mathrm{~m}$. The instrument is made astatic by using two sets of moving and fixed coils, one placed slightly above the other, the direction of winding of one set of coils being reversed with respect to the direction of winding of the other. Air damping is provided by mounting a rectangular damping vane on the moving system and having it-rotate within a fixed damping box. In order to obtain the most nearly linear relation between deflection and power being measured, the moving coils are held near the position of zero mutual inductance between the fixed and moving coils when the moving coils are deflected to their full-scale position. This is accomplished by resetting the series resistor in the voltage circuit for each nominal value of watts being measured. The mechanical zero of the instrument need not be disturbed, and the deflection of the moving system is thus approximately the same, regardless of the nominal value of watts being measured. The result is to minimize the zero shift, on steady deflections, resulting from the inelastic yielding of the spring and suspensions. This means, in turn, that it is permissible to make less frequent calibrations of the instrument on direct current when it is being used as a standard a-c wattmeter or ammeter.

\section{DETAILS OF DESIGN AND CONSTRUCTION}

(a) SUPPORTING FRAMEWORK

One of the chief difficulties encountered with previous transfer instruments has been warping of the supporting framework, which resulted in sticlsing of the moving system. The difficulty of locating and removing the causes of this misalinement, and the consequent sticking, led to the conclusion that the framework of the new instrument should be made of materials less subject to warping than wood and which preferably allowed some degree of vision within the instrument. Since all metallic materials are out of the question because of eddy-current or magnetic errors, the field of choice was very limited. After some discussion it was decided to use glass for the supporting framework, since it is nonwarping and nonmagnetic, permits a certain amount of vision within the instrument, can be obtained readily in slabs of almost any reasonable size, and can be readily worked if care is used.

The fixed coils and the damping box are each split into two halves in a vertical plane, each half being mounted in a rectangular glass panel. 
These glass panels are hinged to each other, one being stationary and the other opening and closing like a door. In the closed position the corresponding halves of the fixed coils and the two halves of the damping box fit snugly together to form two complete fixed coils and one damping box.

The difficulty of attaching hinges directly to the glass forced the use of supporting frames of mahogany for the two glass panels. The hinges are attached to these frames. These frames also allow the lack of squareness of the corners of the glass panels to be corrected for and concealed. The use of any wood at all is an undoubted disadvantage. However, half of each fixed coil, half of the damping box, and the entire moving system are supported entirely by the stationary glass panel. The other half of the fixed-coil system and the other half of the damping box are supported by the second glass panel, which is attached to the first panel by the hinges and the mahogany frame. Any warping of the wooden frames can result only in a displacement of the entire second panel with respect to the first panel. The possibility of warping was reduced to a minimum by carefully varnishing the mahogany inside and out.

In order to provide an immediate and visible check on any gross misalinement of the two glass panels with respect to each other, two groups of fiducial marks were etched on the panels. Those groups were placed at widely separated intervals on the panels. Each group consists of a large cross etched on the rear surface of the rear panel, a smaller cross on the rear surface of the front panel, and a still smaller cross on the front surface of the front panel. With the instrument as originally assembled, the panels properly alined and the moving system free from sticking, the three crosses constituting each group lie accurately on a straight line perpendicular to the face of the panels. They may therefore be lined up by eye so as to fall one upon the other. Any subsequent displacement of one of the panels with respect to the other will be evident as a displacement of these fiducial marks from a straight line. It will be possible to bring the panels back into their original alinement by inserting suitable shims between the glass panels and the wooden frames.

The glass panels were made of plate glass of rectangular shape, 1 in. thich and 8 in. wide by 44 in. long. The only machine work required was to cut the glass to size and drill holes through it for mounting the wattmeter parts. Round holes are readily put through glass by using a tubular tool in a drill press with carborundum and water as a grinding compound. Holes of other shapes can be made by drilling a series of small round holes around the outline desired and then smoothing down the edges with a flat brass tool worked by hand with carborundum. The only prerequisite in working this glass was found to be patience. The location and size of the holes drilled in the glass panels are shown on the assembly drawing of the wattmeter in figure 1.

The fixed coils were mounted on the glass panels by first embedding them in bakelite holders which could be inserted into round holes in the glass panels. An attempt was made to mold the fixed coils in to the Bakelite holders under heat and pressure by constructing a die which was used in a vulcanizing press. The heat and pressure of the press destroyed the insulation of the coils and the attempt had to be abondoned. The die was remade for molding cylindrically shaped 
coil holders closed at one end. The fixed coils were cemented into these holders with sealing wax. Coils thus made have been in service 5 years now and still appear to be very solid. The coils in their holders were fixed in the glass panels with fiber shims pressed and cemented in place with a cellulose-acetate cement. The Bakelite holders were slightly smaller than the holes, and the holes in the glass were slightly conical to permit this construction. In constructing another instrument it might be desirable to make the holes even more conical deliberately for this purpose.

The damping box was made of molded Bakelite. It was molded in two pieces, the same die being used for each piece. Each half of the damping box fits into an opening in the bottom of each glass panel, as is shown in figure 1, and was fixed firmly in position by small wedges cemented in place. An opening was also made in the upper half of the movable glass panel to allow the free passage of a beam of light to and from the mirror attached to the moving system. The trim surrounding this opening was also made of molded Bakelite. A thin piece of cover glass, cemented on the rear surface of this piece of trim, allows the passage of light but protects the interior of the instrument from the stray air currents of the room.

\section{(b) FIXED COILS}

To obtain a high sensitivity for the completed instrument, the flux density at the moving coils, due to current flowing through the fixed coils, should be as great as possible. This would require a maximum number of ampere turns concentrated in a small space as a fixed coil. A limit as to the size of wire for the fixed coils, and thus to the allowable current flow through the instrument, is set by the introduction of eddy currents and skin effects in the larger sizes of wire. Number 16 copper wire, for which eddy currents and skin effects are negligible at power frequencies, was used in winding the fixed coils. A limit to the number of turns in the fixed coils is set by the introduction of capacitance currents. To keep these capacitance currents low, and to obtain some flexibility in current rating, four No. 16 silk-enamel copper wires were formed into a cable. These cables were then wound into tight coils (32 turns of cable per coil) on a coil-winding machine. A piece of fiber tubing, turned to the correct inside diameter, was slipped over the coil after completion of the winding; the coil and tubing were lashed together and repeatedly soaked in insulating varnish and baked. After the final baking, the fiber tubing was stripped from the coil and the coil mounted in the molded coil holders previously mentioned, with the aid of sealing wax. These coil holders were in turn mounted in the glass panels as previously indicated (see fig. 1).

Two different types of fixed coil were made (see fig. 2 for the shapo and dimensions of each style of coil). Tests on the completed wattmeter showed that style $B$ gave the better performance, and coils of this style are permanently mounted on the glass panels.

Four of these coils are required for the completed instrument, two mounted in each glass panel in such a manner that with the panels closed and face to face two complete fixed coils are formed, one above the other. Sufficient space is left to allow a moving coil to be suspended in the center of each of the two fixed coils so formed. The cables of the four individual coils were connected in series in such a manner that the flux of the upper coil is in the direction opposite 


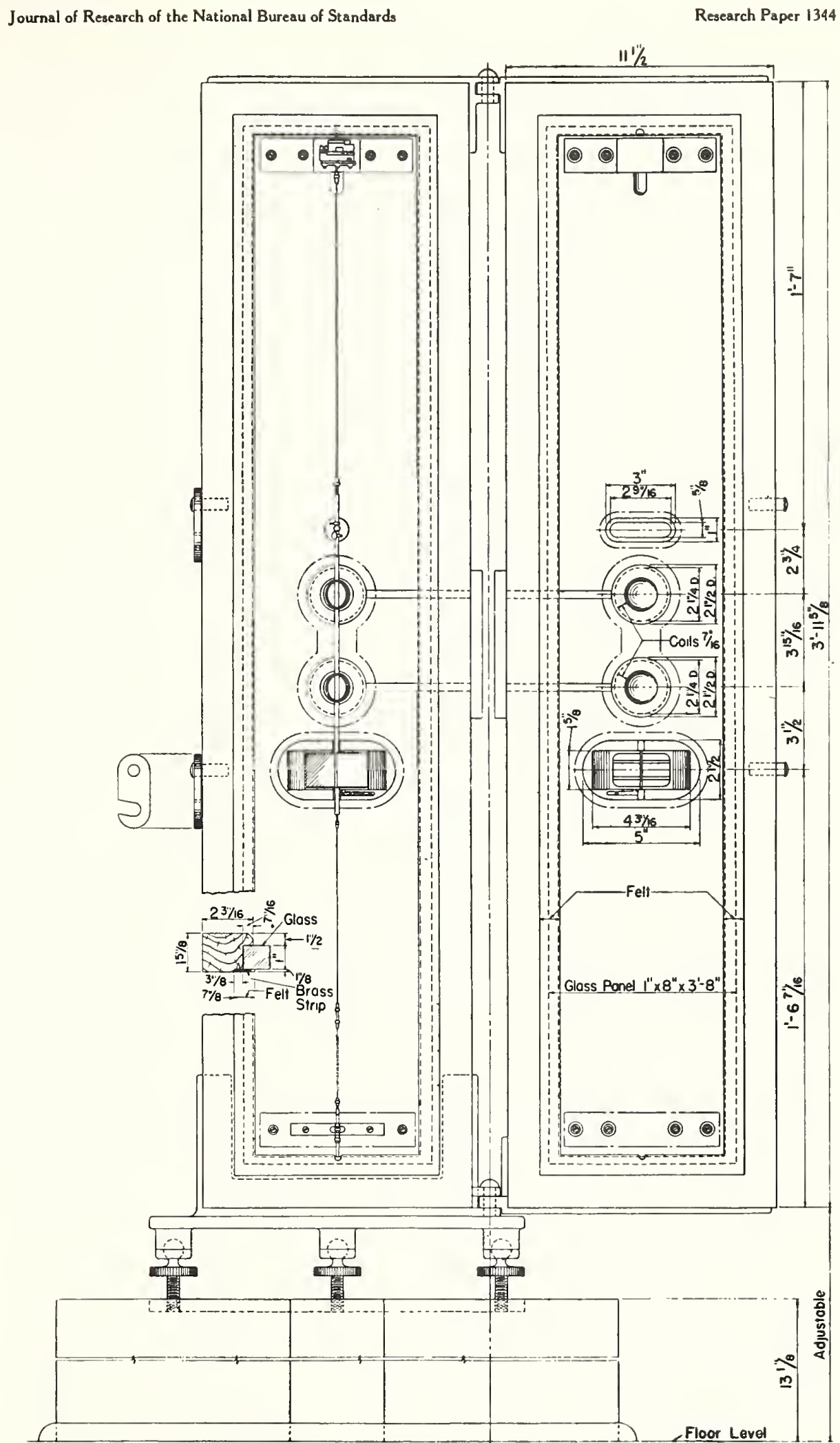

FIGURE 1.-Drawing of the complete wattmeter, with front panel open. 


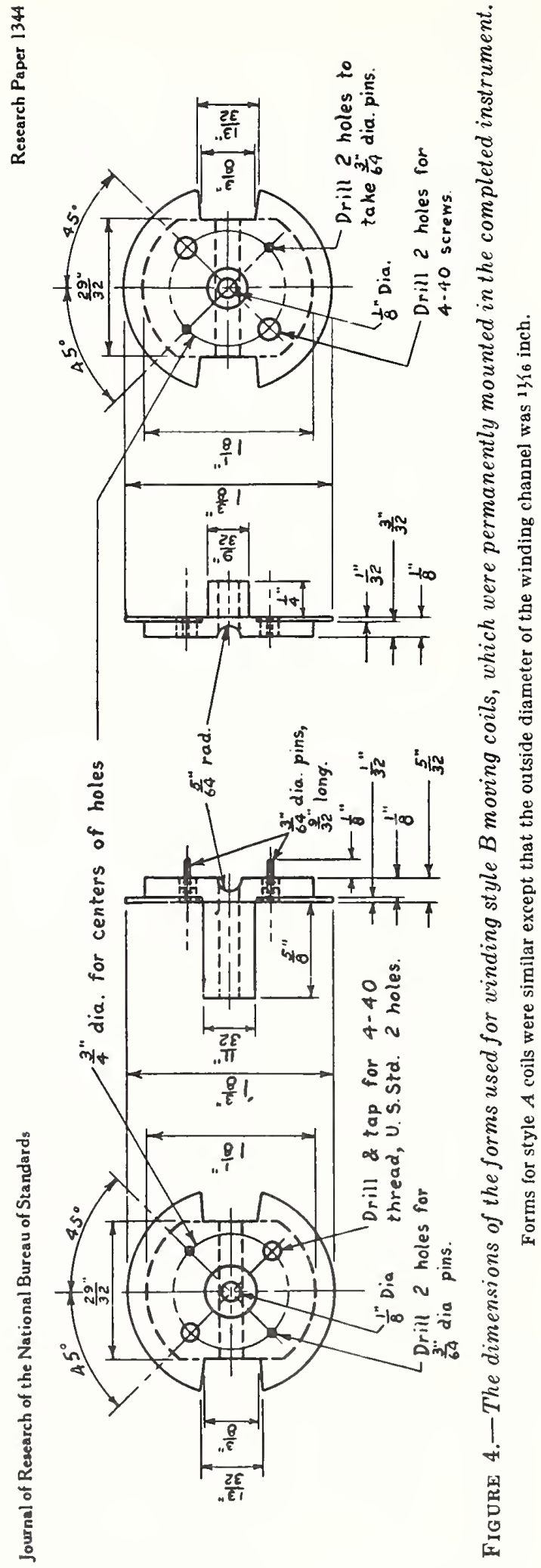


to the flux of the lower coil, thus giving astatic construction. The two ends of the fixed-coil cable were brought out to a terminal board located on the wall near the wattmeter. The terminal board is arranged so that the four coils formed by the four wires in the fixed coil cable can be connected in series, series-parallel, or parallel.

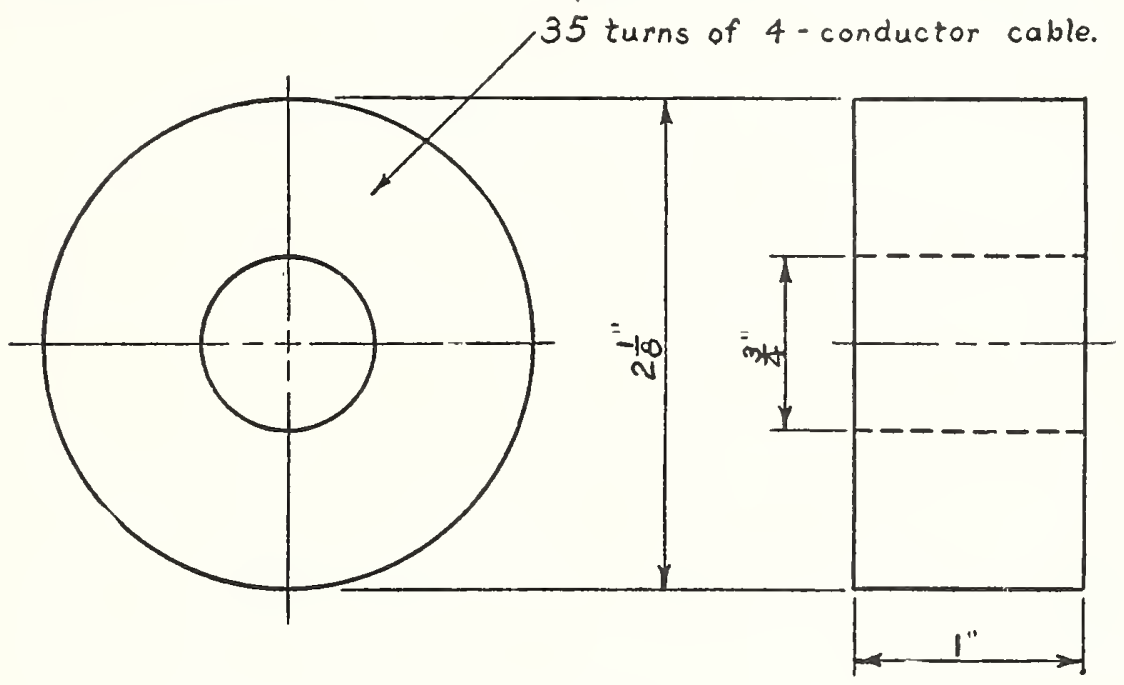

Style A

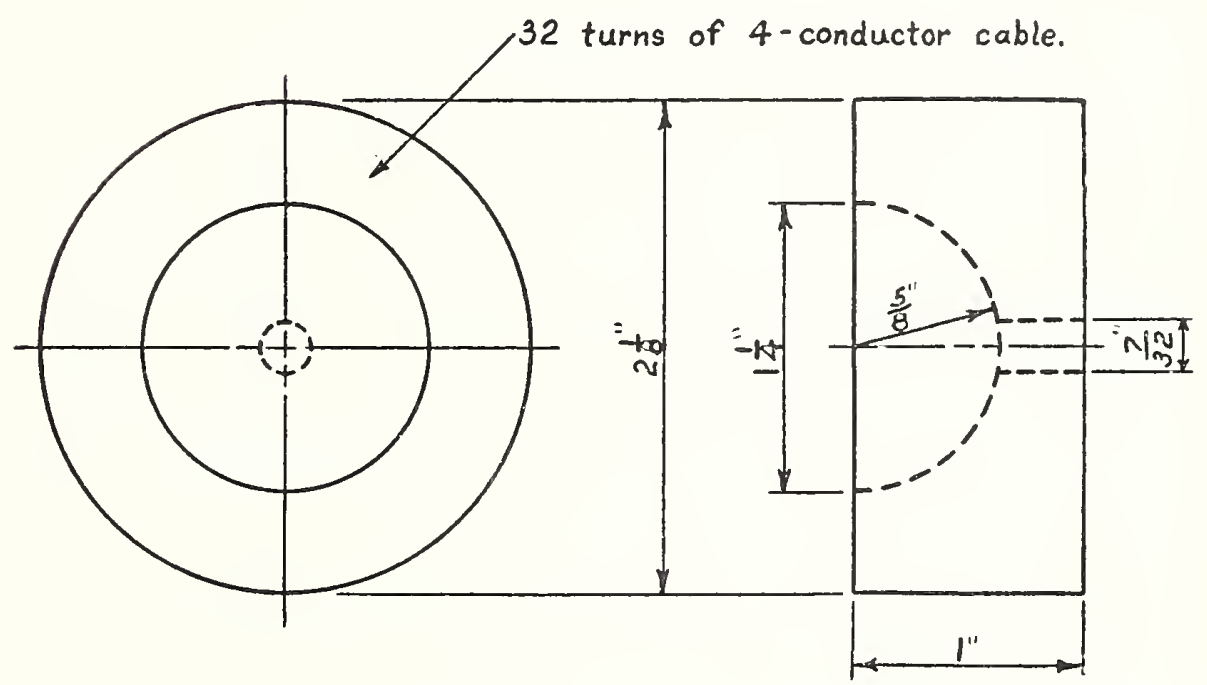

Style $B$

FIGURE 2.-The dimensions of the two styles of fixed coils. Style $B$ coils were permanently mounted in the wattmeter. 
The current rating of the instrument is limited by the heating of the fixed coils. Each wire of the fixed-coil cable has a resistance of $0.25 \mathrm{ohm}$. Experimental heating runs were made with the fixed coils mounted in their working positions and with 2.5 amperes flowing in each wire of the fixed-coil cable. Under these conditions the fixed-coil system experienced a temperature rise of about $10^{\circ} \mathrm{C}$ when the current had been flowing for about $2 \frac{1}{2}$ hours. Previous experience has indicated that the position of the moving coils becomes uncertain, because of convection currents in the air, if the temperature rise in the fixed coils is much more than $10^{\circ} \mathrm{C}$. 'The current rating of the fixed coils has therefore been set at $2.5 \mathrm{amp}$ with the four wires in series, $5 \mathrm{amp}$ with the four wires in series-parallel, and $10 \mathrm{amp}$ with the four wires in parallel. These current ratings are conservative on a self-heating basis and might be exceeded by moderate amounts with some sacrifice in accuracy if necessary.

\section{(c) MOVING SYSTEM}

In order to provide a rigid connection between the various parts of the moving system, ${ }^{5}$ all the components of the moving system are mounted on a stiff Bakelite tube, $3 / 10$ in. in outside diameter and $13 \frac{1}{2}$ in. long. This tube is suspended in a vertical position by means of two phosphor-bronze suspension ribbons. These ribbons, 1.5 mils thick and 20 mils wide, are mechanically attached to the tube by means of special brass and aluminum chucks fastened on the ends of the tube. The shank of each chuck is so milled as to allow the suspensions to come through under the shell. Thus the moving coil can be soldered directly to the end of the ribbon to form a definite electric contact, and the chuck jaws make a definite mechanical connection on a portion of the suspension which has not been softened by heating with a soldering iron. This construction minimizes zero shift and the failure of soldered joints in the moving system.

The upper suspension ribbon extends $12 \mathrm{in}$. above the chuck on the top end of the Bakelite tube, and there it is fastened to a similar chuck which is supported by an adjustable head attached to the top of the stationary glass panel. This head is so built that the chuck attached to it can be moved independently along any one of the three coordinate axes and can also be rotated about the vertical axis. The mechanism of this adjustable head is shown in figure 3 .

The lower suspension ribbon extends 8 in. below the chuck on the bottom end of the Bakelite tube, and there it is coupled by means of a double-end chuck, similar to those already described, to the top of a phosphor-bronze coil spring. The bottom of this coil spring is attached to another chuck which is supported from the bottom of the stationary glass panel by means of a machine screw that allows a vertical adjustment for setting the tension on the suspension. This tension is adjusted to about $230 \mathrm{~g}$, but the wattmeter performance is not greatly affected by changes in this value.

In order to make the mechanical zero of the instrument relatively insensitive to small alterations in the leveling of the instrument, it is

\footnotetext{
${ }^{5}$ If the entire moving system is not rigidly interconnected, it is possible, particularly at very low power factors, for forced internal vibrations to take place between its component parts. The result is a broadening of the reflected spot of light. Beeause of the varying scale law of the instrument, particularly if the moving coils are not at the position of zero mutual, the truc posittion of the spot of llght will not correspond to the midpoint of this broadened band of light. The apparent indication of the instrument will thesefore be in error.
} 
desirable to have the center of mass of the moving system coincide with the line of suspension of the moving system. Weights for balancing the moving system are therefore attached to the Bakelite support rod by the use of a brass collar with three small threaded rods (120 degrees apart) projecting from the collar. The balancing weights consist of sniall thumb nuts fitting these threaded rods.

Two sizes of moving coils were made originally, one with a 13/16-in. outside diameter to fit style $A$ fixed coils, and the other with a $18 / 1$. in. outside diameter to fit style $B$ fixed coils. Because of the greater sensitivity of the wattmeter with this latter combination, as explained in section $d$, Preliminary Tests of Wattmeter, style $B$ fixed coils and

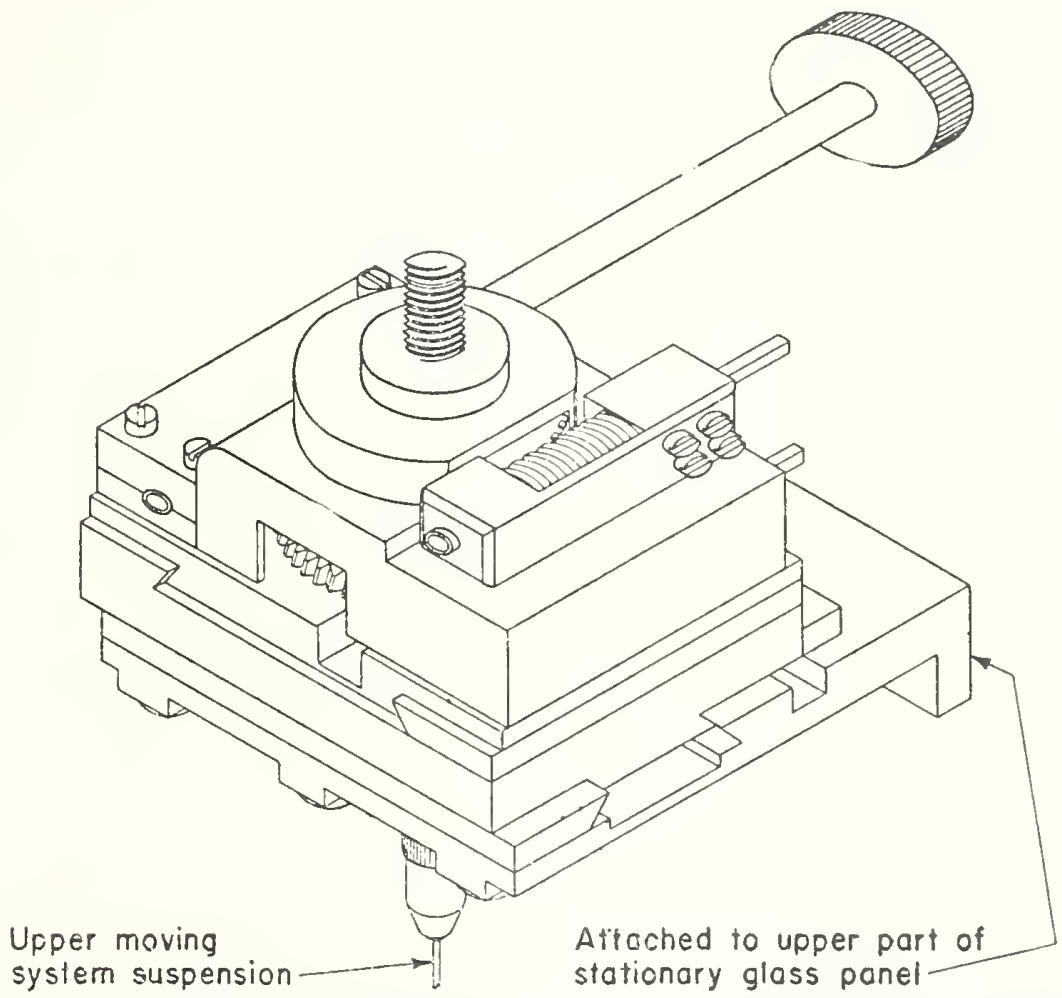

Figdre 3.-Details of the movable head to which the upper end of the suspension is attached.

the larger moving coils were chosen for permanent mounting in the wattmeter.

The details and dimensions of the brass forms used for winding these coils are given in figure 4. Each coil contains 100.5 turns of No. 32 silk-enamel copper wire. After each layer of a coil was wound (about 19 turns), a coat of thin shellac was applied and allowed to dry. This served to hold the completed coil together after removing the form. The completed coil was then dipped in thin insulating varnish and baked at about $120^{\circ} \mathrm{C}$ for 24 hours. This procedure was repeated four times to form a tough protective coating over the coil and to give it rigidity. The coils were mounted on the moving system by inserting the suspension tube through the two holes in the 
top and bottom of the coil. After the moving coils were located on the tube so as to fit into the hollow centers of the fixed coils and the two moving coils were twisted into the same plane, they were lashed to the tube with silk thread and shellac was applied to hold them rigidly to the tube. The two moving coils were connected in series in such a manner that the direction of winding on the upper coil is reversed with respect to the direction of winding on the lower coil. The two remaining free ends of the coils were then soldered to the upper and lower suspensions.

The damping vane, $1 \frac{1}{2}$ in. ligh by 4 in. wide, is made of $10-\mathrm{mil}$ sheet aluminum with the edges rolled for stiffness. This damping vane is riveted to the suspension tube at the proper distance below the lower moving coil to fit into the damping box when the moving system is in place.

Two mirrors are attached to the suspension tube above the upper moving coil by the use of brass collars and spiders. One mirror $(3 / 8$ in. in diameter) is located so that with the moving coils in the position of zero mutual inductance with the fixed coils it reflects a beam of light from a straight filament lamp onto the center of a $50-\mathrm{cm}$ groundglass scale located $2 \mathrm{~m}$ directly in front of the wattmeter. The other mirror ( $1 / 4$ in. in diameter) is located on the tube so that its plane makes an angle of 60 degrees with the plane of the first mirror. The mechanical zero of the moving system is normally set so that the lightbeam reflected from this smaller mirror falls on the center of the groundglass scale when the instrument circuits are open. This allows any shifts in the mechanical zero to be readily detected.

\section{(d) PRELIMINARY TESTS}

Before designing a serıes resistor for the voltage circuit of the wattmeter, it was necessary to know the maximum allowable moving-coil current and the wattmeter sensitivity. The wattmeter was therefore set up in its operating position and the optical system adjusted until the spot of light from the $3 / 8$-in. mirror fell on the center of the groundglass scale when the moving coils were in their position of zero mutual inductance with the fixed coils. The position of zero mutual inductance is readily determined by passing an alternating current through the fixed coils and shorting across the moving-coil terminals. If the moving coils are in the zero mutual position, there will be no deflection of the moving system. If they are not at the position of zero mutual, there will be an emf induced in them and a current will flow when their terminals are shorted, causing a deflection towards the position of zero mutual. This test can be made as sensitive as desired by increasing the frequency of the current in the fixed coils.

A preliminary value of the sensitivity of the wattmeter with the moving coils in the position of zero mutual was determined by passing rated direct current ( $2.5 \mathrm{amp}$ per wire) through the fixed coils and measuring the direct current through the moving coils necessary to obtain a deflection of about $10 \mathrm{~cm}$ with a scale distance of $2 \mathrm{~m}$. The sensitivity with style $A$ fixed coils and the smaller moving coils was found to be $34 \mathrm{~cm} / \mathrm{ma}$ in the moving coil. The sensitivity for style $B$ fixed coils and the larger moving coils was found to be $125 \mathrm{~cm} / \mathrm{ma}$ in the moving coils. These latter were therefore permanently mounted in the instrument. This latter sensitivity might alternatively be 
expressed as 2.4 milliradians per ampere turn in the fixed system per ampere turn in the moving system.

The maximum allowable current through the moving coils is limited by the heating due to this current. A heating test was made with 2.5 amp through the fixed coils (all connected in series) and 0.1 amp through the moving coils. Under these conditions the moving coils showed a temperature rise of $9.5^{\circ} \mathrm{C}$ at the end of $2 \frac{1}{2}$ hours. A current of $0.1 \mathrm{amp}$ is therefore considered a safe value for the moving system. Higher current values might be used, but their use is not anticipated at this time.

(e) SERIES RESISTOR

The resistors to be used with the moving coils of this instrument to form the voltage circuit were designed to be adjustable over such a range that by means of this adjustment the instrument deflection could be brought to the same value for any nominal value of watts to be measured. A total deflection of $400 \mathrm{~cm}$ was chosen, thus allowing a change in deflection of 0.01 percent $(0.4 \mathrm{~mm})$ to be observed easily. With a sensitivity of $125 \mathrm{~cm} / \mathrm{ma}$ in the moving coils for rated current in the fixed coils, a plain series resistor must have a maximum resistance of $337 \mathrm{ohms}$ per volt; that is, about 100,000 ohms for the maximum voltage range of $300 \mathrm{\nabla}$. The minimum fixed-coil current (with fixed coils connected in series) at which the wattmeter is expected to be used is $0.25 \mathrm{amp}$ (25-percent load on the 1-amp range of a watthour meter). This requires a maximum moving-coil current of $0.03 \mathrm{amp}$ to obtain a deflection of $400 \mathrm{~cm}$. It was decided to design the resistor for a maximum current of $0.06 \mathrm{amp}$ and to provide a circuit arrangement which would give a moving-coil current equivalent to that which would be obtained if a plain series resistor were used with a maximum resistance of $200,000 \mathrm{ohms}$.

The arrangement of resistors and their electric connections designed to meet these requirements is shown in figure 5. A fixed resistance of 985 ohms is connected in series with the wattmeter moving coil, and a capacitance of 0.001 microfarad is connected across a $937-0 h m$ section of this resistor to compensate for the inductance of the moving coils. The "series resistance" consists of nine $1-\mathrm{ohm}$, nine $10-\mathrm{ohm}$, nine 100-ohm, and nine 1,000-ohm coils connected to four 10-step selector switches so that any resistance from zero to 9,999 ohms, in 1-ohm steps, may be connected. The "shunt resistance" is connected in parallel with the moving coil and the $985-\mathrm{ohm}$ resistor. The value of this shunt resistance may be set at $\infty, 1,000,400,200,100$, or $50 \mathrm{ohms}$ by means of a 6 -step selector switch. The purpose of the shunt resistance is to decrease the current through the moving coil, which, as regards voltage sensitivity, is the equivalent of increasing the series resistance. This allows an effective series resistance of $200,000 \mathrm{ohms}$ to be attained while the actual value of the series resistor is kept below 10,000 ohms. The coils used in the shunt resistance (1,000 ohms and lower) are much cheaper and less apt to give trouble than the 10,000-ohm coils that would be needed in the series resistance if a shunt resistance were not used. In addition the capacitance errors of the $1,000-\mathrm{ohm}$ coils will be much less than the capacitance errors of $10,000-$ ohm coils.

A 1- $\mu$ f paper capacitor can be connected across the 985-ohm resistance which is in series with the wattmeter moving coils by means of the push-button switch marked "P. F. test." When this capacitor is 
connected, the current through the moving coils is made to lead in phase with respect to the supply voltage. Thus, if the current supplied to the wattmeter fixed coils is lagging in phase with respect to the supply voltage, pushing the "P. $\mathrm{F}$. test" button will cause the

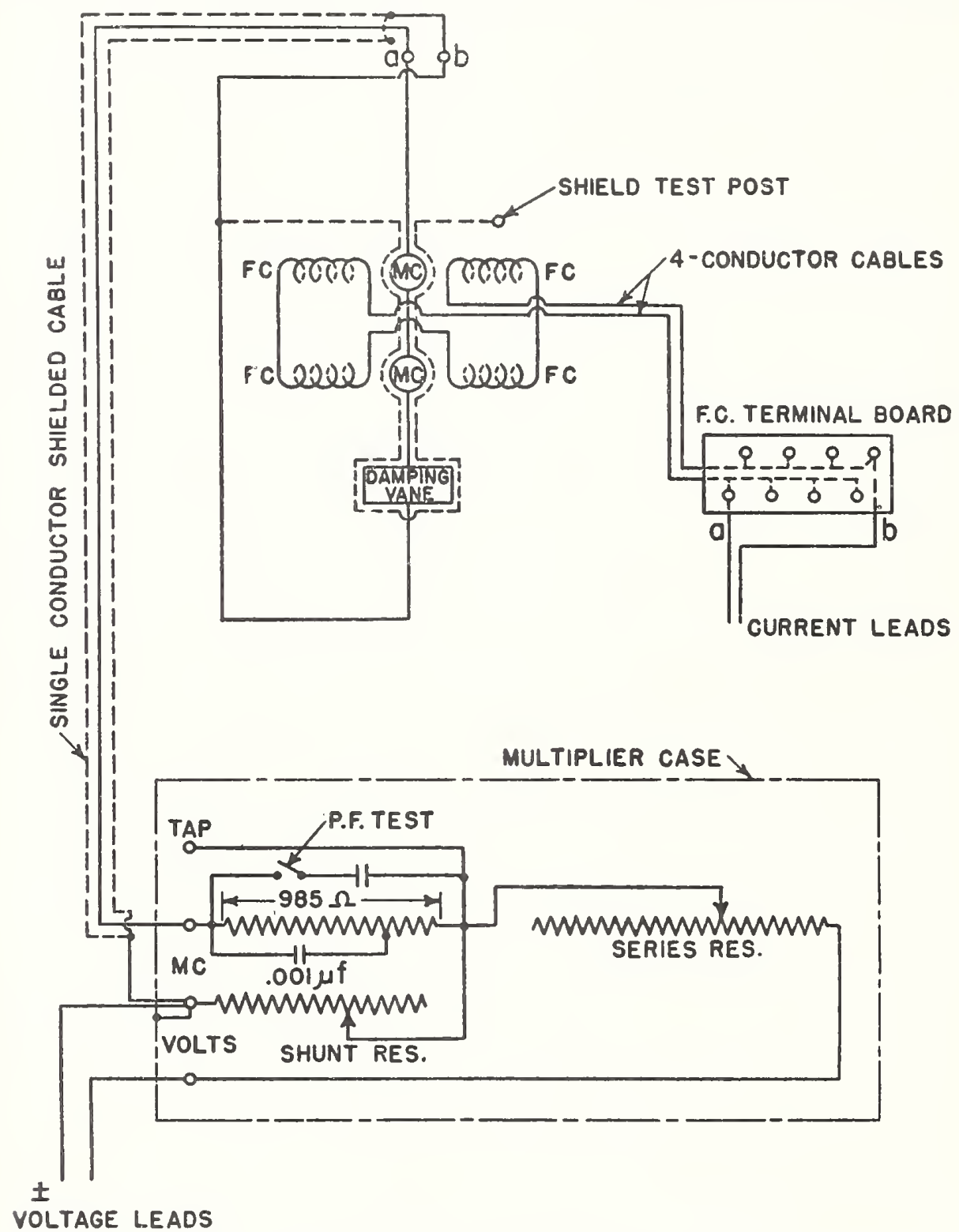

FigURE 5.-Schematic diagram of the wattmeter and multiplier connected for measuring power, showing shield circuils.

wattmeter deflection to decrease; if the current is leading, pushing this button will cruse the wattmeter deflection to increase. Because of the slight decrease in impedance of the wattmeter voltage circuit when the 1- $\mu$ f capacitor is connected, this "leading" or "lagging" test is ambiguous when the power factor is greater than 0.9 . 
The resistance units used in this multiplier are all of commercial construction, except an 85-ohm section of the 985-ohm resistor connected in series with the moving coils. This $85-\mathrm{ohm}$ resistor was made in this laboratory. It is of bifilar construction in two sections, allowing a tap to be brought out at the proper point for connecting

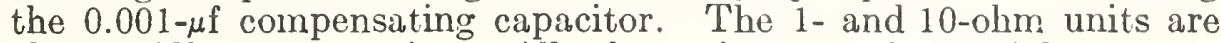
also of bifilar construction. All other units were of a special "woven" construction which reduces the time constant to the lowest value attainable for precision resistors. They are mounted on porcelain tubes $7 / 8$ in. in diameter and $3 \frac{1}{2}$ in. long and then dipped in "Ceresin" wax. The units are all made of manganin wire, and with a current of 60 ma their maximum temperature rise was specified to be less than $25^{\circ} \mathrm{C}$. In order to obtain this current rating, the $1,000-\mathrm{ohm}$ units are each made up of four 4,000-ohm windings mounted on separate spools and connected in parallel.

All the component parts of the multiplier are mounted on a laardrubber panel $1 / 2$ by $11 \frac{1}{2}$ by $201 / 2$ in. This hard-rubber subpanel is concealed from exposure to light by an aluminum panel 1/8-in. thick placed $1 / 2$ in. in front of it and attached to it by brass screws and hardrubber spacers. The shafts of the selector switches and the binding posts protrude through the aluminum panel without touching it. These two panels are screwed to an aluminum base $1 / 4$ by 5 by $20 \% \frac{1}{2}$. A one-piece brass cover is bent up to fit over the top, back, and two sides. The completed multiplier box is fastened in an upright position at the back of the observer's table, the ground-glass scale being located 5 in. in front and 3 in. above it.

\section{(f) SHIELDING}

In order to minimize the effect of capacitance currents between the moving and fixed coils and to prevent the accumulation of electrostatic charges near the moving coils or damping vane, it is usual to provicle a conducting screen or shield between the fixed and moving parts. In the present instrument conducting paint was used in making this shield. Four $3 / 8$-in. stripes are painted along the axes of great circles on the inside of the hemispherical depression in each fixed coil so that they cross each other at the deepest point in the depression. The inside surface of each half of the damping box is coated with this paint, the glass windows being left clear except for two $3 / 8$-in. stripes painted across them. All sections of the shield are connected in series by a $1 / 8$-in. stripe of conducting paint, the connection from the stationary glass panel to the hinged pancl being made by a spring contact between the two halves of the damping box. One end of this series circuit is connected to the moving-coil lead going to the bottom suspension (the damping vane is soldered to the lead from this suspension to the lower moving coil). The other end of the series circuit is brought out to a binding post on the mahogany frame of the hinged panel. A measurement of the resistance between this binding post and a moving-coil terminal thus serves as a check on the continuity of the shield. This resistance as measured by a $1 \frac{11}{2}-\mathrm{v}$ ohmmeter is about 50,000 ohms.

A schematic diagram of the location and interconnection of the shields used in the instrument is shown in figure 5 . The wattmeter moving coil is connected to the multiplier by a single-conductor 
shielded lead with rubber insulation over the shield (the capacitance between conductor and shield being $710 \mu \mu \mathrm{f}$ ). At the wattmeter end, the shield of this lead is connected through a binding post to the lower moving-coil suspension, which is also tied to the wattmeter shield. The central wire of this lead is connected through a binding post to the upper moving-coil suspension. At the multiplier end, the shield of this lead is connected to the lower moving-coil "MC" binding post (the " \pm " supply voltage lead to which the electrostatic tie between current and voltage circuits is attached is also connected to this binding post). The central wire of the shielded lead is connected to the upper moving-coil binding post. The multiplier metal case is tied to the lower moving-coil binding post and thus acts as a shield for the multiplier.

(g) WATTMETER CONSTANTS

(1) Fixed coils.

1. Connected in series $(2.5 \mathrm{amp})$, resistance $0.848 \mathrm{ohm}$, self inductance $1,300 \mu \mathrm{h}$.

2. Connected in series-parallel (5 amp), resistance $0.212 \mathrm{ohm}$, self inductance $304 \mu \mathrm{h}$.

3. Connected in parallel (10 amp), resistance $0.053 \mathrm{ohm}$, self inductance $72 \mu \mathrm{h}$.

4. Mutual inductance between any two of the four fixed coils, $85 \mu \mathrm{h}$.

5. Average capacitance between two of the four fixed coils, $0.003 \mu f$.

6. Capacitance from fixed coils to shield, $200 \mu \mu \mathrm{f}$.

7. Maximum heating of fixed coils with rated current, $10^{\circ} \mathrm{C}$.

8. Flux density at moving coils with rated current flowing in fixed coils, 110 gauss.

(2) Moving system.

1. Resistance of single moving coil, $5.3 \mathrm{ohms}$.

2. Resistance of entire moving system (between terminals on back of wattmeter), $15.30 \mathrm{ohms}$.

3. Self inductance of moving system, $830 \mu \mathrm{h}$.

4. Mutual inductance between fixed coils (connected in seriesparallel) and moving coils at position of mechanical zero (58 degrees from position of zero mutual inductance between fixed and moving coils), $135 \mu \mathrm{h}$.

5. Capacitance between moving system and shield, $52 \mu \mu \mathrm{f}$.

6 . Capacitance between moving system and fixed coils (with shield at same potential as moving system), $9 \mu \mu \mathrm{f}$.

7. Maximum heating of moving coils with $0.1 \mathrm{amp}$ flowing through them and rated current through fixed coils, $10^{\circ} \mathrm{C}$.

8. Total weight of moving system, $18 \mathrm{~g}$.

9. Period of moving system, 8 sec.

10. Torque required to deflect moving system from mechanical zero to full scale (position of zero mutual), 30 dyne $\mathrm{cm}$.

11. Stiffness of suspension, 0.5 dyne $\mathrm{cm} / \mathrm{deg}$.

12. Natural frequency of internal resonance; with direct current on fixed coils and alternating current on moving coils maximum vibration occurs at $80 \mathrm{c} / \mathrm{s}$. 


\section{PERFORMANCE TESTS}

\section{DIRECT-CURRENT ACCURACY}

Before the ac-dc errors were determined, the accuracy of the instrument assuming no ac-dc errors, that is d-c accuracy, was investigated. The factors effecting the d-c accuracy are (1) drift of deflection with time for a constant value of power, (2) variation of wattmeter sensitivity for small changes in deflection, and (3) imperfection of astatic construction.

With a constant value of current in the fixed coils and a constant voltage across the moving-coil circuit, the deflection of the wattmeter was noted during a period of 2 hours. The drift in deflection was found to be very nearly a linear function of time and equal to 0.1 $\mathrm{mm} / \mathrm{min}$. In ac-dc transfer tests the effect of this drift is cancelled in the average a-c and d-c readings by the sequence in which the readings are taken. When the instrument is used as a standard watimeter, it is calibrated on direct current before and after the a-c readings are taken so that here also the effects of drift tend to cancel. Even if the drift were not cancelled by the sequence of taking readings, the maximum error caused by it would be only 0.025 percent in 10 minutes.

In an ac-dc transfer test of a wattmeter that is neither magnetically shielded nor of astatic construction, the reading of the standard instrument may be several percent different for one direction of current and voltage than for the reversed direction; also, the a-c reading may be slightly different from the d-c readings. Since the scale of the standard instrument is uniform, if its sensitivity is different for the various readings of the standard instrument in one set of data, an error will be introduced which may be as large as the percentage difference in sensitivity.

The sensitivity of the wattmeter can be derived from the following quantities:

$G=$ torque on moving system in dyne-centimeters per milliampere in the moving coils with rated current in the fixed coils.

$\alpha=$ angular deflection of moving system from the mechanical zero position as measured by the travel in centimeters of the working spot of light at a $2-\mathrm{m}$ radius from the mirror.

$U=$ restoring torque of suspension in dyne-centimeters per unit of angular deflection, $\alpha$.

$i=$ moving-coil current in milliamperes.

Since the summation of the torques on the moving system is zero, for any fixed position of the moving system

$$
i G-\alpha U=0 \text { or } \alpha=i \frac{G}{U},
$$

that is, the wattmeter sensitivity as deflection in centimeters per milliampere in the moving coils is $G$ divided by $U$. From Hooke's Law $U$ is constant but

$$
G=K \frac{d M}{d \alpha}
$$


where $M$ is the mutual inductance between the moving and fixed coils. Thus sensitivity is a function of the position of the moving coils with respect to the fixed coils but is independent of the mechanical zero or total deflection of the moving system.

The true value of sensitivity is obtained by measuring $\alpha$ for a given value of $i$ and dividing $\alpha$ by $i$. In the present wattmeter with a 50-cm scale and a total normal deflection from mechanical zero position of $400 \mathrm{~cm}$, it is not feasible to obtain an accurate value of $\alpha$ at normal deflection. However, the changes in $\alpha$ over the range of the $50-\mathrm{cm}$ scale can be accurately measured, and if the total deflection is known to a fairly close approximation for one point on the scale, the changes in sensitivity on the scale can be accurately determined.

The values of relative sensitivity (which are absolute values within 0.3 percent and which show changes in sensitivity over the scale range within 0.01 percent) were obtained by holding rated current through the fixed coils and measuring the moving-coil currents required to deflect the moving system from the normal mechanical zero position (beam of light from small mirror on center of scale) to various positions of the working spot on the scale at 5 -cm intervals. Since the sensitivity is very nearly constant at the point of zero mutual inductance, a reference value of sensitivity at this point was computed from the values of $\alpha$ and $i$ at points $5 \mathrm{~cm}$ to the right and left of this point, that is,

$$
\alpha_{B}=\frac{G_{0}}{U} i_{B} \quad \alpha_{L}=\frac{G_{0}}{U} i_{L}
$$

from which

$$
\frac{G_{0}^{Y}}{U}=\frac{\alpha_{R}-\alpha_{L}}{i_{B}-i_{L}}
$$

and the total deflection at the point of zero mutual inductance is

$$
\alpha_{0}=\frac{G_{0}}{U} i_{0}
$$

The total deflection at any other point on the scale was found by adding the difference between the scale reading at this point and the scale reading at the point of zero mutual to the total deflection at the point of zero mutual. This gave total relative deflections for various points on the scale which were as accurate as the scale could be read.

From these data the relative wattmeter sensitivity at any point on the scale was computed by dividing the total deflection at the point by the moving-coil current required to-deflect the wattmeter to this point. The relative sensitivity this obtained is in deflection (in centimeters) per milliampere in the moving coils with rated current in the fixed coils. A plot of this sensitivity against scale reading is shown in figure 6. This plot shows that there is a range of $10 \mathrm{~cm}$ near the point of zero mutual within which the sensitivity is constant to within 0.01 percent. Thus no corrections for wattmeter sensitivity are required for readings within this range. For readings outside this range corrections ${ }^{6}$ can be applied.

As seen from the curve of figure 6 , the point of zero mutual inductance between the fixed and moving coils (as indicated by the arrow)

\footnotetext{
6 For a computation of these corrections see appendix I.
} 
does not exactly coincide with the point of maximum sensitivity. If the flux density due to the fixed coils were uniform throughout the volume occupied by the moving coils, maximum sensitivity, that is maximum $d M / d \alpha$, would 'come at the point where $M=0$. The con-

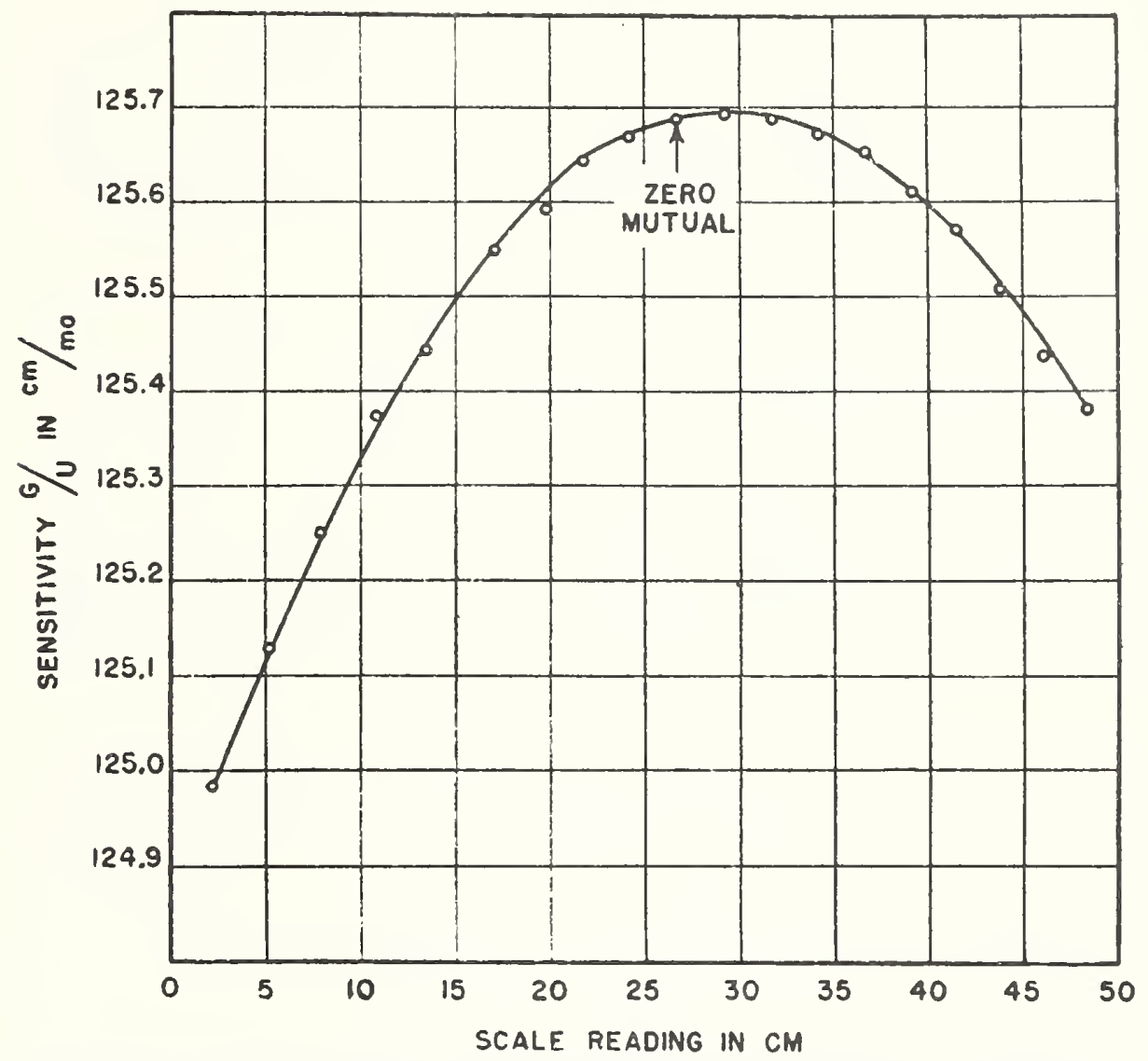

FIGURE 6.-Relative wattmeter sensitivily for various positions of working spot on the ground-glass scale in centimeters of deflections per milliampere in the moving coils with rated current in the fixed.coils.

The absolute wattmeter sensitivity is equa! to the relative sensitivity to within 0.3 percent.

clusion is that the flux density is not strictly uniform in the present instrument, and this is probably the case in most instruments of this type.

Imperfection of the astatic construction of the wattmeter results in a small deflection of the moving system with direct current through the moving coils only, because of interaction with the earth's magnetic field. This deflection was measured with $60 \mathrm{ma}$ (the maximum value to be used) through the moving coils for various positions of the moving system near the point of zero mutual inductance between the fixed and moving coils. These deflections as measured by the movement of the light beam on the ground-glass scale were found to be +0.07 $\mathrm{cm}$ for one direction of moving-coil current ard $-0.07 \mathrm{~cm}$ for the other direction, and they were the same for all positions of the light beam on the scale within $\pm 15 \mathrm{~cm}$ of the point of zero mutual inductance. Thus in using the instrument for an ac-de transfer test, even though 
the two $d-c$ readings are quite different, the small deflection due to lack of astaticism will be cancelled out in the average $d-c$ reading.

\section{ANALYSIS OF AC-DC ERRORS}

The present instrument was found to have ample sensitivity and its errors on direct current, as explained above, were not greater than 0.01 percent. However, this instrument was intended for use as an ac-dc transfer instrument and for this purpose its deflection for a given value of power must be the same on alternating current as on direct current. Before devising the actual tests for determining the ac-dc errors, it is advisable to analyze the various sources of such errors. They may be listed as follows:

1. Interaction between moving coils and other parts of the voltage circuit.

2. Magnetic impurities in the moving system.

3. Induced and capacitance currents in the moving system.

4. Eddy current or skin effects in the fixed coils.

5. Capacitance currents in the fixed coils.

6. Phase defect in the voltage circuit. ${ }^{7}$

The average deflection of a wattmeter for direct and reversed directions of a given direct voltage, $E_{d}$, across its moving-coil circuit and a given direct current, $I_{d}$, through its fixed coils may be expressed as

$$
\alpha_{d}=K E_{d} I_{d}
$$

If the direct current and voltage are replaced by an alternating current, $I_{a}$, and an alternating voltage, $E_{a}$, such that $E_{a} I_{a} \cos \theta=E_{d} I_{d}$, were $E_{a}$ and $I_{a}$ are simple sine functions of time and $\theta$ is the angle of lag of $I_{a}$ with respect to $E_{a}$, and if the wattmeter is free of ac-dc errors, the deflection on alternating current would be

$$
\alpha_{a}=K E_{a} I_{a} \cos \theta=K E_{d} I_{d}=\alpha_{d} .
$$

However, as noted above, there are a number of possible sources of ac-dc errors and these errors enter into the above equation as (1) " $\Delta m$ ", a percentage change in magnitude of $E_{a} I_{a},(2)$ " $\tau_{w}$ ", in effect, a change in the angle $\theta$, and (3) " $\Delta \alpha$ ", a change in $\alpha_{a}$, which is a function of $I_{a}$ or $E_{a}$ but not of the product $E_{a} I_{a}$. When these three factors are put into the equation, the deflection on alternating current becomes

$$
\alpha^{\prime}{ }_{a}=K E_{a} I_{a}(1+\Delta m) \cos \left(\theta+\tau_{v o}\right)+\Delta \alpha .
$$

and the difference between the average d-c deflection and the a-c deflection is

$$
\alpha^{\prime}{ }_{a}-\alpha_{a}=\Delta m K E_{a} I_{a} \cos \theta-K E_{a} I_{a} \sin \theta \sin \tau_{2}+\Delta \alpha
$$

neglecting second-order terms.

The sources of ac-dc error listed above may now be separated into three groups according to their entrance in eq 1 as " $\Delta m$ ", " $\tau_{w}$ ", or

\footnotetext{
7 In uncompensated electrod yamic instruments the largest source of this phase defect is the inductance of the moving coils. In the present instrument this inductance is compensated by a capacitor connected across a section of the scries resistor. Thus, this phasc defect (difference in phase between the moving-coil current and the voltage across the voltage circuit) depends upon (1) the efficacy of the compensation, (2) the small residual inductance or capacitance in the scries resistor, and (3) the small capacitance currents between various sections of the series resistor or from any of these sections to the multiplier caso.
} 
" $\Delta \alpha$ ". Those entering as " $\Delta \alpha$ " will be present either with voltage only or with current only applied to the wattmeter, and by properly choosing the experimental conditions each one of these errors can be measured separately. The errors entering as " $\Delta m$ " may be measured as a group by determining the ac-dc errors of the wattmeter at unity power factor (making $\sin \theta=0$ ), and the errors entering as " $\tau_{10}$ " may be measured as a group by determining the ac-dc errors of the wattmeter at zero power factor (making $\cos \theta=0$ ). The " $\Delta \alpha$ " errors will also be present in measurements at unity and zero power factor; but since the " $\Delta \alpha$ " errors can be determined independently, corrections can be applied for them.

\section{ERRORS DEPENDING ON E OR I SEPARATELY- " $\Delta \alpha$ "}

(a) INTERACTION BETWEEN PARTS OF VOLTAGE CIRCUIT

If there were appreciable interaction between the moving coils and other parts of the voltage circuit, it would cause a deflection of the moving system when voltage only (either alternating current or direct current) is applied to the wattmeter. In order to detect this effect if present, 100 volts alternating current (a d-c voltage would cause a deflection by reaction with the earth's magnetic field as explained above) were applied to the voltage circuit. No deflections were observed for values of series resistor from 2,000 to 10,000 ohms, and it was concluded that this interaction effect is negligible.

\section{(b) MAGNETIC IMPURITIES IN THE MOVING SYSTEM}

Although the utmost care was exercised in selecting materials for and construction of the wattmeter moving system, there is a possibility that the resulting system may contain magnetic impurities. If the magnetic impurity is not symmetrically distributed around the moving-system axis, it will cause the moving system to deflect with current through the fixed coils only. With magnetic impurities, such us "hard iron" filings, which are capable of retaining some magnetism, the deflection of the moving system would not be the same for alternating current in the fixed coils as for direct current and in the case of direct current it would depend on the direction of the current. With magnetic impurities of zero retentivity, the deflection of the moving system would be the same for alternating current as for direct current through the fixed coils but would vary as the square of the magnitude of this current. In measuring a given value of watts, the alternating current will be different from the direct current if (1) the alternating and direct voltages are different or (2) the power factor is other than unity. Thus, even though the magnetic impurities have zero retentivity, the deflection caused by them will not necessarily be the same for equal magnitudes of a-c and d-c watts bcing measured.

When the present wattmeter was first assembled, one of the tests consisted in putting current through the fixed coils, the moving-coil circuit being open, and noting the deflection of the moving system. Deflections as large as $2 \mathrm{~mm}$ (0.05 percent of normal full-scale deflection) were observed. The magnitude of these deflections varied with the orientation of the moving system with respect to the fixed coils but was independent of the frequency of the current through the fixed coils, indicating that the deflections were caused by magnetic impurities in the moving system. 
The component parts of the moving systen (1, moving coils; 2 , mirror; 3, collars and clamps for holding mirrors; 4, collar with adjustable weights for balancing the system; 5 , damping vane; and 6 , Bakelite tube upon which all other parts were mounted) were all made of materials that are normally considered to be nonmagnetic. With the moving system assembled it was inpracticable to determine which of the component parts contained magnetic impurities. A new moving system was constructed and each component part was tested for magnetic impurities before assembly. This test consisted in suspending the part, with a small mirror attached to it for reflecting a beam of light onto a scale, in the field of a strong electromagnet and noting the deflection of the beam of light when the coil of the electromagnet was energized. These tests showed that a Bakelite tube, obtained from the same source as the tube used in the first moving system, contained considerable magnetic impurities which appeared to be concentrated in a plane containing the central axis of the tube. This would account for the results obtained with the first moving system in the wattmeter. In searching for a substitute for this tube, samples of glass, porcelain, clear Bakelite (no filler), and other resins were tested for magnetic impurities. It was found that tliese samples contained only very slight magnetic impurities in comparison with a sample of the Bakelite tube used in constructing the first moving system. Thus it was concluded that the filler used in making the Bakelite tube and not the Bakelite itself contained the magnetic impurity. Four samples of Bakelite tube were obtained from separate sources and tested for magnetic impurities. Of these four, one was chosen which contained about the same amount of magnetic impurity as the glass or porcelain, and a new moving system was constructed using a tube of this material for mounting the moving-system parts. Before mounting each component part on the new moving system, the system was suspended in the wattmeter and its deflection was noted with current through the fixed coils only. At each stage of construction this deflection was just barely noticeable with rated current through the fixed coils. When the moving system was completed, twice-rated current was passed through the fixed coils and measurable deflections of the moving system were obtained. The moving coils were then twisted around on the Bakelite tube until the position of zero mutual inductance between the fixed and moving coils (the position near which the moving coils are held when the wattmeter is in use) coincided with the position for minimum deflection of the moving system with current through the fixed coils only. The deflection of the moving system at this position was found to be about 0.2 $\mathrm{mm}(0.005$ percent of normal wattmeter deflection) with twice-rated current through the fixed coils. Thus it was concluded that the error due to magnetic impurities would be negligible with rated current through the fixed coils.

\section{c) INDUCED AND CAPACITANCE CURRENTS IN THE MOVING SYSTEM}

'The effects of induced and capacitance currents in the moving system increase as the frequency of the fixed-coil current is iucreased, and although they are entirely negligible at power frequencies (as shown in the tests made to detect magnetic impurities in the moving system) they may become measurable at frequencies above $1,000 \mathrm{c} / \mathrm{s}$. They may be detected by passing high-frequency current through the 
fixed coils with the moving coils open-circuited and noting any deflection of the moving system. When rated current at a frequency of $2,000 \mathrm{c} / \mathrm{s}$ was passed through the fixed coils, deflections of the moving system as large as $1 \mathrm{~cm}$ were obtained. These deflections are the result of a combination of the following effects: (1) eddy currents within the thickness of the wires forming the individual turns of the moving coils, (2) eddy currents in other parts of the moving system, such as the damping vane, and (3) capacitance currents flowing in some of the moving-coil turns and through the capacitance existing between the fixed and moving coils.

The summation of the torques produced by eddy currents in individual turns of the moving coils tends toward zero and would be exactly zero with the moving coils in the position of zero mutual inductance with the fixed coils if the fixed-coil flux were uniform throughout the area occupied by the moving coils. Any torque produced in the present wattmeter by the eddy currents in the moving coils may be combined with the torques produced by eddy currents in other moving parts of the wattmeter, since the torques due to all these eddy currents are similar functions of the same variables.

The three effects causing the deflections noted above may now be considered as two: (1) eddy currents -in moving system, and (2) capacitance currents flowing in some of the moving-coil turns. The deflection due to eddy currents will (a) increase approximately as the square of the frequency of the fixed-coil current, (b) increase as the square of the current through the fixed coils; but it will be independent of (a) the efficacy of the shield between the fixed and moving coils, (b) the location of the electrostatic tie between the fixed and moving coils, and (c) the connection of the fixed coils (series, series-parallel, or parallel). The deflection due to capacitance currents will (a) increase approximately as the square of the frequency of the fixedcoil current, (b) increase as the square of the current through the fixed coils, (c) depend upon the efficacy of the shield between the fixed and moving coils, (d) be approximately unchanged in magnitude but reversed in direction when the electrostatic tie between fixed and moving coils is changed from one terminal of the fixed coils to the other terminal, and (e) depend upon the fixed-coil connections, being largest for series and least for parallel. The total deflection caused by eddy currents and capacitance currents may be scparated into its two components by use of relation (d). For a given magnitude and frequency of fixed-coil current the deflection of the moving system was noted with (1) the \pm or $b$ terminal of the moving coils connected to terminal $a$ (see fig. 5 ) of the fixed coils, (2) the $b$ terminal of the moving coils connected to terminal $b$ of the fixed coils. The algebraic mean of these two deflections is the deflection caused by eddy currents, and one-half the algebraic difference between these two deflections is the deflection caused by capacitance currents.

From tests performed on the wattmeter with rated current at a frequency of $2,000 \mathrm{c} / \mathrm{s}$ through the fixed coils, the deflections due to eddy currents in the moving system and capacity currents in the moving coils were determined and the results are shown in table 1 . These tests were performed with the shield between the fixed and moving coils tied to the \pm side of the moving-coil circuit as shown in figure 5 . With this shield "floating," the deflections due to capacitance currents were found to be about three times those given in the table. 
TABLE 1.-Wattmeter deflections due to eddy currents and capacity currents

[Moving-coil circuit opened. Fixed-coil current frequency, 2,000 cycles. Shield tied to moving coil terminal $b$.]

\begin{tabular}{|c|c|c|c|c|c|}
\hline Fixed coil-connections &.$I_{0}$ & $\mathrm{MC}$ terminal $b$ tied to & $\begin{array}{l}\text { Total watt- } \\
\text { meter de- } \\
\text { lection }\end{array}$ & $\begin{array}{l}\text { Deflection } \\
\text { due to } \\
\text { eddy cur- } \\
\text { rents }\end{array}$ & $\begin{array}{l}\text { Deflection } \\
\text { due to } \\
\text { capaci- } \\
\text { tance } \\
\text { currents }\end{array}$ \\
\hline Series............. & $\begin{array}{r}a m p \\
2.5\end{array}$ & $\begin{cases}\mathrm{FC} & a \\
\mathrm{FC} & b\end{cases}$ & $\begin{array}{l}c m \\
0.47 \\
1.04\end{array}$ & $\begin{array}{l}\mathrm{cm} \\
0.76\end{array}$ & $\begin{array}{l}\mathrm{cm} \\
0.28\end{array}$ \\
\hline Series-parallel. . & 5 & $\left\{\begin{array}{l}\mathrm{FC} \quad a_{1} \\
\mathrm{FC} b\end{array}\right.$ & $\begin{array}{r}0.50 \\
.86\end{array}$ & .68 & .18 \\
\hline Parallel ... & 10 & $\left\{\begin{array}{l}\mathrm{FC} a \\
\mathrm{FC} b\end{array}\right.$ & $\begin{array}{l}.56 \\
.75\end{array}$ & .86 & .09 \\
\hline
\end{tabular}

The deflection of about $0.70 \mathrm{~cm}$ given in table 1 as due to eddy currents in the moving system seemed quite large. In order to determine which part of the moving system contained these eddy currents, a coil was placed near the wattmeter in such a position that, with a given value of current at $2,000 \mathrm{c} / \mathrm{s}$ passing through this coil, the magnetic field strength at the damping vane would be about the same as that produced by the wattmeter fixed coils at rated current and the magnetic field strength at other parts of the moving system would be low. With current flowing through this external coil and no current through the wattmeter coils, the deflection of the moving system was found to be about $0.50 \mathrm{~cm}$. Thus it appears that most of the deflection due to eddy currents in the moving system is caused by eddy currents in the damping vane. In constructing another wattmeter, it would be advisable either to increase the distance between the damping vane and wattmeter coils or to use as a domping vane some nonconducting material, coated with a very thin conducting film to eliminate accumulation of static charge.

Another possible source of induced currents in the moving system is the mutual inductance between the fixed and moving coils. These induced currents will flow only when the moving-coil circuit is closed and when the position of the moving system is not at the point of zero mutual inductance. With the moving system displaced 2 degrees of arc (a deflection of $15 \mathrm{~cm}$ on the scale) from the point of zero mutual inductance and with rated current at a frequency of $2,000 \mathrm{c} / \mathrm{s}$ through the fixed coil a deflection of $0.50 \mathrm{~cm}$ was noted when the moving-coil circuit was closed through an external resistance of 1,000 ohms (without a compensating capacitance). With positions of the moving system at which the wattmeter is generally used (within about $2 \mathrm{~cm}$ of the deflection at zero mutual inductance), no noticeable deflection was obtained when the moving-coil circuit was closed through a resistance of 1,000 ohms.

The only appreciable error, entering eq 1 as " $\Delta a$ ", was found to be that due to eddy currents in the moving system and capacitance currents in the moving coils. The magnitude of this error was measured at various frequencies up to $3,000 \mathrm{c} / \mathrm{s}$ with $5 \mathrm{amp}$ through the fixed coils connected in series-parallel and with the moving-coil circuit open, the shield and moving-coil terminal $b$ being tied to fixedcoil terminal $b$. The results of these measurements are shown by the curve plotted in figure 8. By means of these results the " $\Delta \alpha$ " 
errors can be separated from the total ac-dc errors measured in the experiments to be described.

\section{ERRORS DEPENDING UPON EI-TEST AT UNITY POWER-FACTOR}

(a) METHOD

A simple and convenient way to obtain unity power-factor conditions on the wattmeter is to connect it for use as an ammeter, as shown in figure 7. For these connections, the current in the moving

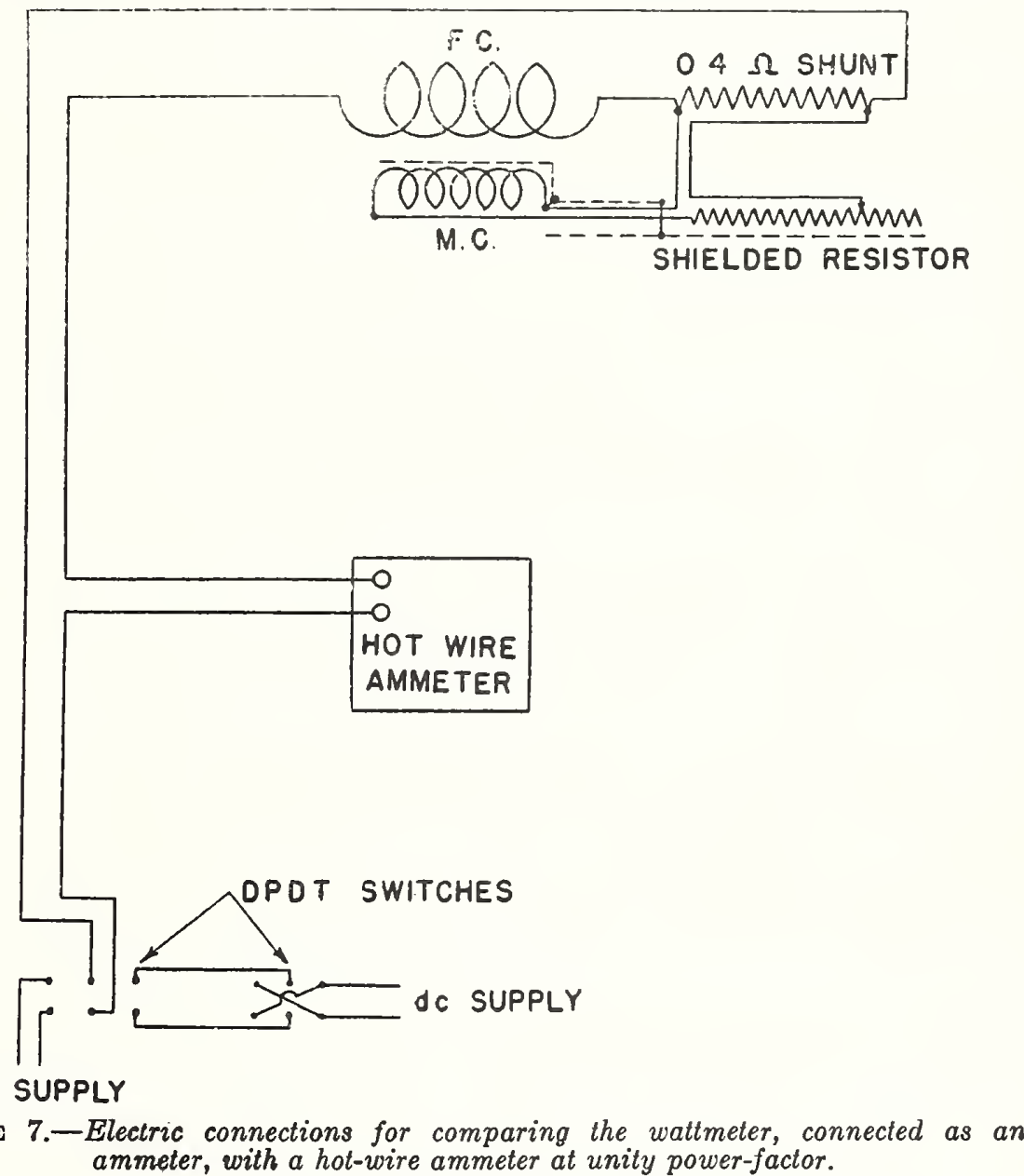

coil may be considered to be in phase with the current through the fixed coil. Any slight difference in phase $(\tau)$ between these currents will enter into the results as an error equal to $100(1-\cos \tau)$ in percent, and the conditions to be fulfilled in order to make the error negligible are easily attainable. Thus the only ac-dc errors entering this unity power-factor test will be those designated as " $\Delta m$ " and " $\Delta \alpha$ " in eq 1 , and these errors will be equivalent to the errors of the wattmeter when it is used to measure power at unity power-factor, provided the moving-coil circuit is properly shielded. 
The main reason for connecting the wattmeter as an ammeter to measure its ac-dc errors at unity power-factor ${ }^{8}$ was that a 5 -amp Hartman \& Braun hot-wire ammeter, which was constructed for the accurate measurement of radio-frequency currents, was readily obtainable and convenient to use as a standard. It was assumed that the ac-dc errors of the ammeter are negligible for frequencies up to $3,000 \mathrm{c} / \mathrm{s}$.

\section{(b) PROCEDURE}

The procedure consisted in adjusting the alternating- and directcurrent supplies independently, so that full-scale deflection of the hot-wire ammeter was obtained for both positions of the ac-dc

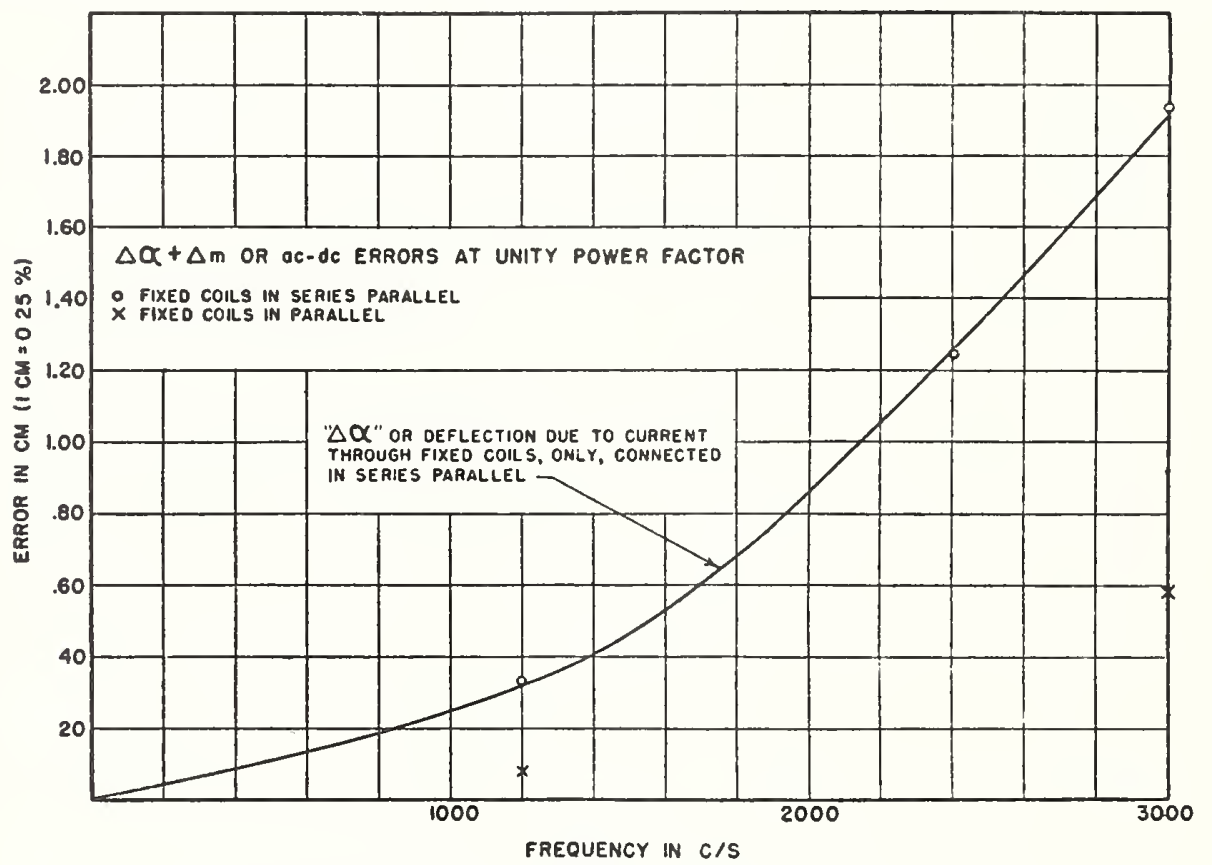

FIGURE 8.- Waltmeter ac-dc errors at unity power-factor.

Fixed-coll curtent equals 6 amp. Moving-coll terminai $b$ connected to fixed-coil tcrminal $b$ (see fig. 5).

The curve is a plot of " $\Delta \alpha$ " errors only". The piotted points include " $\Delta \alpha$ " and " $\Delta m$ " errors.

switch (see fig. 7). The series resistor in the moving-coil circuit of the wattmeter was adjusted to bring the wattmeter deflection to normal full-scale position (this required about $1,300 \mathrm{ohms}$ in the moving-coil circuit), and the current was left on for one-half hour to allow both instruments to come to temperature equilibrium. The test data were taken in the following order: (1) with alternating current of the desired frequency on, the value of current was adjusted to give full-scale deflection on the hot-wire ammeter and the deflection of the wattmeter was read;(2) with direct current on and adjusted to duplicate the setting on the hot-wire ammeter, the deflection of the wattmeter was read; (3) with direct current reversed, another reading of wattmeter deflection was obtained; and (4) with alternating current on, the first step was repeated. The dffference between the average a-c (steps 1 and 4 above) and the average d-c

${ }^{8}$ A quadrant electrometcr connected to measure power could bc usod as a standard inctrument for this purposo, but a conslderable amount of tlme and patlence would be required to set up and calibrate an instrument of this type so that the desired accuracy could be attalned. 
(steps 2 and 3 above) wattmeter deflections is a measure of the wattmeter ac-dc errors at unity power-factor.

The accuracy of the measurements is limited by the ability of the observer to repeat settings on the hot-wire ammeter. By using an instrument-reading camera focused directly on the pointer and adjusting current until the image of the pointer edge coincides with a fine line on the ground-glass screen of the camera, settings can be repeated to within 0.025 of a scale division. Since each value of ac-dc difference was the average of several sets of data, the accuracy of these average values was considered to be within about 0.01 of a scale division or 0.01 percent (settings were all made at the 100division point).

(c) RESULTS

For the purpose of comparison the results of these tests have been entered in figure 8. As already noted, the curve in this figure is a plot of the wattmeter deflection " $\triangle \alpha$ " against frequency, obtained on a previous test with $5 \mathrm{amp}$ through the wattmeter fixed coils connected in series-parallel and the moving-coil circuit open (this is the deflection due to eddy currents in the moving system and capacitance currents in the moving coils). The plotted points on this figure represent the values of ac-dc errors of the wattmeter at unity power-factor obtained by the tests just described. The agreement of the plotted points with the curve (with the fixed coils connected in parallel only one-half of rated current was used and the ac-dc error is correspondingly less) indicates that for frequencies up to $3,000 \mathrm{c} / \mathrm{s}$ the ac-dc errors of the wattmeter at unity power-factor are due only to eddy currents in the moving system and capacitance currents in the moving coils. These errors enter into eq 1 as the term " $\triangle \alpha$ ". The error designated as " $\triangle m$ " in this equation is thus found to be negligible (less than 0.01 percent) for frequencies up to $3,000 \mathrm{c} / \mathrm{s}$.

\section{ERRORS DEPENDING UPON PHASE ANGLE O-TESTS AT ZERO POWER-FACTOR}

The ac-dc errors of the wattmeter entering eq 1 as a phase defect " $\tau_{w}$ " will appear only when the wattmeter is used at power factors other than unity, and their effect will be maximum at zero powerfactor. Possible sources of phase defect are (1) eddy current or skin effects in the fixed coils, (2) capacitance currents in the fixed coils, and (3) phase defect in the voltage circuit. Since the phase defect introduced by these sources of error increases as the frequency increases, it was decided to determine the phase defect at several frequencies. Tests were made at $60 \mathrm{c} / \mathrm{s}$ using a quadrant electrometer and at $500,1,000$, and $2,000 \mathrm{c} / \mathrm{s}$ using mica capacitors.

(a) AT 60 CYCLES PER SECOND, USING QUADRANT ELECTROMETER

(1) Method.

The source of current and voltage consisted of two alternators driven by a direct-current inotor, the shafts of all these machines being coupled together. The motor was supplied from storage batteries, and the speed was adjusted to give a 60 -cycle output from each generator. The output voltage of each alternator and the phase angle between their voltages could be varied independently. One alternator was used to supply the voltage circuit of the standard wattmeter, and this 
same voltage was connected between the case and the needle of the quadrant electrometer. The other alternator was used to supply the current through the fixed coils of the wattmeter, and by also passing this current through a shunt and then stepping-up the drop across the shunt by means of a voltage transformer whose secondary was connected to the quadrants of the electrometer, a voltage was applied to the quadrants which bore a fixed relation to the current through the wattmeter fixed coils.

The voltage, $E$, from one of the alternators and the current, $I$, from the other alternator correspond to a value of power $E I \cos \theta$ which

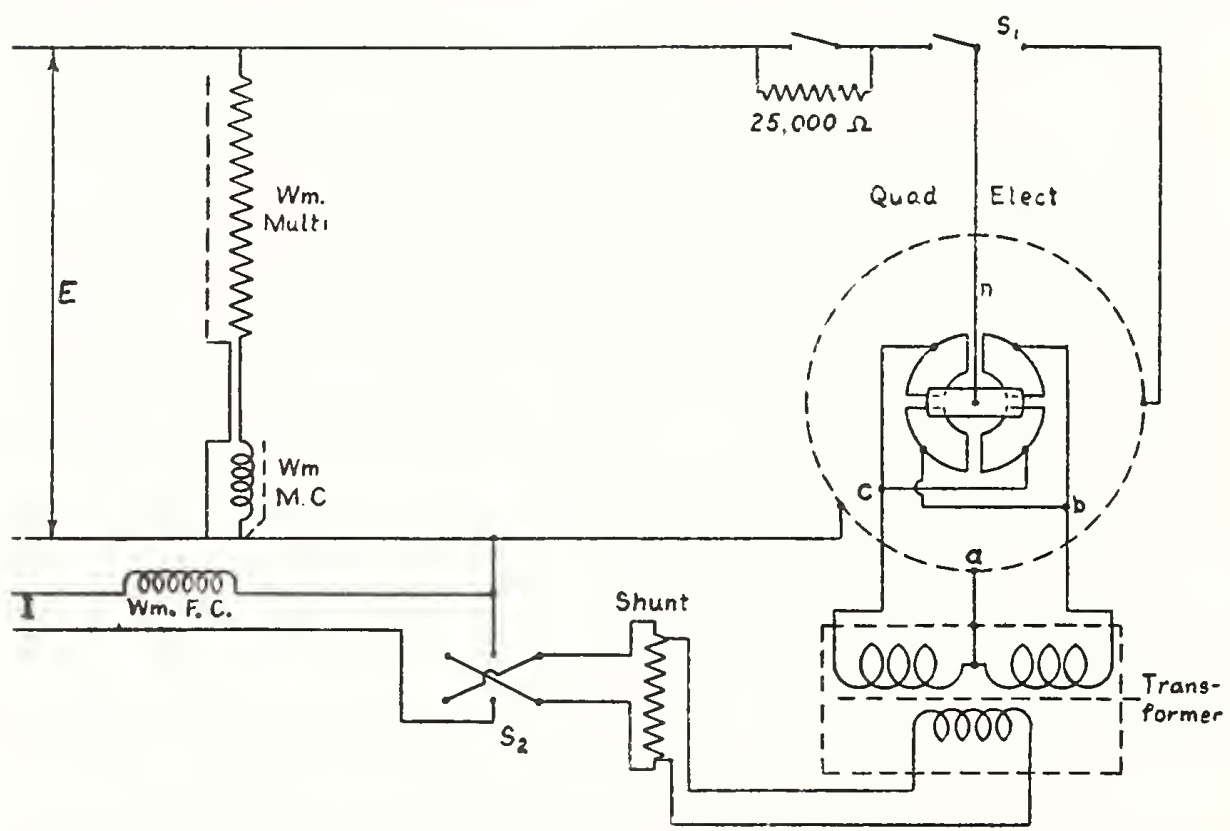

FigJRe 9.-Electric connections for comparing the wattmeler with a quadrant electrometer at zero power-factor.

$R=$ resistance of shunt.

$L=$ inductanco of shunt.

$r=$ resistanco of shunt potential leads.

$R_{t}=$ effective resistance of transformer primary
winding. $L_{\mathrm{t}}=$ effective inductance of transformer primary
winding.

$I_{t}=$ transformer primary current

$E_{1}=$ transformer primary voltage.

$E_{0}=$ transformer secondary voltage from $a$ to $c$.

$E_{\mathrm{b}}=$ transformer secondary voltage from $a$ to $b$.

can be measured independently by the wattmeter and by the electrometer. The phase angle, $\theta$; between the voltage and current was adjusted until the electrometer indicated zero power. The wattmeter would then be expected to indicate the power corresponding to 90 degrees minus the phase difference between the current through the fixed coils of the wattmeter and the quadrant voltage of the electrometer. A derivation of the value of this phase difference is given in appendix II. Any discrepancy between the actual power indicated by the wattmeter and the power computed from this phase difference is considered to be caused by a phase defect in the wattmeter, and the wattmeter phase-defect was computed on this basis.

(2) Procedure.

The wiring diagram in figure 9 was followed in connecting the wattmeter and electrometer for the test at zero power-factor. The voltage, $E$, was set at 150 (within 0.2 of a volt) by adjusting the field of the 
alternator. The current, $I$, was set at $2.5,5$, or $10 \mathrm{amp}$ (within 0.2 percent), depending upon whether the series, series-parallel, or parallel connection, respectively, of the wattmeter fixed coils was being used. The nominal value of the shunt corresponding to these currents was $0.8,0.4$, or $0.2 \mathrm{ohm}$, respectively, thus keeping the primary voltage of the transformer 2 volts for all tests. The secondary voltage across each half of the winding was approximately 25 volts. The connection from the transformer secondary to the quadrant of the electrometer was a two-conductor shielded lead, the shield being connected to the transformer shield and the midpoint of the winding at one end and to the electrometer case at the other end. The same leads were used in measuring the ratio and phase angle of the transformer so that any capacitance load imposed by the leads would be the same in both the test and the use of this transformer. The phase angle, $\theta$, between $E$ and $I$ was adjusted to approximately 90 degrees by rotating the stator of the alternator supplying the current. A fine control of this phase angle was obtained by inserting a variable self-inductor in the current circuit. The 25,000-ohm resistor in series with the lead to the electrometer needle was inserted to protect the needle suspension from possible short circuits between needle and quadrant during preliminary adjustments. For final readings this-resistor was shorted out to reduce to a minimum any voltage drop in this lead due to capacitance currents. A switching arrangement was placed in the current and voltage supply such that the alternating current and voltage could be disconnected and a direct current and voltage substituted. The d-c supply was used to calibrate the wattmeter.

The detailed procedure in checking the wattmeter against the electrometer at zero power-factor will now be described in chronological order. The electrometer was set on a stable platform with the needle centrally located with respect to the quadrants, as indicated in figure 9. A light source was located in such a position that the beam reflected from the mirror mounted on the electrometer suspension was focused on a ground-glass scale located about $2 \mathrm{~m}$ from the electrometer. The leveling screws of the electrometer were adjusted until the mechanical and electrical zeros of the electrometer coincided (to within $2 \mathrm{~mm}$ ), that is, with current off and a-c voltage adjusted to $150 \mathrm{v}$ the electrometer reading did not change when switch $S_{1}$ was thrown from right to left. The zero readings of both the electrometer and the wattmeter were recorded with no current flowing and $E=150$ $\mathrm{v}$ a-c. The current supply was connected and current adjusted to $2.5,5$, or $10 \mathrm{amp}$ a-c depending on the fixed-coil connections of the wattmeter. Frequency was set to $60 \mathrm{c} / \mathrm{s}$ and voltage was reset if necessary. The phase angle between $E$ and $I$ was adjusted until the electrometer reading was the same as its zero, and the reading of the wattmeter was recorded. The current supply was disconnected and zero readings of electrometer and wattmeter were recorded. The setting of the electrometer with current on should agree with the average of the two electrometer zero readings (in the few cases where this was not true the readings were discarded). The above procedure was repeated with switch $S_{2}$ in the reversed position. The a-c supply was disconnected and the d-c supply was connected, the voltage being adjusted to $150 \mathrm{v}$. The direct current was adjusted until the wattmeter reading was equal to the average of the two readings obtained 
with alternating current. The value of direct current was recorded, direct voltage and current were reversed, and another value of direct current was obtained and recorded. The entire procedure outlined above was repeated with the alternating voltage supply connections reversed. The average of the four values of direct current thus obtained divided by the alternating current gives $\cos \theta$, which should equal $1 / 2 \sin \left(2 \delta+\beta_{c}+\beta_{b}\right)^{9}$ if there is no phase defect in the wattmeter.

(3) Results.

TABLE 2.-Wattmeter phase defect at $60 \mathrm{c} / \mathrm{s}$

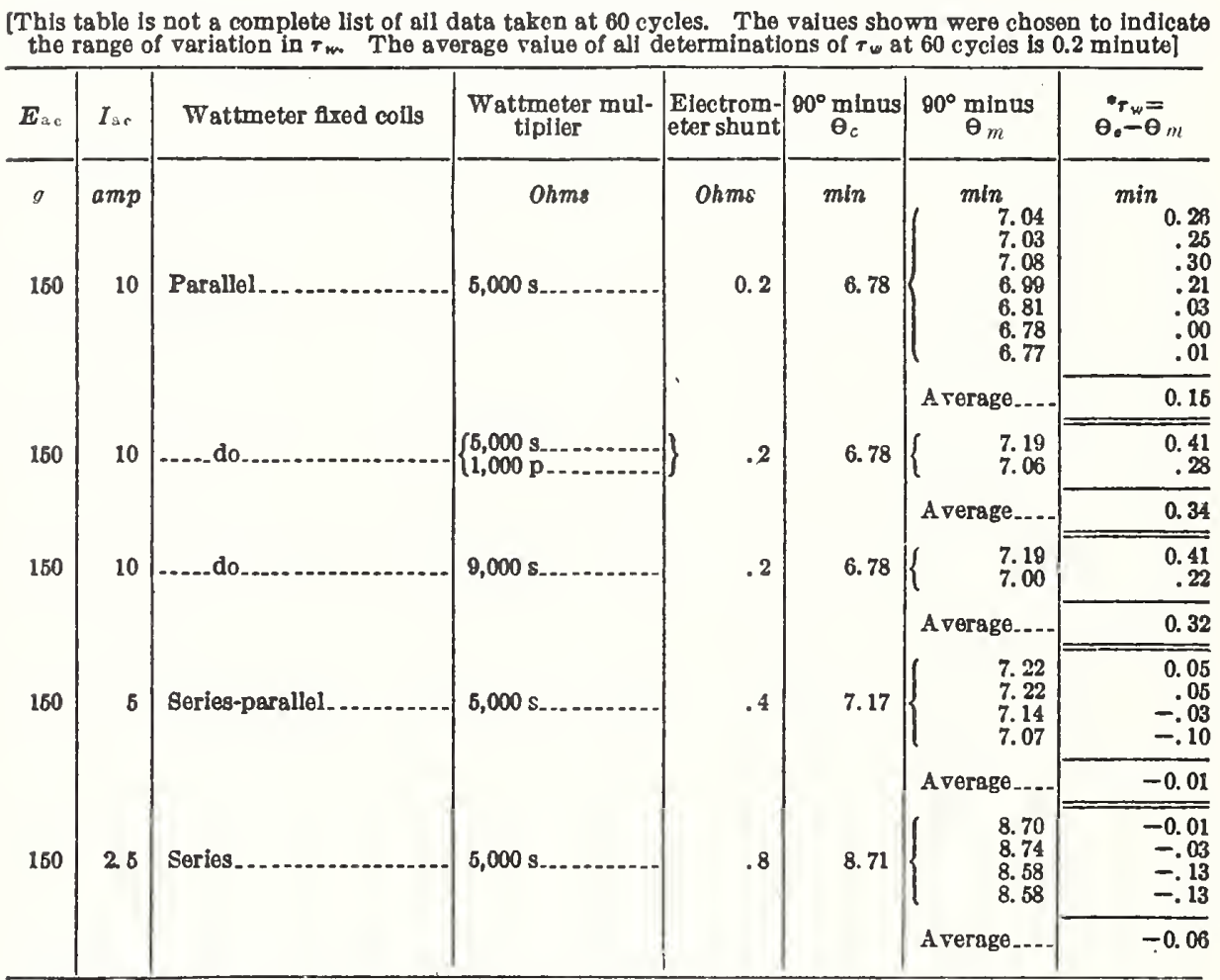

- A positive value of $\tau_{w}$ indicates that the moving coll current leads the supply voltage.

The results of tests at zero power-factor comparing the wattmeter with a quadrant electrometer are tabluated in table 2. The phase defect of the wattmeter is indicated as the difference between two phase angles (1) $\theta_{c}$ which is 90 degrees minus the computed phase angle between the current through the wattmeter fixed coils and the voltage across the quadrants of the electrometer, and (2) $\theta_{m}$ which is the power-factor angle measured with the wattmeter while the quadrant electrometer indicated zero watts. The wattmeter phase defect, $\tau_{w}$, probably consists of two components (1) a phase displacement between the moving-coil current and the voltage applied to the wattmeter voltage circuit, and (2) a phase displacement between the magnetic flux due to the fixed coils and the current supplied to the fixed coils. For convenience in discussing the phase defect, the two components are added and this total phase defect is treated as if it were a phase displacement between the moving-coil current and the wattmeter supply voltage; a positive value of $\tau_{w}$ indicates that the moving-coil current leads the supply voltage. The computed phase angle, $\theta_{c}$,

\footnotetext{
$\checkmark$ For the derivation of this relation see appondix II.
} 
was primarily dependent upon the phase angle of the potential transformer. The phase angle of this transformer had an uncertainty of \pm 0.1 minute, probably due to magnetic instability of the core. The experimental error in comparing the wattmeter and the electrometer was \pm 0.1 minute. Thus the total uncertainty in $\tau_{w}$ was \pm 0.2 minute, which is sufficient to account for the variations of the phase defect of the wattmeter as indicated in the last column of table 2 . The conclusion is that the phase defect of the wattmeter at a frequency of $60 \mathrm{c} / \mathrm{s}$ is very probably less than 0.4 minute for any fixed-coil connection and for any arrangement of the multiplier circuit as normally used.

(b) AT HIGH FREQUENCIES, USING CAPACITANCE LOAD

(1) Method and Procedure.

By passing current through the fixed coils of the wattmeter and a capacitor, connected in series, and applying the voltage drop across the capacitor to the voltage circuit of the wattmeter, the current and voltage of the wattmeter will be very nearly 90 degrees out of phase and the wattmeter deflection should be about zero. The small value of watts indicated by the wattmeter will be due to (1) any phase defect in the capacitor used as a load; (2) the current taken by the voltage circuit of the wattmeter, which necessarily flows through the wattmeter fixed coils; (3) the current taken by the voltmeter used to measure the drop across the capacitor; and (4) any phase defect in the wattmeter. Since the total watts indicated by the wattmeter can be determined and the first three of the above factors making up the total watts can be computed or measured, the fourth factor (wattmeter phase defect) can be deduced.

The capacitance used as a load on the wattmeter must have a value such that with the normal wattmeter voltage across it (about $150 \mathrm{v})$, the current taken will be equal to the rated current of the wattmeter fixed coils $(2.5,5$, or $10 \mathrm{amp})$. It would be almost impossible to build up enough capitance by using air capacitors. Paper capacitors have losses too large and too unstable. Mica capacitors were considered feasible for this work, and the largest capacitance which could be built up from all of the mica capacitors readily available was about $10 \mu \mathrm{f}$. With these capacitors it was possible to test the wattmeter at frequencies of 500 to $2,000 \mathrm{c} / \mathrm{s}$. It was decided to measure the phase defect of the wattmeter at 500,1,006, and 2,000 $\mathrm{c} / \mathrm{s}$ and then if possible to extrapolate the results back to lower frequencies.

The wiring diagram for the equipment used in this test is shown in figure 10. A Kelvin voltmeter of the multicellular electrostatic type was used to measure the voltage across the wattmeter voltage circuit, because the deflection of this type of voltmeter is independent of frequency over a wide range. A resistance voltage divider was used in conjunction with this voltmeter to extend its range, since the maximum voltage which could be read on the voltmeter alone was $120 \mathrm{v}$. An attempt was made to extend the range of this voltmeter by putting an air capacitor in series with it, but this plan had to be abandoned because of an error introduced by dielectric losses in the voltmeter.

With switch $S_{2}$ (see fig. 10) thrown to the right-hand position the wattmeter measures the power loss in capacitor $C$, the power consumed in $r_{m}$ and $r_{v}$, and an additional quantity $\omega C E^{2} \sin \tau_{w}$, where $\tau_{w}$ is 
the phase defect of the wattmeter. With switch $S_{2}$ thrown to the left-hand position the wattmeter measures the power consumed in $r_{m}, r_{v}$, and $R$. Under these conditions the current through the wattmeter fixed coils is small in value compared to the large capacitance current taken by $C$ and is in phase with the voltage, $E$, across the wattmeter voltage circuit, so that the effect of the wattmeter phase defect is negligible. The procedure followed in measuring the wattmeter phase defect, $\tau_{w}$, was first to set the supply frequency and voltage with switch $S_{2}$ in the right-hand position and read the wattmeter deflection, then to duplicate the setting of supply frequency

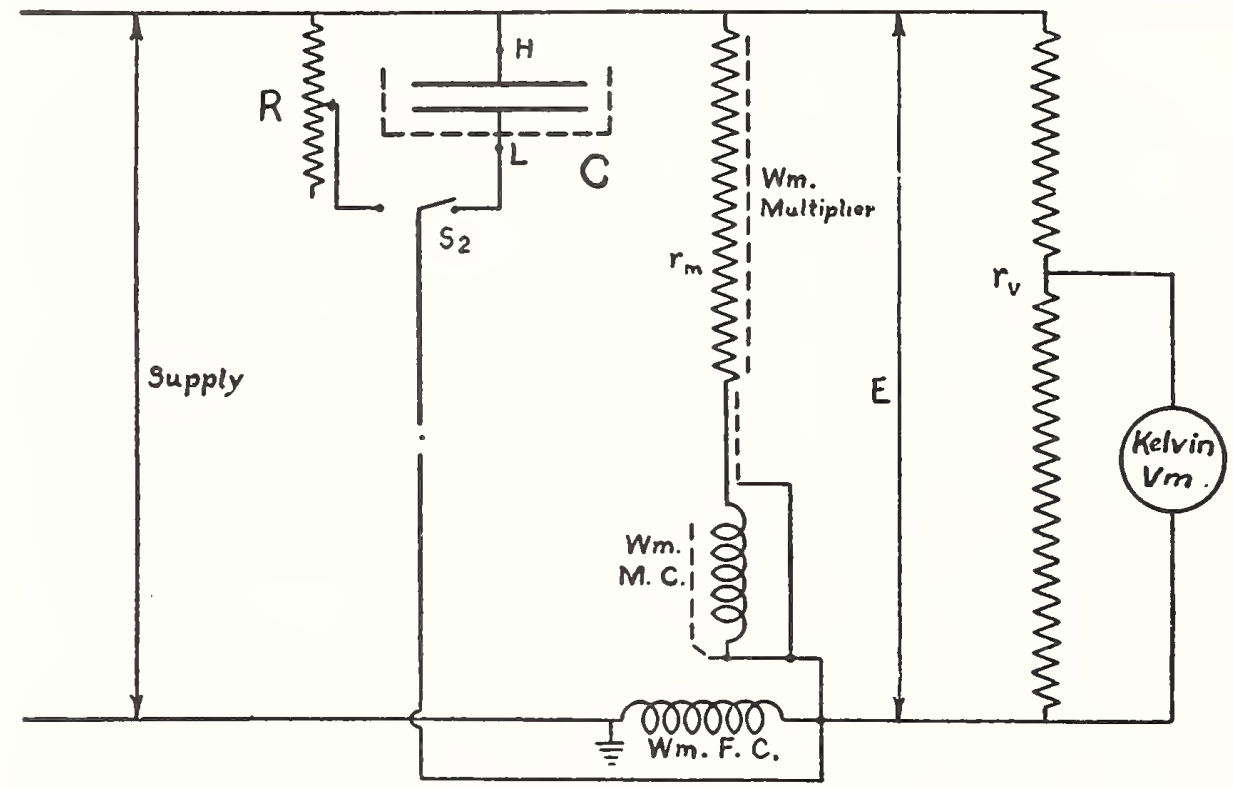

FIGURE 10.-Electric connections for measuring wattmeter phase defect at high frequencies using a capacitance load.

$C=$ mica capacitor.

$R=$ adjustable resistor.

$r_{\mathrm{v}}=$ resistance of voltage divider for electrostatlo

$r_{m}=$ resistance of wattmeter voltage circuit.

$E=\begin{aligned} & \text { voltmeter. } \\ & \text { voltage applied to wat tmeter voltage circuit. }\end{aligned}$

and voltage with $S_{2}$ in the left-hand position and adjust the resistor, $R$, until the wattmeter deflection was the same as just read. The wattmeter phase defect can then be deduced from the value of the resistor, $R$, capacitor, $C$, and phase defect, $\tau_{c}$, of capacitor $C$.

(2) Derivation of Equation.

$\mathrm{By}$ referring to the wiring diagram in figure 10 and using the notation indicated on the diagram, two equations for the quantity measured by the wattmeter can be written. When the capacitor, $C$, is used as a load on the wattmeter (switch $S_{2}$ to the right) the quantity is

$$
P_{\mathrm{n}}=\omega C E^{2} \sin \left(\tau_{c}+\tau_{w}\right)+\frac{E^{2}}{r_{m}}+\frac{E^{2}}{r_{v}} .
$$

When the resistor, $R$, is used as a load on the wattmeter (switch $S_{2}$ to the left), this quantity is

$$
P_{R}=\frac{E^{2}}{r_{n}}+\frac{E^{2}}{r_{v}}+\frac{E^{2}}{R}
$$


During the tests, the voltage, $E$, and the deflection of the wattmeter were held constant for the two loads on the wattmeter. Thus $P_{c}=P_{R}$, and the two equations give

$$
\omega C \sin \left(\tau_{c}+\tau_{w}\right)=\frac{1}{R}
$$

and since $\tau_{c}$ and $\tau_{w}$ are small angles,

$$
\sin \tau_{w}=\frac{1}{R \omega C}-\sin \tau_{c} \text {. }
$$

(3) Results.

The results of these tests are shown in figure 11 as a plot of wattmeter phase defect against frequency. The error $(\Delta \alpha)$ due to induced currents in the moving system and capacitance currents in the moving coils is present in the results shown, but its magnitude (a maximum of 2 minutes at $2,000 \mathrm{c} / \mathrm{s}$ ) was not sufficient to be taken into account. The possible sources of this phase defect are (1) eddy current or skin effects in the fixed coils, (2) phase defect in the voltage circuit, and (3) capacitance currents between turns of the fixed coils and from fixed coils to shield. Possible source of error (3) would cause a phase defect proportional to the square of the frequency, but the results show the phase defect to be directly proportional to frequency; also, when performing the tests, a $0.005-\mu f$ capacitor connected across the fixed coils was found to have no noticeable effect on the phase defect. Therefore, it may be concluded that source of error (3) is negligible for frequencies up to $2,000 \mathrm{c} / \mathrm{s}$. Sources of error (1) and (2) would cause a phase defect directly proportional to frequency. Since the observed phase defect was found to vary in this manner (up to $2,000 \mathrm{c} / \mathrm{s}$ ), it may be concluded that it is the result of one or both of these two effects.

A computation of the part of this phase defect caused by source (2), based on the estimated values of capacitance between parts of the voltage circuit, indicated that part of the wattmeter phase defect can be accounted for by the capacitance currents in the series resistor. The remainder of the wattmeter phase defect must be due to "skin effects" in the fixed coils. The relative magnitude of these two components of the total wattmeter phase defect cannot be definitely stated.

Since the results shown in figure 11 indicate that the wattmeter phase defect is directly proportional to frequency between 500 and $2,000 \mathrm{c} / \mathrm{s}$, it is fairly safe to extend this curve to $60 \mathrm{c} / \mathrm{s}$ and obtain an extrapolated value of phase defect of 0.38 minute. This extrapolated value checks the actual value $(0.2 \text { minute })^{10}$ obtained at $60 \mathrm{c} / \mathrm{s}$, using the quadrant electrometer, within the limits of accuracy $( \pm 0.2$ minute) of the measured value.

\section{SUMMARY}

The electrodynamic instrument described in this paper can be used as a standard wattmeter or ammeter and as a standard ac-dc transfer instrument in testing wattmeters or ammeters. The current range is from 0.25 to $10 \mathrm{amp}$. The voltage range is from 25 to $300 \mathrm{v}$. The

\footnotetext{
$10 \mathrm{This}$ value is an average of all the data taken at $60 \mathrm{c} / \mathrm{s}$.
} 
results obtained from this instrument are accurate within 0.01 percent when it is used at power frequencies. The only correction which must be applied to obtain this accuracy is the one due to the phase defect

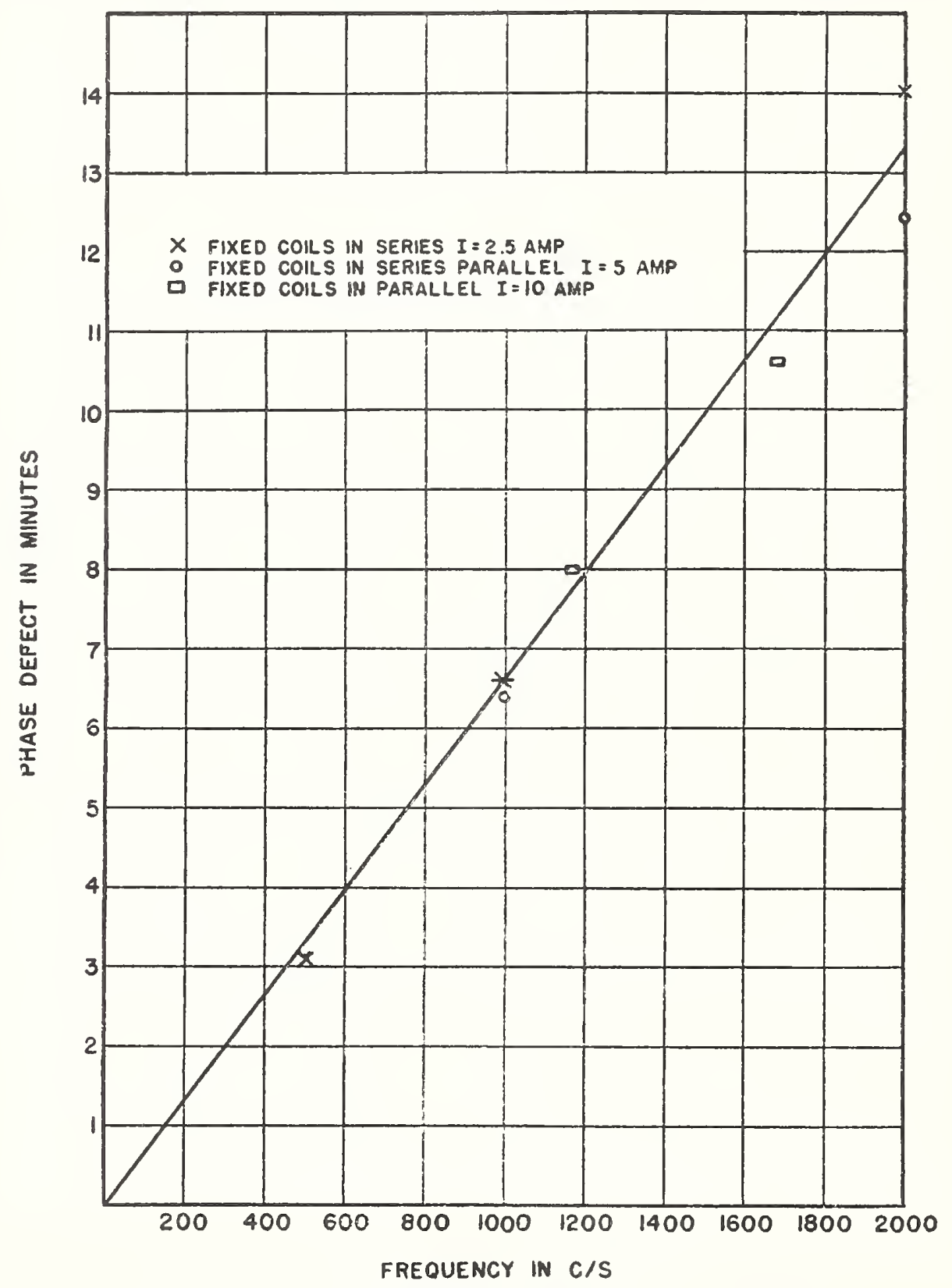

FIGURE 11.-Plot of wattmeter phase defect against frequency.

Results obtained from tests using a capacitance load.

$(0.38$ minute at $60 \mathrm{c} / \mathrm{s})$. This correction will be negligible for power factors greater than 0.7. The instrument can also be used at frequencies up to $2,000 \mathrm{c} / \mathrm{s}$, provided the necessary corrections as described in this paper are applied. 
Almost all the construction of this transfer instrument was done by E. A. Tibbals, one of the Bureau's mechanicians, who is responsible for many valuable suggestions relating to the design of important details of the instrument.

\section{APPENDIX I}

\section{Computation of the Effect of Variation in Wattmeter Sensitivity}

The wattmeter deflection in terms of $S$, wattmeter sensitivity (equal to $G / U$ as defined on page 559$) ; i$, moving-coil current in milliamperes; and $I$, fixed-coil current in amperes is

$$
\alpha=S i \frac{I}{I \text { (rated). }}
$$

The power being measured is proportional to $I i$, and $I$ (rated) is constant for a given connection of the fixed coils. Thus the deflection may be expressed as

$$
\alpha=K S W,
$$

where $K$ is a constant and $W$ is the power being measured. If $\alpha_{a}, S_{a}$, and $W_{a}$ denote valucs with alternating current and voltage; $\alpha_{d}, S_{d}, W_{d}$ denote values with direct current and voltage; $\alpha_{r}, S_{r}, W_{r}$ denote values with direct current and voltage in the reverse direction

$$
\begin{aligned}
& \alpha_{a}=K S_{a} W_{a}, \text { or } W_{a}=\frac{\alpha_{a}}{K S_{a}} ; \\
& \alpha_{d}=K S_{d} W_{d}, \text { or } W_{d}=\frac{\alpha_{d}}{K S_{d}^{\prime}} ; \\
& \alpha_{r}=K S_{r} W_{r}, \text { or } W_{r}=\frac{\alpha_{r}}{K S_{r}} .
\end{aligned}
$$

The relation to be obtained is the ratio of $\frac{1}{2} W_{a}^{r}$ to the average d-c power in terms of the $\alpha$ 's and S's.

$$
\frac{2 W_{a}}{W_{d}+\bar{W}_{r}}=1+\frac{W_{a}-\frac{1}{2}\left(W_{d}+W_{r}\right)}{\frac{1}{2}\left(W_{d}+W_{r}\right)}=1+\delta_{0}
$$

Substituting values of $\alpha$ and $S, \delta$ becomes

$$
\delta=\frac{\frac{\alpha_{a}}{K S_{a}}-\frac{1}{2 K}\left(\frac{\alpha_{d}}{S_{d}}+\frac{\alpha_{r}}{S_{r}}\right)}{\frac{1}{2 K}\left(\begin{array}{l}
\alpha_{d} \\
S_{d}^{-}
\end{array}+\frac{\alpha_{\tau}}{S_{r}}\right)} .
$$

Since $S_{d}$ and $S_{r}$ differ but slightly from $S_{a}$, by putting $S_{d}=S_{a}+s_{d}$ and $S_{r}=$ $S_{a}+s$, the above equation simplifies to

$$
\delta=\frac{\alpha_{u}-\frac{\alpha_{d}+\alpha_{r}}{2}}{\frac{\alpha_{d}+\alpha_{r}}{2}}+\frac{1}{2}\left(\frac{s_{r}+s_{d}}{S_{a}}\right) .
$$

One hundred times the first term on the right side of the above equation gives the percentage excess of a-c power over average d-c power as normally computed assuming constant sensitivity. One hundred times the second term gives the correction in percentage to be applied when the sensitivity for $\alpha_{d}$ and $\alpha_{r}$ are different from the sensitivity for $\alpha_{a}$. Values of $S_{a}, s_{r}$, and $s_{d}$ are obtained from the curves of sensitivity against deflection in figure 6 . 


\section{APPENDIX II}

\section{Phase Difference Between the Fixed-Coil Current and the Quadrant Voltage on the Electrometer and the Relation of This Phase Difference to $\theta$, the Phase Angle Between $E$ and $I$}

The diagram in figure 9 shows the connections of the.wattmeter and the electrometer for measuring the power $E I \cos \theta$. The first step is to derive the relation between $I$ and $E_{c}$ or $E_{b}$ in terms of the known constants of the apparatus. By inspection of the diagram and using the notation given under it, the following equation can be written

$$
I_{\ell}\left(R_{t}+j \omega L_{t}\right)=-I_{t} 2 r+\left(I-I_{t}\right)(R+j \omega L) .
$$

The currents in this equation are all complex quantities, but $I$ may be assumed to have no imaginary component. By equating the real and imaginary components of eq 3 and solving for the two components of $I_{t}$, these may be substituted in

to obtain:

$$
E_{1}=I_{\imath}\left(R_{t}+j \omega L_{\imath}\right)
$$

$$
\begin{aligned}
E_{1}= & I\left\{\frac{\left(R_{t} R-\omega^{2} L_{t} L\right)\left(R_{t}+R+2 r\right)+\left(\omega L R_{t}+\omega L_{t} R\right)\left(\omega L_{1}+\omega L\right)}{\left(R_{t}+R+2 r\right)^{2}+\left(\omega L+\omega L_{t}\right)^{2}}\right. \\
& \left.\frac{ \pm j\left[\left(\omega L_{t} R+\omega L R_{t}\right)\left(R_{t}+R+2 r\right)+\left(\omega^{2} L_{t} L-R_{t} R\right)\left(\omega L_{t}+\omega L\right)\right]}{\left(R_{t}+R+2 r\right)^{2}+\left(\omega L+\omega L_{t}\right)^{2}}\right\} .
\end{aligned}
$$

For the present work the approximate values of the constants in the above equation are $R=0.4 \mathrm{ohm}, R \geqq 400 \mathrm{ohms}, r=0.03 \mathrm{ohm}, L=30 \times 10^{-0} h, \omega L_{\imath} \geqq 600$ ohms.

Thus with sufficient accuracy the equation may be reduced to

$$
E_{1}=I R\left[1-(2 r+R) \frac{R_{\imath}}{R_{\imath}^{2}+\omega^{2} L_{\imath}^{2}}+j \frac{\omega L}{R}+j(R+2 r) \frac{\omega L_{\imath}}{R_{\imath}^{2}+\omega^{2} L_{\imath}^{2}}\right]
$$

and putting

$$
g=(2 r+R) \frac{R_{t}}{R_{t}+\omega^{2} L_{\imath}^{2}}
$$

and

$$
\begin{gathered}
\delta=\sin ^{-1}\left[\frac{\omega L}{R}+(R+2 r) \frac{\omega L_{t}}{R_{t}+\omega^{2} L_{t}^{2}}\right], \\
E_{1}=I R(1-g+j \sin \delta) .
\end{gathered}
$$

If the ratios and phase angles for the transformer are $n_{c}, \beta_{c}$ and $n_{b}, \beta_{b}$ for the $a c$ and $a b$ coils respectively,

$$
E_{c}=n_{c} E_{1}\left(1+j \sin \beta_{c}\right)
$$

and

$$
E_{b}=n_{b} E_{1}\left(1+j \sin \beta_{b}\right) .
$$

Substituting the value of $E_{1}$ given in eq 4 and neglecting the products of the sines of the small angles $\delta, \beta_{c}$, and $\beta_{b}$,

$$
E_{c}=n_{c} I R\left[1-g+j\left(\sin \delta+\sin \beta_{c}\right)\right]
$$

and

$$
E_{b}=n_{b} I R\left[1-g+j\left(\sin \delta+\sin \beta_{b}\right)\right] .
$$

For the connections of the quadrant electrometer shown in figure 9 , if $V_{n}$ is the voltage from the case to needle, $V_{c}$ from case to quadrant $c$, and $V_{b}$ from case to quadrant $b$, the general equation for the torque is

$$
T=\lambda V_{n}^{2}+\rho_{c} V_{c}^{2}+\rho_{b} V_{b}^{2}+\gamma_{c} V_{n} V_{c}+\gamma_{b} \cdot V_{n} V_{b}+\eta V_{c} V_{b}+\nu_{n} V_{n}+\nu_{c} V_{c}+\nu_{b} V_{b}+\nu_{0},
$$


where $\lambda, \rho_{c}, \rho_{b}, \gamma_{c}, \gamma_{b}, \eta, \nu_{n}, \nu_{c}, \nu_{b}$, and $\nu_{0}$ are constants of the electrometer. Take the mechanical zero to be the reading when $c, b$, and $n$ are all tied to the case, that is, with current circuit open and $S_{1}$ to the right. This makes $\nu_{0}=0$. For a-o work $\nu_{n}, \nu_{c}$, and $\nu_{b}$ can also be ignored, because the mean values of $V_{n}, V_{o}$, and $V_{b}$ over any number of complete cycles is zero. The electrical zero is adjusted by tilting or rotating the needle until no deflection occurs when $S_{1}$ is thrown to the left. This makes $\lambda=0$. With both current and voltage on, the torque then becomes

$$
T_{1}=\rho_{c} V_{c}^{2}+\rho_{b} V_{b}^{2}+\gamma_{c} V_{n} V_{c}+\gamma_{b} V_{n} V_{b}+\eta V_{c} V_{b}
$$

The phase angle, $\theta$, between the current and the voltage is adjusted until the average torque becomes zero, that is, until the electrometer reading is the same as its electrical zero, then

$$
\begin{aligned}
0= & \rho_{b} n_{b}^{2} R^{2} I^{2}+\rho_{b} n_{b}^{2} R^{2} I^{2}+\gamma_{c} n_{c} E I R\left[\cos \left(\theta-\delta-\beta_{c}\right)\right] \\
& -\gamma_{b} n_{b} E I R\left[\cos \left(\theta-\delta-\beta_{b}\right)\right]-\eta n_{c} n_{b} R^{2} I^{2}
\end{aligned}
$$

With switch $S_{2}$ reversed, that is $E_{c}$ and $E_{b}$ reversed, $\theta$ is again adjusted until the average torque becomes zero. Then

$$
\begin{aligned}
0= & \rho_{c} n_{c}^{2} R^{2} I^{2}+\rho_{b} n_{b}^{2} R^{2} I^{2}-\gamma_{c} n_{c} E I R\left[\cos \left(\theta^{\prime}-\delta-\beta_{c}\right)\right] \\
& +\gamma_{b} n_{b} E I R\left[\cos \left(\theta^{\prime}-\delta-\beta_{b}\right)-\eta n_{c} n_{b} R^{2} I^{2} .\right.
\end{aligned}
$$

Subtract eq 8 from eq 7

$$
\begin{gathered}
0=\gamma_{c} n_{c} E I R\left[\cos \left(\theta-\delta-\beta_{c}\right)+\cos \left(\theta^{\prime}-\delta-\beta_{c}\right)\right] \\
-\gamma_{b} n_{b} E I R\left[\cos \left(\theta-\delta-\beta_{b}\right)+\cos \left(\theta^{\prime}-\delta-\beta_{b}\right)\right] .
\end{gathered}
$$

Expanding the cosines in this equation and neglecting the departure from unity of $\cos \left(\delta+\beta_{0}\right), \cos \left(\delta+\beta_{b}\right), \sin \theta$, and $\sin \theta^{\prime}$ since $\delta+\beta_{c}$ or $\delta+\beta_{b}<10$ minutes and $\theta$ or $\theta^{\prime}$ is within 10 minutes of $\pi / 2$

$$
\begin{aligned}
& \gamma_{c} n_{c}\left[\cos \theta+\sin \left(\delta+\beta_{c}\right)+\cos \theta^{\prime}+\sin \left(\delta+\beta_{c}\right)\right] \\
= & \gamma_{b} n_{b}\left[\cos \theta+\sin \left(\delta+\beta_{b}\right)+\cos \theta^{\prime}+\sin \left(\delta+\beta_{b}\right)\right]
\end{aligned}
$$

Since $\gamma_{0}$ very nearly equals $-\gamma_{b}$ and $n_{0}$ nearly equals $n_{b},-\gamma_{c} n_{e}(1+h)$ may be substituted for $\gamma_{b} n_{b}$; then

$\cos \theta+\cos \theta(1+h)+\cos \theta^{\prime}+\cos \theta^{\prime}(1+h)=-2 \sin \left(\delta+\beta_{c}\right)-2\left[\sin \left(\delta+\beta_{b}\right)\right](1+h)$, and this reduces to

$$
\cos \theta+\cos \theta^{\prime}=-\left[\sin \left(\delta+\beta_{c}\right)\right]\left(1-\frac{1}{2} h\right)-\left[\sin \left(\delta+\beta_{b}\right)\right]\left(1+\frac{1}{2} h\right)
$$

Since $\beta_{c}$ nearly equals $\beta_{b}$ the $h$ terms cancel, and dividing by 2 to get the average $\cos \theta$

$$
\frac{\cos \theta+\cos \theta^{\prime}}{2}=-\frac{1}{2} \sin \left(2 \delta+\beta_{c}+\beta_{b}\right) \text {. }
$$

This equation yields the average value of the cosine of the angle between current $I$ and voltage $E$, for the two positions of switch $S_{2}$, in terms of the constants of the apparatus in the electrometer circuit. The cosine of this angle can also be obtained from the expression

$$
W=E I \cos \theta \text {, }
$$

where $W$ is the average power indicated by the wattmeter for the two positions of switch $S_{2}$. Any difference between the two values of $\cos \theta$ thus obtained is considered to be caused by the wattmeter phase defect, $\tau_{w}$.

Washington, July 25, 1940.

For sale by the Superintendent of Documents, Washington, D. C. . . . . Price 10 cents 


\title{
Precise Comparison Method of Testing Alternating- Current Watthour Meters
}

\author{
A. W. Spinks and T. L. Zapf
}

\begin{abstract}
A brief description of the basic method of testing alternating current watthour meters at the National Bureau of Standards is given, followed by a description of equipinent for a faster and less laborious method.

Equipment with several novel features has been assembled for making precise tests of alternating-current watthour meters by a comparison method employing a group of caref ully selected alternating-current watthour meters, which serve as a secondary standard group. One of this group, designated the "Standard Watthour Meter", is used with multirange instrument transformers as a reference standard to test other watthour meters with good precision. The testing procedure is explained, and the formulas used in computing the results of the tests are derived.

An analysis of the possible errors of measurement and data from numerous tests indicate that the measurement of energy applied to a watthour meter under test can be relied upon to better than 0.06 percent.
\end{abstract}

\section{Introduction}

For many years the National Bureau of Standards has employed a method of testing alternating-current watthour meters, which, although very precise and accurate, is tedious and time consuming. By this method, the energy indicated by the watthour meter under test is compared with the true value of energy as measured in terms of a constant and accurately known power and an accurately determined time interval. The power is held constant with the aid of a standard electrodynamic wattmeter ${ }^{1}$ which is calibrated on reversed direct current before and after each run. For this test, a very stable, manually controlled alternating-current source is required to insure a steady value of power.

In the midthirties it was observed that repeated tests on particular watthour meters showed remarkably small deviations from the initial test values. This suggested the possibility of using a group of watthour meters of demonstrated good repeatability as secondary standards. The register and gear train could be removed from each meter to reduce friction. Disk revolutions would be counted photoelectrically.

Four such meters were obtained, but unfortunately, work on this project was interrupted, and construction of a permanent setup and the apparatus for intercomparing the meters was delayed. In 1940 the paper of Goss and Hansen ${ }^{2}$ describing the excellent performance of a group of watthour meters caused a revival of interest in this project. Further cxperimental work led to detailed plans, but actual shop construction was interrupted by duties imposed by World War II.

Work was resumed after the war, and the equipment, except for minor modifications, was constructed as originally planned. 'This apparatus has been under observation for several vears, and its reliable performance has been verified. 'The new

1 John H. Park and Arthur B. Lewis, Standard electrodynomic wattmeter and ac-de transfer instrument, J. Research NBS 2.5, 545 (1940) RP1344.

$2 J$. H. Goss and A. Hansen, $J_{r}$, A nrecision rotating standar 1 for the measurement of kilowatt hours. Trans. A1E $\mathbf{5 9}, 412(1940)$. equipment is now used at the National Bureau of Standards in preference to the older equipment for practically all of the testing of a-c watthour meters. This paper describes the equipment, the procedures followed in its calibration and use, and the results obtained.

\section{Equipment}

Basically, the complete equipment consists of a group of modified commercially available portable watthour meters maintained at a constant temperature, and the means for their calibration and intcrcomparison with a high degrec of precision. In order to eliminate friction except at the top and bottom bearings, the meters are not equipped with registers. Instead, phototubes serve for counting revolutions of these meters, which are opcrated continuously during a test. For any direct intercomparison, some means of reading the registration of at least one metcr of the group to a fraction of a revolution is extremely desirable. This is accomplished by providing a light-beam pointer and a spccial circular phosphorescent scale for one of the meters. At the beginning and again at the end of each run the light source for the pointer (a mercury-rapor lamp) is flashed, lcaving spots on the phosphorescent scale that persist for several scconds. The standard meters are always operated at the same voltage and current. Other ranges are provided by special voltage and current transformers.

Originally it was intended to use four house-type meters, one from cach of four American manufacturers. Each of the manufacturcrs was consulted, and asked for advice in the selection of the meters. Two of the manufacturers recommended their portable standards rather than sclected house-type meters. As a result, the original setup was designed to accommodate two portable standards and two (or more) house-type meters. More recently, however, a different type of watthour metcr has proved to be so much more stable than the older house-type meters originally procured, that the latter have been replaced by two of the newcr type. 
The two original portable standard meters were individually housed in separate temperature-controlled enclosures, each enclosure consisting essentially of a Bakelite housing completely surrounding the aluminum case of the meter, a mereury thermoregulator to control the temperature, and four small lamps distributed inside the almninum case to serve as heaters. The temperature is regulated at approximately $35^{\circ} \mathrm{C}$ to better than $\pm 0.2 \mathrm{deg} \mathrm{C}$. The upper end of the meter shaft projects through the top of the Bakelite enclosure. The mirror for the lightbeam pointer is attached to this end of the shaft at an angle of $45^{\circ}$ to the axis of the shaft. Each meter cnclosure is assembled on a brass base plate to make a complete unit for interchangeable mounting in the housing that holds the phosphorescent scale. The particular portable watthour meter that is used with the circular scale is referred to in this discussion as the Standard Watthour Meter, as distinguished from the Comparison Standard Watthour Meters.

The cast-alumimum base of the circular-scale housing is equipped with leveling serews. The eircular scale, approximately 26 inches in diameter, is assembled on brackets attached to the base of the housing. The meter enclosure rests on screws for leveling the meter and adjusting its height for proper scale alinement. These screws, in turn, are mounted on an adjustable centering plate, which permits centering the axis of the meter shaft with respect to the scale.

The mercury-rapor lamp is mounted on a hinged arm above the meter. I cliaphragm below the lamp has a $1 / 2-m m$ aperture that serves as a point souree of light. Adjustments are provided for centering the light beam. The light path is vortically downward from the aperture through a lens to the milror on the end of the meter shaft where it is reflected horizontally to the circular seale, a cur'ved brass strip coated with phosphoreseent paint. The dark-blue component of light from the aperture is focused by the lens to provide a well-defined image of 1-min diameter when a flash occurs. Flush with this strip and directly below it is a circular strip of Lucite engraved with 1,000 small divisions (about $2 \mathrm{~mm}$ per division). Every tenth division is numbered. Effective use of the phosphorescent scale requires that the room be semidark; therefore, the Lucite scale is provided with edge illumination by 12 small panel lamps spaced at regular intervals. The zero mark on the scale, which is also the 1,000 division mark, is centered on the bracket supporting the mercury lamp. There is a narrow slit cut through the scale and bracket at this point. A phototube, which is used with auxiliary apparatus for coumting revolutions, is mounted directly behind this slit. Figure 1, a photograph of the Standard Watthour Nieter, shows most of these features.

The temperature-controlled cabinet originally designed for the house-type meters is now being used to house the newer type watthour meters, which serve as comparison standards. Its dimensions are approximately $112 \mathrm{~cm}$ long, $37.5 \mathrm{~cm}$ wide, and 30 $\mathrm{cm}$ high. It consists of a wooden cabinet with a removable glass top, a thermoregulator, electric

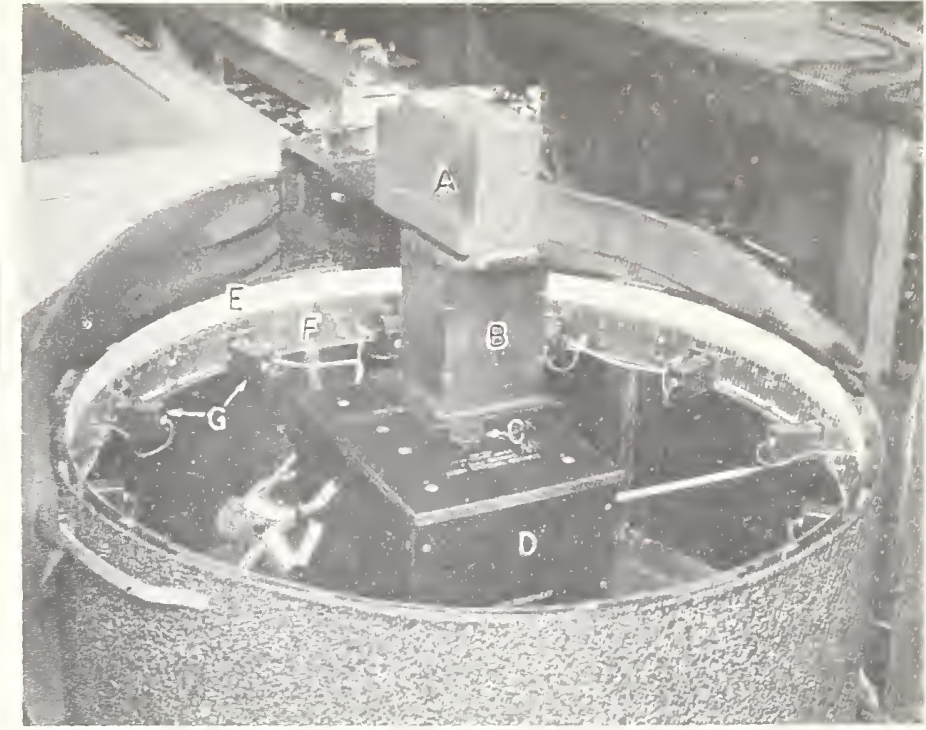

Frrere 1. Standend II athour Meter with circular phosphorescent scale used as a reference standard for the testing of wathour meters by a comparison method.

The light beam "pointer" is produeed by a mereury vapor lamp and lamp combination located above the standard Wathour Meter

a, Mreury velur lamp housing; b, lens hox; c, mirror; d, standard Watthour Meter; (", phosphotescent scalle; i, engraved plastice scale; g, liamps.

heating coil, and a small blower for circulating the air within the cabinet. Temperature is maintained at approximately $40^{\circ} \mathrm{C}$ to within $\pm 1 \mathrm{deg} \mathrm{C}$.

The accessory electronic equipment performs three functions. The first function, that of counting, is aceomplished by a relay pulse-counting circuit by means of which either a predetermined length of time or a predetermined number of revolutions of a watthour meter may be used to make or break electric circuits. The counting circuit may be preset to count seconds or revoltuions from 1 to 999 . When calibrating the Standard Watthour Meter, using the Standard Wattmeter to aid in maintaining constant power, the counting circuit is nperated by pulses accurately spaced 1 second apart. These pulses, or seconds signals, are derived from the quartz-crystalcontrolled frequency standards maintained at the Nationa! Bureau of Standards, and, as a basis for timing, are reliable to better than a part in a million. With the aid of phototubes the pulse counter can be used for counting the revolutions of the disk of cither a comparison standard, the reference standard, or a watthour meter under test.

The second function of the accessory electronic. equipment is that of flashing the mercury-rapor lamp. The pulse counter completes a circuit for the starting pulse to trigger a thyratron whose plate circuit discharges a capacitor through the mereuryvapor lamp. The capacitor is chareed to approximately $1,000 \mathrm{v}$ by means of a eomventional highvoltage power supply. The lamp is normally supplied with a current of 0.7 amp from a $240-v$ d-c powere eireuit. Normal voltage provides sufficient light intensity in the beam to trigger the phototube each time it passes orer the slit in the seale. A large choke prevents the capacitor discharge from en tering 
the power circuit. Just before the end of the preset time interval (or preset number of revolutions of a meter) the pulse counter again completes the circuit, so that the last or stopping pulse fires the thyratron. Thus the flashing is entirely automatic; the timing of the flash depends on the signal pulse, which in the case of the seconds signals is accurate to better than a part in a million.

The third function of the accessory electronic equipment is that of counting the disk revolutions of the Standard Watthour Meter. This, too, is automatic. During a run the constant light actuates the phototube behind the slit in the scale. This operates an electromagnetic counter by means of an amplifier and a small thyratron. An electronic switch opens the cathode circuit of the thyratron tube to prevent counting except during the run. The pulse that flashes the mercury lamp is also fed to the grids of the electronic-switch tubes. 'The first, or starting, pulse that flashes the lamp also enables conduction in the comnter thyratron and allows counting to start. The second, or stopping, pulse that flashes the lamp again at the end of the preset timing or counting interval, disables the thyratron and stops the counting.

\section{Testing}

The accuracy of a test of a watthour meter, using the apparatus described is directly dependent upon the latest previous calibration of the reference standard, the stability of which is substantiated by more recent intercomparison tests. Phantom loading is employed in all tests. Correct cireuit connections are made rapidly by means of links on an otherwise permanently wired circuit board.

\subsection{Calibration of the Standard Watthour Meter}

'The word "calibration" usually implies not only a carefully made test; but also an initial careful adjustment or marking of the apparatus under test. For the purpose of this diseussion, however, a calibration of the Standard Watthour Meter implies a test to letermine aceurately its percentage registration and possibly, but not necessarily, an adjustment of its mechanism in order to bring the percentage registration within certain limits.

The Standard Watthour Meter is periodically calibrated with $120 \mathrm{v}, 5 \mathrm{amp}$ applied, at unity power factor, and at 0.5 power factor with the current lagging the voltage. During calibration, a measured value of a-c power is maintained constant for an accurately determined time interval.

The testing circuit is shown in figure 2. In the potential circuit it is important that the effective terminals of the Standard Wattmeter be located precisely at the Standard Watthour Meter terminals on alternating current, and at the rolt-box terminals on direct current. Hence the lead resistance from the a-c-d-e switch to the Standard Watthour Meter potential terminals and to the volt-box terminals must be equal to prevent errors that would otherwise be caused by a difference in IR drops in

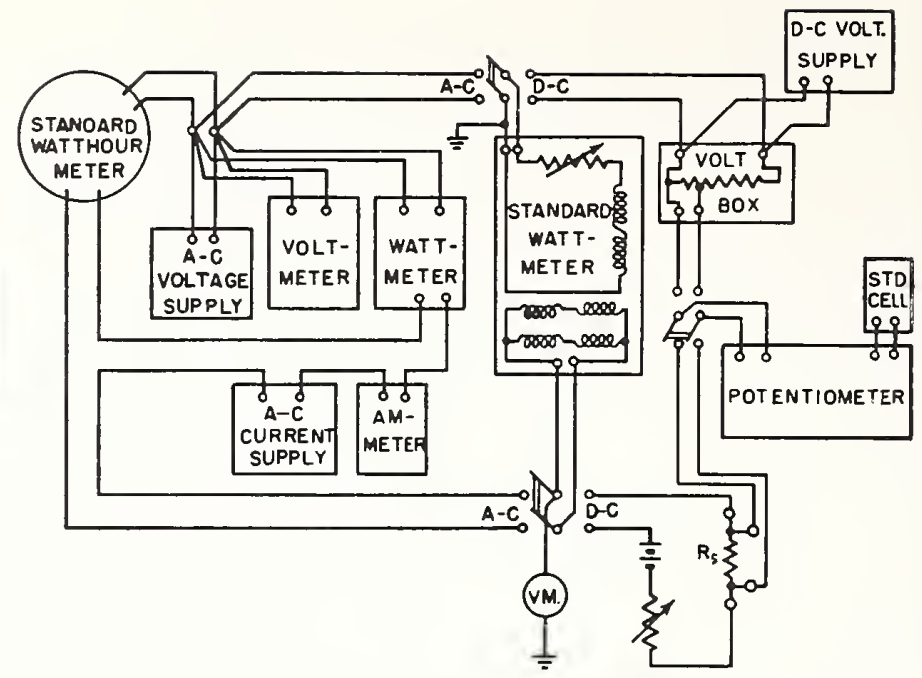

Hatre 2. Calibration of Standard Wathour Meter.

the leads. It is also important that the voltage supplies be connected to these same terminals, otherwise a phase-angle error in the watthour-moterpotential circuit might result from an IR drop in the leads. Lead resistances are chocked and adjusted, if necessary, just before each calibration. Note also in figure 2 that the moving-coil side of the Standard Wattmeter potential circuit is grounded, and that the current eircuit is connected to ground through a rectifier-type voltmeter, a 300-v instrument having a total resistance of 300,000 ohms. This serves as an electrostatic tie to ground, which minimizes electrostatic forces between fixed and moving coils of the wattmeter, and provides an indication of the presenee of leakage eurrent and its magnitude.

In calibrating the Standard Watthour Meter the procedure is as follows. First, temperature equilibrium of the entire equipment is established by applying a-c power arljusted (in the case of the wattmeter and watthour-meter circuits) to approximately the values required for the test and then waiting for an hour or two before proceeding. After temperature equilibrium has been attained, the Standard Wattmeter is calibrated on reversed direct current to indicate the desired value of power. Alternating-current power is restored to the Standard Wattmeter and Standard Watthour Meter circuits and adjusted to give the same deflection of the Standard Wattmeter as that obtained on direct current. The a-c power is held substantially constant throughout the run.

The pulse-counting circuit is then energized. The next seconds signal flashes the mercury-vapor lamp and starts the run. The position of the spot on the phosphorescent scale is recorded to the nearest division. At the end of the preset interval (usually 100 seconds) the final seconds pulse again flashes the mercury-rapor lamp, thereby ending the run. The position of this last spot is recorded as well as the reading of the revolution counter, which indicates the number of complete revolutions. Thus, the number of revolutions of the Standard Watthour Meter's disk equals the reading of the revolution counter plus the scale reading at the end of the run 
minus the scale reading at the beginning of the rum. For example, if the reading at the start of the run were 872 divisions, and at the end of the run, 493 divisions, and the revolution counter read 10 , the number of revolutions would be $10+0.493-0.872=$ 9.621. After the a-c run, two additional readings of the Standard Wattmeter on reversed d-c power are obtained in the same manner as at the start, giving a total of four d-c readings. Care is taken to insure a minimum of delay between the d-c readings and the run with alternating current. If this is done, any small linear drift that may be present in the deflection of the Standard Wattmeter causes no significant error in the result. The d-c readings are averaged and a correction applied for the difference between the a-c setting and the average $d-c$ reading. Corrections are also applied for known errors in the resistance standard, volt box, wattmeter (phasedefect angle), and for deviations from normal of the average frequency of the source, if significant.

The formula used in this calibration for computing percentage registration is

$\mathscr{R}=100\left[\frac{K R_{s} n 3600}{E_{p} N_{v b} E_{c} t}+\frac{D_{d c}-D_{a c}}{D_{d c}}+c_{s}-c_{v b}+c_{f}-T_{w} \tan \theta\right]$

where

$K=$ disk constant of the watthour meter, in wat thours per revolution.

$R_{s}=$ nominal resistance of resistance standard, in ohms.

$n=$ number of disk revolutions of watthour meter.

$E_{p}=$ potentiometer setting, in volts, when adjusting the d-c voltage.

$N_{v b}=$ nominal volt box ratio.

$E_{c}=$ potentiometer setting, in volts, when adjusting the d-c current.

$t=$ time of run in seconds.

$D_{d c}=$ average of the four deflections of the Standard Wattmeter, in centimeters, observed during the d-c calibrations before and after each run.

$D_{a c}=$ average deflection of Standard Wattmeter, in centimeters, maintained during the a-c run.

$c_{s}=$ correction to the resistance standard, in parts per unit.

$c_{v b}=$ correction to the volt box, in parts per unit.

$c_{f}=$ frequency correction to the Standard Watthour Meter, determined from the reading of the synchronous timer, the time duration of the rum, and a frequency correction factor obtained in a separate test of the Standard Watthour Meter.

$T_{20}=$ phase defect angle of the Standard Wattmeter, in radians, positive if the moving coil current leads the supply voltage.

$\theta=$ power factor angle, which is the angular phase difference between the voltage, $E$, applied to the Standard Wattmeter, and the current, $I$, in the Standard Wattmeter current coils, and is positive if the current lags the voltage.
A complete derivation of this equation appears in the appendix.

\subsection{Tests of the Comparison Standard Watthour Meters}

The watthour meters used as Comparison Standards are tested with $120 \mathrm{v}$ and 5 amp applied, at unity power factor, and at 0.5 power factor, with the current lagging the voltage. The method of testing a Comparison Standard Watthour Meter involves a comparison with the Standard Watthour Meter immediately following or during its calibration and periodically between calibrations. Figure 3 is a diagram of the circuit involved in the intercomparison of the standard wathour meters.

The similarity between the circuit shown in figure 3 and the alternating-current portion of figure 2 should be noticed. When a calibration of the Standard Watthour Meter is in progress the Comparison Standard Watthour Meters shown in figure 3 are incorporated in the circuit of figure 2, although in the interest of simplicity, this modification is not shown in the figure. With the circuit revised in this manner, the Standard Watthour Meter, the Comparison Standard Watthour Meters, and the Standard Wattmeter are energized simultaneously, and the calibration runs on the reference standard can be performed alternately with intercomparison test runs, thereby insuring nearly identical conditions for both tests. Truly simultaneous testing of the reference standard (having the circular plosphorescent scale) and the comparison standards is possible with the addition of more equipment, but it is believed that any improvement that might accrue from simultaneous testing would be too small to detect with certainty.

There is a phototube-lamp combination associated with each Comparison Standard, but only one of these combinations is energized at a time. The phototube produces a pulse for each revolution of the disk of the Comparison Standard under test. This pulse is amplified and used in conjunction with the pulse-counting circuit to start and stop the run.

To start the run, a pulse from the phototube associated with the Comparison Standard Wathour

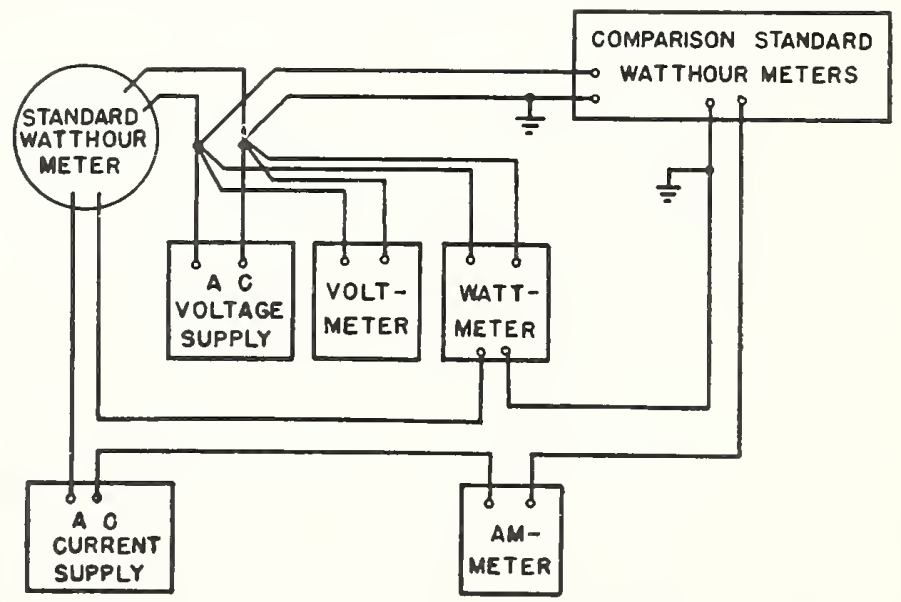

Figure 3. Intercomparison of Standard Wathour Meters. 
Meter is used to flash the mercury-vapor lamp and a spot is left on the phosphorescent scale of the Standard Watthour Meter. The position of this spot is recorded on the data sheet. When the preset number of turns have been completed, the mercuryvapor lamp again flashes; another spot is left, and its position is recorded together with the reading of the revolution counter. From these readings the number of revolutions of the Standard Watthour Meter's disk is computed. The average percentage registration of the Comparison Standard obtained from several runs is taken as the most probable value. The other Comparison Standard Watthour Meters are tested in the same way.

The formula used in this test for computing the percentage registration of the Comparison Standards is

where

$$
\mathscr{R}_{c}=\frac{n_{c} K_{c}}{K_{s}} \frac{\mathscr{R}_{s}}{n_{s}}
$$

$n_{c}=$ number of disk revolutions of the Comparison Standard Watthour Meter.

$n_{s}=$ number of disk revolutions of the Standard Watthour Meter.

$K_{s}=$ disk constant of the Standard Watthour Meter, in wat thours per revolution.

$K_{c}=$ disk constant of the Comparison Standard Watthour Meter, in watthours per revolution.

$\mathscr{T}_{s}=$ percentage registration of the Standard Watthour Meter.

A derivation of this equation appears in the appendix.

\subsection{Tests of Other Watthour Meters, Using the Standard Watthour Meter}

Alternating-current wathour meters that are sent to the National Bureau of Standards for test are compared with the Standard Watthour Meter in the following manner. The current and potential circuits of the Standard Watthour Meter are energized from the secondaries of a current transformer and a potential transformer having multiple ranges. The primary windings of these transformers and the wathour meter under test are excited by the a-c power supplies, as shown in figure 4 .

The resistance of the leads that connect the potential circuit of the wathour meter under test to the primary of the voltage transformer is made small enough to render negligible the error in phase angle caused by the voltage drop in the leads. A voltmeter, ammeter, and a wattmeter of good quality are connected in the secondary circuit, and they enable an observer to set the secondary voltage, current, and power factor.

A phototube-lamp combination is arranged to produce a pulse at each revolution of the disk of the wathour meter under test if this is conveniently possible. The pulses are amplified, ond, in conjunction with the pulse counter, are used to determine the duration of the run.

An alternative method of testing meters, particu- larly those that have no good means of operating a phototube, is to let the duration of the run be determined by the Standard Watthour Meter. This is accomplished by connecting the pulse counter to the output of the phototube located behind the slit in the scale of the Standard Watthour Meter. The potential circuit of the watthour meter under test is then closed at the beginning of the run and opened at the end by relays operated by the pulse counter. Contacts on one of the relays, when energized, close the potential circuit; while normally closed, contacts on the other relay, when energized, open the circuit. The time lags in the operation of these relays are carefully equalized to reduce timing errors to a negligible amount.

As the percentage registration of the Standard Watthour Meter is accurately known, the percentage registration of the watthour meter under test can be computed. Corrections are applied for the ratio and phase-angle errors of the current and potential transformers.

The procedure followed in making a test of this sort is practically identical to that foilowed in testing a Comparison Standard Watthour Meter.

The formula used in this test for computing the percentage registration of the test watthour meter is

$$
\mathscr{R}_{t}=\frac{K_{t} n_{t} \mathscr{R}_{s}}{K_{s} N_{p t} N_{c t} n_{s}}+100\left[c_{\theta}-c_{c t}-c_{p t}\right]
$$

where

$K_{t}=$ disk constant of the watthour meter under test, in watthours per revolution.

$n_{t}=$ number of disk revolutions of the test wat $t$ hour meter.

$\mathscr{R}_{s}=$ percentage registration of the Standard Watthour Meter.

$K_{s}=$ disk constant of the Standard Watthour Meter, in watthours per revolution.

$N_{p l}=$ nominal potential transformer ratio.

$N_{c t}=$ nominal current transformer ratio.

$n_{s}=$ number of disk revolutions of Standard Watthour Meter.

$c_{c t}=$ correction for the current transformer ratio (= ratio factor -1 ), in parts per unit.

$c_{\phi}=$ correction for the potential transformer ratio (= ratio factor -1 ), in parts per. unit.

$c_{\varphi}=$ colvection for the phase angle, in parts per unit. It is equal to $(\beta-\gamma)$ tan $\theta_{s}$.

$\beta=$ phase angle of the current transformer, in radians, considered positive when the reversed secondary-current vector leads the primary-current vector.

$\gamma=$ phase angle of the potential transformer, in radians, considered positive when the reversed secondary-voltage vector leads the primary-voltage vector.

$\theta_{s}=$ power-factor angle at the Standard Watthour Meter, considered positive when the current vector lags the voltage vector.

A complete derivation of this equation appears in the appendix. 


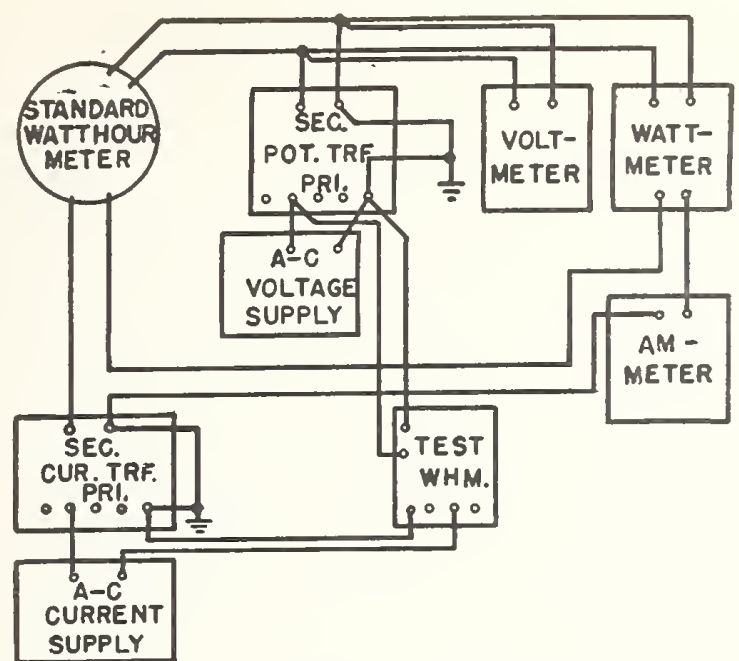

Figure 4. Testing wathour meters, using the standara Watthour Meter.

\section{Calibration and Testing Schedule}

The periodic primary calibrations of the Standard Watthour Meter serve to accurately ascertain its percentage registration. Primary calibrations performed at monthly intervals for a period of time have indicated a long-time stability of the reference standard that will permit an increase in the interval between calibrations to 6 months or longer. A graphical record is kept of the intercomparison tests, which are performed at more frequent intervals, to detect any appreciable drifts or erratic changes in registration of any one of the group relative to the others, and possibly, to form a basis for a revision of the registration assigned to the Standard Watthour Meter if an observed change is not large enough to warrant a primary calibration. When a revision of the registration of reference standard is warranted on the basis of intercomparison tests and the evidence does not indicate gross instability in any one particular meter, the computation of registration is performed on the assumption that the mean registration of all meters remains unchanged between calibrations. With this assumption, the entire group of meters is useful in maintaining the unit of energy, although transfer of the unit to a watthour meter under test is accomplished through only one member of the group.

\section{Precision and Accuracy Attainable}

Both systematic and random errors limit the accuracy of test results obtained with the Standard Watthour Meter and its associated apparatus. Systematic errors, those which cannot be considered accidental, can be further classified in to several types. First, there are the errors that have escaped the attention of the observers. After a careful analysis of all possible sources of error and the comparison of the results of tests made by several methods, it is believed that unknown systematic errors of magnitudes larger than about 0.01 percent are highly improbable.

Second, there are residual errors from the application of imperfect corrections. These elrors are sys- tematic in nature and have been minimized in the present work by carefully: calibrating each piece of apparatus for which a correction must be applied in terms of the National Electrical Standards, which are readily available. The largest systematic errors of this type result from the uncertainty in the ratio and phase-angle corrections of the current and potential transformers. Other residual errors of this type, such as those associated with the standard cell, potentiometer, and volt box, occur in the primary calibrations of the Standard Watthour Meter, and are rather small. The history of the components used in the measurements circuit is well known. The maximum net change in value of the components between their periodic tests, for the last several years, is listed in table 1.

The third type of systematic error is, in a sense, similar to the second type, in that it arises from the imperfect application of corrections, but in this case the corrections are for the compensation of fluctuating errors. Such elrors may vary with certain parameters in a definite though unknown manner. On the other hand, the fact that an error exists and that it is a function of a certain parameter may be known, but its effect may be negligibly small (and a

TABLE 1. Observed maximum net change in the value of the components necessary for the accurate standardization of the Standard Electrodynamic Wattmeter.

\begin{tabular}{|c|c|}
\hline Component & $\begin{array}{l}\text { Maximum net } \\
\text { change in value }\end{array}$ \\
\hline $\begin{array}{l}\text { Standard cell } \\
\text { Potentiometer } \\
\text { Volt box }\end{array}$ & $\begin{array}{l}p p m \\
10 \\
10 \\
11\end{array}$ \\
\hline Total $\ldots . . . . . .$. & 31 \\
\hline $\begin{array}{l}\text { Standard cell } \\
\text { Potentiometer } \\
\text { Resistance standard }\end{array}$ & $\begin{array}{r}10 \\
10 \\
6\end{array}$ \\
\hline Total & 26 \\
\hline Grand total $\ldots$ & 57 \\
\hline
\end{tabular}

correction impractical to apply) compared with that of the other errors. Varying systematic elrors are evident in the Standard Watthour Meter as well as the Comparison Standard Watthour Meters and, in fact, sharply limit the accuracy of which any watthour meter is capable. Reference is here made to the effect on the percentage registration of a watthour meter of such factors as incorrect magnitude of applied voltage and current, incorrect phase angle, incorrect frequency, or the presence of harmonics in the applied voltage or current. Studies have been made of the effect of each of these variables on the meters used as standards. It has been found feasible to use a good quality voltmeter, ammeter, and wattmeter, to facilitate setting the voltage, current, and phase angle. The power supplies are electronically regulated to maintain the initial adjustment of voltage and current, and are operated and loaded in such a manner as to minimize harmonics.

A third harmonic of as little as 1 percent of the fundamental in the current circuit of a watthour 
meter may cause a registration error of several hundredths of a percent if certain phase relationships exist. This error arises from the existence of a third harmonic flux in the air gap of the electromagnet even if a purely sinusoidal voltage is applied to the potential coil. It is important to realize that even if a watthour meter is tested using alternating current of practically sine wave form it will register inaccurately if used in a circuit in which the wave form is nonsinusoidal. Although it would be unfair to impute errors due to nonsinusoidal wave form to watthour meters, which are designed for use on a sinusoidal waveform, the possibility of an appreciable error arising from this cause should not be overlooked if accurate measurements are desired. Odd harmonics which are present in the wave form of the current and voltage supplied to the Standard Watthour Meter and associated apparatus at the time of this writing are, under most load conditions, considerably less than 1 percent, much less than usually found in commercial power sources.

During calibrations of the Standard Watthour Meter, average frequency of the applied power during a run is measured and a correction applied for any deviation from $60 \mathrm{c} / \mathrm{s}$. In most other tests a measurement of average frequency, and a correction for errors therein, is not conveniently possible, and this probably contributes the greatest share of systematic errors that vary in a definite, but unknown, manner. However, it has been determined that errors arising from customarily observed deviations in frequency are of the order of a few thousandths of a percent. The effect of temperature changes on the watthour meter standards is reduced to a small value by keeping all meters in temperature-controlled enclosures. The standards are not removed from the position in which they are calibrated. 'Throughout all tests the Standard Watthour Meter is operated continuously, thus eliminating those errors associated with starting and stopping the disk.

Since the scale of the Standard Watthour Meter is marked with 1,000 small divisions, any. small variations in the angular speed of the disk which would be undetectable in other meters may very likely be easily discernible. To determine the nature and the magnitude of elrors due to angular speed variations, the standard was centered as accurately as feasible in its scale and calefully levelled. It was operated at a reduced speed, and seconds pulses were used to flash the mercury vapor lamp once every second. The applied power was held constant at a value sufficient to cause successive spots from the mercuryvapor lamp to appear on the phosphorescent scale at four points as the disk revolved. Scale readings corresponding to spot positions indicated a regular (nearly sinusoidal) variation of disk speed during each revolution. The variations were such as to cause a maximum shift in scale reading of about 0.005 revolution. A method was devised for aligning the wathour meter in its scale to reduce this error to less than 0.001 revolution, thereby partially compensating for the angular speed variations. An error of 0.001 revolution would cause a scale reading error of only 0.001 percent if the watthour meter is operated for 100 revolutions, as is usually done.

Some of the residual errors thus far discussed will be positive, others will be negative, and the magnitude of many of these systematic errors will appear to vary in a random manner as more and more tests are performed. To this must be added the truly random errors, the distribution of which is entirely by chance, and to which the theory of errors may be applied. The values listed in table 2 include an estimate of the standard deviation of random errors based upon numerous test data, and half the maximum systematic error that it is believed may possibly be present in any one measurement of energy. It is recognized that the errors listed do not represent truly random variations, yet their individual contribution (some being positive and some negative) to the final result partakes of randomness and probably justifies the computation of the total propagated error from the square root of the sum of the squares of the component errors. There is one exception. The error in the standard cell and the error in balancing the potentiometer against the electromotive force of the standard cell occur twice in a single measurement of d-c power; hence, these component errors are not independent, and the propagation of these errors is by simple addition. The potentiometer error also occurs twice, but these are independent, since different resistance sections of the potentiometer are used for the measurement of voltage and current. The total propagated error provides an estimate of expected accuracy with which the applied energy is known, when the Standard Watthour Meter and its associated apparatus is used to test other meters. It is not to be inferred that the other meters are capable of calibration or maintaining their calibration to the degree of accuracy indicated at the bottom of the table.

$A$ test was made to determine the repeatability of results in the comparison testing of the Comparison Standard Watthour Meters against the Standard Watthour Meter. Individual runs of about 100 seconds duration were made about 10 minutes apart until a group of 10 runs had been made. This was repeated the next day, and again 4 days later. The relative percentage registrations of the two Comparison Standards are shown in table 3 . The values listed are the means of the groups of 10 runs, and the measure of precision is the standard deviation of a single run of the group.

The precision indices listed in table 3 result from a combination of random errors inherent to (1) the Comparison Standard Watthour Meter being tested, and (2) the Standard Watthour Meter, including observational error's in reading its scale. If it is assumed that each meter contributes equally to the total error, but that the contributions are random, then an index of precision for each meter may be computed. If the total error is considered as 0.012 percent, the individual errors which produce this errol are each $0.012 / \sqrt{2}=0.008$ percent. From these data it is estimated that the precision of each of the watthour meter's in the setup over a period of a fow hour's is about 0.008 percent. 
TARLE 2. Effect of residual errors on the test of a wathour meter

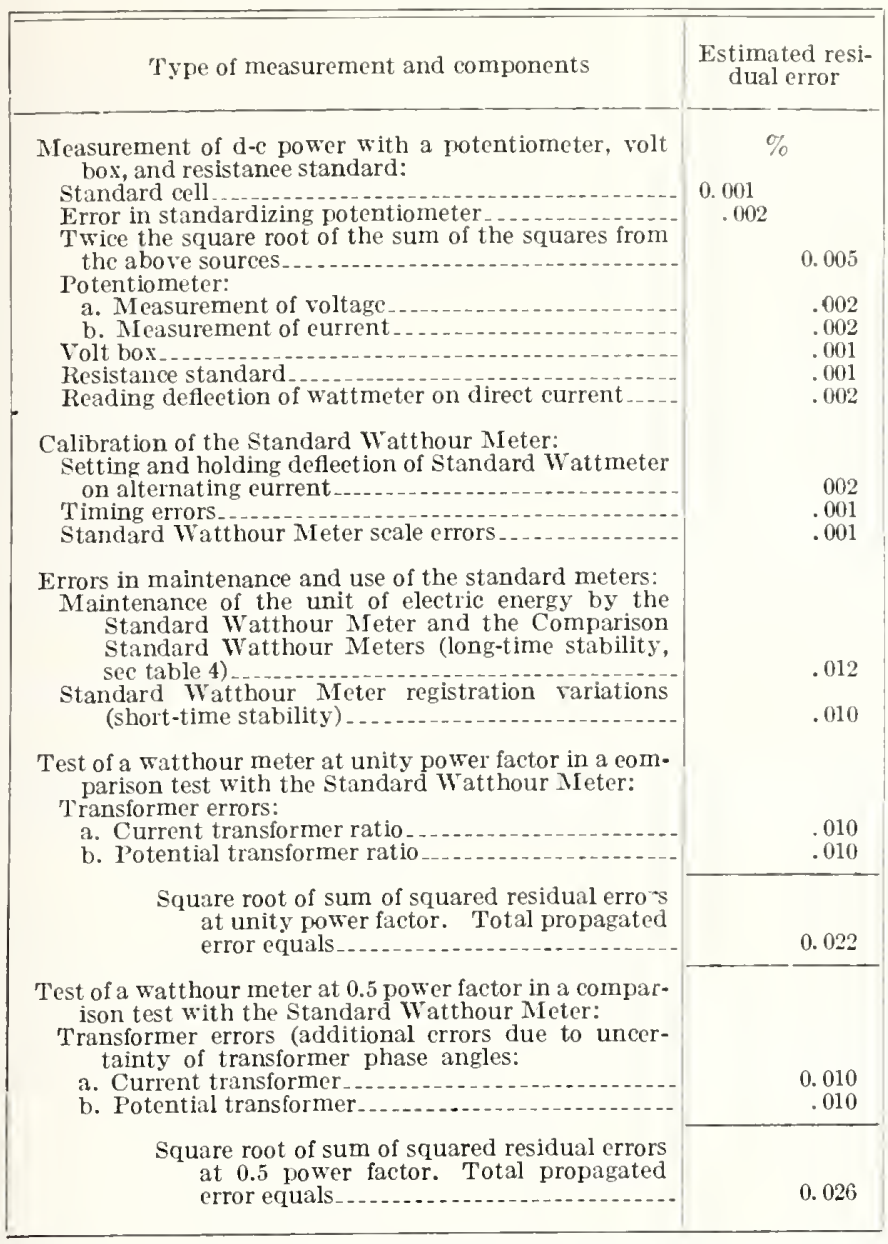

TABLE 3. Short-time repeatability of intercomparison tests on the Comparison Standard Watthour Meters against the reference Standard Watthour Meter

At 0.5 power factor, the eurrent lagged the voltage.

\begin{tabular}{|c|c|c|c|c|c|c|c|c|}
\hline \multirow{3}{*}{ Group } & \multicolumn{4}{|c|}{ Comparison Standard No. 1} & \multicolumn{4}{|c|}{ Comparison Standard No. 2} \\
\hline & \multicolumn{2}{|c|}{1 power factor } & \multicolumn{2}{|c|}{0.5 power factor } & \multicolumn{2}{|c|}{1 power factor } & \multicolumn{2}{|c|}{0.5 power factor } \\
\hline & $\begin{array}{l}\text { Regis- } \\
\text { tration }\end{array}$ & $\begin{array}{l}\text { Stand- } \\
\text { ard } \\
\text { devia- } \\
\text { tion }\end{array}$ & $\begin{array}{l}\text { Regis- } \\
\text { tration }\end{array}$ & $\begin{array}{l}\text { Stand- } \\
\text { ard } \\
\text { devia- } \\
\text { tion }\end{array}$ & $\begin{array}{l}\text { Regis- } \\
\text { tration }\end{array}$ & $\begin{array}{l}\text { Stand- } \\
\text { ard } \\
\text { devia- } \\
\text { tion }\end{array}$ & $\begin{array}{l}\text { Regis- } \\
\text { tration }\end{array}$ & $\begin{array}{l}\text { Stand- } \\
\text { ard } \\
\text { devia- } \\
\text { tion }\end{array}$ \\
\hline $\begin{array}{l}\mathrm{A} \\
\mathrm{B} \\
\mathrm{C}\end{array}$ & $\begin{array}{c}\% \\
100.067 \\
100.085 \\
100.056\end{array}$ & $\begin{array}{c}\% \\
0.014 \\
.001 \\
.010\end{array}$ & $\begin{array}{c}\% \\
99.923 \\
99.932 \\
99.936\end{array}$ & $\begin{array}{c}\% \\
0.012 \\
.016 \\
.016\end{array}$ & $\begin{array}{c}\% \\
100.140 \\
100.160 \\
100.135\end{array}$ & $\begin{array}{r}\% \\
0.011 \\
.010 \\
.015\end{array}$ & $\begin{array}{c}\% \\
100.068 \\
100.064 \\
100.082\end{array}$ & $\begin{array}{r}c \% \\
0.007 \\
.010 \\
.018\end{array}$ \\
\hline
\end{tabular}

TABLE 4. Precision of intercomparison test results over a period of several months (from same data as figure 5)

At 0.5 power factor, the current lagged the voltage.

\begin{tabular}{|c|c|c|}
\hline \multirow{2}{*}{ Wathour meter } & \multicolumn{2}{|c|}{ Standard deviation } \\
\hline & 1.0 power factor & 0.5 power faetor \\
\hline standard Watthour Meter & $\begin{array}{l}\text { Percent } \\
0.012\end{array}$ & $\begin{array}{l}\text { Percent } \\
0.014\end{array}$ \\
\hline $\begin{array}{l}\text { Comparison Standard Watt- } \\
\text { hour Meter No. }\end{array}$ & .008 & .007 \\
\hline $\begin{array}{l}\text { Comparison Standard Watt- } \\
\text { hour Meter No.2. }\end{array}$ & .007 & .012 \\
\hline
\end{tabular}

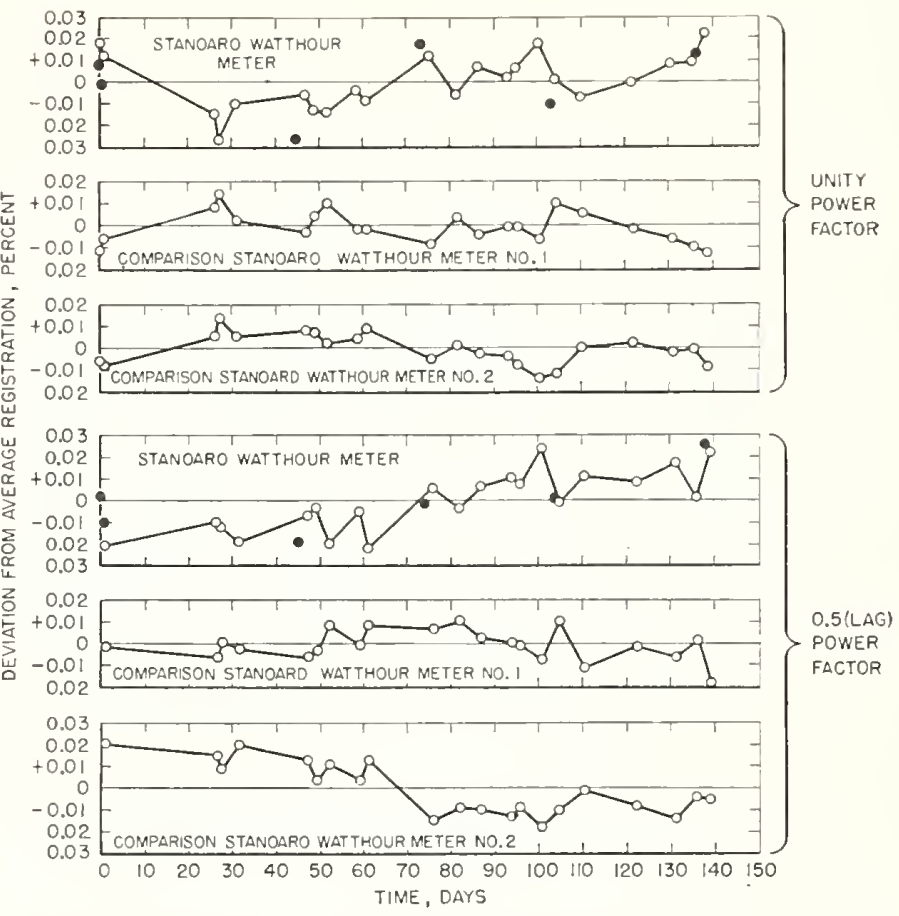

Fintre 5. Percentage registration deviations of the Standard Watthour Meter and Comparison Standard Watthour Meters over a period of several months.

Connected points are results of intercomparison tests; isolated points are results of calibrations of the Standard Watthour Meter.

As these meters are used to maintain the unit over much longer periods of time than were used in the test described, it might be expected that the longtime stability of the meters should not be as good as that for short intervals because of varying systematic errors. On the other hand, the use of a multiplicity of meters improves the over-all precision. A measurement of the precision with which the unit is maintained is obtained from the records of calibration and intercomparison tests. Figure 5, for example, shows the test results over a period of several months. The points connected by straight lines represent the results of intercomparisons, and are based on an assumed constant mean registration of all three meters. The validity of this assumption is proved by the proximity of the intercomparison test results to the isolated points representing calibrations of the Standard Watthour Meter by the wattmeter method. The standard deviation for each of the meters (assuming all deviations are normally distributed and random) computed from the data shown in figure 5 is given in table 4 . From data such as these it is estimated that the unit of the watthour can be maintained over a period of several months with precision represented by a standard deviation of 0.012 percent or better. Many tests that have been run in rapid succession, a few minutes apart, indicate drifts in registration in one direction for a period, then a reversal or rapid change of some sort. Such drifts or changes have been very small (few thousandths of a percent). However, an abnormal distribution of this sort reveals the presence of those 
varying systematic errors, discussed previously, which depend upon other parameters. A comparison of tables 3 and 4 shows that the stability over several months is very nearly the same magnitude as that over a period of an hour or two. This implies that any varying systematic errors that may exist vary rapidly and in such a manner that their effect on the mean of a number of observations of percentage registration taken several hours or more apart is negligibly small. Accordingly, when calibrations of the Standard Watthour Meter are performed, the individual runs are taken at time intervals sufficient to extend the duration of the test to several hours.

When the apparatus is used for testing other watthour meters it is believed that the measurement of the energy applied to the test watthour meter is valid and can be relied upon to better than 0.06 percent.

\section{Conclusion}

At the National Bureau of Standards a set of several carefully selected and prepared watthour meters has been arranged as a standard group for testing alternating-current wat thour meters by a comparison inethod.

Although the initial cost of designing, constructing, and assembling this apparatus would ordinarily deter the general adoption of such an elaborate system of testing watthour meters, the use of the apparatus described has yiclded a definite saving in time and labor, without significant loss of accuracy when compared with the primary method used in the past.

Accurate initial calibration of the apparatus is accomplished by averaging the results of a number of primary calibrations of the Standard Watthour Meter, using (1) a transfer wattmeter and potentiometer for measurements in terms of the fundamental electrical units of electromotive force and resistance, and (2) standard seconds signals for time measurements. Assurance of the continued accuracy of the reference standard watthour meter is obtained by intercomparison tests with other members of the standard group. The reference standard, supplemented by calibrated instrument transformers, accuratcly measures the energy supplied to a watthour meter under test.

J. B. Dempsey initiated the early work in setting up selected wat thour meters for secondary standards. F. B. Silsbee proposed the light-beam pointer and phosphorescent scale. F. J. Gross carried out the developmental work and the original design of the comparison apparatus. Most of the mechanical construction of this apparatus was done by C. H. Hochgesang of the Bureau's Instrument Shop. Robert W. Balcom assembled most of the electronic equipment and provided many valuable suggestions. Thomas W. Cushing carried the project forward for a time and designed and supervised the construction of the constant-temperature cabinet for the comparison standards.

\section{Apppendix}

The derivations of eq (1), (2), and (3) proceed most easily from the definition of percentage registration, which is the ratio of the indicated energy as obtained from the reading of the wathour meter to the true energy, expressed in percent.

$$
\mathscr{R}=100 \frac{\text { Indicated energy }}{\text { True energy }}
$$

In a test of the Standard Watthour Meter, using the Standard Wattmeter, the indicated energy in watthours is $K n$. The true energy is determined by the readings and settings of the Standard Wattmeter. Because this instrument is very nearly astatic, its sensitivity, $s$, will be the same for both directions of the direct current, and the average deflection is

$$
D_{d c}=s W_{d c}^{\top}
$$

where $W_{d c}$ is the true power impressed on the Standard Wattmeter." The true power in terms of the potentiometer settings and circuit constants is

$$
W_{d c}=\frac{E_{p} N_{v b} E_{c}}{R_{s}}+c
$$

where $c$ represents the correction terms for the circuit components. The average a-c deflection of the Standard Wattmeter is

$$
D_{a c}=s E I \cos \left(\theta+T_{w}\right),
$$

where $E$ is the a-c voltage between the Standard Wattmeter potential terminals causing a potential coil current, $I_{p}$, see figure 6 . It is the magnetic field from this current that reacts with the magnetic field from the current, $I$, in the fixed coils of the Standard Wattmeter to produce the deflection, $D_{a c}$.

The wattmeter sensitivity, $s$, will be the same on alternating current as on direct current provided all readings are grouped closely on the Standard Wattmeter's scale.

Dividing (5) by (7)

$$
\frac{D_{d c}}{D_{a c}}=\frac{s W_{d c}}{s E I \cos \left(\theta+T_{u c}\right)} .
$$

Solving for $E I$

$$
E I=W_{d c} \frac{D_{a c}}{D_{d c} \cos \left(\theta+T_{w}\right)} .
$$

The a-c power impressed on the Standard Watthour Meter is

$$
W_{a c}=E I \cos \theta
$$

and substituting for $E I$ from eq (9)

$$
W_{a c}=W_{d c} \frac{D_{a c} \cos \theta}{D_{d c} \cos \left(\theta+T_{t o}\right)} .
$$

4 The symbols not specifically defined in the appendix have been previously defined in the text. 


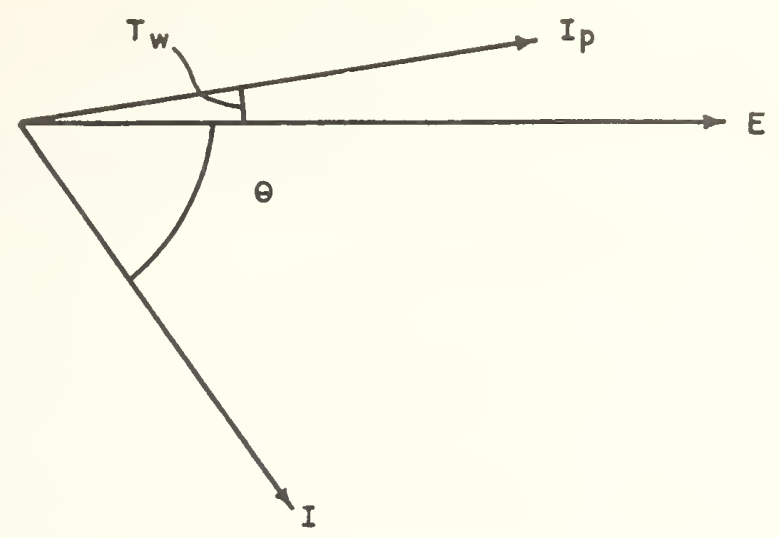

FIgURE 6. Vector relationships at the Standard Wattmeter terminals during a calibration of the Standard Watthour Meter.

No scale is intended and the angles indicated are grossly exaggerated for clarity.

This can be rearrangeci in the form

$W_{a c}=W_{d c}\left[1+\frac{D_{a c}}{D_{d c}}-\frac{D_{d c}}{D_{d c}}\right]\left[1+\frac{\cos \theta}{\cos \left(\theta+T_{v o}\right)}-\frac{\cos \left(\theta+T_{w}\right)}{\cos \left(\theta+T_{w}\right)}\right]$

$W_{a c}=W_{d c}\left[1+\frac{D_{a c}-D_{d c}}{D_{d c}}\right]$

$$
\left[1+\frac{\cos \theta-\cos \theta \cos T_{w}+\sin \theta \sin T_{w}}{\cos \theta \cos T_{w}-\sin \theta \sin T_{w}}\right]
$$

$W_{a c}=W_{d c}\left[1+\frac{D_{a c}-D_{d c}}{D_{d c}}\right]$

$$
\left[1+\frac{\cos \theta\left(1-\cos T_{w}\right)+\sin \theta \sin T_{w}}{\cos \theta \cos T_{w}-\sin \theta \sin T_{w}}\right]
$$

and since $T_{w}$ is a very small angle (116 microradians at $60 \mathrm{c} / \mathrm{s})$, sin $T_{w}$ very nearly equals $T_{2}$ in radians and $\cos T_{2}$ is very nearly 1 ; therefore, the equation can be simplified by neglecting the extremely small terms.

$$
W_{a c}=W_{d c}\left[1+\frac{D_{a c}-D_{d c}}{D_{d c}}\right]\left[1+T_{w} \tan \theta\right]
$$

and since the second terms in both pairs of brackets are much smaller than 1 , the equation may be further simplified by multiplying and then neglecting: the smaller second-order terms.

$$
W_{a c}=W_{d c}\left[1+\frac{D_{a c}-D_{d c}}{D_{d c}}+T_{w} \tan \theta\right]
$$

When $W_{d c}$ from eq (6) is substituted and the result is multiplied by $t / 3600$ to obtain energy in watthours, the true energy thus obtained may be used in eq (4), and the results, showing all corrections as correction terms, is eq (1) shown in section 3.

The derivation of eq (2), which is used in intercomparison tests, follows logically from eq (4). The indicated energy equals $K_{c} n_{c}$. Hence the percentage registration of the Comparison Standard Watthour Meter is

$$
\mathscr{R}_{c}=100 \frac{K_{c} n_{s}}{\text { True energy }}
$$

and that of the Standard Watthour Meter is

$$
\mathscr{R}_{s} \frac{K_{s} n_{s}}{\text { True energy }} \text {. }
$$

Since, jn this test, the true energy is identical for both watthour meters, eq (15) and (16) can be combined to yield eq (2), as shown in section 3.2.

In the derivation of eq (3) the following additional symbols will be used:

$\theta=$ power factor angle at the test watthour meter, considered positive when the current vector lags the voltage vector. It is equal to $\theta_{s}+\beta-\gamma$.

$E_{p}=$ primary voltage applied to the test watthour meter.

$I_{p}=$ primary current applied to the test watthour meter.

$E_{s}=$ secondary voltage applied to the Standard Watthour meter.

- $I_{s}=$ secondary current applied to the Standard Watthour meter.

$t=$ time of run, in seconds.

$N^{\prime}{ }_{p t}=$ true potential transformer ratio $=$
$N_{p t}\left(1+c_{p t}\right)$.

$N^{\prime}{ }_{c t}=$ true current transformer ratio $=$ $N_{c t}\left(1+c_{c t}\right)$.

The indicated energy of the watthour meter under test is $K_{t} n_{t}$. The true energy that should be measured by the test watthour meter is $E_{p} I_{p} t \cos \theta$, or, if $E_{p}$ and $I_{p}$ are expressed in terms of secondary voltage and secondary current, the true energy is $E_{s} N_{p t}^{\prime} I_{s} N_{c t}^{\prime} t \cos \theta$. Substituting these factors in eq (4), the percentage registration of the test watthour meter is

$$
\mathscr{R}_{t}=100 \frac{K_{t} n_{t}}{E_{s} N_{p t}^{\prime} I_{s} N_{c t}^{\prime} t \cos \theta} .
$$

Now consider the energy measured by the Standard Watthour Meter. Since the percentage registration of the Standard Watthour Meter is known, and the indicated energy is $K_{s} n_{s}$, then the true energy measured by the Standard Watthour Meter is

$$
\frac{100 K_{s} n_{s}}{\mathscr{R}_{s}}=E_{s} I_{s} t \cos \theta_{s}
$$

or

$$
\frac{1}{E_{s} I_{s} t}=\frac{\mathscr{R} \cos \theta_{s}}{100 K_{s} n_{s}}
$$

and substituting eq (19) in eq (17)

$$
\mathscr{R}_{t}=\frac{K_{t} n_{t} \cdot \mathscr{R}_{s}}{K_{s} N_{p l}^{\prime} N_{c \ell}^{\prime} n_{s}} \frac{\cos \theta_{s}}{\cos \theta}
$$


In this equation $\cos \theta_{s} / \cos \theta$ is a correction factor for the phase angles of the current and voltage transformers. To simplify this correction the denominator may be expanded

$$
\frac{\cos \theta_{s}}{\cos \left(\theta_{s}+\beta-\gamma\right)}=\frac{\cos \theta_{s}}{\cos \theta_{s} \cos (\beta-\gamma)-\sin \theta_{s} \sin (\beta-\gamma)}
$$

Dividing both numerator and denominator by cos $\theta_{s}$ the correction factor becomes

$$
\frac{1}{\cos (\beta-\gamma)-\sin (\beta-\gamma) \tan \theta_{s}} .
$$

Since $(\beta-\gamma)$ is a small angle, $\cos (\beta-\gamma)$ is very nearly 1 and $\sin (\beta-\gamma)$ is very nearly $(\beta-\gamma)$, heuce the correction factor is

$$
\frac{1}{1-(\beta-\gamma) \tan \theta_{s}}
$$

and when the indicated division is performed, neglecting the extremely small second-order terms, the result is

$$
1+(\beta-\gamma) \tan \theta_{s}
$$

Thus the correction tel'm for the phase angle in parts per unit is $c_{\varphi}=(\beta-\gamma)$ tan $\theta_{s}$, and when the correction terms for the current transformer ratio, potential transformel ratio, and phase angles are shown, the final form of eq (20) is that of eq (3) in part 3.3.

Washington, March 5, 1954. 
Preprint of paper to be dellvered at the Eighth Natlonal Conference of the Instrument Soclety of America, September 21-25, 1953, Hotel Murrison, Chlcago, 111.

Prlce to members 25 cents, to non-members 50 cents,

Please Note: Statements and opinions advanced in this paper are to be understood as Indlvidual expressions of the author(s) and not those of the Soctety.

\title{
The Testing of Electrical Instruments
}

\author{
By FRANCIS L. HERMACH*
}

\begin{abstract}
This paper describes the basic methods and procedures for testing elec trical instruments at power and audio frequencies, discusses the standards used and their tests, and the power sources and control consoles. It also outlines the facilities and testing services of the National Bureau of Standards to science and industry in this fleld, and describes some new developnents which may be useful in instument testing.
\end{abstract}

TNCREASING demands for greater accuracy of electrical measurements in industrial and military development and production are reflected in the increasing number of laboratory installations in which electrical instruments are tested or standardized. The equipment in each such installation depends on the type, range and accuracy of the measurements to be made. It may include potentiometers for testing laboratorystandard instruments of 0.1 per cent accuracy; or may consist only of multipurpose instruments for testing electronic or panel instruments of the 2 per cent and 3 per cent accuracy class. Certain principles and methods underlie all electrical instrument testing, however, and it is the purpose of this paper to discuss the basic methods and equipment for standardizing ${ }^{* 1}$ instruments at power and audio frequencies. The services and equipment at the National Bureau of Standards, in this field, will also be described.

\section{TESTS OF D-C INSTRUMENTS}

The basic electrical standards are groups of standard cells and resistors maintained at NBS and corresponding standardizing laboratories in other large countries. Their values are determined in absolute volts and ohms by measurement based upon the standards of length, mass and time.

- Electrlcal Fingineer, Electrical Instruments Section, Natlonal Bureau of Standards.

orTo standardize an instrument is to compare lts lndicutlons with those of an Instrument of known accuracy. To calibrate an instrument is literaliy to mark its scale. In each cese this requires precise ineasure ments of the electrical quantity involved. The speclal techniques that simplity the The speclal techniques that simplify the
factory callbration of instrumelitp will not be dlscussed here.

These standard cells may be compared with others by means of potentiometers, and the unit of voltage may thus be disseminated. Similarly, by means of bridges, resistors may be intercompared, potentiometers, and indeed bridges themselves, standardized. The potentiometer, which serves the same purpose for voltage comparisons as a chemist's balance serves for mass comparisons, thus becomes the basic instrument for making d-c measurements of voltage, current and power and, therefore, for testing $d-c$ instruments.

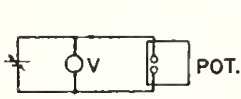

Fig.la

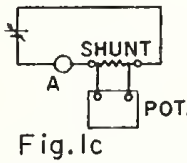

Fig. 1. Circuits for Testing Instrument With the Potentioneter

\section{The Potentiometer Method}

The basic principle of the potentiometer is well known and will not be discussed here. ${ }^{1}$ The elementary diagrams for the use of the potentiometer in testing instruments are shown in Fig. 1. The test of a voltmeter of ranges less than that of the potentiometer $(1.6$ volts) is shown in Fig. 1a, the use of a volt box for tests of voltmeters of higher range in $1 \mathrm{~b}$, and the use of a shunt for tests of ammeters in 1c. In each case, the d-c source is adjusted for the desired deflection of the test instrument; and the true voltage or current is determined from the potentiometer reading and the volt-box ratio or shunt resistance.

Over a hundred years of development have resulted in many forms and refinements of potentiometer's; but in general, only three types are useful in instrument testing. These are the 2- or 3range general-purpose potentiometer, the more convenient but more limited

Superior numbers refer to similarly numbered relerences in the Bibliography at the end of paper. deflection potentiometer, and the newer forms of the portable potentiometer. The general-purpose potentiometer is more accurate than required for instrument testing and is somewhat slow and cumbersome to use, but it is very stable, flexible and reasonable in cost. Although it is less fiexible and stable, the deflection potentiometer overcomes some of these disadvantages and is well-suited for instrument testing. However, it requires a special volt box and is not readily adapted for low-current or low-voltage measurements (such as for'tests of microammeters and millivoltmeters). Inexpensive portable potentiometers of 0.05 to 0.1 per cent accuracy are now commercially available. They do not require external galvanometers, standard cells, or storage batteries, and are very convenient. It seems likely that slight improvements may further increase their usefulness, for instrument testing.

\section{Comparison Tests}

Well-designed and well-constructed instruments may retain their calibration for months or even years. Such instruments of suitable accuracy, which have been properly tested, may be used as reference standards to test other instruments with greater speed and convenience but more limited accuracy than potentiometer methods. Laboratory standard instruments of 0.1 per cent accuracy are often used and are well suited for testing other instruments of lesser accuracy. However, they are somewhat limited in range and comparatively expensive so that multirange 0.25 or 0.5 per cent instruments are often chosen to supplement a potentiometer installation. The potentiometer is then used for tests of instruments of 0.5 per cent accuracy class or better and for periodically testing the standard instruments.

For comparison tests, the instruments are connected in series if ammeters, and in parallel if voltmeters; and the current or voltage is adjusted for the desired deflection of the test instrument. The standard is then read (preferably in divisions and tenths of a division) and its multiplying factor and correction applied to give the true voltage or current corresponding to that indication. This is preferable to the alternate procedure of setting the standard. It is 
desirable to specify two or three extra scale divisions at the top of the scale of each standard instrument; i.e., a 153volt instead of a 150 -volt instrunient.

\section{TESTS OF A-C INSTRUMENTS}

Since the basic electrical standards can be used only for direct-current measurements, all a-c measurement.s of voltage, current, and power depend fundamentally on certain types of instruments which ideally have the same response on alternating and direct current. Such instruments can be calibrated on direct current and then used for alternatingcurrent measurements. There are three basic types of such instruments: electrodynamic instruments, which depend on the forces between current-carrying conductors; electrostatic instruments, which depend on the force between charged conductors; and electrothermic instruments, which depend on the heating of a current-carrying conductor.

There are three distinct types of tests of a-c instruments; an a-c test, a reversed d-c test, and a transfer test. An a-c test is a direct comparison of the instrument with another a-c instrument of known accuracy. Alternatively, instruments of the types mentioned can be tested on reversed direct current and then given a transfer test to determine their ac-dc difference. Since this difference is relatively permanent and generally small, a transfer test need ordinarily be made only once for each in strument. Thus, this latter procedure not only separates the errors but also makes it feasiblc to confine subsequent periodic tests to direct current. Moving iron, rectifier and electronic instruments must be tested on alternating current only.

\section{A-C Tests}

Comparison tests on alternating current are similar to those already discussed for direct current. Ordinarily, electro-dynamic instruments of the 0.1 or 0.25 per cent accuracy classes are used as standards at power frequencies and can now be compensated for frequencies up to about $2000 \mathrm{c} / \mathrm{s}$. Multirange current transformers of high accuracy can be obtained with primary ranges from 0.25 to 100 amperes, and special three-winding voltage transformers with ranges from 1.5 to 300 volts, so that one ammeter and one voltmeter can suffice for most measurements at power frequencies.

For tests at higher frequencies, multirange thermocouple instruments are preferred. However, they have unusually large temperature influences (frequently 0.2 per cent per deg. C) and are not as stable as electrodynamic instruments.

If the ac-dc difference of the standard instrument is known and the self-heating error is negligible, an a-c test may be made with greatly increased acculacy by observing the response of the standard under a low-power microseope, switching only the standard to direct current, adjusting the current or voltage for the same deflcetion of the standard and then measuring it with a d-c potentiometer in the usual way." The direct current should then be reversed, reset and remeasured. The true value of alternating voltage or current is then simply $Q_{u c}=Q_{u c}\left(1+S_{0}\right)$, where $Q_{u c}$ is the average value of the measured d-c voltage or current and $S_{0}$ the proportional ac-dc difference of the standard at the frequency used.

\section{Reversed D-C Tests}

D-c tests of a-c instruments may be made with the potentiometer as already described, using the circuits of F'ig. 1 with the addition of a reversing switch in the input lcads to the potentiometer. 'I'wo readings should be taken at each scale point to be tested with the supply voltage reversed between readings, and the average of the potentiometer readings should be multiplicd by the volt-box ratio or shunt resistance to obtain the true voltage or current. Such a reversed $d$-c test greatly reduees effects, such as residual magnetism in the shield of the test instrument, which depend on the direction of the current. Wattmeter's are tested on reversed direct current by the circuit of $1 \mathrm{~d}$. Here, the separate sources for the voltage and current circuits are independently adjustable and need supply only the power taken by the instruments, not the fictitious power indicated by the wattmeter. If the sources are stable, one potentiometer. may be uscd, as shown, to measure voltage and current in succession. The indicated power is the product of the two, and no correction for instrument losses is necessary.

\section{Transfer Tests}

In a transfer test to directly determine the ac-de diffcrence, the test instrument and a standard instrument of known or negligible ac-dc difference at the frequencies to be tested are connected in series or parallel to see the same quantity. They are then in rapid succession connected to (a) alternating, (b) direct, (c) reversed direct and (d) alternating current, which is in each case adjusted to give the same deflection of the test instrument after which the response of the standard is recorded. From the difference between the average of the a-c readings, $D_{a}$, and of the $d-c$ readings, $D_{\mathrm{w}}$, of the standard, the proportional or per-unit ac-de difference of the test instrument, $S$ can be computed by the formula:

$$
S=\frac{D_{a c}-D_{d c}}{D_{d r}}+S_{v}
$$

where $S_{\circ}$ is the known proportional acde difference of the standard.

\section{TESTS OF STANDARDS}

It is obvious that the standards used in iny instrument testing laboratory should themselves be tested periodically to guard against errors due to drifts as well as unsuspected changes due to aecidental misusc. Such tests are of two types: frequent single-point checks and intercomparisons to guard against major change, and less-frequent, formal tests which may in many cases have to be performed in another laboratory. The frequency with which such tests should be made is difficult to specify, ${ }^{* 2}$ since it depends on the type of instruments, the accuracy sought, and the skill and care of the users. A fundamental rule, which should not be violated, is that instruments which are used as standards should not be used for general measurements or any other purpose.

Standard cells are usually purchased with a factory or NBS certificate stating their emf. They are readily susceptible to abuse, so that 2 or preferably 3 , cells should be purchased. The working cell should be compared with a rescrve cell weekly by connecting them in opposition and to the low range of the potentiometcr to measure their difference voltage, which should agree with the calculated value. One cell should be sent to a standardizing laboratory yearly.

Shunts or resistors for current measurements are much less liable to abuse. They can readily be intercompared in the laboratory with the potentiometer by connecting two, of adjacent ranges, in series and measuring their voltage drops in succession at a convenient steady current. The potentiometer can be checked at one point by balancing its standard cell circuit in the usual way and then connecting the cell, without moving it, ${ }^{* 3}$ to the $\mathrm{X}$ terminals of the potentiometer and measuring its emf, which should agree with its certificate value. Resistors and the potentiometer shculd be sent to a standardizing laboratory perhaps every five years.

High-grade d-c instruments and electrodynamic a-c instruments, which are used as standards, should be tested on d-c with the potentiometer perhaps every six months. If a potentiometer is not available, a valuable partial check, to verify that major changes have not occurred, can be made by comparing the full-scale deflection of the highest range of each instrument with the resulting deflection of the lowest range of the next highest instrument (for example, the 5 -ampere range of a $2.5 / 5$-ampere ammeter with the 10 -ampere range of a 10/20-ampere instrument). Additional checks can be made by comparing the a-c instruments with the d-c instruments of corresponding range. Such d-c checks of thermocouple instruments should be made frequently. If possible, the ac-dc differences of all a-c standards should be determined initially at a suit-

\section{- a suggested schedule for testing stand- ards used primarily lu a meter laburatory is riven in reference " 2 . Much valuable de- taild informution on settling up a stand- ardizing luborutory and on the potentl- ometer and other measurlug aplaratus is given in this leferenec.}

"3he emf of a cell muy be changed tenrporarily if the enell is moved. 
ably equipped standardizing laboratory.

A recent commercial development is a line of self-checking $\mathrm{d}$-c and thermocouple instruments in which a single point check and adjustment of the basic dc or ac milliammeter in the instrument can readily be made in terms of an internal standard cell each time the instrument is used.

Instrument transformers are so stable that a test at a standardizing laboratory every five years should be sufficient.

\section{SUPPLY AND CONTROL EQUIPMENT}

Special supply and control circuits are usually required in instrument testing. In order that measurements may be made rapidly and conveniently, the power sources must be free of erratic fluctuations or rapid drifts, must be adjustable in fine increments over wide ranges, and for wattmeter testing, must have two channels independently adjustable in magnitude and phase.

Rectified and electronically stabilized d-c sources can now, in many cases, replace storage batteries for d-c voltmeter testing just as electronic alternating-voltage stabilizers have largely replaced motor generator sets for a-c testing. For testing instruments at audio frequencies, an oscillator and power amplifier followed by suitable matching transformers can be used. An important limitation that must be checked is the waveform distortion of each supply. For a d-c supply used for reversed d-c tests of a-c instruments, the residual ripple voltage must be low enough.so that the form factor is unity to the desired accuracy. For an a-c supply used solely to test $\mathrm{rms}$ instruments, rather large amounts of harmonics of low order are permissible. However, if electronic instruments (which respond to the crest or average value but are marked in rms units) are tested with rms standards, the crest and form factors must be equal to those of a sine wave under all test conditions to the desired accuracy.

A number of control consoles for instrument testing have been described ${ }^{\tilde{\pi}, \theta}$. ${ }^{\top, 8}$ so that it should no longer be -necessary to begin a design from the ground $u p$. Some consoles feature simplicity and convenience of controls, others emphasize safety features so that the instru. ments cannot ordinarly be overloaded.

An important precaution to observe in any design or in the use of a testing console is that the standard and test instrument must always be connected to see exactly the same quantity. Extraordinary care is required to insure that voltage drops, leakage currents, and electromagnetic fields in the console circuits connecting the standard and test instruments do not unwittingly violate this principle.

\section{SERVICES AND EQUIPMENT AT THE NBS}

One of the primary functions of the

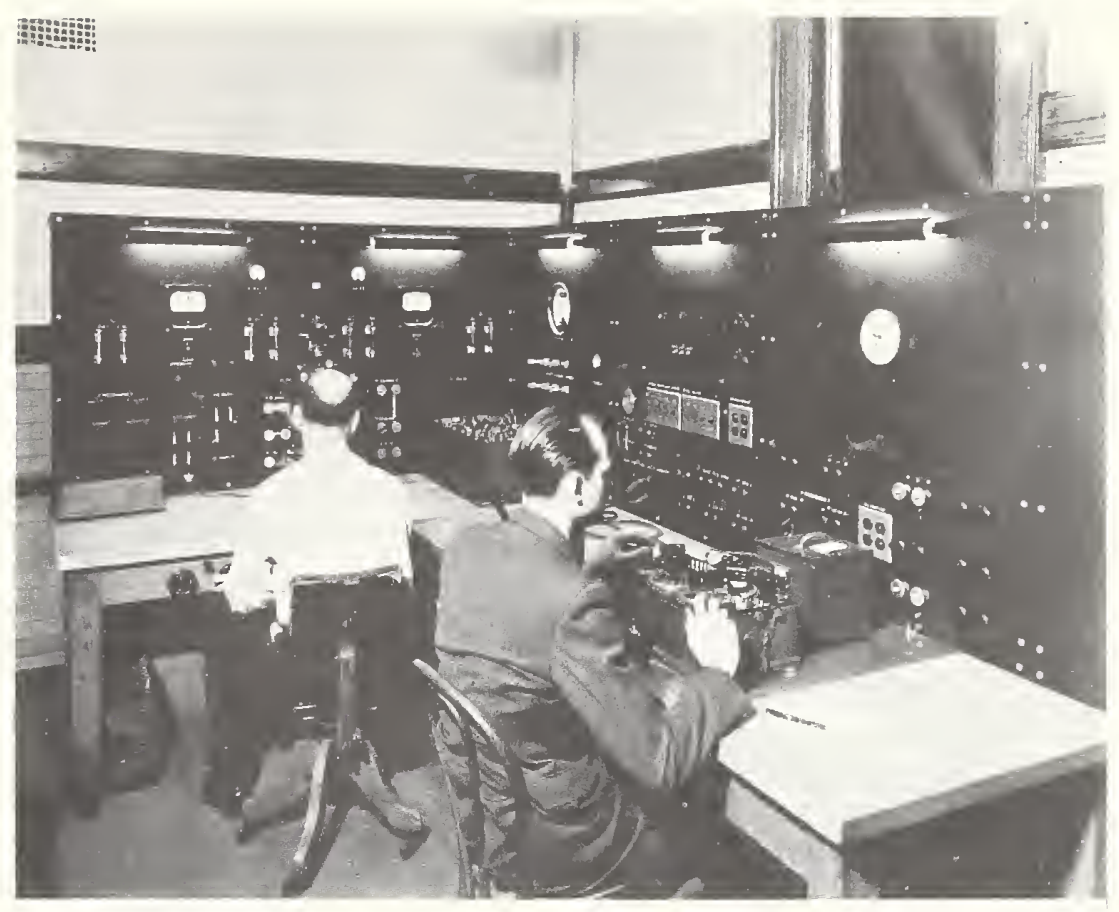

Fig. 2. NBS Instrument Testing Console

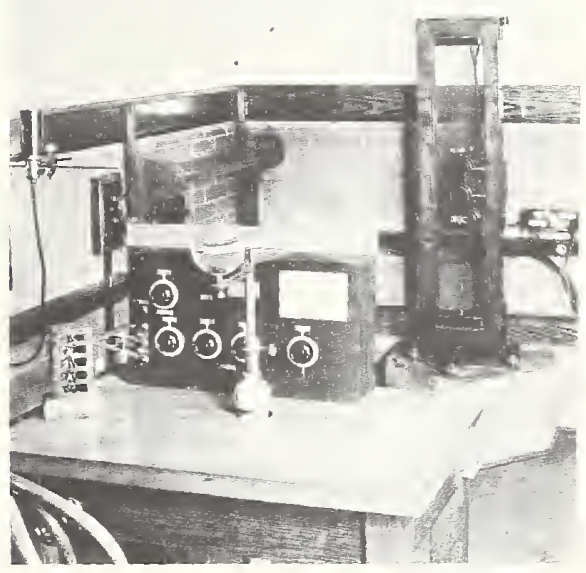

Fig. 3. NBS Standard Electrodynamic Transfer Instrument

NBS is the establishment, maintenance and dissemination of units and standards of measurement. By careful intensive work, satisfactory electrical standards have been established and are being painstakingly maintained." Methods and equipment have been developed for testing potentiometers, standard cells, resistors, bridges and volt boxes to an accuracy of 0.01 per cent or better; for testing d-c and a-c instruments at frequencies up to $20 \mathrm{kc} / \mathrm{s}$ with an accuracy of 0.03 per cent or better, and for accurately testing inductors and capacitors, instrument transformers, and the properties of magnetic materials." Tests of standard cells and resistors serve to physically transfer the units of voltage and current from the Bureau to the laboratory using them and thus to dis-

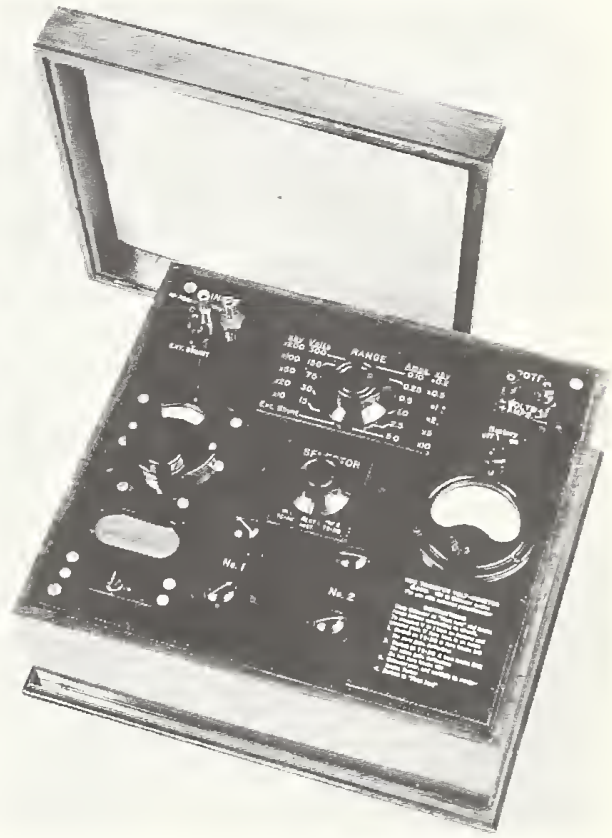

Fig. 4. NBS Volt Ampere Converter

seminate the units with high accuracy. Tests of electrical instruments serve the same purpose with reduced accuracy. Moderate fees are charged to cover the actual cost of these tests.

Fig. 2 shows the main console used for testing electrical instruments and meters at the Bureau, and Fig. 3 shows the basic electrodynamic transfer instrument." Recent work at the Bureau has shown that certain properly specified and selected thermal converters (thermocouples) can be used as transfer standards of high accuracy, and meth- 
ods have been developed for using then to measure current or voltage over wide ranges at audio frequencits. ${ }^{32}$

All instruments sent in to NBS for standardization are tested with a null potentiometer and its accessories, and a-c tests are made with these transfer instruments, which have ac-dc difference of 0.01 per cent or less. The NBS instruments do not have marked scales in the usual sense and must be calibrated with the potentiometer for every a-c measurement. However, they are primarily designed and used for rapidly and conveniently determining ac-de differences of other instruments.

\section{NEW-INSTRUMENT DEVELOPMENTS}

Fig. 4 shows a portable, self-contained, multirange volt-ampere converter that has recently been developed at the Bureau. This converter serves as an accessory to the d-c potentiometer to make possible a-c measurements of voltage and current to an accuracy (without corrections) of 0.05 per cent at frequencies up to $20 \mathrm{kc} / \mathrm{s}$. Its basic principle is shown in Fig. 5. For voltage measurements, as shown in $5 \mathrm{a}$, a thermal converter of negligible ac-dc difference is connected to a series resistor (total resistance $R_{v}$ ), and the resulting output emf of the thermocouple is balanced out by means of an adjustable internal bucking circuit, $B$. The heater of the converter is then switched to an internal $d-c$ circuit which is adjusted for the same output emf and therefore the same heater current. The voltage drop across the resistor, $R_{\text {, }}$, in this circuit is then measured with an external d-c null potentiometer and multiplied by a factor, which, as the equations show, depends on a ratio of resistances and not on the characteristics of the thermal converter. A corresponding arrangement, shown in Fig. 5b, is used for current measurements; and the circuits are combined in the actual instrument. Repeated tests have shown that the desired accuracy of $0.05 \%$ is easily maintained.

The generally poor stability and high temperature-coefficient of the conventional thermocouple instrument often linit its sustained accuracy in service and sometimes make a single-point check at every use desirable. Ordinarily,
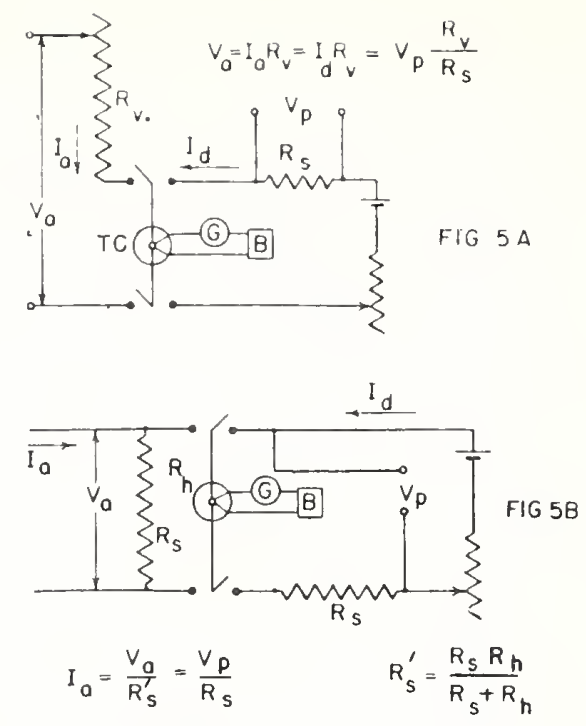

Fig. 5. Elementary Diagram of NBS VoltAmpere Converter

such a check is made by comparison with a standard d-c instrument. A new 0.5 per cent thermocouple instrument has been developed at the Bureau in which the millivoltneter in the instrument, together with appropriate manganin resistor's, serves as this standard. As shown in Fig. 6, with the switch in the $A$ position, the millivoltmeter, $M$, is connected as a nillianmeter of low temperature coefficient to measure the direct current through the heater from an internal source. This is manually adjusted by resistor $A$ for full scale deflection. In the $L^{\prime}$ position of the switch, the millivoltmeter is reconnected to the thermocouple and is adjusted for the same deflection by resistor $B$, thus compensating for any changes due to temperature influence or converter drift. In the actual instrument, the circuits are somewhat more complicated; because the millivoltmeter is also used to check the resistance of the heater of the convertcr (which may be changed by moderate overloads to cause errors when shunted for current measurements).

These developments are mentioned because they are particularly useful in testing other instruments. The first instrument makes accuracies possible over wide ranges heretofore not obtainable in a portable device, and the second is

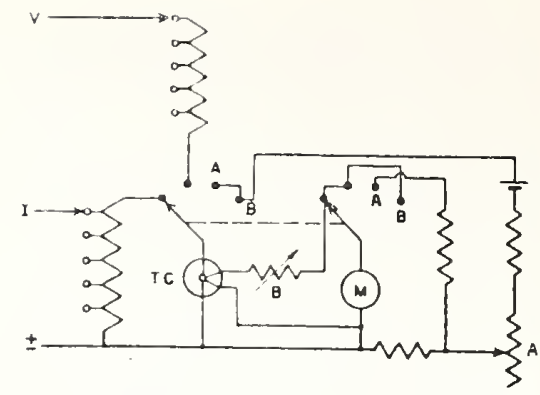

Fig. 6. Elementary Diagram of NBS $1 / 2 \%$ Thermocouple: Volt-Amineter

a conventional 0.5 per cent thermocouple instrument which can manually be compensated for temperature and other changes.

\section{BIBLIOGRAPHY}

1. Harris, F. K., Electrieal Measurements, John Wiley \& Sons, New York City, 1952.

2. Electrical Metermen's Handbook, Edison Elfetric Institute, New York City Sixth Edition, Section 12, 1950.

3. "A-C Measurcments to 10.000 Cycles", Wcaver, F. D., Instruments, v. 25, p. 757,1952

4. "Notes on the Care and Use of Electrical Instruments," Weaver, F. D., Instruments, v. 23, p. 1236, 1950.

5. "An Easily Asscmbled Console for Rapid Testing of Electrical Indicating Instruments," Wcaver, F. D., Instruments, v. 22, p. $396,1949$.

6. "General Purpose Instrument Standardization Console," Gencral Engineering Laboratory, General Electric Company, Schenectady, New York.

7. "Equipment for Instrument Calibra tion," Gilbert, E. E., Electrical Engineering, v. 68, p. 1065, 1949.

8. "Electrical Instrument Calibrating for Industry." Kinsell. W. L., Instruments, v. 21, p. 830,1948 (Contains bibligg. raphy).

9. "Establishment and Maintenance of the Electrical Units," Silsbee, F. B., NBS Circular 475, 1949, Government Printing Offlce ( 25 cents).

10. "Extension and Dissemination of the Electrical and Magnetic Units by the National Bureau of Standards," Silsbee. F. B., NBS Circular 531, Government Printing Offlce (25 cents).

11. "Standard Electrodynamic Wattmeter and AC-DC Transfer Instruments." Park, J. H. and Lewis, A. B., Journal of Reseurch of the National Bureau of itandards, v. 25, p. 545, 1940 (RP 1344)

12. "Thermal Converters as AC-DC Transfer Standards for Current and Voltage Measurements at Audio Frequencies," Hermach. F. L., Journal of Research of the National Bureau of Standards, v. 48 . p. 121, 1952 (RP 2296). 


\title{
Testing Electrical Instruments
}

\author{
By F. D. WEAVER \\ National Bureau of Standards \\ Washington 25, I). C.
}

\begin{abstract}
A few basic reference standards, circuit diagrams, and testing procedures can be utilized by laboratories and plants for standardizing electrical instruments and meters.
\end{abstract}

T

HIS article presents methods, equipment, and simple circuits for standardizing the usual ranges of electrical instruments. All the units can be compactly installed in one small console.

The a-c. power service to the building, which is the source of alternating current and voltage, can be improved by electronic voltage stabilizers. Either a regulated and rectified alternating voltage or a battery is suggested as tho supply for direct voltage. A storage battery is the best source of direct current. As a similar console was described in a published article (see reference 9) this article discusses testing procedures.

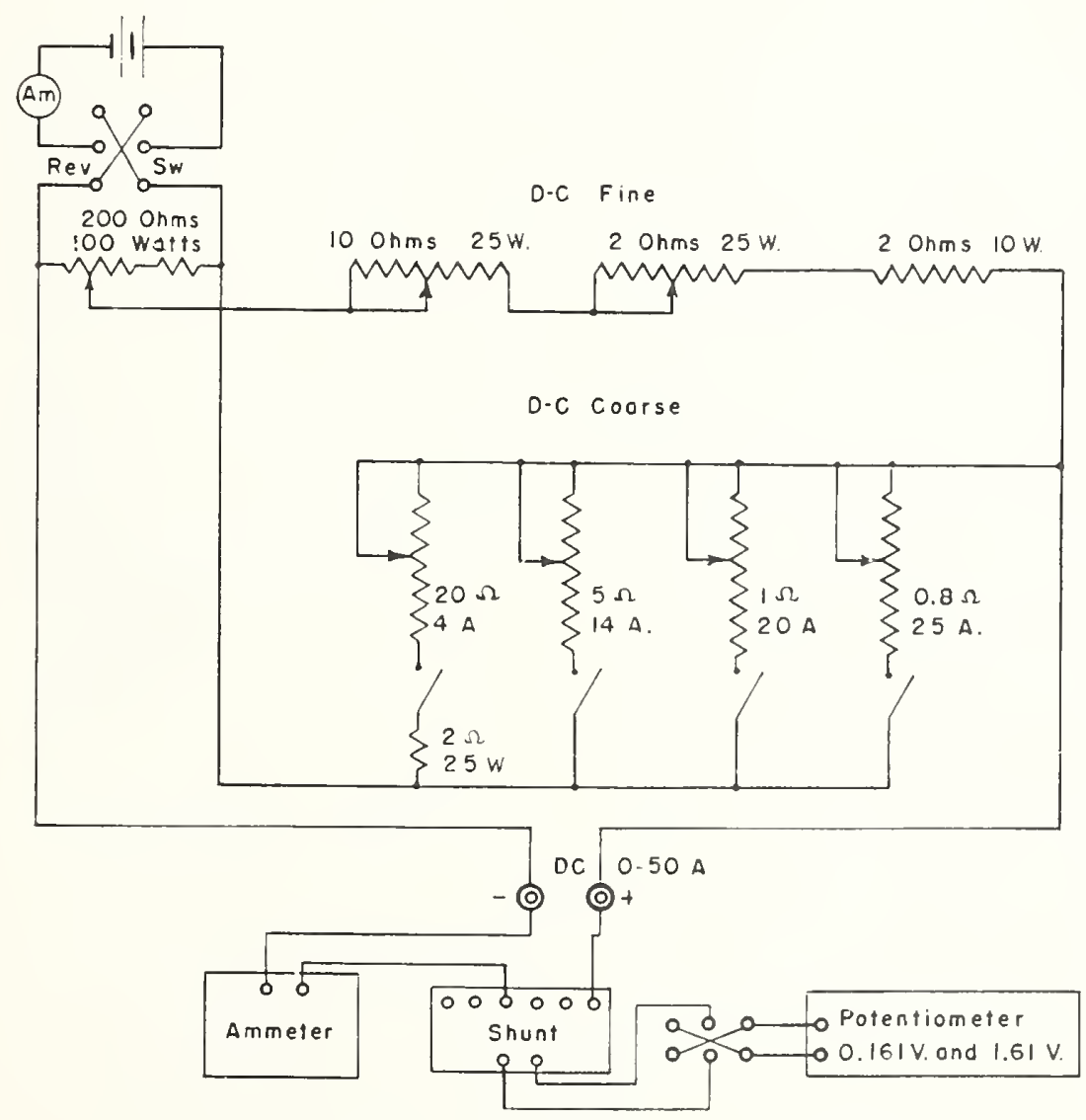

Fig. 1. Arrangement for testing ammeters on DC and reversed DC.
The testing of electrical instruments and meters involves the comparison of the indications of one instrument with those of some suitable standard of bet ter accuracy. For $d-c$. testing of electrical instruments, the basic standards are standard cells and resistors; the basic standard instrument is the potentiometer. The corrections for the standards must be known and their corrections applied if measurements of the highest accuracy are desired. A-c. in struments are tested in terms of these same basic d-c. standards by means of transfer instruments. A transfer instrument must have the same response on a-c. as on d-c, or any difference in its response must be known. Such an instru- ment can be calibrated on $\mathrm{d}$-c. and then used to check a-c. instruments. This in effect transfers the d-c. units of measurement to the measurement of alternating current, voltage, and power. High-grade electrodynamic and thermocouple instruments serve best to make this transfer from a-c. to d-c.

The wiring diagrams (Figs. 1 to 4 ) indicate arrangements for testing electrical instruments and meters. Properly selected sources and controls provide for adjustments fine enough for increments of pointer indication of $0.05 \mathrm{di}$ vision or less on most ranges of ammeters and voltmeters. The suggested sources and controls are adequate to cover (1) direct and alternating voltages from 0.1 to 300 volts, (2) alternating current from 0.01 to $100 \mathrm{am}$ peres, and (3) direct current from 0.01 to 50 amperes.

\section{REFERENCE STANDARDS}

\section{D-c. Reference Instruments}

A potentiometer with its accessory equipment is desirable for direct-current and direct-voltage measurements. A potentiometer having two ranges, $0-0.161$ volt and $0-1.61$ volts, is preferable to one having a single range. Its dial readings should be correct within 0.0005 volts and it should be possible to read the lowest adjustable dial within 0.0001 volt. A good portable potentiometer with a built-in galvanometer that meets these requirements is satisfactory. A resistance voltage divider (volt box) with ranges of $3,7.5,15,30$, 75,150 , and 300 to 1.5 volts, with ratio errors of less than 0.04 percent, is recommended for use with the potentiometer for testing d-c. voltmeters. Current shunts having resistances from 0.002 ohm to 20 ohms are suggested as potentiometer accessories. These shunts should have current ratings from 75 amperes to 0.075 ampere for testing d-c. ammeters, and errors of less than 0.04 percent.

\section{A-c. Reference Instruments}

Two instruments of the electrodynamic type-(1) an a-c. and d-c. voltrneter with ranges of 75,150 , and 300 volts and (2) an a-c. and d-c. ammeter

Reprinted from Instruments, Vol. 26, No. 9, September 1953 


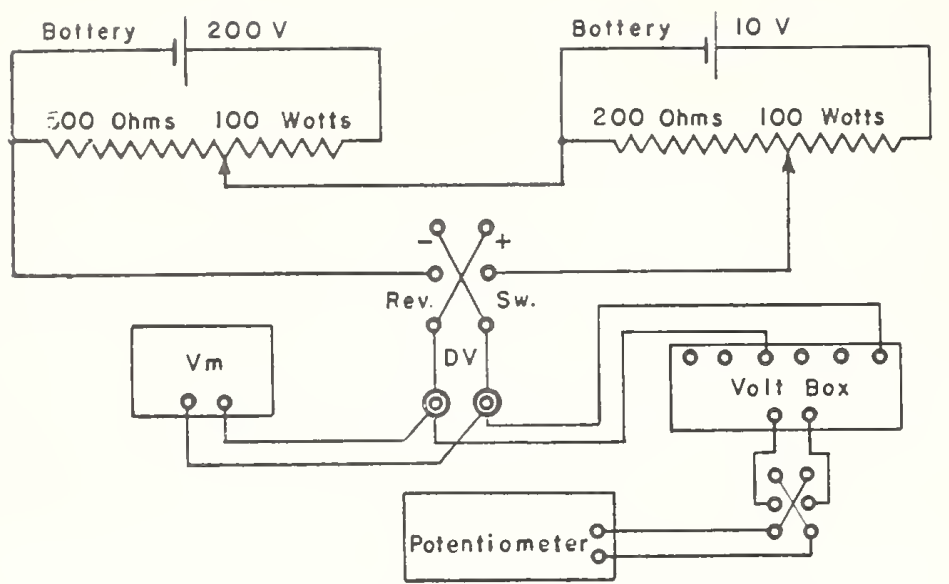

Fig. 2. Arrangement for testing voltmeters on direct voltage and reversed direct voltage.

with 2.5-and 5-ampere ranges-of the 0.25 -percent accuracy class, are suggested as reference instruments for testing other a-c. voltmeters and ammeters. This type of instrument is preferred because its indication is the same on direct current as on alternating current. Each may be tested (corrections accurately determined) on reversed d-c. with the potentiometer and the abovementioned accessories, and then used as a reference instrument for a-c. testing. In the selection of reference instruments the important consideration is that they have the needed ranges and accuracy. They are most likely to fulfill these requirements if they conform to the specifications outlined for their accuracy class in ASA C39.1-1951 (see reference 1). If corrections are known for each of the cardinal points on the scale and the instrument is used on only the upper third of its scale, then the accuracy obtainable from a 0.25 . percent instrument (see reference 8 ) is limited mainly by the ability of the observer to read pointer indications.

A three-winding potential transform. er-with tapped windings for 1.5, 3 , $7.5,15$, and 30 volts on one secondary, a 150 -volt winding for the reference voltmeter on the other secondary, and a 120-volt primary winding-is available for testing a-c. voltmeters for the lower voltage ranges (see reference 6 ). This type is recommended because losses as a result of current in the supply winding, which affect the accuracy of the usual potential transformer, have no effect on the ratios between the two secondary windings that are compared in the normal use of this transformer.

A multi-range current transformerwith primary ranges of $0.25,0.5,1$, $1.5,2,3,4,5,10,15,20,25,30,40,50$, 75 , and 100 amperes and a 5 -ampere secondary to which the reference ammeter is connected-is satisfactory for testing a-c. ammeters. The errors for these two standard transformers on any range for the burden imposed by the usual instrument should be less than 0.1 percent in ratio and 5 minutes in phase angle. It is possible that errors will be so small that corrections need not be applied.

$$
\text { o-C Voltoge supply }
$$

ever, for measurements of the highest accuracy, it may be desirable to have these differences determined in a standardizing laboratory such as the $\mathrm{Na}$ tional Bureau of Standards. Initially, the instrument should be tested at each of the cardinal points on the scale for one rallge and at two of the cardinal points oln each of the other ranges. Thereafter, an annual test at five of the cardinal points on the base range and at the same two cardinal points on each of the other ranges is sufficient.

Transformers, potentiometers, shunts, volt boxes, and reference wathour metel's should be sent at intervals to a standardizing laboratory for highly accurate tests. Shunts and volt boxes should be initially tested at 10 percent and 100 percent of rated current or voltage; infrequent tests at 10 -percent load are ordinarily sufficient thereafter. Ratio factor and phase-angle corrections should be determined for the transformers. However, phase-angle corrections are not necessary unless the transformers are used as accessories in power and energy measurements.

\section{Tests of D-C. A Mmeters}

The diagram of Fig. 1 shows an arrangement for obtaining both fine and coarse control for adjusting currents in testing d-c. ammeters. The ammeter to be tested is connected in series with an appropriate section of the multirange current shunt. The controls are adjusted until the pointer of the ammeter under test is deflected to the desired position. The observer then measures the $I R$ drop across the shunt with the potentiometer. The current necessary to deflect the pointer to the indicated position is determined by the formula $l=E / R$, where $E$ is the voltage at the $\mathrm{X}$ terminals of the potentiometer and $R$ is the actual shunt resistance.

If possible, a section of the nuultirange current shunt that has a slightly higher or the same current rating as the ammeter range to be tested should be chosen. For example, a 10 - or 15 . ampere instrument will likely be tested d-c. difference of this type is ordinarily rather small at power frequencies. How-

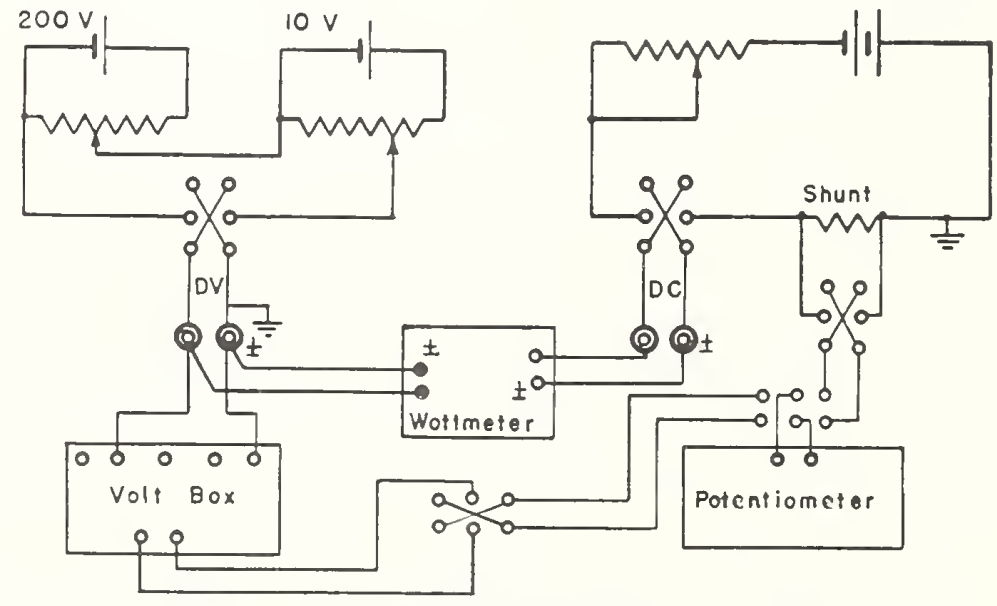

Fig. 3. Arrangement for testing wattmeters on reversed d.c. 


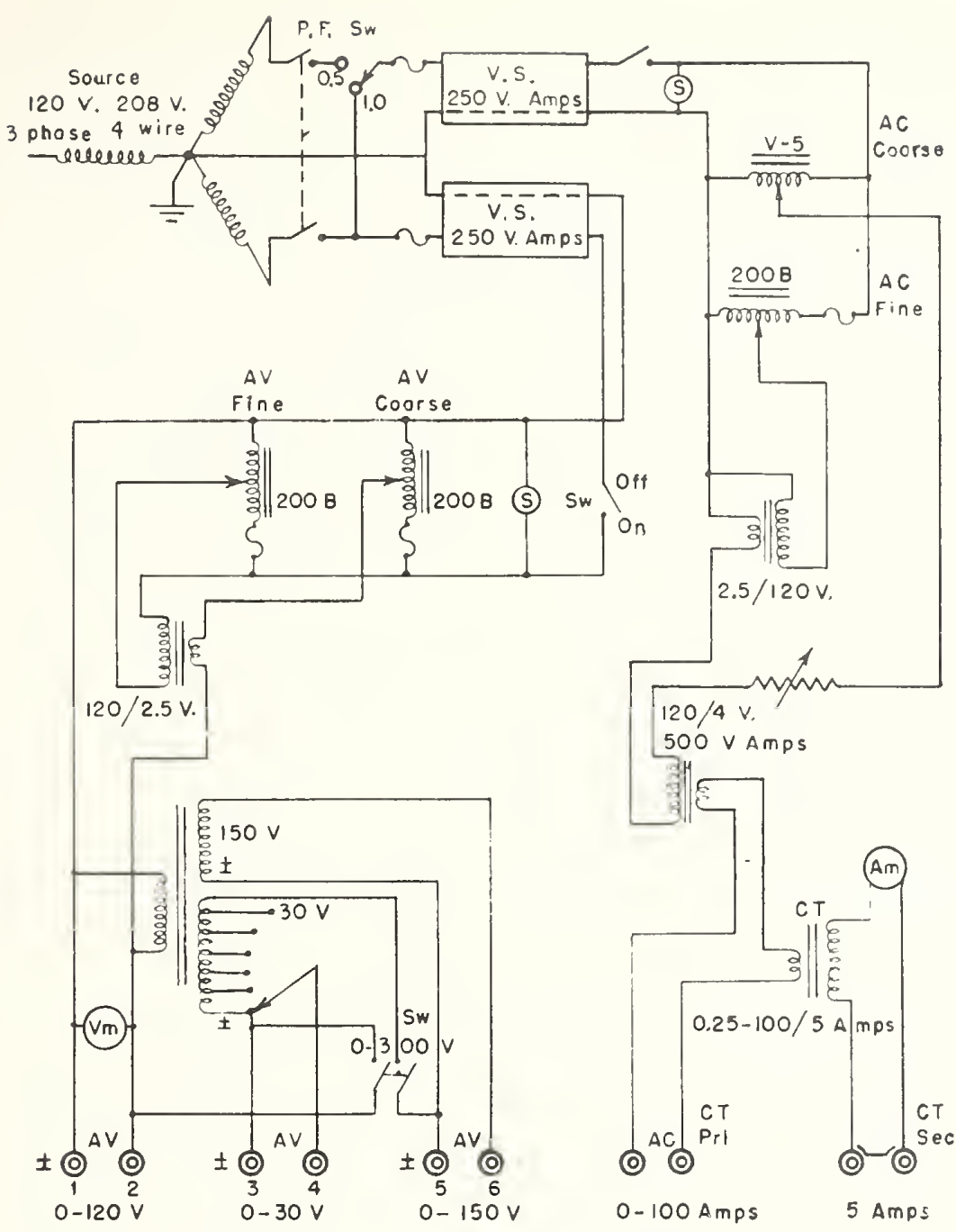

Fig. 4. Arrangement for alternating-voltage and alternating-current testing. thermocouple types on reversed direct current. In such a test, a reading is taken for current in one direction and is averaged with a second reading for current in the opposite direction, thereby minimizing the effects of residual and stray magnetic fields. The direction of current in the instrument is reversed after each reading by means of a reversing switch. In general, this method which makes use of a potentiometer gives more accurate results than a simple a-c. test.

A phantom-loading method is employed in testing wattmeters on reversed direct current. In this method separate sources of voltage serve to energize the voltage and current circuits of the instrument (see Fig. 3). Since the two sources are required to supply only the losses in the instrument and test equipment, they need not be large sources of power. The voltage coil of the instrument and the volt box in parallel are connected to the directvoltage terminals. The current coil and an appropriate range of the multi-range shunt in series are connected to the direct-current terminals. The potentiometer in conjunction with the volt box and the voltage controls are used to set the voltage to the desired value. Then the current is adjusted by means of the current controls to give the desired pointer deflection of the wattmeter, and the same potentiometer is switched over to measure the IR drop across the shunt. If the wattmeter has a compensating coil, the switch on the instrument should be in the uncom. pensated position when the instrument is tested. Care must be taken to prevent overloading either the voltage or current coil. The precaution should be taken to connect the voltage and current terminals on the instrument marked \pm to the respective \pm terminals of the voltage and current supply as or one having a range of 150 volts in shown in the diagram. To avoid the
parallel with the 150 -volt range of the errors caused by electrostatic effects parallel with the 150 -volt range of the errors caused by electrostatic effect of the instrument pointer the potenti- tial of the two circuits of a wattmeter ometer will read approximately 1.0 or without a common connection, the \pm 1.5 volts respectively. A correspond- voltage terminal should be connected ingly desirable selection should be made through a voltmeter to the \pm current for other instrument and volt-box terminal.

ranges. For each point tested the volt- Values of power for each test point age $V$ indicated by the instrument point- are determined by means of the follower will be determined by the formula ing formula: $W=N E_{V} E_{1} / R$, where $V=N E$, where $N$ is the actual ratio $N$ is the actual ratio of the volt box, and $E$ the voltage at the $\mathrm{X}$ terminals $R$ is the corrected resistance of the of the potentiometer.

sh it, $E_{\mathrm{v}}$ is the voltage at low-side

For voltage ranges less than 1.5 terminals of the volt box and $E_{1}$ is the volts the instrument with its leads voltage at the shunt potential termiand the $\mathrm{X}$ terminals of the potention. nals.

eter should be connected in parallel across a low-voltage source. This source may be the voltage drop across a resistor in which the current is controlled by the direct-current controls shown in Fig. 1. In this case $V=E$ provided that no corrections need be applied.

\section{Tests of A-c. Ammeters}

To make a test of an a-c ammeter, the ammeter to be tested is connected to the terminals marked C T Primary (see Fig. 4). It is then in series with the primary of the multi-range current transformer. A primary range should be selected that has the same current Tests on Reversed Direct Current rating as the ammeter to be tested.

The direct-current and direct-voltage The 5-ampere electrodynamic ammeter circuits illustrated in Figs. 1, 2, and 3 mentioned previcusly is connected to serve for testing ammeters, voltmeters, the $\mathrm{C} T$ Secondary terminals. The conand wattmeters of electrodynamic or trols are then adjusted so that the point- 
er of the ammeter under test is deflected to the desired scale mark. The reference instrument is then read in divisions. The current necessary to deflect the pointer of the test instrument to any test point is $I_{\mathrm{pri}}=I_{\mathrm{sec}} \times N \times R F$. Corrections for the reference ammeter and for the current transformer, if significant, should be applied. $I_{\text {sec }}$ is the corrected reading of the reference ammeter, $N$ is the marked ratio of the current transformer and $R F$ is the ratio factor of the transformer.

\section{Tests of A-c Voltmeters}

The three-winding potential transformer mentioned previously is convenient for testing voltmeters of 30 volts or less. It also serves as a supply transformer for voltages up to 300 volts when all windings are connected in series (see reference 6). This is accomplished between terminal AV 1 and AV 6 as illustrated in Fig. 4 by closing the switch marked $0-300 \mathrm{~V}$ SW.

A test voltmeter having ranges of 30 volts or less is connected to terminals marked AV 3 and 4, Fig. 4, with the range switch on the appropriate tap and the reference voltmeter is connected to terminals marked AV 5 and 6 . The supply to the 120 -volt primary is controlled by the usual AV controls on the panel. Voltmeters having ranges greater than 30 volts are tested by comparison with the reference voltmeter on the 75 -, 150 - or 300 -volt range using terminals $\mathrm{AV} 1$ and 6 . The voltage for any given indication of the instrument under test (ranges of 30 volts and less) is computed by multiplying the corrected reading of the reference instrument by the corrected value of the transformer ratio for the selected range.

If the instrument tested is of a rectifier or electronic type, there is a possibility of error unless the test volt age has almost a pure sine-wave form. The recommended reference instrument responds to rms. values of voltage and is not appreciably affected by waveform distortion. The observer should make sure that the output wave form of the setup has a total harmonic distortion of less than 2 percent.

\section{Watthour Meter Testing}

A watthour meter can be tested by either of two methods: (1) using a wattmeter, and (2) using a watthour meter. In the first method, a wattmeter is connected to respond to the same value of power as seen by the watthour meter under test. The power (average active power on a.c.) is held at a constant value as indicated by the pointer of a wattmeter for an accurately measured time interval, after which the registration of the meter is observed. The percentage registration is computed as

$$
100 \times \frac{\text { registered energy }}{\text { measured energy }},
$$

where the measured energy is the product of the measured power and time.

In the second method, a meter of known accuracy is connected to respond to the same value of power as seen by the wathour meter under test. The registrations of the two are observed for the same time interval. This is generally a much simpler method than (1) since power does not have to be held constant and the time interval need not be accurately known. Method (2) is usually to be preferred because of its simplicity. Standard watthour meters of suitable accuracy and stability are now commercially available. It is necessary, of course, to determine corrections for the standard at suitable intervals by either method (1) or (2) or to have such corrections determined at a standardizing laboratory.

If there are several rotating standards in one laboratory, or in one locality, it may be advisable to maintain one or more of these as reference meters to be used only in the testing laboratory. This reference meter could be sent to a standardizing laboratory for accurate tests and then used only in the laboratory for comparison tests with other rotating standards. This could be a cooperative project with several companies in the same area participating. Since stable and accurate watthour meters are now available, they would in this way be assured of energy measurements of high accuracy.

Metel testing in accordance with the proposed wiring diagram follows the plan of method (2). The voltage circuit of the standard is energized for $a$ time corresponding to a desired num. ber of revolutions of the test meter (house type) as counted by visual observation. Tests are made at unity power factor, and 0.5 power factor, current lagging the voltage, using phantom loading. A 3-phase 3- or 4wire source is convenient for testing at the two power factors mentioned. Unity power factor is obtained by connecting the voltage circuits of the two meters to the same phase as that energizing the current coils as illustrated in Fig. 4. A resistance load in the primary of the supply transformer of the current circuit will tend to eliminate any phase difference between the voltage and current circuits at near unity power factor. A power factor of closely 0.5, current lagging the voltage, is obtained by connecting the voltage coils to a selected other phase.

A wattmeter may conveniently be used with a voltmeter and ammeter to check the power factor in watthour meter tests. If the wattmeter has a double voltage range, 75 and 150 volts, a capacitor may be used to determine whether the load current leads or lags the voltage. To check the phase of the current with respect to the voltage with the 150-volt range in the test circuit of such a meter, a 1-microfarad capacitor of good quality is connected between the 75- and 150 -volt posts. If the pointer of the wattmeter goes upscale the load current is leading, if the pointer goes downscale it is lagging. Alternatively, a small inductance of low resistance in the wattmeter potential circuit may be used as a power-factor test. ${ }^{\top}$

Once the phase that gives 0.5 power factor, current lagging the voltage, is determined, the external power leads to the setup should remain fixed. The selected leads may then be wired directly to the test circuit and 0.5 power factor can be obtained by shifting the switch illustrated in the upper lefthand corner of Fig. 4. An adjustment of the resistance in the primary of the supply transformer mentioned above may serve to give more nearly a 0.5 power factor.

Either a housetype or a portable meter may be compared with the rotating standard. The potential terminals of the two are connected in parallel to the AV terminals indicated in the diagram. The current coil of the meter under test is connected to the CT Primary terminals and the standard to the CT Secondary terminals. The current transformer should be connected for the proper range. A voltmeter and ammeter are used with the controls to set the voltage and current. The two meters may be started and stopped by use of the panel voltage on-off switch indicated on Fig. 4. When a correction is to be applied for the readings of the standard, the percentage registration of the test meter is determined as follows:

$$
\begin{gathered}
\text { Percentage Registration }= \\
k_{\mathrm{h}} \times r \times A / K_{\mathrm{b}} \times R
\end{gathered}
$$

where $r$ equals the revolutions of the test meter, $R$ equals revolutions of the standard, $k_{n}$ equals the watthour constant of the test meter, $K_{\mathrm{h}}$ equals the watthour constant of the standard and $A$ equals the percentage registration of the standard.

If transformer corrections are to be anplied, formulae may be found in Electrical Engineer's Handbook.

(1) "Anerican Standard for Electrical In. dicating Instruments, C39.1-1951." Standards Association, New Vork if, N. Y

(2) H. B. Brooks "The Aceuracy of Com nercial Electrical Measurements," Tranutertions of the dinerican Institute of Electrioal Eusiheers. Vol. 39, February 1920, pages 495.615.

(3) I. B. Dowden. "Organizing an Vilectrical Instrument Standardizing Laboratory." kingi. neering Notes, (Weston Electrical Instrument Corp., Newark, N.J.) Vol, 2, June 1947, pages 6-R; Vol. 4, February 1949, pages $3-5$.

(4) Electrical Enginepr Handbook. by len. der and Del Mar, Palt IV, Electrical Power, John Wiley \& Sons, Inc., New York, 1936, pazes 5.02 to 5-112.

(5) Electrical Metermen's Handbook, Edison Electrul Institute, 420 Lexington Avenue, New York, N. Y., sixth edition, 1950. (6) R. Estoppey, "Potential and Current
Transformers for Standardizing A.C Voltmeters Transformers for Standardizing A.C Voltmeters and Ammeters," Engineering Notes, (Weston Vol, 3, October 1948, Pages $7 \cdot 8$.

(7) Frank A. Laws, Electrical Measurements. McGraw-Hill Book Co., Inc., New York, 1938. pages $624-639$.

(8) Frank D. Weatrer, "Notes on the Care and Use of Electrical Instruments," Instrumenes (Instruments Publishing Co., Pittsburgh 13, Pa.) Vol. 23, No. 12, December 1950, pages Pa.) Vol.

(9) Frank D. Weaver, "An Easily Assembled Console for the Rapid Testing of Electrical In. dicating Instrumente," Instruments (Ingtruments P'ublishing Co., l'ittsburgh 12, l'a.) Vol. 22. No. 5, Mar 1949. pages 396-399. 


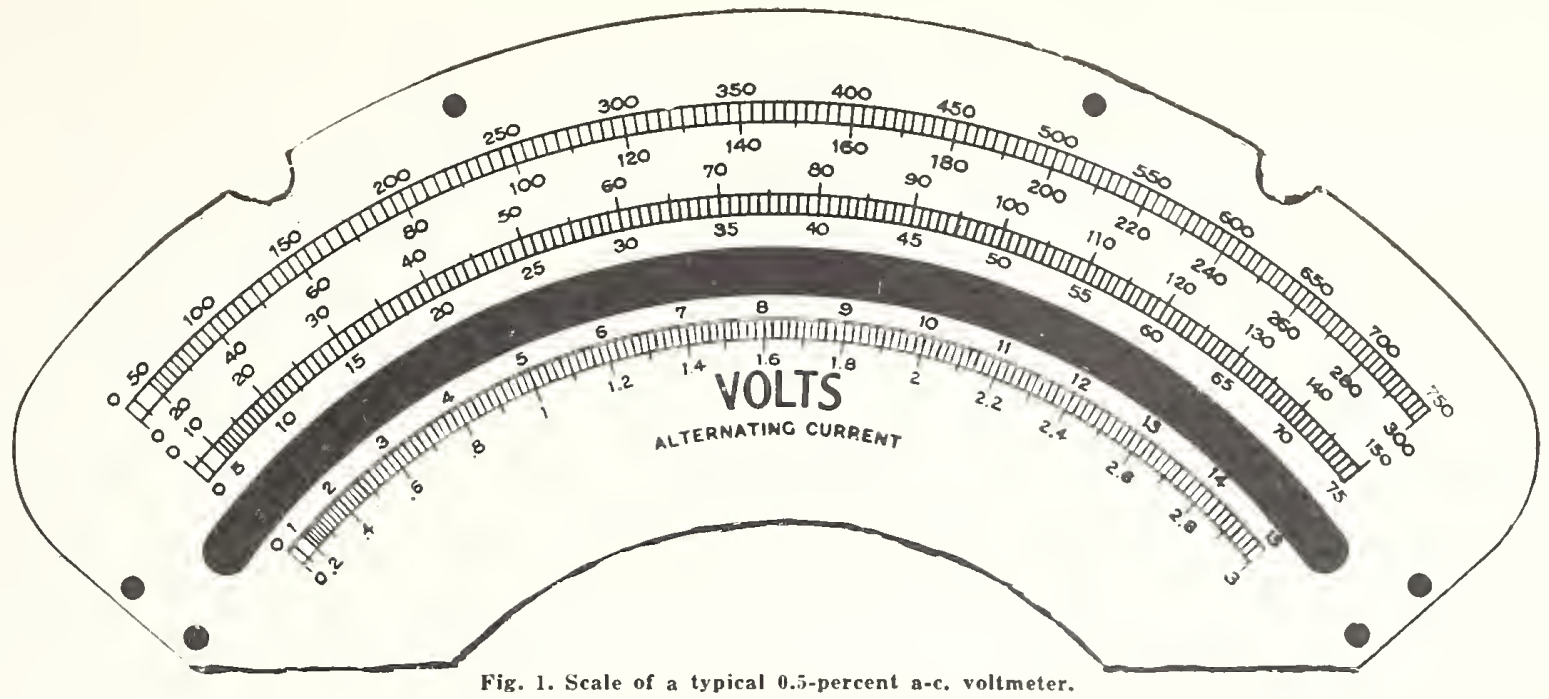

Scale and Reading Errors of

\title{
ELECTRICAL INDICATORS
}

\begin{abstract}
Scale and reading errors limit the accuracy of even the best electrical indicating instrument to about 0.1 percent. Sources of errors are analyzed, and the NBS standard calihration methods are presented.
\end{abstract}

$\mathbf{T}$ HE SCALE of an electrical instrument is an important component; a poor or defective scale can result in errors greater than that claimed by the manufacturer.

An evenly divided scale is desirable. However, if the same percentage accuracy of reading the measured quantity is desired at all points, the divisions should be wider at the beginning than at the end of the scale.

As most types of instruments cannot be designed to have operating and control torques that balance to permit the same percentage of accuracy of indica. tion at all points on the scale, it has become common practice to refer to percent of error and accuracy class in terms of full-scale reading. This convention implies that the indication of an instrument with 0.25-percent accuracy and a 100-division scale may deviate from the true value by $0.25 \mathrm{di}$ vision at any reading along the scale.

One of the prerequisites of a good instrument is that its scale be calibrated accurately-that is, the cardinal scale marks must be located correctly and the intermediate division marks must not be out of position by

\section{FRANK D. WEAVER}

National Bureau of Standards Washington 25, D. C.

more than the desired accuracy. These marks may be out of place because of (1) errors in the reference instrument used originally for calibrating the scale, (2) random errors in locating the cardinal points, (3) failure to subdivide between cardinal marks in ac. cordance with the scale law, (4) erratic spacing of the subdivision marks between two adjacent cardinal points, and (5) changes that have occurred in components since the original calibration.

In order that the pointer indications may be read accurately, a high-grade instrument should have an anti-parallax mirror and a knife-edge pointer with the knife-edge about equal to that of the division lines, and preferably not more than $1 / 10$ that of the distance between two successive scale division marks. These scale division marks should be at least $1 \mathrm{~mm}$. apart.

For the better grades of portable instruments with which this article is concerned it is a customary practice for the manufacturer to fit the scale to each individual instrument. A blank scale is put in place and cardinal points are marked on it to correspond to values determined with an accurate reference standard. After the cardinal marks are ruled on the scale the intermediate division marks between pairs of cardinal marks are located by a mechanical subdivision device. If an accurate dividing machine is not used there may be a noticeable variation in the spacing of subdivision marks. Some instruments show errors of several tenths of a percent because of carelessness in spacing of the ruled subdivision marks.

\section{Seale Errors}

Electrical instruments that come to the National Bureau of Standards for standardization tests are ordinarily tested at five of the cardinal points on the scale $(20,40,60,80$, and 100 division points on a 100 -division scale; $30,60,90,120$, and 150 division points on a 150-division scale) and, upon request, at each of the cardinal points. Unless the customer uses the instru. 


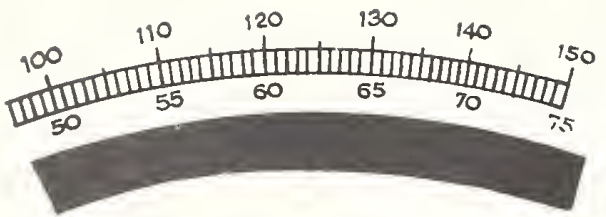

Fig. 2. Enlarged portion of middle acale of rig. 1.

nrent at only the points tested the ques. tion arises as to the corrections for the other scale points. In the absence of further tests it is generally consid. ered that the best value for the other scale points is obtained by linear in. terpolation (graplrically) of the corrections for the five or ten points tested. Then the question arises as to the validity of the interpolated values-in other words, low close is the interpolated value to the true value.

Some electrical instruments that are sent to the laboratory reveal, upon a $v$ isual inspection of the scale, a noticeable inequality in the spacing of adjacent subdivision marks.

'l'o study this a number of instruments of dillerent manufacturers were tested al each scale division throughout the usable or upper part of the scale. Botli angular measurements and a test at each division mark were made on a number of instruments; 39 instruments of 8 different manufacturers were in. cluded in tests. Of these, 15 were of the electrodynamic type, 4 of the moving-iron and 20 of the p-in. m-c. types. Threre were eight laboratory standards (0.1-percent-aciuracy class) and the rest were in the 0.5 -percent-accuracy class or better. Ol these, 22 were tested at each of the scale divisions over the usable part of the scale. The p-m. m-c. and electrodynamic types were tested witl a potentiometer and its accessories; the moving-iron type was tested witl a transfer instrunient. It is believed that for a good instrument with a good scale the values obtained by setting the pointer over a scale division inark in our usual testing procedure are correct within about 0.02 division.

The illustrations show some of the scale cliaracteristics of some of the instruments tested. Fig. 1 shows a scale for one instrument. Fig. 2 is an enlargement of the upper portion of the middle scale of the same instrument. Fig. 3 gives the corrections that must be applied to the several division marks of the sanie voltmeter (150-volt range) to give the true value for each of these points. Fig. 4 is a correction graph for another typical instrument. The broken lines show the errors that result if the common linear interpolating procedure is followed after testing at five of the cardinal points.

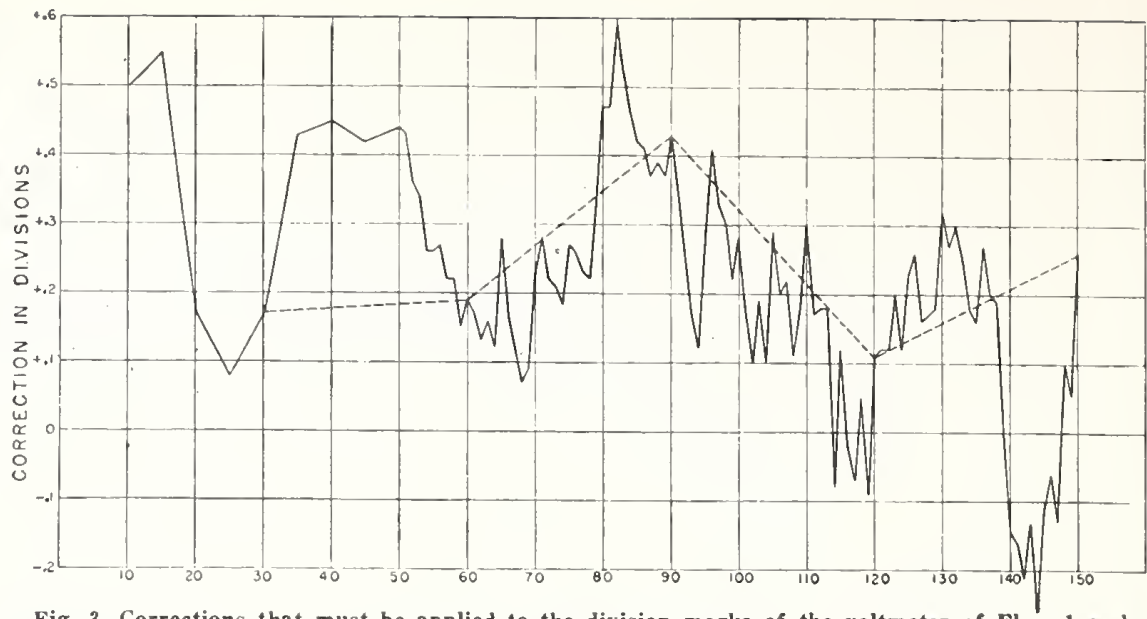

Fig. 3, Corrections that must be applied to the division marks of the voltmeter of Flgr, 1 and 2 to give the true value at each point. Dotted lines show errors that would result if interpolating procedure is followed after basic NBS test at 5 cardinal points.

The magnitude of the irrogularities may in some instances be influenced by the observers incorrect setting of the pointer over the division mark, or by pivot friction. However, it is believed that errors from these two sources have been held to a minimum.

No attempt will be made here to identify the particular influencing fac. tors that cause the large errors indicated by some of the graphs. Self heating errors probably have considerable effect on the general shape of the correction curves in those instruments that are not well temperature-compen. sated.

Figs. 1 and 2 indicate that some of the irregularities in the correction graph of Fig. 3 are due to the irregular spacing of the subdivision marks on the scale of this particular instru. ment.

Note that Fig. 4 also shows the relationship between divisions and degrees of the instrument scale.

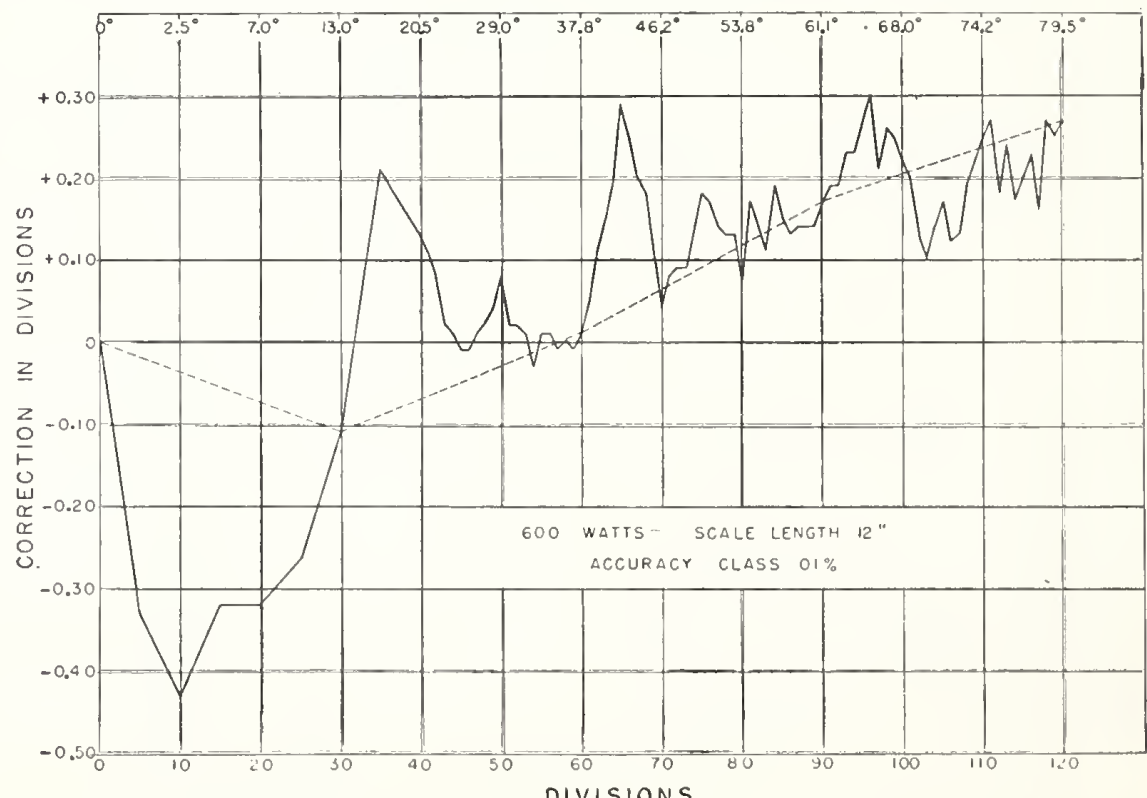

Fig. 4. Scale characteristics of an a-c. and d-c. wattmeter of 0.1-percent accuracy class.

\section{Errors of Observation}

Observational errors usually result failure to eliminate parallax on reading and (3) failure to estimate correctly fractional divisions. The first may be minimized by careful attention on the part of an observer in taking readings. The parallax error is effectively eliminated by the correct use of devices.

A number of studies have been made on the accuracy of estimates of fractional divisions. A Swedish scientist, Backstrom", made one of the most thorough studies on this subject. His conclusions may be summarized as follows: (1) the estimation of any particular observer cannot be depended upon to be accurate to the nearest tenth of a division except at the division

$\star$ Backstrom H. E., Zeit f. Instrumentenkunde, Vol. 50 , p. $561 \cdot 75,609 \cdot 624,665 \cdot 679,1930$, and
Vol. 52 , p. $105-123$ and $260-274,1932$ from (1) misreading the scale, (2) anti-parallax mirrors and other optical 
marks themselves and at the midpoint of the division, (2) an individual will make estimations of tenths of a division in much the same manner over long time intervals, (3) the miniınum error occurred when the line width was equal to the pointer width. and each was roughly 0.1 that of a division width, and (4) the average error of estimation decreased with increasing division width. There was little gain in increasing the width beyond about $2 \mathrm{~mm}$.

In the ordinary procedure of read. ing an instrument scale an observer will estimate the pointer position to the nearest 0.1 scale division. Backstrom's study and our experience indicate that the accuracy "ith which one is able to estinate depends on a number of lle factors. It is assumed that the observer luas normal vision, that the instrument has a good scale and pointer, and that the scale is well illuminated. 'The influencing factors are then (1) width of scale marks and width of pointer, (2) distance hetween scale marks, (3) the particular tenth which is being estimated, (4) fatigue and physical condition of the observer, (5) color cons. binations for scale, pointer and lines, and (6) personal bias.

We tested the ability of our laboratory personnel to estimate pointer positions. A 100-v. d-c. voltmeter in the
0.5 -percent-accuracy class, and an a-c. and d.c. 150-r. voltmeter in $1 / 4$-percentaccuracy class were selected for the test. The interval between the 80 and 81 division marks on the d-c. voltmeter and the 120 and 121 division marks on the a-c. and d-c. voltmeter was selected for the experiment. Each instrument was carefully tested at its two chosen reference division marks with a potentiometer. The olstained values then were used as a basis for dividing the intervening space into tiventy equal in. tervals. An observer at the potentiom. eter adjusted the voltage to set the pointer in randon order to the various tentlis or twentieths of a division. Most of the subjects liad considerable previous experience reading instruments. As indicated in the table, each was asked to estimate to the nearest tenth (and in other tests to the nearest twentieth) of a division. The subject was not aware of the setting being made at the potentiometer nor was he told how close his estinnated values were to the true values until the test was finished.

In obtaining the data for the table, the pointer was set to each 0.05 position and the observer was asked to estimate to the nearest 0.1 . For the $0.05,0.15,0.25,0.35$, and 0.45 positions he most frequently estimated the 0.1 below the value set (for 0.45 he

\section{Table.-Estimation of pointer position of a-c. and d-c. voltmefer to nearest 0.1 division, 14 observers, no lens used.}

Pointer Position

Set in Divisions

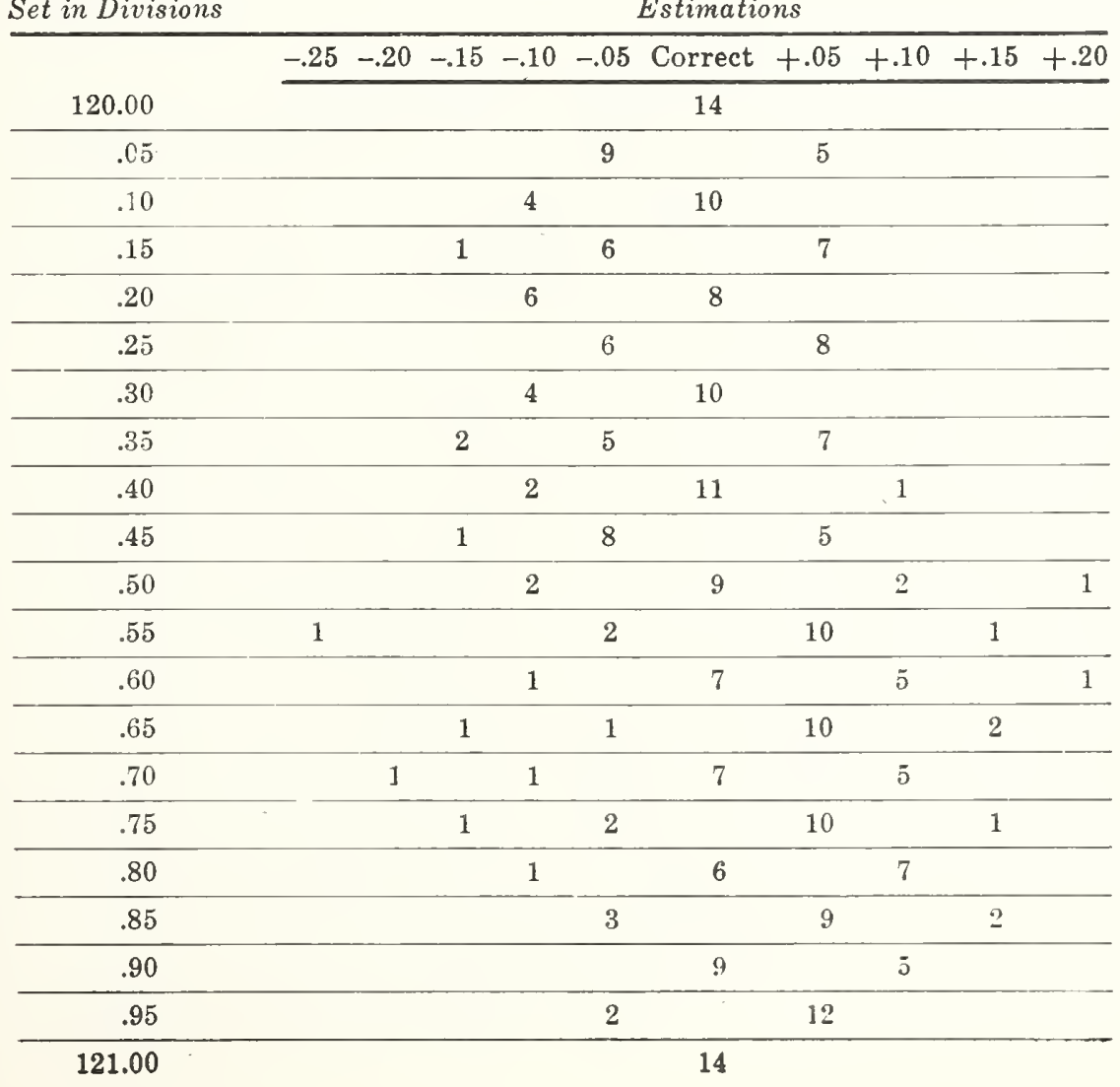

estimated 0.40 ), and for $0.55,0.65$. $0.75,0.85$, and 0.95 positions he most frequently chose the 0.1 above the value set (for 0.85 he estimated 0.90 ).

$\ln$ further tests, a flat-field $x 2$ lens. $1 \% / 5$ inches in diameter, was used, and each observer was asked to estimate to the nearest $1 / 20$ th of the scale interval. For most observers, this lens materially aided estimational accuracy.

The results indicate that an observer generally can estimate on a good scale to within 0.1 scale division. Out of 945 estimations only 25 were in error by as much as 0.15 division, and 123 (about 13 percent) were in error by as much as 0.1 division. The greatest uncertainty of estimation appears to be in the area of 0.3 and 0.7 division.

\section{Conclusion}

The data indicate that sone instruments in the better-accuracy class have inaccurately marked scales. If these instruinents are tested at five of the cardinal points, values obtained by applying corrections to the scale indications at intermediate points may be expected to be in error by several tenths of a division when the corrections are obtained by linear interpolation between values reported at the cardinal points.

It is possible to draw two conclu. sions: (1) If the corrections at five of the cardinal points are plotted on a graph, as in the figures, and the broken line connecting all plotted points is abnormally irregular, it is unlikely that interpolation between lwo adjacent cardinal points will give correct values. (2) If the spacings between adjacent division marks when viewed with the eye appear to be of unequal widths it is also unlikely that an interpolation value will be valid.

If an instrument is tested at every five divisions on its scale and the sepa. ration of intermediate marks appear normal the interpolation procedure should give values that are reasonably correct. Thereafter it should be necessary to test it only occasionally at five of the cardinal points. If the scale does not meet the necessary accuracy requirements (adjacent intermediate division marks are irregularly spaced or cardinal points are incorrectly placed) it may be advisable to return the instrument to the manufacturer for a new scale.

The procedure of estimating tenths of a division is not the weakest link in electrical measurements. However. this factor combined with a large number of other possible sources of error limit the accuracy of even the best instrument under ideal conditions to about 0.1 percent. If greater accuracy is desired one should resort to poten. tiometric measurements. 


\title{
Notes on the Care and Use of Electrical Instruments
}

\author{
By FRANK D. WEAVER, Electrical Instruments Section, National Bureau of Standards, Washington, D. C.
}

This article presents notes on precautions that should be observed by users of electrical instruments to enable them to achieve greater accuracy in measurements and to extend the useful life of their equipment.

\section{INTRODUCTION}

$\mathrm{F}$ ROM the performance and general appearance of most electrical instruments coming to the National Bureau of Standards for standardizing tests, it appears that their users generally employ the best practices in using and handling their measuring equipment. However, instruments occasionally come to our laboratory with bent pointers, damaged coils, dirty and corroded contacts and other evidences of abuse. Had certain precautions been observed it is not likely that these damages would have occurred. The following notes are given with the express purpose of aiding personnel in (1) achieving more accurate and precise measurements, (2) increasing the useful life of instruments, (3) decreasing the cost of maintenance, and (4) promoting safety. The items included are those that, as indicated by the writer's experience with laboratory personnel and with instruments, are most likely to be helpful to individuals who use electrical measuring equipment.

\section{General Precautions}

Certain general precautions should be observed in every laboratory using electrical equipment. These niay be roughly classified into two groups, those pertaining to (a) the general circuit, and (b) component instruments.

\section{A. The Circuit}

In nraking connections for a test, see that contact surfaces are clean, that nuts or binding posts are tightened firmly, that wires or cables have sufficient cross-section for the current in the circuit, and that insulation is appropriate for the voltage in use.

Sliding electrical contacts on apparatus such as bridges, resistance boxes and potentiometers should be cleaned occasionally with a dry cloth or a solvent such as varsol or benzol. If the pressure between the contacting surfaces is high, white vaseline may be used as a lubricant to reduce wear and oxidation at the contacts. For light- pressure contacting surfaces a highgrade light oil, such as Marcol, may be used with beneficial results if the contacts are cleaned periodically and only a sniall amount of oil is applied. In case the contact pressures are so high or edges so sharp that undue wearing is taking place at the contacting surfaces, corrections or adjustments should be made to alleviate this condition.

The laboratory worker is reminded that some of the common cleaning solvents such as benzol and carbon tetrachloride are highly toxic. Such solvents should be used only in small quantities and then in well-ventilated areas. One should not inhale the fumes or let the fluid come in contact with the skin. Some chlorinated solvents, such as carbon tetrachloride, contain traces of free chlorine which, in the presence of water, will form hydrochloric acid. Such solvents in general should not be used on electrical contacts because of the danger of subsequent corrosion from this acid residue.

Only rosin or rosin-in-alcohol should be used as flux for "tinning" and softsoldering parts and wires in circuits in electrical instruments and apparatus. Most of the prepared fluxes contain acid or other corrosive ingredients that are destructive when used on components of delicate instruments. Surfaces to be soldered should be thoroughly cleaned and heated. Silver solder should be used when soldering manganin or for joints in which a solder connection is under stress.

Leads which are attached to instruments should never be left hanging down over a table, stretched between tables or across the floor where they may be accidentally caught with the hand or foot and the instrument pulled to the floor. They should be twisted in pairs to each instrument to reduce the effects from magnetic fields produced by current in the leads.

For measuring alternating currents of 25 amperes or more, the use of a current transformer with suitable primary ranges and a 5-ampere ammeter is generally preferable to a self-contained ammeter. This aids in keeping heavy-current leads away from the instrument and isolates the measuring instrument from the possible high volt- age of the supply circuit. One multirange current transformer with one ammeter can be used to replace a number of separate a-c. ammeters. The transformer should be tested to determine the ratio factor for its various ranges with its ammeter as a burden if the highest accuracy is desired. There are multi-range a-c. ammeters on the market with built-in current transformers that have proved very satisfactory. The primary of a current transformer should never be energized unless there is an ammeter or some other low-resistance burden, such as a shorting link, across the secondary. Neither should the secondary be opened while the primary is energized. Otherwise the secondary winding may have suffciently high voltage induced in it to endanger insulation or the safety of personnel. There is also the possibility that the magnetic condition of the core will be changed and alter the ratio and phase angle until the transformer is thoroughly demagnetized. This condition may also happen if direct current flows through the windings. If the secondary is left open for some time with the primary energized, overheating due to the greatly increased iron loss in the core may damage the insulation.

A potential transformer with a voltmeter connected to its secondary should be used for measuring alternating voltages in excess of a few hund:ed volts. As a safety precaution the secondary (115-volt winding) should be grounded at one point. For highest accuracy the transformer should be tested (ratio and phase angle determined) with the burden of its circuit.

An observer in doubt about a particular setup or the use of a given instrument should inquire until the needed infolmation is obtained. Before a circuit is energized, check all components to make sure that connections are properly made and that ranges are high enough for the magnitude to be measured. Protective resistors should be inserted where necessary. If feasible, the voltage applied shouid have a low initial value and then be increased gradually to the desired value.

When opening a circuit connected to electrical equipnrent, it is generally advisable, where possible, to reduce 
the supply voltage to a low value before throwing the supply switch or circuit breaker. Remove conductors or leads to equipment one at a time, making the first break at the power source tcrminal and next remove the end attached to the equipment. This latter precaution applies when unplugging from a distribution board to a subpanel as well as when disconnecting an instrument, such as a voltmeter, from a line, or a standard cell from a potentiometer. The reverse steps should be taken when placing equipment in a circuit. Some careless observers, when taking an instrument out of a circuit, disconnect the leads at the instrument terminals, remove the instrument, leave the free ends of the two leads dangling while the other two ends are connected to a source of power. This is a dangerous procedure. Power circuits are ordinarily protected with fuses or circuit breakers and switches, but these protective devices do not guarantee that instruments and other equipment will not be damaged if the circuit is shorted by the careless handling of leads or switches.

Laboratory personnel should be particularly careful when working with equipment from which there is a chance of electrical shocks. Currents as small as 10 or 20 milliamperes can be dangerous to life, depending upon such factors as the current path through the body and physical condition of the victim. The value of current will depend upon the resistance of the body and the applied voltage. Under certain conditions one may receive a fatal shock from equipment that operates from the usual 110-volt circuit. If the hands or feet are damp when contact is made, the body resistance may be low enough $(11,000 \mathrm{ohms}$ or less) to permit a dangerous or fatal current. Such an electrical shock may be received if one touches two separate metal components of a piece of equipment, or one metal component and a grounded object so that a portion of the body acts as a parallel path to the electrical circuit. Exposed metal parts should be insulated from the wiring of a piece of electrical equipment (motors, drills, soldering irons, etc.) but sometimes, because of worn or defective insulation, the conductor comes into electrical contact with the exposed metal. The cases of some equipment are provided with ground terminals and if properly connected wili protect the operator from the hazards of electrical shock.

\section{B. The Instrument}

Multi-range instruments are desirable for some applications. Many such instruments show up for repairs with bent pointers and damaged coils because users have not been careful to set the range switch in the proper position before closing the circuit. When some circuits (for example a bank of tungsten filament lamps) are initially closed, the cold-state resistance may be low and the initial surge of current rather high. An ammeter placed in such a circuit to measure the steadystate current may be overloaded by these initial high currents and result in a bent pointer. It may be advisable to short out the ammeter during, the period of high initial currents. In a similar way the insulation of the coils of a voltmeter may be damaged by voltage surges or by distorted waves that have a high peak value, say as much as five times as great as the rms. value to which the instrument responds. This latter situation is especially dangerous for electrostatic voltmetcrs. A protective resistor should be placed in series with the high side of an electrostatic voltmeter to prevent "burning up" the instrument spring in casc of flashover between its plates.

In the laboratory one frequently uses a suspension-type d-c. galvanometer in connection with bridge and potcntiometer measurements. Ordinarily these sensitive instruments are used to determine voltage balances betwecn the arms of a bridge or potentiometer and do not directly measure electrical magnitudes. Such an instrument should have the sensitivity necessary for the precision desired, should have a short period, and should be slightly underdamped in its circuit. If its sensitivity is higher or its period longer than necessary, time will be wasted in getting balances. Since galvanometers are made of delicate parts they should be handled with great care and be protected in a circuit by suitable switches and resistors.

Make it a practice to handle all instruments with care; treat those used as laboratory reference standards with special respect. Pivoted instruments should never be placed or left where they may be exposed to vibrations. One severe shock, such as a hammer blow on a bench or table where instruments are resting, can permanently damage their pivots and jewels.

In making adjustments or repairs on instruments, bear in nind that only the manufacturer is really well qualified to make MAJoR repairs. If it is necessary to open the case, it should be done in a room where there is a minimum of dust and in an area where there are no iron filings.

When instruments are not in use, they should be stored in cases or cabinets free from dust, acid fumes, excessive heat, moisture and vibration.

Shorting the terminals of a galvanometer or microammeter by means of a small conductor before such instruments are moved heavily damps the motion of the moving parts and helps to prevent damage. Locking devices are sometimes provided for moving systems to protect pivots and jewels or suspensions from shock while the instrument is in transit; make proper use of them.
Instruments that are being prepared for shipment should first be wrapped in heavy paper to exclude dust and dirt, surrounded by a cushioning material such as several inches of excelsior, and carefully and securely packed in a strong, properly labelled box. The top of a wooden packing box should be fastened with screws (not nails) to avoid damage resulting from blows with a hammer or nail puller. It may be advisable to invert the instrument in the shipping container so that the upper instead of the lower jewel and pivot receive any damaging blows that occur in shipment. If this is done, the box should carry a "This Side Up" label. These precautions aid in protecting delicate lower jewels and pivots from injurious mechanical shocks during transit.

Precautions should be taken to prevent marring or scratching the finish of an instrument lid or case. Such defacing may occur if an instrument is placed near a hot rheostat or an inverted lid is carelessly pulled along a rough surface. An occasional cleaning of the surface of an instrument case is advisable in order to restore good appearance, as the better the appearance the more respect it is likely to receive.

A relative humidity of less than 65 percent and a temperature of $25 \mathrm{C}$. are desirable for the air of a room where instruments are to be used. If the air is too humid, moisture may condense on insulating surfaces between rosistors and act as a highresistance shunt or in effect reduce the total resistance of that part of the circuit. This effect is ordinarily nore noticeable in high ohms-per-volt instruments. For extremes of humidity, moisture may be absorbed by or evaporated from the insulating fibers surrounding fine resistance wire, causing the threads to change dimensions, thus exerting pressures and stresses on the fine wire and altering the resistance.

It is a good practice to compare working instruments against reference instruments at least once a year. If high precision of measurements is needed, the instruments should be checked against reliable reference standards or sent to a measurements laboratory where precision tests may be made. Such tests will reveal changes in the accuracy of instruments.

Most instrument companies occasionally issue instruction manuals, bulletins and catalogs for the benefit of their customers. These ordinarily give valuable information about their instruments, with diagrams showing how to connect them in a circuit. It is well to consult such manuals in addition to handbooks, textbooks and other useful sources of information.

\section{Sources of Error (INSTRUMENTS and Associated Apparatus)}

A knowledge of the conmon sources of error in instruments and associated 
apparatus may be helpful in eliminating certain errors of measurement and iricreasing the accuracy of results.

Some of the conditions that may interfere with the normal turning of the moving system of an instrument are: a warped or loosened paper scale, thin fibers projecting upward from a paper scale, iron filings in the air gap, a bent pointer, a bent damping vane, a flat pivot, a broken jewel, or a moving system too tight or too loose in its jewels. Each of these possible sources of error should be corrected and the instrument retested where necessary before use if highly accurate results are desired. Obstructions can usually be detected by a "jumping of the pointer" as current through the instrument is slowly increased or decreased to deflect the pointer upscale or downscale. Occasionally during this test, set the pointer to a scale mark and tap the case lightly: pivot friction will be revealed by a slight change in pointer position.

Tapping the case of an instrument while it is being read is usually unnecessary if the instrument is properly constructed and in good condition. However, when slight friction is present from any cause, its effect can usually be temporarily minimized by gently tapping the case. Hard tapping may result in damaged pivots and jewels.

Errors resulting from friction and from mechanical unbalance may be expected if a portable instrument, designed to be operated with the axis of the moving coil in a vertical position, is read while the instrument is so placed that this axis is inclined from the vertical or is in a horizontal position. In general it is good practice to use an instrument with its movingcoil axis only in the position for which it was designed.

Parts of moving systems, including pointers of instruments, tend to vibrate at certain frequencies where they are mechanically resonant. Ordinarily, at the frequency for which the instrument is designed, this should not be expected as the maker tries to avoid this in his design and construction. This phenomenon is revealed by erratic pointer movements, pointer widening or blurring, and sometimes by a rattling noise. Errors should be expected if the instrument is read under these conditions. It is possible to change the resonance frequency by changing the balance weights and rebalancing the moving system.

Some instruments show inherently large zero shifts: After the moving system is deflected up-scale for a few minutes, the pointer does not return to its initially set zero position when the circuit is opened. The observed shift may generally be explained on the basis of inelastic yield in the springs. Such shifts constitute a source of error for which it is not practical to apply a correction. The only remedy if the shifts are large is to have the spring replaced by the manufacturer.
Poor electrical contacts at the switches or in any part of a measurement circuit are a source of considerable annoyance to an observer. In instances where this condition cannot be alleviated by cleaning and oiling the contacts, it may be necessary to have the contacting surfaces plated with nickel, silver or gold. For rugged service, where high currents and greater wear due to higher contact pressures are involved, a silver inlay in the contacting areas has proved highly satisfactory.

An electrical instrument may give inaccurate values because of errors in the original scale calibration. In other words, the cardinal points on the scale may have been improperly placed by reason of uncorrected errors in the test equipment or in procedures used to locate lines on the scales. An inspection of the scales of some instruments reveals a noticeable inequality in the spacing of adjacent subdivision marks. An observer should not place much trust in the indications of an irstrument that has an erratic and carelessly-drawn scale.

To provide the greatest reading accuracy, a portable instrument should have a good scale, a knife-edge pointer and an anti-parallax mirror. The knifeedge width should be about equal to that of the scale division marks, and not greater than one-tenth of the distance between successive scale divisions. For súch an instrument an experienced observer should be able to estimate the pointer indications to better than \pm 0.2 scale division. With a laboratory standard having the major scale divisions subdivided into fifths by means of diagonal lines intersecting six concentric ares, one would be expected to estimate to about 0.05 of the major divisions. Instruments that do not have anti-parallax mirrors are subject to rather large reading errors.

When a wattmeter is initially inserted in a circuit for the measurement of a-c. power, it is a good practice to put an ammeter in series with the current coil and a voltmeter in parallel with the voltage coil. This precaution is advisable because it is possible for either or both coils to be damaged by overload and yet the pointer may not indicate a full-scale reading since a wattmeter measures watts $(V I \cos \theta)$. To avoid the errors caused by electrostatic effects resulting from differences in potential of the two circuits of a wattmeter without a common connection, the \pm voltage terminal should be connected through a voltmeter to the \pm current terminal.

Errors may result from (1) electrostatic forces, and (2) leakage currents, if the moving system of an instrument is at a different voltage from that of its case or panel. Correct electrostatic shielding will eliminate this source of error. Static charges on the glass window or case of an instrument may also cause errors in pointer indications. The effect of a static charge, such as one arising from rubbing the glass with a cleaning cloth, may usually be eliminated by blowing one's breath on the affected area. Trouble from the accumulation of static charges will be more frequent at low humidities because of increased insulation resistance.

Insofar as possible, one should avoid locating instruments in an area where there is a strong magnetic field or adjacent to large masses of metal. Strong fields may be encountered near current-carrying conductors, transformers, Variacs, and even other instruments. Pointer indications of some unshielded portable d-c. instruments may be in error by as much as 2 percent when the instrument is read while resting on a table having a sheet-iron top. Errors resulting from stray fields, eddy currents and the shunting effect of nearby sheets of iron, are generally much less for shielded instruments than for unshielded ones.

A panelboard instrument should not be inserted in a steel panel unless it has been calibrated by the manufacturer for use in such a panel.

There is a tendency for the magnets of $d$-c. instruments to become weaker with time if they have not been properly aged or conditioned, thereby weakeving the flux in the air gap. Such a tendency is indicated when periodic tests show that an increasing value of the measured magnitude is required to deflect the pointer to a given scale mark, other factors remaining constant.

In making measurements with ammeters, voltmeters, and wattmeters, it is well to remember that there may be considerable power losses and voltage drops in the instruments as well as in their leads. These must be taken into consideration in precise measurements.

Voltmeters requiring $0.1,1,10$ and 100 milliamperes for full-scale deflection are in common use. Before a voltmeter is placed in a circuit, one should consider the effect of the current losses of the instrument upon the circuit. Voltmeters having current losses of 0.1 milliampere are desirable for some applications; however, one that requires 10 milliamperes for full-scale deflection is likely to have more rugged components and therefore is preferable where current loss is not an item of importance.

When carrying rated current, shunts and resistors in some instruments are required to dissipate a considerable amount of heat. Unless the resistance material was properly annealed by the manufacturer and has a low temperature coefficient of resistivity, changes in resistance with use may be expected. These changes, accelerated by heating which occurs when the resistor carries rated current, may produce significant errors in instrument indication. Adequate provisions have not been made in some instruments and equipment for dissipating their large heat losses, and this may result in excessively high temperature when in use. 
Some types of instruments not properly compensated for changes in temperature may be in error by as much as 0.4 percent per degree Centigrade. An instrument will normally be at least partly temperature compensated, so the coefficient is more likely to be 0.1 percent or less per degree Centigrade. Since most instruments are calibrated for use at $25 \mathrm{C}$., for precise work it is best to allow them to reach temperature equilibrium in a room maintained at this temperature. This is especially true for thermocouple instruments because they have rather large temperature coefficients. If this precaution is taken, it will not be necessary to apply temperature corrections to pointer indications. It may also insure against errors arising from an unsuspected difference in temperature of the components of an instrument.

Lamps used for scale illumination radiate considerable heat and, if placed too near the coil or spring, may increase the temperature of these components and thereby produce considerable error in the pointer indication.

The values indicated by moving-iron and rectifier-type instruments may be in error by several percent if used on direct current or if used on alternating current at a frequency other than that for which they were designed by the manufacturer. Rectifier instruments are generally calibrated for use on a pure sine wave and their readings may be considerably in error if used for measurements in which the wave-form is distorted.

Electrodynamic (except those having iron cores or built-in transformers), electrostatic, and thermocouple instruments are designed for use either on direct current or on alternating current within specified frequency ranges. The mean of values corresponding to the two directions of current should be used when measuring direct current with either of these three types. When alternating current is measured, the frequency should lie within the range for which the scale was calibrated. Ac.-dc. differences for these three types are ordinarily rather small at power frequencies. However, for measurements of the highest accuracy, it may be desirable to have these differences determined.

Switchboard shunts of large current rating (1000 amperes or more) should be tested in position with the current and potential terminal connections normally used. A test in any other position is not likely to duplicate the current distribution through or the heating of the shunt encountered in normal use and may give values of current that differ from those for normal connection by as much as 2 percent.

Standard cells must be used with care. Unlike electrical instruments they give no visible signs of electrical abuse. They are designed for use as standards of emf. only and should be properly connected to a potentiometer.
The unsaturated type of cells are generally, used in laboratories. Even a small current drain for a short period of time. (100 microamperes for $5 \mathrm{~min}$ utes or more) maý temporarily impair the usefulness of such cells. If used in potentiometers where highly accurate measurements are desired the cells should be tested periodically. It is sometimes advisable to enclose them in thermally. insulated boxes to eliminate errors that arise from sudden changes in temperature or differences in temperature between the two legs of the cell.

A millivoltmeter in combination with a pair of leads and an external shunt (or a group of shunts to provide a number of ampere ranges) is sometimes used for measuring direct currents. To get reliable results, it is necessary to use the particular leads (or leads having equivalent resistance) with which the millivoltmeter and its shunts were tested. Since the millivoltmeter may have a resistance of only a few ohms, it is apparent that a small change in resistance of the leads or their contacts may cause an appreciable error in measurement.

\section{Measurement Procedures}

When making a test, record on the data sheets all pertinent information about the test and the equipment used. A wiring diagram of the test setup, date, humidity of air, room temperature, duration of warm-up period, makes, models, ranges and serial numbers of instruments and equipment used should be listed. Unusual temperature rises as well as defective or unsatisfactory performance of any equipment should be noted. The record should be sufficiently detailed to permit an accurate appraisal of the test results or a later duplication of the setup to permit a satisfactory experimental check. Test records should be complete, in ink, and on a good grade of paper so that they may be filed for future reference. The records should be made unless the observer is certain that they will have no future use. Inadequate test data may be a source of genuine embarrassment at some later date.

Before an observer reads an instrument he should make sure that the scale is properly illuminated. This is of considerable importance for precise readings or if errors resulting from fatigue are to be minimized where consecutive readings are to be made over a period of time. A hand lens having a magnifying power of 5 is a helpful aid in getting accurate pointer readings.

It is a good practice for an observer to read and record the pointer indications of an instrument in divisions estimated to tenths (on the appropriate scale of a multi-range instrument) rather than in measured units. The correction table for a given instrument should also be expressed in divisions. There will be a minimum of confusion if the formula $I+C=T$ (indicated reading plus correction equals true value) is used in applying corrections. The true value (in divisions) may then be converted to measured units.

Assuming that an observer can estimate a pointer deflection on a 100-division scale to 0.2 division on any part of the scale, a reading error of 0.2 division at the 100-division point will be 0.2 percent of the measured magnitude, whereas a reading error of 0.2 division at the midpoint of the scale will be 0.4 percent of the measured magnitude. On the same basis the error at 20 divisions will be 1 percent of the measured magnitude. It is apparent then that if one is to minimize reading errors, only about the upper half of a scale should be used. For lower values of the measured magnitude, one should make use of a lower range instrument.

The laboratory and its equipment should be restored to order after completion of each job. Instruments and other equipment should be returned to their assigned cabinet or storage space where they will be protected and can be readily located by the next user. An instrument that has been damaged or found to be defective should be properly labelled "To be repaired," and should not be returned to its usual shelf space. The person in charge should be notified and steps taken to repair or replace the damaged part.

\section{CONCLUSION}

In conclusion then, to get the most out of an instrument one should take advantage of the information in manufacturers' instruction manuals and any other good sources. The proper connections, correct applications and limitations should be known for each piece of equipment. Such factors as frequency, wave-form, temperature, and stray fields, must be given careful consideration. The instruments should be tested periodically, and when in use precautions should be taken to eliminate or to correct for all possible sources of error. The life of an instrument will be materially increased if a little forethought and respect are given at all times in its care and use.

\section{SELECTED REFERENCES}

1. K. Edgecumbe and F. E. J. Ockenden. "Industrial Electrical Measuring Instruments" (book), Pitman Publishing Corp., New York, 1933.

2. W. H. Lawes, "Precautions in the Use of Standard Instruments," Journal of the Institu. tion of Electrical Engineers, Vol. 67, 1929, pages 541-548.

3. E. S. Lincoln, "Electrical Measurements and Meter Testing in the Power Station" (book), Weston Electrical Instrument Corp., Newark, New Jersey, 1929.

4. A. E. Peterson, "Performance of Portable Electrical Instruments in Magnetic Fields," Transnctions of the American Institute of Elec. trical Engineers, Vol. 67, August 1948, pages 1228-1231.

5. James Spencer, "Maintenance and Serv icing of Electrical Instruments" (book), The Instruments Publishing Company, Inc., Pittsburgh, Pa., 1944. 
F. K. Harris and F. D. Weaver

Electrical Instruments Section

National Bureau of Standards

(Abridged from a Conference Paper presented at the Winter-General Meeting of the American Institute of Electrical Engineers, January 1954)

\section{INTRODUCTION}

An instrument reading camera, or scale projector, is used in the electrical instruments testing laboratory at the National Bureau of Standards as an aid for the observer in accurately setting the pointer of a portable instrument to selected division marks on an instrument scale. The unit (lens head and hood arrangement) makes use of a photographic objective lens system and a front face mirror to project the enlarged image of a portion of the scale of an instrument on a vertical ground glass screen where it may be conveniently viewed by the observer. Standardization testing of indicating instruments requires that the pointer settings be accurately made; the camera with its magnification of $5 \mathrm{x}$, improves the accuracy with which pointer settings can be made. The unit may also be used for accurately reading the pointer indications of an instrument.

It is a common practice to use a hand lens as an aid in setting a pointer to a given scale mark or in reading the pointer position on a scale. A reading lens is often inconvenient to use and is conducive to eye strain and physical fatigue. This is especially true if a large number of observations are to be made consecutively. The arrangement described here eliminates much of the eye strain and physical fatigue involved in reading instrument scales. This is in large part due to the fact that when using the camera the observer sits in a normal restful position viewing the scale on the ground glass screen with both eyes open. Without the aid of a scale projector (to convert binocular to monocular vision) the observer must line up the pointer, the scale division mark and the image of the pointer in the anti-parallax mirror with one eye closed. This procedure requires the observer to sit in an unrestful position and necessarily causes tiring of ${ }^{*}$ both the eyes and the body muscles.

\section{CONSIDERATIONS}

In designing the instrument reading camera certain requirements were set up as essential to its use in a standardizing laboratory. These were (1) adjustable focusing arrangement, (2) scale and pointer of the test instrument must be clearly visible simultaneously, (3) sufficient scale illumination without undue temperature changes in the instrument under test, (4) moderate magnification, (5) ease of reading, to minimize eye strain and physical fatigue, (6) ease of adjustment to accommodate portable instruments of various depths and scale lengths, (7) adjustment so that the pointer can be easily followed as it is deflected up or down scale, and (8) provision for eliminating parallax errors. 
Al1 of these features were incorporated in the instrument reading camera (scale projector) under consideration. /Figure 1/

The optical system consists of a photographic objective lens (Goerz Dogmar) having a focal length of 3-1/8 inches and an associated iris diaphragm adjustable from $\mathbf{f} 4.5$ to $\mathrm{f} 32$, a front surface aluminized mirror and a ground glass screen. All of these components are encased in a lens head and hood arrangement.

The hood is part of a cone $14^{\prime \prime}$ long with a diameter at the viewing end of about 6-3/4" The ground glass screen is located inside the hood about 4" from the viewing end. The distance from the scale to the lens is about 4", lens to center of mirror 6", and from mirror to screen 12".

The instrument to be tested is placed below the lens head so that the instrument scale faces away from the observer. When the instrument is placed properly for observations one sees on the vertical ground glass screen an inverted and enlarged image of a portion of the instrument scale and pointer.

The scale illuminating system consists of seven lamps, each 6 candle power 6 - 8 volts, equally spaced around a circular ring, outside but attached to the lens cone. The reflectors are sections of ellipsoids of revolution with the light source at one focal point, which focus the light at a distance of about 6". The illumination on the instrument scale is concentrated in an area about equal to the field of view of the camera. The lamp housings may be rotated through about 30 degrees in their mountings about either of two mutually perpendicular axes. This allows lateral adjustment of the spots of illumination from each lamp to provide uniform illumination of the part of the scale and pointer being viewed. The illumination on the instrument scale is of high intensity and is completely shadowless. A heat-absorbing glass filter (Corning-Ak1o), mounted in each lamp housing, absorbs about 90 percent of the total energy in the light beam but transmits more than 65 percent of the blue green where visibility is greatest; i.e., the visual brightness is decreased by only about 40 percent while the total energy is only 10 percent of that in the unfiltered beam.

The temperature rise resulting from heat reaching an electrical instrument in position under the camera may be seen in Figure 2. In this test the illuminated spot was held fixed at about the mid-point of the scale. Temperature rise is plotted against time for a number of locations on the face of the instrument and, by means of a thermometer we11, within the interior of the case. The latter temperature rise amounts to only $1.5^{\circ} \mathrm{C}$ after one hour exposure. This temperature change is not objectionable unless one is testing instruments that are poorly temperature compensated. 
The arrangement of components is shown in Figure 3. Note that in this Figure only one of the 7 lamps is shown and that the hood is turned through an angle of 180 degrees from its normal position. All the machine work was done in the shops at the National Bureau of Standards. All the metal parts are made of non-magnetic material: duralumin, brass or bronze. The upright post which supports the camera, and the other bulky metal parts are so located that, when the test instrument is in its proper position, no eddycurrent error can be detected, even in an unshielded moving-iron instrument.

The central portion of the shaft on which the boom slides is threaded. A threaded nut for focusing the lens on an instrument scale fits into an opening in the boom frame. By manually turning this nut the boom may be raised or lowered to accommodate instruments of various heights.

The lens head is supported from a horizontal arm attached to the end of the boom through a hinge joint. The lens head may be moved along the boom arm by a rack and pinion arrangement. If the axis of the moving element of an instrument is placed directly beneath the pivot of the boom arm and the lens is directly above the instrument scale, it is possible to follow the pointer over the scale by manually turning the boom arm on its pivot. The lens moves along the arc of the instrument scale.

By means of two knurled knobs it is possible to alter the plane of the front-surface mirror in the optical train and shift the image of the scale and pointer on the viewing screen. The purpose of this adjustment is to bring the "no-parallax" position of the pointer to the center of the screen. If the adjustment is properly made, parallax will be minimized when the pointer intersects the small circle at the center of the screen. This is shown in Figure 4. Parallax errors will be eliminated for each setting if the boom arm is adjusted until the image of the pointer on the screen coincides with the pointer image reflected from the anti-parallax mirror of the instrument. The optical system of the camera has sufficient depth of focus that both of these images can be seen.

In testing electrical instruments the procedure which we usually follow is to adjust the measured quantity until the instrument pointer is on a chosen scale mark, and then to evaluate the quantity. It is therefore pertinent to inquire how closely an observer can set the pointer to a division mark on an instrument scale. Five of our laboratory people were asked to make settings to a division mark on a voltmeter scale using (1) the unaided eye and (2) the reading camera. In each instance a second observer measured the voltage set, using a potentiometer and volt box. Each observer made a group of five settings, and, an hour later, a second group. The instrument, a high quality d-c voltmeter having a 100 division scale 6.1 inches long, was energized for an hour before the test was started, in order to eliminate errors of spring fatigue and self heating. The results are given in Table 1. 
It may be noted that the range of mean values determined by the various observers, as well as the total spread in their individual observations is about twice as large with the unaided eye as with the camera. If we assume that the true value of voltage corresponding to the chosen scale mark is 60.10 it may be concluded that the average for any of the observers will be within 0.01 division of the true value when the camera is used. Using the unaided eye, the average error in setting may be expected to be as much as 0.03 division.

\section{CONCLUSIONS}

It is realized that the above sampling is too limited to serve as a basis for general conclusions concerning the ability of observers to set particular values on a scale. Many other factors such as the relative width of divisions, scale lines and pointer, uniformity of scale and its overall quality would have to be considered before a general statement could be made. However, the data given here verify our laboratory experience with the reading camera. Its use is of material assistance in the accurate setting or reading of an instrument scale. Of equal or greater importance is the fact that an observer can use it comfortably without eye strain or undue fatigue when observations must be continued over a considerable time.

There are of course factors other than the nature and quality of the scale that have to be considered when one is setting a pointer to a mark or estimating pointer position on a scale. Pivot friction, spring fatigue, self heating, and mechanical unbalance are all possible sources of error that the manufacturer attempts to eliminate through good design and workmanship. Errors of observation can be minimized by the use of a reading aid such as the one we have described.

Table 1

Unaided Eye

Observer Mean Value Maximum Deviation

1

2

3

4

5
60.11

60.07

60.11

60.11

60.09

60.07

60.11

60.11

60.12

60.11
0.01

.03

.05

.02

.03

.05

.01

.01

.05

.03
Mean Value $\underline{\text { Camera }}$ Maximum Deviation

60.10

60.10

0.02

.01

60.11

.01

60.10

.02

60.10

.01

60.09

.02

60.11

.01

60.11

.01

50.09

.03

60.09 


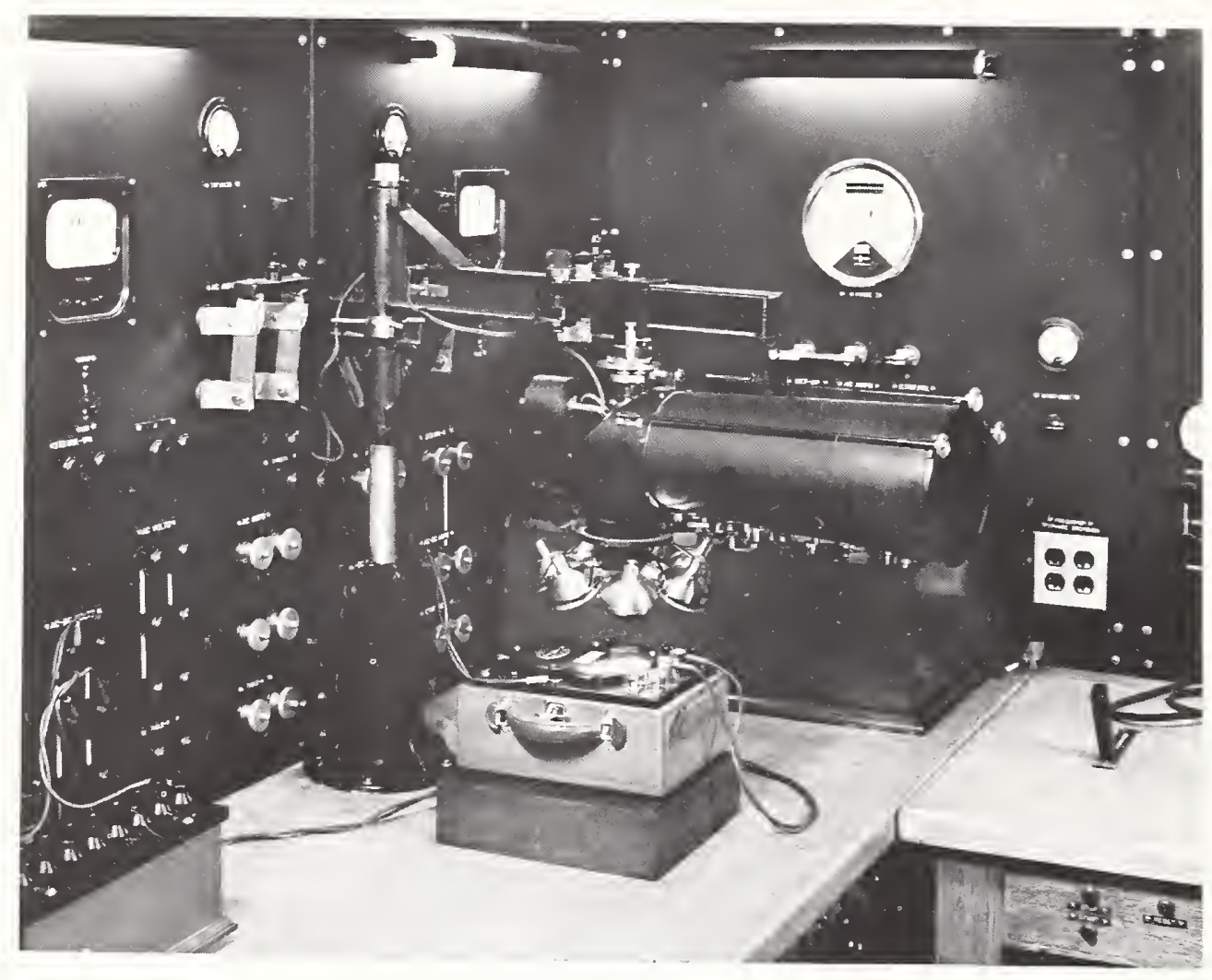

FIG. I

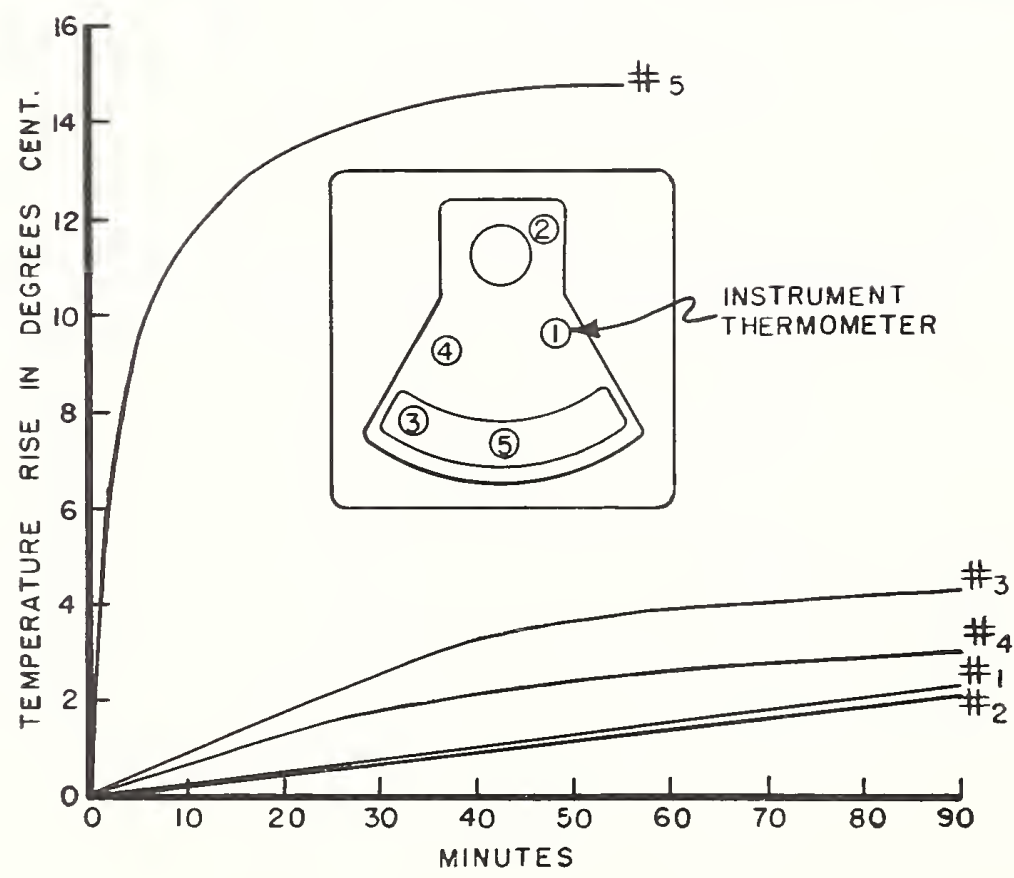

FIG. 2 


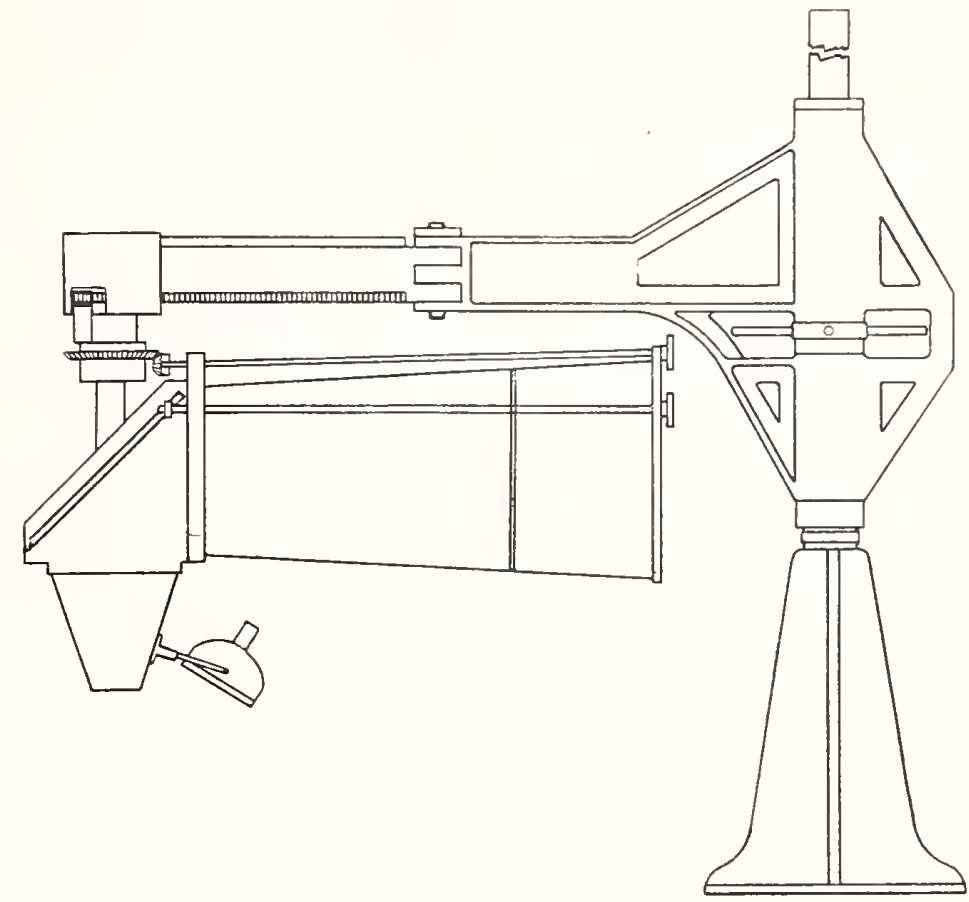

FIG. 3

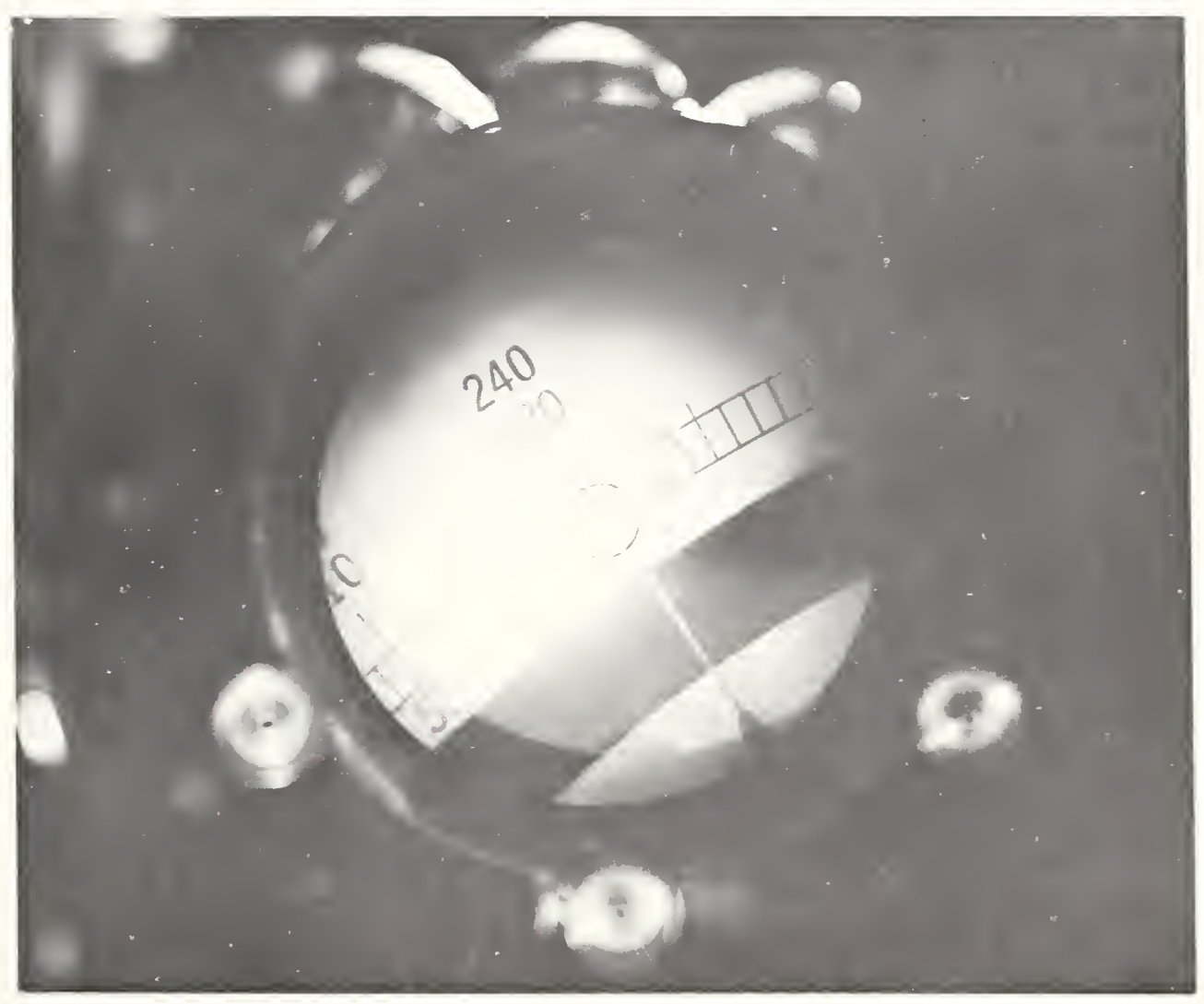

FIG. 4

$437 / 6$ 


\section{Power Supplies for 60-Cycle Tests of Electrical}

\section{Instruments and Meters}

Abstract: A description is given of power supplies used at the National Bureau of Standards in the accurate testing of a-c electrical instruments and meters. The requirements which such supplies must meet and the tests to determine the performance of the voltage stabilizers used in these supplies are also presented.

\section{INTRODUCTION .}

THE standardization testing of a-c ameters and voltmeters requires a power supply having an output of good wave form, exceptionally free of fluctuations, and adjustable in coarse and fine increments over wide ranges of current and voltage. In addition, if watthour meters, wattmeters and phase-angle or powerfactor meters are to be tested, the supply should have" two such outputs of the same frequency, adjustable in phase so that phantom loading may be used. In this arrangement one source supplies the voltage circuits and the other the current circuits of the test and standard instruments or meters, making it possible to stimulate with ease any desired test condition.

The commercial development of electronic 60-cycle voltage stabilizers has made it possible to supplant sine-wave motor-generator sets at the National Bureau of Standards with convenient semi-portable power supplies for instrument and meter testing at currents up to 100 amperes and voltages up to 750 volts. Two such supplies, each consisting of two stabilizers energized from a 3-phase 208-volt distribution system through suitable phase-shift and control circuits, have proven their value over a period of several years. Similar supplies should prove equally valuable in other laboratories. This paper presents an analysis of significant requirements, a brief description of the circuits and components used, and an outline of the tests that were made to determine how we 11 these supplies met the requirements.

\section{REQUIREMENTS FOR SATISFACTORY POWER SUPPLY}

$$
\text { Regulation and Stabilization }
$$

For instrument testing, the most important requirement upon the power supply is that fluctuations of the pointer of the instrument must be unnoticable during a measurement. Since the load impedance during a measurement is constant, such fluctuations are caused chiefly by changes in the applied voltage. Thus the regulationt of the source must be sufficient only to prevent large changes in voltage when loads are switched on and off. In addition, unless the 1 ine voltage fluctuates widely during a measurement, only moderate stabilization is required. If the line-voltage fluctuations do not exceed 1 per cent, the stabilization ratio of the stabilizer, $\Delta V_{i} / \Delta V_{o}$, (for nomially equal input and output voltages, $V_{i}$ and $V_{o}$ ) does not ordinarily need to be more than about 20 .

\section{Recovery Time}

An abrupt change in the supply voltage is initially practically unattenuated by most types of stabilizers. Subsequently, the change in output

*Electrical Engineer, Electrical Instruments Section, Electricity and Electronics Division, National

Bureau of Standards

+Regulation here refers to the effect of load changes on output voltage (with fixed input voltage) and stabilization to the effect of input-voltage changes on output voltage (with fixed load impedance). 
voltage is roughly exponentially reduced to nearly zero by the control action of the stabilizer. The recovery time of this action must be so short that the ballistic kick of the instrument resulting from the abrupt change is negligible.

As shown in Appendix A, the ratio, under these conditions, of the maximum change in deflection, $\triangle D$, to the steady state deflection, $D$, of a critically damped rms instrument having a period, $t_{a}$, is $\triangle D / D=$ $2.3 n t_{c} / t_{a}$ where $n$ is the fractional change in applied voltage and $t_{c}$ is the time constant of recovery of the stabilizer. Thus if a 1 per cent change in voltage is to result in less than 0.1 per cent momentary change in deflection of an instrument having a period of 1 second, the time constant $t_{c}$ of the stabilizer must not be more than $0.04 \mathrm{sec}$. This is about 2.5 cycles for a 60 cps supply, a rather severe requirement.

\section{Wave Form}

Instrument testing imposes several distinct requirements on the wave form of the supply if the results are to be the same as for a purely sinsusoidal wave form.

If an rms instrument (ammeter or voltmeter) is tested with an rms standard, surpris ingly large harmonics of low order can be tolerated. It can be shown (1) that a rms instrument which has an error of $e_{k}$ per cent at the $k$ th harmonic will measure the true rms value of a distorted wave with a precentage error of approximately $e_{r}=\sum e_{k} h_{k}^{2}$, where $h_{k}$ is the ratio of the kth harmonic to the fundamental. (The approximation is reasonably close for each $e_{k}<20$ per cent and $\left.h_{k}<0.2\right)$. For example, an instrument which is correct at the fundamental and has an error of 10 per cent at the 5 th harmonic will measure the rms value of a wave having 10 per cent of that harmonic with an error of only 0.1 per cent. The frequency influence for most a-c instruments is very small at power frequencies and for many types increases roughly as the square of the frequency. Thus harmonics in a 60 cycle supply for testing only rms instruments should not be greater than perhaps $20 / k$ per cent where $k$ is the order of the harmonic.

When the peak or average reading instrument (such as an electronic or rectifier instrument) is tested with an rms instrument, the effect of harmonics depends on the crest and form factors* of the wave, since the scales of such instruments are always marked in rms values for a sinusoidal wave. These factors in turn depend on the phase angle as well as the magnitude of each harmonic. For a voltage wave having a single odd harmonic of amplitude, $V_{k}=h_{k} V_{1}$, the resultant error in per cent may in the worst case be as large as $100 h_{k}$ for a peak reading instrument and $100 h_{k} / k$ for an average reading instrument.

\section{Phase Shift}

From the power equation, $P=V I \cos \theta$, it is evident that the two voltage sources used in testing wattmeters by phantom loading should be stabilized for changes in phase angle as we 11 as magnitude. Unfortunately, even though the output voltage remains constant, a change in input voltage causes a shift in phase of output voltage of most types of stabilizers, resulting in a change in the deflection of a wattmeter connected to the test circuit. From the above equation $\Delta P / V I=\Delta \theta \sin \theta$. Thus the change in wattmeter reading is zero at unity power factor and maximum at zero power factor (for the same volt-amperes). If this change is to be less than 0.1 per cent of the applied volt amperes at any power factor, the phase shift in output voltage resulting from a normal change in input voltage should not be more than 1 milliradian or 3.4 minutes of angle.

\section{Frequency}

The slight deviations in frequency (up to 0.4 cps) which occur in many power systems will not affect the accuracy of commercial indicating instruments, but may be significant in watthour meter testing if accuracies better than 0.1 per cent are desired. Quite apart from this frequency influence on the meter, fluctuations in output voltage of the stabilizer caused by these changes in frequency should be negligible.

\section{Adjustability and Range}

An instrument testing source should be adjustable in increments of 0.02 per cent or less from zero to rated voltage. In addition for wattmeter and wathour meter testing, the phase angle between the two channels should be adjustable in increments of about 1 minute or less over at least $270^{\circ}$, if tests are to be made at any power factor from zero lead to zero lag with the usual range of instrument loads.

*Crest-factor is the crest (peak) value divided by the rms value, and form-factor is the rms value divided by the half-period average value of the wave.

(1) Numbers in parentheses refer to similarly number references in the bibliography at the end of this paper. 
Transformers in the output 1 ines should provide voltages in several ranges up to perhaps 750 volts and currents (at low voltage) up to about 100 amperes. To minimize possible wave-form distortion caused by magnetizing current, each transformer should be operated below the knee of its iron-saturation curve.

\section{Stray Field}

It is not generally realized that stray fields at power frequency have appreciable effects on some a-c instruments. Based on figures given in a recent paper (2) a stray field of only 0.05 gauss may affect some types of moving iron instruments by 0.1 per cent. Thus the stray field from the power supply at the instrament under test must not exceed this value.

\section{DESCRIPTION OF NBS POWER SUPPLIES}

A simplified diagram of the arrangement used in each of the two NBS power supplies, showing, in addition, the load connections for the testing of a wattmeter or watthour meter, is given in Fig. 1. The 3-phase 208-volt $60 \mathrm{cps}$ distribution system energizes two voltage stabilizers, $\mathrm{VS}_{1}$ and $\mathrm{VS}_{2}$, through the adjustable auto-transformer, $A_{1}$, and switch, $S$, which shift the phase angle between the two input voltages to the stabilizers from 0 to $360^{\circ}$.* Auto-transformers, $A_{2}$ and $A_{3}$, and matching transformers, $T_{1}$ and $T_{2}$, control the output voltages for the voltage and current circuits of the test instrument or meter, $P_{t}$ and the standard instrument, $P_{S}$. If an ammeter or voltmeter is to be tested, only one of the two output channels is used.

The connections of the phase shifting transformer. $A_{1}$, are shown diagrammatically in Fig. 2. The length and direction of the dotted line, cd, show the relative magnitude and phase angle of the output voltage with respect to the line voltages $V_{a c}$ or $V_{a b}$. The reduction in magnitude $\left(\mathrm{V}_{\mathrm{cd}}=0.87 \mathrm{~V}_{\text {ac }}\right.$ with tap at midpoint) will not be significant if the voltage remains within the operating range of the stabilizer.

A complete schematic diagram of this type of powe $r$ supply is shown in Fig. 3, and the ratings of the components are given in Table I. Two such supplies, differing essentially only in power rating, have been assembled and rack-mounted. Supply $B$ is shown in Fig. 4. The adjustable auto-transformers, $A_{4}, A_{5}$ and $A_{6}$, and the $120 / 5$ volt transformers, $T_{4}, T_{5}$, and $T_{6}$, shown in Fig. 3, provide fine control of the magni- tudes of the output voltages and of the phase angle between them. The booster transformer, $T_{3}$, keeps the input voltage to stabilizer $V_{1}$ within the proper range. Two impedance matching transformers (not shown in Fig. 3) having rated input voltages of 300 volts, provide seven voltage ranges from 12 to 750 volts and three current ranges of 25, 50 and 100 amperes (at low vol tages).

The most important components are of course the voltage stabilizers. For each supply two commerciallyavailable electronic a-c stabilizers are used, having $1000 \mathrm{v}-\mathrm{a}$ ratings for power supply $\mathrm{A}$, and $250 \mathrm{v}-\mathrm{a}$ ratings for power supply $B$.

Extensive tests have verified the claims by the manufacturers of 0.1 per cent regulation and stabilization, with developed harmonics of less than 3 per cent. Best performance for these stabilizers was obtained, however, for the loads between 5 per cent and perhaps 75 per cent of rated value. Resistors, $R_{1}$ and $R_{2}$, provide the necessary minimum loads to reduce hunting and improve powerfactor.

\section{ESTS AND PERFORMANCE OF NBS POWER SUPPLIES}

\section{Regulation and Stabilization}

Measurements of the regulation and stabilization of the electronic voltage stabilizers used in these power supplies were made under carefully controlled input and load conditions. Output voltages were measured with a reflecting, wall-mounted, suppressedzero electrodynamic voltmeter. (3) With this instrument, which has negligible self-heating drift and zeroshift, voltage changes as small as 0.002 per cent can be detected with certainty by means of a telescope and scale.

The results of representative tests of one of the 1000-va stabilizers are given in Table 2. They show excellent stabilization and regulation. The difference in the output voltages for the two tests at 25 per cent 1 oad and 210 volts are an indication of slow drifts, probably due to self heating and ambient temperature changes, which make such stabilizers unreliable for use as long-time standards of voltage, but in no way affect their suitability in power supplies such as these.

* This use of an adjustable transformer for shifting phase in phantom-loading circuits was first described to us by Mr. Lloyd E. Smith of TVA about 10 years ago. 


\section{Recovery Time}

A number of oscillograms were made of the output voltage of one of the 250 va stabilizers with a 40 va load, to determine the effect of a sudden 10 per cent change in the input voltage. In most of these oscillograms the time constant of recovery was not more than 1/60 second (one cycle). At low input voltage a marked hunting effect persisted that decayed at a much slower rate but would not affect an instrument.

An rms instrument having a one-second period was connected to the output, and a number of observations were made of the momentary changes in deflec$t$ ion produced when the input voltage was suddenly changed by 10 per cent. These changes did not exceed 0.3 per cent, in good agreement with the value of 0.4 per cent computed from the measured time constant of the stabilizer by means of the equation developed in Appendix A.

\section{Wave Form}

Extensive tests of the harmonic content of the output of the stabilizers and of the completed supplies were made with a commercial heterodyne-type harmonic analyzer. Some duplicate tests were made by the separately-excited-wattmeter method (4) which demonstrated that this latter method, which is seldom used, can give results of ample accuracy at these low frequencies ${ }^{+}$. The rms distortion of each of these stabilizers was less than 2 per cent, with 2 nd and 3 rd harmonics predominating.

Measurements of the crest and form factors of the output voltage of one stabilizer of power supply A were made with an electrodynamic voltmeter, a rectifier voltmeter, and a crest-reading electronic voltmeter. All three voltmeters were high quality portable instruments with mirror scales. They were connected in parallel, and in quick succession were connected first to a 60-cycle source having a harmonic distortion less than 0.3 per cent, and then to the output of the power supply, the voltage of which was adjusted to give the same reading of the electrodynamic instrument. The measurements with the crest-reading electronic roltmeter were repeated after the leads to the instrument were reversed, to evaluate any assymetrical "turnover effect" because of the second harmonic component of the voltage of the power supply.* The percentage correction for each type of instrument (valid to about t0.3 per cent) was found to be +0.3 per cent for the rectifier voltmeter (average-reading instrument) and -0.8 per cent and -1.1 per cent for the two connections of the electronic voltmeter (crest-reading) instrument.

\section{Phase Shift}

The phase shifts in output voltage of each stabi1 izer resulting $f r o m$ input voltage changes, were measured with an electrodynamic wat tmeter, voltmeter and ammeter. A circuit similar to Fig. 1 was used, with transforme $r T_{1}$ removed and $A_{2}$ connected ahead of the stabilizer, $V_{1}$, under test. With the power factor seen by the wattmeter near zero and with maximum rated current in the wattmeter current coils, the input voltage, $v_{1}$, to stabilizer $V_{1}$ was changed in 10-volt steps.**

The phase shift for each step was computed from the resultant difference in wattmeter readings by means of the formula previously given, and was then divided by 5 to obtain the average shift for a 2-volt change in $V_{1}$ (roughly 1 per cent). As shown in Fig. 5, the results were found to be dependent on the setting of the output voltage adjustment of the stabilizer as well as on the input voltage and the output load. It is evident that for the usual loads used in instrument testing (about 10 per cent to 25 per cent of rated voltamperes including the fixed resistor) low phase shifts are obtainable for certain combinations of input and output voltages. The input voltage to the stabilizer in the $A C$ ine is relatively constant, fluctuating on $1 y$ between 205 and 215 volts, so that an output-voltage setting which provides optimum performance car be found. However, the input voltage to.the stabilizer in the $A V$ line is dependent on the position of the phase-shift transformer, $A_{1}$, and normally varies from 190 to 230 volts. This could be reduced, if necessary, by means of a rheostat or adjustable auto-transformer ganged to the phase-shift trans forme $r$.

\section{Other Tests}

${ }^{+}$An ordinary $10 w-p o w e r-f a c t o r$ wattmeter with its zero shifted upscale several divisions by means of the zero corrector was used in place of the special center-zero instrument described in reference 4.

- Instruments which respond to the average or rms value are not subject to turnover effects, provided (for half-wave average instruments) that the wave has no $d-c$ component.

* Similar tests with the voltage circuit of the wattmeter connected ahead of the stabilizer verified that the change in phase of the input voltage was negligible. 
quency changes upon these electronic stabilizers because such effects would be expected to be negligible by the nature of the operation of these devices.

The stray field around the 1000-va power supply was measured with a search coil of known area-turns and an electronic voltmeter. (2) The field at table height, $12 \mathrm{in.}$ from the supply, was less than 0.03 gauss, and was therefore negligible.

\section{SUMMARY}

This paper has described 60-cycle power supplies which extensive tests and actual use over a period of several years have shown to be well suited for testing electrical instruments and meters. They contain commercially-available, electronic voltage stabilizers and components. The particular arrangement and the list of components given may simplify the design of such supplies to meet similar needs in other laboritories. The discussion of the requirements and the method of testing such supplies may aid those who wish to evaluate or specify other types of voltage stabilizers or other circuit arrangements. The test methods are simple and most of them can be carried out with ordinary instruments that are readily available. The method of measuring phase changes is particularly simple, sensitive and accurate. The information given should make some of the tests unnecessary for the components and circuit described. A completed supply can be at least partially evaluated under conditions of actual use by observing the residual fluctuations in the deflection of a wattmeter at unity and zero power factors when connected as shown in Fig. 1 .

\section{Appendix A}

\section{Ba11istic Kick of an RUS Instrument}

Let the steady current, $I_{1}$, be changed suddenly by a small amount $n I_{1}$, which is exponentially reduced to zero by a stabilizer having a time constant $t_{c}$. The rms current during this time is then

$$
i=I_{1}\left(1+n e^{-t / t} c\right)
$$

From the basic steady-state torque equation for rms (square law response) instrument, we have

$$
\mathrm{U}_{1}=\mathrm{GI}_{1}^{2}
$$

where $U$ and $G$ are the restoration and motor constants respectively, and $\theta_{1}$ is the deflection of the instrument in radians.

If $t_{c}<<t_{a}$, the period of the instrument, the deflection remains essentially unchanged during the impulse, and we have

$$
\mathrm{T}=-\mathrm{U} \theta_{1}+\mathrm{Gi}_{1}^{2}
$$

where $T$ is the unbalanced torque at any instant during the impulse. Substituting the value of $i^{2}$ from (1) in (3), and neglecting higher order terms since $n<1$, results in

$$
T=2 n G I_{1}^{2} e^{-t / t} c
$$

From the law of impulse and momentum, we have, after the impulse

$$
P \theta^{\prime}=\int \mathrm{Td} t
$$

where $P$ is the moment of inertia of the movable system, $\theta^{\prime}$ is the angular velocity, and the integral is taken over a time which is long compared with $t_{c}$.

Substituting the value of $T$ from (4) in (5) results in

$$
P \theta^{\prime}=2 \mathrm{nGI}_{1}^{2} \mathrm{t}_{\mathrm{c}} \text {. }
$$

At any time thereafter, the differential equation governing the motion of the instrument is

$$
\mathrm{P}^{\prime \prime}+\mathrm{A} \theta^{\prime}+\mathrm{U} \theta=\mathrm{GI}_{1}^{2}
$$

with the initial conditions

$$
\theta_{0}=\theta_{1} \text {, and } \theta_{0}^{\prime}=\frac{2 \mathrm{nGI}_{1}^{2} \mathrm{t}_{\mathrm{c}}}{\mathrm{P}}=\frac{\mathrm{GM}}{\mathrm{P}}
$$


and where $A$ is the damping constant and $\theta^{\prime \prime}$ the angular accelleration. If we let $\phi=\theta-\theta_{1}$ this becomes

$$
P \emptyset^{\prime \prime}+A \emptyset^{\prime}+U \varnothing=0
$$

with $\phi_{0}=0$ and $\phi_{0}^{\prime}=\frac{G M}{P}$.

If the instrument is critically damped, i.e.. if $A^{2}=4 P U$, the solution to this equation for these initial conditions is, (5)

$$
\varnothing=M\left(\frac{\Theta_{1}}{I_{1}^{2}}\right) \frac{4 \pi^{2} t}{t_{a}^{2}} e^{-2 \pi t / t} a
$$

where $t=2 \pi \sqrt{P / U}$, and is undamped period of the instrument.

The maximum value of $\phi\left(\right.$ when $\phi^{\prime}=0$ ) is

$$
\frac{2 \pi M \theta_{1}}{\text { et }_{a} I_{1}^{2}}
$$

Therefore.

$$
\frac{\varnothing_{\mathrm{m}}}{\theta_{1}}=\frac{\theta_{\mathrm{m}}-\theta_{1}}{\theta_{1}}=\frac{\Delta_{1}^{\theta_{1}}}{\theta_{1}}=\frac{2 \pi \mathrm{M}}{\mathrm{t}_{\mathrm{a}} \mathrm{eI}_{1}^{2}}=\frac{4 \pi \mathrm{n} \mathrm{t}_{\mathrm{c}}}{\mathrm{et}_{\mathrm{a}}}
$$

Since the scale of a square-1aw instrument is marked off so that the reading $D=\sqrt{ } \Delta$ we heve $\frac{\Delta D}{D}=$ $\frac{\Delta \theta}{2 \theta}$ if $\frac{\Delta D}{D}<1$.
Thus the maximum ba 11 ist ic kick or change in deflection of the instrument is

$$
\frac{\Delta D}{D}=\frac{2 \pi n t_{c}}{e t_{a}}=2.3 n t_{c} / t_{a}
$$

\section{ACKNOWLEDGEMENTS}

The author acknowledges with thanks the help of E. S. Williams, who constructed both power supplies, and $T$. W. Cushing, who made a number of the measurements described.

\section{References}

(1)

"The Effect of Harmonics on A-C Ammeter and Voltmeter Indications," R. F. Es toppey, Weston Engineering Notes, Weston Electrical Instrument Corporation, Newark, New Jersey, v. 2, No. 5, Oct. 1947.

"Performance of Portable Electrical Instruments in Magnetic Fields," A. E. Peterson, Trans AIEE v. 67,1948, p. 1228 .

(3) "A Suppressed-Zero Electrodynamic Vol tmeter," F. K. Harris, BS J. Research, v. 3, 1929, p. 445 . Electrical Measurents, (book), F. K. Harris, John Wiley and Sons, Inc., New York City, 1952 Chapter 8 . 


\section{Ratings of Components}

\section{Components}

Voltage Stabilizer, $\mathrm{VS}_{1} \& \mathrm{VS}_{2}$

Auto Transformer, $A_{1}$

Auto Transformer, $A_{2}, A_{3}$

Auto Transformer, $A_{4}, A_{5} \& A_{6}$

Transformer $\mathrm{T}_{3}$

Transformers $\mathrm{T}_{4}, \mathrm{~T}_{5} \& \mathrm{~T}_{6}$

Load Resistors $R_{1} \& R_{2}$
Power Supply A

$190-230 v, 1000 v a$

$230 v, 4 a$

$230 v, 3 a$

$115 v, 1 a$

$240 / 24 v, 100 v a$

$120 / 5 v, 25 v a$

1500 ohm, 100w
Power Supply B $190-230 v, 250 v a$

$230 v, 2 a *$

$230 v, 2 a x$

$115 \mathrm{v}, \quad 1 \mathrm{a}$

$240 / 24 v, 25 v a$

$120 / 5 v, 10 v a$

1500 ohm, 100w

*Smallest 230 volt unit available

Table 2

Stabilization and Regulation 1000 v-a Stabilizer, VS 1

\begin{tabular}{|c|c|c|c|}
\hline $\begin{array}{c}\text { Input } \\
\text { Voltage } \\
\text { Volts } \\
\end{array}$ & \multicolumn{2}{|c|}{ Output } & $\begin{array}{c}\text { Output } \\
\text { Voltage } \\
\text { Volts }\end{array}$ \\
\hline 190 & 25 & 1.0 & 230.525 \\
\hline 200 & 25 & 1.0 & .523 \\
\hline 210 & 25 & 1.0 & .513 \\
\hline 220 & 25 & 1.0 & .498 \\
\hline 230 & 25 & 1.0 & .478 \\
\hline 240 & 25 & 1.0 & .448 \\
\hline 210 & 0 & -- & 229.860 \\
\hline 210 & 25 & 1.0 & .860 \\
\hline 210 & 50 & 1.0 & .999 \\
\hline 210 & 75 & 1.0 & 230.080 \\
\hline
\end{tabular}

Stabilization Ratio

5000

1000

700

500

300 


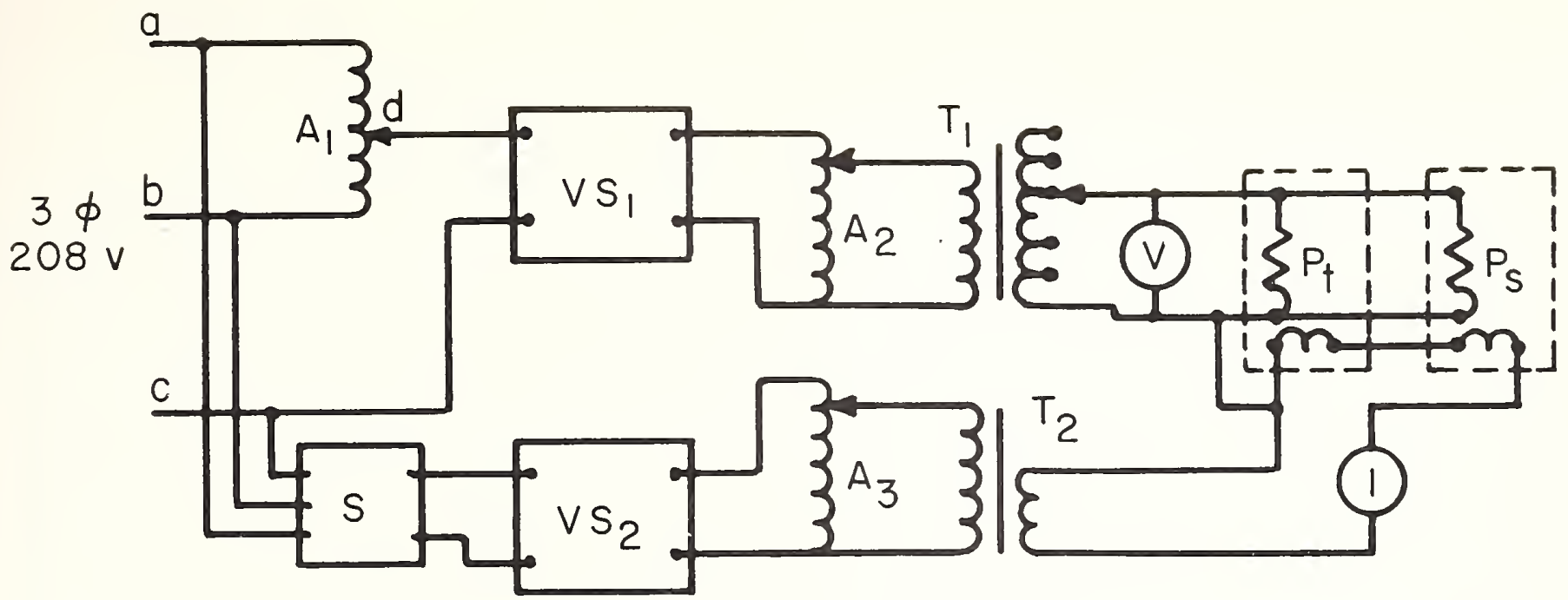

Fig. 1 Elementary Diagram of Power Supply

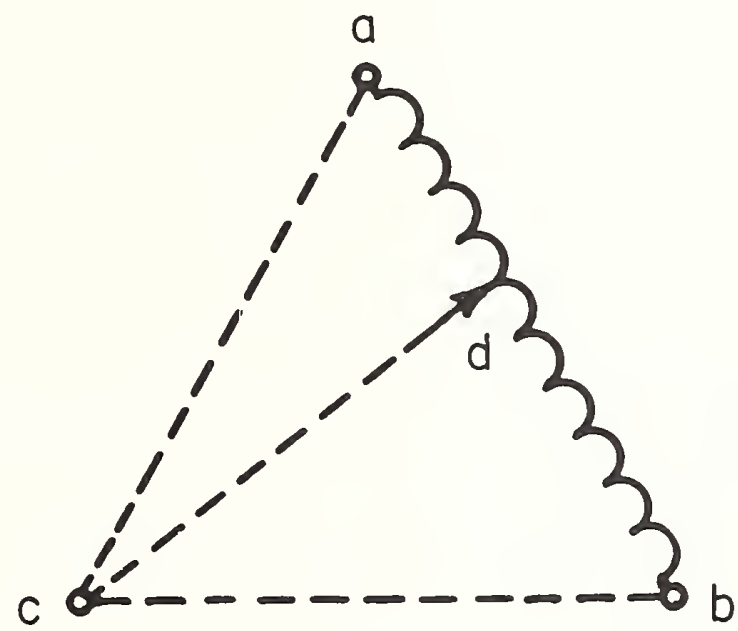

Fig. 2 Phase-Shifting Auto-Transformer

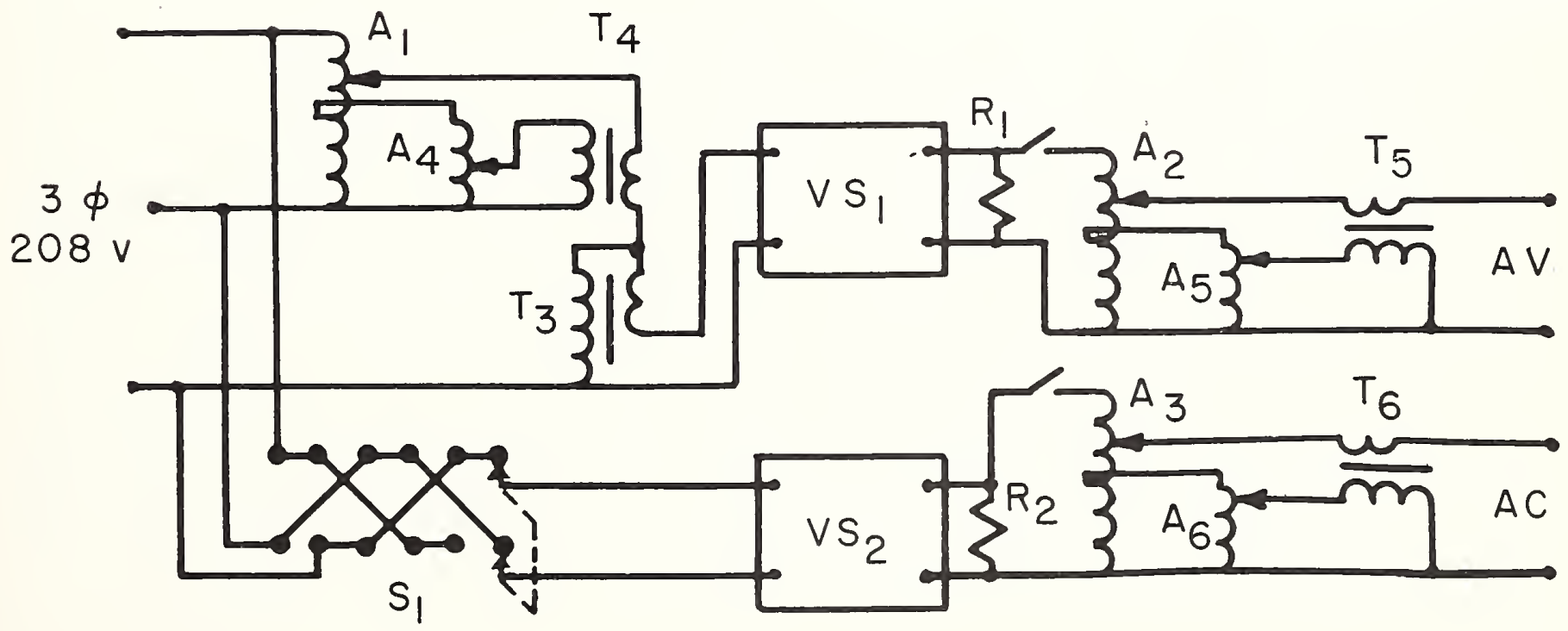

Fig. 3 Complete Diagram of Power Supply

$445 / 8$ 


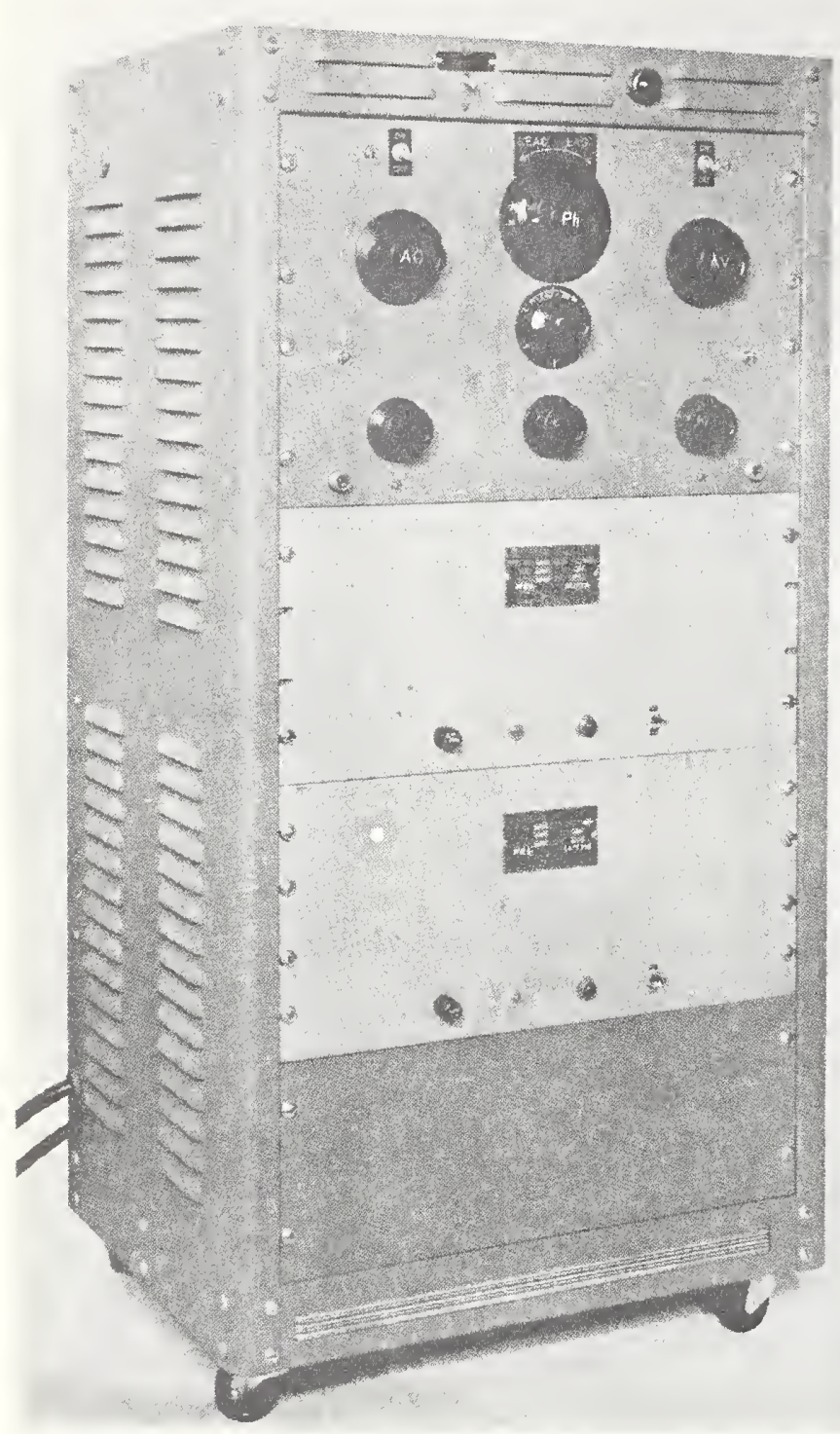

Fig. 4 Photograph of Power Supply B

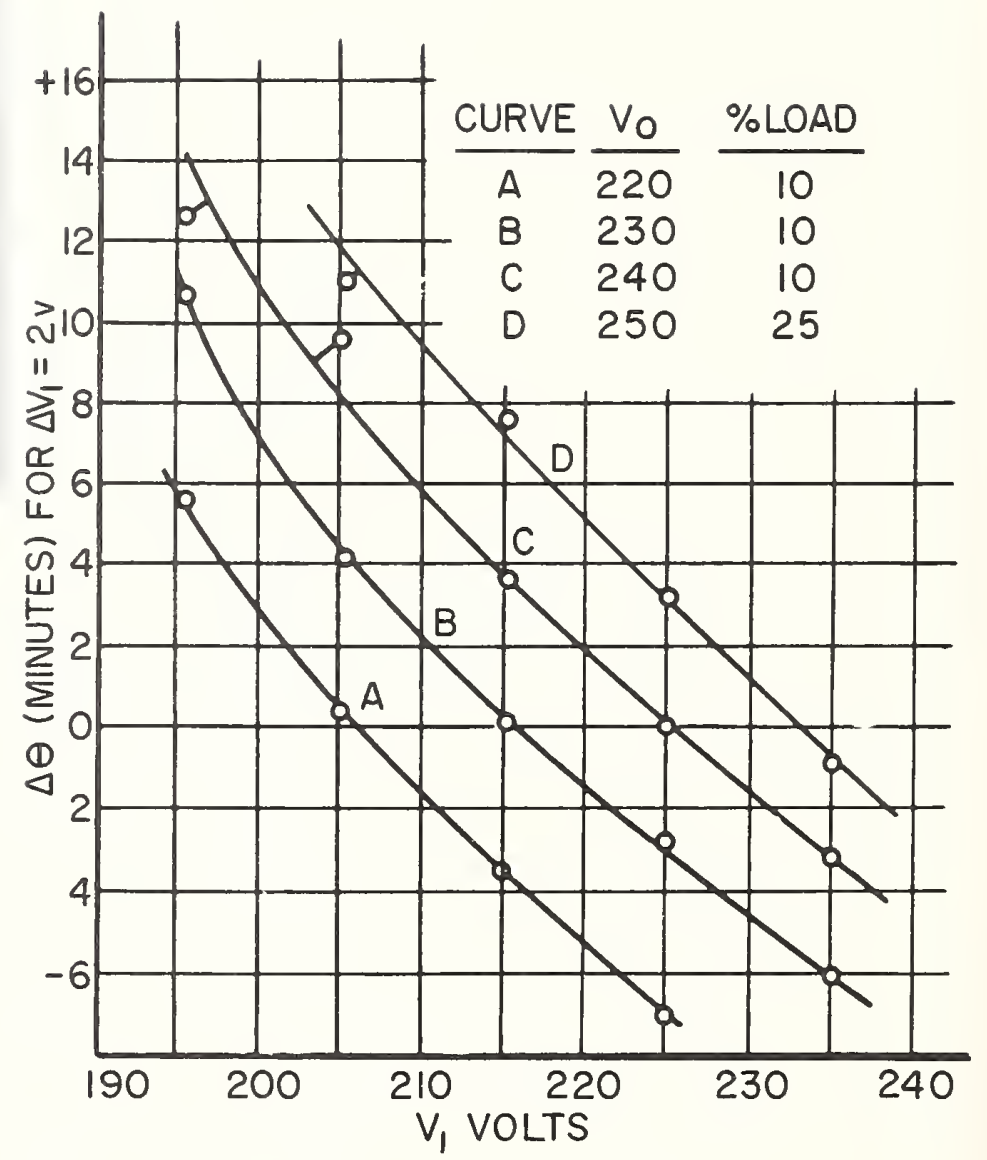

Fig. 5 Phase Shift in Output Voltage of $1000 \mathrm{v}-\mathrm{a}$ Stabilizer, VS 
Electrical Units, Instruments, and Measurements

Publications by the Members of the Staff of the National Bureau of Standards

\section{General Information}

This list of publications is a selection which describes the methods and equipment used in the establishment and maintenance of the electrical units and the standards that have been developed at the National Bureau of Standards for the calibration of measuring apparatus. It includes references to older publications of the Bureau which describe basic principles and methods and are therefore still useful. References to articles on electrical measurements at radio and higher frequencies are not included.

Many inquiries sent to this Bureau can best be answered by reference to a recognized national standard, or by reference to a particular textbook or handbook. Accordingly a few such standards and books are listed that include the information on instruments and measurements most frequently requested.

This Bureau makes no tests on motors, generators, or transformers used for power or lighting service, and has no current publications on their design or performance. It cannot undertake to answer questions concerning the design, construction, repair, or rewinding of such apparatus in cases where the matter is not one of general interest. A number of pertinent references are listed in a folder "Electric Motors and Generators, Basic Information Sources" available on request from the Office of Publications, Department of Commerce, Washington 25, D. C.

Some of the publications by Bureau authors have appeared in the regular series of publications of this Bureau, and others in various scientific and technical journals. Reprints of some of the more recent papers may be available upon request from the authors. NBS Circulars may be obtained by purchase, where the price is stated, from the Superintendent of Documents, Government Printing Office, Washington 25, D. C.

\section{Units and Absolute Electrical Measurements}

By international agreement the absolute system of electrical units was adopted to become effective on January 1, 1948. The following papers concern the measurements and values entailed in the establishment of the values of standards in terms of absolute units. 
1. A determination of the absolute ohm, using an improved self inductor. H. L. Curtis, C. Moon, and C. M. Sparks. J. Research NBS 21, 375 (1938) RP1137.

2. An absolute determination of the ampere, using helical and spiral coils. R. W. Curtis, R. L. Driscoll, and C. L. Critchfield. J. Research NBS 28, 133 (1942) RP1449.

3. Review of recent absolute determinations of the ohm and the ampere. H. L. Curtis, J. Research NBS 33, 235 (1944) RP1606.

4. An absolute measurement of resistance by the Wenner method.

J. L. Thomas, C. Peterson, I. L. Cooter and F. R. Kotter. J. Research NBS 43, 291 (1949) RP2029.

5. Establishment and maintenance of the electrical units. F. B. Silsbee. NBS Circ. 475, 38 p. (1949) 30 cents.

6. Measurement of the proton moment in absolute units. H. A. Thomas, R. L. Driscoll, J. A. Hipple. J. Research NBS 44, 569 (1950) RP2104.

7. A new method for determining the value of the Faraday.

D. N. Craig, J. I. Hoffman. Phys, Rev, 80, No. 3, 487 (1950).

8. Standards for electrical measurement. F. B. Silsbee. Phys. Today $\underline{4}, 19$ (1951).

9. Measure for measure: Some problems and paradoxes of precision. F. B. Silsbee. J. Wash. Acad. Sci. 41, No. 7, 213 (1951).

10. Fundamental units and standards. F. B. Silsbee. Instruments 26, 1520 (1953).

11. Extension and dissemination of the electrical and magnetic units by the National Bureau of Standards. F. B. Silsbee. NBS Cir. 531, 33 p. (1952) 35 cents.

12. Electrochemical constant. NBS Circ. 524 (1953) \$2.00. This circular includes:

a Standard cells and the unit of electromotive force.

W. J. Hamer, L. H. Brickwedde, P. R. Robb. pp 103-118.

$\mathrm{b}$ Determination of the Faraday constant by the electrolytic

oxidation of oxalate ions. D. N. Craig, J. I. Hoffman. pp 13-20.

$c$ The Faraday and the omegatron. H. Sommer, J. A. Hipple. pp 21-26. 
13. Nuclear magnetic resonance and the measurement of magnetic fields. R. L. Driscoll. Natl. Phys. Lab. Symp. of Precision Electrical Measurements (1955).

14. Measurement of current with a pellat-type electrodynamometer. R. L. Driscoll. J. Research NBS 60, 287 (1958)

RP2845.

15. Measurement of current with the National Bure au of Standards current balance. R. L. Driscoll and R. D. Cutkosky. J. Research NBS 60, 297 (1958), RP2846.

16. New apparatus at the National Bureau of Standards for absolute capacitance measurement. M. C. McGregor, J. F. Hersh, R. D. Cutkosky, F. K. Harris, and F. R. Kotter. IRE Trans. Instrumentation, I-7, No.3-4, 253 (Dec. 1958).

17. A free precession determination of the proton gyromagnetic ratio. P. L. Bender and R. L. Driscoll. IRE Trans Instrumentation, I-7, No. 3-4, 176 (Dec. 1958).

18. The ampere. F. B. Silsbee. Proc. IRE 47, No. 5, 643 (May, 1959).

19. Simplification of systems of units. F. B. Silsbee. AAAS Symposium, 1959.

20. Some results on the cross capacitances per unit length of cylindrical three-terminal capacitors with thin dielectric films on their electrodes. D. G. Lampard and R. D. Cutkosky. Inst. Elec. Engs. (London) Mono. No. $351 \mathrm{M}$, January 1960.

21. Determination of the value of the Faraday with a silverperchloric acid coulometer. D. Norman Craig, C. A. Law, J. I. Hoffman, and W. J. Hamer. J. Research NBS 64A5, $381(1960)$.

\section{Standard Cells}

1. Effect of service temperature conditions on the electromotive force of unsaturated portable standard cells. J. H. Park. B.S. J. Research NBS 10, 89 (1933) RP518.

2. Standard cells and the unit of electromotive force. W. J. Hamer, L. H. Brickwedde, and P. R. Robb. NBS Circ. 524, p 103-118 (1953) \$2.00. (-see Section 2).

3. A temperature-control box for saturated standard cells. E. F. Mueller and H. F. Stimson. J. Research NBS 13, 699 (1934) RP739. 
4. Standards of electromotive force. G. W. Vinal, D. N. Craig, and L. H. Brickwedde. Trans. Electrochem. Soc. 68, 139 (1935).

5. Metastability of cadmium sulfate and its effect on electromotive force of saturated standard cells. G. W. Vinal and L. H. Brickwedde. J. Research NBS 26, 455 (1941) RP1389.

6. Standard cells and the change from international to absolute electrical units. G. W. Vinal. J. Electrochem. Soc. 93, 95 (1948).

7. New quartz container for standard cells at the National Bureau of Standards. G. W. Vinal, L. H. Brickwedde, and W. J. Hamer. Compt. Rendu. Sept. 5-10, 92 (1949).

\section{Resistors}

1. The four-terminal conductor and the Thomson bridge. F. Wenner. Bul. BS 8, 559 (1912) S181.

2. Adjustments of the Thoms on bridge in the measurement of very low resistances. F. Wenner and E. Weibel. Bul. BS 11, 65 (1915) S225.

3. A study of the inductance of four-terminal resistance standards. F. B. Silsbee. Bul. BS 13, 375 (1916-17) S281.

4. Notes on the design of four-terminal resistance standards for alternating currents. F. B. Silsbee. BS J. Research 4. 73. (1930) RP133.

5. A method of adjusting the temperature coefficient and resistance of low-valued resistance standards. F. Wenner and J. L. Thomas. BS J. Research 12, 147 (1934) RP639.

6. Methods, apparatus, and procedures for the comparison of precision standard resistors. F. Wenner. J. Research NBS 25, 229 (1940) RP1323.

7. Stability of double-walled manganin resistors. J. L. Thomas, J. Research NBS 36, 107 (1946) RPl692.

8. Precision resistors and their measurement. J. L. Thomas. NBS Circ. 470,32 p.(1948) 30 cents.

9. Best arrangement of resistors in a series group. H. B. Brooks. Rev. Sci. Instr. 21, No. 5, 491 (1950).

10. Measurement of multimegohm resistors. A. H. Scott. J. Research NBS 50, 147 (1953) RP2402.

$$
450 / 4
$$


11. Alloys for precision resistors. C. Peterson. National Physical Laboratory Symposium of Precision Electrical Measurements, (1955).

12. Rack for standard resistors, P. H. Lowrie, Jr, Rev. Sci. Instr. 30, 291-292 (April 1959).

13. A method of controlling the effects of resistance in the link circuit of the Thomson or Kelvin double bridge. D. Ramaley. J. Research NBS 64C4, 267 (1960).

\section{Inductors}

(See also Section 2)

1. Design of standards of inductance, and the proposed use of models in the design of air-core and iron-core reactors. H. B. Brooks. BS J. Research 7, 289 (1931) RP342.

2. Improved continuously variable self and mutual inductor.

H. B. Brooks and A. B. Lewis. J. Research NBS 19, 493 (1937) RP1040.

3. Formulas for computing capacitance and inductance. C. Snow. NBS Circ. 544, 64 p. (1954) 40 cents.

4. A study of absolute standards of mutual inductance and in particular the three-section National Bureau of Standards type. F. W. Grover. J. Research NBS 53, 297 (1954) RP2548.

5. Methods for measuring the $Q$ of large reactors. C. Peterson, B. L. Dunfee, F. L. Hermach. Am. Inst. Elec. Engrs. Trans. Paper 56-87 (1956).

6. Inductive efficiency of reactive coils. H. B. Brooks. Elec. Engr. (May 1956).

\section{Capacitors}

(See also Section 2)

1. Measurement of relative and true power factors of air capacitors. A. V. Astin. J. Research NBS 21, 425 (1938) RP1138.

2. Nature of energy losses in air capacitors at low frequencies. A. V. Astin. J. Research NBS 22, 673 (1939) RPl212. 
3. Standards for low values of direct capacitance. C. Moon and C. M. Sparks. J. Research NBS 41, 497 (1948) RP1935.

4. A standard of small capacitance. C. Snow. J. Research NBS 42, 287 (1949) RP1970.

5. Formulas for computing capacitance and inductance. C. Snow. NBS Circ. 544, 69 p. (1954) 40 cents.

6. Variable capacitor calibration with an inductive voltage divider bridge. T. L. Zapf. NBS Tech. Note 57 (PB161558) 50 cents. (Available only by purchase from the Office of Technical Services, Department of Commerce, Washington 25, D. C.)

7. Capacitor calibration by step-up methods. T. L. Zapf. (informal communication).

8. New apparatus at the National Bureau of Standards for absolute capacitance measurement. M. C. McGregor, J. F. Hersh, R. D. Cutkosky, F. K. Harris, and F. R. Kotter. IRE Trans. Instrumentation, I-7, No. 3-4, 253 (Dec. 1958).

7. Potentiometers, Volt Boxes, and Bridges

1. Deflection potentiometers for current and voltage measurements. H. B. Brooks. Bul. BS $\underline{8}, 395$ (1912) S172.

2. A multi-range potentiometer and its application to the measurement of small temperature differences. H. B. Brooks and A. W. Spinks. BS J. Research 9, 781 (1932) R P506.

3. The standard-cell comparator, a specialized potentiometer. H. B. Brooks. BS J. Research 11, 211 (1933) RP586.

4. The Waidner-Wolff and other adjustable electrical-resistance elements. E. F. Mueller and F. Wenner. J. Research NBS 15, 477 (1935) RP842.

5. Les Potentiometres. H. B. Brooks. Cong. Ind. d'Elec. (Paris) 3, 275 (1932).

6. Testing and performance of volt boxes. F. B. Silsbee and F. J. Gross. J. Research NBS 27, 269 (1941) RP1419.

7. Portable potentiometer and thermostatted container for standard cells. A. W. Spinks, and F. L. Hermach. Rev. Sci. Instr. 26, No. 8, 770 (1955). 
8. Lindeck potentiometer. D. W. Oliver. Rev. Sci. Instr. 26, No. 11, 1078 (1955).

9. An a-c Kelvin bridge for the audio frequency range. B. L. Dunfee. Am. Inst. Elec. Engrs. Trans. Paper 56-25 (1956).

10. The use of an a-c bridge to measure core loss at high inductions. I. L. Cooter, W. P. Harris. Am. Inst. Elec. Engrs. Trans. Paper 56-26 (1956).

11. A low-cost microvolt potentiometer. W. H. Wood. Rev. Sci. Instr. (1957).

8. Instruments and Meters and Their Testing

1. Accuracy of commercial electrical measurements. H. B. Brooks. Trans. Am. Inst. Elec. Engrs. 39, 495 (1920).

2. A suppressed-zero electrodynamic voltmeter. F. K. Harris. BS J. Research 3, 445 (1929) RPl05.

3. Composite-coil electrodynamic instruments. F.B. Silsbee. BS J. Research $\underline{8}, 217$ (1932) RP411.

4. Temperature compensation of millivoltmeters. H. B. Brooks. J. Research NBS 17, 497 (1936) RP926.

5. Standard electrodynamic wattmeter and ac-dc transfer instrument. J. H. Park and A. B. Lewis. J. Research NBS 25, 545 (1940) RP1344.

6. Basis of standardization of electrical instruments and meters. F. B. Silsbee. Elec. World 130, No. 21, 90 (1948).

7. Performance of portable electrical instruments in magnetic fields. A. E. Peterson. Trans. Am. Inst. Elec. Engrs. 67, 1228 (1948).

8. A precision electrothermic voltmeter for measurement between 20 and 20,000 cycles. F. L. Hermach. Trans. Am. Inst. Elec. Engrs. 67, 1224 (1948).

9. An easily assembled console for rapid testing of electrical indicating instruments. F. D. Weaver. Instruments 22, 396 (1949).

10. Notes on the care and use of electrical instruments. F. D. Weaver. Instruments 23, No. 12, 1236 (1950). 
11. Thermal converters as ac-dc transfer standards for current and voltage measurements at audio frequencies. F. L. Hermach. J. Research NBS 48, 121 (1952) RP2296.

12. A-C measurements to $10,000 \mathrm{cps}$. F. D. Weaver. Instruments 25, No. 6, 757 (1952).

13. The testing of electrical instruments. F. L. Hermach. Proc. Instr. Soc. Am. 8, 18 (1953).

14. Testing electrical instruments. F. D. Weaver. Instruments 26, No. 9, 1362 (1953).

15. Electrical indicating instruments used in early Edison central stations. H. B. Brooks. J. Franklin Inst. 256, No. 5, 401 (1953).

16. Lead resistance errors in watthour meter tests. F. L. Hermach and T. L. Zapf. Elec. World 141, No. 16, 113 (1954).

17. Multirange, audiofrequency thermocouple instruments of high accuracy. F. L. Hermach and E. S. Williams. J. Research NBS 52, 227 (1954) RP2494.

18. Precise comparison method of testing alternating-current watthour meters. A. W. Spinks and T. L. Zapf. J. Research NBS 53, 95 (1954) RP2521.

19. Scale \& reading errors of electrical indicators. F. D. Weaver. Instr. \& Automation 27, No. 11 (Nov. 1954).

20. Suggested practices for electrical standardizing laboratories, F. B. Silsbee. NBS Circ. 578, 9 p. (1956) 15 cents.

21. Power supplies for 60-cycle tests of electrical instruments and meters. F. L. Hermach. Proc. Instru. Soc. Am. 11, Paper No. 56-21-3 (1956).

22. AC-DC transfer instruments for current and voltage measurements. F. L. Hermach. IRE Trans. Instrumentation. I-8, 235 (1958).

23. The definition and measurement of the time constant and response time of thermal converters. F. L. Hermach. Trans. Am. Inst. Elec. Engrs. 77, 277 (1958).

24. A wide range volt-ampere converter for current and voltage measurements. F. L. Hermach and E. S. Williams. Communications \& Electronics, Am. Inst. Elec. Engrs. Paper 59-161 (1959). 
25. Thermal voltage converters for accurate voltage measurements to 30 megacycles. F. L. Hermach and E. S. Williams. Communications \& Electronics, Am. Inst. Elec. Engrs., Paper 60-135 (1960).

\section{Transformers}

1. A method for testing current transformers. F. B. Silsbee. Bul. BS 14, 317 (1918-19) S309.

2. Lead resistance for current transformers. F. B. Silsbee. Elec. World 81, 1082 (1923).

3. Methods for testing current transformers. F. B. Silsbee. Trans. Am. Inst. Elec. Engrs. 43, 282 (1924).

4. A shielded resistor for voltage transformer testing. F. B. Silsbee. BS Sci. Pap. 20, 489 (1924-26) S516.

5. Equipment for testing current transformers. F. B. Silsbee, R. L. Smith, N. L. Forman, and J. H. Park. BS J Research 11,93 (1933) RP580.

6. Accuracy of high-range current transformers. J. H. Park. NBS J. Research 14, 367 (1935) RP775.

7. Information for the amateur designer of transformers for 25- to 60-cycle circuits. H. B. Brooks. NBS Circ. 408, 25 p. (1935).

8. Effect of wave form upon the performance of current transformers. J. H. Park. NBS J. Research 19, 517 (1937) RP1041.

9. Measurement of voltage ratio at audio frequencies. W. C. Sze. Communications \& Electronics, Am. Inst. Elec. Engrs., Paper 57-648 (1957).

10. A standard transformer and calibration method--A basis for establishing ratios of currents at audio frequencies. B. L. Dunfee. IRE Trans. Instrumentation (1960).

11. The Precision measurement of transformer ratios.

R. D. Cutkosky and J. Q. Shields. IRE Trans. Instrumentation (1960).

\section{Galvanometers}

1. A theoretical and experimental study of the vibration galvanometer. F. Wenner. Bul. BS 6, 347 (1909-10) S134. 
2. General design of critically damped galvanometers. F. Wenner. Bul. BS 13, $211(1916-17)$ S273.

3. A study of electromagnet moving coil galvanometers for use in alternating-current measurements. E. Weibel. Bul. BS 14, $23(1918-19)$ S297.

4. A new form of vibration galvanometer. P. G. Agnew. BS Sci. Pap. 16, 37 (1920) S370.

5. Sensitivity of a galvanometer as a function of its resistance. H. B. Brooks. BS J. Research $\underline{4}, 297$ (1930) RPl50.

6. Galvanometer efficiency as a design parameter. F. K. Harris. Trans. Am. Inst. Elec. Engrs. 56-60 (1956).

\section{High-Voltage and Surge Measurements}

1. An experimental study of the corona voltmeter. H. B. Brcoks and F. M. Defandorf. BS J. Research $\underline{1}, 589$ (1928) RP2l.

2. Calculations of electrical surge-generator circuits. A. B. Lewis. J. Research NBS 17, 585 (1936) RP929.

3. An absolute electrometer for the measurement of high alternating voltages. H. B. Brooks, F. M. Defandorf, and F. B. Silsbee. J. Research NBS 20, 253 (1938) RP1078.

4. A transformer method for measuring high alternating voltages and its comparison with an absolute electrometer. F. B. Silsbee and F. M. Defandorf. J. Research NBS 20, 317 (1938) RP1079.

5. Shunts and inductors for surge-current measurements. J. H. Park. J. Research NBS 39, 191 (1947) RPl823.

6. The measurement of high voltage. F. M. Defandorf. J. Wash. Acad. Sci. 38, No. 2, 33 (1948).

7. A fifty-fold momentary beam intensification for a high voltage cold-cathode oscillograph. J. H. Park. J. Research NBS 47, 87 (1951) RP2231.

8. Surge voltage breakdown of air in a non-uniform field. J. H. Park and H. N. Cones. J. Research NBS 56, 201 (1956) RP2669.

9. High voltage pulse generator and tests on an improved deflecting system of a cold-cathode oscillograph. H. N. Cones. J. Research NBS 57, 143 (1956) RP2704. 
12. Dielectric Measurements

(See also Section 6)

1. Edge correction in the determination of dielectric constant. A. H. Scott and H. L. Curtis. J. Research NBS 22, 747 (1939) RP1217.

2. Dielectric constant, power factor and corductivity of the system rubber-calcium carbonate. A. H. Scott and A. T. McPherson. J. Research NBS 28, 279 (1942) RP1457. (Describes measurements by a three-terminal bridge).

3. Measurements of dielectric properties at temperatures up to $500^{\circ} \mathrm{C}$. A. H. Scott, P. Ehrlich and J. F. Richardson. Symp. on Temperature Stability of Electrical Insulating Materials, Am. Soc. Testing Materials Spec. Tech. Publ. No. 161 (1954).

4. Precise measurements of dielectric constant over a wide range of frequencies and temperatures. A. H. Scott. Proc. Instr. Soc. Am. 1l, Paper No. 56-8-2 (1956).

\section{Magnetic Measurements}

1. An apparatus for magnetic testing at magnetizing forces up to 5000 oesterds. R. L. Sanford and E. G. Bennett. J. Research NBS 23, 415 (1939) RP1242.

2. Permanent magnets. R. L. Sanf̣ord. NBS Circ. 448, 39 p. (1944).

3. Magnetic testing. R. L. Sanford. NBS Circ, 456, 39 p. (1946) (Revision Scheduled for 1961).

4. A permeameter for magnetic testing at magnetizing forces up to 300 oersteds. R. L. Sanford and P. H. Winter. J. Research NBS 45, 17 (1950) RP2109.

5. The use of an AC bridge to measure core loss at high inductance. I. L. Cooter and W. P. Harris. Communications \& Electronics, Am. Inst. Elec. Engrs. (May 1956).

6. Investigation of an alternating current bridge for the measurement of core losses in ferromagnetic materials at high flux densities. I. L. Cooter and W. P. Harris. J. Research NBS 57, 103 (1956) RP2699.

7. Improved bridge method for the measurement of core losses on ferromagnetic materials at high flux density W. P. Harris and I. L. Cooter. J. Research NBS 60, 509 (1958) RP2865. 
8. A feedback amplifier with negative output resistance for magnetic measurements. I. L. Cotter and W. P. Harris. IRE Natl. Conv. Record, Pt. 5, 217 (1958).

\section{Miscellaneous}

1. Accuracy tests for meggers. H. B. Brooks. Elec. World 85, 973 (1925).

2. Precautions against stray magnetic fields in measurements with large alternating currents. F. B. Silsbee. Trans. Am. Inst. Elec. Engrs. 48, 1301 (1929).

3. A new cathode-ray oscillograph and its application to the study of power los $\mathbf{s}$ in dielectric materials. F. K. Harris. BS J. Research 12, 87 (1934) RP636.

4. Suggested practices for electrical standardizing laboratories. F. B. Silsbee. NBS Circ. 578, 9 p. (1956) 15 cents.

5. Copper wire tables. NBS Circ. 31, 4th Ed., 36 p. (1956).

6. Measurement of the resistance-strain relation and Poisson's ratio for copper wires. T. E. Wells. Proc. Instr. Soc. Am. 11 (1956).

7. Environment control of electronic calibration laboratories. W. F. Snyder. (informal communication).

8. Facilities and services of the electronic calibration center. H. W. Lance and W. F. Snyder. (informal communication).

9. Electrical measurement in the core curriculum. F. R. Kotter. Proc. Am. Inst. Elec. Engrs, Analog \& Digital Instrument Conf. (April 1959).

10. An analysis of the accumulated error in a hierarchy of calibration. E. L. Crow. IRE Trans. Instrumentation (1960).

11. Phase angle master standard for 400 cycles per second. J. H. Park and H. N. Cones. J. Research NBS 64C3, 229 (1960).

12. The nation's electronic standardization program: Where do we

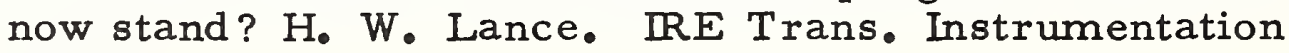
(1960). 
The National Bureau of Standards receives frequent inquiries for information on electrical instruments and measurements which is not specifically covered in its publications. To meet the needs of such inquiries a list of books and standards is given below.

15.1 Textbooks

1. Electrical measurements, F. K. Harris. (John Wiley \& Sons, New York, N. Y.).

2. Electrical measuring instruments, Drysdale \& Jolley - revised by Tagg (John Wiley \& Sons, New York, N. Y.).

3. Die technik elektrischer messgerate, George Keinath (Oldenbourg).

4. Alternating-current bridge methods, B. Hague. (Pitman Publishing Co., New York, N. Y.).

5. Electrical measurements \& measuring instruments, E. W. Golding. (Pitman Publishing Co., New York, N. Y॰).

6. Electrical measurements, H. L. Curtis, (McGraw-Hill Book Co., New York, N. Y.).

7. Electrical measurements \& their application, E. Frank. (McGraw-Hill Book Co., New York, N. Y.).

8. Elektrische messgerate und einrichtungen, A. Palm (Springer).

9. Electronic measurements, F. E. Terman \& J. M. Pettit. (McGraw-Hill Book Co., New York, N. Y.).

10. Principles of electronic instruments, G. R. Partridge (Prentice Hall, Anglewood Cliffs, New Jersey).

11. Basic electrical measurements, M. B. Stout. (Prentice Hall, Anglewood Cliffs, New Jersey).

12. Electrical measurements, F. A. Laws (McGraw-Hill Book Co., New York, N. Y.).

13. Industrial instrumentation, D. P. Eckman. (John Wiley \& Sons, New York, N. Y.).

14. Applied electrical measurements, I. F. Kinnard. (John Wiley \& Sons, New York, N. Y.). 
15. Principles of electrical measurements, H. Buckingham \& E. M. Price (Philosophical Library).

16. Primary batteries, G. W. Vinal. (John Wiley \& Sons, New York, N. Y.).

17. Electric power meeting, A. E. Knowlton. (McGraw-Hill Book Co., New York, N. Y.).

18. Measurement of inductance, capacitance, and frequency, A. Campbell and E. C. Childs. (D. Van Nostrand \& Co., Princeton, N. J.).

19. Inductance calculations, F. W. Grover (D. Van Nostrand \& Co., Princeton, N. J.).

20. Instrument transformers, B. Hague. (Pitman Publishing Co., New York, N. Y.).

21. Die messwandler, I. Goldstein (Springer).

22. Wechselstrom-leistungsmessungen, W. Skirl (Springer). 15. 2 Standards and Handbooks

Standards and handbooks are frequently revised, and the current edition should always be consulted.

1. American standard for electrical indicating instruments. (Am. Standards Association, 70 East 45th Street, New York, N. Y., C39.1). (Supersedes AIEE Standard No.33).

2. American standard for instrument transformers. (Am. Standards Assoc., New York City, C57.13).

3. Code for electricity meters. (Edison Elect. Institute, New York, N. Y.).

4. Standard handbook for electrical engineers. (McGraw-Hill Book Co., New York, N. Y.).

5. Electrical engineers' handbook. (John Wiley \& Sons, New York, N. Y.).

6. Electrical metermen's handbook. (Edison Elect. Institute, New York City).

7. Master test code for resistance measurements. (Am. Inst. Elec. Engrs., New York, N. Y., AIEE publication No. 550). 
Selected Papers on

\section{Electronics}

(Contents on page V) 



\title{
High-Frequency Standards of the Electronic Calibration Center, NBSBL*
}

\author{
M. C. SELBY $\dagger$
}

\section{INTRODUCTION}

$\mathrm{T}$ YHE equipment described below was developed by personnel of the High-Frequency Electrical Standards Section, Radio Standards Division of the National Bureau of Standards. The purposes are to provide a necessary link between the national standards, maintained at the NBS Radio Standards Laboratory and laboratories throughout the country, and to do this at minimum possible cost to the public.

The reference standards and techniques to be used in this link must meet two basic requirements. The first is to retain the accuracy of the NBS primary standards as closely as possible. The second is to reduce the time necessary for accurate calibrations of "unknowns" to a reasonable minimum. It is, of course, clear that the major consideration in developing a primary standard and technique for a certain quantity is the realization of a magnitude as closely as possible to its true or "absolute" value. The time element, convenience to user, and other considerations are of secondary importance and are given weight only when choosing one of two methods rendering equal basic accuracy and reliability. That is why primary standards and associated techniques are, in many cases, not practical for calibrating even limited numbers of reference standards. This is particularly true as the radio frequency involved increases. At the time of initiation of this undertaking the calibration, for example, of an RF voltmeter at points up to several hundred megacycles was a job requiring a number of weeks and at a nearly prohibitive cost; this was particularly discouraging in view of the limited ability of the "unknowns" to retain their calibrations for a reasonable length of time. The new reference standards and techniques incorporated, as described below, permit calibration of the same voltmeter in a matter of days with relatively little loss in accuracy as compared directly with the previous calibration in terms of the primary standard.

There seems to be little doubt that the ranges and accuracies quoted below are inadequate to meet present needs, in view of the considerable deterioration in accuracy from the initial calibrated instrument to the final one because the equipment is calibrated through several steps starting with the National Bureau of Standards and ending with the working bench equipment. The present minimum NBS accuracy requirements of many RF quantities are still largely unknown.

\footnotetext{
* Manuscript received by the PGI, August 7, 1958.

$\dagger$ Boulder Labs., National Bureau of Standards, Boulder, Colo.
}

In an effort to assist in the realization of a more complete national radio-electronic standardization program, one of the IRE Subcommittees (entitled "Basic Standards and Calibration Methods") has embarked on a program, one of the objectives of which is to determine and maintain an up-to-date list of the RF quantities needed and the minimum required accuracies. It is believed that these findings will be useful as a guide for further development and improvement of the NBS standardization equipment now available for RF power, voltage, attenuation, and field strength and that equipment for other quantities may also be made available. For example, pulse power, pulsed voltage, balanced voltages, and balanced attenuation must be standardized, whereas the equipment and techniques developed so far are generally applicable only to $\mathrm{CW}$ unbalanced conditions. Other quantities such as current, peak power, phase shift, thermal noise, modulation, antenna gain, RF interference and, indeed, any measurable RF quantity need standardization for the simple reason that the "measurements" without a stated accuracy are utterly useless.

\section{Power Standards}

At the time of this writing, three different types of high-frequency power reference standards have been installed at the Electronic Calibration Center: the lowlevel thermistor bridge, the dry static calorimeter, and the liquid flow calorimeter. The first covers the approximate range of $100 \mu \mathrm{w}$ to $100 \mathrm{mw}$; the second, $20 \mathrm{mw}$ to 12 watts; and the third, 10 to 350 watts. The dry static calorimeter is fully described by Hudson and Allred ${ }^{1}$ and will, therefore, not be mentioned further here.

\section{Bolometer Bridge}

The operation of the power-measuring bridge is conventional and is based on the equivalent heating effects of dc power and RF power when each is in turn dissipated in a purely resistive load. A thermistor forms one arm of a Wheatstone bridge circuit which is biased with dc power until the bridge is balanced. When RF power is fed into the thermistor simultaneously with the do bias power, the bridge becomes unbalanced because of the change in the resistance of the thermistor; a change in the dc power must then be marle to restore the initial balance. The quantity of $\mathrm{dc}$ power withdrawn is equated to the RF power present (for the case of an equal arm bridge) as follows:

${ }^{1}$ P. A. Hudson and C. M. Allred, "A dry, static calorimeter for RF power measurement," this issue, pp. 292-296. 


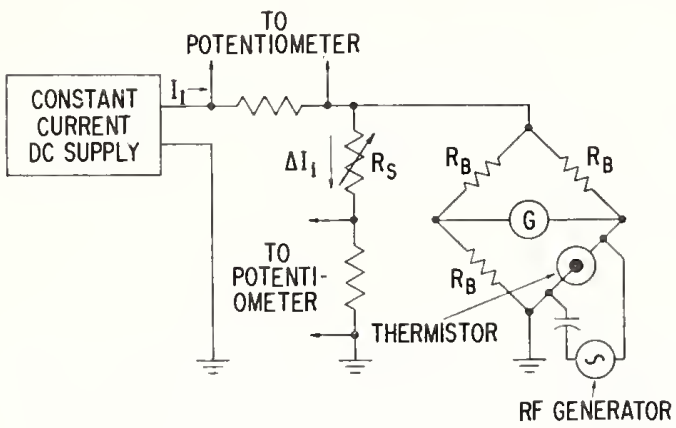

(a)

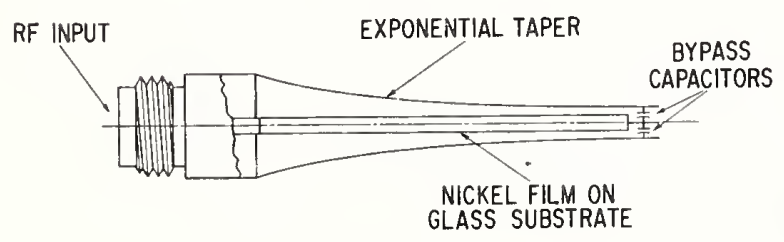

(b)

Fig. 1-Schematic diagram of power bolometer bridge. (a) Block diagram of RF power-measuring thermistor bridge. Power range: $100 \mu \mathrm{w}$ to $100 \mathrm{mw}$, dc accuracy: $\pm(0.05$ per cent $+2 \mu \mathrm{w})$, equation: $P_{\mathrm{RF}}=R_{\mathrm{B}}\left(2 I_{1}-\Delta I_{1}\right) \Delta I_{1} / 4$. A similar bridge has been constructed for use with the mount shown in (b). (b) Detail of mount construction. Power range: $10 \mathrm{mw}$ to $8 \mathrm{w}$, de accuracy: $(0.25$ per cent $+10 \mu \mathrm{W}$ ), maximum VSWR of mount: 1.15 to $300 \mathrm{mc}$.

$$
P_{\mathrm{RF}}=\frac{1}{4}\left(I_{1}^{2}-I_{2}^{2}\right) R=\frac{1}{4}\left(2 I_{1}-\Delta I\right) \Delta I R
$$

where $I_{1}$ and $I_{2}$ are, respectively, the dc bias currents before and after RF power is ted into the thermistor; $\Delta I=\left(I_{1}-I_{2}\right)$, and $R$ is the bridge arm resistance at balance.

Sometimes direct measurements of $I_{1}$ and $I_{2}$ are made. This results in large errors when the RF power level is small because the above equation involves the difference between two relatively large quantities, $I_{1}{ }^{2}$ and $I_{2}{ }^{2}$. To alleviate this problem, it is the usual practice (an inconvenient one) to use several bolometer or thermistor elements with various sensitivities to cover the power range from microwatts to milliwatts.

The approach used in the present bridge eliminates this difficulty by measuring the difference, $\Delta I$, directly and at the same time by making use of a very simple and relatively foolproof circuit arrangement. The principle is to use a fixed total amount of direct current, furnished by a constant current source, and to divert a known fraction of this current from the bolometer bridge into a shunt resistor where it can be measured directly. By using a highly stable and constant current source, a greater accuracy can be achieved, especially at low power levels, and thus the usable power range may be increased. Fig. 1 shows a schematic diagram of this arrangement.

A simple constant current source can be improvised from a laboratory regulated dc power supply and a series resistor whose value is about 100 times that of the bridge resistance. The maximum change in the total current due to load (bridge) impedance changes will then be only about 1 per cent. Accuracies of the order of 5 per cent

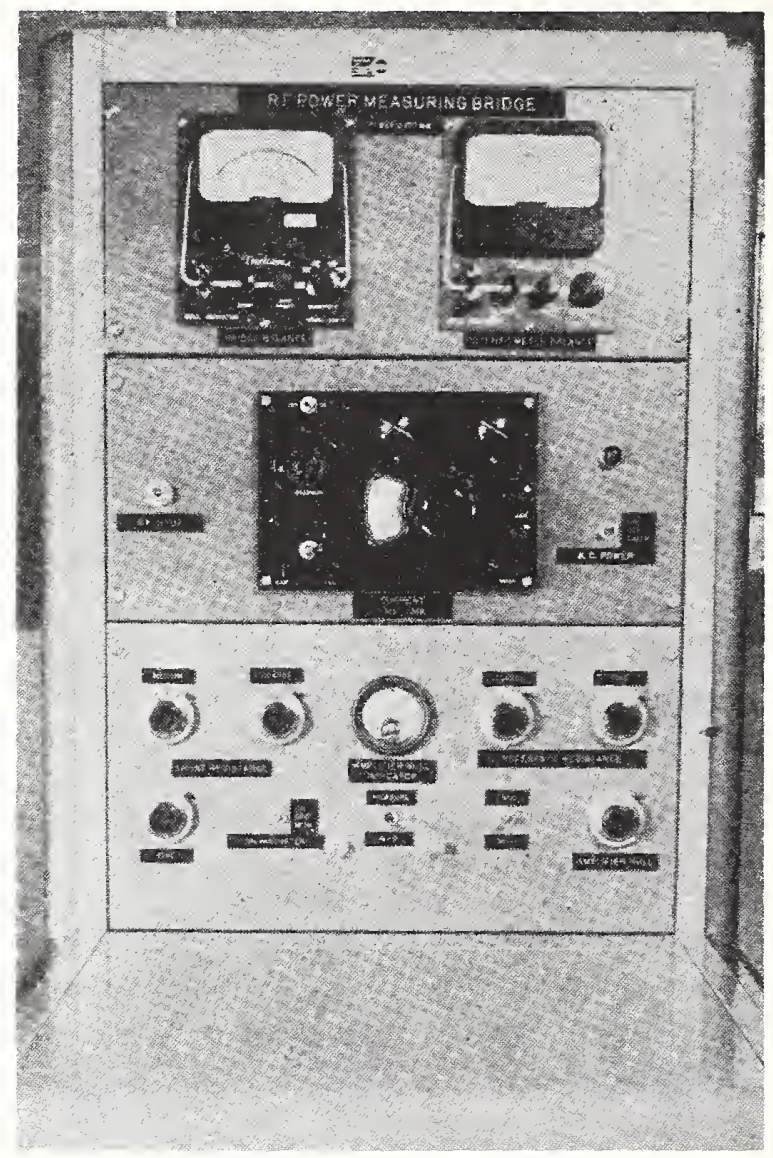

Fig. 2-Power bolometer bridge.

or better may be obtained using such a source with a 50 -ohm single-thermistor bridge at power levels ranging from about 1 to $100 \mathrm{mw}$.

Excellent current regulation as well as stability is obtained in this new bridge by using a well-designed constant current source. The one in use (a special electronically regulated power supply) has a regulation of 1 part in $10^{5}$ for load variation from zero to $100 \mathrm{ohms}$ and the stability is \pm 1 part in $10^{5}$ per hour. Stabilities of \pm 1 part in $10^{6}$ have been observed over periods of from 5 to 10 minutes. With this source, RF power levels at the thermistor from $100 \mathrm{mw}$ down to as low as $100 \mu \mathrm{w}$ were measured with accuracies ranging from 0.05 to 5 per cent.

The design of this bridge circuit readily permits incorporating a self-balancing feature by having the amplified output of the bridge control the amount of shunt current. The improvement over present-day commercial bridges is about 10 to 1 in the power range and as much as 100 to 1 in percentage accuracy, with the greatest improvement at the lower levels. Fig. 2 shows a photograph of this equipment.

\section{Liquid Flow Calorimetric RF Wattmeter}

Power levels in the range 10 to 350 watts to be measured in the Electronic Calibration Center will be done with a liquid flow type calorimeter which employs silicone oil as the coolant. The RF power to be measured 
is dissipated in a cylindrical resistor of the evaporated thin film type, which serves as the inner conductor in a coaxial system. The outer conductor is a thin-walled tractorial taper ${ }^{2}$ jacket made of copper. This load has a VSWR of less than 1.15 at all frequencies from 0 to 2500 mc.

Operation of the calorimeter may best be understood by reference to the block diagram of the equipment shown in Fig. 3. Oil is pumped from the constant temperature reservoir through a flow meter to an input connection on the load where it passes through an outer copper jacket surrounding the load. Inside the load heat is transferred from the cylindrical resistor to the oil which is returned to the reservoir. In the reservoir means are provided to cool the oil to its initial temperature. The temperature rise of the oil, caused by its passage through the load, is measured by a 10 -junction differential thermopile, the cold junctions of which are located in the oil stream immediately ahead of the load. The hot junctions are located at the point where the oil leaves the load, and mixing orifices are inserted in the line ahead of both sets of junctions to insure that the true average temperature of the oil stream is the quantity measured. The RF load and thermopile are enclosed in a dewar flask to prevent heat interchange with the surroundings. The oil bath temperature is kept at or near ambient temperature.

In operation, RF power is fed into the load through the type- $\mathrm{N}$ connector and the flow rate $\mathrm{F}$ is adjusted to give a steady-state differential temperature $\Delta T$ of from $3^{\circ} \mathrm{C}$ to $6^{\circ} \mathrm{C}$. When thermal steady state is reached in the system, the values of $\Delta T$ and $F$ are observed and the power absorbed is calculated by means of

$$
P=C \times F \times \Delta T
$$

where $C$ is the specific heat of the oil and $F$ is in units of oil mass flow per second. Thus, the wattmeter does not require calibration since power is measured in terms of fundamental quantities and the known properties of a material. The performance of the calorimeter has, however, been checked, using known values of dc or low-frequency power.

Measurement accuracy of the wattmeter is limited primarily by the accuracy of the flowmeter which is \pm 0.5 per cent over a dynamic range of 10 to 1 . Higher accuracies $( \pm 0.25$ per cent) are obtainable if the range of the flowmeter were limited to say 2 to 1 . Some sacrifice, however, would then be necessary in the dynamic range of the wattmeter. The value of $C$ is known to an accuracy of \pm 0.1 per cent while $\Delta T$ is measured to an accuracy of \pm 0.2 per cent. Thus the over-all accuracy of the wattmeter is better than \pm 1 per cent; this estimate has been checked and confirmed using dc power.

The time necessary for the calorimeter to reach thermal equilibrium after the RF power is turned on

${ }^{2}$ C. T. Kohn, "Radio frequency coaxial resistor using tractorial jacket," Proc. IRE, vol. 3, pp. 951-960; August, 1955.
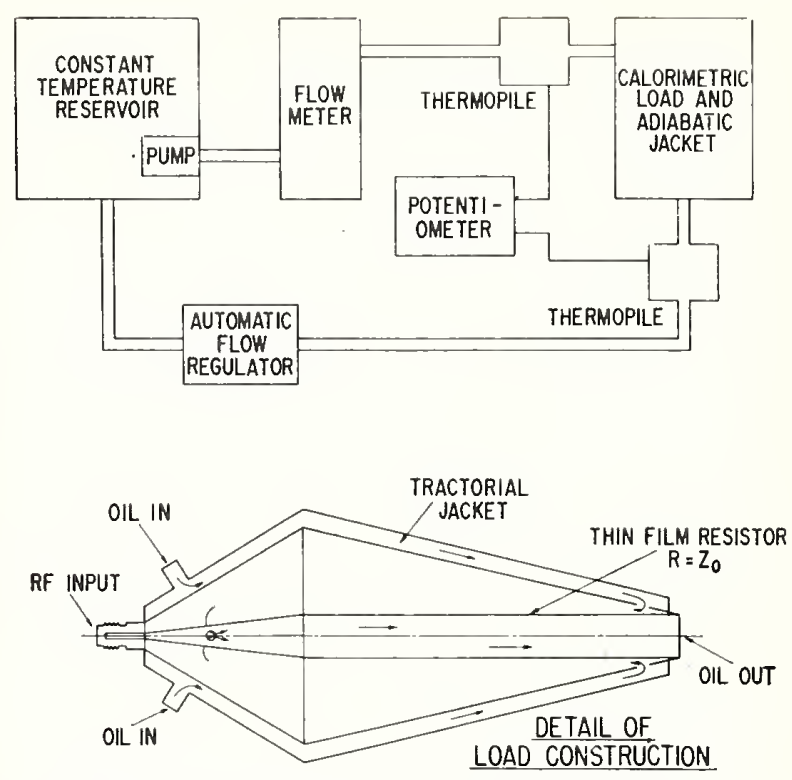

Fig. 3-Block diagram of liquid flow type calorimetric RF power meter. Frequency range: 0 to $2500 \mathrm{mc}$, power range: $10 \mathrm{w}$ to $350 \mathrm{w}, \mathrm{RF}$ accuracy: \pm 1 per cent or better, maximum VSWR: below $400 \mathrm{mc}-1.05$, below $2500 \mathrm{mc}^{-1}-1.16$.

ranges from 5 to 10 minutes depending on the power level. This relatively short-time constant is realized because the oil enters the load at a fixed temperature. Thus, only the load need reach equilibrium rather than the entire system as, would be the case were not the reservoir temperature kept constant. Another advantage in using a constant reference temperature is obvious from (2). The power is computed in terms of mass flow, whereas the flow meter readings are given in terms of volume flow. Since, for a fixed volume, the mass is a function of density which is in turn a function of absolute temperature, computations are simplified by maintaining the reference temperature constant.

A photograph of the equipment is shown in Fig. 4. The upper half of the left-hand console houses the reservoir and flowmeter while in the lower section is the dewar flask containing the load. The right-hand console houses the potentiometer used in determining $\Delta T$.

\section{RF Voltage Standards}

The purpose of the RF voltmeter consoles is to provide as rapidly as possible means of calibrating all types of voltmeters in discrete steps ranging from $30 \mathrm{kc}$ to $700 \mathrm{mc}$ with the maximum accuracy available at the present time. Two major steps made the above possible. The first was the use of the AT voltmeters; ${ }^{3}$ the second was the decision to calibrate at discrete frequencies. The latter decision is justified because the frequency response curves for present-day RF voltmeters seldom have serious discontinuities. However, frequencies other than those selected may be necessary. At the time of this writing two of these consoles have been completed and

\footnotetext{
3 "Stable radiofrequency voltmeters," NBS Tech. News Bull., vol.
} 40 , pp. 29-30; February, 1956. 


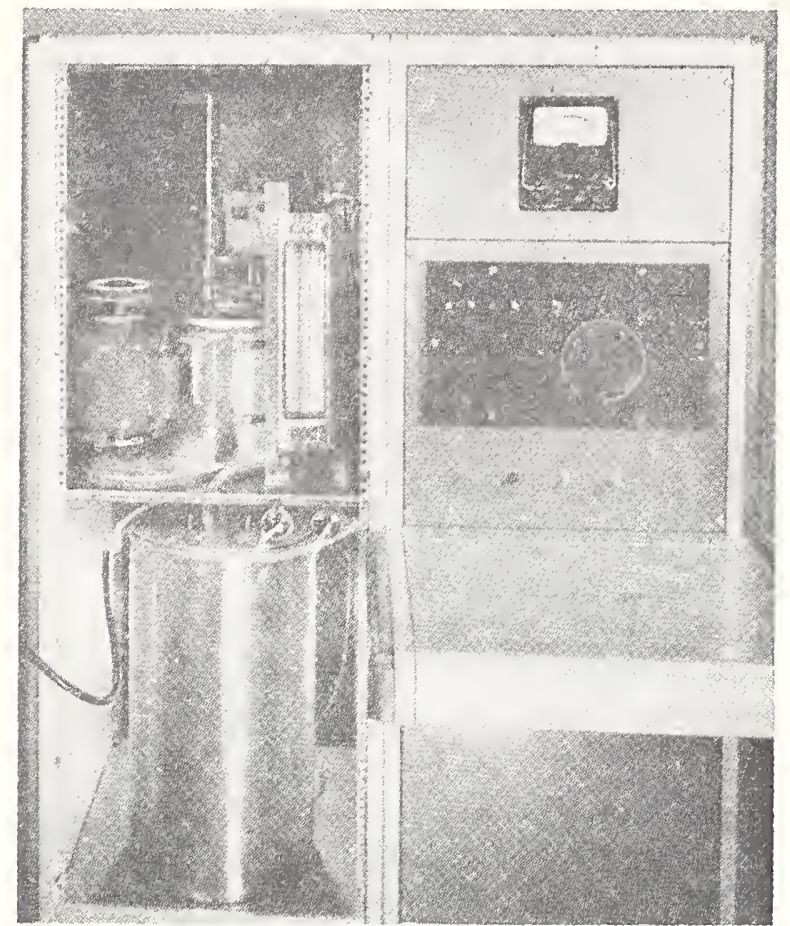

Fig. 4-Liquid flow calorimeter.

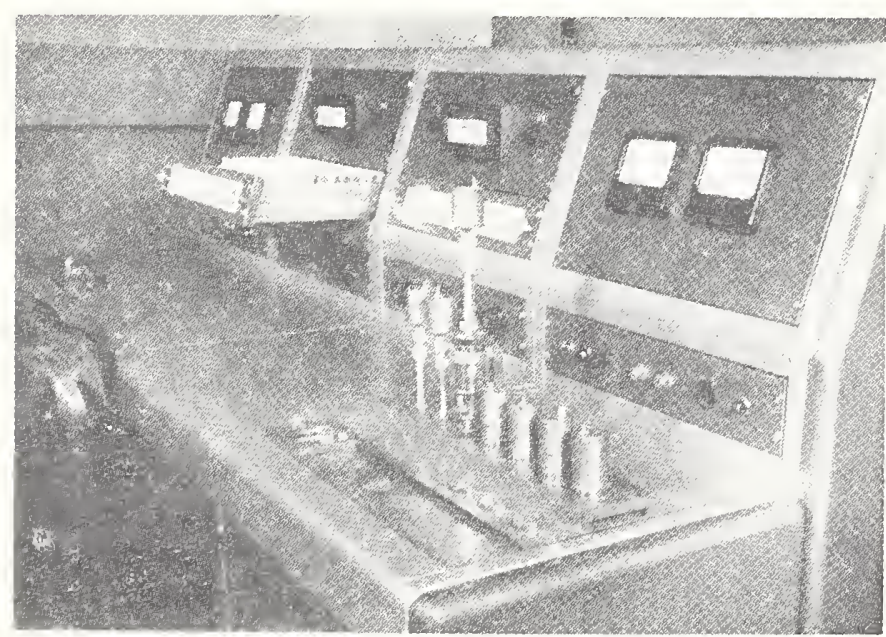

Fig. 5-RF high-voltage calibration console.

placed in service, one for $30,100,300$, and $1000 \mathrm{kc}$ and the other for 5, 10, 30, and $100 \mathrm{mc}$. The third partially finished unit will operate at $300,400,500$, and $700 \mathrm{mc}$. The placement of components and controls is essentially the same for each of the consoles thus presenting a uniform appearance to facilitate servicing and to provide for their most efficient use.

Fig. 5 illustrates the arrangement of the controls and indicators of one of the consoles. A specially designed coupling plate for connecting the voltmeter under calibration in parallel with the reference standards is mounted at the front of each of the two pedestals of the console. The indicator lights in the narrow panels above the pedestals are for the overload protective circuits, the crystal oven heaters and the ac power lines. The meters

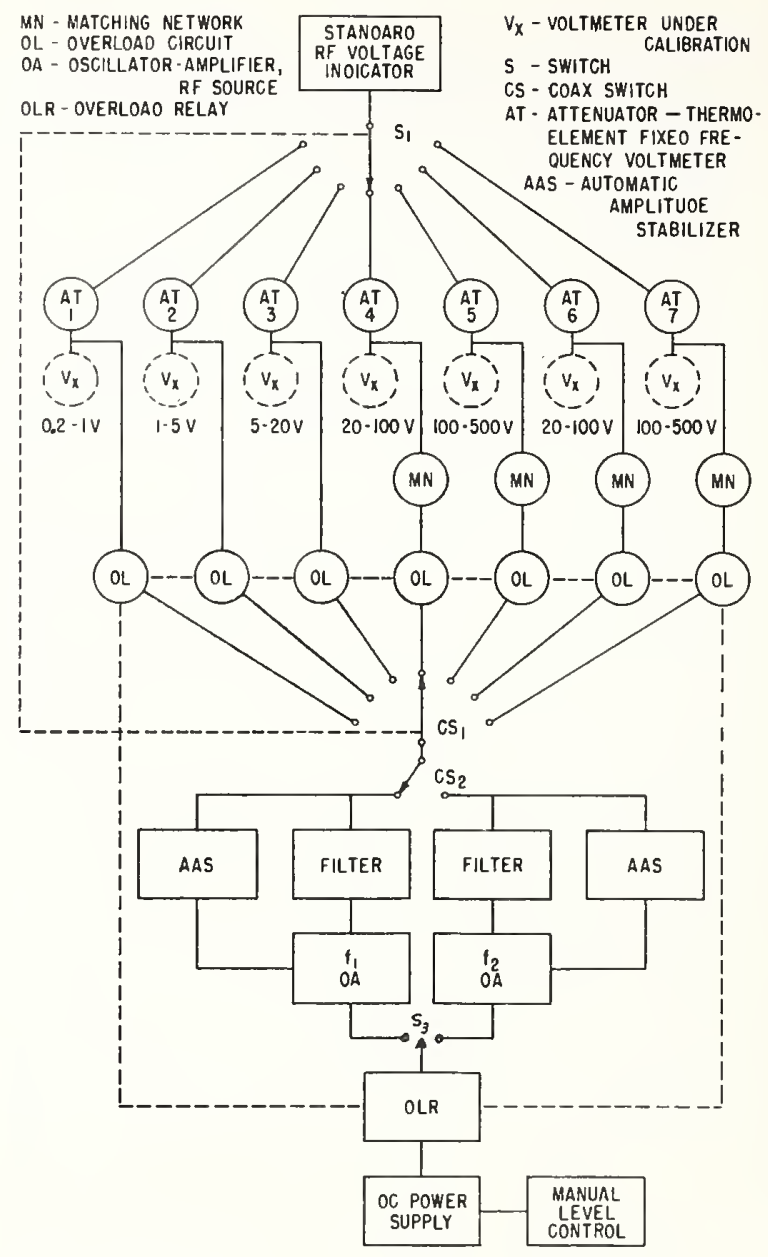

Fig. 6-Block diagram of RF high-voltage calibration console.

above the lights are in the dc power supply circuits to monitor the operation of the RF sources. The millivoltmeters in the adjacent panels indicate the thermocouple output from the AT voltmeters; and the line charts, displayed in the windows of the storage drawers beneath them, are used to convert these indications quickly into the actual RF voltages applied. Controls for operating the corresponding half of the console are located on a panel below each storage drawer. The controls in the center of the panel are for the adjustment of the RF voltage level. Referring to Fig. 6, a sevenposition switch, $C S_{1}$, connects the RF source to the appropriate reference standard and also connects the millivoltmeter to its thermocouple output through switch $S_{1}$. The remaining control, switch $S_{3}$, connects the dc high voltage to the RF voltage source of the appropriate frequency and, by means of a solenoidoperated coaxial switch, $C S_{2}$, connects all the reference standards to that RF voltage source. These controls and the four crystal controlled RF voltage sources-two in each half of the console - are permanently installed together with low-pass RF filters, impedance matching networks, and overload protective circuits. All of the RF components are carefully shielded to eliminate stray radiation. 
Rapid connection of the "unknown" voltmeter to a reference standard is achieved with the RF voltmeter coupling plate, Fig. 7. Reference standards, seven for each half of a console, are mounted on the under side of each plate. To connect the "unknown" in parallel with a standard, the operator attaches a suitable adaptor ring to the probe of the "unknown," inserts this ring in a sliding carriage on the top side of the plate, centers it over the reference standard to be used and clamps it in place. The "unknown" and the standard are now connected in parallel through a $\mathrm{T}$ connection of approximately $\frac{3}{16}$-inch long, with the RF voltage applied midway between the two. Such a $\mathrm{T}$ exists at each of the seven standards. The operator then rotates the sevenposition switch until the number below the index corresponds to the position of the standard in the plate and closes the switch. The RF level is adjusted with the manual level controls and the dc thermocouple output in millivolts, as indicated on the panel meter, is converted immediately into actual applied RF voltage with the aid of the line chart displayed in the window.

As already indicated above, fixed type AT voltmeters have been chosen in preference to the adjustable type used in the laboratory because of their simplicity and superior stability. The mechanism required to operate the piston of the continuously adjustable type increases their complexity of construction and operation. Thiree reference standards, having dissipative fixed attenuator input pads, cover the voltage range 0.2 to 20 volts in ranges of $0.2-1.0,1.0-5.0$, and $5.0-20$ volts, respectively. These are used at both frequencies provicled in the part of the console in which they are located. The voltage ranges of $20-100$ and $100-500$ volts at each frequency are covered by single-frequency standards using fixed "capacitive" attenuators, bringing the total number of reference standards in each half of a console to seven. The series reactance of this type of "capacitive" attenuator is many times larger than the resistance of the thermoelement heater terminating the attenuator. Therefore, in analyzing the effect of a change in heater resistance with a given $\mathrm{RF}$ voltage input to the voltmeter on heater current, the attenuator can be represented at its output terminals by a constant current source. Changing the heater resistance even by as much as 50 per cent has no effect on heater current.

Consequently, once an RF voltage calibration of a given reference standard has been made, replacement of its thermoelement does not necessitate an RF recalibration. Only a dc calibration to determine the relationship of heater current to dc millivolts output need be made, and if the characteristics of the replaced couple match those of the original closely enough, even this calibration is unnecessary. When matching can be achieved, the same calibration chart can remain in service. For the voltmeters using dissipative attenuators, however, a complete recalibration of the voltmeter is required when thermoelements are replaced. The overall accuracy is \pm 3 per cent or better.

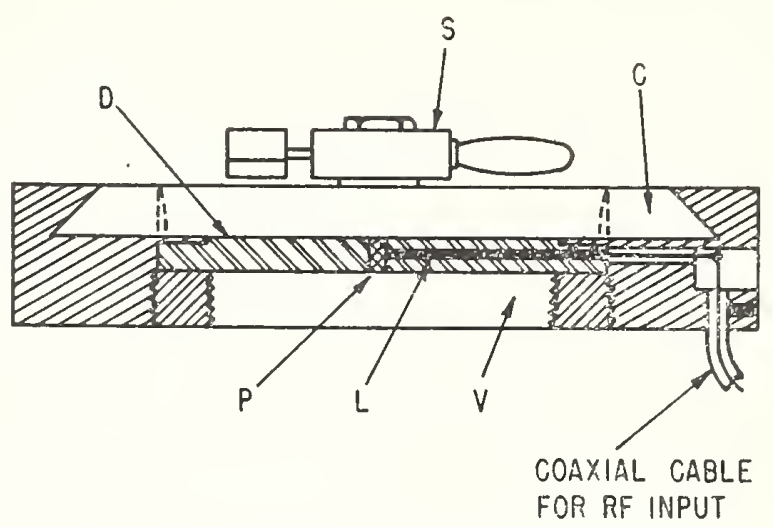

Fig. 7 -Cross section of voltmeter coupling plate. Note: carriage not sectioned.

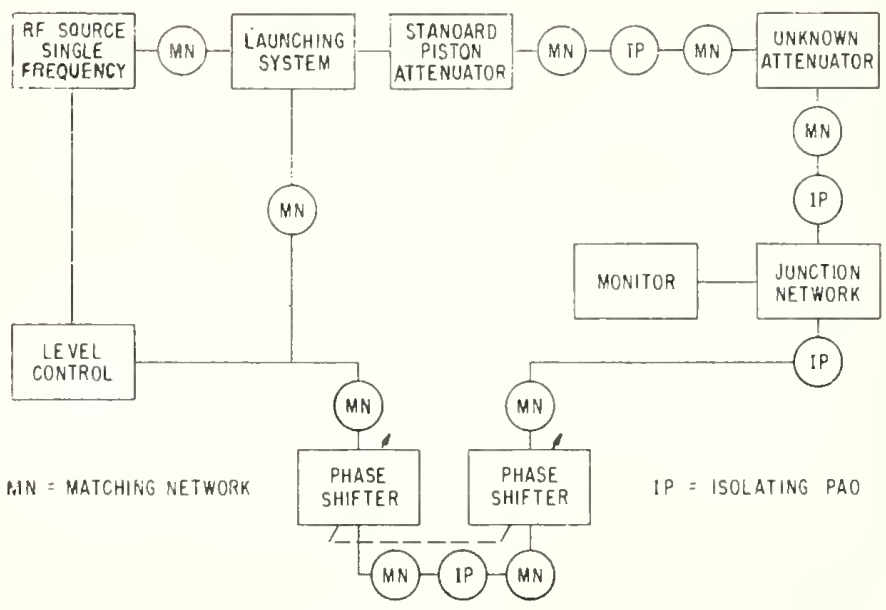

Fig. 8-Block diagram of the duo-channel null balance RF attenuation system.

\section{Attenuation Standards}

The present plans call for 8 or 9 independent attenuation consoles, one each for 9 discrete frequencies. At least 6 and possibly 7 will employ new precision, 3 -inch ID, waveguide-below-cutoff attenuators. The other 2 , for 30 and $100 \mathrm{kc}$, will probably employ dissipative attenuators. One of these consoles, for $30 \mathrm{mc}$, is approaching completion. The block diagram, Fig. 8 , shows the general method and basic equipment which will most likely be used in all cases.

The system consists of a series substitution branch where the stanclard and unknown attenuators are connected in the conventional way. This branch is shunted by another branch incorporating an impedance phase shifter. The vectoral sum of the outputs of the two branches is detected by a common monitor or null detector. The procedure, as usual, is to adjust the attenuator and phase shifter for a null balance both before and after inserting the unknown into the circuit. The clifference between the two positions of the attenuator ancl phase shifter gives the results desired. For maximum sensitivity and minimum noise it was found best to work with output levels of each channel as high as possible. 


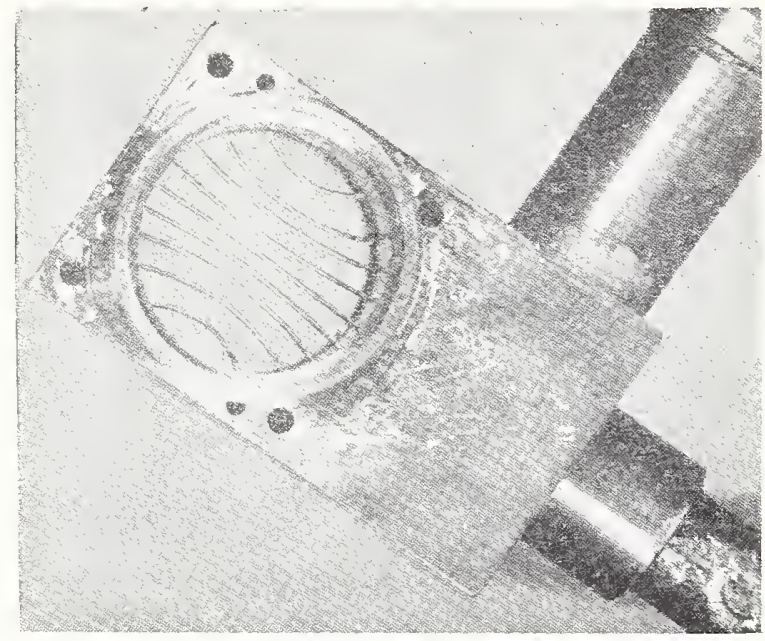

Fig. 9-Constant current launching coil assembly.

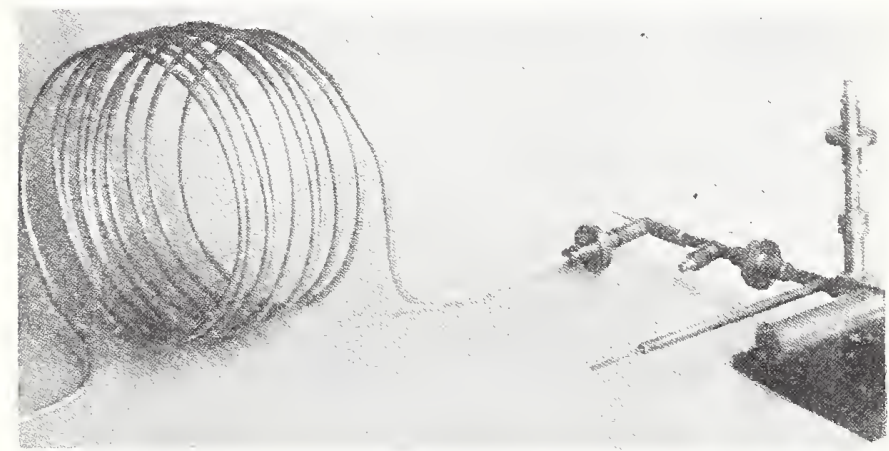

Fig. 10-Launching and piston end assembly of a precision waveguide-below-cutoff attenuator.

A serious difficulty in the construction of the phase shifter was eliminated by the adoption of fixed frequency consoles. Use of a wide band and a high precision requires phase shifters that have a constant characteristic impedance vs frequency, a feature difficult to accomplish mechanically. At a single frequency the system may be readily matched and the problem is solved.

Another interesting feature of the equipment is the novel launching system which makes available a constant current in the launching coil essentially free from effect of proximity of the pick-up coil in the standard attenuator, thus reducing the initial insertion loss of the attenuator and improving the range and efficiency of the setup. This feature is obtained by sampling the launching signal and automatically compensating the input level from the RF source.

The launching coil itself (shown in Fig. 9) is, to the best of our knowledge, a new type and has important advantages. The coil is a combination-mode filter and relatively high current carrying, low-loss conductor which shorts the input line of the piston attenuator. It eliminates the need of a mode filter, allows the pick-up coil to be in closer proximity to the launching coil and thus considerably reduces the initial insertion loss. It also permits high current input without excessive heat-

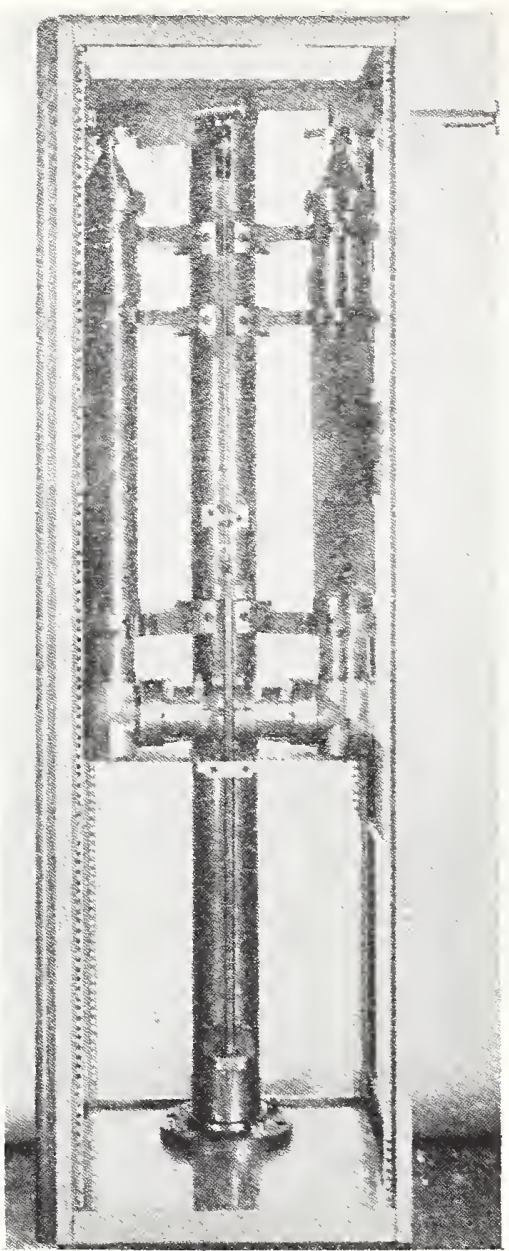

Fig. 11-Phase shifter and housing.

ing of the attenuator, thus increasing the dynamic range of the system.

Fig. 10 shows one end of a precision piston attenuator wherein the above launching coil is mounted. The solenoidally wound large diameter coil is a solid aluminum-shield semiflexible coaxial transmission line feeding RF power to the launching coil. A section of line is seen at right angles to this main feed line and is used to sample the launching coil current. The other tubes at right angles to the main line are for matching purposes and to attenuate the wave reflected from the launching coil. Winding the main line into a solenoidal shape along an axis parallel to the motion of the piston of the standard attenuator is an important innovation, eliminating the considerable interference with precision measurements caused by bending (and impedance variation) of conventional flexible cables as the piston of the attenuator is moved.

The phase shifter, housed in a closed rack (seè Fig. 11 ) is motor driven and has a range of 20 inches, or $36^{\circ}$, at $30 \mathrm{mc}$. The coaxial attenuation, which will be mounted in another cabinet next to the one shown, will have a 3 -inch ID and a bore uniformity of \pm 0.0001 inch over a length of 20 inches. The piston will be motor driven and its displacements measured by means of a 
micrometer projector to an accuracy of \pm 0.0001 inch in 6 inches.

The attenuation range of this console at $30 \mathrm{mc}$, based on preliminary measurements of provisional setups and design analysis, is expected to be 0.0 to $80 \mathrm{db}$ at an estimated accuracy of $\pm(0.005 \mathrm{db}+0.03$ per cent of the indicated value in $\mathrm{db}$ ). The maximum sensitivity of the experimental setup (for unknowns of up to $10 \mathrm{db}$ ) was of the order of $0.00001 \mathrm{db}$.

\section{Field-Strength Standards}

The National Bureau of Standards offers a calibration service for radio field-strength meters employing loop-type antennas at frequencies from $30 \mathrm{c}$ to $30 \mathrm{mc}$ and for meters employing dipole-type antennas from 30 to $300 \mathrm{mc}$. The calibration consists of measuring the over-all linearity of the instrument at one or more frequencies and power levels, measuring the internal attenuator ratios at one or more frequencies and measuring the so-called antenna coefficient or correlation factor relating field strength to the output meter reading under specified conditions of set gain at specified frequencies. All measurements are made in terms of sinusoidal voltages or currents.

The portions of the calibrations involving only RF voltage ratios are made in terms of precision dissipative step attenuators which comprise "Pi" or "T" coaxial sections of evaporated or deposited metal film for frequencies up to $300 \mathrm{mc}$.

Since the over-all linearity of a field-strength meter will, in general, be a function of both frequency and RF input level, it is necessary to determine this dependence. Fortunately, this effect is usually limited to the highest attenuator steps in those types of sets having intermediate-frequency attenuators only. The departure from linearity has been found, in general, to be less than 2 per cent if the signal voltage applied to the mixer grid does not exceed about 5 per cent of the injected local oscillator voltage. For practical and economical reasons it is necessary to limit linearity checks on the attenuator steps in question to one or two frequencies as specified by the user.

For those sets also employing RF attenuators in the antenna input circuit it is necessary to insure that these ratios are measured under actual operating conditions. Those RF attenuator steps which may be affected by the changing source impedance of the antenna, or the changing input admittance of the first RF stage with frequency, are measured with the antenna placed in a field which can be attenuated in steps of known ratios.

The attenuation linearity is certified to an accuracy of $\pm(0.1 \mathrm{db}+0.3$ per cent of ratio in db). Fig. 12 shows the equipment installed for these measurements.

At the present time the National Bureau of Standards certifies only field-strength meters using loop antennas below about $30 \mathrm{mc}$. The calibration is made in terms of a standard free-space quasi-static magnetic field produced by a single-turn, unshielded, balanced, transmitting loop of known radius and with a known current flowing with a typical unit as shown in Fig. 13. The receiver loop must, of course, either be shielded or balanced to ground to eliminate pickup from the electric component of the field. In calibrating a field-strength meter the setup is made in a cleared space such that a distance of at least two or three times the loop spacing, $d$, exists to the nearest sizable metallic objects and to the ground.

The magnitude of the field strength produced by a single-turn circular transmitting loop is given by (3) for the case of coaxial transmitting and receiving loops as shown in Fig. 13. The actual value of the quasi-static magnetic field, $H$, produced by the loop is expressed in terms of the equivalent electric component, $E$, that would exist in a free-space radiation field. The relationship used is $E=Z H$, where $Z$ is the impedance of free space $(Z=376.7 \mathrm{ohms})$.

$$
|E| \cong \frac{60 \pi r_{1}^{2} I}{\left(d^{2}+r_{1}^{2}+r_{2}^{2}\right)^{3 / 2}} \sqrt{1+\left(\frac{2 \pi d}{\lambda}\right)^{2}}
$$

where

$E=$ equivalent free-space electric field strength in rms volts per meter,

$r_{1}=$ radius of transmitting loop in meters,

$r_{2}=$ radius of receiving loop in meters,

$d=$ axial spacing (meters) between coaxial loops (make $d>7 r_{1}$ and $d>7 r_{2}$ ).

$I=$ transmitting loop current in rms amperes,

$\lambda=$ free-space wavelength in meters.

The value of field strength used for calibration is of the order of $0.1 \mathrm{v} / \mathrm{m},\left(r_{1}=0.1 \mathrm{~m}, I=0.1 \mathrm{a}\right)$.

The "antenna coefficient," $K$, is evaluated by the relation

$$
K=\frac{E_{1}}{A_{1}} \times \frac{}{M_{1}},
$$

where

$E_{1}=$ standard field given by (3) usually expressed in microvolts per meter,

$A_{1}=$ true attenuator ratio used,

$M_{1}=$ output meter reading corrected from linearity data,

$f=$ frequency, usually in kc or mc.

In using the field-strength meter later for actual measurements, the unknown field in microvolts per meter is given by

$$
E=\frac{K M_{2} A_{2}}{f}
$$

where $K$ is given by (4), and 


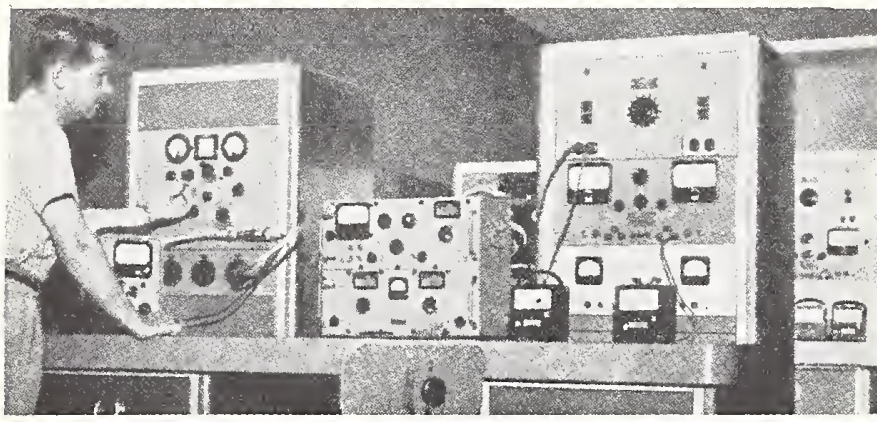

Fig. 12-Equipment to measure linearity and calibrate attenuators of field-strength meters.

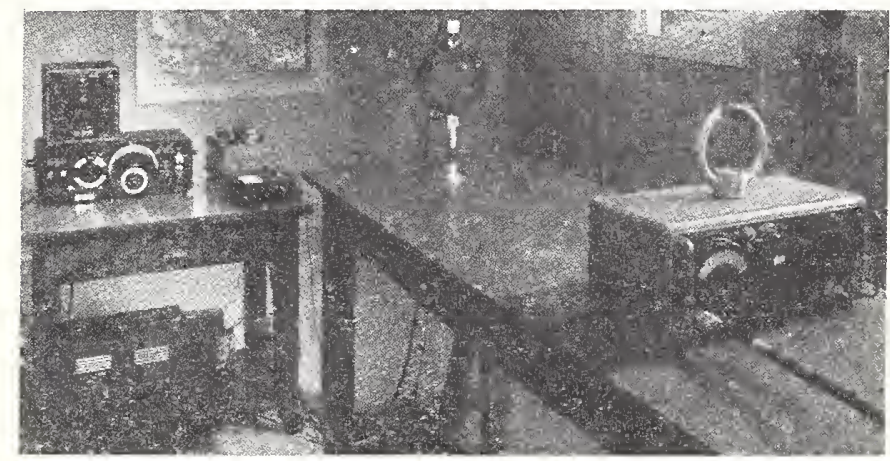

Fig. 13-"Standard-field" equipment assembly to determine loop antenna coefficients.

$M_{2}=$ output meter reading corrected from linearity data,

$A_{2}=$ true attenuator ratio used,

$f=$ frequency in same units as used in (4).

The accuracy of antenna coefficients is usually certified to \pm 3 per cent below $5 \mathrm{mc}$ and \pm 5 per cent between 5 and $30 \mathrm{mc}$ for sets using loop antennas.

At the frequencies in the VHF band, the National Bureau of Standards certifies only field-strength meters using dipole antennas. The determination of antenna coefficients is made in terms of a radiation field by the standard-antenna method using half-wave dipoles and horizontally polarized transmission.

The field strength of a locally generated field is determined by measuring directly the induced EMF in a standard receiving dipole oriented for maximum response and calculated from the relation:

$$
|E|=e / l_{H},
$$

where

$e=$ induced EMF in the receiving dipole in volts,

$l_{I I}=$ effective length of the dipole in meters, $\left(l_{H}=\lambda / \pi\right.$ meters for a half-wave dipole).

The EMF is measured directly by means of a relatively high impedance silicon crystal voltmeter built into the gap at the center of the antenna. This eliminates the necessity for a separate measurement of the antenna

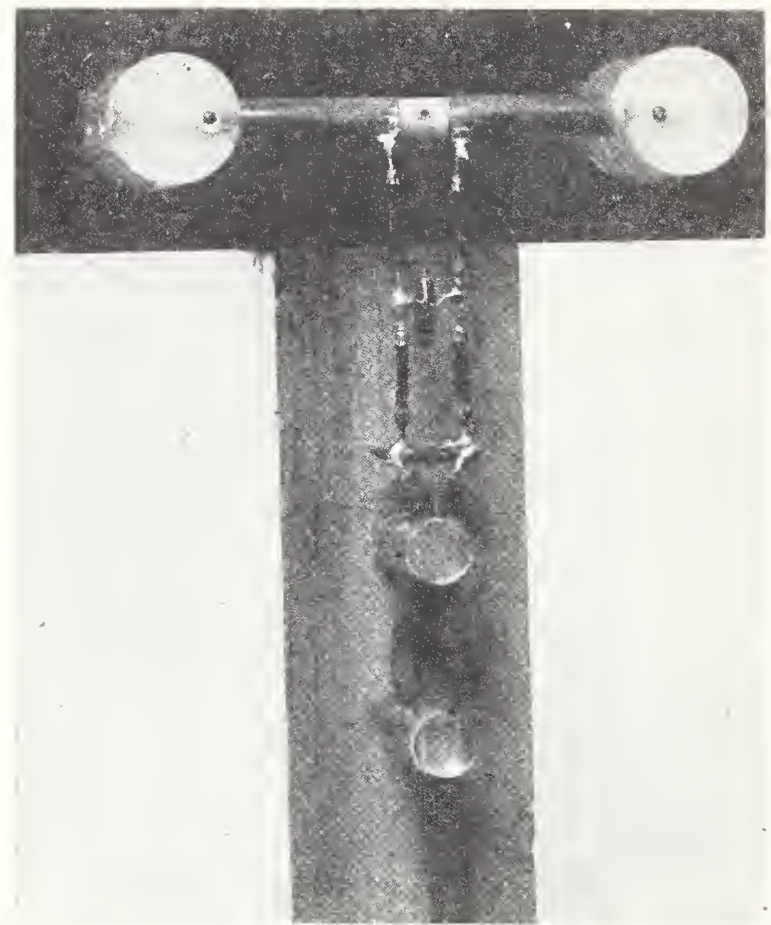

Fig. 14-Crystal voltmeter assembly with interchangeable dipoles to determine field values for standardization of field-strength meters at 30 to $300 \mathrm{mc}$.

impedance, greatly simplifying the problem and improving the over-all accuracy of the measurement. Fig. 14 shows the voltmeter assembly to which dipoles of various lengths may be connected.

Owing to the lack of selectivity in this method as used, it does not lend itself to calibration work in the vicinity of strong interfering fields that may often be encountered.

While it is possible to use either the standard-field or the standard-antenna method for determining the antenna coefficients, the latter method is used at the National Bureau of Standards at the present time. Field tests have shown the excellent agreement possible between the two methods throughout the VHF band. The choice was largely one of convenience, as it was found somewhat more convenient to provide an accurate calibration of the silicon crystal voltmeter than to insure an accurate measurement of the transmitting antenna current throughout the VHF band.

The "antenna coefficient" of the field-strength meter under test is determined by placing its dipole antenna at a specified height, usually 10 feet, in the radiation field previously measured using the standard receiving dipole and the relationship given by (6). Use of the meter at a later time at other antenna heights or over ground having appreciably different constants may require corrections for heights less than about one wavelength.

The accuracy of the antenna coefficients certified by the National Bureau of Standards depends largely upon 
the quality of the individual meter under test. In general, these coefficients are certified at present to \pm 10 per cent for frequencies between 30 and $150 \mathrm{mc}$ and to \pm 15 per cent for frequencies between 150 and $300 \mathrm{mc}$.

\section{Conclusion}

The equipment described above embodies techniques and design features arrived at during the last several years. Because requirements for better accuracy are already in evidence, there is a strong likelihood that considerable modifications in the above units may be introduced and perhaps entire setups may be replaced within the near future. The technical phase of the Electronic Calibration Center should of necessity remain fluid in order to keep services, as closely as possible, up to date. Complete papers are expected to be published on individual equipments as soon as possible. However, additional information may be obtained from previous $\mathrm{Na}$ tional Bureau of Standards publications on these sub- jects. ${ }^{4-8}$ The individuals to be credited with the development, design, and construction of the equipment are the project leaders, C. M. Allred, L. F. Behrent, F. M. Greene, P. A. Hudson, A. H. Morgan, M. C. Selby, and E. C. Wolzien, and every member of the HighFrequency Electrical Standards Section including those assigned to the Electronic Calibration Center as well as a number of machine shop personnel who contributed valuable ideas and skill throughout this activity.

${ }^{4}$ F. M. Greene, "Calibration of commercial radio field-strength meters at the National Bureau of Standards," NBS Circular 517; December, 1951.

${ }^{5}$ C. C. Cook, "Calibration of field-strength meters," Tele-Tech., vol. 11, pp. 44-46, 96, 99-101; October, 1952.

${ }^{6} \mathrm{~F}$. M. Greene and M. Solow, "Development of very high-frequency field-intensity standards," J. Res. NBS, vol. 44, pp. 527-547; May, 1950. RP2100.

7 C. M. Allred, "Precision piston attenuators," NBS Rep. 5078; May $1,1957$.

${ }_{8}^{8}$ M. C. Selby, L. F. Behrent, and F. X. Ries, "RF-voltage calibration consoles," 1958 IRE NATIONal Convention Record, pt. 5, pp. 251-257.

\section{Reprinted from IRE TRANSACTIONS \\ ON INSTRUMENTATION}

Volume I-7, Numbers 3 \& 4, December, 1958 
National Standards of Time and
Frequency in the United States*

TIME

Time is one of the independent quantities chosen as a basis for the measuring system of science. To establish a time scale, that is, to specify a time coordinate, we must first establish an origin and a constant unit of time and lay off the unit at least as far as all points of interest, in exact analogy to the specification of position. The orbital motion of the earth about the sun provides a time scale, called Ephemeris Time (ET) which is suitable in these respects, and also constitutes a standard from which the unit may always be obtained. In October, 1956, the International Comnittee on Weights and Measures, with representation from the United States, effectively adopted the second of ET as the fundamental unit of time by resolving that "the second is the fraction $1 / 31,556,925.9747$ of the tropical year for January 0, 1900 at 12 hours Ephemeris Time." 1 The tropical year for any given epoch (or moment) is the interval, taken symmetrically about that epoch, necessary for the mean longitude of the sun to increase by $360^{\circ}$, as measured along the ecliptic from the vernal equinox. This definition of the second has been one of many attempts to define a unit of time 1 ) which remains constant with epoch, 2) which is physically realizable to high precision, and 3 ) which is permanently available for observation.

Ephemeris Time is obtained in practice by observations on the position of the moon and reference to tables giving this position as a function of ET. As the fundamental unit, the second of ET replaced the second of Universal Time (UT), a time scale based on the rotation of the earth. The delay in the determination of UT from the astronomical observations is of the order of a month; the delay in the determination of ET to any useful degree of accuracy is of the order of several years.

The maintaining of a national standard of time consists of the process of determining the time scale in the United States from the international standard and making this information available to the user. Often, however, as with position, it is only time differences or time intervals which are of interest. This need for convenient interval determination has led to the common use of other time scales with easier realizability than ET.

Measurements of ten have been, and still are, expressed in terms of time scales other than ET. These other scales are all related or relatable to ET and to each other through conversion factors with various degrees of precision as to size of unit at least, and in

* Received by the IRE, O-tober 15, 1959 .

1 Comite International des Poids et Mesures, "Prores-Verbaux de Seances," 1950 Session, Ser. 12 vol. 25, Gauthier-Villars, Paris; 1957 some cases as to origin also. The situation is analogous to using a measuring rod of different length than the standard rod but of known calibration. For example, UT0 is the astronomically observed Universal Time or mean solar time, uncorrected for polar variation and annual fluctuation in the earth's speed of rotation; UT1 is UT0 corrected for polar variation; UT2 is UT1 corrected for annual variation. None of the UT seconds is constant. They are variously determined by several nations. Various other nominal "seconds" are defined by the duration of a specified number of periods of various oscillators or resonators, such as quartz or atomic devices. These all have the outstanding characteristic that they are more readily observable than ET. The atomic time unit, furthermore, is assumed for the present to be as constant as the unit of ET.

\section{The United States Frequexcy STANDARD}

A national standard of frequency called the United States Frequency Standard (USFS) is maintained at the Boulder Laboratories, National Bureau of Standards, for the purpose of making immediately and continuously arailable, through the Standard Frequency Broadcasts discussed below, a provisional time scale with which to make measurements. This provisional scale is sufficiently accurate for all civil and most scientific uses. Nevertheless, for the highest possible accuracy, this scale must be continually corrected to or calibrated against the national time scale as finally determined, and it must be relatable to other frequency and time scales in common use.

The USFS in general consists of the weighted value of the outputs (reduced of course to a common basis) of several actual oscillators and resonators maintained at the Boulder Laboratories, these devices being among the best available at any current state of the frequency control art. In particular, the USFS is presently stable to 2 parts in $10^{10}$ or better over intervals from about 1 to $10^{3}$ minutes; over longer intervals, its value has been maintained as constant as possible prior to October 9, 1957 with respect to the UT2 second as determined by the U. S. Naval Observatory, and since October 9, 1957 with respect to atomic frequency standards. These atomic standards have been compared with other atomic standards via a network comparison. Such atomic standards have been shown to be in agreement and to remain constant with respect to each other to 5 parts in $10^{10}$ or better, ${ }^{2,3}$ 2Essen, Parry, Holloway, Mainburger, Reder, and
Winkler, "Comparison of cesium frequency standards Winkler, "Comparison of cesium frequency standards
of different construction," Nature, vol. 182, pp. 41-42; of different
July, 1958.

3ockler, Beehler, and Barnes, "An evaluation of a cesium beam frequency standard." Symposium Record, Office of Naval Research Symposium on Quantum Electronics, Bloomingburg, N. Y.; September $14-16,1959$. 


\section{STANDARD FREQUENCY BROADCASTS}

The United States Frequency Standard is distributed to interested users by means of standard broadcasts of Radio Stations IVWV and IVWVH. The frequencies of these stations are kept in agreement with respect to each other and have been maintained as constant as possible with respect to the United States Frequency Standard since December 1, 1957. The nominal broadcast frequencies should, for the purpose of highly accurate scientific measurements, for establishing high uniformity among frequencies, or for removing unavoidable variations in the broadcast frequencies, be corrected either to the United States Frequency Standard, as indicated in the monthly tables published in the IRE, ${ }^{4}$ or to a particular time scale as determined by the Naval Observatory, with adequate limits assigned for propagation errors.

\section{Time Signals}

Time signals, sufficiently accurate for all civil and most scientific uses, are also carried by the standard broadcasts. The WVVV and WWVH time signals are also kept in agreement with each other. They are locked to the nominal frequency of the transmissions, and consequently may depart continuously from other time scales such as UT2. Corrections expressed as the times of reception of the WWV time signals on the UT2 scale are determined and distributed by the U. S. Naval Observatory. ${ }^{5}$ Recently, the Observatory has established a particular atomic time scale, A.1, with unit determined by $9,192,-$ 631,770 periods of $\mathrm{Cs}$ at zero field and origin coinciding with UT2 on January 1, 1958, and has published times of reception of WWV on this scale also. ${ }^{6}$ Agreement with time on the UT2 scale, or simply agreement with UT2, within \pm 30 msec at all times, has been maintained by making step adjustments in time of precisely plus or minus twenty milliseconds on Wednesdays at 1900 UT. when necessary. Beginning January 1, 1960, the broadcast frequencies will be offset from the United States frequency standard by a different amount than heretofore in order to establish a unit in substantial agreement with the current value of the unit of UT2. Thus the time signals, locked to the broadcast frequency, will require less frequent adjustment than in the past.

\section{Corrections of THE USFS AND THE} Standard Frequency Broadcasts

The method of final correction of the ${ }_{4}^{4}$ W. D. George, "IVWV standard frequency transmissions," Proc. IRE, vol. 46, pp. 910-911; May, 1958, and subsequent months.

5 "U. S. Naval Observatory Time Signals, I. Preliminary Times of Reception, UT2," Bulletin B, U. S. Naval Observatory, Washington, D. C. (unpublished).

"U. S. Naval Observatory Time Signals, Final Times of Reception, UT2," Bulletin A, U. S. Naval Observatory, Washington, D. C. (unpublished).

6 "Time Service Notice No. 6 " U. S. Naval Observatory, Washington, D. C. January 1, 1959 (unpublished).
USFS and the Standard Frequency Broadcasts to the national time scale has changed over the years as improvements in the measurement of time and frequency have been made.

Fig. 1 shows schematically the relation between the frequencies (i.e., cycles per common unit of time) of hypothetical oscillators which oscillate such as to mark off the unit of the time scales indicated after exactly the same number of cycles in each case. The frequencies are shown relative to the value of the hypothetical oscillator marking off the defined unit of ET. The figure is equivalently interpreted as the reciprocal of the size of the units of the indicated time scale relative to the unit of ET.

The Standard Frequency Broadcasts and Time Signals have been and are related to the UT and A.1 time scales as explained above. From these observations, frequency corrections of the Standard Frequency Broadcasts to these scales are easily made; and in addition, conversion from the UT scale at a given epoch to the ET scale is available after reduction of astronomical observations. ${ }^{7-9}$

Prior to October 9, 1957, the final value of the USFS was assigned retrospectively on the UT scale from the determination of the frequency of the Standard Frequency Broadcasts on this scale and the known relation between the Standard Frequency Broadcasts and the USFS. These values have not been published, but are a matter of scientific record at the National Bureau of Standards.

With improvement in stability and constancy realized in the maintenance of the USFS by the availability of atomic frequency standards, monthly publications of corrections of the Standard Frequency Broadcasts to the USFS were made from October 9, 1957, to realize the advantage of rapid correction to a more constant frequency than that hased on the unit of UT2.

It is thus also necessary to relate the USFS and Standard Frequency Broadcasts to frequencies provided by atomic frequency standards such as cesium. Since the relation of an atomic time unit defined by a given number of periods, $N$, of an atomic oscillation to the unit of ET has not yet been adopted internationally, we are required to state explicitly an assumed value of $N$ when comparing frequencies or times to an atomic scale. In comparing the USFS to an atomic time scale, the following equality was adopted as of October 9, 1957, and holds to a precision of 1 part in $10^{10} ; 100,000.000 \ldots$ periods of the USFS equals $N_{1}$ periods of the zero-field $(4,0) \leftrightarrow(3,0)$ Cs transition, where $N_{1}$ equals $9,192,631,838$.

If $N_{0}$ is the number of Cs oscillations per unit of ET, then the ratio $N_{0} / N_{1}$ may be used to convert the USFS to the ET scale as soon as $N_{0}$ is determined. Markowitz, et al. ${ }^{8}$ have measured $N_{0}$ to be $9,192,631,770$ 
\pm 20 as the number of $\mathrm{Cs}$ oscillations per unit of ET, providing the best and only value expected for several years. Effective January 1,1960, the value of the USFS will be corrected so that $N_{1}$ equals $9,192,631,770$, and the published corrections to the Standard Frequency Brcadcasts will thus be given with respect to the unit of ET as realized by atomic standards to a precision of the intercomparison of atomic standards (a few parts in $10^{10}$ ) and to an accuracy determined by the work of Markowitz, et al. ( \pm 22 parts in $\left.10^{10}\right)$.

The final correction of the Standard Frequency Broadcasts, and hence the USFS, to the national time scale, will be effected by the Naval Observatory by the observation of the times of reception as described.
In summary, to express a given time interval or frequency in terms of the international standard, it must be referred to a standard broadcast, or some atomic standard with proper regard for the precision of this comparison, and the latter standard must be referred to the ephemeris second as indicated herein with proper regard for the uncertainties involved.

\section{National Bureau of Standards} Boulder, Colo.

7 Essen, Parry, Markowitz, and Hall, "Variation in the speed of rotation of the earth since June, 1955," Nature, vol. 181, p. 1054; April, 1958.

8 Markowitz, Hall, Essen, and Parry, "Frequency of cesium in terms of Ephemeris Time," Phys. Rev. Letters, vol. 1, pp. 105-106: August, 1958.

9 Brouwer. "A study of the changes in the rate rotation of the A stu $n$ 146; September, 1952.

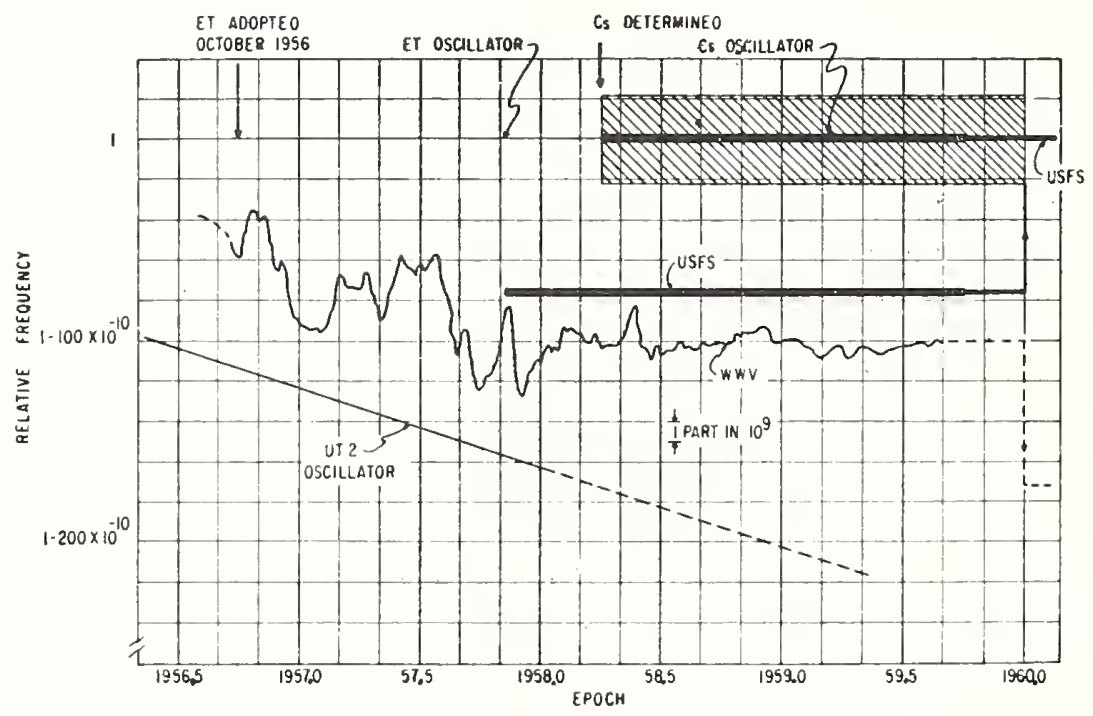

Fig. 1-Relative frequencies of oscillators keeping the time scales indicated; or equivalently, reciprocals of the lengths of unit intervals on the indicated time scales. Width of $\mathrm{C}_{S}$ and USFS lines indicates precision; cross hatching shows assigned accuracy of $\mathrm{Cs}$ frequency. The UT2 scale is derived from smoothed data. ${ }^{8}$ The UT2 unit is considerably less precise than the other units. The WWV scale is taken from a U. S. Naval Observatory Notice. ${ }^{6}$ Values of USFS and WWV transmissions will be altered as indicated on January 1, 1960. 


\section{Standard Frequencies and Time Signals from NBS Stations WWV and WWVH}

\section{Contents}

1. Technical services and related information

1.1. Standard radio frequencies .......................... 1

1.2. Standard audio frequencies . . . . . . . . . . .

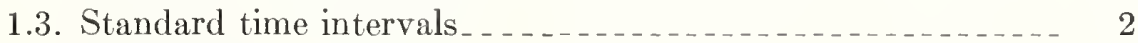

1.4. Standard musical pitch $\ldots$

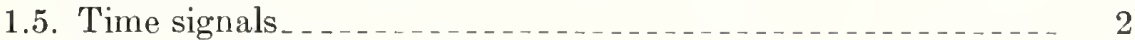

1.6. Radio propagation forecasts _._._.

1.7. Geophysical alerts _............... 4

1.8. Radiated power, transmitting antennas, modulation $\ldots \ldots \ldots$

1.9. Other standard frequency and radio time signal services _..._. 5

2. Stations operating outside the exclusive bands _._. 5

2.1. LF and VLF

2.2. Other broadcasts. $\ldots \ldots \ldots$

\section{National Bureau of Standards Miscellaneous Publication 236}

Issued December 1, 1960

For sale by the Superintendent of Documents, U.S. Government Printing Office, Washington 25, D.C. Price 10 cents 


\title{
Standard Frequencies and Time Signals From NBS Stations WWV and WWVH
}

\begin{abstract}
Detailed descriptions are given of six technical services broadcast by National Bureau of Standards radio stations WWV and WWVH. The services include 1, standard radio frequencies; 2 , standard audio frequencies; 3 , standard time intervals; 4 , standard musical pitch; 5, time signals; and 6, radio propagation forecasts. Other domestic and foreign standard frequency and time signal broadeasts are tabulated.
\end{abstract}

\section{Technical Services and Related Information}

The National Bureau of Standards' radio stations WWV (in operation since 1923) and WWVH (since 1948) broadcast six widely used technical services: 1, Standard radio frequencies; 2, standard audio frequencies; 3 , standard time intervals; 4 , standard musical pitch; 5 , time signals; 6 , radio propagation forecasts. ${ }^{1}$

'The radio stations are located as follows: WWV, Beltsville, Maryłand (Box 182, Route 2, Lanham, Maryland); WWVH, Maui, Hawaii (Box 901, Punnene, Maui). Coordinates of the stations are: WWV (lat. $38^{\circ} 59^{\prime} 33^{\prime \prime} \mathrm{N}$, İong. $76^{\circ} 50^{\prime} 52^{\prime \prime} \mathrm{W}$ ); WWVH (lat. $20^{\circ} 46^{\prime} 02^{\prime \prime} \mathrm{N}$, long. $156^{\circ} 27^{\prime} 42^{\prime \prime} \mathrm{W}$ ).

\subsection{Standard Radio Frequencies}

(a) Program

Station WWV broadcasts on standard radio frequencies of $2.5,5,10,20$, and $25 \mathrm{Mc}$. The broadcasts are continuous, night and day, except WWV is interrupted for approximately $4 \mathrm{~min}$ each hour. The silent period commences at $45 \mathrm{~min}$ (plus 0 to $15 \mathrm{sec}$ ) after each hour. (See fig. 1.)

Station WWVH broadcasts on standard radio frequencies of 5, 10, and $15 \mathrm{Mc}$. The broadcast is interrupted for 3 min commencing on the hour (plus 0 to $15 \mathrm{sec}$ ) and on each quarter hour thereafter, and for periods of 34 min each day beginning at 1900 U'T (Universal Time, UT, is the same as GMT and GCT).

\section{(b) Accuracy}

Since December 1, 1957, the standard radio transmissions from stations WWV and WWVH have been held as nearly constant as possible with respect to the atomic frequency standards which constitute the United States Frequency Standard (USFS), maintained and operated by the Radio Standards Laboratory of the National Bureau of Standards. Carefully made atomic standards have been shown to realize the idealized Cs resonance frequency, $f_{\mathrm{C}_{\theta}}$, to a few parts in $10^{10}$ or better; and the present USFS is believed to realize this resonance to 1.5 parts in $10^{11}$. The frequency $f_{\mathrm{Cs}}$ has been measured in terms of the second ${ }^{2}$ to be $f_{\mathrm{Cs}}=9,192,631,770 \pm 20 \mathrm{cps}$. This uncertainty of 2 parts in $10^{9}$, with which frequency can be expressed in terms of the second, has usually been avoided in practice by provisionally taking

1 All inquiries conccrning the technical radio broadeast services should be addressed to: Radio Standards Laboratory, National Bureau of Standards, Boulder, Colo.

Costz, 1lall, Essen, and Parry, Frequency of cesium in terms of ephemeris time, Phys. Rev. Letters 1, 105 (1958).
$f_{\mathrm{Cs}}$ exactly equal to the above number (or to some other stated number before the above was available).

On January 1, 1960, the USFS was brought into agreement with $f_{\mathrm{Cs}}$ as quoted above by arbitrarily increasing its assigned value by 74 parts in $10^{10}$. Frequencies measured in terms of the USFS between December 1, 1957 and January 1, 1960 may be referred to the above value of $f_{\mathrm{Ca}}$ and to the (Ephemeris) second by means of this relative correction. $^{3}$

The frequency of the transmissions from WWV normally are held stable to 1 part in $10^{9}$, at all times. Deviations at WWV are normally less than 2 parts in $10^{10}$ from day to day. When necessary, frequency adjustments not exceeding 5 parts in $10^{10}$ are made at WWV at 1900 UT. Frequency adjustments at WWVH do not exceed 3 parts in $10^{9}$.

Changes in the propagation medium (causing Doppler effect, etc.) result at times in fluctuations in the carrier frequencies as received which can be very inuch greater than the uncertainties described above.

\section{(c) Corrections}

All carrier and modulation frequencies at WWV are derived from a common 2.5-Mc quartz oscillator, the stability of which is described above. In addition, since January 1, 1960, this oscillator has been intentionally offset from the USFS by a small but precisely known amount in order to reduce departure between the time signals broadcast and astronomical time UT2. For example, the offset for 1960 is about 150 parts in $10^{10}$, and is low with respect to the USFS, since the unit of U'T2 is about this much longer than the (Ephemeris) second. Although UT2 is subject to unpredictable changes observable at this level of precision, it is nevertheless expected that a particular offset can be left unchanged throughout each calendar year, thus providing as constant a frequency as possible for the year.

For the above reasons, corrections to the actual oscillator frequency are continuously determined with respect to the USFS and are published in the Proceedings of the IRE. These were begun in May, 1958 with data extending back to December $1,1957 .{ }^{4}$

${ }^{3}$ National standards of time and frequency in the United States, Proc. 1RE 48,105 (1960). $910(1958)$ and subsequent issues. 


\subsection{Standard Audio Frequencies}

\section{(a) Program}

Two standard audio frequencies, 440 cps and $600 \mathrm{cps}$, are broakcast on each radio carrier frequency. The audio frequencies are given alternately at 5 -min intervals starting on the hour with $600 \mathrm{cps}$, as shown in figure 1. At WWV, the first tone period $(600 \mathrm{cps})$ of each hour is of $3-\mathrm{min}$ duration; the remaining periods are of $2-$ min duration. At WWVH, all tone periods are of 3min duration.

\section{(b) Accuracy}

'The accuracy of the audio frequencies, as transmitted, is the same as for the carriel. The frequency offset mentioned under 1.1(c) applies. Changes in the propagation medium (causing Doppler effect, etc.) result at times in fluctuations in the audio frequencies as received.

\subsection{Standard Time Intervals}

\section{(a) Program}

Seconds pulses at precise intervals are derived from the same oscillator which controls the radio carrier frequencies, e.g., they commence at intervals of 5,000,000 cycles of the nominal $5 \mathrm{Mc} / \mathrm{s}$ carrier. They are given by means of double-sideband amplitude-modulation on each radio carrier frequency. Intervals of $1 \mathrm{~min}$ are marked by omitting the pulse at the beginning of the last second of every minute and by commencing each minute with two pulses spaced by 0.1 sec; the first pulse is used to mark the beginning of the minute. The 2-min, 3-min, and 5-min intervals are synchronized with the seconds pulses and are nuarked by the beginning or ending of the periods when the audio frequencies are off. The pulse duration is 0.005 sec. The pulse wave form is shown in figure 2. At IVWV each pulse consists of five cycles of a 1,000-cps frequency. At WWVH each pulse consists of six cycles of a 1,200-cps frequency. The pulse spectium is composed of discrete frequency components at intervals of 1.0 cps. The components have maxinıum amplitudes at approximately $995 \mathrm{cps}$ and 1,194 cps for the WWV and WWVH pulses, respectively. The tone is interrupted 0.040 sec for each seconds pulse. The pulse starts $0.010 \mathrm{sec}$ after commencement of the interruption.

\section{(b) Accuracy}

The accuracy of the time intervals, as transmitted, is the same as for the carrier with an additional limitation of $\pm 1 \mu$ sec. The frequency offset mentioned under $1.1(\mathrm{c})$ applies. (See note concerning time adjustments under section $1.5(\mathrm{~b})$, Time Signals.)

\subsection{Standard Musical Pitch}

The frequency $440 \mathrm{cps}$ for the note $\mathrm{A}$ above middle $\mathrm{C}$ is the standard in the music industry in many countries and has been in the United States since 1925. The radio broadcast of this standard was commenced by the National Bureau of Standards in 1937. The periods of transmission of 440 cps from WWV and WWVH are shown in figure 1. With this broadcast the standard pitch is maintained, and musical instruments are manufactured and adjusted in terms of an unvarying standard. Practically no instruments are manufactured which cannot be tuned to $440 \mathrm{cps}$. Listeners of music are benefited because there are fewer instruments not in tune.

\subsection{Time Signals}

(a) Program

The audio frequencies are interrupted at precisely 3 min before each hour at $W W V$, and $2 \mathrm{~min}$ before each hour at IVWVH. They are resumed on the hour at WWV and at 5- and 10-minute intervals at both stations as shown in figure 1 .

Universal Time (referenced to the zero meridian) is announced in International Morse Code each 5 min from WWV and WWVH. This provides a quick reference to correct time where a timepiece may be in error by a few minutes. The 0-to-24-hr system is used starting with 0000 at midnight at longitude zero. The first two figures give the hour and the last two figures give the number of minutes past the hour when the tone returns. For example, at $1655 \mathrm{U}^{\prime} \mathrm{T}$, four figures $(1,6,5$, anc 5$)$ are broadcast in code. The time announcement refers to the end of an announcement interval, i.e., to the time when the audio frequencies are resmmed.

At station WWV a voice announcement of Eastern Standard Time is given before and after each International Morse Code amnouncement. For example, at 9:10 a.m., EST, the voice announcement in English is: "National Bureau of Standards, WWV; when the tone returns, Eastern Standard Time is 9:10 a.m."

\section{(b) Corrections}

The time signals are kept in close agreement with UT2 by making step adjustments of precisely 50 msec when necessary. Such adjustment will be made on the first of the month following the month in which the transmitted time departs from UT2 by more than $50 \mathrm{msec}$. Corrections to the time signals are published periodically by the U.S. Naval Observatory.

\subsection{Radio Propagation Forecasts}

A forecast of radio propagation conditions is broadcast in International Morse Code on each of the standard radio carrier frequencies; from WWV at approximately 19.5 and $49.5 \mathrm{~min}$ past each hour, 
and from WWVH at approximately 9.4 and 39.4 min past each hour, as shown in figure 1. Propagation notices were first broadcast from WWV in 1946; the present type of announcement has been broadcast from WVIV since July, 1952, and from IVWVH since January, 1954.

The forecast announcement tells users the condition of the ionosphere at the regular time the forecast is made and how good or bad communication conditions are expected to be in the succeeding six or more hours. The NBS forecasts are based on information obtained from a worldwide network of geophysical and solar observatories, including radio soundings of the upper atmosphere, short wave reception data, and similar information. Trained forecasters digest the information and formulate the predictions.

From WWV the forecasts refer only to North Atlantic radio paths, such as Washington to London or New York to Berlin. The times of issue are 0500,1200 (1100 in summer), 1700, 2300 UT. These are the short-term forecasts prepared by NBS-CRPL North Atlantic Radio Warning Service, Box 178, Ft. Belvoir, Va.

From WWVH the forecasts are for North Pacific radio paths, such as Seattle to Tokyo, or Anchorage to San Francisco. As of March 1, 1959 , the times of issue are 0600 and $1800 \mathrm{U} T$, and the forecasts are first broadcast at 0639 and 1839 UT, respectively. These are the short-term forecasts prepared by NBS-CRPL North Pacific Radio Warning Service, Box 1119, Anchorage, Alaska.

The forecast is broadcast as a letter and a digit. The letter portion of the announcement identifies the radio quality at the time the forecast is made. The letter's denoting quality are "N," "U," and "W," signifying that radio propagation conditions are normal, unsettled, or disturbed. The digit portion is the forecast of the radio propagation quality on a typical North Atlantic (from WWV) or a typical North Pacific (from WWVH) transmission path during the 6 or more hours after the forecast is made. Quality is graded in steps ranging from 1 (useless) to 9 (excellent) as follows:

$\begin{array}{ccc}\begin{array}{c}\text { Disturbed } \\ \text { grades }(W)\end{array} & \begin{array}{c}\text { Unsettled } \\ \text { grade }(U)\end{array} & \begin{array}{c}\text { Normal } \\ \text { grades }(N)\end{array} \\ \text { 1-useless } & \text { 5-fair } & \text { 6-fair-to-good } \\ \text { 2-very poor } & & \text { 7-good } \\ \text { 3--poor } & & \text { 8-very good } \\ \text { 4--poor-to-fair } & & \text { 9-excellent }\end{array}$

If, for example, propagation conditions at the time the forecast is made are normal but are expected
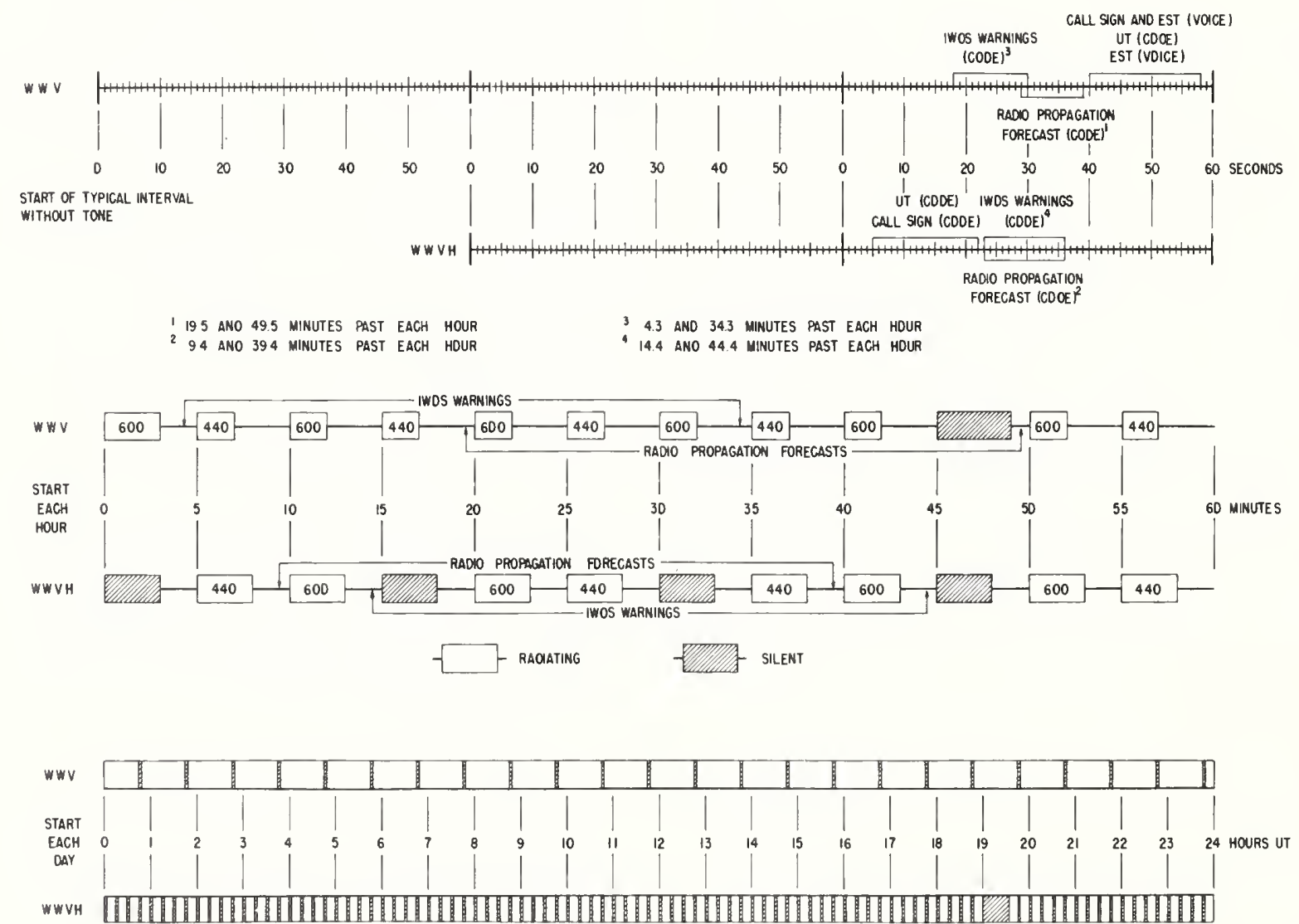

Figure 1. Programs, $W W V$ and $W W V H$. 
to be only "poor-to-fair" within the next 6 or more hours, the announcement would be broadcast as N4 in International Morse code.

\subsection{Geophysical Alerts}

A symbol indicating the geophysical "state of warning" as declared under the international program of the International Council of Scientific Unions, is broadeast in very slow International Morse Code on each of the standard radio carrier frequencies; from WWV at approximately 4.5 and 34.5 min past each hour, and from WWVH at approximately 14.4 and 44.4 min past each hour, as shown in figure 1 . Such notices were first broadcast during the International Geophysical Year 1957-58 and are continuing under the similarly organized progran, International World Day Service (IWDS).

The "state of warning" symbol indicates to experimenters in radio, geophysical, and solar sciences the content of the IWDS Warning Message issued at 1600 UT by the World Warning Agency on days when outstanding geophysical events have occurred in the preceding $24 \mathrm{hr}$. The information in each such message is first broadcast fiom WWV at 1604.5 UT and from WWVH at 1714.4 UT.

If the IWDS Warning Message declares an alert, the symbol AGI AAAA is broadcast for $24 \mathrm{hr}$. This means that a significant magnetic storm has started, with $K$-index reaching 5 or higher at a midlatitude station. It can also mean that an outstanding auroral display has been reported (or inferred, because the $K$-index has leached at least 7) or that an outstanding increase in cosmic ray flux has been observed. The IWDS Warning Message itself, distributed through other channels including the meteorological communication networks coordinated by the World Meteorological Organization, specifies whether the alert is Magnetic Storm, Aurora, or Cosmic Ray; the "state of warning" symbol broadcast from WWV and WWVH does not make these distinctions at the present time.

A Special World Interval in progress is indicated by the symbol AGI and three extra long dashes. This means that an alert, as above, has been declared and, further, that the geophysical activity is of sufficient interest to warrant special attention and intensified observations by experimenters throughout the world. A Special World Interval will usually last two or three davs. The Interval is considered to extend until 2359 UT on the final day even though the symbol "Special World Interval in progress" is not broadcast after $1600 \mathrm{UT}$ (WWV) or $1700 \mathrm{UT}$ (WWVH).
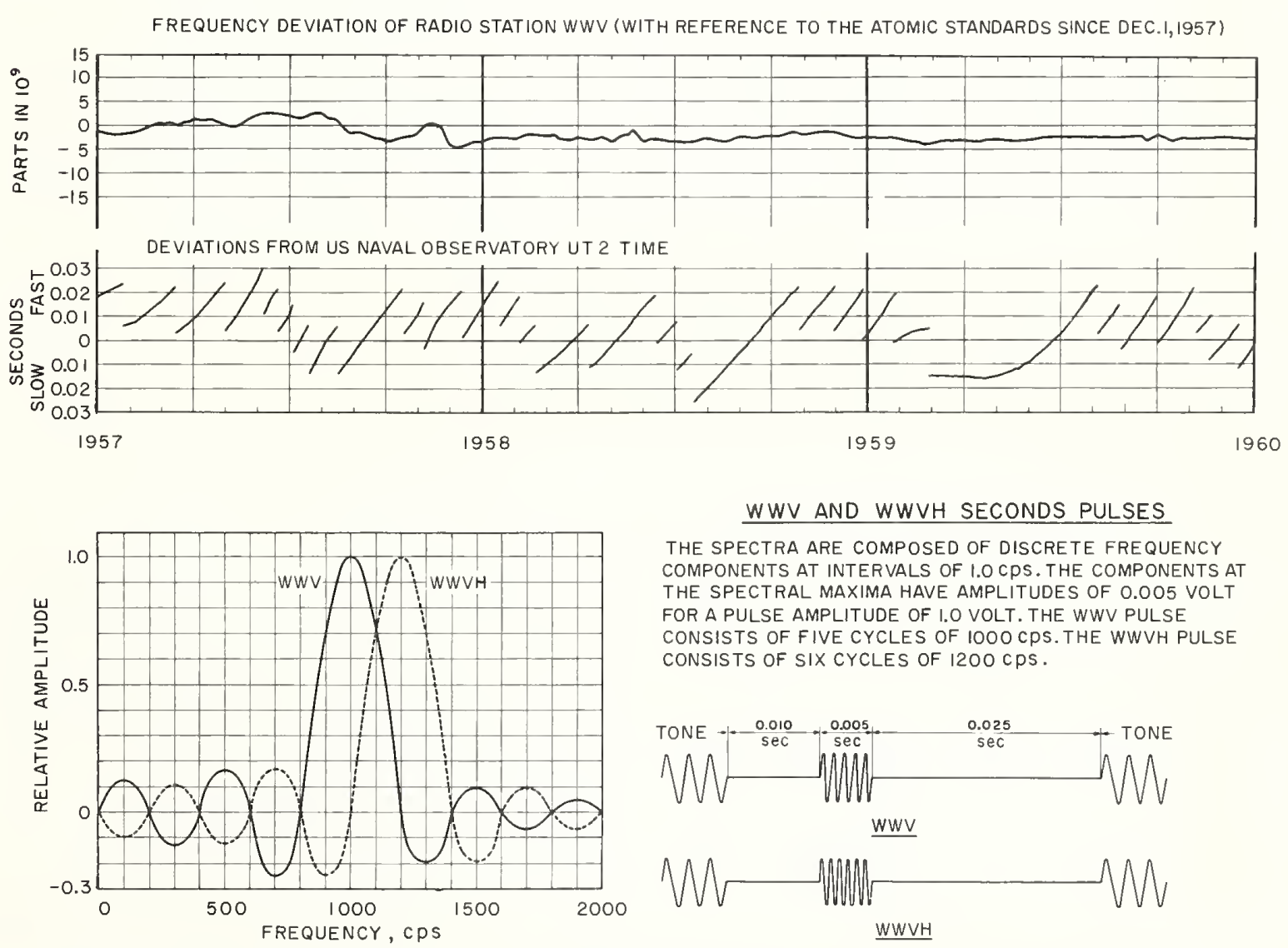

WWV AND WWVH SECONDS PULSES

THE SPECTRA ARE COMPOSED OF DISCRETE FREQUENCY COMPONENTS AT INTERVALS OF I.O CPS.THE COMPONENTS AT THE SPECTRAL MAXIMA HAVE AMPLITUDES OF 0.005 VOLT FOR A PULSE AMPLITUDE OF I.O VOLT. THE WWV PULSE CONSISTS OF FIVE CYCLES OF $1000 \mathrm{cPS}$. THE WWVH PULSE CONSISTS OF SIX CYCLES OF $1200 \mathrm{CPS}$.

FIGURE 2. Sample characteristics of time and frequency broadcasts from NBS stations $W W V$ and $W W V H$. 
When the "state of warning" is neither alert nor Special World Interval in progress, the symbol broadcast is AGI EEEEE.

\subsection{Radiated Power, Transmitting Antennas, Modulation}

(a) Radiated Power

Power, kw
$W H V$
1
8
9
9
1
0.1

\section{Power, kw}

Frequency, $M c$

2.5
5
10
15
20
25

(b) Transmitting Āntennas

The broadeast on 2.5 Me from WWV and on $5 \mathrm{Mc}$ from WWVH is from a vertical quarter-wave antenna. The broadcasts on all other frequencies are from vertical half-wave dipoles. The antennas are omnidirectional.

\section{(c) Modulation}

The amplitude modulation, double sideband, is:

Audio frequencies 440 or $600 \mathrm{cps}-75$ percent, voice and seconds pulses, peak-100 percent.

At WWV, the tone frequency 440 or $600 \mathrm{cps}$, except on $25 \mathrm{Mc}$, is normally operated as a single upper sideband with full carrier. Power output from each sideband transmitter is about one-third the carrier power. Single sideband tone modulation on $25 \mathrm{Mc}$ may be added at a later date. Other signals (announcements and seconds pulses) are double sideband, 100-percent amplitude modulation.

\subsection{Other Standard Frequency and Radio Time Signal Services}

Standard frequencies and time signals broadcast include the stations indicated in the following table:

\begin{tabular}{|c|c|c|c|c|}
\hline Call sign & Location & Carrier frequency & Modulation & $\begin{array}{l}\text { Carrier } \\
\text { power }\end{array}$ \\
\hline $\begin{array}{l}\text { ATA } \\
\text { FHH } \\
\text { IIBN } \\
\text { IAMT }\end{array}$ & $\begin{array}{l}\text { (a) HF Broadcasts } \\
\text { New Delhi, India } \\
\text { Paris, France_- } \\
\text { Neuchatel, Switzerland } \\
\text { Rome, Italy }\end{array}$ & $\begin{array}{l}10 \quad M c \\
2.5,5,10 \\
2.5,5 \\
5\end{array}$ & $\begin{array}{l}1,1000 \\
1,440,1000 \\
1,500 \\
1,440,600,1000\end{array}$ & $\begin{array}{l}k w \\
1 . \\
0.3 \\
0.5 \\
1 .\end{array}$ \\
\hline $\begin{array}{l}\text { IBF } \\
\text { JJY } \\
\text { LOL } \\
\text { MSF }\end{array}$ & $\begin{array}{l}\text { Turin, Italy } \\
\text { Tokyo, Japan } \\
\text { Buenos Aires, Argentina } \\
\text { Rugby, England }\end{array}$ & $\begin{array}{l}5 \\
2.5,5,10,15 \\
2.5,5,10,15,20,25 \\
2.5,5,10\end{array}$ & $\begin{array}{l}1,440,1000 \\
1,1000 \\
1,440,1000 \\
1,1000\end{array}$ & $\begin{array}{l}0.3 \\
2 . \\
2 . \\
0.5\end{array}$ \\
\hline $\begin{array}{l}\text { OMA } \\
\text { ZLFS } \\
\text { ZUO }\end{array}$ & $\begin{array}{l}\text { Prague, Czechoslovakia. } \\
\text { Lower Iutt, New Zealand... } \\
\text { Olifantsfontein, South Africa.- } \\
\text { Moscow, USSR. }\end{array}$ & $\begin{array}{l}2.5 \\
2.5 \\
5 \\
10,15\end{array}$ & 1,1000 & $\begin{array}{l}1 . \\
0.03 \\
4 . \\
20 .\end{array}$ \\
\hline WWVL & $\begin{array}{l}\text { (b) VLF Broadcasts } \\
\text { Sunset, Colorado }\end{array}$ & 20 lic & & 0.02 \\
\hline
\end{tabular}

\section{Stations Operating Outside the Exclusive Bands}

\subsection{LF and VLF}

\begin{tabular}{|c|c|c|c|c|}
\hline Call sign & Location & Carrier frequency & Modulation & $\begin{array}{l}\text { Carriel } \\
\text { power }\end{array}$ \\
\hline $\begin{array}{l}\text { WWVB } \\
\text { DCF77 } \\
\text { CHU } \\
\text { OMA } \\
\text { GBR } \\
\text { MSF } \\
\text { NBA }\end{array}$ & $\begin{array}{l}\text { Boulder, Colorado } \\
\text { Federal German Republic } \\
\text { Ottawa, Canada } \\
\text { Czechoslovakia } \\
\text { Rugby, England } \\
\text { Rugby, England } \\
\text { U.s. Navy, Canal Zone }\end{array}$ & $\begin{array}{l}\quad \text { ke } \\
60 \\
77.5 \\
3330,7335,14670 \\
50 \\
16 \\
60 \\
18\end{array}$ & $\begin{array}{c}c p s \\
1,200,440 \\
1 \\
1\end{array}$ & $\begin{array}{l}\quad k w \\
0.0015 \\
12 \\
0.3,3,5 \\
5 \\
300 \\
10 \\
100\end{array}$ \\
\hline
\end{tabular}

1 National Bureau of Standards station WWVB (formerly K K2XEI) broadeasts continuously and the call letters are keyed approximately on the hour and each 20 min thereafter. The frequeney is normally controlled by an atomie standard. Present plans are to inerease the radiated power to about $3 \mathrm{kw}$ as soon as yossible.

\section{$480 / 5$}




\subsection{Other Broadcasts}

The U.S. Naval Observatory, Department of the Navy, broadcasts time signals continuously or at regular intervals from a number of stations including NSS (Annapolis, Md.), NPG (Mare Island, Calif.), NPM (Pearl Harbor, Hawaii), and NBA (Balboa, C.Z.). Detailed information may be obtained from the U.S. Naval Observatory, Washington 25, D.C.

A comprehensive list of United States and foreign radio time signals is given in chapters 3 and 9 of Radio Navigational Aids, Hydrographic Office Publication No. 117 (formerly H.O. Pub. No. 205), for sale by local and foreign authorized sales agents of the U.S. Navy Hydrographic Office or direct from the U.S. Navy Hydrographic Office, Washington 25, D.C. Price of the publication, with a binder, is $\$ 5.00$; contents only (without binder), $\$ 3.50$.

$$
\text { Boulder, Colo. }
$$

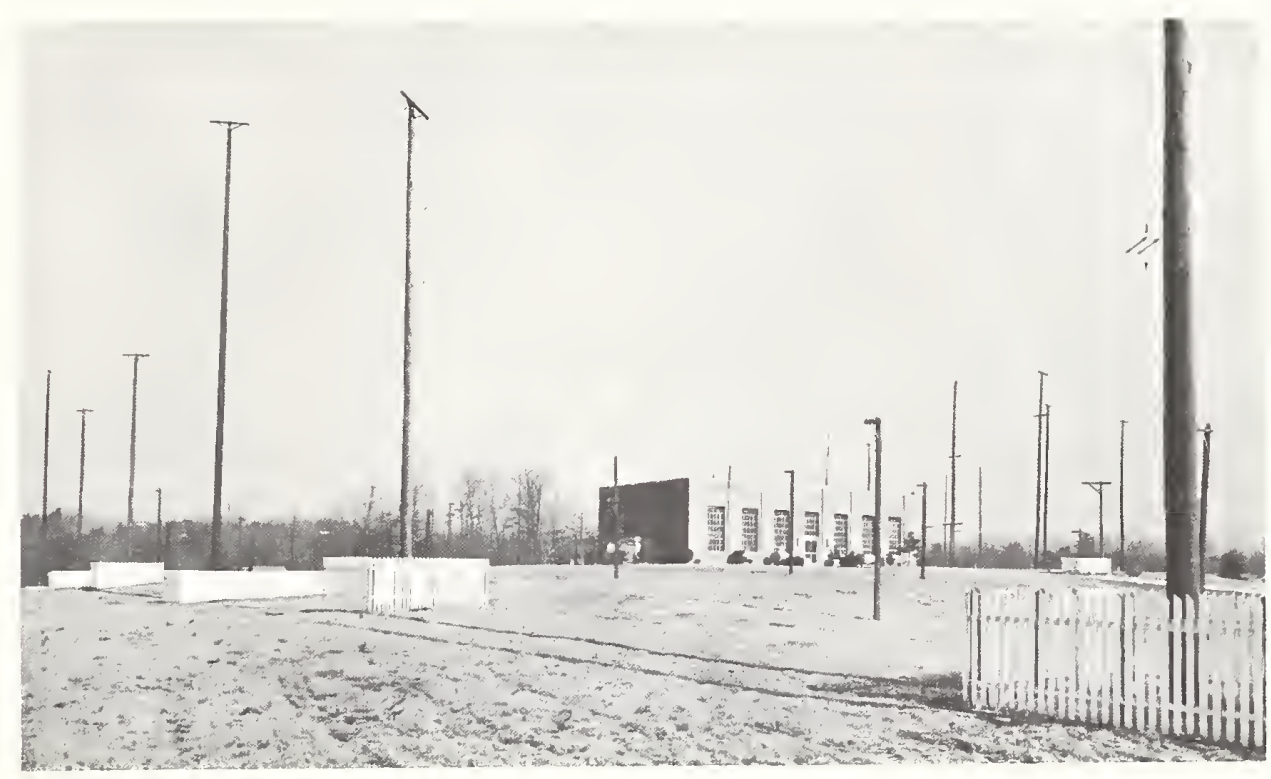

Figure 3. Station $W W V$.

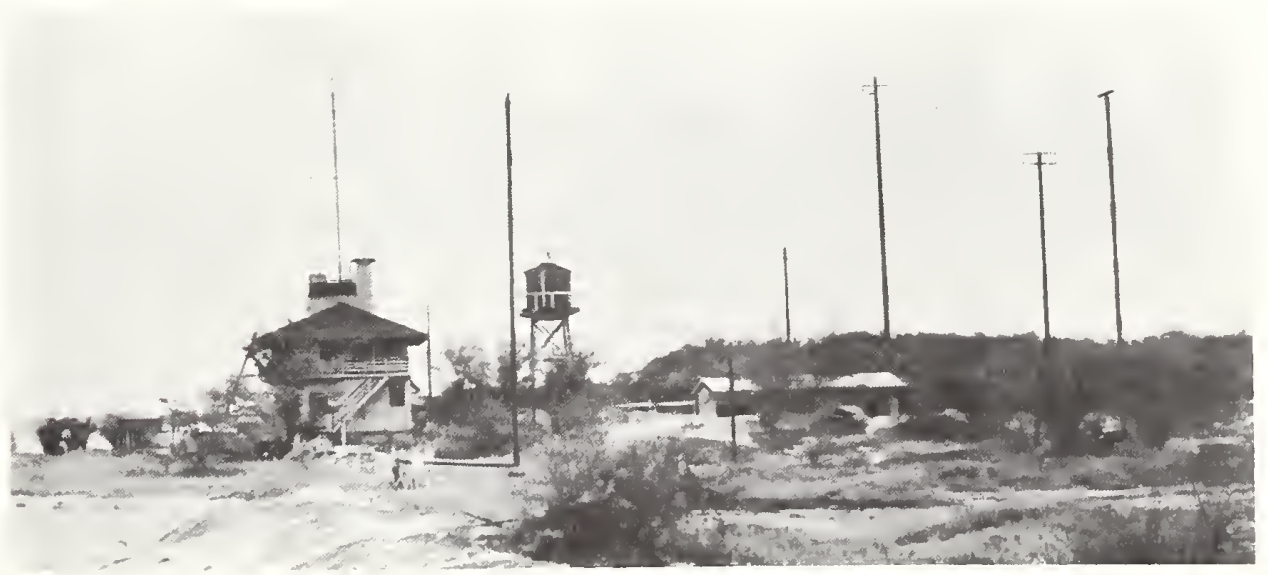

Figure 4. Station WWVH. 


\section{Adjustment of High-Precision Frequency and Time Standards*}

JOHN M. SHAULL $\dagger$

Summary-High-precision frequency and time standards are becoming more and more widely used in many technical fields. The basic equipment used by the Central Radio Propagation Laboratory of the National Bureau of Standards in providing and broadcasting standard frequency and time signals is discussed. Mention is made of the manner in which it is adjusted and the corrections which may be applied to make use of the ultimate accuracy that may be expected in using these signals for measuring and calibrating similar equipment. Several methods are given for checking the frequency of precision oscillators and precision clock performance. Suggestions are given as to methods of recording and evaluating performance data for such standards. Expected improvements in constancy and accuracy and possible future changes in the types of standards used in physical time measurements are considered.

\section{INTRODUCTION}

IGH-PRECISION frequency and time standards are becoming more and more widely used in technical and scientific fields. Among their uses are: control sources for standard-frequency and time broadcasting; time standards for astronomical observatories; synchronization of master and slave stations for pulse navigation systems; investigation of long-distance radio transmission phenomena by pulse techniques; as the heart of frequency synthesizers in large communications systems; and in physical research laboratories for precise calibrations and measurements, such as time-rate phenomena and microwave spectroscopy.

While this paper deals primarily with the adjustment and performance of high-precision frequency and time standards, many of the principles involved and techniques discussed should prove helpful to those using the WWV frequencies or time signals in adjusting or calibrating other high or medium precision equipment.

The accuracies presently required by some users of frequency and time services are approximately as shown in Table I.

\section{TABLE I}

\begin{tabular}{lll}
\hline \hline & $\begin{array}{c}\text { Accu- } \\
\text { racy } \\
\text { Service } \\
\text { quency) }\end{array}$ & $\begin{array}{c}\text { Sec/day } \\
\text { (time) }\end{array}$ \\
\hline $\begin{array}{c}\text { Physical research } \\
\text { Astronomers }\end{array}$ & $1 \times 10^{-8}$ & \pm 0.001 \\
$\begin{array}{c}\text { Monitoring stations } \\
\text { Surveyors }\end{array}$ & $1 \times 10^{-7}$ & \pm 0.01 \\
$\begin{array}{c}\text { Radio broadcasting } \\
\text { Astro-navigators }\end{array}$ & $1 \times 10^{-6}$ & \pm 0.1 \\
$\begin{array}{c}\text { Commercial } \\
\text { communication }\end{array}$ & $1 \times 10^{-5}$ & \\
$\begin{array}{c}\text { Musical instruments } \\
\text { Commercial power } \\
\text { distribution }\end{array}$ & $1 \times 10^{-4}$ & \\
& $1 \times 10^{-3}$ & $( \pm 5)$
\end{tabular}

* Decimal classification: R214. Original manuscript received by the Institute. September 27, 1949. D. C. National Bureau of Standards, Washington,

\section{Equipment and Methods in General Use}

A high-precision frequency standard may be defined as one whose changes in frequency are less than 1 part in $10^{8}$ per day. This degree of precision now requires continuous operation of the standard oscillator, with the control unit and other critical elements operating in a temperature-stabilized conpartment. Very careful shielding, filtering of battery supply leads, and use of buffer amplifier stages are also necessary, especially if more than one oscillator unit is operated at a single location. Such standards now generally employ a GT-cut quartz-crystal operated in a bridge-stabilized oscillator circuit arrangement. ${ }^{2}$

A standard oscilla tor in wide use operates at $100 \mathrm{kc}$ and has a multiposition switch for coarse frequency adjustments of approximately 4 parts in $10^{\circ}$ per step. A precision gear-driven capacitor with a drum dial arrangement of 5,000 dial divisions provides for a control of frequency to approximately 1 part in $10^{9}$ per division. A dial witl such an exp nnded scale is of great advantage in making precise frequency adjustments and interpolations.

A minimum of three standard oscillators is recommended for those installations requiring a reasonable maximum of reliability and continuity of service. By intercomparing three standards locally, either once daily or continuously, short-time stability may be determined to a much higher order than possible through radio transmission comparisons. The most reliable standard may thus be determined and used to supply the desired need, with the other units available for standby duty.

For checking frequencies by the timecomparison method, and for supplying the desired audio frequencies and time intervals, each standard oscillator should be operated continuously, and two or more such oscillators should be provided with frequencydividing and synchronous-clock equipments. The frequency dividers may be pulse counters, fractional frequency generators, locked oscillators, or multivibrators. The choice of type should be influenced by the number of output frequencies and wave form desired, simplicity of adjustment, and reliability of operation required. If seconds intervals are desired for laboratory use, a system similar to that used at IVWVV, wherein the pulse trains are generated electronically and selected mecha nically by a rotating cam, offers certain advantages. An alternate method is to divide the standard frequency down to 1 cps by counter methods, employing an all-electronic system.

A continuously adjustable phase shifter

1W. P. Mason, “A new quartz crystal plate, designated the GT, which produces a very constan requency over a wide temperature range, " Proc. I.R.E., vol. 28 , pp. 220-223; May, 1940.

2 L A Meacham "The bridge stabilized oscillator, Proc. I.R.E., vol. 26, pp. 1278-1294; October, 1938. of the polyphise electrostatic or electromagnetic type is desirable in the divider chain, preferably at the 1,000-cycle stage, to permit the clock or seconds signals to be synchronized. This device also provides a convenient means of measuring small daily differences in time kept by different standard-oscillator clocks, including time signals received by radio. Measurements of time differences may be made by adjusting the phase shifter dial until the pulses coincide on an oscilloscope screen when alternately or simultaneously connected.

Other methods for measuring and recording time differences may employ a spark chronograph, or a polyphase modulator and in tegra ting phasemeter. ${ }^{3}$

A harmonic generator and mixer unit may be conveniently used in rapid frequency intercomparison. This device may be provided with two inputs and means to control the inputs which feed into the crystal diode harmonic generators. The common output is connected to a radio receiver for counting the difierence beats between two local standards. Two frequency multiplier units having outputs of 500 and $2,500 \mathrm{kc}$ or other convenient frequencics, are recommended to facilitate frequency measurements at higher harmonics.

Standard Frequency and Time Broudcasts from $W W V$

The radio and audic frequencies as transmitted from WVWV, near Washington, D. C. ${ }^{4}$ are accurate (with reference to mean solar time) within 1 part in 50 million. The time signals broadcast by IVIVV are maintained in agreement with U. S. Naval Observatory time within several hundredths of a second. This is done by setting the IVWV control oscillator frequency slightly higher or lower than exactly $100 \mathrm{kc}$ by an amount ordinarily not greater than 1 part in $10^{8}$, to advance or retard gradually the broadcast time. For this reason the time broadcast by WWV is uniform, changing by less than 0.001 -second average or 0.002 -second maximum per day. Fig. 1 shows the frequency and time deviations of the WWV transmissions for the years 1947 and 1948 .

The present oscillators at IVIVV have a gradual and fairly constant drift to a higher frequency of from 0.6 to 1.2 parts in $10^{9}$ per day. The control standard at IVWV was adjusted on an average of every twelve days during the past year. Adjustments are usually to a lower frequency but are occasionally made to a higher frequency because of slight discrepancies in the assessment of average drift rate in terms of the Observatory's time determinations. The adjustments

3 W. A. Marrison, "Evolution of quartz crystal clock," Bell Sys. Tech. Jour., vol. 27, pp. 510-588 July, 1948.

Technical radio broadcast services, radio station WWV, "Letter circular LC886, obtainable from Na- 

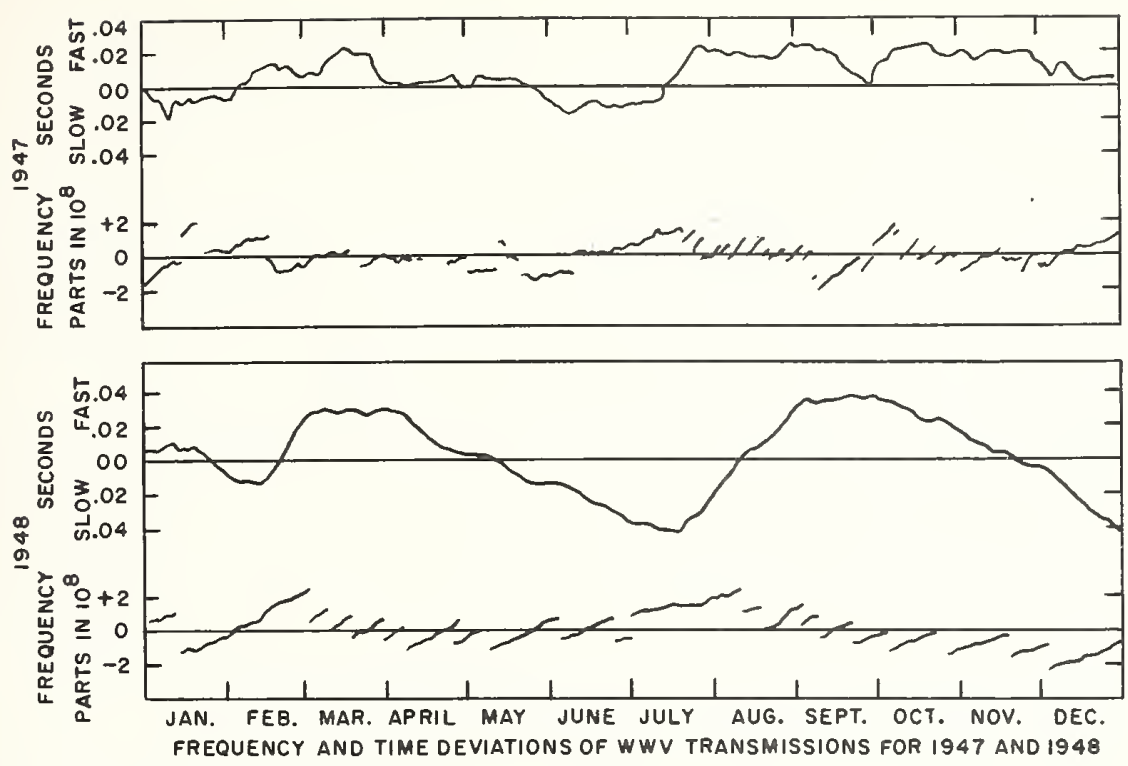

Fig. 1-Frequency and time deviations of WWV transmissions for 1947 and 1948.

are usuallymitde on Fridays between 9 and 10 A.M. ES T but may be made on any day of the week.

A second standard-frequency station WWVH, located in Maui, T. H., has been operating experimentally since November, 1948. The purposes of this station are to learn ways of improving coverage in areas not well served by WWV and to determine the feasibility of operating several standardfrequency stations in different parts of the world on the same frequencies. At present, WWVH provides transmissions on 5,10 , and $15 \mathrm{Mc}$ with carrier powers of 400 watts for each frequency. The carrier frequencies and modulation components are derived from primary frequency standards similar to those at WWV. These oscillators are maintained in agreement with the WWV transmitted frequencies within 2 parts in $10^{8}$ by means of time comparisons over 6-day periods.
The National Primary Standard of Frequency

The present primary standard of frequency consists of three precision frequency standards located at the WWV transmitting site near Greenbelt, Md., and five located at the National Bureau of Standards in the Dis trict of Columbia. These eight standards are automatically compared with each other and with Naval Observatory time determinations. The estimated or daily assigned frequencies, accurate to about 1 part in $10^{8}$, are determined by a weighted extrapolation process in terms of the most reliable standards corrected frequencies are evaluated, over 100-day intervals, in a manner explained under "Interval-derived frequencies" and "Long-interval performance determinations" in terms of the Naval Otservatory time signals for three well-stabilized standard oscillators. The mean of these corrected values applied through the daily beat-frequency comparisons is adopted as the final daily corrected or "absolute" frequency for each standard. This value is obtained some 60 days later and is used as a guide in extrapolating the daily assigned frequencies so as to keep their corrections as small as possible. Agreement of the 100 -day corrected frequencies computed separately in terms of each of the three interval-derived curves is generally within 2 parts in $10^{9}$. The principal limitation on the obtainable accuracy is thus evidently caused by the slight wanderings in the determinations of the earth's mean rate of rotation.

Daily and short-time variations in the individual primary frequency standards are of the order of 1 or 2 parts in $10^{9}$ and 1 or 2 parts in $10^{10}$, respectively. Fig. 2 is a 24 -hour chart recording of the beat frequencies a

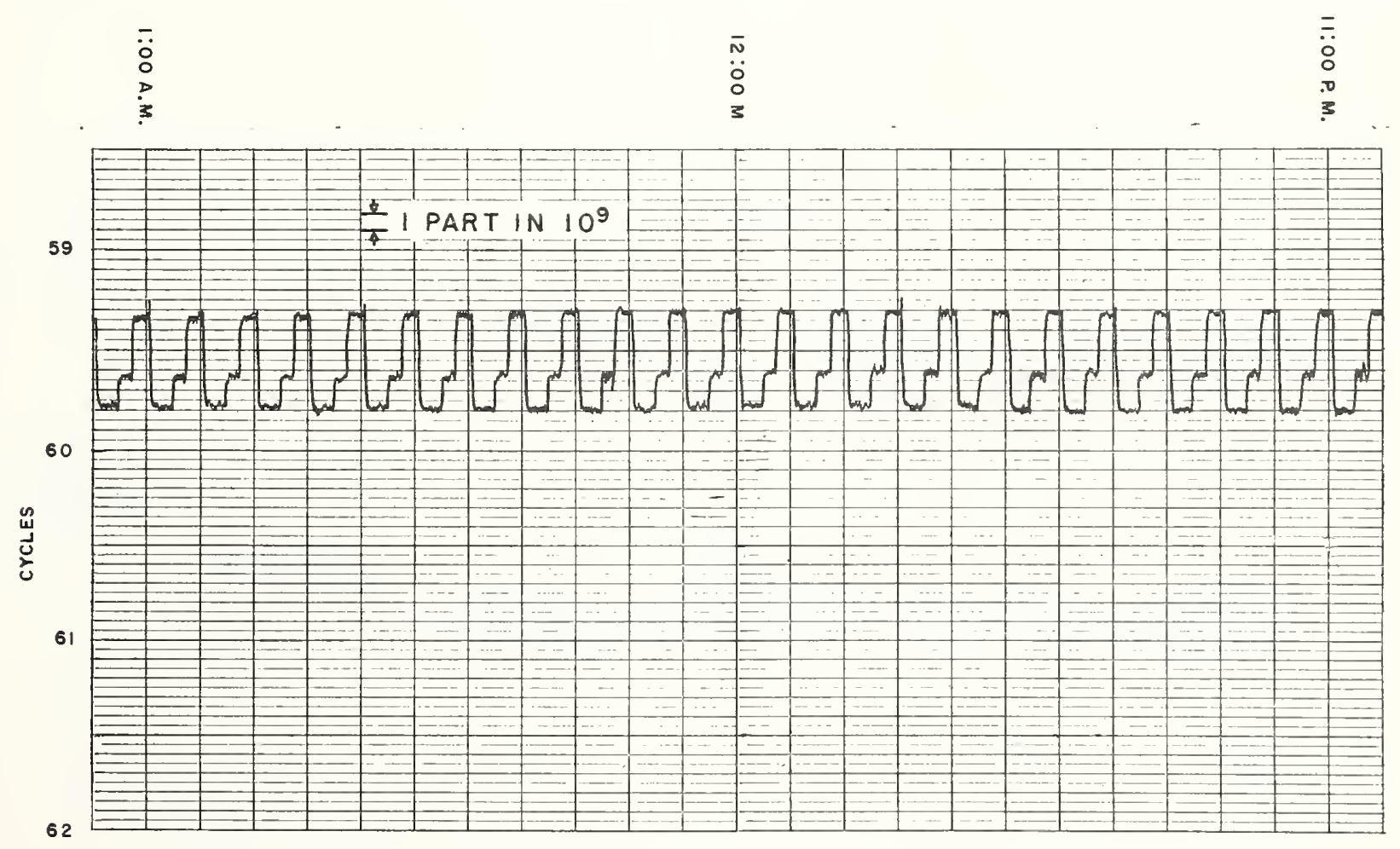

Fig. 2-Chart recording of the beat frequencies at $80 \mathrm{Mc}$ of the three WWV standard oscillators in terms of the monitoring reference oscillator at CRPL. 
80 Mc of the three WWV oscilla tors in terms of the monitoring reference standard, showing the order of these variations. Fig. 3 is a chart recording showing short-time variations in the beat frequency between two standards, on different days, at 80 and 1,280 Mc. The small variations of about 2 parts in $10^{10}$ are attributable to slight $\mathrm{mu}$ tual coupling between the units and to heater thermostat operations. Fig. 4 shows chart plottings of the three WWV standards, primary 25 , primary $W 1$, primary 65 , and the two monitoring reference standards, primary $\mathrm{K} 15$ and primary 35 , for several months.

\section{Measurement of Precision Standard OSCILLATORS BY FREQUENCY COMPARISONS WITH WWV}

For direct frequency measurements, a harmonic of the local frequency standard is compared with the received WWV"signal to determine the difference frequency. Where several standard oscilla tors are available and regularly intercompared, it is necessary to check only one in terms of WWV. Harmonic power from the local standard to the radio receiver should be adjusted to be about equal to the received signal level so as to obtain a maximum modulation or beat. During severe fading, it may be necessary to coun t bea ts that are suppressed but would continue the natural rhythm of those observed. When reception is good, the best results should be obtained by counting beats over a 1- to 2-minute period. When fading is severe, the averaging of a larger number of successful counts of periods of from 10 to 20 seconds may prove most useful.

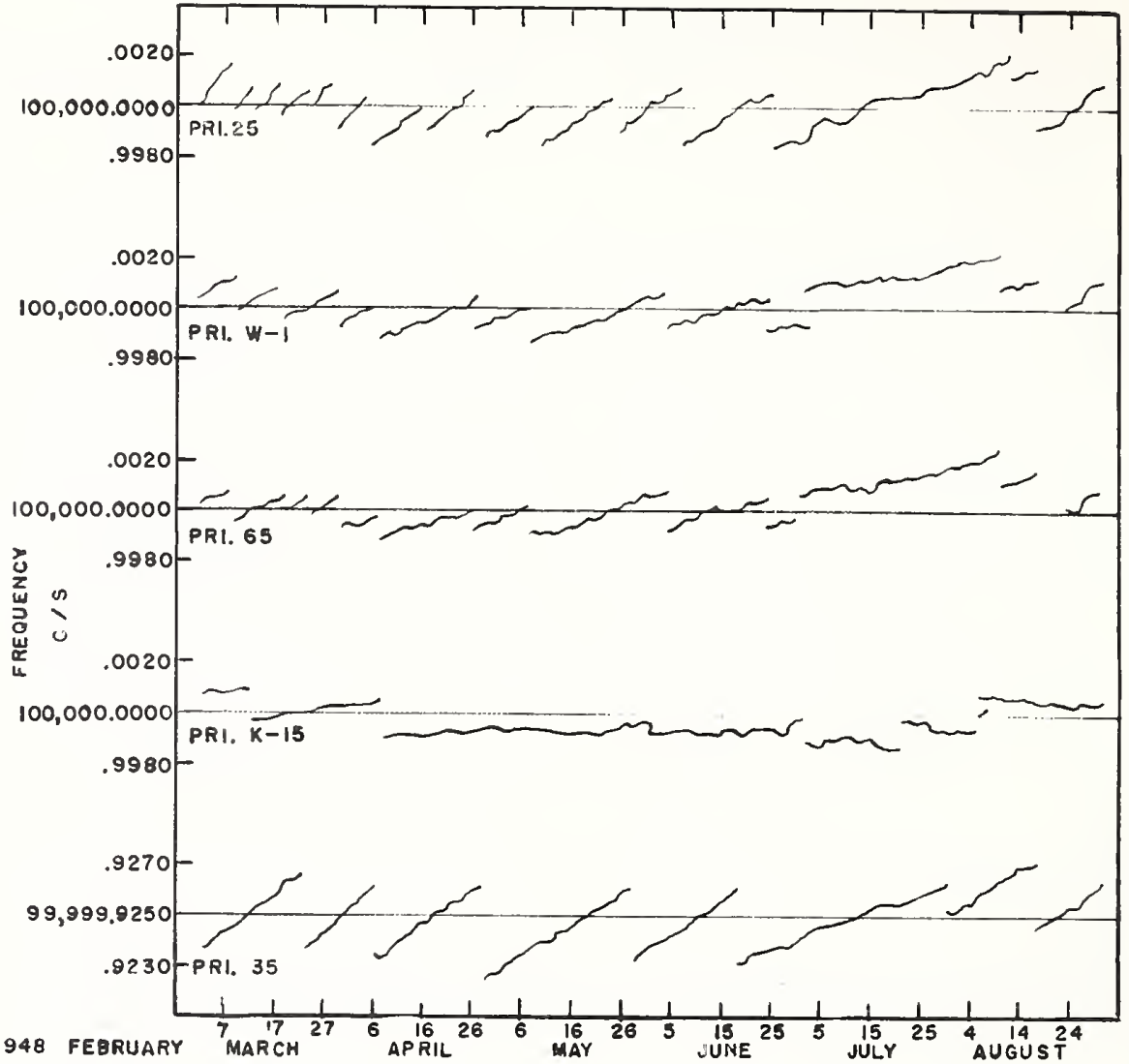

Fig. 4-Chart plottings of the three WWV standard oscillators and the two monitoring reference oscilla tors.

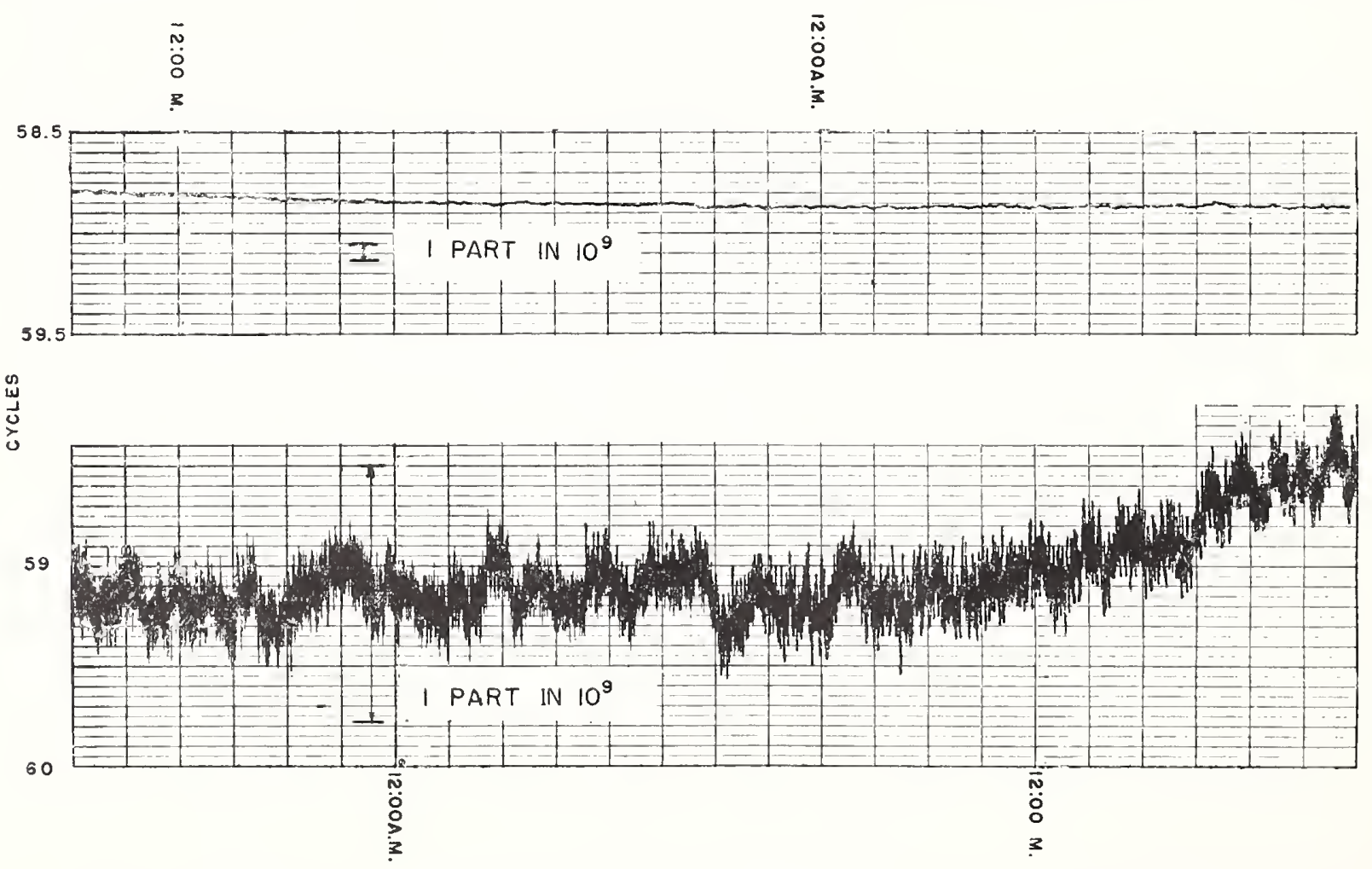

Fig. 3-Chart recording showing short-time variations in the beat frequency between two frequency standards at the. National Bureau of Standards, on different days, at 80 Mc (top) and 1,280 Mc (bottom). 


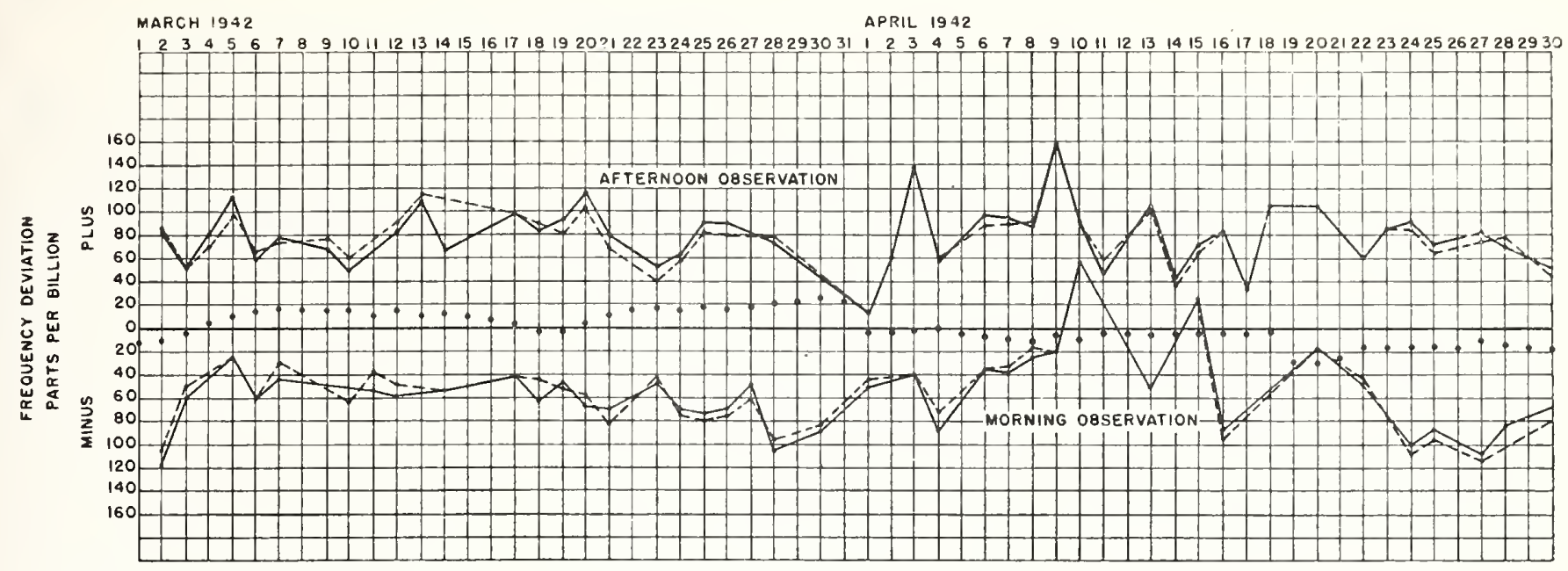

Fig. 5-Illustration of Doppler variation of the WWV received frequencies in terms of two frequency standards at Western Electric's Haw thorne, Chicago, Laboratory. Dotted points are WWV transmitted frequencies.

Experience has shown that it is of ten quite difficult to adjust a local compared oscillator directly to zero beat frequency with WWV at remote locations by either aural or S-meter means. This is generally caused by numerous rapid variations in signal level, or by propagational flutter in the received frequency. Tests at the Na tional Bureau of Standards where a ground-wave signal from WWV prevails, showed no difficulty in reproducing zero-beat settings of an oscillator dial to \pm 1 part in $10^{9}$.

\section{Multiple-Setting Interpolation Method}

Multiple-setting interpolation is best carried out by setting the fine-frequency dial of the compared oscillator slightly off frequency so as to obtain beats with WWV of from 0.1 to 2 beats per second. Several settings should be made on each side of zero beat, choosing values which are readily distinguished from fading, and a number of beat periods measured by means of a stop watch. Values thus obtained are then converted to beat frequencies in cycles per second and are plotted on rectangular co-ordinate paper as ordinates with dial settings as abscissa. By plotting the beat frequencies obtained below zero beat negative and the ones above zero beat positive, and drawing a straight line through the average of these points, an accurate zero beat dial setting will be indicated where this line crosses the $x$ axis. For this method an adjustment dial having good linearity, very little backlash and one dial division equal to about 1 part in $10^{9}$ is required.

\section{Offset Method}

The multiple-setting method is somewhat cumbersome in that it requires a number of separate settings of the dial and computations and involves plotting the data, preferably on rather large graph paper. As the dial setting versus frequency is generally quite linear over a small frequency range, the desired zero-beat setting may be more easily obtained to about the same degree of accuracy by using an offset method. This is done by taking the average of several counts of beats at a single offset point at approximately 1 part in $10^{7}$ from the zero-beat point. The average of these counts in seconds per beat is converted to beats per second which represents the amount the oscilla tor is then off zero beat at the standard frequency to which the receiver is tuned. This is then converted to parts-in- $10^{9}$ deviation from zero beat by dividing the beat in cycles per second by the standard frequency in cycles per second and multiplying by $10^{9}$. To return the oscilla tor to zero beat it is only necessary to advance or retard the dial the indicated number of parts in $10^{9}$ with allowance for the incremental dial correction factor for the region in which it is operating. The dial correction factor (number of parts in $10^{9}$ per dial division) is most readily obtained by comparing the standard with a similar local standard at several dial settings, and thus avoiding radio transmission difficulties and possible errors.

This method has the advantage of requiring only one offset adjustment. With either method the amount of offset should be made small as any adjustments may temporarily disturb the normal drift rate to some ex tent, even though the dial is returned to the original setting. If several units are available in the frequency standard arrangement, the oscillator which is off frequency by a small amount and infrequently adjusted may be used to make the comparison with the transmitted frequency. The others may then be evaluated in terms of this standard so that no changes in the others are necessary. The offset standard should be sufficiently off-frequency so as to be certain that it is higher or lower than the received frequency.

\section{Variations in Received Frequency Arising from the Doppler Effect}

The accuracy of any method using direct comparison of a local frequency standard with a received standard frequency will be affected by fluctuations in transit time of the received signals. This arises from changes in the radio propagation medium during the measurement period, which make the received frequency slightly lower or higher than that transmitted. This well-established Doppler effect could be readily computed for radio propagation if sufficient information on changes in the medium were available. I ts bearing on frequency measurements may be outlined as follow's. The ionospheric reflecting layers vary in height considerably with frequency, time of day, season, geographical location, and phase of sunspot cycle. Average conditions are predictable, but conditions on a given day may depart greatly from the mean. Changes in elfective layer height will cause the frequency. of a received carrier to differ from that transmitted by a fractional amount equal to the rate of change in equivalent path length on kilometers per second divided by the propagation velocity in hilometers per second. For example, if one assumes $F 2$-layer propagation and that the virtual height where reflections occur changes from 300 to $400 \mathrm{~km}$ in 2 hours, then for a "3-hop" transmission from WWV, Washington, to WWVH, Maui, (7,700-km surface distance) an effective change in frequency of the received signal of -3.2 parts in $10^{8}$ for each hop can be computed. On a similar basis the computation for "4-hop" transmission (at a higher angle of departure) gives -3.7 parts in $10^{8}$ per hop so affected. Of course, it is unlikely that changes of this magnitude wil! occur simultaneously at all reflection points of the path considered; thus the error caused by the Doppler elfect will usually be less than the above figures nultiplied by the number of hops.

The WVV frequencies as received at Maui, T. H., in October, 1948, were gener ally slightly higher in the morning and slightly lower in the afternoon. Measure ments based on reception at a time when noon occurred about halfway between transmitter and receiver showed consistent agreement to about 1 part in $10^{8}$, with occasional discrepancies as great as 2 or 3 parts in $10^{8}$.

Observations madein England on IVIVV's $15-\mathrm{Mc}$ received frequencies in December, 1945 , showed errors ranging from 2 to -7 parts in $10^{8}$, with an average variation of -3 
parts in $10^{8}$ when compared with very stable primary frequency standards. ${ }^{5}$ Similar measurements reported by Booth and Gregory showed slightly greater variations. ${ }^{6}$ Fig. 5 shows the variation in IVWV's 5-Mc received frequency at Western Electric's Hawthorne, Chicago, Laboratory in terms of two precision frequency standards. It is evident that high-accuracy measurements of frequency in terms of WWW should be made when ionospheric layer heights are likely to be most stable, i.e., with noon or midnight prevailing at about halfway between transmitter and receiver locations. Long-distance north-south transmission may be expected to show greater variations in the morning and afternoon where all of the reflection points are subject to changing conditions at the same time. Long-distance comparisons should, in most cases, prove most reliable when lower angles and fewer modes of propagation prevail. However, one should particularly avoid use of a frequency and time where a dominant mode of transmission is very near the maximum usable frequency at the propagation angle for any of the reflec. tion points, as the effective layer height changes very rapidly under these conditions. It should be mentioned that errors other than those attributed to the Doppler effect were occasionally found possible.

Publications of the National Bureau of Standards are available which are useful in determining the optimum reception frequencies at a given time and location..$^{7,8}$

\section{Measurement of Precision Standard Oscillators by Daily Time COMPARISONS WITH WIVV}

The average frequency of a standard osciilator may be determined by successively comparing the number of cycles generated (time kept hy its synchronously-operated clock) with any sufficiently reliable time signals. If the standard's drif trate or gradual change in frequency is assumed to be constant, its average frequency during the period will be the same as the instantaneous irequency for the center of the period considered. Departures from a constant or uniform frequency urift can be detected by this method only by successive determinations of frequency, and only then if the reference time source is known to be extremely accurate. When using time signals transmitted by radio over long distances (sky'-wave propagation), slight errors will result because of variations in the time of transmission of as much as several milliseconds under adverse conditions. For this reason frequency determinations based on time comparisons of less than about two days may be in greater error than direct-frequency comparisons with transmitted standard frequencies.

H. V. Griffiths, "Doppler effect in propagation,

Wireless Eng.o vol. 24, pD. 162-167; June, 1947 . pier's principle on the comparison of standard frepier's principle on the comparison of standard reGiencies over a transatlantic radio path, "Post Office lec. Eng. Jour. vol. 40, pp. 153-158; January, 1948. issued June 25,1948 , a vailable from Superintendent of Documents, Government Printing Office, Washington $25, D$. C. (price $\$ 1.00$; foreign $\$ 1.25$ ).

"Basic radio propagation predictions." CRPL-D series (monthly, three months in advance) available on subscription (price $\$ 1.00$ yearly, foreign $\$ 1.25$ ) from Superintendent of Documents. Government Printing Office. Washington 25, D. C.

\section{Interval-Derived Frequency Determinations}

The computation of average frequency by time comparisons may be explained by the following equations:

$$
f_{\mathrm{BV}}=f_{0} \frac{h_{2}-t_{1}}{T_{2}-T_{1}}
$$

where $f_{0}$ is the nominal frequency of the oscillator, i.e., that frequency for which the frequency division ratios were chosen; $t_{1}, l_{2}$ and $T_{1}, T_{2}$ represent the time indicated by the clock, and the correct time represented by the time signals respectively at the beginning and end of the period being averaged.

Equation (1) is usually simplified in actual use by referring all measurements to days or multiples thereof and by using measured errors in time indicated by a crystal clock. For example, the average frequency of a $100-\mathrm{kc}$ standard oscillator during interval $T$ is

$$
f_{\mathrm{BV}}=10^{5}\left(1+\frac{\Delta b_{2}-\Delta l_{1}}{T}\right),
$$

Where $\Delta t_{1}$ and $\Delta l_{2}$ are positive or negative errors in time at beginning and end of the measurement period. The value obtained represents the instantaneous frequency for the center of the period, if uniform drift is assumed, or the value for the entire period if no change in frequency during the entire period is assumed. Instantaneous departures from the average values are generally determined by daily comparisons with other oscillators, involving an averaging based on their predicted daily drifts. This is justified by the probability that the average of the drifts of a number of selected oscillators will be more nearly linear than the drift of any one unit.

Daily measurements of time differences or errors between standard clocks and time signals may be accomplished by adjustment of a calibrated phase shifter dial for pulse coincidence on an oscilloscope screen or by use of calibrated circular or linear sweeps on the oscilloscope. A recording chronograph may also be used to get a continuous record of the time differences of several standard clocks and time signals.

\section{Clock Acceleration}

In order to determine the amount of time a crystal clock will gain or lose over a long period it is necessary to consider the effect of the change in frequency (and thus the change in rate of the clock) over the period. For very long intervals it is even necessary to consider the change in the drift rate of the oscillator (change in the change of rate of the clock).

The time a crystal clock will indicate at some future time $T_{1}$ at $\ell$ day's distant is:

$$
T_{1}=T_{0}+\iota+a l+b t^{2}+c t^{3}
$$

where $a t$ represents the "rate" term, $b t_{2}$ the acceleration term, and $c l^{3}$ the change in acceleration term.

The terms $T_{0}$ and $\ell$ are always positive. Coefficient ' $a$ ' may be positive or negative, and is the principal factor in determining the overall rate of the clock over short periods. Coefficient ' $b$ ' is generally' positive, and must be considered for periods of more than a few day's. Coefficient ' $c$ ' is very small and is generally negative. It may" be neglected in all but very long-time computations.

The above equation may be more readily understood if times $T_{0}, T_{1}, T_{2}$, etc., are considered as marking off an absolute, continuous time scale, with the expression $l+a l+$ $b t^{2}+c t^{3}$ marking off the distance covered by the clock on this time scale in $l$ days. This may be recognized as the well-known equation for linear motion. Usually $l+a t$ is conbined and considered as the velocity term, but for horological purposes it is more convenient to keep these two terms separate. Horologists express the "rate" of a clock as the seconds gained or lost per day.

Fig. 6 shows the computed daily change in time of a crystal clock for a uniform daily change in frequency of 1 part in $10^{9}$ per day. The clocks are usually set slightly fast in such manner as to lose time, and become slightly slow as the oscillator passes through correct frequency. As the oscillator becomes high in frequency, this time is regained to approximately the original setting when the oscillator frequency is about 1 part in $10^{7}$ high. The oscillator is then adjusted low again and the cycle repeated, this adjust-

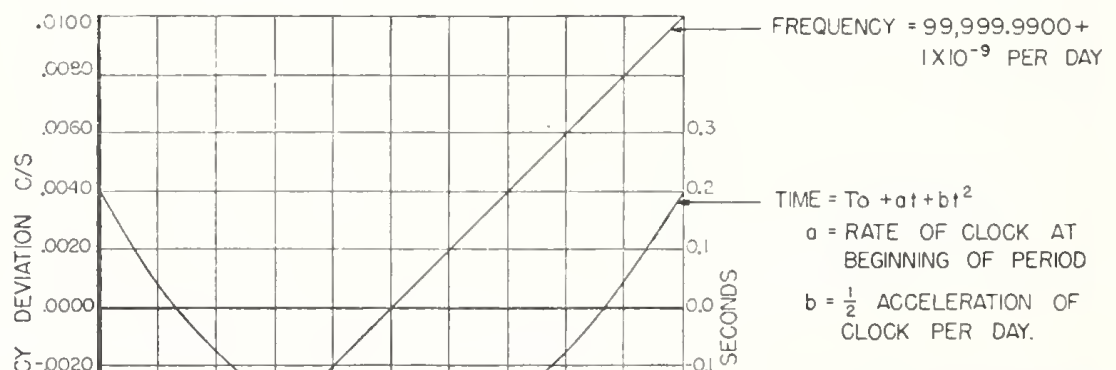

(ACCELERATION = FRACTIONAL DAILY CHANGE IN OSCILLATOR FREQENCY PER DAY.) change of frequency of +1 part in $10^{9}$ 
ment being required about once or twice per year to maintain frequency limits of \pm 1 part in $10^{7}$.

\section{Propagation Delay of Time Signals}

Changes in the height of the ionosphere layers by which time signals or seconds pulses are received, or propagation by different layers or numbers of hops on successive measurement periods will introduce variable delay in the time of reception. The amount of time delay introduced by transmission time may be computed if the mode of transmission is known or assumed. This delay is nearly 1 millisecond per 186 miles of actual transmission path length. The difference in reception time for signals propaga ted by various modes and going only one way around the earth is seldom greater than a few milliseconds. By observing the earliest consistently received pulses over a short period, this may be generally reduced to less than 1 millisecond.

As an illustration of the relative delays to be expected from various modes of propagation, the time lags for a curved earth-ionosphere path for several propagation conditions have been computed. These values for transmission from WWV, Washington, to WWVH, Maui, a great-circle ground-path length of about $7,700 \mathrm{~km}(4,800$ mi) are as shown in Table I. Geometrical one-hop transmission over this path is impossible, and two-hop transmission would be expected to occur less frequently than transmission by the higher modes for this

\section{TABLE I}

\begin{tabular}{ccc}
\hline $\begin{array}{c}\text { No. of } \\
\text { hops }\end{array}$ & $\begin{array}{c}\text { F layer } \\
(210 \mathrm{mi} .)\end{array}$ & $\begin{array}{c}F_{2} \text { layer } \\
(267 \mathrm{mi} .)\end{array}$ \\
\hline & $\begin{array}{c}\text { Milliseconds } \\
\text { delay }\end{array}$ & $\begin{array}{c}\text { Milliseconds } \\
\text { delay }\end{array}$ \\
2 & 26.6 & 27.2 \\
3 & 27.0 & 27.7 \\
4 & 27.9 & 29.1 \\
0 ground path equivalent lag \\
(for comparison purposes) is 25.7
\end{tabular}

path length. If F1-layer transmission is assumed the delay should be generally a little less than for the similar F2-layer mode. These values assume propagation by a number of geometrical hops and normal reflections at the indicated virtual heights. Scattering, interlayer reflections, ionospheric turbulence, and abnormal conditions are of frequent occurrence.

The average variations in delay of the WWV seconds pulses as received at Maui, Hawaii, over a number of one-hour observation periods ranged from 0.1 to 0.2 millisecond for observations on a single frequency, and 0.6 to 1.0 millisecond for observations on the several frequencies receivable at a given time. These variations represent the deviations in a number of loggings of the earliest pulses consistently received over short intervals and not the scatter of multiple pulses received at the same time or consecutively. Variation of transmission delay time of about 2 milliseconds has been reported on high-frequency time signals over the North Atlantic path.

9 C. F. Booth ard F. J. M. Laver, "A standard of frequency and its a oplications,
part III, Jour. IEE, vol. 93 ,

$$
\text { beat } C / S=\frac{\text { number of beats counted }}{\text { number of seconds } \times \text { receiver frequency in } \mathrm{Mc} \times 10^{6}} .
$$

When using the WWV or WWVH seconds pulses at locations where both stations are received, one or the other signal should be selected when making time measurements for frequency determination. This may be done by using a directive receiving antenna or by selecting the proper pulses when making observations. Similarly, at certain locations it is necessary to differentiate between pulses received from both ways around the earth. For example, at Maui pulses are consistently received in the morning from WWV on 15 and $20 \mathrm{Mc}$ by paths going both ways around the earth, with delays of approximately 0.027 and 0.113 second. Quite often, for brief periods, the pulses received over the longer path are stronger than those received over the shorter path because of differences in absor ption. When checking a wellstabilized standard clock, the expected time of arrival will be known to a few milliseconds, which helps to discriminate against untesired signals. As with the frequency comparisons, it is desirable to make these

\section{Local Frequency Intercomparison by Use of Oscilloscope}

The beat frequency between two 100 -kc frequency standards may be readily obtained to a high degree of precision by observing high-order Lissajous patterns on an oscilloscope. This may be conveniently done by connecting the output from a frequency multiplier controlled by one standard to the vertical deflection plates or their wideband amplifier input, and $100 \mathrm{kc}$ from the other standard through the horizontal amplifier to the horizontal deflection plates. The horizontal pattern should be expanded to get sufficient resolution of the multiple pattern. Phase changes, caused by any difference in frequency, will cause the pattern to close and open in continuous sequence. A point near the center of the screen may be chosen where the lines close and the time for an even number of closures measured with a stop watch. is:

The difference frequency in parts in $10^{9}$ measurements when reception conditions are optimum and the ionosphere is stable.

Interval-derived frequency determinations covering periods of 1 or 2 days may not prove much more accurate than those made by direct frequency comparisons under optimum conditions. However, if highly stable oscillators are considered, and the measurement period is increased to about 6 to 10 days, comparison accuracies of a few parts in $10^{9}$ may be obtained.

\section{INTERCOMPARISON OF LOCAL STANDARDS}

When more than one frequency standard is available, it is generally desirable to check each local standard at regular intervals or continuously with the local reference oscillator (one checked in terms of WWV). The beat frequencies between the reference and each of the other oscillators are then determined for plotting purposes at the time chosen for evalua ting the reference oscillator. This may be done by one of the following methods.

Local Frequency Intercomparison by Use of Harmonic Mixer and Receiver

The harmonic mixer unit has already been mentioned in connection with WWV frequency comparisons. Measurements are made by adjusting the $100-\mathrm{kc}$ in put of each source separately by means of the level controls to about S-4 on the radio receiver. Both oscillators are then connected, and the beat counted on the S-meter at any convenient frequency multiple of $100 \mathrm{kc}$. To get the desired accuracy the beats should be counted using a stop watch for a minimum of 2 beats, or a convenient even number of beats which can be counted in about $2 \mathrm{~min}$ utes. The difference frequency in cycles per second at $100 \mathrm{kc}$ is then
A minimum of two closures or a minimum counting time of at least two minutes should be used for the desired accuracy. These observations will not indicate which of the two frequencies is higher. However, by using the frequency from one oscillator to lock in the linear-sweep oscillator in the oscilloscope, observation of the direction of the pattern drift will indicate which of the two frequencies is higher.

Other Precision Frequency Intercomparison Methods

For a complete installacion of standard oscillators, some method should be provided for continuously and automatically recording the difference or beat frequencies between the reference oscilla tor and the others. Several methods will be described, although others may be employed. The direct-reading, or easily computable sensitivity, should be 1 part in $10^{\circ}$ or better. The maximum deviation capability of the recorder need not be greater than a few parts in $10^{8}$. The instrument should be capable of indicating short-interval stability conforming to a sampling or reading completed in a minute or less time.

One very satisfactory but somewhat elaborate method, which is direct-reading and unambiguous with respect to beat sign, uses a commercial power-frequency 60 -cycle recorder to record the beat frequencies at $100 \mathrm{Mc}$. Two $100-\mathrm{kc}$ to $100-\mathrm{Mc}$ frequency multipliers are used. A continuously adjustable phase shif ter of the rotating or electronic type is used to subtract exactly $60 \mathrm{cps}$ (obtained from the standard) from the output of the reference multiplier at $100 \mathrm{Mc}$. The resultant frequency and the output from the other multiplier are supplied to a converter and the difference frequency of approximately $60 \mathrm{cps}$ obtained. The difference fre- 
quency is amplified and supplied to the 58 to $62 \mathrm{cps}$ frequency recorder so that the 60 cycle point represents zero difference frequency. This gives a continuous record of the difference frequency to a sensitivity of a few parts in $10^{10}$ with a range of \pm 2 parts in $10^{8}$. The other oscillators can be switched on successively in rotation to record the deviation of each one in terms of the reference oscillator.

A variation of this method which is currently used at National Bureau of Standards is to set the reference oscilla tor a pproximately 60 parts low in 100 million, which gives a recordable difference frequency without the use of the phase-shifting mechanism. This method has the disadvantage of putting the reference off frequency by an amount making it unusable for many purposes.

The difference frequency between two standard oscillators may be determined by accurately measuring the time required to complete one beat at the fundamental or a multiplied harmonic of each standard. A method for doing this has been described by H. B. Law, and is used by the British Post Office and the National Physical Laboratory for the comparison of precision frequency standards.10 A balanced phase discriminator is supplied with two $100-\mathrm{kc}$ frequencies and arranged to trigger a counter chronometer at the beginning and ending of one beat. The chronometer counts a convenient standard frequency (100 or $10 \mathrm{kc}$ ) and thus indicates the elapsed time of one beat. The instrument accuracy under ideal conditions is estimated to be 1 part in $10^{11}$.

Two electronic counter chronometers may be used in a number of ways to obtain accurate comparison data between standard oscillators. A general method uses one instrument to count the difference or beat frequency between high harmonics of the two compared frequencies, while a second instrument counts cycles of appropriate standard frequency. One unit may be adjusted to serve as a predetermined counter to stop the other unit after a definite count and thus increase the accuracy somewhat. This method of frequency measurement is very flexible and can be used to measure frequencies which fall outside the limits of other very sensitive measurement devices.

A very simple but accurate method of recording low beat frequencies such as obtained from the harmonic mixer and receiver comparison method is to use a recording milliammeter or recording oscillograph. The beats are then evaluated in terms of uniform chart speed or time markers recorded simultaneously. This method offers comparative simplicity for temporary or experimental use, but is quite laborious where a number of readings are required over a long period of time.

\section{Recording and Evaluation of Data}

The amount of statistical data taken on each standard should be kept to the minimum necessary to determine the probable performance of each individual unit over a continuous period. The chief reason for

${ }^{10} \mathrm{H}$. B. Law, "An instrument for short-period frequency comparisons of great accuracy, Jour. IEE vol. 94, part III, pp. 38-41: January, 1947. taking such data on a number of standards is to determine the most suitable one for use, and to determine its short-time stability in terms of the other available standards. When such a standard is used to control standard-frequency transmissions or to make high-precision calibrations, it should be monitored continuously against one or more similar standards.

As a result of experience gained in the operation of frequency-standard oscillators at the National Bureau of Standards and in particular at the WWVH, Maui, T. H., transmitting station, it may be helpful to outline maintenance, procedures and techniques that have been developed and found useful in this work.

\section{Logging and Plotting of Data}

At the NBS it has been found necessary to keep a daily record which includes all frequency and time readings and measurements, as well as the final results of all computations of frequency and time. Changes in control standard, methods, or equipment are always noted. These data are conveniently kept in a record book having a number of ruled columns, or on specially prepared mineographed sheets carrying the desired notations for a particular installation.

The daily computed frequencies for each. oscillator are plotted on continuous crosssection paper in a manner as shown in Fig. 4. These data are of considerable aid in studying the relative performance of the individual standards, and in graphically extrapolating predicted values during periods when reception conditions do not permit making the daily frequency or time checks.

It has been found desirable to keep periodic (weekly) instrument readings which include the readings of all battery chargers and power supplies, voltmeter readings on each switch position for the frequency standards and frequency dividers, temperature readings of each standard's oven where applicable, and room temperature at time of readings. These readings are useful in anticipating tube or equipment failure in that adjustment or repair can of ten be made before complete breakdown occurs.

\section{Adjustments in Frequency and Time}

Periodic adjustments in frequency and time of each standard oscillator and time equipment are required to keep within the desired accuracy tolerance and to simplify the measuring and plotting requirements. The frequency and range of these adjustments are influenced considerably by the type of service and standard. For greatest constancy of drift rate and greatest ease in computation, frequency adjustments should be held to a minimum. For many uses the calculation of and allowance for the slight error in frequency or time presents no great difficulty. In other cases, where numerous measurements are being made and services given, it is helpful to establish high initial accuracy so as to eliminate corrections.

Where the working standard (one used or distributed) is held to very close accuracy tolerance, the continuity of service required will determine if the second or standby standard need be held to a similar close toler- ance. If continuity of service is only of moderate importance, two equipments may be infrequently adjusted and only the working unit held within very precise limits. The oscillator and time equipment least adjusted should be generally used as the reference or one by which frequency and time determinations are made with respect to WWV. For most purposes an adjustment of the working standard so as to hold its frequency within 1 or 2 parts in $10^{8}$ of WWV's average frequency should prove satisfactory, with the standby and spare oscilla tors being held to a frequency tolerance of 1 part in $10^{7}$.

Daily phase shifter (time) adjustments may be made if time synchronization is desired, and these daily changes used in computing interval-derived frequencies. Allowance should be made for transmission time lag in setting or using the local time pulses, if extremely precise time synchronization is important.

\section{Short-Interval Performance}

High-precision standard-frequency oscillators now in use have short-period stabilities (intervals of one minute or so) ranging from 1 part in $10^{9}$ to 1 part in $10^{10}$. In addition to the gradual ageing or drift various causes will contribute to these shorttime frequency fluctuations. Mechanical shock may cause an instantaneous effect through easing of stresses or displacements in the crystal unit or associated components. A slow recovery in frequency may or may rot take place. A change in supply voltage produces an immediate effect followed by additional changes as thermal equilibrium is restored. Changes in load impedance will cause frequency variations unless proper decoupling is employed. Unless proper thermal lagging is provided for the crystal compartment cyclic operations of its hea ters will a lso ca use varia tions in the output frequency. The oscillator circuits must be protected from direct or electromagnetic mutual coupling to better than $100 \mathrm{db}$ to reduce frequency "pulling" or tendency to synchronize. Other changes in frequency, of ten of unpredictable source, may be caused by imperfect connections, faulty components or erratic vacuum tubes. These causes of poor frequency stability can generally be elimina ted only by a slow process of substitution and observation.

Where average frequencies are computed for periods of more than a few days, the daily values may be determined by calculating the departure of the reference standard from its average curve in terms of the other reliable standards available. The daily values for the other standards may then be determined by adding the daily beat frequencies for each standard to the computed value for the reference. The mean values of relative frequencies thus obtained represent a weighted extrapolation in terms of the selected group of reliable standards. Considerations involving three independent reliable standards should give a relative daily accuracy within 2 parts in $10^{9}$. The inclusion of six or more standards, as is done in the CRPL primary standard of frequency, results in a relative daily determination of performance of each standard oscillator to within 1 part in $10^{9}$. Very-short period or 
"instantaneous" stability may be determined by intercomparing pairs of standard oscillators by high-precision automatic recording equipment.

\section{Long-Interval Performance Determinations}

The determination of deviations over periods of months and years is complicated and obscured by the fact that the earth's mean rate of rotation (even after application of a number of established corrections) is not uniform. Changes in the length of the a pparent day as large as 4 or 5 milliseconds, equivalent to a frequency change of about 1 part in 20 million, are believed to have occurred on several occasions within the last half-century. These changes occur at rather irregular intervals and in varying amounts and are, as yet, unpredictable. They are evidenced by an accumulation of error in the earth's observed angular position compared with theoretically predictable astronomical events.

Smaller variations, with periods of several weeks to slightly more than a year, are noted when comparing the earth's observed time with high-precision crystal clocks. Until very recently these variations were attributed almost entirely to clock discrepancies. Agreement of a number of precision clocks and technical improvements in methods of observing and recording star transits indicate that the earth's rate of rotation is subject to a number of more or less random small variations which add up to as much as several milliseconds per day at times. The probable error of a single time determination in terms of a number of star sights is believed to be not greater than several milliseconds and such errors are not cumulative. Some of the time variations are caused by rather irregular wanderings of the earth's poles by as much as 30 feet with a principal component having a period of slightly more than a year. Observational errors, caused by this variation in longitude (which would be zero at the equator) amount to as much as \pm 20 and 30 milliseconds at the Washington and Greenwich observatories, respectively. These variations may be corrected by applying results of observations of latitude variation at a number of locations and computing the equivalent changes in longitude. The intricacies of time determination in terms of the earth's rate and the conversion from observed sidereal to mean solar time have been discussed by H. Spencer Jones, British Astronomer Royal. ${ }^{11}$

Another factor, although of no immediate concern in determining frequency, is the gradual slowing down of the earth's rate of rotation, chiefly because of tidal friction. This deceleration over the past 2,000 years averages about 0.0016 second per day per century, which amounts to an accumulated time difference of $29 \mathrm{~T}^{2}$ seconds for a mean solar clock considered as having zero rate at 1900 A.D., where $T$ is the number of centuries from $1900 . .^{12}$ At present the deceleration is estimated at approxima tely 0.001 second/

11 H. Spencer Jones, “The measurement of time," Endeavour, vol. 4, pp. 123-130; October, 1945. $12 \mathrm{G} . \mathrm{M}$. Clemence, "On the system of astronomical
constants, May. 1948.

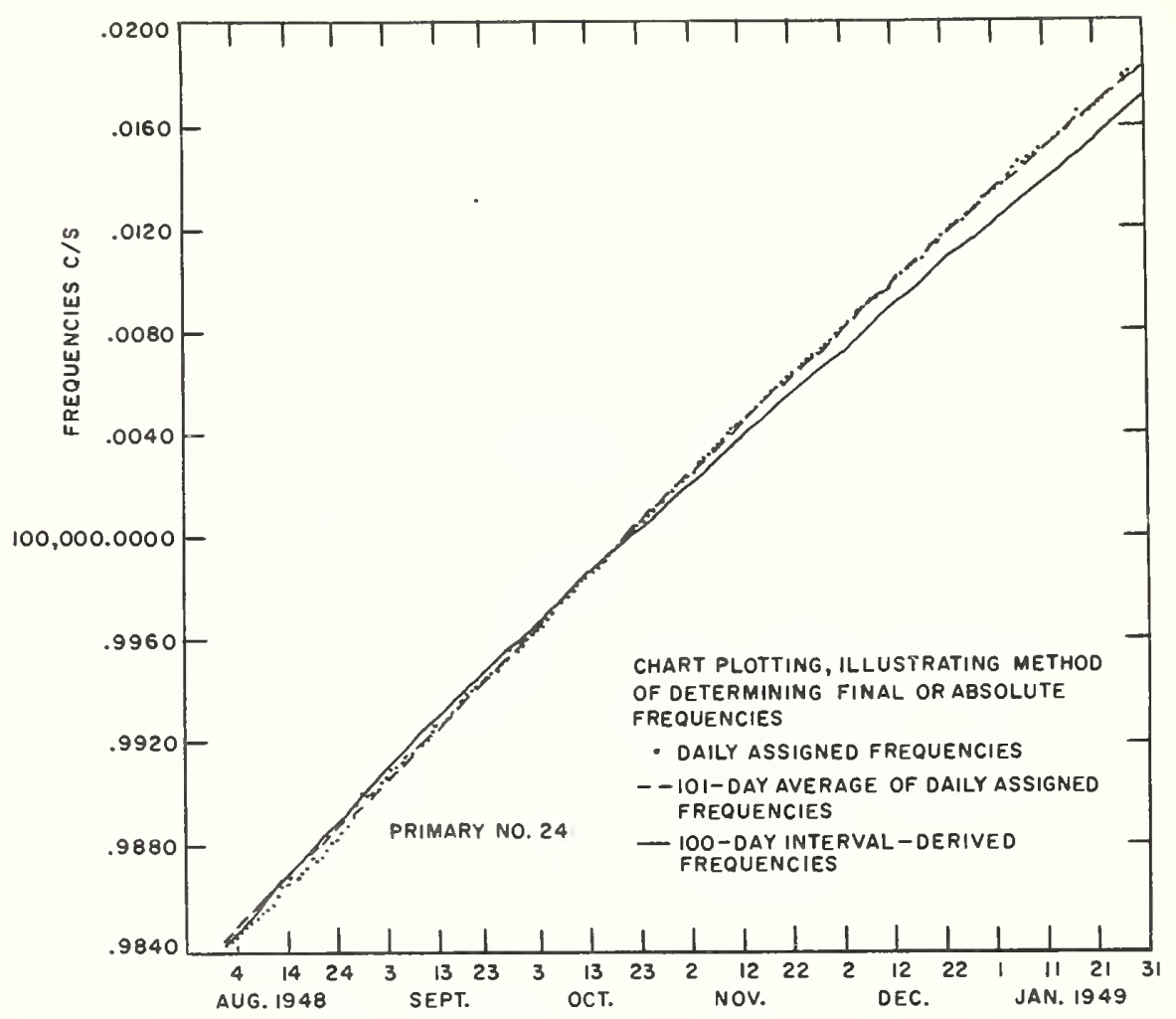

Fig. 7-Chart plottings illustrating graphical method of determining final or absolute frequencies.

day/century, which amounts to an average apparent increase in frequency of about 1 part in $10^{8}$ per century for an absolutely constant oscillator if measured in terms of the earth's rate of rotation.

The GT wire-supported crystals of the type widely used at present have initial drif $t$ rates of 2 to 4 parts in $10^{8}$ per day for unaged units, and 1 to 2 parts in $10^{9}$ per day for units a year old. The drift varies approximately inversely with time for several years, and may thus be predicted and allowances made accordingly.

In the primary standard of frequency of the National Bureau of Standards, three well-stabilized oscillators are evalua ted over 100 -day periods in terms of Naval Observatory time corrections. This is done as shown in Fig. 7. The dotted points for each day represent the departures from the average curve in terms of the entire group of selected standards as explained previously. The dashed curve represents the average of 101 daily values including 50 before and 50 after the day plotted. These are computed each 10 days and connected by a smooth curve. The solid curve represen ts the 100 -day interval-derived average frequency computed for each day. The final corrected frequency, for this standard only, is then the daily plotted value of the interval derived (solid) curve plus the algebraic departure of the daily assigned (dotted point) value from the daily average (dashed) curve. A displacement of more than 1 part in $10^{8}$ between these two curves indicates that the daily drift rates should be reassessed and the dotted points replotted to bring the two curves more nearly into agreement.
For a 100 -day averaging period the daily assigned curves must thus be extrapolated or guided on the basis of past performance for about 60 days in advance of the established average curves. For this reason, a temporary 20-day interval-derived curve is computed as an aid in systematically determining the daily assigned values. Users of the WWV time signal transmissions in the field for average frequency determinations thus have the advantage of the long averaging period applied to the WWV frequency determinations. They may, therefore, approach the full limit of accuracy (1 part in 50 million) by using these signals over intervals of 6 to 10 days to compute average frequencies without knowledge of the time or extent of WWV frequency adjustments. If these adjustment data are known, accuracies several times this order are generally possible.

The corrected daily frequency for the reference oscillator is computed in terms of each of these three oscillators so evaluated by adding algebraically the respective daily beat differences to their corrected values. The mean of these computed frequencies is then taken as the final or "absolute" value of the reference for each day. Should one of the three. values disagree excessively with the other two for explainable reasons, only two values are averaged. The values so computed generally agree within 1 or 2 parts in $10^{9}$, which represents the residual error in the graphical method and the unpredictable random deviation of the daily assigned sampled values from the daily mean frequencies. The daily "absolute" frequencies for each of the other standards are obtained by algebraically adding their daily beat differences 


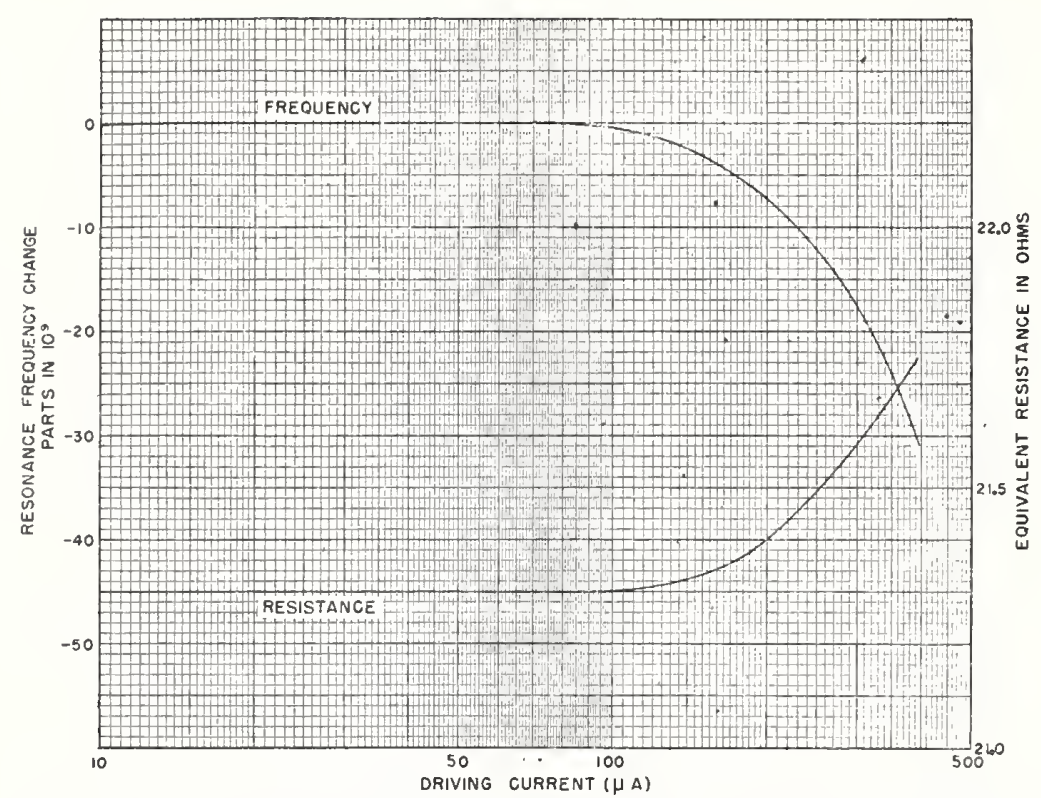

Fig. 8-Graphs showing variations of resonance frequency and series resistance at resonance with changes in driving current for a 100-kc GT-cut quartz-crystal unit.

to the daily "absolute" frequency of the reference standard.

No great difficulty has been experienced in predicting the "absolute" value of frequency for the primary standard in terms of the earth's mean rate of rotation over 100 day in tervals to 1 part in $10^{8}$. The mean solar second, which is by definition the physical standard of time, may be expected to change occasionally by as much as \pm 4 parts in $10^{8}$ in addition to a gradual average change of about 1 part in $10^{8}$ per century. Frequency which is defined in terms of the mean solar second will, of course, deviate from a constant value by a corresponding a mount.

\section{Prospective Improvements in Quartz-Crystal Frequency Standards}

Quartz-crystal units, measured as resonators in a balanced bridge network, have been compared with the primary standard of frequency with a precision of 1 part in $10^{9}$. in this manner, crystal units most suitable for use in future standard oscilla tors are currently selected and studied. The use of crystal-unit resonators as primary frequency reference standards has been tried in this manner to some extent and is being further investigated. This method of use eliminates the variations arising from aging or detuning of tubes and circuit components. It has been found that the crystals generally drif ted about as rapidly in a nonoscillating condition as they do in continuously oscillating frequency standards. Fig. 8 shows the results of bridge measurements on a typical GT-cut, wire-mounted crystal-unit for variation in excitation current. It may be noted that the frequency decreases and the resistance increases slightly with increases in excitation beyond about 100 microamperes through the crystal-unit at resonance. All crystal oscillator standards operating at present at The National Bureau of Standards are working at amplitudes of 500 microamperes or more, which is slightly beyond the maximum shown on the graph. Attempts to operate with appreciably lower amplitudes, by adjusting the bridge arms to closer limits, have resulted in increased short-time instability although the curves indicate greater stability should result. Extreme care in shielding, decoupling, and attention to the reduction of tube and circuit noise may be necessary to gain improvement in this manner.

Ability to operate the amplifier-bridge loop at a higher stable amplification ratio may be made possible by supplying the thermistor element for amplitude stabiliza. tion with direct current obtained from rectified and filtered oscillator output. This may also allow operation of the crystal unit at nearer its natural $Q$ value. The present standards have crystal units with $Q$ values of about 200,000 and operating $Q$ values of about one-half this amount. This gives a bandwidth between half-power points of about $1 \mathrm{cps}$ at $100 \mathrm{kc}$. The short-period stability of about 1 part in $10^{10}$ obtained with several of the standard oscillators means that they hold the frequency constant to about $1 / 100,000$ of the half-power bandwidth. Current development work on improving crystal-units shows promise of greatly reducing the initial aging or drif ting in frequency and of obtaining an operating $Q$ factor of one million or greater.

The use of direct polarizing voltage across the crystal electrodes as a means of fine frequency adjustment may be advantageous in certain cases. A change in frequency of about 1 part in $10^{8}$ per volt occurs for the present type $100-\mathrm{kc}$ units. This change in frequency is very linear over ranges of several hundred volts of either polarity withou t apparent loss of $Q$. It offers an extremely sensitive control method for interpolation or servo applications without the problems that arise from backlash and wear.

Improved temperature control of the crystal inclosure over long periods is needed. A temperature-control method using dual resistance-bridge thermostats is planned for use in several new standard oscillators. The possibility of using magnetic amplifiers in this application to eliminate tube failures is being investigated.

\section{Absolute Frequency and Time Standards}

It is now generally accepted that the earth's mean rotation period is neither absolutely constant nor is it predictable with desirable accuracy over short or long periods. Its long-time period is increasing by an amount sufficient to be slightly disturbing to both physicists and astronomers in this era of high precision measurements.

Astronomers are using what is termed Newtonian time. ${ }^{13}$ This time is consistent with Newton's laws of motion (with slight modifications for relativity) when applied to the movement of astronomical bodies. So far as has been determined, intervals in Newtonian time are invariable.

The Newtonian second for astronomical purposes has been defined as equal to a mean solar second at 1900 A.D. Astronomical events in the distant past are computable in Newtonian time by applying the proper corrections, based on the earth's known variable rate through the period considered. Future corrections, while not accurately predictable, can be observed and adopted as time progresses. The sidereal year (average period of the earth's revolution around the sun) is believed by astronomers to be a better unit of time. With present techniques it is difficult to determine the period of a single sidereal year to the desired accuracy. It has been estimated that in an interval of 100 years a mean value good to 1 part in $10^{9}$ could be established. To subdivide this long interval in to useful physical time units imposes extremely stringent requirements on a standard clock.

Clocks have continued to be improved since the discovery of the escapement mechanism about 1360 A.D., reaching their high degree of dependability in the presentday precision quartz clocks which permit a predictable daily constancy of considerably better than 0.001 second per day. They have an attainable precision of about 10 times that of the best mechanical clocks and are the most precise timekeepers now available, exceeding the constancy of the determination of the earth's rate for periods up to several months. The quartz clock, however, must be set in terms of the earth's mean rate, as it in no way constitutes an absolute standard in itself.

In recent years, aided by the rapid development of microwave techniques, considerable attention has been directed toward use of a tomic resonance effects at microwave frequencies..$^{13}$

The first atomic clock was built at the National Bureau of Standards in 1948. ${ }^{14}$ This clock makes use of the sharp absorption line of ammonia gas at $23,870.1 \mathrm{Mc}$ to maintain a $100-k c$ crystal oscillator at constant frequency. This is done by means of an electronic servosystem consisting of frequency

${ }^{13} \mathrm{~W}$. D. Hershberger and L. E. Norton, " $\mathrm{Fre}$ quency stabilization with microwave spectral lines, CA Rev., vol. 9, pp. 38-49; March, 1948

33. $0.17-24 ;$ ic clock, 
multipliers, auxiliary frequency-modulated search oscillator, and pulse discriminator circuits. The $100 \mathrm{kc}$ is then divided down to audio frequencies in conventional manner and used to operate a synchronous-motor clock as a time standard. A constancy in frequency of 5 parts in $10^{8}$ has been obtained for periods of several days with this experimental clock when compared with WWV frequency standards. Improvement of this type of standard and the development of more constant types of atomic resonance controlled oscillators are to be expected.

It is shown in the references cited that the sharpness of resonance within individual oscillating molecules is extremely great. Because of collisions of molecules with each other and with the gas cell walls, and the Doppler broadening attributable to natural thermal agitation, the practical working $Q$ of an a mmonia gas absorption line ranges between 50,000 and 500,000. This compares favorably with the $Q$ of quartz crystals used in frequency standards which ranges from 100,000 to $1,000,000$. It is thus reasonable to hope that a constancy of 1 part in $10^{8}$ to 1 part in $10^{9}$ may be obtained by proper refinements in circuitry and technique. Whether or not this degree of constancy of absolute value can be maintained without or even with precise temperature and pressure regulation remains to be investiga ted.

Civil time will, no doubt, continue to be defined in terms of the mean solar second, as would ordinary frequency desiguations. After about 2,000 years, if the earth continues to slow down at its present rate, the mean solar second would be about 1 part in 3 million longer than at present and the accumulated time difference between mean solar time and Newtonian time would amount to about three hours.

\section{Conclusions}

Frequency and time standards, using high-precision quartz crystals, are now available which are capable of supplying frequencies and time intervals constant to considerably better than 1 part in $10^{8}$ per day. In order to achieve an accuracy approaching this order, these standards must be frequently checked in terms of standard frequency or time broadcasts. An accuracy of 1 part in $10^{8}$ represents about the limit obtainable in terms of the earth's mean rate of rotation over a 100-day period. Longer periods of averaging can not be expected to give greatly improved accuracy, because of the possibility of slight oscillator frequency deviations and uncertainties in the uniformity of the determinations of the earth's mean rate.

By using ordinary zero beating methods, a remote frequency standard may be adjusted within 1 part in $10^{7}$ to IVIVV's received frequency. Special offset techniques permit this setting error to be reduced to less than 1 part in $10^{8}$. Changes in the radio propagation medium may cause the received frequency to differ from that transmitted by as much as several parts in $10^{7}$. By averaging a number of determinations made when noon or midnight prevails about half way between transmitter and receiver, long-distance frequency comparisons can generally be made with a precision of better than 1 part in $10^{8}$

The intercomparison of two remote oscillators, constant to 1 or 2 parts in $10^{9}$ per day, by means of transmitted time pulses from one or both of the standards, is possible to a precision of a few parts in $10^{\circ}$ through comparisons of average frequencies over periods of 6 or more days.

The development of a tomic or molecularresonance standards of high constancy and absolute accuracy may greatly simplify the maintenance of precise frequency and time standards. The practical realization of such standards, which seems reasonably probable in the near future, will eliminate the necessity of making highly precise physical measurements in terms of the earth's variable rate of rotation. Such a standard would supply a means of studying more precisely the motions of the earth and other astronomical bodies.

Considerable work is being done to $\mathrm{im}$ prove the constancy of quartz-crystal frequency standards, especially with regard to aging or frequency drift and improved temperature control methods.

It is probable that frequency and time standards, which have improved by a factor of ten or more per decade in the last thirty years, will continue to reach new orders of accuracy and constancy. However, these improvements in accuracy will probably be referred to a new kind of standard, rather than to the mean solar second.

\section{ACKNOWLEDGMENTS}

The author wishes to acknowledge his indebtedness for the valuable advice and assistance of W. D. George, Chief of the HighFrequency Standards Section of the National Bureau of Standards, and the many suggestions of V. E. Heaton and E. L. Hall of the same organization.

Reprinted from the PROCEEDINGS OF THE I.R.E. VOL. 38, NO. 1, JANUARY, 1950

PRINTED IN THE U.S.A. 


\title{
Accurate Microwave Wavemeters with Convenient Calibration Tables
}

\author{
H. E. Bussey ANd A. J. Estin \\ National Bureau of Standards, Boulder, Colorado \\ (Received December 3, 1959; and in final form, February 11, 1960)
}

\begin{abstract}
Accurate and convenient microwave cavity wavemeters are described that are suitable for many precise physical measurements. High $Q$ 's were attained by refined construction techniques. At $9000 \mathrm{Mc}$ a precision of $0.02 \mathrm{Mc}$ was obtained. The absolute accuracy also may be very high after strains in the metal have stabilized. A calibration table containing $10^{4}$ entries, easily formed by means of a high speed computer, makes the wavemeters convenient to use. The curve fitting method, accurate to one in $10^{6}$, is described.
\end{abstract}

\section{INTRODUCTION}

$\mathrm{T}$ HE primary function of a wavemeter is to determine the frequency of an electromagnetic wave. Its design can range from that of a coarse search meter to that described here, which is suitable for precise and accurate physical measurements. ${ }^{1}$ The precise determination of frequency differences is of paramount importance in many scientific experiments. For example, the present wavemeters have been used to observe dielectric, magnetic, and other cavity perturbations, and they have been very valuable. They have conveniently replaced more complicated methods of frequency measurements that use heterodyning and frequency multiplication. The precision achieved with the wavemeters is 1 or 2 parts in $10^{6}$, as estimated both from routine use and from the repeatability of settings during original calibration. The absolute accuracy depends of course on the long time mechanical stability, the atmospheric conditions, and the VSWR of the waveguide arms. It was found, for example, that after a year one wavemeter had changed frequency by $0.25 \mathrm{Mc}$ out of $9000 \mathrm{Mc}$. This change was probably due to the relief of strains. It is expected that henceforth the accuracy will be a few parts per million, provided atmospheric corrections are made.

This paper summarizes the principles used in the design of these instruments. The chief features of an accurate wavemeter are a fine micrometer for tuning, an accurate calibration, a convenient and accurate calibration table, a high $Q$, the temperature compensation scheme, and the transmission or absorption coefficient. These will be discussed.

TABLE I.

\begin{tabular}{|c|c|c|c|c|c|c|c|}
\hline Cavity & $\begin{array}{l}\text { Frer. } \\
\text { Mc }\end{array}$ & $\begin{array}{l}\text { Diam } \\
\text { in. }\end{array}$ & $\begin{array}{l}L^{a} \\
\text { in. }\end{array}$ & $\begin{array}{l}2 a \\
\text { in. }\end{array}$ & $R$ & $\begin{array}{c}T \\
\mathrm{db}\end{array}$ & ()$_{1}$ \\
\hline$A$ & $8650-10000$ & 1.88 & $0.8-1.5$ & $0.210^{1}$ & $81 \%$ & 12 & $190(x)$ \\
\hline B & $8500-9500$ & 1.79 & $1.17-2.17$ & $0.201^{\mathrm{b}}$ & 85 & 16 & 230000 \\
\hline $\mathrm{C}$ & $2800-3200$ & 6.42 & $2.5-3.5$ & $\ldots$ & $83 \%$ & 15 & 33000 \\
\hline
\end{tabular}

a Definitions: $L$ is the length; $2 a$ is the iris diameter: $R$ is the ratio of the observed unloaded $O$ to the theoretical $O$ expected from silver with a resistivity of $1.63 \times 10^{-6} \mathrm{ohm} \mathrm{cm} ; T$ is the transmission loss through the resonator. $O_{L}$ is the observed loaded $O$.

$b$ The thickness at the irises was $0.030 \mathrm{in}$

${ }^{1}$ For another paper on accurate wavemetcrs see J. C. Van Den Bosch and F. Bruin, Physica 19, 705 (1953).
The just-mentioned factors are concerned with the cavity itself. The precision may, however, be limited by noise rather than by the sharpness of the response $(Q)$ or the mechanical precision. A discussion of how signal-tonoise ratio limits the ultimate precision of a wavemeter is an important factor in designing a wavemeter and in choosing the coupling factor for optimum performance. An investigation of this subject is in progress.

\section{DESCRIPTION OF WAVEMETERS}

A good choice for the mode of oscillation of a wavemeter is the $\mathrm{TE}_{01 n}$ mode of a right circular cylinder, as was indicated by Wilson, Kinzer, and Schramm. ${ }^{2}$ The wavemeters described here operate in the $\mathrm{TE}_{011}$ mode. All were transmission type cavities. Table I summarizes several characteristics of these wavemeters, designated as $\mathrm{A}, \mathrm{B}$, and $\mathrm{C}$, respectively. Figure 1 is iepresentative of cavities $A$ and $B$. Figure 2 gives dimersions for cavity $\mathrm{B}$, but the perturbation plunger was only used in A. Figure 3 shows cavity C.

Tuning was accomplished in the usual way by moving one end plate with a micrometer movement. The micrometer ${ }^{3}$ had a nonrotating spindle which by a small modification could be keyed so as to be positively nonrotating. The end plate in general is not quite normal to the micrometer

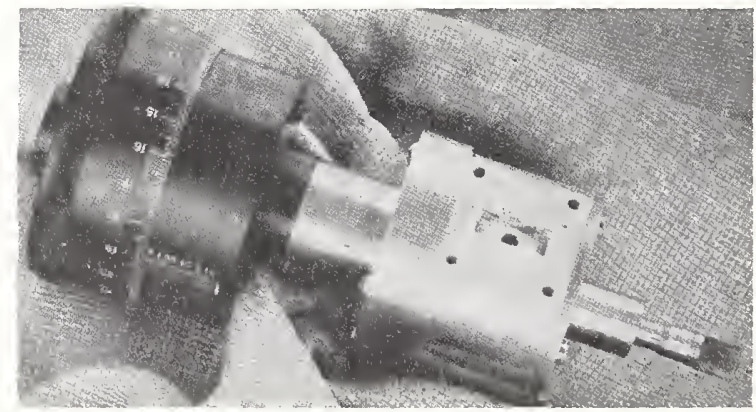

FIg. 1. Invar wavemeter ( 8.6 to $10 \mathrm{kMc}$ ) with a high quality micrometer to move end plate and an ordinary micrometer with needle plunger for small frequency increments. Short waveguide sections were alliccl later to provide isolation from variable screw tension during flange attachment.

${ }^{2}$ I. G. Wilson, C. W. Schramm, and J. P. Kinzer, Bell System Tech. J. 25, $408(1946)$. They also give convenient tables and graphs useful for cavity design.

${ }^{3}$ Arizona Tool and Die Company, Tucson, Arizona. 
spindle, and therefore a rotation would cause variations in the frequency. The micrometer head was mounted on the bottom of cavity $\mathrm{C}$ so that gravity would assist the return spring in holding the end plate against the micrometer.

The manufacturer's specifications indicate that the absolute accuracy of the micrometer is $1.5 \times 10^{-5}$ in. With care the scale may be read to $10^{-5}$ in. Ideally the precision of the micrometer should just correspond to the inherent. or theoretical precision of the resonator and its detection system as determined by the $Q$ and the signal-to-noise ratio. It may be noted that the length of a cavity can be increased in order to obtain more precision from a given micrometer; a $\mathrm{TE}_{012}$ mode furnishes twice the micrometer precision of a $\mathrm{TE}_{011}$ mode, keeping the same diameter. The precision increases as $L^{3}$, keeping the same mode. However, as the cavity is lengthened, the price paid is a reduced tuning range.

A second way to obtain precision is to use a small perturbation plunger ${ }^{4}$ in the other end of the cavity as illustrated in Figs. 1 and 2. The diameter of the plunger was $0.05 \mathrm{in}$, and the length was centered at half the cavity length. The tuning rate (approximately $0.9 \mathrm{Mc} / \mathrm{mm}$ ) was calculated from perturbation theory. ${ }^{5}$ The result, Eq. (4) to follow, agreed with experiment to within $1 \%$. With this plunger good differential precision was obtained over a useful range of $6 \mathrm{Mc}$. Outside of this range the large micrometer again determined the precision. Still another way
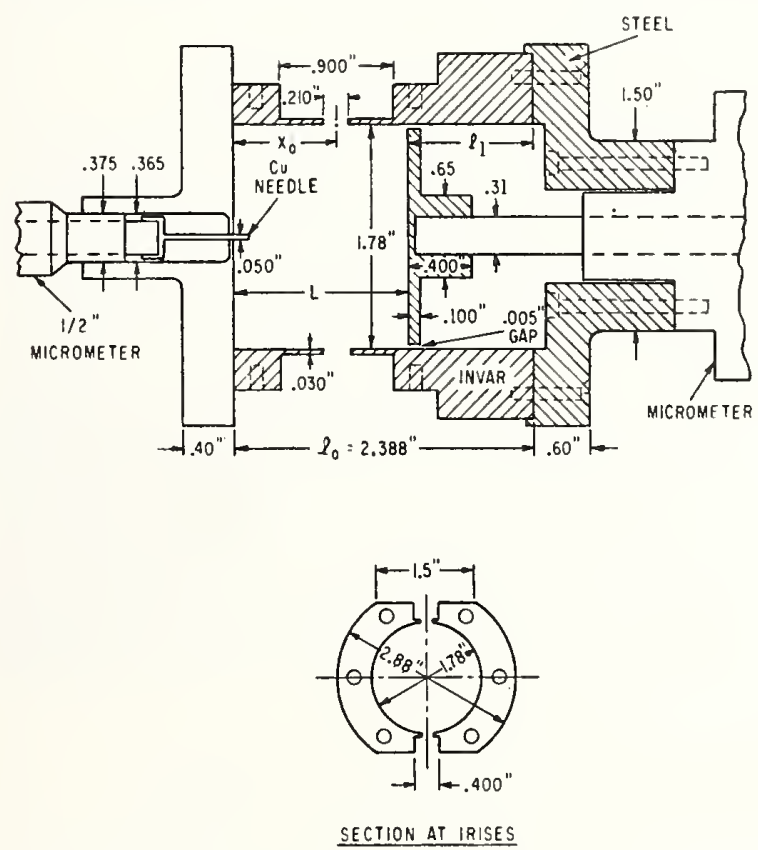

Fıg. 2. Schematic drawing with dimensions for cavity B at about $9000 \mathrm{Mc}$

${ }^{4}$ The use of fine plungers for precision has been reported by G. Birnbaum, Rev. Sci. Instr. 21, 169 (1950).

${ }^{5} \mathrm{H}$. A. Bethe and J. Schwinger, "Perturbation theory for resonant cavities," NDRC Rept. D1 117, March, 1943.

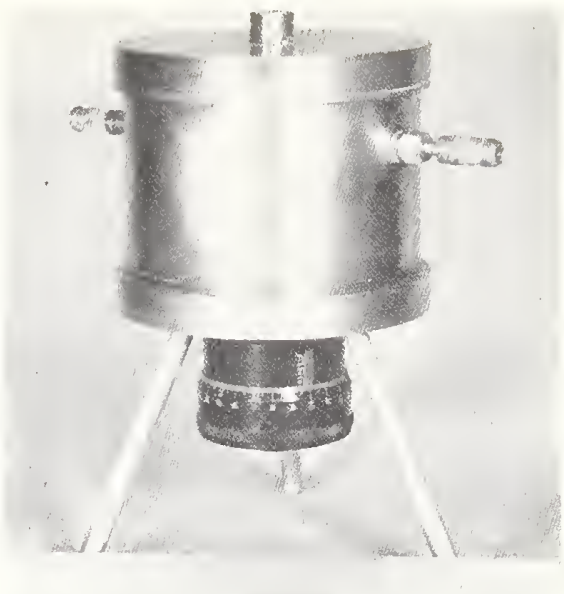

FIs. 3. Wavemeter for the range 2.8 to $3.2 \mathrm{kMc}$.

to obtain differential precision is to attach a long lever arm to the main micrometer so that small rotations can be introduced and measured.

\section{ERRORS CAUSED BY TEMPERATURE AND OTHER SYSTEMATIC EFFECTS}

The variation of the irequency $f$ of a wavemeter with temperature $T$ may be expressed as

$$
\partial f / \partial T=g-\partial u / \partial T
$$

where $g$ represents metallic thermal expansion effects ${ }^{6}$ and $u$ is the refractive index ${ }^{7}$ of the dielectric. $\partial u / \partial T$ is usually negative, which makes the contribution of the last term positive. This term is zero if the cavity is sealed and contains either a nonpolar gas or a vacuum. The cylindrical barrels of cavities A and B were of Invar which was very pure and was heat treated ${ }^{8}$ in order to lower its thermal expansion coefficient. The expansion of the reentrant part of the micrometer shaft ( $l_{1}$ in Fig. 2) and the effect of $\partial u / \partial T$ gives a temperature compensation. For example, at 8500 Mc where $l_{1}$ was small the observed frequency change was $+0.1 \mathrm{ppm} /{ }^{\circ} \mathrm{C}$. The wavemeter was not sealed, therefore the contribution of the air was $1.3 \mathrm{ppm} /{ }^{\circ} \mathrm{C}$, and the cffect $g$ of the metal is found to be $-1.2 \mathrm{ppm} /{ }^{\circ} \mathrm{C}$. Thus, the effect of unsealed air is very significant when good compensation is to be attained.

At higher frequencies $l_{1}$ is longer, $g$ becomes positive, and the compensation is poorer. Compensation over a frequency band would require special techniques. ${ }^{9,1,6}$ Cavity $\mathrm{C}$, made of brass, with its large thermal expansion has to

${ }^{6}$ A discussion of thermal expansion effects and possible temperature compensation is given in C. G. Montgomery (Ed.), Technique of IIicrowave Measurements (McGraw-Hill Book Company, Inc., New York, 1947), p. 384 .

${ }^{7}$ E. K. Smith and S. Weintraub, Proc. Inst. Radio Engrs. 41, 1035 (1953).

${ }^{8}$ B. S. Lement, B. L. Averback, and M. Cohen, Trans. Am. Soc. Metals, 43, 1072 (1951).

${ }_{9}$ T. S. Saad, IRE Trans. on Microwave Theory Tech. MTT-1, 25 (1953). 
be corrected for temperature and insulated to decrease the temperature changes.

For absolute frequency determinations as in spectroscopy a wavemeter should be sealed. Convenient seals for 50-ohm coaxial leads to a cavity were described by Bussey and Birnbaum, ${ }^{10}$ and seals for irises also were discussed. For observations on unsealed resonant cavities perturbed by dielectric or magnetic solid samples, however, the results may be more accurate if the wavemeter is left open since pressure changes will affect both cavities in the same way and gradual humidity changes of the laboratory will diffuse into the two cavities, and thus compensate. Corrections for atmospheric changes can be applied."

Recalibration of one of the Invar wavemeters after a year indicated that the cavity dimensions had decreased resulting in a frequency increase of 0.15 to $0.3 \mathrm{Mc}$. The reason for this change is not definitely known. A possible cause is dimensional changes due to creep. Invar for meter bars can exhibit a creep of 1 or more parts in $10^{6} /$ year. $^{12}$ The heat treatment may enhance this effect. ${ }^{8}$ The room was air conditioned which eliminated ambient conditions as a significant factor.

There are other systematic errors. Small variable strains are introduced by waveguide connections and by routine handling. The Invar cavities had $0.5 \mathrm{in}$. wall thickness to reduce such effects, nevertheless, when waveguide flanges (perhaps distorted) were screwed very tightly to cavity A (Fig. 1) a small frequency change was observed. Therefore permanent waveguide sections were attached to cavities $\mathrm{A}$ and $\mathrm{B}$ to provide mechanical isolation. After this no frequency change was observed due to loosening or tightening a flange. A bending moment on the waveguide of about 250 oz-in. changed the frequency by less than $0.01 \mathrm{Mc}$. The strains are not so variable with cavity $\mathrm{C}$ because the connecting cables are flexible. Thinner walls, such as the $\frac{1}{4}$-in. walls used, are then satisfactory.

\section{Q FACTOR}

The percentages of theoretical unloaded $Q, 81$ to $85 \%$, indicated in Table I, are fairly high. A few auxiliary experiments at $9200 \mathrm{Mc}$ have shown that our particular silver plating facility can furnish a deposit which attains up to $90 \%$ of the theoretical $Q$ of bulk silver.

The $Q$ or conductivity of the silver depends upon the density of the deposit, which was beyond our control, and the smoothness of the finish, which was here obtained by means of a sharp diamond tool. The machining and finishing proceeds approximately as follows: (a) rough out to within 0.01 in., (b) heat treat and anneal, (c) finish the Invar by grinding and the brass with a diamond tool,

\footnotetext{
${ }^{10}$ H. E. Bussey and G. Birnbaum, Rev. Sci. Instr. 30, 800 (1959). ${ }^{11} f_{1} u_{1}=f_{2} u_{2}$ where $f$ is frequency and $u$ is refractive index, given, for example, in footnote 7.

${ }^{12}$ B. L. Page, J. Research NBS, 54, 1 (1955).
}

(d) deposit 0.007 in, of silver, and (e) remove about 0.005in. silver with sharp diamond tool in a lathe adjusted for best possible finish. Steps $d$ and e may, however, be replaced by simply depositing, say, 0.0004 in. of silver on a base that is as smooth as possible.

The $Q$ also depends on the precision of construction, i.e., the geometrical perfection of the shape. For example, a tilted end plate or almost any distortion will couple the $\mathrm{TE}_{011}$ mode to its companion $\mathrm{TM}_{111}$ mode which would lower the $Q$. The gap around the moving end plate may split this degeneracy sufficiently, but this depends on the degree of imperfection present. A concave end plate can introduce sufficient splitting to decouple the two modes even in severe cases. ${ }^{13,10}$ The $Q$ did not improve when concave end plates were tried, which proved that the construction was sufficiently precise. While design with a concave end plate might reduce machining tolerances, some increase in the labor of fitting the calibration would occur because the frequency formula could become more complicated.

\section{COUPLING}

The adjustable loops of cavity $\mathrm{C}$ provide a functional design that allows the coupling to be adjusted easily to any desired value, which is thereafter left fixed. The coupling factor of an iris is given in reference 2. Starting with the iris sizes given in Table $I$ for A or B, changes in coupling may be made on the basis that the power transmitted by each iris is proportional to the sixth power of the iris diameter.

\section{CALIBRATION TABLES}

After constructing a wavemeter which could resolve over its range some $10^{5}$ individual frequencies it was, for reasons of economy, only calibrated ${ }^{14}$ at 21 equally spaced frequency points over the band. (The needle plunger was held stationary for this calibration.) In order to use the instrument conveniently, however, it is necessary to construct a calibration table containing, perhaps, some $10^{4}$ points. Such a table may be constructed easily with a high speed computer from any expression which fits the calibration points sufficiently closely.

The fitting of the calibration points may proceed as follows: The observed frequency $f^{\prime}$ at any micrometer reading $M$ may be considered to be made up of several contributions as follows,

$$
f^{\prime}=f_{0}\left(b, L_{0}, M\right)+\sum \Delta f(M)+R(f)+S,
$$

where $f_{0}$ is the theoretical frequency associated with the

${ }^{13}$ A. Banos, "Design of an improved X-band echo box," MIT RL Rept. 631, December 7, 1944. See also footnote 10 for experience with this method.

14 Calibration was performed by the Electronic Calibration Center of the National Bureau of Standards, Boulder, Colorado. 
dimensions, $\sum \Delta f$ represents the sum of all known perturbations, $R$ represents the residual deviations remaining after $f_{0}$ and $\Delta f$ have been fitted, and $S$ represents stochastic deviations that remain unexplained. The theoretical expression for $f_{0}$ as a function of length $L$ and radius $b$ for a right circular cylinder is

$$
\left(2 \pi f_{0} / c\right)^{2}=(n \pi / L)^{2}+\left(r_{L m} / b\right)^{2},
$$

where $c$ is the velocity of light, $n$ is the number of half wavelength along the axis, and $r_{L m}$ is 3.8317 for $\mathrm{TE}_{01 n}$ modes. The micrometer reading $M$ enters from the fact that $L=L_{0}+M$ where $L_{0}$ is the length when $M$ is zero.

The residuals for cavities $A$ and $B$ could not be fitted to high accuracy even with a high-degree polynomial when perturbations were neglected. With perturbations taken into account, however, a second-degree polynomial was adequate for $\mathrm{B}$ and a small sine wave for $\mathrm{A}$.

The perturbations considered were those due to the irises, the perturbation plunger, the finite $Q$, and the gap around the moving end plate. Based on perturbation theory, ${ }^{5}$ the frequency shift due to one iris in the cylindrical wall of a $\mathrm{TE}_{01 n}$ cavity should be

$$
\Delta f=-\frac{a^{3} r_{L m}{ }^{2} c^{2} \sin ^{2}\left(n \pi x_{0} / L\right)}{3 \pi^{3} b^{4} L f},
$$

where $a$ is the iris radius and $x_{0}$ is the distance from the stationary end plate to the center of the iris. This theoretical result was multiplied by an empirical factor, of the order of 0.8 , which had been determined in other experiments. The contribution from Eq. (3) was then 1.7 to 2.1 Mc/iris for cavity $\mathrm{A}$ and 1.3 to $2 \mathrm{Mc}$ for cavity $\mathrm{B}$. The perturbation due to a small axial plunger that reaches into the strong magnetic field is given quite accurately by

$$
\Delta f=\frac{c^{2} r_{L m^{2}} p^{2} l}{8 \pi^{2} b^{4} L f J_{0}^{2}\left(r_{L m}\right)}\left[1-\frac{\sin 2 n \pi l / L}{2 n \pi l / L}\right],
$$

where $p$ and $l$ are, respectively, the radius and length of the plunger. Perturbations due to the finite $Q,(2 \Delta f=f / Q)$, and the small gap around the end plate were unimportant.

The fitting procedure used was to calculate $\sum \Delta f$ (using nominal values for $L_{0}, b$, and $f$ ) at two points about $\frac{1}{4}$ and $\frac{3}{4}$ of the way through the tuning range, and then to fit $L_{0}$

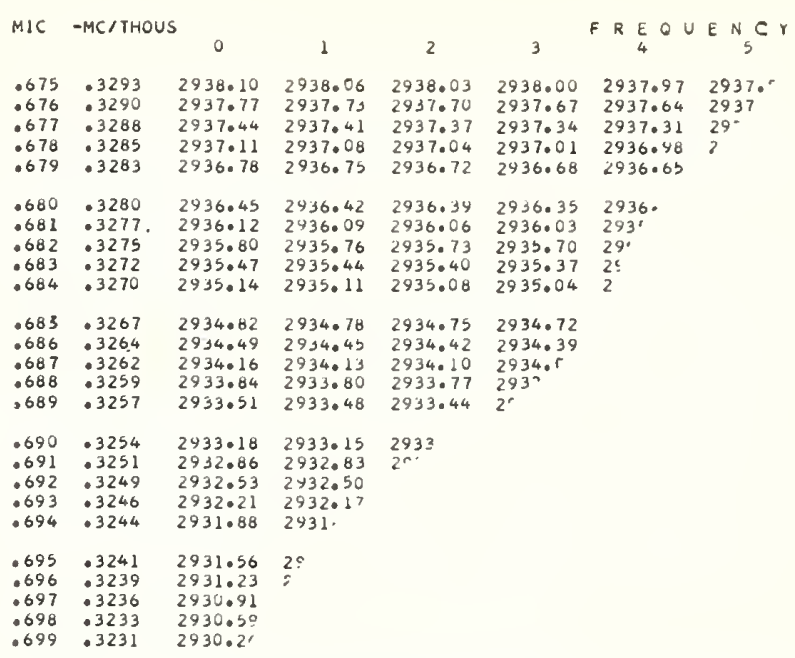

Fig. 4. Page from calibration table of cavity $\mathrm{C}$ containing an. entry for each $10^{-4}$-in. increment.

and $b$ to $f^{\prime}-\sum \Delta f$ at these two points using Eq. (2). The residuals to be fitted are the values of $f^{\prime}-f_{0}-\sum \Delta f$ at each calibrated point. Because these residuals must be small, it is valid to write $R(f)$ rather than $R(M)$ in Eq. (1) where $f$ is, say, $f_{0}+\sum \Delta f$. For example, in cavity $A$ the residuals from 8650 to $9650 \mathrm{Mc}$ formed roughly a sine wave with an amplitude of $0.04 \mathrm{Mc}$. With $R$ as $R(f)$ the data points are usually equally spaced, which allows some very convenient tables $^{15}$ to be used for fitting a polynomial to $R$. The perturbations for cavity $\mathrm{C}$ were evidently very small or very smooth, and $R$ was fitted without benefit of $\sum \Delta f$.

Figure 4 shows part of a page from the calibration table of cavity $\mathrm{C}$ as run off on the computer. The first column is $M$ in units of inches, the second column is the first difference in $\mathrm{Mc} / 10^{-3}$ in., and the succeeding columns are frequencies for each $10^{-4}$-in. increment.

\section{ACKNOWLEDGMENTS}

The authors are indebted to Dr. P. F. Wacker for his guidance in the method of curve fitting by orthogonal polynomials.

${ }^{15}$ R. L. Anderson and E. E. Houseman, "Tables of orthogonal polynominal values extended to $N=104$," Agricultural Exptl. Station, Iowa State College, Research Bull. 297 (1942). For background see also R. A. Fisher and F. Yates, Stalistical Tables for Biological, Agricultural and Medical Research (Oliver and Boyd, Edinburgh, 1938). 


\section{Short-Time Stability of a Quartz- Crystal Oscillator as Measured with an Ammonia Maser*}

There are many applications, such as that required with atomic standards, where the very short time (second-to-second) stability of a quartz oscillator is important. Work at the National Bureau of Standards Boulder Laboratories on a high-precision oscillator, operated with the quartz crystal immersed in liquid helium, gave the results shown in Fig. 2. This may be compared with the short-time stability (Fig. 1) of another quartz oscillator with the crystal at about $40^{\circ} \mathrm{C}$.

Temperature variations of the quartz crystal immersed in the liquid helium are reduced by controlling the pressure of the helium gas above the liquid. Apparentlycompare Figs. 2 and 3-the regulator is adversely affecting the short-time stability of the oscillator. The pressure regulator, however, does provide satisfactory long-time stability.

About one hour trace was taken like that of Fig. 2, and about four hours like that of Fig. 3. The results were very consistent. The first run was made with the pressure regulator in operation for a period of over two hours and showed a drift of less than $\sim 2$ parts in $10^{11}$. The following day, a trace was made without the pressure regulator. After about one hour of this recording, the pressure regulator was activated and the transition from the stability illustrated by Fig. 2 to that in Fig. 3 was observed.

The larger frequency fluctuations when the pressure regulator is used may be attributed to either the temperature change associated with pressure fluctuations or to mechanical vibrations introduced by the regulator-crystals at very low temperatures are rather microphonic.

The quartz crystal was enclosed in an evacuated glass bulb and this was placed inside a brass cylinder. Liquid helium was in direct contact with the outside of the cylinder. A double dewar was used, with liquid nitrogen in the outer jacket and the helium in the inner container. In this system the pressure was regulated at about 650 $\mathrm{mm}$ of mercury.

* Received by the IRE, April 9, 1959.

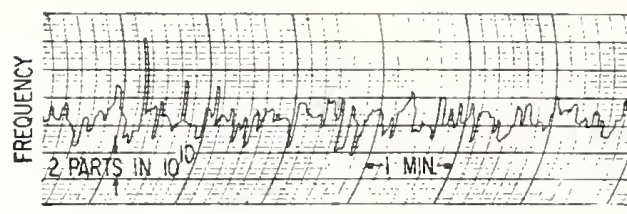

Fig. 1-Oscillator vs maser (approx. $40^{\circ} \mathrm{C}$ ).

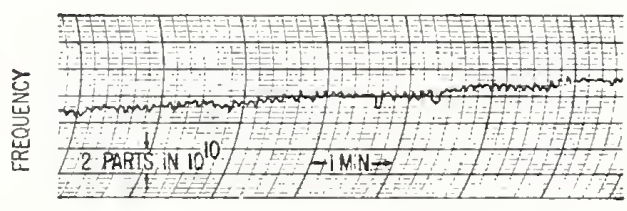

Fig. 2-Helium oscillator vs maser (no pressure control).

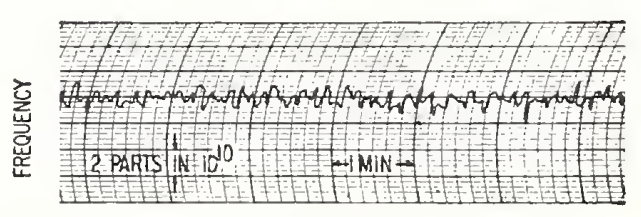

Fig. 3-Helium oscillator vs maser (pressure control).

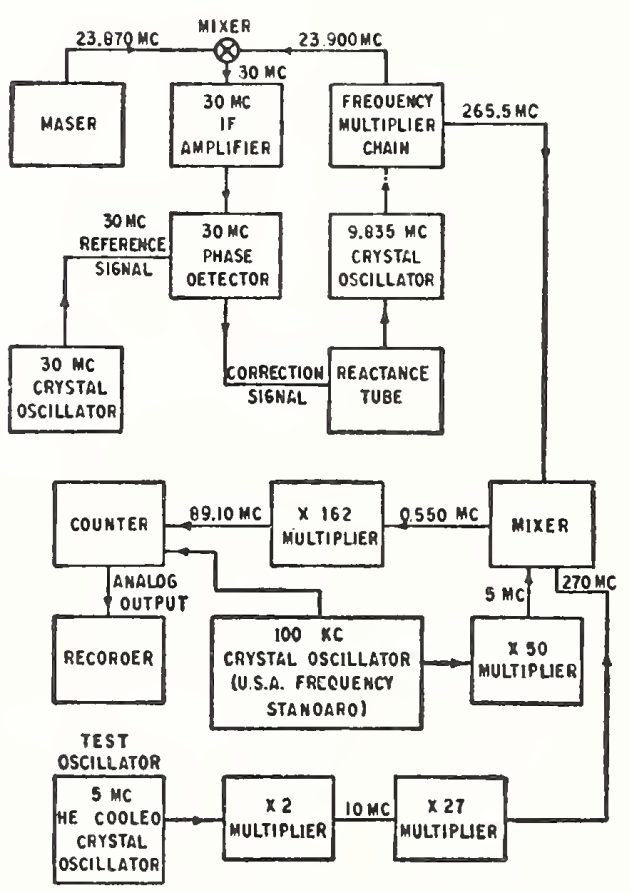
Fig. 4-Maser stabilized frequency-multiplier chain
(all frequencies are nominal).
The scheme used in comparing the helium-cooled oscillator with a maserstabilized multiplier chain is shown in Fig. 4. The traces, of which Figs. 2 and 3 are samples, were derived from the analog output of the counter. The counter was set to count for one second and display for one second. Fluctuations at shorter time intervals - to 0.001 second $^{1}$ - could be observed with this maser apparatus with a somewhat different scheme of comparison. The minimum time interval in the above experiment was limited to one second by the electronic counter.

The authors wish to acknowledge the contribution of Dr. R. C. Mockler, who supervised the development of the maser, and also the helpful assistance of P. A. Simpson and J. B. Milton, who were responsib!e for the cryostat development and the construction and operation of the oscillator.

A. H. Morgan J. A. BARNES National Bureau of Standards Boulder, Colo.

10.001 second is the time constant of the maser servo-system. 


\title{
AN EVALUATION OF A CESIUM BEAM FREQUENCY STANDARD
}

\author{
R. C. MOCKLER, R. E. BEEHLER, AND J. A. BARNES \\ National Bureau of Standards, Boulder, Colorado
}

A CESIUM atomic beam frequency standard constructed at the National Bureau of Standards has been tested for reproducibility and accuracy. The estimated standard deviation of the frequency measurements is 8.5 parts in $10^{11}$. Measurements and control of the various parameters affecting the measured frequency indicate that the accuracy of the machine falls within the precision, i.e., within $8.5 \times 10^{-11}$.

Comparisons with other cesium standards have been made. Agreement is satisfactory in view of the uncertainties incurred by the method and circumstances of comparison. Relative frequency excursions of several parts in $10^{10}$ between Atomichrons introduce uncertainties in these comparisons.

An unsymmetrical power spectrum of the radiation exciting the cesium transition would, in general, give a different frequency measurement for a spectral line than would monochromatic radiation. Power spectra of the multiplied frequencies of several crystal oscillators were observed. In some cases the power spectrum displayed large asymmetries. Furthermore, the spectral asymmetry was observed to change in time for one particular oscillator. It seems essential-for a reliable standard-that the exciting radiation be without frequency modulation, or-if frequency modulation is needed for servo purposes - the modulating signal should be introduced into an otherwise clean spectrum. Multiple frequency modulating signals introduce asymmetrical character to the power spectrum.

THE EXPERIMENTAL APPARATUS

The NBS cesium beam frequency standard (hereafter referred to as NBS-1) employs Ramsey type excitation, has a spectral 
R. C. MOCKLER, R. E. BEEHLER, AND J. A. BARNES line width of $300 \mathrm{cps}$ (at $9192.631 \mathrm{Mc}$ ), and provides a signalto-noise ratio in the range 100 to 400 . The atomic beam is produced by heating pure cesium metal to $150^{\circ} \mathrm{C}$ and allowing it to effuse from the oven through a channel 0.038 inch long with a cross section of $0.003 \times 0.100$ inch $^{2}$. The beam is detected by a surface ionization detector. The hot wire is made of a platinum-iridium alloy ( 80 percent $\mathrm{Pt} ; 20$ percent Ir). The ion current is measured with an electrometer. Typical values of the undeflected beam current fall in the range 1 to $3 \times 10^{-11}$ a mperes.

The beam excitation is derived from a quartz crystal oscillator $(10.317 \ldots . \mathrm{Mc})$. The output of this oscillator is multiplied in frequency up to the cesium transition by the scheme shown schematically in Fig. 1. The exciting radiation is swept in frequency over the width of the spectral line by tuning the $10.317 \ldots$... crystal oscillator by means of voltage sensitive capacitors in the crystal circuit. The detected beam signal is applied to the $y$-axis and the analog output of a frequency counter is applied to the $x$-axis of a $x-y$ plotter. A Ramsey line shape plotted in this manner is shown in Fig. 2. The frequency scale on the $x$ axis is linear. This allows the position of the peak of the line to be determined by taking the average of the frequency of two points on opposite sides of the central peak, both points having the same value of $y$. Several averages of this sort are made for each line trace. The line is swept in both directions and a linear interpolation is made. This compensates for delays that occur in the detecting circuit.

The Ramsey excitation structure consists of a long electroformed resonant cavity of rectangular cross section bent into the shape of a $U$. The $Q$ of this cavity is about 6000. It is symmetric about the coupling iris and operates in the $\mathrm{TE}_{1,0,60}$ mode. The beam passes through the two ends of the cavity - which are separated by $56 \mathrm{~cm}$-and just grazes the end surfaces. Frequency shifts incurred through frequency "pulling" of the cavity are given approximately by

$$
\Delta \nu_{R}=\left(\frac{Q_{\text {cavity }}}{Q_{\text {line }}}\right)^{2} \Delta \nu_{c}
$$

where $\Delta \nu_{R}$ is the shift in the peak of the atomic resonance re- 
A CESIUM BEAM FREQUENCY STANDARD

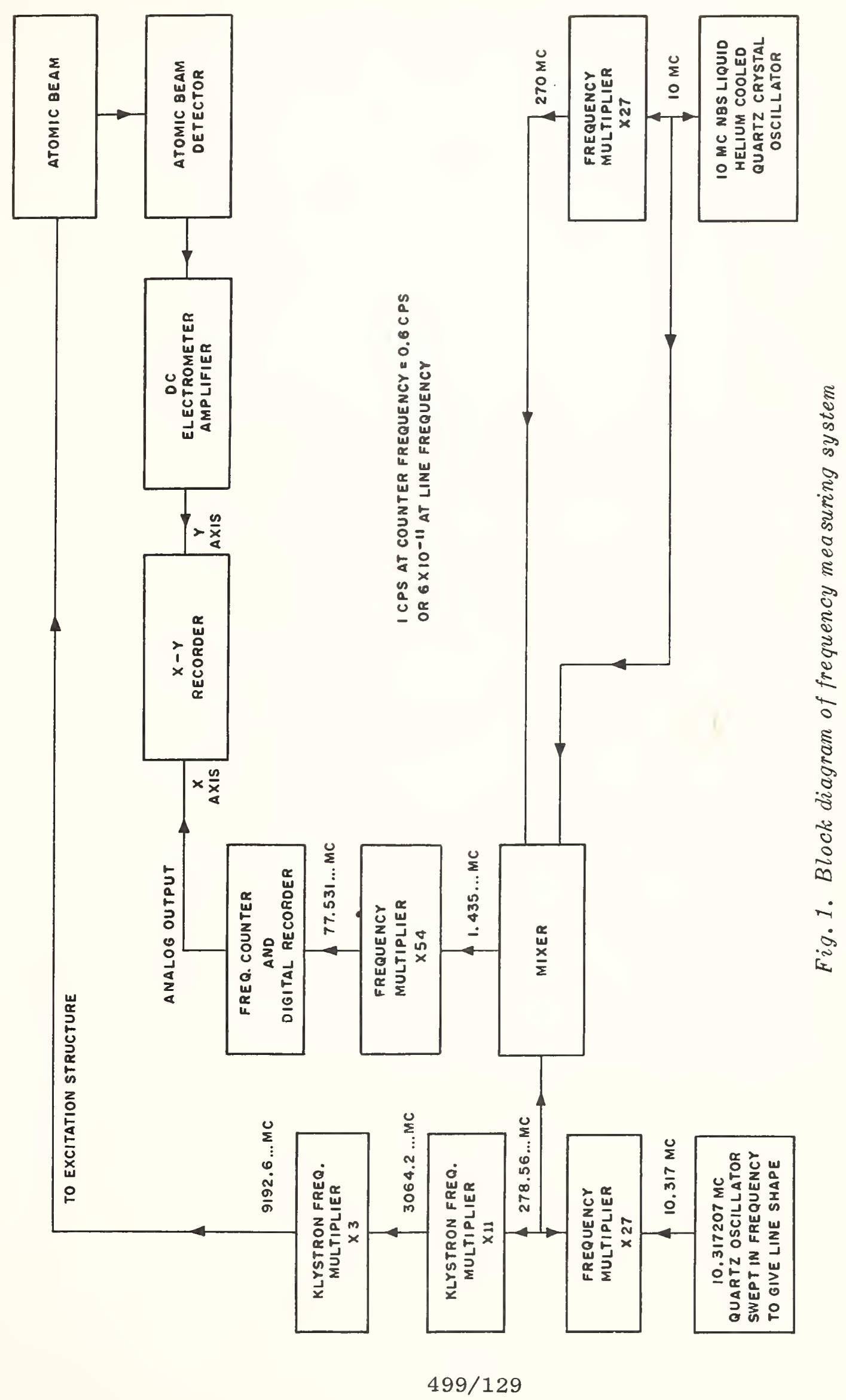


R. C. MOCKLER, R. E. BEEHLER, AND J. A. BARNES

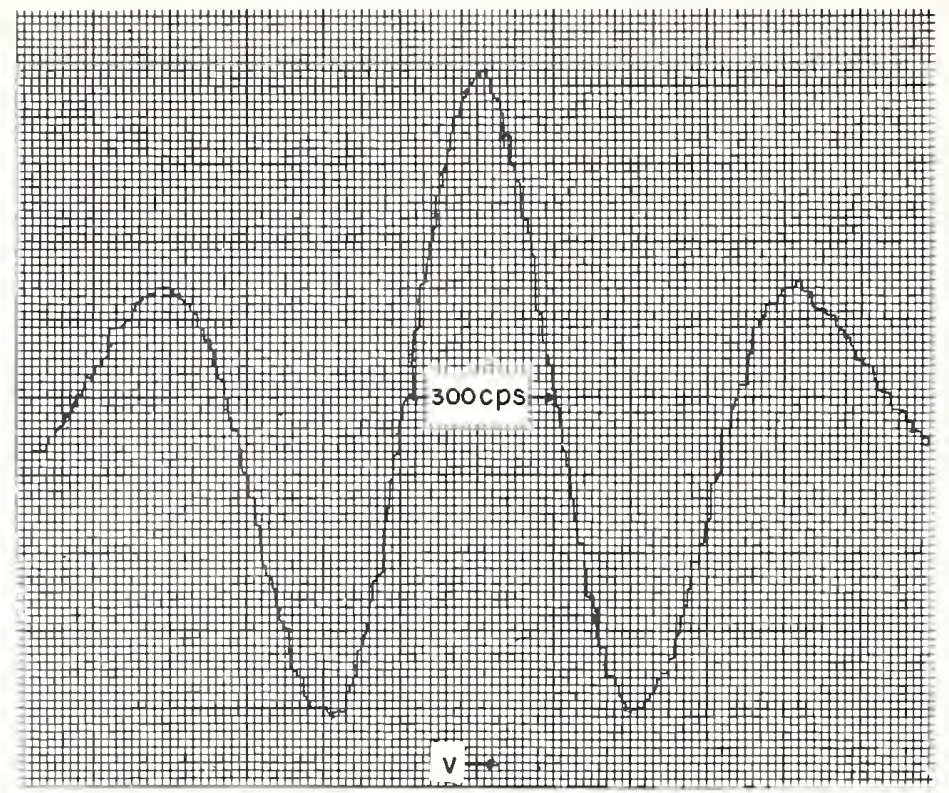

Fig. 2. Ramseyline shape of the $\left(F=4, M_{F}=0\right) \leftrightarrow(F=3$, $\mathrm{M}_{\mathrm{F}}=0$ ) transition

The trace was made on an $x-y$ plotter. The $x$-axis sweep is derived from the analog output of a frequency counter. The step variations in the curve occur because of the step behavior of the counter output.

sponse, and $\Delta \nu_{c}$ is the difference in frequency between the peak of the cavity response and the peak of the atomic response. $Q_{1 \text { ine }}$ is the $Q$ of the atomic response. $\Delta \nu_{R}$ is negligible for this machine for reasonable values of $\Delta \nu_{c}$. Consequently, temperature variations which shift the cavity resonance only tend to vary the intensity of the exciting radiation. No measurable shift is observable for variations in radiation intensity.

The "C" field is ordinarily adjusted to .080 oersted and is produced by a brass strip parallel to the atomic beam through which a current is passed. The uniformity of the field is determined by measuring the low frequency transitions in the beam $(\sim 28 \mathrm{kc})$ induced by small coils placed at different positions along the " $\mathrm{C}$ " field. The measured uniformity is within \pm .003 oersted. The " $\mathrm{C}$ " field region is magnetically shielded from external fields by a mu-metal shield. 
The accuracy of the machine depends upon the precision to which certain parameters can be controlled (and/or measured).

The uncertainty in the measured magnitude of the " $\mathrm{C}$ " field is \pm 0.003 oersted. This corresponds to an uncertainty in the microwave frequency measurements of $2 \times 10^{-11}$ - well within the precision.

There is an uncertainty introduced in the measured frequency if the phase relation between the two oscillating fields of the Ramsey exciting structure is not precisely known. In the machine described here, a single resonant cavity is used. Consequently, the phases are precisely the same at the two cavity ends. The beam passes through these two regions of identical phase. As further evidence of phase identity, the cavity can be rotated $180^{\circ}$ (except for the shorted ends) and the two measured transition frequencies compared. A lack of phase identity in the two oscillating field regions can also be detected by observing the symmetry of the line shape. The absence of perfect line symmetry implies unequal phases in the two regions. The degree to which the inaccuracy can be determined by this kind of observation is limited by the signal-to-noise ratio to a greater extent than by comparing the frequencies for one orientation of the waveguide structure and the inverted orientation. However, if the line breadth is $300 \mathrm{cps}$ and the signal-to-noise ratio is 400 , this method permits the inaccuracy in the frequency to be specified within about $1.4 \times 10^{-10}$. No asymmetry is observable in the line traces.

Any frequency shift caused by electric fields has been shown by Haum and Zacharias ${ }^{(1)}$ to be negligible for magnitudes of the electric field intensity that would be expected in the " $\mathrm{C}$ " field region. They have found that the frequency shift in the cesium line is

$$
\Delta \nu_{0}=1.89 \times 10^{-6} \mathrm{E}^{2} \mathrm{cps},
$$

where $\mathrm{E}$ is the electric field intensity in volts $/ \mathrm{cm}$.

The microwave frequency magnetic field is polarized parallel to the static " $\mathrm{C}$ " field. Under these circumstances, the most important selection rule is $\Delta M_{F}=0$. There are seven transitions 
R. C. MOCKLER, R. E. BEEHLER, AND J. A. BARNES in cesium for which this selection rule applies. It can be shown that these are sufficiently well resolved at .080 oersted so that the resonant peak of interest is not shifted beyond the present uncertainties in measurement by the overlapping of neighboring lines.

Uncertainties can be introduced by the exciting radiation. If this radiation consists of more than one signal and if these signals are not symmetrical in frequency and amplitude about a frequency that is an integer multiple of the primary crystal oscillator driving the multiplier chain, then erroneous line frequencies will obtain. (2) It is not unusual to find unsymmetric power spectra of this sort from frequency multiplier chains and large errors could result. The square root of the power spectrum of an oscillator identical to that used in NBS-1 is shown in Fig. 3. Notice that the spectrum has no sidebands and any asymmetry of the central peak will be of no consequence

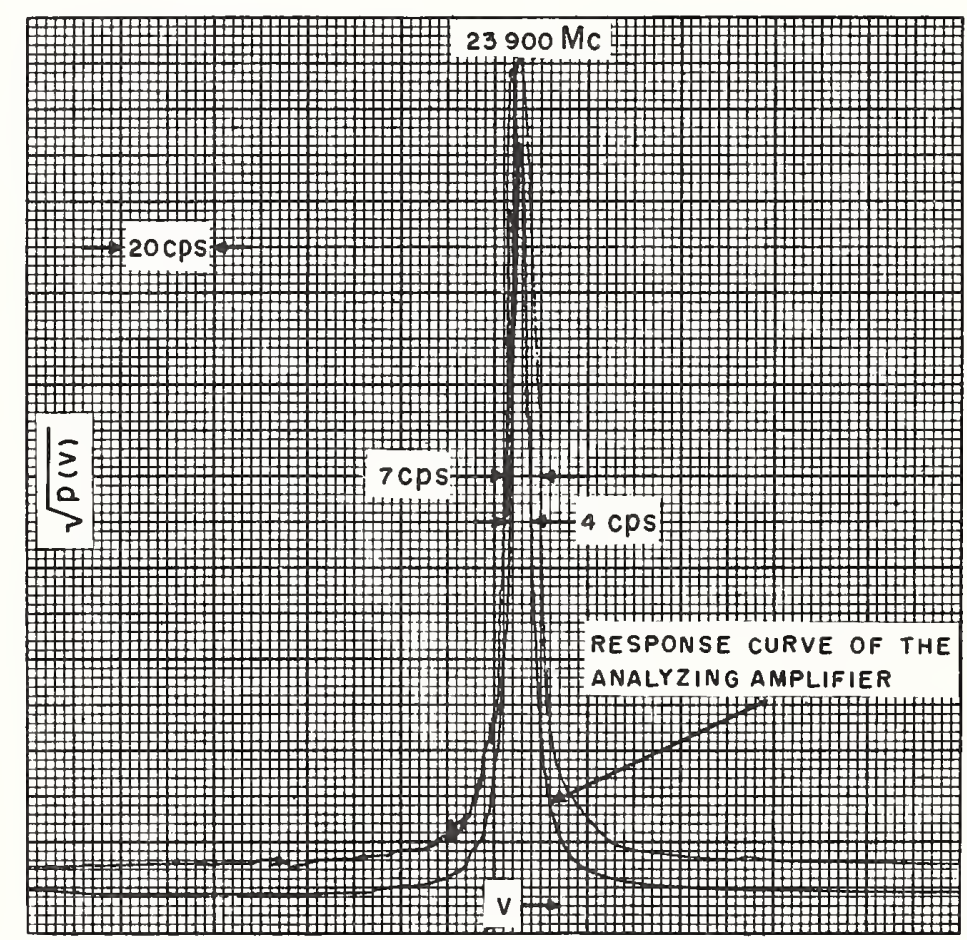

Fig. 3. $\sqrt{\mathrm{p}(\nu)}$ versus frequency for a 9.835 Mc crystal oscillator multiplied in frequency 2430 times 
since this peak is much narrower than the spectral line breadth. Other tests were made on the NBS-1 oscillator to show that its spectrum was similar to that of its twin (Fig. 3)-in particular, no low-frequency sidebands were detected in the multiplier chain output. This spectral character will provide accurate frequency comparisons. The usual assumption that the median frequency of a frequency multiplier is an exact integer multiple of the primary oscillator is an accurate assumption for the sort of spectrum displayed in Fig. 3.

Considering the various sources of inaccuracy and the results of their study, we believe-with some confidence-that the accuracy of the machine falls within the precision or $\pm 8.5 \times 10^{-11}$.

\section{THE MEASUREMENTS}

The reproducibility of the cesium standard (NBS-1) was determined by comparison with a $10 \mathrm{Mc}$ quartz crystal oscillator in which the quartz crystal and associated crystal circuit are immersed in liquid helium. The stability of this oscillator over periods of several days is 3 to $5 \times 10^{-11 *}$ - a figure determined from a continuous comparison with an Atomichron (106) during periods of good behavior. This Atomichron, even though large excursions in frequency occur in short time intervals (Fig. 4b), has excellent stability when averaged over time intervals of 1 minute or more. Periods during which the helium-cooled oscillator and Atomichron (106) showed no significant variations relative to each other were considered best to determine a reproducibility figure (precision) for NBS.1.

The helium oscillator has also been demonstrated to be stable to $2-4 \times 10^{-11}$ over periods of $\frac{1}{2} \sec$ to 6 hours by comparison with a maser stabilized frequency. multiplier chain (Fig. 4a).

In handling the data, the mean of a single day's measurements is considered one piece of data. An estimate of the standard deviation was obtained from successive differences. Let

$$
\delta^{2}=\sum_{i=1}^{n-1} \frac{\left(x_{i}-x_{i+1}\right)^{2}}{n}
$$

*The aging rate of the helium-cooled oscillator appears to be less than $1 \times 10^{-11}$ per day. 
R. C. MOCKLER, R. E. BEEHLER, AND J. A. BARNES

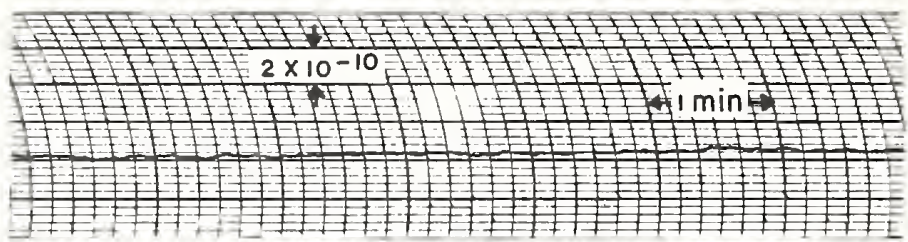

(a)

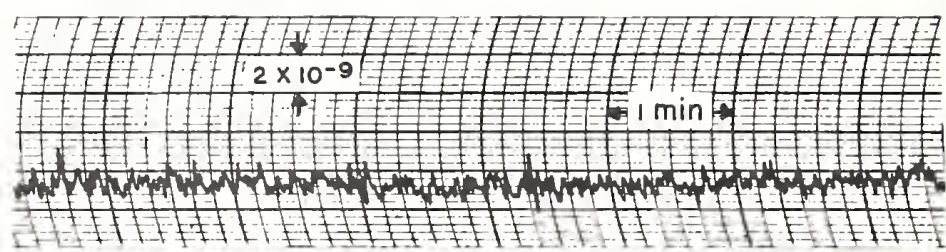

(b)

Fig. 4 .

(a) Maser stabilized chain compared with 10 Mc helium-cooled crystal oscillator. The recorder plots the analog output of a frequency counter versus time. The counting period is 1 sec and the display time is 1 sec.

(b) Maser stabilized chain compared with 5 Mc Atomichron crystal oscillator. In this trace, the difference signal is measured with a frequency meter and not with a counter. The time constant for the meter is about $\frac{1}{2} \mathrm{sec}$. Note the change in frequency scale between (a) and (b).

where $x_{i}$ is the frequency measurement for the ith day and $x_{i+1}$ is the frequency measurement for the $(i+1)$ day; $n$ is the total number of data points (or days). The estimate of the standard deviation by successive differences is given by

$$
\sigma(\delta)=\sqrt{\frac{\delta^{2}}{2}}
$$

For our set of data $\delta=1.1 \mathrm{cps} \cdot$ and $\sigma(\delta)=0.77 \mathrm{cps}$ which is $8.5 \times 10^{-11}$ of the cesium frequency. Successive differences are likely to be random even though certain systematic changes may occur. These systematic changes are small when data is taken on succeeding days but nevertheless still exist. It is impossible to attribute these variations to the Atomichron, the helium-cooled oscillator, or NBS-1, or to a combination when the variations are small. Actually the standard deviation estimated from suc-

${ }^{*}$ R. H. Kent and J. VonNeumann have shown that $\sigma^{2}(\delta)=\left(\sigma^{\prime}\right)^{2}$ where $\sigma^{\prime}$ is the true standard deviation. 
cessive differences is almost the same as that estimated in the usual way. In fact, this standard deviation,

$$
\sigma=\sqrt{\sum_{i=1}^{n} \frac{\left(\bar{x}-x_{i}\right)^{2}}{n}}
$$

is $.5 \mathrm{cps}$ or $8 \times 10^{-11}$.

The data show Atomichron (106) to be lower than NBS-1 by $3.6 \mathrm{cps}$ for the week of August 30. The mean of all the data taken over the months of July and August show about the same difference. Data of one day was deleted from the average because the Atomichron (106) made a large excursion, $8 \times 10^{-10}$, just prior to a general retuning. The excursion was verified by NBS-1, the helium-cooled oscillator, the masers, and Cruft Laboratory data.

In addition to the tests carried on at the Boulder Laboratories to determine precision and accuracy, frequency comparisons were made between NBS-1 and other cesium standards in the United States and England. The comparisons were made through propagated signals between the different locations.

In the discussion that follows, the following designations will be used to identify the various cesium standards:

Designation
NBS-1
106
109
110
112
M4
NPL

\section{Location}

Cesium resonator, NBS, Boulder, Colo.

Atomichron, NBS, Boulder, Colo.

Atomichron, Station WWV, Beltsville, Md.

Atomichron, Naval Research Laboratories, Washington, D. C.

Atomichron, Cruft Laboratory, Cambridge, Mass.

The mean of 106, 109, 110, 112

British cesium resonator, Teddington, England

Table 1. (1 unit $\left.=1 \times 10^{-10}\right)$

1. $($ NBS- 1$)-106=+3.9^{*}$,

2. $\mathrm{M} 4-106=+4.2$,

3. $\mathrm{M} 4-(\mathrm{NBS}-1)=+0.3$

4. $\mathrm{NPL}-\mathrm{M} 4=+1.5$,

5. NPL-(NBS- 1$)=+1.8$ mean over July and August, 1959

6 -month mean for the period

Sept. 1958 to March 1, 1959

6 -month mean for the period

Sept. 1958 to March 1, 1959

* A positive sign means that the first standard is higher in frequency than the second. 
R. C. MOCKLER, R. E. BEEHLER, AND J. A. BARNES

It should be emphasized that this comparison assumed that 106 had the same mean frequency during July and August 1959 as it had during the 6-month period September, 1958, to March, 1959. The accumulated data over the past two years indicate that the Atomichrons tend to wander in frequency. In fact, Atomichrons $112,106,110$ were in rather good agreement during March 1959differing by probably less than $2 \times 1.0^{-10}$. This comparison was made by a single Atomichron transported by air to each of the locations to provide a more direct comparison between the different units.* The relative frequencies of Atomichrons 112, 106, 110, 109 have changed considerably between March and July according to the propagation data of July. During the month of July, 1959,112 differed from 106 by about $1 \times 10^{-9}$ and it differed from 110 by several parts in $10^{10}$. Other variations of this nature are evident from the various data including the direct comparison data made at Boulder between 106, NBS-1, and the helium-cooled oscillator.

\begin{tabular}{lccccc}
\multicolumn{7}{c}{ Table 2. $\left(1\right.$ unit $\left.=1 \times 10^{-10}\right)$} \\
Date & (NBS-1) - 106 & $112-106$ & $112-109$ & $112-110$ & $($ NBS- 1$)-M 4$ \\
July 8 & +4.5 & +8.6 & +4 & +7.6 & +0.9 \\
July 9 & +2.5 & +9.3 & +5 & +8.4 & -1.1
\end{tabular}

The link between Boulder and Cruft Laboratory is through the $60 \mathrm{kc}$ transmission of station KK2XEI at Boulder. This transmission is reported to be very weak, and comparison with it difficult. Recently, new alternatives for comparison through radio signals have come into being. The Navy VLF stations in San Diego and Hawaii are now transmitting signals of high power and of suitable stability measurements. These strong signals measured at Boulder and at Cruft are expected to provide a more satisfactory comparison between standards at the Boulder Laboratories and the east coast.

THE POWER SPECTRUM OF THE EXCITATION RADIATIONITS EFFECT ON THE MEASURED FREQUENCY

For the purpose of understanding the detailed nature of extremely precise microwave frequency measurements, it is neces-

* The experiment was performed under the auspices of the U.S. Signal Corps by Dr. J. H. Holloway from the National Company. 
A CESIUM BEAM FREQUENCY STANDARD

sary to investigate the power spectra of the radiation from frequency multiplier chains used in such measurements. This is especially important in atomic frequency standards where a quartz oscillator is compared with an atomic resonance through a frequency multiplication process. The multiplier chain measured output frequency would be some sort of average-depending upon the method of measurement. If the power spectrum were unsymmetric, this average would not be an exact integral multiple of the primary crystal oscillator frequency.

In the method of observing the power spectrum used here, the output signal of the multiplier chain to be investigated is mixed with an essentially monochromatic signal. This relatively pure signal is obtained from an ammonia maser stabilized chain (Fig. 11). In this fashion the power spectrum is shifted to low frequencies - in fact, audio frequencies-where it can be conveniently examined with a variable frequency narrow band filter

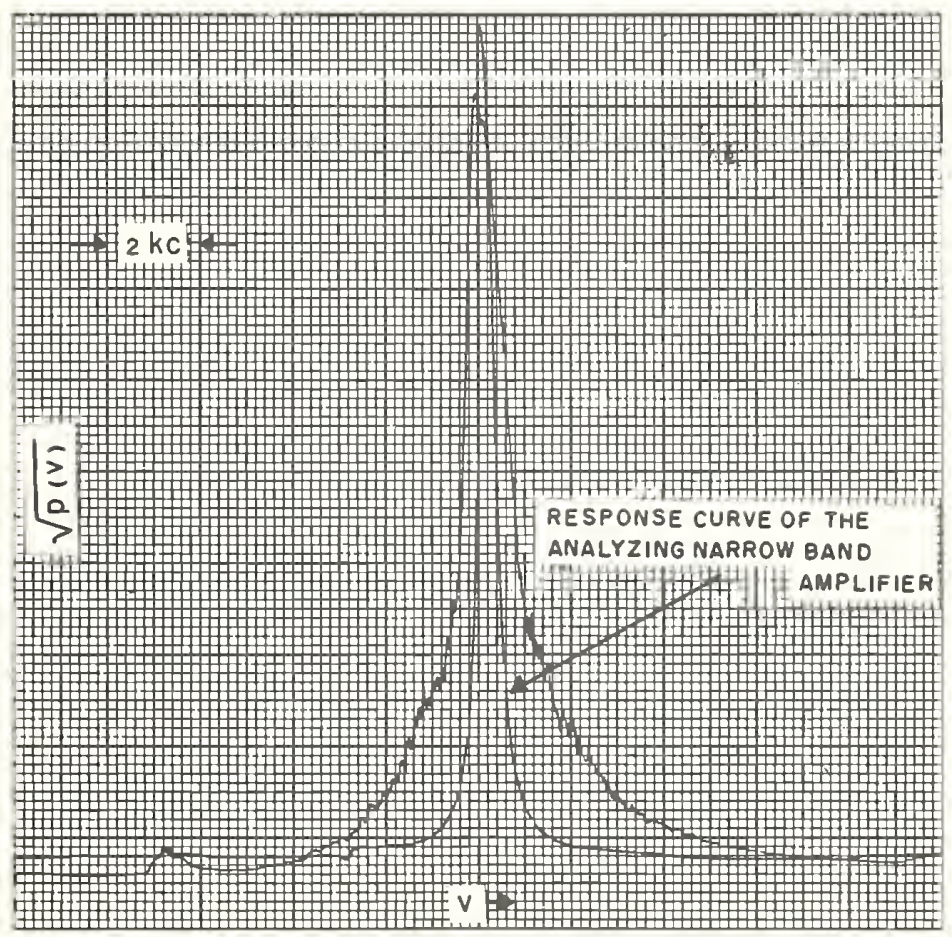

Fig. 5. $\sqrt{\mathrm{p}(v)}$ versus frequency for a 10 Mc helium-cooled crystal oscillator multiplied in frequency 1458 times 
R. C. MOCKLER, R. E. BEEHLER, AND J. A. BARNES

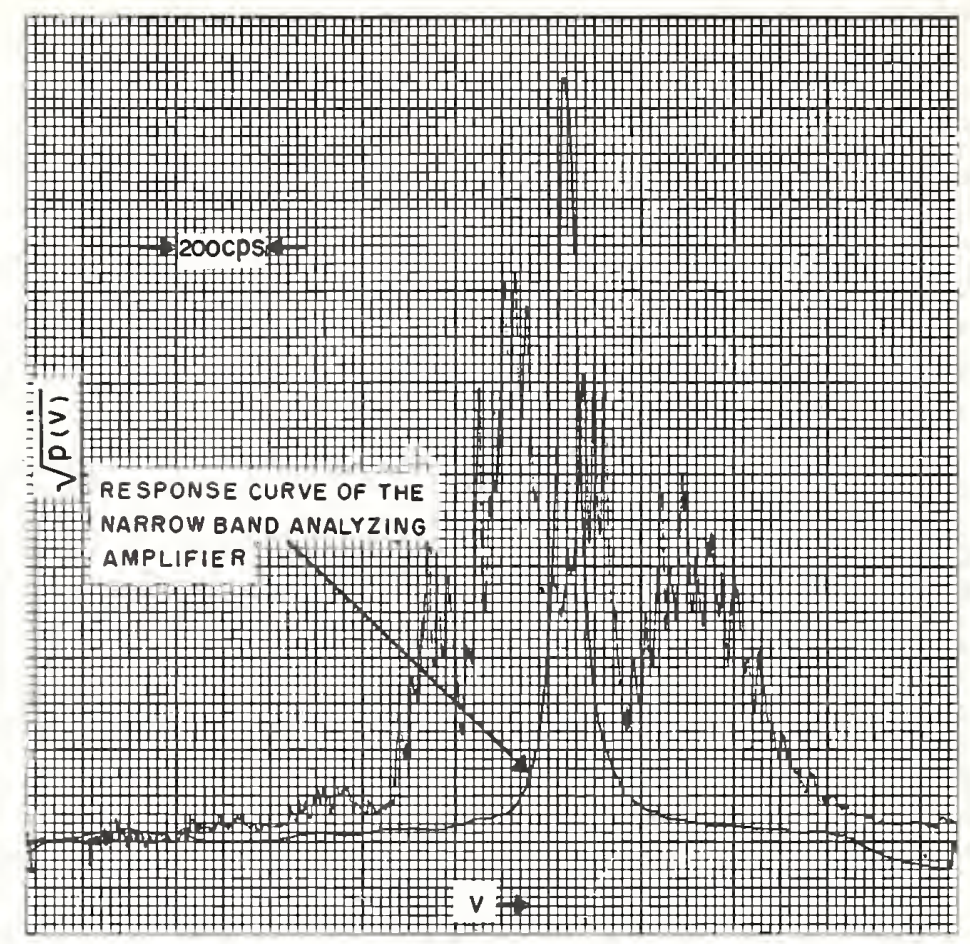

Fig. 6. $\sqrt{\mathrm{p}(\nu)}$ versus frequency for a 10 Mc helium-cooled crystal oscillator multiplied in frequency 1458 times

(amplifier). In the experiments, $100 \mathrm{kc}, 10 \mathrm{Mc}$, and $5 \mathrm{Mc}$ crystal oscillators-and chains-were investigated (Figs. 3, 5, 6, 7, 8, $9,10)$. The frequency multiplication factors used were 1458 for the $10 \mathrm{Mc}$ oscillators, 2916 for the 5 Mc oscillators, and 145,800 for the $100 \mathrm{kc}$ oscillators. Direct multiplication of these oscillators by these factors would give the frequency $14,580 \mathrm{Mc}$. The most prominent features of the observed power spectra are the $60 \mathrm{cps}$ (the commercial power frequency) and the harmonics of $60 \mathrm{cps}$ sidebands. These sidebands are enhanced very significantly by the multiplication process - in fact, by a factor of roughly the frequency multiplication. These sidebands are apparently introduced through frequency modulation in the crystal oscillator, buffer amplifiers, and the first stages of frequency multiplication. The existence of limiters in the frequency multipliers removes practically all of the amplitude modulation. One would expect the $60 \mathrm{cps}$ (and harmonics of $60 \mathrm{cps}$ ) sidebands of 
A CESIUM BEAM FREQUENCY STANDARD

an amplitude-modulated signal to be symmetric in their amplitude about the central peak. This is not necessarily true of frequencymodulated signals - provided that the primary signal is modulated with two or more modulating signals. Thus unsymmetric power spectra are expected-under certain circumstances-when frequency modulation occurs in frequency multipliers. Suppose that the output frequency of such a frequency-modulated chain were measured. Suppose this is done by beating it with a known and relatively monochromatic signal, and the beat note is measured with a counter. The counter would measure the center of gravity of the power spectrum and this frequency will not be an integer multiple of the basic quartz oscillator (unless the power spectrum is symmetric). Furthermore, if this radiation were used to excite the atomic transition in an atomic beam frequency standard, the measured frequency of the spectral line would be different than that measured if the multiplier chain output were monochromatic. In general, the frequency measured by the spectral line will be different than that measured by the counter under

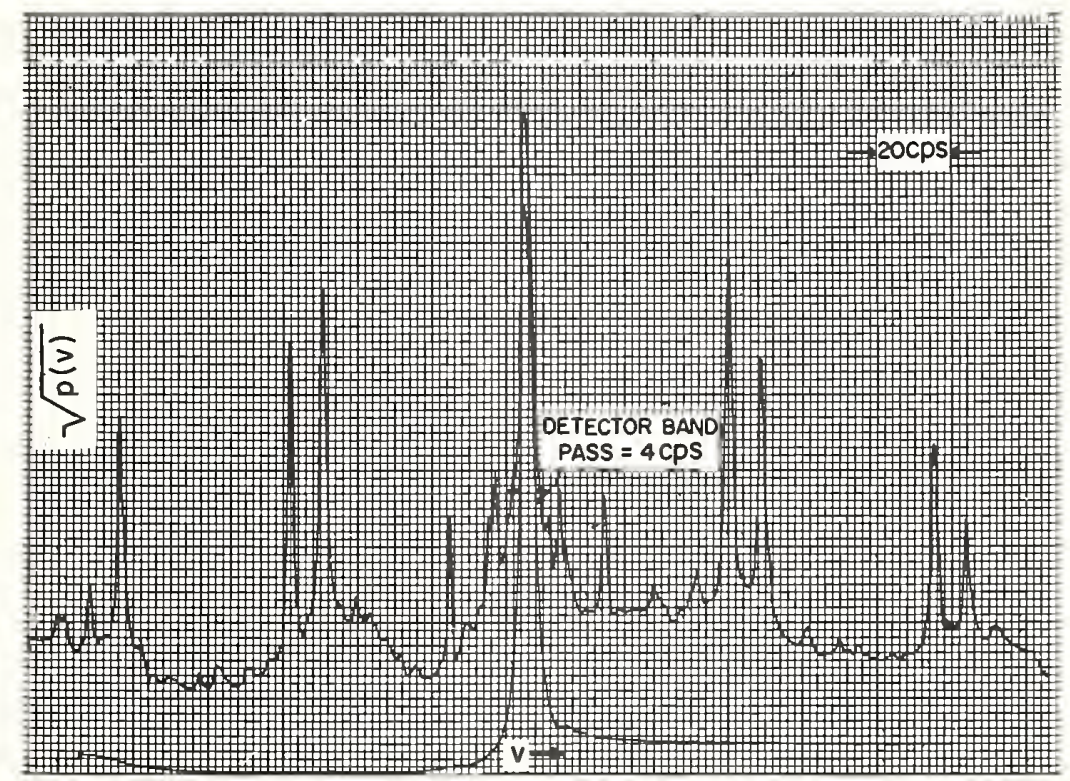

Fig. 7. 5 Mchelium-cooled crystal oscillator power spectrum

Multiplication factor $=2916$.

The detector band pass $\sim 4 \mathrm{cps}$.

The individual peaks in the spectrum have a width less than $1 \mathrm{cps}$. 
R. C. MOCKLER, R. E. BEEHLER, AND J. A. BARNES the unfavorable - but not unusual-conditions discussed above. Elimination of the sidebands and the reduction in the frequency multiplying factor is the best cure-and also a possible cure for these difficulties.* If the power spectrum is symmetric, regardless of whether the frequency is measured by a spectral line or a counter, the measured frequencies will be the same so long as the receiver amplifier is not extremely narrow banded.

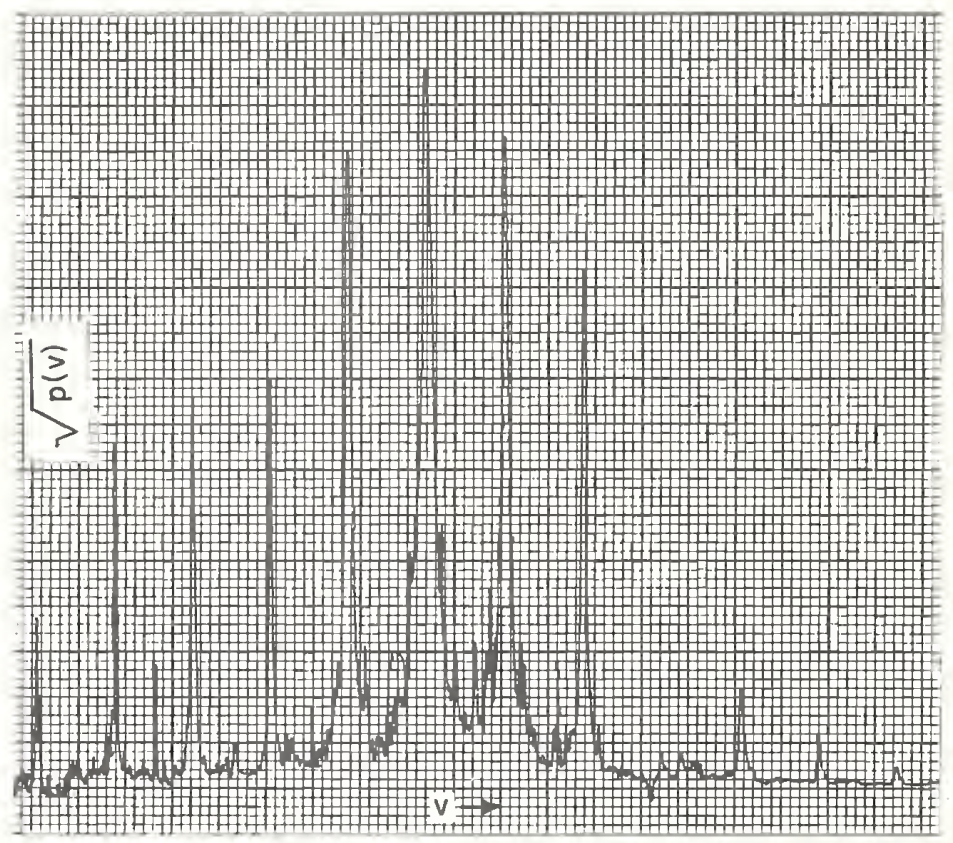

Fig. 8. The pouer spectrum of a $5 \mathrm{Mc}$ helium-cooled quartz crystal oscillator multiplied in frequency 2915 times

The prominent sidebands are due to frequency modulation of 60 . cps and harmonics of $60 \mathrm{cps}$. Note that the spectrum is unsymmetric. The center of gravity of the spectrum has been shifted 41 cps in the multiplication process from the values it would have had, had there been no sidebands.

In the investigations described here, power spectra for the 5 Mc helium-cooled quartz crystal oscillator, taken at different times, showed large changes in appearance and symmetry for no known reason. See Figs. 7 and 8 -notice that one of the sidebands is missing in Fig. 8. This particular oscillator has been shown to

*See Fig, 3. 
A CESIUM BEAM FREQUENCY STANDARD

have a maximum deviation in frequency of about $2 \times 10^{-11}$ over a six-hour interval (by comparison with the maser stabilized chain) during periods of a fixed power spectrum. Variations of this sort in the power spectrum would undoubtedly produce a corresponding change in the "fixed" frequency of an atomic frequency standard.

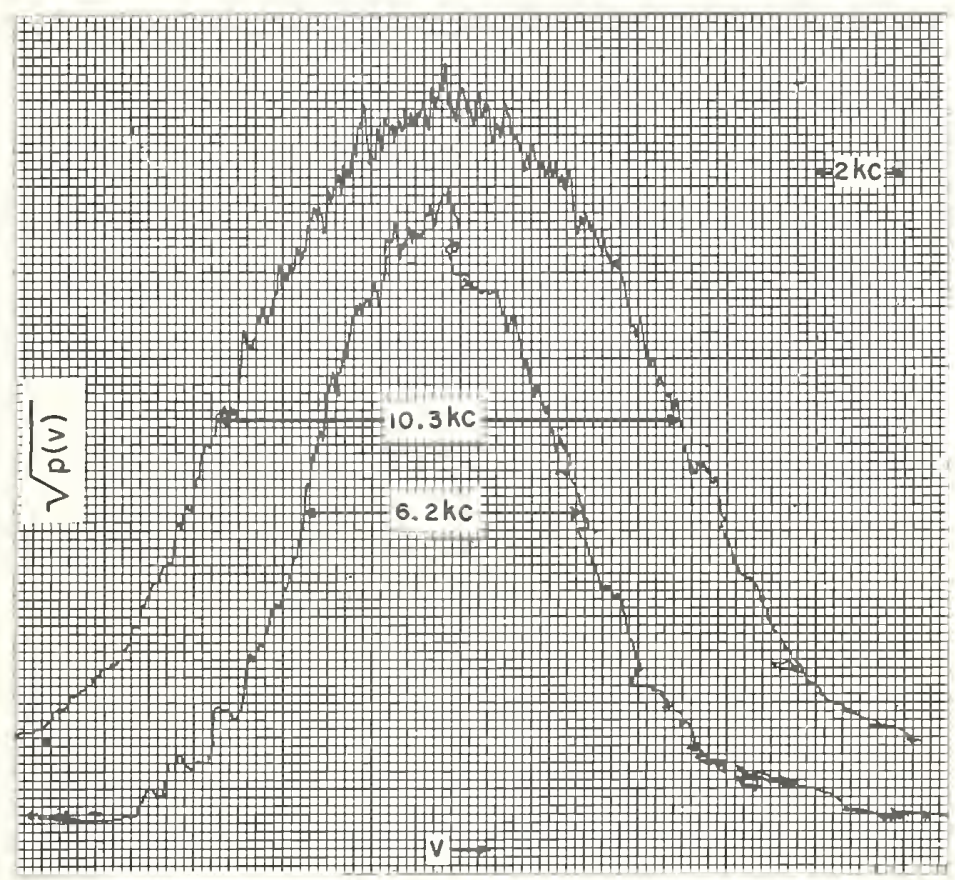

Fig. 9. $100 \mathrm{kc}$ GT crystal oscillator

Multiplication factor $=145,800$. The two traces differ in that the upper trace was obtained 1 hour after the multiplier chain was switched on, and the lower trace was obtained 6 hours after the chain was switched on.

The extremely sharp spectrum ( 1 cps or less at $14,580 \mathrm{Mc}$ ) shown by the $5 \mathrm{Mc}$ crystal oscillator-disregarding the $60 \mathrm{cps}$ sidebands-suggests that the high stability feature of quartz crystal oscillators has not been completely exploited. The width of the peaks in the spectrum of the $5 \mathrm{Mc}$ helium-cooled oscillators is not perceptible with the dispersion used in our experiments. However, the width is observable in the square root of the power spectrum of the $9.835 \mathrm{Mc}$ oscillator shown in 
R. C. MOCKLER, R. E. BEEHLER, AND J. A. BARNES

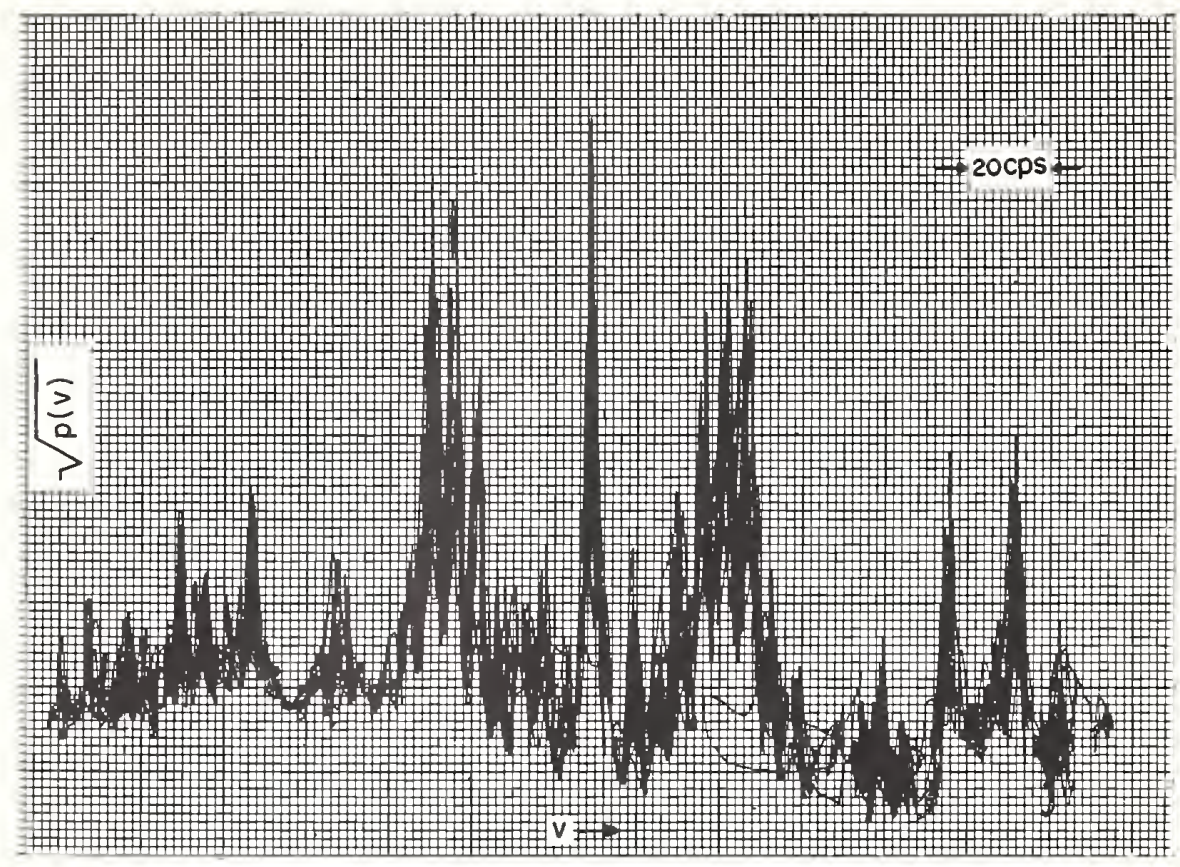

Fig. 10. Power spectrum of the 5 Mc crystal oscillator of an Atomichron (multiplied by 2916)

Fig. 3 in which the crystal circuit is maintained at $46^{\circ} \mathrm{C}$. Perhaps this suggests that this width is due to fundamental noise in the quartz crystal and crystal circuit. The extremely narrow spectrum of a helium-cooled oscillator $(<1 \mathrm{cps})$ implies that crystal oscillators could be used in atomic beam frequency standards for line widths less than $10 \mathrm{cps}$. Evidently, when the breadth of a spectral line is less than the breadth of the power spectrum of the exciting radiation, a plotted resonance curve will have a width determined primarily by the breadth of the radiation spectrum. Fig. 9 shows the square root of the power spectrum for a $100 \mathrm{kc}$ oscillator and multipler chain. If this spectrum were used to excite the transition in NBS-1) (line breadth $=300 \mathrm{cps}$ ), the Ramsey pattern would be completely obliterated leaving only the broad Rabi line shape. This has actually been observed to occur. ${ }^{(3)}$ It is for this reason that higher frequency oscillators are used in atomic standards. As a matter of interest, this $100 \mathrm{kc}$ oscillator has a frequency stability of $1-2 \times 10^{-10}$ per day. Figs. 5 and 6 display the square root of the power spectrum of the $10 \mathrm{Mc}$ 


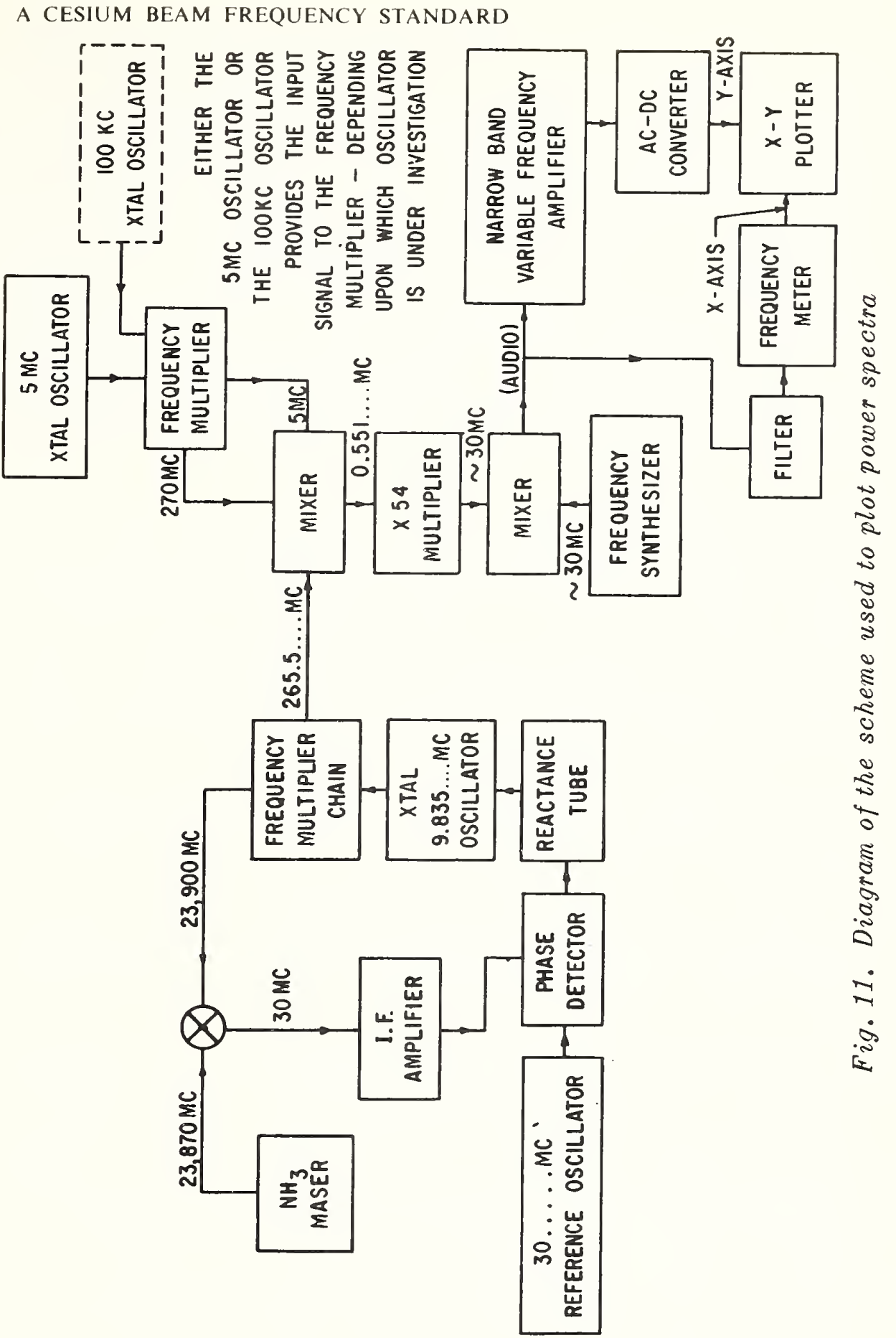


R. C. MOCKLER, R. E. BEEHLER, AND J. A. BARNES helium-cooled crystal oscillator used in the frequency comparison with NBS-1. The spectrum is quite complex and is also unsymmetric. It provides a superb signal for frequency comparisons (Fig. 4a) but would not be useful as a signal source to induce the atomic transition. (4) The square root of the power spectrum of the $5 \mathrm{Mc}$ oscillator belonging to the NBS Atomichron (106) is shown in Fig. 10. The spectrum is symmetric. In spite of its complex character it provides a uniform averaged frequency over long periods of time.

In establishing the performance of an atomic standard it is important to know the spectral character of the excitation. A clean spectrum is likely to give the most reliable behavior.

\section{CONCLUSION}

The agreement between the NBS cesium atomic standard frequency and a mean frequency of four Atomichrons (M4) is rather good-within $\pm 1 \times 10^{-10}$. This, agreement may be fortuitous, however, because there is considerable disagreement among the Atomichrons used in determining the mean frequency $M-4$. The comparison between the NBS atomic standard and that of England $[\mathrm{NPL}-(\mathrm{NBS}-1)=+1.8]$ may be more meaningful.

The variations between atomic standards seem to be quite real and remain unexplained. The source of disagreement can only be determined by test experiments on the individual machines.

At the present time a new cesium resonator is being put into operation at the Boulder Laboratories. This machine will have a line breadth of $90-100 \mathrm{cps}$.

The authors acknowledge with gratitude a large amount of work on the cesium beam by Mr. Lowell Fey during the initial stages of the experiment. Mr. Henry Salazar constructed most of the electronic apparatus. Mr. John Carlson and Mr. Donald Harriman constructed the cesium beam and maser apparatus. We further recognize the invaluable cooperation of Messrs. A. H. Morgan, J. Shoaf, V. E. Heaton, and especially, P. Simpson of the Radio Broadcast Service Section to whom belong the heliumcooled oscillators and Atomichron 106 used in the experiments. 
1. R. D. Haun, Jr., and J. R. Zacharias, Phys, Rev. 10\%, 107 (1957).

2. N. F. Ramsey, Phys. Rev. 100, 1191 (1955).

3. R. C. Mockler, R. E. Beehler, and J. A. Barnes, "A Practical Limitation to the Length of an Atomic Beam Machine," to be published.

4. A more detailed report on the power spectra is in preparation by two of the authors of this paper.

Reprinted from Quantum Electronics, Pages 127-145 (1960)

Columbia University Press, New York, N. Y. 
PRECISE TIME SYNCHRONIZATION

OF WIDELY SEPARATED CLOCKS

\section{SUMMARY}

In many fields of science, such as in astronautics, missiles, astronomy, etc., which are being intensely investigated, the needs for new orders of magnitude of timing precisions have grown. For instance, the need has been greatly increased for more precise determination of the time of initiation and termination of certain events, which may -occur several hundred miles apart. Or, in the location of bodies in space flight, the precise determination of their transit time at known sites is of major importance.

This paper describes known precise methods of setting a group of widely separated clocks to precisely the same time and keeping them in close agreement indefinitely; most of the proposed methods are now available. An estimate of the accuracies of each method are given. Also, there is some discussion of high frequency radio propagation theory pertinent to two of the methods and a few sets of measurements of the propagation delay time of high frequency signals from WWV to WWVH are given. Several graphs and tables are included to simplify some of the calculations.

\footnotetext{
Published as NBS Téchnical Note 22.
} 
Precise Time Synchronization

Of Widely Separated Clocks

Alvin H. Morgan

\section{INTRODUCTION}

The need for higher precision of synchronization of highly accurate clocks, which are widely spaced from each other, has increased in many fields of science such as physics, astronomy, astronautics, etc. Only a few years ago the highest precision required was of the order of 10 milliseconds for most physical experiments. Today, the need for a precision of better than 100 microseconds is fairly common, and in a few instances a precision of 1 to $10 \mathrm{microseconds}$ has been required.

At the present time quartz crystal clocks are the best wo riking time standards for purposes such as those mentioned above. Atomic and molecular resonances are us eful as frequency standards but not directly as time standards because instruments utilizing them are not able to run reliably for long periods of time. However, they are invaluable in maintaining the crystal clocks at a highly uniform rate and thus in effect making "atomic-time" available.

The problem we are concerned with here is not how to obtain uniform astronomical or atomic time, but how to precisely set widely spaced clocks to the same time and keep them in very close agreement, indefinitely. Of course, this requires very accurate and reliable clocks and a method of setting and continuously checking them relative to each other. 


\section{METHODS OF SYNCHRONIZING}

WIDELY SEPARATED CLOCKS

There are several known methods whereby it is possible to adjust the time of several clocks to a certain degree of agreement even when they are widely separated. However, most of these methods are not able to render the required accuracy when it is necessary to set the clocks to agree to better than one or two hundred microseconds.

One-precise method is to transport a very accurate master clock around to each of the slave clocks, and set each one to it, in turn. The master clock would, of necessity, have to be controlled during the experiment by some very stable oscillations in order to achieve the required accuracy. A second method, which requires considerable apparatus, would be to transmit by radio a high resolution timing signal from the master clock to each slave clock and they in turn would immediately retransmit the timing signal back to the master clock. This would enable one to determine very accurately the signal propagation time and the great circle distance to each clock, without prior knowledge of the path lengths. A third method, which is less precise but which involves considerably less apparatus than either of the first two methods, is to transmit high resolution timing signals from the master to each slave clock. It requires knowledge of their exact longitude and latitude and that of the master clock, and the calculation of the great circle distance to each from the master clock. Using this information calculations are made of the delay time of the signal from the master to each slave clock, which is used to determine their corrections. This system may employ either ground wave or sky wave signals. Each is considered separately. 


\section{TRANSPORTING A MASTER}

\section{TO EACH SLAVE CLOCK}

a. General

This method of synchronizing widely separated clocks is limited now to clocks at rest on or near the surface of the earth. However, when space travel becomes a reality it may be possible to adapt it to use in outer space. The method at present requires considerable apparatus and is cumbersome, time consuming, and expensive. However, it appears to be the most accurate method of setting clocks, especially when really portable long running atomic clocks become available.

An appreciable period of time generally will elapse between the time the master clock is initially calibrated and the time it is used to correct the time of each of the slave clocks. Unless the master clock keeps time over long intervals to a precision better than that desired in the synchronization of the slave clocks, some corrections must be applied to it at the time of the setting of each clock. In order to do this, the initial rate of the master clock must be known to a high precision and it must be constant or predictable during the whole measuring interval, i.e., from the setting of the first to the last slave clock.

\section{b. Clock Errors}

The error observed in the master clock in $\mu$ sec, at the end of a time interval $\mathrm{P}$, in days, in terms of quantities observed at a previous time $T_{0}$ ' is approximated by:

$$
\varepsilon_{t}=\left(\varepsilon_{o}+\Delta \varepsilon\right)+P\left(r_{o}+\propto \frac{P}{2}\right)+\Delta P
$$

where: $\varepsilon_{0}=$ error in clock at $t=T_{0}, \mu s e c$, fast or slow

$r_{0}=$ rate of clock at $t=T_{0}, \mu s e c /$ day. 
$\infty=$ accel. of clock, $\mu$ sec/day/day.

$\Delta \varepsilon=$ error made by observer in reading clock, $\mu \mathrm{sec}$.

$\Delta P=$ error made in determining $P, \mu s e c$.

Unless $P$ is a very long interval of time, a may be considered to be zero in really high precision clocks, such as present-day quartz crystal clocks. In case an atomically controlled clock were used as the master clock, both the $r_{0}$ and $\propto$ terms would (so far as is known now) be equal to zero, regardless of the length of the time interval.

For quartz clocks, in present day high precision units, the $r_{0}$ term is of the order of $10^{-10}$ per day (or less) which, in time increments, is around $\pm 10 \mu \mathrm{sec} / \mathrm{day}$. Some special crystal units have been developed which have a relative rate, $r_{0}$, of 1 jsec/day, or less. When a high quality master clock is used, in many timing applications a period of from 10 to 30 hours may elapse between the setting of the first and the last slave clocks without the necessity of making any time corrections to the master clock.

\section{c. Time Kept by a Clock}

The time shown by a clock, at a time $(T)$, may be expressed in terms of its rate $r_{0}$ at $T_{0}$, acceleration $\propto$, change in acceleration $\beta$, elapsed time $P$, and the clock reading $A_{0}$ at a time $T_{0}$, as:

$$
T=A_{0}+P+r_{0} P+\frac{\alpha P^{2}}{2}+\frac{\beta P^{3}}{6}
$$

In high precision quartz crystal clocks, $r_{0}$ is generally positive because of the aging characteristics of quartz crystals, although it may sometimes be negative; $\propto$ may be either positive or negative; and, $\beta$, which is usually negative, may be neglected in all but very, very long time intervals, such as years. 


\section{a. HF Timing Signals}

This method of synchronization has two attractive features inherent in it that may, in some instances, outweigh its disadvantages. First, because time signals are transmitted both ways over the given path, the one-way propagation time may be quite accurately determined without prior knowledge of the precise length of the path. Second, from this measured propagation time, it is then possible to calculate the great circle distance with quite high accuracy.

In Appendix C, it is shown that the propagation time, $t$, from the master clock to a given slave clock, is given, in terms of two measurements $\Delta t$ and $\delta t$, thus:

$$
t_{p}=\left(\frac{\Delta t-\delta t}{2}\right)
$$

where: $\delta t$ is the delay time a time pulse undergoes from its reception at the slave clock until its retransmission, and $\Delta t$ is the delay time, measured at the master clock, between a transmitted pulse and its return to the master clock. From this, the path length is, obviously:

$$
d_{p}=t_{p} c
$$

where: $d_{p}$ is the apparent path length, at the time of the measurement, from the master to the slave clock, and $c$ is the velocity of light. Also, it may be easily shown, Equation (13), Appendix B, that the great circle distance, $\mathrm{d}_{\mathrm{g}}$, for an $\mathrm{n}$-hop mode, is given in terms of $\mathrm{d}_{\mathrm{p}}$, as:

$$
\mathrm{d}_{\mathrm{g}}=\left[\frac{\mathrm{d}}{\left(\frac{\sin (\theta / 2)}{\theta / 2}\right) \sqrt{(1+\gamma)\left(\frac{\gamma}{2 \sin \theta / 2}\right)^{2}}}\right]
$$


where: the symbols are as defined in Appendix B. Obviously, it is not precisely known a priori what the mode of propagation will be at the time of a given measurement. For a given great circle distance and a given ionospheric layer height there is, in general, a definite minimum number of hops possible and a practical maximum number that would furnish a usable signal. In practice, the most consistently received pulse with the least delay time, as observed on an oscilloscope or similar timing device, would be the mode to use in the measurements indicated above. A brief discussion of ionospheric propagation is given below. 


\section{a. Ground Wave Timing Signals}

There are at present some LF radio navigation systems which use sharp pulses and, therefore, have very good time resolution. These systems transmit ground wave as well as sky wave propagated signals, but they can be used to obtain very stable timing signals. By gating to accept the ground wave part of the signal at the receiving end, it is possible to synchronize clocks to a high precision, even when they are separated by distances of several hundred miles.

Figure 1, Section XV, may be used to determine the ground wave propagation delay time. Of course, it is necessary to know, quite precisely, the distances from the master to each slave clock. This may be obtained as shown in Appendix A.

Usually, only one-way propagation of the ground wave signals would be necessary because their path length is very nearly constant. However, if higher accuracies are desired, two-way propagation of the signals would be necessary.

\section{b. Ground Wave Propagation Delay}

Although it is relatively easy to calculate the ground wave propagation delay time for a given great circle distance, a chart is included (Fig. 1) to simplify this work.

The distance is given, for convenience, in paralleled scales in miles and kilometers versus propagation delay time.

\section{c. HF Propagation}

This method of synchronizing widely separated clocks requires a precise knowledge of the signal path lengths between the master and each slave clock, which may be calculated from other known data. The longitude and latitude at the master and each slave clock must be accurately known along with the average ionospheric layer height at the 
time of day at which the calculation is to be made. In addition, the mode of the signal being propagated between the master and each slave clock is required.

This method first requires a calculation of the great circle distance between the two points on the earth for which the signal propagation time is desired. Although the calculations are, in principle, rather simple they are quite lengthy, and are best explained by means of an example. This is done in Appendix A. After the great circle distance, $d_{g}$, between the master and each slave clock is calculated, the propagation time for a ground wave signal may then be easily determined from Figure 1 or by the well-known relationship:

$$
\mathrm{t}_{\mathrm{g}}=\mathrm{d}_{\mathrm{g}} / \mathrm{c}
$$

where: $d_{g}$ is the great circle distance and $c$ is the velocity of the ground wave and may be taken equal to that of light. The next step is to calculate the difference in propagation times between a given mode sky wave signal and a ground wave signal over the path in question. In Appendix $B$, this is shown to be given by the expression:

$$
\Delta t=\left(t_{p}-t_{g}\right)=t_{g}\left(\frac{\sin \theta / 2}{\theta / 2}\right) \sqrt{\left[(1+\gamma)+\left(\frac{\gamma}{2 \sin \theta / 2}\right)^{2}\right]}-1
$$

$$
\mathrm{d}
$$

where: $\theta=\frac{g}{4 r}$ and $\gamma=h^{1} / r$. This may be further simplified (e.g. , Appendix B) to:

$$
\Delta \mathrm{t}=\mathrm{t} g\left(\mathrm{y} \sqrt{\mathrm{h}+\mathrm{p}^{2}}-1\right)
$$


where: $y=\left(\frac{\sin \theta / 2}{\theta / 2}\right), h=(1+\gamma)$ and $p=\left(\frac{\gamma}{2 \sin \theta / 2}\right)$.

Either equation (7) or (8) would normally be evaluated for the given path in terms of various ionospheric layer heights, $h^{\prime}$, and angles ( $\left.\theta / 2\right)$, and the mode of the signal, n. This involves considerable calculations for even one path.

To reduce this labor, a set of curves, Figures 10 through 15 , are included in Section $X V$, from which values of $\Delta t$ may be taken directly. Each chart has a given fixed layer height, ${ }^{\dagger}$, and the curves are in terms of the great circle distances, $\mathrm{d}_{\mathrm{g}}$, the modes of propagation, from $n=1$ to $n=4$, and the differential propagation time, $\Delta t$.

After the differential time, $\Delta t$, is determined from the charts in Figs. 10-15, for a given path and mode, it is added to the ground wave propagation time, $t_{g}$, to obtain the total propagation time, $t_{p}$, for the path.

\section{ACCURACY OF METHODS GIVEN}

\section{a. Transporting a Master Clock}

The limits of the accuracy of this method lie in two factors:

(a) long term stability or constancy of the master clock, and (b) resolution of the system ${ }^{1,2}$ used to compare the master and slave clocks. If the master clock is controlled by atomic or molecular resonances, the error due to the long term clock stability is negligible. In case the master clock is controlled by a quartz oscillator of high quality, the error would be quite small: from 10 to $100 \mu \mathrm{sec} /$ day is easily attainable.

The resolution of the time comparison system can be made very good: from $0.001 \mu \mathrm{sec}$ to $0.01 \mu \mathrm{sec}$ is not too difficult to achieve with good signal-to-noise ratio.

Thus, the overall accuracy of this method would be from 0.001 $\mu \mathrm{sec}$ to $0.1 \mu \mathrm{sec}$, depending on the clocks and the time comparison 
system used. With care and very high quality apparatus and rapid transportation of the master or reference clock, it is conceivable that the accuracy could be extended one to two orders better.

b. Transmission of Time Signals (one- and two-way)

(1) General

The systems of synchronizing clocks by either one- or two-way transmission of timing signals is not as accurate as the one described above, but they are obviously more convenient, especially when the clocks are many thousands of miles apart. Because of the different nature of the problems, the hf and the ground wave timing signals will be considered separately.

(2) HF Timing Signals

(a) One Way Transmissions

Results of measurements, described in Appendix $D$, indicate that the stability of the HF delay time is dependent on several factors. Among these the following are susceptible to control by the observer: (a) time of day that the measurements are made, (b) method of observing the received timing signals, i. e., whether a zero crossing or a peak of the signal is used, (c) the extent to which the antenna pattern favors the optimum mode of propagation, (d) whether or not the propagation mode with the least delay time is used, (e) whether or not the measurements are related to a precise slave clock at the receiving site so that advantage may be taken of the average (over several days), (f) the actual frequency used, i.e., in general, the higher the frequency (but still below the MUF) the more stable the delay time, and (g) accuracy of knowledge of path lengths involved.

Other factors which may affect the stability of the relative delay times of the signals are: (h) epoch of the sunspot cycle; (i) whether or not the radio path passes near or through one of the auroral zones, (j) whether or not the path is all in darkness or all in daylight at the 
time of the measurement, (k) signal-to-noise ratio at the receiving site, and (1) number of hops involved in the propagation of the signal. Ideally, the following should prevail:

\section{Factor}

(a) time of day fo'r observation

(b) part of signal to measure

(c) directivity of antenna

(d) propagation mode to use

(e) rneasurements

(f) choice of frequency

(g) accuracy of knowledge of pa.th lengths

(h) epoch of sunspot cycle

(i) location of radio path

(j) length of longest ideal path

(k) signal-to-noise

(1) number of hops of path

\section{Ideal Condition}

near noon or midnight at the center of the path.

zero crossing time of the least delayed pulses.

favorable to mode with the least delay time.

the one with least, consistent delay time.

be relative to a very precise slave clock at the receiving site.

highest available at which a consistent signal can be obtained at the time of the measurements (see item (a) above).

as accurate as possible.

near minimum.

well away from both auroral zones. short enough to be at least 2 hours all in daylight or all in darkness. as high as possible. equal to next integer greater than the quotient of the length of the great circle path expressed in $\mathrm{km}$ divided by 4.000 .

The accuracy of the synchronization of the slave clock using this method obviously then depends on how near to ideal conditions the actual conditions are. Under conditions approaching the ideal, the accuracy may be better than \pm 0.1 millisecond, even for very long paths; however, it will usually be less than this by a factor of from 2 to 5 , or more. 
At the other extreme, for example if the path passed near or through an auroral zone, or was so long that it was never in all darkness or all daylight, etc., the accuracy might be only about \pm 10 milli seconds, or worse.

More definite statements of accuracy are not possible at this time, but it is hoped that further measurements in the future will make this possible.

Further data on this may be obtained by reference to Appendix D.

(b) Two-Way Transmissions

If a transponder, or similar device, is used at the slave clock, it is possible to:

1) determine the apparent length of the path, the long term average of which may be quite precise.

2) increase the knowledge of the accuracy of each measurement over that obtained with one-way transmissions.

The method of calculating the path delay time from the measurement data is given in Fig. 4. From this, and other considerations, it is quite apparent that the actual conditions of the measurements will not need to be as near the ideal, as in one-way transmissions, to produce quite good results. This is also apparent from the data given in Appen$\operatorname{dix}$ D.

Using this method, and under quite favorable conditions, as defined in paragraph (a) above, an accuracy of time synchronization of slave clocks, at distances approaching half-way around the world, might be about 10 microseconds; however, a more conservative estimate would be from 0.1 to 1 millisecond.

(3) Ground-Wave Timing Signals

(a) One-Way

Because the delay times of ground wave signals are rela = tively constant, it is possible to achieve. with them, a high accuracy in 
synchronization of widely separated clocks. Within the distance range wherein the signal-to-noise ratio is sufficiently good, an accuracy of from 0.01 to $0.1 \mu$ sec is possible. This requires a narrow-band receiver: gating circuits to accept the ground. wave component of the timing pulses, and associated circuitry to permit pulse-locking of a highly precise local oscillator, which drives the clock to be synchronized. It is assumed, of course, that the timing pulses are sufficiently sharp to meet the accuracy quoted.

(b) Two-Way

One distinct advantage of using a transponder, or similar device, with ground wave timing signals is that it is possible to determine the great circle path length between the master and slave clock to better than 10 feet up to distances of several thousand miles by using the results of the measurements. This fact may be of considerable importance in geodetic or similar work and in missile tracking systems.

Of course the above implies: (1) a method of signal gating so as to receive only the ground-wave component of the timing pulses; (2) precise measurements of the signal delay in the transponder; and (3) use of timing signals with very fast rise-time to permit (1) above.

The accuracy of this method of time synchronization of the slave clocks using the latest techniques, appears to be limited only by the signal-to-noise ratio in the receiving systems.

\section{MODES OF PROPAGATION OF HF SIGNALS}

\section{a. Introduction}

High frequency signals are propagated between two points on the earth's surface by means of one or more reflections from one of the ionospheric layers and the surface of the earth. One reflection from the ionosphere is called a one-hop mode, two reflections, with a single reflection from the earth's surface, is called a two-hop mode, and so forth. 
Only two of the reflecting layers will be considered here, the F-2 and E-layers, because other layers ( $F-1$ and $E s$ ) may usually be neglected in considering the propagation of hf timing signals over long periods of time. Signals reflected by the F-2 or E-layer are called F-2 and E-layer modes, respectively.

\section{b. Maximum Usable Frequencies (MUF)}

In the hf range, where signals are propagated by ionospheric reflection, there is an upper limit of frequency which may be transmitted over a path of a given length. At frequencies above this limit, which is called the "maximum usable frequency" (MUF), the wave is said to "skip," or pass over the receiving end of the path.

\section{c. One-Hop Modes}

Over relatively short distances, sky wave signals are propagated by means of a single reflection from an ionosphere layer; either F-2 or E-layer. Remembering that both the earth's surface and the ionosphere are curved, it is quite clear that there will be a limiting distance for a one-hop mode. For frequencies equal to, or lower than, the MUF (explained above) for the path, the limiting distance may range from 3500 to $5000 \mathrm{~km}$ (2200 to $3000 \mathrm{mi}$.) for the F-2 layer mode, depending on the reflecting layer height, and is an approximately constant distance of about $2400 \mathrm{~km}$ (1500 mi.) for the E-layer mode. However, it has been found in practice that the average limiting distance for the F-2 layer mode is about $4000 \mathrm{~km}$ (2500 mi.), which will be the value used here.

Thus, for distances less than $4000 \mathrm{~km}$ (2500 mi.) but greater than $2400 \mathrm{~km}$ (1500 mi.), the F-2 layer single-hop mode would be predominant. For shorter distances, either the E- or F-2 layer mode could exist, if the frequency used were equal to, or below, the MUF for that layer. 
d. Multihop Modes

For great circle paths longer than about $4000 \mathrm{~km}$, it is obvious that more than one reflection from the ionospheric layer usually must occur. The minimum number of hops is approximately the next integer greater than the quotient of the total great circle distance expressed in kilometers divided by 4000 . This mode will be observed during the time the signal frequency is below the MUF for the path.

As an example, assume the path length is 9, $000 \mathrm{~km}$ (6800 $\mathrm{mi}$.$) .$ It is quite obvious that two hops of maximum limit (4000 km each), laid end-to-end, would not cover this distance. Therefore, the simple geometric picture of the minimum mode of propagation would be threehops, each of $3000 \mathrm{~km}$ length, laid end-to-end.

e. Control Points (see Fig. 9)

Over very long paths, the MUF for the signal may be estimated by considering the condition of the reflecting layer at only two reflecting or control points, i. e., one a distance "d" from the transmitter, call it point "a", and the other a distance "d" from the receiver, which will be called point " $k$ ". The distance " $d$ " is one-half the limiting distance for a one-hop mode (i.e., one-half of $4000 \mathrm{~km}$ ), or $2000 \mathrm{~km}$ (1250 mi.) for F-2 propagation. The MUF for the path is taken to be the lower of the 4000-km MUF's for the points "a" and "k".

Unfortunately, the MUF for a given path of any length, varies from hour-to-hour, day-to-day and year-to-year. Diurnal variations in the reflecting layers are a consequence of the rotation of the earth, and seasonal variations are associated with the movement of the earth in its orbit around the sun. In addition, there are longer period variations that have been correlated quite well with the sunspot cycle.

Further information on the above may be found in standard treatises in the subject, such as Reference 5, Section XIII. 
VIII. VARIATIONS IN PROPAGATION DELAY OF HF SIGNALS

a. Introduction

The propagation delay timo of hí timing signals over a given path are not constant from hour-to-hour or day-to-day, but undergo some variations. In many timing problems, such as the ones under discussion, it is necessary cither to measure the variations or determine the maximum variations that are likely to occur at the given time over the given path.

b. Factors Affecting the Propagation Delay

This subject can only be very sketchily treated here because of its relatively complex nature. Among the many factors affecting the. velocity of propagation of an hf signal, only one will be discussed here. This is the variation in the virtual height of the $F-2$ ionospheric layer. Besides its quite regular diurnal and seasonal variations, the F-2 layer also undergoes severe changes during magnetic or ionospheric disturbances. The ion density of the F-2 layer usually decreases and then slowly returns to normal over a period of 1 to 3 days. The virtual height $\left(h^{\prime} F_{2}\right)$ suffers rapid changes during such periods, sometimes by a factor of nearly two.

This, of course, will introduce unknown but relatively large variations in the propagation time of the signals and thus seriously affect high precision work. Data taken during such periods are, obviously, useless in the precision timing problems under consideration here, and should be discarded; only data taken during normal conditions should be used.

c. Estimation of. Effect of Normal Variations of Virtual Height

The effect of diurnal changes in the virtual height of the F-2 layer may be minimized by: (a) measuring the timing signals at the same time every day, and (b) selecting this time of day so that the whole path, from the master clock to the slave clock, is all in daylight 
or all in darkness. In other words, neither the sunrise nor sunset

poriod should occur anywhere over the path during the time the measurements are being made. On fairly short paths, for instance, the measm uring period should be selected when it is approximately noon at the center of the path.

There remains, however, some small and irregular changes in the $F-2$ layer height which are unpredictable. To obtain an accurate value for these changes would require some detailed measurements of the ionospheric height versus time at the reflecting points of the given path. This is obviously not a practical undertaking for those engaged in timing problems.

Alternatively, a knowledge of the effect of changes of the F-2 virtual height on the propagation delay time, for a given path, might be useful. To make this information available in an easily usable form, Fig. 5 was devised. This is a plot of virtual height, $h^{\prime}$, (in $\mathrm{km}$ ) versus $(\Delta t / n)(\mu s e c)$, (where $n$ is the number of hops in the path in question), along with a third parameter which is the great circle path length, $d_{g}$ divided by $n$. To simplify its use, only five discrete path lengths are plotted; 500, 1000, 1500, 2000 and $3000 \mathrm{~km}$; the latter curve being suitable also for the $4000 \mathrm{~km}$ path.

To use the chart, determine the desired stability, $\delta \dot{t}$, in propagation delay time $(\Delta t)$ and the nominal virtual height $\left(h^{\prime}\right)$ for the path. For example, say $h^{\prime}$ is $300 \mathrm{~km}$, the desired propagation delay time stability $(\Delta t / n)$ is $10 \mu s e c$, the great circle path length $\left(\mathrm{d}_{\mathrm{g}} / \mathrm{n}\right)$ is 3000 $\mathrm{km}$ and $\mathrm{n}=1$ (one-hop mode) (see Fig. 5). Then from the curve, the variations in virtual height, $\delta h^{\prime}$, must be less than $5 \mathrm{~km}$. 
IX. CHART FOR DETERMINING TOTAI

HE PROPAGATION DELAY

a. Introduction

In many timing problems, the use of the detailed curves given in Fig. 10 through Fig. 15 along with the chart in Fig. 1 is not necessary, if a lower precision than given in these charts is acceptable. In this case, the chart given in Fig. 6 may be used.

b. Use of Chart

This chart gives the total propagation delay time, per hop, for a given path. That js, the delay time in Fig. 6 includes the ground wave delay time in Fig, 1 plus the additional delay time in Figs. 10-15 caused by one reflection of the signal from the ionospheric layer.

It is assumed, in preparing the chart, that the total propagation time was that of one hop multiplied by the number of hops. Thus, the distance is given as $\left(\mathrm{d}_{\mathrm{g}} / \mathrm{n}\right)$, where $\mathrm{n}$ is the number of hops, and the path delay time as $\left(t_{p} / n\right)$.

For example, if the great circle distance, $\mathrm{d}_{\mathrm{g}}$, is $9,900 \mathrm{~km}$, then assuming a three-hop path, $\left(\mathrm{d}_{\mathrm{g}} / \mathrm{n}\right)$ would be $3300 \mathrm{~km}$, and the path delay time, as read on the chart, assuming a layer height of $350 \mathrm{~km}$, would be $\left(t_{p} / n\right)=11.5 \mathrm{~ms}$, which when multiplied by $n=3$, is $34.5 \mathrm{~ms}$.

\section{ASTRONOMICAL AND "ATOMIC" TIME}

a. Introduction

Until very recently the only unit of time available was that derived from the movements of the earth and it was called solar time. This is the time obtained by an observer on a given meridian marking the passages of the sun. Because of variations in the length of a "solar day" throughout the year, the mean solar day was used as the time standard; it is the mean of all the solar days throughout a given solar year. 
In 1955 a more uniform astronomical time was adopted as the international time standard and is called Ephemeris Time. It is defined by the orbital motion of the earth about the sun but in practice it is determined from the orbital motion of the moon about the earth.

The latest uniform time, as yet officially undefined, is based on the motions of the atoms or molecules subject to electrical and nuclear forces. Several standards are now in use employing either the cesium atorn or the ammonia molecule.

Definitions of a few quantities of interest to those using time signals or engaged in timing problems are given below.

b. Mean Solar Time ${ }^{8}$

(1) Mean Solar Second

The unit of mean solar time is the mean solar second and it is defined as the $(1 / 86,400)$ part of a mean solar day.

(2) Mean Solar Day

A mean solar day is defined as the mean of all the solar days in a solar year; there are 365 solar days in one solar year.

(3) Uniform Solar Time UT

(a) UT -0

This was the same as mean solar time; it was uncorrected for perturbations that are known to occur from year to year.

(b) UT - 1

Is mean solar time corrected for polar variations.

(c) UT-2

Is mean solar time corrected for polar variations and estimated annual fluctuations.

c. Ephemeris Time 8

The Ephemeris Second is defined as the fraction $1 / 31,556,925,9747$ of the tropical year for 1900 January 0 at 1200 hours Ephemeris Time. 
d. Atomic Time $\mathrm{e}^{8}$

The atomic second is at present not defined. However, if one maintains a frequency standard whose frequency is derived from the atom, then a properly geared clock driven by some submultiple of this frequency would keep "atomic time", its main merit being its uniformity.

e. Standard HF Time Signals

There are at present many nations broadcasting hf standard time signals, including the two USA stations (WWV, Washington, D. C., and WWVH, Maui, Hawaii), JJY (Tokyo, Japan), and MSF (Rugby, England). All known stations, as listed by the CCIR are given in Appendix E.

\section{USE OF TIME SIGNALS FROM MORE THAN ONE STATION}

If all the wor $1 d^{\prime} s$ hf standard time signals, as broadcast, were precisely synchronized, it would be possible to use any one of them in any timing problems and then when necessary switch to another one without loss of timing accuracy. Unfortunately, this is not the case at present. Except for WWV and WWVH, none of the transmitted standard hf time signals are synchronized.

This should be taken into account, for instance, when a missile tracking and timing problem arises wherein, say, the time signals from WWVH are used initially and then later a switch is made to the JJY standard time signais. Errors up to several milliseconds may occur, in this case, if suitable corrections are not made.

\section{ACKNOWIEDGMENTS}

The data used in plotting the curves in Fig. 10 to Fig. 15, inclusive, was obtained from Mr.A.G. Jean, who kindly consented to their use in this paper. Also, many helpful discussions were held with Mr.T.N. Gautier. 
1. "Comparing outputs from precision time standards," J.M. Shaull and C. M. Kortman, Electronics, p. 102 (April 1951).

2. "Measurement of time interval," chap. VI, Handbook of Electronic Measurements Vol. II, Polytechnical Inst. of Brooklyn, edited by Moe Wind (40 references given at end of chapter).

3. Measurements were made by Mr. H.F. Hastings, of the U.S. Naval Research Laboratory.

4. "Reference data for radio engineers," International Telephone and Telegraph Corp., pp. 732-735.

5. "Ionospheric radio propagation," Circular 462, NBS.

6. "Standard frequencies and time signals WWV and WWVH," Letter Circular LC 1023, June 1956.

7. "Ionospheric data, part A," CRPL F-Series, CRPL, National Bureau of Standards, Boulder Laboratories.

8. "Astronomical and atomic times," Wm. Markowitz, U.S. Naval Observatory, Washington 25, D.C. (March 1959). 
XIV. APPENDICES

APPENDIX A

GREAT CIRCLE DISTANCE, CALCULATIONS

1. Data Given (see Fig. 7)

\begin{tabular}{|c|c|c|}
\hline Site & Latitude & Longitude \\
\hline B & $38^{\circ} 59^{\prime} 33.16^{\prime \prime} \mathrm{N}$. & $76^{\circ} 50^{\prime} 52.35^{\prime \prime} \mathrm{w}$ \\
\hline A & $34^{\circ} 56^{\prime} 43.19^{\prime \prime} \mathrm{N}$. & $117^{\circ} 55^{\prime} 01.57^{\prime \prime}$ \\
\hline
\end{tabular}

3. Great Circle Distance (Z) Calculations 4

a. Equations Used

$$
\begin{aligned}
& \tan \left(\frac{y-x}{2}\right)=\cot (c / 2)\left[\frac{\sin \left(\frac{L_{B}-L_{A}}{2}\right)}{\cos \left(\frac{L_{B}+L_{A}}{2}\right)}\right] \\
& \tan \left(\frac{y+x}{2}\right)=\cot (c / 2)\left[\frac{\cos \left(\frac{L_{B}-L_{A}}{2}\right)}{\sin \left(\frac{L_{B}+L_{A}}{2}\right)}\right] \\
& \tan \left(\frac{Z}{2}\right)=\tan \left(\frac{L_{B}-L_{A}}{2}\right)\left[\frac{\sin \frac{y+x}{2}}{\sin \frac{y-x}{2}}\right]
\end{aligned}
$$

Angle $\mathrm{C}=$ longitude $\mathrm{A}$ - longitude $\mathrm{B}$ 
b. Calculation of $\left(\frac{L_{B}-L_{A}}{2}\right)$ and $\left(\frac{L_{B}+L_{A}}{2}\right)$

Sites

$B, A$

$$
\frac{\left(\mathrm{L}_{\mathrm{B}}-\mathrm{L}_{\mathrm{A}}\right)}{4^{\circ} 02^{\prime} 49.97^{\prime \prime}} \frac{\left(\frac{\mathrm{L}_{\mathrm{B}}-\mathrm{L}_{\mathrm{A}}}{2}\right)}{2^{\mathrm{O}} \mathrm{O} \mathrm{1}^{\prime} 24.98^{\prime \prime}}
$$

$\frac{\left(\mathrm{L}_{\mathrm{B}}+\mathrm{L}_{\mathrm{A}}\right)}{73^{\mathrm{O}} 56^{\prime} 16.35^{\prime \prime}}$

$$
\left(\frac{L_{B}+L_{A}}{2}\right)
$$

$36^{\circ} 58^{\prime} 08.17^{\prime \prime}$ $36^{\circ} 58.14^{\prime}$

\begin{tabular}{|c|c|c|c|}
\hline Sites & c & $c / 2$ & $\cot \mathrm{c} / 2$ \\
\hline$B$, & $41^{\circ} 04^{\prime} 09.22^{\prime \prime}$ & $\begin{array}{l}20^{\circ} 32.04,61^{\prime \prime} \\
20^{\circ} 32.077^{\prime}\end{array}$ & $2.6698^{\prime}$ \\
\hline
\end{tabular}

c. Determining Angle (c) and (c/2) and $\cot (\mathrm{c} / 2)$

d. Tabulation of Trigonometric Functions Needed

$$
\begin{array}{cccc}
\underline{\text { Angles }} & \underline{\cos } & \underline{\sin } & \underline{\tan } \\
\left(\frac{\mathrm{L}_{\mathrm{B}}-\mathrm{L}_{A}}{2}\right)=2^{\circ} 01.416^{\circ} & 0.99931 & 0.03531 & 0.03531 \\
\left(\frac{\mathrm{L}_{\mathrm{B}}+\mathrm{L}_{\mathrm{A}}}{2}\right)=36^{\circ} 58.14^{\prime} & 0.79896 & 0.60138 &
\end{array}
$$

e. Calculation of $(Z)$ in degrees

$$
\begin{gathered}
\tan \left(\frac{y-x}{2}\right)=2.6699\left(\frac{0.03531}{0.79896}\right)=(0.11799) \\
\left(\frac{y-x}{2}\right)=\tan ^{-1}(0.11799)=6^{\circ} 43.76^{\prime} \\
\sin \left(\frac{y-x}{2}\right)=\sin 6^{\circ} 43.76^{\prime}=0.11718 \\
\tan \left(\frac{y+x}{2}\right)=2.6699\left(\frac{0.99938}{0.60138}\right)=4.436
\end{gathered}
$$




$$
\begin{gathered}
\left(\frac{y+x}{2}\right)=\tan ^{-1}(4.4369)=77^{\circ} 17.93^{\prime} \\
\sin \left(\frac{y+x}{2}\right)=\sin 77^{\circ} 17.93^{\prime}=0.9755 \\
\tan \left(\frac{z^{9}}{2}\right)=0.03533\left(\frac{0.97552}{0.11703}\right)=0.2942 \\
\left(\frac{z^{9}}{2}\right)=\tan ^{-1}(0.29429)=16^{\circ} 23.6^{\prime} \\
z^{\circ}=32^{\circ} 47.2^{\prime}=32.79^{\circ}
\end{gathered}
$$

f. Converting $(Z)^{\circ}$ to $(Z)$ kilometers (or miles)

$$
\begin{gathered}
z_{\mathrm{km}}=\mathrm{z}^{\circ} \times 111.195 \mathrm{~km} / \mathrm{deg}=32.79 \times 111.195=\underline{\underline{3642 \mathrm{~km}}} \\
\mathrm{z}_{\mathrm{mi}}=0.6214 \mathrm{mi} / \mathrm{km} \times \mathrm{km}=0.6214 \times 3642=\underline{\underline{262 \mathrm{mi} .}}
\end{gathered}
$$

g. Determining the Mode of Propagation

For a great circle distance of 3650 miles, the minimum mode of propagation would be one-hop, i. e., the distance is less than the maximum of $4000 \mathrm{kms}$ for the one-hop mode. 
1. Definitions (Figure 8)

(a) $\mathrm{d}_{\mathrm{g}}^{\prime}=\mathrm{ac}=\mathrm{ce}$, great circle distance of one-hop path.

(b) $\mathrm{d}_{\mathrm{g}}=\mathrm{nd}_{\mathrm{g}}^{\prime}=$ aceg.... great circle distance of $\mathrm{n}$-hop path.

(c) $\mathrm{d}_{\mathrm{p}}^{\prime}=\mathrm{abc}=\mathrm{cde}$, sky wave distance of one-hop path.

(d) $\mathrm{d}_{\mathrm{p}}=\mathrm{nd}_{\mathrm{p}}^{\prime}=$ abcde, .... sky wave distance of $\mathrm{n}$-hop path.

2. Sky Wave Propagation Time (Based on Geometric Paths)

From Figure 8 , it is easily shown that, for path abc,

$$
d_{p}^{\prime}=2 \sqrt{2 r\left(r+h^{\prime}\right)(1-\cos \theta)+\left(h^{\prime}\right)^{2}}
$$

For simplification, let:

$$
h^{\prime} / r=\gamma, \text { and } 2(1-\cos \theta)=(2 \sin \theta / 2)^{2}
$$

Then, using (2) in (1), and factoring:

$$
d_{p}^{g}=4 r \sin (\theta / 2) \sqrt{(1+\gamma)+\left(\frac{\gamma}{2 \sin \theta / 2}\right)^{2}}
$$

Then, the propagation delay time, for the path $d_{p}^{\prime}$, is:

$$
\mathrm{t}_{\mathrm{p}}^{\prime}=\frac{\mathrm{d}_{\mathrm{p}}^{\mathrm{p}}}{\mathrm{c}}=\frac{4 \mathrm{r} \sin \theta / 2}{\mathrm{c}} \sqrt{(1+\gamma)+\left(\frac{\gamma}{2 \sin \theta / 2}\right)^{2}}
$$

Now, the ground wave propagation delay time for the great circle distance, $d_{g}^{\prime}=a c$, is:

$$
\mathrm{t}_{\mathrm{g}}^{\prime}=\frac{\mathrm{d}_{\mathrm{g}}^{\prime}}{\mathrm{c}}
$$


Now, from trigonometry, we know that:

$$
d_{g}^{\prime}=2 r \theta
$$

so,

$$
t_{g}^{\prime}=\frac{2 r \theta}{c}
$$

Then, using (7) in (4), we get:

$$
t_{p}^{\prime}=t_{g}^{\prime}\left[\left(\frac{\sin \theta / 2}{\theta / 2}\right) \sqrt{(1+\gamma)+\left(\frac{\gamma}{2 \sin \theta / 2}\right)^{2}}\right]
$$

This is the sky wave propagation delay time in terms of the great circle distance delay time. For more than one hop, say n-hops both sides of equation (8) are multiplied by $n$.

3. Difference in Sky Wave and Ground Wave Propagation Times

From (8) and (2):

$$
\Delta t=\left(t_{p}-t_{g}\right)=t_{g}\left(\frac{\sin \theta / 2}{\theta / 2}\right) \sqrt{\left[(1+\gamma)+\left(\frac{\gamma}{2 \sin \theta / 2}\right)^{2}\right]}-1
$$

If we let:

$$
(1+\gamma)=\mathrm{h},\left(\frac{\gamma}{2 \sin \theta / 2}\right)=\mathrm{p}, \text { and }\left(\frac{\sin \theta / 2}{\theta / 2}\right)=\mathrm{y}
$$

we may write (9) as:

$$
\Delta t=t_{g}\left(y \sqrt{h+p^{2}}-1\right)
$$


4. Great Circle Distance Determined from Path Distance

Multiply equation (8) by $n$, and solve for $t_{g}$ :

$$
t_{g}=\left[\frac{t_{p}(\theta / 2)}{\sin \theta / 2 \sqrt{(1+\gamma)+\left(\frac{\gamma}{\sin \theta / 2}\right)^{2}}}\right]
$$

from which it is obvious that:

$$
d_{g}=t_{g} c=\left[\frac{d_{p}}{\left(\frac{\sin (\theta / 2)}{\theta / 2}\right) \sqrt{(1+\gamma)+\left(\frac{\gamma}{2 \sin \theta / 2}\right)^{2}}}\right]
$$

5. Tables for Use with Equations (9) and (11)

TABLE I

(See Fig. 2)

$$
\left(\frac{\mathrm{d} g}{\mathrm{n}} \operatorname{versus} \theta / 2\right)
$$

$\left(\frac{\mathrm{d}}{\mathrm{n}}\right) \mathrm{km}$

444.4

888.9

1333.3

1777.8

2222.2

2666.7

3111.5

3555.5

4000.0 $\underline{(\theta / 2) \text { deg. }} \quad(\theta / 2) \mathrm{rad}$.

1

0.01745

2

0.03491

3

0.05236

4

0.06981

5

0.08727

6

0. 10472

7

0. 12217

8

0.13963

9

0.15708 
TABLE II

(y versus $\theta / 2)$

\begin{tabular}{|c|c|c|c|c|}
\hline$(\theta / 2)^{\circ}$ & $(\theta / 2) \mathrm{rad}$. & $\sin \theta / 2$ & $y$ & where: \\
\hline 1 & 0.01745 & 0.01745 & 1.0000 & \multirow{2}{*}{$y=\frac{\sin \theta / 2}{\theta / 2}$} \\
\hline 3 & 0.05236 & 0.05234 & 0.99962 & \\
\hline 6 & 0.10472 & 0.10453 & 0.99818 & \multirow{2}{*}{$\theta / 2=\frac{\phi}{4 n}$} \\
\hline 9 & 0.15708 & 0.15643 & 0.99586 & \\
\hline
\end{tabular}

TABLE III

(See Fig. 3)

$\left(h\right.$ versus $\left.h^{2}\right)$

$\begin{array}{cccc}\mathrm{h}^{8} & \underline{\gamma} & \underline{\mathrm{h}} & \text { where: } \\ 100 & 0.0157 & 1.0157 & \mathrm{~h}=(1+\mathrm{r}) \\ 150 & 0.0236 & 1.0236 & \\ 200 & 0.0314 & 1.0314 & \\ 250 & 0.0392 & 1.0392 & \gamma=\frac{\mathrm{h}^{\prime}}{\mathrm{r}} \\ 300 & 0.0471 & 1.0471 & \\ 350 & 0.0550 & 1.0550 & \mathrm{r}=6368 \mathrm{~km} \\ 400 & 0.0628 & 1.0628 & \end{array}$


1. Definition of Symbols Used (Fig. 9)

(a) $\mathrm{d}_{\mathrm{g}}=$ great circle path, A bd ... B.

(b) $\mathrm{d}_{\mathrm{p}}=\mathrm{n}$-hop path, Aabcd ...kB.

(c) $\delta \mathrm{t}=$ delay time signal suffers, at clock B, from its reception to its retransmission.

(d) $\Delta t=$ time difference between a transmitted and received pulse at $A$.

(e) $t_{0}=$ time first pulse leaves $\mathrm{A}$.

(f) $t_{1}=$ time first pulse arrives at $B$.

(g) $t_{2}=$ time retransmitted pulse leaves $B$.

(h) $t_{3}=$ time retransmitted pulse reaches $A$.

(i) $t_{p}=$ apparent path length.

Now:

$$
t_{1}=t_{0}+\left(\frac{d}{c}\right)=t_{0}+t_{p}
$$

and:

$$
t_{2}=t_{1}+\delta t=t_{0}+2 t_{p}+\delta t
$$

so:

$$
\Delta t=t_{3}-t_{0}=t_{0}+2 t_{p}+\delta t-t_{0}=2 t_{p}+\delta t
$$

Therefore:

$$
t_{p}=\left(\frac{\Delta t-\delta t}{2}\right)
$$

and:

$$
d_{p}=c t_{p}
$$




\section{MEASUR EMENT OF HF TIMING SIGNALS}

1. A Note on the Measurements

(a) Acknowledgments

The measurements of the propagation delay time of the hf signals ${ }^{6}$ from WWV to WWVH and return, and at Boulder, Colorado, were made by personnel of the National Bureau of Standards Boulder Laboratories at Maui, Hawaii, and Boulder, Colorado, of the Naval Research Laboratory, and of the U.S. Naval Observatory. In all cases, the measurement precision was at least 0.1 millisecond for each individual observation.

(b) Definitions of Symbols Used in Appendix D

$\mathrm{d}_{\mathrm{g}}=$ great circle path length.

$\mathrm{t}_{\mathrm{g}}=$ propagation delay time of a signal over a given $\mathrm{d}_{\mathrm{g}} \cdot$

$r=$ mean radius of earth; taken as $6368 \mathrm{~km}$.

$c=$ velocity of light in free space; taken as 300,000 $\mathrm{km} / \mathrm{sec}$.

$d_{p}=\begin{aligned} & \text { length of path of sky wave (ionospherically reflected) } \\ & \text { signal. }\end{aligned}$

$t_{\mathrm{p}}=$ propagation delay time of a signal over a given $\mathrm{d}_{\mathrm{p}}$.

$\sigma=$ standard deviation of a single observation.

$h^{7}=$ virtual height of the reflecting ionospheric layer.

$\mathrm{n}$ = number of hops (mode) of the signal propagated over a given $d_{p}$.

2. Measurement of Propagation Delay Times

(a) Data on Following Paths

$\underline{\mathrm{d}}$

(1) WWV to WWVH $7687 \mathrm{~km}$

(2) $W W V$ to Boulder $2430 \mathrm{~km}$

(3) WWVIH to

Boulder

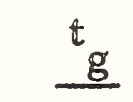

$25.62 \mathrm{~ms}$

$8.10 \mathrm{~ms}$

$17.56 \mathrm{~ms}$ min.

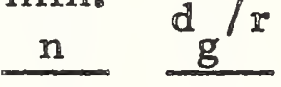

21.205

10.382

20.825 
(b) Calculated Propagation Delay Times (on above paths) versus Number of Hops (n)

(1) Figure $16-W W V$ to $W W V H$

(2) Figure 17 - WWV to Boulder

(3) Figure $18-W W V H$ to Boulder

(c) Measurements Made on Above Paths (September 1955)

$\underline{\text { Signals }}$

(1) WWV vs. WWVH

(2) WWVH vs. WWV

\begin{tabular}{cccc}
$\begin{array}{c}\text { Meas. } \\
\text { Made at }\end{array}$ & $\begin{array}{c}\text { Freq. } \\
\text { Mc }\end{array}$ & $\begin{array}{c}{ }_{\mathrm{p}} \\
\mathrm{ms}\end{array}$ & $\begin{array}{c}\sigma \\
\mathrm{ms}\end{array}$ \\
\cline { 5 - 5 } Maui & & & \\
& 5 & 27.77 & 0.26 \\
& 10 & 27.32 & 0.24 \\
& 15 & 27.13 & 0.22 \\
\cline { 2 - 4 } & ave. & 27.40 & 0.22
\end{tabular}

Wash. D. C.

\begin{tabular}{rrr}
5 & $-\cdots-$ & --- \\
10 & 27.16 & 0.19 \\
15 & $-\cdots--$ & $-\cdots$ \\
\hline ave. & 27.16 & 0.19
\end{tabular}

(3) WWV vs. WWVH Boulder

\begin{tabular}{rrr}
5 & 10.95 & 0.27 \\
10 & 11.31 & 0.28 \\
15 & 11.67 & 0.37 \\
\hline ave. & 11.31 & 0.31
\end{tabular}

(4) Actual Propagation Delay Time, Based on $a$ and $b$, above (round trip)

$\underline{\text { Path }}$

WWV to WWVH

(or vice versa)

\begin{tabular}{rrr}
5 & $\ldots--$ & --- \\
10 & 27.24 & 0.20 \\
15 & ---- & ---- \\
\hline 27.24 & 0.20
\end{tabular}


(5) Calculations of Actual Propagation Delay Times, Based on Above Measurements, at $10 \mathrm{Mc}$

Path $\quad \underline{\text { Freq., Mc }} \quad \underline{{ }_{\mathrm{p}}^{\mathrm{t}} \mathrm{ms}}$

WWV to Boulder WWVH to Boulder WWV to WWVH
10

10

10
8. 14

19. 10

27.24

16) Use of Charts in Figure 16,17 and 18 (with $\mathrm{h}^{\circ}=350 \mathrm{~km}$ ) to Determine Propagation Delay of Signals on

\begin{tabular}{|c|c|c|c|}
\hline & & $t$ & ${ }_{\mathrm{p}}^{\mathrm{t}}$ \\
\hline Path & $\mathrm{km}$ & $\mathrm{g}$ & $\mathrm{ms}$ \\
\hline
\end{tabular}

WWV to WWVH

$W W V$ to Boulder

WWVH to Boulder
7687

2430

5270
25.62

8. 10

17. 57
27.19

8.63

19.00
3

1

3

(7) Summary of Above Data at $10 \mathrm{Mc}$

\begin{tabular}{|c|c|c|c|c|}
\hline Path & $\begin{array}{l}\text { Meas. } \\
t_{p}, \mathrm{~ms}\end{array}$ & $\begin{array}{l}\text { Calc. } \\
t_{p}, \mathrm{~ms}\end{array}$ & $\begin{array}{c}\text { Difference } \\
\text { ms }\end{array}$ & $\mathrm{n}$ \\
\hline WWV to WWVH & 27.24 & 27.19 & 0.05 & 3 \\
\hline WWV to Boulder & 8.14 & 8.63 & 0.49 & 1 \\
\hline WWVH to Boulder & 19. 10 & 19.00 & 0.10 & 3 \\
\hline
\end{tabular}

(d) Second-to-Second Measurements, at $10 \mathrm{Mc}$, of Actual Delay Times of Path, WWV to WWVH (Based on Round Trip)

(1) Figure 20-WWV to WWVH, December 19, 1956

(2) Figure 19-WWV to WWVH, December 21, 1956

(3) Summary of Above 


\begin{tabular}{|c|c|c|c|c|c|c|c|}
\hline Fig. & Date & $\begin{array}{c}\text { UT } \\
\text { Time } \\
\end{array}$ & $\begin{array}{c}\text { No. } \\
\text { Observ. }\end{array}$ & $\begin{array}{l}\text { Ave. } \\
\mathrm{t} \\
\mathrm{p}, \mathrm{ms} \\
\end{array}$ & $\begin{array}{c}\text { Max. dev. } \\
\text { from ave., } \\
\text { ms } \\
\end{array}$ & $\begin{array}{c}\text { Min. } \\
\mathrm{t}_{\mathrm{p}}, \mathrm{ms} \\
\end{array}$ & $\sigma$ \\
\hline \multirow[t]{3}{*}{20} & $12-19$ & 0200 & 6 & 27.50 & 0.1 & 27.10 & 0.10 \\
\hline & & 0500 & 30 & 27.70 & 0.3 & 27.45 & 0.15 \\
\hline & & 1100 & 92 & 27.40 & 0.5 & 27.00 & 0.15 \\
\hline \multirow[t]{4}{*}{21} & $12-20$ & 0200 & 26 & 27.50 & 0.4 & 27.20 & 0.17 \\
\hline & & 0500 & 18 & 27.30 & 0.2 & 27.10 & 0.11 \\
\hline & & 0800 & 33 & 27.25 & 0.3 & 27.05 & 0.10 \\
\hline & Signif & Value & & 27.41 & 0.5 & 27.00 & 0.17 \\
\hline
\end{tabular}

(4) Conclusions Based on Above and Figure 16

\begin{tabular}{|c|c|c|c|c|c|}
\hline $\begin{array}{c}\text { Ave. of } \\
\text { ave. } t_{p}, \mathrm{~ms} \\
\end{array}$ & $\begin{array}{l}\text { Max. } \\
\sigma, \mathrm{ms} \\
\end{array}$ & $\begin{array}{l}\text { Min. } \\
\mathrm{t}, \mathrm{ms}\end{array}$ & $\begin{array}{c}\text { Probable } \\
\mathrm{n} \\
\end{array}$ & $\begin{array}{c}\text { Ave. } \\
\mathrm{d}_{\mathrm{p}}, \mathrm{km}\end{array}$ & $\begin{array}{c}\text { Probable } \\
\mathrm{h}^{\prime}, \mathrm{km} \\
\end{array}$ \\
\hline 27.41 & 0.17 & 27.00 & 3 & 8223 & 350 \\
\hline
\end{tabular}

(e) Hourly Measurements, at $10 \mathrm{Mc}$, on Path WWV to WWVH, June 1956

(1) Measurements, June 20

Meas.

$\underline{\text { Signals }}$

WWV vs. WWVH

WWVH vs. WWV made at

Wash.

D. C.

Maui
UT

Time

0600

0900

1200

0600

0900

1200
No.

Ave.

Observ. Diff., ms

29.01

28.72

29. 15

26.43

27.08

10

-.... $\sigma$

$\underline{m s}$

0.43

0.24

0.21

0.13

0.15 
(2) Average, Actual Propagation Delay Times (June 20)

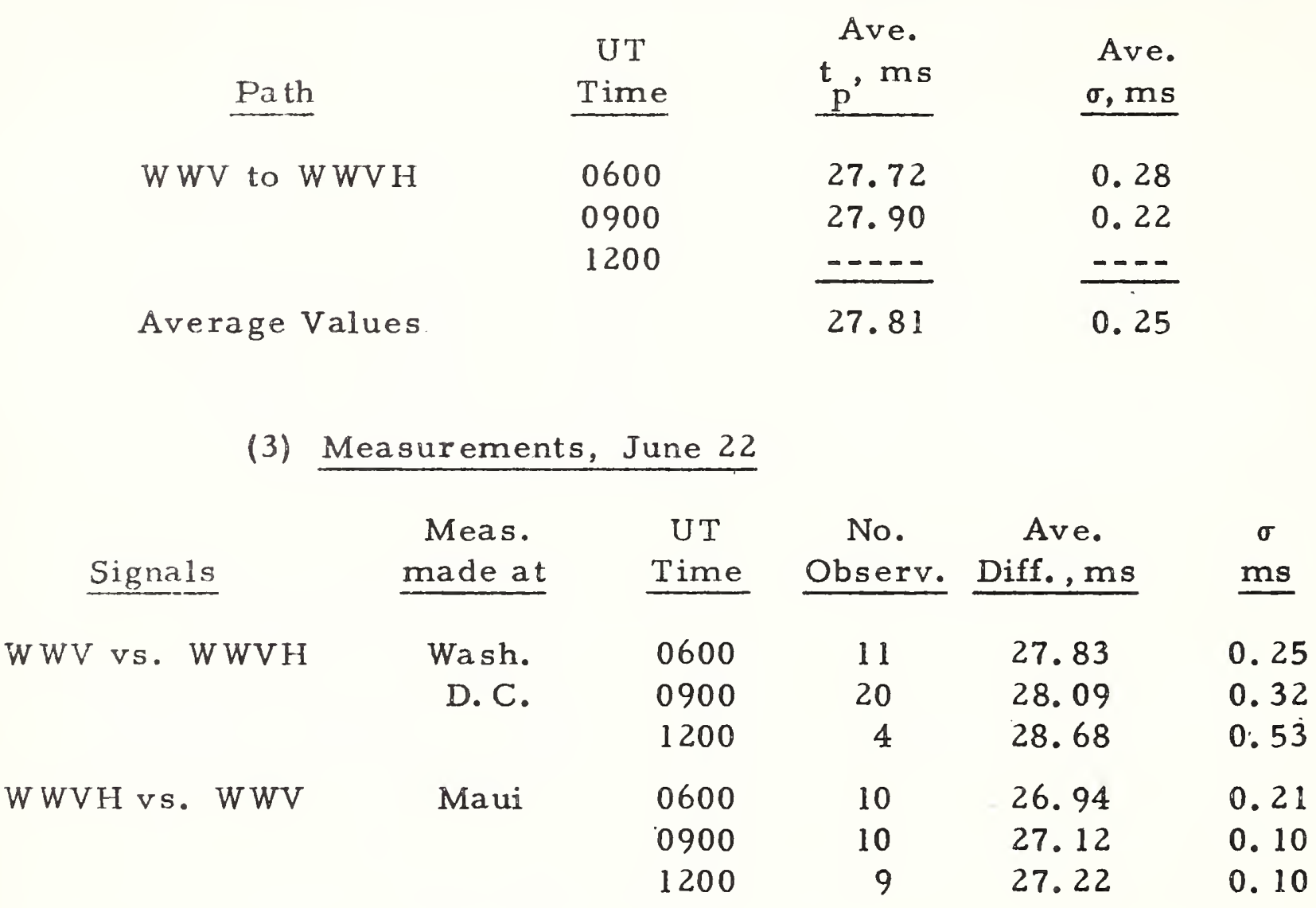

(4) Average Actual Propagation Delay Times (June 22)

\begin{tabular}{cccc} 
& UT & Ave. & Ave. \\
Path & $\underline{\mathrm{t}_{\mathrm{p}}, \mathrm{ms}}$ & $\underline{\sigma, \mathrm{ms}}$ \\
\hline
\end{tabular}

WWV to WWVH

0600

27.38

0.23

0900

27.60

0.21

1200

27.95

0.31

Average Values

27.64

0.25

(5) Summary of Above (Based on Round Trip Measurements)

\begin{tabular}{|c|c|c|c|c|}
\hline Day & Path & $\begin{array}{c}(3 \text { hour }) \\
\text { Ave. } t_{p}, \mathrm{~ms} \\
\end{array}$ & $\begin{array}{r}\text { (3 hour) } \\
\text { Ave, } \sigma, \mathrm{ms} \\
\end{array}$ & $\begin{array}{r}\text { (3 hour) } \\
\text { ave.path } \\
\text { length, km }\end{array}$ \\
\hline une 20 & $W W V$ to $W W V H$ & 27.81 & 0.25 & 8343 \\
\hline une 22 & WWV to WWVH & 27.64 & 0.25 & 8292 \\
\hline
\end{tabular}


(6) Conclusions from Above and Figure 16

\begin{tabular}{|c|c|c|c|c|}
\hline $\begin{array}{l}\text { Ave. } \\
t_{p}, \mathrm{~ms}\end{array}$ & $\begin{array}{l}\text { Ave. } \\
\sigma, \mathrm{ms}\end{array}$ & $\begin{array}{c}\text { Probable } \\
n\end{array}$ & $\begin{array}{c}\text { Ave. } \\
\mathrm{d}_{\mathrm{p}}, \mathrm{km}\end{array}$ & $\begin{array}{c}\text { Approx. } \\
h^{\prime}, \mathrm{km}\end{array}$ \\
\hline 27.75 & 0.25 & 4 & 8317 & 350 \\
\hline
\end{tabular}

Month

(b) Daily Measured Values of Actual Delay Times on Path WWV to $\mathrm{WWVH}, 1958$

(1) Figure 21 - January 1958 to September 1958 (Based on

(2) Summary of Figure 21 (WWV to WWVH)

Jan.

Min.

$\mathrm{t}$, ms

27.20

26.85

27. 10

27.35

27.25

27.45

27.20

27. 20

27. 30
Ave.

$t_{p}, \mathrm{~ms}$

27.52

27. 24

27.46

27.63

28. 12

27.85

27.65

27.59

27.67

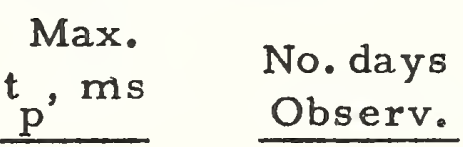

17

11

18

9

12

9

14

16

11
Ave.

$\mathrm{d}, \mathrm{km}$

8256

8172

8238

8289

8436

8355

8295

8277

8291

(3) Considerations from Above and Figure 16

a) Estimated Values of $n$ and $h^{\prime}$

Min.

Month

Jan.

Feb.

Mar.

Apr.

May

Jun.

Jul.

Aug.

Sep. Prob.

$\mathrm{t}_{\mathrm{p}}, \mathrm{ms}$

27.20

26.85

27. 10

27.35

27.25

27.45

27. 20

27.20

27.30

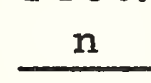

3

2

3

3

$\dot{3}$

3

3

3

3
Ave.

$\underline{\mathrm{t}}$, ms

27.52

27. 24

27.46

27.63

28. 12

27.85

27.65

27.59

27.67
Prob. Approx.

$\mathrm{n} \quad \mathrm{h}^{\prime}, \mathrm{km}$

325

355

380

330

375

350

330

330

330 
b) Summary of Data (Over a 9-Month Period)

\begin{tabular}{|c|c|c|c|c|c|c|}
\hline $\begin{array}{l}\text { Ave. } \\
\text { to ms }\end{array}$ & $\begin{array}{l}\text { Max. } \\
\mathrm{t}_{\mathrm{p}} \text {, ms } \\
\end{array}$ & $\begin{array}{l}\text { Min. } \\
\mathrm{t}, \mathrm{ms} \\
\end{array}$ & $\begin{array}{c}\text { Max. Dev. } \\
\text { from Ave., ms } \\
\end{array}$ & $\begin{array}{c}\text { Prob. } \\
\underline{\text { n }}\end{array}$ & $\begin{array}{r}\text { Ave. } \\
\mathrm{h}^{1}, \mathrm{~km} \\
\end{array}$ & $\begin{array}{r}\text { Ave. } \\
\mathrm{d}_{\mathrm{p}}, \mathrm{km}\end{array}$ \\
\hline 27. & 28.85 & 26.85 & 1.22 & 4 & 345 & 8289 \\
\hline
\end{tabular}

c) Conclusions (10 Mc)

1) The actual delay time variations from dayto-day (data taken same time every day) were taken about the average, about 1 millisecond or less.

4 hops.

2) In general, the mode of propagation was

near $350 \mathrm{~km}$.

3) Average virtual height of the layer was

(g) Summary of Above (Items c through $\mathrm{f}$ )

\begin{tabular}{|c|c|c|c|c|c|c|}
\hline Item & $\begin{array}{c}\text { Ave. } \\
\mathrm{t}, \mathrm{ms} \\
\end{array}$ & $\begin{array}{l}\text { Min. } \\
\mathrm{t}, \mathrm{ms} \\
\mathrm{p}\end{array}$ & $\begin{array}{c}\text { Prob. } \\
\mathrm{n}\end{array}$ & $\begin{array}{r}\text { Ave. } \\
\mathrm{d}_{\mathrm{p}}, \mathrm{km} \\
\end{array}$ & $\begin{array}{c}\text { Ave, or Prob. } \\
\mathrm{h}^{1}, \mathrm{~km}\end{array}$ & $\begin{array}{c}\text { Ave. } \\
\sigma \\
\end{array}$ \\
\hline (c) & 27.24 & $\ldots-$ & 3 & 8172 & 350 & $\ldots$ \\
\hline (d) & 27.41 & 27.00 & 3 & 8223 & 350 & 0.17 \\
\hline (e) & 27.75 & ---- & 4 & 8317 & 350 & 0.25 \\
\hline (f) & 27.63 & 26.85 & 4 & 8289 & 345 & $\ldots$ \\
\hline Average & 27.50 & 26.92 & & 8250 & 350 & 0.21 \\
\hline
\end{tabular}




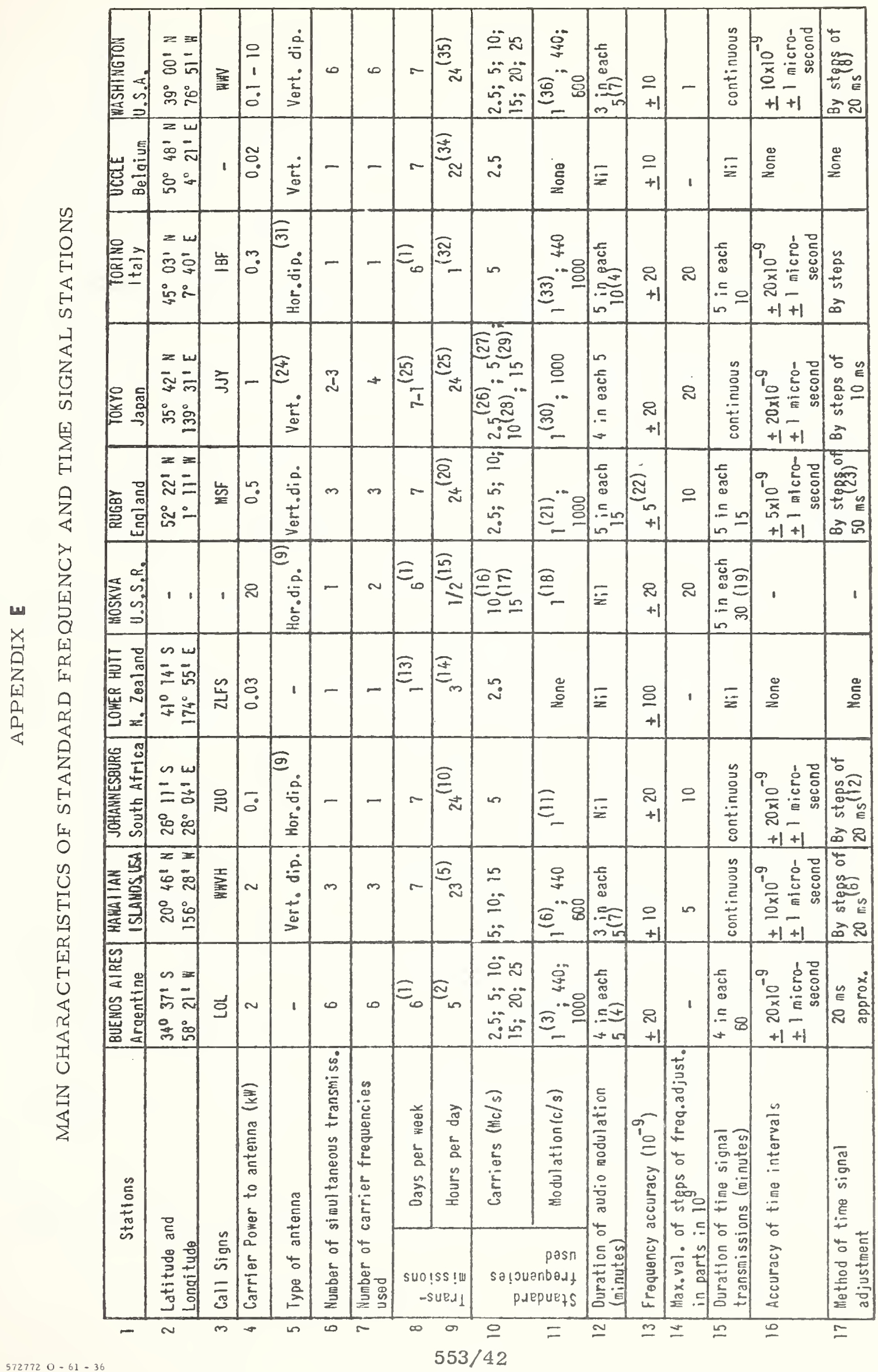


1. Weekdays.

2. From 11.00 to 12.00 , from 14.00 to 15.00 , from 17.00 to 18.00 , from 20.00 to 21.00 , and from 23.00 to $24.00 \mathrm{U}$. T.

3. Pulses of 5 cycles of $1000 \mathrm{c} / \mathrm{s}$ tone; no 59 th pulse of each minute.

4. Alternately 440 or $1000 \mathrm{c} / \mathrm{s}$.

5. Interruptions from minute 0 to minute 4 , and from minute 30 to minute 34 of each hour, as well as from 19.00 to 19.34 U. T.

6. Pulses of 6 cycles of $1200 \mathrm{c} / \mathrm{s}$ tone; no 59 th pulse of each minute.

7. Alternately 440 and $600 \mathrm{c} / \mathrm{s}$.

8. Adjustments are made on Wednesdays at 19.00 U. T. when necessary.

9. Maximum radiation $\mathrm{N}-\mathrm{S}$.

10. Inter ruption from 06.30 to $07.00 \mathrm{U} . \mathrm{T}$.

11. Pulses of 10 cycles of $1000 \mathrm{c} / \mathrm{s}$ tone; the first pulse of each minute is prolonged (500 ms).

12. If required, the first Monday of each month.

13. Tuesdays.

14. From 01.00 to 04.00 U.T.

15. From 07.15 to $07.45 \mathrm{U} . \mathrm{T}$.

16. Even days.

17. Odd days.

18. Signals Al keyed. Duration of each signal $100 \mathrm{~ms}$; the first signal of each minute is prolonged.

19. From 07.15 to 07.18 , and from 07.43 to 07.45 U.T.

20. Interruption from minute 15 to minute 20 of each hour.

21. Pulses of 5 cycles of $1000 \mathrm{c} / \mathrm{s}$ tone; the first pulse of each minute is prolonged (100 $\mathrm{ms})$.

22. Relative to an atomic standard.

23. Adjustments are made the first day of the month, when necessary. 
24. Two half-wave dipoles on $15 \mathrm{Mc} / \mathrm{s}$; one half-dipole on 2.5 and $5 \mathrm{Mr} / \mathrm{s}$.

25. See (26) to (29).

26. From 07.00 to 23.00 U. T.; interruption from minute 29 to minute 39 of each hour.

2.7. Mondays. Inter ruption from minute 9 to minute 19, from minute 29 to minute 39, and from minute 49 to minute 59 of each hour.

28. Wednesdays. Interruptions as for (27).

29. From 21.00 to 11.00 U. T. Interruptions as for (26).

30. Transmission suspended for $20 \mathrm{~ms}$; the suppression before second 0 lasts $200 \mathrm{~ms}$.

31. Maximum radiation NW-SE.

32. From 07.00 to 07.30 and from 11.00 to 11.30 U. T.

33. Pulses of 5 cycles of $1000 \mathrm{c} / \mathrm{s}$ tone; the first pulse of each minute is repeated 7 times at intervals of $10 \mathrm{~ms}$.

34. Interruptions from 11.30 to 12.30, and from 20.30 to 21.30 U. T.

35. Inter ruption from minute 45 to minute 49 of each hour.

36. Pulses of 5 cycles of $1000 \mathrm{c} / \mathrm{s}$ tone; no 59 th pulse of each minute. The first pulse of each minute is repeated $100 \mathrm{~ms}$ later. 


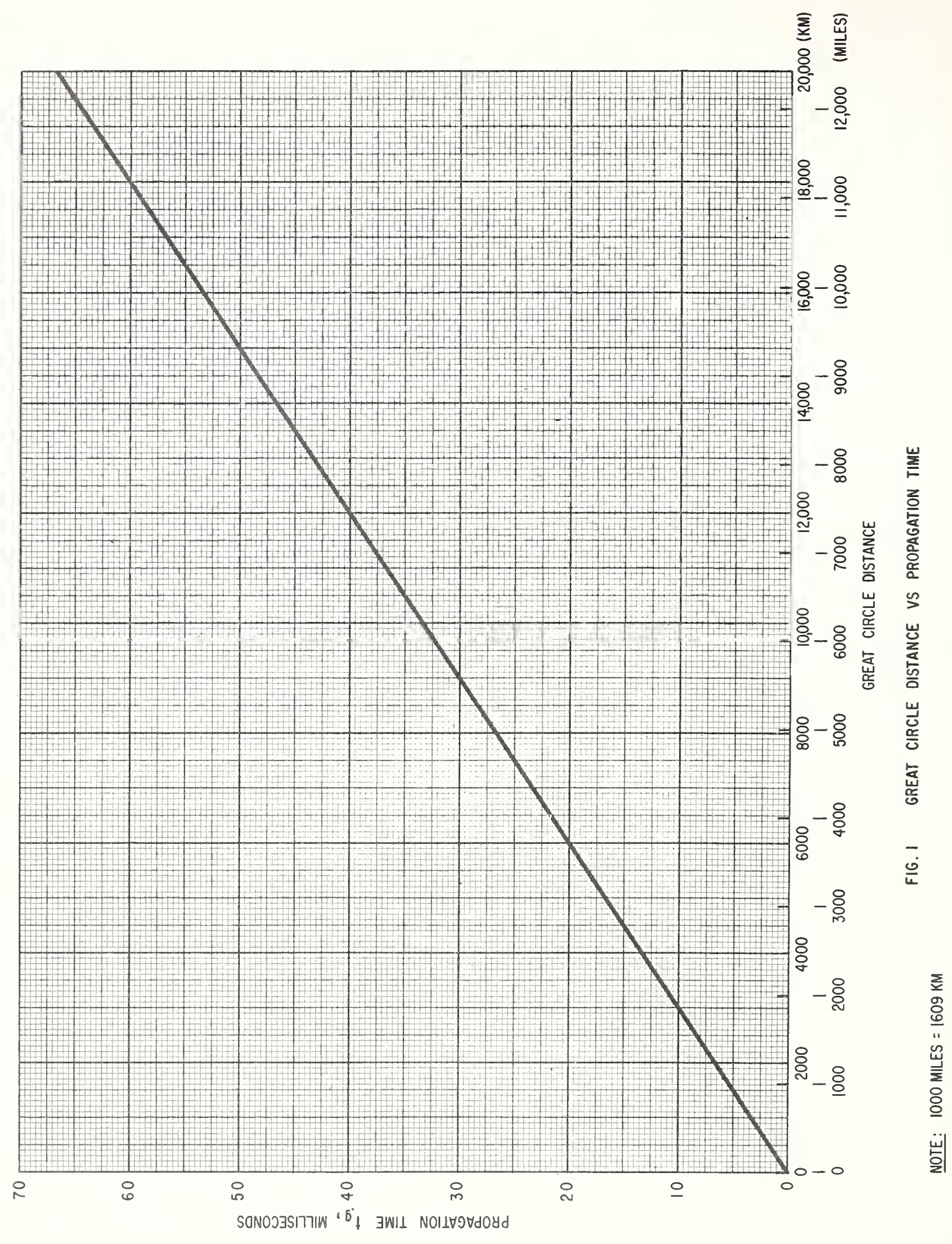




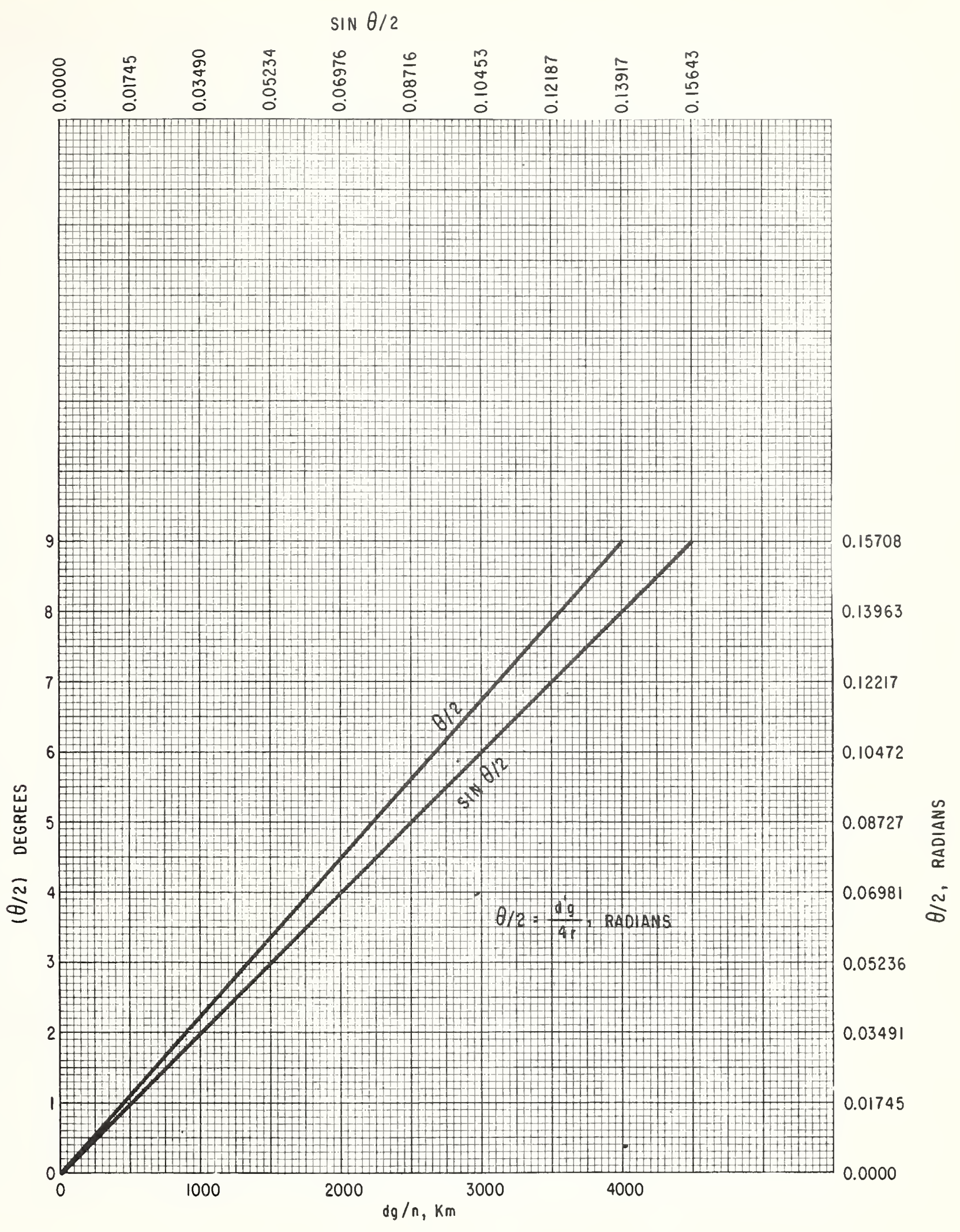

FIG. 2 PLOT OF TABLE I 


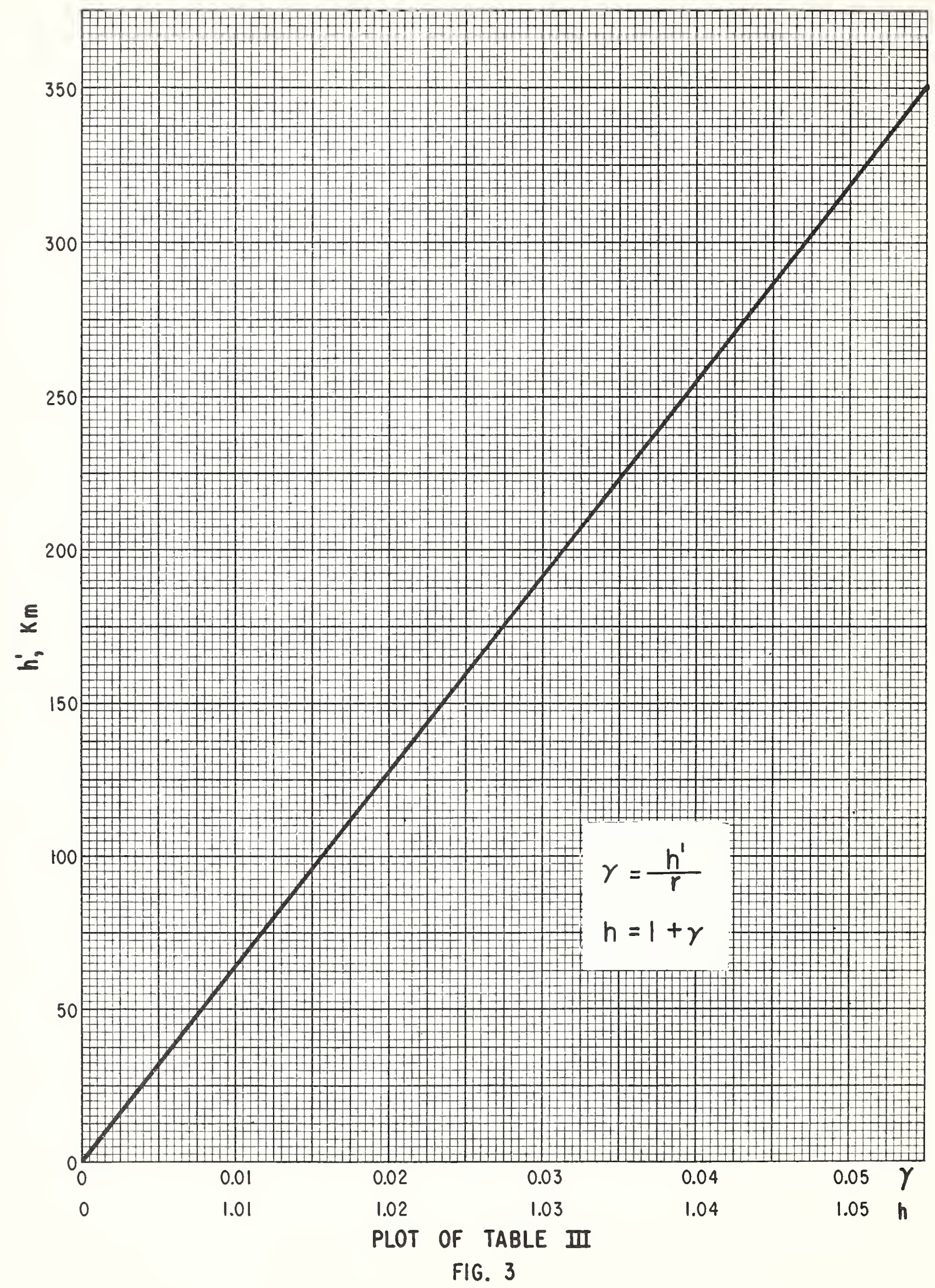




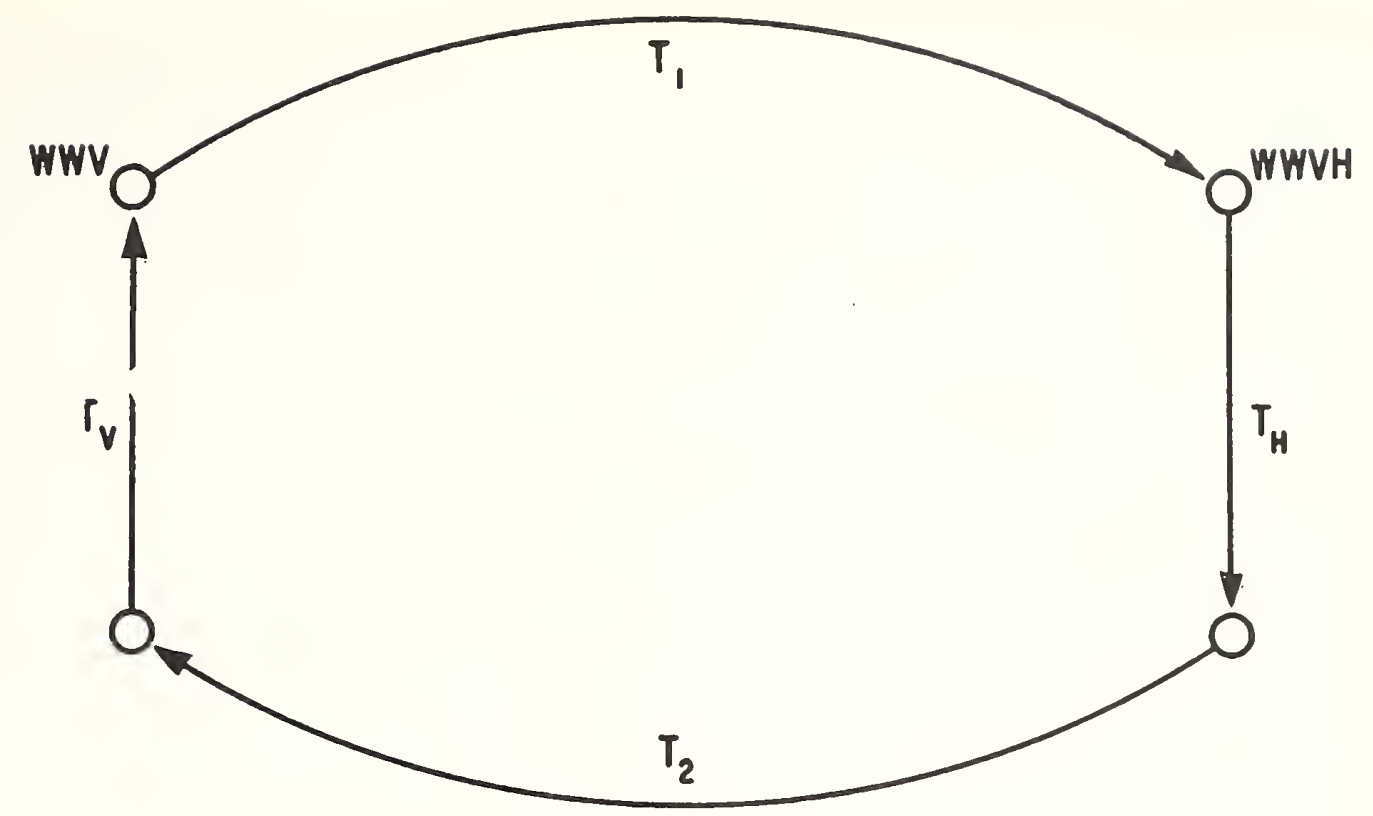

FIG.4 ILLUSTRATION OF DELAY TIMES, WWV AND WWVH

$T_{1}=$ delay time of signal from $W W V$ to $W W V H$

$\mathrm{T}_{2}=$ delay time of signal from $\mathrm{WWVH}$ to $\mathrm{WWV}$

$\mathrm{T}_{\mathrm{H}}=$ delay time introduced into pulses transmitted from WWVH

$\mathrm{T}_{\mathrm{V}}=$ delay time measured at $\mathrm{WWV}$ of received $\mathrm{WWVH}$ time signals

Then:

and

$$
\text { (1) } \quad\left(T_{1}-T_{H}+T_{2}\right)=T_{v} \text {, }
$$

(2) $\mathrm{T}_{1}+\mathrm{T}_{2}=\mathrm{T}_{\mathrm{V}} \mathrm{T}_{\mathrm{H}}$,

therefore, $\mathrm{T}_{\mathrm{p}}=\frac{\mathrm{T}_{1}+\mathrm{T}_{2}}{2}=\frac{\mathrm{T}_{\mathrm{v}}+\mathrm{T}_{\mathrm{H}}}{2}$ 


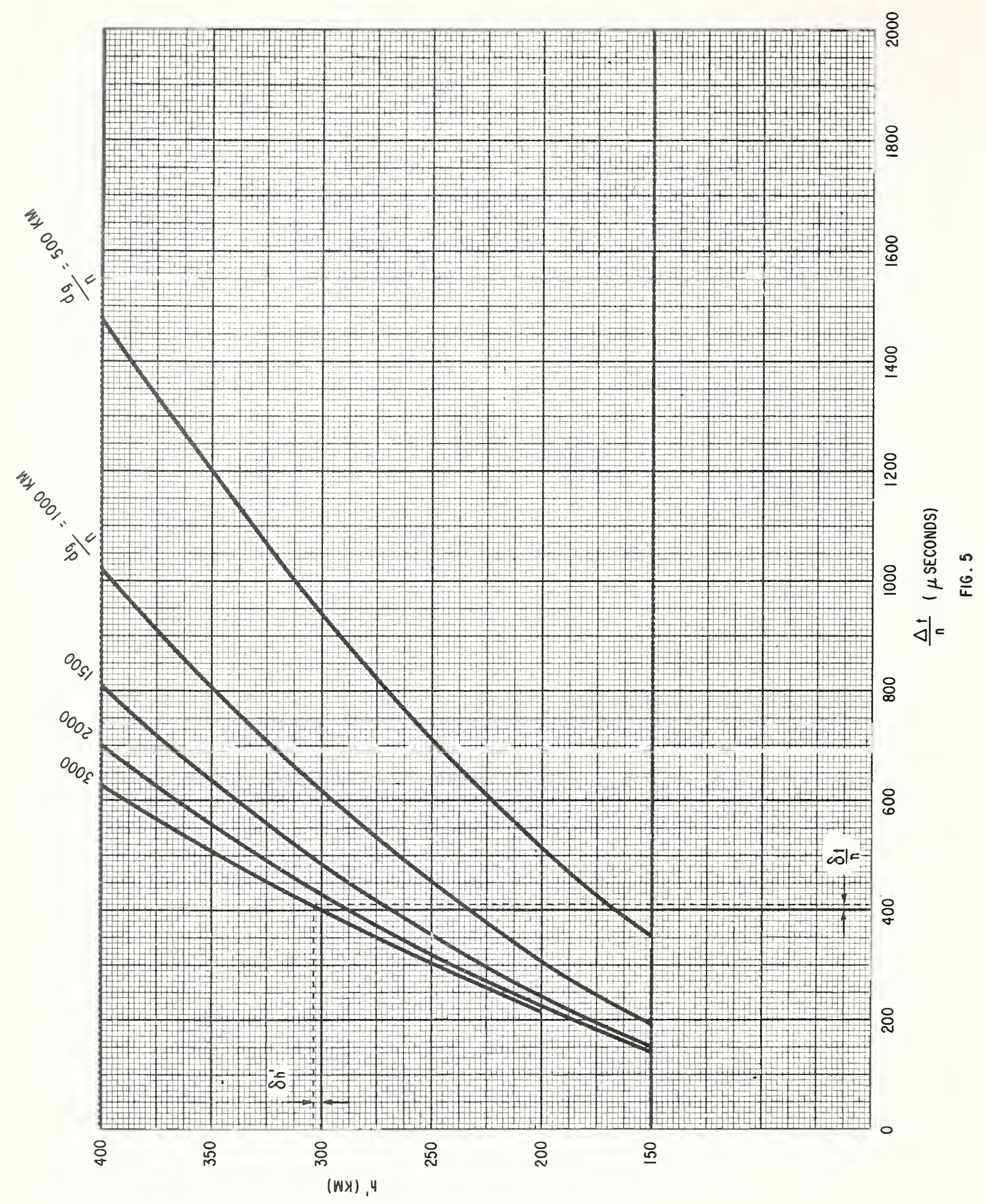




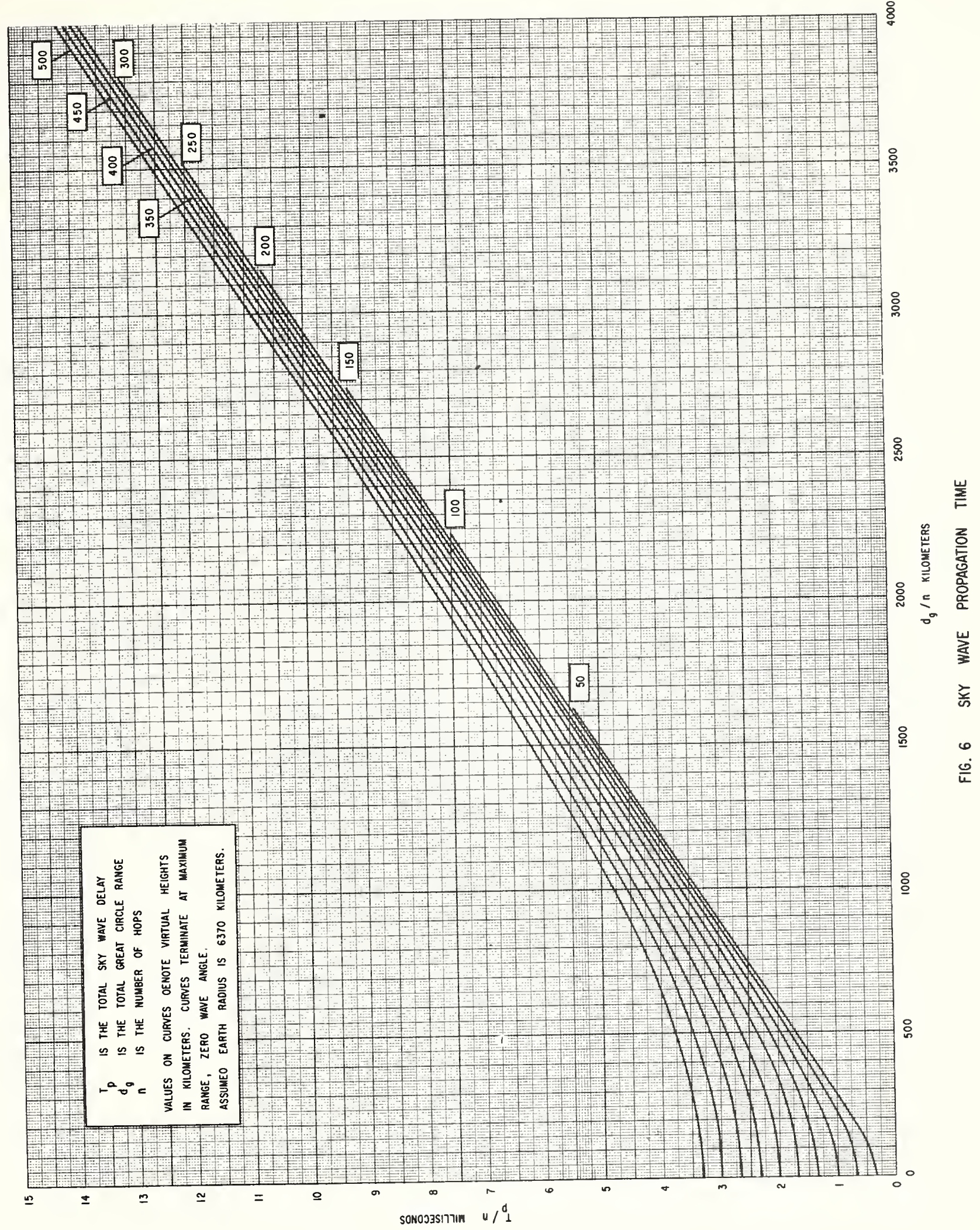




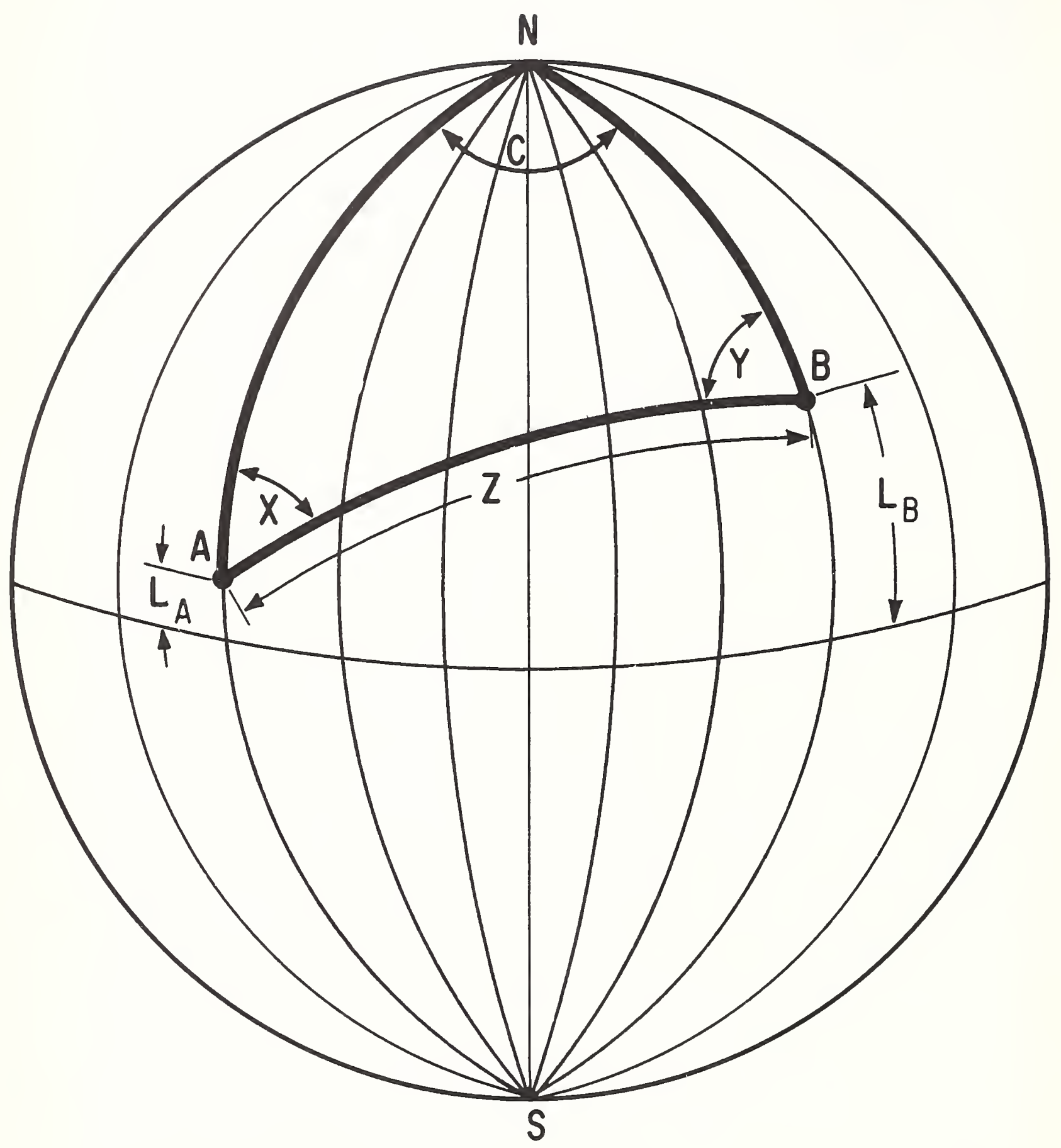

FIG. 7 GREAT CIRCLE DISTANCE CALCULATIONS 


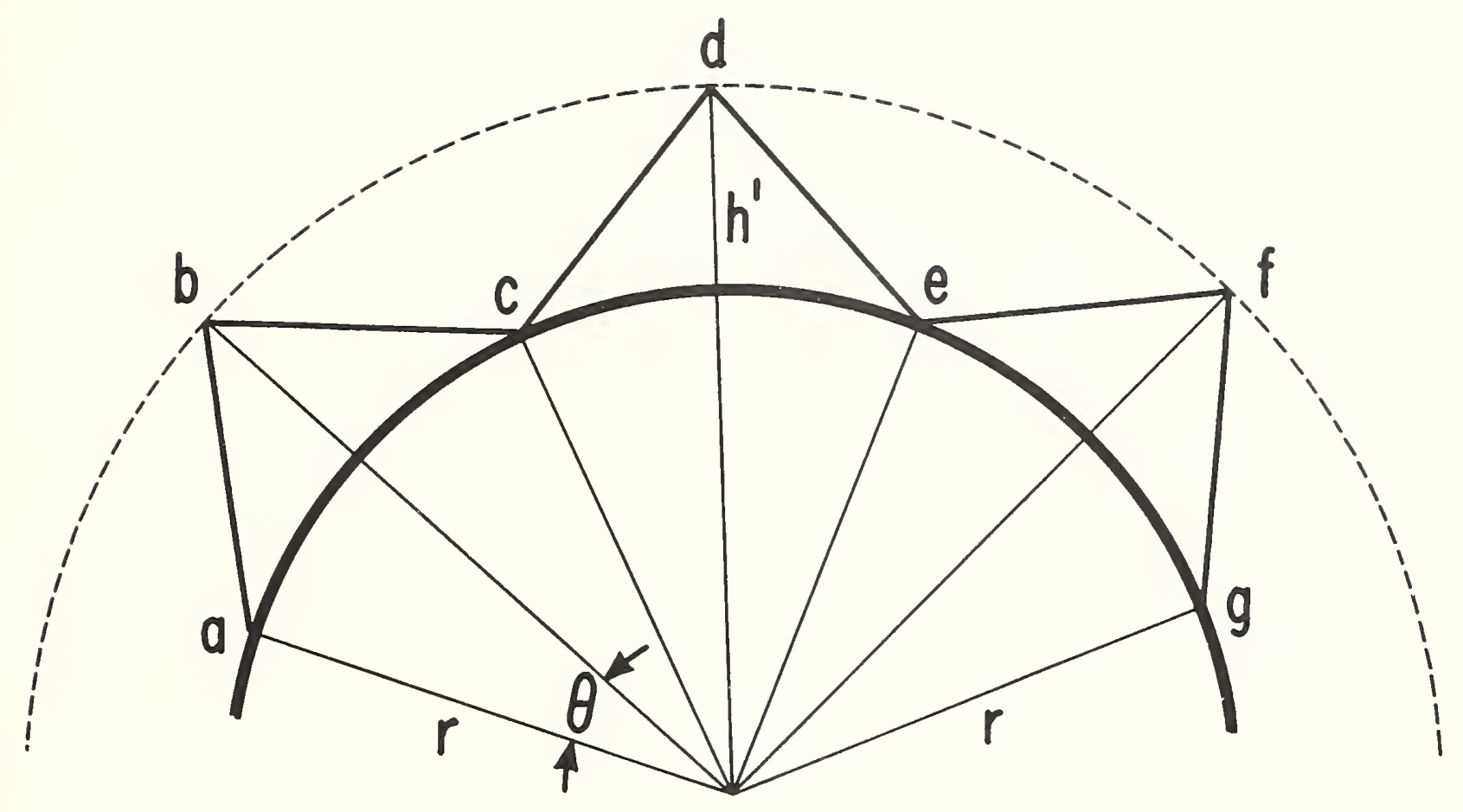

FIG.8 GEOMETRY OF THREE - HOP MODE 


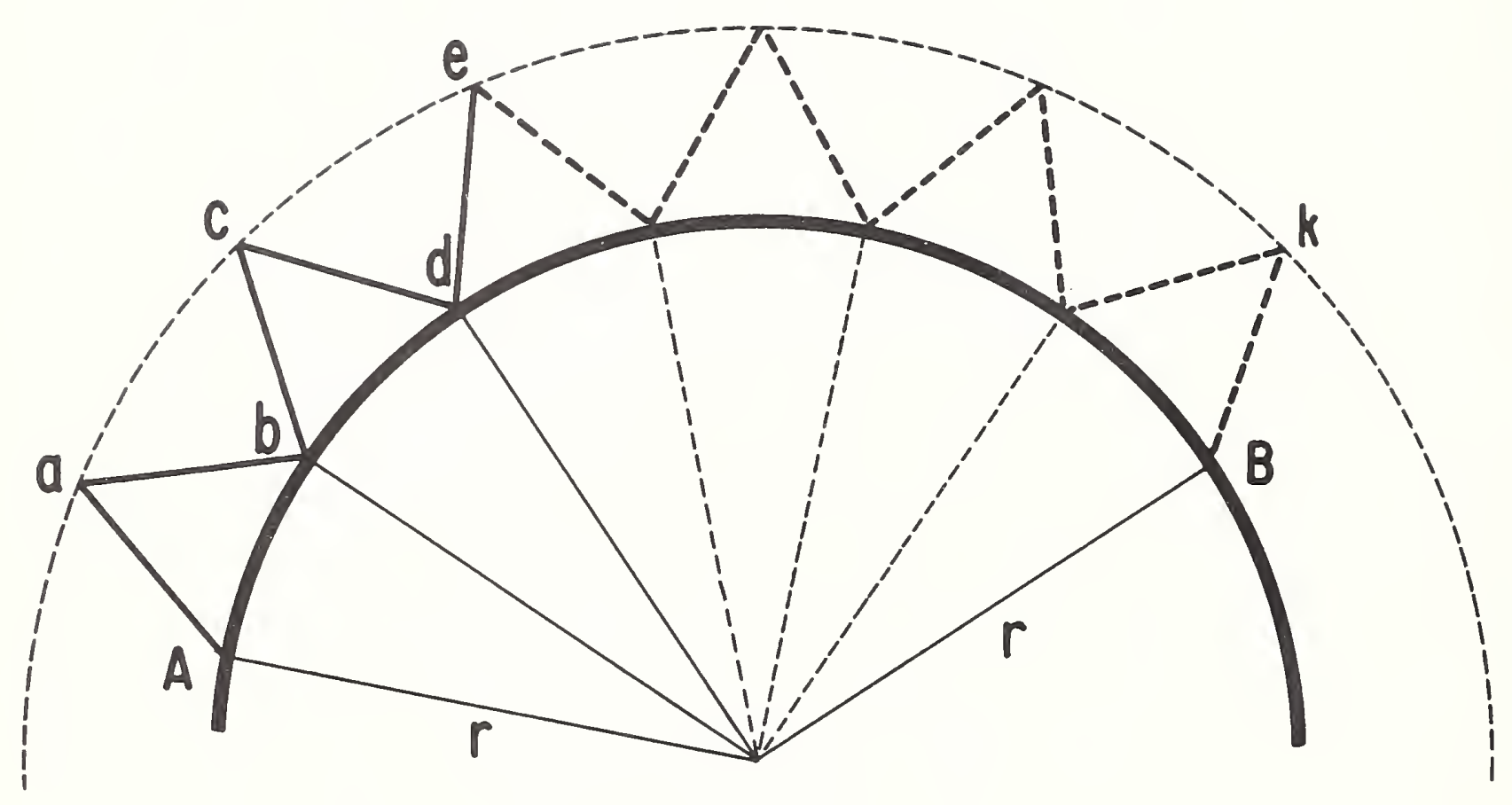

FIG.9 GEOMETRY OF $n$-HOP MODE 


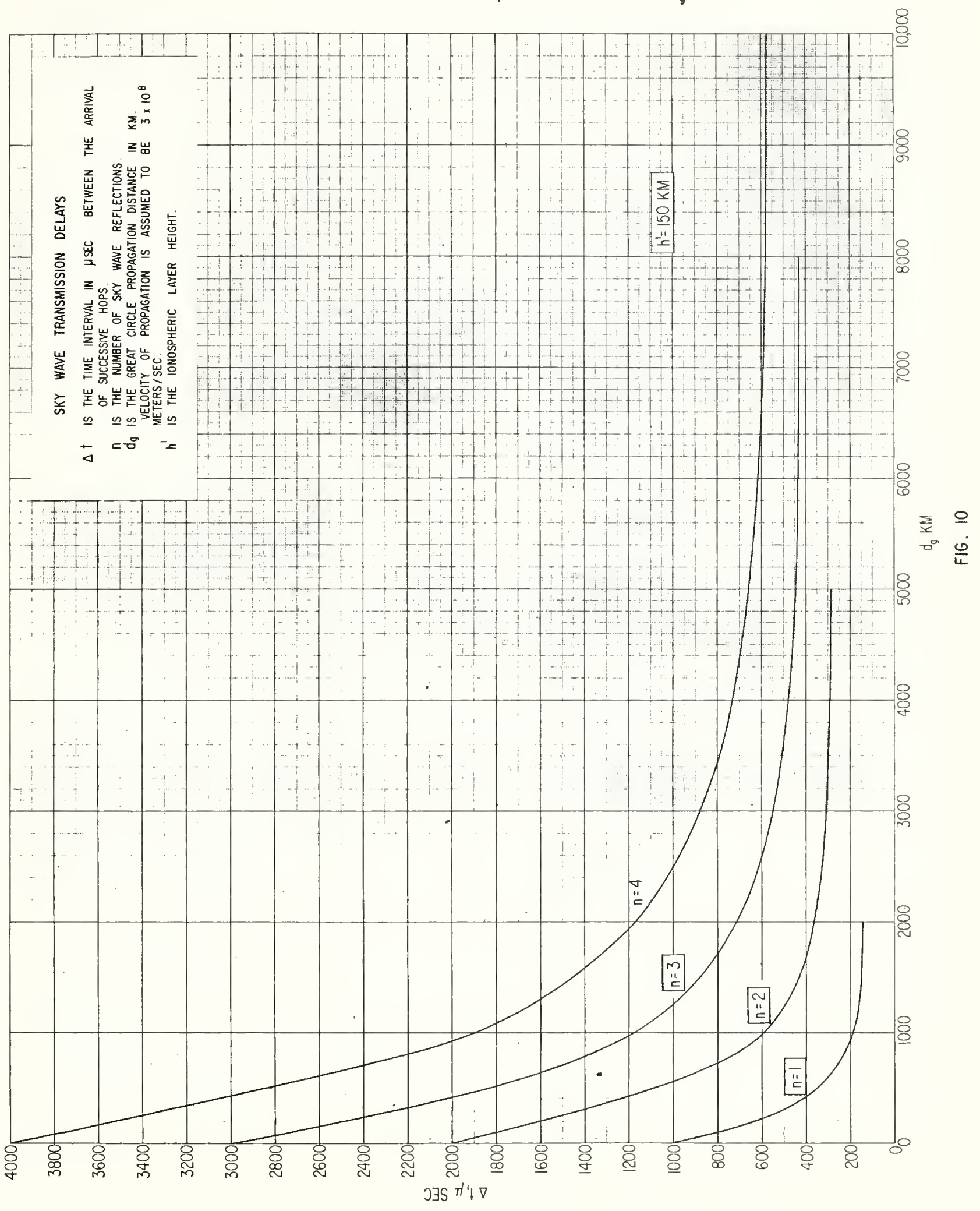




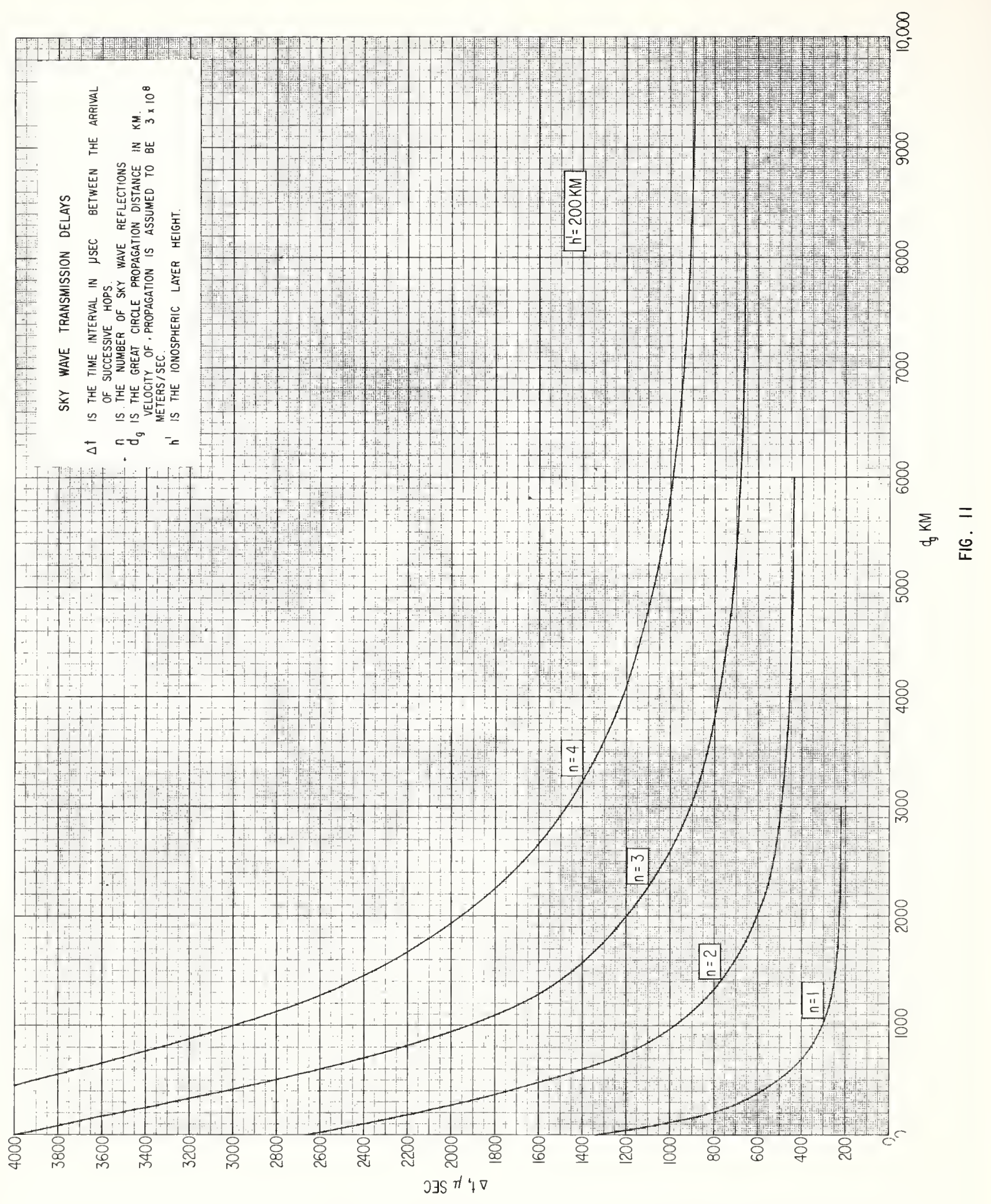




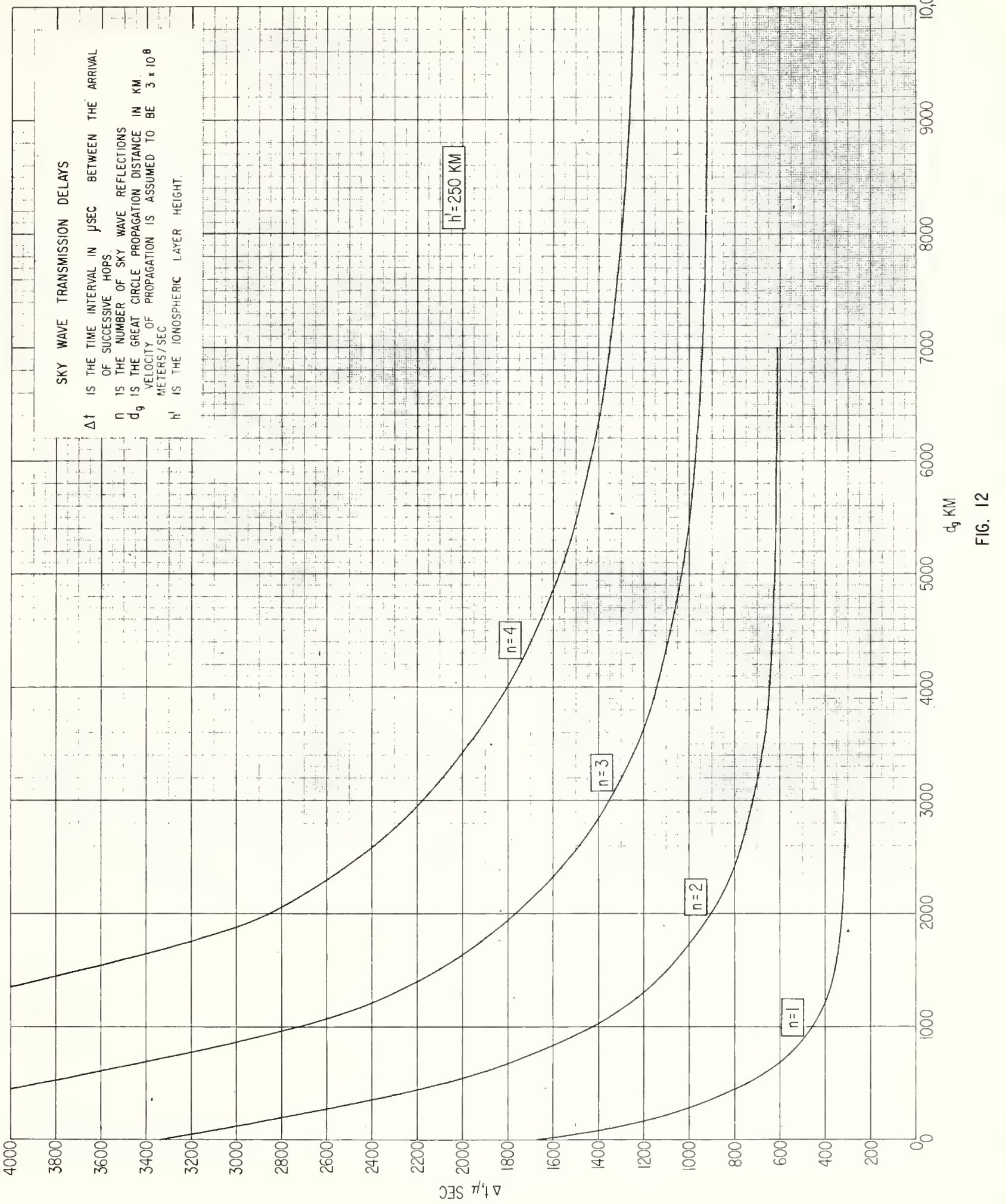




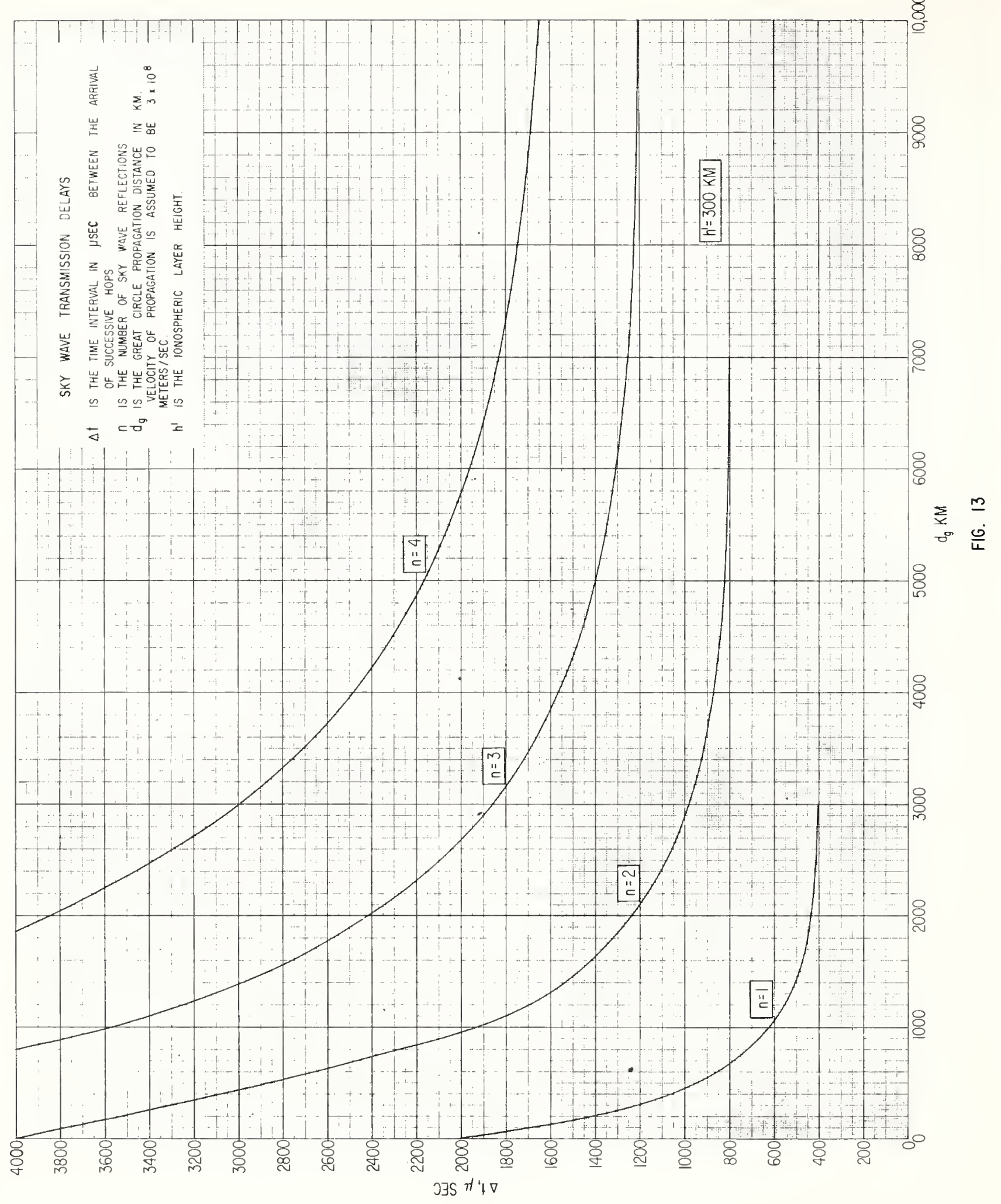




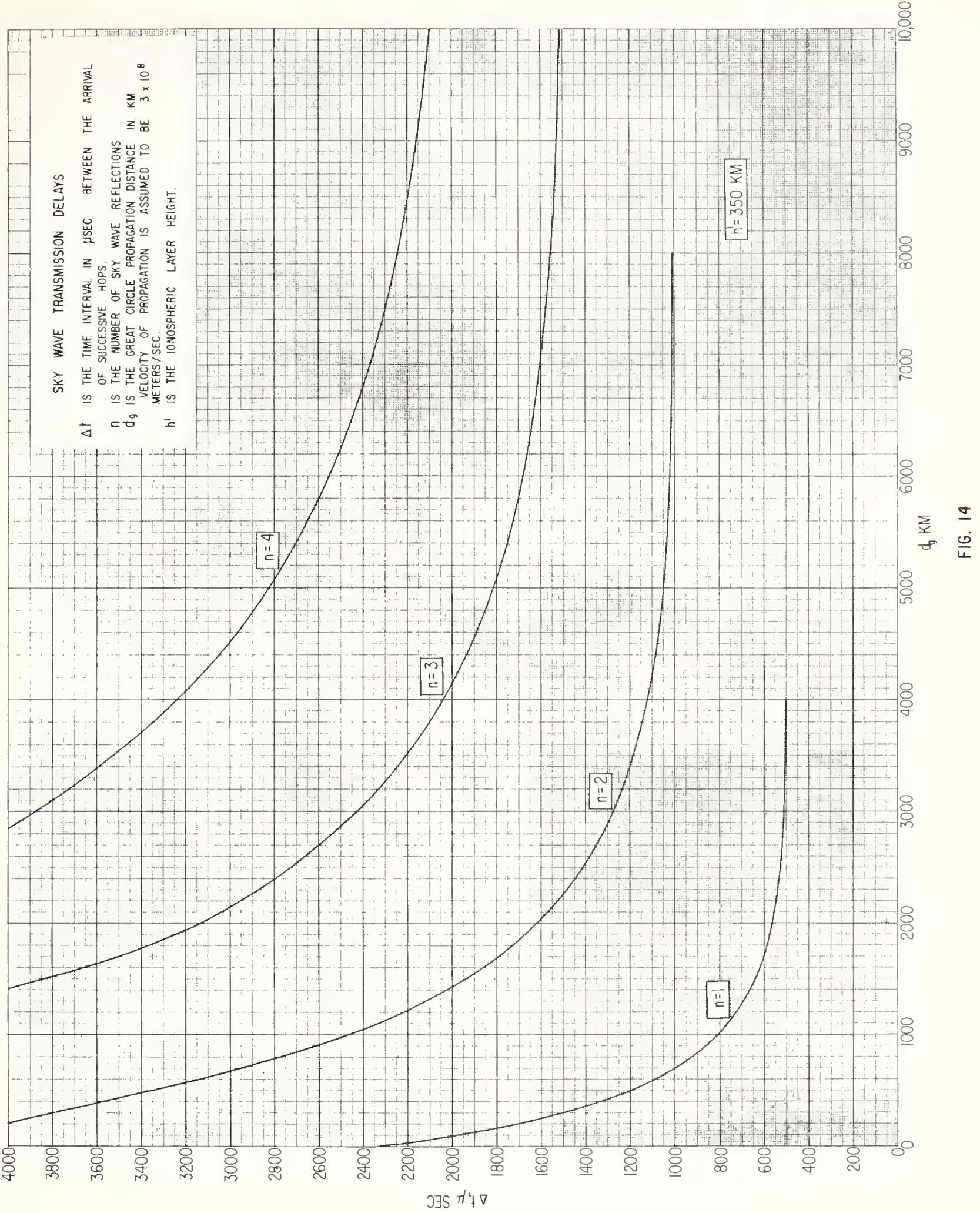




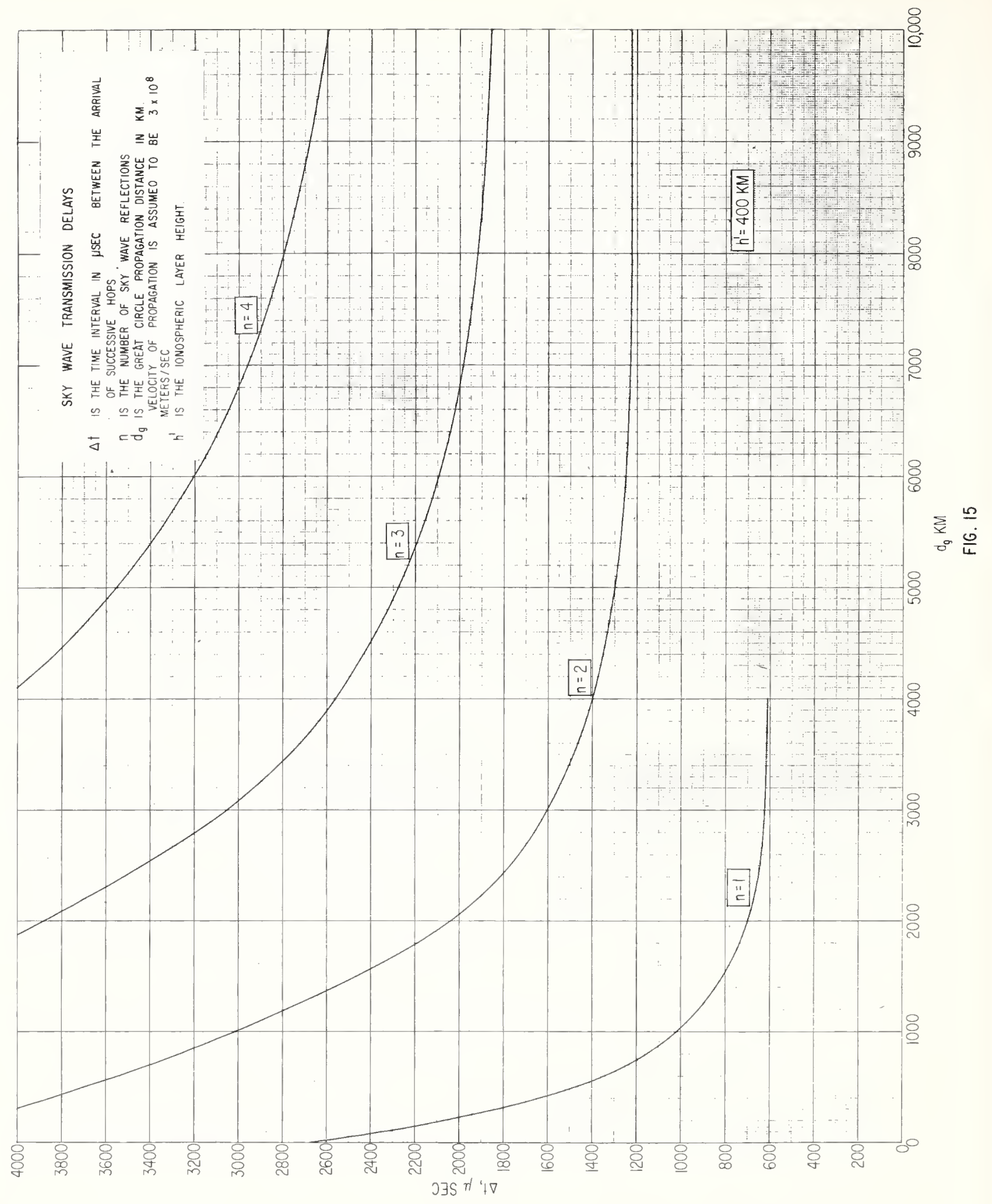




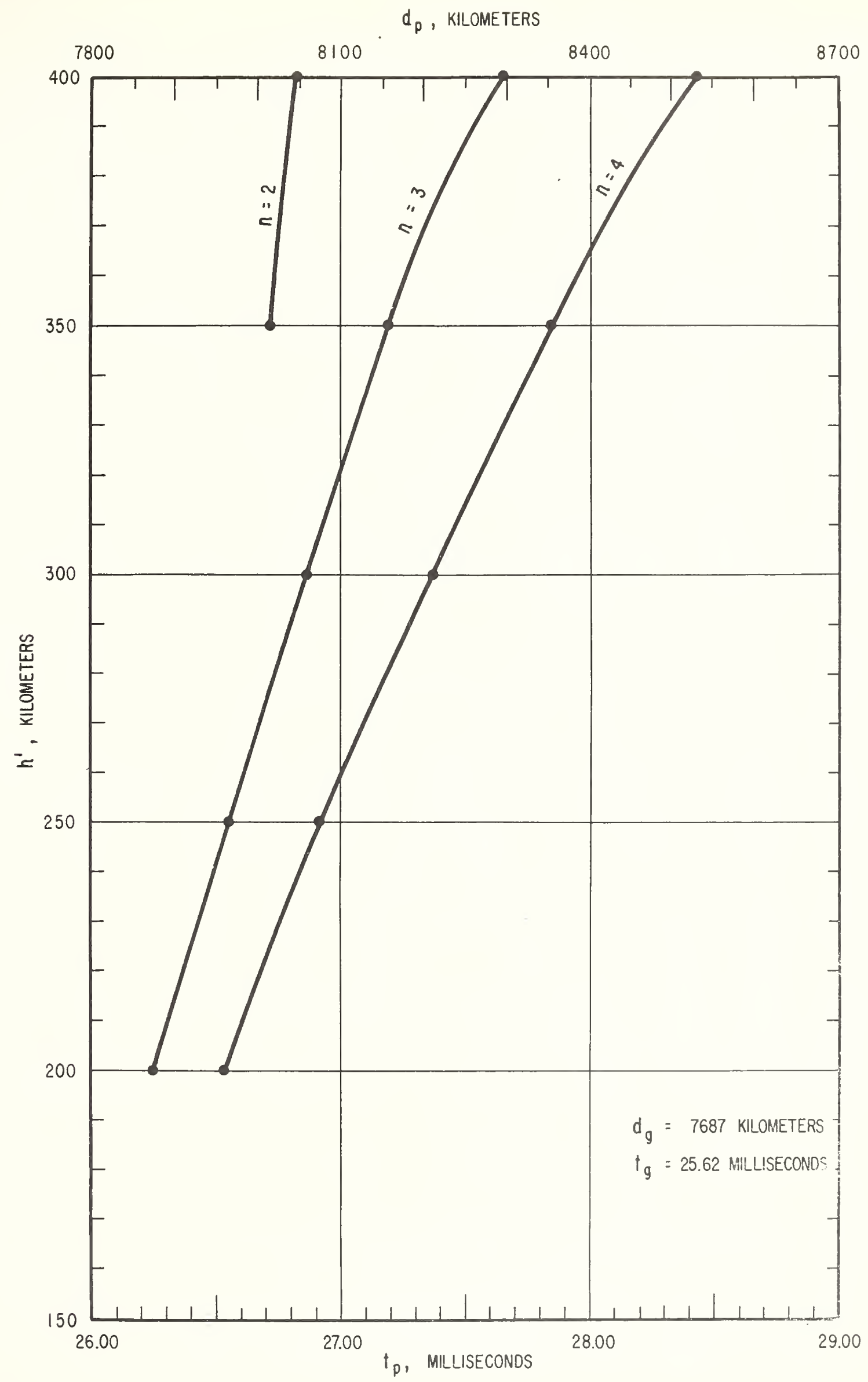

FIG. 16 CALCULATED PROPAGATION DELAY TIME WWV TO WWVH 


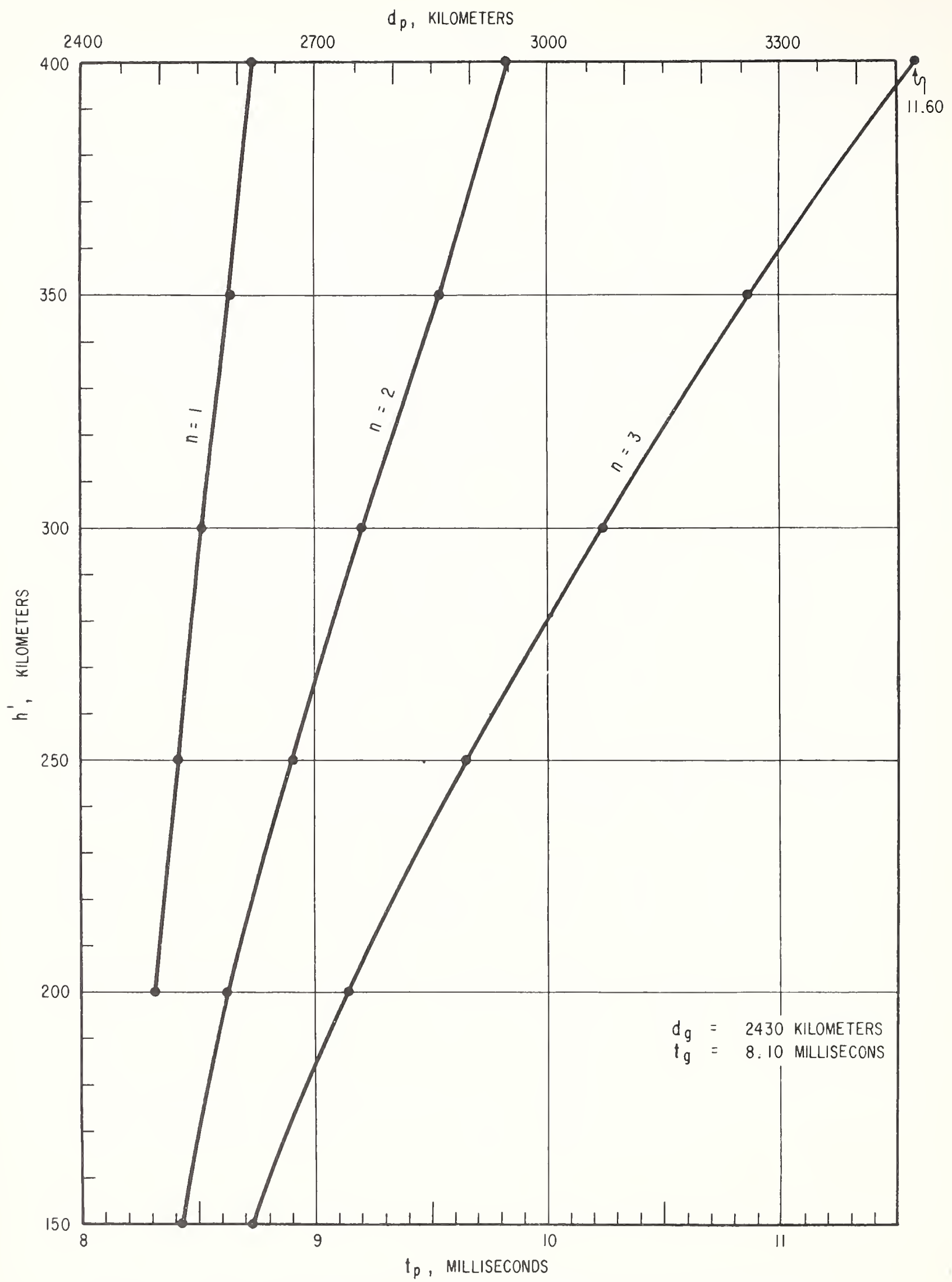

FIG. 17 CALCULATEd PROPAGATION DELAY TIME WWV TO BOULDER 


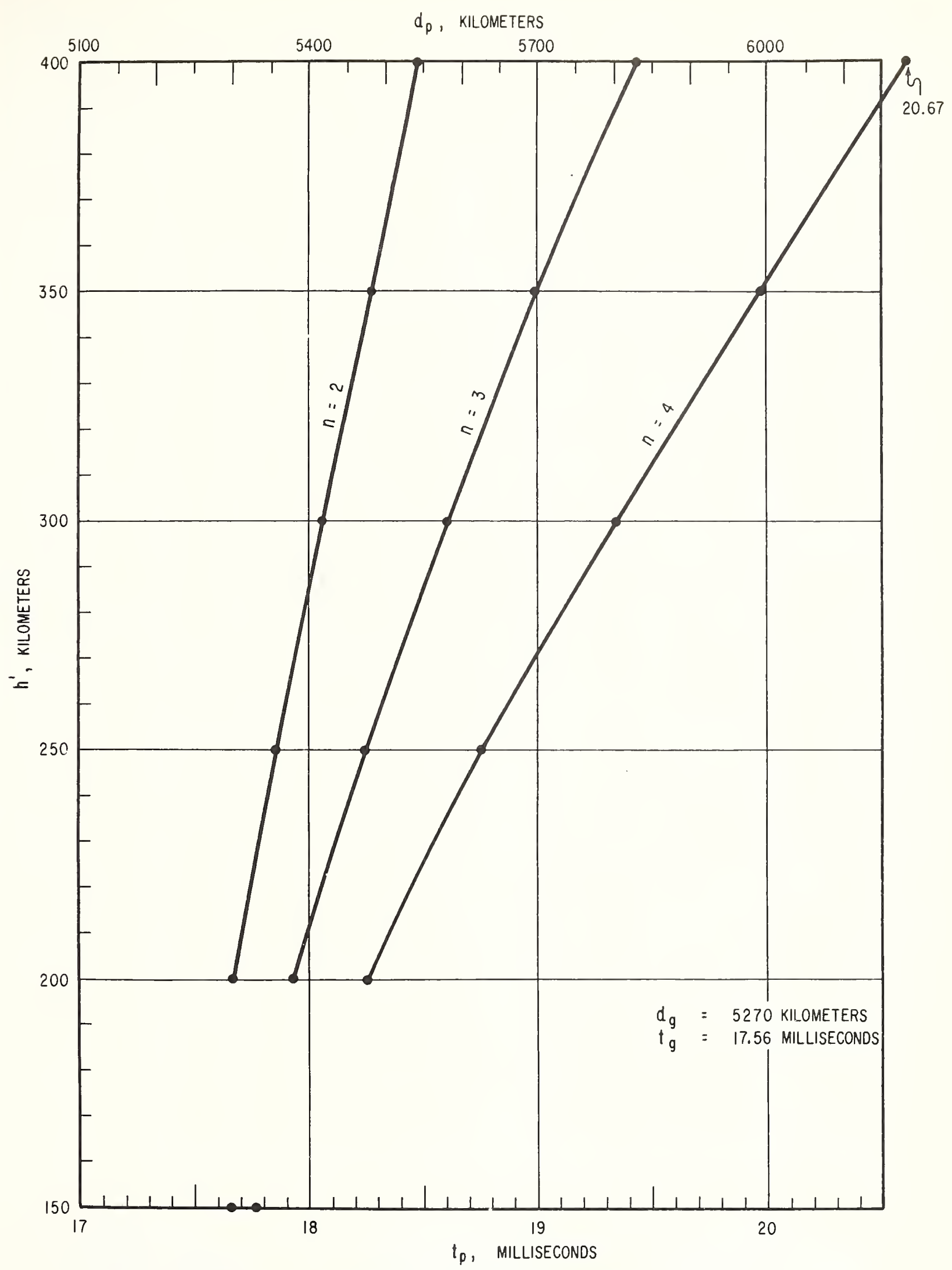

FIG. 18 CALCULATED PROPAGATION DELAY TIME WWVH TO BOULDER 


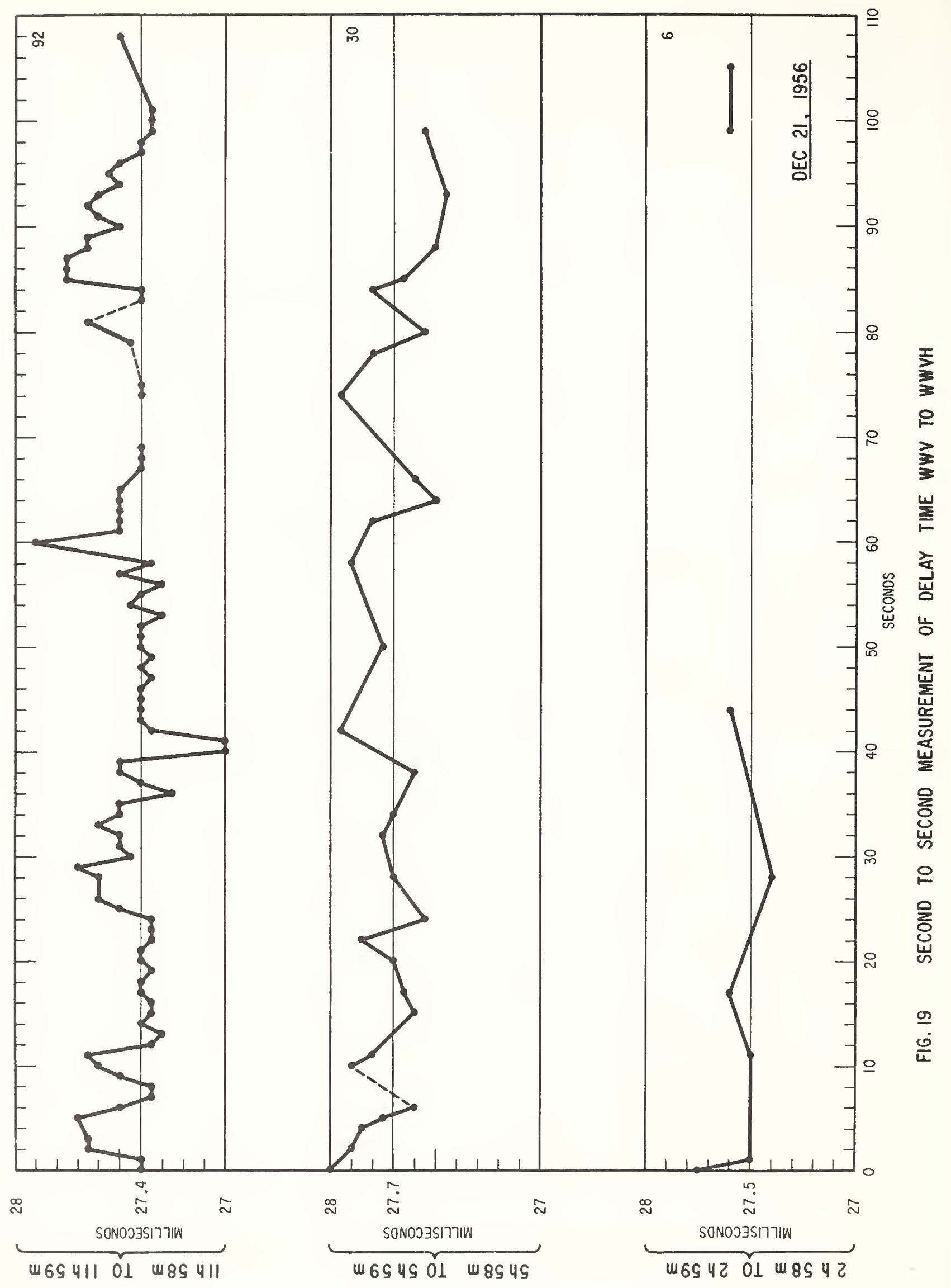




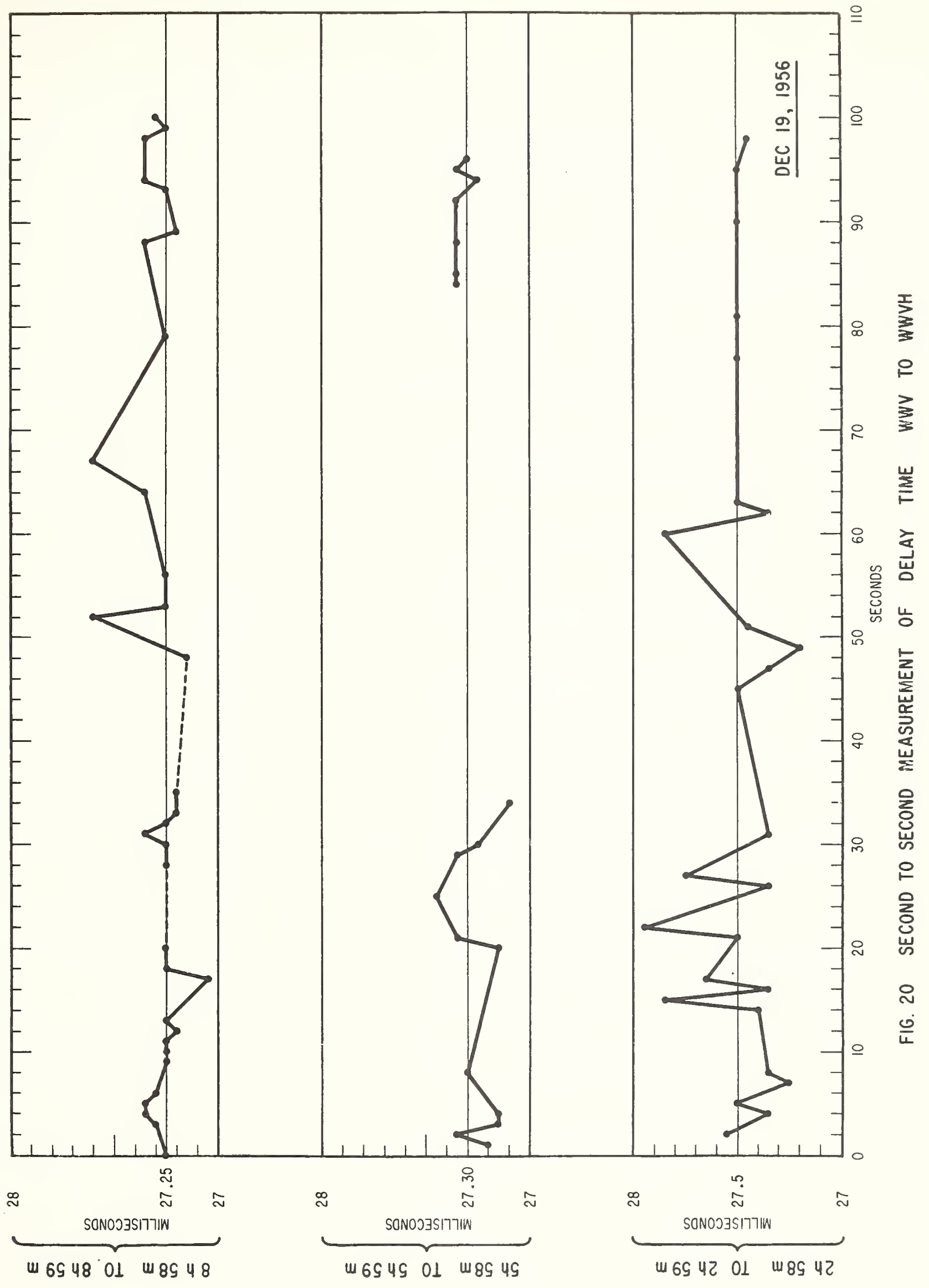




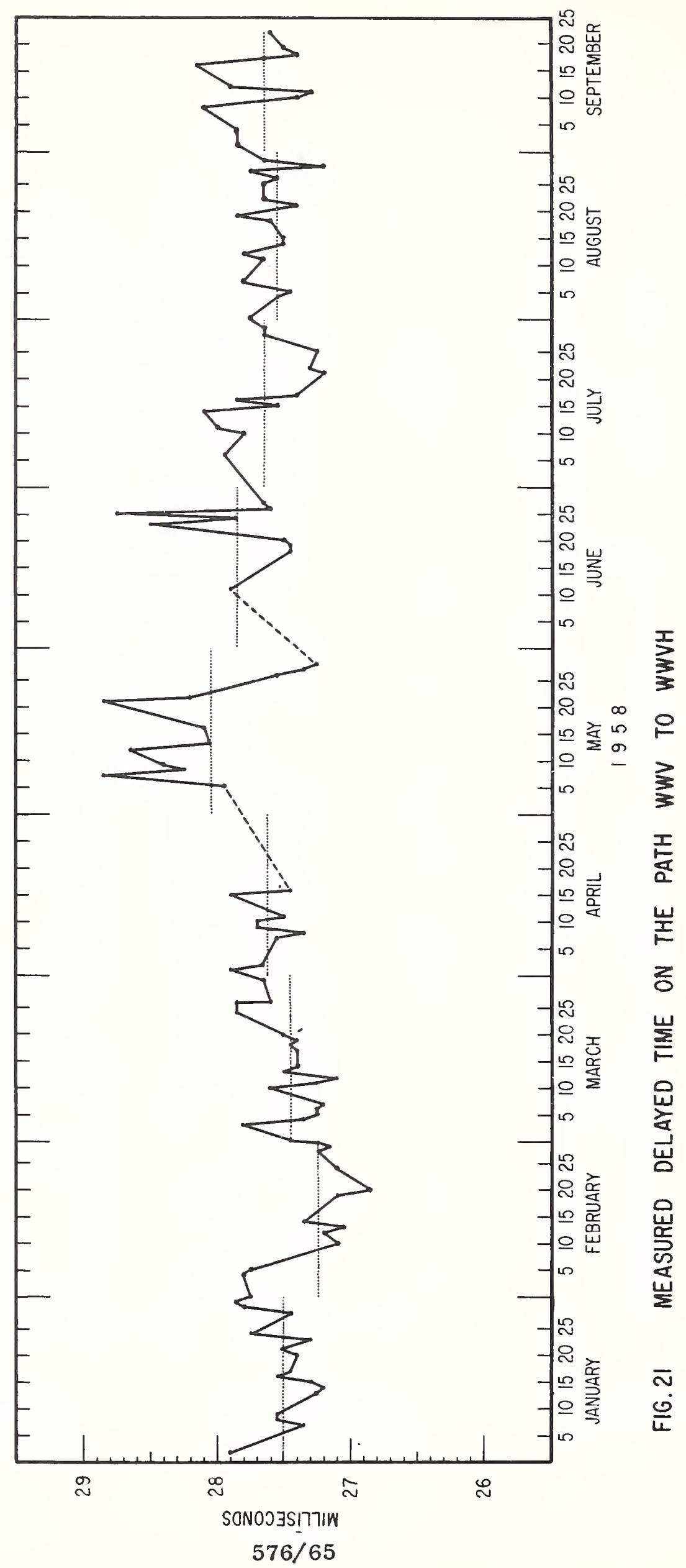


ERRATA SHEET

for

TECHNICAL NOTE NO. 22

Precise Time Synchronization

Of Widely Separated Clocks

1. Page 8. Equation 9: Braces should be used to make factor $t_{g}$ be multiplied by all rest of rhs of equation including term -1 .

2. Page 9. Last line: Units should be milliseconds (msec), not microseconds ( $\mu \mathrm{sec}$ ).

3. Page 13. Line 4, paragraph (b): should read "100 feet" not

4. Page 23. Last line: should be "Angle c" not "Angle C."

5. Page 28. Equation 9: should have braces as indicated in item 1 above.

6. Page 30. Table III, right column: should read "h $=(1+\gamma) . "$

7. Page 39. Fifth line: should be paragraphed as "(f)" not "(b)."

8. Fig.10-15. The " $\Delta \mathrm{t}$ " is the time interval between arrival of ground-wave and arrival of sky-wave signals after $n$ hops. 
Reprinted from the PROCEEDINGS OF THE I.R.E.

VOL. 41, NO. 1, JANUARY, 1953

PRINTED IN THE U.S.A.

\title{
A UHF and Microwave Matching Termination*
}

\author{
ROBERT C. ELLENWOOD $\dagger$, AND WILLIAM E. RYAN $\ddagger$, ASSOCIATE, IRE
}

\begin{abstract}
Summary-Coaxial transmission line and waveguide terminations are described which employ a double-slug transformer and a lossy dielectric load to provide an impedance match. Electromagnetic waves reflected from the load are canceled out by adjusting the positions of the two dielectric transformer slugs relative to the load, by means of bakelite rods which extend axially through the end of the termination. The terminations can be matched over wide frequency ranges; they are simple in operation and construction, having no critical dimensions.
\end{abstract}

* Decimal classification: R117.3 $\times$ R310. Original manuscript received by the Institute, February 4, 1952; revised manuscript received September 11, 1952.

† Bureau of Ships, Navy Dept., Washington, D. C. Formerly with Central Radio Propagation Laboratory.

$\ddagger$ Central Radio Propagation Laboratory, National Bureau of Standards, Washington, D. C.

\section{INTRODUCTION}

T N ULTRA-HIGH-FREQUENCY (uhf) and microwave measurements, transmission-line terminations are needed which can be adjusted to match the impedance of the line or to provide a given voltagestanding-wave ratio (vswr) in the line. These loads are useful, for example, in the evaluation of discontinuities in transmission-line components, such as connectors or slotted-line standing-wave machines. Other applications of these adjustable terminations are power and attenuation measurements and the calibration of probes under matched-load conditions.

This paper describes a simple termination composed of two low-loss dielectric slugs and one lossy dielectric, 
which are moved back and forth in a waveguide or coaxial line by means of bakelite rods parallel to the axis of the guide. ${ }^{1}$ The two low-loss dielectrics operate together as a double-slug transformer to cancel out the multiple reflections, and the lossy dielectric which is backed by a brass short absorbs the electromagnętic energy. The termination (used with a fixed probe) is adjusted for an impedance match by varying the distances between the three dielectrics with the rods which extend out the end of the guide. A match is indicated if no standing wave is observed as the three dielectrics are slid back and forth together with the spacing between them fixed.

The dimensions of the components of the termination are not critical and most of them were chosen arbitrarily. The major requirement is that both transformer slugs be a quarter wavelength long at some suitable frequency, as is explained later.

\section{Coaxial Termination}

This design was adopted especially for a 7/8-inch coaxial termination (Fig. 1) for use in the uhf range from 300 to $3,000 \mathrm{mc}$. The $7 / 8$-inch coaxial model is

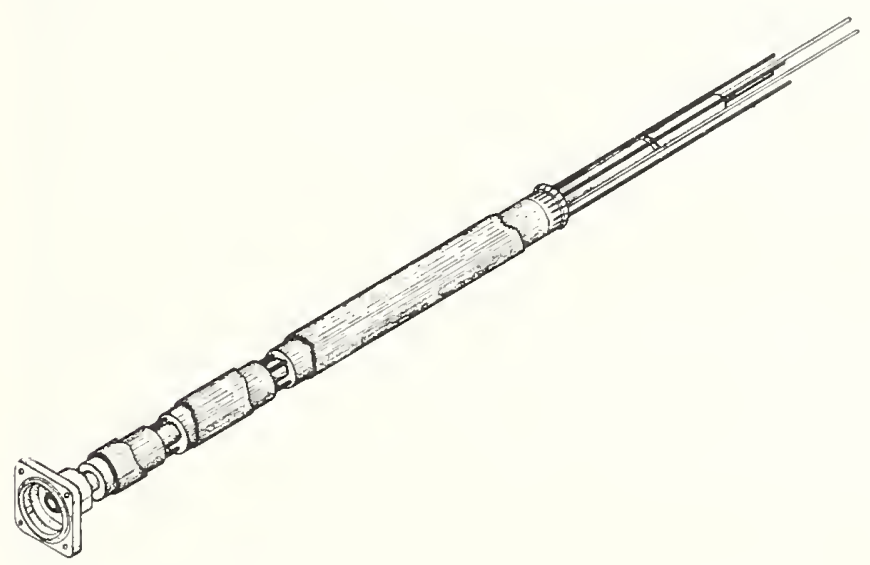

Fig. 1-Cutaway view of $\frac{7}{8}$-inch coaxial-line termination.

built of 4-foot conductors from ordinary machine brass stock, a type which has nominal tolerances of 0.003 to 0.005 inch. To ensure free movement of the dielectric cylinders, about 0.008 -inch clearance was allowed between them and the conductors.

The brass short attached to the end of the lossy dielectric has bronze fingers with a spring fit in order to prevent leakage, help to center the inner conductor, and maintain a fairly constant short circuit as the slugs are slid back and forth in the line. Three pairs of long rods of 0.113 -inch diameter extending through 0.125inch holes in the short are used to position the three dielectric slugs as it was found that two rods were necessary for proper mechanical control of each slug. One pair of rods, made of brass, is screwed through the short into the lossy dielectric load in order to fasten them together, and is used to position not only the lossy

1 R. E. Grantham, in "A reflectionless waveguide termination," Rev. Sci. Instr., vol. 22, pp. 828-834; November, 1951, describes another matching termination of somewhat different design. dielectric load but also the complete termination after the transformer has been tuned. A brass slug beyond the'end of the conductors (not shown in Fig. 1) with holes through which the rods may slide and screws to tighten on the rods, was used to fix the relative positions of the slugs once the transformer was tuned. The other two pairs of rods, which must be dielectric (bakelite was chosen for mechanical strength), extend through holes in the load and screw into their respective transformer slugs. The pair of rods attached to the slug furthest from the load passes also through 0.125-inch diameter holes in the other transformer slug.

Because of the presence of these bakelite drive rods and the loose fit of the slugs in the transmission line, accurate calculations of the electrical properties of the load are impractical. It is simpler and more accurate to determine the vswr or reflection coefficient of the termination by direct measurement. The general approximation for the attenuation of a lossy dielectric in a coaxial transmission line is ${ }^{2}$

$$
\alpha=\frac{8.686 \pi \sqrt{\epsilon} \tan \delta}{\lambda} \mathrm{db} \text { per inch, }
$$

where $\lambda$ is the wavelength in air in inches, $\epsilon$ the real part of the complex dielectric constant relative to air, and $\tan \delta$ the loss tangent, or ratio of the imaginary to the real component of the complex dielectric constant.

A plastic, Catalin (700 base), was chosen as the lossy dielectric because of its high loss-tangent and excellent machinability. Its loss tangent is 0.15 to 0.20 and its dielectric constant approximately 5 . This results in an attenuation of about $\frac{1}{2}$ to $3 \mathrm{db}$ per inch in the uhf range. After the measurement of the vswr of various lengths of Catalin loads, a 13 -inch length was chosen as a suitable compromise for a load with a small reflection coefficient and a reasonably short length. VSWR of Catalin load is shown as a function of frequency in Fig. 2(a).

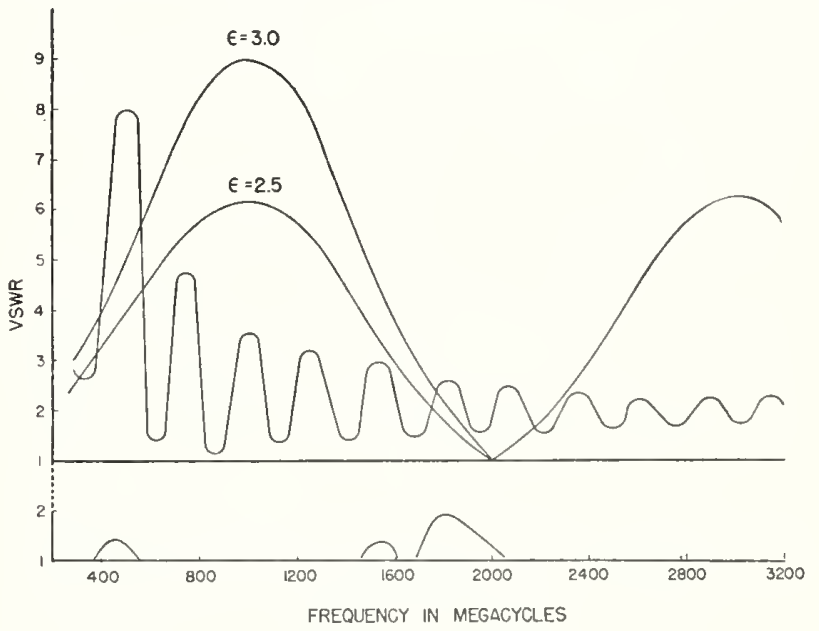

Fig. 2-(a) at the top. Measured vswr before matching of 13-inch length of Catalin in $\frac{7}{8}$-inch coaxial-line termination, together with curves showing theoretical matching capabilities of transformer slugs with dielectric constants of 2.5 and 3.0 . (b) at the bottom. VSWR after matching using $\epsilon=2.5$ transformer slugs.

2 T. Moreno, "Microwave Transmission Design Data," McGrawHill Book Co., Inc., New York, N. Y. first ed., p. 65; 1948. 
The vswr's can be matched to the transmission line by a double-slug transformer, a type which is effective over wide frequency ranges for fairly large vswr's. This transformer can match any impedance whose vswr does not exceed $\epsilon^{2}$ for the dielectric of which the slugs are made. Though the transformer will match a vswr of this magnitude only at frequencies for which the slugs are an odd number of quarter-wavelengths long, it is sufficiently "broad-band" to match any vswr approaching this values at other frequencies according to the following equation, ${ }^{3}$

$$
\operatorname{vswr}_{\max }=1+\frac{b}{2}+\sqrt{b+\frac{b^{2}}{4}}
$$

where

$$
\begin{aligned}
b=\left[1 / 2\left(\epsilon-\frac{1}{\epsilon}\right)\right. & \left(1-\cos 2 \beta_{a} l_{a}\right) \cos \beta_{0} l_{0} \\
& \left.+\sqrt{\epsilon}\left(1-\frac{1}{\epsilon}\right) \sin 2 \beta_{a} l_{a} \sin \beta_{0} l_{0}\right]^{2},
\end{aligned}
$$

and

$\beta_{a} l_{a}=$ electrical length of each slug,

$\beta_{0} l_{0}=$ electrical distance between the slugs,

$\epsilon=$ dielectric constant of the slugs.

The curves marked $\epsilon=2.5$ and $\epsilon=3.0$ in Fig. 2(a) are plots of the above equation for slugs a quarter-wavelength long at $1,000 \mathrm{mc}$ and indicate the maximum vswr's that can be matched at each frequency with slugs having these dielectric constants. It can be seen that, if the vswr's are not large, there is little increase in the matching band to be gained by using transformer slugs of large dielectric constant. Moreover, high dielectric-constant slugs were found to be more difficult to adjust for match than low dielectric-constant slugs, such as polystyrene, whose dielectric-constant is about 2.5. Transformer slugs of polystyrene are capable of matching the vswr of the Catalin termination over all but the lower end of the uhf range. The measured vswr of the 13-inch Catalin termination is also shown in Fig. 2(a).

The experimental performance of the adjustable coaxial termination is shown in Fig. 2(b). With the polystyrene slugs $(\epsilon=2.5)$ of Fig. 2(a) which were 1.87 inches long it was possible to obtain a match over more than 80 per cent of the uhf range. It is seen that the frequencies at which one could not obtain a match with this set of slugs (that is, where the vswr of the Catalin load exceeds the vswr matching capability of the transformer) are somewhat lower than the curves of Fig. 2(a) predict. This is probably caused by an effective increase in the dielectric constant of the transformer slugs due to the presence of the bakelite rods, so that the slugs were a quarter wavelength long at a frequency somewhat less than $1,000 \mathrm{mc}$.

${ }^{3}$ R. C. Ellenwood and E. C. Hurlburt, "The determination of impedance with a double-slug transformer," to be published in a forthcoming issue of the PROC. I.R.E.
With this coaxial termination it is possible to obtain a vswr measured with a differential vacuum-tube voltmeter less than 1.004 (reflection coefficient <0.002) without any difficulty. This small limitation in making a perfect match is apparently caused by a slight warping of the conductors so that, as the load moves along the line, the conductors go in and out of concentric positions, thus changing the characteristic impedance of the line.

At the lower frequencies the lossy termination results in larger vswr's. In order to match a vswr of the order of 6 to 10 , it is necessary to use slugs of such material as bakelite or Dielectene, which have dielectric constants of 3 or more. The more critical adjustment of these high dielectric-constant slugs is partially compensated for by the fact that in this case they are being employed at the longer wavelengths. Of course, some decrease in the vswr at low frequencies can be obtained by the use of longer pieces of Catalin.

As the first set of polystyrene slugs could not match any appreciable vswr's near 2,000 mc, a second pair was made which was a quarter wavelength at $2,000 \mathrm{mc}$ to cover that frequency range. Thus, with two or three pairs of transformer slugs the entire uhf band can be covered. With only one pair a match can be obtained over most of the range. In order that the sets may be easily changed, the slugs have threaded holes into which their respective drive rods may be screwed.

\section{Waveguide Models}

Though this termination was designed primarily for use with coaxial transmission lines, waveguide models were also built for the purpose of determining their performance at $X$-band $(8,200-12,400 \mathrm{mc})$ and at $K$-band $(18,000-26,500 \mathrm{mc})$.

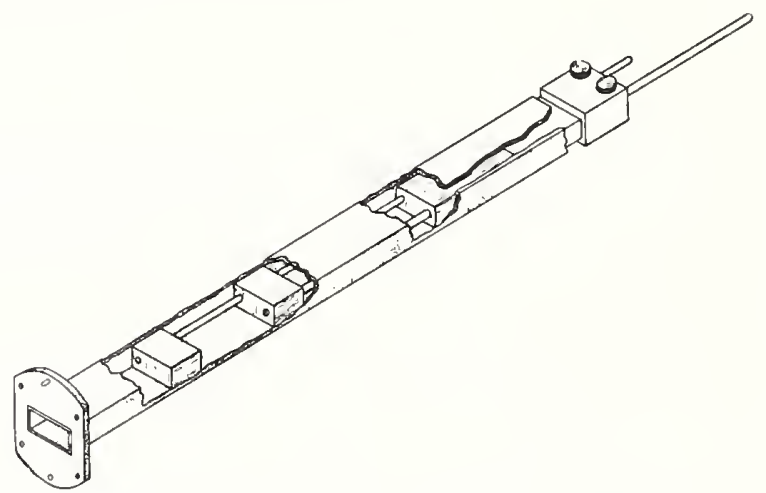

Fig. 3-Cutaway view of waveguide termination.

The waveguide model which is shown in Fig. 3 uses 2-inch lengths of Catalin for the lossy terminations in both the 0.900 - by 0.400 -inch $X$-band guide and the 0.420 - by 0.170 -inch $K$-band guide. These lengths are sufficiently lossy to reduce the vswr in the waveguide to a value less than three. At these frequencies this vswr is due mostly to the impedance mismatch at the air-Catalin interface. The Catalin in the waveguide is fastened to a 1.5 -inch sliding brass block which serves 
as a short circuit and as a centering device. The slugs and two 0.113 -inch diameter drive rods in botlı waveguide models are made of polystyrene. In the $X$-band model the slugs are 0.675 inch long, three-quarters wavelength at $9,380 \mathrm{mc}$, and in the $K$-band model 0.475 inch long, five-quarters wavelength at 23,000 $\mathrm{mc}$, in order to improve their rigidity and stability within the guide. The two thumb screws shown in Fig. 3 are tightened on the drive rods to anchor the slugs in position once they have been properly adjusted. In the waveguides a single drive rod was sufficient for mechanical control of each transformer slug. With each waveguide termination it was found that a match could be obtained to a vswr of 1.01 (reflection coefficient $<0.005$ ).

\section{Conclusion}

This instrument is capable of covering wide frequency ranges. The coaxial termination will provide a match to a vswr of 1.004 with two pair of slugs over the entire uhf range. Similarly waveguide models will provide matches to 1.01 over their particular frequency ranges. Not only can these terminations provide a match, but they may also be adjusted to given values of vswr of the order of one to ten when for various reasons such a vswr is desired.

The outstanding features of this termination are its simplicity of construction and ease of tuning. There are no critical dimensions and the essential requirements are three dielectric slugs (one lossy) to fit loosely within a transmission line and the rods by which they may be moved. It takes but a short time to reduce the vswr to less than 1.01. The vswr could be reduced still further if closer tolerances and more refined components were used, such as a gearing arrangement to adjust the position of the slugs, with a sacrifice, of course, of some of the simplicity of construction. 


\title{
National Bureau of Standards Circular 481
}

Issued September 1, 1949

\section{High-Frequency Voltage Measurements}

\author{
By Myron G. Selby
}

\begin{abstract}
The paper presents an up-to-date account of fundamental principles and techniques used for voltage measurements primarily for frequencies in the upper audio- and radiofrequency ranges and including part of the ultrahigh frequency range. Subject matter is limited to principles and methods that have met with some degree of success for both high and moderate precision, emphasizing those developed and applied for primary standard work in this frequency range at the National Bureau of Standards. This paper is intended to give professional workers and graduate students a more comprehensive picture of the methods employed with regard to this subject than is presently available in textbooks and handbooks.
\end{abstract}

\section{Introduction}

Standard procedures of $r-f$ voltage measurements are at the present time based upon direct-voltage calibrations with the voltage of a standard cell as the primary reference. For maximum accuracy, direct substitution is made of $\mathrm{d}-\mathrm{c}$ for $\mathrm{r}-\mathrm{f}$ voltage having negligible harmonic components. Reliability is insured by crosschecking results with one or more independent calibration methods based on different principles. In the light of present-day experience at these frequencies, measurements to accuracies of approximately \pm 1 percent may be considered of high precision, whereas accuracies of the order of \pm 5 percent are of moderate precision. Direct voltage measurements are readily made to 0.1 percent so that transfer methods, employing measurements made directly in terms of direct voltage without needing corrections for frequency, may be called primary-standard methods. Reproducibility of results, as well as agreement between individual primary-standard methods, is expected to be within \pm 1 percent or better. In this paper, methods that in terms of primary-standard techniques are only reproducible to \pm 5 percent will be referred to as "moderate" precision methods of measurement. At this point one should differentiate between accuracy and precision. An accuracy of \pm 1 percent implies that the value of the quantity measured is within \pm 1 percent of its true or absolute value. Precision on the other hand refers primarily to sensitivity and repeatability incidental to relative measurements. Thus precise repeatable indications that can be obtained using different instruments may be extremely useful in making circuit adjustments and for experimental purposes, but they need not agree among themselves to the extent of being individually accurate. Accuracy implies ample sensitivity and precision of measurement to establish the extent of agreement with accepted theory or standards.
The most suitable primary-standard $r-f$ voltage measurement methods seem to be those employing:

(a) Power substitution.

(b) Measurement of current tlirough a known resistance.

(c) Deflection of cathode-ray beam.

(d) The electrostatic voltmeter.

Not suitable for $d-c$ calibration are methods employing:

(e) Vacuum tube voltmeters.

(f) Rectifiers (nonthermionic).

\section{High-Precision Methods Based on D-C Measurements}

\section{(a) Power Substitution}

In this method bolometers are made use of with the assumption that the same temperature or heating effect is produced by an equal amount of power whether produced by radio frequency or by direct current, and that the resistance of the bolorimetric element is independent of frequency and is reliably dependent upon its temperature.

Thermistor and Wollaston type bolometers are in general use. A thermistor is a semiconductor, such as uranium oxide $\left(\mathrm{U}_{3} \mathrm{O}_{8}\right)$, or a mixture of nickel oxide ( $\mathrm{NiO})$. having a large negative resistance-temperature coefficient $[1,2]$. A Wollaston wire is a platinum wire of the order of 0.001 $\mathrm{mm}$ in diameter drawn inside a silver wire; this silver coat is removed over a small section by etching with a solution of nitric acid $\left(\mathrm{HNO}_{3}\right)$; the exposed platinum core constitutes the active section of the bolometer. Typical thermistors have a resistance versus temperature sensitivity approximately 10 times larger than Wollaston wire, will carry considerably ligher overloads, are superior in mechanical ruggedness, have a larger thermal time constant [53], and seem more adapt- 
able for voltage measurements because of their relatively small physical dimensions. Precautions must be observed in mounting the bolometer so that the voltmeter under calibration is connected directly across an $r-f$ current-carrying circuit clement having negligible series reactance, since a fundamental assumption of this method is that the $\mathrm{r}$-f resistance of a particular bolometer is equal to its d-c resistance.

Figure 1 shows an elementary circuit diagram of a bridge employing two thermistors. In order to determine the value of a radio-frequency

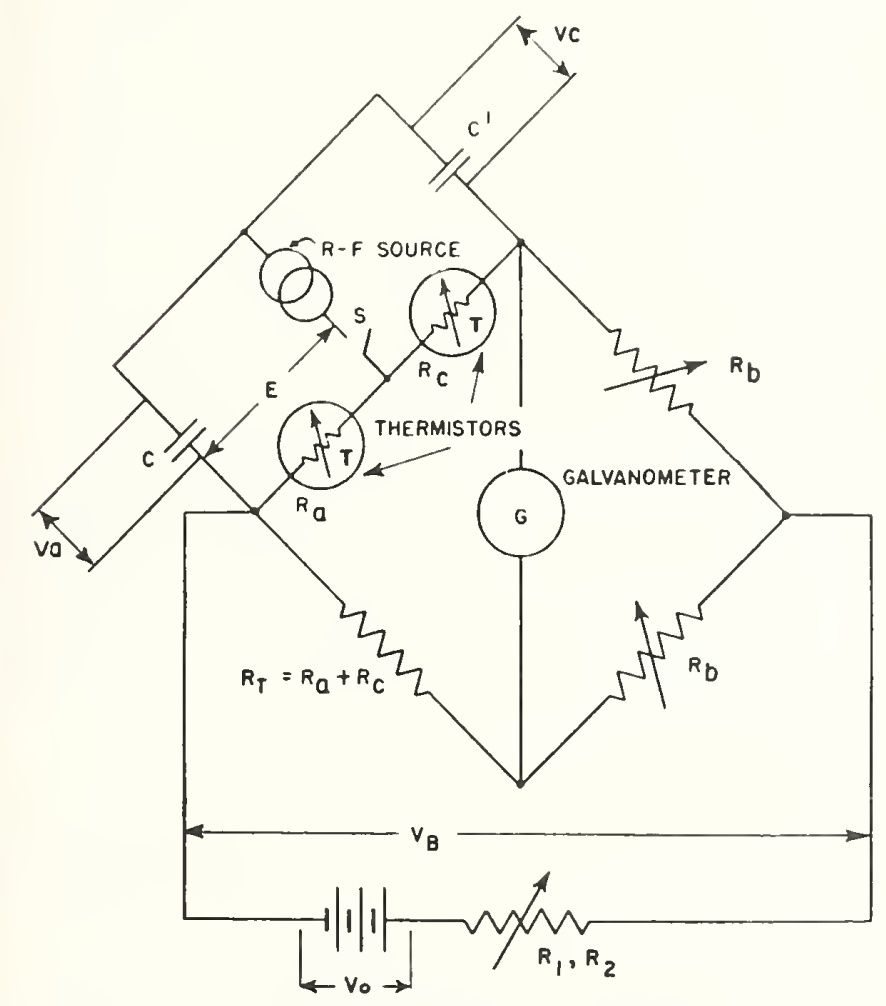

FiGURE 1. Ceneral bolometer-bridge circuit employing two thermistors.

voltage, $E$, the bridge is first balanced using only the direct voltage sour'ce, $V_{0}$, which for a selected value of $R_{1}$ establishes a definite supply of powel to the two thermistors and their corresponding values of resistance, $R_{a}$ and $R_{b}$. The r-f voltage $E$, is then applied and the bridge is restored to balance by adjusting $R_{1}$ to a new value $R_{2}$. In this manner radio-frequency power is substituted for a portion of the power initially entirely supplied to the thermistors from the battery source. Since $R_{a}$ and $R_{b}$ are effectively the same for both balances, the following equations arising from the bridge constants for the two balanced conditions permit an evaluation of $E$. This method is applicable to several hundred and possibly to 1,000 Me over an approximate range of 0.02 to $1.5 \mathrm{v}$.
The general expression for the $r$-f voltage is

$E=$

$\frac{R_{T}}{\left(R_{T}+R_{b}\right)(1+\alpha)}\left[\alpha\left(V_{R_{2}}-V_{R_{1}}\right)\left(2 V_{0}-V_{R_{2}}-V_{R_{1}}\right)\right]^{1 / 2}$,

and for thermistors matched to have identical resistances and characteristics,

$$
E=\frac{R_{T}}{2\left(R_{T}+R_{b}\right)}\left[\left(V_{R_{2}}-V_{R_{1}}\right)\left(2 V_{0}-V_{R_{2}}-V_{R_{1}}\right)\right]^{1 / 2} .
$$

$E=$ the root-mean-square value of the r-f voltage across the two thermistors in parallel; $V^{r}$ designates d-c voltages with the proper subseript. $V_{0}$, the voltage across the battery, is assumed constant with load variations.

Subscripts 1 and $2=$ respective values before and after the r-f voltage is applied.

$\left(R_{a} R_{c}\right) /\left(R_{a}+R_{c}\right)=$ load presented to the $\mathrm{r}-\mathrm{f}$ source.

$R_{a}$ and $R_{c}=$ individual thermistor resistances.

$\alpha=R_{a} / R_{c}=V_{a} / V_{c}$. For matched thermistors $\alpha=1$.

$R_{T}=R_{a}+R_{c}$

$V_{a}$ and $V_{c}=$ the d-c individual thermistor voltage drops with switch $S$ (fig 1) closed.

$C, C^{\prime}=\mathrm{d}$-c blocking condensers.

When unmatehed thermistor's are used it is necessary to provide a d-c path through the r-f source (or in shunt with it) to measure $I_{a}$ and $V_{c}$ with the r-f voltage applied to the thermistors.

The following special cases are of practical significance:

1. Natched bolometers $\left(R_{a}=R_{c}\right)$, unequal-arm bridge $\left(R_{b} \neq R_{T}\right)$, relatively low $V_{0},\left(R_{1}=0\right)$ :

This seems to be the most convenient arrangement for the following reasons: It makes it unnecessary to measure $\alpha$; no decoupling chokes (to keep r-f power out of the bridge) are required; greater accuracy is obtained by measuring $V_{R_{2}}$ alone (as compared with measuring a small difference between two relatively large voltages), especially when $E$ is low in magnitude.

$$
E=\frac{R_{T}}{2\left(R_{T}+R_{b}\right)}\left[V_{R_{2}}\left(2 V_{0}-V_{R_{2}}\right)\right]^{1 / 2} .
$$

2. Matched bolometers, unequal-arm bridge, relatively high $V_{0}$ :

It is sometimes advisable to maintain bridgearm resistances of the same order of magnitude, thereby assuring high bridge sensitivity. $V_{0}$ is usually not continuously adjustable. $R_{1} \neq 0$. In this case, eq 2 may be used.

3. Single bolometer $\left(R_{c}=0, C^{\prime}=0\right)$; unequal-arm bridge, $R_{1}=0$ : 
This arrangement eliminates the necessity of matching bolometers and removes the possibility of only one of the two earrying the entire r-f load. This could occur, for example, when operation takes place over a negative resistance portion of thermistor characteristics. Means are required, however, to keep the r-f power out of the d-e bridge.

$$
E=\frac{R_{T}}{\left(R_{T}+R_{b}\right)}\left[V_{R_{2}}\left(2 V_{0}-V_{R_{2}}\right)\right]^{1 / 2} .
$$

4. Single bolometer, equal-arm bridge:

$$
E=1 / 2\left[\left(V_{R_{2}}-V_{R_{1}}\right)\left(2 V_{0}-V_{R_{2}}-V_{R_{1}}\right)\right]^{1 / 2} .
$$

The major advantage of having $R_{b} \neq R_{T}$ arises when a relatively small value of $\left(V_{R_{2}}-V_{R_{1}}\right)$, as compared with $V_{R_{2}}$, has to be measured, as is the case at very low values of $E$. Other modifications may occasionally be desirable, such as a singlebolometer unequal-arm bridge with high $V_{0}$, $\left(R_{1} \neq 0\right)$, or an unmatehed two-bolometer bridge when $R_{a}$ and $R_{c}$ are known individually and are expected to remain stable in either equal- or unequalarm bridges. When unmatched thermistors are used, the degree of departure of $R_{a}$ from $R_{c}$ may increase considerably with increasing $r-f$ power fed to the two in parallel as a result of their regative resistance vs. temperature coefficient [62].

To obtain maximum accuracy, precision potentiometers and a galvanometer of proper sensitivity are desirable. Among the precautionary requirements of this method, especially in measuring small voltages, are the following: Constant ambient temperature, stable $V_{0}$, stability of the resistances in the circuit and accurate determination of their values, an $r-f$ voltage source relatively free from harmonics.

\section{(b) Resistor in series with current measuring device}

In employing this method to obtain aceuracies of about 1 percent, the d-c calibration of the current indicating device and the value of the series resistor must be known to 0.5 pereent. Either changes in current indication or resistance depending on frequency should be negligible, or they should be small and known, so that corrections may be applied. The physical design and arrangement of compenents must insure that all of and only the current entering one end of the resistor passes out the otber end and through the current measuring element [62]. Unless the impedance of the current-measuring element is negligible, it must be taken into account and should, in any event, be preferably resistive if the voltage drop across it is part of the total voltage being measured.
Thermockements (heater with thermocouple for current indieation) may be used in series with special resistors $[3,4]$ up to a frequeney of several megacycles to measure voltage ranging from several millivolts to approximately $50 \mathrm{v}$. Errors at high frequencies are likely to be introduced by stray capacitances associated with either the resistor or thermocouple eireuit and by stray fiekls coupling with thermocouple cireuit. Currents induced in the thermocouple cireuit whose resistance is low in comparison with that of the $r-f$ current-carrying heater element may introduce spurious heating effeets not present during ealibration of the thermoclement on direet current [5] and be a large souree of error. When the couple is not insulated from the heater, calibration of the thermoetement is usually made on leversed direct current to reduce crror eaused by the section of the conduetor common to both clements.

Instead of using a thermoclement, a heater element alone may be used, depending on other. means of correlating the heating effect of $\mathrm{r}-\mathrm{f}$ current through the heater with a suitable indicating device. An arrangement, applicable to approximately $30 \mathrm{Mc}$ [4], utilizes a photoclectric alibration of the glow from the eurent-carlying heater element. Under conditions of use the impedance across the heater terminals must be known as a function of voltage and should be essentially resistive.

Skin effect, inductance, and power dissipation limit both the frequeney and voltage ranges.

\section{(c) Cathode-ray beam deflection}

This method makes use of the deflection of an eleetron beam by an electrie field. An elementary circuit arrangement is shown in fig. 2 .

The leflection is

$$
D=E_{\max } \frac{l y}{2} \frac{y}{V_{a} d},
$$

where $r_{a}$ is the accelerenting potential of the beam, $l$ is the effective length of the deflecting field, $y$ is the effective length of the beam between this field and screen, and $d$ is the distance between the deflection plates.

The procedure in measuring an $r$-f voltage $E$ (fig. 2) is to adjust first the zero spot position by setting $V_{1}$ with $V=0$ and $E=0$. The $\mathrm{r}-\mathrm{f}$ voltage is then applied and the position of either end of the deflection trace on the sereen is restored to the original position of the spot by applying the proper value of $V$; this value of $T^{\prime}$ is then equal to $E$ peak, provided the transit time and other frequency errors are negligible. For maximum aceuracy at low roltage levels it is best to line up the edge of the spot against a fine hair-line with a low-power microscope.

As a result of transit-time effect, the voltage and 


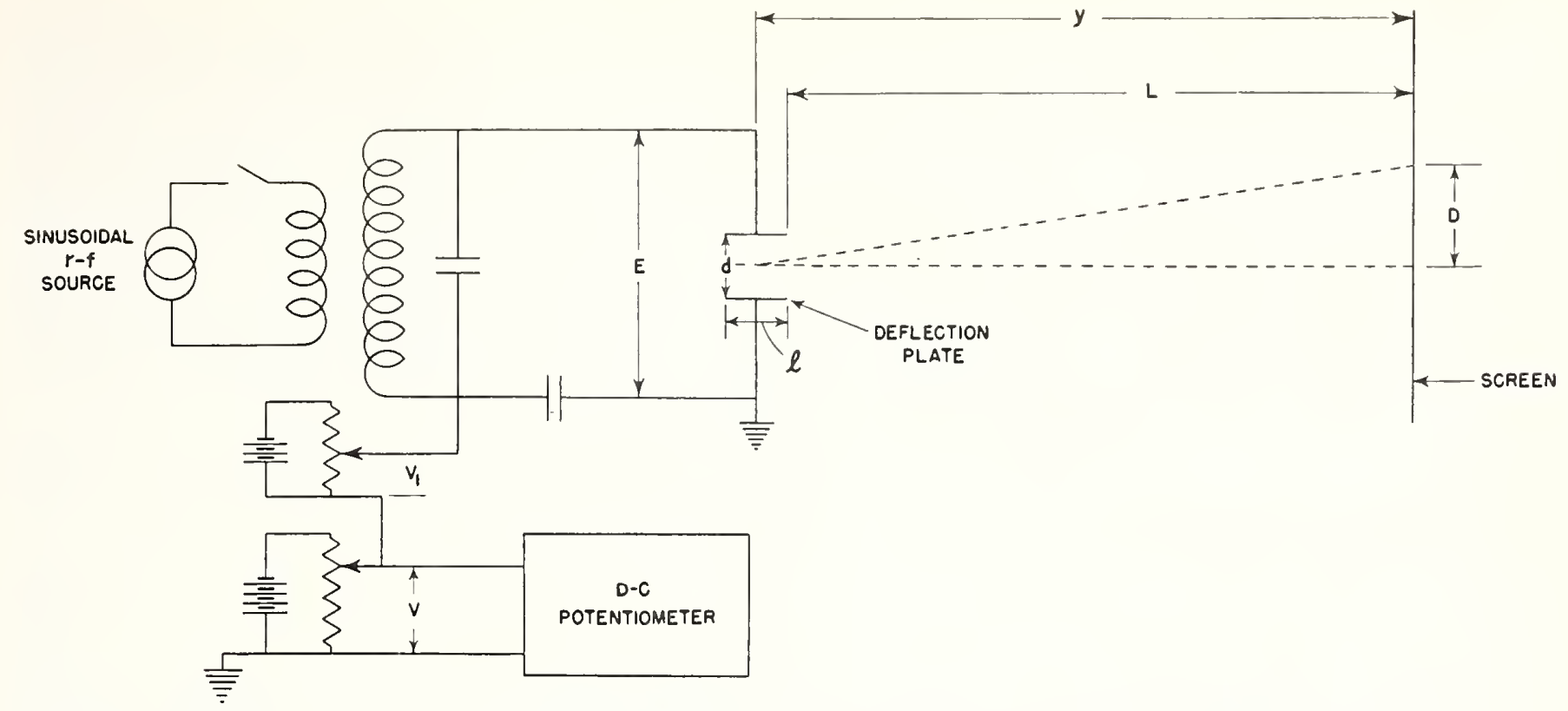

FIGURE 2. Essential components of $r$-f voltage-mcasuring circuit arrangement employing deflection of cathode-ray beam.

frequeney ranges are limited and are interdependent. This effeet takes place when the phase of the deflecting 'voltage begins to reverse before the eleetrons in the beam have sufficient time to cross the defleeting field. The transit time thus reduces the magnitude of the deflection unless it is smaller than a lialf-period of the highest frequency to be used. The error caused by this effect is usually expressed as a ratio, $C$, of dynamic to statie sensitivity, the sensitivity being the linear defleetion per volt. This ratio is expressed as $[6,7,8]$

$$
C=\frac{\left.\sqrt{2(1-\cos \phi)+\phi^{2}-2 \phi \sin \phi+2 \phi^{2} \frac{L}{l}\left[1+\frac{L}{l}(1-\cos \phi)\right.}\right]}{\phi^{2}\left(1 / 2+\frac{L}{l}\right)},
$$

where

$$
\begin{aligned}
\phi & =\frac{\omega l}{v_{0}} \\
\omega & =2 \pi f \\
v_{0} & =5.97 \times 10^{7} \sqrt{V_{a}}=\text { beam velocity }
\end{aligned}
$$

$V_{a}=$ d-e voltage accelerating the beam within the deflection field for $V_{a} \leqslant 10,000 \mathrm{v}$. For higher values of $T_{a}$, Einstein's eorreetion for the increase in mass of the electron must be applied.

Equation 7 takes into aeeount the beam displaeement parallel to the axis. This displaeement is usually negligible for large ratios of the $L / l$, where $L$ is the effeetive distanee between the screen and the defleetion field as shown in figure 2. In the latter ease, for sinusoidal input,

$$
C=\frac{\sin \phi / 2}{\phi_{/ 2}}
$$

Figure 3 shows $C$ as a function of $\phi / 2$, given by eq $10[9,10]$.

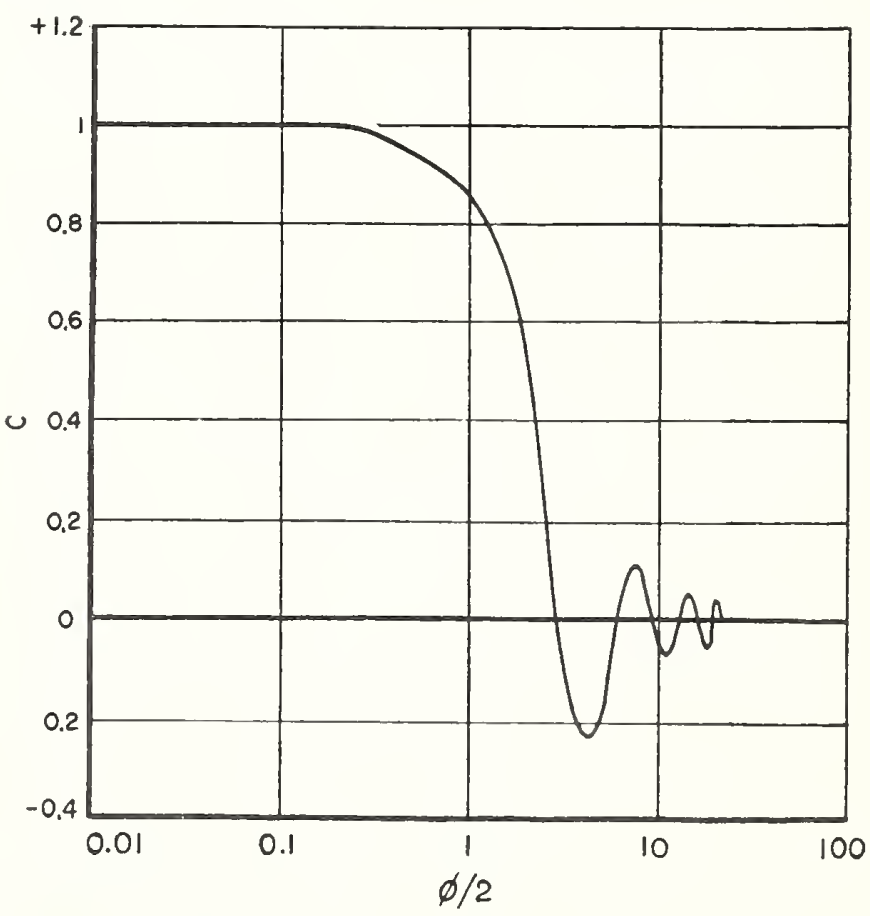

FIGURE 3. Efject of electron transit time on sensitivity of cathode-ray tube [9]. 
Another source of error is the effect on the deflection field of the lead inductance and deflection plate capacity. The voltage between the plates is larger than the voltage applied to the outside terminals of the cathode-ray tube (where the voltmeter under calibration is connected), as given by the following expression [5]:

$$
E^{1}=E \frac{1}{1-\left(f / f_{r}\right)^{2}}
$$

where

$E^{1}=$ deflecting voltage

$E=$ applied voltage

$f=$ operating frequency

$f_{r}=$ plate and lead series resonance frequency.

The design and construction of present-day commercially available cathode-ray tubes is such that they may be used with negligible frequency error up to $75 \mathrm{Mc}$ for voltages ranging from 5 to several hundred volts. Their suitability and range is dependent upon electrode and lead arrangement, spacing, and insulation.

Deflection sensitivity may be increased by reducing accelerating voltage as is evident from eq 6, permitting satisfactory measurements at less than 5v radio frequency but only at the expense of a reduction of the upper limit of frequency, and an increase in spurious effects from magnetic fields and normally minor voltage fluctuations. Similarly, the upper frequency limit of satisfactory response may be increased by increasing the accelerating voltage (within its rated limits) at the expense of a reduction in deflection sensitivity.

Modified designs of oscilloscope tubes, with proper precautions in circuit connections, may be used for frequencies above $75 \mathrm{Mc}$. One modification is the replacement of the conventional deflecting plates of an oscillosope by a section of a two-wire transmission line (in a plane normal to the axis of the tube) extending through the tube [11]. The beam passing through the spacing between the conductors is deflected in proportion to the voltage at this point of the line. The voltmeter under calibration is placed either as close as possible to the tube or a distance $\lambda / 2$ away along the extended transmission line, where the voltage across the line is the same as at the cathode ray. This method is shown in figure 4 . The deflection angle $\theta$ is given by

$$
\theta=\frac{E_{\max }}{2 V_{a}} \frac{\pi}{\cosh ^{-1}(d / 2 r)} .
$$

For large values of $d / r$

$$
\theta \cong \frac{E}{2 V_{a}} \frac{\pi}{\ln (d / r)}
$$

where $d$ and $r$ are the separation and wire radius, respectively.

The effective deflecting field is largely concentrated within a space between the wires approximately equal in width to the wire separation; the distance responsible for a transit time error is therefore approximately equal to the wire separation.

A line having a $3-\mathrm{mm}$ wire diameter and a $6-\mathrm{mm}$ center-to-center separation results in a sensitivity of $0.3 \mathrm{~mm} / \mathrm{v}$ for $V_{a}=1,000 \mathrm{v}$ at a screen distance of $25 \mathrm{~cm}$; this may be compared with a sensitivity of approximately $0.63 \mathrm{~mm}$ under the same conditions with deflecting plates having a transit time distance nearly five times as large.

Experimental work, now in progress at the National Bureau of Standards, with these types

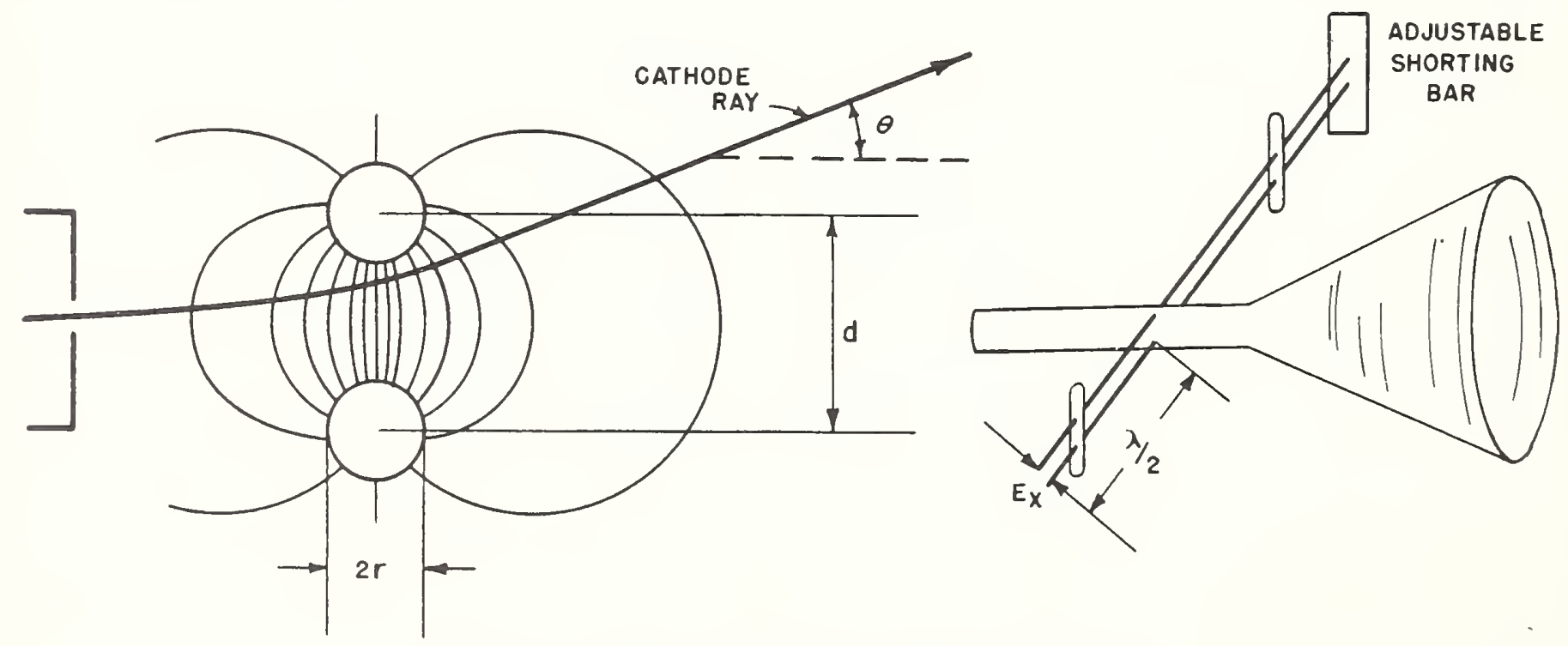

Figure 4. Tuned transmission-line deflection system [11]. 
of tubes resulted in precision measurements of voltages up to $150 \mathrm{Mc}$.

Another modification consists of a specially constructed cathode-ray tube employing a short focus lens and very small deflecting plates $[6,9]$. Figure 5 shows a diagram of this device referred to as a "micro" oscillograph. The initial cross section of the beam is reduced to a degree resulting in a spot size of $10^{-2}$ to $10^{-3} \mathrm{~mm}$ in diameter. The beam passes through the deflecting plates and into the short-focus electrostatic lens. This oscillograph depends on optical magnification for increased initial deflection. Using a $50 \times$ optical magnifier and $V_{a}=10,000 \mathrm{v}$, an effective deflection sensitivity of approximately $0.5 \mathrm{~mm} / \mathrm{v}$ is obtained; the plate length and separation are about 0.2 inch, and the transit time effect is negligible at 1,500 Mc. These two modified applications of the cathode-ray tube seem to indicate that this method can be used for precision wide-range voltage measurements to at least $300 \mathrm{Mc}$ and perhaps to much higher frequencies simply by further reduction in size of cathode-ray tube elements.
The voltage range of present-day commercial electrostatic voltmeters is approximately 20 to $50,000 \mathrm{v}$.

\section{Moderate precision methods}

\section{(a) Vacuum-tube voltmeters}

The first vacuum-tube (v-t) voltmeter was patented by R. A. Heising in 1917 [36]. For a number of years prior to that date and up to the present time, $v$-t voltmeters have been universally used to measure $r-f$ voltages of the order of one millivolt to several kilovolts. Vacuum-tube voltmeters are made in a variety of forms and in various voltage ranges. The useful frequency range in each basic design is limited primarily by the adapted circuit arrangement and characteristics of such components as tubes, amplifiers, voltage dividers, etc. The $\mathrm{v}$-t voltmeter is not satisfactory as a high-precision standard, because it is difficult to determine precisely the law of its voltage-current characteristic as well as to maintain its operation sufficiently constant over a

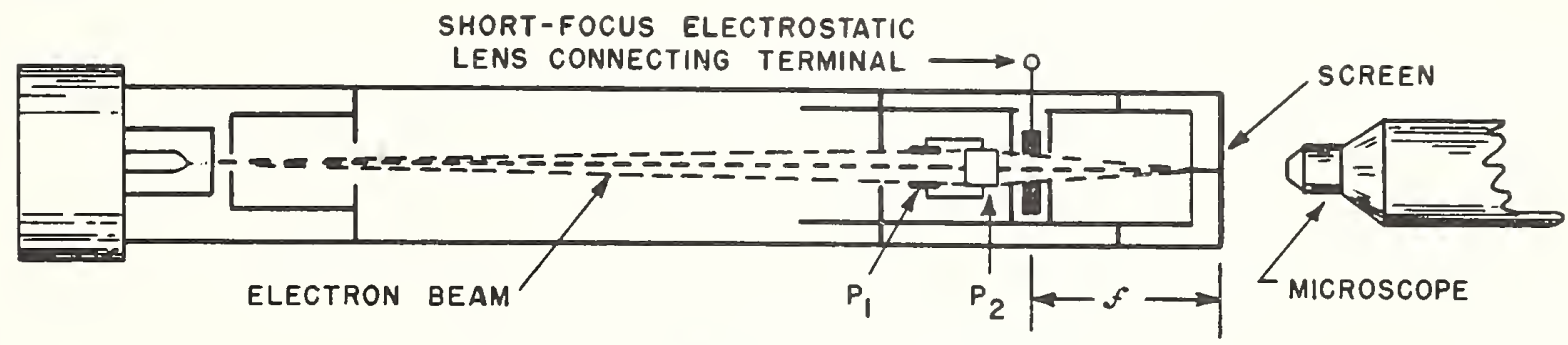

Figure 5. Diagram of micro oscillograph [6].

(d) Electrostatic voltmeter

Electrometers and electrostatic voltmeters make use of the force existing between charged conductors. These instruments are essentially capacitors with the rotor, either fiber suspended or jewelpivoted. One set of the conductors is usually fixed in position and the other one (the set corresponding to the rotor of a variable capacitor) is fiber-suspended or jewel-pivoted, and so restrained by the suspension or a coiled spring that its displacement is indicative of the voltage applied. Commercial electrostatic voltmeters have a scale (usually nonlinear unless the plates are especially shaped) that is graduated to read r'ms values of voltage, and have a high input resistance and low power consumption at frequencies up to approximately 5 Mc [12].

The major disadvantages of this type of voltmeter are low sensitivity and high input capacity (a few micromicrofarads to several hundred micromicrofarads), with the capacity a function of the instrument deflection. Thus certain difficulties and limitations are introduced when the instrument is connected across a tuned circuit. reasonable length of time. In addition, in the case of voltmeters employing the usual form of grid-leak-condenser diode circuits, the analytically derived output contains factors depending upon the nature of the impedance across which the voltage is being measured. Finally the input impedance of the voltmeter is a function of the voltage applied to it [13].

Tubes with more than three electrodes are seldom used for voltages above 0.5 v. Pentodes connected as triodes are desirable for some voltage and frequency ranges as a result of spacing and shielding of the electrodes. Triodes connected as diodes are used for higher voltages.

Vacuum-tube voltmeters are usually calibrated at all frequencies using one of the above standard methods. They may also be calibrated in terms of power or audio-frequency standard instruments, in which case freedom from frequency correction should be verified. For higher accuracy, calibration at the operating frequency is preferred. The major performance desiderata of a $v$ - $t$ voltmeter for freuencies up to several hundred megacycles are:

1. Low-input capacitance. 
Table 1. Elementary circuits and characteristics of vacuum-tube voltmeters

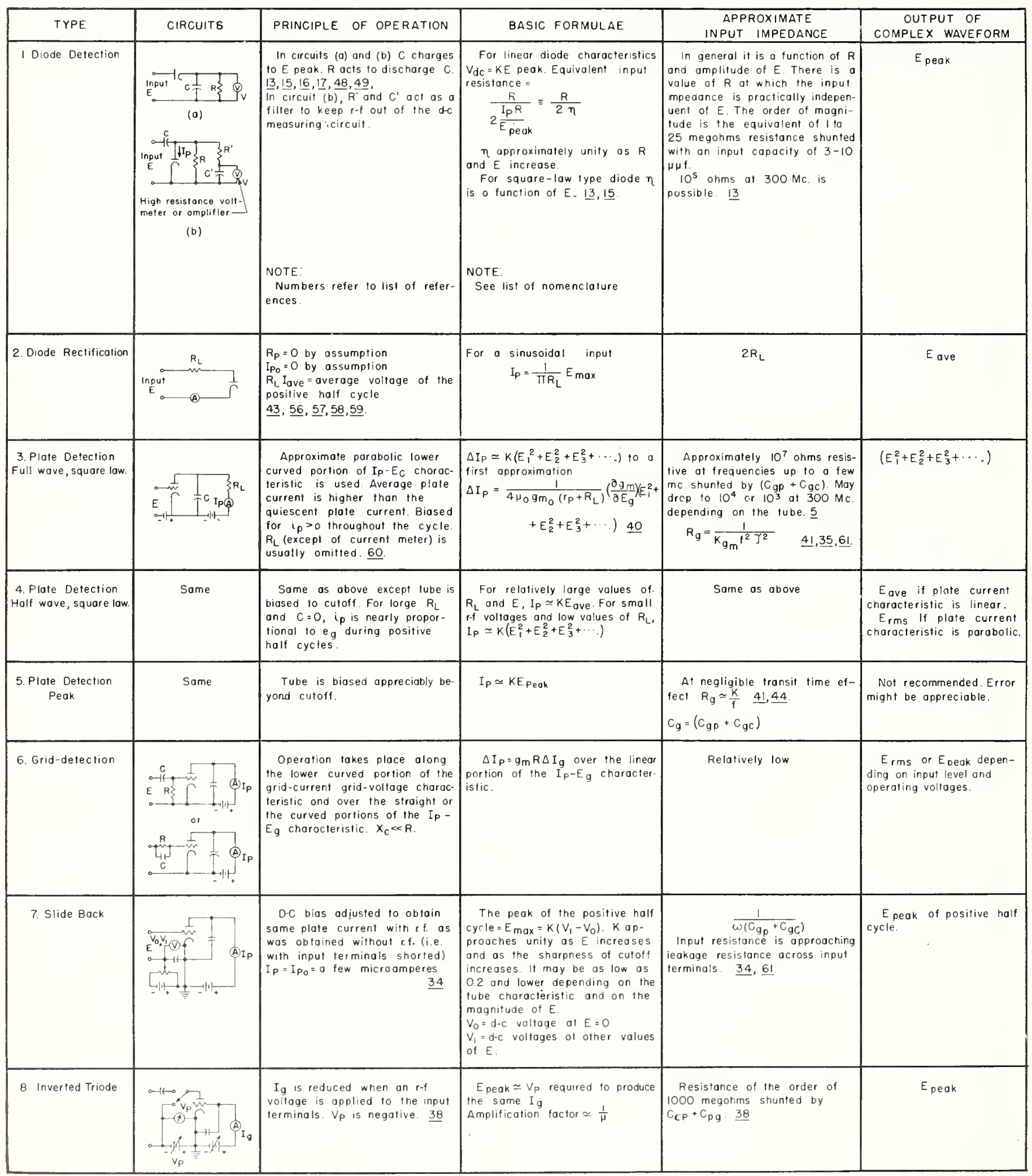

* See footnotes at end of this paper. 
Table l. Elementary circuits and characteristics of vacuum-tube voltmeters - continued

\begin{tabular}{|c|c|c|c|c|c|}
\hline TYPE & EFFECT OF WAVEFORM & $\begin{array}{c}\text { APPROXIMATE } \\
\text { VOLTAGE RANGE }\end{array}$ & $\begin{array}{c}\text { FREQUENCY } \\
\text { RANGE AND ERROR }\end{array}$ & $\begin{array}{l}\text { CALIBRATION } \\
\text { STABILITY } \\
\end{array}$ & REMARKS \\
\hline I Diode Defection & $\begin{array}{l}\text { The snurce impedance must } \\
\text { be negligible at oll hormonics, } \\
\text { and the level of harmonics } \\
\text { must be low otherwise error } \\
\text { may be as large os the per } \\
\text { centage hormonics present. I3 }\end{array}$ & $\begin{array}{l}\text { The upper limit depends on } \\
\text { tube ruting. With sensitive } \\
\text { decvoltmeter or with d-c am- } \\
\text { plifier the lower limit is } \\
\text { froction of a valt. Range } \\
\text { depends on frequency; the } \\
\text { higher the frequency, the } \\
\text { narrower the ronge; correction } \\
\text { may be opplied. }\end{array}$ & $\begin{array}{l}\text { Upper limit is affected by } \\
\text { (1) Series resononce of in- } \\
\text { put } L \text { and } C \text { anode to cath- } \\
\text { ode r-f voltage = } \\
E \frac{1}{1-\left(\frac{f}{f f}\right)^{2}} \\
2 \% \text { for o type } 955 \text { vocuum } \\
\text { tube of } 100 \mathrm{Mc} \text {. } \\
\text { (2) Transit time error = } \\
\frac{\Delta V}{V}=\frac{K d 0}{\lambda \sqrt{E} \text { peok }} \\
3 \% \text { at } 100 \text { Mc with } 955 \\
\text { "ocorn" of } 10 v \text {. Ronge is } \\
\text { therefore o function of vol- } \\
\text { tage opplied. Correction } \\
\text { curves moy be used to } 2 x- \\
\text { tend the ronge Overall } \pm \\
12 \% \text { is obtainable without } \\
\text { correction for } E>0.5 v \text {. } 16, \underline{5} \text {. }\end{array}$ & $\begin{array}{l}\text { Good Depends on the } \\
\text { constoncy of filoment } \\
\text { voltage ond emission. } \\
\text { Moy require yeorly coli- } \\
\text { brotions. }\end{array}$ & $\begin{array}{l}\text { This circuit, followed by a self-biosed d c ompli- } \\
\text { fier seems to be the prefered type most suit- } \\
\text { able for the widest frequency ronge } \\
\text { To eliminate low-frequency discrimination, } \\
\text { the product of RC should equal at least } 100 \\
\text { at the lowest volue of frequency The effect } \\
\text { of series resononce of the input to the tube } \\
\text { is to: } \\
\text { (1) Increose the apporent input voltage of the } \\
\text { fundamental frequency } \\
\text { (2) Emphasize hormonics more thon the fun- } \\
\text { damentol. For high voltoges, triodes con- } \\
\text { nected as diodes or power rectifiers are } \\
\text { used }\end{array}$ \\
\hline 2. Diode Rectification & $\begin{array}{l}\text { Not subject to turnover. } 45 \\
\text { (i.e. no error is coused by re- } \\
\text { versing input teminols even in } \\
\text { the cose of unsymetricol } \\
\text { wove-form consisting only of } \\
\text { fundomentol ond its hormonics.) }\end{array}$ & $\begin{array}{l}\text { A froction of o volt to a } \\
\text { few hundred volts and high- } \\
\text { ef depending on the value } \\
\text { of } R_{L} \text { and tube voltage rot- } \\
\text { ings. }\end{array}$ & $\begin{array}{l}\text { Should be colibrated at } \\
\text { the operoting frequency } \\
\text { when used in the neighbor- } \\
\text { hood of I MC and higher. } \\
\text { Probable useful ronge up } \\
\text { to severol Mc. }\end{array}$ & Some & $\begin{array}{l}R_{L} \text { may vary from } 0 \text { to I megohm. For } R_{L} \\
\geq 100,000 \Omega \text {, error coused by slight curvature } \\
\text { of stotic tube characteristic is negligible. } 51 \text {. }\end{array}$ \\
\hline $\begin{array}{l}\text { 3. Plote Detection } \\
\text { Full wave, square low. }\end{array}$ & $\begin{array}{l}\text { In practice } \Delta \mathrm{I}_{\mathrm{p}} \text { will depend } \\
\text { to some degree upon wove } \\
\text { form. Theoretically there is no } \\
\text { lurnover. Phose of harmonic } \\
\text { hos no effect. }\end{array}$ & $\begin{array}{l}\text { Froction of o volt to top } \\
\text { limit within squore low ronge } \\
\text { of tube (a few volts for } \\
\text { commerciol tubes) }\end{array}$ & $\begin{array}{l}\text { With present commercial } \\
\text { type tubes, a low-frequency } \\
\text { calibration will hold within } \\
5-10 \% \text { to } 20 \text { or } 30 \mathrm{Mc} \text {. At } \\
\text { higher frequencies colibro- } \\
\text { tion at each frequency is } \\
\text { necessory. } 39\end{array}$ & $\begin{array}{l}\text { Poor as a result of } \\
\text { tube ogeing ond vori- } \\
\text { ations in the } d-c \text { volt- } \\
\text { ages. }\end{array}$ & $\begin{array}{l}\text { Noise output can be corrected far by subtrac- } \\
\text { ting it from tolal output, } 40 \\
\text { i.e. } \Delta I_{x}=\Delta I_{\text {Total }}-\Delta I_{\text {Noise }}\end{array}$ \\
\hline $\begin{array}{l}\text { 4. Plate Detection } \\
\text { Half wave, square law. }\end{array}$ & $\begin{array}{l}\text { Subject to turnover and phose } \\
\text { of hormonics. }\end{array}$ & $\begin{array}{l}\text { Fraction of a volt to a } \\
\text { volue of } E \text { cousing grid cur- } \\
\text { rent flow }\end{array}$ & Some & Some & \\
\hline $\begin{array}{l}\text { 5. Plote Detection } \\
\text { Peak }\end{array}$ & $\begin{array}{l}\text { Subject to furnover and } \\
\text { phose of harmonics. }\end{array}$ & $\begin{array}{l}\text { From } E_{\text {max }}=V_{g} \text { to volues } \\
\text { cousing flow of grid currents. }\end{array}$ & Same & Some & \\
\hline 6. Grid-detection & Error moy be opprecioble. & $\begin{array}{l}\text { Fraction of a valt to a } \\
\text { few volts with receiving type } \\
\text { lubes. }\end{array}$ & Approximately to $10 \mathrm{Mc}$. & Very poor. & $\begin{array}{l}\text { When plote rectificotion tokes ploce in oddi- } \\
\text { tion to grid rectificotion, } \Delta I p \text { moy equal } \\
\text { zero of o certoin level of } E \text {. } \underline{51}\end{array}$ \\
\hline 7. Slide Back & Subject to turnover. & $\begin{array}{l}\text { Fraction of a volt to o few } \\
\text { hundred volts. Colibration is } \\
\text { indispensible especially for } \\
\text { voltages below oppraximately } \\
\text { IOvolts; calibration should be } \\
\text { made for a given Ip. }\end{array}$ & $\begin{array}{l}\text { Approximotely to } 10 \text { or } \\
20 \text { MC depending on input } \\
\text { capocity }\end{array}$ & $\begin{array}{l}\text { Good. Proctically inde- } \\
\text { pendant of ogeing and } \\
\text { operoting voltoge vori- } \\
\text { ations. }\end{array}$ & $\begin{array}{l}\text { Shorp cut off is obtained with pentodes } \\
\text { connected os triodes with screen grid used os } \\
\text { the control element. } 37\end{array}$ \\
\hline 8. Inverted Triode & Subject to turnover & $\begin{array}{l}\text { Lorge voltages, depending } \\
\text { on tube design. }\end{array}$ & $\begin{array}{l}\text { Possibly to } 10 \mathrm{Mc} \text {. Theo- } \\
\text { retically limited by the in- } \\
\text { put capacity No experimen- } \\
\text { tal dato ovoiloble. }\end{array}$ & $\begin{array}{l}\text { Probably good Na } \\
\text { experimental dolo ovoil- } \\
\text { oble. }\end{array}$ & \\
\hline
\end{tabular}

"See footnotes at end of this paper. 
2. High-input resistance.

3. Short-input terminals.

4. High series-resonance frequency of inputlead inductance and capacity.

5. Freedom from transit-time correction.

6. Calibration must not be affected by ordinary line-voltage variations, aging and temperature and humidity changes, and must have negligible zero-setting drift. Vacuum-tube voltmeters must not generate disturbing voltages and must give steady indications.

7. Calibration must hold over a reasonable length of time and shall not be affected by tube replacément.

8. Maximum voltage range with minimum auxiliary equipment like amplifiers and voltage dividers.

9. Peak voltage calibration for nonsinusoidal waves; rms for sinusoidal waves.

10. Linear scale or ]arge number of overlapping scales for square-law indications.

Some relative merits of triodes versus diodes for voltmeter' applications are listed below.

Triodes are preferable at frequencies below approximately $20 \mathrm{Mc}$.

1. For high sensitivity to small applied voltages,

2. For lower loading effect on circuit being measured,

3. For greater reliability of calibration at a power frequency.

Their major disadvantages are:

1. Input voltage is usually limited to values low enough to keep the grid from going positive.

2. The d-c plate current has to be stably balanced out to obtain maximum sensitivity.

3. Null point shifts as a result of supply voltage variations, aging and warm-up period required.

4. Triodes may have a shorter life and may require more frequent calibrations as compared with diodes.

5. The input resistance at frequencies of about $100 \mathrm{Mc}$ is lower than that of a diode by a factor of $10[5]$.

6. It is difficult to construct a triode having the small interelectrode spacing required to keep transit time and resonance errors to a minimum $[5,15,23]$.

A special diode construction was reported employing in indirectly heated cathode having the shape of a cylinder with a sealed-off end. A similar indirectly heated sealed-off cylinder is used as an anorle. Both cylinders are lined up end-to-end in such a manner that the two sealed ends face each other, and the gap between them forms the electron transit distance from catbode to anode. The width of this gap, and consequently the transit time, is controlled and is held at a minimum by varying the heating current fed to the anode, thereby causing its expansion or contraction [54]. Diodes were recently developed with an interelectrode spacing and element structure permitting their application without frequency correction for frequencies well above several humdred megacycles.

Table 1 lists fundamental detecting circuit elements of vacuum-tube voltmeters and their major functional characteristics. Associated circuits including regular and feedback amplifiers, current balancing circuits, voltage divider's, voltage stabilizing elements, etc., are equally important in determining sensitivity, linearity, stability, and range of the meter $[14,16,17,18,19]$. Voltage dividers, specially constructed to fit given mechanical and electrical requirements, may be used to measure high voltages at high frequencies. One arrangement is shown in figure 6 [20].

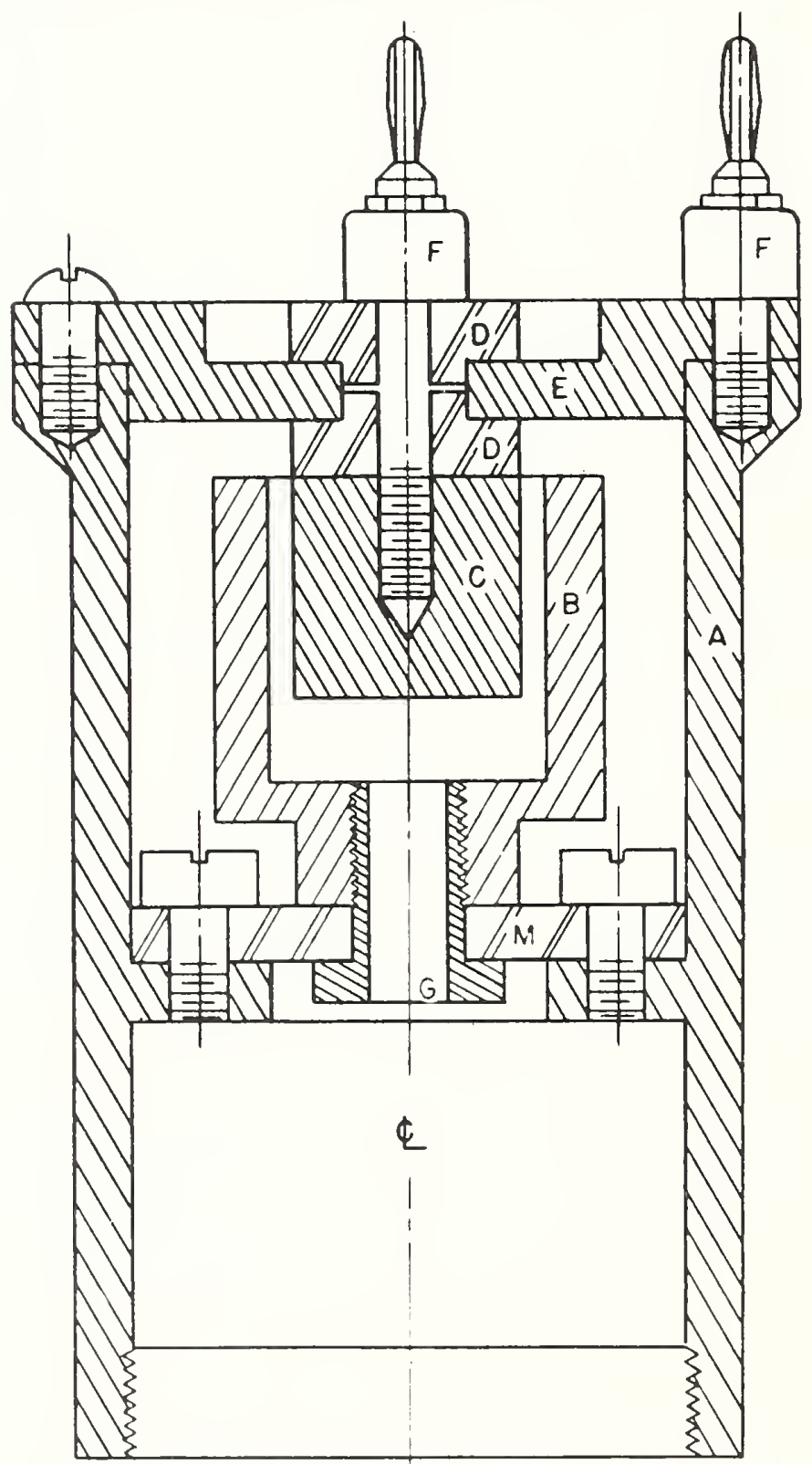

Figure 6. Toltage divider for high $r-f$ voltage measurements

$G$ is the terminal conneeted to a calibrated low voltage meter. The high $G$ is the terminal conneeted to a calibratcd low voltage meter. The high
voltage is eoupled through the capacity between parts $B$ and $C$. Various sizes of part $C$ are provided to obtain different voltage ratios. 
(b) Nonthermionic rectifiers

In addition to thermionic diodes, other rectifiers are used as $r-f$ voltmeter diode elements. These may be broadly subdivided into two classes, copper-oxide or selenium rectifiers and crystal diodes.

Copper-oxide and selenium rectifiers have good overload characteristics and ruggedness. They are, however, affected by temperature and aging, have a relatively large shunt capacitance and high forward resistance. The approximate equivalent circuit is given in figure $7[21,22]$. The capacitance is approximately $0.02 \mu \mathrm{f} / \mathrm{cm}^{2}$ of contact surface. $R_{1} \cong 2$ ohms for $1 \mathrm{~cm},{ }^{2}$ and $R_{2}$ with polarity connections for maximum resistance (that is, backward resistance) is approximately 11,000 ohms for $1 \mathrm{~cm}^{2}$ at applied voltages of -0.25 to -3: this holds over a frequency range of $50 \mathrm{kc}$ to $5 \mathrm{Mc}$. The forward resistance is several ohms per square centimeter at $26^{\circ} \mathrm{C}$ and decreases slightly with increasing temperature. Backward resistance is considerably affected by temperature changes. Figure 8 shows direct-current characteristics of some of these rectifiers [22]. The rectified current depends on temperature, load resistance, frequency, and current density. This type of rectifier is manufactured in all sizes down to a small fraction of an inch in cross-sectional area for currents of a few milliamperes.

Copper-oxide rectifiers are preferable to selenium types for instrument application because of their lower resistance. Selenium types may be operated up to about $10 \mathrm{v}$ per element as against about $2 \mathrm{v}$ for copper oxide.

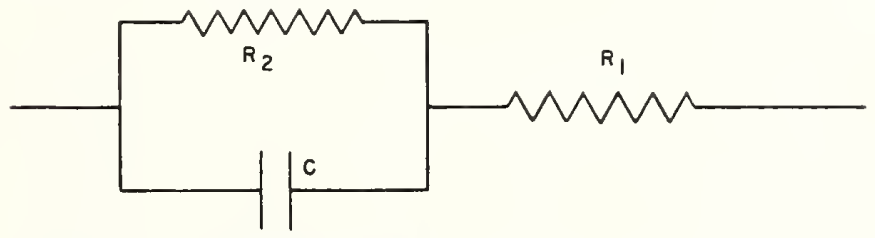

Figure 7. Approximate equivalent circuit of copper-oxide rectifier in the $50 \mathrm{kc}$ to $5 \mathrm{Mc}$ range.

$R_{1}$ represents the resistance of the body of the oxide. $R_{2}$ represents the resistance of the oxide-copper interface and is a function of applied voltage.

Copper-oxide-rectifier-type voltmeter's are available for frequencies to approximately $30 \mathrm{kc}$ and can be designed up to 1 Mc [22]. The major frequency-limiting element is the shunt capacitance, $C$. The effect of the wave form of the applied voltage is appreciable; the error in the indicated output, calibrated in terms of voltage free of harmonics, may approach in magnitude the percentage of harmonic content of the voltage measured. These rectifiers are used in series with resistors in $1,000-\mathrm{olhm} / \mathrm{v}$ instruments. They are very much higher in sensitivity and draw considerably less current from the source than the iron-vane or thermocouple type instruments. When the power of the voltage source exceeds $1 \mathrm{w}$ and a high impedance instrument is not necessary it is preferable to employ an instrument having a higher inherent order of accuracy than that of the rectifiertype voltmeter [22].

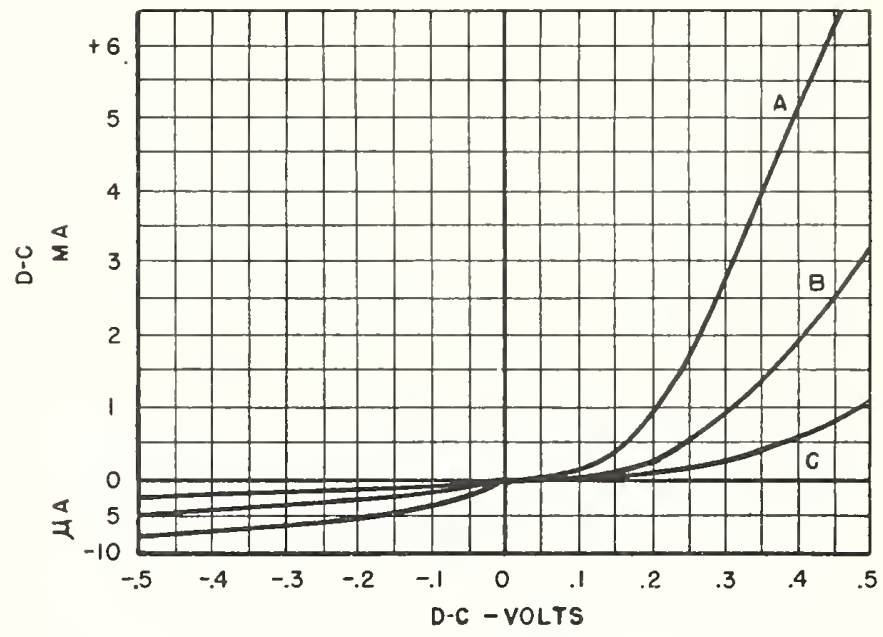

FIGURE 8. Direct-current characteristics of copper-oxide rectifiers.

A, 0.200 disk; B, 0.130 disk; C, 0.008 disk.

Whereas copper-oxide rectifiers are applicable as voltage indicators only at the lowest frequencies considered here, modern crystal rectifiers are useful up to $300 \mathrm{Mc}$ and higher.

The crystals most commonly used at present are silicon and germanium.

Table 2 shows the chemical constituents of some of these crystals [26]. Other useful crystals are galena, iron-pyrites and carborundum. In measurement applications involving present-day dry rectifiers, one should recognize the following difference in design: the contact area, and thus power-handling capacity, of crystal diodes is much smaller than for copper-oxide rectifiers.

TABLE 2. Composition of crystal rectifiers

\begin{tabular}{|c|c|c|c|}
\hline \multirow{2}{*}{ Bulk material } & \multicolumn{3}{|c|}{ Impurities added } \\
\hline & $\begin{array}{l}\text { High-frequency } \\
\text { mixer crystals }\end{array}$ & $\begin{array}{c}\text { High-back roltage } \\
\text { erystals }\end{array}$ & $\begin{array}{c}\text { Low-frequency } \\
\text { rectifiers }\end{array}$ \\
\hline Silicon & $\begin{array}{l}\text { Aluninum } \\
\text { Boron }\end{array}$ & $\begin{array}{l}\text { Germanium } \\
\text { Also } \\
\mathrm{Ni} \\
\mathrm{Sn} \\
\mathrm{Bi} \\
\mathrm{Ca}\end{array}$ & $\begin{array}{ll}\text { Aluminum } \\
\text { Boron } \\
\text { Germanium } \\
\text { Also } \\
\begin{array}{ll}\text { Mo } & \text { Ta } \\
\mathrm{Zr} & \mathrm{Co} \\
\text { WV } & \mathrm{Re} \\
\mathrm{Be} & \mathrm{Fe}\end{array}\end{array}$ \\
\hline Germanium..... & $\begin{array}{l}\text { Antimony } \\
\mathrm{P} \text { Also } \\
\mathrm{Fe}\end{array}$ & $\begin{array}{l}\text { Tin } \\
\mathrm{Also} \\
\mathrm{Ca} \\
\mathrm{Ni} \\
\mathrm{Sr} \\
\mathrm{Bi} \\
\mathrm{N}\end{array}$ & $\begin{array}{l}\text { Antimony } \\
\text { Tin }\end{array}$ \\
\hline
\end{tabular}


Figure 9 shows the mechanical construction of a modern crystal diode; figure 10 shows typical static characteristics for three germanium diodes, and figure 11 shows the rectification-efficiency characteristic of a germanium diode for different loads and frequencies; figure 12 shows the rectification efficiency of an iron-pyrites rectifier $[24,25,26,27]$

Figure 13 shows the equivalent circuit of a crystal diode where $R_{e}$ and $C_{b}$ are the nonlinear resistance and shunt capacity of the barrier layer, and $R_{s}$ is the resistance of the body of the semiconductor $[27,28]$.

Relative merits of crystal versus thermionic diodes for $r-f$ voltage measurements are listed as follows.

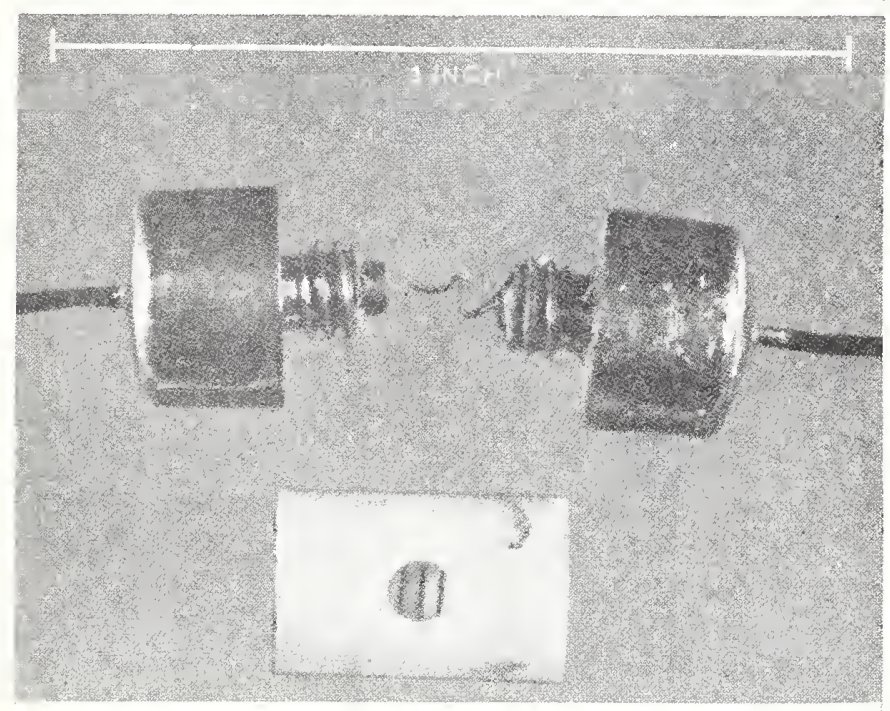

Figure 9. Components of a 1 N34 germanium crystal.

The wire "whisker" points toward the small germanium block mounted on the lower threaded terminal.

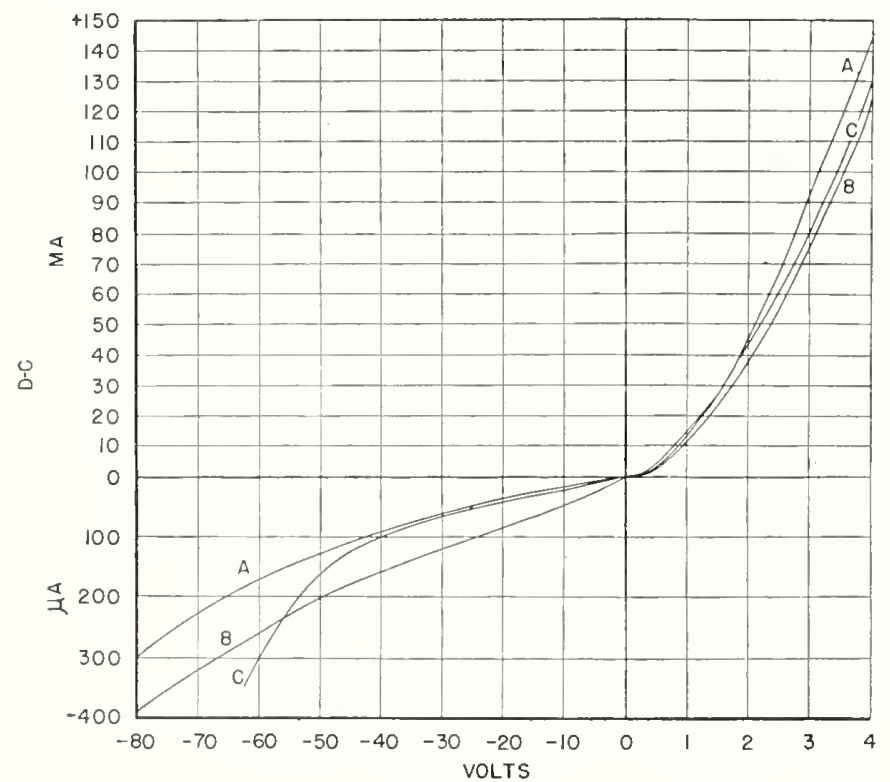

FIGYRE 10. Individual static characteristics of three small germanium diodes $A, B$, and $C$.

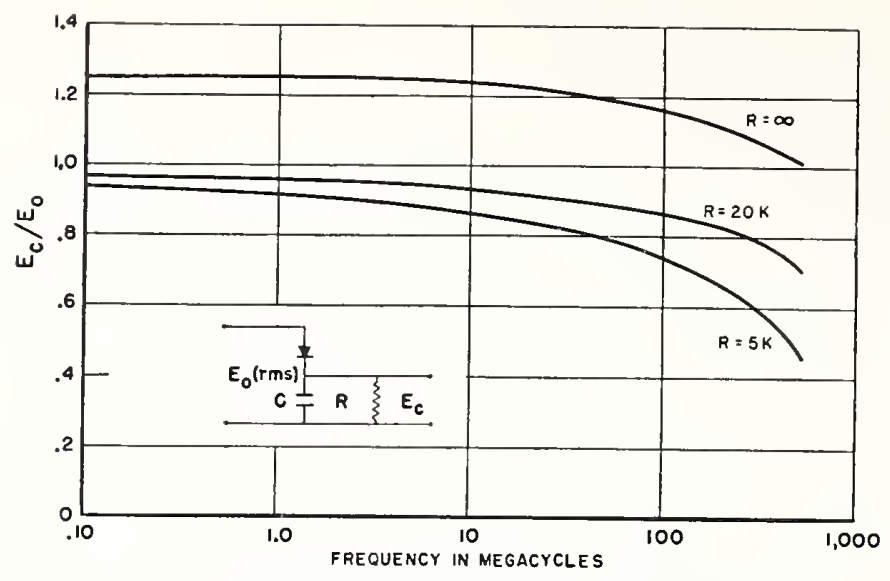

FIGURE 11. Rectification-eflciency characteristics of a germanium crystal.

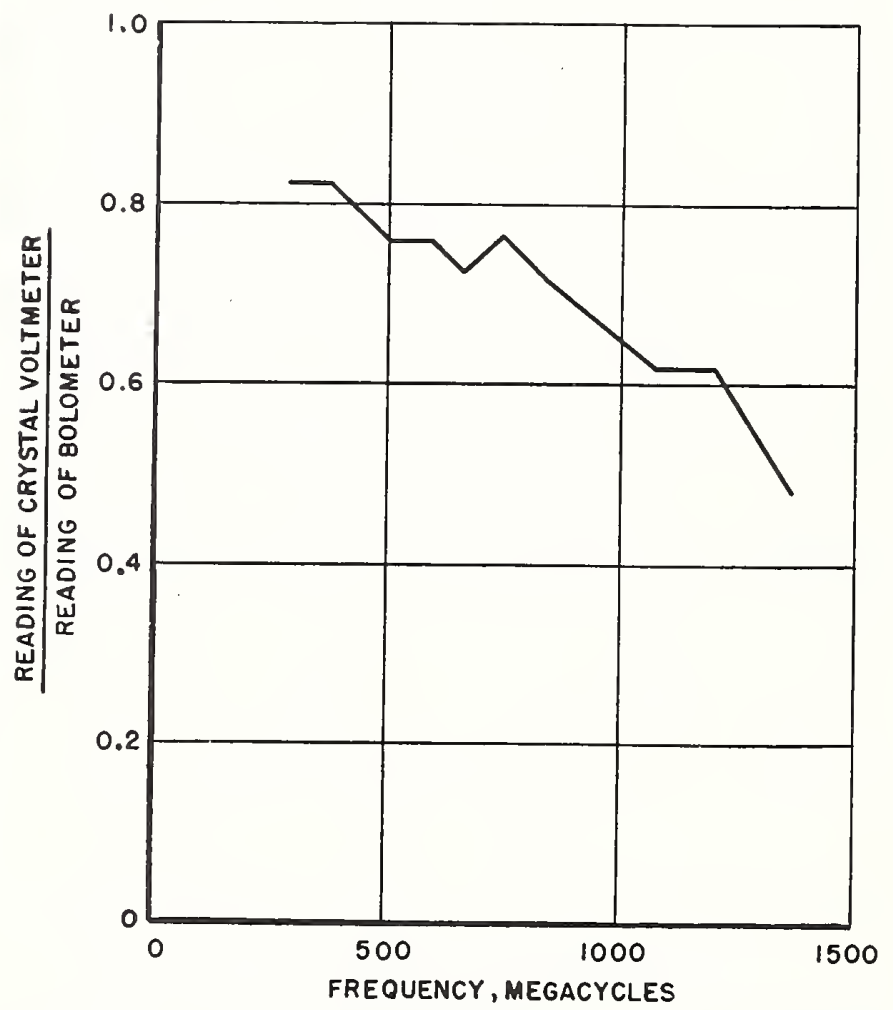

FIGURE 12. Rectification efficiency of an iron-pyrites rectifier.

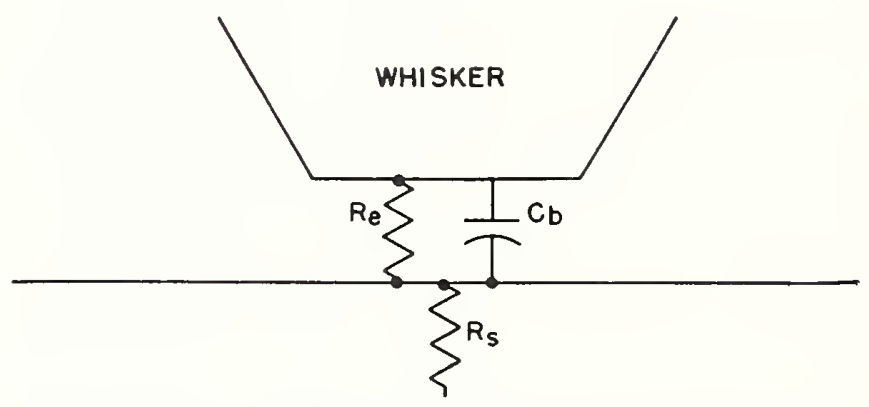

FIGURE 13, Equivalent circuit of a crystal rectifier. 
Relative advantages of crystal diodes:

1. Transit-time effect is negligible. For comparison the rectification efficiency in acom-type thermionic diodes as a result of transit-time effect begins to drop off at $30 \mathrm{Ne}$ for voltage levels of $0.5 \mathrm{v}$. This reduction is 30 percent at $500 \mathrm{Ne}$

2. The crystal has smaller physical dimensions and therefore a higher input resonant frequency. This is approximately $3,500 \mathrm{Mc}$ as against 1,500 IC for the smallest commereial thermionic diode and 2,800 Me claimed for the latest special type. diode (Eimac type 2-01C).

3. The cathode does not have to be maintained at constant temperature, an added requirement in the case of thermionic tubes to insure constant cminsion.

4. Crystals are useful in rectifying lower voltages than vareum diodes.

Relative disadvantages of crystals:

1. They have poorer stability, are less rugged, and show greater variation in characteristics for units of the same type.

2. They are frequeney-sensitive partly for the following reasons: the capacity $C_{b}$ (fig. 13) $\left(C_{b}\right.$ ranged from 0.2 to $0.6 \mu \mu$ for goorl commercial units) shunts the "reverse" resistance of the barricr"; this produces a drop in rectification effiriency. The effective l'esistance and capacity of the barrier laver are functions of the voltage applied across this barrier [25, 28]; the magnitude of this voltage is in turn a function of $C_{b}, R_{e}$, and $R_{s}$ acting as a voltage divider; $R_{s}$ varies between 5 to 100 olmms for different types of crystals. 'This effect is negligible, however, for some units at frequencies below $500 \mathrm{Nc}$ in circuits having relatively high crystal-load resistance. Figures 11 and 12 show typical frequency characteristies of two commercial types of crystals.

3. Reverse rectification at the contact between the crystal and its supporting electrode and the relatively large shunting capacity at this contact introduces another error. In the particular case of an iron-pyrites crystal this error amounts to a 50-percent increase in the total output at 10 Me as compared with the output at $1 \mathrm{Me}$ [27]. This takes place because the $r-f$ voltage across these contacts is a function of the ratio of the contact resistance to the capacitive reactance shunting it; at 10 Me the $\mathrm{r}-\mathrm{f}$ voltage rectified in the reverse direction is therefore lower than that of $1 \mathrm{Mc}$; consequently the total rectified output at $10 \mathrm{Mc}$ is higher. Plating or fusing the crystal in place largely eliminates this effect.

4. The input impedance of crystal and probe is comparable with that of a thermionic diode and its probe at ultrahigh frequencies. At lower frequencies the $\mathrm{v}$-t diode has a higher input resistance than the crystal.

5. The voltage range of commercially available crystal units designed for high back voltage and for frequencies up to $100 \mathrm{Mc}$ is limited to a maximum of approximately $30 \mathrm{v}$ rms. Those recommended for higher frequencies have a maximum rating of approximately $1 \mathrm{v}$ r'ms $[25,26]$. Overloading causes a change in characteristics or permanent damage to the contacts.

6. Resistance and sensitivity vary with temperature as shown in figure 14 [26].

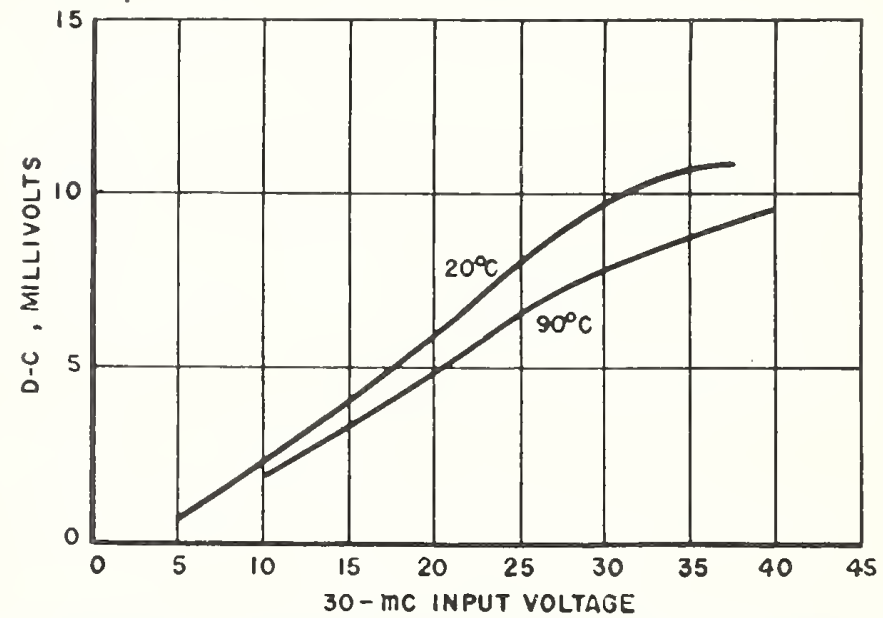

Figure 14. Rectified current through a 1,000-ohm load on a high-back-voltage silicon-crystal rectifier as a function of input voltage at $20^{\circ}$ and $90^{\circ} \mathrm{C}$.

A crystal-type voltage indicator was recently placed on the market [28]. It has a range of 0.1 to $1 \mathrm{v}$ with a \pm 5 percent claimed accuracy from 10 to $300 \mathrm{Mc}$. The "forward" resistance of the crystal is of the order of a few hundred ohms; in the "reverse" direction it is 15,000 to 100,000 ohms. The crystal is used in a peak-reading circuit. The input resistance of the meter is approximately one-third of the "reverse" resistance. An improved construction of a germanium crystal was announced having an optically polished face of specially processed germanium and a platinum "whisker" point welded to that plane; stability and constancy of performance superior to that employing pressure-type contact is claimed [29].

\section{Pulse-peak voltage measurement}

(a) Cathode-ray beam deflection

The most accurate method of measuring peaks of voltage-pulses employs a cathode-ray oscilloscope. Proper synchronization of the sweep circuit of an oscilloscope with the pulse source facilitates detailed measurement of the pulse shape including the evaluation of the erest voltage of the pulse. Deflections can be measured directly on the screen, or a d-c voltage slide-back circuit arrangement similar to the one shown in figure 2 may be used. Resistance or capacitance dividers are frequently used with oscilloscopes in making high peak measurements. The design of 
the divider should be such that in reducing the relative magnitude of the voltage pulse it does not alter the shape of the pulse applied to the oscilloscope.

\section{(b) Diode peak voltmeters}

Diode peak voltmeters are generally used as convenient moderate-precision indicators [30, 31]. However, the discrepancy between the voltmeter reading and true peak value may be very large. Figure 15 shows the response of a diode-type vacuum-tube voltmeter as a function of pulserepetition frequency for a rectangular pulse shape and pulse duration of 3.5 microseconds. An approximate expression is given by Easton [30] for the rectangular pulse peak in terms of the d-c diode output as follows:

$$
e_{0} \cong E_{d c}\left[1+\frac{T}{t_{1}} \frac{R_{1}}{R_{2}}\right]
$$

where

$e_{0}=$ the peak voltage of a rectangular pulse $E_{d c}=$ the d-c voltage across $\mathrm{R}_{2}$

$T=$ the duration. of the discharging interval

$t_{1}=$ the duration of the charging interval

$R_{1}=$ the total resistance during charging

$R_{2}=$ the total resistance during discharging.

One of the curves of figure 15 shows values computed on the basis of this expression. The effective input impedance of this type of voltmeter for pulse voltages may be very low. The directvoltage output (measured across $R_{2}$ ) decreases with increasing rate of pulse repetition, with increasing values of $R_{2}$ and with decreasing values of $R_{1}$. $\quad R_{1}$ is a function of the combined source and diode resistances at the particular operating conditions. Improved performance may be obtained by means of auxiliary circuits like cathode followers [30] and automatic slideback arrangements [31].

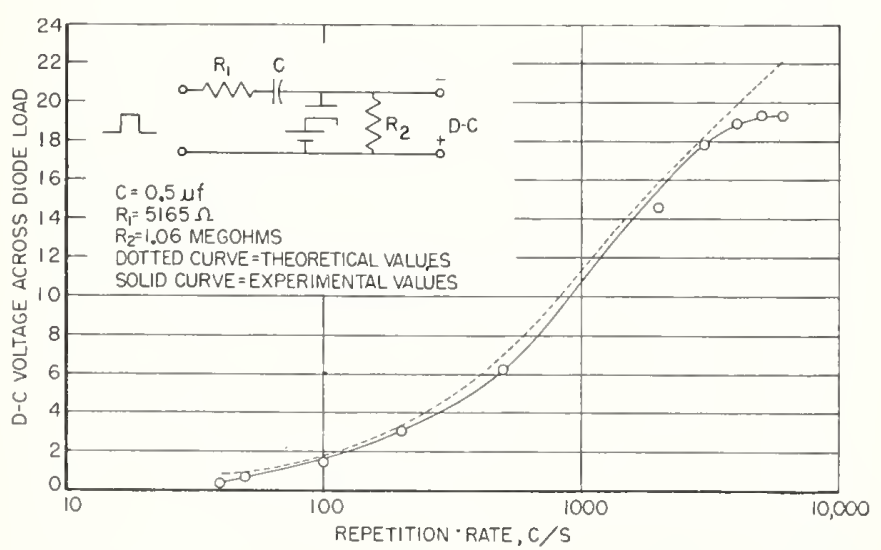

Figure 15. Response of a diode-type T'TlM as a function of pulse-repetition frequency for a charging-interval duration of 3.5 microseconds and a peak of $26 v$.

\section{Miscellaneous Methods}

The following voltage-measuring methods are of interest as relatively independent and useful for certain applications.

\section{(a) Heterodyne Method of Extending the Voltage Range [32]}

This principle is illustrated in figure $16, \mathrm{~A}$

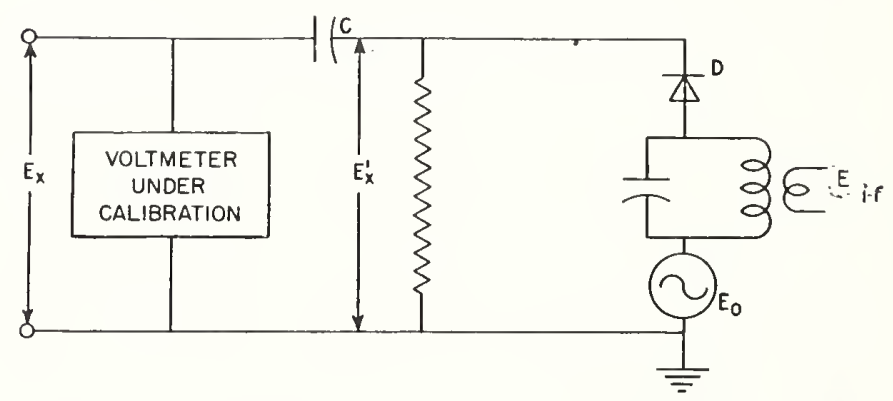

Figure 16. Heterodyne principle of calibrating $r-f$ roltmeters.

linear diode frequency changer, $D$, mixes voltages, $E_{0}$, supplied from an auxiliary source, with $E_{x 1}$, a fraction of the unknown voltage $E_{x}$ used to calibrate the voltmeter. The intermediate-frequency output voltage is directly proportional to $E_{x}$ for large ratios of $E_{0} / E_{x 1}$. This proportionality holds for values of $E_{r 1}$ not exceeding the order of magnitude of 1 volt. The capacitor, $C$, is therefore indicated as an element of a linear voltage dividing network used to maintain $E_{x 1}$ considerably below $E_{x}$. The interelectrode capacity of the diode, shunted by other circuit capacities (not shown in the figure), constitutes the second element of the voltage divider. One can thus use a single calibration point of the same voltmeter determined at a low voltage (for example, 1 volt obtained by means of the bolometer or any of the other standard methods listed above) and proceed with calibrating it at higher voltages. 'The advantage of this method is that it can be used to calibrate high voltage levels at high frequencies in terms of a standard attenuator used at a relatively low intermediate frequeney. Let, for example, $C$ be chosen at such a value that $E_{1-\varepsilon}$, corresponding to the calibrated $E_{x 1}$ (of, let us say, 1 volt), is some small value (say $\left.Y_{1} m v\right) . \quad E_{x}$ is then increased until a new value of $E_{1-1}$ (say $X_{2} m \mathrm{v}$ ) is obtained; the new higher value of $E_{x}$ is then equal the previous times the ratio of these i-f voltages (that is, $E_{x 2}=$ $\left(X_{2} / X_{1}\right) E_{x 1}$ volts). This latter ratio can be accuratcly determined with a standard attenuator placed in series with the i-f output terminals. The use of a low transit-time diode may eliminate the transit time error at frequencies up to several hundred megacycles. 


\section{(b) Spark-gap method}

Spark gaps may be used to measure peak voltages of the order of 1 to $30 \mathrm{kv}$ at all frequencies up to about 100 ke [33]. The sphere spark gap is preferred to other eleetrodes because the breakdown voltage ehanges little up to about 25 ke. For a symmetrical sphere-gap voltmeter the peak voltage is given approximately by

$$
E=\sqrt{2} \epsilon \frac{l}{m}
$$

where

$$
\text { ' } \epsilon=19.3 \rho[1+0.76 / \sqrt{p D}] \mathrm{kv} / \mathrm{cm}
$$

$\rho=3.92 p / T=$ relative air density

$D=$ sphere diameter in centimeters

$l=$ maximum gap-length in centimeters at which spark-over may take place

$p=$ atmospheric pressure in centimeter's of a mercury column

$T=\left(273+N^{\circ} \mathrm{C}\right)=$ absolute tempcrature in $\mathrm{deg}$ $\mathrm{K}$, and$$
m=0.25\left[2 l / D+1+\sqrt{(2 l / D+1)^{2}+8}\right] .
$$

Actual voltages at $100 \mathrm{kc}$ are 10 percent lower than eorresponding power frequency voltages normally used to calibrate the gap voltmeter.

\section{(c) Glow-discharge voltmeter}

A method applicable for peak voltages up to about $15 \mathrm{kv}$ and frequencies up to $1 \mathrm{Mc}$ makes use of a glow tube as shown in figure 17 [33]. The

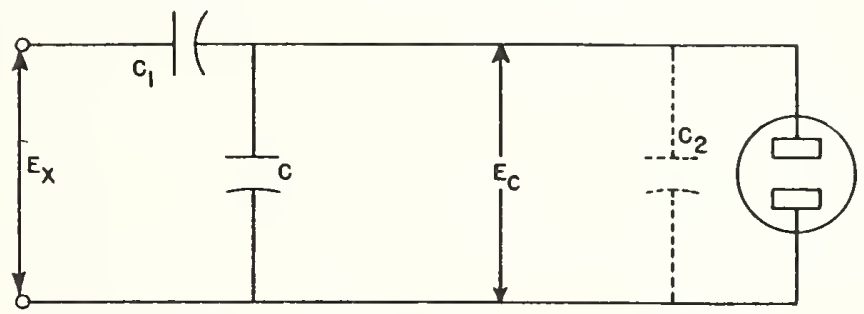

Figure 17. Glow-discharge tube voltmeter.

value of $C$ is continuously decreased until the peak value of the voltage across it is just equal to a predetermined critical value $E_{c}$ that is causing the tube to glow. The value of $E_{x}$ may then be computed from known values, of $C, C_{1}$, and $C_{2}$, where $C_{2}$ is the tube and distributed imput lead capacity.

\section{(d) Electrometer employing miniature open- wire line}

An electrometer consisting of a short platinum open parallel-wire line is reported applicable for measurement accuracies of 1.5 percent over a frequeney range of 30 to $100 \mathrm{Me}$ and voltage range of 10 to 70 volts effective [42]. The wire diameter is $0.01 \mathrm{~mm}$, line separation $1 \mathrm{~mm}$, and line length approximately $5 \mathrm{~cm}$. One of the lines is tightly mounted whereas the other is kept under relatively low tension. The deflection of the latter is observed under a microscope and calibrated at 100 ke and at direct eurrent, the two calibrations yielding identical results. Varying the tension of the wire under obsel'vation provides a eontrol of the voltage range that could be increased to $300-v$ effective value. A major adrantage of this metlod is claimed to be the high input impedance, the capacity of the electrometer being less than $0.5 \mu \mu$ f. The maximum reduction in line separation (with a consequent effect on the characteristic impedance and voltage (listribution) is 10 pereent; this contributes an error of less than 0.1 percent to the voltage measurements at all frequencies up to $100 \mathrm{Me}$.

\section{(e) Electrometer employing suspended wire}

An electrostatic voltmeter employing a suspended wire described by Peterson [55] may be used for frequeneies up to 1,000 Me or higher. It consists of a 3.5-em-long, 0.0013-em-diameter platinum wire suspended inside a 2.5 by $1.1-\mathrm{cm}$ opening of a brass block. The wire is spaced $0.16 \mathrm{~cm}$ from the $1.1-\mathrm{cm}$ side and the deflection of its free end is measured when the r-f voltage is applied between the insulated wire-suspension terminal and the brass block. A deflection of about $2 \mathrm{~cm}$ may be obtained for $10 \mathrm{v}$ when the shadow of the wire is projected optically on a screen with an effective defteetion magnifieation of 1,000 . The deflection is increased by about 3 percent at $300 \mathrm{Mc}$ and 33 pereent at 1,000 Mc as compared with that of direct current. The top value of the voltage range is limited by the corresponding charging eurrent that might cause the fine platinum wire to fuse.

The listed difficulties eneountered with this .voltmeter were:

1. A darkened room may be required.

2. The electrometer is very sensitive to motions of the building and should preferably be used in the clead of night.

3. The heat from the projector lamp causes a drift of the wirc position.

4. It cannot be used at low frequencies where the low inertia of the wire is insufficient to prevent wire vibration.

5. The input capacity is a function of the roltage applied, which may sometimes be objectionable.

The author welcomes an opportunity to express his appreciation of the large amount of careful work covered by the references cited in this paper and for the kind permission of some of those authors to quote and include data and reproduee some of their curves and drawings and acknowleges helpful suggestions of some of his eolleagues at the National Bureau of Standards during the course of preparation of this paper. 


\section{References}

[1] G. L. Pearson, Thermistors, their characteristies and uses, a pamphlet on varistors by Western Electric Co., Radio Division, WECO, T2096B, p. 22 (1945).

[2] J. A. Becker, C. B. Green, and G. L. Pearson, Properties and uses of thermistors - thermally sensitive resistors, Trans. Am. Inst. Elect. Engrs. 65, No. 11, $711(1946)$

[3] D. B. Sinclair, The type 663 resistor, General Radio Experimenter, 13, No. 8, 6 (1939).

[4] P. M. Lamps, technical data (Sylvania Electrical Products Inc., Feb. 5, 1945).

[5] L. S. Nergaard, Electrical measurements at wavelengths less than two meters, Proc. Inst. Radio Engrs. 24, 1207 (1936).

[6] H. E. Hollmann, Ultra-high-frequency oscillography, Proc. Inst. Radio Engrs. \$8, No. 5, 213 (1940).

[7] F. M. Gager, Cathode-ray electron ballistics, Communications, p. 10, (1938).

[8] H. E. Hollmann, The use of the cathode-ray oscillograph at ultra-high frequencies, W. Eng. and Exp. IW. 10, 430,48方 (1933).

[9] G. M. Lee, A three beam oscillograph for recording at frequencies up to $10,000 \mathrm{Mc}$., Proc. Inst. Radio Enors. 34, No. 3, $121 \mathrm{~W}$ (1946).

[10] L. K. Libby, Cathode rays for the uhf, Electronics 3, No. 9,15 (1936)

[11] H. G. Rudenberg, Deflection sensitivity of parallelwire lines in cathode-ray oscillographs, J. Applied Phys, 16, 279 (1945).

[12] Electrical measurements, 13, No. 1 (Scientific Research Instrument Corp., New York, N. Y., Jan. 1946).

[13] C. B. Aiken, Theory of the diode voltmeter, Proc. Inst. Radio Engrs. 26, 859 (1938)

[14] J. F. Rider, Vacuum tube voltmeters (J. F. Rider Publisher Inc., 404 4th Ave., New York, N. Y., 1941).

[15] E C. S. Megaw, Voltage measurement at very high frequencies, W. Eng. 13, 65, 135, 201 (1936)

[16] C. A. Woodward, Jr., A new vacuum tube voltmeter, General Radio Experimenter, 22, No. 4 (1946)

[17] W. N. Tuttle, Type $726-\mathrm{A}$ vacuum-tube voltmeter, General Radio Experimenter, 11, No. 12 (1937)

[18] T. G. Faston, Radio-frequency characteristics of the type 726-A vacuum-tube voltmeter, General Radio Experimenter, 15, No. 11 (1941).

[19] The use of the 954 as a vacuum-tube voltmeter, RCA Radiotron Division, Application note, No. 47 (May $20,1935)$

[20] Radio-frequency voltmeter, Bell Labs. Record 21, No. $5,126(1943)$

[21] W. H. Brattain, The copper oxide varistor, a pamphlet on varistors by Western Electric Co., Radio Division, p. 9, WEECO, T2096B (1945).

[22] J. H. Miller, Copper oxide rectifiers as used in measuring instruments, Weston Eng", notes, 1, No. 1. (1946).

[23] B. J. Thompson, G. M. Rose, Jr., Vacuum tubes of small dimensions for use at extremely high frequencies, Proc. Inst. Radio Engrs. 21, No. 12, 1707 (1933).

[24] Sylvania Electric Products Inc., bulletin on erystal diode IN34, No. EC-22, Jan. 1946.
[25] E. C. Cornelius, Germanium crystal diodes, Electronics 19, No. 2, 118 (1946)

[26] W. E. Stephens, Crystal rectifiers, Electronies 19, No. 7, 112 (1946).

[27] A. Peterson, Vacuum tubes and crystal rectifiers as galvanometers and voltmeters at ultra-highfrequencies, General Radio Experimenter 19, No. 12 (1945).

[28] A. Peterson, A peak reading voltmeter for uhf ranges, General Radio Experimenter 21, No. 5 (1946).

[29] The new germanium erystal diode, G. E. Electronic Dept., bulletin ESD-88, Feb. 24, 1947.

[30] A. Easton, Pulse response of diode voltmeters, Flectronies 19, No. 1, 146 (1946).

[31] C. J. Creveling, L. Mantner, An automatic slideback peak voltmeter for measuring pulses, Proc. Inst. Radio Engrs. 35, No. 2, 208 (1947).

[32] This method is at present under investigation at the Central Radio Propagation Laboratory of the NBS.

[33] A. Hund, High-frequency measurements, 1st ed., p. 134 to 136 (MeGraw-Hill Book Co., Ine., New York, N. Y.; 1933).

[34] C. B. Aiken, L. C. Birdsall, Sharp cutoff in vacuum tubes, with applications to the slide-back voltmeter, Trans. Am. Inst. Elec. Engrs. 57, 171 (1938).

[35] I. M. Miller, Dependence of the input impedance of a three-electrode vacuum tube upon the load in the plate cireuit, BS Sci. Pap. 15, (1919-20) S351.

[36] R. A. Heising, Thermionic voltmeters, Patent No. $1,232,919$ (1917).

[37] H. J. Reich, G. S. Marvin, K. Stall, Vacuum tube voltmeter of high sensitivity, Electronics 3, 109 (1931).

[38] F. E. Terman, The inverted vacuum tube, a voltagereducing power amplifier, Proc. Inst. Radio Engrs. 16, No. 4, 447 (1928).

[39] RCA Radiotron Division, Harrison, N. J., Application note on the use of the 954 as a vacuum tube voltmeter. Application note No. 47, May 20, (1938).

[40] F. E. Terman, Radio Eng., 2d cd., p. 269 (McGrawHill Book Co., Ine., New York, N. Y., 1937).

[41] IV. R. Ferris, Input resistance of vacuum tubes as ultra-high frequency amplifiers, Proc. Inst. Radio Engrs. 24, 82 (1936).

[42] A. L. Chodakov, An electrometer for measuring voltages in the meter wave range, J. Tech. Phys. 11, No. 8, 767 (1941). (In Russian).

[43] H. J. Reich, Theory and application of electron tubes, 2d cd., p. 597 to 612 (MeGraw-Hill Book Co., Inc., New York, N. Y., 1944).

[44] J. G. Chaffee, The determination of dielectric properties at very high frequencies, Proc. Inst. Radio Engrs. 22, No. 8, 1009 (1934).

[45] H. A. Brown, Radio-frequency elertrical measurements, 2d ed., p. 274 and 281 (MeGraw-Hill Book Co., Ine., New York, N. Y., 1938).

[46] A valve voltmeter for auchio frequencies, IV. Eng. 10, 310 (1933)

[47] L. S. Nergaard, A survey of ultra-high-frequency measurements, RCA Rev. 3, 156 (1938). 
[48] M. J: O. Strutt, K. S. Knol, Measurement of current and voltages down to a wavelength of 20 centimeters, Proc. Inst. Radio Engrs. 27, 783 (1939).

[49] R. Davis, G. W. Bowdler, W. G. Standring, The measurement of high voltages with special reference to the measurement of peak, J. Inst. Elec. Engrs. (London) 68, 1222 (1930).

[50] C. E. Kilgour, J. M. Glissner, Diode detection analysis Proc. Inst. Radio Engrs. 21, No. 7, 930 (1933).

[51] A. Hund, High-frequency measurements, 1st ed., p. 149 to 153 (McGraw-Hill Book Co., Inc., New York, N. Y., 1933).

[52] J. T. Kendall, The rectifying property of carborundum, Proc. Phys. Soc. 56, part 2, No. 314, 123 (1944).

[53] T. MIoreno, O. C. Lundstrom, Microwave power measurement, Proc. Inst. Radio Engrs. 35, 514 (1947).

[54] Report on measurement voltage at high frequencies, Research Laboratories, Electric and Musical Industries, Ltd., Hayes, Middix, Dec. 28, 1940 (unpublished).

[55] A. P. G. Peterson, The measurement of voltages at ultra-high-frequencies, Thesis, submitted to Massachusetts Institute of Technology, May 15, 1941).
[56] I. Wolff, Alternating-current measuring instruments as discriminators against harmonics, Proc. Inst. Radio Engrs. 19, No. 4, 647 (1931).

[57] F. E. Terman, Multirange rectifier instruments having the same scale graduation for all ranges, l'roc. Inst. Radio Engrs. 23, No. 3, 234 (1935).

[58] A valve voltmeter for audio frequencies, The wireless engineer and experimental wireless 10, 310 (1933).

[59] J. Taylor, An application of the diode to the measurement of a-c voltages, J. Sci. Instruments 3, 113 $(1925-26)$.

[60] F. E. Terman, Measurements in Radio Engineering, 1st ed., p. 18 to 30 (McGraw-Hill Book Co., Inc., New York, N. Y., 1935).

[61] H. J. Reich, Theory and applications of electron tubes, 2d ed., chapter 4 (McGraw-Hill Book Co., Inc., New York, N. Y., 1944).

[62] F. L. Hermach, A precision electrothermic voltmeter for measurements between 20 and 20,000 cycles, Trans. Am. Inst. Elec. Engrs. 67, paper 48-218 (1948).

Washington, March 2, 1949.

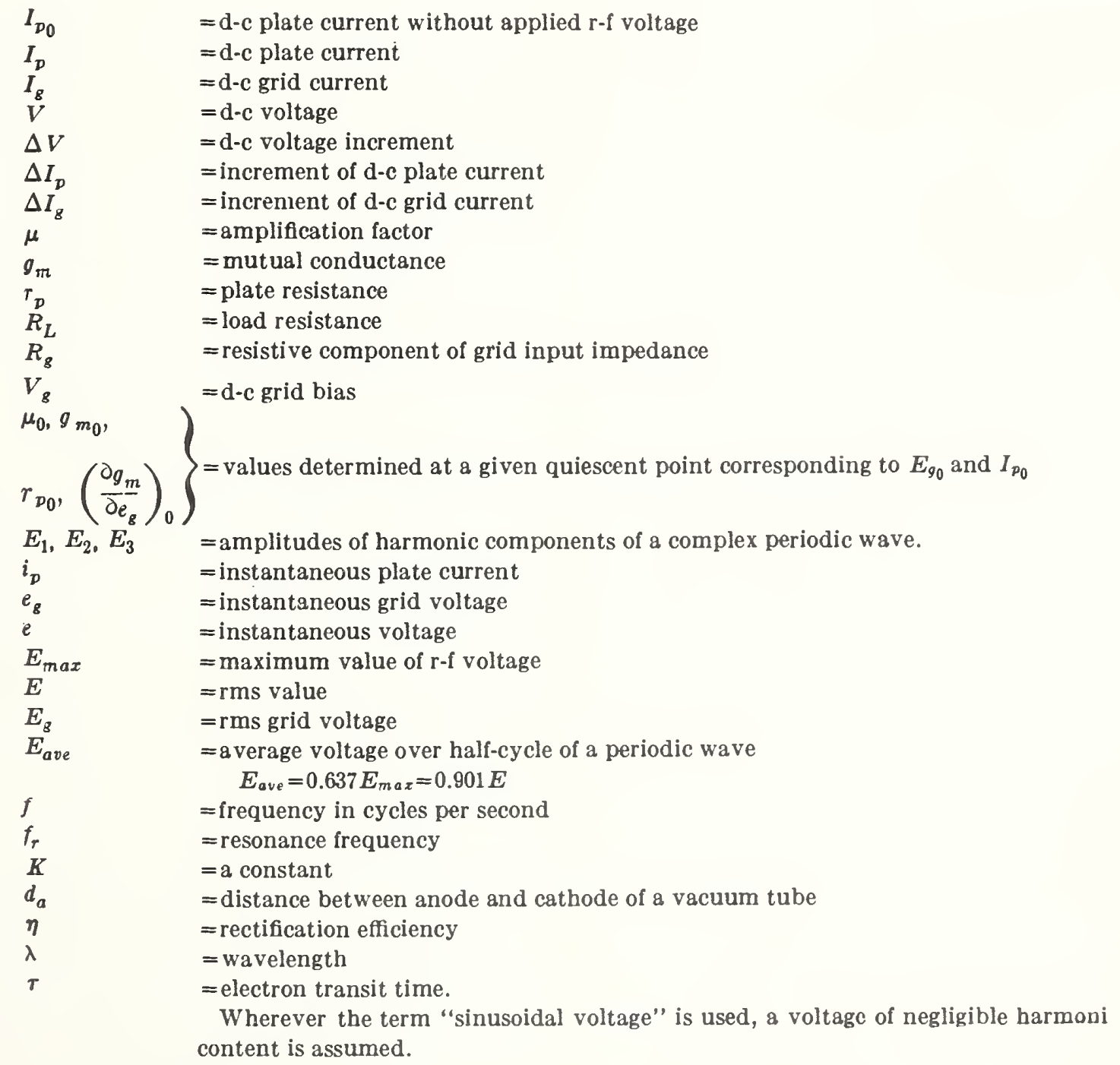




\author{
M. C. Selby, L. F. Behrent and F. X. Ries \\ National Bureau of Standards \\ Boulder Laboratories \\ Boulder, Colorado
}

Consoles to calibrate $\mathrm{rf}$ voltmeters for science and industry were developed at the National Bureau of Standards Boulder Laboratories. Very accurate calibrations will be performed in a fraction of the time heretofore required at the Bureau at any practical voltage level starting with 0.2 volt at twelve discrete frequencies between $30 \mathrm{kc}$ and $700 \mathrm{Mc}$. New type AT voltmeters, the most stable rf-voltage reference standards known to date are used. These voltmeters can reproduce calibration data to $\pm 1 \%$ or better over a period of one year or longer. The major components, in addition to the reference voltmeters, are extremely stable rf sources, automatic and manual level controls and protective and indicating circuits.

$\underline{\text { Introduction }}$

Since 1952 the National Bureau of Standards has been engaged in planning and installation of a Calibration Center to house at one location electrical measurement standards covering the range from dc to the millimeter wave region. The purpose is to provide a necessary link between the national standards, maintained at the NBS, and the laboratories throughout the country, and to do this at a minimum possible cost to the public. The reference standards and techniques to be used in this link must meet two basic requirements. The first is to retain the accuracy of the NBS primary standards as closely as possible. The second is to reduce the time necessary for accurate calibrations of "unknowns" to a reasonable minimum. It is, of course, clear that the major consideration in developing a primary standard and technique for a certain quantity is the realization of a magnitude as closely as possible to its true or "absolute" value. Time element, convenience and other considerations are of 'secondary importance and are given weight only when comparing two methods rendering equal basic accuracy and reliability. That is why primary standards and associated techniques are in many cases not practical for calibrating even limited numbers of reference standards. This is increasingly the case as the radio frequency involved increases. It thus turned out at the time of initiation of this undertaking, that the calibration of an $\mathrm{rf}$ voltmeter to several hundred megacycles was a job requiring a number of weeks and a nearly prohibitive cost; this was particularly discouraging in view of the limited ability of the "unknowns" to retain their calibration for a reasonable length of time. The new reference standards and techniques incorporated in the consoles described below enable calibration of the same voltmeter in a matter of days with relatively little loss in accuracy as compared with a calibration directly in terms of primary standards.

\section{NBS Primary Standards}

The NBS primary standards of rf voltages for levels currently used with popular commercial voltmeters are a cathode-ray tube in a "slideback" system and a special rf-voltage thermistor bridge .

The cathode-ray tube is used at frequencies from $30 \mathrm{kc}$ to $40 \mathrm{Mc}$ for all voltage levels between 5 and 300 volts. The voltmeter under test is connected directly across the vertical deflection plates; the cathode ray is used as a balance indicator having equal deflection sensitivity to dc and $\mathrm{rf}$ within the frequency limits specified. In operation, a fixed edge of the fluorescent spot is first set to a reference point on the screen with the aid of a microscope. Sinusoidal rf voltage of approximately the desired amplitude is then applied to the plates and one end of the resulting trace is returned to the same reference point with a dc "slide-back" voltage. The dc voltage is measured with a potentiometer in terms of a standard cell, its magnitude being equal to the peak value of the rf applied in the absence of resonance or transittime effects.

The other primary voltage standard, the bolometer bridge , uses two matched thermistors as bolometer elements in one arm of the bridge. The voltmeter under test is connected across these thermistors in a special mount, illustrated in figure 1, and the $\mathrm{rf}$ power applied to them is measured by dc power substitution. Because of the small physical dimensions and the special manner of mounting the thermistors their rf resistances are essentially equal to their dc values and the series reactances are negligible at all frequencies to $1000 \mathrm{Mc}$. Any other impedances shunting the thermistors do not affect the 
value of the voltage appearing across the thermistors. Consequently, knowing the rf power and the resistance of the thermistors, the rf voltage may be calculated. In this manner voltage levels from about 20 millivolts to 1.5 volts can be obtained at all frequencies from dc up to $1000 \mathrm{Mc}$. The voltage range is extended to higher levels by inserting accurately known amounts of rf attenuation between the voltmeter under test and the bridge.

The accuracy of the voltages obtained directly with the cathode-ray tube and the bridge as checked in terms of each other and several independent methods is $\pm 1 \%$ or better. The attenuator is introducing another possible error of $\pm 1 \%$ or less.

\section{Reference Standards}

To make efficient use of the above primary standards it was necessary to develop a reference instrument capable of fairly quick response in indicating a given value of voltage and above all, capable of reproducing the same indication over long periods of time. The latter critical quality may be referred to as "calibration stability". After a long search for suitable components, waveguide below-cutoff attenuators operating in the $\mathrm{TM}_{01}$ mode and capacitive type attenuators in combination with thermoelements proved most promising. Attenuator-thermoelement (AT) voltmeters are, therefore, currently being used as rf voltage reference standards. After calibration in terms of the primary standards, they retain their calibrations to $\pm 1 \%$ for more than a year.

There are two general types of $\mathbf{A T}$ voltmeters, "fixed" types having voltage range of about 5 to 1 and adjustable types. Figure 2 illustrates the basic configuration of the adjustable types, having continuously adjustable attenuators. Two such voltmeters were developed at the NBS for voltages from 20 to 1000 volts at frequencies between 0.1 and $1000 \mathrm{Mc}$. One of them is using a wave-guidebelow-cutoff capacitive type attenuator with disk electrodes for frequencies from 10 to $1000 \mathrm{Mc}$. The other, having conical electrodes as the adjustable elements in a capacitive voltage divider, covers the low-frequency end of the range. Another type employs a fixed attenuator of high input impedance. Voltages between 0.2 and 20 volts at all frequencies are measured with a third "fixed" type, $50 \mathrm{ohm} \mathrm{AT}$ voltmeter; fixed interchangeable attenuation pads are used tc obtain voltage ranges of $0.2-1.0,1.0-5.0$, and $5-20$ volts, respectively. The adjustable AT voltmeters have input impedances comparable to that of vacuum-tube voltmeters.
All AT voltmeters used as reference standards with the rf consoles described below are of the high and low impedance "fixed" type because these are rendering highest efficiency of operation and maximum calibration stability.

Description of the Consoles

The purpose of the rf-voltmeter calibrating consoles is to provide means of calibrating all types of voltmeters from $30 \mathrm{kc}$ to $700 \mathrm{Mc}$ and higher frequencies with maximum accuracy available at present and in the shortest possible time. Two major steps made this possible; the first was the application of the AT voltmeters; the second was the decision to calibrate the meters at discrete frequencies. The latter decision is justified because the frequency response curves for present day rf voltmeters seldom have serious discontinuities. However, frequencies other than those selected may be necessary. At the time of this writing two of these consoles have been completed and placed in service, one for 30 , 100,300 and $1000 \mathrm{kc}$ and the other for 5, 10,30 and $100 \mathrm{Mc}$. The third partially finished unit will operate at $300,400,500$ and $700 \mathrm{Mc}$. The placement of components and controls is essentially the same for all consoles to present a uniform appearance, to facilitate servicing and for efficient operation.

Figure 3 illustrates the arrangement of the controls and indicators of one of the consoles. A specially designed coupling plate for connecting the voltmeter under calibration in parallel with the reference standards is mounted at the front of each of the two pedestals of the console. The indicator lights, in the narrow panels above the pedestals, are for the overload protective cir cuits, the crystal oven heaters and the ac power lines. The meters above the lights are in the dc power supply circuits to monitor the operation of the rf sources. The millivoltmeters in the adjacent panels indicate the thermocouple output from the $\mathrm{AT}$ voltmeters, and the line charts, displayed in the windows of the storage drawers beneath them, are used to convert these indications quickly into the actual rf voltages applied. Controls for operating the corresponding half of the console are located on a panel below each storage drawer. The controls in the center of the panel, figure 3 , are for the adjustment of the rf voltage level. Referring to figure 4, a seven position switch, CS, connects the rf source to the appropriate reference standard and also connects the millivoltmeter to its thermocouple output through switch $\mathrm{S}_{1}$. The remaining control, switch $\mathrm{S}_{3}$, connects the dc high voltage to the rf voltage source of the appropriate frequency 
and by means of a solenoid-operated coaxial switch, CS, connects all the reference standards to that rf voltage source. These controls and the four crystal controlled $r f$ voltage sources - -two in each half of the console--are permanently installed together with low-pass rf filters, impedance matching networks and overload protective circuits. All of the rf components are carefully shielded to eliminate stray radiation.

Each half of a console may be operated independently of the other, permitting the simultaneous calibration of $\mathrm{rf}$ voltmeters at two frequencies per console. In this manner a large volume of calibration work can be accomodated.

The time required to calibrate a voltmeter, including every step in performing the calibration of a voltage point on a typical rf voltmeter, has been analyzed. Then the components and their arrangement in the consoles were designed to reduce the time wherever possible. Rapid connection of the "unknown" voltmeter to a reference standard is achieved with the RF Voltmeter Coupling Plate, figures 5 and 6. Reference standards, seven for each half of a console, are mounted on the under side of each plate. To connect the "unknown" in parallel with a standard, the operator attaches a suitable adaptor ring to the probe of the "unknown", inserts this ring in a sliding carriage on the top side of the plate, centers it over the reference standard to be used and clamps it in place. The "unk nown" and the standard are now connected in parallel through a " $\mathrm{T}$ " connection of approximately $3 / 16$ inch length, with the $r f$ voltage applied midway between the two. Such a "T" exists at each of the seven standards. The operator then rotates the sevenposition switch until the number below the index corresponds to the position of the standard in the plate, and closes the switch. The rf level is adjusted with the manual level controls and the dc thermocouple output in millivolts indicated on the panel meter is converted immediately into actual applied $\mathrm{rf}$ voltage with the line chart displayed in the window.

As already indicated above, fixed type AT voltmeters have been chosen in preference to the adjustable type used in the laboratory because of their simplicity and superior stability. The mechanism required to operate the piston of the continuously adjustable type increases their complexity of construction and operation. Three reference standards having dissipative fixed attenuator input pads cover the voltage range 0.2 to 20 volts in ranges of $0.2-1.0,1.0-5.0$ and 5.0 - 20 volts, respectively. These are used at both frequencies provided in the part of the con- sole in which they are located. The voltage ranges of 20 - 100 and $100-500$ volts at each frequency are covered by single-frequency standards using fixed "capacitive" attenuators, bringing the total number of reference standards in each half of a console to seven. The series reactance of this type of "capacitive" attenuator is many times larger than the resistance of the thermoelement heater terminating the attenuator. Therefore, in analyzing the effect on heater current of a change in heater resistance with a given rf voltage input to the voltmeter, the attenuator can be represented at its output terminals by a constant current source. Changing the heater resistance even by as much as $50 \%$ has no effect on heater current.

Consequently, once an rf voltage calibration of a given reference standard has been made, replacement of its thermoelement does not necessitaie an rf recalibration. Only a dc calibration to determine the relationship of heater current to dc millivolts output need be made, and if the characteristics of the replaced couple match those of the original closely enough, even this calibration is unnecessary. When matching can be achieved, the same calibration chari can remain in service. For the voltmeters using dissipative attenuators, however, a complete recalibration of the voltmeter is required when thermoelements are replaced.

The designing of special labor saving equipment and the streamlining of procedures to reduce time is of little value if the amplitude of the applied $\mathrm{rf}$ voltage does not remain stable to at least $\pm 0.1 \%$ during the course of a measurement. It was found, from experimental tests, that regulation of the dc screen and plate voltages applied to the rf voltage sources produces sufficient output-amplitude stability, except in the case of the $100 \mathrm{M}$ 'c source, where additional stabilization has been provided thr qugh an automatic amplitude stabilizing circuit ${ }^{4}$. Figure 7 shows one of the circuits used. $V_{1}$ is a temperature limited diode. Its plate current is very sensitive to cathode temperature variations. Raising the temperature of the cathode increases the plate current, causing the drop through $R_{1}$ to increase and reducing the dc voltage at the diode plate. As this voltage decreases, the positive voltage on the grid of $\mathrm{V}_{3}$ decreases, resulting in an increased voltage drop through $\mathrm{V}_{3}$. The voltage drop across $V_{3}$ added to the constant voltage drop through $\mathrm{V}_{2}$ determines the screen voltage on the final stage of the rf source. Therefore, if a sample of the rf output power is fed to the diode, an increase in the rf output level causes an automatic decrease in the screen voltage of the final 
rf stage which ip turn reduces rf output. With an open circuit gain of approximately 2000, the $r f$ voltage amplitude stability is considerably improved. The setting of $C_{1}$ determines the value of the $\mathrm{rf}$ output level. $\mathrm{C}_{2}{ }^{1}$ is adjusted during installation of the equipment so that at any operating level, i.e. at any setting of $C_{1}$, the diode is never extinguished. From experimental observations, the amplitude remains stable to better than $0.1 \%$ for a period much longer than is required to make a measurement.

For the $100 \mathrm{Mc}$ circuit, smooth manual adjustment of the $\mathrm{rf}$ voltage amplitude is obtained by varying an element in the circuit of the automatic amplitude stabilizing circuit, described below, and by varying the plate and screen voltages on the rf signal source. Final adjustment of $r f$ voltage level is made by using the dc voltage control as the vernier element. For the other frequencies, all manual adjustment of rf voltage level is achieved simply by varying the dc plate and screen voltages.

Any of the reference standards might accidentally be subjected to voltages considerably beyond its maximum rating, damaging the standard and necessitating its removal for repair and recalibration. Protective circuits, therefore, were incorporated. Figure 8 shows, in block diagram form, the method of protection used. A crystal diode associated with each standard (except the one for the top voltage levels) monitors the $\mathrm{rf}$ voltage applied. When the level reaches $120 \%$ of a standard's maximum rated level, relays are activated to remove the high dc voltage from the rf voltage source, thus, instantaneously reducing the rf voltage at. the voltmeter to zero. Returning the manual level control to zero operates a reset switch, which restores the relays to their original condition. Attenuator sections $\mathrm{A}_{10}, \mathrm{~A}_{11}, \mathrm{~A}_{20}$, $\mathrm{A}_{21}, \mathrm{~A}_{22}, \mathrm{~A}_{23^{\prime}}$ and $\mathrm{A}_{24}$ are used to enable mum and maximum levels with maximum stability and at safe plate dissipations of the $\mathrm{r} f$-source output tubes. Since the output is reduced by dropping the plate and screen operating voltages, the latter must be kept above a certain minimum value; operation at lower values introduces instabilities in the frequency and amplitude.

In addition to protection against overload damage to the reference standards, every possible precaution has been taken in designing the system to minimize the man-hours required to keep the consoles functioning properly. All components and equipment used in a console are operated well within the ratings specified by the manufacturers. To prevent the accumulation of heat or "hot spots" which might adversely effect components or cause thermal emfs to appear in metering circuits, equipment containing vacuumtubes, which generate heat, has been carefully located where there is adequate ventilation.

\section{Accuracy}

Since in most cases, rf voltmeters calibrated by means of these consoles are to be used as primary standards in a laboratory outside the NBS, it is imperative that the error in measurements be as low as possible. With the calibration of the AT standards good to \pm 1 or $2 \%$, the other factors effecting measurement accuracy have to be very carefully studied. As the thermoelements are used in the AT voltmeters, the dc output from the thermocouples range approximately trom $200 \mu \mathrm{v}$ to 7 millivolts. Even a small amount of thermal emf in the circuit from the couple to the millivoltmeter introduces error. Consequently, all wiring of the metering circuits is located well away from heat sources and junctions of dissimilar metals have been eliminated wherever possible.

The millivoltmeter selected to indicate the thermocouple output has an internal resistance of the same magnitude as the junction of the thermocouple itself ( $10 \mathrm{ohms}$ ) to eliminate sluggishiness in the meter due to overdamping and to get maximum meter deflection. Since each AT voltmeter is calibrated individually, reproducability may seem to be more important than the accuracy of the thermocouple output indication. However, in case of replacing a thermoelement or if it is more expedient to check an AT voltmeter with a millivoltmeter other than the one used in the console, accuracy is as important as reproducibility. Using a multiplier, the millivoltmeter indicates thermocouple output in two ranges. The calibration of actual applied rf voltage at the input to the AT voltmeter vs, the millivoltmeter indication for both ranges is presented on separate line charts mounted back to back in the same slide holder. Splitting each of these charts, a total scale length of 24 inches is obtained which enables expansion of the scales for more precise reading .

\section{Conclusion}

The development of the consoles has resulted in reduction in the time to calibrate rf voltmeters to a fraction of that previously required at the NBS. RF voltmeters have been calibrated using both the consoles and primary standards; the agreement between the calibrations ranged from 1 to $3 \%$. If the workload should increase to the 
point where the system needs to be expanded, the proper solution would be to duplicate the present consoles. Some improvement in measurement accuracy may be possible by substituting a more accurate instrument such as a potentiometer for the panel millivoltmeters. However. this may result in a sacrifice in efficiency.

$\underline{\text { References }}$

1. M. C. Selby, High-frequency voltage measurements, NBS Circular 481, (1949).
2. M. C. Selby and L. F. Behrent, A bolometer bridge for standardizing radio-frequency voltmeters, J. Research, NBS 44, pp. $15-30$, (Jan, 1950).

3. Stable radiofrequency voltmeters, NBS Tech. News Bul. 40, No. 2, 29 - 30, (Feb. 1956).

4. Patent pending. Publication pending by C. M. Allred and P. A. Hudson.

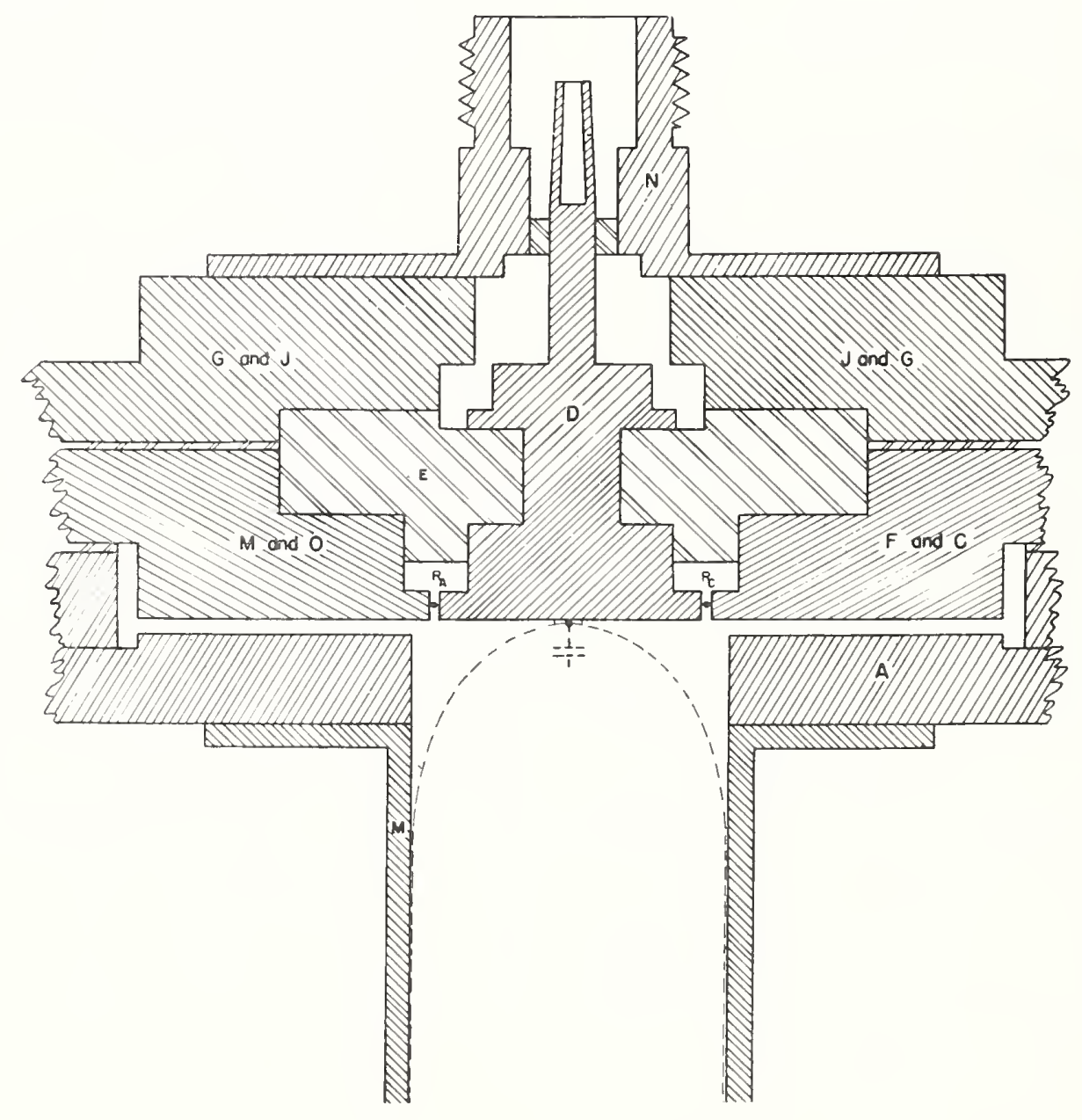

Fig. 1 Thermistor mount for standard $\mathrm{rf}$ voltage bridge showing the heart of the bridge, the core of the mount andlocation of the thermistor beads $R_{A}$ and $R_{C}$. A dashed outline is shown of a typical VTVM probe in position for calibration. RF power is fed into fitting $N$. 

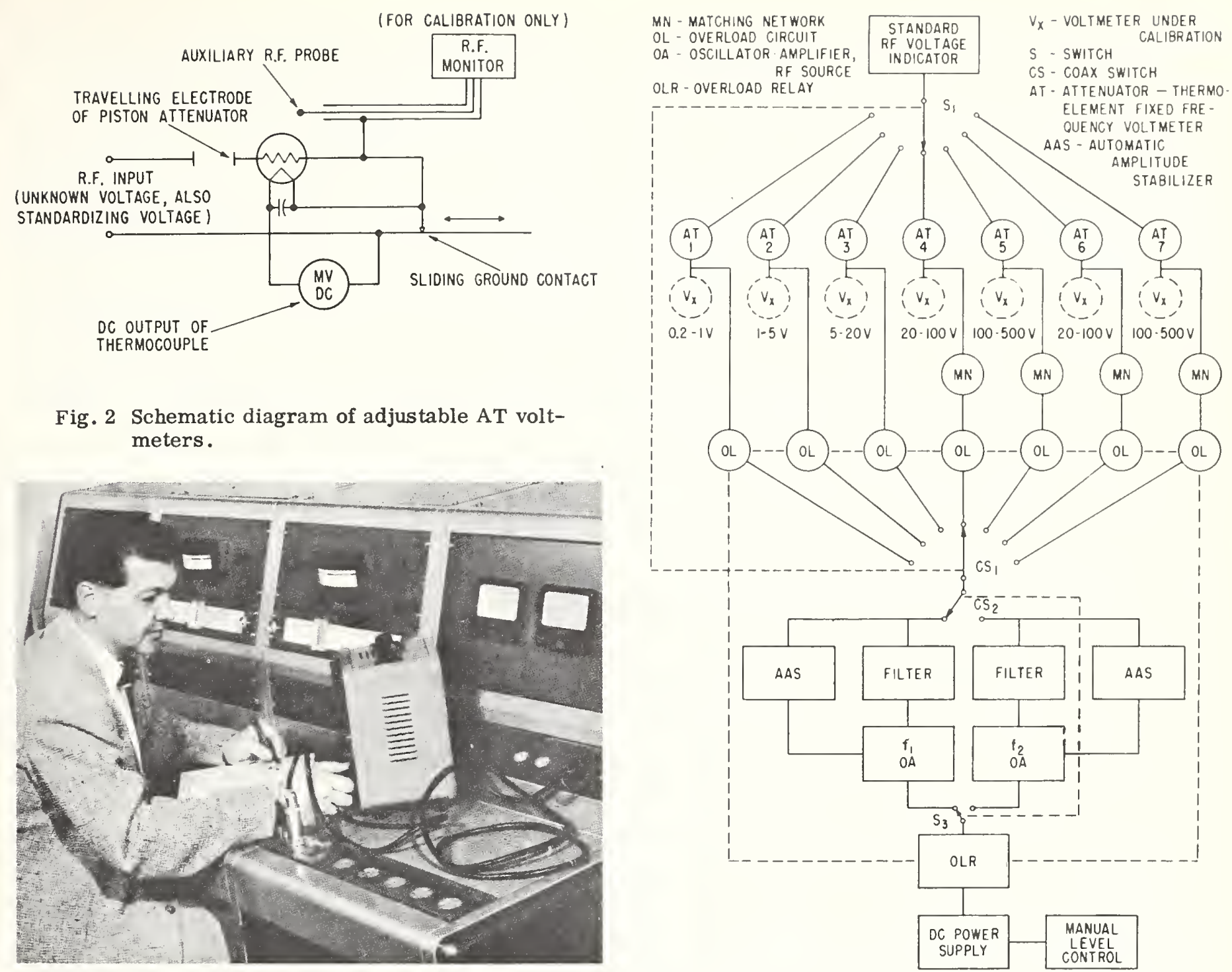

Fig. 2 Schematic diagram of adjustable AT voltmeters.

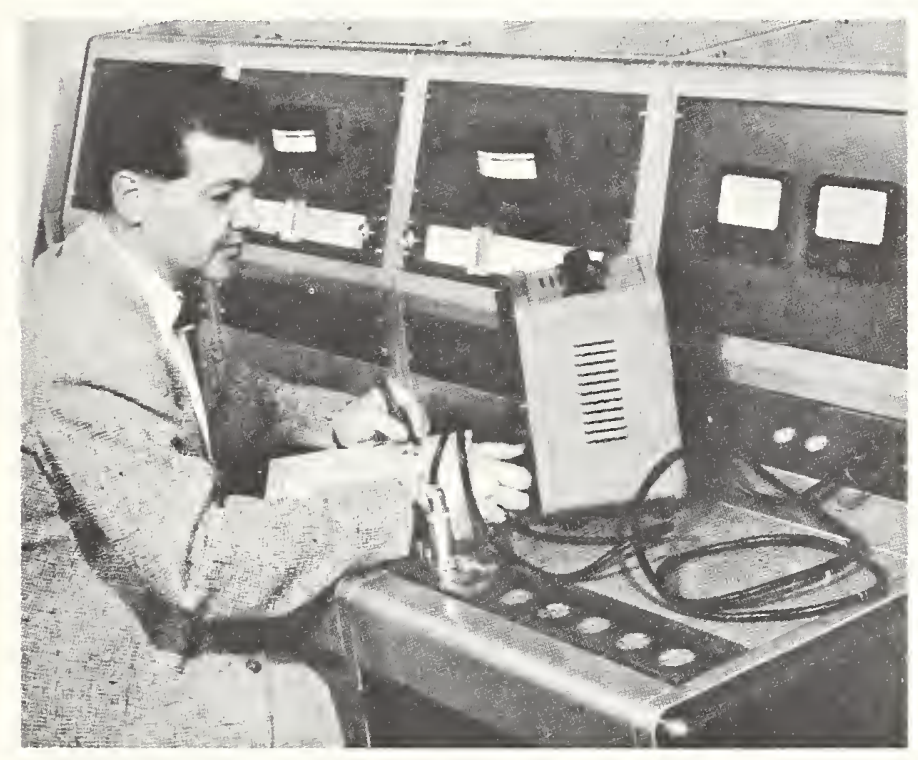

Fig. 4 Block diagram of half of rf voltage console.

Fig. 3 RF voltage calibration console.

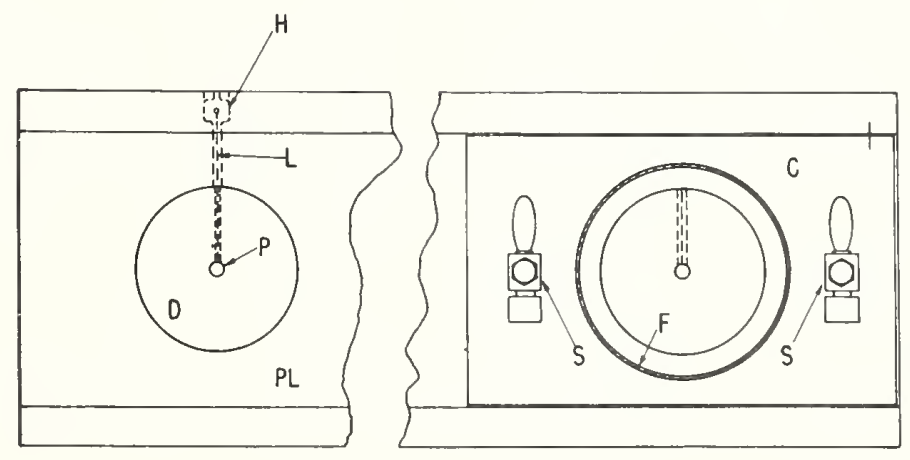

Fig. 5 Top view of voltmeter coupling plate and sliding carriage.

Reprinted from the 1958 IRE NATIONAL CONVENTION RECORD, Part 5 


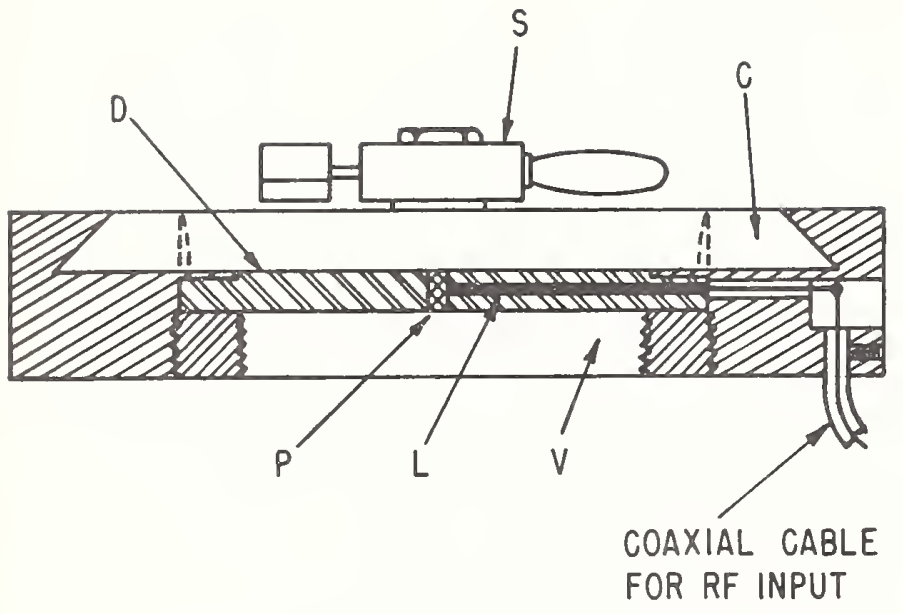

NOTE: CARRIAGE NOT SECTIONED

Fig. 6 Cross section of $\mathrm{rf}$ voltmeter coupling plate.

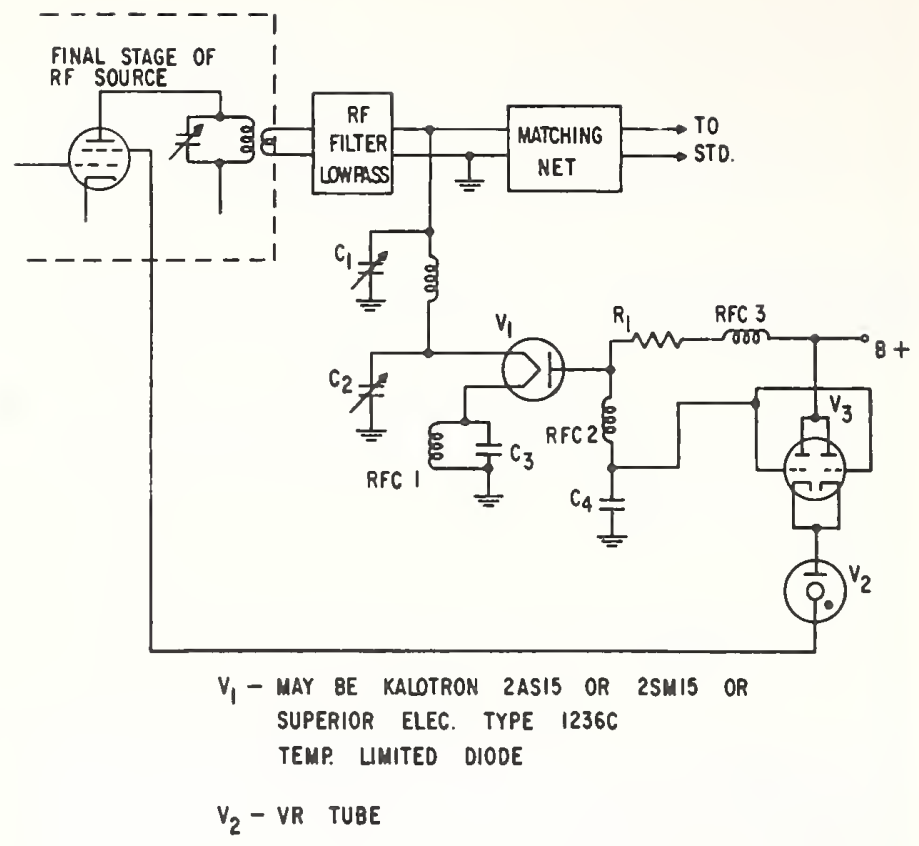

Fig. 7 Automatic amplitude stabilizing circuit.

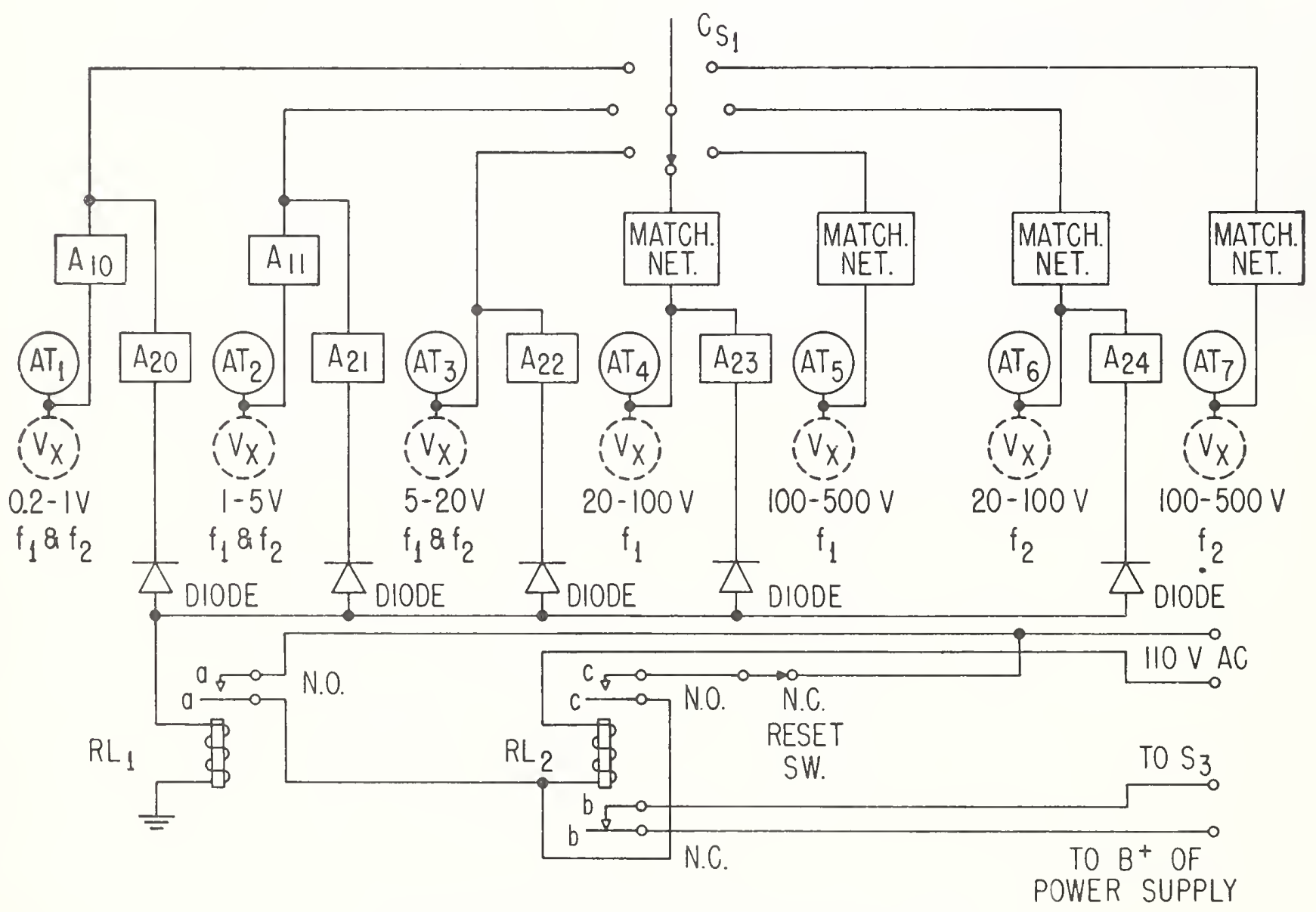

Fig. 8 AT voltmeter overload protection circuit. 


\title{
A Bolometer Bridge for Standardizing Radio-Frequency Voltmeters
}

\author{
By Myron C. Selby and Lewis F. Behrent
}

\begin{abstract}
In the course of work on $r-f$ standardization projects of the National Burear of Standards, practical equipment and techniques were developed for measurement of 20 millivolts to 1.9 volts at radio frequencies below 700 megacycles, with further broadening of these ranges still in progress. The devices employed do not require frequency corrections, so that results are based on direct-current measurements, which can be made quite accurately. Reproducibility and agreement with independent methods was found to be \pm 1 percent or better. The primary function of the equipment is to make available a standard voltage of any desired value and frequency within the above range that would be required for a highly accurate and at the same time practical voltmeter-calibration procedure. In addition, the equipment may be used for direct measurement of $r-f$ generator voltages; as a known standard of $\mathrm{r}-\mathrm{f}$ impedance; and for accurate power measurements of 20 microwatts to 100 milliwatts. An analytical study of the range-limiting factors of this technique is presented, appropriabe curves and illustrations are shown, and major steps of procedure are listed.

An independent method and appropriately designed equipment for accurate $r-f$ voltage determinations based on measurements of direct current and of linear dimensions along a transmission line of known characteristic impedance are also described.
\end{abstract}

\section{Introduction}

In searching for voltage standardization metnods, at frequencies from $10 \mathrm{kc}$ to $300 \mathrm{Mc},{ }^{1}$ promising results were obtained in the early part of 1946 by using the bolometer bridge. This eventually led to the development of the equipment and technique described herein. To meet requirements the methods and equipment were to be practical, reliable, of high precision, and were to approach an accuracy of 1 percent. Reliability was to be assured by cross-checking results of two or more independent methods. Individual methods and techniques were to be adapted for regular use on the basis of accuracy, speed of measurement, and of their individual maximum ranges of voltage and frequency.

In the light of present experience, measurements at radio frequencies to accuracies of 1 percent

1 M. C. Selby, High-frequency voltage measurements, NBS Circular C481. Central Radio Propagation Laboratory Report CRPL-8-2 issued A pril 14, 1948. were considered of high precision. Reproducibility of results, as well as agreement between individual primary methods, was to be within \pm 1 percent, or better. Frequency errors were to be negligible. Herein "precision" is used in reference to sensitivity, incidental variations, scalereading facilities, and other errors of observation; "accuracy" refers to the true value of the quantity measured. Results may thus be precise and not accurate, but not vice versa, i. e., once a value is stated to be accurate to a certain degree it is necessary that measurements be precise to the same degree. The term "systematic error" is frequently used in place of "accuracy" as defined here, and "accidental error" is used in place of "precision."

Among the devices suitable for primary measurements of $r-f$ voltages in the sense stated above, the most practical operating principles employed were: the bolometer-bridge using the substitution of $\mathrm{r}-\mathrm{f}$ for $\mathrm{d}-\mathrm{c}$ power, measurement of current 
through a known resistance, cathode-ray beam deflection, and the electrometer. Each of these principles is useful over a considerable range of voltage and frequency. The bolometer bridge method and the extent of its agreement with other methods will be described here.

\section{Basic Principle}

The principle of $\mathrm{r}-\mathrm{f}$ power measurement by means of a bolometer bridge is widely known. It consists bricfly of balancing a d-e wheatstone bridge, having a bolometer (a device the resistance of which is a function of the power dissipated in it) in one of its arms, and of rebalancing it again after some of the d-c power in the bolometer is replaced by $r-f$ power. Under these two conditions the difference in the d-c power consumed by the bridge gives a precise indication of $\mathrm{r}-\mathrm{f}$ power applied. An elementary circuit diagram of such a bridge is shown in figure 1. As used here, the term "bolometer" refers to r-f power detectors having resistance elements of high temperature coefficient of resistivity.

The fundamental difference bctween $r$-f power bolometer-bridges and those for $\mathrm{r}-\mathrm{f}$ voltage rests in the fact that the reactive component of the bolometer impedance has no great bearing on the

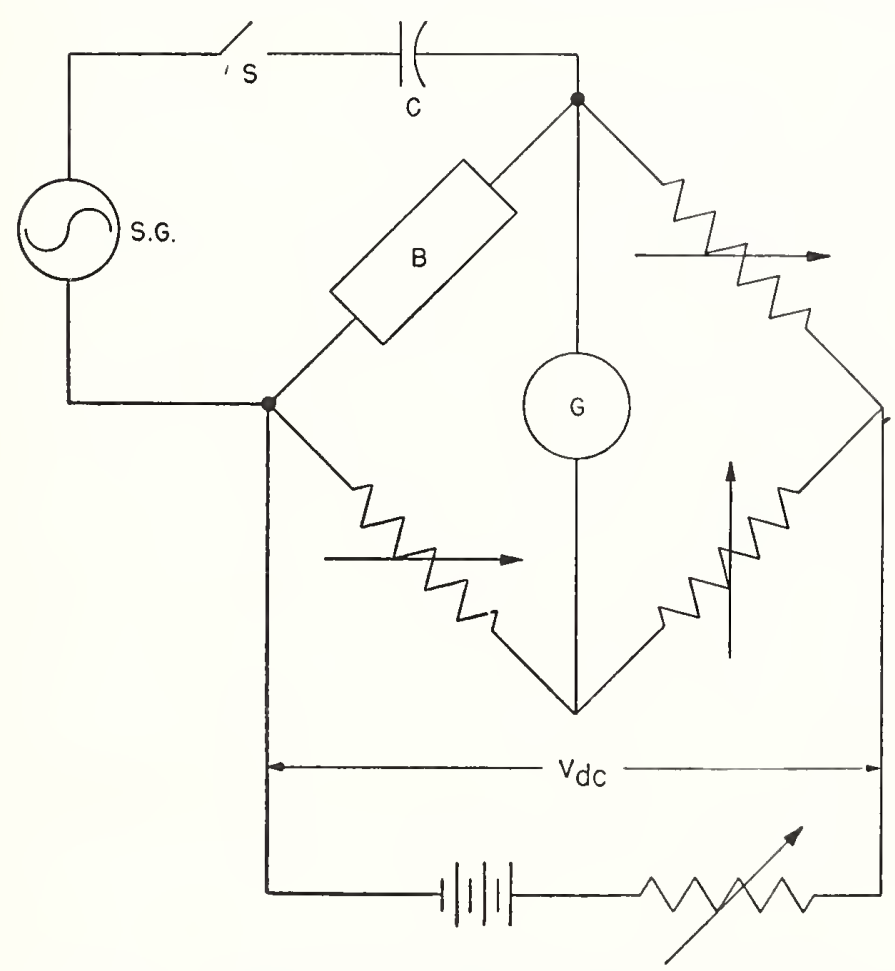

FIgure 1. Elementary circuit diagram of a bolomete bridge. aceuracy of the power measured, whereas it is of prime importanee for voltage measurements. For the present purpose, the bolometer impedance configuration must meet the condition that the d-e voltage across it be practically equal to the rms value at all frequencies considered. This condition can be met only if the bolometer admittance consists essentially of a pure conductance shunted by any value of positive or negative susceptance. Appropriate types of bolometers were therefore chosen, and a mounting for them was designed to meet the above condition over a wide frequency range. The equivalent cireuit of the assembly is shown in figure 2. It is evident that $X_{T}=\omega L_{7}$

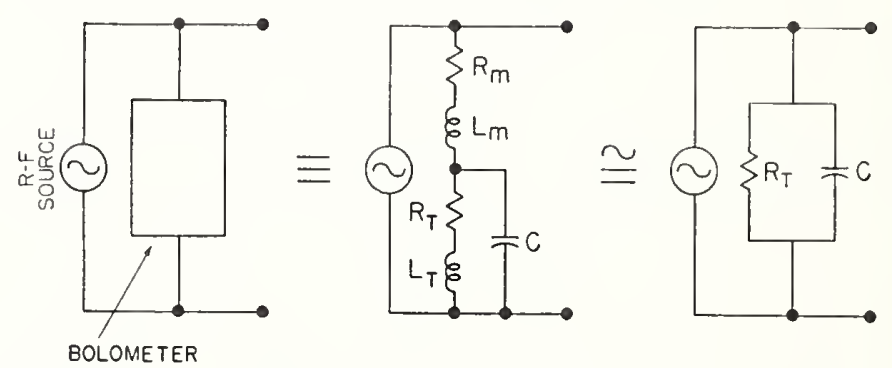

FIGtRE 2. Equivalent rircuit of a bolometer assembly applicable for $r$-f voltage measurements.

$R_{m}$ and $L_{m}$, the ineidental series resistance and inductance of the boloneter mount and $I_{i}$, the series induetance of the bolometer are assumed to be negligible. $R_{T}$ is the bolometer resistance.

and $Y_{m}\left(=\omega L_{m}\right)$ must be negligible, although the valuc of $C$ is of no direct significance except when the bolometer assembly is used for power output measurements, in which case it may be tured out. $R_{m}$ may have a finite value, but it should be negligible as compared with $R_{T}$ because of its variation with frequency as a result of skin effect.

\section{Technique and Its Limitations}

\section{Bolometers and Bridge Circuity}

The choiee of the type of bolometer and the design of its mount seemed to be the most eritical features in establishing $r-f$ voltages of known value. Two types were available for consideration, namely, the thermistor and the wollaston-wire (or "little-fuse") unit. A glance at figure 3 will show that the physical dimensions of the former were more adaptable for the purpose, whereas the series inductance and size of the available wollaston-wire units limited their usefulness only to relatively low frequencies. Other advantages of the thermistor are indicated below. 


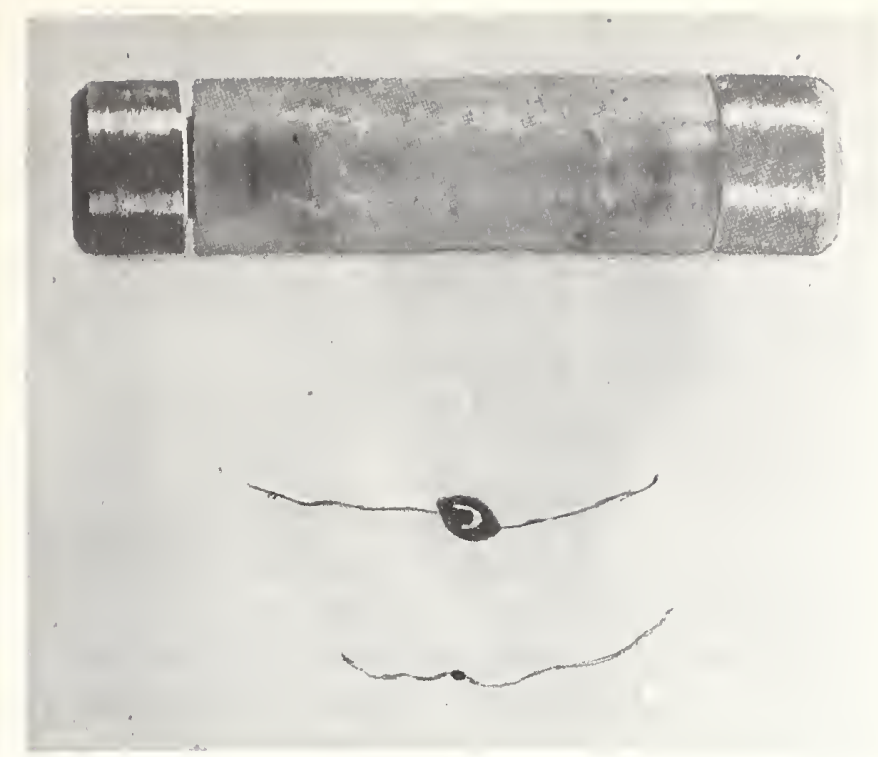

FIGURE 3. Two thermistor beads and a barretter in its mount.

A thermistor ${ }^{2}$ is a semiconductor such as uranium oxide $\left(\mathrm{U}_{3} \mathrm{O}_{8}\right)$, or a mixture of nickel oxide $(\mathrm{NiO})$ and manganese oxide $\left(\mathrm{Mn}_{2} \mathrm{O}_{3}\right)$, having a large negative resistance-temperature coefficient. The beads used in this work are glass-coated and differ in diameter depending on characteristies desired. The smallest applied in this case had an approximate over-all outside diameter of $0.015 \mathrm{in}$. with platinum connecting-lead diameter of 0.001 in. and a single lead length of several thousandths in. A wollaston-wire is a platinum wire of the order of $0.0004 \mathrm{in}$. in diameter drawn inside a silver wire; this silver coat is removed over a small section by etching with a solution of nitric acid $\left(\mathrm{HNO}_{3}\right)$; the exposed platinum core constitutes the active section of the bolometer and may be 0.1 in. long. With mounting provisions the final over-all dimensions are usually greater than those of thermistors and consequently prove less desirable for some applications. Greater overload handling capacity is a major factor in favor of the thermistor. The power sensitivity of a commercial unit wollaston-wire type bolometer (such as the Sperry type barretter $)^{3}$ is approximately 5 $\mathrm{ohms} / \mathrm{mw}$ (for 200-ohm initial condition at $25^{\circ} \mathrm{C}$ ). A corresponding figure for the unmounted thermistor mentioned is approximately $15 \mathrm{ohms} / \mathrm{mw}$. It will be shown later that the thermistor sensi-

2 J. A. Becker, C. B. Green, and G. L. Pearson, Properties and uses of thermistors-thermally sensitive resistors. Trans. AIEE 65, No. 11, p. 711 (Nov, 1946).

${ }^{3}$ Sperry Gyroscope Co., Great Neck, N. Y., Model 821 barretter. tivity is reduced when mounted close to bodies having good heat conductivity.

Schematic circuit diagrams of a single-and twothermistor bridge are shown in figures 4 and 5 . The single thermistor bridge (fig. 4) requires chokes to keep r-f current out of the d-c circuit, whereas in the two-thermistor bridge (fig. 5) the d-c circuit has no $\mathrm{r}-\mathrm{f}$ potential across it, thus eliminating the need for chokes.

The general expression for the rms roltage $T$, for the two-thermistor biridge, as derived in appendix $I$, is:

$$
\begin{aligned}
V= & \frac{R_{T}}{\left(R_{T}+R_{b}\right)(1+\alpha)} \\
& {\left[\alpha\left(V_{R 2}-V_{R 1}\right)\left(2 V_{0}-V_{R 2}-V_{R 1}\right)\right]^{\hat{\lambda}}, }
\end{aligned}
$$

where

$\alpha=R_{T 1} / R_{T 2}=$ constant ratio of the individual thermistor resistances when the bridge is balanced with or without r-f applied to it.

$R_{T}=R_{T 1}+R_{T 2} ; R_{T 1}, R_{T 2}$, and $R_{b}$ are as shown in -figure 5 .

$T_{R 1}^{r}=$ voltage drop across the resistor in series with the battery at initial d-c bridge balance (switch $S$ open).

$T_{R 2}=$ voltage drop across that resistor at second balance (switch $S$ closed)

$T_{0}=$ battery voltage, which is assumed to remain constant with load variation.

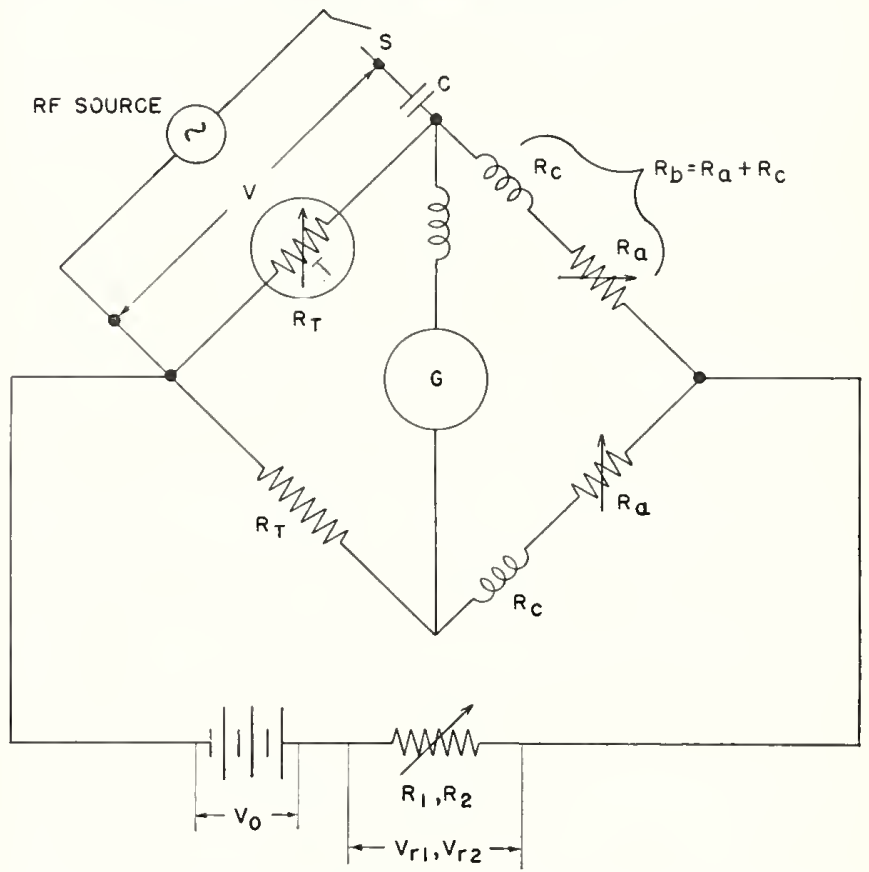

FIGURE 4. Schematic diagram of a single thermistor bridge. $R_{T}=$ thermistor resistance; $R_{a}=$ resistance-ratio arms; $R_{\varepsilon}=$ resistance of $r$-f chokes in bridge arms. 


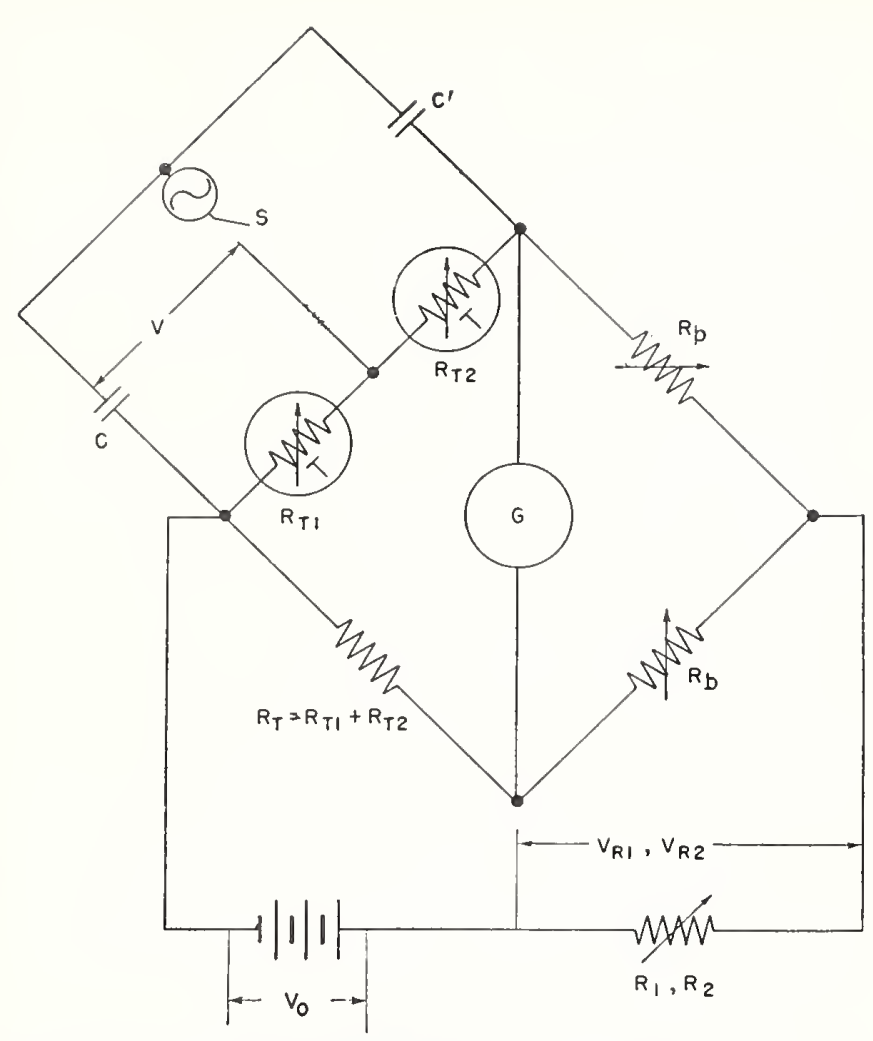

Figure 5. Schematic diagram of two-thermistor bridge. $R_{T 1}$ and $R_{T 2}$ are individual thermistor resistances.

Condensers $C$ and $C^{\prime}$ prevent direct current flowing to or from the $r$-f source or device connected to the measurement terminals. $C$ and $C^{\prime}$ are chosen large enough so that the effective load presented to the $\mathrm{r}-\mathrm{f}$ source is essentially the $\mathrm{d}-\mathrm{c}$ resistance of the two thermistors connected in parallel.

For the single-thermistor bridge, and for the two-thermistor bridge when $R_{T 1} \simeq R_{T 2}$ the 1-f voltage is:

$V=\frac{R_{T}}{2\left(R_{T}+R_{b}\right)}\left[\left(V_{R 2}-V_{R 1}\right)\left(2 V_{0}-V_{R 2}-V_{R 1}\right)\right]^{\frac{1}{3}}$.

$\mathrm{R}$-f chokes in the single-thermistor bridge reduce errors caused by incidental rectification of $r-f$ voltages entering the $d-c$ circuit. They also reduce the loading effect of the bridge on the r-f source. A choke in series with each of the $R_{b}$ arms is shown in figure 4 . This simplifies operation and computation when the chokes are matched. The chokes must be shielded, properly installed, and must have stable resistance and a sufficiently high impedance over the entire frequency range of application. Difficulties in meeting these requirements make the two-thermistor bridge preferable.
Equation 2 is further simplified when $R_{1}$ can be made negligibly small as compared with $R_{2}$. In this case:

$$
V=\frac{R_{T}}{2\left(R_{T}+R_{b}\right)}\left[V_{R 2}\left(2 V_{0}-V_{R 2}\right)\right]^{\frac{1}{2}}
$$

If, in addition, $R_{b}=R_{T}$, the simplest case of an equal-arm bridge is obtained and

$$
V=\frac{1}{4}\left[V_{R 2}\left(2 V_{0}-V_{R 2}\right)\right]^{\frac{1}{2}} .
$$

Typical thermistor characteristic curves (see footnote 2), one of which is illustrated in figure 15 , show that there are two possible values of thermistor current for each value of voltage across it over part of the range. The voltage maximum will be referred to as the "turning point." In order to assure operation over the high temperature coefficient portion of the curve (low $R_{T}$ values), $V_{0}$ must be equal to or larger than the turning-point voltage. If $V_{0}$ is lower than the turning-point voltage, other means, such as application of external heat, of $r-f$ or a-f power, may be used to obtain an initial bridge balance at the desired $R_{r}$ value. However, this procedure is unsatisfactory, because $R_{T}$ may assume a high value during the process of rebalancing the bridge for a measurement; this instability may necessitate several time-consuming balancing attempts. Another difficulty may be encountered with parallel operation of thermistor's as a result of the presence of two possible values of thermistor resistance for every value of applied voltage. It is conceivable (and this was observed in practice) that one of the two thermistors will operate in its high-resistance region, whereas the other will be in its low-resistance region and will therefore carry most of the $r-f$ current. This may take place when the thermistors have widely different values of turning-point voltages and resistance-temperature coefficients. It can be avoided by approximately matching the thermistors and by choosing operating values reasonably removed from the turning point. The reader' may at this point wonder how it is at all possible to obtain stable operation with negative temperature-coefficient resistors connected in parallel to the same power source, because a slight decrease in the resistance of one of them would cause consecutive reactions decreasing its resistance further, and reducing the current in the other shunt ele- 
ments. The answer is that the above reaction may in fact take place up to a certain equilibrium point at which the voltage drop in the source impedance reduces the voltage applied to the thermistors to a value necessary to maintain the thermistor resistances constant. The difference in operation of negative temperature-resistancecoefficient elements as against zero or positive temperature-resistance units is that the former cannot be indiscriminately connected to a power source of insufficient internal (or series external current-limiting) impedance without damaging either the load or the power source, or both.

As will be shown later, batteries are preferable to rectified n-c power sources for wide voltagerange and accuracy. Once batteries are chosen, $V_{0}$ cannot be readily varied except in steps of single cells. This feature makes it difficult to use an equal-arm bridge at a negligible value of $R_{1}$. One might mention here the desirability of having $R_{T}$ as low as possible. A lower $R_{T}$ corresponds to a higher thermistor temperature with a lower consequent interference from ambient temperature variations.

The error in $V$ as a result of thermistor mismatch (i. e., the elror caused by assuming $\alpha=1$ ) is given by

$$
\frac{\Delta V}{V}=1-2 \alpha^{\frac{1}{2}}(1+\alpha)^{-1}
$$

Appendix II gives the derivation of this error, and figure 6 shows its magnitude versus $\alpha$. Here, and throughout the discussion below, $\Delta$ designates a relatively small finite increment of a quantity. There is, however, no difficulty in measuring $\alpha$, as will be shown in the discussion of the final equipment. It may be seen from figure 6 that a mismatch of about 5 percent can be safely neglected, because the error in $V$ will thereby not exceed 0.1 percent. Equation 1 must therefore be used for values of $\alpha$ larger than 1.05, whereas for values of $\alpha$ closer to 1 , eq 3 (or its equivalent when $R_{1} \neq 0$ ) is sufficient. Factors contributing individually an error of 0.1 percent in $V$ may be neglected on the assumption that the total accumulated error will not exceed 1 percent.

The accuracy with which $\alpha$ must be determined, to not exceed a 0.1 -percent error in $V$, is derived in appendix III and is shown in figure 6. A lower value of $\alpha$ requires less accuracy in its determination.

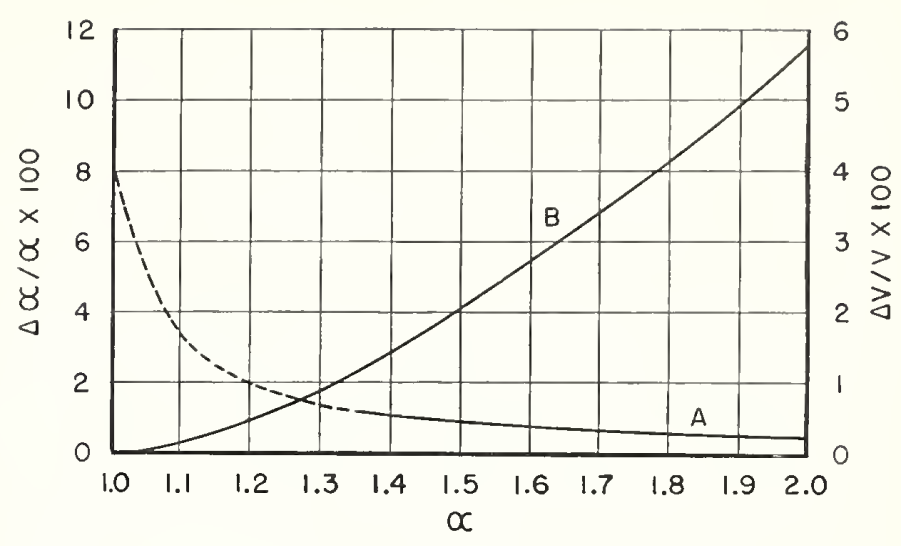

Figure 6. (A) Permissible error in determining $\alpha$ for a 0.1 -percent error in the $r$-f voltage, $V$. Broken-line section represents eq 27, appendix III. Broken line,

$$
\frac{\Delta \alpha}{\alpha}=\frac{-\left(2 \sigma-1.002 \sigma^{2}\right) \pm \sqrt{\left(2 \sigma-1.002 \sigma^{2}\right)^{2}+0.008 \sigma^{2}}}{2} .
$$

Solid line, $\frac{\Delta \alpha}{\alpha}=\frac{2(1+\alpha)}{1-\alpha} 10^{-3}$. (B) Error in V when mismatch between the thermistors of a two-thermistor bridge is neglected. $\frac{\Delta V}{V}=\left(1-\frac{2 \sqrt{\alpha}}{1+\alpha}\right)$

It is assumed throughout the text that $\alpha$ remains constant for all values of $d-c$ and $r-f$ voltages within the working limits of the bridge. Experimental evidence indicated this to be essentially true for the great majority of individual pairs of thermistors of the type used when their lead lengths and position in the gap of the mount were closely alike. The variation of $\alpha$ in these cases did not exceed 1.1 and the agreement with independent methods was well within \pm 1 percent. ${ }^{4}$ In some cases, however, (as shown in fig. 16) $\alpha$ increased considerably with applied $r$-f voltage. For these cases eq 1 does not hold. It is therefore advisable to make sure that $\alpha$ remains constant to at least 10 percent within the working voltage range required. This is in fact a requirement of matching the resistance, versus power functions of the thermistors under operating conditions and can be accomplished by comparing the value of $\alpha$ without $\mathrm{r}-\mathrm{f}$ voltage to that with maximum $\mathrm{r}-\mathrm{f}$ voltage at any low frequency, e. g., $100 \mathrm{kc}$ or lower. The potential error from this source increases with $V$.

\footnotetext{
4 Equation 10 on page 92 of the Technique of microwave measurements 11, Radiation Laboratory Series (McGraw-Hill Book Co., New York, N. Y., 1947), gives the thermistor resistance as a function of power dissipated in it. Computations made after the original manuscript was written indicate that this equation elosely represents the characteristics of the thermistors in the mount described. The initial $\alpha$ of a pair of thermistors was computed and measured to be 1.04 . It increased to about 1.1 at an $r-f$ voltage of $1.5 \mathrm{v}$. The potential error in this case was computed to be about $0.04 \%$.
} 
The required precision of bridge resistors must be of the same order of magnitude as that desired for $V$, because as shown in appendix $I V$,

$$
\frac{\Delta R_{T^{\prime}}}{R_{T}}=\frac{\Delta R_{b}}{R_{b}}=-\frac{\Delta V}{V} \frac{\left(R_{T}+R_{b}\right)}{R_{b}},
$$

The factor $\left(R_{T}+R_{b}\right) / R_{b}$ is fixed by the operating thermistor characteristies and as used was around 1.7 .

\section{Factors Limiting High End of Voltage Range}

The top value of the standard r-f voltage that can be determined with either the singte- or the two-thermistor bridge is limited by the thermistor characteristics in a specifie mount. With the smallest thermistors having dimensions mentioned above, this value was between 1.3 and $1.9 \mathrm{v}$, depending upon the temperature of components to which the bridge was connected. With thermistor beads having a diameter of about $y_{1} 6 \mathrm{in}$, voltages as high as $10 \mathrm{v}$ may be obtained. When proper capacity-type voltage dividers are employed, considerably higher voltages may be determined. These dividers will not be dealt with here.

\section{Factors Limiting Low End of Voltage Range}

The low limit of r-f voltages is of major importance. The lower it is, the more accurately voltages at the critically meeded microvolt levels can be determined with the help of standard atteinators. Because of imperfections of standard attemuators, especially in the VHF and UHF regions, a considerably more accurate microvolt may be realized by using, for example, a stanclard millivolt and a 60-db attenuator, ra ther than a standard volt and a $120-\mathrm{db}$ attemuator. This was the primary reason why considerable efforts were made to obtain as low a known voltage as possible. In doing so, another important objective was altained, namely, the opportunity of measuring the attenuation of standard attenuators directly in terms of voltage ratios. This is especially desirable where the permissible input power into an attenuator is limited.

The low end of the vorlage range is limited by (a) the sensitivity of the thermistors and of the d-c bridge, (b) the ease with which low values of $V_{R 2}$ can be adjusted and the accuracy with which $V_{R 2}$ and $V_{R 1}$ can be measured, (c) stability and accuracy of $V_{0}$, (d) thermal voltages and incidental voltage drops in circuit leads and connections, and (e) ambient temperature stability.

\section{(a) Thermistor and Bridge Sensitivity}

The bridge sensitivity is proportional to the sensitivity of the thermistor arm to $r$-f inputvol tage changes. For the single-thermist or bridge, this sensitivity (as indicated in the characteristic curves of fig. 16) is about $0.18 \mathrm{olm} / \mathrm{mv}$ for operating values of thermistor resistances of from 50 to 100 ohms. For the matched two-thermistor bridge, the thermistor arm sensitivity is twice the single-thermist or sensitivity, i. e., $0.36 \mathrm{ohm} / \mathrm{mv}$, because the same $r-f$ voltage will cause an equal resistance increment in each thermistor.

The characteristies of the small-beat thermistors are such that an $R_{T}$ of $200 \mathrm{ohms}$ and $R_{b}$ of about 300 ohms were found most suitable for a twothermistor bridge arrangement. With the bridge and galvanometer used, changes in $R_{T}$ of the order of $0.01 \mathrm{ohm}$ in $200 \mathrm{ohms}$ could readily be detected. For the stated two-thermistor bridge sensitivity of $0.36 \mathrm{ohm} / \mathrm{mv}$, an $\mathrm{r}$-f voltage change of $14 \mathrm{mv}$ can be detected. The resulting uncertainty is therefore 0.1 percent for an applied voltage of $14 \mathrm{mv}$ and, of course, correspondingly less for higher applied voltages.

\section{(b) Accuracy of $\mathrm{V}_{R 1}$ and $\mathrm{V}_{R 2}$}

As already mentioned, a perfect voltage regulation of the souree $r_{0}$ was assumed. A storage battery does approach this requirement, although any other monitored source could be applied if it meets stability requirements. One must keep in mind two difficulties when batteries are used. The first is the one stated before, namely, that $V_{n}$ cannot be conveniently adjusted except in approximate 2 -r steps. The second is the poor accuracy obtainable in trying to determine a small quintity when this small quantity is the differenec betwern two measured relatively large quantities. This would be the case when both $V_{R 2}$ and $T_{R 1}$ were large. These two difficulties may be largely climinated (as was done in this case) by the use of an unequal arm bridge. The initial bridge balance was obtained by varying $R_{b}$ with the adjustable resistor $\left(R_{1}, R_{2}\right)$ shorted. $T_{R 1}$ is therefore the voltage drop across the shorting switch. It can thereby be kept at a minimum and for relatively 


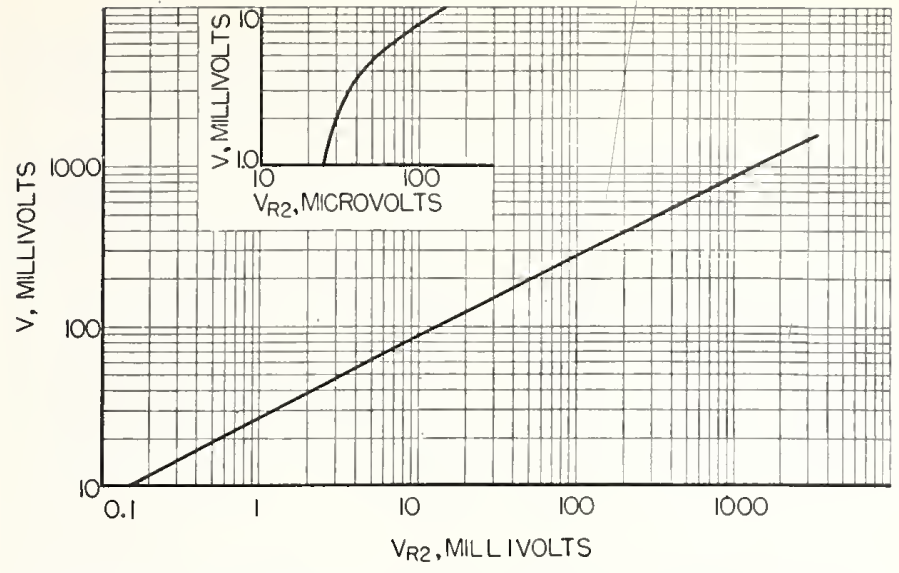

FiguRE 7. Values of $V_{R 2}$ corresponding to various values of $\mathrm{V}$.

$V_{0}=10$ volts; $V_{R_{1}}=25$ microvolts; $R_{T}=200 \mathrm{ohms} ; R_{b}=300 \mathrm{ohms} ; \alpha=1.0$

$$
V=\frac{R_{T}}{\left(R_{T}+R_{b}\right)(1+\alpha)}\left(\alpha\left[V_{R 2}-V_{R_{1}}\right]\left[2 V_{0}-V_{R 2}-V_{R 1}\right]\right)^{\frac{1}{2}} .
$$

large measured voltages may be assumed equal to zero. As used, $V_{R 1}$ was of the order of $25 \mu \mathrm{v}$.

Figure 7 shows nominal d-c voltages $\left(V_{R 2}\right)$ that one must measure for the entire $r-f$ range at a given set of values of the bridge circuit. The curve is only nominal because $R_{b}$ is in practice somewhat different from the value chosen for this curve, depending on the characteristics of the particular thermistors in use. The case presented here is in many respects only typical. Modifications are possible in order to extend the low range; for example, a mount employing a large number of thermistors, all of them connected in series for direct current and in parallel for r-f current, may be used, which would considerably increase the ratio of $R_{T}$ to the equivalent $\mathrm{r}-\mathrm{f}$ load resistance; lower values of $V$ may then be obtained for corresponding $V_{R 2}$ values. The greater part of the curve of figure 7 is linear on log-log paper. This may be expected from the interrelation (eq 2) when $V_{R 1} \cong 0$ and $2 V_{0}>>V_{R 2}$. Under these conditions the expression assumes the form

or by

$$
V=K\left(2 V_{R 2} V_{0}\right)^{\frac{1}{2}}=K_{1}\left(V_{R 2}\right)^{\frac{1}{2}},
$$

$$
\log V=\log K_{1}+\frac{1}{2} \log V_{R 2}
$$

The degree of departure of $V$ from linearity below about $10 \mathrm{mv}$ is a function of the magnitude of $V_{R 1}$.
The effect of inaccuracy in measuring $V_{R 2}$ is shown in appendix $V$. An error of 0.1 percent in $V$ is assumed, and the corresponding "permissible error" in determining $V_{R 2}$ is derived and plotted in figure 8 for values of $V$ from several millivolts to $1.5 \mathrm{v}$. The "potential error" is shown in figure 8 for values of $V$ from several millivolts to $1.5 \mathrm{v}$. The potential error shown in figure 8 is based on the performance of the particular potentiometer on hand with the present equipment. It was capable of measuring $V_{R 2}$ to $1 \mu \mathrm{V}$ or to 0.015 percent, whichever is greater. The intersection point of the two curves in figure 8 indicates that the minimum value of $V$ obtainable to 0.1 percent because of inaccuracy in measuring $V_{R}$ is approximately $20 \mathrm{mv}$. Lower levels of $V$ may no doubt be measured to the same accuracy with other potentiometers. The low limit may in that case be computed in a similar manner.

It is shown in appendix VI that, with the present equipment, a value of $V$ of $20 \mathrm{mv}$ can be measured to within 0.1 percent as a result of the inaccuracy in determining a voltage $V_{R l}$ of the order of $25 \mu \mathrm{v}$.

Difficulties in adjusting $V_{R z}$ will be discussed in the next section where the equipment components are described.

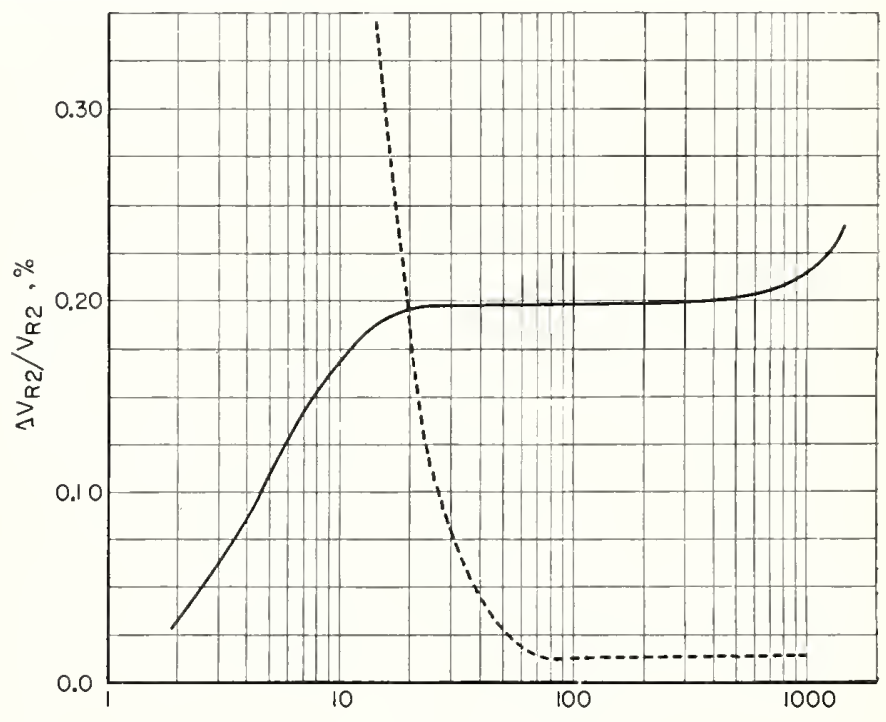

V. MILLIVOLTS

FIGURE 8. Required $V_{R 2}$ accuracy for $V$ accuracy of 0.1 percent.

Solid curve shows permissible error for $\Delta V / V$ equal 0.1 percent. Broken line shows potential error in measurement of $V_{R_{2}}$ as a result of limitations in d-c measuring equipment. $\quad V_{0}=10$ volts; $V R_{1}=25$ microvolts: $R_{T}=200 \mathrm{ohms}$; $R_{b}=300$ olims. 
(c) Accuracy and Stability of $V$

It can be shown that the accuracy with which $V_{0}$ must be measured for a desirable accuracy in $V$ is expressed by

$$
\frac{\Delta V_{0}}{V_{0}}=\frac{\Delta V}{V} \frac{V^{2}}{K V_{0}\left(V_{R_{2}}-V_{R_{1}}\right)}
$$

where $K=\left[R_{T} / 2\left(R_{T}+R_{b}\right)\right]$. For the present case of $\Delta V / V=10-{ }^{3}$ and $K=0.04$, the required accuracy of $V_{0}$ is 0.17 to 0.20 percent over the voltage range of about $1.5 \mathrm{v}$ to $20 \mathrm{mv}$, respectively. This accuracy is well within the limits of the equipment used.

The discharge rate of the storage batteries interferes with obtaining a bridge balance when the galvanometer circuit is adjusted for the high sensitivity required at low-voltage measurements.

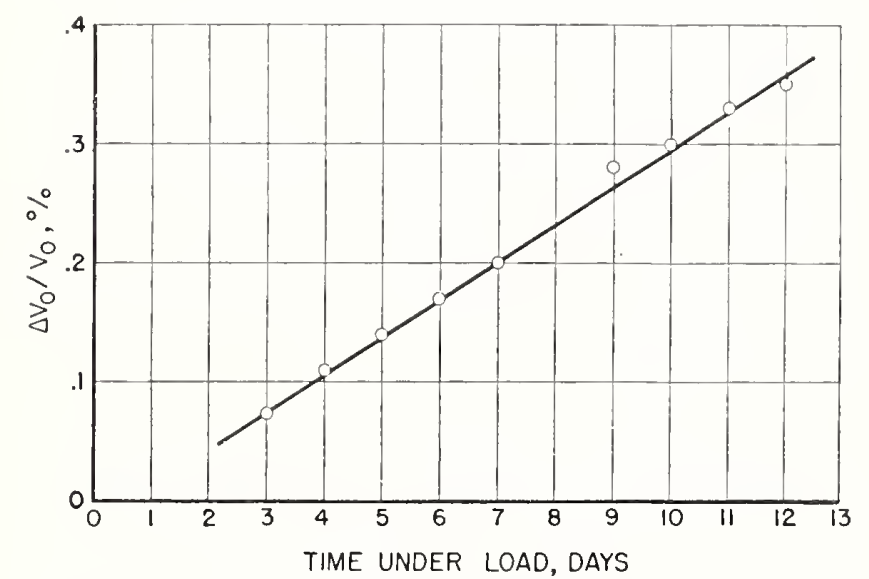

FIGURE 9. Drop in terminal voltage with time of special batteries under a constant load of 45 milliamperes.

Figure 9 shows the drift of $V_{0}$ (under the present load) to be approximately 0.001 percent per hour. To evaluate this quantitatively one may assume that the drift is equivalent to a readjustment of $V_{R 2}$ required to restore bridge balance during a time interval of a single $r$-f voltage measurement. The application of a special $\mathrm{r}-\mathrm{f}$ and $\mathrm{d}-\mathrm{c}$ switch (described in the next section) reduces this interval essentially to the time required for a measurement of $V_{R 2}$. It may be seen from the curves given and the indicated drift that $V_{R 2}$ for a $V$ of $20 \mathrm{mv}$ will have to be determined in about $40 \mathrm{sec}$ if the drift is to be neglected. The steps leading to this value are as follows: The actual drift in $V_{0}=10 \mathrm{v}$ is $100 \mu \mathrm{v} / \mathrm{hr}$ (fig. 9). From figure 7 a voltage $V_{R 2}$ of about $550 \mu \mathrm{v}$ corresponds to a $V$ of $20 \mathrm{mv}$. The permissible error in this value of $V_{R 2}$ for a
0.1 percent error in $V$ is close to 0.2 percent or about $1.1 \mu \mathrm{v}$. The time allowed for a drift of not more than $1.1 \mu \mathrm{v}$ is therefore about $40 \mathrm{sec}$. Experimental observations indicate that common storage batteries have a drift of 5 to 10 times larger than the batteries used in our case. In addition they are considerably less stable. (The rate of voltage drop with time varied about 10 to 1 over a short time of observation.) It would thus be necessary to measure $V_{R 2}$ for a $V$ of $20 \mathrm{mv}$ in a fraction of a second. The importance of using low-discharge batteries of high stability and low internal resistance for very low voltage measurements is therefore apparent.

Summarizing the errors affecting the low limit of $V$, it is seen that a conservative estimate of the total possible error for all values down to $20 \mathrm{mv}$ caused by the determination of $\alpha, R_{T}, R_{b}, V_{R 1}$, $V_{R 2}, V_{0}$, and by limited bridge sensitivity and instability of $V_{0}$ will not exceed 0.8 percent assuming the individual 0.1 percent errors to be all positive or all negative. It is shown in section IV that errors contributed by incidental thermal voltages, connecting-lead voltage-drops, and ambient temperature variations of $\pm 1^{\circ} \mathrm{F}$. are negligible in comparison with others. An extension of $V$ below $20 \mathrm{mv}$ without sacrificing accuracy will require first further improvement of $V_{0}$ stability and, second, the improvement of the accuracy of measuring $V_{R 1}$ and $V_{R 2}$.

\section{Measuring Equipment and Results}

\section{Description of Equipment}

The complete arrangement of the two-thermistor bridge is shown in figures 10 and 11 . Figure 10 is a view of the experimental setup including a vacuum-tube voltmeter under calibration. A final working arrangement could no doubt be consolidated to a great extent. Figure 11 is a combination schematic and block diagram in which the designations of the components correspond with those of figure 10 . The source of $\mathrm{r}-\mathrm{f}$ voltage consisting of a generator, filter, and matching networks is not shown in figure 10 .

The major components of the equipment were:

(a) Resistance boxes comprising the legs $R_{T}$ and $R_{b}$ of the bridge.

(b) Resistance boxes and a helical type singlewire adjustable resistance comprising the resistance $R$ in series with the battery 


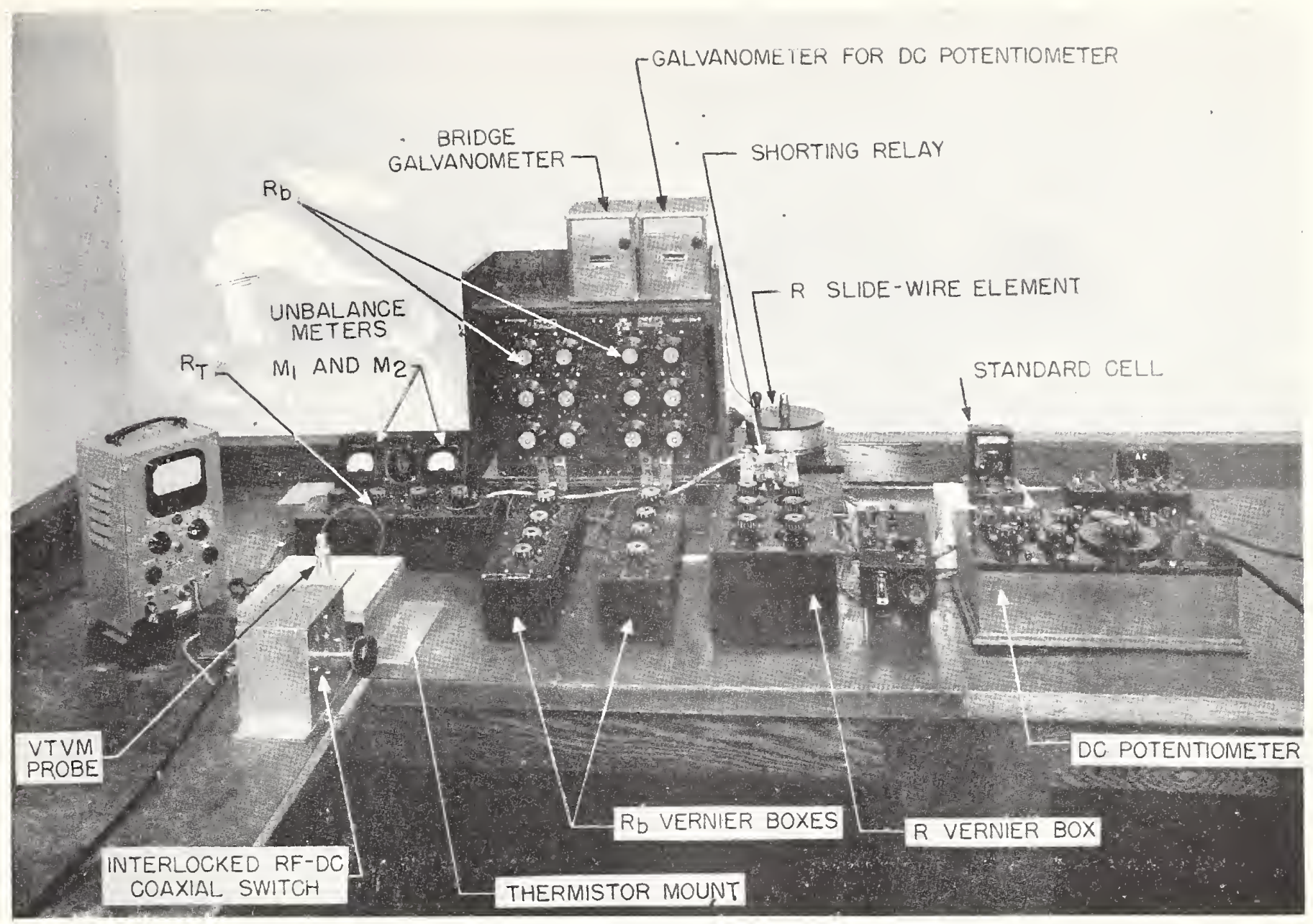

FIgURE 10. Radio-frequency voltage thermistor-bridge equipmen.

$V_{0}$ used to vary the voltage applied to the bridge. Voltage drops $V_{R 1}$ and $V_{R 2}$ (used in eq 1, 2, 3, and 4) are measured across this resistance.

(c) D-c potentiometer, associated resistors, $R_{p 1}$ and $R_{p 2}$ to increase its voltage range, a plug box used for conveniently connecting the potentiometer for measurement of $V_{0}, V_{R 1}, V_{R 2}$, and of $\left(V_{0}-V_{R}\right)$ when desirable, and a standard cell:

(d) An interlocked, r-f, d-c switch used to replace instantaneously the r-f power fed to the bridge by its equivalent $\mathrm{d}$-c power or vice versa without upsetting the bridge balance.

(e) A shorting relay controlled by the interlocked $\mathrm{r}-\mathrm{f}$, d-c switch, mentioned in item (d); this relay shorts, the resistor $R$ in series with the bridge battery, thereby increasing the voltage applied to the bridge while the $\mathrm{r}$-f power is removed from the bridge.

(f) The thermistor mount.

(g) High-resistance d-c voltmeters to indicate the ratio of $R_{T 1}$ to $R_{T 2}$.

(h) Switch $S_{0}$ used to connect the battery $V_{0}$ to the bridge.

(i) Switch $S_{D}$ used to connect a standby load $R_{D}$ to the battery $V_{0}$ in place of the bridge. This load was necessary to prevent a slight increase in battery voltage when standing idle for several hours. On comnecting a load to an idle battery the rate at which the terminal voltage decreases is relatively high during the initial discharging period.

The operational procedure was briefly as follows. Referring to figures 10 and 11 , with no voltage applied to the bridge, $R_{T}$ and both $R_{b}$ arms were set to predetermined values that would approx- 


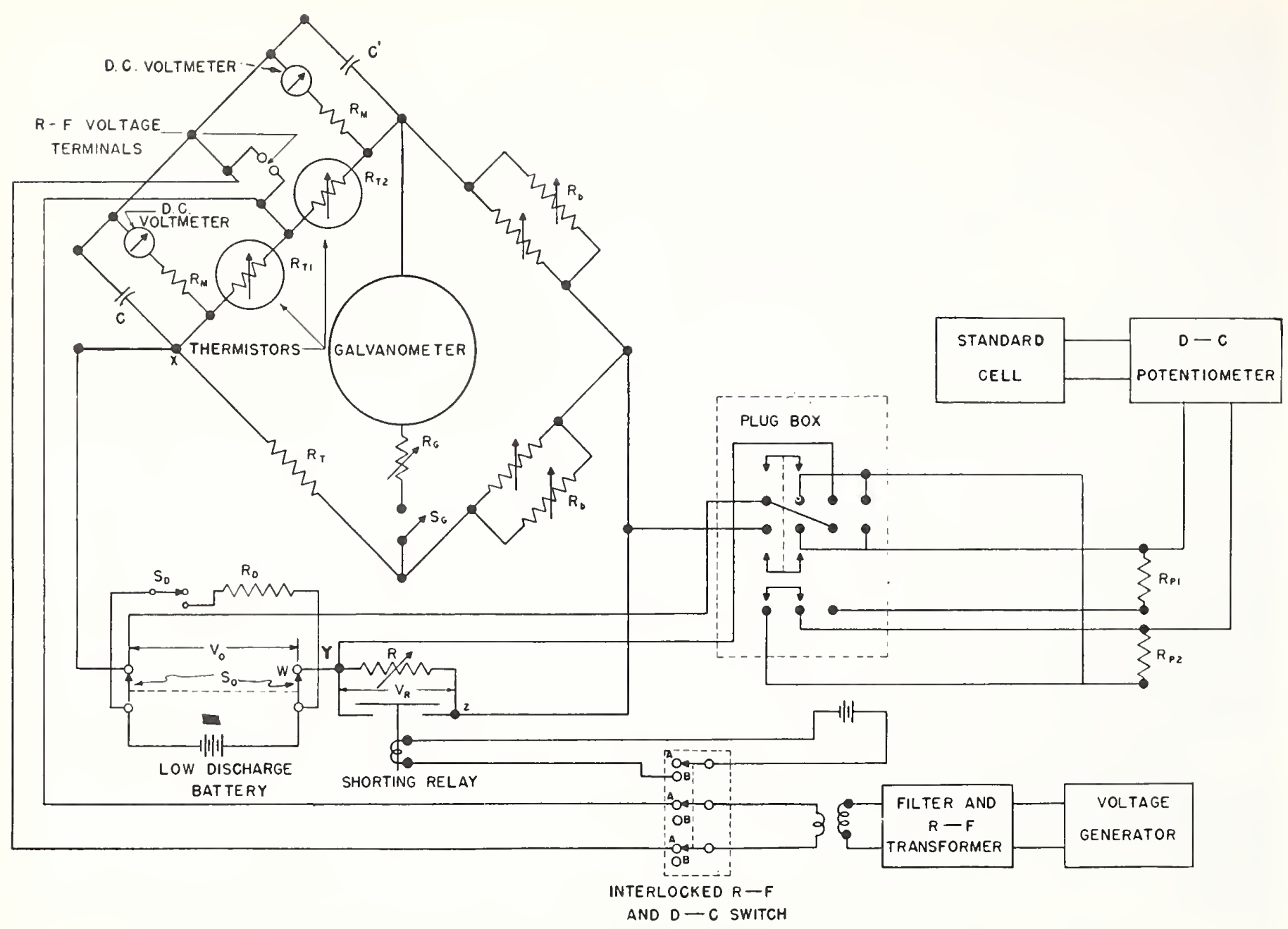

FIGURE 11. Operational schematic and block diagram of the r-f voltage thermistor-bridge equipment.

$C, C^{\prime}=$ mica 0.01 mierofarad eondensers; $R_{T}, R_{b}=$ bridge ratio arms; $R=$ series dropping resistors; $R_{P_{1}}, R_{P_{2}}=$ d-e voltage divider userl with d-e potentiometer; $R_{G}=$ galvanometer sensilivity eontrol; $S_{Q}=$ galvanometer switeh.

imately balance the bridge at the specific d-c voltage to be applied. With the shorting relay closed, the interlocked $r-f$, d-c switch in position $B$ (fig, 11), and the sensitivity of the galvanometer reduced, switch $S_{0}$ was closed. Both $R_{b}$ arms were adjusted simultancously until a rough balance was obtained. After increasing galvanometer sensitivity by reducing $R_{g}$, further adjustments in $R_{b}$ were made until a satisfactory balance resulted. R-f was then applied in small increments after closing the coaxial switch and equilibrium restored by increasing the value of $R$. Several operations of the interlock switch were necessary until a desirable balance was observed. In calibrating a vacuum-tube voltmeter, the amount of $r-f$ applied depended upon the meter deflection desired. The amount of $\mathrm{d}-\mathrm{c} \cdot$ voltage removed from the bridge to restore balance was then measured by the potentiometer.
Cross sections of the thermistor mount are slown figures 12 and 13 . The plate, $A$, and the pin, $L$ (fig. 12) are removable, as is illustrated in figure 14, to permit means of connecting various probes of voltmeters under calibration to the mount. The line drawing in the lower corner of figure 14 is an enlargement of the gap in which the thermistors

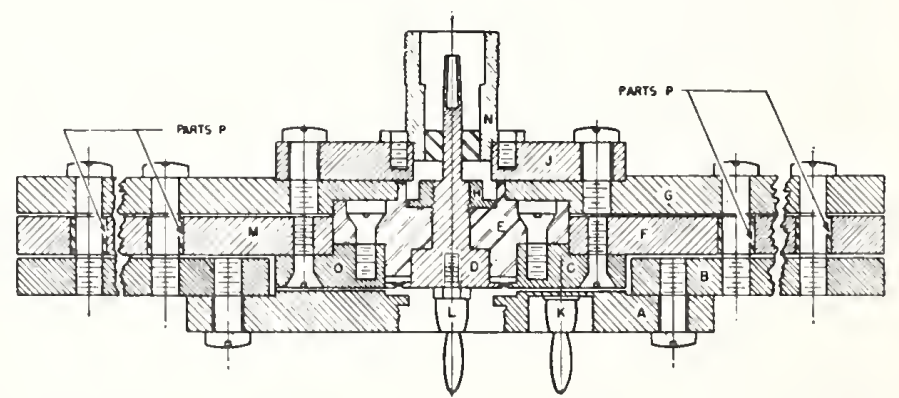

Figurf 12. Cross-sectional drawing showing the construction of the thermistor mount. 


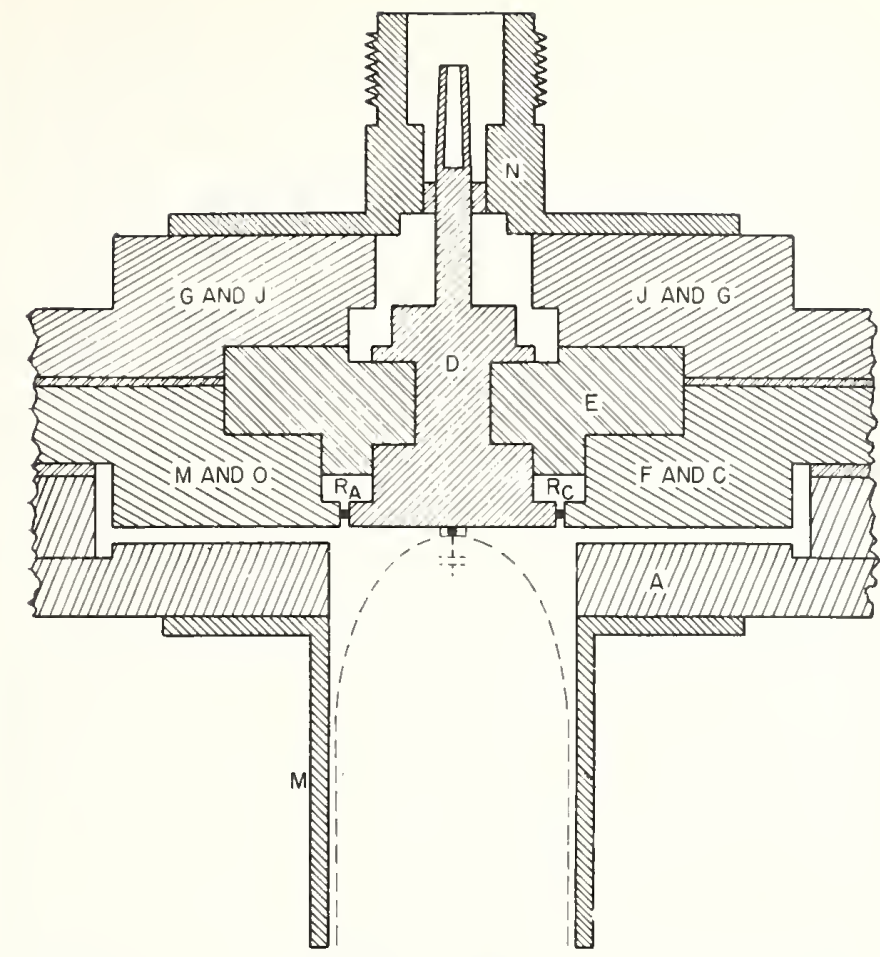

Figure 13. Cross-sectional drawing of the core of the mount showing the location of the thermistors and their proximity to the point where external connections are made for calibration purposes.

A dashed outline is shown of a typical VTVMI probe in position for ealihration. R-f power is fed into fitting $N$.

are mounted, whereas the part in the upper right corner (fig. 14) shows the other side of the core assembly. The remainder of the mount consists

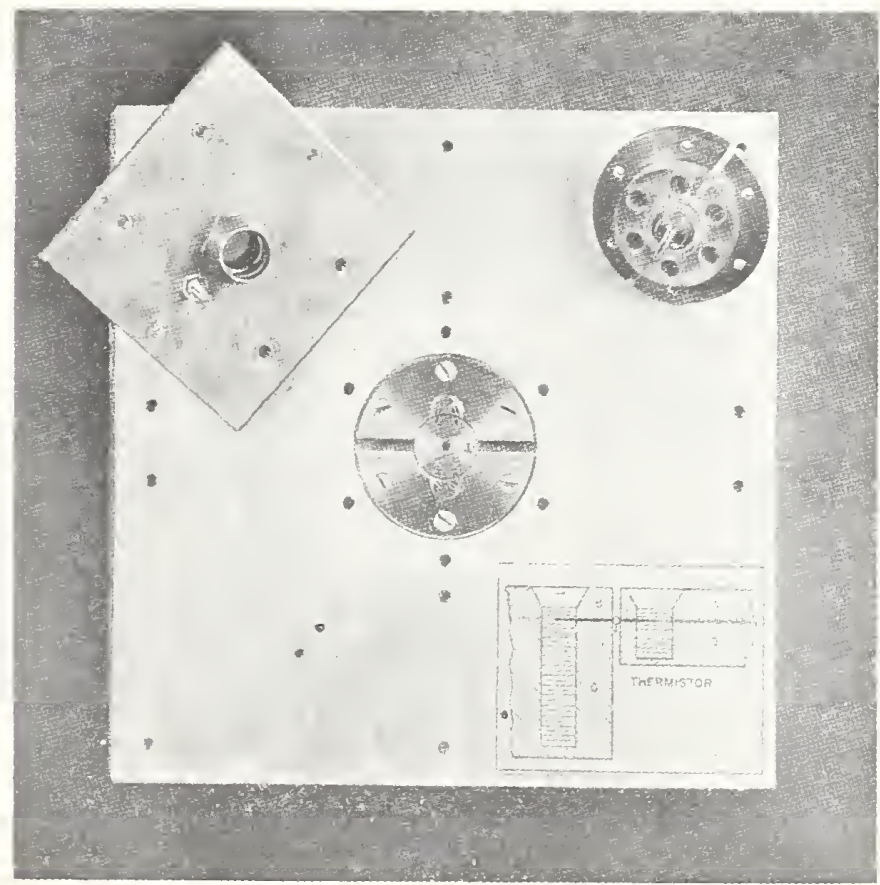

FIGCRE 14. Thermistor mount partly disassembled. of an assembly of the mica blocking condensers $C$ and $C^{\prime}$ shown in figures 5 and 11 . One thermistor is connected to parts $D$ and $O-M$ (fig. 12); the other to $D$ and $C-F$. Parts $B, F$, and $G$ and $B, M$, and $G$, figure 12 , comprise the condensers $C$ and $C^{\prime}$ of about $0.01 \mu \mathrm{f}$ each. At low frequencies where the reactance of $C$ and $C^{\prime}$ becomes appreciable, additional external capactance was added in parallel with them.

The $r$-f admittance of the thermistor bridge was measured in the VHF range employing a slotted coaxial transmission line. The input conductance was found to equal the d-c value of the two thermistors in parallel within the limits of accuracy of the measuring equipment.

Figure 15 illustrates the effect the large metal mass of the mount had on the thermistor characteristics. The broken line curve is a typical

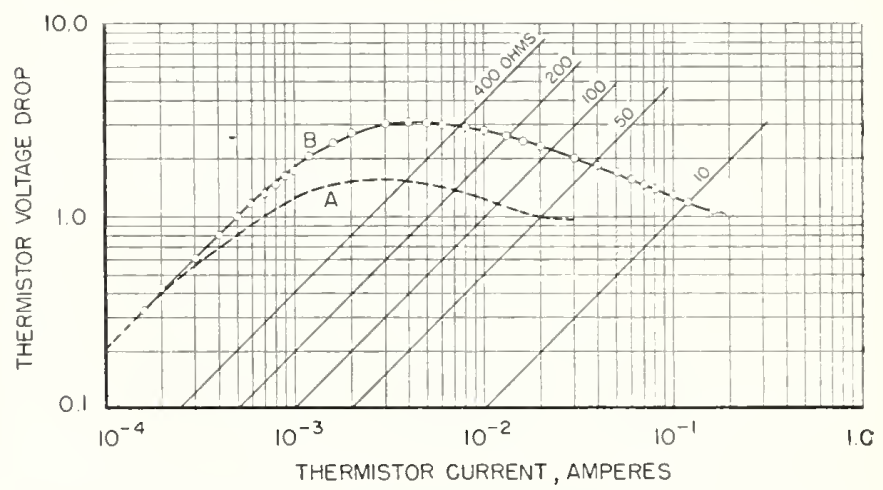

Figure 15. Thermistor characteristic curves.

A, thermistor unmounted (manufacturers data); B. thermistor mounted close to large heat-conducting surfaces of mount (fig. 14).

characteristic of the thermistor, unmounted, at $25^{\circ} \mathrm{C}$. whereas the solid curve is that obtained with the thermistors in the mount. The difference was due to the large heat dissipation of the metal plates to which the thermistors were connected. The decrease in sensitivity that resulted was more than compensated for by a saving of about 80 percent of the time required to obtain thermal equilibrium of the thermistors during initial balance adjustments.

It was found experimentally that $\alpha$ increased as the $r$-f volt age level was increased $(\alpha \geqslant 1)$. Figure 16 shows $R_{T 1}$ and $R_{T 2}$ at different values of $I^{2}$ for an appreciably mismatched pair of thermistors. This effect was found to be independent of frequency. As was previously explained, individual pairs of thermistors were chosen having characteristics sufficiently similar to eliminate large 
values of $\alpha$ requiring high-precision $d-c$ voltmeters, as well as large ratios of $\alpha_{2} / \alpha_{1}$, where $\alpha_{1}$ is the ratio of the two thermistor resistances without $r-f$ applied to the bridge, and $\alpha_{2}$ is the ratio with $r-f$ applied. For $\alpha_{2} / \alpha_{1}$ of 2.0, an error in $V$ of 4 percent resulted. Two precautions were necessary. in connection with the use of these voltmeters for measuring $\alpha$. The first was that the $d$-c resistance of these instruments be large compared with $R_{T 1}$ and $R_{T 2}$ so as not to introduce an appreciable error in the thermistor resistance values. 'The second was that there be a constant low-resistance $\mathrm{d}$-c path through the $\mathrm{r}-\mathrm{f}$ source if the $\mathrm{d}-\mathrm{c}$ instruments were to measure the drop across $R_{T 1}$ and $R_{T 2}$.

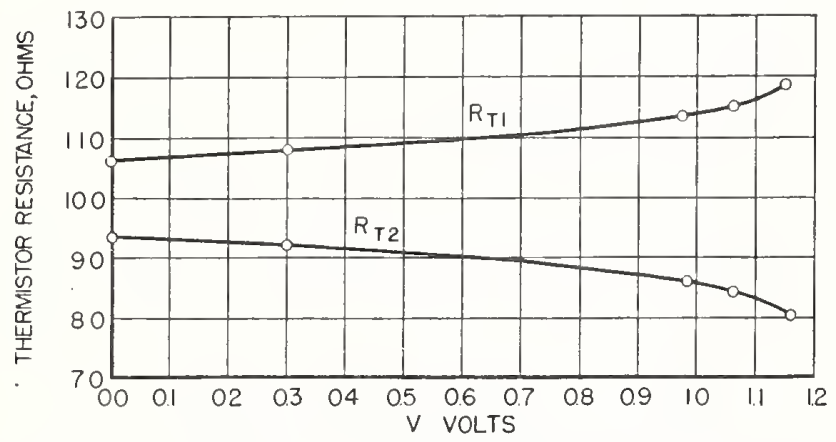

Figure 16. Resistance of individual thermistors, connected in parallel, versus $V$

$R_{T_{1}}+R_{T_{3}}=R_{T}=200 \mathrm{ohms}$ (fig. 11).

The voltage $V_{R 2}$ was controlled by varying the resistance $R$ (fig. 11) in series with the bridge (fig. 10). The greatest difficulty occurred at low values of $V$, where the resistance $R$ was of the order of $1 \mathrm{ohm}$ or less. As shown in figure 11, a low-resistance slide-wire in parallel with a resistance box of high value was used, the box serving as a vernier on the slide-wire.

The shorting relay (fig. 11) is a special relay with mirror-smooth silver-plated contact surfaces. Such construction was necessary to insure a low, constant voltage drop when the relay was closed; otherwise measurement of $V_{R_{1}}$ would have been necessary each time the relay was operated. The value of $V_{R 1}$ for typical, bridge-operating conditions ( $R_{T}=200 \mathrm{ohms} ; R_{b}=300 \mathrm{olhms}$ ) was found to be about $25 \mu v$ and remained stable to within the measuring accuracy of the potentiometer used.

The voltages $V_{0}, V_{R 2}$, and $V_{R 1}$ were measured by the d-c potentiometer, $P$, in terms of the standard cell (fig. 11) at points $x, y$, and $z$. To reduce the resistance of leads, wherever advisable, wide low-resistance straps were employed for interconnecting the resistance arms of the bridge. The average resistance contributed to any arm by the leads was no more than $0.005 \mathrm{ohm}$, which was negligible compared with 200 to 300 ohms. With the present setup, it was found that the internal resistance of the battery and the resistance of the leads used to connect the battery to the bridge totaled less than $0.05 \mathrm{ohm}$ and justified the assumption of negligible voltage regulation for the loads used.

The equipment was installed in an air-conditioned room with the temperature controlled to within $1 \operatorname{deg} \mathrm{C}$. This precaution served the dual purpose of, first, eliminating the effect of ambient temperature variations on the thermistors, and, second, reducing variations of $V_{0}$ and thermal voltages within the circuit. To reduce further the effect of thermal emf's, every effort was made to remove contacts of dissimilar metals.

\section{Agreement With Other Methods}

Voltage measurements by independent methods, including the use of a single-thermistor bridge, were used as a check on the two-thermistor bridge. The results obtained are presented in table 1.

The principles involved in methods 1 and 2 of table 1 are indicated by their titles in the table and have been described in previous literature (see footnote 1). The agreement between the two-thermistor and the single-thermistor bridges

TABle 1.

\begin{tabular}{|c|c|c|c|}
\hline Method & 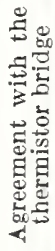 & $\begin{array}{c}\text { Frequency range } \\
\text { of agreement }\end{array}$ & Range-extension devices \\
\hline $\begin{array}{l}\text { Deflection of cathode- } \\
\text { ray heam. }\end{array}$ & $\begin{array}{r}\text { Per- } \\
\text { cent } \\
1\end{array}$ & $100 \mathrm{kc}$ to $200 \mathrm{Mc}$ & $\begin{array}{l}\text { Capacity-type voltage } \\
\text { dividers of about } \\
10: 1 \text {, and r-f trans- } \\
\text { formers. }\end{array}$ \\
\hline $\begin{array}{l}\text { Measurement of eur- } \\
\text { rent through a } \\
\text { known resistance. }\end{array}$ & 1 & $100 \mathrm{kc}$ to $30 \mathrm{Mc}$. & None. \\
\hline $\begin{array}{l}\text { Transmission-line } \\
\text { voltage-distribu- } \\
\text { tion. }\end{array}$ & ] & $100 \mathrm{Mc}$ to $600 \mathrm{Mc}$ & Do. \\
\hline $\begin{array}{l}\text { Single-thermistor } \\
\text { bridge. }\end{array}$ & 1 & $100 \mathrm{kc}$ to $30 \mathrm{Mc}$ & Do. \\
\hline
\end{tabular}


was.verified to $30 \mathrm{Mc}$. The difficulties encountered with the single-thermistor bridge for frequencies above $30 \mathrm{Mc}$ were previously pointed out in section III.

\section{(a) Transmission-Line-Voltage-Distribution Method}

The third listed independent method is based upon the interrelation between the $\mathrm{r}$-f power propagated along a transmission line (having a known characteristic impedance and negligible attenuation) and the voltage distribution along this line. A slotted transmission line is used, and the voltage ratios at any two given points are accurately determined by moving a probe along the slot. The conventional way to determine the ratios is either by using a standard attenuator or a calibrated probe-output detector. A more direct, and therefore a more accurate, way is to determine these voltage ratios from measurements of line distances translated in turn by computation into equivalent electrical lengths. Thus absolute values of voltages at a given point along the line may be computed from: (a) the characteristic impedance of the line, (b) power, determined by d-c measurements, and (c) distances measured along the line. The interfering factor in this otherwise potentially highly accurate method is the nonavailability of slotted transmission lines with sufficiently uniformly distributed constants. The frequency range is limited at the low end by the physical length of the line.

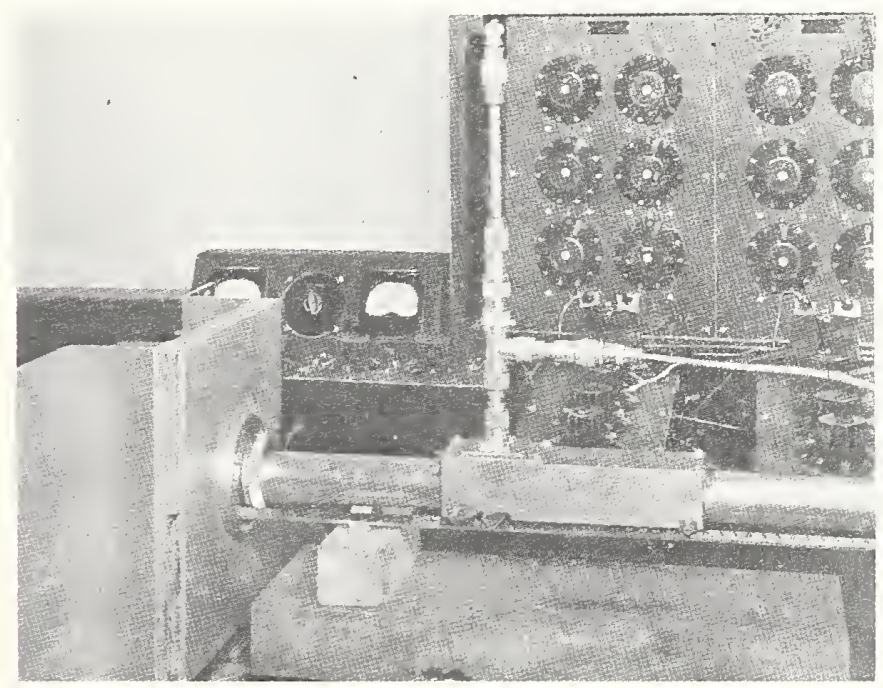

Figure 17. Thermistor mount connected to a slotted transmission line for measurement of agreement between two independent methods of voltage measurements and for standard impedance measurements.
For purposes of comparison with the bridge measurements, the thermistor mount was conneeted to a slotted transmission line as illustrated in figure 17. A line extension designed for use with the line was so connected to the mount that the uniform line was carried to within $0.003 \mathrm{in}$. of the core of the mount. Therefore the voltage at the end of the line was essentially the same as that at the thermistors.

The measurement procedure was as follows:

(1) The thermistor mount, having been internally shorted where the thermistors connect, was placed directly at the end of the slotted transmission line, and the position $\lambda / 2$ from the load determined (step $A$, fig. 18).

(2) With the short removed, the thermistor bridge was used as an r-f load and power meter. The voltage standing-wave ratio $\left(V_{\max } / V_{\mathrm{mln}}=\rho\right)$, the voltage ratio $\left(V_{A} / V_{\mathrm{max}}=K_{1}\right)$, and the power delivered to the load, were measured (steps $B$ and $C$, fig. 18).

The magnitude of the standard voltage, $V$, was calculated from eq 9. (See derivation in appendix VII).

$$
V=K_{r}\left(Z_{0} P_{R F} \rho\right)^{\frac{1}{2}},
$$

where $K_{1}=V_{A} / V_{\max }$, the ratio of the voltage $\lambda / 2$ from the load to the maximum voltage in the line. $Z_{0}=$ characteristic impedance of the line.

$\rho=$ ratio of maximum to minimum voltage in the line.

$P_{R F}=$ r-f power transmitted to the load=bridged-c power decrement.

The agreement between the two methods up to $600 \mathrm{Mc}$ was 1 percent or better, whereas up to $700 \mathrm{Mc}$ it was better than 2 percent (fig. 19).

The transmission-line voltage-distribution method was the only one used to verify the accuracy of the bridge at frequencies above $200 \mathrm{Mc}$. Because the transmission line used was known to have an insufficiently uniform distribution of constants, the bridge measurements were considered the more reliable of the two. One may apply the transmission-line method for direct calibration of voltmeters with some sacrifice in accuracy using an arrangement similar to that illustrated in figure 20. The probe of the voltmeter under calibration is connected at the end of the slotted line in place of the thermistor mount. In parallel with this probe and physically as close as possible to it, a cable is connected to feed the $\mathrm{r}-\mathrm{f}$ power 

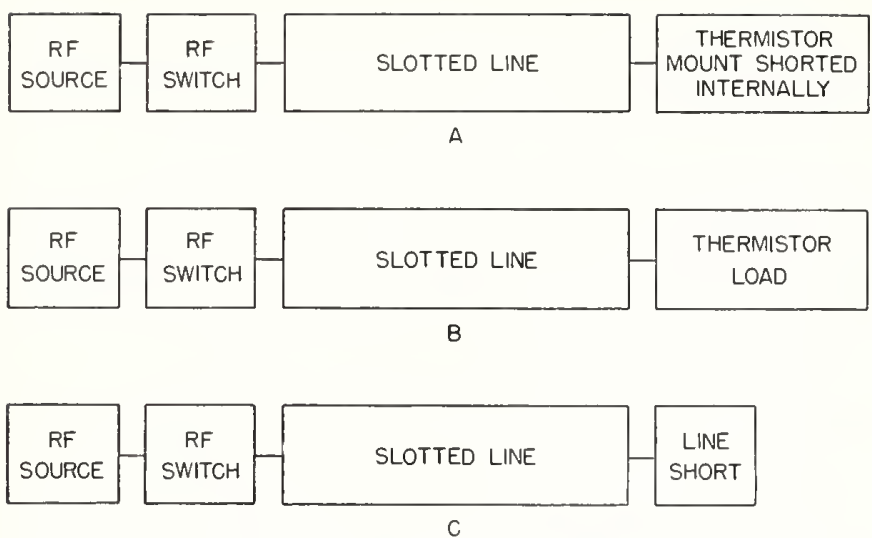

Figure 18. Block diagram of equipment and steps to ascertain agreement between bridge method and voltagedistribution-in-a-slotted-line method of voltage measurements.

from the line to any available accurate $r-f$ powermeasuring device. In figure 20 the thermistor bridge is shown as such a device. The procedure and computations may be the same as described above. One must correct for possible power losses in the voltmeter probe if these are appreciable.

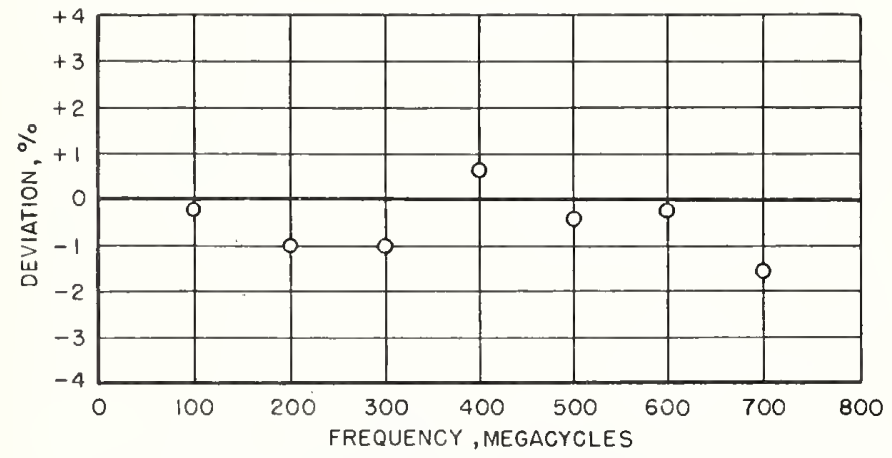

Figure 19. Deviation in percentage of $\mathrm{T}_{p}$ from $\mathrm{T}_{T}$.

$V_{p}=V$ obtained from power and voltage-distribution measurement; $V_{T}=V$ obtained with bridge measurement.

\section{Standardization of a V-T Voltmeter}

A calibration curve of a V-T voltmeter obtained with the bridge from 100 ke to $800 \mathrm{Mc}$ is shown in figure 21 . The applied standard voltage required to obtain a fixed deflection of the voltmeter had to be increased in the frequency range of about 50 to $400 \mathrm{Mc}$ as a result of the transit-time effect in the VTVMI probe diode. At higher frequencies the standard voltage had to be decreased because of the natural resonance of the diode and input terminals of the probe.

In conclusion, we fecl confident that standard voltages could be obtained with this two-thermis- tor bridge at frequencies considerably above 800 Mc with accuracies equal to those at the lower frequencies.

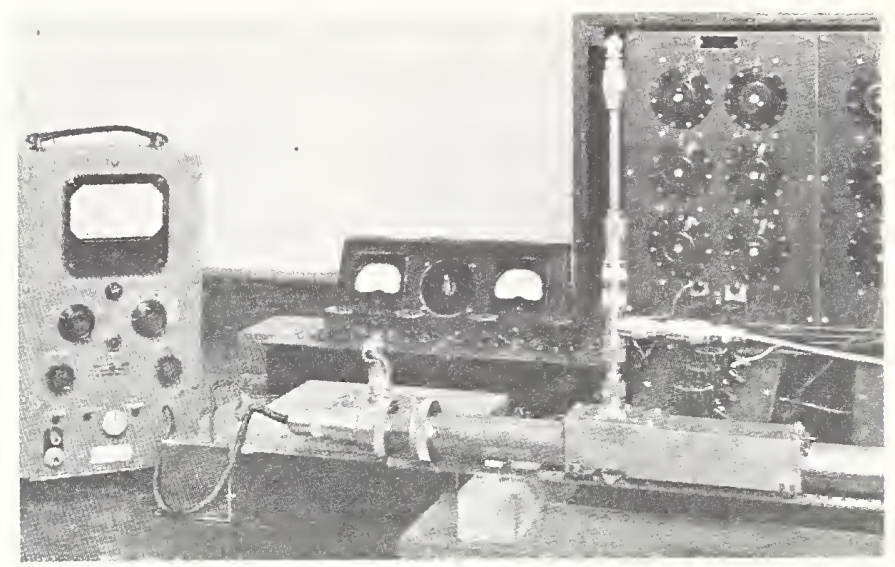

Figure 20. VTIM-probe connected to the slotted transmission line for calibration using power and voltagedistribution technique.

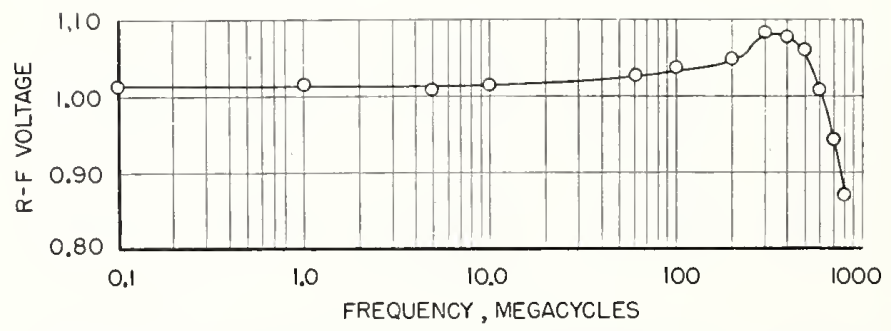

FIGURE 21. Calibration curve of a vacuum-tube voltmeter obtained with the bridge.

\section{Appendix I. General Expression for Bo- lometer Bridge r-f Voltage}

$$
W_{1}=\frac{V_{b 1}^{2} R_{T}}{\left(R_{T}+R_{b}\right)^{2}}
$$

$=\mathrm{d}-\mathrm{c}$ power dissipated in the thermistors with nor-f applied. (See fig, 5 and definition of terms below eq 1 of text).

$$
W_{2}=\frac{V_{b 2}^{2} R_{T}}{\left(R_{T}+R_{b}\right)^{2}}
$$

$=\mathrm{d}-\mathrm{e}$ power dissipated in the thermistors with $\mathrm{r}-\mathrm{f}$ applied, where $V_{b 2}=V_{0}-V_{R 2}$ and $V_{b 1}=V_{0}-V_{R 1}$

Substituting for $V_{b 2}$ and $V_{b 1}$ in eq 10 and 11 .

$$
\begin{gathered}
W_{1}=\frac{R_{T}}{\left(R_{T}+R_{b}\right)^{2}}\left(V_{0}-V_{R 1}\right)^{2} \\
W_{2}=\frac{R_{T}}{\left(R_{T}+R_{b}\right)^{2}}\left(V_{0}-Y_{R 2}\right)^{2} . \\
\text { If } W=W_{1}-W_{2}, \\
\text { and } W=\frac{\varphi^{2}}{R_{L}} \text { where } \frac{1}{R_{L}}=\frac{R_{T}}{R_{T 1} R_{T 2}},
\end{gathered}
$$


substituting for $W_{1}$ and $W_{2}$ in eq 14 and solving for $V$ yields:

$$
V=\frac{1}{R_{T}+R_{b}} \sqrt{R_{T} R_{L}}\left(V_{R 2}-V_{H 1}\right)\left(2 V_{\mathrm{n}}-\overline{V_{R 2}-V_{R 1}}\right) .
$$

Let $R_{T 1}=\alpha R_{T 2}$, where $\alpha \geq 1$ and remains constant for all values of applied $r-f$ voltage. It can be shown that eq 14 holds in this case.

Then $R_{L}=[\alpha /(1+\alpha)] R_{T 2}$ or $R_{L}=\left[a /(1+\alpha)^{2}\right] R_{T}$

Substituting in equation eq $\mathbf{1 6}$

$$
V=\frac{R_{T}}{\left(R_{T}+R_{b}\right)(1+\alpha)} \sqrt{\alpha\left(V_{R 2}-V_{R 1}\right)\left(2 V_{0}-V_{R 2}-V_{R 1}\right)} .
$$

\section{Appendix II. Error in $V$ When $\alpha=R_{T 1} / R_{T 2}$ Is Neglected}

In ey 1 let

$$
\frac{R_{T}}{\left(R_{T}+R_{b}\right)}\left[\left(V_{R 2}-V_{R 1}\right)\left(2 V_{0}-V_{R 2}-V_{R 1}\right)\right]^{\frac{1}{2}}=K,
$$

then

$$
V=K \alpha^{\frac{1}{2}}(1+\alpha)^{-1}
$$

Let also $V$ for $\alpha=1$ be designated as $(V)_{1}$

then

and

$$
(V)_{1}=\frac{1}{2} K
$$

$$
\frac{\Delta V}{V}=\frac{(V)_{1}-V}{(V)_{1}}=\frac{\frac{1}{2} K-K \alpha^{\frac{1}{2}}(1+\alpha)^{-1}}{\frac{1}{2} K}=1-2 \alpha^{\frac{1}{2}}(1+\alpha)^{-1}
$$

Substituting values of $\alpha$ from 1 to 2 , the error in percent of $V$ is computed and is shown in figure 6 .

\section{Appendix III. Error in Measured $V$ as a Result of Inaccuracy in Measuring $\alpha$}

From eq 18, appendix II,

$$
\frac{\Delta V}{V}=\Delta \alpha\left[\frac{1-\alpha}{2 \alpha(1+\alpha)}\right]
$$

which holds for $\Delta \alpha \leqq 0.02$

Tlius

$$
\frac{\Delta \alpha}{\alpha}=\frac{\Delta V}{V} \frac{2(1+\alpha)}{1-\alpha}
$$

and for an error of 0.1 percent in $V$ caused by an inaccuracy in measuring $\alpha$ this becomes

$$
\frac{\Delta \alpha}{\alpha}=0.001\left[\frac{2(1+\alpha)}{(1-\alpha)}\right]
$$

which is plotted in figure 6 versus $\alpha$.

For larger values of $\alpha$, the dfferential approach is incorrect. From eq 18, appendix II

$$
V=K \alpha^{\frac{1}{2}}(1+\alpha)^{-1}
$$

As $\alpha$ increases, $V$ decreases. $\frac{V}{V_{1}}>1$, where $V_{1}=$ the cal- culated value of the $\mathrm{r}-\mathrm{f}$ voltage when $\alpha>1$ for the condition where $\Delta V / V=10^{-3}$.

$$
\frac{V}{V_{1}}-1=10^{-3} \text {. }
$$

Substituting for $V$ and $V_{1}$ in terms of $\alpha$

$$
\frac{\alpha^{\frac{1}{2}}(1+\alpha)^{-1}}{(\alpha+\Delta \alpha)^{\frac{1}{2}}(1+\alpha+\Delta \alpha)^{-1}}-1=10^{-3} .
$$

Reducing to a common denominator and substituting

$$
\left(\frac{1}{\alpha}+1\right)=\sigma \text { and } \frac{\Delta \alpha}{\alpha}=y .
$$

Then $\frac{(\sigma+y)^{2}}{\sigma^{2}(1+y)}=1.002$.

Solving for $y$ in terms of $\sigma$

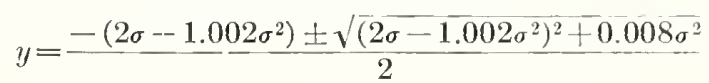

\section{Appendix IV. Accuracy of $V$ as a func- tion of accuracy of $R_{T}$ and $R_{b}$}

In eq 1 let the factor in the square bracket $=K$ and $\alpha=1$, then

and

$$
V=\frac{1}{2} K R_{T}\left(R_{T}+R_{b}\right)^{-1},
$$

$$
\Delta V=\Delta R_{T}=\frac{1}{2} K^{r}\left[\left(R_{T}+R_{b}\right)^{-1}-R_{T}\left(R_{T}+R_{b}\right)^{-2}\right] .
$$

Therefore

$$
\frac{\Delta R_{T}}{R_{T}}=\frac{\Delta V}{V} \frac{R_{T}+R_{b}}{R_{b}} .
$$

Similarly

$$
\frac{\Delta R_{b}}{R_{b}}=-\frac{\Delta V}{V} \frac{R_{T}+R_{b}}{R_{b}} .
$$

For the apparatus presently in use, $\left(R_{T}+R_{b}\right) / R_{b}$ is approximately $5 / 3, R_{T}=200$ ohms and $R_{b}=300$ ohms.

Dropping the mimus sign as of no significance (errors will be added),

$$
\frac{\Delta R_{T}}{R_{T}}=\frac{\Delta R_{b}}{R_{b}}=1.7 \frac{\Delta V}{V} .
$$

Appendix V. Derivation of $\Delta V_{R 2} / V_{R 2}$ in terms of $V$

From eq 1

$$
V^{2}=K\left(2 V_{0}-V_{R 2}-V_{H 1}\right)\left(V_{H 2}-V_{H 1}\right),
$$

where

$$
\begin{gathered}
K=\left[\frac{R_{T}}{2\left(R_{T}+R_{b}\right)}\right]^{2} \text { when } \alpha=1, \\
\therefore V \delta V=K\left(V_{0}-V_{R 2}\right) \delta V_{R 2 .} . \\
\Delta V_{R 2}^{r}=\Delta V \frac{\delta V_{R 2}}{\delta V}=\Delta V \frac{V^{r}}{K\left(V_{0}-V_{R 2}\right)}, \\
\frac{\Delta V_{R 2}}{V_{R 2}}=\frac{\Delta V}{V} \frac{V^{2}}{K V_{R 2}\left(V_{0}-\right.} \overline{\left.V_{R 2}\right)} .
\end{gathered}
$$

Figure 8 (solid line) is a plot of this equation for

$$
\frac{\Delta V}{V}=0.1 \% \text {. }
$$




\section{Appendix VI. Error in $V$ Caused by Inaccuracy of Measuring $V_{R 1}$}

From eq 33

$$
\begin{gathered}
\Delta V_{R 1}=\Delta V \frac{\partial V_{R 1}}{\partial V}=\Delta V \frac{V}{K\left(V_{R 1}-V_{0}\right)}, \\
\therefore \frac{\Delta V_{R 1}}{V_{R 1}}=\frac{\Delta V}{V} \frac{V^{2}}{K V_{R 1}\left(V_{R 1}-V_{0}\right)} \cdot
\end{gathered}
$$

In the case under consideration $V_{0} \gg V_{R 1}$, because $V_{0}=10$ $\mathrm{v}$ and $V_{R 1}=25 \times 10^{-6} \mathrm{v} . \quad V_{R 1}$ can be measured to $1 \mu \mathrm{v}$ or $\Delta V_{R 1} / V_{R 1}= \pm 1 / 25$

$$
\therefore \frac{\Delta V_{R 1}}{V_{R 1}} \doteq-\frac{\Delta V}{V} \frac{V^{2}}{10 K V_{R 1}} .
$$

Neglecting the minus sign because the error can be positive or negative and assuming $\Delta V / V=10^{-3}$ we have

$$
\begin{aligned}
\frac{1}{25} & =10^{-3} \frac{V^{2}}{10 K \times 25 \times 10^{-6}}, \\
K & =0.04 \\
\therefore V & =2 \times 10^{-2} \mathrm{v}=20 \mathrm{mv}
\end{aligned}
$$

\section{Appendix VII. Relation of the R-F Load Voltage to the Power and VSWR in $\dot{\alpha}$ Slotted Transmission Line}

Equation 9 is derived as follows:

$$
\begin{gathered}
\supset_{R F}=\frac{V_{\max } V_{\min }{ }^{5}}{Z_{0}}, \\
\rho=\frac{V_{\max }}{V_{\min }} .
\end{gathered}
$$

Substituting for $V_{\mathrm{min}}$ in eq 39 yields

Let

$$
P_{R F}=\frac{V_{\max }^{2}}{\rho Z_{0}}
$$

$$
\frac{V_{A}}{V_{\max }}=K_{1}
$$

${ }^{3}$ Principles of radar, by the staff of the MIT Radar School, pp. 8 to 45 (The Technology Press, Massachusetts Institute of Technology, Cambridge, Mass., 1944).
$=$ ratio of the voltage $\lambda / 2$ from the load to the maximum voltage in the line.

Then

and

$$
P_{R F}=\frac{V_{A}^{2}}{K_{1}^{2} \rho Z_{0}}
$$

$$
V_{A}=K_{1}\left(Z_{0} P_{R F} \rho\right)^{\frac{1}{2}}
$$

The ratio $V_{\max } / T_{A}$ was determined in two steps. First, when the line was loaded, the probe on the line was located where the voltage was a maximum. The probe output $D_{m}$ was recorded. The probe was then moved to the position corresponding to $\lambda / 2$ from the load, and the probe output $D_{A}$ was noted. The line was then shorted and the probe moved to where the voltage was a maximum in the line. The $\mathrm{r}-\mathrm{f}$ level was adjusted until a $T_{S \max }$ corresponding to the maximum indication, $D_{m}$, was reproduced ( $T_{S}$ designates shorted line conditions). Then two positions were found on the line that produced the indication $D_{A}$, and the distance in centimeters between these points (both on the same side of the maximum) was recorded. This distance was called $\Delta_{S}$. The distance between a maximum and a minimum corresponded to $\lambda / 4$ ( $\pi / 2$ radians). Since the voltage distribution in the line on short circuit was very nearly sinusoidal, $D_{A}=D_{M}$ $\cos \theta$, where $\theta$ was the angle in radians between $D_{M}$ and $D_{A}$. But $2 \theta / \pi=l /(\lambda / 4)$, where $l$ is the distance in centimeters from $V_{S \max }$ to $V_{A}$ on the line. $l$ also equaled $\left(\lambda / 4-\Delta_{S^{\prime}} / 2\right)$. Substituting and solving for $\theta$ yields $\theta=\pi\left(0.5-\Delta_{S} / \lambda\right)$. Therefore

$$
K_{1}=\frac{V_{A}}{V_{\mathrm{max}}}=\cos \theta=\cos \left(0.5-\frac{\Delta_{S}}{\lambda}\right) \pi
$$

The VSWR is determined in a similar manner, i. e., by reproducing the probe outputs corresponding to $I_{\max }$ and $V_{\min }$ at two positions, one of them at a voltage maximum of the probe with the line shorted. The distance between these two positions in electrical degrees is then the angle, the cosine of which is equal to $\rho$. Sequence of steps suitable for various standing-wave ratios, as well as a general analysis of this method of measuring voltage ratios along a transmission line is presented by Winzemer. ${ }^{6}$

${ }^{6}$ A. M. Winzemer, Method for obtaining the voltage standing-wave ratio on transmission lines independently of the detector characteristics. Thesis presented to the Polytechnic Institute of Brooklyn (May 1948).

Washington, April 18, 1949. 


\title{
NATIONAL BUREAU OF STANDARDS Eechnical Note
} 37

JANUARY 1960

\author{
APPLICATION OF RF MICROPOTENTIOMETERS \\ FOR \\ CALIBRATION OF SIGNAL GENERATORS TO $1000 \mathrm{MC}$ \\ by \\ L. F. Behrent
}

SUMMARY

With the RF Micropotentiometer, signal generator output voltage can be calibrated to $1000 \mathrm{Mc}$ simply and accurately if the procedures outlined in this paper are carefully followed. The sources of error are discussed and methods for minimizing or eliminating them are described. Topics discussed include: rf shielding, selection of a suitable rf detector, impedance matching and the proper selection of voltage reference planes. 
APPLICATION OF RF MICROPOTENTIOMETERS FOR CALIBRATION OF SIGNAL GENERATORS TO $1000 \mathrm{MC}$

by

\section{F. Behrent}

\section{INTRODUCTION}

The RF Micropotentiometer ${ }^{1}$ is a simple and accurate standard for calibrating the rf voltage output of signal generators. However, from the inquiries received it seems that some precautions basic to high-frequency measurements are being overlooked or given insufficient consideration. By discussing in detail some difficulties which have been brought to the attention of the RF Voltage Measurement Standards Group at the National Bureau of Standards, and describing the necess zry corrective measures, it is hoped that this material will help improve techniques so that higher accuracies will be obtained.

\section{CALIBRATION PROCEDURE}

The block diagram of Figure \& lilustrates how the RF Micropotentiometer is used. $G_{1}$ is the $r f$ generator to be calibrated (the unknown); $\mathrm{G}_{2}$ energizes the reference standard - the RF Micropotentiometer $S$ - and $D$ is the detector, usually a sensitive receiver, for comparing the $r$ output voltage of the unknown with the reference standard.

The usual procedure in calibrating the unknown is to preset its output level to an arbitrary indicated value. This output is compared with the standard by switching the detector alternately between them. 
The level of the standard is adiusted until the detector shows its amplitude is equal to the unknown. Since the rf resistance of the RF Micropotentiometer output is known from previous comparison with the Primary Standara, and the rf current flowing in it can be determined from a d-c calibration of the thermoelement, the magnitude of the rf voltage may now be calculated.

One possible combination of thermoelements and RF Micropotentiometer resistance elements which will provide standardized $\mathrm{rf}$ microvoltages at any level between 1 and 100,000 microvolts is presented in Appendix I. This combination of units permits a ready check of each RF Micropotentiometer against those covering adjacent ranges.

\section{PRECAUTIONS}

Stray radiation is the most serious source of error in low level measurements and the most difficult to eliminate. Even at $\mathrm{rf}$ levels as high as $10 \mathrm{k} \mu \mathrm{v}$ errors greater than 100 percent have been traced to this cause, with even greater errors at lower rf levels. In the circuit illustrated in Figure 1 there are several possible sources of radiation. Between the $\mathrm{rf}$ input and output of the RF Micropotentiometer there is sometimes as much as $100 \mathrm{db}$ of attenuation. Therefore, the metal box containing the reference standard must be well constructed to prevent leakage. One very satisfactory and economical design is illustrated in Figures 2 and 3. Heavy-walled construction is used to provide large contact surfaces between the box and the removable cover, the body of the coaxial rf input connector, and the outer electrode of the RF Micropotentiometer resistance element. The most effective by-passing of the thermoelement's $d-c$ leads can 
be obtained by mounting button type mica capacitors in recesses in the heavy side wall of the box. (See Figure 3.)

How extensively the signal generators and the detector must be shielded can best be determined by connecting an antenna to the detector and probing the system for radiation. Often it is necessary to place each of these pieces of equipment into individual doublescreened boxes (Figure 4).

Commercial double-screened cabinets can be used to shield each of the signal generators and the rf detector. The shielding will be most effective if the inner screened box is grounded to the outer only where the coaxial rf cable connecting the rf equipment in the screened cabinct to the outside circuitry passes through the side of the screened boxes. All other connections into the cabinets required for energizing and controlling the enclosed equipment must be properly filtered to prevent $\mathrm{rf}$ leakage. There is ample published literature on this phase of the shielding problem ${ }^{2}$. The rf input cable to the detector and the cable connecting signal generator $G_{2}$ to the standard $S$ should be double-shielded coaxial cables, preferably with an additional external shielding braid. With the equipment arranged as shown in Figure 4, the most effective shielding against rf leakage was obtained when external braid was placed over each of the rf cables, with the braid connected to the internal shield by clamping it tightly to the rf connectors at the cable ends with adjustable metal hose clamps.

For some calibration work a crystal rectifier and microammeter or an electronic rf millivoltmeter will be an adequate detector, but in most instances a radio receiver sensitive to 1 microvolt or less will be required. In any case, there must be enough sensitivity to resolve incremental changes in the $r f$ input of 1 percent or less. 
Any receiver so used should be tested to determine the optimum oper ating conditions and maximum $r$ input level for which maximum resolution is obtained. For rf voltages above this, the receiver must be decoupled to prevent los s of sensitivity due to receiver overloading.

The amplitude of the voltage output from an $r f$ source depends upon how the external load impedance, whether an rf voltmeter, radio receiver or other impedance, is connected to it. With a perfect trans mission line terminated in its characteristic impedance connected to the output, the amplitude of the $r$ voltage would be the same at all points along the line and it would be immaterial how long a line section was used. However, because of imperfections in practical rf cables and connectors, discontinuities will exist which will introduce voltage variations along the line. At very-high and ultra-high frequencies large changes in the amplitude of the output voltages may result from changing the length of the coaxial connection between the output and the load even by a fraction of an inch. The plane through the rf connection at which the voltage output is measured should be properly identified. In using the rf source as a reference standard, the magnitude of the voltage is accurately known only at this Voltage Reference Plane. The ideal reference plane for the RF Micropotentiometer would be the plane in which the resistive annular ring lies, a-a Figure 3. However, because it was necessary to provide a coaxial output connection, the best practical reference plane is essentially at $b-b$ Figure 3. The rf voltage $a t b-b$ is compared with the Primary Voltage Standard at all frequencies when determining dc-rf characteristics of the RF Micropotentiometer.

The output voltage of a signal generator should be measured at the output connector provided. Where this connector is similar to that of the RF Micropotentiometer, the recommended voltage reference 
plane would pass through the connector essentially at the tip of the female center pin (b-b Figure 5). Where other types of output connectors are found on the generator output, an adaptor should be used which henceforth should be considered a part of the generator.

The output of the unknown must be terminated with an accurately known rf impedance connected at the voltage reference plane. The detector can be used as the termination by adjusting its input impedance with a suitable matching network. (See Figure 6) However, it is not safe to assume that impedance measurements of the input to the detector made with an impedance bridge will be valid at low rf levels. Such bridges impress from 0.1 to 1 volt or more across the unknown impedance. For a radio receiver, such a level would result in complete saturation, causing the input impedance to change greatly from what it would be at relatively low levels. Moreover, the impedance is a function of the tuning of the receiver. One method of adjusting a receiver's input impedance to $50 \mathrm{ohms}$ with a matching network is to connect the input of the matching network to an rf source through a $20 \mathrm{db}, 50 \mathrm{ohm}$ pad of high quality. With only a few microvolts applied to the receiver input, and the receiver tuned to the source frequency, adjust the network for maximum receiver output indication. Once the matching is completed, the receiver tuning must not be changed. Where the sensitivity of the detector permits, a high quality attenuator pad may be used in place of the matching network, thus terminating the unknown in an impedance essentially that of the attenuator pad. 


\section{CONCLUSIONS}

Observance of the precautions described in the text cannot be overemphasized. In a recent case brought to the attention of the RF Voltage Standards Group, insufficient attention to these details resulted in measurement errors of approximately $14 \mathrm{db}$ at $1000 \mathrm{Mc}$.

\section{REFERENCES}

(1) M. C. Selby, Accurate radio-frequency microvoltages, Communications and Electronics, AIEE 6: 158-164 (May 1953)

(2) E. E. Zepler, The technique of radio design, 240-264 (John Wiley \& Sons, Inc., New York, 1949)

\section{APPENDIX I}

Typical Values of RF Micropotentiometer Components for the Range of 1 to 100,000 Microvolts

\begin{tabular}{ccc}
$\begin{array}{c}\text { Thermoelement } \\
\text { rating, ma }\end{array}$ & $\begin{array}{c}\text { Resistance } \\
\text { (RF Micropotentiometer), } \\
\text { milliohms }\end{array}$ & $\begin{array}{c}\text { Standardized } \\
\text { rf voltage, } \\
\text { microvolts }\end{array}$ \\
\hline 5 & 1 & $1-5$ \\
25 & 1 & $5-25$ \\
100 & 1 & $20-100$ \\
50 & 10 & $100-500$ \\
50 & 50 & $500-2500$ \\
50 & 100 & $1000-5000$ \\
25 & 1000 & $5000-25000$ \\
100 & 1000 & $20000-100000$
\end{tabular}




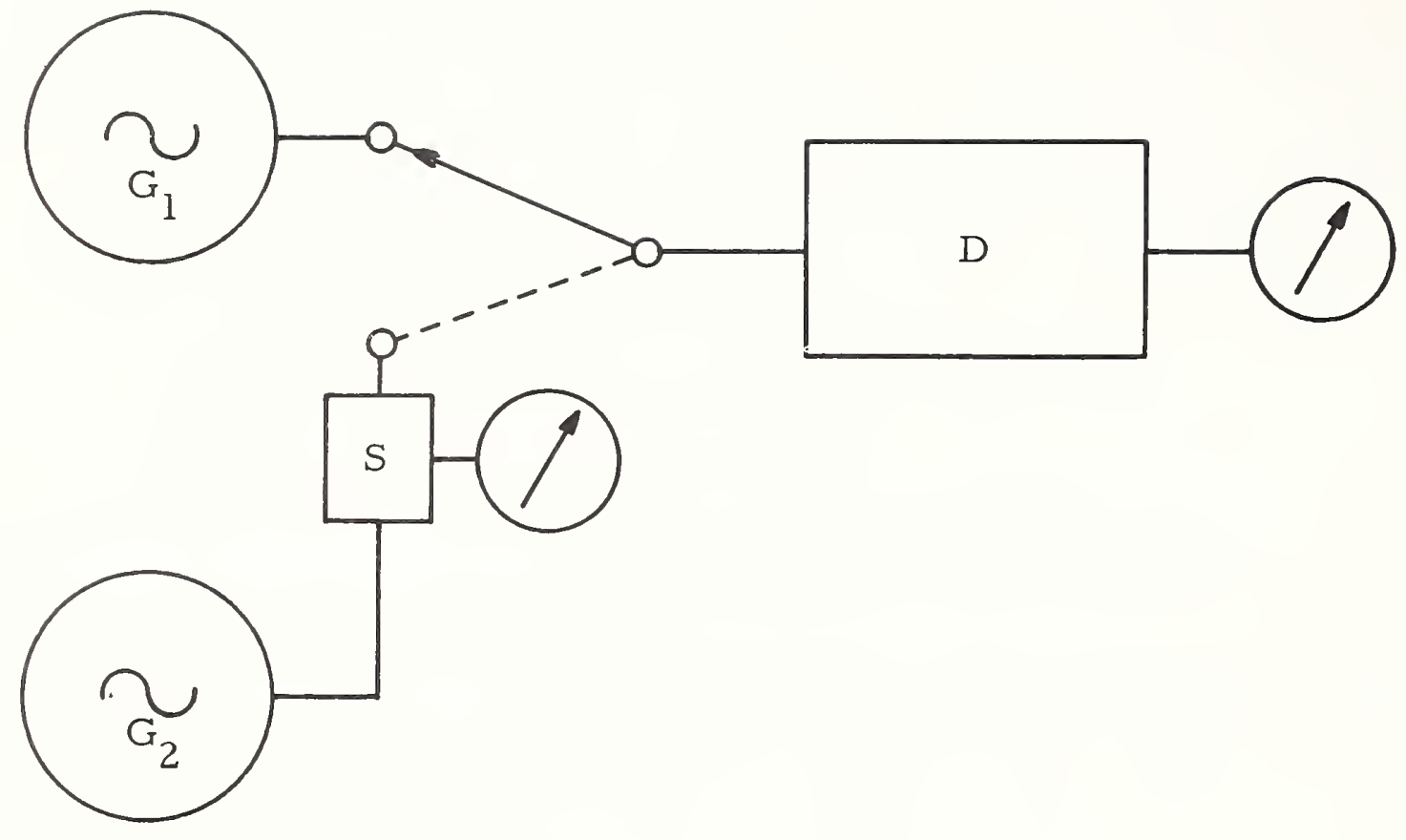

$\mathrm{G}_{1}$ is the $\mathrm{rf}$ generator to be calibrated.

$S$ is the transfer standard, the RF Micropotentiometer.

$G_{2}$ is the signal generator energizing the RF Micropotentiometer.

$D$ is the rf detector.

Figure 1 

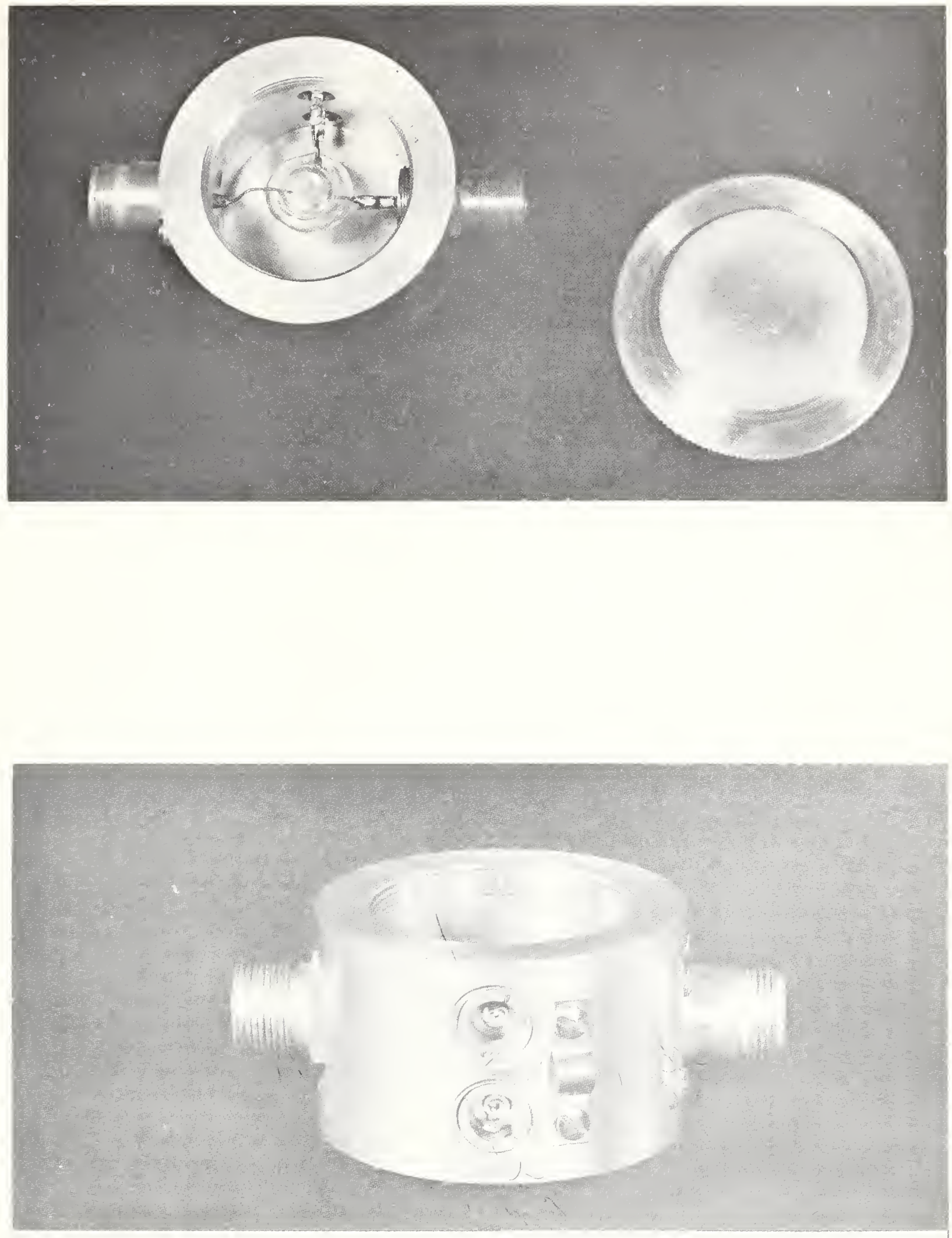

Figure 2

$629 / 8$ 


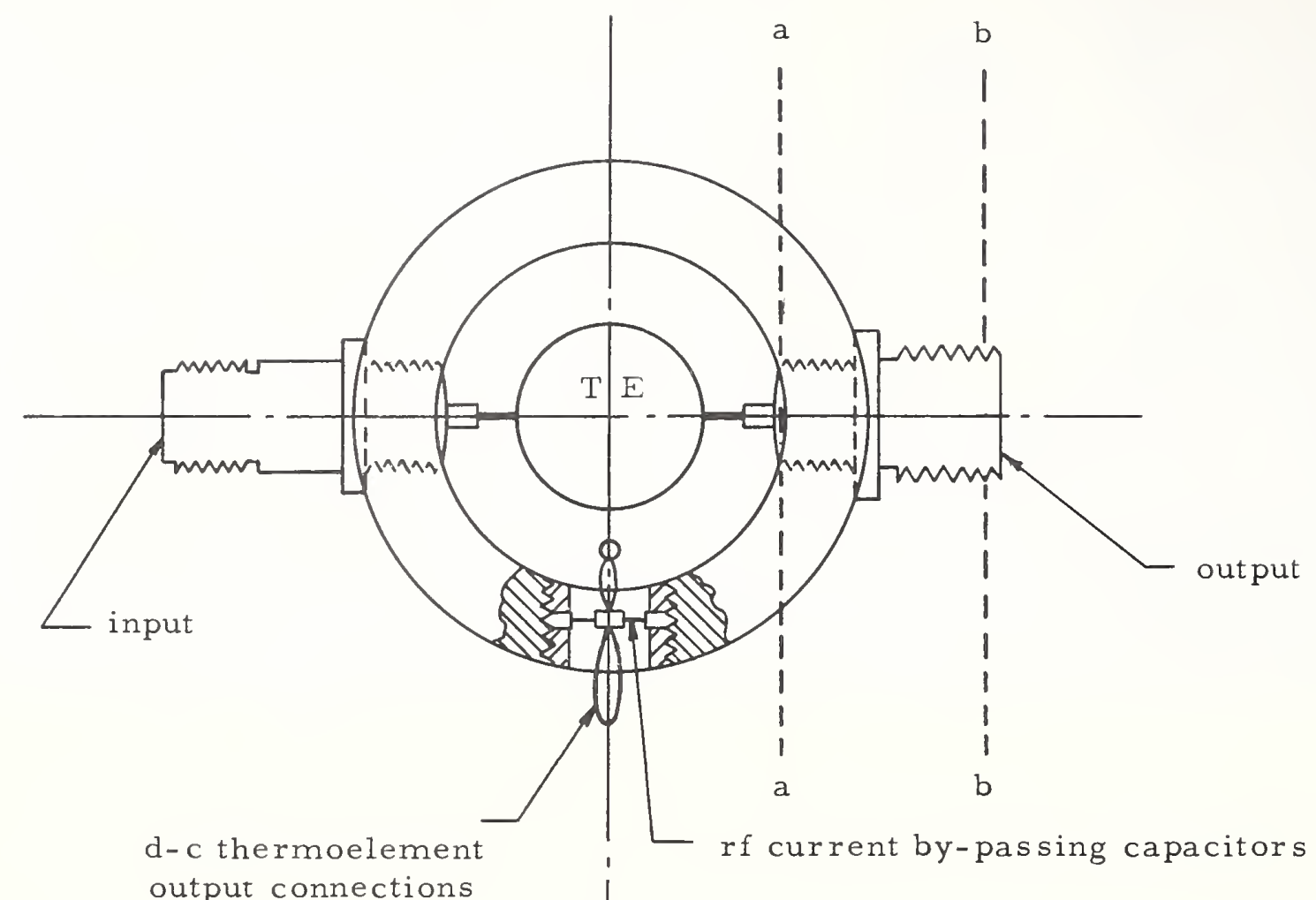
output connections

d-c output connections

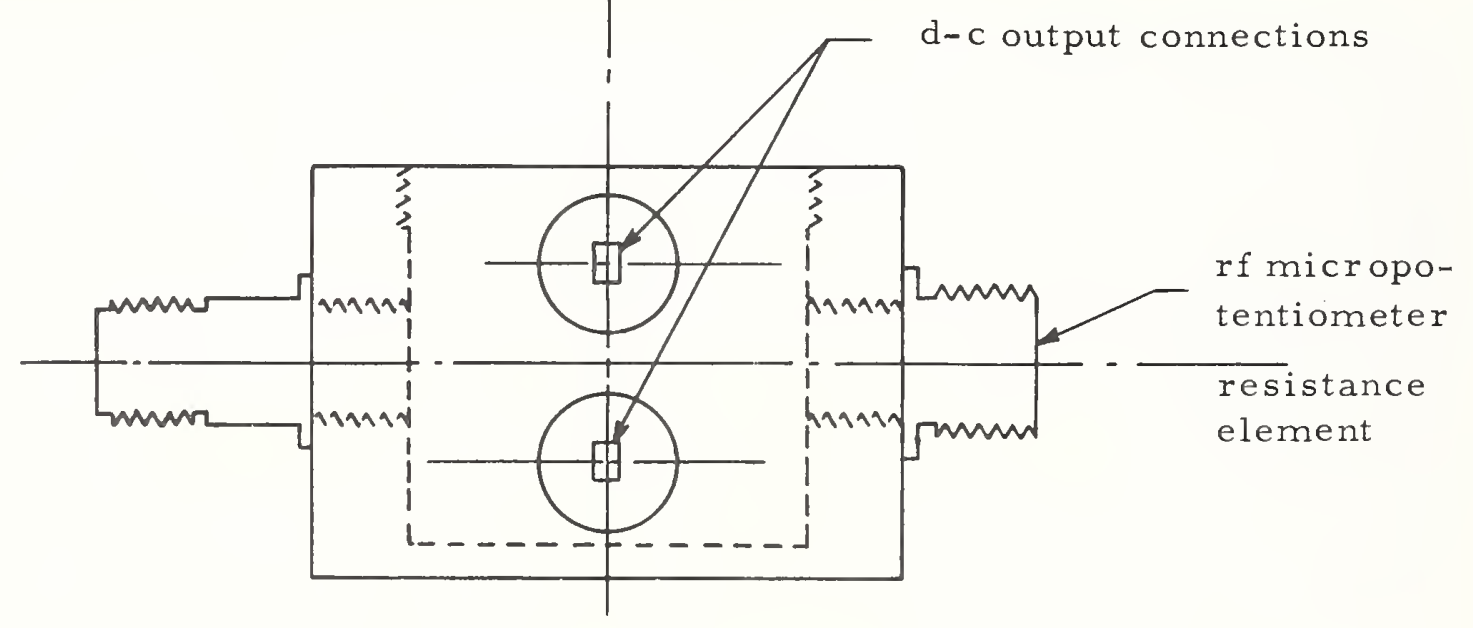

a-a is the plane in which the standardized reference voltage exists.

$\mathrm{b}-\mathrm{b}$ is the plane in which the $\mathrm{rf}$ voltage is compared with the primary rf voltage standard.

Figure 3

$630 / 9$ 

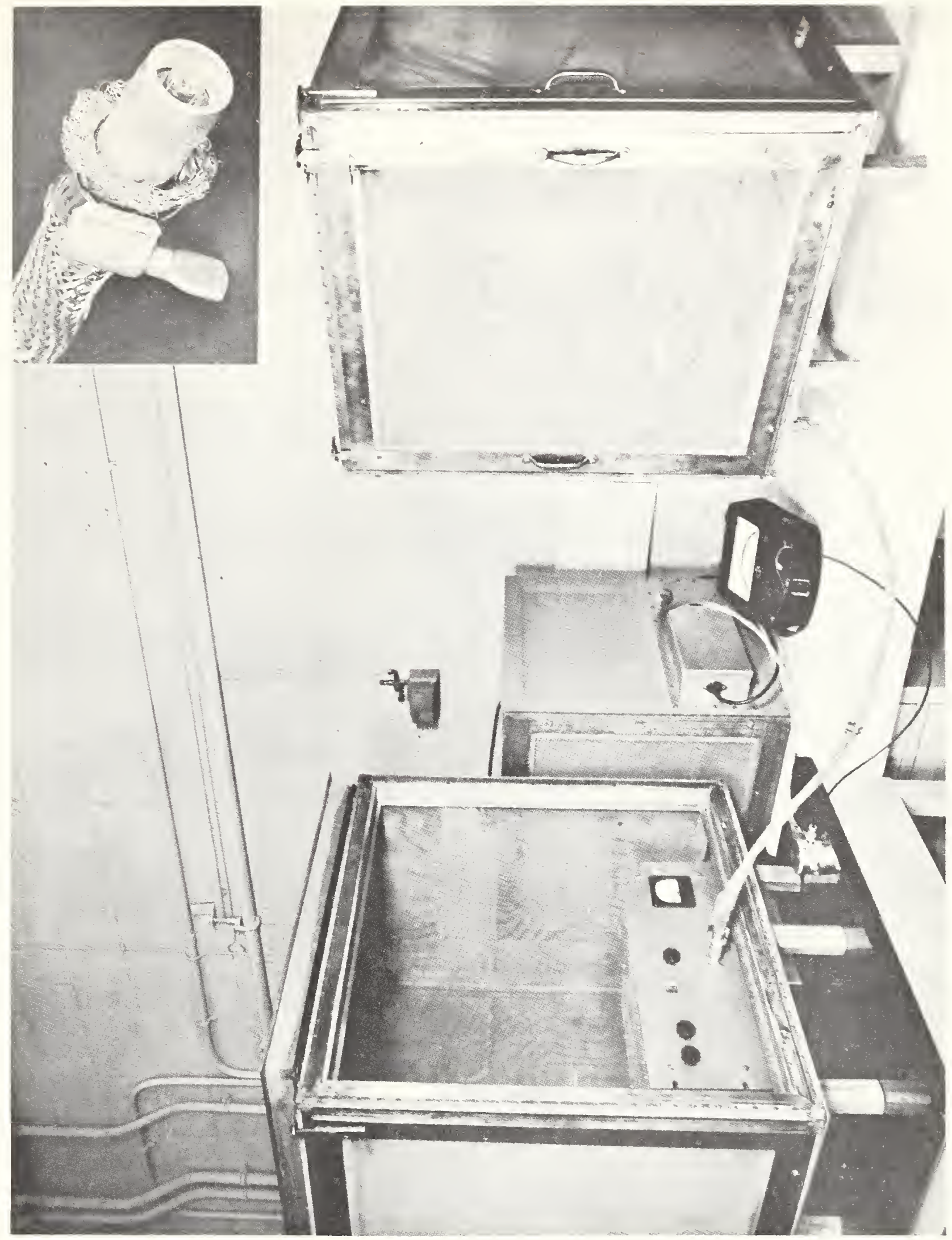

$631 / 10$ 

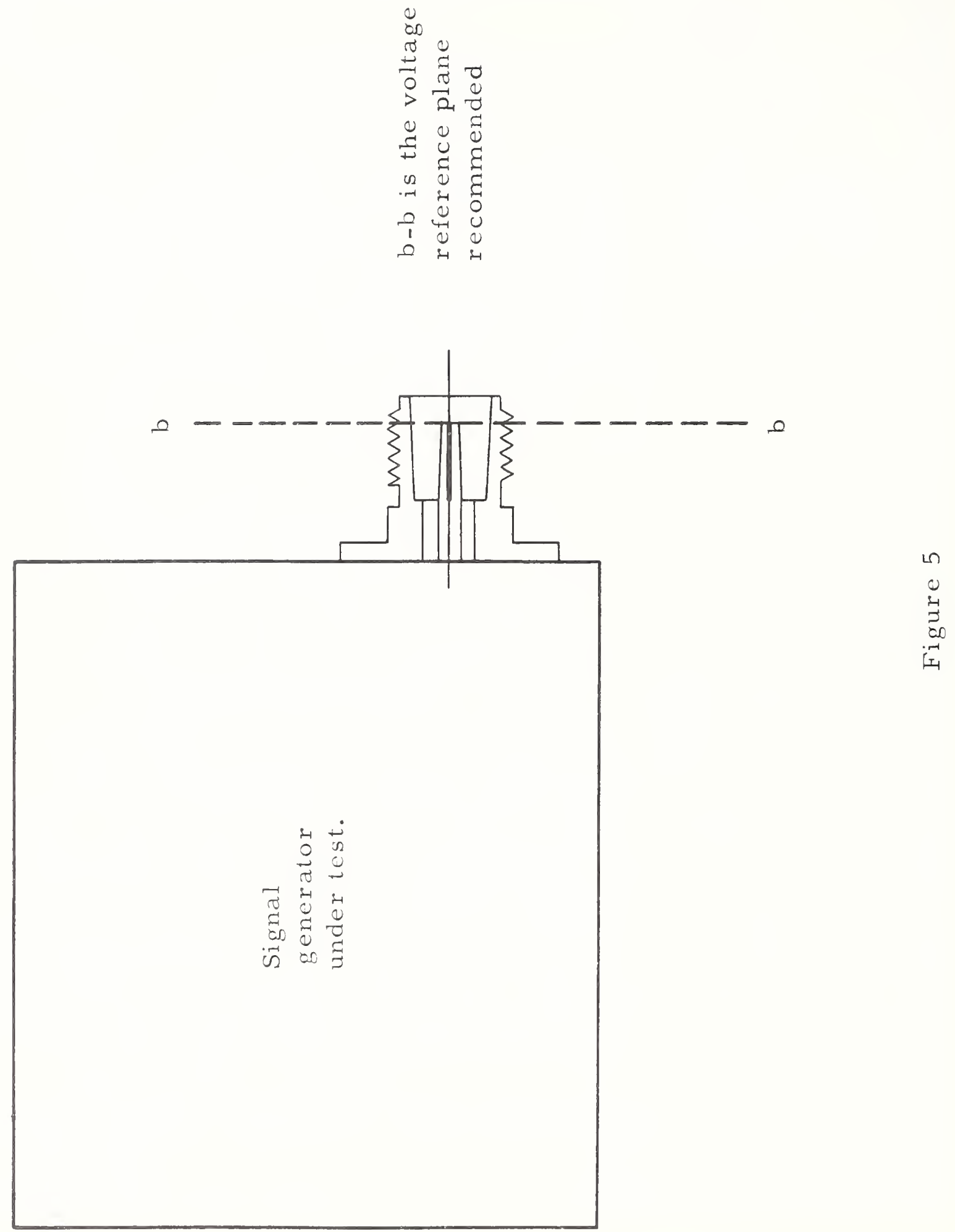

$632 / 11$ 


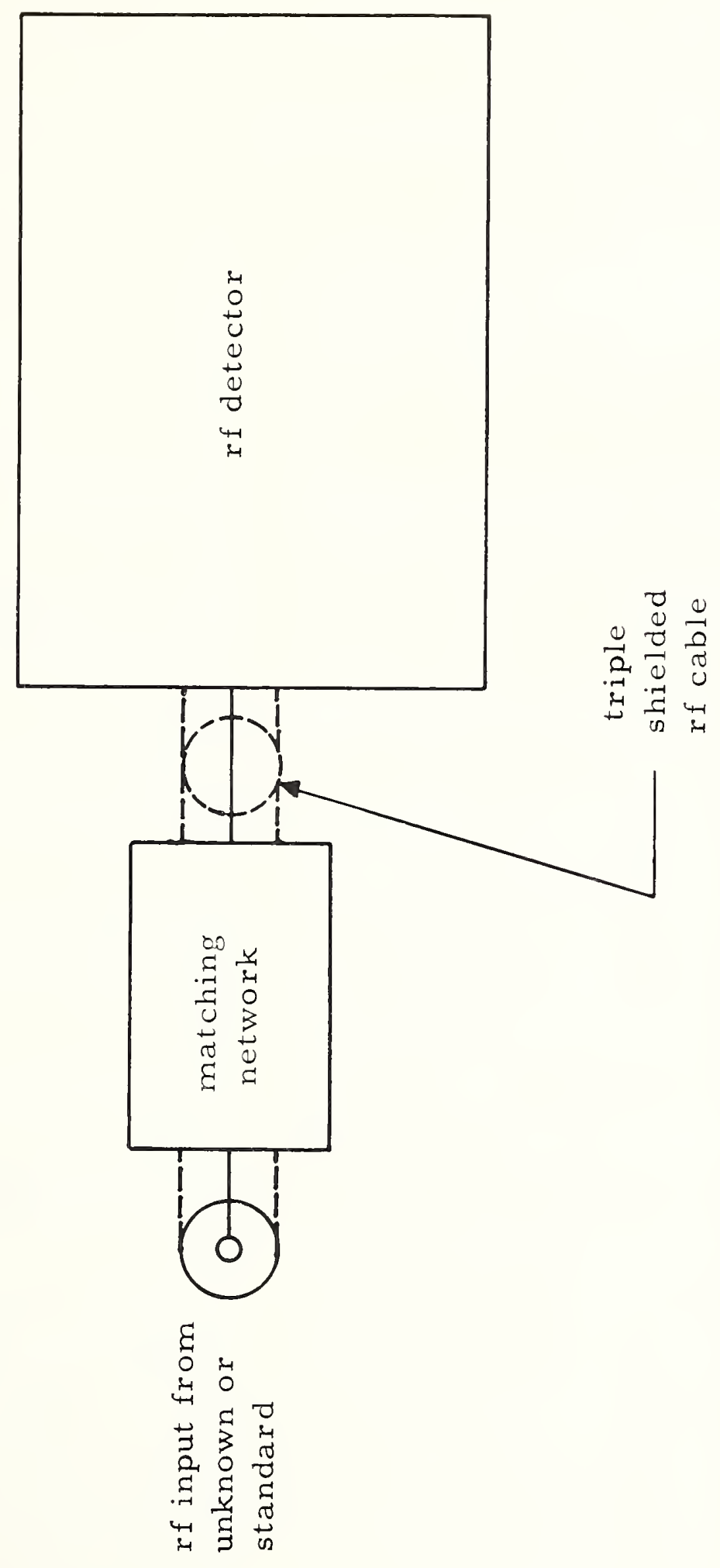

0
0
3
3
00
01
41

$633 / 12$ 


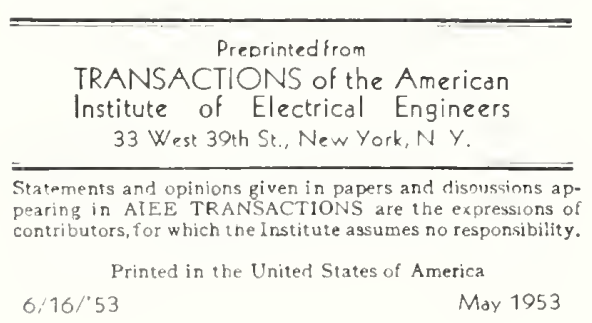

\section{Accurate Radio-Frequency Microvoltages}

\author{
MYRON C. SELBY \\ NONMEMBER AIEE
}

\begin{abstract}
Synopsis: The questionable accuracy of radio-frequency microvoltages has been of great concern to the radio field for many years. There is an urgent need for a simple, yet reliable, source of microvolts for measurements in general and for radio receiver sensitivity determinations in particular. Extremely simple devices, which seem to satisfy that need most adequately, were recently developed. They are sources of potcntial drop obtained across a known resistance through which known currents flow. These devices provide constant voltage sources of accurate microvolts over a range of 1 to $10^{5}$ and wider, at all frequencies to 300 megacycles and higher. They are adaptable for balanced as well as for unbalanced sources. Their electrical constants are simply detcrmined by using known direct voltages and currents. Basic principles, design features, and applications are discussed
\end{abstract}

$\mathbf{T}$ HE RADIO and electronics ficld has been facing the problem of accuracy of radio-frequency ( $r-f)$ microvoltages since sensitivity of radio receivers came into prominence as a compctitive index of performance. The rcasons for the continued existence of this problem are too well known. There is no nced to discuss them other than perhaps to indicate the 111ajor ones: namely, 1. the uncertainty i1 voltage source and load (receiver input) impedance values, 2 . the unccrtainty in performance of attenuators over wide frequency ranges and attenuations of up to 100 decibcls and in many cases of ligher values, and 3. the uncertainty in the accuracy and stability of derices monitoring the input voltages to the attenuators.

Considerable progress was apparently made as time went on, and a large section of the field has managed to attain high accuracies despite these difficulties. A recent poll of the field, instigated by the National Bureau of Standards in $195 \mathrm{l}$ to determine the urgency of this problem, revealed the following status, Of about 70 standard voltage-generator manufacturing and applying laboratories (six of them in United Kingdom and France) about one-half believed they had available 1 microvolt at frequencies to 1,000 megacycles with an absolute accuracy of 30 per cent or better. The indicated accuracy was naturally better at lower frequencies. The other half was uncertain of their values in various degrees, in many cases exceeding 100 and in some 200 per cent. About one-half of them desired absolute accuracics of 0.5 to 6 per cent for all frequencies to 1,000 inegacycles; the rest desired accuracies of 50 per cent or better.

The critical need of reliable tools to supply or measure microvolts accurately was therefore still very much in eridence. Deviccs apparently fulfilling this need most satisfactorily were recently developed. They seem to meet the most persistently demanded feature of a standard of inicrovolts, namely, extreme simplicity and reliability. These devices, referred to, for want of a better name, as "Micropotentiometers."' are described later.

\section{General Description of the Micropotentiometer}

\section{Basic Requirements and Principle OpERATION}

In searching for a source of accurate and reliable $r$-f microwolts the following basic requircments seemed indispensable or highly desirable:

1. The output voltages of the source had to be known irrespective of loading conditions, that is, a constant voltage source was necessary.

2. Freedom from frequency corrections was essential at least over reasonable frequency ranges.

3. A reliable, simple, and rugged physical construction with a very minimum of component parts was most desirable.

4. The simplest and a very minimum of calibration requirements were essential.

All of these requirements seem to be satisfactorily achieved with the Micropotentiometer. In basic principle it is a source of potential drops obtained across a known resistance through which known currents flow. These resistances are of the order of 111illiohms (for microvolt levels), and therefore constitute an essentially zcro source impedance (constant voltage source) for all practical presentday needs. A cardinal requirement of

Paper 53-22, recommended by the A1EE İstruments and Measurements Committee and approved by the AIEE Committee on Technical Operations for presentation at the AlEE Winter General Meeting, New York, N. Y., Jannary 19-23, 1953. Manuscript submitted August 29, 1952; made available for printing November 10, 1952.

MYron C. Selay is with the National Bureau of Standards, Washington, D. C. 


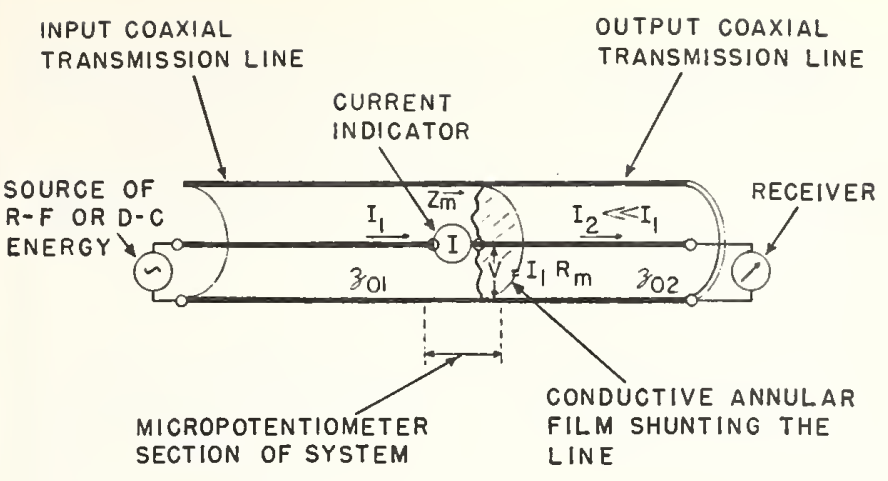

Figure 1 (left). Equivalent circuit diagram of system employing a Micropotentiom. eter

Figure 2 (right). Section of Micropotentiometer showing position of annular element in a low dielectric coaxial line this impedance, as previously implied, was that its reactive component be negligible compared with its resistive component. This suggested the annular type of resistance element construction. The equivalent circuit of the Micropotentiometer together with the input and output circuits is that of a coaxial line shunted by a conductive film in a plane normal to the axis of the line, as shown in Figure 1. A source of $r-f$ energy supplies current to the Micropotentiometer section of the system through a coaxial line and the voltagc output $V$, from the Micropotentioneter, available across the annulus, is fed to the receiver either directly or through an output line. To a very good approximation the output voltage $V$ is simply the product of the input line current entering the annulus and the $d-c$ resistance of the annulus.

To show that this is true the transfer impedance of a thin conducting film placed as already indicated (see Figure 2) will be considered under the following assumptions:

1. The characteristic impedance of the coaxial lines is of practical magnitude, for example, any valuc between 20 and 200 ohms.

2. The bridging film has a finite high conductivity approaching that of silver, copper, platinum, and so forth. Perfect contact is assumed betwcen the film and the coaxial conductors.

3. The diametcr values of the coaxial lincs are very small compared with a wave length.

4. The coaxial line terminating impedance (that is, the receiver input impedance) is of such a value that the impedances along the line, beyond the film, remain fairly high, that is, $1 \mathrm{ohm}$ or higher.

The general field-theory approach ${ }^{2,3}$ treats the solid metallic disk (or annulus) as a section of a coaxial line with an intrinsic inpedance corresponding to that of the particular metallic medium. Under the preceding assumptions the fields in all the coaxial linc sections are that of the dominant transverse electromagnetic (TEM) planc wave modc of propagation.
Let $\epsilon_{m}, \mu_{m}, \sigma_{m}$ and $\eta_{m}$ designate respectively the permittivity, permeability, conductivity, and intrinsic impedance of an infinite metallic medium. Under the preceding assumptions

$\omega \epsilon_{m}<<\sigma_{m}$

where

$\omega=2 \pi f$

For copper at 1,000 megacycles

$\omega \epsilon_{m}=0.17$ mhos per meter

as against

$\sigma_{m}=5.7 \times 10^{7}$ mhos per meter

Therefore $^{3}$

$\eta_{m l} \cong(1+j) \sqrt{\frac{\omega \mu_{m}}{2 \sigma_{m}}}$

The propagation constant in the same medium is

$\gamma_{m} \cong(1+j) \sqrt{\frac{\omega \mu_{m} \sigma_{m}}{2}}$

The characteristic impedance of a coaxial line with a solid conductor as a propagation medium is

$Z_{0}=\eta_{m} \frac{1}{2 \pi} \ln \frac{r_{1}}{r_{2}} \cong(1+j) \frac{\left(\omega \mu_{m}\right)^{1 / 2}}{2 \sigma_{m}} \frac{1}{2 \pi} \ln \frac{r_{1}}{r_{2}}$

where $r_{1}$ and $r_{2}$ are respectively the large and small radii of the conductors.

The $d-c$ resistance of the annulus of thickness $d$ is

$R_{m}=\frac{1}{\sigma_{m} 2 \pi d} \ln \frac{r_{1}}{r_{2}}$

and the depth of penetration is

$\delta=\left(\frac{1}{\sigma_{m} \pi f \mu_{m}}\right)^{1 / 2}$

Therefore

$Z_{0} \cong(1+j) R_{m} \frac{d}{\delta}$

and

$\gamma_{m}=(1+j) \frac{]}{\delta}$

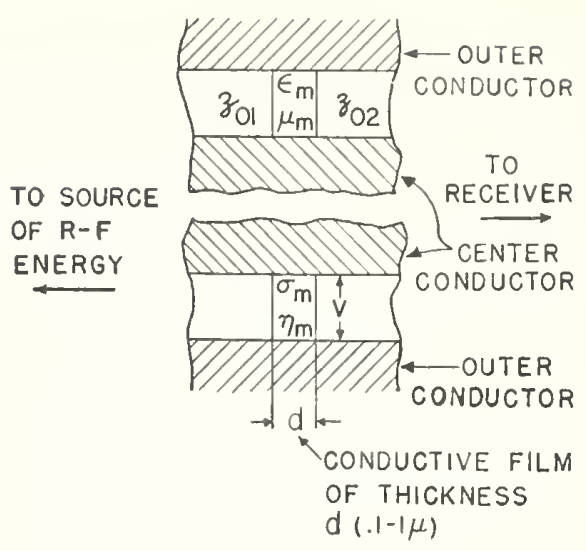

Using well-known transmision line theory, the output voltage $V$ of a line section equivalent to the annulus terminated by an impedance $Z_{r}$ is given in terms of the input current $I_{1}$ by

$$
V_{r}=\frac{I_{1}}{\frac{1}{Z_{r}} \cosh \gamma d+\frac{1}{Z_{0}} \sinh \gamma d}
$$

and the transfer impedance is

$$
\begin{aligned}
Z_{m}=\frac{V_{T}}{I_{1}}=\frac{1}{\frac{1}{Z_{T}} \cosh \left[(1+j) \frac{d}{\delta}\right]+} \\
\frac{1}{R_{m}} \frac{\sinh [(1+j) d / \delta]}{(1+j) d / \delta}
\end{aligned}
$$

In this application $Z_{r}>>R_{m}$, therefore for all practical purposes

$Z_{m}=R_{m}(1+j)(d / \delta) \operatorname{csch}\{(1+j) d / \hat{i}]$

or taking the first threc terms of the expansion $^{4}$

$\left|Z_{m}\right|=R_{m}\left|1-j \frac{d^{2}}{3 \delta^{2}}-\frac{7}{90} \frac{d^{4}}{\delta^{4}}+\ldots\right|$

The absolute value of the transfer impedance is therefore equal to the $\mathrm{d}-\mathrm{c}$ resistance of the annulus to better than

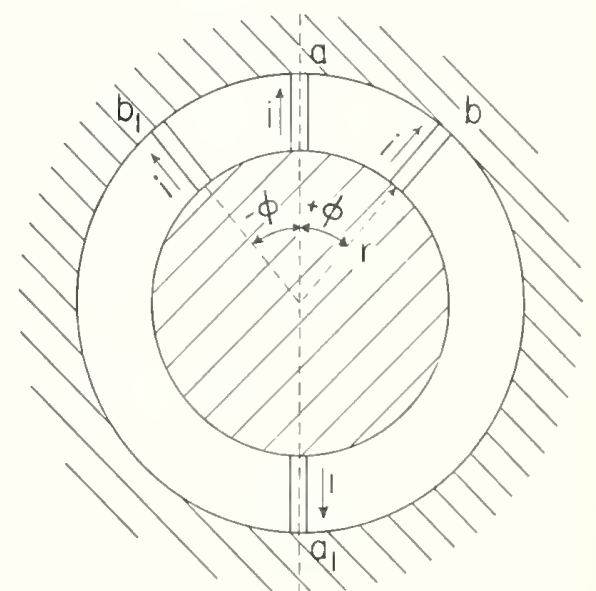

Figure 3. Radial conductor structure approaching that of a solid annular ring 


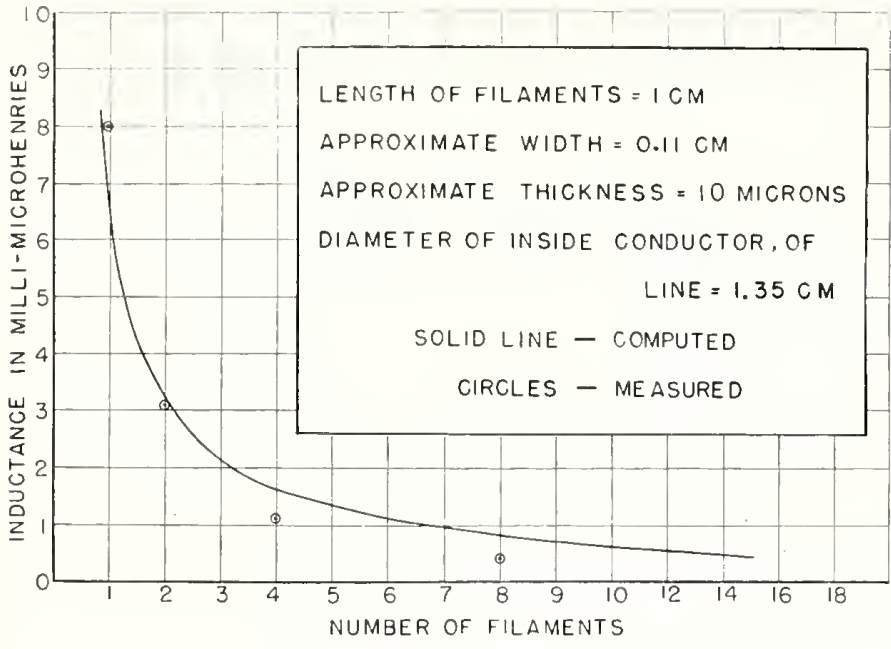

Figure 4 (left). Inductance of a system of tin $\left(S_{n}\right)$ radial filaments short-circuiting the output end of a coaxial transmission line

Figure 6 (right.) Cross sections of interchangeable Micropotentiometer resistance element assemblies. Solid black line indicates the metallic film, part of which is the annular resistor

A. Reversible unit using ceramic insulator. When used with resistance film on the outside of the Micropotentiometer housing, maximum accuracy is assured. When reversed, type $N$ connector is applicable)

B. Unit using glass bushing as insulator

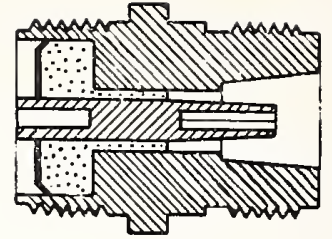

(A)

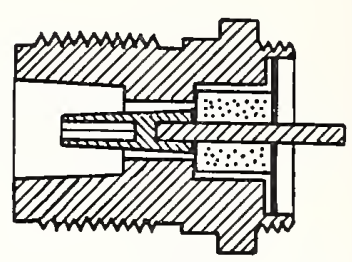

(B)
1 per cent for $d / \delta \leq 0.5$, and

$\left|V_{T}\right|=I_{1}\left|Z_{m}\right| \cong I_{1} R_{m}$

That this is true can also be slown in a simpler but less rigorous manner, as follows.

The input impedance $Z_{m}{ }^{\prime}$ of the line section (Figure 1) having a metal as a dielectric and terminated in an open circuit or in an impedance $Z_{T}>>Z_{0}$ is

$Z_{m}^{\prime} \cong \frac{Z_{0}}{\operatorname{tgh} \gamma_{m} d}=\frac{R_{m}(1+j)^{d / \delta}}{\operatorname{tgh}\left[(1+j)^{d / \delta}\right]}$

For sufficiently small values of $d / \delta$ therefore

$Z_{m}^{\prime} \cong R_{m}$

Since for small values of $d / \delta$ the current in the annulus is uniform over its thickness to better than 1 per cent, the annulus can be trcated as a resistance element is ordinarily treated at direct current, and the voltage drop across it is simply the product of its d-c resistance and $I_{1}$.

As was already mentioned, the succcss or failure of this device depended on the requirement of negligible reactance in the annulus. Therefore all possible substantiating evidence was investigated both analytically and experimentally.

In applying the lumped-circuit approach, the top limiting value of the inductance of an annular conductor carrying radial currents was derived as follows.

The annular ring can be looked upon as consisting of a large number of equal radial conductors of finite length and thickness, all connected in parallel. A square cross section may be chosen for these conductors and a maximum possi-

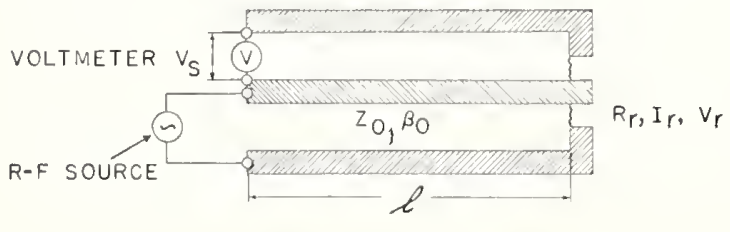

Figure 5. Schematic representation of "coaxial" type Micropotentiometer

inductance of all the elements in parallel, or it may be considerably less than this value because of the additional parallel elements not accounted for, which complete the solid annular ring. Thus

$L_{A} \leqq \frac{d}{2 \pi r}\left(L_{a}{ }^{\prime}+M a a_{1}\right)$ conductors are at right angles to the radial elements and therefore have no mutual inductance with them. The return path for the current in the annulus is assumed far enough away to avoid any influence on the radial elements. Complete penetration of the current, that is, uniform current density in these elements is assumcd at all frequencies. Consequently all deductions based on lowfrequency analysis may be used at the higher frequencies at which current density remains essentially uniform.

Each radial element has self-inductance and mutual inductance with all the other elements. Assuming an even number of elemcnts, for each element $b$ on one side of a given element $a$ there is another element $b_{1}$ located at an angular symmetry which will cancel the mutual effect of $b$ with $a$. Thus the effective inductance of $a$ will be

$$
\begin{array}{r}
L_{a}=L_{a}{ }^{\prime}+M_{a b}+M_{a c}+\ldots+M_{a b_{1}}+ \\
M_{a c_{1}}+\ldots+M_{a a_{1}}=L_{a}{ }^{\prime}+M_{a a_{\mathrm{s}}}
\end{array}
$$

where $L_{a}{ }^{1}$ is the self-inductance of element $a$ and $M_{a a_{1}}$ is the mutual inductance between clement $a$ and $a_{1}$ located diametrically opposite $a$.

Let $d$ be the thickness of the element, and $r$ be the radius of the inner coaxial conductor. Then the total inductance of the annulus, $L_{A}$, will be equal to the

The self-inductance of a square bar is ${ }^{5}$

$$
L_{a}{ }^{\prime}=0.002 l\left[\log \frac{2 l}{0.447 d}-1+\frac{0.447 d}{l}\right]
$$

where $d=$ thickness of the square bar in centimeters and $l=$ its length in centimeters.

The mutual inductance betwcen elements $a$ and $a_{1}$ will have a negative sign. because they are connected in series bucking, and is given by ${ }^{6}$

$$
\begin{array}{r}
M_{a a_{1}}=-0.002[(2 l+2 r) \log (2 l+2 r)+2 r \times \\
\log 2 r-2(l+2 r) \log (l+2 r)] \\
\text { microhenrys }
\end{array}
$$

Computations for a typical annular ring for resistances of the order of one milliohm, having

$l=0.1$ centimeter

$d=2.5 \times 10^{-4}$ centimeters

$r=0.5$ centimeter, result in

$L_{a} \cong 0.45$ millimicrohenry

$M_{a a_{1}} \cong 8$ micromicrohenrys

$L_{A} \leqq 0.035$ micromicrohenry

The series rcactance of this inductance is about 22 microhms at 100 megacycles which seems entirely negligible even at considerably higher frequencies, it being in quadrature with the resistive component. The actual series inductive reactance may be much lowcr, as already indicated, and seems still less important as the annular resistance is increased. The validity of the lumped circuit approacl was verified experimentally Equations similar to 16,17 , and 18 were used to compute the inductances of 
symmetrical systems of radial tin ( $\mathrm{Sn}$ ) foil filaments of a centineter in length and approximate width and thickness of 0.11 centimeter and 10 microns respectively, shorting the output end of a slotted measuring transmission line. The inductances were measured at 200 and 400 megacycles. Figure 4 shows a fairly good agreement between computed and measured values of inductances of these conductors, with a tendency of the measured values to diminish somewhat faster than the computed, with increasing number of filaments.

The only conclusive experimental evidence verifying the preceding analytical predictions was obtained by comparing voltage drops across annular elements with those of a voltage standardization bolometer bridge. ${ }^{7}$ These results will be discussed later. Some further supporting evidence was found in the apparent failure to detect inductance in shorting disks of precision slotted transmission lines. None seemed to have been reported in the past anywhere in the field of slotted lines, resonance lines, nor in coaxial resonant cavities. However the impedance values here under consideration require a precision of measurement of voltage-node displacements of the order of a micron or better; such a precision was hardly available to anyone to date.

\section{Ranges of Voltage and Frequency}

The general conclusion that can be drawn from the preceding is that annular resistance elements of the type described can be used without frequency corrections over the entire ultrahigh-frequency range and higher, and indeed up to the frequency for which the metal-film thickness required is insufficient to maintain a homogeneous, continous metallic medium. This limit (about 0.05 of a micron) indicates that the top frequency of the Micropotentiometer application is dictated by the current indicators and higher modes of propagation rather than by the resistance elements.

At any frequency the resistance of the annulus will remain the same as at direct current to within 1 per cent or better as long as the thickness of the conductive film does not exceed one-half the depth of penetration at that frequency for a thick conductor of the same material. If the film thickness is equal to the full depth of penetration, there may be an error of about 3 per cent in the $r-f$ output voltage. It is necessary to select materials of proper conductivity and choose annular diameters of proper ratios and practical values to obtain various resist- ances desired to a given top frequency. One might call attention to the fact that the resistance is a function of diameter ratios and not of dianeter values. Thus annular resistances of a fraction of a milliohm to $1 \mathrm{ohm}$ and even higher may be used over very wide frequency ranges.

The voltage range of a given resistance element is clearly a function of the power it can dissipate in a particular physical arrangement. However, the power levels in question are generally of such a low level and the annular films are in such close proximity to large and efficient heat conductors that the voltage range of a Micropotentioneter with a given resistance element seems limited at the present time by the current monitoring elements rather than by power dissipation of the resistance films. No appreciable resistance changes were observed on 1- to 10-milliohm silver elements for currents up to an ampere.

Any means to indicate current accurately and independentiy of frequency are usable with the Micropotentiometers provided they can be physically located in such a manner that the same current is passing through the annular resistor as through the current monitor. This condition hecomes more difficult to fulfill as frequency is increased. However, the fact that the annular resistances are very low renders the application of conventional $r-f$ current indicators considerably less critical than in their application with high resistance elements. Thus thermoelements and thermistors were successfully used, as will be shown later, and other bolometer type and thermoelectric elements are applicable for frequencies approaching 1,000 megacycles and perhaps higher.

The low limit of the voltage range is controlled by the smallest practical diameter ratios of the annular elements, by the conductivity of the materials, by the accuracy with which low d-c resistance values can be determined as well as by the accuracy with which low current values can be measured. For frequencies up to the very-high-frequency range the low limit to date seemed of the order of 0.1 of a microvolt; for higher frequencies it was of the order of 1 microvolt.

\section{General Design Features}

In designing assemblies of components and housing of Micropotentiometers only one somewhat critical requirement has to be fulfilled. The resistance films comprising the annular elements and having various thicknesses down to a micron and lower have to be in perfect continuous contact with the outside and inside conductors and must remain mechanically rugged and stable. Other requirements are of the ordinary variety which can be readily met. One of these requirements concerns the proximity of the current monitoring element to the annulus and is not critical because there is a current loop at the annulus; the current falls off as a cosine function with distance towards the current monitor (or $\mathrm{r}$-f power source). This feature tends to equalize the current distribution along heaters of thermoelements as well, and thus extends the frequency range. For example, a distance of 2 centimeters from the resistive film to the current indicator may cause a 1-per-cent error at 300 megacycles. With thermistors as current monitors this question seems of no consequence, even over the entire ultraligh-frequency range since thermistor beads having diameters of 15 mils can be placed right at the annulus.

Another noncritical requirement concerns the length of the output coaxial connector of the Micropotentiometers. This connector may be considered an integral part of the feeder supplying the standardizing voltage to a receiver or any other monitor. For best results the annular resistance film should be placed in the output plane of the Micropotentiometer and the connectors to the monitor and to the equipment being calibrated in terms of the Micropotentiometers should be mechanically matched. In case this cannot be done, then the annular resistance film should be placed as close as possible to the output plane. The accuracy will then depend on the length of the Micropotentiometer connectors and the input impedance to the monitoring feeder. To be more specific, the output of the Micropotentiometer may be affected in two ways. The first is the actual increase of the shunt admittance across the annulus; since the resistance of the latter is very low the probability of trouble from this cause is remote. The second is the transformation action of the short connector from the resistance film to the physical output plane which may contribute an error of several per cent in the ultrahigh-frequency range, depending on the monitor-feeder impedance. In the extreme case, if that impedance is infinite, there will be a cosinusoidal reduction of the voltage along the short connector as one travels from the output plane towards the annular film; for cxample, at 300 megacycles a connector 1.5 centimeters in length will have a voltage about 1 per cent higher at the output plane as compared with 


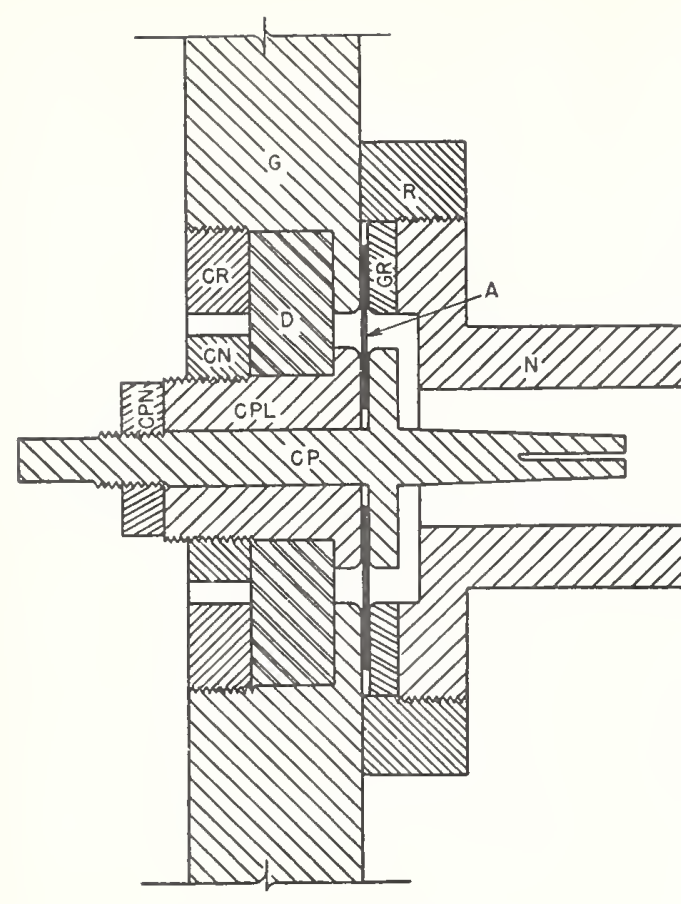

the standardizing voltage across the annulus. As the monitoring-feeder input impedance decreases, this error will be reduced and will be zero when this impedance is equal to the characteristic impedance of the connector.

One might point out here a practical advantage of these devices for universal standardization purposes. A known resistor of reliable $r-f$ characteristics may be used as the coaxial center conductor of the output terminal. This would form a well-defined readily reproducible impedance of the source of standardizing $r-f$ voltages when reliable voltage generators with given ontput impedances are required. Conventional standard "dummy antennas" may of course be used instead, with full certainty that the source impedance in this case is that of the dummy antenna only.

\section{Types of Micropotentiometers}

The basic principle of the Micropotentiometers lends itself to balanced circuit
Figure 7 (left). Cross section of interchangeable Micropotentiometer resistance element assembly. Solid black line indicates resistance ring clamped between electrodes

Figure 9 (right). Micropotentiometer employing a thermoelement as a current indicator

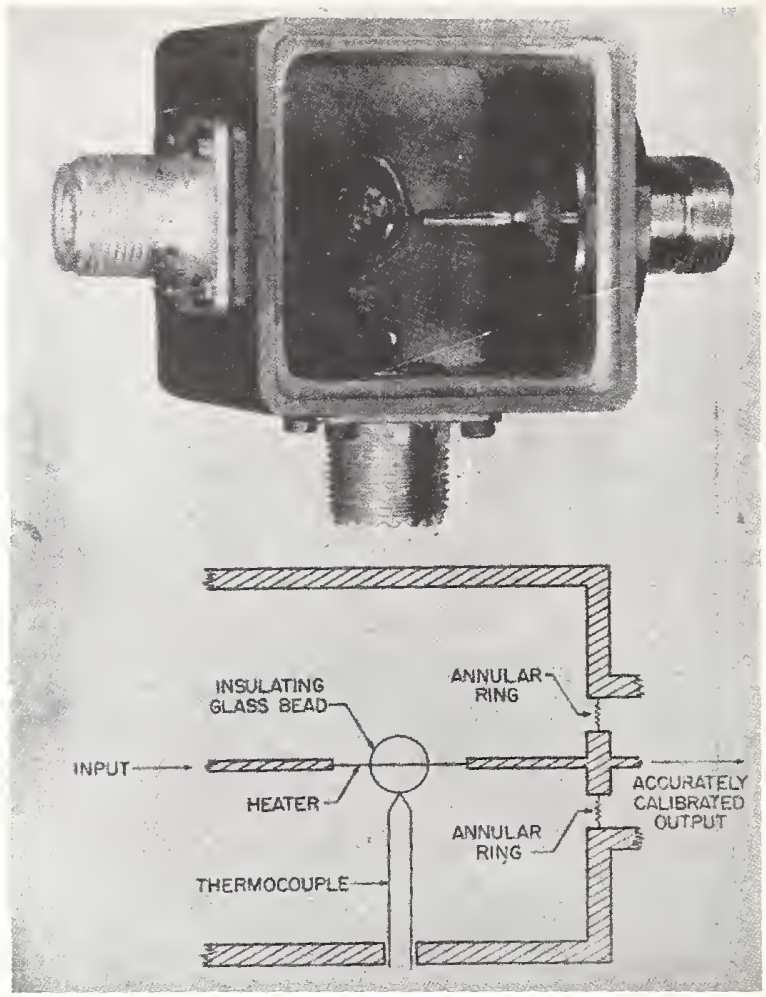

applications as well as to unbalanced. Since development work, so far, has been limited to unbalanced requirements, only the latter are discussed here.

The only other classification of these devices as to general type, aside from the preceding, is based on current measuring methods. These affect the basic physical structure of the units. All units using thermoelements may be essentially of the same construction and may have interchangeable annular and current indicating elements to cover a wider voltage range. Insulated-type thermoelements preferably should be used. In this type the structure requires only the thermocouple output terminals in addition to $r-f$ input and output terminals. On the other hand, types employing bolometers (for example thermistors) or directly heated thermo-
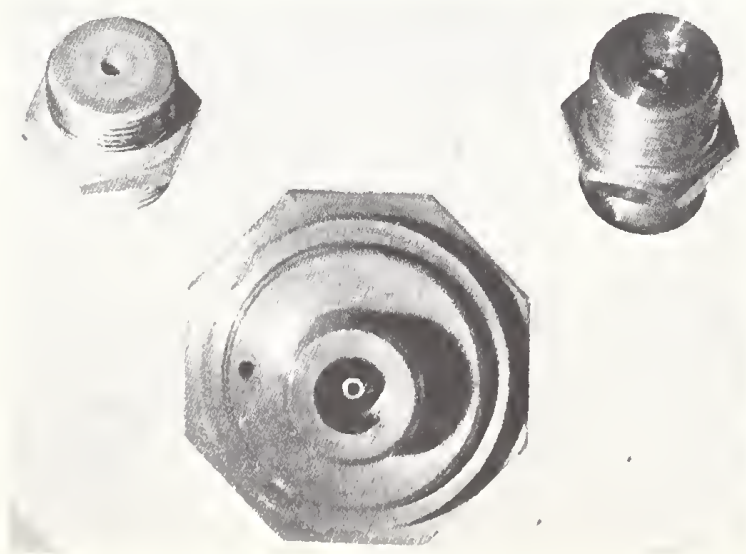

Figure 8. Micropotentiometer interchangeable resistance assemblies

A, B. Units using glass or ceramic bushings and evaporated and plated or fired-on films

C. Unit using clamped carbon disk

couples require either $\mathrm{r}-\mathrm{f}$ chokes or $\mathrm{d}-\mathrm{c}$ blocking condensers, and in some cases, both.

One type, radically departing from the preceding and having some desirable features, is a Micropotentiometer using a reliable voltmeter instead of a current indicator. Figure 5 shows this modification schematically. R-f power is fed into a section of an air or solid dielectric coaxial transmission line having negligible losses. A calibrated vacuum-tube voltmeter connected at the input to the line measures the input voltage. The output end of the line is terminated in a solid metallic disk containing an annulus of one-half the penetration thickness at the highest frequency of interest. Then, to a very good approximation

$V_{s}=I_{\tau} Z_{0} \sin \beta_{0} l$

and

$$
\begin{aligned}
V_{T} & =I_{T} R_{T}=V_{3} \frac{R_{\tau}}{Z_{0}} \csc \beta_{0} l \\
& =V_{S} K_{1} \csc K_{2} f
\end{aligned}
$$

where

$R=$ the resistance of the annulus, ohms

$V_{T}$ and $V_{S}=$ the output and input voltages respectively, volts

$I_{r}=$ the current in the short circuit, amperes $Z_{0}=$ the characteristic impedance of the line, ohms

$\lambda_{0}=$ the wave length in the line, meters

$\beta_{0}=2 \pi / \lambda_{0}=$ phase constant of the line

$l=$ length of the line in meters

$f=$ frequency in cycles per second

Since $K_{1}$ and $K_{2}$ are both constants 
for a given air or solid dielectric line, one can compute and plot $V_{\text {r versus fre- }}$ quency for $V_{s}=1$ volt to facilitate the determination of $V_{r}$ over wide frequency and voltage ranges. A further simplification is obtained by using check frequencies for which the line length is an odd multiple of $\lambda_{0} / 4$, in which case

$$
V_{T}=\frac{R_{r}}{Z_{0}} V_{S}
$$

It can be shown that equation 21 holds for any value of $R_{r}$, thus a wide range of voltages is available at the output of this type of Micropotentiometer: for example, with $Z_{0}=50$ ohms, $V_{s}=0.1$ volt and intcrchangeable $R_{T}$ elements of 0.5 to 100 milliohms, one can obtain $V_{r}$ values of 1 to 200 microvolts.

\section{Practical Micropotentiometers and Verification of Performance}

The ideal Micropotentiometer should have a construction whereby the annular resistance element constitutes an integral part of the inner or outer surface of the relatively heavy metallic enclosure. There are numerous ways to approach this ideal and many of them were tried with various degrees of success. Details of construction and fabrication processes are beyond the scope of this discussion. However, three different satisfactory methods will be indicated on the basis of up-to-date experience. Low resistance elements, of the order of 0.2 to several hundred milliohms, were constructed in one of two ways. First, ceramic or glass cylinders were sealed as insulators between the two concentric conductors. For this sealing, metallic paints or pastes (silver, platinum, gold) were fired on the cylindrical surfaces of these insulators; the actual sealing was accomplished by soldering. An annular resistance element was then formed at one end of the coaxial assembly by evaporating and plating a given metal over its entire surface. Second, the same process was used as in the first case except that the metallic paints or pastes were also fired on one of the ends of the glass or ceramic insulators to form the annular resistor; evaporation and plating were thereby eliminated. A third method was applied successfully only to elements of the order of 1 ohms in resistance; carbon (deposited on bakelite) disks were clamped in a solid coaxial assembly; silver painted rings were used as conducting electrodes for the disk at the clamped surfaces. One modification of the low resistance elements made use of commercial Kovarglass sealed terminals; metallic films fired on, or evaporated and plated over one sicle of the terminals formed the resistance elements; the terminals were then soldered into an appropriate housing. Figures 6,7 , and 8 show cross-sectional and photographic views of these elements and Figure 9 shows an assembled unit employing a thermoelement.

Once a Micropotentiometer is assembled, all that is necessary in order to determine its voltage output is to measure the $\mathrm{d}-\mathrm{c}$ resistance of the annular film and to calibrate the current indicating element on direct current. However, in the up-todate stages of this development, it was necessary to verify the results in terms of other independent methods at all frequencies. Reliability of new units can, of course, in the future be checked against older units with little difficulty. Agreement tests were conducted against the voltage standardizing bolometer bridge, mentioned previously ${ }^{7}$ and precision wave guide below cutoff attenuators. These tests indicated agreement well within over-all experimental errors, that is \pm 1 per cent to about 50 megacycles and \pm 3 per cent to 300 megacycles. Measurements at higher frequencies have been conducted so far only on the clamped 1ohm units and resulted in agreements of \pm 5 per cent to 900 megacycles.

\section{Application of Micropotentiometers}

Though the primary objective of these Micropotentiometers is to eradicate the wide uncertainty in the absolute values of voltages in the microvolt range, they can be used for numerous other purposes. The more obvious applications may be briefly indicated as follows:

1. Reference standard for accurate voltages in the range previously indicated for calibration of voltmeters, signal generators, field intensity meters, and so forth. The voltage and frequency range seems to be limited primarily by current-indicating facilities.

2. Sources of accurate voltages for direct use in place of conventional signal generators. The user has complete freedom to vary the internal impcdanec of these sourees for various requirements with a high degree of certainty in the values of this impedanec. A compaet assembly incorporating an oseillator and Mieropotentiometer may be constructed as a standard voltage (signal) generator.

3. Calibration of attenuators direetly in terms of voltage ratios.

4. Determination of performance of current indicators, such as of various types of thermoelements.

5. Determination of output impedanees of $r-f$ sources. Resistanee elements of the Mieropotentiometers may be used to shortcircuit the output of a source; the voltage output of these elements is then a measure of the short-circuited eurrent of the sourcc and eonsequently of its internal impedance.

6. Calibration of modulation meters. This applieation is common to all devices having an accurately known eorrelation between output and percent modulation, for example, the output of the eouple of the thernoelement. Attention may be called here to the fact that presence of harmonics in the $r$-f carrier will normally have a negligible effeet on the conple output, whereas the effcet may bc very appreeiable on a vaeuumtube voltmeter usually employed with conventional voltage generators.

7. Applications where voltages in the mierovolt range are desired without the usual Johnson noise of higher imperlancc sources present.

8. Applications where eonstant voltage sources are required, for example for $Q$ measurement circuitry.

9. Applications where a single device to cover the entire frequency range from zero to 300 megaeyeles and higher is esscntial.

\section{References}

1. Radio-Frequency Micropotentiometers. Technical News Bulletin, National Bureau of Standards (Washington, D. C.), volume 35 , March 1951 , pages $33-3 t$

2. Electromagnetic Wayes (book), S. A. Schelkunoff. D. Van Nostrand Company, Inc., New York, N. Y., 1943, page 280.

3. Networks, Lines and Fields (book), J. D. Ryder. Prentice Hall, Inc, New York, N. Y., chapters 8 and 10,1949

4. Tables of Complex Hyperbolic and Circi lar Functions, A. E. Kennelly. Harvard Univer sity Press (Cambridge, Mass.), second edition, 1927, page 231

5. Inductance of Linear Inductors, E. B. Rosa. Bulletin, National Bureau of Standiards (Washington, D. C.), volume 4, number $2,1907-08$ page 313 .

6. Circular C77, National Bureau of Stanciards (Washington, D. C.), 1937. page 270.

7. A Bolometer Bridge for Standardi\%ing Radio-Frequency Voltmeters, M. C. Selby, L. F. Behrent. RP 2055, Journal of Research, National Bureau of Standards (Washington, D. C.) volume 44, 1950, page 15.

\section{Discussion}

Donald M. Hill (Boonton Radio Corporation, Boonton, N. J.): The micropotenti- ometer appears to be a worth-while contribution. Some time after the micropotentiometer was announced we had an application for it as the coupling impedance in a $Q$ meter. After conferring with Mr. Selby concerning the methods of construction, we constructed a number of the resistors by firing a platinum-gold alloy on ceramie dises. These were tested over the frequency range from zero to 50 megaeyeles and found to be 
surtably free of inductance.

The temperature coefficient of the combination of an annular resistor and a thermocouple was found to be 0.11 per cent per degree centigrade

One point which has perhaps not been sufficiently emphasized by Mr. Selby is that in order for the inductance to be negligibly low the resistive film must have complete axial symmetry. Until a manufacturing method can be devised which insures perfect symmetry, it will be necessary to test each resistor individually.

Myron C. Selby: The author wishes to thank Dr. Hill for his encouraging discussion. One can hardly question Dr. Hill's emphasis of the axial symmetry of the resistive film because it is a basic requirement of this development. Unfortunately we at the National Bureau of Standards have had no opportunity as yet to correlate degree of departure from symmetry with accuracy. Annular elements of superior symmetry were fabricated by means of evaporation and plating. However, there was no evidence to date that this fabrication resulted in units of higher average accuracy compared with others. 


\title{
Development of Very-High-Frequency Field-Intensity Standards*
}

\author{
By Frank M. Greene and Max Solow
}

\begin{abstract}
A description is given of the development of two very-high-frequency field-intensity standards, which are being used at this Bureau for the calibration of commercial fieldintensity sets in the range 30 to 300 megacycles. These standards are employed to establish known values of field intensity by either of two methods: (a) the standard-antenna method in which the open-circuit voltage at the center of a receiving dipole is measured directly; (b) the standard-field method in which the current at the center of a transmitting dipole is accurately known. The techniques used for determining the antenna current and voltage are described.

The current distribution on the antenna is determined theoretically, using Schelkunoff's method, which gives the effective length. These values are compared with those obtained by measurement.

Results of field tests at 100 megacycles are presented in which the above two methods were directly intercompared using horizontal polarization. Their accuracy and limitations are discussed.
\end{abstract}

\section{Introduction}

For a number of years the National Bureau of Standards has maintained among its other public services, the calibration of commercial field-intensity meters. These instruments are largely used by engineering consultants and broadcast engineers, in order to determine a radio station's antenna efficiency and coverage area, acceptable values of which are specified by the Federal Communications Commission. The frequency range of this calibration service has in the past extended to approximately $30 \mathrm{Mc}$ and is maintained by the Bureau's Central Radio Propagation Laboratọry.

More recently, as a result of the greatly increased use of the very-high-frequency (VHF) band (30 to $300 \mathrm{Mc}$ ) for frequency-modulation and television broadcasting and other communication purposes, the Central Radio Propagation Laboratory has undertaken to extend the frequency range of this calibration service to include these frequencies.
This necessitated the development of accurate field-intensity standards for calibration purposes. Such standards are usually employed to determine a value of field intensity in terms of either a known voltage or current in an antenna, together with certain geometrical relationships involved, such as the antenna length, distance of separation, etc. The standards developed are similar in principle to those already in use at the lower frequencies. However, it was necessary to modify the techniques employed for the measurement of antenna current and voltage as well as the over-all technique in the use of the standards in order to adapt them to use at the higher frequencies.

In many instances the accuracy with which the value of a physical quantity may be determined increases with the simplicity of the method used to make the determination. Such measurements usually involve a theoretical calculation, or an experimental observation, or both. In establishing the field-intensity standards discussed herein, the methods adopted for current and voltage measure-

*A summary of this paper was presented at the joint meeting of the International Scientific Radio Union and the Institute of Radio Engineers at Washington, D. C , on May 2, 1949. 
ment were purposely made as elementary as possible for this reason. It is believed that this enables the achievement of the maximum aceuracy possible or practicable at the present time. This of necessity results in some limitation of the range of measurement and flexibility of use, but the premium paid results in increased accuracy.

The standards or methods used to establish accurately known values of VHF ficld-intensity are (a) the standard-antenna method and (b) the standard-field method.

It is the purpose of this paper to describe two such experimental standards in some detail, the techniques involved for measuring the antenna voltage or current, as well as the propagation tests made to intercompare the field-intensity values obtained by both methods. The accuracy and limitations of the two methods are also discussed.

This work done by the authors represents one phase of a program for the development of accurate VHF ficld-intensity calibration standards. Although these standards were developed to cover the entire VHF band, the initial propagation tests described herein were made at a frequency of $100 \mathrm{Mc}$, using horizontal polarization only; and the standard antennas were horizontal halfwave self-resonant dipoles in all cases.

No claims are made as to the originality of the individual methods or techniques used, as most of these have been reported elsewhore. However, the standards diseussed here are novel at least in their simplicity and in the accuracy obtainable. Practical rationalized mks units are used throughout this paper.

\section{Standard-Antenna Method}

\section{Relation Between Field-Intensity and Induced Voltage}

The magnitude of the electric component of VHF field-intensity existing at a given point in space may be determined in terms of the voltage, $\mathrm{I}_{\text {oc }}$, indueed in a standard receiving dipole immersed in the field, together with the antenna geometry. It will be assumed in the following that the antemua is a horizontal half-wave dipole oriented for maximum response, and that the voltage is referred to the center terminals. In case the antenna is not open-circuited, the voltage measured, $V_{L}$, is of course not the induced voltage but is related to it by the voltage-transfer ratio,

$$
\eta \equiv\left|\begin{array}{c}
V_{L} \\
V_{o c}
\end{array}\right|=\frac{Z_{L}}{Z_{A}+Z_{L}},
$$

where $\eta_{L}$ is the load imperlance, in ohms, connected to the antenna terminals, and $Z_{A}$ the antenna input impedance in olims.

The magnitude of the electric component of field-intensity (in volts per meter) is related to the induced voltage by

$$
|E|=\frac{V_{o c}}{l_{H}},
$$

where $l_{H}$ is the effective length of the antenna in meters. ${ }^{1}$

\section{Effective Length of the Antenna}

Assuming a sinusoidal current distribution on the antenna, the effective length (in meters) is

$$
l_{H}=\frac{\lambda}{\pi} \tan \frac{\pi l}{\lambda},
$$

where $\lambda$ is the wavelength in meters, and $l$ the antenna half-length in meters.

For a half-wave dipole the effective length (in meters) reduces to

$$
l_{H}=\frac{\lambda}{\pi}
$$

As is known, the current distribution on an antenna is not exactly sinusoidal except for an infinitely thin filament. For cylindrical antennas of radius $a$ and half-length $l$, the departure from a sinusoidal distribution becomes progressively greater as the ratio $2 l / a$ decreases (i. e., as the antenna gets fatter).

An approximate solution for the free-space current distribution on a cylindrical transmitting antenna ${ }^{2}$ has been obtained by Schelkunoff [1] ${ }^{3}$ among others, and is given by the sum of eq 34 and 35 in the appendix. The relative distribution

\footnotetext{
'It is assumed here (and our results seem to indicate) that the relative eurrent distribution and hence the eflective length is not (to a first approximation) a function of either the height of the antenna above the ground or the terminating impedance, $Z_{\boldsymbol{l}}$.

${ }^{2}$ How this is handled in the case of the receiving antenna has not been made too clear in the literature, but the difference between the two cases is apparently small for moderately thin antennas. The reader is referred, for instance, to J. C. Slater, Microwave transmission, pp. 219 to 230 (McGraw-Hill Book Co., Inc., New York, N. Y., 1942).

3 Figures in brackets indicate the literature references at the end of this paper.
} 


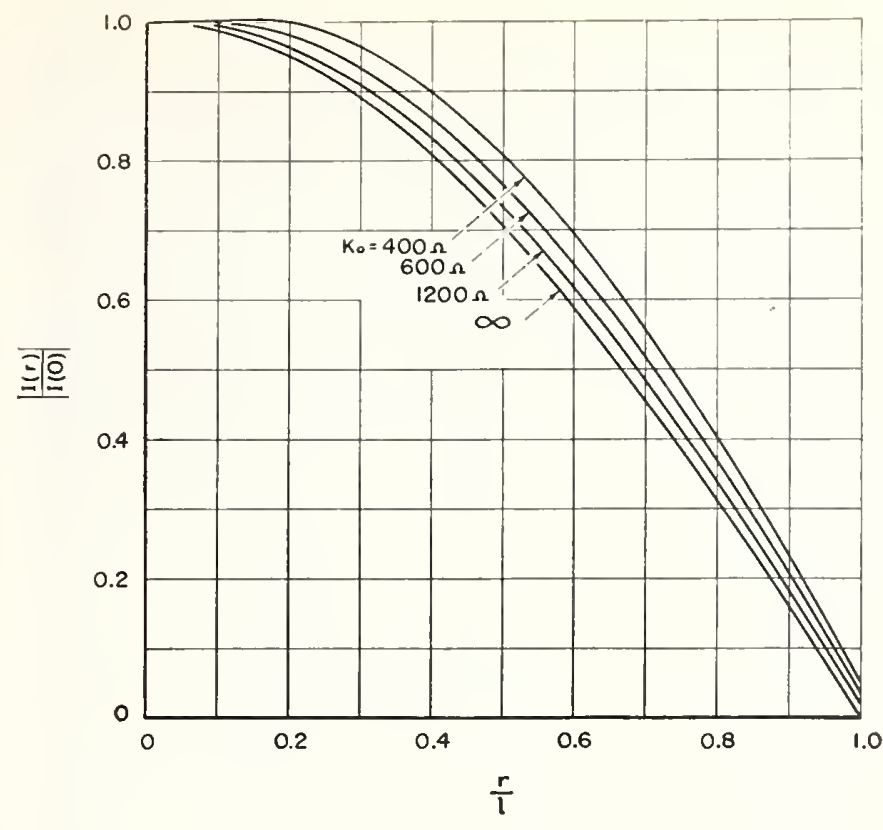

FIGURE 1. Theoretical free-space relative current distributions on a cylindrical half-wave dipole as determined from Schelkunoff's anolysis.

The relative current $I(r) / I(0)$ is shown versus the fractional balf-length $r / l$ for several values of the average characteristic impedance, $K_{0}=120\left[\log _{\diamond}(2 / / a)-1\right]$, as determined from the sum of eq 34 and 35 in the appendix.

is a function of both the phase length $2 \beta l$, and the ratio $2 l / a$, and may be represented by

$$
\frac{I(r)}{I(0)}=f\left(\beta l, K_{0}\right)
$$

where

$I(r)=$ current in amperes at a distance $r$ meter's from the center of the antenna $I(0)=$ current in amperes at the center of the antenna $(r=0)$

$K_{0} \equiv 120\left(\log _{e} \frac{2 l}{a}-1\right)$ ohms, and is the average cliaracteristic impedance over the half-length $l$

$\beta=2 \pi / \lambda$.

The relative current distributions as determined from the magnitude of the sum of eq 34 and 35 are shown in figure 1 for half-wave dipoles for several values of $K_{0}$. Because of the increasing complexity of the higher order Bessel functions in the infinite series of eq 35, only the first two terms of this series were evaluated. This accounts for the fact that the current as shown in figure 1 does not reduce quite to zero as it should at the end of the antenna, $r / l=1.0$. The contribution from the neglected terms in this series is appreciable only near the ends of the antenna, so that this approximation does not appreciably affect our results here.

The effective length of the antenna (in meters) may be determined from the relative current distribution by the relation

$$
l_{H}=\int_{-l}^{l}\left|\frac{I(r)}{I(0)}\right| d r
$$

The magnitude of the sum of eq 34 and 35 may be substituted in eq 6 and the integration performed either mathematically or mechanically. We preferred the latter as being less involved.

In this manner a number of values of effective length, $l_{H}$, were determined for various values of the average characteristic impedance, $K_{0}$, between 400 and 1,200 ohms. The percentage increase in $l_{H}$ over that of an infinitely thin filament, $K_{0}=\infty$, is shown versus $K_{0}$ in figure 2 for lialf-wave dipoles. ${ }^{*}$

The agreement between calculated and observed values of $l_{H}$ for the antenna used will be discussed in section IV.

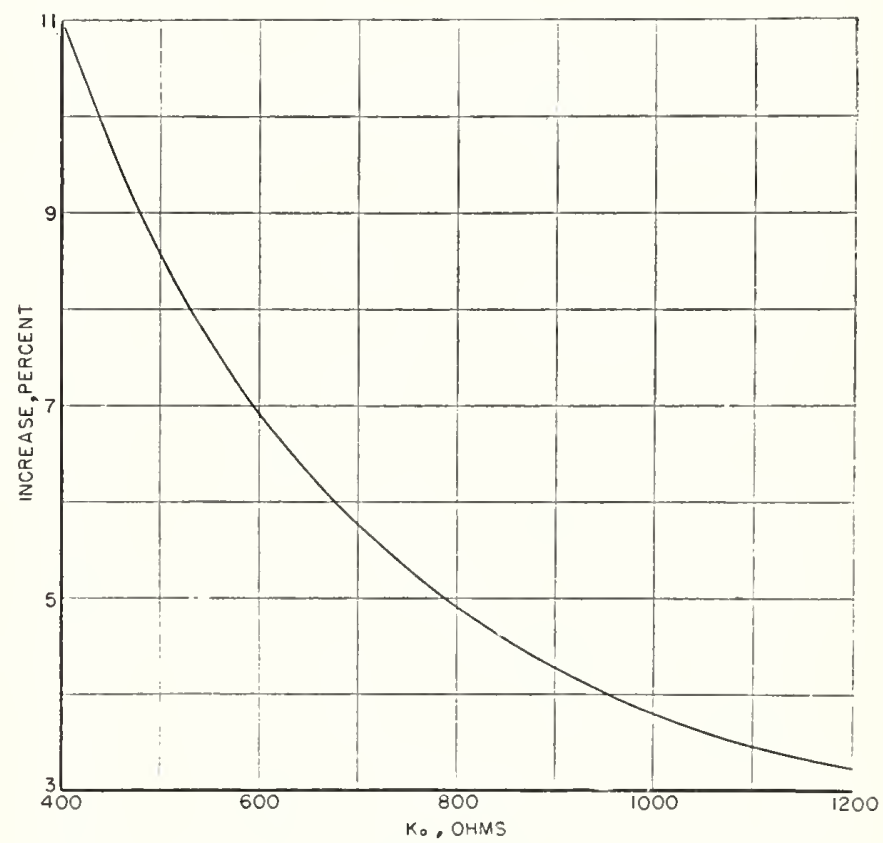

FIGURE 2. Percentage increase in effective length of a cylindrical half-wave dipole (over that of an infinitely thin filament) versus the average characteristic impedance, $K_{0}$, as determined by substituting the sum of eq 34 and 35 in 6.

4 The percentige increase in $/ H$ for the case of thin self-resonant dipfle antennas was found to be rery nearly the same as that for full half-wave dipoles for the ralues of ho usually encountered. 
In order to operate a half-wave dipole at selfresonance it is necessary, as is known, to shorten its length somewhat from a full half-wavelength.

Schelkunoff [2] and King [3] have obtained independent approximate solutions yiclding the required slortening for self-resonant operation of thin cylindrical antemnas. Although obtained by radically different methods, the ralue of the required shortening as determined from Schelkunofl"s solution is in substantial agreement with that obtained from King's second-order solution, and is given by

$$
\frac{4 l}{\lambda}=1-\frac{60 S i(2 \pi)}{\pi K_{0}}=1-\frac{27.0 S}{K_{0}}
$$

where $\operatorname{Si}(x) \equiv \int_{0}^{x}(\sin t / t) d t$ and $\operatorname{Si}(2 \pi)=1.41815$.

Talues of the percentage shortening determined from eq 7 are shown versus the average characteristic impedance of the antenna $K_{0}$, in figure 3 .

\section{Antenna Voltmeter}

The standard receiving antenna used was a selfresonant (half-wave) dipole shortened approximately 4 percent from a full half-wavelength ( $f=$ $100.0 \mathrm{Mc}$ ) (as determined from fig. 3) to make it self-resonant. Dural tubing $3 / 16 \mathrm{in}$. in diameter was used, giving a ratio $2 l / a \cong 600$, and an average characteristic impedance $K_{0}=120 \quad\left[\log _{e}(2 l / a)-1\right]$ $\cong 650 \mathrm{ohm}$. The induced voltage referred to the center terminals of the antenna was measured directly by means of a relatively high-impedance balanced roltmeter comnected across the gap at the center. In this manner the necessity for a separate measurement of the antemna input impedance was eliminated, which greatly simplified. the problem. The roltmeter consisted of a modified type $1 \times 2 S$ silicon-crystal rectifier built into the gap as shown in figure 4 . The crystal output was filtered by means of a balanced resistancecapacitance network, and the d-c output voltage was measured directly against a standard cell on a special precision slide-wire potentiometer. The central portion of an experimental receiving antenna assembly minus the antenna rods is shown in figure 5. The input impedance of the resistance-capacitance filter was approximately 10,000 ohms in shunt with $0.05 \mu \mu f$. Highfrequency resistors were cmployed, which were a vailable commercially.

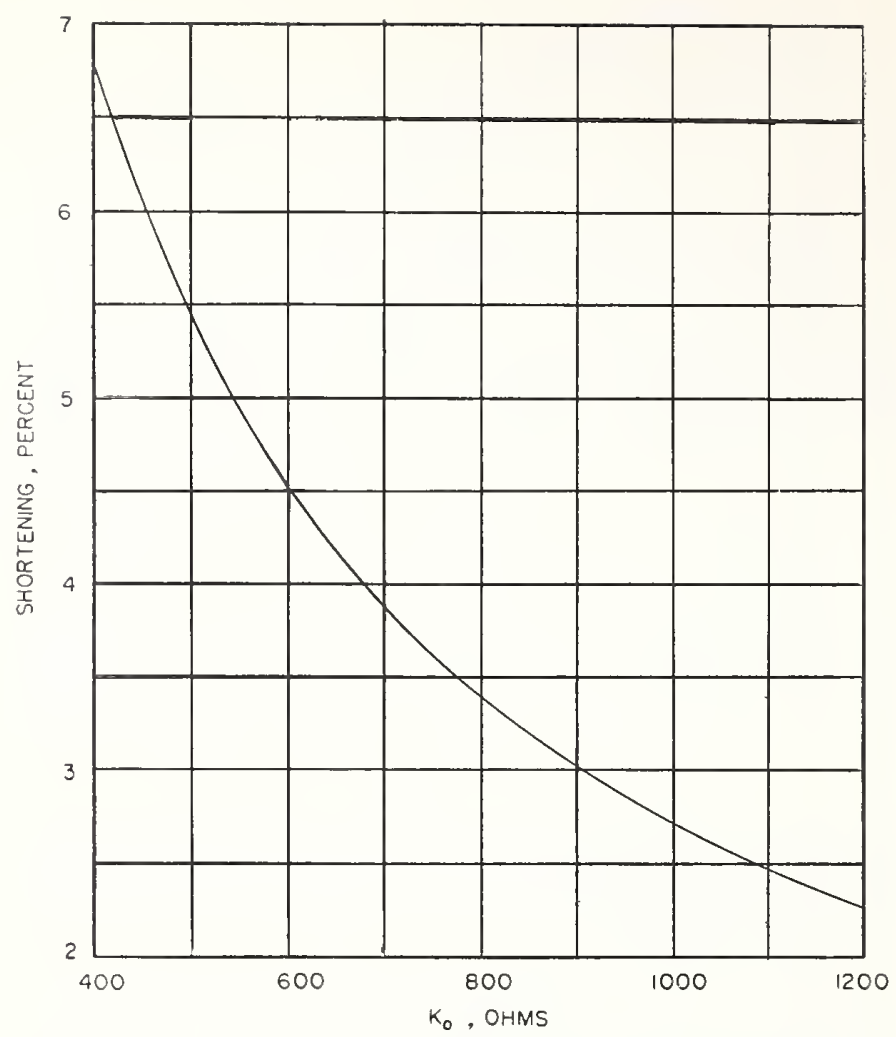

Figure 3. Pereentage shortening of a eylindrieal half-waie dipole required for self-resonant operation versus the average characteristic impedanee, $K_{0}$, as determined from eq.

The effective shunt capacity of the crystal diode was less than $0.75 \mu \mu \mathrm{f}$. Howerer, since the resulting reactance of some 2,000 ohms was in quadrature with the radiation resistance of the antenna, the resulting shunting error from this source was a small fraction of 1 pereent.

In selecting the crystal rectifier it was necessary to consider both its r-f (input) resistance and its
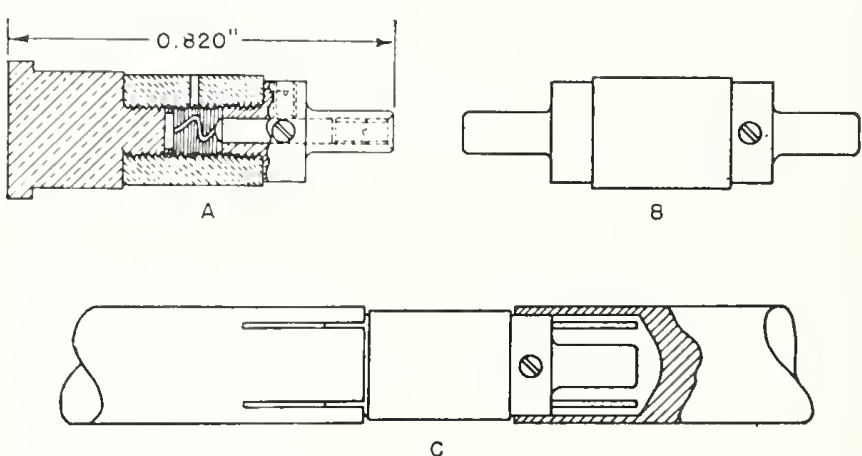

Figure 4. Method used to mount the type 1 N2S silicon crystal rectifier in the gap at the center of the half-uave standard receiving antenna.

$A$, Western Electric 1 N28 silicon crystal rectifier; $B, 1 N 28$ as modified; $C$, modified $1 \Upsilon 28$ as mounted in the antenna gap. 


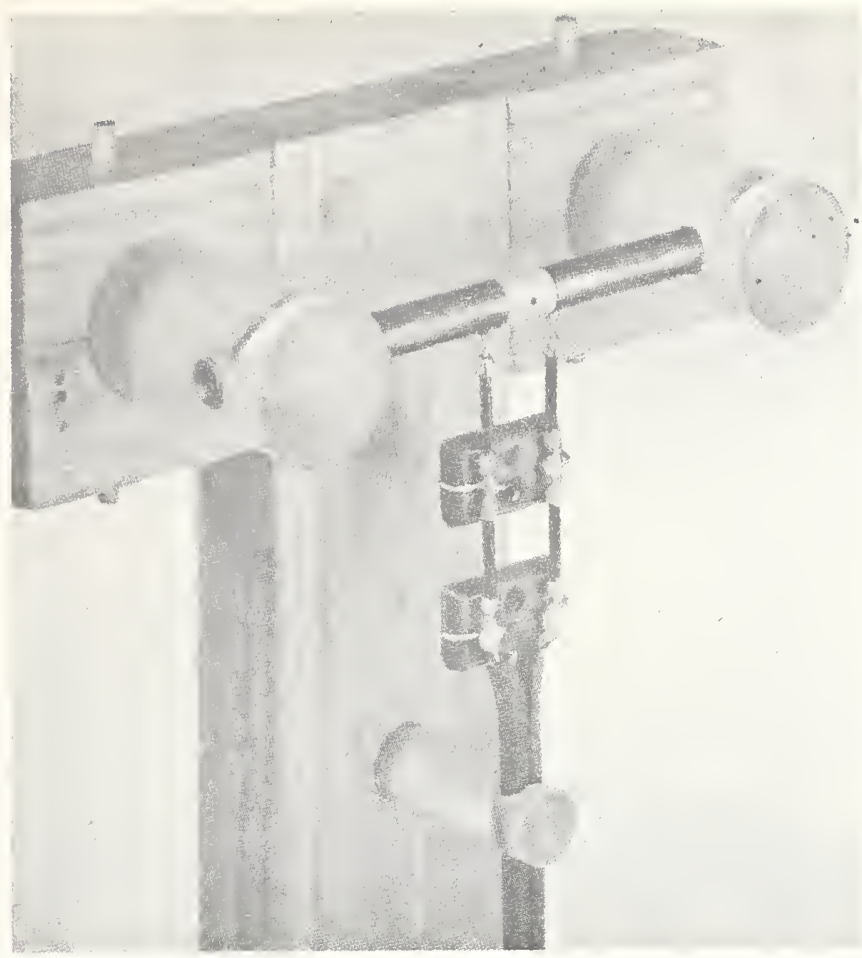

Figure 5. Tiew of the central portion of an experimental standard receiving antenna minus the antenna rods showing the crystal rectifier and resistance-capacity filter network.

(internal) d-c-output resistance. Both are a function of the applied r-f-roltage level, decreasing with increasing level, although not necessarily at the same rate.

In order to limit the shunting caused by the presence of the crystal voltmeter in determining the open-circuit antenna voltage, it was necessary to select a crystal having as high a value of $r-f$ resistance as possible. On the other hand, to maintain as high a sensitivity as possible in measuring the d-c-output roltage of the crystal, it was necessary to select a crystal with a relatively low value of $d$-c-output resistance.

A satisfactory compromise was obtained by selecting a crystal having an $r-f$ resistance between 8.000 and 10,000 ohms when measured at an $r-f$ lerel of $0.1 \mathrm{r}$ and self-biased. This resulted in a shunting error of less than 2 percent in determining the induced antenna voltage. It was found in gencral that the type $1 \mathrm{~N} 28$ crystals came closest to meeting these requirements.

The variation of both the $\mathrm{r}-\mathrm{f}$ resistance and the I-c-output resistance versus applied $r-f$ voltage at $100 \mathrm{Nc}$ is shown in figure 6 for the $1 \times 28$ crystal selected when operated self-biased. These crystal characteristics are not representative of the $1 \times 28$ crystals in general, as it was possible to find many with considerably higher or lower values of $\mathrm{r}-\mathrm{f}$ and $d-c$ resistance than the values shown. The variation among other crystals of types $1 \mathrm{~N} 21$ to $1 \mathrm{~N} 27$ was found to be even greater.

The $r-f$ input resistance of the 1 N28 crystal was measured by a resistance-variation method [4] using several high-frequency resistors having widely separated values as cross checks in determining each value of crystal resistance. The crystal was connected in series with a $200-\mu \mu \mathrm{f} \mathrm{d-c-}$ blocking condenser across an antiresonant circuit during these measurements. The d-c-output voltage acting as self-bias on the crystal was developed across this condenser.

The $d$-c-output resistance of the crystal is the equivalent internal dynamic source resistance of the d-c-output circuit. This is sometimes referred to as the $\mathrm{d}-\mathrm{c}$, or video, impedance [5] and may be defined (in ohms) as

$$
R_{i} \equiv \frac{\Delta e}{\Delta i}
$$

where $\Delta e=$ a small change in the d-c balancing voltage of the slidewire potentiometer and $\Delta i=$ the resulting change in the $\mathrm{d}-\mathrm{c}$ current in the output circuit. If $\Delta e$ is kept smaller than about $5 \mathrm{mv}$, when measuring $R_{i}$, either a positive or a negative value of $\Delta e$ will result in very nearly the same value of $R_{i}$.

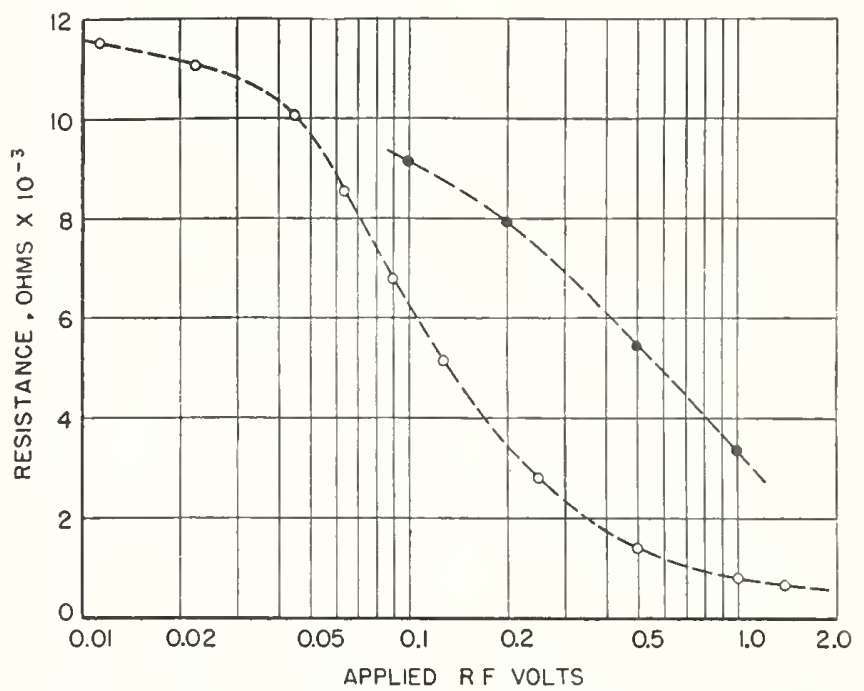

Figure 6. Tariation of the $r-f$ input resistance and dynamic $d-c$ output resistance as a function of the applied $r-f$ voltage level for a selected type 1 N28 silicon crystal rectifier.

The measurements were made at 100 Mc with full self $\mathrm{d}$-c bias applied to the crystal. These curves are not representative of the type $1 \mathrm{~N} 28$ crystals in general. $\bigcirc$, d-c output resistance; $0, r-f$ resistance. 
The d-c output resistance of the crystal was determiner in this manner for a number of $\mathrm{r}-\mathrm{f}$ voltage levels. Thr crystal was removed from the antenua and placed in a coaxial mount fed by a 25-ohm r-f source. The d-c output voltage was measured across a $200-\mu \mu \mathrm{f}$ blocking condenser in the ground-return circuit.

As the d-c output voltage of the crystal was measured on a precision slidewire potentiometer, it is necessary to define the sensitivity of this instrument. This determined the useful range of (l-e output voltages that could be measured, and hence the resulting range of applied $r-f$ voltages that could be determined.

The voltage sensitivity of the potentioneter (in volts) is

$$
\Delta e=R \Delta i
$$

where

$\Delta e=$ smallest change in the balancing voltage that can be set on the slidewire dial of the potentiometer.

$\Delta i=$ smallest unbalance current readable on the galvanometer.

$R=$ total series d-c resistance of the measuring circuit and crystal.

The potentiometer used in these experiments was a commercially available portable type, weighing approximately $15 \mathrm{lb}$. It housed a standard cell, dry cells for comparison purposes, and a sensitive light-beam galvanometer, together with the slidewire dial and standard resisters all in a portable carrying case. The instrument had a useful measuring range of 0.5 to $161 \mathrm{mv}$. Other instruments were available having a range up to $1.6 \mathrm{v}$.

The most sensitive portable light-beam galvanometer available was used for measuring the output voltage of the crystal. Its sensitivity was approximately $0.03 \mu \mathrm{a}$ per scale division $(1 \mathrm{~mm})$. Assuming that one could detect a change of current, $\Delta i$, of 0.1 division, and that the total series d-c resistance of the crvstal, filter, and potentiometer was approximately $20,000 \mathrm{ohms}$, the voltage sensitivity was $\Delta \epsilon=R \Delta i=2 \times 10^{2} \times 3 \times 10^{-9}=$ $60 \times 10^{-6} \mathrm{v}$. Thus, neglecting the other errors in the instrument, which were small, d-c output voltages of the crystal could be determined to an accuracy of better than 2 percent from something less than $3 \mathrm{mv}$ to orer $1 \mathrm{r}$ (the safe upper voltage limit of the crystal). This corresponded to a range of $r-f$ input voltage to the crystal of roughly 0.02 to $1.0 \mathrm{v}$ as will be shown.

The silicon crystal voltmeter was calibrated in terms of a standard roltmeter. One type used at 100 Me comprised a quarter-warelenenth balanced transmission line of accurately known characteristic impedance terminated in a previously "alibrated VHF racuum thermocouple. $\Lambda s$ is known, the mitguitule of the transfer impedance of a quarter-wave length uniform lowloss transmission line is equal to its characteristic impedance [6], i. e.,

$$
Z_{T}|\cong| \frac{V_{s}}{I_{R}} \mid \cong Z_{0}
$$

where

$T_{S}=$ sending-end voltage

$I_{R}=$ receiving-end current

$Z_{0}=$ characteristic impedance.

Thus the sonding-end voltage may be determined in terms of the receiving-end current and the characteristic impedance of the transmission line. This relationship is independent of the magnitude or phase-angle of the terminating impedance (in this case the heater of the VHF vacuum thermocouple). The line losses involved were found to be small enough so as not to appreciably affect the results.

The frequency error of the thermocouple was determined in terms of small bead-type thermistors used as current standards. These are known to maintain their $d-c$ value of resistance up to frequencies well above the VHF band when biased to low values of resistance. The circuit used to determine the frequency error is shown in figure 7. The bridge circuit measured the $\mathrm{d}-\mathrm{c}$ resistance of the series combination of the thermistors and thermocouple heater biased with either $\mathrm{d}-\mathrm{c}$ or $r-f$ current. A measure of the $r-f$ current was thus provided in terms of a known d-c current. A negligibly small d-c measuring current was used in the presence of the r-f current so as not to appreciably alter the thermistor resistance. Two matched thermistors were used to preserve the symmetry, as the frequency correction of the thermocouple will in general be considerably different if used in an unbalanced circuit.

The desired value of direct current was first established in the thermocouple heater. Resist-

\footnotetext{
s The VHF thermocouple referred to herein has a separate heater insulated from the couple by means of a small glass bead.
} 


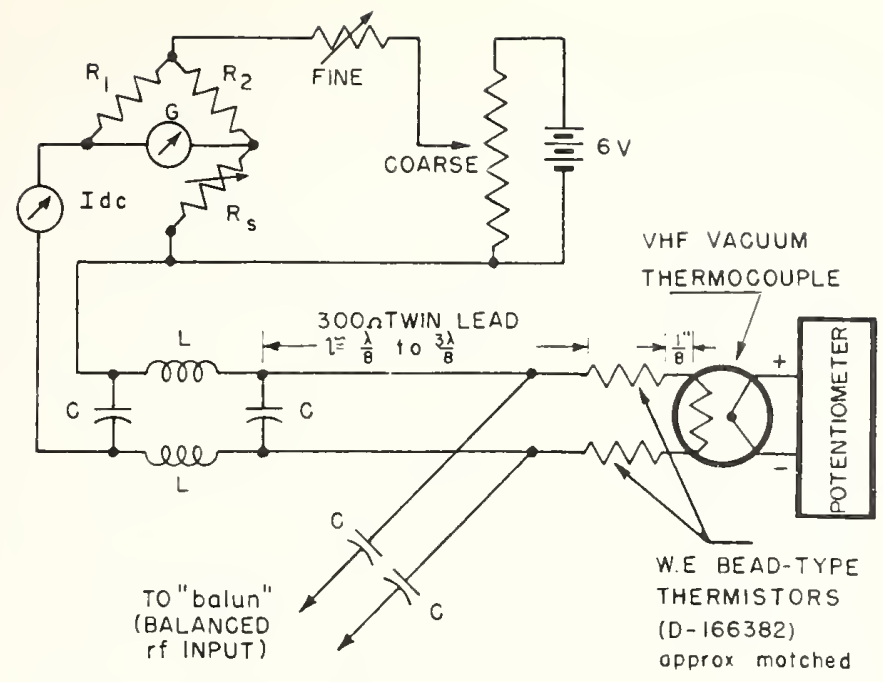

Firitre 7. C'ircuit diagram of the apparatus used to determine the frequency correction of THF vacuum thermoelements when used in a balanced circuit arrangement.

$\mathrm{L} \cong 1 / 1 \mu \mathrm{h} ; \mathrm{C} \cong 200 \mu \mu \mathrm{f}(\mathrm{f}=100 \mathrm{Mc})$.

ance $R_{s}$ was then adjusted to balance the bridge, at the same time maintaining the value of current by readjusting the battery voltage if necessary. After measuring the d-c voltage output of the themocouple, the direct current was reduced in value to 5 percent or less. The $r-f$ voltage of desired frequency was then applied, adjusted in value until the bridge was rebalanced, and the d-c voltage output of the thermocouple measured. The $r-f$ voltage was then removed and the value of direct current determined to reproduce this value of thermocouple output. The frequency correction was obtained from the two values of direct current. With sufficient sensitivity in the bridge and associated controls, it was found possible to reproduce these frequency corrections to a small fraction of a percent.

Generally speaking, for a given accuracy, a current standard is somewhat easier to provide at these frequencies than is a voltage standard. Fortunatcly, the relation given by eq 10 enibles one to provide an $r$-f voltage standard lraving an accuracy approximating that of the current standard used. 'The difficulty, however, in this case is in the restricted frequency range over which the standard may be used. Nomally a continnous voltage calibration is not required over the frequency range of the standard antema. A number of individual calibrations spaced at frequerey intervals of approximately 25 or 50 percent over the desirerl range is usually sufficient when the response is broadband.
Probably one of the most accurate methods of determining the characteristic impedance of the balanced low-loss transmission line used in this voltage standard is from the relation

$$
Z_{0}=\frac{1}{V_{p} C}
$$

where

$$
\begin{aligned}
& Z_{0}=\text { characteristic impedance in ohms, } \\
& V_{p}=\text { velocity of propagation in meters per } \\
& \text { second, and } \\
& C=\text { mutual capacity between conductors in } \\
& \text { farads per meter. }
\end{aligned}
$$

The type of transmission line used was commercially available unshicleled twin-lead having a nominal characteristic impedance of 75 ohms. The velocity of propagetion was determined to an accuracy of better than 0.25 percent at $100 \mathrm{Mc}$ by measuring the resonant frequency of a hilfwavelength section of line short circuited at. both ends. The length of the short circuit used was such as to introduce a negligible error in the measurement. The calculated effect of the line loss on the velocity of propagation was also found to be negligible. The balanced mutual capacity, $C$, per unit length was determined on a special precision Schering bridge at a frequency of 1,000 cycles to the same order of accuracy. There is no reason to suspect that the capacity per unit length should change with frequency, at least up to frequencies of several hundred megacycles. Thus the characteristic impedance was probably determined to better than 0.5 percent. The characteristic impedance of a number of samples $1 \mathrm{~m}$ in length cut from the same piece of twin-lead varied less than \pm 0.1 percent from the average value of the lot. This would seem to indicate the exceedingly small departure from uniformity existing within a given sample of the line. The average value of $Z_{0}$ for the lengths measured was 83.0 ohms. The variations between samples of different production-runs of twin-lead was quite large, being as high as 10 to 15 percent, as might be expected. The aging of the twin-lead seems to be negligible if not exposed to the weather or undue stress. This was indicated by having a value of the velocity of propagation of a particular sample repeat to within 0.1 percent after several inonths. 
Another type of balanced voltage standard used consisted simply of a sensitive thermocouple previously calibrated in a manner similar to that already described and shown in figure 7. However, in this case a single thermistor was used in shunt with the thermocouple heater. A 1,000$\mu \mu \mathrm{f}$ ceramic disk condenser was used in series with each thermocouple heater lead to provide dc isolation and preserve the symmetry. The leads were kept as short as physically possible (1/8 in. in most cases).

The voltage standards described above were used at only one voltage level (approximately $1 \mathrm{v}$ ), that corresponding to the rated current through the terminating thermocouple. The voltage range was extended downward by means of a precision piston (mutual inductance) attenuator [6a] having an accuracy better than $0.1 \mathrm{db}$ (approximately $1.0 \%$ ) over a range of $60 \mathrm{db}$. The total rms harmonic content of the r-f generator output was less than 1 percent, so that any resulting error in the voltage standard from this cause was negligible. Pi matching networks were used in both the input and output circuits of the standard attenuator to decrease the insertion loss.

Figure 8 is a block diagram showing the equipment and its arrangement for one of the methods used to calibrate the balanced crystal voltmeter. The antenna rods were removed during calibration. A broadband balun [7] was used to transform from the unbalanced output of the standard attenuator to the balanced circuit of

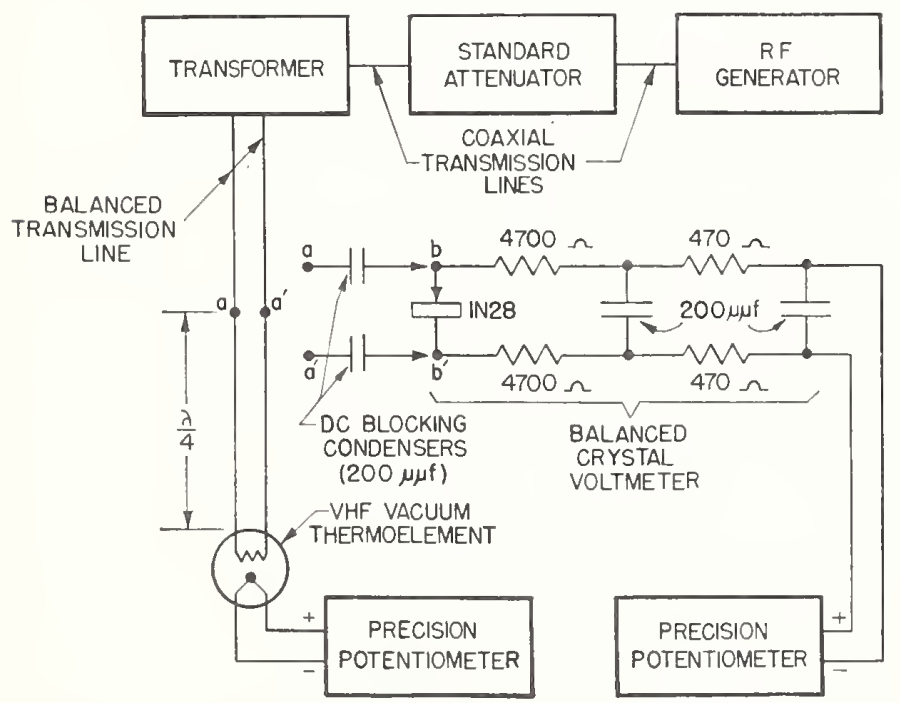

FIGURE 8. Diagram showing the equipment and method used to calibrate the balanced criystal voltmeter used in the standard reeeiving antenna.

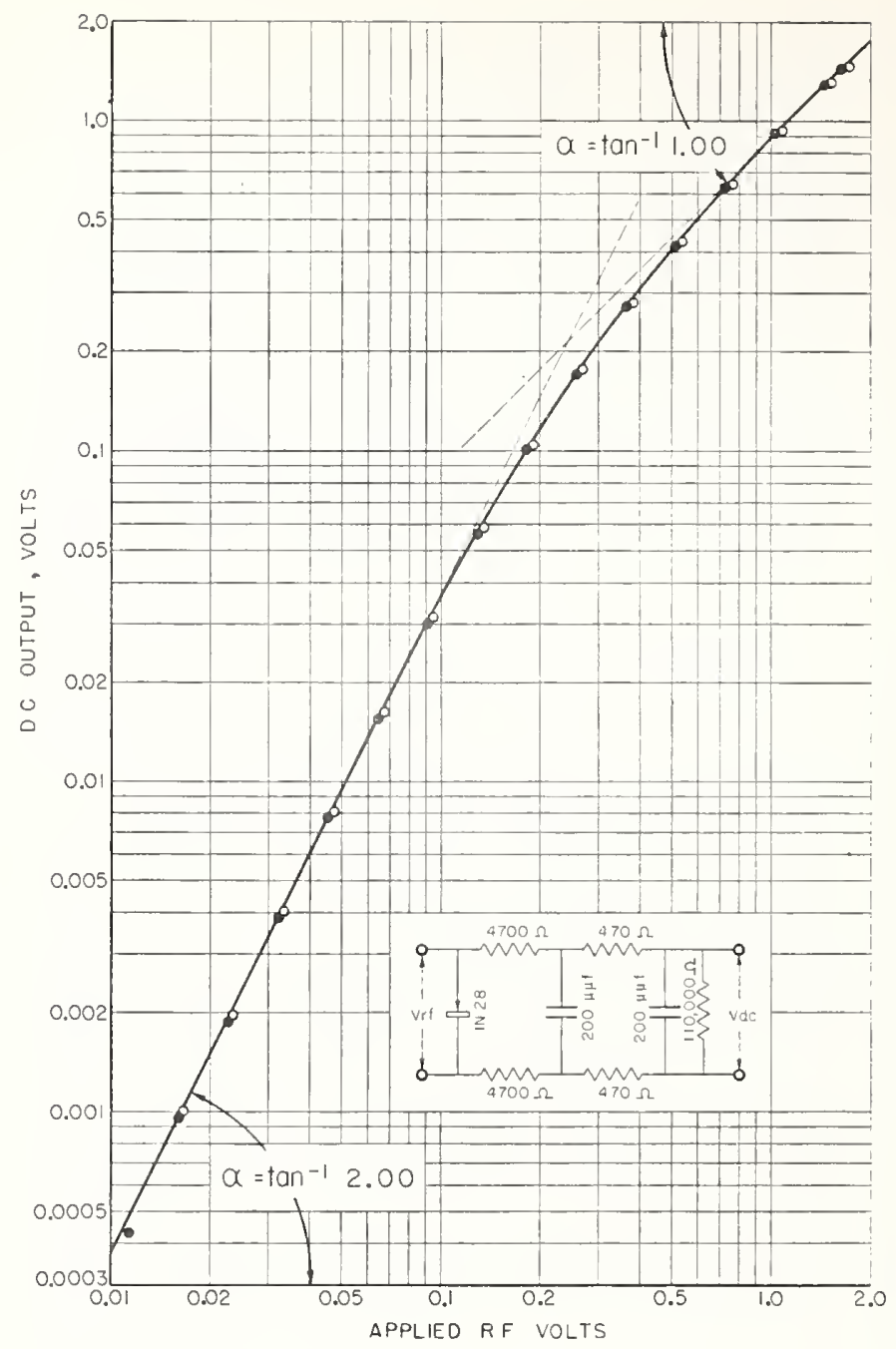

Figure 9. Typical calibration curves of the crystal voltmeter, showing the variation in $d-c$ output voltage versus applied $r$-f voltage for the circuit shown.

Calibrations "A" and "B" were made 2 days apart at approximately the same ambient temperature. ,Calibration A; O, calibration B. f-100 Me.

the antcnna and voltmeter. "The "Pi" matching networks are not shown. Two 200- $\mu \mu \mathrm{f} d-\mathrm{c}$ blocking condensers were used to prevent shortcircuiting the d-c output of the antenna crystal.

Typical calibration curves of the antenna voltmeter made at $100 \mathrm{Mc}$ are shown in figure 9. The usable range of $r-f$ input voltage was from 0.02 to $1.0 \mathrm{v}$ and the probably accuracy about \pm 2 percent over most of the range. The solid points and curve joining them represent the first of two calibrations. The circles represent a repeat calibration 2 days later at a temperature $1 \mathrm{deg} \mathrm{C}$ lower.

The data of figure 9 show the order of reproducibility possible with such a crystal voltmeter under partially controlled temperature conditions. The 
diameter of the individual points represents approximately 5 percent of the voltage, so that any point touching the curve is in agreement to within $\pm 2 \frac{1}{2}$ percent.

It was usually possible to repeat a crystal calibration to within 1 percent if made within a few hours at the same ambient temperature. In no case was the calibration relied on for more than a day.

In making the field-intensity measurements described in section IV, using this standard-antenna, the crystal voltmeter was always calibrated in the field within an hour of the time the tests were completed. A record of the temperature was kept and usually did not vary more than $2 \mathrm{deg} \mathrm{C}$ during any test. Thus all that was required of the crystal voltmeter was a good short-time stability.

Although the effect of variations in temperature and the accompanying hysteresis are known to contribute largely to the instability in a crystal voltmeter, no data were accumulated on the exact magnitude of these effects. We have so far avoided this by operating under as small a temperature variation as possible. Thus it would probably not be feasible to calibrate the crystal in a laboratory and use it for measurements outside at a temperature as much as $10 \mathrm{or} 15 \mathrm{deg} \mathrm{C}$ different.

The response of the antenna crystal roltmeter was found to be essentially independent of frequency up to $200 \mathrm{Mc}$ witl a rising response beyond (output indication high) due to series resonance within the crystal and mount. The series-resonant. frequency for this voltmeter was of the order of 2,000 Mc.

\section{Standard-Field Method}

\section{Theory}

A predetermined value of field intensity may be established at a given point in space in terms of the current distribution in a transmitting antenna, the effect of the ground, and the geometry involved.

In the development of the standards being reported here, only horizontally polarized transmission over plane homogeneous earth having finite values of relative dielectric constant, $\epsilon_{\tau}$, and conductivity, $\sigma$, was considered. The distance of separation used between the transmitting and receiving antenuas was from 2 to 20 wavelengths. These conditions simplify the resulting formulas somewhat and for the accuracy desired are quite justified. This range of distances is usually of little interest to the propagationist, at these frequencies, and many of the transmission vagaries of concern to him are ignored here. At these smaller separations a more exact knowledge of the actual angle of incidence and ground reflection coefficient is usually needed in order to produce the required degree of accuracy in predicting the actual value of field intensity existing. The geometry of the transmission system is shown in figure 10 .

The root-mean-square value of the electric component of field intensity produced by a horizontal transmitting dipole at distances greater than about $2 \lambda$ over plane homogeneous earth is $[8,9,10]$ (in volts per meter)

$$
E \cong-j \frac{60 \pi l_{H} I}{\lambda}\left[\frac{e^{-j k R_{1}}}{R_{1}}+\frac{\Gamma e^{-j k P_{2}}}{R_{2}}+\frac{(1-\Gamma)}{R_{2}}+\frac{\substack{\text { Ground- } \\ \text { wave } \\ \text { reflected } \\ \text { wave }}}{R_{2}} A(R) e^{-j k R_{2}}\right] .
$$

in which

$$
\begin{aligned}
& l_{H}=\text { effective length of the antenna in } \\
& \text { meters } \\
& \lambda=\text { wavelength in meters } \\
& R_{1}=\text { direct-ray path lengtl in meters } \\
& R_{2}=\text { ground-reflected-ray path-length in } \\
& \text { meter's } \\
& \Gamma=\rho e^{-j_{\phi}}=\text { complex plane-wave reflec- } \\
& \text { tion coefficient (horizontal polar- } \\
& \text { ization) } \\
& A(R)=\text { complex surface-ware attenuation } \\
& \text { factor } \\
& I=\text { l'ms current in amperes at the center } \\
& \text { of the transmitting antenna } \\
& k=2 \pi / \lambda \\
& j=\sqrt{-1}
\end{aligned}
$$

The first term of eq 12 represents the field intensity that would exist if the transmitting and receiving antennas were located in free space. The remaining terms take into account the presence of the earth. The second term represents the ground-reflected wave which, when added vectorially to the first term of eq 12 , comprises the space wave. The third term of eq 12 represents the surface wave associated with the r-f currents actually flowing in the ground.

The total field given by eq 12 is usually referred to as the ground wave. Propagation via the 


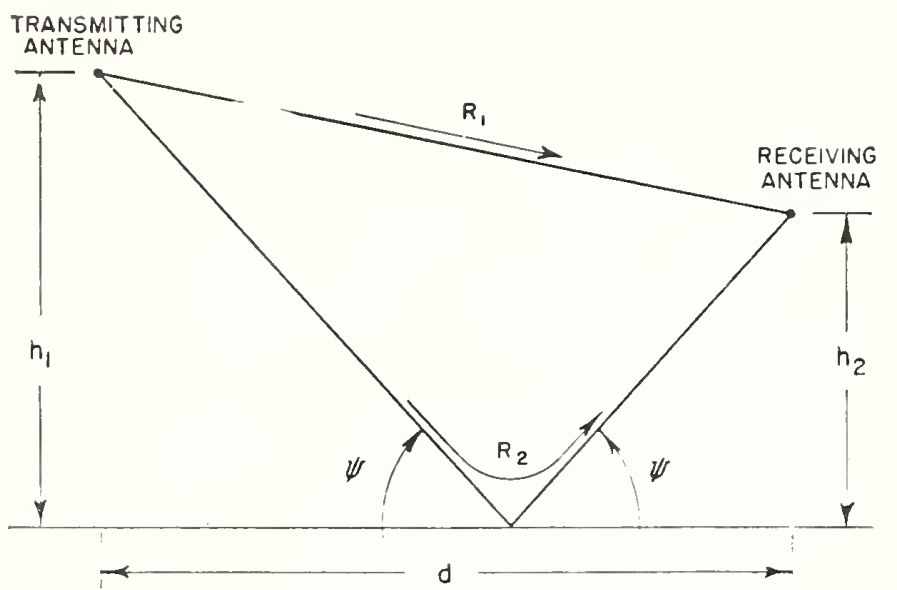

FigCRE 10. Ray-path diagram showing: (a) direct ray along $R_{1}$; (b) ground-reflected ray along $R_{2}$ making an angle $\psi=\tan ^{-1}\left(h_{1}+h_{2}\right) / d$ with the earth.

Heights of the transmitting and receiving antennas are $h_{1}$ and $h_{2}$, respectively, and $d$ is the horizontal distance of separation.

ionosphere or troposphere, which is subject to changing solar or meteorological conditions is being ignored here.

The magnitude of the surface wave is usually negligibly small for horizontally polarized transmission at these frequencies [11], and in the remaining formulas used herein we shall neglect its presence entirely.

The complex reflection coefficient of the ground, $\Gamma$, may be expressed in terms of the angle $\psi=$ $\tan ^{-1}\left(h_{1}+h_{2}\right) / d$ and a complex dielectric constant $\epsilon_{0}$, as follows, for horizontal polarization [12],

$$
\Gamma=\frac{\sin \psi-\sqrt{\epsilon_{0}-\cos ^{2} \psi}}{\sin \psi+\sqrt{\epsilon_{0}-\cos ^{2} \psi}}
$$

where

$$
\begin{aligned}
& \epsilon_{n} \equiv \epsilon_{T}\left(1-j \frac{\sigma}{\epsilon \omega}\right)=\epsilon_{\tau}-j 60 \lambda \sigma \\
& \epsilon_{\tau}=\text { relative dielectric constant of the ground } \\
& \text { (referred to free space as unity) } \\
& \epsilon \equiv \epsilon_{r} \epsilon_{\tau} \text {, where } \epsilon_{\tau} \text { is the permittivity of evac- } \\
& \text { uated free space } \\
& \epsilon_{r} \cong \frac{1}{36 \pi} \times 10^{-9} \text { farad per meter } \\
& \sigma=\text { ground conductivity, in mhos per meter } \\
& \omega=2 \pi f \\
& \lambda=\text { wavelength, in meters } \\
& f=\text { frequency, in cycles per second }
\end{aligned}
$$

Talues of the magnitude of eq $13, \rho$, are shown rersus $\tan \psi=\left(h_{1}+h_{2}\right) / d$ in figure 11 for various values of the relative dielectric constant, $\epsilon_{r}$, for low-loss dielectries $(\sigma / \epsilon \omega \ll 1)$. Many types of ground may be treated as low-loss dielectrics over a large portion of the VHF band as far as their reflecting properties are concerned. This is particularly true at the higher frequencies above 50 or $75 \mathrm{Mc}$.

In this case the phase shift on reflection $\phi$, is very nearly $180^{\circ}$ for horizontal polarization, so that the value of the reflection cocfficient is given approximately by

$$
\Gamma \cong-\rho
$$

Ignoring the surface wave altogether, the magnitude of eq 12 may be written (in volts per meter)

$$
|E| \cong \frac{60 \pi l_{H} I}{\lambda}\left|\frac{1}{R_{1}}+\frac{\Gamma e^{-j k\left(R_{2}-R_{1}\right)}}{R_{2}}\right| .
$$

Equation 15 may be expanded without further approximation, giving (in volts per meter)

$$
\begin{aligned}
& \mid E_{\mid} \cong \frac{60 \pi l_{H} I}{\lambda} \\
& \left\{\left(\frac{1}{R_{1}}+\frac{\rho}{R_{2}}\right)^{2}-\frac{4 \rho}{R_{1} R_{2}} \sin ^{2}\left[\frac{k\left(R_{2}-R_{1}\right)+\phi}{2}\right]\right\}^{\frac{1}{2}},
\end{aligned}
$$

where $\rho$ is the magnitude of the reflection coefficient, $\Gamma$, and $\phi$ is its phase lag in radians. Equation 16 is in a form somewhat more suitable for computation, since no loss of accuracy is involved as a result of the taking of small differences between large computed quantities.

In case the approximation given by eq 14 applies, eq 16 may be written (in volts per meter)

$$
|E| \cong \frac{60 \pi l_{H} I}{\lambda}\left\{\left(\frac{1}{R_{1}}-\frac{\rho}{R_{2}}\right)^{2}+\frac{4 \rho}{R_{1} R_{2}} \sin ^{2}\left[\frac{k\left(R_{2}-R_{1}\right)}{2}\right]\right\}^{1 / 2} .
$$

The quantity $\left[\left(1 / R_{1}\right)-\left(\rho / R_{2}\right)\right]^{2}$ within the radical becomes negligibly small when $d \gg\left(h_{1}+h_{2}\right)$, since $R_{1} \rightarrow R_{2} \rightarrow d, o \rightarrow 1$, and $R_{2}-R_{1} \cong 2 h_{1} h_{2} / d$. Under these conditions, eq 17 becomes (in volts per meter)

$$
|E| \cong \frac{120 \pi l_{H} I}{\lambda d}\left|\sin \left(\frac{2 \pi h_{1} h_{2}}{\lambda d}\right)\right| .
$$

Equation 18 is obviously not valid in the vicinity of the zeros of the sine term, since the first term of eq 17 is not then negligible. Therefore use of eq 18 should preferably be restricted to values of $h_{1} h_{2} / \lambda d<0.5$. If either the wavelength, $\lambda$, or the distance, $d$, is increased, eq 18 will reach its last maximum value when $h_{1} h_{2} / \lambda d=0.3229 \ldots$, after which it will steadily decrease. If the antenna 


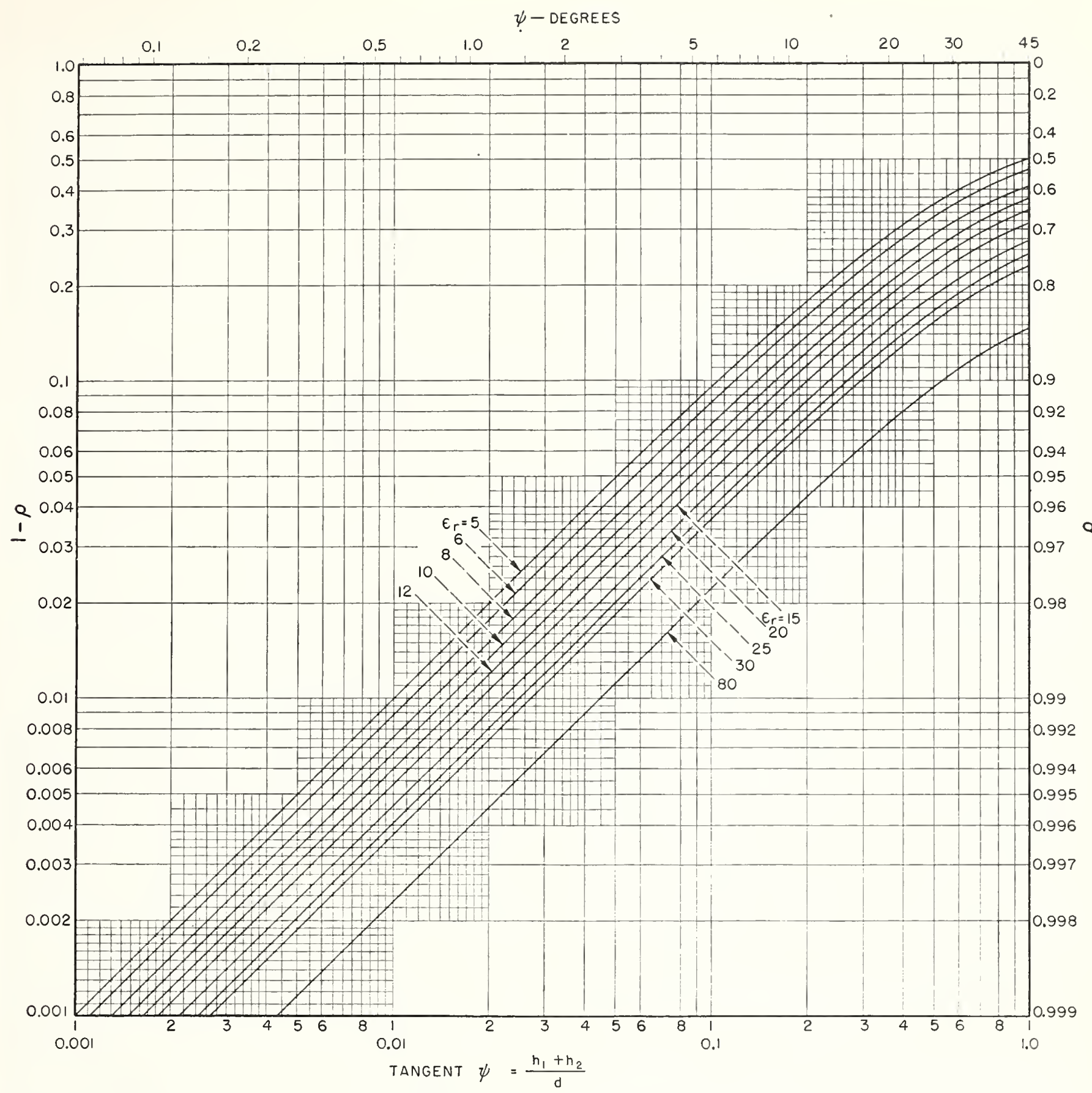

FIGURE 11. Variation of the magitude, $\rho$, of the complex plane-wave reflection coeffcient, $\Gamma$, versus tan $\psi=\left(h_{1}-h_{2}\right) / d$ for several values of relative dielectric constant, $\epsilon_{r}$.

The curves apply to low-loss dielectries, $(\sigma / \epsilon \omega \ll 1)$, and borizontal polarization onfy and were determined from eq 13.

heights $h_{1}$ or $h_{2}$ above are raried, this maximum will occur when $h_{1} h_{2} / \lambda d=0.25$.

When $2 \pi h_{1} h_{2} / \lambda d \leqq 1 / 4$ the field intensity ja given (in rolts per meter) to within 1 percent by

$$
|E| \cong \frac{240 \pi^{2} l_{H} I}{d^{2}}\left(\frac{h_{1} h_{2}}{\lambda^{2}}\right),
$$

since $\sin \theta \cong \theta$ to within the stated accuracy for $\theta \leq 1 / 4$ radian.

When attempting to establish values of standard field intensity, however, one should rely on eq 16 or 17 until the assumptions applying to eq 18 and 19 are found to be fully justified. 


\section{Description of the Standard Transmitting Antenna}

The transmitting antenna used was similar in many respects to the standard receiving antenna described in section II. A horizontal half-wave dipole was used shortened 4 percent for selfresonant operation at 100.0 Mc. Dural tubing $3 / 16$ in. in diameter was used, giving a ratio $2 l / a \cong 600$ and an average characteristic impedance $K_{0}=120\left[\log _{e}(2 l / a)-1\right] \cong 650$ ohms.

The current at the center of the antenna was measured by means of a previously calibrated VHF vacuum thermocouple and a section of balanced twin-lead transmission line one-half wavelength long. The antenna and thermocouple terminated the transmission line at opposite ends, and a balanced feed-line from the $\mathrm{r}-\mathrm{f}$ generator was tapped in at the midpoint. Thus the currents flowing at the opposite ends of the half-wave line were identical in value. The magnitude of the antenna current was thus indicated by the thermocouple $\lambda / 2$ distant and was entirely independent of the magnitude and phase angle of the terminations, namely, the antenna input impedance, and the impedance of the thermocouple heater. A broadband balun [7] was used to transform the unbalanced output of the r-f generator to the balanced transmission system. A block diagram of the equipment and its arrangement are shown in figure 12 . The thermocouple was calibrated in a similar manner to that described earlier for the receiving antenna voltmeter, and was found to have a frequency error of 0.5 percent at $100 \mathrm{Mc}$.

\section{Propagation Tests}

\section{General}

In order to determine the probable accuracy of the VHF field-intensity determinations made by the standard-antenna and standard-field methods previously described, actual propagation tests were made at a frequency of 100.0 Mc, using horizontal polarization only. The purpose was to actually intercompare values of field-intensity determined with the two standards.

For instance, a predetcrmined value of field intensity was established by means of the standardfield method, and simultaneously measured using the standard (receiving)-antenna method, and the results compared. However, instead of relying on

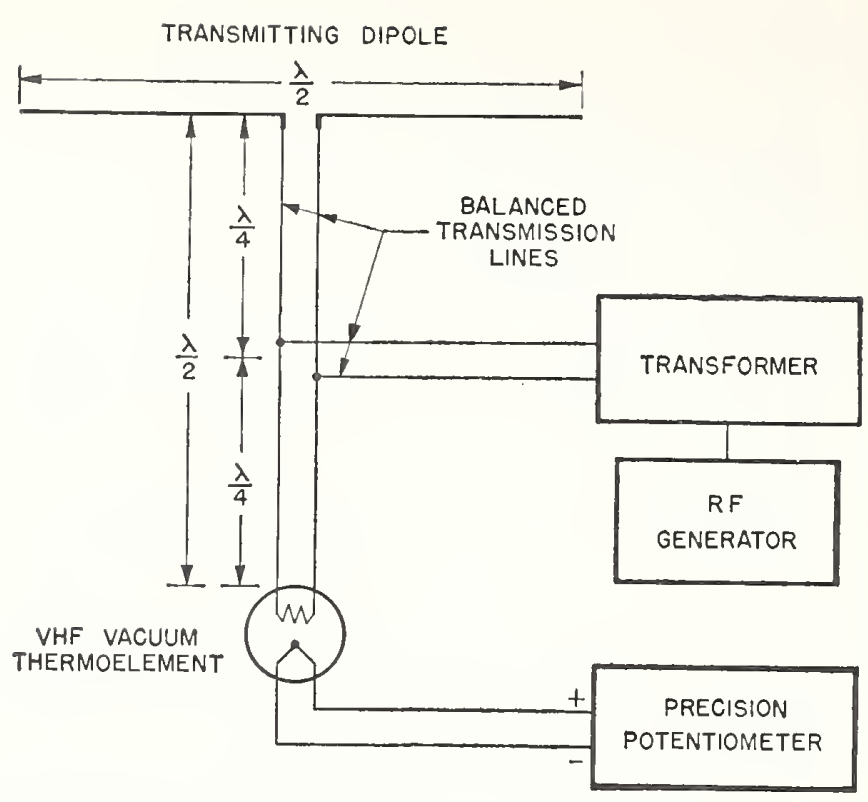

Figure 12. Diagram showing the method used for determining the $r-f$ current at the center of the transmitting dipole.

isolated pairs of such measurements to determine the probable accuracy of the two methods, more or less complete propagation runs were made involving some 15 or 20 pairs of determinations in each, in order to determine the trend with varying antenna height or distance of separation. In this manner it was possible to determine the range of antenna heights and distances of separation between the antennas over which the previous assumptions applying to the earth were valid, and to what extent we were dealing with a uniform field.

Three gencral types of tests were made as follows:

(a) Vertical-incidence standing-wave measurements, in which the standard field beneath the horizontal transmitting antenna was probed vertically using the standard (receiving) antenna.

(b) Tariable-height runs, in which the heights of the transmitting and receiving antennas were varied separately for a fixed horizontal distance of separation.

(c) Tariable-distance runs, in which the horizontal distance betreen the transmitting and receiving antennas was varicd for several fixed antenna heights.

In order to conduct these tests it was necessary to select a special site. The usual requirements specify that the location be relatively flat and free of such reflecting objects as trees, buildings, wires, 
etc. in order to approximate the conditions assumed to exist in deriving the standard field formulas in section III.

A site of approximately 100 acres was made arailable at the Beltsville, Mcl. Airport for these tests. The land had been cleared and graded and met the above requirements. Interference from aircraft was negligible, inasnuch as the airport was officially closed and used only for emergency landings.

One other requirement, dictated by the lack of selectivity in the standard antenna, made it necessary to conduct such tests at a location where the field intensity of frequency-modulation and television broadcast stations was negligible compared to the standard field being used. Such interference was further minimized (since the test site was sufficiently free of reflecting objects) by properly orienting the two antennas. This was possible in our case since the interfering stations, some 20 miles distant, were all approximately in the same direction from the test site.

\section{Vertical-Incidence Standing-Wave Measure- ments}

This run was originally intended to be used for determining the reflection coefficicnt and hence the apparent dielectric constant, and conductivity of the ground at the test site. However, it was also found to be uscful later in determining the effective length of the transmitting and receiving antennas, as will be shown.

For this test the horizontal transmitting dipole was located at the top of an all-wood ladder-mast at a fixed height $h_{1}=9.27 \mathrm{~m}$. The balanced unshielded 75 -ohm $\mathrm{r}$-f transmission line ran at right angles to the antemna and approximately $10 \mathrm{deg}$ below the horizontal. The unshielded balanced d-c output lead from the thermocouple paralleled the $\mathrm{r}$-f line and was spaced approximately $3 \mathrm{ft}$ with negligible $r-f$ pickup. The $r-f$ power generator and associated equipment were located on the ground approximately $200 \mathrm{ft}$ distant. The transmitting-antenna current was continuously monitored by an operator at the generator and was held constant to within \pm 0.1 percent. The d-c output of the thermocouple was measured on a portable precision potentiometer. The probable accuracy of the antenna-current measurement was about 1 percent. The frequency of the $r-f$ generator was also continuously monitored by means of a calibrated heterolyne frequeney meter, and held to $100.0 \mathrm{Nc} \pm 0.1$ pereent.

The standard (receiving) antema (oriented parallel to the transmitting antenna), was mounted on a movable carriage on the same mast, and was readily acljustaline in height by means of a continuous rope passing through nonmetallic pulleys at the base and top of the mast. Certain predetermined heights were marked directly on the side of the mast and read by a second operator located at some distance off the end of the antenna in a null. The actual height of the antenna above ground could be thus measured to better than 0.5 $\mathrm{cm}$. The balanced $\mathrm{d}-\mathrm{c}$ output of the receiving antenna crystal-rectifier was carried over rubbercovered lamp cord, which passed vertically down the mast to the ground. From this point the d-c line went directly to the operator's position where the voltage was measured on the precision slidewire potentiometer previously mentioned. Tests were made for $r-f$ pickup on the d-c line, and in the resistance-capacitance filter at the antenna, with the antenna rods removed, but was found to be negligible even with the d-c line running the entire height of the mast.

Tests were made for stray radiation from the transmittingsystem caused by any slight unbalance in the feed line to the antenna or by leakage. The transmitting antenna rods were removed and a $65-0 h m$ high-frequency resistor substituted to terminate the transmission line. With the normal $\mathrm{r}$-f current of $0.1 \mathrm{amp}$ flowing, the resulting field was too small to be measured for any height of the receiving antenna. The vertical component of field existing at the receiving antenna was more than $40 \mathrm{db}$ below the main field, with the horizontal transmitting antenna excited.

Returning to the description of the verticalincidence measurements, the resulting interference between the direct and ground-reflected waves, set up a standing-wave in the space beneath the transmitting antenna, as shown in figure 13. The standard (receiving) antenna was moved vertically up or down the mast to measure the resulting value of field intensity. The reflection coefficien $t$ was accurately determined from the standingwave ratio, as will be shown. Once this was known, the expressions for the field intensity for the standard-antenna and standard-field methods, eq 2 and 15, were equated and solved for the remaining unknown, the effective length of the 
antennas. This was deemed permissible since both antennas were physically identical.

The field intensity as determined by the standard-field methor was obtained by substituting $R_{1}=h_{1}-h_{2}, R_{2}=h_{1}+h_{2}, d=0$ in eq 15 , giving (in volts per meter)

$$
|E| \cong \frac{60 \pi l_{H} I}{\lambda}\left|\frac{1}{h_{1}-h_{2}}+\frac{\Gamma e^{-j 2 k h_{2}}}{h_{1}+h_{2}}\right| .
$$

If $h_{1} \gg h_{2}$, the value of field intensity given by eq 20 will have a maximum value if $\left(2 k h_{2}+\phi\right)=2 n \pi$, and a minimum value if $\left(2 k h_{2}+\phi\right)=(2 n-1) \pi$, where $n=1,2,3, \ldots$. The magnitude of the ratio of the maximrum to minimum values of field intensity is then

$$
S W R=\left|\frac{E_{\mathrm{max}}}{E_{\mathrm{m} \ln }^{\prime}}\right| \cong \frac{\frac{1}{h_{1}-h_{2 \mathrm{max}}}+\frac{\rho}{h_{1}+h_{2 \mathrm{max}}}}{\frac{\rho}{h_{1}-h_{2 \mathrm{~m} \ln }}-\frac{\rho}{h_{1}+h_{2 \mathrm{~m} \ln }}} .
$$

The magnitude, $\rho$, of the complex reflection coefficient, $\Gamma$, of the ground may be determined from the SWR and the heights, $h_{2}$, of the receiving antenna corresponding to the maximum and the minimum values of field intensity measured plus the height of the transmitting antenna, thus

$$
\rho \simeq \frac{\frac{S W R}{h_{1}-h_{2 \min }}-\frac{1}{h_{1}-h_{2 \max }}}{\frac{S W R}{h_{1}+h_{2 \min }}+\frac{1}{h_{1}+h_{2 \max }}}
$$

The angle of phase lag, $\phi$, of the reflection coefficient may be determined from the shift in the positions of the maxima or minima from the positions corresponding to $\phi=0$. For the maxima this gives

$$
\phi=2\left[\frac{2 \pi h_{2 \max }}{\lambda}-(n-1) \pi\right]
$$

where $n=1,2,3, \ldots$ is the number of the maximum used to calculate $\phi$, counting from the ground upward.

In using the minima to determine $\phi$, the expression is

$$
\phi=2\left[\frac{(2 n-1) \pi}{2}-\frac{2 \pi h_{2 m 1 n}}{\lambda}\right]
$$

$h_{2 \min }$ and $h_{2 \max }$ are the actual heights, in meters, at which the particular minimum or maximum occurs.

In determining the value of the reflection co-

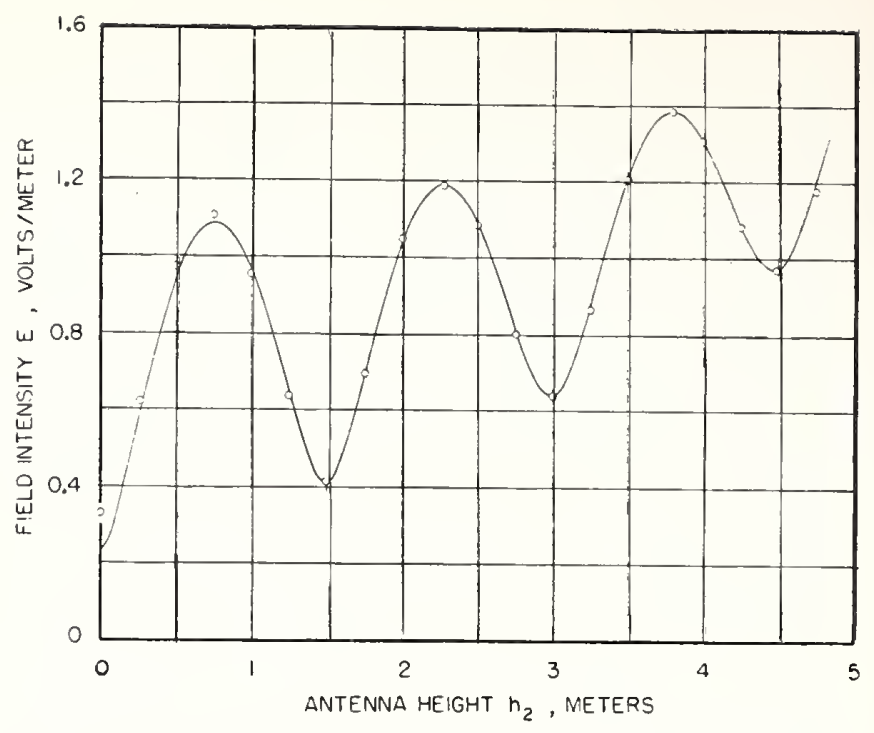

FIgURE 13. Tariation of the obserted and calculated values of electric field intensity, $|E|$ in volts per meter, versus the height, $h_{2}$, in meters, of the standard receiving antenna.

The transmitting antenna was located above and parallel to the receiving antenna at a fixed height, $h_{1}=9.27 \mathrm{~m}, d=0, f=100.0 \mathrm{Xc}, I=0.100 \mathrm{amp}$. O, observed, - - calculated.

efficient of the ground at the test site, the heights, $h_{2}$, of the various maxima and minima were carefully measured. It was found possible to detelmine these to within $\pm 1.0 \mathrm{~cm}$. To within this accuracy the positions corresponded to a phase shift, $\phi$, on reflection of $180 \mathrm{deg}$. The possible error in determining $\phi$ thus amounts to approximately $2 \mathrm{deg}$. It is interesting to note that the calculated phase shift, $\phi$, for average ground $\left(\epsilon_{r}=\right.$ $15, \sigma=5 \times 10^{-3}$ mho per meter) at $100 \mathrm{Mc}$ is very nearly $179 \mathrm{deg}$ at normal incidence. An error of 2 deg in determining $\phi$ usually has a negligible effect on the magnitude, $\rho$, of the reflection coefficient, and on the resulting value of field intensity calculated.

Although the standincr-wave method cannot be used to determine even approximately the ground conductivity at these frequencies, such information is usually not required if the reflection coefficient can be accurately determined.

An example will be given of the method of determining the magnitude, $\rho$, of the reflection coefficient, and the dielectric constant, $\epsilon_{T}$, of the ground from the observel data presented in figure 13. Three different values of standing-wave ratio were determined from the three maxima and the three succeeding minima. The minimum occurring at the ground $\left(h_{2}=0\right)$ was ignored because of its questionable value. 
The value of $\rho$ corresponding to each SWR was then determined from eq 22 , and the resulting value of the relative dielectric constant $\epsilon_{\tau}$ determined from the relation

$$
\epsilon_{T}=\left(\frac{1+\rho}{1-\rho}\right)^{2}
$$

which was obtained from eq 13 by substituting $\psi=\pi / 2$, and $\Gamma=-\rho$ and solving for $\epsilon_{\tau},(\sigma / \epsilon \omega \ll 1)$. The results are given in table 1.

TABLE 1. Talues of $\rho$ and $\epsilon_{T}$, as determined from standingwave measurements

\begin{tabular}{|l|r|r|}
\hline SWR & \multicolumn{1}{c|}{$\rho$} & \multicolumn{1}{c|}{$\epsilon_{r}$} \\
\hline & & \multicolumn{1}{c|}{} \\
\hline 2.67 & 0.648 & 21.9 \\
1.85 & .635 & 20.1 \\
1.42 & .636 & 20.2 \\
\hline Average & 640 & 20.7 \\
\hline
\end{tabular}

The values of $\rho$ and $\epsilon_{r}$ measured are determined largely by the moisture content of the ground. Consequently, their values fluctuate somewhat from day to day with changing moisture content. Four values of $\epsilon_{r}$ obtained in a similar manner over a period of a few months are shown in figure 14, together with values of daily rainfall. It can be seen that the variation in the dielectric constant is quite large. Normally, this is not of much concern to the propagationist, who is usually interested in grazing incidence, but it is apt to affect the accuracy of standard-field measurements at very short distances if the incident angle is large. Near the surface the moisture content and consequently the ground constants themselves may vary with depth. Since the skin depth in turn varies with frequency and angle of incidence (among other factors), the (apparent) values determined for these constants may also depend on these parameters.

If desired, the reflection coefficient of the ground for vertical incidence may be calculated from eq 13 upon substituting $\psi=\pi / 2$, giving

$$
\Gamma=\frac{1-\sqrt{\epsilon_{\tau}\left(1-j \frac{\sigma}{\epsilon \omega}\right)}}{1+\sqrt{\epsilon_{\tau}\left(1-j \frac{\sigma}{\epsilon \omega}\right)}} .
$$

Values of the magnitude of eq 26 are plotted versus $\epsilon_{T}$ in figure 15 for low-loss dielectries, $(\sigma / \epsilon \omega \ll 1)$, remembering that $\Gamma \cong-\rho$ for this condition.

Determining the value of the reflection coefficient, $\Gamma$, of the ground from the observed data of figure 13 actually required only relative values of the measured field intensity. With the value of $\Gamma$ now accurately determined, the only remaining unknown in eq 20 is the effective length, $l_{H}$, of the transmitting antenna. Since the standard transmitting and receiving antemnas used are geometrically identical, the assumption is made that their effective lengths are equal. There has been some indication in the works of King [13] that such is not excatly the case, and further that the value of $l_{H}$ is also somewhat dependent upon the antenna termination. However, it is shown that for self-resonant antennas these differences are small. Shelkunoff does not elaborate on these points, but our experimental values of

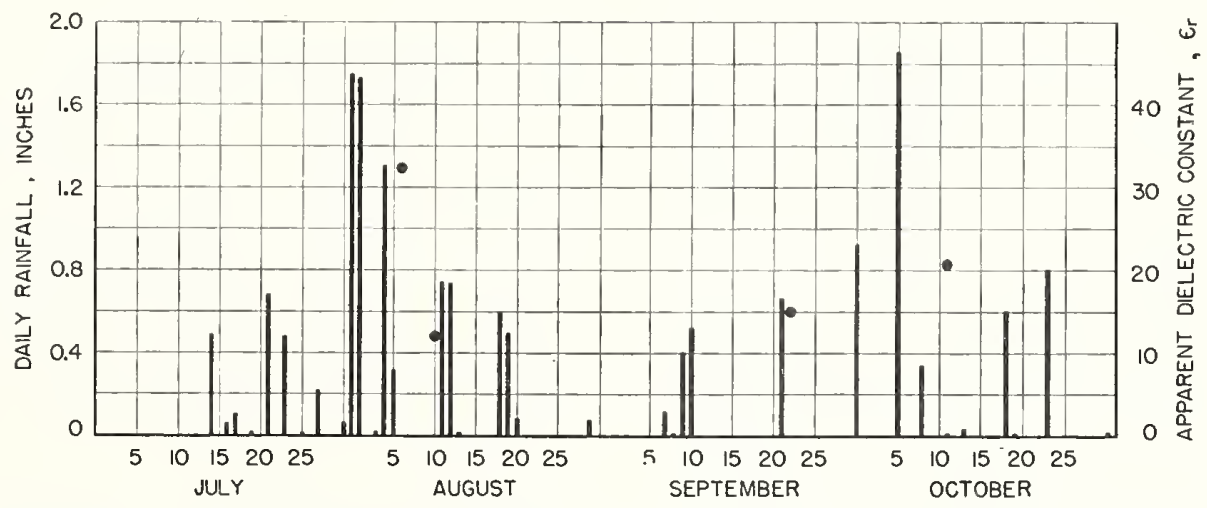

FIGURE 14. Chart showing four measured values of the apparent relative dielectric constant, $\epsilon_{r}$, of the ground at one particular site, as affected by rainfall. $f=100.0 \mathrm{Mc}$.

- Dielectic constant; |, daily rainfall. 
effeetive length agree well with those ealeulated from his analysis of eylindrieal antennas, as will be shown. The value of the effeetive length, $l_{H}$, may be determined by equating eq 2 and 20, and solving for $l_{H}$.

The data shown in figure 13 provide 19 separate determinations of $l_{H}$ (ignoring the observed value eorresponding to $h_{2}=0$ beeause of its questionable value). The average of these gives a measured value of $l_{H}=0.960 \mathrm{~m}$ for the self-resonant antennas used.

Figure 16 shows the pereentage difference between eaeh pair of determinations as presented in figure 13 , versus the height, $h_{2}$, of the reeeiving antenna. In this information is included experimental error, differenees caused by ehanges in the reeeiving antenna eurrent distribution due to the presenee of the ground, and differenees eaused by the proximity of the transmitting and reeeiving antennas, of whieh the latter should be small in view of the faet that the reeeiving antenna was operated open-eireuited. These differences, in general, are less than 3 pereent, and give a good indieation of the agreement possible between the standard-antenna and standard-field methods at least for the ease of normal ineidenee where the refleetion eoeffieient of the ground eould be aeeurately determined.

It is also indicated that any varying effeet (with antenna height) of the presenee of the ground on

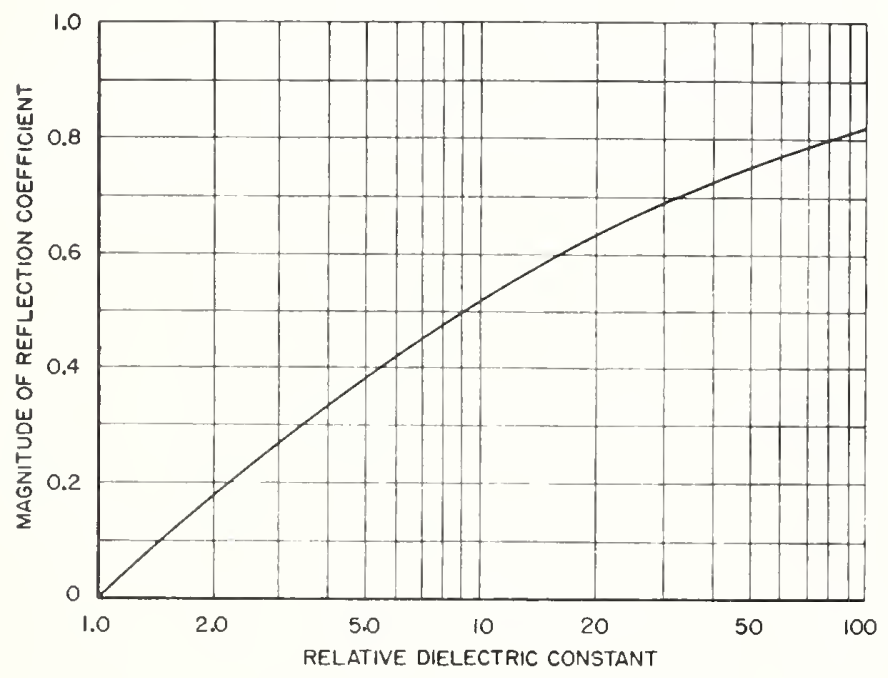

FIGURE 15. Calculated variation of the magnitude, $\rho$, of the complcx plane-wave reflection coefficient of the ground $\Gamma$, versus the rclative dielcctric constant, $\epsilon_{r}$, for vertical incidence, $\psi=\pi / 2$.

The curve applies to low-loss dielectrics only $\left(\sigma^{\prime} / \epsilon \omega \ll 1\right)$, and was determined from eq 26.

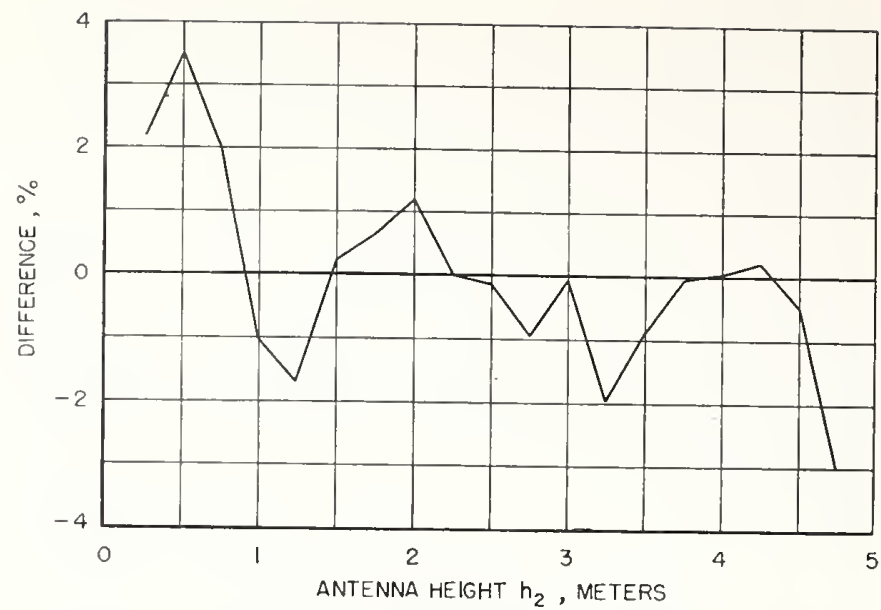

FiguRE 16. Variation of the percentage difference between the observed and calculated values of field intcnsity of figure 13, with height, $h_{2}$, of the receiving antenna.

the reeeiving antenna eurrent distribution and henee upon its effeetive length is negligible, at least for the present purpose for antenna heights in exeess of 0.1 wavelength.

The ealeulated value of the effeetive length is obtained from the product of the following values: (a) the classical value of effeetive length for a half-wave dipole given by eq 4 ; (b) the pereentage shortening for self-resonant operation from figure 3 ; and (e) the pereentage inerease for eylindrieal antennas from figure 2 . The resulting value is $l_{H} \cong \lambda / \pi \times 0.96 \times 1.06 \cong 0.97 \mathrm{~m}$ for operation at 100 $\mathrm{Me}$, and agrees to within about 1 pereent with the above measured value.

\section{Variable Height Runs}

The purpose of the tests deseribed in this seetion was to determine the probable measurement error existing in the two field-intensity standards under discussion. Determinations made by the two standards were direetly intereompared, the maximum probable error (in the absence of other referenee standards) being evaluated as one-half the percentage differenee between the two measurements. Parallel horizontal self-resonant dipoles were used at a frequeney of $100.0 \mathrm{Me}$.

Values of field intensity established by means of the standard-field method were measured using the standard (reeeiving)-antenna method and the results compared.

These runs consisted of varying separately the heights of either the reeeiving or transmitting antennas for a fixed height of the other antenna, and for a fixed distance of separation. 


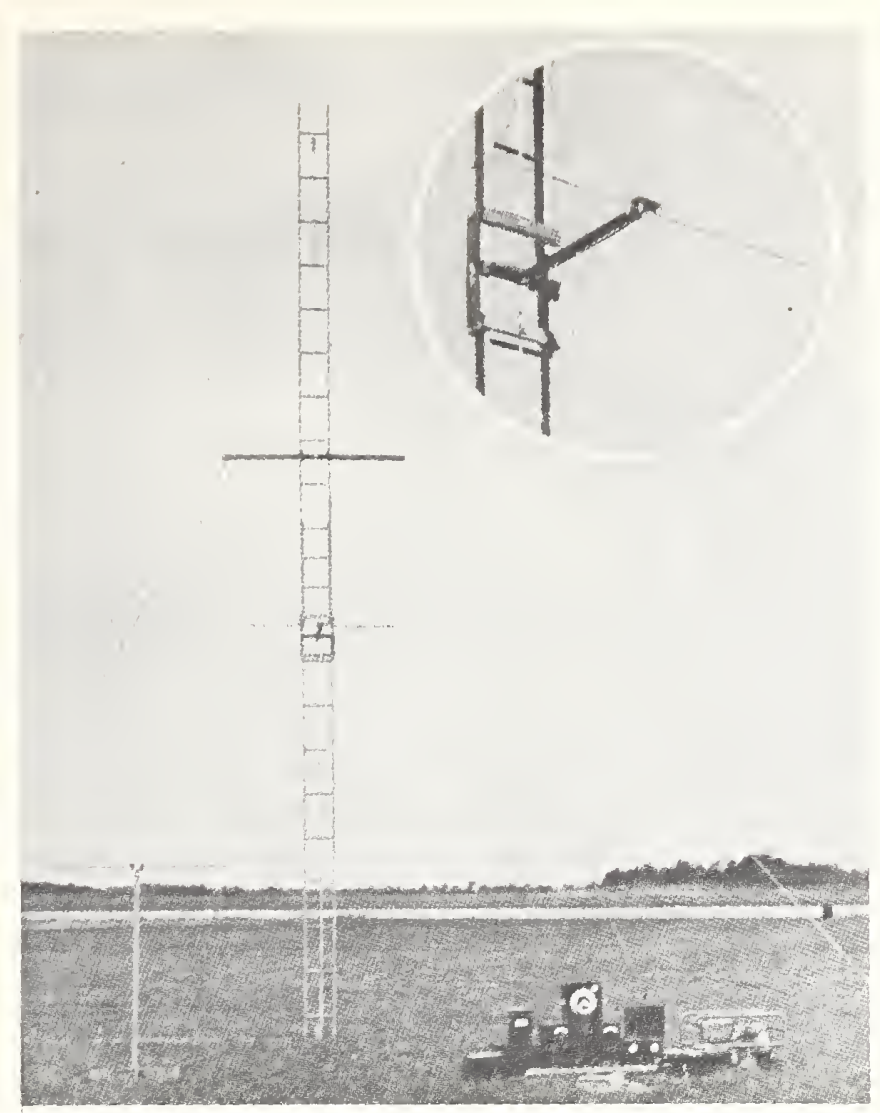

FIGURE 17. View of the various pieces of equipment used in obtaining the measured data of figures 13,14,18,19, and 20 .

In the baekground and insert are shown the lidder nuast and earriage for the receiving dipole. The transmitting antenna, $r-f$ generating equipment, and an engine-driven alternator are shown in the foreground. The location is the Beltsville (Md.) Airport.

In the first two runs the reeeiving antenna was varied in height, $h_{2}$, from 0.265 to $9.0 \mathrm{~m}$, using the ladder-mast assembly shown in figure 17. The heights, $h_{1}$, of the transmitting antenna were 1.58 and $3.05 \mathrm{~m}$, respeetively, and the horizontal distance of separation, $d$, was $30.5 \mathrm{~m}$. The measured value of the effeetive length of the antennas determined in the standing-wave test was used, namely, $l_{H} \cong 0.96 \mathrm{~m}$.

The results are shown in figure 18. In determining the field by the standard-antenna method, eq 2 was used (in volts per meter)

$$
|E|=\frac{V_{o c}}{l_{H}}
$$

where $V_{o c}$ is the measured value of open-eircuit voltage.

The value of field determined by the standardfield method was obtained (in volts per meter) from eq 17 ,

$$
|E| \cong \frac{60 \pi l_{H} I}{\lambda}\left\{\left(\frac{1}{R_{1}}-\frac{\rho}{R_{2}}\right)^{2}+\frac{4 \rho}{R_{1} R_{2}} \sin ^{2}\left[\frac{k\left(R_{2}-R_{1}\right)}{2}\right]\right\}^{1 / 2}
$$

where

$$
\begin{aligned}
l_{H} & =0.96 \mathrm{~m} \\
I & =0.100 \mathrm{amp} \\
\lambda & =3.00 \mathrm{~m} \\
k & =2 \pi / \lambda \\
R_{1} & =\left[\left(h_{1}-h_{2}\right)^{2}+d^{2}\right]^{1 / 2} \\
R_{2} & =\left[\left(h_{1}+h_{2}\right)^{2}+d^{2}\right]^{1 / 2} .
\end{aligned}
$$

Values of the magnitude, $\rho$, of the reflection coeffieient were determined by taking the magnitude of eq 13, assuming low-loss dieleetries, $(\sigma / \epsilon \omega \ll 1)$. Values of $\rho$ may also be taken from figure 11 .

It was not found practical to measure the dielectrie constant of the ground before each run beeause of the speeial setup required and the time eonsumed. Previous measurements showed that on the average the dieleetrie eonstant had a value around 15 , exeept after a rain or after a prolonged dry spell. This value of $\epsilon_{r}$ was therefore used in ealculating $\rho$ from eq 13 .

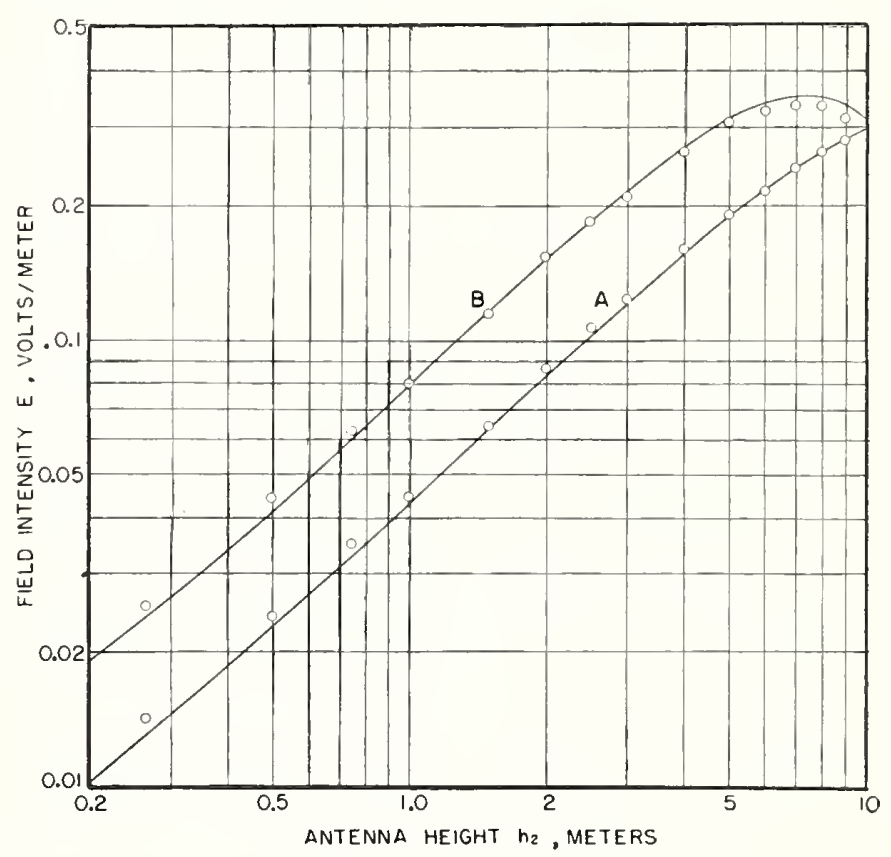

FIGURE 18. Variation of the magnitude of the electric component of field intensity, $|E|$ in volts per meter as determined by the standard-antenna and standard-field methods vs the height, $h_{2}$ in meters, of the standard receiving antenna.

Fixed heights $h_{1}=1.58 \mathrm{~m}(\mathrm{~A})$, and $3.05 \mathrm{~m}$ (B) of the transmitting antenna were used, and $d=30.5 \mathrm{~m}$. $f=100.0 \mathrm{Me}, I=0.100 \mathrm{amp}, \epsilon_{\mathrm{r}}=15, \sigma=0$. Horizontal self-resonant half-wave dipoles were used having effeetive lengths of $0.960 \mathrm{~m}$. $\bigcirc$. Standard-antenna method; --, standard-field method. 
The diameter of the circles representing the standard-antenna data in figure 18 is approximately 5 percent of the value of field intensity. Thus the difference is 2.5 percent or less for those points that touch the solid curves. Over most of the range of heights, $h_{2}$, the agreement is within about 5 percent.

A similar run is shown in figure 19 , except that the height of the transmitting antenna was varied for a fixed height of the receiving antenna. The antenna current, $I$, was maintained constant at 0.100 amp. The other conditions are the same as those applying to figure 18 . The agreement between the two standards is about the same as in the two previous runs.

\section{Variable-Distance Runs}

In these last tests the field intensity versus the horizontal distance of separation, $d$, was determined for fixed heights of the transmitting and receiving antemnas.

The receiving antenna was maintained at a fixed height $h_{2}=3.05 \mathrm{~m}$, and the distance, $d$, varied from 6 io $60 \mathrm{~m}$ for each of two heights of

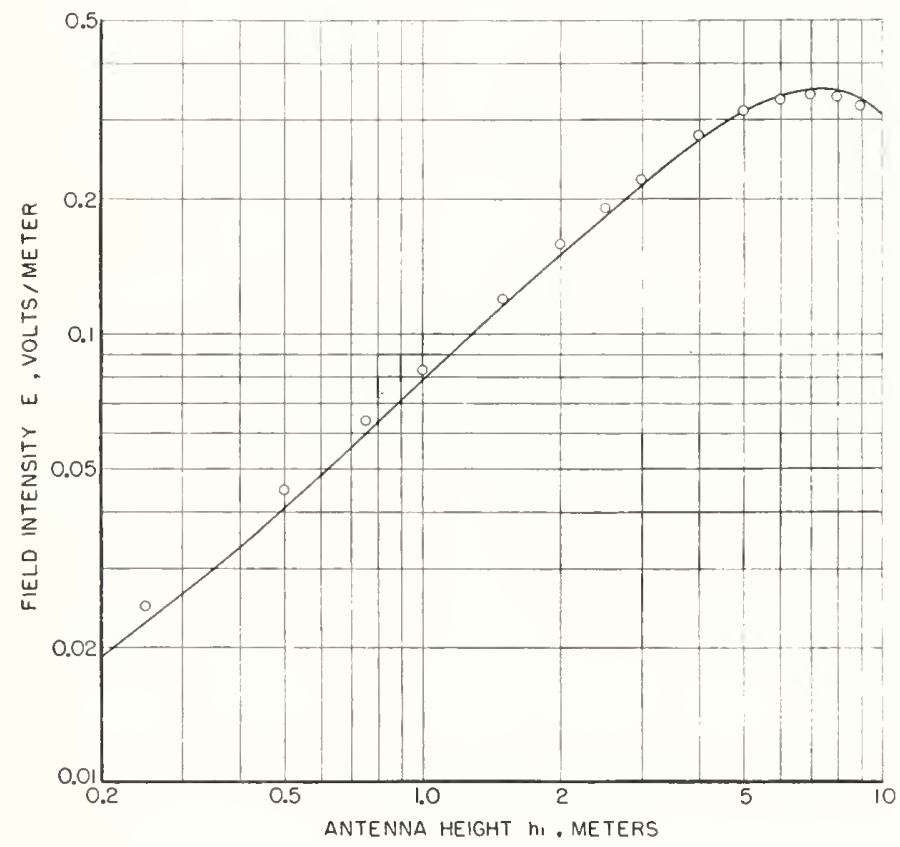

FIGURE 19. Variation of the magnitude of the elertric component of field intensity, $|E|$ in volts per meter, as determined by the Standard-Antenna and Standard-Field methods versus the height, $h_{1}$ in meters, of the transmitting antenna.

A fixed height, $h_{2}=3.05 \mathrm{~m}$, of the receiving antenna was used. The remaining conditions are identical to those applying to figure 18.0 , standardantenua method; --, standard-field method.

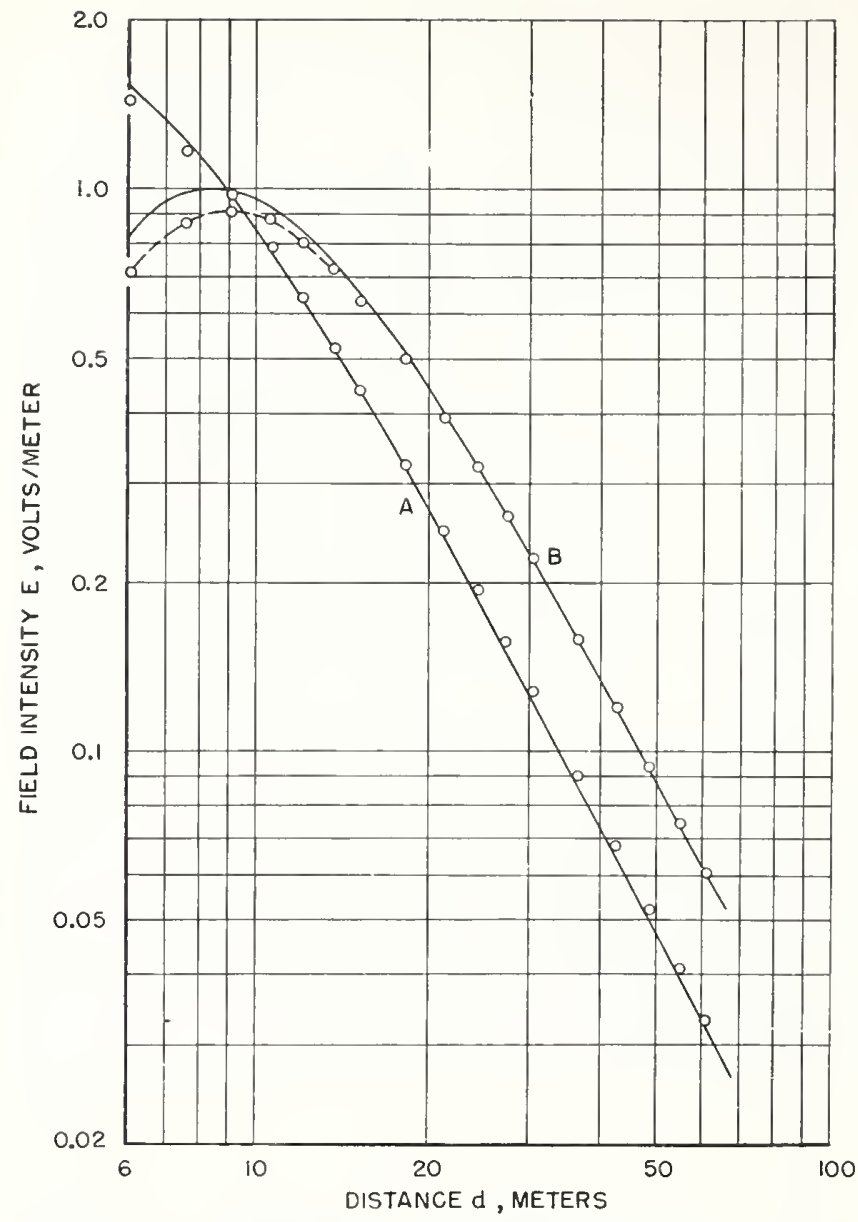

FIGURE 20. Variation of the magnitude of the electric component of field intensity, $|E|$ in volts per meter, as determined by the Standard-Antenna and Standard-Field methods versus the horizontal distance of separation, $d$ in meters, between the antennas.

Fixed heights of the transmitting antenna $h_{1}=1.58 \mathrm{~m} \mathrm{(A)}$, and $3.05 \mathrm{~m}(\mathrm{~B})$, and a fixed height of the receiving antenna, $h_{2}=3.05 \mathrm{~m}$ were used. The remaining conditions are identical to those applying to figure 18. O. Standard-antenna method; -, standard-field method.

the transmitting antenna $h_{1}=1.58$ and $3.05 \mathrm{~m}$, respectively.

Horizontal self-resonant half-wave dipoles were used as before. The field intensity was determined for various distances of separation using both the standard-antemna and standard-ficld methods. The remaining conditions are the same as those in the previous section. The results are shown in figure 20.

The values of field intensity determined by both the standard-antenna and standard-field methods (figs. 13, 18, 19, and 20) seem to be in fairly good agreement over most of the range of antenna. heights or distance of separation involved. It is reasonable to assume that the effective length of both antennas remains fixed throughout the entire 
range. The larger disagrecments noted are therefore probably a result of errors in calculating the standard ficld because of the accuracy with which the actual ground constants are known for the various incident angles involved.

\section{Conclusions}

A description has been given of two standard methods of establishing known values of VHF field intensity used at the National Bureau of Standards for the calibration of commercial fieldintensity meters. Methods and techniques used to calibrate and verify the accuracy of the standards were described. Values of standard field intensity can be established in the range from 20 $\mathrm{mv} / \mathrm{m}$ to $1 \mathrm{v} / \mathrm{m}$ at $100 \mathrm{Mc}$. This range can be considerably increased by rarying the transmitting-antenna current in known amounts by means of the standard attenuator previously mentioned. The propagation tests described indicate that the probable accuracy of the standards is about 5 percent under most of the conditions used.

The limitations in the use of the standards lie in the somewhat restricted range of ficld intensity available, the temperature effect of the crystal rectifier, and for some uses the lack of selectivity in the standard receiving antenna. However, for the purposes reported here and under the conditions used these factor's presented little difficulty

The initial work described in this report was confuned to the use of horizontal polarization at a irequency of $100 \mathrm{Mle}$. Further tests are in progress to verify the accuracy of these calibration standarels at frequencies up to $300 \mathrm{Mc}$ under conditions nol'mally used in the field. The additional use of rertical as well as circular polarization will be incluted in this program.

Unter most conditions normally employed for calibrating VHF ficlel-intensity meters, the value of the antema constant ${ }^{6}$ as determined by meaus of leorizontally polarized transmission would probably be substantially the same if vertical polarization were employed. This would be approximately true provided the antenma were immersed in a uniform field in both iustances, and that its

\footnotetext{
sThe antemua constant may be defund as $\kappa^{\circ}=1 / \eta l_{H}$, where $\eta$ is the voltagetriusfor ratio defined by equ 1 .
}

height above ground was greater than about a half wavelength. The antenna constant can be shown to be influenced by the effect of the presence of the ground on the current distribution along the antenna, as well as on its input impedance. Although, these effects are different for horizontal polarization than for vertical, they are probably relatively small for antenna heights above approximately a half wavelength.

Results presented herein indicate that for the case of horizontal polarization any possible effect of the ground on the antenna current distribution probably results in less than about a 2-percent change in the antenna constant for antenna heights greater than 0.1 wavelength.

The effect of the presence of the ground on the antenna constant as caused by changes in the antenna input impedance has been shown to be less than about $\tilde{\partial}$ percent for antenna heights greater than 0.65 wavelength above average ground $\left(\epsilon_{r}=15, \sigma=5 \times 10^{-3} \mathrm{mho} / \mathrm{m}\right)[14]$.

In the calibration of commercial VHF fieldintensity meter's the accuracy of calibration as certified by this Bureau at the present time would probably be fiom twice to several times the accuracy of the standards described, depending upon the merits of the individual meter under test.

\section{Appendix. A Summary of Schelku- noff's Analysis of the Current Distribu- tion on a Thin Cylindrical Antenna}

Schelkunoff [1] obtained an approximate solution for the free-space current distribution on a hollow cylindrical antenna by analogy with a thin biconical antenna, together with the theory of the nonmiform transmision line.

The antenna region and the space beyond are considered as two multiple transmission lines or wareguides in tanden, each with it - own distinct set of transmission modes.

In the antenna region (that space within a geometrical boundary sphere passing through the ends of the antenna) the field is composed of a principal or dominant mode plus a complementary set of an infinite number of higher order modes. The principal mode or ware, is that usually as. sociated with a two-conductor transmission line. The complementary waves are generated in order to match the field (at the boundary sphere) to the field in the externat region where no principal mode of transmission exists. Because of the nomexistence of physical conductors in the external region, the field consists solely of an infinite set of higher-order transmission modes. While these sets 
are distmct in their respective regions, they approach equality at the boundary, thus preserving the continuity of the field.

Associated with the principal and complementary waves in the antcnna region are the principal current $I_{0}(r)$, and the complementary current flowing on the antenna given by

$$
\bar{I}(r)=I_{1}(r)+I_{3}(r)+I_{5}(r) \ldots
$$

where the cven-order mordo: ranish because of the symnetry involved.

At the ends of the hollow cylindrical doublet antenna $(r=l)$ the total current is assontially zero or

$$
I(l)=I_{0}(l)+\bar{I}(l)=0 .
$$

Therefore,

$$
I_{0}(l)=-\bar{I}(l) .
$$

Since it can be shown that the total voltage asrociated with any complementary wave is zero,

$$
V(r)=V_{0}(r) \text {. }
$$

It can be seen that the presence of the complementary waves affect the amplitudes of the principal waves as would an impedance

$$
Z_{\imath}=\frac{V_{0}(l)}{I_{0}(l)}
$$

tcrminating the antenna.

As far as the principal waves are concerned, then, the antenna may be considered as a nonuniform transmission linc terminated by an impcdance $Z_{\mathrm{t}}=R_{\mathrm{t}}+j X_{\mathrm{t}}$. The real part, $R_{t}$, is the radiation resistance of outer spacc as seen from the ends of the antenna, and the imaginary part, $X_{t}$, represents the reactive field associated with the complementary waves. These two components represent the end effect usually associated with radiation from an antenna.

The current distribution on the antenna is therefore determined by the actual distribution of $L$ and $C$ along the antenna and by the presence of the complcmentary currents generated by reflection at the ends.

In a uniform parallel-wire transmission line, $L$ and $C$ per unit length, and consequently $Z_{0}$, are constant throughout. In a transmission line composed of a straight wire of length $2 l$, driven at the center, $L$ and $C$ vary continwously along its length. The potential difference betwcen any two points equidistant from the center is simply the integral of the electric field intensity along a line of con- stant intensity terminating at the two points. The cylindrical doublet may be compared to such a nonuniform transmission line. $L$ and $C$ usually vary only slowly with $r$ in such a line, and by analogy to a thin biconical antenna with slowly varying cone angle, $\psi$, the characteristic impedance as a function of $r$ can be expressed approximately as

$$
K(r) \cong 120 \log _{e} \frac{2 r}{a}(r \gg a)
$$

The average characteristic impedance over length $l$ is

$$
K_{0} \cong \frac{1}{l} \int_{0}^{l} K(r) d r \cong 120\left(\log _{e} \frac{2 l}{a}-\right)
$$

From the theory of the nonuniform transmission line, the relative distribution of the principal current is given for the case in which the variation in $K(r)$ is small

$$
\begin{aligned}
\frac{I(r)}{I(0)}= & {\left[\cos \beta r-\frac{M(r)}{K_{0}} \cos \beta r+\frac{N(r)}{K_{0}} \sin \beta r\right] } \\
& \frac{-j Z(0)}{K_{0}}\left[\sin \beta r+\frac{M(r)}{K_{0}} \sin \beta r+\frac{N(r)}{K_{0}} \cos \beta r\right],
\end{aligned}
$$

where

$$
\begin{aligned}
\beta= & 2 \pi / \lambda, \text { and } Z(0) \text { is the input impedance of } \\
& \text { the antenna } \\
M(r)= & \beta \int_{0}^{r}\left[K_{0}-K(r)\right] \sin 2 \beta r d r \\
= & 60(1-\cos 2 \beta r)\left[\log _{e}(l / r)-1\right]+60\left(\log _{e} 2 \beta r\right. \\
& -C i 2 \beta r+C) \\
N(r)= & \beta \int_{0}^{r}\left[K_{0}-K(r)\right] \cos 2 \beta r d r \\
= & 60\left(\log \frac{l}{r}-1\right) \sin 2 \beta r+60 S i 2 \beta r, \\
S i(x) \equiv & \int_{0}^{x} \frac{\sin t}{t} d t \\
C i(x) \equiv & \int_{\infty}^{x} \frac{\cos t}{t} d t \\
C= & 0.5772 \ldots \text { (Euler's constant). }
\end{aligned}
$$

The relative complcmentary current for a biconical antenna and to a second approximation for any thin antenna is given by

$$
\frac{\bar{I}(r)}{I_{0}}=\frac{60}{K_{0}} \sum_{m=0}^{\infty} \frac{4 m+3}{(m+1)(2 m+1)}\left[\hat{N}_{2 m+1}(\beta l)+j \hat{J}_{2 m+1}(\beta l)\right] \hat{J}_{2 m+1}(\beta r)
$$


$I_{0}$ is the principal current amplitude on the transmission line, and $m$ is an integer. For $(2 n+1) \lambda / 2$ doublets in which we are interested (where $n=0,1,2,3, \ldots$. ), $I_{0} \cong I(0) . \quad \hat{J}_{2 \mathrm{~m}+1}(\beta l)$ and $\hat{N}_{2 \mathrm{~m}+1}(\beta l)$ are special functions related to $J$ and $N$ Bessel functions of order $n+1 / 2$ $[1$, p. 52].

The total relative current on the antenna is given by the sum of eq 34 and 35 . For an antenna composed of an infinitely thin filament $\left(K_{0}=\infty\right)$ the relative current distribution. reduces to $\cos \beta r$, as is usually assumed for such a case.

\section{References}

[1] S. A. Schelkunoff, Electromagnetic waves, p. 290 to 292 and 441 to 479 (D. Van Nostrand Co., Inc., New York, N. Y., 1943).

[2] S. A. Schelkunoff, Theory of antennas of arbitrary size and shape, Proc. IRE 29, 508 (Sept. 1941).

[3] R. King, Graphical representation of the characteristics of cylindrical antennas, Cruft Laboratory, Tech. Rept. No. 20 (ONR) (Oct. 1, 1947).

[4] L. S. Nergaard, A survey of ultra-high-frequency measurements, RCA Rev. 3, 183 (Oct. 1938).

[5] MIT Radiation Laboratory Series-Crystal rectificrs, Louis N. Ridenour, editor, p. 333 to 360 (McGrawHiil Book Co., New York, N. Y., 1948).

[6] Ernst A. Guillemin, Communication Networks II, 64 (John Wiley \& Sons Inc., New York, N. Y., 1935). [6a] R. E. Granthanı, and J. J. Frceman, A Standard of Attenuation for Microwave Measurements, Trans. A. I. E. E., 67, p. 535, 1948.

[7] Nathand Marchand, Transmission-line converion transformers, Electronics p. 142 to 145 (Dec. 1944).

[8] K. A. Norton, The propagation of radio waves over the earth and in the upper atmosphere, Proc. IRE 25, 1217 (Sept. 1937).

[9] K. A. Norton, The calculation of ground-wave field intensity over a finitely-conducting spherical carth, Proc. IRE 29, 623 to 639 (Dec. 1941).

[10] C. R. Burrows, Radio propagation over planc earthField strength curves, Bell System Tcclı. J. 16, 45 to 75 (Jan. 1937).

[11] K. Bullington, Radio propagation at frequencies above 30 megacycles, Proc. IRE 35, 1122 to 1136 (Oct. 1947).

[12] J. A. Stratton, Electromagnetic theory, p. 493 (McGraw-Hill Book Co., Inc., New York, N. Y., 1941).

[13] R. King, H. R. Mimno, A. H. Wing, Transmission lines, antennas, and waveguides, p. 160 (McGrawHill Book Co., Inc., New York, N. Y., 1945).

[14] Frank M. Greene, The influence of the ground on the calibration and use of VHF ficld-intensity meters, J. Research NBS 44, 123 (1950) RP2062.

Washington, October 3, 1949. 


\title{
Influence of the Ground on the Calibration and Use of VHF Field-Intensity Meters
}

\author{
By Frank M. Greene
}

\begin{abstract}
One type of error known to be present in VHF field-intensity meters (30 to $300 \mathrm{Mc}$ ) is caused by the influence of the ground on the value of the antenna voltage-transfer ratio. This is a result of fluctuation of the receiving-antenna input impedance with height and changing ground conditions. An approximate method is presented for calculating the input impedance of horizontal dipole antennas over earth having finite values of dielectric constant and conductivity. The effect of both changes in ground conditions and antenna terminating impedance on the above error is calculated as a function of the antenna height. Measured values are presented in support of the above method, and the results are discussed.
\end{abstract}

\section{Introduction}

As is known, THF field-intensity measurements will be generally in cror if made at antenna heights other than that for which the antenna constant was determined when the ficld-intensity meter was calibrated. An error will likewise exist if the ground constants at the site chosen to make measurements are appreciably different from those existing at the time or place of calibration.

At present most VHF ficld-intensity meters use a doublet receiving antenna, which is usurlly terminated at its center terminals in an impedance roughly equal in value to its free-space input impedance. The crror referred to exists because of the change of the antenna-input impedance with height above ground or with changing ground conditions ${ }^{1}$. This results in a corresponding fluctuation in the proportion of the induced roltage that appears across the icminals at the center of the artuma. Consequently the ralue of the antemia constant determined at the time of the calibration is in general not the same if the height or ground conditions are altered.

An approxirate expression for the input inpedance at various heights above a finitely condueting ground nua be easily obtained for the ease of a horizontal antemna. The ground is assumed

It is assumed here that the field-intensity meter is calibrated and used at such locations that the distances to the nearest reflecting objects such as trees or inilungs are very much grater than the heights of the receising antenna above the ground. to be plane, homogeneous, and with finite valuc's of the relative dielectric constant $\epsilon_{\mathrm{r}}$, and conductivity $\sigma$. Once the antcmna-input impedance is known, the effect of the earth on the antenna constant may be determined.

Although the solution attempted here is not rigorous, it can be shown to yield the limiting value of the input impedance of a horizontal antemna if its height above ground is increased sufficiently. The results, howerer, are useful in obtaining approximate values of the imput impedance corresponding to antenua heights of a fraction of a. wavelength.

Theoretical values of the measurement crror are reasonably well supported by measurement at one particular site for antenna heights down to onetenth wavelength at $100 \mathrm{Mc}$. The effect both of changes in ground conditions and of the value of the antenna terminating impedance upon this error are determined. Practical rationalized mks units are uscd throughout.

\section{Theory}

In formulating the following solution, the usual system will be considered, comprising a transmitting and receiving antenna at heights $h_{1}$ and $h_{z}$, respectively, above ground. The ground is assumed to be plane, homogeneous, and of infinite extent, having finite values of relative dielectric constant $\epsilon_{r}$ and ronductirity $\sigma$. Although the 
method is applicable to horizontal antennas of any length, the results will be evaluated only for the case of parallel horizontal half-wave dipoles. Their locations and the geometry involved are shown in figure 1.

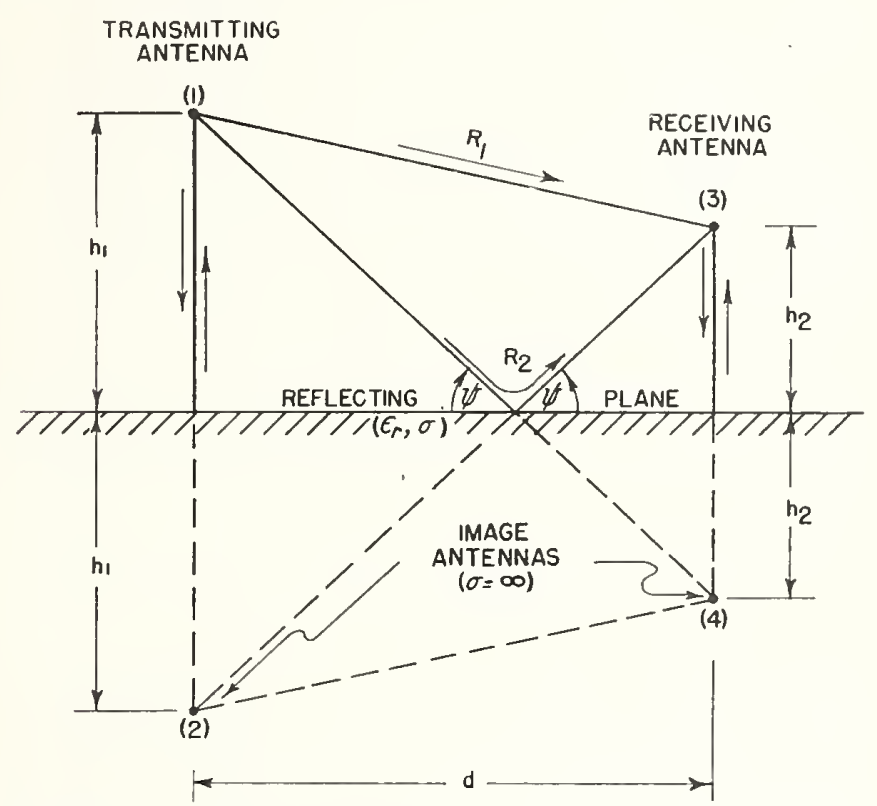

Figure 1. Ray-path diagram showing: direct ray along $R_{1}$; ground-reflected ray along $R_{2}$; rays from both horizontal transmitting and receiving antennas reflected at normal incidence from the ground back to the antenna.

Heights of the transmitting and receiving antenns are $h_{1}$ and $h_{2}$ respectively, and $d$ is the horizontal distance of separation.

In addition to the direct and ground-reflected rays along $R_{1}$ and $R_{2}$, respectively, a ray will be considered that leaves each antenna and is reflected at normal incidence from the ground back to the antenna.

\section{Perfectly Conducting Ground}

Perfectly conducting ground will be considered first. Its effect may be simulated in the usual manner by postulating the image antennas (2) and (4), each located at a distance below the perfect reflecting plane equal to the height of the actual antenna. The two antennas and their images may be treated as four coupled antennas. The resulting voltage-current relationship will have exactly the same form as would exist in a linear four-mesh network. For this case ${ }^{2}$ the resulting four equations reduce to the following two:

\footnotetext{
${ }^{2} \mathrm{P}$. R. Karr, The influence of the ground upon the voltage induced in $\mathbf{a}$
} receiving antenna, Report OD-2-348R (NBS) (July 23, 1947).

$$
\left.\begin{array}{l}
V \stackrel{\bullet}{=} I_{1}\left(Z_{11}-Z_{12}\right)+I_{3}\left(Z_{13}-Z_{14}\right), \\
O=I_{1}\left(Z_{31}-Z_{32}\right)+I_{3}\left(Z_{33}-Z_{34}\right),
\end{array}\right\}
$$

where $V$ is the impressed emf at the center of the transmitting antenna, and $I_{1}$, and $I_{3}$, are the respective currents at the centers of the transmitting and receiving antennas. The terms $Z_{11}$ and $Z_{33}$ are the free-space self impedances, respectively, of the transmitting and receiving antennas referred to the center terminals. Also

$$
Z_{m n}=Z_{n m}=-\frac{V_{m n}}{I_{n}}
$$

where $Z_{m n}$ is the mutual impedance between antennas $m$ and $n$, and $V_{m n}$ is the emf induced in antenna $m$ (referred to the center terminals) by the current, $I_{n}$, at the center of antenna $n$.

\section{Finitely Conducting Ground}

In considering the case involving a finite earth, the equations for meshes (2) and (4) of the previous system become meaningless. However, by benefit of analogy with eq 1 and with the aid of expcrimental evidence, one may write a similar set of equations involving antennas (1) and (3) and the ground, which under certain conditions, describe this transmission system to a first approximation at least. The equations are:

$$
\left.\begin{array}{c}
\dot{V}=I_{1}\left(Z_{11}+\Gamma_{1} Z_{12}\right)+I_{3}\left(Z_{13}+\Gamma_{2} Z_{14}\right), \\
U=I_{1}\left(Z_{31}+\Gamma_{2} Z_{32}\right)+I_{3}\left(Z_{33}+\Gamma_{1} Z_{34}\right),
\end{array}\right\}
$$

where $\Gamma_{1} \equiv \rho_{1} e^{-\jmath_{\phi}}=$ complex plane-wave reflection coefficient for vertical incidence; $\Gamma_{2} \equiv \rho_{2} e^{-j_{\phi_{2}}}=$ complex plane-wave reflection coefficient (horizontal polarization) for the angle $\psi=\tan ^{-1}\left(h_{1}+h_{2}\right) / d$ made with the eartl by the principal groundreflected ray (along $R_{2}$ ).

The reflection coefficient, $\Gamma_{2}$, may be expressed in terms of the angle $\psi$ and a complex dielectric constant $\epsilon_{0}$ as follows ${ }^{3}$ (for horizontal polari :ation):

$$
\mathrm{I}_{2}=\frac{\sin \psi-\sqrt{\epsilon_{0}-\cos ^{2} \psi}}{\sin \psi+\sqrt{\epsilon_{0}-\cos ^{2} \psi}}
$$

where $\epsilon_{0}=\epsilon_{r}\left(1-j \frac{\sigma}{\epsilon \omega}\right)$,

$$
=\epsilon_{r}-j 60 \lambda \sigma
$$

3 J. A. Stratton, Electromagnetic theory, B. 493 (McGraw-Hill Book Co., Inc., New York, N. Y., 1941). 
$\epsilon_{r} \doteq$ relative dielectric constant of the ground (refenred to free-spacc as unity),

$\epsilon=\epsilon_{\ell} \epsilon_{r}$, wlicre $\epsilon_{v}$ is the permittivity of eracuated free-space,

$\epsilon_{n} \simeq \frac{1}{36 \pi} \times 10^{-9}$ farads/meter

$\sigma=$ ground conductivity is mhos/neter,

$\omega=2 \pi f$,

$\lambda=$ wave hongth in meters,

$j=\sqrt{-1}$.

Equations 3 will reduce to eq 1 if the ground conductivity $\sigma$ is allowed to increase without limit, since in this case $\Gamma_{1}=\Gamma_{2}=-1$ for all angles of incidence, as can be seen from eq 4.

\section{Evaluating the Self and Mutual Impedances}

Before practiral use can be made of eq :3, the various self and mutual impedances must be evaluated. Schelliunofl ${ }^{4}$ las determined the freespace input impedance of cylindrical antennas in general. Values may be obtained graphically from figs. 11.21 and 11.22 of this reference for antennas of several length-to-diameter ratios. A value of $73.2+j 42.5$ (ohms) may be used if desired, corresponding to a thin $\lambda / 2$ dipole in free-space, without substantially affecting the resulting valuc of the measurement error. Carter ${ }^{5}$ has evaluated the nutual impedance between ant ennas of various configurations. For the case of parallel half-wave dipoles in frec-space the mutual impedance iu olıms is:

$$
\begin{aligned}
Z= & 30\left\{2 \operatorname{Ei}(-j k R)-\operatorname{Ei}\left[-j k\left(\sqrt{\left.\left.R^{2}+l^{2}+l\right)\right]}\right.\right.\right. \\
& \left.-\operatorname{Ei}\left[-j k\left(\sqrt{ } R^{2}+l^{2}-l\right)\right]\right\},
\end{aligned}
$$

where $l=a n t e m n$ length in meter's,

$R=$ distance betweon antennas in meters,

$\mathrm{Ei}(-j x) \equiv \mathrm{Ci}(x)-j \mathrm{Si}(x)$,

$$
\begin{aligned}
\mathrm{Ci}(x) & \equiv-\int_{x}^{\infty} \frac{\cos t}{t} d t, \\
\mathrm{Si}(x) & \equiv \int_{0}^{x} \sin t d t \\
k & \equiv 2 \pi / \lambda .
\end{aligned}
$$

4S. A. Sehelkunoff, Electromagnetic waves, pp. 441 to 479 (1). Van Nos. trind Co., New York, N. Y., 1943).

s. S. Carter, Cirenit relations in radiating systens and applications to antonna problems, I'lec. IRE 20, INP. 1004 to 1041 (June 1932).
Values of eq 5 are shown plotted in figures 11 and 12 of the reference given in footnote 5 for spacings from 0 to 7.5 wavelengths.

It is possible to derive a more simplc expression than eq 5, valid for distances of separation in excess of about $2 \lambda$. At this distance from an antenna, only the radiation component of the clectric field-intensity usually necd be considered. For a half-wave transmitting dipole in free-space, oriented normal to a line from its center to the point of observation, the field intensity is (in volts/ meter)

$$
E \cong-j \frac{60 I}{R} e^{\frac{-j 2 \pi R}{\lambda}},
$$

where $R=$ distance in meters,

$I=$ current in amperes at the conter of the antenna.

The voltage (refcrred to the center terminals) induced in a half-wave dipole placed in the field given by eq 6 and oriented parallel to the transmitting antenna is (in volts)

$$
V \cong E l_{H} \cong-j \frac{60 \lambda I}{\pi R} e^{\frac{-j 2 \pi R}{\lambda}}
$$

where $l_{H}=$ effective length of the dipole in meters,

$l_{H}=\lambda / \pi$ meters for a half-wave dipole assuming sinusoidal current distribution.

From eq 7 and 2 the mutual imperance betwcen the two parallel half-wave dipole antennas is (in ohms)

$$
Z=-\frac{V}{I} \cong j \frac{60 \lambda}{\pi R} e^{\frac{-j 2 \pi R}{\lambda}}
$$

It can be shown that eq 8 is the limiting value of eq 5 for sufficiently large values of the distance of separation, $R$. In fact, for separations in excess of about $2 \lambda$, the value of mutual impedance given by eq 8 is sufficiently accuratc for many purposes ${ }^{6}$ and will cause less than 0.1-percent error in the final results in which we are interested here. For smaller values of separations between the antennas than $2 \lambda$, eq 5 , or figures 11 and 12 of the reference given in footnote 5 must be used to craluate the mutual impedance.

${ }^{6}$ Losmo J. Affanasiev, Simplifications in the consideration of mutual effects between half wave dipoles, Proc. IRE 34, pp. 635 to 638 (Sept. 1946).

\section{Effect of Ground on VHF Field-Intensity Meters}




\section{Relations Existing in the Receiving Antenna}

\section{Antenna Current}

Equations 3 may now be solved for the current $I_{3}$ at the center of the receiving antenna. The problem will be simplified if it is assumed that the distance between transmitting and receiving antennas is sufficiently large that the presence of the receiving antenna does not measurably affect the current flowing in the transmitting antenna. This is usually the case in practice, and the assumption is certainly justified if the spacing is at least several wavelengths. In this case the current in the receiving antenna terminated at its center in a load impedance $Z_{L}$ is, from eq 3 :

$$
I_{3}=-\frac{\left(Z_{31}+\Gamma_{2} Z_{32}\right) I_{1}}{Z_{L}+Z_{33}+\Gamma_{1} Z_{34}}
$$

\section{Input Impedance}

The numerator of eq 9 is the induced emf in the receiving antenna, and the denominator is the input impedance (in the presence of the ground) plus the terminating impedance $Z_{L}$ connected at the center, the input impedance being

$$
Z_{i} \equiv Z_{33}+\Gamma_{1} Z_{34}
$$

The effect of the ground in the immediate vicinity of the receiving antenna is accounted for by the second term on the right of eq $10, \Gamma_{1} Z_{34}$. $Z_{34}$ is the mutual impedance that would exist between the receiving antenna and its image if the ground were perfectly conducting, and is given by eq 5 or eq. 8 upon substituting $R=2 h_{2}$. $\Gamma_{1}$ is of course the actual reflection coefficient of the ground for normal incidence obtained from eq. 4 by placing $\psi=\pi / 2$, which gives

$$
\Gamma_{1}=\frac{1-\sqrt{\epsilon_{r}\left(1-j \frac{\sigma}{\epsilon \omega}\right)}}{1+\sqrt{\epsilon_{r}\left(1-j \frac{\sigma}{\epsilon \omega}\right)}}
$$

Values of the magnitude of eq. 11, $\rho_{1}$, are shown plotted vs $\epsilon_{\tau}$ in figure 2 for low-loss dielectrics, $(\sigma / \epsilon \omega<<1)$. Many types of ground may be treated as low-loss dielectrics over a large portion of the VHF band as far as their reflecting properties are concerned. This is particularly true at the higher frequencies above 50 or 75 Mc. Under these conditions the phase shift on reflection, $\phi$, is very nearly $180^{\circ}$, so that $\Gamma_{1} \cong-\rho_{1}$.

\section{Voltage Relations}

The terminal voltage of the receiving antenna terminated in an impedance $Z_{L}$ is, from eq. 9

$$
V_{L}=-\frac{\left(Z_{31}+\Gamma_{2} Z_{32}\right) Z_{L} I_{1}}{\left(Z_{L}+Z_{33}+\Gamma_{1} Z_{34}\right)}
$$

and the open-circuit voltage is, letting $?_{L} \rightarrow \infty$,

$$
V_{o c}=-\left(Z_{31}+\Gamma_{2} Z_{32}\right) I_{1} \text {. }
$$

If the receiving antenna is sufficiently high above the ground, $Z_{34}$ may be considered negligible compared to $\left(Z_{L}+Z_{33}\right)$, in which rase the terminal voltage will be, from eq 12 ,

$$
V_{L}=-\frac{\left(Z_{31}+\Gamma_{2} Z_{32}\right) Z_{L} I_{1}}{Z_{L}+Z_{33}}
$$

The -true value of the electric component of field intensity at any antenna height, $h_{2}$, above the ground is, from eq 7 and 13 :

$$
E_{i}=\frac{V_{o c}}{l_{H}}=-\frac{\left(Z_{31}+\Gamma_{2} Z_{32}\right) I_{1}}{l_{H}}
$$

The value of field intensity that would be indicated by a field-intensity meter previously calibrated in the presence of the ground is, from eq 12 :

$$
E_{i}=K V_{L}=-\frac{K\left(Z_{31}+\Gamma_{2} Z_{32}\right) Z_{L} I_{1}}{\left(Z_{L}+Z_{33}+\Gamma_{1} Z_{34}\right)}
$$

$K$ may be defined as the antenna constani and may be evaluated at any desired height of the receiving antenna. If a height $h_{2}$ is chosen such that $Z_{34} \ll\left(Z_{L}+Z_{33}\right), K$ might then be termed the free-space antenna constant and in such a case its value would be

$$
K \cong \frac{1}{l_{H}}\left(\frac{Z_{L}+Z_{33}}{Z_{L}}\right)
$$

since $E_{t} \cong E_{i}$ at this height. The antenna constant is seen to be the reciprocal of the product of the effective length, $l_{H}$, and the voltage-transfer ratio, $V_{L} / V_{o c}=Z_{L} /\left(Z_{L}+Z_{33}\right)$.

The assumption is made here that the relative current distribution and hence the effective lengtl of the half-wave receiving dipole is not (to $x$ 
first approximation) a function of either the terminating impedance, $Z_{L}$, or the height of the antemna above ground. Although a complete solution to the general problem of the receiving antenna has unfortunately not yet been achieved, this assumption is supported by a number of our measurements.

\section{Evaluation of the Measurement Error}

The difference between the true value of field intensity existing at some antenna height $h_{2}$, and that indicated by a field-intensity meter with a previously determined antenna constant is (in percent)

$$
\delta=\left(\left|\frac{E_{i}}{E_{t}}\right|-1\right) \times 100 .
$$

For the case in which the antenna constant, $K$, was determined at a sufficient antenna height that it may be considered to have a free-space value, the above difference, or measurement error, may be obtained by substituting eq 15, 16, and 17 in eq 18 , giving (in percent)

$$
\delta=\left(\left|\frac{Z_{L}+Z_{33}}{Z_{L}+Z_{33}+\Gamma_{1} Z_{34}}\right|-1\right) \times 100
$$

$Z_{L}=$ load impedance connected to the center terininals of the receiving antenna.

$Z_{33}=$ input impedance (in free space) of the receiving antenna. $Z_{33}$ may be evaluated from figures 11.21 and 11.22 of the reference given in footnote 4 or if desired may be taken as $73.2+j 42.5$ (ohms) corresponding to a thin $\lambda / 2$ dipole in free-space, without substani.ially affecting the resulting value of the measurement error.

$Z_{34}$ may be evaluated from eq 5 , or from figures 11 and 12 of the reference given in footnote 5 . For heights of the receiving antenna $h_{2} \geqq \lambda$, $Z_{34}$ may be evaluated from eq 8 , placing $R=2 h_{2}$. 'This gives (in ohms)

$$
Z_{3 \dot{4}}=j \frac{30 \lambda}{\pi h_{2}} e^{-j} \frac{i \pi h_{2}}{\lambda} .
$$

$\Gamma_{1}=$ planc-wave reflection coefficient for normal incidence. $\Gamma_{1}$ may be evaluated from eq 11, ol

\footnotetext{
- Further details are contained in a forthcoming Bureau paper entitled,

"Development of Vif field-intensity standards", by F. M. Greene and M. Solow.
}

Effect of Ground on VHF Field-Intensity Meters in the case of low-loss dielectrics, from figure 2, since $\Gamma_{1} \cong-\rho_{1}$.

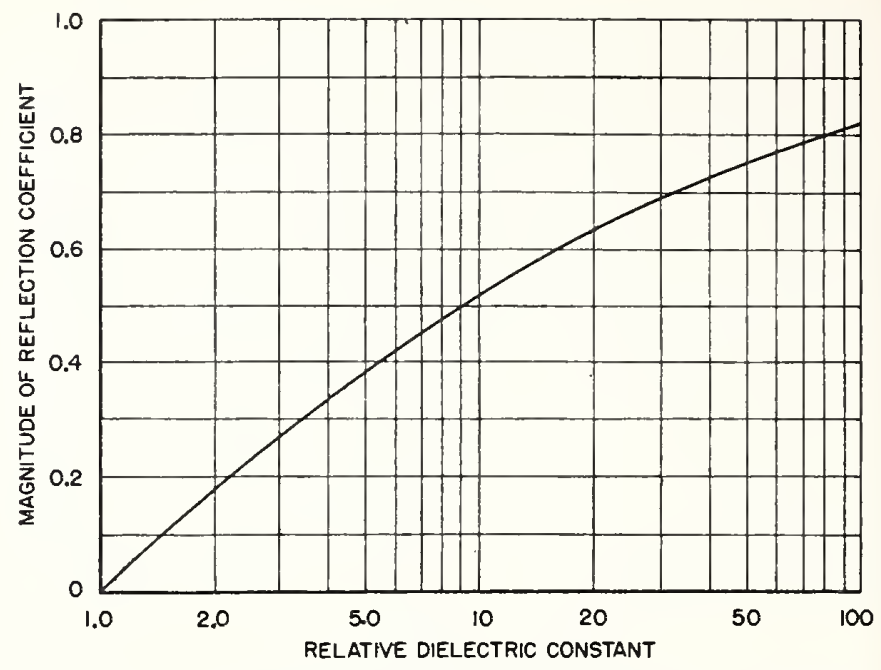

FIGURE 2. Magnitude of the plane-wave reflection coefficient, $\rho_{1}$ (at normal incidence) vs the relative dielectric constant, $\epsilon_{r}$.

Low-loss dielectrics are assumed $(\sigma / \epsilon \omega<<1) . \quad \Gamma_{1}=-\rho_{1}$.

\section{Discussion of Results}

The measurement error to be discussed is that existing in a field-intensity meter whose antenna constant was determined under free-space conditions. This error or difference (as calculated) is given by eq 19 and is shown in figures 3 and 4 vs $h_{2} / \lambda$ for various values of the parameters $\Gamma_{1}$ and $Z_{L}$. Measured values of the error determined at one particular site as well as the corresponding calculated values $(f=100.0 \mathrm{Mc})$ are shown in figure 5 .

Figure 3 shows the effect of changes in the ground constants on the measurement error calculated for an antenna terminated in an impedance $Z_{L}=73+j 0$ ohms. The self-impedance of the antenna was assumed to be $73.2+j 42.5 \mathrm{ohms}$. Curves are showll for (A) $\sigma=\infty$, (B) $\epsilon_{\tau}=30$. (C) $\epsilon_{r}=15$, and (D) $\epsilon_{r}=9$. Low-loss dielectrics were assumed in the last three cases.

The high and low values of the relative dielectric constant chosen represent the approximate extremes neasured at one particular site $(f=100.0$ Mc) during the summer of 1948 (see footnote 7). The value, $\epsilon_{r}=15$, is usually assigned to average ground, along with a value of conductivity $\sigma=5 \times 10^{-3}$ mhos/meter. Values of conductivity of this order of magnitude can be ignored, at least for frequencies above $50 \mathrm{Mc}$ as far as the 


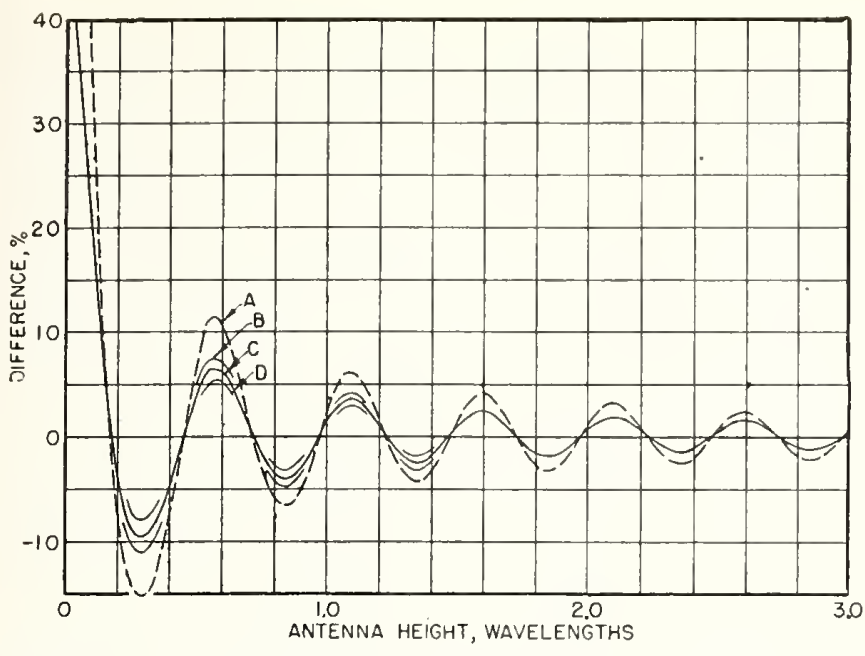

Figdre 3.-Calculated percentage difference $\left(\left|E_{i} / E_{t}\right|-1\right) \times$ 100 vs receiving antenna height, $h_{2} / \lambda$, in wavelengths.

$E_{1}$ is the true value of feld intensity, and $E_{i}$ is the value indicated by a field-Intensity meter with a previously determined free space value of antenna constant (horizontal polarization). Curves are shown for four values of ground constants: (A) $\sigma=\infty$; (B) $\epsilon_{r}=30$; (C) $\epsilon_{r}=15$; and (D) $\epsilon_{r}=9$ (for low-loss dielectrics $\sigma / \epsilon \omega<<1$ ). Antenna length $l=\lambda / 2$. The free-space antenna input impedance is taken as $Z_{33}=73.2+j 42.5$, and the terminating impedance $Z_{L}=73+j 0$ obms.

effect on the reflection coefficient $(\psi=\pi / 2)$ is concerned.

Apparently the usual changes in the ground constants experienced (due to changing moisture content) have but little effect upon the measurement error as presented here. The total variation from average ground conditions $\left(\epsilon_{r}=15\right)$ does not exceed 1.5 percent, except for values of $h_{2} / \lambda$ $<0.15$.

As shown by figure 3, a field-intensity. meter

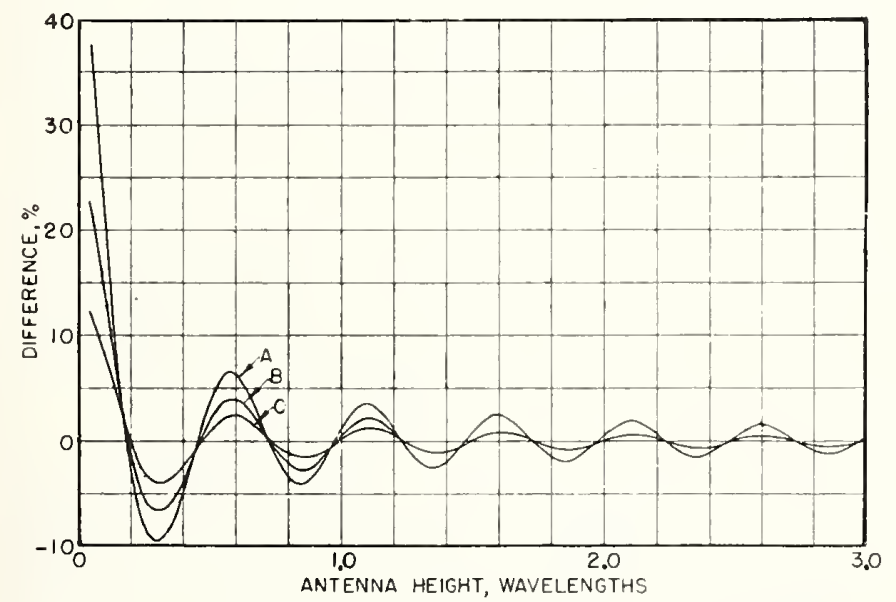

FIgURE 4. Calculated percentage difference in field intensity (horizontal polarization) $\left(\left|E_{i} / E_{t}\right|-1\right) \times 100$ vs receiving antenna height, $h_{2} / \lambda$, in wavelengths for three v'alues of antenna terminating impedance.

(A) $Z_{L}=73$; (B) 150, and (C) 300 ohms, over averige ground $\epsilon_{r}=35$, $\sigma / \epsilon \omega<<1 . l=\mathrm{\lambda} / 2, Z_{33}=73.2+j 42.5$
$\left(Z_{L}=73 \Omega\right)$ calibrated under free-space conditions may indicate values of field intensity that are in error by as much as 10 percent for values of $h_{2} / \lambda$ near 0.3 , and 7.5 percent for values of $h_{2} / \lambda$ near 0.6 . If this error is to be held to values less than 5 percent, antenna heights greater than about 0.65 wavelength should be used for field-intensity measurements under these conditions.

It is somewhat doubtful at the present state of the art just what maximum values of measurement error of this type should be permitted. One method of redueing the error, obviously, is to increase the value of the antenna terminating impedance, $Z_{L}$.

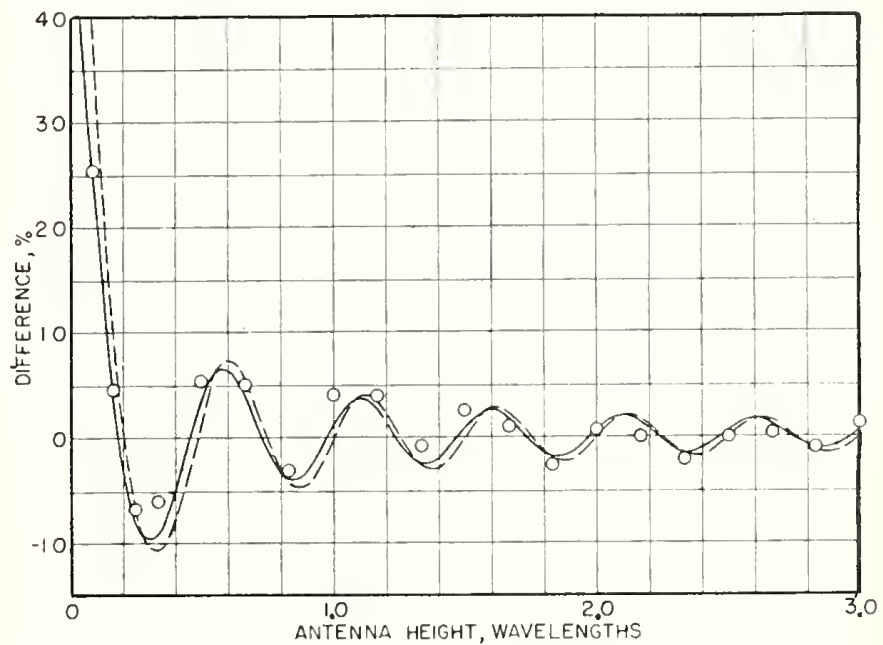

FIGURE 5.-Calculated percentage difference in field intensity (horizontal polarization) $\left(\left|E_{i} / E_{t}\right|-1\right) \times 100$ vs receiving antenna height, $h_{2} / \lambda$, in wavelengths over average ground, $\epsilon_{r}=15, \sigma / \epsilon \omega<<1$, for both a half-wavelength dipole and a self-resonant dipole $(l \cong \lambda / 2)$.

The measured points were determined at $100.0 \mathrm{Mc}$ and were obtained from the data presented in figure $7 . \ldots . . . .$. , self-resonant dipole; ___ . . $\lambda / 2$ dipole; $O$ observed for self-resonant dipole.

Figure 4 shows the calculated measurement error vs $h_{2} / \lambda$ for values of $Z_{L}=73,150$, and 300 ohms for average ground, $\epsilon_{r}=15,(\sigma=0)$. For the case of $Z_{L}=73 \mathrm{ohms}$, the error does not exceed 10 percent for heights of the receiving antenna in excess of 0.15 wavelength. If $Z_{L}$ is increased to 150 , and $300 \mathrm{ohms}$, this error is reduced to 7 and 4 percent, respectively, and approaches zero as $Z_{L}$ approaches infinity.

Figure 5 shows the computed measul' ment elrol for beth a $\lambda / 2$ dipole and a self-resonant dipole, as well as measured values for the latter case $(f=$ $100 \mathrm{Mc})$. In the case of the $\lambda / 2$ dipole, $Z_{33}=73.2$ + -j 42.5 ohms, and $Z_{I}=73+j 0$ ohms. For the 


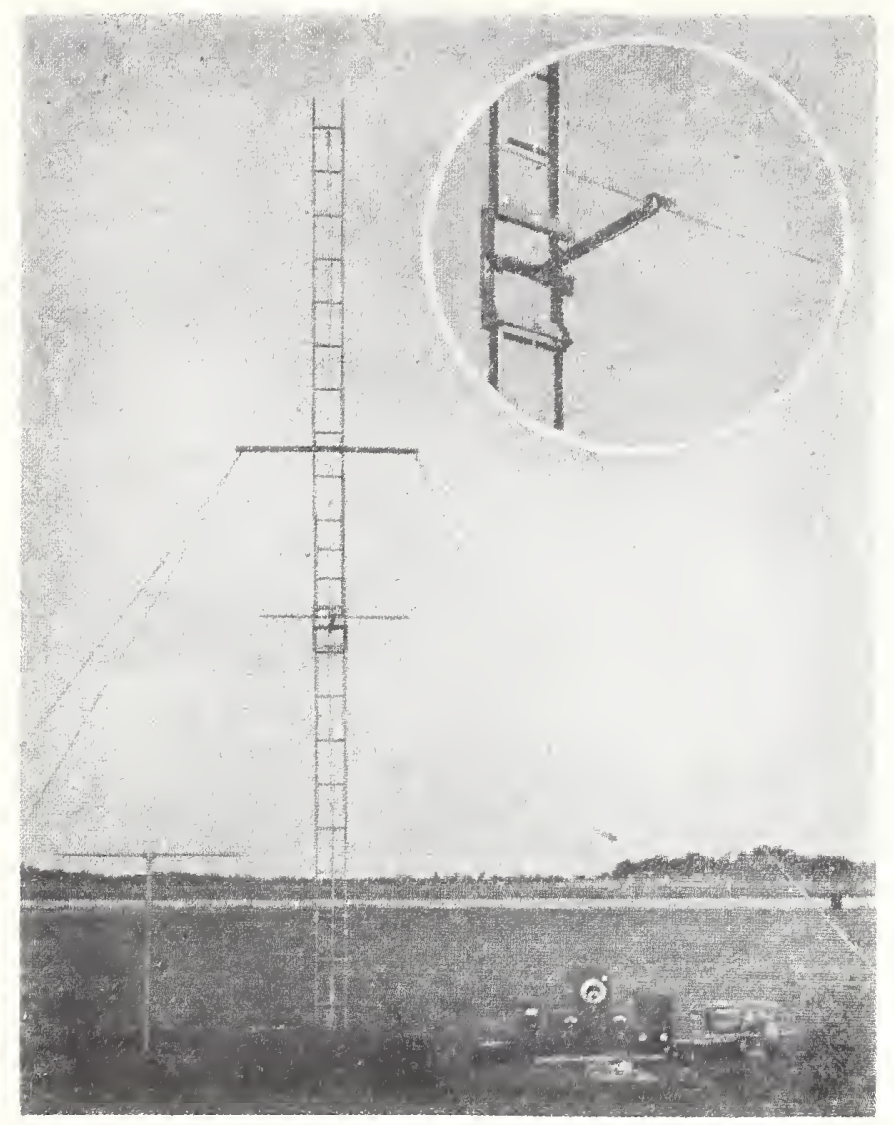

FIgure 6. View of the various pieces of transmitting and receiving equipment used in obtaining the measured data of figure 7 .

In the background is shown the ladder-mast and carriage for the receiving dipole. The location is the Beltsville, Md., airport.

self-resonant dipole, $Z_{33}=65+j \quad 0$ ohms, and $Z_{L}=62+j 0$ ohms.

The latter values were chosen as representing the approximate impedances of the self-resonant antenna actually used for obtaining the measured points of figure 5 . The terminating impedance, $\mathrm{Z}_{L}=62+j 0$ ohms, was the closest value to 65 ohms available at the time the measurements were made. As might be expected, there is no substantial difference between the calculated values of the measurement error for the full $\lambda / 2$ dipole and for the self-resonant dipole. The measured points support the theory reasonably well. The difference does not exceed 3 percent for antenna heights above 0.1 wavelength. Various pieces of the transmitting and receiving equipment used in making these measurements are shown in figure 6 .

The measured values of figure 5 were obtained from the data presented in figure 7 . In the latter, the receiving antenna terminal voltage (horizontal polarization) is shown vs height, $h_{2}$, in meters over ground having a measured relative dielectric constant $\epsilon_{r} \cong 15, \quad(\sigma / \epsilon \omega<<1)$ for: (A) antenna "open-circuited"; and (B) antenna terminated in $\mathrm{Z}_{L}=62+j 0 \mathrm{ohms}$. The measured percentage difference shown in the upper curve of figure 7 was determined from the data with the aid of eq 18 which, upon substituting eq 15 and 16, gives (in percent)

$$
\delta=\left(K l_{H}\left|\frac{V_{L}}{V_{o c}}\right|-1\right) \times 100 .
$$

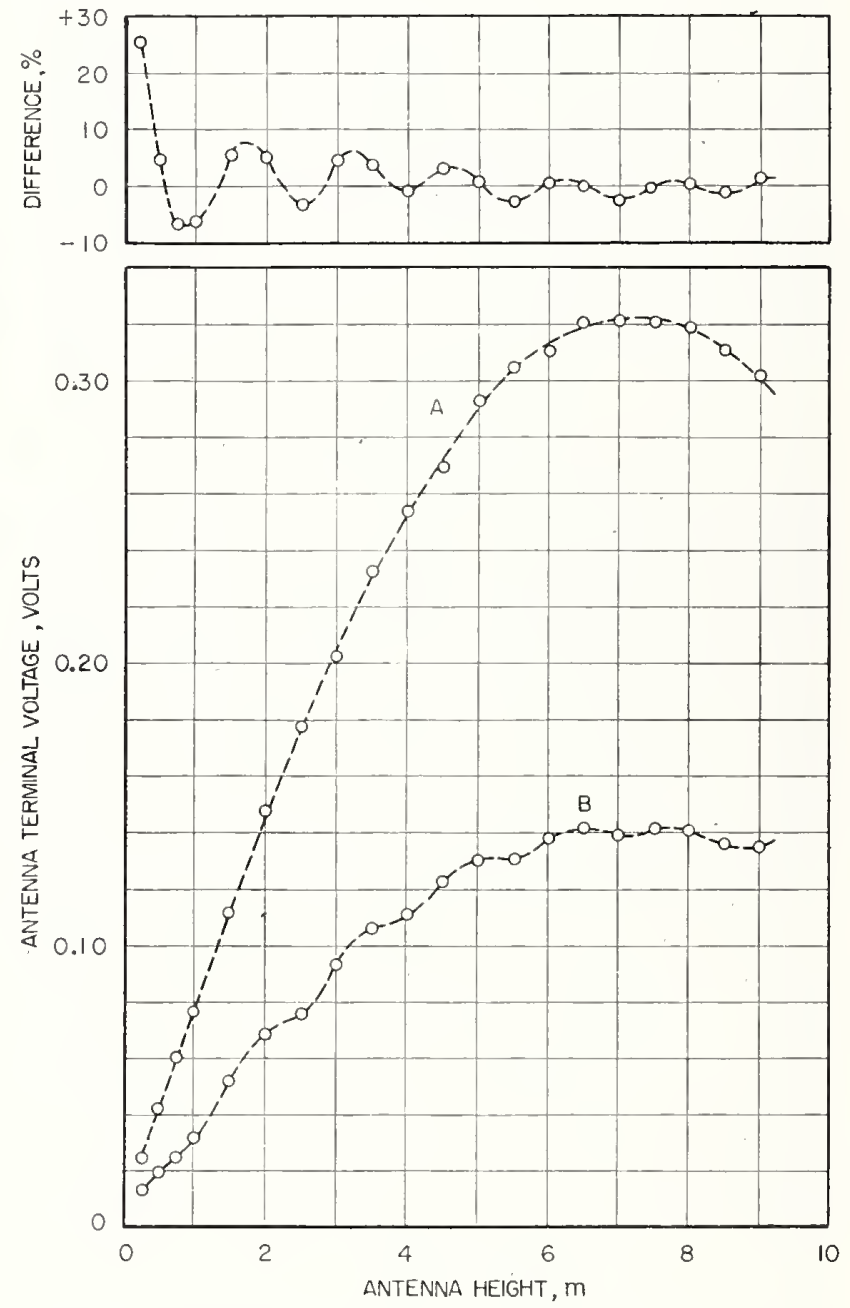

FIGURE 7.--Measured values of receiving antenna terminalvoltage (horizontal polarization) vs height in metcrs over ground having a measured relative dielectric constant $\epsilon_{\tau}=15,(\sigma / \epsilon \omega<<1)$ for: $(A)$ antenna "open-circuited"; and $(B)$ antenna terminated in $Z_{L}=62+j 0$ ohms

The measured percentage difference was determined with the aid of eq 21 , $\delta=\left(K l_{H} V_{L} / V_{o e}-1\right) \times 100$. The free space value of the factor $K l_{B}=\left(Z_{L}+Z_{2 s}\right)$ $\mid Z_{L}=V_{o c} / V_{L}$ (see eq 17 ) was estimated from the data, and is the reciprocal of the voltage-transfer ratio. $f=100.0 \mathrm{Mc}, d=30.5 \mathrm{~m}, h_{1}=3.05 \mathrm{~m}, I=0.100$ amp.

\section{Effect of Ground on VHF Field-Intensity Meters}


The free-space value of the factor, $K l_{H}=\left(Z_{L}+\right.$ $\left.Z_{33}\right) / Z_{L}=V_{\text {oc }} / V_{L}$, used, was estimated from the data, and is the reciprocal of the voltage-transfor ratio previously mentioned.

For the open-circuited condition referred to above, the receiving antenna was actually terminated in a special balanced voltmeter of the silicon crystal-rectifier type. This crystal rectifier, together with the balanced RC network used to take off the direct-current output voltage, presented a resistance of approximately $4,000 \mathrm{ohms}$ in shunt with $0.75 \mu \mu \mathrm{f}$ across the gap at the center of the antenna. This accounts for the slight oscillation of the points around the averaging curve, but introduced an error of less than 1 percent in the final results, as the shunting was present during both the open-circuited and terminated runs.

\section{Conclusions}

An approximate method has been presented for determining the effect of finitely conducting ground beneath a horizontal receiving dipole on the value of the antenna constant as used for measuring VHF field intensity. Three variables are mainly involved in this effect: (a) the antenna height, in wavelengths, $h_{2} / \lambda$, (b) the ground constants $\epsilon_{\tau}$ and $\sigma$; (c) the antenna terminating impedance $Z_{\boldsymbol{L}}$.

Changes in antenna height probably have the greatest effect on the antenna constant, as can be seen from figure 3 , and are of primary concern here. Normal variations in the ground constants encountered in practice apparently have only a minor effect. Under most conditions and to within the probable accuracy of this method, these variations can probably be neglected.
This error ${ }^{8}$ in measurement caused by the ground may be reduced by increasing the value of $Z_{L}$. The error vs height is shown in figure 4 for three values of $Z_{L}$, viz, 73, 150, and 300 ohms. The error approaches zero as $Z_{L}$ approaches infinity.

In figure 5, measured values of the error are compared with theoretical values calculated as previously described. The agreement is reasonably good for antenna heights above 0.1 wavelength.

In view of the approximations involved it is felt that the curves shown in figures 3,4 , and 5 probably should not be used for actually applying corrections to field-intensity measurements. Rather they might be used to estimate the maximum probable error (due to ground effect) existing in measurements made below a given antenna height.

. Figure 3 shows the variations in the error with antenna height occurring over perfectly conducting ground. ${ }^{9}$ 'The error is appreciably larger in this case than for finitely conducting ground. This would seem to indicate the inadvisability of using or calibrating a VHF field-intensity meter over a perfectly conducting plane unless the antenna heights were carefully chosen so as to result in a low value of error.

\footnotetext{
The error, as previously defined, is the percentage-difference between the true value of field intensity existing at a given antenna height, in wavelengths, $h_{2} / \lambda$ and that indicated by a field-intensity meter whose antenna constant was determined under free-space conditions.

${ }^{\ominus}$ Perfectly conducting ground and a solid metallic ground plane would have escentially the same reflecting properties for the present purpose.
}

Washington, October 18, 1949. 


\title{
Calibration of Commercial Radio Field-Strength Meters at the National Bureau of Standards
}

\author{
By Frank M. Greene
}

\begin{abstract}
A brief description is given of the standards and methods used in the calibration of commercial radio field-strength meters at the National Bureau of Standards in the frequency range $10 \mathrm{kc}$ to $300 \mathrm{Mc}$. A calibration consists in part of ineasuring the over-all linearity of the field-strength meter at one or more frequencies and radio-frequency input voltage levels, and in measuring the internal attenuator ratios at one or more frequencies in terms of precision dissipative-type step attenuators, as well as precision mutual-inductance attenuators, depending upon the frequency being used. The remander of the calibration consists in determining the so-called antenna coefficient or correlation factor of the set relating field strength to the output meter reading. Below about $30 \mathrm{Mc}$ this is done only for sets using loop antennas in terms of a quasi-static magnetic field produced by a single-turn balanced transmitting loop. Above this frequency for sets using only dipole antennas a locally generated radiation field is used and is evaluated in terms of the electromotive force induced in a horizontal receiving dipole. The accuracies of the various parts of the calibration are discussed for different portions of the above frequency range.
\end{abstract}

\section{General}

The National Bureau of Standards offers a calibration service ${ }^{1}$ for certain types of radio field-strength meters in the frequency range $10 \mathrm{kc}$ to $300 \mathrm{Mc}$. The calibration consists in measuring the over-all linearity of the instrument at one or more frequencies and radio-frequency ( $\mathrm{r}-\mathrm{f}$ ) levels, measuring the internal attenuator ratios at one or more frequencies, and measuring the so-called antenna coefficient or correlation factor relating field strength to the output meter reading under specified conditions of set gain at specified frequencies. All measurements are made in terms of sinusoidal voltages or currents.

In developing the equations used in this paper. for determining field strength, the practical rationalized mks units have been used. This is in agreement with recent international action.

1 For further information and calibration fees, write to Director, National Bureau of Standards, Washington 25, D. C.

\section{Measurement of Linearity and Attenuator Ratios}

'Those quantities involving only 1 -f voltage ratios are measured in terms of precision dissipative step attenuator's or precision mutual-inductance (waveguide-below-cutoff) attenuators, depending on frequency. Use is made of two types of dissipative attenuators comprising "Pi" or " $\mathrm{T}$ " sections, one employing wire-wound resistance elements for frequencies below 1 or $2 \mathrm{Mc}$, the other using coaxial sections of evaporated or deposited metal film for frequencies up to $300 \mathrm{Mc}$. These step attenuators are standardized on direct current and their ratios determined to within 0.1 percent. Frequency corrections are determined by comparing one standard attenuator used at signal frequency against another used at the intermediate frequency in a receiver. Cross checks are made against the standard mutual-inductance attenuator at frequencies above about five megacycles.
'This type of attemuator ${ }^{2}$ serves as a primary reference standard, since the attenuation is a funci ion only of its linear dimensions which can be precisely determined. 'The $\mathrm{TE}_{1,1}$ mode of excitaiion is used, the higher order unwanted modes being relluced by means of a strip filter: Errors resulting from the finite conductivity of the cylinder wall, and from the proximity of the operating frequency to the cut-off frequency are less than $0.1 \mathrm{db}$ in a $60-\mathrm{db}$ range in the HF band $(3$ to $30 \mathrm{Mc})$ and the VHF band $(30$ to $300 \mathrm{Mc})$ and can usually be neglected.

Since the over-all linearity of a field-strength meter will in general be a function of both frequency and $r-f$ input level, it is necessary to determine this dependence. Fortunately, this effect

2 R. E. Grantham and J. J. Freeman, A standard of attenuation for micro wave measurements, Trans. AIE E 67, 535 (1948). 


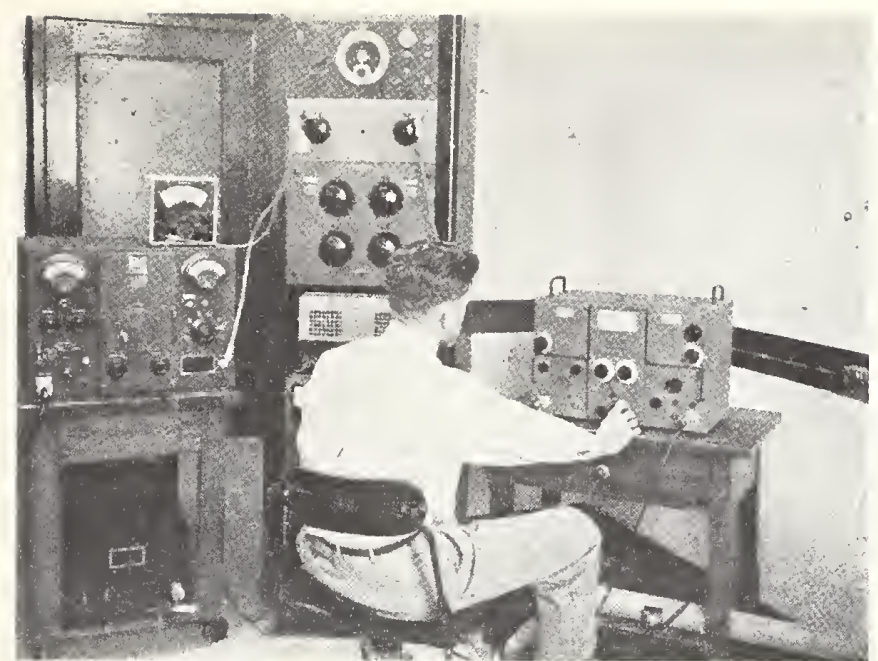

Frgure 1. Calibration of the internal attenuators and linearity of a commercial field-strength meter.

is usually limited to the highest attenuator steps in those types of sets having intermediate- frequency attenuators only. The departure from. linearity has been found in general to be less than 2 percent if the signal voltage applied to the mixer grid does not exceed about 5 pereent of the injected local oscillator voltage. For practical and cconomical reasons it is necessary to limit linearity rhecks on the attenuator steps in question 10 one or two frequencies as specified by the usel'.

For those sets also employing 1 -f attenuators in the anterma input ciremit it is necessaly to chlsure that these ratios are measured under actual operating anditions. 'Those r-f attenuator steps which naty bo aflected by the changing somere impedanee of the antema, or the changing imput admittance of the first 1 -f stage with frequenery, are measured with the antenna placed in a ficid which can be attenuated in known ratios.

The voltage linearity and attenuator ratio measurements made on field-strength meters are usually certified to \pm 2 percent. 'The equipment used is shown in figure 1.

\section{Measurement of Antenna Coefficients From $10 \mathrm{kc}$ to $30 \mathrm{Mc}$}

At the present time the National Bureau of Standards certifies only field-strengtl nneters using loop antennas below about $30 \mathrm{Mc}$. The calibration is made in terms of a standard free-space quasi-static magnetic field produced by a singleturn, unshielded, balanced, transmitting loop of known radius and with a known current flowing. The current is measured by means of a vacuum thermocouple ${ }^{3}$ at the loop center, which was previously standardized on direet eurrent, the (l-c output of the thermocouple being measured by means of a precision slide-wire potentiometel. The frequency error of the type of thermocouple used for this purpose has been found to be less than 1 percent at frequencies even as high as 100 Mc when used in a balanced circuit with the thermocouple at essentially ground potential.

The magnitude of the ficld strength produced by a single-turn circular transmitting loop is given by eq (1) for the case of coaxial transmitting and receiving loops as shown in figure 2. The actual value of the quasi-static magnetic field, $I I$, produced by the loop is expressed in terms of the equivalent electric component, $E$, that would exist in a free-space radiation field. The relationship used is $E=Z H$, where $Z$ is the impedance of free space $(Z=376.7 \mathrm{olims})$.

where

$$
|E| \cong \frac{60 \pi r_{1}^{2} I}{\left(d^{2}+r_{1}^{2}+r_{2}^{2}\right)^{3 / 2}} \sqrt{1+\left(\frac{2 \pi d}{\lambda}\right)^{2}}
$$

$E=$ equivalent free-space electric field strength in r'ms volts per meter

$r_{1}=$ radius of transmitting loop, meters. 'To keep a uniform current in the transmitting loop make $2 \pi r_{1}<\lambda / 8$

3 This refers to a four-terminal unit having an 1 -f heater electrically insulated from the thermocouple. $r_{2}=$ radius of receiving loop, meters. If receiving loop is rectangular use equivalent radius of circle having same area

$d=$ axial spacing (neters) between coaxia] loops (make $d>7 r_{1}$ and $d>7 r_{2}$ )

$I=$ transmit ting loop current, I'ms amperes

$\lambda=$ free-space wavelength in meters.

The value of field given by eq (1) is essentially independent of frequency up to about $5 \mathrm{Me}$, above which the frequency correction term under the radical (the induction-field component) begins to become appreciable for the spacing used in the NBS standard $(d \cong 1.25 \mathrm{~m})$. 'The value of field strength used for calibration is of the order of 0.1 $\mathrm{v} / \mathrm{m},\left(r_{1}=0.1 \mathrm{~m}, I=0.1 \mathrm{amp}\right)$.

The magnitude of the field, $|E|$, predicted by eq (1) was verified at several frequencies up to $30 \mathrm{Me}$ by measuring the electromotive force, $e$, induced in a single-turn untuned balanced receiving loop immersed in the field. The relationship used was

$$
|E|=\frac{e}{l_{L}}
$$

where $l_{L}$ is the effective length of the single-turn loop in meters.

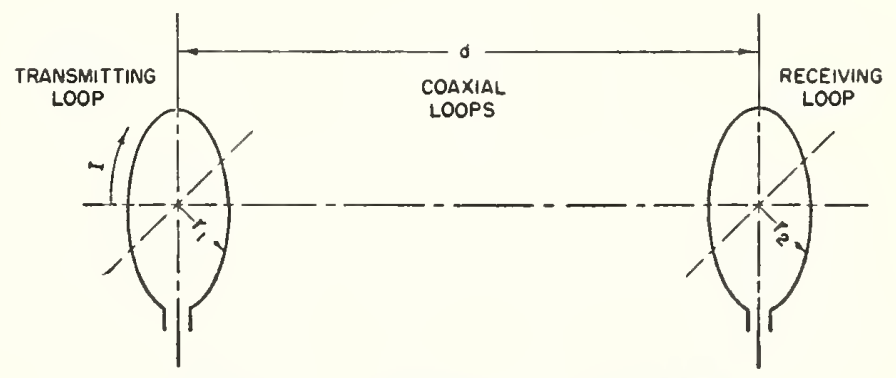

Figure 2. Loop-antenna calibration. 


$$
l_{L}=\frac{2 \pi A}{\lambda}
$$

where $A$ is the loop area in square meters, and $\lambda$ is the operating wavelength in meters. Agreements were obtained between these two methods of determining the field to within 3 percent.

It is known that the case of the ficld-strength meter will distort the field being measured. 'There is reason to believe that this distortion will not be the same when the instrument is placed in the above quasi-static field $\left(E \propto 1 / d^{3}\right)$ as when placed near the ground in a radiation field, $\left(E \propto 1 / d^{x}\right)$, where usually $1 \leq x \leq 2$ for plane earth depending upon frequency, distance, and the ground constants. ${ }^{4}$ It is believed that this difference is probably not great. Previous comparisons of instruments calibrated in both types of fields have agreed to within considerably better than 5 percent at broadcast frequencies. However, orientation of the receiving loop antenna relative to the instrument case must be specified since if later used in other positions an error of as much as 5 to 10 percent may be introduced into subsequent measurements depending upon the height of the loop above the case. ${ }^{5}$ This error is usually negligible if this height is greater than the loop diameter. It does not appear feasible to attempt to establish standard radiation fields using the standard-field method at frequencies much below $30 \mathrm{Mc}$ because of the difficulties in accurately taking into account the ground effects.

In calibrating a field-strength meter by means of eq (1), the setup is made in a cleared space as shown in figure 3 such that a distance of at least two or three times the loop spacing, $d$, exists to the nearest sizable metallic objects and to the ground. Their effect in distorting the field can be estimated by moving up metal objects of similar size and noting the effect on the value of the received field. For the above distance and spacing the effect of small objects on the value of field was usually found to be less than 1 percent.

It may be found necessary to shield thoroughly the $\mathrm{r}$-f generator and transmission line to the loop to reduce leakage fields, also to correct the current calibration of the thermocouple for harmonics in the $r-f$ supply. It is preferable that the $r-f$ generator output be balanced to ground at least for frequencies above 1 or $2 \mathrm{Mc}$.

The "antenna coefficient," $K$, is evaluated by the relation

where

$$
K=\frac{E_{1}}{A_{1}} \times \frac{f}{M_{1}},
$$

$$
\begin{gathered}
E_{1}=\text { standard field given by eq (1) usually } \\
\text { expressed in microvolts per meter }
\end{gathered}
$$

${ }^{4} \mathrm{~K}$. A. Norton, The calculation of ground-wave field intensity over a finitely -conducting spherical earth, Proc. IRE 29, 623 to 639 (Dec. 1941).

fi. Diamond, K. A. Norton, E. G. Lapham, On the accuracy of radio 795 (1938) RP1156.

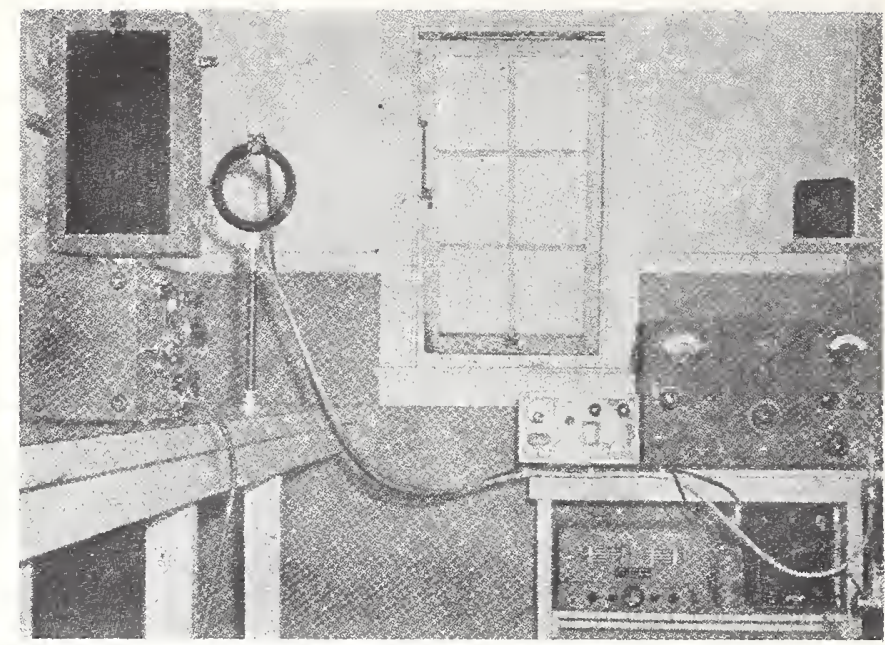

Figure 3. Determining the loop-antenna coefficient of a commercial field-strength meter.

$A_{1}=$ true attenuator ratio used

$M_{1}=$ output meter reading corrected from linearity data

$f=$ frequency, usually in kc or Mc. This frequency factor is introduced arbitrarily as a convenience to make $K$ (theoretically) independent of frequency, since $M_{1} \widetilde{\propto} f$.

In using the field-strength meter later for actual measurements, the unknown field in microvolts per meter is given by

$$
E=\frac{K M_{2} A_{2}}{f}
$$

where $K$ is given by eq (4), and

$M_{2}=$ output meter reading corrected from linearity data

$A_{2}=$ true attenuator ratio used

$f=$ frequency in same units as used in eq (4).

While the instrument actually measures the magnetic component, $I I$, of the unknown field, the indication is given in terms of the electric component, $E$, that would exist if the measurement were being made in free space, from the relationship, $E=Z H$, previously given. When the meter is used to measure field strength near the ground,. however, this relationship is in general no longer valid. Consequently, the indicated value for the electric component may be subject to question in some cases unless the effect of the ground at the measurement site is considered; the magnetic field is however always correctly given by converting the electric field strength reading by the above simple free-space relation.

The accuracy of antenna coefficients is usually certified to \pm 3 percent below $5 \mathrm{Mc}$ and \pm 5 percent between 5 and $30 \mathrm{Mc}$ for sets using loop antennas. 


\section{Measurement of Antenna Coefficients From 30 to $300 \mathrm{Mc}$}

At the frequencies in the VHF band, the National Bureau of Standards certifies only fieldstrength meters using dipole antennas. The determination of antenna cocfficients is made in terms of a radiation field by either the standardfield method or the standard-antenna inethod using half-wave dipoles and horizontally polarized transmission in either case. 'These two methods were intercompared and excellent agreement was obtained over most of the range of antenna heights and distances of separation used between transmitting and receiving antennas. ${ }^{6}$

In using the VHF standard-field method it was necessary to select a flat graded site free of any reflecting objects such as trees, buildings or wires within a radius of at least several hundred feet around the transmitting and receiving antennas. With these conditions approaching the ideal, the absolute magnitude of the field at the receiving antenna was accurately computed at these frequencies by considering only the direct and ground-reflected waves from the transmitting antenna, as indicated in figure 4. Both horizontal half-wave dipoles are assumed oriented normal to a line joining their centers, and spaced a horizontal distance, $d$, greater than $2 \lambda$. The resulting rms field strength ${ }^{7}$ in volts per meter at the receiving antenna is

$$
\begin{aligned}
|E| \cong \frac{60 \pi l_{H} I}{\lambda} & {\left[\left(\frac{1}{R_{1}}+\frac{\rho}{R_{2}}\right)^{2}\right.} \\
& \left.-\frac{4 \rho}{R_{1} R_{2}} \sin ^{2}\left(\frac{k\left(R_{2}-R_{1}\right)+\phi}{2}\right)\right]^{\frac{1}{2}}
\end{aligned}
$$

where

$l_{H}=$ effective length of antenna in meters $\cong \lambda / \pi$ meters for a half-wave dipole

$\lambda=$ wavelength in meters

$R_{1}=\left[\left(h_{1}-h_{2}\right)^{2}+d^{2}\right]^{\frac{1}{2}}=$ direct-ray path-length in meters

$$
\begin{gathered}
R_{2}=\left[\left(h_{1}+h_{2}\right)^{2}+d^{2}\right]^{\frac{2}{2}}=\text { ground - reflected-rayo } \\
\text { path-length in meters } \\
d=\begin{array}{c}
\text { horizontal distance of . separation, } \\
\text { meters }
\end{array}
\end{gathered}
$$

$h_{1}$ and $h_{2}=$ the heights in meters above ground of the transmitting and receiving antennas, respectively

$\rho=$ magnitude of the plane-wave reflection coefficient of the ground (horizontal polarization), and $\phi$ is the angle of phase lag on reflection

$I=$ rms current in amperes at the center $k=2 \pi / \lambda$. of the transmitting antenna

The phase shift on reflection, $\phi$, is very nearly 180 degrees for many types of ground over a large

\footnotetext{
B F. M. Greene and M. Solow, Development of very high-frequency field-

intensity standards, J. Research NBS 44, 527 (1950) RP2100.
7 Based on equation derived from reference 4 in mks units.
}

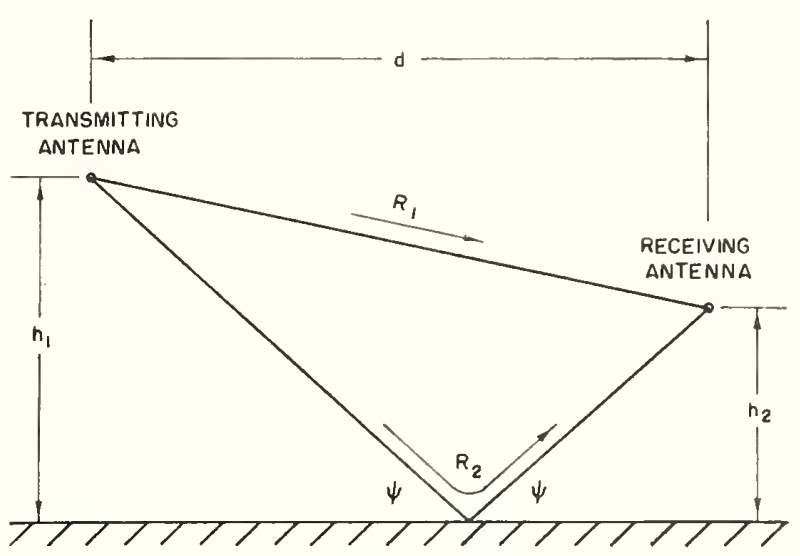

Figure 4. Dipole-antenna calibration.

portion of the VHF band. In this case, eq (6) may be written

$$
\begin{aligned}
|E| \simeq \frac{60 \pi l_{H} I}{\lambda} & {\left[\left(\frac{1}{R_{1}}-\frac{\rho}{R_{2}}\right)^{2}\right.} \\
& \left.+\frac{4 \rho}{R_{1} R_{2}} \sin ^{2}\left(\frac{k\left(R_{2}-R_{1}\right)}{2}\right)\right]^{\frac{1}{2}}
\end{aligned}
$$

Unless $\left(h_{1}+h_{2}\right) / d<0.05$, it may be $n$ ecessary to determine accurately the actual dielectric constant or reflection coefficient of the ground. For instance, if $\left(h_{1}+h_{2}\right) / d=0.2$, the magnitude of the reflection coefficient may have any value in the range 0.85 to 0.93 , depending upon themoisture content of the top layers of the soil at the measurement site. If $\left(h_{1}+h_{2}\right) / d=0.1$, this range may bc roughly 0.93 to 0.96 . For $\left(h_{1}+h_{2}\right) / d<0.05$, the reflection coefficient will usually be greater than 0.96. For this condition, and if further $2 \pi h_{1} h_{2} / \lambda d<1 / 4$, eq ( 7$)$ will reduce to

$$
|E| \simeq \frac{240 \pi^{2} l_{H} I}{d^{2}}-\left(\frac{h_{1} h_{2}}{\lambda^{2}}\right)
$$

giving the field to within \pm 2 percent of the value obtained from eq (7) for these conditions.

In using the standard-antenna method the field strength of a locally generated field is determined by measuring directly the induced emf in a standard receiving dipole oriented for maximum response from the relation

where

$$
|E|=e / l_{H}
$$

$e=$ induced emf in the receiving dipole in volts

$l_{H}=$ effective length of the dipole, meters, $l_{H} \cong \lambda / \pi$ meters for a half-wave dipole.

The emf is measured directly by means of a relatively high impedance silicon crystal voltmeter built into the gap at the center of the antenna. This eliminates the necessity for a separate meas- 
mement of the antema impedance, which greatly simplifies the problem, improving the over-ail aceuracy of the measurement.

Owing to the lack of selectivity in this method as used, it does not lend itself to ealibration work in the vicinity of strong interfering fiekts that may often be elleountered. The site used for VHF calibration work at the National Bureau of Standards is in a partially shielded valley somo 1.5 miles from the nearest interfering station.

While it is possible to use either the standardfield or the standard-antenna method for determining the antenna rocfficients, the latter method is used at the National Bureau of Stamelards at the present time. Field tests have shown the exeeltent agresmont possible botwern the two methods throughout the VHF bame. The choice was largely one of ronvenience, as it was found somewhat more enomenient to provide an ale'urate ralibution of the sition crystal voltmeter than to ensure an aleculate mosurement of the transmitting antema current throughout the VHF band.
The "antenna coefficient" of the fich-strength meter under test is determined by placing its dipole antenna at a specified height, usually 10 feet, in the radiation field previously measured using the standard receiving dipole and the relationship given by eq (9). Fuluations (4) and (5) are used at at lower frequencies, except that the frequency term, $f$, is msually omitted. I'se of the meter later at other antenna heights or over ground having appreciably different constants may require corrections for heights less than about one wavelength. ${ }^{8}$

The acenracy of the antenma coeflicients certilied by the National Burean of Stanclarels depends: largely upon the merits of the individual meter under test. In general, these coefficients are rertified at present to \pm 10 pereent for frecfuencies breween 30 and 150 . Me and to \pm 15 pereent for frequencies between 150 and 300 Me.

$$
\text { Wasmington, May 9, } 1951 .
$$

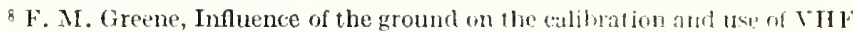
field-intensity meters, J. Research NBS 44, 123-130 (196i) R I'20ri?. 
Reprinted from IRE TRANSACTIONS

ON INSTRUMENTATION

Volume I-7, Numbers 3 \& 4, December, 1958

PRINTED IN THE U.S.A.

\title{
High-Frequency Impedance Standards at the National Bureau of Standards
}

\author{
R. C. POWElt $\dagger$, R. M. JICKLING $\dagger$, AND A. E. HESS $†$
}

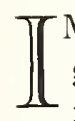
MPEDANCE can be defined either passively as a geometrical configuration of materials whose magnitude depends only on a length and the electromagnetic properties of free space, or actively as the ratio of potential and current at a given point.

The highest two-terminal impedance that could be constructed would be a capacitor, the magnitude of its impedance being inversely proportional to its physical size, and its value approaching infinity as frequency approaches zero. How high an impedance can be made and still be evaluated depends only on how short a length can be measured. If we assume $1 / 200$ th of a wavelength of visible light, or about $3 \times 10^{-9}$ meter, to be the limit to which a length can presently be measured, we can see that our small capacitor could be no smaller than the permittivity of free space times this small length, or about $3 \times 10^{-20}$ farad. Actively, this capacitance represents the charge of one electron at a potential of about 5 volts. Since this capacitance can be determined to only \pm 100 per cent, a capacitor 10 times as large could be determined to \pm 10 per cent and the accuracy improved to about a part per million as the capacitor is

* Manuscript received by the PGI, August 14, 1958.

$\uparrow$ Boulder Labs., National Bureau of Standards, Boulder, Colo. made larger until it reaches about $3 \times 10^{-14}$ farad, where the accuracy is limited by the accuracy of free space permittivity which is defined as the reciprocal of the product of the permeability of free space and the square of the speed of light, and hence limited by the accuracy to which the speed of light is known.

If the capacitance is calculated in electrostatic units, the accuracy could be improved to about a part in 100 million as the capacitor is made larger until it reaches about $30 \mathrm{~cm}$, where the accuracy is limited by the accuracy to which length can be measured. Therefore, the only present advantage in making a standard capacitor larger than about $3 \times 10^{-14}$ farad in mks units or $30 \mathrm{~cm}$ in electrostatic units would be to increase the ease of measurement both electrically and mechanically. This increase in ease, however, is offset by a decrease in the maximum frequency to which it can be used, this maximum frequency being determined roughly as that frequency at which the impedance of the associated inductance becomes about 10 per cent of that of the capacitor. At this point errors in the calculated values of inductance and resistance reduce any accuracy gained by increasing physical dimensions.

The lowest two-terminal impedance may similarly be determined to be an inductor with a value in the 
order of the permeability of free space times' our smallest increment of length, or about $4 \times 10^{-15}$ henry. The largest inductor that can be made without loss of ac-' curacy is not limited by the free space permeability, which is an implicitly defined quantity, but rather by the longest length which can be measured to the accuracy of $1 / 200$ th of a wavelength of visible light. Since this length is on the order of $3 \times 10^{-1}$ meter, the inductance would be about $4 \times 10^{-7}$ henry for a possible part per 100 million accuracy or two orders of magnitude less if free space permittivity rather than the permeability is defined. Therefore, a standard of impedance must be between the limits of $4 \times 10^{-7}$ henry and $3 \times 10^{-12}$ farad for a part in 100 million accuracy as shown in Fig. 1. It is interesting to note that these values converge at about $100 \mathrm{mc}$ to a value approximately equal to the characteristic impedance of free space. This is, of course; true since this value is the square root of the ratio of the free space permeability and permittivity, and both have been multiplied by the same factor in determining our limits of $L$ and $C$. This is important in that it shows that a part per 100 million accuracy in impedance can be attained to about $10^{8} \mathrm{cps}$ and at this frequency only with an impedance equal to the characteristic impedance of free space. Above this frequency the maximum possible accuracy decreases until about $10^{16} \mathrm{cps}$ in the ultraviolet where $3 \times 10^{-9}$ meter can no longer be considered small compared to a wavelength.

Now that the limits of impedances that can be constructed to maximum accuracy have been determined, the next problem is to choose a single impedance to which all the other values can be related most effectively at radio frequencies. At our maximum frequency of about $10^{8} \mathrm{cps}$ at which maximum accuracy can still be obtained, there are only three choices: a $3 \times 10^{-12}$ farad capacitor, a $4 \times 10^{-7}$ henry inductor, or a 377 -ohm re-. sistor. Of these three, an air capacitor has many advantages. Since most of its energy is stored in free space, the material properties have little effect, so the capacitance can be calculated simply and directly in terms of length and free space permittivity only. To calculate the impedance of a resistor, its material properties must be known to the same accuracy as its impedance. Since the current in an inductor does not flow only on the surface, the material properties come in again to a second order correction which at best requires an approximation. We have chosen for a derived impedance standard at radio frequencies to $300 \mathrm{mc}$ a capacitor with about $10^{-12}$ farad. This value allows lengths to be determined more surely while the inductance correction at $10^{9} \mathrm{cps}$ is only 10 per cent. Such a capacitor need consist simply of a short section of coaxial line made sufficiently uniform for its dimensions to be accurately measured. Not only can the impedance added to a matching coaxial line due to the addition of such a coaxial capacitor be accurately derived from its shape and dimensions, but with an appropriate adapter it can be intercompared with the low-frequency

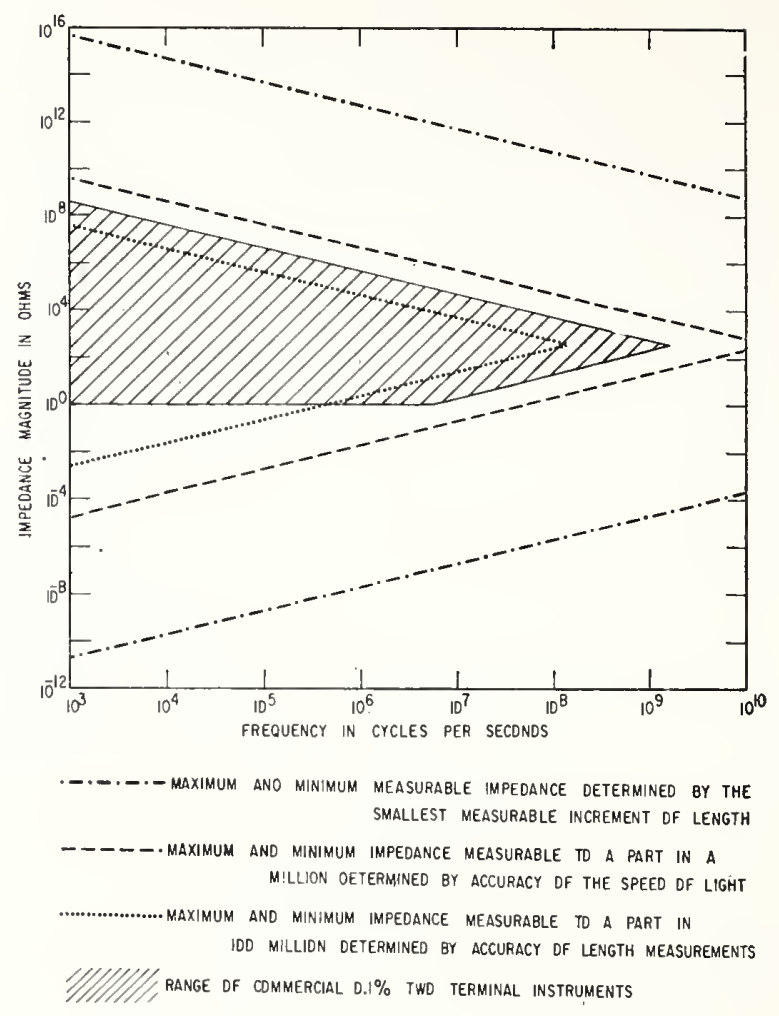

Fig. 1-Range of impedance standards.

Thompson and Lampard type three-terminal standard capacitors. Also, its high-frequency characteristics may be checked by using microwave coaxial measuring techniques. Such an impedance is only useful in calibrating other impedances which are exact multiples of its value; to provide calibrations of intermediate values, an incremental coaxial capacitor with a range of $10^{-12}$ farad is also required. It is so constructed that its capacitance change can be calculated, and hence can be used for interpolation. This capacitor and a model of a $10^{-12}$ coaxial fixed capacitor are shown in Fig. 2. Supplementing these is a series of stable incremental and decade capacitors whose impedances cannot be accurately calculated but which are calibrated by substitution techniques, thus giving capacitive impedances of all practical values over the radio frequency spectra. These capacitors are then used to calibrate the equipment of the high-frequency impedance measurements group in the Electronic Calibration Center.

A similar analysis can be made of the impedance instruments and standards which are to be calibrated at the Center. Of these, only the large number of commercially constructed devices presents a problem, since the very accurate custom-made devices are few enough in number to be specially handled. If we assume the average effective part of the device to be on the order of one inch in dimensions and made with a tolerance of onethousandth of an inch, we would calculate the maximum accuracy to be about 0.1 per cent with a maximum range from $3 \times 10^{-8}$ henry to $2 \times 10^{-13}$ farad. An additional restriction need also be made since it is difficult 


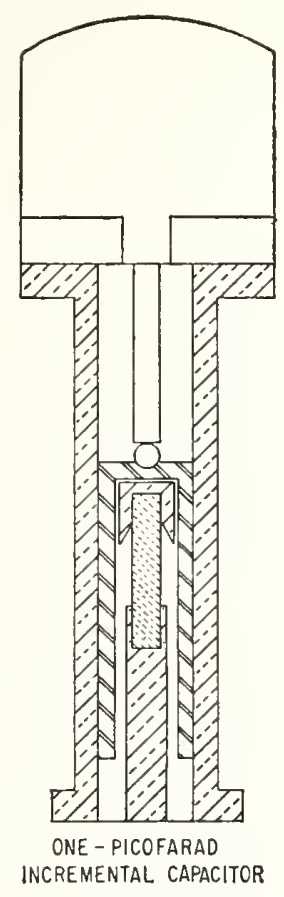

Fig. 2-Derived impedance standards.

to reduce the contact resistance of a two-terminal device much below $10^{-3} \mathrm{ohm}$. Therefore, $1 \mathrm{ohm}$ becomes the minimum two-terminal impedance which can be calibrated to 0.1 per cent when $1 \mathrm{ohm}$ exceeds the impedance of $3 \times 10^{-8}$ henry below about $5 \mathrm{mc}$. This range is also shown in Fig. 1. To efficiently calibrate these instruments and standards, the Center is now being equipped with four sets of working standards of impedance, each covering the range from $1 \mathrm{ohm}$ or $10^{-8}$ henry to $10^{-13}$ farad. Each set consists of about 60 standards composed of resistors, capacitors, and inductors which are stable to better than 0.1 per cent per year. Their nominal values are in decimal multiples of 1,2 , and 5 . Each is calibrated over a frequency range within which its value does not deviate by more than 10 per cent from nominal. These, together with special mounts as outlined in Fig. 3 for combining standards, yield the values of complex impedance necessary to calibrate nearly all radio frequency impedance measuring instruments made today. Two systems are used for calibrating these standards. The first is a resonance method for calibrating the high- $Q$ components. The working standard inductors are resonated on a special mount with the standard incremental capacitors which were calibrated against the derived standards. This mount consists of one and one-half inches of coaxial line in which current and voltage probes are symmetrically arranged to excite and detect resonance. The working standard capacitors are calibrated on the same mount by substitution still using the resonance technique. The low- $Q$ components and resistors are calibrated on a similar mount with the standard incremental capacitors using a susceptance variation method. Both of these methods require the use of either a standard piston at-
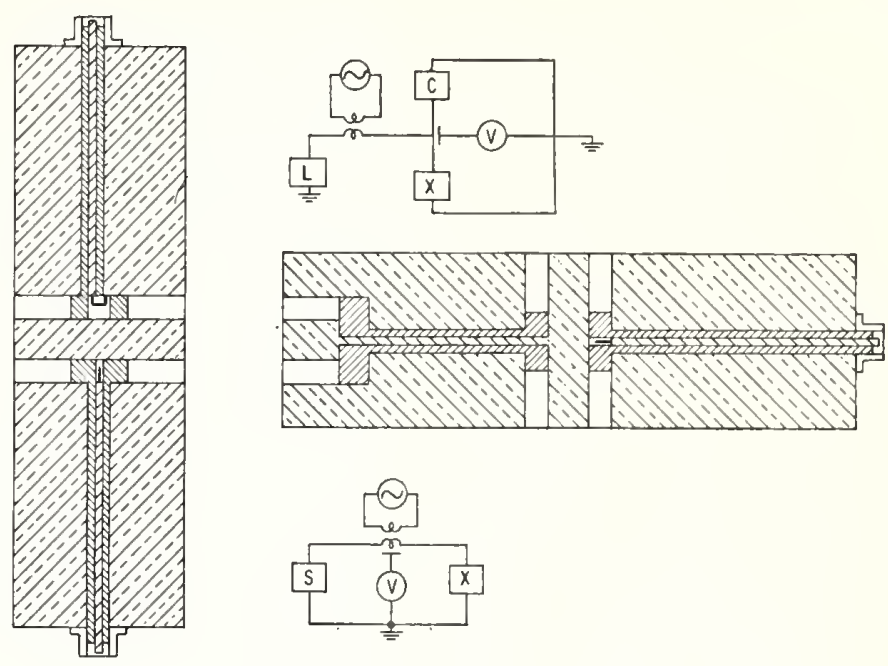

Fig. 3-Calibration mounts.

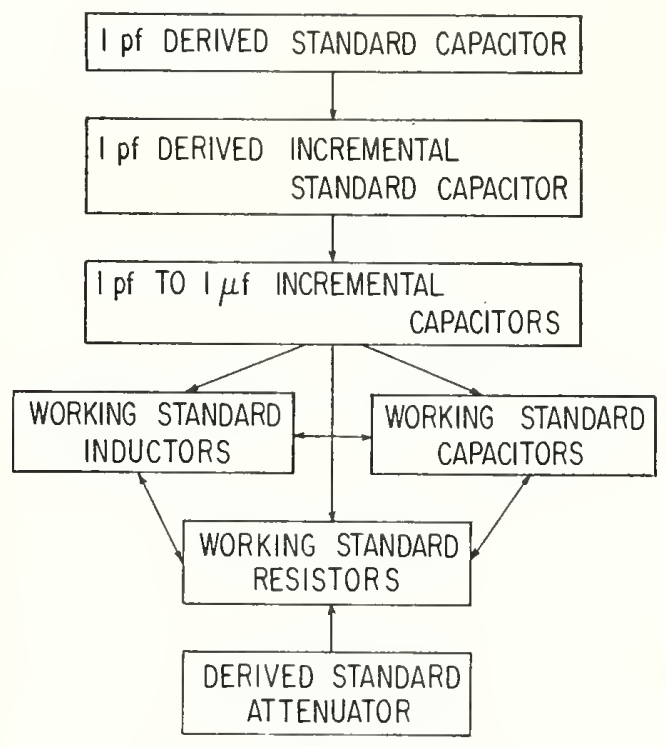

Fig. 4-Calibration sequence.

tenuator or a calibrated thermoelement to determine the shape of the resonant curve as a function of frequency or capacitance in order to determine the relative power loss and, hence, the resistance. Using these methods the required 0.1 per cent accuracy can be obtained on all the standards at radio frequencies.

These standards can then be intercompared on the same mounts to increase the accuracy at certain points and to detect possible calibration errors. The system is diagrammed in Fig. 4 and illustrated in Figs. 5-7.

It should be noted here that when accuracy is given for complex impedance, it refers to error in the absolute magnitude of impedance rather than to the separate components of $R, L$, and $C$. While some measuring methods allow high precision on the resistive or reactive components individually, careful analysis will show that the absolute accuracy on these components is not justified beyond that implied in the accuracy of $|Z|$.

When an impedance measuring instrument is submitted to the Center for calibration, sufficient combina- 


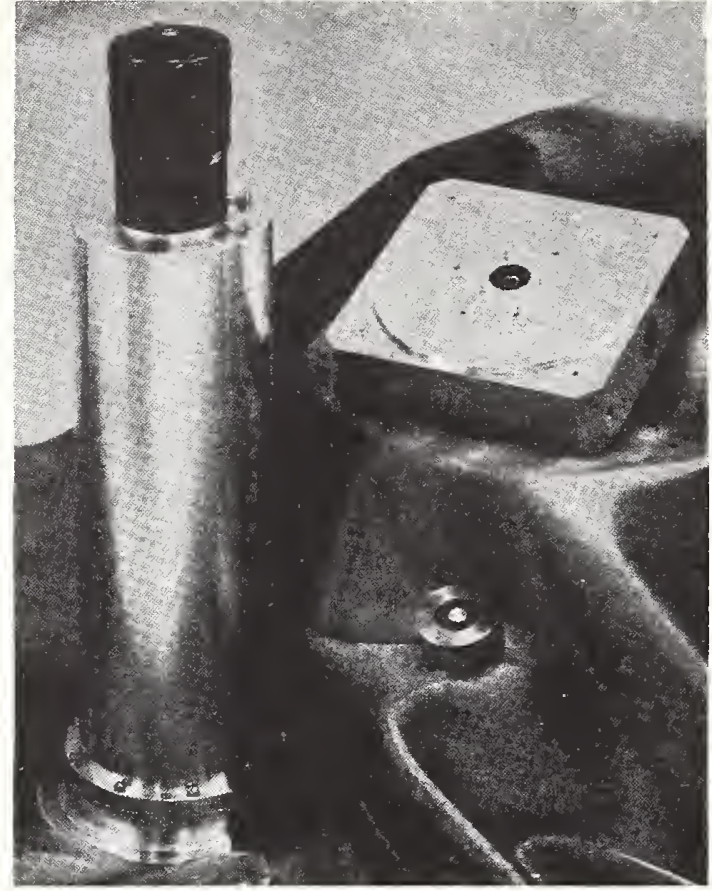

Fig. 5-Derived standards.

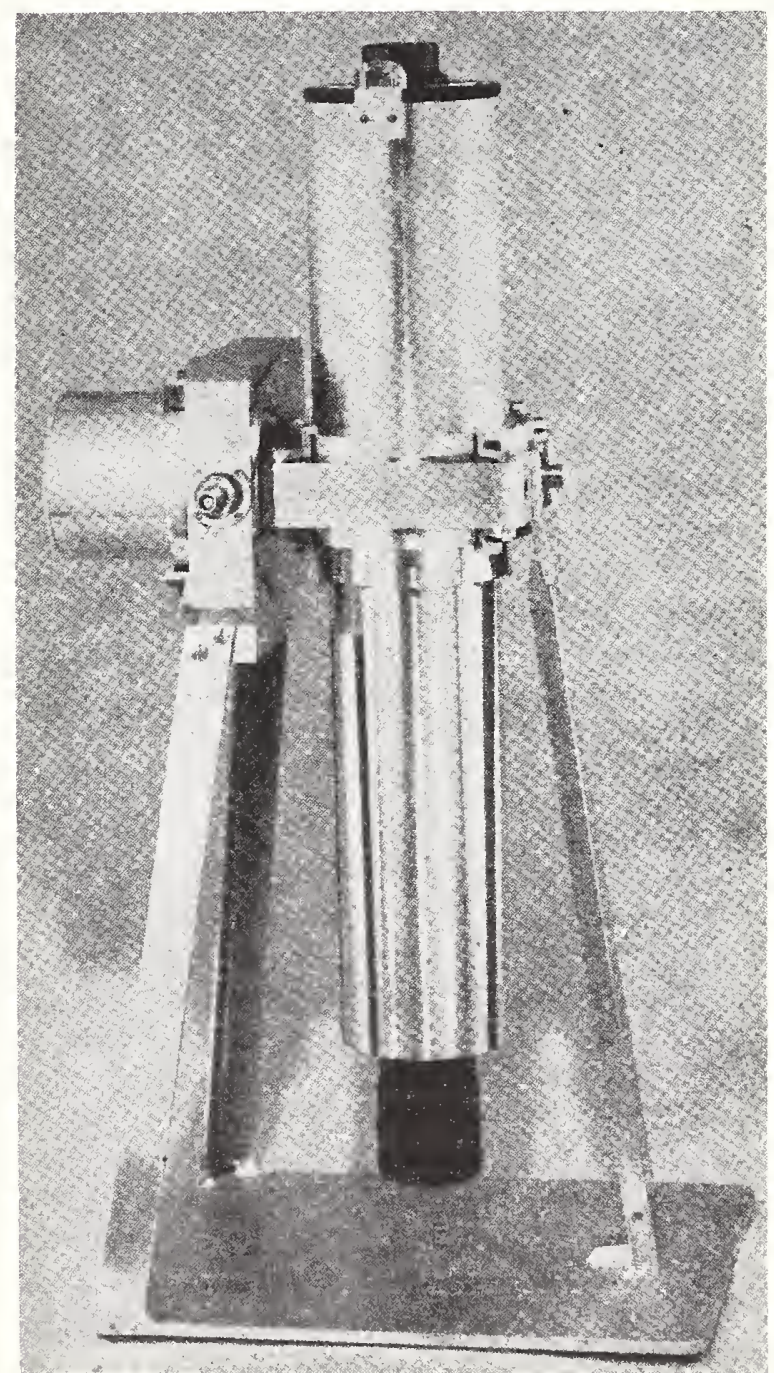

Fir 6-Calibration of incremental capacitor using a derived standard.

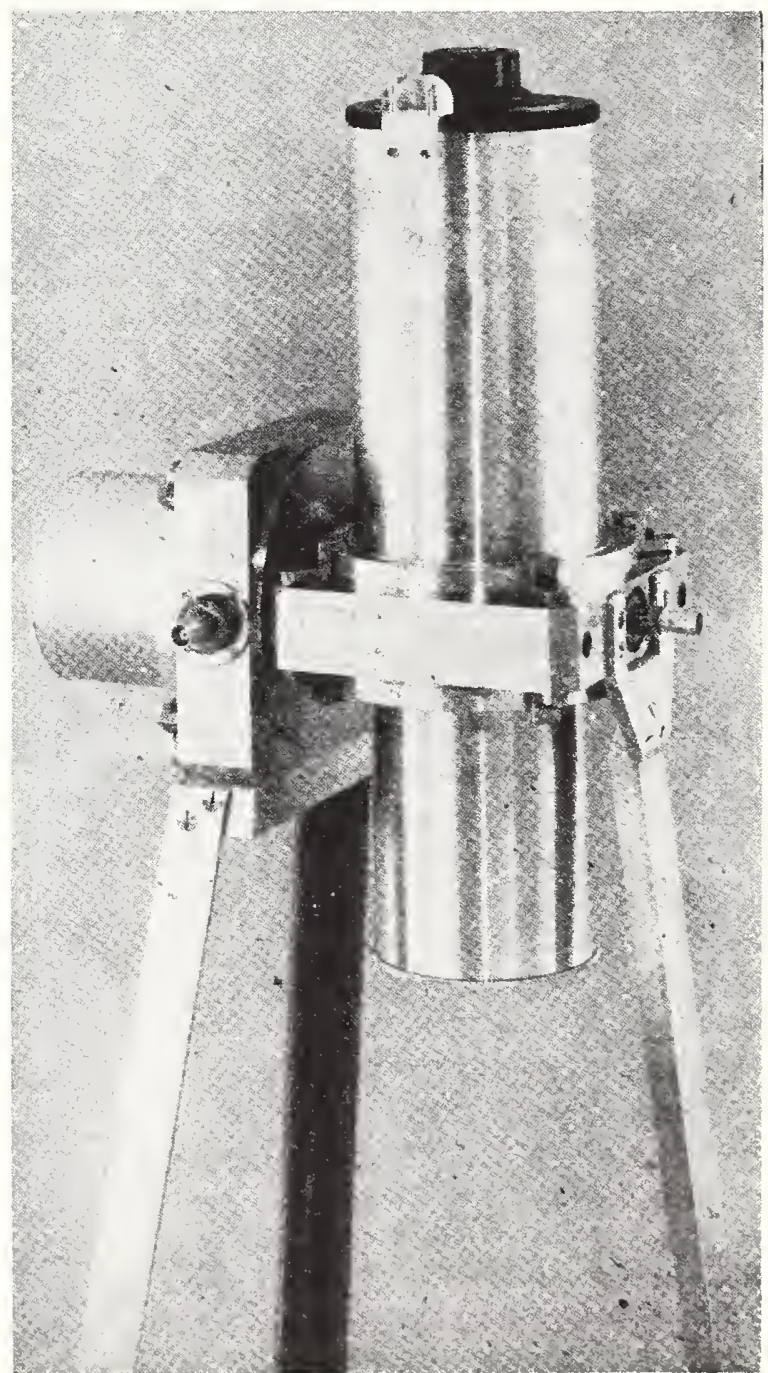

Fig. 7-Calibration of a working standard against an incremental capacitor. 


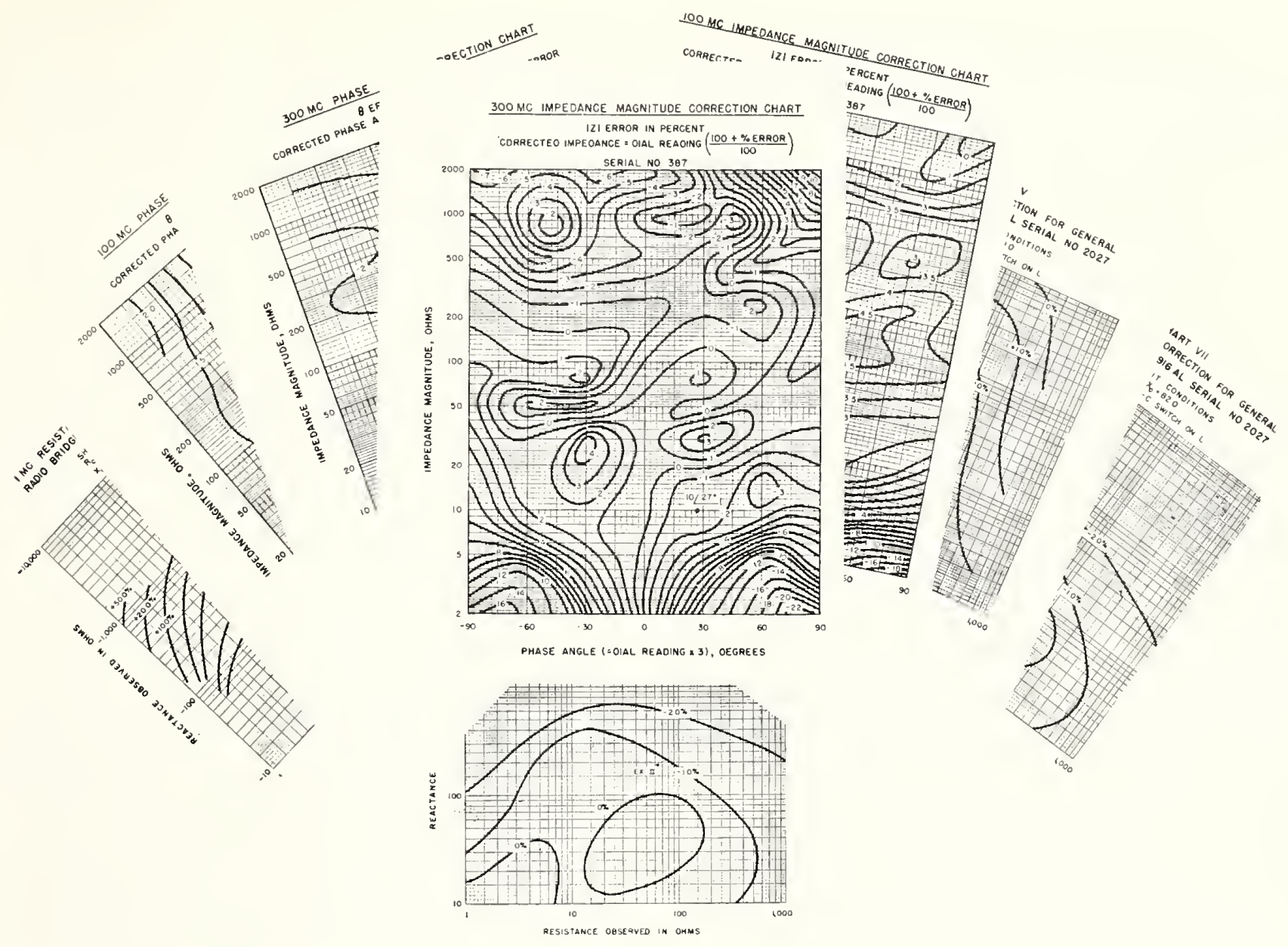

Fig. 8-Bridge calibration charts.

tions of the working standards are measured on the instrument so that the error in each dial reading of the instrument can be plotted as a function of the readings of all dials on the instrument as shown in Fig. 8. This method makes it unnecessary for the calibration persomnel to completely understand the construction of all models of all instruments made and also gives the operator a set of calibration charts which can be quickly used with a minimum of calculation.

For comparing standards submitted for calibration, the Center is being equipped with an admittance and an impedance bridge at each of nine fixed frequencies. These 18 bridges are adjusted to be direct reading to 0.1 per cent so that comparison with the working standards can be done with minimum loss of accuracy. Such acljustment is possible since each bridge is operated in conjunction with a crystal-controlled generator and receiver so that the frequency does not deviate more than one part per million from nominal.

With such a system nearly every existing radio frequency impedance instrument or stanclard can be calibrated efficiently to better than its rated accuracy. Still, each piece of equipment presents its own individual problems which are too numerous to detail in this piper. The most important of these, however, is the matter of terminals. There are hundreds of different types of terminals in use today and it would be impractical to maintain impedance standards with each type; therefore, all equipment in this group is constructed with a terminal that will allow the highest accuracy of the primary standard while giving the least loss of accuracy in transfer to calibrated instruments. This terminal is a 50-ohm air dielectric coaxial line with an outer conductor inside diameter of $\frac{3}{4}$ inch, the faces being flat and perpendicular to the axis. In order to accommodate all types of terminals, adapters are designed and cilibrated to give minimum loss of accuracy to a defined plane in the commercial connectors.

All of the dozens of instruments and hundreds of standards used in this system are newly constructed, and it will not be possible to certily mexinnm accuracy until a certain amount of history is obtained on each unit.

Since high accuracy in radio Irecuency imperanter measurements is relatively new, and much technifue is required to utilize it effectively, there is still much work to be done, and we hope that the linits we have placed on possible impedinnes and accuracies as a guide for designing the facilities in the Center will serve as a chatlengerather than the barrier it is implied to be. 


\title{
Recently Developed Microwave Impedance Standards and Methods of Measurement*
}

\author{
R. W. BEATTY $\dagger$ AND D. M. KERNS $\dagger$
}

\section{INTRODUCTION}

$\pi$ $\mathrm{N}$ the following, some of the recent developments at the Boulder Laboratories of the National Bureau of Standards in the field of microwave impedance standards and measurements will be briefly described.

The majority of the experimental work has been done in WR-90 ( $X$-band) rectangular waveguide systems although the results are applicable to any size of waveguide and with increased difficulty, perhaps, to coaxial systems.

These developments cannot be described in detail in a paper of this length, but it is expected that the details will be published elsewhere in separate papers.

\section{STANDARDS OF IMPEDANCE FOR Rectangular Waveguide}

Fixed impedance, or reflection, standards consist of terminated waveguides, ${ }^{\perp}$ having practically no reflection; short-circuits, having almost total reflection; and discontinuities $^{2,3}$ of fairly simple geometry, having a reflection calculable from the essential dimensions and wavelength.

An adjustable sliding termination, having a VSWR (voltage standing-wave ratio) of less than 1.0002 , or a return loss greater than $80 \mathrm{db}$, has been constructed using a well-known principle. ${ }^{1}$ In order to provide fine adjustment and minimum variation in reflection as the termination is slid, a number of refinements were necessary. These included construction of a uniform waveguide section with a dimensional tolerance of approximately 0.0001 inch in the cross-sectional dimensions, a spring-loaded ball bearing alignment plunger, a resistive strip tapered from both sides toward the center of the waveguide, and independent fine controls for the required mechanical movements.

Short-circuited sections of waveguide have been constructed in which the input flange is a quarter wavelength from the short circuit. Flange losses are minimized since there is no longitudinal current in the wave-

* Manuscript received by the PGI, September 5, 1958.

$\dagger$ Natl. Bur. of Standards Boulder Labs., Boulder, Colo.

${ }^{1} \mathrm{R}$. W. Beatty, "An adjustable sliding termination for rectangular waveguide," IRE Trans. ON Microwave Theory and TechNiques, vol. MTT-5, pp. 192-194; July, 1957.

2 N. Marcuvitz (ed.), "Waveguide Handbook," M.I.T. Rad. Lab. Ser., McGraw-Hill Book Co., Inc., New York, N.Y., vol. 10; 1951.

${ }^{3}$ D. M. Kerns, "Half-round inductive obstacles in rectangular waveguide as standards of impedance," (in preparation). guide walls at the flange. Electroforming techniques have been used in order to obtain great mechanical accuracy and freedom from working of the interior surfaces. The VSWR $\left(\sigma_{s}\right)$ of a short-circuited quarter wavelength section of waveguide operating in its dominant $T E_{1,0}$ mode can be shown to be approximated closely by

$$
\mathrm{VSWR} \approx \frac{1}{\frac{\pi \delta}{\lambda_{G}} \sqrt{\frac{\mu_{m}}{\mu}}+\left(\alpha \frac{\lambda_{G}}{4}\right)}
$$

where $\mu$ and $\mu_{m}$ are, respectively, the permeabilities of the medium within the waveguide and the metal of which the waveguide is constructed. $\delta$ is the skin depth in the metal; $\lambda_{G}$ is the guide wavelength; $\alpha$ is the attenuation $^{4}$ per unit length of the waveguide.

For example, such a short-circuited section of WR-90 ( $X$-band) copper waveguide has a calculated VSWR at $10 \mathrm{kmc}$ of approximately 5680 corresponding to a voltage reflection coefficient having a magnitude of approximately 0.99965 .

Even though the effective conductivity may not be known to great accuracy, the difference between the actual and calculated reflection coefficient magnitude will probably show up only in the 4 th or 5 th significant figure. This will cause errors of less than 0.01 per cent in measured reflection coefficient magnitudes when the short-circuited section is used as a reference.

Inductive half-round obstacles have been investigated for use as impedance standards in rectangular waveguide. These obstacles consist of either one or two opposed semicircular cylindrical indentations extending across the narrow sides of the waveguide. Standards employing half-round obstacles may be fabricated by electroforming or by machining, do not require unduly close tolerances, and avoid a change in the waveguide cross section. They seem especially suitable for use as independent, absolute standards of reflection or waveguide impedance. Accurate values have been calculated for the lowest-mode lumped-element representation using well-known methods to obtain stationary expressions for the desired reactance elements as functionals of the obstacle currents. The Rayleigh-Ritz process with one-term, two-term, and three-term Fourier expansions for the obstacle current as an "extremaliz-

${ }^{4}$ See, e.g., E. C. Jordan, "Electromagnetic Waves and Radiating Systems," Prentice-Hall, Inc., New York, N. Y., p. 290; 1950. 
ing" sequence yields rapidly convergent numerical results. Accuracy of the results is estimated at five figures or better for VSWR's up to three. Tables were calculated of VSWR and reflection coefficient. Values are obtained for 15 obstacle sizes and for 41 frequencies within the recommended operating range of the waveguide. The results are based upon normalized parameters and are applicable to any size of rectangular waveguide.

Further theoretical and numerical work is being done to enable corrections to be made for finite conductivity and for dimensional imperfections in the half-round standards. These corrections must be adequately known in order to give the half-round obstacles full status as standards and to enable assessment of the significance of the agreement between calculated and méasured values.

\section{Measurement Techniques}

It is useful to measure the reflection from carefully constructed impedance standards and compare with the calculated results. The degree of agreement is an overall indication of measurement errors and errors in the actual standard. It is necessary to use measurement techniques in order to obtain the reflection of an unknown termination in terms of an impedance standard.

In the following, some methods for the accurate determination of VSWR will be discussed.

A number of measuring systems can be represented by a three-arm junction with arms connected to a generator, detector, and the unknown.

The variation in detector signal (square root of detector power) as one changes the relative phase of the reflection coefficient of the unknown is called the response of the system. One can obtain different types of response by choosing junctions having certain properties to be discussed. A careful analysis ${ }^{5}$ of this type of measuring system reveals that the amplitude $b_{3}$ of the output of the third arm (connected to the detector) may be expressed in the form

$$
b_{3}=b_{G} \frac{\left|\begin{array}{cc}
S_{21} & S_{22} \\
S_{31} & S_{32}
\end{array}\right| \Gamma_{L}+S_{31}}{\left|\begin{array}{cc}
\left(1-S_{11} \Gamma_{G}\right) & S_{13} \Gamma_{D} \\
S_{32} \Gamma_{G} & \left(1-S_{33} \Gamma_{D}\right)
\end{array}\right|\left(1-\Gamma_{2 i} \Gamma_{L}\right)},
$$

or

$$
b_{3}=b_{G} \frac{S_{31}}{\left|\begin{array}{cc}
\left(1-S_{11} \Gamma_{G}\right) & S_{13} \Gamma_{D} \\
S_{31} \Gamma_{G} & \left(1-S_{33} \Gamma_{D}\right)
\end{array}\right|} \cdot \frac{1+\mathrm{K} \Gamma_{L}}{1-\Gamma_{2 i} \Gamma_{L}},
$$

where terms of the form $S_{m, n}$ are the scattering coefficients of the junction, and the voltage reflection coefficients of the generator, detector, and load are represented by $\Gamma_{G}, \Gamma_{D}$, and $\Gamma_{L}$, respectively. The term $\Gamma_{2 i}$

${ }^{5}$ For background material, see A. C. Macpherson and D. M. Kerns, "A new technique for the measurement of microwave standing-wave ratios," Proc. IRE, vol. 44, pp. 1024-1030; August, 1956. denotes the reflection coefficien $t^{6}$ corresponding to the internal impedance of the equivalent generator connected directly to the load.

In impedance measuring systems such as the slotted line, it is approximately true that $|K|=1$ and $\Gamma_{2 i}=0$. For these systems,

$$
\left|b_{3}\right|=C|1+| \Gamma_{L}\left|e^{j\left(\psi_{K}+\psi_{L}\right)}\right|,
$$

and as the phase $\psi_{L}$ of $\Gamma_{L}$ varies,

$$
\frac{\left|b_{3}\right|_{\max }}{\left|b_{3}\right|_{\min }}=\frac{1+\left|\Gamma_{L}\right|}{1-\left|\Gamma_{L}\right|}=\sigma_{L}, \text { the VSWR. }
$$

It is possible to construct a "lossless" junction for which $|K| \approx 1$ and $\left|\Gamma_{2 i}\right| \approx 1$. This can yield a squared VSWR response; for as the phase of $\Gamma_{L}$ varies,

$$
\frac{\left|b_{3}\right|_{\max }}{\left|b_{3}\right|_{\min }} \approx\left(\frac{1+\left|\Gamma_{L}\right|}{1-\left|\Gamma_{L}\right|}\right)^{2}=\sigma_{L}^{2}
$$

It is necessary to tune the junction in order to obtain the correct phase relationship between $K$ and $\Gamma_{2 i}$. This response is not only a curiosity, but may find applications in measuring techniques.

Of more promise is the class of junctions for which $|K| \gg 1, \Gamma_{2 i} \approx 0$. Inspection of (2) and (3) reveals that $|K|$ may be approximated closely by $\left|S_{32} / S_{31}\right|$, the directivity ratio of a directional coupler.

It is well known ${ }^{7}$ that the effective directivity of a given directional coupler may be increased (or decreased) by adjustment of a tuner connected to the coupler output. By this means, one can adjust $|K|$ to any desired value. Then another tuner connected to the coupler input may be adjusted to make $\Gamma_{2 i}=0$. This yields a response similar to that of (4) except that $\Gamma_{L}$ is multiplied by $K$. Since $|K|$ may be greater than unity, this can be called a magnified response.

One technique for impedance measurement consists of connecting the unknown $\left(\Gamma_{L}=\Gamma_{U}\right)$, tuning for $b_{3}=0$, $\left(K \Gamma_{U}=-1\right)$, then tuning for $\Gamma_{2 i}=0$. If the unknown is removed and a standard $\left(\Gamma_{L}=\Gamma_{S}\right)$ is connected, the response is

$$
\left|b_{3}\right|=C\left|1-\frac{\Gamma_{S}}{\Gamma_{U}}\right|
$$

If a variable standard were available, one could adjust it until $b_{3}=0$, then $\Gamma_{U}=\Gamma_{S}$. If only fixed standards are available, the following procedure may be used to obtain the magnitude of $\Gamma_{U}$.

$$
\Gamma_{2 i}=\frac{\left|\begin{array}{ccc}
\left(1-S_{11} \Gamma_{G}\right) & S_{12} & S_{13} \Gamma_{D} \\
S_{21} \Gamma_{G} & S_{22} & S_{23} \Gamma_{D} \\
S_{31} \Gamma_{G} & S_{32} & \left(1-S_{33} \Gamma_{D}\right)
\end{array}\right|}{\left|\begin{array}{cc}
\left(1-S_{11} \Gamma_{G}\right) & S_{13} \Gamma_{D} \\
S_{32} \Gamma_{G} & \left(1-S_{33} \Gamma_{D}\right)
\end{array}\right|} .
$$

7 E. F. Barnett, "More about the hp precision directional cou. plers," Hewlett-Packard J., vol. 4, no. 5-6; January/Februar 
If one varies the phase of $\Gamma_{S}$ (a line stretcher, not necessarily of the constant impedance type, may be used), the ratio of maximum to minimum response is

$$
\frac{\left|b_{3}\right|_{\max }}{\left|b_{3}\right|_{\min }}=\left|\frac{\left|\Gamma_{U}\right|+\left|\Gamma_{S}\right|}{\left|\Gamma_{U}\right|-\left|\Gamma_{S}\right|}\right| \text {. }
$$

This type of response is very sensitive to small differences in $\left|\Gamma_{U}\right|$ and $\left|\Gamma_{S}\right|$. One can choose a suitable half-round inductive obstacle impedance standard so that $\left|\Gamma_{S}\right|$ is close to $\left|\Gamma_{U}\right|$. (It is interesting to note that the attenuation of a short section of waveguide connected to an impedance standard could be determined by this technique.) If a short circuit is used as the standard $\left|\Gamma_{S}\right| \approx 1$, the ratio of (8) very nearly equals the VSWR of the unknown. Variations of these techniques based upon (8) are possible.

It is especially interesting to consider the response when the tuners are adjusted ${ }^{8}$ for the conditions $\Gamma_{2 i}=0$, and $|K|=\infty$ (or $S_{31}=0$ ). Eq. (2) becomes

$$
\begin{gathered}
\left|b_{3}\right|=\left|b_{G} \frac{S_{21} S_{32}}{\left(1-S_{11} \Gamma_{G}\right)\left(1-S_{33} \Gamma_{D}\right)}\right| \cdot\left|\Gamma_{L}\right| \\
=C^{\prime}\left|\Gamma_{L}\right| .
\end{gathered}
$$

With this type of response it is not necessary to vary the phase of $\Gamma_{L}$, but merely to observe $\left|b_{3}\right|$ when one

${ }^{8}$ G. F. Engen, private communication. alternately connects the unknown and the standard to the output. In this case

$$
\frac{\left|b_{3}\right|_{U}}{\left|b_{3}\right|_{S}}=\frac{\left|\Gamma_{U}\right|}{\left|\Gamma_{S}\right|}=r \text {, or }\left|\Gamma_{U}\right|=r\left|\Gamma_{S}\right| \text {. }
$$

Any standard of known reflection coefficient $\left|\Gamma_{S}\right|$, such as the ones previously mentioned (except $\Gamma_{S}=0$ ), may be used. If a short circuit is used, the measuring system can easily be arranged so that return loss

$$
\left(20 \log _{10} \frac{1}{\left|\Gamma_{U}\right|}\right)
$$

is measured directly.

Other systems for the measurement of microwave impedance can be represented by four-, five-, and six-arm junctions. The consideration of the accuracy with which impedance measurements can be made with these systems has been partially deferred pending the investigation of simpler systems.

\section{Acknowledgment}

The following personnel of the NBS Boulder Laboratories contributed to the work described above: D. F. Wait, W. W. Longley, Jr., P. F. Wacker, D. Nash, W. Anson, E. Risley, R. Manka, E. Niesen, R. Blixt, and W. E. McNaney.

\author{
Reprinted from IRE TRANSACTIONS \\ ON INSTRUMENTATION \\ Volume I-7, Numbers 3 \& 4, December, 1958
}




\title{
Coaxial Radio-Frequency Connectors and Their Electrical Quality
}

\author{
M. C. Selby, E. C. Wolzien, and R. M. Jickling
}

\begin{abstract}
The wiskely aecepted mamer of evaluating the quality of coaxial radio-frequency connectors was in the past linnted to a single ease, namely, to the condition when the loait trminating the system was equal to the characteristic impedance of the line on the output rod of the commetor. The quality was expressed as the voltage standing-wave ratio in the input line.

'To broalen this method of eraluation a "connector" is redefined, and several methorls are given to find the corrections of varions types of connectors with any termination. Typical results of measurements made by these methods are given for frecuencies from 100 to 900 megacreles. Application of these methods are also indicated in determining the quality of transmission lines in general.
\end{abstract}

\section{Introduction}

The areuracy of impelance measurement depends, among other things, upon the frequency at which it is to be measured. It is generally admitted that the frequency range of 30 to about $1,000 \mathrm{Mc} / \mathrm{s}$, which is under consideration throughout this paper, happens to be one of the more difficult ranges from the standpoint of impedance measurements. 'This is the transition range where components cease to act as lumped-constant elements. Dimensions and distributed constants must be considered, particularly when optimum accuracies are desired. Unfortumately, it is difficult to speeify quantitatively the aceuracy sought because the aceuracy of an impedance measurement is generally a function of botl the masnitude and phase angle of the mlinown. One may howerer indicate its order of manniturle as 1 perecent or betler for "mateher" conditions. In searching for reliable measurement techniques and equipment for this frequener runge to meed the needs of the National Bureau of Standards, the wollknown slotedelline mothod was found the most promisine. After considerable effort, slotterl lines having the highest available mechanical and electrieal precision were realized. 'These lines, usable down to :30 $\mathrm{M} / \mathrm{c} / \mathrm{s}$, had a miformity of probre-rollage output of 0.25 pereent or betted ored the entire working range at all usoful frequencius, and a potential aceurary comsiderably higher than any other available aquipment. 'T'o makle use of that potential aceourary, it was necossary to minimize the corors

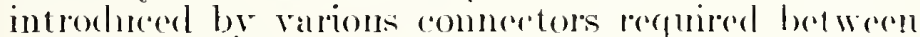
unknown impedanees and the uniforn section of the precision s!otted line. 'These errors, asually nexligible with equipment of lowre acemeracy, could no fonger be disregarded for full ntilization of the quality of the new lines. 'This leel 10 an inrestigation of methosls of determining comnector quality in general with results presented below.

The determination of arrors introduced by connecetimer rements has beren a problem for a nminber of years, particularly when used with measuring or controlling equipment, e.g., attenuators, torminating loads, matching indicators, power monitors, bridges, etc. In most eases these connecting elements consist of rigid coaxial structures, referred to as "connectors," with or without a short solisl-rliclectric cable or air-dielectric rigid transmission line. 'The' characteristic impedances of the components of these connecting elements usually differ from each other by several percent. In addition, the comnectors may have a nonmniform internal structure and discontinuities of their own. The losses are usually negligible and are assumed to be so throughout this treatment.

\section{Definition of Connector and of Its Quality}

The tendency has been to interpret a "conneder" as the physical body of a connecting element, such as an adapter, a baliun, a taper, a jack, or a plug, by itself or in combinations without recard to discontinnities located immediately at the terminating plancs. 'Thus, some indefinitely located planc bretwoen a trpe N plug and jack was taken as a referenee planc for all purposes, e. gr., the plane where a short circuit was to be loeated, the plane where the unknown impedance, voltage, or current was to be measured, atc. When accurate knowtedge of intperlance is desired, this interpretation of a connertor is not alwars aecoptable, because for 'TEM propacation (considered here) impedance is basically drefined and treated as the ratio of voltage to current or of clectric to magnetic fields in a cross sectional plann of a uniform distributed-constant system. 'Tlere are no known commercial connectors merting 1 he requirements of such a system; all have approciabla discontimuities affecting the field distribution in : manner difficult to compute, predict, or measure.

'The term "comnector" as used here inclurles all lossless rircuit arrangements used to interconneet. two transmission lines of any configuration and rharacteristic impedance. An example of such a 

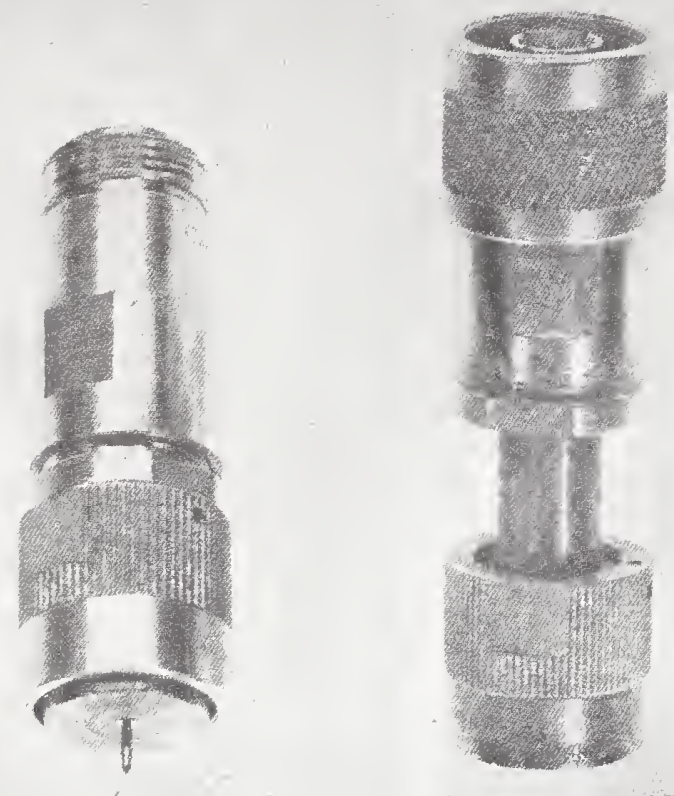

FIGURE 1. Typical connector showing one of terminating planes.

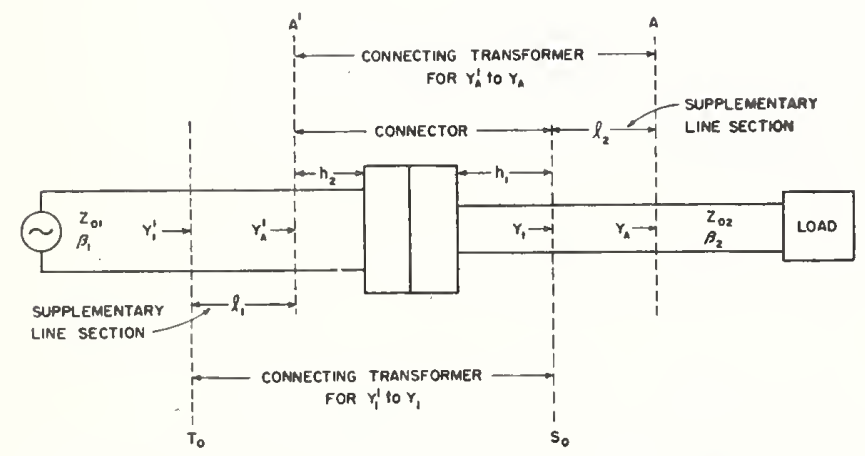

Frgure 2. Representation of connector and connecting transformers.

connector is shown in figure 1. The transmission lines are assumed to have uniform distributed constants up to the connector. 'To complete the definition of a connector, it is necessary to fix the minimum allowable distance between its terminating planes and its adjacent discontinuities (e. g., $h$, fig. 2 ). This distance depends on the particular configuration of the transmission lines and the attenuation rate of the higher modes generated at the discontinuities. For coaxial lines, $h$ (fig. 2) should be equal to or larger than the diameter of the outer conductor [1]. ${ }^{1}$ When used in this sense, connectors can be readily treated as four-terminal networks and their constants defined and measured.

Another accepted convention is to indicate the quality of a connector by the voltage standing-wave

\footnotetext{
1 Figures in brackets indicate the literature references at the cnd of this paper.
}

ratio (VSWR) introduced on a uniform transmission line feeding the connector when the latter is terminated in a load equal to the characteristic impedance of the line. This convention can only be used to estimate the maximum error in a measured VSWR caused by connector reflections. It does not furnish necessary corrections for all impedance values. The approach in this paper is to treat the connector as a component part of a transformer; the figures of merit of this transformer are two transformation corrections for the components of its terminating impedances. The problem is to establish relationships between the connector input and output impedances or admittances. Admittance relationships prove to be more convenient for computation simplicity.

Let, in figure 2, a uniform transmission line having a characteristic impedance $Z_{01}$ be joined with another uniform line, $Z_{02}$, by means of a connector terminated in the planes $A^{\prime}$, at its input end, and $S_{0}$, at its output end. Also, let $T_{0}$ be the location of a voltage node when a short circuit is placed at $S_{0}$. It can be shown [2] that simple expressions interrelate admittances on opposite side of the connector; e. g., the admittances $Y_{A}=G_{A}+j B_{A}$ and $Y_{A^{\prime}}=G_{A^{\prime}}+j B_{A^{\prime}}$ at planes $A$ and $A^{\prime}$, where $A$ and $A^{\prime}$ are effectively an integral number of half wavelengths apart. Two planes in a transmission-line system are effectively an integral number of half wavelengths apart when a voltage node placed in one of these planes (closer to the load) will cause a voltage node to appear in the other plane irrespective of what the physical distance is between them. A supplementary line section, $l_{2}$, is added to fulfill the above requirement. The relationships of these admittance components (fig. 2), as shown by Oliver [2], are given by

and

$$
G_{1}^{\prime}=a_{1}^{2} G_{1}
$$

$$
B_{1}^{\prime}=a_{1}^{2} B_{1}+a_{1} b_{1}^{\prime},
$$

where $a_{1}{ }^{2}$ is a dimensionless constant, and $a_{1} b_{1}^{\prime}$ is an additive constant in mhos. Similarly,

and

$$
G_{A}^{\prime}=a_{A}^{2} G_{A}
$$

$$
B_{A}^{\prime}=a_{\Delta}^{2} B_{\Delta}+a_{A} b_{A}^{\prime} \text {. }
$$

If desired, one can treat the connector shown in figure 2 between $A^{\prime}$ and $S_{0}$ (minus the supplementary line section $l_{2}$ ) as a transformer by expressing the input admittance in terms of the output admittance. From well-known interrelations

$$
\begin{gathered}
g_{1}=\frac{g_{A}\left(1+\cot ^{2} \beta_{2} l_{2}\right)}{\left(\cot \beta_{2} l_{2}-b_{A}\right)^{2}+g_{A}^{2}} \\
b_{1}=\frac{b_{A} \cot ^{2} \beta_{2} l_{2}+\left(1-g_{A}^{2}-b_{A}^{2}\right) \cot \beta_{2} l_{2}-b_{A}}{\left(\cot \beta_{2} l_{2}-b_{A}\right)^{2}+g_{A}^{2}}
\end{gathered}
$$

where: $\beta_{2}$ is the phase constant and the admittance components are normalized with respect to $Y_{02}$. 
The termination admittance of the connector may also be obtained, using various charts and diagrams and thus avoiding computation of eq (5) and (6). However, there is no great advantage in knowing the admittance at the plane $S_{0}$ in preference to that at any other plane. The additional computation work involved is therefore seldom justified.

The definition of a "connecting transformer" may here be introduced as follows:

\begin{abstract}
A connecting transformer is any linear bilateral passive four-terminal lossless network terminated at both ends in uniform line sections; the latter are electrically and mechanically identical to the corresponding adjacent lines of the system. When a short circuit is placed at either end of the connecting transformer, a voltage node appears at its other end.
\end{abstract}

Thus the connecting transformer consists of the conmector and 1 or 2 supplementary line sections. However, there is generally no practical advantage of having more than one supplementary section; the disadvantage is, of course, the need of two (instead of one) admittance transformations when one is interested in the actual input and output admittance of the connector. A connecting transformer, as defined above, therefore consists of the connector proper and one supplementary line section at its output or at its input end. In figure $2, l_{1}$ is the input-end supplementary line section of the connecting transformer correlating $Y_{1}^{\prime}$ and $Y_{1}$ through a set of corrections $a_{1}^{2}$ and $a_{1} b_{1}^{\prime}$. If the output supplementary line section $l_{2}$ is chosen, a set of corrections, $a_{A}^{2}$ and $a_{A} b_{A}^{\prime}$, is required, and the correlation between $Y_{A}^{\prime}$ and $Y_{A}$ is established. It is convenient to use $l_{2}$ in preference to $l_{1}$ when the quality of connectors is studied. On the other hand, $l_{1}$ is used when the connector is an integral part of an admittance measuring system used to determine $Y_{1}$ with optimum accuracy. The justification for these preférences will be further clarified below when the methods of obtaining the transformation corrections are discussed. Special problems may indicate a different choice of supplementary sections and may justify the use of two sections simultaneously (i. e., one at the input, the other at the output). However, the two major groups of problems anticipated at present are impedance and connector measurements; therefore the discussion below will be limited to connecting transformers using either $l_{1}$ or $l_{2}$.

The interpretation of $a^{2}$ and $a b^{\prime}$ in the case of impedance measurements is straightforward. For connector measurements the interpretation is as follows: With a perfect connector (referring to fig. 2) normalized admittance $Y_{A}^{\prime} / Y_{01}$ will always be equal to the normalized $Y_{A} / Y_{02}$; otherwise the values of $a^{2}$ and $a b^{\prime}$ will indicate the errors introduced by the connector discontinuities, i. e., the relative quality of various connectors for all values of admittances. For a connector mounted on the panel of an enclosed network like an attenuator, voltage generator, etc., one may simply consider the connector as a part of the enclosed network with the reference plane at $A^{\prime}$. Otherwise, required reference impedance-planes like. $S_{0}$ and $A$ may be assumed inside the network.
'To summarize, the definitions of connector', connecting transformer, and connector quality, as applied here, are as follows: (a) A connector is a lossless linear bilateral passive four-terminal network terminated at each end in a uniform line section of a minimum length required to attenuate higher modes likely to appear at these ends. These line sections are identical electrically and mechanically to the respective lines of the interconnected system. (b) A connecting transformer is a connector as defined in (a), terminated at one end in a supplementary lossless line section. This supplementary line section is less than half a wavelength long and is identical electrically and mechanically to that at the connector end. When a short circuit is placed at either end of a connecting transformer (with $\mathrm{r}$-f power fed from the opposite end), a voltage node will appear at the other end of the transformer. With perfect connectors a connecting transformer is an ideal transformer; the normalized input admittance components of this transformer are equal to its normalized output admittance components. (Normalization is with reference to input and output lines, respectively.) (c) With imperfect connectors one must apply corrections to obtain accurate termination admittances. These "transformation corrections" $a^{2}$ and $a b^{\prime}$ indicate the quality of the connectors. The simplest form of the corrections are given in eq (1) to (4). These transformation corrections hold only for the chosen combination of connector and supplementary line section.

\section{Determination of Connector Quality}

\subsection{Analytical Background}

The inadequacy of specifying the VSWR of a "matched" connector as its quality index has been pointed out above. For systems of equal characteristic impedances the effective characteristic impedance of the connector is frequently taken as the square root of the product of the measured open and shorted input impedances (i. e., $Z_{0}=\sqrt{Z_{O C} Z_{S C}}$ ). This yields, strictly speaking, an inaccurate parameter, because the approach assumes infinite and zero impedances at open- and short-circuited conditions, respectively, and uniformly distributed constants, none of which are true in practice. In addition, this approach neglects likely discontinuities at the termination planes of the connector as the latter is conventionally referred to. Therefore, the value of $Z_{0}$ so obtained cannot be used as a simple transformation factor. A reliable way to find quantitative values of the transformation corrections of connectors is the one employing the node-shift method $[2,3,4,5]$. Frequency-variation methods can be used only to find qualitative data:

The node-shift (n-s) method is well described in the references cited and consists briefly in measuring corresponding shifts of voltage-minima at both ends of the connecting transformer. The original application of this method involved a less efficient and 
less accurate handling of measurement data, requiring the determination of tangents to curves of uncertain regularity. An improvement, introduced by Oliver, largely eliminated this difficulty, because the transformation corrections are obtained from a curve that is analytically shown to be a straight line; measurement errors can be readily detected in the plot of a straight line. Techniques developed for the practical application of this method in systems emploving equal or unequal characteristic impedances are described in section 3.3 Several variations in these techniques may be used, depending on conditions and requirements.

The essential analytical steps underlying this method of approach are as follows: Let $a, b, c, d$ represent, at one frequency, linear parameters [2] of a four-terminal network equivalent to either one of the two connecting transformers shown in figure 2. As the elements of the network are assumed passive and linear, and a zero termination impedance is transformed into a zero input imedpance,

$$
\begin{gathered}
a d-b c=1 \\
c=0 \\
Z_{1}^{\prime}=\frac{Z_{1}}{A^{2}+a b Z_{1}} \\
Y_{1}^{\prime}=a^{2} Y_{1}+a b .
\end{gathered}
$$

In the last two expressions $a$ and $b$ are the parameters of the transformer between $Y_{1}^{\prime}$ and $Y_{1}$ (i. e., between planes $T_{0}$ and $S_{0}$ ).

As pointed out above, a voltage node at $S_{0}$ will cause a voltage node at $T_{0}$. The plane $T_{0}$ may be located at the imput to the connecting transformer or at any other plane an integral number of half wavelengths away from it. Let the voltage node now be moved a distance $s$ from $S_{0}$ by means of a movable short circuit. 'This is equivalent to varying the reactance of $Z_{1}$ by a given amount. The node at $T_{0}$ will therefore shift in turn by some distance $t$, not necessarily equal to $s$, as shown in figure 3 . Here the transformer input plane is shown at $K$ and $T_{0}$ is rhosen a half wavelength away from $K$; this is neces-

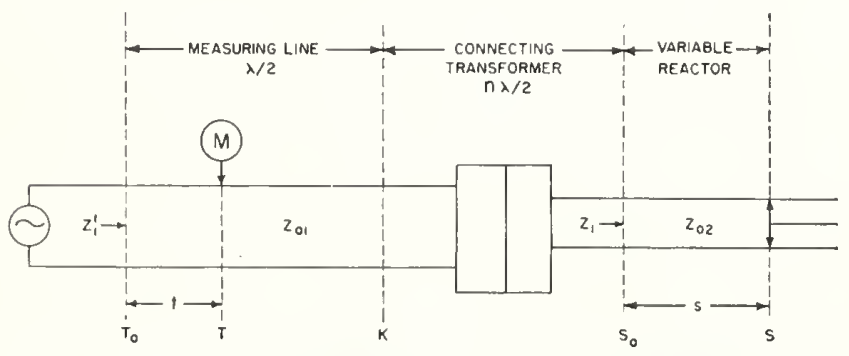

Firure 3. Basic arrangement to measure quality of a connecting transformer. sary to permit measurements along a uniform line section. With the nodes in these positions,

$$
\begin{aligned}
& Z_{1}{ }^{\prime}=j Z_{01} \tan \beta_{1} t \\
& Z_{1}=j Z_{02} \tan \beta_{2} s
\end{aligned}
$$

where $\beta=2 \pi / \lambda$ is the phase constant of the respective lines.

Using eq (9),

$$
\frac{Z_{01}}{Z_{02}} \tan \beta_{1} t=\frac{\tan \beta_{2} s}{a^{2}+j a b Z_{02} \tan \beta_{2} s},
$$

since the network is lossless, $b$ is an imaginary quantity [2] and one may substitute $-b^{\prime}$ for $j b$. Rewriting the terms,

$$
\cot \beta_{1} t=\frac{Z_{01}}{Z_{02}}\left(a^{2} \cot \beta_{2} s-a b^{\prime} Z_{02}\right) ;
$$

therefore,

$\cot \beta_{1} t-\cot \beta_{2} s=\left(\frac{Z_{01}}{Z_{02}} a^{2}-1\right) \cot \beta_{2} s-a b^{\prime} Z_{01}$.

One can thus obtain a number of $\cot \beta_{1} t$ values corresponding to chosen $\cot \beta_{2} s$ values and the plot of $\left(\cot \beta_{1} t-\cot \beta_{2} s\right)$ versus $\cot \beta_{2} s$ will be a straight line with $\left[\left(Z_{01} / Z_{02}\right) a^{2}-1\right]$ as its slope and $\left(-a b^{\prime} Z_{01}\right)$ as its intercept on the $\left(\cot \beta_{1} t-\cot \beta_{2} s\right)$ axis. As $Z_{01}$ and $Z_{02}$ are assumed to be known, $a^{2}$ and $a b^{\prime}$ can be determined for use in eq (1) to (4). In addition, relationships for impedance components can be derived to show how the load impedance, $R+j X$, of a slotted line, for example, is determined from its measured value, $R^{\prime}+j X^{\prime}$, when the transformation colrections of its connecting transformer are known. Thus, from eq (9), again writing $-b^{\prime}=j b$,

$$
R+j X=\frac{a^{2}\left(R^{\prime}+j X^{\prime}\right)}{1-j a b^{\prime}\left(R^{\prime}+j X^{\prime}\right)}
$$

or

$$
\begin{aligned}
& R=\frac{a^{2} R^{\prime}}{1+2 a b^{\prime} X^{\prime}+\left(a b^{\prime}\right)^{2}\left(R^{\prime 2}+X^{\prime 2}\right)} \\
& X=\frac{a^{2}\left[X^{\prime}+a b^{\prime}\left(R^{\prime 2}+X^{\prime 2}\right)\right]}{1+2 a b^{\prime} X^{\prime}+\left(a b^{\prime}\right)^{2}\left(R^{\prime 2}+X^{\prime 2}\right)} .
\end{aligned}
$$

It may be of interest to note at this point the interrelation between the parameters of the same connecting transformer in the forward and reversed directions. A case may arise where it is necessary to reverse the transformer end for end. For example, because of external structural irregularities, one may wish to connect the transformer into a circuit in a direction opposite to that used for the measurement of its constants. The termination impedances must then be corrected by a new set of parameters, because the system is not necessarily symmetrical. There is a simple correlation between the "forward" and 
"reversed" sets of corrections, obviating the necessity of additional measurements. It can be shown $[\dot{6}]$ that

$$
a_{f}=\frac{1}{a_{r}}
$$

and

$$
b_{f}^{\prime}=b_{r}^{\prime}=b^{\prime},
$$

where the subscripts $f$ and $r$ refer to the forward and reversed conditions, respectively.

Let the intercept of the straight-line plot measured in the forward direction be $n$; then

$$
a_{f} b^{\prime}=-\frac{n}{Z_{01}}
$$

Let also the slope obtained in the forward direction be $m$; then

$$
a_{f}^{2}=\frac{Z_{02}}{Z_{01}}(m+1)
$$

For at reversed systom

$$
a_{r} b^{\prime}=\frac{b^{\prime}}{a_{f}}=\frac{a_{f} b^{\prime}}{a_{f}^{2}}=\frac{-n}{Z_{02}(m+1)} \cdot
$$

Equations (22) and (23) niay be used to compute the output imperlance of a connectine transformer. The impedance at the input end of the connereting transformer is assumed to be known.

\subsection{Connectors in Tandem}

There is frequently the need of measuring the quality of two comnectors in tandem in order to obtain the quality of one of thens. A trpical example is as follows: Precision slotted lines of special mechanical and electrical design are frequently constructed at a considerable cost; special diameters are chosen to obtain optimum quality of the slotted line. It is therefore necessary to employ adapters, tapers, ete. to connect this line to conventional connectors of various dianeters. There is a simple relationship between the transformation corrections of a connecting transformer composed of two transformers in tandem and the corrections of the individual transformer's.

In figure 4 let a precision slotted line be connected through commector 1 and connector 2 to an umbinown load in plane $S_{02}$ at the cond of eomecting transformer 2. 'The supplementary line section of transformer 1 is $\dot{l}_{1}$, a section of the precision slotted line. From the eq (1) to (4),

$$
a_{12} b_{12}^{\prime}=a_{1}^{2} a_{2} b_{2}^{\prime}+a_{1} b_{1}^{\prime}
$$

where subseripts 1,2 , and 12 correspond, respectively, to comecting transformers 1,2 , and $1-2$, and where planes $S_{01}$ and $T_{02}$ coincide.

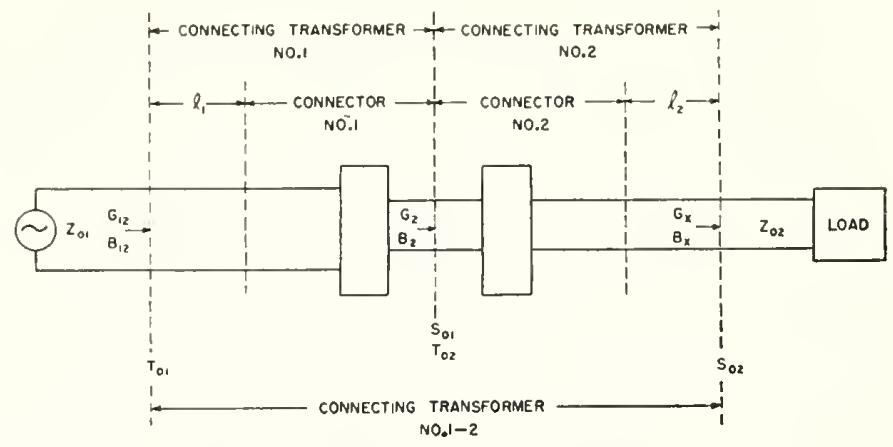

FinURE 4. Representation of two connecting transformers in tandem.

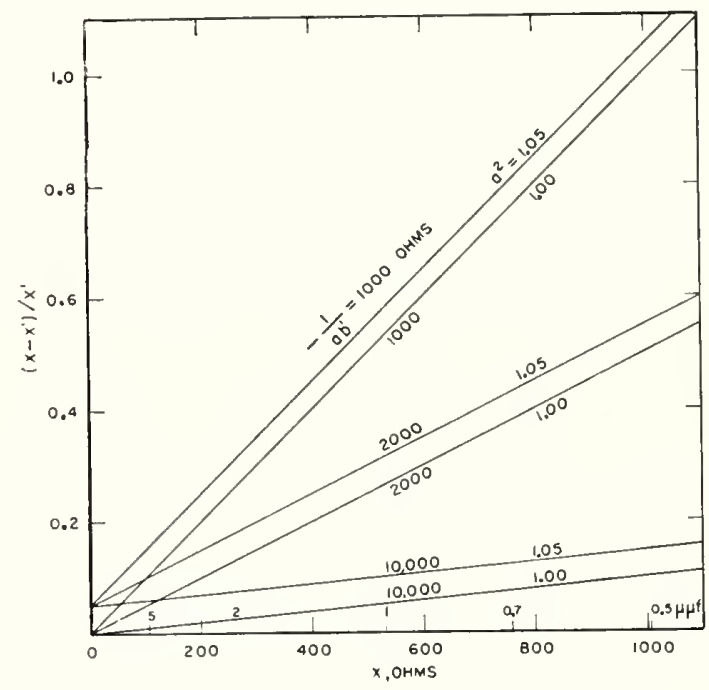

FreURE 5. Fructional error in measured shunt reactance ve'sus. shuml capacitive reaclance lerminating the connecting transformer.

The values in micomieroluals are the equivalent shunt capacities at 300

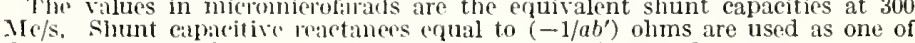
the parameters to be eonsistent with the values used along the axes.

It is thus seen that one can obtain the individual transformation corrections of transformer 2 from the corrections of the combined transformer 1-2. This is a great advantage, as it allows the use of slotted lines of the highest precision to accurately measure convector quality and unknown impedance, even when the latter do not match the line mechanically or electrically.

The signifieance of the magnitudes of the transformation corrections $a^{2}$ and $a b^{\prime}$ may be demonstrated ans follows: Equation (1) indicates that the error in a measured conductance is a function of $a^{2}$, whereas eq (2) shows that the error in a measured susceptance is a function of $a, b^{\prime}$, and of the magnitude of that susceptance. From eq (2) the fractional error in this susceptance is

$$
\frac{B-B^{\prime}}{B}=\left(1-a^{2}\right)-\frac{a b^{\prime}}{B}
$$

or

$$
\frac{x-x^{\prime}}{x^{\prime}}=-a \cdot b^{\prime} x+\left(a^{2}-1\right)
$$


where $B=-1 / x, B^{\prime}=-1 / x^{\prime} ; x$ and $x^{\prime}$ are the respective equivalent shunt reactances of the unknown and measured impedances at the ends of the connecting transformer.

Equation (26) thus gives the errors in the measured shunt reactances, which can be plotted versus $x$ as straight lines with shunt capacitive reactances equivalent to $\left(-a b^{\prime}\right)$ mhos as slopes, and $\left(a^{2}-1\right)$ as intercepts. Representative values usually encountered in practice at frequencies to $1,000^{\circ} \mathrm{Mc} / \mathrm{s}$ are shown in figure 5.

\subsection{Techniques and Procedures}

The node-shift method of connector-quality determination may utilize one of the following reactors, depending on the problem and facilities at hand: (a) An adjustable stub, (b) fixed line sections, (c) a slotted line, or (d) a perforated line. Steps of procedure and precautions that are common to the use of all of these reactors will first be discussed; then features of each device and its associated technique will be described individually.

\section{a. Equipment}

In general, the equipment requirements are these: The known reactor, connected as shown in figure 3 at $S_{0}$ (the end of the unknown connecting transformer) must be lossless, uniform, and adjustable or variable over at least half a wavelength. The junction at $S_{0}$ must be free of discontinuities; thus, for best results, the cross-sectional dimensions and dielectric material of the reactor and the connecting transformer must be the same at this plane: In addition, this uniformity must be maintained on the input side of $S_{0}$ for an axial distance equal to at least the diameter of the outer conductor. Sometimes, as mentioned in section g, a small amount of mismatch may be tolerated without invalidating the measured data. Good mechanical alinement and contact at this plane are essential. All discontinuities should lie between $S_{0}$ and $K$, the lattel being a plane of reference on the measuring line, effectively an integral number of half wavelengths from $S_{0}$.

\section{b. General Procedure}

The measurement procedure consists in first placing a short circuit at $S_{0}$ and locating $T_{0}$, a position on the measuring line where a voltage node occurs, one or more half wavelengths from $K$. Whenever possible, the first $T_{0}$ beyond $K$ should be used so as not to introduce extra losses into the measuring system. As the short circuit is moved from $S_{0}$ to $S$, a distance $s$, the voltage node on the measuring line moves from $T_{0}$ to $T$, a distance $t$. From 5 to 10 colresponding positions of $S$ and $T$ are recorded. This number of points has been found desirable to identify any irregularities in the data. Values of $s$ are chosen so that the distribution of $\cot \beta_{2} s$ will be fairly evenly spaced from -2 to +2 . These limits are selected because usable data, for the rapidly changing cotangent function outside this interval, require equipment precision which is usually beyond practical achievement. When the magnitude of the $s$ values is limited by the usable length of the reactor, lengths of $s$ corresponding to $\cot \beta_{2} s$ values from 0 to +2 may be adequate. Each of the $S$ and $T$ nodes are located by determining the midpoint between two positions of equal detector response one on each side of the minimum. However, when structural defects in the slotted line produce nonuniformity in the voltage distribution along the line, errors may be caused in the determination of the nodal positions. To minimize these errors, it has been shown [7] that the optimum equal-response voltage in the determination of a nodal position should be approximately $3 \mathrm{db}$ (within $\pm 0.5 \mathrm{db}$ ) above the minimum voltage when the VSWR is above 7 . In addition to structural defects, the presence of losses in the measuring system may introduce errors in making accurate nodal measurements. When such is the case, one may minimize these errors by following the procedure outlined later in section $\mathrm{g}$.

The difference, $\cot \beta_{1} t-\cot \beta_{2} s$, is then plotted versus cot $\beta_{2} s$ and the resulting straight line interpreted as shown in section 3.1. The values of $a^{2}$ and $a b^{\prime}$ thus obtained are the transformation corrections of the unknown connecting transformer at the test frequency.

As the magnitudes of $a^{2}$ and $a b^{\prime}$ are functions of the relative positions of the discontinuities in the connecting transformer, and as these positions are scattered and unknown, the effect of these discontinuities may cancel at some frequencies and increase at others. Therefore, the number of frequencies at which measurements must be made in order to find the quality of a connector over a given frequency range will be determined by the extent of the frequency range and the accuracy desired. Measurements indicated that the transformation corrections for commercial connectors varied rather slowly with frequency up to $1,000 \mathrm{Mc} / \mathrm{s}$, and that measurements at $100-\mathrm{Mc} / \mathrm{s}$ intervals in this range would be sufficient to determine the frequency characteristies of a given connector.

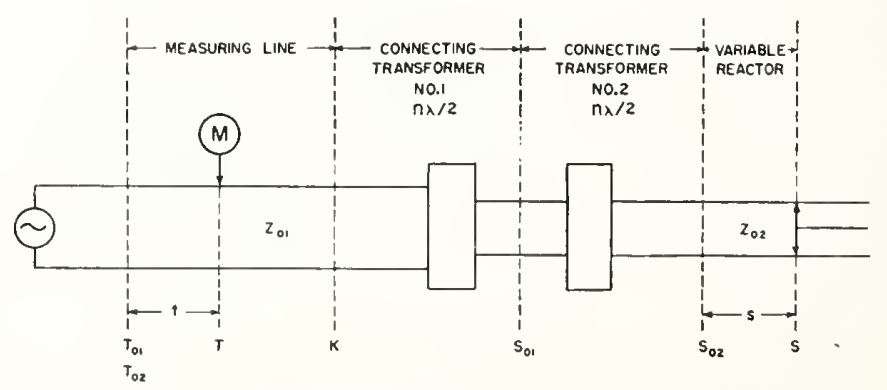

Figure 6. Basic arrangement to measure quality of two connecting transformers in tandem. 


\section{c. Connectors in Tandem}

When the measuring line does not match the unknown connector in size or characteristic impedance, or both, an adapter is required between them. Then it is necessary to separate the corrections of the unknown connector from those of the adapter according to the procedure described above in section 3.2. In this situation $S_{02}$ (see fig. 6) must be so placed that a voltage node appears at $S_{01}$. This condition can be met by first placing a short circuit at $S_{01}$, the output end of connecting transformer No. 1 , and by locating a corresponding $T_{01}$ on the measuring line. Then the short circuit is replaced by the unknown connector and adjustable reactor, and the latter is varied until a voltage node again appears at $T_{01}$. Thus a voltage node is also assured at $S_{01}$. The values of $s$ and $t$ are then obtained as described previously. In some cases the voltage node at $S_{02}$ cannot be moved continuously because of the type of variable reactor being employed or because connecting transformer No. 2 has a fixed length. The procedure to place a voltage node at $S_{01}$ under such conditions will be described in section $\mathrm{h}$.

\section{d. Curvature in Plot of Data}

It was occasionally observed that the plot of measurement data substituted in eq. (15) resulted in a curve rather than a straight line. The following precautions were found to prevent such curvature. (1) The phase constants of the measuring line and the reactor must be known to an accuracy of \pm 0.01 percent. This required that the corresponding frequency stability be maintained to an accuracy of at least \pm 0.01 percent. (2) The positions of $S_{0}$ and $T_{0}$ should be measured, for example, to $\pm 0.1 \mathrm{~mm}$ at 300 $\mathrm{Mc} / \mathrm{s}$ for lines with dielectrics having constants of $\cdot 1$ to 3. The presence of any appreciable discontinuity at these planes, such as a poor contact at $S_{0}$, may cause an excessive error. (3) When the voltage distribution near a node is nonsymmetrical because of losses in the measuring system, proper precautions must be taken to locate the positions of $S$ and $T$ colrectly. These precautions are described in section $\mathrm{g}$. (4) The accuracy of finding voltage-node positions may be improved by keeping the input voltage high and the probe penetration deep (for low input voltages), probe reflections being negligible near voltage nodes, by filtering out harmonics from the input voltage, and by operating probe output indicators at the most sensitive positions of the meters and circuit action. Means of minimizing errors caused by structural defects in slotted lines were indicated under section b.

\section{e. Use of an Adjustable Stub as a Reactor}

This type of reactor is the most obvious one to select for use in n-s measurements of air-dielectric elements and is quite simple to operate. A 5- $\mathrm{ft} 50-$ ohm stub of this type was found particularly useful in measuring $7 / 8$-in. connectors in the VHF range.
To determme values of $s$, relative stub positions may be measured with the required precision by means of large vernier calipers, gage blocks, or a carefully engraved scale attached to the adjustable stub. An advantage of this type of reactor is that one stub of sufficient length can be used to obtain convenient values of $s$ over a wide frequency range.

In the construction of the stub it is essential to use the least amount of solid support for the center conductor near the input end. Whatever support may be required should be small in mass and have the lowest possible dielectric constant. When it is possible to support the center conductor by the connector being measured, the omission of any additional support is desirable. Another critical construction feature is that the movable shorting device should locate a nearly lossless short circuit in positively defined planes. For small-sized connectors it is very difficult to build a stub long enough for use in lowfrequency $n-s$ measurements without an objectionable sag in the center conductor. An adjustable stub is unsuitable for measurements of solid-dielectric connectors, because of unavoidable discontinuities of the junction of the two dielectric media.

\section{f. Use of Fixed-Line Sections as Reactors}

The use of accurately constructed line sections for reactors simplifies $\mathrm{n}$ s measurements. First, a short circuit is placed at $S_{0}$; this is then replaced by shorted fixed-line sections of different lengths corresponding to selected $s$ values. Alternatively, sections of equal length open at both ends may be connected in tandeni; a short circuit is then placed at the output end of the combination. In both cases it is essential that a good mechanical contact exist at $S_{0}$ and that no discontinuities be introduced in this plane. In using combined sections, these conditions must also be maintained at the junctions. Otherwise, a curvature or excessive random scatter will result in the final plot of the data.

Although the procedure followed with this type of reactor is simple, a number of disadvantages are involved. First, the careful matching and alinement referred to above requires precision machining. Thus when $n$-s measurements are to be made over a wide frequency range, numerous costly precision sections are required. Second, if an adapter is required between the unknown connector and the measuring line, it may be awkward to use fixed sections as reactors (particularly at low frequencies) because of the requirement that the ends of the unknown connecting transformer be an effective half wavelength apart (see section 3.2). Third, although reactors of this type can be constructed to match any present-day air-dielectric connector, this can not easily be done for solid-dielectric components because it is difficult to determine with sufficient accuracy the phase constant of solid-dielectric sections. In coaxial lines having solid insulation the phase constant is very closely equal to $2 \pi f(k)^{1 / 2} /\left(2.998 \times 10^{8}\right)$ radians/meter. where $k$ is the dielectric constant of 
the insulation. Tariations from 1 to 3 pereent may be found in the dielectrie constants of commonly used insulators, depending on the batch or manufacturer. In adclition, these constants may be affected by a change in volume (i. e., density) eaused during fabrieation steps and by the application of excessive heat. 'The phase-eonstant accuraey is also limited by the accuracy ( \pm 0.1 percent) to which the caparity of line sections can be measured, by effects of fre. queney on the dielectric constant, and by dimensional tolerances of the conductors. 'Thus it appears that the best prospect of obtaining the required aceuracy of \pm 0.01 pereent in the phase constant is to measure the electrical lengths directly in the finished line sections. For example, a scetion of line can be resonated by varying the frequency within the range in question and obscrving the output of a fixed probe located at a precisely known distanee approximately a half wavelength from the shorted end. Assuming that the dielectric and dimensional uniformity was maintained, this line section can then be cut into a number of slort sections. This fixed probe method sugrests the application of a perforated solid-dielectric line; it also suggests an accurate way to measure the diclectric constant of a coaxially fabricated material.

A type of reactor combining the features of the adjustable shorting stub and fixed-line sections might be used to alleviate some of the disadvantages mentioned above. First, an air-dielectric stub is required, in which the shorting deviee can be removed from the eoaxial line. Coaxial sections of any desired solid insulator are then inserted in the coaxial line, and the shorting device is replaeed so as to produce a short circuit at the end of the newly formed solid-dieleetric eoaxial line. It is neeessary that each of the insulator sections be accurately maelined to fit the coaxial line and to liave end planes perpendicular to its axis. The lengtlis of these sections depend on the desired values of eot $\beta_{2} s$. Advantages of this type of reactor are (a) the conductors are not broken; (b) numerous s values are readily available, because it is simpler to machine any desired number of insulators rather than to construet an equal number of individual line sections; (c) the terminiating plane of the unknown connecting transformer (solid-dielectric type) can casily be placed at any approximate point in the reactor" when required; (d) any number of different dielectric materials mar be used with the same air stub for various desired $Z_{02}$ values; (e) the phase eonstants may be determined with an auxiliary fixed probe, as described above.

\section{g. Use of a Slotted Line as a Variable Reactor}

In emploring a slotted line as a known reactor, an auxiliary line stretcher or adjustable stub is connected at the output end of the slotted line to move a voltage mininum along the slotted line where it is detected by a probe (see fig. 7). The method recommended for accurately locating this voltage node is the same

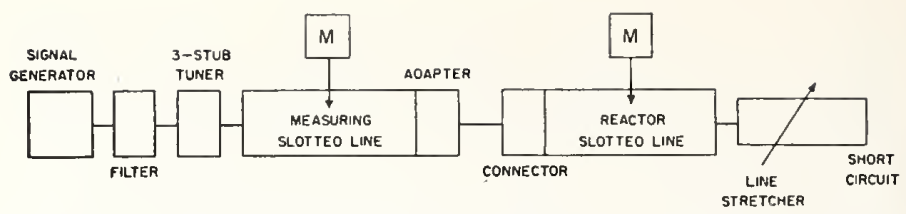

FICURE 7. System for node-shift measurements using a slotted line as a reactor.

as that described in section b. It is not essential that the voltage node be moved to the exact $S$ Jocation previously selected; it is possible to loeate the position of the voltage minimum veryaeeurately and thus know the corresponding value of $s$.

At higher frequencies the presence of losses in the measuring system may make accurate measurements of $S$ and $T$ more difficult. As the voltagc curve in the region of a minimum closely approaches a parabola, the voltage nodes may be located acecurately by joining the midpoints of three horiziontal chords through each selected trough of the voltage standing-wave [8]. However, this proeedure is quite tedious; in most cases the following simplor method may be employed, with results of sufficient aecuracy; (a) place a short circuit at $S_{0}$ and find the true $T_{0}$; (b) conneet the slotted-line reactor at $S_{0}$; this will introduce losses (especially when a soliddiclectric line is used), reducing the VSWR and upsetting the svmmetry of the voltage distribution in the measuring line particularly; (c) locate a norle at $T_{0}$ as follows: Adjust the auxiliary variable reactor to obtain a node near $T_{0}$, using a voltage increment, $\Delta E_{t}$, about $3 \mathrm{db}$ above the minimum; readjust the variable reaetor to move the node toward the desired location; repeat these steps until a node is obtained exactly at the true $T_{0}$; (d) then loeate the corresponcling minimum for $S_{0}^{\prime}$ (an effective half wavelength from $S_{0}$ toward the short circuit). Use a eertain $\Delta E_{s}$ approximately $3 \mathrm{db}$ above the voltage minimum. (e) Finally, locate the selected $S$ and corresponding $T$ positions, using the same $\Delta E_{s}$ and $\Delta E_{t}$ in each ease. as was used to locate the $S_{0}^{\prime}$ and $T_{0}$, respectively.

Relative merits of this technique are: (a) This trpe of reactor is useful wherever a slotted line is available to match the connector being measured. The technique was found particularly applicable in determining the quality of $3 / 8$-in. connectors, the sizc in which solid-dieleetric slotted lines of fairly good precision have been made. (b) Only one slotted line is needed as a reaetor for n-s measirements orer a wide frequency range, e. g., from 100 to 1,000 Mrc/s. (c) Beeause of the precision with which voltage nodes ean be located, the veloeity of propagation, and henee the phase constant of this type of reactor, can be very accurately determined. (d) The use of this reactor is more convenient than the others for the n-s measurement of connectors in tandem, beeause of the ease with which $S_{02}$ can be located whenever it must be placed in the reactor to meet the half-wavelength requirement referred to previously. 
In turn, this technique possesses a few disadvantages. Measuring the $s$ values in a slotted line is somewhat more time consuming that adjusting them, as described in the previous methods. A slotted line is considerably more expensive than reactor's of the other types for use over the same frequency range. Because of the slot, it is impossible to match perfectly a slotted line to a closed line, a compromise necessarily being required either in the conductor dimensions or in the characteristic in.pedances. However, the use of a slotted line as a reactor is practical if the resulting discontinuitr at the junction of the line and the unknown connector is kept as small as possible. For example, satisfactory data were obtained by using such a slotted line to measure the quality of adapters on precision slotted lines.

\section{h. Use of a Perforated Line as a Variable Reactor}

Although applicable to making n-s measurements of connectors in all sizes, this technique is especially recommended for use in determining the quality of large-sized connectors because of the economy made possible in the construction of the variable reactor. As an example, a 50-ohm 31/8-in. perforated airdielectric line, shown in figure 8, was constructed for the quality measurements of connectors and lines having that diameter and characteristic impedance. The line consisted of a 4 -ft brass outer conductor with a duralumin center conductor supported at the output end by a Teflon dise $1 / 4$ in. thick. At the input end no dise was used; the center conductor was supported by the connector being measured so as to minimize the discontinuity at the junction of the connector and reactor. Stock tubing was used for the conductors. Probe holes, $1 / 8$ in. in diameter, spaced $1 \mathrm{in}$. apart, were bored in the outer conductor. This spacing permitted the choice of convenient $s$ values over a wide frequency range. Surrounding each hole was a milled "flat" to help center the probe. The probe was a stiff copper wire, 0.020 in. in diameter, soldered to a BNC connector, and was shielded by a short cylinder that fitted the probe hole. An external adjustable stub was used to tune the probe (see fig. 9). As with the slotted line, a short-

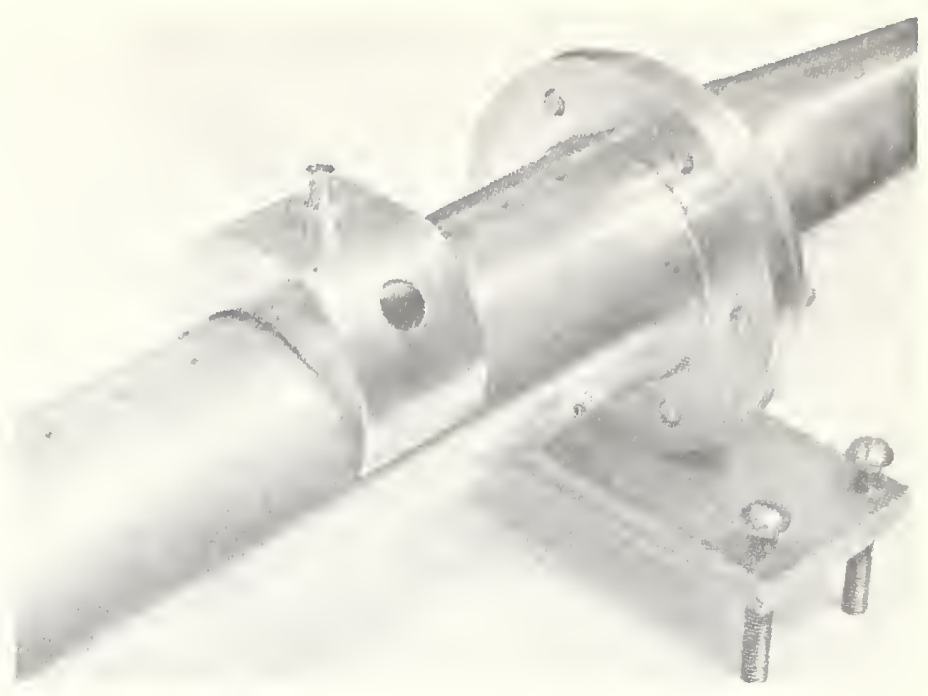

FIGURE 8. Details of a perforated line used as a variable reactor in node-shift measurements.

circuited line stretcher was used at the output end to move the voltage node along the perforated line. To facilitate placing the voltage node at a preselected hole, a line stretcher that can be moved by very small increments in a positive fashion is desirable. If difficulty is experienced in this operation (as indicated by a lack of reproducibility), the following method may be used: (a) Shift the voltage node slightly to one side of the selected hole. Let the probe output voltage at this hole be $V_{1}$ and the position of a corresponding voltage node on the measuring line be $T_{1}$. (b) Move the voltage node to the other side of the selected hole so that the new probe voltage $V_{2}$ equals $V_{1}$. Let the position of the corresponding voltage node on the measuring line be $T_{2}$. (c) Then the true $T$ for a voltage node placed at the desired hole will be midway between $T_{1}$ and $T_{2}$. This procedure assumes that the voltage curves at $S$ and $T$ are not distorted by excessive losses. This is not always true at $T$. If such is the case, the precautions discussed in section $g$ must be observed in finding $T$. In all cases, when the movable short circuit is being adjusted, it is advisable to have the source impedance equal to $Z_{0}$ of the line connected

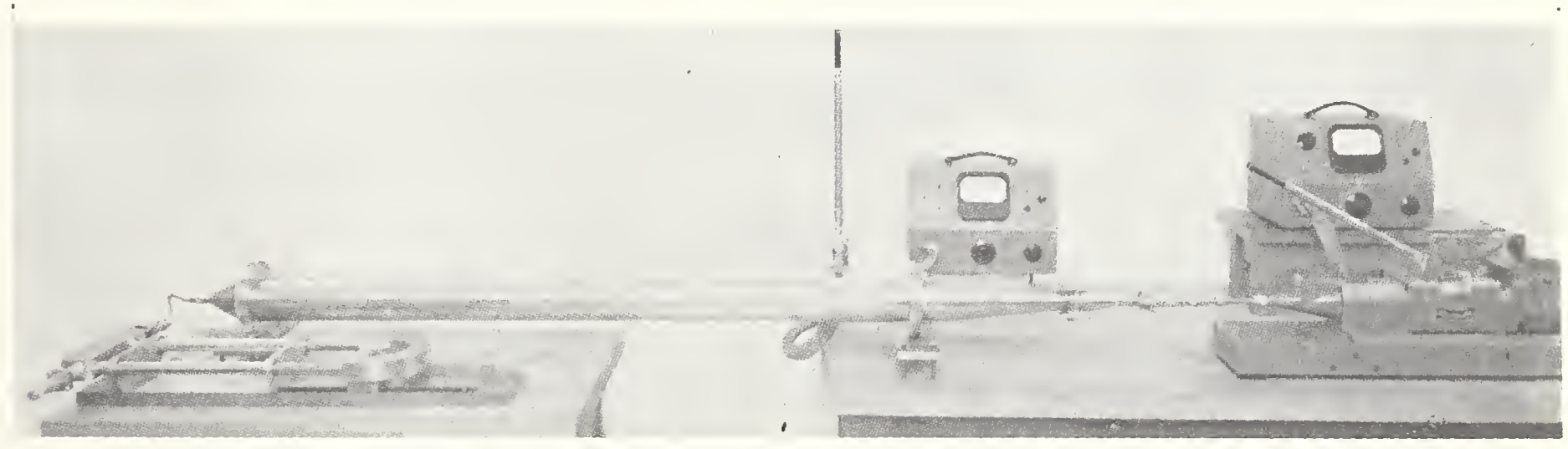

Figure 9. Perforated line used as a variable reactor to measure the rorrections of a transformer. 
to it; this assures a constancy of voltage amplitudes along the system.

When it is necessary to use an adapter between the unknown connector and the measuring line, the corrections of the two connectors must be separated, as previously explained. Because of the fixed probe positions of the perforated line, a special step must usually be employed here to determine the exact frequency at which the unknown connecting transformer is effectively equal to a half wavelcngth. A short circuit is first placed at the end of the adapter to the measuring line, and three values of $T_{01}$ are found for three different frequencies around the estimated frequency at which the unknown connecting transformer meets the length requirement. Then the short circuit is removed, and the unknown connector and perforated line are attached, the probe of the perforated line is placed in a selected hole, and three values at $T_{02}$ are found for three similar frequencies. With these data two curves of frequency versus $T_{0}$ are plotted. The intersection of these curves determines the desired frequency. If the effective discontinuity of the adapter is small (so that the transformation corrections remain essentially the same for small displacements of $S_{01}$ ), it is permissible to. use an approximate frequency, e. g., the integral number of megacycles per second nearest the intersection of the above curves. Then the original corrections of the adapter, predetermined at a shightly different frequency, can still be used in the separation procedure. When it is necessary to know the desired frequency more precisely, the above procedure is repeated over a more narrow frequency range bracketing the frequency located above. The frequency used in this technique must be known and kept stable to 0.01 percent. The corrections of the measuring-line adapter may, of course, be remeasured at the new exact frequency if optimum accuracy is to be assured.

\section{Typical Measurement Results}

In all the techniques described above, a precision slotted line has been used to measure the input reactance variations of the connecting transformer corresponding to known output-reactance variations. Obviously, impedance-measuring devices other than slotted lines may be used for this purpose; e. g., bridges, Q-meters, instruments employing directional couplers, admittance comparators, etc. However, present experience indicates a need of precision in measuring input-reactance variations considerably greater than that provided by currently available commercial instruments other than slotted lines. Also, when these other impedance-measuring devices are employed for this purpose, the steps of procedure in the n-s method must be modified. A discussion of the use of such devices is reserved for another treatment of the subject.

The results of some n-s measurements of typical connecting transformers, using a precision slotted line as the measuring instrument, are compiled in table 1. The transformation correction's given do not necessarily represent average values for the particular types of commercial units listed, but rather demonstrate the typical magnitudes of these corrections. Each connecting transformer, of course, terminates in uniform line sections and, when required, includes mating connectors. For type N connectors and adapters the mating connectors are assembled from $\mathrm{UG}-21 \mathrm{~B} / \mathrm{U}$ plugs and $\mathrm{UG}-23 \mathrm{~B} / \mathrm{U}$ jacks, combined with 11/2-in. uniform sections of $50-$ ohm Teflon-dielectric rigid line. For a particular connector the transformation corrections vary, depending on the position of the connector along the transmission-line type of transformer. The corrections for the cable connectors and adapters, Nos. 3 to 11 , in table 1 represent the parameters of each transformer, with the test unit placed at the input

TABLE 1. Transformation corrections of typical connecting transformers

\begin{tabular}{|c|c|c|c|c|c|}
\hline \multicolumn{2}{|r|}{ Connecting transformer } & \multirow{2}{*}{ Frequency } & \multirow{2}{*}{$a^{2}$} & \multirow{2}{*}{$a b^{\prime}$} & \multirow{2}{*}{ Variable reactor } \\
\hline Number & Components & & & & \\
\hline 1 & $\begin{array}{l}\text { 5/16-in.-diam line; taper to } 21 / 2 \text {-in.-diam solid-dielectric precision slotted } \\
\text { line. }\end{array}$ & $\begin{array}{l}M c / s \\
300 \\
600\end{array}$ & $\begin{array}{l}1.010 \\
1.028\end{array}$ & $\begin{array}{l}\text { Micromhos } \\
\quad-410 \\
\quad-260\end{array}$ & \\
\hline 2 & 5/16-in.-diam line; taper to $15 / 8$-in.-diam solid-dielectric slotted line.... & $\begin{array}{l}300 \\
600 \\
900\end{array}$ & $\begin{array}{r}0.998 \\
.991 \\
.992\end{array}$ & $\begin{array}{r}-60 \\
-430 \\
-360\end{array}$ & \\
\hline $\begin{array}{r}3 \\
4 \\
5 \\
6 \\
7 \\
8 \\
9 \\
10 \\
11\end{array}$ & 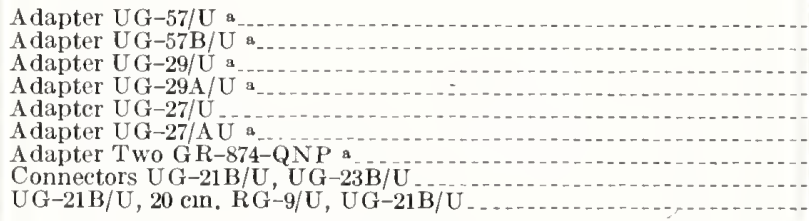 & 300 & $\begin{array}{r}.982 \\
1.014 \\
1.004 \\
0.988 \\
1.026 \\
0.981 \\
.981 \\
.991 \\
1.005\end{array}$ & $\begin{array}{r}-160 \\
110 \\
50 \\
-130 \\
0 \\
-250 \\
-20 \\
-50 \\
-680\end{array}$ & $\begin{array}{l}\text { 5/16-in.-diam solid-dielectric slotted line. } \\
Z_{0}=49.9 \mathrm{ohms} \text {. }\end{array}$ \\
\hline $\begin{array}{l}12 \\
13 \\
14\end{array}$ & $\begin{array}{l}\text { Step adapter type } N \text { to } 7 / 8 \text {-in. diam.b } \\
\text { 7/8-in.-diam, 140-cm partial-air-dielectric linc- } \\
\text { 7/8-in.-diam, 150-cm Teflon-bead line. }\end{array}$ & 100 & $\begin{array}{l}\text { 1. } 054 \\
\text { 1. } 032 \\
1.005\end{array}$ & $\begin{array}{r}-90 \\
-500 \\
100\end{array}$ & $\left\{\begin{array}{l}\text { z,s-in.-diam air-dielectric adjustable } \\
\text { stub. } Z_{0}=50.0 \text { ohms. }\end{array}\right.$ \\
\hline $\begin{array}{l}15 \\
16\end{array}$ & $\begin{array}{l}\text { 31/6-in.-diam, 140-cm partial-air-dielectric line } \\
\text { 31,6-in.-diam, 150-cm air-dielectric line. }\end{array}$ & 100 & $\begin{array}{r}0.996 \\
.999\end{array}$ & $\begin{array}{r}130 \\
70\end{array}$ & $\begin{array}{l}31 \& \text {-in.-diam air-dielectric perforated } \\
\text { line. } Z_{0}=50.9 \text { ohms. }\end{array}$ \\
\hline
\end{tabular}

a $\mathrm{UG}-21 \mathrm{~B} / \mathrm{U}, \mathrm{UG}-23 \mathrm{~B} / \mathrm{U}$ mating connectors werc used; $h=1.5 \mathrm{in}$.

t $\mathrm{UG}-21 \mathrm{~B} / \mathrm{U}^{\prime}$ mating connector at input end. 


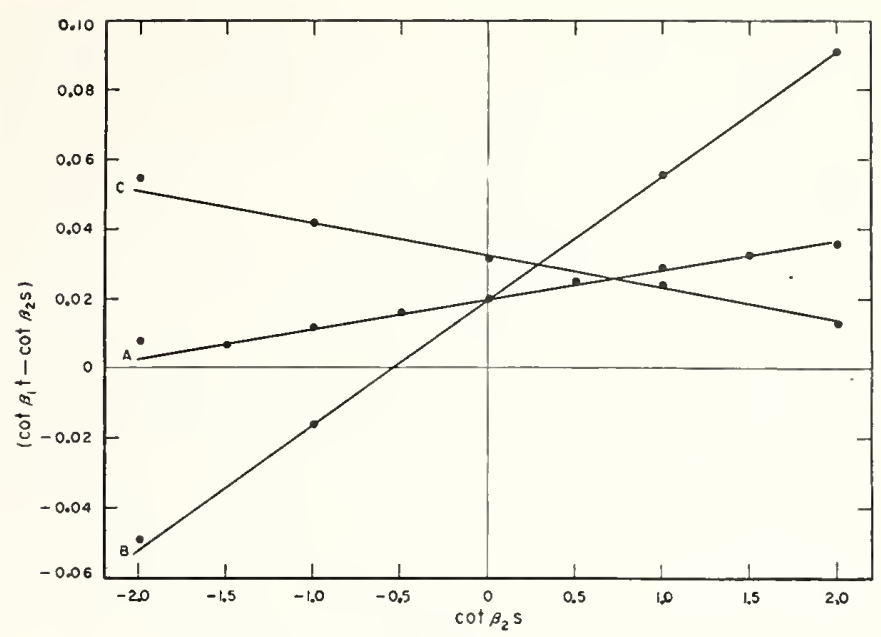

Figure 10. Measurement results.

Plots of equation (15) for connecting transformers listed in table 1: A, transformer $1 ; \mathrm{B}$, transformer 7 in tandem with transformer 1 ; and $\mathrm{C}$, transformer 8 in tandem with transformer 1 .

end. The magnitude of correction that can be produced by a given transformer may be estimated by considering, for example, transformer $8 ; a^{2}=0.981$, $a b^{\prime}=-250$ micromhos. Using eq (17) and (18), it can be shown that a measured impedance of $300-j 10.0$ ohms at the input end of this connecting transformer corresponds to an actual load impedance of $291-j 31.5$ ohms at its output end. Thus the error in the resistive component is 3 percent, and the reactive component is in error by 3 to 1 .

The accuracy with which the values of $a^{2}$ and $a b^{\prime}$ can be determined depends first on the absence of curvature in the plot of eq (15) as represented in figure 10. With no observable curvature, the precision with which the straight line can be drawn depends on the scatter of the plotted points. The scatter, in turn depends on the accuracy with which the $s$ and $t$ values are measured. The accuracy of the transformation corrections was \pm 0.5 percent or better for $a^{2}$, and $\pm(5$ percent +0.01 micromicrofarad) for $a b^{\prime}$.

\section{Measurement of the Quality of Slotted Lines}

The n-s method may advantageously be applied to determine the uniformity of a slotted line and thus indicate the order of its utility, either as a measuring line or as a variable reactor. The auxiliary equipment required in performing this quality measurement is an adjustable stub or a short-circuited line stretcher connected to the output end of the slotted line. This stub need not be of high accuracy; its purpose is only to move a voltage node along the slotted line. The procedure follows the general $n$-s technique. At a half wavelength from input and output ends of slotted line, voltage nodes are selected to represent, respectively, $S_{0}$ and $T_{0}$. It is desirable that the measurement frequency be high enough so that most of the line lies between $S_{0}$ and $T_{0}$. The movable short circuit is then used to place a voltage node at selected $S$ locations, and the corresponding values of $s$ and $t$ are accurately measured. The data are then plotted and the values of $a^{2}$ and $a b^{\prime}$ calculated, by the usual procedure.

These resulting corrections represent the total transformation produced by all discontinuities between $S_{0}$ and $T_{0}$. Usually it is found that the magnitude of these corrections is less than would be deduced from the nonuniformity of the probe output voltage, indicated as the probe is moved along the perfectly terminated line. The choice of whether to use the n-s technique or the voltage uniformity method to determine the quality of a slotted line depends on the information desired. Variations in probe coupling do not necessarily affect the accuracy with which nodal positions can be located, but do result in probe output irregularities along the line. Hence, the n-s method (depending only on accurate nodal measurements) determines rather accurately the electrical uniformity of a slotted line, and voltage uniformity measurements (depending on line quality and probe coupling) determine the combined effect of probe penetration variations and electrical nonuniformity and establish the error limitations of complete impedance measurements. "Thus, the n-s method offers means of separating the two major causes of slotted-line errors. It also follows that slotted lines, which are poor as impedance-measuring instruments, often may serve satisfactorily as variable reactors.

A way in which the uniformity of a slotted line can be qualitatively evaluated is by using it as a variable reactor in the $\mathrm{n}$-s measurement of some discontinuity placed outside this line. Fixed discontinuities in the line, which change the electrical lengths of the $s$ values, will cause curvature in the plotted results; when no curvature is obtained, the line is free from serious discontinuities.

\section{Conclusions}

The work described above was limited to lossless connectors. Transmission-line sections may also be considered connectors as long as their losses are negligible. A long lossless line may, of course, be looked upon as consisting of several connecting transformers in tandem; one can then obtain the transformation corrections of representative sections and calculate the transformations likely to result from the long line.

The measurements were made on connectors having ideal junctions with uniform rigid-line sections; therefore, some of the transformation corrections cited are probably better than those for connectors joined to commercial cables having braided outer conductors. One may, of course, measure a number of the latter samples and get comparative data for various types and makes of connectors, cables, and adapters, as well as for any combinations of these. The transformation corrections are obtained for discrete frequencies; it is pointed out, however, that in cases of rather small 
distributed discontinuities these corrections do not vary sharply with frequency. Thus, measurements at several well-selected frequencies may be sufficient to cover a wide frequency range.

\section{References}

[1] J. R. Whinnery, M. W. Jamieson, and Theo. Eloise Robbins, Coaxial line discontinuities, Proc. Inst. Radio Engrs. 32, 695-709 (Nov. 1944).

[2] M. H. Oliver, Discontinuities in concentric-line impedance measuring apparatus, Proc. Inst. Elec. Eng. 97, part III, p. 29-38 (Jan. 1950); also discussion, p. 242 (July 1950).

[3] R. W. King, Absolute method for measuring dielectric constants of fluids at ultra-high frequencies, Rev. Sci. Instr. 8, 201 (June 1937).
[4] Albert Weisfloch, Ein Transformationssatz über verlustlose Vierpole und seine Amvendung auf die experimentelle Untersuchung von Dezimeter und Zentimeterwellen-Schaltungen, Hochfrequenztechnik und Elektroakustik 60, 67 (Sept. 1942).

[5] E. Feenberg, The relation between nodal position and standing wave ratio in a composite transmission system, J. Appl. Phys. 17, 530-532. (June 1946).

[6] A. T. Starr, Electric circuits and wave filters, p. 171, 2d ed. (Pitman \& Sons Ltd., London, 1948).

[7] H. F. Sorrows, W. E. Ryan, and R. C. Ellenwood, Evaluation of coaxial slotted-line impedance measurements, Proc. Inst. Radio Engrs. [2] 39, 162-168 (Feb. 1951).

[8] F. D. Bennett, P. D. Coleman, and A. S. Meier, The design of broad-band aircraft antenna systems, Proc. Inst. Radio Engis. p. 671-700 (Oct. 1945).

Washington, July 21, 1953. 
11 August, 1958

TABLES OF

FREQUENCY, VSWR, AND $|\Gamma|$

FOR SELECTED HALF-ROUND INDUCTIVE OBSTACLE

IMPEDANCE STANDARDS IN WR-90 (X-BAND)

RECTANGULAR WAVEGUIDE

by

Members of the Microwave Impedance

Standards Project and Consultants 
The following graph and tables pertain to four half-round inductive obstacle impedance standards for WR-90 (X-band) rectangular waveguide.

They were prepared primarily for the use of the NBS Electronic Calibration Center, Boulder, Colorado and represent the first information available on this type of standard. It is expected that complete information will be published later in a forthcoming paper by D. M. Kerns.

Two of the standards employ single half-round obstacles of radii 0.0720 inch and 0.1080 inch. The other two standards each use two halfround obstacles as shown in the sketch below. The radii of the se pairs are 0.0900 inch and 0.1080 inch.

Although not all of the standards give useful reflections at all $\mathrm{X}$-band frequencies, this combination of standards will give at least two wellspaced values of VSWR between 1.05 and 1.5 at each $\mathrm{X}$-band frequency. Errors in these values will be due to finite conductivity, mechanical tolerances, and approximations in the original calculation. The error in the calculations is probably beyond the 5 th significant figure in both VSWR and $|\Gamma|$. Corrections for dimensional perturbations and finite conductivity are in the process of calculation at this time (August, 1958). The accuracy of the corrected $|\Gamma|$ may be as good as $0.01 \%$.

The personnel responsible for the preparation of these tables are the following:

Theory and Calculations by: D. M. Kerns, D.F. Wait, P.F. Wacker, W. W. Longley, Jr., and D. Payne.

Tables by: R.W. Beatty, R.H. Manka, and W.E. Boulton. 


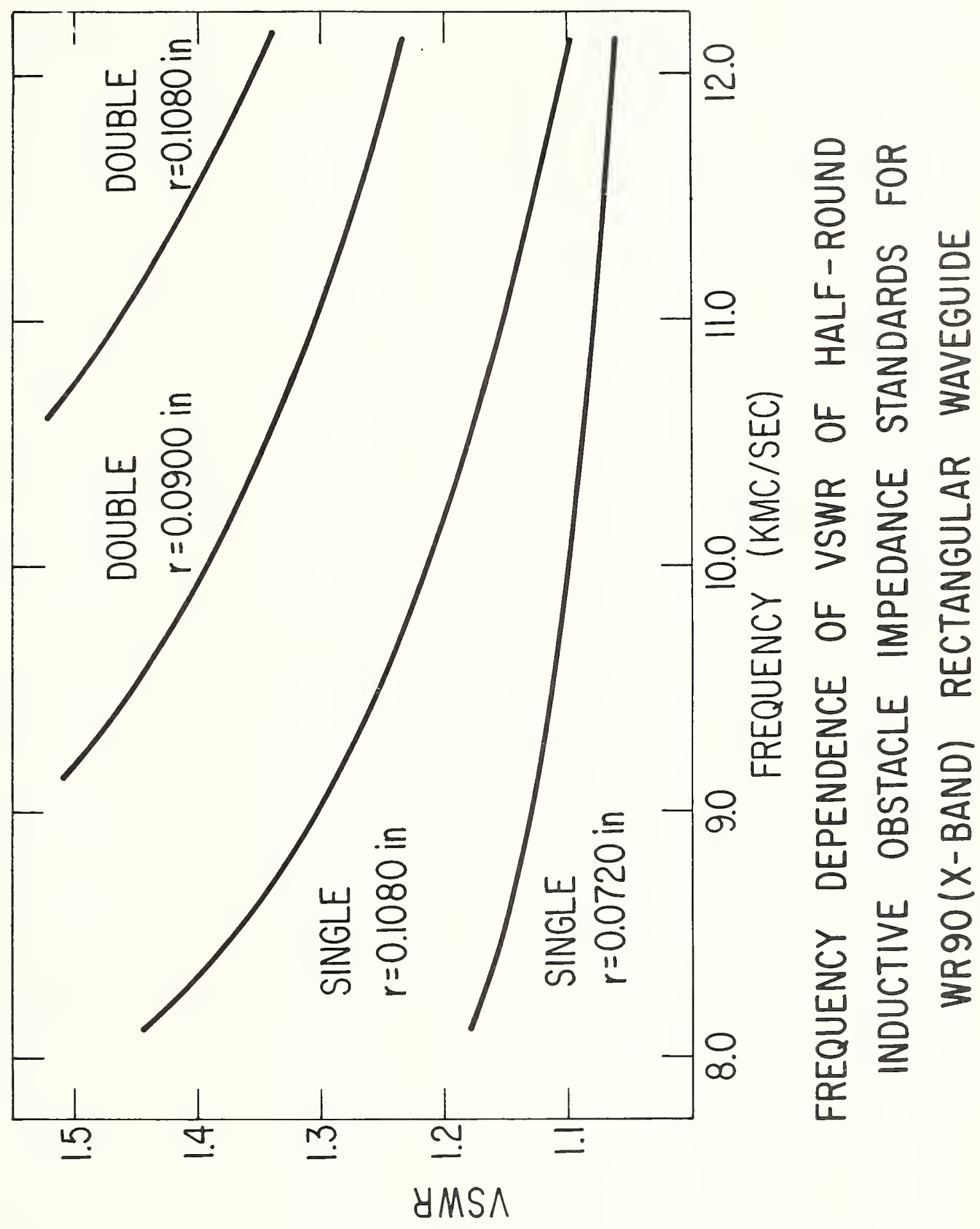


IN WR 90 RECTANGULAR WAVEGUIDE

Radius $=0.0720 \mathrm{inch}$

\begin{tabular}{|c|c|c|c|}
\hline $\begin{array}{l}\text { Frequency (f) } \\
\qquad(\mathrm{KMc} / \mathrm{s})\end{array}$ & $\left|\Gamma_{s}\right|=\left|s_{11}\right|$ & $\operatorname{VSWR}\left(\sigma_{\mathrm{S}}\right)$ & $\frac{d \sigma}{\sigma} / \frac{d}{f}$ \\
\hline 8.2421 & 0.07883 & 1.17115 & \\
\hline 8.3464 & 0.07592 & 1.16432 & 0.450 \\
\hline 8.4508 & 0.07324 & 1. 15805 & \\
\hline 8.5551 & 0.07075 & 1.15227 & 0.395 \\
\hline 8.6594 & 0.06843 & 1. 14692 & \\
\hline 8.7638 & 0.06626 & 1.14193 & 0.360 \\
\hline 8.8681 & 0.06423 & 1.13728 & \\
\hline 8.9724 & 0.06232 & 1.13292 & 0.320 \\
\hline 9.0768 & 0.06051 & 1. 12882 & \\
\hline 9.1811 & 0.05880 & 1. 12496 & 0.292 \\
\hline 9.2854 & 0.05718 & 1. 12130 & \\
\hline 9.3897 & 0.05564 & 1.11783 & 0.272 \\
\hline 9.4941 & 0.05417 & 1. 11453 & \\
\hline 9.5984 & 0.05276 & 1.11139 & 0.254 \\
\hline 9.7027 & 0.05141 & 1.10840 & \\
\hline $9.807 \mathrm{i}$ & 0.05012 & 1.10553 & 0.239 \\
\hline 9.9114 & 0.04888 & 1. 10278 & \\
\hline 10.0157 & 0.04768 & 1.10013 & 0.226 \\
\hline 10.1201 & 0.04653 & 1.09759 & \\
\hline 10.2244 & 0.04541 & 1.09514 & 0.215 \\
\hline 10.3287 & 0.04433 & 1.09278 & \\
\hline 10.4330 & 0.04328 & 1.09049 & 0.208 \\
\hline 10.5374 & 0.04227 & 1.08827 & \\
\hline 10.6417 & 0.04128 & 1.08611 & 0.199 \\
\hline 10.7460 & 0.04031 & 1.08402 & \\
\hline 10.8504 & 0.03937 & 1.08197 & 0.194 \\
\hline 10.9547 & 0.03845 & 1.07998 & \\
\hline 11.0590 & 0.03755 & 1.07803 & 0.190 \\
\hline 11.1634 & 0.03666 & 1.07611 & \\
\hline 11.2677 & 0.03579 & 1.07423 & 0.188 \\
\hline 11.3720 & 0.03492 & 1.07237 & \\
\hline 11.4764 & 0.03407 & 1.07054 & 0.188 \\
\hline 11.5807 & 0.03322 & 1.06872 & \\
\hline 11.6850 & 0.03237 & 1.06691 & 0.190 \\
\hline 11.7893 & 0.03152 & 1.06509 & \\
\hline 11.8937 & 0.03067 & 1.06327 & 0.196 \\
\hline 11.9980 & 0.02980 & 1.06143 & \\
\hline 12. 1023 & 0.02891 & 1.05955 & \\
\hline
\end{tabular}


IN WR 90 RECTANGULAR WAVEGUIDE

$$
\text { Radius }=0.10801 \mathrm{nch}
$$

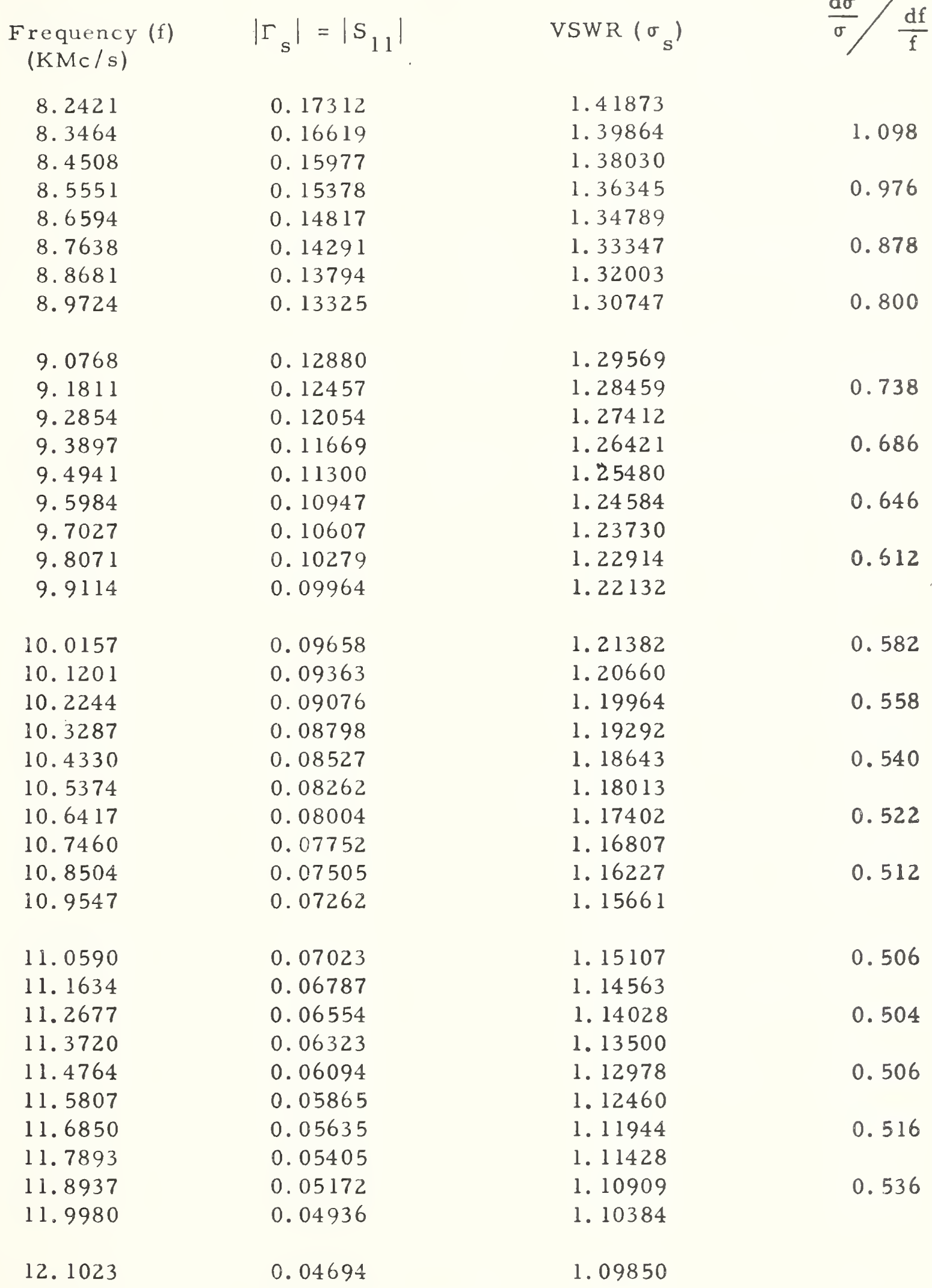


DOUBLE HALF-ROUND INDUCTIVE OBSTACLE

IN WR 90 RECTANGULAR WAVEGUIDE

\begin{tabular}{|c|c|c|c|}
\hline $\begin{array}{l}\text { Frequency (f) } \\
(\mathrm{KMc} / \mathrm{s})\end{array}$ & $\begin{array}{l}\text { Radius }=0.09 \\
\left|\Gamma_{s}\right|=\left|S_{11}\right|\end{array}$ & $\operatorname{VSWR}\left(\sigma_{s}\right)$ & $\frac{\mathrm{d} \sigma}{\sigma}$ \\
\hline 8.2421 & 0.26561 & 1.72336 & \\
\hline 8.3464 & 0.25630 & 1.68924 & 1.54 \\
\hline 8.4508 & 0.24764 & 1.65831 & \\
\hline 8.5551 & 0.23957 & 1.63011 & 1.36 \\
\hline 8.6594 & 0.23203 & 1.60425 & \\
\hline 8.7638 & 0.22494 & 1.58044 & 1.21 \\
\hline 8.8681 & 0.21826 & l. $5584 \mathrm{l}$ & \\
\hline 8.9724 & 0.21196 & 1.53795 & 1.11 \\
\hline 9.0768 & 0.20600 & 1.51888 & \\
\hline 9.1811 & 0.20034 & 1.50106 & 1.01 \\
\hline 9.2854 & 0.19496 & 1.48434 & \\
\hline 9.3897 & 0.18983 & 1.46862 & 0.935 \\
\hline 9.4941 & 0.18494 & 1.45381 & \\
\hline 9.5984 & 0.18026 & 1.43981 & 0.872 \\
\hline 9.7027 & 0.17578 & 1.42655 & \\
\hline 9.8071 & 0.17149 & 1.41397 & 0.804 \\
\hline 9.9114 & 0.16737 & 1.40201 & \\
\hline 10.0157 & 0.16340 & 1.39063 & 0.768 \\
\hline 10.1201 & 0.15958 & 1.37976 & \\
\hline 10.2244 & 0.15590 & 1.36939 & 0.726 \\
\hline 10.3287 & 0.15235 & 1.35946 & \\
\hline 10.4330 & 0.14892 & 1.34994 & 0.690 \\
\hline 10.5374 & 0.14560 & 1.34082 & \\
\hline 10.6417 & 0.14239 & 1.33205 & 0.652 \\
\hline 10.7460 & 0.13927 & 1.32362 & \\
\hline 10.8504 & 0.13626 & 1.31550 & 0.629 \\
\hline 10.9547 & 0.13333 & 1.30768 & \\
\hline 11.0590 & 0.13048 & 1.30013 & 0.605 \\
\hline 11.1634 & 0.12772 & 1.29284 & \\
\hline 11.2677 & 0.12503 & 1.28580 & 0.581 \\
\hline 11.3720 & 0.12242 & 1.27898 & \\
\hline 11.4764 & 0.11987 & 1.27238 & 0.562 \\
\hline 11.5807 & 0.11738 & 1.26599 & \\
\hline 11.6850 & 0.11496 & 1. 25979 & 0.543 \\
\hline 11.7893 & 0.11260 & 1.25377 & \\
\hline 11.8937 & 0.11029 & 1.24793 & 0.526 \\
\hline 11.9980 & 0.10804 & 1. 24225 & \\
\hline 12.1023 & 0.10584 & 1.23672 & \\
\hline \multicolumn{4}{|c|}{$700 / 6$} \\
\hline
\end{tabular}


DOUBLE HALF-ROUND INDUCTIVE OBSTACLE

IN WR 90 RECTANGULAR WAVEGUIDE

$$
\text { Radius }=0.1080 \text { inch }
$$

Frequenc
(KMc/s
8.2421
8.3464
8.4508
8.5551
8.6594
8.7638
8.8681
8.9724

9.0768
9.1811
9.2854
9.3897
9.4941
9.5984
9.7027
9.8071
9.9114

$$
\left|\Gamma_{s}\right|=\left|S_{11}\right| \quad \operatorname{vSwR}\left(\sigma_{s}\right)
$$

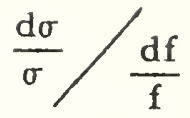
2421
3464
8.5551
8.7638
8.8681
9724

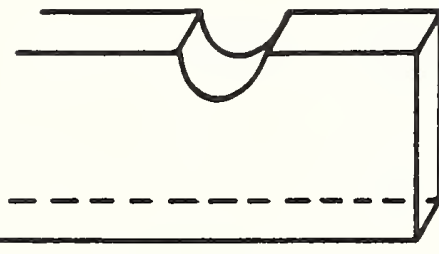

SINGLE

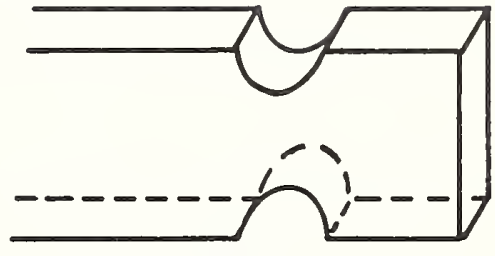

DOUBLE

HALF-ROUND INDUCTIVE OBSTACLES

10.0157

10. 1201

10.2244

10.3287

10.4330

0.22021

1. 56481

0.21492

1. 54752

0.20979

1. 53099

10.6417

0.20482

1. 51515

1.093

10. 7460

0. 19999

1. 49996

10.8504

0. 19529

1. 48538

10.9547

0. 19073

1. 47137

11.0590

0. 18629

11. 1634

0. 18197

1.45789

1. 44490

0. 17776

1. 43238

0.17366

1. 42030

0.16966

1. 40864

0.16575

1. 39737

0.16194

1. 38646

0.15822

1. 37591

0.15458

1. 36568

0. 15102

1. 35577

0.962

1. 044

1.000

11.8937

11.9980

0.14754

1. 34616

0.927

0.896

0.865

0.841

12.1023 
December 17,1958

TABLES OF

FREQUENCY, VSWR, AND $|\Gamma|$

FOR SELECTED HALF-ROUND INDUCTIVE OBSTACLE

IMPEDANCE STANDARDS IN WR-284 (S-BAND)

RECTANGULAR WAVEGUIDE

by

Members of the Microwave Impedance

Standards Project and Consultants 
The following graph and tables describe the reflections from four half-round inductive obstacle impedance standards for WR-284 (S-band) rectangular waveguide. These values correspond to those for WR-90 waveguide given in NBS Memorandum Report of 11 August, 1958 titled "Tables of Frequency, VSWR, and $|\Gamma|$ for Selected Half-Round Inductive Obstacle Impedance Standards in WR-90 (X-Band) Rectangular Waveguide".

These calculations are based on ideal conditions and the only corrections made were in the index of refraction of air using $23^{\circ} \mathrm{C}, 50 \%$ relative humidity, and $625 \mathrm{~mm} \mathrm{Hg}$ barometric pressure. Errors will be due to finite conductivity, mechanical tolerances, and approximations in the calculating process. The error in the calculating process is probably beyond the 5 th significant figure in both VSWR and $|\Gamma|$. Corrections for dimensional perturbations and finite conductivity are in the process of calculation at this time (December, 1958). The accuracy of the corrected $|\Gamma|$ may be as good as $0.01 \%$.

The personnel responsible for the preparation of the se tables are the following:

Theory and Calculations by: D.M. Kerns, D.F. Wait, P.F. Wacker, W. W. Longley, Jr., and D. Nash.

Tables by: R.W. Beatty, R.H. Manka, Ed Niesen, and W.J. Anson. 

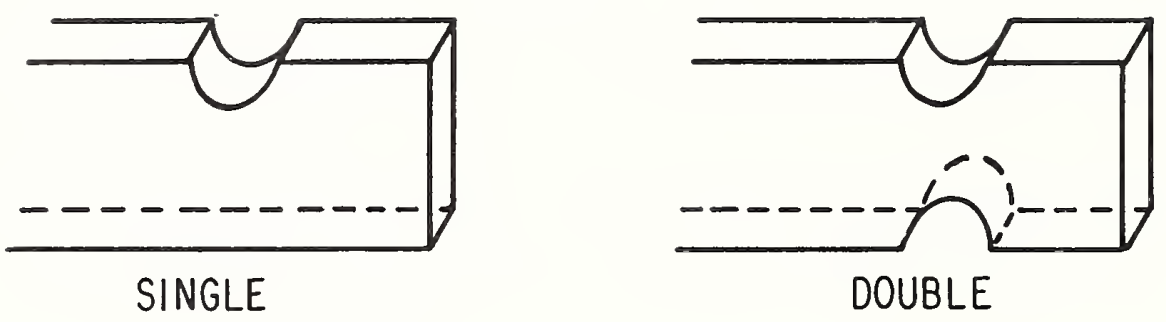

HALF-ROUND INDUCTIVE OBSTACLES 


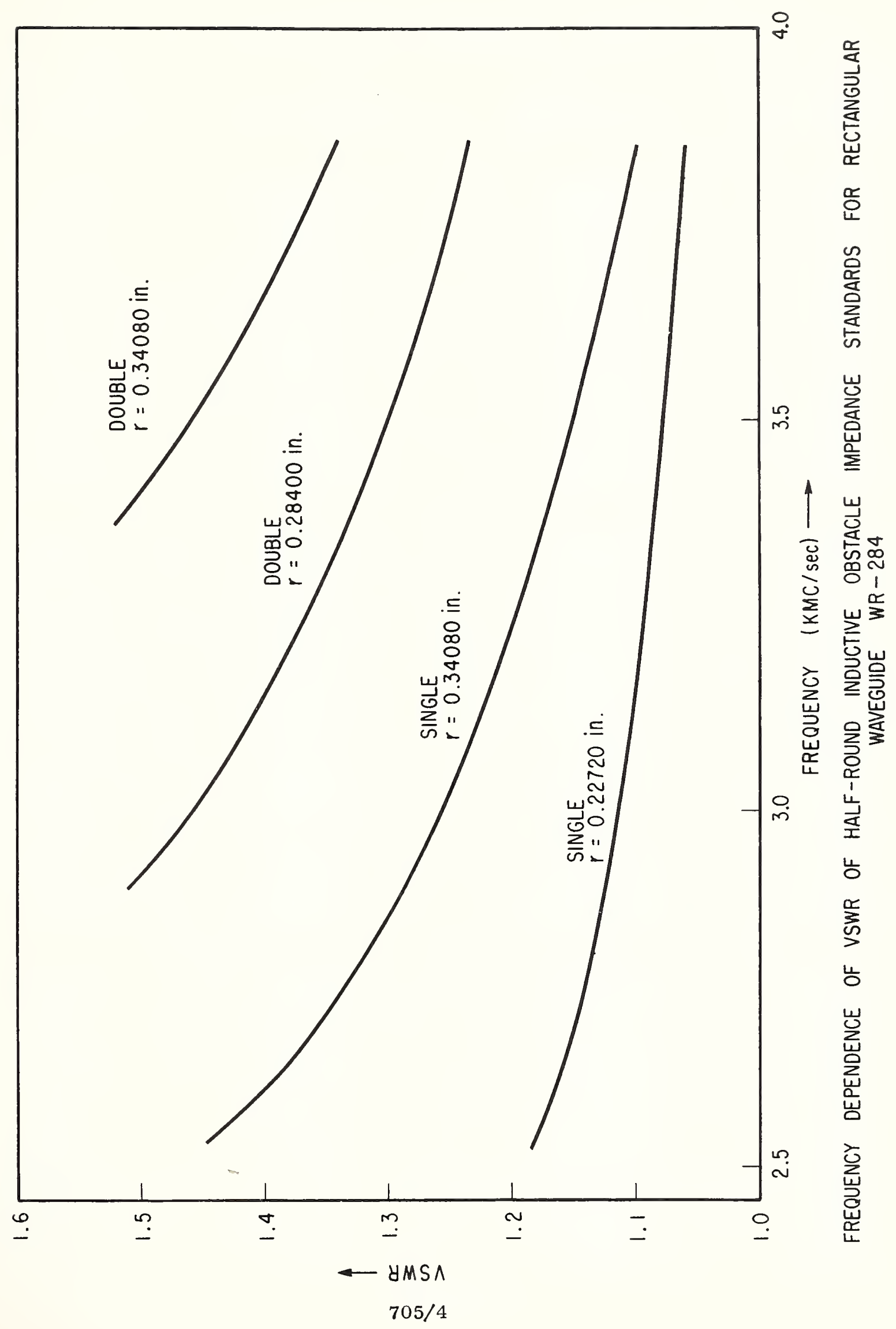


Radius $=0.22720$ inch

\begin{tabular}{|c|c|c|c|}
\hline $\begin{array}{l}\text { Frequency (f) } \\
\qquad(\mathrm{KMc} / \mathrm{s})\end{array}$ & $\left|\Gamma_{s}\right|=\left|S_{11}\right|$ & $\operatorname{VSWR}\left(\sigma_{S}\right)$ & $\frac{d \sigma}{\sigma}$ \\
\hline 2.6119 & 0.07883 & 1.17115 & \\
\hline 2.6450 & 0.07592 & 1. 16432 & 0.450 \\
\hline 2.6781 & 0.07324 & 1. 15805 & \\
\hline 2.7111 & 0.07075 & 1. 15227 & 0.395 \\
\hline 2.7442 & 0.06843 & 1. 14692 & \\
\hline 2.7773 & 0.06626 & 1. 1419 & 0.360 \\
\hline 2'. 8103 & 0.06423 & 1. 13728 & \\
\hline 2.8434 & 0.06232 & 1.13292 & 0.320 \\
\hline 2.8764 & 0.06051 & 1. 12882 & \\
\hline 2.9095 & 0.05880 & 1. 12496 & 0.292 \\
\hline 2.9426 & 0.05718 & 1.12130 & \\
\hline 2.9756 & 0.05564 & 1.11783 & 0.272 \\
\hline 3.0087 & 0.05417 & 1.11453 & \\
\hline 3.0418 & 0.05276 & 1.11139 & 0.254 \\
\hline 3.0748 & 0.05141 & 1. 10840 & \\
\hline 3.1079 & 0.05012 & 1.10553 & 0.239 \\
\hline 3.1409 & 0.04888 & 1. 10278 & \\
\hline 3.1740 & 0.04768 & 1.10013 & 0.226 \\
\hline 3.2071 & 0.04653 & 1.09759 & \\
\hline 3.2401 & 0.04541 & 1.09514 & 0.215 \\
\hline 3.2732 & 0.04433 & 1.09278 & \\
\hline 3.3063 & 0.04328 & 1.09049 & 0.208 \\
\hline 3.3393 & 0.04227 & 1.08827 & \\
\hline 3.3724 & 0.04128 & 1.08611 & 0.199 \\
\hline 3.4054 & 0.04031 & 1.08402 & \\
\hline 3.4385 & 0.03937 & 1.08197 & 0.194 \\
\hline 3.4716 & 0.03845 & 1.07998 & \\
\hline 3.5046 & 0.03755 & 1.07803 & 0.190 \\
\hline 3.5377 & 0.03666 & 1.07611 & \\
\hline 3.5708 & 0.03579 & 1.07423 & 0.188 \\
\hline 3.6038 & 0.03492 & 1.07237 & \\
\hline 3.6369 & 0.03407 & 1. 07054 & 0.188 \\
\hline 3.6699 & 0.03322 & 1.06872 & \\
\hline 3.7030 & 0.03237 & 1.06691 & 0.190 \\
\hline 3.7361 & 0.03152 & 1.06509 & \\
\hline 3.7691 & 0.03067 & 1.06327 & 0.196 \\
\hline 3.8022 & 0.02980 & 1.06143 & \\
\hline 3.8353 & 0.02891 & 1.05955 & \\
\hline
\end{tabular}


SINGLE HALF-ROUND INDUCTIVE OBSTACLE

IN WR-284 RECTANGULAR WAVEGUIDE

Radius $=0.34080$ inch

$\underset{\substack{\text { Frequency (f) } \\(\mathrm{KMc} / \mathrm{s})}}{\left|\Gamma_{\mathrm{s}}\right|=\left|\mathrm{S}_{11}\right|} \quad \operatorname{VSWR}\left(\sigma_{\mathrm{s}}\right)$

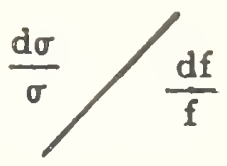
2.6119
0.17312
2.6450
0.16619
0.15977
2. 6781
0.15378
0.14817
2. 7447
0.14291
0.13794
2.8103
0.13325
0.12880
2. 8764
0.12457
2. 9095
0. 12054
2. 9426
0.11669

1. 41873

1. 39864

1. 38030

1. 36345

1. 34789

1. 33347

1. 32003

1. 30747

1. 29569

1. 28459

1. 27412

1. 26421

1. 098

0.976

0.878

0.800

0.738

0.686

3. 0087

0.11300

1. 25480

3. 0418

0.10947

1. 24584

0.10607

1. 23730

3. 0748

0. 10279

1. 22914

0.09964

1. 22132

3. 1409

0.09658

3. 1740

0.09363

1. 21382

1. 20660

0.09076

1. 19964

0.08798

1. 19292

0.08527

1. 18643

0.08262

1. 18013

0.08004

1. 17402

0.07752

1. 16807

0.07505

1. 16227

0.07262

0.646

0.612

0.582

0.558

0.540

0.522

3. 4054

3. 4385

1. 15661

0.512

3.4716

0.07023

1. 15107

0.506

3. 5377

0.06787

1. 14563

0.06554

1. 14028

0.504

3. 5708

0.06323

1. 13500

0.06094

1. 12978

0.506

0.05865

1. 12460

0.05635

1. 11944

0.516

3. 7030

0.05405

1. 11428

0.05172

1. 10909

1. 10384

0.04936

1. 09850

0.536

3.8022

0.04694 
DOUBLE HALF - ROUND INDUCTIVE OBSTACLE

IN WR-284 RECTANGULAR WAVEGUIDE

Radius $=0.28400$ inch

Frequency (f)

$(\mathrm{KMc} / \mathrm{s})$

$\left|\Gamma_{s}\right|=\left|s_{11}\right|$

$\operatorname{VSWR}\left(\sigma_{S}\right)$

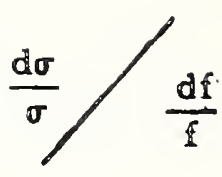

2.6119

0.26561

1.72336

2.6450

0.25630

1.68924

1.54

2.6781

0.24764

1.65831

2. 7111

0.23957

2. 7442

0.23203

1.63011

1.36

2. 7773

0.22494

1. 60425

2. 8103

0.21826

1. 58044

1.21

2. 8434

0.21196

1. 55841

1. 53795

1.11

2. 8764

0. 20600

1. 51888

2. 9095

0. 20034

2. 9426

0. 19496

1. 50106

1. 48434

2. 9756

0. 18983

1. 46862

1.01

0.935

3.0087

0. 18494

0.18026

1. 45381

3. 0418

0. 17578

1. 43981

3. 0748

0.17149

3. 1079

0.16737

1. 42655

1. 41397

0.872

3. 1409

0. 16340

1. 40201

3. 1740

0.15958

1. 39063

1. 37976

0.15590

1. 36939

0.15235

3. 2732

0. 14892

1. 35946

1. 34994

0. 14560

1. 34082

0.14239

0.13927

1. 33205

1. 32362

0.13626

3. 4385

0.13333

1. 31550

1. 30768

0.804

0.768

0.726

0.690

0.652

0.629

3.4716

0.13048

3. 5046

0. 12772

1. 30013

0.605

3. 5377

0. 12503

1. 29284

1. 28580

0.581

3. 6038

0. 12242

1. 27898

0.11987

1. 27238

0.562

3.6699

0.11738

1. 26599

0.11496

1. 25979

0.543

0.11260

1. 25377

0.11029

1. 24793

0.526

3. 7691

0. 10804

1. 24225

3.8353
3.

0. 10584

1. 23672 
DOUBLE HALF - ROUND INDUCTIVE OBSTACLE

IN WR-284 RECTANGULAR WAVEGUIDE

Radius $=0.34080$ inch

\begin{tabular}{|c|c|c|c|}
\hline $\begin{array}{l}\text { Frequency (f) } \\
(\mathrm{KMc} / \mathrm{s})\end{array}$ & $\left|\Gamma_{8}\right|=\left|S_{1 i}\right|$ & $\operatorname{VSWR}\left(\sigma_{s}\right)$ & \\
\hline 2.6119 & 0.38621 & 2.25846 & \\
\hline 2.6450 & 0.37326 & 2.19110 & 2.333 \\
\hline 2.6781 & 0.36111 & 2. 13041 & \\
\hline 2.7111 & 0.34967 & 2. 07538 & 2.076 \\
\hline 2.7442 & 0.33889 & 2.02520 & \\
\hline 2.7773 & 0.32869 & 1.97923 & 1.872 \\
\hline 2.8103 & 0.31901 & 1.93691 & \\
\hline 2. 8434 & 0.30982 & 1.89779 & 1.707 \\
\hline 2.8764 & 0.30107 & 1.86150 & \\
\hline 2.9095 & 0.29272 & 1.82772 & 1. 572 \\
\hline 2.9426 & 0.28474 & 1.79617 & \\
\hline 2.9756 & 0.27710 & 1. 76662 & 1.459 \\
\hline 3.0087 & 0.26977 & 1.73888 & \\
\hline 3.0418 & 0.26274 & 1.71276 & 1.362 \\
\hline 3.0748 & 0.25599 & 1.68812 & \\
\hline 3.1079 & 0.24948 & 1.66482 & 1.281 \\
\hline 3. 1409 & 0.24321 & 1.64275 & \\
\hline 3.1740 & 0.23717 & 1.62180 & 1.209 \\
\hline 3.2071 & 0.23132 & 1.60188 & \\
\hline 3.2401 & 0.22568 & 1.58291 & 1. 118 \\
\hline 3.2732 & 0.22021 & 1. 56481 & \\
\hline 3.3063 & 0.21492 & 1. 54752 & 1.093 \\
\hline 3.3393 & 0.20979 & 1.53099 & \\
\hline 3.3724 & 0.20482 & 1. 51515 & 1.044 \\
\hline 3.4054 & 0.19999 & 1.49996 & \\
\hline 3.4385 & 0.19529 & 1.48538 & 1.000 \\
\hline 3.4716 & 0.19073 & 1.47137 & \\
\hline 3.5046 & 0.18629 & 1.45789 & 0.962 \\
\hline 3.5377 & 0.18197 & 1.44490 & \\
\hline 3.5708 & 0.17776 & 1.43238 & 0.927 \\
\hline 3.6038 & 0.17366 & 1.42030 & \\
\hline 3.6369 & 0.16966 & 1.40864 & 0.896 \\
\hline 3.6699 & 0.16575 & 1.39737 & \\
\hline 3.7030 & 0.16194 & 1. 38646 & 0.865 \\
\hline 3.7361 & 0.15822 & 1.37591 & \\
\hline 3.7691 & 0.15458 & 1. 36568 & 0.841 \\
\hline 3.8022 & 0.15102 & 1.35577 & \\
\hline 3.8353 & 0.14754 & 1. 34616 & \\
\hline
\end{tabular}


December 17,1958

\author{
TABLES OF \\ FREQUENCY, VSWR, AND $|\Gamma|$ \\ FOR SELECTED HALF-ROUND INDUCTIVE OBSTACLE \\ IMPEDANCE STANDARDS IN WR-187 \\ RECTANGULAR WAVEGUIDE
}

by

Members of the Microwave Impedance

Standards Project and Consultants 
The following graph and tables describe the reflections from four half-round inductive obstacle impedance standards for WR-187 rectangular waveguide. These values correspond to those for WR-90 waveguide given in NBS Memorandum Report of 11 August, 1958 titled "Tables of Frequency, VSWR, and $|\Gamma|$ for Selected Half-Round Inductive Obstacle Impedance Standards in WR-90 (X-Band) Rectangular Waveguide".

These calculations are based on ideal conditions and the oniy corrections made were for the index of refraction of air using $23^{\circ} \mathrm{C}, 50 \%$ relative humidity, and $625 \mathrm{~mm} \mathrm{Hg}$ barometric pressure. Errors will be due to finite conductivity, mechanical tolerances, and approximations in the calculating process. The error in the calculating process is probably beyond the 5 th significant figure in both VSWR and $|\Gamma|$. Corrections for dimensional perturbations and finite conductivity are in the process of calculation at this time (December, 1958). The accuracy of the corrected $|\Gamma|$ may be as good as $0.01 \%$.

The personnel responsible for the preparation of these tables are the following:

Theory and Calculations by: D. M. Kerns, D.F. Wait, P.F. Wacker, W. W. Longley, Jr., and D. Nash.

Tables by: R.W. Beatty, R.H. Manka, Ed Niesen, and W.J. Anson. 

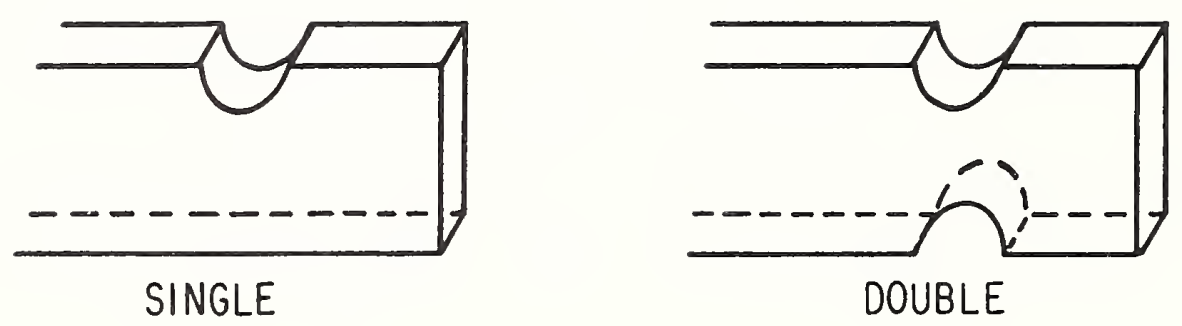

HALF-ROUND INDUCTIVE OBSTACLES 


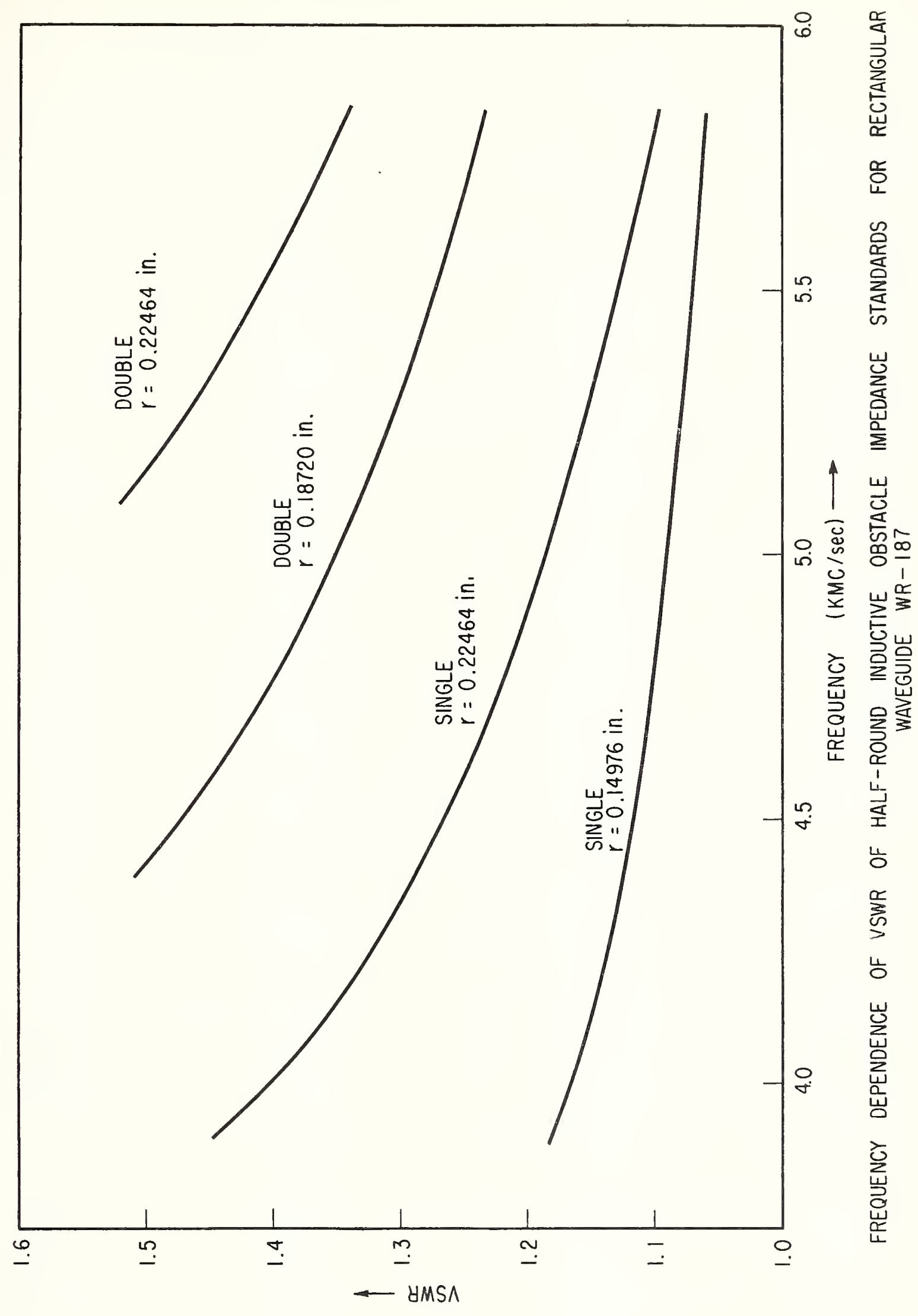

$5727720-61-46$ 
IN WR - 187 RECTANGULAR WAVEGUIDE

Radius $=0.14976$ inch

Frequency (f)

( $\mathrm{KMc} / \mathrm{s})$

$$
\left|\Gamma_{s}\right|=\left|s_{11}\right| \quad \operatorname{VSwR}\left(\sigma_{s}\right)
$$

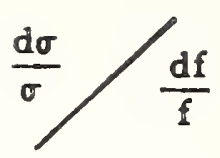

\subsection{6}

4.0127

4.0629

4.1130

4. 1632

4.2134

4.2635

4.3137

4.3638

4.4140

4.4642

4.5143

4. 5645

4.6146

4.6648

4.7149

4.7651

4.8153

4.8654

4.9156

4.9657

5.0159

5. 0661

5. 1162

5. 1664

5. 2165

5. 2667

5. 3169

5. 3670

5.4172

5.4673

5. 5175

5. 5676

5. 6178

5.6680

5. 7181

5. 7683

5. 8184
0.07883

0.07592

0.07324

0.07075

0.06843

0.06626

0.06423

0.06232

0.06051

0.05880

0.05718

0.05564

0.05417

0.05276

0.05141

0.05012

0.04888

0.04768

0.04653

0.04541

0.04433

0.04328

0.04227

0.04128

0.04031

0.03937

0.03845

0.03755

0.03666

0.03579

0.03492

0.03407

0.03322

0.03237

0.03152

0.03067

0.02980

0.02891
1. 17115

1. 16432

1. 15805

1. 15227

1. 14692

1. 14193

1. 13728

1. 13292

1. 12882

1. 12496

1. 12130

1. 11783

1. 11453

1. 11139

1. 10840

1. 10553

1. 10278

1. 10013

1.09759

1. 09514

1. 09278

1.09049

1.08827

1.08611

1. 08402

1. 08197

1.07998

1.07803

1.07611

1. 07423

1. 07237

1.07054

1. 06872

1.06691

1.06509

1.06327

1.06143

1. 05955
0.450

0.395

0.360

0.320

0.292

0.272

0.254

0.239

0.226

0.215

0.208

0.199

0.194

0.190

0.188

0.188

0.190

0.196 
IN WR-187 RECTANGULAR WAVEGUIDE

Radius $=0.22464$ inch

\begin{tabular}{|c|c|c|c|}
\hline $\begin{array}{c}\text { Frequency (f) } \\
(\mathrm{KMc} / \mathrm{s})\end{array}$ & $\left|\Gamma_{\mathbf{s}}\right|=\left|\mathrm{S}_{11}\right|$ & $\operatorname{VSWR}\left(\sigma_{\mathrm{s}}\right)$ & $\frac{\mathrm{d} \sigma}{\sigma}$ \\
\hline 3.9626 & 0.17312 & 1.41873 & \\
\hline 4.0127 & 0.16619 & 1. 39864 & 1.098 \\
\hline 4.0629 & 0.15977 & 1.38030 & \\
\hline 4.1130 & 0.15378 & 1.36345 & 0.976 \\
\hline 4.1632 & 0.14817 & 1.34789 & \\
\hline 4.2134 & 0.14291 & 1.33347 & 0.878 \\
\hline 4.2635 & 0.13794 & 1.32003 & \\
\hline 4.3137 & 0.13325 & 1.30747 & 0.800 \\
\hline 4.3638 & 0.12880 & 1.29569 & \\
\hline 4.4140 & 0.12457 & 1.28459 & 0.738 \\
\hline 4.4642 & 0.12054 & 1.27412 & \\
\hline 4.5143 & 0.11669 & 1.26421 & 0.686 \\
\hline 4.5645 & 0.11300 & 1.25480 & \\
\hline 4.6146 & 0.10947 & 1. 24584 & 0.646 \\
\hline 4.6648 & 0.10607 & 1.23730 & \\
\hline 4.7149 & 0.10279 & 1. 22914 & 0.612 \\
\hline 4.7651 & 0.09964 & 1.22132 & \\
\hline 4.8153 & 0.09658 & 1.21382 & 0.582 \\
\hline 4.8654 & 0.09363 & 1.20660 & \\
\hline 4.9156 & 0.09076 & 1. 19964 & 0.558 \\
\hline 4.9657 & 0.08798 & 1.19292 & \\
\hline 5.0159 & 0.08527 & 1. 18643 & 0.540 \\
\hline 5.0661 & 0.08262 & 1.18013 & \\
\hline 5.1162 & 0.08004 & 1.17402 & 0.522 \\
\hline 5.1664 & 0.07752 & 1. 16807 & \\
\hline 5.2165 & 0.07505 & 1.16227 & 0.512 \\
\hline 5. 2667 & 0.07262 & 1.15661 & \\
\hline 5.3169 & 0.07023 & 1.15107 & 0.506 \\
\hline 5.3670 & 0.06787 & 1.14563 & \\
\hline 5.4172 & 0.06554 & 1. 14028 & 0.504 \\
\hline 5.4673 & 0.06323 & 1.13500 & \\
\hline 5.5175 & 0.06094 & 1. 12978 & 0.506 \\
\hline 5.5676 & 0.05865 & 1. 12460 & \\
\hline 5.6178 & 0.05635 & 1.11944 & 0.516 \\
\hline 5.6680 & 0.05405 & 1.11428 & \\
\hline 5.7181 & 0.05172 & 1.10909 & 0.536 \\
\hline 5.7683 & 0.04936 & 1. 10384 & \\
\hline 5.8184 & 0.04694 & 1.09850 & \\
\hline
\end{tabular}


DOUBLE HALF - ROUND INDUCTIVE OBSTACLE

IN WR - 187 RECTANGULAR WAVEGUIDE

Radius $=0.18720$ inch

Frequency (f)
$(\mathrm{KMc} / \mathrm{s})$

$\left|\Gamma_{s}\right|=\left|s_{11}\right|$

$\operatorname{VSWR}\left(\sigma_{s}\right)$

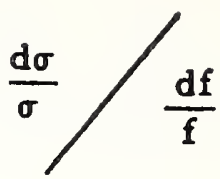

3.9626

0.26561

1.72336

4.0127

0.25630

1.68924

1.54

4.0629

0.24764

1.65831

4.1130

0.23957

1.63011

0.23203

1. 60425

4.1632

0.22494

1. 58044

1. 55841

4.2635

0.21826

1. 53795

0.20600

1. 51888

0.20034

1. 50106

0.19496

1. 48434

4. 5143

0.18983

1. 46862

0.935

4. 5645

0.18494

1. 45381

4.6146

0.18026

1. 43981

0.872

4. 6648

0.17578

1. 42655

0.17149

1.41397

0.804

4.7651

0.16737

1.40201

0.16340

1.39063

1.36

1.21

1.11

1.01

4.8153

0.15958

1. 37976

0.15590

1. 36939

0. 15235

1. 35946

4.9657

0.14892

1. 34994

0.690

5. 0661

0.14560

1. 34082

o. 14239

1. 33205

0.652

5. 1664

0.13927

1. 32362

0. 13626

1. 31550

0.629

5.2667

0. 13333

1. 30768

0. 13048

1. 30013

0.605

0. 12772

1. 29284

0. 12503

1. 28580

0.768

0.726

5.3670

0.12242

1. 27898

0.581

5.4673

0.11987

1. 27238

0.562

5. 5676

0. 11738

1. 26599

0. 11496

1. 25979

0.543

5.6680

0.11260

1. 25377

0.11029

1. 24793

0.526

5.7683

0. 10804

1. 24225

5. 8184 .

0. 10584

1. 23672 
DOUBLE HALF-ROUND INDUCTIVE OBSTACLE

IN WR-187 RECTANGULAR WAVEGUIDE

Radius $=0.22464$ inch

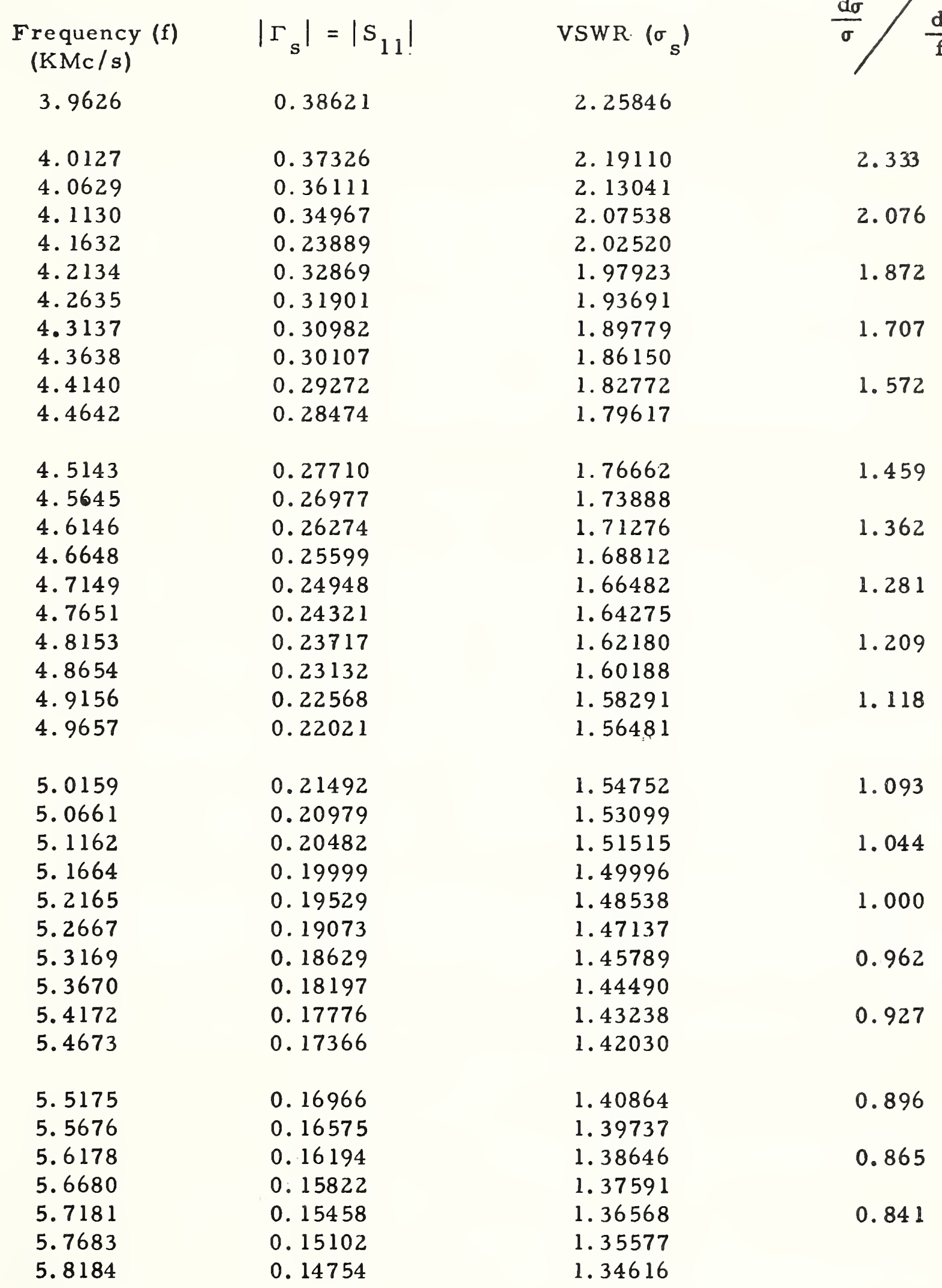




\title{
Half-Round Inductive Obstacles in Rectangular Waveguide
}

\author{
D. M. Kerns \\ (January 30, 1960)
}

\begin{abstract}
Formulas are derived for the accurate calculation of the lowest-mode, lumped-eiement representation of perfectly conducting half-round inductive obstacles in rectangular waveguide. These obstacles consist of either one or two opposed semicircular cylindrical indentations extending across the narrow sides of the waveguide. They seem especially suitable for use as precise calculable standards of reflection or impedance in waveguide. Schwinger's integral equation approach $[1,2]^{1}$ is used to obtain stationary expressions for the desired parameters as functionals of the surface currents on the obstacles. Upper bounds are obtained for one of the two parameters. Explicit formulas are derived for the values of the parameters under the assumption of $n$-term Fouriel sine-series expansions for the obstacle currents. Rapid convergence is indicated by numerical evaluations for $n=1,2$, and 3 . In the process of obtaining expressions suitable for numerical calculation, an expansion (believed to be new) of the Green's furction of the problem is obtained and the sums of certain infinite series of Bessel's functions occurring in this expansion are expressed in terms of definite integrals. A brief numerical table of these sums, sufficient for the evaluation of the $n=1$ approximation, is included.
\end{abstract}

\section{Introduction}

In this paper formulas are derived for the accurate calculation of lowest-mode, lumpedelement parameters for what may be identified as "single half-round" and "double half-round" inductive obstacles in rectangular waveguide. As shown in figure 1, the obstacles consist of semicircular cylindrical indentations extending across the narrow sides of the waveguides.

The particular geometry considered seems especially suitable for obstacles to be used as calculable standards of reflection or impedance in waveguide. "The geometry is well suited to electroforming, so that obstacles may be fabricated by this process as well as by machining. For obstacles producing standing-wave ratios of moderate values, the obstacle radius is large compared to high-standard machining tolerances, so that unduly close tolerances are not required. A change in waveguide cross section before and after the obstacle is avoided, eliminating the need for waveguide components in odd sizes and permitting maximum flexibility of interconnection.

In the present work the obstacle and waveguide surfaces are assumed perfectly conducting.

The Waveguide Handbook [3] contains approximate formulas for semi-elliptical obstacles, ${ }^{2}$ which can be specialized to apply to the present problem. However, for the contemplated use in standards work, formulas with very much greater accuracy $\left(10^{3}\right.$ or $10^{4}$ times greater) are wanted.

Brief descriptions of the present and some related work have been given previously [4].

\section{Formulation of Problem}

We employ the customary complex electric and magnetic field vectors $\boldsymbol{E}=\boldsymbol{E}(\boldsymbol{r}), \boldsymbol{H}=\boldsymbol{H}(\boldsymbol{r})$, which satisfy Maxwell's equations in the form

$$
\left.\begin{array}{r}
\nabla \times \boldsymbol{E}=-j \omega \mu \boldsymbol{H}, \\
\nabla \times \boldsymbol{H}=j \omega \epsilon \boldsymbol{E}+\boldsymbol{J},
\end{array}\right\}
$$

\footnotetext{
Figures in brackets indicate the literature references at the end of this paper.

2 The pertinent formulas are afflicted with a number of misprints; correct forms of these results for half-round obstacles are given later in this paper.
} 
under the assumption of time-dependence represented by the (omitted) factor exp ( $j \omega t)$. Here $t$ is the time, $\omega /(2 \pi)$ is the frequency, $j$ is the imaginary unit, $\boldsymbol{J}$ is the complex current-density vector, and $\mu$ and $\epsilon$ are respectively the permeability and the permittivity of the homogeneous, isotropic, nondissipative medium in the wareguide. The MKS system of units is employed.

As shown in figure 1 , we choose a rectangular coordinate system Oxyz such that the interior of the waveguide is the space $0<x<a, \quad 0<y<b, \quad-\infty<z<\infty$ and the surfaces of the obstacles are given by the loci

$$
\left.\begin{array}{l}
R^{2}=x^{2}+z^{2}, \quad 0 \overline{<} x \overline{<} R \\
R^{2}=(a-x)^{2}+z^{2}, \quad 0 \overline{<} a-x \overline{<} R
\end{array}\right\}
$$

in the double half-round case and by

in the single half-round case.

$$
R^{2}=x^{2}+z^{2}, \quad 0 \overline{<} x \overline{<} R
$$
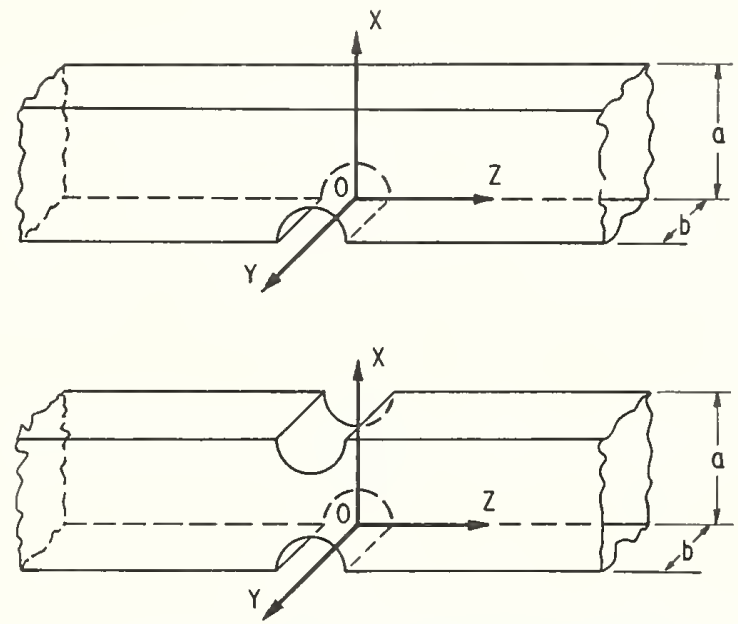

FIGURE 1. Single and double half-round inductive obstacles in rectangular waveguide.

The fields incident on the obstacles are, by hypothesis, to be in the $\mathrm{TE}_{10}$ mode. Travelingwave forms for this mode may be written

$$
\left.\begin{array}{l}
\boldsymbol{E}^{ \pm}=\sin (\pi x / a) e^{\mp_{j \beta z}} \boldsymbol{e}_{y}, \\
\boldsymbol{H}^{ \pm}=\left[\mp \eta \sin (\pi x / a) \boldsymbol{e}_{x}+j \pi(\omega \mu a)^{-1} \cos (\pi x / a) \boldsymbol{e}_{z}\right] e^{\mp_{j \beta z} \cdot}
\end{array}\right\}
$$

The upper and lower signs refer respectively to waves going in the positive and the negative $z$-directions; the phase constant $\beta$ is equal to $\left[k^{2}-(\pi / a)^{2}\right]^{1 / 2}$, where $k=\omega(\mu \epsilon)^{1 / 2}$; the wave-admittance $\eta=\beta /(\omega \mu)$; and $\boldsymbol{e}_{x}, \boldsymbol{e}_{y}, \boldsymbol{e}_{z}$ are the unit vectors of the system Oxyz. It is assumed that $\pi<k a<2 \pi$, so that the $\mathrm{TE}_{10}$ mode, but no higher mode of the type $\mathrm{TE}_{n 0}$, will have real propagation.

We define "voltage" and "current" $v_{m}\left(z_{m}\right), i_{m}\left(z_{m}\right)$ for the TE 10 mode by means of the equations

$$
\left.\begin{array}{l}
E_{m y}=v_{m}\left(z_{m}\right) \sin (\pi x / a), \\
H_{m x}=(-)^{m} i_{m}\left(z_{m}\right) \eta \sin (\pi x / a) .
\end{array}\right\} m=1,2
$$

Here the indices $m=1,2$ refer respectively to the "left-hand" side $\left(z_{1}<-R\right)$ and the "righthand". $\left(z_{2}>R\right)$ of the obstacle considered, and $E_{m y}, H_{m x}$ are components (identified by the subscripts) of the $\mathrm{TE}_{10}$-mode part of whatever total electromagnetic field may be present in the waveguide at $z=z_{m}$. From (2.6) and (2.4) it follows that the waveguide characteristic impedance, defined as the value of $v_{m}\left(z_{m}\right) / i_{m}\left(z_{m}\right)$ for a pure traveling wave incident on the side $m$ of the obstacle, is equal to unity for $m=1,2$. 
The desired lowest-mode, lumped-clement description of an obstarle is rontaned in its impedance matrix 7 , which characterizes the linear relations

$$
r_{1}=Z_{11} i_{1}+Z_{12} i_{2}, \quad r_{2}=Z_{121} i_{1}+Z_{22} i_{2}
$$

imposed by the obstack on the terminal variables $v_{m}, i_{m}$. The values of the $Z_{m n}$ depend upon the location of the terminal planes $z=z_{1}$ and $z=z_{2}$; in the present problem it turns out to be convenient to extrapolate these planes to $z_{1}=z_{2}=0$.

Now, the equality $Z_{11}=Z_{22}$ follows from the structural symmetry (and the symmetrical disposition of the terminal planes) with respect to the plane $z=0 ; Z_{12}=Z_{21}$ is assured by both symmetry and reciprocity; and, since losslessness has been assumed, $Z$ is pure inaginary. 'Thus $Z$ is of the form

$$
\left(\begin{array}{ll}
j X_{11} & j X_{12} \\
j X_{12} & j X_{11}
\end{array}\right)
$$

and there are only two independent parameters to be determined.

We do not obtain formulas for $X_{11}$ and $X_{12}$ directly; but rather, to exploit the symmetry of the problem more fully, we consider the impedances obtaining under modes of excitation in which the electromagnetic field is either symmetric or antisymmetric with respect to the plane $z=0$. Thus in the symmetric ase, $r_{1}(0)=r_{2}(0), i_{1}(0)=i_{2}(0)$, and the imperlanee may be written

$$
Z_{\rho e}=r_{1, e}(0) / i_{1, e}(0)
$$

where we have added a subscript distinguishing the "even" case. In the antisymmetric ("odd") case, $r_{1}(0)=-i_{2}(0), i_{1}(0)=-i_{2}(0)$, and the impedance may be written

$$
Z_{00}=v_{1,0}(0) / i_{1,0}(0)
$$

again adrling a distinguishing subscript. The relations

$$
2 j X_{11}=Z_{e e}+Z_{00}, \quad 2 j X_{12}=Z_{c e}-Z_{00}
$$

follow directly from (2.7), (2.8), and (2.9). (Lt may be noted that the symmetric and antisymmetric field distributions correspond to the eigenvectors of the natrix (2.8) and the quantities $Z_{e e}$ and $Z_{00}$ are the eigenvalues of this matrix.)

An equivalent network for the obstacles is of interest and is presented here for convenient reference. For a T-network, using the sign conventions shown in figure 2, we find

$$
Z_{1}=Z_{00}, \quad 2 Z_{2}^{\circ}=Z_{e e}-Z_{00}
$$

where $Z_{1}$ and $Z_{2}$ are respectively the series and the shunt elements of the symmetrical $T$. It may be remarked that the obstacles considered are ealled "induetive" because for small radii the important element, $Z_{2}$, is a positive reactance.

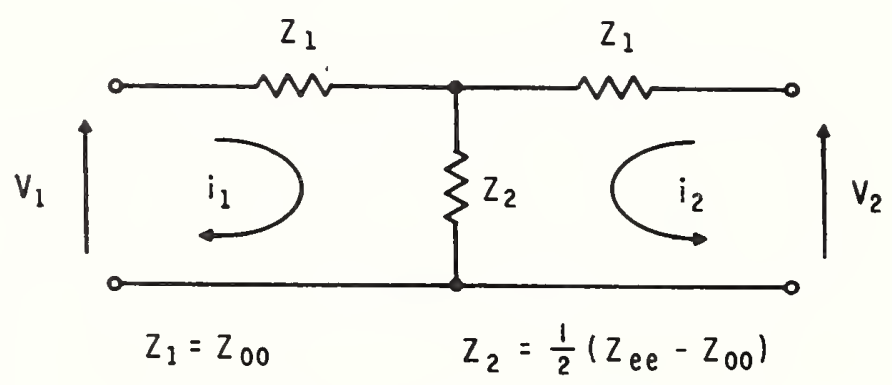

Figure 2. Equivalent network. 


\section{Analysis}

\subsection{Reduction to Scalar Form}

Maxwedl's equations directly imply the general differential equation for $\boldsymbol{E}$,

$$
\nabla \times \nabla \times \boldsymbol{E}=k^{2} \boldsymbol{E}-j \omega \mu \boldsymbol{J}
$$

which must be sperialized appropriately for the present problem. $\boldsymbol{E}$ is moreover subject to the boundary condition that its tangential components vanish on perfectly condueting surfaces.

In the present problem both the exciting field and the waveguide-obstacle structure are independent of $y$ in the range $0 \overline{<} y \overline{<} b$, and it follows that the whole field, secondary as well as primary, will be independent of $y$ in the same range. Further, since the $x$ - and z-components of $\boldsymbol{E}$ must vanish in particular on the $y=0$ surface of the waveguide, these components must vanish for all values of $y$ involved. Thus the electrie field is of the form $\boldsymbol{E}=\phi(x, z) \boldsymbol{e}_{\boldsymbol{y}}$; ค. (3.1) (with $\boldsymbol{J} \equiv 0$ ) reduces 10

$$
\nabla^{2} \phi+k^{2} \phi=0 \quad \text { in } S,
$$

and the boundary condition becomes

$$
\phi=0 \quad \text { oll } \quad C^{\prime},
$$

where $S$ is the cross section and $C$ is the boundary of the strueture in a plane $y=$ (e)nstant (lig. 3).
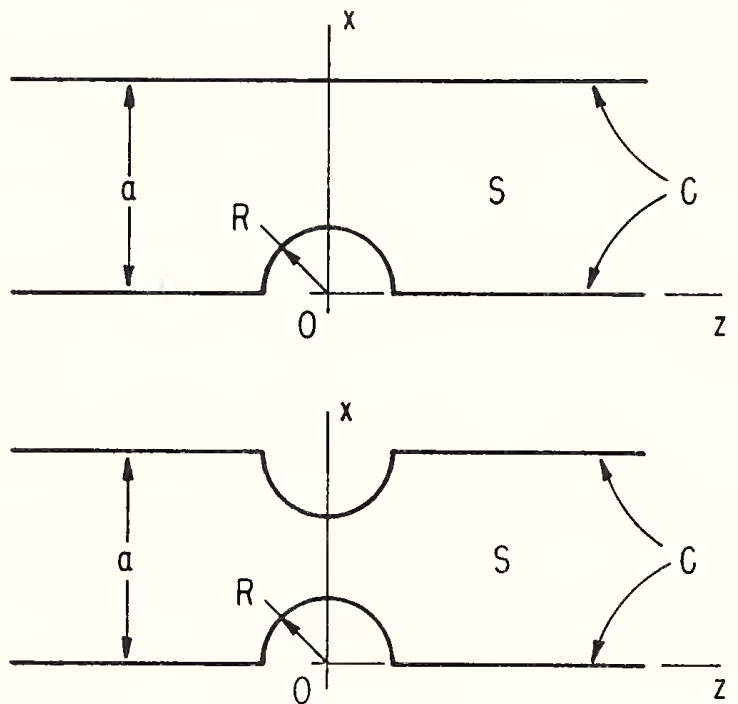

Figitre 3. Relevant geometry and coordinates for the singlt and double half-round problems.

The magnetie field corresponding to $\boldsymbol{E}$ of the above form is

$$
\boldsymbol{H}=(j \omega \mu)^{-1}\left(\frac{\partial \phi}{\partial z} \boldsymbol{e}_{x}-\frac{\partial \phi}{\partial x} \boldsymbol{e}_{z}\right)
$$

The surface current $\boldsymbol{K}=\boldsymbol{H} \times \boldsymbol{n}$ on $\boldsymbol{C}^{\prime}$ is thus given by

$$
\boldsymbol{K}=-(j \omega \mu)^{-1} \boldsymbol{n} \cdot \nabla \phi \boldsymbol{e}_{\boldsymbol{y}}
$$

evaluated on $C$, where $\boldsymbol{n}$ is the outward nolmal unit vector on C. 'Thus $\boldsymbol{K}$ hals only al $y$-compoinent and may be written as $K(x, z) \boldsymbol{e}_{y}$.

Inasmuch as the whole problem ean be dealt with in terms of $\phi$ and $K$, the problem is essentially a two-dimensional scalar one. In what follows it will be eonvenient to refer to $\phi$ simply as "the field" 


\subsection{Integral Equation Formulation}

We now introduce a Green's function $\Gamma=\Gamma\left(x, z, x^{\prime}, z^{\prime}\right)$ such that $\Gamma$ is the field that would be produced in the unperturbed waveguide at the point $(x, z)$ by a unit current filament parallel to $\boldsymbol{e}_{y}$ at the point $\left(x^{\prime}, z^{\prime}\right)$. As a function of $(x, z)$, $\Gamma$ satisfies the differential equation

$$
\nabla^{2} \Gamma+k^{2} \Gamma=j \omega \mu \delta\left(x-x^{\prime}\right) \delta\left(z-z^{\prime}\right)
$$

obtained from (3.1) with $\boldsymbol{J}=\delta\left(x-x^{\prime}\right) \delta\left(z-z^{\prime}\right) \boldsymbol{e}_{y}$, where $\delta$ denotes the Dirac delta-function. $\Gamma$ further satisfies the boundary condition $\Gamma=0$ for $x=0, a$ and represents outgoing waves for $\left|z-z^{\prime}\right| \rightarrow \infty$. An explicit form for $\Gamma$ is [5]

$$
\begin{aligned}
\Gamma\left(x, z, x^{\prime}, z^{\prime}\right)=-\omega \mu(\beta a)^{-1} \sin (\pi x / a) \sin \left(\pi x^{\prime} / a\right) e^{-j \beta\left|z-z^{\prime}\right|} & \\
& -(j \omega \mu / a) \sum_{n=2}^{\infty} \alpha_{n}^{-1} \sin (n \pi x / a) \sin \left(n \pi x^{\prime} / a\right) e^{-\alpha_{n}\left|z-z^{\prime}\right|},
\end{aligned}
$$

where $\alpha_{n}=\sqrt{(n \pi / a)^{2}-k^{2}}$ for $n>2$ and the other symbols are as already defined.

It is convenient to put

$$
\Gamma=-\omega \mu(F+j G)
$$

where $F$ and $G$ are real. We observe that $F$ can be written in the form

$$
F=(\beta a)^{-1}\left[\psi_{e}(x, z) \psi_{e}\left(x^{\prime}, z^{\prime}\right)+\psi_{0}(x, z) \psi_{0}\left(x^{\prime}, z^{\prime}\right)\right],
$$

with the definitions

$$
\left.\begin{array}{l}
\psi_{e}(x, z)=\cos \beta z \sin (\pi x / a), \\
\psi_{0}(x, z)=\sin \beta z \sin (\pi x / a) .
\end{array}\right\}
$$

The functions $\psi_{\mathrm{e}}$ and $\psi_{0}$ are respectively symmetric and antisymmetric with respect to reflection in the plane $z=0$.

With the aid of the Green's function we can set up the fundamental equations of the problem in integral-equation form. Let $\psi=\psi(x, z)$ denote the exciting field-i.e., the field, due to remote sources, that would exist in the absence of obstacles. Then, with an obstacle present, the total field $\phi$ can be written as the sum of the exciting field and the secondary field due to the current on the obstacle:

$$
\phi(x, z)=\psi(x, z)+\int_{C_{0}} \Gamma\left(x, z, x^{\prime}, z^{\prime}\right) K\left(x^{\prime}, z^{\prime}\right) d C^{\prime},
$$

where $K$ is the surface current on the obstacle and the integral is a line integral going over the contour $C_{0}$ of the obstacle ( $C_{0}$ consists of two parts in the case of the double half-rounds). If the point $(x, z)$ is taken on $C_{0}, \phi$ must vanish and we have

$$
0=\psi(x, z)+\int_{C_{0}} \Gamma\left(x, z, x^{\prime}, z^{\prime}\right) K\left(x^{\prime}, z^{\prime}\right) d C^{\prime}, \quad(x, z) \text { on } C_{0} .
$$

This is an inhomogeneous integral equation of the first kind determining $K$ for a given $\psi$. The equation reduces to slightly different forms in the symmetric and antisymmetric cases, which we now consider.

$$
\text { a. Symmetric Case }
$$

Let the exciting field be $A \psi_{e}(x, z)$, where $A$ is an arbitrary amplitude and $\psi_{e}$, as defined in (3.6), is a symmetric function of $z$. The surface current on the obstacle will then also be a symmetric function of $z$; we denote it by $K_{e}$. We now examine (3.7) for $z<<z^{\prime}$, say, to find the lowest-mode component and thus to find $v_{1, e}(z)$ and $i_{1, e}(z)$. For $z<<z^{\prime},(3.7)$ becomes

$$
\phi(x, z)=A \cos \beta z \sin (\pi x / a)-(\beta a)^{-1} \omega \mu \sin (\pi x / a) e^{j \beta z} \int_{C_{0}} \psi_{e} K_{e} d C,
$$


since integrals of the type $\int_{C_{0}} \psi_{0} K_{e} d C$ vanish because of the mutual orthogonality of symmetric and antisymmetric functions. By comparison with (2.6) it is seen that

$$
v_{1, e}(z)=A \cos \beta z-(\beta a)^{-1} \omega \mu e^{\mathrm{j} \beta z} \int_{C_{0}} \psi_{e} K_{e} d C .
$$

Using (3.2a) to obtain the $x$-component of the magnetic field associated with (3.9) and again referring to (2.6) one finds

$$
i_{1, e}(z)=-j A \sin \beta z+(\beta a)^{-1} \omega \mu e^{j \beta z} \int_{C_{0}} \psi_{e} K_{e} d C
$$

Equations (3.10) and (3.11a) hold directly from the definitions for $z<-R$ and by extrapolation for $z=0$. In particular (3.11a) yields

$$
i_{1, e}(0)=(\cdot \beta a)^{-1} \omega \mu \int_{C_{0}} \psi_{e} K_{e} d C .
$$

Next, in the integral eq (3.8), we separate the Green's function into real and imaginary parts as in (3.5), use the orthogonality property of even and odd functions, and thus find

$$
\psi_{e}(x, z) v_{1, e}(0)=j \omega \mu \int_{C_{0}} G\left(x, z, x^{\prime} z^{\prime}\right) K_{e}\left(x^{\prime}, z^{\prime}\right) d C^{\prime} .
$$

This equation and (3.11b) together furnish a definitive mathematical statement of the symmetric part of our problem.

\section{b. Antisymmetric Case}

In this case we let the exciting field be $A \psi_{0}(x, z)$, where $A$ is again an arbitrary constant and $\psi_{0}$, defined in (3.6), is an antisymmetric function of $z$. The current, $K_{0}$, on the obstacle will then also be antisymmetric with respect to $z$. In the same manner as in the symmetric case one finds, for $z \overline{<} 0$,

$$
v_{1,0}(z)=A \sin \beta z+(\beta a)^{-1} j \omega \mu e^{j \beta z} \int_{G_{0}} \psi_{0} K_{0} d C
$$

In particular,

$$
v_{1,0}(0)=(\beta a)^{-1} j \omega \mu \int_{C_{0}} \psi_{0} K_{0} d C
$$

Further,

$$
i_{1,0}(z)=j A \cos \beta z-(\beta a)^{-1} j \omega \mu e^{j \beta z} \int_{C_{0}} \psi_{0} K_{0} d C .
$$

and the integral equation becomes

$$
\psi_{0}(x, z) i_{1,0}(0)=-\omega \mu \int_{C_{0}} G\left(x, z, x^{\prime}, z^{\prime}\right) K_{0}\left(x^{\prime}, z^{\prime}\right) d C^{\prime} .
$$

This equation and (3.14b) together furnish a definitive statement of the antisymmetric cases of our problem.

\subsection{Summary of all Cases}

In the case of the double half-rounds the above integrations go over a two-part contour consisting of an upper semicircle $C_{u}$ and a lower semicircle $C_{l}$. An equivalent problem involving only one of the parts of the contours, say $C_{l}$, can be formulated using the fact that the exciting field as well as the structure is symmetric with respect to the plane $x=a / 2$, so that the current on the obstacles must also be symmetric with respect to this plane. This symmetry is quite 
independent of the symmetries with respect to $z=0$ distinguished by the subscripts " $e$ " and "o," and so these subseripts will be dropped for the moment. Thus, it is clear that

$$
\int_{C_{o}} \psi K d C=\int_{C_{u}} \psi K d C+\int_{C_{l}} \psi K d C=2 \int_{C_{l}} \psi K d C
$$

Further,

$$
\int_{C_{o}} G\left(x, z, x^{\prime}, z^{\prime}\right) K\left(x^{\prime}, z^{\prime}\right) d C^{\prime}=\int_{C_{l}} G^{(2)}\left(x, z, x^{\prime}, z^{\prime}\right) K\left(x^{\prime}, z^{\prime}\right) d C^{\prime}
$$

where $G^{(2)}$ is defined by

$$
G^{(2)}\left(x, z, x^{\prime}, z^{\prime}\right)=G\left(x, z, x^{\prime}, z^{\prime}\right)+G\left(x, z, a-x^{\prime}, z^{\prime}\right) .
$$

Equations (3.18) and (3.19) enable the desired restatement of the double half-round problems.

In what follows, superseripts 1,2 will bo used when it is desired to distinguish quantities associated with the single and the double half-round problems, respectively (this moans in particular $\left.G \equiv G^{(1)}\right)$. Integrals will be indicated by means of the convenient scalar product notation, e.g.,

$$
(\psi, K)=\int_{C_{l}} \psi K d C
$$

and by the operator notation

$$
G K=\int_{C_{l}} G\left(x, z, x^{\prime}, z^{\prime}\right) K\left(x^{\prime}, z^{\prime}\right) d C^{\prime} .
$$

As indicated, all such integrals are to be taken over $r_{l}$, the lower semicircle. As a further notational convenience, we introduce the "normalized" surface currents,

$$
I_{e}=j \omega \mu K_{e} / v_{1, e}(0), \quad I_{o}=-\omega \mu K_{o} / i_{1, o}(0),
$$

for both the single and the double problems.

Our results thus far may now be summarized as follows. For the symmetric cases we have

$$
\psi_{e}=G^{(h)} I_{e}, \quad 1 / X_{e e}^{(h)}=h\left(\psi_{e}, I_{e}\right) /(\beta a)
$$

and for the antisymmetric cases

$$
\psi_{o}=G^{(h)} I_{o,} \quad X_{o o}^{(h)}=-h\left(\psi_{o}, I_{o}\right) /(\beta a),
$$

where $h=1$ or 2 for the single or the double half-rounds, respectively, and $X_{e e}, X_{o o}$ are the reactances corresponding to the (pure imaginary) in pedances $Z_{e e}, Z_{o o}$ defined in (2.9).

\section{Solution for the Reactances}

\subsection{Application of Rayleigh-Ritz Method}

In each of the problems specified by (3.22) and (3.23), an unknown reactance or susceptance is proportional to a scalar product

$$
M=(\psi, I)
$$

where the function $I$ is determined by an integral equation,

$$
G I=\psi
$$

in which $\psi$ and $G$ are given and $G$ is symmetric. From these equations one may easily construct the "stationary representation" [1,2]

$$
M=(\psi, I)^{2} /(I, G I)
$$


for M. This constitutes the starting point for the method of Rayleigh and Ritz [6], which we wish to use to obtain an approximate expression for $M$. One assumes in (4.2) an approximation for the surface current in the form

$$
I_{n}=x_{i} f_{i}
$$

where the $x_{i}$ are coefficients to be determined, the $f_{i}$ are members of a suitable set of basis functions (to be chosen explicitly), and summation from 1 to $n$ over repeated indices $i, j$, . ., is understood. Expression (4.2) becomes an ordinary function of the $x_{i}$ and is to be subjected to the conditions of stationarity $\partial M / \partial x_{i}=0, \quad i=1,2, \ldots, n$. 'This leads to the system of equations

where

$$
G_{i j x_{j}}=\left(x_{i} c_{k} / M_{n}\right) c_{i}
$$

$$
c_{i} \equiv\left(\psi, f_{i}\right), \quad G_{i j} \equiv\left(f_{i}, G f_{i}\right),
$$

and $M_{n}$ denotes the now-determinate approximate value for $M$. If the a bitrary normalization of the $x_{i}$ is chosen so that $x_{k} c_{k}=M M_{n}$, then

$$
x_{i}=\left(G^{-1}\right)_{i j} c_{j}
$$

where $\left(G^{-1}\right)_{i j}$ denotes an element of the inverse of the $n \times n$ matrix of the $G_{i j}$, and it immediately follows that

$$
M_{n}=\left(G^{-1}\right)_{i j} c_{i} c_{j}
$$

Finally, we write this quadratic form as a ratio of determinants,

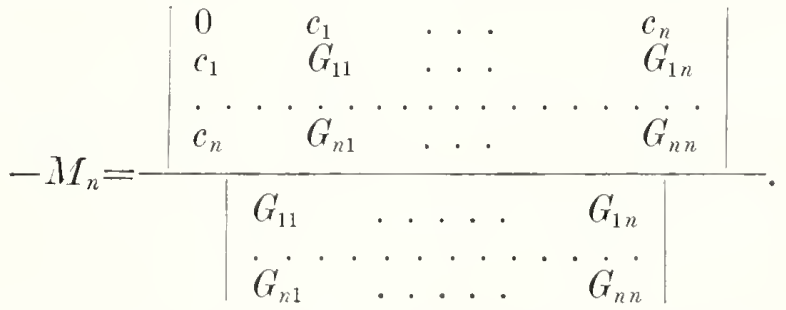

Thus $M_{n}$ is expressed in terms of the known quantities (4.4); if the objective is only to calculate the reactances, it is not necessary to calculate the $x_{i}$.

\subsection{Definiteness of $G$; Uppér Bounds for $X_{00}$}

Although in our problem $G$ is not a definite kernel, it does have definite character with respect to a suitably restricted class of admissible functions. In fact, $G$ is positive definite with respect to functions that are antisymmetric with respect to $z$ and vanish for $|z|>\lambda_{g} / 4$, where $\lambda_{g}=2 \pi / \beta$ is the "guide wavelength" of the singte propagating mode. In terms of the integrals of interest here, this means that if $\tilde{I}_{0}$ is any assumed surface current distribution, continuous, not identically equal to zero, and antisymmetric with respect to $z$, then

provided $R<\lambda_{g} / 4$.

$$
\left(\tilde{I}_{0}, G \tilde{I}_{0}\right)>0
$$

Now, for a positive definite kernel it is well known that the right-hand side of (4.2) is not only stationary, but also a maximum for the true $I$. For this result we offer the following brief proof based directly on the Schwarz inequality. Assuming $G$ to be positive definite, we wish to compare the approximate vahe for $M /$ given by

$$
\tilde{I}=(\tilde{I}, \psi)^{2} /(\tilde{I}, G \tilde{I})
$$

3 'This result is suggested by qualitative physical considerations and has been verified analytically by the authol. It has also been rerified that $G$ is not definite with respect to functions symmetrie with respect to $z$.

4 Proofs of this result in the literatme known to the author, e.g., [1,2], employ the so-called bilinear expansion for the kernel involved and do not yicht the "only" if" part of the result $(4.7)$. 
where $\tilde{I}$ is an admissible approximation for the surface current, with the true value given by (4.1a) (as well as by (4.2)) when $\tilde{I}$ satisfies (4.1b). From (4.1b) it follows that $(\tilde{I}, \psi)=(\tilde{I}, G I)$; the Schwarz inequality for positive-definite symmetric transformations [7] gives

$$
(\tilde{I}, G I)^{2} \overline{<}(\tilde{I}, G \tilde{I})(I, G I) \text {. }
$$

Since $(I, G I)=(I, \psi)=M$, we have the result

$$
\tilde{M} \overline{<} M
$$

Moreover the sign of equality holds in the Schwarz inequality and hence in this result if and only if $\tilde{I}$ is proportional to $I$.

Clearly, (4.7) is applicable when $G$ is merely positive definite with respect to all functions that need be admitted; by (4.6) this will be the case in the antisymmetric parts of our problem provided $R<\lambda_{g} / 4$. Hence, subject to this inequality, in the antisymmetric cases we shall have $M_{n}<M$ (we do not anticipate an exact result for finite $n$ ); this in turn implies that numerical values for $X_{00}$ will be (algebraic) upper bounds for the true values-assuming, of course, that numerical evaluations involved are sufficiently precise.

Unfortunately one can not obtain a bound for $X_{e e}$ in this simple manner. Furthermore, derived quantities of practical interest (such as (4.17)) will usually depend upon both $X_{e e}$ and $X_{00}$. Thus in general no bounds for such derived quantities are determined.

\subsection{Basis Functions}

We introduce plane polar coordinates $r, \theta$ such that

$$
x=r \sin \theta, \quad z=r \cos \theta .
$$

The path of integration $C_{l}$ is then given parametrically by

$$
x=R \sin \theta, \quad z=R \cos \theta,
$$

where $R$ is the obstacle radius and $0 \overline{<} \theta \overline{<} \pi$. On this path the surface current $I=I(\mathrm{R} \sin \theta$, $R \cos \theta$ ) becomes a function of $\theta$, which we denote simply by $I(\theta)$. We observe that symmetries with respect to $z=0$ are equivalent to symmetries with respect to $\theta=\pi / 2$.

As basis functions we take

$$
f_{i}=2(\pi R)^{-1} \sin (2 i-1) \theta
$$

for the symmetric cases (expansion of $I_{\varepsilon}$ ), and

$$
f_{i}=2(\pi R)^{-1} \sin 2 i \theta
$$

for the antisymmetric cases (expansion of $I_{0}$ ). 'The two sets of functions are complete for the expansion of symmetric and antisymmetric functions, lespectively, in the interval $(0, \pi)$. The completeness gives good assurance that for sufficiently large $n, M_{n}$ will approach arbitrarily close to the true $M$. For practical numerical calculation it is vital that the convergence of $M_{n}$ be rapid. In the present instance rapid convergence might be anticipated on the grounds that the true $I_{e}$ and $I_{0}$ may be expected to be smooth (possibly infinitely smooth) functions of $\theta$ vanishing at 0 and $\pi$, so that their Fouricr sine series should converge rapidly. ${ }^{5}$

\footnotetext{
${ }_{5}$ The points $(R, 0)$ and $(R, \pi)$ are the vertices of the corners formed where the half-round centered at $O$ meets the waveguide wall. The vanishing of the surface current in these vertices is equivalent, by (3.2), to the vanishing of the components of the surface magnetic field normal to the vertices. That these field components should vanish in the limit as a vertex is approached follows from properties of Sommerfeld's solution [14] of the problem of diffraction by a wedge of arbitrary angle.
} 


\subsection{Evaluation of Integrals}

Integrals of the type $\left(\psi, f_{i}\right)$ may be obtained relatively easily with the aid of the well-known expansion [8]

$$
e^{j \zeta \sin \alpha}=\sum_{n=-\infty}^{\infty} J_{n}(\zeta) e^{j n \alpha},
$$

wherein $J_{n}$ is the $n^{\text {th }}$ order Bessel function of the first kind. In this expansion we put $\zeta=k R$ and $\alpha= \pm \theta-\chi+(\pi / 2)$, with $k \sin \chi=\pi / \alpha, \quad k \cos \chi=\beta$, and employ (4.9). The expansiori

$$
e^{j \beta z} \sin (\pi x / a)=2 \sum_{n=1}^{\infty} j^{n-1} J_{n}(k R) \sin n \theta \sin n \chi
$$

is then easily obtained. The integrations yielding the desired quantities are now elementary; one obtains

$$
c_{i}=2(-)^{(p-1) / 2} J_{p}(k R) \sin p \chi, \quad p=2 i-1,
$$

for the symmetric cases, and

$$
c_{i}=2(-)^{(p-2) / 2} J_{p}(k R) \sin p \chi, \quad p=2 i,
$$

for the antisymmetric cases. (It is encouraging that these quantities decrease rapidly with increasing index.)

The evaluation of the integrals of the type $\left(f_{i}, G f_{j}\right)=G_{i j}$ is considerably more complicated than the evaluation of the $c_{i}$, and the task has been relegated to the appendix The results obtained may be summarized conveniently in the following form, which comprehends four cases:

$$
\frac{G_{j j}^{(h)}}{c_{i} c_{j}}=-\frac{1}{4 s_{p} s_{q}}\left[\rho_{p}\left(l_{R} R\right) \delta_{p q}+\sigma_{p q}^{(h)}(k a)\right]
$$

Here $s_{p} \equiv \sin p \chi ; \rho_{p}(k R) \equiv Y_{p}(k R) / J_{p}(k R)$; in the symmetric cases, $p=2 i-1$ and $q=2 j-1$; in the antisymmetric cases, $p=2 i$ and $q=2 j ;$ the functions $\sigma_{p q}^{(h)}$ are defined in the appendix (and tabulated briefly in table 2); $Y_{p}$ denotes the Bessel function of the second kind; and all other symbols involved have been defined previously.

\subsection{Results}

The value of a reactance element corresponding to an $n$-term expansion (4.3) will be called an " $n^{\text {th }}$ approximation" and will be written simply $X_{e e}^{(h)}$ or $X_{00}^{(h)}$ without special notation indicating the value of $n$ being considered. An explicit expression, in determinantal form, for $\boldsymbol{X}_{e e}^{(h)}$ in the $n^{\text {th }}$ approximation may be obtained by combining (3.22), (4.5), and (4.14); viz,

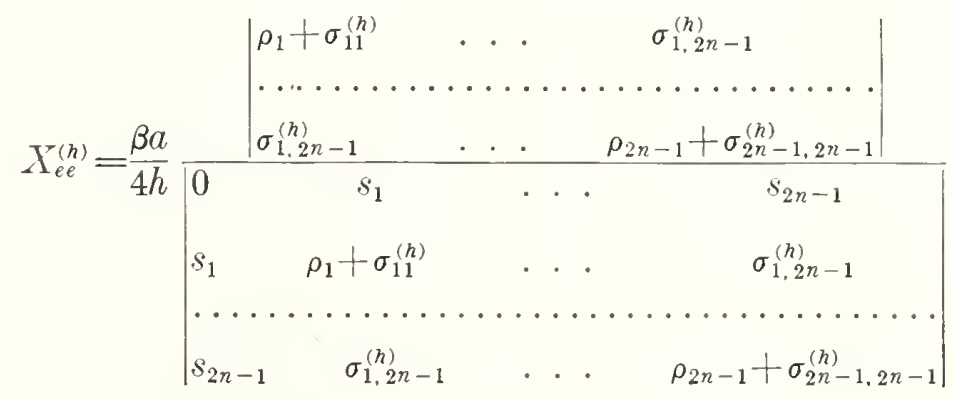


(In this and in the following equation the arguments $k R$ and $k a$ are understood.) Similarly, (3.23), (4.5), and (4.14) yield

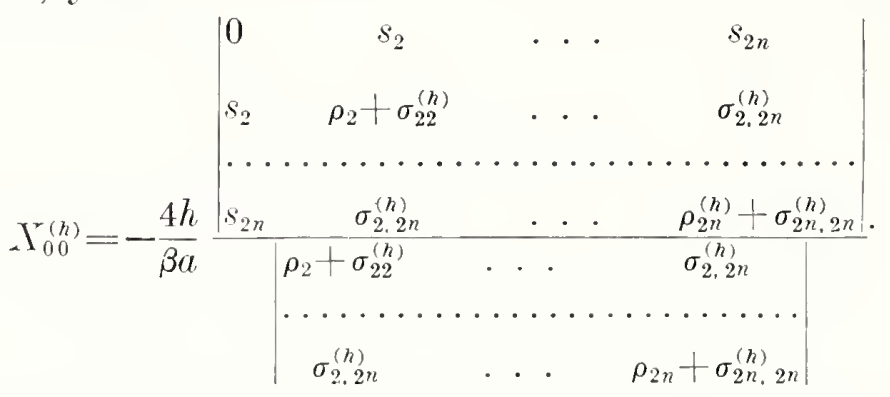

The determinantal lorm of these expressions secms convenient enough for practical calculations with $n$ at least as large as 3 . However, it may be remarked that, because of the special form of the diagonal clements, the determinants can be expanded in the same way as certain determinants occurring in the Fredholm theory of integral equations. This expansion may be convenient for the consideration of arbitrarily large $n$, but is not needed here.

Once the leactances $X_{e e}$ and $X_{00}$ are determined, other quantities or parameters associated with the representation of an obstacle are of course also determined. In particular, the "voltage" standing-wave ratio secm on one side of an obstacle when the other side is terminated in a matched (reflectionless) load is a familiar quantity having immediate physical signifieance, and it is convenient to discuss some of the results in terms of this quantity. Recalling that waveguide characteristic impedances were chosen equal to mity and reforing to the equivalent network, figure 2 , it is easily found that the standing-wave ratio in question is given by

$$
\eta=(1+|S|) /(1-|S|),
$$

where $|S|$, the magnitude of the associated reflection coefficient, is

$$
|S|=\left[1+\left(\frac{X_{c t}-X_{00}}{X_{c e} X_{00}+1}\right)^{2}\right]^{-1 / 2} \text {. }
$$

In order to get some idea of the behavior of the sequence of approximations, the reactance clements and the related VSTRR (4.17) have been calculated for $n=1,2$, and 3 and for a range of values of $k a$ and $k R$. Table 1 presents more or less typieal results in terms of the values of TSWR. 'The convergence of the sequence of' approximations, judged on the basis of numerical results exemplified in the table, appears to be vely rapid; by the same token, the first approximation fumishes a rather good result for TSWR's up to about 2.

'Thus far it has not proved l'asible to deterninc the manner of convergence of the sequence of approximations by theoretical means. In view of the character of the particular problem at hand, it seems probabte that the numerieal evidence may be relied upon.

TABLE 1. Convergence of TSWR

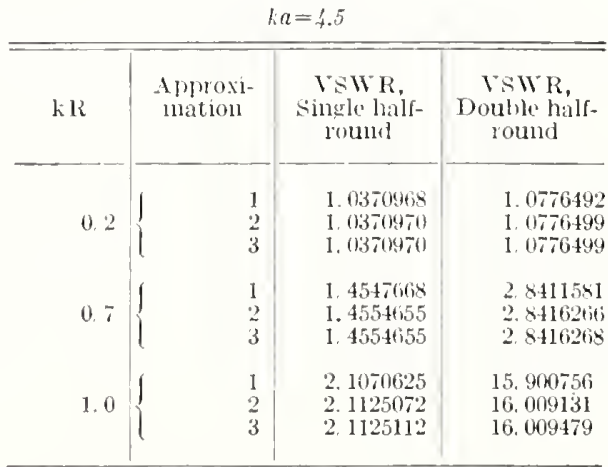

Cote: Maximum computational inaccuracy in the above figures is estimated to be approximately \pm 1 in the sixth significant figure; eight figures, as given, may be significant in indicating the behavior of successive approximations. 
Further calculations have been made regarding $k a$ and $R / a$ as independent variables. This choice of variables corresponds to the practical situation where one has a given obstacle (having a given value of $R / a$ ) that is to be operated at various frequencies. Figures 4 and 5 show the matched-termination VSWR (4.17) resulting for various values of $R / a$ and $f / f_{c}$, where $f$ is the operating frequency and $f_{c}$ is the cut-off frequency for the $T E_{10}$ mode (and $\left.f / f_{c}=k a / \pi\right)$. (Precise tables (based on the third approximations) giving basic parameters and possibly some derived quantities are being prepared for separate publication.)

The first approximations appear to be of useful accuracy for some purposes and represent an appreciable extension of previously available results. Consequently, a brief tabulation of the $\sigma$-functions, sufficient for the calculation of first approximations, is presented as table 2. For convenience of reference we write down (4.15) and (4.16) for the special case $n=1$,

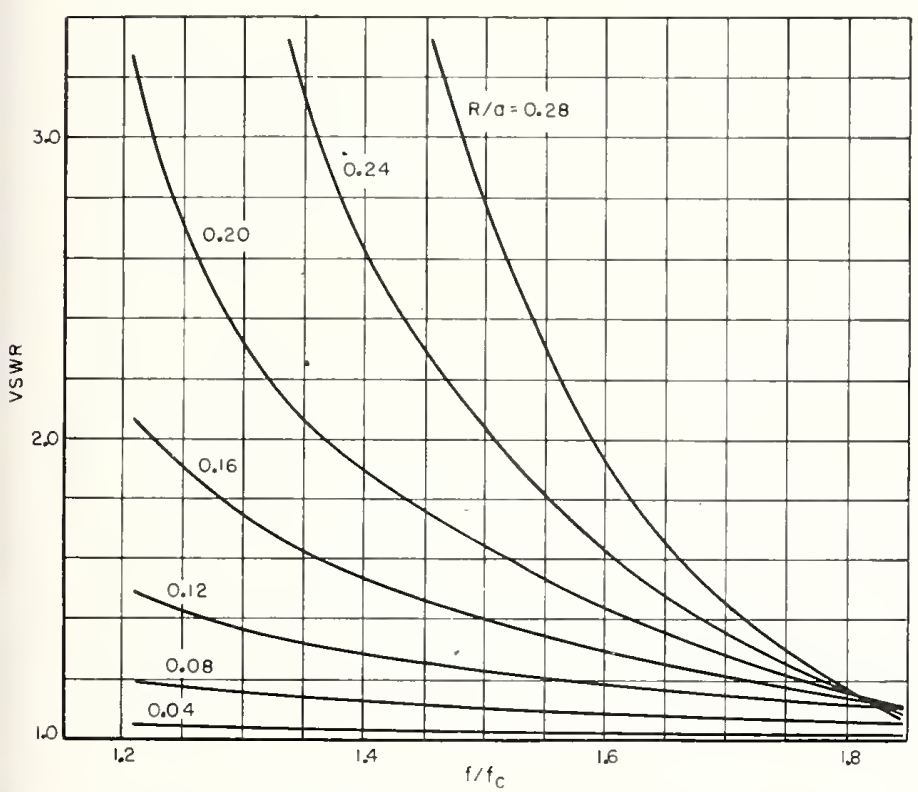

FIGURE 4. VSWR versus $f / f_{c}$ for single half-rounds.

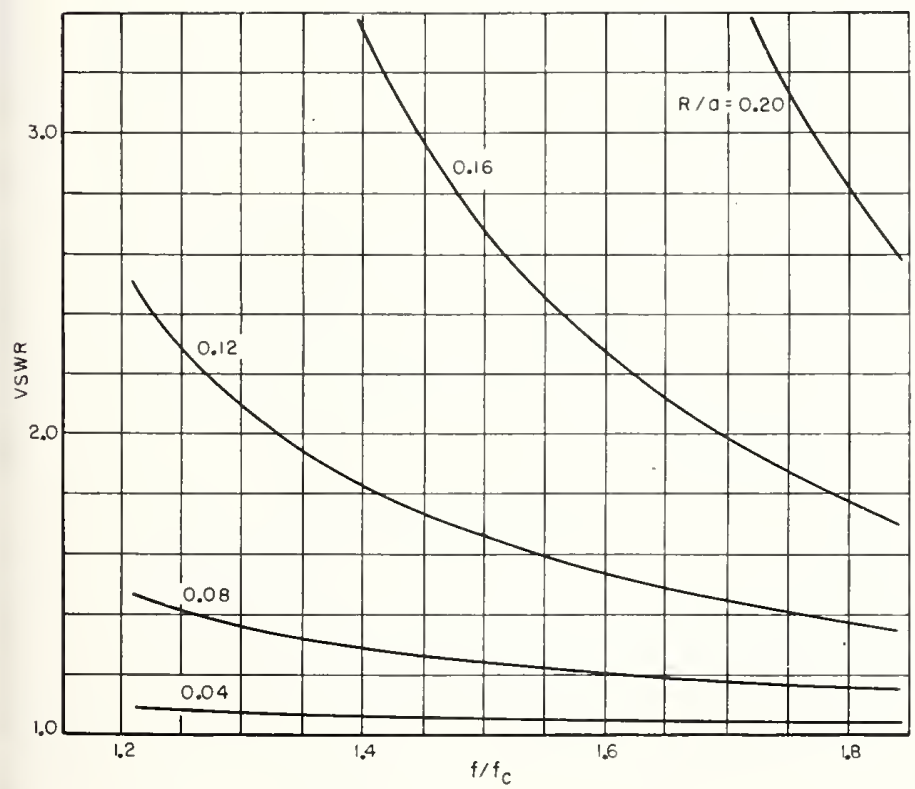

FIGURE 5. VSWR versus $f / f_{c}$ for double half-rounds.
$X_{e e}^{(h)}=-\frac{\beta a}{4 h s_{1}^{2}}\left[\rho_{1}(k R)+\sigma_{11}^{(h)}(k a)\right]$,

$X_{00}^{(h)}=\frac{4 h s_{2}^{2}}{\beta a}\left[\rho_{2}(k R)+\sigma_{22}^{(h)}(k a)\right]^{-1}$,

and note that $s_{1}=\pi /(k a)$ and $s_{2}=2 \pi \beta /\left(k^{2} a\right)$.

TABLE 2. Values of $\sigma_{p p}^{(h)}(x)$

\begin{tabular}{|c|c|c|c|c|}
\hline$x$ & $\sigma_{11}^{(1)}(x)$ & $\sigma_{22}^{(1)}(x)$ & $\sigma_{1 \mathrm{I}}^{(2)}(x)$ & $\sigma_{22}^{(2)}(x)$ \\
\hline $\begin{array}{l}3.80 \\
3.85 \\
3.90 \\
3.95\end{array}$ & $\begin{array}{l}2.055435 \\
1.976710 \\
1.899606 \\
1.823995\end{array}$ & $\begin{array}{c}-0.3170852 \\
-.1982432 \\
-.09078960 \\
006197600\end{array}$ & $\begin{array}{l}3.960997 \\
3.857142 \\
3.756554 \\
3.659060\end{array}$ & $\begin{array}{l}-2.544410 \\
-2.215670 \\
-1.912736 \\
-1.633535\end{array}$ \\
\hline $\begin{array}{l}4.00 \\
4.05 \\
4.10 \\
4.15\end{array}$ & $\begin{array}{l}1.749764 \\
1.676799 \\
1.604988 \\
1.534222\end{array}$ & $\begin{array}{l}.09356160 \\
.1720644 \\
.2424036 \\
.3052036\end{array}$ & $\begin{array}{l}3.564510 \\
3.472757 \\
3.383659 \\
3.297087\end{array}$ & $\begin{array}{r}-1.376168 \\
-1.138910 \\
-0.9201812 \\
-.7185580\end{array}$ \\
\hline $\begin{array}{l}4.20 \\
4.25 \\
4.30 \\
4.35\end{array}$ & $\begin{array}{l}1.464400 \\
1.395414 \\
1.327159 \\
1.259533\end{array}$ & $\begin{array}{l}.3610476 \\
.4104564 \\
.4539056 \\
.4918312\end{array}$ & $\begin{array}{l}3.212923 \\
3.131048 \\
3.051353 \\
2.973735\end{array}$ & $\begin{array}{l}-.5327088 \\
-.3614372 \\
-.2036444 \\
-.05831720\end{array}$ \\
\hline $\begin{array}{l}4.40 \\
4.45 \\
4.50 \\
\text { 4. } 55\end{array}$ & $\begin{array}{l}1.192430 \\
1.125746 \\
1.059372 \\
0.9931968\end{array}$ & $\begin{array}{l}.5246272 \\
.5526568 \\
.5762488 \\
.5956964\end{array}$ & $\begin{array}{l}2.898094 \\
2.824341 \\
2.752387 \\
2.682146\end{array}$ & $\begin{array}{l}.07546600 \\
.1985600 \\
.3117472 \\
.4157360\end{array}$ \\
\hline $\begin{array}{l}4.60 \\
4.65 \\
4.70 \\
4.75\end{array}$ & $\begin{array}{l}.9271072 \\
.8609836 \\
.7946988 \\
.7281208\end{array}$ & $\begin{array}{l}.6112796 \\
.6232416 \\
.6318024 \\
.6371680\end{array}$ & $\begin{array}{l}2.613542 \\
2.546500 \\
2.480946 \\
2.416812\end{array}$ & $\begin{array}{r}.5112028 \\
.5987500 \\
.6789328 \\
.7522756\end{array}$ \\
\hline $\begin{array}{l}4.80 \\
4.85 \\
4.90 \\
4.95\end{array}$ & $\begin{array}{l}.6611076 \\
.5935064 \\
.5251500 \\
.4558572\end{array}$ & $\begin{array}{l}.6395192 \\
.6390196 \\
.6358108 \\
.6300236\end{array}$ & $\begin{array}{l}2.354034 \\
2.292551 \\
2.232301 \\
2.173228\end{array}$ & $\begin{array}{l}.8192564 \\
.8803180 \\
.9358596 \\
.9862656\end{array}$ \\
\hline $\begin{array}{l}5.00 \\
5.05 \\
5.10 \\
5.15\end{array}$ & $\begin{array}{l}.3854276 \\
.3136376 \\
.2402376 \\
.1649416\end{array}$ & $\begin{array}{l}.6217692 \\
.6111440 \\
.5982284 \\
.5830836\end{array}$ & $\begin{array}{l}2.115279 \\
2.058400 \\
2.002545 \\
1.947661\end{array}$ & $\begin{array}{l}1.031884 \\
1.073038 \\
1.110027 \\
1.143125\end{array}$ \\
\hline $\begin{array}{l}5.20 \\
5.25 \\
5.30 \\
5.35\end{array}$ & $\begin{array}{c}.08742720 \\
.007320000 \\
-.07581400 \\
-.1624856\end{array}$ & $\begin{array}{l}.5657628 \\
.5462944 \\
.5246972 \\
.5009664\end{array}$ & $\begin{array}{l}1.893705 \\
1.840631 \\
1.788397 \\
1.736963\end{array}$ & $\begin{array}{l}1.172594 \\
1.198667 \\
1.221571 \\
1.241508\end{array}$ \\
\hline $\begin{array}{l}\text { 5. } 40 \\
\text { 5. } 45 \\
\text { B. } 50 \\
\text { B. } 55\end{array}$ & $\begin{array}{l}-.2533004 \\
-.3489884 \\
-.4504328 \\
-.4587224\end{array}$ & $\begin{array}{r}.4750804 \\
.4469912 \\
.4166292 \\
.3838912\end{array}$ & $\begin{array}{l}1.686288 \\
1.636332 \\
1.587059 \\
1.538434\end{array}$ & $\begin{array}{l}1.258672 \\
1.273236 \\
1.285370 \\
1.295225\end{array}$ \\
\hline $\begin{array}{l}\text { 5. } 60 \\
\text { 5. } 65 \\
\text { 5. } 70 \\
\text { 5. } 75\end{array}$ & $\begin{array}{c}-.6752200 \\
-.8016548 \\
-.9402632 \\
-1.094004\end{array}$ & $\begin{array}{l}.3486376 \\
.3106864 \\
.2697956 \\
.2256488\end{array}$ & $\begin{array}{l}1.490420 \\
1.442981 \\
1.396088 \\
1.34970 \text { 1 }\end{array}$ & $\begin{array}{l}1.302940 \\
1.308654 \\
1.312488 \\
1.314555\end{array}$ \\
\hline 5. 80 & -1.2668887 & .1778340 & 1.303798 & 1.314964 \\
\hline
\end{tabular}

Note: Accuracy of the above figures is estimated to be approximately one part per million. 
For sufficiently small $k R$, the expressions just given may be simplified still further. For small $k R$, the quantities $\rho_{1}(k R)$ and $\rho_{2}(k R)$ become large, in accordance with the formula

$$
\rho_{q}(k R) \cong-\frac{1}{\pi} q !(q-1) !\left(\frac{2}{k R}\right)^{2 q} \quad(q>1),
$$

and thus determine the qualitative behavior of $X_{e e}$ and $X_{00}$. Using this approximation for the $\rho$ 's, neglecting the $\sigma^{\prime} s$, and introducing the guide wavelength $\lambda_{g}=2 \pi / \beta$, one obtains

$$
\begin{aligned}
& X_{e e}^{(h)}=\frac{2 a}{h \lambda_{g}}\left(\frac{a}{\pi R}\right)^{2}, \\
& X_{00}^{(h)}=-\frac{h a}{\lambda_{g}}\left(\frac{\pi R}{a}\right)^{4} .
\end{aligned}
$$

('These are the correct forms of the results in the order of approximation of the more general formulas in the Waveguide Handbook [3].) Although these formulas give a rather good picture of the behavior of the reactances, they fail to reflect the interesting qualitative feature that $X_{e e}^{(h)}$ and $X_{00}^{(h)}$ must eventually become equal to each other as $R$ approaches $a / h$ (as can be seen physically).

\section{Appendix}

\subsection{Expansion of Green's Function in Terms of Cylindrical Wave Functions}

In order to arrive conveniently at the respective types of Green's functions needed for the single and the double half-round problems, we consider a waveguide of width $w$ (instead of $a$ ), bounded by the lines $x=0$ and $x=w$; and, in addition to the electric boundary condition at $x=0$, we consider both electric and magnetic boundary conditions at $x=w$ (by "electric" or "magnetic" boundary conditions it is meant that the Green's function or its normal derivative, respectively, vanishes on the boundary). The radius vectors of the source-point and the field-point will be denoted by $\boldsymbol{r}^{\prime}$ and $\boldsymbol{r}$, respectively.

Now, it is well known that the two-dimensional "free-space" Green's function, satisfying (3.3) and representing outgoing waves at infinity, is $-(\omega \mu / 4) H_{0}\left(k\left|\boldsymbol{r}^{\prime}-\boldsymbol{r}\right|\right)$, where $H_{0}$ denotes the Hankel function of the second kind (as is appropriate for exp $(j \omega t)$ time dependence). It is also well known that the desired Green's function, $\Phi$, satisfying (3.3) and the required boundary conditions at $x=0, x=w$, and at $z= \pm \infty$, can be obtained in the form of a sum of free-space Green's functions by the method of images [9]. In fact

$$
\Phi=-(\omega \mu / 4) \sum_{n=-\infty}^{\infty} s^{n}\left[H_{0}\left(k\left|\boldsymbol{\rho}_{n}^{+}-\boldsymbol{r}\right|\right)-H_{0}\left(k\left|\boldsymbol{\rho}_{n}^{-}-\boldsymbol{r}\right|\right)\right],
$$

where $\boldsymbol{\rho}_{n}^{ \pm}=\left(2 n w \pm x^{\prime}\right) \boldsymbol{e}_{x}+z^{\prime} \boldsymbol{e}_{z}$ and $s=+1$ or -1 according to whether the boundary condition at $x=w$ is electric or magnetic. Br inspection of the array of sources (fig. 6) it can be seen that this function has the proper symmetry to satisfy the boundary conditions: it is antisymmetric with respect to $x=0$ and it is antisymmetric or symmetric with respect to $x=w$ according to whether $s=+1$ or -1 .

Equation (5.1) can be transformed into a sum of elementary wave-functions of polar coordinates by repeated application of Graf's addition theorem for Bessel functions [10]. We introduce polar coordinates $(r, \theta),\left(r^{\prime}, \theta^{\prime}\right)$, and $\left(\rho_{n}, \theta_{n}\right)$ for $\boldsymbol{r}, \boldsymbol{r}^{\prime}$, and $\boldsymbol{\rho}_{n}$ in the manner shown in (4.8), and we assume $r$ and $r^{\prime}$ both to be less than $w$, so that $\rho_{n}>w$ for $n \neq 0$. Considering the triangles with sides $\boldsymbol{\rho}_{n}, \boldsymbol{r}$, and $\boldsymbol{\rho}_{n}-\boldsymbol{r}$ and applying the addition theorem, we obtain

$$
\begin{aligned}
-4(\omega \mu)^{-1} \Phi= & \sum_{p=-\infty}^{\infty} \begin{array}{l}
J_{p}(k r) H_{p}\left(k r^{\prime}\right) \\
J_{p}\left(k r^{\prime}\right) H_{p}(k r)
\end{array} e^{j p \theta\left(e^{-j p \theta \prime}-e^{j p \theta^{\prime}}\right)} \\
& \quad+\sum_{n=-\infty}^{\infty} s^{n} \sum_{p=-\infty}^{\infty} J_{p}(k r) e^{j p \theta}\left[H_{p}\left(k \rho_{n}^{+}\right) e^{-j \theta+}-H_{p}\left(k \rho_{n}^{-}\right) e^{-j p \theta-}\right],
\end{aligned}
$$




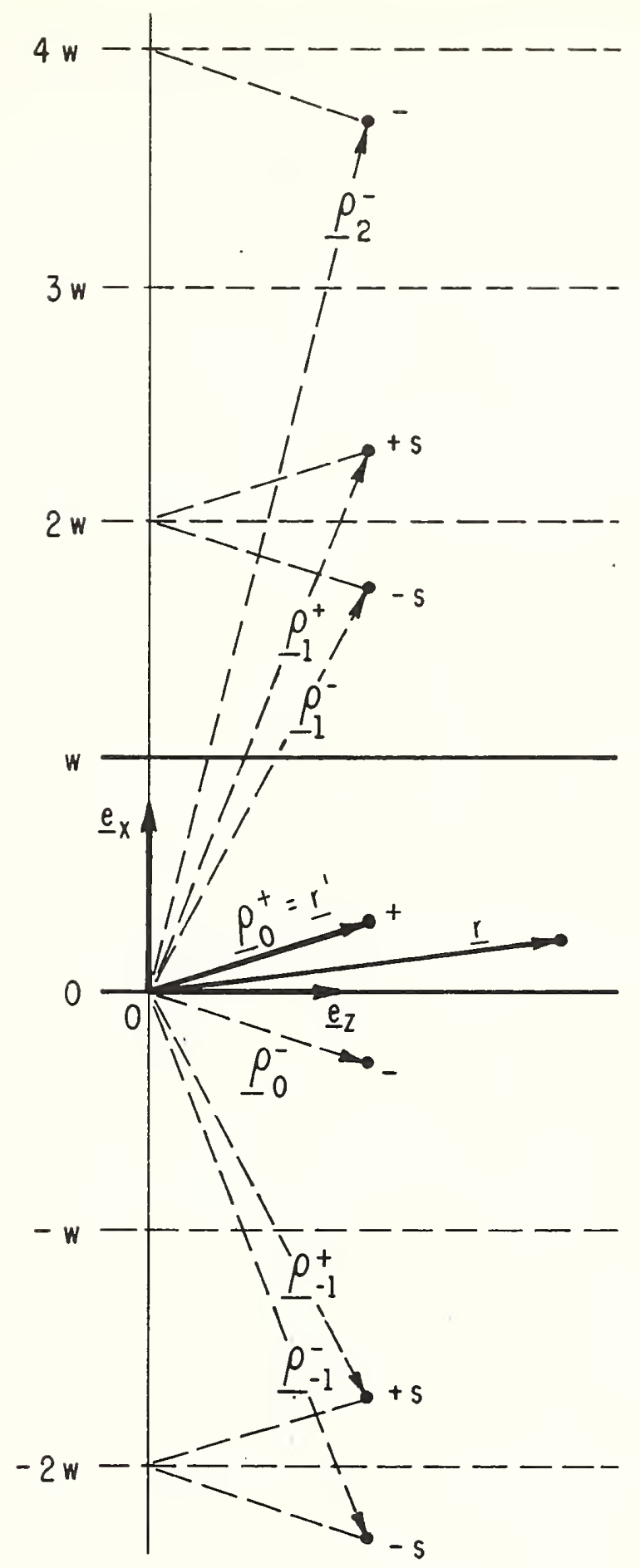

Figure 6. Source points in the method of images.

Relative signs of sources are indicated by the adjacent symbols,+- , $+s$, and $-s$; the role of $s$ is defined in connection with $(5.1)$.

where the terms arising from $n=0$ appear in the first line and are to be omitted from the primed summation in the second line; the upper and lower alternatives in the first line apply according as $r<r^{\prime}$ or $r>r^{\prime}$, respectively. Again, by the addition theorem,

$$
H_{p}\left(k \rho_{n}^{ \pm}\right) e^{-j p \theta_{n}^{ \pm}}=\sum_{q=-\infty}^{\infty} J_{q}\left(k r^{\prime}\right) H_{p+q}(|2 n k w|) \exp \left[j(\pi / 2)(q-p) \operatorname{sgn} n \pm j q \theta^{\prime}\right]
$$


Combining the last two equations and rearranging terms, one finds that (5.1) can be written in the form

$$
\begin{aligned}
\Phi=-\omega \mu \sum_{p=1}^{\infty} \sum_{q=1}^{\infty} \sin p \theta \sin q \theta^{\prime}\left\{\begin{array}{l}
J_{p}(k r) H_{p}\left(k r^{\prime}\right) \\
J_{p}\left(k r^{\prime}\right) H_{p}(k r)
\end{array} \delta_{p q}\right. \\
\left.+2 J_{p}(k r) J_{q}\left(k r^{\prime}\right) \cos [(\pi / 2)(p-q)] \sum_{n=1}^{\infty} s^{n}\left[H_{p-q}(2 n k w)-H_{p+q}(2 n k w)\right]\right\} .
\end{aligned}
$$

If now one puts $w=a$ and $s=+1$, this expression becomes the desired representation of $\Gamma$ of the text; similarly, if $w=a / 2$ and $s=-1,(5.2)$ becomes the desired representation of a Green's function $\Gamma^{(2)}$ related to $G^{(2)}$ in the same way as $\Gamma$ is related to $G$ (cf. 3.5). Thus, employing the relation (3.5), the relation $H_{p}=J_{p}-j Y_{p}$, the notation (3.20), the basis functions $(4.10,4.11)$, and carrying out the integrations with respect to $\theta, \theta^{\prime}$, one obtains

$$
\left(\frac{2}{\pi R}\right)^{2}\left(\sin \mathrm{p} \theta, G^{(h)} \sin q \theta^{\prime}\right)=-J_{p}(k R) Y_{p}(k R) \delta_{p q}-J_{p}(k R) J_{q}(k R) \cos [(\pi / 2)(p-q)] \sigma_{p q}^{(h)}(k a),
$$

where the functions $\sigma_{p q}^{(h)}$ are defined as

$$
\begin{aligned}
& \sigma_{p q}^{(1)}(k a)=2 \sum_{n=1}^{\infty}\left[Y_{p-q}(2 n k a)-Y_{p+q}(2 n k a)\right], \\
& \sigma_{p q}^{(2)}(k a)=2 \sum_{n=1}^{\infty}(-)^{n}\left[Y_{p-q}(n k a)-Y_{p+q}(n k a)\right] .
\end{aligned}
$$

We note that the above expressions are needed only for positive integer indices $p, q$ that are both even or both odd. We observe also that the form of the expressions is such that those with unequal subscript indices can be evaluated simply in terms of those with equal subscript indices. Further, in view of the easily-verified relation

$$
\sigma_{p q}^{(2)}(k a)=2 \sigma_{p q}^{(1)}(k a)-\sigma_{p q}^{(1)}(k a / 2),
$$

values or formulas for the $\sigma$ 's "of the second kind" can be derived readily from the same for the $\sigma$ 's "of the first kind." Thus, the evaluation of the $G_{p q}^{(h)}$, which, on the face of it, originally involved double integrals with $k a, k R, p, q$, and $\dot{h}$ as independent parameters, has been reduced essentially to the evaluation of sums of the form $\sum_{n=1}^{\infty} Y_{2 s}(n x)$ with $\pi<x<2 \pi$ or $2 \pi<x<4 \pi$ and $s=0,1,2, \ldots$.. It should be observed, however, that these sums converge extremely slowly: for the accuracy desired in the present circumstances the number of terms required would be of the order of $10^{10}$ or more, at least for the most important $\sigma$ 's. Needless to say, some method of evaluation other than direct summation is indicated.

\subsection{Evaluation of the Bessel-Function Series}

Two methods of evaluating the series defined in (5.4) will be discussed briefly here.

a. Conversion to Definite Integrals

An evaluation of the desired series may be obtained in terms of definite integrals as follows. We start with the series of Hankel functions (of the second kind)

$$
S_{\lambda}(x)=\sum_{n=1}^{\infty} H_{\lambda}(n x)
$$

where $x$ is real, positive, and not an integer multiple of $2 \pi$. For the Hankel functions we use the integral representation [11]

$$
H_{\lambda}(x)=\frac{1}{\pi} \int_{C} e^{-j x \sin \zeta+\lambda \lambda \zeta} d \zeta
$$


where $\zeta=\xi+\jmath \eta$ and $C$ is a suitable path, to be chosen in a moment, between the limits $-j \infty$ and $\pi+j \infty$. With the aid of the well-known expression for the sum of a finite number of terms of a geometric series, one finds that the $M$-term partial sum of (5.6) can be written

$$
\sum_{n=1}^{M} H_{\lambda}(n x)=\frac{1}{\pi} \int_{C} \frac{e^{j \lambda \zeta} d \zeta}{e^{j x \sin \zeta}-1}-\frac{1}{\pi} \int_{C} \frac{e^{-j M x \sin \zeta}}{e^{j x \sin \zeta}-1} e^{j \lambda \zeta} d \zeta .
$$

Among the infinitely many poles of the integrands in these integrals, we note in particular $\xi_{m}^{\prime}$ and $\xi_{m}^{\prime \prime}=\pi-\xi_{m}^{\prime}$, where $\xi_{m}^{\prime}$ is the principal value of $\sin ^{-1}(2 m \pi / x), m=0,1,2, \ldots, Q$, and $Q$ is the largest integer such that $2 Q \pi / x<1$. The path $C$ is now chosen to comprise (except for indentations) the negative imaginary axis, the real axis between 0 and $\pi$, and the upper half of the line $\pi+j \eta$; indentations, of radius $\rho$, are made at each of the above identified poles in the manner shown in figure 7 , which illustrates $C$ for the case $Q=\dot{1}$. Taken on this path, the second integral in (5.7) approaches 0 as $M$ increases indefinitely; as a detailed examination shows, this happens because on the various parts of the path the integrand approaches 0 or it becomes increasingly oscillatory (or both) as $M \rightarrow \infty$. Hence we have

$$
S_{\lambda \cdot}(x)=\frac{1}{\pi} \int_{C} \frac{e^{j \lambda \zeta} d \zeta}{e^{j x \sin \zeta}-1}
$$

This result can be expressed in terms of real integrations plus contributions from the indentations. In the limit as $\rho \rightarrow 0$, the indentations yield

$$
R_{o}^{\prime}=\frac{1}{2 x}, \quad R_{o}^{\prime \prime}=\frac{e^{j \lambda \pi}}{2 x}
$$

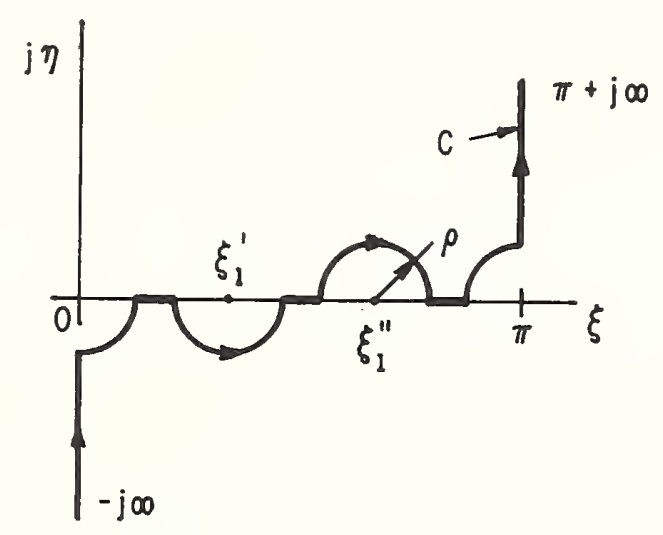

FIGURe 7. Path of integration.

and for $m>1$,

$$
R_{m}^{\prime}=\frac{e^{j \lambda \xi_{m}^{\prime}}}{\sqrt{x^{2}-(2 m \pi)^{2}}}, \quad R_{m}^{\prime \prime}=\frac{e^{j \lambda\left(\pi-\xi_{m}^{\prime}\right)}}{\sqrt{x^{2}-(2 m \pi)^{2}}} \cdot
$$

Here the notation corresponds to that used for the respective poles and the factor $1 / \pi$ standing in front of the integral is included. Provided $\lambda$ is an even integer, the straight-line segments of the path of integration yield"

$$
-\frac{1}{2} \delta_{0 \lambda}+\lim _{\rho \rightarrow 0} \frac{j}{\pi}\left[2 \int_{\rho}^{\infty} \frac{\cosh \lambda \eta d \eta}{e^{x \sinh \eta}-1}-P \int_{\rho}^{\pi / 2} \cos \lambda \xi \cot \left(\frac{1}{2} x \sin \xi\right) d \xi\right],
$$

where $\delta_{o \lambda}$ is the Kronecker delta. It should be noted that when the integrand in the finite integral is singular, the Cauchy principal value is to be taken, as indicated by the symbol $P$ (the indicated limit as $\rho \rightarrow 0$ essentially is also a Cauchy value).

Upon comparing (5.4) and (5.6) it is seen that

$$
\sigma_{p p}^{(1)}(k a)=2 \operatorname{Im}\left[S_{2 p}(2 k a)-S_{0}(2 k a)\right] .
$$


It happens that for $\lambda=2 p$ (where $p=0,1,2, \ldots$ ) the net imaginary contribution of (5.8) to $S_{2 p}$ vanishes. Consequently (5.9) yields

$$
\sigma_{p p}^{(1)}(k a)=\frac{8}{\pi} \int_{0}^{\infty} \frac{\sinh ^{2} p \eta d \eta}{e^{2 k a \sinh \eta}-1}+\frac{4}{\pi} P \int_{0}^{\pi / 2} \sin ^{2} p \xi \cot (k a \sin \xi) d \xi
$$

wherein the integrands are now well-behaved at their lower limits and thus we were permitted to put $\rho=0$. The corresponding result for $\sigma_{p p}^{(2)}$ is

$$
\sigma_{p p}^{(2)}(k a)=-\frac{8}{\pi} \int_{0}^{\infty} \frac{\sinh ^{2} p \eta d \eta}{e^{k a \sinh \eta}+1}-\frac{4}{\pi} P \int_{0}^{\pi / 2} \sin ^{2} p \xi \tan \left(\frac{k a}{2} \sin \xi\right) d \xi,
$$

which is easily obtained from (5.10) with the aid of (5.5).

(An interesting by-product of the above development, obtained by taking the real parts of $(5.6),(5.8)$, and $(5.9)$, is

$$
\frac{1}{2} \delta_{o p}+\sum_{n=1}^{\infty} J_{2 p}(n x)=\frac{1}{x}+2 \sum_{m=1}^{Q} \frac{\cos \left[2 p \sin ^{-1}(2 m \pi / x)\right]}{\sqrt{x^{2}-(2 m \pi)^{2}}},
$$

where $p=0,1,2, \ldots ; x$ and $Q$ are as specified above. This result was obtained by Ignatowsky [12] by a different method.)

Thus far numerical results for the $\sigma$ 's have been obtained primarily from (5.10). The integrals were evaluated numerically by Paul F. Wacker and William W. Longley, Jr., using Legendre-Gaussian quadrature formulas programed for machine computation. ${ }^{6}$ Values for $\sigma_{p p}^{(2)}$ were calculated from values of $\sigma_{p p}^{(1)}$ using (5.5). Results for the two lowest values of $p$ are given in table 2. (More extensive tables are planned for the previously-mentioned separate publication.)

\section{b. Conversion to More Rapidly Convergent Series}

An alternative method of evaluating the $\sigma$ 's is afforded by a transformation of the Besselfunction series involved into more rapidly convergent series. This was accomplished a good many years ago by W. von Ignatowsky [12] in connection with his work on diffraction by gratings-a subject that will be recognized as being rather closely related to the subject of the present paper. Ignatowsky obtained

$$
\begin{gathered}
\pi \sum_{n=1}^{\infty} Y_{0}(n x)=\log \frac{2 \kappa}{\gamma}+\sum_{n=1}^{Q} \frac{1}{n}+\sum_{n=Q+1}^{\infty}\left(\frac{1}{n}-\frac{\kappa}{\sqrt{n^{2} \kappa^{2}-1}}\right) \\
\pi \sum_{n=1}^{\infty} Y_{2 p}(n x)=\frac{1}{2 p}-\frac{1}{2} \sum_{\mu=1}^{p} \frac{(2 \kappa)^{2 \mu}(p+\mu-1) ! B_{2 \mu}}{(2 \mu) !(p-\mu) !} \\
-\kappa \sum_{n=1}^{Q} \frac{\sin \left[2 p \sin ^{-1}(n \kappa)\right]}{\sqrt{1-n^{2} \kappa^{2}}}-(-)^{p} \kappa \sum_{n=Q+1}^{\infty} \frac{\left(n \kappa-\sqrt{n^{2} \kappa^{2}-1}\right)^{2 p}}{\sqrt{n^{2} \kappa^{2}-1}} .
\end{gathered}
$$

In these expressions $\kappa=2 \pi / x ; p=1,2,3, \ldots ; Q$ is as defined above; $\log \dot{\gamma}=0.57721566 \ldots$. . is the Euler-Mascheroni constant; and the $B_{2 \mu}$ are the Bernoulli numbers, here so labeled that $B_{2}=1 / 6, B_{4}=1 / 30, B_{6}=1 / 42, B_{8}=1 / 30, B_{10}=5 / 66$, etc. Equation (5.12a) is fairly well known at the present time. (A few numerical values are given for the series $\sum_{n=1}^{\infty}(-)^{n} Y_{0}(n x)$ in

\footnotetext{
B The estimates of accuracy of computed quantities given in this paper were made by Mr. Longley, who was responsible for most of the detail of the calculations.
} 
[13]:) The series for $\sigma_{11}^{(2)}$ obtained from (5.12a) and (5.12b) has been duplicated by an independent mode of derivation; this represents a partial check of the complicated expression $(5.13 \mathrm{~b})$. Thus far in the present work, Ignatowsky's series have been used only to spot check some of the numerical results obtained from the definite integrals. The series are especially suitable for either estimation or calculation of the $\sigma$ 's when $p$ is large.

The author has benefited from the discussion of many points with Paul F. Wacker and is indebted to David F. Wait for assistance with some of the theory and computations.

\section{References}

[1] D. S. Saxon, Notes on lectures by Julian Schwinger: Discontinuities in waveguide, Mass. Inst. Tech. Rad. Lab. report $43-7 / 4 / 44$.

[2] F. E. Borgnis and C. H. Papas, Randwertprobleme der Mikrowellenphysik (Springer-Verlag, 1955).

[3] N. Marcuvitz, ed., Mass. Inst. Tech. Rad. Lab. Series 10, 255-257 (McGraw-Hill Book Co., New York, N.Y. 1951).

[4] R. W. Beatty and D. M. Kerns, Recently developed microwave impedance standards and methods of measurement, IRE Trans. on Instrumentation I-7, 319 (1958).

[5] See [2], p. 24.

[6] R. Courant and D. Hilbert, Methods of mathematical physics, 1st Eng. Ed., p. 175 (Interscience, X.Y., 1953).

[7] F. Riesz - B. Sz.-Nagy, Functional analysis, p. 262 (Frederick Ungar Publishing Co., New York, N. Y., 1955).

[8] See [6], p. 474.

[9] P. M. Morse and H. Feshback, Methods of theoretical physics, p. 81. (McGraw-Hill Book Co., Inc., New York, N.Y., 1953).

[10] G. X. Watson, A treatise on the theory of Bessel functions, p. 361 (The University Press, Cambridge, England, 19.44).

[11] See [6], p. 468.

[12] W. von Ignatowsky, Über Reihen mit Zylinderfunktionen nach dem Vielfachen des Argumentes, Arch. der Math. u. Phys. 23, 193 (1914).

[13] V. G. Smith, I. Infeld, and W. J. Chien, On some series of Bessel functions, J. Math. and Phys. 20, 22 (1947).

[14] P. Frank and R. v. Mises, Die Differential- und Integralgleichungen der Mechanik und Physik, Vol. 2, p. 808ff (Mary Rosenberg, New York, N.Y., 1943).

Boulder, Colo.

(Paper 64B2-29) 


\title{
An Adjustable Sliding Termination for Rectangular Waveguide*
}

\author{
ROBERT W. BEATTY $\dagger$
}

\begin{abstract}
Summary-A new adjustable sliding termination for rectangular waveguide has been developed. The termination is of simple design and can easily be adjusted to have reflection coefficients from zero to nearly unity in magnitude and any desired phase. In addition to the usual applications of adjustable sliding terminations for rectangular waveguide, it provides a suitable design for an adjustable transfer or secondary standard of impedance for rectangular waveguide systems.
\end{abstract}

\section{INTRODUCTION}

$\Lambda^{\mathrm{D}}$ DJUSTABLE sliding terminations for rectangular waveguide are useful for a variety of purposes, including calibration of microwave impedance measuring devices, obtaining the directivity of directional couplers, and determining the parameters of waveguide junctions.

An adjustable sliding termination for rectangular waveguide was described by Grantham in $1951 .{ }^{1}$ Several years later, a similar termination was produced commercially. This termination ${ }^{2}$ differed from its predecessor in details of construction of the dissipative element and the reflecting antenna. Both of these terminations were designed primarily to provide minimum reflection and could not be adjusted over a wide range of vswr (voltage standing-wave ratio). The commercial termination has a vswr range of 1.005 to 1.15 . The principle of the double slug tuner was used ${ }^{3}$ to obtain an adjustable sliding termination with a somewhat greater range of adjustment. An adjustable sliding termination having a wide range of vswr was described by Kato and Sakai, ${ }^{4}$ but it was not possible to adjust this termination for cancellation of reflections.

\section{Principle of Operation}

As shown in the diagram of Fig. 1, this termination slides inside a rectangular waveguide and consists of a short-circuiting piston to which is attached a dissipative strip supported by a dielectric rod, which can rotate and slide relative to the piston. The phase of the reflection from the strip can be varied by sliding the strip, while the magnitude of the net reflection from the short cir-

* Manuscript received by the PGMTT, November 19, 1956.

† Natl. Bur. of Standards, Boulder, Colo.

'R. E. Grantham, "A reflectionless wave-guide termination," Rev. Sci. Instr., vol. 22, pp. 828-834; November, 1951.

${ }^{2}$ W. A. Andrews, U. S. Patent No. 2,701,861.

${ }^{3}$ R. C. Ellenwood and W. E. Ryan, "A uhf and microwave matching termination," PROC. IRE, vol. 41, pp. 104-107; January, 1953.

${ }^{4}$ N. Kato and T. Sakai, "Waveguide Type Variable Impedance Circuit and its Application for Rieke Diagram," Rep. of the Microwave Communication Res. Committee in Japan, p. 7; December, 1955. cuit can be varied by rotating ${ }^{5}$ the strip. With independent control of these two motions, complete cancellation of reflections can be obtained. On the other hand, with the strip surface perpendicular to the electric field, minimum losses occur in the strip and almost perfect reflection is obtained. It is possible to adjust the termina ion to any intermediate condition, then to slide the entire assembly, to obtain almost any reflection coefficient desirable.

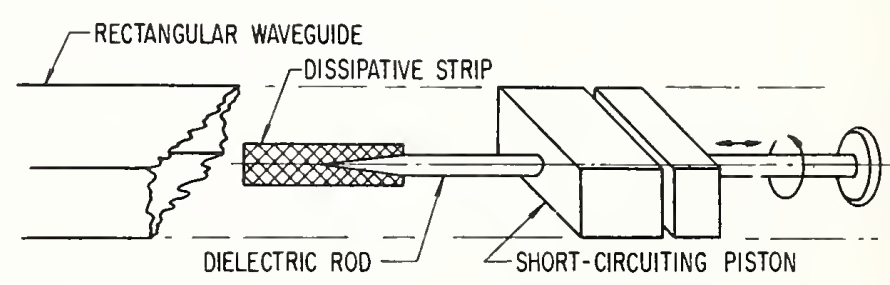

Fig. 1-Essential features of termination.

\section{THEORY}

Apparently, the analysis of the fields inside a rectangular waveguide containing an arbitrarily positioned dissipative strip has not yet been published. Lacking this basic information, a rigorous analysis of the action of this termination has not been attempted. An approximate study based upon microwave circuit theory is given below.

As shown in Fig. 2, the termination can be regarded as an attenuator terminated in a short-circuited line of variable length. In terms of the scattering coefficients ${ }^{6}$ $S_{11}, S_{12}$, and $S_{22}$ of the attenuator, the input voltage reflection coefficient is

$$
\Gamma_{1}=S_{11}-\frac{S_{12}{ }^{2}}{S_{22}+e^{j 2 \beta l}},
$$

where $\beta=2 \pi / \lambda_{G}$, and $\lambda_{G}$ equals the wavelength in the waveguide. The condition for cancellation of reflections $\left(\Gamma_{1}=0\right)$ is

$$
S_{12}^{2}=S_{11} e^{j 2 \beta l}\left(1+S_{22} e^{-j 2 \beta l}\right) .
$$

${ }^{5}$ G. C. Southworth, "Principles and Applications of Waveguide Transmission," D. Van Nostrand Co., Inc., New York, N.. Y,, pp. $374-376 ; 1950$. An attenuator employing a rotating strip is described.

${ }^{6}$ Scattering coefficients are the elements of the scattering matrix. See C. G. Montgomery, R. H. Dicke, and E. M. Purcell, "Principles of Microwave Circuits," M.I.T. Rad. Lab. Ser., McGraw-Hill Book Co., Inc., New York, N. Y., vol. 8, pp. 146-149; 1948. 


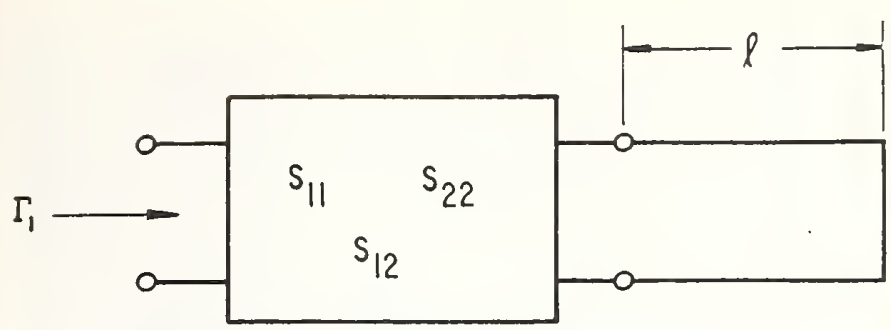

Fig. 2-Approximate equivalent circuit representation.

Normally, $\left|S_{11}\right|$ and $\left|S_{22}\right|$ are much less than unity, and the approximate condition for cancellation of reflections is

$$
S_{12}^{2} \approx S_{11} e^{i 2 \beta l} .
$$

Rotation of the strip will, in general, change both $\left|S_{12}{ }^{2}\right|$ and $\left|S_{11}\right|$. A typical variation of these quantities with $\theta$ (the angle between the electric field direction and the normal to the surface of the strip) is shown in Fig. 3(a). At the angle $\theta c$, the magnitudes of $S_{12}{ }^{2}$ and $S_{11}$ are equal, and it is possible to obtain cancellation of reflections by sliding the strip, varying $l$. If the strip is too short, $\left|S_{12}{ }^{2}\right|$ will not decrease enough to equal $\left|S_{11}\right|$, as shown in Fig. 3(b). However, it is possible to increase $\left|S_{11}\right|$ by adding a reflecting object at the end of the strip, so that cancellation of reflections can again be obtained as in Fig. 3(c).

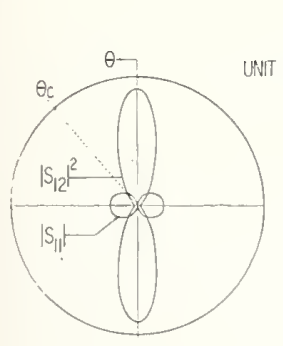

(a)

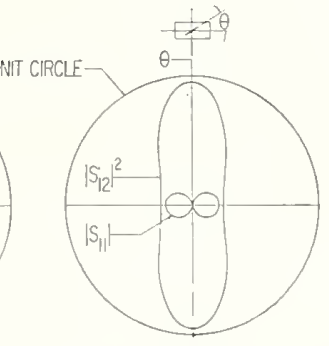

(b)

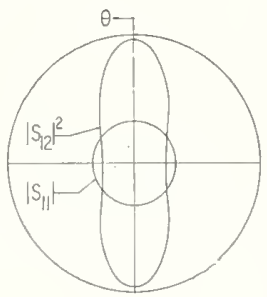

(c)
Fig. 3-Dependence of $\left|S_{11}\right|$ and $\left|S_{12}{ }^{2}\right|$ upon rotation of thin rectangular strip: (a) rectangular strip 0.35 inch $\times 1.5$ inches, (b) rectangular strip $0.35 \mathrm{inch} \times 0.75 \mathrm{inch}$, and (c) metal reflector added to increase $\left|S_{11}\right|$.

\section{DESIGN}

A number of considerations can influence the design of the termination. If the primary need is for a reflection-free termination, with no need for a wide range of vswr, it seems advisable to use a thicker strip of dissipative material such as Synthane. The extra thickness can give added strength to a long, tapered strip, which will require less rotation to achieve cancellation of reflection, and give a smoother adjustment which is less frequency sensitive than with shorter strips. Examples of such a design are shown in Fig. 4, and a diagram of typical variation of $\left|S_{12}{ }^{2}\right|$ and $\left|S_{11}\right|$ is shown in Fig. 5.

If a wide range of adjustment of vswr is desired, a thin strip is required. IRC resistance strip may be used

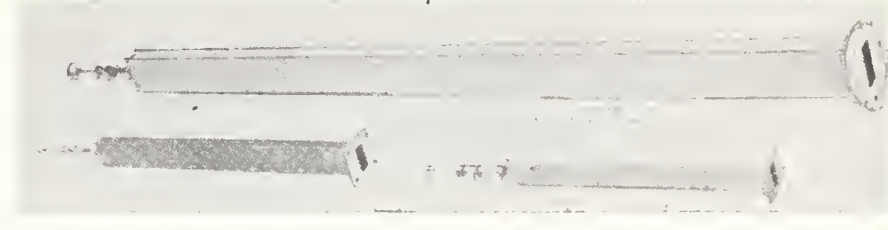

Fig. 4-Adjustable sliding loads for different waveguide sizes.

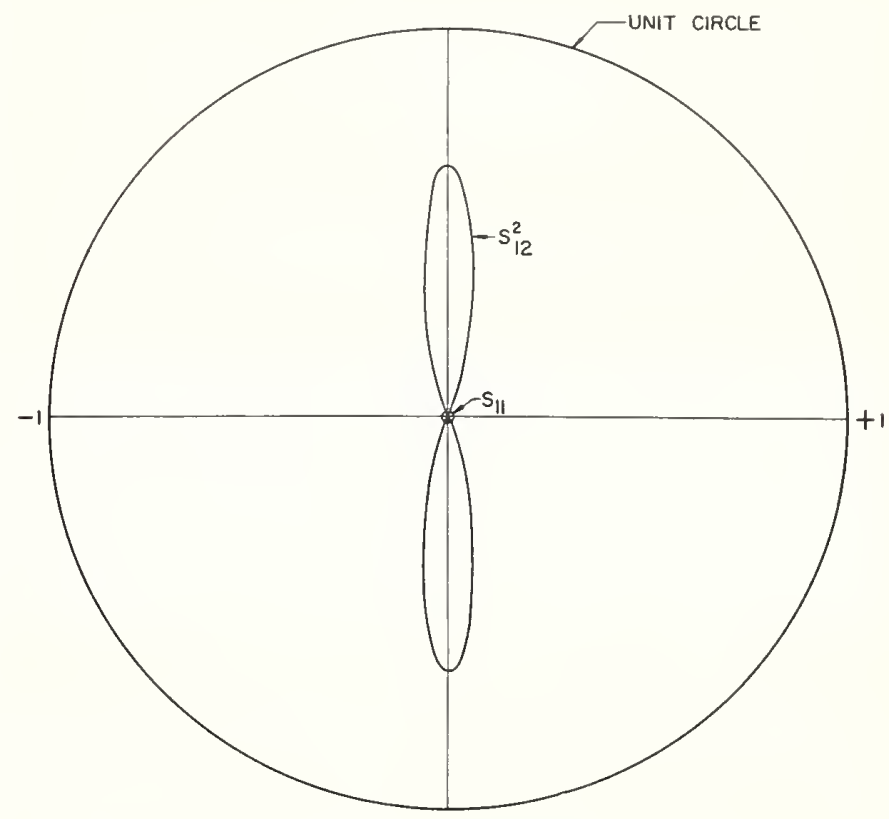

Fig. 5-Variation of $\left|S_{11}\right|$ and $\left|S_{12}{ }^{2}\right|$ with rotation of thick tapered strip.

to obtain fairly high ranges, but a dissipative film on a thin mica strip is capable of greater range. A thin film has little strength and it may be desirable to add a reflecting disk or rod as shown in Fig. 6. The disk or rod permits a shorter strip to be used, while retaining the ability to cancel reflections.

Control of the mechanical motion required may be achieved by the arrangements shown in Figs. 7 and 8, next page. Independent control of the sliding and rotation of the strip is achieved in both arrangements, but in Fig. 8, the strip may be rotated and slid by hand until the clamping screw is used, which clamps the dielectric rod supporting the strip so that it can only be moved by the limited fine adjustments $A$ and $B$. One adjustment of sliding (knob $A$ ) and an independent adjustment of rotation $(\mathrm{knob} B)$ are provided.

As a matter of practical interest, an adjustable load having satisfactory performance over the recommended frequency range of $\mathrm{RG}-52 / \mathrm{U}$ waveguide was constructed, using a rectangular IRC resistance strip 200 ohms per square, 0.35 inch $\times 1.50$ inches. The strip was not tapered, but mounted on a dielectric rod as was shown in Fig. 1. 


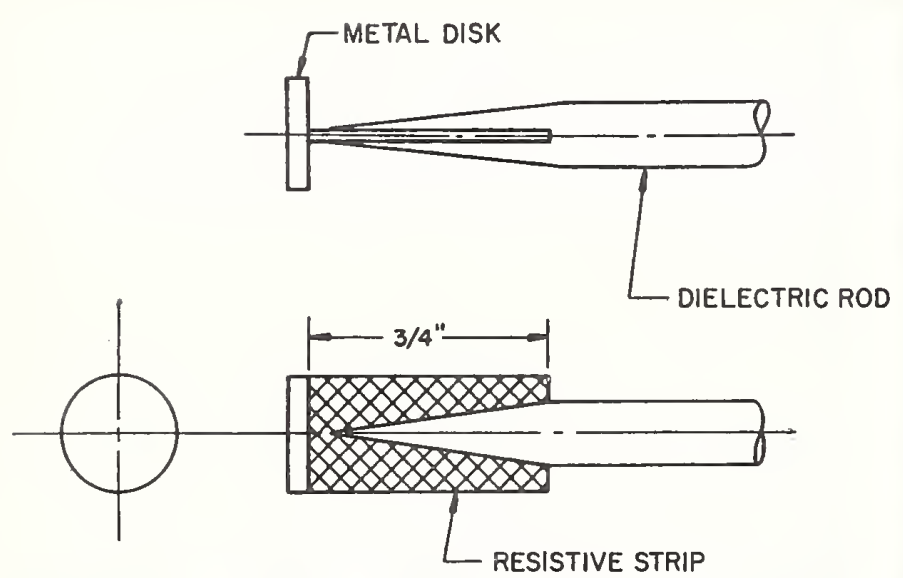

Fig. 6-Short strip and reflecting disk.

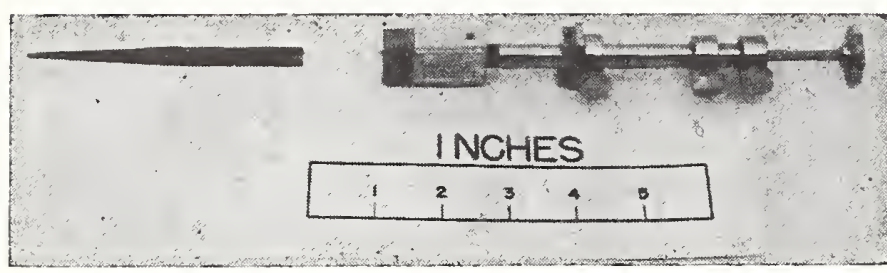

Fig. 7-Mechanical controls permitting independent adjustment of rotation and sliding, with control locking.

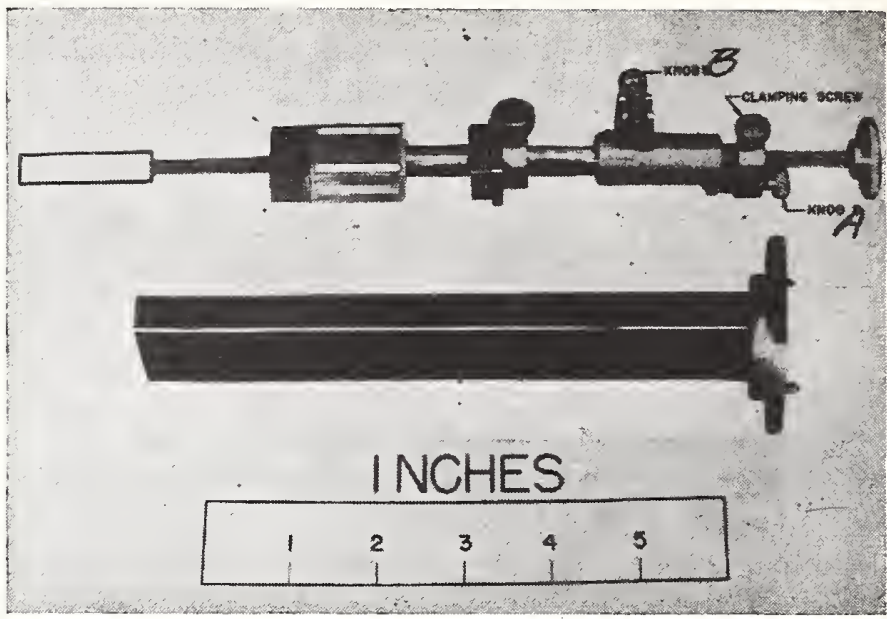

Fig. 8-Mechanical controls permitting coarse manual adjustment and locking, followed by fine independent adjustments of rotation and sliḍing.

\section{ACKNOWLEDGMENT}

The writer is indebted to J. Lowenbach, W. Foote, and $\mathrm{W}$. Clore of the Instrument Shop, who made experimental models and contributed to the mechanical design, to E. Niesen, who made the measurements upon which Figs. 3 and 5 are based, and to Dr. D. M. Kerns and Dr. D. G. Burkhard, who reviewed the paper.

Reprinted from IRE TRANSACTIONs

ON MICROWAVE THEORY AND TECHNIQUES

Volume MTT-5, Number 3, July, 1957

PRINTED IN THE U.S.A. 


\title{
A New Technique for the Measurement of Microwave Standing-Wave Ratios*
}

\author{
A. C. MACPHERSON† AND D. M. KERNS $\ddagger$
}

\begin{abstract}
Summary-A new microwave standing-wave-ratio (or reflection coefficient) measurement technique, apparently suitable for standards and other high precision work, is described. The technique requires that the phase angle of the unknown reflecticn coefficient be subjected to arbitrary, known variations-as is possible with a sliding load, for example. Accurate measurement of suitable sliding loads furnishes standards, and with the aid of comparison techniques, enables indirect measurement of arbitrary unknowns.

Generator and detector are connected to two arms of a threearm waveguide junction. Tuning elements in the junction are (desirably) adjusted for small reflection looking into the third arm, to which the unknown connects. Observation of the detector response vs - variation of phase of the unknown yields a curve, similar to a standing-wave pattern, from which the unknown is determinable by procedures that are given.

The technique has the advantages of 1) being amenable to rigorous theoretical analysis, 2) enabling the attainment of heavy coupling to the detector without simultaneous severe distortion of the response pattern, and 3 ) being, in a sense explained in the text, fundamentally simpler than the conventional slotted-line technique.
\end{abstract}

\section{INTRODUCTION}

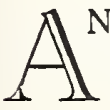

NEW MICROWAVE standing-wave-ratio (or reflection coefficient) measurement technique, which appears to be suitable for standards and

* Original manuscript received by the IRE October 6, 1955: revised manuscript received, April 23, 1956.

† Naval Research Lab., Washington, D. C., formerly with Natl. Bur. of Standards, Boulder, Colo.

$\ddagger$ National Bureau of Standards, Boulder, Colo. other high-precision work, is described. An earlier stage in the development of this technique has been reported previously by the authors. ${ }^{1}$

The technique requires that the phase angle of the unknown reflection coefficient be subjected to arbitrary, known variations. (A reflection coefficient whose phase can be subjected to such variations will be termed "phasable.") It is clear that this phasability requirement would be no limitation if an ideal phase-shift device were available.

Thus far the technique has been used primarily with specially constructed "sliding-loads" (terminating structures arranged to slide in the waveguide in which usedsee Fig. 5). One other phasing method has been tried and will be mentioned. However, the sliding-load technique in itself offers a solution to most problems of standingwave-ratio (swr) standardization. A suitable sliding load whose swr has been accurately measured constitutes a standard of swr. A series of such standards could be used, for example, to calibrate a conventional sţanding-wave machine. With the aid of sliding loads of adjustable swr and microwave-bridge comparison techniques, arbitrary unknowns can be measured indirectly.

'A. C. Macpherson and D. M Kerns, "A New SWR Measurement Technique," URSI-IRE Meeting, Washington. D. C.. April 27. 1953. 
The experimental work done thus far has been concerned only with the determination of the magnitude of reflection coefficients. There is no reason why angles cannot also be determined, however, and the necessary theory and equations for this will be given.

The basic arrangement (see Fig. 1) consists essentially of a detector and a generator connected to two of the arms of a three-arm waveguide junction. This arrangement (generator and detector included) constitutes an instrument for the measurement of any phasable reflection coefficient presented at the reference plane in the third arm of the junction. The operating procedure consists essentially of observation of detector response as a function of the variation of phase of the unknown reflection coefficient. This yields a curve, similar to a standing-wave pattern, from which the unknown reflection coefficient is determinable by procedures to be given below.

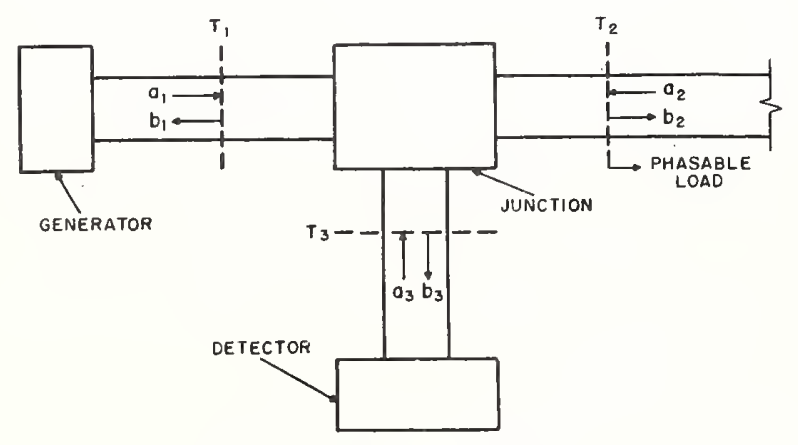

Fig. 1-Basic arrangement.

The junction is provided with tuning elements that are adjusted for zero reflection coefficient as seen looking into the load arm of the junction (with the detector and inactive generator connected). This matching operation is not critical; the technique does not assume that a zero or negligible reflection coefficient is actually obtained. The matching need be performed only once for a given setup operating within a limited frequency range. The matching operation reduces (or may practically eliminate) distortion of the response pattern and enables one to have heavy coupling to the detector without at the same time having a highly distorted response pattern.

It should be emphasized that the waveguide junction used in this technique is not subject to special requirements, such as symmetry or losslessness. The junction may indeed be of a rather general type, in that the waveguide leads may individually be of arbitrary cross section and one or more of them may be two-conductor systems, such as coaxial line. The main assumptions are that the electromagnetic field in a portion of each waveguide lead consists of a single waveguide mode and that the variation of the phase of the unknown is accomplished without affecting its magnitude. These two assumptions are only mildly unrealistic. The further requirement that the system (apart from the if source itself, which must be well padded) be linear and passive is hardly restrictive. Reciprocity is not used in the main part of the analysis. It may be well to mention explicitly that it is not required that the detector or generator ${ }^{2}$ present matched loads to the junction and that; by the same token, the joints in the waveguides connecting detector and generator to the junction are not required to be perfect.

The idea of using a fixed detector and a phasable load is not in itself new. ${ }^{3}$ It is felt that the present work represents an advance in three main respects: 1) In significantly increased generality and rigor. Apparently it has not been realized that a much more thorough treatment could be carried out with very little additional complication in the results. 2) In enabling the attainment of heavy coupling to the detector without simultaneous distortion of the response pattern. In the present work the heavy coupling permitted the use of a bolometric substitution method of power measurement as the detector, virtually eliminating the question of detector linearity. Probably the principal advantage of having small distortion is that it permits simplification of the general procedure for the determination of the unknown reflection coefficient from the observed data; it may also enable increased accuracy. 3) In the application of an analysis (developed in a different context by Altar, Marshall, and Hunter ${ }^{4}$ ) which derives the desired information from the response pattern whether or not the pattern is highly distorted. It is remarkable that the analysis applies more rigorously in the present problem than it does in its original context.

The characteristic equation of the instrument-the analytical expression of detector power as a function of phase of the unknown reflection coefficient-is obtained by a rigorous analysis under the above hypotheses. The characteristic equation is of a simple form and contains effectively just two complex constants. In general both constants must be evaluated or accounted for empirically; the necessary procedures are relatively simple.

The new technique should also be compared with the conventional slotted-line standing-wave measurement technique. In Appendix I the slotted-line instrument is analyzed with rigor comparable to that applied in the text to the new technique, and it is shown that the characteristic equation for the slotted-line instrument (neglecting slot-end effects) contains four complex constants and is of a fairly complicated form. It is considered that the new technique has a fundamental and important advantage in the relative simplicity of its characteristic equation.

\section{THEORY}

Our first theoretical task is to obtain and discuss the characteristic equation of the system.

2 Here and subsequently the "generator" is to be understood as the equivalent generator as seen at the junction reference plane.

${ }^{3}$ C. G. Montgomery, "Technique of Microwave Measurements," M.I.T. Rad. Lab. Ser., The McGraw-Hill Book Co., Inc., New York, N.Y., Vol. 11, p. 507; 1947.

${ }_{4}$ Wm. Altar, F. B. Marshall, and L. P. Hunter, "Probe error in standing wave detectors," Proc. IRE, vol. 34, [3. 33, January, 1946. 
Let reference planes $T_{1}, T_{2}$, and $T_{3}$ be chiosen in the generator, load, and detector waveguide leads, respectively, as indicated in Fig. 1. For the purpose of analy: sis, the definitions of the "junction" and the "terminations" are, of course, relative to the choice of reference planes. (It should be noted that any discontinuities introduced by flanged joints in the generator and detector leads will be taken care of implicitly by inclusion either in the junction or in the terminations.) It is convenient to characterize the junction (as defined by the choice of reference planes) by means of the inverse of its scattering matrix. Letting $a_{1}, a_{2}, a_{3}$ measure the amplitudes of the incident traveling-wave componients of the field on the respective terminal surfaces, and letting $b_{1}, b_{2}, b_{3}$ similarly measure the amplitudes of the emergent traveling-wave components, we then have

$$
\begin{aligned}
& a_{1}=G_{11} b_{1}+G_{12} b_{2}+G_{13} b_{3} \\
& a_{2}=G_{21} b_{1}+G_{22} b_{2}+G_{23} b_{3} \\
& a_{3}=G_{31} b_{1}+G_{32} b_{2}+G_{33} b_{3}
\end{aligned}
$$

where the $G_{i j}$ are the elements of the inverse scattering matrix $G$ ( $G$ will be called the "gathering matrix" of the junction). The relations imposed at the terminal surfaces by the terminations we express in the form

$$
\begin{aligned}
& a_{1}=S_{g} b_{1}+b_{g} \\
& a_{2}=S e^{-2 j \theta} b_{2} \\
& a_{3}=S_{d} b_{3}
\end{aligned}
$$

Here the reflection coefficient $S_{\theta}$ and the fixed waveamplitude $b_{g}$ characterize the equivalent generator at the reference plane $T_{1}, S$ is the unknowr reflection coefficient, $-2 \theta$ is the phase shift imposed upon $S$ (so that $S e^{-2 z^{\theta}}$ is the reflection coefficient presented at reference plane $T_{2}$ ), and $S_{d}$ is the reflection coefficient of the detector at $T_{3}$. (In the case of a sliding-load unknown, $\theta$ may be interpreted as the variable electrical distance between $T_{2}$ and the reference plane of the unknown.) Of these quantities only $S$ and $\theta$ will be explicitly involved in the final results.

Combining (1) and (2) one obtains a set of three equations in three unknowns,

$$
\begin{aligned}
b_{o} & =\left(G_{11}-S_{\theta}\right) b_{1}+G_{12} b_{2}+G_{13} b_{3}, \\
0 & =G_{21} b_{1}+\left(G_{22}-S e^{-2 j \theta}\right) b_{2}+G_{23} b_{3}, \\
0 & =G_{31} b_{1}+G_{32} b_{2}+\left(G_{33}-S_{d}\right) b_{3}
\end{aligned}
$$

which determines $b_{1}, b_{2}$, and $b_{3}$. The desired quantity, the power $P$ delivered to the detector, is proportional to the square of the magnitude of $b_{3}$. We introduce the determinant

$$
\Delta=\left|\begin{array}{ccc}
G_{11}-S_{o} & G_{12} & G_{13} \\
G_{21} & G_{22} & G_{23} \\
G_{31} & G_{32} & G_{33}-S_{d}
\end{array}\right|
$$

(note that this is not exactly the determinant of the above system of equations) and the two minors

$$
\begin{aligned}
& M_{13}=G_{21} G_{32}-G_{31} G_{22} \\
& M_{22}=\left(G_{11}-S_{\vartheta}\right)\left(G_{33}-S_{d}\right)-G_{31} G_{13} .
\end{aligned}
$$

The power $P$ can be expressed in the form

$$
P=C|1+y+Y(K S, \theta)|-2
$$

where $C$ is a constant of proportionality,

$$
\left.\begin{array}{rl}
K & =G_{31} / M_{13} \\
y & =\frac{-2\left(M_{22} / \Delta\right)}{K+\left(M_{22} / \Delta\right)} \\
Y(K S, \theta) & =\frac{1-K S \exp (-2 j \theta)}{1+K S \exp (-2 j \theta)}
\end{array}\right\}
$$

the last being a functional notation. The significance of expressing $K$ and $y$ in terms of the quantities (4), (5), and (6) will become apparent. The "constants" $C, y$, and $K$ are of course frequency-dependent. Eq. (.7) is the desired characteristic equation.

The value of the constant $C$ is immaterial as far as the analysis of the response curves is concerned, inasmuch as only relative values of $P$ will be involved. Thus the equation contains effectively just two parameters, $y$ and $K$, that characterize the instrument. Of course $y$ and $K$ are in general complex so that four real parameters are involved.

We observe that the characteristic constant $K$ and the unknown reflection coefficient $S$ appear in the characteristic equation only in the combination $K S$. In what follows it will be convenient to deal with the product $K S$ as an intermediate unknown and to postpone the question of determining $S$ itself. The notation $S^{\prime}=K S$ will be used.

As a function of $S^{\prime}$ and $\theta$, the characteristic equation is of the same form as the highly simplified equation for the slotted-line standing-wave machine given in Appendix II. (This is the basis of our application of the Altar, Marshall, and Hunter analysis; see below and Appendix II.) Thus it is recognized that in general the graph of the characteristic equation will have the form of a distorted standing-wave pattern, the distortion being introduced by the presence of the quantity $y$ and vanishing for $y=0$. An experimentally obtained graph in which the distortion is rather small is shown in Fig. 2. It is interesting to note that the quantity $y$ can be regarded as a thoroughgoing generalization of the "probe admittance" $y_{p}$ appearing in $(16) ; y$ depends on $S_{\theta}$, $S_{d}$, and on all the elements of the matrix $G$ and will in general be nonzero whether or not the system actually contains a probe.

If an ordinary waveguide junction (for which reciprocity holds and in which dissipation is small) is employed, qualitative statements can be made about the magnitudes of $K$ and $y$ :

1) The magnitude of $K$ will be approximately equal to unity. This follows from the fact that under the assumed conditions the gathering matrix will be symmetric 


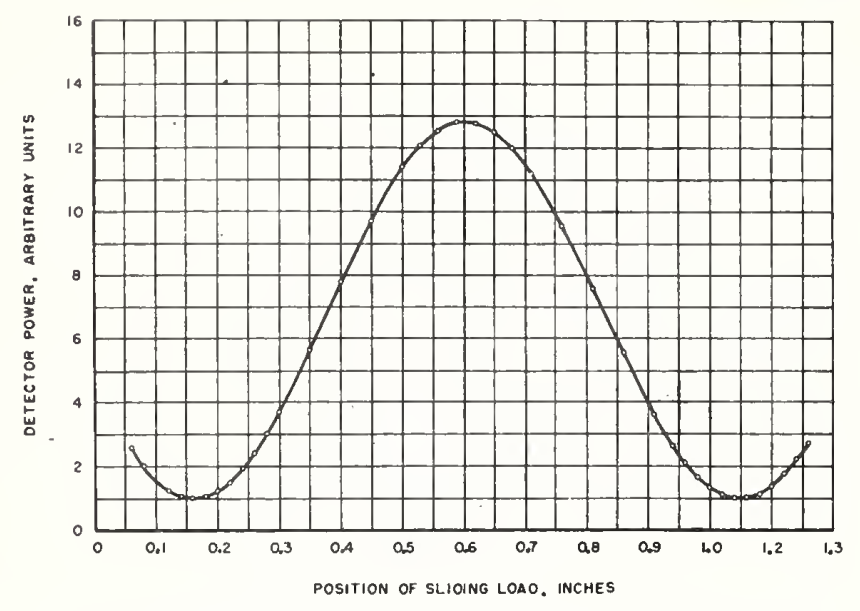

Fig. 2-Detector power vs load position-experimental.

and nearly unitary, ${ }^{5} K$ becomes equal to the ratio of the element $G_{31}$ to its cofactor in $G$, and (arguing by continuity from a well-known property of unitary matrices) the magnitude of this ratio will be approximately equal to unity. ${ }^{6}$

2) The matching operation described-previously will make $y$ small (relative to unity). This may be established as follows. Let $S_{2 i}$ denote the reflection coefficient that is observed and minimized in the matching operation; it may be shown without difficulty that

$$
S_{2 i}=M_{22} / \Delta
$$

where $\Delta$ and $M_{22}$ are defined in (4) and (6). Hence the expression for $y$ may be written

$$
y=-2 S_{2 i} /\left(K+S_{2 i}\right) .
$$

Now, although $K$ as well as $S_{2 i}$ will be affected by the matching operation, the magnitude of $K$ will remain approximately equal to unity (as shown in the preceding paragraph). Thus it is seen that making $S_{2 i}$ small will make $y$ small.

A small or vanishing value for the distuituon term $y$ is not incompatible with heavy coupling to the detector. To establish this it is sufficient to consider an ideal case. Assume that a symmetrical lossless $H$-plane $T$ junction is used in the arrangement of Fig. 4, and assume further that $S_{\theta}=S_{d}=S_{2 i}=S=0$. Then it can be shown with little difficulty that of the power from the generator incident on the junction 25 per cent is reflected, 25 per cent goes to the load, and 50 per cent to the detector.

We turn now to the second main theoretical task, which is to provide techniques for handling the following

${ }^{5}$ The argument assumes that the characteristic impedances in the three waveguide leads are chosen equal, as is always possible; the result established is independent of the choice of characteristic impedances.

${ }^{6}$ It is worth noting that a $K$ wholly similar to the above $K$ appears in the rigorous characteristic equation for the slotted-line standing-wave machine (appendix I). In slotted line work it has generally been assumed (in effect) that $K=1$. In the present technique the use of an ordinary junction would permit the assumption of the magnitude of $K$ equal to unity with about the same justifiability as in the slotted line case. This assumption is not made in the present work, which is concerned with the development of a measuring technique under minimum assumptions. problem: given an empirically determined graph of $P$ vs $\theta$ for a given $S^{\prime}$, together with the form of the characteristic equation, to determine $S^{\prime}$ and $y$. It happens that techniques developed by Altar, Marshall, and Hunter ${ }^{7}$ $(\mathrm{AMH})$ in connection with an approximate treatment of probe error in standing-wave machines are rigorously applicable in the present problem. ${ }^{8}$ Both graphical and analytical techniques were provided; here we shall give a selection of the analytical results. These results have been checked by employing an independent method of derivation and are presented with minor emendations and modifications in form.

The magnitude of $S^{\prime}$ may be determined as follows. Let $P_{\mathrm{max}}, P_{\mathrm{min}}$, and $P_{\mathrm{mid}}$, respectively, denote detector power readings at maxima, minima, and "midpoints" of the $P$ vs $\theta$ curve; $P_{\mathrm{mid}}$ being defined by

$$
P_{\mathrm{mid}}=\frac{2 P_{\max } P_{\min }}{P_{\max }+P_{\min }} .
$$

Let $\theta_{\max }, \theta_{\min }$, and $\theta_{\text {mid }}$ denote angular positions corresponding to $P_{\max }, P_{\min }$, and $P_{\text {mid }}$, respectively. In order to avoid ambiguities in sign and in multiples of $\pi$, the angles may be chosen in the manner shown in Fig. 3 .

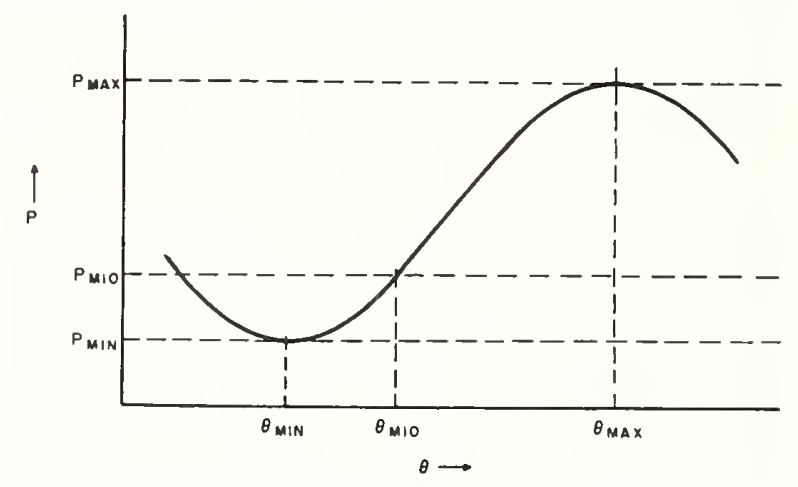

Fig. 3-Illustrating choice of $\theta_{\min }, \theta_{\max }$, and $\theta_{\text {mid. }}$.

Further, let

$$
\begin{aligned}
\eta & =\theta_{\max }-\theta_{\min } \\
\epsilon & =\theta_{\max }+\theta_{\min }-2 \theta_{\mathrm{mid}}
\end{aligned}
$$

and define the auxiliary quantity $\bar{\eta}$ by

$$
\sin \bar{\eta}=\frac{\sin \eta}{\sqrt{1+\left(\frac{\sin \epsilon \sin \eta}{\cos \epsilon-\cos \eta}\right)^{2}}}
$$

with the proviso that $\bar{\eta}$ is to be taken in the first quadrant. Then the magnitude of the intermediate reflection coefficient is

$$
\left|S^{\prime}\right|=\frac{1-\tan (\bar{\eta} / 2)}{1+\tan (\bar{\eta} / 2)}
$$

[The voltage standing-wave-ratio (vswr) corresponding to $S^{\prime}$ is $\rho^{\prime}=\cot (\bar{\eta} / 2)$.]

${ }^{7}$ Montgomery, loc, cit.

${ }^{8}$ The relation between their problem and our problem is explained in Appendix II. 
The angle $\sigma^{\prime}$ of $S^{\prime}$ may be determined as follows. The shift of the minima corresponding to the presence of the term $y$ in the characteristic equation may be defined as

$$
\delta=\theta_{\min }-\theta_{\min }^{(t)}
$$

where $\theta_{\text {min }}{ }^{(t)}$, the "true" minimum position, is the value that $\theta_{\min }$ would have if $\operatorname{Im}(y)$ were zero. It may easily be determined that

$$
\sigma^{\prime}=2\left(\theta_{\min }-\delta\right)+\pi
$$

apart from multiples of $2 \pi$. The value of $\delta$ is given in terms of $\eta$ and $\epsilon$ by?

$$
\delta=\frac{1}{2} \sin ^{-1}\left(\frac{\sin \eta}{\sqrt{1+\cos ^{2} \eta\left(\frac{\cos \epsilon-\cos \eta}{\sin \epsilon \sin \eta}\right)^{2}}}\right)-\frac{1}{2} \eta .
$$

Here the inverse sine is to be taken in the 1 st or 2 nd quadrant according to whether $\eta$ is in the 1 st or 2 nd quadrant.

It will be observed that the above formulas do not involve the characteristic constant $y$ explicitly and are applicable whether or not $y$ is small. However, when $y$ is small, certain approximate formulas involving the real and imaginary parts of $y$ explicitly may be preferable. The value of $y$ is obtainable from an expression given by $\mathrm{AMH}$ (p. 38P):

$$
y=\frac{2}{\left(1-\left|S^{\prime}\right|{ }^{2}\right)}\left(\left|\frac{S^{\prime}}{S_{0}}\right| e^{j \psi}-1\right) .
$$

Here $S^{\prime}$ is as already defined, $\left|S_{0}\right|$ is defined in terms of the directly observed vswr $\rho_{0}=\sqrt{P_{\max } / P_{\min }}$ by $\left|S_{0}\right|$ $=\left(\rho_{0}-1\right) /\left(\rho_{0}+1\right)$, and $\psi$ (which is to be taken between $-\pi / 2$ and $+\pi / 2)$ is given by $\tan \psi=\csc \epsilon \cot \eta \cdot(\cos$ $\epsilon-\cos \eta)$. Let us denote the real and the imaginary parts of $y$ by $g$ and $b$, respectively. It can be shown that, to the first order in $g$ and $b$, the intermediate vswr is given by ${ }^{10}$

$$
\rho^{\prime}=\rho_{0}+\rho_{0} g \frac{\rho_{0}-1}{\rho_{0}+1}
$$

and the shift of the minima by

$$
\delta=\frac{-b}{\left(\rho_{0}+1\right)^{2}} .
$$

Both of these expressions furnish small corrections to be applied to the directly observed quantities.

Thus far we have provided formulas for the determination of $S^{\prime}$; in order to be able to obtain the actual unknown reflection coefficient, $S$, the value of the characteristic constant $K$ must be known. Inasmuch as $K$

${ }^{\theta}$ In $\mathrm{AMH}$ the quantity measuring the shift of the minima is $\mu$, and $\mu=-2 \delta$. The factor of 2 is accounted for by the different angular measure used by AMH and the negative sign is presumably due to an opposite definition of the shift. (The approximate formula for $u$ given by $\mathrm{AMH}$ is in error.)

10 The expressions given here can be derived along the lines indicated by Montgomery, op. cit., pp. 483-488. (The result given for the shift of the minima in this reference should be multiplied by $2 \pi$ ). and $S$ appear in the characteristic equation only in the combination $S^{\prime}=K S$, the empirical evaluation of $K$ requires that $S^{\prime}$ be determined for at least one phasable load whose reflection coefficient $\left(S=S_{8}\right.$, say) is known independently ( $K$ being then given simply by $K=S^{\prime} / S_{s}$ ). Probably the type of load for which the reflection coefficient can best be determined independently is a "short." A sliding short was used by the authors and the means of evaluating it are described briefly in the following section.

As soon as $K$ is known, the value of any $S$ is of course at once obtainable from the corresponding value of $S^{\prime}$

\section{EXPERIMENTAL}

For purposes of illustration, some of the equipment and practices employed in the experimental part of the present work will be described. The arrangement used was designed with the idea of obtaining the highest possible accuracy. Much attention was given to problems of generator stability, detector linearity, detector noise and drift, and mechanical precision. The complete set-up is shown in Fig. 4. The rf source is an X-21 klystron in $\mathrm{cw}$ operation. This tube yields about 5 watts output, which easily permits the use of adequate padding, and in addition is very stable in power level and frequency. For ordinary work the X-21 has the disadvantage of being essentially nontunable. (An ar-

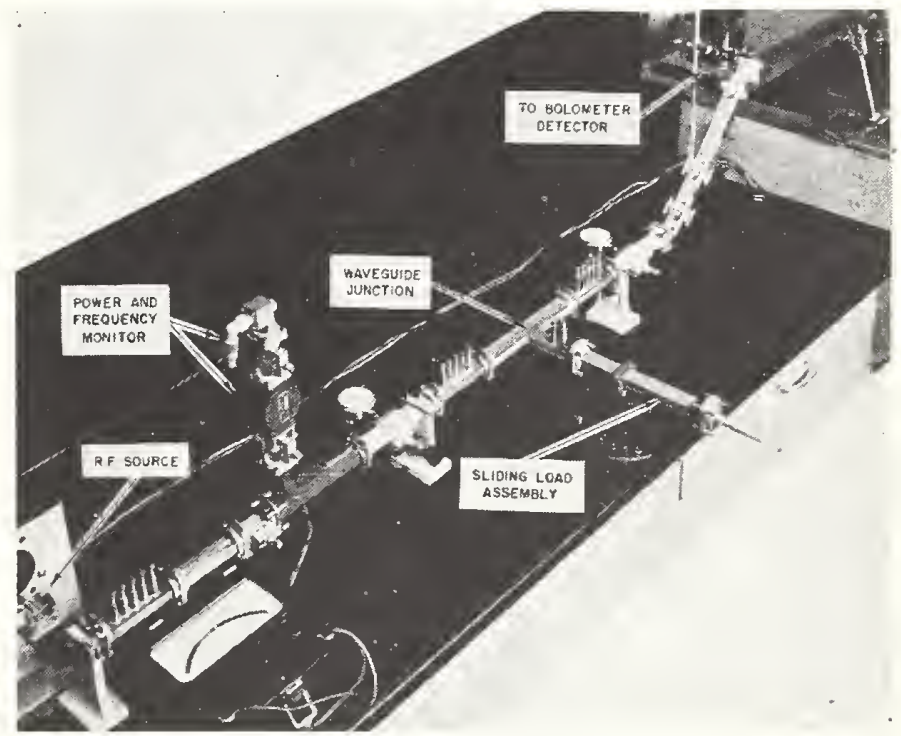

Fig. 4-Laboratory set-up.

rangement that would probably be sufficiently stable and yet adjustable in frequency would be to use a tunable reflex klystron operating in an oil bath, with a unidirectional device for padding.) Means are provided for adjusting the power level and monitoring the frequency. The waveguide junction used is an $H$-plane tee provided with built-in tuning elements. The detector is a commercial platinum-wire barretter in a commercial mount operated in an oil bath. The barretter is connected into a manually-balanced dc wheatstone bridge. 
Since the bridge is balanced each time a reading is made, the $\mathrm{rf}$ impedance of the detector is virtually independent of the rf power level. Power generation and detection are relatively simple since no modulator is needed and the detector requires only a simple dc network and uses no vacuum tubes.

Three more or less typical sliding loads are shown in Fig. 5. The top one in the figure is hand driven and consists of a steel slug (which is a close sliding fit in the waveguide) to which is attached a tapered resistance strip. The load shown in the middle of the figure is similar except that the lossy material is a slug of Catalin and it is driven by a micrometer screw. The load at the bottom, designed by Ivan K. Munson, uses Polyiron as the dissipative material and employs a short transverse wire mounted on the end of an axial dielectric rod that can be rotated. This permits adjustment of the swr. The waveguides in which the loads slide must be of highly uniform cross section; they were made by electro-forming copper on precision mandrels. It should be noted that whatever discontinuity there may be at the flange connection between the junction and the guide in which the load slides is automatically associated with the junction and not with the sliding load.

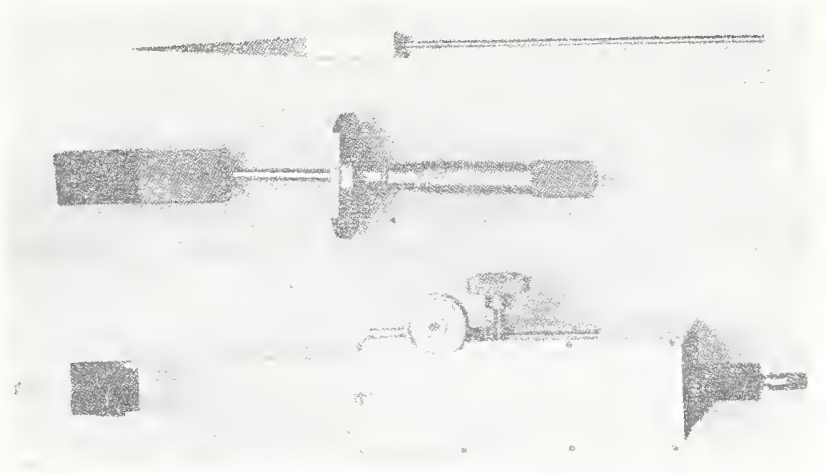

Fig. 5-A selection of sliding loads.

A sleeve-type "line-stretcher" for phase shifting purposes is shown in Figs. 6 and 7. This device permits the direct measurement of portable loads (that are not too bulky or heavy). It is the outcome of a suggestion by H. Lyons and was developed by A. J. Couvillion. An important point to be brought out in connection with this phase shifter is that discontinuities such as those at $A$ and $B$ are quite innocuous (if they are constant). These discontinuities are spatially fixed with respect to the $H$-plane junction when the sleeve is moved and therefore are automatically taken care of in the measurement procedure (cf previous section). The effective unknown is that seen between $B$ and the joint $F$ and thus the effects of the joint are associated with the unknown in the present instance. Some tests were made using sliding-load assemblies as loads on the "linestretcher." Preliminary measurements using the "linestretcher" are encouraging but much more thorough

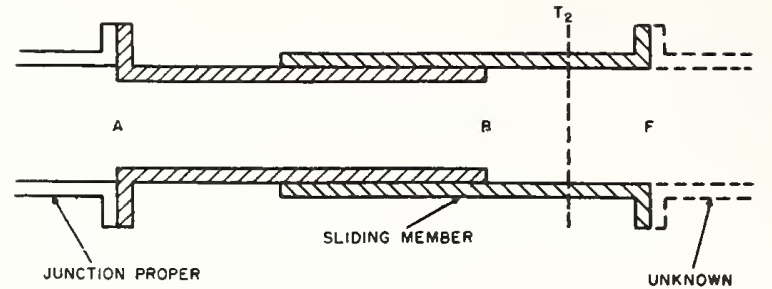

Fig. 6-Sleeve-type "line-stretcher"-schematic; sliding member is waveguide of standard internal dimensions. Slides over fixed member of reduced size attached to junction proper. $T_{2}$ is the junction reference plane; it is fixed in position relative to the junction.

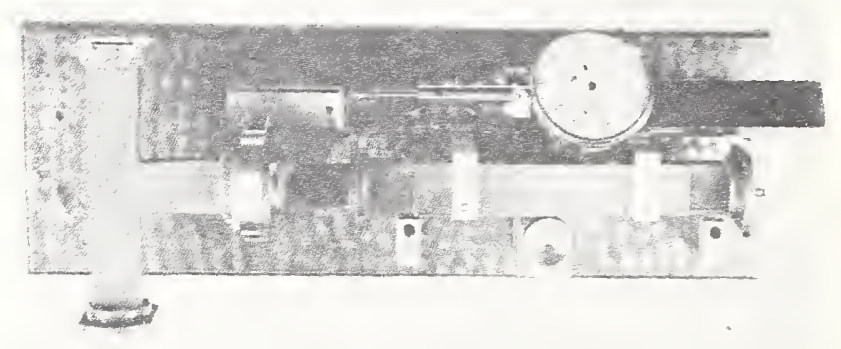

Fig. 7-Sleeve-type "line-strecher"-mechanical.

evaluation is necessary. If mechanically feasible, an 'rf choke at $B$ would seem desirable in order to reduce the effect of variable sliding contacts.

As mentioned in the previous section a load whose reflection coefficient $S_{s}$ is known is needed for the evaluation of $K$. In the experimental work only the magnitude of $S_{s}$ was considered. A choked sliding short was chosen and two independent methods of evaluating $\left|S_{s}\right|$ were employed. 1) A cavity was formed from a straight section of waveguide terminated at both ends with shorts of the type being evaluated. The $Q$ of this cavity was measured and $\left|S_{s}\right|$ was calculated assuming that the two shorts were identical and that the waveguide was lossless. This is a sensitive method giving a lower limit for $\left|S_{s}\right|$. (Inasmuch as $\left|S_{s}\right|$ cannot exceed 1, the problem can be regarded largely as that of determining lower bounds for $\left|S_{8}\right|$.) 2) Using well-known microwave bridge techniques, the sliding short was compared with a fixed, soldered, silver plate short whose reflection coefficient was calculated from the geometry and the dc conductivity involved. The two methods gave good agreement, and it is considered that $\left|S_{s}\right|$ for the load measured was almost certainly within the range $(0.990,1.000)$.

In the arrangement described above there are two main sources of random error. Under good conditions, the effects of electrical noise and drift due to both the generator and the detector amount to about one microwatt during the time necessary to make one powerratio measurement. Since the detector power level for a nearly matched load is about 10 milliwatts, the power resolution is about one part in ten-thousand, and the corresponding minimum detectable vswr is about 1.00005. This figure is not realized because of the addi- 
tional "noise" associated with the mechanics of the phase shifting process. Some measurements made on a sliding load of nominal vswr 1.01 give an idea of the mechanical stability possible. Six vswr values were increases and that on the other decreases in such a way that $\theta_{1}+\theta_{2}$ remains constant.

Solving for $b_{3}$ in the problem just set up yields a result expressible in the form

$$
b_{3}=\frac{c \exp \left(-j \theta_{1}\right)}{1+y+m S_{o} \exp \left(-2 j \theta_{1}\right)+\left[1+n S_{0} \exp \left(-2 j \theta_{1}\right)\right] Y\left(K S, \theta_{2}\right)}
$$

computed from readings of maxima and minima with the load at 7 different positions in the waveguide; the mean was 1.0166 and the greatest deviation from the mean was 0.0003 . With loads of higher vswr, and with the "line-stretcher" phase shifter, the reproducibility in terms of vswr was poorer in general. Nevertheless, the mechanical problem for a sliding load seems considerably easier than for a slotted line.

\section{Appendix I}

\section{Characteristic Equation for Standing-Wave MACHINE}

We give a brief but rigorous derivation of the characteristic equation for a conventional standing-wave machine. The general approach and much of the notation used in the second section will be applied here.

Let reference planes $T_{1}, T_{2}$ be chosen on the generator side and on the load side of the probe assembly, respectively (see Fig. 8). These two reference planes,

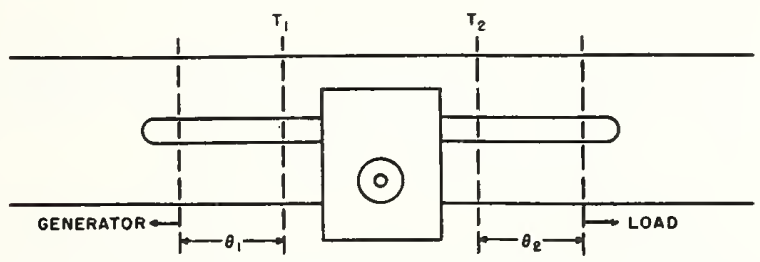

Fig. 8-Notation for standing-wave machine; coaxial line for probe output is indicated in the sketch.

which are to move with the probe assembly, plus a third one $T_{3}$ in the rf probe output, define a 3 -arm waveguide junction. We denote the gathering matrix of this junction by $G$ and use the notation $a_{1}, a_{2}, a_{3}$, and $b_{1}, b_{2}$, $b_{3}$ for the incident and emergent wave amplitudes, as in the second section. The relations imposed by the terminations are

$$
\begin{aligned}
& a_{1}=S_{g} e^{-2 j \theta_{1}} b_{1}+e^{-j \theta_{1}} b_{\theta} \\
& a_{2}=S e^{-2 j \theta_{2}} b_{2} \\
& a_{3}=S_{d} b_{3}
\end{aligned}
$$

[cf. (2)], where $\theta_{1}$ is the electrical distance between $T_{1}$ and the generator reference plane and $\theta_{2}$ is the same between $T_{2}$ and the load reference plane. The "generator" and the "load" dealt with here are, of course, as seen within the slotted portion of the slotted line. When the probe assembly is moved, the line length on one side where $c, y, m, n$, and $K$ are constants independent of $\theta_{1}$ and $\theta_{2}$, and $Y\left(K S, \theta_{2}\right)$ is the function defined in (8). The characteristic equation, which is an expression for the rf power $P$ delivered to a detector on arm 3, may be written

$$
\begin{aligned}
P=C \mid 1+y & +m^{\prime} \exp \left(-2 j \theta_{1}\right) \\
& +\left.\left[1+n^{\prime} \exp \left(-2 j \theta_{1}\right)\right] Y\left(K S, \theta_{2}\right)\right|^{-2},
\end{aligned}
$$

where we have put $m^{\prime}=m S_{g}$ and $n^{\prime}=n S_{g}$. The four characteristic constants are $y, m^{\prime}, n^{\prime}$, and $K$. This $K$ is the same function of the elements of $G$ as the $K$ of the text and thus shares the property $|K|=1$ for a lossless junction fulfilling the reciprocity condition. It is worth noting that if the generator is matched $\left(S_{g}=0\right)$, then $m^{\prime}$ and $n^{\prime}$ vanish and (14) becomes formally identical to (7), as it should.

\section{ApPENDix II}

\section{Problem Treated by AMH}

In the treatment of the conventional standing-wave machine given by AMH the two main assumptions involved are 1) that the generator is matched (this was tacit), and 2) that the effects of the probe assembly may be represented by a pure shunt element in a lossless transmission line. These assumptions lead at once to an expression for the voltage $V$ across the equivalent transmission line at the probe position,

$$
V=\frac{c}{1+y_{p}+Y(S, \theta)}
$$

where $c$ is a constant, $y_{p}$ is the admittance associated with the probe, and $Y(S, \theta)$ is the normalized loadadmittance referred to the probe position. If it is assumed that the power delivered to a detector is proportionl to $|V|^{2}$, (15) leads to the characteristic equation

$$
P=C\left|1+y_{p}+Y(S, \theta)\right|^{-2} \text {. }
$$

Although AMH do not actually write down equations of the form of (15) and (16), their analysis does in fact pertain to such equations. Upon comparing (16) with the characteristic equation of the text (7), the applicability of the analysis given by AMH becomes obvious.

\section{ACKNOWLEDGMENT}

The authors are grateful to Robert Sullivan, for help in many measurements and calculations, and to Harold Lyons, for his interest and encouragement in this work. 


\title{
Magnified and Squared VSWR Responses for Microwave Reflection Coefficient Measurements*
}

\author{
R. W. BEATTY $\dagger$
}

\begin{abstract}
Summary-In conventional microwave impedance' measuring instruments, the measured ratio of maximum to minimum detector signal level is ideally equal to the voltage standing-wave ratio (VSWR) of the termination. In this paper, it is shown how radically different types of response are obtainable in which the observed ratio may approximately equal the square of the VSWR or may be magnified any desired amount. Theory is given enabling accurate measurements by interesting techniques. Accuracies of 0.1 per cent in VSWR to 2.0 have been achieved using magnified response techniques.
\end{abstract}

\section{INTRODUCTION}

【 $\mathrm{N}$ most microwave impedance measuring instruments, such as the idealized slotted line, the resonance line, and rotary standing-wave indicators, the ratio of the maximum to the minimum amplitude of the output to the detector is ideally equal to the voltage standing-wave ratio (VSWR) of the termination subjected to measurement.

* Manuscript received by the PGMTT, February 20, 1959.

$\dagger$ Radio Standards Lab., Nat. Bur. of Standards, Boulder, Colo
Other radically different types of response are obtainable. The two responses to be discussed in this paper have been called magnified and squared VSWR responses for reasons which will become apparent.

A simplified explanation will first be given, followed by a more complete mathematical description.

The differences among responses are shown in Fig. 1, three response curves calculated for the same termination.

\section{Simplified Explanations}

Squared VSWR Response

A simplified explanation can be given for one system yielding squared VSWR response. Other systems which have been devised apparently do not permit simplified explanations and will not be thoroughly analyzed. Enough theory will be given however, to permit their use as measurement systems.

The system shown in the diagram in Fig. 2 consists 
of a straight section of uniform lossless waveguide (which may be either coaxial line or rectangular waveguide, for example) with oppositely located coupling probes for generator and detector. A short circuit which may be adjusted in position terminates one end of the uniform waveguide section while the other end is terminated in the sliding load to be measured. In this system it is necessary to vary the phase of the load by sliding it inside the waveguide, but in other systems to be described, this is not always required.

Referring to the simplified model of Fig. 2(b),

$$
E=e \frac{Z_{p}}{Z_{G}+Z_{p}} \approx i_{G} Z_{p}=i_{G} \frac{1}{\frac{1}{Z_{s}^{\prime}}+\frac{1}{Z_{L}^{\prime}}}
$$

If the short circuit is located $\lambda_{G} / 4$ from the probes, $Z^{\prime}{ }_{8}=\infty$, and

$$
E \approx i_{G} Z_{L}^{\prime}=i_{G} \frac{1+\Gamma_{L} e^{-j 2 \beta l}}{1-\Gamma_{L} e^{-j 2 \beta l}} .
$$

As $l$ varies, $|E|$ goes through maxima and minima. The ratio $\sigma_{A}$ of the maxima to minima is

$$
\sigma_{A}=\left(\frac{1+\left|\Gamma_{L}\right|}{1-\left|\Gamma_{L}\right|}\right)^{2}=\sigma_{L}{ }^{2} .
$$

Where $\sigma_{L}$ is the VSWR of the load. The meaning of other symbols used above should become clear upon reference to Fig. 2(b).

\section{Magnified Response}

A system yielding magnified response is shown in Fig. 3. A directional coupler is connected to respond mainly to the wave reflected from a-phasable or sliding termination whose VSWR is to be measured. For simplicity, it is assus ned that the generator and detector do not produce reflections $\left(\Gamma_{G}=\Gamma_{D}=0\right)$, and that no reflections are produced in arm 2 by the directional coupler $\left(S_{22}=0\right)$.

The signal coupled to the detector has two components. One is fixed and exists because the directivity is not infinite. The other is from the load reflection and varies in phase as the load is slid inside the waveguide. As the relative phase of the two components vary, the magnitude of the resultant varies. If the components are of approximately equal magnitudes, the range of variation of the resultant may be large even though the reflection from the termination may be small.

Inspection of the diagram of Fig. 3 leads to the following equations describing the response. ${ }^{1}$

$b_{3} \approx b_{G}\left(S_{31}+S_{21} S_{32} \Gamma_{L} e^{-j 2 \beta l}\right)=b_{G} S_{31}\left(1+K \Gamma_{L} e^{-j 2 \beta l}\right)$.

The response is of the same form as that of the idealized slotted line (see Fig. 1) excepting that $\Gamma_{L}$ is multiplied by the factor $K$. Since $|K|$ may be very large (it

${ }^{1}$ In this equation, $b$ represents a wave amplitude, $S$ a scattering coefficient of the directional coupler, and $\Gamma_{L}$ the voltage reflection coefficient of the load.

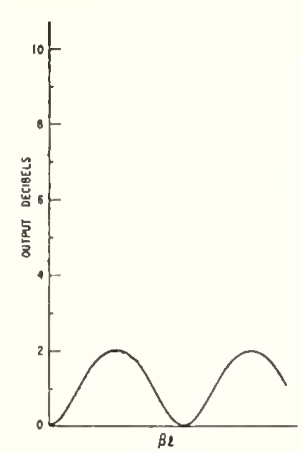

(a)

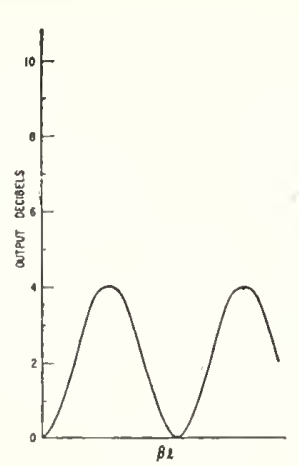

(b)

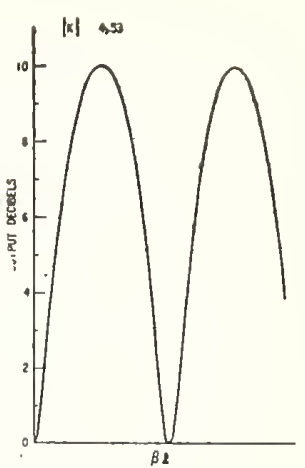

(c)
Fig. 1-Response curves of three measurement systems with termination having a VSWR of 1.26;

(a) idealized slotted line,

$$
d=c\left[1+K \Gamma_{1} e^{-j 2 \beta l}\right]
$$

(b) squared VSWR response,

(c) magnified response,

$$
d=c^{\prime}\left[\frac{1+\Gamma_{L} e^{-j 2 \beta l}}{1-\Gamma_{L} e^{-j 2 \beta l}}\right],
$$

$$
d=c^{\prime \prime}\left[1+K \Gamma_{L} e^{-j 2 \beta l}\right] .
$$

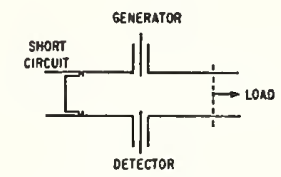

(a)

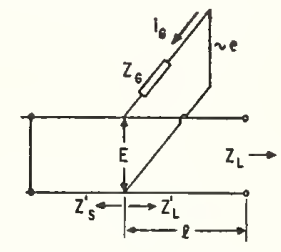

(b)
Fig. 2-Diagram and simplified model of one system yielding squared VSWR response; (a) diagram of system, (b) simplified model.

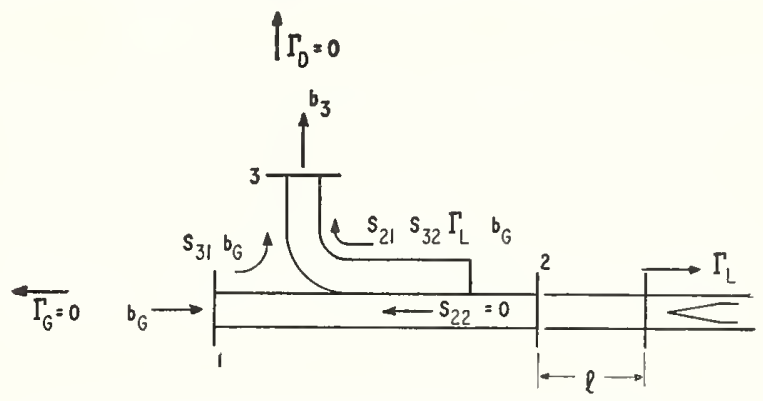

Fig. 3-Diagram of simplified system to illustrate magnified response.

approximately equals the directivity ratio of the directional coupler), one may consider $\Gamma_{L}$ to be magnified by the factor $|K|$, leading to the term magnified response. It should be noted however that the response variation will increase as one increases $|K|$ up to the point where $\left|K \mathrm{~T}_{L}\right|=1$, and then will decrease with further increasé in $|K|$.

\section{AnAlysis}

Both magnified and squared VSWR responses may be analyzed by considering the generalized treatmen $\mathrm{t}^{2}$

${ }^{2}$ A. C. MacPherson and D. M. Kerns, "A new technique for the measurement of microwave standing-wave ratios," Proc. IRE, vol. 44, pp. 1024-1030, August, 1956. 
of MacPherson and Kerns of a 3-arm junction measurement system for phasable loads. Such a junction is shown in Fig. 4. where it has been assumed for convenience that the necessary variation in phase is obtained by changing the length $l$ of uniform, lossless waveguide.

Instead of using the gathering coefficients employed by MacPherson and Kerns, the solution ${ }^{3}$ for $b_{3}$, is obtained in terms of the more familiar scattering coefficients and may be expressed as follows:

$$
\begin{aligned}
b_{3} & =C \frac{1+K \Gamma_{L} e^{-j 2 \beta l}}{1-\Gamma_{2 i} \Gamma_{L} e^{-j 2 \beta l}} \\
& =C K \Gamma_{L} e^{-j 2 \beta l} \frac{1+\frac{1}{\hbar \Gamma_{L}} e^{j 2 \beta l}}{1-\Gamma_{2 i} \Gamma_{L} e^{-j 2 \beta l}}
\end{aligned}
$$

where

$$
\begin{aligned}
C & =\frac{b_{G} S_{31}}{\left|\begin{array}{rr}
\left(1-S_{11} \Gamma_{G}\right) & S_{13} \Gamma_{D} \\
S_{31} \Gamma_{G} & \left(1-S_{33} \Gamma_{D}\right)
\end{array}\right|}, \\
K & =\frac{S_{21} S_{32}}{S_{31}}-S_{22},
\end{aligned}
$$

and

$$
\Gamma_{2 i}=\frac{\left|\begin{array}{ccc}
\left(1-S_{11} \Gamma_{G}\right) & S_{12} & -S_{13} \Gamma_{D} \\
-S_{21} \Gamma_{G} & S_{22} & -S_{23} \Gamma_{D} \\
-S_{31} \Gamma_{G} & S_{32} & \left(1-S_{33} \Gamma_{D}\right)
\end{array}\right|}{\left|\begin{array}{rr}
\left(1-S_{11} \Gamma_{G}\right) & S_{13} \Gamma_{D} \\
S_{31} \Gamma_{G} & \left(1-S_{33} \Gamma_{D}\right)
\end{array}\right|}
$$

In the above expressions, the component of the emergent wave amplitude supplied by the generator is $b_{G}=a_{1}-b_{1} \Gamma_{G}$, where $a_{1}$ represents the amplitude of the wave incident on the junction in arm 1. Symbols of the form $S_{m, n}$ are the scattering coefficients of the junction, and $\Gamma_{G}, \Gamma_{D}$, and $\Gamma_{L}$ are the voltage reflection coefficients of the generator, detector, and load, respectively, as indicated in Fig. 4.

The reflection coefficient $\Gamma_{2 i}$ is that which would be obtained "looking into" arm 2 if the generator was turned off and its impedance (as observed at $T_{1}$ ) was unchanged in so doing.

The variation in $\left|b_{3}\right|$ as we vary the phase $\left(\psi_{L}\right)$ of $\Gamma_{L}$ is defined to be the response of the systems represented by Fig. 4, and is determined by (5).

The properties of (5) will be examined in an effort to classify types of responses obtainable. It is evident that the parameter $C$ affects only the level of the response, while the form of the response curve $\left(\left|b_{3}\right|\right.$ vs $\left.\psi_{L}\right)$ is affected by the parameters $K$ and $\Gamma_{2 i}$.

We may consider the response for the conditions $\Gamma_{2 i}=0,|K|=1$, the usual or normal type of response,

${ }^{3}$ G. E. Schafer and R. W. Beatty, "A method for measuring the directivity of directional couplers, "IRE Trans. ON MiCrowave TheORY And Technioues, vol. MTT-6, pp. 419-422; October, 1958.

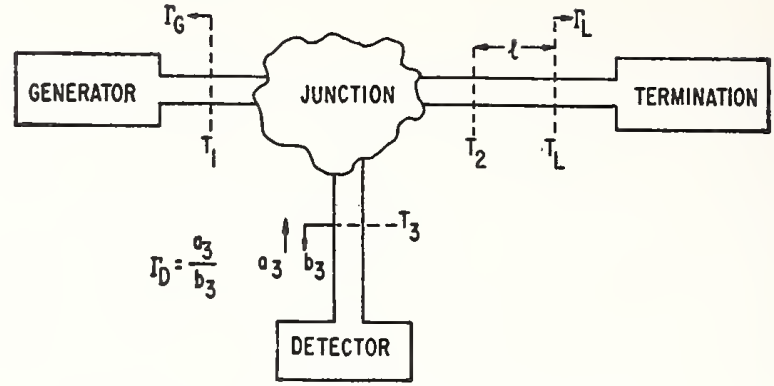

Fig. 4-Three-arm junction with phasable load.

since it leads to the form obtained in the case of an idealized slotted line.

The response form previously referred to as magnified response is obtained when $\Gamma_{2 i}=0$, and $|K|$ is unrestricted. However values of $|K|$ may range from zero to infinity, and it is possible to have a magnification factor $|K|$ greater or less than unity. The case where $|K|$ is less than one is of dubious interest, but the possibility of $|K|$ greater than unity appears especially attractive for the measurement of small reflections.

If $\Gamma_{2 i}$ differs from zero, it will cause distortion in the response curve, which is considered undesirable. However, a distinctly different type of response may be obtained if $\left|\Gamma_{2 i}\right| \approx 1$, and if the phases of $|K|$ and $\left|\Gamma_{2 i}\right|$ are equal. This leads to squared VSWR response if $|K| \approx 1$. This type of response is not only curious, but may prove useful in some measurement applications.

Actually $\left|\Gamma_{2 i}\right|$ is less than unity in actual (not lossless) systems, so that the ideal squared VSWR response may be closely approached with an actual system, but never quite reached.

A fourth type of response is obtained if the phases of $K$ and $\Gamma_{2 i}$ are the same, $\left|\Gamma_{2 i}\right| \approx 1$, and $|K|$ is unrerestricted. The ratio of maximum to minimum detector signal level corresponding to (3) is

$$
\sigma_{A}=\frac{1+\left|K \Gamma_{L}\right|}{1-\left|K \Gamma_{L}\right|} \sigma_{L} .
$$

It seems appropriate to call this a magnified squared VSWR response, and it may have applications in the measurement of large VSWR.

This completes the classification of responses, since conditions other than those mentioned may be regarded as causing distortions of the types described above.

\section{Means of Obtaining Various Responses}

Examples have already been given (Figs. 2 and 3) of junctions permitting magnified and squared VSWR responses. However other types of arrangements are possible and offer a variety of measurement systems, each with its possible advantages and disadvantages.

In order to closely approach squared VSWR response $\left(\left|\Gamma_{2 i}\right| \approx 1,|K| \approx 1\right)$ it becomes evident that the 3 -arm junction should have low loss and low coupling to the load. (This may be shown from a consideration of the conditions imposed upon the scattering coefficients by 
losslessness.) These conditions are not sufficient however, as one may conclude after trying junctions which satisfy only these conditions. It is necessary for $K$ and $\Gamma_{2 i}$ to have the same phase, and this is obtained by some tuning device, such as the adjustable short-circuit in Fig. 2. There may be some difficulty in obtaining the desired response in some cases, because it is not always possible to obtain the correct phase relationship, but the junction forms represented in Fig. 5(a) to (d) have all been found experimentally to permit a close approach to squared VSWR response by proper adjustment of the tuner. The arrangement of $5(\mathrm{e})$ should also permit squared VSWR response, but has not been constructed or tested.

Magnified Response occurs upon making $|K|$ greater than unity while $\Gamma_{2 i}=0$. It evidently cannot be obtained with a lossless junction, for then $|K|=1$. If it is assumed that we can always make $\Gamma_{G}=0$, then $\Gamma_{2 i}$ would equal $S_{22}$, and this would vanish, so that $K=S_{21} S_{32} / S_{31}$. The directional coupler connected as shown in Fig. 3 evidently permits magnified response since $\left|S_{32} / S_{31}\right|$ is the directivity ratio and may be quite large while $\left|S_{21}\right|$ is usually between 0.7 and 1.0. The use of auxiliary tuners ${ }^{4}$ with a directional coupler permits greater versatility since one may adjust the directivity ratio upwards or downwards with one tuner, then adjust the other tuner to make $\Gamma_{2 i}=0$. These adjustments are independent only if made in the order described. Referring to Fig. 6, the tuner in arm 2 is adjusted first in order to obtain the desired value of $|K|$, then the tuner in arm 1 is adjusted to make $\Gamma_{2 i}=0$.

\section{Measurements Using Souared VSWR Response}

Any of the junctions of Fig. 5 or their equivalents may be used if the unknown is phasable. This requirement is satisfied if the unknown termination slides inside the waveguide. In principle, a phase shifter or line stretcher may also be used to provide the phase variation, but in practice, they are less than perfect, leading to additional errors in measurement. If the unknown termination does not slide within the waveguide, the arrangements of Fig. 5(d) and (e) may be used: Either flexible cables must be used to couple the generator and detector to the moving junction, or the generator and detector may be arranged to move with the junction. If the termination is not too large, it and the waveguide section could be moved, keeping everything else fixed. The arrangement shown for rectangular waveguide is not readily adaptable to operation with coaxial lines. However the arrangement of Fig. 5(e) should be satisfactory for operation with coaxial line.

The correct adjustment of the tuner is made with $\Gamma_{L}=0$ and corresponds to maximum detector output for Figs. 2 and 5(a) and to minimum detector output for the others shown. When the correct adjustment has

${ }^{4}$ G. F. Engen and R. W. Beatty, "Microwave Reflectometer Techniques," this issue, p. 351 .
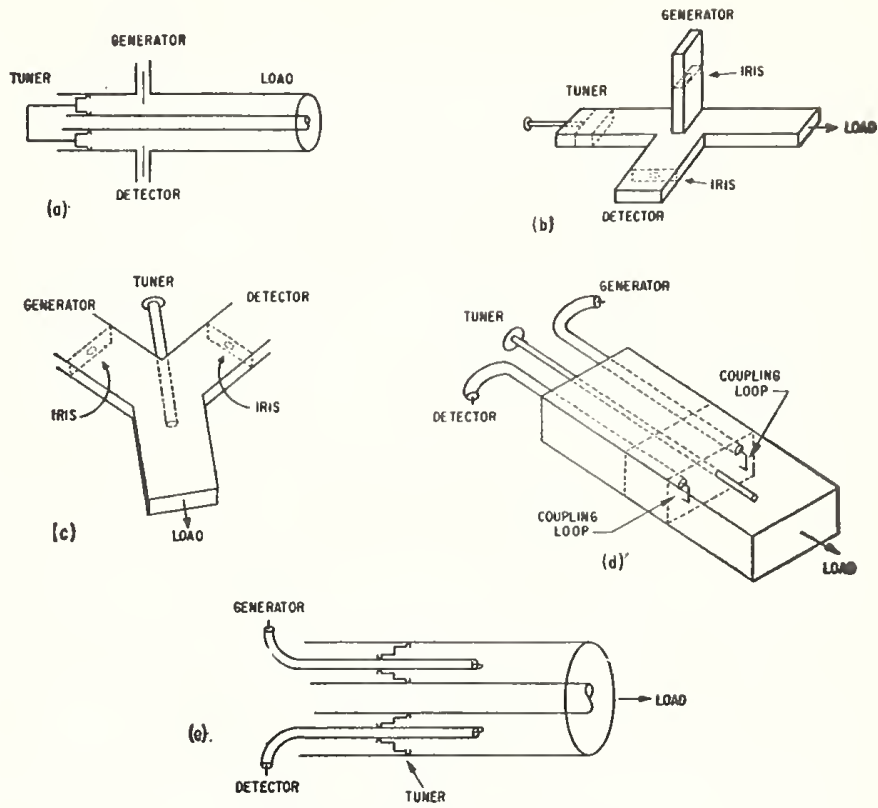

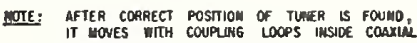

IT Hor ch

Fig. 5-Schematic drawings of junctions permitting squared VSWR response. (a), (b), (c), (d), (e).

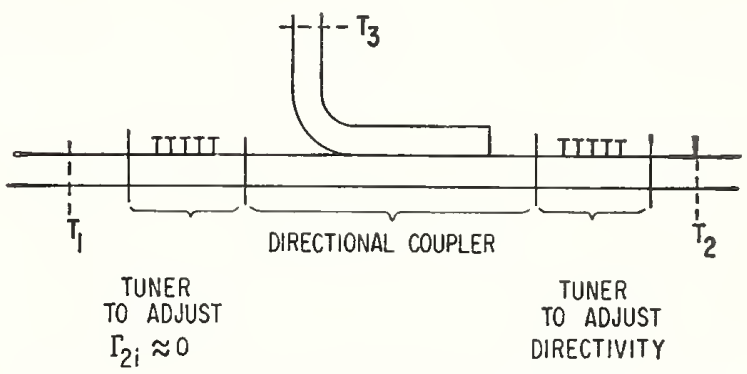

Fig. 6-Directional coupler with auxiliary tuners.

been made, the response curves will be symmetrical about the maxima and minima.

A correction may be made for deviations from the ideal conditions $|K| \approx 1,\left|\Gamma_{2 i}\right| \approx 1$, using the methods indicated $^{2}$ by MacPherson and Kerns. Instead of analyzing the response curve to obtain the parameter $y$ required for the correction, a shorter method is as follows. Only the real part $(g)$ of $y$ is needed for a correction to the VSWR. It can be shown that to a good approximation (to the first order in $g$ and $b$ ),

$$
\sigma_{I}=\frac{1+\left|K \Gamma_{L}\right|}{1-\left|K \Gamma_{L}\right|}=\sqrt{\sigma_{A}}+\frac{1}{2}(g+1)\left(\sigma_{A}-1\right),
$$

where

$$
(g+1) \approx \frac{\left|b_{3}\right|\left(\Gamma_{L}=0\right)}{\left|b_{3}\right|_{\max }\left(\left|\Gamma_{L}\right|=1\right)}
$$

It is still necessary to determine $|K|$ in order to obtain $\left|\Gamma_{L}\right|$ or $\sigma_{L}$. This may be done by measuring $\sigma_{1}$ when a termination of known $\left|\Gamma_{L}\right|$ is connected. 


\section{Measurements Using Magnified Response}

The arrangement of Fig. 6 may be used to measure the voltage reflection coefficient $\Gamma_{U}$ of an unknown termination. Two basic methods ${ }^{4,5}$ will be outlined.

In the first method, the auxiliary tuners are adjusted for the conditions $\Gamma_{2 i}=0$ and $K=\infty$. Inspection of (5) shows that $\left|b_{3}\right|$, the magnitude of the detector arm wave amplitude will then be proportional to $\left|\Gamma_{L}\right|$. One then measures the ratio $r$ of the $\left|b_{3}\right|$ values obtained when the load is first unknown $\left(\Gamma_{U}\right)$, then a standard of known reflection coefficient magnitude $\left|\Gamma_{S}\right|$. Then

$$
\left|\Gamma_{U}\right|=r\left|\Gamma_{S}\right| \text {. }
$$

The adjustments of the tuners preceding the measurement is as follows. ${ }^{4}$ One adjusts the tuner in arm 2 until no variation is observed in $\left|b_{3}\right|$ as one slides a termination of low reflection inside the output waveguide. Then the tuner in arm 1 is adjusted until no variation in $\left|b_{3}\right|$ is observed as one slides a termination of high reflection inside the output waveguide. If necessary, the above operations are repeated in sequence until no variation in $\left|b_{3}\right|$ is observed as either termination is slid.

In the second method, the tuner in arm 2 is adjusted (with the unknown connected to arm 2) until the detector output is zero. Then $K \Gamma_{U}=-1$. The tuner in arm 1 is then adjusted until $\Gamma_{2 i}=0$. A reflection standard of known $\left|\Gamma_{S}\right|$ is then connected to arm 2, and the phase of $\Gamma_{S}$ is varied. Substitution of the above conditions in to (5) leads to

$$
\sigma_{A}=\frac{\left|b_{3}\right|_{\max }}{\left|b_{3}\right|_{\min }}=\frac{\left|\Gamma_{U}\right|+\left|\Gamma_{S}\right|}{|| \Gamma_{U}|-| \Gamma_{S}||} .
$$

In the event that $\left|\Gamma_{S}\right| \approx 1$, (approximately true for a sliding short-circuit), then $\sigma_{A}=\sigma_{U}$.

Note that it is unnecessary to vary the phase of $\Gamma_{U}$, the reflection coefficient of the unknown termination in either method. In the second method one needs to vary the phase of $\Gamma_{S}$, but this is easily done if a sliding shortcircuit is used.

Alternatively a fixed reflection standard may be used if a suitable line stretcher is incorporated into arm 2 of the measuring instrument.

${ }^{5}$ R. W. Beatty and D. M. Kerns, "Recently developed microwave impedance standards and methods of measurement," IRE, Trans. ON Instrumentation, vol. I-7, pp. 319-321; December, 1958.
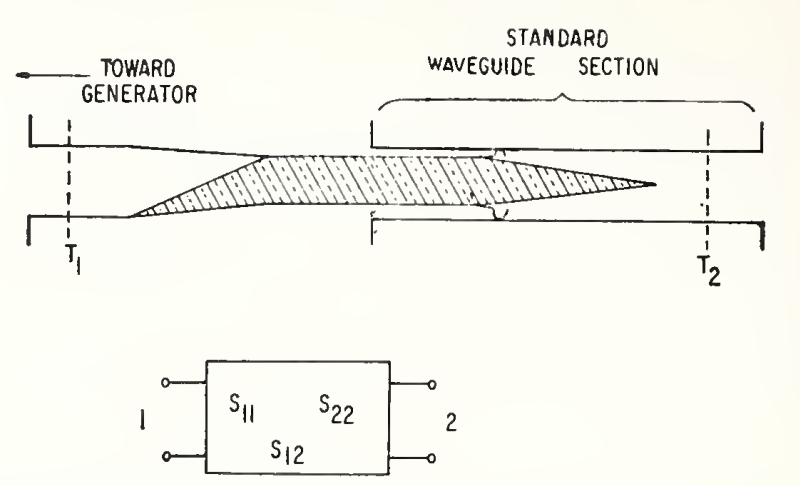

Fig. 7-Line stretcher and scattering coefficient representation.

Because of the special condition $\Gamma_{2 i}=0$, the line stretcher need not be of the constant impedance type, since reflections that it may introduce may be cancelled by reflections from the tuner in arm 1 . Also, the reference plane for arm 2 may be located in the uniform waveguide section of the line stretcher between the source of, its reflections and the load. As shown in Fig. 7 , the reference plane $T_{2}$ remains fixed although the output waveguide and load move. The line stretcher must be stable however, so that the parameters $S_{11}, S_{12}$, and $S_{22}$ with respect to the reference planes $T_{1}$ and $T_{2}$ do not vary as it operates.

With the addition of the line stretcher, the second method described above may be called a magnified difference method, since the smaller the difference between $\left|\Gamma_{S}\right|$ and $\left|\Gamma_{U}\right|$, the greater the variation in $\left|b_{3}\right|$ as the phase is changed (9).

\section{Evaluation}

It is too early to make a conclusive evaluation of the worth of the responses described above and their applications in measurement systems. However, the techniques employing magnified response give promise of increased accuracy in the measurement of low and intermediate VSWR. Accuracies of approximately 0.1 per cent in VSWR to 2.0 have been achieved, and perhaps an order of magnitude better than that is possible.

\section{ACKNOWLEDGMENT}

Dr. D. M. Kerns made many helpful suggestions, including the junction of Fig. 5(b), and W. J. Anson and E. Niesen made measurements to verify the techniques described above. 


\title{
Microwave Reflectometer Techniques*
}

\author{
G. F. ENGEN† AND R. W. BEATTY $\dagger$
}

Summary - A rigorous analysis of the microwave reflectometer is presented for what is believed to be the first time. By means of this analysis, the correct adjustment of auxiliary tuners is described, and the errors resulting from incorrect adjustments are treated in a quantitative manner.

It is shown how the reflectometer technique may be further simplified while preserving the accuracy of measurement. A cunvenient method of adjusting the auxiliary tuners is described, sources of error are discussed, and an example is given of the calculation of error limits.

\section{INTRODUCTION}

$\mathrm{T}$ THE microwave reflectometer in its usual form consists of a pair of directional couplers so arranged that one couples to the forward, and the other to the reverse wave. The ratio of the sidearm outputs is, in the ideal case, equal or at least proportional to the magnitude of the reflection coefficient of the termination, from which one can calculate the standing wave ratio.

In practice, this relationship is oniy approximately realized because of imperfections in the directional couplers and other factors. However, directional couplers having high directivity (40 $\mathrm{db}$ ö more) and low main guide VSWR (less than 1.05) are commercially available which permit good accuracy to be realized over a large (1.5 to 1 ) frequency range, while at a given frequency, further improvements may, be realized by the use of auxiliary tuning. ${ }^{1}$

The use of auxiliary, tuners has not been fully exploited or completely treated however, and in this paper a more general and rigorous analysis of the microwave reflectometer will be presented leading to the introduction of additional tuning elements. Procedures for the adjustment of these transformers will be described and a particularly simple form of the reflectometer developed. A quantitative treatment of the errors in these techniques will be presented.

\section{General Theory}

The basic form of the reflectometer is shown in Fig. 1. If $b_{3}$ and $b_{4}$ represent the voltage amplitudes of the signals at the respective detectors, the desired response is

$$
\left|\frac{b_{3}}{b_{4}}\right|=\left|\Gamma_{l}\right| \quad \text { or } \quad\left|\frac{b_{3}}{b_{4}}\right|=K\left|\Gamma_{l}\right|
$$

* Manuscript received by the PGMTT, January 13, 1959; revised manuscript received, February 20, 1959.

$\dagger$ Radio Standards Lab., Nat. Bur. of Standards, Boulder, Colo.

"J. K. Hunton and N. L. Pappas, "The $-h p$ - microwave reflectometers," Hewlett-Packard J., vol. 6; September-October, 1954.

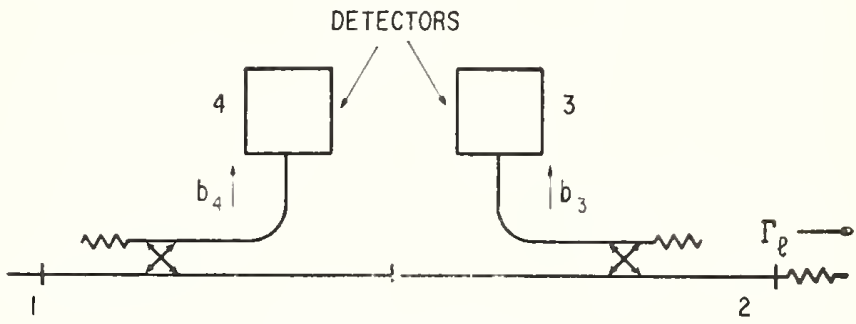

Fig. 1-Basic reflectometer.

where $\Gamma_{l}$ is the voltage reflection coefficient of the termination on arm 2, and $K$ is a constant whose value must be determined.

A mathematical treatment of the four arm junction and detectors shows (assuming linearity) that the response will in general be of the form:

$$
\frac{b_{3}}{b_{4}}=\frac{A \Gamma_{l}+B}{C \Gamma_{l}+D}
$$

where the $A, B, C$, and $D$ are functions of the parameters of the four arm junction and detectors.

A particularly simple and convenient group of expressions for the terms $A, B, C$ and $D$, may be obtained by the following procedure. The performance of the reflectometer of Fig. 1 is characterized by the functional relationships imposed upon the terminal variables $a_{1}$, $b_{1}, a_{2}$, and $b_{2}$, which represent the incident and emergent voltage wave amplitudes at arms 1 and 2 respectively and the responses of the detection systems (usually power) employed at arms 3 and 4 . The main interest is in the relationship between the detector responses and the ratio

$$
\frac{a_{2}}{b_{2}}=\Gamma_{l}
$$

as given in (1). A variety of equivalent circuit representations may be substituted for that shown in Fig. 1 provided the relationships of interest among the terminal variables are preserved. A convenient choice for the present purpose is as follows:

Assuming that the detector impedance is constant (as will be true for example of a barretter operated at a constant resistance) mathematical models for the detectors may be constructed of lossless and matched detectors preceded by lossy fourpoles of the required parameters to produce the externally observed behavior. Reference planes in arms 3 and 4 are then chosen between these ideal detectors and the lossy discontinuities such that the latter become part of the four arm junc- 
tion as shown in Fig. 2. The remainder of the system may be represented in the usual manner. This permits one to analyze the general behavior of the reflectometer as a four arm junction under the materially simplifying assumption of matched detectors on arms 3 and 4 .

The main effects of this type of formulation are those of modifying the values of the scattering coefficients from those which would obtain were the reference planes in arms 3 and 4 chosen to coincide with the physical junction between the detector mounts and the associated four arm junction; and of placing the reference planes in arms 3 and 4 in a physically inaccessible position, but this is of no concern since the subsequent measurements or adjustments of the four arm junction to be described do not require access to these planes. In addition, as noted, the detector impedance is assumed to be constant. There is no further loss in generality.

An analysis of the reflectometer of Fig. 2 yields:

$$
\begin{aligned}
& A=S_{21} S_{32}-S_{31} S_{22} \\
& B=S_{31} \\
& C=S_{21} S_{42}-S_{41} S_{22} \\
& D=S_{41}
\end{aligned}
$$

where the $S_{m, n}$ are the scattering coefficients of the four arm junction comprised of the directional couplers and lossy fourpoles.

It is evident that the desired response will be realized if $B=C=0$. For ideal couplers of infinite directivity and a main guide VSWR of unity (and matched detectors) the terms $S_{31}, S_{42}$, and $S_{22}$ are all zero, and

$$
\left|\frac{b_{3}}{b_{4}}\right|=\left|\frac{A}{D} \Gamma_{l}\right| \text {. }
$$

as required.

In practice, the failure of the directional couplers and detectors to meet these criteria may be compensated or corrected for by the introduction of tuners at positions $X$ and $Y$ as shown in Fig. 3 (the tuiner at $Z$ serves an auxiliary role to be described later $)$. The adjustment of $T_{X}$ and $T_{Y}$ to produce the conditions $B=C=0$ may be carried out in a variety of ways, two of which will be described. The first of these tuning procedures perhaps gives one a better feel for the conditions under which the adjustments may be physically realized, and is included for the sake of completeness, while the second method, the one which is recommended, is more convenient and potentially more accurate.

\section{Adjustment of Tuners}

In the first method, arm 2 is terminated in a matched load and $T_{X}$ adjusted for a null in arm 3 (with arm 1 connected to the generator). This produces the condition $S_{31}=0$ (by definition of the scattering coefficient). The generator is then connected to arm 2; a load (not necessarily matched) connected to $\operatorname{arm} 1$, and $T_{Z}$ ad-

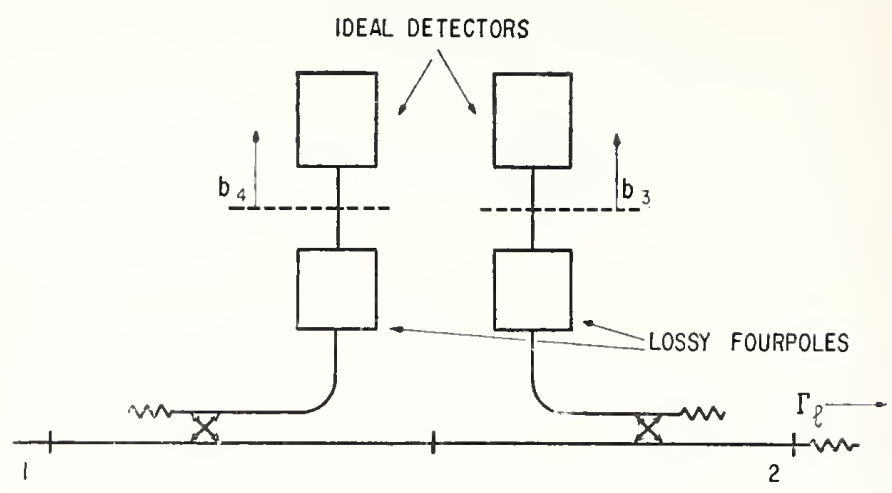

Fig. 2--Equivalent reflectometer.

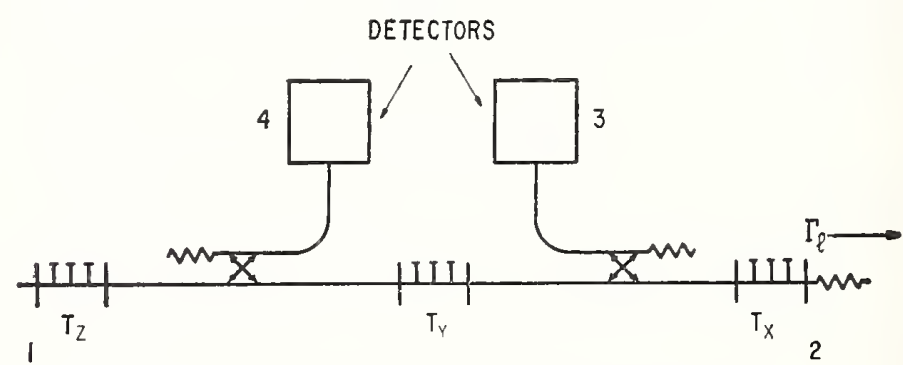

Fig. 3-Reflectometer with auxiliary tuners.

justed to produce a null in arm 4 . If $T_{Y}$ is now adjusted such that the reflection coefficient

$$
\Gamma_{2 i}=\frac{b_{2}}{a_{2}}
$$

observed at arm 2 vanishes, it can be shown ${ }^{2}$ that

$$
S_{21} S_{42}-S_{41} S_{22}=0,
$$

and the desired operating conditions have been realized. ${ }^{3}$

It will be noted ${ }^{2}$ that the first adjustment (of $T_{X}$ ) is independent of the second, while the converse is not true, thus the adjustments should be made in the order indicated. In addition, while $T_{Z}$ is employed in the second tuning operation, once the proper adjustment of $T_{Y}$ has been realized, $T_{Z}$ may be readjusted (as will subsequently prove desirable) without affecting this result obtained by adjustment of $T_{Y}{ }^{4}$

The alternative procedure will now be described. Referring again to (1), it will be evident that even before the introduction of tuning transformers, the terms $B$ and $C$ (for a reflectometer assembled from commercial

2 The proof of this statement and several of those to follow may be effected by solving the scattering equations for the indicated quantities under the stated conditions, or by obtaining the scattering parameters of the individual components, i.e., tuners and directional couplers. The proofs are generally straightforward but tedious.

${ }^{3}$ The minimum requirements which must be satisfied by the two couplers in order that these adjustments may be physically realized have not been determined at the time of this writing, but would appear to be well satisfied by commercially available components.

4 The expression $\left(S_{21} S_{42}-S_{41} S_{22}\right)$ may also be written

$$
S_{41}\left(\frac{S_{21} S_{42}}{S_{41}}-S_{22}\right)
$$

It can be shown (see note 2) that the second factor is invariant to the adjustment of tuner $T \%$. 
components) are quite small with regard to $A$ and $D$ respectively. Consider the system response to a phasable or sliding load of such magnitude that $\left|A \Gamma_{l}\right| \approx|B|$. From inspection it is evident that the numerator of (1) will undergo marked changes in amplitude, while the denominator remains relatively constant as the phase of the load is varied; and if $T_{X}$ is adjusted to minimize the variation in the ratio

$$
\left|\frac{b_{3}}{b_{4}}\right|
$$

as the phase of $\Gamma_{l}$ is varied, the condition $S_{31}=0$ will be approximately realized. The sliding load of low VSWR is then replaced by one of large VSWR (a sliding short). Variations in output as the position of the short is changed will now be predominantly due to variations in the denominator, and if $T_{Y}$ is adjusted such that $\left|b_{3} / b_{4}\right|$ is again constant, the condition $S_{21} S_{42}-S_{41} S_{22}=0$ will also be very nearly realized.

A more complete mathematical treatment of the above procedure yields three solutions for a constant magnitude of $b_{3} / b_{4}$ as the phase of $\Gamma_{l}$ is varied. The first solution, $A / C=B / D$ is trivial since it gives for $b_{3} / b_{4}$ a value which is entirely independent of $\Gamma_{l}$. The second solution, the one of interest, is:

$$
\frac{B}{A}=\left(\frac{C}{D}\right)^{*}\left|\Gamma_{l}\right|^{2}
$$

where $\left({ }^{*}\right)$ denotes the complex conjugate.

In practice, $A$ and $D$ are nominally of the same order of magnitude so when (3) is satisfied, $|B|$ is of the order of $\left|C \Gamma_{t}{ }^{2}\right|$. Thus if the sliding load has a VSWR of 1.02 $\left(\left|\Gamma_{l}\right|=0.01\right)$, it is evident that the first tuning operation will make $|B|$ smaller than $|C|$ by a factor of approximately $10^{4}$, while the second operation $\left(\left|\Gamma_{l}\right| \approx 1\right)$ will reduce $|C|$ to the nominal size of $|B|$. Thus a series of these operations rapidly converges to the desired conditions $B=C=0$.

The third solution referred to above is the limiting one of a matched load $\left(\Gamma_{\iota}=0\right)$ which also yields a constant ratio of $b_{3} / b_{4}$. The ideal or perfect match is, of course, never achieved in practice, while the closest approach to this ideal is usually by means of a variable sliding load which is adjusted to produce the minimum change of signal in an appropriate associated measuring system as the position of the load is varied. If a matched load is available, it is only necessary to adjust $T_{X}$ until the output at arm 3 vanishes, while if an adjustable sliding load is used, it is convenient to carry out the operations as required to make $\Gamma_{\iota}$ and $b^{3}$ vanish simultaneously. The technique described earlier, however, does not require a matched (reflection free) sliding load, but only that its reflection coefficient be small.

If the initial conditions are such that $|B| \gg\left|A \Gamma_{l}\right|$, the variation in the ratio

$$
\left|\frac{b_{3}}{b_{4}}\right|
$$

would be quite small, so as a first step (where only the phase of $\Gamma_{l}$ is adjustable), it is usually desirable to first adjust for a null in arm 3 for an arbitrary position of the load and then adjust for a constant ratio

$$
\left|\frac{b_{3}}{b_{4}}\right|
$$

as the phase is varied. It will be noted that the magnitude of $b_{3} / b_{4}$ depends upon the magnitude of $\Gamma_{l}$.

It is thus of interest to note that the technique requires neither a perfectly matched load or ideal short, but only two phasable loads of different reflection coefficient magnitudes. The more closely these ideals are realized however, the more rapidly will a series of these operations converge to the desired conditions, which in practice can usually be realized to the required accuracy by only one adjustment each of $T_{X}$ and $T_{Y}$. It is also of interest to note that if a perfectly matched load $\left(\Gamma_{l}=0\right)$, an ideal sliding short $\left(\Gamma_{l}=e^{j \theta}\right)$, and a dissipation free transformer at $X$ are assumed, the two tuning operations, in this method, are completely independent of one another. That is, the adjustment of $T_{X}$ to yield the condition $B=0$, as noted earlier, is independent of the adjustment of $T_{Y}$, while the adjustment of $T_{Y}$ for the condition $B / A=(C / D) *$ is independent of $T_{X} \cdot{ }^{5}$

Having completed these adjustments, the response becomes:

$$
\left|\frac{b_{3}}{b_{4}}\right|=\left|\frac{A}{D} \Gamma_{l}\right| .
$$

The magnitude of the ratio $A / D$ may be conveniently determined by observing the response to a load of known reflection, a convenient example being a fixed short for which $\Gamma_{l}$ has the nominal magnitude 1 . Thus if the response to the short is

$$
\left|\frac{b_{3}}{b_{4}}\right|_{8}
$$

one has for the unknown reflection coefficient $\Gamma_{u}$.

$$
\left|\Gamma_{u}\right|=\frac{\left|\frac{b_{3}}{b_{4}}\right|_{u}}{\left|\frac{b_{3}}{b_{4}}\right|_{8}}
$$

Once the reflection coefficient has been determined, the VSWR may, of course, be obtained by the usual formula

$$
\sigma_{u}=\frac{1+\left|\Gamma_{u}\right|}{1-\left|\Gamma_{u}\right|} .
$$

- A formal proof of this statement is somewhat lengthy, but may be recognized intuitively in the following way. An ideal sliding short preceded by a dissipation free transformer still appears as a load of $\left|\Gamma_{l}\right|=1$ and variable phase angle, thus the condition $B / A$ $=(C / D)^{*}$ is invariant to the addition (or removal) of a lossless tuning transformer at arm 2. 
A variety of techniques are available for measuring the ratio

$$
\left|\frac{b_{3}}{b_{4}}\right|
$$

In general, both $b_{3}$ and $b_{4}$ will change with generator output and load impedance, requiring a ratio type meter such that

$$
\left|\frac{b_{3}}{b_{4}}\right|
$$

is indicated directly, or a pair of instruments to determine $\left|b_{3}\right|$ and $\left|b_{4}\right|$ individually. Alternatively, a feedback servo loop may be employed to keep $\left|b_{4}\right|$ constant, requiring only the observations of the values of $\left|b_{3}\right|$.

The functional dependence of $b_{4}$ upon the load impedance may be substantially eliminated by adjusting transformer $T_{Z}$ such that the value of $\left|b_{4}\right|$ is independent of a sliding short at arm 2. This will reduce the amount of correction required of the servo loop, if one is employed, or the signal $b_{4}$ might be then applied to the automatic gain control channel if a standing wave amplifier were employed to measure $\left|b_{3}\right|$. These are only several of a number of possibilities.

If the adjustment to make $\left|b_{4}\right|$ independent of the load impedance has been made with sufficient care, $\left|b_{4}\right|$ will depend only upon the generator level, and if the generator is sufficiently stable, the signal $\left|b_{4}\right|$ will be constant and thus no longer contain any useful information. This first coupler with its associated detector and transformer $T_{Z}$ may then be eliminated, resulting in a simplified system. This particular case appears to be enough of interest to warrant separate treatment.

\section{A Modified Form of Reflectometer}

Referring to the three arm junction of Fig. 4 , the wave amplitude $b_{3}$ incident upon the detector in arm 3 can be written:

$$
b_{3}=b_{v} \frac{E \Gamma_{l}+F}{G \Gamma_{l}+H},
$$

where

$$
\begin{aligned}
& E=\left|\begin{array}{cc}
S_{21} & S_{22} \\
S_{31} \cdot S_{32}
\end{array}\right|, \\
& F=S_{31} \\
& G=-\left|\begin{array}{ccc}
-\left(1-S_{11} \Gamma_{g}\right) & S_{12} & S_{13} \Gamma_{d} \\
S_{21} \Gamma_{g} & S_{22} & S_{23} \Gamma_{d} \\
S_{31} \Gamma_{g} & S_{32} & -\left(1-S_{33} \Gamma_{d}\right)
\end{array}\right| \\
& H=\left|\begin{array}{ccc}
\left(1-S_{11} \Gamma_{g}\right) & S_{13} \Gamma_{d} \\
S_{31} \Gamma_{g} & \left(1-S_{33} \Gamma_{d}\right)
\end{array}\right|,
\end{aligned}
$$

$b_{o}$ is the equivalent generator voltage wave amplitude, and $\Gamma_{a}$ and $\Gamma_{d}$ are the generator and detector reflection coefficients at reference planes 1 and 3 respectively. It will be noted that the reference planes have been chosen to coincide with the terminal surfaces of the directional coupler, thus exhibiting the dependence on the detector impedance explicitly.

Eq. (5) is of the same form as (1) and the adjustment of $T_{X}{ }^{\prime}$ and $T_{Y}{ }^{\prime}$ to make $F$ and $G$ vanish may be carried out in the manner already described except that in step two of the first method $\Gamma_{2 i}=b_{2} / a_{2}$ is made to vanish by adjustment of $T_{Y}{ }^{\prime}$ with arm 1 terminated in the generator impedance, instead of the procedure described earlier.

Measurement of the ratio

$$
r=\frac{\left|b_{3}\right|_{u}}{\left|b_{3}\right|_{s}}=\frac{\left|\Gamma_{u}\right|}{\left|\Gamma_{s}\right|}
$$

and a knowledge of $\left|\Gamma_{s}\right|$ again permits the calculation of $\left|\Gamma_{u}\right|$ or $\sigma_{u}$ and again, a variety of techniques such as power, audio, or heterodyne detection may be employed, or a calibrated standard attenuator may be placed in the system and the changes in attenuation required to keep $\left|b_{3}\right|$ constant observed.

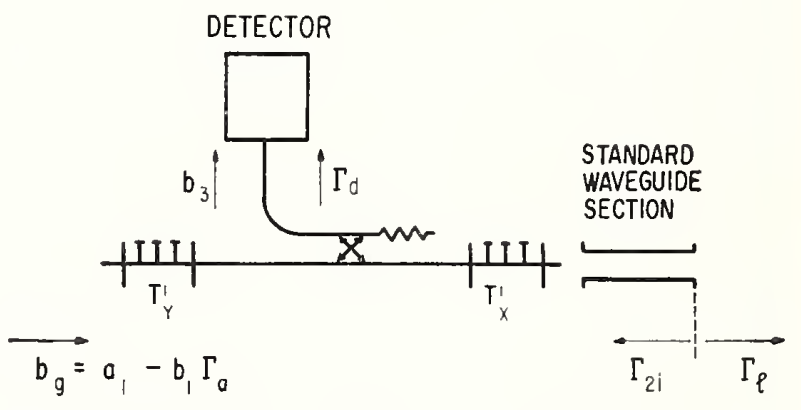

Fig. 4-Modified reflectometer.

The modified technique thus provides a reduction in the complexity of both the waveguide plumbing and associated detection equipment, but requires a signal source of stable amplitude, while the more conventional system is ideally independent of the generator level. Both systems are adaptable to either rectangular waveguide or coaxial systems, and may usually be assembled from commercially available components although it is desirable to use a precision waveguide section to terminate arm 2 in which the sliding loads may be inserted, as shown in Fig. 4. The importance of good flanges or connectors at arm 2 should also be recognized.

\section{ANALYSIS OF ERRORS ${ }^{6}$}

The sources of error, in determining $\left|\Gamma_{u}\right|$ and $\sigma_{u}$ by these methods to be discussed in this section include: a) incorrect measurement of $r$, and uncertainty in value of $\left|\Gamma_{s}\right|$, and b) improper adjustment of the tuners, such that $B$ and $C$ do not vanish.

The consideration of other sources of error is outside the scope of this present paper, but will be treated more

- The analysis also applies to the modified reflectometer if the quantities $E, F, G, H$ are substituted for $A, B, C, D$. 
fully in a subsequent paper (in preparation) on the application of the technique to measurement of bolometer mount efficiency.

The error due to a) may be determined by inspection. If the equation for $\left|\Gamma_{u}\right|$ is written in the form:

$$
\left|\Gamma_{u}\right|=\left|\Gamma_{s}\right| \frac{\left|\frac{b_{3}}{b_{4}}\right|_{u}}{\left|\frac{b_{3}}{b_{4}}\right|_{s}}=\left|\Gamma_{8}\right| r,
$$

it is evident that the fractional error in $\left|\Gamma_{u}\right|$ will equal the sum of the fractional errors in $\left|\Gamma_{s}\right|$ and $r$ if the latter are small.

With regard to the second item b) it will be recalled that the condition for constant output as the phase of the load is varied is:

$$
\frac{B}{A}=\left(\frac{C}{D}\right)^{*}\left|\Gamma_{l}\right|^{2}
$$

which establishes a theoretical upper limit to the accuracy with which a particular tuning adjustment may be made.

In practice, however, the limitation usually stems from improper adjustment of the tuning transformers such that the output variations are not completely eliminated. In the discussion to follow it will be assumed that such is the case, that is, it is assumed that $B$ and $C$ have been reduced to the point where the variations in the expression

$$
\left|\frac{b_{3}}{b_{4}}\right|=\frac{\left|A \Gamma_{l}+B\right|}{\left|C \Gamma_{l}+D\right|}
$$

are due entirely to variations in the numerator or denominator as the loads of small and large VSWR are employed respectively.

A first order correction to (6) may be written as follows:

$$
\begin{aligned}
\left|\Gamma_{u}\right|=\left|\Gamma_{s}\right| \frac{\left|b_{3}\right|_{u}}{\left|b_{3}\right|_{s}} 1+ & \frac{B}{A} \frac{\Gamma_{s}-\Gamma_{u}}{\Gamma_{s} \Gamma_{u}} \\
& +\frac{C}{D}\left(\Gamma_{s}-\Gamma_{u}\right)+\cdots \mid
\end{aligned}
$$

The ratios $|B / A|$ and $|C / D|$ may be determined from the expressions:

$$
K_{1}=20 \log \left[1+2\left|\frac{B}{A \Gamma_{l}}\right|\right]
$$

and

$$
K_{2}=20 \log \left[1+2\left|\frac{C}{D}\right|\right]
$$

where $K_{1}$ and $K_{2}$ are the ratios in decibels of the maximum to minimum outputs with the sliding loads of small and large VSIVR respectively, $\left|\Gamma_{l}\right|$ is the reflec- tion coefficient of the load of small VSWR, and a reflection coefficient of unity has been assumed for the load of large VSWR.

Except for the presence of the factor $\Gamma_{l}$, these equations for $K_{1}$ and $K_{2}$ are of the same form, and values for $|B / A|$ and $|C / D|$ may be obtained from Fig. 5 where the value of $|C / D|$ is taken from the line $\left|\mathrm{\Gamma}_{l}\right|=1$. It will be noted that the evaluation of the right hand side of (7) further presupposes a knowledge of $\Gamma_{u}$ but for the present purpose of assigning a limit of error, an accurate value is not required.

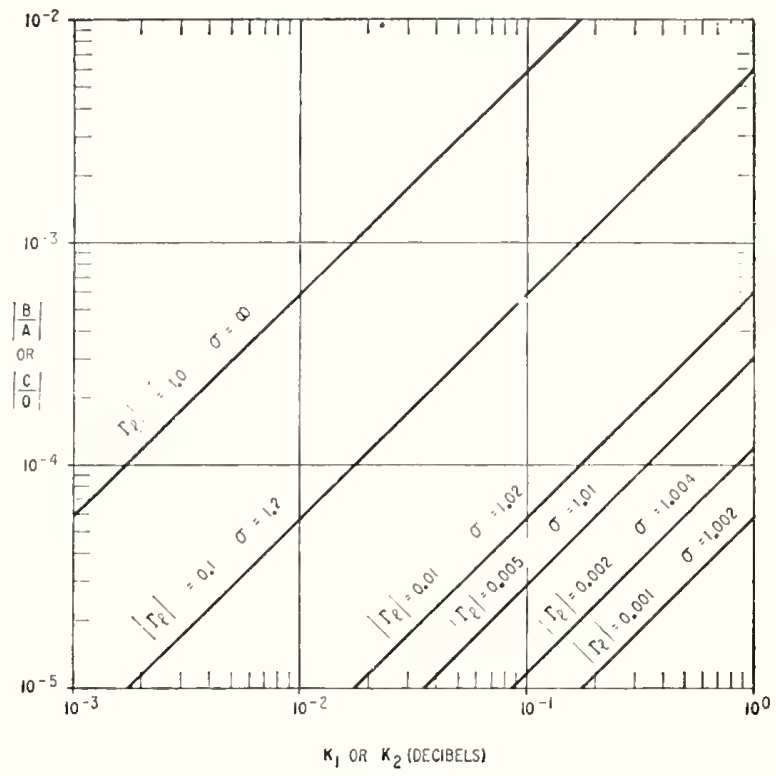

Fig. 5-Graph for the determization of $|B / A|$ and $|C / D|$.

As an example, if a sliding load of VSWR $=1.005$ is employed in the first tuning operation, and the variation in output is reduced to $1 \mathrm{db},|B / A|$ will have a value of approximately $1.6 \times 10^{-4}$. If the variation in the second step with the sliding short is reduced to 0.02 $\mathrm{db}$, the value for $|C / D|$ will be approximately $1.2 \times 10^{-3}$. Assuming that the unknown load has a value $\left|\Gamma_{u}\right| \approx 0.2$, $(\sigma \approx 1.5),\left|\Gamma_{s}\right|=1.000$ (corresponding to a short-circuit) and assuming the terms in the right hand factor of (7) combine in the worst phase, values of 6 and 1.2 for

$$
\left|\frac{\Gamma_{s}-\Gamma_{u}}{\Gamma_{s} \Gamma_{u}}\right|
$$

and $\left|\Gamma_{s}-\Gamma_{u}\right|$ obtain respectively, for a total error of $\pm\left(1.6 \times 10^{-4} \times 6+1.2 \times 10^{-3} \times 1.2\right) \approx \pm 2.4 \times 10^{-3}$, or \pm 0.24 per cent.

\section{ACKNOWLEDGMENT}

The authors extend their thanks to David F. Wait who contributed to the error analysis, to Dr. David M. Kerns for his helpful suggestions in reviewing the manuscript, and to Wilbur J. Anson and Edward Niesen who provided experimental demonstrations of the tech niques described. 
NATIONAL PHYSICAL LABORATORY

TEDDINGTON, MIDDLESEX, ENGLAND

PAPER $4-3$

PRECISION MILLIMETER WAVE INTERFEROMETRY

AT THE U.S. NATIONAL BUREAU OF STANDARDS

by

W. CULSHAW, T. M. RICHARDSON, and D. M. KERNS

Tc be presented at a symposium on Interferometry, whlch will be held at the National Physical Laboratory, Teddington, Middlesex, from 9th11th June, 1959. The papers and the discussions are to be published by H.M.S.0. In the Proceedings of the Symposium. This paper should not be reproduced without the permission of the authors and of the Secretary, National Physical Laboratory.

(40305)

$756 / 1822$ 
PRECISION MILLIMETER WAVE INTERFEROMETRY

AT THE U.S. NATIONAL BUREAU OF STANDARDS

by

W. CULSHAW, J. M. RICHARDSON, AND D. M. KERNS

\begin{abstract}
VARIOUS techniques of precision millimeter wave interferometry are being developed at the U.S. National Bureau of Standards for the purposes of wavelength determination, determination of the velocity of light, and length determination, bearing in mind the extreme coherence and sensitivity avallable in the microwave region. Assuming the reflectors are very large compared with the wavelength, a solution is given for the diffraction correction applicable to the Fresnel region in terms of the continuous scattering and transmission matrices of the microwave antennnas, distances, and reflectors involved in the interferometers. Modifications to this solution due to ilnite reflector dimensions are also discussed. Precision carrlages are being constructed for a Michelson interferometer sultable for measuring length and the velocity of light. A millimeter wave Fabry-Perot interferometer is also being investigated for these applications. The present status of experimentation and the results are described.
\end{abstract}

\title{
1. INTRODUCTION
}

THE high degree of coherence and detection sensitivity possible in the microwave region, suggests the use of microwave interferometers for the measurement of long lengths, and for precision measurements such as the determination of the velocity of light. Such microwave interferometers are usually variants of optical types such as the Michelson and FabryPerot, and operate in the same way. However, in optical interferometers the apertures used are so large compared with the wavelength, that the effect of diffraction on the wavelength measured with them is negligible. In micro-wave optics the dimensions of radiators and reflectors, etc., are of necessity much smaller in terms of wavelength than in optics, and

(40305) 
hence radiate a relatively wide angular spectrum of plane waves, instead of the ideal single plane wave along the axis of the interferometer. The angular spectrum of plane waves radiated by a given aperture may be determined by measurements of the distant fleld, as in antenna pattem measurements.

Wavelengths measured on a microwave interferometer are in general too large due to the resultant interference effects of plane waves radiated by the aperture, and to modiflcations of these by the finite. size of reflectors used. The magnltude of the error depends on the path lengths used in the interferometer, and on the aperture and reflector dimensions. When these are some 15 wavelengths in extent, the error may be as much as a few parts in $10^{4}$ in a microwave Michelson interferometer (ref.1). A similar remark applies to the microwave Fabry-Perot interferometer, ( ref. 2), although further work is necessary to determine the magnitude of the error.

In the application of precision microwave interferometry to the determination of ' $\mathrm{C}$ ', and to metrology, a diffraction correction to the wavelength measured on the interferometer must be considered. Froome ( refs. 3 , 4. 5) In the precision determinations of ' $c$ ' by variants of the Michelson interferometer, operated in the distant field, or Fraunhofer region, of the radiator where diffraction errors are smaller, and corrected for them by integration of the fleld over the recelving aperture. In contrast the work at the National Bureau of Standards is directed towards a precision determination of ' $\mathrm{c}$ ', and to metrology, with $\mathrm{mlcrowave}$ interferometers operating in the near fleld, or Fresnel region, of large radiating apertures. The diffraction correction is more complicated, since the complete plane wave spectrum radiated by the aperture must be considered. However, the effects of stray radiation are considerably reduced, since the beam is well collimated in this region, and the attenuation of the signal in the open arm of the interferometer is also considerably less than when operating in the Fraunhofer region. Much more precise information on the system 1s, however, required. The radiation patterns of the apertures used must be measured accurately, and the use of large reflectors becomes desirable in order that charges in the radiation pattern on reflection, due to the finite size of reflector, may be kept small.

\section{MICROWAVE MICHELSON INTERFEROMETER}

A perspective view of the reflector and mount of the proposed interferometer for use in the Fresnel region, is shown in figure 1. The reflector is $5 \mathrm{ft}$. square, and rests on a steel ball as shown. Provision is made for alignment of the reflector in the carriage, which then moves on 


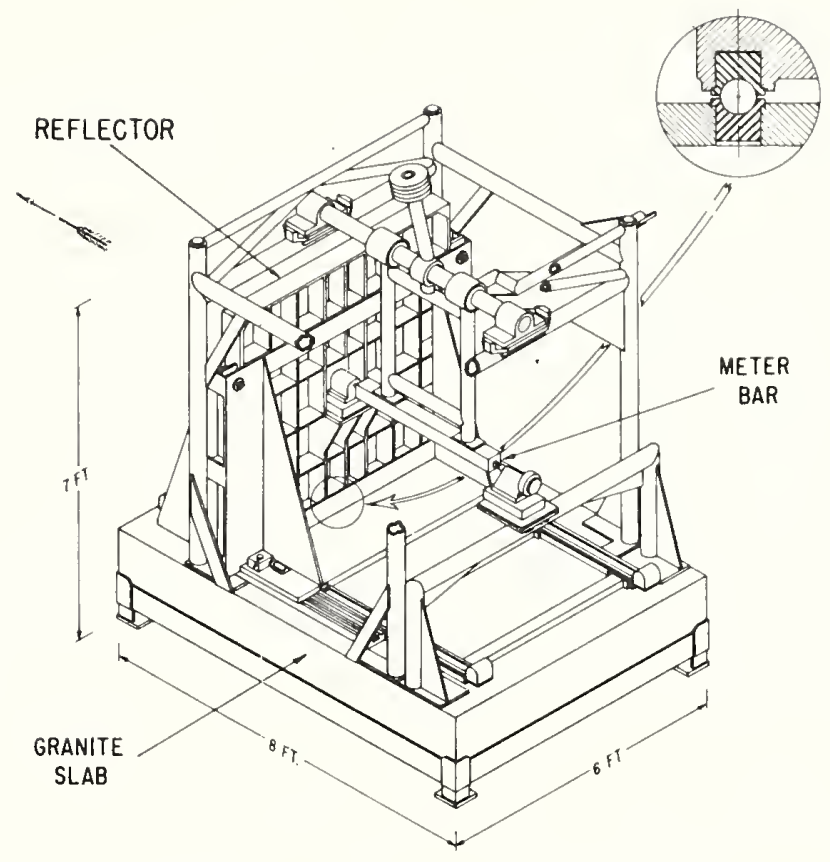

F1g.1. Microwave Interferometer Reflector and Mount

balls running in two $V$. grooves on the base plate. This rests on a substantial granite block to give stability to the system. A displacement of the reflector of the order of a meter can be made, and the meter bar, which is supported above by ball bushings on the steel rod, can be swung into position to measure the displacement. The small differences in length between an integral number of fringes and the meter bar, will be measured by a sultable gauge such as an optimeter. This also has sufficient measuring range to allow for path length changes due to expected variations in refractive index of the air. Faclilties for accurate lining up of the measuring anvils and the reflector are provided, and auto coll1mators will be used to see that the orientation of the reflector and the meter bar alignment are maintained during the course of the experiment.

Figure 2 is a diagram of the complete interferometer. Two alternative radiating apertures $30 \mathrm{cin}$ and $60 \mathrm{~cm}$ square respectively will be used, and are each formed in the usual way by tapering up from rectangular waveguide. Matched polystyrene lenses are used in the apertures in order to reduce the beam widths radiated, which are around 2 degrees and 1 degree respectively for the present operating frequency of 47,730 Mc. Radiation 
at this frequency is obtained by multiplying up from a 5 Mc crystal controlled oscillator, a power output of 1 - $2 \mathrm{mw}$ being finally obtained, with a frequency stability of a few parts in $10^{8}$. The microwave radiation is divided by the hybrid junction shown in figure 2; half of the energy passes down the reference waveguide arm, and hal $f$ passes through the radiating horn out into the open, where it is ultimately returned after reflection from the movable reflector. The returning waves from the reference arm and reflector are then, combined in the detector arm, and suitable adjustments on the reference level can be made to equalize the amplitudes of the two signals. The fringes are thus due to the interference of sinusoidal wave trains which have traversed different paths and apart from the effects of diffraction, the shape of the recorded iringes will depend on the type of detector employed. Sharp minima at displacement intervals of $\lambda / 2$ may be obtained by the use of linear detection with large amplification and precise balancing of the beams, the fringe shape then varying as $|\cos \phi / 2|$, where $\phi$ is the phase angle between the two interfering beams. The balance of the beams changes with distance ' $d$ ' due to diffraction, and if the minima are not sharp enough over the region of operation used, some adjustment for this must be used.

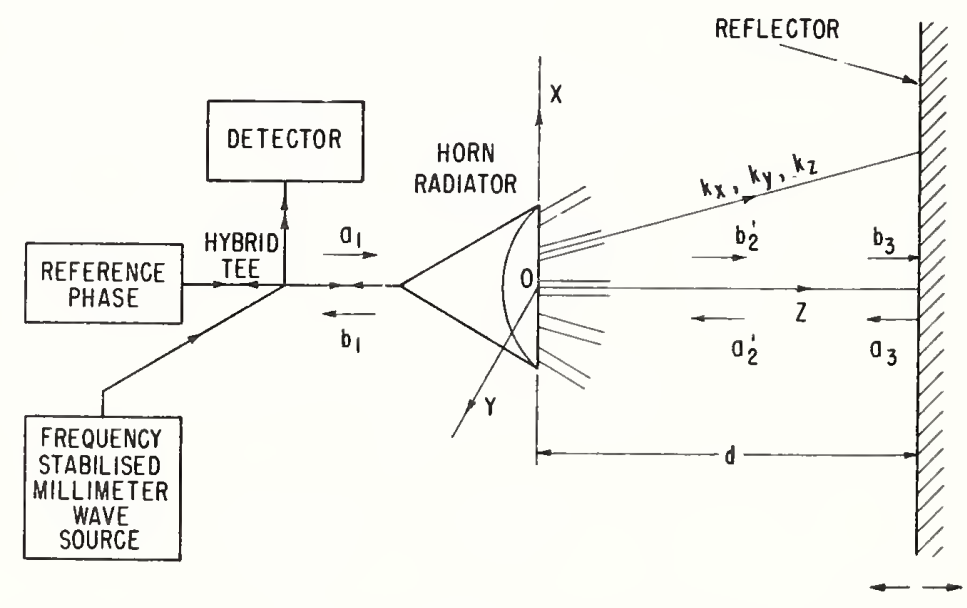

Fig.2. Schemat1c diagram or M11limeter Wave Michelson. Interferometer. 
Operation in the Fresnel region has the advantage that the beams are well collimated in this region, and the effects of stray radiation, which are troublesome in distant field operation, are considerably reduced. Also the attenuation in the open arm is less in this region. The diffraction correction, however, is more difficult, especlaliy if the reflector size must be considered. We require the effective reflection coefficient of the open arm when the reflector is in the Fresnel region of the radiating aperture, and hence the manner in which the amplitude and phase of this vary with the distance 'd' of the reflector from the radiator. Deviations from the 1 deal phase change with displacement, $v 1 z 2$ kd radians where $k=2 \pi / \lambda$, can then be found and corrections applied. By thus correcting for diffraction, and also measuring the refractive index of the medium surrounding the interferometer, the wavelength in vacuo may be found from the number of fringes in a given displacement. The refractive index can be observed to an accuracy of around 1 part in $10^{7}$ by observing the shift in the resonant frequency of a high $q$ microwave cavity, when evacuated and when filled with the medium (refs.6,7). The velocity of electromagnetic waves in vacuo is then determined from the known frequency and measured wavelength.

An outiline of a derivation of the diffraction correction may be given as follows*. The fleld at any point in eront of the antenna is due to the superposition of the angular spectrum of plane waves radiated by 1 t, and this leads to the representation of an anterina in terms of a scattering

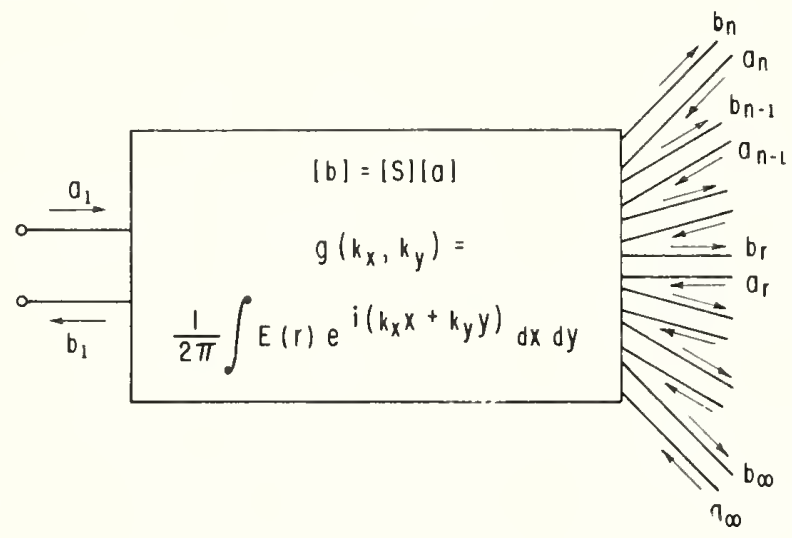

F1g.3. Scattering Matrix of Antenna

matrix (ref.8). Referring to figure 3 which shows this representation, we may write the scattering matrix for the system as

* A more rigorous treatment of the diffraction correction wiii be given in \& paper, "Diflraction Correction in Microwave Interferometry" by D. M. Kerns and E. S. Dayhoff, which is now in preparation.

$(40305)$ 


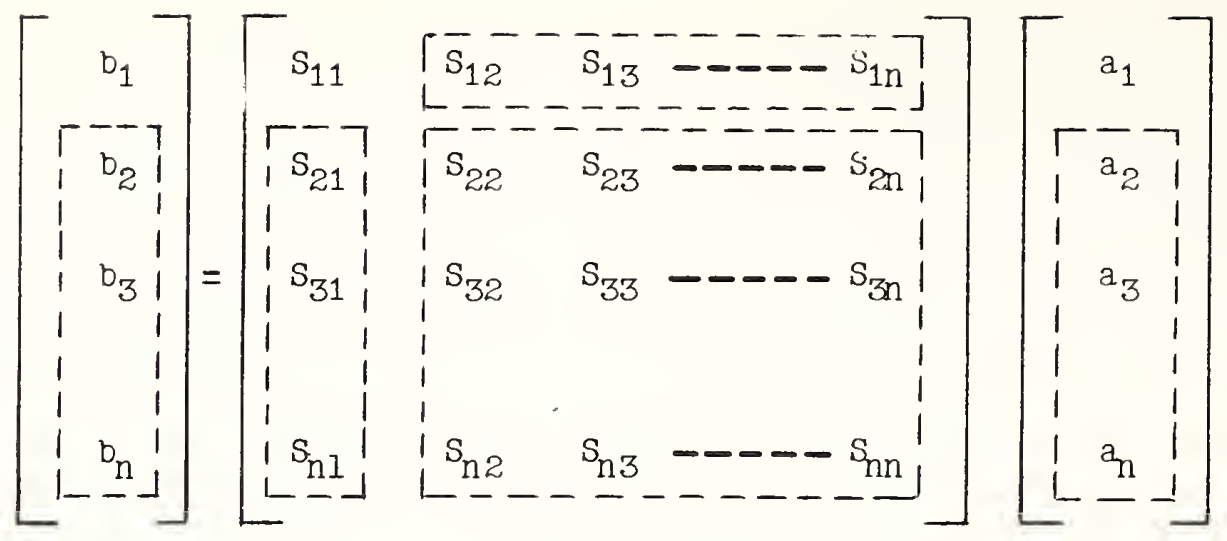

where $n \rightarrow \infty, a_{r}, b_{r}$ are complex numbers representing the amplitude and phase of the electric field in the incident and reflected waves at the $r^{\text {th }}$ terminals, and we Ignore the dashed lines for the moment. Thus if $a_{1}=1$, and $a_{r}=0$ for $r=2,3-\cdots, n$, the radiated spectrum of plane waves is determined by $S_{j}$, where $j=2,3,-\cdots, n$. Similarly, 1 i a plane wave determined by $a_{r}$ is incident on the $r^{\text {th }}$ terminals, the waves developed in the various terminals are determined by $S_{j r}$, where $j=1,2,--n$. Thus a portion of the incident wave is received by the detector, and a portion is scattered, or re-radiated, into all the other terminals on the right-hand side.

Proceeding to a continuous distribution of radiated plane waves, and using the Fourier transform, (refs. 9,10$)$ then

$$
g\left(k_{x}, k_{y}\right)=12 \pi \int \vec{E}(x, y) \exp \left[1\left(k_{x} x+k_{y} y\right)\right] d x d y
$$

where $\mathrm{k}_{\mathrm{x}}, \mathrm{k}_{\mathrm{y}}, \mathrm{k}_{\mathrm{z}}$ are the rectangular components of the propagation vector $k$ in the interferometer, $g\left(k_{x}, k_{y}\right)$ represents the radiated plane wave spectrum, the $x$ and $y$ components of which determine the fleld completely. In passing from the discrete to the continuous plane wave distribution, $S_{j 1}$ in equation (1) with $f>1$ corresponds to $g\left(k_{x}, k_{y}\right)$, and $a_{2}, b_{2}$ correspond respect1vely to $\mathrm{a}_{2}\left(\mathrm{k}_{\mathrm{x}}, \mathrm{k}_{\mathrm{y}}\right), \mathrm{b}_{2}\left(\mathrm{k}_{\mathrm{x}}, \mathrm{k}_{\mathrm{y}}\right)$. Referring to figure 2 , as for oblique incidence on a plane boundary between two media, the phase changes due to propagation of the plane waves in the interferometer are determined by the axial propagation constant $\mathrm{k}_{\mathrm{z}}=\mathrm{k} \cos \theta_{0}$

The vector nature of the plane electromagnetic waves can also be considered by resolving the electric vector of the plane wave into components in and perpendicular to the plane of incidence, which is determined by the vector $k$ and the $z$ axis (ref. 11). These polarizations can now be considered separately as regards propagation through the interferometer, 
and then recombined on entering the horn again. The appropriate impedances to use are those for propagation in the $\mathrm{z}$ direction and are given by (ref. 11).

$$
\eta \cos \theta=\frac{\mathrm{k}_{\mathrm{z}}}{\omega \epsilon} \text { for } \mathrm{E} \text { in plane of incidence }
$$

and

$$
\eta \sec \theta=\frac{\omega \mu}{\mathrm{k}_{\mathrm{z}}} \text { for } \mathrm{E} \text { perp. to plane of incidence }
$$

where $\eta=(\mu / \epsilon)^{\frac{1}{2}}$ is the intrinsic impedance, $\mu$ the permeability, and $\epsilon$ the permittivity of the homogeneous medium in the interferometer, and $\theta$ is the polar angle, corresponding to the angle of incidence. For small angles $\theta$ these two impedances become very nearly equal to $\eta$, corresponding to the scalar solution which is thus adequate for large apertures. Since we need only consider the $x$ and $y$ components of the electric field, the effective transmission line system into which the antenna radiates has impedances which vary as $\eta \cos \theta$, and $\eta \sec \theta$ for the two polarizations respectively. The scattering matrix is thus not symmetrical, and to solve the problem of reception in the interferometer, the appropriate relation between $S_{f 1}$ and $S_{1 j}$ must be found. This is done by normalizing the scattering matrix so that $1 \mathrm{t}$ becomes symmetrical, the normalized element $\overline{\mathrm{S}}_{1 \mathrm{j}}$ being given by (refs. 12,13)

$$
\bar{s}_{1 j}=s_{1 j} \frac{z_{0 j}}{z_{01}}
$$

where $\bar{s}_{j 1}=\bar{s}_{1 j}$. The reciprocity relation between the radiated and recelved angular spectra is then

$$
z_{01} s_{j 1}=z_{0 j} s_{1 \jmath}, \quad j=2,3,-\infty
$$

Here $z_{01}$ is the characteristic impedance of the wavegulde feeding the antenna, and $z_{0 j}$ is elther $k_{z} / \omega \in$ or $\omega \mu / k_{z}$ depending on the polarization. other matrix elements $S_{j k}$ with both $j$ and $k>1$ can similarly be normalized, and represent the scattering of the retuming waves by the antenna, which will give rise to multiple reflections in the interferometers. The term $S_{11}$ is the reflection co-efficient when only wave $a_{1}$ is present, it will be zero for a matched antenna.

To determine the reflection coefficient froin the open arm of the interferometer, substitute the relation $a_{2}^{\prime}\left(k_{x}, k_{y}\right)=-\exp \left(-21 k_{z} d\right) \cdot b \frac{1}{2}\left(k_{x}, k_{y}\right)$, which is imposed by the reflector at distance 'd', into equation (1) and 
solve for $b_{1} / a_{1}$. One obtains

$$
b_{1}=s_{11}-s_{12} \exp (-21 \psi)\left[1+s_{22} \exp (-21 \psi)\right]^{-1} s_{21}^{\prime}
$$

where $\psi=k_{\mathrm{z}} \mathrm{d}$. Here the primes denote the column vectors, row vectors, etc., corresponding to the partitioning of the scattering matrix shown in equation (1). The term $S_{22}^{\prime}$ is quite complicated and gives rise to multiple reflections between antenna and reflector; this effect will usually be small in a well designed interferometer, and can be reduced by the insertion of attenuation in the beam. It will therefore be assumed negligible.

The fleld distribution in the horns to be used approximates that of the $\mathrm{H}_{10}$ rectangular wavegulde mode, whence the radiated angular spectrum is

$$
g\left(k_{x}, k_{y}\right)=c_{1} e_{y} \frac{\cos \left(k_{x} a / 2\right)}{(\pi / a)^{2}-k_{x}^{2}} \frac{\sin \left(k_{y} b / 2\right)}{k_{y}}
$$

where ' $a$ ' and ' $b$ ' are the aperture dimensions, $C_{1}$ is a constant, and the electric fleld in the aperture is in the $\mathrm{y}$ direction. Resolving this fleld into components in and perpendicular to the plane of incidence, using the reciprocity relation given by equation (6), and in the limit of a continuous distribution identifying

$$
\begin{aligned}
& \sum_{j} S_{j 1}^{2} \text { with } \iint\left[g\left(k_{X}, k_{y}\right)\right]^{2} d k_{X} d k_{y} \text {, then w1th } S_{11}=0 \text { we have } \\
& b_{1}=k \exp (-12 k d) \iint Y\left[g\left(k_{X}, k_{Y}\right)\right]^{2} \exp [12 k d(1-\cos \theta)] d k_{X} d k_{y} \\
& a_{1}
\end{aligned}
$$

where $g\left(k_{X}, k_{y}\right)$ is given by equation (8), $K$ is a constant, and $Y$ is an admittance due to the vector nature of the fleld, given by

$$
Y=\left(k_{y}^{2} \omega \epsilon / k_{z}+k_{X}^{2} k_{z} / \omega \mu\right) /\left(k_{X}^{2}+k_{y}^{2}\right)
$$

which in the scalar approximation reduces to a constant, $1 / \eta_{0}$

Equation (9) assumes that the radiated angular spectrum is a symmetrical function of both $k_{x}$ and $k_{y}$. Since the wave retumed after reflection approaches the antenna from the direction in wh1ch the wave $g\left(-k_{x},-k_{y}\right)$ is radiated, this must be considered by replacing $\left[\mathrm{g}\left(\mathrm{k}_{\mathrm{x}}, \mathrm{k}_{\mathrm{y}}\right)\right]^{2}$ by 
$\left[g\left(k_{x}, k_{y}\right) g\left(-k_{x},-k_{y}\right)\right]$ when $g\left(k_{x}, k_{y}\right)$ is not symmetrical. In a similar way the effect of mirror tilt can be considered by inserting the value of $g\left(-k_{x}^{\prime},-k_{y}^{\prime}\right)$ for the retuming waves where $k_{x}^{\prime}, k_{y}^{1}$ account for the tilt. The integral in equation (9) may be written as

$$
|\rho(d, a)| e^{+i \psi(d, a)}
$$

for a square aperture, where for large apertures and hence sharp radiation patterns, $\psi(\mathrm{d}, \mathrm{a})$ is a small phase change due to diffraction, and If $(d, a) \mid$ corresponds to the modulus of the reflection coefficient and is nearly constant for distances ' $d$ ' well within the Fresnel region. In the operation of the interferometer we note the reflector displacement $\mathrm{D}=\mathrm{d}_{2}-\mathrm{d}_{1}$ for which the phase of the reflection coefficient changes by 2n $\pi$, and hence

$$
\mathrm{D}=\mathrm{n} \lambda / 2+\left(\psi_{2}-\psi_{1}\right) \lambda / 4 \pi
$$

where $\psi_{1}$ and $\psi_{2}$ correspond to the value of $\psi$ at the initial and final distances 'd' used. Equations (9) and (12) give the diffraction correction to the simple interferometer equation. Table I shows results of some calculations made using equation (9), the apertures being square with sides of dimensions 'a', ' $d$ ' is the distance between horn and

\begin{tabular}{|c|c|c|c|c|}
\hline \multirow{2}{*}{$\begin{array}{c}\lambda \\
\mathrm{mm}\end{array}$} & \multirow{2}{*}{$\begin{array}{l}\text { ' } \mathrm{a} \text { ' } \\
\mathrm{cm}\end{array}$} & \multirow{2}{*}{$\begin{array}{l}\mathrm{d} \\
\mathrm{m}\end{array}$} & \multicolumn{2}{|c|}{$\begin{array}{l}\text { diffraction correction } \\
\text { to ' } d^{\prime} \text { in microns }\end{array}$} \\
\hline & & & & Scalar \\
\hline 6.278 & 60 & 2 & 56.96 & 56.96 \\
\hline 6.278 & 60 & 10 & 193.80 & 193.80 \\
\hline 6.278 & 30 & 2 & 155.93 & 155.93 \\
\hline 6.278 & 30 & 10 & 503.28 & 503.28 \\
\hline 1.0 & 60 & 2 & 2.32 & 2.32 \\
\hline 1.0 & 60 & 10 & 7.56 & 7.56 \\
\hline
\end{tabular}
reflector and $\lambda$ the wavelength.

Table I Diffraction corrections for Michelson interferometer in the Fresnel region. Aperture field that of $\mathrm{H}_{10}$ rectangular wave-guide mode.

For the apertures and wavelengths envisaged in the interferometer the scalar treatment is adequate to the accuracy quoted in Table I, since the radiation is confined to relatively small angles. Considerations involving the vector field are, however, important in the precise treatment of the problem. 
Equation (9) is applicable when the reflector may be considered as infinite in extent, so that the incident angular spectrum is reflected unchanged. If its dimensions are finite compared with the wavelength, the radiated spectrum is modified on reflection, and this must be considered. Let $\mathrm{k}_{\mathrm{x}}^{\prime}$, $\mathrm{k}_{\mathrm{y}}^{\prime}$ be components of a propagation vector for the radiation from the reflector, then neglecting edge effects the incident angular spectrum $\mathrm{g}\left(\mathrm{k}_{\mathrm{x}}, \mathrm{k}_{\mathrm{y}}\right)$ is modified according to the formula

$$
G\left(k_{x}^{\prime}, k_{y}^{\prime}\right)=\frac{1}{\pi^{2}} \iint g\left(k_{x}, k_{y}\right) \frac{\sin \left(K_{X}^{\prime} b / 2\right)}{K^{\prime}{ }_{x}} \frac{\sin \left(K_{y}^{\prime} b / 2\right)}{K_{y}^{\prime}} \exp \left(-1 k_{z} d\right) d k_{x} d k_{y}
$$

where $K_{x}^{\prime}=\left(k_{x}^{\prime}-k_{x}\right), K_{y}^{\prime}=\left(k_{y}^{\prime}-k_{y}\right)$, ' $b$ ' is the side of the square reflector, $d$ the distance from the radiating aperture, and integration is over the radiated spectrum $g\left(\mathrm{k}_{\mathrm{x}}, \mathrm{k}_{\mathrm{y}}\right)$. Equation (13) is obtained by applying the relation corresponding to equation (2) to the distribution of induced electric field over the reflector face due to the incident field. The consequence of this is that equation (13) replaces a factor $g\left(k_{x}, k_{y}\right)$ $\exp \left(-1 k_{z} d\right)$ in equation (9). It is thus advantageous to use a large reflector when operating in the Fresnel region, since the diffraction correction becomes quite complicated otherwise.

\section{MICROWAVE FABRY-PEROT INTERFEROMETER}

In this interferometer the fringes are made very sharp by multiple reflections between two highly reflecting surfaces, and it corresponds to the free space analogue of the cavity resonator. The narrow bright rings in the optical interferometer show the increased resolution possible, the microwave form being analogous except that silver films would not be used at all for the replectors since they would seriously attenuate the microwave radiation.

A microwave Fabry-Perot arrangenent is shown in figure 4. As before horn radiators with lenses in the apertures are used, the radiated energy passing between the reflectors represented by the composite structures shown. The sharpness of the fringe as ' $d$ ' is varied depends on the reflectivity obtained, and on the angular width of the radiated and recelved spectra, since ' $d$ ' can be optimized for each plane wave. This interferometer is basically quite simple, it does not require any form of beam divider, and the problem of preserving a balance with displacement between two intefering beams does not arise. It 1s, however, necessary to use highly reflecting devices for the reflectors, as the setting accuracy and the diffraction correction will depend on this. 


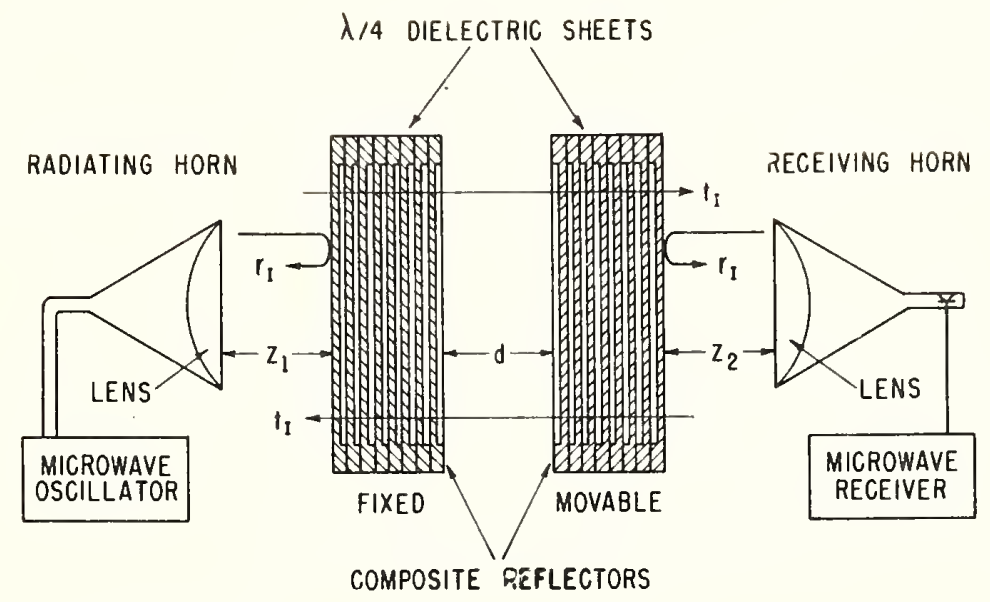

F1g.4. Microwave Fabry-Perot Interferometer

One way of obtaining high reflectivity (ref.2) is to use quarter wave plates of dielectric spaced quarter wavelength apart. Values of amplitude reflection coefficient $r_{n}$ calculated for polystyrene are given in Table II, $\mathrm{n}$ being the number of quarter wave plates. Due to finite dielectric loss the limiting value of reflectivity would be 0.9982. For a dielectric with smaller loss and higher dielectric constant, such as fused quartz, higher values of reflectivity can be obtained. Such reflectors would be very costly due to the required size, except at very short wavelengths, but here the reflector plates become very thin. Odd multiples of quarter wavelength may be used, but the ultimate reflectivity obtained, and the bandwidth of the reflectors will decrease.

\begin{tabular}{|c|c|c|c|c|c|c|c|c|}
\hline $\mathrm{n}$ & 1 & 2 & 3 & 4 & 5 & 6 & 7 & 8 \\
\hline $\mathrm{r}_{\mathrm{n}}$ & 0.4378 & 0.7340 & 0.8861 & 0.9528 & 0.9806 & 0.9913 & 0.9961 & 0.9977 \\
\hline
\end{tabular}

TABLE II Values of the amplitude reflection coefficient obtained from $n$ quarter wave plates of polystyrene with $\lambda / 4$ air space be tween them.

This leads to the consideration of other types of reflectors, such as a stacked system of gratings, consisting or plane layers of inductive, or capacitive irises, or rods placed symmetrically behind each other. 
Figure 5 shows the basic element of such a structure, the particular grating being represented by a discontinulty transmission matrix syminetrically located on a length ' $l$ ' of parallel plate transmission 11ne. The matrix representing the discontinulty may be derived from the equivalent circult of the grating, and the reflectivity from a finite number of such elements must be determined. Only TFM mode interaction between the gratings 1 s considered, and the spacing between the grating elements is less than $\lambda / 2$ so that all other posslble modes on the structure are highly attenuated.

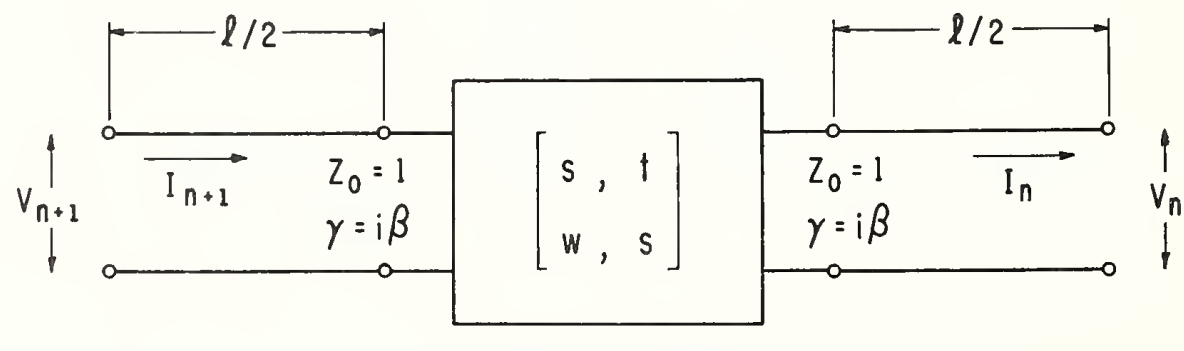

GRATING ELEMENT F1g.5. Line Symmetrically Loaded by Transmission Matrix of
Grating Element.

Neglecting losses the equation for the basic element shown in figure 5 may be written

$$
\left[\begin{array}{l}
V_{n+1} \\
I_{n+1}
\end{array}\right]=\left[\begin{array}{ll}
A B \\
\\
\text { C A }
\end{array}\right]\left[\begin{array}{l}
V_{n} \\
I_{n}
\end{array}\right]
$$

where $A=s \cos \theta+[i(w+t) / 2] \sin \theta, B=[(w+t) / 2] \cos \theta+i s \sin \theta-$ $(w-t) / 2, c=[(w+t) / 2] \cos \theta+1 s \sin \theta+(w-t) / 2$, and $\theta=2 \pi 1 / \lambda$. The reflectivity from a number of such elements, and the spacing ' $l$ ' for optimum reflection can then be determined from the effective propagation constant $\Gamma$ per section, and characteristic impedance $Z$ of the element. These are given by (ref.14)

$$
\cosh \Gamma=A \text { and } z^{2}=B / C
$$


and may be determined from equation (14) for any particular structure. The overall matrix for $n$ such structures in cascade is then given by

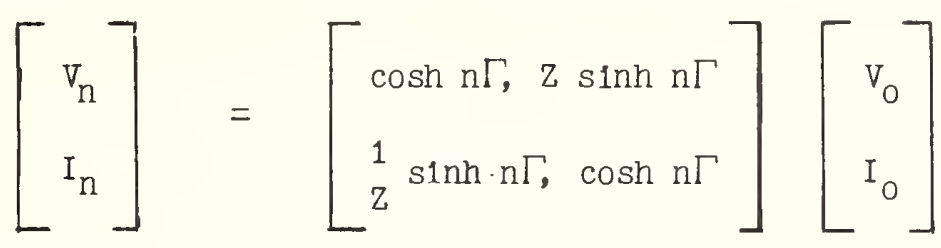

Consider the propagation constant of the element, then

$$
\cosh \Gamma=A=\operatorname{sos} \theta+[1(w+t) / 2] \sin \theta
$$

and since for purely reactive elements cosh $\Gamma$ must be real, the stop bands of the structure are given by $\sin \beta=0$, and the pass bands by $\sinh \alpha=0$, where $\Gamma=\alpha+1 \beta$. For the pass bands

$$
\cos \beta=A \text { where }|A|<1
$$

and for the stop bands

$$
\cosh \alpha=|\mathrm{A}| \text { where }|\mathrm{A}|>1
$$

From equation (16), if the spacing ' 1 ' between gratings corresponds to a pass band, then the reflection coefficient from $\mathrm{n}$ elements $1 \mathrm{~s}$,

$$
r_{n}=\frac{1\left(z^{2}-1\right) \sin n \beta}{2 z \cos n \beta+1\left(z^{2}+1\right) \sin n \beta}
$$

wh1lst for spacings corresponding to a stop band

$$
r_{n}=\frac{\left(z^{2}-1\right) \sinh n a}{2 Z \cosh n a+\left(z^{2}+1\right) \sinh n a}
$$

For spacings such that $\theta$ lies within the pass band, the reflection coepflcient $r_{n}$ will oscillate with increasing $n$, and becomes zero when $\sin \mathrm{n} \beta=0$. When ' $\mathrm{l}$ ' corresponds to a stop band, the reflectlvity will increase monotonlcally with $n$, and 1 t can be shown that the spacing for maximum reflectivity corresponds to the value of $\theta$ which gives the maximum value of $\cosh \alpha=|A|$ within the stop band.

As an example consider the capac1tive rod grating shown in figure 6 , the electric vector being perpendicular to the rods. Values of the appropriate reactances are avallable (ref. 15) and for rods of diameter $0.063 \mathrm{in}$., a wavelength of $6.28 \mathrm{~mm}$, and a spacing of $\mathrm{a} / \lambda=0.417$, we 

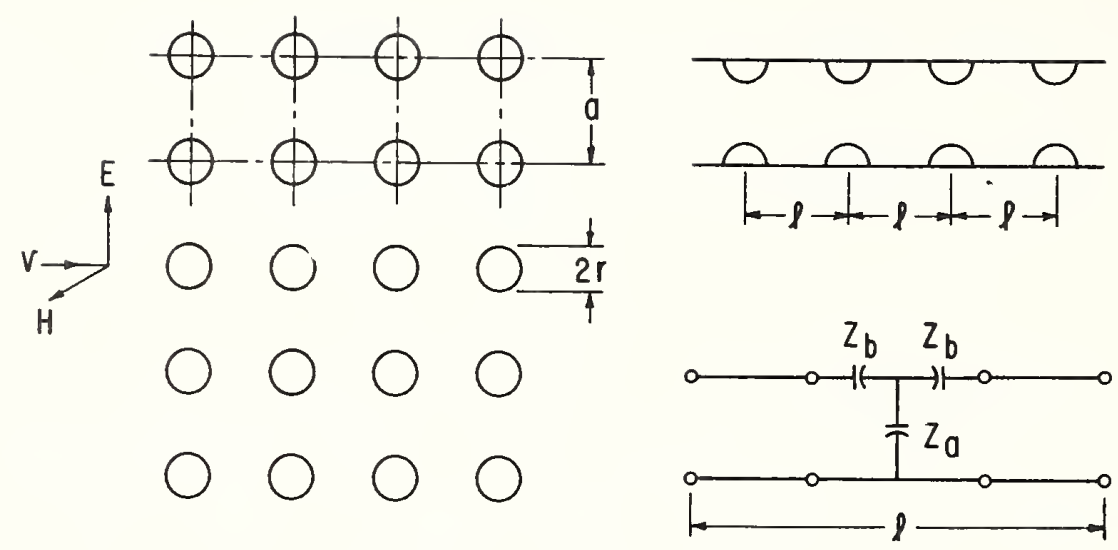

F1g.6. Stacked Capacitive Rod Gratings and Equivalent Circuits.

obtain $s=1.3916, t=-0.8901$, and $w=1.0531$. Hence the effective propagation constant is

$$
\cosh \Gamma=A=1.3916 \cos \theta-0.0815 \sin \theta
$$

Figure 7 shows the variation of $\mathrm{A}$ with $\theta$, the optimum value of 1 being close to $\lambda / 2$ for this grating. Values of reflectivity $\left|r_{n}\right|^{2}$ are given in Table III for this spacing. At a wavelength of $6 \mathrm{~mm}$ the bulk reflectivity of silver is around 0.99958 , and thus the reflectivity obtained by stacking 5 or 6 such capacitive gratings should be comparable.

\begin{tabular}{|c|c|c|c|c|c|c|}
\hline$n$ & 1 & 2 & 3 & 4 & 5 & 6 \\
\hline$\left|r_{n}\right|^{2}$ & 0.48542 & 0.87963 & 0.97699 & 0.99583 & 0.99925 & 0.99986 \\
\hline
\end{tabular}

Table III. Calculated reflectivity $\left|r_{n}\right|^{2}$ from a number $n$ of stacked capacitive rod gratings at $\lambda / 2$ spacings. Rod diameter 0.063 in., spacing 'a' 0.103 in., $\lambda=6.28 \mathrm{~mm}$.

Similarly other types of gratings can be considered, and figure 7 shows the prodagation constant for the element of an inductive type of rod grating, (ref.16) and for comparison the propagation constant for the basic element of the dielectric sheet reflector. An indication of the bandwidth of the various structures is given by these curves, since provided a sufficient number of gratings is used the reflectivity will be high over the region of the stop band. 


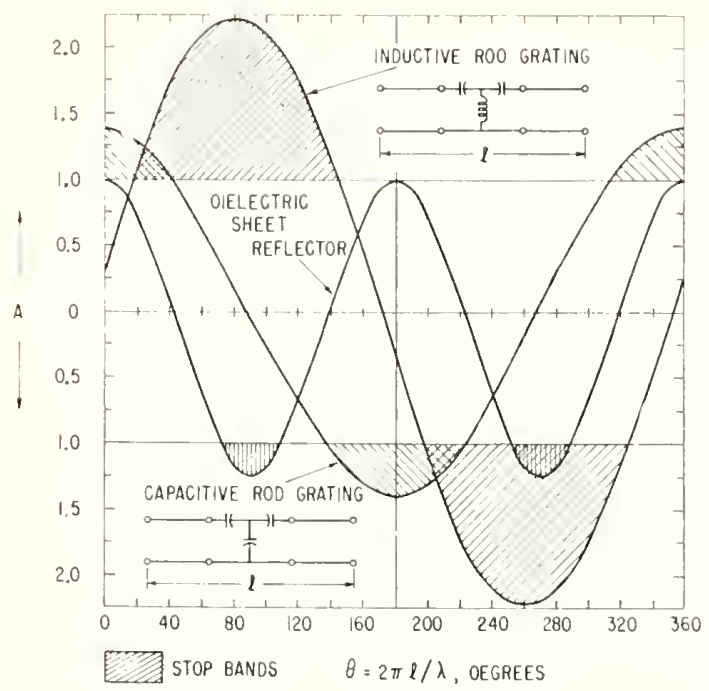

F1g.7. Pass and Stop Bands for Th1ck Gratings

A pilot Fabry-Perot interferometer has been operated with reflectors of the capacitive rod type discussed above. Using horn-lens apertures $6 "$ square, with reflector dimensions $71 / 2^{*}$ square, the fringes shown in figure 8 were obtained, the wavelength being $6.28 \mathrm{~mm}$.

In the derivation of the diffraction correction in this interferometer it is assumed that the reflectivity of the composite reflectors is constant for the range of angles concerned in the plane wave spectrum incident on them. They will also be assumed infinite in extent, so that the radiation pattern is not modified on reflection. Proceeding in the manner used in the discussion of the Michelson interferometer, and again neglecting interaction terms containing $S_{22}^{\prime}$ it is found that the amplitude reflection and transmission coefficients of the Fabry-Perot interferometer are given by

$$
\begin{aligned}
& r_{F}=c_{2} \iint\left[g\left(k_{x}, k_{y}\right)\right]^{2} \exp \left(-12 k_{z} z_{1}\right) r_{I} d k_{x} d k_{y} \\
& t_{F}=c_{3} \iint\left[g\left(k_{x}, k_{y}\right)\right]^{2} \exp \left[-1 k_{z}\left(z_{1}+z_{2}\right)\right] t_{I} d k_{x} d k_{y}
\end{aligned}
$$

where $\mathrm{C}_{2}$ and $\mathrm{C}_{3}$ are constants, $\mathrm{g}\left(\mathrm{k}_{\mathrm{x}}, \mathrm{k}_{\mathrm{y}}\right)$ is again the radiated and recelved angular spectra of the apertures, here assumed identical, $z_{1}$ and $z_{2}$ are 


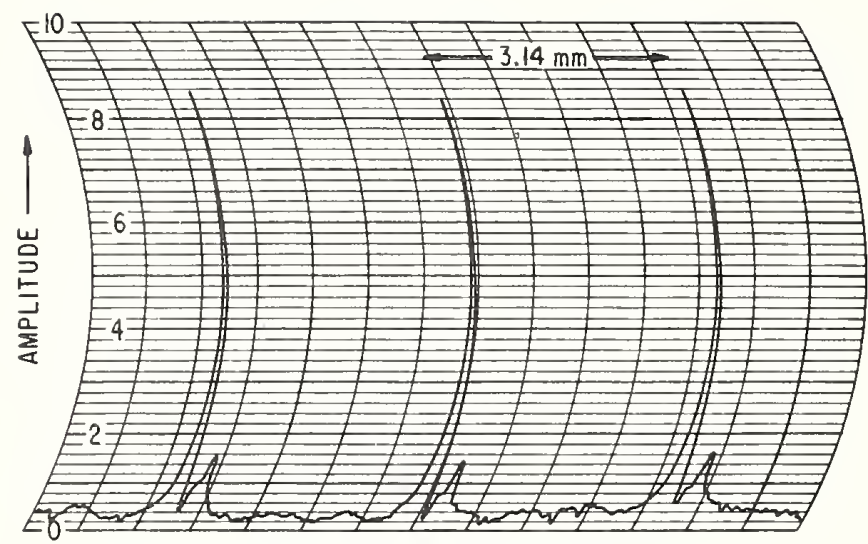

DISTANCE $\triangle$ BETWEEN REFLECTORS INCREASING $\longrightarrow$

Fig.8. Fringes Recorded on Microwave Fabry-Perot Interferometer with Capacitive Rod Grating Reflectors. $\lambda=6.28 \mathrm{~mm}$.

the distances of the radiating and recelving apertures from the reflectors, and $r_{I}$ and $t_{I}$ are the amplitude reflection and transmission coefficients of the interferometer section shown in figure 4. Assuming no losses in the reflector elements, these are given by

$$
\begin{aligned}
& r_{I}=\frac{\left\{r_{v} 1-\exp \left[-12\left(k_{z} d+\phi\right)\right]\right\}}{1-R \exp \left[-12\left(k_{Z} d+\phi\right)\right]} \\
& t_{I}=\frac{(R-1) \exp \left[-1\left(k_{Z} d+2 \phi\right)\right]}{1-R \exp \left[-12\left(k_{Z} d+\phi\right)\right]}
\end{aligned}
$$

where $r_{v}=|r|$ exp $(-1 \phi)$ is the voltage reflection coefficient of a single reflector given by equation (20) or (21), and $R=|r|^{2}$. From equation (26) we obtain the usual transmission formula used in optics viz.,

$$
\left|t_{I}\right|^{2}=\frac{(1-R)^{2}}{1-2 R \cos 2\left(k_{Z} d+\phi\right)+R^{2}}
$$

which for high reflectivity is a sharp function of $\mathrm{kz}_{\mathrm{z}} \mathrm{d}$.

Equations (23) and (24) are derived on the basis of scalar diffraction theory, and as before would be a good approximation for apertures larger than about 50 wavelengths. Comparing these equations with equation (9) 
for the Michelson interferometer, the only change is that the reflection and transmission coefficients of the reflector system are now introduced. The shape of the reflected and transmltted fringes thus depends on the radiation patterm $\mathrm{g}\left(\mathrm{k}_{\mathrm{X}}, \mathrm{k}_{\mathrm{y}}\right)$, and provided the reflectivity achieved is high enough, this is effectively scanned by the flltering action of the reflector system, as the distance 'd' varies. Also for high reflectivity and discrimination in the refiector system, the reflected power will always be quite high, even at the spacings for optlmum transmission, since only a few of the incldent plane waves will be transmltted by the reflectors at a given spacing.

The setting accuracy in this inteferometer thus depends on the reflectivity, and on the radiation pattern, and these must be such that the fringes become very sharp, especlally for preclsion work. The maximum transmitted fringe intensity will tend to occur when ' $d$ ' 1 s such that the portion of the radiated angular spectrum centered about the maximum amplitude is transmltted, and for very large reflectors the diffraction correction should be small if the reflectivity is extremely high so that only a small portion of the radiated angular spectrum is effective between the reflectors. Further work is required to establish the precision possible with this interferometer, and to determine the magnitude of the diffraction correction for various reflectivities. This is especlally so when the reflectors must be considered as finfte compared with the wavelength, as the radiated angular spectrum will be modified in a complicated way by the multiple reflections, and this will affect the diffraction correction.

\section{CONCLUSIONS}

The theory given for the diffraction correction in the Michelson interferometer operating in the Fresnel region represents the solution of the problem for a reflector of infinite size. Table I, which applies for the stated aperture fleld distribution, shows that for apertures some hundred wavelengths in extent, the correction amounts to around two parts in $10^{5}$. Thus if $1 \mathrm{t}$ is known to $1 \%$ the results should be accurate to a few parts in $10^{7}$. In practice the antenna radiation pattern will have to be measured quite accurately, and the measured pattern used to determine the diffraction correction. Both the amplitude and phase of the angular spectrum $\mathrm{g}\left(\mathrm{k}_{\mathrm{x}}, \mathrm{k}_{\mathrm{y}}\right)$ are required, and measurements of the antenna pattern should thus include phase as well as amplitude measurements, or else the phase must be deduced from information on the phase distribution in the aperture. As indicated, for these aperture sizes the scalar diffraction theory approximation is adequate to at least this order of accuracy. 
These results are valuable, since applications of microwave interferometry to metrology would most likely require operation in the Fresnel region of large radiating apertures.

Advantages of operation in this region, are that the radiation is well collimated, and effects of stray radiation are less troublesome. Also the attenuation in the open arm of the interferometer is considerably less, with a consequent increase in avallable signal power; an extremely important consideration at millimeter wavelengths. To alleviate multiple reflection troubles in the interferometer, some attenuation in this arm may be necessary, but 1 t is not expected that large values will be required. The main disadvantage is that for precise work large radiators and reflectors are required for the wavelengths presently avallable, and this leads to some mechanical problems as indicated by the Michelson interferometer now under consideration. The work also represents a different approach to the diffraction correction than that used by Froome, and is important for this reason. As in his work, it is expected that the accuracy in the determination of ' $\mathrm{c}$ ' will be limited by the length measurements, and by the determination of the refractive index of the a1r.

The Fabry-Perot interferometer with 1 ts sharp fringes and basic simplicity, represents a very promising instrument for application to the determination of ' $\mathrm{C}$ ', and the reflector designs discussed should adequately meet the problem of obtaining high reflectivity in the microwat region. The diffraction correction is more difficult especially if the reflectors are finite compared with the wavelengths, but calculations assuming infinite reflectors will give an indication of the magnitude of this. There are also possibilities of using evacuated "etalons" in the microwave region, with the consequent elimination of the difficult measurement of refractive index at short wavelengths. The high wavelength resolution due to sharp radiation patterns and high reflectivity, could also prove useful in the wavelength analysis of millimeter wavelength sources too high in Prequency to be measured conventionally.

Finally, the general complexity of the formula involved in the diffraction correction for the Fresnel region, especially in the microwave FabryPerot interferometer, indicates the great advantages of using very short wavelengths, preferably in the one millimeter region or below. At these wavelengths apertures of dimensions approaching 1000 times the wavelength could be used, with a considerable reduction in diffaction effects. 
The authors would like to acknowledge the assistance derlved from the inftial work of Dr. E. S. Dayhoff on the diffractioncorrection in the

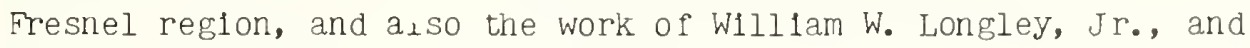
Dr. Paul F. Wacker on the computation of the integrals representing the diffraction correction.

\section{PEFERENCES}

1. Culshlaw, W., Proc. Phys. Soc. B, 1950, 63, 939.

2. Culshaw, W., Proc. Phys. Soc. B, 1953, 66, 597.

3. FROOME, K. D., Proc. roy. Soc. A, 1952, 2/3, 123.

4. FROOME, K. D., Proc. roy. Soc. A, 1954, 223, 195.

5. FROOME, K. D., Proc. roy. Soc. A, 1958, 247, 109.

6. BI RNBAUM, G., KRYDER, S. J., and LYONS, H., J. appl. Phys. 1951, $22,95$.

7. ESSEN, L., and FROOME, K. D., Proc. Phys. Soc. B, 1951, 64, 862.

8. MONTGOMERY, C. G., DICKE, R. H., and PURCELL, E. M., Principles of Microwave Circuits. McGraw-Hill Book Co. New York: 1948, p. 317.

9. BOOKER, H. G., and rLErriow, P. C., Proc. I.E.E., 195097 Part III, 11. 10. SILVER, S., Microwave Antenna Theory and Design: McGraw-Hill Book Co., New York: 1949, p.174.

11. SCHFLKUNOFF, S. A., Electromagnet1c Waves, D. Van Nostrand Book Co., New York: 1943, p.251.

12. KERNS, D. M., J. Research of N. B.S., 1949, 42, 515.

13. CARLIN: H. J., Report R-366-54 Polytechn1c Institute of Brooklyn - 300, June 1954.

14. BRILLOUIN, I., Wave Propagation in Periodic Structures: Dover Publications Inc., 1953, p.193.

15. LEWIN, L., Advanced Theory of Waveguides, Iliffe and Sons Ltd., London: 1951, p. 37.

16. MARCUVITZ, N., Waveguide Handbook, McGraw Hill Book Co., New York: 1951, p. 285. 


\section{A Standard of Attenuation for Microwave Measurements}

\author{
R. E. GRANTHAM \\ NONMEMBER AIEE
}

\author{
J. J. FREEMAN \\ NONMEMBER AIEE
}

$\Gamma^{\mathrm{N}}$ $\mathrm{N}$ LINE with the program of the $\mathrm{Na}$ tional Bureau of Standards for extending the standards of electrical quantities up through microwaves, the establishment of a standard of attenuation was undertaken. For such a standard to be useful, it must be readily adaptable to the calibration of commercial attenuators over the entire frequency range. Also, for self-consistency, such a standard must be capable of being checked by $\mathrm{d}-\mathrm{c}$ measurements, thus relating the radiofrequency ( $r-f$ ) standard to the more accurate primary standards.

A standard of any physical quantity consists not merely in the unique specification of that particular quantity, but equally important, in the specification of the operations, or procedures, through which an unknown quantity is measured in terms of it. The purpose of this paper is to describe such a standard, and the preliminary experiments employed in its evaluation and development.

\section{General Description}

Because of the extremely wide frequency range over which attenuators must be calibrated, the construction of a series of standard microwave attenuators to cover the entire spectrum, and the use

Paper 48-50, recommended by the AIEE instruments and measurements committee and approved by the technical program committee for presentaby the technical program conner tion at the AlEE winter general meeting. Pittsburgh, Pa., January $26-30,1948$. Manuscript sub-
mitted November 12, 1947; made available for printing December 15, 1947.

R. E. GRantham and J. J. FreEman are both with the National Bureau of Standards, Washington, D. C.

The authors wish to express appreciation for the valuatle services of R. H. McCracken who constructed much of the electronic equipment and performed many of the experiments, and A. A Feldmann who designed the mechanical features of the wave-guide-below-cutoff attenuator. of the direct substitution method of calibration, is impracticable. A modification of direct substitution, namely, the heterodyne or intermediate-frequency (i-f) substitution method, has been developed in various laboratories, ${ }^{1,2}$ and permits the comparison of an unknown attenuator, operating at an arbitrary frequency, with a standard attenuator, operating at a fixed frequency. Thus, one standard attenuator, operating at a convenient frequency, may be used to calibrate attenuators over the entire microwave spectrum.

Essentially, the heterodyne method operates as follows. A r $\mathrm{f}$ generator feeds power through an unknown attenuator into a linear frequency converter, which converts the microwave frequency into the intermediate frequency. The converter feeds through an $\mathrm{i}-\mathrm{f}$ standard attenuator into an amplifier followed by a detector and meter. The unknown attenuator is then removed, and the standard attenuator adjusted to give the same meter reading. The attenuation of the unknown is then equal to the increase of attenuation of the standard.

This method assumes that the frequency converter is linear, that is, that the $\mathrm{i}-\mathrm{f}$ power from the converter is proportional to the input microwave power. For small enough input power this is true for a crystal converter, and the operating conditions for a prescribed departure from linearity may be determined experimentally.

The range of attenuation which may be measured by the heterodyne method is less than that measurable by direct substitution, since in the heterodyne method the maximum permissible power input into the converter is limited by the advent of converter nonlinearity. However, a combination of direct substitution, us- ing a previously calibrated $r$ - $f$ attenuator, together with the heterodyne method, extends the heterodyne attenuation range to that of the direct substitution method, although with a decrease in accuracy.

\section{Detailed Description of the Equipment Used to Evaluate the Heterodyne Method at $\mathrm{X}-\mathrm{Band}$}

The block diagram of the equipment used to evaluate the heterodyne method is shown in Figure 1. The $r-f$ generator is a klystron providing at least $250 \mathrm{milli}$ watts of $r-f$ power. The $d-c$ power supplies for the klystron and amplifying circuits are battery-stabilized, rather than voltage-regulator-tube stabilized, and stabilized, and special shock mountings are used to prevent noise modulation of the $r$-f signal by the blower used to cool the klystron. A very high degree of amplitude stability is necessary in all of the components of the system, because the output meter of the system is sensitive to a 0.2 per cent $(0.01$ decibel) change in power level.

The frequency of the $r-f$ generator is measured by a calibrated wavemeter in the spectrum analyzer. The amplitude of the $r-f$ output of the generator is monitored by a unit as shown in Figure 2. A sensitive $d$-c galvanometer in the power monitor gives a unit scale deflection for a 0.05 per cent $(0.002 \mathrm{db})$ change in power level. Variable attenuator $A$, in Figure 1 , is used to calibrate the power monitor.

The $\mathrm{r}-\mathrm{f}$ generator klystron is followed by buffer attenuator $B$ which prevents changes in impedance caused by inserting and removing the unknown attenuator from changing the load impedance presented to the klystron. Thus the power output and frequency of the $r-f$ generator are maintained constant throughout a measurement. A twenty-db attenuator provides an adequate amount of buffing, as evidenced by the fact that the introduction of a 10 to 1 voltage standingwave ratio at transformer $A$ resulted in no change of generator output, as indicated by the power monitor.

When measuring the insertion loss of 


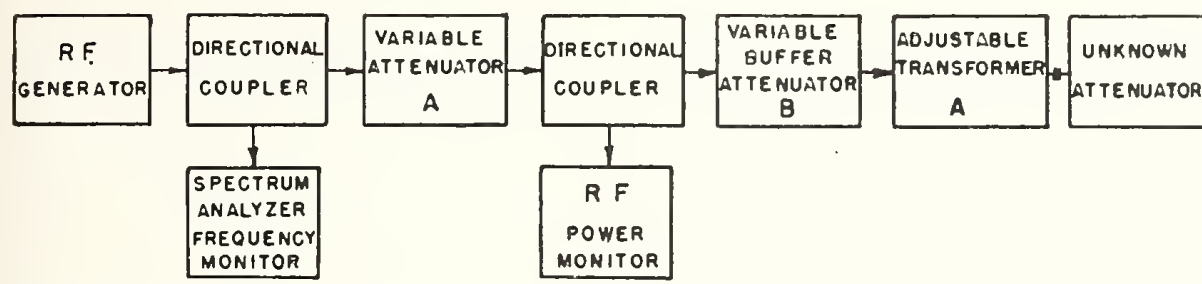

Figure 2. Radio-frequency power monitor schematic

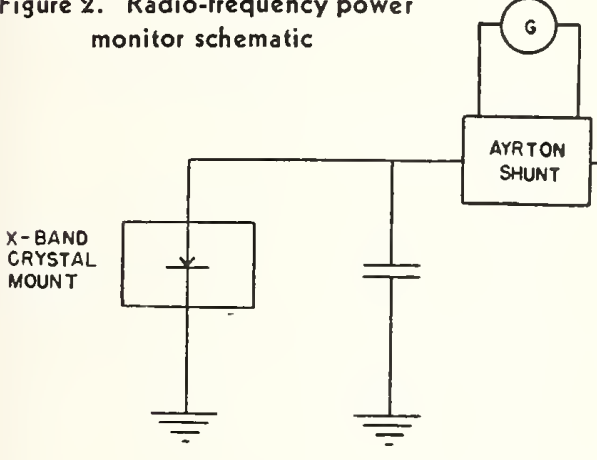

an attenuator, it is necessary to insert the attenuator in a transmission line which is matched looking both ways from the point of insertion. To meet this requirement it is necessary to use adjustable transformers $A$ and $B$ to match attenuators $B$ and $C$ respectively as accurately as possible to the characteristic impedance of the wave guide.

Buffer attenuator $C$ is needed to isolate the local oscillator from the changes in the impedance of its load which would occur when the unknown attenuator is inserted and removed. The conversion efficiency of the crystal changes appreciably with local-oscillator power. Hence, the local-oscillator power output must be maintained as constant as possible. Twenty $\mathrm{db}$ is the minimum attenuation used for buffer attenuator $C$.

The repeller voltage of the local-oscillator klystron is swept by a linear sawtooth voltage so that the beat-frequency output of the mixer sweeps over about 0.5 megacycles per second. The i-f amplifier band width is about 50 kilocycles per second, so the i-f response curve is plotted on the oscilloscope as in the usual spectrum analyzer, but with much more than the usual frequency dispersion. The peak voltage of the pulse on the oscilloscope is measured by a differential vacuum-tube voltmeter, which has a sensitivity of one scale division per 0.2 per cent $(0.01 \mathrm{db})$ change in power level. The reason for sweeping the local-oscillator frequency rather than operating it continuous wave $(c-w)$ is that small shifts in the frequency of the $r$-f generator or the

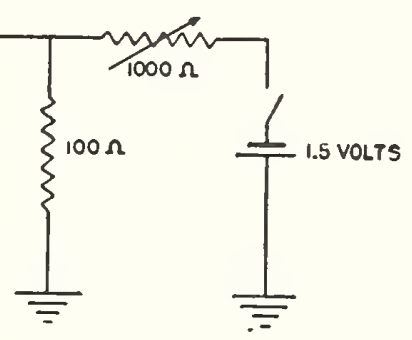

local oscillator may occur up to about 200 kilocycles per second without causing appreciable variations in the output differential voltmeter. If the local oscillator were operated $\mathrm{c}-\mathrm{w}$, the beat frequency could not vary more than about plus or minus 3 kilocycles per second for the same variations in output.

\section{Range of Measurement and Errors of Heterodyne Method}

The attenuation range measurable by the heterodyne method is determined by the maximum input power consistent with crystal linearity, and by the crystal noise power. The range of crystal linearity was determined by measuring a fixed value of attenuation as a function of crystal input power, using the apparatus indicated in Figure 3 . The measured values of attenuation of a 30- $\mathrm{db}$ pad as a function of $\mathrm{r}-\mathrm{f}$ buffing, and hence crystal input power, are depicted in Figure 4. For 100 milliwatts of $r-f$ input power, it appears that $40 \mathrm{db}$ of buffing attenuation (corresponding to a crystal input power of 10 microwatts) suffices for accuracies of plus or minus $0.02 \mathrm{db}$.

The crystal noise power was measured to be less than one micromicrowatt. Accordingly, the maximum range of attenuation measurement corresponding to an error of $0.05 \mathrm{db}$ due to crystal noise, is 50 $\mathrm{db}$.

In addition to possible crystal nonlinearity, errors in the heterodyne method may occur through r-f or i-f leakage, mismatch on either side of the $r-f$ or $i-f$ at-

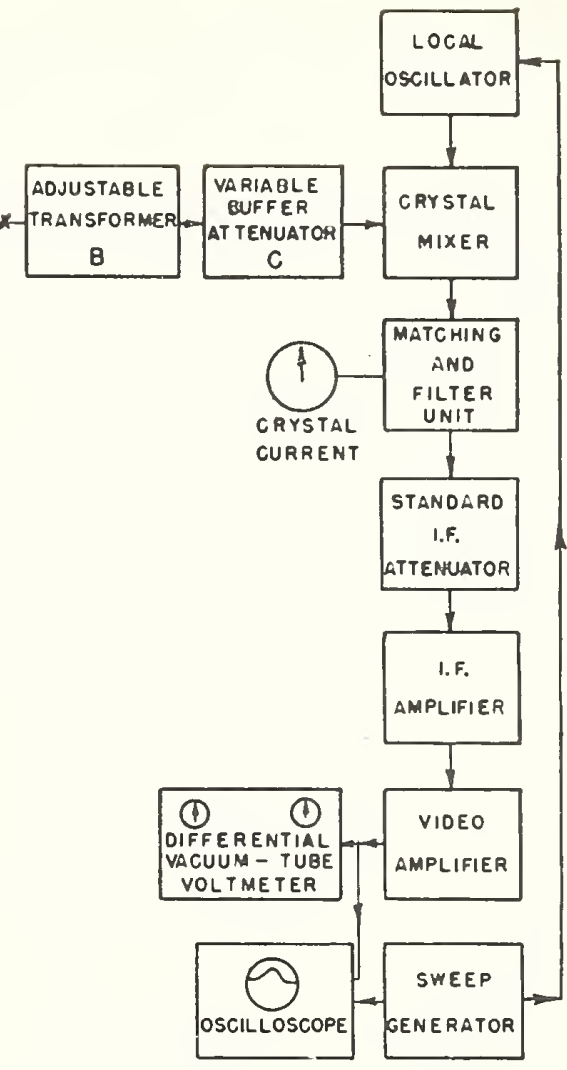

tenuators, frequency and amplitude instability and errors in the i-f standard attenuator. Errors of the i-f standard will be discussed in another paragraph, and the question of errors due to mismatch is too lengthy and detailed to consider at this time. If we neglect the last two sources of error, the over-all accuracy of attenuation measurements with the equipment described herein is estimated to be within plus or minus $0.02 \mathrm{db}$ in the $0-10 \mathrm{db}$ range, and plus or minus 0.2 per cent of the attenuation value in $\mathrm{db}$ for the $10-50$ db range.

\section{Theoretical Factors Affecting the Choice and Design of the Attenuator}

To be suitable as a standard, the i-f attenuator must be able to yield known values of attenuation in terms of an accurately measurable parameter, such as length, or it must be capable of calibration in terms of $\mathrm{d}-\mathrm{c}$ measurements. First designed by Harnett and Case, ${ }^{3}$ the cutoff attenuator, Figure 6 , which consists of a hollow tube excited at one end below its cutoff frequency, and a coil or condenser which picks up the attenuated field at the other end, fulfills the former requirement. Since the generated field falls off exponentially with distance from the exciting source, and since the attenuation constant may be computed from the dimensions of the tube, the ratio of any two voltages, or 
the attenuation introduced by a given length of tube, is reduced to a measurement of length.

\section{Cross-Section of Tube}

However, it is well known that the electromagnetic field generated within the tube consists of the superposition of an infinite number of modes, each of which is attenuated along the axis of the tube as $e^{-\alpha_{n m} z}$, where $\alpha_{n m}$ is a function of the geometry of cross section, and the particular mode. The higher the mode, the greater is its attenuation constant $\alpha_{n m}$, so that only for large enough values of $z$ do the higher modes become negligible. Obviously, then, the accuracy of the attenuator increases as the ratios of the amplitudes of the higher modes to that of the lowest one decrease, and as the ratios of the attenuation constants of the higher modes to that of the lowest one increase. The comparison of these factors for tubes of circular and rectangular cross section is given in Tablc $\mathrm{I}$.

Circular Cross Section. The fifth column of Table I enumerates the ratio of attenuation constants, $\alpha_{n m}$, of the first few modes to that of the lowest mode, for circular cross-section.

From Table I, it is apparent that a measure of purity of mode can be achieved by exciting the $T E_{11}$ mode, and by eliminating the $T E_{01}, T E_{21}$, and $T M_{01}$ modes through symmetry of excitation. Just as a violin string plucked in the center does not vibrate with even harmonic modes similarly, a proper symmetry of the exciting current distribution in a cutoff attenuator will not excite certain classes of modes. In particular, if one considers the cross section divided into four symmetrical quadrants, and if the current distribution in the right-hand quadrants is the negative mirror image of that in the left hand quadrants, and if the current dis. tribution in the upper quadrants is the positive mirror image of that in the lower quadrants, then only those nodes will bc excited for which $n$, (the mode index in dicating angular dependence) is odd, eliminating five of the seven undesired modes listed in the column. However, if one uses an unbalanced generator to excitc the attenuator coil, the distributed capacitance of the coil makes the achievement of this symmetry difficult.

The obvious type of exciting symmetry associated with a circular tube is, of course, circular symmetry. In particular, circularly-symmetric capacitative disk excitation, described by Harnett and Case $^{3}$ and W. O. Smith ${ }^{4}$ will excite only transverse magnetic modes, for which $n=$
0 ; of these the mode with the lowest at tenuation constant is the $T M_{01}$. The danger with this type of attenuator is that any asymmetry will produce a $T E_{11}$ modo which has a lower attenuation constant, and hence, for a large enough value of at tenuation, can lead to appreciable error.

Rectangular Cross Section. The first four columns of Table I enumerate the ratios of attenuation constants, $\alpha_{n m}$, of the first few modes to that of the lowest mode, for several ratios of rectangular crosssection dimensions, $a$ and $b$. It will be noticed that for $a=b / 2$, or less, the unwanted modes decay much faster than for circular cross-section. Also, if the exciting-current distribution is chosen so that to each element of current corresponds its negative mirror image with respect to the plane $x=a / 2$, and its positive mirror image with respect to the plane $y=b / 2$, then it may be shown that only those modes are excited for which $m$ is even and $n$ is odd. Although this type of symmetry is difficult to achieve at lower frequencies, using coil structures, at microwave frequencies this type of symmetry should be easily obtainable using symmetric windows as the exciting structures.

\section{REACTION EFFECT}

The reaction of the receiver circuit on the exciting current becomes the limiting factor affecting the closeness of spacing between exciter and receiver, when precautions have been taken to reduce the unwanted modes, since exponential decay with spacing implies constant current or voltage excitation, for the $T E_{11}$ and $T M_{01}$ modes respectively. As already mentioned by Harnett and Case, ${ }^{3}$ the impedance coupled into the exciter circuit by the receiver decreases as $e^{-2 \alpha_{n} z}$ for the
$T E_{11}$ mode, and as $e^{-\alpha 0_{1} z}$ for the $T M_{0}$ mode, where $z$ is the separation between exciter and receiver. Accordingly, a further advantage of the $T E_{11}$ mode over the $T M_{01}$ mode attenuator is the smaller interaction between exciting and receiving circuits for a given attenuation

\section{Skin EFfect}

For a perfectly conducting circular tube, of radius $a$,

$$
\begin{aligned}
& \alpha_{11}=\frac{1.841}{a}\left[1-\left(\frac{2 \pi}{1.841} \frac{a}{\lambda}\right)^{2}\right]^{1 / 2} \\
& \text { for the } T E_{11} \text { mode } \\
& \alpha_{01}=\frac{2.405}{a}\left[1-\left(\frac{2 \pi}{2.405} \frac{a}{\lambda}\right)^{2}\right]^{1 / 2}
\end{aligned}
$$

for the $T M_{01}$ mode

If one takes into account the effect of finite conductivity, $\sigma$, following the method of Stratton, ${ }^{6}$ one gets easily, for the attenuation constans.

$\alpha^{\prime}{ }_{11}=\frac{1.841}{a}\left[1-\frac{\delta}{a}+\left(\frac{2 \pi}{1.841} \frac{a}{\lambda}\right)^{2}\right]^{1 / 2}$ for the $T E_{11}$ mode

$\alpha_{01}^{\prime}=\frac{2.405}{a}\left[1-\left(\frac{2 \pi}{2.405} \frac{a}{\lambda}\right)^{2}\left(1+\frac{\delta}{a}\right)\right]^{1 / 2}$

for the $T M_{01}$ mode

where $\delta=\left(\frac{2}{\omega \mu \sigma}\right)^{1 / 2}$ is thd skin depth.

For an i-f attenuator, $a / \lambda<<1$, and it is seen that there is no correction for the $T M_{01}$ mode, whereas the effects of finite conductivity are such as to increase the effective radius from $a$ to $a+(\delta / 2)$, for the $T E_{11}$ mode.

Figure 3. Buffing block diagram for measuring crystal nonlinearity

\begin{tabular}{|c|c|c|c|c|c|c|}
\hline \multicolumn{5}{|c|}{ Rectangular Wave Guide } & \multicolumn{2}{|c|}{ Circular Wave Guide } \\
\hline Mode & $\frac{\alpha}{\alpha T E_{01}}$ & $\frac{\alpha}{\alpha T E_{01}}$ & $\frac{\alpha}{\alpha T E_{0 r}}$ & $\frac{\alpha}{\alpha T E_{01}}$ & $\frac{\alpha}{\alpha T E_{11}}$ & Mode \\
\hline & $a=b$ & $a=0.9 b$ & $a=b / 2$ & $a=b / 3$ & & \\
\hline $\begin{array}{l}T E_{01} \ldots \\
T E_{02} \ldots \\
T E_{03} \ldots \\
T E_{10} \ldots \\
T E_{20} \ldots \\
T E_{11}, T M_{1} \\
T E_{21}, T M\end{array}$ & $\begin{array}{l}1.0 \\
2.0 \\
3.0 \\
1.0 \\
2.0 \\
2.0 \\
1.41 \\
2.2 .\end{array}$ & $\begin{array}{l}\ldots 1.0 \ldots \\
\ldots 2.0 \ldots \\
\ldots 3.0 \ldots \\
\ldots 1.1 \ldots \\
\ldots 1.5 \ldots \\
\ldots 2.4 \ldots\end{array}$ & $\begin{array}{l}\ldots 1.0 \ldots \\
\ldots 2.0 \ldots \\
\ldots 3.0 \ldots \\
\ldots 2.0 \ldots \\
\ldots 2.0 \ldots \\
\ldots 4.2 \ldots \\
\ldots 4.1 \ldots\end{array}$ & $\begin{array}{r}\ldots 1.0 \\
\ldots 2.0 \\
\ldots 3.0 \\
\ldots 3.0 \\
\ldots 6.0 \\
\ldots 3.1 \\
\ldots 6.1\end{array}$ & $\begin{array}{l}1.0 \\
1.31 \\
1.66 \\
2.08 \\
2.79 \\
2.90 \\
3.0\end{array}$ & $\begin{array}{l}T E_{11} \\
T M_{01} \\
T E_{21} \\
T E_{11}, T M_{11} \\
T M_{21} \\
T E_{12} \\
W_{01}\end{array}$ \\
\hline
\end{tabular}

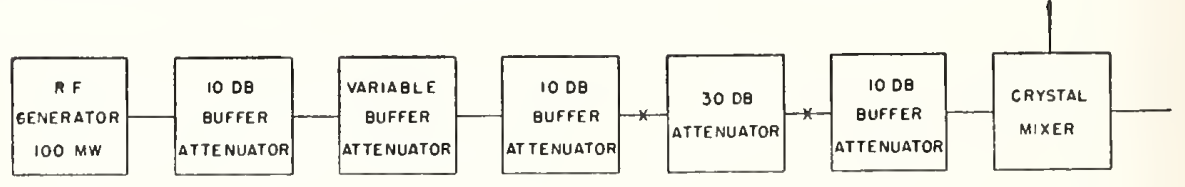

Table I 


\section{Experimental Evaluation of Cutoff Attenuator}

\section{Mechanical DEscription}

A circular waveguide-below-cutoff attenuator was built for operation at 20 megacycles per second, with the $T E_{11}$ mode having an attenuation rate of $21 \mathrm{db}$ per inch. The distance between coils is adjusted by a mechanical screw drive, and a mechanical counter reads the attenuation directly in units of $0.01 \mathrm{db}$. Rectangular coils mounted on precision-made bakelite forms are used in an effort to secure symmetry in the generation of the $T E_{11}$ mode, and hence reduce the unwanted modes. The mechanical precision of construction is such that the accuracy of attenuation reading is \pm 0.01 db per inch.

Early experiments with the attenuator revealed that it could not be set to a given attenuation value within plus or minus $0.1 \mathrm{db}$. The fingers on the moving plunger, as shown in Figure 6, were replaced by helical spring fingers, as described by Sydoriak ${ }^{2}$ but with no improvement. Finally, the contacts were removed entirely, and the plunger which supported the coil was constructed of bakelite, eliminating the variable attenuation value for a given setting.

\section{Linearity of AtTenuator}

Direct Measurement of Attenuator Linearity. In order to check the linearity of the attenuator, that is, the deviation of its rate of attenuation from $e^{-\alpha_{11} t^{2}}$, the measused. The exciting coil in Figure 6 was connected to the i-f signal generator, and the receiving coil was matched to $50 \mathrm{ohms}$ by inserting a resistor and capacitor in series with the coil, and tuning for resonance.

Measurements of the attenuation of a particular 5-db step of the Leeds and Northrup carbon attenuator were made for different separations of the coils in the cutoff attenuator, and were found to urement setup illustrated in Figure 5 was
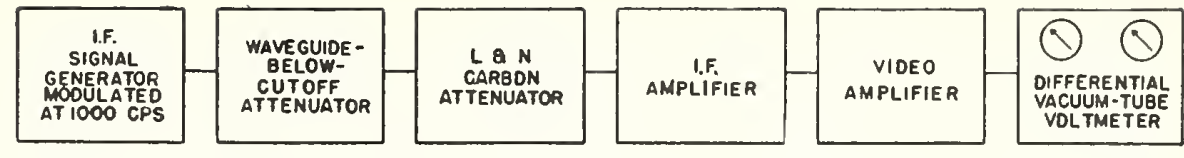

be constant within experimental error (plus or minus $0.01 \mathrm{db}$ ), for coil separations greater than $20 \mathrm{db}$ (about 1 inch). For smaller separations, the measured values of attenuation were variable, depending upon the angle between the axes of the coils. This departure from linearity was caused partly by the change in exciting current due to coupled impedance of the secondary coil, and partly by the presence of higher order modes.

Measurement of Relative Amplitudes of Higher Modes. The furst few higher modes, the $T M_{01}, T E_{21}, T M_{11}$, and $T E_{01}$, have attenuation rates of $28,35,44$, and $44 \mathrm{db}$ per inch, respectively, in the experimental cutoff attenuator. For large enough coil separations the preponderant higher mode will be the $T M_{01}$, since its attenuation rate is not so very different from that of the $T E_{11}$. For this condition, a method of measuring the relative amplitudes of the $T E_{11}$ and $T M_{01}$ modes by utilizing their different angular dependence was described by Sydoriak, ${ }^{2}$ and developed by Griesheimer. ${ }^{5}$ This method assumes that

1. Only the $T M_{01}$ and $T E_{11}$ modes are present in sufficient strength to be of importance.

2. Transfer impedance effects are negligible.

3. The tube cross section has no elliptical eccentricity.

It follows from waveguide theory that the voltage induced in the receiving coil from the $T E_{11}$ mode varies as the cosine of the angle, $\theta$, between the axes of the coils, and that induced from the $T E_{01}$ mode is independent of angle. Then, let

$V_{T E}=A_{1} e^{-\alpha_{11} z} \cos \theta$

$V_{T M}=A_{2} e^{-\alpha 0_{12}}$

represent the voltage induced in the re-

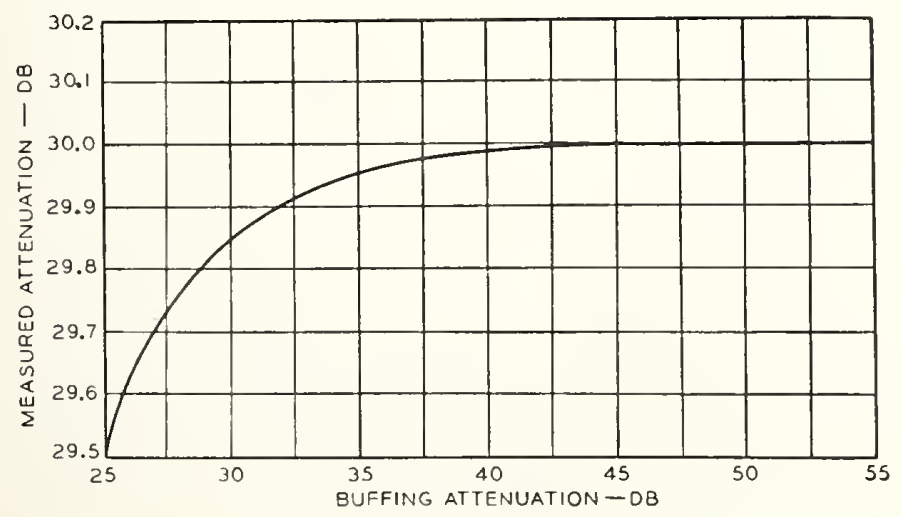

Figure 4. Measured attenuation of nominal 30-db radio-frequency attenuator as a function of total buffing attenuation using 100 milliwatts of input power

A buffing attenuation of 40-db corresponds to a crystal input power of 10 microwatts
Figure 5. Intermediate-frequency measurement setup for comparing wave-guide-belowcarbon attenuator

ceiving coil by the $T E_{11}$ and $T M_{01}$ modes, respectively, where $z$ represents the dic tance between the coils.

Since these voltages are separated in time phase by an angle $\zeta$ which is approximately 180 degrees, the resultant voltage amplitude is given by

$V^{2}=V_{T E}{ }^{2}+V_{T M}{ }^{2}+2 V_{T E} V_{T M} \cos \zeta$

This may also be written

$V^{2}=\left(A_{1} e^{-\alpha_{11} z}\right)^{2}\left[1+r^{2} e^{-2 b z}+2 r e^{-b z}\right.$ $\left.\cos \theta \cos \zeta-\sin ^{2} \theta\right]$

where

$r=\frac{A_{2}}{A_{1}}$

and

$b=\alpha_{01}-\alpha_{11}$

- If we keep $z$ constant, and vary $\theta, V^{r_{2}}$ will have a maximum, $V_{\text {m }}^{2}$, at $\theta= \pm \pi$, and a secondary maximum, $V^{2}{ }_{\text {submax }}$ at $\theta=0$, and a minimum, $V^{2}{ }_{\min }$, at $\cos \theta^{\prime}=-r e^{-b z}$ $\cos \zeta$. If we assume the coils are adjusted for maximum power transfer, $\theta=\pi$, then it follows from equation 8 that the attenuation in decibels introduced by displacing the secondary coil from an initial separation, $z_{0}$, between coils to a final separation $z$, is given by

$A_{d b}=10 \log _{10} \exp \left(-2 \alpha_{11}\left[z_{0}-z\right]\right)+10 \log _{10}$

$\frac{1+r^{2} \exp \left(-2 b z_{0}\right)-2 r \exp \left(-b z_{0}\right) \cos \zeta}{1+r^{2} \exp (-2 b z)-2 r \exp (-b \tau) \cos \zeta}$

From equation 11 , it is seen that the presence of the $T M_{01}$ mode introduces a deviation from the exponential law of decay which obtains when only the $T E_{11}$ mode is present. If we define this deviation of the actual attenuation in decibels from the theoretical exponential value as the error, $E_{d b}$, due to the $T M_{01}$ mode, it follows from equation 11 that the maximum error in a measurement of attenuation where the minimum separation between coils is $z_{\theta}$, is

$E_{d b}=10 \log _{10}$

$\left[1+r^{2} \exp \left(-2 b z_{0}\right)-2 r \exp \left(-b z_{0}\right) \cos \zeta\right]$

The value of $E_{d b}$ for a given minimum cutoff attenuator with Leeds and Northrup 

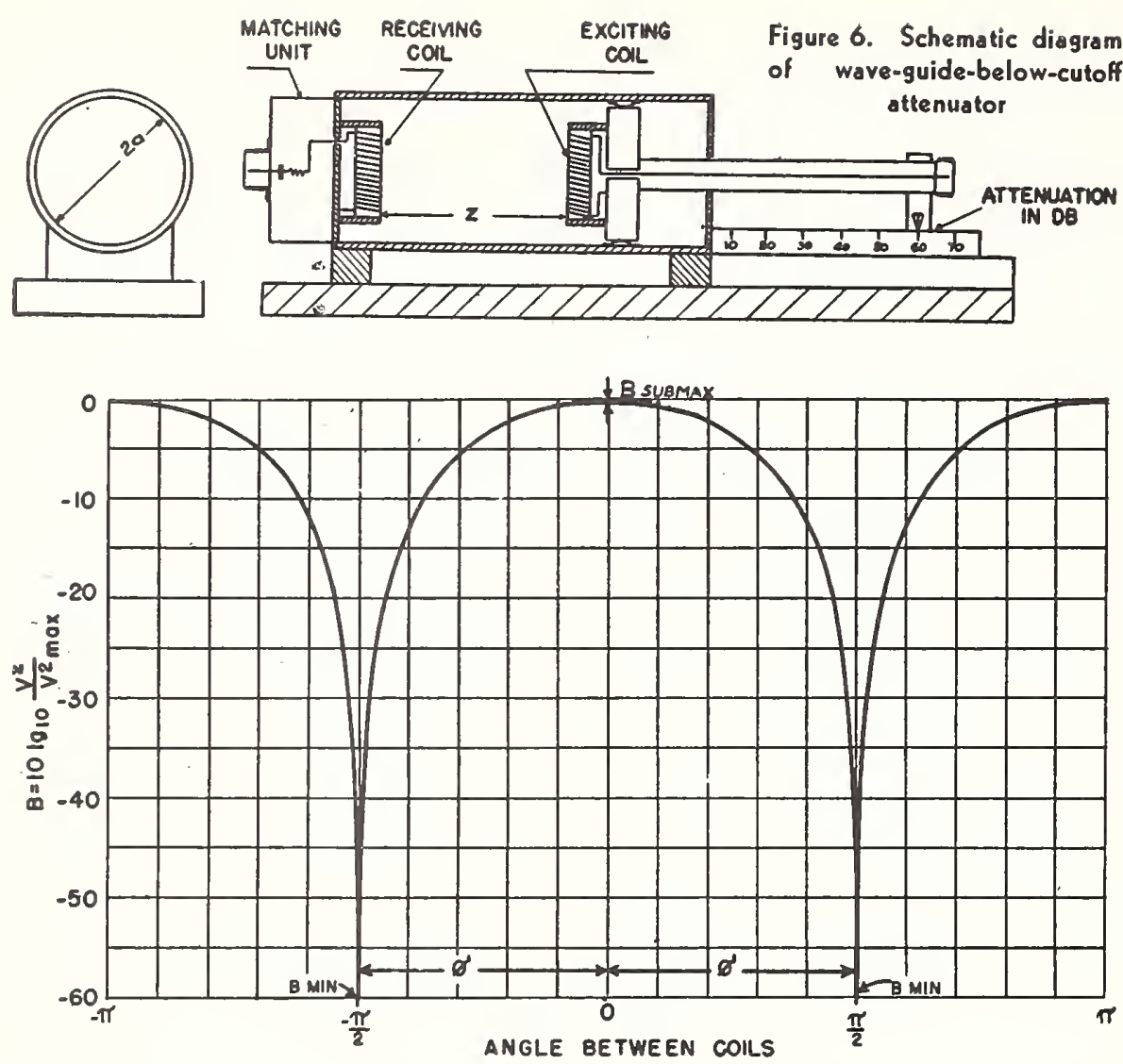

Figure 7. Graph of $B=10 \log \left(V^{2} / V^{2}\right.$ max as a function of angular rotation of coils fol $\mathrm{TE}_{11}$ mode circular attenuator

separation between coils may be determined experimentally by measuring

$\frac{V_{\text {submax }}^{2}}{V^{2}{ }_{\max }}$

and

$\frac{V^{2} \min }{V^{2} \max }$

at the coil separation $z_{0}$.

It is convenient to define the following quantities:

$B=10 \log _{10} \frac{V^{2}}{V^{2} \max }$

$B_{\text {submax }}=10 \log \frac{V_{\text {submax }}^{2_{\text {max }}}}{V^{2}{ }_{\max }}$

$B_{\min }=10 \log _{10} \frac{V^{2}{ }_{\min }}{V^{2}{ }_{\max }}$

$\frac{V^{2}{ }_{\text {submax }}}{V^{2}{ }_{\max }}=h^{2}$

$\frac{V^{2} \min }{V^{2} \max }=\beta^{2}$

Figure 7 is a typical plot of $B$ as a function of angle between the axes of the coils, and illustrates the power coupled into the receiving coil as a function of angle of rotation, betweels the coils. If the two values of $B_{\min }$ in Figure 7 are not the same, then a third mode must be present. Also if the minimum points are not displaced by equal angles to either side of
Figure 8. Calculated insertion loss of barium titanate disk for $\mathrm{TM}_{01}$ mode versus thickness of disk
$B_{\text {submax }}$, a third mode must be present. Using the measured values of $B_{\text {submax }}$ and $B_{\min }$ as defined herein, the values for $r$ and $\zeta$ may be determined from the following equations, as derived by Griesheimer. ${ }^{5}$

$J=\left[\frac{h^{2}-\beta^{2}}{1-\beta^{2}}\right]^{1 / 2}$

$r e^{-b z_{0}}=\frac{1-J}{1+J}\left[1+\frac{4 \beta^{2}}{1-h^{2}} \frac{(1+J)}{(1-J)}\right]^{1 / 2}$

$\cos \zeta=-\left[1+\frac{4 \beta^{2}}{1-h^{2}} \frac{(1+J)}{(1-J)}\right]^{-1 / 2}$

$\cos \theta^{\prime}=\frac{(1-J)}{(1+J)}$

Measurements on the experimental $T E_{11}$ mode cutoff attenuator, using rectangular coils, showed that the angular calibration was symmetrical within the accuracy of tie measurement, and it was concluded that there was no appreciable coupling due to modes higher than the $T M_{01}$. The value of $B_{\text {summax }}$ was found to be -0.30 , and the value of $B_{\min }$ to be -60 . From equations $16-20$ the following values of $r, \zeta$, and $\theta^{\prime}$ were obtained $r e^{-b z_{0}}=0.017, \zeta=176.5$ degrees, and $\theta^{\prime}=$ 89 degrees.

The maximum error in attenuation, $E_{d b}$, for this separation was therefore, from equation $12,0.14 \mathrm{db}$. For a minimum coil separation corresponding to an attenuation of $20 \mathrm{db}$, the maximum error

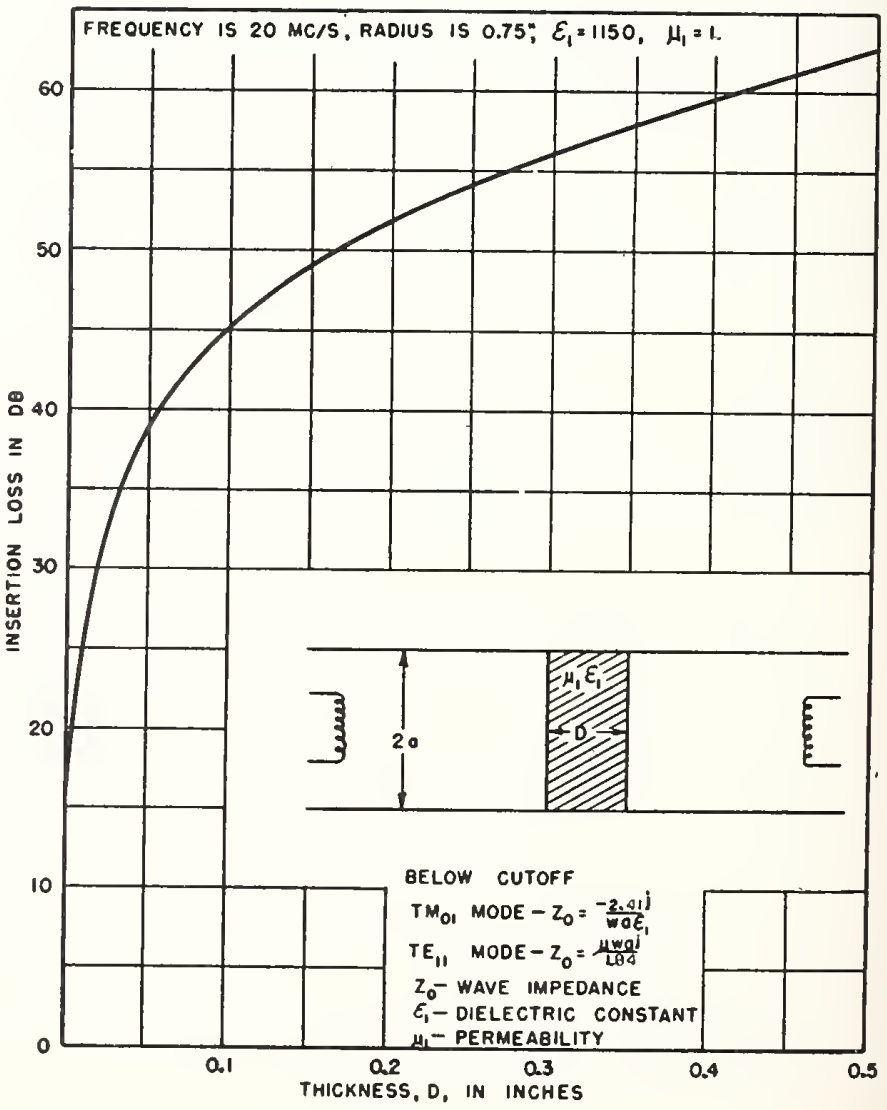


in attenuation was $0.08 \mathrm{db}$. The latter error was not detected by measuring the 5 -db step of the Leeds and Northrup attenuator because the error was spread over such a wide range of attenuation that the amount included in a 5 -db interval was smaller than the experimental error.

Dielectric Filters. The equation for the $T M$-mode wave impedance, $Z_{n m}$, of a circular hollow tube of radius $a$, containing a medium of permeability, $\mu_{1}$, and dielectric constant of $\xi_{1}$, is given by

$Z_{n m}=\left[\left(1-\frac{f_{n m^{2}}}{f^{2}}\right)\left(\frac{\mu_{1}}{\xi_{1}}\right)\right]^{1 / 2}$

This simplifies, at frequencies far below cutoff, to

$Z_{n m}-\frac{-U_{n m} j}{2 \pi f a \xi_{1}}$

For $T E$ waves, the wave impedance simplifies to:

$Z_{n m}=\frac{a \mu_{1} 2 \pi f j}{v_{n m}}$

Here, $f_{n m}$ and $f$ are the cutofi and im. pressed frequencies, $j=\sqrt{-1}$, and $U_{n m}$ and $v_{n m}$ are numerical factors which depend upon the mode. Hence, a material of high dielectric constant will reflect the $T M$ wave and pass unchanged the $T E$ wave. The high dielectric constant material has a wave impedance, for the $T M$ mode, inversely proportional to the dielectric constant so that a large discontinuity between air and the material would exist and cause reflections of the $T M$ mode. The TE mode, on the other hand, would not be reflected because its wave impedance is independent of $\xi_{1}$. Similarly, a material of high permeability could be inserted in the guide to reflect the $T E$ wave, but not reflect $T M$ wave. The theoretical insertion loss for the $T M_{01}$ mode of a barium titanate disk with a dielectric constant of 1,150 is plotted in Figure 8 , as a function of its thickness.

The measured insertion loss of a barium titanate disk of 0.25 inches in thickness, having a dielectric constant of 1,150 , was $40 \mathrm{db}$ for the $T M_{01}$ mode, and $0.05 \mathrm{db}$ for the $T E_{11}$ mode. To insure an intimate contact between the barium titanate and the inside surface of the cutoff attenuator, the rim of the disk was silvered.

The insertion loss of the barium titanate disk for the $T M_{01}$ mode, and of other types of $T M_{01}$ mode filters described in the following paragraph was measured by replacing the exciting coil in the circular attenuator by a capacitative exciting disk, which excited the $T M_{01}$ mode primarily. At a fairly close spacing between the receiving coil and exciting disk, the

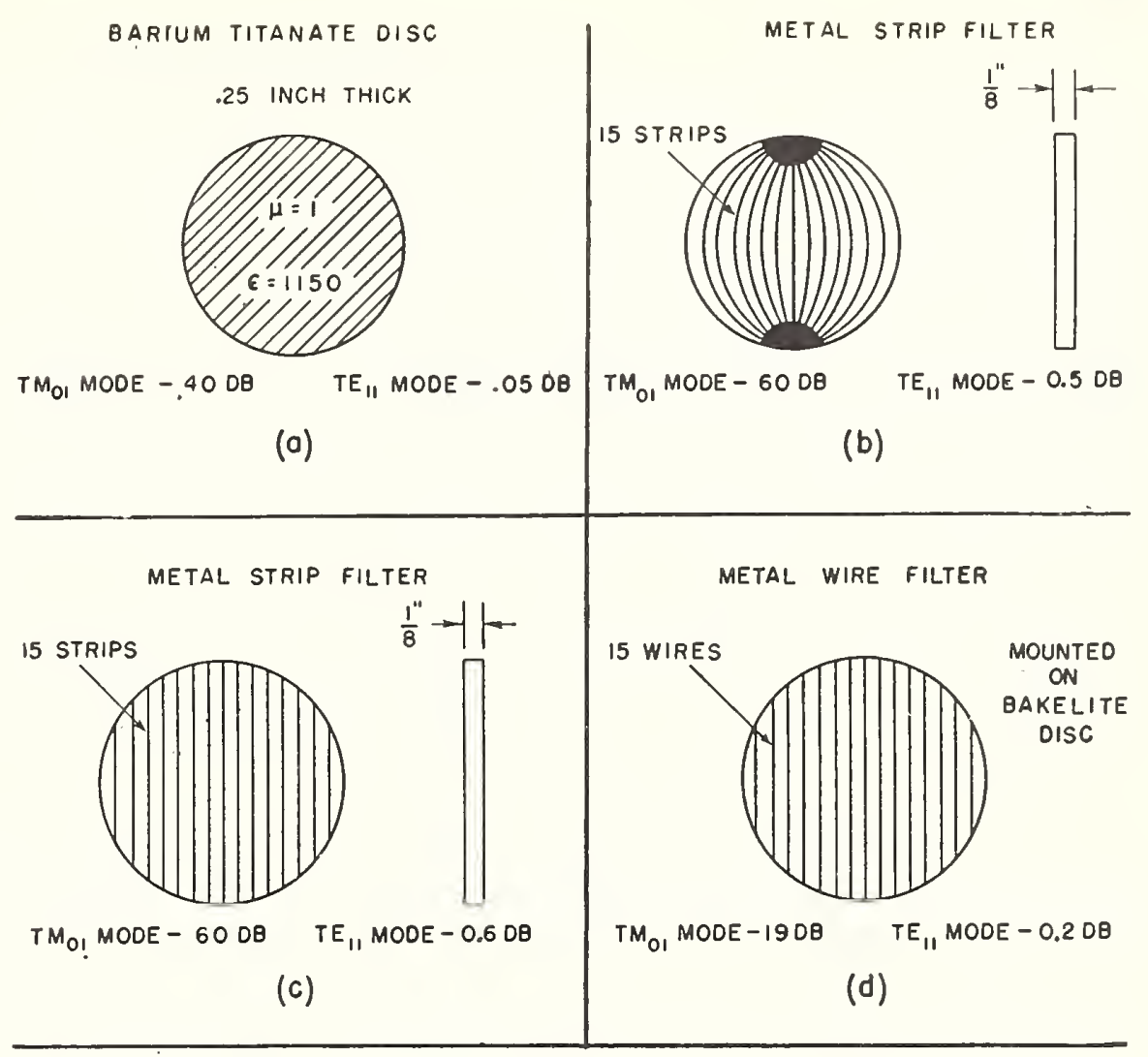

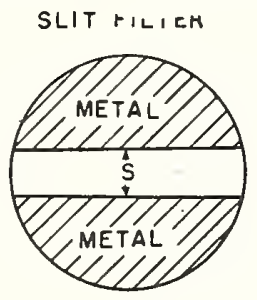

(e)

amplitude of the $T E_{01}$ mode was measured to be less than $1 / 1,000$ that of the $T M_{01}$ mode. Thus there was ample range for measurement of $T M_{01}$ insertion loss for various filtẹs.

Metal Mode Filters. Various other types of filters illustrated in Figure 9 were tried. The slit filter, Figure-9e, was used by Sydoriak ${ }^{2}$ at higher frequencies, but it is not the best type for $\mathrm{i}-\mathrm{f}$ frequencies, as the data on Figure 9 indicate, because the slit filter reflects a considerable amount of the $T E_{11}$ mode as well as the $T M_{01}$ mode.

Gainsborough ${ }^{1}$ developed the wire filter as shown in Figure 9d. It is not as effective as the copper-strip filter shown in Figure 9c. A little less insertion loss for the $T E_{1}$ mode is obtained if the copper strips are bent so as to be approximately perpendicular to the electric lines of the $T E_{11}$ mode as is shown in Figure $9 \mathrm{~b}$. Of course the filters in Figure 9b, c, d, and e must be properly oriented or they will reflect the $T E_{1}$ mode as well as the $T M_{01}$ mode.

\begin{tabular}{c|c|c}
$S$ & $T M_{01} M O D E$ & $T E_{11} M O D E$ \\
\hline INCHES & $D \theta$ & $D Q$ \\
\hline$\frac{3}{16}$ & 25 & 5 \\
\hline$\frac{1}{4}$ & 20 & 5 \\
\hline$\frac{3}{8}$ & 12 & 4.8 \\
\hline$\frac{1}{2}$ & 9 & 3.6
\end{tabular}

Figure 9. Insertion loss of various mode filters for $\mathrm{TE}_{11}$ and $T \mathrm{TM}_{01}$ modes in cutoff attenuator

Using the metal strip filter of Figure $9 \mathrm{~b}$ an angular calibration of the cutoff attenuator was made. The value of $B_{\text {submax }}$ was zero within limits of measurement, and $B_{\min }$ was less than -80 .

Comparison With Leeds and Northrup Carbon Attenuator. A calibration of the Leeds and Northrup carbon attenuator was made over a 40 -db range in attenuation at 20 megacycles per record. The resulting attenuation calibration checked within $0.03 \mathrm{db}$ the calibration made with direct current. A check was then made by operating the cutoff attenuator as a $T M_{01}$ attenuator as described previously for $T M_{01}$ mode-filter insertion loss. The $T M_{01}$ mode calibration was made at only two values of attenuation, $10 \mathrm{db}$ and 20 $\mathrm{db}$, and the values obtained checked the previous calibration within plus or minus $0.01 \mathrm{db}$. 


\section{Conclusion}

The heterodyne or i-f substitution method of calibrating microwave attenuators has been investigated, and preliminary equipment has been built to utilize the method. The accuracy obtained is estimated to be within plus or minus 0.02 $\mathrm{db}$ in the $0-10 \mathrm{db}$ attenuation range, and plus or minus 0.2 per cent of the attenuation value in $\mathrm{db}$ for the $10-50 \mathrm{db}$ range, assuming unity standing-wave ratio presented to both sides of the attenuator under calibration. The basic standard of the heterodyne method is a waveguidebelow-cutoff attenuator operated at an intermediate frequency. A preliminary model of an i-f standard cutoff attenuator has been built, tested, and improved until its accuracy is plus or minus $0.01 \mathrm{db}$ per $20 \mathrm{db}$ unit of attenuation.

\section{References}

1. A Method of Calibrating Standard-Signal Generators and Radio-Frequency Attenuators, G. F. Gainsborough. Journal, Institution of Electrical Engineers (London, England), volume 94, part 3, number 29, May 1947.
2. Radio-Frequency Attenuators, S. G. Sydoriak. Radiation Laboratory Report 404 (Massachusetts Institute of Technology (Cambridge, Mass.), September 7, 1943.

3. The Design and Testing of Multirange Receivers, D. E. Harnett, N. P. Case. Proceedings, Institute of Radio Engineers (New York, N. Y.), volume 23 , number 6 , June 1935 .

4. Capacitance Type Radio-Frequency AttenuATORS, W. O. Smirh. Radiation Laboratory Report 995, Massachusetts 1nstitute of Technology (Cambridge, Mass.), January 18, 1946.

5. Notes on TE 11 MOde Cutoff Attenuators, R. N. Griesheimer. Radiation Laboratory Report 55, Massachusetts Institute of Technology (Cambridge, Mass.), November 16,1944 .

6. Electromagnetic Theory (book), J. A Stratton. McGraw-Hill Book Company, New York, N. Y., 1941, page 543.

\title{
Determination of Attenuation from Impedance Measurements*
}

\author{
R. W. BEATTY $\dagger$, MEMBER, IRE
}

Summary-Heretofore, the determination of attenuation from impedance measurements has been applied to cases in which the reflections from the attenuator terminals were negligibly small. In the proposed method no restrictions are placed upon the attenuator except the requirement that it be a linear, passive, four-terminal network.

The dissipative and reflective components of attenuation $A_{D}$ and $A_{R}$ are measured separately. $A_{D}$ is shown to be a function of the efficiency of the attenuator. The efficiency is determined from reflection coefficient measurements of the short-circuited attenuator. $A_{R}$ is determined from a single voltage-standing-wave ratio measurement of the attenuator when terminated in a matched load.

Experimental data show close agreement with an independent method of determining attenuation.

\section{INTRODUCTION}

$\mathrm{T}$ THE DETERMINATION of attenuation from impedance measurements of a short-circuited attenuator is well known. ${ }^{1}$ It is usually assumed that the reflections caused by mismatch at the attenuator terminals are negligibly small. The purpose of this paper is to present an impedance method in which no restrictions are placed upon the attenuator except the requirement that it be a passive, linear four-terminal network.

Although the method is of general application, it has been developed for use with ultra-high-frequency and microwave measuring equipment. At lower frequencies

* Decimal classification: $\mathrm{R} 247 \times \mathrm{R} 244$. Original manuscript received by the Institute, October 14, 1949; revised manuscript received, April 24, 1950.

$\dagger$ National Bureau of Standards, Washington, D. C.

'C. G. Montgomery, "Technique of Microwave Measurements," McGraw-Hill Book Co., Inc., New York, N. Y., vol. 11, p. 818; 1947. the method may be used, if measured impedances are converted to reflection coefficients.

\section{THEORY}

Attenuation is defined as the insertion loss which occurs when an attenuator is placed in a matched system.? In the matched transmission-line system shown in Fig. 1 , the generator and load impedances are equal to the characteristic impedance of the line. The generator
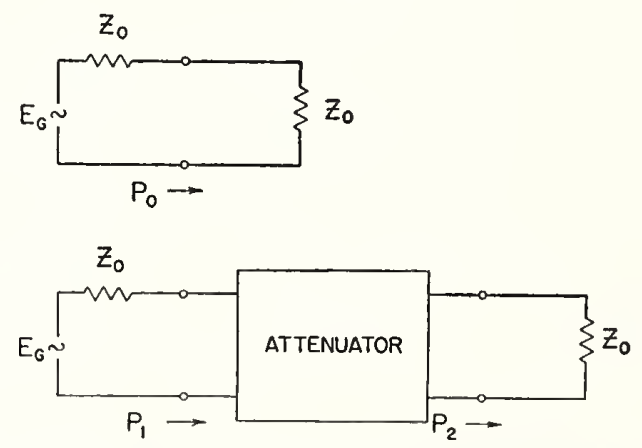

Fig. 1 -Insertion loss in a matched system. $A_{T}=10 \log _{10}\left(P / P_{2}\right)$.

voltage $E_{\theta}$ is assumed to remain constant when the attenuator is inserted.

Referring to Fig. 1, it is apparent that the attenuation in decibels is

$$
A_{T}=10 \log _{10} \frac{P_{0}}{P_{2}}
$$

${ }^{2}$ See page 680 of footnote reference 1. 
The total attenuation may be separated into two components by the following steps:

$$
\begin{aligned}
& \frac{P_{0}}{P_{2}}=\frac{P_{0}}{P_{1}} \cdot \frac{P_{1}}{P_{2}} \\
& A_{T}=10 \log _{10} \frac{P_{0}}{P_{1}}+10 \log _{10} \frac{P_{1}}{P_{2}} .
\end{aligned}
$$

The first component $A_{R}$ is caused by reflection and the second component, $A_{D}$ is caused by dissipation of energy. It has been shown ${ }^{3}$ that $A_{R}$ is given by

$$
A_{R}=10 \log _{10} \frac{\left(\sigma_{1 m}+1\right)^{2}}{4 \sigma_{1 m}},
$$

where $\sigma_{1 m}$ equals the voltage-standing-wave ratio measured at the input terminals of the attenuator when terminated in a matched load.

The determination of $A_{D}$ involves the measurement of the power ratio $P_{1} / P_{2}$ or its reciprocal, the efficiency $\eta_{m}$ of the attenuator when terminated in a matched load. The efficiency of a network can be determined from reflection coefficient measurements. ${ }^{4-6}$ In this method, the attenuator is reversed and its normal input terminals are connected to a variable reactance usually consisting of a short-circuited section of transmission line of variable length. The reflection coefficient measured at the other terminal pair is a function of the terminating reactance and has a circular locus. The radius $R_{2}$ of this reflection coefficient circle is equal to the efficiency $\eta_{m}$. The equation for $A_{D}$ is:

$$
A_{D}=10 \log _{10} \frac{1}{\eta_{m}}=10 \log _{10} \frac{1}{R_{2}}
$$

\section{Measurement Procedure.}

The arrangement of apparatus for the individual measurement of $A_{R}$ and $A_{D}$ is shown in Fig. 2. In the measurement of $A_{R}$, it is necessary to match the load as closely as possible. The measured value of $\sigma_{1 m}$ is then substituted in (4) to obtain $A_{R}$.

The determination of $A_{D}$ involves reversal of the attenuator and termination of its normal input terminals in a lossless variable reactance. The voltage-standing-wave ratio $\sigma_{N}$ and the position $l_{N}$ of the voltage node are measured with the standing-wave machire for selected positions of the short-circuiting plunger as it travels a total distance of half-wavelength. The reflection coefficient $\Gamma_{N}$ may be calculated in each case from the following equation:?

${ }^{3}$ See page 681 of footnote reference 1 .

4 William Altar, "Measurement of dielectric properties of lossy materials," Westinghouse Lab. Res. Report R-94318-E, September $13,1944$.

5 William Altar, "Q-circles," Proc. I.R.E., vol. 35, pp. 355-351, 1947; and pp. 478-485, May, 1947.

- A. L. Cullen, "Measurement of microwave-transmission efficiency," Wireless Eng., vol. 26, pp. 255-258; August, 1949.

7 See page 476 of footnote reference 1 .

$$
\left|\Gamma_{N}\right|\left|\underline{\psi_{N}}=\frac{\dot{\sigma_{N}}-1}{\sigma_{N}+1}\right| \underline{2 \beta l_{N} \pm \pi}
$$

The measured values of reflection coefficient are plotted as in Figs. 3 and 4. The circle is drawn which best fits the measured points and the radius $R_{2}$ of this circle yields $A_{D}$ when substituted in (5).

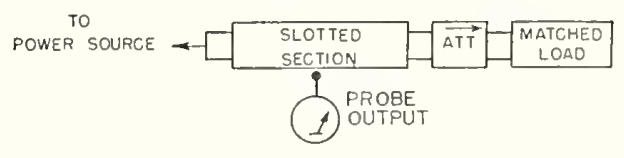

MEASURE VOLTAGE STANDING-WAVE RATIO $\left(\sigma_{M}\right)$

(a)

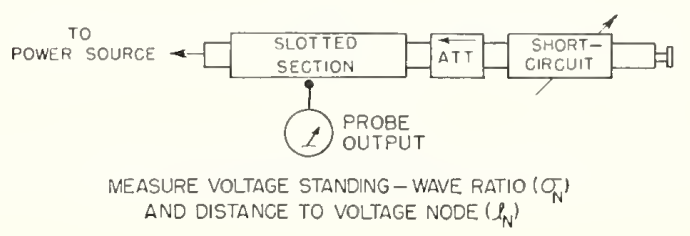

(b)

Fig. 2-Block diagram of apparatus used for measuring (a) reflective component of attenuation, and (b) dissipative component of attenuation.

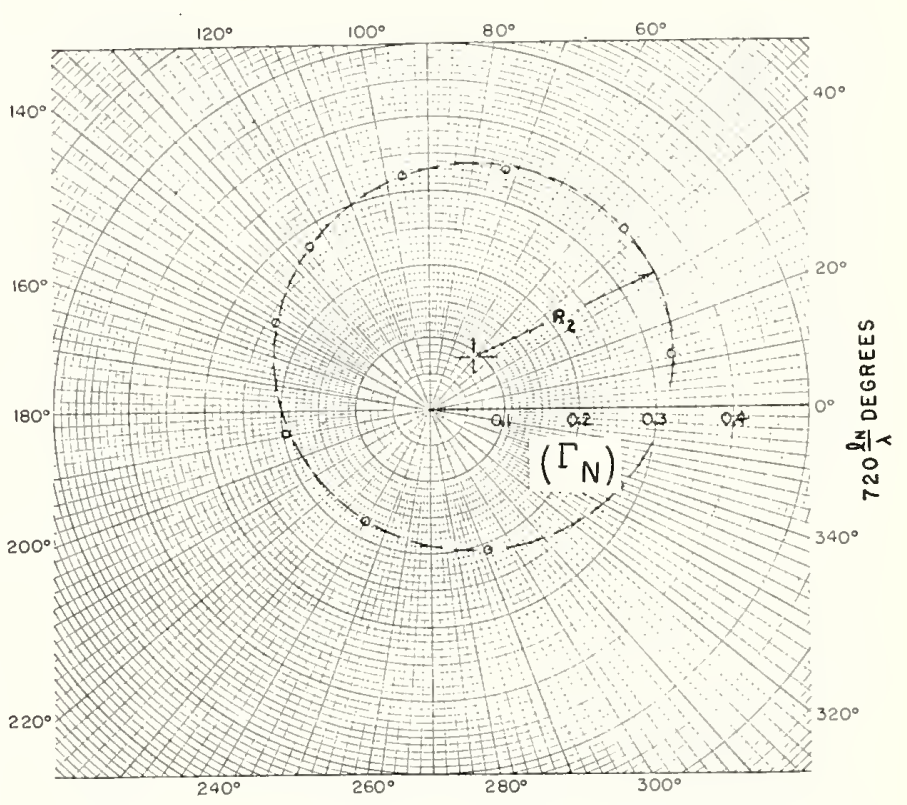

Fig. 3-Measured reflection coefficients of 6-db attenuator, $A_{D}=5.74 \mathrm{db}, 1,000 \mathrm{Mc}, R_{2}=0.266$.

The total attenuation $A_{T}$ is

$$
A_{T}=A_{R}+A_{D}=10 \log _{10} \frac{\left(\sigma_{1 m}+1\right)^{2}}{4 R_{2} \sigma_{1 m}} .
$$

This equation is represented by the nomogram of Fig. 5 .

\section{Experimental Data}

In order to further illustrate the method, experimental data were obtained on commercially available coaxial attenuators.

A standard 7/8-inch rigid coaxial transmission line was used with a Microline Model $361 \mathrm{~A}$ impedance meter. Measurements were made at $1,000 \mathrm{M}$ on four attenuators having nominal values of $3,6,10$, and 20 


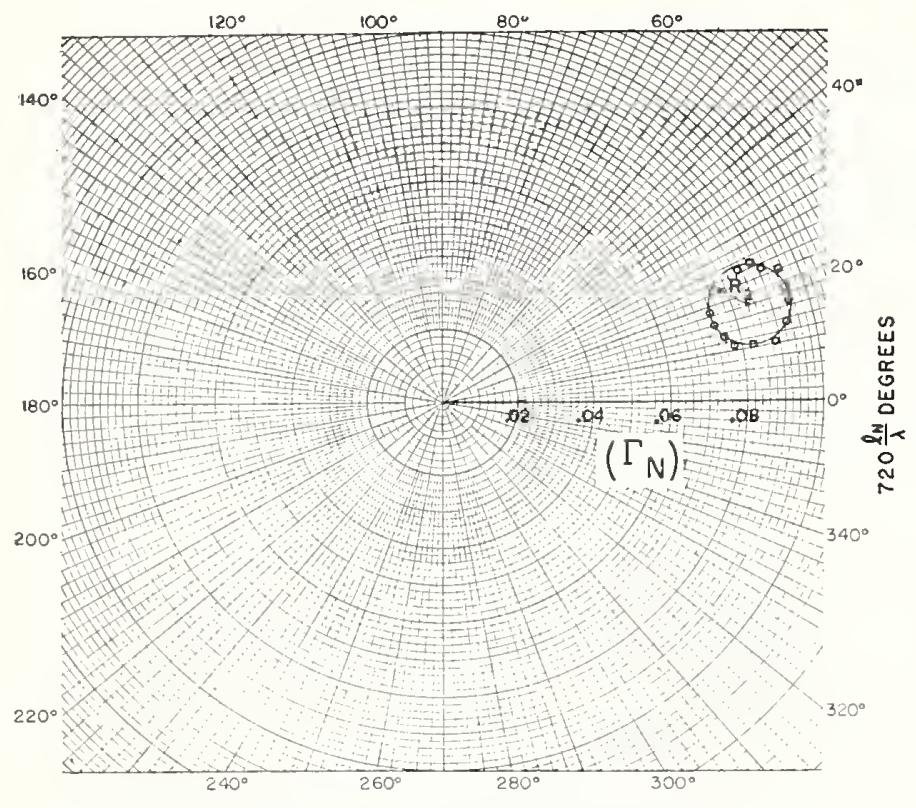

Fig. 4-Measured reflection coefficients of 20-db attenuator, $A_{D}=19.74 \mathrm{db}, 1,000 \mathrm{Mc}, R_{2}=0.0106$.

decibels. The observed data in the measurement of $A_{D}$ are shown in Figs. 3 and 4. (Lack of space prohibits showing more data.) It is apparent that the measured circles either enclose the origin or lie completely outside. In each case, $R_{2}$ may be expressed in terms of the minimum and maximum value of voltage-standing-wave ratio measured as the position of the short-circuit changes.

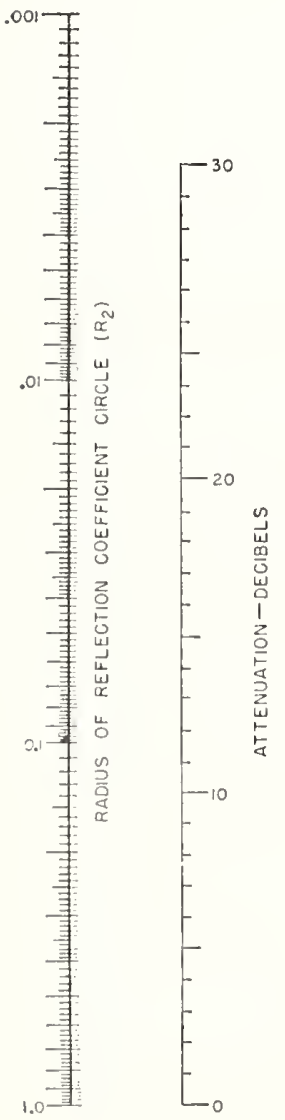

Fig. 5-Nomogram of equation $A_{T}=10 \log _{10}\left(\sigma_{1 m}+1\right)^{2} / 4 R_{2} \sigma_{1 m}$.
If the circle encloses the origin,

$$
2 R_{2}=|\Gamma|_{\max }+|\Gamma|_{\min }=\frac{2\left(\sigma_{\max } \sigma_{\min }-1\right)}{\left(\sigma_{\max }+1\right)\left(\sigma_{\min }+1\right)} .
$$

If the circle lies outside the origin,

$$
2 R_{2}=|\Gamma|_{\max }-|\Gamma|_{\min }=\frac{2\left(\sigma_{\max }-\sigma_{\min }\right)}{\left(\sigma_{\max }+1\right)\left(\sigma_{\min }+1\right)} .
$$

Although the radius $R_{2}$ can be obtained from the measurement of $\sigma_{\max }$ and $\sigma_{\min }$, improved accuracy can be obtained by measuring a number of other points on the reflection coefficient circle.

In the measurement of $A_{R}$, the load impedance had a measured voltage-standing-wave ratio (VSWR) of 1.02. The data obtained in the measurement of $A_{R}$ are shown in Fig. 6.

\begin{tabular}{|c|c|c|c|c|c|c|}
\hline \multirow{2}{*}{$\begin{array}{c}\text { Nominal } \\
\text { Volue of } \\
\text { Attenuation }\end{array}$} & InswR & $A_{R}$ & $A_{D}$ & $A_{T}$ & $\begin{array}{c}\text { MEASURED ATTENUATION } \\
\text { Power } \\
\text { Rotío } \\
\text { Method }\end{array}$ & Difference \\
\hline $3 \mathrm{db}$ & 1.070 & $.005 \mathrm{db}$ & $2.87 \mathrm{db}$ & $288 \mathrm{db}$ & $2.85 \mathrm{db}$ & $.03 \mathrm{db}$ \\
$6 \mathrm{db}$ & 1.235 & .048 & 5.74 & 5.79 & $5.75 \mathrm{db}$ & $.04 \mathrm{db}$ \\
$10 \mathrm{db}$ & 1.180 & .030 & 9.50 & 9.53 & $9.53 \mathrm{db}$ & $.00 \mathrm{db}$ \\
$20 \mathrm{db}$ & 1.240 & .050 & 19.74 & 19.79 & $19.81 \mathrm{db}$ & $.02 \mathrm{db}$ \\
\hline
\end{tabular}

Fig. 6-Observed attenuation data.

The total attenuation $A_{T}$ was also measured at 1,000 Mc by a power-ratio method using a bolometer detector $^{8}$ which permits an accuracy better than $\pm 0.15 \mathrm{db}$. The results of both methods are shown in Fig. 6 .

\section{Discussion of Errors}

The evaluation of the absolute accuracy of the proposed method has not been completed because of the complexity of the problem in the case of $A_{D}$.

However, the limited amount of experimental data obtained indicates that the impedance method accuracy is comparable with that of power ratio methods over the range of values considered. The very close agreement of the two methods is felt to be partly fortuitous, especially in the case of the $20-\mathrm{db}$ attenuator.

The errors in the measurement of VSIVR and nodal position have been related ${ }^{9}$ to the error in measurement of relative voltage in a slotted-section impedance machine. From these studies, it is to be expected that best accuracy is obtained for intermediate values of attenuation.

Besides these errors in measurement, the fact that it is difficult to obtain a perfectly matched load and a lossless variable reactance contribute to the over-all error.

8 Referred to as the "Ballantine Voltmeter Method" in the book "Technique of Microwave Measurements," by C. G. Montgomery, pp. 806, 841, as cited in footnote reference 1 .

${ }^{9}$ Errors of this nature were discussed in a paper by H. E. Sorrows, W.E. Ryan, and R. C. Ellenwood, "Evaluation of the accuracy of impedance measurements made with a slotted section of transmission line," presented, URSI-IRE Meeting, Washington, D. C., May 2, 1949. 


\title{
Microwave Attenuation Measurements With Accuracies From 0.0001 to 0.06 Decibel Over a Range of 0.01 to 50 Decibels
}

\author{
G. F. Engen and R. W. Beatty
}

(January 15, 1960)

\begin{abstract}
The application of certain power stabilization and measurement techniques to the problem of attenuation measurement has yielded a measurement system with a stability and resolution of the order of 0.0001 decibel. A practical application for this technique was recently provided in the calibration of a rotary vane type of variable microwave attenuator. In order to take complete advantage of this increased stability it was necessary to apply refined techniques to the evaluation and reduction of mismatch error.

This proved to be by far the most exacting practical application of the cited techniques encountered to date, but the results of this calibration showed excellent agreement with the mathematically predicted values used in marking the attenuator dial.
\end{abstract}

\section{Introduction}

The following is a report on some recent work at the Radio Standards Laboratories of the National Bureau of Standards at Boulder, Colo. It is believed that this work represents perhaps the most accurate measurements of microwave attenuation ret made.

In the measurement of microwave attenuation, the stability or resolution of the associated measuring system places a limit on the accuracy with which small values of attentuation may be measured, because the reduction of other sources of error (such as mismatch) will tend to make the total error approach this value. In practice this limit has been typically of the order of $\pm 0.01 \mathrm{db}$.

The application of certain power stabilization ${ }^{1}$ and measurement ${ }^{2}$ techniques to the problem of attenuation measurement has yielded a measurement system with a nominal hundredfold increase in resolution and stability. A practical application for demonstrating the capabilities of this technique was recently provided in the calibration of a rotary vane type of variable microwave attenuator which is characterized, in part, by a high degree of resolution for small values of attenuation. Thus when a recent application called for this characteristic in a variable attenuator, and when a preliminary study of the mismatch and stability characteristics of an attenuator of this type indicated that such a step was warranted, it proved desirable to experimentally confirm the mathematically predicted values of attenuation which were engraved on the attenuator dial. This proved to be by far the most exacting practical application of the cited techniques encountered to date.

In oreler to take complete advantage of the improved stability, refined techniques were applied to the evaluation and reduction of the mismatch error. 'The capability of the system is indicated by the tabulated results of the attenuation calibration, and the estimate of the limits of error is supported by an analytic and experimental treatment.

1 Glenn F. Engen, Amplitude stabilization of a mierowave signal source, IRE Trans. on Misrowave Theory \& Tech. MTT-6, No. 2, 202-206 (Apr. 1958).

2 Glenn F. Engen, A self-balancing direct-current bridge for accurate bolometric power measurements, J. Research NBS 39, No. 2, 101-105 (Aug. 1957) RP2776. 


\section{The Calibration System}

A simplified diagram of the calibration system is shown in figure 1. The attenuator under test is placed between an amplitude stabilized microwave signal source ${ }^{1}$ and a bolometer mount and power meter. The power meter consists of a self-balancing d-c bolometer bridge ${ }^{2}$ with provisions for measuring and recording the d-c bias power required to maintain the bolometer at its operating resistance of 200 ohms. The two bolometer mounts $M_{1}$ and $M_{2}$ shown in the temperature stabilized water bath are for power measurement and signal source stabilization, respectively. A reasonable amount of care was exercised to obtain good performance from each item of equipment, with a resultant system performance as shown in figures 2 a and 2b. Figure 2a shows the stability and repeatability with the attenuator alternately set at the 0.00 - and $0.01-\mathrm{db}$ positions. It will be noted that the stability and repeatability are better than $0.0001 \mathrm{db}$ (10 microbels). A recording of the long term system stability is given in figure $2 \mathrm{~b}$ where the maximum variation is of the order of \pm 10 microbels.

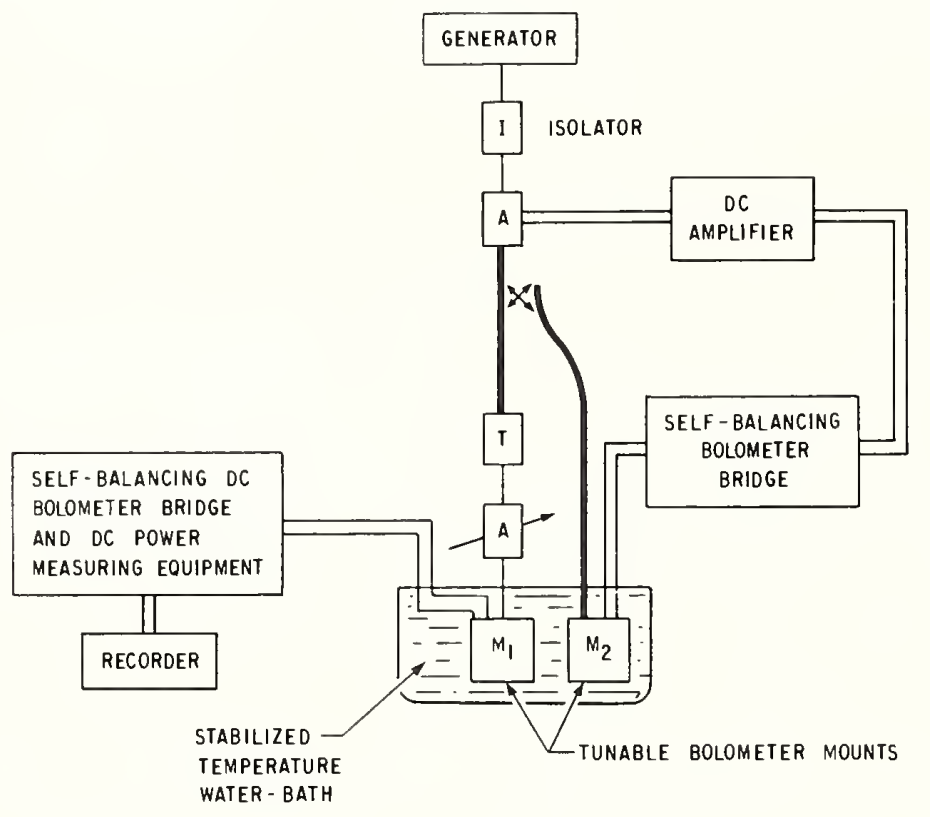

FiguRE 1. Simplified diagram of calibration system.

The absolute accuracy of measurement of small changes in d-c power at this level is estimated to be of the order of $0.02 \mu \mathrm{w}$. This indicates that further improvement could be expected in the results if the system stability could be improved in some manner, perhaps by use of a frequeney-stabilized signal source.

\section{Theory of Measurement}

Ope could make an attenuation measurement by measuring the microwave powers $P_{1}$ and $P_{2}$ absorbed by the bolometer mount $M_{1}$ when the attenuator is set first on zero, then to some other setting. The relative attennation $A$ at this setting is

$$
1=10 \log _{10} \frac{P_{1}}{P_{2}}
$$




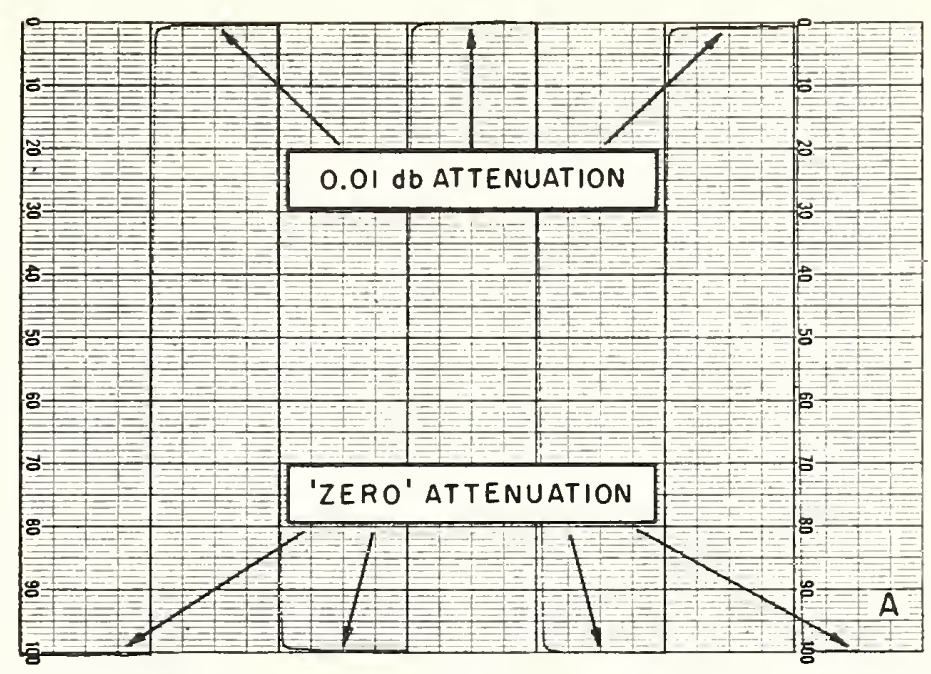

Firure 2a. System response to a 0.01 db change in attenuation.

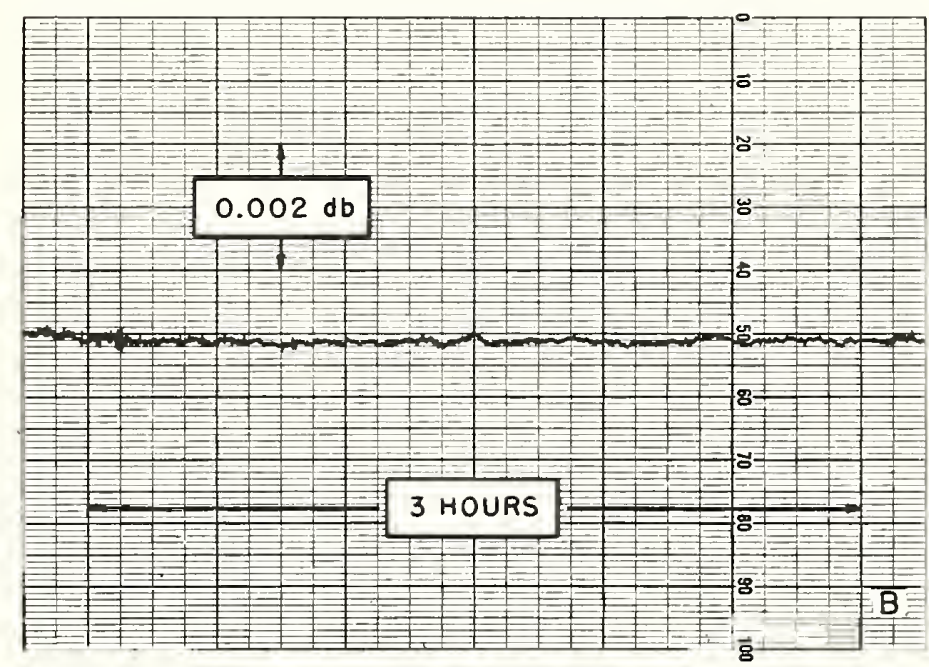

Figure 2b. Long-term system stability.

In making such a measurement, one assumes that the microwave power $P$ absorbed by the bolometer element is proportional ${ }^{3}$ to the $d-c$ power $I^{r}$ withdrawn in order to keep the bolometer resistance constant. Letting $W_{0}$ represent the d-e power required to bias the bolometer at its operating resistance when $P$ equals zero,

$$
A=10 \log _{10} \frac{W_{0}^{\prime}-W_{1}^{*}}{H_{0}^{\prime}-W_{2}^{\top}}
$$

where $W_{1}$ and $W_{2}$ are the d-c bias powers corresponding to $P_{1}$ and $P_{2}$. (For the bolometer used, $\mathrm{H}_{0} \approx 15 \mathrm{mw}$ and $P_{1} \approx 10 \mathrm{mw}$.)

The apparatus employed permitted direct measurements of differences in d-c power, a procedure permitting greater accuracy and convenience than calculation of differences from separate measurements. The changes in d-c power level during an attenuation measurement are shown in figure 3.

3 The constant of proportionality is determined by the substitution error of the bolometer which is known from other experimental data to be independent of power level. 

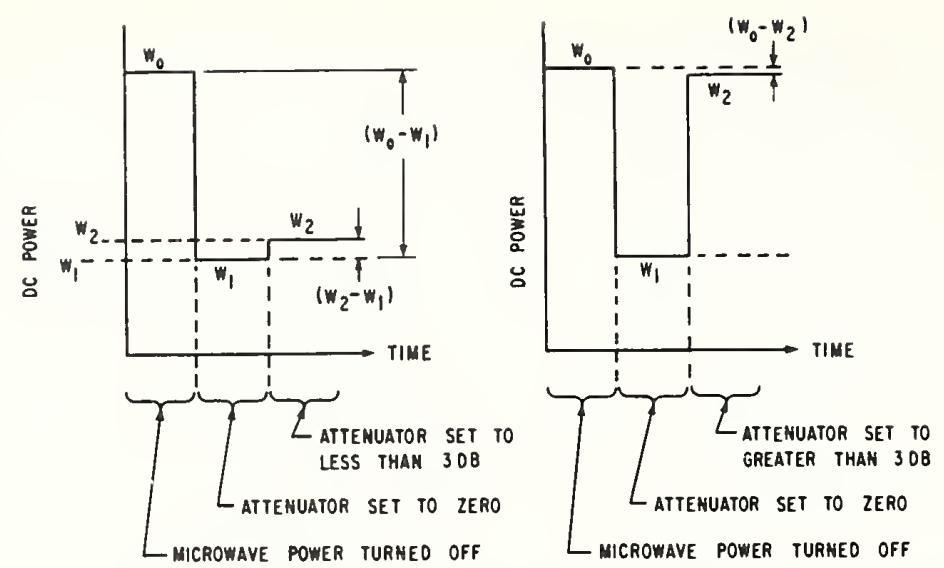

FIGURE 3. Changes in d-c power level during an attenuation measurement.

For attenuations less than approximately $3 \mathrm{db}$, the power difference $W_{2}-W_{1}$ was measured directly. If we let $W_{21}=W_{2}-W_{1}$, and $W_{01}=W_{0}-W_{1}$, eq (2) may be written

$$
A=10 \log _{10}\left(\frac{1}{1-\frac{W_{21}}{W_{01}}}\right) .
$$

For attenuations greater than approximately $3 \mathrm{db}$, the power difference $W_{0}^{r}-W_{2}^{r}$ or $W_{02}^{r}$ was measured directly. Equation (2) becomes

$$
A=10 \log _{10} \frac{W_{01}^{\tau}}{W_{02}^{\top}}
$$

\section{Propagation of Error in Measuring D-C Power Differences}

It is estimated that the error in measuring $d$-c power differences is within 0.1 percent $+0.1 \mu \mathrm{w}$. When $W_{2}-W_{1}$ is measured, it can be shown that the limit of error in determining the attenuation is

$$
\epsilon=10 \log _{10}\left[\frac{1}{1-\frac{1}{1-\frac{W_{21}^{2}}{W_{01}^{2}}}\left(\frac{0.1 \mu \mathrm{W}}{W_{01}^{*}}+0.001 \frac{W_{21}^{r}}{W_{01}^{r}}\right)}\right]
$$

and when $W_{z}-W_{0}^{r}$ is measured,

$$
\epsilon=10 \log _{10}\left[\frac{1}{1-\frac{0.1 \mu \mathrm{W}+0.001 W_{02}^{*}}{W_{02}^{+}}}\right]
$$

'The calculated limits of error are shown in figure 4.

\section{Mismatch Errors}

The mismatch error ${ }^{4}$ in calibrating a variable attenuator depends upon the reflections from the system in which the at tenuator is placed and upon the changes in characteristies of the attenuator as its dial is moved from the reference position. 'The graph of figure 4 shows calculated

4 R. W. Beatty, Mismatch errors in the measurement of ultrahigh-lrequency and microwave variable attenuators, J. Research XBS $\mathbf{5 2}$, No. 1, 7-9 (1954) R P 2465 . 


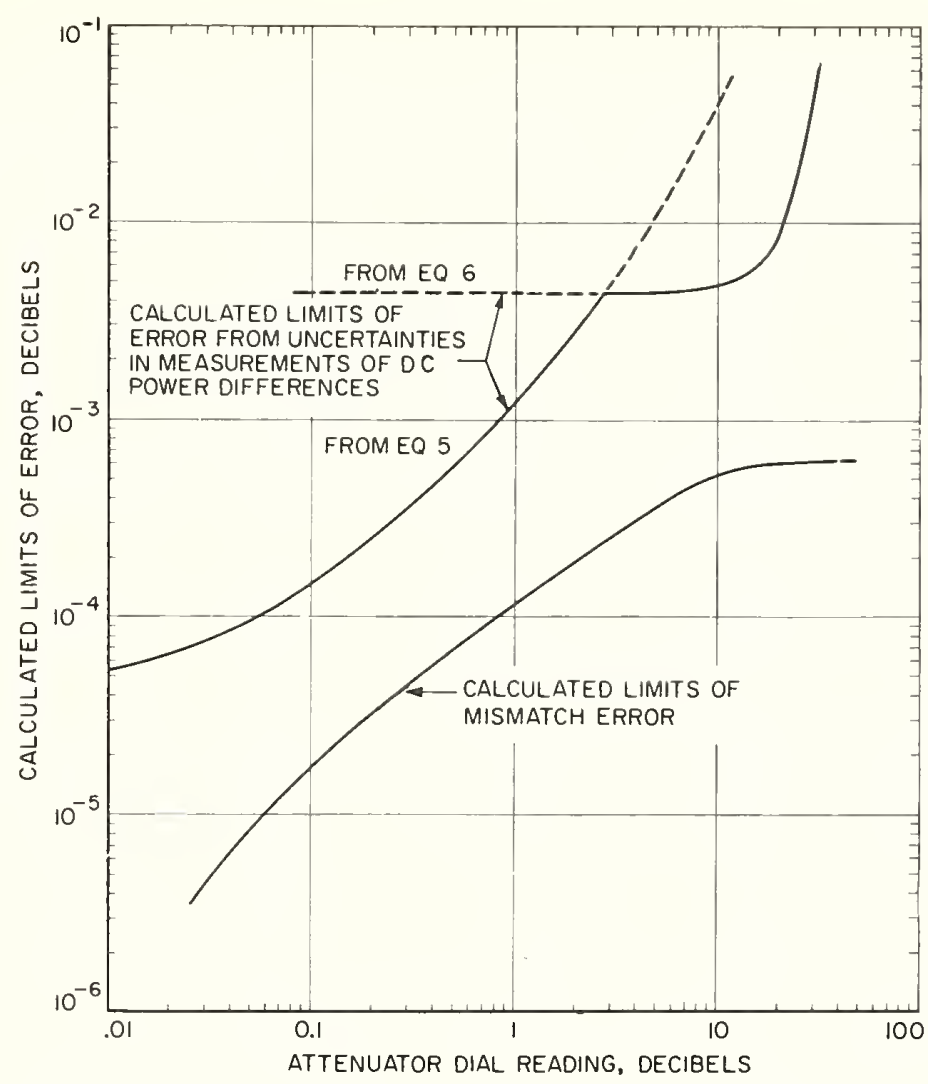

FIGURE 4. Limits of error as a function of attenuation.

limits of error ${ }^{5}$. for the attenuator used, based upon measurements of the magnitude of the changes in the scattering coefficients $S_{11}$ and $S_{22}$ of the attenuator. It was assumed that there was no phase change in $S_{21}$. The mismatch error is below $0.0001 \mathrm{db}$ for attenuator settings up to $0.1 \mathrm{db}$, and remains below $0.001 \mathrm{db}$ for higher settings.

\section{Results}

The calibration data taken at $9.3897 \mathrm{kMc}$ is shown in table 1 . Three sets of data are shown in order to give an idea of the reset tability of the attenuator, and more significant figures are given than one can normally use when interpolating between the marked dial divisions. It should be noted that the calibration is not necessarily representative of this type of attenuator, because only one such attenuator was calibrated by this procedure, and this was done at only one frequency. It should also be noted that this type of calibration is not available on a routine basis, because there are as yet no commercially available attenuators which can be read and are repeatable to $0.0001 \mathrm{db}$.

The estimated limit of error for the complete range of the attenuator, as determined from. figure 4 , is also shown in table 1. Above $20 \mathrm{db}$, the calibration was made in two parts: Measurement of the $20 \mathrm{db}$ step, and measurement of the additional attenuation referred to this step. For these values, the quoted limit of error is the sum of the errors in the individual steps. 'The accuracy of setting the attenuator dial on the marks is not as good as the accuracy of the measurements.

It is noted that at the low end, the estimated accuracy of the measurement is also better than the repeatability of setting the attenuator on the mark. One concludes that further improvements in attenuators need to be made if we are to take full advantage of the most accurate measurement techniques.

\footnotetext{
${ }^{5}$ See appendix.
} 
TABLE 1. Results of three sets of measurements and calculated errors in single measurements

\begin{tabular}{|c|c|c|c|c|c|c|}
\hline \multirow{2}{*}{$\begin{array}{l}\text { Attenuator } \\
\text { dial } \\
\text { reading }\end{array}$} & \multicolumn{4}{|c|}{ Measured attenuations corresponding to dial reading } & \multirow{2}{*}{$\begin{array}{l}\text { Maximum devia- } \\
\text { tion from average }\end{array}$} & \multirow{2}{*}{$\begin{array}{l}\text { Calculated limit } \\
\text { of error in single } \\
\text { measurements }\end{array}$} \\
\hline & 1 & 2 & 3 & Avg & & \\
\hline $\begin{array}{c}d b \\
0.01 \\
.02 \\
.03 \\
.04 \\
.05\end{array}$ & $\begin{array}{c}d b \\
0.01077 \\
.02123 \\
.03015 \\
.04043 \\
.05181\end{array}$ & $\begin{array}{c}d b \\
0.01083 \\
.02145 \\
.03035 \\
.04088 \\
.05216\end{array}$ & $\begin{array}{c}d b \\
0.01044 \\
.02145 \\
.03038 \\
.04074 \\
.05226\end{array}$ & $\begin{array}{c}d b \\
0.0107 \\
.0214 \\
.0303 \\
.0407 \\
.0521\end{array}$ & $\begin{array}{c}d b \\
0.0003 \\
.0002 \\
.0001 \\
.0003 \\
.0003\end{array}$ & $\begin{array}{c}d b \\
0.000055 \\
.000068 \\
.000080 \\
.000091 \\
.00010\end{array}$ \\
\hline $\begin{array}{l}.06 \\
.07 \\
.08 \\
.09 \\
.1\end{array}$ & $\begin{array}{l}.06102 \\
.06992 \\
.07991 \\
.09075 \\
.10226\end{array}$ & $\begin{array}{l}.06067 \\
.07027 \\
.08056 \\
.09104 \\
.10203\end{array}$ & $\begin{array}{l}.06086 \\
.06985 \\
.08017 \\
.09078 \\
.10210\end{array}$ & $\begin{array}{l}.0609 \\
.0700 \\
.0802 \\
.0909 \\
.1021\end{array}$ & $\begin{array}{l}.0002 \\
.0003 \\
.0004 \\
.0001 \\
.0002\end{array}$ & $\begin{array}{l}.00011 \\
.00013 \\
.00014 \\
.00015 \\
.00016\end{array}$ \\
\hline $\begin{array}{l}.12 \\
.14 \\
.16 \\
.18 \\
.2\end{array}$ & $\begin{array}{l}.11886 \\
.13681 \\
.15737 \\
.17806 \\
.20071\end{array}$ & $\begin{array}{l}.11884 \\
.13760 \\
.15765 \\
.17792 \\
.20089\end{array}$ & $\begin{array}{l}.11951 \\
.13819 \\
.15703 \\
.17888 \\
.20041\end{array}$ & $\begin{array}{l}.1191 \\
.1375 \\
.1573 \\
.1783 \\
.2007\end{array}$ & $\begin{array}{l}.0004 \\
.0007 \\
.0003 \\
.0006 \\
.0003\end{array}$ & $\begin{array}{l}.00019 \\
.00021 \\
.00024 \\
.00026 \\
.00029\end{array}$ \\
\hline $\begin{array}{l}.25 \\
1^{.5} \\
3\end{array}$ & $\begin{array}{l}.24702 \\
.49799 \\
1.0037 \\
1.9994 \\
2.9968\end{array}$ & $\begin{array}{l}.24709 \\
.49762 \\
1.0037 \\
1.9972 \\
2.9975\end{array}$ & $\begin{array}{l}.24724 \\
.49795 \\
1.0037 \\
1.9998 \\
2.9993\end{array}$ & $\begin{array}{l}.2471 \\
.4979 \\
1.004 \\
1.996 \\
2.998\end{array}$ & $\begin{array}{l}.0001 \\
.0003 \\
.0000 \\
.0010 \\
.0015\end{array}$ & $\begin{array}{l}.00035 \\
.00065 \\
.0013 \\
.0029 \\
.0047\end{array}$ \\
\hline $\begin{array}{r}5 \\
10 \\
15 \\
20 \\
25\end{array}$ & $\begin{array}{l}4.9841 \\
9.9624 \\
14.991 \\
19.963 \\
24.999\end{array}$ & $\begin{array}{c}4.9923 \\
9.9671 \\
14.988 \\
19.956 \\
25.031\end{array}$ & $\begin{array}{c}4.9927 \\
9.9647 \\
15.001 \\
19.945 \\
24.987\end{array}$ & $\begin{array}{c}4.990 \\
9.965 \\
14.99 \\
19.95 \\
25.01\end{array}$ & $\begin{array}{l}.006 \\
.003 \\
.000 \\
.01 \\
.02\end{array}$ & $\begin{array}{l}.0048 \\
.0053 \\
.0063 \\
.0093 \\
.014\end{array}$ \\
\hline $\begin{array}{l}30 \\
40 \\
50\end{array}$ & $\begin{array}{l}30.080 \\
40.354 \\
52.338\end{array}$ & $\begin{array}{l}30.049 \\
40.281 \\
52.041\end{array}$ & $\begin{array}{l}30.074 \\
40.367 \\
52.336\end{array}$ & $\begin{array}{l}30.07 \\
40.33 \\
52.24\end{array}$ & $\begin{array}{l}.02 \\
.05 \\
.20\end{array}$ & $\begin{array}{l}.015 \\
.02 \\
.06\end{array}$ \\
\hline
\end{tabular}

\section{Appendix}

The analysis (see footnote 4 ) of mismatch errors in the calibration of variable attenuator's yielded an equation for the error in terms of the scattering coefficients of two fourpoles corresponding to two settings of the attenuator dial, and the reflection coefficients of the system in which the attenuator was inserted. The measurement of all these quantities may be tedious or difficult, and an approximate method has been developed. One obtains reasonably close limits within which the error lies from a fairly simple experimental procedure.

The complete expression for the mismatch error is

$$
\epsilon=20 \log _{10}\left|\frac{\left(1-S_{11}^{\prime} \Gamma_{G}\right)\left(1-S_{22}^{\prime} \Gamma_{L}\right)-\left(S_{21}^{\prime}\right)^{2} \Gamma_{G} \Gamma_{L}}{\left(1-S_{11} \Gamma_{G}\right)\left(1-S_{22} \Gamma_{L}\right)-\left(S_{21}\right)^{2} \Gamma_{G} \Gamma_{L}}\right|
$$

where scattering coefficients are denoted by $S_{m n}$, and $\Gamma_{G}, \Gamma_{L}$ represent, respectively, the reflection cocfficients of the system "looking towards" the generator and load. Primes are used to designate a setting of the attenuator other than the zero or reference setting.

For small reflections, the following expression is derived from (1a):

$$
\epsilon \approx 20 \log _{10}\left|1-\left(S_{11}^{\prime}-S_{11}\right) \Gamma_{G}-\left(S_{22}^{\prime}-S_{22}\right) \Gamma_{L}+\left[S_{11}^{\prime} S_{22}^{\prime}-S_{11} S_{22}-\left(S_{21}^{\prime}\right)^{2}+S_{21}^{2}\right] \Gamma_{G} \Gamma_{L}\right| .
$$

If the attenuator VSWR is small, the products $S^{\prime}{ }_{11} S^{\prime}{ }_{22}$ and $S_{11} S_{22}$ may be neglected. Then (2a) becomes

$$
\epsilon \approx 20 \log _{10}\left|1+\left(S_{11}-S_{11}^{\prime}\right) \Gamma_{G}+\left(S_{22}-S_{22}^{\prime}\right) \Gamma_{L}+\left[S_{21}^{2}-\left(S_{21}^{\prime}\right)^{2}\right] \Gamma_{G} \Gamma_{L}\right|
$$

It is convenient to determine the magnitudes of the individual terms but not their phases, so that the limit of error, allowing random phase variations in $S_{11}$ and $S_{22}$, but none in $S_{21}$ is

$$
\epsilon \approx 20 \log _{10}\left[1+\left|S_{11}^{\prime}-S_{11}\right|\left|\Gamma_{G}\right|+\left|S_{22}^{\prime}-S_{22}\right|\left|\Gamma_{L}\right|+\left(\left|S_{21}\right|^{2}-\left|S_{21}^{\prime}\right|^{2}\right)\left|\Gamma_{G} \Gamma_{L}\right|\right]
$$


The quantity $\left|S_{11}-S_{11}^{\prime}\right|$ is determined as follows. With the attenuator under test connected as shown in figure 5 , and set to its zero or reference position, tuner $\mathrm{A}$ is adjusted for a detector null, and tuner $\mathrm{B}$ is then adjusted until the reflection coefficient $\Gamma_{2 i}$ of the equivalent generator at terminal plane 2 vanishes. (This condition may be recognized by means of an auxiliary reflectometer.)

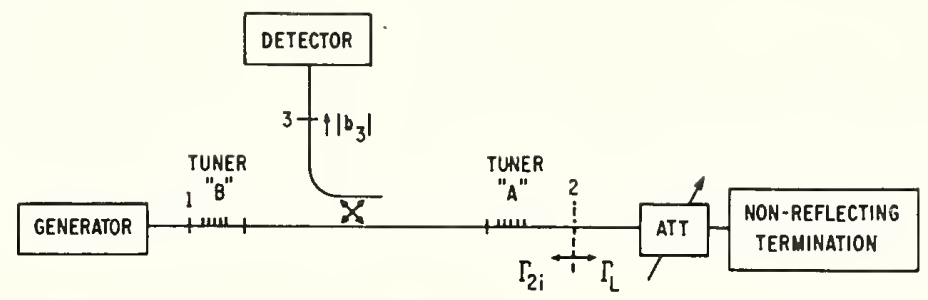

FIGURE 5. Schematic of system for measuring $\left|S_{11}-S_{11}^{\prime}\right|$.

Movement of the attenuator dial to some other setting will then give an observable detector output. Using the theory ${ }^{6}$ of a directional coupler with auxiliary tuners, one can obtain $\left|S_{11}-S_{11}^{\prime}\right|$ in the following way.

The amplitude of the output power from the sidearm of the directional coupler in figure 5 has the magnitude $\left|b_{3}\right|=k\left|\frac{1+K \Gamma_{L}}{1-\Gamma_{2 i} \Gamma_{L}}\right|$, where $k$ is a constant for a given stable generator operating level.

With the attenuator set first on zero, $\Gamma_{L}=S_{11}$. Adjusting tuner $\mathrm{A}$ for $\left|b_{3}\right|=0$ changes $K$ so that $K S_{11}=-1$. Adjusting tuner $\mathrm{B}$ for $\Gamma_{2 i}=0$ makes the dependence of $\left|b_{3}\right|$ on $\Gamma_{L}$ take the simple form:

$$
\left|b_{3}\right|=k\left|1-\frac{\Gamma_{L}}{S_{11}}\right|
$$

Suppose the attenuator dial is moved to a new position such that $\Gamma_{L}$ now equals $S_{11}^{\prime}$; then $\left|b_{3}\right|=k\left|1-\frac{S_{11}^{\prime}}{S_{11}}\right|=\frac{k}{\left|S_{11}\right|}\left|S_{11}-S_{11}^{\prime}\right| \cdot \quad$ The factor $k /\left|S_{11}\right|$ is obtained by replacing the attenuator by a waveguide section containing a sliding short. Upon' sliding the short, $\left|b_{3}\right|$ goes through small variations so that one may observe $\left|b_{3}\right|_{\max }$ and $\left|b_{3}\right|_{\min }$. It is easily shown that

$$
\frac{k}{\left|S_{11}\right|}=\frac{1}{2}\left(\left|b_{3}\right|_{\max }+\left|b_{3}\right|_{\min }\right)
$$

One can assume that $\left|b_{3}\right|_{\max }=\left|b_{3}\right|_{\min }$ and employ a fixed short circuit with negligible error if the VSWR corresponding to $S_{11}$ is less than 1.15. It may be that this error is tolerable for higher VSWR's since it is not important to know $\left|S_{11}-S_{11}^{\prime}\right|$ to great accuracy.

The quantity $\left|S_{22}-S_{22}^{\prime}\right|$ is found in the same way as above with the attenuator turned end for end.

The authors acknowledge the assistance of James E. Gilbert and Morris E. Harvey in making the attenuation measurements and of Wilbur J. Anson and Ronald Ridge in matching the system and making measurements to evaluate the limits of mismatch error. G. E. Schafer read the manuscript and provided information on phase shifts in variable attenuators.

${ }^{6}$ R. W. Beatty and D. M. Ferns, Recently developed microwave impedance standards and methods of measurement, IR E Trans. on Instr., I-7, Nos. 3 \& 4, 319-321 (Dec. 1958).

Boulder, Colo.

(Paper 64C2-33) 


\title{
Mismatch Errors in the Measurement of Ultrahigh-Frequency and Microwave Variable Attenuators
}

\author{
R. W. Beatty
}

\begin{abstract}
The mismatch error in the measurement of ultrahigh-frequency and microwave variable attemuators is analyzed and expressions are derived. An example is given to show that the mismatch error in measuring the difference in attenuation between two attenuators is les: than the sum of the mismatch errors obtained when measuring each attenuator individually.
\end{abstract}

\section{Introduction}

The error resulting from mismatched generator and detector sections in the measurement of the attenuation of a single attenuator is well known. ${ }^{1}$ The corresponding error in the measurement of changes in attenuation is important in the calibration of variable attenuators and in the calibration of large attenuators, using known paels as gage blocks or fixed attemuation standards.

The calibration of a variable attenuator consists in measuring the change in the insertion loss as the attenuator dial moves from a zero or reference position to another position that is marked or ean be read on a scale. The change in the insertion loss equals the change in attenuation if the attenuator is placed in a reflectionless, or matched, system. There is always a degree of uncertainty regarding the match, depending upon the accuracy of the instruments used to indicate or recognize matched conditions and upon the reflections from comnectors. For this reason the change in the insertion loss cannot be considered to be exactly equal to the change in attenuation, and the difference is called the mismatch error.

\section{Expression for Mismatch Error}

A change in a variable attenuator from the reference position to another position is equivalent to removing one attenuator and inserting another attenuator in the circuit. The insertion loss, in decibels, of the attenuator ${ }^{2}$ corresponding to the reference position of the variable attemuator is

$$
L=20 \log _{10}\left|\frac{\left(1-S_{11} \Gamma_{\mathrm{G}}\right)\left(1-S_{22} \Gamma_{\mathrm{L}}\right)-S_{12}^{2} \Gamma_{\mathrm{G}} \Gamma_{\mathrm{L}}}{S_{12}\left(1-\Gamma_{\mathrm{G}} \Gamma_{\mathrm{L}}\right)}\right|
$$

The voltage-reflection coefficients $\Gamma_{G}$ and $\Gamma_{L}$ refer to the generator and load, respectively, and are measured at the terminals where the attenuator is inserted. The scattering coefficients ${ }^{3} S_{11}, S_{12}$, and

\footnotetext{
1 C. G. Montgomery, Technique of microwave measurements, p. 824 (MeGrawHill Book Co., Ine., New York, N. Y., 1947).

2 See Appendix.

3 The seattering coeffieients $S_{11}, S_{12}$, and $S_{22}$ are in effect defined by eq 12 and figure 2. See also, C. G. Montgomery, R. 1I. Dicke, and E. M. Purcell, Prin ciples of microwave eircuits, p. 146-151 (MeGraw-Hill Book Co., lne., New Fork, N. Y., 1948).
}

$S_{22}$ refer to the attenuator, colresponding to the reference or zero position of the rariable at tenuator. The corresponding insertion loss for a different setting of the variable attenuator is

$$
L^{\prime}=20 \log _{10}\left|\frac{\left(1-S_{11}^{\prime} \Gamma_{\mathrm{G}}\right)\left(1-S_{22}^{\prime} \Gamma_{\mathrm{L}}\right)-\left(S_{12}^{\prime}\right)^{2} \Gamma_{\mathrm{G}} \Gamma_{\mathrm{L}}}{S_{12}^{\prime}\left(1-\Gamma_{\mathrm{G}} \Gamma_{\mathrm{L}}\right)}\right|
$$

The change in the insertion loss is

$$
\begin{aligned}
\Delta L & =L^{\prime}-L=20 \log _{10}\left|\frac{1}{S_{12}^{\prime}}\right|-20 \log _{10}\left|\frac{1}{S_{12}}\right| \\
& +20 \log _{10}\left|\frac{\left(1-S_{11}^{\prime} \Gamma_{\mathrm{G}}\right)\left(1-S_{22}^{\prime} \Gamma_{\mathrm{L}}\right)-\left(S_{12}^{\prime}\right)^{2} \Gamma_{\mathrm{G}} \Gamma_{\mathrm{L}}}{\left(1-S_{11} \Gamma_{\mathrm{G}}\right)\left(1-S_{22} \Gamma_{\mathrm{L}}\right)-S_{12}^{2} \Gamma_{\mathrm{G}} \Gamma_{\mathrm{L}}}\right|,
\end{aligned}
$$

or

$$
\Delta L=A^{\prime}-A+\epsilon,
$$

where $A^{\prime}, A$, and $\epsilon$ in eq (4) correspond to the three terms in eq (3). The error $\epsilon$ must be subtracted from the change in the insertion loss to obtain the change in attenuation, $A^{\prime}-A$.

'The error term can also be written ${ }^{4}$

$$
\epsilon=20 \log _{10}\left|\frac{\left(1-\Gamma_{1}^{\prime} \Gamma_{G}\right)\left(1-S_{22}^{\prime} \Gamma_{L}^{\prime}\right)}{\left(1-\Gamma_{1} \Gamma_{G}\right)\left(1-S_{22} \Gamma_{L}\right)}\right|,
$$

where $\Gamma_{1}$ and $\Gamma_{1}^{\prime}$ are the input-voltage reflection coefficients of the attemutor terminated in a load having a voltage reflection coefficient $\Gamma_{L}$.

'The mismatch error in the measurement of a single attenuator can be obtained as a special case of $\mathrm{eq}$ (5). The reference attenuator vanishes in this case, changing $\Gamma_{1}$ to $\Gamma_{\mathrm{L}}, S_{12}$ to unity, and $S_{11}$ and $S_{22}$ to zero. Substituting these values for $\Gamma_{1}$ and $S_{22}$ into eq (5) yields

$$
\epsilon=20 \log _{10}\left|\frac{\left(1-\Gamma_{1}^{\prime} \Gamma_{G}\right)\left(1-S_{22}^{\prime} \Gamma_{L}\right)}{1-\Gamma_{G} \Gamma_{L}}\right|,
$$

which corresponds to eq (24), page 826 of the ieference of footnote 1.

4 Substitute $\Gamma_{1}=S_{11}+\frac{S_{12}^{2} \Gamma_{L}}{1-S_{22} \Gamma_{L}}$ and $\Gamma_{1}^{\prime}=S_{11}^{\prime}+\frac{\left(S_{12}^{\prime}\right)^{2} \Gamma_{L}}{1-S_{22}^{\prime} \Gamma_{L}}$ in eq (3). 


\section{Evaluation of the Mismatch Errors}

$1 \mathrm{t}$ is possible in principle to evaluate the mismatch "ror by measuring the voltage reflection coefficients $\Gamma_{(\mathrm{i}}, \Gamma_{\mathrm{L}}^{\prime}, \Gamma_{1}, \Gamma_{1}^{\prime}, S_{22}$, and $S_{22}^{\prime}$, and substituting them into eq (5).

In many cases, the magnitudes of the reflection roefficients can be determined, hut their phases ramnot be conveniently determined because of the limitations of a particular measuring apparatus. Equation (5) can then be used to find the limits of the mismatch error, permitting the phases of the reffection coefficients to have all possible values. The limit of error ean be expressed in the form

$$
\epsilon_{\text {limalt }}=20 \log _{10} \frac{\left(1 \pm\left|\Gamma_{1}^{\prime} \Gamma_{G}\right|\right)\left(1 \pm\left|S_{22}^{\prime} \Gamma_{L}\right|\right)}{\left(1 \mp\left|\Gamma_{1} \Gamma_{G}\right|\right)\left(1 \mp\left|S_{22} \Gamma_{L}\right|\right)}
$$

and the corresponding limit of error for single attenuators is

$$
\epsilon_{11 \mathrm{~m} ! \mathrm{t}}=20 \log _{10} \frac{\left(1 \pm\left|\Gamma_{1} \Gamma_{\mathrm{G}}\right|\right)\left(1 \pm\left|S_{22} \Gamma_{\mathrm{L}}\right|\right)}{1 \mp\left|\Gamma_{\mathrm{G}} \Gamma_{\mathrm{L}}\right|} .
$$

For rxample, in order to reduce mismatch errors in the calibration of attenuators, the magnitudes of $\Gamma_{G}$ and $\Gamma_{L}$ are made as small as possible, and their probable amplitude is estimated from the accuracy of the apparatus used to recognize matched conditions and from the known connector characteristics. It is difficult to accurately determine the phases of these small reflection coefficients, and the mismatch crror can generally be determined not exactly, but within limits.

An example will illustrate the determination of mismatch error. If the voltage standing-wave ratios $\sigma_{\mathrm{G}}, \sigma_{\mathrm{L}}, \sigma_{1}, \sigma_{1}^{\prime}, \sigma_{22}$, and $\sigma_{22}^{\prime}[\sigma=(1+|\Gamma|) /(1-|\Gamma|)]$ corresponding to $\left|\Gamma_{\mathrm{G}}\right|,\left|\Gamma_{\mathrm{L}}\right|,\left|\Gamma_{1}\right|,\left|\Gamma_{1}^{\prime}\right|,\left|S_{22}\right|$, and $\left|S_{22}^{\prime}\right|$ are $1.1,1.1,1.2,1.5,1.2$, and 1.5 , the limits of error for the initial attenuator calculated from eq (8) are approximately \pm 0.095 decibel. 'The corresponding limits of error for the final attenuator are approximately \pm 0.185 decibel. The limits of error for the change in attenuation calculated from eq (7) are approximately \pm 0.242 decibel. It is seen that the mismatch error for the change in attenuation is less than the sum of the mismatch errors in measuring each attenuator individually.

It is often possible to determine the phases of $\Gamma_{1}, \Gamma_{1}^{\prime}, S_{22}$, and $S_{22}^{\prime}$, enabling one to calculate the mismatch error within narrower limits. The error in measuring voltage standing-wave ratios and phase angles produces an error in determining the limits of mismatch crror but is in general a second-order effect that can be neglected.

\section{Appendix. Derivation of Expression for Insertion Loss}

The insertion loss of a four-terminal network is defined by the equation

$$
L=10 \log _{10} \frac{P_{\mathrm{L}}}{P_{2}}
$$

where $P_{\mathrm{L}}$ denotes the power delivered directly to a load, and $P_{2}$ denotes the power delivered to that load when the network is inserted between the load and the generator. It is assumed that the generator is stable and unaffected by changes in loading.

The insertion loss of a four-terminal network can be expressed in terms of its scattering coefficients $S_{11}, S_{12}$, and $S_{22}$, and the voltage reflection coefficients $\Gamma_{\mathrm{G}}$ and $\Gamma_{\mathrm{L}}$ of the generator and the load.

Referring to figures 1 and 2, the insertion loss is

$$
L=10 \log _{10} \frac{P_{\mathrm{L}}}{P_{1}} \cdot P_{1} P_{2}=10 \log _{10} \frac{P_{\mathrm{L}}}{P_{1}} \cdot \frac{1}{\eta}
$$

where $\eta$ is the efficiency of the network. If $A$ and $B$ denote the incident and reflected voltage waves shown in figure 2. The efficiency is ${ }^{5}$

$$
\eta=P_{2}=\frac{E_{2} \cdot\left(-I_{2}\right)}{E_{1} \cdot I_{1}}=\frac{\left|B_{2}\right|^{2}-\left|A_{2}\right|^{2}}{\left|A_{1}\right|^{2}-\left|B_{1}\right|^{2}}=\left|\frac{B_{2}}{A_{1}}\right|^{2} \frac{1-\left|\Gamma_{\mathbf{L}}\right|}{1-\left|\Gamma_{1}\right|^{2}} .
$$

The scattering equations of the network are

$$
\left.\begin{array}{l}
B_{1}=S_{11} A_{1}+S_{12} A_{2} \\
B_{2}=S_{12} A_{1}+S_{22} A_{2}
\end{array}\right\},
$$

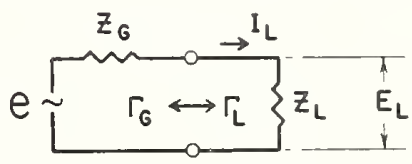

$$
\begin{aligned}
& \Gamma_{G}=\frac{z_{G}-z_{0}}{z_{G}+z_{0}} ; \quad \Gamma_{L}=\frac{z_{L}-z_{0}}{z_{L}+z_{0}} \\
& E_{L}=e \frac{Z_{L}}{Z_{G}+Z_{L}}=\frac{e}{2} \frac{\left(1-\Gamma_{G}\right)\left(1+\Gamma_{L}\right)}{\left(1-\Gamma_{G} \Gamma_{L}\right)} \\
& I_{L}=\frac{E_{L}}{Z_{L}}=\frac{e}{2 Z_{0}} \frac{\left(1-\Gamma_{G}\right)\left(1-\Gamma_{L}\right)}{\left(1-\Gamma_{G} \Gamma_{L}\right)} \\
& P_{L}=E_{L} \cdot I_{L}=\frac{e^{2}}{4 Z_{0}}\left|\frac{1-\Gamma_{G}}{1-\Gamma_{G} \Gamma_{L}}\right|^{2}\left\{1-\left|\Gamma_{L}\right|^{2}\right\}
\end{aligned}
$$

Figure 1. Load connected directly to generator.

3 Note that expressions of the form $E \cdot I$ represent the dot, or scalar, product of two vector quantities. 


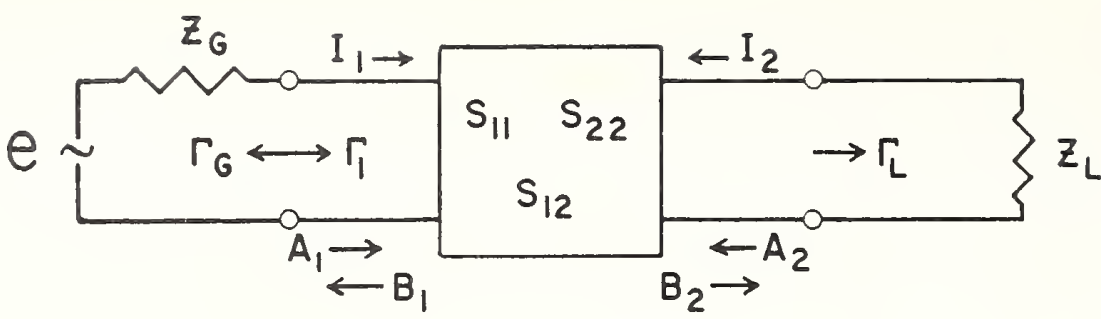

$$
\begin{aligned}
& \left\{\begin{array} { l } 
{ E _ { 1 } = A _ { 1 } + B _ { 1 } } \\
{ I _ { 1 } = \frac { 1 } { Z _ { 0 } } ( A _ { 1 } - B _ { 1 } ) }
\end{array} \quad \left\{\begin{array} { l } 
{ E _ { 2 } = A _ { 2 } + B _ { 2 } } \\
{ I _ { 2 } = \frac { 1 } { Z _ { 0 } } ( A _ { 2 } - B _ { 2 } ) }
\end{array} \quad \left\{\begin{array}{l}
\Gamma_{1}=\frac{B_{1}}{A_{1}} \\
\Gamma_{L}=\frac{A_{2}}{B_{2}}
\end{array}\right.\right.\right. \\
& P_{1}=E_{1} \cdot I_{1}=\frac{\left|A_{1}\right|^{2}-\left|B_{1}\right|^{2}}{Z_{0}}=\frac{\left|A_{1}\right|^{2}}{Z_{0}}\left\{1-\left|\Gamma_{1}\right|^{2}\right\} \\
& P_{2}=E_{2} \cdot\left(-I_{2}\right)=\frac{\left|B_{2}\right|^{2}-\left|A_{2}\right|^{2}}{Z_{0}}=\frac{\left|B_{2}\right|^{2}}{Z_{0}}\left\{1-\left|\Gamma_{L}\right|^{2}\right\}
\end{aligned}
$$

Figure 2. Network inserted between generator and load.

where $\Gamma_{1=B_{1} / A_{1}}$ and $\Gamma_{L}=A_{2} / B_{2}$. But

therefore,

$$
\frac{B_{2}}{A_{1}}=\frac{S_{12}}{1-S_{22} \Gamma_{\mathrm{L}}^{\prime}}
$$

$$
\eta=\left|\frac{S_{12}}{1-S_{22} \Gamma_{\mathrm{L}}}\right|^{2} \frac{1-\left|\Gamma_{\mathrm{L}}\right|^{2}}{1-\left|\Gamma_{1}\right|^{2}}
$$

Inspection of figure 1 leads to the conclusion

$$
\frac{P_{\mathrm{L}}}{P_{1}}=\left|\frac{1-\Gamma_{\mathrm{G}} \Gamma_{1}}{1-\Gamma_{\mathrm{G}} \Gamma_{\mathrm{L}}}\right|^{2} \cdot \frac{1-\left|\Gamma_{\mathrm{L}}\right|^{2}}{1-\left|\Gamma_{1}\right|^{2}}
$$

Substitution of these equations into eq (8) yiekds

$$
L=20 \log _{10} \frac{\left(1-\Gamma_{\mathrm{G}} \Gamma_{1}\right)\left(1-S_{22} \Gamma_{\mathrm{L}}\right)}{S_{12}\left(1-\Gamma_{\mathrm{G}} \Gamma_{\mathrm{L}}\right)} .
$$

Since

$$
\Gamma_{1}=S_{11}+\frac{S_{12}^{2} \Gamma_{\mathrm{L}}}{1-S_{22} \Gamma_{\mathrm{L}}},
$$

the insertion loss may also be written

$$
L=20 \log _{10}\left|\frac{\left(1-S_{11} \Gamma_{\mathrm{G}}\right)\left(1-S_{22} \Gamma_{\mathrm{L}}\right)-S_{12}^{2} \Gamma_{\mathrm{G}} \Gamma_{\mathrm{L}}}{S_{12}\left(1-\Gamma_{\mathrm{G}} \Gamma_{\mathrm{L}}\right)}\right|
$$




\title{
Mismatch Errors in Cascade-Connected Variable Attenuators*
}

\author{
G. E. SCHAFER AND A. Y. RUMFELT $\dagger$
}

Summory-The treatment of mismatch errors is extended to cover variable attenuators cascade-connected in a system which is not free from reflections. The method of analysis is applicable to any number of cascaded attenuators, but only the analysis of two and three variable attenuators in cascade is presented. Graphs are given to aid in estimating the limits of mismatch error.

In an example, which is considered representative of rigid rectangular waveguide systems, the limits of error are: for two attenuators in cascade, $0.19 \mathrm{db}$ in a 3-db measurement, and $0.17 \mathrm{db}$ in a 40-db measurement; and for three attenuators in cascade, $0.25 \mathrm{db}$ in a $40-\mathrm{db}$ measurement, and $0.23 \mathrm{db}$ in a $75-\mathrm{db}$ measurement.

\section{INTRODUCTION}

$\mathbb{E}^{n}$ RRORS in attenuation caused by the interaction of reflections from generator, attenuator, and load mismatches are termed mismatch errors. Previous treatments of mismatch errors have considered variable or fixed single attenuators in systems with reflections $^{1,2}$ and cascaded fixed attenuators in reflectionfree systems. ${ }^{3}$ In cases where the variable attenuator to be calibrated has a very wide range (greater than 45 $\mathrm{db})$, measurements are often made by using one or two previously calibrated attenuators cascade-connected with the test attenuator (a direct series substitution method). Therefore, the analysis is extended to cascaded variable attenuators in a system which is not free from reflections. Although the method of analysis employed is applicable to any number. of variable attenuators in cascade, only the cases of two or three variable attenuators in cascade are presented. Graphs are presented which may be used to estimate the limits of mismatch error in these two cases. An example is given of the use of the graphs. Other sources of error such as the accuracy of the original calibrations, leakage, noise, etc., are not considered here but must be taken into account to obtain total limits of error in an actual calibration.

\section{THEORY}

In a direct series-substitution method of measuring the attenuation ${ }^{4}$ of a variable attenuator, the test attenuator is connected in series with a reference (previ-

* Manuscript received by the PGMTT, May 4, 1959; revised manuscript received, June 17,1959

$\dagger$ U. S. Dept. of Commerce, Natl. Bur. Standards, Boulder Labs., Boulder, Colo.

${ }_{1} \mathrm{R}$. W. Beatty, "Mismatch errors in the measurement of ultrahigh frequency and microwave variable attenuators," J. Research Natl. Bur. Standards, vol. 52, pp. 7-9; January, 1954.

${ }_{2}$ C. G. Montgomery, ed., "Technique of Microwave Measurements," Mass. Inst. Tech. Rad. Lab. Ser., McGraw-Hill Book Co., Inc., New York, N. Y., vol. 11, Ch. 13; 1947.

'R. W. Beatty, "Cascade-connected attenuators," J. Research Natl. Bur. Standards, vol. 45, pp. 231-235; September, 1950.

1 In this paper, attenuation is defined as the insertion loss in a reflection-free system. Thus, in measuring attenuation, one attempts to eliminate the reflections in the system, and then one measures the insertion loss. ously calibrated or standard) attenuator. The total insertion loss of the series-connected pair of attenuators is adjusted to be the same at two different settings. The relative attenuation of the test attenuator is taken to be equal in magnitude to the relative attenuation of the reference attenuator. This is in error because the standard attenuator is connected to a mismatched attenuator, and also because the pair of attenuators are inserted in a mismatched system.

An expression can be derived for the insertion loss of the pair of attenuators connected in cascade. From this, an expression may be written for the difference between the total insertion loss and the sum of the individual attenuations. Inspection of this reveals that the error can be separated in to two convenient parts. One of these has been previously worked out ${ }^{3}$ in detail for fixed attenuators and is readily extended to variable attenuators, and the other is evaluated here by calculation of certain quantities associated with the combination of attenuators. This separation is performed in the following analysis.

At the initial settings of the attenuators, one may write the total insertion loss as

$$
L_{i}=A_{t i}+A_{r i}+\epsilon_{i},
$$

where $L_{i}$ is the total insertion loss at the initial settings of the attenuators, $A_{t i}$ and $A_{r i}$ are the attenuations of the test and reference attenuators at the initial settings, and $\epsilon_{i}$ is the difference between the insertion loss of the pair in cascade and the sum of the individual attenuations. At the final settings of the attenuators, a similar expression for the total insertion loss may be written as

$$
L_{f}=A_{t f}+A_{r f}+\epsilon_{f},
$$

where the symbols have the same meaning as in (1) except that the subscript $f$ refers to the final settings. The adjustments of the attenuators are made in this way to result in the initial and final total insertion losses being equal, $L_{i}=L_{f}$, from which

$$
\left(A_{t j}-A_{t i}\right)=\left(A_{\tau i}-A_{\tau f}\right)+\epsilon_{T},
$$

where $\epsilon_{T}$ has been written for $\epsilon_{i}-\epsilon_{f}$. This means that the relative attenuation of the test attenuator may differ from the relative attenuation of the reference attenuator by $\epsilon_{T}$. This is the desired error. However, a convenient separation may be made as follows. Rewrite (3) as

$$
\left(A_{t j}+A_{\tau j}\right)-\left(A_{t i}+A_{r i}\right)=\epsilon_{T} .
$$

Let $A_{i}$ and $A_{f}$ be the attenuation at the initial and final settings, respectively, of the combination of series-con- 
nected attenuators considered as a single attenuator (called the combination attenuator in the remainder of the paper). These may differ from the sums of the individual attenuations by $\epsilon_{c i}$ and $\epsilon_{c f}$, respectively, and therefore one may write

$$
\begin{aligned}
& A_{f}=A_{t f}+A_{r f}+\epsilon_{c f} \\
& A_{i}=A_{t i}+A_{r i}+\epsilon_{c i} .
\end{aligned}
$$

Substituting (5) and (6) into (4) yields

$$
A_{f}-A_{i}+\epsilon_{2}=\epsilon_{T},
$$

where $\epsilon_{2}$ has been written for $\epsilon_{c i}-\epsilon_{i f}$. The component $\epsilon_{2}$ of the mismatch error is the difference between the attenuation of the combination attenuator and the sums of the attenuations of the individual attenuators. This component may be evaluated by an extension of the results of Ref. 3 to include the case of variable attenuators connected in cascade, and may be written as

$$
\epsilon_{2}=20 \log _{10}\left|\frac{1-{ }^{(f)} S_{11^{\prime \prime}}{ }^{\prime(f)} S_{22}{ }^{\prime}}{1-{ }^{(i)} S_{11}{ }^{\prime(i)} S_{22}}\right|,
$$

where the front superscripts $(i)$ and $(f)$ refer to the initial and final values, respectively; the primed $S^{\prime}$ s are elements of the scattering matrices ${ }^{5}$ of the individual attenuators, and the number of primes indicate the position of the attenuator as counted from the generator.

Eq. (7) may be written as

$$
A_{f}-A_{i}=\epsilon_{T}-\epsilon_{2}=\epsilon_{1} .
$$

This form is equivalent to considering the combination attenuator as a single attenuator at two different attenuation settings, and $\epsilon_{1}$ can therefore be evaluated as the mismatch error in a relative attenuation measurement with a single attenuator. This component of mismatch error has been treated by Beatty ${ }^{1}$ and for this application may be written as

$$
\epsilon_{1}=20 \log _{10}\left|\frac{\left(1-{ }^{(f)} \Gamma_{1} \Gamma_{9}\right)\left(1-{ }^{(f)} S_{22} \Gamma_{L}\right)}{\left(1-{ }^{(i)} \Gamma_{1} \Gamma_{9}\right)\left(1-{ }^{(i)} S_{22} \Gamma_{L}\right)}\right|,
$$

where the front superscripts $(i)$ and $(f)$ refer to the initial and final values, respectively, and where $\Gamma_{1}$ is the input reflection coefficient of the combination attenuator when terminated with an equivalent detector having à reflection coefficient $\Gamma_{L}$. Also, $\Gamma_{G}$ is the reflection coeffi- smaller limits of mismatch error than the addition of the separate limits of mismatch error for attenuation at the initial and final settings.

The total error in cascading variable ${ }^{6}$ attenuators in a mismatched system is then $\epsilon_{1}+\epsilon_{2}$, where $\epsilon_{1}$ is the error caused by interactions of reflections from the generator, combination attenuator, and the load, and $\epsilon_{2}$ is the error caused by interactions of reflections from the individual attenuators.

The scattering matrix of the combination attenuator is used to evaluate the component $\epsilon_{1}$ of the mismatch error according to (10). This is readily obtained in terms of the scattering matrices of the individual attenuators through use of the $T$ matrices. ${ }^{7}$ The $T$ matrix for a combination of $n$ cascade-connected attenuators may be written as

$$
T=\prod_{k=n}^{k=1} T_{k}
$$

where the $T_{k}$ are the matrices for the individual attenuators. This $T$ matrix is the inverse of the $A$ matrix used in Ref. 3. The scattering matrix, $S$, for the two-arm junction is related to the $T$ matrix as follows:

$$
S=\frac{1}{T_{22}}\left|\begin{array}{cc}
-T_{21} & 1 \\
1 & T_{12}
\end{array}\right| \text { and } T=\frac{1}{S_{12}}\left|\begin{array}{cc}
S_{12}{ }^{2}-S_{22} S_{11} & S_{22} \\
-S_{11} & 1
\end{array}\right|,
$$

when reciprocity in the form $S_{p q}=S_{q p}$ is assumed. The necessary characteristics of the combination of attenuators needed to evaluate the error by (10) are $\Gamma_{1}$ and $S_{22}$. These may be obtained by use of (11) and (12), and the expression

$$
\Gamma_{1}=S_{11}+\frac{S_{12}^{2} \Gamma_{L}}{1-S_{22} \Gamma_{L}}
$$

The results for two attenuators in cascade are

$$
\Gamma_{1}=S_{11}{ }^{\prime}+\frac{\left(S_{12}{ }^{\prime}\right)^{2} S_{11}{ }^{\prime \prime}\left(1-S_{22}{ }^{\prime \prime} \Gamma_{L}\right)+\left(S_{12}{ }^{\prime}\right)^{2}\left(S_{12}{ }^{\prime \prime}\right)^{2} \Gamma_{L}}{\left(1-S_{22}{ }^{\prime \prime} \Gamma_{L}\right)\left(1-S_{11}{ }^{\prime \prime} S_{22}{ }^{\prime}\right)-S_{22}{ }^{\prime}\left(S_{12}{ }^{\prime \prime}\right)^{2} \Gamma_{L}},
$$

and

$$
S_{22}=S_{22}^{\prime \prime}+\frac{S_{22}{ }^{\prime}\left(S_{12}{ }^{\prime \prime}\right)^{2}}{1-S_{11}^{\prime \prime} S_{22}{ }^{\prime}}
$$

and for three attenuators in cascade, they are

$\Gamma_{1}=S_{11}{ }^{\prime}+\frac{\left(S_{12}{ }^{\prime}\right)^{2}\left\{S_{11}{ }^{\prime \prime}\left[\left(1-S_{22}{ }^{\prime \prime} S_{11}{ }^{\prime \prime \prime}\right)\left(1-S_{22}{ }^{\prime \prime \prime} \Gamma_{L}\right)-S_{22}{ }^{\prime \prime}\left(S_{12}{ }^{\prime \prime \prime}\right)^{2} \Gamma_{L}\right]+S_{11}{ }^{\prime \prime \prime}\left(S_{12}{ }^{\prime \prime}\right)^{2}\left(1-S_{22}{ }^{\prime \prime \prime} \Gamma_{L}\right)+\left(S_{12}{ }^{\prime \prime}\right)^{2}\left(S_{12}{ }^{\prime \prime \prime}\right)^{2} \Gamma_{L}\right\}}{\left(1-S_{22}{ }^{\prime \prime \prime} \Gamma_{L}\right)\left[\left(1-S_{22}{ }^{\prime} S_{11}{ }^{\prime \prime}\right)\left(1-S_{22}{ }^{\prime \prime} S_{11}{ }^{\prime \prime \prime}\right)-S_{22}{ }^{\prime}\left(S_{12}{ }^{\prime \prime}\right)^{2} S_{11}{ }^{\prime \prime \prime}\right]-\left(S_{12}{ }^{\prime \prime \prime}\right)^{2} \Gamma_{L}\left[S_{22}{ }^{\prime \prime}\left(1-S_{11}{ }^{\prime \prime} S_{22}{ }^{\prime}\right)+S_{22}{ }^{\prime}\left(S_{12}{ }^{\prime \prime}\right)^{2}\right]}$,

and

$$
S_{22}=S_{22}{ }^{\prime \prime \prime}+\frac{\left(S_{12}{ }^{\prime \prime \prime}\right)^{2}\left\{S_{22}{ }^{\prime}\left(S_{12}{ }^{\prime \prime}\right)^{2}+S_{22}{ }^{\prime \prime}\left(1-S_{11}{ }^{\prime \prime} S_{22}{ }^{\prime}\right)\right\}}{\left(1-S_{22}{ }^{\prime} S_{11}{ }^{\prime \prime}\right)\left(1-S_{11}{ }^{\prime \prime} S_{22}{ }^{\prime \prime}\right)-S_{22}{ }^{\prime} S_{11}{ }^{\prime \prime}\left(S_{12}{ }^{\prime \prime}\right)^{2}}
$$

cient of the equivalent generator, and $S_{22}$ is an element of the scattering matrix, $S$, of the combination attenuator. It should be recalled that this expression gives

${ }^{5}$ C. G. Montgomery, op. cit., Ch. 14.
${ }^{6}$ The same analysis applies to cascaded-fixed, or a combination of fixed and variable attenuators in a mismatched system by using the appropriate forms of (8) and (10).

${ }^{7}$ C. G. Montgomery, R. H. Dicke, and E. M. Purcell, "Frinciples of Microwave Circuits," Mass. Inst. Tech. Rad. Lab. Ser., McGrawHill Book Co., Inc., New York, N. Y., vol. 8, Ch. 5; 1948. 
where the primed $S$ 's are the scattering coefficients of the individual attenuators, where the number of primes indicates the position of the attenuator as counted from the generator end where $\Gamma_{L}$ is the equivalent reflection coefficient of the detector, and where reciprocity in the form $S_{p q}=S_{q p}$ has been assumed.

Substitution of (14) and (15) into (10) gives the expression for the component $\epsilon_{1}$ of the mismatch error for two variable attenuators in cascade. Similarly, substitution of (16) and (17) gives the corresponding expression for the case of three variable attenuators in cascade.

Assuming that the phases of the individual coefficients of (8) and (10) are not known and that they can have any possible value, the actual values of the error components cannot be determined, but limits may be found. Such a limit is a conservative figure, and a closer estimate of the actual error may be obtained if one determines the phases of the individual coefficients. The limits of $\epsilon_{1}$ and $\epsilon_{2}$ may be added to obtain limits of the mismatch error, $\epsilon_{T}$, since the phases of the coefficients in (8) and (10) can take on values so that each component of mismatch error is simultaneously at its maximum possible value.

\section{Graphical Presentation of Results}

One of the factors taken into consideration, in constructing the graphs, was the ease of obtaining an esti-

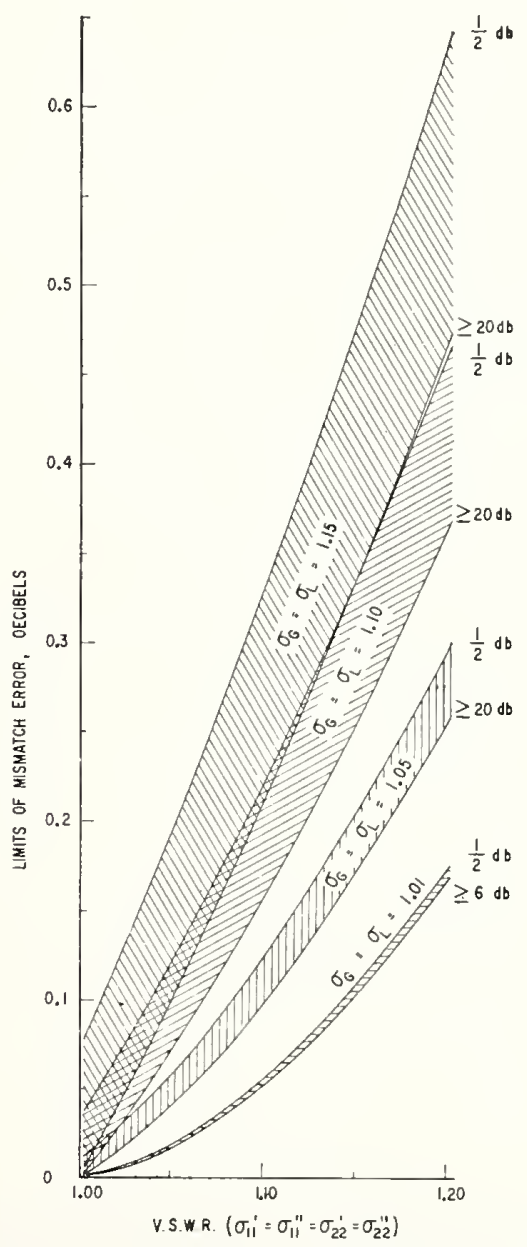

Fig. 1-Variation with range of measurement in limits of mismatch error, $\epsilon_{T}$, for two variable attenuators in cascade.
Cascade-Connected Variable Attenuators

mate of the limits of the mismatch error. For this purpose, certain assumptions to be discussed were made concerning the scattering coefficients of the attenuators. Since many commercially available attenuators have similar voltage-standing-wave ratio (VSWR) characteristics, it will become evident that these assumptions caused very little loss in the generality of application. The following equalities were assumed:

$$
\begin{aligned}
\left|S_{11}{ }^{\prime}\right| & =\left|S_{11}{ }^{\prime \prime}\right|=\left|S_{11}{ }^{\prime \prime \prime}\right|=\left|S_{22}{ }^{\prime}\right| \\
& =\left|S_{22}{ }^{\prime \prime}\right|=\left|S_{22}{ }^{\prime \prime}\right|
\end{aligned}
$$

and

$$
\left|\Gamma_{G}\right|=\left|\Gamma_{L}\right|
$$

Furthermore, it was assumed that the phases of each coefficient in (14)-(17) and the $S_{12}$ 's took on values at the initial and final settings of the attenuators which would give the maximum possible mismatch error (limits of mismatch error). This assumption yields a conservative estimate of the mismatch error.

The range of the limits of the mismatch error, $\epsilon_{T}$, for two attenuators in cascade and for three attenuators in cascade are shown in Figs. 1 and 2, respectively. The sectors indicate how the limits of error vary with the number of decibels to be measured and with the VSWR of the attenuators for a number of specified equivalent generator and detector mismatches. Eq. (18) implies

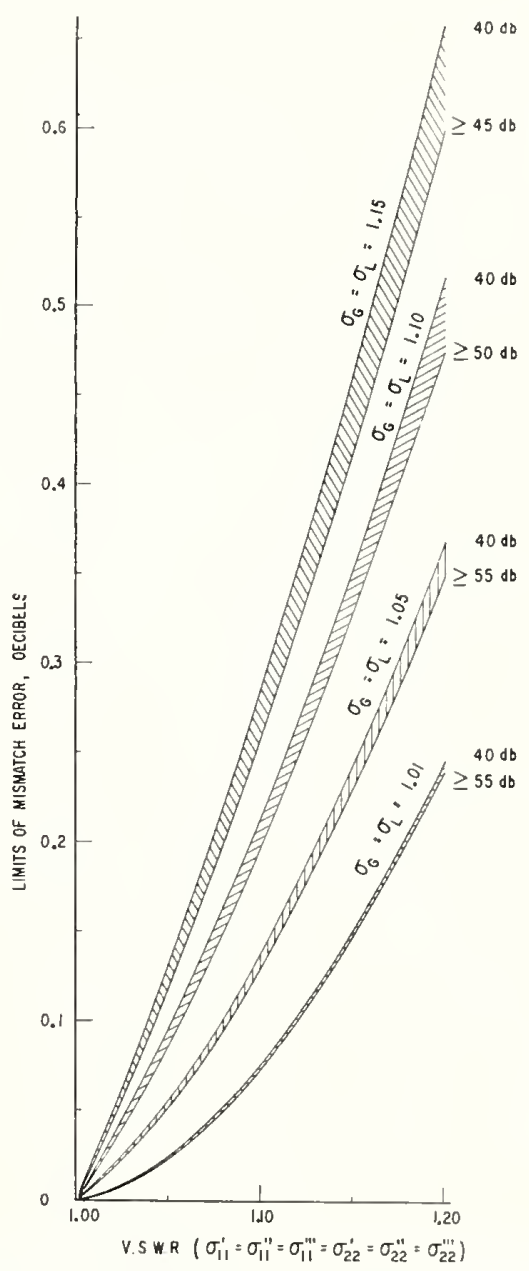

Fig. 2-Variation with range of measurement in limits of mismatch error, $\epsilon T$, for three variable attenuators in cascade. 


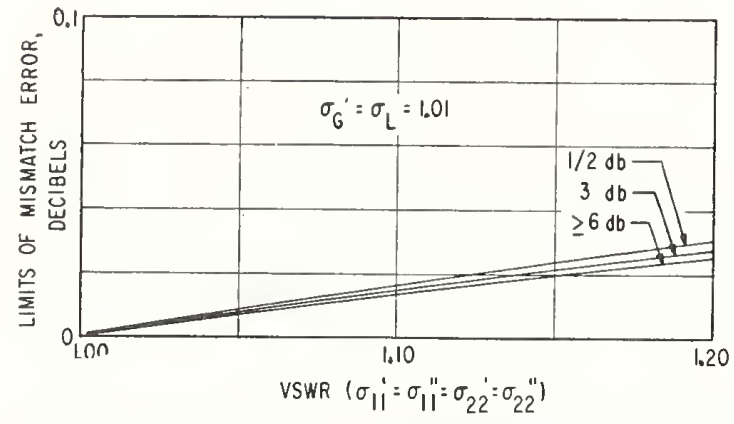

Fig. 3-Limits of component $\epsilon_{1}$ of mismatch error for two attenuators with equal magnitudes of $S_{11}$ and $S_{22}$ cascaded in a system where $\sigma_{G}=\sigma_{L}=1.01$.

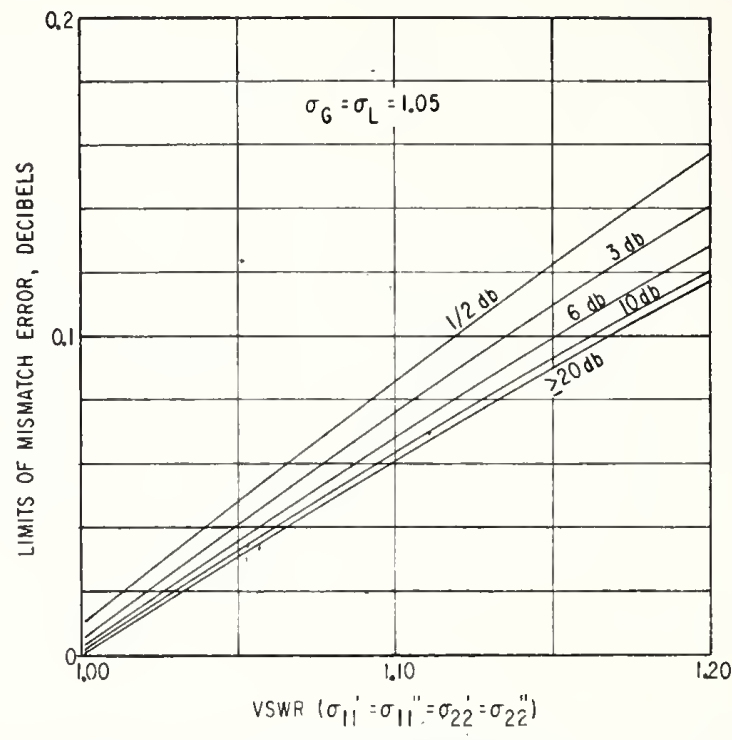

Fig. 4-Limits of component $\epsilon_{1}$ of mismatch error for two at tenuators with equal magnitudes of $S_{11}$ and $S_{22}$ cascaded in a system where $\sigma_{G}=\sigma_{L}=1.05$

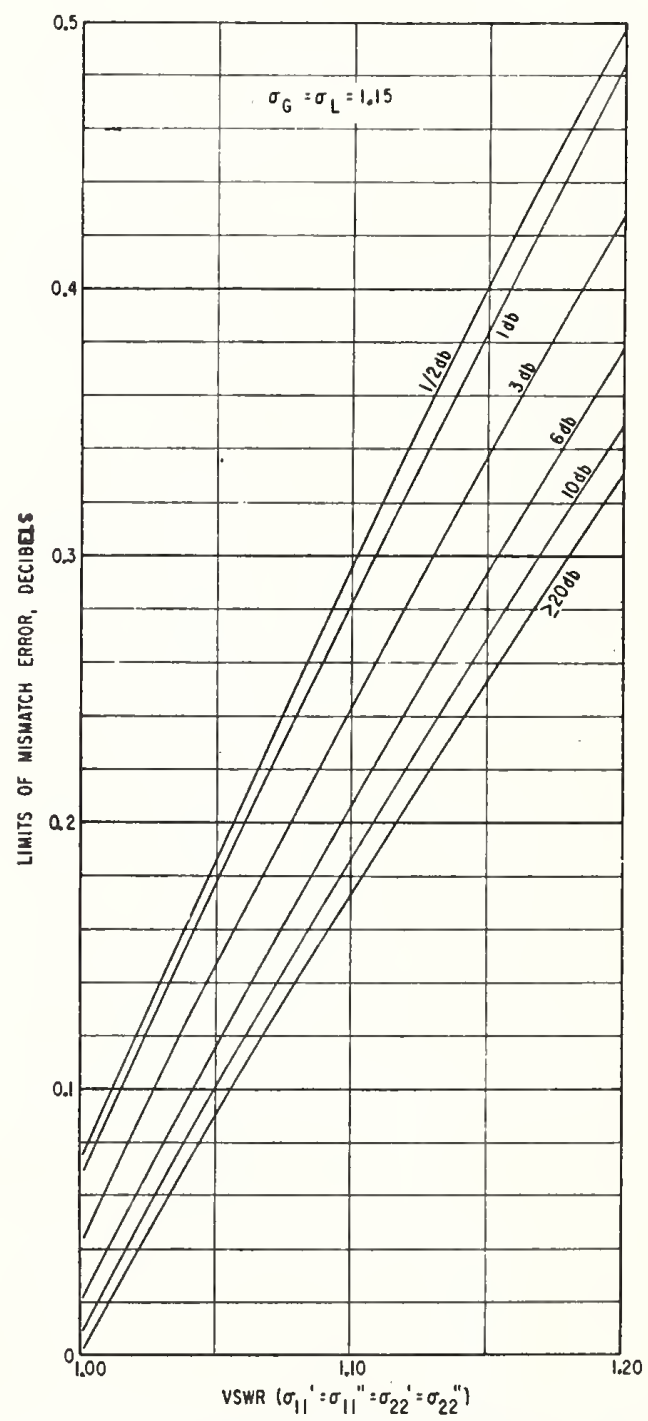

Fig. 6-Limits of component $\epsilon_{1}$ of mismatch error for two attenuators with equal magnitudes of $S_{11}$ and $S_{22}$ cascaded in a system where $\sigma_{G}=\sigma_{L}=1.15$ with equal magnitudes of $S_{11}$ and $S_{22}$ cascaded in a system where $\sigma_{G}=\sigma_{L}=1.10$ 


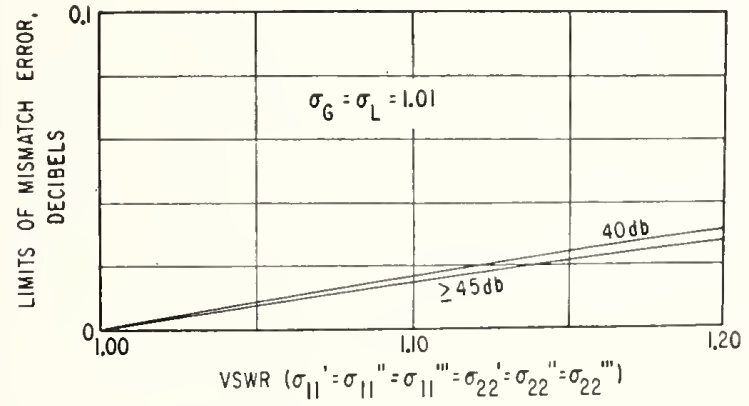

Fig. 7-Limits of component $\epsilon_{1}$ of mismatch error for three attenuators with equal magnitudes of $S_{11}$ and $S_{22}$ cascaded in a system where $\sigma_{\theta}=\sigma_{L}=1.01$.

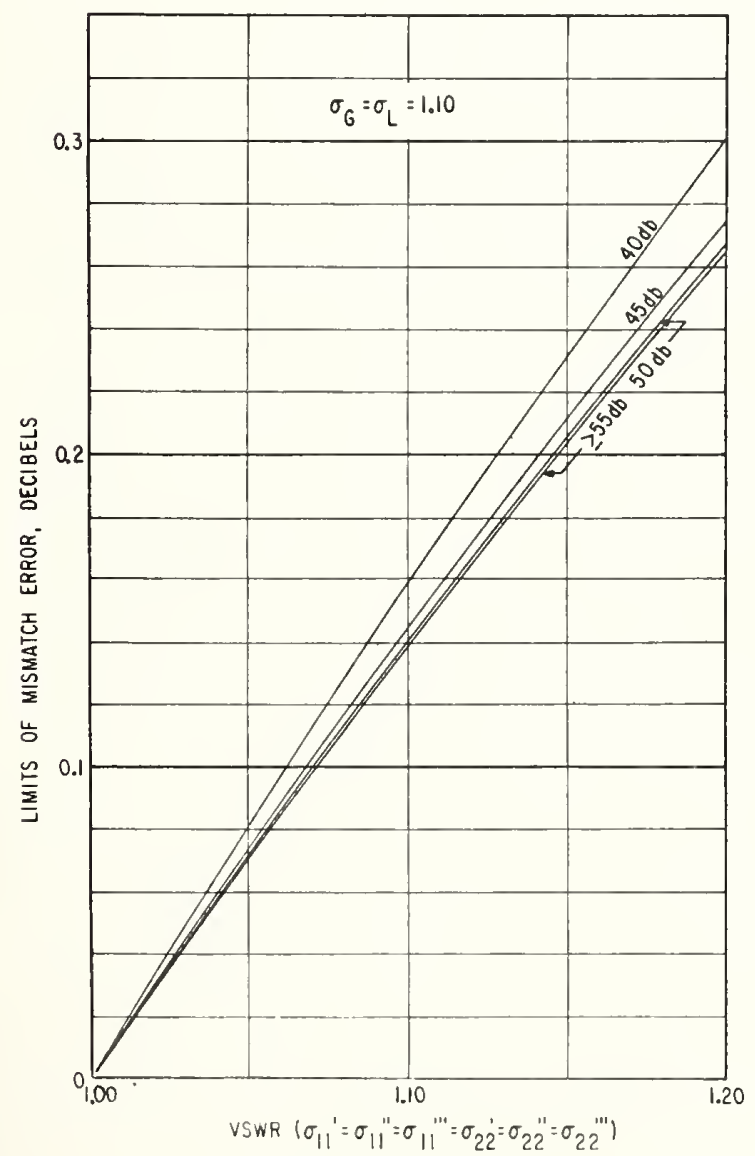

Fig. 9-Limits of component $\epsilon_{1}$ of mismatch error for three attenuators with equal magnitudes of $S_{11}$ and $S_{22}$ cascaded in a system where $\sigma_{\theta}=\sigma_{L}=1.10$.

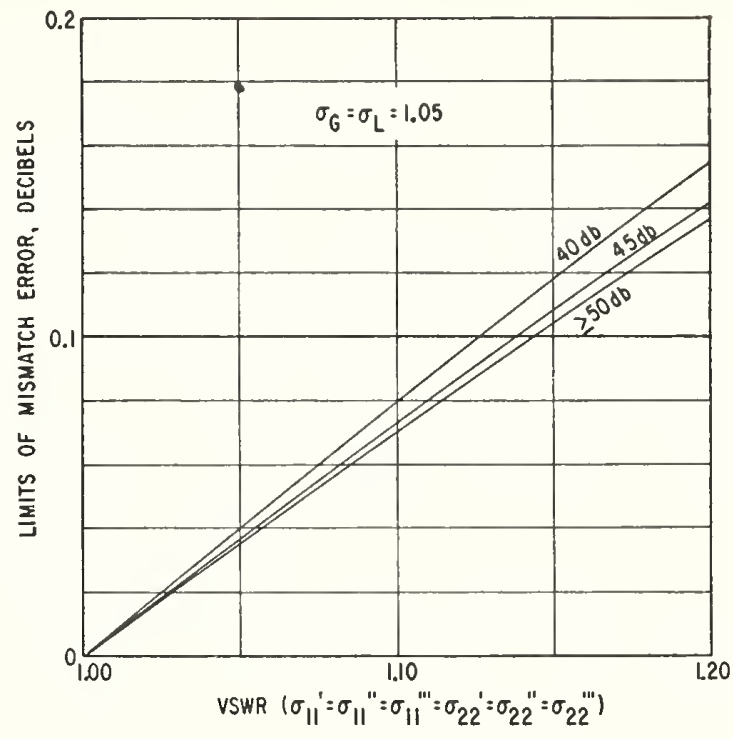

Fig. 8- Limits of component $\epsilon_{1}$ of mismatch error for three attenuators with equal magnitudes of $S_{11}$ and $S_{22}$ cascaded in a system where $\sigma_{G}=\sigma_{L}=1.05$.

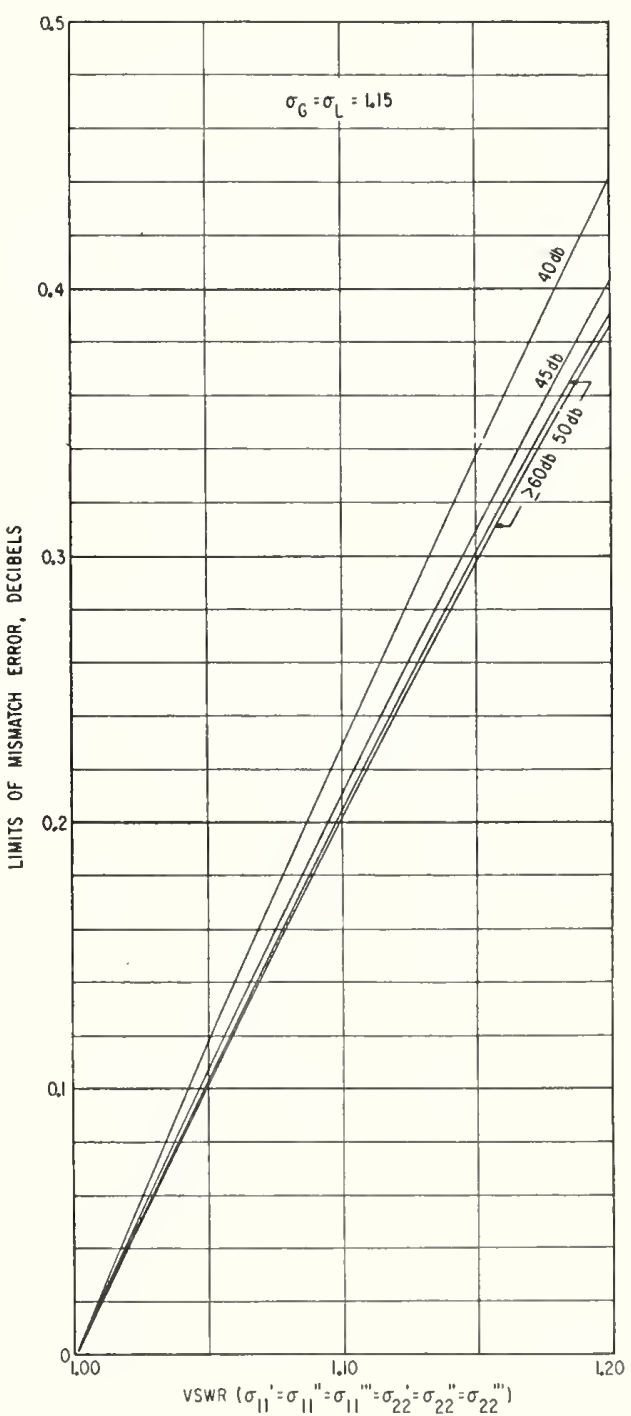

Fig. 10-Limits of component $\epsilon_{1}$ of mismatch error for three attenuators with equal magnitudes of $S_{11}$ and $S_{2 \mathrm{l}}$ cascaded in a system where $\sigma_{G}=\sigma_{L}=1.15$. 
that the VSWR corresponding to $S_{11}$ or $S_{22}$ of the attenuators, which is the abscissa, has been assumed to be the same and equal for the input and output of each attenuator. Each sector is labeled with the appropriate values of the VSWR associated with the equivalent generator and detector reflection coefficients which were assumed to be equal in (19). The ordinates are the limits of mismatch error. These figures are presented to illustrate the range of limits of mismatch error to be expected in the assumed situations.

Figs. 3-6 are a series of graphs of the limits of the component $\epsilon_{1}$ of mismatch error for two attenuators in cascade with $\sigma_{G}\left(=\sigma_{L}\right)$ of $1.01,1.05,1.10$, and 1.15 , respectively. $\sigma_{G}$ and $\sigma_{L}$ are the VSWR's associated with the equivalent generator and detector reflection coefficients, respectively. The VSWR's used as the abscissas are the input and output VSWR's of the variable attenuators. It is assumed that these VSWR's are all equal. The parameter for the family of curves is the number of decibels to be measured by this technique.

Figs. 7-10 are an equivalent series of graphs of the limits of the component $\epsilon_{1}$ of the mismatch error for the case of three attenuators in cascade. These are applicable for measurements of attenuation of $40 \mathrm{db}$ or more.

Figs. 11 and 12 are graphs of the limits of the component $\epsilon_{2}$ of the mismatch error for the cases of two and three attenuators in cascade. The abscissas are the VSWR's of the attenuators. The limits of error in this case are independent of the load and generator mismatches. For the case of three attenuators in cascade, Fig. 12 is constructed on the assumption that at least 20 $\mathrm{db}$ attenuation is in the middle attenuator at one of the attenuation settings, either the initial or the final.

\section{UsE OF THE GraphS}

In order to use the graphs of Figs. 3-12 to estimate the limit of error, one must know the VSWR's of the attenuators and of the equivalent generator and detector, and the approximate number of decibels to be measured. For an example, consider a case where 1)

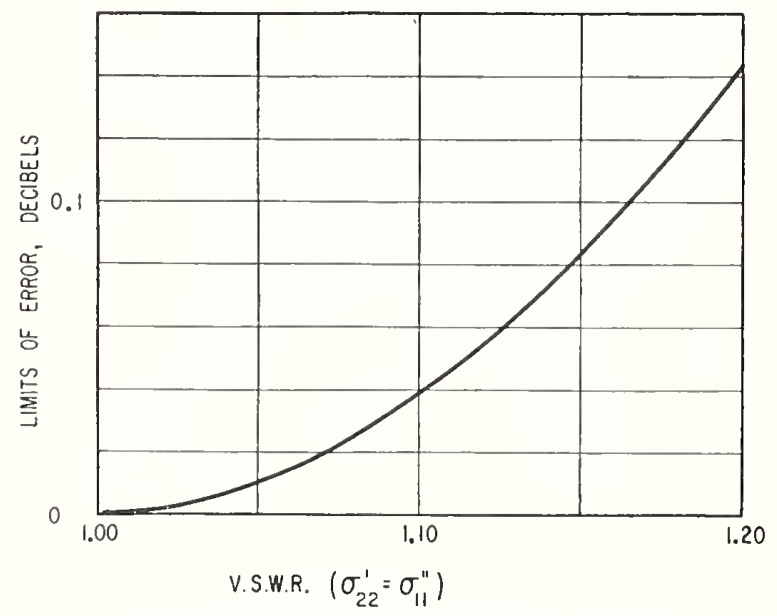

Fig. 11-Limits of component $\epsilon_{2}$ of mismatch error for two attenuators with equal magnitudes of $S_{11}$ and $S_{22}$. the equivalent generator and detector VSWR's are 1.03 and 1.04 , respectively; 2) the three attenuators have input and output VSWR's of 1.15 or less; and 3) the desired attenuation measurements are $3 \mathrm{db}, 40 \mathrm{db}$, and $75 \mathrm{db}$, and the reference attenuators are calibrated up to $40 \mathrm{db}$. The values of the generator and detector VSWR's are used to determine which of the graphs of $\epsilon_{1}$ is to be used. One selects the graph with the nearest available value of $\sigma_{G}\left(=\sigma_{L}\right)$ which is equal to, or greater than, the larger value of the actual load or generator VSWR. This will give a conservative estimate of the limits of error. In this example, the actual VSWR's of the generator and the detector are 1.03 and 1.04 , respectively, and for the case of two attenuators in cascade, Fig. 4 would be selected, since it is constructed on the assumption that both of these are 1.05. Having selected the graph, the largest value of the VSWR at the input or output of the attenuators is the abscissa. Typical commercially-available attenuators for rectangular waveguide systems have maximum VSWR of 1.15 over their entire frequency and attenuation ranges. For a conservative estimate, then, one could use an abscissa of 1.15 unless the actual values of the VSWR are known to be different. The value of $\epsilon_{1}$ is different for different values of attenuation. For a 3 -db measurement it has the value $0.11 \mathrm{db}$, and for a $40-\mathrm{db}$ measurement it is $0.09 \mathrm{db}$. Note that for measurements of attenuation of $20 \mathrm{db}$ or greater the component $\epsilon_{1}$ of the limits of error does not change within the resolution of the graphs.

One determines $\epsilon_{2}$ from Fig. 11, and it depends only on the VSWR's of the attenuators, and not on the amount of attenuation or on the generator and load mismatches. For a VSWR of $1.15, \epsilon_{2}$ is $0.08 \mathrm{db}$. Addition of these two components, as determined from Figs. 4 and 11 , yields the limits of mismatch error, $\epsilon_{T}$, of 0.19

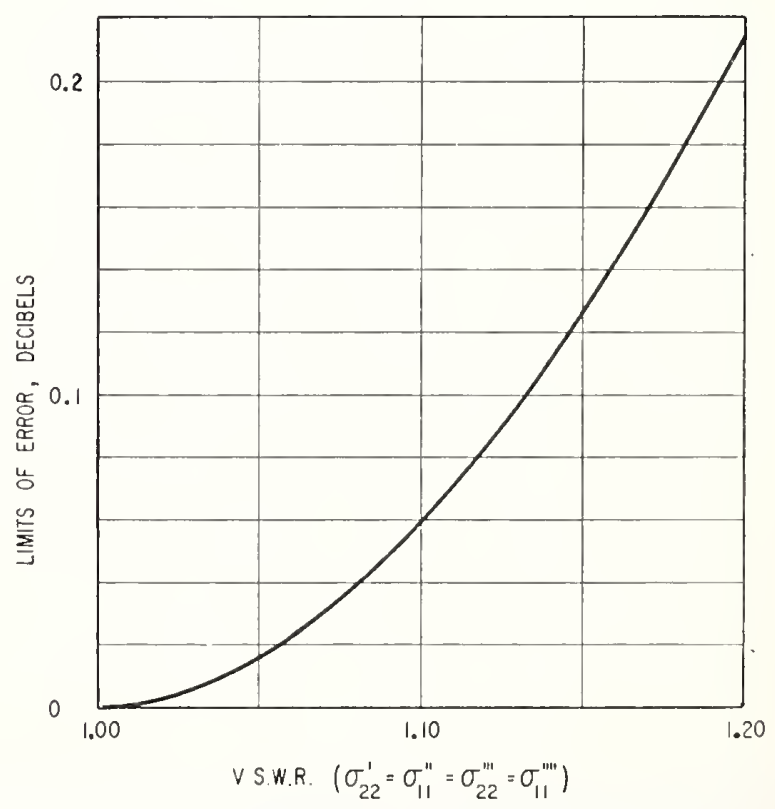

Fig. 12-Limits of component $\epsilon_{2}$ of mismatch error for three attenuators with equal magnitudes of $S_{11}$ and $S_{22}$. 
$\mathrm{db}$ for a 3 -db measurement and $0.17 \mathrm{db}$ for a 40 -db measurement.

If the three similar attenuators are connected' in cascade and inserted in the system, Fig. 8 shows that $\epsilon_{1}$ is now $0.12 \mathrm{db}$ for a 40 -db measurement and $0.10 \mathrm{db}$ for a 75 -db measurement, and Fig. 12 shows that $\epsilon_{2}$ is 0.13 $\mathrm{db}$ for both measurements. Addition of these yields the limits of mismatch error, $\epsilon_{T}$, of $0.25 \mathrm{db}$ for a 40 -db measurement and $0.23 \mathrm{db}$ for a $75-\mathrm{db}$ measurement. These examples are felt to be representative of conditions met $\mathrm{n}$ typical rectangular waveguide systems.

\section{Conclusion}

It can be seen that if maximum possible error is assumed, the mismatch error increases for smaller relative attenuation measurements.

The limit of mismatch error estimated by this method is a conservative figure since it is based on the assumption that all values of the coefficients have phases at the initial and final settings which give the maximum possible error. Thus, in an actual application, the mismatch errors are very probably less than those estimated in the examples. '

Reprinied from IKE TRANSACTIONS

ON MICROWAVE THEORY AND TECHNIQUES

Volume MTT-7, Number 4, October, I959

PRINTED IN THE U.S.A. 


\title{
A Method for Measuring the Directivity of Directional Couplers*
}

\author{
G. E. SCHAFER $†$ AND R. W. BEATTY $\dagger$
}

\begin{abstract}
Summary - This method of measuring directivity requires the measurement of the ratio of powers delivered to the side arm when the normal input arm is connected alternately to an adjustable sliding termination and a sliding short circuit. The short circuit is phased to yield maximum and minimum responses and the amplitudes are averaged. Two techniques of adjusting the termination may be used. One procedure requires zero reflection from the termination. The other procedure requires adjustment for a null at the detector and then measurement of the maximum response due to changing the phase of the termination. The inherent errors of the method are analyzed and found to be within the limits -0.01 to $0.00 \mathrm{db}$ in a specific example.
\end{abstract}

\section{INTRODUCTION}

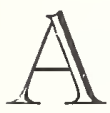
METHOD to measure directivity of a directional coupler is described. The errors in the method are evaluated and graphs are presented for estimating the total error.

Previously described methods ${ }^{1,2}$ required measurement of the combined attenuation of coupling and directivity, the impedance of an auxiliary component, the reversal of the directional coupler, or a combination of these. This method permits measurement of the directivity values up to the entire dynamic range of the attenuation measurement system and completes a measurement by attaching first a short circuit and then an adjustable sliding termination to the same terminal.

\section{Procedure}

The arrangement of equipment is indicated in Fig. 1, with the coupler oriented as in Fig. 2. Preliminary adjustments ${ }^{3}$ are made to the tuners shown in Fig. 1 so that: 1) the calibrated attenuator is operated in a matched system, (the condition under which it was calibrated) and 2) the reflection coefficient $\Gamma_{2 i}$ (measured at terminal surface 2 of the directional coupler with the normal signal source inactive) has a magnitude less than 0.01 .

Procedure 1) is to attach the short circuit and obtain a maximum and minimum amplitude reading by

* Manuscript received by the PGMTT, May 1, 1958; revised manuscript received, June 13, 1958.

+ Natl. Bureau of Standards, Boulder, Colo.

${ }^{1}$ C. G. Montgomery, ed., "Techniques of Microwave Measurements," M.I.T. Rad. Lab. Ser., McGraw-Hill Book Co., Inc., New York, N. Y., ch. $14 ; 1947$.

2 M. Wind and H. Rapaport, "Handbook of Microwave Measurements," Polytechnic Institute of Brooklyn, Microwave Res. Inst., Brooklyn, N. Y., 2nd ed.; 1955.

${ }^{3}$ If the detecting system has sufficient gain, the tuners may be replaced by well-matched broad-band pads with little loss in accuracy. This would simplify the procedure, especially in the case where measurements were to be made at a number of different frequencies.

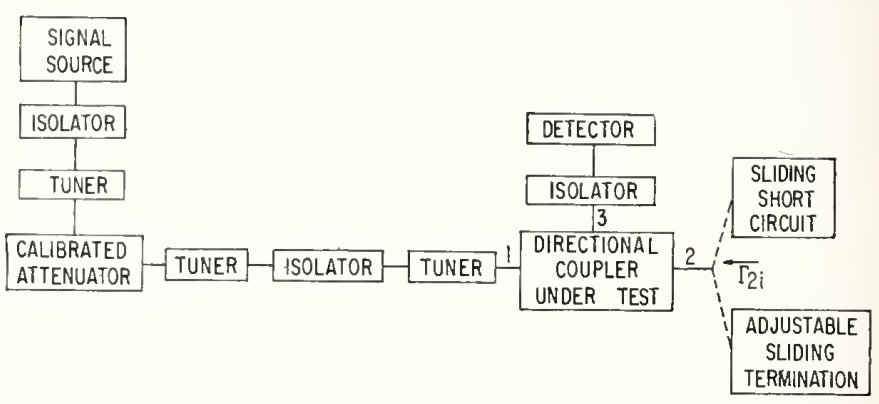

Fig. 1-Arrangement of equipment.

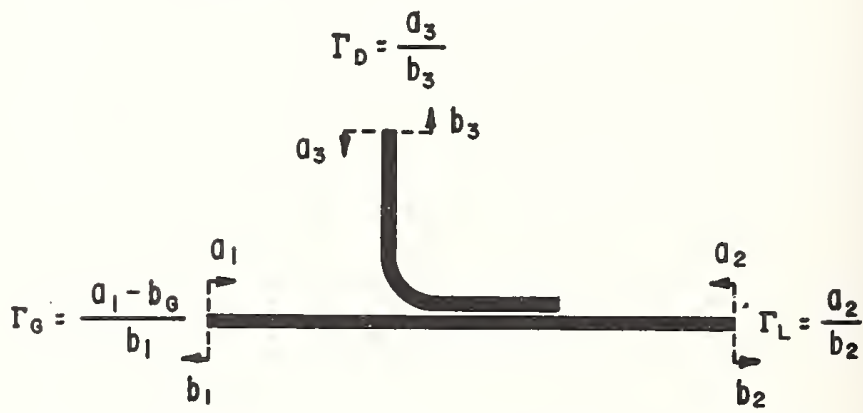

Fig. 2-Representation of directional coupler as a 3-arm junction.

adjusting the phase of the short circuit. The average of these two readings is a reference level and is denoted by $\left|b_{3}\right|_{\text {av. }}$. An adjustable sliding termination (abbreviated AST in the remainder of the paper) is then attached in place of the sliding short. This AST must be capable of providing zero reflection ${ }^{4}$ at the frequency being used. The condition of zero reflection is indicated by no fluctuation in the output level of the detector as the termination is moved along the waveguide. This level is called $\left|b_{3}\right|_{0}$ and is determined either by the calibrated attenuator or the detector.

$\left|b_{3}\right|_{\text {av }}$ is approximately equal to the amplitude of the forward coupled wave, while $\left|b_{3}\right|_{0}$ is the amplitude of the reverse coupled wave. Therefore, the directivity can be determined to a good approximation by the expression

$$
D \approx 20 \log _{10} \frac{\left|b_{3}\right|_{\mathrm{av}}}{\left|b_{3}\right|_{0}}
$$

The adjustment of the AST for $\Gamma_{L}=0$ [in procedure 1)] is of ten tedious. Procedure 2) eliminates this opera-

${ }^{4} \mathrm{R}$. W. Beatty, "An adjustable sliding termination for rectangular waveguide," IRE Trans. ON Microwave Theory and TeCHNIQUES, vol. MTT-5, pp. 192-194; July, 1957. 
tion and substitutes instead a null adjustment which is readily obtained. ${ }^{5}$ (The short circuit measurement remains unchanged from the first procedure and the significance of $\left|b_{3}\right|_{\mathrm{av}}$ is the same.) The AST is manipulated to yield a null in the output of $\operatorname{arm} 3$, and is then moved along the waveguide until a maximum response, $\left|b_{3}\right|_{T}$, occurs. This is approximately twice $\left|b_{3}\right|_{0}$. The ratio

$$
\frac{\left|b_{3}\right|_{\mathrm{av}}}{\left|b_{3}\right|_{T}}
$$

can also be used to determine the directivity to a good approximation by the expression

$$
D \approx 20 \log _{10} \frac{2\left|b_{3}\right|_{\mathrm{BV}}}{\left|b_{3}\right|_{T}} .
$$

\section{THEORY}

Analysis of this method is accomplished by writing a matrix equation for the three-arm junction representation of a directional coupler

$$
b=S a,
$$

(where $S$ is the scattering matrix, and $b$ and $a$ refer respectively to outgoing and incoming wave amplitudes), and solving for the above ratios. The elements of $a$ and $b$ in (1) are displayed in Fig. 2.

The substitution of the appropriate elements into matrix (1) yields a solution for the amplitude of the emergent wave from arm 3,

$$
b_{3}=-b_{0} \frac{\left|\begin{array}{rrr}
S_{12} & 1-S_{22} \Gamma_{L} \\
S_{13} & -S_{23} \Gamma_{L}
\end{array}\right|}{\left|\begin{array}{rrr}
1-S_{11} \Gamma_{G} & -S_{12} \Gamma_{L} & -S_{13} \Gamma_{D} \\
-S_{12} \Gamma_{G} & 1-S_{22} \Gamma_{L} & -S_{23} \Gamma_{D} \\
-S_{13} \Gamma_{G} & -S_{23} \Gamma_{L} & 1-S_{33} \Gamma_{D}
\end{array}\right|},
$$

where $b_{g}$ is the fixed wave amplitude characteristic of the equivalent generator as designated in Fig. 2, and where reciprocity in the form $S_{i j}=S_{j i}$ has been assumed. This may be written in a convenient partition suggested by the form derived by MacPherson and Kerns, ${ }^{\circ}$

$$
\left|b_{3}\right|=\frac{\left|b_{0} \frac{k}{2}\right|}{\left|\frac{y}{2}+R+r \exp (j \phi)\right|},
$$

where

$$
R=\frac{1}{1-\left|K \Gamma_{L}\right|^{2}}, \quad r=\left|K \Gamma_{L}\right| R
$$

"A similar technique is described by H. C. Poulter in "A note on measuring coaxial coupler directivity," Hewlett-Packard J., vol. 8, pp. 1-4; May-June, 1957.

A A. C. MacPherson and D. M. Kerns, "A new technique for the measurement of microwave standing-wave ratios," PROC. IRE, vol. 44, pp. 1024-1030; August, 1956.

$$
\begin{array}{ll}
y=\frac{2 \Delta}{K M_{22}-\Delta}, & K=\frac{\left|\begin{array}{ll}
S_{12} & S_{22} \\
S_{13} & S_{23}
\end{array}\right|}{S_{13}}, \\
k=\frac{2 S_{13} K}{K M_{22}-\Delta}, & M_{22}=\left|\begin{array}{rr}
1-S_{11} \Gamma_{G} & -S_{13} \Gamma_{D} \\
-S_{13} \Gamma_{G} & 1-S_{33} \Gamma_{D}
\end{array}\right|,
\end{array}
$$

and

$$
\Delta=\left|\begin{array}{rrr}
1-S_{11} \Gamma_{G} & -S_{12} & -S_{13} \Gamma_{D} \\
-S_{12} \Gamma_{G} & -S_{22} & -S_{23} \Gamma_{D} \\
-S_{13} \Gamma_{G} & -S_{23} & 1-S_{33} \Gamma_{D}
\end{array}\right| .
$$

For the arrangement with the short circuit attached, maximum and minimum responses are obtained as the phase of $\Gamma_{L}$ is shifted. These responses are given by

$$
\left|b_{3}\right| \max =\frac{\left|b_{\theta} \frac{k}{2}\right|}{|| r|-| \frac{y}{2}+R||},
$$

and

$$
\left|b_{3}\right| \min =\frac{\left|b_{g} \frac{k}{2}\right|}{|| r|+| \frac{y}{2}+R||}
$$

The average value of these two responses may be written

$$
\begin{aligned}
\left|b_{3}\right|_{\mathrm{av}} & =1 / 2\left(\left|b_{3}\right|_{\max }+\left|b_{3}\right|_{\min }\right) \\
& =\left|b_{0} \frac{k}{2}\right| \frac{|r|}{\left|r^{2}-\right| \frac{y}{2}+\left.R\right|^{2} \mid} .
\end{aligned}
$$

For procedure 1 ), the AST is adjusted for zero reflection $\left(\Gamma_{L}=0\right)$, and the response may be written

$$
\left|b_{3}\right|_{0}=\left|b_{0} \frac{k}{2}\right| \frac{2}{|y+2|} .
$$

The ratio of these two responses is a measure of the directivity and the apparent directivity is given by

$$
\begin{aligned}
D_{A 1} & =20 \log _{10} \frac{\left|b_{3}\right|_{\mathrm{av}}}{\left|b_{3}\right|_{0}} \\
& =20 \log _{10} \frac{|y+2|}{2}\left(\frac{|r|}{\left|r^{2}-\right| \frac{y}{2}+\left.R\right|^{2} \mid}\right) .
\end{aligned}
$$

In procedure 2), it is convenient to express the response as

$$
\left|b_{3}\right|=\left|b_{9}\right| \frac{|k|}{\left|y+\frac{2}{1+K \Gamma_{L} e^{-2 j \beta l}}\right|},
$$




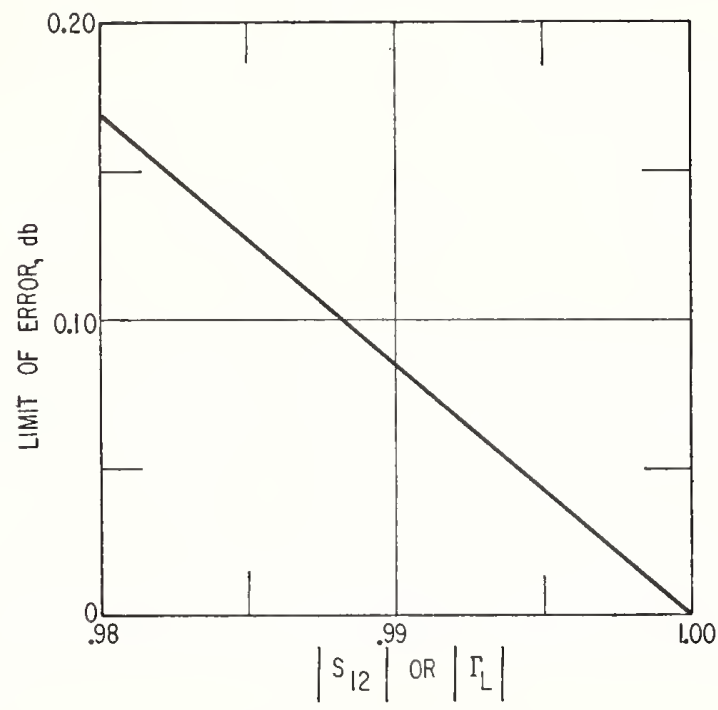

Fig. 3-Limit of error calculated from (17a) (Term 1) and (20).

where $\beta l$ is the electrical length between reference planes of the load and the junction. One adjusts $\left|\Gamma_{L}\right|$ and $l$ to give a null response and it is apparent from (9) that $K \Gamma_{L} e^{-2 i \beta l}=-1$. In deriving the equation for maximum response as $l$ is varied, one considers that the locus of

$$
\frac{2}{1+K \Gamma_{L} e^{-2 j \beta l}}
$$

when $\left|K \Gamma_{L}\right|=1$, is a straight line parallel to the imaginary axis through the point $(1,0)$ in the complex plane. The maximum response may be written as

$$
\left|b_{3}\right|_{T}=\frac{\left|b_{g} k\right|}{g+1}
$$

where $g$ is the real part of $y$. Therefore, the apparent directivity may be written as

$$
\begin{aligned}
D_{A 2} & =20 \log _{10} \frac{2\left|b_{3}\right|_{\mathrm{av}}}{\left|b_{3}\right|_{r}} \\
& =20 \log _{10}(g+1)\left(\frac{|r|}{\left|r^{2}-\right| \frac{y}{2}+\left.R\right|^{2} \mid}\right) .
\end{aligned}
$$

\section{Error ANatysis}

The sources of error can be evaluated by considering the true directivity, defined by

$$
D_{T}=20 \log _{10} \frac{\left|S_{23}\right|}{\left|S_{13}\right|},
$$

and evaluating the error in $\mathrm{db}$ as

$$
\epsilon=D_{T}-D_{A}
$$

where $D_{A}$ is given by (8) and (11) for procedures 1) and 2), respectively.

The following three conditions are sufficient to reduce the error to zero:

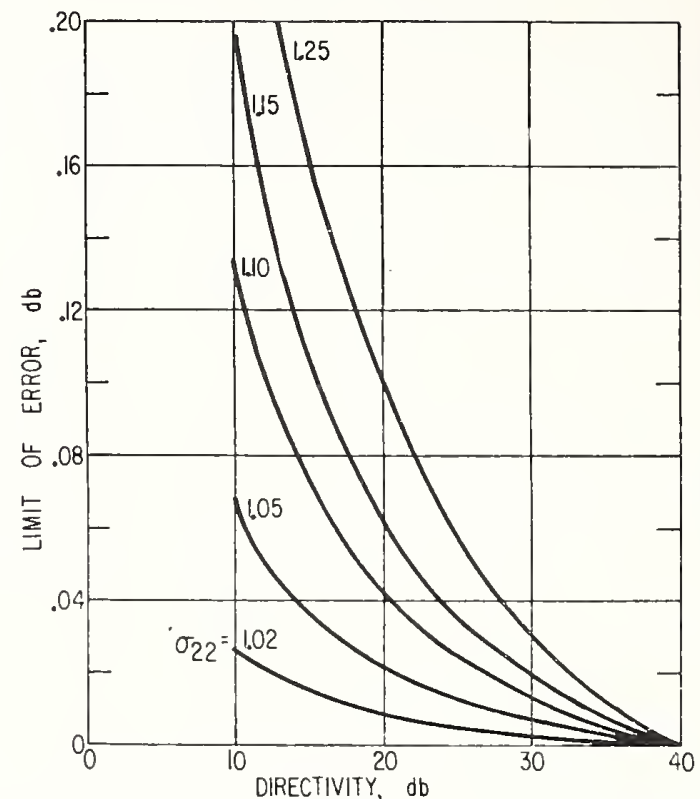

Fig. 4-Limit of error calculated from (17a) (Term 2).

$y=0$, which reduces the ratios in (8) and (11)

$$
\text { to }\left|K \Gamma_{L}\right| \text {; }
$$

$\left|\Gamma_{L}\right|=1$, which reduces (14) to $|K|$;

$S_{22}=0, S_{12}=1$, which reduce (15) to $\frac{\left|S_{23}\right|}{\left|S_{13}\right|}$.

It is assumed that departures from these conditions will be small and are therefore considered as individual cases.

\section{Case I}

$S_{22} \neq 0$ and $S_{12} \neq 1$, but other conditions are satisfied. Since $D_{A 1}$ and $D_{A 2}$ reduce to $20 \log _{10}|K|$ in Case I, the error is

$$
\begin{aligned}
\epsilon_{I} & =20 \log _{10}\left|\frac{S_{23}}{S_{12} S_{23}-S_{13} S_{22}}\right| \\
& =20 \log _{10}\left|\frac{1}{S_{12}\left(1-\frac{S_{13} S_{22}}{S_{12} S_{23}}\right)}\right| .
\end{aligned}
$$

One can separate (17) into two terms. If the coupling in decibels is $10 \mathrm{db}$ or more, $S_{12}$ may be eliminated from the second term with little loss of accuracy:

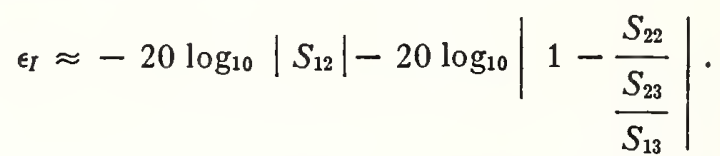

The magnitude of the contribution from the first and second term is shown in Figs. 3 and 4, respectively. If the coupling in decibels is less than $10 \mathrm{db}$, the contribution of the second term is appreciably larger than shown in Fig. 4 and it is advisable to employ (17) for a more accurate estimate of the error. (In these cases 
where the error caused by assuming $\left|S_{12}\right|=1$ would be large, it would probably be desirable to determine $\left|S_{12}\right|$ and make a correction to the measured ratio.)

\section{Case 11}

$y \neq 0$, but ther conditions are satisfied. Considerable manipulation of (12) minus (8) and use of the approximation

$$
\frac{y}{2} \approx \frac{\Gamma_{2 i}}{K}
$$

yields for procedure 1),

$$
\begin{aligned}
20 \log _{10} \frac{1-2\left|\frac{\Gamma_{2 i}}{K}\right|-4\left|\Gamma_{2 i}\right|^{2}}{1+\left|\frac{\Gamma_{2 i}}{K}\right|} \leq \epsilon_{I I, 1} \\
\leq 20 \log _{10} \frac{1+2\left|\frac{\Gamma_{2 i}}{K}\right|-4\left|\Gamma_{2 i}\right|^{2}}{\left|\frac{\Gamma_{2 i}}{K}\right|}
\end{aligned}
$$

where $\Gamma_{2 i}$ is the reflection coefficient of arm 2, with the inactive generator and detector connected to arms 1 and 3. For the second procedure the limits of error after similar manipulation of (12) minus (11) may be expressed as

$$
\begin{aligned}
20 \log _{10} \frac{1-2\left|\frac{\Gamma_{2 i}}{K}\right|-4\left|\Gamma_{2 i}\right|^{2}}{\left|\frac{\Gamma_{2 i}}{K}\right|} \leqq \epsilon_{I I, 2} \\
\leq 20 \log _{10} \frac{1+2\left|\frac{\Gamma_{2 i}}{K}\right|-4\left|\Gamma_{2 i}\right|^{2}}{1-2\left|\frac{\Gamma_{2 i}}{K}\right|} .
\end{aligned}
$$

This expression differs from $\epsilon_{I I, 1}$ only in the denominator.

Fig. 5 shows the limits of error for both procedures 1) and 2) as a function $\sigma_{2 i}$, (the VSWR corresponding to $\left.\Gamma_{2 i}\right)$. The solid lines indicate the limits of error for procedure 1) and the dashed lines the limits of error for procedure 2). The limiting values for the error as the directivity becomes infinite are indicated on the right-hand side of the graph.

\section{Case III}

An imperfect short circuit, $\left|\Gamma_{L}\right| \neq 1$, but other conditions are satisfied.

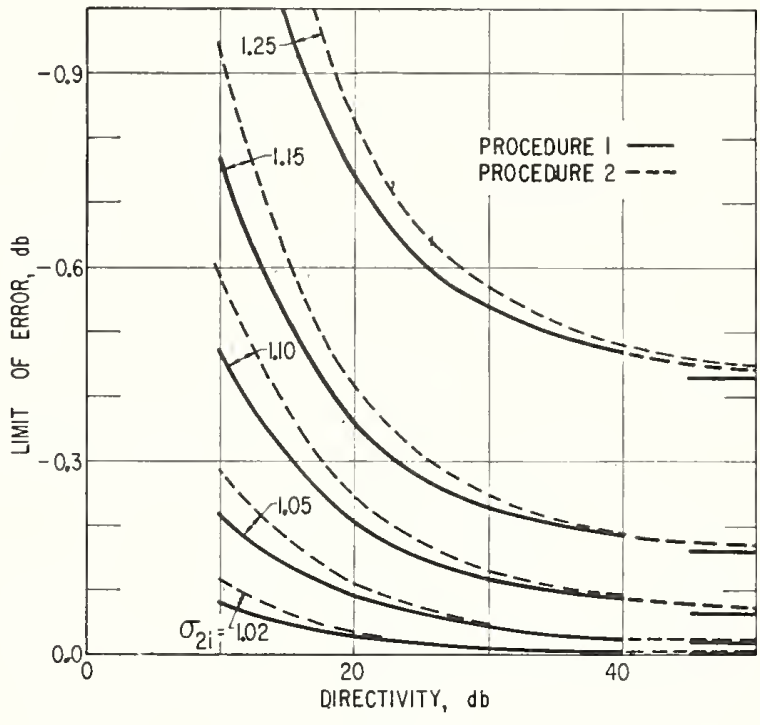

Fig. 5-Limit of error calculated from (18) and (19).

By use of (16) and implications of (17), the error may be written

$$
\epsilon_{I I I}=-20 \log _{10}\left|\Gamma_{L}\right| .
$$

This is of the same form as the first term of (17a) and is included in Fig. 3 as an alternate abscissa. This formula applies to both procedures.

The following example shows the limits of error for a case that might be considered typical. For a well-constructed, high directivity, 20-db coupler $\left(\left|S_{23}\right|=0.1\right)$, the absolute values of the other coefficients are usually

$$
\begin{array}{ll}
\left|S_{11}\right| \leq 0.025, & \left|S_{12}\right| \approx 0.995 \\
\left|S_{22}\right| \leq 0.025, & \left|S_{13}\right|<0.001 . \\
\left|S_{33}^{\prime}\right| \leq 0.025, &
\end{array}
$$

With the preliminary adjustment of $\left|\Gamma_{2 i}\right|$ to less than 0.01 , and use of a short circuit with a reflection coefficient of magnitude greater than 0.995 , the limits of error from the above sources are -0.01 to +0.08 . By measuring $\left|S_{12}\right|$ and $\left|\Gamma_{L}\right|$, and applying corrections for their departures from unity, the limits of error are reduced to -0.01 to $0.00 \mathrm{db}$. One must also consider the error made in measuring the ratio

$$
\frac{\left|b_{3}\right|_{\mathrm{av}}}{\left|b_{3}\right|_{0}} \text { or } \frac{\left|b_{3}\right|_{\mathrm{av}}}{\left|b_{3}\right|_{T}}
$$

Using IF substitution techniques with a below-cutoff standard attenuator, the error may be held to \pm 0.05 $\mathrm{db}$ with normal precautions. Unless this error is reduced to $\pm 0.01 \mathrm{db}$ or less, the error in measuring directivity in this example would be limited by the error in'measuring the above ratios. 


\title{
Recent Developments in the Field of Microwave Power Measurements at the National Bureau of Standards*
}

\author{
GLENN F. ENGEN†
}

\section{INTRODUCTION}

$\mathrm{T}$ HE bolometric technique, by means of which the heating effect of an unknown amount of RF or microwave power is compared with that of a measured amount of dc or audio-frequency power through a temperature-sensitive resistive element, is a well-known and extensively employed method in the low-level measurement of microwave power.

The measurement of this bolometric or substituted dc bias power has been the object of refinement until the measurement can be carried out to an accuracy of 0.1 per cent. ${ }^{1}$

The use of the value thus obtained as a measure of the microwave power involves, however, two well-recognized sources of systematic error. First, the microwave and dc bias currents take different distributions within the bolometer element which means that the functional dependence of the resistance will in general be different for the two sources of power, giving rise to a dc-RF substitution error; and second, the bolometric method at best measures only the microwave power dissipated within the bolometer element, whereas one is usually interested in the power dissipated in the entire terminating waveguide structure or bolometer mount. The ratio of these quantities is, by definition, the bolometer mount efficiency.

The evaluation and measurement of these two phenomena has been the object and goal of a large percentage of the effort by the Microwave Power Standards Project of the Boulder Laboratories, National Bureau of Standards, resulting recently in several sig ificant contributions to the existing art. Because these developments will be reported in detail by papers now in preparation, the present paper will only attempt to summarize these results.

\section{MicRoCALORIMETER}

The microwave microcalorimeter described by Macpherson and Kerns ${ }^{2}$ gave the details of a calorimetric method for the determination of the combined effect of

* Manuscript received by the PGI, September 2, 1958.

$\dagger$ Radio Standards Lab., Nat. Bur. of Standards, Boulder, Colo.

${ }^{1}$ G. F. Engen, "A self-balancing dc bridge for accurate bolometric measurements," J. Res. NBS, vol. 59, R.P. 2776, pp. 101-105; August, 1957.

${ }_{2}$ A. C. Macpherson and D. M. Kerns, "A microwave microcalorimeter," Rev. Sci. Instr., vol. 26, pp. 27-33; January, 1955. these two sources of error, and an improved instrument based on this method was recently placed in operation.

The basic theory of the microcalorimeter operation has been presented in detail in the earlier work. In brief, however, the instrumentation is such as to permit a calorimetric determination of the total power input to a bolometer mount while a simultaneous bolometric determination is made. The difference between the two measurements is ascribed to the mount efficiency and the substitution error. The method, in its present state at least, permits only a determination of the combined effect of these phenomena, also referred to as "effective efficiency."

The problem of making a calorimetric determination of power (or energy) may be subdivided as follows: 1) selection of a calorimetric body or object in which to dissipate the power to be measured, 2) choice of a suitable technique to measure the temperature rise of this body or object, and 3) determination of the proportionality factor, or functional dependence of the thermometer response upon the power input. In the microcalorimeter technique, the bolometer mount serves as the calorimetric body or object in which the power is dissipated and whose temperature rise is subsequently measured by means of a suitable thermopile, while calibration is effected by observing the thermopile response to a measured amount of dc power dissipated in the bolometer element.

Refinements in the technique include: 1) greatly improved ambient temperature control, permitting higher sensitivity and resolution, 2) improved dc instrumentation, 3) improved mechanical construction giving better repeatability, 4) relocation of the thermopile such that it is no longer attached directly to the bolometer mount, thus providing flexibility in the chonce of termination, and 5) a more comprehensive error analysis. These features permit a determination of the effective efficiency of a bolometer mount to an absolute accuracy of better than 0.2 per cent.

\section{Impedance Method}

A second contribution relates to the so-called "impedance" method of determining bolometer mount efficiency devised by Kerns. ${ }^{3}$ As originally outlined, the

${ }^{3}$ D. M. Kerns, "Determination of efficiency of microwave bolometer mounts from impedance data," J. Res. NBS, vol. 42, R.P. 1995, pp. 579-000; June, 1949. 
accuracy which could be achieved by this technique was rather severely limited by the state of the impedance measuring art, and this led in turn to the development of a number of modifications with the objective of improving the over-all accuracy. Beatty, ${ }^{4}$ for example, proposed a modification based on certain mathematical approximations and restrictions in generality which provided improved accuracy, but the associated operating procedures were nonetheless time-consuming and exacting, and the accessory instrumentation was never developed or refined to the point where a great deal of confidence could be placed in the results. Further refinements or modifications of the technique have been proposed by Weinschel, ${ }^{6}$ Ginzton, ${ }^{6}$ Lane, ${ }^{7}$ and perhaps others, but it is probably safe to say that none of these proposals have yet come into widespread use.

Another variation of the impedance method has been recently developed at the Boulder Laboratories, employing directional coupler techniques, which provides improved accuracy and simplified operational procedures, but unlike the earlier modifications, involves neither mathematical approximations nor restrictions in generality, although still limited to barretter type bolometers. A particularly attractive feature of the new method is its independence (assuming dissipationless connectors) of an impedance discontinuity at the input connector, which has been a particularly troublesome source of error in coaxial systems. Another advantage is its direct applicability to either matched or unmatched mounts. A preliminary implementation of the method in waveguide has given agreement of one half of one per cent and better with results obtained by the microcalorimetric method. The details of this technique will be presented in a later paper.

\section{Comparison of Power Meters}

The mismatch or impedance measurement problems involved in intercomparing power meters have been treated in detail by Beatty and Macpherson. ${ }^{8}$ One of the simpler intercomparison techniques is that of alternate connection to a stable generator, and it was shown that the power, $P_{m}$, dissipated in the meter to be calibrated is given in terms of the power, $P_{s}$, absorbed by the standard meter by the expression:

$$
P_{m}=P_{s}\left|\frac{1-\Gamma_{g} \Gamma_{s}}{1-\Gamma_{g} \Gamma_{m}}\right|^{2} \frac{1-\left|\Gamma_{m}\right|^{2}}{1-\left|\Gamma_{s}\right|^{2}},
$$

${ }^{4} \mathrm{R}$. W. Beatty and F. Reggia, "An improved method of measuring efficiencies of ultra-high-frequency and microwave bolometer mounts," J. Res. NBS, vol. 54, R.P. 2594, pp. 321-327; June, 1955.

${ }^{5}$ B. O. Weinschel, "Phase Engineering Rep. No. 2 on Standard Signal Generator Output Power, Voltage, and Attenuation Calibration Assembly," Contract AF33 (600)-25238, E.O. No. C-30047 SR-6J3, pp. 32-43; May, 1956.

6 E. L. Ginzton, "Microwave Measurements," McGraw-Hill Book Co., Inc., New York, N. Y., pp. 185-186; 1957.

7 J. A. Lane, "Measurements of efficiency of bolometer and thermistor mounts by impedance methods," Proc. IEE, pt. B, no. 17, pp. 485-486; September, 1957.

${ }_{8}$ R. W. Beatty and A. C. Macpherson, "Mismatch errors in microwave power measurements," PROC. IRE, vol. 41, pp. 1112-1119; Si'ptember, 1953. where the $\Gamma_{\theta}, \Gamma_{s}$, and $\Gamma_{m}$ are the reflection coefficients of the generator, standard power meter, and meter under calibration at the point or plane of connection respectively.

It will be noted that a determination of the middle factor requires, in general, a knowledge of the reflection coefficients in both magnitude and phase. A considerable simplification in the expression may be effected by adjusting the generator for an impedance match so that $\Gamma_{g}$ vanishes. Under these conditions the expression reduces to the form:

$$
P_{m}=P_{s} \frac{1-\left|\Gamma_{m}\right|^{2}}{1-\left|\Gamma_{s}\right|^{2}}
$$

requiring a knowledge of only the magnitudes of $\Gamma_{s}$ and $\Gamma_{m}$.

It has been shown ${ }^{9}$ that a generator match may be simulated by means of a high directivity directional coupler with appropriate sidearm monitor or detector and suitable auxiliary apparatus. While the equivalent generator match was achieved by Engen ${ }^{9}$ by means of a servo loop which held the sidearm power at a constant level, the same result may also be effectively realized with a stable generator by a manual attenuation adjustment or by simply noting the change in sidearm power and making an appropriate correction.

Viewed in this manner, the directional coupler and sidearm power detector assembly becomes an output monitor for the signal source in addition to providing the generator impedance match. Thus, in practice, one determines a calibration factor $K$ for the directional coupler such that

$$
K=\frac{P_{s}}{P_{c}\left(1-\left|\Gamma_{s}\right|^{2}\right)}
$$

where $P_{c}$ is the power indicated by the sidearm detector, and the other terms have been previously defined.

Then

$$
P_{m}=K P_{c}\left(1-\left|\Gamma_{m}\right|^{2}\right),
$$

which gives the power, $P_{m}$, dissipated in the load of reflection coefficient, $\Gamma_{m}$, in terms of the sidearm power, $P_{c},\left|\Gamma_{m}\right|$, and the measured constant $K$.

Eqs. (3) and (4) have been obtained from (2) which assumes a matched generator. In general:

$$
K=\frac{P_{s}}{P_{c}} \frac{\left|1-\Gamma_{\theta} \Gamma_{s}\right|^{2}}{1-\left|\Gamma_{s}\right|^{2}}
$$

and

$$
P_{m}=K P_{c} \frac{1-\left|\Gamma_{m}\right|^{2}}{\left|1-\Gamma_{\vartheta} \Gamma_{m}\right|^{2}} .
$$

The procedure for determining the equivalent $\Gamma_{g}$

${ }^{9}$ G. F. Engen, "Amplitude stabilization of a microwave signal source," IRE TRANS. ON Microwave THEORY AND TECHNIOUES, vol. MTT-6, pp. 202-206; April, 1958. 
achieved by this technique is discussed by Engen ${ }^{9}$ who shows that, in practice, a broad-band match of $\left|\Gamma_{g}\right|$ in the range of 0.025 to 0.035 may be readily realized. If the meter under calibration and standard are also nearly matched, the additional factors in (5) and (6) are sraall and their simpler counterparts may be used, while for larger generator or load mismatches these more complete expressions should be employed if accurate results are to be achieved.

Provided that the sources of error associated with the bolometric measurement as discussed earlier, and the properties of the coupler are independent of power level, the technique provides a convenient and accurate method of comparing power meters of different as well as equal power levels. For example, if it were required to compare a $100-\mathrm{mw}$ meter against a $10-\mathrm{mw}$ standard, a nominal $10-\mathrm{db}$ coupler would be chosen and the measurement required for (3) carried out at nominal values of 1 and $10 \mathrm{mw}$ for $P_{c}$ and $P_{s}$, respectively. The power input to the coupler would then be increased by $10 \mathrm{db}$, yielding nominal. values of 10 and $100 \mathrm{mw}$ for $P_{c}$ and $P_{m}$ in (4). If calibration of a $1-m w$ meter were required, the procedure is similar except that the connections to the main and sidearms are reversed.

It should be noted that this technique of extending the operating range is different from; and potentially more accurate than the usual method where the parameters of the individual components are measured. The present method treats the coupler and power detector as a unit assembly and measures a single parameter for the entire system. (A second measurement is required if the equivalent generator impedance is desired.) The extension of range is thus based upon the linearity of the system and the ability to make accurate bolometric type measurements over a wide dynamic range. The bolometric measurement may be easily carried out over a $10-\mathrm{db}$ range and can be extended to $20^{\circ} \mathrm{db}$ with little loss in accuracy if suitable precautions are taken. Regarding the assumed linearity, it may be noted that the parameters of interest in the coupler and associated bolometer mount detector depend primarily upon the geometry and surface impedance of the waveguide structure which are substantially, if not entirely, independent of power level.

The other potential source of nonlinearity is in the dc-RF substitution error, but here it will be noted that a conservative upper limit of 1-2 per cent has been established analytically ${ }^{10}$ for this error, and the close

${ }^{10} \mathrm{H}$. J. Carlin and M. Sucher, "Accuracy of bolometric power measurements," PRoc. IRE, vol.40, pp. 1042-1048; September, 1952. agreement achieved in the microcalorimetric and impedance methods of bolometer mount evaluation, in many cases to 0.2 per cent or so, implies that this error is probably no greater than a few tenths of a per cent. (The difference between these two determinations should in principle give the substitution error.) Even if the larger value of 1-2 per cent is assumed, the cooling process is still approximately linear, and thus even if a strong dependence of the substitution error upon this cooling were postulated, a few tenths of a per cent would still appear to be a conservative estimate of the limit of this error.

Confirmation of this estimate is rather strongly implied by the results of an experiment in which a barretter mount was connected to one arm and a thermistor mount to the other arm of a 3-db coupler. The ratio of power indicated by the barretter to that of the thermistor was found to be constant, within the limit ( 0.1 per cent) of experimental error, over a 20 -db range. This result may be interpreted as implying that there is no change in substitution error within this limit and over this range, or if there is a change, it is at least the same for both elements. Because of the marked difference in the nature of the barretter and thermistor elements, the first implication is strongly favored. In another experiment the effective efficiency of a thermistor mount was determined by the microcalorimetric method using arst unmodulated $\mathrm{CW}$ microwave energy, and then pulse energy in which the peak power exceeded the average by approximately $40 \mathrm{db}$. Again the results were equivalent within the limits of experimental error, lending further support to the above conclusion.

A directional coupler with appropriate sidearm bolometric detector thus provides a useful technique for realizing a calibrated and matched signal source, and permits an extension of the range (in both directions) of a given power standard by $20 \mathrm{db}$ or so. For example, accurate signals in the range 1 microwatt-1 watt may be derived from a calibrated bolometer mount which operates in the 100-microwatt-10-milliwatt range. In order to realize these results, however, care must be used in the associated bolometric measurements, and the techniques described by Engen ${ }^{1}$ are suggested.

\section{ACKNOWLEDGMENT}

The author extends his thanks to the members of the Microwave Power Standards Project, particularly Morris Harvey and William Case, for their contributions to the developments discussed in this paper. 


\title{
A Dry, Static Calorimeter for RF Power Measurement*
}

\author{
P. A. HUDSON $\dagger$ AND C. M. ALLRED $\dagger$
}

\section{INTRODUCTION}

T $\checkmark \mathrm{HE}$ measurement of RF power at the National Bureau of Standards at frequencies below about $500 \mathrm{mc}$ with accuracies of \pm 1 per cent or better has been chiefly limited, in the past, to the dynamic range which can be measured with bolometer or thermistor bridges. At the present time such bridges have an upper limit of about $100 \mathrm{mw}$. A thermistor bridge with a range of $100 \mu \mathrm{w}$ to $100 \mathrm{mw}$ and a dc accuracy of $\pm(0.05$ per cent $+2 \mu \mathrm{w})$ has recently been constructed at the National Bureau of Standards Boulder Laboratories and is presently being used as a standard. ${ }^{1}$

To extend the power range upward, at least three possibilities were considered at the National Bureau of Standards. First, the range of the $100-\mathrm{mw}$ thermistor bridge could be extended by the use of suitable attenuator pads or with calibrated directional couplers used as power dividers. The second alternative was to develop a bolometer bridge with a higher power range than the present model. Thirdly, a power standard operating on a principle different from that of the thermistor bridge could be developed. Actually, all three of these alternatives have been pursued simultaneously. For example, high-power, high-quality attenuator pads have been constructed in the laboratory and their use with the thermistor bridge has been partially investigated. Also, a bolometer bridge capable of measuring RF power up to 8 watts has recently been built and a report covering its design and performance is planned for the near future.

In pursuing the third alternative, that of developing a standard independent of the bolometer bridge method, a dry, static calorimeter-type wattmeter was chosen as being the most desirable from the standpoint of accuracy, high sensitivity, and wide dynamic range. ${ }^{2}$ Because intercomparison of independent methods of measurement is highly desirable in standards work, it was decided that the range of the calorimeter should extend well below $100 \mathrm{mw}$ and up to about 10 watts to permit its comparison with both the thermistor bridge and a liquid flow type calorimetric power meter currently under development. The power range of the latter extends from 10 to 350 watts.

* Manuscript received by the PGI, August 23, 1958.

$\uparrow$ National Bureau of Standards, Boulder, Colo.

1 "Improved thermistor bridge for RF power measurements," NBS Tech. News Bull., vol. 40, pp. 134-135; September, 1956.

2 M. Wind, ed. "Handbook of Electronic Measurements," vol. I, pp. $3-35$ to $3-41 ; 1956$.

\section{THEORY}

The calorimetric method of measuring power involves the determination of the rate of conversion of electrical energy into heat in a resistive load. The load may consist of an extended lossy medium or a finite resistive element. The heat generated may be measured in various ways, such as in a liquid flow calorimeter, where the temperature rise of a coolant fluid is observed. The heat may be absorbed in the fluid by means of a heat exchanger or the fluid may absorb the energy directly if it is sufficiently lossy. The phenomena of change of state may also be utilized. A third method is the use of a system in which the dissipating medium or element is fixed in position and in addition there is no change of state. That is, the system is static and the temperature rise of some part of the system is observed. Calorimeters operating with the latter properties are termed "static calorimeters." They are usually simply instrumented, may have high accuracy, but are usually slow in response.

In the static calorimeter where high accuracy is desired, the temperature rise is usually measured at conditions of thermal steady state. Since a measurement of temperature rise only is desired, the steady-state temperature $T_{1}$ may be measured with respect to some fixed arbitrarily chosen reference temperature $T_{0}$.

In this method $T_{1}$ is a function of power input as well as the degree of thermal isolation of the body from its surroundings. Ideally, $T_{1}$ would be independent of frequency, and the calorimeter could conveniently be calibrated with dc power and subsequently used to measure power at any frequency. Thus the calorimeter is actually a transfer standard measuring RF power in terms of accurately known dc power. The validity of the assumption that the equilibrium temperature $\left(T_{1}\right)$ is independent of frequency will be discussed later.

An insight into the physical phenomena involved as well as the prediction of the approximate system behavior may be gained by representing the calorimeter by its electrical analog shown in Fig. 1. It should be noted here that the accuracy of the calorimeter is not dependent on this analogy which is, of course, only approximate.

When power is fed into the input of the calorimeter, at a fixed level, heat is generated in the load resistor at the same rate as the absorption of the electrical energy. The heat is first manifested in a temperature rise of the load resistor which has a certain thermal capacitance $C$. Sub- 


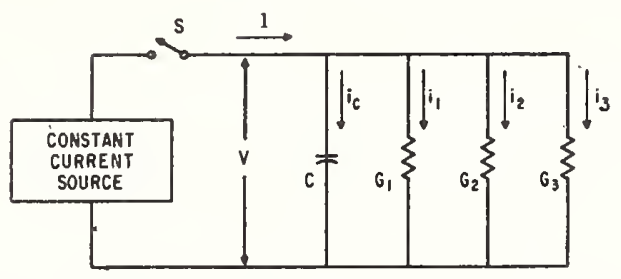

Fig. 1-Electric analog of the calorimetric system.

sequently, heat flows form the load to the surroundings by conduction, convection, and radiation. The analogy between the circuit of Fig. 1 and the thermal system can be readily seen as follows: capacitance $C$ is analogous to the heat capacity of the thermal mass whose temperature rises from $T_{0}$ to $T_{1}$ and $G_{1}, G_{2}$, and $G_{3}$ represent, respectively, the "conductances" for heat from the mass due to conduction, convection, and radiation. A constant current source is used in the circuit because $I$ is analogous to the rate of heat generation in the load which is held constant because the input power level is assumed constant. Finally, the temperature difference $T=\left(T_{1}-T_{0}\right)$ between the thermal mass and the fixed reference temperature is represented by a voltage $V=\left(V_{1}-V_{0}\right)$. The total conductance, $G$, is obviously the sum of the individual conductances. It should be noted that $G_{2}$ and $G_{3}$ are nonlinear because convective heat loss is assumed proportional to $\left(T_{1}-T_{0}\right)^{1.25}$ $\left(Z_{\text {emansky }}\right)$ and radiation heat loss is proportional to $\left(T_{1}{ }^{4}-T_{0}{ }^{4}\right)$. Thus, a nonlinear relationship should be expected between $V$ and $I$. That such is the case will be shown when performance characteristics are discussed.

Even though the values of the $G$ 's are very difficult to predict, it is possible, nevertheless, to gain some information on the likely behavior of the system by analyzing the circuit in Fig. 1. Now if $Q=0$ when $t=0$

$$
V=\frac{Q}{C}=\frac{1}{C} \int i_{c} d t
$$

where $Q=$ charge and $t=$ time, in seconds; also

$$
\begin{gathered}
i_{c}=\frac{d Q}{d t}=C \frac{d V}{d t} \\
I=i_{c}+i_{1}+i_{2}+i_{3}=C \frac{d V}{d t}+G_{1}\left(V_{1}-V_{0}\right) \\
+G_{2}\left(V_{1}-V_{0}\right)^{1.25}+G_{3}\left(V_{1}{ }^{4}-V_{0}^{4}\right) .
\end{gathered}
$$

Now if $G_{2}$ and $G_{3}$ can be neglected, then

$$
I=C \frac{d V}{d t}+V G_{1} .
$$

Eq. (4) is a linear differential equation with constant coefficients whose solution is well known. It is satisfied by

${ }^{3}$ M. W. Zemansky, "Heat and Thermodynamics," McGraw-Hill Book Co., Inc., New York, N. Y., pp. 94-95; 1951.

$$
V=I / G_{1}\left(I-e^{-G_{1} t / C}\right) .
$$

The steady-state solution of (4) is

$$
V=I / G_{1} \text { or } I=V G_{1},
$$

which is obtained by allowing $t$ to approach infinity. Referring to (3), at steady state $d V / d t$ is zero and the equation for $I$ becomes

$$
I=G_{1}\left(V_{1}-V_{0}\right)+G_{2}\left(V_{1}-V_{0}\right)^{1.25}+G_{3}\left(V_{1}^{4}-V_{0}^{4}\right) .
$$

The time constant from (5) is given by $C / G_{1}$, while the sensitivity is proportional to $I / G_{1}$. Since the smallest possible time constant is desired so that the steadystate condition' is reached in a reasonable time, $G_{1}$ should be large. On the other hand, a large $G_{1}$ reduces the sensitivity and thus it would seem that a compromise is necessary. Actually, in the design of the calorimeter the method used to measure $\left(T_{1}-T_{0}\right)$ allowed an increase in $G_{1}$ without seriously affecting the sensitivity. The method used will be described in the next section.

\section{Description of Calorimeter}

A section drawing of the calorimeter is shown in Fig. 2. Power is fed into the calorimeter through the type- $N$ connector at the top. By means of a constant impedance taper the line diameter is increased to $1 \frac{1}{2}$ inches. A 4 inch length of silver-coated glass line is soldered to the large end of the taper and the line is terminated by a 50-ohm disk-type resistor which constitutes the load or heat source.

The section of silvered glass line provides a fair degree of thermal isolation for the load resistor. The latter was made by evaporating a thin film of a low temperature coefficient alloy onto a $\frac{3}{8}$-inch thick glass substrate. Contact to the film is made by means of fired silver electrodes. The thickness of the film is of the order of $10^{-6}$ $\mathrm{cm}$ and its temperature coefficient of resistance is about 20 parts per million per degree $C$. Hence, at frequencies up to at least $500 \mathrm{mc}$, skin effect is negligible, and at temperatures up to $100^{\circ} \mathrm{C}$ the resistance changes by only 0.2 per cent. The change in reflected power due to such a small change in resistance is negligible in comparison to other uncertainties in the system.

The load resistor and its coaxial mount are housed inside a thick-walled ( $\frac{1}{2}$-inch) cylindrical aluminum container 4 inches in diameter by 6 inches in length. The entire assembly is placed inside a thermally-insulated chamber containing a refrigeration coil over which air is continually recirculated. The temperature inside the chamber is $0^{\circ} \mathrm{C}$ and is kept constant to within $\pm 0.002^{\circ} \mathrm{C}$ by means of a sensitive electronic control circuit. Thus, the thick-walled aluminum container is kept at the temperature of the chamber which serves as the reference temperature $T_{0}$. In the absence of power input the equilibrium temperature of the entire calorimeter will, of course, be $T_{0}$ also.

A measure of the load resistor temperature rise, which 


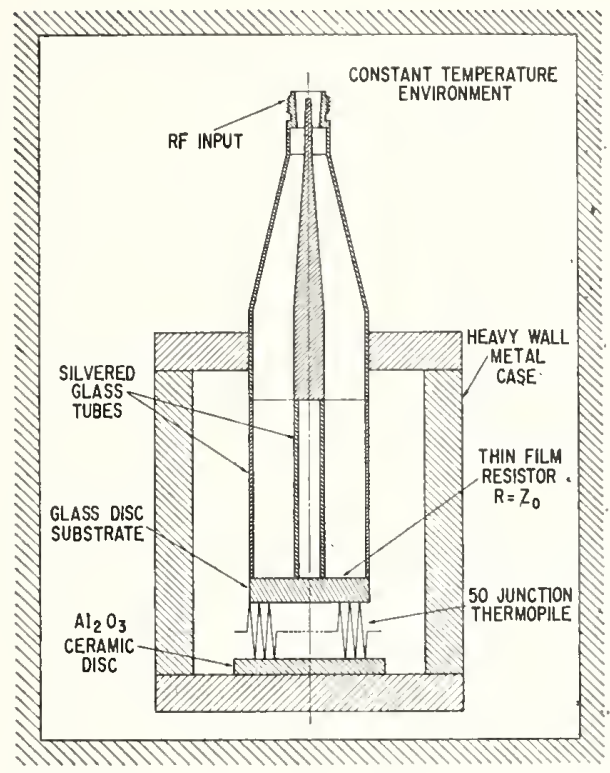

Fig. 2-Section drawing of the dry, static calorimeter for RF power measurement.

$$
\begin{aligned}
& \text { Range: Frequency } 0-300 \mathrm{mc} \\
& \text { Power } 20 \mathrm{mw} \text { to } 12 \text { watts } \\
& \text { RF Accuracy: } 0.5 \text { per cent }+0.2 \mathrm{mw}
\end{aligned}
$$

is produced by the dissipation of power in the load, is obtained by means of a 50 -junction copper-constantan thermopile. The hot junctions are soldered to small, electrically isolated squares on the back side of the disk resistor while the cold junctions are cemented to a ceramic disk of large area which in is turn cemented to the aluminum container. A 1-inch length of wire separates the hot and cold junctions, and hence, for each junction added, the magnitude of $G_{1}$ increases while at the same time the sensitivity is also increased.

On the assumption that all the heat developed is conducted away by the thermopile leads alone, the total thermal conductivity $G_{1}$ would be

$$
G_{1}=n g
$$

where $g$ is the conductivity of one pair of thermocouple leads and $n$ is the number of pairs of leads or junctions in the thermopile. Since the open-circuit thermopile output voltage $e$ is proportional to the temperature difference, $\left(T_{1}-T_{0}\right)$, and to the number of junctions, $n$, we have

$$
e=k\left(T_{1}-T_{0}\right) n
$$

where $k$ is a constant of proportionality. The temperature difference in turn is related to the power absorbed by

$$
\left(T_{1}-T_{0}\right)=k^{\prime} \frac{P}{G_{1}}
$$

where $k^{\prime}$ is another constant and convection and radiation losses are neglected under the simplifying assumption that $G_{1}$ is the only heat loss path. Substituting (10) in (9) gives

$$
e=k k^{\prime} \frac{P}{G_{1}} n
$$

The sensitivity $S_{1}$ is given by

$$
S_{1}=\frac{e}{\cdot P}=\frac{k k^{\prime}}{G_{1}} n=\frac{k^{\prime \prime}}{G_{1}} n=\frac{k^{\prime \prime} n}{n g_{1}}=\frac{k^{\prime \prime}}{g_{1}} .
$$

Thus, the sensitivity is independent of the number of junctions. The time constant, however, is inversely proportional to $G_{1}$ and hence to $n$.

The assumption that the thermocouple leads are the sole conductors of heat is, of course, not valid, since an appreciable portion of the heat is conducted away through the coaxial mount and an additional portion is lost by convection and radiation. The net effect of adding more thermocouples is to increase the sensitivity and also the thermal conductance, but by an amount less than the theoretical maximum of $G_{1}=n g_{1}$. The number of junctions was limited to 50 for practical reasons.

\section{The Substitution Principle}

As mentioned earlier this calorimetric wattmeter is not an absolute instrument, but rather the response of the temperature-sensing device must be calibrated using accurately known values of dc or low-frequency power. When the calorimeter is subsequently used to measure RF power in terms of the response at dc, the question arises as to whether or not the response varies with frequency. If the response is frequency sensitive then all RF power measurements would contain an error which is usually referred to as substitution error or equivalence error and arises principally from the fact that the current distribution, even in a uniform resistor, may not be the same at RF as at dc. Thus, the distribution of heat sources in the load may be a function of frequency.

If a large disk resistor whose value at dc is $R=Z_{0}$ is used to terminate a coaxial transmission line at RF, a reactive component will be present in the termination. The reactance is capacitive and is primarily due to the fringing fields which extend beyond the termination. Insofar as the reactive component is concerned, the fringing fields are a first-order effect because the termination can be made almost purely resistive by the addition of a quarter-wave shorted length of line beyond the disk resistor. ${ }^{4}$ Also, because the fringing fields exist beyond the resistive film they will have little if any effect on the current distribution in the disk resistor. Thus, it is reasonable to expect that changes in the RF current distribution with frequency would be due to secondorder effects and would, for this reason, be quite small.

It would be possible of course, to calculate the RF current distribution in an idealized disk resistor. However, due to departure of the actual resistor (in a manner that is difficult to predict), from the assumed conditions the effort involved in such a calculation is not considered worthwhile. It is possible, however, to obtain an indication of the current distribution by measuring the temperature distribution on the back side of the disk

4 "The Matching of Transmission Lines and Waveguides with Resistive Films," Telecommun. Res. Est. (England), Rep. DWF/13; February 28, 1941. 
substrate as a function of frequency. Results of some measurements made in this laboratory indicate that the temperature distribution does change when, for example, RF power at $300 \mathrm{mc}$ is substituted for dc power. These measurements have also shown that if the RF and $\mathrm{dc}$ power are made equal then the integrated temperatures are almost identical. This means that if the disk temperature were measured at only one point on its surface then a significant transfer error could result. On the other hand, if the integrated temperature is measured, as with the 50 -junction thermopile, the substitution error would be kept small.

Although it is extremely difficult to evaluate the exact value of the error involved in the substitution method, it is possible to estimate its upper bound from some special measurements. For the case of the disk resistor an experiment was carried out wherein the response of a 12 -junction thermopile uniformly spaced on the back of a 1-inch diameter disk resistor was measured at two different dc power levels. Initially the resistor was made as uniform as possible and was subsequently made nonuniform by the step-wise removal of wedge-shaped radial sections of the film. At the beginning and after each removal, the response of the thermopile was measured at each of the same two power levels. The results are shown in Fig. 3, where the average relative thermopile response is plotted against $R / R_{0}$. The value of $R / R_{0}$ $=2$ corresponds to removal of about $\frac{1}{2}$ of the resistive film.

The results of this experiment indicate the ability of the thermopile to accurately integrate the disk nonuniform temperature. As can be seen from Fig. 3, removal of the resistive film from $\frac{1}{2}$ of the disk surface resulted in a decrease in response of only 0.25 per cent. The integration is considered very good in view of the drastic changes made in the dc current distribution. These changes are probably much more pronounced than the changes in current distribution that occur in a uniform disk resistor when RF power is substituted for $\mathrm{dc}$ power. Therefore, the maximum substitution error may be conservatively estimated at 0.25 per cent.

\section{Performance of the Calorimeter}

The measured response of the thermopile, in millivolts, is plotted in Fig. 4 as a function of dc power input in watts. Experimental data show that below 0.2 watt the curve is approximately linear, while between 0.2 watt and 1 watt the curve is defined quite well by the simple empirical equation

$$
e=K_{1} P^{0.951}
$$

and plots as a straight line on log-log paper. Above 1 watt the equation of the curve is

$$
e=K_{2} P^{0.927}
$$

which gives another straight line on a log-log plot. Thus the nonlinear relationship between $e$ and $P$, mentioned in the section entitled "Theory," is shown to exist.

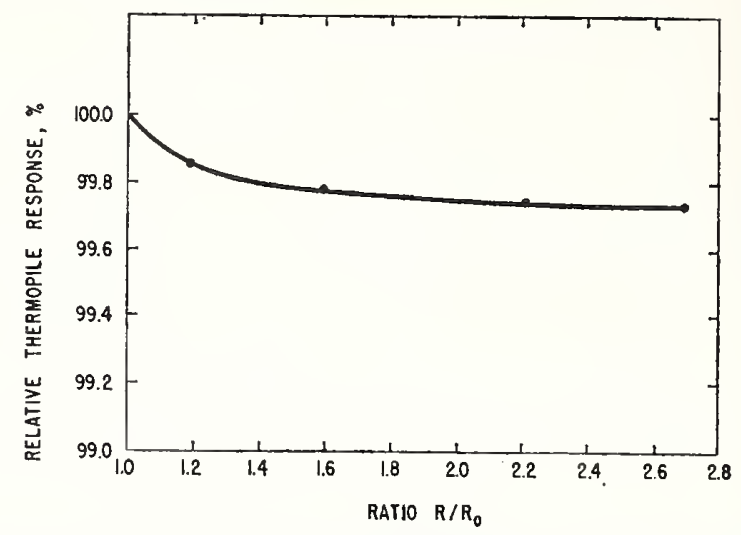

Fig. 3-Relative response of the thermopile as sections of the resistive film are removed from the disk resistor. $R / R_{0}=2$, for example, corresponds to removal of one-half of the film from the disk surface.

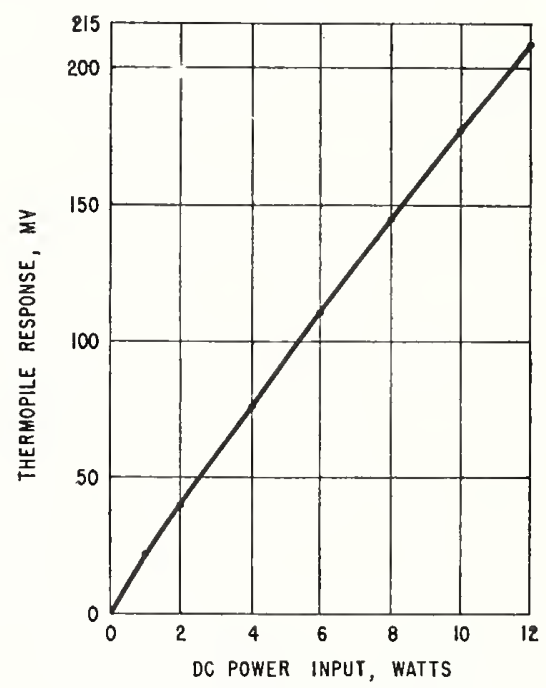

Fig. 4-Thermopile output plotted as a function of dc power input to the calorimeter.

It is interesting to note that the thermal equivalent to the assumed electrical circuit whose response is given by (6) also fits the curve of Fig. 4. This equation is based on the physical properties of the system, namely that at thermal steady state the rate of heat loss is equal to the rate of heat input. That is

$P=g_{1}\left(T_{1}-T_{0}\right)+g_{2}\left(T_{1}-T_{0}\right)^{1.25}+g_{3}\left(T_{1}^{4}-T_{0}{ }^{4}\right)$

where $g_{1}, g_{2}$, and $g_{3}$ are the coefficients of heat conduction, convection, and radiation, respectively. Referring to $(9)$

$$
e(\mathrm{mv})=k\left(T_{1}-T_{0}\right),
$$

where $k$ is the constant of the thermopile in $\mathrm{mv} /{ }^{\circ} \mathrm{C}$. Hence,

$$
\left(T_{1}-T_{0}\right)=e / k \text { and } T_{1}=e / k+T_{0} .
$$

Thus:

$$
P=g_{1}(e / k)+g_{2}(e / k)^{1.2}+g_{3}\left[\left(e / k+T_{0}\right)^{4}-T_{0}^{4}\right] .
$$

It is logical to assume that for power levels below 0.2 watt the second and third terms of (18) are negligible 
because the experimental curve of power vs thermopile output is linear. This means that the coefficient $g_{1}$ can be evaluated at low powers. The other two coefficients $g_{2}$ and $g_{3}$ can also be obtained experimentally by solving simultaneous equations at two different values of $P$. Carrying out the above computation the final equation is:

$$
\begin{aligned}
P= & 7.6 \times 10^{-2}(e / k)+2.44 \times 10^{-3}(e / k)^{1.2} \\
& +1.56 \times 10^{-10}\left[\left(e / k+T_{0}{ }^{4}\right)-T_{0}{ }^{4}\right] .
\end{aligned}
$$

Above 1 watt this equation fits the curve in Fig. 4 to better than \pm 1 per cent, again showing that the actual system is fairly well represented by the simple equivalent circuit of Fig. 1.

The sensitivity of the calorimeter is $23.50 \mathrm{mv}$ per watt below 0.2 watt and decreases, due to convection and radiation losses, at higher power levels as shown in Fig. 5 . The equation of the curve is $S=S_{0} P^{-0.08}$, where $S$ is the sensitivity at any power level, $S_{0}$ is the initial sensitiviky, and $P$ is power in watts. At 12 watts the value of $S$ is about 17 per cent below $S_{0}$.

Since the calorimeter is housed in a temperature controlled environment ordinary room temperature variations have no noticeable effect on the thermopile output. There is, however, a small residual output from the thermopile of $\pm 5 \mu \mathrm{v}$ with zero power input to the calorimeter. This residual EMF is equivalent to $\pm 0.2 \mathrm{mw}$ and causes this much uncertainty in the measurements at all power levels.

The time constant of the calorimeter, which is defined as the time necessary for the thermal EMF to reach 63 per cent of its final value, is approximately 4 minutes. To obtain maximum accuracy, however, a period of about 40 minutes must be allowed between measurements to permit the system to reach steady-state conditions.

The calorimeter was calibrated with dc power using a laboratory standard type voltmeter and ammeter. The accuracy of each of these instruments is 0.1 per cent and hence the accuracy of the dc power was 0.2 per cent. At frequencies up to $300 \mathrm{mc}$ losses in the coaxial mount were estimated to be $0.0025 \mathrm{db}$ or 0.05 per cent. This estimate is based on values given in tables for $1 \frac{1}{2}$-inch diameter rigid coaxial line with air dielectric. Thus, in using the calorimeter to measure RF power the uncertainty in the measurements amounts to $\pm(0.5$ per cent $+0.2 \mathrm{mw}$ ). To repeat, this figure includes the substitution error, the dc calibration error, and losses in the mount. Errors due to the VSWR being different from unity are not included. The calorimeter measures, of

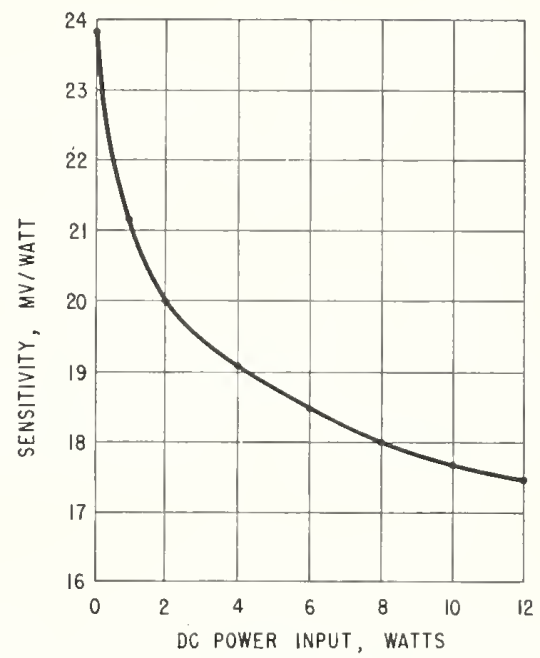

Fig. 5-Thermopile sensitivity vs power input level.

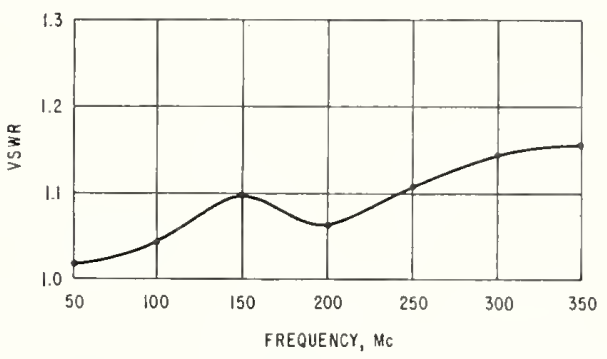

Fig. 6-VSWR of the calorimeter as a function of frequency.

course, only the power it absorbs. Some energy is reflected at RF since the VSWR is not unity. Because the VSWR is known, corrections due to the reflected power can be made in those cases where necessary. The VSWR varies with frequency, as shown in Fig. 6.

The performance of the calorimeter was further evaluated by making comparison RF power measurements between it and other independent methods. These included the low-power thermistor bridge, and the medium-power bolometer bridge whose power-sensitive element is a nickel film deposited on a glass tube $\frac{1}{8}$ inch in diameter by $2 \frac{1}{2}$ inches long. In all cases agreement was equal to or better than the sum of the uncertainties of the instruments involved. Comparison was also made with a second calorimeter of the dry, static type which employs two identical cylindrical loads. Accuracy of the second calorimeter is also 0.5 per cent and again good agreement was obtained.

The results of these comparison measurements are considered important since they give added confidence in the accuracy of individual instruments. 


\title{
A Self-Balancing Direct-Current Bridge for Accurate Bolometric Power Measurements
}

\author{
Glenn F. Engen
}

\begin{abstract}
Until recently, the most accurate microwave power measurements of the bolometric type have required the use of a manual d-c bridge. A self-balancing d-c bridge has been developed that preserves the inherent accuracy of the manual bridge, extends the dynamic range of operation, and greatly simplifies the operating procedure. A general description of the equipment and operating techniques is given, followed by a comprehensive survey of the sources of error accompanying the method and the accuracy achieved.
\end{abstract}

\section{Introduction}

A large amount of the research effort expended in the fietd of low level microwave power measurement in the past few years has been directed toward a determination of bolometer-mount efficiencies, or evaluation of the validity of the $r-f-d-c$ substitution principle. ${ }^{1,2,3,4}$ Until recently the only - accessory instrument of sufficient accuracy to utilize the results of these investigations has been a manually operated d.e Wheatstone bolometer bridge. The use of this device is, however, both tedious and time consuming; the dynamic range over which the desired accuracy is achieved is rather small, and the danger of barretter burnout due to a misstep on the part of the operator is ever present.

A self-balancing d-c bolometer bridge has been developed at the NBS Boulder Laboratories, which to a large measure relieves these problems, while preserving the accuracy and extending the dynamic range of operation. 'To date, the technique has been employed primarily with barretter-type elements, although on the basis of preliminary results, the technique should prove equally useful with thermistors.

\section{Manual Bridge}

One of the simplest forms of the manual bridge previously employed at the National Bureau of Standards is shown in figure 1 . The $r-f$ power $^{5}$ is given by

$$
P_{\tau f}=\frac{r_{0}}{4}\left(i_{1}^{2}-i_{2}^{2}\right)
$$

where $i_{1}$ and $i_{2}$ are the total currents required to balance the bridge without and with $\mathrm{r}-\mathrm{f}$ power present, respectively. A precision potentiometerstandard resistor combination is used to measure $i_{1}$ and $i_{2}$. If it is assumed that this measurement

\footnotetext{
${ }^{1}$ R. W. Beatty and Frank Reggia, An improved method of measuring effieieneies of ultra-high-frequeney and mierowave bolometer mounts, J. Researeh NBS 54, 321 (1955) RP2594.

${ }^{2}$ A. C. Macpherson and D. M. Kerns, A mierowave microealorimeter, Rev. Sci. Instr. 26, 27 (1955)

${ }_{3}$ H. J. Carlin and Max Sueher, Aeeuraey of bolometrie power measurements, Proe. Inst. Radio Engrs. 40, 1042 (Sept. 1952)

${ }_{4}$ D. M. Kerns, Determination of effieicney of mierowave bolometer mounts from impedanee data, J. Researeh NBS 42, 579 (1949) R P1995.
}

5 More eorreetly, "the retraeted d-c power". contains a random error of \pm 0.005 percent, it can be shown that for a typical bolometer operating at $200 \mathrm{ohms}$ and requiring $8.5 \mathrm{ma}$ of bias $\left(i_{1}=17 \mathrm{ma}\right)$ the error in measuring $P_{\tau f}$ may be as large as 0.02 , 0.3 , and 3 percent at the 10,1 , and 0.1 mw levels, respectively, if all other sources of error are neglected. The small difference between $i_{1}$ and $i_{2}$ at low levels accounts for the deterioration in accuracy. A variety of alternative techniques have been proposed and employed with some degree of success, but it is not within the scope of this paper to examine the manual technique in detail or to establish the maximum accuracy obtainable. In practice, this is often determined by the stability requirements imposed on the generator and measuring system by the time-consuming nature of the technique, rather than by more fundamental limitations.

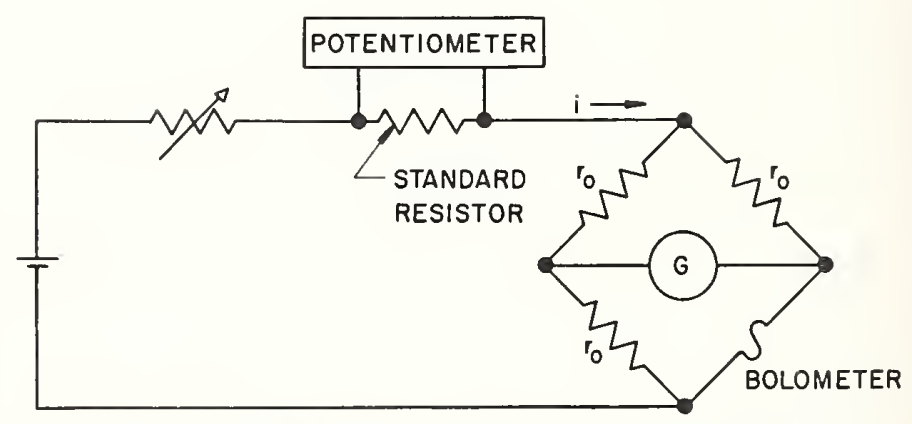

Figure 1. Manual direct-current bolometer bridge.

\section{Self-Balancing Bridge}

The self-balancing d-c bolometer bridge employs a d-c amplifior in a feedback loop to balance the bolometer bridge. The amplifier is connected in such a manner that the bridge umbalance signal is amplified to provide the bridge current (fig. 2). 'The use of this self-balancing circuit eliminates many of the time-consuming features of the technique, but the power-measurement problem is essentially the same. This problem, at low power levels, is basically one of trying to accurately measure a small change in a comparatively large $d-c$ power. 


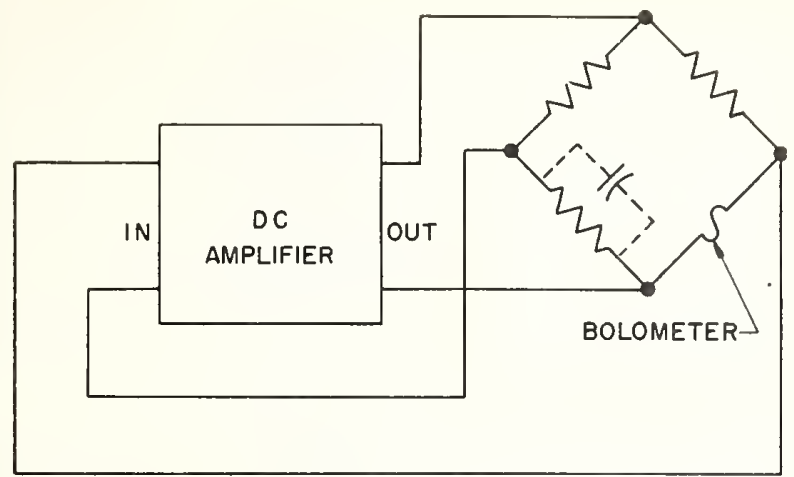

Figure 2. Basic circuit of self-balancing bridge.

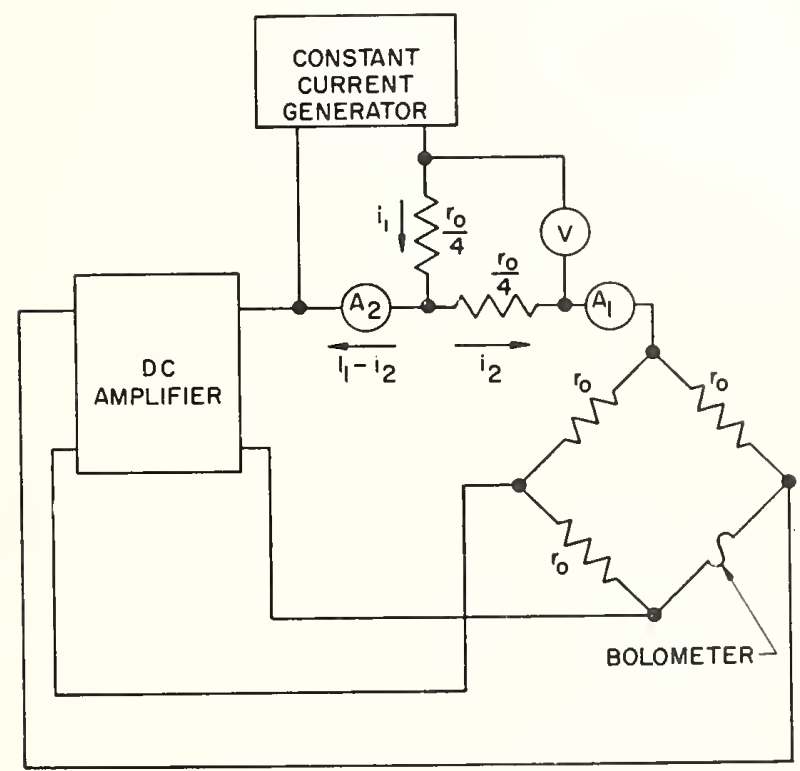

Figure 3. Basic circuit for the determination of $\Delta i$ and power.

The formula for the r-f power may be rewritten

$$
\begin{aligned}
P_{r f} & =\frac{r_{0}}{4}\left(i_{1}+i_{2}\right)\left(i_{1}-i_{2}\right) \\
& =\frac{r_{0}}{4}\left(2 i_{1}-\Delta i\right) \Delta i,
\end{aligned}
$$

where $\Delta i=i_{1}-i_{2}$.

The difficult factor to measure accurately is $\Delta i$. A constant-current generator of high stability has been developed for use in conjunction with a differential current-measuring technique, which enables a direct and accurate determination of $\Delta i$. The circuit is illustrated in figure 3 and operates as follows: First, with no microwave power present in the bolometer, $i_{1}$ is determined by ammeter $A_{1}$, and the output of the constant-current generator is adjusted witl the aid of ammeter $A_{2}$ to equal $i_{1}$ (ammeter $A_{2}$ reads zero). 'Then, with microwave power' applied, $A_{2}$ will read $\Delta i$ directly, and the power may be determined from eq (3). In practice, a potenti- ometer and standard resistor are employed in place of $A_{1}$ and $A_{2}$ to achieve high accuracy.

Alternatively, a voltmeter, $Y$, connected as shown, will read $\left(r_{0} / 4\right)\left(i_{1}+i_{2}\right)$, and the power is given by the product of " and $A_{2}$. These operations may also be combined in a single instrument of the drinamometer type to achieve a direct reading of the power.

The degree of success obtained with this technique is, of course, contingent upon the degree of stability aclieved in the constant-current generator. In this case the change in generator output for a complete cutoff of bridge current is less than 1 part in $10^{5}$, resulting in a negligible error from this source.

\section{Performance}

It is shown in a following section that the over-all accuracy in power measurement achieved, using a potentiometer to measure the current, is about 0.1 percent, whereas substitution of a suitable dynamometer to achieve direct reading is expected to yield an accuracy of about 0.3 percent. The instrument thus provides an increase in accuracy of 10 to 50 times that provided by the commercially avaiablel audiobridges. Other advantages over the tylpica audiobridge include: (1) Comparatively simple techniques are available for establishing, verifying, and monitoring the specified accuracy. (For example, the bridge balance may be monitored by a suitable galvanometer.) (2) The performance is, to a large degree, independent of the nominal deterioriation of the vacuum tubes and other electronic components. (3) The elimination of the audio bias power avoids its interaction with the pulse-repetition frequency in the nieasurement of pulse power.

At low power levels the $r-f$ power is approximately proportional to $\Delta i$ (eq 3$)$, and the low-level performance may be displayed by recording this current with the $r-f$ power alternately on and off. The system response to a $1-\mu \mathrm{W}$ signal is given in figure 4 .

Barring failure of the electronic components, the self-balancing operation eliminates the danger of bolometer burnout due to excessive bias current, whereas protection against an $\mathrm{r}-\mathrm{f}$ overload may also

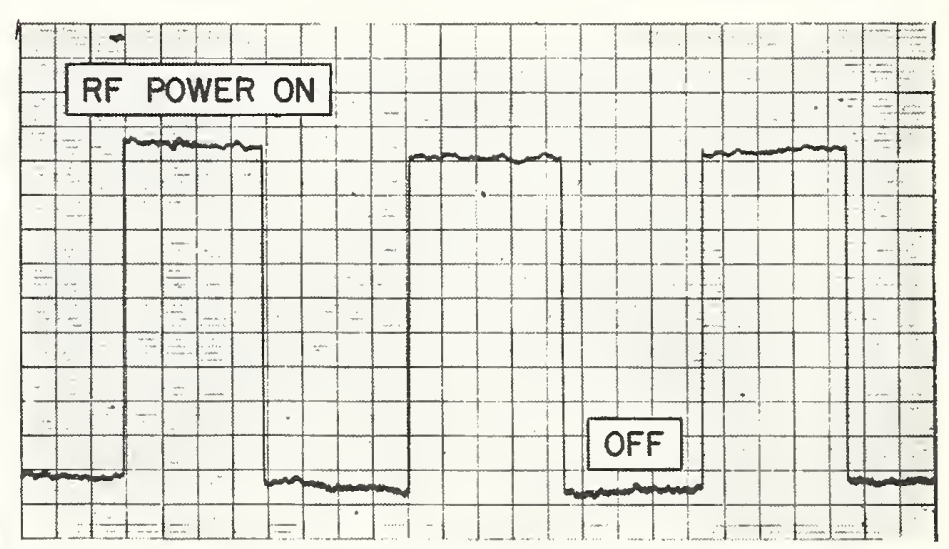

Figure 4. Bridge response to a 1-microwatt signal. (Radiofrequency on and off for 1-minute interval- 

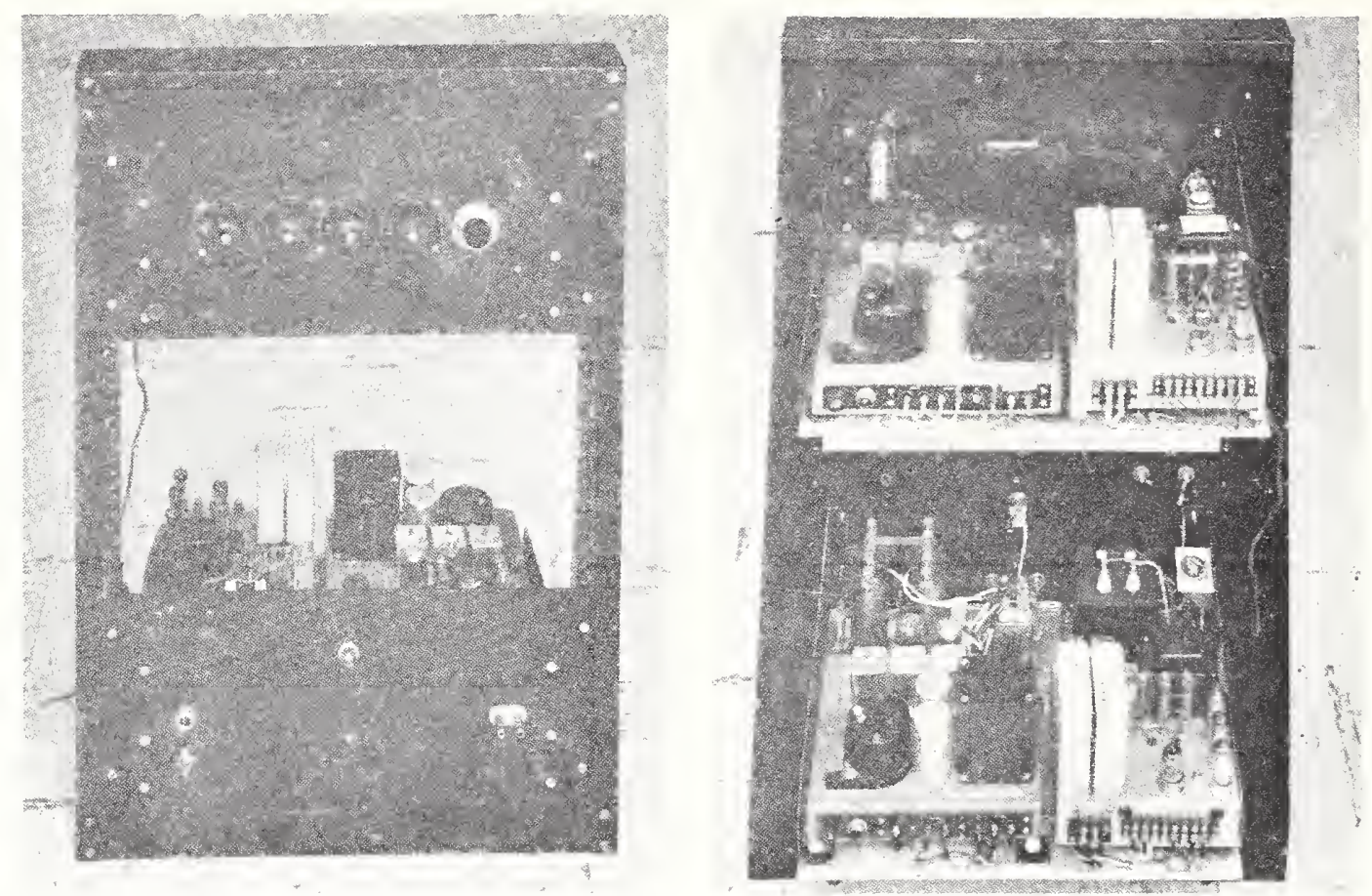

Frat RE 5. Prototype model of self-balancing bridge, front and rear views.

be provided through use of a suitable relay to turn off the $r^{-f}$ souree when the power input to the bolometer exeecels a preseribed amount.

The ol-e amplifier employed in this application is a commeretally arailable instrument of exerptional gain, zero stabilitr, and phase lesponse, which was modified by the addition of a cathode follower to provicte the recunired output amrent. 'The enrent amplification of the amplifier, as thus modified, is approximately 100,000. Compensation for the thermal hay of the bolometer is provided in the feedback loop by mons of a condenser, as indicated in figure 2, but the achievement of stable operation with this larese amplifieation is primarily a tribute to the amplifier' phase response. 'The constant-rurrent generator employs a second amplifier of the same type, which, with modification, mantains a constantoutput eurent by reference against a mereury cell. A prototrpe model of this acfuipment is shown in figure 5

\section{Analysis of Errors}

The practical problems involved in making an accurate determination of the power, using eq (1) to (3), have been noted. But use of these formulas implies that the bridge is exactiy balaneed, the bridge arms are equal to $r_{0}$, ete. It is shown in the appendix that a more complete expression for the power is

$$
\begin{aligned}
P_{r f} \cong & \frac{r_{0}}{4}\left(i_{b 1}^{2}-i_{b 2}^{2}\right)\left[1+Z-\frac{r_{2}}{r_{0}}+\frac{2 \rho}{r_{0}\left(i_{b 1}+i_{b 2}\right)}\left(1+\frac{8}{\gamma i_{b 1} i_{b 2}}\right)\right. \\
& \left.+\frac{16(1+g)}{\gamma i_{b 2}\left(i_{b 1}+i_{b 2}\right)}\left(\frac{1}{\alpha}-\frac{i_{k 1}}{i_{b 1}}\right)-\frac{2 g}{\left(i_{b 1}+i_{b 2}\right)}\left(\frac{i_{b 1}}{\alpha}+i_{g 2}\right)\right],
\end{aligned}
$$

where

$\alpha=\left(i_{b 1}-i_{b 2}\right) /\left(i_{g 1}-i_{g^{2}}\right)=$ current amplification of amplifier

Z. = fractional deviation of the lower bridge arm from the value $r_{0}$

$r_{l}=$ resistann of bolometer leads

$e=$ thermal (or other) emf in amplifier input

$i_{b}=$ total britge eurrent

$i_{g}=$ amplifier input emrent

tr: $r_{0}=$ amplifier input resistance

$\gamma=$ "ohms per" watt" bolometer" eosfficiont.

For a typical bolometer operating in the 0 to 10 mw revion, the foilowing values may be assigned:

$$
\begin{aligned}
r_{0} & =200 \mathrm{ohms} \\
\gamma & =4.5 \mathrm{ohms} / \mathrm{mw} \\
i_{b 1} & =17 \mathrm{ma} \\
i_{b 2} & >8 \mathrm{ma}
\end{aligned}
$$

Substituting these values in the above expression vields the result

$$
\begin{aligned}
P_{r f} \cong \frac{r_{0}}{4}\left(i_{b 1}^{2}-i_{b 2}^{2}\right)\left[1+Z-\frac{r_{1}}{r_{0}}+5.6 \rho+18\right. & (1+!)\left(\frac{1}{\alpha}-\frac{i_{g 1}}{i_{b 1}}\right) \\
& \left.-80 \vartheta\left(\frac{i_{b 1}}{\alpha}+i_{g 2}\right)\right],
\end{aligned}
$$

where the last three terms in the bracket reflect the inability of the d-e amplifier to balance the bridge exactly.

'The eharacteristies of the d-e amplifier' employed in this application are such that the following values 
obtain:

$$
g=1 / 4 \quad \text { (amplifier input resistance }
$$

$$
\alpha>10^{5}
$$

(current amplification)

$$
\left.\begin{array}{l}
0<i_{g 1} / i_{b 1}<2 \times 10^{-5} \\
0<i_{g 2}<10^{-i} \\
|e|<10^{-}
\end{array}\right\} \text {(amplifier zero stability). }
$$

Substituting these values in the last three terms gives a value for their sum of about 0.0002 .

The bolometer lead resistance can be measured with little effort and the correction applied, so the error from this source is negligible, whereas the value of $|Z|$ can ordinarily be held to 0.0001 or less.

Finally, it is possible to measure current by means of a precision potentiometer-standard resistor combination to an accuracy of a few parts in $10^{4}$; thus the first factor in eq (4) may be determined by the technique discussed earlicr to an accuracy of about 0.06 percent.

In summary, the total limits of error are as follows:

$$
\begin{aligned}
& \text { Deviation of bridge arms from } \\
& \text { value } r_{0 \ldots \ldots} \ldots .01 \text { percent. } \\
& \text { Failure of bridge to be exactly } \\
& \text { balanced............... } 02 \text { percent. } \\
& \text { Measurement of } i_{b 1}+i_{b 2} \ldots \ldots .03 \text { percent. } \\
& \text { Measurement of } i_{b 1}-i_{b 2} \ldots \ldots .03 \text { percent. } \\
& \text { Bolometer lead resistance _.... . .00 percent. } \\
& \text { Amplifier noise and instability } \\
& \text { (experimentally observed) - - } \\
& \text { Total }
\end{aligned}
$$

\section{Appendix}

From figure 6 the power may be expressed in the form

$$
P_{r f}=\frac{r_{0}}{4}\left(i_{b 1}^{2}-i_{b 2}^{2}\right)+f\left(X, Y, Z, i_{g}, e, r_{l}\right)
$$

where $X, Y$, and $Z$ are the deviations of each of the bridge arms from. the value $r_{0}, i_{g}$ and $e$ are the detector current and thermal (or other) emf, respectively, and $r_{l}$ the resistance of the bolometer leads.

Assuming a knowledge of the bolometer resistance law, an exact expression for the second term could be derived, but the labor required suggests the following approximate treatment. By hypothesis, the variables $X \ldots r_{l}$ and $f\left(X \ldots \dot{r}_{l}\right)$ are small, and $f\left(X \ldots r_{l}\right)$ may, to a good apploximation, be writ ten as the sum of the first-order corrections for each of these quantities, where the correction for each of the variables is ketermined with the other variables equal to zero.

Application of this technique to the circuit in figure 6 richls the following first-order expressions for the d-ce power dissipated in the bolometer, and the boloneter resistance

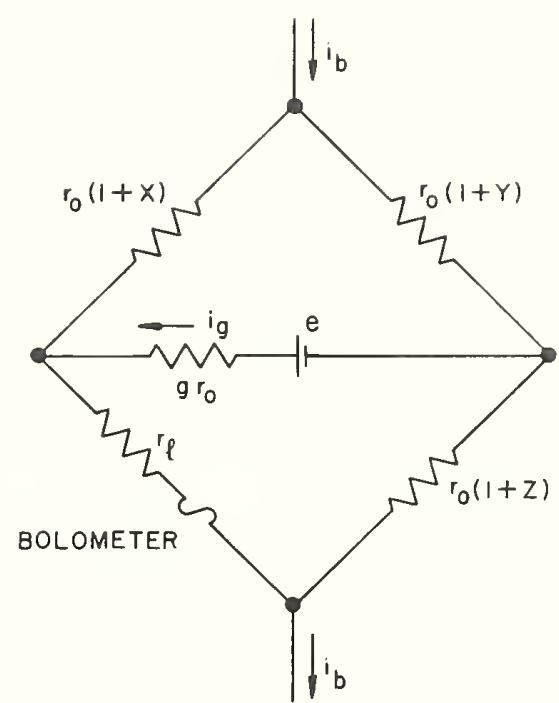

FIGCRE 6. Bridge circuit used in the evaluation of errors.

$$
\begin{gathered}
P_{a c} \cong \frac{i_{b}^{2} r_{0}}{4}\left[1+Z-\frac{r_{l}}{r_{0}}+\frac{2 e}{r_{0} i_{b}}-\frac{2 g i_{g}}{i_{b}}\right] \\
r \cong r_{0}\left[1+x-y+Z-\frac{r_{l}}{r_{0}}+\frac{4 e}{i_{b} r_{o}}-\frac{4(1+g) i_{g}}{i_{b}}\right]
\end{gathered}
$$

The failure of the expression for power to have a first-order dependence on $Y$ or $Y$ is explained as follows: A prescribed, small value for $I$ or $Y$ will cause a first-order variation in bolometer resistance $r$, as indicated in eq (7), but also produces an uncyual division of eurrent in the two sides of the bridge. These two sources of error cancel to a first approximation. The only general requirement on the upper bridge arms is that they be equal, and if ther have a nominal value of $r_{0}$, it can be shown that they may differ from each other by as much as 2 percent and produce an error in the power measurement of only 0.01 percent.

At a constant ambient temperature and assuming equivalence of $d-c$ and $r-f$ heating, ${ }^{6}$ the power, $P$, dissipated in the bolometer will be a function of its resistance, $r$ :

$$
P=f(r)
$$

Because the bolometer is nearly a square-law detector, one may in the reighborhood of an albitrarily selected operating resistance $r_{0}$, write

$$
P \cong P_{0}+\frac{\partial P}{\partial r}\left(r-r_{0}\right)
$$

where $P_{0}$ is the power required to bring the bolometer to the resistance $r_{0}$ at the given ambient temperature,

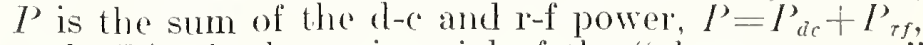
and $\partial P / \partial r$ is the reciprocial of the "ohms-per-watt"

6 The r-f-d-e strbstitution error is assumed to he negligible in this discussion. For a treatment of this problem, see lootnotes 2 and 3. 
bolometer coefficient, i. e., $(\partial P / \partial r)=(1 / \gamma)$. Then

$$
P_{d c}+P_{r f} \cong P_{0}+\frac{1}{\gamma}\left(r-r_{0}\right)
$$

$P_{0}$ may be determined by measurements in the absence of $\mathrm{r}$-f power:

$$
P_{0} \cong P_{d c}-\frac{1}{\gamma}\left(r_{1}-r_{0}\right)
$$

where the subscript 1 has been used to indicate the value of quantities measured in the absence of $\mathrm{r}-\mathrm{f}$ power, whereas the subscript 2 will indicate values with 1 -f present.

The formula for r-f power is then

$$
P_{\gamma f}=P_{0}-P_{d c}+\frac{1}{\gamma}\left(r_{z}-r_{0}\right)=P_{d c 1}-P_{d c 2}-\frac{1}{\gamma}\left(r_{1}-r_{2}\right) .
$$

In the given application, only $i_{g}$ of the variables $I . . r_{l}$ has a functional dependence upon $i_{b}$, or is subject to change with the application of $\mathrm{r}-\mathrm{f}$ power. Substituting eq (6) and (7) in eq (8) gives the desired result

$$
\begin{aligned}
P_{r f} \cong \frac{r_{0}}{4}\left(i_{b 1}^{2}-i_{b 2}^{2}\right)\left[1+Z-\frac{r_{l}}{r_{0}}+\frac{2 e}{r_{0}\left(i_{b 1}+i_{b 2}\right)}\left(1+\frac{8}{\gamma i_{b 1} i_{b 2}}\right)\right. \\
\left.+\frac{16(1+g)}{\gamma i_{b 2}\left(i_{b 1}+i_{b 2}\right)}\left(\frac{1}{\alpha}-\frac{i_{g 1}}{i_{b 1}}\right)-\frac{2 g}{\left(i_{b 1}+i_{b 2}\right)}\left(\frac{i_{b 1}}{\alpha}+i_{g 2}\right)\right]
\end{aligned}
$$

where

$$
\alpha=\frac{i_{b 1}-i_{b 2}}{i_{g 1}-i_{g 2}}
$$

The author extends his thanks to R. W. Beatty and John E. Lauer for their valuable criticisms and suggestions in the preparation of this paper, and to Ray R. Rumfelt for his assistance in the design and construction of this equipment.

Boulder, Colo., November 28, 1956. 


\title{
A Refined X-Band Microwave Microcalorimeter
}

\author{
Glenn F. Engen
}

(May 1, 1959)

\begin{abstract}
The microcalorimetric method for evaluation of the efficiency and substitution error of a bolometer mount proposed by Macpherson and Kerns has been the object of further study and refinement at the Boulder Laboratories of the National Bureau of Standards, and an improved instrument based on this technique has been recently placed in operation.

The new microcalorimeter design features are: (1) Greatly improved ambient temperature control, permitting higher sensitivity and resolution; (2) improved d-c instrumentation; (3) improved mechanical construction giving better repeatability; (4) relocation of the thermopile so that it no longer is attached directly to the bolometer mount, thus providing flexibility in the choice of termination; and (5) a more comprehensive treatment of the calorimetric substitution or equivalence error. These features permit the determination of the effective efficiency of a bolometer mount to an accuracy of better than 0.2 percent.
\end{abstract}

\section{Introduction}

The bolometric technique, by means of which the heating effect of an unknown amount of rf or microwave power is compared with that of a measured amount of d-c or audiofrequency power through a temperature-sensitive resistive clement, is a wellknown and extensively employed method in the low-level measurement of microwave power.

In a commonly used arrangement, the bolometer element forms one arm of a Wheatstone bridge, the parameters of which are chosen in such a manner that the bridge is balanced when the bolometer is at its nominal operating resistance. 'The bolometer is then provided with the required bias power to bring its resistance to this operating value, and the reduction in bias power required to maintain the bridge balance following the application of a microwave signal, is taken as a measure of the microwave power. The measurement of this bolometric or substituted d-c bias power has been the object of refinement until the measurement can be carried out to an accuracy of 0.1 percent [1].

The use of the value thus obtained as a measure of the microwave power involves, however, two well-recognized sources of systematic error. First, the microwave and $\mathrm{d}-\mathrm{c}$ bias currents take different distributions within the bolometer element, which means that the functional dependence of the bolometer resistance will, in general, be different for the two sources of power, giving rise to a de-rf substitution error. Second, the bolometric method at best measures only the microwave power dissipated within the bolometer element, whereas one is usually interested in the power dissipated in the entire termina ting wa veguide structure or bolometer mount. The ratio of these quantities is, by definition, the bolometer mount efficiency. In addition, if the mount is not properly tuned there may be a net reflection of power at its input terminals, but the methods of dealing with this phase of the problem are well-recognized [2] and will not be considered explicitly in this paper.

\footnotetext{
1 Figures in braekets indicate the literature referenees at the end of this paper.
}

Macpherson and Kerns [3] gave the details of a calorimetric method for the determination of the combined effect of these two sources of error. This technique has been the object of further study and refinement at the Boulder Laboratories, and an improved instrument based on this method was recently placed in operation. A number of calorimetric-type power meters have also been developed $[4,5,6]$ in other laboratories which utilize the same general techniques employed by Macpherson and Kerns, but the further development at the NBS has retained the original objective of obtaining a correction factor for the bolometrically-determined power.

\section{2; General Description}

The basic theory of operation has been presented in detail in the cited references and need not be repeated here. In brief, lowever, the instrumentation is such as to permit a calorimetric determination of the total power input to a bolometer mount while a simultaneous bolometric determination is made. The difference between the two measurements is ascribed to the mount efficiency and substitution error. The method, in its present state at least, permits only a determination of the combined effect of these phenomena, referred to as "effective efficiency."

The problem of making a calorimetric determination of power (or energy) may be subdivided as follows: (1) Selection of a calorimetric body or object in which to dissipate the power to be measured, (2) choice of a suitable technique to measure the temperature rise of this body or object, and (3) determination of the proportionality factor or functional dependence of the thermometer response upon the power input. In the microcalorimeter technique, the bolometer mount serves as the calorimetric body or object in which the power is dissipated and whose temperature rise is measured by means of a suitable thermopile, while calibration is effected by observing the thermopile response to a measured amount of d-c power dissipated in the bolometer element. 
The thermopile consists of 34 constantanchromel-P junctions with an approximate senstivity of $2,000 \mu \mathrm{v} /{ }^{\circ} \mathrm{C} \%$ The instrument is designed to yield a nominal output at equilibrium of $100 \mu \mathrm{v}$ corresponding to a temperature rise of $0.05^{\circ} \mathrm{C}$. Since this potential mar be measured by the associated instrumentation to an accuracy of a few parts in $10^{+}$, a temporature background which is stable to a few millionths of a degree is indicated for the application.

In order to achieve this background, the twin Joule arrangenent is employed as shown in figures 1 and 2 , along with a temperature-regulated water hath. The advantage of this method stems from the fart that if symmetry exists with respect to a plane normal to the plane of the illustration as shown, and if no thermal gradients exist within the thermal shiclds, then the actual temperature of these shichls may fluctuate without producing a temperature difl'erence between the bolometer' mounts. 'Thus, hy using a tripte thermal shich (the mmermost one of which is a heavy copper casting), a simmetrical waveguide input system, and suitable thermal shumts, a deglee of simmotry is achicved such that the dependenee of the temperature difference between the boloneter mounts upon the thermal head existine between the imner themal shield and the external water hath, is only a factor of approximately one part in $10^{\text {t. }}$. Since a bath regulation of $\pm 0.005^{\circ} \mathrm{C}$ mia be obtained with little difficulty, adequate isolation from the bath fluctuations is thus achieved. 'There still exist in the eurent model, however, temperature aludients whose origin is ret undetermined such that the thermal background is stable to only 5 to 10 millionths of a degree, but this is satisfactory for the application.

In order to function in the required manner, thermal isolation is required between the bolometer mounts and the remainder of the system. (Isolation is also required between the thermal shields.) 'This rules out the possibility of using conventional waveguide to convey microwave encrey to the boloneter mounts, since this would also readily conduet away the heat gencrated in the mount. In the earlier instrument, this isolation took the form of choke-flange-air-gap combination, but such an

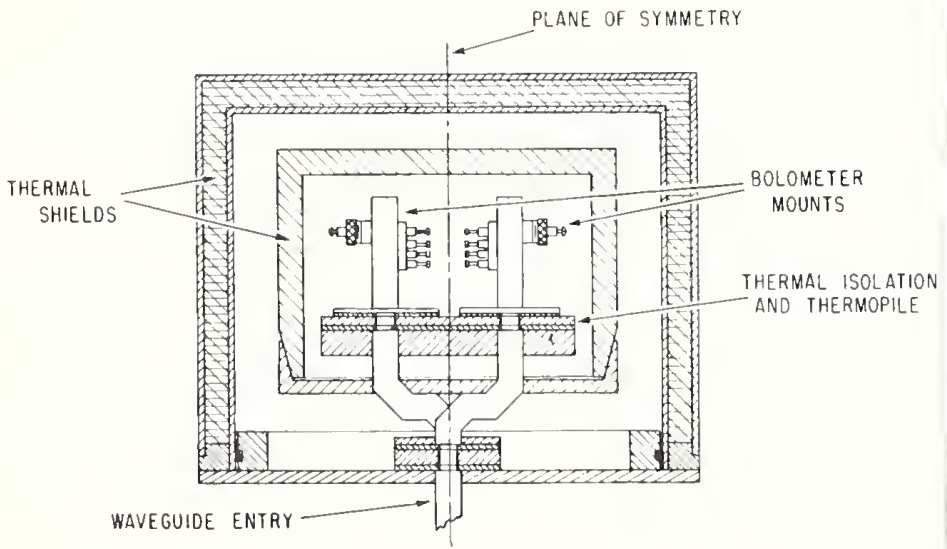

FIGURE 1. Cross section of microcalorimeter.

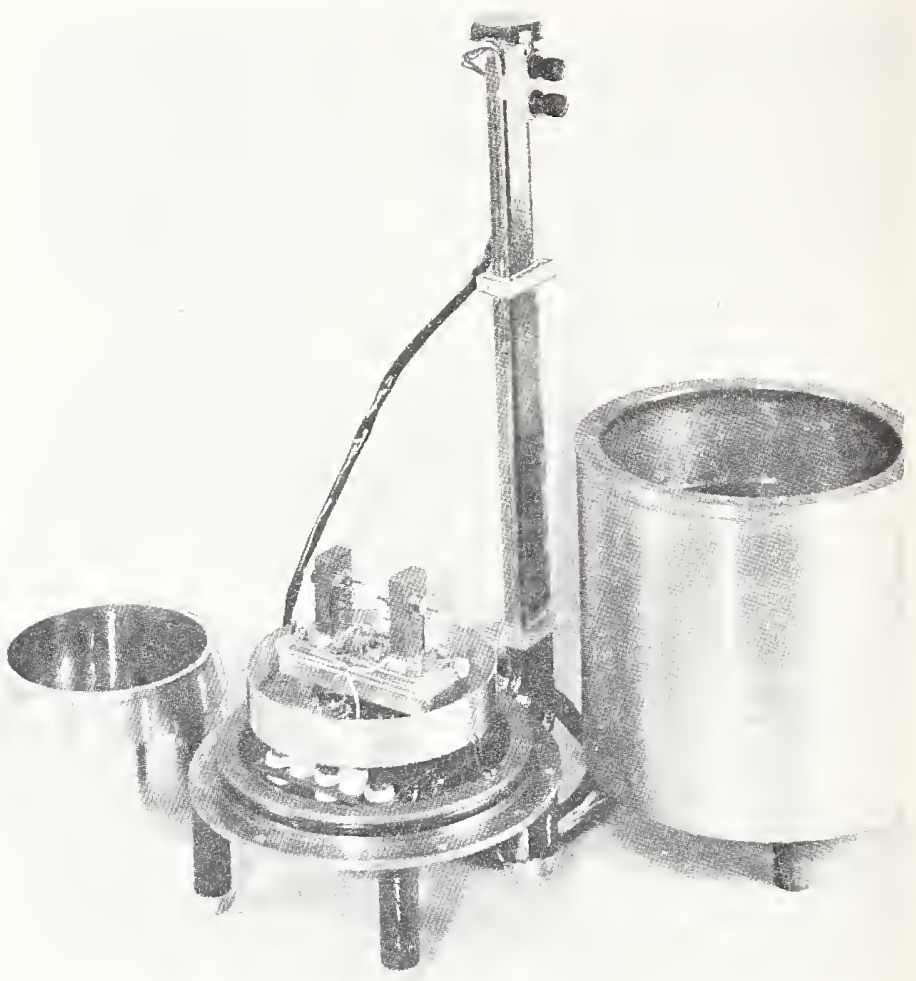

FIgURE 2. Microcalorimeter and thermal shields.

arrangement at best is frequency sensitive, and at worst, may introduce systematic error's whose evaluation is quite difficult. Tn the present model, this isolation is provided by a short section of wareguide, the body or sides of which are fabricated from 0.001-in. copper shim with the addition of suitable plastic to provide the required mechanical rigidity.

A significant increase in the flexibility and operating convenience of the instrument has been realized through relocation of the thermopile. Instead of being a taiched (lirectly to the bolometer mounts as in the former model, the thermopile is built in to the thermal isolation sections in such a way that it senses the temperature difference between the flanges which mate with the bolometer mount flanges as shown in figures 3 and 4 . 'The obvious advantage of this arrangement is that removal of the four screws which hold the bolometer mount in place in the colorimeter also serves to remove the mount from the thermopile assembly, thus permitting flexibility in the choice of termination and enabling the ready duplication or multiplication of the number of bolometer mounts which may be so calibrated.

There are a number of design criteria which the bolometer mounts employed in the calorimeter must satisfy. First, a high degree of stability in those parameters which determine the mount efficiency is obviously desimble, while the ability to tume the mount for an impedance match (no reflection) at the operating frequency is also desirable if comparison with other mounts is anticipated. The choice of the tuning dements which may be employed in achieving this impedance match is severely limited by the necessity of keeping the mass (heat capacity) of the entire mount within prescribed limits. In 


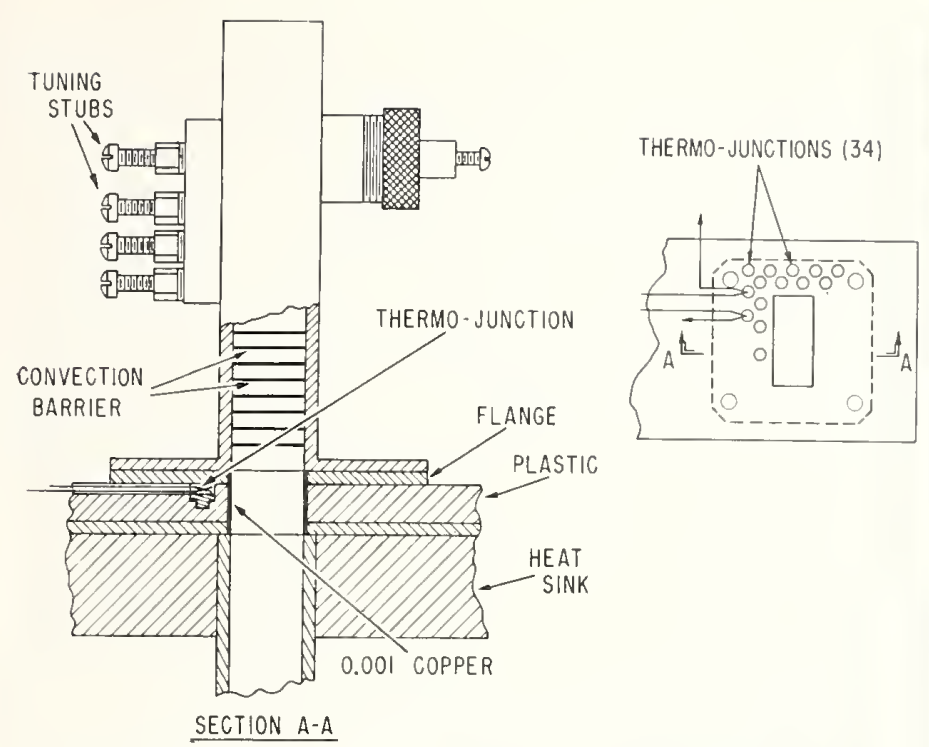

Figure 3. Detail of thermopile construciion.

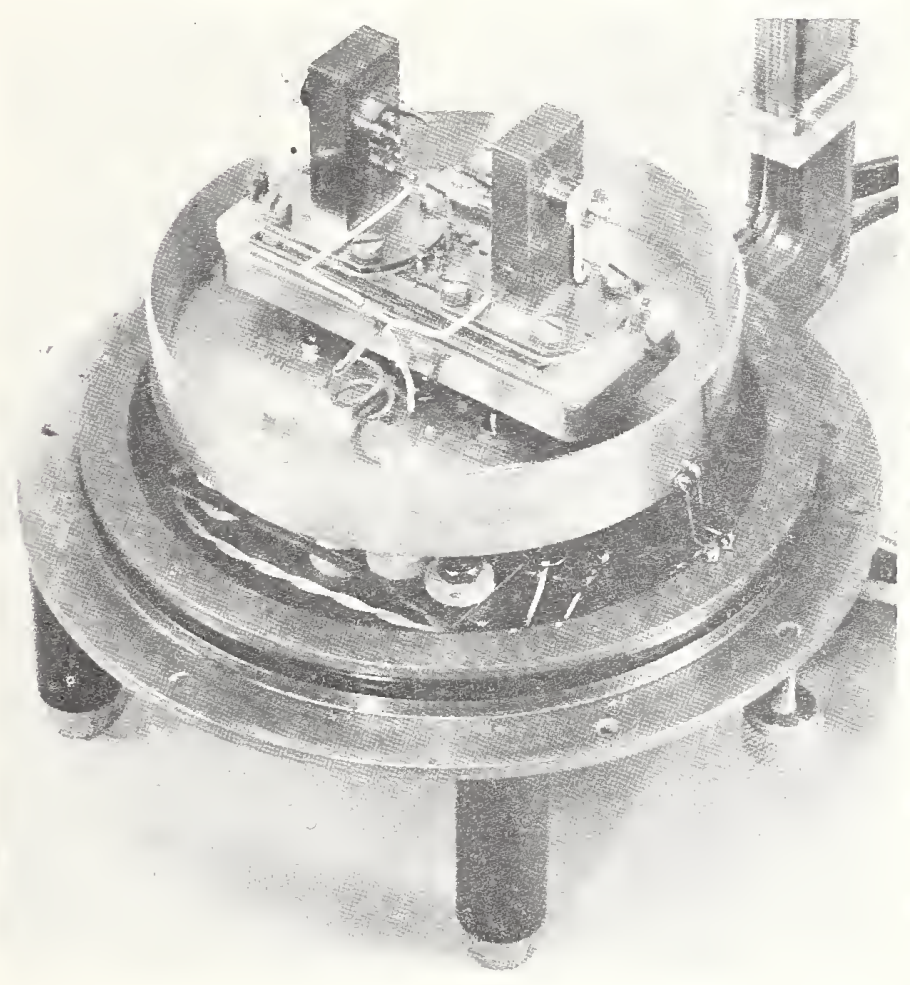

FIGURE 4. Closeup of microculorimeter interior.

addition, white comparatively little is yet known of the distribution of losses within a bolometer mount, many of the commonly employed tuning elements would appear to be potential sources of instability in the mount effieiency.

Another requirement on the mount design is that the calorimetric equivalence error (to be discussed later) be held to a small value; that is, the thermopile response should be essentially independent of the distribution of the heat sources within the mount. This will be true if the mount is of uniform temperature overall, which suggests the use of thick walls and keeping the length short. The design should also provide a high value for the efficiency as this will also reduce the error from ealorimetric nonequivalence. Some of these problems will be discussed in greater detail in later sections, but it will be recognized from the present discussion that the design must effect a compromise among a variety of conflicting requirements.

\section{Operational Procedure}

The operational procedure associated with the instrument is comparatively simple and straightforward. Using the terminology introduced by Macpherson and Kerns [3] an effective efficiency, $\eta_{e}$, naay be defined,

Substituted or retracted bias power as measured by the bolometric technique.

$$
\eta_{e}=\frac{\text { as meal microwave power dissipated within }}{\text { Total }} \text {. }
$$

In practice the bolometer is operated in conjunction with a selfbalancing $d-c$ bridge [1]. The d-c bias power is first applied and the thermopile response allowed to effectively reach an equilibrium value (which takes $45 \mathrm{~min}$ ). Microwave power is then applied, d-c power is withdrawn as required to maintain bridge balance, and the thermopile response again permitted to reach equilibrium, at which time the microwave source is removed and the thermopile response to d-c power only once inore observed. The procedme thus consists of eneroizine the bolometer element from the $d-c$ bridge and observing the thermopile response and bridge curl'ent, without and with microwave encrgy present in 45-min intervals. Ideally, only one cycle of microware power off and on would be required, but in practice areraging the results of two "off" intervals occurring before and after an "on" interval permits further suppression of the thermal backeround drift. Typical thermopile response curves are shown in figures 5 and 6.

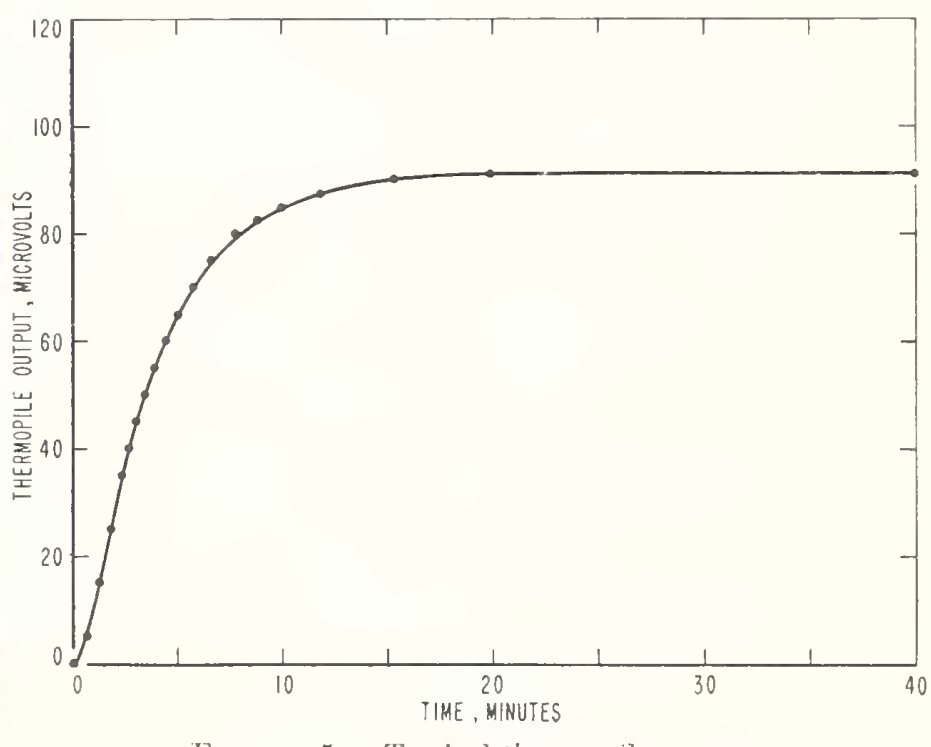

Figure j. Typical thermopite response. 


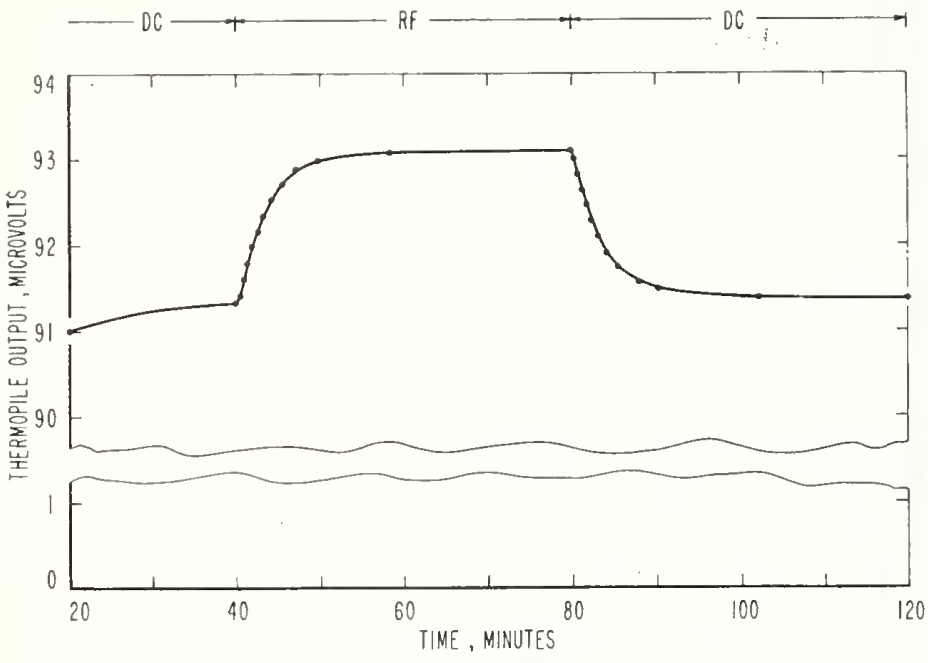

Figure 6.-Response curve for determination of effective efflciency.

Let $i_{1}$ and $i_{2}$ represent the bolometer bridge curlents without and with microwave encrgy present in the bolometer and, respectivclv, $e_{1}$ and $e_{2}$ the corresponding thermopile responses. Then for the equal arm bridge in common use, the bolometric power is given by the usual formula,

$$
P_{b}=\frac{r_{0}}{4}\left(i_{1}^{2}-i_{2}^{2}\right)
$$

where $r_{0}$ is the resistance of the bolometer element and bridge arms.

The total power input to the bolometer mount (microwave and (d-c) during the microwave "on" interval ideally will be proportional to the thermopile response, $e_{2}$, the proportionality factor being $r_{0} i_{1}^{2} / 4 e_{1}$. The microwave power is the difference between this total input and the d-e component, thus

$$
P_{r f}=\frac{r_{0}}{4}\left(\frac{e_{2}}{e_{1}} i_{1}^{2}-i_{2}^{2}\right)
$$

and

$$
\eta_{e}^{\prime}=\frac{1-\left(i_{2} / i_{1}\right)^{2}}{e_{2} / e_{1}-\left(i_{2} / i_{1}\right)^{2}}
$$

which gives the effective efficiency $\left(\eta_{e}^{\prime}\right)$ in terms of the thermopile response and bolometer-bridge eurlents. The prime is used to indicate that the equation is based on the assumptions that the thermopile response is linear and independent of the distribution of the power dissipation within the mount. The validity of these assumptions will be examined below.

\section{Error Analysis}

Equation (2) for $\eta_{e}^{\prime}$ has been obtained under certain simplifying assumptions; a more general approach to the problem will now be presented. Within the limits of experimental crror (to be discussed below), the thermopile response and cooling mechanisms are both linear, and the thermopile response $e_{2}$ is of the form:

$$
e_{2}=k\left(P_{2_{d c}}+g P_{r f}\right)
$$

where $k$ and $g$ are constants, and $P_{2_{d c}}$ and $P_{r f}$ are the d-c power dissipated in the bolometer element and the total microwave power dissipated in the bolometer mount, respectively. Equation (3) expresses the fact that a given amount of microwave power will, in general, produce a different thermopile response than an equal amount of $d$-c power through the factor $g$.

The constant $k$ may be determined in the manner already discussed so that $k=e_{1} / P_{1_{d c}}$. Substituting this result and solving eq (3) for $P_{r f}$ gives,

and

$$
P_{r f}=\frac{1}{g}\left(\frac{e_{2}}{e_{1}} P_{1_{d c}}-P_{2_{d c}}\right)
$$

$$
\eta_{e}=g \frac{P_{1_{d c}}-P_{2_{d c}}}{\frac{e_{2}}{e_{1}} P_{1_{d c}}-P_{2_{d c}}}=g \frac{1-\left(i_{2} / i_{1}\right)^{2}}{e_{2} / e_{1}-\left(i_{2} / i_{1}\right)^{2}}
$$

The more complete analysis thus gives a factor $g$ by which the right-hand side of $\mathrm{eq}(2)$ is to bc nultiplied in determining $\eta_{e}$. The error in determining $\eta_{e}$ thus consists of the uncertainty in the value of $g$, and the experimental error in determining the value of this second factor, $\frac{1-\left(i_{2} / i_{1}\right)^{2}}{e_{2} / e_{1}-\left(i_{2} / i_{1}\right)^{2}}$. These will be referred to as the calorimetric equivalence and instrumentation errors, respectively.

The instrumentation error may be evaluated with little difficulty. If the second factor is differentiated with respect to $i_{1}$ and $i_{2}$, it is found that for typical values of the ratio $e_{2} / e_{1} ;$ i.e., $1<e_{2} / e_{1}<1.05$, the error in $\eta_{e}$ is only 5 percent or less of the error in $i_{1}$ or $i_{2}$. In practice, $i_{1}$ and $i_{2}$ are measured by a precision potentiometer-standard resistor combination to an accuracy of about 0.03 pereent; thus, the total error due to the uncertainty in $i_{1}$ or $i_{2}$ is only of the order of 0.01 percent and may be neglected.

The error due to the uncertainty in $e_{2} / e_{1}$ may be found in a similar manner, and if the ratio of $i_{2}$ to $i_{1}$ is $1 / 2$ or less, the crror in $\eta_{e}$ is in the range 1.0 to 1.3 times the error in $e_{2} / e_{1}$.

In order for eq (3) to be valid, the thermopile response must be a linear function of the $d-c$ and microwave powers. The error in the ratio $e_{2} / e_{1}$ may conveniently be defined to include, in addition to the instrumentation error in measuring the thermopile output, the error due to nonideal performance of the calorimeter caused by such things as nonlinear thermopile response and cooling, thermal-background drift, cte., which might affect the validity of eq (3). The crror in measuring the ratio $e_{2} / \ell_{1}$ was determined in the following manner. The measurement procedure outlined in the foregoing section was followed except that instead of applying microwave power at the end of the first 45-min interval, the d-c power applied to the bolometer was increased by a nominal 10 percent. In this way the actual operating conditions were simulated, and the measured value of this second d-c power was compared with that predicted on the basis of a linear response from the obselvation of $e_{1}, e_{2}$ and the first value of $d-c$ power. 
In ten measurements of this type, the maximum error observed was 0.04 percent, with an average error of 0.013 percent. A figure of 0.1 percent, corresponding to an error in $e_{2} / e_{1}$ of 0.07 percent, has bcen arbitrarily assigned as a conservative limit to the error from this source, in which the probable error is perhaps 0.02 percent or less.

With regard to the equivalence error, $g$ is a number which expresses the relative effectivcness of microwave power as compared with d-c power in producing a thermopile response, that is, for a given thermopile output, $g=P_{d c} / P_{r f}$. Idcally, $g=1$ but in practice differs from this value due to the following phenomena:

1. The d-c and microwave powers take different distributions within the bolometer elcment yielding different thermopile responses.

2. Some of the microwave energy will be dissipated in places other than the bolometer element and the thermopile response will again differ from that produced by an cqual amount of $d-c$ power in the bolometer.

3. The thermopile will respond to the dissipation of microwave cnergy in the waveguide lcad-in which does not comprise part of the bolometer mount.

It is just these first and second phenomena, of course, which arc responsible for the dc-rf substitution crror and mount inefficicncy.

With reference to the first of these, the tempcrature-sensing device in the calorimcter is relatively far removed from the main heat source and it might be estimated conservatively that the error in the calorimetric method due to this phenomena would be at least an order of magnitude or more bclow the bolometric substitution error which according to the information available. [7], does not exceed a few percent. ${ }^{2}$

In order to check the validity of this assumption experimentally, the $\mathrm{d}$-c calibration factor, $k$, was determined for several different bolometer elements (barretter and thermistor). The initial results of this experiment gave values which differed from one another by as much as 2 percent with a repeatability of 0.1 percent. This anomaly at first appeared to challenge the validity of the above assumption, but further investigation showed that the insertion of a $0.05 \mathrm{in}$. copper plate at the bolonetcr-mount input flange (thus blocking the waveguide input), would climinate this difference. This was interpreted to mean that air convection was playing a significant role in cooling the bolometer capsule (not to be confused with the cooling of the bolometer element within the capsule), and the variations in the value for $k$ were ascribed to the variablc role, occasioned by the differences in the capsules associated with the bolometcrs, played by air convection in conveying this lieat from the capsule to the external heat sink via the interior of the waveguide.

By inverting the bolometer mounts such that the closed end was up, it had been hoped that the need

2 Recent advances in the impedance method of measuring bolometer mount efficiency have demonstrated that the substitution error at frequencies near 10 $\mathrm{kMC}$ is probably no greater than a few tenths of a percent. for a convection shield or barrier could be eliminated, but these results clearly demonstrated the need for such a shield. In order to provide a convection barrier, in which the microwave dissipation would be negliqible, a series of eight 0.002 in. Teflon membranes spaced at 0.05 in. intervals and transverse to the waveguide axis was arranged at the waveguide input. This effectively eliminated the convection problem and gave repeated determinations of $k$ in a refined bolometer mount which agreed to 0.05 percent, which is about the limit of experimental error for this particular measurement. Since the power distributions in the barretter and thermistor are markedly different, it may be safely assuined that the small difference in distribution of the $\mathrm{d}-\mathrm{c}$ and microwave powers which occurs in practice when the same element is employed, will have a negligible effect.

With regard to the degree of equivalcnce realized in the thermopile output for power dissipated in the bolometer mount walls as compared with an equal amount in the bolometer clement, the problem, once again, is primarily that of attempting to prevent the escape of heat from the mount via air convection. The effectiveness of the convection barrier described in the previous paragraph was demonstrated by temporarily inserting two other heat sources within the mount such that they were in good thermal contact with the side walls. The first of these was located just inside the mount before the teflon barrier, the second on the other side of the barrier, close to the bolometer element as shown in figure 7 . The thermopile response for equal amounts of power in the two positions was then compared with that for the bolometer element, and it was found that the heat sources at positions 1 and 2 were approximatcly 1.5 and 0.5 percent more effective respectively. A conservative figure for the increased effectiveness of the power dissipated in the walls is thus 1 pcrcent with an crror of \pm 1 percent. It should be noted that this figure applies only to that fraction of the power which is so dissipated. In practice a lower limit of 97 percent may bc taken for the efficiency of the mounts designed for use in this calorimeter, which leaves only 3 percent of the energy to be dissipated other than in the bolometer element.

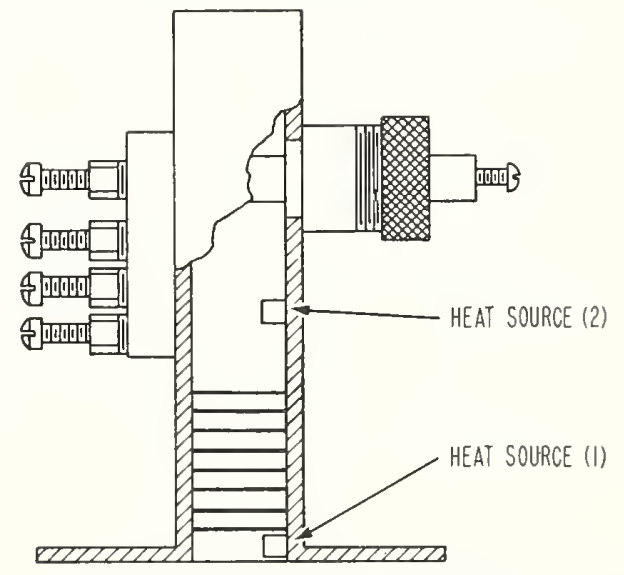

FIGURE 7. Bolometer mount with auxiliary heat sources. 
Finally, as noted, the thermopile will respond to the dissipation of microware energy in the thermal isolation section which is not part of the bolometer mount. If an electrical conductivity of 50 pereent of thr d-c ralue is assumed for this $1 / 4 \mathrm{in}$. length of copper waveguide, the theorotical value of powel dissipation will be apploximately 0.05 pereent, of which the thermopile will probably measme somewhat suore than half. A ralue of 0.04 pereent t- 0.04 pereent thus represents a conservative estimate for this contribution.

Tha results of this seretion are summalized in lable 1.

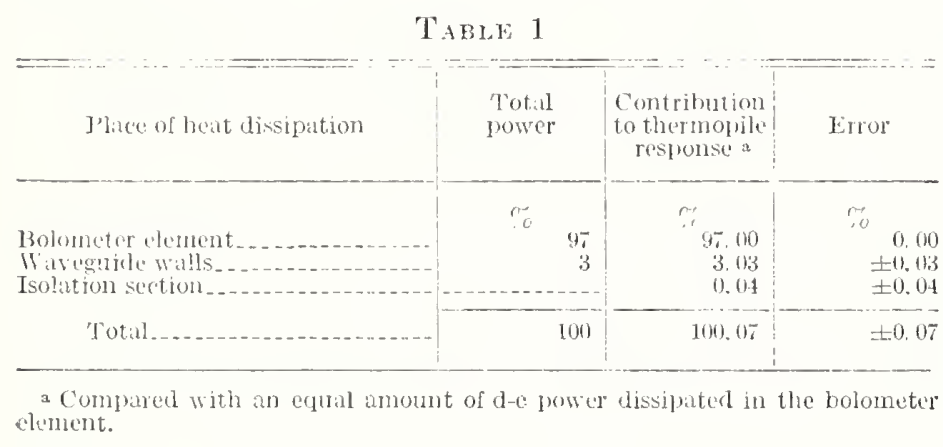

Rounding off these results gives for $g$ a value 1.001 with a possible erol of \pm 0.1 pereent; and this, combined with the instrumentation cror of \pm 0.1 pereent, gives for the total crror \pm 0.2 percent.

\section{Bolometer Mount Design and Experi- mental Results}

As may be inferred from the foregoing description, the design of the bolometer mounts employed in the microcalorimoter has undergone a serio's of transitions as required to cope with the rarious engineering problems encountered in the evaluation of the device. While no claim is made as to the extent of their applicability, certain features of the construction are believed to be of enough interest to merit further description.

The general features of the construction are shown in figures 4 and 7 . 'The mount is machined in two halves from a solid piece of tellurium copper, and the two halves hard-soldered together along the center of the wider side. The brass flange is then attached by soft solder. Except for these two applications, the use of solder has been completely avoider in the construction. The incentive for this methou of fabrication was provided by the observation of efficiencies of the order of 85 percent for mounts in which the back plate was fastened with soft solder, while the use of hard solder increased these ralues to $95-98$ percent.

The design emplovs fire tuning stubs as shown, three in front of the bolometer and two at the side. These side stubs provide additional susceptance at the plane of the bolometer element, and thus, orer a limited range, serve the same function as a moreable back plate, while the other three stubs serve the usual purpose.

In the fire mounts which have been built to this general pattern, effective efficieneies in the vicinity of 98 to 99 percent have been realized. The result of additional measurements to determine the place of this resichal dissipation seem to imply that most of it occurs in the plastic capsule surromuling the clement and/or in the microwave bypass mechanism, at the d-c lead-in to the bolometer. While ardelitional research will be required to complotely resolve these questions, it appears likely, on the basis of present information, that effective officiences of 99.5 pereent and perhaps higher may be realized by proper design and fabrication techniques.

Finaly, table 2 shows the results of intereomparisons with commercially arailable mounts.

TABLE 2

\begin{tabular}{|c|c|c|}
\hline & Model & $\begin{array}{l}\text { Firective } \\
\text { elficieney }\end{array}$ \\
\hline 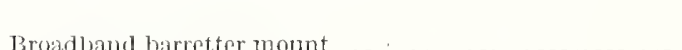 & $A$ & $r$ \\
\hline $\begin{array}{l}\text { B roadband harretter mount } \\
\text { B }\end{array}$ & 13 & 96. 2 \\
\hline Jiroadband barretter mount & C & 95.4 \\
\hline Brourbind thermistor mount & 1) & 95.2 \\
\hline
\end{tabular}

The author acknowledges the assistance of Morris E. Harrey, to whose care in making the experimental observations a large amount of credit for the success of this project is due, and cxtends his thanks to R. IV. Beatty and D. M. Kerns for their helpful suggestions in the preparation of the manuscript.

\section{References}

[1] G. F. Engen, A self-balancing d-c bridge for accurate bolometric power measurements, J. Research NBS $\mathbf{5 9}$ 101 (1957) RP2776.

[2] R. W. Beatty and A. C. Macpherson, Mismatch errors in microwave power measurements, Proc. IRE 41, 1112 (1953).

[3] A. C. Macplierson and D. M. Kerns, A microwave microcalorimeter, Rev. Sci. Instr. 26, 27 (1955).

[4] MI. Sucher and H. J. Carlin, Broad-band calorimeters for the measurement of low and medium level microwave power. I. Analysis and design. IRE Trans. on Microw. Theory Tech.6, 188 (1958).

[5] A. V. James and L. O. Sweet, Broad-band calorimeters for the measurement of low and medium level microwave power. II. Construction and performance, IRE Trans. on Microw. Theory Tech. 6, 195 (1958).

[6] IT. M. Sharpless, A calorimeter for power measurements at millimeter wavelengths, IRE Trans. on Microw. Theory Tech. 2, 45 (1954)

[7] H. J. Carlin and M. Sucher, Accuracy of bolometric power measurements, Proc. IRE 40, 1042 (1952).

Boulder, Colo.

(Paper 63C1-9) 


\section{2,883,620 \\ HIGH FREQUENCY POWER MEASURING BRIDGE CIRCUIT}

Myron C. Selby, Charles M. Allred, Paul A. Hudson, and Ira S. Berry, all of Boulder, Colo., assignors to the United States of America as represented by the Secretary of Commerce

\section{Application September 6, 1957, Serial No. 682,560 \\ 3 Claims. (Cl. 324-106)}

This invention relates to $\mathrm{R}-\mathrm{F}$ or microwave power measurements and more particularly is concerned with a bridge including a temperature-sensitive thermistor or bolometer mount for measuring low-level R-F power.

Resistance-type bridges have been used almost exclusively for the measurement of low-level R-F powers for the past several years. As compared with calorimetry equipment, bridges have the advantage of high sensitivity, fast response and higher accuracy. Prior art measuring circuits have almost invariably been of the Wheatstone bridge type in one arm of which is included a temperaturesensitive resistor such as a thermistor or barretter. Thermistors have negative temperature coefficients while barretters have generally positive ones. The prior art bridges include a source of low frequency. or D.C. bias current having means for varying the magnitude of the current. Since this bias source must be extremely stable, batteries operated under conditions of constant current drain have usually been used. To meet the condition of constant current drain, constant impedance attenuators are generally placed in series with the bridge to vary the bias power. The computation of R-F power is then obtained from the equation $P_{r f}=1 / 4\left(I_{1}^{2}-I_{2}^{2}\right) R$ for the case of an equal arm bridge, where $\mathrm{R}$ is the bridge resistance and $I_{1}$ and $I_{2}$ are the total bridge currents before and after the application of R-F power, respectively. The validity of this equation is based on the equivalent heating effect of D.C. power and R-F power when both are dissipated in a purely resistive load.

Batteries as used in the prior art bridges need frequent recharging and/or replacement and constant impedance attenuators are both bulky and expensive. The chief disadvantage, however, is that if the R-F power is small then $I_{1}-I_{2}$ may well be of the same order of magnitude as the accuracy with which $I_{1}$ and $I_{2}$ are measured. Such a condition can lead to large errors and the only alternative is to use special high sensitivity, low power capacity barretters or thermistors for low power level; thus several elements are necessary to cover the range from microwatts to milliwatts.

The bridge circuit of the present invention avoids the abovementioned difficulties by employing a variable resistance in parallel across the bridge. A conventional constant current D.C. supply source provides a singlevalued total current at all times. The bridge is rebalanced by shunting a portion of the bridge current through the parallel variable resistor. The change in current necessary to rebalance the bridge can then be directly measured in the shunt circuit.

It is therefore one object of this invention to provide an improved R-F power measuring bridge.

Another object of this invention is to provide an $\mathrm{R}-\mathrm{F}$ power measuring bridge in which the change in bridge current may be measured directly.

Still another object of this invention is to provide a simple, inexpensive R-F power measuring bridge having improved accuracy and greater range.

An additional object of this invention is to provide an
2

improved R-F power measuring bridge in which a single thermo-sensitive unit may be used to measure R-F powers over a range of approximately 30 decibels.

A better understanding of the invention can be had 5 with reference to the single figure of the drawing showing the improved R-F power measuring bridge circuit of the present invention.

Referring to the drawing, a conventional Wheatstone bridge indicated generally at $\mathbf{1}$ includes equal resistance J0 arms 2, 3, and 4. The fourth arm of bridge 1 contains a thermo-sensitive element 6 which may be in the form of a thermistor or barretter mount. An R-F generator 7 supplies high-frequency energy to element 6 through a coupling capacitor 8 . Bridge balance may be detected

15 by a galvanometer 9 connected across two arms of the bridge.

Connected in parallel with bridge 1 are a pair of series resistors $\mathbf{1 1}$ and $\mathbf{1 2}$. Adjustment of the variable resistor 11 controls the amount of current shunted around 20 bridge 1. A switch 13 provides means for disconnecting resistors $\mathbf{1 1}$ and $\mathbf{1 2}$ from the remainder of the circuit. The magnitude of the shunt current flowing through resistors 11 and 12 may be measured by voltmeter 14 connected across measuring resistor 12 .

25 Bias current for bridge 1 is derived from a constant current D.C. supply 16 . Source 16 maintains a steady flow of constant magnitude current at all times. The magnitude of this current is measured by means of a voltmeter 17 connected across a second measuring resistor 8 30 in series with the D.C. supply source.

In operation the bridge is first balanced by varying the current $I_{1}$ from the constant current source 16. When bridge 1 is balanced, the current from source 16 is set and remains constant throughout the remainder of the 35 measuring period. During this original balancing operation switch 13 in the shunt circuit is open so that the shunt current $\Delta \mathrm{I}_{1}$ is zero. The R-F power to be measured from $\mathrm{R}-\mathrm{F}$ generator 7 is then fed into the temperaturesensitive element 6 with switch 13 in the closed posito tion. Shunt resistor $\mathbf{1 1}$ is then adjusted until the bridge is rebalanced. The two currents $I_{1}$ and $\Delta \mathrm{I}_{1}$ may be measured on voltmeters 17 and 14 , respectively, and the R-F power may then be simply computed.

By using a constant current bias source 16 the shunt current $\Delta I_{1}$ is exactly equal to the differential current $\left(I_{1}-I_{2}\right)$ in the equation as given above for computing $\mathrm{R}-\mathrm{F}$ power. This shunt current may be measured directly to the desired degree of accuracy regardless of the magnitude of the current $I_{1}$. The power equation

\section{0}

$$
P_{r f}=1 / 4\left(I_{1}^{2}-I_{2}^{2}\right) R
$$

is used in the factored form $P_{r f}=1 / 4\left(2 I_{1}-\Delta I_{1}\right) \Delta I_{1} R$ where $\Delta I_{1}=\left(I_{1}-I_{2}\right)$. The shunt current $\Delta \mathrm{I}_{1}$ can be substituted directly into the equation. In this way a single high-power thermistor, for example, can be used to cover the range from about 10 microwatts to around 100 milliwatts of radio frequency power.

Constant current supply source $\mathbf{1 6}$ may be any of the well-known constant current D.C. generators. A special electronically regulated power supply has been constructed having a regulation of one part in $10^{5}$ for load variations from 0 to $100 \mathrm{ohms}$ with a stability of plus or minus one part in $10^{5}$ per hour. With this source and using a thermistor for temperature-sensitive element $\mathbf{6}$, R-F lower levels from 100 milliwatts down to 100 microwatts 65 have been measured with respective accuracies of 0.05 to $5 \%$.

A low-cost bridge with reduced accuracy may' be constructed by substituting a resistor having a value of about 100 times that of the bridge resistance for the constant current control circuit. In such a modification a large resistor is placed in series with the bridge and fed from 
a conventional voltage regulated power supply. In this case changes in the shunt resistance from zero to infinity will result in a maximum change of total Bridge current $\mathrm{I}_{1}$ of only $1 \%$.

As can be seen, the present invention provides a novel inexpensive bridge circuit for measuring low-level, highfrequency power limited in frequency range only by the frequency limitations of a thermistor or bolometer mount. Expensive batteries and constant impedance attenuators are eliminated through the use of a shunt circuit making it possible to measure directly the differential current involved in rebalancing the bridge. The design of this bridge circuit readily permits incorporating a self-balancing feature by having the amplified output of the bridge control the amount of shunt current. Improvement over present day commercial bridges is about 10 to 1 in power range and as much as 100 to 1 in percentage accuracy with the greatest improvement at low power levels.

It will be apparent that the embodiment shown is only exemplary and that various modifications can be made in construction and arrangement within the scope of invention as defined in the appended claims.

What is claimed is:

1. A high-frequency power measuring circuit compris- ing an electrical balancing bridge having a variable im. pedance in one arm sensitive to high-frequency energy, a constant direct-current source connected to said bridge for supplying a bias current of fixed amplitude, means 5 for applying the high-frequency energy the power of which is to be measured to said sensitive impedance to produce unbalance in said bridge, means for varying the amount of said fixed-amplitude bias current applied to said bridge comprising variable shunt impedance means 10 connected across said bridge and said constant directcurrent source for restoring the balance of said bridge, and means for measuring the current flow through said shunt impedance means.

2. The invention of claim 1 in which said balancing 15 bridge is a Wheatstone bridge and said variable impedance arm comprises a thermal-resistive resistor.

3. The invention of claim 2 in which said variable impedance arm is a thermistor.

\section{References Cited in the file of this patent}

\section{UNITED STATES PATENTS}

Houghton Feb. 18, 1947
Ginzton

Carlin 
HIGH FREQUENCY POWER MEASURING BRIDGE CIRCUIT

Filed Sept. 6, 1957

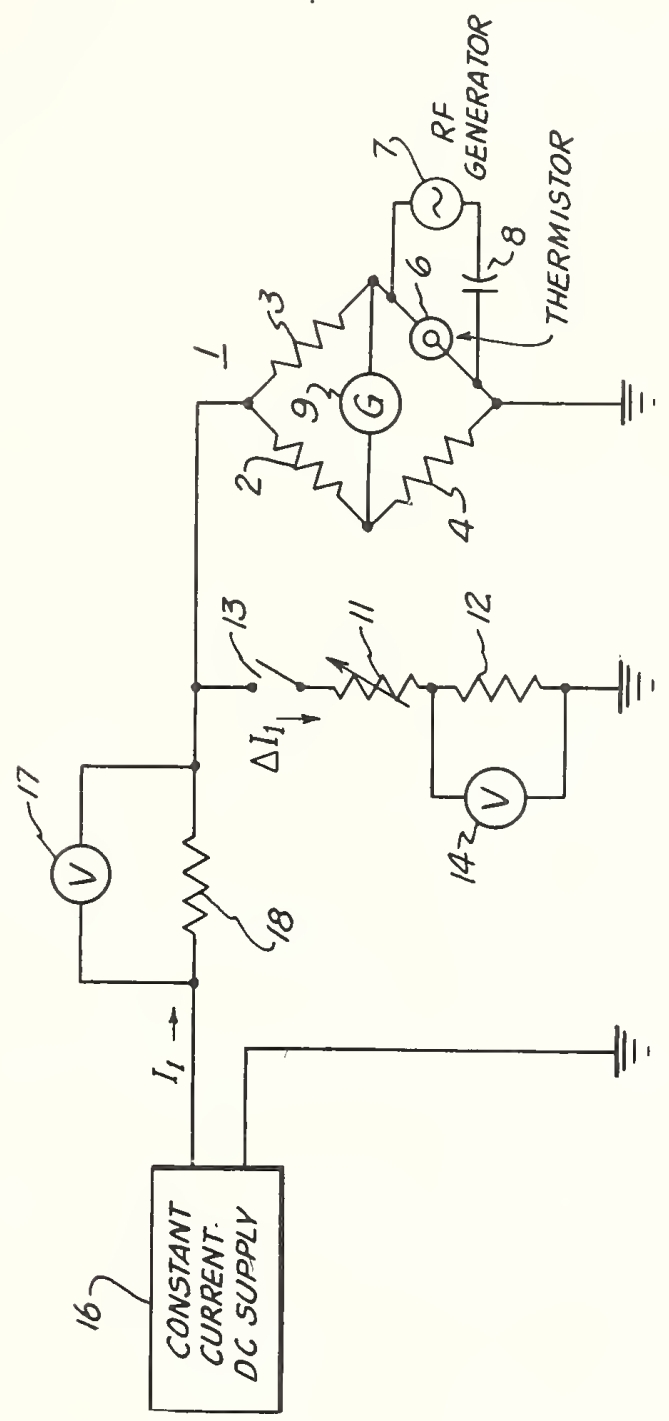

Myron C. Selby

Charles M. Allred

Paul A. Hudson

BY

Cattur Ira S. Berry

Lomand $\pi$. stoll ATTORNEYS 


\section{A Technique for Reducing Errors in Permeability Measurements with Coils*}

A recent note ${ }^{1}$ gave a summary of the results of an investigation carried on at the National Bureau of Standards by Kostyshyn and $\mathrm{Haas}^{2}$ for the purpose of studying the sources of error that are associated with the measurement of initial permeability using coils wound on toroidal samples of rectangular cross section. It was pointed out that various laboratories are, in general, using one of the following formulas for evaluating the permeability of a magnetic material.

$$
\mu^{\prime}=\frac{L_{m}}{L_{a}}
$$

or

$$
\mu^{\prime}=\frac{L_{m}}{L_{s}}
$$

where

$\mu^{\prime}=$ real part of the relitive initial complex permeability,

$L_{m}=$ measured inductance of the coil wound on the magnetic sample,

$L_{a}=$ the calculated inductance of an equivalent air core coil assuming a thin uniform current sheet,

$L_{s}=$ measured value of inductance of an identical coil wound on a polystyrene core.

Neither formula, as given above, can be expected to give a correct answer. Presumably, this is due to the fact that flux leakage effects and current sheet corrections are not considered in these equations. Various correction factors are usually applied to (1), and an attempt was made to evaluate the validity of these correction terms in the previously mentioned work at the National Bureau of Standards. However, one result that came out of this study, which has not been sufficiently emphasized, was that the errors in these measurements appeared to be largely independent of the magnitude of the permeability. This is apparently true even for a permeability of the order of unity which would be the case for the polystyrene

* Received by the IRE. September 14, 1959

1 R. D. Harrington and R. C. Powell, "High-frequency magnetic permeability measurements using

2 B. Kostyshyn and P. H. Haas, "Discussion of current-sheet approximations in reference to highStendords, vol 52, Do. 279-287; J , J 1954. core. It thus follows that errors in permeability measurements should subtract out if $L_{m}$ and $L_{s}$ are both measured and the results are substituted in an equation of the following form:

$$
\mu^{\prime}=\frac{L_{m}-L_{s}}{L_{a}}+1
$$

This equation follows readily from (1) if we let $\Delta L$ be the error in the measured value of both $L_{m}$ and $L_{s}$. Since $\Delta L$ is independent of permeability, we should write for the correct values of $L_{m}$ and $L_{s}$ :

$$
L_{m}=\mu^{\prime} L_{a}+\Delta L
$$

and

$$
L_{s}=L_{a}+\Delta L
$$

Subtraction of (5) from (4) will lead directly to $(3)$.

In order to obtain a comparison of (1) through (3), a series of measurements were made at $50 \mathrm{kc}$ using a Maxwell-type inductance bridge. A powdered iron core was used having a permeability of 7.05 as determined by a radio-frequency permeameter. The resulting data giving permeability as a function of number of turns used on the coil, are given in Fig. 1.

The increase in accuracy obtained using (3) is readily apparent. It was also observed that a curve similar to that resulting from (3) could be obtained by using (1) alone with corrections which account for finite wire size and departure of the wire from a uniform current sheet. On the other hand, the calculation of these corrections is usually quite tedious. As such, utilization of (3) offers a distinct advantage even though it does make it necessary to wind a separate coil on a polystyrene core. However, this must be done anyway whenever a measurement of magnetic loss is desired.

The above measurements were made using coils wound directly on the samples. Data are also given in Fig. 1 using (3) for the case where the sample has been reduced in size and a 0.05-inch layer of polystyrene lies between the core and the winding. The over-all cross section dimensions of the coil are the same as used in the previous measurements. A slight modification in $L_{a}$ must, of conrse, be made in order to account for the reduced size of the core. As can be seen in the graph, removing the wire somewhat from the surface of the sample improves the accuracy of permeability measurements for

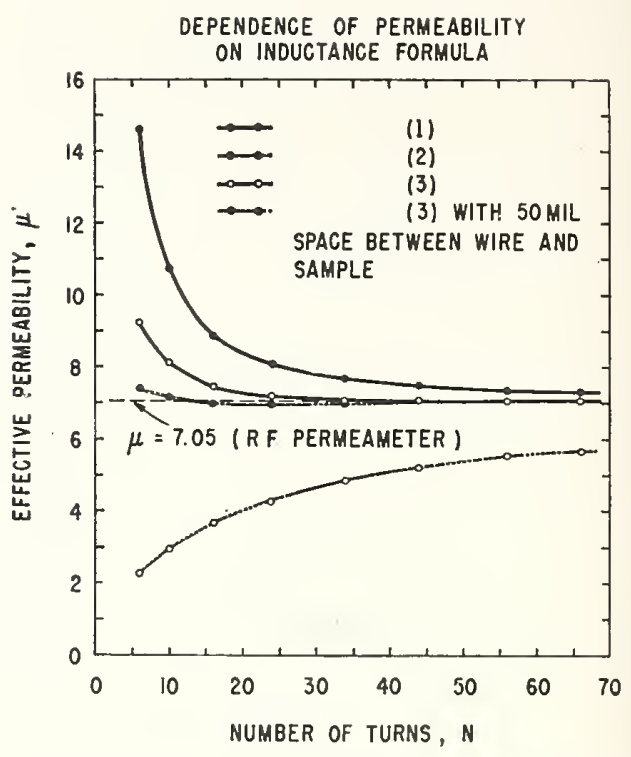

Fig. 1-Relationship between permeability, inductance formula, and number of turns on toroidal coil $\mathrm{O} . \mathrm{D}=1.37$ inche $:$, height $=0.30 \mathrm{inch}$, No. 36 wire),

a small number of turns. Presumably this is due to the creation of a more uniform flux inside the sample. The technique of separating the winding from the sample has been used in the past by other workers for obtaining more accurate loss measurements: however, the resulting improvement in real permeability apparently has not been adequately emphasized.

The above results indicate that coils with a small number of turns can be constructed which can be opened for sample insertion and which will take advantage of the accuracy and convenience of inductance measurements obtained in connection with (3). Such coils have been described in the literature. ${ }^{3}$ Similar work at the National Bureau of Standards has shown that coils of this type are satisfactory for precise measurements of both $\mu^{\prime}$ and magnetic loss in the frequency range up to several mc.

B. L. DANiELSON

R. 1). HARRINGTON Natl. Bur. of Standards Boulder, Colo.

${ }^{3}$ I. Epelboin, "A study of metals with Hertzian waves with the aid of demountable winding perme ameters," L'Onde Electrique, vol. 28, pp. 322-327. August-September, 1948 . 


\section{Supplementary References}

\section{Electricity, d-c and Low-Frequency}

F. K. Harris, Electrical measurement (John Wiley \& Sons, Inc., New York, N.Y., 1952).

G. W. Vinal, Standard cells, standards of electromotive force, ch. 6 in book, Primary Batteries (John Wiley \& Sons, Inc., New York, N.Y., 1958).

G. D. Vincent, The construction and characteristics of standard cells, IRE Trans. Instr. I- 7, 22 (1958).

C. Peterson, Alloys for precision resistors (National Physical Laboratories Symp. Precision Electrical Measurement, 1955).

F. R. Kotter, Location of the galvanometer branch for maximum sensitivity of the Wheatstone bridge, J. Research NBS 40, 401-404 (1948) RP1884.

A. M. Thompson, The precise measurement of small capacitances, IRE Trans. Instr. I- 7,245 (1958).

J. F. Hersh, A close look at connection errors in capacitance measurements, General Radio Experimenter 33, No. 7 (1959).

W. H. Wood, Low-cost microvolt potentiometer, Rev. Sci. Instr. 28, 202-203 (1957).

F. B. Silsbee, R. L. Smith, et al., Equipment for testing current transformers, NBS J. Research 11, 93-122 (1933) RP580.

B. L. Dunfee, An a-c Kelvin bridge for the audio-frequency range, Comm. and Electronics (AIEE, Paper No. 56-25, $1956)$.

\section{Amplifiers and Mixers}

G. F. Montgomery, Intermediate-frequency gain stabilization with inverse feedback, Proc. IRE 38, 662-667 (1950).

G. E. Boggs, Improvement in gain stability of the superheterodyne mixer through the application of negative feedback, Proc IRE 40, 202-207 (1952).

N. C. Hekimian, Chart speeds design of feedback amplifiers, Electronics 25, 153-155 (1952).

N. C. Hekimian, Feedback amplifiers with stabilized output impedances, Tele-Tech 12, 103-105, 148-149 (1953).

G. E. Boggs, Gain stable mixers and amplifiers with current feedback, IRE Conv. Record 1953, Part 4, 140-145; Proc. IRE 42, 1144-1147 (1954).

G. E. Schafer, A modulator for microwave mixers, IRE Trans. on Microwave Theory Tech. M TT-6, 333-334 (1958).

\section{Āttenuation, RF to Microwave}

J. J. Freeman, Theory and design of a cavity attenuator, J. Research NBS 40, 235-243 (1948) RP1868; Theory and design of a waveguide below cutoff attenuator, CRPL-9-2 (Mar. 1948)

R. W. Beatty, Cascade-connected attenuators, J. Research NBS 45, 231-235 (1950); Proc. IRE 38, 1190 (1950); CR PI, Preprint 50-17.

NBS magnetic attenuator, NBS Tech. News Bul. 35, 109-111 (1951).

F. Reggia, Design details of a linear magnetic uhf oscillator attenuator, FN-TV Radio Comm. 11, 16 (1951).

C. W. Allred, Chart for the $T E_{11}$ mode piston attenuator, J. Research NBS 48, 109-110 (1952); Electronics 26, No. 1 (1953)

F. Reggia and R. W. Beatty, Characteristics of the magnetic attenuator of uhf, Proc. IRE 41, 93-100 (1953).

R. W. Beatty and G. E. Huber, Lossy cable attenuator pads, Rev. Sci. Instr. 24, 1002-1003 (1953).

C. M. Allred, Precision piston attenuator (second and third quarters) (unpublished report, Jan. 1954).

G. E. Schafer and R. R. Bowman, Preliminary report on a inodulated subcarrier technique of measuring microwave attenuation (unpublished report, April 28, 1960).
C. M. Allred, Precision piston attenuators (umpublished report, May 1957).

Self-calibrating method of measuring insertion ratio, NBS Tech. News Bul. 41, 147 (1957).

A guide to the operation of microwave attenuation measurement systems developed for the Electronic Calibration Center (Unpubl. rep't. by the staff, Microwave Attenuation Standards, SM-1008, Nov, 1958).

A. Y. Rumfelt and R. J. Como, Rapid insertion device for coaxial attenuators, Rev. Sci. Instr. 30, 687-688 (1959).

Ultraprecise attenuation measurement, NBS Tech. News Bul. 43, 230-231 (1959).

A supplement to a guide to the operation of microwave attenuation measurement systems developed for the Electronic Calibration Center (unpublished report by the staff of Project 84183, 1959).

\section{Calibration Services}

Facilities and services of the Electronic Calibration Center (unpublished report by the staff of the Electronic Calibration Center, Aug. 1958; rev. Jan. 1959).

\section{Dielectrics}

J. L. Preston and E. L. Hall, A study of the seasonal variation of radio frequency phase difference of laminated phenolic insulating materials, Tech. Paper BS 19, 225 -235 (1925).

A. B. Lewis, E. L. Hall, and F. R. Caldwell, Some electrical properties of foreign and domestic micas and the effect of elevated temperatures on micas, BS J. Research 7, 403$418(1931)$.

G. Birnbaum and J. Franeau, Measurement of the dielectric constant and loss of solids and liquids by a cavity perturbation method, J. Appl. Phys. 20, 816-818 (1949).

G. Birnbaum, A recording microwave ref ractometer, Rev. Sci. Instr. 21, 169-176 (1950).

J. L. Dalke and R. C. Powell, Measuring power factor of lowloss dielectrics, Electronics 24, 224 (1951).

G. Birnbaum and S. K. Chatterjee, The dielectric constant of water vapor in the microwave region, J. Appl. Phys. 23, 220-223 (1952)

G. Birnbaum, S. J. Kryder, and H. Lyons, Microwave measurements of the dielectric properties of gases, J. Appl. Phys. 22, 95-102 (1951).

J. H. Beardsley, A variable length re-entrant cavity for dielectric measurements from 100 to $400 \mathrm{Mc}$, Rev. Sci. Instr. 24, 180-181 (1953).

Dielectric research, NBS Tech. News Bul. 43, 168 (1959).

H. E. Bussey and G. Birnbaum, Cavity resonators for dielectric spectroscopy of compressed gases, Rev. Sci. Instr. 30, 800-804 (1959).

H. E. Bussey, Cavity resonator dielectric measurements of rod samples, Insulation, 26-27 (Nov. 1959); Electr. Ifgr. 65-66 (Jan. 1960).

\section{Field-Strength, RF to Microwave}

F. M. Greene, A source of error in the measurement of radiated harmonics, Proc. IRE 40, 486-487 (1952); CR PL Preprint $50-23$.

C. C. Cook, Calibration of commercial field-strength meters, Tele-Tech 11, 44 (1952).

D. M. Kerns and E. S. Dayhoff, Theory of diffraction in microwave interferometry, J. Research NBS 64B, 1 (1960).

\section{Frequency}

P. G. Sulzer, Modified locked-oscillator frequency dividers, Proc IRE 39, 1535-1537 (1951).

L. J. Rueger, A. E. Wilson, The microwave frequency standard, Radio and Television New's 49, 5-7, 40-41 (1953). 
J. M. Shaull, High precision automatic frequency comparator and recorder, Tele-Tech 14, 58-59 (1955).

P. G. Sulzer, One-megacycle high-stability frequency standard, Radio-Elec. Engr. 24, 14, 38 (1955).

J. M. Shaull, Frequency multipliers and converters for measurement and control, Tele-Tech 14, 86-89, 120-122, $142-146,148,159-160$ (1955).

P. G. Sulzer, High stability frequency standard, Tele-Tech 14, $108-109,420-423(1955)$.

M. C. Thompson, M. J. Vetter, and D. M. Waters, SHF frequency standard uses double conversion, Electronics 31, $100-101(1958)$

\section{Frequency and Time, Atomic}

C. H. Townes, Atomic clock and frequency stabilization in microwave spectral lines, J. Appl. Phys. 22, 1365-1372 (1951).

H. Lyons, Spectral lines as frequency standards, Ann. N.Y. Acad. Sci. 55, 831-871 (1952).

J. M. Richardson, Experimental evaluation of the oxygen microwave absorption as a possible atomic frequency standard, J. Appl. Phys. 29, 137-145 (1958)

R. C. Mockler, J. A. Barnes, R. E. Beehler, H. F. Salazar, and R. L. Fey, The ammonia maser as an atomic frequency and time standard, IRE Trans. on Instr. I-7, 201-202, 311-312 (1958).

R. C. Mockler, R. E. Beehler, and J. A. Barnes, A practical limitation to the length of an atomic beam machine (unpublished report, May 25, 1959).

N. Tepley, Ultimate noise limitation of electron multipliers and vacuum tube electrometers as used for the measurement of beam current in a cesium clock (unpublished report, Sept. 29, 1959)

W. Culshaw and R. C. Mockler, A millimeter wave FabryPerot maser (unpublished report, Nov. 16, 1959)

\section{Frequency and Time Broadcasts}

Experimental standard frequency broadcast on 60 kilocycles, NBS Tech. News Bul. 41, 99-100 (1957).

A. D. Watt and W. W. Brown, Anticipated coverage of a vlf standard frequency broadcasting station located near Fort Collins, Colo. (umpublished rcport, Aug. 1959)

A. D. Watt, R. W. Plush, W. W. Brown, and A. H. Morgan, World-wide standard frequency and time signal broadcasting (unpublished report, Mar. 8, 1960).

\section{Generators, Oscillators, Detectors, and Receivers}

P. G, Sulzer, Frequency and amplitude stability of the cathode-coupled oscillator, Proc. IRE 38, 540-542 (1950).

P. G. Sulzer, Wide-range R-C oscillator, Electronics 23, 88-89(1950)

P. G. Sulzer, Precision transistor oscillator, Radio and Television News, Radio Engincering Sec. 49, 18-19 (1953).

P. G. Sulzer, High-stability, bridge balancing oscillator, Proc. IRE 43, 701-707 (1955).

P. G. Sulzer, An oscillator with direct-feedback frequency correction (unpublished report, Feb. 10, 1956)

A. Y. Rumfelt, List of commercially available klystron oscillator tubes, backward wave oscillator tubes and travcling wave amplifier tubes (unpublished report, May 1957).

G. F. Engen, Amplitude stabilization of a microwave signal source, IRE Trans. on Microwave Theory Tech. MTT-6, 202-206 (1958)

F. Niesen, R. W. Beatty, and W. J. Anson, Water-cooling of low-power klystrons used in the laboratory, Rev. Sci. Instr. 29, 791-792 (1958)

A. H. Morgan and P. A. Hudson, Vhf and uhf power generators for rf instrumentation (unpublished report, Mar. 17, 1960)

\section{Impedance, $\mathrm{RF}$ to Microwave}

E. I. Hall, Resistance of conductors of various types and sizes of windings of single-layer coils at 150 to 6000 kilocycles, Tech. Paper BS 21, 109-119 (1926)

G. R. Waite, F. G. Brickwedde, and E. L. Hall, Electrical resistance and magnetic permeability of iron wire at radio frequencies, Phys. Rev. 32, 967-973 (1928).
A. C. MacPherson and D. M. Kerns, Accuracy with which two loads can be matched on a magic tee, Electronics 23, 190-192(1950).

H. E. Sorrows, W. E. Ryan, and R. C. Ellenwood, Evaluation of coaxial slotted-line impedance measurements, Proc. IRE 39, 162-168 (1951).

R. E. Grantham, a reflectionless waveguide termination, Rev. Sci. Instr. 22, 828-834 (1951).

R. C. Ellenwood and E. H. Hurlburt, The determination of impedance with a double-slug transformer, Proc. IRE 40, 1690-1693 (1952).

W. J. Anson, A guide to the use of the modified reflectometer techniques of VSWR measurement (unpublished report, April 13, 1960).

\section{Magnetism, Low-Frequency}

Methods of testing magnetic materials, ASTM A 34-55.

Methods of test for normal induction and hysteresis of magnetic materials, ASTM A 341-55.

Methods of test for permability of feebly magnetic materıals, ASTM A 342-53.

Methods of test for alternating current core loss and permeaability of magnetic materials, ASTM A 343-54.

Methods of test for electrical and mechanical properties of magnetic materials, ASTM A 344-52.

R. L. Sanford, Magnetic testing, NBS Circ. 456 (1946).

W. P. Harris and I. L. Cooter, Improved bridge method for the measturement of core losses in ferromagnetic materials at high flux densities, J. Research NBS 60, 509 (1958) RP2865.

\section{Magnetism, RF to Microwave}

A. A. Feldmann, The machining of powdered iron materials and ferromagnetic ferrites (unpublished report, Mar. 27, 1952).

High frequency calibration of magnetic materials, NBS Tech. News Bul. 36, 158-160 (1952).

P. H. Haas, A radio-frequency permeameter, J. Research NBS 51, 221-228 (1953) RP2454.

B. Kostyshyn and P. H. Haas, Discussion of current-sheet approximations in reference to high-frequency magnetic measurements, J. Research NBS 52, 279-287 (1954) RP2501.

P. H. Haas, R. D. Harrington, R. C. Powell, and A. I. Rasmussen, Instruments for magnetic high frequency measurements, WADC Tech. Report 54-238 (1954).

R. D. Harrington, Cavity techniques for permeability measurements in the vhf region, 6th Electronics Components Conf. Proc., 27-28 (1955)

R. D. Harrington, R. C. Powell, and P. H. Haas, A re-entrant cavity for measurement of complex permeability in the veryhigh-frequency region, J. Research NBS 56, 129-134 (1956).

R. D. Harrington, Characteristics and present requirements of ferrites, Proc. 13th Ann. Meeting Metal Powder Assoc., Chicago, pp. 177-188 (1957).

R. D. Harrington and R. C. Powell, High-frequency magnetic permeability measurcments using toroidal coils, Proc. IRE 46, 784 (1958)

A. L. Rasmussen and A. E. Hess, Rf permeameter techniques for testing ferrite cores, Elec, Mfg 61, 86-91, 308 (1958).

A. L. Rasmussen, A. W. Enfield, and A. Hess, Advances in the design and application of the radiof requency permeameter, J. Research NBS 56, 261-268 (1956)

H. E. Bussey and L. A. Stcinert, Exact solution for a gyromagnetic sample and measurements of a ferrite, IRE Trans. on Microwave Theory Tech. MTT-6, $72-76$ (1958).

V. E. Bottom, Dynamic measurements of the magnetoelastic properties of ferrites, NBS Tech. Note 49 (1960).

H. E. Bussey, Tensor permeability data for seven fcrrites at $9200 \mathrm{Mc}$, (umpublished report, Dec. 29, 1958).

R. D. Harrington and A. L. Rasmussen, Initial and remanent permeability spectra of yttrium iron garnet, Proc. IRF (Jan. 1959).

A. L. Rasmussen, R. D. Harrington, R. C. Powell, and J. L. Dalke, A summary of the investigation of ferromagnetic matcrials - ferrites (unpublished report, June 1959). 
L. A. Steinert, Geometrical anistropy of magnetic materials in wave guides and cavities, J. Appl. Phys. 30, 1109 (1959).

Magnetic materials; measurement and evaluation laboratory (unpublished report by the radio and microwave materials section, NBS, Sept. 1959).

H. E. Bussey, Tensor permeability measurements on commercial ferrites (umpublished report, Sept. 30, 1959).

Electromagnetic materials work at NBSBL (unpublished report by the radio and microwave materials section, NBS, Oct. 22,1959$)$.

\section{Microwave Optics}

Applications of optical techniques of millimeter wavelengths, NBS Tech. News Bul. 41, 24-28 (1957).

W. Culshaw, Reflectors for a microwave Fabry-Perot interferometer, IRE Trans. on Microwave Theory Tech. MTT-7 (April 1959)

W. Culshaw, Diffraction corrections in microwave optics (unpublished report, July 1, 1958).

J. M. Richardson, Provisional alinement requirement for Michelson microwave interferometer carriage (unpublished report, Sept. 1958).

W. Culshaw, High resolution millimeter wave Fabry-Perot interferometer, IRE Trans. (Mar. 1960).

D. M. Kerns and E. S. Dayhoff, Theory of diffraction in microwave interferometry, J. Research NBS 64B, 1-13 (1960).

II. Culshaw and R. D. Mockler, A millimeter wave FabryPerot maser (unpublished report, Nov. 16, 1959).

\section{Microwave Theory}

D. M. Kerns, Basis of the application of network equations to waveguide problems, J. Research NBS 42, 515-540 (1949) RP1990.

J. J. Freeman, The field generated by an arbitrary current distribution within a waveguide, J. Research NBS 44, 193-198 (1950) RP2070.

D. M. Kerns, Analysis of symmetrical waveguide junctions, J. Research NBS 46, 267-282 (1951).

R. IV. Hedberg and D. M. Kerns, Propagation constant in rectangular wave guide of finite conductivity, J. Appl Phys. 25, 1550-1551 (1954).

H. E. Bussey and L. A. Steinert, An exact solution for a cylindrical cavity containing a gyromagnetic material, Proc. IRE 45, 693-694 (1957).

D. M. Kerns and E. S. Dayhoff, Theory of diffraction in microwave interferometry, J. Research NBS 64B, 1 (1960).

\section{Power}

D. M. Kerms, Determination of efficiency of microwave bolometer mounts from impedance data, j. Research NBS 42, 579-585 (1949).

D. M. Kerns, Analysis of the direct-current bolometer bridge, J. Research NBS 43,581-589 (1949).
R. A. Schrack, Radio-frequency power measurements, NBS Circ. 536 (1953).

R. W. Beatty and A. C. MacPherson, Mismatch errors in microwave power measurements, Proc. IRE 41, 1112-1119 (1953).

A. C. MacPherson and D. M. Kerns, A microwave microcalorimeter, Rev. Sci. Instr. 26, 27-33 (1955).

R. W. Beatty and F. Reggia, An improved method of measuring efficiencies of ultra-high-frequency and microwave bolometer mounts, J. Research NBS 54, 321-327 (1955)

Improved thermistor bridge for rf power measurements, NBS Tech. News Bul. 40, 134-135 (1956).

H. A. Thomas, Microwave power measurements employing electron beam techniques, Proc. IRE 45, 205-211 (1957).

A. C. MacPherson, Calorimetric measurements of microwave power (unpublished report, April 1, 1957).

Recent international comparisons of microwave power standards, NBS Tech. News Bul. 43, 155 (1959).

G. F. Engen, A bolometer mount efficiency measurement technique (unpublished report, June 20, 1960).

\section{Quartz}

W. D. Geolge, M. C. Selby, and R. Scolnik, Electrical characteristics of quartz-crystal units and their measurement, J. Research NBS 38,309-328 (1947) RP1774.

L. T. Sogn and C. Barclay, Fundamental techniques in the frequency adjustment of quartz crystals, NBs Circ. 480 (1949).

IV. D. George, M. C. Selby, and R. Scolnik, Precision measurement of electrical characteristics of quartz crystal units, Proc. IRE 36, 1122-1131 (1948)

C. Barclay and L. T. Sogn, Reference data for orienting quartz plates by X-ray diffraction, NBS Circ. 543 (1953).

C. Barclay, Quartz X-ray standards (tentative specifications for, and notes on their use) (unpublished report, March 1958).

P. A. Simpson and A. H. Morgan, Investigation of stability of quartz resonators at low temperatures (final report) (unpublished report, Oct. 1959).

S. A. Basri, A method for the dynamic determination of the elastic, dielectric and piezo-electric constants of quartz, NBS Mono. 9 (1960).

\section{$\mathrm{RF}$ and Microwave Standards and Measurements, General}

Radio instruments and measurements, 1st ed. (1918), 2d ed. (1924, reprinted 1937).

Electromagnetic materials work at NBSBL (unpublished report by the radio and microwave materials section, NBS, Oct. 22,1959 ).

Standards and calibration in radio and electronics, NBS Tech. News Bul. 43, 226-230 (1959). 


\section{Subject Index}

Combined subject index for the three volumes of NBS Handbook 77

A

Absolute temperatures below $1^{\circ} \mathrm{K}$

Absolute viscosity of water at $20^{\circ} \mathrm{C}$

Absorption of X-rays in air

Accurate radio frequency microvoltages

Acoustic impedance of a right circular cylindrical enclosure

Acoustic method for measurement of vibration amplitudes

Acoustics, list of publications

Adiabatic demagnetization experiments in low temperature investigations

Adjustment of high-precision frequency and time standards

Airplane camera calibration, sources of error

Ammonia vapor, super heated, specific heat of

Angle blocks, assembled polygon for the calibration of

Attenuation, microwave measurements, standard

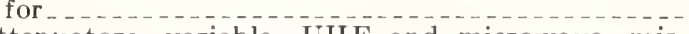

Attenuators, variable, $\mathrm{UHF}$ and microwave, mismatch errors in the measurements of

Audiometers, calibration of

\section{B}

Balance, density, for liquid hydrocarbons, assembly and calibration

Balance, quick-weighing, testing

Benzoic acid, heat of combustion and density

Beryllium oxide, thermal conductivity, $40^{\circ}$ to $750^{\circ}$

ibliography, electrical instruments and measure-

Bibliography, electrical instruments and measure-

Blocks, angle, calibration of

Bolometer bridge for standardizing radio frequency voltmeters _._.

Bolometric power measurements, accurate, selfbalancing, d-c, bridge for

Bomb calorimeter measurements

Branching ratio in the decay of polonium-210...

Bridge, bolometer, for standardizing $R F$ volt-

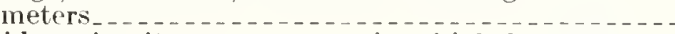

Bridge, circuit, power measuring, high-frequency --

Bridge equations.

Bridge, establishment of known ratios

Bridge, insulation $\ldots \ldots \ldots$

Bridge, NBS precision

Bridge, precision and accuracy

Bridge, self-balancing, d-c, for accurate bolometric power measurements.

Bridge, thermoelectromotive forces...............

Bridge, Thomson

Bridge, Thomson, adjustments of

Bridge, Wagner branches

Bridge, Wheatstone

Bridges, index to subjects

Bunsen-type calorimeter.

1,3-butadiene, thermodynamic properties

\section{C}

Calibrating consoles, voltmeter, $\mathrm{RF}$

$\begin{array}{ll}\text { Calibrating testing machines with proving rings }-- & \text { II-598 } \\ \text { II-573 }\end{array}$

Calibration and testing of hygrometers _... Il-698, II-741

Calibration and use of $\mathrm{VHF}$ field-intensity meters,

influence of the ground on standards NBSBL

Calibration of a density balance for liquid hydrocarbons

Calibration of audiometers $\ldots \ldots$

Calibration of commercial radio field-strength meters at the National Bureau of Standards.-

Volume

II-10

II -523

III -900

$\mathrm{I}-634$

II -858

II -802

II -952

II-22

I-482

III-747

I I-460

III-318

I-776

I-792

II-852

II -691

III -740

II-410

I I-508

III-381

I-605

I-814

I I-361

II-1014

I-605

I-825

I-162

I- 168

I-158

I- 172

I-814

I-182

I -153

I -171

I-188

I-210

I I-222

I I-41.3

\section{I-662}

I-46.3 II-852

I-670
3, I-447

II-691 Dead-weight machines, 111,000 and 10,000 pound

Volume

I I -153

II -573

II -131

$\mathrm{I}-621$ III-907

Calibration of thimble-chamber, soft X-rays......

Calibration of vibration pickups by the reciprocity method

Calibration, pressure, of condenser microphones above $10000 \mathrm{cps}$

Calibration techniques of liquid-flowmeters

Calorimeter, adiabatic, range $30^{\circ}$ to $500^{\circ} \mathrm{C} \ldots$

Calorimeter, bunsen-type

Calorimeter, dry, static, for RF power measurement

Calorimeter for measuring heat capacity and heat of vaporization of water, $0^{\circ}$ to $100^{\circ} \mathrm{C}$

Calorimeter for precise measurement of heat content and heat capacity --

Calorimeter (micro), microwave, X-band

Calorimetry, precision adiabatic, automatic temperature regulation and recording ............

Calorimetry, temperature correction methods

Camera, airplane, errors in calibration.

Camera obscura, for instrument reading

Capacitance measurement, absolute ...............

Capacitor, calibration by step-up methods........

Capacity standards

Carbon dioxide, gaseous, heat capacity of

a function of wall material _ _ II-933

Cells, standard, effect of temperature........... I-252

Cells, standard, effect of temperature inequality in

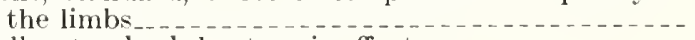

Cells, standard, hysteresis effects ..............

Cells, standard, international comparisons........

Cells, standard, modifications

Cells. standard, unit of electromotive force.

Chamber, ionization, standard, requirements for

250- to 500-kilovolt X-rays _...................

Chamber, reverberation, study of the sound power

output of subsonic air jets .

Chambers, ionization, free-air, design of
Chambers, ionization, free-air, soft X-ray region,

design of lonization, free-air, soft X-ray region,

Chromic methylammonium alum as a thermometric

substance

Color temperature, lamp standards- II -10

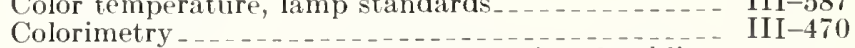

Colorimetry and spectrophotometry, list of publica-

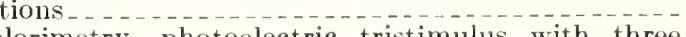

Colorimetry, photoelectric tristimulus with three

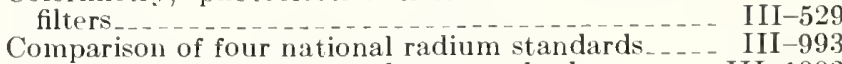

Comparisons of national radium standards____ III-1002

Converter, volt-ampere, wide-range _......... I-352

Converters, thermal, as a-c-d-c transfer standards.. $\quad$ I-317

Converters, voltage, theory, construction, tests_... I-336,

Converters, voltage, thermal

Couplers, directional, method of measuring the directivity of

Current, absolute measurement of

urrent and voltage measurements at audio frequencies Current balance, NBS
Current ineasurement $\ldots \ldots$

$\mathrm{I}-802$

$\mathrm{I}-41$

$-317$

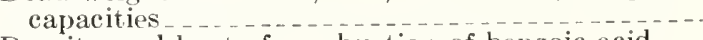

Density and heat of combustion of benzoic acid...-

I I -596

II -410

Density and volumetric determinations _........ III-719 
Density balance for liquid hydrocarbons, assembly and calibration

Density of solids and liquids

Design of free-air ionization chambers

Design of free-air ionization chambers for the soft $\mathrm{X}$-ray region $(20-100 \mathrm{kv})$

Directional couplers, method of measuring the directivity of _............................

Double freezing-point method of determining styrene purity ............................

Earphones, response in ears and couplers

Electric hygrometers semination of, by NBS

Electrical and magnetic units, international relations

Electrical instruments, care and use

Electrical units, derivation

Electrical units, establishment and maintenance

Electrodynamometer, Pellat-type

Electrometer, Brooks attracted disk $\ldots \ldots$ I-283

Electrometer, compressed gas ...................

Electrometers

Electromotive force; history of USA unit
Electrothermic transfer standards, development and description

Engineering mechanics, list of publications on

Expansion, thermal, of solids

\section{$\mathbf{F}$}

Facility for the evaluation of resistance strain gages

Field intensity meters, calibration and use of, influence of the ground on

Field-intensity standards, VHF, development of ...

Field strength meters, commercial, radio, calibration of

Free-air ionization chambers for soft X-ray region.

Freezing points of hydrocarbons, a measurement of purity

Frequencies and time signals, standard

Frequency and time, national standards in the U.S

Frequency and time standards, high precision, adjustment of

Frequency, VSIVR and $|\Gamma|$ for certain impedance standards, tables............ I-695, I-702, I-7 10

G

Gage blocks

I I I-164, II I-280

Gage blocks, precision interference methods for

testing -

Gage blocks, surface characteristics........ III-249

Gages, all types _. III-80

Galvanometer, used with NBS precision bridge _.. I I-158

Galvanometers, damping of _................ I-146

Galvanometers, optical systems of $\ldots$ I-190

Glass volumetric apparatus, testing _........ III-723

Ground (influence of the), on the calibration and use of VHF field-intensity meters ...........

\section{$\mathbf{H}$}

Heat capacity and heat of vaporization of water in the ringe $0^{\circ}$ to $100^{\circ} \mathrm{C}$, measurements

Heat capacity of gaseous carbon dioxide

Heat capacity of sodium between $0^{\circ}$ and $900^{\circ} \mathrm{C}$, the riple point and heit of tisionl-

Heat content and heat capacity, precise measireinent, $0^{\circ}$ to $1500^{\circ} \mathrm{C}$

Heat of combustion and density of benzoic acid

Heat of combustion of benzoic acid, standardization of bomb calorimeters - -

Heat of combustion, precise neasurement with bomb calorimeter.

Heat of fusion, triple point, and heat capacity of sodium between $0^{\circ}$ and $900^{\circ} \mathrm{C}$
$11-691$
II -659

II -870

II -889

$1-802$

$\mathrm{I}-195$

$\mathrm{II}-869$

$\mathrm{I}-718$

$\mathrm{I}-5.3$

$\mathrm{I}-43$

I-58

I -89

$\mathrm{I}-284$

$\mathrm{I}-277$

I-318

II -913

I I -349

I I -638

$-662$

I-670

I I I-889

II -168

$\mathrm{I}-475$

I -182

$11-72$.

I- 662
II $-27 \%$

I I-352

II -341

II $-2+1$

II -410

II -386

I I -361

I I-3. 341

High frequency impedance standards at the Nationa I3urean of Standards.

High frequency standards of the Electronic Calibration Center, NBSBL adjustinent of

High temperature materials, physical properties

Humidity, measuring

Humidity-pressure apparatus.

Humidity (relative)-temperature relations

Humidity test apparatus, low-temperature

Hydrocarbons, deternination of purity by measurement of freezing points

Hydrometers, testing of

Hygrometers, electric _............... II-718

Hy grometers, testing

Hygrometers, testing, recirculation apparatus for .. II -774

I

Impedance standards, higl frequency, at the National Bureau of Standards . . . . . .

Impedance standards, microwave, methods of measurement

Impedance tube method of measuring sound absorption coefficient

Indicators, electrical, scale and reading errors of

Indices of refraction, precision, rapid determination of

Influence of the ground on the calibration and use of Infrared high-resolution grating spectrometer.

Infrared measurements with a small grating from 100 to 300 microns

Instrument reading, a camera obscura for $\ldots$

Instruments, electrical, testing-

I-482

II -241

II $\mathrm{I}-698$

I I -794

II -780

Instruments, spectrophotometric

Interferometer for large surface testing

Interferometer, parallel testing

Interferometer, stroboscopic for vibration measurement.

Interferometry, precision millimeter wave, at the National Bureau of Standards.

International Bureau of Weights and Measures International temperature scale of 1948

Ionization (cavity) as a function of wall material

Ionization chamber, standard, requirements for 250 to 500-kilovolt X-rays

Ionization chambers, free-air, design of

Ionization chambers, free-air, soft X-ray region.

III-381

III -467

III -459

II-808

I-756

I II-28

II-1

I I I -9.33

I I I-926

I I I -870

I II -889

\section{$\mathbf{L}$}

Laboratories, electrical, st:undardizing, suggested practices for Lamp standards, color temperature Lamps, thorium-halide, wavelengths from $\ldots \ldots \ldots$ III-861 Length standards................ III-3 1, III-3.3 Lenses, photographic, testing at NBS $\ldots \ldots$ III-761 Line standards, calibration of Liquid fowmeter calibration techniques....... II-648 Liquids and solids, density of $\ldots \ldots$ II 659

\section{$\mathbf{M}$}

Machines, dead-weight, 111,000 and 10,000 pound capacities. Magnetic and electrical units, extension and dissemination of, by NBS
Iagnetic thermometer to examine the helium Magnetic thermometer to examine the helium
vapor-pressure scale of temperature

Mass standards.........

Mass standards, precision termination, UHF and microwave

Ieasurement, methods, nicrowave impedance

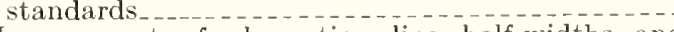

Measurement of absorption line half-widths and intensities, slit function effects...............
II -596

$\mathbf{I}-53$

$\mathrm{I}-680$

II -118 
Measurement of correlation coefficients in reverberant sound fields.

Measurement of microwave $S I V R$, new technique

Measurement of $\mathrm{UHF}$ and microwave variable attenuators, mismatch errors in the

Measurement of vibration amplitudes, acoustic method

Measurement of vibration by stroboscopic interferometer

Measurement, power, RF, calorimeter for

Measurement, thickness

Measurement, voltage, high-frequency

Measurements, electrical, transfer from d-c to a-c

Measurements, infrared

Measurements, microwave reflection coefficients, magnified and squared, VSWR responses.

Measurements, microwave, standard of attenuation for

Measurements of acoustic impedance to determine reverberant sound absorption coefficients.

Measurements of heat of combustion, precise, with bomb calorimeter

Teasurements of pressure list of publications-- -

II-939

Measurements, power, self-balancing d-c bridge

Measurements, power, microwave, at NBS

Measurements, precise, with Bingham and Cannon master viscometers

Measurements, temperature, list of publications...

Measuring, bridge circuit, power, high-frequency - -

Measuring humidity

Measuring sound absorption coefficient

Measuring system, the basis of our

Measuring the directivity of directional couplers.

Mechanics, list of publications

Meter bar \#27, United States Prototype

Meter line standards, ealibration at $\mathrm{N}$

Meters, electrical, reading errors of calibration of condenser microphone

Methods of calibration and testing of hygrometers

Methods of measuring humidity and testing hygrometers

Micrographs, interference, interpretation of

Microinterferometer, description

Microphones, calibration of

Microphones, method of measurement

Microphones, condenser, pressure calibration of -

Micropotentiometers, RF, for calibration of signal generators to $1000 \mathrm{Mc}$

Microwave impedance standards, methods of measurement

Microwave measurements, standard of attenuation for

Microwave microcalorimeter, X-band

Microwave power measurements at NBS

Microwave reflection coefficient measurements magnified and squared VSWR responses

Microwave reflectometer techniques

Microwave SWR, new technique for the measurement of

Mismatch errors in the measurement of UHF and microwave variable attenuators - - - - - - - - -

\section{$\mathbf{N}$}

National radium standards, comparison of four

National radium standards, comparisons of

National standards of time and frequency in the United States

New technique for the measurement of microwave SWR

Nitrogen, thermal conducitivity, $50^{\circ}$ to $500^{\circ} \mathrm{C}, 1$ to 100 atm.

O

Ohm, maintenance of, at NBS

Optical flats, determination of planeness and bind-

Optical instruments, list of publications on

Optical surfaces, large, testing $\ldots \ldots$
II-808

I -809

III -80

I -582

I-65

III-838

$\mathrm{I}-746$

I-776

II-814

I -814
I-806

II -554

II-200

II-200
I-825

II 698

II-812

III-1 4

I-802

II -913

III -53

III -67

I-425

II-893

II-741

II-698

III-328

III-324

II-894

II-893

II-862

I-621

I-680

I -776

I -819

I-806

I-746

I -751

I-739

II I-993

III-1002

I- 172

$\mathrm{I}-739$

II-515

I-20

III-336

III -778

III-343
I-792
Parallelism.

Photoelectric tristimulus colorimetry

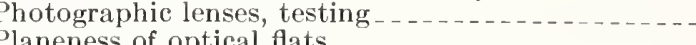

Polonium-210, branching ratio in the decay of .... III-1014

Potentiometer, lindeck

Power measurements, $\mathrm{RF}$, calorimeter for $\ldots$ I-809

Power measurements, microwave, at NBS

Power measuring bridge circuit, high frequency ... $\quad$ I-825

Power supplies for meters and instruments, 60 cycle tests_

Practices, suggested, for electrical standardizing

laboratories

Pressure-humidity apparatus

Pressure measurements, list of publications-.....

Pressure measurements, list of publications

Proving rings, temperature coefficients for

Publications, list, optical instruments

Publications on electrical units, instruments and

measurements

Pyrometric cones, calibration of

\section{$\mathbf{R}$}

Radiation, cavity ionization, wall material

Radiation physies, list of publications

Radioactivity, standards of, preparation, mainte-

nance and application

Radium, national standards, comparison of four

Radium standards, national, comparisons of

Radium standards, preparation of new solutions.-

Reflection coefficient measurements VSWR responses .

$\mathrm{I}-438$

I-1

II-788

II-939

II -573

II-612

III-778

$\mathrm{I}-447$

II-131

Reflectometer techniques, microwave

Refraction, indices of, and dispersion by immersion,

precision, determination

Relative humidity-temperature relationships of some saturated salt solutions saturated salt solutions ---
Resistance, index to subjects

Resistance thermometry, precision, and fixed points

Resistors, commercial, aging and voltage of

Resistors, load coefficients _.....................

Resistors, multimegohm, measurement

Resistors, precision, measurement $\ldots \ldots$

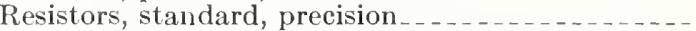

Response of earphones in ears and couplers.......

Reverberation chamber method of measuring sound absorption coefficient

Reverberation chamber, output of a sound source R F micropotentiometers for calibration of signal generators to $1000 \mathrm{Mc}$ $\mathrm{RF}$ microvoltages, accurate

RF voltmeter calibrating consoles

\section{S}

Seintillation spectrometry of low-energy bremsSlit function effects in the direct measurement of absorption line half-widths and intensities.

Solids and liquids, density of Solids and liquids, density of $\ldots+\ldots$
Sound absorption coefficient, measuring Sound absorption (reverberant) coefficients determined by acoustic measurements

Sound power output of subsonic air jets _._._. II-825

Sound source, output of, in a reverberation chamber $\quad$ II-833

Specific and latent heat of water, $0^{\circ}$ to $100^{\circ} \mathrm{C}_{\ldots} \ldots$ II- 277

Specific heat of superheated ammonia vapor

Spectral absorption method for determining popu-

lation "temperatures" in hot gases . . . .

Spectral radiant intensities, procedure

Spectral standards

Spectrometer, infrared, high resolution-

Spectrometers _.......................

Spectrometers, wavelength calibration of, application of vibration-rotation structure

Spectrometry, scintillation, low energy bremsstrahlung

Spectrophotometers

II $\rightarrow 139$

III -458

III-381

III-835

III-390

III-841

III-9 13

III-381 
Spectrophotometry and colorimetry, list of publications

Standard conditions for refractometry

III -432

Standard ionization-chamber requirements for 250 to 500-kilovolt X-rays

Standard of attenuation for microwave measurements _.....................................

Standardization of bomb calorimeters, heat of combustion of benzoic acid ...................

Standards, high frequency impedance, at the National Bureau of Standards

Standards, high frequency, of the Electronic Cali-

bration Center, NBSBL
Standards, impedance, microwave, methods of measurement

Standards, lamp, color temperature

Standards, length, commercial

Standards, length, reference.

Standards, length, working -

Standards, national of time and frequency in the U.S

Standards, national radium, comparisons of

Standards, national radium, comparison of four._. III-993

Standards, national radium, statistical analysis... - III-999

Standards of mass, design and test . . . . . . . . _ III -615

Standards of mass, precision _................. III-591

Standards of radioactivity, preparation, maintenance and application of ....................

Standards or radium, preparation of new solutions -

Standards, spectral energy $\ldots \ldots \ldots \ldots \ldots$

Standards, spectral reflection

Standards, spectral transmission

Standards, threshold, probe tube method for the transfer of

Standards, VHF field intensity, development of

Strain gages, evaluation of elevated temperatures

Styrene, determination of purity by double freezing point method

Surface roughness, measurement

SWR, microwave, new technique for the measurement of

$\mathrm{T}$

Tables of frequency, VSWR and $|\Gamma|$ for selected halfround inductive obstacle impedance standards.

$\overline{\mathrm{I}}-702, \mathrm{I}-710$

Tapes, measuring, calibration of

III -942

II-1008

III-424

III -427

III-425

II-865

I-641

II -638

II- 195

III-324

$\mathrm{I}-739$

Technique (new) for the measurement of microwave SWR.

Temperature coefficients for proving rings

Temperature, effects on refractive media

Temperature measurements, list of publications.

Temperature measurements of hot gases spectral absorption method

Temperature regulation and recording, automatic in precision adiabatic calorimetry ...........

Temperature scale, international, $1948 \ldots \ldots$

Termination, matching. UHF and microwave

Test apparatus, humidity, low-temperature $\ldots . .$. II-780

Testing and calibration of hygrometers $\ldots$ II -698 , II-741

Testing electrical instruments $\ldots$ I-417, I-421

Testing hydrometers $\ldots \ldots \ldots$ III-707

Testing hygrometers

Testing hygrometers, recirculation apparatus for - - II-774

Testing of glass volumetric apparatus _......... III-723

Testing, quick-weighing balance...... III-740

Thermal conductivity of beryllium oxide from $40^{\circ}$ to $750^{\circ} \mathrm{C}$

Thermal conductivity of nitrogen from $50^{\circ}$ to $500^{\circ}$ $\mathrm{C}$ and 1 to 100 atmospheres

III-349

Thermocouple materials, methods of testing _... II-88

Thermocouples in air from $800^{\circ}$ to $2,200^{\circ} \mathrm{F}$, stability of base-metal

Thermocouples, methods of testing

Thermodynamic properties of 1,3-butadiene in the solid, liquid, and vapor states.......

Thermoelectric thermometry $\ldots$

II -111

II-88

II -68
Volume

$\begin{array}{lr}\text { Thermometers, liquid-in-glass, calibration } \ldots \ldots \ldots & \text { II }-153 \\ \text { Thermometry, precision resistance........... } & \text { II }-40\end{array}$

Thermometry, thermoelectric $\ldots \ldots$ II

Thickness measurement III-80

Thimble-chamber calibration on soft X-rays..... III-907

Thorium-halide lamps, wavelengths from_..... III-861

Time and frequency, national standards in the U.S - I-472

Time and frequency standards, high precision, adjustment of

signals and frequeney, standard ........... I-475

Time standards .....

Transfer instrument, ac-dc

\section{$\mathrm{U}$}

Units and systems of weights and measures, their origin, development, and status

Units, electrical, capacitance, derivation

Units, electrical, current, power, energy, derived.-

Units, electrical, establishment and maintenance.-

Units, electrical, inductance, derived

Units, magnetic, derived .

Units, measurement, tables of equivalents

Units of measurement, interrelation

V

Vapor-pressure scale of temperature, helium, using a magnetic thermometer

VHF field-intensity standards, development of

Vibration amplitudes, measurement of, acoustic method

Vibration measurement, stroboscopic interferometer for

Viblation pickups, calibration of

Vibration-rotation structure in absorption bands for the calibration of spectrometers from 2 to 16 microns.

Viscometers, Bingham and Cannon master, precise measurements with._._._.

Viscosity, precise measurements

Volt box, standard

Volt boxes, testing and performance.

Volt, maintenance of, at NBS

Voltage and current measurements at audio frequencies

Voltage converters, thermal $\ldots \ldots \ldots$

Voltage, high, measurement of $\ldots$

Voltage, high, measurements by transformer methods.

Voltage measurement, high-frequency $\ldots \ldots$

Voltage, measurement of crest and surge

Voltage measurements, to $30 \mathrm{Mc}_{\ldots} \ldots \ldots$

Voltage ratio, measurement, audio frequency

Voltages, micro, RF, accurate.

Voltmeter consoles, calibrating, $\mathrm{RF} \ldots$

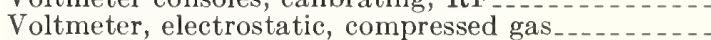

Voltmeter, ellipsoidal

Voltmeters, electrostatic

Voltmeters, RF, bolometer bridge for standardizing I-605

Volumetric and density determinations......... III-719

Volumetric apparatus, glass, testing - _- III-723

VSWR, frequency and $|\Gamma|$, tables for impedance

standards $\ldots \ldots$ I-695, I-702, I-710

W

Water, absolute viscosity at $20^{\circ} \mathrm{C}$

Watthour meter, standard, calibration of

Watthour meters, a-c, precise comparison method of testing.

Wattmeter, standard, design and construction

Wattmeter, standard, electrodynamic .

Wattmeter, standard, performance tests

Wavelengths from thorium-halide lamps

Weights and measures tables.

Weights, laboratory.

$\mathrm{I}-406$

$\mathrm{I}-372$

I- 369

I-385

III-861

III-41

\section{$\mathrm{X}$}

II-413 X-band microwave microcalorimeter

I-739
$\mathrm{X}$-rays, absorption in air 


\section{Author Index}

\section{Combined author index for the three volumes of NBS Handbook 77}

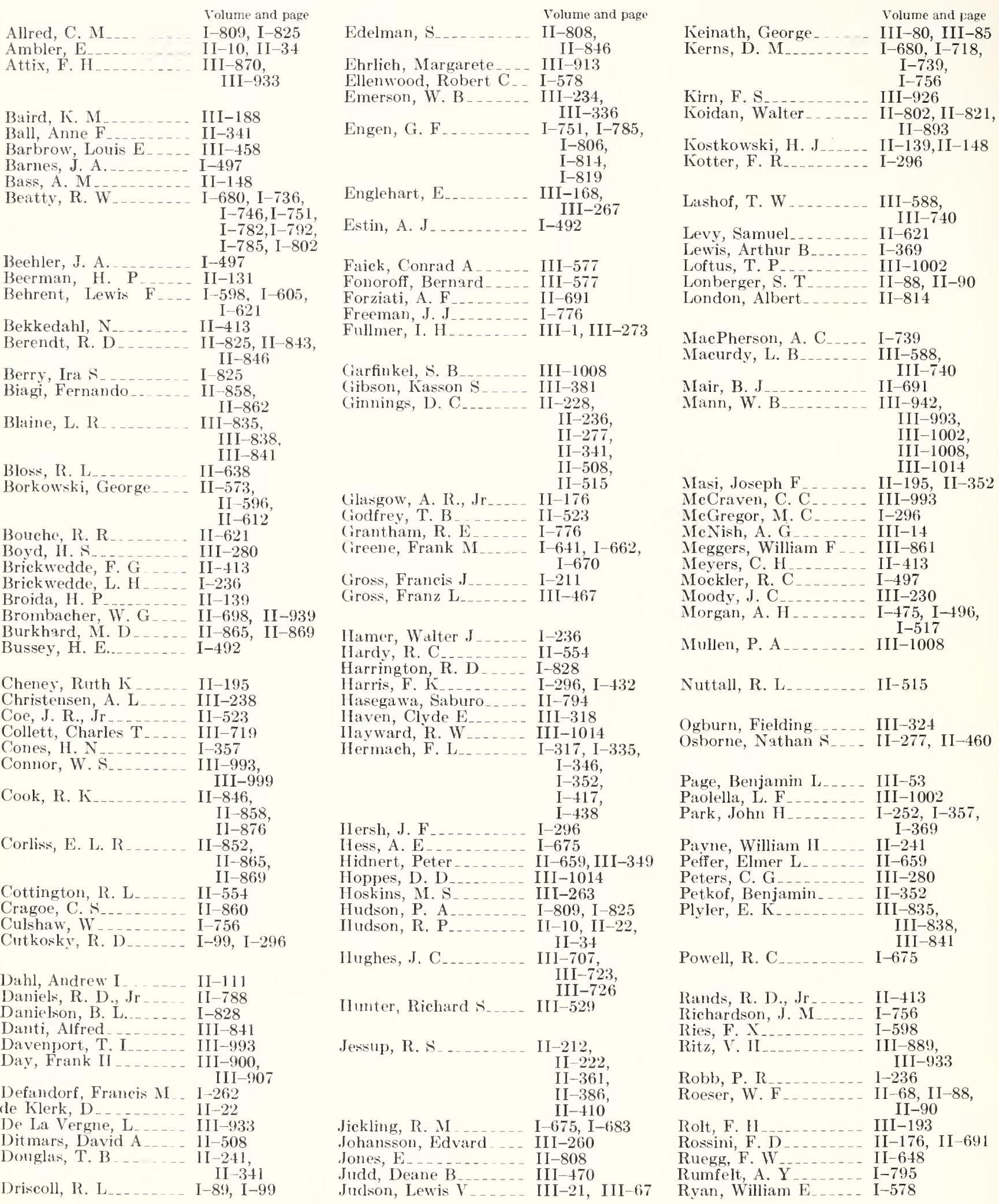


Volume and page

Saunders, J. B II-216,

I I I-343,

I I I-459,

III -467

Schafer, G. E

$\mathrm{I}-795, \mathrm{I}-802$

Schmidt, $V$.
Schwebel, A.

II-808

III-1008

Scott, Arnold $\mathrm{H}=-$ I-230

Scott, R. B

II -413

Selby, M. C

Seliger, H. H

I-463, I-582,

I-598, I-605,

I $-634, \mathrm{I}-683$

I-825

Shafer, M. R I II-942,

$$
\text { III }-945
$$

Shaull, John

I I-648

Silsbee, Francis B

I-1, I-12, I-15.

Simmons, B. I I $-53, \mathrm{I}-211$

- II-862

Smith, C. C . . . . III-993

Smith, E. R . . . I I-808

Snyder, W. F _...... II -852

Solow, Max_._........ I-641

Souder, Wilmer ...... III-349

Spinks, A. IV _ I-406

Stanley, R. W. . . . . III-861
Volume and page

Stimson, H. F II-1, II-40,

II -277,
II -460

Stockmann, L. I

III-1002, III-1008

Strang, A. G I I I-249, III -318 , II I-324, I I I -349

Streiff, Anton J ...... II-176

Sugg, R. E _...... III-225

Swindells, James F_ _ _ II-153, II-155, I I-523,

Sze, Wilbur C

Tate, D.

$\mathrm{I}-573, \mathrm{II}-596$

Taylor, I. S II 612

Thomas, James L_... I-108

Thompson, M. C., Jr - II-846

Tidwell, E. D

I I I -84

Tilton, Leroy $\mathrm{W}_{\ldots}$.

III-432

Victory, F. C

III-243

Washer, Francis $\mathbf{E}$

III-747,

II I-761
Volume and page

Waterhouse, R. V I-825, II -83.3

$\mathrm{I}-421, \mathrm{I}-425$

I-428, I-432

Wenner, Frank I 143

West, E. D_....... II-228, II-236

Wexler, Arnold _... II-698, II-718,

II-741,

II -774

II -780 ,

II-788,

II -794

Williams, E. S ....... I-335, I-352

Wilson, B. L_....... II-573, II -596 , II -612

Wolzein, E. C

I -683

Wvckoff, H. O

III-870,

I I -926

Youden, W. J

III-993, I I -999 , III -1002 ,

Young, 'T. R

III-208

Zapf, Thomas I

$\mathrm{I}-305, \mathrm{I}-310$ 


\section{PUBLICATIONS PROGRAM OF THE NATIONAL BUREAU OF STANDARDS}

Results of fundamental and applied research at the National Bureau of Standards in physics, chemistry, engineering, and mathematics are published in the Bureau's own series of publications. Papers are also published in the journals of professional and scientific societies and in technical and trade publications.

\section{JOURNAL OF RESEARCH}

reports National Bureau of Standards research and development in physics, mathematics, chemistry, and engineering. Comprehensive scientific papers give complete details of the work, including laboratory data, experimental procedures, and theoretical and mathematical analyses. Illustrated with photographs, drawings, and charts.

The Journal presents review articles by recognized authorities and compilations of information on subjects closely related to the Bureau's technical program.

Selected NBS nonperiodical publications and articles by the Bureau staff in professional journals are abstracted in the appropriate section of the Journal. In addition, each section carries a complete listing of all Bureau publications that are not abstracted in that section.

Four Sections .......................... Separately Available

\section{A. PHYSICS AND CHEMISTRY}

Papers of interest primarily to scientists working in these fields. This section covers a broad range of physical and chemical research, with major emphasis on standards of physical measurement, fundamental constants, and properties of matter. Issued six times a year. Annual subscription: Domestic, $\$ 4.00$; foreign, $\$ 4.75$.

\section{B. MATHEMATICS AND MATHEMATTCAL PHYSICS}

Studies and compilations designed mainly for the mathematician and theoretical physicist. Topies in mathematical statistics, theory of experiment design, numerical analysis, theoretical physics and chemistry, logical design and programing of computers and computer systems. Short numerical tables. Issued quarterly. Annual subscription: Domestic, $\$ 2.25$; foreign, $\$ 2.75$. *

\section{ENGINEERING AND INSTRUMENTATION}

Reporting results of interest chiefly to the engineer and the applied scientist. This section includes many of the new developments in instrumentation resulting from the Bureau's work in physical measurement, data processing, and development of test methods. It also covers some of the work in acoustics, applied mechanies, building research, and cryogenic engineering. Issued quarterly. Annual subscription: Domestic, $\$ 2.25$; foreign, $\$ 2.75$ * $^{*}$

\section{RADIO PROPAGATION}

Reporting research in radio propagation, communications, and upper atmospheric physics. Topics covered include propagation in ionized media, scattering by turbulence, effect of irregular terrain on propagation, diffraction and scattering by solid obstacles, propagation through time-varying media, surface waves, and antennas. Issued six times a year. Annual subscription: Domestic, \$4.00; foreign, $\$ 4.75 .^{*}$

*Difference in price is due to extra cost of foreign mailing. 


\section{OTHER PERIODICALS}

The following periodicals may be obtained on a 1-, 2-, or 3-year subscription basis (although no reduction in rates can be made) from the Superintendent of Documents, U.S. Government Printing Office, Washington 25, D.C.:

Technical News Bulletin. Summaries of current research at the National Bureau of Standards are published in the Bulletin. 'The articles are brief, with emphasis on the results of research, chosen on the basis of their scientific or technologic importance. All publications by Bureau staff are announced in the Bulletin. Annual subscription: Domestic, $\$ 1.50$; foreign, $\$ 2.25$.

Basic Radio Propagation Predictions. The Predictions provide the information necessary for calculating the best frequencies for communication between any two points in the world at any time during the given month. The data are important to users of long-range radio communication and navigation, including broadcasting, airline, steamship, and wireless services, and to investigators of radio propagation and ionosphere. Each issue, covering a period of 1 month, is released 3 months in advance. Annual subscription: Domestic, \$1.50; foreign, $\$ 2.00$.

\section{NONPERIODICALS}

Applied Mathematics Series. Mathematical tables, manuals, and studies. Monographs. Major contributions to the technical literature on various subjects related to the Bureau's scientific and technical activities. These comprise much of the type of material previously published in the larger National Bureau of Standards Circulars.

Handbooks. Recommended codes of engineering and industrial practice (including safety codes) developed in cooperation with interested incustries, professional organizations, and regulatory bodies.

Miscellaneous Publications. Charts, administrative pamphlets, annual reports of the Bureau, conference reports, etc.

Technical Notes. This series consists of communications and reports (covering both other agency and NBS-sponsored work) of limited or transitory interest. NBS Technical Notes are designed to supplement the Bureau's regular publications program. They are for sale by the Office of Technical Services, U.S. Department of Commerce, Washington 25, D.C.

\section{Catalog of NBS Publications}

A complete listing of National Bureau of Standards publications is given in NBS Circular 460, Publications of the National Bureau of Standards, issued from 1901 to June 30, 1947, and its Supplement issued from July 1, 1947, to June 30, 1957. Technical abstracts are given for the publications issued since January 1, 1942. The price of the Circular is $\$ 1.25$ and the Supplement is $\$ 1.50$. These documents may be purchased from the Superintendent of Documents, Government Printing Office, Washington 25, D.C. (see "Purchase Procedure" below).

\section{Announcements of New Publications}

The Bureau's Technical News Bulletin and the Journal of Research contain monthly announcements of new NBS publications. Besides listing the Bureau's own publications, the Bulletin and the Journal announce papers by Bureau staff members published in non-Bureau periodicals.

\section{Other Government Sources of Information on NBS Publications}

Business Service Checklist. Weekly announcement of publications of the Dopartment of Commerce. Lists titles and prices of National Bureau of Standards publications, and those of other parts of the Department of Commerce. Available from the Superintendent of Documents. $\$ 1.50$ a year (foreign $\$ 3.50$ ). 
Monthly Cataloy of United States Government Publications. Issued by the Superintendent of Documents. $\$ 3.00$ a year (foreign $\$ 4.50$ ).

Government Printing Office Price Lists. Lists of Government publications on particular subjects. Free from the Superintendent of Documents.

In addition, many of the technical journals carry notices of new Bureau publications of interest in their respective fields.

\section{Purchase Procedure}

Most NBS publications are for sale ONLY by the Superintendent of Documents, Government Printing Office, Washington 25, D.C. (PLEASE DO NOT SEND ORDERS TO THE NATIONAL BUREAU OF STANDARDS.) Payment in advance is required, and may be made by coupons sold in sets of 20 for $\$ 1.00$ and good until used, postal money order, express order, check, or by currency (at sender's risk). A discount of 25 percent is allowed to purchasers of 100 or more copies of a single publication to be mailed to one address. Postage is not required for orders mailed to the United States and possessions. Remittances from foreign countries should be made by international money order or draft on an American bank, and should include an additional one-fourth of the purchase price for postage.

Some purchasers of Government publications prefer to use the "deposit account" service provided by the Superintendent of Documents. Deposits of $\$ 10$ or more are accepted, against which orders may be placed without making individual remittances or first obtaining quotations. Order blanks are provided by the Superintendent of Documents for the convenience of users of the deposit account arrangement. Orders on these blanks are returned with notations slrowing the publications supplied, explanations regarding those not sent, the amount charged, and the balance on deposit.

Out of print NBS publications are available for reference use in many scientific, technical, and public libraries. The Bureau's Catalog of Publications, NBS Circular 460, contains a list of Government Depository Libraries that receive selected Bureau publications. Some libraries are equipped to provide photostat or microfilm copies of out of print documents. 


